





CATALOGUE

$\mathrm{OF}$

SCIENTIFIC PAPERS

1800-1900

SUBJECT INDEX

VOLUME I

\section{PURE MATHEMATICS}


CAMBRIDGE UNIVERSITY PRESS WAREHOUSE, C. F. Clay, Manager.

Zlondon: FETTER LANE, E.C.

Glaggow: 50, WELLINGTON STREET.

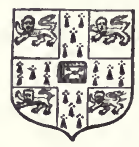

Zleipsig: F. A. BROCKHAUS.

fecw gork: G. P. PUTNAM'S SONS.

Bomban and Calcutta: MACMILLAN AND CO., LTd. 


\title{
ROYAL SOCIETY OF LONDON
}

\author{
CATALOGUE \\ $\mathrm{OF}$ \\ SCIENTIFIC PAPERS \\ 1800-1900
}

\section{SUBJECT INDEX}

VOLUME I

PURE MATHEMATICS

CAMBRIDGE :

AT THE UNIVERSITY PRESS

1908 
ARRANGED FOR A COMMITTEE OF THE ROYAL SOCIETY UNDER THE SUPERINTENDENCE OF

\section{HERBERT M'LEOD, LL.D., F.R.S.}

DIRECTOR OF THE CATALOGUE

with the assistance of

R. J. DALLAS, M.A.

KING'S COLLEGE, CAMBRIDGE 


\section{PREFACE}

$\mathrm{W}^{\mathrm{H}}$

HEN the plan for a Catalogue of Scientific Papers was drawn up in 1857 by the Royal Society, it was also contemplated that a Subject Index to the papers should be made. During the compilation of the well known twelve volumes of the Catalogue of Authors (1800-1883) much material was collected for the Subject Index by the Committee of the Society which had charge of the work, but nothing was printed.

In 1898 it was determined to undertake the continuation for the period 1884-1900, and at the same time to prepare material for a Subject Index for these last seventeen years of the century. It was afterwards decided that one Index should be made to the papers of the whole of the Nineteenth Century; and to bring this Index into relation with the International Catalogue of Scientific Literature, which deals with publications subsequent to 1900 , the Index was to be arranged in accordance with the schedules of the different sciences which form the basis of the International Catalogue.

The extent of the work for the period 1884-1900 proved to be so great that the resources of the Royal Society would have been quite inadequate for its completion. Its continuation has been made possible by liberal donations received from various sources. The Catalogue is especially indebted to Dr Ludwig Mond, F.R.S., a member of the Committee entrusted with its preparation, who has encouraged and forwarded its progress in every way for many years past; in particular, in addition to similar generous donations at earlier stages, Dr Mond recently undertook to provide an extremely liberal subsidy during the three years 1907 -9, which has enabled the work to be pressed forward rapidly.

The Subject Index will be published as separate Index-volumes for each of the seventeen Sciences of the Schedules of the International Catalogue, viz. Mathematics, Mechanics, Physics, Chemistry, Astronomy, Meteorology, Mineralogy, Geology, Geography, Palaeontology, Biology, Botany, Zoology, Anatomy, Anthropology, Physiology, and Bacteriology.

The practical advantage which will accrue from thus classifying the literature of each Science seems to be amply sufficient, in spite of unavoidable imperfections, to justify the great expense that has been involved. In the past a student interested in a single branch of the subject would probably consult special Treatises and Reports from which he could obtain references to the more important memoirs. But it often happens that such references are transferred simply from one treatise to another, and 


\section{Preface}

experience shows that important contributions that have once dropped out of sight may for a long time, or indeed ever afterwards, be overlooked. When the whole literature is set out systematically and exhaustively under appropriate headings as in the present volume, such accidents are guarded against so far as seems to be possible.

The present volume dealing with Pure Mathematics is the first instalment of the Subject Index : it contains 38748 entries referring to 700 serials. It is believed that these entries cover all the contents of the serials indexed with the exception of about 750 short notes (mostly falling under 6810 and 7210 ) which were not thought of sufficient permanent interest to be entered.

The Index titles for papers published from 1884 to 1900 inclusive have been made by Referees familiar with the subjects, who have consulted the original papers and have made the titles from the contents of the papers and not merely from their headings. It was intended that the Index entries for the papers from 1800 to 1883 should be made from the titles as printed in the Catalogue of Authors, but it was soon found that many of the titles were too indefinite for such minute classification, and reference has had to be made to the original papers in a large number of cases. The latter part of this work was commenced by the late Mr George Griffith of Harrow, whose untimely death was a great loss to the Committee.

The entries in the Index are arranged so that reference can be made to the complete titles in the Catalogue of Scientific Papers. Generally the author's name together with the date will indicate the volume in which the title of the paper may be found. These clues are insufficient when the paper is anonymous or occurs in Volume XII or in the additions to Volume VI. They are also at fault for titles marked with an asterisk showing that they belong to previous volumes; in these cases the number of the volume is given in the Index entry in small Roman numerals within brackets.

When an error has been found in an author's name in the Catalogue, it is corrected in the Index by a reference made to the error.

The Index contains references to some papers of dates earlier than 1884 which were omitted in previous volumes of the Catalogue: these are indicated by an asterisk placed before the date; the full titles of these papers will be given in the continuation of the Catalogue of Authors.

When an author's personal name does not appear in the heading of an original paper, no attempt has been made to find the name for the Index, but this will be done for the Catalogue of Authors.

The Catalogue of Authors for the period 1884 to 1900 is in preparation; the material will not however be quite complete until the Subject Indexes for the various Sciences have been reduced into order.

Entries on the same subject are arranged, so far as possible, in order of vi 


\section{Preface}

date irrespective of the authors' names, with the endeavour to present the subject in the historical form. This grouping of the entries, involving modifications of titles prepared by different Referees, or by the same Referee at different times, has been one of the most difficult problems in the preparation of the Index.

The abbreviations of the names of the serials used in the Royal Society Catalogue have been further shortened for the Index. As the abbreviations are not uniform in all the volumes, it will be found that the same journal may be indicated by several different abbreviations, but in each case the one selected is that which was used in the volume in which the title of the paper occurs.

In the case of serials commencing since 1883, the abbreviations adopted in the International Catalogue have been used as a guide.

The list of serials will, it is hoped, be a valuable feature of the Index. It has been drawn up by the Director and contains the names of 701 serials from which the entries in the Index have been taken. Each title is preceded by the abbreviation which represents the serial in the Index; the date of commencement of the serial is given, and if it is extinct the date of the last volume is added. There are appended symbols representing the names of twenty-three British Libraries in some of which the serials may be found; where the set is incomplete the symbol is followed by $i$. The information from which this list has been compiled was obtained, in the first instance, from published catalogues; subsequently the list was submitted to the custodians of many of the libraries, who kindly marked many serials which had not been found in the catalogues used. The thanks of the Committee for this valuable assistance are due to Mr F. Jenkinson of the Cambridge University Library, Mr E. W. B. Nicholson of the Bodleian Library, the Librarian of the Radcliffe Library, the Librarian of the Patent Office, Dr B. Daydon Jackson and Mr A. W. Kappel of the Linnean Society, Mr W. H. Wesley of the Royal Astronomical Society, Mr C. V. Crook of the Geological Museum, Mr F. W. Clifford of the Chemical Society, and Mr R. W. Chambers of University College, London; Mr R. Lloyd Praeger obtained the information from the five libraries in Dublin, and Dr Hugh Marshall, F.R.S., from two libraries in Edinburgh and two in Glasgow.

Although much care has been expended in making this list as accurate as possible, it is probable that some errors will still be found and the Director will be thankful to any one who will send corrections: portions of the list will be required for the subsequent volumes of the Index.

The subjects are arranged under the registration numbers adopted in the International Catalogue; a copy of Schedule A, Mathematics, is prefixed to the Index, with indication of the pages on which the titles for the 


\section{Preface}

different sections occur. It has occasionally been found convenient, in order to save repetition in printing, to group entries under a sub-heading which is not contained in the International Catalogue Schedule. Where this has been done the sub-heading is printed in italics. In some of these cases the words of the sub-heading are understood to exist before the entries following them, and consequently these entries commence with small letters. These minor classifications, being often made mechanically on the basis of the explicit mention of the sub-heading, are not to be taken as exhaustive; cognate entries may be found elsewhere under the same main heading. The unit of classification is thus the complete numbered heading.

The first portion of the present volume of the Index having been sent to press before the whole of the material had been sorted, some slips belonging to earlier sections were discovered during the arrangement of the later ones. These titles have been placed in an Appendix under their appropriate registration numbers.

The following referees have assisted at various times in the preparation of the Subject Index in Pure Mathematics :-the late Professor J. D. Everett, F.R.S., Mr R. J. Dallas, Miss Alice Everett, Mr R. Hargreaves, Miss W. M. Hudson, Mr H. Knapman, Miss E. Perrin and Mr G. Harold Wilson. The Committee is indebted to them for much valuable help.

The arrangement of the contents of this volume for the press has been made by Mr R. J. Dallas, M.A., of King's College, Cambridge. To him and to Miss Bremner and the members of the Catalogue Staff thanks are due for careful and conscientious work.

At the request of the other members of the Committee, Mr G. B. Mathews, F.R.S., examined the whole of the proof-sheets, and made suggestions for their improvement: he was also consulted from time to time about questions of arrangement, head lines and sub-headings.

When the time for going to press approached, the Committee learned with much satisfaction that the Syndics of the Cambridge University Press were willing to undertake the complete risk of printing and publishing, as regards both the Catalogue of Scientific Papers and the Subject Index. It will be the care of the Committee, and it is hoped of the Scientific world generally, to use their best endeavours that this public-spirited action shall not result in financial loss.

Finally the thanks of the Committee are due to the officials of the Cambridge Press for their unfailing courtesy in the discharge of a complex task.

March 1908. 


\section{SUBJECT INDEX OF PURE MATHEMATICS}

\section{LIST OF SERIAL PUBLICATIONS}

\section{WITH THE ABBREVIATIONS OF THEIR TITLES USED IN THE INDEX, AND LIBRARIES WHERE THE SERIALS CAN BE CONSULTED.}

The date following the title of a serial indicates the year of its commencement; if a second date is given it marks the termination of the serial.

The letters following the dates indicate libraries where the serials are to be found: if the serial is incomplete, the symbol of the library is followed by $i$.
B.M. British Museum.
Camb.P.S. Cambridge Philosophical Library.
Camb.U. Cambridge University Library.
Chem.S. Chemical Society.
Glasg.P.S. Royal Philosophical Society of
Dub.R.C.S. Royal College of Science, Dublin.
Dub.R.D.S. Royal Dublin Society.
Dub.R.I.A. Royal Irish Academy, Dublin.
Dub.T.C. Trinity College, Dublin.
$\begin{array}{ll}\text { Glasg.U. } & \text { Glasgow. } \\ \text { Linn.S. } & \text { Linnean University. }\end{array}$
Math.S. Mathematical Society.
N.H.M. Natural History Museum.
Oxon.B. Bodleian, Oxford.
Edinb.R.S. Royal Society of Edinburgh.
Oxon.B.(R.) Deposited in Radcliffe.
Oxon.R. Radcliffe, Oxford.
Edinb.U. Edinburgh University.
P.0. Patent Office, London.
Geol.M. Geological Survey Museum, Jer- myn St.
R.A.S. Royal Astronomical Society.
R.S. Royal Society.
U.C.L. University College, London.

Abv. Mm. 8. ím.

Abv. B. Mm.

A. C.

Ac. Cxs. Ieop. N. Acta..

Acireale Ac. At.

A. Cond. Pon. Chauss. ... Annales des Conducteurs des Ponts et Chaussées; recueil de

A. Cons. Arts et Ḿ́t. ... Annales du Conservatoire des Arts et Métiers. Paris.

Ac. Nt. C. N. Acta

Acta Mth. mémoires, etc., concernant le Service de Conducteurs des Ponts et Chaussées. Paris.

1857- P.O.

1861 - B.M.; Camb.U.; Glasg.P.S.i.; Oxon.B.; P.O.; R.S.

See Par. A. Cons.

(Mémoires de la Société d'Émulation d'Abbeville. Abbeville.

1833 - B.M.; Camb.U.i. ; Dub.T.C. ; Edinb.R.S.i.; Linn.S.i.; N.H.M.i.; Oxon.B.i.; R.S.i.

Annales de Chimie, ou Recueil de Mémoires concernant la Chimie et les Arts qui en dépendent. Paris.

1789 - B.M. ; Camb.U.; Chem.S. ; Dub.R.D.S.i. ; Dub.T.C.i. ; Edinb.R.S. ; Edinb.U.; N.H.M.; Oxon.B.i.(R.) ; P.O.; R.S. ; U.C.L.

Na physico-medica Academiæ Cæs. Leopoldino-Carolinæ Erlangen, Bonn, Breslau.

1758 - Camb.P.S.; Camb.U.; Chem.S.i.; Dub.T.C.; Edinb.R.S.i.; R.S.; U.C.L.i.

See Ac. Nt. C. N. Acta and Cæs. Ieop. Ac. N. Acta.

Atti e Rendiconti dell' Accademia di Scienze, Lettere e Arti dei Zelanti e PP. dello Studio di Acireale.

1890 - Camb.P.S. $i$; N.H.M.i.; R.S. $i$.

See Ac. Crs. Ieop. N. Acta and Cæs. Ieop. Ac. N. Acta.

Acta Mathematica. Stockholm.

1882- B.M. ; Camb.P.S.; Camb.U.; Dub.T.C.; Edinb.R.S. ; Edinb.U.; Glasg.U.; Math.S.; Oxon.R.; R.A.S.; R.S.; U.C.L. 


\section{List of Serial Publications}

\section{A. der Fydrog.}

A. di C.

Aer. J.

A. Gén. Clv.

\section{A. Iydrog.}

Aix Ac. Mm.

Aix $\mathrm{Mm}$.

Aix Mm, Ac.

Alb. I. T.

Allelod. 8. T.

Am. Ac. $\mathbf{m m}$.

Am. Ac. $\mathbf{P}$.

Am. A.. $\mathbf{P}$.

Am. c.

Am. Ing. \& Railroad J.

Amiene Ac. $\mathbf{M m}$.

Amiens Mm.

Amiens $\mathbf{M m}$. A............ Amiens $\mathbf{m m}$. Ac. Bc. ... A. Mines

Am. I. T.

Am. J. Mth.

Am. J. Bc.

Am. Th. 8. $\mathbf{P}$.

Am. Ph. 8. T.
Annalen der Hydrographie und Maritimen Meteorologie. Herausgegeben von der Deutschen Seewarte in Hamburg. Berlin. 1873 - B.M.; P.O.i.

Annali di Chimica. Milano.

1845-1900. B.M.; Chem.S.i.; P.O.i.

The Aeronautical Journal. London.

1897 - B.M.; Camb.U.i.; P.O.; R.S.

Annales du Génie Civil; Recueil de Mémoires sur les Mathématiques pures et appliquées; l'Astronomie, la Chimie, la Physique, etc. Paris.

1862-80. B.M. ; Camb.U.; Dub.R.C.S.i.; P.O.

Annales Hydrographiques. Recueil d'avis, instructions, documents, et mémoires relatifs à l'Hydrographie et à la Navigation. Paris.

1849 - B.M.; Ebinb.R.S.i.; Oxon.B.; R.A.S.i.; R.S.i.

(Mémoires de l'Académie des Sciences, Agriculture, Arts et Belleslettres, Aix.

1819- B.M.; Dub.R.I.A. ; N.H.M.i.; Oxon.B.i.; R.S.i.

Transactions of the Albany Institute. Albany.

1830 - B.M.; N.H.M.; R.S.

The Transactions of the Allelodidactic Society. London.

1848. R.S.

Memoirs of the American Academy of Arts and Sciences. Cambridge and Boston.

1785 - B.M.i.; Camb.P.S. ; Camb.U. ; Dub.R.D.S.i.; Dub.R.I.A.i.; Dub.T.C.i.; Edinb.R.S.; Linn.S.; N.H.M.; Oxon.R.; P.O.i.; R.A.S.; R.S.; U.C.L.i.

See Bost. Am. Ac. Mm. and Bost. $\mathbf{M m}$. Am. Ac.

Proceedings of the American Academy of Arts and Sciences. Boston. 1846- B.M.; Camb.P.S.; Camb.U.; Chem.S.i.; Dub.R.D.S.; Edinb.R.S.; Glasg.P.S.; Glasg.U.i.; Linn.S.; N.H.M.; Oxon.R.; P.O.; R.A.S.; R.S.; U.C.L.i.

Proceedings of the American Association for the Advancement of Science. Washington, Salem.

1848- B.M.i.; Camb.P.S.; Camb.U.; Dub.R.I.A.; Edinb.R.S.i. ; Glasg.P.S.i.; Glasg.U.i.; Linn.S.; N.H.M.; Oxon.B.; Oxon.R.; P.O.; R.A.S.i.; R.S.

The American Chemist, a monthly Journal of theoretical Chemistry. New York.

1871-77. Chem.S.i.; N.H.M. ; P.O.

American Engineer and Railroad Journal. New York.

1893-95. B.M.; P.O.

(Mémoires de l'Académie des Sciences, Agriculture, Commerce, Belles-lettres, et Arts du département de la Somme. Amiens.

1835- B.M.; Camb.U. ; Dub.T.C.i. ; N.H.M.i.; Oxon.B.i. ; R.S.i.

Annales des Mines, ou Recueil des Mémoires sur l'exploitation des Mines, et sur les Sciences et les Arts qui s'y rapportent. Paris.

1817- B.M.; Camb.U.; Chem.S.i.; Dub.R.I.A.; Edinb.R.S.; Edinb.U.i.; Glasg.P.S.i.; N.H.M.; Oxon.B.(R.); P.O.; R.S.

[Reports and Transactions] of the American Institute of the City of New York. Albany.

1841 - B.M.i.; P.O.i.; R.S.i.

American Journal of Mathematics. Baltimore.

1878- B.M.; Camb.P.S.; Camb.U.; Dub.N.L.I. ; Dub.R.I.A. ; Dub.T.C.; Edinb.R.S.; Edinb.U.; Glasg.U.i.; Math.S. ; Oxon.B.; Oxon.R.; R.A.S.; R.S.; U.C.L.

The American Journal of Science and Arts; Silliman. New Haven. 1818 - B.M.; Camb.P.S.i.; Camb.U.; Chem.S.i.; Dub.N.L.I.i.; Dub.R.C.S.i. ; Dub.T.C.i. ; Edinb.R.S.; Edinb.U.; Geol.M.; Glasg.P.S.; Glasg.U.i.; N.H.M.; Oxon.B.; Oxon.R.; P.O.; R.A.S.i.; R.S.

\section{See silliman $\mathbf{J}$.}

Proceedings of the American Philosophical Society. Philadelphia.

1840 - Camb.P.S.; Camb.U.i.; Chem.S.i.; Dub.R.I.A.; Edinb. R.S.; Glasg.P.S.i.; Glasg.U.i.; Linn.S.; Math.S.i.; N.H.M.i.; Oxon.B.; Oxon.R.i.; P.O.; R.A.S.; R.S.; U.C.L.

Transactions of the American Philosophical Society. Philadelphia. 1771 - B.M.i.; Camb.P.S.; Camb.U.i.; Chem.S.i.; Dub.R.I.A.; 


\section{List of Serial Publications}

Edinb.R.S.; Linn.S.; N.H.M.i.; Oxon.B.; Oxon.R.i.; P.O.; R.A.S.i.; R.S.; U.C.L.i.

Am. 8. CE. T.

Amst. Ak. Jb.

Amst. Ak. $\mathbf{P}$.

Amst. Ak. Vh.

Amst. Ak. Vs.

Amst. Ak, Vs, M

Amst. Ak. Wet. P.

Amst. Arch. Wisk. Gn...

Amst. I

Amst. Jb.

Amst. Jb. Ak. ...............

Amst. Mengelwerk

Amst. N. Th.

Amst. N. Ws. Ntk. Vh...

Amst. Ts, Ws, Nt. Wet.

Amst. Vh.

Amst. Vh. Ak.

Amst. V8. Ak.

A. mt.
Transactions of the American Society of Civil Engineers. New York. 1871 - P.O.

Jaarboek van de Koninklijke Akademie van Wetenschappen gevestigd te Amsterdam. Amsterdam.

1857- B.M.; Camb.P.S.; Dub.R.D.S.; Dub.T.C.; Edinb.R.S.i.; Glasg.P.S.i.; Linn.S.; N.H.M.; R.A.S.i.; R.S.; U.C.L.i.

See Amst. Jb. and Amst. Jb. Ak.

Koninklijke Akademie van Wetenschappen te Amsterdam. Proceedings of the Section of Sciences. Amsterdam.

1899 - Camb.P.S. ; Camb.U. ; Chem.S. ; Dub.R.I.A.; Dub.T.C. ; Edinb.R.S.; Glasg.P.S.; Glasg.U.; Oxon.B.; R.A.S.; R.S.

Verhandelingen der Koninklijke Akademie van Wetenschappen. Amsterdam.

1854 - Camb.P.S.; Camb.U.; Dub.N.L.I.; Dub.R.I.A.i.; Edinb. R.S. ; Glasg.P.S.i. ; Glasg.U. ; Linn.S. ; N.H.M. ; Oxon.B. ; R.A.S.; R.S.; U.C.L.i.

Verslagen der Zittingen van de Wis- en Natuurkundige Afdeeling der Koninklijke Akademie van Wetenschappen. 1893, 1894.

Verslagen van de Zittingen der Wis- en Natuurkundige Afdeeling van de Koninklijke Akademie van Wetenschappen. 1895, 1896.

Koninklijke Akademie van Wetenschappen te Amsterdam. Verslagen van de Gewone Vergaderingen der Wis- en Natuurkundige Afdeeling. Amsterdam.

1897 - B.M.; Camb.P.S.; Camb.U. ; Dub.T.C. ; Edinb.R.S. ; Glasg.P.S.; Glasg.U.; N.H.M. ; R.A.S. ; R.S.

Verslagen en Mededeelingen der Koninklijke Akademie van Wetenschappen. Afdeeling Natuurkunde. Amsterdam.

1853-92. B.M.; Camb.P.S. ; Camb.U.; Dub.T.C. ; Edinb.R.S. ; Glasg.P.S.; Linn.S.; N.H.M.; Oxon.B.i.; R.A.S.i.; R.S.; U.C.L.i.

See Amst. Vs. Ak.

Processen-Verbaal van de Gewone Vergaderingen der Koninklijke Akademie van Wetenschappen, Afdeeling Natuurkunde.

1865-84. Dub.R.D.S.; Linn.S.i.; R.A.S.; R.S.

Archief uitgegeven door het Wiskundig Genootschap. Amsterdam.

1856- B.M.; R.S.i.; U.C.L.i.

See Arch. Wisk. Gn.

Het Instituut. Amsterdam.

1841-46. B.M.; Edinb.R.S.i.

See Amst. Ak. Jb.

Mengelwerk van uitgeleezene en andere Wis- en Natuurkundige Verhandelingen; door het Genootschap der Mathematische Wetenschappen, etc. Amsterdam.

1796-1816. R.S.

See Mengelwerk wisk. Th.

Nieuwe Verhandelingen der eerste Klasse van het Koninklijk Nederlandsche Instituut van Wetenschappen, Letterkunde, en Schoone Kunsten te Amsterdam. Amsterdam.

1827-52. B.M. ; Camb.U.; Dub.T.C. ; Edinb.R.S.; Linn.S. ; N.H.M. ; R.S.

Nieuwe Wis- en Natuurkundige Verhandelingen van het Genootschap te Amsterdam. Amsterdam.

1844-54. B.M.; R.S.

Tijdschrift voor de Wis- en Natuurkundige Wetenschappen, Letterkunde, en Schoone Kunsten te Amsterdam. Amsterdam.

1847-52. B.M.; Camb.U.; Dub.T.C.; Edinb.R.S.i.; Linn.S. ; Oxon.B.; R.S.

, Verhandelingen der Eerste Klasse van het Koninklijk Nederlandsche

( Instituut vah Wetenschappen, Letterkunde, en Schoone Kunsten te Amsterdam. Amsterdam.

1812-25. B.M. ; Camb.U; Dub.T.C. ; Edinb.R.S.; N.H.M.; Oxon.B.; R.S.

See Amst. Ak. Vs. M.

Annali di Matematica pura ed applicata...; Tortolini. Roma, Milano. 1858 - B.M.; Camb.U.i.; Dub.R.D.S.; Dub.T.C.; Edinb.U.; Glasg.U.i.; Math.S.i. ; Oxon.B.(R.); R.S.; U.C.L.

See Tortolini A. 


\section{List of Serial Publications}

A. Mth

Amt. Mch. s. J.

Angers Mm. S. Ag.

Angers s. 8c. Bu.

A. Pon. Chanss.

A. Ps. C.

Arch. f. Ap.

Arch. Inth. Ntvd.

Arch. Mth. Ps.

Arch. Néerl.

Arch. Sc. Ps. Nt.

Arch. Wisk. Gn.

Arg. 8. Ci. A.

As.

As. \& Asps.

A. Bc.

A. 8c. Lomb. Ven.

A. Sc. $\mathbf{N}$.

A8. Fr. C. $\mathbf{R}$.

Ashmol. 8. $\mathbf{P}$.
Annals of Mathematics. University of Virginia. Charlottesville, Va. 1884- Camb.P.S.; Camb.U.; Dub.R.I.A.i.; Edinb.R.S.; Math.S.i.; Oxon. B.

The [Quarterly] Journal of the Amateur Mechanical Society. London. 1872-79. B.M.; P.O.

Mémoires de la Société d'Agriculture, Sciences, et Arts. Angers. 1831 - B.M.; N.H.M.i.; Oxon.B.i.; R.S.i.

Bulletin de la Société d'Etudes Seientifiques d'Angers. Angers. 1872 - B.M.; N.H.M.

Annales des Ponts et Chaussées. Mémoires et documents relatifs à l'Art des Constructions et au Service de l'Ingénieur. Paris. 1831- B.M.; Camb.U.; Edinb.U.i.; Glasg.P.S.i.; Glasg.U.i.; P.O.; R.S.i.

See Par. A. Pon. Chauss.

Annalen der Physik und Chemie; Poggendorff. Leipzig.

1824 - B.M.; Camb.P.S.i.; Camb.U.; Chem.S.; Dub.R.I.A.; Dub.T.C.; Edinb.R.S.; Edinb.U.; Glasg.P.S.i.; Glasg.U.i.; N.H.M. ; Oxon.B.(R.); P.O.; R.S. ; U.C.L.i.

See Pogg. A.

Archiv für Anthropologie. Zeitschrift für Naturgeschichte und Urgeschichte des Menschen. Braunschweig.

1867- B.M. ; Camb.U.; Dub.N.L.I. ; N.H.M.i. ; Oxon.R.; R.S.

Archiv for Mathematik og Naturvidenskab. Kristiania.

1876- B.M.; Camb.U.; Dub.R.I.A.i.; Edinb.R.S.; Math.S.i. ; N.H.M.; Oxon.B.; Oxon.R.i.; R.S.

Archiv der Mathematik und Physik; Grunert. Greifswald, Leipzig. 1841 - B.M. ; Camb.U.; Dub.N.L.I. ; Dub.R.C.S. ; Edinb.U.; Glasg.U.; Math.S.i.; Oxon.B.(R.); R.S.; U.C.L. $i$.

See Grunert Arch.

Archives Néerlandaises des Sciences Exactes et Naturelles. La Haye, Harlem.

1866- B.M. ; Camb.P.S. ; Camb.U.; Chem.S.; Dub.R.I.A.; Dub.T.C.; Edinb.R.S.; Geol.M.i.; Glasg.P.S.; Glasg.U.; Linn.S.; Math.S.; N.H.M. ; Oxon.R.; P.O.; R.S.; U.C.L.i.

Bibliothèque Universelle. Archives des Sciences Physiques et Naturelles. Genève.

1846- B.M.; Camb.U.; Chem.S.i.; Dub.N.L.I.i.; Dub.R.D.S.i.; Dub.R.I.A.i.; Edinb.R.S.; Edinb.U.; Glasg.U.; N.H.M.; Oxon. B.; P.O.; R.S.

See Bb. Un. Arch.

See Amst. Arch. Wisk. Gn.

Anales de la Sociedad Científica Argentina. Buenos Aires.

1876 - B.M.i.; N.H.M.

L'Astronomie. Revue d'Astronomie populaire, de Météorologie et de Physique du Globe. Paris.

1882-94. B.M.; Camb.U.i.; Edinb.R.S.; R.A.S.; R.S. $i$.

Astronomy and Astrophysics. Northfield, Minn.

1892-94. B.M.; Camb.P.S.i.; Dub.N.L.I.i.; R.A.S.; R.S.

Annaes das Sciencias e Lettras, publicados debaixo dos auspicios da Academia Real das Sciencias; Sciencias mathematicas, physicas, historico-naturaes e medicas. Lisboa.

1857- B.M. ; Dub.R.I.A. ; Edinb.R.S.i. ; N.H.M. ; R.A.S.i. ; R.S. $i$.

See Iisb. A.

Annali delle Scienze del Regno Lombardo-Veneto. Padova e Venezia. 1831 -45. B.M.; Camb.U.; Dub.T.C.i.; Oxon.B.

Annales des Sciences Naturelles, comprenant la Physiologie animale et végétale, l'Anatomie comparée des deux règnes, la Zoologie, la Botanique, la Minéralogie et la Géologie. Paris.

1824 - B.M. ; Camb.P.S. ; Camb.U.; Dub.R.D.S.; Dub.T.C. ; Geol.M.i.; Glasg.P.S.i.; Linn.S. ; N.H.M.; Oxon.R.; P.O.i.; R.S.; U.C.L.i.

Association Française pour l'Avancement des Sciences. Compte Rendu. Paris, etc.

1872- B.M.; Camb.U.; Edinb.R.S. ; Edinb.U.; Geol.M.; Glasg.

P.S. ; Glasg.U.; N.H.M.; P.O. ; R.A.S.i.; R.S.

Abstracts of the Proceedings of the Ashmolean Society. Oxford.

1844-81. Camb.U.; Dub.R.D.S. ; Edinb.R.S.i.; N.H.M. ; Oxon. B.i.; Oxon.R.; P.O.i.; R.S. 


\section{List of Serial Publications}

Ashmol. S. T.

As. J.

As. Nr.

Asps. J.

As. Researches

As. \$. J.

As. s. IMm.

As. s. M. Not.

As. S. Pac. Pb.

A8. \$. $\boldsymbol{T}$

Assur. Mg.

At. Ac. It.

A. Tél.

\section{Atlantis.}

At. Sc. It.

Aube IIm. S. Ag.

Ausl.

Aust. As. Rp.

Barcel. Ac. $\mathbf{B} 1$.

Barcel. Ac. IMm.
Transactions of the Ashmolean Society. Oxford.

1834-76. Camb.U.; Dub.R.D.S. ; Edinb.R.S.; N.H.M.i. ; Oxon. B.i.; Oxon.R.; P.O.i.; R.S.i.

The Astronomical Journal. Boston.

1851-61. 1888- B.M.; Camb.U.; Glasg.U.i.; Oxon.B.; Oxon.R.i.;

R.A.S.; R.S.i.

See Gould As. J.

Astronomische Nachrichten; Schumacher. Altona.

1823- B.M.; Camb.U.; Dub.R.I.A.i.; Edinb.R.S.; Edinb.U.i.; Glasg.U.i.; Oxon.B.(R.); R.A.S.; R.S.; U.C.L.i.

The Astrophysical Journal. Chicago.

1895- B.M.; Camb.P.S. ; Camb.U.; Dub.N.L.I. ; Dub.R.D.S. ; Glasg.U.; Oxon.R.i.; R.A.S.; U.C.L.

Asiatick Researches; or Transactions of the [Bengal] Society instituted for inquiring into the History and Antiquities, Arts, Sciences, and Literature of Asia. Calcutta.

1788-1836. B.M. ; Camb.U.; Edinb.R.S.i. ; Edinb.U. ; Linn.S.; N.H.M. ; Oxon.B.; Oxon.R.; R.A.S.; R.S.; U.C.L.i.

Journal of the Royal Asiatic Society. London.

1834- B.M.; Camb.U.; Dub.R.D.S.; Edinb.R.S.i.; Edinb.U.i.; Linn.S.; Oxon.B.; Oxon.R.; P.O.i.; R.A.S.i.; R.S.i.; U.C.L.i.

Memoirs of the Astronomical Society of London. London.

1822- B.M.; Camb.P.S. ; Camb.U.; Chem.S.i.; Dub.R.I.A.; Dub.T.C.; Edinb.R.S. ; Edinb.U. ; Glasg.P.S.i. ; Glasg.U. ; Oxon.B.; Oxon.R.; P.O.i.; R.A.S.; R.S.; U.C.L.

Monthly Notices of the Astronomical Society of London. London.

1827- B.M. ; Camb.P.S. ; Camb.U. ; Chem.S.i.; Dub.R.I.A. ; Edinb.R.S.; Glasg.P.S.i.; Glasg.U.; Oxon.B.i.; Oxon.R.i.; P.O.i.; R.A.S.; R.S.; U.C.L.

Publications of the Astronomical Society of the Pacific. San Francisco.

1889- B.M.; Camb.U.i.; Dub.R.D.S.i.; R.A.S.; R.S.i.

Transactions of the Royal Asiatic Society of Great Britain and Ireland. London.

1827-35. B.M. ; Camb.U.; Dub.R.I.A. ; Edinb.R.S. ; Edinb.U. ; Linn.S.; Oxon.B.; Oxon.R.; R.A.S.; R.S.; U.C.L.

The Assurance Magazine and Journal of the Institute of Actuaries. London.

1830-67. B.M.; Camb.U.i.; Edinb.R.S.i.; R.A.S.i.; R.S.i.; U.C.L. $i$.

Atti dell' Accademia Italiana di Scienze, etc. Livorno.

1810. B.M.; Camb.U.; Dub.T.C.; N.H.M.; Oxon.B.

Annales Télégraphiques, publiées sous le patronage du Directeur Général des Lignes Télégraphiques. Paris.

1855- B.M.i.; Camb.U.i.; P.O.

The Atlantis, or Register of Literature and Science, conducted by members of the Catholic University of Ireland. London.

1858-63. B.M.; Camb.U.; Dub.N.L.I.; Dub.R.I.A.i.; Edinb.R.S.i.; Linn.S.; Oxon.B.; R.S.

Riunione degli Scienziati Italiani. Atti. Pisa, etc.

1839 - B.M.; Camb.U.; N.H.M.; R.S.

Mémoires de la Société d'Agriculture, des Sciences, et des Lettres du département de l'Aube. Troyes.

1823 - B.M.; Camb.U.i.; Dub.T.C.i.; Oxon.B.; R.S.i.

Das Ausland. München und Stuttgart.

1828-93. B.M.; Camb.U.i.; N.H.M.i.; Oxon.B.i.

Report of the.......... Meeting of the Australasian Association for the Advancement of Science. Sydney.

1888 - Camb.P.S.; Camb.U.; Chem.S.; Dub.R.D.S.; Dub.R.I.A.; Edinb.R.S.; Edinb.U.i.; Geol.M.; Glasg.U.; Linn.S.i.; N.H.M.; Oxon.B.; Oxon.R.; P.O.; R.A.S.; R.S.

Boletín de la Real Academia de Ciencias y Artes de Barcelona. Barcelona. 1892- N.H.M.

Memorias de la Real Academia de Ciencias Naturales y Artes de Barcelona. Barcelona.

[1st Series cannot be traced.] 1876- N.H.M. 


\section{List of Serial Publications}

B. A. Rp

Basel $\mathbf{B}$

Basel Th.

Batav. Irtk. Ts.

Baumgartner $z$.

Bb. Brit.

Bb. It.

Bb. Mth.

Bb. $\mathbf{U n}$.

Bb. Un. Arch.

Belfast NI. S. $\mathbf{P}$. Belfast NI. B. Rp. \& $\mathbf{P}$.

Beng. As. S. J.

Beng. As. S. $\mathbf{P}$.

Beng. J. As. $\mathbf{S}$.

Berg-IIm. Ztg.

Berg. Ms, Aarb.

Berl. Ab.

Berl. Ak. Ab.

Berl. Ak. Mib.
Report of the.........Meeting of the British Association for the Advancement of Science. London.

1831- B.M.; Camb.P.S.; Camb.U.; Chem.S.i.; Dub.R.D.S.; Dub.R.I.A.; Dub.T.C.; Edinb.R.S.; Edinb.U.; Geol.M.; Glasg. P.S.; Glasg.U.; Linn.S.; N.H.M.; Oxon.B.i.; Oxon.R.; P.O.; R.A.S.; R.S.; U.C.L.

Bericht über die Verhandlungen der Naturforschenden Gesellschaft in Basel. Basel.

1835-52. B.M.; Camb.P.S.; Dub.T.C.; Linn.S.; N.H.M.; R.S.

Verhandlungen der Naturforschenden Gesellschaft in Basel. Basel.

1857- B.M.; Camb.P.S.; Camb.U.; Chem.S.; Dub.R.D.S.i.; Dub.R.I.A.i.; Dub.T.C.i.; Edinb.R.S. ; Linn.S.; N.H.M. ; Oxon.R.; R.A.S.i.; R.S.; U.C.L. $i$.

Natuurkundig Tijdschrift voor Nederlandsch-Indië. Batavia.

1850- Camb.P.S.; Camb.U.; Edinb.R.S.i.; Linn.S.; N.H.M.; R.A.S.i.; R.S.i.; U.C.L.i.

Zeitschrift für Physik, Mathematik, und verwandte Wissenschaften; Baumgartner und von Ettingshausen. Wien.

1826-42. B.M.; Camb.U.i.; Oxon.B.i.(R.); R.S.i.; U.C.L.i.

Bibliothèque Britannique, ou Recueil extrait des Ouvrages Anglais périodiques et autres: partie des Sciences et Arts. Genève.

1796-1815. B.M.; Edinb.U.; N.H.M.; Oxon.B.; P.O.; R.S.

Biblioteca Italiana, ossia Giornale di Letteratura, Scienze, etc. Milano.

1816-56. B.M.; Edinb.R.S.i.; Oxon.B.

Bibliotheea Mathematica. Stockholm and Leipzig.

1887- B.M. ; Camb.U.; Glasg.U.; Oxon.B.; Oxon.R.; R.S. ; U.C.L.

Bibliothèque Universelle des Sciences, Belles-Lettres, et Arts, faisant suite à la Bibliothèque Britannique rédigée à Genève. Partie des Sciences. Genève.

1816-45. B.M.; Camb.U.; Dub.R.I.A.i.; Edinb.R.S.i.; Edinb.U.; N.H.M.; Oxon.B.; P.O.; R.S.

Bibliothèque Universelle. Archives des Sciences Physiques et Naturelles. Genève.

1846- B.M.; Camb.U.; Chem.S.i.; Dub.N.L.I.i.; Dub.R.D.S.i.; Dub.R.I.A.i.; Edinb.R.S.; Edinb.U.; Glasg.U.; N.H.M.; Oxon.B.; P.O.; R.S.

See Arch. Sc. Ps. Nt.

Report and Proceedings of the Belfast Natural History and Philosophical Society. Belfast.

\{1852- B.M.i.; Camb.P.S.; Dub.N.L.I.; Dub.R.D.S.; Dub.T.C.; Edinb.R.S.i.; Glasg.P.S.i.; N.H.M.; P.O.i.; R.A.S.

Journal of the Asiatic Society of Bengal. Calcutta.

1832 - B.M.; Camb.P.S.i.; Camb.U.; Dub.R.D.S.; Dub.R.I.A.; Dub.T.C.; Edinb.R.S.i.; Linn.S.; N.H.M.; Oxon.B.; Oxon.R.i.; R.A.S.i.; R.S.; U.C.L.

See Beng. J. As. s.

Proceedings of the Asiatic Society of Bengal. Calcutta.

1865- B.M.; Camb.P.S.i.; Camb.U.; Dub.T.C.; Edinb.R.S.i.; Edinb.U.i.; Linn.S.; N.H.M.; Oxon.B.; R.A.S.i.; R.S.; U.C.L. See Beng. As. s. J.

Berg- und hüttenmännische Zeitung; mit besonderer Berücksichtigung der Mineralogie und Geologie; Hartmann. Nordhausen und Leipzig.

1842- B.M.; N.H.M.; P.O.

Bergens Museums Aarbog for......Afhandlinger og Aarsberetning udgivne af Bergens Museum. Bergen.

1892- B.M.; Camb.P.S.; Edinb.R.S.; Linn.S.; N.H.M.; Oxon.R.;

R.S.

Abhandlungen der Königlichen Akademie der Wissenschaften zu Berlin. Berlin.

1804- B.M. ; Camb.P.S.; Camb.U.; Dub.R.D.S.; Edinb.R.S.; Edinb.U.; Glasg.U.; Linn.S.i.; N.H.M.; Oxon.B; Oxon.R.; P.O.i.; R.A.S.i.; R.S.; U.C.L. $i$.

Monatsberichte der K. Preuss. Akademie der Wissenschaften zu Berlin. Berlin.

1856-81. B.M.; Camb.P.S.; Camb.U.; Chem.S.i.; Dub.R.D.S.i.; xiv 


\section{List of Serial Publications}

Berl. Ak. Sb.

Berl. As. Jb.

Berl. B.

Berl. B.

Berl. Mb.

Berl. Mm

Berl. Mm. Ac.

Ber1. Pol. Gs. B.

Berl. Pol. Gs. Vh

Berl. Ps. Gs. Wh.

Berl. Sm. D. A.b.

Berl. z. Frdk.

Bern Mt.

Birm. Ph. \$. $\mathbf{P}$.

B11. As.

Bll. Sc. Inth.

Bu. Sc. Irth. As.

Bil. sc. Nord.

Bode As. Jb.

Bode $\mathbf{J b}$.

Böhm. Gs. Ab.

Böhm. Gs. Ws. Jbr.
Dub.R.I.A.i.; Dub.T.C.i.; Edinb.R.S.; Glasg.P.S.i.; Glasg.U.i.; Linn.S.; Math.S.i.; N.H.M.; Oxon.B.; Oxon.R.; P.O.; R.A.S.i.; R.S.; U.C.L.

See Berl. Imb.

Sitzungsberichte der K. Preussischen Akademie der Wissenschaften zu Berlin. Berlin.

1882- B.M.; Camb.P.S. ; Camb.U.; Chem.S. ; Dub.R.D.S. ; Dub.R.I.A.; Dub.T.C.; Edinb.R.S.; Edinb.U.i.; Glasg.P.S.; Glasg.U.; Linn.S.; Math.S. ; N.H.M.i.; Oxon.B.; Oxon.R.; P.O.; R.A.S.; R.S.; U.C.L.

Berliner Astronomisches Jahrbuch; Encke. Berlin.

1776- Camb.U.; R.A.S.; R.S.i.

Bericht über die zur Bekanntmachung geeigneten Verhandlungen der K. Preuss. Akademie der Wissenschaften zu Berlin. Berlin.

1836-55. B.M.; Dub.R.I.A.i.; Edinb.R.S.; Linn.S.; N.H.M. ; Oxon.B.; P.O.; R.A.S.; R.S.

Berichte der Deutschen Chemischen Gesellschaft. Berlin.

1868 - B.M.; Camb.P.S.; Camb.U.; Chem.S.; Dub.N.L.I.; Glasg. P.S.; Glasg.U.; N.H.M.; Oxon.R.; P.O.; R.S.; U.C.L.i.

See Berl. Ak. Mrb.

Mémoires de l'Académie Royale des Sciences de Berlin. Berlin.

\{1770-1804. B.M.i.; Camb.U.; Dub.R.D.S.i.; Dub.T.C.i.; Edinb. R.S.; Edinb.U.; N.H.M.; Oxon.B.; P.O.; R.S.; U.C.L.

Bericht über die Verhältnisse und die Wirksamkeit der Polytechnischen Gesellschaft zu Berlin. Berlin.

1839-51. R.S.

Verhandlungen der Polytechnischen Gesellschaft. Berlin.

1851 - R.S. $i$

Verhandlungen der Physikalischen Gesellschaft in Berlin. Berlin.

1882- Camb.P.S.i.; Camb.U.; N.H.M.; Oxon.B.; P.O.; R.A.S.; R.S.

Sammlung der Deutschen Abhandlungen, welche in der K. Akademie der Wissenschaften $\mathrm{zu}$ Berlin vorgelesen worden in den Jahren 1788-1803. Berlin.

1789-1803. B.M.; N.H.M.; R.S.

Zeitschrift der Gesellschaft für Erdkunde zu Berlin; Koner. Berlin.

1866- B.M.; Camb.U.; Dub.R.I.A.i.; N.H.M.; Oxon.B.; R.S.i.

Mittheilungen der Naturforschenden Gesellschaft in Bern. Bern.

1843 - B.M.; Camb.P.S.i.; Dub.R.D.S.i.; Dub.R.I.A.i.; Edinb. R.S.i.; N.H.M.; R.S.

Proceedings of the Birmingham Philosophical Society. Birmingham. 1876- B.M.; Camb.P.S.; Camb.U.; Dub.R.I.A.i.; Dub.T.C.i.; Edinb.R.S.; Edinb.U.i.; Geol.M.; Glasg.P.S.; Linn.S.i.; N.H.M.; Oxon.B.; Oxon.R.; P.O.; R.A.S.i.; R.S.; U.C.L.i.

Bulletin Astronomique publié sous les Auspices de l'Observatoire de Paris. Paris.

1884- B.M.; Camb.U.; Edinb.R.S.; Oxon.R.; R.A.S.

Bulletin des Sciences Mathématiques. Paris.

1885- Camb.U.; Dub.T.C. ; Edinb.R.S.; Glasg.U.; Math.S.; Oxon.B.; Oxon.R.; R.A.S.; R.S.; U.C.L.

Bulletin des Sciences Mathématiques et Astronomiques. Paris.

1870-84. B.M.; Camb.U.; Edinb.R.S.; Math.S.; Oxon.R. ; R.A.S.i.; R.S.; U.C.L.i.

Bulletin Scientifique, Historique et Littéraire du Département du Nord et des pays voisins. Lille.

1869 - Camb.U.; Linn.S.; N.H.M.

Astronomisches Jahrbuch, nebst einer Sammlung der neuesten in

die astronomischen Wissenschaften einschlagenden Abhandlungen, Beobachtungen, und Nachrichten; Bode. Berlin.

1776-1829. Dub.T.C.i.; R.A.S.; R.S.i.

Abhandlungen der K. Böhmischen Gesellschaft der Wissenschaften. Prag.

1804-92. B.M.i.; Camb.P.S.; Camb.U.i.; Dub.R.I.A.i. ; Edinb. R.S.i.; Linn.S.i.; N.H.M.; Oxon.B.; R.S.i.

See Prag Ab.

Jahresbericht der Königl. Böhm. Gesellschaft der Wissenschaften. Prag.

1876- B.M.i.; Edinb.R.S.i.; Linn.S.i.; N.H.M.; R.S. 


\section{List of Serial Publications}

Bologna Ac. Mm. ......... (Memorie della Accademia delle Scienze dell' Istituto di Bologna. Bologna Ac. Sc. $\mathbf{M m}$. ...

Bologna Imm. Ac. ......... $\{1850$ - B.M. ; Camb.U. ; Dub.R.I.A.; Edinb.R.S.; N.H.M. ; Bologna $\mathbf{m m}$. Ac. sc. ... ( Oxon.B.; R.A.S.; R.S.; U.C.L.i.

Bologna mm. I. It. ...... Memorie dell' Istituto Nazionale Italiano: Classe di Fisica e di Matematica. Bologna.

1806-13. B.M.; Camb.U.; Dub.R.I.A.; Dub.T.C.; Edinb.R.S.i.; N.H.M.; Oxon.B.i.; R.S.i.

Bologna N. A.

Nuovi Annali delle Scienze Naturali; Alessandrini, Bertolini, Gherardi, e Ranzani. Bologna.

1838-54. Camb.U.; N.H.M.; Oxon.B.i.; R.S.

See N. A. Sc. Nt.

Bologna N. Cm.

Novi Commentarii Academiæ Scientiarum Instituti Bononiensis. Bononiæ.

1834-49. Camb.U.; Edinb.R.S.; N.H.M.; Oxon.B.; R.S.

Bologna Opusc. sc. ...... Opuscoli Scientifici. Bologna.

1817-23. B.M.; Camb.U.; Edinb.R.S.i.; N.H.M.

Bologna Opusc. sc. N. Nuova collezione d' Opuscoli Scientifici. Bologna.

Col.

Bologna Rd.

1824-25. Camb.U.

Rendiconto delle Sessioni dell' Accademia delle Scienze dell' Istituto di Bologna. Bologna.

1851- B.M.; Camb.U.i.; Dub.T.C.; Edinb.R.S.i.; N.H.M.i.; Oxon.B.i.; R.A.S.i.; R.S.i.; U.C.L.i.

Bône Ac. Fip. Bll.

Bulletin de l'Académie d'Hippone. Bône.

1865- Camb.U.; N.H.M.i.

Bonn Niedr. Gs. sb. ...... Sitzungsberichte der Niederrheinischen Gesellschaft für Natur- und Heilkunde zu Bonn. Bonn.

1854 - B.M.i.; Camb.U.i.; Edinb.R.S.i.; Linn.S.i.; N.H.M. ; Oxon.R.; R.S.i.

Bordeaux Ac. Act.

Recueil des Actes de l'Académie des Sciences, Belles-lettres, et Arts de Bordeaux. Bordeaux.

1839 - B.M.i.; Dub.R.I.A.i.; Dub.T.C.i.; N.H.M.; Oxon.B.i.; R.S. $i$.

See Bordeaux Act. Ac. Sc.

Bordeaux Ac. Sc. Sé. Pbl. |Séances publiques de l'Académie Royale des Sciences, Belles-lettres, Bordeaux Ac. Sé. Pbl....

et Arts de Bordeaux. Bordeaux.

$1819-37$. N.H.M.

Bordeaux Act. Ac. Sc. ... See Bordeaux Ac. Act.

Bordeaux Mm. s. Sc. ... (Mémoires de la Société des Sciences Physiques et Naturelles de Bordeaux $\mathbf{M m}$. s. sc. Ps. $\{$ Bordeaux. Bordeaux.

1855- Camb.P.S.; Dub.R.D.S.; Dub.T.C.; Edinb.R.S.; Glasg.P.S.; Linn.S.; Math.S.; N.H.M.; Oxon.B.; R.A.S.; R.S.

See Bordeaux s. Sc. IMm.

Bordeaux Obs. A.

Annales de l'Observatoire de Bordeaux. Paris, Bordeaux.

1885- Dub.R.D.S.; R.A.S.; R.S.

Bordeaux s. Sc. Imm. ... See Bordeaux Mm. S. Sc.

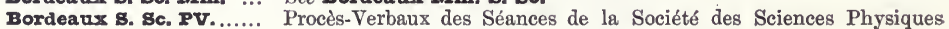
et Naturelles de Bordeaux. Paris, Bordeaux.

1894- Camb.P.S.; Dub.R.D.S.i.; Edinb.R.S.; Math.S.; N.H.M.; R.A.S.; R.S.

Bost. Am. Ac. Mm.

Memoirs of the American Academy of Arts and Sciences. Cambridge and Boston.

1785 - B.M.i.; Camb.P.S.; Camb.U.; Dub.R.D.S.i.; Dub.R.I.A.i.; Dub.T.C.i.; Edinb.R.S.; Linn.S.; N.H.M.; Oxon.R.; P.O.i.; R.A.S.; R.S.; U.C.L.i.

See Am. Ac. Mm. and Bost. Mm. Am. Ac.

Bost. J. Ph.

The Boston Journal of Philosophy and the Arts; Webster, etc. Boston. 1824-26. B.M.

Bost. Imm. Am. Ac.

See Am. Ac. Mm. and Bost. Am. Ac. Mm.

Braunschw. Vr. Nt. Jbr.

Jahresbericht des Vereins fïr Naturwissenschaft zu Braunschweig. Braunschweig, Altenburg.

1879 - Dub.R.I.A.i.; Edinb.R.S.; Linn.S.; N.H.M.; R.S.

Brem. Ab.

Abhandlungen herausgegeben vom Naturwissenschaftlichen Vereine zu Bremen. Bremen.

1868- B.M.; Camb.U.; Dub.R.D.S.i.; Dub.R.I.A.i.; Edinb.R.S.i.; Linn.S.; N.H.M.; R.S.

Brescia At. Cm.

(Commentarj della Accademia di Scienze, Lettere, ed Arti dell' Ateneo di Brescia. Brescia.

1808- B.M.; Camb.U.; N.H.M.i.; Oxon.B.i.; R.S.i. 


\section{List of Serial Publications}

Bresl, 51. Gs. Jbr.

Brest 8. Ac. Bu

Brugnatelli G.

Brünn Vh.

Brux. Ac. Bu.

Brux, Ac. Cent. Anniv...

Brux. Ac. IIm.

Brux. Ac. 8c. $\mathbf{m m}$.

Brax. A. Tr. Pbl.

Brux. A. Un.

Brux. Bll. Ac.

Brux. Mm. Ac. Bc.

Brux. Mm. Cour.

Brux. Mm. Coux. 4

Brux. Mm. Cour. $\mathbf{8}^{\circ}$.

Brux. Obs. A. (As.)

Brux. 8. As, Bu

Brux. s. Sc. A.

Bucarest Ac. Rom. A. ...

Bncarest s. Sc, Bl.

Cadiz Period. M. Ci.

Caen Ac. Mm.

Cæs. Ieop. Ac. N. Acta.
Jahresbericht der Schlesischen Gesellschaft für vaterländische Cultur. Breslau.

1850 - Dub.R.D.S.i.; Dub.R.I.A.i.; N.H.M.; R.S.

Bulletin de la Société Académique de Brest. Brest.

1858- B.M.; Camb.U.i.

Giornale di Fisica, Chimica, e Storia Naturale; Brugnatelli, etc. Pavia.

1808-27. B.M.; Camb.U.; Dub.T.C.; N.H.M.i.; Oxon.B.; P.O.; R.S.

Verhandlungen des Naturforschenden Vereins zu Brünn. Brünn.

1863 - Camb.U.i.; Dub.R.I.A.; Linn.S.; N.H.M.; R.S.

Bulletins de l'Académie Royale des Sciences, etc., de Belgique. Bruxelles.

1834 - B.M.i.; Camb.U.; Chem.S.i.; Dub.R.I.A.; Edinb.R.S.; Glasg.P.S.i.; Glasg.U.i.; Linn.S.; Math.S.i.; N.H.M.; Oxon.B.; Oxon.R.; P.O.; R.A.S.; R.S.

See Brux. B1l. Ac.

Centième Anniversaire de Fondation (1772-1872) de l'Académie Royale de Belgique. Bruxelles.

1872. B.M.; Camb.U.; Edinb.R.S.; Glasg.P.S.; Linn.S.; N.H.M.; P.O.; R.A.S.; R.S.

(Mémoires de l'Académie Royale des Sciences, des Lettres et des Beaux-Arts de Belgique. Bruxelles.

1820 - B.M.; Camb.P.S. ; Camb.U.; Dub.R.I.A.; Dub.T.C.; Edinb.R.S.i.; Edinb.U.; Linn.S.i.; N.H.M.; Oxon.B.(R.); P.O.i.; R.A.S.i.; R.S.

See Brux. IMm. Ac. Sc.

Annales des Travaux Publics de Belgique. Bruxelles.

1843 - B.M.; P.O.

Annales des Universités de Belgique. Bruxelles.

1842 -59. Camb.U.; Oxon.B.; P.O.; R.S.i.

See Brux. Ac. Bu.

See Brux. Ac. Sc. Mm.

(Mémoires Couronnés et Mémoires des Savants Etrangers, publ. par l'Acad. Roy. des Sciences, etc. de Belgique. 4to. Bruxelles.

1818 - B.M.i.; Camb.P.S.; Camb.U.; Dub.T.C.; Edinb.R.S.i.; Edinb.U.; Glasg.U.i.; Linn.S.i.; N.H.M.; Oxon.B.; P.O.i.; R.A.S.i.; R.S.

Mémoires Couronnés et autres Mémoires, publ. par l'Acad. Roy. des Sciences, etc. de Belgique. 8vo. Bruxelles.

1840 - B.M.; Camb.P.S. ; Camb.U.; Dub.T.C.; Edinb.R.S. Glasg.U.i.; Linn.S.i.; N.H.M.; Oxon.B.; P.O.; R.A.S.i.; R.S.

Annales de l'Observatoire Royal de Belgique. Bruxelles.

1834 - B.M.; Camb.U.; Dub.R.D.S.; Dub.R.I.A.; Edinb.R.S.; Glasg.P.S.i.; Glasg.U.i.; Oxon.B.i.; R.A.S.; R.S.; U.C.L.i.

Bulletin de la Société Belge d'Astronomie. Comptes Rendus des Séances mensuelles de la Société et Revue des Ściences d'Observation. Astronomie, Météorologie, Géodésie et Physique du Globe. Bruxelles.

1896- R.A.S.

Annales de la Société Scientifique de Bruxelles. Bruxelles.

1877 - B.M.; Dub.N.L.I.i.; Edinb.R.S.; N.H.M.

Analele Academiei Romane. Bucuresci.

1880 - B.M.; Camb.U.i.; N.H.M.i.

Buletinul Societătii de Sciinte Fizice (Fizica, Chimia si Mineralogia) din Bucuresci-România.

[1892]-[1896].

Buletinul Societătii de Sciinte din Bucuresci-România. Bucuresci. (Bulletin de la Société des Sciences Bucarest-Roumanie.)

[1897]- Glasg.P.S.; Glasg.U.; N.H.M.; R.S.i.; U.C.L.i.

Periódico mensual de Ciencias matemáticas y fisicas. Cadiz.

1848. B.M.; R.S.

Mémoires de l'Académie des Sciences, Arts et Belles-Lettres de Caen. Caen.

1811 - B.M.i.; Camb.U.i.; Dub.T.C.i.; N.H.M.i.; Oxon.B.i.; R.S.i. Nova Acta physico-medica Academiæ Cæs. Leopoldino-Carolinæ naturæ Curiosorum. Erlangen, Bonn, Breslau. 


\section{List of Serial Publications}

1758- Camb.P.S.; Camb.U.; Chem.S.i.; Dub.T.C.; Edinb.R.S.i.; Edinb. U.; Linn.S.i.; Math.S.i.; N.H.M.; Oxon.R.; R.A.S.i.; R.S.

See Ac. Cas. Ieop. N. Acta.

Calif. Ac. $\mathbf{P}$.

Proceedings of the California Academy of Natural Sciences. San Francisco.

1854 - B.M.i.; Camb.P.S.i.; Dub.R.I.A.; Edinb.R.S.i.; Glasg.P.S.; Linn.S.i.; N.H.M.; P.O.i.; R.S.i.

Camb. and Dubl. mth. J. The Cambridge and Dublin Mathematical Journal; Thomson and Ferrers. Cambridge.

1846-54. B.M.; Camb.P.S.i.; Camb.U.; Dub.T.C.i.; Edinb.R.S.; Edinb.U.; N.H.M.; Oxon.B.; R.S.; U.C.L.

Camb. Imm. Anal. s...... Memoirs of the Cambridge Analytical Society. Cambridge.

1813. B.M.; Camb.U.; Edinb.R.S.; P.O.; R.A.S.; R.S.; U.C.L. The Mathematical Monthly; Runkle. Cambridge (Massachusetts). 1859-61. B.M.; Camb.U.; Oxon.B.; P.O.; R.A.S.i.; R.S.; U.C.L. See Camb. (U.s.) Mth. M.

Camb. IMth. J.

The Cambridge Mathematical Journal. London.

1839-45. B.M.; Camb.P.S.; Camb.U.; Dub.T.C.; Edinb.U.; Glasg.U.; Math.S.i.; Oxon.B.i.; R.S.; U.C.L.

Camb. Ph. s. $\mathbf{P}$.

Proceedings of the Cambridge Philosophical Society. Cambridge.

1866- B.M.i; Camb.P.S.; Camb.U.; Chem.S.i.; Dub.R.D.S.; Edinb.R.S.; Edinb.U.; Glasg.P.S.i.; Glasg.U.i.; Linn.S.i.; Math.S.i.; N.H.M.; Oxon.B.i.; Oxon.R.i.; P.O.; R.A.S.i.; R.S.; U.C.L.

Camb. Ph. 8. T.

Transactions of the Cambridge Philosophical Society. Cambridge. 1822- B.M.; Camb.P.S.; Camb.U.; Chem.S.i.; Dub.T.C.i.; Edinb.R.S.; Edinb.U.; Glasg.P.S.i.; Glasg.U.; Linn.S.; Math. S.i.; N.H.M.; Oxon.B.; Oxon.R.; P.O.; R.A.S.; R.S.; U.C.L.

Camb. (U.8.) Mth. M.

Card. Nt. s. $\mathbf{x}$.

See Camb. (M.) Mth. Mr.

Cardiff Naturalists' Society. Reports and Transactions. Cardiff.

1868- B.M.i.; Camb.U.i.; Dub.R.D.S.; Geol.M.i.; Glasg.P.S.i.; Linn.S.; N.H.M.; Oxon.B.i.; R.S.i.

Carl Rpm.

Repertorium für physikalische Technik, für mathematische und astronomische Instrumentenkunde; Carl. München.

1865-91. B.M.; Camb.U.i.; Dub.N.L.I.i.; Oxon.R.; P.O.; R.S.

See Ixner Rpm.

Časopis

Časopis pro Pèstováni Mathematiky a Fysiky. Prag.

1872 - B.M.

Catania Ac. Gioen. At... Atti dell' Accademia Gioenia di Scienze Naturali in Catania. Catania.

1825- B.M.; Camb.U.i.; Dub.R.I.A.; Edinb.R.S.i.; Linn.S.i.; N.H.M.; Oxon.B.; R.S.

Catania Ac. Gioen. Bu. Bulletino mensile della Accademia Gioenia di Scienze Naturali in Catania. Catania.

1888 - Dub.R.I.A.; N.H.M.; R.S.

Catania At. Ac. Gioen...

CE. I. $\mathbf{P}$.

\section{See Catania Ac. Gioen. At.}

Minutes of Proceedings of the Institution of Civil Engineers, containing Abstracts of the Papers and of the Discussions. London.

1837- B.M.; Camb.P.S.; Camb.U.; Dub.R.C.S.; Dub.R.D.S.; Dub.R.I.A.; Edinb.R.S.; Edinb.U.; Glasg.P.S.i.; Glasg.U.; Oxon.B.; Oxon.R.i.; P.O.; R.S.; U.C.L.

See $\mathbf{I}$. Cr. $\mathbf{P}$.

Chambéry Mm. Ac. sav. Mémoires de la Société Académique de Savoie. Chambéry.

1825- Camb.U.; Dub.R.I.A.; Dub.T.C.; N.H.M.; Oxon.B.; R.S.i.

See Sav. Ac. Mm. and Sav. Mm. Ac.

Charleston Md. J. ........ Charleston Medical Journal and Review; Gaillard, de Saussure, etc. Charleston.

$1846-60$. B.M.

Chemnitz $\mathbf{B}$.

Bericht der Naturwiss. Gesellsch. zu Chemnitz. Chemnitz.

1859- Edinb.R.S.i.; N.H.M.; R.S.i.

Cherb. Mm. s. Ac.

Mémoires de la Société Académique de Cherbourg. Cherbourg.

1833 - B.M.; Camb.U.i.; Edinb.R.S.i.; N.H.M.i.; Oxon.B.i.

Cherb. Mm. s. Sc. Mémoires de la Société Impériale des Sciences Naturelles de Cherbourg. Cherbourg.

Cherb. 8. Sc. Imm. .........

Cherb. s. Sc. Nt. $\mathbf{M m}$.

\{1852- B.M.; Camb.P.S.; Camb.U.; Dub.R.I.A.; Edinb.R.S.i.; Linn.S.; N.H.M.; R.A.S.i.; R.S.

Anales de la Universidad de Chile. Santiago de Chile.

1843 - B.M.i.; Dub.T.C.; N.H.M.i.; Oxon.B.i.

See Santiago de Chile Un. A. 


\section{List of Serial Publications}

Chili s. sc. Act.

Christiania $\mathbf{r}$.

Christiania skr. (Mth.$N t . K l$.)

Ciel et Terre

Civing.

c. $\mathbf{N}$.

Cn. I. $\mathbf{P}$

Cn. I. T

Cn. J.

Cn. R. \$. P. \& T.

\section{Coimbra I}

Con. des Temps

Conn. Ac. T.

Conn. IIm. Ac.

Cornwall Pol. s. Rp.

Cornwall Pol. S. T.

Cornwall R. I. J.

C. $\mathbf{R}$.

Crc. Ac. Sc. Bll

Crelle J.

Crelle J. Mth.
Actes de la Société Scientifique du Chili (Sociedad científica de Chile). Santiago.

1892 - B.M.; Dub.R.D.S.i.; Dub.R.I.A.i.; Edinb.R.S.i.; Linn.S.i.; N.H.M.; R.S.i.

Forhandlinger i Videnskabs-Selskabet i Christiania. Christiania.

1859 - B.M. ; Camb.P.S.; Camb.U.; Dub.R.I.A. ; Edinb.R.S.; Glasg.P.S.; N.H.M.; Oxon.B.; R.S.; U.C.L.i.

Skrifter udgivne af Videnskabsselskabet i Christiania. Mathematisk-Naturvidenskabelig Klasse. Christiania.

1894- B.M.; Camb.P.S.; Dub.R.I.A.i.; Edinb.R.S.; Glasg.P.S.; N.H.M.; Oxon.B.; R.S.; U.C.L.i.

Ciel et Terre. Revue populaire d'Astronomie, de Météorologie et de Physique du Globe. Bruxelles.

1881 - B.M.; Edinb.R.S.i.; R.A.S.

Der Civilingenieur: Zeitschrift für das Ingenieurwesen. Freiberg, Leipzig.

1854 - B.M.; Camb.U.i.; Dub.R.I.A.i.; P.O.

The Chemical News and Journal of Physical Science. London.

1860 - Camb.P.S.; Camb.U.i.; Chem.S.; Dub.N.L.I.i.; Dub.R.D.S.i.; Dub.R.C.S.i.; Edinb.U.; Geol.M.; Glasg.P.S.; N.H.M.; Oxon.B.i.; Oxon.R.; P.O.; R.S.; U.C.L.i.

Proceedings of the Canadian Institute, Toronto. Toronto.

1879-90; 1897- B.M.; Camb.P.S.i.; Dub.R.D.S.; Edinb.R.S.i.; Edinb.U.; Glasg.P.S.; Math.S.i.; N.H.M.; Oxon.B.; P.O.; R.A.S.i.; R.S.

Transactions of the Canadian Institute. Toronto.

1889 - B.M.; Camb.P.S.; Dub.R.D.S.; Edinb.R.S.; Edinb.U.; Glasg.P.S.; Linn.S.; Math.S.i.; N.H.M.; P.O.; R.A.S.; R.S.

The Canadian Journal of Industry, Science, and Art. Toronto.

1853-78. B.M.i.; Dub.R.I.A.; Edinb.R.S.; N.H.M.; P.O.; R.A.S.i.; R.S.

Proceedings and Transactions of the Royal Society of Canada. Montreal.

1883 - Camb.P.S.; Camb.U.; Chem.S.; Dub.R.D.S.i.; Dub.R.I.A.i.; Dub.T.C.i.; Edinb.R.S.; Geol.M.i.; Glasg.P.S.; Linn.S.; N.H.M.; Oxon.B.; Oxon.R.; P.O.; R.A.S.; R.S.; U.C.L.

O Instituto, jornal scientifico e litterario; Forjaz. Coimbra.

1853 - B.M.

Connaissance des Temps, à l'usage des Astronomes et des Navigateurs. Paris.

1679 - B.M.i.; Camb.U.; Dub.T.C.; Oxon.B.; R.A.S.i.; R.S.

Transactions of the Connecticut Academy of Arts and Sciences. New Haven.

1866- B.M.; Camb.P.S.; Camb.U.; Dub.R.I.A.; Edinb.R.S. ; Glasg.P.S.; Linn.S.; Math.S.i.; N.H.M.; Oxon.R.; P.O.; R.A.S.; R.S.

Memoirs of the Connecticut Academy of Arts and Sciences. New Haven.

1810-16. Linn.S.i.; N.H.M.i.; R.S.

(Reports and Transactions of the Royal Polytechnic Society of Cornwall. Falmouth.

1833 - B.M.; Camb.U.i.; Dub.R.D.S.; Edinb.R.S.i.; Glasg.P.S.i.; Glasg.U.i.; Linn.S.; N.H.M.; Oxon.B.i.; P.O.; R.S.; U.C.L.i. Journal of the Royal Institution of Cornwall. Truro.

1864- B.M.; Camb.U.i.; Dub.R.D.S.; Edinb.R.S.; Glasg.P.S.i.; N.H.M.; Oxon.B.i.; P.O.; R.A.S.i.; R.S.i.; U.C.L.i.

Comptes Rendus hebdomadaires des Séances de l'Académie des Sciences. Paris.

1835- B.M. ; Camb.P.S. ; Camb.U.; Chem.S.; Dub.R.D.S.; Edinb.R.S.i.; Edinb.U.; Geol.M.i.; Glasg.P.S.i.; Linn.S.; N.H.M.; Oxon.B.; Oxon.R.i.; P.O.; R.A.S.; R.S.; U.C.L.

Bulletin International de l'Académie des Sciences de Cracovie. Cracovie.

1889- B.M.; Camb.U.; Chem.S.; Dub.R.I.A.i.; Edinb.R.S. ; Glasg.U.; N.H.M.; Oxon.B.; Oxon.R.i.; R.A.S.i.; R.S.; U.C.L.i.

Journal für die reine und angewandte Mathematik; Crelle. Berlin. 1826- B.M. ; Camb.U. ; Dub.N.L.I. ; Dub.T.C.; Edinb.R.S. ; Edinb.U.; Glasg.U.; Math.S.i.; Oxon.B.(R.); R.S.; U.C.L.

xix 


\section{List of Serial Publications}

C. 8. $\boldsymbol{~}$.

Cuyper Rv. Un.

Cztg. Opt.

D. Alpvr. Z.

Danzig N. Schr.

Danzig Schr.

Delft گ̇́c. Pol. A.

Des Molnes Anal.

Devon. As. $T$.

D. Gs. Ostas. Mt.

Dijon Ac. Mm.

Dijon Ac. Sc. Mm.

Dijon Mm. Ac.

Dijon Ac. sé.

Dijon sé. Ac.

Dinglex

D. Ith. Vr. Jbr.

D. Nf. $\mathbf{B}$.

D. Nf. Thl.

D. NE, $\mathbf{T h}$.

D. NE. Vsm. $\mathbf{B}$.

Dn. vd. Belak. Skr.
The Quarterly Journal of the Chemical Society of London. London. 1849- B.M.; Camb.P.S.; Camb.U.; Chem.S.; Dub.R.C.S.; Dub.R.D.S.; Dub.R.I.A.; Dub.T.C.; Edinb.R.S.; Geol.M.i.; Glasg.P.S. ; N.H.M.i.; Oxon.B.; Oxon.R.i.; P.O. ; R.S.; U.C.L.

Revue Universelle des Mines, de la Métallurgie, des Travaux Publics, des Sciences, et des Arts, appliqués à l'Industrie; de Cuyper. Paris et Liège.

1857- B.M.; Camb.U.; Dub.R.I.A.i.; Glasg.P.S.i.; Glasg.U.i.; N.H.M.; P.O.

See Rv. Un. Mines.

Central-Zeitung für Optik und Mechanik. Leipzig.

1880- Edinb.U.i.; P.O.i.; R.S.i.

Zeitschrift des Deutschen [und des Oesterreichischen] Alpenvereins. München.

1870 - B.M.; Camb.U.; Oxon.B.

Neueste Schriften der Naturforschenden Gesellschaft in Danzig. Danzig.

1820-62. B.M.i.; Dub.R.I.A.i.; N.H.M.; Oxon.R.; R.S.

Schriften der Naturforschenden Gesellschaft in Danzig. Danzig.

1863- Camb.P.S.; Camb.U.i.; Dub.R.D.S.i.; Dub.R.I.A.i.;

Edinb.R.S.i.; Linn.S.i.; N.H.M.; Oxon.R.i.; R.S.

Annales de l'École Polytechnique de Delft. Leide.

1885-97. Camb.P.S.; Camb.U.; Chem.S.i.; Dub.R.D.S.; Dub. R.I.A.; Edinb.R.S.; Math.S.; R.A.S.; R.S.

The Analyst: a monthly Journal of Pure and Applied Mathematics. Des Moines, Iowa.

1874-83. Camb.U.; Edinb.R.S.; R.S.

Reports and Transactions of the Devonshire Association for the Advancement of Science, Literature, and Art. Plymouth and London.

1862- Camb.U.i.; Geol.M.; Linn.S.; N.H.M.; Oxon.B.; Oxon. R.i.; P.O.; R.S.

Mittheilungen der Deutschen Gesellschaft für Natur- und Völkerkunde Ostasiens. Yokohama.

1873- B.M.; Edinb.R.S.i

Mémoires de l'Académie des Sciences, Arts, et Belles-lettres de Dijon. Dijon.

1769- B.M.i.; Camb.U.; Dub.R.D.S.i.; Dub.R.I.A.i.; Dub.T.C.i.; Edinb.R.S.i.; N.H.M.; Oxon.B.i.; R.A.S.; R.S.i.

(Séances publiques de l'Académie des Sciences, Arts, et Belles-lettres de Dijon. Dijon.

1810-29. B.M.i.; N.H.M.

Polytechnisches Journal; Dingler. Stuttgart.

1820- B.M.; Camb.U.; Chem.S.i.; Dub.N.L.I.i.; Dub.R.C.S.i.; Dub.R.D.S.i.; Edinb.R.S.; Glasg.P.S.i.; Glasg.U.i.; P.O.; R.S.i.

Jahresbericht der deutschen Mathematiker-Vereinigung. Berlin, Leipzig.

1890 - Camb.U.; Dub.R.I.A.; Edinb.R.S.i.; Math.S.i.; Oxon.B.; R.S.

Bericht über die Versammlung der Deutschen Naturforscher und Aerzte.

1822-83. Irregular, see Tageblatt. Camb.U.i.; N.H.M.i.; Oxon. R.i.; R.S. $i$.

See D. Nf. Vsm. B.

Tageblatt der...Versammlung Deutscher Naturforscher und Aerzte.

1836 - Irregular, see Ber. and Verh. Camb.U.; N.H.M.; Oxon. R.i.

Verhandlungen der Gesellschaft Deutscher Naturforscher und Aerzte. Leipzig.

1890- Camb.U.; N.H.M.; Oxon.R.

See D. Xf. B.

Det Kongelige DanskeVidenskabernes Selskabs Skrivter. Kiöbenhavn. 1801-18. B.M.; Camb.P.S.i.; Camb.U.; Edinb.R.S.; N.H.M.; Oxon.B.; R.S.

See Kdöb. Dn. vd. selsk. skr. 


\section{List of Serial Publications}

Dorpat sb.

Douai Mm. S. Ag.

Doubs s. $\mathbf{M m}$.

Dresden Isis $\mathbf{S b}$

Dresden Sb. Isis

Dubl. Ph. J.

Dubl. R. S. J.

Dubl. s. Sc. $\mathbf{P}$.

Dnbl. s. Sc. T.

Dunkerque $\mathbf{M m}$. S. Incour.

zdinb. J. 8c.

Fdinb. Mth. S. P.

Fdinb. N. Ph. J.

Fdinb. Ph. J.

Fdinb. P. R. S

Fdinb. R. $\mathbf{s}$. $\mathbf{P}$

Fdinb. R. \$. T.

Fdinb. Sc. S. Arts T.
Sitzungsberichte der Naturforscher-Gesellschaft zu Dorpat. Dorpat. 1861 - Dub.R.I.A.i.; Edinb.R.S.i.; Geol.S.; N.H.M.; R.S.i.

Mémoires de la Société d'Agriculture, de Sciences, et d'Arts, séant à Douai. Douai.

1826- B.M.; Camb.U.i.; Oxon.B.i.; R.S.i.

Mémoires et Comptes Rendus de la Société [Libre] d'Émulation du Doubs. Besançon.

1841 - B.M.; N.H.M. $i$.

(Sitzungsberichte der Naturwissenschaftlichen Gesellschaft Isis in Dresden. Dresden.

1861 - Camb.U.i.; Dub.T.C.; Geol.S.; N.H.M.

The Dublin Philosophical Journal and Scientific Review. Dublin. 1825-26. B.M.; Dub.R.D.S.; Dub.T.C.; Edinb.R.S.i.; N.H.M.; - Oxon.B.; Oxon.R.; R.S.i.

Journal of the Royal Dublin Society. Dublin.

1856-75. B.M.; Camb.P.S.; Camb.U.; Chem.S.i.; Dub.N.L.I.; Dub.R.C.S.; Dub.R.D.S.; Dub.R.I.A.; Geol.M.; Glasg.U.i.; Linn.S.; N.H.M.; Oxon.B.; Oxon.R.i.; P.O.; R.A.S.; R.S.

The Scientific Proceedings of the Royal Dublin Society. Dublin.

1877- B.M.; Camb.P.S.; Camb.U.; Chem.S.; Dub.N.L.I.; Dub. R.C.S.; Dub.R.D.S.; Dub.R.I.A.; Edinb.R.S.; Geol.M.; Glasg. P.S. ; Linn.S.; Math.S.i.; N.H.M.; Oxon.B.; Oxon.R.; P.O.; R.A.S.; R.S.; U.C.L.i.

The Scientific Transactions of the Royal Dublin Society. Dublin.

1877- B.M.; Camb.P.S.; Camb.U.; Chem.S.; Dub.N.L.I.; Dub. R.C.S.; Dub.R.D.S.; Dub.R.I.A.; Dub.T.C.; Edinb.R.S.; Geol.M.; Glasg.P.S.; Linn.S.; Math.S.; N.H.M.; Oxon.B.; Oxon.R.; P.O.; R.A.S.; R.S.; U.C.L.i.

Mémoires de la Société Dunkerquoise pour l'Encouragement des Sciences, des Lettres, et des Arts. Dunkerque.

1853 - B.M.; Oxon.B.; R.S.i.

The Edinburgh Journal of Science, exhibiting a view of the progress of discovery in Natural Philosophy, Chemistry, Mineralogy, Geology, Botany, etc. ; David Brewster. Edinburgh.

1824-1832. B.M.; Camb.U.; Dub.T.C.; Edinb.R.S.; Edinb.U.; Glasg.P.S.i.; Glasg.U.; N.H.M.; Oxon.B.i.; Oxon.R.; P.O.; R.S.

Proceedings of the Edinburgh Mathematical Society. London and Edinburgh.

1883- B.M.; Camb.P.S.; Camb.U.; Edinb.R.S.; Edinb.U.; Glasg.U.; Math.S.; R.S.i.

The Edinburgh New Philosophical Journal, exhibiting a view of the progressive Improvements, etc. in the Sciences, etc.; Robert Jameson. Edinburgh.

1826-64. B.M.; Camb.P.S.; Camb.U.; Chem.S.i.; Dub.T.C.i.; Edinb.R.S.; Edinb.U.; Glasg.P.S.; Linn.S.i.; N.H.M.; Oxon.B.; Oxon.R.; P.O.; R.A.S.i.; R.S.

The Edinburgh Philosophical Journal, exhibiting a view of the Progress of Discovery in Natural Philosophy, etc.; David Brewster and Robert Jameson. Edinburgh.

1819-26. B.M.; Camb.P.S.; Camb.U.; Chem.S.; Dub.T.C. ; Edinb.R.S.; Edinb.U.; Glasg.P.S.; Linn.S.; N.H.M.; Oxon.B.i.; Oxon.R.; P.O.; R.A.S.; R.S.; U.C.L. $i$.

(Proceedings of the Royal Society of Edinburgh. Edinburgh.

1845- B.M.i.; Camb.P.S.;- Camb.U.; Chem.S.i.; Dub.N.L.I.; Edinb.R.S.; Edinb.U.; Geol.M.i.; Glasg.P.S.; Linn.S.; Math.S.i.; N.H.M.; Oxon.B.i.; Oxon.R.; P.O.i.; R.A.S.; R.S.; U.C.L.i.

Transactions of the Royal Society of Edinburgh. Edinburgh.

1788 - B.M.; Camb.P.S.; Camb.U.; Chem.S.; Dub.N.L.I.; Dub. R.I.A.; Edinb.R.S.; Edinb.U.; Geol.M.i.; Glasg.P.S.; Glasg.U.; Linn.S.; Math.S.i.; N.H.M.; Oxon.B.i.; Oxon.R.; P.O.i.; R.A.S.; R.S.; U.C.L.

See Fdinb. T. R. 8.

Transactions of the Royal Scottish Society of Arts. Edinburgh.

1841 - B.M.i.; Camb.U.; Dub.R.D.S.; Edinb.R.S.; Edinb.U.; Glasg.P.S.; Glasg.U.; P.O.; R.S.

See Fdinb. T. sc. 8. Arts and s. Sc. Arts T. 


\section{List of Serial Publications}

Edinb. T. R. S rdinb, T. Sc. S. Arts..... 2duc. Times

Flect.

Ilektech. Z.

Fng. 8. T.

Ins. Ith.

Ípinal (Vosg.) A.

Frdm. J. Pr. C.

Erfurt Ak. Jb.

Erfurt N. Acta

Frlang. Ps. Md. s. sb. Frlang. sb. Ps. Md. \$.

Erman Arch. Rs.

Fure J. d'Ag.

Fure Rec. 8. Ag.

Pure Rec. Tr.

Fure 8. Ag. Rec. Évk.

Ixner Rpm.

Férussac Bll. Sc. Mrth.

Finist. s. sc. Bu.

Firenze Ac. Georg. At... Firenze At. Ac. Georg... Firenze Opusc. Sc.

Förster Al. Banztg.

Frankilin I. J.

\section{See Fdinb. R. s. T.}

See Idinb. Sc. s. Arts T. and Sc. s. Arts T.

The Educational Times, and Journal of the College of Preceptors. London.

1847- B.M.; Camb.P.S.i.; Camb.U.i.; Dub.N.L.I.; Math.S.i. ; Oxon.B.i.; Oxon.R.i.; R.S.i.

The Electrician. London.

1862- B.M.i.; Camb.P.S.i.; Camb.U.i.; Dub.N.L.I.i.; Dub. R.C.S.i.; Edinb.R.S.i.; Edinb.U.i.; Glasg.P.S.i.; Glasg.U.i. ; Oxon.B.i.; Oxon.R.i.; P.O.; R.S.i.; U.C.L.i.

Elektrotechnische Zeitschrift. Berlin, München.

1880 - B.M.; Glasg. U.; P.O.

Transactions of the Society of Engineers. London.

1860 - B.M.; Camb.U.; Dub.N.L.I.; Dub.R.C.S.; Glasg.P.S.i.; Oxon.B.; P.O.; R.S.i.

L'Enseignement Mathématique. Revue Internationale. Paris. 1899- Math.S.

Annales de la Société d'Émulation du département des Vosges. Épinal. 1831- B.M.; R.S.i.

Journal für praktische Chemie; Erdman, etc. Leipzig.

1834 - B.M.; Camb.U.; Chem.S.; Dub.N.L.I.i.; Dub.R.C.S.i.; Dub.R.D.S.i.; N.H.M.; Oxon.B.; Oxon.R.; R.S.; U.C.L.i.

Jahrbücher der königlichen Akademie gemeinnütziger Wissenschaften zu Erfurt. Erfurt.

1860 - B.M.; N.H.M.

Nova Acta Academiæ Electoralis Moguntinæ Scientiarum utilium quæ Erfurti est. Erfurti.

1799-1809. B.M.i.; Camb.U.; N.H.M.; Oxon.B.i.; R.S.

(Sitzungsberichte der Physikalisch-Medicinischen Societät zu Er$\{$ langen. Erlangen.

1864- B.M.; Camb.P.S.; Dub.R.D.S.; Edinb.R.S.i.; Glasg.U.i.; Linn.S.i.; Math.S.i.; N.H.M.; R.S.i.

Archiv für wissenschaftliche Kunde von Russland; Erman. Berlin. 1841-67. B.M.; Camb.U.; N.H.M.; Oxon.B.; R.S.i.

Journal d'Agriculture, de Médecine et des Ściences accessoires. Evreux.

1824-29. B.M.; R.S.

Recueil de la Société d'Agriculture, Sciences, Arts, et Belles-lettres du département de l'Eure. Evreux.

1830-39. B.M.; Camb.U.; Oxon.B.; R.S.

Recueil des Travaux de la Société Libre d'Agriculture, des Sciences, des Arts et des Belles-Lettres du département de l'Eure. Evreux. 1841 - B.M.; Camb.U.; R.S.

See rure Rec. 8. Ag.

A'Magyar Tudós Társaság' Évkőnyvei. Pest. Continued as: A'Magyar Tudományos Ảkademia Evkőnyvei. Budá.

1833-89. B.M.; Edinb.R.S.i.; N.H.M.; Oxon.B.; R.A.S.i.; R.S.; U.C.L. $i$.

Repertorium der Physik; Exner. München, Leipzig.

1865-91. B.M.; Camb.U.i.; Dub.N.L.I.i.; Edinb.U.; Oxon.R.; P.O.; R.S.

See Carl Rpm.

Bulletin des Sciences Mathématiques, Astronomiques, Physiques, et Chimiques; Baron de Férussac. Paris.

1824-31. B.M.; Edinb.U.i.; Oxon.R.; P.O.; U.C.L.

Bulletin de la Société d'Études Scientifiques du Finistère. Morlaix. 1879 - N.H.M.

IAtti della R. Accademia economico-agraria dei Georgofili. Firenze.

1818 - Camb.U.; Dub.T.C.i.; Edinb.R.S.i.; Oxon.B.

Collezione d' Opuscoli scientifici, etc. Firenze.

1807-18. B.M.; N.H.M.

Allgemeine Bauzeitung; Förster. Wien.

1836- B.M.; Camb.U.; P.O.

Journal of the Franklin Institute of the State of Pennsylvania. Philadelphia.

1828 - B.M. ; Camb.U.; Chem.S.i.; Dub.R.I.A.; Glasg.P.S.i.; Oxon.B.; P.O.; R.A.S.i.; R.S.; U.C.L.i.

xxii 


\section{List of Serial Publications}

Fr. Cg. Ird. Chir.

Fr. $\mathrm{Cg}$. Sc.

Freiburg B.

Fr. S. Ag. Mm.

Fr. S. Mn. B11.

Fschr. Mth.

Fschr. Ps.

Gand. A. Ac.

G. Arcad.

Gard Aperçn Tr.

Gard Mm. Ac.

Gard Not. Tr. Ac.

Gard Tr. Ac.

Gen. I. Nt. Mm.

Gen. $\mathbf{M m}$. $\mathbf{s} . \mathbf{P s}$.

Genova $\mathbf{M m}$. Ac.

Genova IMm. I. Iigure

Genova Un. At.

Gen. S. Ps. IMm.

Gergonne A. Mth.

Gg. \$. J.

Gg. s. $\mathbf{P}$

Giamb. Vico

Gee $\mathbf{n}$ Giamb. Vico.

Berichte der Oberhessischen Gesellschaft für Natur- und Heilkunde. Giessen.

1847- B.M.i.; Camb.P.S.; Dub.R.D.S.i.; Dub.R.I.A.i.; Edinb. R.S.i.; Glasg.P.S.i.; Linn.S.i.; N.H.M.; P.O.i.; R.S.i.

xxiii

Congrès Médico-Chirurgical de France. Paris.

1863 - B.M.

Sessions des Congrès Scientifiques de France.

Berichte über die Verhandlungen der Naturforschenden Gesellschaft zu Freiburg i. B. Freiburg i. B.

1855- B.M. ; Camb.U.i. ; Dub.R.I.A. ; Linn.S.i. ; N.H.M. ; Oxon.R.; R.S.

Mémoires d'Agriculture, d'Économie rurale et domestique publiés par la Société d'Agriculture. Paris.

1801- B.M.; Edinb.R.S.i.; Oxon.B.

Bulletin de la Société Minéralogique de France. Meulan, Paris.

1878- B.M.; Dub.T.C.; Geol.M.; N.H.M.; Oxon.R.; R.S.

Jahrbuch über die Fortschritte der Mathematik. Berlin.

1868 - B.M.; Camb.U.; Dub.N.L.I. ; Dub.R.C.S.; Edinb.U. ; Glasg.P.S.i.; Glasg.U.; Math.S.; Oxon.R.; R.S.; U.C.L.

Die Fortschritte der Physik. Berlin.

1845- Camb.P.S.; Camb.U.; Chem.S.; Dub.N.L.I.i.; Dub.R.I.A.i.; Edinb.R.S.i.; Edinb.U.; Glasg.U.; Oxon.B.(R.); P.O.; R.A.S.i.; R.S.; U.C.L.

Annales Academiæ Gandavensis. Gandavi. (Ghent.)

1819-31. B.M.; Camb.U.; N.H.M.; Oxon.B.

Giornale Arcadico di Scienze, etc. Roma.

1819 - B.M.; N.H.M.i.; Oxon.B.

Notice [ou Aperęu analytique] des Travaux de l'Académie Royale du Gard. Nîmes.

1807-? B.M.; Camb.U.; Oxon.B.

See Gard Not. Tr. Ac. and Gard Tr. Ac.

Mémoires de l'Académie du Gard. Nîmes.

1832 - B.M.; Camb.U.; Oxon.B.; R.S.i.

See Gard Aperçu Tr.

Mémoires de l'Institut National Génevois. Genève.

1854 - B.M.; Camb.U.; Dub.R.D.S.; N.H.M.i.; Oxon.B.; R.S.

Mémoires de la Société de Physique et d'Histoire Naturelle de Genève. Genève.

1821 - B.M.; Camb.P.S.; Camb.U.; Dub.R.I.A.; Dub.T.C. ; Edinb.R.S.; Glasg.U.i.; Linn.S.; N.H.M.; Oxon.B.; Oxon.R.i.; R.A.S.i.; R.S.; U.C.L. $i$.

See Gen. S. Ps. IMm.

Memorie dell' Accademia Imperiale delle Scienze di Genova. Genova. 1809-14. Camb.U.i.; N.H.M.i.; R.S.

Memorie dell' Istituto Ligure. Genova.

1806. B.M.; Camb.U.; R.S.

Atti della R. Università di Genova. Genova.

1869- B.M.; Camb.U.; N.H.M.

$\mathrm{See}$ Gen. $\mathbf{M m}$. S. Ps.

Annales de Mathématiques, pures et appliquées; Gergonne. Nîmes et Paris.

1810-31. B.M.; Dub.T.C.; Edinb.U.i.; Glasg.U.; Oxon.B.(R.); R.A.S.i.; R.S.; U.C.L.

Journal of the Royal Geographical Society of London. London.

1832-80. B.M.; Camb.P.S.i.; Camb.U.; Dub.R.D.S.; Dub.R.I.A.;

Edinb.R.S.; Edinb.U.; Geol.M.; Glasg.P.S.; Glasg.U.; Linn.S.;

N.H.M.; Oxon.B.; Oxon.R.; P.O.; R.S.

Proceedings of the Royal Geographical Society of London. London. 857-92. Camb.P.S.i.; Camb.U.; Dub.T.C.; Edinb.R.S.; Geol.M.; Glasg.P.S.i.; Glasg.U.; Linn.S.; N.H.M.; Oxon.B.; Oxon.R.; P.O.i.; R.A.S.i.; R.S.

Il Giambattista-Vico, giornale scientifico. Napoli.

1857. N.H.M.; Oxon.B.i 


\section{List of Serial Publications}

Gilbert A

Giu Tech. Rep.

Glasg. I. Jng. T

Glass. P. Ph. 8 .

Glasg. Ph. S. P.

Gleanings 8c.

c1. $M g$

G1. 8. QJ

G. Mt.

Görl. Ab.

Göteb. Indl.

Gött. Ab

Gött. Cm.

Gött. Nr.

Gött. stud.

Gould As. J.

's Gravenh. I. Ing. Ts. ..

Grenoble Ac. Delph. BL.

Groningen A. Ac.

Gruithuisen N. Analekt.

Grunert Arch.
Annalen der Physik; Gilbert. Halle und Leipzig.

1799_1824. Camb.U.; Chem.S.; Edinb.U.; N.H.M.; Oxon.B.(R.); P.O.; R.S.

The Technical Repository; Gill. London.

1822-27. B.M.; Camb.U.; Edinb.R.S.i.; Oxon.B.; P.O.; R.S.i.

Transactions of the Institution of Engineers [and Shipbuiders] in Scotland. Glasgow.

1857- Camb.U.i.; P.O.; U.C.L.i.

Proceedings of the Philosophical Society of Glasgow. Glasgow.

1841 - B.M.; Camb.P.S.; Camb.U.; Dub.R.D.S.; Dub.R.I.A.; Edinb.R.S.; Geol.M.i.; Glasg.P.S.; Glasg.U.i.; N.H.M.; Oxon.B.; P.O.i.; R.A.S.; R.S.

Gleanings in Science. Calcutta.

1829-31. B.M.; Edinb.R.S.i.; N.H.M.; U.C.L.i.

The Geological Magazine or Monthly Journal of Geology. London.

1864 B.M.; Camb.U.; Dub.N.L.I.; Dub.R.C.S.; Geol.M. ; Glasg.P.S.; Glasg.U.; Linn.S.; N.H.M.; Oxon.R.; P.O.i.; U.C.L. The Quarterly Journal of the Geological Society of London. London. 1845 - B.M.; Camb.P.S.; Camb.U.; Chem.S.i.; Dub.N.L.I.; Geol.M.; Glasg.U.; Linn.S.; N.H.M.; Oxon.B.; Oxon.R.; P.O.; R.A.S.; R.S.; U.C.L.

Giornale di Matematiche ad uso degli Studenti delle Università Italiane; Battaglini. Napoli.

1863 - B.M.; Camb.U.; Dub.R.C.S.i.; Dub.R.I.A.i.; Math.S.i.; Oxon.B.; R.S.; U.C.L.i.

Abhandlungen der Naturforschenden Gesellschaft zu Görlitz. Görlitz. 1827- B.M.; Camb.U.i.; Dub.R.D.S.i.; Dub.R.I.A.i.; N.H.M.; R.S.

Göteborgs Kongl. Vetenskaps och Vitterhets Samhälles Handlingar. Göteborg.

1850 - B.M.; Camb.P.S.i.; Dub.R.D.S.i.; Dub.R.I.A.i.; Edinb. R.S.i.; N.H.M.; R.S.i.

Abhandlungen der k. Gesellschaft der Wissenschaften. Göttingen.

1843- B.M.; Camb.P.S.; Camb.U.; Dub.R.I.A.; Edinb.R.S.i.; Glasg.U.; Linn.S.; N.H.M.; Oxon.B.; Oxon.R.; R.S.; U.C.L.i.

Commentationes Societatis Regiæ Scientiarum Göttingensis. Gottingæ.

1778-1808. B.M.i.; Camb.U.; Dub.R.I.A.i.; Edinb.R.S.; Glasg. U.i.; N.H.M.; Oxon.B.; R.S.; U.C.L.

Commentationes recentiores Societatis, etc. Gottingæ.

1808-37. B.M.; Camb.U.; Edinb.R.S.i.; N.H.M.; Oxon.B. ; Oxon.R.; R.A.S.i.; R.S.; U.C.L.

Nachrichten von der $k$. Gesellschaft der Wissenschaften und der Georg-Augusts-Universität zu Göttingen. Göttingen.

1845- B.M.; Camb.P.S.; Camb.U.i.; Dub.R.D.S.i.; Dub.R.I.A.i.; Dub.T.C.i.; Edinb.R.S.; Glasg.U.; Linn.S.; Math.S.i.; N.H.M.; Oxon.B.; Oxon.R.; R.A.S.i.; R.S.

Göttinger Studien; Krische. Göttingen.

1845-47. N.H.M.; Oxon.B.i.; R.S.i.

The Astronomical Journal; Gould. Cambridge, Mass.

1851-61. B.M.; Camb.U.; Glasg.U.i.; Oxon.B.; Oxon.R.i. ; R.A.S.; R.S.

See As. J.

Tijdschrift van het Koninklijk Instituut van Ingenieurs. 's Gravenhage.

1869- B.M.

Bulletin de l'Académie Delphinale, ou Société des Sciences et Arts de Grenoble. Grenoble.

1846 - B.M.; Camb.U.i.; Oxon.B.; R.S.i.

Annales Academiæ Groninganæ. Groningæ.

1815-37. B.M.i.; Camb.U.; Dub.T.C.; Oxon.B.; R.S.

Neue Analekten für Erd- und Himmelskunde. Gruithuisen. München.

1832-36. B.M.; R.A.S.; R.S.

Archiv der Mathematik und Physik; Grunert. Greifswald, Leipzig. 1841 - B.M. ; Camb.U.; Dub.N.L.I.; Dub.R.C.S.; Edinb.U. ; Glasg. U.; Math.S.i.; Oxon.B.(R.); R.S.; U.C.L.i.

See Arch. Mrth. Ps. 


\section{List of Serial Publications}

G. Teix. J. Sc.

Inarl. Ms. Teyl. Arch.... Archives du Musée Teyler. Haarlem.

1866- B.M.; Camb.P.S.; Camb.U.; Dub.R.I.A.i.; Edinb.R.S.; Glasg.P.S.; N.H.M.; Oxon.R.; R.A.S.; R.S.

Eaarl. Ntk. Vh. Mtsch... Natuurkundige Verhandelingen van de [Bataafsche] Hollandsche Maatschappij der Wetenschappen te Haarlem. Haarlem.

1799 - B.M.; Camb.U.i.; Dub.R.D.S.; N.H.M.; R.S.

Inaidinger Ab.

Naturwissenschaftliche Abhandlungen; Haidinger. Wien.

1847-51. Camb.U.; Chem.S.i.; Edinb.R.S.i.; Linn.S.; N.H.M.; R.A.S.i.; R.S.

Faidinger $\mathbf{B}$.

Berichte über die Mittheilungen von Freunden der Naturwissenschaften in Wien; Haidinger. Wien.

1847-51. Camb.U.; Chem.S.i.; Edinb.R.S.; Linn.S.; N.H.M.; R.A.S.; R.S.

Hain. Mm. $\mathbf{s}$. Mémoires et Publications de la Société des Sciences, des Arts et des ) Lettres du Hainaut. Mons.

1839 - B.M.; Dub.T.C.i.; N.H.M.; Oxon.B.i.; R.S.i.

Fall Bij.

Bijdragen tot de Natuurkundige Wetenschappen; Hall, etc. Amsterdam.

1826-32. B.M.; Camb.U.; N.H.M.; R.S.

Falle Ab. Nt. Vr.

Abhandlungen des Naturwissenschaftlichen Vereins für Sachsen und Thüringen in Halle; Giebel. Berlin.

1860- B.M.; Camb.U.; N.H.M.i.; R.S.i.

Bericht der Naturforschenden Gesellschaft zu Halle. Halle.

Ialle Nf. Gs. B.

1856- Camb.U.i.; Dub.R.D.S.i.; Dub.R.I.A.i.; Oxon.R.i.; R.S

rialle z.

Falle Z. Nw.

Zeitschrift für die gesammten Naturwissenschaften; herausgegeben von dem Naturwissenschaftlichen Vereine für Sachsen und Thüringen in Halle; Giebel. Berlin.

1853- B.M.; Camb.U.i.; Dub.R.D.S.i.; Dub.R.I.A.i.; Dub.T.C.i.; Edinb.R.S.; Linn.S.; N.H.M.; Oxon.B.; Oxon.R.; R.S.

See $\mathbf{Z}$. Nw.

Iamb. Mth. Gs. Mt. ..... Mitteilungen der Mathematischen Gesellschaft in Hamburg. Leipzig. 1889 - Math.S.

Allgemeine Oesterreichische Zeitschrift für den Landwirth, Forstmann und Gärtner; Hammerschmidt. Wien.

Frammerschmidt Al. Oestr. z.

Tamn. Archt. Fann. Z. Archt.-Vr.

\{Zeitschr. des Architekten- und Ingenieur-Vereins zu Hannover. Hannover.

1855- Camb.U.i.; P.O.

Feid. Nt. IId. Vh.

Freidl. Vh. Nt. Ima.

Verhandlungen des Naturhistorisch-Medicinischen Vereins zu Heidelberg. Heidelberg.

1857- Camb.U.i.; Chem.S.i.; Dub.R.I.A.; Linn.S.i.; N.H.M.i.; R.S.i.

Frelsingf. Acta.

Acta Societatis Scientiarum Fennicæ. Helsingfors.

1842- B.M.; Camb.P.S.; Camb.U.i.; Dub.R.I.A.; Edinb.R.S.i.; N.H.M.; Oxon.B.; R.A.S.; R.S.

Helsingf. Bd.

Bidrag till Kännedomen af Finlands Natur och Folk, utgifna af Finska Vetenskaps-Societeten. Helsingfors.

18.5 - B.M.; Camb.P.S.i.; Camb.U.; Dub.R.I.A.; Edinb.R.S.i.; N.H.M.; Oxon.B.; R.A.S.; R.S.

Helsingf. Öfv.

Öfversigt af Finska Vetenskaps-Societetens Förhandlingar. Helsingfors.

1853 - B.M.; Camb.P.S.i.; Camb.U.i.; Dub.R.D.S.; Dub.R.I.A.; Edinb.R.S.i.; Glasg.U.i.; N.H.M.; Oxon.B.; R.A.S.; R.S.

Fermbstädt Ms.

Museum des Neuesten und Wissenswürdigsten aus dem Gebiete der Naturwissenschaft, der Künste, der Fabriken, der Manufakturen, der technischen Gewerbe, der Landwirthschaft, der Produktenwaaren und Handelskunde, und der bürgerlichen Haushaltung, etc.; Hermbstädt. Berlin.

1814-18. B.M.; R.S.

Hertha, Zeitschrift für Erd-, Völker-, und Staaten-Kunde; Berghaus und Hofimann. Stuttgart und Tübingen.

1825-29. B.M.; Camb.U.; Oxon.B.; R.S.; U.C.L.i.

Journal of the Institute of Actuaries. London.

1869 - B.M.; Camb.U.; Dub.N.L.I.i.; Oxon.B.; R.A.S.i. 


\section{List of Serial Publications}

I. C. $\mathbf{P}$.

I. జ́gypt. Bu.

I. tgrypt. $\mathbf{~ m m}$.

I. Flect. .. J.

n Cim.

n Giamb. Vico

n Polit.

I. MIr. $\mathbf{P}$

I. Mn. Mtl. T.

n Progresso

Ing.

Inghirami Opusc.

Innsb. Nt. Md. B.

Intell. Obs.

Iowa Ac. Sc. $\mathbf{P}$.

Ir. Ac. Cunningham Mm.

Ir. Ac. $\mathbf{P}$.

Ir. Ac. $\boldsymbol{T}$.

I. $\mathbf{R} \mathbf{v}$.
Minutes of Proceedings of the Institution of Civil Engineers, containing Abstracts of the Papers and of the Discussions. London.

1837- B.M.; Camb.P.S.; Camb.U.; Dub.R.C.S.; Dub.R.D.S.; Dub.R.I.A.; Edinb.R.S.; Edinb.U.; Glasg.P.S.i.; Glasg.U.; Oxon.B.; Oxon.R.i.; P.O.; R.S.; U.C.L.

See CE. I. $\mathbf{P}$.

Bulletin de l'Institut Egyptien. Caire.

1859 - Camb.P.S.i.; Camb.U.i.; N.H.M.; U.C.L.i.

Mémoires ou Travaux Originaux présentés et lus à l'Institut Égyptien publiés sous les auspices de S. A. Mohammed Said, vice-roi d'Egypte, sous la direction de M. le Docteur B. Schnepp. Paris $\&$ Caire.

1862 - Camb.P.S.i.; Camb. U.; N.H.M.

Journal of the Institution of Electrical Engineers. London.

1889- B.M.; Camb.U.; Dub.R.D.S.; Edinb.R.S.i.; Edinb.U.; Glasg.U.i.; Oxon.R.i.; P.O.; R.S.; U.C.L.

Il Cimento, rivista di Scienze, Lettere, ed Arti. Torino.

1852-55. B.M.

Il Giambattista-Vico, giornale scientifico. Napoli.

1857. N.H.M.; Oxon.B.i.

See Giamb. Vico.

Il Politecnico; repertorio mensile di studj applicati alla prosperità e coltura sociale.

Il Politecnico; repertorio di studj letterarj, scientifici e tecnici. Milano. $1839-44$; $1860-$ B.M.; P.O.

Institution of Mechanical Engineers. Proceedings. Birmingham, London.

1847 - B.M.; Camb.P.S.i.; Camb.U.; Dub.R.D.S.; Glasg.U.; P.O.; R.S.; U.C.L.

Transactions of the Institution of Mining and Metallurgy, London. London.

1892- B.M.; Camb.U.i.; Geol.S.; N.H.M.; P.O.

Il Progresso delle Scienze, Lettere, ed Arti. Napoli.

First series undated; Second series 1832- Camb.U.; Oxon.B.

Der Ingenieur; Zeitschrift für das gesammte Ingenieurwesen; Bornemann. Freiberg.

1848-50. B.M.; P.O.

Nuova Collezione di Opuscoli e Notizie di Scienze; Inghirami. Fiesole.

$1820-23$. B.M.

Berichte des Naturwissenschaftlich-Medizinischen Vereins in Innsbruck. Innsbruck.

1870 - B.M.; Camb.U.; Dub.R.D.S.i.; Dub.R.I.A.i.; Linn.S.i. ; N.H.M.; Oxon.R.; R.S.

The Intellectual Observer; a Review of Natural History, Microscopic Research, and Recreative Science. London.

1862-68. B.M.; Camb.U.; Linn.S.; N.H.M.; Oxon.B.i.; Oxon.R.; P.O.; R.A.S.; R.S.i.

Proceedings of the Iowa Academy of Sciences. Des Moines.

1875- B.M.i.; Edinb.R.S.i.; N.H.M.; Oxon.B.i.; P.O.; R.S.i.; U.C.L. $i$.

Royal Irish Academy. " Cunningham Memoirs." Dublin.

1880- B.M. ; Camb.P.S.; Camb.U.; Chem.S.i.; Dub.N.L.I. ; Dub.R.C.S. ; Dub.R.D.S. ; Dub.R.I.A. ; Dub.T.C.; Edinb.R.S.i.; Edinb.U.; Glasg.P.S.; Linn.S.; N.H.M.i.; Oxon.B.; Oxon.R.; R.A.S.; R.S.i.; U.C.L.

Proceedings of the Royal Irish Academy. (Science.) Dublin.

1841 - B.M.; Camb.P.S. ; Camb.U.; Chem.S.i.; Dub.N.L.I. ; Dub.R.C.S.; Dub.R.I.A.; Dub.T.C.; Edinb.R.S. ; Edinb.U.; Glasg.P.S.i.; Glasg.U.; Linn.S.; Math.S.i.; N.H.M.; Oxon.B.i.; Oxon.R.; P.O.i.; R.A.S.; R.S.; U.C.L.i.

Transactions of the Royal Irish Academy. Science. Dublin.

1787- B.M.; Camb.P.S.; Camb.U.; Chem.S.i. ; Dub.N.L.I. ; Dub.R.D.S.; Dub.R.I.A. ; Edinb.R.S.; Edinb.U.; Glasg.P.S.; Glasg.U.i.; Linn.S.; Math.S.i.; N.H.M. ; Oxon.B.i.; Oxon.R.; P.O.i.; R.A.S.; R.S.; U.C.L.

India Review and Journal of Foreign Science and the Arts. Calcutta. 1837-38. B.M.; N.H.M.i. 


\section{List of Serial Publications}

Isère S. BL.

I. \& S. I. J.

Jap. As. S. T.

Jb. Berg- I $\mathbf{w}$.

J. Bt.

J. de $\mathbf{P} \mathbf{s}$.

J. de $\mathbf{P s}$.

Jena. Sb.

Jena. Z.

J. Gén. Civ.

J. Ir. Un. Cir.

J. Mcr. Sc.

\section{J. Mines}

J. Ps. C.

J. Saจ.

J. Tél.

Kan. Ac, Sc, $\boldsymbol{T}$.

Kan. Un. Q.

Karlsruhe Nt. Vr. Vh.

Karsten Arch.

VOL. I.
Bulletin de la Société de Statistique, des Sciences Naturelles, et des Arts Industriels du département de l'Isère. Grenoble.

1838- B.M.i.; N.H.M.; Oxon.B.; R.S.i.

The Journal of the Iron and Steel Institute. London.

1872 - Camb.U.; Chem.S.i.; Dub.R.D.S.; Dub.R.I.A.; Dub.T.C.; Edinb.U.; Geol.M.i.; Glasg.P.S.i.; Glasg.U.i.; Oxon.B.; P.O.; R.S.; U.C.L.

Transactions of the Asiatic Society of Japan. Yokohama.

1872_ B.M.; Camb.U.; Edinb.R.S.; N.H.M.; Oxon.B. ; Oxon.R.; P.O.i.; R.S.

Jahrbuch für das Berg- und Hüttenwesen im Königreiche Sachsen. Freiberg.

1873- B.M.; N.H.M.i.; P.O.

The Journal of Botany. London.

1863 - B.M.; Camb.U.; Dub.N.L.I.i.; Dub.R.C.S. ; Glasg.U. ; Linn.S.; N.H.M.; P.O.i.

Journal de Physique, de Chimie, et de l'Histoire Naturelle; de Lamétherie ete. Paris.

1794-1823. B.M.; Camb.U.; Glasg.U.i.; N.H.M.i.; Oxon.B.; Oxon.R.; R.S.; U.C.L.i.

Journal de Physique Théorique et Appliquée; D'Almeida. Paris.

1872- Camb.U.; Dub.R.C.S.; Glasg.U.i.; Oxon.R.; P.O.; R.S. Sitzungsberichte der Jenaischen Gesellschaft für Medicin und Naturwissenschaft. Jena.

1877-86. Edinb.R.S.i.; Linn.S.i.; Oxon.R.; R.S.

Jenaische Zeitschrift für Naturwissenschaft, herausg. von der Medicinisch-Naturwissenschaftlichen Gesellschaft zu Jena. Jena.

1864 - B.M.; Camb.P.S.i.; Camb.U.; Chem.S.i.; Dub.N.L.I.i.; Dub.R.D.S.i.; Edinb.R.S. ; Edinb.U.i.; Glasg.U.i.; Linn.S.; N.H.M. ; Oxon.B.; Oxon.R.; R.S.; U.C.L.

Journal du Génie Civil des Sciences et des Arts. Paris.

1828-48. B.M.i.; Camb.U.; P.O.

The Johns Hopkins University Circulars. Baltimore.

1879- Camb.P.S. ; Camb.U. ; Dub.N.L.I.i.; Dub.R.I.A.i. ;

Edinb.R.S.i. ; Edinb.U.; Glasg.P.S. ; Math.S.i. ; N.H.M.;

Oxon.B.; Oxon.R.; R.A.S.i.; R.S.; U.C.L.i.

Quarterly Journal of Microscopical Science; Lankester and Busk. London.

1853- B.M.; Camb.P.S.; Camb.U.; Chem.S.i.; Dub.N.L.I.; Dub.R.C.S. ; Edinb.R.S. ; Edinb.U. ; Glasg.P.S. ; Linn.S. ; N.H.M.; Oxon.B.i.; Oxon.R.; P.O.; R.S.; U.C.L.

Journal des Mines, ou Recueil de Mémoires sur l'exploitation des Mines, et sur les Sciences et les Arts qui s'y rapportent. Paris.

1794-1815. B.M.; Camb.U.; Dub.T.C.; Edinb.R.S.; Linn.S.i.; N.H.M.; Oxon.B.(R.); R.S.i.

The Journal of Physical Chemistry. Ithaca, N.Y.

1896- B.M. ; Camb.P.S.; Camb.U. ; Chem.S. ; Edinb.R.S. ; Edinb.U.; Glasg.U.; Oxon.R.; P.O.; R.S.i.

Journal des Savants. Paris.

1816- B.M.; Camb.U.; Dub.N.L.I.; Dub.T.C.; Edinb.R.S.i.; Glasg.U.i.; Oxon.B.; Oxon.R.; P.O.i.; R.S.

Journal Télégraphique publié par le Bureau International des Administrations Télégraphiques. Berne. 1869 - P.O.

Transactions of the Kansas Academy of Science. Topeka, Kansas. 1872- Camb.P.S.i.; Dub.R.I.A.; Edinb.R.S.i.; Glasg.P.S.i.; Linn.S.i; N.H.M.; Oxon.B.i.; R.S.i.

The Kansas University Quarterly. Laurence, Kansas.

1893- B.M.i.; Dub.R.D.S.i.; Dub.R.I.A.i.; Edinb.R.S.i.; Glasg. P.S.i.; Math.S.; N.H.M.; R.S.

Verhandlungen des Naturwissenschaftlichen Vereins in Karlsruhe.

Karlsruhe.

1864- B.M.i.; Dub.R.I.A.; N.H.M.

Archiv für Mineralogie, Geognosie, Bergbau, und Hüttenkunde;

Karsten. Berlin.

1829-55. B.M.; Edinb.R.S.i.; Geol.M.; N.H.M.; P.O.; R.S. 


\section{List of Serial Publications}

Kastner Arch. Nt1.

Fazan Mm. Un.
Archiv für die gesammte Naturlehre; Kastner. Nürnberg.

1824 35. B.M.; N.H.M.; P.O.

Scientific Memoirs published by the Imperial University of Kazan. Kazan.

1834 - B.M.i.; Camb.U.; Dub.R.I.A.; Edinb.R..S.i.; Glasg.P.S.i.; Linn.S.i.; R.S. $i$.

See Kazan Un. Mm.

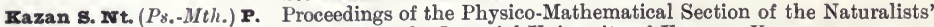
Society of the Imperial University of Kazan. Kazan.

1883-90. R.S.

Kazan 8. Ps.-Mrth. Bu. ... Bulletin de la Société Physico-Mathématique de Kasan. Kasan.

1891- Dub.R.I.A.i.; Edinb.R.S.i.; R.S.i.

Bulletin of the Imperial University of Kazan. Kazan.

1865- B.M.i.; Camb.U.i.; Edinb.R.S.i.; Linn.S.i.

See Kazan Mm. Un.

Kazan Un. Mm. ........... Tharkov Irth. S. Com...

ICiel Schr.

Communications and Proceedings of the Mathematical Society of the Imperial University of Kharkov. Kharkov.

1879 ? - R.S. $i$.

Schriften der Universität zu Kiel. Kiel.

1855-80. B.M. ; Camb.U.; Dub.R.I.A.; Edinb.R.S.i.; N.H.M.i.; Oxon.B.; R.S.

Det Kongelige Danske Videnskabernes Selskabs naturvidenskabelige og mathematiske Afhandlinger. Kiöbenhavn.

Käb. Dn. vd. Selsk. Afh. Kjфb. Dn. Vd. Selsk. Afh.

Iöb. Dn. Vd. Selsk. Skr. Ijöb. Dn. Vd. Belsk. Skr.

1824-46. B.M. ; Dub.T.C.; Edinb.R.S. ; Linn.S.i.; N.H.M. ; R.S.

Det Kongelige Danske Videnskabernes Selskab Skrivter. Kiöbenhavn.

1801-18. B.M.; Camb.P.S.i. ; Camb.U.; Edinb.R.S.; N.H.M. ; Oxon.B.; R.S.

See Dn. vd. Selsk. skr.

Det Kongelige Danske Videnskabernes Selskabs Skrifter. Naturvidenskabelig og Mathematisk Afdeling. Kjфbenhavn.

1849 - B.M.; Camb.U.i.; Edinb.R.S.; Linn.S.; N.H.M. ; R.A.S.; R.S.; U.C.L.i.

Td, Selsk. Skr. riöb. skr.

Kjøb. skr.

Kiöb. Ov.

Kjob. Ov.

Kjöb. Ov.

Königsb. Arch.

Königsb. Schr.

Kosmos (Lw.)

Oversigt over det Kongelige Danske Videnskabernes Selskabs Forhandlinger. Kjobenhavn.

1806- Camb.P.S.; Camb.U.i.; Chem.S.i.; Dub.R.D.S.i. ; Dub. R.I.A.i.; Dub.T.C.i.; Edinb.R.S.i.; Glasg.U.i.; Linn.S.i.; N.H.M.i.; Oxon.R.; P.O.i.; R.A.S.i.; R.S.

Königsberger Archiv für Naturwissenschaften und Mathematik; Bessell, etc. Königsberg.

1812. B.M. ; Camb. U.; Linn.S.

Schriften der königlichen Physikalisch-Oekonomischen Gesellschaft zu Königsberg. Königsberg.

1860 - B.M. ; Camb.P.S.; Dub.R.I.A.; Edinb.R.S.i.; Linn.S.; N.H.M. ; P.O.i.; R.S.

Kosmos. Czasopismo polskiego Towarzystwa przyrodnik6w imienia Kopernika. [Cosmos. The Journal of the Polish Society of Naturalists founded in honour of Copernicus.] Lwow.

1876- B.M.; N.H.M

Krk. Ak. (Mt.-Prz.) Pam. Pamiętnik Akademii Umiejętności w Krakowie. Wydział Matematyczno-Przyrodniczy. [Memoirs of the Academy of Science in Cracow. Section of Mathematics and Natural Science.] Kraków.

1874 - B.M.; Edinb.R.S.i.; N.H.M.

(Rozprawy.... Wydziatu Matematyczno-Przyrodniczego Akademii

Krk. Ak. (Mt.-Pŕz.) Rz...

Krk. Ak. (Mt.-Prz.) Rz.

\& sp. Umiejętności. [Proceedings of the Section of Mathematics and Natural Science of the Academy of Science.] Krak6w.

1874 - B.M. ; Edinb.R.S.i.; N.H.M.

Rocznik Towarzystwa Naukowego z Uniwersytetem Krakowskim Polačzonego. Krakowie. [Annals of the Scientific Society of the Polish University of Krakow. Krakow.]

1817-72. B.M.

Ianc. Fist, $\mathbf{s}$. $\mathbf{T}$ Ianc. T. Hist. $\mathbf{s}$
Proceedings and Papers of the Lancashire and Cheshire Historic Society. Liverpool.

1849-54. Continued as: Transactions, etc., 1855- B.M. ; Camb.U.i.; Dub.R.I.A.i.; Edinb.R.S.i.; Glasg.P.S.i.; Oxon.B.i.; R.S. 


\section{List of Serial Publications}

Iaus. Bll. s. Vd.

Laus. s. Vd. Bu.

Ieic. $\mathbf{s .} \boldsymbol{T}$.

I.eijd. A. Ac.

Ieip. Ab. Jablon. Gs.

Leip. Ab. Mth. Ps.

Leip. As. Gs. Vjschr.

Leip. $\mathbf{B}$.

Leip. Jablon. Preisschr.

Leip. Mth. Ps. Ab.

Ieip. Inth. Ps. $\mathbf{B}$.

Ie Mans $\mathbf{8}$. R. Tr.

Ies Mondes

I'I.

Iniège A. Ac.

Iiège $\mathbf{M m}$. $\mathbf{s . ~ s c . ~}$ Liége s. Sc. $\mathbf{M m}$.

Iille $\mathbf{M m}$.

Iille I/m. 8 .

Iille s. Imm.

rille $\mathbf{T r}$.

Lille Tr. $\mathbf{m m}$.

Iindenau Z.
(Bulletin des Séances de la Société Vaudoise des Sciences Naturelles. Lausanne.

1842 - Dub.R.D.S.i.; Dub.R.I.A.i.; Edinb.R.S.i.; Linn.S. ; N.H.M.; Oxon.B.i.; R.S.

The Transactions of the Leicester Literary and Philosophical Society. Leicester.

1835- Camb.U.; Dub.R.D.S.; Geol.S.; Glasg.P.S. ; Linn.S.i. ; N.H.M.i. ; Oxon.B.; P.O.; U.C.L.

Annales Academiæ Lugduno-Batavæ. Leijden.

1815-75. B.M.; Camb.U.; Dub.T.C.i.; N.H.M.; Oxon.B.; R.S.i.; U.C.L.i.

Abhandlungen bei Begründung der k. Sächsischen Gesellschaft der Wissenschaften am Tage der zweihundertjährigen Geburtsfeier Leibnizens; herausg. v. d. Jablonowski'schen Gesellschaft zu Leipzig. Leipzig.

1846. Camb.U.; Dub.R.I.A.; Edinb.R.S.; N.H.M.; R.A.S.; R.S.

Abhandlungen der Mathematisch-Physischen Classe der Königlich Sächsischen Gesellschaft der Wissenschaften. Leipzig.

1852 - B.M. ; Camb.P.S.; Camb.U.; Dub.R.I.A.; Edinb.R.S. ; Glasg.U.; Math.S.i.; N.H.M.; Oxon.B.; P.O.; R.A.S.; R.S.; U.C.L.i.

See Leip. Irth. Ps. Ab.

Vierteljahrsschrift der Astronomischen Gesellschaft. Leipzig.

1866- B.M.; Camb.P.S.i.; Camb.U.; Dub.R.I.A.; Edinb.R.S.; Oxon.R.; R.A.S.; R.S.

Berichte über die Verhandlungen (Math.-Phys. Classe) der Königlich Sächsischen Gesellschaft der Wissenschaften zu Leipzig. Leipzig.

1846- B.M.; Camb.P.S. ; Camb.U.; Dub.R.I.A.; Edinb.R.S.; Glasg.U.; Math.S.i.; N.H.M.; Oxon.B.; Oxon.R.; R.A.S.; R.S.; U.C.L. $i$.

See Ieip. Mth. Ps. B.

Preisschriften gekrönt und herausgegeben von der Fürstlich Jablonowski'schen Gesellschaft zu Leipzig. Leipzig.

1847- B.M. ; Camb.U.; Dub.R.I.A.; Edinb.R.S.i. ; N.H.M. ; Oxon.B.; R.A.S.i.; R.S.i.; U.C.L.i.

See Ieip. Ab. Inth. Ps.

See reip. $\mathbf{3}$.

Analyse des Travaux de la Société [Royale] des Arts du Mans. Le Mans.

1820. N.H.M.; R.S.

Les Mondes, Revue hebdomadaire des Sciences et de leurs Applications aux Arts, et à l'Industrie; l'Abbé F. Moigno. Paris.

1863 - B.M.; Camb.U.i.; Dub.N.L.I.i.; Glasg.P.S.i.; Oxon.R.; P.O.; R.S.i.

L'Institut; Journal des Académies et Sociétés Scientifiques de la France et de l'Étranger. Paris.

1833 - B.M.i.; Camb.U.; Dub.T.C.; Edinb.R.S.i.; Glasg.P.S.i.; N.H.M.; Oxon.B.(R.); P.O.i.; R.S.i.

Annales Academiæ Leodiensis. Liège.

1817-27. B.M.; Camb.U.; Dub.T.C.; N.H.M.; Oxon.B.; R.S.

(Mémoires de la Société [Royale] des Sciences, de l'Agriculture, et $\{$ des Arts à Liège. Liège.

1843 - B.M.; Camb.U.; Dub.T.C.; Edinb.R.S.i.; Glasg.P.S.i.; Glasg.U.i.; Linn.S.i.; N.H.M.; Oxon.B.; P.O.; R.S.

(Mémoires de la Société [Royale] des Sciences, etc. à Lille. Lille.

1827 - B.M.; Camb.U.; Dub.T.C.; N.H.M.; Oxon.B.; Oxon.R. ; R.S. $i$.

Recueil des Travaux de la Société d'Amateurs des Sciences, de l'Agriculture, et des Arts à Lille. Lille.

1819-27. B.M.; Camb.U.; Dub.T.C.; N.H.M.; Oxon.B.; Oxon.R.; R.S.

Travaux et Mémoires de I'Université de Lille. Lille.

1889 - Camb.P.S.; Camb.U.; Dub.R.D.S.; Dub.R.I.A.; N.H.M.; R.S.

Zeitschrift für Astronomie und verwandte Wissenschaften; Lindenau. Tübingen.

1816-18. B.M.; Camb.U.; R.A.S.; R.S.

xxix 


\section{List of Serial Publications}

Liouv. J.

Iiouv. J. IIth.

Isisb. A.

Iisb. Ac. Sc. $\mathbf{M m}$.

Iisb. J. Sc. Mth.

Iisb. IMm.

Iisb. $\mathbf{M m}$. Ac. Sc. $\}$.....

I. Mth. S. P.

Indw. V.-st.

Iotos

Ionvain A. Ac.

Ipldina

Ipool It. Ph. S. P.

I. Pg. $\mathbf{s} . \mathbf{P}$.

Iucca At. Ac.

Ium. Hlect.

Iund. Acta Un.

Iund Phys. Sälsk. Ts...

Irand. Un. Acta

Inx. I. R. $\mathbf{P b}$.

Lux. S. sc. $\mathbf{~ m m}$

Inx. s. Sc. Nt.

Iyon Ac. Mm. (Bc.)

Iyon Ac. sc. Mm.
(Journal de Mathématiques pures et appliqueés, fondé par Joseph Liouville. Paris.

1836- B.M.; Camb.U.; Dub.R.I.A. ; Edinb.R.S.i.; Edinb.U. ; Glasg.P.S.i.; Glasg.U.; Math.S.i.; Oxon.B.(R.); R.S.; U.C.L.

Annaes das Sciencias e Lettras, publicados debaixo dos auspicios da Academia Real das Sciencias:-Sciencias mathematicas, physicas, historico-naturaes, e medicas. Lisboa.

1857- B.M.; Dub.R.I.A.; Edinb.R.S.i.; N.H.M.; R.A.S.i.; R.S.i. See A. sc.

Historia e Memorias da Academia Real das Sciencias de Lisboa. Lisboa.

1797- B.M.; Camb.U.; Edinb.R.S.; N.H.M.; Oxon.B.; R.A.S.; R.S. $i$.

See Iisb. Mm., Iisb. Mm. Ac. Sc. and Iisb. Mm. Sc.

Jornal de Sciencias mathematicas, physicas e naturaes. Publicado sob os auspicios da Academia R. das Sciencias de Lisboa. Lisboa.

1868 - B.M.; Camb.U.; Dub.R.D.S.i.; Edinb.R.S.i.; Linn.S.; Math.S.i.; N.H.M.; Oxon.B.; R.A.S.; R.S.; U.C.L.i.

\section{See Iisb. Ac. Sc. $\mathbf{~ M m}$.}

Proceedings of the London Mathematical Society. London.

1865- B.M. ; Camb.P.S. ; Camb.U.; Dub.R.I.A. ; Dub.T.C. ; Edinb.R.S.; Edinb.U.; Glasg.U.; Math.S.; Oxon.B.i.; Oxon.R.; R.S.; U.C.L.

Die landwirthschaftlichen Versuchs-Stationen. Organ für wissenschaftliche Forschungen auf dem Gebiete der Landwirthschaft. Dresden, Chemnitz.

1859- B.M.i.; Camb.U.; Chem.S.i.; Oxon.B.; P.O.i.; R.S.i.

Lotos. Zeitschrift für Naturwissenschaften. Prag.

1851-95. B.M.; Camb.U.; Dub.R.I.A.i.; N.H.M.

Annales Academiæ Lovaniensis. Bruxelles, Louvain.

1821-27. B.M.; Camb.U.; Dub.T.C.; Oxon.B.; R.S.

Leopoldina: amtliches Organ der Kaiserlichen Leopoldino-Carolinischen Deutschen Akademie der Naturforscher. Dresden.

1859- B.M.; Camb.P.S.; Camb.U.i.; Edinb.R.S.i.; Linn.S. ; N.H.M.; R.A.S.i.; R.S.

Proceedings of the Literary and Philosophical Society of Liverpool. London, Liverpool.

1844 - B.M.; Camb.U.i.; Chem.S.i.; Dub.R.I.A.; Edinb.R.S.i.; Glasg.P.S.; Linn.S.; N.H.M.; Oxon.B.i.; P.O.i.; R.A.S.i.; R.S.; U.C.L.i.

Proceedings of the Physical Society of London. London.

1874 B.M.; Camb.P.S.; Camb.U.; Chem.S.; Dub.R.C.S.; Dub. R.D.S.; Dub.R.I.A.; Dub.T.C.; Edinb.R.S.i.; Glasg.P.S.i.; Glasg.U.i.; Math.S.; Oxon.B.; Oxon.R.; P.O.; R.A.S.; R.S.; U.C.L. $i$.

Atti della R. Accademia Lucchese di Scienze, Lettere, ed Arti. Lucca. 1821 - B.M.; Camb.U.; Dub.T.C.i.; Oxon.B.i.

La Lumière Électrique. Journal universel d'Électricité. Paris. 1879-94. B.M.; Glasg.U.i.; P.O.

Acta Universitatis Lundensis. Lunds Universitets Års-skrift. Afdelningen för Mathematik och Naturvetenskap. Lund.

1864- B.M.; Camb.P.S.; Camb.U.; Dub.R.I.A.; Edinb.R.S.i.; Linn.S.i.; N.H.M.; Oxon.B.; R.S.

See Iund. Un. Acta.

Physiografiska Sällskapets Tidskrift. Lund.

1837-38. Camb.U.; N.H.M.; R.S.

See Innd. Acta Un.

Publications de l'Institut Royal Grand-Ducal de Luxembourg. Section des Sciences Naturelles et Mathématiques: ci-devant "Société des Sciences Naturelles." Luxembourg.

1870 - Dub.R.D.S.; Dub.R.I.A.; Edinb.R.S.i.; N.H.M.; R.S. $i$.

(Société des Sciences Naturelles du Grand-Duché de Luxembourg. Luxembourg.

1853-69. Dub.R.I.A.; R.S.

jMémoires de l'Académie des Sciences, Belles-Lettres et Arts de Lyon. Classe des Sciences. Lyon, Paris. 


\section{List of Serial Publications}

1845- B.M.; Camb.U.; Edinb.R.S.i.; Linn.S.i.; N.H.M.; Oxon.B.; R.S. $i$.

See Iyon Mm. Ac. and Iyon Mm. Ac. Sc.

Iyon A. S. Ag.

Iyon Mm. Ac.

Iyon $\mathbf{M m}$. Ac. Sc.

Iyon \$. A. Ag.

Lyon s. Ag. A.

Iyon Un. A.

Maclurian Iyceum Ct...

Mâcon Ac. A.

Mâcon s. C. $\mathbf{R}$.

Madras Eng. Rp.

Madras J.

Madrid Ac. Ci. $\mathrm{Mm}$. Madrid IMm.

Madrid Rv.

Mag. Ak. ists.

Mag. Ak. Łts. (Mth.Term.)

Magdeb. Nt. Vr. Jbr. $u$. Ab.

Mag. Tud. Ak. 2́tk. (Mth.)

Mag. Tud. Ak. Ĺts.

Majocchi A. Fis. C.

Manch. It. Ph. S. Mm...

Manch. It. Ph. s. Mm. $\& \mathbf{P}$.
Annales des Sciences physiques et naturelles, d'Agriculture, et d'Industrie, publiées par la Société d'Agriculture, etc.

Annales de la Société d'Agriculture, Histoire Naturelle et Arts Utiles de Lyon. Lyon.

1838 - B.M.; Camb.U.; Dub.R.I.A.; Linn.S.; N.H.M.; Oxon.B.; P.O.; R.S.

See Iyon s. A. Ag. and Iyon s. Ag. A.

See Iyon Ac. Mm. (Sc.) and Iyon Ac. Sc. $\mathbf{M m}$.

See ryon A. 8. Ag.

Annales de l'Université de Lyon. Paris, Lyon.

1891 - B.M.; Edinb.R.S.; N.H.M.i.; R.S.i.

Contributions of the Maclurian Lyceum to the Arts and Sciences. Philadelphia.

1827-29. B.M.; Linn.S.i.; N.H.M.

Annales de l'Académie de Mâcon, Société des Arts, Sciences, BellesLettres et d'Agriculture. Mâcon.

1851 - B.M. R.S.i.

Compte Rendu des Travaux de la Société (d'Agriculture,) des Sciences, Arts et Belles-Lettres, de Mâcon. Mâcon.

1807-52. B.M.i.; R.S.i.

Reports, etc. on various professional subjects connected with the duties of the Corps of Engineers of the Madras Presidency; Capt. J. T. Smith, F.R.S. Madras.

$1839-46$. P.O.; R.S.

The Madras Journal of Literature and Science. Madras.

1833 - B.M.i.; Camb.U.; Dub.N.L.I.i.; Linn.S.i.; N.H.M.; Oxon. B.i.; P.O.; R.A.S.i.; R.S.i.; U.C.L.i.

Memorias de la Real Academia de Ciencias. Madrid.

$\{1850$ - B.M.; Camb.U.i.; Dub.R.I.A.; Dub.T.C.; Edinb.R.S.; Linn.S.i.; N.H.M.; Oxon.B.; R.A.S.i.; R.S.i.; U.C.L.i.

Revista de los Progresos de las Ciencias exactas, fisicas, y naturales. Madrid.

1850-86. B.M.; Dub.R.D.S.i.; Edinb.R.S.i.; N.H.M.; Oxon.R.i.; R.A.S.i.; R.S.i.

Magyarakademiai Ertesítő. [Report of the Hungarian Academy.] Pest. 1840 -59. B.M.

Magyar akademiai Értesítö. A mathematikai és természettudományi osztalyok közlönye. [Report of the Hungarian Academy. Communications of the Mathematical and Natural Science sections.] Pest.

1860-65. B.M.; Camb.P.S.i.; R.S.

Jahresbericht und Abhandlungen des Naturwissenschaftlichen Vereins zu Magdeburg. Magdeburg.

1869 - B.M.; R.S.i.

Értekezések a Mathematikai Osztály kőréből. Kiadja a Magyar Tudományos Akadémia. [Memoirs on Mathematical subjects. Published by the Hungarian Academy of Science.] Pest.

1867-94. B.M.; Edinb.R.S.i.; R.S.

A Magyar Tudományos Akadémia Ertesitoje. [Report of the Hungarian Academy of Science.] Pest.

1867- B.M.

Annali di Fisica, Chimica, e Matematiche, col Bulletino dell' Industria meccanica e chimica; Majocchi. Milano.

$1841-50$. B.M.; R.S.

Memoirs of the Literary and Philosophical Society of Manchester. London and Manchester.

1785-1887. B.M.; Camb.P.S.; Camb.U.; Chem.S.i.; Dub.R.D.S.; Dub.R.I.A.; Glasg.P.S.i.; Linn.S.; Math.S.i.; N.H.M.; Oxon.B.; Oxon.R.; P.O.; R.A.S.i.; R.S.; U.C.L.i.

See Manch. Mm. Ph. S., Manch. Ph. \$. Mm. and $\mathbf{M a n c h}$. s. $\mathbf{M m}$.

Memoirs and Proceedings of the Manchester Literary and Philosophical Society. Manchester. 


\section{List of Serial Publications}

1888 - B.M.; Camb.P.S.; Camb.U.; Chem.S.; Dub.R.I.A.i. ; Edinb.R.S.; Glasg.P.S.; Linn.S.; Math.S.; N.H.M.; Oxon.B.; Oxon R.; P.O.; R.A.S.; R.S.; U.C.L.i.

Manch. It. Ph. s. P. ..... Proceedings of the Literary and Philosophical Society of Manchester. Manchester.

1857-87. B.M.; Camb.P.S.; Camb.U.; Chem.S.; Dub.R.D.S.; Dub.R.I.A.; Dub.T.C.; Edinb.R.S.; Glasg.P.S.; Linn.S.; Math. S.i.; N.H.M.; Oxon.B.; P.O.; R.A.S.; R.S.; U.C.L.i.

See Manch. Ph. S. $\mathbf{P}$.

Manch. Mm. $\mathbf{P h}$. S. Manch, Ph. 8. Mm. ...... Manch. Ph. 8. $\mathbf{P}$. manch. 8. Mm. Manch. $8 . P$.

Marb. Schr.

Mars, Fac. Sc. A.

Mathematician

Mathesis

mbl. Nt.

Medley I. Ing.

Mengelwerk wisk. Vh.

Mess. Mth.

M.-et-I. Mm. S. Ac.

Met. s. QJ.

Met. $\mathbf{z}$.

Metz Ac. Mm.

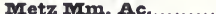

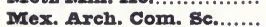

Méx. Reg. Trim.

Méx. 8. "Alzate" MIm.

Mg. NTtva.

Mh. Mth. Ps.

midl. Ntist.

Mil. At. I. Tomb.
See Manch. It. Ph. S. Mm.

See Manch. It. Ph. s. $\mathbf{P}$.

See Manch. It. $\mathbf{P h}$. 8. Mm.

See Manch. It. $\mathbf{P h}$. 8. $\mathbf{P}$.

Schriften der Gesellschaft zur Beförderung der gesammten Naturwissenschaften zu Marbarg. Marburg.

1823 - B.M.i.; Camb.U.; N.H.M.; Oxon.R.; R.S.

Annales de la Faculté des Sciences de Marseille. Marseille, Paris.

1891- B.M.; Camb.P.S.; Dub.R.I.A.; Edinb.R.S.; Glasg.P.S.; Linn.S.; Math.S.i.; N.H.M.; R.A.S.; R.S.

The Mathematician; Davies, etc. London.

1845-50. B.M.; Camb.U.; Dub.T.C.; Oxon.B.; R.S.

Mathesis. Recueil Mathématique.... Gand, Paris.

1881- B.M.; Camb.U.

Maanblad voor Natuurwetenschappen, uitgegeven door de Sectie voor Natuurwetenschappen van het Genootschap ter Bevordering van Natuur-, Genees- en Heelkunde. Amsterdam.

1871 - N.H.M.

Professional Papers on Indian Engineering; Major J. G. Medley. Roorkee.

$1864-68$. P.O.i.; R.S.

Mengelwerk van uitgeleezene en andere Wis- en Natuurkundige Verhandelingen; door het Genootschap der Mathematische Wetenschappen, etc. Amsterdam.

1796-1816. R.S.

See Amst. Mengelwerk.

The Messenger of Mathematics. Cambridge, London.

1862_-B.M.; Camb.P.S.; Camb.U.; Dub.N.L.I.i.; Dub.R.C.S.i.;

Dub.R.D.S.i.; Edinb.R.S.i.; Edinb.U.; Glasg.U.; Math.S.i.; Oxon.B.; Oxon.R.; R.S.; U.C.L.

Mémoires de la Société Académique de Maine et Loire. Angers. 1857- B.M.; Camb.U.; N.H.M.; R.S.i.

Quarterly Journal of the Royal Meteorological Society. London.

1873- Camb.U.; Dub.R.I.A.i.; Dub.T.C.i.; Edinb.R.S.; Linn. S.i.; Oxon.R.; P.O.; R.A.S.; R.S.

Meteorologische Zeitschrift. Berlin.

1884 - Camb.U.; Edinb.R.S.; P.O.; R.S.

I Mémoires de l'Académie (Royale, Impériale) de Metz. Metz.

$\{1821$ - B.M.; Camb.U.; Dub.T.C.; N.H.M.; Oxon.B.; R.S.i.

Archives de la Commission Scientifique du Mexique, publiées sous les auspices du Ministère de l'Instruction Publique. Paris.

1865-69. Oxon.B.; R.S.

Registro trimestre, $\delta$ Coleccion de Memorias de Historia, Literatura, Ciencias, etc., por una Sociedad de Literatos. México.

$1832-33$.

Memorias de la Sociedad Cientifica "Antonio Alzate." México.

1887 - B.M.i.; Camb.P.S.; Dub.R.I.A.; Edinb.R.S.; Linn.S.i.; Math.S.i.; N.H.M.i.; R.A.S.; R.S.; U.C.L.i.

Magazin for Naturvidenskaberne; Lundh, etc. Christiania.

1823-36. B.M.; N.H.M.i.; R.S.

Monatshefte für Mathematik und Physik. Wien.

1890 - B.M.; Camb.U.; Edinb.U.; Math.S.i.; N.H.M.

The Midland Naturalist. London, Birmingham.

1878-93. Camb.U.; Geol.M.; Linn.S.; P.O.

Atti dell' I. R. Istituto Lombardo di Scienze, Lettere, ed Arti. Milano.

1858-64. B.M.; Camb.U.; Edinb.R.S.; N.H.M.; Oxon.B.; R.S. xxxii 


\section{List of Serial Publications}

Mil. Dffem.

Mil. Effem. As.

Mil. Ephem. As.

Mil. G. I. Iomb.

Mil. G. s. Inc.

Mil. I. Iomb. G.

Mil. I. Iomb. $\mathrm{Mm}$.

Mil. I. Iomb. Rd.

Mi1. Mm. I. Iomb.

Mil. Mm. I. Iomb. Ven.

Mitau Arb. Kurländ. Gs.

Mitau Quatember.

Mitan Send. Kurländ. Gs.

Mn. $\mathbf{M g}$.

Mntp. Ac. $\mathbf{M m}$.

Mntp. Ac. PV.

Mntp. Ac. Sc. Mm.

Mntp. Mm. Ac. Intp. Mm. Ac. Sect. Sc. Mntp. Mm. Ac. Sect. Md.

Mntp. Rec. Bu.

Mod. Ac. Sc. $\mathbf{m m}$.

Mod. Mm. Ac. sc.

Mod. Im. S. It. Mod. s. It. $\mathbf{M m}$.
(Effemeridi Astronomiche di Milano. Con Appendice di Osservazioni e Memorie Astronomiche. Milano.

1806- Camb.U.; Oxon.B.; R.A.S.i.

Ephemerides Astronomicæ, ad Meridianum Mediolanensem supputatæ ab Angelo de Cesaris. Accedit Appendix cum Observationibus et Opusculis. Mediolani. (Milan.)

1774-1805. Oxon.B.

Giornale dell' I. R. Istituto Lombardo di Scienze, Lettere, ed Arti, e Biblioteca Italiana; compilata da varj dotti nazionali e stranieri. Milano.

1841-56. B.M.; N.H.M.; Oxon.B.; R.S.

See Mil. I. Iomb. $\mathbf{G}$.

Giornale della Società d' Incorragiamento delle Scienze, etc. stabilità in Milano. Milano.

1808-65. B.M.; Camb.U.

See Mil. G. I. Iomb.

Memorie dell' I. R. Istituto Lombardo di Scienze, etc. Milano.

1843 - B.M.; Camb.P.S.; Camb.U.; Dub.R.I.A.; Dub.T.C. ; Edinb.R.S.i.; Math.S.i.; N.H.M.; Oxon.B.; R.A.S.i.; R.S.; U.C.L.i.

See Mil. Mm. I. Iomb.

Reale Istituto Lombardo di Scienze e Lettere. Rendiconti. Milano. 1864 - B.M.; Camb.P.S.; Camb.U.; Dub.R.I.A.; Edinb.R.S.i.; Math.S.i.; N.H.M.; Oxon.B.i.; R.A.S.i.; R.S.; U.C.L.i.

See $\mathbf{M i l}$. I. Tomb. $\mathbf{M m}$.

Memorie dell' I. R. Istituto del regno Lombardo-Veneto. Milano. 1819-38. B.M.; Camb.U.; N.H.M.; Oxon.B.i.; R.S.

Arbeiten der Kurländischen Gesellschaft für Literatur und Kunst. Mitau.

1847-51. B.M.; Camb.U.

Die Quatember; Zeitschrift für naturwissenschaftl., geschichtl., philolog. und gemischte Gegenstände; von Trautvetter. Mitau. 1829-30. B.M.

Sendungen der Kurländischen Gesellschaft für Literatur und Kunst. Mitau.

1840-47. B.M.

The Mineralogical Magazine and Journal of the Mineralogical Society of Great Britain and Ireland. Truro, London.

1876 - B.M.; Camb.U.; Chem.S.i.; Dub.N.L.I.; Geol.M.; N.H.M.; Oxon.B.(R.); P.O.; R.S.

Académie des Sciences et Lettres de Montpellier. Mémoires de la Section des Sciences. Montpellier.

1847- B.M.; Camb.U.; Dub.R.D.S.; Dub.R.I.A.; Edinb.R.S.; Linn.S.i.; N.H.M.; Oxon.B.; R.A.S.; R.S.; U.C.L.i.

See Mntp. Ac. Sc. Mm., Mntp. Mm. Ac. and Mntp. Mm. Ac. sect. Sc.

Extraits des Procès-Verbaux des Séances de l'Académie des Sciences et Lettres, établie à Montpellier. Montpellier.

$1847-54$. N.H.M.i.

See Mntp. Ac. $\mathbf{M m}$.

Mémoires de l'Académie des Sciences et Lettres: Section de la Médecine. Montpellier.

1849 - B.M.; Camb.U.; Dub.R.D.S.; Dub.R.I.A.; Edinb.R.S.i.; Oxon.B.; R.S.

Recueil des Bulletins publiés par la Société Libre des Sciences, etc., de Montpellier. Montpellier.

1803-14. B.M.; Camb.U.; Oxon.B.i.

(Memorie della Regia Accademia di Scienze, Lettere ed Arti di

\{ Modena. Modena.

1833 - B.M.; Dub.R.I.A.i.; Dub.T.C.i.; Edinb.R.S.; Math.S.i.; N.H.M.; Oxon.B.i.; U.C.L.i.

Memorie di Matematica e di Fisica della Società Italiana delle Scienze. Modena.

1782 - B.M.i.; Camb.P.S.; Camb.U.i.; Dub.R.I.A.; Edinb.R.S.i.; Linn.S.i.; Oxon.B.i.; R.A.S.i.; R.S.; U.C.L.i.

See $\mathbf{R m}$. s. It. Mm., Verona $\mathbf{M m}$. S. It. and Verona s. It. $\mathbf{M m}$. $\mathrm{x} \times \mathrm{xiii}$ 


\section{List of Serial Publications}

Mod. s. Nt. At.

Moigno An. Cosmos

Moigno Cosmos

Mondes (1es)

Mon. sc.

Mosc. Bu. s. Nt.

Mosc. Obs. A.

Mosc. s. INt. Bul.

Mosc. S. Sc. Bll.

Mosc. Un. $\mathbf{M m}$.

Mosc. Un. Mm. (Ps.-Mth.)

mth. A.

Mth. Gz.

Mth. Misc.

Mth. Nt. B. Ung.

Mth. Term. Hts.

Mth. Termt. 'its.

Mth. Ts.

Mulhouse $\mathbf{B l l}$.

Mulhouge $\mathbf{B l 1}$. $\mathbf{8}$. In.......

Mulhouse s. In. Bll. ......

Münch. Ab.

Münch. Ak. Ab.

Münch. Ak. 8b.

Münch. D.

Münch. sb.
Atti della Società dei Naturalisti di Modena. Modena.

1883 - Camb.U.; Dub.R.I.A.i.; N.H.M.

Annuaire du Cosmos; Moigno. Paris.

$1859-70$. B.M.

(Cosmos. Révue Encyclopédique Hebdomadaire des Progrès des Sciences; Moigno. Paris.

1852- B.M.; Camb.U.; Dub.T.C.; Edinb.R.S.i.; N.H.M.i. ; Oxon.B.; P.O. $i$; R.A.S.i.; R.S.

Le Moniteur Scientifique du Chimiste et du Manufacturier; Quesneville. Paris.

1857- B.M.; Chem.S.i.; Dub.R.C.S.i.; Oxon.B.; P.O.; R.A.S.i. Bulletin de la Société Impériale des Naturalistes. Moscou.

1829 - B.M. ; Camb.P.S.; Camb.U.; Chem.S.i.; Dub.R.D.S.; Dub.R.I.A.; Edinb.R.S.i.; Glasg.U.i.;Linn.S.;N.H.M.;Oxon.B.i.; Oxon.R.; P.O.i.; R.A.S.i.; R.S.

See Mosc. s. Nt. Bll.

Annales de l'Observatoire de Moscou; Bredichin. Moscou.

1874 - B.M.i.; Camb.U.; R.A.S.; R.S.

See Mosc. Bu. s. Nt.

Bulletin of the Imperial Society of Lovers of Natural Science, Anthropology and Ethnography, in connection with the Imperial University of Moscow. [In Russian.] Moscow.

1865- B.M.i.; Edinb.R.S.i.; N.H.M.i.

Scientific Memoirs of the Imperial University of Moscow. [In Russian.] Moscow.

B.M.i.; Chem.S.i.; N.H.M.i.

Scientific Memoirs of the Imperial University of Moscow. Section Phys.-Math. Moscow.

1880-96. Chem.S.

Mathematische Annalen; Clebsch. Leipzig.

1869- B.M.; Camb.P.S.; Camb.U.; Dub.N.L.I.i.; Dub.R.C.S.i.; Dub.R.D.S.i.; Dub.T.C.i.; Edinb.U.; Glasg.U.; Math.S.; Oxon.R.; R.S.; U.C.L.

The Mathematical Gazette. London.

1894- B.M.; Camb.U.; Math.S.; U.C.L.i.

The Mathematical Miscellany; Gill. New York.

1836-39. U.C.L.

Mathematische und naturwissenschaftliche Berichte aus Ungarn. Berlin.

1882 - Camb.P.S.; Chem.S.; Edinb.R.S.; R.A.S.i.; R.S.

Mathematikai és természettudományi Ertesitö. Kiadja a Magyar Tudományos Akadémia. [Mathematical and Natural Science Report, published by the Hungarian Academy of Science.] Budapest.

1883 - B.M.i.; Edinb.R.S.; N.H.M.; R.S.

Mathematisk Tidsskrift. Kjøbenhavn.

1859- B.M.; Camb.U.; Oxon.B.; R.S.i.

(Bulletin de la Société Industrielle de Mulhouse. Mulhouse.

1828- B.M.i.; Camb.U.i.; Chem.S.i.; Dub.R.C.S.i.; Dub.T.C.i.; Glasg.P.S.i.; Glasg.U.i.; Oxon.B.i.; P.O.

Abhandlungen der mathematisch-physikalischen Classe der Königl. Bayerischen Akademie der Wissenschaften. München.

1832 - B.M. ; Camb.P.S. ; Camb.U.; Chem.S.i. ; Dub.T.C. ; Edinb.R.S.; Edinb.U.; Glasg.U.; Linn.S.; Oxon.B.; Oxon.R.; P.O.; R.A.S.i.; R.S.

Sitzungsberichte der Mathematisch-Physikalischen Classe der K. B. Akademie der Wissenschaften zu München. Muinchen.

1871- B.M. ; Camb.P.S. ; Camb.U.; Chem.S. ; Dub.T.C. ; Edinb.R.S.; Glasg.U.i.; Linn.S.; Oxon.B.; Oxon.R.; P.O.; R.A.S.; R.S.; U.C.L.i.

Denkschriften der Königl. Bayerischen Akademie der Wissenschaften zu München. München u. Salzbach.

1808-24. B.M.; Camb.P.S.; Camb.U.; N.H.M.; Oxon.R.; P.O.; R.S.

Sitzungsberichte der Königl. Bayerischen Akademie der Wissenschaften zu München. München.

1860-70. B.M.; Camb.P.S.; Camb.U.; Chem.S.; Dub.R.D.S.i.; Dub.R.I.A.i.; Dub.T.C.i.; Edinb.R.S.; Linn.S. ; N.H.M.; Oxon.B.; P.O.i.; R.A.S.; R.S. 


\section{List of Serial Publications}

N. A. Mth.

Nancy Mm. s. Sc.

Nancy s. Sc. BL.

N. Antol. Sc.

Nap. Ac. At.

Nap. Ac. Pont. At.

Nap. Ac. Sc. IMm.

Nap. At. Ac.

Nap. At. Ac. sc.

Nap. At. I. Inc.

Nap. At. s. Pont.

Nap. I. Inc. At.

Nap. Mm. Ac. Sc.

rap. Ms.

Nap. Rd.

Nap. s. Pont. At.

N. Arch. Wisk.

N. A. Sc. Nt.

N. Cim.

Nebr. Un. Stud.

N. Ing. I. Mn. ․ T.

Neuch. Bul.

Neuch. \$. Sc. Bu.

NF. RV.
Nouvelles Annales de Mathématiques. Paris.

1842 - B.M.; Camb.U.; Dub.T.C.; Edinb.U.; Glasg.U.; Math.S.i.; Oxon.B.(R.); R.S.; U.C.L.i.

Mémoires de la Société [Royale] des Sciences, Lettres, et Arts de Nancy. Nancy.

1833- B.M.; Camb.U.i.; N.H.M.i.; Oxon.B.; R.S.i.

Bulletin de la Société des Sciences de Nancy. Nancy, Paris.

1873 - B.M.; N.H.M.; R.S.

Nuova Antologia di Scienze, Lettere ed Arti. Firenze e Roma.

1866- B.M.; Dub.N.L.I.i.; N.H.M.

Atti della Reale Accademia delle Scienze e Belle Lettere; Sezione della Società R. Barbonica. Napoli.

1819-51. B.M.; Camb.U.; Dub.R.D.S.; N.H.M. ; Oxon.B.; R.A.S.i.; R.S.

Atti della R. Accademia delle Scienze Fisiche e Matematiche. Napoli.

1863-82; 1888 - B.M. ; Camb.U. ; Dub.R.I.A. ; Edinb.R.S. ; Glasg.U.i.; Linn.S.i.; Math.S.i.; N.H.M.; Oxon.B.i.; Oxon.R.; R.A.S.i.; R.S.

See Nap. At. Ac. and Nap. At. Ac. Sc.

Atti dell' Accademia Pontaniana di Napoli. Napoli.

1832- B.M.; Camb.U.; Dub.R.D.S.i.; N.H.M.; R.S.i.; U.C.L. $i$.

Memorie della R. Accademia delle Scienze, etc. Napoli.

1852-57. B.M.; Camb.U.; Dub.R.D.S.; Edinb.R.S.; Linn.S. ; N.H.M.; Oxon.B.; Oxon.R.; R.A.S.; R.S.

See Nap. IMm. Ac. Sc.

See Nap. Ac. At.

Atti del Real Istituto d' Incorraggiamento alle Scienze Naturali di Napoli. Napoli.

1811- B.M.; Camb.U.; Edinb.R.S.i.; N.H.M.; Oxon.B.; P.O.; R.S.i.

See Irap. I. Inc. At.

Atti della Società Pontaniana di Napoli. Napoli.

1810-47. B.M.; Camb.U.; N.H.M.; R.S.

See Nap. s. Pont. At.

See Nap. At. I. Inc.

See Nap. Ac. Sc. $\mathbf{M m}$.

Museo di Letteratura e Filosofia; Gatti. Napoli.

1842-62. B.M.; Oxon.B.

Rendiconto dell' Accademia delle Scienze Fisiche e Matematiche. Napoli.

1862 - Camb.U.; Dub.R.I.A.; Edinb.R.S.; Glasg.U.i.; Linn.S.i.; Math.S.; N.H.M.; Oxon.R.i.; P.O.; R.A.S.; R.S.; U.C.L.i.

See Nap. At. s. Pont.

Nieuw Archief voor Wiskunde. Amsterdam.

1875 - Camb.P.S.i.; Edinb.R.S.i.; Math.S.

Nuovi Annali delle Scienze naturali; Alessandrini, Bertoloni, Gherardi, e Ranzani. Bologna.

1838-54. Camb.U.; N.H.M.; Oxon.B.i.; R.S.

See Bologna N. A.

Il Nuovo Cimento, Giornale di Fisica, Chimica e Storia Naturale. Pisa.

1855- B.M. ; Camb.P.S.; Camb.U.; Chem.S.i.; Edinb.R.S.i.; N.H.M.; Oxon.R.i.; P.O.i.; R.S.

University Studies. Published by the University of Nebraska. Lincoln, Nebraska.

1888- B.M.; Camb.P.S.; Camb.U.; Dub.R.I.A.; Edinb.R.S. ; Oxon.B.; R.S.

Transactions of the North of England Institute of Mining Engineers. Newcastle-upon-Tyne.

1852- B.M.; Camb.U.; Edinb.R.S.i.; Oxon.B.i.; P.O.i.; R.S.

(Bulletin de la Société des Sciences Naturelles de Neuchâtel.

Neuchâtel.

1844- B.M.i. ; Camb.U.; Dub.R.I.A. ; Edinb.R.S.i.; N.H.M. ; Oxon.B.i.; R.A.S.i.; R.S.i.

The Natural History Review and Quarterly Journal of Science. London and Dublin. 


\section{List of Serial Publications}

1854-60. B.M.; Camb.U.; Dub.R.D.S.; Dub.T.C. ; Glasg.P.S. ; Linn.S.; N.H.M.; Oxon.R.; P.O.; U.C.L.i.

The Natural History Review; a Quarterly Journal of Biological Science. London.

1861 -65. B.M.; Camb.P.S. ; Camb.U.; Dub.R.D.S.; Dub.T.C. ; Edinb.R.S.; Glasg.P.S.; Glasg.U.; Linn.S.; N.H.M.; Oxon.B.i.; Oxon.R.; P.O.; R.S.

Nicholson J.

Niort Mm. de l'Athénée

N. Jb. Mn.

N. Mg. Ntvd.

Nrorske Vd. skr.

N. Rs. s. $\mathbf{N t . ~} \mathbf{m m}$. (Mth.)

N. 8. W. Ph. s. T.

N. s. W. R. S. J.

N. 8. w. R. S. T.

$\mathbf{N}$.

N. Ts. Mth

Nüirnb. Nt. Gr. Ab.

Nv. Archt. T.

N.-Vorp. IMt.

N. Y. Am, Inth. 8. Bu.

N. Y. Am. Mth. S. T. ...
Journal of Natural Philosophy, Chemistry, and the Arts; Nicholson. London.

1797-1813. B.M. ; Camb.U. ; Chem.S.i. ; Dub.R.I.A.i. ; Dub. T.C.i.; Edinb.R.S.; Edinb.U.; Glasg.P.S.i.; Glasg.U.; N.H.M.; Oxon.B.; Oxon.R.; P.O.; R.A.S.i.; R.S.; U.C.L.

Mémoires de l'Athénée de Niort, Société Libre des Sciences et des Arts du département des Deux-Sèvres. Niort.

1808 (?). B.M.; Oxon.B.; R.S.i.

Neues Jahrbuch für Mineralogie, Geologie und Palaontologie. Stuttgart.

1833 - B.M.; Camb.U.; Chem.S.i.; Dub.N.L.I.i.; Dub.R.D.S.i.; Geol.M.; N.H.M.; Oxon.R.; R.S.

Nyt Magazin for Naturvidenskaberne. Christiania.

1838 Camb.U.i.; Edinb.R.S.i.; Linn.S.i.; N.H.M.; R.S.

Det Kongelige Norske Videnskabersselskabs Skrifter i det 19de Aarhundrede. Kjøbenhavn og Trondhjem.

1817 - B.M.; Camb.U.i.; Dub.T.C.i.; Edinb.R.S.i.; N.H.M.; R.S. See Thrond. skr.

Memoirs of the Mathematical Section of the New Russian Society of Naturalists. [In Russian.] Odessa.

1878- B.M.i.; Dub.R.I.A.; Math.S.i.; R.S.i.

Transactions of the Philosophical Society of New South Wales. Sydney.

1866. Camb.P.S.; Camb.U.; Chem.S.; Edinb.R.S.i.; Glasg.U. ; Linn.S.; Oxon.B.; R.A.S.; R.S.

Journal and Proceedings of the Royal Society of New South Wales. Sydney.

1876- B.M. ; Camb.P.S. ; Camb.U. ; Chem.S.i.; Dub.T.C. ; Edinb.R.S.i.; Geol.M.i.; Glasg.P.S.i.; Glasg.U.i.; Linn.S.i.; N.H.M.; Oxon.B.; Oxon.R.i.; P.O.i.; R.A.S.; R.S.

Transactions of the Royal Society of New South Wales. Sydney.

1867-75. B.M.; Camb.P.S.; Camb.U.; Chem.S.i.; Dub.R.D.S.; Dub.R.I.A.; Dub.T.C.; Edinb.R.S.i.; Glasg.U.i.; N.H.M.; R.A.S.; R.S.

Nature: a weekly illustrated Journal of Science. London.

1870- B.M.; Camb.P.S.; Camb.U.; Chem.S.; Dub.N.L.I.; Dub. R.C.S.; Dub.R.D.S.; Dub.R.I.A.; Dub.T.C.; Edinb.R.S.; Edinb.U.; Geol.M.; Glasg.P.S.; Glasg.U.i.; Linn.S.; Oxon.B.; Oxon.R.; P.O.; R.A.S.; R.S.; U.C.L.

Nyt Tidsskrift for Mathematik. Kjøbenhavn.

1890 - B.M.; Math.S.i.

Abhandlungen der Naturhistorischen Gesellschaft zu Nürnberg. Nürnberg.

1852- B.M.i.; Camb.U.; Dub.R.I.A.; N.H.M.; R.S.i.

Transactions of the Institution of Naval Architects. London.

1860 - B.M. ; Camb.U. ; Dub.R.I.A. ; Edinb.U. ; P.O. ; R.S. ; U.C.L. $i$.

Mittheilungen aus dem Naturwissenschaftlichen Vereine von NeuVorpommern und Rügen. Berlin.

1869 - B.M.; Camb.U.; Dub.R.D.S.; Dub.R.I.A.; N.H.M.

Bulletin of the American Mathematical Society. New York.

1895- B.M. ; Camb.P.S. ; Camb.U.; Dub.T.C. ; Edinb.R.S. ; Edinb.U.; Glasg.P.S.; Glasg.U.; Math.S.; Oxon.B.; Oxon.R.; R.S.i.

Transactions of the American Mathematical Society. Lancaster, Pa. and New York.

1900 - Camb.P.S.; Camb.U.; Dub.R.I.A.; Dub.T.C.; Edinb.R.S.; Edinb.U.; Glasg.U.; Math.S.; Oxon.B.; Oxon.R.; R.S.

Bulletin of the New York Mathematical Society. New York.

1892-94. B.M.; Camb.P.S.; Camb.U.; Edinb.R.S.; Glasg.P.S.; Math.S.; Oxon.B.; Oxon.R.; R.A.S.

xxxvi 


\section{List of Serial Publications}

N. z. I. T.

Obs.

Oestr. Z. Brgw.

Oken Isis

Opusc. Mt. Fis.

Orv.-Termt. מ́ts. (Termt. Szak)

Transactions and Proceedings of the New Zealand Institute. Wellington.

1868 - B.M. ; Camb.P.S.i. ; Camb.U.; Dub.R.D.S. ; Dub.R.I.A. ; Edinb.R.S.; Edinb.U.; Geol.M.i.; Glasg.P.S.i.; Linn.S.; N.H.M.; Oxon.B.; Oxon.R.; P.O.; R.S.; U.C.L.i.

The Observatory. A monthly Review of Astronomy. London.

1878- Camb.P.S.; Camb.U.; Dub.T.C.i.; Edinb.R.S.; Oxon.R.; P.O.; R.A.S.

Oesterreichische Zeitschrift für Berg- und Hüttenwesen; von Otto Freiherrn von Hingenau. Wien.

1853- B.M.; P.O.

Isis, oder Encyclopädische Zeitung; Oken. Jena.

1817-48. B.M.i.; Camb.U.; Edinb.U. ; Linn.S.i.; N.H.M. ; Oxon.B.(R.); R.S.

Opuscoli matematici e fisici di diversi Autori. Milano.

1832-34. R.S.

Orvos-Természettudományi Értesitő a Kolozsvári Orvos-Természettudományi Társulat és az Erdélyi Muzeum-Egylet Természettudományi Szakosztályának....... [Medical and Natural History Proceedings of the Sections of the Klausenburg Medical and Natural History Society and of the Natural History Section of the Museum Association of Transylvania.] Kolozsvár [Klausenburg]. 1879 - N.H.M.; R.S.i.

Padora Ac. At. e Mm.

Atti e Memorie della R. Accademia di Scienze, Lettere ed Arti in Padova. Nuova serie. Padova.

1885- Edinb.R.S.; N.H.M.

Padova Mm. Ac.

Memorie dell' Accademia di Scienze, Lettere, ed Arti di Padova. Padova.

1809. B.M.; Camb.U.; N.H.M.; Oxon.B.; R.S.

Padova IN. Sag.

Nuovi Saggi dell' Accademia di Scienze, Lettere, ed Arti di Padova. Padova.

1817-83, B.M.i.; Camb.U.i.; Dub.R.I.A.i.; Dub.T.C.i.; Edinb. R.S.; N.H.M.; Oxon.B.i.; R.S.i.

Padova Rv. Period.

Revista Periodica dei Lavori della I. R. Accademia di Scienze, Lettere, ed Arti di Padova. Padova.

1851- B.M.; Edinb.R.S.i.; N.H.M.; R.S.i.

Palermo Ac. At.

Palermo At.

(Atti dell' Accademia di Scienze, Lettere ed Arti di Palermo. Palermo.

1845- B.M.; Camb.U.i.; Dub.R.I.A.; Dub.T.C.; N.H.M.; Oxon. B.i.; R.A.S.i.; R.S.

Palermo Cir. Mt. Rd. ... Rendiconti del Circolo Matematico di Palermo. Palermo.

1887- B.M. ; Camb.P.S. ; Camb.U. ; Dub.R.I.A. ; Dub.T.C. ; Math.S.; R.S.

Palermo G. Sc. Nt.

Giornale di Scienze naturali ed economiche, pubblicato per cura del Consiglio di Perfezionamento annesso al R. Istituto Tecnico di Palermo. Palermo.

1865- B.M.; Camb.U.; Dub.R.D.S.i.; R.S.

Palomba Rac.

Raccolta di Lettere, etc. intorno alla Fisica ed alle Mathematiche; Palomba. Roma.

1845-48. B.M.i.

Par. A. Cons.

Annales du Conservatoire des Arts et Métiers. Paris.

1861 - B.M.; Camb.U.; Glasg.P.S.i.; Oxon.B.; P.O.; R.S.

See A. Cons. Arts et mét.

Par. Ac. Sc. Mm.

Mémoires de l'Académie des Sciences de l'Institut de France. Paris.

1816 - B.M.; Camb.U.; Dub.N.L.I.i.; Dub.R.D.S.i.; Dub.T.C.i.; Edinb.R.S.i.; Edinb.U.; N.H.M. ; Oxon.B.; Oxon.R. ; P.O.i.; R.A.S.i.; R.S.; U.C.L.

See Par. Mm. Ac. Sc.

Par. A. Éc. Norm. ........ Annales scientifiques de l'École Normale Supérieure. Paris.

1864 - B.M.; Camb.P.S.i.; Camb.U.; Dub.N.L.I.i.; Dub.R.C.S.i.; Dub.R.D.S.i.; Edinb.R.S.i.; Edinb.U.i.; Glasg.U.i.; Oxon.B.; R.S.

See Par. Éc. Norm. A.

Par. An. Mrét.

Annuaire de la Société Météorologique de France. Paris.

Par. An. S. Mét.

Par. A. Obs.

1849 - B.M.; Camb.U.i.; Dub.T.C.i.; Edinb.R.S.i.; R.S.i.

Annales de l'Observatoire de Paris; mémoires publiées par U. J. Le Verrier. Paris. 


\section{List of Serial Publications}

1855- B.M.; Camb.U.; Dub.N.L.I. ; Dub.T.C. ; Edinb.R.S. ; Oxon.B.; R.A.S.; R.S.

Par. A. Pon. Chanss.

See Par. Obs. A. and Par. Obs. A. (Mm.)

Annales des Ponts et Chaussées. Mémoires et documents relatifs à l'Art des Constructions et au Service de l'Ingénieur. Paris.

1831- B.M.; Camb.U.; Edinb.U.i.; Glasg.P.S.i.; Glasg.U.i.; P.O.; R.S.i.

See A. Pon. Chauss.

Par. Bu. 8. Encour. ...... Bulletin de la Société d'Encouragement pour l'Industrie Nationale. Paris.

1802- Camb.U.; Dub.R.C.S.i.; Dub.T.C.i.; Edinb.R.S.i.; Glasg. P.S.i.; Oxon.B.; P.O.; R.S.

Par. Bul. s. Gg.

Bulletin de la Société de Géographie. Paris.

1822- B.M.; Camb.U.; Dub.R.I.A.; Edinb.R.S.i.; N.H.M.; Oxon.B.; R.S.; U.C.L.i.

See Par. Gg. S. BLl. and Par. s. Gg. Bul.

Par. Bu. s. Phlm.

Bulletin des Sciences de la Société Philomathique de Paris. Paris.

1791-1805; 1814-24; 1864- B.M.i.; Camb.U.; Dub.T.C.i.; Edinb.R.S.i.; Glasg.U.i.; Math.S.i.; N.H.M.; Oxon.R.i.; P.O.i.; R.A.S.i.; R.S.; U.C.L.

See Par. s. Phlm. Bu.

Par. F́c. Norm. A.

See Par. A. Éc. Norm.

Par. Éc. Pol. Cor.

Correspondance sur l'École Polytechnique, à l'usage des Élèves de cette École; Hachette. Paris.

1808-16. B.M.i.; Oxon.B.; R.S.; U.C.L.

Par. x́c. Pol. J.

Journal de l'École Polytechnique. Paris.

1795- B.M. ; Camb.P.S. ; Camb.U.; Dub.R.I.A.; Dub.T.C. ; Edinb.R.S. ; Edinb.U.; Glasg.P.S.i.; Glasg.U.; Linn.S.i.; Math.S.i.; Oxon.B.(R.); P.O.; R.A.S.i.; R.S.; U.C.L.i.

See Par. J. Éc. Pol.

Par. Gg. s. Bu.

See Par. Bu. s. Gg.

Par. Ing. Civ. Mm.

Mémoires et Comptes Rendus des Travaux de la Société des Ingénieurs Civils. Paris.

1848 - B.M.; Glasg.U.i.; P.O.

See Par. Mm. Ing. Civ.

Par. J. źc. Pol.

Par. Mm. Ac. Sc.

See Par. Éc. Pol. J.

See Par. Ac. sc. $\mathbf{M} \mathbf{m}$.

Par. Mm. de 1'r.

Mémoires de la Classe des Sciences mathématiques et physiques de l'Institut. Paris.

1798-1815. B.M.; Edinb.R.S.; N.H.M.; Oxon.B.; Oxon.R.; P.O.; R.A.S.i.; U.C.L.

Par. Mm. Ing. Civ.

See Par. Ing. Civ. Mm.

Par. Mm. Sav. źtr.

Mémoires présentés à l'Institut des Sciences, Lettres et Arts par divers Savans, et lus dans ses Assemblées: Sciences Mathématiques et Physiques. Paris.

1806-11. B.M.; Camb.U.; Dub.R.D.S.; Dub.T.C.; Edinb.R.S.; N.H.M.; Oxon.R.; P.O.; R.A.S.; R.S.; U.C.L.

Mémoires présentés par divers Savans à l'Académie des Sciences de l'Institut de France. Paris.

1827- B.M. ; Camb.U.; Dub.T.C. ; Edinb.R.S.i.; Linn.S.i. ; N.H.M.; Oxon.B.; Oxon.R.; P.O.i.; R.A.S.i.; R.S.

Par. $\mathbf{M m}$. s. sav.

Mémoires des Sociétés Savantes et Littéraires de la République Française. Recueillis et rédigés par les Citoyens Prony, etc. Paris.

1801-02. B.M.; Oxon.B.; R.S.

Par, Obs. A.

Par. Obs. A. (Mm.) .........

Pax. Poids et Mes. PV. .

See Par. A. Obs.

Comité International des Poids et Mesures. Procès-Verbaux des Séances. Paris.

1875- Camb.P.S. ; Camb.U.i.; Dub.R.D.S.; Oxon.R.; P.O.; R.A.S.; R.S.

Par. S. Amat. Tr.

Notices des Travaux de la Société des Amateurs des Sciences physiques et naturelles de Paris. Paris.

$1807-08$.

See Par. Tr. s. Amat.

Par. s. Ap. Bu.

Bulletin de la Société d'Anthropologie de Paris. Paris.

1860- B.M.; Camb.U.; Dub.R.I.A.; Edinb.R.S.i.; N.H.M.i.; Oxon.R.; R.S. 


\section{List of Serial Publications}

Par. sé. 1̇c. Norm.

Par. s. Gg. Bll.

Par. s. Inth. Bu.

Pax. s. Phlm. Bll.

Par. S. Phlm. Mm. Cent.

Par. 8. Phlm. N. Bu.

Par. S. Phlm. PV.

Par. T. Nauk Sc. Pam. ...

Par. Tr. S. Amat.

Perpignan Bll. s. Ag. Pyr. Orient.

Peterm. mt.

Phil. Trans.

Ph. $\mathbf{M g}$.

Phm. J.

Ph. Stud.

Pisa A. Scuola Norm.

Pisa A. Un. Tosc.

Pisa A. Un. Tosc. Sc. Cosm.

Pisa N. G.

Pisa s. Tosc. At. $(P V$.$) ...$

Pistoja At. Ac.

Pogg. A.

\section{Poligrafo}

Séances des Écoles Normales. Paris.

1800-01. R.S.; U.C.L.

See Par. Bul. s. Gg. and Par. Gg. s. Bu.

Bulletin de la Société Mathématique de France. Paris.

1873- B.M.; Camb.P.S.; Camb.U.; Edinb.R.S. ; Math.S. ; Oxon.R.; R.A.S.; R.S.

See Par. Bu. s. Phim.

Mémoires publiés par la Société Philomathique à l'occasion du Centenaire de sa Fondation. Paris.

1888. B.M.; Edinb.R.S.; N.H.M.; R.A.S.; R.S.

Nouveau Bulletin des Sciences de la Société Philomathique de Paris. Paris.

1807-1813; 1825-26; 1832_33. B.M.i.; Camb.U. ; Dub.T.C. ; N.H.M.; P.O.i.; R.S.; U.C.L.

Extraits des Procès-Verbaux des Séances de la Société Philomathique. Paris.

1836-63. N.H.M.; R.S.

Pamiętnik Towarzystwa Nauk Ścisłych w Paryzu. Paris.

1871-82. B.M.; N.H.M.

See Par. s. Amat. Tr.

[Bulletin de la] Société Agricole, Scientifique, et Littéraire des Pyrénées-Orientales. Perpignan.

1834 - N.H.M.; R.S.i.

Mittheilungen aus Justus Perthes' Geographischer Anstalt über wichtige neue Erforschungen auf dem Gesammtgebiete der Geographie; Petermann. Gotha.

1855- B.M.; Camb.U.; Dub.R.C.S.; Geol.M.i.; Glasg.P.S.i.; Glasg.U.; N.H.M.i.; Oxon.B.; Oxon.R.; R.S.; U.C.L.i.

Philosophical Transactions of the Royal Society of London. London.

1665- B.M. ; Camb.P.S.i. ; Camb.U.; Chem.S.i.; Dub.R.C.S. ; Dub.R.I.A.; Dub.T.C.; Edinb.R.S.; Edinb.U.; Geol.M.; Glasg. P.S. ; Glasg.U.i.; Linn.S.i.; Math.S.i.; N.H.M.; Oxon.B.; Oxon.R.; P.O.; R.A.S.i.; R.S.; U.C.L.i.

The London, Edinburgh, and Dublin Philosophical Magazine and Journal of Science. London.

1827- B.M.; Camb.P.S.; Camb.U.; Chem.S.; Dub.R.D.S.; Dub. R.I.A.; Edinb.R.S.; Edinb.U.; Geol.M.i.; Glasg.P.S.i.; Glasg.U.; Linn.S.i.; Math.S.i.; N.H.M.; Oxon.B.; Oxon.R.; P.O.; R.A.S.; R.S.; U.C.L.

The Pharmaceutical Journal and Transactions. London.

1841- B.M.; Camb.U.; Chem.S.; Dub.N.L.I.i.; Dub.T.C.i.; Glasg.P.S.; Glasg.U.i.; N.H.M.; Oxon.B.; Oxon.B.(R.); R.S.i.; U.C.L.

Philosophische Studien herausgegeben von Wilhelm Wundt. Leipzig.

1883- Camb.U.; Dub.T.C.; Edinb.U.; Oxon.B.; R.S.; U.C.L.

Annali della R. Scuola Normale Superiore di Pisa. Scienze Fisiche e Matematiche. Pisa.

1871. Oxon.B.; Oxon.R.; R.S.

(Annali delle Università Toscane. (Parte 2da.) Scienze Cosmologiche. Pisa.

1846- Camb.U.i.; N.H.M.; R.S.i.

Nuovo Giornale de' Letterati. Pisa.

1822-39. B.M.; Camb.U.; Oxon.B.

Atti della Società Toscana di Scienze Naturali residente in Pisa. Processi Verbali. Pisa.

1875- B.M.; Camb.P.S.i.; Dub.T.C.; N.H.M.; R.S.

Atti della R. Accademia Pistojese di Scienze, Lettere, ed Arti: Memorie di Matematica e Fisica, per l' anno 1816. Pistoja.

1816. B.M.; Camb.U.; N.H.M.; Oxon.B.; R.S.

Annalen der Physik und Chemie; Poggendorff. Leipzig.

1824 - B.M.; Camb.P.S.i.; Camb.U.; Chem.S.; Dub.R.I.A. ; Dub.T.C.; Edinb.R.S.; Edinb.U.; Glasg.P.S.i.; Glasg.U.i.; N.H.M.; Oxon.B.(R.); P.O.; R.S.; U.C.L.i.

See A. Ps. C.

Il Poligrafo: Giornale di Scienze, Lettere, ed Arti; Orti. Verona. 1830-45. B.M.; Oxon.B. 


\section{List of Serial Publications}

Pol. mt.

Pop. As.

Prace $\mathbf{M} \mathbf{t}_{\text {。 }}-\mathbf{F i z}$.

Prag Ab.

Prag České Ak. Fr. Jos. Pam.

Prag České Ak. Fr. Jos. R7.

Prag Fr. Jos. Ac. Sc. Bll. (Mth. Nt.)

Prag Jb. Böhm. Ms.

Prag sb.

Presse sc

Ps. Rv.

Ps. z.

Queb. T. It. $\mathbf{8}$

Quetelet Cor. Imth.

QJ. Mth.

QJ. Sc.

Railroad \& Eng. J.

Ranuzzi An. Gg.
Polytechnische Mittheilungen, unter Mitwirkung von Professoren höherer technischer Lehranstalten. Tübingen.

1844-46. B.M.; R.S.

Popular Astronomy. Northfield, Minnesota.

1894 - B.M.; Glasg.U.; R.A.S.

Prace Matematyczno-Fizyczne. Warsaw.

1888 - Camb.P.S.; Camb.U.; Chem.S.i.; Dub.R.I.A.i.; Math.S.; R.S.i.

Abhandlungen der k. Böhmischen Gesellschaft der Wissenschaften. Prag.

1804-92. B.M.i.; Camb.P.S.; Camb.U.i.; Dub.R.I.A.i.; Edinb. R.S.i.; Linn.S.i.; N.H.M.; Oxon.B.; R.S.i.

See Böhm. Gs. Ab.

Památnik na oslavu padesátileténo panovnického jubilea jeho veličenstva císaře a krále Františka Josefa I. Vydala Ceská Akademie Císaře Františka Josefa pro Vẻdy, Slovesnost a Umění. [Memoirs in honour of the jubilee of his Imperial and Royal Majesty Franz Joseph I. Edited by the Imperial Bohemian Franz-Joseph Academy of Sciences, Literature and Art.] Praze. (Prag.)

1898. Camb.P.S.; N.H.M.

Rozprawy České Akademie Císaře Františka Josefa Pro Vědy, Slovesnost a Umění. [Memoirs of the Imperial Bohemian FranzJoseph Academy of Sciences, Literature and Art.] Prag.

1891- B.M.; Edinb.R.S.; N.H.M.i.

Académie des Sciences de l'Empereur François Joseph I (Česká Akademie Císaře Františka Josefa I). Bulletin International. Résumé des Travaux présentés. Sciences Mathématiques et Naturelles. Prag.

1897 - Edinb.R.S.; N.H.M.i.

Jahrbücher des Böhmischen Museums für Natur- und Länderkunde, etc. Prag.

1830 -31. B.M.; N.H.M.

Sitzungsberichte der k. Böhmischen Gesellschaft der Wissenschaften in Prag. Prag.

1859 - Camb.P.S.; Camb.U.i.; Dub.R.D.S.; Dub.R.I.A.; Edinb. R.S.i.; Linn.S.i.; N.H.M.; R.S.; U.C.L.i.

Presse Scientifique des Deux Mondes. Paris.

1860-66. B.M.; R.S.i.

The Physical Review. New York, London, Berlin.

1894 B.M.; Camb.P.S.; Camb.U.; Dub.R.C.S.; Edinb.R.S. ; Edinb.U.i.; Oxon.R.; P.O.; R.S.

Physikalische Zeitschrift. Leipzig.

1899 - Camb.P.S.; Edinb.U.; Oxon.R.; R.S.

Transactions of the Literary and Historical Society of Quebec. Quebec.

1829 - B.M.i.; Dub.R.I.A.; Edinb.R.S.i.; Glasg.P.S.i.; Linn.S.i.; N.H.M.; R.S.i.

Correspondance Mathématique et Physique; publiée par MM. Garnier et Quetelet. Gand et Bruxelles.

1825-39. B.M.; Camb.U.; R.A.S.i.; R.S.; U.C.L.

The Quarterly Journal of Pure and Applied Mathematics. London. 1855- B.M.; Camb.P.S.; Camb.U.; Dub.N.L.I.; Dub.T.C. ; Edinb.R.S.i.; Edinb.U.; Glasg.P.S.i.; Glasg.U.; Math.S.i.; Oxon.B.; Oxon.R.; P.O.; R.A.S.i.; R.S.; U.C.L.

The Journal of Science and the Arts; edited at the Royal Institution of Great Britain. London. Continued as: Quarterly Journal of Science, Literature and Arts. London.

1816-30. B.M.; Camb.U.; Chem.S.; Dub.T.C.; Edinb.R.S. ; Glasg.U.; Oxon.B.; Oxon.R.; R.S.; U.C.L.

The Railroad and Engineering Journal. New York. 1887-92. B.M.; P.O.

Annuario geografico Italiano; Ranuzzi. Bologna. 1844-45. B.M.; Camb.U. 


\section{List of Serial Publications}

Rec. Mth. (Moscou)

R. .. Pp.

Rheinl. Westphal. Sb.

Riga Cor.-Bl.

R. I. J.

Rio Arch. Palestr.

R. I. $\mathbf{P}$.

Rm. At.

Rm. At. $\mathbf{~ r . ~ I i n c . ~}$

Rm. At. R. Ac.

Rm. Cor. Sc.

Rm. Mm. Osserv.

Rm. N. Iinc. At.

Rm. N. Iinc. $\mathbf{M m}$.

Rm. R. Ac. Iinc. At. Rm. R. Ac. Linc. $\mathbf{M m}$. ...

Rm. R. Ac. Iinc. Rd.

Rm. R. Ac. Iinc. T.

Rm. S. Cor.

Rm. s. It. $\mathbf{M m}$.

Rochester (I. Y.) Ac. sc. $\mathbf{P}$.

Rot. N. Vh.
Recueil mathématique. Publié par la Société Mathématique de Moscou. [In Russian.] Moscou.

1866- R.S.

Papers on subjects connected with the duties of the Corps of Royal Engineers. London.

1844- Camb.U.; Geol.M.i.; Glasg.U.i.; P.O.i.

Sitzungsbericht des Naturhistorischen Vereins der Preussischen Rheinlande und Westphalens. Bonn.

1844- Dub.R.D.S.i.; Dub.R.I.A.i.; Edinb.R.S.; Linn.S.i.; N.H.M. ; Oxon.R.; R.S.i.

Correspondenzblatt des Naturforscher-Vereins zu Riga. Riga.

1846- B.M.; Dub.R.I.A.i.; N.H.M.; R.S.i.

Journal of the Royal Institution of Great Britain. London.

1802-03; 1830-31. Camb.U.i.; Chem.S.i.; Dub.R.D.S.; Edinb. R.S.i.; Glasg.P.S.i.; Linn.S.i.; N.H.M.i.; Oxon.R.; P.O.i.; R.A.S.i.; R.S.; U.C.L.i.

Archivos da Palestra Scientifica do Rio de Janeiro. Rio de Janeiro. 1858. N.H.M.; R.S.

Notice of the Proceedings at the meetings of the members of the Royal Institution, with Abstracts of the Discourses delivered at the Evening Meetings. London.

1851- B.M. ; Camb.U.; Chem.S.; Dub.R.I.A. ; Dub.T.C. ; Edinb.R.S.; Geol.M.; Glasg.P.S.; Glasg.U.; Linn.S.; N.H.M.; Oxon.R.; P.O.; R.A.S.; R.S.; U.C.L.

(Atti dell' Accademia Pontificia dei Nuovi Lincei. Roma.

\{1847- B.M.; Dub.R.I.A. ; Edinb.R.S.; N.H.M.; Oxon.B.i. ; R.A.S.i.; R.S.

See Rm. N. Iinc. At.

Atti della Reale Accademia dei Lincei. Roma.

1870-83. B.M.; Camb.P.S.; Camb.U.i.; Chem.S.i.; Dub.R.D.S.; Dub.R.I.A.; Linn.S.; Math.S.; N.H.M.; Oxon.B.; Oxon.R.i.; R.A.S.i.; R.S.; U.C.L.i.

See Rm. R. Ac. Iinc. At.

Corrispondenza Scientifica in Roma per le avanzamento delle Scienze, etc. Roma.

1848

See $\mathbf{R m}$. s. Cor.

Memorie dell' Osservatorio dell' Università Gregoriana del Collegio Romano, diretto dai PP. della Compagnia di Gesu. Roma.

1850-63. B.M.; R.A.S.; R.S.

See Rm. At. and $\mathbf{R m}$. At. N. Iinc.

Memorie della Pontificia Accademia dei Nuovi Lincei. Roma.

1887- Dub.R.D.S.; Dub.R.I.A.; Edinb.R.S.; N.H.M.; R.S.

See Rm. At. R. Ac.

Atti della R. Accademia dei Lincei. Memorie della Classe di Scienze fisiche, matematiche e naturali. Roma.

1877- B.M.i.; Camb.P.S.; Camb.U.; Chem.S.; Dub.R.I.A.i.; Edinb.R.S.; Glasg.P.S.i.; Glasg.U.i.; Linn.S.; Math.S.; N.H.M.; Oxon.B.; Oxon.R.; P.O.i.; R.A.S.; R.S.; U.C.L.

Atti della R. Accademia dei Lincei. Rendiconti. Roma.

1885- B.M.; Camb.P.S.; Camb.U.; Chem.S.; Dub.T.C.; Edinb. R.S.; Glasg.U.i.; Linn.S.; Math.S.; N.H.M.; Oxon.B.; Oxon.R.; R.A.S.; R.S.; U.C.L.

Atti della R. Accademia dei Lincei. Transunti. Roma.

1877-84. B.M.; Camb.P.S.; Camb.U.; Chem.S.; Dub.R.I.A. ; Edinb.R.S.; Glasg.P.S.i.; Linn.S.; Math.S.; N.H.M.; Oxon.B.; Oxon.R.; R.A.S.; R.S.; U.C.L.

See Rm. Cor. sc.

Memorie di Matematica e di Fisica, della Società Italiana delle Scienze. Napoli e Roma.

1782 - B.M.i.; Camb.P.S.; Camb.U.i.; Dub.R.I.A.; Edinb.R.S.i.; Linn.S.i.; Oxon.B.i.; R.A.S.i.; R.S.; U.C.L.i.

See Mod. Mm. s. It., Verona $\mathbf{M m}$. s. It, and Verona s. It. Mm.

Proceedings of the Rochester Academy of Sciences. Rochester, N.Y. 1890 - B.M.; Camb.P.S.; Edinb.R.S.i.; Linn.S.; N.H.M.; R.S.; U.C.L.i.

Nieuwe Verhandelingen van het Bataafsch Genootschap der Proefondervindelijke Wijsbegeerte te Rotterdam. Rotterdam. 


\section{List of Serial Publications}

Ronen Bll. s. Ém.

Rouen Ac. Tr.

Ronen Tr. Ac.

Rpm. Ith.

Rpm. Ps.

Rs. Gg. Gs. D.

R. \$. $\mathbf{P}$.

Rs, P8.-C. S. J.

R. 8. Yearbook

Rv. Artl.

Rv. Brazil.

Rv. Cours. BC.

Rv. Itgure

Rv. Irax.

Rv. Mar. et Col.

Rv. mt.

Rv. Mth.

Rv. 8c.

Rv. Sc.-Ind.

Rv. Un. Mines

B. Afr. Ph. 8. T.

8. Afr. QJ.

Santlago de Chlle Un. A

Sarthe 8. Bu.
1800 - B.M.i.; Camb.U.i.; Chem.S.i.; Dub.R.D.S.; Edinb.R.S.i.; Oxon.B.; R.S.

Bulletins [des travaux] de la Société Libre d'Émulation de Rouen. Rouen.

1837- B.M.; Oxon.B.

Précis analytique des Travaux de l'Académie des Sciences, BellesLettres, et Arts de Rouen. Rouen.

1804- B.M.; Camb.U.; Dub.R.I.A. ; Dub.T.C.; N.H.M.i.; Oxon.B.; R.S.i.

Repertorium der literarischen Arbeiten aus dem Gebiete der reinen und angewandten Mathematik. Leipzig.

1877-79. Camb. U.; R.S.

Repertorium der Physik. Enthaltend eine vollständige Zusammenstellung der neuern Fortschritte dieser Wissenschaft. Berlin.

1837-49. Chem.S.; Glasg.P.S.i.; P.O.; R.S.; U.C.I.

Denkschriften der Russischen Geographischen Gesellschaft zu St Petersburg. Weimar.

1849. B.M.; Camb.U.; R.S.

Proceedings of the Royal Society of London. London.

1832- B.M.; Camb.P.S.; Camb.U.; Chem.S.; Dub.N.L.I.i. ; Dub.R.C.S.; Dub.R.D.S.; Dub.R.I.A. ; Dub.T.C.; Edinb.R.S.; Edinb.U.; Geol.M.; Glasg.P.S.; Glasg.U.i.; Linn.S.i.; Math.S.i.; N.H.M.; Oxon.B.i.; Oxon.R.; P.O.; R.A.S.; R.S.; U.C.L.

Journal of the Russian Physico-Chemical Society of the Imperial University of St Petersburg. [In Russian.] St Petersburg.

1869 - Camb.P.S.i.; Chem.S.; Edinb.R.S.i.; N.H.M.

Yearbook of the Royal Society of London. (Biography. 1900.)

Revue d'Artillerie. Paris, Naney.

1872- B.M.; P.O.

Revista Brazileira, Jornal de Sciencias, Lettras, e Artes; Oliveira. Rio de Janeiro.

1857-61. B.M.; N.H.M.; R.S.i.

Revue des Cours Scientifiques de la France et de l'Étranger; Eug. Yung et Ém. Alglave. Paris.

1863-70. B.M.; Edinb.R.S.i.; Edinb.U.; N.H.M.; Oxon.R. ; P.O.; R.S.

Rivista Ligure, giornale di Lettere, Scienze, etc. Genova.

1843. B.M.; N.H.M.; Oxon.B.

(Revue maritime et coloniale. Paris.

\{1861 - B.M.; Oxon.B.; Oxon.R.; P.O.

Rivista di Matematica. Torino.

1891-95. Camb.U.; Oxon.B.; R.S.

Revue de Mathématiques. (Rivista di Matematica.) Turin.

1896- Camb.U.; Oxon.B.; Oxon.R.; R.S.

La Revue Scientifique de la France et de l'Étranger. Paris.

1871- B.M.; Camb.U.; Edinb.R.S.; Edinb.U.; N.H.M.; Oxon.R.; P.O.; R.A.S.i.; R.S.

Rivista Scientifico-Industriale delle principali scoperte ed invenzioni fatte nelle scienze e nelle industrie. Firenze.

1869 - P.O.

Revue Universelle des Mines, de la Métallurgie, etc. Liége \& Paris. 1857 - B.M.; Camb.U.; Dub.R.I.A.i.; Glasg.P.S.i.; Glasg.U.i.; N.H.M.; P.O.

See Cuyper Rv. Un.

The Transactions of the South African Philosophical Society. Cape Town.

1878- B.M.; Camb.P.S.; Camb.U.i.; Chem.S.; Edinb.R.S.; Edinb.U.; Glasg.P.S.; Linn.S.i.; N.H.M.; Oxon.B.; Oxon.R.; P.O.; R.A.S.; R.S.

The South African Quarterly Journal; edited at the African Institution. Cape Town.

1830-35. B.M.i.; Edinb.R.S.i.; N.H.M.

Anales de la Universidad de Chile. Santiago de Chile.

1843 - B.M.i.; Dub.T.C.; N.H.M.i.; Oxon.B.i.

See Chlle A. Un.

Bulletin de la Société d'Agriculture, etc., de la Sarthe. Le Mans. 1833 - R.S.i. 


\section{List of Serial Publications}

S. Aust. R, s. T.

Sav. Ac. $\mathbf{M m}$. Sav, Mm. Ac.

Sc. Abs.

Sch. Gs. N. D.

Sch. Gr. Vh

Schlömilch Z

Sch. Nf. Gs. Wh.

Schumacher As. Ab......

Schweigger J.

Science

Sc. 8. Arts T.

Sid. Mess.

Siena At. Ac.

Silliman $\mathbf{J}$.

Skandia

Sk. Nf. $\mathbf{~} . \ldots . . .$.
sk. Nt, Mód. $\mathbf{.}$
Sk. Nt. Möt. $\mathbf{~}$.

Smiths. Ct.

Smiths, Misc. Col.
Transactions and Proceedings and Report of the Royal Society of South Australia. Adelaide.

1877- Camb.P.S.i.; Camb.U.i.; Chem.S.i.; Dub.R.I.A.i.; Edinb. R.S.; Linn.S.i.; N.H.M.; P.O.; R.A.S.; R.S.i.

Mémoires de la Société Académique de Savoie. Chambéry.

1825- Camb.U.; Dub.R.I.A.; Dub.T.C.; N.H.M.; Oxon.B.; R.S. $i$. See Chambéry $\mathbf{M m}$. Ac. Sav.

Science Abstracts. Physics and Electrical Engineering. London.

1898- Camb.P.S.; Camb.U.; Chem.S.; Edinb.R.S.i.; Edinb.U.; Glasg.P.S.; Oxon.R.; P.O.; R.A.S.i.; R.S.; U.C.L.

Neue Denkschriften der allgemeinen Schweizerischen Gesellschaft für die gesammten Naturwissenschaften. Neuchatel, Zürich, etc. 1837- B.M.; Camb.P.S.; Camb.U.; Dub.R.D.S.i.; Dub.R.I.A.i.; Edinb.R.S.; Linn.S.i.; N.H.M.; Oxon.B.; R.S.

See Zutr. N. D. Sch. Gs.

Verhandlungen der Schweizerischen Gesellschaft für die gesammten

Naturwissenschaften. Aarau, etc.

1823 - B.M.i.; Edinb.R.S.i.; Linn.S.i.; N.H.M.; R.S.

$\mathrm{See}$ sch. No. Gs. Vh.

Zeitschrift für Mathematik und Physik; Schlömilch. Leipzig.

1856 - B.M.; Camb.U.; Dub.N.L.I.i.; Dub.R.D.S.i.; Dub.R.I.A.i.; Dub.T.C.i.; Edinb.U.; Glasg.U.i.; Math.S.i.; Oxon.B.(R.); R.S.; U.C.L.i.

See Z. IMth. Ps.

See $\mathbf{S c h}$. Gs. Vh.

Astronomische Abhandlungen; Schumacher. Altona.

1823-25. B.M.; Camb.U.; Dub.R.D.S.; Edinb.R.S.; R.A.S.; R.S. Journal für Chemie und Physik; Schweigger. Nürnberg.

1811-29. Edinb.R.S.; N.H.M.; Oxon.R.; P.O. Continued as: Neues Jahrbuch der Chemie und Physik; Schweigger-Seidel. Berlin. 1831-33. B.M.; Chem.S.; Edinb.R.S.; N.H.M.; Oxon.R.; P.O.; R.S.

Science. Cambridge, Mass., and New York.

1883- B.M.; Dub.N.L.I.i.; Dub.R.C.S.i. ; Edinb.R.S. ; Edinb. U.i.; Glasg.P.S.i.; N.H.M.; Oxon.R.i.; P.O.; R.A.S.i.

Transactions of the Royal Scottish Society of Arts. Edinburgh.

1841 - B.M.i.; Camb.U.; Dub.R.D.S.; Edinb.R.S.; Edinb.U. ; Glasg.P.S.; Glasg. U.; P.O.; R.S.

See Fdinb. Sc. S. Arts $\mathbf{r}$. and rdinb. T. Sc. 8. Arts.

The Sidereal Messenger. Northfield, Minn.

1883-91. B.M.; R.A.S.

Atti del. Accademia delle Scienze di Siena detta de' Fisio-critici. Siena.

1761- B.M.; Camb.U.i.; Dub.R.I.A.i.; Dub.T.C.i.; N.H.M.i.; Oxon.B.; R.S.i.

The American Journal of Science and Arts; Silliman. New Haven. 1818 - B.M.; Camb.P.S.i.; Camb.U.; Chem.S.i.; Dub.N.L.I.i.; Dub.R.C.S.i.; Dub.T.C.i.; Edinb.R.S.; Edinb.U.; Geol.M.; Glasg.P.S.; Glasg.U.i.; N.H.M. ; Oxon.B.; Oxon.R.; P.O.; R.A.S.i.; R.S.

See Am. J. Sc.

Skandia. Tidskrift för Vetenskap och Konst; utgifven af Svenska Litteratur-Föreningen. Upsala.

1833-37. B.M.

Förhandlingar vid det af Skandinaviska Naturforskare och Läkare hållna Möte....... Götheborg, etc.

1839 - Oxon.B.i.; R.S.i.

Forhandlingerne ved de Skandinaviske Naturforskeres...Møde.... Götheborg, etc.

1839 - B.M.; N.H.M.; R.S.i.

Smithsonian Contributions to Knowledge. Washington.

1848 - B.M.; Camb.P.S.; Camb.U.; Chem.S.; Dub.T.C.; Edinb.

R.S.; Edinb.U.; Geol.M.i.; Glasg.P.S.; Glasg.U.i.; Linn.S.;

N.H.M.; Oxon.B.; Oxon.R.i.; P.O.i.; R.A.S.; R.S.; U.C.L.i.

Smithsonian Miscellaneous Collections. Washington.

1862 - B.M.; Camb.P.S.; Camb.U.; Chem.S.; Dub.T.C.; Edinb. R.S.; Edinb.U.; Geol.M.; Glasg.P.S.; Glasg.U.; Linn.S. ;

N.H.M.; Oxon.B.; Oxon.R.i.; P.O.i.; R.A.S.; R.S.; U.C.L.i. 


\section{List of Serial Publications}

Smiths. Rp.

Spet. It. IMm.

Stelerm. Mt.

st. Gal. B.

St. Louis Ac. T.

Stockh. Ac. Indl. Stockh. Ak. Indl.

Stockh. Ak. Indl. Bh. ...

stockh. Öfv.

stockh. Vt. Ak. Lefn.

st. Pét. Ac. IMm.

St. Pét. Ac. sc. Bu.

st. Pét. Ac. sc. Mm.

st. Pet. Ac. Sc. IMm. (Rs.)

st. Pet. Ac. Sc. N. Acta...

St. Pét. Ac. Sc. Rec.

st. Pét. Bll. Ac. Bc.

st. Pét. Bu. sc.

st. Pét. $\mathbf{M m}$.

st. Pót Imm Sav. tir.
Annual Report of the Board of Regents of the Smithsonian Institution. Washington.

1846- B.M.i.; Camb.P.S.; Camb.U.; Dub.T.C.; Edinb.R.S.i.; Geol.M.; Glasg.P.S.i.; Glasg.U.; Linn.S.i.; Math.S.i.; N.H.M.i.; Oxon.B.; Oxon.R.i.; P.O.i.; R.A.S.i.; R.S.i.; U.C.L.i.

Memorie della Società degli Spettroscopisti Italiani, raccolte e pubblicate per cura del Prof. P. Tacchini. Palermo.

1872- B.M.i.; Camb.U.; Edinb.R.S.i.; P.O.; R.A.S.; R.S.

Mittheilungen des Naturwissenschaftlichen Vereins für Steiermark. Graz.

1863- B.M.; Camb.U.i.; Dub.R.I.A.; Edinb.R.S.i.; Linn.S.i.; N.H.M.; R.S.; U.C.L. $i$.

Bericht über die Thätigkeit der St. Gallischen Naturwissenschaftlichen Gesellschaft. St. Gallen.

1860 - N.H.M.; R.S. $i$.

The Transactions of the Academy of Science of St. Louis. St. Louis.

1856 - B.M.; Dub.R.I.A.; Edinb.R.S.; Glasg.P.S. ; Linn.S.i.; N.H.M.; Oxon.B.; P.O.i.; R.S.

Kongliga Svenska Vetenskaps-Akademiens Handlingar. Stockholm. 1739- B.M. ; Camb.P.S. ; Camb.U.; Chem.S.i.; Dub.R.I.A.; Edinb.R.S.i.; Glasg.P.S.i.; Linn.S.i.; N.H.M.; R.A.S.; R.S.

Bihang till Kongl. Svenska Vetenskaps-Akademiens Handlingar. Stockholm.

1872- B.M. ; Camb.P.S. ; Camb.U.; Chem.S. ; Dub.R.I.A. ; Edinb.R.S.; Glasg.P.S.; Linn.S.i.; N.H.M.; R.A.S.; R.S.

Öfversigt af Kongl. Vetenskaps-Akademiens Förhandlingar. Stockholm.

1844 - B.M. ; Camb.P.S. ; Camb.U.; Chem.S.i. ; Dub.R.I.A.; Edinb.R.S.i.; Glasg.P.S.i.; Glasg.U.i.; Linn.S.i.; N.H.M.; Oxon.R.; R.A.S.; R.S.; U.C.L.i.

Lefnadsteckningar öfver Kongl. Svenska Vetenskaps Akademien... ledamöter. Stockholm.

1869-73. Chem.S.i.; Dub.R.I.A. ; Edinb.R.S. ; Glasg.P.S. ; Linn.S.i.; R.A.S.; R.S.

Mémoires de l'Académie Impériale des Sciences de St. Pétersbourg. St. Pétersbourg.

1803- B.M.; Camb.P.S.; Camb.U.; Chem.S.i.; Dub.R.D.S. ; Dub.R.I.A. ; Dub.T.C.; Edinb.R.S.; Edinb.U.; Glasg.U.i.; N.H.M.; Oxon.B.; Oxon.R.; P.O.i.; R.S.; U.C.L.i.

See $\mathbf{s t}$. Pét. Ac. Sc. Mm., st. Pét. IMm. and $\mathbf{~ s t . ~ P e ́ t . ~} \mathbf{M m}$. Ac. sc.

Bulletin Scientifique publié par l'Académie Impériale des Sciences de St. Pétersbourg. St. Pétersbourg.

$1836-42$.

Bulletin de la Classe Physico-mathématique de l'Académie Impériale des Sciences de St. Pétersbourg. St. Pétersbourg and Leipzig.

$1843-59$.

Bulletin de l'Académie des Sciences de St.Pétersbourg. St.Pétersbourg.

1860- B.M.i.; Camb.P.S. ; Camb.U.; Chem.S.i.; Dub.R.I.A.; Edinb.R.S.; Glasg.P.S.i.; Glasg.U.; Linn.S.i.; N.H.M.; Oxon.B.; Oxon.R.i.; P.O.i.; R.A.S.i.; R.S.

See st. Pét. Bll. Ac. Sc. and st. Pét. Bull. sc.

See st. Pét. Ac. IMm.

Memoirs of the Imperial Academy of Science. [In Russian.] St. Petersburg. Not the same as st. Pét. Ac. $\mathbf{~ M m}$.

1862 -95. B.M.; Dub.R.I.A.

Nova Acta Academiæ Scientiarum Imperialis Petropolitanæ. Petropoli.

1783-1802. B.M.; Camb.U.; Edinb.R.S.; Linn.S.i.; N.H.M.; Oxon.B.; Oxon.R.; P.O.; R.A.S.i.; R.S.; U.C.L.

Recueil des Actes des Séances Publiques de l'Académie Impériale des Sciences de St. Pétersbourg. St. Pétersbourg.

1827-48. B.M.; Camb.U.; Chem.S.i.; Dub.R.I.A.i.; Edinb.R.S.; N.H.M.; Oxon.B.; Oxon.R.i.; R.A.S.; R.S.

See st. Pét. Ac. Bc. Bu.

See st. P6́t. Ac. $\mathbf{m m}$.

Mémoires présentés à l'Académie Impériale des Sciences de St. Pétersbourg par divers Savans. St. Pétersbourg. 


\section{List of Serial Publications}

Strasb. Mm. s. Sc.

Strasb. S. Sc. N. Mm.

Srea

Sym. Iret. Mg.

Tasm, R, S. $\mathbf{P}$

Taylor Sc. Irm.

Tel. 2.

Terr. Mag.

Texas Ac. Sc. I.

Thomson A. Ph.

Thrond. Bkr.

Tilloch $\mathbf{P h}$. Mg.

TбK. Coll. Sc. J.

Tok. Un. Irm.

Tox. Ac. Mm.

Tor. Ac. Sc. At.
1831-59. B.M.; Camb.U.; Edinb.R.S.; Glasg.U.; Linn.S.; N.H.M.; R.A.S.; R.S.; U.C.L. $i$.

Mémoires de la Société des Sciences Naturelles de Strasbourg. Strasbourg.

1830-70. B.M.; Dub.R.I.A.i.; Dub.T.C.i.; N.H.M.

Nouveaux Mémoires de la Société des Sciences, Agriculture, et Arts du Département du Bas-Rhin. Strasbourg.

1832-42; 1859-68. Camb.U.i.; N.H.M.; Oxon.B.; R.S.i.

Svea. Tijdskrift för Vetenskap och Konst. Upsala.

$1818-31$.

Symons's Monthly Meteorological Magazine. London.

1866- Camb.U.; P.O.; R.S.

Monthly Notices of Papers and Proceedings of the Royal Society of Tasmania. Hobart.

1863- B.M.i.; Camb.P.S.i.; Dub.R.D.S.; Edinb.R.S.i.; Linn.S.i.; N.H.M.; R.A.S.; R.S.

Scientific Memoirs, selected from the Transactions of Foreign Academies and Learned Societies and from Foreign Journals; Taylor. London.

1837-52. B.M.; Camb.U.; Chem.S.i.; Edinb.R.S.; Linn.S.i. ; N.H.M.; Oxon.B.(R.); P.O.; R.A.S.i.; R.S.; U.C.L.

Journal of the Society of Telegraph Engineers. London.

1872- B.M.; Camb.P.S.; Camb.U.i.; Dub.T.C.i.; Oxon.B.; Oxon.R.; P.O.; R.S.; U.C.L.

Terrestrial Magnetism [and Atmospheric Electricity]. An International Quarterly Journal. Chicago, Cincinnati, Baltimore.

1896- Camb.U.i.; R.S.

Transactions of the Texas Academy of Science. Austin.

1892- Camb.P.S.; Edinb.R.S.; Glasg.P.S.; Math.S.i.; N.H.M.; R.S.

Annals of Philosophy, or Magazine of Chemistry, Mineralogy, Mechanics, and the Arts; Thomson. London.

1813-26. B.M.; Camb.P.S.; Camb.U.; Chem.S.i.; Edinb.R.S.i.; Linn.S.; N.H.M.; Oxon.B.; Oxon.R.; P.O.; R.A.S.; R.S.; U.C.L. $i$.

Det Kongelige Norske Videnskabersselskabs Skrifter i det 19de Aarhundrede. Kjøbenhavn og Throndhjem.

1817- B.M.; Camb.U.i.; Dub.T.C.i.; Edinb.R.S.i.; N.H.M.; R.S.

See Norske vd. skr.

The Philosophical Magazine, comprehending the various branches of Science, the Liberal and Fine Arts, Geology, Agriculture, Manufactures, and Commerce. London.

1798-1826. B.M.; Camb.P.S.; Camb.U.; Chem.S.i.; Edinb. R.S.i.; Edinb.U.; Glasg.P.S.; Glasg.U.i.; Linn.S.i.; N.H.M.; Oxon.B.; Oxon.R.; P.O.; R.A.S.; R.S.; U.C.L.

The Journal of the College of Science, Imperial University, Japan. Tōkio, Japan.

1887- B.M.; Camb.P.S.; Camb.U.; Chem.S.; Dub.T.C.; Edinb. R.S.; Edinb.U.i.; Glasg.P.S.i.; Linn.S.i.; Math.S.; N.H.M.; Oxon.B.; Oxon.R.; P.O.; R.S.; U.C.L.

Memoirs of the Science Department, University of Tokio, Japan. Tokio, Japan.

1879-85. Camb.U.; Chem.S.i.; Dub.R.D.S.; Dub.R.I.A.; Edinb. R.S.i.; Glasg.P.S.; Linn.S.i.; N.H.M.; Oxon.B.; Oxon.R.; R.A.S.i.; R.S.; U.C.L.i.

Memorie della R. Accademia delle Scienze di Torino. Torino.

1818 - B.M.i.; Camb.P.S.; Camb.U.; Dub.R.I.A.; Edinb.R.S.; Glasg.U.i.; Linn.S.; N.H.M.; Oxon.B.; P.O.; R.A.S.; R.S.; U.C.L. $i$.

See Tor. Ac. Sc. Mm., Tor. Mm. Ac., Turin Ac. Mm. and Turin Mm. Ac.

Atti della R. Accademia delle Scienze di Torino. Torino.

1865- B.M.; Camb.P.S.; Camb.U.; Chem.S.i.; Dub.R.I.A.; Edinb.R.S.; Glasg.U.i.; Linn.S.; N.H.M. ; Oxon.B.; P.O.i.; R.A.S.; R.S.; U.C.L.i.

See Tor. At. Ac. Sc.

See Tor. Ac. $\mathbf{m m}$. 


\section{List of Serial Publications}

Tor. At. Ac. Sc.

rox. Mm, Ac.

Tortolini A.

Toul. Ac. Sc. BL.

Toul. Ac, Sc, Mm.

Toul. Iac. Sc. A.

Toul. Mm. Ac.

Toul. Mm. Ac. Sc.

Toul. Obs. A.

Toul. B. I. Nt. B11.

Ts. Mth.

Ts. Mt. Eys.

Turin Ac. Mm.

Turin Mm. Ac.
See For. Ac. sc. At.

Notizia storica dei lavori fatti dalla Classe di Scienze Fisiche e Matematiche della R. Accademia delle Scienze negli anni 1864 65. Torino.

1869. Linn.S.; R.A.S.; R.S.

See rox. Ac. Im.

Annali di Scienze, Matematiche, e Fisiche; Tortolini. Roma.

1850-57. B.M.; Camb.U.i.; Dub.R.D.S.; Dub.T.C.; Edinb.U.; Glasg.U.i.; Math.S.i.; Oxon.B.(R.); R.S.; U.C.L.

\section{See A. Int.}

Bulletin de l'Académie des Sciences, Inscriptions et Belles-lettres de Toulouse. Toulouse.

1898-99. Dub.R.I.A.; Edinb.R.S.; N.H.M.; R.S.

Mémoires de l'Académie des Sciences, Inscriptions et Belles-Lettres de Toulouse. Toulouse.

1782- B.M.; Camb.U.; Dub.R.I.A.; Edinb.R.S.i.; N.H.M. ; Oxon.B.i.; R.A.S.i.; R.S.

See Toul. Mm. Ac, and Toul. Mm. Ac. Sc.

Annales de la Faculté des Sciences de Toulouse pour les Sciences Mathématiques et les Sciences Physiques. Paris.

1887- Camb.P.S.; Camb.U.; Edinb.R.S.; Math.S.; Oxon.R.; R.S.

See Toul. Ac. Sc. Mm.

Annales de 1'Observatoire Astronomique, Magnétique et Météorolo. gique de Toulouse. Paris.

1880 - B.M.; Edinb.R.S.; Math.S.i.; R.A.S.; R.S.

Bulletin de la Société d'Histoire Naturelle de Toulouse. Toulouse.

1867 - N.H.M.

Tidsskrift for Mathematik. Kjøbenhavn.

1859-89. B.M.; Camb.U.; Math.S.i.; Oxon.B.

Tidskrift för Matematik och Fysik, tillegnad den Svenska Elementar. Undervisningen. Upsala.

1868-74. B.M.; R.S.i.

(Mémoires de l'Académie Royale des Sciences de Turin. Turin.

1784-1816. B.M.; Dub.R.I.A.i.; Edinb.R.S.; Linn.S.; Oxon.B.; P.O.; R.A.S.; R.S.; U.C.L.

See ror. Ac. Mm.

Un. Serv. I. J.

Journal of the Royal United Service Institution. London.

1858 - B.M. ; Camb.U. ; Dub.N.L.I.i. ; Edinb.U. ; Oxon.B.i. ; P.O.; R.S.

Ups. Arsk.

Upsala Universitetets ̊̈rsskrift. Upsala.

1861 - B.M.; Camb.U.; Dub.R.D.S.i.; Dub.R.I.A.i.; Edinb.R.S.; Linn.S.i.; Math.S.i.; N.H.M.; Oxon.B.; R.A.S.i.; R.S. Nova Acta Regiæ Societatis Scientiarum Upsaliensis. Upsaliæ.

Ups. IT. Acta S. Sc.

Ups. 8. 8c. N. Acta

Ups. vet. s. Arsk.

R.S.i.; U.C.L.i.

Årsskrift utgifven af Kongl. Vetenskaps-Societeten i Upsala. Upsala. 1860-61. B.M.; Camb.U.; Edinb.R.S.; N.H.M.; R.S.

U. S. Coast Sv. Rp.

Reports of the Superintendent of the Coast Survey, showing the Progress of the Survey from year to year. Washington.

1851 Camb.U.; Dub.R.I.A.i.; Edinb.R.S.; N.H.M.; R.A.S.; R.S.; U.C.L. $i$.

Utr. A. Ac.

Annales AcademiæRheno-Trajectinæ. Trajectiad Rhenum. (Utrecht.)

1815-37. B.M.; Camb.U.; N.H.M.; Oxon.B.; R.S.i.

Aanteekeningen van het Verhandelde in de Sectie-Vergaderingen van het Provinciaal Utrechtsch Genootschap van Kunsten en Wetenschappen. Utrecht.

1846 - Dub.R.D.S.; Edinb.R.S.; R.S.

Vars. s. It. Tr. (C. R., Travaux de la Société des Naturalistes de Varsovie. Comptes P8. C.) .............. Rendus de la Section de physique et de chimie. Varsovie. [In Russian.]

1889 - Math.S.; N.H.M.

Vars. 8. Nt. Tr. $(M m$.$) ... Travaux de la Société des Naturalistes de Varsovie. Mémoires.$ Varsovie. [In Russian.]

1891-96. Math.S.; N.H.M.

xlvi 


\section{List of Serial Publications}

ven, At.

Ven. Aten.

Ven. Aten. At.

Ven. Aten. Fsercit.

Ven. At. I.

Ven. I. At. A............

$\checkmark e$ ercit.

Ven.

Verona Mm. s. It.

Verona $\mathbf{S}$. It. IMm.

Vict. R. S. $\mathbf{P}$.

Vict. R. 8. T.

Vict. T. Ph. I.

Vict. T. R. $\mathbf{s .}$

v. Nost. Fng. Mg.

Voigt $\mathbf{M g}$.

Wash. Nat. Ac. IMm

wash. Ph. 8. Bu.

w. Ing. J.

westf. Vr. Jbr.

wet. Gs. Jbr.

wiad. Int

Wrien Ak. D.
Atti delle Adnnanze dell' I. R. Istituto Veneto di Scienze, Lettere, ed Arti. Venezia.

1841 - B.M.; Dub.R.D.S.i.; Dub.R.I.A.i.; Edinb.R.S.i.; Linn.S.i.; R.S. $i$.

L'Ateneo Veneto: Rivista mensile di Scienze, Lettere ed Arti. Venezia. 1881? - Dub.R.D.S.i.; R.S.i.

Atti dell' Ateneo Veneto. Venezia.

1864- Dub.R.D.S.; R.S.i.

Esercitazioni Scientifiche e Letterarie dell' Ateneo di Venezia. Venezia.

1837-60. B.M.i.; Dub.T.C.i.; Oxon. B.i.; R.S.i.

See Ven. Fsercit. Aten.

(Atti del Reale Istituto Veneto di Scienze, Lettere ed Arti. Venezia.

1841- B.M.; Edinb.R.S.i.; Linn.S.i.; Math.S.i.; N.H.M.; R.S.i. See Ven. Aten. Fsercit.

(Memorie del Reale Istituto Veneto di Scienze, Lettere, ed Arti. Venezia.

1843- B.M.; Camb.U. ; Dub.R.I.A.i. ; Linn.S.i. ; N.H.M. ; Oxon.B.i.; R.S.

(Memorie di Matematica e di Fisica della Società Italiana delle Scienze. Modena e Verona.

1782 - B.M.i.; Camb.P.S.; Camb.U.i.; Dub.R.I.A.; Edinb.R.S.i.; Linn.S.i.; Oxon.B.i.; R.A.S.i.; R.S.; U.C.L.i.

See Mod, Irm. s. It. and $\mathbf{R m}$. s. It. $\mathbf{M m}$.

Proceedings of the Royal Society of Victoria. Melbourne.

1889 - B.M. ; Camb.P.S. ; Camb.U.; Dub.R.I.A. ; Dub.T.C. Edinb.R.S.; Edinb.U.; Glasg.P.S.; N.H.M.; Oxon.B.; Oxon.R. P.O.; R.S.; U.C.L.i.

Transactions and Proceedings of the Royal Society of Victoria. Melbourne.

1861- B.M.; Camb.P.S. ; Camb.U.; Dub.R.D.S.; Dub.R.I.A.; Edinb.R.S.; Edinb.U.; Glasg.P.S.i.; Glasg.U.i.; Linn.S.i.; N.H.M.; Oxon.B.; Oxon.R.i.; P.O.; R.A.S.; R.S.

See vict. T. R. s.

Transactions of the Philosophical Institute (afterwards Royal Society) of Victoria. Melbourne.

1855-60. B.M.; Camb.U.i.; Dub.R.I.A. ; Edinb.R.S.; Glasg. P.S.i.; Linn.S.; N.H.M.; Oxon.B.; P.O.; R.A.S.; R.S.

See Vict. R. 8. T.

Van Nostrand's Engineering Magazine. New York.

1869-85. B.M.; P.O.; R.S.i.

Magazin für den neuesten Zustand der Naturkunden, mit Rücksicht auf die dazu gehörigen Hülfswissenschaften; Voigt. Jena und Weimar.

1797-1806. B.M.; Camb.U.; N.H.M.; R.S.

Memoirs of the National Academy of Sciences. Washington.

1866- B.M.i.; Camb.P.S.; Camb.U.i.; Dub.R.I.A.; Edinb.R.S. ; Math.S.i.; N.H.M.; Oxon.B.i.; Oxon.R.; P.O.; R.S.; U.C.L.i. Bulletin of the Philosophical Society of Washington. Washington. 1874- B.M.; Camb.P.S.; Edinb.R.S.; Linn.S.; N.H.M.; Oxon.B.; P.O.; R.A.S.; R.S.

The West of England Journal of Science and Literature. Bristol.

1835-36. B.M.; Camb.U.; Edinb.R.S.; N.H.M.; Oxon.B.; P.O.

Jahres-Bericht des Westfälischen Provinzialvereins für Wissenschaft und Kunst. Münster.

1873 - N.H.M.

Jahresbericht der Wetterauischen Gesellschaft für die gesammte Naturkunde. Hanau.

1843 - Dub.R.I.A.; R.S.i.

Wiadomości Matematyczne. Warsaw.

1897- Camb.P.S.; Math.S.

Denkschriften der Kaiserlichen Akademie der Wissenschaften. Mathematisch-Naturwissenschaftliche Classe. Wien.

1850 - B.M.; Camb.P.S.i.; Camb.U.; Chem.S.i.; Dub.R.I.A.; Edinb.R.S.; Edinb.U.; Linn.S.; N.H.M.; Oxon.B.(R); P.O.i.; R.A.S.; R.S.; U.C.L.i.

See wien $\mathbf{D}$. 


\section{List of Serial Publications}

Wien Ak. Sb.

Wrien AIm.

Wien Az.

Wien Berg-Im. Jb.

Wien D.

Wien Jb. Pol. $\mathbf{I}$.

Wien Jbr. Ober-Realsch. Inn. Stadt

Wien Met. $\mathbf{z}$.

Wien Mt. Gg. Gs.

Wien SB.

wien sb.

Wisc. Ac. T.

witurtb. Jh.

Würzb. Ps. Md. sb.

Würzb. P\&. Md. Vh. würzb. Vh.

Zach Cor.

Zach Mr. Cor.

Z. Bauw,

z. Instk.

Z. Mth. $\mathbf{P a}$.

Z. $\mathbf{x w}$.
Sitzungsberichte der Mathematisch-Naturwissenschaftlichen Classe der Kaiserlichen Akademie der Wissenschaften. Wien.

1848- B.M.; Camb.P.S.i.; Camb.U.; Chem.S.i.; Dub.R.I.A.; Dub.T.C.; Edinb.R.S.i.; Glasg.U.i.; Linn.S.; N.H.M.; Oxon.B.; Oxon.R.; P.O.i.; R.A.S.i.; R.S.; U.C.L.i.

See wien sB, and wien $\mathbf{s b}$.

Almanach der Kaiserlichen Akademie der Wissenschaften. Wien.

1851 - B.M.; Camb.P.S.i.; Camb.U.; Dub.R.I.A.i.; Edinb.R.S.i.; Oxon.B.; P.O.i.; R.A.S.i.; R.S.i.; U.C.L.i.

Anzeiger der Kaiserlichen Akademie der Wissenschaften: Math.Naturwiss. Classe. Wien.

1864 - Camb.U.; Linn.S.; N.H.M.; Oxon B.; R.S.i.

Berg- und Hüttenmännisches Jahrbuch der k. k. Schemnitzer Bergakademie und der k. k. Montan-Lehranstalten zu Leoben und Pruibram; Gustav Faller. Wien.

1851 - B.M.i.; P.O.i.

See Wien Ak. D.

Jahrbuch des k. k. Polytechnischen Instituts in Wien; Prechtl. Wien.

1819-39. B.M.; Camb.U.; Oxon.B.; P.O.

Jahresbericht der öffentlichen Ober-Realschule in der innern Stadt. Wien.

$1859-63$.

Zeitschrift der Oesterreichischen Gesellschaft für Meteorologie. Wien. 1866-85. Dub.R.D.S.; Edinb.R.S.; P.O.; R.S.

Mittheilungen der k. k. Geographischen Gesellschaft. Wien.

1857- B.M.; Dub.R.I.A.i.; Dub.T.C.i.; N.H.M.; Oxon B.; R.S.

See wien Ak. sb.

Transactions of the Wisconsin Academy of Sciences, Arts and Letters. Madison.

1872- B.M. ; Camb.P.S.; Dub.R.I.A.; Edinb.R.S.; N.H.M.; Oxon.R.i.; P.O.i.; R.S.

Jahreshefte des Vereins für vaterländische Naturkunde in Württemberg. Stuttgart.

1845- B.M.; Camb.U.; Dub.R.D.S.i.; Dub.T.C.i.; Linn.S.i.; N.H.M.; R.S.

Sitzungsberichte der Physikalisch-Medicinischen Gesellschaft zu Würzburg. Würzburg.

1859-62. 1881- Camb.P.S.; Camb.U.; Chem.S.i.; Dub.R.I.A.; Linn.S.i.; Oxon.R.i.; R.S.

(Verhandlungen der Physikalisch-Medicinischen Gesellschaft. Würzburg.

1850-60. 1868- B.M.i. ; Camb.P.S. ; Camb.U. ; Chem.S.i. ; Dub.R.I.A.; Linn.S.; N.H.M.; Oxon.R.; R.S.; U.C.L.i.

Correspondance Astronomique, Géographique, Hydrographique, et Statistique; von Zach. Génes.

1818-26. B.M.; R.A.S.; R.S.

Monatliche Correspondenz zur Beförderung der Erd- und HimmelsKunde; von Zach. Gotha.

1800-13. Oxon.B.; R.A.S.; R.S.; U.C.L.

Zeitschrift für Bauwesen; herausg. unter Mitwirkung der königl. technischen Bau-Deputation und des Architecten-Vereins zu Berlin. Berlin.

1851 - B.M.; Camb.U.i.; P.O.

Zeitschrift für Instrumentenkunde. Organ für Mittheilungen aus dem gesammten Gebiete der wissenschaftlichen Technik. Berlin.

1881- B.M.; Camb.U.; Chem.S.; Edinb.U.; Oxon.R.; P.O.; R.A.S.; R.S.

Zeitschrift für Mathematik und Physik; Schlömilch. Leipzig.

1856 - B.M.; Camb.U.; Dub.R.D.S.i.; Dub.R.I.A.i.; Dub.T.C.i.; Edinb.U.; Glasg.U.i.; Math.S.i.; Oxon.B.(R.); R.S.; U.C.L.i.

\section{See schlömilch z.}

Zeitschrift für die gesammten Naturwissenschaften; herausgegeben von dem Naturwissenschaftlichen Vereine für Sachsen und Thüringen in Halle; Giebel. Berlin.

$$
\text { xlviii }
$$




\section{List of Serial Publications}

1853 - B.M. ; Camb.U.i.; Dub.N.L.I.i. ; Dub.R.D.S.i. ; Dub. R.I.A.i.; Dub.T.C.i.; Edinb.R.S.; Linn.S.; N.H.M.; Oxon.B.; Oxon.R.; R.S.

See rralle z. and Halle z. Nw.

z. Pøychol.

Zür. Mschr.

zür. Mnt.

Zür. N. D. Sch. Gs.

Zür. Vjschr.

Zwick. Vr. INt. Jbr.
Zeitschrift für Psychologie und Physiologie der Sinnesorgane. Hamburg, Leipzig.

1890 - B.M.; Camb.U.; Edinb.U.; Oxon.B.; Oxon.R.; R.S.; U.C.L. Monatsschrift des Wissenschaftlichen Vereins in Zürich; Hitzig, etc. Zürich.

1856-59. B.M.; Camb.U.; N.H.M.; Oxon.B.; R.S.

Mittheilungen der Naturforschenden Gesellschaft in Zürich. Zürich. 1847-56. Chem.S.i.; Dub.R.I.A.; Edinb.R.S.i.; Linn.S.; N.H.M.; R.A.S.; R.S.

Neue Denkschriften der allgemeinen Schweizerischen Gesellschaft für die gesammten Naturwissenschaften Neuchâtel. Zürich, etc. 1837 - B.M.; Camb.P.S.; Camb.U.; Dub.R.D.S.i.; Dub.R.I.A.i.; Edinb.R.S.; Linn.S.i.; N.H.M.; Oxon.B.; R.S.

See $\mathbf{s c h}$. Gs. N. D.

Vierteljahrsschrift der Naturforschenden Gesellschaft in Zürich. Zürich.

1856- B.M.; Camb.P.S.; Camb.U.i.; Chem.S.i.; Dub.R.I.A.; Edinb.R.S.; Linn.S.i.; Math.S.i.; N.H.M.; R.A.S.; R.S.

Jahresbericht des Vereins für Naturkunde zu Zwickau. Zwickau. 1874 - N.H.M.; R.S.i. 



\section{PURE MATHEMATICS}

\section{SCHEDULE OF CLASSIFICATION}

\section{ADAPTED FROM SCHEDULE A OF THE INTERNATIONAL CATALOGUE OF SCIENTIFIC LITERATURE.}

0000

0000

$\begin{array}{cccr}\text { Philosophy } & & & \text { PAGE } \\ \text { History. Biography } & \ldots & \ldots & 1 \\ \text { History } & \ldots & \ldots & 3 \\ \text { Arabian Mathematics } & \ldots & -17 \\ \text { Greek Mathematics } & \ldots & 4 \\ \text { Indian Mathematics } & \ldots & 9-10 \\ \text { Biography } \ldots & \ldots & & 17-25\end{array}$

0020 Periodicals. Reports of Institutions, Societies, Congresses,

0030 Getc. $\dddot{\text { Teneral }}$ Treatises, Text Books, Dictionaries,Tables, Collected

$\begin{array}{lllll}\text { Works } & \ldots & \ldots & \ldots & 25\end{array}$

0032 Bibliographies ... $\quad \ldots . \quad \ldots . \quad 26$

0035 Tables of Mathematical Functions ... ... ... ...

0040 Addresses, Lectures, etc., of $\ldots$ general character ... $\quad$... 29

$\begin{array}{llllll}0050 & \text { Pedagogy } & \ldots & \ldots & \ldots & 30\end{array}$

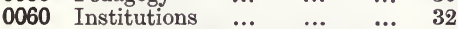

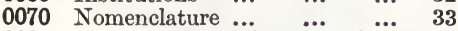

0080 Instruments, including Calculating Machines. Models 34 Instruments ... ... $34-38$ Calculating Machines 38-39 Models $\quad . . \quad \ldots \quad \quad 39-40$

0090 Aids to Calculation, Graphical Processes $\quad . . \quad \ldots \quad \ldots \quad 40$

\section{ARITHMETIC AND ALGEBRA.}

Foundations of Arithmetic.

0400 General ... $\quad . . \quad \ldots \quad \ldots \quad 42$

0410 Rational numbers; arithmetical operations $\quad \ldots \quad \quad \ldots \quad 43$ Scales of Notation $\quad{ }_{45-46}$

0420 Existence of irrational and transcendental numbers ; infinite processes adapted to rational numbers ... $\quad$... 46

0430

\section{Universal Algebra.}

0800 General ... $\quad \ldots \quad \ldots \quad \ldots \quad \ldots \quad 50$

0810 Calculus of Operations $\quad \ldots \quad 51$

0820 General theory of complex numbers. (See also 2870.) 53

0830 Quaternions $\ldots . \quad \ldots . \quad \ldots \quad 56$

0840 Ausdehnungslehre; vector an$\begin{array}{lllll}\text { alysis ... } & \ldots & \ldots & \ldots & 59\end{array}$

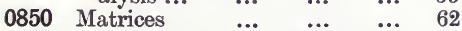

0860 Other special sorts of complex $\begin{array}{lllll}\text { numbers } & \ldots & \ldots & \ldots & 63\end{array}$

$\begin{array}{lllll}0870 & \text { Algebra of Logic } & \ldots & \ldots & 64\end{array}$

\section{Theory of Groups.}

1200 General ... $\quad \ldots \quad$... $\quad \ldots .65$

1210 Discrete groups of finite and of infinite order (including groups of permutations). (See also 2450, 4440.) $\quad$... 66

1230 Continuous groups of finite and of infinite order $\quad \ldots \quad 72$

\section{ALGEBRA AND THEORY OF NUMBERS.}

1590 Genèral ... $\quad \ldots \quad \ldots \quad \ldots \quad \ldots \quad 75$

\section{Elements of Algebra.}

1600 General ... ... 1610 Rational polynomials ; divisibility ; reducibility... ...

$\begin{array}{llll}1615 & \text { Algebraic inequalities ... } & \ldots & 77\end{array}$

1620 Permutations, combinations, partitions, distributions. Binomial and multinomial coefficients $\quad$... $\quad$... 80

Binomial coefficients $\quad{ }^{\prime} \quad 80-81$ Chess $\quad \ldots \quad \ldots \quad$ 81-82 $\begin{array}{lll}\text { Combinations } & \ldots & 82-83\end{array}$ Magic squares $\quad \ldots . . \quad 84-85$ Partition of numbers $\quad 85-86$ 


\section{Schedule of Classification}

\begin{tabular}{|c|c|c|}
\hline & $\begin{array}{l}\text { Partitions .... } \\
\text { Permutations }\end{array}$ & $\begin{array}{r}\text { PAGE } \\
86-87 \\
87-88\end{array}$ \\
\hline 25 & FiniteSummation. Recurring & \\
\hline & $\begin{array}{l}\text { series } \\
\text { Fiqurate }\end{array}$ & $\begin{array}{l}89 \\
89\end{array}$ \\
\hline & Special series ... & 91-92 \\
\hline & $\begin{array}{l}\text { Probabilities (including com- } \\
\text { bination of observations). }\end{array}$ & \\
\hline & $\begin{array}{l}\text { Theory of errors } \\
\text { Least Squares Method }\end{array}$ & $\begin{array}{r}92 \\
97-98\end{array}$ \\
\hline & Probabilities $\quad \ldots$ & $100-103$ \\
\hline & Problems & $101-102$ \\
\hline & $\begin{array}{c}\text { Theory of statistics. Actu- } \\
\text { arial mathematics }\end{array}$ & 104 \\
\hline & Mortality & $114-116$ \\
\hline & Population & $117-118$ \\
\hline
\end{tabular}

1640 Calculus of differences; inter-

$$
\text { polation ... ... ... }
$$$$
\text { Interpolation } \quad \ldots \quad 123-125
$$

\section{Linear Substitutions.}

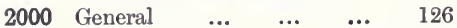

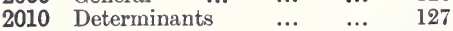

2020 Discriminants and resultants 136

$$
\text { Elimination ... 137-139 }
$$

2030 Characteristic properties of

linear substitutions ; types of linear substitutious.

(See also 1210.) ... ...

2040 General theory of quantics

2050 Binary forms $\quad \ldots \quad$...

2060 Ternary forms ... ...

2070 Special developments associated with forms in more than three variables $\quad . .$.

\section{Theory of Equations.}

2400 General

2410 Elements of $\dddot{m}$ the theory $\dddot{\text { of }}$ algebraic equations; existence of roots, symnietric functions; rational fractions ; partial fractions

Existence of roots Symmetric functions $\quad{ }_{162-163}$

2420 Reality, multiplicity, separation, of roots

142

146

153

155

quations of the second, third and fourth orders: other particular equations ... Equations, 2nd order .... Equations, 2nd, 3rd and 4 th orders

2430 Equations, 3 rdo Irreducible case Equations, 3rd and 4 th orders... ...

Equations, 4th order

$-172$ Trinomial Equations 173-174

2440 Numerical solution of equations (including graphical methods) ...
Numerical solution of equations in general

PAGE

Approximate determination of roots

Graphical methods

174-175

$177-178$

2450 General resolution of equa-

tions; theory of Galois.

(See also 1210.) ... $\quad$... 178 Equations, 5th order 180-181 Equations, 6th order ... 181

2460 Simultaneous equations; including linear equations 184

Linear equations ... 185-186

2470 Transcendental equations ... 187

Theory of Numbers.

2800 General $\quad \ldots \quad \ldots \quad \ldots . \quad \ldots \quad 188$

2810 Divisibility ; ${ }^{2}$ linear congruences $\quad$... $\quad \ldots \quad 193$

Divisibility $\quad \ldots . \quad \cdots 193-194$

2815 Continued fractions and indeterminate equations

Continued fractions

Indeterminate equations 201-204

$$
\text { lst order } \quad \text {... 202-203 }
$$

2 nd order, 3 rd order, 4 th order, 5 th order $\quad$...

203

2820 Quadratic residues ... ... 204

2830 Quadratic binary forms ... 205

2840 Quadratic forms of three or more variables; bilinear forms. (See also 2060, 2070.) (.. ... ... Decomposition of numbers into sums of squares 209-210

Bilinear forms ... 213-214

2850 Congruences other than linear; cubic and higher residues ... ... ...

2860 Forms of higher degree which cannot be considered as products of linear factors. (See also 2060, 2070.) ... Fermat's theorem on $x^{n}+y^{n}=z^{n} \quad \ldots$
of higher degree which

2870 Forms of higher degree which
can be considered as products of linear factors; algebraic numbers; ideals. (See also 0820.) ... ...

2880 Application of trigonometrical functions to arithmetic; $\begin{array}{llll}\text { cyclotomy } . . . & \ldots & \ldots & 222\end{array}$ Binomial equations $\quad \ldots . \quad 222$

2890 Application of other transcendental functions to arithmetic... $\quad \ldots \quad \ldots$

2900 Distribution of prime numbers

2910 Special numbers and $\ldots$ numerical functions $\ldots$... $_{228} 228$ 


\section{Schedule of Classification}

2920 Irrationality and transcendence of particular numbers, such as $e$ and $\pi$. (See also 6810.) ... ... $\begin{array}{rrrrr}e \ldots & \ldots & \ldots & & 232-233 \\ \pi \ldots & \ldots & \ldots & \ldots & 233\end{array}$ ANALYSIS.

3190 General $\quad$... $\quad$... $\quad$... $\quad 233$

Foundations of Analysis.

3200 General

3210 Theory of functions of real variables $\ldots \quad \ldots \quad \ldots$

3220 Infinite series; infinite products and other infinite processes. (See also 5610, 5620.) ... ... ... Infinite series $\quad \ldots$ Binomial theorem Convergence ...

238-252

238-239

239-241

Expansion … 242-244

Power series $\quad \ldots . \quad 245-246$

Summation $\quad . . . \quad 248-250$

Trigonometric series $\quad$ 250-251

Infinite products ... $\quad 252-253$

Other infinite processes ... 253

3230 Principles and elements of the differential calculus

3240 Taylor's series. Maxima and

minima ; other analytical applications of the differential calculus $\quad \ldots \quad$... Lagrange's series $\quad$... 261 Other series $\quad \ldots \quad \ldots \quad 261$

Maxima and Minima 262-263 $\begin{array}{llll}M a x i m a . . . & \ldots & \ldots & 264\end{array}$ $\begin{array}{llll}\text { Minima ... } & \ldots & \ldots & 264\end{array}$

Taylor's series $\quad \ldots \quad 265-266$

3250 Principles and elements of the integral calculus. Approximate integration. Mechanical quadrature Integration $\quad \ldots \quad 270-272$

3260 Definite integrals (simple) ... $\quad 274$ Evaluation … 277-278 Transformation $\quad \ldots \quad 280$

3270 Multiple integrals ... $\quad \ldots \quad 283$ Double integrals $\quad$ 284-285

3280 Calculus of variations 286

\section{Theory of Functions of Complex} Variables.

3600 General $\ldots . \quad \ldots \quad \ldots$

3610 Uniform functions of one variable $\quad \ldots \quad \ldots \quad \ldots$

3620 Multiform functions of one variable ; Riemann surfaces. (See also 4060, 6420,8075 .) $\quad \ldots \quad \ldots$

Riemann surfaces $\quad \cdots 296-297$
3630 Expansions in series of functions, other than powers of the variable $\quad \ldots \quad \ldots \quad 297$

3640 Functions of several variables 298

Algebraic Functions and their Integrals.

4000 General $\ldots . \quad \ldots \quad$... 299

4010 Algebraic functions of one $\begin{array}{lllll}\text { variable } & \ldots & \ldots & \ldots & 301\end{array}$

4020 Algebraic functions of several variables $\ldots . \quad \ldots \quad \ldots . \quad 302$

4030 Logarithmic, " circular, exponential functions ... 303

Circular functions $\quad 303-305$ Expansion $\quad$... 303-304

Logarithmic and exponential functions Logarithmic tables
...

4040 General properties of elliptic functions and single theta functions ; addition-theorem. (See also 8050, 8060.) Addition 311

Addition-theorem $\quad{ }^{3}$ 311-312 Doubly periodic functions $\ldots$. $\quad \ldots \quad 312-313$

Elliptic functions... $\quad 313-319$ Elliptic integrals $\quad 319-321$

Theta functions ... 323-324

4050 Multiplication, division,transformation of elliptic functions; modular functions. (See also 4440.)... ... 325

Division $\quad \ldots \quad$... $\quad \ldots \quad 325$ Modular equations $\quad \ldots . \quad 326$

Modular functions $\quad \ldots . \quad 326$ Multiplication $\quad \ldots \quad \ldots \quad$... 327

Transformation ... $\quad 327-329$

4060 Abelian integrals. (See also 8050,8060 .) $\quad \ldots \quad \quad \ldots \quad 329$ Abelian functions $\quad 329-331$

Abelian integrals ... 331-333 Abel's theorem ... $\quad 333-334$

4070 Periodic functions of several variables; general theta functions ... $\quad$... $\quad$... 335

\section{Other Special Functions.}

4400 General $\quad . . \quad \ldots . \quad \ldots \quad 338$

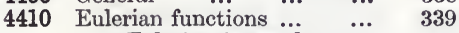
Eulerian integrals $\quad \ldots \quad 340$ Gamma functions $\quad 340-342$

4420 Legendre's functions; Bessel's functions ; hypergeometric functions $\quad$... 342

Bessel's functions $\quad{ }_{343-344}$ Cylindrical functions ... $\quad 344$

Hypergeometric functions 344-345 Hypergeometric series $\quad 345-346$

Legendre's functions $\quad 346-347$ Legendre's polynomials $\quad 347$ Spherical harmonics $\quad 347-348$ 


\section{Schedule of Classification}

4430 Polymorphicfunctions. Other functions which may be defined by definite integrals. (See also 4860.) ... ...

4440 Automorphic functions. (See also 1210, 4050.) ... ... Fuchsian functions $\quad 350-351$

4450 Other functions which may be defined by linear differential equations. Lamé's functions ... ... Lamés functions ...

351

352

4460 Functions which may be defined by functional equations. (See also 6030.) ...

4470 Integral functions ... ... Differential Equations.

4800 General. (See also 8080 Connexes.) $\quad \ldots \quad \ldots . . .$.

$4810 \begin{gathered}\text { Existence theorems for } \\ \text { ordinary and partial }\end{gathered}$ differential equations ... Integrability ... ...

4820 Methods of solution and reduction of ordinary differential equations $\quad \ldots \quad \ldots$

$\begin{array}{lllr}\text { Equations } & \ldots & \ldots & 357 \\ \text { Integrals } & \ldots & \ldots & 358 \\ \text { Integration } & \ldots & \ldots & 359 \\ \text { Solution... } & \ldots & & 359-362 \\ & & \end{array}$

4830 Methods of solution and reduction of partial differential equations of first order, including the differential equations of theoretical dynamics ... $\quad \ldots \quad \ldots$ \begin{tabular}{llrr} 
Equations & $\ldots$ & $\ldots$ & 364 \\
Integration & $\ldots$ & \multicolumn{3}{r}{$365-367$} \\
Solution & $\ldots$ & \multicolumn{2}{r}{$367-368$} \\
Theory & $\ldots$ & $\ldots$ & \multicolumn{2}{c}{... } & 368
\end{tabular} duction of partial differential equatious of the second and higher orders Equations $\quad \cdots \quad{ }^{369-371}$ Integrals $\quad \ldots \quad \ldots \quad 371$ Integration $\quad \ldots \quad 371-373$ Solution, ... $\quad \ldots . \quad \ldots \quad 374$

4850 General theory of ordinary linear equations. (See also 4450.)

$$
\begin{array}{llr}
\text { Equations } & \ldots & 377-380 \\
\text { Integrals } & \ldots & 381-382 \\
\text { Integration } & \ldots & \mathbf{3 8 3 - 3 8 6} \\
\text { Solution ... } & \ldots & \mathbf{3 8 6}-\mathbf{3 8 7}
\end{array}
$$

4860 Integration of ordinary linear equations by definite integrals. (See also 4430.) ...

4870 General theory of ordinary equations, not linear, of the first order
4840 Methods of solution and re-

\section{Riccati's equation}

Solutions

$390-391$

4880 General theory of ordinary equations, not linear, of order higher than first ...

392

\section{Differential Forms and Differential Invariants.}

5200 General $\quad \ldots \quad \ldots \quad \ldots . \quad 393$

5210 Linear differential forms; Pfaffians ... ... ...

5220 Differential forms of the second and higher orders. (See also 8450.) ... ...

5230 Transformation of differential forms, including tangential (or contact) transformations $\quad \ldots \quad \ldots \quad 396$

Transformation ...

5240 Differential invariants. (See also 1230.)... ... ...

\section{Analytical Methods connected with} Physical Problems.

$\begin{array}{llllll}5600 & \text { General } & \ldots & \ldots & \ldots & 400\end{array}$

5610 Harmonic Analysis; Fourier's series. (See also 3220.) 400

Fourier's series ... 400-401

5620 Harmonic Analysis; series other than Fourier's. Spherical and ellipsoidal harmonics. (See also 3220.)

5630 Generalities on the differential equations of mathematical physics ... .... equations of mathematical physics by series ...

5650 Integration of the differential equations of mathematical physics by definiteintegrals logous problems affected by boundary conditions

Difference Equations and Functional Equations.

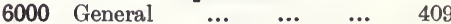

6020 Solution of equations of finite differences... $\quad \ldots \quad \ldots$

6030 Solution of functional equations. (See also 4460.) ...

\section{GEOMETRY.}

6390 General

\section{Foundations.}

6400 General

6410 Principles of geometry; nonEuclidean geometries ; ... ...
5640 Integration of the differential

5660 Dirichlet's problem and ana- 


\section{Schedule of Classification}

PAGE

4-Dimensional Space

413-414

n-Dimensional Space 414-415

Fundamental Principles 415-416

Non-Euclidean geometry 417-418

Theory of Parallels 419-420

6420 Topology of space and hyperspace. (See also 3620 , 8075.) $\quad \ldots \quad \ldots \quad \ldots$

6430 Methods of analytical geometry. (See also 0840.)

Coordinates

423

Elementary Geometry.

6800 General ... ... ...

6810 Planimetry; straight lines, and circles...

Circles. (See also 2880.) 431-435

Circle touching 3 given circles. (Apollonian problem.)

432

Quadrature of the Circle

Ratio of Diameter to Circumference. (See also 2920.) ...

Polygons. (See also 7210.)... ... 437-438

Regular polygons

438-439

Porisms. (See also 4050 and 7210.) ... 439-440

Pythagorean theorem ... 440

Quadrilaterals. (See also 7210.)... ... ...

Straight lines $\quad .$.

Triangles (including Cir(For Conic Sections related to Triangles see 7210.) ...

Circles of the Triangle

Malfatti's problem .... Circle... ... ...

$442-449$

$443-444$

444

Remarkable Points of the Triangle

$446-447$

6820 Stereometry; straight lines,

planes, and spheres; poly-

hedra

.

Mensuration of volumes 450-451

Polyhedra. (See also 8075.) 452-456 Kegular Polyhedra ... 454 Tetrahedra ... 455-456

Spheres ... ... $\quad 457-460$ Contacts... ... 458-459

6830 Trigonometry, plane and spherical ...

Circular Arcs

461

462-463

Trisection

$462-463$

Formule of Plane Trigo-

nometry

463-465

Plane Triangles

$466-467$

Spherical Trigonometry
$468-471$
Formulae of Spherical Trigonometry

PAGE

Spherical Triangles 470-471

6840 Descriptive geometry; perspective $\quad \ldots \quad \ldots . \quad \ldots$ Anharmonic or CrossRatio ... ... $\quad 472-473$

Axonometry $\quad$... $\quad 473-474$

Involution $\quad$... $\quad . . \quad 475$

Perspective $\quad$... $\quad 475-476$

Practical Solid Geometry 477-478

Projective Geometry $\quad 478-480$

Stereogruphic Projections, and other Projections of the Sphere. (See also 8840 and Geography 83.) ... 480

Transversals ... 480-481

\section{Geometry of Conics and Quadrics.}

7200. General

Equations relating to
Conics and Quadrics

481

7210 Metrical and projective properties of conics ...

Conics deternined by given Points or Tangents, and Methods of Determination

Contacts...

Curvature of Conics. (See also 8430.) $\quad$... $\quad 485-486$

Ellipses ... $\quad . . . \quad 486-487$

Equations and Coordi-

nates ... ... 487-488

Foci $\quad \ldots \quad$... $\quad 488-489$

Hyperbolas $\quad$... $\quad$... 489

Normals $\quad$... $\quad 490-491$

Parabola $\quad$... 491-492

Pascal's hexagram, Brianchon's kexagon, and connected figures. (See also 8075.)

492-493

Polars and Polar Reciprocals $\quad$... $\quad 493-494$

Polygons related to Conics. (See also 6810.)... ... ...

Poncelet's Polygons, Porisms. (See also 4050 and 6810.)

Quadrilaterals, Quadrangles, and related Conics. (See also 6810.)... ... ... 496

Tangents $\quad \ldots \quad 496-497$

Triangles, and related Conics. (See also 6810.)... ... 497-499

7230 Systems of conics. (See also 8070 and 8090.) ... ... 


\section{Schedule of Classification}

\section{Conics with Common} Focus or Foci

7240 Metrical and projective properties of quadric surfaces

Axes of Quadric Surfaces 502-503 Ellipsoid Equations of Quadrics. (See also 2040 and 7200.)...

504-505

Foci and Focal Properties of Quadrics ...

Generation and Genera
tors of Quadrics

Hyperboloid and Hyperbolic Paraboloid

Inscribed, Circumscribed, and Conjugate Figures. (See also 7210 and 8075.).

505-506 506

Intersections of Quadrics. (See also 7660, 8440.) 508-509 Normals to Quadrics ... Plane Sections of Quadrics ... .... Quadrics determined by
Points, Lines and Tangent Planes, and Methods of Determination

7260 Systems of quadric surfaces. (See also 8070, 8090.) ... Confocal Quadrics ...

513

Algebraic Curves and Surfaces of degree higher than the second. (See also 8070.) 7600 General $\quad \ldots \quad \ldots . \quad \ldots \quad 514$

7610 Metrical and $\ldots$ projective properties of algebraic plane curves of degree higher than the second. (See also 8030, 8075,8430 .)

$\begin{array}{ccc}\text { Curves, } 2 \text { nd } & \text { and } & 3 \mathrm{rd} \\ \text { degrees } & \ldots & \ldots\end{array}$

Curves, 3rd degree

Curves, 3 rd and 4 th, 3 rd and 5 th degrees ...

Curves, 4th degree $529-524$

Curves, 5th, 5th and 6 th, 6 th, $14 t h$, mth degrees

Curves, nth degree

Curves, nth and pth degrees

7630 Special plane algebraic curves. (See also 8030.)

530

Curves, 3rd degree 531-532

Curves, 3rd and 4th degrees ... ... ...

Curves, 4 th degree $\quad 532-533$

Curves, 5th, 6 th degrees
Lemniscates

PAQE

533-534

7640 Algebraic surfaces of degree higher than the second. (See also 8040.) ... ...

Contacts, and Tangent Lines and Planes. (See also 8450.)

Surfaces, 2nd and $3 r d$, 2nd, $3 r d$ and $4 t h$, degrees ... $\quad . . \quad$... 539

Surfaces, $3 r d$ degree $\quad 539-540$

Configurations. (See also 8075.) ... … .

Surfaces, 3rd and 4th, $4 t h$, 4 th and 5 th, 5th, $(m+n)$ th, nth degrees

7650 Special algebraic surfaces ... Anallagmatic surfaces. (See also 8020.) ...

Kummer's surface. (See also 8060 and 8080.) 542-543

Steiner's surface $\quad$... $\quad 544$

Surfaces, 3rd degree, $3 r d$ and 4 th degrees $\quad$... $\quad 545$

Surfaces, 4 th degree $\quad 545-546$

Surfaces, 5th, 6th, 7 th, 8 th, nth, $(n+2)$ th degrees ... $\quad$... $\quad . . \quad 546$

Tore or Anchor-ring $\quad 546-547$

Wave surface $\quad \ldots \quad$... 547

7660 Skew algebraic curves. (See also $7240,7660,8030,8050$.) $\quad 548$

Curves, 3rd degree $\quad 548-549$

Curves, 3 rd and 4 th de$\begin{array}{lllll}\text { grees } & . . & \ldots & \ldots & 549\end{array}$

Curves, 4 th degree $\quad{ }_{549-550}$

Curves, 5th and higher degrees $\quad \ldots \quad \ldots \quad 550$

Transformations and General Methods for Algebraic Configurations.

(References to papers dealing with enumerative geometry may be found in sections 8000 to 8100 .)

8000 General $\quad \ldots . \quad \ldots . \quad \ldots .552$

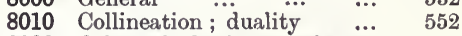

8020 Other algebraic transformations. (See also 6810, 7650.)

556

8030 Groups of points on an algebraic curve; genus of curves ; principle of correspondence. (See also 7610, 7630,7660 .) $\quad \ldots \quad \ldots$ Groups of points $\quad 562-563$ Involutions $\quad \ldots \quad 563-564$ Principle of correspondence $\quad . . \quad$... 564 $564-565$

8040 Groups of curves and points on an algebraic surface; genus of surfaces. (See also 7640.) $\quad$... ... 


\section{Schedule of Classification}

8050 Application of transcendental functions to algebraic curves. (See also 4040, 4060,7660 .)

8060 Application of transcendental functions to algebraic surfaces. (See also 4040, 4060, 7650.) $\quad \ldots \quad \ldots \quad \ldots$

8070 Enumerative geometry. (See also $7230,7260,7600-7660$.)

8075 Special configurations of points, lines, planes or other elements. Space partitioning. (See also 3620, 6820, 7210, 7610, 7640.) $\quad \ldots \quad \ldots \quad \ldots$

Special configurations of points, lines, planes or other elements...

573-575

Space partitioning

$575-576$

8080 Line geometry. Connexes, complexes, congruences ; higher elements of space. (See also 7650.).... ...

Line geometry ... 576-57

Connexes ... $\quad \ldots \quad \ldots \quad 577$

Complexes... $\quad \ldots \quad{ }_{577-580}$

Congruences $\quad \ldots . \quad 580-582$

Higher elements of space $\quad 582$

8090 Systems (linear, and not linear) of curves and surfaces ... ... $\ldots$.

Systems of curves. (See also 7230.) (...

Systems of surfaces

583-585

$585-586$

8100 Algebraic configurations in $\underset{4 \text {-dimensional space }}{\text { hyperspace }}$

586

589

Infinitesimal Geometry; applications of Differential and Integral Calculus to Geometry.

8410 Principles of infinitesimal geometry ... ...

8420 Kinematic geometry. ( also Mechanics 0420.) ...

Curve of pursuit ...

Cycloids s...

Displacement $\quad \cdots \quad{ }_{592-593}$

Epicycloids $\quad \ldots . \quad \ldots \quad 593$

Hypocycloids $\quad \ldots \quad$... 594

Motion ... ... $\quad 595-596$

$\begin{array}{llll}\text { Roulettes } & \ldots & \ldots & 597\end{array}$

8430 Curvature of plane curves;

other applications of the differential calculus to plane curves. (See also 7210.) $\quad \ldots \quad \ldots \quad \ldots$ Curves. (ee also 7610, 7630.)...

...

600-602

$$
\begin{array}{llrr}
\text { Envelopes } & & & \text { PAGE } \\
\text { Evolutes ... } & \ldots & & 602 \\
\text { Pedals ... } & \ldots & 602-603 \\
\text { Radii of curvature } & & 604-605 \\
\text { Trajectories } & \text {... } & \text {... } & 605-606 \\
\hline
\end{array}
$$

8440 Curvature of skew curves: other applications of the differential calculus to skew curves. (Seealso 7240,8420.)

8450 Curvature of surfaces ; curvilinear coordinates, and other applications of the differential calculus to surfaces. (See also 5220, $8810,8840,8850$.) , ... 610

Curvature of surfaces $\quad 610-616$ Indicatrix $\quad . . . \quad 611-612$ Lines of curvature 612-613 Measure of curvature 613-614 Kadii of curvature $\quad$... 614 Surface of centres $\quad$... $\quad 615$ Surfaces... $\quad$... $\quad 615-616$ Theorems $\quad \ldots \quad \ldots \quad 616$ Curvilinear coordinates 616-618 Other applications of the differential calculus to surfaces $\quad$... 618-621 Surfaces... $\quad \ldots . \quad 619-621$

8455 Differential geometry of congruences and other applications of the differential calculus to elements of space

8460 Rectification and quadrature of curves ; areas and volumes of surfaces. Other applications of the integral calculus to geometry ...

$\begin{array}{llr}\text { Areas ... } & \ldots & 622-624 \\ \text { Quadrature } & \ldots & 626-627 \\ \text { Rectification } & \ldots & 627-629 \\ \text { Volumes } & \ldots & 629-630\end{array}$

8470 Special transcendental curves. (For cycloids, epicycloids and trochoids see 8420.) ... ... ... Spirals ... … $\quad \cdots \quad 639$

8480 Special transcendental surfaces $\quad . . \quad \ldots . \quad \ldots$

8490 Hypergeometric configurations and higher elements of hyperspace ...

631

Differential Geometry; applications of Differential Equations to Geometry.

8800 General $\quad \ldots . \quad \ldots \quad$... $\quad 634$

8810 Determination of curves on surfaces $\ldots \quad \ldots \quad \ldots \quad 634$ Curves on surfaces $\quad 635-636$ Geodesics $\quad$... $\quad 636-638$ 


\section{Schedule of Classification}

\begin{tabular}{cccc} 
& \multicolumn{2}{c}{$\begin{array}{c}\text { Geodesics on } \\
\text { (See also }\end{array}$} & Geography \\
70)$. & $\ldots$ & $\ldots$ & $\ldots$ \\
8820 & Loxodromes & $\ldots$ & $\ldots$ \\
8830 & Minimal surfaces & $\ldots$ & $\ldots$ \\
Surfaces determined by rela- \\
tions of curvature and by \\
other differential proper- \\
ties ... \\
$\begin{array}{cccc}\text { Surfaces of constant cur- } \\
\text { vature }\end{array}$ & $\ldots$
\end{tabular}

8840 Conformal and other representations of surfaces on others. (See also 3600, 6840 and Geography 7095.)
PAGE

638

639

640

643

8870 Hypergeometric configurations and higher elements of hyperspace ...

PAGE

Conformal representation $645-646$ Projection ... 646-647 Representation ... $\quad 647-648$

8850 Deformation of surfaces ... 648

Deformation $\quad \ldots \quad 649-650$ Surfaces... $\quad$... 650-651

8860 Orthogonal and isothermic surfaces. (See also 5630.) 651

Isothermic surfaces ... 651

$651-653$ 653

\section{APPENDIX}

\begin{abstract}
$0000,0010,0032,0035,0050$
$0080,0090,0400,0410 \quad \ldots \quad \ldots$

$0420,0430,0830,0840,0850,0870$, 1200,1210

$1230,1600,1620,1625,1630 \quad \ldots$

$1635,2000,2010,2020,2030,2040$, 2050,2060

... ...

$2070,2400,2420,2430,2440,2450$,

$2460,2800,2810,2815,2830$

$2840,2850,2880,2890,2900,2920$, $3200,3220,3230,3240,3250$
\end{abstract}

654

655

656

657

658

659

660
$3260,3270,3280,3600,4010,4040$, $4050,4060 \quad \ldots \quad \ldots \quad \ldots$

661

$4420,4440,4800,4830,4840,4850$, $4870,5200,5210,5230 \quad \ldots$

662

$5240,5600,5610,5620,5630,5660$, $6000,6400,6410,6420,6430$, $6800,6810 \quad \ldots \quad \ldots 6 \quad \ldots \quad 663$

$6820,6830,6840 \quad \ldots \quad \ldots . \quad \ldots \quad 664$

$7210,7230,7240,7610,7630,7640 \quad 665$

$7660,8010,8020,8075,8080,8090$, $8420,8430,8460,8820$

666 


\section{INDEX TO SCIENTIFIC PAPERS FOR THE NINETEENTH CENTURY.}

\section{PURE MATHEMATICS.}

\section{Philosophy.}

A posteriori truth. Bing, F. (xIr) Ts. Mth. 3 (1879) 1-.

- - (Bing). Lorenz, L. V. (xrr) Ts. Mth. 3 (1879) 57-.

- - Bing, F. (xII) Ts. Mth. 3 (1879) $66-$

- Lorenz, L. V. (хп) Ts. Mth. 3 (1879) 118-.

- - Bing, F. (xII) Ts. Mth. 3 (1879) $122-$

Aesthetic value of line forms. Wieher, C. Karlsruhe Nt. Vr. Vh. 11 (1896) (Ab.) 47-.

Algebra of relations, principles. Schröder, $F$. [1900] N. Y. Am. Mth. S. Bll. 7 (1901) 176 -

Analysis, pure, metaphysics. Piola, G. Bb. It. 97 (1840) 315-

Aristotle, difference between natural philosophy and mathematics according to. Dutordoir, -. Brux. S. Sc. A. 24 (1900) (Pt. 1), 52-.

Arithmetization of mathematics. Pierpont, $J$. N. Y. Am. Mth. S. Bll. 5 (1899) 394-.

Axioms. Zehender, W. von. Z. Psychol. 19 (1899) 41-.

"Begriffsschrift," notation in algebra of logic. Frege, G. [1879-82] Jena. Sb. (1879) 29-; (1883) 1-.

- of Peano and Frege. Frege, G. Leip. Mth. Ps. B. 48 (1896) 361-.

Choice of forms, importance in mathematics. Clariana y Ricart, L. Fschr. Mth. (1891) 50 .

Comte's views on philosophy of mathematics. Vasiljev, A. V. Kazan S. Ps.-Mth. Bll. 8 (1899) (Prot.) 11-; Ens. Mth. 2 (1900). 157-. Conception of direction. Dixon, E. T. B. A. Rp. (1891) 572.

$-\frac{\text { space. Hoppe, -. D. Nf. Tbl. }\left({ }^{*} 1875\right)}{}$ $142-$.

- - an extension of conception of length. Graberg, F. Zür. Vjschr. 31 (1886) 339-.

- - further development. Graberg, $F$. Zür. Vjschr. 32 (1887) 191-.

Converse of a theorem, truth of ; Hauber's criterion, applications and generalisations. Ginther, S. Arch. Mth. Ps. 56 (1874) 26-.

VOL. I.
Correlation of the different branches of elementary mathematics. Hayward, R. B. Nt. 33 (1886) 543-.

Definition of real number. Burali-Forti, $C$. [1900] N. Y. Am. Mth. S. Bll., 7 (1901) 158,170 -

Definitions. Laurent, $H$. Ens. Mth. $2(1900)$ 194-.

- Peano, G. [1900] N. Y. Am. Mth. S. Bll. 7 (1901) 158, 168-.

—, theory. Gergonne, J.D. Gergonne A. Mth. 9 (1818-19) 1-.

-, Whewell's views. Hennessy, J.P. B. A. Rp. (1858) (Pt. 2) 3-.

Demonstration, indirect. De Morgan, $A$. $\mathrm{Ph}$. Mg. 4 (1852) 435-.

Demonstrations, reduction to simplest form. Barré de Saint-Venant, - . Par. S. Phlm. PV. (1849) 45-.

Equality, notion of. Burali-Forti, C. Ens. Mth. 1 (1899) 246-.

Equation of the beautiful. Lagout, E. A. Gén. Civ. 2 (1863) 141-.

Ex absurdo proof. Brunel, - - Bordeaux S. Sc. Mm. 4 (1894) xxxii-.

Failure of attempt to deduce inductive principles from the theory of probabilities. Bryant, $S$. Ph. Mg. 17 (1884) 510-

Foundations of the theory of analytical functions. Timčenko, I. N.Rs. S. Nt. Mm. (Mth.) 12 (1892) 256 pp. ; 16 (1899) 216 pp. ; 19 (1899) xv + 183 pp.; Fschr. Mth. (1899) 48-.

Freedom of the will and physical determinism. Hoppe, R. Arch. Mth. Ps. 11 (1892) 339-.

Function of algebra, chief, formulation and simplification of formulæ. Ermakow, W.P. Fschr. Mth. (1896) 47.

Fundamental operations of arithmetic, the five. Kupffer, $K$. Dorpat Sb. 10 (1895) 359-. _ Nap. Rd. 39 (1900) 138.

- principles of human knowledge. Laurent, $H$. Ens. Mth. 1 (1899) 381-.

General philosophy, aid rendered by mathematics. Cauchy, A. L. C. R. 21 (1845) 134 . Genius in mathematics. Studnicka, $F$. $J$. Časopis 2 ("1873) 57-; 8 ("1879), 85-; Fschr. Mth. ("1873) 54; ("1879) 56. 


\section{Philosophy}

Geometric calculus, principles of. Macfarlane, A. [1900] N. Y. Am. Mth. S. Bll. 7 (1901) 158,178 .

- methods considered from the philosophical and historical points of view. Galdeano, Z. G. de. Fschr. Mth. (1892) 500.

Geometry applied to practical life. Pearson, $K$. Nt. 43 (1891) 273-.

- as a purely logical system. Pieri, M. [1900] N. Y. Am. Mth. S. Bll. 7 (1901) 158, 171-, 234.

Hegel and metaphysics of fluxional calculus. Smith, W. R. Edinb. R. S. T. 25 (1869) 491-.

Induction, Aristotle's account. Whewell, $W$. [1850]. Camb. Ph. S. T. 9 (1856) 63-.

- and deduction. Jones, E. E. C. Nt. 46 (1892) 293-.

- - - Russell, F. C. Nt. 46 (1892) 586-.

- - Jones, E. E. C. Nt. 46 (1892) $587-$.

- - Dixon, E. T. [1892] Nt. $47(1892$ 93) 10 -

- - - Jones, E. E. C. Nt. 47 (1892-93) 78

- mathematical. Colecchi, O. Il Progresso, 17 (1837) 55-.

- - W Wundt, W. Ph. Stud. $1\left({ }^{*} 1883\right) 90$

-, -, applications. Valeriani, V. G. Mt. 15 (1877) 34-.

- in mathematics. Hadamard, J. [1900] N. Y. Am. Mth. S. Bll. 7 (1901) 158, 181.

Inference, theory of mathematical. Stokes, G. J. Am. As. P. (1899) 71.

Infinite and infinitesimal quantities. Doolittle, -. Smiths. Misc. Col. 33 (1888) Art. 1, 133-. (Wash. Ph. S. Bll. 6 (1884).)

numbers, various opinions. Cantor, $G$. Stockh. Ak. Hndl. Bh. 11 (1887) No. 19, $10 \mathrm{pp}$.

- - - - Cantor's article. Eneström, $G$. Stockh. Öfv. (1885) No. 10, 69-

- real integers, and their relation to each other. Cantor, - . D. Nf. [B.] (*1883) 57.

Infinitesimal calculus, metaphysic of. $\mathrm{Sa}$ poretti, A. Bologna Ac. Sc. Mm. 5 (1895-96) 309-.

Infinity, conception of in mathematics and natural science. Cranz, C. Ph. Stud. 11 (1895) 1-.

-, mathematical and metaphysical. Franceschinis, F. M. Ven. Aten. Esercit. 1 (1827) 317-.

Introduction to course of mathematics. Gergonne, J. D. Gergonne A. Mth. 21 (1830-31) 305-.

Intuition and logic in mathematics. Poincaré, H. N. Y. Am. Mth. S. Bll. 7 (1901) 71-。

Iteration as the fundamental mathematical operation. Schapira, H. D. Nf. Vh. (1893) (Th. 2, Hälfte 1), 8-; D. Mth. Vr. Jbr. 3 (1894) 88-.

Language, scientific basis of an artificial. Macfarlane, A. N. Y. Mth. S. Bll. 1 (1892) 189-.

Law of continuity. An apparent violation. Roget, P. M. Ph. Mg. 3 (1828) 118-, 203-.

Logic and intuition in mathematics and in teaching. Poincare, $H$. Ens. Mth. 1 (1899) 157-.

\section{Philosophy 0000}

Logic, mathematical. Peano, G. As. Fr. C. R. (1894) (Pt. 2) 222-

Logical equalities with three terms. Poretsky, P. [1900] N. Y. Am. Mth. S. Bll. 7 (1901) $158,172-$.

Mathematical form, theory. Kempe, A. B. R. S. P. 38 (1885) 393-; Phil. Trans. 177 (1887) 1-; R. S. P. 42 (1887) 193-.

- problems, and the development of mathematics. Hilbert, $D$. Ens. Mth. $2(1900)$ 349 -.

- reasoning, nature of. Poincaré, $H$. [1894] Kazan S. Ps.-Mth. Bll. 8 (1899) (Prot.) 74-. - science, relation of parts to fundamental idea. Price, B. Ashmol. S. T. 2 (1849) $23 \mathrm{pp}$.

- sciences, modern classifications. Dickstein, S. Wiad. Mt. 2 (1898) 181-; Fschr. Mth. (1898) 42.

- studies and deductive inference. Pérez, $E$. Méx. S. "Alzate" Mm. 8 (1894) 315-.

- theory of composition of sensations. De La Rive, L. Gen. S. Ps. Mm. 30 (1890) No. 4, $99 \mathrm{pp}$.

- thought. Newcomb, S. [1893] N. Y. Mth. S. Bll. 3 (1894) 95-.

Mathematics applied to natural history. $B u$ quoy, G. von. Oken Isis (1825) 1266-.

-, basis of. Lipps, G. F. Ph. Stud. 9 (1894) 151-, 358-; 10 (1894) 169-; 11 (1895) 254-; 14 (1898) 157-.

-, certainty in. Burja, - Berl. Mm. Ac. (1802) 3-.

-, experimental groundwork. Hoppe, $R$. D. Nf. Tbl. (*1876) (Beil.) 60-

-, first notions. Liotard, - Gard Aperęu Tr. (1822) 171-.

-, fundamental notions. Cugnin, E. Rv. So. 6 (1896) 193-, 264.

-, - principles. Alexander, S. Silliman J. 7 (1849) 178-, 329-.

Herbart's view of place in education. Greenstreet, W.J. Mth. Gz. No. 2 (1894) 9-. - and " natural history" \&c., common methods of reasoning. Heron, $R$. Tilloch $\mathrm{Ph} . \mathrm{Mg}$. 11 (1801) 294 .

- and natural science, mutual relations. Wild, J. St Gal. B. (1885-86) 101-.

- - - sciences, relation. Zech, J. D. Nf. Vsm. B. (1844) $(A b .2) 12-$

- and physics, relation to other sciences. Jellett, J. H. B. A. Rp. (1874) (Sect.) 1-.

-, study of, influence. Györy, S. Evk. 2 (1835) (Pt. 2) 23-

- and theory of knowledge. Milhaud, G. Rv. Sc. 39 (1887) 195-

-, usefulness to knowledge and mankind. Schüibler, $E$. Oken Isis (1842) 167-.

Mean values, principle. De Morgan, A. (Ix) [1853] Camb. Ph. S. P. 1 (1866) 123-.

Mill's theory of geometrical reasoning mathematically tested. Smith, W. R. Edinb. R. S. P. 6 (1869) $477-$.

Most probable existence, principle of. Gosiewski, $W$. Prace Mt.-Fiz. 3 (1892) 55-; Fschr. Mth. (1892) 73-.

Multiplication and division of concrete quantities. Lodge, A. Nt. 38 (1888) 281-. 


\section{History}

Natural laws, mathematical expression. Breton, D. [1881] Rv. Sc. 2 (1882) 87-.

New mathematics, interpretation of old by Halsted, G. B. Texas Ac. Sc. T. 1 (No. 2) (1893) 89-

Observation in mathematics. Gaillard, $G$. Rv. Sc. 8 (1897) 584 .

Philosophy of hyperspace. Newcomb, S. [1897] N. Y. Am. Mth. S. Bll. 4 (1898) 187-.

- mathematics. Ladame, $H$. Neuch. Bll. 2 (1846-47) 213-.

- - - Ehrenfels, C. von. Z. Psychol. 6 (1894) 44, 56-

- and teaching of mathematics, reduction of proofs to simplest form. St Venant, A.J.C.B. de. C. R., 83 (1876) 102-, 256-.

Positive integers, history and philosophy of conception. Vasiljev, A. V. Kazan S. Ps.Mth. Bll. 1 (1891) 1- ; Fschr. Mth. (1891) 34.

- psychology and metaphysics, mathematical applications. Arnoux, G. As. Fr. C. R. (1891) (Pt. 2) 241-; (1896) (Pt. 2) 45-.

Principles underlying any deductive theory. Padoa, A. [1900] N. Y. Am. Mth. S. Bll. 7 (1901) 158, 171.

Probability and its logical significance. Aall, $A$. Arch. Mth. Ntvd. 19 (1897) No. 9, 24 pp.

Psychological elements of the physico-mathematical sciences. Smirnov, A. [1895] Kazan Un. Mm. 1896 (Pt. 3) 113-, (Pt. 4) 211-, (Pt. 5) 147-.

Pure mathematical sciences, system. Papperitz, E. D. Mth. Vr. Jbr. 1 (1892) 36-, 40.

- mathematics, principles. Thiele, T. N. (xII) Ts. Mth. 4 (1880) 33-.

- and mixed mathematics, relation. Dyck,W. Wiad. Mt. 1 (1897) 139-.

Rôle of mathematics in physics. Bloch-Lyon, E. Rv. Sc. 10 (1898) 682-.

Simplicity, measure of in mathematics. Lemoine, ${ }_{E}$. As. Fr. C. R. (1888) (Pt. 2) 75-.

Space, philosophic bearing of speculations concerning realities of. Roberts, S. [1882] L. Mth. S. P. 14 (1882-83) 5-.

- in relation to mathematical laws. Raciborski, A. Kosmos (Lw.) 10 (1885) 493-

Subject-matter of exact thought. Kempe, A. B. [1890] Nt. 43 (1891) 156-.

Synthetic evolution. Taylor, C. Mess. Mth. 4 (1868) 181-.

Theory of numbers, logical investigation. Brix, $W$. Ph. Stud. 5 (1889) 632-; 6 (1891) 104-, 261-.

Time as a fourth dimension (of space). $S$. Nt. 31 (1885) 481.

Truth, necessary and contingent, in regard to principles of science. Powell, B. [1849] Ashmol. S. T. 2 (1838-52) 41 pp.

Zero and infinity. Judson, C. H. Des Moines Anal. 8 (1881) 105-; 9 (1882) 9-.

\section{History. Biography. HISTORY.}

III. century, value of position for expression of numbers known to Romans in. Vincent, A. J. H. C. R. 14 (1842) 43-.
History 0010

X. century mathematics, Harib and Garib. Steinschneider, M. Z. Mth.Ps. 11 (1866) 235-.

XII. century mathematics, Abraham Judaeus (Savasorda), and Abraham ibn Ezra. Steinschneider, M. [1866]. Z. Mth. Ps. 12 (1867) 1-.

_- - Abraham ibn Ezra. Steinschneider, $M$. Z. Mth. Ps. 25 (1880) (Suppl.) 57-.

XIV. century arithmetical problems. Curtze, M. Bb. Mth. (1895) 77-.

XIV. and XV. centuries, mathematics. Curtze, $M$. Bb. Mth. (1894) 107-; (1895) 1-.

XV. century mathematics, history. Wappler, E. Z. Mth. Ps. 45 (1900) (H.-lt. Ab.) 47-.

XVI. century, arithmetic of Estienne de la Roche. Anon. Rv. Sc. 33 (1884) 561-.

- - books of arithmetic and algebra. Fontès, -. Toul. Ac. Sc. Bll. 2 (1899) 202-

_ - solution of Euclid's problems with ruler and circle of fixed radius, by Italian geometers. Mackay, J. S. Edinb. Mth. S. P. 5 (1887) 2-.

XVII. century, mathematics at beginning of. Wolf, R. Bb. Mth. (1889) 33-.

- - mathematics, history. Dickstein, S. Bb. Math. (1894) 24.

- -, methods of reckoning annuities. Eneström, G. Stockh. Öfv. (1896) 41-; Fschr. Mth. (1896) 187.

XVIII. century, historical development of mathematics as an exact science to end of. Zeuthen, H. G. Fschr. Mth. (1896) 29.

- mathematics: "Il Giornale de' Letterati d' Italia " and "La Raccolta Calogerà" as sources of history. Loria, G. Z. Mth. Ps. 44 (1899) (Suppl.) 241-.

XIX. century mathematics, historical sketch. Clariana y Ricart, L. Barcel. Ac. Mm. 2 (1892-1900) 539-.

Abacus, ancient traces. Chasles, M. C. R. 64 (1867) 1059-.

- Gerbert's treatise, \&c. Chasles, M. C. R. 16 (1843) 156-, 218-, 281-, 1393-; 17, 143-.

-, two 12 th century treatises. Marducci, $E$. Rm. R. Ac. Linc. T. 6 (1882) 324-.

Addition-machines. Unger, F. A. Z. Mth. Ps. 44 (1899) (Suppl.) 515-.

Albert of Saxony: "De proportione dynametri quadrati ad costam ejusdem," question of. Suter, H. Z. Mth. Ps. 32 (1887) (H.-lt. Ab.) 41-.

- - - Treatise "De quadratura circuli." Suter, H. Z. Mth. Ps. 29 (1884) (H.-lt. Ab.) 81-.

d'Alembert. Letter to Lagrange. Liouville, $J$. Liouv. J. Mth. 2 (1837) 245-.

Algebra, early history. Strachey, E. As. Researches, 12 (1816) 158-.

-, evolution. Hyde, E. W. Am. As. P. (1891) 51-.

-, fundamental principles. Macfarlane, $A$. Am. As. P. (1899) 21-.

-, in Germany in 15th century. Curtze, $M$. Z. Mth. Ps. 40 (1895) (Suppl.) 31-.

-, history. Franchini, $P$. Lucca At. Ac. 3 (1827) 211-.

- introduction into Europe, and terminology. Chasles, M. C. R. 13 (1841) 497-, 601-. 
Algebra, introduction into Europe, and terminology. Libri, G. C. R. 13 (1841) 559-.

- in Italy since Fibonacci. Gerhardt, C. I. Grunert Arch. 3 (1843) 284 -.

-, various names for. Nesselmann, G. H. F. N. A. Mth. 14 (1855) 445-.

Algebraic notation, history. Starkov, A. P. Kazan S. Nt. (Ps.-Mth.) P. 3 (1885) 107-.

Algebraical functions, development of theory down to present time. Brill, A., \& Noether, M. D. Mth. Vr. Jbr. 3 (1894) 107-.

Algorithm, Bolyai's. Farkas, G. Mag. Tud. Ak. Étk. (Mth.) 8 (1881) No. 3, 8 pp.

- of Christan of Prachatic. Studnička, F. J. Prag Sb. (1892) (Mth. Nt.) 100-; (1893) (Mth. Nt.), No. 6, 14 pp.

_, Sacrobosco's, nine "limits" in. Eneström, G. Bb. Mth. (1897) 97-.

Algorithms, ancient. Jacobi, C. G.J. Berl. B. (1850) 426-.

-, work on, 12th century, and on the spread of Indo-Arabic arithmetic and ciphers in the West. Nagl, A. Z. Mth. Ps. 34 (1889) (H.-lt. Ab.) 129-, 161-.

-, writing on, 12th century. Curtze, M. Z. Mth. Ps. 42 (1897) (Suppl.) 1-.

Algorithmus linealis of H. Stromer (1512). Günther, S. [1880] Prag Ab. 10 (1881) (Mth.) No. 4, $18 \mathrm{pp}$.

Alhazen's problem. Baker, M. Am. J. Mth. 4 (1881) 327-.

Alternants, history. Muir, T. Edinb. R. S. P. 23 (1902) 93-.

America (U.S.), study of mathematics. Cajori, E. Bb. Mth. (1891) 74-.

Analytical geometry, discovery, and work of Ghetaldi, 1630. Gelcich, E. Z. Mth. Ps. 27 (1882) (Suppl.) 191-.

Ancient problems, two. Saalschüitz, L. Königsb. Schr. 39 (1898) [8]-.

Ancients, numerals of. Saalschütz, L. Königsb. Schr. 33 (1892) [4]-.

Angle-measurements by means of Hipparchus' dioptra. Hultsch, F. Z. Mth. Ps. 44 (1899) (Suppl.) 191-.

Apparatus for calculation, arithmetical puzzles. Lucas, É. Rv. Sc. 45 (1890) 1-.

Approximation methods, ancient. Ginther, $S$. D. Nf. [B.] (*1877) 95-.

$-\ldots,-$, in light of modern mathematics. Giinther, S. Prag Ab. 9 (1878) (Mth.), No. 4, 44 pp.

\section{Arabian Mathematics.}

Ahmed ibn Yusuf. Liber de similibus arcubus. Curtze, M. Bb. Mth. (1889) 15-.

Ahmed and his book on proportion. Cantor, $M$. Bb. Mth. (1888) 7-.

Algebraic geometry of Mûsa al-Kharizmi. Marre, $A$. N. A. Mth. 5 (1846) 557-.

Arabian algebra, Ms. of Al-Khayyami with geometric construction for cubic equations. Woepcke, F. Crelle J. 40 (1850) 160-.

- -. "Messâhat" of Mûsa al-Kharizmi. Marre, A. A. Mt. 7 (1865) 269-

- algebraic notations. Woepcke, F. C. R. 39 (1854) 162-.
Arabian Mathematics, continued

Arabian arithmetic, history. Vaux, G. de. Bb. Mth. (1899) 33-.

- astronomers and mathematicians. Suter, $H$. Bb. Mth. (1897) 83-; (1899) 86-.

- astronomy and arithmetic. Ms. in possession of Chasles. Marre, A. Rm. At. N. Linc. 19 (1866) 362-.

- books of the viceregal library in Cairo, 5th vol. of catalogue. Suter, H. Z. Mth. Ps. 38 (1893) (H.-lt. Ab.) 1-, 41-, 161-.

- calculation. "Talkhis" of Ibn al-Bannah. Marre, A. Rm. At. N. Linc. 17 (1864) 289-. - Mss., historical extracts. Woepcke, F. C. R. 31 (1850) 715-.

- - two, in Berlin Royal Library. Suter, $H$. Bb. Mth, (1898) 73-.

- methods for finding $\sin 1^{\circ}$ approximately. Woepcke, F. Liouv. J. Mth. 19 (1854) 153-, $302-$.

- numerals. Cantor, M. (vi. Adds.) Heidl. Vh. Nt. Md. 3 (1862-63) 5-.

Arabic numerals, geom. form. Dubois-Maisonneuve, - Par. Tr. S. Amat. 2 (1808) 51-.

Arabs, binomial theorem, as known to. Tytler, $J$. As. Researches 13 (1820) 457-.

-, books of. Steinschneider, M. Z. Mth. Ps. 10 (1865) 456-.

-, geometrical construction of equations of $4 \mathrm{th}$ order. Woepcke, F. Liouv. J. Mth. 8 (1863) $57-$.

-, progressions among. Günther, S. Z. Mth. Ps. 21 (1876) (Suppl.) 57-.

- and root extraction. Tytler, J. As. Researches 17 (1832) 51-.

Biography of Ibn al-Bannah (13th cent.). Marre, A. Rm. At. N. Linc. 19 (1866) 1-.

Diophantus among Arabs of 9 th century. Steinschneider, M. Z. Mth. Ps. 10 (1865) 499.

Euclid, bibliographical study. Steinschneider, M. Z. Mth. Ps. 31 (1886) (H.-lt. Ab.) 81-.

Hero's square-root formula in Arabian mathematics and in Regiomontanus's writings. Curtze, M. Z. Mth. Ps. 42 (1897) (H.-lt. Ab.) 145-.

Historical notes. Chasles, M. C. R. 40 (1855) 782 -; 60 (1865) 601-.

Ibn-al-Haitam, quadrature of the circle. Suter, $H$. Z. Mth. Ps. 44 (1899) (H.-lt. Ab.) 33-..

Indian numerals, employment by Arabs. Woepcke, F. Tortolini A. 6 (1855) 321-.

Yusuf ibn Ibrahim and Ahmed ibn Yusuf. Steinschneider, M. Bb. Mth. (1888) 49-, 111-.

Khelâsat al Hisâb. Marre, A. N. A. Mth. 5 (1846) 263-.

Multiplication and division. Latin abridgement of an Arabian work on numbers, written by N. O'Creat to Adelhard of Bath in 12th century (fragment). Henry, C. Z. Mth. Ps. 25 (1880) (Suppl.) 129-.

Mûsa ibn Shakir, sons of, 10th century. Steinschneider, M. Bb. Mth. (1887) 44-, 71-.

Radical axis probably discovered by Arabs. Merrifield, C. W. L. Mth. S. P. $2(1869)$ 175 -. 


\section{History}

Arbogast's rule, history of. Tanner, H.W. L. Mess. Mth. 20 (1891) 83-.

Arithmetic book, oldest German. Unger, $F$. Z. Mth. Ps. 33 (1888) (H.-lt. Ab.) 125-.

-, historical. Ferrari, G. Mil. I. Lomb. Rd. 8 (1875) 5-, 227-, 289-, 624-, 1006-; 9 (1876) 199-.

- primitive. Tylor, E. B. Midl. Ntlist. 15 (1892) 192.

- teaching, beginnings of, in middle ages. Cantor, M. D. Nf. Tbl. (1889) 190.

- treatise (1478). Boncompagni, B. Rm. At. 16 (1862-63) 1-, 101-, 300-, 389-, 503-.

Arithmetical questions, primitive solutions. Bobynin, $V . V$. Bb. Mth. (1894) 55-.

- triangle, history. Fontès, - As. Fr. C. R. (1893) (Pt. 2) 236-.

- - and other notes from mediæval mathematics. Steinschneider, M. Bb. Mth. (1889) 35-.

Assurance, history. Farren, E.J. Assur. Mg. [1 (*1851)] 40-.

- -. Hendriks, F. Assur. Mg. $2\left({ }^{*} 1852\right)$ 121-, 222-; 3 (1853) 93-.

- - first Parliamentary Committee. Hendriks, F. Assur. Mg. 4 (*1854) 58-, 119-, 300 -.

Babylonian mathematics. Cantor, M. D. Nf. Tbl. $\left({ }^{*} 1879\right) 75$ -

Barrett, G., and F. Baily, correspondence between. De Morgan, A. Assur. Mg. 4 (* 1854) 185

Barrow, Isaac. Zeuthen, H. G. Kjøb. Ov. (1897) 565-

Barycentric calculus of Möbius. Allardice, $R$. E. Edinb. Mth. S. P. 10 (1892) 2-.

Bernoulli, Daniel, life and works. Basel Vh. 7 (1885) (Anhang) 3-.

-, John 1st, three letters to Euler in library of Stockholm Academy. Eneström, $G$. [1879] Stockh. Ak. Hndl. Bh. 5 (1880) No. 21, $24 \mathrm{pp}$.

Besson, Jacques $(+1569)$, work of. (Mathematical instruments.) Beck, $T$. Civing. 36 (1890) 191-.

Betti, Brioschi, and Casorati, comparison of work of. Volterra, V. [1900] N. Y. Am. Mth. S. Bll. 7 (1901) 60 .

Binomial theorem, demonstration (Pascal). Delègue, -. Dunkerque Mm. S. Encour. 14 (1869) 163-.

- -, historical paradox. Bourgoin, L. Rv. Sc. 7 (1874) 453-.

- - before Newton. Marre, A. N. A. Mth. 5 (1846) 488.

Boëtius, Ms. of Alexandrine Library containing apices of, without abacus and with values of position. Narducci, $E$. Rm. R. Ac. Linc. Mm. 1 (1877) 503-.

- notes on. Steinschneider, M. Bb. Mth. (1891) 113.

-, pseudo-geometry. Tannery, $P$. Bb. Mth. 1 (1900) 39-.

- question. Weissenborn, H. Z. Mth. Ps. 24 (1879) (Suppl.) 167-, 185-.

Bohemian mathematicians, some. Teige, $K$. Časopis 22 (1893) 244-; Fschr. Mth. (189394) 15 .

\section{History 0010}

Bologna University, mathematical faculty. Gherardi, S. [1844]. Bologna N. Cm. 8 (1846) 519-.

- _-, - - historical notes. Gherardi, S. Arch. Mth. Ps. 52 (1871) 65-.

Bolyai, János, investigations in non-Euclidean geometry. Stäckel, $P$. Mth. Termt. Éts. 18 (1900) 241-; Mth. Nt. B. Ung. 17 (1901) $1-$.

-, - , letter, 1823, theory of parallels. Szily, K. Mth. Termt. Éts. 5 (1887) 187-; Mth. Nt. B. Ung. 5 (1886-87) 189-.

-, 一, work. Schmidt, F. D. Mth. Vr. Jbr. 4 (1897) 107-.

Bolyai's theory of imaginaries. Stäckel, $P$. Mth. Termt. Ets. 17 (1899) 259-; Mth. Nt. B. Ung. 16 (1899) $263-$ -

Boncompagni, Baldassarre, history of physics and mathematics. Favaro, A. Ven. I. At. 4 (1894-95) 509-.

Boskovič, Roger Josef. Seydler, A. Časopis 16 (1887) 267-.

Brachistochrone problem, an historical essay. Compère, C. Liège S. Sc. Mm. 1 (1899) No. 2, 128 pp.

Byzantine analecta. Heiberg, J.L. Z. Mth. Ps. 44 (1899) (Suppl.) 161-.

- mathematics. Heiberg, J. L. Kjøb. Ov. (1887) 88-; Z. Mth. Ps. 33 (1888) (H.-lt. Ab.) $161-$.

Calculating genius. Colburn, Zerah (American boy). Saint, $W$. Nicholson J. 34 (1813) 291-.

J. $35(1813)$ 9-

- - Inaudi. Flammarion, C. As. 11 (1892) 114-.

_ - Touraine herd-boy's calculating feats. Cauchy, A. L. C. R. 11 (1840) 952-

- machines, history. Mehmke, R. D. Mth. Vr. Jbr. 3 (1894) 59-

Calculation in columns before 10th century. Friedlein, G. Z. Mth. Ps. 9 (1864) 297-.

_- -, evolution. Friedlein, G. Z. Mth. Ps. 10 (1865) 241-

_, "Gobâri" method, 14th century Ms. in Vatican on method borrowed from. Nar ducci, E. Bll. Sc. Mth. As. 7 (1883) 247-

- in 16th century. Treutlein, P. Z. Mth. Ps. 22 (1877) (Suppl.) 1-.

Calculus, infinitesimal, invention. Bertrand, J. J. Sav. (1862) 465-.

- of probabilities, historical notes. Gabba, A. Brescia Cm. (1840) 203-.

- - - - Cantor, G. Halle Nf. Gs. B. (1873) 34-.

- - origin and progress. Brown, $S$. Assur. Mg. 6 (* 1857) 134-.

"- Victorii" (Victorius of Aquitaine in 5th century). Tables of weights and measures, squares of numbers \&c. Kinkelin, H. [1868] Basel Vh. 5 (1873) 147-.

- of Victorius. Friedlein, G. Z. Mth. Ps. 16 (1871) 42-, 253-.

Cambridge Phil. Soc., history. Clark, J.W. Camb. Ph. S. P. 7 (1892) i-.

Cantor's history: 13th century solution of a cubic. Zeuthen, H. G. Kjøb. Ov. (1893) 1-. 


\section{History}

Cantor's history: 13th century solution of a cubic, comment on. Gram, J. P. Kjøb. Ov. (1893) 18-.

- mathematical works, memoirs and critiques. Curtze, M. Z. Mth. Ps. 44 (1899) (Suppl.) 625-.

Caporali, Ettore, scientific work. Loria, $G$. G. Mt. 27 (1889) 1-.

Cardan's formula, historic note. Forestier, $C$. Toul. Ac. Sc. Mm. 6 (1874) 254-

Cardanus, Hieronymus (1501-1576), work of. $B e c k, T$. Civing. 35 (1889) 511-.

Carnot, Lazare. Arago, D. F. J. Par. Mm. de l'I. 22 (1850) (H.) 1-.

Casanova de Seingalt. Henry, C. Rv. Sc. 33 (1884) 503-

Case book of John Rowe, London and Exeter, 1775 to 1790 . Hendriks, $F$. Assur. Mg. 7 (*1858) 136-.

Cataldi, inventor of continued fractions. Grunert, J. A. Grunert Arch. 30 (1858) 275-.

Cauchy as founder of theory of determinants. Studnička, F. J. [1876] Prag Ab. 8 (1877) (Mth.) No. 3, 39 pp.

Cauchy's "Lecons sur le Calcul Différentiel" criticism. Matzka, $W$. Grunert Arch. 4 (1844) 357-.

- works. David, -. Toul. Ac. Sc. Mm. 4 (1882) 178-.

Čebyšev's (Tchebisheff) works on the theory of linkages. Delaunay, N. Z. Mth. Ps. 44 (1899) (H. -lt. Ab.) 101-.

Chasles, Michel, scientific work. Ligin, $V$. N. Rs. S. Nt. Mm. (Mth.) 3 (*1881):

Chinese mathematics. Bertrand, J. J. Sav. (1869) 317-, 464-.

- -, algebra. Matthiessen, H. F. L. Z. Mth. Ps. 19 (1874) 270-.

- - arithmetic. Biernatzki, K.L. Crelle J. 52 (1856) 59-.

- - - and algebra. Biernatzki, K. L. N. A. Mth. 2 (1863) 529-.

- - problem of Sun-tsze and Yih-hing on remainders. Matthiessen, H. F. L. [1880-81] Crelle J. Mth. 91 (1881) 254-; Z. Mth. Ps. 26 (1881) (H.-lt. Ab.) 33-

Circle, quadrature, A.D. 1580-1630. Glaisher, J. W. L. (vII) [1873] Mess. Mth. 3 (1874) 27 -

-, - (historical). Schubert, H. Smiths. Rp. (1890) 97-

-, - - Fricke, R. Braunschw. Vr. Nt. Jbr. 11 (1899) 184

-, - . S. van der Eycke. Bierens de Haan, D. N. Arch. Wisk. $1\left({ }^{*} 1875\right) 70-206$ -

Clairaut, Alessio Claudio, letters. Boncom pagni, B. Rm. N. Linc. At. 45 (1892) 157-.

Codex (Liber Algorizmi) in Salem monastery. Cantor, $M$. Z. Mth. Ps. 10 (1865) 1-.

Complex quantities, history. Brunel, $W$. Bordeaux S. Sc. Mm. 5 (1890) lxxvi-.

Condorcet, Marie Jean Antoine Nicolas Caritat, Marquis de. Arago, D.F.J. Par. Mm. de l'I. 20 (1849) (H.) 1-.

-, - - - - - - 1743-94. Robinet, - Rv. Sc. 52 (1893) 554 .
History 0010

Conic section compass, the first. Braunmithl, A. von. Z. Mth. Ps. 35 (1890) (H.-lt. Ab.) 161-.

- sections (historical account). Zeuthen, $H$. G. Kjøb. Dn. Vd. Selsk. Skr. 3 (1885-86) 1-.

Continuity, historical note on. Taylor, (Rev.) C. B. A. Rp. (1884) 645-.

Coordinate geometry in antiquity. Zeuthen, H. G. Kjøb. Ov. (1888) 127-.

Copernicus, biographical sketch. Studnička, F. J. Ćasopis $2\left({ }^{*} 1873\right)$ 1-

Cotes, Roger, historical note. Terquem, $O$. N. A. Mth. 9 (1850) 195-.

Counting with fingers \&c., ancient methods. Marre, A. A. Mt. 6 (1864) 93-.

Cube, duplication, ancient methods for. Mackay, J. S. [1885]. Edinb. Mth. S. P. 4 (1886) 2-.

-, 一, and Delphic oracle. Schweigger, J.S.C. Grunert Arch. 9 (1847) 115-.

Curves and surfaces, history. Tannery, $P$. Bll. Se. Mth. As. 7 (1883) (Pt. 1) 278-.

Cusa, Nikolas de, in connection with mathematical and physical geography. Günther, S. Z. Mth. Ps. 44 (1899) (Suppl.) 123-.

Cyphers, holocryptic. Chase, P. E. Camb. (U. S.) Mth. M. 1 (1859) 194-.

Dalton's early writings. Wilkinson, T. $T$. Manch. Ph. S. Mm. 12 (1855) 1-.

Decimal fractions, history of. Hunrath, $K$. Z. Mth. Ps. 38 (1893) (H.-lt. Ab.) 25-.

- - invention of. Grosse, - Brem. Ab. 14 (1898) 168-.

- numeration among the ancients. Chasles, M. C. R. 6 (1838) 678-; 8 (1839) 72-; 9 (1839) 447-, 463-.

- point, introduction in arithmetic. Glaisher, J. W. L. B. A. Rp. 43 (1873) (Sect.) $13-$.

Decline of mathematical study and sciences. Toplis, J. Tilloch Ph. Mg. 20 (1805) 25-.

Decomposition of fraction into quantièmes (fractions with unity for numerator). Bobynin, V. V. Z. Mth. Ps. 44 (1899) (Suppl.) $1-$.

Definite integrals, historical note. Escher, $R$. $J$. N. Arch. Wisk. 18 (1891) $142-$.

"De inquisicione capacitatis figurarum," anonymous work of the 15th century. Curtze, M. Z. Mth. Ps. 42 (1897) (Suppl.) 29 -.

Desargues, works. Chasles, $M$. C. R. 20 (1845) 1550 -

- and Pascal on conic sections. Gerhardt, $C$. I. Berl. Ak. Sb. (1892) 183-

Descartes. "Calcul de Mons. Des Cartes ou Introduction à sa Géométrie, 1638." Adam, H. Bll. Sc. Mth. 20 (1896) 221-.

-. "Excerpta ex Mss. R. Des-Cartes." Tannery, P. Z. Mth. Ps. 44 (1899) (Suppl.) 501-.

- and Huygens: unpublished letters. Bierens de Haan, D. Z. Mth. Ps. 32 (1887) (H.-lt. Ab.) 161-.

- and Mss. of Snellius. Korteweg, D.J. N. Arch. Wisk. 3 (1898) 57-.

-, a neglected memoir of. Jonquières, - de. C. R. 110 (1890) 261-. 
0010 History

Descartes, philosophical method. Jacobi, C. G.J. [1846] Liouv. J. Mth. 12 (1847) 97-. -, posthumous work on polyhedra. Jonquières, - de. C. R. 110 (1890) 315-.

-, services to the exact sciences. Studnicka, F. J. Časopis 26 (1897) 73-; Fschr. Mth. (1897) 7-.

- work hitherto unpublished: "De Solidorum Elementis." Jonquières, - de. Bb. Mth. (1890) 43-; Par. Ac. Sc. Mm. 45 (1899) 325-.

Descriptive geometry, Monge's (Ikonognosie). Tilser, F. Prag Ab. 9 (1878) (Mth.) No. 3, $88 \mathrm{pp}$.

Determinants, history and theory. Gïnther, - D. Nf. Tbl. $\left.{ }^{*} 1874\right) 78$.

-, an overlooked discoverer in the theory. Muir, T. Ph. Mg. 18 (1884) 416-.

-, skew, history. Muir, T. [1900] Edinb. R. S. P. 23 (1902) 181-.

-, theory, in the historical order of its development. Muir, T. Edinb. R. S. P. 13 (1886) 547-; 14 (1888) 452-; 15 (1889) 481-; 16 (1890) 207-, 389-, 748-.

-, -, origin and development. Studnička, F. J. Časopis 5 (*1876) 1-, 88-, 193-, 279-.

Differential calculus. Eneström, G. [1878] Ups. Årsk. (1879) 71 pp.

- -. Eneström, G. Stockh. Öfv. (1894) 297-; Fschr. Mth. (1893-94) 66-.

- - history and philosophy. Simon, M. Z Z. Mth. Ps. 42 (1897) (Suppl.) 113-.

- -, inventor, \&c. Gerhardt, C.I. Grunert Arch. 27 (1856) 125-.

- - , supposed discovery by Indian astronomer. Spottiswoode, W. As. S. J. 17 (1860) 221-.

- - , work of Taylor and Nicole. Eneström, G. Stockh. Öfv. (1894) 177-; Fschr. Mth. (1893-94) 65.

- and integral calculus, history. Studnickka, F. J. Č́asopis 8 (*1879) 1-, 97-, 272-; Fschr. Mth. (1879) 39.

- - - - - and philosophy. Simon, $M$. D. Nf. Vh. (1896) (Th. 2, Hälfte 1) 257-.

- - - origin and progress. Gabba, A. Brescia Cm. (1825) 89-.

Digits introduced in Europe. Cantor, $M$. Schlömilch Z. 1 (1856) 65-; 3 (1858) 325-. - and numeration, history. Friedlein, G. Z. Mth. Ps. 9 (1864) 73-.

-, origin. . Sédillot, L. A. Rm. At. N. Linc. 18 (1865) 316-.

- - , and the abacus. Vincent, A.J. H. Liouv. J. Mth. 4 (1839) 261-.

- _. _ - - (Vincent's paper). GersonLevy, -. Metz Mm. Ac. 21 (1839-40) 336-.

-, origination. Gerhardt, C.I. Grunert Arch. 2 (1842) 427-.

Directions of modern mathematical investigations. Żorawski, $K$. Wiad. Mt. 3 (1899) 48-; Fschr. Mth. (1899) 69.

Dirichlet and Kronecker, correspondence. Gött. Nr. (1885) 361-.

Division in extreme and mean ratio, historic note on expression. Terquem, $O$. Liouv. J. Mth. 3 (1838) 97-.

\section{History 0010}

Dominicus Parisiensis. Curtze, $M$. Bb. Mth. (1895) 107-.

Double periodicity, Jacobi's share in discovery. Gundelfinger, S. Berl. Ak. Sb. (1898) 342-.

Dresden, Royal Public Library, Ms. in. Curtze, M. Z. Mth. Ps. 28 (1883) (H.-lt. Ab.) 1-.

Egypt, arithmetic in ancient. Loria, G. Bb. Mth. (1892) 97-.

Egypt, mathematics and astronomy in ancient. Isely, L. Neuch. S. Sc. Bll. 23 (1895) 23-.

Egyptian arithmetic. Berg, F. J. van den. N. Arch. Wisk. 19 (1892) 211-.

- geometry, history of ancient. Weyr, $E$. Wien Alm. 34 (1884) 213-.

- papyrus, calculator's manual. Rodet, $L$. Par. S. Mth. Bll. 6 (1878) 139-.

"Encyklopädie der mathematischen Wissenschaften." Meyer, W. F. Z. Mth. Ps. 44 (1899) (Suppl.) 293-.

Epitaphs of mathematicians. Isely, L. Neuch. S. Sc. Bll. 27 (1899) 167-.

Equation problem of A. Romain solved by Vieta, letter of Fermat. Ritter, $F$. Bll. Sc. Mth. As. 4 (1880) (Pt. 1) 171-.

Equations of 3rd order, general solution, historical note. Verdam, G. J. Amst. I. (1846) 163-.

Errors, mathematical. Epstein, S. S. Bern Mt. (1893) 183-.

Euler, extract referring to memoir of. Liouville, J. Liouv. J. Mth. 19 (1874) 189-.

- and his works. Bertrand, J. J. Sav. (1868) 133-.

Euler's constant, history. Glaisher, J. W. L. [1871] Mess. Mth. 1 (1872) 25-; 2 (1873) 64.

- correspondence with Pontoppidan. Hansted, B. Bll. Sc. Mth. As. 3 (1879) 26-.

- letters to Jean Bernoulli. Eneström, $G$. Bb. Mth. (1897) 51-.

- use of $i$. Beman, W. W. N. Y. Am. Mth. S. Bll. 4 (1898) 274, 551.

- works, new list. Hagen, J. G. D. Nf. Vh. (1896) (Th. 2, Hälfte 1) 43-.

Exact sciences, history of, and the need of its study. Rosenberger, $F$. Z. Mth. Ps. 44 (1899) (Suppl.) 359-.

Exponents, Cartesian notation. Vincent, $A$. J. $H$. N. A. Mth. 6 (1847) 35-.

Fergola, Nicola, and his school of mathematics. Loria, G. Genova Un. At. [11] (1892) 363-.

Fermat, date of principal discoveries. Tannery, $P$. Bll. Sc. Mth. As. 7 (1883) (Pt. 1) 116-.

-, a demonstration by. Wertheim, G. Z. Mth. Ps. 44 (1899) (H.-lt. Ab.) 4-.

-, us. by. Genocchi, A. (xII) Rv. Sc.-Ind. 15 (1883) 148-.

Fermat's "Observatio" to Nikomachus' proposition. Wertheim, G. Z. Mth. Ps. 43 (1898) (H.-lt. Ab.) 41-.

- theorem and an Arabian Ms. Kummer, E. E. N. A. Mth. 9 (1850) 386-.

- 'works. Brassinne, E. Toul. Mm. Ac. 3 (1853) 1-.

Ferrari-Cardan, solution of reduced equation of 4 th order. Hunrath, K. Z. Mth. Ps. 30 (1885) (H.-lt. Ab.) 41-.

Fibonacci, algebraic operations wrongly attributed to. Chasles, $M$. C. R. 12 (1841) 741-. 


\section{History}

Fibonacci's treatise on algebra. Gerhardt, $\boldsymbol{C} . \boldsymbol{I}$. Grunert Arch. 2 (1842) 423-.

"Fihrist" of Ibn Abu Ya'kub an-Nadîm, bibliographical notices of mathematicians in. Suter, H. Z. Mth. Ps. 37 (1892) (Suppl.) 1-; 38 (1893) (H.-lt. Ab.) 126-.

Florentine problem (ænigma Florentinum). Drobisch, M. W. Leip. Ab. Mth. Ps. 1 (1852) 431-.

Forcadel, Pierre, circa 1560. Fontés, - Toul. Ac. Sc. Mm. 6 (1894) 282-; 7 (1895) 316-; 8 (1896) 361-

Fourier, Jean Baptiste Joseph. Arago, D. F.J. Par. Mm. de l'I. 14 (1838) lxix-; A. C. 67 (1838) 337-; Smiths. Rp. (1871) 137-.

- series, history of. Gibson, G.A. Edinb. Mth. S. P. 11 (1893) 137-

Fourier's statistical tables. De Morgan, A. I. Act. J. 14 (" 1869) 89-.

Fractional arithmetic in middle ages. Guérard, -. Liouv. J. Mth. 3 (1838) 483-.

Fractions, history of conception and treatment. Bobynin, V. V. Bb. Mth. (1896) 97-.

French mathematical progress since 1830 . Dupin, C. C. R. 1 (1835) 564-.

Galileo Galilei. Favaro, A. Ven. I. Mm. 24 (1891) $430 \mathrm{pp}$.

-, letter. Duhamel, J. M. C. $\quad$ C. R. 69 (1869) 231-.

-, and the mathematicians of the Roman College, 1611. Govi, G. [1873] Rm. R. Ac. Linc. At. 2 (1875) 230-.

一, six letters. Govi, G. Rv. Sc. 33 (1884) 85-.

Gauss and Bolyai, W. (F.), correspondence. Stäckel, P. Gött. Nr. (1897) 1-.

- - - - - - - König, G. Mag. Tud. Ak. Ets. 10 (1899) 617-.

- - - , - - and J., correspondence (non. Euclidean geometry). Stäckel, P., \& Engel, F. Mth. A. 49 (1897) 149-.

- on elliptic functions. Pepin, T. Rm. N. Linc. Mm. 9 (1893) (Pt. 2) 1-; 10 (1894) 125-.

- and Germain, correspondence. Genocchi, $\boldsymbol{A}$. [1880] Tor. Ac. Sc. At. 15 (1879) 795-.

-, influence on geometrical ideas of Lobachevskiî. Vasil'ev, A. V. (xII) [1883] Kazan S. Nt. (P8.-Mth.) P. 2 (1884) 9-.

-, - - - - - Reìngardt, N. V. (xir) [1883] Kazan S. Nt. (P8.-Mth.) P. 2 (1884) 11-.

Gauss's investigations in elliptic functions. Gïnther, P. Gött. Nr. (1894) 92-.

Gauss, letter. Jonquieres, - de. C. R. 122 (1896) 829-

-, letter to Gerling. Stäckel, P. Gött. Nr. (1896) 40-

-, unpublished letter. Jonquières, - de. C. R. 122 (1896) 857-.

Geodesy of Chaldeans. Oppert, J. As. Fr. C. R. (1896) (Pt. 1) 133-.

Geodetic lines, history. Stäckel, $P$. Leip. Mth. Ps. B. 45 (1893) 444-.

Geometers, methods of ancient. Flauti, $V$. Nap. At. Ac. Sc. 1 (1852-54) 17-

Geometric solution of problems of 2 nd degree before Euclid. Tannery, $P$. Bordeaux S. Sc. Mm. 4 (1882) 395-.
History 0010

Geometrical character of ancient astronomy. Mansion, P. Z. Mth. Ps. 44 (1899) (Suppl.) 275-.

- theories, past and present of important. Loria, G. Tor. Ac. Sc. Mm. 38 (1888) $327-$.

Geometry and analysis at different periods. Zeuthen, - . Sk. Nf. F. (1886) 44-.

- of the ancients, in particular an axiom of Archimedes. Stolz, O. Mth.A.22 (1883) 504-.

- with circle of fixed radius. Kutta, W. M. Ac. Nt. C. N. Acta 71 (1898) 69-.

-, development of methods and ideas. $E a$ zarski, - . Kosmos (Lw.) 14 (1889) 271-.

-, - of synthetie from Monge to 1847. Kötter, E. D. Mth. Vr. Jbr. 5 (1901) (Heft 2) xxviii $+486 \mathrm{pp}$.

—, infinitesimal, Pascal's works on. Delègue,-. Dunkerque Mm. S. Encour. 14 (1869) $137-$.

- of Kepler and Newton. Taylor, C. Camb. Ph. S. T. 18 (1900) 197-.

- Le Clere and Ozanam. Graf, J. H. Z. Mth. Ps. 44 (1899) (Suppl.) 113-.

- in $n$ dimensions, history and present state. Schlegel, V. Lpldina. 22 (1886) 92-, 108-, 133-, 149-, 160-; Ens. Mth. 2 (1900) 77-.

- of Polifilo. Barca, A. Padova Mm. Ac. (1809) 222-.

- triangle. (Euler.) Mackay,J.S. Edinb. Mth. S. P. 4 (1886) 51-.

- - (Historical sketch.) Vigarie; $\hat{E}$. As. Fr. C. R. (1889) (Pt. 2) 117-.

- triangles in 1890 . Vigarie, $\hat{E}$. Fschr. Mth. (1891) 38.

Georgius de Hungaria, arithmetic of, 1499. Szily, C. von, \& Heller, A. [1894] Mth. Nt. B. Ung. 12 (1895) 134-.

German algebra (Coss) of 15th and 16th centuries. Treutlein, P. Z. Mth. Ps. 24 (1879) (Suppl.) 1-.

- -, history. Wappler, E. Z. Mth. Ps. 44 (1899) (Suppl.) 537-.

arithmetical problems, old. Curtze, M. Bb. Mth. (1895) 110-

- mathematics. Herwart ab Hohenburg's "Tabulæ Arithmeticæ $\pi \rho \circ \sigma \theta a \phi a \iota p \in \sigma \epsilon \omega s$ universales," (1610). Glaisher, J. W. L. [1875] Camb. Ph. S. P. 2 (1876), 386-.

- universities, 17 th cent., mathematics at the. Bartholomai (Dr). Z. Mth. Ps. 13 (1868) (Suppl.) 1-.

Germany, history of algebra in. Gerhardt, C. J. Berl. Mb. (1867) 41-; (1870) 141-.

- mathematical teaching in, history. Günther, S. Ens. Mth. $2(1900) 237-$.

Gompertz, unfair suppression of acknowledgment to. De Morgan, A. Assur. Mg. 9 $\left({ }^{*} 1861\right)$ 86-.

Graeco-Egyptian arithmetic. Loria, G. Bb. Mth. (1893) 79-; G. Mt. 32 (1894) 28-.

- papyrus of the VII.-IX. century, treating mainly of fractions. Bobynin, W.W. $[V . V$. Fschr. Mth. (1895) 47-.

Graeco-Indian researches. Cantor, M. Mil. I. Lomb. Rd. 9 (1876) 818-.

Graeco-Italian Golden Synthesis' from Ms. of Viviani. Ferroni, P. Mod. Mm. S. It. 19 (1821) 187-. 


\section{History}

Grassmann's "Ausdehnungslehre." Schlegel, V. Z. Mth. Ps. 41 (1896) (H.-lt. Ab.) 1-, 41-.

\section{Greek Mathematics.}

Aegidius de Columna. Steinschneider, M. Bb. Mth. (1891) 113-.

Analysis, ancient Greek compared with modern. Matthes, C. J. Leijd. A. Ac. (1832-33), $36 \mathrm{pp}$.

Anaritius, commentary on the elements of Euclid. Mansion, - Brux. S. Sc. A. 24 (1900) (Pt. 1) 47-.

Apollonius, "Conica" Book V, problem. Drobisch, M. W. Leip. B. 8 (1856) 103-.

-, conics. Housel, -. Liouv. J. Mth. 3 (1858) 153-.

-, -. Milne, J.J. Mth. Gz. No. 6 (1895) 49-. -, fragments. Tannery, P. Bll. Sc. Mth. As. 5 (1881) (Pt. 1) 124-

-, Inclinations. Flauti, V. [1850] Mod. S. It. Mm. 25 (1852) 223-.

-, - , Bk. I., solution of problem in. Sangro, $G$. Nap. At. Ac. 2 (1825) 45-

-, 一, - - , - - IV. Giannattasio, $F$. Nap. At. Ac. 2 (1825) 317-

-, lost works on irrationals (Wöpcke). Chasles, M. $\quad$ C. R. 37 (1853) 553-.

,$--\ldots-\ldots$, reconstructed from Arabian Ms. Woepcke, F. Par. Mm. Sav. Etr. 14 (1856) 658-.

-, problems and porismatic developments. Gardiner, M. Vict. T. R. S. 5 (1860) $19-$.

Archimedes, ancient criticism of a demonstration of. Tannery, P. [1881] Bordeaux S. Sc. Mm. 5 (1883) 49-.

- and conics. Heiberg, J. L. Z. Mth. Ps. 25 (1880) (H.-lt. Ab.) 41-

-, cyclometry. Hultsch, F. Z. Mth. Ps. 39 (1894) (H.-lt. Ab.) 121-, 161-.

-, De numero arenæ. Chasles, $M$. C. R. 14 (1842) 547-.

-, Ephodikon. Schmidt, W. Bb. Mth.1 (1900) 13-.

-, Greek manuscripts used in translation by W. von Moerbek. Heiberg, J.L. Z. Mth. Ps. 37 (1892) (H.-lt. Ab.) 81-.

-: "Loculus Archimedius" or the "Syntemachion," publication of translation from two Arabic Mss. Suter, $H$. Z. Mth. Ps. 44 (1899) (Suppl.) 491-.

-, measurement of circle. Tannery, $P$. Bordeaux S. Sc. Mm. 4 (1882) 313-.

-, new studies. Heiberg, J. L. Z. Mth. Ps. 34 (1889) (Suppl.) 1-.

- and problem of oxen. Amthor, A., \& Krum. biegel, B. Z. Mth. Ps. 25 (1880) (H.-lt. Ab.) 121-, 153-.

---1 Tannery, P. Bll. Sc. Mth. As. 5 (1881) (Pt. 1) 25-.

-, solution of cubic. Zeuthen, H. G. Bb. Mth. (1893) 97-.

-, theorem for the surface of a cylinder. Cayley, A. Mess. Mth. 13 (1884) 107-.

- , theorems. Heiberg, J. L. Z. Mth. Ps. 24 (1879) (H.-lt. Ab.) 177-.

-, treatment of arithmetic progression. Gibson, G. A. Edinb. Mth. S. P. 16 (1898) 2-.
History 0010

Greek Mathematics, continued

Aristarchus of Samos. Tannery, P. Bordeaux S. Sc. Mm. 5 (1883) 237 -

Aristotle, on a construction for parallels. Heath, T. L. Z. Mth. Ps. 44 (1899) (Suppl.) 153-.

-, - difference between natural philosophy and mathematics. Dutordoir, - . Brux. S. Sc. A. 24 (1900) (Pt. 1) 52-.

-, geometrical theorem in. Heiberg, J. L. Kjøb. Ov. (1888) 1-.

Diophantus codex, a re-found. Gollob, E. Z Z. Mth. Ps. 44 (1899) (H.-lt. Ab.) 137-.

- and composition of numbers from 2 squares. Jacobi, C. G. J. Berl. B. (1847) 265-.

-, German translation, annotations. Nesselmann, G. H. F. Z. Mth. Ps. 37 (1892) (H.it. Ab.) 121-, 161-.

- , methods of calculation of irrational square roots. Schoenborn, $W$. Z. Mth. Ps. 30 (1885) (H.-lt. Ab.) 81-.

-, polygonal numbers, treatise, concluding proposition. Wertheim, G. Z. Mth. Ps. 42 (1897) (H.-lt. Ab.) 121-.

-, problems of, proofs. Zeuthen, H. G. (xi) Ts. Mth. 6 (1876) 168-, 181-; 6 (1882) 97-; 1 (1883) 145-; Fschr. Mth. (1884) 32.

-, Psellus on. Tannery, P. Z. Mth. Ps. 37 (1892) (H.-lt. Ab.) 41-.

-, work. Tannery, P. Bb. Mth. (1887) 37-, 81-, 103-; (1888) 3-.

Euclid, Bk. I. 32. Corollary. Tucker, R. Nt. 63 (1900-01), 58.

- - - - - Allman, G.J. [1900] Nt. 63 (1900-01) 106-.

-, - - - - Eumorfopoulos, S. [1900] Nt. 63 (1900-01) 157.

-, - X., equations of 4 th order. Christensen, S. A. Z. Mth. Ps. 34 (1889) (H.-lt. Ab.) 201-. -, - XII., and Archimedes on measurement of circle, \&c. Ovidio, E. d'. G. Mt. 9 (1871) 122-.

- and his century. Cantor, M. Z. Mth. Ps. 12 (1867) (Suppl.) 1-.

-, porisms. Chasles, M. Quetelet Cor. Mth. 10 (1838) 1-.

-, - . Breton [de Champ], P. C. R. 29 (1849) 479-; Liouv. J. Mth. 2 (1857), 185-.

-, - C Cantor, M. Schlömilch Z. 2 (1857) 17-. - - Breton [de Champ], P. Liouv. J. Mth. 3 (1858) 89-.

-, -, reply to Breton. Chasles, M. C. R. 51 (1860) 1043-

-, - restoration. Chasles, M. C. R. 48 (1859) 1033-; 50 (1860) 940, 997, 1007-.

_- - . Wilkinson, T. T. [1867] Manch. Lt. Ph. S. P. 7 (1868) 68-.

-, postulates and axioms. Mansion, P. Brux. S. Sc. A. 14 (1890) (Pt. 2) 35-.

-, problems, Ms. in library of Royal Gym. Thorn. Curtze, M. Z. Mth. Ps. 13 (1868) (Suppl.), 45-.

-, translation from Arabic into Latin by Adelhard of Bath, according to Mss. at Erfurt. Weissenborn, H. Z. Mth. Ps. 25 (1880) (Suppl.) 141-.

Euclid's elements, Arabic version. Heiberg, J. L. Z. Mth. Ps. 29 (1884) (H.-lt. Ab.) 1-. 


\section{History}

Greek Mathematics, continued

Euclid's elements, Nasr ad-Din's edition. Suter, $H$. Bb. Mth. (1892) 3-.

- text, alterations. Vincent, A. J. H. N. A. Mth. 3 (1844) 5-.

Grecian page-numbers and diametral numbers. Bergh, P. Z. Mth. Ps. 31 (1886) (H.-lt. Ab.) 135.

Greek abacus, methods of reckoning on. Nagl, A. Z. Mth. Ps. 44 (1899) (Suppl.) 335-.

- arithmetic. Hawkes, H. E. N. Y. Am. Mth. S. Bll. 4 (1898) 530-.

- geometry. Treutlein, $P$. (1883) (H.-lt. Ab.) 209-.

- - the golden period of. Loria, $G$. Tor. Ac. Sc. Mm. 40 (1890) 369-.

- - higher. Valat, -. Bordeaux Act. Ac. Sc. 24 (1862) 223-.

- mathematical fragment in Bobbio, Cod. Ambrosianus. Heiberg, J. L. Z. Mth. Ps. 28 (1883) (H.-lt. Ab.) 121-.

- mathematics, remarks on history. Sturm, A. Z. Mth. Ps. 44 (1899) (Suppl.) 485-.

-- , surviving history. Heiberg, J.L. Kјøb. Ov. (1896) 77-.

- methods of calculating square-root. Schoenborn, W. Z. Mth. Ps. 28 (1883) (H.-lt. Ab.) 169 -.

Greeks, mathematical discoveries. Ofterdinger, L. F. Grunert Arch. 5 (1844) 102-.

-, theory of numbers among. Cantor, $M$. (vr. Adds.) [1863] Heidl. Vh. Nt. Md. 3 (1862 63) $61-$

Hero of Alexandria. Tannery, $P$. Bordeaux S. Sc. Mm. 5 (1883) 347-.

- - fragments preserved by Proclus. Tannery, P. Bll. Sc. Mth. As. 6 (1882) (Pt. 1) 99 .

- - Greek arithmetic. Tannery, $P$. Bordeaux S. Sc. Mm. 4 (1882) 161-.

- _ - influence on 17 th century. Schmidt, W. Z. Mth. Ps. 42 (1897) (Suppl.) 195-.

- - - stereometry. Tannery, $P$. Bordeaux S. Sc. Mm. 5 (1883) 305-.

-, extraction of irrational cubic-roots. Wertheim, G. Z. Mth. Ps. 44 (1899) (H.-lt. Ab.) 1-.

- metrics, a fragment. Tannery, $P$. Z. Mth. Ps. 39 (1894) (H.-lt. Ab.) 13-.

Lunes, quadrature, fragment of Eudemus. Tannery, $P$. Bordeaux S. Sc. Mm. 5 (1883) 211-.

- - , and Hippocrates of Chio. Tannery, $P$. Bordeaux S. Sc. Mm. 2 (1878) 179-

Pappus, Greek arithmetic. Tannery, $P$. Bordeaux S. Sc. Mm. 3 (1880) 351-.

-, - text, Mss. in Great Britain. Rigaud, S. P. Edinb. Ph. J. 7 (1822) 56-, 219-.

- passage. Heiberg, J. L. Z. Mth. Ps. 23 (1878) (H.-lt. Ab.) 117-.

- on progressions. Mackay, J. S. Edinb. Mth. S. P. 6 (1888) 48-.

-, theorem, restoration. Barnwell, $C . F$. Edinb. J. Sc. 6 (1832) 90-.

Perspective, "Was it known to Greek geometers?" Taylor, C. [1880] Mess. Mth. 10 (1881) 112-.
History 0010

Greek Mathematics, continued

Planudes, calculation book. Gerhardt, C. I. Berl. Mb. (1867) 38-.

Plato's geometry in Meno and Pythagoras' parabola in Plutarch. Wex, F. C. Arch. Mth. Ps. 47 (1867) 131-.

Platonic number. Dupuis, J. As. Fr. C. R. (1886) (Pt. 2), 135-

- Demme, C. Z. Mth. Ps. 32 (1887) (H.lt. Ab.) 81-, 121-.

Problem of Delos, solutions by Archytas and Eudoxus. Tannery, $P$. Bordeaux S. Sc. Mm. 2 (1878) 277-。

Ptolemy, "De Analemmate." Heiberg, J. L. Z. Mth. Ps. 40 (1895) (Suppl.) 1-.

-, geometrical knowledge. Ottema, J. G. (viii) Amst. N. Ws. Ntk. Vh. 1 (1844) 235-. - on ratio of diameter to circumference. Terquem, $0 . \quad$ N. A. Mth. 13 (1854) 253-.

-, theories of movements of the planets. Haebler, A. Z. Mth. Ps. 45 (1900) (H.-lt. Ab.) $161-$.

Pythagoras, mathematics of. Cantor, $M$. Heidl. Vh. Nt. Md. (1857-59) 164-.

Root extraction according to Hero. Curtze, M. Z. Mth. Ps. 42 (1897) (H.-lt. Ab.) 113-. Science in Ancient Greece. Loria, G. Mod. Ac. Sc. Mm. 10 (1894) 3-; 11 (1895) 3-.

Serenus of Antissa. Tannery, P. Bll. Sc. Mth. As. 7 (1883) (Pt. 1) 237-.

-, birthplace. Heiberg, J. L. Bb. Mth. (1894) 97-.

Simplicius. Steinschneider, M. Bb. Mth. (1892) 7-.

-, commentary on Euclid's elements. Besthorn, R. O. Bb. Mth. (1892) 65-.

Spheres, homocentric, of Eudoxus, Calippus and Aristotle. Schiaparelli, G. V. [1874] Mil. I. Lomb. Mm. 13 (1877) 117-.

Spherical trigonometry and Ptolemy. Schubert, F. T. [1796] St Pét. Ac. Sc. N. Acta 12 (1801) 165-.

Square roots, Archimedes' approximate values. Heilermann, H. Z. Mth. Ps. 26 (1881) (H.lt. $A b$.) 121-

_- Weissenborn, H. Z. Mth. Ps. 28 (1883) (H.-lt. Ab.) 81-.

,$---1-$. Hultsch, $\dot{F}$. Gött. Nr. (1893) $367-$.

- calculation, Archimedes. Zeuthen, H. G. Ts. Mth. 3 (1879) 145-.

- - - - - and Hero. Demme, C. Z. Mth. Ps. 31 (1886) (H.-lt. Ab.) 1-.

- extraction in ancient Greece. Bobynin, $V$. V. Z. Mth. Ps. 41 (1896) (H.-lt. Ab.) 193-.

Stobæus on series. Bienaymé, -. C. R. 71 (1870) 460-.

Tactions of Apollonius, modern solution. Brennecke $(D r)$. B. A. Rp. (1860) (Pt. 2$) 4$.

Thales and geometry of Egyptians, ancient references. Hugo, L. C. R. 108 (1889) 767-.

Work of Greeks in development of mathematics. Bobynin, V.V. Bb. Mth. (1892) 1-.

Gyldén's scientific methods. Bohlin, $K$. Acta Mth. 20 (1897) 397-. 
Hamburg Math. Ges., Dutch members, history. Bierens de Haan, D. Hamb. Mth. Gs. Mt. 2 (1890) (Festschr., Tl. 1) 79-.

- - history, 1690-1890. Bubendey, J.F. Hamb. Mth. Gs. Mt. 1 (1889) 8-; 2 (1890) (Festschr., Tl. 1), 8-

Harriot's papers. Zach, F.X. von. Zach Cor. 7 (1822) 105-.

- - Rigaud, S. P. R. I. J. 2 (1831) $267-$.

Hebrew Ms. of Ibn-Ezra, on arithmetic. Terquem, O. Liouv. J. Mth. 6 (1841) 275-.

- on geometry (Mischnath Hammidoth) in Munich library, translation. Schapira, $H$. Z. Mth. Ps. 25 (1880) (Suppl.) 1-.

-- , notes on. Steinschneider, M. Bb. Mth. (1893) 73-.

Helmholtz's investigations on the fundamental principles of mathematics and mechanics. Koenigsberger, L. [1895] Smiths. Rp. (1896) 93-.

Historical notes. Luca, F. de. Il Progresso, 1 (1832) 163-; 2, 3-, 169- ; 3, 235-.

- - Hill, C. J. D. Sk. Nf. F. 5 (1847) 360 -.

-. Luca, F. de. Il Giamb. Vico, 4 (1857) 451-.

- - Baltzer, $R$. Leip. B. 17 (1865) 1-. - Cockle, (Sir) J. [1875] Manch. Lt. Ph. S. P. 15 (1876) 6-.

History of $e$ and $\pi$. Dikstein, S. Kosmos (Lw.) 20 (1895) 359-; Fschr. Mth. (1895) 56.

Holland, history of mathematical and natural sciences in. Bierens de Haan, D. Amst. Ak. Vs. M. 8 (1874) 57-, 163-; 9 (1876) 1-, 90-, 322-; 10 (1876) 161-; 12 (1878) 1-; 14 (1879) 180-; 16 (1881) 1-; 18 (1883) 218-; 19 (1884) 1-, 78-, 249-; 20 (1884) 102-, 197-; 1 (1885) 224-; 3 (1887) 69-; 4 (1888) 65-; 6 (1889) 103-; 9 (1892) 4-; Fschr. Mth. (1884) 21-; (1885) 12-; (1886) 10-; (1887) 14 ; $(1889)$ 12-.

Hudde, Johannes, year of birth. Korteweg, D.J. Z. Mth. Ps. 41 (1896) (H.-lt. Ab.) 22-.

Huygens, Christian. Bosscha, J. Rv. Sc. 4 (1895) 609-; Arch. Néerl. 29 (1896) 352-; Ciel et Terre 17 (1896-97), 173-, 202-, 289-'

- - - discoveries and documents published by Chasles. Kaiser, F. Arch. Mth. Ps. 49 (1869) 81-.

-, Constantijn, and Descartes. Korteweg, D.J. Arch. Néerl. 22 (1888) 422-.

Hypergeometric series, Gauss' works on, errors in. Simon, H. Z. Mth. Ps. 32 (1887) (H.lt.-Ab.) 99-.

-- , history. Schulze, K. Hamb. Mth. Gs. Mt. 1 (1889) 110-.

Imaginaries, geometric representation. Hoüel, $J$. Bordeaux Mm. S. Sc. 5 (1867) i-.

Imaginary elements in geometry, historical monograph on. Ramorino, A. G. Mt. 35 (1897) 242-; 36 (1898) 317-.

- numbers, history. Studnička, F.J. Časopis 13 (1884) 49-, 254-; Fschr. Mth. (1884) 37-.

- quantities in analysis, development. Kiessling, K. J. Hamb. Mth. Gs. Mt. 1 (1889) 24-.

As. P. (1897) 33-.
Imaginary roots, historical note. Haan, $D$. B. de. Amst. Vs. Ak. 8 (1858) 248-.

Indeterminate equation $a x+b y=c$, ancient process for solution in integers. Rodet, $L$. Par. S. Mth. Bll. 7 (1879) 171-.

\section{Indian Mathematics.}

Ahmes and Baudhâyana, rules regarding quadrature of circle. Demme, C. Z. Mth. Ps. 31 (1886) (H.-lt. Ab.) 132-.

Binomial theorem known to Hindoos. Burrono, R. As. Researches, 2 (1790) 487-.

Hindu arithmetic. Burrow, $R$. As. Researches, 3 (1792) 145-.

- geometry. Oppermann, L. H.F. (хп) Ts. Mth. 1 (1871) 55-, 124-

- quadrature of circle. Whish, C. M. [1832] As. S. T. 3 (1835) 509-.

Indian calculation of the sine, and the word Kardaga. Woepcke, F. N. A. Mth. 13 (1854) $386-$.

Mathematics in the East Indies. Delbos, $L$. Bll. Sc. Mth. 16 (1892) 93-; 17 (1893) 145-.

Quadrilateral in circle whose parts are all rational. Terquem, $O$. N. A. Mth. 5 (1846) 636.

Square-root approximation method known before Alexander's conquest. Rodet, L. Par. S. Mth. Bll. 7 (1879) 98-, 159-.

Trigonometrical tables of the Brahmins. Playfair, J. [1795] Edinb. R. S. T. 4 (1798) 83-.

Two problems by Bāpu Deva Shāstri. Hall, B. H. Silliman J. 11 (1851) 194 -

Infinitesimal calculus and Bolzano. Stolz, $O$. [1880] Mth. A. 18 (1881) 255-.

- - , history of. Dickstein, S. Prace Mt.Fiz. 10 (1899-1900) 178-.

- - invention. Zeuthen, H. G. Kjøb. Ov. (1895) 193-, 257-.

- - , origin. Milhaud, G. [1900] N. Y. Am. Mth. S. Bll. 7 (1901) 157, 158.

- - - Cantor, M. [1900] N. Y. Am. Mth. S. Bll. 7 (1901) 158, 159-.

_- principles, history. Critics of Lagrange's "Théorie des fonctions analytiques." Dickstein, S. Z. Mth. Ps. 44 (1899) (Suppl.) 65-. - - text books from Euler to present time. Bohlmann, G. D. Mth. Vr. Jbr. 6 (1899) (Heft 2) 91-.

Infinitesimals, early history in England. De Morgan, A. (vi Adds.) Ph. Mg. 4 (1852) 321-.

Integral $(1+n \cos \phi)^{r} d \phi$, Ms. memoir on. Gauss, C. F. Gött. Nr. (1893) 617-.

"Introduction Arithmétique" (de Gérase) Livre II, ch. 9 and 20. Martin, H. Tortolini A. 8 (1857) 429-.

Invariant theory, history of development and account of present condition. Meyer, $F$. D. Mth. Vr. Jbr. 1 (1892) 79-.

"Invention nouvelle en l'Algèbre" (reproduction of 16th century work by Albert Girard). Bierens de Haan, D. N. Arch. Wisk. 11 (1884) 83-.

Irrational square-roots, calculation of, and the invention of continued fractions. Wertheim, G. Z. Mth. Ps. 42 (1897) (Suppl.) 147-. 


\section{History}

Irrationality of $e$ and $\pi$, on early proofs of. Pringsheim, A. Münch. Ak. Sb. 28(1899) 325-.

Irrationals, quadratic, of ancients and their methorls of development. Giinther, S. Z. Mth. Ps. 27 (1882) (Suppl.) 1-.

Italian contributions to mathematics. Riccardi, P. Bologna Ac. Sc. Mm. 6 (1896-97) 755-; 7 (1897) 371-.

- mathematical works 1800-16. Anon. (vI. 138.) Bb. It. 1 (1816) 225-, 358-; 2, 60-, - sources for historico-mathematical knowledge. Favaro, A. Bb. Mth. (1889) 113-.

Jacobi's correspondence with Legendre. Jacobi, C. G. J. Bll. Sc. Mth. As. 8 (1875) 287-; 9 (1875) 38-, 51-, 126-.

- posthumous works. Lejeune-Dirichlet, $G$. Crelle J. 42 (1851) 91-.

- theorem, extension, priority claim. Meyer, U. H. Grunert Arch. 22 (1854) 474-.

- theta formulæ, date and origin. Kronecker, L. Berl. Ak. Sb. (1891) 653-.

Japan, history of mathematics in. Westphal, $A$. (xII) [1876] D. Gs. Ostas. Mt. 1 (1873-76) (Heft 9) 54 .

Japanese arithmetic, use of geometry in. Berson, - . Toul. Ac. Sc. Mm. 3 (1891) 268-. - mathematics. Fujisawa, - [1900] N. Y. Am. Mth. S. Bll. 7 (1901) 69 .

Jaquemet, Père, two letters. Marre de Marin, E. A. Bll. Sc. Mth. As. 4 (1880) (Pt. 1) 200-.

Jewish mathematics. Steinschneider, $M$. Bb. Mth. (1893) 65-, 105-; (1894) 37-, 79-, 99-; (1895) 19-, 43-, 97-; (1896) 32-, 77-, 109-; (1897) 13-, 35-, 73-, 103-; (1898) 5-, 33-, 79-; (1899) 1-, 37-, 97-.

- - (1501-50). Steinschneider, M. Z. Mth. Ps. 44 (1899) (Suppl.) 471-.

Johann von Gemunden. Curtze, $M$. Bb. Mth. (1896) 4.

Johannes de Lineriis, nationality. Curtze, $M$. Bb. Mth. (1895) 105-.

Johannes de Muris, "Das Quadripartitum," and practical arithmetic in the 14 th century. Nagl, A. Z. Mth. Ps. 34 (1889) (Suppl.) 135-.

- Saxonia's commentary on Introductio Alcabitii. Steinschneider, $M$. Bb. Mth. (1891) 114.

Jordanus Nemorarius, certain algebra problems. Wertheim, G. Bb. Mth. 1 (1900) 417-.

- -, "Tractatus de numeris datis." Treutlein, P. Z. Mth. Ps. 24 (1879) (Suppl.) 125-.

- - - - - - Curtze, M. [1880-91] Lpldina. 18 (1882) 26-; Z. Mth. Ps. 36 (1891) (H.-lt. Ab.) 1-, 41-, 81-, 121-.

----1. Daublebsky von Sterneck, $R$. Mh. Mth. Ps. 7 (1896) 165-

Josephus Sapiens or Hispanus, Gerbert on. Weissenborn, $H$. Bb. Mth. (1893) 21-.

- - Hispanus, Gerbert on. Curtze, $M$. Bb. Mth. (1894) 13-

- - (reply to Curtze). Suter, H. Bb. Mth. (1894) 84.

Kepler's logarithms, and letters of Kepler. Frisch, -. Grunert Arch. 24 (1855) 286-.

Kochanski and Leibnitz, correspondence between. Dickstein, S. Fschr. Mth. (1897) 9-; Krk. Ak. (Mt.-Prz.) Rz. 13 (1898) 1-.
History 0010

Lagrange and Euler's correspondence. Cantor, M. Z. Mth. Ps. 23 (1878) (H.-lt. Ab.) 1-, letters. Genocchi, A. Tor. Ac. So. At. 9 (1874) 746-.

-, letters to Euler, published by Boncompagni. Genocchi, A. [1877] Tor. Ac. Sc. At. 12 (1876) 350 -

-, -, four. Lagrange, J. L. (x) Tor. Ac. Sc. At. 9 (1873-74) 756-.

-, works. Bertrand, J. J. Sav. (1869) 257-. Lancashire geometers. Wilkinson, T. T. Manch. Ph. S. Mm. 11 (1854) 123-

- - and ancient geometrical analysis. Wilkinson, T. T. Lanc. T. Hist. S. 8 (1856) 75 -

Least squares method, historical note. Abbe, $C$. Am. J. Sc. 1 (1871) 411-.

- - - history. Merriman, M. Des Moines Anal. 4 (1877) 33-, 140-

- - - priority claims. Gerling, C. L. Gött. Nr. (1861) 273-.

Legendre, Adrien Marie. $\quad M ., F . \quad B b$. Un. 52 (1833) 45-.

Legendre's correspondence with Jacobi. Legendre, A. M. Bll. Sc. Mth. As. 8 (1875) 287-; 9 (1875) 38-, 51-, 126-.

- "Theory of numbers," error in. Dujardin, -. C. R. 119 (1894) 843-, 934.

Leibnitz, correspondence. Jacobi, C. G. J. Berl. B. (1850) 426-.

- on determinants. Gerhardt, K. I. Berl. Ak. Sb. (1891) 407-.

- Mss. published by Gerhardt. Weissenborn, $H$. Schlömilch Z. 1 (1856) 240-.

- and Pascal. Gerhardt, C. I. Berl. Ak. Sb. (1891) 1053-

- theorem; and a letter of Lagrange. Genocchi, A. Tor. At. Ac. Sc. 4 (1868-69) 263-.

Leibnitz's discovery of algorithm of higher analysis, 200th anniversary of. Gerhardt, C. I. Berl. Ac. Mb. (1875) 588-

Leo, Byzantine mathematician of 9th century. Heiberg, J.L. Bb. Mth. (1887) 33-.

Leonardo da Vinci, geometrical constructions by. Cantor, M. Hamb. Mth. Gs. Mt. 2 (1890) (Festschr., Tl. 1) 8-.

- - Vinci's geometrical construction for ellipse. Rulf, W. Mh. Mth. Ps. 9 (1898) 30 -.

Leonardo Pisano, life and works. Boncompagni, B. Rm. At. 5 (1851-52) 5-, 208-.

- - three of his writings published by Boncompagni. Genocchi, A. Tortolini A. 6 (1855) 161-, 218-, 273-, 345-.

- - , - - _ - - - Terquem, 0 . Tortolini A. 7 (1856) 106-.

- - treatise on square numbers. Woepcke, F. Liouv. J. Mth. 20 (1885) 54 .

- - work on cubic equations. Woepcke, F. Liouv. J. Mth. 19 (1854) 401-.

- Pisano's “Liber Quadratorum," problems. Genocchi, A. Tortolini A. 6 (1855) 186-, 251-.

- - writings, and their relation to Arabian works. Woepcke, F. Rm. At. 10 (1856-57) 236-; 12 (1858-59) 230-, 399-; 14 (1860-61) 211-, 241-, 301-, 343-. 


\section{History}

Letter " $x$," origin of denoting the unknown by. Wertheim, G. Z. Mth. Ps. 44 (1899) (H.-lt. Ab.) 48.

Liber karastonis sive de statera. Steinschneider, M. (vIII) [1862] A. Mt. 5 (1863) 54-.

Liber trium fratrum. Kinkelin, $H$. Basel Vh. 3 (1863) 511-。

- - - (9th century work on geometry). Curtze, M. Ac. Nt. C. N. Acta 49 (1887) 105-.

_- - proposition of an approximate calculation in. Vaux, C. de. Bb. Mth. (1898) 1-.

Lie, Marius Sophus, communications from, 1869-71. Sylow, L. Christiania Skr. (Mth.Nt. Kl.) (1899) No. 9, $15 \mathrm{pp}$.

_, _ _, scientific work. Żorawski, K. Wiad. Mt. 3 (1899) 85-.

Limits of error of $\frac{a}{b}$ etc. deduced from the known limits of error of $a, b$, respectively. Mehmke, R. Fschr. Mth. (1888) 1289-.

Linear differential equations. Radelfinger, F. G. [1899] Wash. Ph. S. Bll. 14 (1906) 21-.

Lobačevskij as algebraist. Vasiliev, A. N. Y. Mth. S. Bll. 3 (1894) 231-.

-, centenary of birth, celebration at Imperial University, Kasan. Weyr, E. Časopis 25 (1896) 1-; Fschr. Mth. (1896) 15.

Logarithm of sum or difference, and solution of quadratic equation by circular functions, Cavalieri's methods, 1639. Govi, G. Rm. R. Ac. Linc. At. 3 (1876) (Pte. 2) 173-.

Logarithmic tables, early history. Glaisher, J. W. L. Ph. Mg. 44 (1872) 291-, 500-.

- - - , and their calculators. Glaisher, J. W. L. Ph. Mg. 45 (1873) 376-

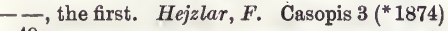
49 -

- - progress to accuracy. Glaisher, J.W.L. As. S. M. Not. 33 (1873) 330-.

Logarithms, Gaussian, origin. Bouniakowsky, V. [1862] St Pét. Ac. Sc. Bll. 5 (1863) 471-.

-, history, critique. Matzka, W. Grunert Arch. 34 (1860) 341-.

-, - - and literature. Gieswald, -. Grunert Arch. 26 (1856) 316-.

-, -, notes. Cajori, F. Z. Mth. Ps. 44 (1899) (Suppl.) 31-.

-, notation of. Draenert, -. D. Nf. Tbl. (*1876) (Suppl.) 173-.

Logic, precursors in mathematical. Peano, $G$. Rv. Mt. 4 (1894) 120.

_, _- - Vacca, G. Rv. Mth. 6 (1896-99) 121-.

, - - Gergonne, J. D. Rv. Mth. 6 (1896-99) 183-.

Longitude determination at sea, history. Gelcich, E. Z. Mth. Ps. 44 (1899) (Suppl.) 105-.

Malfatti's problem, history. Baker, M. [1877] Smiths. Misc. Col. 20 (1881) Art. 2, 113-. (Wash. Ph. S. Bll. 2 (1880).)

MS. No. 14836 of the K. Hof-u. Staatsbibliothek, München. Curtze, M. Z. Mth. Ps. 40 (1895) (Suppl.) 75-.

MSS., some old. Wittstein, A. Z. Mth. Ps. 40 (1895) (H.-lt. Ab.) 121-.

\section{History 0010}

Marci, Joannes Marcus (Jan Marck). Studnička, F. J. Böhm. Gs. Ws. Jbr. (1890) xxxii pp.

Martinus Rex and his geometry. Dickstein, $S$. Fschr. Mth. (1895) 10.

Mascheroni's geometry of the compass. Cay. ley, A. Mess. Mth. 14 (1885) 179-.

Mathematical analysis, development of theories in, during 19th century. Picard, É. [1899] Wiad. Mt. 4 (1900) 173-.

- anecdotes. Ferroni, $P$. Verona S. It. Mm. 7 (1794) 319-.

- historical research, purpose and results of recent. Günther, - D. D. Tbl. ( $\left.{ }^{*} 1875\right) 130$ -

- history, present problems. Loria, G. [1892] Bb. Mth. (1893) 39-.

- instruments of Count G. Suardi of Brescia. Gelcich, E. Z. Mth. Ps. 30 (1885) (H.-lt. Ab.) 1-.

- knowledge previous to science. Bobynin, V. V. Bb. Mth. (1889) 104-.

- papyrus in Greek language. Cantor, $M$. Z. Mth. Ps. 38 (1893) (H.-lt. Ab.) 81-.

- and physical disputations at Leipzig, 1512 26. Suter, $H$. Bb. Mth. (1889) 17-.

- - science, history (Günther). Favaro, $A$. Ven. I. At. 3 (1877) 913-.

- science in Russia, development. Bobynin, W. W. [V. V.]. Fschr. Mth. (1890) 3.

- sciences, progress of. Zegers, $J$. Chile A. Un. (1852) 35-

- - - among European nations. Bobynin, V. V. Bb. Mth. (1892) 110-.

- - , - since 1789. Delambre, J. B. J. Par. Mm. de l'I. (1808) (H.) 171-.

- theory of life assurance (historical). Samot, $D$. N. Arch. Wisk. 5 ( $\left.{ }^{*} 1879\right)$ 35-.

Mathematics, development from the most remote times to 15th century. Vashchenko-Zakharchenko, M. [1882] Bordeaux S. Sc. Mm.5 (1883) 259-.

-, historiography of. Cantor, - . [1900] N. Y. Am. Mth. S. Bll. 7 (1901) 57-。

-, history. Piani, D. (viri) Bologna Opusc. Sc. N. Col. (1824) 191-.

-, -. Eneström, G. (xII) Ts. Mth. 3 (1879) 113-, 161-.

- - - in antiquity, series of articles. Tannery, P. Bll. Sc. Mth. As. 8 (1884); Bll. Sc. Mth. 9 (1885); 10 (1886); 11 (1887).

-, - , applied to the solution and statement of some questions. Bobynin, W.W. [V.V.]. Fschr. Mth. (1892) 77.

- - - by Boncompagni. Chasles, M. C. R. 34 (1852) 889-.

-, - contribution to. Wappler, E. Z. Mth. Ps. 34 (1889) (Suppl.) 147-; 45 (1900) (H.-lt. Ab.) 7 -.

-, - for high-schools. Eneström, G. (xII) Ts. Mth. 4 (1880) 62-.

-, - , in Middle Ages, contributions to. Heiberg, J. L. Z. Mth. Ps. 35 (1890) (H.-lt. Ab.) $41-, 81-$.

-, - , a question of. Rubini, R. G. Mt. 17 (1879) 149-.

- - , recent researches. Gïnther, S. D. Nf. [B.] $\left.{ }^{*} 1877\right) 83$-.

-,$--\cdots$ Eneström, G. Bb. Mth. (1887) 
0010 History

Mathematics, \&c., history, proposed method of writing. Luca, $F$. de. Il Giamb. Vico 4 (1857) 451-.

-, present state of some branches. Smith, H. J. S. [1876] L. Mth. S. P. $8(1876-77)$ 6 -

-, use of history in teaching. Heppel, G. Nt. 48 (1893) 16-.

Matthew Stewart's theorem. Mackay, J.S. Edinb. Mth. S. P. 10 (1892) 90-.

Maurolico, Francesco, on Archimedes and Apollonius. Flauti, $V$. Nap. Rd. 5 (1856) 112-.

-, -, arithmetic, historical notes. Fontana, M. Bologna Mm. I. It. 2 (1808) 275-.

Meaning, traditional, of word "geometry." Zeuthen, H. G. Kjøb. Ov. (1893) 330-.

Mediæval translations of Euclid's elements. Curtze, M. Bb. Mth. (1896) 1-.

Middle ages, progressions in. Curtze, M. Bb. Mth. (1895) 113-.

Modern organization of mathematics. Galdeano, Z. G. de. Fschr. Mth. (1899) 68.

Möbius and Weiske, recollections of. Baltzer, R. Leip. Mth. Ps. B. 37 (1885) 1-.

Monge, Gaspard, life and works. Mercadier,- . Par. Mm. de l'I. 24 (1854) (H.) 1-; Rv. Sc. 1 (1894) 779-

Mortality tables before Halley, history. Eneström, G. Stockh. Öfv. (1896) 157-; Fschr. Mth. (1896) 187.

Moscow Math. Soc., historical sketch. Mlodzèevskij, B. K. [1894] Rec. Mth. (Moscou) 18 (1896) xxx-

Multiplication, abridged. Curtze, M. Z. Mth. Ps. 40 (1895) (H.-lt. Ab.) 7-.

Napier, John, biography and works. Gravelaar, N. L. W. A. Amst. Ak. Vh. (Sect. 1) 6 (1899) No. 6, 159 pp.

- - - inventor of logarithms. Biot, J. B. J. Sav. (1835) 354-; Con. des Temps (1838) 3-.

-, 一, review of memoirs on. Biot, J.B. J. Sav. (1835) 257-

Nasr ad-Din and Regiomontanus. Braunmiihl, A. von. Ac. Nt. C. N. Actr 71 (1898) 31-.

National mathematics, memorials. Iznoskov, I. Kazan S. Nt. (P8.-Mth.) P. 3 (1885) 88-.

Negative numbers, introduction. Fouche, $M$. N. A. Mth. 12 (1893) 164 .

Newton, (Sir) Isaac, Brewster's Life of. Biot, J. B. J. Sav. (1832) 193-, 263-

,,---- Memoirs of. Biot, J. B. J. Sav. (1855) 589-, 662-.

- and his correspondence with Cotes. Biot, J. B. J. Sav. (1852) 133-, 217-, 269-.

- Pascal, forged correspondence between. Chasles, $M$. C. R. 65-72 (1867-71) passim.

,---- Brewster, (Sir) D. C. R. 65 (1867) 261-, 537-, 653-, 717-, 769-, 770-, 825-, 925-.

- Chevreul, M. E. C. R. 69

-, portraits of. Crompton, S. [1866-67] Manch. Lt. Ph. S. P. 6 (1867) 1-; 7 (1868) 3-.

Newton's classification of cubic curves. Ball, $W . W . R$. L. Mth. S. P. 22 (1891) 104

\section{History 0010}

Newton's influence on modern geometry. Graham, R. H. Nt. 42 (1890) 139-.

- Principia, a section of, in relation to modern geometry. Taylor, C. Camb. Ph. S. P. 3 (1880) 359-.

- - singular passage in. Tait, P. G. Edinb. R. S. P. 13 (1886) 72-.

Nine-point circle, history. Mackay, J. S. Edinb. Mth. S. P. 11 (1893) 19-.

Nipsus, rule, and new theorem. Poinsot, $L$. C. R. 28 (1849) 581-.

Non-Euclidean geometry, brief historical sketch. Prosper, V. R. Fschr. Mth. (1893-94) 78.

- -, progress in. Halsted, G. B. Am. As. P. (1899) 53-

Notation, systems used by various races. Humboldt, F. H. A von. Crelle J. 4 (1829) 205-.

Numerals, earliest history. Cantor, M. (vx. Adds.) D. Nf. Vsm. B. 34 (1858) 135-.

-, transition from Roman to Arabic. Herford, B. Manch. Lt. Ph. S. P. 12 (1873) 91-.

Numeration of ancient Mexicans. Siméon, Mex. Arch. Com. Sc. 3 (1867) 523.

-, origin. Savary, A. Par. Tr. S. Amat. 2 (1808) 71-.

Oppermann and Steen. Zeuthen, H. G. N. Ts. Mth. 10 (A) (1899) 33-; Fschr. Mth. (1899) 80.

Optic and catoptric anamorphoses, history. Ruoss, H. Z. Mth. Ps. 39 (1894) (H.-lt. Ab.) 1 -.

Oresme, Nicole, works. Curtze, M. Bll. Sc. Mth. As. 6 (1874) 57-

Oriental and occidental mathematics, connection. Günther, S. Lpldina. 13 (1877) 38-.

- origin of $\pi=3$. Mahler, E. Z. Mth. Ps. 27 (1882) (H.-lt. Ab.) 207-

Painvin, L., works. Bll. Sc. Mth. As. 9 (1875) 188 .

Paoli, Pietro. Mod. Ac. Sc. Mm. 1 (1898) 105-.

Pappus and Kepler, relation between. Günther, S. Bb. Mth. (1888) 81-.

Papyri recently discovered, dealing with mathematics. Heiberg, J. L. Kjøb. Ov. (1900) $147-$.

Pascal denies authorship of certain papers presented to Academy. Breton (de Champ), - . C. R. 68 (1869) 710-, 862-, 969-.

- and Lalouvère. Tannery, $P$. Bordeaux $\mathrm{S}$. Sc. Mm. 5 (1890) 55-; 4 (1894) 251-.

Pascal's "Essais pour les coniques." Macdonald, W. J. Edinb. Mth. S. P. 2 (1884) 19 .

- letters, supposed. Lindelöf, L. L. [1867] Helsingf. Öfv. 10 (1868) 17-.

- Ms. notes. Faugère, - . C. R. 65 (1867) 202-, 340-, 455-, 643-, 702; 67 (1868) 497.

- - Bénard, - . C. R. 65 (1867) 203-

Persian work on mathematics and astronomy. Tytler, J. As. S. J. 4 (1837) 254.

Petrus de Dacia and his writings. Eneström, $G$. Stockh. Öfv. (1885) No. 3, 15-, No. 8, 65-; (1886) 57-; Fschr. Mth. (1885) 4 -

Pitiscus: "Thesaurus mathematicus." Burckhardt, $F$. [1868] Basel Vh. 5 (1873) 159-.

Pitiscus' "Trigonometria" (A.D. 1595), account of. Gravelaar, N.L.W.A. N. Arch. Wisk. 3 (1898) 253-; Fschr. Mth. (1898) 32-. 


\section{History}

Plane curves, history of investigation on. Loria, G. Wiad. Mt. 2 (1898) 203-.

- Roberval's method. Geer, P. van. N. Arch. Wisk. 11 (1884) 28-; Fschr. Ps. (1884) (Ab. 1) 217-.

Platonic number. Günther, S. Lpldina. 18 (1882) 149-.

Pliny, remarks on passage in. Günther, $S$. Arch. Mth. Ps. 55 (1873) 147-.

Plücker, works. Bertrand, J. J. Sav. (1867) 269-.

Polish mathematical literature, 1873-92. Dikstein, S. Kosmos (Lw.) 20 (1895) 352-.

Polyhedra and numerals, history. Lindemann, F. Münch. Ak. Sb. 26 (1897) 625-.

Popular account of new fields of mathematics. Miller, G. A. Science 11 (1900) 528-.

Porism, old. Davies, T. S. Mathematician 3 (1850) 75-, 140-, 225-, 311-; (Suppl.) 42-.

Poristic triangles and polygons, historical notes. Mackay, J. S. Edinb. Mth. S. P. 5 (1887) 62-

"Practica Geometriæ" (12th century tract). Curtze, M. Mh. Mth. Ps. 8 (1897) 193-; 9 (1898) 266-.

Problem by V. Viviani. Geoghegan, $E$. Nt. 38 (1888) 78.

Problems on numbers from Fermat, some. Pepin, T. Rm. N. Linc. Mm. 8 (1892) 85-.

-, some inverted (an old arithmetic book). C., R. A. Educ. Times 48 (1895) 29-.

Projectile curve, discovery of parabolic form. Wohlwill, E. Z. Mth. Ps. 44 (1899) (Suppl.) $577-$.

Properties of figure composed of a triangle and the squares on its sides. Mackay, J.S. Edinb. Mth. S. P. 6 (1888) 2 -.

Prosthaphæresis method in trigonometry, history. Braunmühl, A. von. Z. Mth. Ps. 44 (1899) (Suppl.) 15-.

Quadratic irrationalities, approximate calculation, history. Hunrath, $K$. Z. Mth. Ps. 33 (1888) (H.-lt. Ab.) 1-.

- residues, historical account of law of reciprocity. Baumgart, O. Z. Mth. Ps. 30 (1885) (H.-lt. Ab.) 169-, 241-.

- - - - study. Pepin, T. Rm. N. Linc. Mm. 16 (1899) 229-.

Quadratum geometricum, anonymous work on. Curtze, M. Z. Mth. Ps. 40 (1895) (H.-lt. Ab.) 161-.

Quadratures before integral calculus, especially Fermat's. Zeuthen, H. G. Kjøb. Ov. (1895) 37-.

Quaternions, Gauss' claim to invention of. Tait, P. G. [1899] Edinb. R. S. P. $23(1902)$ 17-.

- Klein's view of. Knott, C. G. [1899] Edinb. R. S. P. 23 (1902) 24 .

Quintic equation, history of theory. Pierpont, $J$. Mh. Mth. Ps. 6 (1895) 15-.

Rabbis, irrationalities. Mahler, $E$. Z. Mth. Ps. 29 (1884) (H.-lt. Ab.) 41-.

Raoul de Laon, arithmetical treatise. Nagl, $A$. Z. Mth. Ps. 34 (1889) (Suppl.) 85-

Rates of interest in ancient and modern times. Hodge, W. B. Assur. Mg. 6 (*1857) 301-; 7 ( $\left.{ }^{*} 1858\right) 311-; 8\left({ }^{*} 1860\right) 68-; 9\left({ }^{*} 1861\right) 61-$.
History 0010

Reckoning of chances (reproduction of 16 th century work by Benedict Spinoza). Bierens de Haan, D. N. Arch. Wisk. 11 (1884) 75-.

Rheticus, "Canon Doctrinæ Triangulorum" and Vieta's "Canon Mathematicus." Hunrath, K. Z. Mth. Ps. 44 (1899) (Suppl.) 211-.

Rhind papyrus, interpretation. Favaro, $A$. Mod. Ac. Sc. Mm. 19 (1879) 89-.

- - on reducing $\frac{2}{2 n+1}$ to fractions with numerator 1. Bobynin, V. $V$. Bb. Mth. (1890) 109-.

- -, a table in the. Mansion, - Brux. S. Sc. A. 12 (1888) (Pt. 1) 44-.

Rhythmomachy. Wappler, E. Z. Mth. Ps. 37 (1892) (H.-lt. Ab.) 1-.

Richelot's posthumous papers, extract. Königsberger, $L$. Rpm. Mth. 1 (1877) 191-, 340-.

Riemann and his significance for modern mathematics. Klein, $F$. D. Nf. Vh. (1894) (Th. 1) 57-.

Robertus (? Johannes) Anglicus and his quadrant. Steinschneider, M. Bb. Mth. (1896) 102-.

(1897) 3-.

_ - - "Tractatus Quadrantis," Germ. transl., 1477. Curtze, $M$. Z. Mth. Ps. 44 (1899) (Suppl.) 41-; 45 (1900) (H.-lt.Ab.) 41-.

Ruffini, Paolo, and the theory of groups. Burkhardt, H. Z. Mth. Ps. 37 (1892) (Suppl.) 119-.

Russia, mathematical teaching, history. Bobynin, $V . V$. Ens. Mth. 1 (1899) 77-.

-, sketch of development of physico-mathematical sciences in. Bobynin, $V . V$. [1886] Fschr. Mth. (1887) 2.

Saccheri, a precursor of Legendre and Lobačevskij. Beltrami, E. Rm. R. Ac. Linc. Rd. 5 (1889) (Sem. 1) 441-.

- - - - Lobačevskij. Vasiljev, A. V. Kazan S. Ps.-Mth. Bll. 3 (1893) (Prot.) 53-. - researches on postulate of parallels. Mansion, P. Brux. S. Sc. A. 14 (1890) (Pt. 2) 46-.

Scheubel, Johannes, German algebraist of 16 th century. Staigmuiller, $H$. Z. Mth. Ps. 44 (1899) (Suppl.) 429-.

Schläfli (Ludwig) and Steiner (Jakob), correspondence between. Graf, $J . H$. Bern Mt. (1896) 61-.

"Seqt," so-called, of Egyptian mathematicians. Cantor, $M$. Wien Ak. Sb. 90 (1885) (Ab. 2) 475-.

Signs of arithmetical operations, origin of some. Le Paige, C. Brux. S. Sc. A. $16(1892)$ (Pt. 2) 70-.

-, + and -, history. De Morgan, A. [1864] Camb. Ph. S. T. 11 (Pt. 1) (1866) 203-.

-, - - - - Clifton, R. B. [1865] Camb. Ph. S. T. 11 (Pt. 1) (1866) 213-.

- _ - invention, and use by Leonardo da Vinci. De Morgan, A. (vi. Adds.) Ph. Mg. 20 (1842) 135-, 230-.

- + and -, use to denote "plus" and " minus." Eneström, G. Stockh. Öfv. (1894) 243-; Fschr. Mth. (1893-94) 59-. 


\section{History}

Simson line, historical note on so-called. Muir, T. Edinb. Mth. S. P. 3 (1885) 104 .

"Sinus," origin of word. Ruska, J. Z. Mth. Ps. 40 (1895) (H.-lt. Ab.) 126-.

Sluse, Réné Francois de, geometrician of 17th century. Le Paige, $C$. Ciel et Terre 7 (1886-87) 536-, 570-.

-, - - letter. Marre, A. Lisb. J. Sc. Mth. 10 (1885) 81-.

Snellius, problem of. Verdam, G.J. Arch. Mth. Ps. 2 (1842) 210-.

Solution of cubic, claims of Cardan and Tartaglia. Zeuthen, H. G. Kjøb. Ov. (1893) 303-.

Sovero, Bartolomeo, mathematician of 17th century. Favaro, -. Rv. Sc. 33 (1884) 502.

Spain, history of mathematics, during 16th century. Eneström, G. Bb. Mth. (1894) 33-.

-, mathematics in. Galdeano,Z.G.de. Ens. Mth. 1 (1899) 6-.

Spanish writings of 16 th and 17th centuries, certain. Vicuña, G. Bb. Mth. (1890) 33-.

Spherical trigonometry formulæ, history. Todhunter, I. Ph. Mg. 45 (1873) 98-.

- - historical sketch. Poréckij, P.S. Kazan S. Nt. (Ps.-Mth.) P. 5 (1887) 152-.

Square root of quadrinomial surd. Bierens de Haan, D. N. Arch. Wisk. 3 (* 1877) 208-.

Steiner (Jakob) and Schläfli (Ludwig), correspondence between. Graf, J. H. Bern Mt. (1896) 61-.

Stereographic projection, history. Günther, - . D. Nf. Tbl. (1887) 227-.

Stevin, Spinoza and Girard, certain works of. Eneström, G. Stockh. Öfv. (1884) No. 9, 191-; Fschr. Mth. (1884) 16-.

Styrian mathematicians before Kepler. Peinlich, R. Arch. Mth. Ps. 54 (1872) 470-

Summation of series of cubes (passages from Mss.). Woepcke, F. (vIII) A. Mt. 5 (1863) 147-; Liouv. J. Mth. 9 (1864) 337-; 10 (1865) 83-.

Sweden, history of arithmetic. Hultman, F.W. Ts. Mt. Fys. 1 (1868) 1-, 53-, 149-, 245-; 2 (1869) 57-, 105-; 3 (1870) 7-, 49-, 241-; 4 (1871) 5-, 97-, 209-.

-, - - mathematical sciences before 1679 . Dahlin, E. M. Ups. Årsk. (1875) iv + $146 \mathrm{pp}$.

-, - - mathematics up to 1600 . Eneström, G. Stockh. Öfy. (1889) 489-; Fschr. Mth. (1889) 3-.

-, mathematics, Peder Månsson, three small treatises. Eneström, G. Bb. Mth. (1888) 17-.

Swedenborg, Emanuel, as a mathematician. Eneström, G. [1889] Stockh. Ak. Hndl. Bh. 15 (Afd. 1) (1890) No. 12, 29 pp.; Fschr. Mth. (1890) 20.

Swedenborg's mathematical works. Eneström, G. Stockh. Öfv. (1889) 529-.

Switzerland, history of mathematics. Wolf, R. Bern Mt. (1845) 121-, 137-; (1846) 161-, 209-; (1847) 101-, 129-, 161-; (1848) 46-, 217-, 269-.
History 0010

Switzerland, history of mathematics and physics. Wolf, R. Bern Mt. (1847) 68-.

Symbols, origin. Davis, H. S. Pop. As. 5 (1898) 402-.

Symmedian point, early history. Mackay, J.S. Edinb. Mth. S. P. 11 (1893) 92-.

"Ta Yen," so-called rule, in Europe. Curtze, M. Z. Mth. Ps. 41 (1896) (H.-lt. Ab.) 81-.

Talmud, mathematics. Mahler, E. Z. Mth. Ps. 31 (1886) (H.-lt. Ab.) 121-.

- as source for mathematical history. Mahler, -. D. Nf. Tbl. (*1882) 125-.

Taurinus, Franz Adolph. Contribution to the previous history of non-Euclidian geometry. Stäckel, P. Z. Mth. Ps. 44 (1899) (Suppl.) 399-.

Terminology of the oldest mathematical writings in the German language. Muiller, $F$. Z . Mth. Ps. 44 (1899) (Suppl.) 301-.

Tests for divisibility of numbers (historical). Fontés, - . Toul. Ac. Sc. Mm. 5 (1893) 459 .

“Thabit Ben Korra," Steinschneider, remarks. Curtze, M. (vII) [1873] Z. Mth. Ps. 19 (1874) 95-.

Theory of numbers in XV. century. Curtze, $M$. Bb. Mth. (1895) 37-.

- - - Fermat's contention with Wallis. Wertheim, G. Z. Mth. Ps. 44 (1899) (Suppl.) 555-.

- - - origin and development. Studnička, F. J. Časopis 4 (* 1874) 1-.

Torricelli and first rectification of a curve. Loria, G. Rm. R. Ac. Linc. Rd. 6 (1897) (Sem. 2) 318-.

---1 Bordiga, G. Rm. R. Ac. Linc. Rd. 7 (1898) (Sem. 1) 28 -

Towarzystwo Nauk Ścisłych (Society of the exact sciences) in Paris, 1870-82, origin and development. Folkierski, $W$. Prace Mt.Fiz. 6 (1895) 151-; Fschr. Mth. (1895) 43.

"Tractatus de Abaco," end of 12th or beginning of 13 th century. Curtze, M. Z. Mth. Ps. 43 (1898) (H.-lt. Ab.) 122-.

Trapezium, Brahmegupta's. Zeuthen, H. G. (xII) Ts. Mth. 6 (1876) 168-, 181-; 6 (1882) 97-; 1 (1883) 145-.

- in Euclid, Hero, and Brahmegupta. Weissenborn, H. Z. Mth. Ps. 24 (1879) (Suppl.) 167-, 185-

Trigonometrical canon, earliest. De Morgan, $A$. As. S. M. Not. 6 (1843-45) 221-.

Trigonometry in antiquity. Ideler, C. L. Zach M. Cor. 26 (1812) 3-.

- - Zeuthen, H. G. Bb. Mth. 1 (1900) 20 -

- Christian middle ages. Curtze, M. $\mathrm{Bb}$. Mth. 1 (1900) 321-.

-, history. Braunmühl, A. von. Ac. Nt. C. N. Acta 71 (1898) 1-.

Trisection of angle, classification of solutions. Baker, M. Smiths. Misc. Col. 33 (1888) Art. 4, 96-. (Wash. Ph. S. Bll. 10 (1888).)

Trochoids, history. Hoza, $F$. Casopis $1\left(^{*} 1872\right)$ 54 .

Twilight, problem of the shortest. Zelbr, K. Z. Mth. Ps. 41 (1896) (H.-lt. Ab.) 121-, 153 - 


\section{Biography}

Tycho Brahe's table of sines. Studnička, F.J. Prag Sb. (1899) (Mth.-Nt.) No. 39, 4 pp.

Unverzagt's linear coordinates. Rudio, $F$. Z. Mth. Ps. 44 (1899) (Suppl.) 383-.

Vandermonde, Charles Auguste. Lacépède, B. G. Ḱ. (le comte de). Par. Mm. de l'I. 1 (1798) (H.) xix-.

- Christian names. Simon, $H$. Z. Mth. Ps. 41 (1896) (H.-lt. Ab.) 83-.

Viete, Francois, discoveries. (Quadrature of circle, \&c.) Ritter, F. As. Fr. C. R. (1879) 143-.

-, , inventor of modern algebra. Ritter, $F$. As. Fr. C. R. (1892) (Pt. 2) 17-.

-, -, new algebra. Ritter, $F$. As. Fr. C. R. (1892) (Pt. 2) 177-.

-, - trigonometry. Ritter, F. As. Fr. C. R. (1892) (Pt. 2) 208-

Wallis's investigation of expression for $\pi$. Cayley, A. QJ. Mth. 23 (1889) 165-.

Wargentin, P. W., and the so-called Halley method in mathematical statistics. Eneström, G. Z. Mth. Ps. 44 (1899) (Suppl.) 81-.

Weierstrass, his significance for pure mathematics. Vasiljev, A. V. Kazan S. Nt. (Ps.Mth.) P. 4 (1886) 21-.

-, mathematical work. Poincaré, $H$. Acta Mth. 22 (1899) 3-

Wronski, mathematical discoveries. Dickstein, S. Bb. Mth. (1892) 48-, 85-; (1893) 9-; (1894) 49-, 85-; (1896) 5-.

Ziegler's commentary on "Saphea" of Zarkali. Eneström, G. Bb. Mth. (1896) 53-.

\section{BIOGRAPHY.}

Abel, Niels Henrik. Mon. Sc. 12 (1870) 660-; Bordeaux S. Sc. Mm. 1 (1884) 368 pp.

Abria, Jérémie Joseph Benoit. Bordeaux S. Sc. Mm. 3 (1893) 301-.

Adams, John Couch. Am. J. Sc. 43 (1892) 248; As. J. 11 (1892) 112 ; Educ. Times 45 (1892) 70-; Eng. S. T. (1892) 251-; Lpldina. 28 (1892) 56; L. Ps. S. P. 11 (1892) (Ann. Meet.) 11-; Manch. Lt. Ph. S. Mm. \& P. 5 (1892) 186-; Nt. 45 (1892) 301-; Obs. 15 (1892) 173-; Am. Ac. P. 27 (1893) 444-; As. S. M. Not. 53 (1893) 184-, [348]; Edinb. R. S. P. 20 (1895) i-.

Airy, (Sir) George Biddell. Am. J. Sc. 43 (1892) 248 ; A. Ps. C. (Berl. Ps. Gs. Vh. 11) 45 (1892) 601-; As. J. 11 (1892) 96, 168; As. Nr. 129 (1892) 33-; As. S. M. Not. 52 (1892) 212-; C.R. 114 (1892) 91-; 115 (1892) 1117-; Eng. S. T. (1892) 244-; I. CE. P. 108 (1892) 391-; Lpldina. 28 (1892) 54 ; Manch. Lt. Ph. S. Mm. \& P. 5 (1892) 185-; N. S. W. R. S. J. 26 (1892) 2-; Nt. 45 (1892) 232-; Obs. 15 (1892) 74-; R. S. P. 51 (1892) i-; Science 19 (1892) 64-; Am. Ac. P. 27 (1893) 446-; Edinb. R. S. P. 19 (1893) i-; Kazan S. Ps.Mth. Bll. 2 (1893) (Prot.) 24-; Met. S. QJ. 19 (1893) 97.

Albeggiani, Giuseppe. $\quad$ Palermo Cir. Mt. Rd. 7 (1893) 39-

Alberti, Leon Battista. Bb. Mth. (1895) 9-.

\section{Biography 0010}

Alvord, (Gen.) Benjamin. Smiths. Misc. Col. 33 (1888) Art. 2, 127-. (Wash. Ph. S. Bll. 7 (1885).)

Ameseder, Adolf. Mh. Mth. Ps. 2 (1891) 479.

Ampère, André Marie. Smiths. Rp. (1872) 111-; Amiens Ac. Mm. 7 (1881) 261-; A. Tél. 16 (1889) 84-.

Audouard, Étienne Prosper. Brest S. Ac. Bll. 20 (1895) 475-.

August, Friedrich Wilhelm Oscar. L Lldina. 36 (1900) 46-.

Avery, Robert Stanton. Wash. Ph. S. Bll. 12 (1895) 435-.

Azzarelli, Mattia. Rm. N. Linc. At. 51 (1897) 49 -

Babbage, Charles. As. S. M. Not. 32 (1872) 101-; Devon. As. T. 5 (1872) 30-; Am. Ac. P. 8 (1873) 455-; Smiths. Rp. (1873) 162-.

Babinet, Jacques. Rv. Cours. Sc. 3 (1872) 409-; Rv. Sc.-Ind. 4 (1872) 294.

Bach, - . Nancy S. Sc. Bll. (1885) xxxi-.

Badon-Ghijben, Jacob. Amst. Jb. Ak. (1873)20-

Baehr, George Frederik Willem. Amst. Ak. Vs. 7 (1899) 131-.

Battaglini, Giuseppe. Ven. I. At. (1893-94) 1419-; G. Mt. 32 (1894) 205-; Nap. Rd. 33 (1894) 49-; Palermo Cir. Mt. Rd. 8 (1894) 180-; Rm. R. Ac. Linc. Mm. 1 (1894) 558665 ; Rv. Mt. 4 (1894) 91-; Tor. Ac. Sc. At. 29 (1894) 678-.

Bauernfeind, Carl Maximilian von. Lpldina. 30 (1894) 161 ; 31 (1895) 62-, 78-, 94-; Förster Al. Bauztg. 60 (1895) 31-; Münch. Ak. Sb. 25 (1896) 161-.

Bellavitis, Giusto. Bll. Sc. Mth. As. 4 (1880) 343-; Rm. R. Ac. Linc. T. 5 (1881) 15-; Z. Mth. Ps. 26 (1881) (H.-lt. Ab.) 153-; Ven. I. At. 8 (1882) 395-; Nap. Ac. Pont. At. 15 1883 (Pt. 1) 5-; Rm. S. It. Mm. 6 (1887) Ixxiii-.

Beltrami, Eugenio. Nt. 61 (1899-1900) 568-; Ven. I. At. $(1899-1900)$ (Pt. 1) 72-; A. Mt. 4 (1900) 151-; Bologna Rd. 4 (1900) 91-; C. R. 130 (1900) 677-; 131 (1900) 1037-; Ens. Mth. 2 (1900) 173-; G. Mt. 38 (1900) 355-; Mil. I. Lomb. Rd. 33 (1900) 241-; Nap. Rd. 39 (1900) 74-; Palermo Cir. Mt. Rd. 14 (1900) 275-; Rm. R. Ac. Linc. Rd. 9 (1900) (Sem. 1) 139-; Tor. Ac. Sc. At. 35 (1900) 355- or 541-; Wiad. Mt. 4 (1900) 266-; Kazan S. Ps.-Mth. Bll. 10 (1901) (Prot.) 32-; Münch. Ak. Sb. 30 (1901) 345-

Berg, Franciscus Johannes van den. 's Gravenh. I. Ing. Ts. (1891-92) (Verg.) 133; N. Arch. Wisk. 1 (1895) 1-; Amst. Ak. Jb. (1897) 97-.

Bertrand, Joseph Louis François. Nt. 61 (1899 1900) 614-; C. R. 130 (1900) 961-; 131 (1900) 1033-; G. Mt. 38 (1900) 171-; J. Sav. (1900) 257-, 312-; Lpldina. 36 (1900) 129-; Science 11 (1900) 637; St Pét. Ac. Sc. Bll. 12 (1900) xxxiv; Wiad. Mt. 4 (1900) 267-.

Betti, Enrico. Nap. Rd. 31 (1892) 143-; N. Cim. 32 (1892) 5-; Palermo Cir. Mt. Rd. 6 (1892) 245-; Rv. Mt. 2 (1892) 151-; A. Mt. 20 (1892-93) 256 ; Ven. I. At. (1892-93) 609-.

Biervliet, Albert Marie Joseph van. Brux. S. Sc. A. 15 (1891) (Pt. 1) 86.
B 


\section{Biography}

Björling, Emanuel Gabriel. Stockh. Vt. Ak. Lefn. 2 (1878-85) 89-.

Bobek, Karl. Mh. Mth. Ps. 11 (1900) 97-.

Boltshauser, Giovanni Adamo. Sav. Ac. Mm. 6 (1897) lxxxiii-.

Bolyai de Bolya, Johann. Bordeaux Mm. S. Sc. 5 (1867) 203-; Z. Mth. Ps. 42 (1897) (Suppl.) 133-.

Bolyai de Bolya, (Farkas) Wolfgang. Bordeaux Mm. S. Sc. 5 (1867) 192-; Mag. Tud. Ak. Étk. (Mth.) 11 (1885) No. 9, 36 pp.

Bolzani, Josef Fortunatus. Kazan Un. Bll. 12 (1876) 208-.

Boncompagni, (il principe) Baldassarre. $\mathrm{Rm}$. N. Linc. At. 47 (1894) 161-; Z. Mth. Ps. 39 (1894) (H.-lt. Ab.) 201-.

Bonnaire, Antoine François Donat. Caen Ac. Mm. (1891) (Pt. 2) 95, 95-.

Bonnet, Pierre Ossian. C. R. 115 (1892) 1115-; 117 (1893) 1014-; Lpldina. 28 (1892) 157.

Boole, George. $\quad$ R. S. P. 15 (1867) vi-; Leic. S. T. (1835-79) 272-.

Booth, (Rev.) James. Educ. Times 31 (1878) 126-.

Boschi, Pietro. Bologna Rd. (1887-88) 10-

Bouquet, Jean Claude. Bll. Sc. Mth. 9 (1885) 301-; C. R. 101 (1885) 585-; 102 (1886) 1267-; N. Antol. Sc. 83 (1885) 404.

Bour, Jacques Edmond Émile. Par. S. Phlm. Bll. 3 (1866) 119-; N. A. Mth. 6 (1867) 145-; Rec. Mth. (Moscou) 2 (1867) (Pt. 2) 44-.

Brassai, Samuel. Mag. Tud. Ak. Ets. 10 (1899) 276-.

Brassinne, Philippe Émile. Toul. Ac. Sc. Mm. 7 (Sem. 2) (1885) 25-.

Bravais, Auguste. Par. Ac. Sc. Mm. 35 (1866) xxiii-; Smiths. Rp. (1869) 145-.

Breton, Philippe. Isère S. Bll. 27 (1892) 391-.

Bretschneider, Carl Anton. Z. Mth. Ps. 24 (1879) (H.-lt. Ab.) 73-.

Brianchon, Charles Julien. Rv. Sc. 1 (1894) 592 .

Brioschi, Francesco. A. Mt. 26 (1897) 343-; C. R. 125 (1897) 1139-; Rm. R. Ac. Linc. Rd. 6 (1897) (Sem. 2) 353-; Nt. 57 (1897-98) 279 ; Crelle J. Mth. 119 (1898) 259; G. Mt. 36 (1898) 51-; L. Mth. S. P. 29 (1898) 721-; Manch. Lt. Ph. S. Mm. \& P. 42 (1898) xxxix; Mth. A. 50 (1898) 477-; N. Antol. Sc. 157 (1898) 170-; Nap. Rd. 37 (1898) 3-; Rv. Sc.-Ind. 30 (1898) 28; St. Pét. Ac. Sc. Bll. 8 (1898) xxvii-; Wiad. Mt. 2 (1898) 66-; Wien. Alm. 48 (1898) 332-; Mil. I. Lomb. Rd. 32 (1899) 108-; Münch. Ak. Sb. 28 (1899) 449-; Mathesis 20 (1900) 112-; Palermo Cir. Mt. Rd. 14 (1900) 262-.

Brisse, Charles Michel. J. de Ps. 7 (1898) [637] ; Nt. 59 (1898-99) 80 ; N. Y. Am. Mth. S. Bll. 5 (1899) 211-.

Broch, Ole Jacob. Christiania F. (1889) (Ov.) 12-; Nt. 39 (1889) 375 ; Rv. Sc. 43 (1889) 218.

Bubendey, Georg Heinrich (sen.). Lpldina. 25 (1889) 215.

Buchheim, Arthur. Nt. 38 (1888) 515-.

Buka, Felix. Lpldina. 33 (1897) 49 ; D. Mth. Vr. Jbr. 6 (1899) (Heft 1) 23-.

\section{Biography 0010}

Buniakovskij, Viktor Jakovlevič. Lpldina. 25 (1889) 219 ; Fschr. Mth. (1890) 30; Kharkov Mth. S. Com. 2 (1891) 149-.

Buys-Ballot, Christoph Heinrich Diederich. Brux. Ac. Bll. 19 (1890) 180-; Lpldina. 26 (1890) 58 ; Manch. Lt. Ph. S. Mm. \& P. 3 (1890) 167-; Nt. 41 (1890) 371; Met. S. QJ. 17 (1891) 61-; Met. Z. 8 (1891) 1-; Sym. Met. Mg. 25 (1891) 8; Amst. Ak. Jb. (1899) 59 -.

Casey, John. L. Mth. S. P. 22 (1891) 477-; Lpldina. 27 (1891) 155; Mathesis 11 (1891) 13 ; R. S. P. 49 (1891) xxiv-.

Casorati, Felice. Bb. Mth. (1891) 1-; Lpldina. 27 (1891) 52 ; Palermo Cir. Mt. Rd. 5 (1891) 236-; Mil. I. Lomb. Rd. 25 (1892) 1206-.

Catalan, Eugène Charles. Nt. 49 (1893-94) 437; Brux. Ac. Bll. 27 (1894) 326-, 330-; Lpldina. 30 (1894) 112 ; Mathesis 14 (1894) 33 ; Kazan S. Ps.-Mth. Bll. 4 (1895) (Prot.) 84.

-, - - , letters to some mathematicians. Liège S. Sc. Mm. 17 (1892) No. 5, 22 pp.; 18 (1895) No. 5, 36 pp.

_, _ - mathematical work. Liège $\mathrm{S}$. Sc. Mm. 12 (1885) No. 1, 38 pp.

Cauchy, (le baron) Augustin Louis. J. Sav. (1869) 205-; Rv. Sc. 9 (1898) 97-.

Cayley, Arthur. J. H. Un. Cir. [14 (1894-95)] 51 ; Nt. 51 (1894-95) 323 ; Bll. Sc. Mth. 19 (1895) 189-; C. R. 120 (1895) 233-; Crelle J. Mth. 115 (1895) 349-; L. Mth. S. P. 26 (1895) 546-; Manch. Lt. Ph. S. Mm. \& P. 9 (1895) 235-; Mth. A. 46 (1895) 462-; N. Y. Am. Mth. S. Bll. 1 (1895) 133-; Obs. 18 (1895) 112-; Rm. R. Ac. Linc. Rd. 4, 1895 (Sem. 1) 177-; R. S. P. 58 (1895) i-; Rv. Sc. 3 (1895) 188; As. S. M. Not. 56 (1896) 191-; Kazan S. Ps.-Mth. Bll. 5 (1896) (Prot.) 29-.

Čebyšev (Tchebichef), Pafnutij Lvović. C. R. 119 (1894) 1048-; Kharkov Mth. S. Com. 4 (1895) 263-; Lpldina. 31 (1895) 55; Nt. 52 (1895) 345 ; Science 1 (1895) 129-; St Pét. Ac. Sc. Bll. 2 (1895) x-, 189-; Fschr. Mth. (1899) 15-; Mathesis 20 (1900) 67-.

Cellérier, Charles. Gen. S. Ps. Mm. 31 (189093) xviii-.

Challis, James. As. S. M. Not. 43 (1883) 160-; Obs. 6 (1883) 23.

Charles, Jacques Alexandre César. Par. Mm. Ac. Sc. 8 (1829) (H.) 73-.

Chasles, Michel. Bologna Rd. (1881) 37-; R. S. P. 32 (1881) i-; Kharkov Mth. S. Com. (1881) 23-; Kosmos (Lw.) 6 (1881) 555-; Rv. Sc. 50 (1892) 801-.

Chelini, Domenico. Bll. Sc. Mth. As. 3 (1879) 228-; Rm. N. Linc. At. 32 (1879) 152-; Rm. R. Ac. Linc. T. 3 (1879) 54-; Rm. S. It. Mm. 6 (1887) lviii-.

Chiò, Felice. Rv. Sc.-Ind. 3 (1872) 61.

Clebsch, Rudolf Friedrich Alfred. Gött. Nr. (1872) 550-; Mil. I. Lomb. Rd. 5 (1872) 1041-; Ts. Mth. 2 (1872) 190-; G. Mt. 11 (1873) 44-; 12 (1874) 29-; Mth. A. 6 (1873) 197-; 7 (1874) 1-; A. Mt. 6 (1875) 153-.

Close, (Maj.-Gen.) Frederick. L. Mth. S. P. $31(1900) 488$ -

Coakley, George W. Am. J. Sc. 46 (1893) 484; Lpldina. 29 (1893) 204; Nt. 48 (1893) 398. 


\section{Biography}

Cockle, (Sir) James. As. S. M. Not. 55 (1895) 192 ; L. Mth. S. P. 26 (1895) 551'-; Manch. Lt. Ph. S. Mm. \& P. 9 (1895) 215-; R. S. P. 59 (1896) xxx-.

Coffin, John Huntington Crane. Am. Ac. P. 25 (1890) 312-; Am. J. Sc. 39 (1890) 246.

Correnti, Cesare. Firenze Ac. Georg. At. 11 (1888) xxxix-.

Cotterill, Thomas. L. Mth. S. P. 12 (1880-81) 217-.

Craig, Thomas. J. H. Un. Cir. [19 (18991900)] 67.

Dalgas, Gustavo. Firenze Ac. Georg. At. 11 (1888) xxxv-

Dauge, Félix. Mathesis 19 (1899) 177-.

David, Claude Alexandre. Lille S. Mm. 1 (1865) 452-.

David, Jean Marie. Toul. Ac. Sc. Mm. 2 (1890) 528 -

Davidov, Avgust Julievič. Rec. Mth. (Moscou) 15 [1891] 1-; Fschr. Mth. (1890) 29.

Davies, Griffith. Assur. Mg. 5 (1855) 337-.

Decrue, David. Sch. Nf. Gs. Vh. (1892) 208-.

De la Gournerie, Jules Antoine René Maillard. Par. Ac. Sc. Mm. 44 (1888) cxxxvii-; A. Cons. Arts et Mét. 8 (1896) 23-.

Delsaulx, (le rév. père) Joseph. Brux. S. Sc. A. 15 (1891) (Pt. 1) 86-

De Morgan, Augustus. Ts. Mth. 1 (1871) 94-; As. S. M. Not. 32 (1872) 112-.

Despeyrous, Théodore. Toul. Ac. Sc. Mm. 7 (Sem. 2) (1885) 100-.

Dienger, Josef. Crelle J. Mth. 115 (1895) 350.

Dodgson, (Rev.) Charles Lutwidge (Pseudonym: Lewis Carroll). Nt. 57 (1897-98) 279-, 303; N. Y. Am. Mth. S. Bll. 4 (1898) 241.

Dodson, James. I. Act. J. 14 (1869) 341-.

Drechsler, Hermann Adolf. Dresden Isis Sb. (1888) 43 ; Lpldina. 24 (1888) 171 ; 26 (1890) $193-$.

Drobisch, Moritz Wilhelm. Leip. Mth. Ps. B. 48 (1896) 697-

Du Bois-Reymond, Paul. Crelle J. Mth. 104 (1889) 352-; Lpldina. 25 (1889) 112; Mth. A. 35 (1890) 457-; Münch. Ak. Sb. 20 (1891) 415-.

Duhamel, Jean Marie Constant. Ts. Mth. 2 (1872) 143-.

Dupin, (le baron) Pierre Charles Francois. A. Cons. Arts et Mét. 10 (1873) 233-; Ts. Mth. 3 (1873) 64; Par. Ac. Sc. Mm. 44 (1888) i-.

Durège, Heinrich. Lpldina. 29 (1893) 110 ; Wien Alm. 43 (1893) 264-

Eisenstein, Gotthold. Z. Mth. Ps. 40 (1895) (Suppl.) 143-.

Elliott, Ezekiel Brown. Science 11 (1888) 261 ; Wash. Ph. S. Bll. 11 (1892) 470-

Ellis (Sharpe), Alexander John. L. Mth. S. P. 21 (1891) 457-; Nt. 43 (1891) 20 ; R. S. P. 49 (1891) i-.

Euler, Leonhard. Basel Vh. 7 (1885) (Anhang) 39-; Barcel. Ac. Mm. 1 (1892-1900) 241-.

Fambri, Paulo. N. Antol. Sc. 153 (1897) 131-.

Fedorenko, Ivan. As. Nr. 120 (1889) 319-; Lpldina. 25 (1889) 52.

Ferroni, Pietro. Firenze Ac. Georg. At. 7 (1830) 33-.

\section{Biography 0010}

Fink, Karl. Lpldina. 24 (1888) 58; D. Mth. Vr. Jbr. 7 (1899) (Heft 1) 33-.

Finlaison, John. Assur. Mg. 10 (1863) 147-.

Fleck, (l'abbé) Joseph Martin. Metz Ac. Mm. 79 (1900) 193-.

Fowler, (Sir) Robert Nicholas. L. Mth. S. P. 22 (1891) 476-.

Frost, (Rev.) Percival. L. Mth. S. P. 29 (1898) 726-; Nt. 58 (1898) 131 ; N. Y. Am. Mth. S. Bll. 4 (1898) 557; Science 7 (1898) 858 ; R. S. P. 64 (1899) vii-.

Gabba, Alberto. Brescia At. Cm. (1876) 100 .

Gabelli, Pasquale. Ven. Aten. 1 (1886) 72-, 209-.

Gadolin, Axel Wilhelm. Nt. 47 (1892-93) 232 ; Helsingf. Acta 19 (1893) No. 18, 24 pp.

Galois, Évariste. Par. Éc. Norm. A. 13 (1896) 197-.

Gascheau, Gabriel. Toul. Ac. Sc. Mm. 6 (Sem. 2) (1884) 17-.

Gascó, Luis Gonzaga. Bb. Mth. 1 (1900) 225-; D. Mth. Vr. Jbr. 8 (1900) (Heft 1) 26-.

Gaskin, (Rev.) Thomas. As. S. M. Not. 48 (1888) 161-; R. S. P. 46 (1890) i-

Gasparis, Annibale de. Acireale Ac. At. 3 (1891) viii; As. Nr. 129 (1892) 247-; Nap. Ac. Pont. At. 22 (1892) (Append.) 37-; 28 (1898) No. 1, 7 pp.; Nap. I. Inc. At. 5 (1892) 9; Nap. Rd. 31 (1892) 65-; Nt. 45 (1892) 612 ; Obs. 15 (1892) 231-; As. S. M. Not. 53 (1893) 225-.

Gauss, Johann Friedrich Karl. R. S. P. 7 (1856) 589-; Časopis 6 (1877) 145-; Nt. 15 (1877) 533-; 28 (1883) 272-; Science 9 (1899) 697-.

Genocchi, Angelus Joannes Bartholomaeus Aloysius. Nap. Rd. 28 (1889) 79; Tor. Ac. Sc. Mm. 39 (1889) 463-.

Gergonne, Joseph Diez. Mntp. Mm. Ac. Sect. Md. 3 (1862) 191-.

Gerhardt, Karl Immanuel. Lpldina. 35 (1899) 136; Bb. Mth. 1 (1900) 205-; D. Mth. Vr. Jbr. 8 (1900) (Heft 1) 28 -.

Germain, (Mlle.) Sophie. Ausl. 5 (1832) 623Gibbes, Lewis R. Am. J. Sc. 49 (1895) 80.

Gierster, Josef. D. Mth. Vr. Jbr. 2 (1893) 44

Gilbert, Louis Philippe. Brux. S. Sc. A. 16 (1892) (Pt. 1) 102-; Mathesis 12 (1892) 57; Par. S. Phlm. Bll. 4 (1892) 138 -

Gill, Charles. Assur. Mg. 6 (1857) 216-.

Giorgini, Gaetano. G. Mt. 31 (1893) 23-.

Gogu, Constantin. Bucarest S. Sc. Bll. (1897) 103-, 109-.

Gompertz, Benjamin. As. S. M. Not. 26 (1866) 104-; Assur. Mg. 13 (1867) 1-.

Graindorge, Joseph. Mathesis 16 (1896) 48.

Grassmann, Hermann Günther. Z. Mth. Ps. 23 (1878) (H.-lt. Ab.) 69-; Mth. A. 14 (1879) 1-

Graves, (Rt. Rev.) Charles. Nt. 60 (1899) 277.

Graves, John Thomas. R. S. P. 19 (1871) xxvii-.

Gray, Peter. I. Act. J. 26 (1887) 301-; As. S. M. Not. 48 (1888) 163-.

Gretschel, Heinrich Friedrich. Lpldina. 28 (1892) . 57; D. Mth. Vr. Jbr. 2 (1893) 42-. Grofe, Gustav von. Dorpat Sb. 11 (1896) 186-. 


\section{Biography}

Gromeka, Ippolit Stepanovĩc. Kazan S. Nt. (Ps. Mth.) P. 8 (1890) 163 (bis)-, 168-, 181-; Fschr. Mth. (1890) 30.

Gronau, Johann Friedrich Wilhelm. Danzig Schr. 7 (Heft 1) (1888) i-.

Grunert, Johann August. Arch. Mth. Ps. 55 (1873) 1-; Wien Alm. (1873) 145-.

Günther, Paul. D. Mth. Vr. Jbr. 1 (1892) 10; Z. Mth. Ps. 37 (1892) (H.-lt. Ab.) 46-.

Guthrie, Francis. J. Bt. 37 (1899) 528; Nt. 61 (1899-1900) 84.

Gyldén, Johann August Hugo. C. R. 123 (1896 771-; Lpldina. 32 (1896) 189; Obs. 19 (1896) 446 ; Nt. 55 (1896-97) 38, 158-; Acta Mth. 20 (1897) 397-; As. Nr. 142 (1897) 49-; As. S. M. Not. 57 (1897) 222-; Bll. As. 14 (1897) 289-; Wiad. Mt. 1 (1897) 31-.

Hachette, Jean Nicolas Pierre. Fr. S. Ag. Mm. (1834) 143-

Hallauer, Octave René. Mulhouse S. In. Bll. 54 (1884) 139-; Science 4 (1884) 306-.

Halphen, Georges Henri. C. R. 109 (1889) 994-; 110 (1890) 489-; Liouv. J. Mth. 5 (1889) 345-; Palermo Cir. Mt. Rd. 3 (1889) 210-; Rm. R. Ac. Linc. Rd. 5 (1889) (Sem. 1) 815-; J. H. Un. Cir. [9 (1889-90)] 26; Bll. Sc. Mth. 14 (1890) 62-; Par. Éc. Pol. J. 60 (1890) 137-.

Hamilton, (Sir) William Rovan. Am. J. Sc. 42 (1866) 293-; As. S. M. Not. 26 (1866) 109-; Ir. Ac. P. 9 (1867) 307-.

Hankel, Hermann. Mth. A. 7 (1874) 583-.

Hargreave, Charles James. R. S. P. 16 (1868) xvii-.

Harnack, Carl Gustav Axel. Dresden Isis Sb. (1888) (Ab.) 3-; Lpldina. 24 (1888) 110-; Mth. A. 32 (1888) 161-; Z. Mth. Ps. 33 (1888) (H.-lt. Ab.) 121-.

Haughton, (Rev.) Samuel. Gl. Mg. 4 (1897) 573-; I. ME. P. (1897) 514-; Nt. 57 (189798) 55-; Gl. S. QJ. 54 (1898) lxvi-; R. S. P. 62 (1898) xxix-.

Haydon, Frank Scott. L. Mth. S. P. 18 (188687) 392.

Heilermann, Hermann. $\quad$ Z. Mth. Ps. 45 (1900) (H.-lt. Ab.) 57.

Hellwig, Karl Franz. Erfurt Ak. Jb. 24 (1898) 292.

Heppel, George Hastings. Assur. Mg. 10 (1863) 83-.

Hermite, Charles. Manch. Lt. Ph. S. Mm. \& P. 45 (1901) xxxviii-.

Herr, Josef Philipp. Lpldina. 20 (1884) 221-; As. Nr. 111 (1885) 79-.

Herschel, (Sir) John F. W. Brux. Ac. Bll. 31 (1871) 478-; Smiths. Rp. (1871) 109-; Am. Ph. S. P. 12 (1872) 217-; As. S. M. Not. 32 (1872) 122-; Edinb. R. S. P. 7 (1872) 543-; R. S. P. 20 (1872) xvii-; Am. Ac. P. 8 (1873) 461-; Wien Alm. (1873) 147-.

Hesse, Ludwig Otto. Ts. Mth. 4 (1874) 190-; Crelle J. Mth. 79 (1875) 345-.

Hilgard, Julius Erasmus. Am. Ac. P. 26 (1891) 370-; Am. J. Sc. 41 (1891) 526; Lpldina. 27 (1891) 108; Nt. 44 (1891) 87; Railroad \& Eng. J. 65 (1891) 283-; Science 17 (1891) 272; Wash. Ph. S. Bll. 12 (1895)

\section{Biography 0010}

Hill, Thomas. Am. Ac. P. 27 (1893) 426-

Hirst, Thomas Archer. Lpldina. 28 (1892) 59; Nt. 45 (1892) 399-; As. S. M. Not. 53 (1893) 218-; R. S. P. 52 (1893) xii-; L. Ps. S. P. 12 (1894) (Ann. Meet. 1893) 9.

Hochheim, Karl Adolf. Lpldina. 34 (1898) 130.

Holmgren, Hjalmar Josef. Acta Mth. 7 (1885) 392.

Homans, Sheppard. I. Act. J. 34 (1899) 144 -

Hopkinson, John. Elektech. Z. 19 (1898) 617; I. ME. P. (1898) 534-; L. Mth. S. P. 29 (1898) 727-; Nt. 58 (1898) 419-; I. CE. P. 135 (1899) 338-; L. Ps. S. P. 16 (1899) (Ann. Meet.) 9 ; R. S. P. 64 (1899) xvii-.

Hoppe, Ernst Reinhold Eduard. Lpldina. 36 (1900) 132.

Horner, Johann Caspar. Zür. Vjschr. 41 (1896) (Festschr. Th. 1) 79-.

Horner, Johann Jakob. Zür. Vjschr. 41 (1896) (Festschr. Th. 1) 227-.

Hoüel, Guillaume Jules. Lpldina. 22 (1886) 167-; Bordeaux S. Sc. Mm. 4 (1888) 1-.

Hunyady, Jenö. Mag. Tud. Ak. Éts. 2 (1891) 1 -.

Imšeneckij, Vasilij Grigorjevič. Kazan S. Ps.Mth. Bll. 2 (1893) (Prot.) 15-; 3 (1893) 37-; Rec. Mth. (Moscou) 18 (1896) 347-; Fschr. Mth. (1896) 19.

Isbister, Alexander Kennedy. Educ. Times 36 (1883) 189-; Peterm. Mt. 30 (1884) 101-.

Jacobi, Carl Gustav Jacob. Grunert Arch. 22 (1853) 158-.

Janni, Vincenzo. Nap. Ac. Pont. At. 22 (1892) (App.) 83-

Jeffery, Henry Martyn. Cornwall Pol. S. Rp. (1891) 16-; L. Mth. S. P. 22 (1891) 479-; R. S. P. 50 (1892) vii-; Cornwall R. I. J. 11 (1895) 208-

Jellett, (Rev.) John Hewitt. Nt. 37 (1888) 396-.

Jellicoe, Charles. I. Act. J. 24 (1884) 17-.

Kelland, Philip. R. S. P. $29(1879)$ vii-; Edinb. R. S. P. 10 (1880) 321-.

Kendall, E. Otis. Nt. 59 (1898-99) 300; N. Y. Am. Mth. S. Bll. 5 (1899) 272.

Kieseritzky, Johann Georg Gustav. Lpldina. 32 (1896) 180-

Kirchhoff, Gustav Robert. A. di C. 6 (1887) 380; Am. J. Sc. 34 (1887) 496; Berl. B. 20 (1887) 2771-; Elektech. Z. 8 (1887) 457-; Lpldina. 23 (1887) 216; Nt. 36 (1887) 606-; Obs. 10 (1887) 396; Tor. Ac. Sc. At. 23 (1887-88) 2-; Am. Ac. P. 23 (1888) 370-; As. Nr. 118 (1888) 47-; A. Tél. 15 (1888) 96; L. Ps. S. P. 9 (1888) (Ann. Meet.) 12-; Phm. J. 18 (1888) 376; Wien Alm. 38 (1888) 193-; Gött. Ab. 35 (1889) (Mth.) 10 pp.; Smiths. Rp. (1889) 527-; R. S. P. 46 (1890) vi-.

Kirkman, (Rev.) Thomas Penyngton. Manch. Lt. Ph. S. Mm. \& P. 9 (1895) 238-.

Klein, Benno. D. Mth. Vr. Jhr. 1 (1892) 9.

Koch, Johann Rudolf. Bern. Mt. (1891) xxii-.

Kornuch-Trockij, Jakov Petrovič. As. Nr. 145 (1898) 127-; Kazan S. Ps.-Mth. Bll. 7 (1898) (Prot.) 115-.

Kossak, Ernst. $\quad$ Lpldina. 28 (1892) 56. 


\section{Biography}

Kotelǐnikov, Petr Ivañovič. Kazan S. Nt. (Ps. Mth.) P. 5 (1887) 225-; Fschr. Mth. (1887) 20.

Kovalevskij, Sofja Vasiljevna. Bll. Sc. Mth. 15 (1891) 212-; Christiania F. (1891) (Ov.) 8-; Crelle J. Mth. 108 (1891) 88; Lpldina. 27 (1891) 59-; Nt. 43 (1891) 375-; Palermo Cir. Mt. Rd. 5 (1891) 121-; Rv. Mt. 1 (1891) 21-; A. Mt. 19 (1891-92) 201-; As. \& Asps. 11 (1892) 281-; Acta Mth. 16 (1892-93) 385-; Rec. Mth. (Moscou) 16 (1893) 1-.

Kraus, Ludwig. Časopis 15 (1886) 49-; Fschr. Mth. (1886) 22.

Kronecker, Leopold. Ipldina. 27 (1891) 207; 28 (1892) 94-; A. Ps. C. (Berl. P8. Gs. Vh. 11) 45 (1892) 595-; C. R. 114 (1892) 19-; Mathesis 12 (1892) 18; N. Y. Mth. S. Bll. 1 (1892) 173-; Berl. Ak. Ab. (1893) 22 pp.; D. Mth. Vr. Jbr. 2 (1893) 5-; Mth. A. 43 (1893) 1-; Münch. Ak. Sb. 22 (1893) 202.

Kummell, Charles Hugo. Wash. Ph. S. Bll. 13 (1900) 404-.

Kummer, Ernst Eduard. C. R. 116 (1893) 1163-; Lpldina. 29 (1893) 110; D. Mth. Vr. Jbr. 3 (1894) 13-; Mathesis 14 (1894) 40-; Münch. Ak. Sb. 24 (1895) 140-.

Kunze, Karl Ludwig Albrecht. Lpldina. 27 (1891) 78-, 94-

Laborie, Edmond. Angers S. Sc. Bll. (1891) 236.

Lagrange, Joseph Louis (comte) de. Par. Mm. de l'I. (1812) (H.) xxvii-; Ts. Mth. 1 (1877) 129-.

Laguerre, Edmond Nicolas. C. R. 103 (1886) 424-; 104 (1887) 1643-; Par. Éc. Pol. J. 56 (1886) 213-; N. A. Mth. 6 (1887) 105-; 7 (1888) 352.

Lalanne, Léon Louis Chrétien. C. R. 114 (1892) 569-; 115 (1892) 1118-.

Lamarle, Anatole Henri Ernest. Brux. Ac. Bll. 39 (1875) 360-

Lamé, Gabriel. A. Mines 1 (1872) 271-; Rv. Sc. 14 (1878) 720 -

Langren, Michel-Florent van. Ciel et Terre 12 (1891-92) 241-, 297-.

Laplace, Pierre Simon (le marquis) de. Hall Bij. 2 (1826) 284-; Par. Mm. Ac. Sc. 10 (1831) (H.) 81-.

Lavagna, Giovanni Maria. Rv. Sc.-Ind. 2 (1871) 40-.

Le Besgue, Victor Amédée. N. A. Mth. 16 (1877) 116-

Lefavour, Edward Brown. Wash. Ph. S. Bll. 11 (1892) 488-.

Legendre, Adrien Marie. Smiths. Rp. (1867) 137-.

Lejeune Dirichlet, Gustave Pierre. Tortolini A. 3 (1860) 221-, 283-.

Lětnikov, Aleksěj Vasiljevič. Rec. Mth. (Moscou) 14 (1890) i-; Fschr. Mth. (1889) 21.

Liagre, Jean Baptiste Joseph. Ciel et Terre 11 (1890-91) 541-; Brux. Ac. Bll. 21 (1891) 77-.

Lie, Marius Sophus. Tor. Ac. Sc. At. 34 (1898) 235- or 363-; Nt. 59 (1898-99) 445- ; Arch. Mth. Ntvd. 21 (1899) No. 1, xxiii pp.; C. R. 128 (1899) 525-; 129 (1899) 1062; D. Nf. Vh. (1899) (Th. 2, Hälfte 1) 4-; Ens. Mth. 1
Biography 0010

(1899) 156; Leip. Mth. Ps. B. 51 (1899) xi-; L. Mth. S. P. 30 (1899) 334-; Lpldina. 35 (1899) 60; N. Ts. Mth. 10 (B) (1899) 48; N. Y. Am. Mth. S. Bll. 5 (1899) 367-; Rm. R. Ac. Linc. Rd. 8 (1899) (Sem. 1) 281-, 360-; Science 9 (1899) 447-; St. Pét. Ac. Sc. Bll. 10 (1899) xxxiii-; Bb. Mth. 1 (1900) 166-; D. Mth. Vr. Jbr. 8 (1900) (Heft 1) 30-; Kazan S. Ps.-Mth. Bll. 9 (1900) (App.) 32 pp.; Manch. Lt. Ph. S. Mm. \& P. 43 (1900) xxxiii-; Mathesis 20 (1900) 228-; Mth. A. 53 (1900) 1-; Münch. Ak. Sb. 30 (1901) 339-.

Ligin, W. N. Fschr. Mth. (1900) 29.

Ligowski, Wilhelm. D. Mth. Vr. Jbr. 4 (1897) 46.

Liouville, Joseph. Edinb. R. S. P. 14 (1888) 83 (bis)-.

Lobačevskij, Nikolaj Ivanovič. Kazan Un. Mm. (1857) (Bk. 4) 153-; Bll. Sc. Mth. As. 1 (1870) 66-, 324-, 384-; Mathesis 13 (1893) 117-, 193; Rv. Sc. 52 (1893) 636-; Kazan S. Ps.-Mth. Bll. 3 (1893) (Prot.) 27-; 4 (1895) (Prot.) 4-; Rec. Mth. (Moscou) 17 (1895) 474-; Z. Mth. Ps. 40 (1895) (Suppl.) 205-; Bordeaux S. Sc. Mm. 1 (1896) 307-.

Lobatto, Rehuel. Amst. Jb. Ak. (1866) 25-; Arch. Mth. Ps. 49 (1869) 332-.

Lommel, Eugen Cornelius Joseph von. Elektech. Z. 20 (1899) 458; Lpldina. 35 (1899) 160-; D. Mth. Vr. Jbr. 8 (1900) (Heft 1) 47-. Loomis, Elias. Lpldina. 25 (1889) 214; Met. Z. 6 (1889) 466-; Nt. 40 (1889) 401; Obs. 12 (1889) 358; Railroad \& Eng. J. 63 (1889) 435; Am. Ac. P. 25 (1890) 324-; Am. J. Sc. 39 (1890) 427-; Met. S. QJ. 16 (1890) 101; Sid. Mess. 9 (1890) 241-; Smiths. Rp. (1890) 741-; Sym. Met. Mg. 24 (1890) 122.

Lovering, Joseph. Am. J. Sc. 43 (1892) 167; Lpldina. 28 (1892) 102; Am. Ac. P. 27 (1893) 372-.

Lubbock, (Sir) John William. As. S. M. Not. 26 (1866) 118-; R. S. P. 15 (1867) xxxii-.

Luca, Ferdinando de. Il Polit. 18 (1870) 612-.

Lucas, Édouard. Mathesis 11 (1891) 217.

Luther, Eduard. Lpldina. 23 (1887) 216; 24 (1888) 52; Obs. 10 (1887) 432-; As. Nr. 118 (1888) 31-; As. S. M. Not. 48 (1888) 169-; Königsb. Schr. 28 (1888) (Ab.) 105-.

Lutter, Nándor (Ferdinand). Lpldina. 28 (1892) 53; Mag. Tud. Ak. Éts. 3 (1892) $531-$.

Luvini, Giovanni. Acireale Ac. At. 3 (1891) vii.

M'Cann, H. W. Beng. As. S. P. (1884) 113-.

Machovec, Franz. Mh. Mth. Ps. 3 (1892) 403.

Maddem, Lorenzo. Acireale Ac. At. 2 (1891) xv.

Mainardi, Gaspare. $\mathrm{Rm}$. R. Ac. Linc. T. 3 (1879) 126-.

Majer, Józef. Wiad. Mt. 3 (1899) 302-.

Makeham, William Matthew. I. Act. J. 30 (1893) 1-.

Maksimovič, Vladimir Pavlovič. Kazan S. Nt. (Ps.-Mth.) P. 8 (1890) 53-; Fschr. Mth. (1890) 30 . 


\section{Biography}

Malmsten, Carl Johan. Acta Mth. 7 (1885) 394; Göteb. Hndl. 26 (1891) 67-.

Marsilly, (le gén.) Louis Joseph Auguste de Commines de. Bône Ac. Hip. Bll. 24 (1891) (C. R. 1890) 1-.

Masdę, Giuliano. Nap. I. Inc. At. 1 (1899) 20.

Mathieu, Émile Léomard. Lpldina. 26 (1890) 215; Nancy S. Sc. Bll. (1891) 1-; N. Y. Mth. S. Bll. 1 (1892) 156-, 236.

Matthes, Carel Johannes. Amst. Ak. Jb. (1882) 1-.

Maus, Henri. Brux. Ac. Bll. 26 (1893) 173-.

Maxwell, James Clerk. Edinb. R. S. P. 10 (1880) 331-; Kosmos (Lw.) 6 (1881) 129-; R. S. P. 33 (1882) i-.

Mayr, Alois. L L Lldina. 26 (1890) 217.

Mehler, Gustav Ferdinand. Lpldinء. 31 (1895) 170; Mth. A. 48 (1897) 603-.

Meissel, Ernst Daniel Friedrich. As. Nr. 137 (1895) 239-; Lpldina. 31 (1895) 102.

Melĩnikov, Michail Ivanović. Kazan S. Nt. (Ps.-Mth.) P. 4 (1886) 49-

Merrifield, Charles Watkins. L. Ps. S. P. 5 (1884) (Ann. Meet.) 13-; Nt. 29 (1884) 270; Nv. Archt. T. 25 (1884) 309-; R. S. P. 36 (1884) $\mathrm{i}-$

Meyer, Arnold. Zür. Vjschr. 42 (1897) 65-; D. Mth. Vr. Jbr. 5 (1901) (Heft 1) 18-

Meyer, Friedrich. D. Mth. Vr. Jbr. 8 (1900) (Heft 1) 59-.

Miller, Thomas. Edinb. R. S. P. 19 (1893) liv-.

Minding, Ernst Ferdinand Adolf. Z. Mth. Ps. 45 (1900) (H.-lt. Ab.) 113-.

Minich, Serafino Rafaele. Ven. I. At. 1 (188283) 1095-.

Minnigerode, $B$. Lpldina. 32 (1896) 143-.

Mister, Jean Nicolas. Mathesis 18 (1898) 241.

Mitchell, Oscar Howard. J. H. Un. Cir. 8 (1888-89) 110.

Möbius, August Ferdinand. Arch. Mth. Ps, 49 (1869) (Lt. B.) clxxxxv.

Möllinger, Otto. Lpldina. 23 (1887) 54.

Moigno, (l'abbé) Francois Napoléon Marie. A. Tél. 11 (1884) 289; Les Mondes 8 (1884) 443-; Nt. 30 (1884) 291-.

Moseley, (Canon) Henry. Nv. Archt. T. 13 (1872) 328-

Mossotti, Ottaviano Fabrizio. As. S. M. Not. 24 (1864) 87-

Moth, Franz Xaver. Wien Alm. (1879) 172-.

Nagel, Christian Heinrich. Mathesis 7 (1887) 114 -

Nash, Alfred Moses. L. Mth. S. P. 26 (1895) 557 -

Neumann, Franz Ernst. C. R. 120 (1895) 1189-; D. Nf. Vh. (1895) (Th. 1) 27-, (Th. 2, Hälfte 1) 5-; Elektech. Z. 16 (1895) 353; Gött. Nr. (1895) 248-; Nt. 52 (1895) 176; Lpldina. 32 (1896) 51-, 63-; Wien Alm. 46 (1896) 271-; D. Mth. Vr. Jbr. 4 (1897) 54-; R. S. P. 60 (1897) viii-.

Newton, Hubert Anson. As. Nr. 141 (1896) 407-; Asps. J. 4 (1896) 237; Nt. 54 (1896) 394; Am. J. Sc. 3 (1897) 359-; As. S. M Not. 57 (1897) 228-; N. Y. Am. Mth. S. Bll. 3 (1897) 169-; Pop. As. 4 (1897) 236-; R. S. P. 63 (1898) i-

\section{Biography 0010}

Nobert, Friedrich Adolph. N.-Vorp. Mt. 15 (1884) 38-.

Novarese, Enrico. Rv. Mt. 2 (1892) 35.

Ofterdinger, Felix Ludwig. Bb. Mth. (1896) 50-; Lpldina. 32 (1896) 103.

Oliver, James Edward. Nt. 51 (1894-95) 587; Science 1 (1895) 544-; Am. Ac. P. 31 (1896) $367-$

Olivier, Théodore. A. Cons. Arts et Mét. 8 (1896) 21-.

Omerique, Antonio Hugo de. Fschr. Mth. (1895) 15.

Oppermann, Ludvig Henrik Ferdinand. Ts. Mth. 1 (1883) 137-.

Orlov, Aleksandr Petrovič. Kazan S. Nt. (Ps.Mth.) P. 7 (1889) 396-.

Paciuolo, Lucas. Z. Mth. Ps. 34 (1889) (H.-lt. Ab.) 81-, 121-.

Padova, Ernesto. Rm. R. Ac. Linc. Rd. 5 (1896) (Sem. 1) 284-.

Padula, Fortunato. Rm. R. Ac. Linc. T. 6 (1882) 30 -

Palmieri, Luigi. Lpldina. 32 (1896) 147; Nap. Ac. Pont. At. 26 (1896) (Necrol. No. 5) 15 pp.; Nap. I. Inc. At. 9 (1896) 12-; Nap. Rd. 35 (1896) 236-.

Pankiewicz, Jan. Wiad. Mt. 3 (1899) 302.

Paolis, Riccardo de. Palermo Cir. Mt. Rd. 6 (1892) 208-.

Parkinson, (Rev.) Stephen. R. S. P. 45 (1889) i-; As. S. M. Not. 50 (1890) 167-.

Peck, William Grey. Railroad \& Eng. J. 66 (1892) 146.

Peirce, Benjamin. Pop. As. 3 (1896) 49-.

Penot, Jean Fleury Achille. Mulhouse S. In. Bll. 56 (1886) 505-.

Perigal, Henry. Aer. J. 2 (1898) 67; L. Mth. S. P. 29 (1898) 732-; Nt. 58 (1898) 131; Obs. 21 (1898) 282-; Science 7 (1898) 858; As. S. M. Not. 59 (1899) 226-; Met. S. QJ. 25 (1899) 223-.

Pestalozzi, Hans Jakob. Zür. Vjschr. 41 (1896) (Festschr. Th. 1) 123-.

Pettko, Jânos. Mag. Tud. Ak. Ets. 3 (1892) 429-.

Petzval, Josef. Mh. Mth. Ps. 2 (1891) 480; Wien Alm. 42 (1892) 182-.

Piani, Domenico. Bologna Ac. Sc. Mm. 1 (1871) 3-.

Plana, Giovanni Antonio Amedeo. As. S. M. Not. 24 (1864) 89-; Edinb. R. S. P. 5 (1866) 293; Bll. Sc. Mth. As. 5 (1873) 65-; Par. Ac. Sc. Mm. 38 (1873) cvii-; Rm. S. It. Mm. 6 (1887) lxxxvi-.

Plarr, Gustav. Nt. 45 (1892) 419.

Plch, Kornel. Časopis 19 (1890) 51-.

Plücker, Julius. R. S. P. 17 (1869) 1xxxi-; Bll. Sc. Mth. As. 3 (1872) 59-; 5 (1873) 313-; Gött. Ab. 16 (1872) 40 pp.; G. Mt. 11 (1873) 153-.

Poinsot, Louis. Par. Ac. Sc. Mm. 45 (1899) lxxiii-.

Poncelet, Jean Victor. A. Cons. Arts et Mét. 7 (1895) 1-.

Ponte Horta, Francisco da. G. Teix. J. Sc. 14 (1900) 3-.

Popov, Aleksandr Thedorovich. Kazan S. Nt. (P8.-Mth.) P. 1 (1883) (No. 9) 13-. 


\section{Biography}

Prediger, Carl. $\quad$ D. Mth. Vr. Jbr. 4 (1897) 51-. Price, (Rev.) Bartholomew. Nt. 59 (1898-99) 229-; As. S. M. Not. 59 (1899) 228-; L. Mth. S. P. 30 (1899) 332-; L. Ps. S. P. 16 (1899) (Ann. Meet.) 10-; Science 9 (1899) 188 ; R. S. Yearbook (1900) 185-.

Prowe, Leopold Friedrich. Z. Mth. Ps. 33 (1888) (H.-lt. Ab.) 89-.

Puchewicz, Wtadystaw. Wiad. Mt. 3 (1899) 302.

Puiseux, Victor Alexandre. Bll. Sc. Mth. As. 8 (1884) 234-; Par. Ac. Sc. Mm. 44 (1888) lxvii-.

Quet, Antoine. Les Mondes 9 (1881) 582-.

Quetelet, Lambert Adolphe Jacques. Amst. Ak. Wet. P. (1873-74) (No. 9) 1-; Arch. Sc. Ps. Nt. 49 (1874) 350-; Bll. Sc. Nord 6 (1874) 62-; Brux. Ac. Bll. 37 (1874) 245-; 38 (1874) 816-; Rv. Sc.-Ind. 6 (1874) 38-; As. S. M. Not. 35 (1875) 176-; Edinb. R. S. P. 8 (1875) 474-; Mag. Tud. Ak. Éts. 8 (No. 16) (1875) 241-; R. S. P. 23 (1875) xi-. Quick, (Rev.) Robert Herbert. Educ. Times 44 (1891) 185-.

Raynard, Arthur Cowper. L. Mth. S. P. 26 (1895) 554-.

Réalis, Savin. N. A. Mth. 5 (1886) 200-.

Renjifo, Ismael. Santiago de Chile Un. A. 100 (1898) 527-.

Resal, Henri Amé. A. Mines 10 (1896) 625-; C. R. 123 (1896) 435-; Liouv. J. Mth. 2 (1896) 455-; Lpldina. 32 (1896) 147-; Manch. Lt. Ph. S. Mm. \& P. 41 (1897) liii.

Reuschle, Carl Gustav. Leip. As. Gs. Vjschr. 10 (1875) 178-; Z. Mth. Ps. 21 (1876) (H.-lt. Ab.) 1 -

Rhodes, Edward Hawksley. $\quad$ L. Mth. S. P. 26 (1895) 558

Ribaucour, Albert. Mathesis 13 (1893) 270-.

Ribeiro de Sousa Pinto, Rodrigo. G. Teix. J. Sc. 12 (1895) $2-$.

Riccardi, Pietro. Mod. Ac. Sc. Mm. 2 (1900) xxii-.

Riemann, Georg Friedrich Bernhard. Gött. Nr. (1867) 305-; Rec. Mth. (Moscou) 3 (1868) (Pt. 2) 153-; R. S. P. 16 (1868) lxix-.

Ritter, Ernst. Lpldina. 31 (1895) 218; Nt. 52 (1895) 600 ; D. Mth. Vr. Jbr. 4 (1897) 52-.

Roberts, Samuel Oliver. L. Mth. S. P. 31 (1900) 282-; Mth. Gz. 1 (1900) 278.

Roche, Édonard Albert. C. R. 96 (1883) 1171-; Lille S. Mm. 14 (1885) 17-.

Rogers, William Augustus. Am. J. Sc. 5 (1898) 322; As. Nr. 146 (1898) 343-; Asps. J. 7 (1898) 390-; Ps. Rv. 6 (1898) 315-; Science 7 (1898) 447-

Rosenberger, Johann Carl F'erdinand. Elektech. Z. 20 (1899) 690; Lpldina. 35 (1899) 182 ; Bb. Mth. 1 (1900) 217-

Rossi Re, Vincenzo de. Rm. N. Linc. At. 42 (1889) 83-.

Rubini, Raffaele. Nap. Rd. 29 (1890) 134-; Nap. Ac. Pont. At. 21 (1891) 275-.

Sagastume, Demetrio. Arg. S. Ci. A. 47 (1899) 97-.

Saint-Venant, Adhêmar Jean Claude Barré de. A. Pon. Chauss. 12 (1886) 557-; Brux. S. Sc.
Biography 0010

A. 10 (1886) (Pt. 1) 77-; C. R. 102 (1886) 141-; Nt. 33 (1886) 319-

Sandeman, Archibald. Manch. Lt. Ph. S. Mm. \& P. 8 (1894) 215-

Sang, Edward. Edinb. R. S. P. 21 (1897) xvii-.

Sannia, Achille. Nap. I. Inc. At. 5 (1892) 8-; Palermo Cir. Mt. Rd. 6 (1892) 48-; Nap. Ac. Pont. At. 24 (1894) (Necrol. No. 2) 6 pp.

Sarrus, Pierre Frédéric. Strasb. Mm. S. Sc. 6 (1866-70) livr. 1, 12 pp.

Schäffer, Karl Julius Traugott Hermann. Z. Nw. 72 (1899) 393-; Lpldina. 36 (1900) 78-.

Schapira, Hermann. Lpldina. 34 (1898) 112 ; D. Mth. Vr. Jbr. 8 (1900) (Heft 1) 61-

Scheeffer, Ludwig. Lpldina. 22 (1886) 56; Z. Mth. Ps. 31 (1886) (H.-lt. Ab.) 50-.

Schellbach, Carl Heinrich. Z. Instk. 12 (1892) 253 ; Lpldina. 29 (1893) 49-, 75-, 90-, 104-, $125-$.

Schering, Ernst Christian Julius. Lpldina. 33 (1897) 161-; Nt. 57 (1897-98) 85, 416; As. Nr. 145 (1898) 31-; Crelle J. Mth. 119 (1898) 86 ; D. Mth. Vr. Jbr. 6 (1899) (Heft 1) 25-.

Scherk, Heinrich Ferdinand. Lpldina. 21 (1885) 212-.

Schjellerup, Hans Carl Frederik Christian. Lpldina. 23 (1887) 217; Obs. 10 (1887) 438-; Ts. Mth. 5 (1887) 148-; As. Nr. 118 (1888) 95-; As. S. M. Not. 48 (1888) 171-; Nt. 37 (1888) 154-.

Schläfli, Ludwig. Bern Mt. (1895) 120-; Crelle J. Mth. 115 (1895) 350; Lpldina. 31 (1895) 103-; Rm. R. Ac. Linc. Rd. 4 (1895) (Sem. 1) 310 -

Schober, Karl. D. Mth. Vr. Jbr. 8 (1900) (Heft 1) 66-.

Schols, Charles Mathieu. 's Gravenh. I. Ing. Ts. (1896-97) (Verg.) 85-; Amst. Ak. Vs. 5 (1897) 415-.

Schrentzel, Wilhelm. Z. Mth. Ps. 42 (1897) (H.-lt. Ab.) 1-.

Schroeter, Heinrich Eduard. Crelle J. Mth. 109 (1892) 358-; Lpldina. 28 (1892) 54; D. Mth. Vr. Jbr. 2 (1893) 32-.

Schultén, Nathanael Gerhard af. Helsingf. Acta 6 (1861) 19 pp.

Seidel, Philipp Ludwig (Ritter) von. As. Nr. 141 (1896) 319-; Lpldina. 32 (1896) 138; D. Mth. Vr. Jbr. 7 (1899) (Heft 1) 23-.

Serret, Joseph Alfred. As. Fr. C. R. (1885) (Pt. 1) 27; Bll. Sc. Mth. 9 (1885) 123-; C. R. 100 (1885) 673-; N. Antol. Sc. 80 (1885) 388.

Serret, Paul. C. R. 127 (1898) 37-.

Servois, François Joseph. Doubs S. Mm. 9 (1895) 305-.

Seydler, August. As. Nr. 128 (1891) 15-; Lpldina. 27 (1891) 156; Časopis 21 (1892) 193-.

Silva, J. A. Martins da. G. Teix. J. Sc. 6 (1885) 194-.

Šimerka, Václav. Časopis 17 (1888) 253-; Fschr. Mth. (1888) 19-.

Sinram, Heinrich Theodor. D. Mth. Vr. Jbr. 5 (1901) (Heft 1) 17-.

Sludskij, Fedor Aleksěevič. Rec. Mth. (Moscou) 20 (1899) 337-. 
0010 Biography

Smith, Archibald. R. S. P. 22 (1874) i-; Edinb. R. S. P. 8 (1875) 282-.

Smith, Henry John Stephen. Educ. Times 36 (1883) 96; L. Ps. S. P. 5 (1884) (Ann. Meet. 1883) 8-; Obs. 6 (1883) 91-; As. S. M. Not. 44 (1884) 138-; Met. S. QJ. 10 (1884) 103-.

Snel van Royen, Willebrord. Mathesis 4 (1884) 64.

Snell, Karl. Lpldina. 22 (1886) 171.

Sondhauss, Karl Friedrich Julius. Bresl. Sl. Gs. Jbr. (1886) 324; Lpldina. 22 (1886) 215.

Spottiswoode, William. Nt. 27 (1883) 597-; Obs. 6 (1883) 231-; Science 2 (1883) 27-; As. S. M. Not. 44 (1884) 150-; C. S. J. 45 (1884) 628-; L. Ps. S. P. 5 (1884) (Ann. Meet. 1884) 8-; Peterm. Mt. 30 (1884) 105; R. S. P. 38 (1885) xxxiv-; L. Mth. S. P. 31 (1900) 283-.

Stahl, Wilhelm. Lpldina. 30 (1894) 110; Crelle J. Mth. 114 (1895) 45-; D. Mth. Vr. Jbr. 4 (1897) 36-

Stanecki, Tomasz. Kosmos (Lw.) 16 (1891) 25-.

Staudigl, Rudolf. Mh. Mth. Ps. 2 (1891) 480.

Staudt, Karl Georg Christian von. Kharkov Mth. S. Com. (1880) 167-.

Steen, Adolph. Lpldina. 22 (1886) 219 ; Ts. Mth. 4 (1886) 65-.

Steichen, Michel. Brux. Ac. Bll. 21 (1891) 308-.

Steiner, Jacob. Sch. Nf. Gs. Vh. 56 (1873) 215-; A. Mt. 7 (1875-76) 65-; Bb. Mth. 1 (1900) 129-.

Stern, Moriz Abraham. Zür. Vjschr. 39 (1894) 131-; Münch. Ak. Sb. 24 (1895) 142; D. Mth. Vr. Jbr. 4 (1897) 34-.

Stieltjes, Thomas Johannes (jun.). Lpldina. 31 (1895) 56; Toul. Fac. Sc. A. 9 (1895) [1]-.

Storchi, Felice. Mod. Ac. Sc. Mm. 8 (1892) xiii-.

Strehlke, Friedrich Samuel. Danzig Schr. 6 (Heft 4) (1887) 173-.

Streintz, Heinrich. Lpldina. 28 (1892) 210; Steierm. Mt. (1892) 233-.

Strong, Theodore. Am. Ac. P. 8 (1873) 228-.

Svanberg, Adolf Ferdinand. Stockh. Vt. Ak. Lefn. 1 (1869-73) 111-.

Sylvester, James Joseph. J. H. Un. Cir. 8 (1888-89) 23-; [16 (1896-97)] 25-, 53-; [18 (1898-99)] 29; Nt. 55 (1896-97) 492-; Am. J. Mth. 19 (1897) (Suppl.) 1 p.; Lpldina. 33 (1897) 90-; Manch. Lt. Ph. S. Mm. \& P. 41 (1897) liii-; Mathesis 17 (1897) 245-; Nap. Rd. 36 (1897) 165-; N. Y. Am. Mth. S. Bll. 3 (1897) 299-; Science 5 (1897) 597-; Wiad. Mt. 1 (1897) 175-; I. Act. J. 33 (1898) 345-; Kazan S. Ps.-Mth. Bll. 7 (1898) (Prot.) 89-; Mth. A. 50 (1898) 133-; R. S. P. 63 (1898) ix-.

Tartaglia, Nicold, biographical sketch. TonniBazza, V. Brescia At. Cm. (1900) 160-.

Taylor, William Bower. Wash. Ph. S. Bll. 13 (1900) 418-.

Terrot, (Bishop) Charles Hughes. Edinb. R. S. P. 8 (1873) 9-.

Tesla, Nikola. Časopis 19 (1890) 155-.

\section{Biography 0010}

Tillol, Jules Jérôme. Toul. Ac. Sc. Mm. 3 (1891) 525-.

Timmermans, Jean Alexis. Aroh. Mth. Ps. 49 (1869) Lt. B. 294.

Todhunter, Isaac. Nt. 29 (1884) 436; R. S. P. 37 (1884) xxvii-; As. S. M. Not. 45 (1885) 194

Tortolini, Barnaba. Arch. Mth. Ps. 44 (1865) clxxv-; 45 (1866) clxxvii; Bll. Sc. Mth. As. 8 (1875) 272-; Rm. N. Linc. At. 28 (1875) 93-; A. Mt. 7 (1876) 63-; Rm. S. It. Mm. 6 (1887) $\mathrm{lxv}$ -

Transon, Abel Étienne Louis. A. Mines 14 (1878) 433-

Trudi, Nicola. G. Mt. 22 (1884) 304-; Nap. I. Inc. At. 3 (1884) 5-; Nap. Rd. 23 (1884) 149-; Nap. Ac. Pont. At. 16 (Pt. 1) (1885) $243-$.

Turazza, Domenico. Ven. I. At. 1 (1898-99) (Pt. 1) 69-.

Ullhers, Johann Konrad. Lpldina. 23 (1887) 215.

Unverzagt, Wilhelm. Z. Mth. Ps. 31 (1886) (H.-lt. Ab.) 41-.

Vallas, Antal. Mag. Tud. Ak. Ets. 8 (No. 17) (1875) 260-

Vega, Georg von. Z. Mth. Ps. 39 (1894) (H.-lt. Ab.) 204 -

Venturoli, Giuseppe. N. A. Sc. Nt. 8 (1847) 5-, 185-.

Verdam, Gideon Jan. Amst. Jb. Ak. (1866) 56-; Arch. Mth. Ps. 46 (1866) Lt. B. clxxxiii.

Vervaet, Julian. Časopis 15 (1886) 25-.

Victorica y Soneyra, José. Arg. S. Ci. A. 36 (1893) 265-.

Vicuña, Gumersindo. Bb. Mth. (1891) 33-.

Vincent, Alexandre Joseph Hidulphe. Lille S. Mm. 6 (1869) 561-.

Volpicelli, Paolo. Rm. R. Ac. Linc. T. 3 (1879) 160-.

Vydra, Stanislav. Časopis 1 (1872) 1-, 49-.

Walker, John James. Nt. 61 (1899-1900) 618.

Wappler, Hermann Emil. Bb. Mth. 1 (1900) 225.

Weierstrass, Karl Theodor Wilhelm. Nt. 55 (1896-97) 443; Acta Mth. 21 (1897) 79-; Bologna Rd. 1 (1897) 101-; C. R. 124 (1897) 430-; Crelle J. Mth. 117 (1897) 357; Lpldina. 33 (1897) 54-; Nap. Rd. 36 (1897) 63-; St. Pét. Ac. Sc. Bll. 6 (1897) xxx - ; Wiad. Mt. 1 (1897) 53-; Wien Alm. 47 (1897) 304-; Münch. Ak. Sb. 27 (1898) 402-; D. Mth. Vr. Jbr. 6 (1899) (Heft 1) 27-.

Weninger, Vincze. Mag. Tud. Ak. Étk. (Mth.) 7 (1881) (No. 10) $22 \mathrm{pp}$.

Weyer, Georg Daniel Eduard. Nt. 55 (1896-97) 299; As. Nr. 142 (1897) 343-; Lpldina. 33 (1897) 49; D. Mth. Vr. Jbr. 6 (1899) (Heft 1) 44-.

Weyr, Emil. Lpldina. 30 (1894) 57; Wien Alm. 44 (1894) 244-; Časopis 24 (1895) 163-; Fschr. Mth. (1895) 32-; Mh. Mth. Ps. 6 (1895) 1-; Palermo Cir. Mt. Rd. 9 (1895) 260-; D. Mth. Vr. Jbr. 4 (1897) 24-.

Whewell, (Dr) William. Ausl. 39 (1866) 304-; Gg. S. J. 36 (1866) exxii-; As. S. M. Not. 27 (1867) 110-; R. S. P. 16 (1868) li-. 


\section{Periodicals}

Wiener, Ludwig Christian. L Lpldina, 32 (1896) 136-, 155-, 166-; D. Mth. Vr. Jbr. 6 (1899) (Heft 1) 46-.

Williams, J. Hill. I. Act. J. 26 (1887) 218-.

Wiltheiss, Ernst Eduard. Lpldina. 36 (1900) 135.

Winckler, Anton. Lpldina. 28 (1892) 162; Mh. Mth. Ps. 3 (1892) 403-; Wien Alm. 43 (1893) 257-.

Wolf, Joh. Rudolf. Bern Mt. (1893) 193-; Lpldina. 29 (1893) 211 ; Nt. 49 (1893-94) 163, 266-; As. J. 13 (1894) 181-; As. Nr. 134 (1894) 183-; As. S. M. Not. 54 (1894) 206-; Zür. Vjschr. 39 (1894) 1-.

Wolstenholme, Joseph. Nt. 45 (1892) 85.

Wood, De Volson. Science 6 (1897) 204-.

Woolhouse, Wesley Stoker Barker. As. S. M. Not. 54 (1894) 204-; I. Act. J. 31 (1895) 362-.

Woolley, Joseph. Nt. 39 (1889) 517; Nv. Archt. T. 30 (1889) 463-.

Worpitzky, Julius. D. Mth. Vr. Jbr. 4 (1897) 47-.

Wren, Walter. L. Mth. S. P. 29 (1898) 731-.

Yvon-Villarceau, Antoine Joseph Francois. Ciel et Terre 5 (1885) 20-, 72 ; Par. Ac. Sc. Mm. 45 (1899) i-.

Zajaczkowski, Wtadystaw. Wiad. Mt. 2 (1898) 258 -

Žbikovskij, Anton Kraverjevičt. Wiad. Mt. 4 (1900) 268; Kazan S. Ps.-Mth. Bll. 10 (1901) (Prot.) 39-; Fschr. Mth. (1900) 31.

Zeipel, Ewald Victor Ehrenhold von. Lpldina. 29 (1893) 111.

Zetzsche, Karl Eduard. Dresden Isis Sb. (1894) 17-; Elektech. Z. 15 (1894) 297-; Civing. 41 (1895) 185-.

Zillmer, August. D. Mth. Vr. Jbr. 4 (1897) 23-.

Żmurko, Wawrzyniec (Laurent). Kosmos (Lw.) 14 (1889) 169-; Lpldina. 26 (1890) 52 ; Prace Mt.-Fiz. 2 (1890) 423-.

\section{Periodicals. Reports of In- stitutions, Societies, Congresses, etc.}

Belgian Royal Academy, secular report. 17721872. Tilly, J. M. de. Brux. Ac. Cent. Anniv. 2 (1872) $200 \mathrm{pp}$.

British Association. Elliptic and hyper-elliptic functions, reports on progress. Russell, W. H. L. B. A. Rp. 39 (1869) 334-; 40 (1870) 102-; 42 (1872) 334-; 43 (1873) 307-.

- Fundamental invariants of algebraic forms, reports of committee for calculating tables. B. A. Rp. (1880) 38-; (1881) 55-; (1882) 37-.

- - Geometrical elementary instruction, report of Committee. B. A. Rp. (1876) 8-.

- - Mathematical notation and printing, report of Committee. B. A. Rp. (1875) 337-.

- - - Sciences in Belgium, report. Quetelet, L. A. J. B. A. Rp. (1835) 35-; Quetelet Cor. Mth. 9 (1837) 1-.

- - - tables, reports. B. A. Rp. (1873) 1-; (1875) 305-; (1878) 172-; (1879) 46-; (1880) 30-; (1881) 303-; (1883) 118-.

\section{General Treatises 0030}

British Association. Mathematics and physics, progress, report of Committee. B. A. Rp. (1879) 37-.

Marburg University Math.-phys. Institute. Gerling, C. L. Grunert Arch. 2 (1842) 212-.

Paris Academy of Sciences, reports on papers, \&c. 1816-19, 1821. Delambre, J. B. J. Par. Mm. Ac. Sc. 1 (1816) (H.) 1-; 2 (1817) (H.) 1-; 3 (1818) (H.) 1-; 4 (1819-20) (H.) 1-, 127-; 5 (1821-22) (H.) 1-. $---,--\ldots,-$ 1822-28. Fourier J. B. J. Par. Mm. Ac. Sc. 5 (1826) (H.) 231-; 6 (1827) (H.) 1-; 7 (1827) (H.) 1-; 8 (1829) (H.) 1-; 9 (1830) (H.) 1-; $10(1831)$ (H.) 1-; 11 (1832) (H.) 1-.

-, L'Institut, reports in mathematics and physics. Delambre, J. B. J. J. de Ps. 53 (1801) 380-; 61 (1804) 81-; 82 (1816) 189-. _, - , - - _ - 1805-1815. Delambre, J. B. J. Par. Mm. de l'I. (1806) Sem. 2 (H.) 1-; (1807) Sem. 2 (H.) 1-; (1808) (H.) 1-; (1809) (H.) 1-; (1810) (pte. 2) (H.) i-; (1811) (H.) i-; (1812) (H.) i-; (1815) (H.) 1-.

Philosophical Congress, Mathematies at the. Couturat, L. Ens. Mth. 2 (1900) 397-.

Statistical Congress, Fifth and sixth International, reports. Brown, S. Assur. Mg. 11 (*1864) 195-; I. Act. J. 14 (*1869) 165-.

\section{General Treatises, Text Books, Dictionaries, Tables, Collected Works.}

Analysis, report. Peacock, G. B. A. Rp. (1833) 185-.

Bombelli, Rafael, supposed 2nd edition of his algebra. Favaro, A. Bb. Mth. (1893) 15-. ,,$----\ldots-$ Riccardi, $P, \mathrm{Bb}$ Mth. (1893) 64.

Books of arithmetic and algebra of 16 th century. Fontès, - Toul. Ac. Sc. Bll. 2 (1899) 202-.

" Geometrical Researches," Introduction. Gardiner, $M$. N. S. W. Ph. S. T. 1 (1866) 61-.

Hebrew codex, Mathematical and astronomical writings. Riccardi, $P$. Bb. Mth. (1893) $54-$.

- translations of mathematical works. Steinschneider, M. Bb. Mth. (1893) 51-.

Hug's “ Mathematik," remarks. Schläfi, $L$. Zür. Vjschr. 7 (1862) 366-; 8 (1863) 79.

Huygens, "Exercitationes Mathematicæ." Biot, J. B. J. Sav. (1834) 291-。

Infinitesimal calculus, account of text books from Euler to present time. Bohlmann, $G$. D. Mth. Vr. Jbr. 6 (1899) (Heft 2) 91-.

Journalism, mathematical, in England. Mackay, J. S. As. Fr. C. R. (1893) (Pt. 2) 303-.

Lagrange's " Mécanique Analytique," Poinsot's corrections criticised. Breton (de Champ), $P$. Liouv. J. Mth. 1 (1875) 81-, 263-.

- - - , - justified. Bertrand, J. Liouv. J. Mth. 1 (1875) 181-.

Lhuilier's “Élements d'analyse, \&c." Gergonne, J. D. Gard Not. Tr. Ac. (1810) 196-. 


\section{Bibliographies}

Mathematical text books. Feyerabendt, D. Nf. Tbl. ("1880) 138.

Military text book of trigonometry, note on a. Ostrogradsky, - . [1851] St Pét. Ac. Sc. Bll. 10 (1852) 11-.

Raabe's, "Math. Mittheilungen," remarks and additions. Schläfli, L. [1857] Zür. Vjschr. 3 (1858) 23-.

Ramsing's and Holten's works, remarks. Lorenz, L. Mth. Ts. 3 (1861) 161-.

Tabulation of $l \sin \left(\frac{a \pi}{4}\right)$. Lindman, C. F. Ups. S. Sc. N. Acta 14 (1891) No. 1, 15 pp.

Treatise on Use of Higher Mathematics. Euler, L. [1741] Crelle J. 35 (1847) 106-.

Trisection of the angle, rare book on. Valentin, G. Bb. Mth. (1893) 113-.

Vega's "Thesaurus Logarithmorum," notes on. Gauss, C. F. As. Nr. 32 (1851) 181-.

Wronski's theory of numbers. Dickstein, $S$. Krk. Ak. (Mt.-Prz.) Rz. 4 (1893) 73-;396; Crc. Ac. Sc. Bll. 1892, 64-.

\section{Bibliographies.}

Aggregates, progress of theory 1893-99. Vivanti, G. Bb. Mth. 1 (1900) 160-.

Algebraic equations, history of proof of root existence. Loria, G. Rv. Mt. 1 (1891) 185-; 2 (1892) 37-; 3 (1893) 105-.

- - investigations concerning existence of roots. Loria, G. Bb. Mth. (1891) 99-.

Bernoulli's numbers. Ely, G. S. Am. J. Mth. 5 (1882) 228-.

"Biblioteca Matematica Italiana." Favaro, A. [1880] Ven. I. At. 7 (1881) 47-.

- - Riccardi, $P$. Bologna Ac. Sc. Mm. 10 (1889) 635-.

Decimal calculation. Terquem, O. N. A. Mth. 12 (1853) 195-.

Determinants. Muir, $T$. QJ. Mth. 18 (1882) 110 - ; 21 (1886) 299-.

Differential equations. Mansion, - Brux. S. Sc. A. 15 (1891) (Pt. 1) 32-, 60, 105.

Distributive operations. Pincherle, S. Bb. Mth. (1899) 13-.

Euclid. Riccardi, P. Bologna Ac. Sc. Mm. 8 (1887) 401-; 9 (1888) 321- or 111-; 1 (1890) 27- ; 3 (1892) 639-.

-, two editions of 1482. Valentin, $G$. Bb. Mth. (1893) 33-.

Euler's works, new list. Hagen, J. G. D. Mth. Vr. Jbr. 5 (1901) (Heft 1) 82-.

- writings. Valentin, G. Bb. Mth. (1898) 41-.

Geometry in $n$ dimensions, index of memoirs. Schlegel, $V$. Lpldina. 22 (1886) 160-; Ens. Mth. 2 (1900) 77-

一, triangle. Vigarié, $\dot{E}$. As. Fr. C. R. (1895) (Pt. 2) 50 -.

"Histoire de la perspective," certain Italian works omitted. Riccardi, P. Bb. Mth. (1889) 39-.

History of mathematics, Danish. Christensen, S. A., \& Heiberg, J. L. Bb. Mth. (1889) 75-.
Tables of Functions 0035

History of mathematics, Dutch. Bierens de Haan, D. Bb. Mth. (1891) 13-.

- - Italian. Favaro, A. Bb. Mth. (1892) $67-$.

- - - Norwegian. Holst, E. Bb. Mth. (1889) 97-.

- _ Polish. Dickstein, S. Bb. Mth. (1889) 43-; Prace Mt.-Fiz. 2 (1890) 247-; 3 (1892) 184-.

- - Portuguese. Teixeira, F. G. Bb. Mth. (1890) 91-.

- - - Spanish. Vicuña, G. Bb. Mth. (1890) 13-.

- - - Swedish, 1667-1888. Eneström, G. Bb. Mth. (1889) 1-.

$--\ldots$, Swiss. Suter, H. Bb. Mth. (1890) 97-.

Hyperspace and non-Euclidean geometry. Halsted, G. B. Am. J. Mth. 1 (1878) 261-, 384-; 2 (1879) 65-.

Imšenec̄kij, Vasilij Grigorjevič, list of works. Kharkov Mth. S. Com. 3 (1893) 290-.

Line geometry and its applications. Koenigs, $G$. Toul. Fac. Sc. A. 3 (1889) 24 pp.; 6 (1892) 67 pp.; 7 (1893) 55 pp.

Linear differential equations. Nixon, H. B. \& Fields, J. C. Am. J. Mth. 7 (1885) 353-.

Mathematical bibliographies. Eneström, $G$. Bb. Mth. (1890) 37-.

- bibliography, latest work in. Eneström, $G$. Bb. Mth. (1897) 65-.

- Mss. in Amplonian collection. Stein. schneider, $M$. Bb. Mth. (1890) 65-; (1891) 41-, 65-.

- publications (review). Favaro, A. Ven. I. At. (1892-93) 829-.

- work, extracts from, with complete list of author's publications. Catalan, $E$. C. Liège S. Sc. Mm. 12 (1885) No. 2, 407 pp.; 13 (1886) 404 pp. ; 15 (1888) No. 1, 275 pp.

Numbers, theory. Stieltjes, T.J. Toul. Fac. Sc. A. 4 (1890) 103 pp.

Nuncius Sidereus, Prague edition. Wohlwill, E. Bb. Mth. (1887) 100-

Russian physics and mathematics. Bobynin, V. V. Fschr. Mth. (1887) 2.

Sacrobosco's algorithm. Curtze, $M$. Bb. Mth. (1895) 36-.

- - certain editions. Riccardi, $P$. Bb. Mth. (1894) 73-.

Surfaces and twisted curves. Hill, J. E. N. Y. Am. Mth. S. Bll. 3 (1897) 133-.

Women and the exact sciences. Valentin, $G$. Bb. Mth. (1895) 65-.

- - - - Eneström, G. Bb. Mth. (1896) 73-.

\section{Tables of Mathematical Functions.}

Abscissae and ordinates of points on an arc of a circle $1 \mathrm{~m}$. radius, for intervals of $1 \mathrm{~cm}$. Cretin, -. (vII) A. Cond. Pon. Chauss. 6 (1862) 251-.

Advantages in using tables. Buzengeiger, K. H.J. Lindenau Z. 2 (1816) 23-. 
Annuity-value coefficients. Searle, T. J. I. Act. J. 28 (1890) 192-.

Arc radius and chord. Sigaud, -. (vm) A. Cond. Pon. Chauss, 1 (1857) 132-.

Arcs, tangents and chords. Sigaud, - - (vir) A. Cond. Pon. Chauss. 2 (1858) 122-.

Bernoulli's numbers, first 40 logarithms. Thoman, F. C. R. 50 (1860) 905-.

- - - 62. Adams, J. C. [1877] Crelle J. Mth. 85 (1878) 269-.

- - 250, and their logarithms. Glaisher, J. W. L. [1871-72] Camb. Ph. S. T. 12 (1873) 384-.

Bessel equation, roots. Willson, $R$. $W ., \&$ Peirce, B. O. N. Y. Am. Mth. S. Bll. 3 (1897) 153-.

- -, solution. Aldis, W. S. R. S. P. 64 (1899) 203-.

- functions $\dot{J}_{0}(k)$ and $J_{1}(k)$ (or $I_{k}^{0}$ and $I_{k}^{\prime}$ ) from $k=0$ to $k=15 \cdot 5$. Meissel, $E$. Berl. Ak. Ab. (1888) (Anh. Mth.) 23 pp.

- -, (2nd and 3rd Reports.) Brit. As8. Comm. B. A. Rp. (1893) 227-; (1896) 98-.

Binary duodecimic, generating functions and groundforms. Sylvester, J.J. Am.J.Mth. 4 (1881) 41-.

- sextic. Cayley, A. Am. J. Mth. 4 (1881) 379-.

Brigg's log. $n$ ! Jarolimek, $\check{C}$. Časopis 15 (1886) 70-; Fschr. Mth. (1886) 1123.

Calza's "tables of constants" to facilitate multiplication and division. Baravelli, G. C. Z. Mth. Ps. 44 (1899) 50-.

Complex prime factors, formed from 5th roots of unity, of primes of form $5 \mu+1$. Reuschle [C. G.]. Berl. Mb. (1859) 488-.

- $-1,-7$ th roots of unity, of primes up to 1000. Reuschle [C.G.]. Berl. Mb. (1859) $694-$.

- - - , - 11th roots of unity, of primes up to 1000. Reuschle [C. G.]. Berl. Mb. (1860) 190-.

- $-1,-13$ th roots of unity, of primes up to 1000 . Reuschle [C. G.]. Berl. Mb. (1860) 194-.

- - - , - - 17th roots of unity, of primes up to 1000. Reuschle [C. G.]. Berl. Mb. (1860) 714-.

,----19 th roots of unity, of primes up to 1000 . Reuschle [C. G.]. Berl. Mb. (1860) 719-.

- - - $-1-23 \mathrm{rd}$ roots of unity, of primes up to 1000 . Reuschle [C. G.]. Berl. Mb. (1860) 725-.

$-\ldots-1,-29$ th roots of unity, of primes up to 1000. Reuschle [C. G.]. Berl. Mb. (1860) 729-.

Compound interest. Weninger, V. (xiI) Mag. Ak. Éts. (Mth. Term.) 6 (1865) 207-.

Congruence $M \equiv a^{\alpha} b^{\beta}$ (Mod. $N$ ), specimen table. Cayley, A. QJ. Mth. 9 (1868) 95-.

Connecting curves (on railroads). Ribette, (viri) A. Cond. Pon. Chauss. 5 (1861) 108-.

Cremona's transformation between two planes. Roberts, S. [1872] L. Mth. S. P. 4 (187173) 121-.
Cube roots of primes to 31 places. Drach, S. M. [1877] Mess. Mth. 7 (1878) 86-.

Definite integrals. Bierens de Haan, D. Amst. Vh. 5 (1858) 1-.

- -, (Bierens de Haan's). Bellavitis, G. Ven. At. (1858-59) 423-.

- -, supplement. Bierens de Haan, D. [1861] Amst. Vh. Ak. 10 (1864) 92 pp.

$\Delta^{m} \mathrm{O}^{n} \div \Pi(m)$. Cayley, A. [1879] Camb. $\mathrm{Ph}$. S. T. 13 (1883) 1-.

Differential equations. Adams, Jas. Thomson A. Ph. 10 (1817) 116-.

Elliptic ares, Schmidt's. Verdam, G.J. Amst. I. (1842) 57-.

Empirical formulae, tables for facilitating determination. Hale, A. W. Am. J. Mth. 5 (1882) 342-.

Equation $m \sin ^{4} z=\sin (z-q)$, table for solving. Gasparis, A. de. As. Nr. 78 (1872) 177-.

Errata in several tables. Stanley, $A$. D. Silliman J. 5 (1848) 398-.

Exponential function $e^{x}$, to 12 places of decimals. Newman, F. W. [1887] Camb. Ph. S. T. 14 (1889) 237-.

Factor tables and formation of table for fourth million. Glaisher, J.W. L. [1878] Camb. Ph. S. P. 3 (1880) 99-, 228-.

- - Kulik's (report). Petzval, J. Wien Sb. 53 (1866) ( $A b .2) 460$-.

Formulae and numerical tables, collection. Hoüel, J. Bordeaux Mm. S. Sc. 4 (cah. 2) (1866) i-.

Fractions, with note on number of divisions required to find G.c.M. Hill, C.J.D. Lund Acta Un. 2 (1865) (Mth.) No. 1, 16 pp.

Function, transcendental. Buzengeiger, K.H.J. Zach M. Cor. 24 (1811) 583-.

- $\sigma(n)$ in theory of numbers. Mertens, $F$. Wien Ak. Sb. 106 (1897) (Ab. 2a) 761-.

$G(r, v)$-integrals. Brit. Ass. Comm. (Pearson, $K$.$) . B. A. Rp. (1899) 65-.$

Gaussian. Gould, B. A. Am. As. P. (1849) 362-.

-. Grunert, J. A. Grunert Arch. 30 (1858) 233-.

Gauss's antilogarithmic tables, statements concerning. Gray, P. Assur. Mg. 7 (*1858) 350-.

Geometry of numbers, with a table of squares of numbers up to 10,000 . Preobraschensky, P. W. Fschr. Mth. (1886) 135.

Goldbach's law concerning prime numbers. Haussner, R. Ac. Nt. C. N. Ácta 72 (1899) 1-.

Graphic, for computation. Gilbert, $G$. $K$. Smiths. Misc. Col. 25 (1883) Art. 2, 3 pp.; (Wash. Ph. S. Bll. 5 (1883).)

-, examples. Mehmke, R. Z. Mth. Ps. 44 (1899) 56-.

Hyperbolic functions, some errors in tables. Lampa, E. [1892] Tor. Ac. Sc. At. 32 (1896) 350 - or 530-.

- sectors, and logs. of their sines and cosines. Gronau, J. F. W. Danzig Schr. 6 (Heft 4) (1862).

Improvement, suggestion for. Macdonald, W. J. Edinb. Mth. S. P. 13 (1895) 144-.

Integrals, appendix. Bierens de Haan, $D$. Amst. Ak. Vh. 22 (1883) (No. 2) 225 pp. 


\section{Tables of Functions}

Interest, Smart's. Wilding, $R$. I. Act. J. 22 (*1881) 129-.

-, universal. Goodwyn, H. Nicholson J. 4 (1801) 433-.

Least primitive roots of all odd primes under 3000. Wertheim, G. Acta Mth. 17 (1893) 315-; 20 (1897) 157; 22 (1899) 200.

- _ - primes between 3000 and 5000 . Wertheim, G. Acta Mth. 20 (1897) 153-; 22 (1899) 200.

- primitives of prime numbers. Le Besgue, V. A. Bordeaux Mm. S. Sc. 3 (cah. 2) (1865) 231-.

Log. sin. and log. tan. of small ares. Drach, S. M. As. S. M. Not. 21 (1861) 147-.

Logarithm-integral, exponential integral, and other Eulerian integrals. Bellavitis, $G$. [1873] Ven. I. Mm. 18 (1874) 125-.

- Ms., 12 figure, from 1 to 120,000 , by Thomson, presented to R.A.S. Glaisher, J.W.L. As. S. M. Not. 34 (1874) 447-; 35 (1875) 135.

Logarithmic. Shortrede, R. Beng. J. As. S. 1 (1842) 40-.

- Glaisher, J. W. L. As. S. M. Not. 33 (1873) 440-.

abridged, Burnier's simplification of Flower's method. Hoüel, $J$. Bordeaux Mm. S. Sc. $8(1870) 188-$

-, Callet's, errors in. Lefort, $F$. $\quad$ C. R. 44 (1857) 1097-

-, - , - - Secretan, -. C. R. 44 (1857) 1276-, -, - - Dupuis, -. C. R. 45 (1857)

3 (1887) 130-.

- - , 2nd, correction. Herrmann, - Wien SB. (1848) 113-.

- errata in. Galbraith, W. As. Nr. 23 (1846) 109-.

-, - - Wackerbarth, A. D. As. S. M. Not. 27 (1867) 253-.

-, - - certain. Mendizábal Tamborrel, J. de. Méx. S. "Alzate" Mm. 5 (1891) (Rv.) 9-

-, facilities of certain. Gray, $P$. Assur. Mg. 11 (*1864) 230-

- and factor, with especial reference to Drach's suggestion. Glaisher, J. W. L. (vII) [1873] Mess. Mth. 3 (1874) 7 -。

-, formulae for. Merrifield, C. W. Assur. Mg. 6 (*1857) 298-.

-, index of sketch for. Bierens de Haan, D. Amst. Ak. Vh. 15 (1875) 35 pp.

- to more than 7 places, utility. Govi, G. Tor. At. Ac. Sc. 8 (1872-73) 157-

-, note on some. Mendizábal Tamborrel, $J$. de. Méx. S. "Alzate" Mm. 1 (1887) 223-.

-, of 1849 , errata. Shortrede, R. As. S. M. Not. 27 (1867) 272-.

- sines, construction of canon. Sang, $E$. Edinb. R. S. P. 12 (1884) 601-.

-, suggestions. Hanlon, G. O. (vI) [1873] Mess. Mth. 3 (1874) 100-.

- and trigonometric. Folie, F. Liége Mm. S. Sc. 1 (1866) 89-.

-, Vega, errata. Kulik, J. P. As. Nr. 3 (1825) 191-.
Tables of Functions 0035

Logarithmic, Vega, errata. Bremiker, (Prof.) -. As. Nr. 85 (1875) 373-.

-, Vlacq's (or Briggs'), errors in. Glaisher, J. W. L. As. S. M. Not. 32 (1872) 255-, 288-.

-, - (- - ), - - Sang, E. Edinb. R. S. P. 8 (1875) 371-; Nt. 42 (1890) 593.

Logarithms and anti-logarithms to ten places, formation. McKenzie, D. J. McG. I. Act. J. 24 (1884) 243-.

- $-1,-,-$, (note on McKenzie's paper). Hardy, G. F. I. Act. J. 24 (1884) $252-$.

- - - - twelve places. Gray, P. Assur. Mg. 12 (" 1866) 71-, 121-, 212-, 252-.

-, to calculate. Perry, J. Nt. 59 (1898-99) $393,439$.

-, - - Dufton, A. Nt. 61 (1899-1900) 415. -, - - Perry, J. Nt. 61 (1899-1900) 415-.

- of circular and hyperbolic functions. Forti, A. Pisa A. Un. Tosc. Sc. Cosm. 6 (1861) 5 -.

-, error in a seven figure set. Sang, $E$. I. Act. J. 17 (*1873) 142, 300-.

- of sum and difference of two numbers. Hill, N. Franklin I. J. 140 (1895) 130-.

-, ten place. Chisholm, J. I. Act. J. 26 (1887) 55 -

- of vulgar fractions, with numerator and denominator not over 100 . Airy, G. B. I. CE. P. 65 (1881) 271-.

Manuscript table of Napierian logarithms, sines \&c. Pearson, J.B. [1878] Camb. Ṕh.S. P. 3 (1880) 146-.

Mathematical functions, certain. Brit. Ass. Comm. B. A. Rp. (1898) 145.

Method for extending accuracy of tables. Sheppard, W. F. [1899] L. Mth. S. P. 31 (1900) 423-.

Multiplication by table of single entry. Sylvester, J.J. Assur. Mg. $4\left({ }^{*} 1854\right) 236$-.

- - - - - Glaisher, J.W.L. Ph. Mg. 6 (1878) 331-.

- table, oldest. Ebert, $R$. Dresden Isis Sb. (1896) (Ab.) 44-.

Napierian logarithm of $\pi$ to 30 decimal places. Glaisher, J.W.L. QJ. Mth. 25 (1891) 362-, 384 .

- logarithms of 2, 3, 5, 7 and 10. Adams, J. C. R. S. P. $42(1887) 22-$.

Natural logarithms of Nos. 1-25 to 81 places. Vietz, -. As. Nr. 4 (1826) 53-.

- trigonometrical ratios, and inverses of numbers. Folie, $F$. Liége S. Sc. Mm. 9 (1882) No. 2, $11 \mathrm{pp}$.

Numbers 1-4100 partitioned into 2 squares. Bretschneider, C. A. [1851] Crelle J. 46 (1853) 1-.

Numerical table, and application to certain transcendentals. Catalan, $E$. Brux. Ac. Mm. 47 (1889) No. 3, $26 \mathrm{pp}$.

- tables to assist in change of coordinates. Vinot, -. C. R. 101 (1885) 938-.

- - new method of arranging. Dittmar, $W$. Ph. Mg. 21 (1861) 137-.

- value of $\int_{0}^{h} e^{x z} d x$. Daxoson, H. G. L. Mth. S. P. 29 (1898) 519-. 


\section{Tables of Functions}

Odd numbers 1-191 divided into square numbers. Pollock, (Sir) F. [1853] Phil Trans. (1854) 311-.

- - products of all, from 11 to 99 to 3 figures. Anon. Brux. Obs. A. (A8.) 7 (1896) [at end of vol.].

Prime factors, decomposition of numbers into. Le Besgue, V. A. Bordeaux Mm. S. Sc. 3 (cah. 1) (1864) 1-.

- -, discovery. Hill, C. J. D. Crelle J. 12 (1834) 355-.

Primitive roots. Kulik, J. P. Crelle J. 45 (1853) 55-.

- - of primes from 3 to 101. Crelle, A. L. Crelle J. 9 (1832) 27-.

- - of prime numbers. Ostrogradsky, M. A. [1836] St Pét. Ac. Sc. Mm. 3 (1838) 359-.

Prony's logarithmic and trigonometrical tables. Sang, E. [1874] Edinb. R. S. P. 8 (1875) 421-.

- table of logarithms (Reply to Lefort). Sang, E. Edinb. R. S. P. 8 (1875) 581-.

Proportional logarithms. Shortrede, $R$. Beng. J. As. S. 10 (1841) 713-.

- parts, Vega's. Hartmann, J. Grunert Arch. 31 (1858) 63-.

Pythagorean triangle. Bretschneider, C. A. Grunert Arch. 1 (1841) 96-.

Quadratic binary forms. Cayley, A. [1860] Crelle J. 60 (1862) 357-.

- equations with real roots. Mehmke, $R$. Z. Mth. Ps. 43 (1898) 80-.

- ternary forms, reduced positive. Eisenstein, G. Crelle J. 41 (1851) 141-, 227-.

Quarter squares, method of using table. Laundy, S. L. Assur. Mg. 9 ("1861) 112-. Report of Commission on tables. Mehmke, $R$. D. Mth. Vr. Jbr. 7 (1899) (Heft 1) 123-.

Rhind papyrus. Mansion, - Brux. S. Sc. A. 12 (1888) (Pt. 1) 44-.

Sang's seven-figure logarithms. Ellis, A. J. I. Act. J. 17 ("1873) 298.

- - - Glaisher, J. W. L. I. Act. J. 17 ("1873) 298-. -299-. Tucker, R. I. Act. J. 17 (*1873)

Signs of trigonometrical lines. Henri, M. N. A. Mth. 7 (1848) 285-.

Sine-integral, cosine-integral, exponential integral. Glaisher, J.W.L. Phil. Trans. 160 (1870) 367-.

-, tangent, etc. of small angles from 5 figure tables by using secants. Pfeil, L. von. Arch. Mth. Ps. 42 (1864) 305-.

Sines, for use in plotting mines. La Chabeaussière, A. J. M. P. J. Mines 35 (1814) 321-.

Smallest number of entries necessary in a 7 figure log. table. Steggall, J.E. A. Edinb. Mith. S. P. 10 (1892) 35-.

Squares and cubes. G., $\dot{H}$. Nicholson J. 8 (1804) 150-; 9 (1804) 123-.

_- - calculation. O., E. Nicholson J. 9 (1804) 4-, 171-.

- - , (Jahn), errors. Kulik, J. P. Böhm. Gs. Ab. 2 (1843) (Sect. B. 1841) 19-.

Sum or difference of two numbers whose logs. are given. Gauss, C. F. Zach M. Cor. 26 (1812) 498-.
Addresses, Lectures, etc. 0040

Syzygies, irreducible, of quantics. Sylvester, J. J. Am. J. Mth. 4 (1881) 41-.

Trigonometric functions of cyclic and hyperbolic sectors. Gronau, J. F. M. [1863-65] Danzig Schr. 1 (1866) (Heft 1) viii + 151 pp.; (Heft 2) $4 \mathrm{pp}$.

-, history question. Prony, R.de. Par. Mm. de l'I. 5 (1802) (H.) 67-.

-, new system. Bouché, $A$. M.-et-L. Mm. S. Ac. 8 (1860) 145-.

-, note. Hill, C. J. D. Lund Phys. Sällsk. Ts. 1 (1838) 283-; Grunert Arch. 1 (1841) 191-.

Trigonometry and astronomy, fundamental tables. Sang, E. Edinb. R. S. P. 16 (1890) 249-.

Two old tables. Pendlebury, $R$. Mess. Mth. 4 (1875) 8-.

Values of $\Delta^{n} \mathrm{O}^{m}$. Herschel, $J$. [attriluted to Lacroix in (III)]. N. A. Mth. 13 (1854) 272-.

- $S_{k}=\sum n^{-k}$. Stieltjes, T. J. Acta Mth. 10 (1887) 299-.

- - 1.2.4 ...(x-1)/2.3.5 ...x, denominators being primes. Glaisher, J. W. L. Mess. Mth. 28 (1899) 1-.

Zonal harmonics. Perry, J. [1890] L. Ps. S. P. 11 (1892) 221-; Ph. Mg. 32 (1891) 512-.

\section{Addresses, Lectures, etc., of a general character.}

American Mathematical Society, past and future. McClintock, E. [1894] N. Y. Am. Mth. S. Bll. 1 (1895) 85-.

Astronomy, geometry, and arithmetic, three discourses. Gqrnier, J. G. (vI Add8.) Gand A. Ac. (1817-18) $83 \mathrm{pp.}$

Chicago, inaugural address, Aug. 21, 1893. Klein, $F$. N. Y. Mth. S. Bll. 3 (1894) 1-.

Collaboration in mathematics. Greenhill, $A . G$. [1892] L. Mth. S. P. 24 (1893) 5-.

Combinatory analysis: a review. MacMahon, P. A. [1896] L. Mth. S. P. 28 (1897) 5-.

Confluences and bifurcations of certain theories. (Presidential address, 1888.) Cockle, (Sir) $J$. L. Mth. S. P. 20 (1889) 4-.

"Deutsche Naturforscher," address at meeting, 1858. Eisenlohr, $W$. Grunert Arch. 32 (1859) 140 -

Differential equations and applied mathematics, historical sketch. Naetsch, E. Civing. 41 (1895) 655-

General considerations. Glaisher, J. W. L. B. A. Rp. (1890) 719-.

Geometrical method. (Presidential address.) Larmor, J. Mth. Gz. 1 (1900) 1-.

- researches, comparative reflections on recent. Klein, F. A. Mt. 17 (1889-90) 307-.

Geometry applied to practical life. Pearson, $K$. Nt. 43 (1891) 273-.

Grassmann's "Ausdehnungslehre" applied to mechanics. Schlegel, $V$. Civing. 40 (1894) 59 -.

Historiography of mathematics. Cantor, [1900] N. Y. Am. Mth. S. Bll. 7 (1901) $57-$. 
0040 Addresses, Lectures, etc.

History of mathematics and physics. (Anniversary address.) Knibbs, G. H. N. S. W. R. S. J. 33 (1899) 1-.

Influence of applied, on the progress of pure, mathematics. (Presidential address.) Walker, J. J. [1890] L. Mth. S. P. 22 (1891) 4-.

Invariants of motion, and elementary geometry. Study, E. Leip. Mth. Ps. B. 48 (1896) 649-.

Lectures at École Normale. La Place, P. S. (le marquis) de. Par. Sé. Éc. Norm. 1 (1800) 16-, 268-, 381-; (pte. 2) 3-, 130-; 2 (1800) 116-, 302-; 3 (1800) 24-; 4 (1800) 32-, 223-; 5 (1800) 201-; 6 (1800) 32-.

- - - Lagrange, J. L. de. Par. Sé. Ec. Norm. 1 (1800) (pte. 2) 3-, 34-; 3 (1800) 227-, 276-, 463-; 4 (1800) 401-; 10 (1801) $5-$.

- - - - (elementary), 1795. Lagrange, J. L. de. Par. Éc. Pol. J. 2 (1812) $\left(7^{\circ}\right.$ et $8^{\circ}$ cah.) 173-.

Mathematical problems. Hilbert, D. Gött. Nr. (1900) 253-.

- theories, etc. (Presidential address, 1883.) Cayley, A. B. A. Rp. (1883) 3-.

- tripos, the. (Presidential address.) Glaisher, J. W. L. L. Mth. S. P. $18(1886-87) 4-$

Mathematics. Kempe, A. B. [1894] L. Mth. S. P. 26 (1895) 5-.

_, influence on physical science. Bastarrica, J. Chile A. Ûn. (1852) 41-.

- and natural science, mutual relations. Wild, J. St Gal. B. (1885-86) 101-.

- Phys. Sect. Address, 1865. Spottiswoode, $W$. B. A. Rp. 35 (1865) (Sect.) 1-.

- - - - 1869. Sylvester, J. J. B. A. Rp. 39 (1869) (Sect.) 1-.

,,---- 1870 . Maxwell, J. C. B. A. Rp. 40 (1870) (Sect.) 1-.

,,---- 1871 . Tait, P. G. B. A. Rp. 41 (1871) (Sect.) 1-.

43 (1873) (Sect.) 1-.

- _ - Presidential address, 1883. Henrici, O. M. F. E. B. A. Rp. (1883) 393-.

- $-,-\ldots, 1897$. Pure mathematics. Forsyth, A. R. B. A. Rp. (1897) 541-.

Mission of science, address. Thurston, $R . H$. Am. As. P. (1884) 227-.

Modern function theory, development. Krause, -. Civing. 34 (1888) 639-.

Moscow Math. Soc., Jubilee, address. Bugaev, N. V. [1894] Rec. Mth. (Moscou) 18 (1896) vi-.

- - Presidential address, March 21st, 1900. Bugaev, N. V. Rec. Mth. (Moscou) 21 (1901) 537-.

Purposes of Brit. Assn., and external aspects of mathematics. (Presidential address, 1878.) Spottiswoode, W. B. A. Rp. (1878) 1-.

Riemann's surfaces. Hypergeometric function. Differential equations. Theory of numbers. (Lectures.) Klein, F. [1891-96] Mth. A. 45 (1894) 140-; 46 (1895) 77-; 48 (1897) 562-.

Secondary needs and opportunities of English mathematicians. (Presidential address.) Elliott, E. B. [1898] L. Mth. S. P. 30 (1899) 5-.
Pedagogy 0050

Universities and technical schools, address. Klein, E. D. Mth. Vr. Jbr. 7 (1889) (Heft 1) 39-.

\section{Pedagogy.}

Actuarial competence, requisites. Sutton, $W$. I. Act. J. 16 (*1872) 434-.

Actuaries, importance of mathematical studies. Sprague, T. B. I. Act. J. $18\left({ }^{*} 1875\right) 403-$.

-, mathematical education. Kiepert, $L$. D. Nf. Vh. (1894) (Th. 2, Hälfte 1) 16-.

-, - - Fehr, H. Ens. Mth. 1 (1899) $447-$.

Actuary, education. Porter, H. W. Assur. Mg. $4\left({ }^{*} 1854\right)$ 108-.

Algebra, the first chapter. Anon. Educ. Times 39 (1886) 326-.

- in schools. Heppel, G. Mth. Gz. No. 4 (1895) 25-.

-, teaching, in 1676. (Henrion's "Euclid.") Mansion, - Brux. S. Sc. A. 19 (1895) (Pt. 1) 101-

American colleges, mathematics. $E d d y, H . T$. Am. As. P. (1884) 37-.

Analytical geometry, combination of planimetry and stereometry in teaching. Ripert, $L$. As. Fr. C. R. (1900) (Pt. 2) 1036-.

Application of mathematical and mechanical principles to technical problems. Heun, $K$. D. Mth. Vr. Jbr. 5 (1901) (Heft 1) 91-.

Arithmetic and algebra, elementary teaching. Recknagel, G. D. Nf. Vh. (1899) (Th. 2, Hälfte 1) 279-.

-, the first stage. Quick, (Rev.) R. H. Educ. Times 41 (1888) 227-.

- , the five fundamental operations. Kupffer, $K$. Dorpat Sb. 10 (1895) 359-

-, teaching. Edinb. Mth. S. Comm. Edinb. Mth. S. P. 6 (1888) 89-.

-, -, method. Elowson, G. Ts. Mt. Fys. 2 (1869) 40 -

_, - - Moesta, C. W. Chile A. Un. (1852) 322-.

Arithmetical problems in secondary schools, treatment. Burali-Forti, C. Rv. Mt. 1 (1891) 31-

Blind, teaching of the congenitally. Penjon, -. Gergonne A. Mth. 3 (1812-13) 308-.

Combination of solid with plane geometry in teaching. Candido, G. Ens. Mth. 1 (1899) $204-$.

Descriptive geometry, first lesson. Budelot, $G$. Ens. Mth. 1 (1899) 286-.

- - , lacuna in the teaching. Tilly, (Maj.) de. Brux. S. Sc. A. 9 (1885) (Pt. 2) 95-.

Educational testimony concerning the calculus. Meech, L. W. Des Moines Anal. 1 (1874) 133-.

Elliptic functions, teaching of theory. Mansion, -. Brux. S. Sc. A. 24 (1900) (Pt. 1) 54-

Equations, choice of examples in teaching. Breton [de Champ], P. N. A. Mth. 2 (1843) 539 -.

Errors in mathematical text books. Beyer, - . Grunert Arch. 3 (1843) 113-.

Euclid and teaching of geometry. Magnus, $P$. Educ. Times 33 (*1880) 81-. 


\section{Pedagogy}

Examination questions, composition. Laisant, C. A. Ens. Mth. 1 (1899) 120-

- - solutions. Sprague, T. B. Assur. Mg. 10 ( $^{*} 1863$ ) 45-; 13 (*1867) 253- ; I. Act. J. 14 (* 1869) 147-.

- - - - (a correction). Adler, M. N. I. Act. J. 14 (*1869) 242.

Examinations, judging in mathematical. Bordoni, A. [1841] Mil. Mm. I. Lomb. 1 (1843) 189-.

-, problems suitable. Wittstein, T. Grunert Arch. 7 (1846) 27-.

Exposition of pure mathematics. Delarue, D. M. (xп) [1869] Rec. Mth. (Moscou) 4 (1869-70) (Pt. 2) 81-.

Formulæ, special application of mnemonics. Hoffmann, L. Förster Al. Bauztg. 10 (1845) 362-.

Geometrical drawing, teaching. Henrici, 0 . B. A. Rp. (1895) 608 .

- teaching, reform. Fiedler, W. Zür. Vjschr. 22 (1877) 82-.

- - - - Wittek, H. D. Nf. Vh. (1894) (Th. 2, Hälfte 1) 317-.

Geometry and analysis, higher, Naples University, 1870-71. Bernardinis, G. de, \& Fuortes, T. G. Mt. 9 (1871) 233-.

-, elementary, new trend in. Ciamberlini, $C$. Rv. Sc.-Ind. 30 (1898) 99-, 113-, 153-.

-, - teaching. Develey, - Bb. Un. 8 (1818) 257-.

-, -, -. Barclay, A. J. G. Edinb. Mth. S. P. 2 (1884) 24 -.

-, 一, -. Diekmann, -. D. Nf. Tbl. (1888) $342-$.

-, experiment in teaching. Fraser, A. $Y$. Edinb. Mth. S. P. 5 (1887) 94-.

-, first teaching. Laisant, C. A. Ens. Mth. 1 (1899) 339-.

-, higher, teaching. Mth. 12 (1847) 1-.

-, natural. Mault, A. Tasm. R. S. P. (1886) 103-.

-, practical, development. (Graphics.) Anon. Elect. 28 (1892) 298-.

-, -, -. (-.) Thompson, S. P. Elect. 28 (1892) 332-.

-, - solid, teaching in higher schools. Hildebrandt, C. D. Nf. Vh. (1897) (Th. 2, Hälfte 1) 34-.

- - - - - at Strassburg. Krazer, A. D. Mth. Vr. Jbr. 8 (1900) (Heft 1) 119-.

- in schools. Heppel, $G$. Educ. Times 50 (1897) 181-

-, teaching. Dallas, R. J. Nt. 59 (189899) 416.

-, - Reinhardt, N. V. Kazan S. Ps.-Mth. Bll. 8 (1899) (Prot.) 19-.

- versus Euclid. Minchin, G. M. Nt. 59 (1898-99) 369-.

Graduated system of teaching, especially in geometry. Bazala, J. D. Nf. Vh. (1894) (Th. 2, Hälfte 1) 313-.

Higher mathematics at Italian Universities, teaching. Pascal, E. Rv. Mt. 3 (1893) 170-.

Hyperbolic functions in teaching. Barbarin, $\bar{P}$. Ens. Mth. 2 (1900) 443-.
Pedagogy 0050

Imaginaries, treatment in higher school teaching. Pietzker, F. [1898] D. Mth. Vr. Jbr. 7 (1899) (Heft 1) 154-.

Indices and surds, teaching. Genese, $R . W$. Mth. Gz. 1 (1900) 339-.

Infinitesimal calculus, course. Obrecht, $A$. Santiago de Chile Un. A. 87 (1894) 533-, 657-, 715-; 88 (1894) 39-; 90 (1895) 45-; 91 (1895) 677-; 93 (1896) 105-, 241-; 103 (1899) 699-, 887-; 104 (1899) 39-, 201-, 359-, 747-, 1061-; 106 (1900) 79-, 375-, 545-, 949-, 1097-.

Infinity in elementary mathematics. Lamarle, E. [1851] Brux. Ac. Sc. Mm. 27 (1853) 31 pp.

Inverted problems (an old arithmetic book). C., H. A. Educ. Times 48 (1895) 29 .

Languages and mathematics in state schools of modern Germany. Baumann, H. Educ. Times 37 (1884) 98-.

Leibnitz, calculus of, and its teaching. Arnoux, R. As. Fr. C. R. (1900) (Pt. 2) 1089-.

Mathematical history, 8 years of teaching. Favaro, A. Bb. Mth. (1887) 49-.

- -, lectures in Moscow University, programme of course. Bobynin, $V . V$. Fschr. Mth. (1890) 87; Bb. Mth. (1891) 79-.

- - - at Munich. Steinschneider, $\dot{M}$. Bb. Mth. (1897) 113-.

- -, university course. Eneström, G. Bb. Mth. (1890) 1-.

- instruction in France. Pierpont, J. N. Y. Am. Mth. S. Bll. 6 (1900) 225-.

- problems, and the development of mathematics. Hilbert, D. Ens. Mth. 2 (1900) 349-.

- teaching. Hudson, W. H. H. Educ. Times 46 (1893) 135-.

- - Biraben, F. Arg. S. Ci. A. 48 (1899) 106-, 156-.

- -. Duclaux, E. Rv. Sc. 11 (1899) 353-. -. Garcia de Galdeano, Z. As. Fr. C. R. (1900) (Pt. 2) 1052-

- -. Perry, J. Nt. 62 (1900) 317-.

- -. Heaviside, O. Nt. 62 (1900) 548-.

- changes. Piola, G. (viri) [1840] Bb. It. 2 (1841) 159-.

-, curiosities in early English. Heppel, G. Educ. Times 38 (1885) 145-.

- - elementary. Laisant, -. Rv. Sc. 11 (1899) 358-.

- - general principles. Galdeano, Z. G. de. Ens. Mth. 1 (1899) 195-.

- at German Universities, especially Göttingen. Fano, G. Rv. Mt. 4 (1894) 170-.

- - in Germany, history. Günther, S. Ens. Mth. 2 (1900) 237-.

- - in higher grade schools (gymnasiums and lyceums). Catania, $S$. Rv. Mt. 5 (1895) 33-.

- - Holland. Cardinaal, J. Ens. Mth. 2 (1900) 317-.

- -, methods. Grunert, J.A. Arch. Mth. Ps. 42 (1864) 348-.

- - in preparation for the "École Polytechnique." Laurent, $H$. Ens. Mth. 1 (1899) 38-. 
Mathematical teaching, reform. Mair, D. Nt. 62 (1900) 389.

- - - -. Woollen, H. Nt. 62 (1900) 436.

- -, -. Beard, W. F. Nt. $62(1900) 466$.

- - - -. Stromeyer, C.E. Nt. 62 (1900) 523.

- in Russia. Bobynin, V. V. Ens. Mth. 1 (1899) 420-.

- _ - - history. Bobynin, $V$. V. Ens. Mth. 1 (1899) 77-.

- - - schools. Lie, S. Christiania F.

(1884) No. 16, 8 pp.; (1885) No. 11, 10 pp. - - -. Tafelmacher, A. Santiago de Chile Un. A. 85 (1893) 35-.

- - , simplification of elementary. Heppel, $G$. Educ. Times 44 (1891) 463-.

- - in United States. Safford, T. H. N. Y. Mth. S. Bll. 3 (1894) 4-.

- - - universities in 1894. Galdeano, Z. G. de. Fschr. Mth. (1893-94) 118.

- _ - - problems and methods. Klein, F. D. Mth. Vr. Jbr. 7 (1899) (Heft 1) 126-. - - use of history. Heppel, $G$. Nt. 48 (1893) 16-.

- - , - - practical applications. Bettazzi, R. Ens. Mth. 2 (1900) 14-.

Mathematics for astronomy and navigation. Wilson, $T$. Mth. Gz. No. 4 (1895) 27-.

- at certain German Universities. Dillner, G. Ups. Årsk. (1876) $37 \mathrm{pp}$.

-, elementary, economy of thought. Meyer, W. F. D. Mth. Vr. Jbr. 7 (1899) (Heft 1) 147-.

- in girls' schools. Bryant, (Mrs.) -. Educ. Times $31\left(^{*} 1878\right)$ 145-.

-, how to study. Degen, C. F. Kiob. Dn. Vd. Selsk. Skr. 2 (1801-2) 75-.

- relations between university and high school in domain of. Schotten, H. D. Nf. Vh. (1898) (Th. 2, Hälfte 1) 20-.

- in Russia, history of study. Bobynin, $V$. Bb. Mth. (1888) 103-.

- schools. Heppel, G. Educ. Times 39 (1886) 385-.

- as a scientific and pedagogic aid. Bugaev, N. V. (xm) [1869] Rec. Mth. (Moscou) 3 (1868) (Pt. 2) 183-.

- in Spain. Galdeano, Z. G. de. Ens. Mth. 1 (1899) 6-.

- - - in 1893-94. Galdeano, Z. G. de. Fschr. Mth. (1893-94) 117.

- - teaching of physics, importance. Calzecchi, T. Rv. Sc.-Ind. 30 (1898) 65-.

Mensuration, note. Lefevre, $A$. Texas Ac. Sc. T. 2 (No. 2) (1899) 39-.

Multiplication, method of performing. Berdellé, C. As. Fr. C. R. (1884) (Pt. 2) 584-.

Notion of the infinite in teaching elementary geometry. Ripert, L. Ens. Mth. 2 (1900) 127-.

- - - - - - - - (Correction of error in Ripert's paper, p. 129.) Niewenglowski, B. Ens. Mth. 2 (1900) 219.

- - a limit in teaching. Clasen, Braunschw. Vr. Nt. Jbr. (11) (1899) 159-.

- on number and limit in teaching. Pringsheim, A. D. Mth. Vr. Jbr. 6 (1899) (Heft 1) $73-$.
Problem solving as a discipline. Hall, $A$. Wash. Ph. S. Bll. 11 (1892) 598-.

Pure mathematics for engineering students. Hathaway, A. S. [1900] N. Y. Am. Mth. S. Bll. 7 (1901) 266-.

Quadratic equation, theory. Macfarlane, $A$. Ens. Mth. 2 (1900) 363-.

- equations, solution. Mathews, G.B. Nt. 57 (1897-98) 463-.

Reducing results of measurement. Rudel, $K$. D. Nf. Vh. (1893) (Th. 2, Hälfte 1) 217-。

Role of mathematics in general education. Budelot, G. Ens. Mth. 1 (1899) 222.

Rules of multiplication, their nature and teaching. Baron, R. Ens. Mth. 1 (1899) 317-.

Scientific method in teaching. Binet, $A$. Ens. Mth. 1 (1899) 29-.

Smith's-Prize papers. Cayley, A. Mess. Mth. 3 (1874) 165-; 4 (1875) 6-; 6 (1877) 173-.

Statutes for testing teachers, and Mathematical course. Hauck, G. D. Nf. Vh. (1899) (Th. 2, Hälfte 1) 17-.

- - - (Prussian.) Weber, H. D. Nf. Vh. (1899) (Th. 2, Hälfte 1) 9-.

- - - (-.) Schotten, H. D. Nf. Vh. (1899) (Th. 2, Hälfte 1) 26-.

Statutory tests for teachers in Bavaria. Rudel, K. D. Nf. Vh. (1899) (Th. 2, Hälfte 1) 29-.

Synthetic and projective geometry, need of, in analytical geometry. Davidescu, $D . A$. Bucarest S. Sc. Bll. (1900) 19-.

Systematisation of mathematics. Ellis, A.J. R. S. P. 10 (1859-60) 85-.

Technical High Schools in America. Engels, $H$. Civing. 40 (1894) 13-.

instruction, especially its geometry. Paganini, C. Mil. G. I. Lomb. 1 (1841) 291-.

- teaching in Chili. Noguès, A. F. [189394] Santiago de Chile Un. A. 85 (1893) 277-, 345-, 515-.

Training of teachers of science in higher schools, in view of modern requirements. Schwalbe, B. D. Nf. Vh. (1896) (Th. 2, Hälfte 1) 15-.

Trigonometry, teaching. Aragón, A. Méx. S. "Alzate" Mm. 5 (1891) 69-.

-, - of elements. Fehr, H. Ens. Mth. 1 (1899) 45-.

'Unitary' method in teaching proportion. Beta. Educ. Times 32 (* 1879) 259-.

Universities and technical schools, address. Klein, F. D. Mth. Vr. Jbr. 7 (1899) (Heft 1) 39-.

University lectures on infinitesimal calculus. Pringsheim, A. D. Mth. Vr. Jbr. 7 (1899) (Heft 1) 138-.

Vector theory, teaching. Fontené, G. Ens. Mth. 1 (1899) 50-.

\section{Institutions.}

Franklin Institute during 75 years. Thurston, R. H. Franklin I. J. 149 (1900) 81-. 


\section{Nomenclature.}

"Al-djebr," exact meaning of word. Vaux, C. de. Bb. Mth. (1897) 1-.

Algorithm, meaning of term. Hunrath, $K$. Bb. Mth. (1887) 70.

Antiparallels. Langley, E. M. Nt. 40 (1889) 460 -.

"Autotomic" curves. Basset, A. B. Nt. 62 (1900) 572.

- - Orchard, H. L. [1900] Nt. 63 (190001) 7 .

- - Thorn, A. S. [1900] Nt. $63(1900-$ 01) 7 .

- - Basset, A. B. [1900] Nt. 63 (190001) 82 .

Chinese arithmetical notation. Edkins, Nt. 37 (1888) 65.

"Conjugate angles," new geometrical term. Worthington, A. M. Nt. 47 (1892-93) 8.

Cuneiform numerical signs, transmission. Bobynin, V. Bb. Mth. (1893) 18-.

Curves without double points. Richmond, $H$. [1900] Nt. 63 (1900-01) 58.

Decimal division of circle (i.e. of right angle). Hatzfeld, A. Rv. Sc. 48 (1891) 655-.

Determinant notation. Nanson, $E, J . \mathrm{Ph}$. Mg. 44 (1897) 396-.

Differential notation and teaching. Poincaré, $H$. Ens. Mth. 1 (1899) 106-.

Duodecimal system. Heaviside, $O$. Elect. 37 (1896) 489.

Functions, growth of notation for. Eneström, G. Bb. Mth. (1891) 89-.

General and logico-mathematical notation. Oliver, J. E. A. Mth. 4 (1888) 186-.

Geometrical square, nomenclature. Weissenborn, $H$. Bb. Mth. (1888) 37.

Geometrography or systematic notation for geometric constructions. Lemoine, $E$. G. Teix. J. Sc. 12 (1895) 114-.

Gnomon theorem (Euc. I. 43). Allman, G. $J$. Bb. Mth. (1887) 22.

Kardaga. Steinschneider, M. Bb. Mth. (1891) 114.

Language, mathematical and scientific. Anon. (vi 543). Gergonne A. Mth. 12 (1821-22) 322-.

Logarithms, notation. Draenert, -. D. Nf. Tbl. (*1876) (Suppl.) 173-.

Nomenclature. Dantas Pereira, J. M. Lisb. Mm. Ac. Sc. 10 (1830) (pte. 2) 197-.

-, geometrical. Fontené, G. Ens. Mth. 2 (1900) 134-

of metric system. Rautenfeld-Lindenruh, H. von. Riga Cor.-Bl. 38 (1895) 85.

Notation. Lubbock, J. W. [1829] As. S. Mm. 4 (1831) 471-.

-. Sylvester, J. J. Ph. Mg. 7 (1854) 50-.

-. Houzeau, J. C. Brux. Ac. Bll. 39 (1875) 487-.

-, algebraic. Jarrett, T. [1827] Camb. $\mathrm{Ph}$. S. T. 3 (1830) 65-.

-, -. Collins, J. V. Science '20 (1892) 359.

VOL. I.
Notation, arithmetical. McColl, H. Educ. Times 33 (*1880) 111.

- calculus. Conto, M. V. do, \& Simões Margiochi, F. Lisb. Mm. Ac. Sc. 3 (pte. 2) (1814) 48-.

- of decimal fractions. Gravelaar, N. L.W.A. N. Arch. Wisk. 4 (1899) 54-; Fschr. Mth. (1899) 37.

-, numerical. Prevost, P. Bb. Un. 3 (1816) 306-.

-, questions concerning. Ripert, L. Ens. Mth. 1 (1899) 139.

- useful in analysis. Ocagne, M. d'. Par. S. Mth. Bll. 15 (1887) 156-.

$\pi$ to denote $3 \cdot 14159 \ldots$, first use. Eneström, $G$. Bb. Mth. (1889) 28.

- - - - Ball, W. W. R. Bb. Mth. (1894) 106.

Printing mathematical symbols. Mitchell, C. T., and others. Elect. 29 (1892) 652, et seq.

- - - Thompson, S. P. Nt. 46 (1892) 513.

- mathematics. Cassie, W. [1892] Nt. 47 (1892-93) 8-.

- -. Jackson, M. J. Nt. 47 (1892-93) 227.

Quantitative comparisons, a common error in speech. Johnson, G. H. Science 22 (1893) 352.

Roulettes. Willson, F. N. Am. As. P. (1887) 59 -.

Signs (for functions, operations, \&o.). Hoppe, R. Arch. Mth. Ps. 61 (1877) 323-.

Spoken numeration, from international point of view. Berdellé, C. Ens. Mth. 1 (1899) 269-.

Symbols. Hall, A. [1889] A. Mth. 5 (188990) 19 .

- of non-existence. Doolittle, M. H. Wash. Ph. S. Bll. 11 (1892) 603.

Terminology. Laisant, C. A. Ens. Mth. I (1899) 22-.

- and conceptions. Györy, S. (xII) Mag.

Tud. Ak. Éts. 3 (No. 12) (1869) 131-.

-, mathematical. Roberts, S. [1882] L. Mth. S. P. 14 (1882-83) 5-.

-, suggestions. Muirhead, R. F. Edinb. Mth. S. P. 13 (1895) 143.

Trigonometry, development of notation. Braunmühl, A. von. Bb. Mth. 1 (1900) 64-.

Umbral notation. Harley, (Rev.) $R$. B. A. Rp. (1887) 600-.

Uniform notation for mathematical and physical quantities. Winkler, E., \& Keck, -. Civing. 30 (1884) 285-.

- - - - - - Keck, -. Hann. Archt. . Vr. Z. 30 (1884) 285-.

Use of + and - to denote "plus" and "minus." Eneström, G. Stockh. Öfv. (1894) 243-; Fschr. Mth. (1893-94) 59-; Bb. Mth. (1899) 105-.

- - terms "square" and "cube" in Algebra. Taylor, $W . B$. Wash. Ph. S. Bll. 11 (1892) 590-.

Variation, variable, \&c., remarks on the words. Strauch, G. W. Grunert Arch. 7 (1846) 221-. 


\section{Instruments, including Calculating Machines. Models.}

\section{INSTRUMENTS.}

Algebraical machines. Torres, L. As. Fr. C. R. (1895) (Pt. 2) 90-.

Angle, correction for tilt of horizontal circle. Matzka, W. Grunert Arch. 13 (1849) 113-.

_, instrument for dividing into equal parts. Gouin, E. Les Mondes 12 (1866) 470-.

- measurer. Heineken, N.J. Intell. Obs. 8 (1886) 197-.

- for schools. Fischer, F. W. [1876] Arch. Mth. Ps. 61 (1877) 99-.

-, mechanical division. Perrin, R. [1875] Par. S. Mth. Bll. 4 (1876) 85-.

-, - - Brocard, H. [1876] Par. S. Mth. Bll. 5 (1877) 43-.

Antigraph, a reversing drawing instrument. Marcus, S. Wien SB. 17 (1855) 282-.

Arcs, instrument for drawing. Alderson, Jas. Gill Tech. Rep. 10 (1829) 128-.

Area, moment of inertia, and centre of gravity of a plane area, apparatus for giving. Collignon, É. (1x) A. Pon. Chauss. 3 (1872) $223-$.

Arithmoplanimeter. Lalanne, L. Par. A. Pon. Chauss. 20 (1840) 3-.

Axonometric drawing, protractors for, preparation. Anon. (vi 316) Civing. 7 (1861) 83-.

Besson, Jacques, (†1569), (Mathematical instruments). Beck, T. Civing. 36 (1890) 191-.

Calculating machine. Cunq's "machine à débits," for calculating quantity of water flowing through an orifice. Sornin, J. Toul. Mm. Ac. 6 (1856) 346-.

Calculimeter (sort of slide rule). Fenton, J. J. Vict. R. S. P. 2 (1890) 84-.

Chinese abacus or souan-pan. Rodet, L. Par. S. Mth. Bll. 8 (1880) 158-

Circles, instrument for dividing on paper. Balfour, J. M. (vi Adds.) [1859] Edinb. T. Sc. S. Arts 5 (1861) 149-.

- with remote centres, construction. Fischer, E. Dingler 294 (1894) 133-.

Circular arcs, instrument for use of mechanicians for comparison of. Sipos, $P$. (vi $A d d s$.). Berl. Sm. D. Ab. 2 (1796) 201-.

Compass, bicentric. Maze (l'ab̉é) C. Les Mondes 8 (1884) 703-.

- for drawing spirals. Hartung, A. Dingler 266 (1887) 12-.

-, elliptic, new. Jost, $K$. Wien Ak. Sb. 95 (1887) (Ab. 2) 251-.

-, hyperbolic. Reichelt, K. F. Cztg. Opt. 17 (1896) 208.

Compasses, copying -, a simple form of pantograph. Sedlaczek, $J$. Wien SB. 16 (1855) 180 -

-, elliptic. Burchard, L. Carl Rpm. 13 (1877) 528-.

-, improvement. Möllinger, O. Sch. Gs. Vh. (1850) 52-.

-, proportional. Narrien, $J$. Thomson A. Ph. 8 (1816) 338-.
Compasses, proportional. Butkevīch, F.S. (xII) [1873] Mosc. S. Sc. Bll. 39 (No. 2) (1880) 128 -

-, - Palacios, D. Méx. S. "Alzate" Mm. 7 (1893) 193-.

, trisecting, Laisant. Brocard, H. Par. S. Mth. Bll. 3 (1875) 47-.

Conic section compass. Meyn, $R$. Civing. 8 (1862) 247-.

- compasses. Frizell, J. P. Camb. (M.) Mth. M. 1 (1859) 262-.

_ - - Drzewiecki's for direct construction of conics with mathematical pen. $K u h n, M$. Carl Rpm. 10 (1874) 420-.

- sections, instrument for drawing. Rebiček, $G$. Carl Rpm. 11 (1875) 64-.

Curve description; kinograph. Engelmeier, P. K. [1886-89] Mosc. S. Sc. Bll. 65 (No. 1) (1890) 89-.

- for drawing curves (elastic lamina) "tiracurve-opisometer." Rossi $R e, V$. de. Rm. N. Linc. At. 33 (1880) 232-.

- tracer. Pirani, E. Arch. Mth. Ps. 1 (1884) 113-.

- - and root finder. Bashforth, F. B. A. Rp. (1845) (pt. 2) 3-.

- tracing apparatus. Cayley, A. L. Mth. S. P. 4 (1871-73) 345-.

- - , designing of apparatus for. Thallmayer, V. Carl Rpm. 14 (1878) 713-.

- machine. Dechevrens, M. C. R. 130 (1900) 1616-.

- - mechanical. Jessop, C. M. QJ. Mth. 22 (1887) 151-.

Curves described by Jopling's apparatus, nature. Tredgold, T. Tilloch Ph. Mg. 62 (1823) 211-. -, mechanical description. Russell, W. H. L. [1869] R. S. P. 18 (1870) 72-

Curvimeter (for finding length of curves). Crane, W. R. Kan. Un. Q. 4 (1896) 121-.

Cycloids, description. Kracht, $H$. Dingler 289 (1893) 108-.

Descriptive geometry, apparatus for illustrating. Hoza, F. Arch. Mth. Ps. 53 (1871) 188-.

$-\ldots,-1$ teaching. Weissand, -. N. A. Mth. 14 (1855) 47-

Diagonal scale principle applied to angular measurement in circular slide-rule. Campbell, J. R. L. Mth. S. P. 6 (1875) 73-.

Diagrammometer, cypher. Kozlov, V.S. Rs. Ps.-C. S. J. 23 (Ps.) (1891) 180-; C. R. 111 (1890) 166-.

Difference engine, new. Grant, G.B. Am. J. Sc. 2 (1871) 113-.

Division of angles and arcs of circles. Murphy, I. J. Belfast NH. S. Rp. \& P. (1892-93) 47-.

- straight line into equal parts, machine for. Soave, $F$. Verona Mm. S. It. 8 (1799) 56 -.

- - toothed wheels, machine for. Glasser, A. von. Dingler 252 (1884) 56-.

Drawing instruments, especially ellipsographs in theory and practice. Fischer, $E$. Dingler 255 (1885) 188-, 217-, 261-.

Egg-oval, perfect, machine for drawing; and advantageous method of producing curvilineal figures. Hay, D. R. Edinb. N. Ph. J. 40 (1846) 331-. 


\section{Instruments}

Ellipses, paradox in tracing. Lametz, -. Metz Ac. Mm. 79 (1900) 69-.

Ellipsograph. Lêninn, $M$. St Pét. Ac. Sc. Bll. 5 (1839) 377-.

-. Thallmayer, $V$. Dingler 226 (1877) 237-; 227 (1878) 337-.

-. Sidersky, D. Arch. Mth. Ps. 65 (1880) 420 -

-. Hendricks, J. E. Des Moines Anal. 10 (1883) 161-.

-. Grant, G. B. Franklin I. J. 122 (1886) 301 .

-. Schromm, F. Z. Instk. 12 (1892) 139-.

-. Delone, N. Mosc. S. Sc. Bll. 83 (No. 2) (1894) 11-; Fschr. Ps. (1895) (Ab. 1) 351.

-. Riefler, C. Z. Instk. 16 (1896) 115-.

- of Proclus. Blake, E. M. [1898] Am. J. Mth. 22 (1900) 146-.

- and protractor. Burnham, S. W. Pop. As. 4 (1897) 181-, 336.

Ellipsographs. Zistl, M. Cztg. Opt. 5 (1884) 245-, 258-, 265-.

-. Hartmann, W. Z. Instk. 11 (1891) 285-.

Ellipsometer. Henry, $F$. (x) A. Pon. Chauss. 3 (1872) 459-

-. Jannettaz, E. Fr. S. Mn. Bll. 15 (1892) 237-; 16 (1893) 205-.

Elliptographs, and a mechanical rocker for detecting oscillations. Alexander, T., \& Thomson, A. W. Ir. Ac. T. 29 (1887-92) 673-.

Epi-and hypo-cycloids, apparatus for tracing. Thallmayer, $V$. Dingler 228 (1878) 312-.

Equations, apparatus for graphic solution. Lill, E. Les Mondes 16 (1868) 325-.

- of form $\lambda^{n}+a \lambda^{2}+b \lambda+c=0$, Mehmke's apparatus for solution. Mlodzěevskij, $B . K$. Mosc. S. Sc. Bll. 92 (No. 1) (1896) 36-; Fschr. Mth. (1896) 72.

- with imaginary roots, apparatus for graphic solution. Lill, E. N. A. Mth. 7 (1868) 363-.

-, machine for solution. Girard, P. von. Wien Jb. Pol. I. 14 (1829) 62-.

-, - - - Bashforth, F. B. A. Rp. (1845) (pt. 2) 3-.

- - - Boys, C. V. L. Ps. S. P. 7 (1886) 355-; Ph. Mg. 21 (1886) 241-.

- - - Cunynghame, $H$. L. Ps. S. P. 7 (1886) 360-; Ph. Mg. 21 (1886) 260-.

-, _ - - Ocagne, M. d'. J. de Ps. 5 (1896) 310-

- 888 -

-, - - - of linear. Veltmann, W. Z. Instk. 4 (1884) 338-.

- , - - - simultaneous linear. Thomson, (Sir) W. (Lord Kelvin). [1878] R. S. P. 28 (1879) 111-.

-, - - - transcendental. Herschel (Sir) J.F.W. [1832] Camb.Ph.S.T.4 (1833) 425-.

-, mechanical solution. Kempe, A. B. [1872] Mess. Mth. 2 (1873) 51-.

-, - - Guarducci, $F$. Rm. R. Ac. Linc. Mm. 7 (1891) 219-.

-, 3rd order, mechanical solution by a quadrilateral linkage. Greenhill, A. G. Mess. Mth. 5 (1876) 162-
Instruments 0080

Equations, 3rd order, solution by slide-rule. Bour, E. [J. E. E.] C. R. 44 (1857) 22-.

-, 4th and 5th orders, solution by moving mechanisms. Ameseder, $A$. Wien Ak. Sb. 93 (1886) (Ab. 2) 380-.

- to 7th order, machine for solution. Lalanne, L. C. R. 11 (1840) 859-

- - - - - (Lalanne). Cauchy, A.L. C. R. 11 (1840) 959-.

Generation of orthogonal projections of plane curves, mechanical. Delaunay, N. Z. Mth. Ps. 40 (1895) 242-.

Genesis of quantity demonstrated by means of two mechanical instruments. Araldi, (Capitano) A. (vII) [1828] Mod. Mm. Ac. Sc. 1 (1858) (pte. 4) 90-.

Geometrical drawing, apparatus for. $\dot{Z}$ murko, W. [L.] (xIr) Kosmos (Lw.) 5 (1880) 44-.

Geometrography applied to different solutions of a problem. Lemoine, E. Par. S. Mth. Bll. 20 (1892) 132-.

Harmonic analyser. Henrici, O. [1894] L. Ps. S. P. 13 (1895) 77-; Ph. Mg. 38 (1894) 110-. - LeConte, J. N. Ps. Rv. 7 (1898) 27-.

- - Michelson, A. A. \& Stratton, S. W. Am. J. Sc. 5 (1898) 1-.

- - for graphic determination of coefficients in Fourier series. Bervy, N. Mosc. Obs. A. 3 (Pt. 2) (1896) 109-.

- Henrici and Coradi's. Grabowski, L. Wiad. Mt. 4 (1900) 236-.

- - , reading amplitude and epoch directly. Sharp, A. [1894] L. Ps. S. P. 13 (1895) 89-; Ph. Mg. 38 (1894) 121-.

_ - remarks on Henrici's. Perry, J. [1894] L. Ps. S. P. 13 (1895) 97-; Ph. Mg. 38 (1894) 125-.

- - simple form. Yule, G. U. L. Ps. S. P. 13 (1895) 403-; Ph. Mg. 39 (1895) 367-.

Helical epicycloids, instrument for describing. Burnaby, S. B. Amt. Mch. S. J. 3 (1879) 115-, 160-, 194-, 228-.

Herpolhode of Poinsot, and an apparatus of Darboux and Koenigs. Gilbert, $P$. Brux. S. Sc. A. 14 (1890) (Pt. 2) 25-.

Hydraulic integration of differential equations. Petrovitch, M. Am. J. Mth. 20 (1898) 293-.

Hyperbolas, apparatus for drawing. Thallmayer, $V$. Dingler 228 (1878) 106-.

-, method of drawing. Burch, G.J. $\mathrm{Ph}$. Mg. 41 (1896) 72-.

- - - - Burch's. Wadsworth, F. L. O. Ph. Mg. 41 (1896) 372-.

Hyperbolograph. Cunynghame, $H$. [1886] L. Ps. S. P. 8 (1887) 67-; Ph. Mg. 22 (1886) 138-.

Instruments for ovals and for drawing in perspective. $B ., R$. Nicholson J. 9 (1804) 122-.

Integral of product of $\mathbf{2}$ given functions, instrument for calculating. Thomson, (Sir) W. (Lord Kelvin). R. S. P. 24 (1876) 266--

Integraph, new. Barker, A. H. Sc. Abs. 1 (1898) 381.

Integrating differential equations of first order, Petrovich's apparatus. Price, W. A. Ph. Mg. 49 (1900) 487-.

Integration, mechanical. Abdank-Abakanowicz, B. C. R. 94 (1882) 783- 


\section{Instruments}

Integration, mechanical. Nehls, C. Civing. 20 (1874) 71-; 21 (1875) 131-, 199-, 261-.

- , - , applied to three bodies' problem. Strömgren, $E$. Stockh. Öfv. (1900) 443-.

-, - , of general linear differential equation of any order with variable coefficients. Thomson, (Sir) W. (Lord Kelvin). R. S. P. 24 (1876) 271-.

,,--- linear differential equations of $2 \mathrm{nd}$ order with variable coefficients. Thomson, (Sir) W. (Lord Kelvin). R. S. P. 24 (1876) 269-.

,,--- the product of two functions. Sutherland, $W$. Ph. Mg. 20 (1885) 175-; 21 (1886) 141-.

Integrator, Amsler's. Nehls, C. Hann. Z. Archt. Vr. 18 (1872) 534-.

-. Abakanowicz, B. (xII) Krk. Ak. (Mt.-Prz.) Rz. \& Sp. 7 (1880) 75-.

-, cubical, description. McElroy, G.W. A. Mth. 3 (1887) 105-

-, disk-globe-and-cylinder. Thomson, (Prof.) Jas. R. S. P. 24 (1876) 262-.

-, instrument for graphic integration. AbdankAbakanowicz, B. C. R. 92 (1881) 402-, 515-.

-, mechanical, Amsler-Laffon's. Merrifield, C. W. Nv. Archt. T. 21 (1880) 252-.

-, - , for areas, etc. Deprez, M. C. R. 73 (1871) 785-; Les Mondes 27 (1872) 10-.

-, 一, - $\int(X d x+Y d y)$. Cayley, A. B. A. Rp. (1877) (Sect.) 18-.

- - - - - work done by a force. Fuchs, $K$. Mth. Termt. Ets. 13 (1895) 239-; Mth. Nt. B. Ung. 13 (1897) 144-.

-, new form. Marinovitch, B. Lum. Élect. 17 (1885) 529-.

-, - model. Napoli, D. \& Abdank-Abakanowicz, -. C. R. 101 (1885) 592-.

-, Żmurko's. Skibinski, P.K. Kosmos (Lw.) 9 (1884) 185-; Wien Ak. D. 53 (1887) (Ab.2) $35-$.

Integrators. Hummel, - Dingler 275 (1890) 17-.

-, mechanical. Abdank-Abakanowicz, B. Lum. Élect. 18 (1885) 49-, 110-, 161-, 249-, 306-, 393-, 535-, 589-; 24 (1887) 3-, 54-, 161-.

-, two mechanical. Fraser, A. Y. Edinb. Mth. S. P. 4 (1886) 29-.

Integrometer, new. Abdank-Abakanowicz, $B$. C. R. 95 (1882) 1047-.

Involution and evolution of numbers, instrument for. Roget, P.M. [1814] Phil. Trans. (1815) 8-.

Least squares, instrument for. Bouniakowsky, V. Pogg. A. 107 (1859) 463-.

Linear differential equation of 2 nd order, general mechanism for integrating. Tait, P. G. [1876] Edinb. R. S. P. 9 (1878) 118-.

Link-work apparatus for describing curves. Janse, L. N. Arch. Wisk. 9 ("1882) 61-.

Linkages for tracing conic sections. Strong, W. M. A. Mth. 8 (1893-94) 181-.

- $x^{m}$. Freeland, F.T. [1879] Am. J. Mth. 3 (1880) 316-.

Logical machine, Jevons'. Sonnenschein, $\boldsymbol{E} . A$. (xI) [1883] Birm. Ph. S. P. 4 (1885) 72-.

\section{Instruments 0080}

Logical machine, new. Marquand, A. Am. Ac. P. 21 (1886) 303-.

Mathematical instruments, models etc., exhibition in Munich. Dyck, W. D. Mth. $\nabla r$ 。 Jbr. 3 (1894) 39-.

Mechanisms, new: projector, ellipsograph, ... Delaunay, N. Bll. Sc. Mth. 19 (1895) 240-

Mensurator and cœlometer. Adams, M. L. Mth. S. P. 5 (1874) 141-.

Military surveying circle. Delanges, $P$. Bologna Mm. I. It. 1 (pte. 2) (1806) 63-.

Mirror for drawing in perspective. Schönemann, P. Z. Nw. 10 (1874) 102-。

Multiplication by a mechanical device. Berdellế, C. As. Fr. C. R. (1887) (Pt. 2) 210-.

Nautical instruments, and calculating apparatus. Gelcich, E. Cztg. Opt. 5 (1884) 242-, 253-, 268-, 277-; 6 (1885) 9; 7 (1886) 265-.

Nicolas's gyrograph for tracing ares of large circles. Gobin, A. Lyon A.S. Ag. 8 (1864) $520-$.

Nürnberg, German Museum, mathematical collection. Günther, S. Lpldina. 14 (1878) 93-, 108-.

- - - - - instruments. Günther, S. D. Nf. Vh. (1893) (Th. 2, Hälfte 1) 32.

Observations, mechanical calculation of results. Favaro, A. Ven. I. At. 2 (1876) 559-.

Pantograph; proof of chief theorem, etc. Haussner, A. Arch. Mth. Ps. 65 (1880) 334-.

-, scenographic, for projecting geometric figures. Magistrini, G. B. Bologna Opusc. Sc. 1 (1817) 289-.

Pantographs. Ott, A. Cztg. Opt. 17 (1896) 161-.

-, proof of properties $\left(1^{r}+2^{r}+3^{r} \ldots \ldots x^{r}\right) . \quad L i$ gowski, W. [1879] Arch. Mth. Ps. 65 (1880) 329-.

Parabola, apparatus for drawing. Thallmayer, $V$. Dingler 227 (1878) 430-.

Parabolograph. Delone, N. B. Rs. Ps.-C. S. J. 29 (P8.) (1897) 76.

Parallel lines, instrument for drawing. Nichol8on, W. Nicholson J. 2 (1799) 429-.

- - , - - . Suverkrop, J. P. [1883] Sc. S. Arts T. 11 (1887) 73-.

- rule, new. B., $R$. Nicholson J. 9 (1804) $212-$.

- -, - Boswell, J. W. Nicholson J. 13 (1806) 196-.

"Perspecteur-calquer," instrument for drawing in perspective. Breton, - . Isère S. Bll. 27 (1892) 659-.

Perspective drawer. Brauer, E. Z. Mth. Ps. 43 (1898) 163-.

- drawing instrument. Brunel-Barennes, de. Dingler 34 (1829) 325-.

- - Verly's instrument, report. Rodolphe, Metz Mm. Ac. 20 (1838-39) 258-.

-, scale for dividing vanishing lines. Cumberland, G. Nicholson J. 16 (1807) 1-.

Plagiograph aliter skew pantigraph. Sylvester, $J . J$. Nt. 12 (1875) 168.

-, history. Sylvester, J.J. Nt. 12 (1875) 214-.

Plane surface, formation, law of progress of accuracy. Airy, (Sir) G. B. Ph. Mg. 42 (1871) 107-. 
Planigraph, projecting tortuous curve on plane. Delone, N. B. [1895] Vars. S. Nt. Tr. (1895-96) (C. R., Ps. C.) No. 2, 3 pp.

Planimeter. Amsler, $J$. Zür. Vjschr. 1 (1856) 41-, 101-.

-, Amsler's. Decher, G. Dingler 140 (1856) 27- ; 141 (1856) 29-.

-, -. Weisbach, J. Civing. 4 (1858) 1-.

-, - Wittstein, T. Hann. Z. Archt. Vr. 7 (1861) 289-.

- - - Petsch, - A. Pon. Chauss. 16 (1868) 124 -

一, - Combes, C. P. M. A. Mines 19 (1871) $278-$

-, -. Bramwell, F. J. B. A. Rp. 42 (1872) 401-.

-, -. Purvis, F. P. Ph. Mg. 48 (1874) 11-. -, - Rateau, A. As. Fr. C. R. (1896) (Pt. 2) 130 -

-, - , proof of principle. Steggall, -. Edinb. Mth. S. P. 7 (1889) 25-.

-, -, theory. Zhbikovskir, A. K. (хп) [1865] Kazan Un. Mm. (1864, Ps.-Mth. No. 1), 219-.

-, - - - Aldis, W. S. N. Eng. I. Mn. E. T. 28 (1879) 211-. -, - - - Thiré, A. A. Mines 11 (1887)

-, Bouniakovsky's and modification. Decher, G. Dingler 140 (1856) 27-.

-, construction. Schultén, N. G. af. (viI) Helsingf. Öfv. 5 (1863) 245-.

-, Coradi's. ? Coradi, -. V. Nost. Eng. Mg. 32 (1885) 465-.

-, hatchet. Hill, F. W. [1894] L. Ps. S. P. 13 (1895) 229-; Ph. Mg. 38 (1894) 265-.

-, history. Favaro, A. Förster Al. Bauztg. 38 (1873) 68-, 93-.

-, - Wolf, R. Zür. Vjschr. 37 (1892) 111-; 38 (1893) 3-.

- of Hohmann and Coradi. Fischer, G. Cztg. Opt. 5 (1884) 74-.

-, Lippincott. Greenhill, A. G. [1899] Sc. Abs. 3 (1900) 234.

-, Maass-, description. Günther, F. Cztg. Opt. 7 (1886) 247-.

-, new. Eckert, -, \& Hamann, -. Cztg. Opt. 18 (1897) 125.

-, -, description. Maevskij, J. A. [1889] Vars. S. Nt. Tr. (1889-90) (C. R., Ps. C.) No. 2, 7-

-, - form. Maxwell, J. C. Edinb. T. Sc. S. Arts 4 (1856) 420-.

-, - - (Beverley). Balfour, J. M. [1865] Edinb. Sc. S. Arts T. 7 (1868) 198-.

-, - (1852). Baranoffsky, E. Helsingf. Acta 4 (1856) 3-.

-, - (1855). Bouniakonosky, V. [1855] St Pét. Ac. Sc. Bll. 14 (1856) 152-.

- polar. Amsler, J. Dingler 140 (1856) 321-; 141 (1856) 326-.

-, _, Amsler's. Decher, G. Dingler 141 (1856) 330-.

-, -, -. Schinz, E. Bern Mt. (1857) 152-.

-, -, - . Nehls, C. Civing. 20 (1874) 71-.

-, - - -, results. Hartogh Heys van Zouteven, $H$. Arch. Néerl. 5 (1870) 440-.

\section{Instruments 0080}

Planimeter, polar, determination of constant. Schell, $A$. Wien SB. 56 (1867) (Ab. 2) 325-. ,-- , general theory. Schell, $A$. Wien SB. 58 (1868) (Ab. 2) 189-; Carl Rpm. 11 (1875) 406-.

-, - , , note. Resal, $H$. C. R. 77

-, Richard. ?Anon. Sc. Abs. 1 (1898) 635

-, rolling, theory. Fenner, P. Cztg. Opt. 7 (1886) 133-.

-, solutions of electric problems. Atkinson, L. B. Elect. 22 (1889) 702-; 23 (1889) 29-.

-, Trunk's. Huibner, A. Arch. Mth. Ps. 44 (1865) 337-

, Wetli's. Stampfer, S. Wien SB. (1850) (Ab. 1) 134-.

Planimeters. Moberg, A. (vII) [1859] Helsingf. Öfv. 5 (1863) 53-.

- Beck, A. Riga Cor.-Bl. 40 (1898) 99-.

-, free. Bouniakowsky, V. St Pét. Ac. Sc. Bll. 2 (1860) 567-.

- and similar instruments. Kohlmorgen, $O$. Westf. Vr. Jbr. (1890) 126-.

- of Wetli, Decher, and Amsler. Miller, W. H. Ph. Mg. 16 (1858) 230-.

Problems of 3rd degree, instrument for solution. Simon, C. Les Mondes 4 (1864) 547-.

Proportion-table, equivalent to sliding-rule $13^{\prime} 4^{\prime \prime}$ long. Everett, J. D. Ph. Mg. 32 (1866) 350-.

Protractor, semicircular, improved form. Stewart, J. N. Z. I. T. 3 (1870) 316-.

- for trisecting angles. Alexander, J. $H$. Silliman J. 7 (1849) 243-.

Quadrature, instrument for. Boileau, P. C. R. 15 (1842) 594-.

Refraction through prism; apparatus to illustrate deviation of ray. Marcucci, S. Rv. Sc.-Ind. 27 (1895) 101-.

Scale, accuracy of, and limit of perception. Neovius, E. Helsingf. Öfv. 23 (1881) 71-.

" Scoliograph, unicursal," for drawing algebraic curves. Boguslawsky, A. [1881] Mosc. S. Nt. Bll. 57 (1882) (Pt. 2) 72-.

Screw, use in mathematical instruments. Carl, P. Carl Rpm. 2 (1867) 37-.

Sectograph (for dividing lines and angles, \&c.). Jones, T. Tilloch Ph. Mg. 42 (1813) 401-.

Sine and cosine quadrant. Schreiber, C. Cztg. Opt. 7 (1886) 38-.

Spherical integrator, modification of a form of. Smith, F.J. Ph. Mg. 22 (1886) 147-.

- triangles, instrument for determining, by mechanical action. Penrose, F. C. As. S. M. Not. 36 (1876) 281-.

Spherometer. Mayer, A. M. Am. J. Sc. 32 (1886) 61-.

-, new. Krïss, H. Cztg. Opt. 12 (1891) 63-.

Spirals, Johnson's volutor for drawing. Booth, J. B. A. Rp. (1860) (Pt. 2) 60-.

Square with parallel lines. Chaussenot, (l'Aîné). Par. Bll. S. Encour. 43 (1844) 4 -.

Stanhope logical and arithmetical machine. Harley, (Rev.) R. N. S. W. R. S. J. 24 (1890) 121-. 


\section{Calculating Machines}

Stereoscopic drawing apparatus. Jensen, $J$. [1870] Arch. f. Ap. 4 (1871) 233-.

Straight line apparatus, Hart's. Darboux, $G$. Bll. Sc. Mth. As. 3 (1879) 144-.

Stupakoff's comparometer. Basford, G. M. Am. Eng. \& Railroad J. 73 (1899) 59, 169.

Tachymeter, Cairo's. Puissant, L. C. R. 3 (1836) 140, 200, 245-, 384.

Tractoriograph (instrument for describing curves of "tractory" nature). Kleritj, L. Dingler 305 (1897) 234-, 260-.

- of Kleritj, and rod-planimeter. Korselt, A. Z. Mth. Ps. 43 (1898) 312-.

Trigonometer, instrument for solving spherical triangles. Braun, $K$. (xII) Mth. Term. Éts. 1 (1883) 313-; Mth. Nt. B. Ung. 1 (1882-83) 283-.

-, Savy's. Le Grand, G. Aube Mm. S. Ag. 5 (1854) 235-.

Trisection of angle, instrument for. Burt, T.S. Beng. As. S. J. 1 (1832) 499-.

-,$- \ldots$ Tate, T. Ph. Mg. $19(1860)$ $261-$.

1 (1878) 163 -.

- - - - Longchamps, G. de. As. Fr. C. R. (1893) (Pt. 2) 190-.

- - mechanical. Russell, A.H. Nt. 43 (1891) 547-.

- - angles, test. Rönisch, A. Cztg. Opt. 16 (1895) 253-.

Vernier for line or curve with unequal divisions. Artur, J. F. $\quad$ Par. Bll. S. Encour. 50 (1851) 676-.

-, new form. Ludlow, H. H. V. Nost. Eng. Mg. 27 (1882) 196.

- or Nonius, priority. Breusing, A. [1879] As. Nr. 96 (1880) 129-.

Verniers. Matzka, $W$. Grunert Arch. 34 (1860) 334-.

\section{CALCULATING MACHINES.}

Addition machine. Goss, J. Tilloch $\mathrm{Ph} . \mathrm{Mg}$. 41 (1813) 166-.

Arithmetical machine, Čebyšev's. Bool, $V . G$. von. Mosc. S. Sc. Bll. 91 (No. 1) (1894) 12-.

- with continuous motion. Tchebitchef, P. L. Rv. Sc. 4 (1882) 402-.

- -, supplementary explanation. Čebyšv, P. L. Mosc. S. Sc. Bll. 91 (No. 1) (1894) 22.

- machines, history. Mehmke, $R$. Prace Mt.Fiz. 6 (1895) 177-.

Arithmometer, Thomas's. Ettingshausen, $A$. von. Wien SB. 24 (1857) 16-.

-, - Reuleaux, $F$. Civing. 8 (1862) $182-$

-, - Hirn, G. A. Les Mondes 3 (1863) 433-.

-, -. Junge, A. Z. Mth. Ps. 9 (1864)

198-.

, -. Zillmer, -. I. Act. J. 15 (*1870)

25-.

-, -. Cavallero, A. Cuyper Rv. Un. 8 (1880) 309-.

\section{Calculating Machines 0080}

Arithmometer, Thomas's, actuarial use. Gray, P. I. Act. J. 17 ( $\left.{ }^{*} 1873\right) 249-; 18\left({ }^{*} 1875\right)$ 20 -, 123-.

-, - , adaptation of assurance formulæ to. Hannyngton, J. Assur. Mg. $12\left({ }^{*} 1866\right)$ 184.

-, _, use. Hannyngton, (Maj.-Gen.). I. Act. J. $16\left({ }^{*} 1872\right) 244-$.

-, use. Hirn, G. A. A. Gén. Civ. 2 (1863) (pte. 2) 113-, 153-.

-, - Hancock, W.J. I. Act. J. $16\left({ }^{*} 1872\right)$ $265-$.

-, - in constructing tables. Carment, $D$. I. Act. J. 22 (*1881) 368-; 23 (*1882) 232.

Calculating apparatus, arithmetical puzzles. Lucas, E. Rv. Sc. 45 (1890) 1-.

- - based on Napier's rods. Bridge, J. $\mathrm{Ph}$. Mg. 9 (1880) 191-.

- circle. Weiss, P. C. R. 131 (1900) 1289-.

-instruments and machines. (2) Logarithmic scales. (3) Graphic processes. Ocagne, M. $d$ '. A. Cons. Arts et Mét. 5 (1893) 231-, 323-; 6 (1894) $42-$.

- machine. Babbage, C. Edinb. Ph. J. 8 (1823) 122-.

- - Olivier, T. Par. Bll. S. Encour. 45 (1846) 153-.

- - Nystrom, J. W. Franklin I. J. 21 (1851) 262-.

- - Selling, E. D. Nf. Tbl. (1887) 234-; Würzb. Ps. Md. Sb. (1888) 148-; Cztg. Opt. 9 (1888) 3-.

- -. Levänen, S. Helsingf. Öfv. 38 (1896) 26-.

- -. Siedentopf, H. N.-Vorp. Mt. 30 (1899) xvii-.

- - or arithmometer. Hoyan, - . (vi Adds.) Par. Bll. S. Encour. 21 (1822) 355-.

- - , - Thomas, - . Moigno Cosmos 4 (1854) 72-, 186-

- - Babbage's. Baily, $F$. Tilloch Ph. Mg. 63 (1824) 355-.

- - - - Ménabréa, L. F. Taylor Sc. Mm. 3 (1843) 666-

- - - Brit. Ass. Comm. B. A. Rp. (1878) $92-$.

_-, - Menabrea, (le gèn.) L. F. C. R. 99 (1884) 179-.

$-\ldots,-$ mechanical arrangements. Babbage, (Maj.Gen.) H. P. B. A. Rp. (1888) 616-. - - for definite integrals. Moseley, H. B. A. Rp. (1841) (Pt. 2) 35-.

- -, duplex. Küttner, $W$. Dingler 300 (1896) 199-.

- -, \&c. Ramsing, H. M. Mth. Ts. 2 (1860) 65 -.

- Fowler's. De Morgan, A. R. S. P. 4 (1840) 243-.

- - Henri Genaille's. Genaille, H. As. Fr. C. R. (1894) (Pt. 2) 272-.

- -, new. Lalanne, L. C. R. 9 (1839) 319 -

-, -. Fowler, -. B. A. Rp. (1841) (Pt. 2) 39-.

277-. Grant, G. B. Am. J. Sc. 8 (1874) 
Calculating machine, new. Bollée, L. C. R. 109 (1889) 737-.

- - - - Strehl, K. Cztg. Opt. 11 (1890) 242-.

- - old. Cadell, W. A. Thomson A. Ph. 3 (1814) 251-.

- - for products, quotients, logs., and powers of numbers. Moseley, H. Ph. Mg. 30 (1847) 171-.

- - prospectus, \&c. Slonimsky, C.Z. Crelle J. 28 (1844) 184-.

- - for real roots of numerical equations. Moth, F. X. Wien D. 1 (1850) 105-.

- -, report. Wiberg, -. C. R. 56 (1863) 330 -

- - Scheutz's. Meidinger, H. Dingler 156 (1860) 241-, 321-.

- -, Selling's. Poppe, A. Dingler 271 (1889) 193-.

- machines. Hélie, -. Eure Rec. Tr. 2 (1842) 170-.

- - Olivier, T. Par. Bll. S. Encour. 42 (1843) 411-.

- -. Jomard, E. F. Par. Bll. S. Encour. 42 (1843) 548-.

- -. Lauterburg, -. Bern Mt. (1863) 20-.

- - Warren, T. P. B. Ph. Mg. 43 (1872) 396-.

- - Edmondson, J. [1885] L. Ps. S. P. 7 (1886) 81-; Ph. Mg. 20 (1885) 15-.

- -. Davis, H. S. Pop. As. 4 (1897) 20-.

- - Torres, L. C. R. 130 (1900) 472-.

- - arithmograph. Gattey, -. Par. Bll. S. Encour. 15 (1816) 50-.

- -, continuous. Hele-Shaw, H. S. Phil. Trans. 176 (1886) 367-.

- - history. Mehmke, $R$. D. Mth. Vr. Jbr. 3 (1894) 59-.

- - and instruments. Ocagne, M. d'. Brux. S. Sc. A. 17 (1893) (Pt. 1) 84-.

- - invented by Russians. Bool, V. von. Mosc. S. Sc. Bll. 92 (No. 1) (1896) 33-.

- - theory. Stamm, E. Mil. I. Lomb. Rd. 2 (1865) 360 -

Calculation and calculating machines. Lucas, É. As. Fr. C. R. (1884) (Pt. 1) 111-.

-, mechanical aids. Sang, $E$. I. Act. J. 16 (*1872) 253-.

Calculations, instruments to aid. Thomson, $G$. [1896] Sc. S. Arts T. 14 (1898) 172-.

Chinese-Japanese calculating machine, and Chinese Swan-Pan (calculating table). Westphal, A. (xII) D. Gs. Ostas. Mt. 1 (187376) (Heft 8) 27-, (Heft 9) 43-.

Efficiency, apparatus for calculating. Boys, $C . V$. (Ix) [1882] L. Ps. S. P. 5 (1884) 28-; Ph. Mg. 13 (1882) 193-.

Logarithmic calculating rules, Lambert's. Schweigger, J.S. C. Schweigger J. 14 (1815) 115-.

Slide-rule. Burg, A. Wien Jb. Pol, I. 16 (1830) 101-.

- - Burdon, -. C. R. 58 (1864) 573-.

- -. Mackesy, W. H. Medley I. Eng. 4 (1867) 349-.

- -. Schwind, F. von [1873] Innsb. Nt. Md. B. 4 (1874) liv-.
Slide-rule. Boys, C. V. Nt. 32 (1885) 627-. - - employment. Herrmann, E. Oestr. Z. Brgw. 15 (1867) 193-.

- - - Charnoz, $\mathcal{J}$. Rv. Mar. et Col. 57 (1878) 493-.

- -, English. Sedlaczek, E. Hammerschmidt Al. Oestr. Z. (1846) 439-, 451-, 465-, 478-; (1847) 182-, 221-, 318-, 342-, 365-, 390-, 401-,

- - Fuller's. Fenton, J. J. Vict. R. S. T. 22 (1886) 57-.

- - for gauging casks. Young, (Dr) T. QJ. Sc. 16 (1823) 357-.

- -, gunner's. Smith, John T. Madras Eng. Rp. 2 (1846) 14-.

- -, model. Béghin, A. As. Fr. C. R. (1900) (Pt. 1) 127-.

- -, new. Gallice, G. C. R. 128 (1899) 163-. - - - Puller, -. Hann. Archt.-Vr. Z. 46 (1900) 203-.

- - , - calculating. Fuller, G. (xir) [1879] Belfast NH. S. P. (1878-80) 36-.

,--- form of. Beghin, A. As. Fr. C. R. (1899) (Pt. 2) 142-.

- - proposed addition. Schweth, W. D. Nf. Vh. (1900) (Th. 2, Hälfte 1) 66-.

- - proposed forms. Darwin, G. H. L. Mth. S. P. 6 (1875) 113.

_- radial cursor. Lanchester, F. W. [1895] L. Ps. S. P. 14 (1896) 8-; Ph. Mg. 41 (1896) $52-$.

- - , solution of equations in three variables. San Roberto, P. di. [1867] Tor. Mm. Ac. 25 (1871) 53-.

- - , treatment of equation in two variables. San Roberto, P. di. Tor. At. Ac. Sc. 2 (1866-67) 454-.

- - use. Boys, C. V. V. Nost. Eng. Mg. 33 (1885) 512-.

- rules, Everett's proportion-table. Everett, J. D: Ph. Mg. 32 (1866) 350-.

- - Fuller's calculating helix, and historical notes on calculating instruments with logarithmic divisions. Favaro, $A$. Ven. I. At. 5 (1879) 495-.

- - logarithmic. Sedlaczek, E. Hammerschmidt Al. Oestr. Z. (1847) 493-, 507-, 521-, 531-.

- - modified. Mannheim, A. N. A. Mth. 12 (1853) 327-.

\section{MODELS.}

Cubic surface with 27 real lines. Wiener, D. Nf. Tbl. (*1868) 56-.

- $-1-$ Blythe, W. H. Camb. $\mathrm{Ph}$. S. P. 8 (1895) 241-.

_- Taylor, H. M. Camb. $\mathrm{Ph}$. S. T. 18 (1900) 375-.

_ _ _ _ _ - Wiener's model. Cayley, A. [1871] Camb. Ph. S. T. 12 (1879) 366-.

- surfaces, construction of models. Blythe, W. H. QJ. Mth. 29 (1898) 206-.

- - model by Rodenburg. Korteweg, D. J. N. Arch. Wisk. 20 (1893) 63-; Fschr. Mth. (1893-94) 83.

Cylindroid. Ball, R. S. Ph. Mg. 42 (1871) 181-; Am. As. P. (1881) 52-. 
Descriptive geometry, models. Didier,,$- d$ Evrard, -. Par. S. Phlm. Bll. (1833) 177-.

Ellipsoid, model of the centro-surface. Kummer, E. E. Berl. Mb. (1862) 426-.

Goniometrical instruction. Hoza, F. Arch. Mth. Ps. 61 (1877) 108-.

Kinematio models, epicycloids, linkwork. Schilling, F. D. Nf. Vh. (1898) (Th. 2, Hälfte 1) 6-.

Mathematical models, use. Emch, A. Kan. Ac. Sc. T. 14 (1896) 90-.

Minimal surface, model in plaster. Schwarz, H. A. Berl. Mb. (1872) 122-.

Paraboloids, hyperbolic, model. Olivier, $T$. Par. S. Phlm. N. Bll. (1832) 184-.

Pencils, thin, models. Kummer, E. E. Berl. Mb. (1860) 469-.

Ronayne's cubes. Hennessy, H. Ph. Mg. 39 (1895) 183-.

Ruled surface, $3 r d$ order. La Gournerie, J. de. Par. A. Cons. 6 (1866) 205-.

Sections of quadric surfaces. Wiener, -. Karlsruhe Nt. Vr. Vh. 10 (1888) (Sb.) 50-.

Stereographic equatorial projection of a sphere, transformation by means of a form of the Peaucellier cell. Phillips, A. W. B. A. Rp. (1884) 649.

Subdivision of an arbitrary angle by models. Strauss, A. Arch. Mth. Ps. 12 (1894) 177-.

Thread models of developable surfaces. Schoute, P. H. Amst. Ak. Vs. [2] (1894) 8-.

\section{Aids to Calculation, Graphical Processes.}

Abaci adapted to equations with three and four variables. Ocagne, M. d'. Brux. S. Sc. A. 22 (1898) (Pt. 1) 78-.

- graphical calculation by hexagonal. Lallemand, C. C. R. 102 (1886) 816-.

Abacus. Knott, C. G. Jap. As. S. T. 14 (1886) 18-.

-. Duporcq, E. C. R. 127 (1898) 265-.

- for diurnal and semi-diurnal tides. Ocagne, $M . d^{\prime}$. C. R. 122 (1896) 298-.

-, new kind. Goedseels, - Brux. S. Sc. A. 22 (1898) (Pt. 1) 43-.

- with 16 and 18 variables. Lafay, A. C. R. 119 (1894) 1195-.

Algebraic functions, representation. Ott, C.de. Par. A. Cons. 6 (1866) 450-.

Anamorphosis, problem. Lecornu, L. C. R. 102 (1886) 813-.

Arbitrary functions, development. Perry, J., $\&$ Hunt, H. F. [1895] L. Ps. S. P. 14 (1896) 1-; Ph. Mg. 40 (1895) 506-.

Arc, construction when centre is inaccessible. Prevost, P. Bb. Un. 49 (1832) 98-.

Archimedes' screw, applications in graphical calculation. Sayno, A. Mil. I. Lomb. Rd. 7 (1874) 163-.

Arithmetic mean, determination. Ivancich, $J . N$. Wien Berg-Hm. Jb. 24 (1876) 295-.

Calculation, methods of simplification. Goedseels, E. Brux. S. Sc. A. 23 (1899) (Pt. 2) 1-.

Chess-board, use, for arithmetical problems.
Delannoy, -. As. Fr. C. R. (1886) (Pt. 2) 183-.

Circle divider, description. Laussedat, (le col.) - As. Fr. C. R. (1885) (Pt. 1) 97-.

-, division, \&c., graphic solution. Péreaux, $E$. A. Gén. Civ. 17 (1870-71) 735-.

Circular arcs, graphic rectification, and construction of cyclic curves. Schlömilch, $O$. Schlömilch Z. 2 (1857) 330-.

Coefficients of algebraic equations, representation. Nehls, C. [1893] Hamb. Mth. Gs. Mt. 3 (1900) 139-.

Colours in nature, law of multiple proportions. Collins, F. H. Nt. 52 (1895) 438.

- _ - _ - Lovibond, J. W. Nt. 52 (1895) 547, 577.

- - - C - Cattell, J. McK. Nt. $52(1895) 547$.

Complex numbers, representation. Broch, O.J. As. Fr. C. R. 3 (1874) 1174-.

"Compteurs logarithmiques." Cauchy, A. L. C. R. 40 (1855) 1009-; 44 (1857) 257-.

Construction of surfaces by plane sections. Cardinaal, J. Amst. Ak. Vs. M. 6 (1889) 198-; Fschr. Mth. (1889) 665-.

Cube, duplication by geometry. Fontés, Toul. Ac. Sc. Bll. 1 (1898) 129-.

-, -, graphio approximation. Gergonne, J. D. Gergonne A. Mth. 10 (1819-20) 242-. - - - by means of parabola. Longchamps, G. de. Mathesis 16 (1896) 245-.

- - - mechanical construction of curves for. Matthiessen, L. Arch. Mth.Ps. 48 (1868) 229-. - roots, geometrical construction. Montucci, $H$. C. R. 44 (1857) 773-.

Curve systems and their application to differential equations. Láska, $W$. Prag Sb. (1890) 1 (Mth.-Nt.) 222-.

Curves, graphical treatment of experimental. Lehfeldt, R. A. $[1900]$ L. Ps. S. P. 17 (1901) 605-.

Deduction of differential equations from graphs. Warder, R. B. Science 4 (1896) 345.

Development of arc of circumference of given radius, tables for calculations. Prat, -, \& Bancarel, -. (vII) A. Cond. Pon. Chauss. 6 (1862) 49-.

Diagrammatic and mechanical representation of propositions and reasonings. Venn, J. Ph. Mg. 10 (1880) 1-.

Mg. 10 (1880) 168-.

Diagrams for ascertaining percentage relation. Smith, W. B. A. Rp. (1890) 982-.

-, employment for representation of logical propositions. Venn, J. [1880] Camb. Ph. S. P. 4 (1883) 47 -

Drawing of curves by their curvatures. Boys, C. V. [1893] L. Ps. S. P. 12 (1894) 345-; Ph. Mg. 36 (1893) 75-.

Economic problems, monopolies, graphic representation by series of hyperbolas. Marshall, A. (vir) [1873] Camb. Ph. S. P. 2 (1876) 318-.

Ellipse, graphic rectification. Somoff, J. [1850] St Pét. Ac. Sc. Bll. 9 (1851) 97-.

-, spacing the. Grant, G. B. Franklin I. J. 127 (1889) 280-. 
Ellipses, method of describing. WilliamsEllis, J. C. [1870] Camb. Ph. S. P. 2 (1876) 197-.

Empirical formulø, calculation. Hopkinson, J. Mess. Mth. 2 (1873) 65-.

Equation $y=a x^{n}$, determination of $n$. Durand, W. F. Franklin I. J. 143 (1897) 188-.

Fourier series, graphic development in. Perry, $J$. Elect. 35 (1895) 285-.

Functions connected by linear equation and graphic solution. Delafon, $R$. Rv. Mar. et Col. 79 (1883) 305-.

- of several variables, graphical evaluation. Adler, $A$. Wien Ak. Sb. 94 (1887) (Ab.2) 404 -

Graphic arithmetic, applications to theory of numbers. Arnoux, G., \& Laisant, C. A. As. Fr. C. R. (1900) (Pt. 2) 36-.

- calculation, 1st operations. Favaro, $A$. Ven. At. 1 (1871-73) 1391-.

- calculus, note on question. Florov, P.S. (xII) Kharkov Mth. S. Com. (1880) 36-.

- integration. Šolin, J. M. Prag Ab. 5 (1872) $10 \mathrm{pp}$.

- - Pickering, E. C. Am. Ac. P. 10 (1875) 79-.

- -. Šolin, J. M. Prag Sb. (1879) 195-.

- - and application to statics of external forces on a beam. Baldermann, $O$. Förster Al. Bauztg. 46 (1881) 24-, 33-, 49-, 97.

- - - - applications. Massau, J. Rv. Un. Mines 16 (1884) 243-, 579-; 17 (1885) 1-; 20 (1886) 1-, 475-; 21 (1887) 85-, 308-, 572-; 22 (1887) 1-.

- - of certain types of differential equations. Petrovitch, M. Par. S. Mth. Bll. 27 (1899) 200-. - - - - - - (liquid apparatus). Petrovitch, M. Am. J. Mth. 22 (1900) 1-.

- - curves. Krähnass, A. Chili S. Sc. Act. 4 (1894) 3-.

- - differential equations. Petrovitch, $M$. C. R. 124 (1897) 1081-.

- - - partial differential equations. Coulon, J. C. R. 130 (1900) 1378-.

- interpolation. Dittmar, $W$. Ph. Mg. 21 (1861) 139-.

- - and integration. Darwin, G. H. B. A. Rp. (1876) (Sect.) 13; Mess. Mth. 6 (1877) 134 -.

- method, applications. Pickering, E. C. (viI) Am. Ac. P. 9 (1874) 222-.

- methods in geometry. Carballo, J. M. R. (xII) [1881] Barcel. Ac. Mm. 1 (1878) 223-. - - - mechanical science. Hele-Shaw, $H$. S. B. A. Bp. (1892) 373-; (1893) 573-.

- - - research. Gilbert, G.K. Smiths. Misc. Col. 33 (1888) Art. 4, 4 (bis)-; (Wash. Ph. S. Bll. 10 (1888).)

- - of solving practical problems. Reynolds, O. [1875] Manch. Lt. Ph. S.P. 15 (1876) 55- phoronomy. Kapp, G. Z. Mth. Ps. 17 (1872) 419-.

- problem. Tramontini, G. [1806] Mod. S. It. Mm. 13 (1807) 38-

- tables and anamorphic geometry, priority claim. Lalanne, L. L. C. C. R. 85 (1877) 1012-.

- technology, apparatus for separation of polynomial into factors. Arnoux, $G$. Par. S. Mth. Bll. 21 (1893) 87-.

Graphic transformer. Trotter, A. P. Elect. 33 (1894) 463-.

Graphics. Vose, G. L. V. Nost. Eng. Mg. 12 (1875) 529-.

Gunter's scale, new arrangement of. Nicholson, W. Nicholson J. 1 (1797) 372-.

Harmonic curves, graphical analysis. Wedmore, E. B. Elect. 35 (1895) 512-.

Imaginary functions, graphic representation. Siebeck, H. Crelle J. 55 (1858) 221-.

Integral curve applied to division of volumes. Brauer, E. Z. Mth. Ps. 42 (1897) 272-.

- of $r^{n} d \theta$ found graphically. Price, W. A. Elect. 36 (1896) 73-.

Integrals of curves and moments of inertia. Nehls, C. Z. Bauw. 39 (1889) 293-

Interpolation-curves, a correction sometimes required. Rayleigh, (Lord). Ph. Mg. 42 (1871) 441-.

Isomers, theory (ramifications). Cayley, $A$. Ph. Mg. 47 (1874) 444-.

Japanese arithmetic, use of geometry. Berson, -. Toul. Ac. Sc. Mm. 3 (1891) 268-.

Lagrange-Newton analytical parallelogram. Sincov, D. Kazan S. Ps.-Mth. Bll. 9 (1900) 44-; Fschr. Mth. (1899) 49-.

Line construction for solution of system of linear equations, regarded as a configuration. Berg, F. J. van den. Amst. Ak. Vs. M. 5 (1889) 267-; Fschr. Mth. (1888) 76.

Logarithmic charts, scale lines. Boys, C. V. Nt. 52 (1895) 272-.

- computations. Howe, H. A. [1883] Sid. Mess. 2 ( $\left.{ }^{*} 1883-84\right)$ 45-.

- coordinates. Vincent, J. H. B. A. Rp. (1898) 159-.

- paper for engineers. Saussure, R. de. Rv. Sc. 2 (1894) 743-.

Logarithms, mechanical calculation. Pocklington, H. C. Nt. 61 (1899-1900) 469 .

Logical diagrams for $n$ terms. Mairquand, $A$. Ph. Mg. 12 (1881) 266-.

Mechanical geometrical proof. Lang, $V$. von. Exner Rpm. 21 (1885) 355-.

Medium value of set of normal variants, geometric determination. Galton, $F$. [1899] Nt. 61 (1899-1900) 102-.

Moments of inertia of solids of revolution. Lopes Banhos, G. C. G. Teix. J. Sc. 5 (* 1883) 125-; Fschr. Mth. (1884) 792.

Mortality equations and curves. Isely, $L$. Neuch. S. Sc. Bll. 17 (1889) 38-.

Multiplication, method of performing. Berdelle, C. As. Fr. C. R. (1884) (Pt. 2) 584-.

Nomography, graphic calculation. Ocagne, M. d'. Ens. Mth. 2 (1900) 207-.

Order of magnitudes, graphical representation. Erdmann, H. Z. Nw. 66 (1893) 73-.

Perspective drawing, method. Williams-Ellis, J. C. [1870] Camb. Ph. S. P. 2 (1876) 197-

Physical laws deduced from empirical curves. Schiaparelli, G. V. Mil. Effem. (1867) 3-. - - - - - Pucci, E. N. Cim. 27 (1890) 220-; 28 (1890) 111-.

Planimeters and similar instruments. Kohl. morgen, $O$. Westf. Vr. Jbr. (1890) 126-. 
Practical conformal representation by current sheets. Kelvin (Lord). Elect. 29 (1892) 553, 634.

Probable values of constants in $u=a+b y$. Anon. 's Gravenh. I. Ing. Ts. (1884-95) (Verg.) 68-.

Problems, solution by cycloid. Guimarães, $R$. G. Teix. J. Sc. 6 (1885) 85-; Fschr. Mth. (1884) 492.

- of third and fourth degrees, solution. Lamé, G. J. Gén. Civ. 1 (1828) 435-.

Quadrature, graphical method. Collignon, $E$. Par. S. Mth. Bll. 15 (1887) 145-.

Representation of physical properties of substances by surfaces. Peddie, $W$. Edinb. Mth. S. P. 2 (1884) 33-.

$--z=f(x, y)$. Lalanne, L. Par. S. Phlm. P.-V. (1843) 71-.

Saturated carbides $\left(\mathrm{C}^{n} \mathrm{H}^{2 n+2}\right)$, representation. Brunel, -. Bordeaux S. Sc. Mm. 5 (1895) xxxix-.

Series of experiments, graphic representation. Sebelien, $J . \quad$ (хп) Ts. Mth. 1 (1883) 186-; Fschr. Mth. 16 (1887) 182-.

Solutions by construction. Hill, T. Am. As. P. (1855) 62-.

Spherical triangles, graphic method of solution. Smith, C. H. Am. J. Mth. 6 (1884) 175-.

"Spiraligrafico meccanico," instrument for graphical calculations. Sayno, A. Mil. I. Lomb. Rd. 7 (1874) 499-.

Statistical science, new step. Galton, $\boldsymbol{F}$. Nt. 51 (1894-95) 319.

Statistics, graphical presentation. Haupt, L. H. Franklin I. J. 148 (1899) 384-.

-, mathematical representation. Edgeworth, F. $Y$. B. A. Rp. (1898) 791 .

Terms of imprisonment, statistics and curves. Galton, $F$. Nt. 52 (1895) 174-.

Theory of contours and its applications in physical science. Peddie, $W$. Edinb. Mth. S. P. 4 (1886) 25-, 61-.

Transcendent numbers, graphic construction. Laisant, C. A. Par. S. Phlm. Mm. Cent. (1888) 63-.

Triangle for calculation purposes. Chenevier, -. As. Fr. C. R. (1886) (Pt. 1) 85.

-, graphical solution. Weisz, J. A. (xII) Mag. Ak. Éts. (1858) 73-.

Vector diagrams, use, for representation of diurnal variation of earth's magnetism. Chree, C. Nt. 61 (1899-1900) 490.

N.B. For references to other papers belonging to 0000-0090 see Special Sections.

\section{ARITHMETIC AND ALGEBRA.}

\section{Foundations of Arithmetic. 0400 General.}

Additive theory of numbers. Vahlen, $K . T$. Crelle J. Mth. 112 (1893) 1-.

Arithmetic, foundation of modern. Montessus, R. de. Ens. Mth. 1 (1899) 185-.

-, principles. Volkov, M. S. Kazan S. Nt. (Ps.-Mth.) P. 4 (1886) 5-.

-, theories. Frege, - Jena. Sb. (1885) 94.
Arithmetic, two problems. Schlegel, M. Arch. Mth. Ps. 11 (1892) 93-.

Arithmetical puzzle. Göpel, A. Grunert Arch. 6 (1845) 34-.

Arithmology. Terquem, $O$. N. A. Mth. 9 (1850) 174-.

-, elementary. Lalanne, L. C. R. 11 (1840) 903-.

- - application to algebra. Lebesgue, $V$. A. N. A. Mth. 1 (1862) 219-, 254-, 405-.

Axiom of Archimedes. Stolz, $O$. Mth. A. 39 (1891) 107-.

Axioms of arithmetic. Milhaud, G. Rv. Sc. 40 (1887) 46-.

Cipher, value. Vencat-Juggarow, G. Madras J. 2 (1835) 369-.

Code, arithmetical. Claudel, -. (vII) A. Cond. Pon. Chauss. 4 (1860) 92-.

Decimals. Peacock, A. B. A. Rp. (1842) (pt. 2) 10-.

-. Spitzer, S. Grunert Arch. 9 (1847) 117-.

"Dual arithmetic," new, applied to military calculations. Byrne, O. (Ix) [1866] Un. Serv. I. J. 10 (1867) 37-.

Equivalence, arithmetic conception. Minkowski, H. Crelle J. Mth. 100 (1887) 449-.

Euclid's books VII, VIII, IX, summary. Peano, G. Rv. Mt. 1 (1891) 10-.

Fractions, certain. Tucker, $R$. L. Mth. S. P. 10 (1878-79) 123, 225-.

-, vulgar. A., S. (vi Adds.) Tilloch $\mathrm{Ph}$. Mg. 48 (1816) 204.

-, - , a set. Stouvenel, V. Liouv. J. Mth. 5 (1840) 265-.

Geometry, application to arithmetic. Ricour, A. Douai Mm. S. Ag. 11 (1873) 279-.

Infinite quantities. Oltramare, $G$. Gen. I. Nt. Mm. 4 (1856) 1-.

- real integers, and their relation to each other. Cantor, -. D. Nf. B. (*1883) 57.

Lagrange's "Solution d'un probleme d'arithmétique," remarks on preface. Plana, G. Tor. Mm. Ac. 20 (1863) 87-, 109-.

Magnitude: fundamental notions. Calinon, $A$. Nancy S. Sc. Bll. (1897) 39-.

- and its measure. Zaremba, $S$. Wiad. Mt. 1 (1897) 58-.

New mathematics, interpretation of old by. Halsted, G. B. Texas Ac. Sc. T. 1 (No. 2) (1893) 89-.

Number and allied conceptions, definition. Thiele, T. N. Kjøb. Dn. Vd. Selsk. Skr. 2 (*1881-86) 451-; Fschr. Mth. (1887) 64-.

-, conception. Kronecker, L. Crelle J. Mth. 101 (1887) 337-.

-, -. Keferstein, $H$. Hamb. Mth. Gs. Mt. 2 (1890) (Festschr. Tl. 2) 119-.

-, -. Peano, G. Rv. Mt. 1 (1891) 87-, 256-.

-, - Hilbert, D. [1899] D. Mth. Vr. Jbr. 8 (1900) (Heft 1) 180-.

-, definition. Laurent, H. N. A. Mth. 17 (1898) 277-

Numeral and metral systems. Garbett, E. L. Glasg. Ph. S. P. 8 (1873) 329-.

Numeration. Gergonne, J. D. Gergonne A. Mth. 21 (1830-31) 329-.

-. Rocquigny, F. [G.] de. Les Mondes 1 (1881) 266-. 
Numeration, systems. Cantor, G. Z. Mth. Ps. 14 (1869) 121-.

- - comparison. Stein, J. P. Crelle J. 1 (1826) 369-.

Numerical system, new. Eissfeldt, $F$. Des Moines Anal. 2 (1875) 151-.

Proportionality, conception. Bustelli, A. M. G. Mt. 13 (1875) 82-.

Step fractions. Gohierre de Longchamps, G. Mt. 15 (1877) 299-.

Variations of quantity, intensity. Bordoni, $A$. (vi Adds.) Opusc. Mt. Fis. 1 (1832) 97-.

\section{Rational numbers; arith- metical operations.}

Approximate arithmetic. Heppel, G. Educ. Times 40 (1887) 417-.

- methods. Lodge, A. Mth. Gz. No. 1 (1894) 4-, No. 3 (1894) 21-.

Approximate multiplication and division. Robertson, J. A. I. Act. J. 32 (1896) 160-.

- numbers, errors in products and powers. Anon. (vi 540) Gergonne A. Mth. 11 (1820-21) 376-.

Approximation. Anon. (xI 51) N. A. Mth. 5 (1866) 407-.

-, Fontaine's method. Coelho da Maia, M. J. Lisb. Mm. Ac. Sc. 1 (1797) 503-.

Approximations in arithmetic. Kopp, $C$. Neuch. Bll. (1846-47) 352-.

-, numerical. Guilmin, A. N. A. Mth. 1 (1842) 249-, 487-.

-, -. Lionnet, $\dot{E}$. N. A. Mth. 8 (1849) 266-; 12 (1853) 177-.

,$- \ldots$, relative to the four fundamental operations. Bredin, C. (xiI) [1858] Doubs S. Mm. 5 (1861) 361-.

- of roots, powers, \&c. Bourdat, - . Grenoble Ac. Delph. Bll. 3 (1850) 447-.

Arithmetic, first stage. Quick, (Rev.) R. H. Educ. Times 41 (1888) 227-.

-, fourth process. Schulze, $E$. Arch. Mth. Ps. 3 (1886) 302-; 9 (1890) 320-.

-, solutions without proportion. Berard, $-\&$ Gergonne, -. Gergonne A. Mth. 8 (181718) 353-.

Arithmetical operations, \&c. Houzeau, J. C. Brux. Ac. Bll. 40 (1875) 74-; 41 (1876) 961-.

- - , fundamental generalization. Schapira, $H$. D. Nf. Tbl. ( $\left.{ }^{*} 1882\right) 128$ -

- - - - order of precedence. Capelli, $A$. Nap. Rd. 39 (1900) 138-.

- - inverse. Mainardi, G. [1849] Mod. S. It. Mm. 25 (1852) 1-.

- - number of digits in results. Vinot, $J$. A. Gén. Civ. 2 (1863) (pte. 2) 172-.

- - particular cases of more general. Faure, H. Par. S. Phlm. P.-V. (1847) 31-.

- - , signs. Le Paige, C. Brux. S. Sc. A. 16 (1892) (Pt. 2) 70-.

- summations. Ocagne, M. d'. G. Teix. J. Sc. 7 (1886) 117-.

Binary arithmetic. Farquhar, $H$. Seience 2 (* 1883) 755-.
Calculation, Doms' method. Mewes, $R$. Dinglex 315 (1900) 547-.

-, means of avoiding errors. Cauchy, A. L. C. R. 11 (1840) 789-, 826.

-, numerical, notes. Houzeau, J. C. Brux. Ac. Bll. 20 (1865) 614-

Calculations, arithmetical, involving large numbers. Pervusin, I. Kazan S. Ps.-Mth. Bll. 4 (1895) (Prot.) 74-.

-, checking, note. Walenn, W. H. [1879] Ph. Mg. 9 (1880) 56-.

-, instruments to aid. Thomson, G. [1896] Sc. S. Arts T. 14 (1898) 172-.

Computation for scientific purposes. Lupton, $S$. Nt. 37 (1888) 237-, 262-.

_- _ - Ramsay, W., \& Young, S. Nt. 37 (1888) 294.

- - - Scott, E. E. Nt. 37 (1888) 319.

--- -. King, G. Nt. 37 (1888) 319-.

- of sums of products. Orlov, A.P. Kazan S. Nt. (Ps.-Mth.) P. 4 (1886) 59.

- _ - - Preobraženskij, V. V. Kazan S. Nt. (Ps.-Mth.) P. 4 (1886) 72-

Continued product of numbers without use of logs., by formula and table. Sylvester, J.J. Ph. Mg. 7 (1854) 430-.

Cube and cube-root, obtaining by Horner's method. Rusch, M. Arch. Mth. Ps. 67 (1882) 291-.

- root. Nievengloski, G. H. N. A. Mth. 10 (1851) 86-.

- - extraction. Midy, -. N. A. Mth. 3 (1844) 234-.

- - Györy, S. (xm) Mag. Ak. Éts. (1851) 282-; (1852) 62-.

- - Hofmann, F. Grunert Arch. 22 (1854) 240.

- - Forestier, C. N. A. Mth. 17 (1858) 7-.

- - - Gouzy, E. A. Arch. Mth. Ps. 49 (1869) 101-.

- - - abridged. Hill, C. J. D. Crelle J. 11 (1834) 262-.

- - - - Müller, J. H. T. Grunert Arch. 8 (1846) 46-.

- - - - Genocchi, A. N. A. Mth. 17 (1858) 136-.

- - - - Dostor, G. Arch. Mth. Ps. 64 (1879) 321-.

- - - - Bernardi, G. G. Mt. 35 (1897) 112-, 353.

_ - -, integers. Delorme, -. [1838] Amiens Mm. (1839) 251-.

- - - new method. Nicholson, $P$. Tilloch Ph. Mg. 56 (1820) 360-.

- roots by inspection. Evans, J. Tilloch $\mathrm{Ph}$. Mg. 51 (1818) 443-.

Decimal notation, ancient and modern. Piccard, -. Laus. Bll. S. Vd. 6 (1859) 163-.

- numbers, multiplication. Vervaet, $J$. Časopis 15 (1886) 24-; Fschr. Mth. (1886) 127.

- operations, simplifying and checking. Cauchy, A. L. C. R. 11 (1840) 847-.

- places, error from neglected. Hoppe, $R$. [1845] Crelle J. 40 (1850) 141-. 
Decimal point, determination of position in multiplication and division. Jelinek, $V$. Časopis 20 (1891) 35-; Fschr. Mth. (1891) 166.

Decimals; approximation. Amiot, $B$. Rouen Ac. Tr. (1841) 161-; N. A. Mth. 10 (1851) 238-.

-, multiplication. Bernauggi, I. Verona Mm. S. It. 6 (1792) 1-.

- recurring, division by 11 . Transon, $A$. N. A. Mth. 4 (1845) 227-.

- , - , formula for conversion. Trembloy, N. A. Mth. 1 (1842) 522-.

-, reduction to fractions. Ponte-Horta, F. da. Lisb. J. Sc. Mth. 1 (1868) $275-$.

Discontinuous geometry. Bugaev, N. V. [1890] Rec. Mth. (Moscou) 15 (1891) 600-; Fschr. Mth. (1891) 726.

Division, abridged. Finck, B. N. A. Mth. 4 (1845) 328-, 658-; 5 (1846) 599-.

-, -. Crelle, A. L. Crelle J. 31 (1846) 167-.

-, - Crosson, A. N. A. Mth. 5 (1846) $244-$

-, 一. Guy, -. N. A. Mth. 5 (1846) 460 -.

-, - Verhulst, P. F. N. A. Mth. 5 (1846) 629 -

-, - Spitzer, S. [1850] Haidinger B. 7 (1851) 175-.

-, -. Lionnet, E. N. A. Mth. 11 (1852) 148-.

-, - . Housel, - . N. A. Mth. 14 (1855) 129 -.

-, -. Rouché, E. N. A. Mth. 16 (1857) 152-.

-, -. Henry, C. N. A. Mth. 20 (1881) 213-.

-, - long. Dodgson, C. L. Nt. 57 (1897-98) 269-.

-, - -. Christie, R. W. D. Nt. 57 (189798) 390

-, - and ordinary. Turquan, -. N. A. Mth. 13 (1854) 170-.

- - singular case. Adville, -. N. A. Mth. 11 (1852) 100-.

- of approximate numbers, rule. Verhulst, P. $F$. Brux. Ac. Bll. 13 (1846) 696-.

- arithmetical, new rule. Peirce, C. S. Science 2 (*1883) 788-.

-, 一, theory. Faucheux, L. E. N. A. Mth. 14 (1854) 51-.

-, complementary. Terquem, $O$. N. A. Mth. 6 (1847) 425-.

-, -. Meech, L. W. Des Moines Anal. 9 (1882) 97-.

- and evolution, rationale of processes. Niederrist, -. Grunert Arch. 35 (1860) 201-.

-, Fourier's, and application to square root. Schaar, M. Brux. Ac. Bll. 18 (1851) (pte. 2) 144-

-, Gerbert's rules. Friedlein, G. Z. Mth. Ps. 9 (1864) 145-.

-, new method. Kostěnec, A. Časopis 15 (1886) 74-; Fschr. Mth. (1886) 127-.

-, numerical, easy method. Crelle, A. L. Crelle J. 13 (1835) 209-.

-, results in. Langley, E. M. Mth. Gz. 1 (1900) 205-, 275-。

- and subtraction, extended. Callegari, $P$. Bologna N. Cm. 6 (1844) 513-; 7 (1844) 529-.

Divisors, fractional, in arithmetic. Terquem, $O$. N. A. Mth. 8 (1849) 246-.
Evolution by subtraction. Hummel, F. $H$. Ph. Mg. 10 (1880) 190-.

Factorisation and factorial calculation. Pacinotti, L. Pisa A. Un. Tosc. Sc. Cosm. 2 (1851) 143-.

Fraction and quotient, fusion of notions. Bobynin, V. V. Bb. Mth. (1899) 81-.

Fractions, compound, simplification. Euler, $L$. [1779] St Pét. Ac. Sc. Mm. 1 (1803-6) 3-.

-, elementary theory. Méray, C., \& Riquier, C. N. A. Mth. 8 (1889) 421-。

-, estimation. Petrie, W. M. F. [1875] Nt. 13 (1876) 127-.

with numerator 1 , theorems. Fuss, N [1809] St Pét. Ac. Sc. Mm. 10 (1826) 27-. - of $£ 1$, conversion to decimals. De Morgan, A. Assur. Mg. 11 (*1864) 53-.

-, theorem. Posnikov, M.V. Kazan S. Nt. (Ps.-Mth.) P. 4 (1886) 238.

-, transformation. Lagrange, J. L. Par. Ec. Pol. J. 2 (1798) (5 cah.) 93-.

-, -. Penjon, -. Gergonne A. Mth. 4 (1813-14) 265-.

-, - into decimals. Arndt, J.A. Grunert Arch. 1 (1841) 101.

-, - - - Dienger, J. Grunert Arch. 11 (1848) 232.

-, vulgar, expansion in sorites. Sylvester, J. J. Am. J. Mth. 3 (1880) 332-, 388-.

-, , reduced to decimal, number of figures. Sornin, J. N. A. Mth. 8 (1849) 50-.

Frolov's groups of numbers. Studnička, F.J. Časopis 19 (1890) 124-; Fschr. Mth. (1890) 196.

Functions obtained by combining the four fundamental operations. Bianchi, G. Palomba Rac. 2 (1846) 326-.

Geometry, generalisation of arithmetical operations in reference to. Wiegand, $A$. Mathematician 3 (1850) 60-

G. C. M. Finck, B. N. A. Mth. 1 (1842) 353-.

_- Bertrand, J. N. A. Mth. 8 (1849) 358-.

433-.

- - - Gegenbauer, L. Wien Ak. Sb. 91 (1885) (Ab. 2) 333-.

- - , abridged method of finding. Nievengloski, G. H. N. A. Mth. 8 (1849) 447-.

- - - Lamé's theorem. Finck, B. N. A. Mth. 4 (1845) 71-.

- - and L. C. M. Gergonne, J. D. Gergonne A. Mth. 21 (1830-31) 117-.

- - - - - Barrieu, P. (xiI) Mathesis 3 (1883) 217-; N. A. Mth. 14 (1895) 95-, 165-, 214-.

- - mean values. Cesàro, E. A. Mt. 13 (1885) 235-.

- - , new method. Binet, J.P.M. C. R. 13 (1841) 349-.

- - number of divisions. Lamé, G. C.R. 19 (1844) 867-.

(1844) $937-$.

$-1,-\frac{1}{-}$ Nievengloski, G.H. N.A. Mth. 4 (1845) 568-. 
G. C. M., number of divisions. Lionnet, E. N. A. Mth. 4 (1845) 617-.

- - - - - D. Dupré, A. Liouv. J. Mth. 11 (1846) 41-.

- - - - Grunert, J.A. Grunert Arch. 8 (1846) 137-

- - - - - Schultén, N. G. af (fil.). Helsingf. Acta 2 (1847) (pte. 2) 745-.

- - - - - Lionnet, E. N. A. Mth. 10 (1851) 85-.

- _ - Poinsot's method. Grunert, J. A. Grunert Arch. 7 (1846) 153-.

- - of two numbers, expression for. Lerch, M. Prag Sb. (1885) (Mth.-Nt.) 414-.

Harmonic series, property that $(2 m) !\left[\sum_{1}^{2 m} \frac{1}{r}\right]$ is divisible by $2 m+1$. Schiappa Monteiro, A. Lisb. J. Sc. Mth. 5 (1898) 224-.

Imaginary bases for arithmetical notation. Grïmwald, V. Brescia At. Cm. (1886) 43-.

Involution and evolution, improvements. Nicholson, $P$. Tilloch $\mathrm{Ph}$. Mg. 52 (1818) 341- ; 57 (1821) 33-.

- - - by logarithms, general rule. Riddell, J. L. Silliman J. 7 (1849) 232-.

Laisant's theorem on $1,2,3 \ldots(n-1)$ in scale of notation $n$. Sokolow, N. Fschr. Mth. (1896) 141.

Mental arithmetic. Cuthbertson, C. Nt. 45 (1892) 78-.

- Haas, $K$. Nt. 45 (1892) 198-.

- arithmetical feats by Inaudi, report on. Darboux, J. G. C. R. 114 (1892) 1335-.

- multiplication. Oakes, (Lieut.-Col.) W. H. Assur. Mg. 10 (*1863) 326-.

- - process. Saussure, H. de. Laus. Bll. S. Vd. 3 (1849-53) 256-.

Multiplication, abridged, proof. Pedersen, H.A. Mth. Ts. 2 (1860) 50-.

-, -, Thoyer's method. Cauchy, A. L. C. R. 12 (1841) 242-, 941-.

-, - , various means. Gergonne, J.D. Gergonne A. Mth. 7 (1816-17) 157-.

-, arithmetical note. Scott, C. A. Science 12 (1900) 648-.

-, commutative law in. Poussou [non Posson], -. Mntp. Rec. Bll. 2 (1805) 252-.

- and division, numerical. Querret, - Gergonne A. Mth. 13 (1822-23) 277-.

- games, Lucas's "Arithmétique amusante" in particular. Studnička, F. J. Časopis 25 (1896) 289-; Fschr. Mth. (1896) 122.

- of numbers, facts concerning. Azoulay, Par. S. Phlm. Bll. 4 (1892) 71-.

- - - problem. Berg, F.J.van den. N. Arch. Wisk. 5 (*1879) 47-; 10 (1884) 202.

- by quarter squares. Laundy, S. L. Assur. Mg. 6 ( $\left.{ }^{*} 1857\right)$ 121-.

- rules, nature and teaching. Baron, $R$. Ens. Mth. 1 (1899) 317-.

- simplified by negative digits. Cunningham, A. Nt. 48 (1893) 316.

-, various kinds. Grassmann, H. Crelle J. 49 (1855) 123-.

Negative figures, arithmetic with. Longchamps, G. de. As. Fr. C. R. (1893) (Pt. 2) 200-
Nomography (helps in calculation) and need for its introduction in teaching. Pasquier, $E$. Ens. Mth. 1 (1899) 350-.

Numbers, problems in theory. Gelin, E. Mathesis 16 (1896) (Suppl.) $34 \mathrm{pp}$.

-, property depending on scale of their notation. Andreini, A. G. Mt. 26 (1888) 315-; 28 (1890) 43.

-, - - - - - - Gambioli, -. G. Mt. 27 (1889) 334-.

-, - - - - - Maillet, E. As. Fr. C. R. (1894) (Pt. 2) 242-.

G. Mt. 33 (1895) 157-.

Powers, formation, and root extraction. Querret, -. Gergonne A. Mth. 12 (182122) 359-; 13 (1822-23) 163-.

- of integers. Mariantoni, F. Mathesis 18 (1898) 244-.

Proportion, problems. Lucchesini, - C. R. 11 (1840) 476-.

Pseudo-symmetric numbers. Lemoine, $E_{\text {. Par. }}$ S. Mth. Bll. 12 (1884) 155-.

Pythagorean triangles. Safford, T. H. Wisc. Ac. T. 12 (1900) 505-.

Reciprocals of numbers, theorems. Caminati, - As. Fr. C. R. (1887) (Pt. 1) 173.

-, table, construction. Lehmann, J. W. $H$. As. Nr. 52 (1860) 1-.

Root extraction, new method. Györy, S. (xII) Mag. Ak. Éts. (1850) 260-; (1851) 57-, 173-.

- - - - Vazquez Prada, M. C. R. 121 (1895) 635-.

Rule of double-false-position. Ponssou [non Possou ], -. Mntp. Rec. Bll. 3 (1809) 285-.

Scales of Notation.

Berndtson, B. Stockh. Ac. Hndl. 39 (1818) 212-.

best number for radix. Thiele, T. N. Kjøb. Ov. (1889) 25-.

binary and octonary. Berdellé, C. As. Fr. C. R. (1887) (Pt. 2) 206-.

-, theorems. Simony, O. Mth. A. 31 (1888) 549 -.

denary. Oettinger, $L$. Grunert Arch. 26 (1856) 445-.

-, divisibility. Singer, E. Mil. G. I. Lomb. 5 (1853) 141-.

-, -. Hann, J. [1876] Z. Mth. Ps. 22 (1877) 54-.

-, -, criteria. Züge, -. Arch. Mth. Ps. 17 (1900) 45-.

-, -, two rules. Vervaet, $P . J$. Časopis 5 (*1876) 189-; Fschr. Mth. (1876) 84.

division, arithmetical. Fontès, -. As. Fr. C. R. (1892) (Pt. 2) 182-; Toul. Ac. Sc. Mm. 4 (1892) 289-.

method of transformation. Barlow, $\boldsymbol{P}$. Nicholson J. 25 (1810) 181-

with negative base. Grünwald, $\boldsymbol{V}$. G. Mt. 23 (1885) 203-, 367.

number with digits reversed. Lemoine, $\dot{E}$. As. Fr. C. R. (1883) 113-. 


\section{Scales of Notation}

number with digits reversed, problem. Laisant, C. A. Bordeaux S. So. Mm. 1 (1876) 403-.

- - - , theorem. Lemoine, $\hat{E}$. As. Fr. C. R. (1884) (Pt. 2) $58-$.

- - recurring digits, theorem. Glaisher, J. W. L. [1875] Mess. Mth. 5 (1876) 3-. - whose powers terminate in same digits. Tanner, H. W. L. [1877] Mess. Mth. 7 (1878) 63-.

- - square ends in same digits as itself. Palmström, A. Christiania Skr. (Mth.-Nt. Kl.) (1900) No. 3, $16 \mathrm{pp}$.

- 12345679, properties. Plateau, J.A. F. (vii) Brux. Ac. Bll. 16 (1863) 62-.

- - - (Plateau). Hooton, J. J. Intell. Obs. 4 (1864) 439.

- - - Plateau, J. A. F. Brux. Ac. Bll. 38 (1874) 469-.

- _, - , and generalisation. Laisant, C. A. Lisb. J. Sc. Mth. 5 (1898) 203-.

octonary. Johnson, W. W. [1891] N. Y. Mth. S. Bll. 1 (1892) 1-.

-, application to system of weights and measures. Taylor, A. B. Am. Ph. S. P. 24 (1887) 296-.

powers of integers. Drot, - . N. A. Mth. 4 (1845) 637-; 5 (1846) 25-.

- - . Schlömilch, O. Z. Mth. Ps. 12 (1867) 350.

- - André, D. N. A. Mth. 16 (1877) 370-.

problems. Berdellé, C. As. Fr. C. R. (1898) (Pt. 2) 139-.

quinary, denary, and vicenary. Marre, $A$. Liouv. J. Mth. 13 (1848) 233-.

sum of digits. Berton, -. N. A. Mth. 18 (1859) 209-.

- - - of certain numbers. Sanctis, P. de.

Rm. N. Linc. At. 46 (1893) 115-, 183-; 47 (1894) 53-; 50 (1897) 11-; 54 (1901) 18-.

- - - numbers, last. Gelin, $\left(l^{\prime} A b b e ́\right)$ Les Mondes 43 (1877) 430-.

_ _ _ - sum of three consecutive numbers.

Egidi, G. Rm. N. Linc. At. 46 (1893) 63-.

sums of digits, numbers. Terrot, C. H. [1843]

Edinb. R. S. T. (1849) 87-.

theorem. Crelle, A. L. Crelle J. 30 (1846) 215-.

theorems. Sanctis, P. de. Rm. N. Linc. Mm. 11 (1895) 145-.

"Tonal system." Nystrom, J. W. Franklin I. J. 46 (1863) 263-, 337-, 402-.

2n-ary. Studnička, F.J. Prag Sb. (1896) (Mth.-Nt.) No. 7, $10 \mathrm{pp}$.

with variable base. Sokolow, N. Fschr. Mth. (1896) 122.

weights problem. Sornin, $J$. Toul. Mm. Ac. 6 (1856) 37-. - - 10 -. Stahlberger, E. Carl Rpm. 5 (1869)

- - F., E. R. Nt. 42 (1890) 568.

- -. Willis, J. Nt. 43 (1891) 30-, 198.

- - Ev. Everett, J. D. Nt. 43 (1891) 104,

- 113 -

- - B., R. E. Nt. 43 (1891) 198-.

\section{Irrational Numbers 0420}

weights problem, analogues. Müller, H. F. Z. Mth. Ps. 21 (1876) 227-.

Square of large number. Pastorček, J. M. Časopis $6\left({ }^{*} 1877\right) 277-$; Fschr. Mth. $\left({ }^{*} 1877\right)$ 808-.

- property of $48 \ldots 89$, analogous numbers. Laisant, C. A. Par. S. Phlm. Bll. 4 (1892) 77-.

Sum or difference of products of factors. Austin, J. [1815] Am. Ph. S. T. 1 (1818) 181-.

Transformation of product of natural numbers. Frisiani, P. Mil. Effem. As. (1850) 44 -.

"Unitation," new arithmetical operation. Walenn, W. H. Ph. Mg. 36 (1868) 346-; 46 (1873) 36-; 49 (1875) 346-; 50 (1875) 117-, 521-; 1 (1876) 546-; 4 (1877) 375-; 5 (1878) 214-; 9 (1880) 121-, 271-.

\section{Existence of irrational and transcendental numbers; infinite processes adapted to rational numbers.}

Agreement of two methods of approximation to a square root (Newton \& Lagrange). Carrara, B. G. Mt. 28 (1890) 39-.

Algorithm for the calculation of the $n$th root of $a$. Schmidt, C. Mth. A. 45 (1894) 301-.

Approximate evolution, note. Tanner, H.W.L. L. Mth. S. P. 23 (1892) 295-.

- representation of irrationals by rational fractions. Hurwitz, A. Mth. A. 39 (1891) $279-$.

- roots of numbers. Philbrick, - . Iowa Ac. Sc. P. $\left({ }^{*} 1875-80\right) 24$.

- - and quotients, errors. Anom. (vi 546) Gergonne A. Mth. 13 (1822-23) 175-.

Approximation by rational fractions. Hurwitz, A. Mth. A. 44 (1894) 417-.

Archimedes and $\sqrt{3}$. Thiele, T. N. Ts. Mth. 2 (1884) 151-; Fschr. Mth. (1884) 8.

Arithmetic, transcendental. Eisenstein, $G$. Crelle J. 29 (1845) 177-.

Arithmetical theorems, four. Strnad, A. Časopis 17 (1888) 204-; Fschr. Mth. (1888) 185.

Arithmetization of mathematics. Pierpont, $J$. N. Y. Am. Mth. S. Bll. 5 (1899) 394-.

Chuquet, N., extraction of square root, 1484 . Tannery, P. Bb. Mth. (1887) 17-.

Chuquet's work, mean rule. Mansion, $P$. Bb. Mth. (1888) 36.

Comparison of magnitudes; Brouncker's method extended. Sang, E. [1870-90] Edinb. R. S. T. 26 (1872) 59-; Edinb. R. S. P. 18 (1892) 341-.

Continued radicals. Bouché, A. M.-et-L. Mm. S. Ac. 12 (1862) 81-.

Convergents to the roots of a number. Muir, $T$. Edinb. R. S. P. 19 (1893) 15-.

Cube, duplication with any approximation. Popov, A.T. (xII) Kazan Un. Mm. 6 (1871) 233-. 


\section{Irrational Numbers}

Cube, duplication, new geometrical relation serving to solution of problem. Buonofalce, $G$. Les Mondes 41 (1876) 338-.

- root of 2, computation. Martin, Art. [1877] Mess. Mth. 7 (1878) 50-.

- - - - value to 28 places. Gray, $P$. Mess. Mth. 5 (1876) 172-.

- - - - verification and extension of value. Gray, P. [1877] Mess. Mth. 7 (1878) 51-.

- roots of binomials, extraction. Lockhart, $J$. Thomson A. Ph. 6 (1815) $152-$.

- - - - (Lockhart). Horner, W. G. Thomson A. Ph. 8 (1816) 279-, 388-.

Decimals, non-terminating, property. Young, J.R. Ph. Mg. 36 (1850) 15-.

-, periodicity. Looff, $W$. Grunert Arch. 16 (1851) 54-.

-, recurring. Adams, Jas. Thomson A. Ph. 2 (1821) 16-.

-, - Catalan, E. C. N. A. Mth. 1 (1842) $457-$.

-, - . Stammer, W. Grunert Arch. 27 (1856) 123-.

-, - Hansted, B. (xII) Ts. Mth. 2 (1878) 180 -.

-, -. Glaisher, J. W. L.

471-; Nt. 19 (1879) 208-.

B. A. Rp. (1878)

- Schlömilch, O.X. (1880) 416.

-, -, class. Bouniakowsky, $V$. St Pét. Ac. Sc. Bll. 27 (1881) 362-.

-, 一, divisibility. Garnier, F. P. Moigno Cosmos 6 (1855) 238-.

-, - , for $\frac{1}{81}$, general case for any radix. Glaisher, J. W. L. Mess. Mth. 2 (1873) 188.

-, - , and Henry Goodwyn's tables. Glaisher, J. W. L. [1878] Camb. Ph. S. P. 3 (1880) 185-.

-, -, mixed, theory. Broda, K. [1874] Arch. Mth. Ps. 57 (1875) 297-.

-, - new properties. Coninck $G$. N. A. Mth. 13 (1874) 569-.

-, -, periods. Dienger, $J$. Crelle J. 39 (1850) 67-.

-, -, property (Colling). Oliver, J. E. Camb. (M.) Mth. M. 1 (1859) 345-

- , - theory. Sturm, J. B. Grunert Arch. 33 (1859) 94-.

- -, - Broda, K. Arch. Mth. Ps. 56 (1874) 85-.

-, theorem. Hansted, B. G. Teix. J. Sc. 2 (1880) 154-

Equation $x^{m}=a+b \sqrt{ }-1$. Valat, -. Bordeaux Act. Ac. Sc. (1844) 177-.

Euclid, Book X, summary. Peano, G. Rv. Mt. 2 (1892) 7 -.

Fractions approximating to square root of a variable. Čebyšev, $P$. St Pet. Ac. Sc. Mm. (Rs.) 61 (1890) (Suppl.) No. 1, 22 pp.; Acta Mth. 18 (1894) 113-.

-, periodic. Laisant, C. A. Bordeaux S. Sc. Mm. 3 (1880) 213-.

,-- , in given systems of numbers, law of formation. Broda, $K$. Arch. Mth. Ps. 68 (1882) 85-.

\section{Irrational Numbers 0420}

Fractions, periodic, laws. Moret-Blanc, N. A. Mth. 14 (1875) 229-.

,-- , means of finding period. Laisant, $A$. Les Mondes 19 (1869) 331-.

-, -, properties. Beaujeux, É., \& Laisant, A. N. A. Mth. 7 (1868) 289-.

-, -, theorems. Thibault, - . N. A. Mth. 2 (1843) 80-.

Incommensurability, general theorem. Maizière, - de. Gergonne A. Mth. 1 (1810-11) 293-.

-, and its treatment in certain books. Amicis, E. de. Rv. Mt. 5 (1895) 110-.

Incommensurable fraction, development. Young, J. R. Ph. Mg. 36 (1850) 128-.

- lines. Lebesgue, V. A. N. A. Mth. 3 (1844) 436-.

- - $\sqrt{3}: 1$. Bretschneider, C. A. Grunert Arch. 3 (1843) 440-.

- number, meaning to be attached to the term, etc. Méray, C. Par. Éc. Norm. A. 4 (1887) 341-.

- numbers. Wantzel, L. Par. Éc. Pol. J. 15 (1837) (25e cah.) 151-.

- - of algebraic origin. Wantzel, L. N. A. Mth. 2 (1843) 117-; Par S. Phlm. P.-V. (1843) 10-

- - general remarks. Girault, -. Rouen Tr. Ac. (1849-50) 96-.

- - geometrical proof of theorem. Sylvester, J. J. (xII) J. H. Un. Cir. [1] (1882) 179-, 209.

- - and limits. Jablonski, E. N. A. Mth. 20 (1881) 241-.

- - property. Stephanos, C. Par. S. Mth. Bll. 7 (1879) 81-.

- -, theorem of Čebyšev. Le Paige, $C$. Brux. Ac. Bll. 25 (1893) 235-.

- quantities. Léger, $E$. Liouv. J. Mth. 1 (1836) 93-.

- -, elementary theory. Egidi, G. Rm. N. Linc. At. 46 (1893) 149-.

- - , theorem. Lebesgue, V. A. Liouv. J. Mth. 1 (1836) 266-.

- roots and logarithms, definitions. Guilmin, A. N. A. Mth. 6 (1847) 313-.

Irrational formulæ, irreducible. Bouniakonsky, V. [1840] St Pét. Ac. Sc. Mm. 4 (1841) 471-.

- - reciprocals. Canterzani, S. Bologna Mm. I. It. 1 (pte. 2) (1806) 301-.

- numbers. Minnigerode, B. Mth. A. 4 (1871) 497-.

- -. Peano, G. Rv. Mth. 6 (1896-99) 126-. - - arithmetical properties. Christoffel, E. B. A. Mt. 15 (1887-88) 253-.

- - definition. Illigens, E. Mth. A. 35 (1890) 451-.

- - development in rational continued fractions. Schultén, N. G. af (fil.) [1847] Helsingf. Acta 3 (1852) 405-.

- -, introduction. Pasch, M. Mth. A. 40 (1892) 149-.

- - , - with method of contiguous classes. Capelli, A. G. Mt. 35 (1897) 209-.

- - theorems. Ramsay, A. Stockh. Õfv. 39 (1882) No. 9, 21-. 


\section{Irrational Numbers}

Irrational numbers, theory. Dantscher von Kollesberg, V. Innsb. Nt. Md. B. 17 (1887) 1-.

- - Weierstrass-Cantor theory. Iligens, $\boldsymbol{E}$. Mth. A. 33 (1889) 155-.

- quantities, approximate determination, two methods. Günther, S. Erlang. Ps. Md. S. Sb. 6 (1874) 82-.

- - equidifferent multiples. Sang, E. [1880] Edinb. R. S. P. 11 (1882) 2-.

Irrationality, arithmetical. Glaisher, J. W. L. Ph. Mg. 45 (1873) 191-.

- of $\cos \frac{2 \pi}{n}$, except when $n=1,2,3,4$ or 6 . Wendt, C. Mh. Mth. Ps. 10 (1899) 97-.

Irrationals, algebraic, theory. Capelli, $A$. Nap. Rd. 30 (1891) 61-.

Limit-value and irrational numbers. Jensen, J. L. W. V. Ts. Mth. 3 (1885) 33-; Fschr. Mth. (1885) 203.

Limits and irrational numbers, theory. Arzelà, C. (xII) Rv. Sc.-Ind. 10 (1878) 77-, 144-, 177-.

Periods of decimals equivalent to $1 / p$, terminator. Sardi, C. [1868] G. Mt. 7 (1869) 24-.

- - - for $\frac{1}{19}, \frac{1}{19}, \frac{1}{49}$, etc., short process. Glaisher, J. W. L. Mess. Mth. 7 (1878) 190-.

Pythagorean series for side and diagonal of square. Hultsch, F. Bb. Mth. 1 (1900) 8-.

Radicals, calculation. Eggers, $H$. Des Moines Anal. 3 (1876) 100-.

-, extraction, general method. Ruffini, $P$. [1812] Mod. S. It. Mm. 16 (1813) 373-; 17 (1815) 1-.

-, quadratic, approximate value. Poncelet, J. V. Crelle J. 13 (1835) 277-.

-, - , - values. Horvath, -. (xII) Mag. Tud. Ak. Ets. 1 (No. 8) (1867) 150-; (x) Par. S. Phlm. Bll. 4 (1867) 247-.

-, - approximation, Poncelet's theorem. Zeuner, G. Schlömilch Z. 3 (1858) 383-.

$\ldots,-$, Poncelet's approximate linear valuation. Sylvester, J.J. Ph. Mg. 20 (1860) 203-.

-, -, - geometric approximation. Léauté, H. Par. S. Mth. Bll. 8 (1880) 106-

-, - - - theorem, extension to trinomial. Resal, H.A. (xr) Par. S. Mth. Bll. 1 (1873) 155-.

_, - - - theorems for linear representation generalised. Sylvester, J. J. B. A. Rp. (1860) (pt. 2) 7 .

Real numbers, theory of, according to Dedekind's conception. Ricci, G. Ven. I. At. (1892-93) 233-; G. Mt. 35 (1897) 22-.

Root extraction. West, B. Bost. Mm. Am. Ac. 1 (1785) 165-

- -. Anon. (vi 782) Madrid Rv. 2 (1851) 419-.

- - Kinkelin, H. Grunert Arch. 26 (1856) 361-.

- Evans, A. Des Moines Anal. 3 (1876) 10 -

- C. Chokrobarthy, S.N. Edinb.Mth.S.P. 16 (1898) 77.

- -, approximate. Dienger, J. Grunert Arch. 17 (1851) 421-.

\section{Irrational Numbers 0420}

Root extraction, approximate. Heaton, $H$. A. Mth. 1 (1884-85) 14.

- -, cubic, \&c. Young, $M$. Ir. Ac. T. 1 (1787) 59-.

- - of fractions, Ampère's method; application to gamut. Vincent, A.J. H. N. A. Mth. 5 (1846) 5-.

- - of integers by binomial theorem. Martin, Art. Des Moines Anal. 1 [1874]117-. - -, $n$th degree. Eggers, H. Des Moines Anal. 2 (1875) 14-.

Roots of all powers, extraction. Hoare, $C$. Franklin I. J. 39 (1860) 50-.

-, approximate calculation, and applications. Thallmayer, V. Arch. Mth. Ps. 10 (1891) $32-$.

-, - formulæ for calculation. Borgogelli, $M$. Rm. N. Linc. At. 45 (1892) 101-.

-, expression by rational fractions. Thiele, T. N. N. Ts. Mth. 1 (B) (1890) 33-; Fschr. Mth. (1890) 221-.

- higher, extraction. Greene, D. Camb. (M.) Mth. M. 1 (1859) 123-.

$\sqrt[m]{N}$, last $n$ figures by division. Anne, $L$. N. A. Mth. 4 (1845) 561-.

-, numerical, extraction, abridged. Bobillier, -. Gergonne A. Mth. 20 (1829-30) 125-.

- and powers, calculation. Cordeiro-Feyo, $J$. Lisb. Mm. Ac. Sc. 12 (1837) (pte. 1) 1-.

- rapid method of approximation. Collins, M. B. A. Rp. 42 (1872) (Sect.) 13-.

Square and cube root extraction, abridged. Finck, B. N. A. Mth. 5 (1846) 250-.

- - - - degree of approximation. Verhulst, P. F. N. A. Mth. 7 (1848) 46-

- - roots. Fitremann, - N. A. Mth. 10 (1871) 87-

- - - - , rules for contracting processes, incorrectness of. Hill, M. J. M. L. Mth. S. P. 18 (1886-87) 171-.

- - other roots, principal values. Tilly, - de. Mathesis 15 (1895) 177-, 217-; 16 (1896) 5-

- root extraction. Crosson, A. N. A. Mth. 5 (1846) 128-.

- - Alexéeff, N. Par. S. Mth. Bll. 7 (1879) 167-.

- - Darboux, G. Bll. Sc. Mth. 11 (1887) 176-.

- - Pavlov, A. P. Kazan S. Nt. (P8.Mth.) P. 6 (1888) 8.

- - Bougon, -. Rv. Sc. 46 (1890) 283-.

- - - Kleiber, J. Rv. Sc. 46 (1890) 507-.

- - - abridged. Catalan, E. C. N. A. Mth. 17 (1858) 232-.

- -, 一. Hill, M. J. M. B. A. Rp. (1886) 538.

90-.

764.

- - - , history. Cantor, M. N. A. Mth. 20 (1861) 46-.

- - - methods. Grunert, J.A. Grunert Arch. 30 (1858) 275-.

_ _ , rapidly converging series for. Muir,

T. Edinb. R. S. P. 17 (1891) 14-. 


\section{Aggregates}

Square root of polynomial, approximate fractions. Aleksěev, $N$. St Pet. Ac. Sc. Mm. (Rs.) 37 (*1881) 107-.

--12 and of 3 , approximations. Henry, $C$. Bll. Sc. Mth. As. 3 (1879) 515-.

- roots, approximate expressions for. Hauber, C. $F$. Lindenau Z. 4 (1817) 95-.

- - of numbers. Weill, -. Par. S. Mth. Bll. 14 (1886) 128-.

Surd, formula for fraction approximate in value. Whitworth, W. A. [1874] Mess. Mth. 4 (1875) 79-.

Surds and irrationals, classification. Baker, A. L. Rochester (N.Y.) Ac. Sc. P. 2 (1895) 202-.

$\frac{v^{m}-w^{m}}{v-w}$, expansion. Adams, Jas. Thomson A. Ph. 16 (1820) 418-.

\section{Aggregates.}

Absolute convergence of improper integrals, explanation. Stolz, O. Wien Ak. Sb. 107 (1898) (Ab. 2a) 207-; 108 (1899) (Ab. 2a) 1234-.

Aggregate question in billiards. Vivanti, $G$. Rv. Mt. 5 (1895) 87-.

Aggregates of aggregates. Burali-Forti, C. Mth. A. 47 (1896) 20-.

-, extension of enumerable. Garibaldi, $C$. Palermo Cir. Mt. Rd. 8 (1894) 157-.

- formed by special values of a function. Schönfliess, A. Königsb. Schr. 41 (1900)[11]-.

-, foundations of general theory. Cantor, $G$. Acta Mth. 2 (1883) 381-.

-, functional. Arzelì, C. Bologna Ac. Sc. Mm. 5 (1895-96) 225-; 8 (1899-1900) 131-.

- and incommensurable numbers. Amigues, E. Mars. Fac. Sc. A. 2 (1892) 33-.

- of points, and their signification for analysis. Lerch, M. Časopis 15 (1886) 211-; Fschr. Mth. (1886) 194-.

-, single valued (eindeutig) and continuous formation. Jürgens, $E$. D. Nf. Tbl. (*1878) 137-.

-, theory. Cantor, G. [1877] Crelle J. Mth. 84 (1878) 242-.

-, - Netto, E. Crelle J. Mth. 86 (1879) $263-$

-, 一. Baire, R. C. R. 129 (1899) 946-.

-, -, auxiliary theorem. Bendixson, I. Stockh. Ak. Hndl. Bh. 9 (1884-85) No. 7, 7 pp.

-, -, basis. Gruithuisen, $F$. von $P$. Gruithuisen N. Analekt. 1 (1834) (Heft 3) 1-; (Heft 4) 1-; (Heft 6) 45-.

,-- , elementary point in. Cantor, G. D. Mth. Vr. Jbr. 1 (1892) 75-.

,-- , history and bibliography. Vivanti, $G$. Bb. Mth. (1892) 9-.

- , note. Tannery, P. Par. S. Mth. Bll. 12 (1884) 90-.

-, - - - Garibaldi, C. Palermo Cir. Mt. Rd. 9 (1895) 198-.

Algebra, foundations. Ascoli, $G$. Mil. I. Lomb. Rd. 28 (1895) 1060-.

Analytic functions. Vivanti, $G$. Palermo Cir. Mt. Rd. 3 (1889) 38-.

VOL. I.

\section{Aggregates 0430}

Analytic functions, a property of. Poincaré, $H$. Palermo Cir. Mt. Rd. 2 (1888) 197-.

- representation of real functions, given over any aggregate of points. Phragmén, $E$. Palermo Cir. Mt. Rd. 14 (1900) 256-.

Asymptotic arithmetic, contribution to fundamental principles of. Cesàro, $E$. Nap. Ac. At. 6 (1894) No. 11, 23 pp.

Behaviour of certain functions at infinite values of variables. Vivanti, $G$. Palermo Cir. Mt. Rd. 2 (1888) 135-, 150-.

Boundaries of continua. Phragmén, E. Acta Mth. 7 (1885) 43-.

Cantor, a theorem of. Burali-Forti, C. Tor. Ac. Sc. At. 32 (1896) 153- or 229-.

Cantor's theorems. Schröder, E. D. Mth. Vr. Jbr. 5 (1901) (Heft 1) 81-.

Commensurable quantities, distribution. Cesàro, E. A. Mt. 13 (1885) 295-.

Condensation of singularities, Hankel and Dini's principle. Köpke, -. Hamb. Mth. Gs. Mt. 1 (1889) 230-.

Construction of tangents to circles and limitcircles; proof that Lobatschefsky's space contains a doubly infinite manifoldness of spheres of infinite radius. Simon, $M$. D. Mth. Vr. Jbr. 3 (1894) 80-.

Continuity of functions. Pincherle, S. Bologna Rd. 4 (1900) 99-.

- inconsistent with one to one correspondence between different spaces. Milesi, L. Rv. Mt. 2 (1892) 103-.

Continuous aggregate, $n$-fold, conception. Jïrgens, E. D. Mth. Vr. Jbr. 7 (Heft 1) (1899) 50-.

- aggregates, theorem. Cantor, G. Gött. Nr. (1879) 127-.

- and connected aggregates. Maccaferri, $E$. Rv. Mt. 4 (1894) 97-.

- function with discontinuous differential coefficient. Lerch, M. G. Teix. J. Sc. 9 (1889) 97-.

Convergence conditions. Fouret, $G$. N. A. Mth. 9 (1890) 222-.

- Giudice, -. Palermo Cir. Mt. Rd. 4 (1890) 284-.

"Crinkly" curves (i.e. filling a surface). Moore, E. H. N. Y. Am. Mth. S. T. 1 (1900) $72-, 507$.

Definition of magnitudes 0, 1, 2, 3. Schröder, E. Ac. Nt. C. N. Acta 71 (1898) 363-.

- real number. Burali-Forti, C. [1900] N. Y. Am. Mth. S. Bll. 7 (1901) 158, $170-$.

Differentiable function oscillating in every interval. Köpcke, A. Hamb. Mth. Gs. Mt. 2 (1890) (Festschr. Tl. 2) 128-.

Discontinuities of functions of real variable. Bettazzi, R. Palermo Cir. Mt. Rd. 6 (1892) 173-.

Finite classes. Burali-Forti, C. Tor. Ac. Sc. At. 32 (1896) 34-.

- and infinite assemblage. Keyser, C. J. [1900] N. Y. Am. Mth. S. Bll. 7 (1901) 218 -

Finiteness, two definitions of, and Cantor's theorems. Schröder, E. Ac. Nt. C. N. Acta 71 (1898) 301-。 


\section{Aggregates}

Function with symmetries in every interval. Köpcke, A. [1896] Hamb. Mth. Gs. Mt. 3 (1900) 258-.

Functions and aggregates, theory. Levi, $B$. Rm. R. Ac. Linc. Rd. 9 (1900) (Sem. 2) 72-.

- capable of graphic representation and differentiation. Köpcke, $A$. Hamb. Mth. Gs. Mt. 1 (1889) 128-.

- discontinuous in every interval. Peano, $G$. Rv. Mt. 2 (1892) 41-.

- oscillating in every interval, theorem. Köpcke, A. [1899] Hamb. Mth. Gs. Mt. 3 (1900) 376-

Infinite numbers, various opinions. Cantor, G. Stockh. Ak. Hndl. Bh. 11 (1887) No. 19, $10 \mathrm{pp}$.

- - - - Cantor's article. Eneström, $G$. Stockh. Öfv. (1885) No. 10, 69-

- series, convergence conditions. Giudice,Palermo Cir. Mt. Rd. 4 (1890) 275-, 276-, 278-, 279-, 280-, 281-, 283-.

Infinitesimal constant segments, impossibility. Peano, G. Rv. Mt. 2 (1892) 58-.

- segments, remarks on a demonstration. Veronese, G. Palermo Cir. Mt. Rd. 6 (1892) 73-.

Integrability of ordinary differential equations. A rzelà, C. Bologna Ac. Sc. Mm. 5 (189596) 257-.

Integrals of ordinary differential equations, existence of. Arzelà, C. Bologna Ac. Sc. Mm. 6 (1896-97) 131-.

Limit, existence for regular sequences of rational numbers. Chessin, A. S. J. H. Un. Cir. [15 (1895-96)] 37-.

- of variable classes. Burali-Forti, C. Tor. Ac. Sc. At. 30 (1895) 227-.

Limiting value of quotient series. Brodén, $T$. [1897] Stockh. Ak. Hndl. Bh. 23 (Afd. 1) (1898) No. 2, 49 pp.

Linear manifoldness in $n$ dimensions. Amodeo, F. Rv. Mt. 2 (1892) 145-.

Lines composed of rectilinear parts. Gravé, $D$. C. R. 127 (1898) 1005-.

Number, rôle of, in geometry. Calinon, $A$. [1900] N. Y. Am. Mth. S. Bll. 7 (1901) 158, 178-.

Ordered classes and transfinite numbers. Burali-Forti, C. Palermo Cir. Mt. Rd. 8 (1894) 169-.

- types, theory. Vivanti, G. A. Mt. 17 (1889-90) 1-.

Perfect aggregates. Vivanti, G. Palermo Cir. Mt. Rd. 13 (1899) 86-.

Point-aggregate, doubly extended, determination of all groups. Lie, M.S. Arch. Mth. Ntvd. 3 (1878) 93-.

- aggregates. Phragmén, E. Stockh. Öfv. (1884) No. 1, 121-.

- - contents of. Harnack, A. Mth. A. 25 (1885) 241-.

- - development of theory. Schoenflies, A. D. Mth. Vr. Jbr. 8(1900) (Heft 2) vi +251 pp. - - infinite, linear. Cantor, $G$. Mth. A. 15 (1879) 1-; 17 (1880) 355-; 20 (1882) 113-; 21 (1883) 51-, 545-.

- - limiting values. Stolz, $O$. Mth. A. 23 (1884) 152-.
Universal Algebra 0800

Point aggregates, perfect, power of. Cantor, G. Acta Mth. 4 (1884) 381-.

- - , - - - Bendixson, I. Stockh. Ak. Hndl. Bh. 9 (1884-85) No. 6, 15 pp.

- in a plane. Lerch, $M$. Prag Sb. (1884) (Mth.-Nt.) 176-.

- - in space of $n$ dimensions, theory. Cantor, G. Acta Mth. 7 (1885) 105-.

- - theorems. Bendixson, I. Acta Mth. 2 (1883) 415-.

Popular account of new fields of mathematics. Miller, G. A. Science 11 (1900) 528-.

Quadratic aggregates in higher space. Sommer, J. D. Mth. Vr. Jbr. 8 (1900) (Heft 1) 193-.

Representation, uniform and continuous, of aggregates of different dimensions upon one another. Lüroth, J. Erlang. Ps. Md. S. Sb. 10 (1878) 190-.

Segments and transfinite numbers. Veronese, G. Rm. R. Ac. Linc. Rd. 7 (1898) (Sem. 1) 79 -.

Series, positive terms, partial sums forming continuum. Bettazzi, $R$. Tor. Ac. Sc. At. 33 (1897) 199- or 355-.

Subfinite and transfinite numbers from Cantor's point of view. Giudice, - . Palermo Cir. Mt. Rd. 6 (1892) 161-.

Successions. Giudice, - Palermo Cir. Mt. Rd. 5 (1891) 280 -

Surfaces, general theory, extension to theory of aggregates. Mahler, -. D. Nf. Tbl. (*1882) 147-.

Transfinite magnitudes, class of. Janssen van Raay, W. H. L. G. Mt. 32 (1894) 1-; 33 (1895) 329-, 360.

- - theory. Cantor, G. Mth. A. 46 (1895) 481-; 49 (1897) 207-.

- numbers. Cantor, G. Rv. Mt. 5 (1895) 104-.

- - Stolz, O. Rv. Mt. 5 (1895) 166-.

- - Killing, $W$. Mth. A. 48 (1897) 425-.

- Levi-Civita, T. Rm. R. Ac. Linc. Rd. 7 (1898) (Sem. 1) 91-, 113-.

- -, Archimedes' axiom and projective geometry. Schönfies, A. D. Mth. Vr. Jbr. 5 (1901) (Heft 1) 75-.

- - , question on. Burali-Forti, C. Palermo Cir. Mt. Rd. 11 (1897) 154-, 260.

- -, Veronese's. Schöenflies, A. Rm. R. Ac. Linc. Rd. 6 (1897) (Sem. 2) 362-.

Weierstrass-Cantor condensation process. Brodén, T. Stockh. Öfv. (1896) 583-.

\section{Universal Algebra. 0800 General.}

Algebra, foundation; triple algebra. DeMorgan, A. (Ix) [1844] Camb. Ph. S. P. 1 (1866) 13-. -, notion and boundaries. Cayley, A. QJ. Mth. 6 (1864) 382-.

-, principles, fundamental. Macfarlane, $A$. Am. As. P. (1899) 21-.

- as science of pure time. Hamilton, (Sir) W. R. [1835] Ir. Ac. T. 17 (1837) 293-.

Algebraic operations, philosophic deduction. Valente do Couto, M. Lisb. Mm. Ac. Sc. 3 (pte. 2) (1814) 149-. 


\section{Universal Algebra}

Algebraical fundamental operations, generalisations. Simony, O. Wien Ak. Sb. 91 (1885) (Ab. 2) 223-.

Associative algebra, applicable to hyperspace. Joly, C. J. Ir. Ac. P. 5 (1898-1900) 73-.

Calculus of notations. Couto, $M . V$. do, $e$ Simões Margiochi, $F$. Lisb. Mm. Ac. Sc. 3 (pte 2) (1814) 48-.

- - Travassos, F. de P. Lisb. Mm. Ac. Sc. 3 ( pte 2) (1814) 65-.

Double algebra. Cayley, A. L. Mth. S. P. 15 (*1883-84) 185-.

Factorials, calculus. Logan, H. F. C. [1873] R. S. P. 22 (1873-74) 434-

Generalisations of algebra. Spottiswooode, $W$. [1872] L. Mth. S. P. 4 (1871-73) 147-.

Imaginaries in geometry, and tetrads, von Staudt's theory, and its extension. Lüroth, $J$. [1874-76] Mth. A. 8 (1875) 145-; 11 (1877) 84-.

Laws of Motion, three, in world of universal algebra. Sylvester, J.J. (xII) [1883] J. H. Un. Cir. [3] (1884) 33-, 57.

Linear algebra, uses and transformations. Peirce, B. Am. Ac. P. 10 (1875) 395-.

- associative algebra. Peirce, B. [1870] Am. J. Mth. 4 (1881) 97-.

- - note on Peirce's. Cayley, A. J. H. Un. Cir. [3] (1884) 122.

Multiple algebra. Gibbs, J.W. Am. As. P. (1886) 37-.

- - Cayley, A. QJ. Mth. 22 (1887) 270-.

- - application of logical analysis. Peirce, C. S. Am. Ac. P. 10 (1875) 392-

- algebras, class. Peirce, C. S. (xII) [1882] J. H. Un. Cir. [2] (1883) 3-.

Negative numbers. Burali-Forti, C. Rv. Mt. 3 (1893) 138-

- quantities. Flauti, V. [1855] Nap. At. Ac. (1855-57) 3-.

- - addition, Bertrand. Terquem, O. (v Adds.) N. A. Mth. 15 (1856) 172-.

- - conception. Purgotti, S. Palomba Rac. 3 (1847) 171-, 180-.

- - and rule of signs. Guillon, E. L. N.A. Mth. 8 (1849) 28-.

- -, theory. Cach, -. Gergonne A. Mth. 4 (1813-14) 1-.

- - - - Gergonne, J. D. Gergonne A. Mth. 4 (1813-14) 6-

- - - - Transon, A. (Prof.). N. A. Mth. 3 (1844) 318-, 321-.

- - use. Prouhet, E. N. A. Mth. 6 (1867) 337-.

Physics, algebra of, principles. Macfarlane, $A$. Âm. As. P. (1891) 65-

Postulates of algebra. Padoa, $-[1900]$ N. Y. Am. Mth. S. Bll. 7 (1901) 70.

Proportion, theory. Lehmann, J. W. $H$. Grunert Arch. 8 (1846) 113-.

Signs of operation, distributed, in systems of algebra. Graves, C. Ir. Ac. P. 3 (1847) 325-.

Sylvester's third law of motion, proof. Buchheim, A. Ph. Mg. 18 (1884) 459-.

Symbolical algebra. Gregory, D. F. [1838] Edinb. R. S. T. 14 (1840) 208-.

- - Vaněěek, M.N. Prag Sb. (1888) (Mth. Nt.) 137-.
Calculus of Operations 0810

Symbols, algebraic, and tessarines. Cockle, Jas. Ph. Mg. 34 (1849) 406-.

Tessarines, amplitude. Cockle, Jas. $\mathrm{Ph} . \mathrm{Mg}$. 36 (1850) 290-

Tetrads (Würfe) theory. Lüroth, J. Gött. Nr. (1873) 767-.

$-(-)$ von Staudt's theory. Sturm, $R$. Mth. A. 9 (1876) 333-.

- (-), - - Schröder, E. Mth. A. 10 (1876) 289-.

Universal algebra. Brunel, - . Bordeaux S. Sc. Mm. 2 (1891) ix-

- - principles. Sylvester, J. J. Am. J. Mth. 6 (1884) 270-.

\section{Calculus of Operations.}

Algebra of multilinear partial differential operators. MacMahon, P. A. L. Mth. S. P. 19 (1889) 112-.

Algebraical operation. Cayley, A. QJ. Mth. 13 (1875) 369-.

- (repeated) transformation, principle. Schapira, $H$. Heidl. Nt. Md. Vh. 4 (1892) $25-$.

Calculus of chemical operations. Brodie, (Sir) B. C. Phil. Trans. 156 (1866) 781-.

- - - Williamson, A. W. C. N. 16 (1867) 3-.

- - - Brodie, (Sir) B. C. [1876] Phil. Trans. 167 (1877) 35-.

- $-\ldots$, Brodie's. Naquet, A. Ph. Mg. 7 (1879) 418-.

- - functions. Babbage, C. [1815-20] Phil. Trans. (1815) 389-; (1816) 179-; (1817) 197-; QJ. Sc. 2 (1817) 371-; Camb. Ph. S. T. 1 (1822) 63-.

- - Tanner, H. W. L. Mess. Mth. 7 (1878) 156-.

- - Babbage's paradox. Graves, J. T. Ph. Mg. 9 (1836) 334-, 443-

- - of one independent variable. Liventsov, A. I. (xII) [1875] Rec. Mth. (Moscou) 8 (1876) (Pt. 1) 80-.

- linear partial differential operations. MacMahon, P. A. QJ. Mth. 24 (1890) 246-. -, the most comprehensive. Schröder, $E$. B. A. Rp. (1883) 411-.

- of operation symbols, elementary exposition. Cazzaniga, P. G. Mt. 20 (1882) 48-, 194 -.

- - operations. Boole, G. Phil. Trans. (1844) 225-.

- - Graves, C. Ph. Mg. 34 (1849) 60-

- - Paterson, J. Silliman J. 11 (1851) $327-$.

_ - - application to algebraic expansions. Hargreave, C. J. Ph. Mg. 6 (1853) 351-.

- - - , - to transformation of trigonometric series. Donkin, W. F. QJ. Mth. 3 (1860) $1-$.

- - development. Roberts, S. QJ. Mth. 4 (1861) 44-.

- _ - extended form of index symbol. Spottiswoode, $W$. Phil. Trans. (1860) 13-. - - - formulae. Stokes, G. G. [1876] Edinb. R. S. P. 9 (1878) 101-.

- - , , general. Spottiswoode, $W$. Crelle J. 59 (1861) 367-.

D 2 


\section{Calculus of Operations}

Calculus of operations, notes. Studnicka, $\boldsymbol{F}$. Prag Sb. (1871) (pt. 2) 39-.

- - - problem. Westergaard, H. (xiI) Ts. Mth. 6 (1876) 37-.

- - _, supplement. Paterson, J. [1874] Alb. I. T. 8 (1876) 191-.

- - , theorem. Walton, W. QJ. Mth. 3 (1860), 278-, 314-.

- - - - and applications. Greer, H. R. QJ. Mth. 3 (1860) 148-.

,---- , note. Roberts, S. QJ. Mth. 3 (1860) 310-.

- - - theorems. Donkin, W. F. Camb. and Dubl. Mth. J. 5 (1850) 10-.

- - - . Spottiswoode, W. Camb. and Dubl. Mth. J. 8 (1853) 25-.

- - - Carmichael, $R$. Camb. and Dubl. Mth. J. 8 (1853) 165-.

- - - - Crofton, M. W. QJ. Mth. 16 (1879) 323-.

- - - -. Walker, J. J. [1880-81] L. Mth. S. P. $11(1879-80), 108-; 12$ (1880-81) 193-.

- -1, , general, and applications. Warren, J.W. [1861] QJ. Mth. 5 (1862) 29-.

- symbols. Spottiswoode, W. Phil. Trans. (1862) 99-.

- _ - applications to differential equations. Russell, W. H. L. Phil. Trans. (1861) 69-; (1862) 253- ; (1863) 517-.

$--1,-$ to non-linear differential equations. Russell, W. H. L. R. S. P. 13 (1864) 423-.

- - - - - to partial differential equations. Russell, W. H.L. R. S. P. 13 (1864) 432-. - - - division, internal and external. Spratt, T. A. B. R. S. P. $11(1860-62)$ $557-$.

- - , recent discoveries. Russell, W. H.L. B. A. Rp. (1862) (pt. 2) 7-.

Complete system of polar mutually permutative operations. Capelli, A. Nap. Rd. 32 (1893) 29-, 155-.

De Morgan's extension of the algebraic processes (symbolic algebra). Ladd, C. Âm. J. Mth. 3 (1880) 210 -

Determinants, Smith's. Mansion, P. (xII) Brux. S. Sc. A. 2 (1878) (Pt. 2) 211-.

Differentials of symbolic expressions. Lipschitz, R. Berl. Ak. Sb. (1899) 122-.

Differentiation with fractional indices, and differentiation, general. Center, $W$. Camb. and Dubl. Mth. J. 3 (1848) 274-; 4 (1849) 21- ; 5 (1850) 206-.

- generalised. Moritz, R. E. N. Y. Am. Mth. S. Bll. 6 (1900) 184, 185-.

Distributive functional calculus. Pincherle, $S$. Mil. I. Lomb. Rd. 30 (1897) 1031-; Mth. A. 49 (1897) 325-.

- - operations. Pincherle, S. Rm. R. Ac. Linc. Rd. 4 (1895) (Sem. 1) 142-.

- Calo, B. Rm. R. Ac. Linc. Rd. 4 (1895) (Sem. 2) 52-.

- operations differing by permutation from a given operation. Pincherle, $S$. Tor. Ac. Sc. At. 30 (1895) 820-.

Divisibility by a polynomial, extension of the conception. Pincherle, S. Rm. R. Ac. Linc. Rd. 7 (1898) (Sem. 1) 185-.

\section{Calculus of Operations 0810}

Equations analogous to differential equations. Bourlet, C. C. R. 124 (1897) 1431-.

- - - Appell, P. C. R. 124 (1897) 1433-.

Exponential formula giving sum of sines or cosines of angles in arithmetical progression. Horta, F. Lisb. A. 1 (1857) 713-.

- symbolic operators, theorems. Glaisher, J. W. L. QJ. Mth. 15 (1878) 266-.

Exponentials, representation of function. $\mathrm{Ol}$ tramare, G. Sch. Nf. Gs. Vh. (1896) 50-.

Fractional power of $\left(\frac{d}{d x}\right)^{\theta} x^{0}$. Center, $W$. Camb. and Dubl. Mth. J. 3 (1848) 163-.

Functional equations, replacement of arbitrary constants by operative symbols. Tanner, H. W. L. Mess. Mth. 7 (1878) 157-; 8 (1879) $13-$.

- operations, certain. Pincherle, S. Bologna Ac. Sc. Mm. 7 (1886) 393-

- - groups. Levi-Civita, T. Mil. I. Lomb. Rd. 28 (1895) 458-, 864-.

- - , - and the inversion of definite integrals. Levi-Civita, T. Mil. I. Lomb. Rd. 28 (1895) 529-, 565-.

Functions, determination by formal conditions. Schröder, E. Crelle J. Mth. 90 (1881) 189-.

-, symbolic representations. Bronwin, $B$. Camb. and Dubl. Mth. J. 2 (1847) 134-.

General algorithms and iteration. Lémeray, C. R. 126 (1898) 510- ; Par. S. Mth. Bll. 26 (1898) 10-.

Hyperdistributives. Cockle, (Sir) J. Ph. Mg. 43 (1872) 300-.

Integration problems. Rubini, $R$. G. Mt. 19 (1881) 118-.

Iteration. Bourlet, C. C. R. 126 (1898) 583-。 - of functions. Aristov, I. I. Kazan S. Ps.Mth. Bll. 10 (1901) 14-, 85-; Fschr. Mth. (1900) 403-.

- the problem. Bourlet, $C$. Toul. Fac. Sc. A. $12(1898) \mathrm{c}, 12 \mathrm{pp}$.

-, - - Lémeray, 一. C. R. 128 (1899) $278-, 380$.

Iterative calculus, principles. Böttcher, $\boldsymbol{E} . \boldsymbol{E}$. Prace Mt.-Fiz. 10 (1899-1900) 65-.

Laplace's transformation. Amaldi, $U$. Rm. R. Ác. Linc. Rd. 7 (1898) (Sem. 2) 117-.

Law of combination of operators. Campbell, J. E. [1897] L. Mth. S. P. 29 (1898) 14-.

Mathematical operations, especially restricted differentiation. Gruenewald, - . D. Nf. Tbl. $\left({ }^{*} 1868\right)$ 130-.

Multiplication of functional distributive operations. Bortolotti, E. Rm. R. Ac. Linc. Rd. 7 (1898) (Sem. 1) 16-.

- partial differential operators. Sylvester, J. J. Ph. Mg. 33 (1867) 48-.

Notation, "representative." Blissard, J. [1862] QJ. Mth. 6 (1863) 49-.

$\boldsymbol{\Omega}$, a certain differential, determinantal operator. Vivanti, G. Palermo Cir. Mt. Rd. 4 (1890) 261-.

Operation on functions, definition $[\phi n(x)$ for $\phi(x)$ repeated $n$ times, where $n$ is fractional]. Appell, P. Par. S. Phlm. Bll. 3 (1879) 166. 


\section{Calculus of Operations}

Operation; laws. Ellis, A. J. R. S. P. 10 (1859-60) 85-.

Operations equivalent to their adjuncts. Bortolotti, E. Rm. R. Ac. Linc. Rd. 7 (1898) (Sem. 2) 74-.

- in general. Bourlet, C. C. R. 124 (1897) 348 -.

-, remarkable sequence. Brunel, G. Bordeaux S. Sc. P.-V. (1896-97) 86-.

-, theorems. Grolous, J. Par. S. Phlm. Bll. 12 (1875) 81-.

Periodicity, generalisation of notion. Brunel, - . Bordeaux S. Sc. Mm. 4 (1888) xxvi-.

Quotient- and product-calculation. Guldberg, A. S. Ts. Mth. 2 (1884) 84, 161-; Fschr. Mth. (1884) 342.

Reduction of Cayley's operation $\boldsymbol{\Omega}$ to polar operations. Capelli, A. Mth. A. 29 (1887) 331-.

Signs + and -, nature in symbolical algebra. Gregory, D. F. Camb. Mth. J. 3 (1843) 153-.

Symbolic calculus, principles. Cesáro, E. (xII) Mathesis 3 (1883) 10-.

- equation, $\pi_{m} \pi_{n} u+q \rho u=0$, where symbols $\pi_{m}, \pi_{n}, \rho$ combine according to the laws $\pi_{m} \rho=\rho \pi_{m+1}, \quad \pi_{m} \pi_{n}=\pi_{n} \pi_{m}+a(n-m) \rho$. Boole, $G$. Camb. and Dubl. Mth. J. 2 (1847) 7-.

- equations, certain. Pincherle, S. Bologna Ac. Sc. Mm. 5 (1895-96) 663-.

- - employment. Cauchy, A. L. C. R. 17 (1843) 449-.

- , interchange of symbols. Graves, C. Ir. Ac. P. 6 (1853-54) 144-

-, resolution of non-linear. Cauchy, A.L. C. R. 22 (1846) 235 -.

- - theorems and formulae deduced from. Cauchy, A. L. C. R. 17 (1843) 377-

- expansions. Russell, W. H. L. R. S. P. 14 (1865) 329-.

- methods, proofs of (1) formula for $n$th differential coefficient, (2) Euler's theorems on quantics, (3) Staudt's theorem on Bernoulli's numbers. Buchheim, A. Mess. Mth. 11 (1882) 143-.

- relations

$$
\begin{array}{r}
x^{n-r} \frac{d^{n}}{d x^{n}} x^{r} \cdot u=\left(\frac{d}{d \theta}+r\right)\left(\frac{d}{d \theta}+r-1\right) \\
\left(\frac{d}{d \theta}+r-n+1\right) u .
\end{array}
$$

\&c. Johnson, W. W. Mess. Mth. 11 (1882) 191-.

- theorem involving repeated differentiations. Glaisher, J. W. L. [1879] Camb. Ph. S. P. 3 (1880) 269-.

- theorems, Crofton. Glaisher, J. W. L. QJ. Mth. 16 (1879) 257-.

- variability of several dimensions. Formenti, C. Mil. I. Lomb. Rd. 16 (1883) 288-, 351-.

- versus operative expansion. Hathaway, A. S. Des Moines Anal. 5 (1878) 38-.

Symbolical expression, properties and application to attraction of ellipsoid. Cayley, $A$. Camb. Mth. J. 3 (1843) 62-.

Symbols of operation. Crofton, M.W. Am. J. Mth. 4 (1881) 269-.
Complex Numbers 0820

Symbols, relation of certain. Cockle, (Sir) $\mathrm{J}$. [1879] QJ. Mth. 17 (1881) 20-, 208-.

Ternary quadric operators on products of forms of any orders. Walker, J. J. L. Mth. S. P. 20 (1889) 110.

Transmutations. Bourlet, C. Par. S. Mth. Bll. 25 (1897) 132-.

Uniform substitutions and Babbage's problem. Iaggi, E. N. A. Mth. 19 (1900) 483-

- -, convergence. Lémeray, E. M. N. A. Mth. 16 (1897) 306-; 17 (1898) 75-.

$\frac{d^{n} f(x)}{d x^{n}}$ regarded as function of $n$. Krug, A.

Wien. Ak. D. 57 (1890) 151-.

\section{General theory of complex numbers. (See also 2870.)}

d'Alembert's equation

$$
(1+h \sqrt{-1})^{m}=(1-h \sqrt{-1})^{m} .
$$

Cossali, P. [1783] Mod. Mm. S. It. 9 (1802) 231-.

Algebraic couples. Hamilton, (Sir) W. R. [1835] Ir. Ac. T. 17 (1837) 293-.

- - Cayley, A. Ph. Mg. 27 (1845) 38-.

- - Hargreave, C. J. B. A. Rp. (1857) 184

- equations, theory. Gegenbauer, L. Mh. Mth. Ps. 6 (1895) 12-.

- integers. Landsberg, G. D. Mth. Vr. Jbr. 4 (1897) 111-.

- numbers, new foundation for theory. Hensel, K. D. Mth. Vr. Jbr. 6 (1899) (Heft 1) 83-.

- and transcendent equations, complex numbers derived from. Hug, J.C. Zür. Vjschr. 7 (1862) 270 -

- triplets. Graves, C. Ir. Ac. P. 3 (1847) $51-, 57-, 80-, 105-$.

Calculation with $p+q \sqrt{-1}$. Ohm, M. D. Nf. Vsm. B. (1845) 73-.

Chessboard, mathematical theory. Problem of $n$ queens on board of $n^{2}$ spaces. Günther, $S$. Arch. Mth. Ps. 56 (1874) 281-.

Complex and bicomplex numbers, theory. Benthem, A. Arch. Neerl. 12 (1877) 113-.

- coordinates in plane geometry. Berlin, $M$. Lund. Acta Un. 9 (1872) (Mth.) No. 4, $45 \mathrm{pp}$.

- elements in analytical geométry, significance. Stolz, O. Mth. A. 4 (1871) 416-.

- factors. Cauchy, A. L. C. R. 24 (1847) $347-$.

- forms, real representations. Segre, $C$. Mth. A. 40 (1892) 413-

- geometry, contribution. Sforza, G. G. Mt. 30 (1892) 159-.

- integers. Lanavicensis (Sylvester, J. J.). QJ. Mth. 4 (1861) 94-, 124-.

magnitudes, addition-logarithms tor. Mehmke, R. Z. Mth. Ps. 40 (1895) 15-.

- number, cosine, significance in plane geometry. Thiele, T. N. (xII) Ts. Mth. 6 (1876) 177-. 
Complex numbers. Genocchi, A. Liouv. J. Mth. 19 (1854) 281-.

- - Paci, P. G. Mt. 11 (1873) 244-.

- Zolotarev, E. I. [1877] St. Pét. Ac. Sc. Bll. 24 (1878) 310-.

- -, analysis. Denzler, W. Zür. Mt. 3 (1853-55) 560-.

- , with application to equation $x^{\lambda}+y^{\lambda}=z^{\lambda}$. Kummer, E. E. Berl. Ab. (1857) (Mth.) 41-.

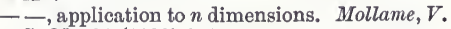
G. Mt. 31 (1893) 271-.

- comprised in formula $a+b \sqrt{-c}$. Pepin, T. Liouv. J. Mth. 1 (1875) 317-.

- - , elementary theory. Hoüel, J. Bordeaux Mm. S. Sc. 5 (1867) 1-; 6 (1868) 1-; 8 (1870) 97-; 1 (1876) 1-.

- - formed from roots of unity. Kummer, E. E. Liouv. J. Mth. 12 (1847) 185-; 16 (1851) 377-; Berl. Mb. (1861) 1051-; (1863) 21-.

Mb. (1863) 340-.

Crelle J. 65 (1866) $\overline{74-}$.

Kronecker, L. Berl.

E. E. Crelle J. 35 (1847) 327-.

- $-\ldots \ldots$ - - and 2 nd factor of class number. Kummer, E. E. Berl. Mb. (1870) 855-.

$-1---1,-$, simplest representation. Kummer, E. E. Berl. Mb. (1870) 409-.

$-----\omega^{n}=1$, ideal prime factors. Kummer, E. E. Berl. Ab. (185̃6) (Mth.) 1-. $---\lambda$ th roots of unity. Kummer, E. E. Crelle J. 40 (1850) 93-, 117-.

$--\ldots-31$ st roots of unity. Kummer, E.E. Berl. Mb. (1870) 755-.

- - Gaussian representation of. Breuer, $A$. Arch. Mth. Ps. 12 (1894) 337-.

- - geometric representation of a new kind. Kotelinikov, A.P. Kazan S. Ps.-Mth. Bll. 6 (1896) (Prot.) 26.

- - - significance (Schlömilch). Unferdinger, $F$. Arch. Mth. Ps. 52 (1871) 252-.

- - introduction. Baltzer, R. [1882] Crelle J. Mth. 94 (1883) 87-.

- - mechanical interpretation. Žbikovskij, A. K. Kazan S. Nt. (Ps.-Mth.) P. 6 (1888) 6.

- - multiplication, geometric significance. Orelli, J. Zür. Vjschr. 20 (1875) 443-.

- - new method for graphical representation. Brill, J. Mess. Mth. 17 (1888) 80-.

- and their functions, theory. Hertz, K. \& Dickstein, S. Par. T. Nauk Sc. Pam. 7 ( $\left.{ }^{*} 1875\right)$ Art. 3, $60 \mathrm{pp}$.

- , theorem of Dedekind, proof. Study, $E$. Gött. Nr. (1898) 1-.

- -, theory. Lejeune-Dirichlet, G. Berl. Ab. (1841) 141-.

- - - Kummer, E. E. Berl. B. (1846) 87-; Crelle J. 35 (1847) 319-. 430- -. Wantzel, L. C. R. 24 (1847)

- - - - Bachmann, P. Crelle J. 67 (1867)

- - - Dedekind, R. C. R. 90 (1880) 1205-.
Complex numbers, theory. Zolotarev, E. J. Liouv. J. Mth. 6 (1880) 51-, 129-.

- units. Kronecker, L. [1845] Crelle J. Mth. 93 (1882) 1-.

- - Lejeune-Dirichlet, G. Berl. B. (1846) 103-.

- - Kronecker, L. Crelle J. 53 (1857) 176- ; C. R. 96 (1883) 93-, 148-, 216-.

- Molk, J. Bll. Sc. Mth. As. 7 (1883) (Pt. 1) 133-.

- - addition to memoir on. Kronecker, $L$. C. R. 99 (1884) 765-.

- - expressions formed from. Kummer, E. E. Crelle J. 50 (1855) 212-

Conjugate functions and imaginary logarithms. Hamilton, (Sir) W. R. B. A. Rp. (1834) 519-.

Cube root of $\sqrt{a}+b i$ in form $x+y i$, simple solution. Simony, O. Arch. Mth. Ps. 55 (1873) $72-$.

- roots, imaginary, number of (Lockhart). Horner, W. G. Thomson A. Ph. 8 (1816) 279-, 388-.

- - of unity, theorem of algebra, geometry deduced from. Appell, P. C. R. 84 (1877) 540 -

Directive numbers, theorems, and proof of Cauchy's theorem on closed contours. Transon, A. N. A. Mth. 7 (1868) 97-.

Discriminants of finite "fields." Dedekind, $R$. Gött. Ab. 29 (1882) Art. 2, 56 pp.

Every equation in one unknown can be satisfied by a complex value of this unknown, geometric proof. Wittstein, $T$. Grunert Arch. 6 (1845) 225-.

Fermat's theorem, generalization for Galois number fields. Moore, E. H. N. Y. Am. Mth. S. Bll. 2 (1896) 189-.

G. C. M. of 2 complex numbers, number of divisions. Dupré, A. Liouv. J. Mth. 13 (1848) 333-.

Geometrical representation of $\sqrt{-1}$, consideration of objections. Warren, $J$. Phil. Trans. (1829) 241-.

Goniometry, application of principles given by Warren. Terrot, C. H. [1847] Edinb. R. S. T. 16 (1849) 345-.

Imaginaries. Buée, - [1805] Phil. Trans. (1806) 23-.

- Francais, J. F. Gergonne A. Mth. 4 (1813-14) 222-, 364-.

-. Volpicelli, P. G. Arcad. 52 (1831) 221-.

-. Bellavitis, $G$. Poligrafo 5 (1831) 15-; 12 (1832) 211-.

-. Arenstein, J. Haidinger B. 3 (1847) 292-.

-. Bellavitis, G. Ven. At. 6 (1847) 459-; Ven. Mm. I. 4 (1852) 243-.

- Arenstein, J. Haidinger Ab. 2 (1848) (Ab. 2) 43-.

-. Cockle, Jas. Ph. Mg. 36 (1850) 281-.

- Grunert, J. A. Grunert Arch. 20 (1853) 121-.

-. Sabatié, J. N. A. Mth. 2 (1863) 206-.

- Cheyrézy, U. N. A. Mth. 3 (1864) 445-.

- Sexe, S.A. N. Mg. Ntvd. 16 (1869) 1-; 17 (1870) 233-; Arch. Mth. Ntvd. 7 (1882) 115-. 
Imaginaries, d'Alembert's theorem. Du Bourguet, - Gergonne A. Mth. 4 (1813-14) 20-. - - - Gergonne, J. D. Gergonne A. Mth. 4 (1813-14) 23-.

-, - - Barsotti, G. Lucca At. Ac. 11 (1842) 393-.

- in algebra. Janssen van Raaij, W. H. L. Haarl. Ms. Teyl. Arch. 4 (1896) 53-.

-, application to mechanics. Durège, -. Zür. Vjschr. 7 (1862) 293-.

- , - - summation of trigonometric series, and calculation of integrals. Michaëlis, J.P. Lux. S. Sc. Mm. 7 (1864) 172-.

-, - - theory of curves. Durège, H. Arch. Mth. Ps. 42 (1864) 1-.

-, bisignal univalent. Kirkman, T. P. Ph. Mg. 37 (1850) 292-.

-, Bolyai's theory. Stäckel, P. Mth. Termt. Ets. 17 (1899) 259-; Mth. Nt. B. Ung. 16 (1899) 263-.

-, calculation with, and geometrical theorems. Zech, P. Grunert Arch. 16 (1851) 354-.

-, calculus. Carmichael, $R$. Ph. Mg. 6 (1853) 273-.

-, construction for. Cayley, A. Mess. Mth. 10 (1881) 1-.

—, defective notation. Mathematicus. [Pseudon.] (vr Adds.) Nicholson J. 29 (1811) 254-; 31 (1812) 193-.

-, doctrine. Walton, W. Camb. and Dubl. Mth. J. 8 (1853) 101-.

- - - in coordinate geometry. Walton, $W$. Camb. and Dubl. Mth. J. 7 (1852) 234-.

, employment in geometry. Laguerre, N. A. Mth. 9 (1870) 163-, 241-.

-, - - - of space. Laguerre, -. N. A. Mth. 11 (1872) 14-, 108-, 241-.

- Fresnel's interpretation. Lippich, $F$. Prag Sb. (1864) (Pt. 2) 73-.

-, geometric application. Schou, (Lieut.) T. V. (xII) Ts. Mth. 1 (1865) 129-

-, - construction. Drobisch, M.W. Leip. B. 2 (1848) 171-.

- - - meaning. Zehfuss, G. Grunert Arch. 32 (1859) 234-.

-, - representation. Argand, - . Gergonne A. Mth. 4 (1813-14) 133-; 5 (1814-15) 197-.

—, - -. Durège, -. Zür. Vjschr. 3 (1858) 358 .

—, - - Lie, S. Crelle J. 70 (1869) 346-; Christiania F. 22 (1870) 16-, 107-.

-,- , applications to cubic equations, etc. Wittstein, T. Grunert Arch. 7 (1846) 402-.

-, - significance of form $a+b \sqrt{-1}$. Müller, G. W. Grunert Arch. 1 (1841) 397-.

- (Grones). Toblini, G. Poligrafo 14 (1833) 37-.

-, history of theory. Beman, W. W. Am. As. P. (1897) 33-.

-, how to avoid. Sexe, S. A. Arch. Mth. Ntvd. 4 (1879) 145-.

-,$-\ldots$, and elementary theory. Sexe, S. A. Arch. Mth. Ntvd. 9 (1884) 205-

- interpretation in mathematical physics. Chancourtois, E. B. de. C. R. 68 (1869) $127-$.
Imaginaries, new theory. Cauchy, A. L. C. R. 24 (1847) 1120-.

-,- , various applications. Cauchy, A.L. C. R. 25 (1847) 129-.

-, octaves. Cayley, A. Ph. Mg. 30 (1847) 257-.

-, paradox resulting from employment. Mathematicus. [Pseudon.] (vI Adds.) Nicholson J. 30 (1812) 209-.

-, real sum of two, Lagrange's equation, proof. Plana, $G$. Tor. Mm. Ac. 16 (1857) 97-.

-, reality. Matzka, W. Böhm. Gs. Ab. 6 (1851) 179-.

- , relation of $\sqrt{-1}$ to geometrical perpendicularity, Paterson, J. Am. As. P. (1851) 1-.

-, representation, etc. Ballauff, $L$. Grunert Arch. 5 (1844) 259-.

-, - method. Duport, - . Par. Ec. Norm. A. 9 (1880) 301-.

,,--- - Amstein, $H$. Laus. S. Vd. Bll. 18 (1882) 1 -

-, - , new. Duport, -. C. R. 88 (1879) 1071-.

-, simplification. Nordmark, Z. Ups. N. Acta S. Sc. 8 (1821) 136-.

-, 8-square. Cayley, A. [1882] Am. J. Mth. 4 (1881) 293-.

-, - - Sylvester, J. J. (xII) J. H. Un. Cir. [1] (1882) 203.

,$- \sqrt{-1}$. Warner, H. S. Ph. Mg. 30 (1847) 185-.

-, 一. Guthrie, F. Ph. Mg. 39 (1870) 282 -. - theory. Francais, J. $F$. Gergonne A. Mth. 4 (1813-14) 364-.

-, 一. Dirksen, E. H. Berl. B. (1839) 6-.

- - Burhenne, H. Grunert Arch. 22 (1854) 43-.

- Laurent, P. A. Par. Éc. Pol. J. 23 (1863) $\left(40^{\circ} \mathrm{cah}.\right) 75-$

- Berger, -. [1864] Mntp. Mm. Ac. Sect. Sc. 6 (1864-66) 1-.

-, - Gomes Teixeira, F. (xir) Brux. S. Sc. A. 7 (1883) (Pt. 1) 59-, (Pt. 2) 417-.

-, - (Teixeira). Mansion, $P$. (xir) Mathesis 3 (1883) (Suppl.) 13-.

-, truth of conclusions obtained. Woodhouse, R. Phil. Trans. (1801) 89-

-, use in integral calculus. Stille, W. A. Des Moines Anal. 2 (1875) 33-.

Imaginary of algebra. Macfarlane, $A$. Am. As. P. (1892) 33-.

- expressions and foreign solutions of equations. Bourdat, -. [1854] Grenoble Ac. Delph. Bll. 5 (1859) 121-.

- limits of some. Tortolini, B. G. Arcad. 87 (1841) 145-

- forms; their interpretation. Vallès, $F$. N. A. Mth. 9 (1870) 20 -

- and negative quantities, nature. Gilbert, $D$. [1830] Phil. Trans. (1831) 91-.

-, a new. Cockle, Jas. Ph. Mg. 34 (1849) 37-. - numerical forms in geometry. Moth, F.X. Münch. Ab. 3 (1837-43) 85-.

- points in plano, a representation. Appell, $P$. Ârch. Mth. Ps. 61 (1877) 359-. 


\section{Complex Numbers}

Imaginary powers of quantities, geometrical representation. Warren, $J$. Phil. Trans. (1829) 339-.

- - $e^{2 k \pi^{\sqrt{-1}}}$, paradox. Catalan, E. C. N. A. Mth. 8 (1869) 456-.

- radicals, principle of fundamental substitution in. Saporetti, A. Bologna Rd. (1878) 126-.

- and real forms, contiguity in approximate solutions. Vallès, F. N.A. Mth. 13 (1854) 449-.

Involution and evolution of imaginaries considered geometrically. Henkle, $W . D$. Des Moines Ånal. 1 (1874) 149.

Logarithms of negative quantities, and on imaginaries. Fontana, $G$. Verona S. It. Mm. 1 (1782) 183-.

Numbers, irreducible with complex numbers. Formenti, $C$. Mil. I. Lomb. Rd. 16 (1883) 981-.

Partitions of complex numbers (theorem contained in theorem of Jacobi's). Sylvester, J. J. C. R. 96 (1883) 1276-.

Position of chessmen defined by complex variables. Gochmann, C. Fschr. Mth. (1897) 426.

Power with complex base and exponent, geometrical representation. Durège, -. Zür. Vjschr. 5 (1860) 297-.

Powers with imaginary exponents. Ballauff, $L$. Grunert Arch. 6 (1845) 409 -

Prime factors, ideal, of complex numbers, formed rationally from roots of an irreducible equation. Selling, E. Z. Mth. Ps. 10 (1865) 17-.

Product of complex factors, reduction. Schiappa Monteiro, A. Lisb. J. Sc. Mth. 5 (1898) 209-.

Quadratic forms, number. Eisenstein, $G$. Crelle J. 27 (1844) 311-.

- - - - belonging to real determinant. Eisenstein, G. Crelle J. 27 (1844) 80.

Quaternions, proof of Frobenius' theorem on their relation to complex numbers. Grissemann, F. X. Mh. Mth. Ps. 11 (1900) 132-.

Reality of quantities called imaginary, of form $a+b \sqrt{ }=1$, etc. Desmonsseaux de Givré, F. X. É. Par. Ing. Civ. Mm. (1877) 389-

Relative Abelian number-fields. Hilbert, $D$. Gött. Nr. (1898) 370-

- - - Wiman, A. Lund. Un. Acta 36 (1900) (S. Psgr. No. 6) $17 \mathrm{pp}$.

- quadratic "fields" (Körper). Hilbert, D. [1897-99] D. Mth. Vr. Jbr. 6 (1899) (Heft 1) 88-; Mth. A. 51 (1899) 1-.

Roots of $\epsilon^{2}=-1$. Plarr, $G$. Edinb. R. S. P. 15 (1889) 93-.

- - unity. Cayley, A. Mess. Mth. 4 (1875) 171.

Seventh roots of unity. Cayley, A. Mess. Mth. 7 (1878) 177-.

Symbols, imaginary, geometrical interpretation. Français, J. $F$. Gergonne A. Mth. 4 (1813-14) 61-.

Theory, means of extending without nse of imaginaries. Saltel, L. C. R. 94 (1882) 166-.

\section{Quaternions}

0830

Triangle, figure of, regarded as a complex quantity. Frege, G. [1878] Jena. Sb. (1879) xviii.

Triple algebra and application to solid geometry. Graves, C. Ph. Mg. 34 (1849) 119 -.

Triplets, functions of two variables employed in interpreting. Graves, C. Ir. Ac. P. 5 (1853) 423-.

Twenty-third roots of unity. Cayley, A. Crelle J. 55 (1858) 192; 56 (1859) 186-.

Units in rationality domain of 5th roots of 1 . Gmeiner, J. A. Mh. Mth. Ps. 9 (1898) 184-

Zero, imaginary and real. Riccati, G. Verona S. It. Mm. 4 (1788) 116-.

-, - - _. Fontana, G. [1798] Mod. S. It. Mm. 8 (1799) 174-

Zeros, imaginary. Young, $J . R . \quad \mathrm{Ph} . \mathrm{Mg} .27$ (1845) 91 -.

\section{Quaternions.}

Analogue to quaternions in 4 dimensions. Wijthoff, W. A. Amst. Ak. Vs. 6 (1898) 520 .

Conditions of integrability of $S u \alpha d \rho, S u \beta d \rho$, $S u \gamma d \rho$, elimination of $\alpha, \beta, \gamma$ from. Plarr, G. [1874] Edinb. R. S. T. 27 (1876) 251-.

Conjugate numbers in general, solution of quadratics by quaternions in particular. Studnička, F. J. Časopis 23 (1894) 72-; Fschr. Mth. (1893-94) 149.

Coordinates versus quaternions. Cayley, Edinb. R. S. P. 20 (1895) 271-.

Coplanarity and homoconicism, quaternion expressions. Spottiswoode, $W$. Ph. Mg. 36 (1850) 379-.

Curves cutting plane into squares, systems. Tait, P. G. Edinb. Mth. S. P. 7 (1889) $2-$.

- of inversion. Raimondi, $R$. G. Mt. 26 (1888) 181-.

Determinants of quaternions. Peirce, J. M. N. Y. Am. Mth. S. Bll. 5 (1899) 335-。

Differentiation of power of a quaternion. M'Aulay, A. [1889] Edinb. R. S. P. 16 (1890) 201-.

- Note on M'Aulay's paper. Tait, -. [1889] Edinb. R. S. P. 16 (1890) 205-.

- in the quaternion analysis. MacFarlane, $A$. Ir. Ac. P. 6 (1900-02) 199-.

Displacements, finite, of system of points whose mutual distances remain invariable; quaternion expression. Plarr, G. (xI) [1883] Edinb. R. S. P. 12 (1884) 151-.

Dupin's theorem [on intersection lines of three systems of orthogonal surfaces] by quaternions. Hamilton, (Sir) W. R. Ir. Ac. P. 6 (1853-54) 86-

- - and its converse, geometrical proof. Sommerfeld, A. D. Mth. Vr. Jbr. 6 (1899) (Heft 1) 123-.

Electrodynamics and magnetism, quaternion investigations. Tait, P. G. QJ. Mth. 3 (1860) 331-.

Elementary universal geometry, new terms. Sylvester, J. J. Nt. 32 (1885) 576-. 


\section{Quaternions}

Ellipsoids, theorem. Hamilton, (Sir) W. R. Ir. Ac. P. 4 (1850) 349-.

Equation $Q=q(w, x, y, z)=w+i x+j y+k z$. Spottiswoode, W. Ph. Mg. 36 (1850) 135-. - V $\rho \phi \rho=0$, graphic solution. Tait, $P$. G. Edinb. R. S. P. 10 (1880) 400-.

- - in quaternions. Elfrinkhof, L. van. N. Arch. Wisk. 1 (1895) 76-; Fschr. Mth. (1893-94) 135.

- - representing a linear vector function, generally not self-conjugate. Plarr, $G$. [1876] Edinb. R. S. T. 28 (1879) 45-

Equations in multiple quantity. Sylvester, J. J. Nt. 31 (1885) 35-.

- - quaternions, solution of a class. Sylvester, J. J. Ph. Mg. 17 (1884) 392-.

Finite quaternion groups, determination. Stringham, W. I. Am. J. Mth. 4 (1881) 345-.

Geometrical calculus, essay. Lasker, E. L. Mth. S. P. 28 (1897) 217-, 500-.

- interpretation of $\log U q$. Macfarlane, $A$. Ph. Mg. 38 (1894) 143-.

Green's formula, application to the study of the electric field. Larose, $H$. A. Tél. 22 (1895) 460-.

Hamilton groups, Dedekind, $R$. Mth. A. 48 (1897) 548-.

- Miller, C. A. N. Y. Am. Mth. S. Bll. 4 (1898) 510 -

- - D'Alessandro, A. G. Mt. 37 (1899) 138 .

Hamiltonian pairs, and generalized theory of complex variable. Maksimovich, V.P. (xII) [1883-84] Kazan S. Nt. (Ps.-Mth.) P. 2 (1884) i-, 95-.

Hamilton's equation in quaternions, explicit solution. Sylvester, J. J. C. R. 99 (1884) 555 -.

- method, attempt at new development. Dillner, G. [1876] Mth. A. 11, (1877) 168-.

- operator $\nabla$, applications in calculus of variations. Joly, C. J. Ir. Ac. P. 5 (18981900) 666.

- quadrantal versors. Wettum, T. B. van. N. Arch. Wisk. 20 (1893) 1-; Fschr. Mth. (1893-94) 225-.

Homogeneous strains. Metzler, W. H. A. Mth. 8 (1893-94) 148-

Homographic divisions of planes, spheres and space (quaternion methods). Joly, C. J. Ir. Ac. P. 4 (1896-98) 515-.

Hyperbolic quaternions. Macfarlane, A. [1900] Edinb. R. S. P. 23 (1902) 169-.

Imaginary or uniaxal geometry, illustrations. Cockle, Jas. Ph. Mg. 34 (1849) 132-.

Infinite and imaginary, use in service of finite and real. Sylvester, J. J. Nt. 32 (1885) $103-, 271$.

Inverting a linear and quaternion function of a quaternion. Hamilton, (Sir) W. R. (m) [1862] Ir. Ac. P. 8 (1864) 182-.

Laplace's equation, application of quaternions to. Brill, J. Camb. Ph. S. P. 7 (1892) 120-, 151-.

-- , quaternion integration. Preobrazenskij, V. V. Kazan S. Nt. (Ps,-Mth.) P. 2 (1884) 46-; Fschr. Mth. (1884) 308-.

\section{Quaternions 0830}

Laplace's equation, quaternions. Carmichael, $R$. Ir. Ac. P. 6 (1853) 216-

Linear bilateral quaternion equation, geometrical interpretation. Stringham, I. Am. As. P. (1884) 54-.

- differential equations in quaternions. Tait, P. G. [1870] Edinb. R. S. P. 7 (1872) 311-.

- equation of quaternions, Hamilton's and the author's methods of solution. Sylvester, J.J. C. R. 99 (1884) $473-$.

- - , solution of general. Sylvester, J.J. C. R. 99 (1884) 502-.

- vector operator of quaternions. Shaw, J.B. Am. J. Mth. 19 (1897) 267-.

Mechanics, applications of quaternions. Laisant, C. A. Liouv. J. Mth. 3 (1877) 325-.

, - -. Genty, -. Liouv. J. Mth. 7 (1881) 49-.

- by quaternions. Hyde, E. W. Des Moines Anal. 7 (1880) 137-, 177-; 8 (1881) 17-, 49-.

Minding's theorem, quaternion investigations connected with. Tait, P. G. [1879] Edinb. R. S. P. 10 (1880) 200.

- - - proof. Walker, J. J. L. Mth. S.P. 10 (1878-79) 100-.

- - - - Tait, P. G. L. Mth. S. P. 10 (1878-79) 101-.

Operations, theorem in. Tait, $P . G$. Edinb. Mth. S. P. 8 (1890) 21-.

Operator $\phi(\nabla)$. Tait, P. G. Edinb. R. S. P. 7 (1872) 607-.

Point and line calculus, with special reference to parallels. Graefe, E. Arch. Mth. Ps. 15 (1897) 34-, vii-.

Potential of closed circuit, quaternion investigation. Tait, P. G. QJ. Mth. 4 (1861) 143-.

Problem of Fermat, to find point, sum of whose distances from 3 given points is least, solution. Tait, P. G. [1867] Edinb. R. S. P. 6 (1869) 165-.

Quaternion analysis, geometrical proofs of theorems by. Hamilton, (Sir) W. R. Ir. Ac. P. 5 (1853) 407-.

- - new geometrical interpretation. Brill, $J$. Camb. Ph. S. P. 6 (1889) 156-.

- associative principle. Hathaway, A.S. [1895] N. Y. Am. Mth. S. Bll. 2 (1896) 43-.

-, definition. Christie, A. S. Wash. Ph. S. Bll. 11 (1892) 579-.

- differences, an equation in. Tait, P. G. Edinb. R. S. P. 12 (1884) 561-.

- differentiation. M'Aulay, A. [1890] Edinb. R. S. P. 18 (1892) 98-.

equations, solution of a large class. Sylvester, J. J. C. R. 98 (1884) 651-.

- forms of propositions in fluid motion. Butcher, J. G. [1876] L. Mth. S. P. 8 (1877) 174-

- formulae. Allardice, R. E. Edinb. Mth. S. P. 7 (1889) 8-.

- - for quantification of curves, surfaces and barycentres. Stringham, W. I. Am. J. Mth. 2 (1879) 205-. 


\section{0}

Quaternion formulae for reduction of multiple integrals. Carvallo, E. Par. S. Mth. Bll. 18 (1890) 80-.

-, function, development. Graves, C. Ir. Ac. P. 6 (1853-54) 385-.

- group, the. Miller, G.A. Am. Ph. S. P. 37 (1898) 312-.

- innovations. Heaviside, O. Nt. 49 (189394) 246.

- integrals, some. Tait, P. G. [1870] Edinb. R. S. P. 7 (1872) 318-, 784-.

- integration, a. Tait, P. G. [1871] Edinb. R. S. P. 7 (1872) 434-.

- - theorems in. McAulay, A. Mess. Mth. 14 (1885) 26-.

- invariants of linear vector functions, and quaternion determinants. Joly, C. J. Ir. Ac. P. 4 (1896-98) 1-.

- and its depreciators. Knott, C. G. Edinb. Mth. S. P. 11 (1893) 62-.

- method, intrinsic nature of. Tait, P. G. [1894] Edinb. R. S. P. 20 (1895) 276-.

- note on a geometrical problem. Tait, P. G. [1889] Edinb. R. S. P. 16 (1890) 315-.

- notes. Tait, P. G. Edinb. R. S. P. 15 (1889) 379-.

-, from number to. Fleuri, $G$. N. S. W. R. S. J. 28 (1894) 65-

" - Path," Tait's, note. Martin, H. [1867] Edinb. R. S. P. 6 (1869) 121-.

- proof of reciprocal curves in space. Hamilton, (Sir) W. R. B. A. Rp. (1862) (pt. 2) 4.

- - Samuel Roberts' theorem of 4 cointersecting spheres. Walker, J. J. L. Mth. S. P. 12 (1880-81) 147-.

- - - triple generation of three-bar motion. Hart, H. [1882] Mess. Mth. 12 (1883) 32-.

- transformation, a. Tait, P. G. Edinb. R. S. P. 5 (1863) 115-

- transformations. Tait, P. G. Edinb. R. S. P. 7 (1872) 501-.

- - Gwyther, R. F. Manch. Lt. Ph. S. P. 19 (1880) 118-.

- treatment of linear complex. Buchheim, A. Mess. Mth. 13 (1884) 120-.

- variables, functions. Studnicka, F. J. Prag Sb. (1894) (Mth.-Nt.) No. 26, 8 pp.

Quaternions. Hamilton, (Sir) W. R. [1843] Ir. Ac. T. 21 (1848) 199-. 208-

-. Tait, P. G. Mess. Mth. 1 (1862) 78-, 140-, 203-.

- Kelland, P. Mess. Mth. 2 (1863) 136-

-. Ladd, C. Des Moines Anal. 4 (1877) $172-$

- Stringham, W. I. Am. Ac. P. 13 (1878) 310-.

- Wood, De V. Des Moines Anal. 7 (1880) 11-, 33-, 65-, 121-.

-. Christie, A. S. Des Moines Anal. 7 (1880) 185-.

一. Ball, R. S. Nt. 48 (1893) 391.

- Studnicka, F. J. Prag Sb. (1893) (Mth.Nt.) No. 47, 10 pp.
Quaternions 0830

Quaternions. Studnicka, F. J. Časopis 23 (1894) 145-, 209-; Fschr. Mth. (1893-94) 135.

- and algebra of vectors. Gibbs, J.W. Nt. 43 (1891) 511-.

- _ - Tait, P. G. Nt. 43 (1891) 608. 93) 463-.

- - - - Gibbs, J. W. Nt. 48 (1893) $364-$

-, application, new. Everett, J. D. B. A. Rp. (1874) (Sect.) 11.

-, applications to exponential and other functions. Studnicka, F. J. Prag Sb. (1894) (Mth.-Nt.) No. 7, 18 pp.

-, - - geometry of straight lines and complexes. Valentiner, $H$. Kjøb. Ov. (1899) $655-$.

-, - - mechanics. Šebuev, G. N. Kazan S. Ps.-Mth. Bll. 2 (1893) 111-; Fschr. Ps. (1893) $(A b .1) 299$.

- $-1-$ and physics. Molenbroek, $P$. Amst. Ak. Vh. (Sect. 1) 2 (1894) No. 3, 38 pp.; Fschr. Mth. (1893-94) 1315-.

-, - - metrical geometry. Versluys, $J$. N. Arch. Wisk. $\left.2{ }^{*} 1876\right)$ 135-.

-, - - physics. Tait, P. G. QJ. Mth. 6 (1864) 279-; Ph. Mg. 29 (1890) 84-

-, - - McAulay, A. [1892] Nt. 47 (1892-93) 151.

-, - - projective geometry. Chapman, C. H. Am. J. Mth. 14 (1892) 115-

-, - - study of quadrics. Papelier, Par. S. Mth. Bll. 17 (1889) 182-.

- , - - theory of linear complex and linear congruence. Buchheim, A. Mess. Mth. 12 (1883) 129-.

-, - - - rotation. Cayley, A. Ph. Mg. 33 (1848) 196-.

- and "Ausdehnungslehre." Gibbs, J. W. Nt. 44 (1891) 79-.

- - - Tait, P. G. Nt. 44 (1891) 105-. - , - - , application to different kinds of uniform space. Cox, H. [1882] Camb. Ph. S. T. 13 (1883) 69-.

-, calculus, theorem, general. Graves, $C$. Ir. Ac. P. (1850) 347-

-, Col. Hime's outlines of. Macfarlane, A. Ph. Mg. 39 (1895) 135-.

-, continued fractions in. Hamilton, (Sir) W. R. Ph. Mg. 3 (1852) 371-; 4 (1852) 303 ; 5 (1853) 117-, 236-, 321-.

-, , and quadratic equations. Hamilton, (Sir) W. R. Ir. Ac. P. 5 (1853) 219-, 299-.

-, development of the $A$-process in. Shaw, J. B. Am. J. Mth. 19 (1897) 193-.

-, elements. Bartlett, W. P. G. Camb. (M.) Mth. M. 2 (1860) 29-, 97-, 128-, 195-.

-, - Peddie, W. Edinb. Mth. S. P. 11 (1893) 130-.

-, establishment of fundamental properties. McAulay, A. Mess. Mth. 18 (1889) 131-.

-, extensions. Hamilton, (Sir) W. R. Ph. Mg. 7 (1854) 492-; 8 (1854) 125-, 261-; 9 (1855) 46-, 280-.

-, and fourfold space. Hathaway, A. S. [1897-1900] N. Y. Am. Mth. S. Bll. 4 (1898) 54-; 7 (1901) 243, 245-. 
Quaternions, functions resembling. Cockle, Jas. Ph. Mg. 33 (1848) 435-.

-, Gauss' claim to invention. Tait, $P . G$. [1899] Edinb. R. S. P. 23 (1902) 17-.

-, general case of. Sylvester, J. J. C. R. 99 (1884) 117-.

-, geometrical interpretation. Donkin, $W . F$. Ph. Mg. 36 (1850) 489-.

-, - - Spottiswoode, W. Ph. Mg. 37 (1850) 108-.

-, geometry of. Paterson, J. Camb. and Dubl. Mth. J. 9 (1854) 241-.

-; - - theory. Hamilton, (Sir) W. R. Ir. Ac. P. 3 (1847) 31-.

-, involution and evolution. Sylvester, J.J. Ph. Mg. 16 (1883) 394-.

- Klein's view. Knott, C. C. [1899] Edinb. R. S. P. 23 (1902) 24-.

-, and method of equipollences. Bellavitis, G. Ven. At. (1857-58) 334-; Mod. Mm. S. It. 1 (1862) 126-.

-, multinomial theorems. Wilkinson, (Rev.) M. M. U. [1890] Edinb. R. S. P. 17 (1891) 149-

-, multiplication, new proof of associative property. Versluys, J. [1871] Arch. Néerl. 7 (1872) 177-.

- nabla $(\nabla)$. Kimura, S. A. Mth. 10 (1895-96) 127-.

-, nonions, sedenions, \&c. Sylvester, J. J. (xII) [1883] J. H. Un. Cir. [3] (1884) 7-.

-, notes. Boole, G. (vi Adds.) Ph. Mg. 33 (1848) 278-.

-, -. Anthony, E. Mess. Mth. 10 (1881) 66-.

-, 一. Kimura, S. Nt. 52 (1895) 366.

-, number-theory. Hurwitz, A. Gött. Nr. (1896) 313-.

- as a practical instrument of physical research. McAulay, A. Ph. Mg. 33 (1892) 477-.

-, principles elementarily explained. Padelletti, D. G. Mt. 20 (1882) 1-.

-, - , elementary, establishment on analytical basis. Plarr, G. [1874-77] Edinb. R. S. T. 27 (1876) 175-; 28 (1879) 37-.

-, proof of Frobenius' theorem on their relation to complex numbers. Grissemann, $F$. $X$. Mh. Mth. Ps. 11 (1900) 132-.

-, property. Mounier, G. J. D. N. Arch. Wisk. $8\left({ }^{*} 1882\right) 81$-.

-, reduced form. Studnička, F. J. Prag Sb. (1875) 183-.

-, relative forms. Peirce, C. S. (xII) J. H. Un. Cir. [1] (1882) 179.

-, and spherical geometry. Möbius, A. F. Leip. B. 11 (1859) 138-.

-, theory. Versluys, J. N. Arch. Wisk. 1 (*1875) 41-, 97-; 2 (*1876) 135-.

-, -. Sarran, É. A. Tél. 8 (1881) 52-, $113-$

589-.

Stephanos, C. Mth. A. 22 (1883)

1320 -

Weyr, E. C. R. 98 (1884) 906-,

-, - Hölder, O. Gött. Nr. (1889) 34.

-, -. Study, E. N.-Vorp. Mt. 31 (1900) 1-.
Quaternions and vector analysis, principles. Peddie, W. Edinb. Mth. S. P. 11 (1893) 85-.

$$
\text { 516-. }
$$

Quotient of vectors in space, product of matrices. Wettum, T. B. van. N. Arch. Wisk. 1 (1895) 68-; Fschr. Mth. (189394) 226.

Quotients of space-directed lines. Elliott, E. B. Smiths. Misc. Col. 33 (1888) Art. 4, 106-; (Wash. Ph. S. Bll. 10 (1888).)

Surfaces, cubic, class treated by quaternions. Chace, A. B. Am. J. Mth. 2 (1879) 315-.

-, quadric, treated by quaternions. Lowell, A. L. Am. Ac. P. 13 (1878) 222-.

-, theorems. Hamilton, (Sir) W. R. Ir. Ac. P. 4 (1850) 306-.

Symbolic cubic, particular case. Plarr, $G$. [1881] Edinb. R. S. P. 11 (1882) 342-.

- equation satisfied by symbol of operation on a quaternion, existence. Hamilton, (Sir) W. R. Ph. Mg. 24 (1862) 127-.

- integration of differential equations in quaternions. Pocklington, H. C. [1899] Camb. Ph. S. P. 10 (1900) 59-.

Tait's elementary treatise, note on paragraphs 439, 440. Plarr, G. C. R. 86 (1878) 1454-.

Trigonometrical coefficients. Dierke, G. $A$. Educ. Times 41 (1888) 171.

Trigonometry, plane, by quaternions. Wood, De $V$. Des Moines Anal. 9 (1882) 19-.

Use of $\sqrt{-1}$ and alternate numbers $i, j, k$ in study of infinitesimal deformations. Perna, A. Palermo Cir. Mt. Rd. 12 (1898) 322-.

Vector expressions for curves. Joly, $C$. $J$. Ir. Ac. P. 4 (1896-98) 374-.

Vectorial equation of the circumference. Raimondi, R. G. Mt. 25 (1887) 219-.

- function of 1st degree $\phi \rho$. Alberti, $V$. G. Mt. 37 (1899) 375-.

Vectors and quaternions, theory. Beez, Z. Mth. Ps. 41 (1896) 35-, 65-.

Wave-surface, electromagnetic, quaternion treatment. Tait, P. G. [1894] Edinb. R. S. P. 21 (1897) 165-.

- - Fresnel's quaternion investigations. Tait, P. G. QJ. Mth. 3 (1860) 190-.

\section{Ausdehnungslehre; vector analysis.}

Analytical geometry, use of imaginaries. Stille, $W$. A. Des Moines Anal. 1 [1874] 181-.

Area of any closed circuit in space. Hayward, R. B. L. Mth. S. P. 4 (1871-73) 289-.

Associated poles, and their representation by products. Grassmann, $H$. Gött. Nr. (1872) 567-.

Ausdehnungen, multiple, simplest theorems. Hoppe, R. Arch. Mth. Ps. 64 (1879) 189-.

" Ausdehnungslehre." Grassmann, H. Grunert Arch. 6 (1845) 337-.

-, geometric representation of imaginaries from standpoint of. Schlegel, S. F. V. Z. Mth. Ps. 23 (1878) 141-. 
"Ausdehnungslehre," Grassmann's. Schlegel, V. Z. Mth. Ps. 41 (1896) (H.-lt. Ab.) 1-, 41-.

-, - , applications. Clifford, W. K. Am. J. Mth. 1 (1878) 350-.

- - - - - to mechanics. Schlegel, $V$. Civing. 40 (1894) 59 .

-,,-- - properties of circles. Cox, H. QJ. Mth. 25 (1891) 1-.

- - - relation to modern geometrical methods. Schlegel, S. F. V. Z. Mth. Ps. 24 (1879) 83-.

- mechanics according to principles of. Grassmann, H. G. Mth. A. 12 (1877) 222-. -, and modern algebra. Grassmann, H. G. Mth. A. 7 (1874) 538-.

-, place in the associative algebra of quaternion type. Joly, C. J. Ir. Ac. P. 6 (1900-02) 13-.

Barycentre of curvature of plane curves. Steiner, J. G. Arcad. 101 (1844) 257-; 102 (1845), 1-, 121-.

Barycentric calculus, application to problems in analytical geometry. Minding, E. F.A. Crelle J. 5 (1829) 397-.

- - based on composition of straight lines. Möbius, A. F. Crelle J. 28 (1844) 1-.

Complex function, construction, theorem of Picard. Stolz, O. [1880] Innsb. Nt. Md. B. 11 (1881) 27-.

functions, geometric representation. Wittstein, T. Grunert Arch. 7 (1846) 411-.

- variables, extension to 3 dimension space. Hall, T. $P$. Iowa Ac. Sc. P. 6 (1899) 202 .

Composition of simultaneous and successive vectors. MacFarlane, $A$. Science 4 (1896) 346.

Crystallography, parameters of, and vectors of higher order. Voigt, W. Gött. Nr. (1900) 355-.

Curves with branches in several planes, existence. (Geometrical interpretation of $\sqrt{-1}$ and fractional powers). Gregory, D. F. Camb. Mth. J. 1 (1839) 259-

Directed magnitudes. Baker, A. L. Rochester (N. Y.) Ac. Sc. P. 3 (1900) 162-

Directions and rotations, arithmetic. Berdellé, C. As. Fr. C. R. (1887) (Pt. 2) 197-

Directive algebra, application. Neuberg, $J$. (xII) Mathesis 1 (1881) 123-.

- - , - to geometry. Transon, A. N. A. Mth. 7 (1868) 145-, 193-, 241-.

- arithmetic. Berdellé, C. As. Fr. C. R. (1888) (Pt. 2) 109-

Distance, theory. Clifford, $W . K$. B. A. Rp. 39 (1869) (Sect.) 9.

Dühring's vector conceptions. Wessely, $K$. Arch. Mth. Ps. 9 (1890) 393-.

Electromagnetism, application of vector functions. Petrini, $H$. Ups. S. Sc. N. Acta 15 (1895) No. 14, $60 \mathrm{pp}$.

Equations in linear and vector functions, note on solution. Tait, P. G. Edinb. R. S. P. 21 (1897) 497-.

Equipollences, calculus, origin. Laquière, E. $M$. As. Fr. C. R. (1881) 76-
Equipollences, graphic solution of geometrical problems. Bellavitis, G. [1842] Ven. Mm. I. 1 (1843) 225-.

-, method. Bellavitis, G. A. Sc. Lomb. Ven. 7 (1837) 243-; 8 (1838) 17-, 85-; Mod. Mm. S. It. 25 (1855) 225-.

- - origin. Bellavitis, G. Ven. I. Mm. 19 (1876) 449-.

- -, solution by. Bellavitis, G. G. Teix. J. Sc. 1 (1878) 145-; 2 (1880) 3-.

proof of theorems by. Bellavitis, $G$. Ven. At. 6 (1847) 53-.

-, solution of a question. Monteiro, A.S. G. Teix. J. Sc. 2 (1880) 97-.

External multiplication (i.e. use of alternate numbers) in algebra. Fehr, H. N. A. Mth. 14 (1895) 74 .

Flat-space coordinates. Young, $W . H$. L. Mth. S. P. 30 (1899) 54-.

Forces, composition, deduced from general principles. De Morgan, A. [1859] Camb. Ph. S. T. 10 (1864) 290-.

Function of complex argument applied to lemniscate geometry, relationship, and kinematics. Holzmüller, F. G. Z. Mth. Ps. 21 (1876) 325-.

Functions of vectors, which are themselves vectors. Burkhardt, H. Gött.' Nr. (1893) 155-.

Geometric calculus, essay. Peano, G. Tor. Ac. Sc. At. 31 (1895) 552- or 952-.

- principles. Macfarlane, A. [1900] N. Y. Am. Mth. S. Bll. 7 (1901) 158, 178.

- multiplication of surfaces. Chessin, A.S. A. Mth. 9 (1894-95) 70-.

- sums and differences. Barré de SaintVenant, - C. R. 21 (1845) 620-.

Geometry of arbitrary magnitudes. Bugaev, N. V. [1888] Rec. Mth. (Moscou) 14 (1890) 394-; Fschr. Mth. (1891) 725-.

Grassmann's geometrical theories. Zivet, $A$. A. Mth. 2 (1885-86) 1-, 25-.

- method. Carvallo, E. N. A. Mth. 11 (1892) 8-; 12 (1893) 454-.

- applied to prove theorems in determinants. Muiller, $E$. Z. Mth. Ps. 44 (1899) 28-.

- - in projective geometry. Burali-Forti, C. Palermo Cir. Mt. Rd. 10 (1896) 177-, 254-; 11 (1897) 64-.

- methods, applications. Mehmke, R. Z. Mth. Ps. 37 (1892) 305-.

- - - - [centroid of quadrilateral and pentagon]. Caspary, F. N. A. Mth. 17 (1898) 389-.

_, - - to elementary mechanics. Genese, R. $W$. As. Fr. C. R. (1894) (Pt. 2) 211- - - - quadric curves and surfaces. Müller, E. Crelle J. Mth. 115 (1895) 234-. -, vectors in a plane. Caspary, $F$. N. A. Mth. 18 (1899) 248-.

- numbers in intrinsic geometry. Cesàro, E. Rm. R. Ac. Linc. Rd. 3 (1894) (Sem. 1) $367-, 621$.

Hertz's and Poynting's equations. Donati, L. Bologna Ac. Sc. Mm. 7 (1897) 633-.

Images in the representation of functions, theory. Harris, R. A. A. Mth. 4 (1888) 65-, 128. 
Imaginary binary straight lines. Thieme, F. E. Arch. Mth. Ps. 59 (1876) 426-.

- geometry (higher). Beyel, C. Zür. Vjschr. 31 (1886) 20 -.

- - of Lobatschewsky. Battaglini, $G$. Nap. Rd. 6 (1867) 157-.

- _ _ - , applications of formulæ. Suvorov, T. M. (xII) [1881] Kazan S. Nt. (Ps.Ith.) P. 1 (1883) [No. 5] 4 -

- or lateral curves, main features of theory. Thieme, F. E. Arch. Mth. Ps. 58 (1876) 185-.

- - - straight lines. Thieme, F. E. Arch. Mth. Ps. 58 (1876) 218-.

- values of a variable, geometric interpretation. Mouchot, - C. R. 61 (1865) 113-.

Line geometry on principles of " Ausdehnungslehre." Miiller, $E$. Mh. Mth. Ps. 2 (1891) 267-.

Lineal multiplication of two factors. Collins, J. V. Am. As. P. (1899) 69-.

Linear vector equation of central pedal of conic. Hart, H. [1882] Mess. Mth. 12 (1883) 32-.

- - equations, solution in special conditions. Elfrinkhof, L. van. N. Arch. Wisk. 19 (1892) 132-; Fschr. Mth. 24 (1895) 84.

- and vector function. Tait, $P$. G. Edinb. R. S. P. 21 (1897) 160-, 310-; 22 (1900) 547-.

- vector functions, scalar invariants of two. Joly, C. J. [1895] Ir. Ac. T. 30 (1892-96) 709-.

- - - theory. Joly, C. J. [1894] Ir. Ac. Tr. 30 (1892-96) 587-.

Lineo-linear vector functions. Hathaway, A. S. Am. As. P. (1892) 59-.

Loci of the equations $p=\phi^{u} e$ and $p=\phi^{u} \psi^{v} e$. Hyde, E. W. Z. Mth. Ps. 42 (1897) 122-.

Magnitudes possessing extension, measurement. Study, E. Wien Ak. Sb. 91 (1885) (Ab. 2) 100 -.

Mixed products. Mueller, E. Mth. A. 48 (1897) 589-.

Moments of inertia (Grassmann's method). Mehmke, R. Mth. A. 23 (1884) 143-.

Non-congruent quantities, geometrical division. Hyde, E. W. A. Mth. 4 (1888) 9-.

Operations on imaginaries considered geometrically. Henkle, $W$. $D$. Des Moines Anal. 1 (1874) 5-.

Parallelogram of forces, analytical proof. Imschenetsky, W. G. Fschr. Ps. (1889) ( $A b$. 1) 290 -

Plane vectors, linear transformation. Peano, G. Tor. Ac. Sc. At. 31 (1895) 113- or 157-.

Point pairs, and circles in space, treated by Grassmann's method. Müller, $E$. Mh. Mth. Ps. 7 (1896) 77-.

Polar affections of geometrical magnitudes in plano. (Direction of lines, etc.) "Lanivicensis," (Sylvester, J. J.). [1864] Mess. Mth. 3 (1866) 11-.

Polars, theory, and algebraic configurations, application of Ausdehnungslehre. Grassmann, H. G. [1877] Crelle J. Mth. 84 (1878) 273-.

Quadruple algebra; and equations of 5 th order. Cockle, Jas. Ph. Mg. 35 (1849) 434-.
Quaternions and "Ausdehnungslehre." Gibb, J. W. Nt. 44 (1891) 79-.

- - - Tait, P. G. Nt. 44 (1891) 105- - complex variable theory, calculus including. Lipschitz, R. O. S. C. R. 91 (1880) 619-, 660-.

-, Hamilton's, place in Ausdehnungslehre. Grassmann, H. G. Mth. A. 12 (1877) 375-.

- and vector analysis. Gibbs, J. W. Nt. 48 (1893) 364-

- - - - principles. Peddie, W. Edinb. Mth. S. P. 11 (1893) 85-.

- vectors. Knott, C. G. Nt. 48 (1893) $516-$.

Quotients of space directed lines. Elliott, E.B. Smiths. Misc. Col. 33 (1888) Art. 4, 106-; (Wash. Ph. S. Bll. 10 (1888).)

Reduction of vectors and metric properties. Andrade, J. C. R. 125 (1897) 394-.

Rigid body, motion of (exercise in Grassmann's methods). Lüroth, J. Z. Mth. Ps. 43 (1898) 243-.

Rotations. Burali Forti, C. Bll. Sc. Mth. 23 (1899) 82-.

Screw as a unit in the Grassmannian system of the sixth order. Hyde, E.W. A. Mth. 8 (1893-94) 38-.

Self-conjugate linear and vector functions, property. Tait, P. G. [1871] Edinb. R. S. P. 7 (1872) 498-.

Spherical geometry according to Grassmann. Miiller, E. Mh. Mth. Ps. 3 (1892) 365-; 4 (1893) 1-, 93 ; 9 (1898) 269-.

Symbolic forms. O'Brien, M. [1851] Phil. Trans. (1852) 161-.

Symbolical geometry and mechanics. O'Brien, M. [1847] Camb. Ph. S. T. 8 (1849) 497-.

Use of $\sqrt{-1}$ and alternate numbers $i, j, k$ in study of infinitesimal deformations. Perna, A. Palermo Cir. Mt. Rd. 12 (1898) 322-.

Vector analysis. Chapman, C. H. J. H. Un. Cir. $[10(1890-91)]$ 46-.

- - Tait, P. G. Nt. 47 (1892-93) 225-.

- - Domati, L. Bologna Ac. Sc. Mm. 7 (1897) 11-.

-. Farkas, G. Mth. Termt. Éts. 16 (1898) 321-; Mth. Nt. B. Ung. 16 (1899) 111-; Orv.-Termt. Ets. (Termt. Szak) (1899) 91-; (1900) 1-, (Rv.) 1-.

- - Burkhardt, H. D. Mth. Vr. Jbr. 5 (1901) (Heft 1) 43-.

- -, direct and logarithmic, \&c. Malcor, (Capt.) -. Rv. Mar. et Col. 76 (1883) 735-; 77 (1883) 44-; 78 (1883) 416-.

- - a notation. Henrici, O. B. A. Rp. (1897) 560-.

-, reality of roots of symbolical cubic. Tait, P. G. [1867] Edinb. R. S. P. 6 (1869) 92-.

conditions, integrability. Tait, $P . G$. [1877] Edinb. R. S. P. 9 (1878) 527-.

-, derivatives of a. Daniels, F. Z. Mth. Ps. 45 (1900) 203-.

differentiation. MacFarlane, A. [1900]

Wash. Ph. S. Bll. 14 (1906) 73-.

- fields and fields of force. Broca, A. C. R. 130 (1900) 109-. 
Vector fields, properties. Donati, L. Bologna Ac. Sc. Mm. 7 (1897) 427-.

- - theory, as preliminary to study of electricity and magnetism. Ferraris, $G$. Tor. Ac. Sc. Mm. 47 (1897) 259-.

- functions. Burkhardt, $\dot{H}$. Mth. A. 43 (1893) 197-.

- quantities, certain. Bjerknes, $V$. Christiania Skr. (Mth.-Nt. Kl.) (1898) No. 4, 27 pp.

- ratios considered as trigonometric functions of angles. Stringham, W. I. (xII) [1879] J. H. Un. Cir. [1] (1882) 35.

Vectorial coordinates. Sylvester, J. J. Ph. Mg. 31 (1866) 287-, 380-.

- geometry on complexes of second order having a centre of figure. Genty, Liouv. J. Mth. 8 (1882) 299-.

Vectors, application to central motion. Castellano, $F$. Tor. Ac. Sc. At. 29 (1894) 80-.

-, calculation with Ptolemy's theorem generalised. Riecke, - . Grunert Arch. 32 (1859) 470 -

- of ellipse and related curves, sums and products. Schlömilch, O. X. Z. Mth. Ps. 26 (1881) 59-.

-, kinematic applications of theory. Castellano, $F$. Rv. Mt. 2 (1892) 19-.

- and linear transformations, with physical applications. Carvallo, $E$. Mh. Mth. Ps. 2 (1891) 177-, 225-, 311-.

,$----\ldots-$, note on Carvallo's paper. Peano, G. Mh. Mth. Ps. 5 (1894) 136.

- quaternions. Knott, C. G. Nt. 48 (1893) 148 .

- - Lodge, A. Nt. 48 (1893) 198.

- 540 -. Macfarlane, $A$. Nt. 48 (1893)

-, relation among four. Tait, P. G. [1889] Edinb. R. S. P. 16 (1890) 88-.

-, scalar relations connecting six. Wilkinson, (Rev.) M. M. U. [1889] Edinb. R. S. P. 16 (1890) 773-.

—, theory. Zeiliger, D.N. N. Rs. S. Nt. Mm. (Mth.) 11 (1890) 1-, 149-; 13 [?1891] 1-; Fschr. Ps. (1890) (Ab. 1) 239.

-, -, analysis. Peano, G. Tor. Ac. Sc. At. 33 (1897) 313- or 513-.

,-- , recent innovations. Knott, $C . G$. [1892] Edinb. R. S. P. 19 (1893) 212-.

-, - teaching. Fontené, G. Ens. Mth. 1 (1899) 50-.

- versus quaternions. Heaviside, $O$. Nt. 47 (1892-93) 533-.

75- -. Macfarlane, A. Nt. 48 (1893)

which can be compounded, theory.

Berruti, G. Tor. Ac. Sc. At. 29 (1894) 115-, 531-.

\section{Matrices.}

Algebraic resolvents. Rados, G. Mth. Termt. Éts. 17 (1899) 421-; Mth. Nt. B. Ung. 18 (1903) 236-.

Birational transformations, prime factors. Kantor, S. Mh. Mth. Ps. 10 (1899) 54.
Characteristics of systems of three dimensions. Suvorov, T. M. (хш) Kazan Un. Mm. 7 (1871) 3-.

Complex numbers. Poincaré, H. C. R. 99 (1884) 740-.

Differential equation to a curve of any order. Sylvester, J. J. Nt. 34 (1886) 365-.

Elementary universal geometry, new terms. Sylvester, J. J. Nt. 32 (1885) 576-.

Equations in matrices. Sylvester, J.J. J.H. Un. Cir. [3] (1884) 122.

- multiple quantity. Sylvester, J.J. Nt. 31 (1885) 35-.

Exchangeable matrices. Frobenius, G. Berl. Ak. Sb. (1896) 601-.

Hamilton's quadratic equation, and the general unilateral equation in matrices. Sylvester, J.J. Ph. Mg. 18 (1884) 454-.

- - - - a general method for unilateral equations in matrices of any order. Sylvester, J.J. C. R. 99 (1884) 621-.

- quaternions and Cayley's matrices. Wettum, T. B. van. N. Arch. Wisk. 17 (1890) 206-; Fschr. Mth. (1890) 699-.

Harriot's law on algebraic equations, extension to matrices. Sylvester, J. J. C. R. 98 (1884) 1026-.

Infinite and imaginary, use in service of finite and real. Sylvester, J.J. Nt. 32 (1885) $103-, 271$.

Interpolation-formula in matrices, extension of Sylvester's. Buchheim, A. Ph. Mg. 22 (1886) 173-.

Invariants, arithmetical, of a rectangular matrix. Smith, H. J. S. L. Mth. S. P. 4 (1871-73) 236-.

Inverse orthogonal matrices, etc. Sylvester, J. J. Ph. Mg. 34 (1867) 461-.

Involutant of two binary matrices. Cayley, $A$. Mess. Mth. 20 (1891) 136-.

Involutants and allied species of invariants to matrix systems. Sylvester, J. J. (xII) [1883-84] J. H. Un. Cir. [3] (1884) 9-, 34 .

Involution of two matrices of second order. Sylvester, J. J. B. A. Rp. (1883) 430-.

Linear equation in matrices of any order, general resolution. Sylvester, J.J. C. R. 99 (1884) 409-, 432-, 481.

- trinomial equation in matrices of any order. Sylvester, J. J. C. R. 99 (1884) 527-, 576.

Matrical equation $p x=x q$. Sylvester, J. J. C. R. 99 (1884) 67-, 115-.

- $q Q-Q q^{\prime}=0$. Cayley, $A$. Mess. Mth. 14 (1885) 176-.

- $\phi \Omega=\Omega \phi$. Taber, H. Am. Ac. P. 26 (1891) 64-.

Matrices. Tyler, H. W. Erlang. Ps. Md. S. Sb. 23 (1891) 33-.

-, application of theory to linear differential equations. Brill, J. Camb. Ph. S. P. 8 (1895) 201-.

- and associated systems of complex quantities. Weyr, E. Prag Sb. (1887) (Mth.-Nt.) 616-.

-, classification. Weyr, E. C. R. 100 (1885) 966-. 
0850 Matrices

Matrices, fundamental proposition. Weyr, E. Prag Sb. (1884) (Mth.-Nt.) 148-; Fschr. Mth. (1885) 107.

-, multiplication of, identical relation. Kretowski, $W$. Krk. Ak. (Mt.-Prz.) Rz. 6 (1893) 151-; Fschr. Mth. (1893-94) 217.

-, note. Brill, J. [1895-97] L. Mth. S. P. 27 (1896) 35-; 28 (1897) 368-

-, product of two. Sardi, C. G. Mt. 5 (1867) 174-.

-, - - - Janni, V. G. Mt. 11 (1873) $357-$.

-, - - - Bonolis, A. G. Mt. 34 (1896) $375-$.

- and quaternions. Elfrinkhof, L. van. N. Arch. Wisk. 1 (1895) 88-; Fschr. Mth. (1893-94) 226-.

- satisfying a certain equation. Brunel, Bordeaux S. Sc. Mm. 4 (1888) xxii.

-, spherical geometry theorem proved by. Chrystal, G. Edinb. Mth. S. P. 2 (1884) 45-. - of substitution. Brunel, - Bordeaux S. Sc. Mm. 4 (1888) viii.

- theorem by Cayley, proof. Forsyth, A.R. Mess. Mth. 13 (1884) 139-.

-, theory. Cayley, A. Phil. Trans. 148 (1858) 17-; 156 (1866) 25-.

-, -. Buchheim, A. L. Mth. S. P. 16 (1884-85) 63-.

-, - Weyr, E. C. R. 100 (1885) 787-; Prag Sb. (1887) (Mth.-Nt.) 358-

-, - Taber, H. Am. J. Mth. 12 (1890) $337-$

-, - , identities in. Taber, H. Am. J. Mth. 13 (1891) 159-

Matrix, a. Ravut, L. N. A. Mth. 17 (1898) 118-.

$-(a, b)$, and function $\frac{a x+b}{c x+d}$. Mess. Mth. 9 (1880) 104.

- of third order, square root of. Cayley, $A$. Edinb. R. S. P. 7 (1872) 675-.

- which represents a vector. Chapman, C. H. Am. J. Mth. 13 (1891) 363-.

Monothetic equations. Sylvester, J.J. C. R. 99 (1884) 13-.

Non-degenerate matrices, Sylvester's theorem. Taber, H. Am. Ac. P. 27 (1893) 46-.

Notation for matrices, etc. Cayley, $A$. Crelle J. 50 (1855) 277-

Quaternion equation $q Q-Q q^{\prime}=0$. Cayley, $A$. Mess. Mth. 14 (1885) 108-.

- matrix. Wettum, T. B. van. N. Arch. Wisk. 18 (1891) 168-; Fschr. Mth. (1891) 77-.

- - Elfrinkhof, L. van. N. Arch. Wisk. 19 (1892) 143-; Fschr. Mth. (1892) 141-.

- symbols, application to matrix. Taber, $H$. [1890] L. Mth. S. P. 22 (1891) 67-.

Roots of matrices. Metzler, W. H. Am. J. Mth. 14 (1892) 326-.

- - unitary matrices. Sylvester, J.J. C. R. 94 (1882) 396-

- - zeroidal matrices. Brunel, G. C. R. 106 (1888) 467-.

Split matrix, properties. Sylvester, J.J. (xII) J. H. Un. Cir. [1] (1882) 210-.

\section{Other Complex Numbers 0860}

Trinomial unilateral quadratic equation in matrices of second order. Sylvester, J. J. QJ. Mth. 20 (1885) 305-.

Vectors and linear transformations, with physical applications. Carvallo, $E$. Mh. Mth. Ps. 2 (1891) 177-, 225-, 311-.

$-{ }_{-}-{ }_{-}-\ldots$, note on Carvallo's paper. Peano, G. Mh. Mth. Ps. 5 (1894) 136.

\section{Other special sorts of complex numbers.}

Associative imaginaries. Cayley, A. (xI) J. H. Un. Cir. [1] (1882) 211-.

Biquaternions. Clifford, $W . \dot{K}$. L. Mth. S. P. 4 (1873) 381-.

-, application to plane geometry. Combebiac, - Par. S. Mth. Bll. 26 (1898) 259-.

- coplanar, or doubly complex quantities, theory. Seydler, A.J. Prag Sb. (1881) 80-. - geometrical interpretation of results. $\mathrm{Ha}$ milton, (Sir) W. R. Ir. Ac. P. 5 (1853) 388-.

-, memoir. Buchheim, A. Am. J. Mth. 7 (1885) 293-.

Classes of numbers derived from real numbers by dexter and sinister operations. BuraliForti, C. Tor. Ac. Sc. At. 29 (1894) 382-.

Complex functions, generalisation of foundations. Scheffers, G. Leip. Mth. Ps. B. 45, (1893) 828- ; 46 (1894) 120-

- numbers extended. Vahlen, T. Königsb. Schr. 38 (1897) [72]-.

- - formed from $n$ principal units. Weier. strass, $K$. Gött. Nr. (1884) 395-.

- - - Schwarz, H. A. Gött. Nr. (1884) 516-.

(1885) 141-.

(1886) 241 .

11 (1887) $205-$

_- _ - _ - Scheffers, G. Leip. Mth. Ps. B. 41 (1889) 290-.

- - - Schur, F. Mth. A. 33 (1889) 49-.

- - - - - Gegenbauer, L. Mh. Mth. Ps. 2 (1891) 429-.

_ _ _ _ - Hilbert, D. Gött. Nr. (1896) 179-.

- -, general. Dedekind, R. Gött. $\mathrm{Nr}$. (1887) 1-.

- - and groups of transformations. Study, E. Leip. Mth. Ps. B. 41 (1889) 177.

- - of $n$ dimensions. Petersen, J. Gött. Nr. (1887) 489-.

- - real systems. Cartan, E. C. R. 124 (1897) 1296-.

- -, systems. Study, E. Gött. Nr. (1889) 237-.

- - - Cartan, E. C. R. 124 (1897) $1217-$.

- - - - of higher. Molien, T. Mth. A. 41 (1893) 83-; 42 (1893) 308-. 
0870 Algebra of Logic

Complex numbers, systems, reducibility. Scheffers, G. Mth. A. 41 (1893) 601-.

- - - - , reduction to typical forms. Scheffers, $G$. Mth. A. 39 (1891) 293-.

,--- , theory. Hausdorf, $F$. Leip. Mth. Ps. B. 52 (1900) 43-.

Elliptic and hyperbolic numbers. Levi-Civita, $T$. Ven. I. At. (1892-93) 1765-.

Nonions analogous to quaternions, quantities forming a group. Sylvester, J.J. C. R. 97 (1883) 1336-; 98 (1884) 273-, 471-.

-, system. Peirce, C. S. (xi) J. H. Un. Cir. [2] (1883) 86-

-, (-, represented by 9 matrices). Sylvester, J.J. (XII) J. H. Un. Cir. [1] (1882) 241-; [2] (1883) 46, 86.

Non-quaternion number-systems containing no skew units. Starkweather, G. P. Am. J. Mth. 21 (1899) 369-.

Octonions. MIcAulay, A. R. S. P. 59 (1896) 169 .

Pluquaternions, and homoid products. Kirkman, T. P. Ph. Mg. 33 (1848) 447-, 494-.

Seven fundamental keys, system. Fontené, $G$. Par: S. Mth. Bll. 27 (1899) 171-.

Symbolical and double numbers. Philippoff, $\boldsymbol{M}$. Z. Mth. Ps. 37 (1892) 298-.

Systems of numbers, calculation. Scheffers, $G$. Leip. Mth. Ps. B. 41 (1889) 400-.

- one-vectors in space of $n$ dimensions. Young, $W . H$. L. Mth. S. P. 29 (1898) 478-.

Trinomial complex numbers. Schwering, $K$. Acta Mth. 10 (1887) 57-

Triquaternions. Combebiac, - Par. S. Mth. Bll. 27 (1899) 180-

Types of algebraical magnitudes arising by composition of two other types. Hensel, $K$. Crelle J. Mth. 105 (1889) 329-.

Units of an $n$-fold space, applications. Chapman. C. H. Am. J. Mth. 10 (1888) 225-.

\section{Algebra of Logic.}

Abacus, logical. Jevons, W. S, Manch. Lt. Ph. S. P. 5 (1866) 161-.

Algebra of binary relatives. Schroeder, $E$. Mth. A. 46 (1895) 144-:

- logic. Peirce, C. S. Am. J. Mth. 3 (1880) 15-.

- _ - , a contribution to the philosophy of notation. Peirce, C. S. Am. J. Mth. 7 (1885) 180-.

- - -, formulae. Peano, G. Rv. Mt. 1 (1891) 24-, 182-.

- - principles. Macfarlane, A. B. A. Rp. (1879) 262-. 1-. -, -. Peano, G. Rv. Mt. 1 (1891)

(1894) 156-.

- relations, principles. Schröder, F. [1900]

N. Y. Am. Mth. S. Bll. 7 (1901) 176-

Boole's analysis of logic. Harley, $R$. B. A. Rp. 36 (1866) (Sect.) 3-.

- laws of thought. Young, G. P. Cn. J. 10 (1865) 161-.
0870 Algebra of Logic

Boole's laws of thought. Ellis, R. L. B. A. Rp. 40 (1870) (Sect.) 12-.

(Sect.) 14 -

Calculus of equivalent statements. MacColl, $H$. [1896-97] L. Mth. S. P. 28 (1897) 156555-; 29 (1898) 98-.

- - and integration limits. $\mathrm{McClll}, H$. L. Mth. S. P. 9 (1877-78) 9-, 177-; 10 (1878-79) 16-; 11 (1879-80) 113-.

- logic. Boole, G. Camb. and Dubl. Mth. J. 3 (1848) 183-.

- - -. Cayley, A. QJ. Mth. 11 (1871) 282-.

_- Boole's, improvement. Peirce, C. S. [1867] Am. Ac. P. 7 (1868) 250-

$-\ldots$, cycle of operations of. Schröder, $E$. Mth. A. 12 (1877) 481-.

- - , functional theorems. Nagy, A. Rv. Mt. 2 (1892) 177-.

Deductive logic, principles. Vailati, $G$. Rv. Mt. 1 (1891) 127-.

Dependence, property of, as a relation. Vailati, G. Rv. Mt. 2 (1892) 161-.

Dialectics, rational. Gergonne, J. D. Gergonne A. Mth. 7 (1816-17) 189-.

Elimination problem in identical calculus. Schröder, E. D. Nf. Tbl. (1885) 353-.

Expressions for hypothetical and disjunctive propositions. Graves, C. Ir. Ac. P. 4 (1850) 147-.

Formal properties of algebraic operations. Burali-Forti, C. Rv. Mth. 6 (1896-99) 141-. Implicational and equational logic. $\mathrm{McCl}$ Cll, $H$. [1880] Ph. Mg. 11 (1881) 40-.

Inverse or inductive logical problem. Jevons, $W$. S. [1871] Manch. Lt. Ph. S. P. 11 (1872) 65-.

Jevons-Clifford problem. Nagy, A. Mh. Mth. Ps. 5 (1894) 331-.

Law of roots in logic. Poretsky, P. Rv. Mth. 6 (1896-99) 5-.

Logic, algebraic analogues. Ellis, A. J. R. S. P. 21 (1873) 497-.

-, application to mathematics. Debrun, $B$. Niórt. Mm. de l'Athénée 1 (1808) 1-.

-, - - - Matzka, W. Grunert Arch. 6 (1845) 353-.

-, formal. Ellis, A. J. R. S. P. 20 (1872) 307-.

- of mathematics. Peirce, C. S. [1867] Am. Ac. P. 7 (1868) 402-.

- number. Peirce, C. S. Am. J. Mth. 4 (1881) 85-.

- relatives, notation. Peirce, C. S. [1870] Am. Ac. Mm. 9 (pt. 2) (1873) 317-.

-, symbolic. MacColl, H. [1900] N. Y. Am. Mth. S. Bll. 7 (1901) 158, 166-.

-, - treatment. Murphy, J.J. Manch. Lt. Ph. S. Mm. \& P. 4 (1891) 201-.

Logical calculus. Porěckij, P. S. Kazan S. Nt. (Ps.-Mth.) P. 7 (1889) 260-.

- - Nagy, A. G. Mt. 28 (1890) 1-.

- equalities, laws. Porěckij, P.S. Kazan S. Ps.-Mth. Bll. 8 (1899) 33-, 129-, 183-; 10 (1901) 50-, 132-, 191-; 11 (1901) $17-$. - equations. Johnson, W. E. $[1900]$ N. Y. Am. Mth. S. Bll. 7 (1901) 158, 177-. 


\section{Algebra of Logic}

Logical equations, methods of solution, and inverse method of mathematical logic. Poryetskin, P. S. (xir) [1882] Kazan S. Nt. (P8.-Mth.) P. 2 (1884) 161-.

- form of geometrical theorems. Macfarlane, A. A. Mth. 3 (1887) 154-.

- functions, theorems concerning. Nagy, A. Rv. Mth. 6 (1896-99) 21-.

- notation, a new system. Murphy, J. J. Manch. Lt. Ph. S. Mm. \& P. 2 (1889) 22-.

propositions, on the transformations. Murphy, J. J. Manch. Lt. Ph. S. Mm. 8 (1884) 132-.

- quantities, graphical representation. Nagy, A. Rm. R. Ac. Linc. Rd. 6 (1890) (Sem. 2) 50-, 373-, 393.

- spectrum. Macfarlane, A. Ph. Mg. 19 (1885) 286-.

- - method, application to Boole's problem. Macfarlane, A. Am. As. P. (1890) 57-.

Magnitudes, theory. Burali-Forti, C. Rv. Mt. 3 (1893) 76-.

Mathematical logic, first principles. Poryetskir, P.S. (xiI) [1880] Kazan S. Nt. (Ps.-Mth.) P. 1 (1883) [(No. 2)] 2-.

- - , some applications in arithmetic. Bunitzkij, E. Fschr. Mth. (1897) 63.

- - studies. Peano, G. Tor. Ac. Sc. At. 32 (1896) 361- or 565-.

Mechanical performance of logical inference. Jevons, W. S. [1869] Phil. Trans. 160 (1870) 497-.

Notations, various, for expressing propositions of logic. Venn, J. [1880] Camb. Ph. S. P. 4 (1883) 36-.

Numerically definite propositions. Boole, $G$. [1868] Camb. Ph. S. T. 11 (pt. 2) (1869) 396-.

- - reasoning, system. Jevons, W. S. [1870] Manch. Lt. Ph. S. Mm. 4 (1871) 330 -

Relation between the logical theory of classes and the geometrical theory of points. Kempe, A. B. $[1890]$ L. Mth. S. P. 21 (1891) 147-.

Relations between logical magnitudes. Nagy, $A$. Mh. Mth. Ps. 4 (1893) 147-.

- - members of a system. Amicis, E. de. Rv. Mt. 2 (1892) 113-.

Straight lines, fundamental geometry of. Vailati, G. Rv. Mt. 2 (1892) 71-.

Syllogism, on the. Harris, J.R. J. H. Un. Cir. [3] (1884) 130-.

Syllogisms of transposed quantity. De Morgan, A. Camb. Ph. S. T. 10 (1864) $355^{*}$-。

Symbolical language, growth and use. McColl, H. [1881] Manch. Lt. Ph. S. Mm. 7 (1882) 225-.

Symbols of logic, and theory of the syllogism. De Morgan, A. [1846-50] Camb. Ph. S. T. 8 (1849) 379- ; 9 (1856) 79-.

Types of compound statement involving 4 classes. Clifford, $W . K$. Manch. Lt. Ph. S. P. 16 (1877) 88-.

\section{Theory of Groups. General}

\section{Theory of Groups. \\ 1200 General}

Adjoint operations. Pincherle, S. Bologna Rd. 2 (1898) 130-.

Anharmonic curves. Bioche, C. Par. S. Phlm. Bll. 5 (1893) (C. R. No. 10) 2.

Constancy of factor-groups, Hölder's theorem. Brown, G. L. N. Y. Am. Mth. S. Bll. 1 (1895) 232-.

Distributive operations and homography. Pincherle, S. Mil. I. Lomb. Rd. 29 (1896) 397-.

Finite and infinite groups. Bettazzi, $R$. Tor. Ac. Sc. At. 31 (1895) 362- or 506-.

Functional substitution equations. Böttcher, $E$. Wiad. Mt. 4 (1900) 233-.

Group-characteristics. Frobenius, $G$. Berl. Ak. Sb. (1896) 985-.

-- , composition. Frobenius, G. Berl. Ak. Sb. (1899) 330-.

- and subgroups, relations. Frobenius, $G$. Berl. Ak. Sb. (1898) 501-.

Group and number. Bettazi, $R$. Tor. Ac. Sc. At. 31 (1895) 304- or 446-.

- theory and its application. Źorawski, $K$. Krk. Ak. (Mt.-Prz.) Rz. 6 (1893) 289-; Crc. Ac. Sc. Bll. (1893) 145-

Groups, complete theory. Kirkman, T. P. [1863] Manch. Ph. S. P. 3 (1862-63) 133-, 161- ; 4 (1865) 171-.

- depending on $\theta^{n}=1$. Cayley, $A$. Ph. Mg. 7 (1854) 40-, 408 ; 18 (1859) 34-.

- of functions. Pincherle, S. Bologna Ac. Sc. Mm. 6 (1884) 205-.

-, functions given by. Kirkman, $T$. $P$. Manch. Lt. Ph. S. Mm. \& P. 4 (1891) 315-; 5 (1892) 23-.

- and generators, systems. Fulco, $P$. Catania Ac. Gioen. At. 12 (1899) Mm. 12, 14 pp.

- of interchangeable elements. Frobenius, G., \& Stickelberger, L. [1878] Crelle J. Mth. 86 (1879) 217-.

- - involution, transformations. Montesano, D. Ven. I. At. (1887-88) 1425-.

- and many-valued functions. Kirkman, $T$. $P$. [1861] Manch. Ph. S. Mm. 1 (1862) 274 -

- of order $8 ! / 2$. Schottenfels, I. M. N. Y. Am. Mth. S. Bll. 6 (1900) 440-.

- related to regular bodies in $n$-dimensional space. Schoute, P. H. B. A. Rp. (1894) $562-$.

-, systems of. Dickson, L. E. N. Y. Am. Mth. S. Bll. 3 (1897) 265-

-, theory. Cayley, A. [1877-89] L. Mth. S. P. 9 (1877-78) 126-; Am. J. Mth. 11 (1889) 139-.

-, - and graphical representation. Cayley, A. Am. J. Mth. 1 (1878) 174-.

Imprimitive groups. Kuhn, $H . W$. N. Y. Am. Mth. S. Bll. 7 (1901) 113, 116-.

- and soluble groups. Söderberg, J. T. [1899] Ups. S. Sc. N. Acta 20 (1904) No. 1, 26 pp.

Infinite order, groups of. Miller, G. A. [1900] N. Y. Am. Mth. S. Bll. 7 (1901) 121-. 


\section{Theory of Groups. General}

Invariants of groups of linear substitutions. Molien, T. Berl. Ak. Sb. (1897) 1152-.

Inversion groups on quadric surfaces. Kasner, E. N. Y. Am. Mth. S. T. 1 (1900) 430-

Linear differential equations, arithmetical developments. Fricke, $R$. D. Mth. Vr. Jbr. 2 (1893) 59-.

- - , form of integrals. Johanson, A. M. Stockh. Öfv. (1889) 583-.

- - - theory. Volterra, V. Rm. S. It. Mm. 6 (1887) No. 8, 104 pp.

- groups of functions. Pincherle, S. Bologna Ac. Sc. Mm. 6 (1884) 101-.

- - report on recent progress. Dickson, L. E. [1899] N. Y. Am. Mth. S. Bll. 6 (1900) 13-.

Maximum groups. Kirkman, T. P. Manch. Ph. S. P. 3 (1862-63) 59-.

Non-isomorphic simple groups of the same order. Schottenfels, I. M. [1898] A. Mth. 1 (1900) 147-.

- -modular groups. Kirkman, T. P. (III) [1862] Manch. Ph. S. Mm. 2 (1865) 204-.

Popular account of new fields of mathematics. Miller, G. A. Science 11 (1900) 528-

Prime factors of the group-determinant. Frobenius, G. Berl. Ak. Sb. (1896) 1343-.

Rational and linear substitutions, the group of. Cordone, G. Tor. Ac. Sc. At. 31 (1895) 472or 804-.

Regions of discontinuity for groups of real linear substitutions of a complex variable. Fricke, R. Gött. Nr. (1895) 360-.

Representation of finite groups by linear substitutions. Frobenius, G. Berl. Ak. Sb. (1897) 995- ; (1899) 482-.

Ruled surfaces admitting homographic automorphic transformation. Bioche, $C$. Par. S. Phlm. Bll. 4 (1892) 130-.

Singular transformations of groups. Taber, $H$. N. Y. Am. Mth. S. Bll. 6 (1900) 199-.

- Newson, H. B. N. Y. Am. Mth. S. Bll. 6 (1900) 431-.

Sub-groups of doubly infinite series of simple groups. Wiman, A. Stockh. Ak. Hndl. Bh. 25 (1900) (Afd. 1) No. 2, 47 pp.

- - - general projective group. Taber, $H$. N. Y. Am. Mth. S. Bll. 2 (1896) 221-, 346.

Substitution groups, fundamental theorem. Miller, G. A. [1895] N. Y. Am. Mth. S. Bll. 2 (1896) 75-.

Substitutions. Zochios, -. C. R. 120 (1895) 766-.

Symmetrical group, characteristics. Frobenius, G. Berl. Ak. Sb. (1900) 516-.

Test of a simple group. Miller, G. A. N. Y. Am. Mth. S. Bll. 1 (1895) 124-.

Uniform substitutions, Koenigs, $G$. Bll. Sc. Mth. As. 7 (1883) 340-

- Podetti, F. G. Mt. 35 (1897) 264 -.

Vanishing groups. Cockle, Jas. Camb. and Dubl. Mth. J. 6 (1851) 174-; 7 (1852) 114-; 8 (1853) 50-.
Discrete Groups 1210

\section{Discrete groups of finite and of infinite order (including groups of permutations). See also 2450, 4440.}

Abelian general linear group, new definition. Dickson, L. E. N. Y. Am. Mth. S. T. 1 (1900) 91-.

- groups. Heffter, L. Crelle J. Mth. 119 (1898) $261-$.

- -. Scarpis, U. G. Mt. 38 (1900) 225-

- and linear modular systems. Pund, $O$. Hamb. Mth. Gs. Mt. 3 (1900) 371-.

- - and their application to number theory. Zsigmondy, $K$. Mh. Mth. Ps. 7 (1896) 185-.

Abstract group isomorphic with the symmetric group on $k$ letters. Dickson, L. E. L. Mth. S. P. 31 (1900) 351-.

- groups of degree 32. Bagnera, G. A. Mt. 2 (1899) 263-。

- - order $k !$ and $\frac{1}{2} k !$. Moore, E. H. [1896] L. Mth. S. P. 28 (1897) 357-.

- simple group of order $2^{7} \cdot 3^{6} \cdot 5 \cdot 7$, a certain. Dickson, $L . E$. N. Y. Am. Mth. S. T. 1 (1900) 353-, 509.

Alternating group, uniform character. Beke, E. Mth. A. 49 (1897) 581-.

- groups. Konig, G. (xII) Mth. Termt. Éts. 1 (1883) 213-; Mth. Nt. B. Ung. 1 (1882-83) $191-$.

Analytical representation of a heptagram. Rogers, L. J. [1890] L. Mth. S. P. 22 (1891) 37-.

Arithmetical group, laws of reciprocity and subgroups. Stouff, X. Par. Ec. Norm. A. 10 (1893) 295-.

Automorphic functions of two variables. Picard, É. Acta Mth. 1 (1882) 297-; C. R. 97 (1883) 1045-.

- modular groups. Fricke, R. Gött. Nr. (1896) 91-.

Binary forms with linear automorphic transformations. Klein, C. F. [1875] Mth. A. 9 (1876) 183-.

Cayley's color-diagrams, representations of finite groups. Maschke, $H$. Am. J. Mth. 18 (1896) 156-.

Characteristics of types $3 \& 4$ and their substitution groups. Pascal, E. A. Mt. 20 (1892-93) 163-; Rm. R. Ac. Linc. Rd. 2 (1893) (Sem. 1) 120-

Commutator groups. Miller, G. A. N. Y. Am. Mth. S. Bll. 4 (1898) 135-.

Commutators of given group. Miller, G. A. [1899] N. Y.Am. Mth. S. Bll. 6 (1900) 105-.

Composite groups. Burnside, $W$. Mess. Mth. 24 (1895) 82-.

- -, formation. Hölder, O. Mth. A. 46 (1895) 321-.

- - theory. Netto, E. Crelle J. Mth. 78 (1874) 81-.

Configuration of 140 straight lines in space. Maschke, H. Mth. A. 36 (1890) 190-. 
Congruence-group contained in the group of linear fractional substitutions. Moore, E. H. Am. As. P. (1892) 62.

- groups of prime rank. Gierster, J. Mth. A. 22 (1883) $177-$.

- -, theorem. Brioschi, F. Mil. I. Lomb. Rd. 12 (1879) 483-

Congruences, class of groups defined by. Burnside, $W$. L. Mth. S. P. 25 (1894) 113-; 26 (1895) 58-.

Conjugate system of linear substitutions, formation. Baraniecki, $M$. A. Par. T. Nauk Śc. Pam. 10 (*1878) Art. 3, 8 pp.

Connexion in series and theory of substitutions. Hoyer, P. Mth. A. 42 (1893) 58- ; 49 (1897) 39-.

Crystalline structure and regular point groups. Rohn, K. Leip. Mth. Ps. B. 51 (1899) (Mth.) 445-.

Cubic surfaces with automorphic collineations. Bobek, K. Mh. Mth. Ps. 10 (1899) 122-, 307-.

- -, polar hexahedra. Cremona, L. D. Nf. B. (*1877) 93-.

Cyclic subgroups of simple ternary linear fractional group in a Galois field. Dickson, L. E. Am. J. Mth. 22 (1900) 231-

Direct product groups, Miller, G. A. N. Y. Am. Mth. S. T. 1 (1900) 66-

Discontinuity in certain homographies. Fricke, $R$. Mth. A. 47 (1896) 557-.

Discontinuous groups. Poincaré, H. C. R. 94 (1882) 840-.

- - in biquadratic field. Fricke, R. Gött. Nr. (1892) 268-.

- and quadratic forms. Picard, É. C. R. 95 (1882) 763-.

- - of real linear substitutions (Poincarés). Kepiñski, S. Krk. Ak. (Mt.-Prz.) Rz. 6 (1893) 37-; Fschr. Mth. (1893-94) 207-.

Doubly transitive groups of degree $n$ and order $n(n-1)$. Burnside, $W$. Mess. Mth. 25 (1896) 147-.

$-----2^{m}$ and order $2^{m}\left(2^{m}-1\right)$. Burn. side, $W$. Mess. Mth. 25 (1896) 187-.

- - - - substitutions of three degrees. Maillet, E. Par. S. Mth. Bll. 25 (1897) 189-.

Exchangeable and decomposable groups. Maillet, E. Par. S. Mth. Bll. 28 (1900) 7-.

Finite group, definition. Bettazzi, $R$. Tor. Ac. Sc. At. 32 (1896) 240 or 352-.

- groups. Frobenius, G. Berl. Ak. Sb. (1895) 163-.

- -. Maillet, E. QJ. Mth. 29 (1898) 250

Bauer, $M$. Mth. Termt. Ets. 17 (1899) 611-; N. A. Mth. 19 (1900) 508-.

- - class of. Burnside, W. Camb. $\mathrm{Ph}$. $\mathrm{S}$. T. 18 (1900) 269-

- contained in group of quadratic homogeneous transformations. Autonne, L. C. R. 97 (1883) 567-

- - - - linear group, determination. Jordan, C. Nap. Ac. At. 8 (1879) No. 11 41 pp.
Finite groups contained in linear groups, class. Jordan, C. Par. S. Mth. Bll. 5 (1877) 175-. - - of correlations. Kantor, $S$. Crelle J. Mth. 116 (1896) 171-.

- - whose degree is the fifth power of a prime. Bagnera, G. A. Mt. 1 (1898) 137-. _- of linear substitutions, fundamental proposition in theory. Burkhardt, H. Mth. A. 41 (1893) 309-.

- - - - , theorem on. Klein, F. D. Nf. Vh. (1896) (Th. 2, Hälfte 1) 5; D. Mth. Vr. Jbr. 5 (1901) (Heft 1) 57.

- - - - transformations of a variable. Gordan, $P$. Mth. A. 12 (1877) 23-.

- - - - - - C. Cayley, A. [1879] Mth. A. 16 (1880) 260-, 439-.

- - - Fano, G. Mh. Mth. Ps. 7 (1896) 297-.

- -, report on. Miller, G. A. [1898] N. Y. Am. Mth. S. Bll. 5 (1899) 227-.

- - , sets of operations in relation to. Whitehead, A. N. R. S. P. 64 (1899) 319-.

-- , theory. Valentiner, $H$. Kjøb. Dn. Vd. Selsk. Skr. 5 (1889-91) 63- (with French résumé).

- - - - Burnside, W. [1893-95] L. Mth. S. P. 25 (1894) 9-; 26 (1895) 191-, 325-, 559.

- -, 一. Bauer, M. N. A. Mth. 19 (1900) 59-.

Functions, cyclic. Dziobek, $O$. Arch. Mth. Ps. 69 (1883) 265-.

-, linear rational, taken according to prime modulus, and substitutions to which they lead. Serret, J. A. C. R. 48 (1859) 112-, 178-, 237-.

- _, of several variables, values. Capelli, $A$. G. Mt. 14 (1876) 141-.

-, number of values, by permutations of letters. Bertrand, J. Par. Éc. Pol. J. $30^{\mathrm{e}} \mathrm{cah}$. (1845) 123-.

-, - - - , - - - (Bertrand). Serret, J. A. Liouv. J. Mth. 14 (1849) 135-。

- - - - - - - - Despeyrons, $C$. Liouv. J. Mth. 10 (1865) 55-

-, polynomial, admitting of reduction in number of variables. Sylvester, J. J. $\mathrm{Ph}$. Mg. 5 (1853) 119-.

- proper to represent substitution of prime number of letters. Rogers, L. J. Mess. Mth. 21 (1892) 44-.

- reproduced by substitution. Cauchy, A.L. C. R. 19 (1844) 1436-

- of several quantities, nature, formation, and substitutions rendering invariable. Mathieu, É. Liouv. J. Mth. 6 (1861) 241- ; N. A. Mth. 1 (1862) 227-.

- 5 or 6 letters, especially those doubly transitive. Cauchy, A. L. C. R. 21 (1845) 1401-; 22 (1846) 1-.

- 4, 5, or 6 letters. Serret, J. A. Liouv. J. Mth. 15 (1850) 45-.

- 6 letters, unsymmetrical, historical origin. Sylvester, J. J. Ph. Mg. 21 (1861) 369-.

- 7 letters. Hermite, C. C. R. 57 (1863) 750 -

$6-\overline{65-}, 81 \overline{-}$, Briosc

C. R. 95 (1882) 
Galoisian groups. Perott, J. Par. S. Mth. Bll. 21 (1893) 61-.

Generators of a symmetric group. Capelli, $A$. G. Mt. 35 (1897) 354-.

Geometrical operations, certain. Fleuri, $G$. N. S. W. R. S. J. 26 (1892) 60-.

Group belonging to irreducible equations of prime degree. Sylow, L. [1867] Christiania F. 10 (1868) 105-.

- of discrete operations, definition. Dyck, $W$. [1881-82] Mth. A. 20 (1882) 1-; 22 (1883) 70 -

- - modular equations. Gierster, $J$. Leip. Mth. Ps. B. 37 (1885) 291-; Mth. A. 26 (1886) 309-.

- theory. Guccia, G. B. Palermo Cir. Mt. Rd. 1 (1887) 388-.

- - and algebraic equations. Foglini, $G$. Rm. N. Linc. Mm. 3 (1888) 3-; 7 (1891) 175-.

- - - Bolza, O. Am. J. Mth. 13 (1891) 59-.

- - Söderberg, J. T. Ups. S. Sc. N. Acta 15 (1895) No. 2, 38 pp.

- - geometrical treatment. Paolis, R. de. Rm. S. It. Mm. 7 (1890) No. 6, 164 pp.

- - investigations. Frobenius, G. Crelle J. Mth. 101 (1887) 273-.

- - and regular solids. Schoute, P. H. N. Arch. Wisk. 20 (1893) 100-.

- of 360 collineations in plane. Fricke, $R$. D. Nf. Vh. (1896) (Th. 2, Hälfte 1) 5.

Groups, Burnside's theory. Miller, G. A. N. Y. Am. Mth. S. Bll. 5 (1899) 249-

- which can be represented as multiply transitive substitution groups. Miller, G.A. Mess. Mth. 28 (1899) 104-.

- of class $n-u$, degree $n$, at least $u-1$ times transitive. Maillet, E. Par. Éc. Pol. J. 5 (1900) 47-

- - degree $p$ and order $p(p+1) \pi, p$ being prime, $\pi$ a divisor of $p-1$. Sylow, $L$. Christiania Skr. (Mth. Nt. Kl.) (1897) No. 9, 19 pp.

-, especially substitutions permutable with given one. Capelli, A. G. Mt. 14 (1876) 66-.

- formed of a finite number of linear substitutions. Jordan, C. C. R. 83 (1876) 1035-; 84 (1877) 1446-.

- of linear substitutions. Bianchi, L. Mth. A. 42 (1893) 30-.

- - - - with coefficients belonging to imaginary quadratic corpora. Bianchi, $L$. Mth. A. 40 (1892) 332-.

- - - - complex coefficients, some classes of. Bianchi, L. Mth. A. 43 (1893) 101-.

- - - - - integral complex coefficients with applications to theory of numbers. Bianchi, L. Rm. R. Ac. Linc. Rd. 6 (1890) (Sem. 1) 331-; Mth. A. 38 (1891) 313-.

- - - - theory. Loewy, A. Mth. A. 53 (1900) 225-.

-, list of systems. Dickson, L. E. N. Y. Am. Mth. S. Bll. 5 (1899) 470-.

- of $n$ dimensions. Frattini, G. Rm. R. Ac. Linc. T. 8 (1884) 260-.
Groups of numbers in algebraic corpora. Weber, $H$. Mth. A. 48 (1897) 433-; 49 (1897) 83-; 50 (1898) 1-.

- whose order is a number without square factors. Hölder, $O$. Gött. Nr. (1895) 211-. - - - power of prime, determination. Young, J. W. A. Am. J. Mth. 15 (1893) 124 .

- - - - - theory. Bauer, $M$. Mth. Termt. Ets. 18 (1900) 133-.

- of order $p^{m} q^{2}$. Jordan, C. Liouv. J. Mth. 4 (1898) 21-.

$---p^{2} q^{2}, p$ and $q$ being primes. Le $V a$ vasseur, $R$. P. C. R. 128 (1899) 1152-.

- - - 16p, $p$ being an odd prime. Le Vavasseur, R. P. C. R. 129 (1899) 26-.

- - $p^{3} q$. Western, A. E. L. Mth. S. P. 30 (1899) 209 .

- whose orders are products of three prime factors. Cole, E. N., \& Glover, J.W. Am. J. Mth. 15 (1893) 191-.

- of orders $p^{3}, p q^{2}, p q r, p^{4}$. Hölder, O. Mth. A. 43 (1893) 301-。

- which $r$ arbitrary points in space of $n$ dimensions can represent. Schlegel, $V$. Arch. Mth. Ps. 10 (1891) 283-.

- several times transitive, elementary properties. Cesàro, E. G. Mt. 22 (1884) 47-.

- and substitutions, new basis for theory. Hoyer, $P^{\prime}$. Mth. A. 51 (1899) 445-.

-, theorem. Cayley, A. Mth. A. 13 (1878) 561-.

-, theorems. Kirkman, T. P. Manch. $\mathrm{Ph}$. S. P. 2 (1860-62) 73-.

-, theory. Burnside, $W$. Mess. Mth. 23 (1894) 50-; 24 (1895) 191-.

Holoedric transformation into itself of a given group. Moore, E. H. [1895] N. Y. Am. Mth. S. Bll. 1 (1895) 61-; 2 (1896) 33-.

- and transitive isomorphs of symmetric or alternant groups. Maillet, $E$. Liouv. J. Mth. 1 (1895) 5-.

Holomorph of the cyclical group, and some subgroups. Miller, G. A. QJ. Mth. 31 (1900) 382-.

Homogeneous substitution groups, theory. Molien, T. Dorpat Sb. 11 (1896) 259-.

Hyperabelian groups, special class. Bourget, $H$. Toul. Fac. Sc. A. 12 (1898) D, 90 pp.

Hyperelliptic curves of class 3, allowing automorphic transformation. Wiman, A. [1895] Stockh. Ak. Hndl. Bh. 21 ( $A f d .1)$ (1896) No. 1, $23 \mathrm{pp}$.

Hypergeometric functions with the branch points $(2,3,7),(2,4,7)$, arithmetical theory. Fricke, R. Mth. A. 41 (1893) 443-.

Hypoabelian group, first, generalised. Dickson, L. E. QJ. Mth. 30 (1899) 1-.

- groups. Dickson, L. E. N. Y. Am. Mth. S. Bll. 4 (1898) 495-.

Icosahedral group, construction. Bagnera, $G$. Palermo Cir. Mt. Rd. 11 (1897) 87-.

- substitutions, the sixty. Cayley, A. QJ. Mth. 27 (1895) 236-.

Imprimitive and soluble groups. Söderberg, J. T. Ups. S. Sc. N. Acta 20 (1904) No. 1, $26 \mathrm{pp}$. 
Imprimitive substitution groups of degree ten. Miller, G. A. N. Y. Am. Mth. S. Bll. 1 (1895) 67-.

Induced linear substitutions, groups. Rados, $G$. Mth. Termt. Éts. 17 (1899) 44-; Mth. Nt. B. Ung. 17 (1901) 227-.

Intransitive groups, construction. Bolza, $O$. Am. J. Mth. 11 (1889) 195-.

- linear substitution-groups. Maschke, H. Mth. A. 52 (1899) 363-.

- substitution groups of ten letters. Miller, G. A. QJ. Mth. 27 (1895) 99-

Invariance of factors of a substitution-group. Pierpont, J. Am. J. Mth. 18 (1896) 153-.

Invariant expression for Poincaré's substitutions. Brun, F. de. Stockh. Öfv. (1890) 265-; Fschr. Mth. (1890) 410.

Invariants attached to Klein's group 168. Boulanger, A. C. R. 130 (1900) 107-.

- of a quadrangle by projective transformation. Lovett, E. O. A. Mth. 12 (1898-99) 79-.

- relative to the Hessian group. Boulanger, -. C. R. 122 (1896) 178-

- of substitutions of a Kleinian group. Bagnera, $G$. Rm. R. Ac. Linc. Rd. 7 (1898) (Sem. 1) 340-.

- of ternary monomial groups. Skinner, E. B. [1900] N. Y. Am. Mth. S. Bll. 7 (1901) 244, 256.

Isomorphism, double, in group theory. Frattini, $G$. Rm. R. Ac. Linc. Rd. 2 (1893) (Sem. 1) 253-.

- of a group with itself. Burnside, W. L. Mth. S. P. 27 (1896) 354-.

- of groups of substitutions. Capelli, A. G. Mt. 16 (1878) 32-.

- of simple linear groups. Dickson, L. E. N. Y. Am. Mth. S. Bll. 6 (1900) 323-.

Isomorphisms, group of. Miller, G. A. N. Y. Am. Mth. S. Bll. 6 (1900) 337-.

-, groups of. Miller, G. A. C. R. 130 (1900) 316-.

-, - with same groups of. Miller, G. A. N. Y. Am. Mth. S. T. 1 (1900) 395-

- of $\left(G_{p}\right)^{3}, p$ a prime greater than 3, study of. Le Vavasseur, R. P. Par. Éc. Norm. A. 16 (1899) 377-.

- - a Hamiltonian group to itself, the simple. Miller, G. A. N. Y. Am. Mth. S. Bll. 5 (1899) 292-.

_ - - substitution group to itself, the simple. Miller, G. A. Ph. Mg. 45 (1898) 234-.

Kirkman's problem, application of theory of groups to. Burnside, $W$. Mess. Mth. 23 (1894) 137-.

Kleinian groups. Poincaré, H. C. R. 93 (1881) 44-; Acta Mth. 3 (1883) 49-.

- - with two variables. Pezzo, P. del. Nap. Rd. 32 (1893) 123-.

Klein's group of $(n+1) ! n$-ary collineations. Moore, E. H. Am. J. Mth. 22 (1900) 336-.

Kummer surface, birational transformations of. Hutchinson, J. I. [1900] N. Y. Am. Mth. S. Bll. 7 (1901) 211-.

Lagrange and theory of groups. Pierpont, $J$. N. Y. Am. Mth. S. Bll. 1 (1895) 196-.
Limit of transitivity of non-alternate groups. Jordan, C. (x) Par. S. Mth. Bll. 1 (1873) 40-; Liouv. J. Mth. 1 (1895) 35-.

Am. Mth. S. Bll. 4 (1898) 140-.

- - - substitution-groups. Bochert, $A$. Mth. A. 29 (1887) 27-; 33 (1889) 572-; 40 (1892) 176-.

Linear differential equations with algebraic integrals. Jordan, C. [1877] Crelle J. Mth. 84 (1878) 89-.

- group of Borchardt's moduli. Maschke, H. Gött. Nr. (1887) 421-; Mth. A. 30 (1887) 496.

- groups of finite order with three variables. Picard, É. $\quad$ Par. S. Mth. Bll. 15 (1887) 152-.

- - including the Abelian group. Dickson, L. E. QJ. Mth. 31 (1900) 60-

- - isomorphic with the simple group of order 25920. Dickson, L. E. C. R. 128 (1899) 873-.

- - as quotient groups. Dickson, L. E. N. Y. Am. Mth. S. T. 1 (1900) 30-.

- homogeneous group. Dickson, L. E. N. Y. Am. Mth. S. Bll. 5 (1899) 120 -

- - groups. Taber, H. N. Y. Am. Mth. S. Bll. 2 (1896) 336-; 3 (1897) 121.

- - - defined by an invariant. Dickson, L. E. Mth. A. 52 (1899) 560 .

- - in a Galois field, etc. Dickson, L. E. Am. J. Mth. 21 (1899) 193-.

- - substitutions on $m q$ variables, etc. Dickson, L. E. L. Mth. S. P. 30 (1899) 200-.

- substitution groups, coefficients. Maschke, H. Mth. A. 50 (1898) 492-.

- - - and quadratic forms of Dirichlet and Hermite. Bianchi, L. Rm. R. Ac. Linc. Rd. 7 (1891) (Sem. 2) 3-.

- substitutions applied to theory of groups. Laurent, H. N. A. Mth. 16 (1897) 149-.

Magic squares, application to group theory. Maillet, $E$. Toul. Ac. Sc. Mm. 6 (1894) 258-.

Metacyclic groups and neighbouring configurations. Heffter, L. Mth. A. 50 (1898) 261-. - _ _ _ _ - and triple systems. Heffer, L. D. Mth. Vr. Jbr. 5 (1901) (Heft 1) 67-.

Multiplication forms with application to theory of automorphic forms. Ritter, $E$. Mth. A. 44 (1894) 261-.

Non-isomorphic simple groups. Dickson, $L, E$. N. Y. Am. Mth. S. Bll. 6 (1900) 268, 276-.

Normal divisors of prime index of a finite group. Bagnera, G. Rm. R. Ac. Linc. Rd. 7 (1898) (Sem. 1) 63-.

Number of values a function can take by permutation of its letters. Cauchy, A. L.

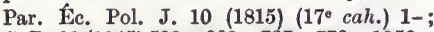
C. R. 21 (1845) 593-, 668-, 727-, 779-, 1356-.

- - _ - _ - _ _ _ - - Spitzer, S. [1848] Haidinger Ab. 3 (1850) $(A b .2)$ 43-. J. A. Liouv. J. Mth. 15 (1850) - 1-. Serret,

- - - - - Mathieu, E. [1858-59] Liouv. J. Mth. 5 (1860) 9-. 
Number of values a function can take by permutation of its letters. Jordan, C. Par. Éc. Pol. J. 22 (1861) (cah. 38) 113-.

- _ - - - Gosiewski, W. Par. T. Nauk Śc. Pam. $2\left(^{*} 1872\right) 1-$; (x) Bll. Sc. Mth. As. 6 (1874) 154-.

- - - - - - _ - - Bochert A. Mth. A. 33 (1889) 584-; 40 (1892) 157-.

E. Par. S. Mth. Bll. 22 (1894) 230-.

- - - - - - - - Bochert, A. Mth. A. 49 (1897) 113-.

- - of rational functions of given number of letters, etc. Spina, C. Rm. At. N. Linc. 21 (1868) 182-.

- variables in irreducible group. Molien, T. Dorpat Sb. 11 (1896) 277-

Octahedron, forms connected with the. Pittarelli, G. Rm. R. Ac. Linc. Rd. 4 (1888) (Sem. 1) 509-.

Operation groups. Cesàro, E. Rm. R. Ac. Linc. Rd. 2 (1886) (Sem. 2) 35-.

- - Le Vavasseur, R. P. C. R. 122 (1896) 180-, 516-, 711-.

- -. Miller, G. A. C. R. 128 (1899) 227-, 628.

- -, generation. Frattini, $G$. Rm. R. Ac. Linc. Rd. 1 (1885) 281-, 455-; 2 (1886) (Sem. 1) 16-.

- - of order $8 p, p$ being any prime number. Miller, G. A. Ph. Mg. 42 (1896) 195-.

- - - - $p^{\alpha}, p$ being any prime number. Miller, G. A. Mess. Mth. 27 (1898) 119-.

- - order less than 64 , and order $2 p^{3}$, $p$ being prime. Miller, G. A. QJ. Mth. 30 (1899) 243-.

- - , theorems on. Miller, G. A. [1896] N. Y. Am. Mth. S. Bll. 3 (1897) 111-.

- symbols involving Galois' imaginaries. Le Vavasseur, R. P. Toul. Ac. Sc. Mm. 9 (1897) 247-.

Operations, a group of. Brunel, G. Bordeaux S. Sc. PV. (1896-97) 30-.

- and substitutions, groups of. Maillet, $E$. As. Fr. C. R. (1897) (Pt. 2) 190-.

Orthogonal group in a Galois field. Dickson, $L$. $E$. N. Y. Am. Mth. S. Bll. 4 (1898) 196-.

- - groups derived from. Dickson, L. E. [1898-99] N. Y. Am. Mth. S. Bll. 4 (1898) 382-, 560; Calif. Ac. P. (Mth. Ps.) 1 (1903) 29-, 47-.

Perfect groups.' Miller, G. A. Am. J. Mth. 20 (1898) 277-.

Permutable substitutions. Baraniecki, M. A. Par. T. Nauk Śc. Pam. 7 (*1875) Art. 7, $35 \mathrm{pp}$.

Permutants, note. Bilenki, H. N. A. Mth. 19 (1900) 213-.

Permutation-groups of 8 things. Wiman, $A$. Gött. Nr. (1897) 55-.

Permutations, relation between number of alternate and quasi-alternate. André, $D$. Par. S. Phlm. Bll. 8 (1896) 5-

-, transformation and classification. Sprague, T. $B$. Edinb. Mth. S. P. 9 (1891) $59-$.
Position of inflection points in a plane cubic. Disteli, M. Zür. Vjschr. 35 (1890) 145-.

Possible types of plane quartics, multiply homological with themselves. Ciani, $E$. Palermo Cir. Mt. Rd. 13 (1899) 347-.

Primitive groups. Jordan, C. Liouv. J. Mth. 16 (1871) 283-; (x) Par. S. Mth. Bll. 1 (1873) 175-.

- - Rudio, F. Crelle J. Mth. 102 (1888) 1-. - of class $n-1$ and degree $n$. Maillet, $E$. Par. S. Mth. Bll. 25 (1897) 16-.

$-\ldots-1-2$ and degree $n$. Maillet, $E$. As. Fr. C. R. (1898) (Pt. 2) 157-.

-- classification. Jordan, C. C. R. 73 (1871) 853-.

- - containing a given substitation, limit of degree. Jordan, C. C. R. 78 (1874) 1217-; Crelle J. Mth. 79 (1875) 248-.

- - of degree less than 17, enumeration. Miller, G. A. C. R. 124 (1897) 1505-; 125 (1897) 673.

- - for first 17 degrees, enumeration. Jordan, C. C. R. 75 (1872) 1754-.

- - , holohedrically isomorphic with groups several times transitive. Maillet, $E$. C. R. 124 (1897) 351-; Liouv. J. Mth. 3 (1897) 277-.

- - mode of formation. Maillet, E. QJ. Mth. 27 (1895) 119-.

- substitution groups of degree 10,15 , and 16. Miller, G. A. L. Mth. S. P. 28 (1897) 533-; Am. J. Mth. 20 (1898) 229-; QJ. Mth. 31 (1900) 228-.

Primitivity of groups. Dyck, W. C. R. 96 (1883) 1024-

- and transitivity of discrete group. Dyck, $W$. [1881-82] Mth. A. 20 (1882) 1-;22 (1883) 70-.

Quartic of Klein, theorem concerning. Ciani, $E$. Mil. I. Lomb. Rd. 33 (1900) 565-.

Quaternary group of 51840 linear substitutions. Morrice, G. G. [1889-92] L. Mth. S. P. 21 (1891) 58-; 23 (1892) 213-.

- _ - - substitutions with ternary Hessian for sub-group. Maschke, $H$. Gött. Nr. (1888) 78-; Mth. A. 33 (1889) 317-. quadratic forms, and polyhedral groups. Bianchi, L. A. Mt. 21 (1893) 237-; 23 (1895) 1-; Rm. R. Ac. Linc. Rd. 3 (1894) (Sem. 1) 3-.

Quotient $G / H$ in the theory of groups, note on so-called. Cayley, A. Am. J. Mth. 15 (1893) 387-.

Rational functions, theory. Beke, $E$. Mth. A. 47 (1896) 441-.

Reduction of the Galois group of an equation by adjunction of arbitrary irrationalities. Bucca, F. Palermo Cir. Mt. Rd. 14 (1900) 122-.

Regular substitution groups of order less than 48. Miller, G. A. QJ. Mth. 28 (1896) 232-.

Representation, analytical, of substitutions. Polignac, C. de. Par. S. Mth. Bll. 9 (1881) 59-.

_, _ , - - for a prime number of elements. Grandi, A. G. Mt. 19 (1881) 238-

-,,$- \ldots-$, theorem generalised. Grandi, A. Mil. I. Lomb. Rd. 16 (1883) 101-. 
Resolvable groups. Frobenius, $G$. Berl. Ak. Sb. (1893) 337-; (1895) 1027-.

Simple group of order 504. Burnside, $W$. Mth. A. 52 (1899) 174-.

_ _ _ between 1092 and 2001, proof of non-existence. Ling, G. H., \& Miller, G. A. Am. J. Mth. 22 (1900) 13-.

- - 360 operations. Fricke, $R$. Gött. Nr. (1896) 199-.

_ _ - plane collineations. Gerbaldi, $F$. Palermo Cir. Mt. Rd. 12 (1898) 23-; 13 (1899) 161-; 14 (1900) 66-.

- - - - homographies. Wiman, $A$. Mth. A. 47 (1896) 531-.

- - 504 operations. Fricke, R. Mth. A. 52 (1899) 321-.

- groups, doubly infinite system of. Moore, E. H. [1893] N. Y. Mth. S. Bll. $3(1894)$ 73-.

- - as far as order 660. Cole, E. N. Am. J. Mth. 15 (1893) 303-.

- - of order less than 200. Hölder, $O$. Mth. A. 40 (1892) 55-.

- - from order 201 to order 500. Cole, E. N. Am. J. Mth. 14 (1892) 378-.

- -, several classes. Miller, G. A. [1899] L. Mth. S. P. 31 (1900) 148-.

- -, some. Miller, G. A. Par. S. Mth. Bll. 28 (1900) 266-.

_ - sub-groups of doubly infinite series. Wiman, A. Stockh. Ak. Hndl. Bh. 25 (1900) (Afd. 1) No. 2, $47 \mathrm{pp}$.

- - triply infinite system. Dickson, L. E. QJ. Mth. 29 (1898) 169-; 30 (1899) 383-.

- linear groups of order 25920, etc. Dickson, L. E. [1899] L. Mth. S. P. 31 (1900) 30 -

- transitive groups, note on. Miller, G. A. [1899] N. Y. Am. Mth. S. Bll. 6 (1900) 103-.

600-cell hyper-polyhedron and its self-corresponding displacements. Os8, S. L. van. [1899] Amst. Ak. Vh. (Sect. 1) 7 (1901) No. 1, 18 pp. ; Nt. 61 (1899-1900) 304.

Space, division by linear transformation groups. Dyck, $W$. Leip. Mth. Ps. B. 35 (1883) 61-.

Special sub-groups of class one. Fricke, $R$. Mth. A. 30 (1887) 345-.

Sub-groups of the Betti-Mathieu group. Dickson, L. E. Am. J. Mth. 22 (1900) 49-.

- - 11th class in the modular group. Rougier, J. Mars. Fac. Sc. A. 6 (1897) Fasc. 1, 112 pp.

Substitution groups. Miller, $G$. A. C. R. 122 (1896) 370-; 123 (1896) 91-, 204.

- -. Maillet, E. Par. S. Mth. Bll. 24 (1896) 85-.

- - composition. Capelli, A. Rm. R. Ac. Linc. Mm. 19 (1884) 262-.

- whose degree does not exceed eight. Miller, G. A. Am. J. Mth. 21 (1899) 287-.

- - general solution of problem of. Hoyer, $P$. Mth. A. 52 (1899) 550-

- - of given order. Maillet, E. Toul. Fac. Sc. A. 9 (1895) D, 22 pp.; 10 (1896) A, 20 pp.

- - with integral coefficients. Viterbi, $A$. G. Mt. 36 (1898) 346-.
Substitution groups whose order $=$ degree. Le Vavasseur, R. P. C. R. 120 (1895) 822-, 899-, 1206-; 121 (1895) 238-.

- - - is 4. Miller, G. A. Ph. Mg. 41 (1896) 431-.

- - - the product of two primes. Miller, G. A. N. Y. Am. Mth. S. Bll. 2 (1896) 332-.

- - producible from given number of elements, list of all. Miller, G.A. N. Y. Am. Mth. S. Bll. 2 (1896) 138-.

- - theorems on. Sylow, L. Mth. A. 5 (1872) 584-.

- - theory of. Netto, E. Crelle J. Mth. 103 (1888) 321-.

$--\frac{-}{-}$, two laws in. Hoyer, B. Mth. A. 46 (1895) 539-.

- for 2, 3, 4, 5, 6, 7 and 8 letters. Cayley, A. QJ. Mth. 25 (1891) 71-, 137-. - - 3, 4, 5, 6 and 7 letters. Askwith, $E$. $H$. QJ. Mth. 24 (1890) 111-.

- of 6, 7 and 8 letters. Cole, F. N. N. Y. Mth. S. Bll. 2 (1893) 184-; 3 (1894) 169. - - 8 letters. Askwith, E. H. QJ. Mth. 24 (1890) 263-.

S. Bll. 3 (1894) 168-.

- - - and 9 letters. Miller, G. A. N. Y. Mth. S. Bll. 3 (1894) 242-.

- - - 9 letters. Askwith, (Rev.) E. H. QJ. Mth. 26 (1893) 79-.

--1 , list. Cole, F. N. QJ. Mth. 26 (1893) 372-.

Substitutions of form

$$
\theta(r) \equiv \epsilon\left(r^{n-2}+a r^{\frac{n-3}{2}}\right),
$$

$n$ being prime. Brioschi, F. Gött. Nr. (1869) 491-.

- $n$ letters which leave $k$ letters unaltered, number of. Durfee, W. P. J. H. Un. Cir. [4] (1885) 23.

$--\ldots$, particularly for $n=3,4,5,6$, in relation to groups of Pascal's hexagram, geometrical interpretation. Veronese, $G$. A. Mt. 11 (1882-83) 93-.

- on $p^{n}$ letters where $p$ is prime. Dickson, L. E. A. Mth. 11 (1896-97) 65-, 161-.

- represented by transpositions. Caldarera, G. Catania Ac. Gioen. At. 9 (1896) Mm. 7, $16 \mathrm{pp}$.

一, theorem in. Cayley, A. C. R. 67 (1868) $784-$. -, - -. Frattini, G. [1884] Rm. R. Ac. Line. Mm. 18 (1883) 487-.

- - - Giudice, $F$. Palermo Cir. Mt. Rd. 1 (1887) 222-.

-, theorems, general. Jordan, C. C. R. 66 (1868) 836-.

-, theory. Betti, E. Tortolini A. 6 (1855) 5-.

-, -. Bachmann, P. Liouv. J. Mth. 10 (1865) 209-.

-, -. Janni, G. G. Mt. 9 (1871) 280-; 10 (1872) 193-; 11 (1873) 1-, 71-, 257-.

-, -. Jordan, C. ' G. Mt. 10 (1872) 116; Liouv. J. Mth. 17 (1872) 351-; C. R. 76 (1873) 952-.

-, - , and applications. Netto, E. Arch. Mth. Ps. 62 (1878) 225-. 


\section{Discrete Groups}

Substitutions, theory, fundamental theorem, new proof. Netto, E. Mth. A. 13 (1878) 249-. - - (Netto). Miller, G. A. A. Mth. 1 (1900) 71-.

, - primitive groups of, etc. Jordan, $C$. C. R. 79 (1874) 1149-

Surfaces admitting an infinite discontinuous group of transformations. Painlevé, P. C.R. 126 (1898) 512-.

Sylow's theorem. Frobenius, $G$. Crelle J. Mth. 100 (1887) 179-.

- - application to symmetric group. Radcig, A. A. Kharkov Mth. S. Com. 5 [1896?] 1-.

- , extension. Miller, G. A. N. Y. Am. Mth. S. Bll. 4 (1898) 323-.

- - generalisation. Frobenius, G. Berl. Ak. Sb. (1895) 981-.

- theorems on groups, illustration. Cayley, A. Mess. Mth. 23 (1894) 59-.

Symmetric and alternant homographic groups. Maschke, $H$. Mth. A. 51 (1899) 253-

- - - -. Wiman, A. Mth. A. 52 (1899) 243-.

- - - permutation-groups of $n$ things.

Wiman, A. Gött. Nr. (1897) 191-.

- group, note. Burnside, W. [1896] L. Mth. S. P. 28 (1897) 119-.

System of linear forms, theorem on. Frattini, G. Rm. R. Ac. Linc. Rd. 2 (1886) (Sem. 1) 132-.

Systems of groups, fundamental theorem relative to two. Cauchy, A. L. C. R. 22 (1846) 630-.

Tactic disclosing existence of 4-valued function. Sylvester, J. J. Ph. Mg. 21 (1861) 515-.

Ternary substitution-groups of finite order, which leave a triangle unchanged. Maschke, H. Am. J. Mth. 17 (1895) 168-.

Theta-fuchsian functions. Ovidio, $E$. $d$ '. Tor. Ac. Sc. At. 29 (1894) 741-.

Transitive functions, reduction to intransitive. Cauchy, A. L. C. R. 21 (1845) 1199-.

- group, G120 of six letters, invariant functions. Dziobek, O. Arch. Mth. Ps. 68 (1882) 225-.

Transitive groups. Maillet, $E$. A. Mt. 25 (1897) 219-.

- - of degree $n$ and class $n-1$. Maillet, $E$. Par. S. Mth. Bll. 26 (1898) 249-

- - whose degree is the square of a prime. Sylow, L. Acta Mth. 11 (1887-88) 201-.

- - isomorphic to alternating group of six. Miller, G. A. Am. Ph. S. P. 36 (1897) 208-.

- - proofs and theorems. Netto, E. Crelle J. Mth. 83 (1877) 43-.

- substitution groups. Bochert, A. Mth. A. 40 (1892) 176-; 49 (1897) 133-.

- - -. Miller, G. A. Mess. Mth. 28 (1899) 84-

- - - of degree 12. Miller, G. A. N. Y. Am. Mth. S. Bll. 1 (1895) 255-.

$----1-$, list. Miller, G. A. QJ. Mth. 28 (1896) 193-.

- - - - 13 and 14. Miller, G. A. QJ. Mth. 29 (1898) 224-.

- - - - 17. Miller, G. A. QJ. Mth. 31 (1900) 49-.

- - - isomorphic to a given group. Miller, G. A. G. Mt. 38 (1900) 63-.
Continuous Groups 1230

Transitive substitution groups of nine letters. Cole, F. N. N. Y. Mth. S. Bll. 2 (1893) 250-.

- - - 10 and of 11 letters, list. Cole, F. N. QJ. Mth. 27 (1895) 39-.

- - 24 letters, etc. Wilkinson, A. C.L. QJ. Mth. 30 (1899) 157-.

- - - whose order is the cube of any prime: the non-regular. Miller, G. A. A. Mth. 10 (1895-96) 156-.

- - - - - product of three primes: the non-regular. Miller, G.A. Zür. Vjschr. 42 (1897) 68-.

- - of order $8 p, p$ being any prime. Miller, G. A. Ph. Mg. 43 (1897) 117-.

- - whose orders are products of three primes. Miller, G. A. N. Y. Am. Mth. S. Bll. 3 (1897) 213-.

- - - of same order and degree. Frattini, G. Rm. R. Ac. Linc. At. 14 (1883) 143-.

Triple systems. Moore, E. $H$. Mth. A. 43 (1893) 271-; Palermo Cir Mt. Rd. 9 (1895) 86.

- - Heffter, L. Mth. A. 49 (1897) 101-。 - Moore, E. H. N. Y. Am. Mth. S. Bll. 4 (1898) 11-.

- -, theory. Netto, E. Mth. A. 42 (1893) $143-$.

- - - Vries, J. de. Palermo Cir. Mt. Rd. 8 (1894) 222-.

- - , transitive. Moore, E. H. Mth. A. 50 (1898) 225-.

- tangent planes of cubic surface, proof of Cayley's theorem. Kohn, G. Mh. Mth. Ps. 2 (1891) 343-.

Two groups in five dimension space. Engel, $\boldsymbol{F}$. D. Mth. Vr. Jbr. 8 (1900) (Heft 1) 196-.

Uniform group and linear transformation. Kneser, A. Mth. A. 28 (1887) 125-

Universal invariant for finite groups of linear substitutions. Moore, E. H. Mth. A. 50 (1898) 213-.

\section{Continuous groups of finite and of infinite order.}

Algebraic groups, certain. Cartan, E. C. R. 120 (1895) 544-.

- - of straight lines and planes. Carda, $K$. Mh. Mth. Ps. 11 (1900) 31-.

- surfaces admitting continuous transitive group of transformations. Fano, $G$. Palermo Cir. Mt. Rd. 10 (1896) 1-.

- - with continuous group of birational transformations into themselves. Castelnuovo, $G$., \& Enriques, F. C. R. 121 (1895) 242-.

- - - - - - Painlevé, $P$. C. R. 121 (1895) 318-

- - - infinite automorphic transformations. Enriques, $F$. Ven. I. At. (1892-93) 1590-; (1893-94) 638-.

Fano, G. Rm. R. Ac. Linc. Rd. 4 (1895) (Sem. 1) 149-; Palermo Cir. Mt. Rd. 11 (1897) 240-

Canonical form, reduction of finite and continuous group of transformations to. Cartan, E. Am. J. Mth. 18 (1896) 1-. 
Canonical form, reduction of group of trans. formations to. Lie, S. Leip. Mth. Ps. B. 41 (1889) 277-.

Complex numbers and groups of transformations. Study, E. Leip. Mth. Ps. B. 41 (1889) 177.

- - , systems of, and transformation groups. Study, E. Mh. Mth. Ps. 1 (1890) 283-.

Conformal representation; Poincaré's theory; substitution groups. Brun, F. de. Stockh. Öfv. (1890) 135-; Fschr. Mth. (1890) 410.

Contact transformations, finite groups contained in the group of linear. Automne, $L$. C. R. 102 (1886) 313-.

$-\ldots$, irreducible groups. Lie, S. Leip. Mth. Ps. B. 41 (1889) 320-

- - theory. Lie, M. S. Arch. Mth. Ntvd. 1 (1876) 194-.

Continuity of infinitesimal transformation groups. Slocum, S. E. Am. Ac. P. 36 (1901) 83-.

Continuous binary linearoid groups, and corresponding differential equations. Wilczynski, E. J. Am. J. Mth. 22 (1900) 191-.

- and discontinuous groups, correspondence between. Bettazzi, R. A. Mt. 16 (1888-89) 49-.

- group of Darboux rotations. Souslow, G. D. Nf. Vh. (1895) (Th. 2, Hälfte 1) 12-.

- - defined by any given group of finite order. Burnside, $W$. L. Mth. S. P. 29 (1898) 207-, 546-.

- of Jonquières' transformations in a plane. Enriques, $F$. Rm. R. Ac. Linc. Rd. 2 (1893) (Sem. 1) 532-.

- in three dimensions. Bianchi, L. Rm. S. It. Mm. 11 (1898) 267-.

- - of transformations with 28 parameters in theory of deformation of surfaces. Staeckel, $P$. C. R. 121 (1895) 396-.

- groups. Lie, S. Christiania F. (1884) No. 8,4 pp., No. 9, 4 pp.; (1885) No. 15, $4 \mathrm{pp}$.

- -. Poincaré, H. C. R. 128 (1899) 1065-; Camb. Ph. S. T. 18 (1900) 220-

- - application to singular points of differential equations. Marotte, $F$. C. R. 123 (1896) 867-.

- - - - - theory of functions. Lie, S. C. R. 114 (1892) 334-. 118 (1894) $\frac{-1}{845-}-$ Painlevé, P. C. R.

- - and differential equations with fixed critical points. Painlevé, P. C. R. 130 (1900) 1171-.

- - Lie's, application. Autonne, L. C. R. 112 (1891) 570-.

- -, - theory. Lovett, E. O. [1897] N. Y. Am. Mth. S. Bll. 4 (1898) 59-.

- - - - - Slocum, S. E. Am. Ac. P. 35 (1900) 237-, 481-.

,$---\ldots$. Rettger, E. W. Am. J. Mth. 22 (1900) 60-

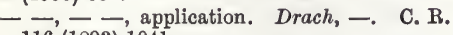
116 (1893) 1041-.

-- , problems in theory. Bouton, C. L. A. Mth. 1 (1900) 93-.
Continuous groups of projective transformations. Newson, H. B. [1895-96] Kan. Un. Q. 4 (1896) 71-, 169-, 243-; 5 (1896) 81-.

- - - quadratic transformations of the plane. Bohlmann, G. Gött. Nr. (1896) 44-.

- - in space of $n$ dimensions, determination. Medolaghi, $P$. Rm. R. Ac. Linc. Rd. 8 (1899) (Sem. 1) 291-.

- - , theorem in. Koch, H. von. Stockh. Öfv. (1894) 311-.

- -, theory. Campbell, J. E. N. Y. Am. Mth. S. Bll. 4 (1898) 407-.

- imprimitive groups of point transformations. Fano, G. Rm. R. Ac. Linc. Rd. 7 (1898) (Sem. 1) 302-.

Cremona cubic group, finite groups. Autonne, L. C. R. 101 (1885) 53-; 105 (1887) 267-.

- group with any number of variables, complete groups contained in a. Del Re, A. Rm. R. Ac. Linc. Rd. 6 (1890) (Sem. 2) 271-.

- - finite groups in. I. Quadratic groups. II. Cubic groups. Autonne, L. Liouv. J. Mth. 1 (1885) 431-; 2 (1886) 49-.

- quadratic group, finite groups in. Autonne, L. C. R. 98 (1884) 565-; Liouv. J. Mth. 4 (1888) 177-, 407-.

- - - irreducible groups of finite order in. Autonne, L. C. R. 103 (1886) 1176-.

- - groups. Autonne, L. C. R. 104 (1887) 1422-.

- transformations, continuous groups of. Noether, M. D. Nf. Vh. (1896) (Th. 2, Hälfte 1) 11-.

,$---\frac{1}{-}$ - Fano, G. Mh. Mth. Ps. 9 (1898) 17-.

- - in plane, continuous groups of. Enriques, $F$. Rm. R. Ac. Linc. Rd. 2 (1893) (Sem. 1) 468-

- - - - - _ _ - , and certain projective transformation groups. Fano, G. Palermo Cir. Mt. Rd. 10 (1896) 16-.

- - of space, continuous primitive groups of. Fano, G. Tor. Ac. Sc. At. 33 (1897) 284-or 480 -.

Differential equations admitting finite groups. Lie, S. Mth. A. 25 (1885) 71-.

- - - a group of transformations. Lie, S. Arch. Mth. Ntvd. 9 (1884) 431-.

- - - infinitesimal transformations. Paczonoski, J. Prace Mt.-Fiz. 7 (1896) 178-.

- - , algebraie and group theory. Loewy, A. Mh. Mth. Ps. 8 (1897) 225-.

- - which possess fundamental integrals. Lie, S. Leip. Mth. Ps. B. 45 (1893) 341-.

invariants of equation of 2 nd order. Tresse, A. Leip. Jablon. Preisschr. 32 (1896) $87 \mathrm{pp}$.

Dynamical problems, whose solutions admit of infinitesimal transformation. Stäckel, $P$. Leip. Mth. Ps. B. 45 (1893) 331-.

Dynamics, differential equations of, application of Lie's transformation groups. Stäckel, $P$. Leip. Mth. Ps. B. 49 (1897) 411-. 
Finite continuous group of transformations, investigation of the finite equations of a. Vessiot, $E$. Toul. Fac. Sc. A. 10 (1896) C, $26 \mathrm{pp}$.

- - groups. Autonne, L. Liouv. J. Mth. 3 (1887) 63-.

_ - - complete systems and differential invariants of. Lindelöf, $E$. Helsingf. Acta 20 (1895) No. 1, $62 \mathrm{pp}$.

- - - determination of equations. Vessiot, E. C. R. 120 (1895) 77-.

- - - of Lie's transformations, decomposition. Maillet, E. C. R. 130 (1900) 1536-.

- - - the plane. Williams, F. B. Am. Ac. P. 35 (1900) 95-.

- - , structure. Cartan, -. C. R. 116 (1893) 962-.

- - of transformations. Engel, $F$. Leip. Mth. Ps. B. 38 (1886) 83-.

- primitive groups of Lie's transformations. Maillet, E. C. R. 130 (1900) 1602-.

- transformations of a projective group, generated by the infinitesimal transformations of the group. Engel, $F$. Leip. Mth. Ps. B. 44 (1892) 279-; 45 (1893) 659-.

General projective group, case of degeneration. Engel, $F$. C. R. 118 (1894) 397-.

Geometry of functional space. Pincherle, $S$. Bologna Rd. 1 (1897) 85-.

Greatest continuous groups of certain systems of differential equations, finite character. Engel, $F$. Leip. Mth. Ps. B. 46 (1894) 297-.

Group and invariant, the conceptions. Lie, S. Leip. Mth. Ps. B. 39 (1887) 83-

- of parameters, canonical form. Schur, F. Leip. Mth. Ps. B. 41 (1889) 229-.

- theory applied to hydrodynamics. Wilczyn$s k i, E . J . \quad$ N. Y. Am. Mth. S. T. 1 (1900) $339-, 509$.

- - for equations of the 2nd degree with $n$ variables. Werner, $H$. Mth. A. 35 (1890) 113-.

- - geometrical treatment. Paolis, R. de. Rm. S. It. Mm. 7 (1890) No. 6, $164 \mathrm{pp}$.

- - minor contributions. Engel, $F$. Leip. Mth. Ps. B. 39 (1887) 89-; 43 (1891) 47-, 308-, 585-; 44 (1892) 43-, 292-; 45 (1893) 360-; 46 (1894) 25-.

- - recent investigations. Lie, S. Leip. Mth. Ps. B. 44 (1892) 297-.

Groups and algebraic surfaces. Picard, $\hat{E}$. Palermo Cir. Mt. Rd. 9 (1895) 244-.

- appearing in the theory of analytical functions. Medolaghi, $P$. C. R. 126 (1898) 1188-.

- of circular transformations. Newson, H. B. [1897] N. Y. Am. Mth. S. Bll. 4 (1898) 107-.

- isomorphous to the group of all the transformations of one variable. Medolaghi, $P$. Palermo Cir. Mt. Rd. 12 (1898) 188 -

Hyperfuchsian groups. Poincaré, H. C. R. 98 (1884) 503-.

Infinite continuous groups. Lie, M. S. Christiania F. (1883) No. 12, 56 pp.; Leip. Mth. Ps. Ab. 21 (1895) 43-.
Infinite continuous groups, theory. Medolaghi, P. A. Mt. 25 (1897) 179-.

- - - of transformations, foundations of theory. Lie, S. Leip. Mth. Ps. B. 43 (1891) 316-, 353-.

- groups, theorem concerning. Lie, S. Christiania F. (1889) No. 7, 6 pp.

- - of transformations. Tresse, A. C. R. 115 (1892) 1003-

- - _ - , and integration of differential equations. Beudon, J. C. R. 125 (1897) 811-.

- ternary automorphic transformations, theorem on. Fano, G. Rm. R. Ac. Linc. Rd. 8 (1899) (Sem. 1) 562-.

Infinitesimal automorphic transformations of a Pfaffian equation. Vivanti, G. Palermo Cir. Mt. Rd. 12 (1898) 1-.

- - - - Weber, E. von. Palermo Cir. Mt. Rd. 12 (1898) 133-.

- groups, singular transformations. Taber, $H$. Am. Ac. P. 35 (1900) 575-.

- transformations of all transitive finite groups, given by quotients of power series. Schur, $F$. Leip. Mth. Ps. B. 42 (1890) 1 -.

Integral invariants of continuous transformation groups. Źoravski, $K$. Krk. Ak. (Mt.Prz.) Rz. 8 (1895) 232-; Crc. Ac. Sc. Bll. (1895) 127-.

Integrals of canonical system, group interpretation. Levi-Civita, T. Rm. R. Ac. Linc. Rd. 8 (1899) (Sem. 2) 235-.

Invariant theory of group of motions. Lie, $S$. Leip. Mth. Ps. B. 48 (1896) 466-.

Invariants of conics and quadric surfaces given by motion and similitude groups. Lovett, E. O. A. Mth. 11 (1896-97) 33-.

Isomorphic groups of substitutions with symmetric or alternate groups. Maillet, -. C. R. 119 (1894) 362-.

Jonquières groups, generalised. Fano, $G$. [1898] Tor. Ac. Sc. Mm. 48 (1899) 221-.

Kummer's configuration, and orthogonal substitutions. Study, E. Leip. Mth. Ps. B. 44 (1892) 122-.

Linear differential equations. Lie, S. Christiania F. (1885) No. 21, 4 pp.

$--\ldots$, groups of transformations. Picard, $\dot{E}$. C. R. 119 (1894) 584-.

- equations, groups. Poincaré, H. Acta Mth. 4 (1884) 201-.

- homogeneous continuous groups. Maurer, L. Münch. Ak. Sb. 24 (1895) 297-.

- - - whose operations are permutable. Burnside, W. L. Mth. S. P. 29 (1898) 325-. - group with invariant Pfaftian. Dickson, $L$. E. N. Y. Am. Mth. S. Bll. 5 (1899) 338-.

- partial differential equations with continuous transformation group. Campbell, J.E. N.Y. Am. Mth. S. T. 1 (1900) 243-, 509.

Monomial group associated with an infinitesimal projective transformation. Torelli, G. $\mathrm{Pa}$ lermo Cir. Mt. Rd. 8 (1894) 41-.

Ordinary differential equations which have fundamental systems of integrals. Lie, S. C. R. 116 (1893) 1233-. 
$m n^{2}$ Parameter group in $m n$ variables, Wil. czynski, E. J. [1899] Calif. Ac. P. (Mth.-Ps.) 1 (1903) 59-.

Parametric groups in the theory of linear substitutions. Maillet, E. A. Mt. 23 (1895) 199-.

Partial differential equations of 1st order, singularities. Marotte, F. C. R. 123 (1896) 933-.

- - - - - with two independent variables, admitting a continuous group of transformations. Tannenberg, $W$. de. Toul. Fac. Sc. A. 5 (1891) B, 150 pp.

Point-aggregate, doubly extended, determination of all groups of. Lie, M. S. Arch. Mth. Ntvd. 3 (1878) 93-.

Primitive transformation groups in five variables. Kowalewski, $G$. Leip. Mth. Ps. B. 51 (1899) (Mth.) 69-

Projective group. Rettger, E. W. Am. Ac. P. 33 (1898) 491-.

Properties of groups of substitutions of given order. Maillet, E. C. R. 118 (1894) 1187-.

Simple finite continuous groups, structure. Cartan, -. C. R. 116 (1893) 784-.

- group with 14 parameters. Engel, $F$. C. R. 116 (1893) 786-.

Simply infinite continuous projective groups in ordinary space. Pittarelli, $G$. A. Mt. 22 (1894) 261-

Simultaneous differential equations with continuous transformation groups. Zindler, $K$. Mh. Mth. Ps. 11 (1900) 289-.

Structure of a group, reduction to canonical form. Cartan, E. C. R. 119 (1894) 639-.

Sub-groups of a group of Lie's transformations. Maillet, E. C. R. 130 (1900) 1449-.

Substitutions, groups of, special class. Ahrens, W. Leip. Mth. Ps. B. 49 (1897) 616-.

Systems of partial differential equations defining a group. Medolaghi, $P$. Rm. R. Ac. Linc. Rd. 6 (1897) (Sem. 1) 575-.

Transformation, general theory. Lie, S. Leip. Mth. Ps. B. 40 (1888) 14-

- groups. Lie, M. S. Gött. Nr. (1874) 529-.

- - Page, J. M. A. Mth. 8 (1893-94) 117-.

- applied to ordinary differential equations. Page, J. M. A. Mth. 9 (1894-95) 59-.

- - determination of all transitive, with $r$ terms. Lie, S. Leip. Mth. Ps. B. 42 (1890) 478-.

- - new proof of fundamental theorem in theory of. Lie, S. Leip. Mth. Ps. B. 42 (1890) 453-.

- - researches. Lie, S. Arch. Mth. Ntvd. 10 (1886) 74-, 353-.

- - simple. Cartan, E. Leip. Mth. Ps. B. 45 (1893) 395-.

- in space of four dimensions. Page, J.M. A. Mth. 9 (1894-95) 11-.

Westfall, J. V. N. Y. Am. Mth. S. Bll. 6 (1900) 185, 191-:

- , theory. Lie, M. S. Arch. Mth. Ntvd. 1 (1876) 19-, 152-; 3 (1878) 93-, 375-; 4 (1879) 232-; 9 (1884) 449-; Mth. A. 16 (1880) 441-; Christiania F. (1888) No. 13, 8 pp.; Leip. Mth. Ps. B. 46 (1894) 322-.
Types of line-sphere transformation. Lovett, E. O. $[1900]$ N. Y. Am. Mth. S. Bll. 7 (1901) 203, 209-.

\section{ALGEBRA AND THEORY OF NUMBERS.}

\section{General.}

Algebra, pure and applied, exercises. Piani, $D$. [1846-47] Bologna N. Cm. 10 (1849) 571-.

Algebraic keys. Cauchy, A. L. C. R. 36 (1853) 70-, 129-, 161-.

- - employment of differentials and variations. Cauchy, A. L. C. R. 37 (1853) 38-, 57-.

Interpretation of algebraic results. Parker, W. H. Camb. (M.) Mth. M. 1 (1859) 160

- - - (Parker). Runkle, J. D. Camb.

(M.) Mth. M. 1 (1859) 178-.

\section{Elements of Algebra.}

\section{General.}

Algebra, elementary developments. Finlay, $R$. Mathematician 3 (1850) 291-.

-, - , theorem. Marcolongo, $R$. G. Mt. 25 (1887) 174-.

-, elements (Lacroix). Oken, L. Oken Isis (1825) 1153-.

- foundation. De Morgan, A. [1839-44] Camb. Ph. S. T. 7 (1842) 173-, 287-; 8 (1849) 139-, 241-.

- foundations. Sexe, S. A. N. Mg. Ntvd. (1866) 1-.

- - - supplementary to Cauchy. Bellavitis, G. A. Sc. Lomb. Ven. 1 (1831) 271-.

-, fundamental notions. Ballauff, L. Grunert Arch. 5 (1844) 259-.

-, laws. De Morgan, A. (Ix) [1853] Camb. Ph. S. P. 1 (1866) 123-.

-, principles. Paque, A.J.N. (vIII) Liége Mm. S. Sc. 18 (1863) 93-.

-, 一, first. Schultén, N. G. af ( $f i l)$. Helsingf. Acta 1 (1840) 395-.

-, - _, - Moigno, $F$. Les Mondes 3 (1863) 254-, 269-, 318-, 358-, 605-, 635-.

-, -, fundamental. 'Schultén, N. G. af (fil).

[1839] Helsingf. Acta 1 (1840) 31-.

-, 一, - Petersen, $J$. Ts. Mth. 3 (1885) 1-; Fschr. Mth. (1885) 123.

Algebraic analysis and the factorial interpretation of powers. Capelli, A. G. Mt. 31 (1893) 291-, 340-; 33 (1895) 361-.

- calculation. Franchini, $P$. Lucca At. Ac. 2 (1823) 193-.

- -, exercises. Realis, S. N. A. Mth. 20 (1881) 501-.

- formulae treated geometrically. Lucas, $\hat{E}$. As. Fr. C. R. (1885) (Pt. 1) 94 .

- and goniometric identities, development of some. Berg, F. J. van den. Amst. Ak. Vs. M. 14 (1879) 340 - 


\section{Algebra. General}

Algebraic quantities, principles of an arithmetical theory of. Kromecker, L. [1881] Crelle J. Mth. 92 (1882) 1-.

Algorithm $[a b c \ldots l]^{(n)}$. Ocagne, $M . d^{\prime}$. N. A. Mth. 5 (1886) 257-.

- of arithmetical means of products of $k$ numbers, taken $r$ at a time. Stieltjes, T. $J$. (jun.). Crelle J. Mth. 89 (1880) 343-.

-, elementary, theory. Simões Margiochi, $F$. Lisb. Ac. Sc. Mm. (Cor.) 3 (pte. 2) (1814) 27-.

- for geometric mean of $\boldsymbol{n}$ quantities. Stieltjes, T. J. (jun.) N. Arch. Wisk. $9\left({ }^{*} 1882\right)$ 198-.

Algorithms of algebra. Séguin, (â̂né). Moigno An. Cosmos (1861) 379-.

- and calculi. Schroeder, E. Arch. Mth. Ps. 5 (1887) 225-, vii-.

Approximations, algebraic. Hermite, $C$. Crelle J. 76 (1873) $342-$

-, calculation (in inserting geometric means). Duterme, - N. A. Mth. 4 (1845) 124-

Arithmetical progressions, new theorem. Jof froy, $J$. N. A. Mth. 8 (1889) 85-.

Decomposition of fractions, problem. Jacobi, C. G.J. Crelle J. 5 (1830) 344-.

Development in ascending powers of $x$ of

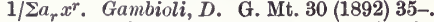

- of numerical quantities analogous to that by continued fractions. Cahen, E. N. A. Mth. 10 (1891) 508-.

$-\left(1+n^{2} x\right)^{\frac{m}{n}}$. Cayley, A. Mess. Mth. 22 (1893) 186-.

Developments of algebraic products. Laisant, C. A. As. Fr. C. R. (1881) 84-.

Equalities in two or three degrees. Frolov, $M$. Par. S. Mth. Bll. 17 (1889) 69-; 20 (1892) $69-$.

Exponents of any order, theory. Terquem, $O$. N. A. Mth. 6 (1847) 106-.

-, fractional. Almqvist, $P . W$. Ts. Mt. Fys. 1 (1868) 264-.

-, laws. Clausen, T. Crelle J. 7 (1831) 234-.

Fraction $\frac{p}{q}$, expression as sum of aliquot parts of unity. Collignon, É. [1881] Par. S. Phlm. Bll. 6 (1882) 88-.

Harmonic relations. Hultman, F. W. Ts. Mt. Fys. 4 (1871) 153-.

Harmony, algebra of. Loquin, A. Bordeaux Ac. Act. 43 (1883) (Suppl.) 1-.

Identities. Hunyady, E. von. Z. Mth. Ps. 12 (1867) 89-.

Identity, algebraic. MacMahon, (Capt.) P.A. Mess. Mth. 13 (1884) 142-.

- connected with theory of parallels. Mal'tsev, M. M. (xII) [1883] Kazan S. Nt. (P8.Mth.) P. 2 (1884) 8.

Index law. Hultman, F.W. Ts. Mt. Fys. 3 (1870) 250-.

Indices, theory. Sexe, S. A. N. Mg. Ntvd. 13 (1864) 155-

-, - Faure, H. N. A. Mth. 15 (1876) 251-, 292-, 339-, 451-, 481-, 529-; 16 (1877) 5-, 160-, 193-, 249-, 289-, 467-, 508-, 541-; 17 (1878) 69-
Algebra. General 1600

Infinity and sign of equality. De Morgan, $A$. Camb. Ph. S. T. 11 (Pt. 1) (1866) 145-.

Limits of the expression $\left(x^{p}-y^{p}\right) /\left(x^{q}-y^{q}\right)$. Knight, $S . R$. Mess. Mth. 22 (1893) 165 -.

(1894) $47-$.

Maximum of product of positive numbers whose sum is given. Darboux, G. Bll. Sc. Mth. 11 (1887) 149-.

- value of $x y$ when $x+y$ is given. Steiner, $J$. Crelle J. 40 (1850) 208.

- - - - - O - Oettinger, L. Crelle J. 57 (1860) 90-.

Mean, arithmetic, error due to its substitution for geometric mean. Dostor, G. Arch. Mth. Ps. 63 (1879) 220.

-, - and geometric. Liouville, $J$. Liouv. J. Mth. 4 (1839) 493-.

- , _ - , comparison. Hurwitz, A. Crelle J. Mth. 108 (1891) 266-.

-,$\ldots$ - - difference, and theorem of integral calculus. Unferdinger, $F$. Wien $\mathrm{Sb}$. $56(1867)(A b .2) 272-$.

-, - greater than geometric: mean of $m$ numbers (Cauchy). Transon, A. N. A. Mth. 11 (1872) 257-.

,,----7 , proof. Thacker, A. Camb. and Dubl. Mth. J. 6 (1851) 81-.

_, _, - - - - Tait, P. G. [1868] Edinb. R. S. P. 6 (1869) 309.

-, arithmetico-geometric, of 4 elements. Borchardt, C. W. Berl. Ak. Mb. (1876) 611-.

Means, arithmetic. Lobatto, R. (vm) Quetelet Cor. Mth. 4 (1828) 169-, 233-.

-, - , geometric and harmonic. Brändli, $H$. Bern Mt. (1849) 178-.

- , - - - , comparison. Schlömilch, O. Schlömilch Z. 3 (1858) 187-.

,,-- of powers of positive quantities. Bienaymé, J. Par. S. Phlm. PV. (1840) 67-.

-, calculation. Laroque, $F$. Toul. Mm. Ac. 6 (1862) 295-

-, a succession of. Neuberg, J. Liège S. Sc. Mm. 11 (1885) No. 5, 12 pp.

Modular systems, applications to elementary algebra. Netto, $E_{\text {. }}$ Crelle J. Mth. 104 (1889) 321-.

Monomial coefficients and coefficients of factorials. Zeipel, E. V. von. Lund Acta Un. 6 (1869) (Mth.) No. 3, $57 \mathrm{pp.}$

Multiplication, algebraic. Englefield, H.C. Tilloch Ph. Mg. 45 (1815) 15-.

-, - . Dillon, J. Tilloch Ph. Mg. 45 (1815) 137 .

- by fractions, interpretation, and principle of permanence of equivalent forms. Taylor, $C$. Mess. Mth. 4 (1868) 159-.

- $-(x+a)$. Andrê, D. Par. Éc. Norm. A. 12 (1883) (Suppl.) 3-.

- - $(x-a)$. André, D. Par. Éc. Norm. A. 12 (1883) (Suppl.) 33-.

Negative quantities and rule of signs. Guillon, $E . L$. N. A. Mth. 8 (1849) 28 -.

- - in solution of equation problems. Greenfield, $W .[1784]$ Edinb. R. S. T. 1 (1788) 131-. 


\section{Algebra. General}

Nomography, problem of algebra relating to. Ocagne, M. $d$. N. A. Mth. 12 (1893) 469-.

Notation, algebraic. Collins, J. V. Science 20 (1892) 359.

-, - and fluxional, principles. Young, $\operatorname{Dr} T$. QJ. Sc. 20 (1826) 321-.

- for printing complicated exponents. Glaisher, J. W. L. [1872] Mess. Mth. 2 (1873) 107-.

Order of factors immaterial. Gergonne, J. $\dot{D}$. Gergonne A. Mth. 1 (1810-11) 52-.

Principles, doubtful points. Györy, S. (xII) Mag. Ak. Éts. (Mth. Term.) 1 (1860) 287-.

-, three which connect algebra with other mathematics. Chelini, D. Palomba Rac. 2 (1846) 57-, 73-.

Problem on a cask of brandy, a given quantity being withdrawn daily, and lighter spirit added. Nürnberger, - Baumgartner Z. 4 (1828) 76-.

-. Persons in a circle passing money to one another. Allégret, A. N. A. Mth. 16 (1857) 136-.

-. Smallest number of weights needed. Gergonne, J. D. Gergonne A. Mth. 8 (1817-18) 305-.

Problems in Nouv. Ann. Mth. Bellavitis, G. Ven. At. 4 (1853) 79-; (1860) 165-.

- for schools. Dienger, J. Grunert Arch. 9 (1847) 113-.

Product of $n$ factors, transformation. Tardy, P. Tortolini A. 2 (1851) 287-.

- - two algebraic expressions. Elliott, $J$. Mth. Gz. 1 (1900) 187-.

Proportions and progressions. Vecchio, A. G. Mt. 7 (1869) 43-.

Quadratic expressions, theorem. Lamb, $H$. [1880] Mess. Mth. 10 (1881) 35-.

Radicals, extraction by binomial theorem and Lambert's formula, accuracy. Schulz von Strasnzitzki, L. C. Baumgartner Z. 6 (1829) $31-$.

Rationalisation. Herth, G. (vI. Adds.) D. Nf. Vsm. B. 34 (1858) 105.

-, case. Ward, P. C. Mess. Mth. 15 (1886) 42 .

- of denominators. Grebe, E.W. Grunert Arch. 13 (1849) 68-.

- - Zehfuss, G. Grunert Arch. 35 (1860) 117-.

- - Janisch, E. Arch. Mth. Ps. 10 (1891) 420

-, note. MacMahon, (Capt.) P. A. Mess. Mth. 15 (1886) 65-.

Ratios of $k$ quantities, approximate representation by ratios of simple integers. Oliver, J.E. [1864] Am. Ac. P. 6 (1866) 288-.

Reduction of Fahrenheit to centigrade. Tiberi, E. Rv. Sc.-Ind. 30 (1898) 216-.

- $\sqrt[n]{a+\sqrt{b}}$ to the sum of two squares. Schmidt, E. Ts. Mth. 2 (1884) 154-; Fschr. Mth. (1884) 146.

Roots, elementary theory. Baltzer, $R$. Grunert Arch. 18 (1852) 405-.

- of higher degree analogous to $B+A \sqrt{ } \vec{a}$. Grebe, E. W. Grunert Arch. 13 (1849) 400-

Rule of three, theory. Gergonne, J.D. Gergonne A. Mth. 7 (1816-17) 117-.

\section{Rational polynomials 1610}

Sign, doubtful, in algebraic formulae, meaning. Stevelly, J. B. A. Rp. (1836) (pt. 2) 5-.

- -symbols, theorems relating to. Cayley, $A$. Mess. Mth. 2 (1873) 17-.

Signs. Schellbach, C. H. Crelle J. 12 (1834) 70-, 148-.

-. Transon, A. N. A. Mth. 6 (1867) 289-.

-, influence in mathematics. Babbage, $C$. [1821] Camb. Ph. S. T. 2 (1827) 325-.

-, + and -, significance. Purgotti, S. Palomba Rac. 3 (1847) 265-, 285-, 324-.

_, - _ - , , algebraic. Massini, L. Palomba Rac. 1 (1845) 73-.

- , _ _ , - before radical $\sqrt{ }$. Judson, C. $H$. Des Moines Anal. 2 (1875) 70-

Simplification of an algebraic expression. Langley, E. M. Mth. Gz. 1 (1900) 129.

Square root of $A+\sqrt{ } B$. Göpel, $A$. Grunert Arch. 3 (1843) 249-.

- - - quadrinomial surd. Bierens de Haan, D. N. Arch. Wisk. $3\left({ }^{*} 1877\right) 208-$.

-- , test for recognizing whether operations $\sqrt[2 m+1]{a \sqrt{v} \pm b \sqrt{v i}}$ etc. can be effected under form $a \sqrt{v} \pm \beta \sqrt{w i}$. Weichold, G. C. R. 96 (1883) 835-.

Surd $\sqrt{a} \pm \sqrt{b}$, explanation of Euler's objection to Newton's rule for extraction of root. Plana, G. Crelle J. 17 (1837) 331-; 20 (1840) 283-.

-, extraction of root. Plana, G. N. A. Mth. 7 (1848) 271-.

Surds. Reduction of $\sqrt[3]{a+b \sqrt{c}}$ to $m+n \sqrt{c}$. Kausler, C. F. [1806] St Pét. Ac. Sc. Mm. 2 (1810) 64-

-, theorems. Treuil, - . Gergonne A. Mth. 12 (1821-22) 104-.

Symbol, new. Whitworth, W.A. Mess. Mth. 5 (1871) 121.

Symbols, algebraic. Baker, A. L. Am. J. Mth. 18 (1896) 62-.

-, new algebraic, in Phil. Trans. 1862. Drach, S. M. (vII) Ph. Mg. 26 (1863) 406-.

Theory of algebra, from arithmetical principles, and nature of signs, etc. Gloesener, $M$. Liège A. Ac. $(1818-19) 64$ pp.

Variations and combinations with fixed totals. Reich, $K$. Arch. Mth. Ps. 11 (1892) 225-。

Viète, François, new algebra of. Ritter, $\boldsymbol{F}$. As. Fr. C. R. (1892) (Pt. 2) 177-.

\section{Rational polynomials; divisibility; reducibility.}

Algebraic calculation and division. Źmurko, $W$. Krk. Ak. (Mt.-Prz.) Pam. 12 (1886) (1)-; Fschr. Mth. (1886) 120.

Approximate expressions, linear in two polynomials. Čebyšev, $P$. St Pet. Ac. Sc. Mm. (Rs.) $30\left({ }^{*} 1877\right.$ ) (Suppl.) No. 4, 24 pp.

- fractional expression for $(1+n)^{n}$ to any odd number of terms. Steggall, J.E.A. Edinb. Mth. S. P. 10 (1892) 21-.

Arithmetic, theorem of Lagrange. Grunert, J. A. Arch. Mth. Ps. 47 (1867) 328-. 
Binomials, cube-roots. Horner, W. G. Thomson A. Ph. 8 (1816) 279-, 388-.

-, cubic, $x^{3} \pm y^{3}$. Lamé, G. C. R. 61 (1865) 921-, 961-.

Common divisors, finding. Buys-Ballot, C.H.D. Amst. Vs. Ak. 13 (1862) 430-.

Condition that $f=A \phi+B \psi$, where $f, A, B, \phi, \psi$ are all rational integral polynomials in two variables. Halphen, G. $H$. Par. S. Mth. Bll: 5 (1877) 160-.

Divisibility of $(x+y)^{n}+(-x)^{n}+(-y)^{n}$, Cauchy's theorem. Muir, T. [1878] Mess. Mth. 8 (1879) 119-.

$-\frac{1}{(1888)}($ Pt. 2) . Lucas, E. As. Fr. C. R. Division, abbreviated algebraic. Reuschle, $C$. Z. Mth. Ps. 41 (1896) 93-.

-, algebraic, applied to homogeneous polynomials. Andoyer, $H$. Liouv. J. Mth. 1 (1895) 61-.

-, - , and new theorem of analysis. Le Cointe, L. A. N. A. Mth. 1 (1842) 409-.

- of integral polynomials, application to integration. Jamet, $V$. Mars. Fac. Sc. A. 8 (1898) 151-.

- - polynomial by another. Rubini, $R$. G. Mt. 4 (1866) 38-.

Divisors, algebraic theorem, with application to curves. Pasch, M. Crelle J. Mth. 89 (1880) 252-.

-, commensurable, 2nd degree. Transon, $A$. (Prof.). N. A. Mth. 6 (1847) 305-.

,,--- Prouhet, E. N. A. Mth. 18 (1859) 257-.

- - given degree of a polynomial. Maley $x, L$. N. A. Mth. 14 (1875) 97-.

- - polynomials with whole numbers for coefficients. Perott, J. Par. S. Mth. Bll. 10 (1882) 250-.

—, rational. Durville, -. N. A. Mth. 4 (1845) 439-.

,-- , 2nd degree. Durville, - . N. A. Mth. 4 (1845) 339-.

- of rational polynomials. Bouniakowsky, $V$. [1854] St Pét. Ac. Sc. Mm. 8 (1857) 305-.

Equations with integral coefficients. Cauchy, A. L. C. R. 24 (1847) 407-.

Expansion of $\left(1+x+x^{2}+\ldots \& c .\right)^{n}$. Euler, $L$. [1778] St Pét. Ac. Sc. N. Acta 12 (1801) 47-.

- $\left(1+x+x^{2}\right)^{n}$. Euler, L. [1778] St Pét. Ac. Sc. N. Acta 14 (1805) 75-.

$--(x+y)^{n}+(-x)^{n}+(-y)^{n}$. Muir, T. QJ. Mth. 16 (1879) 9

Factorials, class.' Gregory, D. F. Camb. Mth. J. 3 (1843) 89-.

- Vandermonde's. Anon. (vi 158) Bb. It. (1830) 407-.

Factorization. Butters, J. W. Edinb. Mth. S. P. 12 (1894) 31-; 16 (1898) 78-.

Factors of integral functions of $n$th degree. Am Ende, -. Grunert Arch. 30 (1858) 442-.

- $a x^{2}+b x y+\ldots+f$. Grunert, J. A. Grunert Arch. 39 (1862) 98-.

- - $(x+y)^{n}-x^{n}-y^{n}$, Cauchy's theorem. Glaisher, J. W. L. QJ. Mth. 15 (1878) 365-; 16 (1879) 89-.

Figurate series. Smyth, B. B. Kan. Ac. Sc. T. 14 (1896) 29-.

Formation-law of coefficients in quotient of two power series. Jezek, $O$. Prag Sb. (1884) (Mth. Nt.) 127-.

Formula giving $x^{m}+y^{m}$ as function of $(x+y)$ and $x y$. Desboves, A. N. A. Mth. 14 (1875) 385-.

H. C. D. Bouverat, - . N. A. Mth. 3 (1844) 329-.

- - - Cirodde, P. L. N. A. Mth. 4 (1845) 497-.

- - of 2 functions of one variable. Trudi, $N$. Nap. Rd. 1 (1862) 153-.

- - - - integral functions. Netto, $E$. [1889] Hamb. Mth. Gs. Mt. 2 (1890) (Festschr. Tl. 2) 36-.

- - - - - polynomials. Pomey, E. N. A. Mth. 7 (1888) 66-, 407-.

- _ - and L. C. M. Barrieu, P. (xII) Mathesis 3 (1883) 217-.

- - of 2 polynomials. Craufurd, A.Q. G. Camb. Mth. J. 4 (1845) 9-.

- $--\ldots-1$, remainder in the process. Faà de Bruno, F. C. R. 42 (1856) 407-.

- - - polynomials, determination by determinants. Zeuthen, H. G. (xII) Ts. Mth. 5 (1881) 45-, 109-.

- $-\ldots-2$ rational integral functions of $x$, method. Falk, M. [1878] Ups. S. Sc. N. Acta 10 (1879) (No. 11) 5 pp.

Homogeneous functions, property. Gukovskij, A. N. Rs. S. Nt. Mm. (Mth.) 11 (1890) 145 -

Identities, algebraic class. Glaisher, J. W. L. [1878] Mess. Mth. 8 (1879) 53-.

$\ldots,-$, relating to sums of squares. Catalan, E. C. Liège S. Sc. Mm. 13 (1886) 33-, 399.

-, connected with $(a+b)^{n}-a^{n}-b^{n}$. Sadun, $E$. Rv. Mt. 4 (1894) 189-; 5 (1895) 19-.

- developed from $\frac{1}{p}=\frac{1}{q}+\frac{q-p}{q} \cdot \frac{1}{p}$. Tait, P. G. [1877] Edinb. R. S. P. 9 (1878) 416-.

Identity, algebraic, on differences of 4 letters. Glaisher, J. W. L. [1878] Mess. Mth. 8 (1879) 45-.

-,,-- reciprocals of cyclic differences. Saint-Germain, - de. Caen Ac. Mm. (1892) (Pt. 1) 15-.

- of polynomials, principle. Roy, A. Mathematician 3 (1850) 1 -.

- Waring's, an. Virieu, J.de. N. A. Mth. 1 (1862) 45-.

-, Waring's analytical proof. Serret, $P . \mathrm{N}$.

A. Mth. 7 (1848) 199-.

-. If $a+b+c=0$,

$$
\begin{aligned}
\left(\frac{b-c}{a}+\frac{c-a}{b}+\frac{a-b}{c}\right) & \\
\left(\frac{a}{b-c}+\frac{b}{c-a}+\frac{c}{a-b}\right) & =9 .
\end{aligned}
$$

Glaisher, J. W. L. [1880] Mess. Mth. 10 (1881) 54-.

Irreducible factors of $x^{n}-1$. Kronecker, $L$. Liouv. J. Mth. 19 (1854) 177-. 


\section{Rational polynomials}

Multiplication and division, algebraic. Salimbeni, L. Verona Mm. S. It. 7 (1794) $482-$.

Polynomial, complete, number of terms. Gergonne, J. D. Gergonne A. Mth. 13 (1822-23) 282 -

-, condition for factor of form $x^{n}-a^{n}$. Zeipel, E. V. von. Ups. N. Acta S. Sc. 4 (1863) $24 \mathrm{pp}$.

-, - - - B Björling, C. F. E. Arch. Mth. Ps. 55 (1873) 429-.

-, determination by integrals of its partial differentials. Mossbrugger, L. Grunert Arch. 4 (1844) 210-.

-, development, case. Weihrauch, K. Z. Mth. Ps. 26 (1881) 127-.

-, - without binomial theorem. Brassinne, $E$. Toul. Mm. Ac. 1 (1851) 184-.

- 4th degree, values of $x$ for which it is a square. Calzolari, L. G. Mt. 7 (1869) 317-.

-, linear factorisation. Küpper, $K$. Časopis 14 (1885) 28-; Fschr. Mth. (1886) 60.

-, nth power. Studnička, F. J. Prag Sb. (1879) 375-; Č́asopis $9\left({ }^{*} 1880\right)$ 49-.

- $\left(x^{2}-1\right)^{n}$, property. Polignac, C. de. [1874] Par. S. Mth. Bll. 3 (1875) 19-.

Polynomials, class. Appell, $P$. Par. Éc. Norm. A. 9 (1880) 119-.

-, - factorisable in linear factors. Moutard, -. C. R. 119 (1894) 42-.

-, division and extraction of roots. Gergonne, J. D. Gergonne A. Mth. 4 (1813-14) 297-.

-, equal factors of. Ostrogradsky, M. A. C. R. 42 (1856) 930-

-,geometrical application of theorem. Laisant, C. A. As. Fr. C. R. (1898) (Pt. 2) 73-.

-, powers. Brianchon, C. J. Par. Ec. Pol. J. 15 (1837) $\left(25^{\circ} \mathrm{cah}\right.$.) 158 -

-, - Casinelli, A. (s. L.) Bologna N. Cm. 4 (1840) 453-.

-, - Lewis, W. J. Franklin I. J. 3 (1842) 44-.

_, - divisibility. Crelle, A. L. Berl. Ab. (1839) 1-, 299-.

-, product of two, (Gauss). Terquem, O. N. A. Mth. 3 (1844) 47-.

-, properties. De Forest, E. L. Des Moines Anal. 7 (1880) 39-, 73-, 105-.

-, - , method of demonstrating. De Forest, E. L. Des Moines Anal. 10 (1883) 97-。

-, rational factors. Gergonne, J.D. Gergonne A. Mth. 12 (1821-22) 309-.

-, - geometric construction. Winckler, A. Wien Sb. 53 (1866) (Ab. 2) 326-.

-, roots. Šebuev, G. N. Kazan S. Nt. (Ps.Mth.) P. 7 (1889) 394-.

- of 3rd degree in one variable. Nordlund, $K$. $P$. Ts. Mt. Fys. 4 (1871) $185-$.

Power of polynomial, expression by symmetric functions. Piarron de Mondesir, E. S. As. Fr. C. R. (1877) 154-.

Powers of $\left(a_{1} x+a_{2} x^{2}+\ldots \ldots\right)^{n}$, theorem. Tralles, $J$. $G$. Berl. Ab. (1816-17) 56-.

Principle of greatest and least exponents. Applications in theory of algebraic functions. Bugaev, N. V. [1889] Rec. Mth. (Moscou) 14 (1890) 553-.
Algebraic inequalities 1615

Quotient and product functions, certain. Guldberg, A. S. Christiania F. (1884) No. 4, $18 \mathrm{pp}$.

- remainder in $(x-1)^{p} / x^{m}$. Catalan, E. C. Liége S. Sc. Mm. 15 (1888) No. 1, 142-.

- - quadrics to sums of squares. Laurent, $H$. N. A. Mth. 19 (1880) 12-; 20 (1881) 38-.

Series. $\Sigma A_{n} \phi(n) x^{n}$. Grunert, J. A. Crelle J. 25 (1843) 240 -.

Square root extraction, of 6-nomials, 5-nomials, etc. Reade, J. Thomson A. Ph. 11 (1818) 31-.

Sum of a series connected with transvectants. Gordan, P. Palermo Cir. Mt. Rd. 12 (1898) 326-.

Sums of like powers of (linear) expressions. Bierens de Haan, D. N. Arch. Wisk. 5 (*1879) 208-.

- - powers of first $n$ numbers, geometrical illustrations. Bochow, $K$. Magdeb. Nt. Vr. Jbr. u. Ab. (1896-98) 165-.

Ternary forms, conditions for linear factors. Brill, A. [1896-98] Mth. A. 50 (1898) 157-; D. Mth. Vr. Jbr. 5 (1901) (Heft 1) 52-.

Theorems of Bezout and Euler. Poussart, A. Ens. Mth. 2 (1900) 136-.

Transformation of expression

$$
(b z-c y)^{2}+(c x-a z)^{2}+(a y-b x)^{2} .
$$

Smith, Arch. Camb. Mth. J. 1 (1839) $155-$.

$-\quad-(c y-b z)^{2}+(a z-c x)^{2}+(b x-a y)^{2}$. N., M. N. Camb. Mth. J. 2 (1841) 9-.

- series $\Sigma\left(\frac{1}{m}\right)=\Sigma\left( \pm \frac{m_{m}}{m}\right)$. Björling,

E. G. Grunert Arch. 27 (1856) 482-.

$x^{n}+y^{n}$ expressed in terms of $x+y$ and $x y$. Valeriani, V. G. Mt. 12 (1874) 208-.

\section{Algebraic inequalities.}

Inequalities, applications. Brashmann, N. D. (xII) Mosc. Un. Mm. 10 (1835) 381-.

calculus. Fourier, J.B.J. Par. S. Phlm. N. Bll. (1826) 99-.

- - Gergonne, J. D. Gergonne A. Mth. 17 (1826-27) 134-.

- extension of theorem in. Rogers, L. J. Mess. Mth. 17 (1888) 145-.

-, generalisation of formula of Tschebyscheff (Čebyšev). Jensen, --. Bll. Sc. Mth. 12 (1888) 134 -

-, means, and imaginaries. Grunert, J. A. Grunert Arch. 1 (1841) 268-.

-, note on some, and their application to integral calculus. La Vallée-Poussin, C. de. Brux. S. Sc. A. 16 (1892) (Pt. 1$) 8-$.

-, notes. Wace, F. C. Mess. Mth. 18 (1889) 90 -

-, number of conditions expressed by. Sludskin, T. A. (xII) [1873] Rec. Mth. (Moseou) 7 (1874-75) (Pt. 1) 419-.

- problem. $\left(x^{m}+y^{m}>\right.$ or $<z^{m}$ when $\left.x^{2}+y^{2}=z^{2}\right)$. Grunert, J. A. Grunert Arch. 20 (1853) 356-. 
Inequalities, theorem. Glaisher, J. W. L. Mess. Mth. 8 (1879) 140-.

- of Tschebyscheff's (Ćebyšev), proof. Markov, A. A. (xII) Kharkov Mth. S. Com. (1883) 105-; Fschr. Mth. (1885) 168, 171.

$-\left\{(n-1) \Sigma a^{2}>2 \Sigma a b\right\}$. Grunert, J.A. Grunert Arch. 26 (1856) 105-.

$-m a^{m-1}>\frac{a^{m}-b^{m}}{a-b}>m b^{m-1}$, proof and applications. Fort, O. Schlömilch Z. 7 (1862) 46-.

Inequality concerning sums of products of numbers. Schiappa Monteiro, A. [1900] Lisb. J. Sc. Mth. 6 (1902) $12-$.

-, conditions, calculus. Navier, C. L. M. H. Par. S. Phlm. Bll. (1825) 66-, 81-.

- - - of, Fourier. Cournot, A. A. (vi Adds.) Férussac Bll. Sc. Mth. 6 (1826) 1-. $-\frac{a^{2 n+2}-1}{a\left(a^{2 n}-1\right)}>\frac{n+1}{n}$. Walton, W. Mess. Mth. 8 (1879) 130-.

$-\frac{x^{p}-y^{p}}{x^{q}-y^{q}}$ lies between $\frac{p}{q} x^{p-q}$ and $\frac{p}{q} y^{p-q}$. Walker, G. F. [1882] Mess. Mth. 12 (1883) 37-.

$-m x^{m-1}(x-1) \gtreqless x^{m}-1 \gtreqless m(x-1)$. Chrystal, G. Edinb. Mth. S. P. 6 (1888) 29 -

- $a^{m} b^{n}+b^{m} c^{n}+c^{m} a^{n}>a^{n} b^{m}+b^{n} c^{m}+c^{n} a^{m}$. Segar, H. W. Mess. Mth. 22 (1893) 47-.

Mean value. Hïlder, $O$. Gött. Nr. (1889) 38-.

\section{Permutations, combinations,} partitions, distributions. $\mathrm{Bi}-$ nomial and multinomial coefficients.

Algorithms of arithmetical functions. Cesìro, E. G. Mt. 23 (1885) 175-.

Arithmetical cube. Laisant, C. A. As. Fr. C. R. (1891) (Pt. 2) 8-.

- tetrahedron. Laisant, C. A. Par. S. Mth. Bll. 19 (1891) 18-.

- triangle, history. Fontès, - As. Fr. C. R. (1893) (Pt. 2) 236-.

- -, properties. Laisant, C. A. As. Fr. C. R. (1891) (Pt. 2) 1-; (1893) (Pt. 2) 206-.

Arrangement of (1) cards, (2) counters: two problems. Crelle, A. L. Crelle J. 44 (1852) 317-.

- - 15 counters in order, generalisation. Malmsten, C.J. Göteb. Hndl. 17 (1882) 77-.

- game (Josephspiel). Curtze, M. Bb. Mth. (1894) 116 ; (1895) 34-.

- - (Josephus's problem), generalisation. Tait, P. G. [1898] Edinb. R. S. P. 22 (1900) 165-.

- and probabilities, question. Tait, P. G. Edinb. R. S. P. 8 (1873) 37-.

- in rows. Brunel, G. Bordeaux S. Sc. PV. (1894-95) 28-.

Arrangements, compound, question in. Sylvester, J. J. R. S. P. 12 (1862-63) $561-$.

-, generalisation of theorem of Cauchy. Sylvester, J. J. Ph. Mg. 22 (1861) 378-.
Arrangements, problem. Bouniakowsky, $V$. [1857] St Pét. Ac. Sc. Bll. 16 (1858) 67-.

-, Cayley, A. [1877] Edinb. R. S. P. 9 (1878) 338-.

- -. Tait, P. G. Edinb. R. S. P. 10 (1880) 400-.

, Tait's problem. Muir, T. [1877] Edinb. R. S. P. 9 (1878) $382-$.

_, - Cayley, A. [1877] Edinb. R. S. P. 9 (1878) 388-.

_, - Muir, T. [1881] Edinb. R. S. P. 11 (1882) 187-.

- of $m$ things of one sort and $n$ things of another sort, under conditions of priority. Whitworth, W. A. [1878] Mess Mth. 8 (1879) 105-

Binomial "calculus." Walsh, $J$. Tilloch $\mathrm{Ph}$. Mg. 63 (1824) 443-.

\section{BINOMIAL COEFFICIENTS.}

Euler, L. [1778] St Pét. Ac. Sc. N. Acta 15 (1806) 33-.

Stockler, F. de B. G. Lisb. Mm. Ac. Sc. 2 (1799) 480-

Pessuti, G. Mod. S. It. Mm. 11 (1804) 446-.

Mazio, N. G. Arcad. 22 (1824) 27-.

Arndt, $F$. Grunert Arch. 3 (1843) 256-.

Horta, F. Lisb. A. 2 (1858) 98-.

Le Taunéac, A. N. A. Mth. 20 (1861) 147-.

Lucas, $E_{\text {. }} \quad$ N. A. Mth. 9 (1870) 308-.

Schlegel, S. F. V. Z. Mth. Ps. 23 (1878) 263-. analytic faculties. Trudi, N. [1873] Nap. Ac. At. 6 (1875) No. 3, 16 pp.

Euler's theorem. Wolfers, J.P. Arch. Mth. Ps. 45 (1866) 411-.

formulæ. Tardy, P. G. Mt. 3 (1865) 1-.

- Delannoy, $H$. As. Fr. C. R. (1890) (Pt. 2) 35-.

a function of, and a series. Siacci, F. G. Mt. 10 (1872) 349-.

and their interpolation. Euler, L. St Pét. Ac. Sc. Mm. 9 (1824) 57-.

note. Busche, E. [1899] Hamb. Mth. Gs. Mt. 3 (1900) 379-.

properties. Schlömilch, $O$. Grunert Arch. 1 (1841) 431-; 2 (1842) 434-.

-. Tirelli, F. G. Mt. 14 (1876) 318-.

-, and application to combinations. Baur, C. W. Z. Mth. Ps. 32 (1887) 218-.

- derived from complex numbers. Studnička, F. J. Prag Sb. (1877) 92-.

property. Zdrahal, A. Časopis 11 (*1882) 47-; Fschr. Mth. ( $\left.{ }^{*} 1882\right) 190$.

-. Ekama, H. N. Arch. Wisk. 19 (1892) 105-; Fschr. Mth. (1892) 156.

in relation to gamma function and figurate numbers. Matthiessen, -. D. Nf. Tbl. $\left({ }^{*} 1868\right)$ 128-

sum of series involving. Bonolis, A. G. Mt. 11 (1873) 233-.

$---1-\frac{n}{2}+\frac{(n-1)(n-2)}{2.3}-$ etc. derived from expansion for $\cos m x$. Schlömilch, $O$. Grunert Arch. 7 (1846) 46-. 


\section{Binomial Coefficients}

sum of squares. Szily, $K$. Mth. Termt. Éts. 11 (1893) 330-; Mth. Nt. B. Ung. 12 (1895) $84-$

by Taylor's series. Crelle, A. L. Berl. Ab. (1843) 1-.

theorem. Graves, C. [1865] Ir. Ac. P. 9 (1867) 297-.

-. Hermes, J. Arch. Mth. Ps. 8 (1890) 269-.

theorems. Catalan, E. C. Liége S. Sc. Mm. 15 (1888) No. 1, 41-, 133-, 250-.

transformation of integral powers into. Trudi, N. [1873] Nap. Ac. At. 6 (1875) No. 4, 13 pp.

Binomial theorem, alternative proof. Schuster, A. Metz Ac. Mm. 72 (1893) 163-.

- - case. Catalan, E. Brux. Ac. Bll. 16 (1888) 194.

- - new form. Mendizábal Tamborrel, J. de. Méx. S. "Alzate" Mm. 4 (1890) 117-; Fschr. Mth. (1890) 256.

- - before Newton. Marre, A. N. A. Mth. 5 (1846) 488-

- - for positive integral index. Bouvier, L. C. Gergonne A. Mth. 14 (1823-24) 205-. _- - _ - Garcet, -. N. A. Mth. 19 (1860) $32-$.

Grunert, J. A. Grunert Arch. 1 (1841) 67-. - - proofs. Woodcock, T. B. A. Rp. (1889) $501-$.

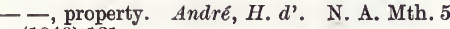
(1846) 121-.

Binomials. Mello, M. P. de. Lisb. Ac. Sc. Mm. (Cor.) 4 (Pte. 1) (1815) 41-.

Card dealing problem, Gergonne's. Dickson, L. E. N. Y. Am. Mth. S. Bll. 1 (1895) 184

- game, question. Le Cointe, J.L.A. (viri) A. Mt. 5 (1863) 108-.

- games of patience. Badoureau, A. [1881] Rv. Sc. 2 (1882) 475-.

- -, problem. Vachette, A. N. A. Mth. 5 (1846) 394-.

- - (i.e. playing cards) and remark on theory of substitutions. Petersen, J.P.C. (xII) Ts. Mth. 1 (1871) 74-.

- trick. Gergonne, J. D. Gergonne A. Mth. 4 (1813-14) 276-.

- puzzle and a law of numbers. Steen, A. (xII) Ts. Mth. 1 (1865) 49-; 2 (1866) 1-; 3 (1867) 1-; 6 (1870) 1-, 181-; 3 (1873) 156-. - - - - Hertzsprung, $S$. (xiI) Ts. Mth. 3 (1867) 97-.

- - - - - - - Lorenz, L. V. (xir) Cards. Theory of whist. Badoureau, - . Rv. Sc. 36 (1885) 587-.

Chess.

Bishop's move. Cherriman, J. B. (xII) Cn. R. S. P. \& T. 1 (1883) (Sect. 3) 19.

Chessboard geometry, and indeterminate equation $a x+b y=c$. Laquière, E. M. Par. S. Mth. Bll. 7 (1879) 85-.

- - , theorem on polygons having vertices at vOL. I.

\section{Chess 1620}

centres of squares. Laisant, C. A. Par. S. Mth. Bll. 6 (1878) 156-

Chessboard geometry, theorem on triangle having vertices at centres of squares. Lucas, E. [1877] Par. S. Mth. Bll. 6 (1878) 9-.

- problem. Lavernède, T. Gard Mm. Ac. (1838-39) 151-.

- - "Entre chiens et loup." Lucas, $\dot{E}$. Rv. Sc. 5 (1883) 555-.

- problems. Lucas, É. Rv. Sc. 17 (1879) 154-; 18 (1880) 948-; 19 (1880) 36-, 375-; 1 (1881) 408-, 783-; 2 (1882) 365-.

- Perott, J. Par. S. Mth. Bll. 11 (1883) 173-.

End games. Cunningham, $\left(L_{t} t_{0}-C_{0} l_{\text {. }}\right) A$. B. A. Rp. (1894) 564.

Knight's move. Brewster, (Sir) D. Edinb. $\mathrm{Ph}$. J. 9 (1823) 236-.

- - Bouvard, A. Dijon Ac. Mm. (1830) 23-.

- Roget, P. M. (vr Adds.) Ph. Mg. 16 (1840) 305-.

- Moon, R. Camb. Mth. J. 3 (1843) 233-.

- -. Minding, E. F. A. [1847] St Pét. Ac. Sc. Bll. 6 (1848) 209-.

- -. Volpicelli, P. C. R. 31 (1850) 314 .

- - Clausen, T. Grunert Arch. 21 (1853)

91-.

- Anon. (vi 883) N. A. Mth. 13 (1854)

181-.

840 .

- - Geynet, A. C. R. 60 (1865) 484.

- -. Urusov, (Prince) S. S. (xII) [1865]

Rec. Mth. (Moscou) 2 (1867) (Pt. 1) 189-.

- - Régnier, A. Les Mondes 32 (1873) $507-$.

-. Frost, A. H. [1876] QJ. Mth. 14 (1877) 123-.

- - Flye Sainte-Marie, C. Par. S. Mth. Bll. 5 (1877) 144-.

_-. Polignac, C. (Prince) de. [1880] Par. S. Mth. Bll. 9 (1881) 17-.

- - Lucas, É. Rv. Sc. 6 (1883) 370-.

- - Gilbert, G. K. [1885] Smiths. Misc. Col. 33 (1888) Art. 2, 88. (Wash. Ph. S. Bll. 7 (1885).)

- - Hofmann, F. N. A. Mth. 5 (1886) 224.

- - Jordan, C. Palermo Cir. Mt. Rd. 2 (1888) 59-.

- - Brunel, -. Bordeaux S. Sc. Mm. 4 (1894) liii.

_- Wolf, R. Zür. Vjschr. 39 (1894) 147-. - - generalisation of problem. Fitting, $F$. Z. Mth. Ps. 45 (1900) 137-.

- - illustrating an electrical problem. Brunel, - Bordeaux S. Sc. Mm. 2 (1891) xxxiv-.

- - number of reentrant moves in halfboards. Laquière, E. M. [1880] Par. S. Mth. Bll. 9 (1881) 11-.

_ - regular solutions of Euler's problem. Laquière, E. M. Par. S. Mth. Bll. 8 (1880) 82-, 132-. 


\section{Chess}

Knight's move, solution by geometry of position. Volpicelli, $P$. Rm. At. R. Ac. 25 (1872) 87-, 364-; 26 (1873) 49-, 241-.

- - - (Volpicelli). Tarry, H. Les Mondes 28 (1872) 60 -

- moves over squares of $5,6,7,8$, and extension to higher squares. Frost, A. $H$. QJ. Mth. 14 (1877) 354-.

Mathematical analysis applied to chess. (Brief résumé.) Jänisch, -. St Pét. Ac. Sc. Bll. 6 (1863) 473-.

Problem, solution by aid of numerical functions. Bugaev, N. V. (xII) [1878] Rec. Mth. (Moscou) 9 (1878-81) (Pt. 1) 355-.

8 queens problem. Glaisher, J. W. L. $\mathrm{Ph}$. Mg. 48 (1874) 457-.

- Rohn, $K$. Dresden Isis Sb. (1889) (Ab.) $89-$.

- - Sprague, T. B. Edinb. Mth. S. P. 8 (1890) 30-; 17 (1899) 43-.

- - - generalisation. Günther, $S$. Arch. Mth. Ps. 56 (1874) 281-.

$n$ queens problem. Parmentier, T. As. Fr. C. R. (1883) 197-。

Sylvester's anallagmatic chess-board pattern. Lucas, Ł́. As. Fr. C. R. (1877) 213-.

Class of numbers arising in certain expansions. Ocagne, M. d'. Am. J. Mth. 9 (1887) 353-.

Classes, division into. Harding, $P . J$. Mth. Gz. No. 6 (1895) 59 .

Coefficients in expansion of $(x+y)^{n}$. Laisant, -. As. Fr. C. R. (1888) (Pt. 2) 72-.

$---\left(\frac{x^{p}-1}{x-1}\right)^{n}$. Mathieu, J. J.A. N. A. Mth. 2 (1863) 509-.

\section{COMBINATIONS.}

Kirkman, T. P. (vi Add8.) Ph. Mg. 5 (1853) 11-.

Buniakovskij, V. Ja. St. Pet. Ac. Sc. Mm. (Rs.) 21 ("1872) 261-.

Mantel, W. N. Arch. Wisk. 11 (1884) 190.

Thiele, T. N. Kjøb. Ov. (1895) 415-, xv-.

application to explanation of Arbogast'smethod. De Morgan, A. Camb. and Dubl. Mth. J. 6 (1851) 35-.

of a certain number of successive integers having a sum below a given limit, number of. Marsano, G.B. G. Mt. 19 (1881) 156-; 20 (1882) 249-.

complete. Levret, - . N. A. Mth. 1 (1842) 87-.

-. Melon, A. G. N. A. Mth. 8 (1869) 168-.

-. Niewenglowski, G. H. N. A. Mth. 13 (1874) 285 -

compound. Cayley, A. Manch. Lt. Ph. S. P. 16 (1877) 113-.

Crocchi's theorem (on sums of combinations of powers and homogeneous products). Sylvester, J. J. (xII) [1882] J. H. Un. Cir. [2] (1883) 2.

- - $\left(-\frac{2}{\text { ranklin }}------\right)$, proof. [2] (1883) 24 .
Combinations 1620

of 28 dominoes. Reiss, M. [1859] A. Mt. 5 (1871-73) 63-.

of elements dispersed in a plane. Mantel, As. Fr. C. R. (1883) 171-.

and factorial numbers, theorems. Catalan, $E$. Brux. Ac. Bll. 25 (1893) 238-, 430-.

formula. Catalan, E. C. Liouv. J. Mth. 7 (1842) 511-.

-, new. Studnička, F. J. Prag Sb. (1879) 295 -

formulæ. Grand, - \& Rochat, - Gergonne A. Mth. 3 (1812-13) 213-.

- for expanding power of binomial. Gergonne, $J$. D. Gergonne A. Mth. 17 (1826-27) $356-$.

a game of. Brianchon, C. J. Par. Éc. Pol. Cor. 3 (1814-16) 387-.

number of given class having a given sum. Boschi, P. Bologna Ac. Sc. Mm. 5 (1883) $805-$.

probabilities and life contingencies. Hardy, $P$. Assur. Mg. 2 ("1852) 151-, 259-.

problem. Euler, L. [1779] St Pét. Ac. Sc. Mm. 3 (1811) 57-.

-. Durrande, J. B. Gergonne A. Mth. 7 (1816-17) 334-.

-. Catalan, E. C. Liouv. J. Mth. 3 (1838) $111-; 5$ (1840) $264 ; 6$ (1841) 74 .

-. Chevillard, A. J. N. A. Mth. 3 (1844) 537 -.

-. Kirkman, T. P. Camb. and Dubl. Mth. J. 2 (1847) 191- ; 5 (1850) 255-.

- Anstice, R. R. Camb. and Dubl. Mth.J. 7 (1852) 279-; 8 (1853) 149-.

- Prouhet, E. N. A. Mth. 12 (1853) 85-.

-. Kelland, P. [1855] Edinb. R. S. T. 21 (1857) 359-.

-. Polignac, (Prince) C.de. [1868] L. Mth. S. P. 2 (1869) 69-.

- Robertson, A. Edinb. Mth. S. P. 4 (1886) 78 -

-. Hertzsprung, S. Ts. Mth. 5 (1887) 13-; Fschr. Mth. (1887) 198-.

-. Brunel, -. Bordeaux S. Sc. Mm. 5 (1895) xiv-.

problems. Encontre, D. Mntp. Rec. Bll. 2 (1805) 68-.

-. Anon. (vi 538) Gergonne A. Mth. 11 (1820-21) 165-

-.Weiss, A. Crelle J. 34 (1847) 255-; 38 (1849) 107-.

-. Sinram, T. Arch. Mth. Ps. 63 (1879) 445-

-. Holtze, A. Arch. Mth. Ps. 11 (1892) 284-.

proof for number of combinations of $n$ things taken $r$ at a time. Scott, W. H. Mess. Mth. 5 (1876) 18-.

- of theorem. Förstemann, W. A. Crelle J. 13 (1835) 237-

- - Crone, C. (xir) Ts. Mth. 1 (1877) 153

questions in. De Morgan, A. Assur. Mg. 5 $\left({ }^{*} 1855\right)$ 93-.

regular, and applications. André,D. Par. Éc. Norm. A. 5 (1876) 155-.

with repetition. Martynowski, $J$. Liège Mm. S. Sc. 16 (1861) 53-.

- - Catalan, E. C. Liége Mm. S. Sc. 2 (1867) 1-. 
Combinations, continued.

with repetition. Souffet, - $\quad$ Les Mondes 17 (1868) 110-; 24 (1871) 337-.

- - Motta Pegado, L. P. da. Lisb. J. Sc. Mth. 8 (1880) 1-.

- - Gelin, -. Mathesis 6 (1886) 175-.

in sets having odd and even numbers of inversions. Metzler, W. H. Am. J. Mth. 22 (1900) 55-.

simple. André, D. N. A. Mth. 10 (1871) 221 -

special class. Bouniakonsky, $V_{\text {. }}$ [1842] $\mathrm{St}$ Pét. Ac. Sc. Mm. 5 (1844) 297-:

- kind. Buniakovskij, V. J. [1871] St Pet. Ac. Sc. Mm. (Rs.) 20 (*1872) (Suppl.) No. 2, $71 \mathrm{pp}$.

theorem. Whitworth, W. A. [1878] Mess. Mth. 8 (1879) 82-.

- Christie, R. W. D. L. Mth. S. P. 20 (1889) 119-.

- of H. F. Baker. Gegenbauer, L. Wien Ak. Sb. 102 (1893) (Ab. $2 a)$ 951-.

theorems. Hessel, J. F. C. Grunert Arch. 7 (1846) 295-.

- Kirkman, T. P. (vi Adds.) Ph. Mg. 4 (1852) 209-; (rir) Camb. and Dubl. Mth. J. 8 (1853) 38-.

- André, D. N. A. Mth. 12 (1873) 84-.

-, new. Oettinger, L. Grunert Arch. 15 (1850) 241-; Crelle J. 53 (1857) 322-.

theory. Baur, C. W. Schlömilch Z. 2 (1857) 267-.

-. Bie, L. H. N. Ts. Mth. 6 (A) (1895) 81-. - and application to theory of numbers. Stern, M. A. Crelle J. 21 (1840) 91-, 177-. and variations, number and sums. Wasmund, C. Grunert Arch. 21 (1853) 228-.

Combinatory aggregation, analysis. Sylvester, J. J. Ph. Mg. 24 (1844) 285-.

- analysis. Jacobi, C. G.J. Crelle J. 22 (1841) 372-.

- - , foundations of new theory. MacMahon, (Maj.) P. A. Phil. Trans. (A) 194 (1900) $361-$.

- - game of mousetrap. Cayley, A. QJ. Mth. 15 (1878) 8-.

$--1,-1$ - Steen, A. QJ. Mth. 15 (1878) 230-.

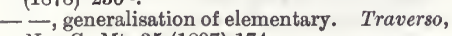
$N$. G. Mt. 35 (1897) 174.

- - inversion of fundamental idea of Hindenburg's. Roth, F. Arch. Mth. Ps. 70 (1884) 427-; 2 (1885) 82-.

- -, Mairan's problem. Cournot, A. A. Férussac Bll. Sc. Mth. 11 (1829) 93-.

- -, new method in. MacMahon, (Maj.)

P. A. Camb. Ph. S. T. 16 (1898) 262-.

- - problem. Spottiswoode, $W$. Ph. Mg. 3 (1852) 349-.

- - - André, D. Par. S. Mth. Bll. 5 (1877) 150-.

- - - - Ocagne, M. d'. N. A. Mth. 19 (1880) 44-.

- - -, solution. Ramus, C. Crelle J. 11 (1834) 353-.
Partitions, Distributions, etc. 1620

Combinatory analysis, summation formulae of Steiner, proof. Gudermann, C. [1829] Crelle J. 5 (1830) 402-.

- - theorems. Scherk, H. F. Crelle J. 11 (1834) 226-; 20 (1840) 360-.

- formula, applications. Gerbaldi, $F$. G. Mt. 18 (1880) 308-.

- numbers, graphic representation. Laisant, C. A. As. Fr. C. R. (1893) (Pt. 2) 298 .

- problem. Vachette, A. N. A. Mth. 7 (1848) 421-.

93-

- - Cantor, M. Schlömilch Z. 2 (1857) $103-$

- - Steiner. Reiss, M. [1856] Crelle J. 56 (1859) 326-.

- - - Brunel, G. Bordeaux S. Sc. PV. (1896-97) 37-.

- problems. Thibault, - . N. A. Mth. 4 (1845) 627-.

- class. Hertzsprung, S. Ts. Mth. 4

(1886) 154-; Fschr. Mth. (1887) 198.

- four. Schröder, E. Z. Mth. Ps. 15 (1870) 361-.

- products, formulae and coefficients for calculation. Wasmund, C. Grunert Arch. 34 (1860) 440-.

- symbol of Euler's, use in analysis. Terquem, O. Liouv. J. Mth. 4 (1839) 177-.

- systems, order and class. Prouhet, $E$. Liouv. J. Mth. 1 (1856) 321-.

- theorem. Stern, M. A. Crelle J. Mth. 95 (1883) 102-.

- - of Stern, proof. Terquem, O. Liouv. J. Mth. 3 (1838) 556-.

Compositions of numbers, theory. MacMahon, P. A. [1892] Phil. Trans. (A) 184 (1894) 835-.

Connected elements, notation for and distribution. Polignac, C. de. As. Fr. C. R. (1884) (Pt. 2) 37-.

Contact and isolation, a problem in permutations. Fortey, H. L. Mth. S. P. 15 (*188384) $98-$.

Cycle, problem of completing a. Waelsch, $E$. Wien Ak. Sb. 90 (1885) (Ab. 2) 160-.

Derangements. Rodrigues, $O$. Liouv. J. Mth. 4 (1839) 236-.

-. Henry, C. N. A. Mth. 20 (1881) 5-

-. Musso, G. Rv. Mt. 4 (1894) 109 -

Dirichlet's function [coefficient of $\sqrt{b}$ in $\left.(x+\sqrt{b})^{n}\right]$. Daublebsky von Sterneck, $R$. Mh. Mth. Ps. 7 (1896) 342-

Distribution of points on a line. Comstock, M. L. Des Moines Anal. 1 (1874) 156-.

Diversities, number of. Lipschitz, - C. R. 102 (1886) 602-.

Double of odd number, partition into two odd numbers. Maria, P. C. G. Mt. 4 (1866) 1-, 65-, 193-.

Dyads formed with 2 elements. Brunel, $G$. Bordeaux S. Sc. PV. (1897-98) 104-.

- - even number of elements. Brunel, $G$. Bordeaux S. Sc. PV. (1894-95) 32-.

- _ $n$ elements, aggregate. Brunel, $G$. Bordeaux S. Sc.. PV. (1895-96) 6-. 
1620 Permutations, Combinations, Partitions, Distributions, etc. 1620

Enumeration of groups of totitives. Tanner, H. W. L. L. Mth. S. P. 27 (1896) 329-.

Equations, linear, symbolic, solution in connection with theory of permutations. Cauchy, A. L. C. R. 21 (1845) 1123-.

Factorial functions. Piani, D. [1847] Bologna Mm. Ac. Sc. 1 (1850) 511-.

- numeration applied to permutations. Laisant, C. A. Par. S. Mth. Bll. 16 (1888) 176-.

- $n ! / r !(n-r) !$ an integer. Miller, W. $H$. Crelle J. 13 (1835) 257-.

$-m(m+1) \ldots(m+n-1)$. Liouville, J. Liouv. J. Mth. 2 (1857) 277-.

Factorials. Bellavitis, G. A. Sc. Lomb. Ven. 4 (1834) 10-.

-. McCulloch, J. F. A. Mth. 4 (1888) 161-.

-, approximate expression for $x$ ! Forsyth, A. R. B. A. Rp. (1883) 407-.

- - value of $n$ ! Liouville, $J$. Liouv. J. Mth. 4 (1839) 317-.

-,- when $n$ is large. Serret, J. A. C. R. 50 (1860) 662-.

- (faculties). Oettinger, L. Crelle J. 33 (1846) 1-, 117-, 226-, 329-; 35 (1847) 13-; 38 (1849) 162-, 216-; 44 (1852) 26-, 147-.

- and faculties, treatment by limits. Ohm, $M$. Crelle J. 39 (1850) 23-.

-, W. G. Horner's method. Horner, J. [1872] QJ. Mth. 12 (1873) 258-.

-, new method. Nicholson, $P$. Tilloch $\mathrm{Ph}$. Mg. 53 (1819) 412-.

-, - theorem. André, D. (Ix) Par. S. Mth. Bll. 1 (1873) 84-.

-, numerical values. Kotel'nikov, P. I. (xII) Kazan Un. Mm. ((1848) Bk. 4) 9-.

-, ratios of reciprocal. Cauchy, A. L. C. R. 17 (1843) 921-, 1159-.

-, theorem. Gomes Teixeira, E. Arch. Mth. Ps. 2 (1885) 265-.

Faculties, geometric, and transformation of logs. into definite integrals. Cauchy, A.L. C. R. 17 (1843) 779-.

- and powers; analogy. Ampère, A.M. Gergonne A. Mth. 15 (1824-25) 369-.

-, quotients of two. Schlömilch, O. Schlömilch Z. 3 (1858) 322-.

-, remarks on some. Guibert, M.P.A. N. A. Mth. 19 (1860) 213-.

-, theorem. Schlömilch, O. Grunert Arch. 7 (1846) 331-.

一, theory. Bessel, F. W. Königsb. Arch. 1 (1812) 241-?

-, -. Kramp, C. Gergonne A. Mth. 3 (181213) 1-, 114-, 325-.

-, -. Müller, A. Crelle J. 11 (1834)

Faculty coefficients. Studnička, F. J. Prag České Ak. Fr. Jos. Rz. (Tř́ida 2) 9 (1900) Art. 17, 10 pp.; Fschr. Mth. (1900) 156-.

- - determination. Schlömilch, $O$. Grunert Arch. (1848) 445-.

- - relations. Schlömilch, O. Grunert Arch. 9 (1847) 333-.

Fractional coefticients. Barsotti, G. Lucca At. Ac. 12 (1843) 411-.
Game of draughts, problem. Lamarle, $E$. [1852] Brux. Ac. Sc. Mm. 27 (1853) 47 pp.

- - Gordian knot (bar and rings), theory. Dassen, C. C. Arg. S. Ci. A. 44 (1897) 337-.

- - jumping frog, arrangement of counters on board. Berg, F. J. van den. N. Arch. Wisk. 12 (1886) 38-; Fschr. Mth. (1885) 121.

- loto. Du Hays, -. Liouv. J. Mth. 7 (1842) 192-.

- played with 15 pieces on square board of 16 holes. Bellavitis, G. Ven. I. At. 6 (1880) 901-.

- of solitaire. Reiss, M. Crelle J. 54 (1857) 344-.

- - Hermary, -. As. Fr. C. R. (1879) 284 .

- - , as problem of analysis and situation. Suremain-de-Missery, A. Dijon Ac. Mm. (1841-42) 58-.

Grouping in games. André, D. Par. S. Phlm. Bll. 1 (1899) 139-; 2 (1900) 45-, 77-.

- of instruments for simultaneous telegraphy. Zetzsche, E. J. Tél. 15 (1891) 201-, 229-, 255-.

- problems, analytical treatment. Hoyer, $P$. Mth. A. 50 (1898) 499-.

Homogeneous products of $n$ things of $r$ dimensions, proof. Walton, W. [1869] QJ. Mth. 10 (1870) 219-.

Hyper-magic arithmetical spaces. Laisant, C. A. Par. S. Mth. Bll. 22 (1894) 28 -

- squares, general limitations affecting. Roberts, S. [1892] L. Mth. S. P. 24 (1893) 37-.

La tour d'Hanoï (problem in permutations). Allardice, R. E., \& Fraser, A. Y. Edinb. Mth. S. P. 2 (1884) 50-.

Latin squares. Cayley, A. Mess. Mth. 19 (1890) 135-.

_-, Euler's. Maillet, $\boldsymbol{E}$. As. Fr. C. R. (1894) (Pt. 2) 244.

Magic cubes in $n$ dimensions, way of representing in plane. Schlegel, - Par. S. Mth. Bll. 20 (1892) 97-.

- cyclovolute. Nulty, E. [1834-44] Am. Ph. S. T. 5 (1837) 205-; 10 (1853) 17-.

- parallelopipeds. Harmuth, T. [1881] Arch. Mth. Ps. 67 (1882) 238-.

- rectangles with odd numbers of sides. Harmuth, T. Arch. Mth. Ps. 66 (1881) 413-.

\section{Magic squares.}

Moon, R. Camb. Mth. J. 4 (1845) 209-. Clausen, T. Grunert Arch. 21 (1853) 97-. Györy, S. (xп) Mag. Ak. Ets. (1856) 77-。 Thompson, W. H. QJ. Mth. 10 (1870) 186-. Drach, S. M. Mess. Mth. 2 (1873) 169-, 187. Cayley, A. Mess. Mth. 6 (1877) 168.

Frisby, $E$. Wash. Ph. S. Bll. 3 (1880) 143-.

Marchand, D. Les Mondes 2 (1882) 106-, 247-.

Rocquigny, F. [G.] de. Les Mondes 2 (1882) 163. 


\section{Magic Squares}

Arnoux, G. As. Fr. C. R. (1885) (Pt. 1$) 94$.

Schoute, P. H. As. Fr. C. R. (1885) (Pt. 2) 152-.

Coccoz, -. As. Fr. C. R. (1886) (Pt. 2) 130-.

Frolow, $M$. As. Fr. C. R. (1886) (Pt. 2) 170 .

Clauss, $F$. Arch. Mth. Ps. 6 (1888) 424-.

Berg, F. J. van den. N. Arch. Wisk. 16 (1889) 1-; Fschr. Mth. (1889) 203.

Parmentier, (le gén.) - As. Fr. C. R. (1890) (Pt. 2) 88-.

Coccoz, -. As. Fr. C. R. (1892) (Pt. 2) 136-, [1213]; (1894) (Pt. 2) 163-, 1219; (1895) (Pt. 2) 102-.

Ball, $W . W: R$. Mess. Mth. 23 (1894) 65-.

Iznoskov, I. Kazan S. Ps.-Mth. Bll. 5 (1896) (Prot.) 48-.

algebra. Horner, J. QJ. Mth. 11 (1871) 57-, 123-, 213-.

application of group theory. Maillet, E. Toul. Ac. Sc. Mm. 6 (1894) 258-.

with base eight. Coccoz,-. As. Fr. C. R. (1893) (Pt. 2) 171-, 1079.

case. Holditch, H. QJ. Mth. 6 (1863) 181-.

- of knight's march. Beverley, W. (vi Adds.) Ph. Mg. 33 (1848) 101-.

- $n^{2}$ numbers. Monteiro, A. S. G. Teix. J. Sc. 3 (1881) 81-.

and cubes. Frost, A. H. QJ. Mth. 7 (1866) $92-$.

- Barnard, F. A. P. Wash. Nat. Ac. Mm. 4 (Pt. 1) (1888) 209-

- - Mayor, P. Laus. S. Vd. Bll. 27 (1892) 243-.

cubes, etc., theory. Moon, R. Camb. and Dubl. Mth. J. 1 (1846) 160 -

doubly magic. Laquière, E. M. As. Fr. C. R. (1880) 243-.

etc. Harmuth, T. Arch. Mth. Ps. 66 (1881) 286-.

Euler's. Boije af Gennäs, C. O. [1898] Stockh. Ak. Hndl. Bh. 24 (Afd. 1) (1899) No. 2, 15 pp.

and Gauss. Günther, S. Z. Mth. Ps. 21 (1876) (Suppl.) 61-.

where highest number is prime, law. Tychsen, C. (xII) Ts. Mth. 1 (1865) 55-.

most perfect forms. McClintock, E. Am. J. Mth. 19 (1897) 99-.

and other such arrangements. Smyth, B. B. Kan. Ac. Sc. T. 14 (1896) 46-

proof of theorem. Ginther, S. Arch. Mth. Ps. 57 (1875) 285-.

Mixtures, problem. Coupy, É. N. A. Mth. 6 (1847) 14.

$n$ 'ads formed with $n^{2}$ elements. Brunel, $G$. Bordeaux S. Sc. PV. (1894-95) 56-.

$\nu$ cycles of $2 \nu+1$ elements. Brunel, $G$. Bordeaux S. Sc. PV. (1898-99) 71-.

Nasik cubes. Frost, A.H. QJ. Mth. 8 (1867) 74.

--, general properties. Frost, A. H. [1877] QJ. Mth. 15 (1878) 93-, 366-.

- squares of any order, construction. Frost, A. H. L. Mth. S. P. 27 (1896) 487-.

\section{Permutations, etc. 1620}

Nasik squares, general properties. Frost, $A$. H. [1877] QJ. Mth. 15 (1878) 34-.

Network representing benzene. Brunel, $G$. Bordeaux S. Sc. Mm. 4 (1894) xviii-.

Notation $\left[\begin{array}{llll}a_{1} & a_{2} & \ldots & a_{p}\end{array}\right]^{(m)}$ to represent expansion of $m$ th power of polynomial $a_{1}+a_{2}+\ldots+a_{p}$. Ocagne, M. d'. N. A. Mth. 2 (1883) 220 .

Number of complete arrangements satisfying given conditions. André, D. C. R. 87 (1878) 838-; Par. S. Mth. Bll. 7 (1879) 43-.

- distributions with repetition of given class and weight. Caldarera, G. G. Mt. 31 (1893) 161-.

- - partitions of a rational integer into sums. Hermes, J. Mth. A. 45 (1894) 371-; 47 (1896) 281-.

- - permutations of $n$ elements presenting $s$ sequences. André, D. C. R. 97 (1883) 1356-.

- substitutions falling into given number of cycles. Schröder, E. Arch. Mth. Ps. 68 (1882) 353-.

- - ways of getting sum not exceeding $n$, from terms of series 1,2,3 ... Platner, $G$. Mil. I. Lomb. Rd. 21 (1888) 690-, 702-.

Numbers, $4 m+2$ as sum of 12 squares. Liouville, J. Liouv. J. Mth. 5 (1860) 143-.

Partition analysis applied to properties of any system of consecutive integers. MacMahon, (Maj.) P. A. [1899] Camb. Ph. S. T. 18 (1900) 12-.

\section{Partition of numbers.}

Warburton, H. [1847] Camb. Ph. S. T. 8 (1849) 471-.

Battaglini, G. Nap. Ac. Sc. Mm. 2 (1855-57) 353-.

Cayley, A. [1855-57] Phil. Trans. (1856) 127-; (1858) 47-; Ph. Mg. 13 (1857) 245-.

Brioschi, F. Tortolini A. 8 (1857) 5-.

Sylvester, J. J. QJ. Mth. 1 (1857) 81-, 141-, 198-; Tortolini A. 8 (1857) 12-.

Volpicelli, P. Tortolini A. 8 (1857) 22-.

Bellavitis, G. Tortolini A. 2 (1859) 137-.

Lanavicensis, (Pseud.) [J. J. Sylvester] $\mathrm{Ph}$. Mg. 18 (1859) 283-.

Trudi, N. (xI) Nap. Ac. At. 2 (1865) No. 23, 50 pp.

Catalan, E. C. N. A. Mth. 8 (1869) 407-.

Buniakovskij, V.Ja. [1874] St Pet. Ac. Sc. Mm. (Rs.) 25 (* 1875) (Suppl.) No. 1, 29 pp. Glaisher, J. W. L. C. R. 80 (1875) 255-.

Laguerre, E. Par. S. Mth. Bll. 5 (1877) 76-.

Faà de Bruno, F. C. R. 86 (1878) 1189-, 1259.

David, 一. C. R. 90 (1880) 1344-; 91 (1880) 621-.

Catalan, E. C. Liège S. Sc. Mm. 12 (1885) No. 2, 19-, 56-, 264-; 13 (1886) 314-.

Pomey, J. B. N. A. Mth. 4 (1885) 408-.

Polignac, C. de. C. R. 104 (1887) 1688-

Glösel, K. Mh. Mth. Ps. 7 (1896) 133-, 290.

Nathews, G. B. L. Mth. S. P. 28 (1897) 486-. 
addition to theory. Csorba, G. Mth. Termt. Éts. 17 (1899) 189-.

application of theorem of Eisenstein. Catalan, - As. Fr. C. R. (1886) (Pt. 1$) 86$.

certain special. MacMahon, (Capt.) P. A. QJ. Mth. 21 (1886) 367-.

formulae. Pepin, -. Liouv. J. Mth. 4 (1888) 83-.

graphic proof of theorem of Euler's. Sylvester, J. J. C. R. 96 (1883) 1110-.

whose graphs possess symmetry. MacMahon, (Maj.) P. A. [1898] Camb. Ph. S. T. 17 (1899) 149-.

number of ways of breaking up a number with one law of subordinate partition. Volpicelli, P. Rm. At. 10 (1856-57) 43-, 122-.

outlines of seven lectures. Sylvester, J. J. L. Mth. S. P. 28 (1897) 33-.

points of analysis depending on. Trudi, N. [1877] Nap. Ac. At. 8 (1879) No. 1, $88 \mathrm{pp}$.

problem. Chrystal, G. Edinb. Mth. S. P. 2 (1884) 49-.

and other problems, application of Arbogast's method of derivations. David, -. Liouv. J. Mth. 8 (1882) 61-.

proof of theorem. Volpicelli, P. C. R. 40 (1855) 1150-.

- - Zuchristian, J. Mh. Mth. Ps. 4 (1893) 185-.

question. Boschi, P. Bologna Ac. Sc. Mm. 1 (1880) 555-.

theory. Maciahon, P. A. [1896] Phil. Trans. (A) 187 (1897) 619-; 192 (1899) 351-.

two theorems. Volpicelli, P. N. A. Mth. 14 (1855) 314-.

verification formulæ. Glaisher, J.W.L. R. S. P. 24 (1876) 250 -.

Partition of pentagonal numbers, graphic proof of Euler's theorem. Sylvester, J.J. C. R. 96 (1883) 743-.

- polygon by diagonals, series for. Binet, J. P. M. Liouv. J. Mth. 8 (1843) 394.

-, problem. Ocagne, M. d'. Par. S. Mth. Bli. 28 (1900) 157-.

- -series, note. Cayley, A. Am. J. Mth. 6 (1884) 63-.

-, solution of problem, and types of abacus. MacMahon, (Maj.) P. A. Par. S. Mth. Bll. 26 (1898) 57-.

- of sum of money, number of ways. Schubert, H. C. H. Hamb. Mth. Gs. Mt. 1 (1889) 269.

- table for binary quantics, specimen. Cayley, A. Am. J. Mth. 4 (1881) 248 -

- tables, non-unitary. Cayley, A. Am. J. Mth. 7 (1885) 57-.

\section{PARTITIONS.}

Cayley, A. QJ. Mth. 1 (1857) 183-.

Franklin, F. Am. J. Mth. 2 (1879) 187-; (xII)

J. H. Un. Cir. [2] (1883) 72.

Ely, G. S. J. H. Un. Cir. [3] (1884) 76-.
Barcroft, D. J. H. Un. Cir. [5] (1885-86) 64.

algebraic expression. Herschel, (Sir) J. F.W. Phil. Trans. (1850) 399-.

- theorems connected with theory. Forsyth, A. R. [1895] L. Mth. S. P. 27 (1896) 18 .

of complex numbers (theorem contained in theorem of Jacobi's). Sylvester, J.J. C. R. 96 (1883) 1276-.

compound, general theory. Sylvester, J. J. Ph. Mg. 16 (1858) 371-.

-, method of graphs applied to. Ely, G.S. Am. J. Mth. 6 (1884) 382-.

constructive theory. Sylvester, J. J. Am. J. Mth. 5 (1882) 251-; 6 (1884) 334-.

- - Sylvester's. Jenkins, M. Am. J. Mth. 7 (1885) 74-.

and derivations. Glaisher, J.W. L. B. A. Rp. (1874) (Sect.) 11-.

double. Cayley, A. Ph. Mg. 20 (1860) 337-.

formulæ of verification. Glaisher, J.W. L.

B. A. Rp. (1875) (Sect.) 11-.

fundamental theorem. Sylvester, J. J. (xII) [1882] J. H. Un. Cir. [2] (1883) 22.

graphical method. Sylvester, J.J. (xII) J. H. Un. Cir. [2] (1883) 70-

isomerism, graphical representation. Brunel, G. Bordeaux S. Sc. PV. (1898-99) 108-.

-, problem (to find number of compounds that can be formed by $n m$-valent atoms and $(m-2) n+2$ univalent atoms). Franklin, $F$. Am. J. Mth. 1 (1878) 365-.

miscellaneous theorems. Glaisher, J. W. L. Mess. Mth. 5 (1876) 164-.

of non-pentagonal number. Jacobi, C. G. J. Crelle J. 32 (1846) 164-.

note. Glaisher, J.W.L. Ph. Mg. 49 (1875) 307 -

- Mitchell, O.H. (xII) J. H. Un. Cir. [1] (1882) 210.

-. Ely, G. S. (xп) J. H. Un. Cir. [1] (1882) 211.

of a polygon. Cayley, A. L. Mth. S. P. 22 (1891) 237-.

problem. Cayley, A. Mess. Mth. 7 (1878) 187-.

-. Brunel, G. Bordeaux S. Sc. PV. (189495) 24 .

-, note. Tait, P. G. Edinb. R. S. T. 32 (1887) 340-.

- and theory of forms. Capelli, A. [1880] G. Mt. 19 (1881) 87-.

product $\overline{1-x} \cdot \overline{1-x^{2}} \cdot \overline{1-x^{3}}$, Franklin's theory. Cayley, A. (xII) J. H. Un. Cir. [2] (1883) 86.

proof of Sylvester's theorem. Roberts, S. QJ. Mth. 4 (1861) 155-.

- - theorem. Jenkins, M. Am. J. Mth. 6 (1884) 331-.

question. Sylvester, J. J. (xп) J. H. Un. Cir. [1] (1882) 179.

of $R$ and of the $\dot{R}$-gon. Kirkman, T. $P$. Manch. Lt. Ph. S. Mm. \& P. 7 (1893) 211-; 8 (1894) 109-.

$r$, of $r^{2}-r+1$. Kirkman, T. P. Lanc. T. Hist. S. 9 (1857) 127-. 
Partitions, continued.

7-, of X. Kirkman, T.P. Manch. Ph. S. Mm. 14 (1857) 137-.

self-opposite, number of. Durfee, W.P. (xII) [1882] J. H. Un. Cir. [2] (1883) 23. - - - - (Durfee). Sylvester, J.J. (xII) J. H. Un. Cir. [2] (1883) 23-, 42-.

theorem. Glaisher, J. W. L. [1879-83] Mess. Mth. 9 (1880) 47-; 12 (1883) 158-.

-. Sylvester, J. J. C. R. 96 (1883) 674-.

-, new. Sylvester, J. J. (xII) J. H. Un. Cir. [2] (1883) 70.

theorems. Mansion, P. Mess. Mth. 5 (1876) 90.

-. Glaisher, J.W. L. Mess. Mth. 5 (1876) 91.

theory, note. Sylvester, J. J. B. A. Rp. 41 (1871) (Sect.) 23-.

Perfect partitions of numbers and compositions of multipartite numbers. Macmahon, (Major) P. A. Mess. Mth. 20 (1891) 103-.

Permutants, theory. Cayley, $A$. Camb. and Dubl. Mth. J. 7 (1852) 40-, 97-.

Permutation, alternate, probability of. André, D. C. R. 97 (1883) 983-.

-, geometrical. Cayley, A. Ph. Mg. 30 (1865) 370-.

- numbers, approximate calculation. Schlömilch, O. Z. Mth. Ps. 10 (1865) 232-.

\section{PERMUTATIONS.}

Conti, C. Padova N. Sag. 3 (1831) 90-

Cayley, A. Ph. Mg. 34 (1849) 527-.

Bourget, J. N. A. Mth. 10 (1871) 254-.

Lucas, E. As. Fr. C. R. (1883) 83-.

alternate. André, D. Liouv. J. Mth. 7 (1881) 167-.

- and quasi-alternate, relation between number of. André, D. Par. S. Phlm. Bll. 8 (1896) 5-.

application of new method to. Sprague, $T . B$. Edinb. R. S. T. 37 (1895) 399-.

a certain species. André, D. Par. S. Phlm. Mm. Cent. (1888) 35-.

circular, applications to theory of numbers. MacMahon, (Maj.) P. A. L. Mth. S. P. 23 (1892) 305-.

- distinct. Moreau, C. N. A. Mth. 11 (1872) 309-.

-, notion of maxima and minima in. André, D. Par. S. Phlm. Bll. 5 (1893) (C. R. No. 11) 2-.

-, sequences. André, D. Par. S. Mth. Bll. 23 (1895) 122-.

and combinations. Pagnini, G. M. Brugnatelli G. 3 (1810) 329-.

- - Warburton, H. [1847] Camb. Ph. S. T. 8 (1849) $471-$.

- - Whitworth, W. A. [1865] Mess. Mth. 3 (1866) 193-

- - problem. Balbo, P. Turin Mm. Ac. 14 (1804-5) 224-. and combinations, proofs of theorems, and of theorem in stereometry. Seidelin, C. J. $L$. (xII) Ts. Mth. 6 (1870) 20-, 22-; 6 (1876) 63-.

- - theory. Hertzsprung, S. (xII) Ts. Mth. 5 (1869) 97-.

continuous, by uniform law. Paradisi, $G$. [1816] Mod. Mm. S. It. 18 (1818) 143-.

and cyclic arrangements, theory. Jablonski, $E$. C. R. 114 (1892) 904-; Liouv. J. Mth. 8 (1892) 331-; Prace Mt.-Fiz. 4 (1893) 71-.

of first $n$ integers, method of deducing consecutively. Renshaw, A. Mess. Mth. 4 (1868) 36-.

- - - numbers, distribution into 4 groups. André, D. Par. S. Phlm. Bll. 5 (1893) 33-.

- $n$ letters, classification in groups. Despeyrous, C. Liouv. J. Mth. 10 (1865) 177-.

$-2 q$ letters alike in pairs. Vachette, $A$. N. A. Mth. 13 (1874) 549-; 14 (1875) 299-, 337-.

- $3 q$ letters alike in threes. Vachette, $A . \mathrm{N}$. A. Mth. 14 (1875) 438-.

- - - Moreau, C. N. A. Mth. 15 (1876) 90 -

- Vachette, A. N. A. Mth. 15 (1876) 114-, 145-, 193-.

limited. Laisant, C. A. C. R. 112 (1891) 1047-.

maxima, minima and sequences. André, $D$. Par. Éc. Norm. A. 1 (1884) 121-.

new calculus, applicable to theory. Cauchy, A. L. C. R. 22 (1846) 53-, 99-.

- demonstration of theorem. André, D. Par. S. Phlm. Bll. 3 (1891) 153-.

notes. Morley, $F$. N. Y. Mth. S. Bll. 3 (1894) $142-$.

of a number, sum. Marre, A. N. A. Mth. 5 (1846) 57-.

- $n$ objects. Bourget, J. N. A. Mth. 2 (1883) 433-.

problem. Barlow, P. Nicholson J. 23 (1809) 203-.

- Arndt, F. Grunert Arch. 3 (1843) 210-.

- Allardice, R. E. Edinb. Mth. S. P. 8 (1890) 64-.

-. Lagrandval, - de. Bordeaux S. Sc. PV. (1895-96) 22-.

_, solution. Drot, -. N. A. Mth. 5 (1846) 97-.

problems, two. Laisant, - . Par. S. Mth. Bll. 19 (1891) 105-.

quasi-alternate. André, D. C. R. 119 (1894) 947-; Liouv. J. Mth. 1 (1895) 315-.

with restrictions as to placing. Kantor, $S$. Z. Mth. Ps. 28 (1883) 379-.

Schubert's solution of Bachet's problem. Busche, E. Mth. A. 47 (1896) 105-.

self-conjugate. Muir, T. Edinb. R. S. P. 17 (1891) 7-.

- MacMahon, (Maj.) P. A. Mess. Mth. 24 (1895) 69-.

with sequences. Morley, F. [1897] N. Y. Am. Mth. S. Bll. 4 (1898) 23-. 


\section{Permutations, Combinations, Partitions, Distributions, etc. 1620}

Permutations, continued.

square, of base 6. Tarry, G. Liège S. Sc. Mm. 2 (1900) No. 7, 10 pp.

successive, problem of Monge's. Bourget, $J$. Liouv. J. Mth. 8 (1882) 413-.

theorem of MacMahon's. Gegenbauer, L. Mh. Mth. Ps. 11 (1900) 287-.

theory. Despeyrous, C. Liouv. J. Mth. 6 (1861) 417 $\rightarrow$

transformation and classification. Sprague, T. B. Edinb. Mth. S. P. 9 (1891) 59-.

Polygons, division into triangles. (Segner's numbers.) Lamé, G. Liouv. J. Mth. 3 (1838) 505-.

Arch. 2 (1842) 117-

- - - - . ( - - .) Catalan, E. C. [1870] Liège S. Sc. Mm. 13 (1886) 54-, 399.

-,- - - . ( - - .) Rove, R. C., d Taylor, H. M. L. Mth. S. P. 13 (1881-82) 102-.

-, - - - . (- - .) Gelin, (l'abbé) E. (xII) Mathesis 3 (1883) 108-.

- , - - ( $(-)$.) Catalan, E. C. [1886] Palermo Cir. Mt. Rd. 1 (1887) 190-.

Polynomial coefficients. Piuma, C.M. G. Mt. 29 (1891) 34-.

- - Klekler, P. Mh. Mth. Ps. 10 (1899) 218 .

- -, properties. Sadun, E. G. Mt. 31 (1893) $119-, 379$.

- theorem. Ladd, C. Des Moines Anal. 5 (1878) 145-

Products of $n$ factors, number of. Rodrigues, $O$. Liouv. J. Mth. 3 (1838) 549.

Quadricycles of $8 n+1$ elements, construction. Brunel, G. Bordeaux S. Sc. PV. (1895-96) $58-$.

- - $n$ elements, systems. Brunel, G. Bordeaux S. Sc. PV. (1898-99) 59-.

"Quotités" of combinations. Soulé, - . Bordeaux S. Sc. Mm. 3 (1893) xiv-, xxv-.

Series for $n !$. Heather, J. F. Mathematician 2 (1847) 296-.

$$
\begin{aligned}
-z^{n}-\left(\begin{array}{c}
m \\
1
\end{array}\right)(z-1)^{n} & +\left(\begin{array}{c}
m \\
2
\end{array}\right)(z-2)^{n}-\ldots \\
& +(-1)^{m}\left(\begin{array}{c}
m \\
m
\end{array}\right)(z-m)^{n},
\end{aligned}
$$

properties and application. Unferdinger, $F$. Wien Sb. 67 (1873) (Ab. 2) 363-.

$-\Sigma \frac{n^{m}}{n !}$ for $m=1,2,3,4,5$, summation. Dobin-

$s k i, G$. Arch. Mth. Ps. 61 (1877) 333-.

Signs, successions of, and application to coloured tiles and Newton's rule, etc. Sylvester, J. J. Ph. Mg. 34 (1867) 461-.

Stifel's bordered squares. Fontès, - . As. Fr. C. R. (1895) (Pt. 2 ) 248-.

Sub-factorial N. Whitworth, W. A. Mess. Mth. 7 (1878) 145-.

Sum of the cubes of the coefficients in $(1-x)^{2 n}$. Richmond, H. W. Mess. Mth. 21 (1892) 77 -
Sum of numbers of all variations $p+1, p+2 \ldots$ to $n$th class inclusive $=n(n-1) \ldots(n-p-1)$ $\{[e(n-p) !]-1\}$. Huber, M. T. Wiad. Mt. 3 (1899) 39-; 4 (1900) 93; Fschr. Mth. (1899) 208.

Symmetric functions of numbers, and their partition. Scott, G. [1865] QJ. Mth. 7 (1866) 81-.

Tactic disclosing existence of 4-valued functions. Sylvester, J. J. Ph. Mg. 21 (1861) 515-; 22 (1861) 45-.

- of 9 elements. Sylvester, J. J. Ph. Mg. 22 (1861) 144-.

Tactical memoranda. Moore, E. H. Am. J. Mth. 18 (1896) 265-.

Ternary systems of 13 elements. Pasquale, $V$. de. Mil. I. Lomb. Rd. 32 (1899) 213-.

Thirty cubes constructed with six differently coloured squares. MacMahon, (Maj.) P. A. L. Mth. S. P. 24 (1893) 145-.

Thirty-six officers, problem. Tarry, G. As. Fr. C. R. (1900) (Pt. 2) 170-.

'Trees.' Cayley, A. Ph. Mg. 13 (1857) 172-; 18 (1859) 374-.

-. Polignac, C. de. Par. S. Mth. Bll. 8 (1880) 120-; 9 (1881) 30-.

-. Cayley, A. (xir) J. H. Un. Cir. [1] (1882) 202.

-. Sylvester, J. J. (хп) J. H. Un. Cir. [1] (1882) 202-.

-, with application to theory of chemical combinations. Cayley, A. B. A. Rp. (1875) 257-; Am. J. Mth. 4 (1881) 266-.

- , - - - - - Delannoy, H. As. Fr. C. R. (1894) (Pt. 2) 102-.

-, isomers, theory. Cayley, A. Ph. Mg. 47 (1874) 444-.

-, number of univalent radicals $\mathrm{C}_{n} \mathrm{H}_{2 n+1}$. Cayley, A. [1876] Ph. Mg. 3 (1877) 34-.

-, theorem. Cayley, A. QJ. Mth. 23 (1889) 376-.

_, yoke-chains and multipartite compositions in connexion with. MacMahom, (Maj.) P.A. L. Mth. S. P. 22 (1891) 330-.

Triads. Fifteen girl problem. Kirkman, T. P. Ph. Mg. 37 (1850) 169; 23 (1862) 199-.

-. - - - (Kirkman). Woolhouse, W. S. B. Ph. Mg. 22 (1861) 510-.

-. - - C Cayley, A. Ph. Mg. 25 (1863) 59-

-. - - -. Power, J. [1865] QJ. Mth. 8 (1867) 236-.

-. - - Frost, A. H. QJ. Mth. 11 (1871) 26-.

2 (1879) 397-.

-. - - Story, W. E. Am. J. Mth. 2 (1879) 399-.

Mth. Ps. 43 (1898) 329-

-. - - - , cyclic solution. Peirce, B. Gould As. J.' 6 (1861) 169-.

-. - - -, geometric picture of. Davis, E. W. A. Mth. 11 (1896-97) 156-.

-. $-\ldots-1,3$ methods and 48 solutions. Frazer, P. (jun.). Am. Ph. S. P. 18 (1880) 505-.

-. - - , note on theory. Tait, P. G. Edinb. R. S. P. 10 (1880) 664-. 


\section{Finite Summation}

Triads. Fifteen girl problem, solution. Carpmael, E. L. Mth. S. P. 12 (1880-81) 148-.

- formed with $6 n+1$ elements, systems. Brunel, G. As. Fr. C. R. (1895) (Pt. 2) 145-.

- - $6 n-1$ and $6 n-2$ elements. Brumel, G. Bordeaux S. Sc. PV. (1895-96) 40-.

- Nine girl problem. Sylvester, J.J. Mess. Mth. 22 (1893) 159-, 192.

- of once-paired elements. Horner, J. [1867] QJ. Mth. 9 (1868) 15-.

--7 and 15 things. Cayley, A. Ph. Mg. 37 (1850) 50-.

Triangle of sequences. André, D. C. R. 118 (1894) 575-, 726.

Vandermonde's theorem. Collins, E. [1839] St Pét. Ac. Sc. Bll. 6 (1840) 81-.

- - Tanner, H.W.L. Mess. Mth. 25 (1896) 71-。

- -, generalisation. Hill, M. J.M. Mess. Mth. 25 (1896) 154-.

Various theorems. Clausen, T. Crelle J. 4 (1829) 278-.

Voting. Macfarlane, A. Edinb. Mth. S. P. 2 (1884) 25-.

\section{Finite Summation. Recur- ring series.}

Arithmetical formula. Netto, E. Mth. A. 49 (1897) 148.

- series. San Bertolo, N. Rm. At. 10 (185657) 78 -

- - Jung, V. Časopis 28 (1899) 281-; Fschr. Mth. (1899) 244.

- - of different orders. Jarolimek, Č. Ča. sopis 6 (*1877) 91-; Fschr. Mth. (*1877) 176.

- - sum of cubes of terms. Shidy, L. P. Des Moines Anal. 3 (1876) 51-.

- - , whose ultimate differences are constant. Pollock, (Sir) F. [1850] R. S. P. 5 (1851) 852-.

Binomial expansion, summation of terms. Querret, - Gergonne A. Mth. 15 (1824-25) 189-.

Combination sums of squares of even and odd numbers, relations. Oppolzer, T. von. Mth. A. 13 (1878) 405-.

Combinatory numbers, certain. Lémeray, As. Fr. C. R. (1899) (Pt. 2) 99-.

Continued product $\prod_{i=0}^{i=n-1}(1+i x)$, expansion in powers of $x$, coefficients. Schläfli, L. C. R. 25 (1847) 381-; Grunert Arch. 10 (1847) 386-; 12 (1849) 53-; Crelle J. 43 (1852) 1 -.

Equations generating recurring series. André, D. Par. S. Mth. Bll. 6 (1878) 166-.

Expansion in recurring series, extension, and physical application. Töpler, A. Steierm. Mt. (1872) 64-.

Factorial expression, summation. Cayley, $A$. Ph. Mg. 13 (1857) 419-.
Figurate Numbers 1625

Factorial expressions, transformations. Plarr, G. B. A. Rp. (1857) 101-.

Factorials, formula involving. Ellis, J. C.W. QJ. Mth. 8 (1867) 256-.

\section{Figurate numbers.}

Barruel, E. Par. Éc. Pol. Cor. 2 (1809-13) 220 -

Gergonne, J. D. Gergonne A. Mth. 4 (1813-14) 115-.

Brassinne, E. N. A. Mth. 6 (1847) 120 -

Transon, A. (Prof.). N. A. Mth. 9 (1850) 255-.

Nicolä̈ès, N. Les Mondes 7 (1865) 693-; 8 (1865) 615-, 708-.

Le Cointe, J. L. A. Les Mondes 8 (1865) 707-.

Souffet, - . Les Mondes 13 (1867) 336-.

Hochheim, A. Arch. Mth. Ps. 55 (1873) 189 -.

formulae, proofs. Stegmann, $F$. Grunert Arch. 5 (1844) 82-.

new method. Nicholson, $P$. Tilloch $\mathrm{Ph} . \mathrm{Mg}$. 53 (1819) 412-.

polygonal numbers. Gough, J. Nicholson J. 20 (1808) 161-.

- - Barlow, P. Nicholson J. 21 (1808) 118 -

- - Gough, J. Nicholson J. 21 (1808) 241-.

- - Barlow, P. Nicholson J. 22 (1809) 33-.

- -. Musso, G. G. Mt. 31 (1893) 173-.

- - Catalan, E. G. Mt. 31 (1893) 227-.

- - Berger, A. Ups. S. Sc. N. Acta 17 (1898) No. 4, $38 \mathrm{pp}$.

- - Fermat's theorem. Pollock, (Sir) F. Phil. Trans. (1861) 409-; R. S. P. 13 (1864) $542-$.

_-, - - Pepin, T. Rm. N. Linc. At. 46 (1893) 119-.

- - - - extension. Pollock, (Sir) $F$. [1850] R. S. P. 5 (1851) 922-.

- - - Maillet, $E$. Par. S. Mth. Bll. 23 (1895) 40-; Liouv. J. Mth. 2 (1896) $363-$.

- - - - general proof. Cauchy, A.L. Par. Mm. de l'I. (1813-15) 177-.

_- formula for. Laisant, C. A. Par. S. Phlm. Bll. 3 (1891) 29-

polyhedral numbers. Giudice, - . Palermo Cir. Mt. Rd. 3 (1889) 231.

sums. Brassinne, E. N. A. Mth. 6 (1847) 120 .

theorem. Björling, E. G. Grunert Arch. 7 (1846) 266-

triangular numbers. Casinelli, A. (8. L.) Bologna N. Cm. 2 (1836) 415-.

- - , summation of like powers of first $n$. Barbette, E. Mathesis 15 (1895) 111-.

- - , theorems. Liouville, J. Liouv. J. Mth. 7 (1862) 407-; 8 (1863) 73-.

- - Glaisher, J. W. L. Mess. Mth. 5 (1876) 164-.

trigonal numbers. Stern, M. A. Crelle J. 69 (1868) 370-. 
Finite series. Verniory, - Mathesis 12 (1892) 217-.

- - , extension by arbitrary parameters. Most, R. Arch. Mth. Ps. 48 (1868) 104-.

-- , sum. Stern, M. A. Crelle J. 33 (1846) 362 .

- - summation. Lindman, C. F. Stockh. Öfv. 17 (1860) 415-; Grunert Arch. 38 (1862) 246-.

- - , - , and application to $\sin ^{n} x$ and $\cos ^{n} x$. Simony, O. Arch. Mth. Ps. 55 (1873) 64 -.

Integral power of integer representable as sum of consecutive odd numbers. Studnička, F. J. Časopis 13 (1884) 30-; Fschr. Mth. (1884) 212.

Multinomial coefficients, sum of all. Jones, $S$. Thomson A. Ph. 4 (1822) 27-.

-- , summation. Masters, $W$. Beng. As. S. J. 1 (1832) 187-.

Numbers, theorem $\Sigma r^{3}=(\Sigma r)^{2}$, generalisation. Liouville, J. Liouv. J. Mth. 2 (1857) 393-.

Piles of shot. Roche, - Quetelet Cor. Mth. 6 (1830) 199-.

- - Guilmin, A. N. A. Mth. 3 (1844) 30 -

-- - elementary calculation. Dostor, $G$. Arch. Mth. Ps. 56 (1874) 298-.

- - - - - theory. Roche, -. Gergonne A. Mth. 18 (1827-28) 19-.

- - - hexagonal. Noel, J. N. Quetelet Cor. Mth. 5 (1829) 347-.

-, triangular, square and oblong, formula. Hubbell, H. Franklin I. J. 34 (1857) 20 -.

Polygons, sums of numbers arranged on. Romero, J. N. A. Mth. 1 (1882) 46-.

Polynomials expressing sums of powers of series of natural numbers. Appell, $P . \mathrm{N}$. A. Mth. 6 (1887) 312-.

Powers, formation from arithmetical progressions. Wheatstone, (Sir) C. R. S. P. 7 (1854-55) 145-.

,$--\frac{1}{-}$ Cantor, M. N. A. Mth. 14 (1855) 229-.

Product of first $n$ numbers. Mandart, $H$. Mathesis 19 (1899) 221-.

- - $n$ quantities expressed in sums of their powers. Day, H. G. Mess. Mth. 5 (1871) 13-.

$-1 \cdot(1+x)(1+2 x) \ldots\{1+\overline{n-1} x\}=\stackrel{n}{\Pi}(x)$, expansion. Schläfi, L. [1866] Crelle J. 67 (1867) 179-.

$-1^{1} \cdot 2^{2} \cdot 3^{3} \ldots n^{n}$. Glaisher, J. W. L. [1877] Mess. Mth. 7 (1878) 43-.

- $n$ ! expression by an integral function. Hadamard, J. Bll. Sc. Mth. 19 (1895) 69-.

Progression, arithmetical, sum of like powers of terms. Ducci, E. G. Mt. 32 (1894) 348-.

-,,-- squares of terms by aid of polygon. Romero, J. N. A. Mth. 1 (1882) 46-.

-, fractional arithmetical, summation of integers. Šimerka, $V$. Časopis $5\left({ }^{*} 1876\right) 39$-, 82- ; Fschr. Mth. (*1876) 86.

-, geometrical, numbers in. Blacklewar, $C$. R. S. P. 3 (1832) 139.

$-,-, 1,3,9, \& \mathrm{c}$. Unferdinger, $F$. Grunert Arch. 33 (1859) 106-.
Progressional series, summation. Benwell, J. B. Tilloch Ph. Mg. 58 (1821) 265-.

Progressions, arithmetical. Elefanti, $F$. R. S. P. 10 (1859-60) 1-.

- - and geometrical. Pedersen, H. A. Mth. Ts. 1 (1859) 17-.

,$- \ldots+$, when $n$ is fractional. Helmes, $J$. Grunert Arch. 35 (1860) 136-.

- by differences. Sardi, C. G. Mt. 11.(1873) 123-.

Rational fraction, formula of Laplace's. Jürgensen, $C$. Crelle J. 11 (1834) 136-.

Recurrence, formula. Thomae, J. Crelle J. Mth. 110 (1892) 78-.

Recurrences, system of combined. Degen, C. F. [1819] St. Pét. Ac. Sc. Mm. 9 (1819-20) 130-.

Recurring series. Malfatti, G. F. Verona S. It. Mm. 3 (1786) 571-.

- - Svanberg, A. F. Ups. N. Acta S. Sc. 11 (1839) 1-.

- Whitworth, W. A. [1865] Mess. Mth. 3 (1866) 117-

- - Ocagne, M. d'. Par. S. Mth. Bll. 14 (1886) 20-; N. A. Mth. 9 (1890) 93-; Par. Éc. Pol. J. 64 (1894) 151-.

- - Netto, $E$. Mh. Mth. Ps. 6 (1895) 285-.

- - and bilinear forms. Study, E. Mh. Mth. Ps. 2 (1891) 23-.

- - conditions that scale should be irreducible. Maillet, E. N. A. Mth. 14 (1895) 152-, 197-.

- - connected with equation $2 x^{2}=y^{2}-1$. Catalan, E. C. As. Fr. C. R. 12 (1883) 98-.

- - , elementary theory. Ocagne, M. $d$ '. N. A. Mth. 3 (1884) 65-.

- - and equations. Laisant, C. A. Bll. Sc. Mth. As. 5 (1881) 218-.

- - general term, and Euler's expression for same. Degen, C. F. [1821] Kiob. Dn. Vd. Selsk. Afh. 1 (1824) 135-.

- - geometric representation. Scherk, H. F. Brem. Ab. 1 (1868) 225-.

- - and indeterminate analysis. Maillet, $E$. As. Fr. C. R. (1895) (Pt. 2) 233-.

- - integration. Longchamps, G. de. As. Fr. C. R. (1885) (Pt. 2) 94 -

- leading to continued fractions. Scheibner, $W$. Leip. B. 16 (1864) 44.

- -, problem. Degen, C.F. [1819] St Pét. Ac. Sc. Mm. (1821-22) 71-.

- - , problems. Weiss, A. Crelle J. 38 (1849) 148-.

- - for rational fractional function. Degen, C. F. (v1 Adds.) Kiöb. Ov. (1818-19) 4-.

- - , and theorem of Lagrange. Gohierre de Longchamps, - - As. Fr. C. R. 9 (1880) 91-.

- -, theory, addition. Vész, J. A. (xIr) Mag. Tud. Ak. Etk. (Mth.) 3 (1875) (No. 1) $15 \mathrm{pp}$.

- - and theory of numbers. Siebeck, $H$. Crelle J. 33 (1846) 71-.

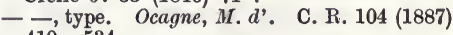
419-, 534. 
Recurring series, use in analysis of equations. Frisiani, $P$. Mil. Effem. As. (1850) 3-.

- systems, generation by a linear differential equation. Pincherle, S. Acta Mth. 16 (189293) 341-.

- - 1st order and 2nd degree. Pincherle, $S$. Rm. R. Ac. Linc. Rd. 5 (1889) (Sem. 1) 8-, $323-$.

Relation $1^{3}+2^{3}+\ldots+n^{3}=(1+2+\ldots+n)^{2}$. Gelin, E. Mathesis 14 (1894) 220-.

Series with alternate law of recurrence. Ocagne, M. d'. Par. S. Mth. Bll. 12 (1884) 78-.

-, doubly recurring. Betti, $E$. Tortolini A. 8 (1857) 48-.

- of Fibonacci, on $\left[u_{n}+1=u_{n}+u_{n}-1\right]$. Cesàro, E. G. Teix. J. Sc. 6 (1885) 17-.

-, Fibonacci, peculiarities. Fontés, - As. Fr. C. R. (1894) (Pt. 2) 217-.

-, general term found as with recurring series. André, D. Par. Éc. Norm. A. 7 (1878) 375-.

-, geometrical, fractional number of terms. Choquet, C. N. A. Mth. 1 (1842) 74-.

- of quotients, Euclid's summation. Zeuthen, H. G. (xII) Ts. Mth. 6 (1876) 168-, 181-; 6 (1882) 97-; 1 (1883) 145-.

-, self-repeating. Warburton, H. Camb. Ph. S. T. 9 (1856) (Pt. 4) 471-.

-, whose series of differences are recurring. Raabe, J. L. Baumgartner Z. 6 (1829) 307-.

-, summation. (Dienger, Grunert Arch. 8 213-214.) Arndt, $F$. Grunert Arch. 8 (1846) 383-.

-, - of certain. Tait, P. G. Edinb. Mth. S. P. 3 (1885) 107-.

,$---\frac{1}{\text { class. }}$ Ocagne, M. d'. C. R. 115 (1892) 790-, 904.

-, -, successive. Dantas Pereira, J. M. Lisb. Mm. Ac. Sc. 2 (1799) 168-.

Sum of a combinatory series, properties. Unferdinger, $F$. Grunert Arch. 26 (1856) $227-$.

- - cubes = a square. Catalan, E. C. [1866] Rm. At. N. Linc. 20 (1867) 1-, 77-.

- - - of secondary numbers, theorem. Barbier, E. Bll. Sc. Mth. As. 7 (1883) 42-.

- - first $n$ products of $p$ consecutive numbers. Laisant, A. N. A. Mth. 6 (1867) 366-.

- - products of $n$ quantities taken $m$ together. Trowbridge, $D$. Des Moines Anal. 1 (1874) $154-$.

- - - - $r$ different terms of series. Hammond, J. L. Mth. S. P. 7 (1875-76) 119-.

- - reciprocals. Bouvier, L. C. Gergonne A. Mth. 15 (1824-25) 39-.

- - - Blissard, J. QJ. Mth. 6 (1863) $242-$

- - Worontzof, M. M. QJ. Mth. 8 (1867) 185-, 310-.

- - of first 1000 integers. Adams, J.C. B. A. Rp. (1877) (Sect.) 14-.

Summation of series, general term of which is $n a^{n}, a$ being a given number. Bertrand, $J$. N. A. Mth. 8 (1849) 354-.
Summation of terms of recurring series, formation of integers by. Maillet, $E$. As. Fr. C. R. (1896) (Pt. 2) 78-.

Summations, successive, $1^{m}+2^{m}+3^{m}+\ldots x^{m}$. Lucas, É. [1877] Mess. Mth. 7 (1878) 84-.

Sums of 4 algebraic series. Glaisher, J.W.L. [1877] Mess. Mth. 7 (1878) 119-.

- - powers of reciprocals of integers. Schlömilch, O. X. Leip. Mth. Ps. B. 29 (1877) 106-.

- - products of an arithmetical progression, taken 2, 3, $4 \ldots$ together, calculation. Lampe, $E$. Fschr. Mth. (1893-94) 409.

- - - natural numbers. Mollame, $V$. N. A. Mth. 5 (1886) 364-.

- - - numbers, theorem. Lerch, G. Teix. J. Sc. 8 (1887) 161-.

- - series $1^{n}+2^{n}+\ldots x^{n}, 1^{n}-2^{n}+\ldots \pm x^{n}$ Glaisher, J. W. L. QJ. Mth. 30 (1899) 166-.

Special series.

$$
\sum_{r=1}^{r=n} r^{k} .
$$

Collins, E. St Pét. Ac. Sc. Mm. 8 (1817-18) 242-.

Schlömilch, O. Grunert Arch. 10 (1847) 342-.

Thacker, A. Camb. and Dubl. Mth. J.5 (1850) 243-.

Mourgues, -. N. A. Mth. 10 (1851) 78-.

Catalan, E. C. N. A. Mth. 15 (1856) 230-.

Siacci, $F$. (vIII) A. Mt. 4 (1861) 46-.

Lucas, É. N. A. Mth. 9 (1870) 49-.

Amigues, $E$. $\quad$ N. A. Mth. 10 (1871) 79-

Dostor, G. Arch. Mth. Ps. 54 (1872) 70-; 57 (1875) 222-.

Lampe, E. [1877] Crelle J. Mth. 84 (1878) 270 -.

Lucas, É. N. A. Mth. 16 (1877) 18-.

Pelnář, M. "Č́asopis 6 ("1877) 279-; Fschr. Mth. (*1877) 110-.

Dostor, G. Arch. Mth. Ps. 63 (1879) 435-; 64 (1879) 310-.

Marchand, D. N. A. Mth. 20 (1881) 140-.

Schier, O. Wien Ak. Sb. 85 (1882) (Ab. 2) 503-.

Boije af Gennäs, C. O. Stockh. Ak. Hndl. Bh. 10 (1885) No. 4, 6 pp.

Slavik, J. Časopis 16 (1887) 121-; Fschr. Mth. (1887) 240.

Locchi, E. Mh. Mth. Ps. 4 (1893) 85-.

Nielsen, $N$. N. Ts. Mth. 4 (B) (1893) 1-; Fschr. Mth. (1893-94) 410-.

Barbette, E. Mathesis 14 (1894) 105-.

Jung, $V$. Časopis 28 (1899) 124-; Fschr. Mth. (1899) 247.

$$
\sum_{r=1}^{r=n} r^{3}
$$

Genocchi, A. A. Mt. 7 (1865) 151-.

Cantor, M. Z. Mth. Ps. 11 (1866) 248-; 12 (1867) 170-. 


\section{Finite Summation}

Unferdinger, $F$. Arch. Mth. Ps. 48 (1868) 361-.

Amigues, É. $\quad$ N. A. Mth. 10 (1871) 117-

Glaisher, J. W. L. Mess. Mth. 4 (1875) 168-

Rocquigny, $F$. [G.] de. Les Mondes 51 (1880) 153-.

geometrical summation. Glaisher, J. W. L. (vII) [1873] Mess. Mth. 3 (1874) 5.

problem of Boncompagni, solution. Matthiessen, L. Z. Mth. Ps. 13 (1868) 348-.

$$
\sum_{r=1}^{r=n}(2 r-1)^{k} \text {. }
$$

Bretschneider, C. A. Grunert Arch. 1 (1841) 415-.

Borchardt, C. W. Berl. Mb. (1857) 301-

Brioschi, F. [1857] Tortolini A. 1 (1858) 43-.

Dostor, G. Arch. Mth. Ps. 64 (1879) 353-.

$$
\sum_{r=1}^{r=n}(2 r-1)^{k} \quad(k=3) \text {. }
$$

Dostor, G. Arch. Mth. Ps. 64 (1879) 356-.

$$
\sum_{r=0}^{r=n-1}(a+r d)^{m} \text {. }
$$

Puiseux, V. Liouv. J. Mth. 11 (1846)477-. Gilbert, $P$. N. A. Mth. 8 (1869) 434-.

formulae. Lionnet, $E$. N. A. Mth. 1 (1842) 175-.

-. Rispal, A. N. A. Mth. 6 (1847) 273-.

$\sum_{n=1}^{n=n}(a+n-1)^{a} h^{n-1}, \sum_{n=1}^{n=n}[a+n-1]^{a} h^{n-1}$.

Pepin (le père). N. N. Ath. 15 (1856) 27-. $\sum_{i=0}^{i=n-1}(x+i h) \mu, \sum_{i=0}^{i=n-1}(-1)_{i}(x+i h)^{\mu}$. Björling, E. G. Stockh. Ak. Hndl. 2 (1857-58) 18 pp. $\sum_{r=1}^{r=n}\left(\frac{1}{r}\right)^{n}$, Jacobian formula involving. Stern, M. A. Crelle J. Mth. 84 (1878) 216$r=2 n+1$

$\sum r^{2}$ or $r^{3}$. Dostor, G. Arch. Mth. Ps. 64 (1879) 361-.

$\sum_{r=1}^{r=n}(r)^{\alpha}$. Dostor, G. N. A. Mth. $18(1879)$ $459-, 513-$.

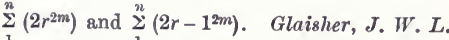
Mess. Mth. 20 (1891) 172-

$\sum_{1}^{n}\left(r^{2 m}\right)$. Glaisher, J. W. L. Mess. Mth. 20 (1891) 120-; 28 (1899) 29 -

\section{Probabilities (including combination of observations). Theory of errors.}

Accuracy of recollection, measurement. Cattell, J. McK. Science 2 (1895) 761-.

Approximate formulae relating to large numbers. La Place, P. S. (Marquis) de. Par. Mm. de 1'I. (1809) 353-, 559-.
Approximations, numerical. Guyou, -. N. A. Mth. 8 (1889) 165-.

Arithmetical events, study. Cesàro, E. Brux. Mm. Cour. 4\%. 47 (1886) No. 9, 14 pp.

Association ratios (of phenomena in observations). Doolittle, M. H. Smiths. Misc. Col. 33 (1888) Art. 4, 83-, 94-. (Wash. Ph. S. Bll. 10 (1888).)

Averages, theory. Local probability. Doolittle, M. H. Wash. Ph. S. Bll. 11 (1892) 596 -

Bernoulli's inverse theorem. Meyer, A. Brux. Ac. Bll. 23 (1856) 148-

- theorem. Mansion, - Brux. S. Sc. A. 16 (1892) (Pt. 1) 85-.

- - Goedseels, -. Brux. S. Sc. A. 19 (1895) (Pt. 1) 4-.

- - Mansion, - Brux. S. Sc. A. 22 (1898) (Pt. 1) 3-.

- - Rayleigh, (Lord). Ph. Mg. 47 (1899) 246-.

- - Eggenberger, J. Z. Mth. Ps. 45 (1900) 43-.

- - and approximate probability. Nekrasov, P. A. [1898] Rec. Mth. (Moscou) 20 (1899) 485-, 535-; Fschr. Mth. (1899) 214, 215.

- - - binomial coefficients. Nasimov, P.S. Fschr. Mth. (1888) 211.

- - gamma-functions and Laplace's integral. Eggenberger, J. Bern. Mt. (1893) 110-, 236. - - inversion. Monro, C.J. (x) L. Mth. S. P. 5 (1873-74) 74-, 145-.

- - - - Nekrasov, P. A. Mosc. S. Sc. Bll. 65 (No. 1) (1890) 45-; Fschr. Mth. (1890) 235.

- - , simple proof. Gosiewski, W. Krk. Ak. (Mt.-Prz.) Rz. 13 (1886) 153-; Fschr. Mth. (1885) 176-.

- - proof by Euler's summational formula. Lagrange, C. Brux. Ac. Bll. 31 (1896) 439-.

- , repeated application. Andrade, J. C. R. 116 (1893) 1281-, 1482.

Best reading of an instrument for measuring a given quantity. Baum, F. G. Ps. Rv. 8 (1899) 181-.

Betting on races. $D u$ Chatenet, $M$. N. A. Mth. 5 (1886) 327-, 380-, 408-.

Bravais's formulæ for regression, etc., with skew correlation. Yule, G.U. R. S. P. 60 (1897) 477-.

Broken diamond, problem. Cesàro, E. G. Mt. 24 (1886) 124-

Buffon's problem of the needle. Barbier, $E$. Liouv. J. 5 (1860) 273-.

- - - - Sylvester, J. J. Acta Mth. 14 (1890-91) 185-

Causation process, results. Paterson, J. Am. As. P. (1856) 29-.

Chance. Whitworth, W. A. [1871] Mess. Mth. 1 (1872) 163-.

- and choice, problems supplementary to. Whitworth, W. A. Mess. Mth. 4 (1868) 152-, 193-.

-, continuity. Davis, E. W. [1897] Nebr. Un. Stud. 2 (1894-1902) 131-.

-, doctrines. Saint, $W$. Nicholson J. 21 (1808) 204-. 
Chance, doctrines. Opsimath. [Pseud.] Nicholson J. 21 (1808) 210-.

-, - doubts respecting. Opsimath. [Pseud.] (vr Adds.) Nicholson J. 21 (1808) 66-.

Chances, determination of $e^{x}$ as a limit in calculation of. Andrade, - Par. Éc. Pol. J. 64 (1894) 225-.

-, doctrine. H., B. (vI Adds.) Nicholson J. 21 (1808) 121-.

,- , calculation in. Young, $\operatorname{Dr} T$. Nicholson J. 18 (1807) 116-

-, paradox in. Galton, F. Nt. 49 (1893-94) 365-.

-, - - Shorter, L. R. Nt. 49 (1893-94) 413.

—, problem. Day, H. G. QJ. Mth. 9 (1868) 354-.

-, - experimental solution. Mendenhall, T. C. Am. As. P. (1879) 190-

-, problems. Wilson, J. M. Ph. Mg. 31 (1866) 170-.

-, theory in Boole's laws of thought. Wilbraham, H. Ph. Mg. 7 (1854) 465-.

-, 一, reply to Wilbraham. Boole, $G$. $\mathrm{Ph}$. Mg. 8 (1854) 87-.

Chessboard, chance of each piece checking king. Taylor, H. M. Ph. Mg. 1 (1876) 221-.

-, use for solution of problems in probability. Delannoy, $H$. As. Fr. C. R. (1889) (Pt. 2) 43-; (1895) (Pt. 2) 70-.

Combination of deviations. Ocagne, M. d'. N. A. Mth. 14 (1895) 133-.

- measures of one magnitude. Bertrand, $J$. C. R. 106 (1888) 701-

- - observations. Frisiani, P. [1859] Mil. I. Lomb. Mm. 11 (1870) 21 pp.

- - Lipschitz, R. C. R. 111 (1890) $163-$.

- - - Pizzetti, P. Genova Un. At. [11] (1892) 113-.

Levänen, S. Helsingf. Öfv. 37 (1895) 19-; Fschr. Mth. (1895) 246.

- - - Equations, linear, resulting from observations of equal weight, solution. Santini, G. [1869] Ven. Mm. I. 14 (1868) 477-.

- - theory. Gauss, C. F. Gött. Cm. 5 (1819-22) 33-, 63-; 6 (1823-27) 57-; 7 (182831) $89-$

- - - Donkin, W. F. Ashmol. S. T. (1844) 71 pp.; Liouv. J. Mth. 15 (1850) 297-.

- - - - Kleiber, I. A. Kazan S. Nt. (Ps.-Mth.) P. 6 (1888) 148-; Fschr. Mth. (1888) 220-.

- - - - Gauss's. Nasimov, P.S. Fschr. Mth. (1889) 218-.

- - - - - —. Krüger, L. Gött. Nr. (1897) 146-.

J. Mth. 8 , (1886) 343-

_ - weights of British noblemen during last three generations. Galton, $F$. Nt. 29 (1884) 266-.

Combinations and factorial numbers, theorems. Catalan, E. Brux. Ac. Bll. 25 (1893) 238-, 430-.
Combining probabilities of same event, possibility. Terrot, C. H. [1856] Edinb. R. S. T. 21 (1857) 369-.

Comparison of probabilities that are functions of one variable. Blaschke, E. Wien Ak, D. 54 (1888) (Ab. 2) 105-.

Compensation, arithmetic mean in calculating. Dorna, A. Tor. At. Ac. Sc. 4 (1868-69) 757-.

- of errors. Lorenz, L. V. (xII) Ts. Mth. 2 (1872) 1-, 125-.

- - Zachariae, G. (xIr) Ts. Mth. 2 (1872) 97-, 182-.

- - Bruns, H. Leip. Mth. Ps. Ab. 13 (1887) 515-.

- - - effect of weights. Paucker, M. G. von. Mitau Arb. Kurländ. Gs. 9 (1851) 183-. _ _ - Laplace's method. Dienger, $J$. [1874] Wien Ak. D. 34 (1875) (Ab. 2) 21-.

$--\ldots$, a law of duality in. Reina, $V$. Tor. Ac. Sc. At. 29 (1894) 433-.

- proportional errors. Jung, G. Mil. I. Lomb. Rd. 13 (1880) 238-.

Conscription, drawing of lots in, problem arising from. Rasch, J.W. N. Arch. Wisk. 1 (1895) 206-; Fschr. Mth. (1893-94) 342-.

Constant aberrations in shooting, precession of shot. Cranz, C. D. Nf. Vh. (1897) (Th. 2, Hälfte 1) 6.

Continued fractions, application to calculation of probabilities. Markov, A. A. Kazan S. Ps.-Mth. Bll. 9 (1900) 29-; Fschr. Mth. (1899) 215.

- -, probability of appearance of given number. Wiman, A. Stockh. Öfv. (1900) 829-.

- - , - - - numbers of given magnitude. Brodén, T. Stockh. Öfv. (1900) 239-.

Correlated averages. Edgeworth, F. Y. Ph. Mg. 34 (1892) 190-.

- - , new method of treating. Edgeworth, F. Y. Ph. Mg. 35 (1893) 63-.

Correlation of averages for four variables, example. Bryant, (Mrs) $\mathrm{S}$. $\mathrm{Ph} . \mathrm{Mg} \cdot 36$ (1893) 372-.

- between organs, note on calculation. Edgeworth, F. Y. Ph. Mg. 36 (1893) 350-.

Criterion that given deviations for correlated variables may have arisen from random sampling. Pearson, $K$. Ph. Mg. 50 (1900) 157-.

Crofton, integral of. Petrini, $H$. Ts. Mth. 6 (1889) 39-; Fschr. Mth. (1888) 305-.

Curve of errors, quadrature. Gram, J. $P$. (xII) Ts. Mth. 1 (1883) 65-; Fschr. Mth. (1884) 242.

Definite integral, evaluation by theory of probabilities. Schoute, P. H. As. Fr. C. R. (1880) 258-.

- - , form for series in theory of probabilities. Carpmael, C. [1885] Cn. R. S. P. \& T. 3 (1886) (Sect. 3) 101-.

- integrals and application to probabilities. La Place, P. S. (Marquis) de. Par. Mm. de I'I. (1810) 279-.

Determination of $\pi$ by needle throwing. Levänen, S. Helsingf. Öfv. 34 (1892) 36-; Fschr. Mth. (1892) 203. 
Determination of some unknowns in geodesy. Berardinis, G. de. G. Mt. 25 (1887) 313-. - - unknowns by a large number of approximate equations of 1 st degree. Cauchy, A.L. C. R. 36 (1853) 1114-.

Development of $[p+(1-p)]^{m}$ approximates to probability curve as $m$ increases. De Forest, E. L. Des Moines Anal. 6 (1879) 65-.

Digits, relative frequency in natural numbers. Newocomb, S. Am. J. Mth. 4 (1881) 39-.

Distribution of frequency of barometric heights. Pearson, $K ., \&$ Lee, A. [1897] Phil. Trans. (A) 190 (1898) 423-.

- points on a line. Bartlett, W. P. G. Camb. (M.) Mth. M. 1 (1859) 84-.

- signs of residual errors. Seeliger, $H$. [1879] As. Nr. 96 (1880) 49-.

Division, eventualities in. Cesàro, $E$. A. Mt. 13 (1885) 269-.

- of a straight line, probability problem. Berg, F. J. van den. N. Arch. Wisk. 18 (1891) 42-; Fschr. Mth. (1891) 233-.

Duration of play. Delannoy, - Par. S. Mth. Bll. 16 (1888) 124-.

- - problem. Rouché, E. C. R. 106 (1888) 47-.

- - - - MacMahon (Maj.) P. A. L. Mth. S. P. 20 (1889) 195-.

- - - - , demonstration. Bertrand, C. R. 106 (1888) 49-

- _ _, some questions of. Rouché, $E$. C. R. 106 (1888) 253-.

Earthquakes, lunar and solar periodicities. Schuster, A. Nt. 56 (1897) 321.

Ellipse of error, theory. Czuber, (or Čuber) $E$. [1880] Wien Ak. Sb. 82 (1881) (Ab. 2) 698-.

- or hyperbola, questions of probability. Cesàro, E. G. Mt. 22 (1884) 44-.

Equation, 2nd order, probability that roots are real. Bouniakowsky, V. [1835] St Pét. Ac. Sc. Mm. 3 (1838) 341-.

Equations, 1st order, in number greater than number of unknowns; geometrical applications. Terquem, O. N. A. Mth. 5 (1846) 551-. -, normal, solution by indirect elimination. Schott, C. A. U. S. Coast Sv. Rp. (1855) 255-.

Error function and symmetric functions. Thiele, T. N. Kjøb. Ov. (1899) 135-; Fschr. Mth. (1899) 216-.

-, law of. Edgeworth, F. Y. Ph. Mg. 16 (1883) 300-.

-, - -. Venn, J. Nt. 36 (1887) 411-.

-, - - Edgeworth, F. Y. Nt. 36 (1887) 482 .

-, - - Backhouse, T. W. Nt. 36 (1887) 531.

-, - -. Schols, C. M. Amst. Ak. Vs. [1] (1893) 194-; Fschr. Ps. (1893) (Ab. 1) 16-. -, - - , in case of correlated variations. Burbury, S. H. B. A. Rp. (1895) 621-.

-, - -, compound. Edgeworth, F. Y. Ph. Mg. 41 (1896) 207-.

- , - - and correlated averages. Edgeworth, F. Y. Ph. Mg. 34 (1892) 429-, 518-.

-,$\ldots$, when differences of observations are large. Oudemans, J. A.C. Amst. Ak. Vs. [2] (1894) 12-.
Error, law of, and elimination of chance. Edgeworth, F. Y. Ph. Mg. 21 (1886) 308-.

- _ - empirical proof. Edgeworth, F. Y. Ph. Mg. 24 (1887) 330-

- - - facility of. Watson, H. W. Birm. Ph. S. P. 7 (1889-91) 289-.

_, - - - for geometric mean. M'Alister, D. B. A. Rp. (1879) 267 .

- , - - - of, in sum of $n$ independent quantities each accurate to nearest degree. Carpmael, C. (xII) [1882] Cn. R. S. P. \& T. 1 (1883) (Sect. 3) 9-.

-, - - frequency. Tait, P. G. [1865] Edinb. R. S. T. 24 (1867) 139-.

- -- - Galton, F. Ph. Mg. 49 (1875) 33-.

- in position of a point in space. De Forest, E. L. Conn. Ac. T. $6\left({ }^{* 1882-85) ~ 123-. ~}\right.$

- - - - - - - theory. De Forest, E. L. Des Moines Anal. 9 (1882) 33-, 65-.

- - target shooting. De Forest, E. L. Conn. Ac. T. 7 (1885-88) 1-.

Errors, accidental, Gauss' law, proof. Gosiewski, W. Prace Mt.-Fiz. 2 (1890) 223-; Fschr. Mth. (1890) 235.

- -, law. Pellerin, - As. Fr. C. R. (1898) (Pt. 2) 156-.

,-- , points in theory. Faye, H. C. R. 106 (1888) 783-

- actual and probable, of interpolated values derived from tables by means of first differences. Woodward, R. S. Des Moines Anal. 9 (1882) 143-, 169-.

-, determination of modulus. Edgeworth, F. Y. Ph. Mg. 21 (1886) 500-

- in 2 dimensions, law of facility. De Forest, E. L. Des Moines Anal. 8 (1881) 3-, 41-, 73-.

—, distribution of residual, after combination. Seeliger, H. Münch. Ak. Sb. 29 (1900) 3-.

-, exercises in calculation. Edgeworth, F. Y. Ph. Mg. 36 (1893) 98-

-, law. Le Maire, A. Brux. S. As. Bll. 1 (1896) 124-, 207-.

- of observation. Damien, B.C. (xII) Bll. Sc. Nord 15 (1883) 121-.

- _ - extension of law of probability. Adan, (Capit.) E. Brux. Ac. Bll. 23 (1867) 148- _ -, formula expressing probability. Pizzetti, P. Rm. R. Ac. Linc. Rd. 5 (1889) (Sem. 1) 191-.

- - formulae for calculation. Maggi, G. A. Mil. I. Lomb. Rd. 15 (1882) 351-.

- - - law. Kummell, C. H. Des Moines Anal. 3 (1876) 133-, 165-; 6 (1879) 80-.

- - - - Bertrand, J. C. R. 106 (1888) 153-.

$--—$, 一. Tisserand, E. C. R. $106(1888)$ 231-.

- - - - Pizzetti, P. Rm. R. Ac. Linc. Rd. 1 (1892) (Sem. 1) 380-.

- - - - - of facility, and method of least squares. Glaisher, J. W. L. As. S. Mm. 39 (1872) 75-.

- - - , proof. Crofton, M. W. [1869] Phil. Trans. 160 (1870) 175-.

- - - mean error of 2 nd order. Paucker, M. G. von. Mitau Arb. Kurländ. Gs. 8 (1850) 104-. 
Errors of observations, probability of sums of powers of, etc. Helmert, F. R. Z. Mth. Ps. 21 (1876) 192-.

- - , signs of residuals. De Forest, E. L. Des Moines Anal. 5 (1878) 65-.

,----- , grouping. De Forest, E.L. Des Moines Anal. 5 (1878) 1-.

_- -, theory. De Morgan, A. [1861] Camb. Ph. S. T. 10 (1864) 409-.

- - - - Lorenzomi, G. Padova Ac. At. e Mm. 5 (1889) 203-.

- - - - Czuber, E. Mh. Mth. Ps. 1 (1890) 457-.

(1893) 1 -

- - - - , theorems. Winckler, A. Wien Sb. 53 (1866) (Ab. 2) 6-.

-, physical basis for theory. Burton, C. V. L. Ps. S. P. 10 (1890) 276-; Ph. Mg. 28 (1889) 480-.

- in plane and space. Schols, C. M. [187486] Amst. Ak. Vh. 15 (1875) 67 pp.; Delft Éc. Pol. A. 2 (1886) 123-.

- _ - - demonstration of law. Schols, C. M. Delft Éc. Pol. A. 3 (1887) 195-.

-, relative and absolute. Gaucherel, E. N. A. Mth. 14 (1855) 145-.

- , 一, theory. Jaufroid, B. N. A. Mth. 15 (1856) 154-.

- from single causes, composition. Kummell, C. $H$. As. Nr. 103 (1882) 177-.

一, theory. Estienne, -. C. R. 130 (1900) 66-.

,- , application to levelling. Ocagne, $M . d$ '. C. R. 120 (1895) 717-.

,-- , correction formulae for series of observations. De Forest, E. L. Des Moines Anal. 4 (1877) 79-, 107-.

-, -, corrections, repeated, limit. De Forest, E. L. Des Moines Anal. 5 (1878) 129-.

-,,,--- , of terms of series. De Forest, E. L. Des Moines Anal. 5 (1878) 65-.

- , - , - unsymmetrical, of terms of series, and their limits. De Forest, E. L. Des Moines Anal. 6 (1879) 140-, 161-; 7 (1880) 1-.

,-- , criticism of formula of Gauss. Czuber, E. Mh. Mth. Ps. 2 (1891) 459-.

-, -, elementary. De Forest, E. L. Des Moines Anal. 8 (1881) 137-.

-, 一, foundations. Müller, $F$. Mh. Mth. Ps. 2 (1891) 61-.

-, - , function occurring in. Moser, C. Sch. Nf. Gs. Vh. (1898) 45-.

,-- , and method of least squares. Goedseels, E. [1896] Brux. S. As. Bll. 1 (1896) 253-; 2 (1896-97) 6-.

- - removal of "side coefficients." Kummell, - Smiths. Misc. Col. 33 (1888) Art. 3, 41. (Wash. Ph. S. Bll. 8 (1885).) -, - , singular law in. Bertrand, J. C. R. 105 (1887) 779-.

-, - , test by target shooting. Kummell, $C$. H. Smiths. Misc. Col. 33 (1888) Art. 1, 138-. (Wash. Ph. S. Bll. 6 (1884).)

Evolution, mathematical theory. Pearson, $K$. [1893] Phil. Trans. (A) 185 (1895) 71-.
Evolution; mathematical theory. (Skew variation in homogeneous material.) Pearson, $K$. [1895] Phil. Trans. (A) 186 (1896) 343-.

-, - - (Regression, heredity, and panmixia.) Pearson, $K$. [1895] Phil. Trans. (A) 187 (1897) 253-.

- - - - (Reproductive selection.) Pearson, K. R. S. P. 59 (1896) 301-.

-, - - (Telegony in man, etc.) Pearson, K. [1896] R. S. P. 60 (1897) 273-.

- - - - (Spurious correlation.) Pearson, $K$. R. S. P. 60 (1897) 489 -

,$--1-(--$.$) Galton, F. R. S. P. 60$ (1897) 498-.

- - - (Relative variation and correlation in civilised and uncivilised races.) Lee, (Miss) A., \& Pearson, $K$. R. S. P. 61 (1897) 343-.

- - - Pearson, K., \& Filon, L. N. G. [1897] Phil. Trans. (A) 191 (1898) 229-.

_. _ - - (Speeds of American trotting horses as hereditary data.) Galton, $F$. [1897] R. S. P. 62 (1898) 310-.

-, - - (Law of ancestral heredity.) Pearson, K. R. S. P. 62 (1898) 386-.

- , - - (Inheritance of cephalic index.) Fawcet, (Miss) C. D., \& Pearson, $K$. R. S. P. 62 (1898) 413-.

- , - - (Reconstruction of stature of prehistoric races.) Pearson, $K$. [1898] Phil. Trans. (A) 192 (1899) 169-.

- - - (Genetic selection.) Pearson, $K$. [1898] Phil. Trans. (A) 192 (1899) 257-.

Pearson, K., \& Lee, A. [1898] Phil. Trans. (A) 192 (1899) 279-.

- - - - (Inheritance of fecundity in thoroughbred racehorses.) Pearson, $K ., \&$ BramleyMoore, L. [1898] Phil. Trans. (A) 192 (1899) 290 -

-, - - (Inheritance of characters not quantitatively measurable.) Pearson, $K ., d$ Lee, A. [1899] Phil.Trans. (A) 195 (1901) 79-. - - - . (Magnitude of certain coefficients of correlation.) Pearson, $K$. [1899] R. S. P. 66 (1900) 23-。

- - - . (Law of reversion.) Pearson, $K$. R. S. P. 66 (1900) 140-.

-, - - (Correlation of characters not quantitatively measurable.) Pearson, $K$. [1900] Phil. Trans. (A) 195 (1901) 1-.

-, - - ( $\quad$ - - - - ) Pearson, K. R. S. P. 66 (1900) 241-.

- - - - (Fertility and homogamy.) Pearson, K. R. S. P. 66 (1900) 316-.

- - - Evolution in man. (Correlation of skull.) Lee, A., \& Pearson, $K$. [1900] Phil. Trans. (A) 196 (1901) 225-.

- _- -. (Homotyposis.) Pearson, K. [1900] Phil. Trans. (A) 197 (1901) 285-.

Factorials, theorems deduced from probabilities. Godfray, $H$. Mathematician 3 (1850) 89-.

Factors for minimum probable error of a mean. Cauchy, A. L. C. R. 37 (1853) 326-.

Finite expansion, with least error. Buchwaldt, F. Ts. Mth. 2 (1884) 33-, 192; Fschr. Mth. (1884) 186.

Forestry problem. Clebsch, R. F. A. Crelle J. 67 (1867) 248-. 
Frequency curve, asymmetrical. Pearson, $K$. Nt. 48 (1893) 615-; 49 (1893-94) 6.

- -distributions, tabulation of certain. Sheppard, W. F. Ph. Mg. 50 (1900) 393-.

- laws of calculus. Cesàro, E. Mil. I. Lomb. Rd. 24 (1891) 101-.

- novel case. Pearson, K. R. S. P. 62 (1898) 287-.

Game of cards, application of probabilities. Dormoy, É. As. Fr. C. R. (1886) (Pt. 1) 83.

- chance, calculation. Trembley, $J$. Berl. Mm. Ac. (1802) 86-.

- - - theory of a. Steen, A. (xIr) Ts. Mth. 3 (1867) 33-.

Games of chance. Nieuport, C. F. F. de. [1818] Brux. Ac. Sc. Mm. 1 (1820). 89-. - - Babbage, C. [1820] Edinb. R. S. T. 9 (1823) 153-.

- - Grebe, E. W. Grunert Arch. 11 (1848) 441-.

$--\frac{1}{-}$. Badoureau, A. Rv. Sc. 1 (1881)

- - Baccarat. Badoureau, A. Rv. Sc. 1 (1881) 239-.

- - - , theory. Bertezène, A. Rv. Sc. 7 (1897) 780-

- - -. "Bell" and "hammer" game, theory. Ekama, H. N. Arch. Wisk. 19 (1892) 107-; Fschr. Mth. (1892) 200-.

- - -. - - - - -. Mounier, G.J.D. N. Arch. Wisk. 19 (1892) 188-; Fschr. Mth. (1892) 201.

- - - Billiards, probabilities at 3-ball game. Peirce, B. [1877] Am. Ac. P. 13 (1878) 141-.

_ - -. "Bouillotte." Dormoy, É. Rv. Sc. 40 (1887) 91-.

- - - - - chances of the "pair royal." Lamé, G. C. R. 28 (1849) 705-.

- - - Dice. Bierens de Haan, D. Amst. Ak. Vs. M. 12 (1878) 371-; Arch. Néerl. 14 (1879) 370-; N. Arch. Wisk. $6\left({ }^{*} 1880\right)$ 49-, 113-.

- - -. - Gomoll, J. Arch. Mth. Ps. 17 (1900) 363-.

- - - - - calculation of probability. Schweigger, F. Z. Nw. 2 (1877) 114-.

- - - - - odds of throwing specified number. Scott, G. Assur. Mg. 4 ("1854) 247-.

- - - - throw with 6. Baur, C. W. Z. Mth. Ps. 12 (1867) 355-.

- - D. Dice-throwing experiments, new set. Wolf, R. Zür. Vjschr. 38 (1893) 10-.

- - Faro, chances for bank. Greisinger, G. A. Baumgartner Z. 4 (1828) 210-.

- - - Piquet, theory. Badoureau, Rv. Sc. 37 (1886) 299-.

- - . Pitch and toss, two ways of finding

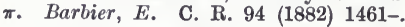

- - - risk. Hausdorf, $F$. Leip. Mth. Ps. B. 49 (1897) 497-.

- - Rouge et noir, advantage of bank. Oettinger, L. Crelle J. 47 (1867) 327-.

- - - Trente-et-quarante, advantage of bank. Poisson, S. D. Gergonne A. Mth. 16 (1825-26) 173-.
Games of chance. Whist, theory. Badoureau, -. Rv. Sc. 36 (1885) 587-.

-, probabilities in connection with. Delannoy, H. As. Fr. C. R. (1890) (Pt. 2) 29-.

Gauss's proposition, proof. Rusjan, C. Prace Mt.-Fiz. 3 (1892) 49-; Fschr. Mth. (1892) 205-.

Geometrical probabilities, theory. Czuber, $E$. Wien Ak. Sb. 90 (1885) (Ab. 2) 719-.

- probability, problem. Panek, A. Časopis 11 (*1882) 121-; Fschr. Mth. (1882) 179.

Geometrography applied to different solutions of a problem. Lemoine, E. Par. S. Mth. Bll. 20 (1892) 132-.

Geometry, application to problems in probability. Simmons, (Rev.) T. C. As. Fr. C. R. (1894) (Pt. 2) 266-.

- - - - - Lemoine, E. As. Fr. C. R. (1896) (Pt. 1) 135-.

- , - - questions of means and probabilities. Lalanne, L. L. Chrétien-. C. R. 87 (1878) 355-; Liouv. J. Mth. 5 (1879) 107-, 123-.

Gravity determination by pendulum, treatment of equations. Venturi, $A$. N. Cim. 11 (1900) 33-.

H-curve, so-called. Boltzmann, L. Mth. A. 50 (1898) 325-.

Hypergeometric series in connexion with observation polygons. Pearson, $K$. $\mathrm{Ph} . \mathrm{Mg}$. 47 (1899) 236-.

Impartiality, postulate of, in theory of errors. Crotti, F. Mil. I. Lomb. Rd. 28 (1895) 271-.

Index numbers, accuracy of proposed calculation. Edgeworth, F. Y. B. A. Rp. (1888) 188-.

Inverse probabilities. Makeham, $W . M$. I. Act. J. 29 (1892) 242-, 444-.

- - Makeham's theory. Stabler, E. L. I. Act. J. 30 (1893) 239-, 580.

- probability. Markow, A. A. Fschr. Mth. (1900) 228.

- - Wilson, J.C. [1900] Nt. 63 (1900-01) $154-, 188$.

- - discussion of Laplace's problem. Catalan, E. C. Liége S. Sc. Mm. 15 (1888) No. 1, 255-.

Large numbers, approximate calculation of functions of. Nekrasov, P. A. [1898-99] Rec. Mth. (Moscou) 20 (1899) 431-; 21 (1901) 379-; Fschr. Mth. (1898) 187-.

- - law, in theory of probabilities. Imśeneckij, $V . G$. Kharkov Mth. S. Com. 1 (1889) 1-; Fschr. Mth. (1888) 211.

- -, and least squares method. Markov, A. A. Kazan S. Ps.-Mth. Bll. 8 (1899) 110-; 9 (1900) 41-; Fschr. Mth. (1898) 188. $-\ldots,-\ldots-\ldots$, Markov's law. Nekrasov, P. A. Kazan S. Ps.-Mth. Bll. 9 (1900) 18-; Fschr. Mth. (1899) 215.

-- , Poisson's law. Mansion, P. Brux. Ac. B1l. 25 (1893) 11-.

- - probabilities depending on, formulae. Poisson, S. D. C. R. 2 (1836) 603-.

Law of the long run. Mendenhall, $T$. $C$. Science 2 (1895) 825-.

Laws of error for situation of points, composition of. Ocagne, M. d'. C. R. 118 (1894) 517-. 


\section{Probabilities, etc.}

Least-square computation, form of. Farquhar, H. Smiths. Misc. Col. 33 (1888) Art. 1, 150-. (Wash. Ph. S. Bll. 6 (1884).)

- fallacies. Safford, T. H. Am. As. P. (1892) 57-.

\section{Least Squares Method.}

Legendre, A. M. Par. Mm. de 1'I. (1810) (pte. 2) 149-.

Ivory, J. Tilloch Ph. Mg. 65 (1825) 3-, 81-, 161-; 68 (1826) 161-.

Nürnberger, -. Görl. Ab. 1 (1827) 22-.

Bitnicz, L. Évk. 3 (1838) 42-.

Bessel, F. W. As. Nr. 17 (1840) 225-, 305-.

Ellis, R. L. [1844] Camb. Ph. S. T. $8(1849)$ 204-.

Wolf, R. Bern Mt. (1849) 140-

Paucker, M. G. von. [1850] St Pét. Ac. Sc. Bll. 9 (1851) 113-; 10 (1852) 33-, 233-, 234-.

Hossard, P. N. A. Mth. 10 (1851) 456-.

Arrest, H. d'. Leip. B. (1854) 133-.

Bertrand, $J$. C. R. 40 (1855) 1190-.

Wrede, F. J. Stockh. Öfv. 14 (1857) 73-.

Kurz, A. Zür. Vjsehr. 8 (1863) 225-

Todhunter, I. [1865] Camb. Ph. S. T. 11 (1871) 219 .

Miller (Miller-Hauenfels), Alb. von. Oestr. Z. Brgw. 15 (1867) 181, 194.

Minding, E. F. A. St Pét. Ac. Sc. Bll. 16 (1871) 305-.

Glaisher, J. W. L. Nt. 6 (1872) 140-.

Jouffret, E. P. Rv. Mar, et Col. 38 (1873) 5-, 523-, $957 ; 42$ (1874) 665-, 986-; 43 (1874) 131-.

Wrede, F. J. Stockh. Öfv. 30 (1873) (No. 8) 3-; (No. 10) 21-.

Faye, H. A. É. C. R. 80 (1875) 352-.

Laurent, $H$. Liouv. J. Mth. 1 (1875) 75-.

Safford, T. H. Am. Ac. P. 11 (1876) 193-.

Lorenz, L. V. (xII) Ts. Mth. 6 (1876) 33-

Adcock, R.J. Des Moines Anal. 4 (1877) 183-; 5 (1878) 21-.

Glaisher, J. W. L. Mess. Mth. $9(1880) 132$.

Edgeworth, F. Y. Ph. Mg. 16 (1883) 360-.

Mansion, P. Brux. Ac. Bll. 9 (1885) 9-.

Bertrand, J. C. R. 106 (1888) 1115-.

Yarochenko, S. Bll. Sc. Mth. 17 (1893) 113-.

Gosiewski, W. Prace Mt.-Fiz. 4 (1894) 103-; Fschr. Mth. (1893-94) 348-.

Andrade, J. C. R. 122 (1896) 1400-.

Goedseels, -. Brux. S. Sc. A. 21 (1897) (Pt. 1) 13-.

alleged proof. Ellis, R. L. Ph. Mg. 37 (1850) $321-, 462$.

application. Gauss, C. F. As. Nr. 5 (1827) 230.

- Hansen, P. A. As. Nr. 8 (1831) 463-.

- Argelander, F. W. A. As. Nr. 21 (1844) 163-.

-. Freuchen, $P$. Mth. Ts. 3 (1861) 81-.

- Carvallo, E. C. R. 106 (1888) 924.

- to the case where the unknown quantity satisfies certain exact conditions. Nasimoff, P. S. Fschr. Mth. (1889) 219.

- cases of systematic error. Thiele, T. N. Kjöb. Dn. Vd. Selsk. Skr. 12 (1880) 381-.

VOL. I.

\section{Least Squares Method 1630}

application to conditioned observations. Steinthal, A. E. Mess. Mth. 10 (1881) 185-.

- of determinants. Geer, P. van. N. Arch. Wisk. 1 (*1875) 179-; 9 ("1882) 180-; Arch. Néerl. 12 (1877) 229-; 18 (1883) 127-.

- Gravelaar, N.L.W.A. N. Arch. Wisk. 10 (1884) 107-

- Zbrożek, D. Krk. Ak. (Mt.-Prz.) Pam. 9 (1884) 199-.

- to geodesy. Bache, A. D. Am. As. P. (1849) 102-.

- - Hansen, P. A. Leip. Ab. Mth. Ps. 8 (1868) 571-; 9 (1871) 1-.

- - - Nagel, A. Civing. 42 (1896) 703-.

- to hydraulic problem. Merriman, $M$. Franklin I. J. 74 (1877) 233-.

approximation. Colding, L. A. Kjöb. Ov. (1857) 52-.

bibliography and notes. Merriman, M. [1877] Conn. Ac. T. 4 (1877-82) 151-.

Bouniakowsky. Erman, A. Erman Arch. Rs. 19 (1860) 1-.

case where number of unknowns is large. Nekrasov, P. A. Rec. Mth. (Moscou) 12 (1885) 189 -.

compensation of errors. Pizzetti, P. Rm. R. Ac. Linc. Rd. 3 (1887) (Sem. 2) 230-, 288-.

correction of observations. Whitaker, H. Sid. Mess. 9 (1890) 458-.

deduction from calculus of probabilities. Reuschle, [C. G.] Crelle J. 26 (1843) 333-; 27 (1844) 182-.

- - - - (Reuschle). Encke, J. F. Crelle J. 28 (1844) 213 -.

determination of point by system of planes. Jung, G. Mil. I. Lomb. Rd. 30 (1897) 1014 -.

elementary proof. Gosiewski, W. Kosmos (Lw.) 20 (1895) 366-; Fschr. Mth. (1895) 245.

— theory. Tsinguer, -. Mosc. Bll. S. Nt. 35 (1862) (pte. 2) 446-.

equivalent factors. Bond, G. P. [1856] Am. Ac. $\mathrm{M}_{\mathrm{n}} .6$ (1857) 179-.

failure, application of method of limiting coefficients. Cauchy, A. L. C. R. 37 (1853) 150 -.

function $\phi$. Landre, C. L. N. Arch. Wisk. 7 (*1881) 211-.

fundamental theorem. Denzler, W. Zür. Mt. $2(1850-52) 110$

Hagen's proof. Merriman, M. Franklin I. J. 74 (1877) 330-.

Laplace's discovery. Bienaymé, J. C. R. 37 (1853) 309-.

- proof. Giaisher, J. W. L. Ph. Mg. 43 (1872) 194-

principle, demonstration. Le Grand Roy, $E$. Neuch. S. Sc. Bll. 27 (1899) 23-.

-, elementary discussion. Merriman, $M$. Franklin I. J. 74 (1877) 173-.

- - - (Merriman). Kummell, C. H. Franklin I. J. 74 (1877) 270 -

- of the mean. Lagrange, C. Brux. Ac. Bll. 32 (1896) 60-.

principles. Giletta, L. [1879] G. Mt. 18 (1880) 159-. 


\section{Least Squares Method}

probable error. Liouville, J. Liouv. J. Mth. 17 (1852) 31-.

- - Bienaymé, J. Liouv. J. Mth. 17 (1852) 33-; C. R. 37 (1853) 5-; Par. Mm. Sav. Étr. 15 (1858) 615-.

problem. Merriman, M. [1891] N. Y. Mth. S. Bll. 1 (1892) 39-.

proof. Matzka, W. Grunert Arch. 11 (1848) 369 -.

- of some relations. Kummell, C. H. Des Moines Anal. 7 (1880) 84.

reduction of experiments. Harvey, G. Edinb. Ph. J. 7 (1822) 292-.

resolution of system of linear equations with three unknowns. Kononowitsch, A.K. Fschr. Mth. (1896) 184.

simplification, in calculation of empiric constants. Bellati, $M$. Ven. I. At. 1 (1875) 849-.

solution of equations. Glaisher, J. W. L. [1874-80] As. S. M. Not. 34 (1874) 311-; 40 (1880) 600-; 41 (1881) 18-.

- - - by successive approximations. Seidel, P. L. von. [1873] Münch. Ak. Ab. 11 (1874) (3 $\left.{ }^{t e} A b.\right) 81-$.

- linear equations. Jacobi, C. G.J. As. Nr. 22 (1845) 297-.

supplement. Wittstein, T. As. Nr. 102 (1882) 339-.

theorem, generalisation. Jordan, C. [1872] Z. Mth. Ps. 18 (1873) 116-.

theory. Encke, J. F. Berl. As. Jb. (1834) 249-; (1835) 253-; (1836) 253-; Berl. B. (1850) 211-.

-. Biver, P.E. Liouv. J. Mth. 18 (1853) 169-.

- Casorati, F. Tortolini A. 1 (1858) 329-.

-. Catalan, E. C. [1878] Brux. Ac. Sc. Mm. 43 (1882) (No. 3) $42 \mathrm{pp}$.

- Lerch, M. Prag České Ak. Fr. Jos. Rz. (Trida 2) 1 (1892) Art. 27, 70 pp.; Fschr. Mth. (1892) 446-

-. Slešinskij, I. V. N. Rs. S. Nt. Mm. (Mth.) 14 (1892) 201-; Fschr. Mth. (1892) 205.

-, formula in. Loudon, W. J. Nt. 41 (1890) 394.

values of constants in equation from the $n+1$ values of $y_{x}$ when $x=0,1,2 \ldots n$. Carpmael, C. As. S. M. Not. 39 (1879) $489-$

weights. Dienger, J. Grunert Arch. 19 (1852) 197-.

- Jordan, W. Z. Mth. Ps. 17 (1872) 350 -

- of 6 elements, general formulæ. Plana, $G$. Lindenau Z. 6 (1818) 249-.

Linguistic resemblances, accidental, probability. Chase, P. E. [1863] Am. Ph. S. T. 13 (1869) 25-.

- -, note. Oliver, J. E. [1864] Am. Ph. S. T. 13 (1869) 69-

Longitude calculations, determination of mean error from differences of double measures. Helmert, F. R. As. Nr. 81 (1873) 49-.

- measures, probable error. Dienger, J. Grunert Arch. 31 (1858) 225-.
Probabilities, etc. 1630

Lottery, state, calculations. Bonicelli, $P$. (vI Adds.) Majocchi A. Fis. C. 20 (1845) 97-.

Marriage, probable duration. Drobisch, $M . W$. Leip. Mth. Ps. B. 32 (1880) 1-.

Mathematical expressions of mean temperature and mean magnetic intensity. Brewster, Sir D. Pogg. A. 21 (1831) 323-.

- and moral expectation. Panek, $A$. Časopis 6 (*1877) 69-, 122-, 218-; 7 (1878) 78-; Fschr. Mth. (1877) 150.

Mayer, Tobias, method. Goedseels, P. J. E. Brux. S. Sc. A. 24 (1900) (Pt. 2) 37-.

Mean, arithmetic, errors likely in finding. Toschi, A. Palomba Rac. 3 (1847) 312 .

- - principle. Schiaparelli, G.V. As. Nr. 87 (1876) 55-; 88 (1876) 141-.

-, - - - Stone, E. J. As. Nr. 88 (1876) 61-.

-, - , -, generalisation. Pizzetti, P. Rm. R. Ac. Linc. Rd. 5 (1889) (Sem. 1) 186-.

-, - , - in observation results. Schiaparelli, G. V. Mil. I. Lomb. Rd. 1 (1868) 771-.

- error. Helmert, F. R. As. Nr. 85 (1875) 353-.

- - of an angle in method of binary combinations. Jadanza, $N$. Tor. Ac. Sc. At. 33 (1897) 643- or 883-; 34 (1898) 698- or 966-.

- - - - - - - Pizzetti, P. Tor. Ac. Sc. At. 34 (1898) 745- or 1013-.

- - chain measures. Winckler, A. Schlömilch Z. 6 (1861) 109-.

- - determination. Zachariae, G. As. Nr. 80 (1873) 67-

- - of a function of observed quantities, theorem. Pizzetti, P. Rm. R. Ac. Linc. Rd. 2 (1886) (Sem. 1) 597-.

- - observations. Gerling, C.L. Grunert Arch. 25 (1855) 219-

- - - , theory. Landrê, C. L. N. Arch. Wisk. 10 (1884) 1 -.

_ _ - Kooten, F. H. van. N. Arch. Wisk. 12 (1886) 94-; Fschr. Mth. (1885) 184.

- - for system of observations. Pizzetti, P. Rm. R. Ac. Linc. Rd. 5 (1889) (Sem. 1) 740 -

-, geometric, law. M'Alister, D. QJ. Mth. 17 (1881) 175-.

- probabilities applied to statistics. Bertrand, J. C. R. 106 (1888) 1311-.

- of successive observations, calculation. Ruffini, F. P. (xI) Mod. Ac. Sc. Mm. 8 (1867) 65-.

-, theorem of the ; the converse of Bernoulli's theorem. Lagrange, $C$. Brux. Ac. Bll. (1899) 428-.

-, theory of errors founded on theorem of. Lagrange, C. Brux. Ac. Bll. 35 (1898) 795-.

- and true value of polynomial, consequences of accepted equality. Bertrand, J. C. R. 106 (1888) 1259-.

- values. Tchébicheff, P. de. Liouv. J. Mth. 12 (1867) 177-.

- -. Scheilmer, W. Leip. B. 25 (1873) 562-.

- - Hayes, E. Science 21 (1893) 333.

- - with reference to plane curves and curved surfaces. Cauber, E. Arch. Mth. Ps. 6 (1888) 294-. 
Mean values, theory. Beeck-Calkoen, J.F.van. Amst. Vh. 2 (1816) 1-.

Means, calculation and scientific applications. Segnitz, E. Schlömilch Z. 7 (1862) 65-.

-, choice of. Edgeworth, F. Y. Ph. Mg. 24 (1887) 268-.

_, method of, applied to transformation, to calculation, and to limits of remainders in series. Poncelet, J. V. [1833] Crelle J. 13 (1835) 1-.

- of observations. Trembley, J. Berl. Mm. Ac. (1801) 29-.

- - Lloyd, H. [1848] Ir. Ac. T. 22 (1855) 61-.

- - -. Cauchy, A. L. C. R. 37 (1853) 198-, 381-.

- - Brassinne, E. Toul. Mm. Ac. 6 (1862) 421-.

Measures, fallible, of variable quantities, and meteorological observations. Darwin, G.H. Ph. Mg. 4 (1877) 1-.

Mechanical rectification of an arc of a curve. Wiener, C. Z. Mth. Ps. 16 (1871) 112-.

Michell's problem (distribution of stars). Lupton, S. Nt. 38 (1888) 272-, 414.

Moral expectation, maxima and minima. Buniakovskij, V. Ja. [1879] St Pet. Ac. Sc. Mm.

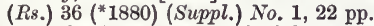

Most probable existence, principle. Gosiewski, W. Prace Mt.-Fiz. 3 (1892) 55-; Fschr. Mth. (1892) 73-.

- - mechanical system of the universe. Gosiewski, W. Crc. Ac. Sc. Bll. (1889) No. 5, xli-.

- - result of number of observations. Wilson, J. M. [1877] As. S. M. Not. 38 (1878) 81-.

- - from observations with assigned weights. Stone, E. J. As. S. M. Not. 36 (1876) 290-.

- - - - - of equal value.' Stone, E. J. As. S. M. Not. 33 (1873) 570-.

- - value of latitude. Flint, A. S. Smiths. Misc. Col. 33 (1888) Art. 4, 91-. (Wash. Ph. S. Bll. 10 (1888).)

- - - quantity from $n$ observations without use of Gauss's postulate. Bervi, N. V. Mosc. S. Sc. Bll. 96 (No. 1) (1899) 41-; Fschr. Ps. (1900) (Ab. 1) 24.

- _ - unknowns connected by equations of condition, calculation. Seidel, P.L. von. As. Nr. 84 (1874) 193-.

- values of frequency-constants, calculation. Sheppard, W. F. L. Mth. S. P. 29 (1898) 353-.

Mountain ranges, application of calculus of probabilities. Spottiswoode, W. [1860] Gg. S. J. 31 (1861) 149-.

Nearness of approximation in theory of observations. Kooten, F. H. van. Batav. Ntk. Ts. 49 (1890) 160-.

Normal distribution and normal correlation. Sheppard, W. F. [1897] Phil. Trans. (A) 192 (1899) 101-.

Observation-equations, special treatment of certain. Woodward, R. S. Smiths. Misc. Col. 33 (1888) Art. 1, 156-. (Wash. Ph. S. Bll. 6 (1884).)
Observation results, numerical, adjustment. Sprague, T. B. B. A. Rp. (1883) 446-.

Observations, a posteriori evaluation of trust. worthy character of a series of. Bertrand, $J$. C. R. 106 (1888) 887-.

-, combination of results. Powalky, C. R. As. S. M. Not. 34 (1874) 476-.

-, compensation and mean error. Paucker, M. G. von. Mitau Arb. Kurländ. Gs. 6 (1849) 41-; 9 (1851) 183-.

, corrections of personal error. Fechner, G. T. Leip. B. 13 (1861) 57-.

-, determination of accuracy. Gauss, C. F. Lindenau Z. 1 (1816) 185-.

- - - - Hauber, C. F. Baumgartner Z. 7 (1830) 286-.

-, direct, calculation of result of system of. Pizzetti, $P$. Liège S. Sc. Mm. 15 (1888) No. 3,32 pp.

-, doubtful. Edgeworth, F. Y. Ph. Mg. 23 (1887) 364-.

,-- , criterion for rejection. Peirce, B. Gould As. J. 2 (1852) 161-.

-, -, Peirce's criterion. Gould, B. A. Gould As. J. 4 (1856) 81-.

-, - - - - Airy, G. B. Gould As. J. 4 (1856) 137-.

- , - - - (Airy's criticism). Winlock, J. Gould As. J. 4 (1856) 145-.

-, - - - Peirce, B. Am. Ac. P. 13 (1878) 348-.

, -, rejection. Stone, E. J. As. S. M. Not. 28 (1868) 165-.

-, - - Glaisher, J.W. L. As. S. M. Not. 33 (1873) 391-.

-, - - - Stone, E. J. [1873] As. S. M. Not. 34 (1874) 9-.

-, - - Doolittle, -. Smiths. Misc. Col. 33 (1888) Art. 1, 152-. (Wash. Ph. S. Bll. 6 (1884).)

-, equations of condition. Grunert, J.A. Grunert Arch. 21 (1853) 453-.

- functions of, accuracy. Gerling, C. $L$. Grunert Arch. 38 (1862) 379-.

_, incomplete, introduction into probability. calculations. Küttner, $W$. Z. Mth. Ps. 29 (1884) 193-.

-, limits of accuracy. Paucker, M. G. von. Mitau Send. Kurländ. Gs. 1 (1840) 73-.

-, - to measure of precision. Adan, (Capitaine) E. Brux. Ac. Bll. 22 (1866) 480-.

-, methods of detecting unknown inequalities in. Stokes, G. G. R. S. P. 29 (1879) 122-.

-, probable existence of a cause of error in a series. Liagre, J. Brux. Ac. Bll. 22 (1855) (pte. 2) 15-.

-, - mean. Svanberg, J. Stockh. Ak. Hndl. (1821) 388-.

-, reduction. Edgeworth, F. Y. Ph. Mg. 17 (1884) 135-.

- relating to several quantities, new method of reducing. Edgeworth, F. Y. Ph. Mg. 25 (1888) 184-.

- - - - - - - - - (Edgeworth's). Turner, H. H. Ph. Mg. 24 (1887) 466-. - represented by diagrams. Earnshaw, S. $\mathrm{Ph}$. Mg. 27 (1845) 127-. 


\section{Probabilities, etc.}

Observations, results, probable mean. Anon. (vi 542) Gergonne A. Mth. 12 (1821-22) 181-.

-, -, properties. Quetelet, L. A. J. Brux. Ac. Bll. 19 (1852) (pte. 2) 303-.

-, series of, translation into continuous formulæ. Merrifield, C. W. [1880] L. Mth. S. P. 12 (1880-81) 4-.

- and statistics. Edgeworth, F. Y. [1885] Camb. Ph. S. T. 14 (1889) 138-; Camb. Ph. S. P. 6 (1889) 101-.

- theory, and method of least squares. Thiele, T. N. Bll. Sc. Mth. 14 (1890) 73-.

—, true average. Smith, R. H. Nt. 37 (1888) 464.

-, values making greatest error a minimum. Cauchy, A. L. [1814] Par. Éc. Pol. J. 13 (1831) (20 cah.) 175-.

Observed points, new method of representing, suitable for explorers. Láska, $V$. Prag Sb. (1895) (Mth.-Nt.) No. 41, 6 pp.; Fschr. Mth. (1895) 248.

Personal equation. Gonnessiat, $F$. Lyon Un. A. 3 (Fasc. 2) (1892) 167 pp.

Petersburg problem. Orchard, $W$. Assur. Mg. 2 (*1852) 185.

- Czuber, (or Čuber) E. Arch. Mth. Ps. 67 (1882) 1-.

- - Catalan, E. C. Liége S. Sc. Mm. 15 (1888) No. 1, 248-.

- - Wolf, R. Zür. Vjschr. 34 (1889) 264 -

- -. Lupton, S. Nt. 41 (1890) 165-.

- - Seydler, - C. R. 110 (1890) 326-.

- paradox analogous to. Bertrand, $J$. C. R. 105 (1887) 831-.

Physical laws deduced from empirical curves. Pucci, E. N. Cim. 27 (1890) 220-; 28 (1890) 111-.

Plane, calculation of mean situation from more than three points. Weisbach, $J$. Civing. 16 (1870) 397-.

Plausible value of a variable magnitude. E8tienne, - C. C. 130 (1900) 393-.

Points problem. Mansion, $P$. Brux. Mm. Cour. $8^{\circ} .21$ (1870) (No. 1) 13 pp.

- - Badoureau, A. Par. Éc. Pol. J. 27 (=cah. 44) (1874) 123-.

- Catalan, E. C. Liège S. Sc. Mm. 12 (1885) No. 2, 65-.

Position of a point determined by many measurements. Hammer, E. Z. Mth. Ps. 43 (1898) 105-.

- - - errors. Dahlander, G.R. Stockh. Öfv. 14 (1857) 405-； 15 (1858) 49- ; 18 (1861) $63-$.

- - - - in a plane, composition of law of errors for. Ocagne, $-d$ '. Par. S. Mth. Bll. 23 (1895) 65-.

- - - probability of error in. Bravais, $A$. Par. Mm. Sav. Etr. 9 (1846) 255-.

Pothenot's problem, theory of errors in. Andra, -. As. Nr. 47 (1358) 193-.

Precision of a system of measurements. Bertrand, J. L. $F$. C. R. 106 (1888) 1195-.

$--\frac{-}{-}-$, determination. Bertrand, J. C. R. 106 (1888) 440-.

\section{Probabilities 1630}

Presumable error in evaluation of the angles of a triangle. Bertrand, J. C. R. 106 (1888) 967-.

Principle of least action and probability theory, connexion between. Gosiewski, W. Prace Mt.-Fiz. 1 (1888) 97-; Fschr. Ps. (1889) (Ab. 1) 253-.

\section{PROBABILITIES.}

Breevilt, C. [1788] Mengelwerk Wisk. Vh. 1 (1796) 81-.

Oettinger, L. Münch. Ab. 2 (1831-36) 199-, 243-.

Poisson, S. D. C. R. 2 (1836) 395-.

Mondésir, É. Liouv. J. Mth. 2 (1837) 3-.

Bienaymê, J. Par. S. Phlm. PV. (1840) 23-.

Oettinger, L. Grunert Arch. 1 (1841) 113-.

Hamilton, (Sir) W. R. B. A. Rp. (1843) (pt. 2) 3-.

Oettinger, L. Crelle J. 26 (1843) 217-, 311-; 30 (1846) 232-, 296-; 34 (1847) 153-; 36 (1848) 221-, 296-; 42 (1852) 213-.

Bordoni, A. Mil. G. I. Lomb. 4 (1852) 31-.

Wolf, $R$. Bern Mt. (1852) 133-.

Cayley, A. Ph. Mg. 6 (1853) 259.

Dedekind, R. Zür. Vjschr. 4 (1859) 346-; 5 (1860) 66-.

Cayley, A. Ph. Mg. 23 (1862) 352-, 470-.

Matthiessen, L. Arch. Mth. Ps. 47 (1867) 457 -

Meyer, A. Liége S. Sc. Mm. 4 (1874) (No. 2) $446 \mathrm{pp}$.

Rocquigny, $F$. [G.] de. Les Mondes 51 (1880) 652-.

a priori. Edgeworth, F. Y. Ph. Mg. 18 (1884) 204 -

- - of errors of observation. Pizzetti, P. G. Mt. 27 (1889) 77-.

accidental errors. Seeliger, $H$. As. Nr. 97 (1880) 289-.

- - Gosiewski, W. Prace Mt.-Fiz. 1 (1888) 1-; Fschr. Mth. (1888) 227.

- -, (Gosiewski). Baraniecki, M. A. Prace Mt.-Fiz. 2 (1890) 220-; Fschr. Mth. (1890) 235-.

- - Russjan, C. N. Rs. S. Nt. Mm. (Mth.) 9 (1889) 134-; Fschr. Mth. (1889) 219-.

- - (Russjan). Somin, N. J. [1889] Vars. S. Nt. Tr. (1889-90) (C.R., Ps. C.) No. 4, 2-. - - , application to educational statistics. Maximowitsch, W. P. Fschr. Mth. (1888) 229.

adjustment and computation. Lazarus, $W$. I. Act. J. 20 (*1978) 410-.

analogy, and least squares. Donkin, W. F. QJ. Mth. 1 (1857) 152-.

analysis. Binet, J. P. M. C. R. 19 (1844) 373-.

application. Brion, L. Abv. Mm. S. Ém. (1838-40) 251-.

-. Ghibaldan, I. Bucarest S. Sc. Bll. 6 (1897) 479-; 7 (1898) 225-; 8 (1899) 18-.

- to comparison of causes favouring a recurring phenomenon. Steen, A. Kjöb. Ov. (1866) 8-. 


\section{Probabilities}

application to dynamical systems. Zermelo, $E$. Ps. Z. 1 (1900) 317-.

- - geodesy. La Place, P. S. (Marquis) de. Con. des Temps (1820) 422-.

_ _ - levelling operations, \&c. La Place, P. S. (Marquis) de. A. C. 12 (1819) 337-. - geodetic measurements. Hansen, P.A. As. Nr. 9 (1831) 189-, 237-.

- - legal subjects. Jerwood, J. Devon. As. T. 2 (pt. 2) (1868) 578-.

- - natural philosophy. La Place, P. S. (Marquis) de. Con. des Temps (1818) 361-.

- - petrography. Cesaro, G. Brux. Ac. Mm. 53 (1895-98) (No. 1) 55 pp.

-, practical. Littrovo, $J$. $J$. von. Baumgartner Z. 9 (1831) 433-.

- to science of humanity. Quetclet, L. A.J. Brux. Ac. Bll. 36 (1873) 19-.

- - variations in the transparency of air. Seidel, L. Münch. Sb. 2 (1863) 320-.

average deviations. Hardy, G. F. I. Act. J. 27 (1889) 214 .

and averages, theory. Nekrasov, P.A. Rec. Mth. (Moscou) 21 (1901) 579-.

correction of a formula. Laquière, $E$. $M$. Par. S. Mth. Bll. 8 (1880) 74-.

criticism of Laplace. Ruffini, $P$. Pisa N. G. 1 (1822) 201-.

definitions and principles of calculus of. Gosiewski, W. Wiad. Mt. 4 (1900) 137-; Fschr. Mth. (1900) 230.

demonstration of Gauss, rigour of a. Bertrand, J. C. R. 106 (1888) 563-.

drawing lots. Jouffret, $E$. Rv. Mar. 38 (1873) 5-, 523-, 957-.

errors of calculations performed in duplicate. Glaisher, J. W. L. (vII) [1873] Mess. Mth.3 (1874) 106-.

events which seldom happen, their possible frequency being unlimited. Seidel, P.L.von. Münch. Ak. Sb. 6 (1876) 44-.

experiments. Baker, A. Cn. I. P. 5 (1888) 194-.

gaming, with application to definite integrals. Mayer, D. E. Par. Éc. Pol. J. 3 (1897) 153-.

general theorem, elementary proof. Tchebicheff, P. Crelle J. 33 (1846) 259-.

generalisation of a problem in. Azzarelli, $M$. Rm. N. Linc. At. 45 (1892) 72-.

integration of recurring series. Ocagne, $M . d^{\prime}$. Par. S. Mth. Bll. 15 (1887) 143-.

judgments. Bertrand, J. Par. S. Phlm. Mm. Cent. (1888) 69-.

- in courts of appeal. Guibert, A. C. R. 7 (1838) 650-.

-, especially in criminal cases. Poisson, S. D. C. R. 1 (1835) 473-.

- and evidence. Bienaymé, J. Par. S. Phlm. PV. (1838) 93-.

judicial errors. Ostrogradsky, M. A. St. Pét. Ac. Sc. Mm. 3 (1838) (Bll. Sc.) xix-.

juries' verdicts. Coste, L. M. P. Liouv. J. Mth. 7 (1842) 169-.

- - proportion of condemnations. Poisson, S. $D$. C. R. 5 (1837) 355-, 459-.

\section{Probabilities: Problems 1630}

legal, decisions of majorities. Guibert, $A$. Liouv. J. Mth. 3 (1838) 25-.

losses in war. Bouniakowsky, $V$. [1846] St. Pét. Ac. Sc. Mm. 6 (Pt. 1) (1850) 233-.

- - - Lafrogne, (le lt.) H. Rv. Mar. et Col. 142 (1899) 275-.

principle, new. Bienaymé, J. Par. S. Phlm. PV. (1840) 37-.

-, - Catalan, E. C. Brux. Ac. Bll. 44 (1877) 463-; 8 (1884) 72-.

\section{Probabilities: Problems.}

Plana, G. Turin Mm. Ac. (1811-12) 355-.

Rangoni, L. (xI) Mod. S. It. Mm. 18 (Mt.) (1820) 518-.

Nieuport, C. F. F. de. Brux. Ac. Sc. Mm. 3 (1826) 143-.

Lubbock, J. W. [1828] Camb. Ph. S. T. 3 (1830) 141-.

Bénard, -. Par. Ec. Pol. J. 15 (cah. 24) (1835) 264-.

Catalan, E. C. Liouv. J. Mth. 2 (1837) 469-; 6 (1841) 75-.

Lévy, A. N. A. Mth. 1 (1842) 179-.

Ostrogradsky, M. A. [1846] St Pét. Ac. Sc. Bll. 6 (1848) 321-.

Terquem, O. N. A. Mth. 5 (1846) 218-, 260.

Dedekind, $R$. Crelle J. 50 (1855) 268-.

Lazarus, W. I. Act. J. 15 (* 1870) 245-.

Sutton, W. I. Act. J. $15\left(^{*} 1870\right) 452-; 16$ (*1872) 151-.

Lemoine, É. (x) Par. S. Mth. Bll. 1 (1873) 39-.

Halphen, G. H. (x) Par. S. Mth. Bll. 1 (1873) 221-.

Jordan, C. (x) Par. S. Mth. Bll. 1 (1873) 256-, 281-.

Bienaymé, J. Par. S. Mth. Bll. 2 (1874) 153-.

Levänen, S. Helsingf. Öfv. 17 (1875) 112-.

Hoppe, R. Arch. Mth. Ps. 61 (1877) 410-.

Bianco, O. Z. G. Mt. 16 (1878) 169-.

Woolhouse, W. S. B. I. Act. J. 21 (*1879) 204-.

Lemoine, $\dot{E}$. As. Fr. C. R. (1880) 183-.

Cesáro, E. (xir) Mathesis 2 (1882) 177-, 232 ; 3 (1883) 233-.

Gosiewski, W. (xп) [1882] Krk. Ak. (Mt.Prz.) Rz. \& Sp. 10 (1883) 137-, liii-.

Lemoine, É. N. A. Mth. 3 (1884) 118-; As. Fr. C. R. (1885) (Pt. 2) 50-.

Edgeworth, F. Y. Ph. Mg. 22 (1886) 371-.

Weill, -. Par. S. Mth. Bll. 14 (1886) 158-

Cesáro, E. Palermo Cir. Mt. Rd. 1 (1887) 299-.

Mayer, E. C. R. 108 (1889) 391-.

Pánek, A. Č́asopis 20 (1891) 105-; Fschr. Mth. (1891) 232.

Makeham, W. M. I. Act. J. 29 (1892) 475-.

Lemoine, É. As. Fr. C. R. (1895) (Pt. 1) 182.

Lindelöf, L. Helsingf. Öfv. 42 (1900) 79-.

Hermite, C. Helsingf. Öfv. 42 (1900) 88-.

analytical. Schlömilch, $O$. Grunert Arch. 9 (1847) 372-.

Bernoulli, etc. Malfatti, G. F. Verona S. It. Mm. 1 (1782) 768-. 


\section{Probabilities: Problems}

Bernoulli and Lagrange. Abbati, $P$. (vr Adds.) Mod. Mm. S. It. 19 (1821) 385-.

Bertrand, generalisation. Barbier, $E$. C. R. 105 (1887) 407.

-, solution. André, D. C. R. 105 (1887) 436-.

cards. Cantor, M. Schlömilch Z. 2 (1857) 410-.

class. Sylvester, J. J. B. A. Rp. 35 (1865) (Sect.) 8-.

collimator for adjustment of azimuth circle. Airy, G. B. B. A. Rp. (1850) (pt. 2) 1 -. competitive examinations. Edgeworth, $F$. $Y$. $\mathrm{Ph}$. Mg. 30 (1890) 171-.

conditions limiting solution. Boole, $G . \mathrm{Ph}$. Mg. 8 (1854) 91-.

election. Korteweg, $D . J . \quad$ N. Arch. Wisk. 1 ( $\left.{ }^{*} 1875\right)$ 157-; 2 (* 1876) 40-.

evidence. Doolittle, M. H. Wash. Ph. S. Bll. 11 (1892) 602.

game of pool. Arcais, F. d'. Padova Ac. At. e Mm. 16 (1900) 219-.

graphic solution. Favaro, $A$. Ven. I. At. 3 (1877) 1025-.

Laurent. Pánek, A. Časopis 20 (1891) 94-; Fschr. Mth. (1891) 231.

note. Laquière, E. M. Par. S. Mth. Bll. 8 (1880) 79-.

and relative values of pieces in game of chess. Lemoine, $\dot{E}$. As. Fr. C. R. (1880) 179-.

solution. Plana, $G$. Mod. S. It. Mm. 18 (1818) 31-.

-. Brashmann, N.D. (xII) Mosc. Un. Mm. 10 (1835) 523-.

-. Poisson, S. D. Liouv. J. Mth. 2 (1837) 373-.

- Hansen, P.A. As. Nr. 16 (1839) 9-, 27-.

- Meyer, A. Brux. Ac. Bll. 15 (1848) (pte. 2) 508 .

-. Roger, $\dot{E}$. Liouv. J. Mth. 17 (1852) 202-.

- Blazejarski, R. N. A. Mth. 1 (1862) 131-.

-. Liagre, J. B. J. Brux. Ac. Bll. 17 (1864) 365-.

- Terrier, (Prof.) -. Neuch. Bll. 9 (1873) 441-.

-. Hendricks, J. E. Des Moines Anal. 3 (1876) 152-.

-. Spitzer, S. D. Nf. B. (*1877) 97-.

-. Piuma, C. M. G. Mt. 17 (1879) 360-.

- Spitzer, $S$. Arch. Mth. Ps. 64 (1879) 74 -

-. Bertrand, J. C. R. 105 (1887) 369, 437-

-, application of. Poréckij, P. S. Kazan S. Nt. (Ps-Mth.) P. 5 (1887) 83-; Fschr. Mth. (1887) 200.

-, geometrical. Lemoine, $\dot{E}$. [1882] Par. S. Mth. Bll. 11 (1883) 13-.

solved by Poisson, indeterminateness. Bertrand, J. C. R. 106 (1888) 636-.

and theorems. Catalan, E. Brux. Ac. Mm. 46 (1886) (No. 2) $16 \mathrm{pp}$.

two. Andrade, -. C. R. 130 (1900) 395-.

question treated by d'Alembert. Maupin, $G$. Par. S. Mth. Bll. 23 (1895) 185-.

- - Delannoy, -. Par. S. Mth. Bll. 23 (1895) 262-.
Probabilities, etc. 1630

repeated trials. Bertrand, J. C. R. 94 (1882) 185-; 105 (1887) 1201-.

subjective, mathematical and objective. Vassilief, A. [1900] N. Y. Am. Mth. S. Bll. 7 (1901) 158, 183.

the surprise ratio. Bostwick, A. E. Science 3 (1896) 66-.

theorem. Luchterhandt, A.R. Grunert Arch. 2 (1842) 65-.

- Dienger, J. Prag Ab. 5 (1872) 44 pp.

-. De Forest, E. L. Des Moines Anal. 7 (1880) 169-.

-. Bertrand, J. C. R. 114 (1892) 701-.

- of Bienaymé, elementary proof. Bertrand, J. C. R. 81 (1875) 458, 491-.

-, demonstration. Wythoff, $\dot{W} . A$. N. Ts. Mth. 4 (B) (1893) 77-.

-, new. Simmons, (Rev.) T. C. L. Mth. S. P. 26 (1895) 290-

-, -, application. Bienaymé, J. C. R. 81 (1875) 417-.

theorems. Franklin, F. (xII) [1881] J. H. Un. Cir. [1] (1882) 128.

-. Ćebyšev, P. St Pet. Ac. Sc. Mm. (Rs.) 55 (1887) (Suppl.) No. 6, 16 pp.; Acta Mth. 14 (1890-91) 305-.

"Théorie Analytique" (Laplace). Glaisher, J. W. L. [1872] Mess. Mth. 2 (1873) 62-. - (-). Laurent, H. N. A. Mth. 12 (1873) 176-.

$-(-)$. Tychsen, C. Kjöb. Ov. (1876) 12-. theory. Encontre, $D$. Mntp. Rec. Bll. 1 (1803) 9-.

-. Bellavitis, G. Poligrafo 4 (1836) 3-.

-. De Morgan, A. [1837] Camb. Ph. S. T. 6 (1838) 423-.

- Ellis, R. L. Camb. Mth. J. 4 (1845) 127-.

- Boole, G. Camb. and Dubl. Mth. J. 6 (1851) 286.

- Donkin, W. F. Ph. Mg. 1 (1851) 353-, 458-; 2 (1851) 55-.

-. Boole, G. Ph. Mg. 2 (1851) 96-; 7 (1854) 29-; 9 (1855) 165-.

-. Bellavitis, G. Ven. At. (1856-57) 299-.

- Newcomb, S. Am. Ac. P. 4 (1857-60) 433-; Camb. (M.) Mth. M. 1 (1859) 136-, 233-, 331-; 2. (1860) 134-, 272-; 3 (1861) 119-, 343-.

-. Boole, G. Phil. Trans. (1862) 225-.

- Prrogov, N. N. Rs. Ps.-C. S. J. 19 (Ps.) (1887) 19-

-. Lindsay, T. Pop. As. 4 (1897) 240 .

- and application to gunnery. Sabudski, $N$. Fschr. Mth. (1898) 197-.

- and applications. Czuber, E. D. Mth. Vr. Jbr. 7 (1899) (Heft 2) viii + 279 pp.

- evidence. De Morgan, A. [1846-50]

Camb. Ph. S. T. 8 (1849) 379-; 9 (1856) 79-.

-, foundations. Ellis, R. L. [1842] Camb. Ph. S. T. 8 (1849) 1-.

-, general method. Boole, G. Ph. Mg. 8 (1854) 431-.

-, geometrical, interpretation of 0 . Simmons, (Rev.) T. C. As. Fr. C. R. (1893) (Pt. 1) 175.

-, -, problems. Vivanti, $G$. Rv. Mt. 1 (1891) 69-. 
theory and practice. Wolf, $R$. Bern Mt. (1849) 97-, 183-; (1850) 85-, 209-; (1851) 17- ; (1853) 25-.

-, principle. Young, J. R. B. A. Rp. (1844) (pt. 2) 1-.

-, proposition. Liapounoff, $A$. St Pét. Ac. Sc. Bll. 13 (1900) 359-.

-. Protection by mimicry. Blakiston, T., \& - Alexander, T. Nt. 29 (1884) 405-.

-, two multiple integrals in. Czuber, $E$. Mh. Mth. Ps. 2 (1891) 119-.

Probability of being hit by a projectile. Keller, o. A. Mines 19 (1891) 622-.

- and certainty, nature and limits. Miller, $G$. [1793] Ir. Ac. T. 5, 199-.

- of composite events. Simmons, (Rev.) T.C. As. Fr. C. R. (1896) (Pt. 2) 1-.

- - - Delannoy, H. Par. S. Mth. Bll. 26 (1898) 64-.

- curve, asymmetrical. De Forest, E. L. Des Moines Anal. 9 (1882) 135-, 161-; 10 (1883) 1-, 67-.

-, -. Edgeworth, F. Y. Ph. Mg. 41 (1896) 90-.

- of error of observation. Schlömilch, O. Z. Mth. Ps. 17 (1872) 87-.

- - - in result of a certain computation. Franklin, F. (xI) [1881] J. H. Un. Cir. [1] (1882) 165.

- - - writing a series of numbers. Nipher, F. E. Am. J. Sc. 12 (1876) 79-. - - errors arising from uncertainty of last figure. Glaisher, J. W. L. (vII) [1873] Mess. Mth. 3 (1874) 59-.

- - - elementary proofs of laws. Laquière, E. M. Par. S. Mth. Bll. 9 (1881) 69-.

_ - - law. Petitcol, (Capt.) _. N. A. Mth. 4 (1885) 441-; Rv. Mar, et Col. 91 (1886) 70 -.

- - - - Ocagne, M. d'. Par. S. Phlm. Bll. 6 (1894) (C. R. No. 9) 2-.

- _ - - Gosiewski, W. Prace Mt.-Fiz. 9

(1898) 117-; Fschr. Mth. (1898) 186-.

- - of observations. Cauchy, A. L. C. R. 37 (1853) 264-.

- - position. Reina, $V$. Rm. R. Ac. Linc. Rd. 6 (1897) (Sem. 1) 107-。

- - - in sum or mean of observations. Jullien, (le père) M. Tortolini A. 1 (1858) 76-, 149-, 227-.

-, exact and popular estimates, comparison. Terrot, C. H. Edinb. R. S. P. 2 (1851) 228 .

- of finding isolated shoals. Littlehales, $G . W$.

A. Mth. 9 (1894-95) 163-.

-, formulae. Jordan, C. C. R. 65 (1867) 993-.

- of hitting a target. Merriman, M. [1897]

N. Y. Am. Mth. S. Bll. 4 (1898) 88, 91-.

- local. Crofton, M. W. [1867] L. Mth. S. P. 2 (1869) 54-.

-, - , applied to straight lines drawn at random in a plane, and to proofs of theorems in integral calculus. Crofton, $M . W$. Phil. Trans. 158 (1868) 181-.

,-- , proof of theorem. Heumann, C. N. Ts. Mth. 4 (B) (1893) 25-; Fschr. Mth. (1893-94) 343.
Probability, local, question in. Berg, F. $J$. van den. N. Arch. Wisk. $9\left({ }^{*} 1882\right) 32-$; 10 (1884) 202.

-, - , theorem of Crofton. Cavallin, C. B.S. Ts. Mth. 2 (1884) 1-; Fschr. Mth. (1884) 181.

- that marriage of man over forty will be fruitful. Sprague, T. B. [1879] Edinb. R. S. P. 10 (1880) 202-.

- of means. Poisson, S. D. Con. des Temps (1827) 273- ; (1832) 3-.

- - - Lobatschewsky, N. Crelle J. 24 (1842) 164-.

- Lobatto, R. Arch. Wisk. Gn. 2 (1860-63) 96-.

_ - Laplace's rule. Bienaymé, J. Par. Mm. Sav. Etr. 5 (1838) 513-; Par. S. Phlm. PV. (1839) 42-; (1840) 19-.

- - Poisson generalised. Hauber, C. F. Baumgartner Z. 7 (1830) 406-.

- that $n$-gon can be formed from $n$ parts of straight line. Berg, F. J. van den. N. Arch. Wisk. 18 (1891) 63-; Fschr. Mth. (1891) 234.

-, physical basis. Edgeworth, F. Y. Ph. Mg. 16 (1883) 433-.

- of system of errors, law of. Gosiewski, $W$. Prace Mt.-Fiz. 3 (1892) 33-; Fschr. Mth. (1892) 206-.

- that two numbers are relatively prime. Cesáro, E: (xп) J. H. Un. Cir. [2] (1883) 85.

Probable error, comparison of two values. Lïroth, $J$. As. Nr. 87 (1876) 209-.

- - of constants. Hagen, G. H. L. Berl. Ak. Sb. (1883) 1169-.

-- , determination. Lüroth, J. As. Nr. 73 (1869) 187-.

- - - - Jordan, W. As. Nr. 74 (1869) 209-.

- - - (Jordan). Andra, C. C. G. As. Nr. 74 (1869) 283-.

- - , - from differences of $m$ equally exact observations. Andre, C. C. G. As. Nr. 79 (1872) 257-.

- - experimental determination of constant. Adcock, R. J. Des Moines Anal. 7 (1880) 189.

-- , Peter's formula. Helmert, F. R. As. Nr. 88 (1876) 113-.

,---1 Johnson, W. W. N. Y. Mth. S. Bll. 2 (1893) 57-.

- - of a series of observations. Guyou, E. C. R. 106 (1888) 1282-.

- - - - - calculation. Mees, R.A. Z. Mth. Ps. 20 (1875) 145-; 21 (1876) 126-. - from sum of variations. Fechner, G. T. A. Ps. C. Jubelbd. (1874) 66-.

- errors, limit, in observations. Cesaris, G. A. de. Mil. Effem. As. (1811) 102-.

- inference. Terrot, C. A. Edinb. R. S. P. 2 (1851) 288 -

- intersection of non-concurrent lines, geometrical determination of point of. Ocagne, M. $d$ '. Par. Éc. Pol. J. 63 (1893) 1-.

- value of least errors in a series of observations. Bertrand, J. C. R. 106 (1888) 786-. 
1630 Probabilities, etc.

Probable value of a series of observations, mechanical representation. Newcomb, $S$. As. S. M. Not. 33 (1873) 573.

- values of constants in $u=a+b y$. Anon. 's Gravenh. I. Ing. Ts. (1884-85) (Verg.) 68-.

Random scattering of points on a surface. Kleiber, J. Ph. Mg. 24 (1887) 439-.

Reckoning of chances (reproduction of 16th century work by Benedict Spinoza). Bierens de Haan, D. N. Arch. Wisk. 11 (1884) 75-.

Reduction of observation equations in more than one observed quantity. Kummell, C.H. Des Moines Anal. 6 (1879) 97-.

- results of experiments. Lupton, $S$. $\mathrm{Ph}$. Mg. 31 (1891) 418-.

- - - - - criticism. Lupton's method. Pickering, S. U. Ph. Mg. 32 (1891) 90-.

$-\ldots-\ldots$, objections raised to Pickering's method. Hayes, E. H. Ph. Mg. 32 (1891) 99-.

Resolution of equations given by method of least squares in a particular case. Port, $A$. C. R. 105 (1887) 491-.

Restricted number of observations, interpretation. Vallier, E. C. R. 128 (1899) 654-.

,---- - Hatt, 一. C. R. 128 (1899) 893-.

Resultant error, law. Schols, C. M. Delft Éc. Pol. A. 3 (1887) 140-.

Roots of the equation $e^{x^{2}} \frac{d^{m}}{d x^{m}}\left(e^{-x^{2}}\right)=0$. Markoff, A. St Pét. Ac. Sc. Bll. 9 (1898) 435-.

Scoring at rifle matches. Mallock, A. Nt. 60 (1899) 366-

- - - Burbury, S. H. Nt. 60 (1899) 412

- - - Mallock, A. Nt. 60 (1899) 412.

Speculation, theory. Bachelier, L. Par. Éc. Norm. A. 17 (1900) 21-.

Stationary motion in an infinite system of molecules. Burbury, S. $H$. L. Mth. S. P. 29 (1898) 225-.

Stock-exchange, probabilities connected with. Dormoy, $\dot{E}$. As. Fr. C. R. (1886) (Pt. 1) 79-; (1887) (Pt. 2) 214-.

Success of prediction, measure of. Peirce, C.S. Science 4 (1884) 453-

Sum of least variations, estimation and utility. Fechner, G. T. [1874] Leip. Mth. Ps. Ab. 11 (1878) 1-.

Surveying, possible error in determination of a point. Sonlages, $E$. Arg. S. Ci. A. 44 (1897) 306-.

- with usual instruments, judging error in. Weisbach, J. Baumgartner Z. 4 (1837) 24-.

Tables of numbers, error due to omitted decimal places. Frischauf, J. Z. Mth. Ps. 16 (1871) 178-.

Target, firing at. Bertrand, J. C. R. 107 (1888) 205-.

- questions, probability applied to. Bertrand, $J$. C. R. 106 (1888) 232-, 387-, 521-.

$-\overline{\text {, }}$ - - - Jung, G. C. R. 106 (1888) 1001-.

Testimonies or judgments, combination. Boole, G. Edinb. R. S. T. 21 (1857) 597-.
Testimony and arguments. Abbott, (Rev.) T.K. Ph. Mg. 28 (1864) 12-.

- for facts contrary to analogy. Young, $M$. [1798] Ir. Ac. T. 7 (1800) 79-.

_, formulæ for value. Bonicelli, $P$. (vi Adds.) Majocchi A. Fis, C. 12 (1843) 167-.

- in legal cases, measure of force. Tozer, $J$. [1843] Camb. Ph. S. T. 8 (1849) 143-.

Todhunter's History of Theory of Probability, passages. Bianco, O. Z. [1877] G. Mt. 16 (1878) 26-.

Tradition and probability. Macfarlane, $A$. I. Act. J. 22 (*1881) 356-.

Transit observation, frequency of occurrence of each digit in 10ths of seconds in chronograph observations. Eastman, J. R. [1873] Wash. Ph. S. Bll. 1 (1874) 85-.

Triangulation, interpolation of a point by method of least squares. Jordan, $W$. Z. Mth. Ps. 16 (1871) 164.

Trigonometric measures, mean error of results. Börsch, $(D r)[A$.$] Arch. Mth. Ps. 46$ (1866) 40 -.

- problem, surveying. Gauss, C. F. As. Nr. 1 (1823) 81-.

- surveys, combination of errors in connexion with. Krüger, L. Gött. Nr. (1900) 1-.

Variability of organs, and Gauss's law of deviation. Ludwig, F. Z. Mth. Ps. 43 (1898) 230 -

Verification of predictions. Doolittle, $M . H$. Smiths. Misc. Col. 33 (1888) Art. 2, 122-. (Wash. Ph. S. Bll. 7 (1885).)

Weighing of errors, theorems. Paucker, M. G. von. Mitau Arb. Kurländ. Gs. 9 (1851) 141-.

Weight of observations where mean of squares of errors is composed of several parts. Helmert, F. R. As. Nr. 89 (1877) 225-, 241-.

- and precision of an observation. Bertrand, $J$. C. R. 105 (1887) 1099-.

Weighted causes, and Bessel's method. Paucker, M. G. Mitau Arb. Kurländ. Gs. 9 (1851) 170 .

\section{Theory of statistics. Actuarial mathematics.}

Accident assurance, and connected questions. Tiselius, H. Stockh. Öfv. (1895) 561-.

Accumulations of capital in United Kingdom, in 1875-85. Giffen, $R$. Nt. 41 (1890) 211-.

Actuarial science, application of mathematics. Stuckey, J. J. Aust. As. Rp. (1893) 280-.

Adjustment of mortality tables. Lazarus, $W$. I. Act. J. 16 (*1872) 424 .

- Woolhouse, W. S. B. I. Act. J. 21 (*1879) 37-, 56-.

- - - Higham, J. A. I. Act. J. 23 (*1882) 335-; 24 (1884) 44-.

_ _ _ Corneille and Landre's method. Danielewicz, B. Wiad. Mt. 1 (1897) 92-.

- - - - Gompertz's method. Gray, P. Assur. Mg. 7 (*1858) 121-.

- - - graphic method. Sprague, T. B. I. Act. J. 26 (1887) 77-. 
Adjustment of mortality tables, graphic method. Woolhouse, W. S. B. I. Act. J. 26 (1887) 420-.

- - - method. M. Assur. Mg. 6 (*1857) 357-.

- - - Woolhouse, W. S. B. I. Act. J. 29 (1892) 236-.

$---\frac{1}{-}$ new method. Woolhouse, W.S.B. I. Act. J. 15 ( $\left.{ }^{*} 1870\right) 389-$.

- _ - _, and weight of observations. McCay, C. F. I. Act. J. 22 (* 1881) 24-.

- - tables, Galloway's method. Watkins, C. Assur. Mg. 6 (*1857) 178, 360.

$-\ldots-$, Woolhouse's formula. Higham, C.D. I. Act. J. 26 (1887) 50-.

Age of parents as influencing sex and number of children. Brown, S. Assur. Mg. $3\left({ }^{*} 1853\right)$ 17-.

Ages at which life assurance is effected. Chandler, S. C. (jun.). I. Act. J. 17 (*1873) 56 -.

Algebra of commercial exchanges. Cossali, $P$. [1812] Mod. Mm. S. It. 16 (1813) 124-.

Allowance during sickness, tables. Higham, J. A. Assur. Mg. 7 (*1858) 112-.

American life, law of. Nichols, W. S. I. Act. J. $19\left(^{*} 1876\right) 28$ -

Amount assured, and sum reserved, connection between. Jellicoe, $C$. Assur. Mg. $5\left(^{*}\right.$ 1855) 100 -.

- of $£ 1$ for fraction of a year. Farren, E. J. Assur. Mg. 3 (* 1853) 335-.

- - - Chisholm, D. Assur. Mg. 3 (* 1853) 336-.

- - - - - - - Filipowski, $H$. Assur. Mg. 3 (*1853) 338-; 4 (1854) 243-, 253.

- and present value of regularly increasing annuity, formulæ. Shaw, C. G. Assur. Mg. 5 (*1855) 152 .

- - - - - - or decreasing annuities, formulæ. Galsworthy, E. H. Assur. Mg. 5 (*1855) 53-.

Annual premium for life assurance. Gray, $P$. Assur. Mg. 2 (* 1852) 95-.

- - - term assurance on two joint lives. Sprague, T. B. Assur. Mg. 8 (* 1860) 59-.

- and single payments, table connecting. Smith, H. A. Assur. Mg. $11\left(^{*} 1864\right)$ 176-. - - premiums for endowment assurance. Smith, H. A. I. Act. J. 22 (*1881) 143-.

- - - - - McDonald, J. I. Act. J. 32 (1896) 447-.

Annuities. Horner, W. G. Thomson A. Ph. 8 (1816) 279-, 388-.

-. Huet, -. N. A. Mth. 5 (1846) 346-.

-. Nievengloski, G. H. N. A. Mth. 6 (1847) 8-.

- Dupain, J. C. N. A. Mth. 2 464-.

-. Guldberg, A. S. (xI) Sk. Nt. Möt. F. (1880) 194-

-. Bryan, G. H. Mth. Gz. 1 (1900) 8-.

- Loney, -. Mth. Gz. I (1900) 38-.

- approximate calculation. Lobatto, $R$. [1861] Amst. Vh. Ak. 10 (1864) 32 pp.

-, - - Bouniakowsky, V. [1877] St Pét.

Ac. Sc. Bll. 24 (1878) 437-.
Annuities, assurance, etc., approximate calculation. Stamkart, F. J. Amst. Vs. Ak. 1 (1866) (Ntk.) 95-.

- and assurances, improved theory. Woolhouse, W. S. B. I. Act. J. 15 (*1870) 95-; 18 (*1875) 151-.

- _ _ - _ - (Woolhouse's). Sutton, W. I. Act. J. 15 (*1870) 307-.

- - - Milne's treatise, correction of error. Hoskins, H. I. Act. J. 17 (* 1873) 192-.

- - on successive lives. Weddle, T. $\mathrm{Ph}$. Mg. 36 (1850) 8-.

- on assured lives, value. Berridge, G. $W$. I. Act. J. $19\left({ }^{*} 1876\right) 351-$.

—, calculation. Lubbock, J.W. [1828] Camb. Ph. S. T. 3 (1830) 141-.

- - Wrede, F. J. [1869] Stockh. Öfv. 26 (1870) 5-.

-, - Fagnart, E. Mathesis 16 (1896) 64-. - under certain conditions, values. Hardy, $P$. Assur. Mg. [1 (*1851)] 1 bis-.

-, certain determining amounts of continuous. Makeham, W. M. I. Act. J. 15 (* 1870) 432-.

Annuities-certain, rate of interest. Hardy, G. F. I. Act. J. 23 (*1882) 266-.

- , theory. Makeham, W. M. I. Act. J. 14 (*1869) 189-.

- -, value. Orchard, W. Assur. Mg. [1 (*1851)] 100 bis-.

-, formula. Hansen, C. N. Ts. Mth. 11 (A) (1900) 65-.

-, formulæ in D. Jones's work. Macfadyen, J. R. Assur. Mg. 13 (* 1867) 200.

,$--1-2-$ McCuaig, C. Assur. Mg. $13\left({ }^{*} 1867\right) 251-$.

involving three lives, approximations. Hardy, G. F. I. Act. J. $\left.23{ }^{*}{ }^{*} 1882\right) 274-$. - and life-assurance. Tychsen, C. (xII) Ts. Mth. 5 (1875) 49-, 97-.

- - - assurances and premiums, calculation of worth. Hultman, F. W. Ts. Mt. Fys. 2 (1869) 63-, 113-, 209-.

- - - expectations. Landré, C. L. N. Arch. Wisk. 12 (1886) 174-; Fschr. Mth. (1885) 194-.

- on lives. Willich, C. M. Assur. Mg. 7 (*1858) 273-.

- on three joint lives, approximate values. Hardy, P. Assur. Mg. 3 (*1853) 330-.

- - - four, five and six joint lives, tables. Shaw, C. G. Assur. Mg. 5 (* 1855) 180-

- on several lives. Quiquet, $A$. C. R. 111 (1890) 337-.

- for long terms, approximations. Brown, $S$. Assur. Mg. [1 (* 1851)] 20-

-, mean annual extinction in chief Portuguese offices. Silva, D. A. da. Lisb. J. Sc. Mth. 1 (1868) 175-.

- payable half yearly or quarterly. Sprague, T. B. Assur. Mg. 13 (* 1867) 188-, 201-, 305-.

$--m$ times a year. Hansen, $C$. N. Ts. Mth. 11 (B) (1900) 58-.

-, question. Lindelöf, L. L. Acta Mth. 3 (1883) 97-.

and rate of interest, theorems for determining. Benwell, J. B. Tilloch $\mathrm{Ph} . \mathrm{Mg}$. 49 (1817) 179-. 
Annuities, tables, comparison. Lubbock, J. W. [1829] Camb. Ph. S. T. 3 (1830) 321-.

-, - of present values. Bowditch, J.I. Am. Ac. Mm. 1 (1833) 73-.

-, theorems for determining. Benwell, J. B. [1816-17] Thomson A. Ph. 8 (1816) 119-; Tilloch Ph. Mg. 48 (1816) 449-; 50 (1817) 164-; 51 (1818) 6-.

-, theory. Landré, C. L. N. Arch. Wisk. 15 (1888) 1-; Fschr. Mth. (1888) 235-.

Annuity, analytic formula and table. Benwell, J. B. Tilloch Ph. Mg. 57 (1821) 161-.

- and assurance formulø, reduction. Woolhouse, W. S. B. I. Act. J. $17\left({ }^{*} 1873\right)$ 171-. - - - tables for three lives. Braid, $W$. Assur. Mg. 6 (*1857) 109, 115-.

- certain, approximation to rate of interest. McKenzie, D. J. McG. I. Act. J. 23 ( ${ }^{*} 1882$ ) 405-.

- - and life annuity, analogy between. King, G. I. Act. J. 20 (*1878) 435-.

$--\frac{-}{-},--$ Ryan, G. H. I. Act. J. 25 (1886) 443-.

- computation on Makeham's hypothesis. McClintock, E. I. Act. J. 18 (*1875) 242-.

- forborn, determination. Hardy, $P$. Assur. Mg. $7\left({ }^{*} 1858\right)$ 1-.

- Lubbock's formula for approximation. Sprague, T. B. I. Act. J. 18 (*1875) 305-.

-, market value. Wilding, R. I. Act. J. 22 (*1881) 61-.

- problem, and Arbogast's development. De Morgan, A. Assur. Mg. 12 (*1866) 206-.

-- , old and new solutions. Gray, $P$. Assur. Mg. $13\left({ }^{*} 1867\right) 60$.

- question. C., J. I. Act. J. 14 (*1869) 71-.

- at one rate deduced from that at another. Meikle, J. A Assur. Mg. 3 ( $\left.{ }^{*} 1853\right)$ 325-.

-, rate of interest. Meikle, $J$. Assur. Mg. 5 (* 1855) 152-.

-, - - - De Morgan, A. Assur. Mg. 8 (*1860) 61-.

-, - -. McLauchlan, J. J. I. Act. J. 18 (* 1875) 290-.

- tables, method of checking. De Morgan, $A$. Assur. Mg. 2 (* 1852) 390-.

- on three lives, application of Gompertz's law. Woolhouse, W. S. B. Assur. Mg. 10 (*1863) 121-.

- _ - note on Woolhouse's paper. De Morgan, A. Assur. Mg. 10 ( $\left.{ }^{*} 1863\right)$ 237-.

- - - - rule for value. De Morgan, $A$. (vi Adds.) Ph. Mg. 15 (1839) 337-.

- value with final payment. Cherriman, J.B. I. Act. J. 21 (*1879) 299-.

Apportionable annuities. Sprague, $T$. $B$. Assur. Mg. 13 (*1867) 358-; 14 (* 1869) 36-; $15\left(^{*} 1870\right) 244$.

Apportionment, problem in. Sprague, $T . B$. I. Act. J. $19\left(^{*} 1876\right) 372$-.

Arbitrary rates of life premium. S., H. A. Assur. Mg. 6 (*1857) 297-.

Association of electors by lot. Bertrand, $J$. C. R. 106 (1888) 17-.

Assurance on adventure so as to cover interest. Mercator. [Pseudon.] (vI Adds.) Bost. Mm. Am. Ac. 1 (1785) 183-.
Assurance, analytical theory. Fourier, J. B.J. A. C. 10 (1819) 177-.

- and annuities, formulø. Eneström, $G$. Stockh. Öfv. (1894) 561-; Fschr. Mth. (1893-94) 364-.

- in another's favour. Dienger, $J$. Arch. Mth. Ps. 42 (1864) 333-.

- of capital and income. Unferdinger, $F$. Grunert Arch. 26 (1856) 408-.

- - in stock. Danielewicz, B. Wiad. Mt. 2 (1898) 32-.

companies, amalgamation. Jellicoe, $C$. Assur. Mg. 7 (*1858) 254-.

+ - - Sprague, T. B. Assur. Mg. 7 (*1858) 355-

- - equitable transference of business between. Sprague, T. B. Assur. Mg. 7 (*1858) 301-.

- company, testing solvency. Younger, $S$. Assur. Mg. 7 (*1858) 183-, 295-.

,---7 . S., H. A. Assur. Mg. 7 ("1858) 294, 353-.

-, comparison of actual with expected experiences. Low, G. M. I. Act. J. 18 (*1875) 195-.

- on continent, progress. Brown, S. Assur. Mg. [1 (*1851)] 293-; 2 (*1852) 16-.

-, demonstration of certain formulæ. Adler, M. N. Assur. Mg. 12 (*1866) 52-.

-, a fallacy concerning. Gray, $P$. I. Act. J. 14 (*1869) 63-.

- of invalid lives. Younger, S. Assur. $\mathrm{Mg}$. 11 (* 1864) 49-.

- - - Younger's plan. Brabrook, E.W. Assur. Mg. 10 (*1863) 349-.

- - - - - . Smith, H. A. Assur. Mg. 10 (*1863) 352-; 11 (* 1864) 180.

,$-----Z$ - Gray, P. Assur. Mg. 10 (*1863) 354.

- against invalidity. Lazarus, W. I. Act. J. $15\left({ }^{*} 1870\right)$ 143-.

- - - and old age, German method. Young, T. E. I. Act. J. 29 (1892) 269-.

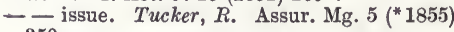
350 -

- - , premiums. Sprague, T. B. I. Act. J. 28 (1890) 350 -.

- - - rate of premium. Day, A. Assur. Mg. 8 (*1860) 127-.

-, life against life, value. Bailey, A. $H$. Assur. Mg. 9 ("1861) 299.

,$- \ldots--$, and for $n$ years longer. Tucker, R. Assur. Mg. 4 (*1854) 250-.

,,$-----\frac{t}{t}$ years longer. Chatham, $J$. I. Act. J. 25 (1886) 439-.

- loan (kind of life assurance). Vész, J.A. (xII) Mag. Tud. Ak. Etk. (Mth.) [1] (No. 3) (1868) 20 pp.

- payable at instant of death. Younger, $S$. Assur. Mg. 7 (*1858) 238.

- pensions, theory. Erieström, G. Stockh. Öfv. (1895) 197-, 243-; Fschr. Mth. (1895) 249-, 250-.

- premiums on ill health. Danielewicz, $B$. Wiad. Mt. 1 (1897) 170-.

-of recently selected lives, premiums. Sprague, T. B. I. Act. J. 20 ( ${ }^{*}$ 1878) 95-. 
Assurance against sickness. Moser, C. Arch. Sc. Ps. Nt. 6 (1898) 364-.

-, some new forms. Fix, - Rv. Sc. 3 (1895) 171-.

-, state-loans, \&c. Oettinger, L. Grunert Arch. 36 (1861) 189-, 453-; 37 (1861) 125-, 365-; 38 (1862) 263-.

- of survivor, formula for premium. Lobatto, R. [1865] Arch. Néerl. 1 (1866) 46-.

- to survivor, new method of calculating. Réboul, E.' Assur. Mg. 9 (*1861) 1-.

- of unsound lives, practice concerning. Humphreys, G. I. Act. J. 18 (* 1875) 178-.

- for widows, theory. Jahn, G. Jb. BergHw. (1888) 42-.

Auxiliary life contingency tables, history. Hendriks, F. Assur. Mg. [1 (*1851)] 1-, $12 \mathrm{bis}$-.

Average duration of life. Merrifield, C. W. Assur. Mg. 6 (*1857) 175-.

- _ calculation for Prussia. Dieterici, C. F. W. Berl. Ab. (1858) (Ph.) 433-.

- life office, determination of an. King, G. I. Act. J. $20\left(^{*} 1878\right) 300$-.

- mortality rates, groupings according to age. Gray, W. T. I. Act. J. 25 (1886) 369-.

- premium, theory. Harvey, C.J. I. Act. J. 24 (1884) 296-.

- prices, official ("mercuriali"). Pareto, $R$. (x) Rm. R. Ac. Linc. At. 26 (1873) 599-; 1 (1875) 3-.

Averages, and application to rate of exchange. Drobisch, M. W. Leip. B. 23 (1871) 25-.

-, general. Morrison, $R$. Assur. Mg. 12 (* 1866) 350-; 13 (* 1867) 39-, 161-.

- and life contingencies, use of integral calculus. Younger, S. Assur. Mg. 7 (*1858) 71-.

-, percentage of. Preece, W. H. (xI) Tel. E. J. 2 (1873) 293-.

Bank-notes, mathematical theory of. Walras, L. [1879] Laus. S. Vd. Bll. 16 (1880) 553-.

Banking, mathematical theory. Edgeworth, F. $Y$. B. A. Rp. (1886) 777-.

Barrett's method and questions of nomenclature. De Morgan, A. Assur. Mg. 10 (*1863) 301-.

Benefits, calculation of certain. Sprague, T. B. I. Act. J. 24 (1884) 327-.

-, complex, calculation. King, G. I. Act. J. 26 (1887) 276-.

- paid several times a year and complete annuities. King, G. I. Act. J. 24 (1884) 418-.

-, present or future, formula. Macfadyen, J. R. I. Act. J. 17 (*1873) 381-.

Births and deaths at Brussels, laws. Quetelet, L. A.J. Brux. Ac. Sc. Mm. 3 (1826) 495-.

- - in Hanau. Moller, (Dr) von. (vI Adds.) Wet. Gs. Jbr. (1861-63) 59-.

- - - Stuttgart 1847. Cless, G. Würtb. Jh. 4 (1849) 120-.

-, deaths and marriages in Prussia, 18201834. Hoffmann, J. G. Berl. Ab. (1841) (Th. 3) 1-.

of different sexes, effect of legitimacy on ratio. Prevost, $P$. Bb. Un. 42 (1829) 139-.
Births, proportion of girls and boys. Poisson, S. D. Par. Mm. Ac. Sc. 9 (1830) 239-.

-, statistics (Versailles). Bérigny, A. Par. An. Mét. (1852) 281-.

- in various states of Europe and particularly Prussia. Dieterici, C. F. W. Berl. Ab. (1855) (Ph.) 321-.

Bond bought at premium, rate of interest. Todhunter, $R$. I. Act. J. 33 (1898) 356-.

Bonus distribution, system. Rothery, H.J. I. Act. J. 30 (1893) 131-.

- - theory. Andras, H. W. I. Act. J. 32 (1896) 320-.

Bonuses as affecting annuities. Macfadyen, J. R. I. Act. J. 14 (*1869) 364-.

Building debts, discharge by capital or rent. Wittstein, $T$. Hann. Z. Archt.-Vr. 7 (1861) 107-.

Calculation in economics. Durini, $G . N$. [1834] Nap. At. I. Inc. 6 (1840) 1-.

Cape of Good Hope Mutual Life Assurance Company, report. Sprague, T. B. I. Act. J. 20 (*1878) 358-.

Carlisle and other tables, relations. Henry, $J$. Assur. Mg. 11 (*1864) 89-.

- $-\ldots, \ldots$ - (Criticism of Henry's paper.) Farr, $W$. Assur. Mg. 11 (*1864) 109-.

- table of mortality, Milne's method in construction. Sutton, W. I. Act. J. 24 (1884) 110-, 186-

Census. Lubbock, (Sir) J. W. (vr Adds.) Ph. Mg. 7 (1830) 416-.

- from 1801-1881, account of. Burridge, A. F. I. Act. J. 25 (1886) 83-.

-, principles of statistics applied. Hough, F. B. Am. As. P. 17 (1868) 145-.

- theorem, applications. Listing, J. B. Gött. Nr. (1867) 430-.

Centenarians in France (census of 1886). Levasseur, E. C. R. 107 (1888) 71-.

Chances, doctrine, and subdivision of risks. Young, Dr T. QJ. Sc. 22 (1827) 84-.

Changes of population of Great Britain of various classes, 1811-1821. Harvey, $G$. QJ. Sc. 16 (1823) 203-.

Chisholm's policy tables, method of using. McKenzie, D. J. McG. I. Act. J. 28 (1890) $247-$.

- tables, advantages. $B ., W . F$. Assur. Mg. $8\left({ }^{*} 1860\right) 110$ -

Civil life, mathematies relating to. Michelsen, J. A. C. (vi Adds.) Berl. Sm. D. Ab. 3 (1799) (Mth.) 36-.

Claim acceleration reserve. Higham, C. D. I. Act. J. 24 (1884) 76; 26 (1887) 478.

Classification of policies to obtain a table of mortality. Brown, S. Assur. Mg. 4 (*1854) 282-.

Coinage; introduction of decimal system. Farey, J. Tilloch Ph. Mg. 49 (1817) 15-.

- and money of account, subsidiary principle applied. Elliott, E. B. Am. As. P. 24 (1875) 87-.

-, termary denominational system, prerogative. Sylvester, J. J. (xIr) [1881] J. H. Un. Cir. [1] (1882) 132.

Coins, nomenclature, \&c. Costaz, -. [1831] Par. Mm. Ac. Sc. 12 (1833) 599-. 
Combined marriage and mortality table. Sprague, T. B. I. Act. J. 21 (*1879) 406-.

Commutation, or $D$ and $N$, tables. Chisholm, J. I. Act. J. 14 (* 1869) 200-.

- formulø, certain. Smith, H. A. Assur. Mg. 8 (*1860) 116-; I. Act. J. 19 (*1876) 143.

- system, use of difference columns. Laundy, S. L. Assur. Mg. $8\left({ }^{*} 1860\right)$ 168-.

- tables of D. Chisholm. Meikle, J. Assur. Mg. 7 (* 1858) 297-.

Complete annuities, formulæ. Sheppard, H.N. I. Act. J. 32 (1896) 458-.

- annuity, formulæ for purchase. Sorley, $J$. I. Act. J. 20 (* 1878) 454-.

- - market value. Baden, A. I. Act. J. 17 (* 1873) 447-. $-\frac{-}{224}$. - Carr, T. I. Act. J. 18 (* 1875)

- payable by instalments, approximate value. Hoskins, $H$. I. Act. J. $19\left({ }^{*} 1876\right)$ 143-.

$--1-m$ equal instalments each year, value. Carr, T. I. Act. J. 18 (* 1875) 247-.

Composition of decremental forces, theory. Makeham, W. M. I. Act. J. $18\left({ }^{*} 1875\right)$ 317-.

- for leave to reside abroad. Campbell, $R$. Assur. Mg. 9 (*1861) 167-.

Compound survivorship annuity. Hardy, G. F. I. Act. J. 34 (1899) 93-.

- - problem, solution. Makeham, W. $M$. Assur. Mg. 10 (*1863) 241-.

Constancy of number of marriages and moral statistics. Quetelet, L. A.J. Brux. Ac. Bll. 5 (1858) 89-.

Constructive total loss, doctrine. Hopkins, $M$. Assur. Mg. [1 (*1851)] 63-.

Consumption in relation to life assurance. Lyon, T. G. I. Act. J. 30 (1893) 120-; 31 (1895) 155.

Contingencies, distinct, problems involving. Makeham, W. M. Assur. Mg. 13 (*1867) 109-.

Contingent assurances, calculation. Colenso, F. E. I. Act. J. 32 (1896) 185-.

- with certain limitations. Otter, W. C. Assur. Mg. 7 (*1858) 239-.

- - premiums. Sunderland, A. W. I. Act. J. 26 (1887) 58-.

- -, problem. Lidstone, G. J. I. Act. J. 35 (1901) 480-.

- parts of terminable annuity. Gray, $P$. Assur. Mg. 11 (*1864) 172-.

- reversionary interests. Sang, E. Assur. Mg. [1 (*1851)] 18 bis-, 151-, 287-. - - - Jellicoe, C. Assur. Mg. 2 (* 1852)

- - - Tucker, R. Assur. Mg. 5 (*1855)

- reversions. Paterson, W. B. I. Act. J. 30 (1893) 536-.

Copyhold, enfranchisement, tables. Smyth, $E$. I. Act. J. 21 (*1879) 381-.

- fine enfranchisement. Mathews, G. S. I. Act. J. 28 (1890) 1-.
Copyhold, fines payable on renewal, formulæ. Ryley, E. Assur. Mg. 4 (* 1854) 367-.

Corn-law sliding scale, expression for discontinuous law. Smith, Arch. Camb. Mth. J. 3 (1841) 152 .

Criminals, decay of morality in relation to number of youthful. Hoffmann, J. G. Berl. B. (1838) 1-.

Cumulative vote. Langley, E. M. Mth. Gz. 1 (1900) 160.

Curves representing statistics. Edgeworth, $F$. $Y$. B. A. Rp. (1899) 825.

$D, N$ columns of a certain company. Morgan, W. Assur. Mg. 12 (*1866) 235-.

- and $N$ formula for term assurance. Smith, H. A. I. Act. J. $19\left({ }^{*} 1876\right) 143$.

Dacea census. Walters, $H$. As. Researches 17 (1832) 534-.

Date of death and payment, period between. Farren, E. J. Assur. Mg. 3 (*1853) 234-.

Death strain in a life office, computation. Ryan, G. H. I. Act. J. 30 (1893) 196-.

- - true measure. Higham, C. D. I. Act. J. 20 (*1878) 153-.

Deaths, excess over births in coloured population of Boston. Boudin, J. C. M. F. J. C. R. 61 (1865) 746-.

Debt, gradual discharge. Drobisch, $M . W$. Leip. B. 2 (1848) 1-.

Decimal systems of money etc. Grubb, $T$. [1855] Dubl. R. S. J. 1 (1856-57) 21-.

Decrement of human life, formula. Young, Dr T. Phil. Trans. (1826) (pt. 3) 281-.

Deductions from sums assured, calculation. Hardy, G. F. I. Act. J. 32 (1896) 153-.

Deferred annuities, tables. Stephenson, J. W. Assur. Mg. 12 (*1866) 178-; 13 (1867) 114-.

- assurances and premiums. Rothery, H. J. I. Act. J. 28 (1890) 483-.

- and other life annuities, approximation. Ivory, H. Assur. Mg. 4 (*1854) 291-.

De Moivre's formula. Gray, $P$. Assur. Mg. 12 (*1866) 232-.

- _ _, method of obtaining. Charlon, I. Act. J. 15 (*1870) 141-.

Deterioration, estimating increase of rate to meet. Smith, H. A. Assur. Mg. 10 ("1863) 120.

Determination of present value of mine on the Rand. Hellmann, $F$. I. Mn. Metall. T. 6 (1898) 229-.

Development and health, estimation from reports of recruiting officers. Bischoff, T.L.W. Münch. Sb. (1867) (I.) 282-.

Dice for statistical experiments. Galton, $F$. Nt. 42 (1890) 13-.

Differential tariffs. Courau, $J$. Arg. S. Ci. A. 50 (1900) 86-.

Direct taxation, true measure of liability. Jellicoe, C. Assur. Mg. 3 (* 1853) 1-.

Discounted bonus premiums, rationale. Moir, H. I. Act. J. 36 (1902) 4-.

Discounting of bills, mathematical theory. Nečaev, N. V. Kazan S. Nt. (Ps.-Mth.) P. 3 (1885) 95-.

- - - - - Preobraženskij, V.V. Kazan S. Nt. (Ps.-Mth.) P. 3 (1885) 97-. 
Diseased lives, valuation reserve necessary. Sorley, J. I. Act. J. 20 (* 1878) 342-.

Dispositions of code of criminal instruction. Gergonne, J. D. Gergonne A. Mth. 9 (181819) $306-$.

Distribution by months of conceptions and births. Villermé, L. A. Sc. Nt. 24 (1831) 130 -. -, pure theory of. Berry, A. B. A. Rp. (1890) 923-.

Doctrine of successive lives. Gray, $P$. Assur. Mg. 2 ( $\left.{ }^{*} 1852\right)$ 1-, 271-.

Doctrines of political economy, mathematical exposition. Whewell, $W$. [1829-31] Camb. Ph. S. T. 3 (1830) 191-; 4 (1833) 155-.

- population. Farr, W. B. A. Rp. (1877) (Sect.) 174-.

Drummond's rule for calculating relative importance of boroughs, attempt to prove. I'Intyre, (Dr). (vI Adds.) Ph. Mg. 11 (1832) 360 -

Drunkenness in towns of England and Wales. Poynting, J. H. Manch. Lt. Ph. S. P. 16 (1377) 211-.

Economic science and statistics section of British Association, address. Duff, M. E. G. B. A. Rp. 37 (1867) (Sect.) 132-.

- - - - - - - - Brown, S. B. A. Rp. 38 (1868) (Sect.) 144-.

- - - - Northcote, (Sir) S. B. A. Rp. 39 (1869) (Sect.) 173-.

- - - - - _. Jevons, W. S. B. A. Rp. 40 (1870) (Sect.) 178-.

- $-1-\ldots-\ldots$ Neaves, (Lord). B. A. Rp. 41 (1871) (Sect.) 191-.

- - - - - - - - Fawcett, (Prof.) H. B. A. Rp. 42 (1872) (Sect.) 217-

$----\ldots-1$, - Campbell, (Sir) $G$. B. A. Rp. (1876) (Sect.) 186-.

- utility of means of transport, especially railways. Perozzo, $L$. Rm. R. Ac. Linc. Mm. 1 (1894) 476-.

Election methods. Nanson, E.J. [1882] Vict. R. S. T. 19 (1883) 197-.

- problem, to secure proportional representation of parties. Phragmén, E. Stockh. Öfv. (1894) 133-.

Elections, counting votes at general. Cauchy, A. L. C. R. 26 (1848) 469 -.

-, multiple, theory. Phragmén, E. Stockh. Öfv. (1896) 181-.

- and representative system. Gergonne, J.D. Gergonne A. Mth. 6 (1815-16) 1-; 10 (181920) 281-.

Electoral districts, problem. Thiele, T.N. N. Ts. Mth. 1 (B) (1890) 56-; Fschr. Mth. (1890) 232-.

- - system; and the minority. Lorenz, $L$. N. Ts. Mth. 1 (B) (1890) 40-; Fschr. Mth. (1890) 232-.

Endowment assurance, demonstration of formula. Sprague, T. B. Assur. Mg. $8\left({ }^{*} 1860\right)$ 111-.

- - , easy method of calculating reserves. Weninger, V. (xII) Mag. Ak. Éts. (Mth. Term.) 6 (1365) 241-.

- , formula. M., A. Assur. Mg. [1 (*1851)] 332.
Endowment assurance policies. Sunderland, A. $W$. I. Act. J. 28 (1890) 257 - .

- - payable at ages $50,55,60,65$, tables. Carment, D. I. Act. J. 23 ( $\left.{ }^{*} 1882\right) 224-$.

- - periodical valuation. Thomson, H.A. I. Act. J. 34 (1899) 1-.

--- , tables. Besant, A. D. I. Act. J. 31 (1895) 366-.

- assurances, grouping for valuation. Altenburger, J. I. Act. J. 34 (1899) 150-; 35 (1901) 332-.

- on two joint lives, approximate calculation. Lidstone, G. J. I. Act. J. 33 (1898) 354-.

- -, valuation in groups. Lidstone, G. J. I. Act. J. 34 (1899) 61-, 510-.

- policies some time in force, valuation. Samot, D. J. A. I. Act. J. $20(* 1878)$ 344-.

- value, deducing annuity and assurance value. Laundy, S. L. Assur. Mg. 11 (*1864) 54-.

Entailed estates, problem concerning. Sprague, T. B. I. Act. J. $19(* 1876)$ 38-.

Equal partition in inheritance, effect of age difference. Mayette, $J$. Mâcon Ac. A. 6 (1388) 360-.

Equated time of payment. Levine, A. I. Act. J. 33 (1898) 539-.

Equation of payments. Walton, W. Camb. Mth. J. 2 (1841) 188-.

- - Schulze, L. $\dot{R}$. Grunert Arch. 36 (1861) 177-.

- - (Malcolm's rule.) Benwell, J. B. Tilloch Ph. Mg. 49 (1817)'269-.

Equitable Society's experience. Young, $\mathrm{Dr} T$. Ph. Mg. 4 (1828) 339-.

Estate duty. Lamé, G. N. A. Mth. 7 (1848) 360 -

- - mathematical law of progression. Lamé, G. C. R. 27 (1848) 125-

$-\ldots,--\ldots$. Dienger, $J$. Grunert Arch. 12 (1849) 401-.

Exchange, mathematical theory. Walras, $L$. [1873] Laus. S. Vd. Bll. 12 (1874) 317-. - value of money, mathematical theory. Vilfredo-Pareto, - . As. Fr. C. R. (1894) (Pt. 2) 1180-

Expectation and estate. Westergaard, H. (xII) Ts. Mth. 6 (1876) 11-.

Expected deaths and expected claims, estimation. Ryan, G. H. I. Act. J. 26 (1887) 249-.

Expenses of life assurance companies, measure. Macfadyen, J. R. I. Act. J. 19 (*1876) 153-, 445-.

$---1-$ - Sprague, T. B. I. Act. J. 19 (*1876) 167-, 305-, 447-.

" Exposed to risk," new formula for deducing. King, G. I. Act. J. 27 (1889) 218-.

Extra mortality. White, F. W., \& Whittall, W. J. H. I. Act. J. 24 (1884) 385-.

- premium. Macfadyen, J.R. I. Act. J. 17 ("1873) 77-。

- -, conditional payments. Younger, $S$. Assur. Mg. 10 (* 1863) 268-.

- - for foreign climates. Meikle, J. I. Act. J. 19 (*1376) 268-. 
Extra premiums on deteriorated lives, dispensing with. Makeham, W. M. I. Act. J. 17 (* 1873) 153-.

- risk, data for fixing premiums. Jellicoe, $C$. Assur. Mg. 7 (*1858) 131-.

- risks, adjustment. Makeham, W. M. I. Act. J. 14 (*1869) 159-, 242-.

- - considered in relation to hypothetical table of mortality. Ryan, G. H. I. Act. J. 24 (1884) 19-.

- - owing to hereditary phthisis. Manly, H. $W$. I. Act. J. 30 (1893) 97-, 338-.

- - - - Macaulay, T. B. I. Act. J. 30 (1893) 335-.

Facts, mode of tabulating, to show risks. Meikle, J. I. Act. J. 31 (1895) 229-.

Family annuities. King, G. I. Act. J. 30 (1893) 291-.

Farr's healthy life table. Davis, W. Assur. Mg. 10 (*1863) 59-.

Fatal accidents in various occupations. Whittall, W. J. H. I. Act. J. $23\left({ }^{*} 1882\right)$ 188-.

Fecundity in Europe at beginning of nineteenth century. Benoiston de Châteauneuf, Par. Mm. Sav. Étr. 3 (1832) 608-.

- of marriages in interior of Cuba. La Sagra, R. de. C. R. 58 (1864) 524-.

Female compared with male lives. Walford, C. I. Act. J. $19\left({ }^{*} 1876\right)$ 174-.

Females of British peerage, adjusted mortality table. Thiele, T. M. I. Act. J. $16\left({ }^{*} 1872\right)$ 43-, 118-.

Finite differences calculus, and its applications. Otter, W. C. Assur. Mg. 7 (*1858) 333-; 8 (*1860) 19-.

Finlaison and English life table. Bailey, $A$. H. Assur. Mg. 9 ("1861) 357-.

- - - - Porter, H. W. Assur. Mg. $10\left({ }^{*} 1863\right) 31$-.

Finlaison's report on mortality of government life annuitants. Porter, H. W. Assur. Mg. 9 (*1861) 277-.

- tables. Smith, H. A. Assur. Mg. 13 (*1867) 58 .

Fire assurance. Barrois, $T$. Lille Mm. S. (1834) 85-.

- - , apportionment of loss in. Miller, $T$. Assur. Mg. 6 ( $\left.{ }^{*} 1857\right) 202-$.

- - divisions of risk in. Miller, T. Assur. Mg. 8 ("1860) 232-.

- - in Great Britain. Brown, S. Assur. Mg. 7 (*1858) 259-, 341-; 8 (*1860) 27 -

- - , scientific application of data. Walford, C. I. Act. J. 21 ( $\left.{ }^{*} 1879\right)$ 1-.

- - , specific and average. Miller, T. Assur. Mg. 8 ("1860) 140-.

- statistics. Miller, T. Assur. Mg. 6 (*1857) 333-; I. Act. J. 22 (*1881) 103-.

- - , settlement under average policies. Atkins, R. Assur. Mg. $3\left({ }^{*} 1853\right)$ 147-.

,---- Lazarus, $W$. Assur. Mg. 4 (*1854) 73-.

7 ( $\left.{ }^{*} 1858\right)$ 24-; 8 . Atkins, $R$. Assur. Mg.

- - - - specific and average policies. Christie, D. Assur. Mg. $8\left({ }^{*} 1860\right)$ 146-.
Fires in London from 1833 to 1849 . Brown, $S$. Assur. Mg. [1 ( $\left.\left.{ }^{*} 1851\right)\right] 31$ bis.

- - - - - - 1856, causes. Fothergill, C. G. Assur. Mg. 7 ( $\left.{ }^{*} 1858\right)$ 91-.

First and subsequent marriage in peerage. Day, A. Assur. Mg. 10 ("1863) 181-.

Force of mortality, formula. De Forest, E. L. I. Act. J. 22 ( $\left.{ }^{*} 1881\right) 231-$.

- - general expression. Sheppard, H.N. I. Act. J. 32 (1896) 295.

- - - - Lidstone, G. J. I. Act. J. 32 (1896) 390-.

- - Makeham's formula. Calderon, H.P. I. Act. J. 35 (1901) 157-.

Foreign loans, rate of interest on, theory. Sutton, W. I. Act. J. 19 ( $\left.{ }^{*} 1876\right) 77-$.

Formula $a_{x}=v p\left(1+a_{x+1}\right)$ and other matters. Gray, $P$. Assur. Mg. 12 ("1866) 176-.

Foundlings, historical and statistical notice. Lepasquier, - Rouen Tr. Ac. (1830) 73-.

French law, succession of illegitimate children. Cournot, A. A. (vi Adds.) Férussac Bll. Sc. Mth. 16 (1831) 3-.

,-----1 Quillet, -. N. A. Mth. 4 (1845) 249

Friendly societies, application of actuarial principles, \&c. Hardy, G. F. I. Act. J. 27 (1889) 245-.

- - experiences. Hardy, R. P. I. Act. $J$. 31 (1895) 86-.

- -, present position. Brown, S. Assur. Mg. 11 (*1864) 333-.

- - sickness and mortality experienced in. Tompkins, H. Assur. Mg. 5 ("1855) 6-. - , tabulation of results. Watson, A.W. I. Act. J. 35 (1901) 268-.

- society levies. King, G. I. Act. J. 26 (1887) 389-.

- - Hardy, G. F. I. Act. J. 26 (1887) 478 -

Fruitful marriages, probability. Sprague, $T$. $B$. I. Act. J. 25 (1886) 160 -

- - relative age of husband and wife. Chatham, J. I. Act. J. 27 (1889) 37-.

Gain or loss from mortality in annuity company. Ryan, G. H. I. Act. J. 30 (1893) 189-.

Geographic statistics, correction of anterior elements. Luca, F. de. Nap. Rd. 5 (1866) 183-.

Gold and silver, consumption in Britain 1810 to 1829. Jacob, W. Edinb. N. Ph. J. 12 (1832) 346-.

Gompertz's formula, and application to duration of life. Simon, $C$. Bll. Sc. Mth. As. 2 (1871) 282-.

- law. De Morgan, A. Assur. Mg. 8 ("1860) 181-; 9 ( $\left.{ }^{*} 1861\right)$ 214-.

- - Sprague, T. B. Assur. Mg. 9 (*1861) 288 .

- - Govan, J. I. Act. J. 34 (1899) 147-.

- - , consideration of alleged inaccuracies. Sprague, T. B. Assur. Mg. 10 (*1863) 32-.

- -, exposition, new. Edmonds, T. $R$. Assur. Mg. 9 (*1861) 327-.

- - further development Makeham, W. M. I. Act. J. 28 (1890) 152-, 185-, 316-, 416.

- - Makeham's extension. Woolhouse, $W$. S. B. I. Act. J. 28 (1890) 481-. 
Gompertz's law, Makeham's modification, application. Colenso, F. E. I. Act. J. 31 (1895) 337-.

_- value. Edmonds, T. R. Assur. Mg. $10\left({ }^{*} 1863\right) 104$.

Gotha life assurance, mortality experience. Hopf, G. Assur. Mg. 5 ( $\left.{ }^{*} 1855\right)$ 324-; 6 (*1857) 1-.

Government annuity and assurance rates. Adler, M. N. Assur. Mg. $12\left({ }^{*} 1866\right)$ 265-.

- life annuities. Adler, M. N. Assur. Mg. $12\left({ }^{*} 1866\right) 3$ -.

Graduated mortality table from limited experience. Chandler, S. C. (jun.). I. Act. J. 17 ( $\left.{ }^{*} 1873\right)$ 161-.

- - - _ - , graphic method. Lidstone, G. J. I. Act. J. 30 (1893) 212-.

Graduation. Higham, J. A. I. Act. J. 31 (1895) 319-.

- of experience, Jellicoe's formula. Sutton, W. I. Act. J. 18 ( $\left.{ }^{*} 1875\right)$ 375-.

- formulae. Higham, J. A. I. Act. J. 27 (1889) 427-.

- - Hardy, G. F. I. Act. J. 32 (1896) 371-.

-, Higham's theory. Levine, A. I. Act. J. 32 (1896) 290 -.

- of mortality table, application of Makeham's method. Sorley, J. I. Act. J. $20\left({ }^{*} 1878\right)$ 340 -

---1 , methods. Sutton, $W$. I. Act. J. 20 (*1878) 170-, 192-.

- _ tables. Sorley, J. I. Act. J. 22 (*1881) 309-.

- - - Ackland, T. G. I. Act. J. 23 (*1882) 352-.

- - - Higham, J. A. I. Act. J. 25 (1886) 15-, 245-.

- _ - Makeham's law. King, G., \& Hardy, G. F. I. Act. J. 22 (*1881) 191-. - - - - -. Sorley, J. I. Act. J. 22 (*1881) 230-.

- _ _ _, Woolhouse's method, Hardy's plan for computation. Carment, D. I. Act. J. 24 (1884) 224-.

- - peerage mortality. Berridge, G. W. Assur. Mg. 12 (*1866) 220-.

- by summation. Todhunter, $R$. I. Act. J. 32 (1896) 378-.

-, Woolhouse's method. Sprague, T. B. I. Act. J. 29 (1892) 59-, 232-.

Grant of policies without further payments (after certain time). Sprague, T. B. Assur. Mg. 7 (*1858) 58-.

Great powers (of Europe). Farr, W. Assur. Mg. $6\left({ }^{*} 1857\right)$ 147-.

Growth of children. Boas, F. Science 19 (1892) 256-, 281-.

-, human, mathematical theory. Lehmann, J. W. H. D. Nf. Vsm. B. (1843) 299-.

Height and weight of man, relation between. Macaulay, T. B. I. Act. J. 23 ( $\left.{ }^{*} 1882\right)$ ) 62-.

Human will and chances, comparison. Guy, W. A. Assur. Mg. 5 ("1855) 315-.

- - - probability theory. Adan, $H$. I. Act. J. 33 (1898) 523-, 527-.

- - - Young, T. E. I. Act. J. 33 (1898) 524-, 529-.
Human will, uniform action. Lazarus, -. Assur. Mg. 3 ( $\left.{ }^{*} 1853\right)$ 169-.

- - Hopf, G. Assur. Mg. 3 (*1853) 255-.

Immediate annuities, series for expressing. Merrifield, C. W. Assur. Mg. $3\left({ }^{*} 1853\right)$ 324-.

Importation of animal, vegetable and mineral substances into the Clyde, 1853-1857. Connal, M., \& Keddie, -. B. A. Rp. 28 (1858) 185-.

_ _ - and vegetable substances (Liverpool, 1851-55). Archer, T. C. B. A. Rp. (1857) 255-.

Incomplete years of exposure, treatment. Lidstone, G. J. I. Act. J. 31 (1895) 304-.

Increasing annuities and assurances, approximate calculation. Lidstone, G.J. I. Act. J. 31 (1895) 68-.

- and decreasing premium scales. Laundy, S. L. Assur. Mg. 10 (*1863) 286-.

- reversionary charges. Paterson, $W$. $B$. I. Act. J. 35 (1901) 245-.

Index, best means of constructing. Curtis, F. A. Assur. Mg. $8\left({ }^{*} 1860\right)$ 54-.

- numbers, use in investigation of trade statistics. Bourne, S. B. A. Rp. (1885) 859-.

Industrial assurances, observations. Rea, $C$. H. E. I. Act. J. 34 (1899) 105-.

Inordinate mortality, how far controllable by human agency. Porter, H. W. Assur. Mg. 9 (*1861) 12-, 89-, 149-.

Insanity in Scotland. Mitchell, (Sir) A. I. Act. J. 28 (1890) 426-, 432-.

Insolvency in life assurance companies. Bailey, A. $H$. I. Act. J. $16\left({ }^{*} 1872\right) 389-$.

Insolvent companies, liquidation and reconstruction. Sprague, T. B. I. Act. J. 16 (*1872) 229-.

Integral for compound survivorship annuity, approximate evaluation. Todhunter, $R$. I. Act. J. 33 (1898) 311-.

Intellectual and moral statistics of France. Fayet, - C. R. 11 (1840) 565-.

Interest, abridged multiplication applied to. Burnier, $F$. [1872] Laus. Bll. S. Vd. 11 (1873) 362-.

- accounts. De Morgan, A. Assur. Mg. 10 (*1863) 281-.

- - T., A. H. Assur. Mg. $10\left({ }^{*} 1863\right) 357-$. - and annuities, demonstration of formulæ. De Morgan, -. Assur. Mg. 4 ( $\left.{ }^{*} 1854\right)$ 277-. -, calculation, method. Perkins, $G . R$. Silliman J. 47 (1844) 51-.

-, capitalising in course of year. Rädell, Grunert Arch. 2 (1842) 68-.

- compound. Adams, Jas. Thomson A. Ph. 4 (1822) 450-.

-, -. Lobatto, R. Quetelet Cor. Mth. 6 (1830) 195-.

-, -. Brassinne, E. N. A. Mth. 9 (1850) 403-.

,-- , and annuities: to find $p$ from relation $E=A(1+p)^{n}+\frac{r\left[(1+p)^{n}-1\right]}{p}$. Simony, $O$. Z. Mth. Ps. 22 (1877) 190-. 
Interest, compound, average amount of sum at. Smith, H. A. I. Act. J. 14 ( $\left.{ }^{*} 1869\right)$ 158-.

,-- , calculated by trigonometrical functions. Vervaet, $P$. Časopis 13 (1884) 264-; Fschr. Mth. (1884) 211.

-, -, continuously reckoned. Studnička, $F$. J. Časopis 2 (*1873) 85-; Fschr. Mth. (*1873) 609 .

-, - , and current accounts. Starkov, $\boldsymbol{A}$. N. Rs. S. Nt. Mm. (Mth.) 3 (*1881).

- - -, curve. Carpenter, F. D. Y. V. Nost. Eng. Mg. 25 (1881) 385-.

-, - , formulae. Scherling, C. Grunert Arch. 2 (1842) 213-.

,- , infinitesimal. Ferriot, - N. A. Mth. 2 (1843) 461.

-, 一, - Dutoit, A. L. Laus. Bll. S. Vd. 5 (1857) 169-.

-, , new formula for calculating. Catalan, E. C. As. Fr. C. R. 1 (1872) 141-.

- , - payable "momently." Young, $(D r) T$. Ph. Ig. 2 (1827) 332-.

-, -, powers, \&c. Berdellé, C. As. Fr. C. R. 8 (1879) 170 -.

,-- , a result of. Bienaymé, $J$. Par. S. Phlm. PV. (1839) 60-.

-, - and simple, equivalence. De Morgan, $\boldsymbol{A}$. Assur. Mg. [1 (*1851)] 335-.

-, - , solution of problems, without tables. Adler, M. N. I. Act. J. 23 ( $\left.{ }^{*} 1882\right)$ 359-.

- , -, theory. Borgogelli, M. Rm. N. Linc. At. 47 (1894) 68-.

,-- , time required to attain given amount. Studnička, F.J. Časopis 13 (1884) 238-; Fschr. Mth. (1884) 210-

-, equation of payment. Schlechter, Grunert Arch. 34 (1860) 291-.

$\ldots,-\ldots-$, extension of term, company's accounts. Schlechter, -. Schlömilch Z. 5 (1860) 214-.

-, fall in rate. Burn, J. I. Act. J. 34 (1899) 474.

-, laws of. Montel, E. de. C. R. 124 (1897) 224-.

- payment, theory. Orlov, A. Kazan S. Nt. (Ps.-Mth.) P. 4 (1886) 250-.

-, question of. Orchard, $\dot{W}$. Assur. Mg. 4 ("1854) 61-.

- - - - Gompertz, B. Assur. Mg. 4 (*1854) 245-.

-, - -. I. Assur. Mg. 4 (*1854) 253-.

- rate, approximations in calculating reduction. Stewart, J. I. Act. J. 32 (1896) 261-.

- - in certain investments, approximation. M. Assur. Mg. 6 (*1857) 54-.

-, short periods. Kelland, $P$. Edinb. R. S. P. 3 (1857) 274-.

-, simple or compound. Wittstein, $T$. Grunert Arch. 40 (1863) 240-.

-,-- - Oettinger, L. Grunert Arch. 40 (1863) 243-.

- at 6 per cent., method of computing. Philomath. [Pseudon.] (vi Adds.) Bost. Mm. Am. Ac. 1 (1785) 174-.

-, theoretical and practical. Crelle, A. L. [1852] Crelle J. 49 (1855) 349-.
Interpolation from irregular series. De Forest, E. L. Smiths. Rp. (1871) 275-; (1873) 319-.

Invalid lives, practice of certain assurance companies. Pinckard, G. H. Assur. Mg. [1 (*1851)] 273-.

Invalidity probabilities, formulæ. Samot, $D$. J. A. I. Act. J. 21 (*1879) 288 .

Investments of funds of assurance companies. Brown, S. Assur. Mg. 7 ( $\left.{ }^{*} 1858\right)$ 241-.

Isolated reversions. Shaw, C. G. Assur. Mg. 2 ("1852) 295-.

Issue of (hitherto) childless marriage, probability. Sprague, T. B. I. Act. J. 22 ("1881) 117-.

Joint life annuity tables, method of finding $D$ numbers. Henry, J. I. Act. J. 14 (*1869) 212-.

- lives' assurance, table. Braid, W. Assur. Mg. 5 (*1855) 350, 363-.

Land, price of, and purchase by the State, mathematical theory. Walras, L. [1880] Laus. S. Vd. Bll. 17 (1881) 189-.

Landed property, theory of yield of soil applied to valuation. Pasini, $V$. Ven. At. 4 (1845) 388 .

Last $r$ survivors of $m$ lives, value of annuity. King, G. I. Act. J. 22 (*1881) 293-.

- survivor of three lives, annuity found by actuaries' tables. Smyth, E. I. Act. J. 17 ( $\left.^{*} 1873\right)$ 379-.

$---\ldots-$, value of annuity. Godward, W. I. Act. J. 17 (*1873) 266-.

Law of large numbers. Poisson, S. D. C. R. 2 (1836) 377-.

_ _ _ - (Quetelet's) in intellectual manifestations of man. Bitnicz, L. (xII) Mag. Ak. Éts. (1851) 241-.

Leaseholds, renewals. Bunyon, C.J. Assur. Mg. 3 (*1853) 280-

Leases; perpetual renewal. Adams, Jas. Thomson A. Ph. 2 (1821) 307-; 3 (1822) 12-.

- Sir Isaac Newton's table. De Morgan, $A$. Assur. Mg. 9 (*1861) 185-.

-, value. B., W. (vi $A d d s$.$) Thomson A.$ Ph. 10 (1825) 335-.

"Leipzieger Krankencasse." Lazarus, W. Assur. Mg. 8 ( $\left.{ }^{*} 1860\right)$ 351-.

Liabilities of assurance companies, estimate. Tucker, R. Assur. Mg. 10 ("1863) 312-.

$---\ldots,-$ Bailey, A. H. Assur. Mg. 11 (*1864) 111-。

- - - - - Bailey's. Sprague, T. B. Assur. Mg. 12 (*1866) 113-.

- _ - - valuation. Sprague, T. B. Assur. Mg. 11 (*1864) 90-

- connected with buildings, estimation of value, with view to settlement by sum of money or annual payment. Emmich, $W$. Z. Bauw. 1 (1851) 195-.

Liability of assurance company, estimation. Sprague, T. B. I. Act. J. 15 (*1870) 411-.

Life assurance. Littrow, J. J. von. Baumgartner Z. 9 (1831) 257-.

- - Sang, E. Edinb. T. Sc. S. Arts 1 (1841) 339-, 349-, 360-, 394-. 
Life assurance. Rieu, A. [1872] Laus. Bll. S. Vd. 11 (1873) 269-.

- - Landré, C. L. N. Arch. Wisk. 12 (1886) 182-; Fschr. Mth. (1885) 194-.

- - applications suggested by 1851 census. Scott, G. Assur. Mg. 6 (*1857) 47-.

- - average risk. Kanner, $M$. I. Act. J. 14 (*1869) 439-.

- - calculations, modern methods of computation. Laundy, S. L. Assur. Mg. 8 $\left({ }^{*} 1860\right) 58$ -

- - - - - -. "Milne, J." Assur. Mg. $8\left({ }^{*} 1860\right)$ 118-.

- companies in Austria. Daninos, S. A. Assur. Mg. 3 (*1853) 121-

- - - - Germany. Hopf, G. Assur. Mg. 3 ("1853) 134-, 220-; 9 ("1861) 42-.

- - -, solvency. Macfadyen, J. R. [1870] Glasg. Ph. S. P. 7 (1871) 221-.

- - compositions, notation for use in. Lazarus, $W$. Assur. Mg. 12 (*1866) 48-.

- contracts, reservations. Paraira, M. C. N. Arch. Wisk. 16 (1889) 215-; Fschr. Mth. (1889) 229.

- - defects in system (1831). Fraser, $W$. Edinb. N. Ph. J. 11 (1831) 118-.

- - formulae connected with. Rubenson, $R$. Stockh. Öfv. (1891) 463-.

- - in France. Curtis, F. A. I. Act. J. 19 (*1876) 414-.

- - fund, progress of profit. Searle, T.J. I. Act. J. 30 (1893) 493-.

- - Hillman's tables, errors in. Laundy, S. $L$. Assur. Mg. 9 (*1861) 239-.

- -, mathematical theory. Samot, D. J.A. N. Arch. Wisk. 5 (" 1879$)$ 35-; $6\left({ }^{*} 1880\right)$ 124-.

- - , - - use of mortality statistics. Landré, C. L. N. Arch. Wisk. 10 (1884) 18-. - - mean gain or loss for whole duration of assurance. Dienger, J. [1877] Rpm. Mth. 2 (1879) 89-.

- -, premium, basis of determination. Weninger, V. (xII) [1861] Mag. Ak. Éts. (Mth. Term.) 2 (1861-62) 193-.

- - - - reserve. Gobbi, U. Mil. I. Lomb. Rd. 29 (1896) 612-.

- - premiums, calculation. Stamkart, F.J. N. Arch. Wisk. $2\left({ }^{*} 1876\right)$ 97-.

- - - renunciation of payment. Rieu, $d$. Laus. Bll. S. Vd. 9 (1866-68) 361-.

- - present worth, and annual payment. Stamkart, F. J. Arch. Wisk. Gn. 2 (186063) 314-.

- -, theory. Malmsten, C. J. Crelle J. Mth. 83 (1877) 245-; Acta Mth. 1 (1882) 63-.

- assurances, mean error in. Gram, J.P. Ts. Mth. 6 (1889) 97-, 184-; Fsch. Mth. (1888) 232-.

- contingencies, old and new methods for finding. Gray, $P$. Assur. Mg. [1 (*1851)] $96 \mathrm{bis}-$.

- -, problem. Gray, P. I. Act. J. 21 (*1879) 67-.

- - valuation. Gompertz, B. Phil. Trans. (1820) 214-.

VOL. I.
Life contingencies, valuation. Hargreave, C.J. Ph. Mg. 5 (1853) 39-.

- contingency calculation, improvement. Farren, E. J. Assur. Mg. 5 (*1855) 185-; $8\left({ }^{*} 1860\right) 121-$.

- - , solution of problems concerning. $F$. E. J. Assur. Mg. [1 (*1851)] 92 bis, 355-.

- expectation. Willich, C. M. Assur. Mg. 7 (*1858) 181-.

- - Biden, W. D. Assur. Mg. 7 (*1858) $352-$.

- Willich, C. A. M. Assur. Mg. 8 (*1860) 139-.

- -, meaning of phrase. Sprague, T. B. Assur. Mg. 13 ( $\left.{ }^{*} 1867\right)$ 381-.

- expectations. Landré, C. L. N. Arch. Wisk. 15 (1888) 22-; Fschr. Mth. (1888) 237-.

- -, formulae connected with. Geer, P. van. N. Arch. Wisk. 12 (1886) 60-; Fsehr. Mth. (1885) 194.

- experience in 1863 , remarks. Tilt, $R . R$. I. Act. J. 32 (1896) 1-。

- interests and reversions, formulø and tables. Nightingale, H. E. I. Act. J. 30 (1893) 9-.

- office, estimation of liability. Carment, $D$. I. Act. J. 21 (*1879) 74-.

- - tabulation of facts in records. Sprague, T. B. I. Act. J. 31 (1895) 205-.

- valuations, principles. McCandlish, J. M. I. Act. J. 20 (*1878) 12-.

-, probability and mean duration, Geneva 1761-1813. Odier, L. Bb. Brit. 55 (1814) 213-.

- risks, assessment. Chisholm, J. I. Act. J. 25 (1886) 408-.

- tables, construction. Farr, W. Phil. Trans. (1859) 837-.

- -, Gray's method of construction. Hannyngton, J. C. I. Act. J. 21 (*1879) 455- ; 22 (*1881) 136-.

- - of Institute of Actuaries. Pell, M. B. I. Act. J. 21 (*1879) 137-.

21 (*1879) 452-.

- -, Philadelphia. Chase, P. E. [1869] Am. Ph. S. P. 11 (1871) 17-.

- tenant and reversioner, apportionment between. Baden, A. I. Act. J. $16\left({ }^{*} 1872\right)$ 269-.

$18\left({ }^{*} 1875\right), \overline{77}-$.

Limitation of risks. Sprague, T. B. Assur. Mg. 13 (*1867) 20-.

Limited and contingent interests in property. Bunyon, C. J. I. Act. J. $18\left({ }^{*} 1875\right)$ 1-.

Lives, and joint lives, values. Young, $(D r) T$. Tilloch Ph. Mg. 47 (1816) 3-.

Loading premiums. Makeham, W. M. I. Act. J. 15 (*1870) 354-.

- -. Smith, H. A. I. Act. J. 20 (*1878) 145-.

- -. Crisford, G. S. I. Act. J. 25 (1886) 184-, 387-.

Loans, arithmetical theory. Ronchetti, F. G. Mt. 24 (1886) 242-.

-, railway. Hultman, F. W. Ts. Mt. Fys. 3 (1870) 278-. 
Loans repayable by instalments. Makeham, W. M. I. Act. J. 18 ( $\left.^{*} 1875\right)$ 132-.

- - - interest rate. Gray, $P$. I. Act. J. 14 (*1869) 91-, 182-, 397-。

Longevity and expectation of life in United States. Worcester, J.E. [1825] Bost. Mm. Am. Ac. 1 (1833) 1-.

-, human. Ponzi, G. Tortolini A. 7 (1856) 417-.

-, increase in France, 1770 to 1845. Dupin, C. C. R. 26 (1848) 585-, 613-.

- of inhabitants of Ipswich and Hingham. Wigglesworth, E. [1782] Bost. Mm. Am. Ac. 1 (1785) 565-.

Lubbock's formula of summation. Sprague, T. B. I. Act. J. $22\left(^{*} 1881\right) 55-$.

Machinery, effect on public wealth, and wages fund. Tozer, J. Camb. Ph. S. T. 6 (1838) 507-.

Makeham's law. Quiquet, A. C. R. 106 (1888) 1465-, 1569.

- -, generalisation. Quiquet, A. C. R. 109 (1889) 794-.

Marine assurance. Flemmich, - Assur. Mg. 2 (*1852) 102-.

- - Lance, $\dot{W}$. Assur. Mg. 2 (*1852) 362-.

- at Hamburg. Lazarus, $W$. Assur. Mg. 5 (* 1855) 221-.

- - principles. Morrison, $R$. Assur. Mg. 11 (*1864) 285-.

- risks, statement of collisions. Rucker, J.A. Assur. Mg. [1 (*1851)] 60-.

Marriage, duration and number of surviving partners. Trembley, J. Berl. Mm. Ac. (1799-1800) 110-.

-, influence on death-rate in Scotland. Stark, J. [1866] Edinb. R. S. P. 6 (1869) 49-.

- and mortality tables combined. Chatham, J. I. Act. J. 28 (1890) 384-.

Marriages, births, and deaths at St. Petersburg, tables. Krafft, W. L. [1798] St Pét. Ac. Sc. N. Acta 12 (1801) 246-.

- at different ages, proportion. Brown, $S$. Assur. Mg. 7 (*1858) 188-.

Married woman's right of dower in real estate if she survives husband, table showing. Bowditch, J. I. Am. Ac. Mm. 1 (1833) 331-.

Mean age, determination. Broca, $P$. Par. S. Ap. Bll. 2 (1879) 298-.

- duration of life. Ivernois, - d'. Quetelet Cor. Mth. 8 (1834) 232-.

- rations of rural population of France. Hervé-Mangon, C. F. C. R. 79 (1874) 931-. Meaning of expression $\frac{1}{1+a}-(1-v)$. Gray, $\boldsymbol{P}$. Assur. Mg. 10 (*1863) 117-.

Median estimates. Galton, $F$. B. A. Rp. (1899) 638-.

Medical statistics of life assurance companies. Porter, H. W. Assur. Mg. 4 (* 1854) 256-.

Metals considered as money. Gergonne, J. $D$. Gergonne A. Mth. 21 (1830-31) 189-.

Military assurance. Fischer, $F$. Hamb. Mth. Gs. Mt. 1 (1889) 4-.

Monetary standard, variations in value. Brit. Ass. Comm. B. A. Rp. (1887) 247-; (1888) 181-; (1889) 133-.
Money and coin, theory. Murhard, F. W. $A$. Hermbstädt Ms. 1 (1814) 374-; 2 (1814) 1-.

- -lending. Weninger, $V$. (xII) Mag. Ak. Éts. (Mth. Term.) 4 (1863) 147-.

-, moral importance, two views. Czuber, (or Čber) E. Arch. Mth. Ps. 62 (1878) 267-.

Moral statistics. Quetelet, L. A. J. Brux. Ac. Sc. Mm. 21 (1848) $112 \mathrm{pp}$.

- values. Henderson, R. [1895] N. Y. Am. Mth. S. Bll. 2 (1896) 46-.

\section{Mortality.}

ages 75 to 81. Thomson, W. T. Assur. Mg. [1 (*1851)] $29 \mathrm{bis}$.

American assured lives. Brown, S. Assur. Mg. 8 (*1860) 184-.

and annuity tables. Brown, S. I. Act. J. 16 (*1872) 428-.

arising from military operations. Hodge, $W . B$. Assur. Mg. 7 (*1858) 80-, 151-, 201-, 275-.

- naval operations. Hodge, W. B. Assur. Mg. 6 (*1857) 254-.

assured lives. King, G. I. Act. J. 19 (*1876) 381 -; 20 (*1878) 233-.

- - in France. Hardy, G. F. I. Act. J. 33 (1898) 485-.

- - West Africa. L., A. I. Act. J. 33 (1898) 516-.

- - - Indies. Hardy, G. F., \& Rothery, H. J. I. Act. J. 27 (1889) 161-.

British Army and Navy. McLauchlan, $J . J$. I. Act. J. 34 (1899) 251-.

canton of Geneva. Espine, M. d'. Bb. Un. 26 (1840) 255-; 55 (1845) 124-.

children, Tasmania. Nowell, E. C. Tasm. R. S. P. (1875) 108-.

the clergy. Stüssi, $H$. I. Act. J. 18 (*1875) 343-.

Danish clergy, 1650-1878. Westergaard, $H$. I. Act. J. 23 (*1882) 29-.

depositors in Norwegian widows fund (18461872). Broch, O. J. N. Mg. Ntvd. 21 (1876) 167-.

determination from population statistics. Knapp, (Dr) [G. F.] Il Polit. 4 (1867) (Tech.) 17-, 91-, 181-, 313-, 452-.

from different diseases, influence of selection. Dovey, $W . R$. I. Act. J. 23 (*1882) 285-.

English clergy, Hodgson's tables. Bowser, W. A. I. Act. J. $17\left({ }^{*} 1873\right) 328-$.

- - - - Brown, S. I. Act. J. 17 (*1873) 339 .

equations and curves. Isely, $L$. Neuch. S. Sc. Bll. 17 (1889) 38-.

estimation of, with migrant elements. Küttner, W. Z. Mth. Ps. 38 (1893) 148-

experience of Scottish Amicable Assurance Society. Spens, W. Assur. Mg. 10 (*1863) 61-, 197-.

Finland. Lindelöf, L. Helsingf. Bd. 49 (1890) 115-.

- and some other countries. Lindelöf, L. L. Helsingf. Öfv. 15 (1873) 129-.

France between 20 and 25 , and 25 and 30 , compared with other countries. Espine, M. d'. Bb. Un. Arch. 5 (1859) 220-. 
Glasgow and other cities. Macfadyen, $J, R$. Glasg. Ph. S. P. 7 (1871) 425-.

government officials on West Coast of Africa. Hart, J. R. I. Act. J. 33 (1898) 307-.

hourly distribution. Lawson, $R$. I. Act. J. 19 (*1876) 110-.

of infants in Scotland. Robertson, $W$. I. Act. J. 22 ("1881) 140-.

influence of high and low prices on rate. Barton, J. (vI Adds.) Ph. Mg. 5 (1834) 278-.

innkeepers, publicans, etc. Stott, $J$. I. Act. J. 20 (*1878) 35-.

law. Edmonds, T. R. Assur. Mg. 9 (*1861) 170 -.

-. Lazarus, W. Assur. Mg. $10\left({ }^{*} 1863\right) 283$ -

-. Makeham. W. M. Assur. Mg. $13\left({ }^{*} 1867\right)$ 325-.

-, answer to De Morgan's criticism. Gompertz, B. Assur. Mg. 9 (* 1861) 296-.

- British India. Henderson, $H$. B. As. Researches 20 (1836) 190-.

- and construction of annuity tables. Makeham, W. M. Assur. Mg. 8 (*1860) 301-.

-, empirical expression. Buniakovskij, V.Ja. St Pet. Ac. Sc. Mm. (Rs.) 15 ( $\left.{ }^{*} 1869\right)$ (Suppl.) No. 4, 20 pp.

-, expectation of life and values of annuities in India and England. Hannyngton, $J, C$. Beng. J. As. S. 12 (1842) 1057-.

-, expression by geometrical series. Woolhouse, W. S. B. Assur. Mg. 11 (*1864) 150-.

-, Makeham's modification of Gompertz's theory. Gray, P. Assur. Mg. 11 (*1864) 236-.

-, mathematical. Young, T. E. I. Act. J. 22 (*1881) 139-.

-, - Wittstein, T. I. Act. J. 24 (1884) 153-; 33 (1898) 399-.

-, mode of determining life contingencies. Gompertz, B. Phil. Trans. (1825) 513-.

- from observations. Hattendorff, $K$. Gött. Nr. (1871) 382-.

laws. Jellicoe, C. R. S. P. 4 (1839) 115.

- of Gompertz and Makeham. Bertrand, $J$. C. R. 106 (1888) 1042-.

Manchester and Salford. Henry, T. [1786] Manch. Ph. S. Mm. 3 (1790) 159-.

and marriage of Europeans in India. Brown, S. [1862] Assur. Mg. 11 (* 1864) 1-.

- - experiences, certain. Hewat, A., \& Chatham, J. I. Act. J. 31 (1895) 428-.

Massachusetts. Elliott, E. B. Am. As. P. (1857) $51-$.

miners in district of Marazion. Couch, R. Q. Cornwall Pol. S. T. (1860) 18-.

- - - St Ives and of agricultural population of St Buryan. Couch, R. Q. Cornwall Pol. S. T. (1859) 30-.

- - - St Just. Couch, R. Q. Cornwall Pol. S. T. (1857) 1-; (1858) 1-.

officers in the army, 1824-1873. Alvord, B. Am. As. P. 23 (1874) ( $p t$. 1.) 57-.

Paris and London. Tite, $W$. B. A. Rp. 34 (1864) (Sect.) 177-.

periodical observation, scheme for. Curtis, F. A. I. Act. J. $19\left({ }^{*} 1876\right) 229$-. from peritonitis (males and females) between fifteen and fifty. Robertson, $W$. I. Act. J. 19 (*1876) 118-.

Philadelphia, 1809-1816. Vaughan, J. [1817] Am. Ph. S. T. 1 (1818) 430-.

and population laws in France. Demonferrand, F. Par. Ec. Pol. J. 16 (26 cah.) (1838) 249-. probabilities. Landré, C. L. N. Arch. Wisk. 10 (1884) 26-.

progressive. Meikle, J. Assur. Mg. 3 (* 1853) 277-.

province of Bologna. Predieri, P. N. A. Sc. Nt. 7 (1847?) 396-; 8 (1847) 31-.

rate on assured lives, effect of withdrawals. Gray, W. T. I. Act. J. 24 (1884) 256-.

- - - - - - Sprague, T. B. I. Act. J. 24 (1884) 293-.

- - in West Indies. Stott, J. I. Act. J. 21 (*1879) 153-.

- in certain parts of Africa. Sprague, A. $E$. I. Act. J. 33 (1898) 285-.

-, De Wit's theory. Orchard, W. Assur. Mg. 2 (*1852) 393-.

- deduced from experience of assured lives. Whittall, W. J. H. I. Act. J. 31 (1895) 161-.

- in early manhood. Bailey, A.H. I. Act. J. 14 ("1869) 247.

- among emigrants. McLauchlan, J.J. I. Act. J. 18 (*1875) 381-.

- Europeans in India. Sprague, T. B. I. Act. J. 19 (* 1876) 295-.

- _ - Jamaica. Marshall, J. Assur. Mg. $4\left({ }^{*} 1854\right) 39$-.

-, experience of an assurance company. Jellicoe, C. Assur. Mag. 4 (* 1854) 199-.

- and its increase. Chatham, J. I. Act. J. 29 (1892) 81-.

- in India. Finlaison, A. J. I. Act. J. 18 (*1875) 153-.

- - infancy and childhood. Bowser, W. A. I. Act. J. 17 (* 1873) 26-.

- as influenced by time of assurance. Sprague, T. B. I. Act. J. $15\left(^{*} 1870\right)$ 328-.

- among Jews. Sprague, T. B. I. Act. J. 27 (1889) 155-.

- in navy. Sprague, T. B. I. Act. J. 25 (1886) 49-.

- - New South Wales. Pell, M. B. N. S. W. R. S. T. 1 (1867) 66-; I. Act. J. 21 (*1879) 257-.

- - peerage during nineteenth century. Bailey, A. H., \& Day, A. Assur. Mg. 9 (*1861) 305-.

- select lives. Spens, W. Assur. Mg. 12 (*1866) 304 .

- throughout life, formula. Thiele, T. N. I. Act. J. 16 (*1872) 313-, 464.

-, Zeuner's method for determining. Danielewicz, B. Prace Mt.-Fiz. 6 (1895) 183-.

rates in Australia. Burridge, A. F. I. Act. J. 24 (1884) 333-.

- as disturbed by migrations. Welton, T. A. I. Act. J. 16 (* 1872) 153-.

-, methods for deducing. Ackland, T. G. I. Act. J. 33 (1898) 68-, 131-. 


\section{Mortality}

rates, methods for deducing discontinuances. Todhunter, R. I. Act. J. 33 (1898) 273-.

- of natives and Europeans in India. Brown, S. I. Act. J. $16(* 1872) 187$ -

- - of India. Hardy, G.F. I. Act. J. 25 (1886) 217-.

- and their causes. Lazarus, W. I. Act. J. 18 (*1875) 54-, 212-.

- in United States. Gill, -. Assur. Mg. 3 $\left({ }^{*} 1853\right) 300$-.

- - Victoria. Burridge, A. F. I. Act. J. 23 (*1882) 309-.

ratio in Europe. Dieterici, C. F.W. Berl. Ab. (1851) (Ph.) 721-.

in relation to age and sex. Bertillon, $(\mathrm{Dr})-$. Fr. Cg. Md. Chir. 3 (1866) 663-.

Salem, 1782 and 1783. Holyoke, E. A. Bost. Mm. Am. Ac. 1 (1785) 546-.

among Sardinian soldiers in times of peace. Bonino, J. J. Tor. Mm. Ac. 35 (1831) 233-.

selected classes. Demonferrand, $F$. C. R. 9 (1839) 244-.

- lives in Germany. Brune, -. Assur. Mg. 3 (*1853) 29-.

- - inadequacy of data. Spens, $W$. Assur. Mg. 4 (* 1854) 1-, 139-.

shifting population. Wittstein, T. Grunert Arch. 39 (1862) 67-.

and sickness among labouring classes. Edmonds, T. R. Assur. Mg. 5 (*1855) 127-.

statistics, collection. Sang, E. I. Act. J. 15 (*1870) 257-.

-, formula. Westergaard, H. (xII) Ts. Mth. 1 (1877) 1-.

-, -. Eneström, G. Stockh. Öfv. (1891) 185-; Fschr. Mth. (1891) 241-.

supplement to two papers in Phil. Trans. 1820, 1825. Gompertz, B. [1861] Phil. Trans. (1862) 511-.

and survival at different ages in Geneva, 18381845. Espine, M. d'. Gen. Mm. S. Ps. 11 (1846) 457-

table for Brabant. Quetelet, L. A.J. Brux. Ac. Bll. 6 (1859) 345-.

- deduced from new experience. Bowser, $W$. A. I. Act. J. 16 (* 1872) 146-

- - whole life premium table. Wylie, $W$. Assur. Mg. 2 (" 1852) 391-.

-, Northampton, mode of construction. Sutton, W. I. Act. J. 18 (* 1875) 107-.

tables. Weninger, V. (xII) [1860] Mag. Ak. Ets. (Mth. Term.) 2 (1861-62) 51-.

- in America. McCay, C. F. I. Act. J. 16 ("1872) 20 -

-, arrangement of data. Meikle, J. Assur. Mg. 13 (*1867) 261-.

--, Bengal Civil Service. Hannyngton, J. $C$. Beng. J. As. S. 19 (1850) 250-.

-, calculation. Quetelet, L. A.J. Brux. Ac. Bll. 19 (1852) (pte. 3) 289-.

-, comparison. Young, (Dr) T. QJ. Sc. 2 (1828) 342-.

-, computation. Thiele, T. N. Kjøb. Ov. (1900) 139-.

- construction. Woolhouse, W. S. B. Assur. Mg. 13 (*1867) 75-.
Theory of Statistics, etc. 1635

Mortality, continued.

tables, construction. Baumhauer, M. M. von. I. Act. J. $16\left({ }^{*} 1872\right) 34$-.

-, - from imperfect data. Makeham, W. M. I. Act. J. $16\left({ }^{*} 1872\right) 344$.

- - - principles of. Makeham, W.M. Assur. Mg. 12.(* 1866) 305-.

- for Finland. Lindelöf, $L$. Helsingf. Öfv. 38 (1896) 113-.

-, formulæ. Eneström, G. Stockh. Öfv (1891) 251-, 343-; Fschr. Mth. (1891) 242-, 243-.

-, - for calculating. Eneström, $G$. Stockh.

Öfv. (1891) 441-; Fschr. Mth. (1891) 244-.

- and policy values. Meikle, J. I. Act. J. 23 (*1882) 385-.

select, construction and use. Sprague, T. B. I. Act. J. 21 (*1879) 229-; $22\left({ }^{*} 1881\right)$ 391-.

-, theory. Samot, D. J.A. N. Arch. Wisk. 3 (*1877) 145-

United States of America. Brown, S. Assur. Mg. 13 (*1867) 272-.

various .classes. Hardy, G. F. I. Act. J. 23 (* 1882) 1-.

countries of Europe. Anon. (vi 354)

Edinb, N. Ph. J. 16 (1834) 259-.

Movement of population, diocese of Maurienne, 1810-1830. Anon. (vi 1123) Sav. Mm. Ac. 5 (1831) 255-.

- - France. Demonferrand, F. C. R. 2 (1836) 437-.

- - Mâcon and environs, 1740-1828. Benon des Chânes, - (xII) Mâcon S. C. R. (1828) 65-.

- - Palermo. Villot, -. A. Sc. Nt. 10 (1827) 442-.

-- , Paris. Villermé, L. A. Sc. Nt. 8 (1826) 423-

--1 , Portugal. Silva, D. A. da. Lisb. J. Sc. Mth. 2 (1870) 255 -

_ - - Spain, 1866. La Sagra, R. de. C. R. 67 (1868) 460 -

Multiple annuities, formation of values and amounts. Gray, P. I. Act. J. 24 (1884) 129 -

Mutual life assurance, theory. Templeton, J. M. I. Act. J. 20 (*1878) 77-.

Negative policy values. Deuchar, J. J. W. I. Act. J. $19\left({ }^{*} 1876\right)$ 97-.

- - Gray, W. T. I. Act. J. 20 (" 1878) 73-, 150-

148.

Net premium and interest rate. Sutton, $W$. I. Act. J. 17 (*1873) 446-.

- mode of valuation, objections to. Makeham, W. M. I. Act. J. $15\left(^{*} 1870\right) 449$ -

- present value of instruments of credit, etc. Ronchetti, F. G. Mt. 26 (1888) 133-.

- profits, possible methods of dividing. Wilbraham, $H$. Assur. Mg. $6 \quad\left(^{*} 1857\right)$ 278-.

Number of white persons in United States, tables. Bowditch, J. I. Am. Ac. Mm. 1 (1333) 345-. 


\section{Theory of Statistics, etc.}

Obligations, contracting and discharge, theory. Boudsot, A. A. (xII) Doubs S. Mm. 2 (1858) 340-.

Obligatory assurance, theory. Eneström, $G$. Stockh. Öfv. (1895) 807-; Fschr. Mth. (1895) 251-.

Occupation, influence on health. Nelson, F. G. P. I. Act. J. 17 (*1873) 95-.

old age and invalid assurance, theory. Lazarus, W. Hamb. Mth. Gs. Mt. 2 (1890) (Festschr. Tl. 2) 158-.

Orchard's tables and a theoretical mortality table. Gray, P. Assur. Mg. 6 (*1857) 181-.

"Paid up" policy as equivalent to policy payable. Macfadyen, J. R. I. Act. J. 15 (*1870) 297-.

Partial commutation of premium. Gray, $P$. I. Act. J. 17 (* 1873) 224-.

Payment of $\frac{1}{m}$ yearly premium. Wiegand, $A$. Assur. Mg. 12 (*1866) 54.

- - L Laundy, S. L. Assur. Mg. 12 (*1866) 55-.

Pension calculation, formula. Eneström, $G$. Stockh. Öfv. (1893) 405-; Fschr. Mth. (1893-94) 360-.

Pensions. Lindeläf, L. Helsingf. Öfv. 26 (1884) 67-; Fschr. Mth. (1884) 189; Helsingf. Acta 14 (1885) 1-; 15 (1888) 209-; Helsingf. Bd. 51 (1892) 43-; 54 (1894) 1-; Acta Mth. 18 (1894) 89-.

- Widows and orphans fund of Leipzig University, 1882. Drobisch, M. W. Leip. Mth. Ps. B. 34 (1882) 51-.

Perpetuity contingent on surviving four lives. M., T. Assur. Mg. $12\left({ }^{*} 1866\right) 301$.

Philosophy of statistics. Woolhouse, W. S. B. I. Act. J. 17 ( $\left.{ }^{*} 1873\right)$ 37-.

Policies of assurance and life interests. Sprague, T. B. Assur. Mg. $8(* 1860)$ 12-.

- - - valuation. Anon. Assur. Mg. 10 (*1863) 252-, 322-.

on recently assured lives, reserve on. Teece, R. I. Act. J. 22 (*1881) 250-

- - - - - - Sprague, T. B. I. Act. J. 22 (*1881) 264 .

Policy on longer of two lives, value. Carr, $T$. I. Act. J. 14 (*1869) 415-.

- valuation, subject to half yearly or quarterly premiums. Sprague, T. B., \& King, G. I. Act. J. 23 ( $\left.{ }^{*} 1882\right)$ 256-, 264-.

- value. Meikle, J. Assur. Mg. 11 (*1864) 241-.

- and rate of interest. Sutton, $W$. I. Act. J. 17 (*1873) 227-. $22(* 1881) 135-$. Walton, W. G. I. Act. J.

- tables, modes of constructing. Manly, H. W. I. Act. J. $15(* 1870) 169$.

- - in terms of premium. Wilding, $R$. I. Act. J. 22 (*1881) 131.

- values, constructing models of. Chisholm, J. I. Act. J. 25 (1886) 141-.

- - effect of increased mortality. Sprague, T. B. I. Act. J. 21 ( $\left.{ }^{*} 1879\right) 77-$.
Population 1635

Policy values found by various methods, com. pared. Manly, H.W. I. Act. J. 14 (* 1869) 249-.

Political arithmetic. Czörnig, K. J. Prag Jb. Böhm. Ms. 2 (1831) 22-.

- economy. Whewell, $W$. [1850] Camb. Ph. S. T. 9 (1856) 128-; (pt. 2) [1]-.

- - and arithmetic, laws. Balbo, $P$. Tor. Mm. Ac. 34 (1830) 47-

- - - assurance, mathematical theory. Wittstein, T. I. Act. J. 17 ( $\left.{ }^{*} 1873\right)$ 178-, 355-, 417-.

- -, mathematics applied to. Rau, Brux. Ac. S. Bll. 8 (1841) (pte. 2) 148-.

- - , - - Helm, G. Dresden Isis Sb. (1887) (Ab.) 3-

$-\frac{-1}{(1889)} 671-$. Edgeworth, F. Y. B. A. Rp.

\section{Population.}

Benares. Prinsep, $J$. As. Researches 17 (1832) 470-

births, etc., in the Netherlands. Quetelet, L. A. J. Brux. Ac. Sc. Mm. 4 (1827) 117-.

Bombay. Macintosh, J. Thomson A. Ph. 14 (1819) 429-.

British Isles. Coquebert de Montbret, (Baron). Par. S. Gg. Bll. 1 (1822) 191-.

density, proposed units of areas. Steinhauser, A. Wien Mt. Gg. Gs. 7 (1863) 129-.

France. Dupin, C. C. R. 28 (1849) 369-.

- in 18th and 19th centuries. Dupin, $C$. C. R. 1 (1835) 103-.

-, influence of price of corn. Dupin, C. C. R. 2 (1836) 585-.

general considerations. Anon. (vi 703) J. de Ps. 93 (1821) 320-, 414-

general laws. Quetelet, L. A. J. Brux. Ac. Bll. 9 (1842) (pte. 2 ) 550-.

- - Pouillet, C. S. M. C. R. 15 (1842) 861-. - (Pouillet). Demonferrand, F. C. R. 15 (1842) 1096-.

Geneva. Mallet, E. Gen. Mm. S. Ps. 7 (1836) 321-.

increase, 1770-1848. Carnot, H. C. R. 31 (1850) 288-

-, apprehensions caused by. Hoffmann, J.G. Berl. Ab. (1835) (Ph.) 121-.

- and decrease in Danish states. Callisen, - . Dn. Vd. Selsk. Skr. 6 (1809-12) (2t. høft.) 169 .

- dependent on abundance of cereals and other foods. Predieri, P. N. A. Sc. Nt. 1 (1850) 58-.

- in Europe and America, decrease of rate. Storer, H. R. [1858] Am. J. Sc. 43 (1867) 141-.

- - kingdom of Naples, decrease of rate. Augustinis, M. de. Ranuzzi An. Gg. (1845) 35-.

- - Prussia in town and country. Dieterici, C. F. W. Berl. Ab. (1857) (Ph.) 99-.

- - relation to means of subsistence. Bourne, S. B. A. Rp. (1877) (Sect.) 165-. law of increase. Verhulst, P. F. Quetelet Cor. Mth. 10 (1838) 113-; Brux. Ac. Sc. Mm. 18 (1845) 38 pp.; 20 (1847) 32 pp. 


\section{Population}

law of increase and decrease. Besnard, $G$. Caen Mm. Ac. (1856) 203-.

- - - duration of families. Bienaymé, $J$. Par. S. Phlm. PV. (1845) 37-.

- - during last 100 years. Edmonds, T.R. Assur. Mg. 2 ( $\left.{ }^{*} 1852\right) 57$ -

mathematical theory. Quetelet, L. A. J. L'I. 2 (1834) 311-.

Mexico, 1840. Galeotti, H. G. Brux. Ac. Bll. 8 (1841) (pte. 2) 95-.

and mortality statisties, emigration and immigration being taken into account. Eneström, G. Stockh. Öfv. (1896) 225-; Fschr. Mth. (1896) 187-.

movement, European birth- and death-rate. Benoiston de Châteauneuf, - . A. Sc. Nt. 7 (1826) 314.

Oceania, Penes Is. Cagnazzi, L. de S. Nap. Rd. 2 (1843) 118-.

statistics, Austria, etc. Ficker, A. [1859] Wien Mt. Gg. Gs. 4 (1860) (Ab.) 71-

-, generalisation from. Eneström, $G$. Stockh. Öfv. (1896) 403-; Fschr. Mth. (1896) 188.

-, theory of pensions. Eneström, $G$. Stockh. Öfv. (1893) 361-; Fschr. Mth. (1893-94) 358-.

theory. Vindé, -. Eure J. d'Ag. 6 (1829) $331-$.

white and slaves, increase in United States. Harvey, G. Edinb. Ph. J. 8 (1823) 41-, 328-; 9 (1823) 63-.

world, ancient and modern. Merritt, - Par. S. Gg. Bll. 9 (1828) 1-.

-, races, religions, etc. Dieterici, C. F.W. Peterm. Mt. (1859) 1-.

Premature death among select lires. Farren, E. J. Assur. Mg. 4 (*1854) 66-, 141.

Premium-obligation, worth for any period. Hultman, $F$. $W$. Ts. Mt. Fys. 4 (1871) 251-.

- payable for life or for term, difference between. Meikle, J. Assur. Mg. 5 (*1855) 154 -

- rates and benefits accruing, incongruities. S., H. A. Assur. Mg. 8 ("1860) 167-.

- - for Bengal. Jellicoe, C. Assur. Mg. [1 (*1851)] 166-.

- to provide certain periodical returns. Tucker, R. Assur. Mg. 9 (*1861) 245-.

- scales, use of reduced. Hardy, G. F. I. Act. J. 31 (1895) 261-.

- values, method of interpolating. Samot, D. J. A. I. Act. J. 20 (*1878) 347-.

- - - - - Carment, D. I. Act. J. 21 (*1879) 213-.

Premiums, calculation by Gompertz's law. Makeham, W. M. Assur. Mg. 9 (*1861) $361-$.

-, half yearly and quarterly, obtained from annual. Laundy, S. L. Assur. Mg. 11 (*1864) 232-.

Present value of payments (when reproductive differs from remunerative rate). Lidstone, G. J. I. Act. J. 33 (1898) 412-.
Theory of Statistics, etc. 1635

Present values, calculation. Heegmann, $A$. Lille Mm. S. (1827-28) 1-.

Price of annuity for $n$ years of life of a person aged $x$. Macfadyen, J. R. I. Act. J. 19 (*1876) 141-.

Probabilities, application to judicial statistics. Cournot, A. A. Liouv. J. Mth. 3 (1838) 257-.

- , - - therapeutic statistics and statistics generally. Hagenbach-Bischoff, E. [1877] Basel Vh. 6 (1878) 516-.

- involved in widows' fund. Gruson, J. P. Berl. Ab. (1812-13) (Mth.) 1-.

-. Values of contingent reversions, 3 lives involved in survivorship. Morgan, $W$. [1799] Phil. Trans. (1800) 22-.

Probability of duration, decrement and expectation of life in New Hampshire and Massachusetts. Wigglesworth, $E$. Bost. Mm. Am. Ac. 2 (1793) 131-.

- - election results. Korteweg, D. J. [1875] Arch. Néerl. 12 (1877) 65-

-, local. Eneström, G. Stockh. Öfv. (1893) 623-; Fschr. Mth. (1893-94) 363.

- of success of candidates for three- or fourcornered constituencies. Hayward, R. B. B. A. Rp. 38 (1868) (Sect.) 9-.

Profit, determination and distribution. Meikle, J. Assur. Mg. 11 (*1864) 251-.

- distribution in mutual assurance societies. Pell, - . I. Act. J. 14 (* 1869) 382-.

Profits, formulø and tables for applying. Manly, H. W. I. Act. J. 27 (1889) 362-; 28 (1890) 333-.

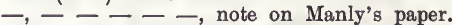
Calderon, H. P. I. Act. J. 28 (1890) 394-.

Progressive £axation, theory. Gobbi, $U$. Mil. I. Lomb. Rd. 30 (1897) 452-.

Property, division between children according to civil code. Catalan, E. C. L'I. 30 (1862) 139-.

- held for life and in reversion, valuation. Jellicoe, C. Assur. Mg. 6 ("1857) 61-.

$--1--\ldots$ - - Wilbraham, $H$. Assur. Mg. 6 ( $\left.{ }^{*} 1857\right)$ 211-.

- and income tax, inequitable distribution. Jellicoe, C. Assur. Mg. 2 (*1852) 213-.

Proportional parliamentary representation. Wasteels, J. Mathesis 20 (1900) 81-.

- representation at elections. Curie, J. As. Fr. C. R. (1889) (Pt. 2) 993-.

- -, methods. Eneström, G. Stockh. Öfv. (1896) 543-; Fschr. Mth. (1896) 189.

- - of minorities. Bayssellance, A. Bordeaux S. Sc. Mm. 3 (1880) 285-.

Prosperity in nations, relation to ethical conditions and mental development. Dieterici, C. F. W. Berl. Ab. (1855) (Ph.) 433-.

Purchasing life assurances as an investment. Day, A. Assur. Mg. 8 (*1860) 326-.

Pure premium method of valuation. Bailey, A. H. I. Act. J. 21 ("1879) 115-.

- $-\ldots$ - - as affected by expense and selection. Sorley, J. I. Act. J. $21\left({ }^{*} 1879\right)$ 192-.

Quinquennial census, deduction of yearly alterations. Eneström, G. Stockh. Öfv. (1893) 541-; Fschr. Mth. (1893-94) 362. 
Railway production and renewal, mathematical theory of cost. Mohr, -. Civing. 37 (1891) 39-.

- travelling, economics. Schneidewind, $A$. Arg. S. Ci. A. 39 (1895) 5-; 40 (1895) 77-; 41 (1896) 185-, 209-.

- - - Soulages, E. Arg. S. Ci. A. 42 (1896) 337-.

Ratio of newly contracted marriages to contemporary population. Dieterici, C. F.W. Berl. Ab. (1856) (Ph.) 559-.

Rationale of some actuarial estimates. Jellicoe, C. Assur. Mg. $8\left({ }^{*} 1860\right) 310$ -

Rejection of fractions of $£ 1$ in extensive valuations. De Morgan, A. Assur. Mg. 10 (*1863) 247-.

Relation of births to deaths, effects of migration and affluence. Benon des Chanes, -. (xII) Mâcon S. C. R. (1824) 89-.

Relationship, human, algebra of. Macfarlane, A. [1879-81] Edinb. R. S. P. 10 (1880) 224-; 11 (1882) 5-, 162-; Ph. Mg. 11 (1881) 436-.

-, - - - Brunel, —. Bordeaux S. Sc. Mm. 4 (1894) xxv-.

-, a problem in. Macfarlane, A. Edinb. R. S. P. 15 (1889) 116-.

Relationships (kinship), Galton's arithmetical notation. Macfarlane, A. Nt. 28 (1883) 588.

Reliability of data, as tested by conclusions. Farren, E. J. Assur. Mg. 3 (* 1853) 204 .

Remarriage rate of widowers. Sprague, $T . B$. I. Act. J. 22 (*1881) 77-.

Rents and value of ground in Rome. Armellini, T. Rm. N. Linc. At. 27 (1874) 111-.

Reproduction table, English. Farr, $W$. Phil. Trans. 171 (1880) 281-.

Reserves of assurances, Keuchel's formula. Danielewicz, B. Wiad. Mt. 2 (1898) 150-. - - with return of premium. Danielewicz, B. Wiad. Mt. 3 (1899) 275-.

- - fixed term assurances, calculation. Danielewicz, B. Wiad. Mt. 2 (1898) 216-.

- on life assurances. Danielewicz, B. Wiad. Mt. 4 (1900) 60 -

- owing to different data of valuation. Valentine, J. I. Act. J. 18 (* 1875) 229-.

Retirants in eighth year. Macaulay, $T$. B. I. Act. J. 22 (* 1881) 134-.

Returnable premiums, calculation. Makeham, W. M. Assur. Mg. $12\left({ }^{*} 1866\right)$ 233-.

Reversionary annuities. King, G. I. Act. J. 22 ( 1881 ) 296-.

- payable half yearly or quarterly. Carr, $T$. Assur. Mg. 7 (*1858) 109-.

- - - - - etc. Sprague, T. B. I. Act. J. 15 (* 1870) 126-; $18\left({ }^{*} 1875\right) 76$.

- - value of second nomination. Hardy, G. F. I. Act. J. 25 (1886) 134-.

- annuity, formula for. M., A. (Editor). Assur. Mg. 5 (* 1855) 52.

,---- Sprague, T. B. Assur. Mg. 7 (*1858) 174-.

- payable oftener than once a year, value. Evans, W. I. Act. J. 19 (*1876) 12-.

- bonus attaching to each policy, method of calculation. Hardy, R. P. I. Act. J. 31 (1895) 330-.
Reversionary interests, certain contingent. Warner, S. G. I. Act. J. 31 (1895) 280 -. - life interests as securities for loans. Sprague, T. B. I. Act. J. $17\left({ }^{*} 1873\right) 229$-. - - - valuation. Sprague, T. B. I. Act. J. $14(* 1869)$ 417-.

- - - - Davies, G. I. Act. J.15 (*1870) 138-.

$--\frac{-}{-}-$ Sprague, T. B. I. Act. J. 27 (1889) 107-.

$--\frac{1}{-}$ - Sunderland, A.W. I. Act. J. 27 (1889) 220 -

- transactions by lifenassurance companies. Macfadyen, J. R. I. Act. J. 20 (*1878) 385-.

Risk premiums for survivorship assurances. Sunderland, A. W. I. Act. J. 27 (1889) 81-.

Risks of assurance, mode of tabulation. Chatham, J. I. Act. J. 32 (1896) 393-.

- in granting life assurance. Bremiker, $C$. I. Act. J. $16\left({ }^{*} 1872\right)$ 216-, 285-.

-, valuation, and division of surplus. Jellicoe, C. Assur. Mg. 10 ("1363) 328-.

Royal Society, effect of limitation of number of fellows elected each year on eventual total. Strachey, (Lt.-Gen.) R. R. S. P. 51 (1892) 463-.

Savings banks. Dupin, C. C. R. 4 (1337) 597-.

- - Crelle, A. L. Crelle J. 39 (1850) 133-. - - progressive development. Dupin, $C$. C. R. 17 (1843) 1008-

$-\ldots$, statistics. Dupin, C. C. R. 20 (1845) 1693-

Second marriages in the peerage. Day, $A$. Assur. Mz. 12 (*1866) 185-.

Selection among assured lives. Higham, J.A. I. Act. J. 20 (*1878) 1-.

- of lives for assurance. Thomson, $S . C$. I. Act. J. $21\left({ }^{*} 1879\right) 170$ -

Sickness and invalidism, in relation to mortality. Makeham, W. M. I. Act. J. 16 (*1872) 408-.

- - mortality among native and European troops in India. Scales, W. H. Assur. Mg. 5 (* 1855) 245-.

- - - present state of information. Tomp. kins, $H$. Assur. Mg. $3(* 1853) 7$-.

- - rate in France. Brown, $S$. Assur. Mg. 5 (*1855) 208-

- tables, Ratcliffe's. Frankland, F.W. I. Act. J. 21 (*1879) 380.

Simpson's rule, generalisation for joint life annuities. Allen, J. M. I. Act. J. 33 (1898) 535-.

Single and annual premium, proof of formulæ. Gray, $P$. Assur. Mg. $10\left(^{*} 1863\right)$ 238-

- _ - _ _ - Wiegard, D.A. Assur. Mg. 10 (*1863) 286.

- life contingencies, calculation. De Morgan, A. Assur. Mg. 12 (*1866) 328-; 13 (*1867) 129-.

Sinking fund assurance. Faulks, J. E. I. Act. J. 34 (1899) 562-.

Smoothing of mortality observations, and Oppermann's formula. Gram, J.P. Ts. Mth. 2 (1884) 113-; Fschr. Mth. (1884) 187-. 
Social statistics and uniform action of human will. Brown, S. Assur. Mg. 2 ("1852) 341-.

Stability of results with reference to number of transactions. Campbell, $R$. Assur. Mg. 9 (*1861) 216-

Standard weight of Cologne mark. Eytelwein, J. A. Berl. Ab. (1816-17) 42-.

Statistical investigation of evolution. Cun. ningham, J. T. Nt. 51 (1894-95) 510.

- method for determining the vitality of a population, that of Sweden in particular. Eneström, G. Stockh. Öfv. (1893) 481-; Fschr. Mth. (1893-94) 361.

- rejection of extreme variations. Sheppard, W. F. L. Mth. S. P. 31 (1900) 70-.

- researches in the Netherlands. Quetelet, L. A. J. Brux. Ac. Sc. Mm. 5 (1829) $\mathrm{vi}+57 \mathrm{pp}$.

- science, new step in. Galton, $F$. Nt. 51 (1894-95) 319.

- study on recruiting for the Polytechnic School. Montessus de Ballore, F. de. Par. Éc. Pol. J. 63 (1893) 27-.

- tables, errors of the argument. Kleiber, $J$. B. A. Rp. (1888) 618 .

$-\frac{-}{-}$ probability of uniformity. Campbell, $R$. Ph. Mg. 18 (1859) 359-.

Statistics. Wittstein, T. D. Nf. B. 40 (1865) 112 .

-. Geddes, P. Nt. 24 (1881) 523-.

-, application of binomial law. Brown, $S$. I. Act. J. 17 (*1873) 340-.

-, association of attributes. Yule, G.U. [1899] Phil. Trans. (A) 194 (1900) 257-.

-, Bengal. Bedford, J. R. Beng. As. S. J. 22 (1853) 387-.

-, Camborne. Lanyon, $R$. Cornwall Pol. S. T. (1841) 99-.

-, classification. Geddes, P. [1881] Edinb. R. S. P. 11 (1882) 295-.

- of conditions of life among working classes. Bodio, L. Rm. R. Ac. Linc. T. 6 (1882) 317-.

-, correction of some documents. Demonferrand, F. Par. Éc. Pol. J. $16\left(27^{\mathrm{e}}\right.$ cah. $)$ (1839) 75-.

- of effect of cholera, 1832 and 1849. Dupin, $C$. C. R. 34 (1852) 65-.

- Egypt, 1837. Duhamel, A. Rs. Gg. Gs. D. 1 (1849) 93-.

-, formula for reduction. Eneström, G. Stockh. Öfv. (1893) 397-; Fschr. Mth. (1893-94) 359-.

- of fuels. Morville, N. [1798] Kiöb. Dn. Vd. Selsk. Skr. 1 (1800) 205-.

- general, of different countries. Quetelet, L. A. J. Brux. Ac. Bll. 12 (1861) 99-.

-, judiciary. Liouville, $\boldsymbol{E}$. Liouv. J. Mth. 18 (1873) 145-.

- and last census, British Empire. Merritt, - QJ. Sc. 2 (1827) 283-.

-, Lot (dept.). Cordier, L. J. Mines 21 (1807) 445- ; 22 (1807) 5-.

-, mathematical. Küttner, W. Z. Mth. Ps. 25 (1880) 11-; 26 (1881) 297-; 32 (1887) 234-.
Actuarial Mathematics

Statistics, States of the world. Babbi, A. Mil. G. I. Lomb. 3 (1842) 387-.

—, use. Delauney, -. C. R. 108 (1889) 909-.

Still-born children, estimation of number. Demonferrand, F. C. R. 3 (1836) 615-.

Suicide in Finland. Westerlund, F.W. Helsingf. Bd. 58 (1900) 111-

-, statistics. Jopling, R. T. Assur. Mg. [1 (*1851)] 308-; 2 (1852) 32-

Summation, approximate, of numerical tables. Buniakovskij, V.Ja. [1867] St Pet. Ac. Sc. Mm. (Rs.) 12 (1868) (Suppl.) No. 4, 41 pp.

Sums of drawings, final law. De Morgan, $A$. I. Act. J. $14\left({ }^{*} 1869\right)$ 175-.

Surplus, conditions giving rise to. Jellicoe, $C$. Assur. Mg. 2 (*1852) 333-.

- determination. Gill, $\dot{C}$. Assur. Mg. [1 (*1851)] 357-.

- - Younger, $S$. Assur. Mg. $4\left({ }^{*} 1854\right)$ 249-.

- and distribution. M., A. (Editor.) Assur. Mg. [1 ("1851)] 22 bis-, 159-, 359-.

$-\frac{-}{-}-$, certain objectionable methods. Jellicoe, C. Assur. Mg. 3 ( $\left.{ }^{*} 1853\right)$ 185-.

- distribution. Homans, S. Assur. Mg. 11 ("1864) 121-.

- - Manly, H. W. I. Act. J. $23\left({ }^{*} 1882\right)$ 233-

- Lidstone, G. J. I. Act. J. 32 (1896) 73-.

- - as defeating object of life assurance. Terry, J. Assur. Mg. 10 ("1863) 130-.

-- methods. Sprague, T. B. Assur. Mg. 6 ("1857) 290-, 344-; 7 ("1858) 61-.

$-\frac{-}{-}$, various. Pattison, W. P. Assur. Mg. $9\left({ }^{*} 1861\right)$ 341-.

-, formula for returning to assured their contributions. Harvey, C. J. I. Act. J. 24 (1884) 173-.

- premiums and interest, bonuses arising from. Sunderland, A. W. I. Act. J. 26 (1887) $357-$.

Surrender of policies, allowance. Jellicoe, $C$. Assur. Mg. [1 ( $\left.\left.{ }^{*} 1851\right)\right] 279$.

,---- Crisford, G. S. I. Act. J. 21 (*1879) 301-.

- values, solution of problem. Marr, T. I. Act. J. 14 ( $\left.^{*} 1869\right)$ 156-.

- - and their calculation. Fulford, F. W. I. Act. J. 35 (1901) 199-.

Survivorship annuities, calculation by columnar method. Meikle, J. Assur. Mg. 11 ("1864) 40-.

- assurance tables, construction. Gray, $P$. Assur. Mg. 5 (*1855) 107-.

- premiums, calculation. Sunderland, A. W. I. Act. J. 27 (1889) 509-.

- between two lives, probabilities. Gray, $P$. Assur. Mg. [1 ("1851)] 137-.

- - - - Ackland, T. G. I. Act. J. 27 (1889) 157-.

- - - - - , method for computing. Chisholm, D. Assur. Mg. 2 ("1852) 305-.

Survivorships, general problems concerning. Makeham, W. M. Assur. Mg. 12 (*1866) 61-. -, three life. Makeham, W. M. Assur. Mg. 12 ("1866) 118-. 


\section{Theory of Statistics, etc.}

Symbol $d_{x}$, origin. Cherriman, J.B. I. Act. J. $21\left({ }^{*} 1879\right) 295$ -

Taxation of temporary versus perpetual annuities. Hardy, P. Assur. Mg. 3 ("1853) 195-.

- and wealth, relation between. Gobbi, U. Mil. I. Lomb. Rd. 32 (1899) 756-.

Taxes, principles regulating incidence. Jenkin, F. Edinb. R. S. P. 7 (1872) 618-.

Ten year non-forfeiture policies. Younger, $S$. I. Act. J. 14 ("1869) 476-; 15 ("1870) 151-.

- - - - American. Cherriman, J. B. I. Act. J. 16 ( $\left.{ }^{*} 1872\right) 384-$

- - - - - McClintock, E. I. Act. J. 17 ("1873) 301-.

Term assurance, life against life, value. Smith, H. A. Assur. Mg. 9 (*1861) 295.

- - for two joint lives. Otter, W. C. Assur. Mg. $8\left({ }^{*} 1860\right) 113$ -

Terms of imprisonment, statistics and curves. Galton, $F^{\prime}$. Nt. 52 (1895) 174-.

Tontine, American, and mutual assessment schemes. Manly, H.W. I. Act. J. 26 (1887) 182-.

-, perpetual. Navier, C. L. M. H. A. C. 12 (1819) 103-

Tontines, early history. Hendriks, $F$. Assur. Ig. 10 ("1863) 205-.

Two life assurances, extra premiums in respect of. Ryan, G. H. I. Act. J. 24 (1884) 305-.

Under-average lives, treatment of. Sunderland, A. W. I. Act. J. 29 (1892) 419-.

Uniform mortality law from birth to old age. Gompertz, B. I. Act. J. 16 ("1872) 329-.

- seniority, law. Henderson, $R$. I. Act. J. 32 (1896) 293-.

United States, eighth census. Brown, S. Assur. Mg. 13 (*1867) 226-.

- - , formula for predicting population. Pritchett, H. S. St Louis Ac. T. 5 (1892) 599-.

Universal notation, necessity for a. Bégault, $A$. I. Act. J. 33 (1898) 1-.

Utility, theory. Gobbi, U. Mil. I. Lomb. Rd. 31 (1898) 237-, 325-, 1300-.

Vaccination, longevity in France before and after its introduction. Dupin, C. C. R. 27 (1848) 565-.

- and small pox, statistics. Sprague, T. B. I. Act. J. 20 ("1878) 216-.

Valuation of absolute reversions. Mountcastle, H. I. Act. J. 15 ("1870) 148-.

-, certain methods. Gray, W. T. I. Act. J. 20 (*1878) 309-.

- methods, notes. Dovey, W.R. I. Act. J. 34 (1899) 346-.

- producing definite reserve. Hardy, R. P. I. Act. J. 21 (1895) 325-.

- reserves, approximate calculation. Chisholm, J. I. Act. J. 27 (1889) 442-.

- returns, with reference to Life Assurance Companies Act. Todhunter, R. I. Act. J. 35 (1961) 1-.

- of whole term policies, tables. Hume, A.S., $\&$ Stott, W. I. Act. J. 34 (1899) 396-.

Value of benefits, on principle of collective assurance. Hardy, R. P. I. Act. J. 30 (1893) 79-.
Calculus of Differences, etc. 1640

Value of contingent reversion under certain limitations. Hardy, P. Assur. Mg. 2 (*1852) 91-, 298.

- options. Stephenson, J. W. Assur. Mg. $12\left({ }^{*} 1866\right) 302-$

- - Younger, S. Assur. Mg. $13\left({ }^{*} 1867\right)$ 55 -

-. Gray, P. Assur. Mg. 13 ("1867)

104-.

-. Younger, S. Assur. Mg. 13 ("1867) 118.

-, Stephenson's theory. Makeham, W.M. Assur. Mg. 12 ("1866) 363-.

- - - - - Smith, H. A. Assur. Mg. 13 ("1867) 103.

- and prices, mathematical investigations. Fisher, I. Conn. Ac. T. 9 (1892-95) 1-.

- of property and conditions which determine it. Buquoy, G. von. Oken Isis (1824) 28-.

- - reversions payable at instant of death. Chisholm, D. Assur. Mg. 4 ("1854) 70-

- - selection as exercised by policy holder. Higham, J. A. .Assur. Mg. [1 ("1851)] 179-.

Values, elementary, special arrangement. Oakes, W. H. Assur. Mg. 12 ("1866) 57-.

Vital statistics; geometric mean. Galton, $F$. R. S. P. 29 (1879) 365-.

- - of Glasgow, 1869 and 1870. Scott, A. Glasg. Ph. S. P. 8 (1873) 78-.

- - Society of Friends. Fryer, A. Manch. Ph. S. Mm. 14 (1857) 153-.

- - towns in Scotland. Sykes, W. H. B. A. Rp. (1842) 121-.

Wealth, social, mathematical theorv. Walras, L. [1876] Laus. S. Vd. Bll. 14 (1877) 365-, 525 -

Whole-life assurance business, approximate valuation. Woolhouse, W. S. B. I. Act. J. 27 (1889) 433-.

- - assurances, a certain conversion of. M., T. Assur. Mg. 13 ("1867) 246-.

- time assurances on single lives, valuation. Coles, J. Assur. Mg. 7 ("1858) 179-.

Widows' pensions, statistics. Eneström, G. Stockh. Öfv. (1894) 479-; Fschr. Mth. (1893-94) 363-.

- and spinsters, marriage rates. Sprague, T.B. I. Act. J. 22 ("18\&1) 352-.

Yearly into fractional yearly payment, transformation. McKenzie, D. J.McG. I. Act. J. 23 ("1882) 162-.

\section{Calculus of differences; interpolation.}

Adjunct of a given linear finite difference form. Bortolotti, E. Rm. R. Ac. Linc. Rd. 5 (1896) (Sem. 1) 349-.

Algebra of difference-tables. Everett, J. D. QJ. Mth. 31 (1900) 357-.

Approximate summation, formulæ. Hardy, G. F. I. Act. J. 24 (1884) 95-.

- - of series. Korteweg, D. J. N. Arch. Wisk. $2\left({ }^{*} 1876\right)$ 161-.

Approximation methods of former times. Günther, S. D. Nf. B. (*1877) 95-. 
Boole's finite differences, notation. Wilding, $R$. I. Act. J. 22 ( $\left.{ }^{*} 1881\right) 60$-.

Calculus of differences, algebraic transformation by. Cesáro, E. N. A. Mth. 5 (1886) 489-.

- _ - finite and infinitesimal. Gregory, D. F. Camb. Mth. J. 1 (1339) 212-.

-- , point in. Tisserand, F. C. R. 70 (1870) 678-.

- - - theorem. Hamilton, (Sir) W. R. B. A. Rp. (1843) (pt. 2) 2-.

- - enlargement (extension of calculus of finite differences). MeClintock, E. Am. J. Mth. 2 (1879) 101-.

- - - theorems. McClintock, E. Am. J. Mth. 17 (1895) 69-.

- - functions derived from limiting ratios. Echols, W. H. [1395] A. Mth. 10 (1895-96) 50 -.

- - generating functions. L., E.J. Camb. Mth. J. 2 (1841) 94-.

Central-difference formulø. Sheppard, $W . F$. [1399] L. Mth. S. P. 31 (1900) 449-.

- interpolation formula Everett, J. D. B. A. Rp. (1900) 648-.

- - - formulø. Spencer, J. I. Act. J. 33 (1898) 349-.

Constant coefficients in finite differences, theory. Naylor, J. I. Act. J. 23 (*1982) 420-.

Convergents applied to Cauchy's interpolation fractions. Padé, $H$. C. R. 130 (1900) 697-.

Cotes' numbers, calculation by approximate quadratures. Blažek, G. Prag Sb. (1879) 167-.

Cotton spinning problem. Woolhouse, W.S. B. Assur. M3. 11 (*1364) 224-.

Cyclical equations, connection with method of least squares. Nekrasov, P.A. Rec. Mth. (Moscou) 12 (1385) 377-.

Deduction of increase rates from tables. Everett, J. D. Nt. 60 (1899) 271.

- - - - - Runge, C. Nt. 60 (1399) $365-$.

$\Delta^{m} 0^{n}$ applied to integration. Herapath, $J$. Tilloch Ph. Mg. 65 (1825) 321-.

- - and Hargreave's form of it. Sums of powers of numbers etc. Scott, G. [1865] QJ. Mth. 8 (1867) 21 -.

Determination of functions from special values of variable. Chebuishev, P.L. (xII) [1369] Rec. Mth. (Moscou) 4 (1369-70) (Pt. 1) 231-.

Difference, $n$ th, deduced from $n$th differential. Picart, A. N. A. Mth. 12 (1373) 419-.

Differences and differential coefficients, connexion between. Nielsen, H. P. N. Ts. Mth. 8 (B) (1897) 86-; Fschr. Mth. (1897) 246.

- - differentials of functions of zero. Hamilton, (Sir) W. R. [1831] Ir. Ac. T. 17 (1937) 235-. - - - nth order. Spitzer, S. Grunert Arch. 33 (1359) 116-.

- method, application to series summed by help of impossible quantities. Gompertz, $B$. Phil. Trans. (1806) 174-.

- - construction of tables by. Gray, $P$. Assur. M3. 13 ("1367) 61-, 119-, 293-; I. Act. J. 11 (*1869) 307-

-, - 2 theorems. Knight, T. Phil. Trans. (1317) 234.

-, $m$ th, of 0. Scott, G. QJ. Mth. 5 (1362) 323-.
Differences, relation between various orders. Harvey, G. Thomson A. Ph. 7 (1816) 475-. -, series of, theory. Werner, 0 . Grunert Arch. 23 (1954) 231-; 24 (1855) 90-.

- - - - - Wallentin, I. G. Arch. Mth. Ps. 63 (1879) 56-.

- and Taylor's theorem. Adams, Jas. Thomson A. Ph. 11 (1818) 275.

-, theory. Sharpe, S. Ph. Mz. 10 (1831) 137-.

- of 0. Alberti, $V$. G. Mt. 38 (1900) $117-$.

Differentiation by aid of finite differences and vice-versa. Lobatto, $R$. Crelle J. 16 (1837) 11 -.

-, arithmetical. Philippono, M. M. Fschr. Mth. (1899) 267.

- under sign of integration, extension to finite differences, with applications. Chio, $F$. Tor. At. Ac. Sc. 6 (1870-71) 194-.

Empirical formulæ. Kleiber, I. A. Rs. Ps.-C. S. J. 22 (Ps.) (1890) 11-; J. de Ps. 10 (1891) 433-.

- - comparison. Runge, C. Z. Mth. Ps. 45 (1900) 78-.

Equation of finite differences, giving sums and differences of $\mathrm{R} x$. Gaudin, (Col.) - Les Mondes 3 (1863) 567-.

Equations, algebraic, theorems. Mathieu, $\dot{E}$. N. A. Mth. 15 (1856) 409-.

- of differences for approximating to differential equation. Coriolis, G. Liouv. J. N'h. 2 (1837) 230-.

Error of linear interpolation. Bary, - . Gergonne A. M h h. 21 (1830-31) 281-.

Errors by interpolation from logarithmic and other tables. Woodward, R.S. A. Mth. 2 (1885-86) 54-.

-, logarithmic. Howe, H. A. A. Mth. 1 (1884-85) 126-; 2 (1885-86) 39-, 77-; 3 (1887) 74.

-, theory, formula in. Makeham, W. $M$. I. Act. J. 28 (1890) 393-.

Euler's summation formula. Landré, C. $L$. N. Arch. Wisk. 6 ("1880) 212-.

Exponential functions and finite differences. Herschel, (Sir) J. F. W. [1815] Phil. Trans. (1816) 25-.

Factorial notation. Elphinstone, H.W. QJ. Mth. 2 (1858) 122-, 254-.

Faculties expressed by $\Delta^{m} 0^{n}$. Jeffery, $H . M$. QJ. Mth. 4 (1861) 364.

Figurate numbers and interpolation, short proofs of theorems. Soufflet, - Les Mondes 13 (1867) 336-.

- series. Smyth, B. B. Kan. Ac. Sc. T. 14 (1896) 29-.

Finite difference equations, partial. Paoli, $P$. Mod. S. It. Mm. 8 (1794) 575-.

- - integrals expressed by definite integrals. Tortolini, B. Tortolini A. 4 (1853) 209-. - differences calculus. Russell, W. H. L. Mass. Mth. 11 (1882) 33-.

- - applied to definite integrals. Paoli, $P$. Mod. S. It. Mm. 20 (1828) 255-.

- - - $\Delta^{n}\left(x^{m}\right)$, factor in general term of. Köhlau, E. Crelle J. 6 (1830) 255-.

- - - determinants of functions in the. Bortolotti, E. Rm. R. Ac. Linc. Rd. 5 (1896) (Sem. 1) 254-. 


\section{Calculus of Differences, etc.}

Finite differences calculus, formula in. Carr, T. I. Act. J. $14\left({ }^{*} 1869\right) 479-$.

$--\longrightarrow,-h u_{x}^{\prime}=\Delta u_{x}-\frac{h}{2} \Delta u_{x}^{\prime}+\frac{B_{1} h^{2}}{1.2} \Delta u^{\prime \prime}{ }_{x}-\& c$. Malmsten, C. J. Stockh. Ak. Hndl. (1844) 363-; Crelle J. 35 (1847) 55-.

- _ - formulæ. Tortolini, B. A. Mt. 5 (1863) 181-.

$--\frac{1}{-}$, , useful. Hill, G.W. Des M jines Anal. 1 (1874) 141-.

- - methods. Carmichael, $R$. NH. Rv. 7 (1360) (P.) 78-, 170-.

- - , theorem, new. Booth, J. Ph. Mg. 19 (1841) 125-.

- - - , theorems, new, based chiefly on researches on complex variability. Casorati, $F$. A. Mt. 10 (1880-82) 10-.

- - - , and theory of numbers, contribution to. Busche, E. Mth. A. 53 (1900) 243-.

- - direct method. Adams, Jas. Thomson A. Ph. 16 (1820) 281-

- -, formula. Cayley, A. QJ. Mth. 2 (1858) 198-.

- -, formulæ. Cauchy, A. L. C. R. 19 (1344) 1179-.

- integrals. Cauchy, A. L. C. R. 39 (1854) 211.

- - - - Bouniakowsky, V. St Pét. Ac. Sc. Mm. 1 (1859) No. 9, 19 pp.

- - - - Barbier, E. Les Mondes 3 (1863) 701-.

- - - - Thoman, F. C. R. 57 (1863) 778-.

- - of powers, conversion to definite integrals. Cauchy, A. L. [1815] Par. Éc. Pol. J. 17 (28 cah.) (1841) 147-.

- - , return to original series from $n$th term of $m$ th series of differences. Müller, G. W. Grunert Arch. 1 (1341) 211-.

- - series connected with. Lukas, F. C. Wien Az. 32 (1895) 269-.

-- , and Taylor's series. Carvallo, E. N. A. Mth. 10 (1891) 24-.

- or mixed differences, uestions depending on. Combescure, $\dot{E}$. Par. Éc. Norm. A. 3 (1874) 305-.

Forms $\Delta^{n} 0^{x}$ and congeners. Horner, J. QJ. Mth. 4 (1861) 111-, $204-$.

Formula for $\Delta^{n} 0^{i} / n^{i}$ when $n$ and $i$ are very large numbers. Cayley, A. [1387] Edinb. R. S. P. 14 (1388) 149-

Functions, approximate forms for representation. Pincherle, S. Bologna Ac. Sc. Mm. 10 (1889) 77-.

Harmonic series, representation by sequences of factors. Rogel, F. Arch. Mth. Ps. 9 (1890) 297-.

Illusory interpolations, new examples. Méray, C. Bll. Sc. M th. 20 (1896) 266-.

Indeterminate analysis, application of calculus of finite differences. Piola, $G$. A. Sc. Lomb. Ven. 1 (1831) 101-; Tortolini A. 1 (1850) 263-.

Integrability of differential and finite difference equations, conditions. Minich, S. R. Tortolini A. 1 (1350) 321-.

Integral, finite, $\Sigma^{n} \phi x$ expressed as a definite integral. Abel, N. H. Mg. Ntvd. 6 (1825) 182-.

\section{Interpolation 1640}

Integral, finite, $\Sigma e^{x} y$. Malmsten, $C . J$. Ups. N. Acta S. Sc. 12 (1844) 293-.

Integration of difference equations reducible to linear form. Oltramare, G. As. Fr. C. R. (1893) (Pt. 2) 106-.

- in finite differences. Trembley, J. Berl. Mm. Ac. (1799-1800) 18-.

- of linear difference-equations. Oltramare, $G$. As. Fr. C. R. (1891) (Pt. 2) 66-; (1895) (Pt. 2) 175-.

-, use in arithmetical calculations. Cesáro, E. Palermo Cir. Mt. Rd. 1 (1887) 293-.

Interpolated multiples. Soulé, - Bordeaux S. Sc. Mm. 4 (1894) iii-.

Interpolating functions. Cauchy, A. L. C. R. 11 (1840) 775-.

- - Genocchi, A. Tor. Ac. Sc. At. 13 (1877) 716-; 16 (1880) 269-; C. R. 86 (1878) 466-.

- Peano, G. Tor. Ac. Sc. At. 18 (1882) $573-$.

- - elementary proof of a fundamental property. Schwarz, C. H. A. Tor. Ac. Sc. At. 17 (1381) 740-.

\section{INTERPOLATION.}

Legendre, A. M. Con. des Temps (1819) 302-.

Degen, C. F. As. Nr. 1 (1823) 231-.

Clausen, T. Crelle J. 5 (1829) 305-.

Encke, J. F. Berl. As. Jb. (1830) 265-.

Cauchy, A. L. [1835] Mod. Mm. S. It. 21 (1836) 374-.

Biot, J. B. C. R. 13 (1844) 545.

Brassinne, E. Liouv. J. Mth. 11 (1846) 177-.

Cauchy, A. L. Con. des Temps (1852) 129-; C. R. 37 (1853) 64-.

Lehmann, J. W. H. As. Nr. 39 (1855) 257-.

Grunert, J. A. Grunert Arch. 32 (1859) 119-.

Hermite, C. C. R. 48 (1559) 62-.

Rouché, E. N. A. Mth. 18 (1859) 26-.

Čebyrev, P. St Pet. Ac. Sc. Mn. (Rs.) 4 (*1864) (Suppl.) No. 5, $23 \mathrm{pp}$.

Tisserand, F. C. R. 68 (1869) 1101-.

Nell, A. M. Arch. Mth. Ps. 61 (1877) 135-.

Bartl, C. [1877] Arch. Mth. Ps. 62 (1378) $202-$.

Laisant, C. A. As. Fr. C. R. (1891) (Pt. 2) 222-.

Pincherle, S. Bologna Ac. Sc. Mm. 3 (1392) 293-.

Borel, $\dot{E}$. C. R. 124 (1897) 673-.

Laisant, - . As. Fr. C. R. (1897) (Pt. 2) 86-.

Markov, A. St Pét. Ac. Sc. Mm. 6 (1898) No. 5, 69 pp.; Fschr. Mth. (1898) 252-.

Veltmann, W. Z. Mth. Ps. 44 (1899) 303-; 45 (1900) 337.

and application to cubic equations. Vieille, $J$. N. A. Mth. 10 (1851) 48-

applied to extraction of square and cube roots. Dolguschin, P. A. Fschr. Mth. (1898) 139.

approximate. Bellavitis, $G$. Ven. At. (185859) 1109-.

- fractions. Györy, S. (xir) Mag. Ak. Éts. (1854) 165-. 


\section{Interpolation}

astronomical formulæ. Le Verrier, U. J.J. (x) Par. Obs. A. 1 (1855) 73-; 2 (1856) $301+$ [167] pp. ; 3 (1857) 199-, and [1]-; 4 (1858) 1-, and [1]-; 5 (1859) 1-; 6 (1861) 435 pp. ; 10 (1874) 1-, and [1]-; 11 (1876) $560+[136]$ pp. ; $12(1876) 76+[178]+$ A. 80 + [A. 286] pp.; 13 (1876) 1-, and [1]-; 14 (1877) Pt. 1, A. 1-, and [A. 1]-; Pt. 2, 1-, and $[1]-$.

and Bernoulli's numbers. Lipschitz, R. O.S. C. R. 86 (1878) 119-.

Briggs' Log. Tables. Legendre, A. M. Con. des Temps (1817) 219-.

and calculus of differences finite or infinite, principles. Ampère, A. M. Gergonne A. Mth. 16 (1825-26) 329-.

certain forms. Bartlett, W. P. G. Bost. Mm. Am. Ac. 8 (1863) 435-.

chemical and physical observations. Bartlett, W. P. G. Silliman J. 34 (1862) 27-.

choice of best ordinate. Kleiber, I. A. Kazan S. Nt. (Ps.-Mth.) P. 8 (1890) 232-; Fschr. Ps. (1890) (Ab. 1) 13.

by circular functions. Gomes Teixeira, $F$. N. A. Mth. 4 (1885) 351-.

- curves of 3 rd and 5 th order. Dupin, C. C. R. 25 (1847) 769-.

- differences. Burckhardt, J. K. Zach M. Cor. 12 (1805) 332-.

at equidistant values. Cebysev, $P$. St Pet. Ac. Sc. Mm. (R8.) 25 (*1874-75) (Suppl.) No. 5, $30 \mathrm{pp}$.

formula. Tchebicheff, $P$. [1854] St Pét. Ac. Sc. Bll. 13 (1855) 210 -

- Brioschi, F. Tortolini A. 2 (1859) 132-.

- Gomes-Teixeira, F. Liége S. Sc. Mm. 10 (1883) (No. 10) $7 \mathrm{pp}$.

-, Cauchy. Schott, C. A. U. S. Coast Sv. Rp. (1860) 392-.

-, Gauss, infinitely extended. Bendixson, $I$. Acta Mth. 9 (1887) 1-.

-, Hermite, expressed algebraically. Schering, E. C. R. 92 (1881) 510 -

-, Lagrange. Dirksen, E. H. Crelle J. 1 (1826) 221-.

-, - Garnier, J. G. Brux. Ac. Bll. 6 (1839) (pte. 2) 19-.

-, - . Studnicka, F. J. Časopis $2\left({ }^{*} 1873\right)$ 82- ; Fschr. Mth. (1873) 122-.

- - Hermite, C. [1877] Crelle J. Mth. 84 (1878) 70-.

-, - Stieltjes, T. J. (jun.). [1881] Amst. Ak. Vs. M. 17 (1882) 239-.

-, 一. Bendixson, I. C. R. 101 (1885) 1050-, 1129 .

-, - Echols, W. H. A. Mth. 8 (1893-94)

-, -. Lewicki, W. Wiad. Mt. 3 (1899)

264-; Fschr. Mth. (1899) 244.

- - , generalisation. Williot, $V$. Bll. Sc. Mth. 14 (1890) 218-.

- - , remainder in. Sonin, N. J. Fschr. Mth. (1899) 242.

-, (mortality-tables). Selling, $E$. Crelle J. Mth. 106 (1890) 193-.

-, new. Alexander, J. H. Silliman J. 7 (1849) 14-.
Interpolation 1640

formula, Newton. Oppermann, L. I. Act. J. 15 (*1870) 145-, 177-.

-, - Jensen, J. L.W. V. Kjøb. Ov. (1894)

-, Pictet. Szily, K. [1879] J. de Ps. 9 (1880) 303-.

-, Prony. Minich, S. R. Ven. At. 13 (186768) 1153-.

, Tchebicheff's (Čebyšev). Tchebicheff, $P$. N. A. Mth. 18 (1859) 193-.

formulæ. Ferrel, $W$. [1865] Am. Ac. P. 7 (1868) 2-.

-. Carvallo, J. C. R. 106 (1888) 346-.

-. Radau, $R$. Bll. As. 8 (1891) 273-, 325-, 376-, 425-.

-, application. Ackland, T. G. I. Act. J. 32 (1896) 286-.

- and application to solution of equations. Olivier, L. Crelle J. 2 (1827) 197-.

- for functions of several variables. Kronecker, L. Berl. Mb. (1865) 686-.

-, Gauss, for $n=7,8$ and 9. Perott, J. QJ. Mth. 25 (1891) 200-

-, Hansen. Mollweide, C. As. Nr. 2 (1824) 259 -.

-, Lagrange. Lewicky, W. Arch. Mth. Ps. 17 (1900) 214-

-, - generalised, applications. Puzyna, J. Krk. Ak. (Mt.-Prz.) Pam. 14 (1888) 1-; Fschr. Mth. (1888) 251-.

-, - and Newton. Gerono, G. C. N. A. Mth. 16 (1857) 317-, 358-.

-, - - -. Transon, A. (Prof.). N. A. Mth. 19 (1860) 248-.

-, new. Astrand, J. J. Christiania F. (1890) (Ov.) 5-.

-, various. Cauchy, A. L. C. R. 12 (1841) 283-.

by fractional rational function. Jacobi, C. G. J. Crelle J. 30 (1846) 127-.

- functions of a variable. Sarrus, F. (vI Adds.) Strasb. S. Sc. N. Mm. 2 (1832) 203-.

in heat. Bary, E. C. R. 3 (1836) 378.

of implicit functions, and calculation of roots. Maksimovich, V. P. (xII) [1881] Kazan S. Nt. (P8.-Mth.) P. 1 (1883) [No. 5] (Suppl.) $23 \mathrm{pp}$.

and integration in series. Buchwaldt, $F$. Ts. Mth. 5 (1887) 79-, 97-; Fschr. Mth. (1887) 281 -

of irrational and logarithmic functions by numerical tables. Lefort, $P . A . F$. [1857] Edinb. R. S. P. 8 (1875) 602-.

inverse. Siebdrat, (Dr.). Dresden Sb. Isis (1871) 233-.

kinematic. Laisant, $C . A$. As. Fr. C. R. (1890) (Pt. 2) 70-.

Lagrange's theorem, generalisation. Sylvester, J. J. Ph. Mg. 6 (1853) 374-.

by least squares. Gergonne, J. D. Gergonne A. Mth. 6 (1815-16) 242-.

- - -. Čeby ̌̌ev, P. L. (Tchebicheff). St Pét. Ac. Sc. Mm. 1 (1859) No. 15, 24 pp.

- - Borchardt, C. W. [1860] Crelle J. 58 (1861) 270 . 


\section{Interpolation}

by least squares, formulæ. Čebyรev, $P$. L. (Chebuishev). (xII) Rec. Mth. (Moscou) 3 (1868) (Pt. 1) 157-.

(Tchebychef). Schols, C. M. [1375] Amst. Ak. Vs. M. 9 (1376) 301-; Arch. Néerl. 12 (1877) 102-.

- - and new method, comparison. Cauchy, A. L. C. R. 37 (1853) 100-.

legitimacy. Méray, C. Par. Ec. Norm. A. 1 (1884) 165-

as means of calculating irrationals. Opper. mann, L. Kjöb. Ov. (1375) 18-.

and mechanical quadrature. Grunert, $J . A$. Grunert Arch. 20 (1853) 361-.

- quadratures in astronomy. Santini, G. Ven. Mm. I. 13 (1366) 177-.

method. Uylenbroek, $P$. J. Leijd. A. Ac. (1820-21) $77 \mathrm{pp}$.

-, Cauchy. Grunert, J.A. Grunert Arch. 2 (1342) 41-.

-, - Seeliger, H. As. Nr. 96 (1880) 235-.

- , - , modified by method of least squares.

Despeyrous, C. Toul. Mm. Ac. 5 (1967) 251-.

-, general. Buquoy, G. von. Oken Isis (1827) 889-.

-, geometrical. Genge, C. Zür. Vjschr. 31 (1886) 268-.

—, new. Aistrand, J. J. Leip. As. Gs. Vjschr. 10 (1875) 279-.

-, - general. McClintock, E. Am. J. Mth. 2 (1879) 307-.

-, Tschebyschew. Backlund, O. St Pét. Ac. Sc. Bll. 29 (1384) 477-.

methods. Le Verrier, U. J. J. (vi Adds.) Par. A. Obs. 1 (1855) 73-; 2 (1856) 1-; 3 (1857) 199-; 4 (1858) 1-; 5 (1359) 1-; 6 (1861).1-。

-, special. Niven, W. D. [1877] Mess. Mth. 7 (1878) 35-.

mixed. Cousinery, B. E. Ing. 1 (1848) 125-. new formula. Sprague, T. B. I. Act. J. 22 (*1881) 270 -.

between observed values. Tchebicheff, $P$. St Pét. Ac. Sc. Bll. 16 (1858) 353-; St Pét. Ac. Sc. Mm. 1 (1859) No. 5, 81 pp.

by parabolic curve. Michal, $A$. C. R. 37 (1853) 185-.

- - curves. Michal, A. A. Pon. Chauss. 10 (1865) 59-

parabolic, by least squares method. Degen, C. F. [1823] St Pét. Mm. Sav. Étr. 1 (1831) 12-.

-, - - - Grave, P. Kazan S. Nt. (Ps.-Mth.) P. 7 (1889) 270-; Fschr. Mth. (1889) 220.

periodic functions. Weyer, G. D. E. Leip. As. Gs. Vjschr. 22 (1887) 292-.

philosophy. Gergonne, J. D. Gergonne A. Mth. 5 (1814-15) 252-.

in physics. Mollweide, C. Gilbert A. 62 (1819) 422-.

problem. Korkine, A. Bll. Sc. Mth. As. 6 (1882) 228-.

-. Radau, R. Bll. As. 4 (1887) 515-.

- Bohlmann, G. Gött. Nr. (1899) 260-.

-. Pincherle, S. Palermo Cir. Mt. Rd. 14 (1900) 142-.
Calculus of Differences, etc. 1640

Interpolation, continued.

problem, Cauchy. Netto, E. Mth. A. 42 (1893) 453-.

, general. Greatheed, S. S. (vi Adds.) Camb. Mth. J. 3 (1843) 97-.

problems, mechanical solution. Jung, G. Mil. I. Lomb. Rd. 13 (1380) 226-.

proof of formula. Berridge, G. W. I. Act. J. 14 (*1869) 244.

with proofs of methods of Briggs, Mouton, and Newton. Maurice, $F$. Con. des Temps (1847) 181-.

questions of fluid motion. Barré de SaintVenant, -. C. R. 17 (1343) 1108-.

recurring series. Maillet, $E$. N. A. Mth. 14 (1895) 473-.

with reference to development and differentiation. Roberts, S. [1865] QJ. Mth. 7 (1866) 184-; 8 (1867) 52-, 139-.

results. Thiele, T. N. (xII) Ts. Mth. 1 (1883) 183- ; Fschr. Mth. (1884) 198-.

to second order without change of sign. Airy, (Sir) G. B. As. S. M. Not. 26 (1866) 236-.

series. Bangma, O. S., \& J. F. Keyser. Amst. Mengelwerk 2 (1316) 42-.

$-A_{1}, A_{1} A_{2}, \ldots A_{1} A_{2} A_{3} \ldots A_{n}$. Svanberg, $A . F$. Stockh. Ak. Hndl. (1828) 1-.

in statistics. Westergaard, $H$. I. Act. J. 32 (1896) 276-.

by successive differences, exactness. Mollweide, C. Zach M. Cor. 21 (1810) 331-.

summation and adjustment of tables. Woolhouse, W. S. B. Assur. Mg. 11 (* 1864) 61-, 301-; 12 (*1866) 136-.

and summation of numerical series, method. Donald, J. Glasg. Ph. S. P. 9 (1875) 272-. symmetric function. Borchardt, C. W. Berl. Ab. (1860) (Mth.) 1-.

table, general. Amante, $F$. (xI) [1844] Nap. Ac. Pont. At. 4 (1351) 251-.

tables. Heawood, P.J. Mess. Mth. 27 (1898) 121-.

-, adequacy. Jelinek, $V$. Časopis 21 (1892) 31- ; Fschr. Mth. (1892) 227.

theorem of Hermite. Gomes-Teixeira, $F$. Crelle J. Mth. 100 (1887) 83-.

theory. Lerch, M. Prag České Ak. Fr. Jos. Rz. (Tř́la 2) 1 (1892) Art. 32, 15 pp.; Fschr. Mth. (1892) 227.

-. Ioachimescu, A. G. Bucarest S. Sc. Bll. 6 (1897) 472-.

of values depending on two variables. Seydler, A. Prag Sb. (1889) 2 (Mth.-Nt.) 337-; Fschr. Mth. (1889) 253-.

Inverse calculus of differences, formula. Prouhet, E. N. A. Mth. 10 (1851) 186-.

- - notes. Combescure, É. $\quad$ C. R. 74 (1872) 454-

- method of finite differences. Brinkley, $J$. Phil. Trans. (1807) 114-.

Least squares method, case where number of unknowns is large. Nekrasov, $P$. A. Rec. Mth. (Moscou) 12 (1885) 189.

Legendre's functions, use of, for interpolation. Seeliger, H. Münch. Ak. Sb. 20 (1891) 499-. 
1640 Calculus of Differences, etc.

Linear difference forms with commutative factors of prime degree. Torelli, G. Nap. Rd. 35 (1896) 238-.

Measurement of cylinder. Rasch, J.W. N. Arch. Wisk. 7 (*1881) 117-.

Mortality tables, theory. Samot, D. J.A. N. Arch. Wisk. $3\left({ }^{*} 1877\right)$ 145-.

Numerical solution and reversion of series, remark on Woolhouse's paper. De Morgan, A. I. Act. J. 15 ("1870) 327-.

Partial differences. Euler, L. [1777] St Pét. Ac. Sc. N. Act̊ 15 (1806) 3-.

- summation. Helm, G. Z. Mth. Ps. 22 (1877) 400-.

Plurality of integrals in calculus of differences. Poisson, S. D. [1800] Par. Éc. Pol. J. 4 (1801-2) (11 $\left.{ }^{\circ} \mathrm{cah}.\right)$ 173-.

Progression, arithmetical, successive differences of powers of terms. Lhuilier, $S$. Gergonne A. Mth. 2 (1811-12) 185-.

Progressions, arithmetical, whose terms are only approximately known. Lucas, $F$. C. R. 96 (1883) 1026-.

- with 2nd and 3rd differences. Jadanza, $N$. G. Mt. 6 (1868) 375-; 7 (1869) 17-.

Quadrature, arithmetical. Danielsson Hill, C. J. Stockh. Ak. Hndl. (1854) 405-.

-, Gauss's formula, and Hermite's formula of interpolation. Mansion, P. C. R. 104 (1887) 488-.

,$--\ldots$, remainder in. Mansion, $P$. Brux. Ac. Bll. 11 (1886) 293-.

Quadratures and interpolation, report on present knowledge of actual application. Merrifield, $C$. W. B. A. Rp. (1880) 321-.

Rational approximation to non-rational homogeneous function. Resal, $H, A$, C. R. 80 (1875) 1185-.

- fractions, integration by finite differences of. Beyer, E. I. von. (xiI) Rec. Mth. (Moscou) 4 (1869-70) (Pt. 1) 297-; 5 (1870) (Pt. 1) 64-, 145-.

Reduction of higher differences when the constant increment is changed. Gudermann, C. As. Nr. 26 (1848) 315-.

Reversion of series and its application to solution of numerical equations. Hagen, J. G. Am. Ph. S. P. 21 (1884) 93-.

Second differences in interpolation, easy method of taking into account. Nell, -. Arch. Mth. Ps. 70 (1884) 302-.

Series of numbers and their interpolation. Ruff, H. Arch. Mth. Ps. 17 (1900) 426-.

-, summatión by differences. Fuss, N. St Pét. Ac. Sc. Mm. 11 (1830) 246-.

Simpson's formula and another. Catalan, E. C. N. A. Mth. 10 (1851) 412-。

- for approximation to areas. Daug, H.T. Stockh. Öfv. 17 (1860) 429-.

- method of interpolation, simplification. Parmentier, $T$. N. A. Mth. 15 (1876) 241-.

- rule, history. Heinrich, G. Bb. Mth. 1 (1900) 90-.

Solution of linear equations with large number of unknowns by successive approximation, Seidel's process. Mehmke, $R$. Rec. Mth. (Moscou) 16 (1893) 342-.
Linear Substitutions 2000

Solution of system of linear equations by successive approximation. Mehmke, R., \& Nekrasov, P. A. [1892] Rec. Mth. (Moscou) 16 (1893) 437-.

Stirling's formula. Serret, J. A. C. R. 50 (1860) 662-.

- - Bonnet, O. C. R. 50 (1860) 862-.

-, elementary proof. Cesáro, E. N. A. Mth. 2 (1883) 43-.

- -, extension. Hermite, C. Mth. A. 41 (1893) 581-.

G. H. Mess. Mth. 19 (1890) 19-.

- series generalised, applications. Raabe, J. L. Zür. Mt. 3 (1853-55) 332-.

- - for interpolation by symmetric differences. Merrifield, C. W. [1866] Mess. Mth. 4 (1868) 110-

Successive differences of observations. Bréger, -. C. R. 93 (1881) 1119-.

Sums and differences, theory, higher examples. Vállas, A. Mag. Ak. Éts. (1850) 381-.

- - - - - (Schlömilch.) Oettinger, L. Grunert Arch. 13 (1849) 36-.

Table of $\Delta^{m} 0^{n} \div \Pi(m)$. Cayley, A. [1879] Camb. Ph. S. T. 13 (1883) 1-.

Time growth equation of trees. Blonay, H. de. Laus. S. Vd. Bll. 28 (1892) 207-.

- - of vegetation in forests, interpolation applied to. Regneault, $E$. E. Nancy Mm. S. Sc. (1838) 169-.

- observations, interpolation. Thiele, $T . N$. Leip. As. Gs. Vjschr. 22 (1887) 302-.

Trigonometrical formulæ of interpolation. Fouret, G. C. R. 99 (1884) 963-, 1011-, 1044, 1062-, 1140-

Trigonometry, finite differences in. Cagnoli, A. Mod. Mm. S. It. 8 (1799) 21 1 -

Value of $\Delta^{n} 0^{m} / n^{m}$ where $m$ and $n$ are very large. Tait, P. G. Edinb. Mth. S. P. 5 (1887) 83-.

Values of $\Delta^{n} 0^{m}$, tables. Herschel, J. lattributed to Lacroix in (III)]. N. A. Mth. 13 (1854) 272-.

Variations, finite, analytical. Lorgna, $A . M$. Verona S. It. Mm. 4 (1788) 156-

\section{Linear Substitutions.}

\section{General.}

Distributive operations and homography. Pincherle, S. Mil. I. Lomb. Rd. 29 (1896) 397-.

Extraction of roots by substitutions. Levänen, S. Helsingf. Öfv. 34 (1892) 28-; Fschr. Mth. (1892) 135.

Formulæ given by Eisenstein and Hesse, note on two. Cayley, A. Crelle J. 29 (1845) $54-$.

Integral functions of a system of $m n$ variables which form $m$ rows and $n$ columns. Mertens, F. Krk. Ak. (Mt.-Prz.) Pam. 17 (1890) 143-; Fschr. Mth. (1890) 186-.

Linear substitutions. Stéphanos, C. Liouv. J. Mth. 6 (1900) 73-. 


\section{Determinants}

Linear substitutions applied to theory of groups. Laurent, H. N. A. Mth. 16 (1897) 149-.

- -, reduction. Jordan, C. Par. Éc. Pol. J. 29 (=Cah. 48) (1880) 151-.

- transformations of quadratic form. Boole, G. Camb. Mth. J. 4 (1845) 167-.

- - general theory. Boole, G. Camb. Mth. J. 3 (1843) 1-, 106-.

- - points in theory. Philippot, I. H. Liège S. Sc. Mm. 20 (1898) No. 9, 14 pp.

Rational reversible substitutions. Kraus, $L$. Prag Sb. (1882) 338-; (1883) (Mth.-Vortr.) 187-.

Representation of binary homographies by points of space, with application to spherical rotations. Stéphanos, C. Mth. A. 22 (1883) 299-.

Salmon's "Higher Algebra." Bellavitis, $G$. Ven. Mm. I. 9 (1860) 237-.

Space, division by linear transformation groups. Dyck, $W$. Leip. Mth. Ps. B. 35 (1883) 61-.

Theorem on two contrary substitutions. Lipschitz, R. O. S. Am. J. Mth. 1 (1878) 336-. - - - Sylvester, J. J. Am. J. Mth. 1 (1878) 341-.

Umbral notation. Harley, (Rev.) R. B. A. Rp. (1887) 600-.

\section{Determinants.}

Adjoint minors. Landsberg, G. Crelle J. Mth. 109 (1892) 225-.

Adjugate determinant. Muir, T. [1894] Edinb. R. S. P. 20 (1895) 323-.

Algebraic determinant, a special. Weihrauch, K. Z. Mth. Ps. 36 (1891) 34-.

- determinants, reduction. Russell, W. H. L. B. A. Rp. (1885) 910-.

- expressions unaltered by certain substitutions. Glaisher, J. W. L. [1880] Mess. Mth. 10 (1881) 60-.

- reversibility, new theorem. André, D. Par. S. Mth. Bll. 24 (1896) 136-.

Alternant of even number of letters, Jacobi's form. Muir, T. [1900] Edinb. R. S. P. 23 (1902) 133-.

-, multiplication by symmetric function. Muir, T. [1899] Edinb. R. S. P. 22 (1900) 539 .

Alternants. Muir, T. [1879] Edinb. R. S. P. 10 (1880) 102-.

一. Starkov, A. N. Rs. S. Nt. Mm. (Mth.) 10 (1889) 143-.

-. Muth, P. Crelle J. Mth. 122 (1900) 89-.

-, a class of. Loria, G. Prag Sb. (1897) (Mth.-Nt.) No. 57, $13 \mathrm{pp}$.

-, constant multiples of the difference product of the variables. Anglin, - . [1888] Edinb. R. S. P. 15 (1889) 468-.

- and elliptic functions, identities connected with. Muir, T. [1900] Edinb. R. S. T. 40 (1905) 187-.

-, history. Muir, T. [1900] Edinb. R. S. P. 23 (1902) 93-.

-, summation of certain series. Anglin, A. H. [1887] Edińb. R. S. P. 14 (1888) 194 -
Determinants 2010

Alternants, theorems connected with. Anglin, A. H. [1886-88] Edinb. R. S. P. 13 (1886) 823-; 15 (1889) 381-.

- of the 3rd order, a formula of reduction for. Johnson, $W . W$. Am. J. Mth. 7 (1885) 347-.

- - - - - geometrical representation. Johnson, W. W. QJ. Mth. 21 (1886) 217-.

- - - 4th order, calculation of co-factors. Johnson, $W . W$. Am. J. Mth. 7 (1885) 380-.

Alternating functions. Cauchy, A. L. [1812] Par. Éc. Pol. J. 10 (1815) (17' cah.) 29-.

- -. Jacobi, C. G. J. Crelle J. 22 (1841) 360 .

- - and applications. Cauchy, A. L. C. R. 10 (1840) 178-; 13 (1841) 939-.

- -, a class of. Muir, T. [1887] Edinb. R. S. T. 33 (1888) 309-.

- - of $n$ variables. Scott, R. F. [1881] Mess. Mth. 11 (1882) 98-.

- , reduction to alternants. Johnson, $W . W$. Am. J. Mth. 7 (1885) 345-.

- - used in elimination. Cauchy, A. L. C. R. 12 (1841) 414-.

- sums. Cauchy, A. L. C. R. 13 (1841) 939-.

Arithmetical determinant, value of certain. Smith, H. J.S. L. Mth. S. P. $7(1875-76)$ 208-.

- determinants, certain. Cesáro, E. G. Mt. 23 (1885) 182-, 367; Rm. R. Ac. Linc. Rd. 1 (1885) 709-, 711-.

- - of higher dimension. Gegenbauer, $L$. Wien Ak. Sb. 101 (1892) (Ab. 2a) 425-.

Bernoullian number expressed as a combinational determinant. Dupuis, N. F. Cn. R. S. P. \& T. 7 (1890) (Sect. 3) 20-.

Bernoulli's and Euler's numbers, representation by determinants. Sachse, A. Arch. Mth. Ps. 68 (1882) 427-.

Bibliography. Muir, T. QJ. Mth. 18 (1882) 110-; 21 (1886) 299-.

Binary determinants. Brioschi, F. Crelle J. 52 (1856) 133-.

Bipartite functions. Muir, T. Edinb. R. S. T. 32 (1887) 461-.

- - and determinants, new relations between. Muir, T. L. Mth. S. P. 16 (1884-85) 276-.

Bordered determinants. Le Paige, C. M. $M$. $H$. H. Par. S. Mth. Bll. 8 (1880) 128-.

- - Arnaldi, M. G. Mt. 34 (1896) 209-.

- skew determinant. Muir, T. Edinb. R. S. P. 21 (1897) 342-.

- symmetric determinant, Cayley's theorem. Muir, T. QJ. Mth. 18 (1882) 46-.

Cauchy's determinant and aleph functions. Crocchi, L. [1878] G. Mt. 17 (1879) 218-, 380.

Circle touching three circles on a sphere. Trzaska, W. Par. T. Nauk. Śc. Pam. 1 (*1871) 123-; Bll. Sc. Mth. As. 6 (*1874) $153-$.

Circulant determinants. Torelli, G. Nap. Rd. 21 (1882) 83-.

Circulants. Studnička, F. J. Mh. Mth. Ps. 10 (1899) 193-.

-, detached theorems on. Muir, T. Edinb. R. S. T. 32 (1887) 639-. 


\section{Determinants}

Circulants, final expansion. Muir, T. Mess. Mth. 14 (1885) 169.

- of odd order. Muir, T. QJ. Mth، 18 (1882) 261-.

-, resolution. Muir, T. Edinb. R. S. P. 21 (1897) 369-.

Co-axial minors of determinant of the fourth order. Muir, T. Edinb. R. S. T. 39 (1900) $323-$

- _ - relations between. Nanson, E. $J$. Ph. Mg. 44 (1897) 362-.

- - expressibility of determinant in terms of its. Muir, T. Ph. Mg. 38 (1894) 537-.

Combination of determinants, theorem. Sylvester, J. J. Camb. and Dubl. Mth. J. 8 (1853) 60-.

Combinations applied to theory of determinants. Picquet, H. Par. Ec. Pol. J. 28 (=Cah. 45) (1878) 201-.

Combinatory analysis of determinants. Deruyts, $J$. Liège S. Sc. Mm. 11 (1885) No. 13, 11 pp. - determinants. Zmurko, L. [1866] Wien D. 27 (1867) (2 $\left.2^{\text {to }} A b.\right) 63-$.

Commutants, property. Cayley, A. Ph. Mg. 30 (1865) 411-.

Composite determinants. Sylvester, J.J. Crelle J. Mth. 88 (1880) 49-.

- _, Sylvester's memoir, remark. Borchardt, C. $W$. Crelle J. Mth. 89 (1880) 82-.

Composition of determinants and bilinear forms, a mode of. Stéphanos, C. G. Mt. 36 (1898) 376-.

- - systems of $n^{2}$ magnitudes with themselves. Kronecker, L. Berl. Ak. Sb. (1890) 1081-.

Compound determinants. Scott, R. F. [1881] Mess. Mth. 11 (1882) 96-.

- - Van Velzer, C. A. (хп) [1881] J. H. Un. Cir. [1] (1882) 132.

- - Scott, R. F. [1882] L. Mth. S. P. 11 (1882-83) 91-.

- -. Van Velzer, C. A. Am. J. Mth. 6 (1884) 164-.

- -. Metzler, W. H. Am. J. Mth. 16 (1894) 131-; 20 (1898) 253-.

- - theorems on a special kind. Hunyady, J. (xII) Mag. Tud. Ak. Étk. (Mth.) 7 (1881) (No. 21) $15 \mathrm{pp}$.

Concomitant determinants, certain. Russell, J. W. L. Mth. S. P. 28 (1897) 430-.

Constituents, elements, and minors of determinant, number of. Cunningham, $A$. Sc. 4 (1874) 212-.

Continuant, condensation of. Muir, T. Edinb. Mth. S. P. 2 (1884) 16-.

-, development. Mollame, $V$. Rv. Mt. 3 (1893) 47-.

- , the discriminant of a special quadratic form. Cesáro, $E$. Mathesis 12 (1892) 5-.

Continuants. Muir, T. [1874] Edinb. R. S. P. 8 (1875) 229-, 380-.

-, note. Sylvester, J.J. Mess. Mth. 8 (1879) 187-.

-, properties. Studnička, F. J. Prag Sb. (1886) (Mth.-Nt.) 3-.

- roots of certain. Segar, $H, W$, Mess. Mth. 22 (1893) 171-.
Determinants 2010

Continuants, theorem. Muir, T. Ph. M3. 3 (1877) 137-, 360-.

Convergence of infinite determinants. Koch, H. von [1896] Stockh. Ak. Hndl. Bh. 22 (Afd. 1) (1897) No. 4, $31 \mathrm{pp.}$

Coordinates, eurvilinear. Combescure, $E^{\prime}$. Par. E. Norm. A. 4 (1867) 93-.

Cramer's determinants. Cantor, M. N. A. Mth. 14 (1855) 113-.

- - or algebraic resultants. Le Cointe, J. L. A. (VIII) A. Mt. 4 (1861) 233-.

Cubic determinants. Armenante, A. [1866] G. Mt. 6 (1868) 175-.

- -. Padova, E. G. Mt. 6 (1368) 182-.

- -, forms of. Scott, K. F. [1881] L. Iith. S. P. 13 (1881-82) 33-.

- - of intinite order. Cazzaniga, T. Mth. A. $53(1900) 272-$.

- - and others of higher class, and determinants of alternate numbers. Scott, $R$. F. [1879] L. Mth. S. P. $11(1879-80)$ 17-.

- - theory. Sziits, M. Mth. Termt. Éts. 8 (1890) 220-; Mth. Nt. B. Ung. 8 (1891) 199 -

Decomposition of systems of $n^{2}$ magnitudes, and application to invariants. Kronecker, $L$. Berl. Ak. Sb. (1889) 479-, 603-.

Deduction of certain determinants from others. Segar, $H$. W. Mess. Mth. 22 (1893) 57 -.

Determinant, a. Stern, M. A. Crelle J. 73 (1871) 374-.

- - Glaisher, J.W. L: As. Fr. C. R. 6 (1877) 177-.

-, -. Minozzi, A. G. Mt. 16 (1878) 118-.

- analysis. Cauchy, A. L. C. R. 42 (1856) 366-.

- of any degree, development. Cadenat, $A$. As. Fr. C. R. (1900) (Pt. 2) 241-.

-, application of a. Zehfuss, G. Schlömilch Z. 7 (1862) 439-.

-, - - Cherriman, J. B. (xII) [1882] Cn. R. S. P. \& T. 1 (1883) (Sect. 3) 13-.

—,-——. Legoux, A. QJ. Mth. 19 (1883) 41 -.

- with binomial elements, development. $A l$ beggiani, $M$. G. Mt. 10 (1872) 279-.

- calculation of a. Lemonnier, H. N. A. Mth. 18 (1879) 518-

-, a certain. Zehfuss, G. Schlömilch Z. 3 (1858) 298-.

-, - - Gegenbauer, L. Wien Ak. Sb. 96 (1888) (Ab. 2) 489-.

-, - - Laisant, C. A. Par. S. Mth. Bll. 17 (1889) 104-.

-, - - Bourlet, G. N. A. Mth. 16 (1897) $369-$.

,$--\frac{-}{-}$, theorem on. Mansion, P. Brux. Ac. Bll. 46 (1878) 892-.

development of a. Brioschi, F. [1857] Tortolini A. 1 (1858) 9-.

-, - - Humbert, $E$. N. A. Mth. 4 (1885) 289-.

- - - - particular. Caldarera, $G$. Catania Ac. Gioen. At. 7 (1894) Mm. 8, 15 pp. 


\section{Determinants}

Determinant, evaluation of a certain. Cayley, A. QJ. Mth. 2 (1358) 163-.

-, - - - Dostor, G. Arch. Mth. Ps. 64 (1879) 57-.

10 (1900) 2-.

- - - - Crawford, L. Edinb. Mth. S. P. 18 (1900) 25-

- expressions for Laplace's coefficients, Bernoulli's and Euler's numbers, etc. Glaisher, J.W.L. [1876-79] Mess. Mth. 6 (1877) 49-; 7 (1878) 160-; 8 (1879) 158-.

- - the sum of a harmonical progression. Muir, T. [1877] Edinb. R. S. P. 9 (1878) $361-$.

- formed by bordering product of two determinants. Muir, T. Mess. Mth. 11 (1882) 161-.

- forms and their applications. Echols, W. H. A. Mth. 6 (1891-92) 105-; 7 (1892-93) 11-, 93-, 109-.

- of four points in plane, with respect to a 5th. Laisant, C. A. Lisb. J. Sc. Mth. 5 (1898) 205-.

-, geometrical and statical interpretation of a. Walton, W. QJ. Mth. 4 (1861) 252-.

- identities in study of perspective triangles. Caspary, F. [1881] Crelle J. Mth. 95 (1883) 36-.

-, maximum value of a certain. Davis, E. W. (xII) [1882] J. H. Un. Cir. [2] (1883) 22.

- minors, certain aggregates of. Muir, $T$. [1900] Edinb. R. S. P. 23 (1902) 142-.

-, new development. Shaw, J. B. [1900] N. Y. Am. Mth. S. Bll. 7 (1901) 244, 256-.

- notations and eváluation of a special determinant. Moore, E. H. A. Mth. 1 (1900) 177-.

- occurring in calculation of symmetric functions. Gubler, E. Zür. Vjschr. 35 (1890) 79-.

- with polynomial elements, development. Albeggiani, M. [1874] G. Mt. 13 (1875) 1-.

-, properties of. Wisselink, D.B. N. Arch. Wisk. 3 (*1877) 84-.

-, property of a. Sardi, C. G. Mt. 2 (1864) 376-.

- of sines and cosines. Scott, R. F. Mess. Mth. 10 (1381) 142-.

- Smith and Mansion. Cesáro, E. Par. Ec. Norm. A. 2 (1885) 425-.

-, special form. Glaisher, J.W. L. [1878] QJ. Mth. 16 (1879) 15-.

- of system compounded of two others, representation. Hensel, $K$. Acta Mth. 14 (189091) 317-.

- - - of functions. Bertrand, J. Liouv. J. Mth. 16 (1851) 212-.

-, vanishing. Heawood, P.J. Mth. Gz. 1 (1900) 344.

- with vanishing diagonal, Baltzer on number of terms. Monro, C. J. [1872] Mess. Mth. 2 (1873) 38-.

- $\left|a_{r s}\right|$, number of terms when $a_{r r}=0$. Hansted, B. G. Teix. J. Sc. 2 (1880) 154-.

- of 4 th order, unique property of an axisymmetric. Muir, T. A. Mth. 1 (188485) 31-.

VOL. I.

\section{Determinants 2010}

Determinant of 6th order, peculiar. Muir, $T$. Ph. Mg. 31 (1891) 429-.

- - - - - - development of a special. Muir, T. Mass. Mth. 13 (1884) 95-.

- mnth order, development. Muir, T. Edinb. R. S. T. 39 (1900) 623-.

Determinantal form of highest common factor. Scheibner, $W$. Leip. Mth. Ps. B. 40 (1888) 1 -.

Determinants. Jacobi, C. G. J. Crelle J. 22 (1841) 285-.

- Catalan, E. C. Brux. Ac. Bll. 13 (1846) 534-.

- Bazin, -. Liouv. J. Mth. 16 (1851) 145 -

-. Terquem, O. N. A. Mth. 10 (1851) 124-.

-. Bazin, -. Liouv. J. Mth. 19 (1854) 209-.

- Newman, F. W. R. S. P. 8 (1856-57) 426-.

-. Oliver, J. E. Camb. (M.) Mth. M. 3 (1860) 86-.

-. Battaglini, G. Nap. Rd. 1 (1862) 101-.

- Tait, P. G. Mess. Mth. 1 (1862) 25-.

- Horner, J. [1865] QJ. Mth. 8 (1867) 157-.

- Battaglini, G. G. Mt. 9 (1871) 136-.

- Baltzer, R. (Ix) Leip. Mth. Ps. B. 25 (1873) 523-.

-. Scott, R. F. Mess. Mth. 8 (1879) 182-; 12 (1883) 105-.

- Lindelöf, L. L. Helsingf. Öfv. 22 (1880) 123-.

-. Young, T. E. I. Act. J. 26 (1887) 149-.

- Gegenbauer, $L$. Wien Ak. Sb. 96 (1888) (Ab. 2) 5-; 97 (1889) (Ab. 2a) 154-.

- Ponte Horta, F. da. [1890] Lisb. J. Sc. Mth. 2 (1892) 67-.

-. Campbell, J. E. [1892] L. M h. S. P. 24 (1893) 67-.

-. Schendel, L. Z. Mth. Ps. 38 (1893) 84-. - of alternate numbers. Spottiswoode, $W$. L. Mth. S. P. 7 (1875-76) 100-.

- in analytical mechanics. Cohen, A. Phil. Trans. (1862) 469-.

-, application. Jamet, $V$. N. A. Mth. 16 (1877) 372-.

-, - to contact of circles and spheres. Bauer, C. $W$. N. A. Mth. 19 (1860) 440-

- , - - curves of 4 th order, etc. Hesse, L. O. [1853] Crelle J. 49 (1855) 243-.

-, - - moments. Durrande, H. N. A. Mth. 12 (1873) 265-.

-, -, note. Gerono, G. C. N. A. Mth. 9 (1870) 392-.

-, applications. Rubini, R. Tortolini A. 8: (1857) 179-.

- - Neuberg, J. J. B. (xп) Mathesis 3 (1383) 29-.

- , - to algebra and geometry. Versluys, $J$. Arch. Mth. Ps. 50 (1869) 157-, 210-; 51 (1870) 49-; 53 (1871) 137-.

- - - geometry. Joachimsthal, $F$. Crelle J. 40 (1850) 21-.

J. 77 (1874) 102-.

_- _ - (metrical). Frobenius, G. [1874] Crelle J. Mth. 79 (1875) 185-. 
Determinants, applications to geometry of $n$ dimensions, Rahusen, A. E. Delft Ec. Pol. A. 4 (1888) 104-.

- arising in cyclotomy. Hermes, $J$. Arch. Mth. Ps. 6 (1888) 276-

- of binomial coefficients. Zeipel, E. V. von. Lund Acta Un. 2 (1865) (Mth.) No. 2, 68 pp. ; 8 (1871) (Mth.) No. 3, $36 \mathrm{pp}$.

- - - Günther, S. Z. Mth. Ps. 24 (1879) 96-.

- - Studnicka, F.J. Prag Sb. (1879) , Borchardt's theory. Lerch, M. Časopis 23 (1894) 76-; Fschr. Mth. (1893-94) 213.

-, calculation. Jürgens, $E$. D. Nf. Vh. (1900) (Th. 2, Hälfte 1) 7-.

-, certain. Eugenio, V. G. Mt. 8 (1870) 285-.

- - Glaisher, J. W. L. B. A. Rp. (1876) (Sect.) 13-.

-, 一. Voss, A. E. Mth. A. 13 (1878) 161-.

-, - Escherich, G. von. Mh. Mth. Ps. 3 (1892) 68-.

- of certain class (Grévy's), theorems on. Böttcher, L. E. Krk. Ak. (Mt.-Prz.) Rz. 18 (1901) 382-; Crc. Ac. Sc. Bll. (1900) 227-.

-, class. Zeipel, E. V. von. Stockh. Öfv. 19 (1862) 439-.

-, 一. Dickson, J. D. H. [1878] Edinb. R. S. T. 28 (1879) 625-.

-, -. Loria, G. G. Mt. 26 (1888) 329-.

-, condensation (a method of calculation). Dodgson, C. L. [1866] R. S. P. 15 (1867) 150-.

- in conics. Pasch, M. Crelle J. Mth. 89 (1880) 247-.

- connected with algebraical expressions having same form as component factors. Roberts, S. Mess. Mth. 8 (1879) 138-.

- and continued fractions, convergence. Koch, H. von. C. R. 120 (1895) 144-.

- of co-variants. Scott, R. F. Mess. Mth. 10 (1881) 142-.

- derived from differential coefficients, new species. Studnička, F.J. Mh. Mth. Ps. 10 (1899) 338-.

- of determinants. Ovidio, E. d'. [1876-90] Tor. Ac. Sc. At. 11 (1875-76) 949-; 12 (1876) 331-; 26 (1891) 131-.

-, development, new method, and application to finding resultant of any two equations. Bonolis, A. [1882] G. Mt. 21 (1883) 336-.

-, developments. Bonolis, A. [1876] G. Mt. 15 (1877) 113-.

-, -. Capelli, A. Nap. Rd. 28 (1889) 58-.

- of differences. Raimondi, R. G. Mt. 26 (1888) 185-.

-, divisors. Frobenius, G. Berl. Ak. Sb. (1894) 31-.

- and duadic disynthemes, note. Sylvester, J.J. Am. J. Mth. 2 (1879) 89-, 214-.

-, elementary divisors of, application to linear differential equations. Sauvage, $L$. Mars. Fac. Sc. A. 6 (1897) Fasc. 5, 9 pp.

- - treatment. Hesse, L. O. G. Mt. 10 (1872) 217-, 325-.
Determinants whose elements are composed of elements of adjoint series, theorems. Hunyady, J. (xIr) Mag. Tud. Ak. Etk. (Mth.) 7 (1881) (No. 19) 27 pp.

- - - rational fractions. Scott, R. F. Mess. Mth. 11 (1882) 165-.

- - - - squares of lines joining vertices of two tetrahedrons. Siebeck, $H$. Crelle J. 62 (1863) 151-.

- - - sums of powers. Studnička, F.J. Prag České Ak. Fr. Jos. Rz. (Třída 2) 9 (1900) Art. 4, 8 pp.; Fschr. Mth. (1900) 156.

- of even order. Brioschi, F. Crelle J. 52 (1856) 133-.

- - figurate numbers. Caldarera, $F$. G. Mt. 9 (1871) 223-.

- - form

$$
Q p=\Sigma \pm\left(a_{0}+b_{0}\right)^{p}\left(a_{1}+b_{1}\right)^{p} \ldots\left(a_{n}+b_{n}\right)^{p} .
$$

Zehfuss, G. Schlömilch Z. 4 (1859) 232-.

- formed of elements with any number of indices. Garbieri, G. $[1876]$ G. Mt. 15 (1877) 89-.

- - from the same elements, relations between. Pascal, $E$. Mil. I. Lomb. Rd. 29 (1896) 436-.

- - - $n^{2}$ elements. Bagnera, $G$. G. Mt. 25 (1887) 228-.

-, formula in. Pincherle, S. Bologna Rd. (1883) 105-.

- - - Mertens, $F$. Wien Ak. Sb. 91 (1885) (Ab. 2) 622-.

- - - Nielsen, $N$. N. Ts. Mth. 7 (B) (1896) 59-; Fschr. Mth. (1896) 111.

- - - Schulze, E. Z. Mth. Ps. 42 (1897) 313-. (1898) 15-.

- - - Schulze, E. Z. Mth. Ps. 44 (1899) $167-$.

—, formulæ. Rubini, R. Nap. Rd. 5 (1866) 109-.

-, - Starkov, A. P. Kazan S. Nt. (Ps.Mth.) P. 3 (1885) 75-.

- and functions, fractional. Dietrich, $M$. Crelle J. 69 (1868) 190-

- of functions and their differentials. Pasch, M. Crelle J. Mth. 80 (1875) 177-.

-, a general property of two. Kretkowski, $W$. (xII) [1882] Krk. Ak. (Mt.-Prz.) Pam. 9 (1884) 45-; Fschr. Mth. (1884) 122-.

-, generalisation of a theorem. Marcolongo, $R$. G. Mt. 25 (1887) 298 -

-, geometrical application. White, H. S. A. Mth. 1 (1900) 103-.

-, - interpretation. [Barré de] Saint-Venant, -. C. R. 36 (1853) 582-.

-, - significance of certain. Bauer, $G$. Münch. Sb. (1872) 345-.

-, - treatment. Waelsch, E. Mh. Mth. Ps. 9 (1898) 207-.

- of higher dimensions. Gegenbauer, $L$. [1880-90] Wien Ak. D. 43 (1882) (Ab. 2) $17-; 46$ (1883) (Ab. 2) 291-; 49 (1885) (Ab. 2) 225-; 50 (1885) (Ab. 1) 145-; 57 (1890) 735-. 


\section{Determinants}

Determinants of higher dimensions and application to formation of invariants. Escherich, G. von. [1880] Wien Ak. D. 43 (1882) (Ab. 2) 1-.

-, history and theory. Günther, -. D. Nf. Tbl. (* 1874) 78-.

-, identical relation between certain. Painlevé, P. Par. S. Mth. Bll. 22 (1894) 116-.

- involving powers of circles. Johnson, $W . W$. J. H. Un. Cir. [10 (1890-91)] 46.

- whose leading elements are in A.P. Cazzaniga, T. Mil. I. Lomb. Rd. 29 (1896) $541-$.

- with magic matrix. Caldarera, F. Palermo G. Sc. Nt. 1 (1866) 173-.

- of a matrix, relations between. Vahlen, K. T. Crelle J. Mth. 112 (1893) 306-.

,$-----\ldots$ Ahrens, W. Z. Mth. Ps. 42 (1897) 65-.

- maximum value. Scarpis, U. Mil. I. Lomb. Rd. 31 (1898) 1441-.

-, method of solving. Macloskie, G. A. Mth. 1 (1900) 74.

- of minor determinants. Franke, E. [1862] Crelle J. 61 (1863) 350-.

- and minors of original and adjoint systems. Studnička, F. J. Časopis 1 (*1872) 6-; Fschr. Mth. (1872) 59.

- - modern algebra in lower mathematics. Prange, A. J. A. N. Arch. Wisk. 17 (1890) 158-; Fschr. Mth. (1890) 181-.

-, multiplication. Gomes Teixeira, $F$. G. Teix. J. Sc. 3 (1881) 185-.

- - Loria, G. G. Teix. J. Sc. 7 (1886) 101-.

-, -. Cazzaniga, T. A. Mt. 2 (1899) 229 -

-, -, application. Souillart, C. N. A. Mth. 19 (1860) 320-.

-, and multiplication of complex quantities. Palmstrøm, A. Arch. Mth. Ntvd. 13 (1890) 128-; Fschr. Mth. (1890) 409.

- multiplication, elementary proof of theorem. Falk, M. B. A. Rp. (1878) 473-.

-, - formula, verification. Weyr, E. Prag Sb. (1880) 55-.

-, - of minors. Zančevskij, I. M. [1894] Rec. Mth. (Moscou) 17 (1395) 587-; Fschr. Mth. (1893-94) 217.

$-;-1 n-1)$ th power of a symmetric determinant by 2 nd power of another. Muir, $T$. Am. J. Mth. 4 (1881) 273-.

-, - rule. Le Paige, C. M. M. H. H. Par. S. Mth. Bll. 9 (1881) 67-.

,$--\ldots$, geometric application. Mertens, $F$. Prag Sb. (1880) 207-.

-, - theorem, proof. König, J. Mth. A. 14 (1879) 507-.

一, - 一, - Falk, M. Gött. Nr. (1885) 181-.

- - - - of two of $n$th degree. Hoza, F. Arch. Mth. Ps. 59 (1376) 403-.

- - of two of same degree. Presle, A. de. Par. S. Mth. Bll. 14 (1886) 157-.

- of $n$ dimensions. Tanner, H. W. L. L. Mth. S. P. $10(1878-79)$ 167-.

\section{Determinants 2010}

Determinants, new formula, with reference to theory of linear differential equations. Anissimoff, W. Mth. A. 51 (1899) 388-.

-, numerical calculation of class, and continued fractions. Dickson, J. D. H. L. Mth. S. P. 10 (1878-79) 226-.

- of $p$ dimensions, theory. Zajaczkowski, $W$. (xII) [1880] Krk. Ak. (Mt.-Prz.) Pam. 6 (1881) (1 ter)-.

- with polynomial elements. Muir, T. Mess. Mth. 13 (1884) 135-.

- of powers. Mitchell, O. H. Am. J. Mth. 4 (1881) 341-.

-, powers of. Weltzien, C. Mth. A. 50 (1898) 282-.

-, product of two. Hunyady, E. von. Z. Mth. Ps. 11 (1866) 359-.

- - - - Weltzien, C. Mth. A. 42 (1893) $598-$

-, proof of formula due to Cauchy. Echols, W. $H$. A. Mth. $8(1893-94)$ 21-.

- , - - geometric theorem by. Mansion, $P$. [1875] Mess. Mth. 5 (1876) 158-

, properties. Dostor, G. (vII) Arch. Mth. Ps. 56 (1374) 238-.

-, property of certain. Burnside, $W$. Mess. Mth. 23 (1894) 112-.

-, - deduced from substitution theory. Scarpis, U. G. Mt. 37 (1899) 73-.

-, -, new. Studnička, F. J. Prag Sb. (1980) 50-.

a proposition in theory of. Drude, $P$. Gött. Nr. (1887) 118-.

-, - - - - Baltzer, R. Gött. Nr. (1887) 389-.

, - - Voss, A. Münch. Ak. Sb. 19 (1890) 329-.

- of quadratic forms. Brioschi, F. N. A. Mth. 11 (1852) 307-.

-, rational proof of first principles. Laquière, $E$. $M$. As. Fr. C. R. (1381) 226-.

- of a rectangular matrix. Pascal, E. A. Mt. 24 (1896) 241-.

-, relation between two. $\operatorname{Del} R e, A$. G. Mt. 19 (1881) 116-

-, relations between certain. Deruyts, $J$. Brux. Ac. Bll. 23 (1392) 507-

- of several functions of one variable. Frobenius, G. (vII) [1873] Crelle J. 77 (1874) 245-.

- - sines and cosines, method of evaluation. Scott, R. F. Mess. Mth. 8 (1379) 155-.

- - Smith. Mansion, P. (xIr) Brux. S. Sc. A. 2 (1878) (Pt. 2) 211-.

-, solution of question relating to. Hadamard, J. Bll. Sc. Mth. 17 (1893) 240

-, some forms. Lerch, M. Prag České Ak. Fr. Jos. Rz. (Trída 2) 8 (1899) Art. 12, 16 pp.; Fschr. Mth. (1899) 151-.

- - hitherto unproved theorems. Muir, $T$. Edinb. R. S. P. 18 (1892) 73-.

-, whose terms are distances of point from 3 points. Cayley, A. QJ. Mth. 5 (1862) 381 -

- _ - - - squared distances of points.

Cayley, A. QJ. Mth. 3 (1860) 275-.

-, theorem. Mirza-Nizam, -. N. A. Mth. 4 (1865) 500-. 


\section{Determinants}

Determinants, theorem. Stern, M. A. [1865] Crelle J. 66 (1866) 285-.

-, - Hesse, L. O. Crelle J. 69 (1868) 319-.

-, -. Sardi, C. G. Mt. 6 (1868) 357-.

-, -. Gundelfinger, S. Z. Mth. Ps. 18 (1873) 312-.

-, -. Cayley, A. QJ. Mth. 15 (1878) 55-.

(1879) 140-.

- -. Faà de Bruno, F. Am. J. Mth. 3 (1880) 154-.

- - Daublebsky von Sterneck, R. Mh. Mth. Ps. 6 (1895) 205-.

- - Metzler, W. H. Am. J. Mth. 20 (1898) 273-

-, 一. Lovett, E. O. A. Mth. 12 (1898-99) $161-$.

-, - Kantor, S. Gött. Nr. (1899) 272-.

- - and applications. Siacci, $F$. Tor. At. Ac. Sc. 7 (1871-72) 772-

-, - - its converse. Puchta, A. [1877]

Wien Ak. D. 38 (1878) (Ab. 2) 215-.

- - , fundamental. Sylvester, J. J. $\mathrm{Ph}$. Mg. 2 (1851) 142-

-, - - Becker, J. C. Z. Mth. Ps. 16 (1871) 526-

- - of Glaisher's. Scott, R. F. QJ. Mth. 17 (1881) 129-.

- - - Jacobi's. Segar, H. W. Mess. Mth. 21 (1892) 148-.

-, - - Netto relating to. Pascal, $\boldsymbol{E} . \quad \mathrm{Rm}$. R. Ac. Linc. Rd. 5 (1896) (Sem. 1) 188-.

-, 一, new. Studnička, F.J. Prag Sb. (1873) 342-; Časopis 9 ( $\left.^{*} 1880\right)$ 97-; Fschr. Mth. (1880) 114

-, - - - Puchta, A. [1881] Wien Ak. D. 44 (1882) (Ab. 2) 277-.

-, - of Sylvester's, proof. Igel, B. Mh. Mth. Ps. 9 (1898) 47-.

-, theorems. Cauchy, A. L. C. R. 21 (1845) 407-.

-, 一. Mogni, A. G. Mt. 3 (1865) 10-

-, - Günther, S. Erlang. Sb. Ps. Md. S. 5 (1873) 88-.

-, 一. Scott, R. F. [1878] Mess. Mth. 8 (1879) 33-.

-, 一. Studnička, F. J. Prag Sb. (1896) (Mth.-Nt.) No. 6, 5 pp.

-, - and application to proofs of Pascal's and Brianchon's theorems. Mertens, $F$. Crelle J. Mth. 84 (1878) 355-

- - - certain. Nanson, -. Crelle J. Mth. $122(1900)-179-$.

-, - elementary. Spottiswoode, W. [1853]

Crelle J. 51 (1856) 209-, 328-.

-, 一, 一. Ferrers, N. M. QJ. Mth. 1 (1857) 364.

-, 一, general. Muir, T. [1879] Edinb. R. S. T. 29 (1880) $47-$.

,-- , homoloidal law, theory of orders, etc. Sylvester, J.J. Ph. Mg. 37 (1850) 363-.

-, - of Mainardi. Bellavitis, G. Ven. At. (1857-58) 623-; (1858-59) 334-.

-, -, new. Studnička, F.J. Časopis 1 (* 1872) 203-.

-, $\frac{-}{142}$, proofs. Janni, $V$. G. Mt. 12 (1874)

\section{Determinants 2010}

Determinants, theorems, two. Zehfuss, $G$. Schlömilch Z. 7 (1862) 436-

-, -, -. Ovidio, E. d'. G. Mt. 1 (1863) 135-. (1884) 180-

-, -, -. Netto, E. Acta Mth. 17 (1893) 199-.

-, theory. Cayley, A. [1843] Camb. Ph. S. T. 8 (1849) 50-

-, -. Bellavitis, G. Ven. Mm. I. 7 (1857) $67-$.

-, -. Cayley, A. Ph. Mg. 21 (1861) 180 .

-, -. Dietrich, M. Arch. Mth. Ps. 44 (1865) $344-$.

(1870) 152-.

-, - Veltmann, W. Z. Mth. Ps. 16 (1871) $516-$

-, - Weyrauch, J. J. [1871] Crelle J. 74 (1872) 273-.

-, -. Studnička, F. J. Prag Sb. (1872) 78-; Časopis 2 ( $\left.{ }^{*} 1873\right)$ 282-; Fschr. Mth. (*1873) 90 .

-, -. Weihrauch, K. Z. Mth. Ps. 19 (1874) $354-$.

-, - Studnička, F. J. Prag Sb. (1877) 120 .

-, 一. Fürstenau, E. [1879] Crelle J. Mth. 89 (1880) 86-.

-, - Igel, B. [1880] Wien Ak. Sb. 82 (1881) (Ab. 2) 1288-

- - Landré, C. L. N. Arch. Wisk. 6 (*1880) 208-.

- - Rados, G. Mth. Termt. Ets. 4 (1886) 268-; Mth. Nt. B. Ung. 8 (1891) 60 -

-, - Szüts, N. von. Mth. A. 33 (1889) 477-.

-, - Igel, B, Mh. Mth. Ps. 3 (1892) 55-.

-, 一. Miller, E. [1892] Kan. Un. Q. 1 (1893) 133-.

-, -. Studnicka, F. J. Časopis 25 (1896) 241-; Fschr. Mth. (1896) 108; Mh. Mth. Ps. 10 (1899) 1-.

-, -, analytical. Schering, E. Gött. Ab. 22 (1877) (Mth.) 41 pp.; C. R. 86 (1878) 1387-.

-, - , application of modular systems to. Netto, E. Crelle J. Mth. 108 (1891) 144-.

- - - based on solution of groups of general linear equations. Matzka, W. [1877] Prag Ab. 9 (1878) (Mth.) (No. 1) 61 pp.

-, - (chess problem). Serdobinskix, V. $E$. (xii) [1877] Rec. Mth. (Moscou) 10 (188283) (Pt. 1) 74-.

-, -, elementary. Gomes Teixeira, F. G. Teix. J. Sc. 1 (1878) 138-.

-, , elements. Hoppe, R. Arch. Mth. Ps. 65 (1880) 65-.

-, - , in the historical order of its development. Muir, T. [1886-89] Edinb. R. S. P. 13 (1886) 547-; 14 (1888) 452-; 15 (1889) 481-; 16 (1890) 207-, 389-, 748-.

-, - , method of stating. Kneser, A. Dorpat Sb. 9 (1892) 522-. 
Determinants, theory, origin and development. Studnička, F. J. Časopis $5\left({ }^{*} 1876\right)$ 1-, 88-, 193-, 279-.

-, - - third fundamental proposition, new method of deduction. Studnička, F. J. Časopis 17 (1888) 193-; Fschr. Mth. (1888) 143.

— of 3rd order, note. Tait, P. G. [1866] Edinb. R. S. P. 6 (1869) 59-.

- 4th order, development. Guimarães, $R$. As. Fr. C. R. (1897) (Pt. 2) 129-.

- $-3,4$ and 5 rows, rules for their calculation by inspection. Teixeira, J. P. G. Teix. J. Sc. 11 (1892) 88-; Fschr. Mth. (1392) 141.

- with triple indices, product expressed as one ordinary determinant. Gasparis, A. de. Rm. R. Ac. Linc. T. 3 (1379) 44-.

- - - and $m$ indices, theorems. Gasparis, A. de. Nap. Rd. 7 (1968) 118-.

- - vector constituents. Chapman, C. $H$. J. H. Un. Cir. [9 (1889-90)] 77.

Determination of four functions to satisfy a certain equation. Vivanti, G. Palermo Cir. Mt. Rd. 6 (1892) 100-.

Dialytic determinant, evaluation of a certain. Taylor, W. W. [1895] L. Mth. S. P. 27 (1396) 60-.

Discriminating symmetrical determinant. Sylvester, J. J. Crelle J. Mth. 88 (1380) 6-.

Distances between points, equation connecting mutual. Muir, T. Edinb. Mth. S. P. 3 (1885) 34-.

- - , relations. Brioschi, F. N. A. Mth. 14 (1855) 172-.

Double series, two, arising from number of terms in determinants. Dickson, J. D. H. L. Mth. S. P. 10 (1878-79) 120-.

Doubly orthosymmetrical determinants. Weihrauch, K. Z. Mth. Ps. 26 (1381) 64-, 132-.

- skew determinants. Sylvester, J.J. C. R. 89 (1879) 24-.

Equation for max. and min. of $n$-ary quadric. Gravelaar, N. L. W. A. N. Arch. Wisk. 4 (*1878) 113-.

Equations, symmetrical linear, with indeterminate coefficients, solution by determinants. Stockwell, J. N, Gould As. J. 6 (1361) 145-.

Factorisation of characteristic equation of induced substitutions. Rados, G. Mth. Termt. Éts. 17 (1899) 66-; Mth. Nt. B. Ung. 17 (1901) 248-.

Factors of a special form of determinant. Glaisher, J. W. L. QJ. Mth. 15 (1878) 347-.

Formula of Lagrange generalised by Cauchy. Barbier, $\dot{E} . \quad$ C. R. 96 (1383) 1345-.

- Liouville's. Lucas, F. C. R. 70 (1370) 1167-.

- Lucas, connecting $n$ quantities and their sum, proof. Albeggiani, $M$. G. Mt. 13 (1875) 107-.

Formulæ, fundamental, of spherical trigonometry derived from theorem in determinants. Studniłka, F. J. Prag Sb. (1375) 1-.
Formulø relative to polar operations. Capelli, A. G. Mt. 32 (1894) 376-.

Functional determinant, property. Brioschi, $F$. QJ. Mth. 1 (1857) 365-.

- determinants. Jacobi, C. G. J. Crelle J. 22 (1341) 319-.

- Combescure, É. Par. Éc. Norm. A. 4 (1367) 93-.

- Casorati, F. [1874] Mil. I. Lomb. Mm. 13 (1377) 181 -.

- - Faà de Bruno, F. Mth. A. 18 (1881) 280 -

- - Kraus, L. Wien Ak. Sb. 90 (1885) (Ab. 2) 813-.

- Torelli, G. Palermo Cir. Mt. Rd. 7 (1893) 75-.

- -, application. Trzaska, W. Par. T. Nauk Śc. Pam. 1 (*1871) 113-; Bll. Sc. Mth. As. 6 (*1874) 153.

- - elementary proof of theorem. Nanson, E. J. [1877] Mess. Mth. 7 (1878) 120-.

- - and Jacobian matrices. Giordano, $G$. G. Mt. 38 (1900) 210 -.

- -, property. Clebsch, R. F. A. Crelle J. 69 (1868) 355-; 70 (1869) 175-.

- - - - and application to implicit functions. Gilbert, P. [1869] Brux. Ac. Sc. Mm. 38 (1871) $12 \mathrm{pp}$.

- - rational. Mansion, P. [1879] Mess. Mth. 9 (1880) 30-

- - - - generalisation. Mertens, $F$. Krk. Ak. (Mt.-Prz.) Pam. 16 (1889) 60-; Fschr. Mth. (1889) 151-.

- - - -, theorems. Anglin, A. H. [1886] Ir. Ac. P. 4 (1884-88) 645-.

- -, theorem (Hesse). $\dot{Z}$ murko, W. Par. T. Nauk Śc. Pam. 1 ("1871) 89-.

- - - - (Jacobi). Donkin, W. F. Camb. and Dubl. Mth. J. 9 (1854) 161-.

- -, theory. Neumann, C. Mth. A. 1 (1869) 208-.

Functions analogous to determinants. Dahlander, G. R. Stockh. Öfv. 20 (1863) 295-.

Fundamental operations of arithmetic, generalisation. Schapira, H. D. Nf. Tbl. ("1882) 128-.

Geometrical series of 2nd order. Hochheim, A. Magdeb. Nt. Vr. Jbr. u. Ab. (1886) 127-; (1887) 25-.

Goniometrical determinants, some. Weihrauch, K. Z. Mth. Ps. 36 (1891) 71-.

Grassmann's method applied to prove theorems in determinants. Müller, E. Z. Mth. Ps. 44 (1899) 28-.

Harmonic determinant, condition for equal roots. Tarleton, F. A. [1887] Ir. Ac. P. 1 (1889-91) 10-.

Hesse, theorem (Hessian determinant). Gordan, $P$. Erlang. Ps. Md. S. Sb. 8 (1876) 89-.

Hessian. Segre, $C$. Rm. R. Ac. Linc. Rd. 4 (1895) (Sem. 2) 143-.

-, application of exponential polygon. Eddy, H. T. Des Moines Anal. 2 (1875) 104-.

- change of independent variables in a. Nanson, F. J. Mess. Mth. 25 (1896) 139-.

- determinant. Voss, A. Mth. A. 30 (1887) 418-. 


\section{Determinants}

Hessian determinant of Hessian of surface of 3rd order. Bauer, G. Münch. Ak. Ab. 14 (1883) (Ab. 3) 77-.

- determinants, theory. Pasch, M. Crelle J. Mth. 80 (1875) 169-.

Higher algebra, and theorems in determinants. Zeipel, E. V. von. Stockh. Ak. Hndl. 3 (1859-60) 32 pp.

Identities, composition, and relation to determinants. Renshaw, A. Mess. Mth. 4 (1868) 34-.

- in determinants. Hunyady, J. [1882] (xi)

Mag. Tud. Ak. Étk. (Mth.) 9, No. 10 (1882)

19 pp. ; (x) Crelle J. Mth. 94 (1883) 171-.

- - rational functional determinants. Prouhet, E. N. A. Mth. 15 (1856) 86-.

Infinite determinants. Poincaré, $H$. Par. S. Mth. Bll. 14 (1886) 77-.

- - Roberts, E. H. A. Mt. 10 (1895-96) $35-$.

- Cazzaniga, T. A. Mt. 26 (1897) 143-. - applied to functional equations. Koch, H. von. Stockh. Ak. Hndl. Bh. 25 (1900) (Afd. 1) No. 5, 24 pp.

- - - - linear homogeneous differential equations. Koch, H. von. Stockh. Öfv. (1890) 225-, 499-; Fschr. Mth. (1890) 316.

- - convergence. Koch, H. von. [1896] Stockh. Ak. Hndl. Bh. 22 (Afd. 1) (1897) No. 4, $31 \mathrm{pp}$.

- - , theory. Koch, H. von. Stockh. Öfv. (1890) 411-; Fschr. Mth. (1890) 185.

Integral elements, reduction of systems of $n^{2}$. Kronecker, L. Crelle J. Mth. 107 (1891) 135-.

Irregular determinants, formation. Perott, $J$. Crelle J. Mth. 95 (1883) 232-; 96 (1884) 327-.

- - and subtriplicate forms. Mathews, G. B. Mess. Mth. 20 (1891) 70-.

Jacobian of functions, extension of theory. Gomes Teixeira, F. Mh. Mth. Ps. 1 (1890) 481-.

-, property of the. Faì de Bruno, $F$. Am. J. Mth. 3 (1880) 154-.

Jacobians, a proposition relating to. Peano, $G$. G. Mt. 27 (1889) 226-.

Kronecker's linear relation among minors of symmetric determinant. White, $H$. S. N. Y. Am. Mth. S. Bll. 2 (1896) 136-.

Lamé, proof of theorem of. Trzaska, $W$. Par. T. Nauk. Śc. Pam. $10\left({ }^{*} 1878\right)$ Art. 9, 3 pp.

Laplace's theorem for the distribution of a determinant, extension. Netto, E. Crelle J. Mth. 114 (1895) 345-.

Linear forms and determinants. Méray, $C$. Liouv. J. Mth. 10 (1884) 181-.

- -, theorem on system of. Frattini, $G$. Rm. R. Ac. Linc. Rd. 2 (1886) (Sem. 1) 132-.

Magic squares, relation to theory of determinants. Studnićka, F.J. Prag Sb. (1876) 269-.

Maximum modulus which a determinant can have. Hadamard, - C. R. 116 (1893) 1500-.
Determinants 2010

Minor determinants. Janni, G. G. Mt. 1 (1863) 270-.

- - of an adjoint determinant. Hoza, $F$. Arch. Mth. Ps. 59 (1876) 401-.

_ - linearly equivalent quadratic functions, relation. Sylvester, J.J. Ph. Mg. 1 (1851) 295-.

- - - symmetrical determinant, property. Hazzidakis, J. N. [1880] Crelle J. Mth. 91 (1881) 238-.

- - - systems. Kronecker, L. Berl. Ak. Sb. (1882) 821-.

- $-1-$ Mehmke, R. Mth. A. 26 (1886) 209-.

- - linear relations. Runge, $C$. Crelle J. Mth. 93 (1882) 319-.

- - theory. Hoza, F. Arch. Mth. Ps. 59 (1876) 387-.

Minors of a determinant of Hankel, relations between. Cazzaniga, T. Mil. I. Lomb. Rd. 31 (1898) 610-.

- _ - - , relation between. Vaněček, M. N. Prag Sb. (1886) (Mth.-Nt.) 21-.

-, law of extensible. Muir, T. [1881] Edinb. R. S. T. 30 (1883) 1-.

-, principal, of a symmetrical determinant. Schultze, E. Bll. Sc. Mth. As. 10 (1876) 242 .

Multinomial determinant. Segar, H. W. Mess. Mth. 21 (1892) 177-.

Multiple determinants, properties. Deruyts, $J$. Liége S. Sc. Mm. 9 (1882) (No. 7) $11 \mathrm{pp}$.

Multiplication of trigonometric and hyperbolic functions. Trzaska, $W$. Par. T. Nauk Śc. Pam. 10 ( $\left.{ }^{*} 1878\right)$ Art. 8, 7 pp.

Normal determinants, reciprocals of. Cazzaniga, T. Tor. Ac. Sc. Ât. 34 (1898) 351or 495 -

Numerical determinant, calculation. Cazzaniga, T. G. Mt. 36 (1898) 362-.

- -, sign of term, rule. Mainardi, $G$. Mil. At. I. Lomb. 1 (1858) 105-.

$\Omega$, a certain differential, determinantal operator. Vivanti, G. Palermo Cir. Mt. Rd. 4 (1890) 261-.

Orders of conditions that minors of different degree of given matrix may vanish. Segre, $C$. Rm. R. Ac. Linc. Rd. 9 (1900) (Sem. 2) 253-.

Orthogonal substitutions. Jacobi, C. G. J. Crelle J. 15 (1836) 309-.

Orthogonants, property. Loria, G. G. Teix. J. Sc. 7 (1886) 129-.

-, theory. Netto, E. Acta Mth. 19 (1895) 105-.

Orthosymmetric determinants. Brunn, $H$. Z. Mth. Ps. 37 (1892) 291-.

Partial compounds. Nanson, E. J. Mess. Mth. 28 (1899) 17-.

- determinants, theorem of Jacobi, generalisation. Barbier, $E$. C. R. 97 (1883) 82-.

- differentials as determinants. Gubkin, I.S. [1880] Mosc. S. Sc. Bll. 73 (No. 1) (1891) 29 -

Pascal's triangle, certain of whose elements constitute a determinant, properties of latter. Pincherle, S. G. Mt. 19 (1881) 385-. 


\section{Determinants}

Permanents and determinants, relation. Muir, $T$. [1897] Edinb. R. S. P. 22 (1900) 134 .

Persymmetric determinants. Muir, T. [1881] Mess. Mth. 11 (1882) 65-.

Pfaffian having a vacant minor, development. Muir, T. [1900] Edinb. R. S. T. 40 (1905) 49-.

Poisson's determinants. Albeggiani, $M$. $L$. Palermo Cir. Mt. Rd. 1 (1887) 6-.

- - generalised. Albeggiani, M. L. Palermo Cir. Mt. Rd. 1 (1887) 9-, 12-, 314-.

Potential determinants, mutual relations. Jonquières, - de. C. R. 120 (1895) 408-, 580.

"Power" and "combination" determinants. Studnička, F.J. Prag Sb. (1897) (Mth.-Nt.) No. 1, 20 pp., No. 16, 16 pp.

- determinants. Studnička, F. J. Časopis 26 (1897) 105 ; Fschr. Mth. (1897) 142-.

- - $\left[S_{\frac{1}{2}}\left(a_{1} a_{2} \ldots a_{n}\right)\right]$. Studnixka, F.J. Prag Sb. (1896) (Mth.-Nt.) No. 22, $8 \mathrm{pp}$.

Powers and determinants, relation. Günther, S. Z. Mth. Ps. 24 (1879) 244 -.

Product of 2 determinants, decomposed. Solution of theorem of Sylvester's. Fà̃ de Bruno, F. [1851] Liouv. J. Mth. 17 (1852) 190-.

Reciprocal determinants. Gruzincev, A. $P$. Kharkov Mth. S. Com. 3 (1893) 94-; Fschr. Mth. (1891) 147.

- -. Musso, G. G. Mt. 31 (1893) 201-; 32 (1894) 81-.

Regular determinants and derived systems. Hensel, $K$. Crelle J. Mth. 114 (1895) 25-.

Resolution of a determinant into quadratic factors. Muir, T. [1881] Mess. Mth. 11 (1882) 105-.

- determinants, general problem. Günther, S. Arch. Mth. Ps. 59 (1876) 130-.

- _ - new method. Teixeira, J. P. G. Teix. J. Sc. 11 (1892) 173-.

- - linear equations, method, and application to determinants. Clasen, (l'abbé) B. I. Brux. S. Sc. A. 12 (1888) (Pt. 2) 251-.

Screws, theory of, note on determinant in. Ball, (Sir) R. S. [1890] Ir. Ac. P. 1 (1889-91) 375-.

Semi-determinants, Scheibner, $W$. Leip. B. 11 (1859) 151-.

- -symmetrical determinants of even order. Le Paige, C. M. M. H. H. Prag Sb. (1880) 125-.

Sets of integers whose determinant is $>$ or $<1$. Hermite, $C$. Liouv. J. Mth. 14 (1849) 21-.

Sign of any term of a determinant. Zehfuss, G. (xI) Z. Mth. Ps. 3 (1858) 247-, 249-.

- - - - Johnson, W. W. [1876] Mess. Mth. 7 (1878) 59.

- - - - - Tanner, H.W. L. [1879] Mess. Mth. 9 (1880) 51-.

- - - - - - Perry, H. M. (xir) [1881] J. H. Un. Cir. [1] (1882) 151.

- - - - - - Muir, T. [1899] Edinb. R. S. P. 22 (1900) 441-.

$-{ }_{-}-{ }_{-}-$, short rule for. Jenkins, M. Mess. Mth. 25 (1896) 60-
Simple alternants. Muir, $T$. [1887] Edinb. R. S. P. 14 (1888) 433-.

- -, class. Muir, T. [1888] Edinb. R. S. P. 15 (1889) 298-.

Skew determinant of infinite order, mean value of numerical coefficients. Sylvester, J.J. C. R. 89 (1879) 497-

- determinants. Cayley, A. Crelle J. 32 (1846) 119-; 38 (1849) 93-.

- - Brioschi, F. Liouv. J. Mth. 19 (1854) 253-.

- Hermite, C. Camb. and Dubl. Mth. J. 9 (1854) 63-.

- Cayley, A. Crelle J. 50 (1855) 277-; 55 (1858) 277-.

- - Muir, T. Ph. Mg. 12 (1881) 391-.

- - Mertens, $F$. Wien Ak. Sb. 96 (1888) (Ab. 2) 1245-.

- Cazzaniga, T. Mil. I. Lomb. Rd. 30 (1897) 1303-.

_ - Cayley's theory applied to displacement of rigid system about a fixed origin. Schläfi, L. [1865] QJ. Mth. 8 (1867) 167-.

- - of higher dimensions. Gegenbauer, $L$. Wien Ak. D. 55 (1889) (Ab. 1) 39-.

- - history. Muir, T. [1900] Edinb. R. S. P. 23 (1902) 181-.

- - partly zero-axial, condensation. Muir, $T$. L. Mth. S. P. 13 (1881-82) 161-.

- - property. Hill, M. J. M. L. Mth. S. P. 26 (1895) 341-.

- or symmetric determinant of infinite order, mean value of coefficients in development of. Sylvester, J. J. C. R. 89 (1879) 24 -

- symmetric determinants. Janni, G. G. Mt. 1 (1863) 275-.

- - - property. Deruyts, F. Liège S. Sc. Mm. 17 (1892) No. 2, 6 pp.

Stieltjes' theorem, twofold generalisation. Taber, H. L. Mth. S. P. 27 (1896) 613-.

Sturm's functions, coefficients expressed as determinants. Heal, W. E. A. Mth. 2 (1885-86) 85-.

- - determination of a series from a single determinant. Vleck, E. B. van. [1399] A. Mth. 1 (1900) 1-。

Sums of determinants. Deruyts, J. Liége S. Sc. Mm. 10 (1883) (No. 4) 11 pp.

- - powers of natural numbers, representation by determinants. Siacci, $F$. A. Mt. 7 (1865) 19-.

Sylvester's determinant. Hesse, L. O. Tortolini A. 2 (1859) 5-.

- transformation to Cauchy's. Biehler, C. N. A. Mth. 19 (1880) 110 -

Symbol analogous to determinants. Ferber, -. Par. S. Mth. Bll. 27 (1399) 285-.

- of positive determinants, application. Ferber, -. Par. S. Mth. Bll. 28 (1900) 128-.

Symbolic determinants. Rusjan, C.K. Fschr. Mth. (1899) 315.

Symmetric determinant connected with Lagrange's interpolation problem. Muir, $T$. L. Mth. S. P. 13 (1881-82) 156-. - - , number of terms. Cayley, A. As. S. M. Not. 34 (1374) 303-, 335. 


\section{Determinants}

Symmetric determinant, special. Gegenbauer, L. [1880] Wien Ak. Sb. 82 (1881) (Ab. 2) 938-.

- - , - note. Muir, T. Des Moines Anal. 8 (1881) 169-

- determinants. Mertens, $F$. Crelle J. Mth. 82 (1877) $207-$.

- - class. Noether, M. Mth. A. 16 (1880) $551-$.

-, , and its use in continued fractions. Studnička, F. Prag Sb. (1872) 74 .

- -, new properties. Muir, T. QJ. Mth. 18 (1882) 166-

- - , theorem relating to. Buchheim, A. Mess. Mth. 14 (1885) 113-.

- forms of determinants. Scott, R. F. Mess. Mth. 8 (1879) 131-, 145-.

-, skew-symmetric, and orthogonal matrices, properties. Taber, H. L. Mth. S. P. 22 (1891) 449-.

-, - - - - - - . Metzler, W. H. Am. J. Nth. 15 (1893) 274-

Systems of moduli applied to theory of determinants. Kronecker, L. Crelle J. Mth. 107 (1891) 254-.

Three-dimensional determinants. Hedrick, E. R. [1899] A. Mth. 1 (1900) 49-.

Transformable system, determinant of a. Deruyts, J. Brux. Ac. Bll. 32 (1896) 433-.

Transformation of determinant into any equivalent determinant. Van Velzer, C. A. Des Moines Anal. 9 (1882) 116-.

- - - - - Muir, T. Des Moines Anal. 10 (1883) 8-.

- - - continuant. Muir, T. [1881] Edinb. R. S. T. 30 (1883) 5-

- - - - Chrystal, G. [1881] Edinb. R. S. T. 30 (1883) 13-.

- - determinants in conic sections. Caspary, F. [1880] Crelle J. Mth. 92 (1882) 123-.

- - - mode of. Fouret, G. Par. S. Mth. Bll. 14 (1886) 146-.

-- , proof. Fiore, $V$. G. Mt. 10 (1872) 170 .

- formulae. Rubini, R. G. Mt. 16 (1878) 198-, 344.

-, a new. Studnička, F.J. Prag Sb. (1879) 489 .

- of product of determinants, Sylvester's theorem, note. Sperling, J.F. de. Liouv. J. Mth. 5 (1860) 121-.

- - rectangular coordinates. Janse, L. (Bz.) N. Arch. Wisk. 12 (1886) 109-; Fschr. Mth. (1885) 676.

Transformations of determinants. Siacci, $F$. [1872] A. Mt. 5 (1871-73) 296-.

Triangle, application of determinants to geometry of. Avillez, J. F. d' (Visconde de Reguengo). [1897] Lisb. J. Sc. Mth. 5 (1898) 14-

-, determinant giving area in terms of sides. Ritsert, E. Z. Mth. Ps. 17 (1872) 513-.

Two-step determinants, theorem. Torelli, $G$. G. Mt. 4 (1866) 294-

Uni-modular determinant, construction. Weihrauch, K. Z. Mth. Ps. 21 (1876) 134-.

Vanishing aggregates of determinants. Muir, T. [1888] Edinb. R. S. P. 15 (1889) 96-.
Discriminants and Resultants 2020

Wronskian determinant. Peano, $G$. Mathesis 9 (1889) 75-, 110-; Rm. R. Ac. Linc. Rd. 6 (1397) (Sem. 1) 413-.

- Vivanti, G. Rm. R. Ac. Linc. Rd. 7 (1898) (Sem. 1) 194-.

- - generalisation of properties. Pincherle, S. Rm. R. Ac. Linc. Rd. 6 (1897) (Sem. 1) $301-$.

- - , - - Bortolotti, E. Rm. R. Ac. Linc. Rd. 7 (1898) (Sem. 1) 45-

- determinants. Lecornu, L. As. Fr. C. R. (1894) (Pt. 2) 282-.

- - properties and some applications. Dickstein, S. Prace Mt.-Fiz. 1 (1888) 5-; Fschr. Mth. (1888) 151.

Wronskians, fundamental property. Demoulin, A. Mathesis 17 (1897) 62-.

Zero-determinants of infinite order. Cazzaniga, T. A. Mt. 1 (1898) 83-.

\section{Discriminants and resultants.}

Algebraic aggregate, discriminant. Hensel, $K$. Gött. Nr. (1897) 247-.

- - , fundamental equation, and non-essential factors of the discriminant. Hensel, $K$. Gött. Nr. (1897) 254-.

- equivalences and elimination. Laurent, $H$. N. A. Mth. 5 (1886) 432-, 456-.

- resolvents. Rados, G. Mth. Termt. Ets. 17 (1899) 421-; Mth. Nt. B. Ung. 18 (1903) 236-.

$C$-discriminant as an envelope. Macdonald, J. A. Edinb. R. S. T. 39 (1900) $27-$.

Continuant, the discriminant of a special quadratic form. Cesáro, E. Mathesis 12 (1892) 5-.

Determinantal equation connected with small oscillations, etc. Muir, T. Mess. Mth. 14 (1885) 141-.

- -, Lagrange's. Muir, T. Ph. Mg. 43 (1897) 220 -

_ - , Sylvester's proof of the reality of the roots of. Muir, T. Am. J. Mth. 19 (1897) $312-$.

- - , reciprocity of roots (Brioschi's theorem). Faà de Bruno, F. Liouv. J. Mth. 19 (1854) 304.

Determination of common roots of given equations by means of their eliminant. Baraniecki, M. A. Par. T. Nauk Śc. Pam. $10\left({ }^{*} 1878\right)$ Art. $5,7 \mathrm{pp}$.

Discriminant of cubic giving axes of a quadric surface, decomposition into sum of squares. Bauer, G. [1868] Crelle J. Mth. 71 (1870) $40-$.

-, degree with respect to a parameter. Roberts, E. [1869] QJ، Mth. 10 (1870) 204-.

- of finite hypergeometric series. Hilbert, $D$. Crelle J. Mth. 103 (1888) 337-.

- - general quadratic. Dupuis, N. F. Cn. R. S. P. \& T. 7 (1890) (Sect. 3) 15-.

- Jacobians of three quadratic forms. Igel, B. [1876] Wien Ak. Sb. 74 (1877) (Ab. 2) 355-; 75 (1877) (Ab. 2) 830-. 
Discriminant of quadratic forms, property. Picquet, H. As. Fr. C. R. (1875) 225-.

- - quartic equation. Weill, M. N. A. Mth. 2 (1883) 265-.

- - quaternary quadratic form, properties. Walker, J. J. [1865] Mess. Mth. 4 (1868) 25-, 187-.

-, special. Cayley, A. QJ. Mth. 11 (1871) 211-.

- surfaces of algebraic equations, coefficients being coordinates. Czuber, $E$. Mh. Mth. Ps. 5 (1894) 62-.

- transformation of characteristic equations by. Brioschi, F. Tortolini A. 7 (1856) 64-.

Discriminantal equation, roots. Brisse, $C$. N. A. Mth. 9 (1890) 367-.

Discriminants. Bellavitis, $G$. Ven. At. (185859) 65-, 82-.

-. Brill, A. Mth. A. 12 (1877) 87-.

- Casorati, E. Mil. I. Lomb. Rd. 18 (1885) 108-.

of algebraic equations, factorisation of. Netto, E. Mth. A. 24 (1884) 579-.

- - functions of one variable. Kronecker, L. Crelle J. Mth. 91 (1881) 301-.

-, arithmetical researches on non-essential factors of. Hensel, $K$. Crelle J. Mth. 113 (1894) 128-.

-, forms of, theorem. Kronecker, L. Crelle J. Mth. 100 (1887) 79-.

-, formulæ, and application to discriminants of discriminants, and to polar curves. Henrici, O. [1868] . L. Mth. S. P. 2 (1869) 104-.

-, invariants, covariants, and hyperdeterminants, elementary notions. Terquem, $O$. N. A. Mth. 18 (1859) 249-, 299-, 446-.

- of resultants. Brill, A. Gött. Nr. (1889) 89-.

- and resultants of equations for singularities. Meyer, F. Gött. Nr. (1888) 74-; (1890) 366- 493-; (1891) 14-.

-, theorem. Sylvester, J. J. R. S. P. 14 (1865) 336-

-, theory. Netto, E. Crelle J. Mth. 90 (1881) 164-; Acta Mth. 1 (1882) 371-

Discriminating cubic; representation of discriminant as sum of squares. Tannery, $J$. Bll. Sc. Mth. As. 7 (1883) (Pt. 1) 103-.

- determinant equations, reality of roots of. Elliott, E. B. QJ. Mth. 31 (1900) 233-.

- equation (formed from symmetric determinant), properties. Kaiser, -. D. Nf. Tbl. (1887) 228.

- equation, reality of roots of, when determinant is not symmetric. Bendixson, $I$. Stockh. Öfv. (1900) 1099-.

Divisibility, notion including, and general theory of elimination. Molk, J. Acta Mth. 6 (1885) 1-.

Eliminant of $f(x)=0, f\left(\frac{1}{x}\right)=0$. Muir, $T$. [1896] Edinb. R. S. P. 21 (1897) 360-.

-, persymmetric. Muir, T. [1899] Edinb. R. S. P. 22 (1900) 543-.

- of set of quaternary quadrics. Muir, $T$. [1896] Edinb. R. S. P. 21 (1897) 328-.
Eliminant of set of ternary quadrics. Muir, $T$. [1896] Edinb. R. S. P. 21 (1897) 220-.

_ _ _ - or quaternary quadrics. Nanson, E. J. [1899] Edinb. R. S. P. 22 (1900) 353-.

-, Sylvester's, form. Gordan, P. D. Nf. Vh. (1893) (Th. 2, Hälfte 1) 4.

- of system of equations, degree. Vahlen, $K$. $T$. Crelle J. Mth. 113 (1894) 348-.

Mth. Ps. 5 (1894) 17-.

- two equations. Mansion, P. Brux. S. Sc. A. 18 (1894) (Pt. 1) 5-.

- - - , degree. Janni, V. G. Mt. 12 (1874) 27.

- - - - - Lachlan, R. Camb. Ph. S. P. 9 (1898) 313-.

_ _ _ - dialytic form. Sylvester, J. J. Ph. Mg. 16 (1840) 132-.

Eliminants and associated roots. Elliott, E. B. L. Mth. S. P. 16 (1884-85) 224-.

-, Sylvester's and Cauchy's, equality. Mansion, P. [1879] Mess. Mth. 9 (1880) 60 -.

\section{Elimination.}

Poisson, S. D. Par. Éc. Pol. J. 4 (1801-2) (11. cah.) 199-.

Badano, -. Genova Mm. Ac. 3 (1814) 125-.

Babbage, C. QJ. Sc. 3 (1817) 355-.

Poncelet, J. V. Gergonne A. Mth. 8 (1817-18) 201-.

Gergonne, J. D. Gergonne A. Mth. 21 (183031) $41-$

Murphy, R. [1832] Camb. Ph. S. T. 5 (1835) 65-.

Sylvester, J. J. Ph. Mg. 17 (1840) 379-.

Liouville, J. Liouv. J. Mth. 6 (1841) 345-.

Terquem, $O$. N. A. Mth. 1 (1842) 125-.

Moon, R. Camb. Mth. J. 3 (1843) 183-.

Finck, B. Liouv. J. Mth. 9 (1814) 334-.

Bouillon, - N. A. Mth. 4 (1845) 120-.

Cockle, Jas. Ph. Mg. 27 (1845) 292-.

Cayley, A. Crelle J. 34 (1847) 30-.

Crelle, A. L. Crelle J. 43 (1852) 37-.

Genocchi, A. N. A. Mth. 14 (1855) 259-.

Cayley, A. [1861] Crelle J. 60 (1862) 373-.

Lemonnier, $H$. C. R. 80 (1875) 252-.

Rouche, E. N. A. Mth. 16 (1877) 105-.

Lemonnier, H. Par. Éc. Norm. A. 7 (1878) 77-, 151-.

Mansion, P. C. R. 87 (1878) 975-; Brux. Ac. Bll. 46 (1878) 899-; 47 (1879) 532-; 48 (1879) 463-, 473-.

Le Paige, C. M. M. H. H. C. R. 90 (1880) 1210-

Biehler, C. N. A. Mth. 1 (1882) 529-。

Laurent, H. N. A. Mth. 11 (1892) 5-.

Worontzoff, - . N. A. Mth. 11 (1892) 291-.

Laurent, $H$. N. A. Mth. 12 (1893) 355-.

Hadamard, J. C. R. 119 (1894) 995-, 1243 ; Acta Mth. 20 (1897) 201-.

Netto, E. Giessen Oberh. Gs. B. 32 (1897-99) 78-.

Bezout-Cauchy method. Falk, $M$. Ups. S. Sc. N. Acta 10 (1879) No. $15,36 \mathrm{pp}$.

- - Hioux, V. N. A. Mth. 18 (1879) 289-. 
Bezout's method. Cayley, A. Crelle J. 53 (1857) 366-; Mess. Mth. 2 (1863) 88-.

- - Painvin, L. N. A. Mth. 13 (1874) 278 .

- -. Stickelberger, 一. D. Nf. B. (*1883) 58.

and continued fractions. Borchardt, $C . W$. Berl. Ak. Ab. (1878) (Mth. 1-).

by cross-multiplication. Smith, Arch. Camb. Mth. J. 1 (1839) 46.

degree of equation resulting from. Minding, E. F. A. Crelle J. 22 (1841) 178-.

- - - - Magnus, L. J. Crelle J. 26 (1843) 365-.

- - - - - expression for. Minding, E. F. A. [1843] St Pét. Ac. Sc. Bll. 2 (1844) 273-.

dialytic method. Sylvester, J.J. Ir. Ac. P. 2 (1840-44) 130-.

- - applied to ternary systems of equations. Sylvester, J.J. Camb. Mth. J. 2 (1841) 232-.

- - extensions. Sylvester, J.J. Ph. Mg. 2 (1851) 221-.

- -, Sylvester's. Madsen, (Kapt.) V. H. O. (xII) Ts. Mth. 5 (1875) 144-.

in equations of 1st order. Gergonne, J. $D$. Gergonne A. Mth. 4 (1813-14) 148-; 12 (1821-22) 281- ; 20 (1829-30) 109-.

---- Majo, L. de. Nap. Mm. Ac. Sc. 1 (1852-54) 101-.

- - - by finding common divisor. Raymond, G. M. Gergonne A. Mth. 2 (1811-12) 38-.

- - higher orders. Kramp, C. Gergonne A. Mth. 1 (1810-11) 321-.

Euler's method. Biehler, $C$. N. A. Mth. 6 (1887) 67-.

example. Anderson, Jas. Mathematician 1 (1845) 181-.

formation of eliminant. Bes, $K$. Amst. Ak. Vs. 8 (1900) 173-; Amst. Ak. P. 2 (1900) 85-.

formula. Cayley, A. I. Mth. S. P. 11 (187980) 139 -

Gauss's method. Hall, A. A. Mth. 8 (189394) 64 .

general theory. Voizot, -. L'I. 3 (1835) 104-.

- - Bes, K. Amst. Ak. Vh. (Sect. 1) 6 (1899) No. 7, $121 \mathrm{pp}$.

by H.C.F. Bret, -. Par. Éc. Pol. J. 8 (1809) (15 eah.) 162- ; Gergonne A. Mth. 3 (1812-13) 13-.

- - Sarrus, F. (vi Adds.) Strasb. S. Sc. N. Mm. 2 (1832) 190-.

- indeterminate multipliers. Smith, Arch. Camb. Mth. J. 1 (1839) 49-.

introduction to a theory. Garbieri, G. G. Mt. 30 (1892) 41-.

linear method for algebraic equations. Sylvester, J. J. Ph. Mg. 18 (1841) 425-.

and linear simultaneous equations, algebraic or differential. Mainardi, $G$. Mil. At. I. Lomb. 1(1858) 95-.

Liouville's theorem, demonstration and application. Fouret, $G^{*}$. N. A. Mth. $9(1890)$ 258-. method. Craufurd, A. Q. G. Camb. Mth. J. 2 (1841) 276-. -397-.

- of obtaining final equation without irrelevant factors. Araldi, A. (Ix) [1858] Mod. Ac. Sc. Mm. 3 (1861) 3-.

methods. Cossali, P. [1812] Mod. Mm. S. It. 16 (1813) 272-.

- of Fontaine, Vandermonde, and Laplace. Terquem, $O$. N. A. Mth. 5 (1846) 153-. new and extended theory. Sylvester, J. $J$. Ph. Mg. 15 (1839) 428-; 16 (1840) 37-.

and polars. Clebsch, A. [1860] Crelle J. 58 (1861) 273-.

problem. Borchardt, C. W. Berl. Mb. (1859) 376-.

-. Krey, H. Mth. A. 12 (1877) 476-.

- Stephanos, C. C. R. 97 (1883) 1050-.

- comprising theory. Ventéjols, -. C. R. 84 (1877) 546-.

- of Sylvester's. Tait, - [1892] Edinb. R. S. P. 19 (1893) 131-.

- - - Muir, T. [1892] Edinb. R. S. P. 20 (1895) 300-.

- - C Cayley, - [1894] Edinb. R. S. P. 20 (1895) 306-.

- - Muir, T. Edinb. R. S. P. 20 (1895) 371-.

- - Nanson, E. J. [1897] Edinb. R. S. P. 22 (1900) 150-.

problems. Cayley, A. QJ. Mth. 8 (1867) 183- ; 11 (1871) 99-.

- on curves satisfying given conditions. Brill, A. Gött. Nr. (1870) 525-.

a process in. Biehler, C. N. A. Mth. 19 (1880) 202-, 332-.

between quadratic and set of linear functions (reply to Boole). Sylvester, J. J. Camb. and Dubl. Mth. J. 6 (1851) 171-.

- quadratics, theorems in. Sylvester, J. $J$. Ph. Mg. 37 (1850) 213-.

result. Salmon, G. Camb. and Dubl. Mth. J. 3 (1848) 169-.

- Cayley, A. Tortolini A. 7 (1856) 454-.

resultants, comparison. Borchardt, $C$. $W$. Crelle J. 57 (1860) 183-.

simple. Drinkwater, J. E. Ph. Mg. 10 (1831) 24 -

by special algorithm. Gruson, J. P. Berl. Ab. (1816-17) 1-.

symbolic process. Clebsch, A. Berl. Mb. (1860) 536-.

by symmetric functions. Liouville, J. N. A. Mth. 6 (1847) 295-.

symmetrical method in simple equations by determinants. Warner, J. D. Am. As. P. (1881) 50 -.

from a system of equations. Brill, $A$. [1871] Mth. A. 5 (1872) 378-.

theorem. Cayley, A. QJ. Mth. 12 (1873) 5-.

theory. Richelot, $F$. J. Crelle J. 21 (1840) 226-.

-. Grunert, J. A. Grunert Arch. 2 (1842) 76-, 337-, 345-.

- Crelle, A. L. Berl. Ab. (1844) 1-; (1845) 1-

-. Chid, F. Palomba Rac. 3 (1847) 193-. 
theory. Cayley, A. Camb. and Dubl. Mth. J. 3 (1848) 116-.

-. Salmon, G. QJ. Mth. 7 (1866) 327-.

- Arzelà, C. [1876] G. Mt. 15 (1877) 62-, 154-.

-. Noether, M. [1876] Erlang. Ps. Md. S. Sb. 9 (1877) 66-.

-. Toeplitz, J. Z. Mth. Ps. 23 (1878) 61-.

-. Laurent, $H . \quad$ N. A. Mth. 2 (1883) 145-.

-. Netto, E. Acta Mth. 7 (1885) 101-.

-. Schmidt, C. Z. Mth. Ps. 31 (1886) 214-.

-. Schendel, L. Z. Mth. Ps. 32 (1887) 46-.

-. Laurent, H. N. A. Mth. 7 (1888) 60-, 116-.

-. Loria, G. G. Teix. J. Sc. 9 (1889) 33-.

-. Kretkonvski, W. Prace Mt.-Fiz. 2 (1890) 21- ; Fschr. Mth. (1890) 174-.

- Pleskot, A. Prag České Ak. Fr. Jos. Rz. (Třida 2) 5 (1896) Art. 25, 13 pp.; Fschr. Mth. (1896) 91.

-. Mertens, F. Wien Ak. Sb. 108 (1899) (Ab. 2a) 1173-, 1344-.

-, and algebraic curves. Brill, A. Mth. A. 4 (1871) 510-.

-, principle of projection in. Meyer, F. D. Nf. Vh. (1890) (Th. 2) 9-.

transformation and canonical forms. Sylvester, J. J. Camb. and Dubl. Mth. J. 6 (1851) $186-$.

of two arbitrary functions. Tanner, $H . W . L$. [1875] Mess. Mth. 5 (1876) 98-.

between two equations. Valerius, $B$. Liège A. Ac. (1826-27) $75 \mathrm{pp}$.

- - Meyer, A. Quetelet Cor. Mth. 5 (1829) 100-.

- - Jacobi, C. G. J. Crelle J. 15 (1836) 101-.

- - Petersen, J. P. C. (xII) [1873] Ts. Mth. 4 (1874) 83-.

- Z Zeuthen, H. G. (xII) Ts. Mth. 4 (1874) 165-

- - Cauchy, -. N. A. Mth. 15 (1876) 385-, 433-.

- - Forestier, C. Toul. Ac. Sc. Mm. 9 (1877) 142-.

- - Mansion, P. Brux. Ac. Bll. 48 (1879) 491-.

(1881) $535-$

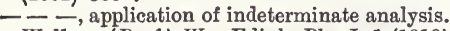
Wallace, (Prof.) W. Edinb. Ph. J. 1 (1819) 29-.

- - , final equation. Hesse, L. O. Crelle J. 27 (1844) 1-.

- - - in one unknown. Darboux, G. (Ix) Bll. Sc. Mth. As. 10 (1867) 56-; 1 (1877) 54-. - - - - two unknowns. Véne, A. A. Brux. Mm. Cour. 4 (1824) (No. 2) 26 pp.

- - - - Finck, B. N. A. Mth. 4 (1845) 198-.

- - - of 2nd order. Horner, W. G. $\mathrm{Ph}$. Mg. 5 (1834) 188-.

_- _ _- 2 unknowns. Merlieux, É. N. A. Mth. 2 (1843) 34-.

- - - - 3rd order. Clebsch, R. F. A. [1864] Crelle J. 64 (1865) 95-.

- three equations of 2 nd order. Hesse, L. O. Crelle J. 28 (1844) 68-.
Eliminations arising in trigonometry. Meyer, F. D. Mth. Vr. Jbr. 4 (1897) 92-.

-, equations resulting from various. Bezout, -. N. A. Mth. 18 (1859) 120-.

Equation for max. and min. of $n$-ary quadric. Gravelaar, N. L. W. A. N. Arch. Wisk. 4 (*1878) 113-.

of squares of differences. Brioschi, $F$. Tortolini A. 7 (1856) 5-.

Equations whose discriminant is a square. Netto, E. Crelle J. Mth. 95 (1883) 237-.

Gauss's method of substitution. Johnson, W.W. A. Mth. 7 (1892-93) 65-.

Order of manifoldness arising from several linear systems. Pieri, M. Palermo Cir. Mt. Rd. 11 (1897) 58-.

$p$-discriminant of differential equation of first order, and its connection with general theory of envelopes. Chrystal, - . Edinb. R. S. T. 38 (1897) 803-.

Power determinant, a. Jung, $V$. Časopis 29 (1900) 41-; Fschr. Mth. (1900) 157.

- determinants. Jung, $V$. Prag České Ak. Fr. Jos. Rz. (Tř́ida 2) 8 (1899) Art. 38, 22 pp.; Fschr. Mth. (1899) 153.

Product of differences of roots of equations, relation of discriminants and resultants to. Sylvester, J.J. Mess. Mth. 9 (1880) 164-.

Property of product of linear factors. Brioschi, F. Camb. and Dubl. Mth. J. 9 (1854) 137-.

Resultant of a covariant and a fundamental form. Kohn, G. Wien Ak. Sb. 100 (1891) (Ab. 2a) 1013-.

-, degree. Isè, E. G. Mt. 11 (1873) 253-.

- of elimination of $z$ from algebraic equations $f(z)=0$ and $\phi(z)=0$, new representation. Rosenhain, G. Crelle J. 30 (1846) 157-.

-, Euler's. Hesse, L. O. Tortolini A. 2 (1859) 5-.

- and G.C.M. of integral algebraical expressions. Szüts, N. von. Mh. Mth. Ps. 9 (1898) 34-.

- of integral functions. Biermann, O. Mh. Mth. Ps. 2 (1891) 143-.

- - multipartite system of equations, degree and "weight." Sylvester, J.J. R.S.P. 12 (1862-63) 674-.

- $n$ equations. Faà de Bruno, $F$. Tortolini A. 7 (1856) 222-.

- an $n$-ic and of a cubic. Pascal, E. G. Mt. 25 (1887) 257-.

-, order of, case of evaporation. Clifford, W. K. [1870] L. Mth. S. P. 3 (1869-71) 80-.

$-R$ of two forms of the $m$ th and $n$th degrees, P.D.E. satisfied by. Gordan, $P$. Gött. Nr. (1870) 427-.

-, the reduced. Brill, A. Münch. Ak. Ab. 17 (1892) 89-.

- of several algebraic equations. Laurent, $H$. N. A. Mth. 12 (1893) 305-.

- - system of equations. Schläfli, $L$. Wien D. 4 (1852) $(A b .2) 1$-.

- - - - (Schläfli). Cayley, A. Crelle J. 50 (1855) 277-.

- _ - - two equations. Cayley, A. [185767] Phil. Trans. 147 (1857) 703-; 158 (1868) 173-. 
Resultant of two congruences. Sylvester, J. J. (xII) [1881] J. H. Un. Cir. [1] (1882) 131. - - equations. Brioschi, $F$. Crelle J. 53 (1857) 372-.

- - -. Isè, E. [1868] G. Mt. 8 (1870) 1-. - - - . Gordan, P. Mth. A. 3 (1871) 355-; C. R. 127 (1898) 539-.

- -1 , application to twisted cubic curve. Waelsch, E. Mh. Mth. Ps. 2 (1891) 421-.

- - - Brioschi's theorem. Faa de Bruno, $F$. Crelle J. 64 (1857) 283-.

- - - of 4 th order. Faà de Bruno, $F$. Tortolini A. 1 (1858) 362-.

- - - forms of the same order, new representation. Stahl, W. Mth. A. 35 (1890) $395-$.

Resultants. Vaněček, M. N. Prag Sb. (1888) (Mth.-Nt.) 152-.

-. Gordan, P. Mth. A. 45 (1894) 405-.

-, anastrophic. Cauchy, A. L. C. R. 44 (1857) 370 .

- corresponding to interpolation. Borchardt, C. W. Crelle J. 57 (1860) 111-。

- of covariants. Gordan, P. Mth. A. 4 (1871) 169-.

_, formation. Reuschle, C. Z. Mth. Ps. 30 (1885) 106-.

-, note. Haskell, M. W. N. Y. Mth. S. Bll. 1 (1892) 223-.

-, property. Brill, A. [1879] Mth. A. 16 (1880) 345-

- of quantics, determination of their numerical coefficients. Vaněček, M.N. Prag Sb. (1888) (Mth.-Nt.) 286-.

-, theorems. Igel, B. [1877] Wien Ak. Sb. 76 (1878) (Ab. 2) 145-

—, theory. Netto, E. Crelle J. Mth. 116 (1896) 33-; 117 (1897) 57-.

-, -, extension. Sylvester, J. C. R. 58 (1864) 1074-, 1130, 1178-.

-, - , note. Netto, E. Giessen Oberh. Gs. B. 32 (1897-99) 37-.

- of two integral functions. Nägelsbach, $H$. Z. Mth. Ps. 17 (1872) 333-.

$--\cdots-$, remark on property. Kowalewski, G. Arch. Mth. Ps. 17 (1900) 202-.

-, two, in theory of rectifiable compound logarithmic waves. Sylvester, J. J. $\mathrm{Ph}$. Mg. 37 (1869) 375-.

Roots common to two complete equations. Hioux, V. Par. Éc. Norm. A. 10 (1881) $383-$.

- - equations. Abel, N.H. Gergonne A. Mth. 17 (1826-27) 204-.

--- Brioschi, $F$. N. A. Mth. 14 (1855) 81-.

- - - - Garbieri, G. Catania Ac. Gioen. At. 6 (1893) Mm. 16, 9 pp.

Sub-discriminant. Perrin, R. Liouv. J. Mth. 10 (1894) 129-.

Sylvester's problem of the three ternary equations, new solution. M'Laren, (Lord). Edinb. R. S. P. 19 (1893) 264-.

Vanishing discriminant, application. Azzarelli, M. Tortolini A. 3 (1860) 16-.

- -, applications to geometry. Azzarelli, $M$. Rm. N. Linc. At. 30 (1877) 290-.

\section{Characteristic properties of linear substitutions; types of linear substitutions. (See also 1210.)}

"Adjoint" substitutions, theory. Rados, G. [1891] Mth. Termt. Ets. 10 (1892) 34-; Mth. Nt. B. Ung. 10 (1893) 98-,

Analytic functions suitable to represent substitutions. Dickson, L. E. Am. J. Mth. 18 (1896) 210-.

Bipartite quadric, automorphic linear transformation. Cayley, A. Phil. Trans. (1858) 39-.

- - - - Muir, T. Am. J. Mth. 20 (1898) 215-.

Canonical form, bi-orthogonal reduction of a lineo-linear form to. Sylvester, J.J. C. R. 108 (1889) 651-.

- - of linear homogeneous substitution in a Galois field. Dickson, L. E. Am. J. Mth. 22 (1900) 121-.

- - - - substitutions. Maschke, $H$. Mth. A. 50 (1898) 220-.

- - - a linear substitution of finite order. Burnside, W. Mess. Mth. 28 (1899) 111-. - - - quadric, proof of reduction by real orthogonal substitution. Sylvester, J. J. Mess. Mth. 19 (1890) 1-.

- - reduction of a linear substitution to. Burnside, $W$. L. Mth. S. P. 30 (1899) 180 -.

- - - - - Dixon, A. C. [1899] L. Mth. S. P. 31 (1900) 170-.

- - - - - - Bromwich, T.J.I'A. [1899] L. Mth. S. P. 31 (1900) 289-.

Characteristic functions of substitutions. Cauchy, A. L. C. R. 21 (1845) 1254-

Equations containing linear functions of rectilinear coordinates. Dieu, T. N. A. Mth. 4 (1865) 165-.

Exchangeable linear substitutions. Amaldi, $U$. Mil. I. Lomb. Rd. 33 (1900) 731-.

- - - Schlesinger, L. Crelle J. Mth. 121 (1900) 177-.

- substitutions, theory. Demeczky, - . C.R. 120 (1895) 39-.

Induced linear substitutions. Franklin, F. Am. J. Mth. 16 (1894) 205-.

- -. Rados, G. Mth. Termt. Éts. 16 (1898) 378-; Mth. Nt. B. Ung. 16 (1899) 241-.

- - Visnya, A. Mth. Nt. B. Ung. 16 (1899) 187-

- - groups. Rados, G. Mth. Termt. Éts. 17 (1899) 44-; Mth. Nt. B. Ung. 17 (1901) $227-$.

- substitutions, rank of determinant in. Kürschâk, J. Mth. Termt. Éts. 18 (1900) 44-; Mth. Nt. B. Ung. 18 (1903) 229-.

Integral linear substitutions. Pick, G. Mth. A. 24 (1884) 588-.

- - - of determinant 1 , composition of. Krazer, A. A. Mt. 12 ("1883-84) 283-. 
Integral linear substitutions not given by algebraical congruences. Pick, G. Mth. A. 28 (1887) 119-.

Invariants of orthogonal substitutions (regarded as rotations). Schläfi, L. [1865] Crelle J. 65 (1866) 185-.

Inverse orthogonal matrices. Sylvester, J. J. Ph. Mg. 34 (1867) 461-.

Kummer's configuration, and orthogonal substitutions. Study, E. Leip. Mth. Ps. B. 44 (1892) 122-.

Linear orthogonal substitutions, special case. Stern, M. A. Crelle J. 67 (1867) 293-.

- substitutions. Polignac, C. de. Par. S. Mth. Bll. 4 (1876) 120-; 5 (1877) 69-.

- - Poincaré, H. C. R. 98 (1884) 349-.

- - Picard, É. C. R. 98 (1884) 416-.

- - Burnside, W. Mess. Mth. 22 (1893) 190 -

- -, certain. Deruyts, J. Brux. Ac. Bll. 24 (1892) 102-.

- - a property. Burnside, W. Mess. Mth. 20 (1891) 163-.

- - of a single variable with periodic coefficients, Ocagne, M. d'. Par. S. Mth. Bll. 19 (1891) 37-.

- -, special groups of. Fricke, R. Mth. A. 38 (1891) 50-, 461-.

- - theory. Netto, E. Acta Mth. 17 (1893) 265-.

- - 一. Loewy, A. Mth. A. 48 (1897) 97-; 49 (1897) 448-.

,--- , new. Laurent, $H$. N. A. Mth. 15 (1896) 345-.

- - by which a quadratic ternary form reproduces itself. Tannery, $J$. Bll. Sc. Mth. As. 11 (1876) 221-.

- transformations. Cayley, A. Camb. Mth. J. 4 (1845) 193-; Camb. and Dubl. Mth. J. 1 (1846) 104-.

- - some properties. Deruyts, J. Brux. Ac. Bll. 16 (1888) 576-

Orthogonal and Boolian invariant systems, - symbolism for. MacMahon, (Maj.) P. A. B. A. Rp. (1900) 647.

-, - related, substitutions. Igel, B. [1877] Wien Ak. D. 39 (1879) (Ab. 2) 29-.

- substitution, properties of coefficients. Bar delli, $G$. Mil. I. Lomb. Rd. 9 (1876) 167-.

- substitutions. Grunert, J.A. Arch. Mth. Ps. 44 (1865) 478-.

- -. Gundelfinger, S. Crelle J. Mth. 99 (1886) 147-.

- - Netto, E. Acta Mth. 9 (1887) 295-.

- Catalan, E. As. Fr. C. R. (1893) (Pt. 2) 19-.

- Taber, H. N. Y. Mth. S. Bll. 3 (1894) 251-.

- -. Rados, G. Mth. Termt. Éts. 18 (1900)

128-; Mth. Nt. B. Ung. 18 (1903) 231-.

-- , conditions among coefficients. Rados, $G$. Mth. Termt. Ets. 16 (1898) 123-; Mth. Nt. B. Ung. 16 (1899) 236-.

- - with determinant (-1). Weill, G. Par. É. Norm. A. 11 (1894) 23-.

- - , equation connected with. Börsch, A. Z. Mth. Ps. 24 (1879) 391-.
Orthogonal substitutions in involution. Prym, F. Gött. Ab. 38 (1892) (Mth.) 42 pp.

- -, parametric expression of coefficients. Hunyady, J. Mag. Tud. Ak. Etk. (Mth.) 14 (1892) No. 2, 43 pp., No. 3, 14 pp.; Mth. Nt. B. Ung. 7 (1890) 233-.

- - and regular divisions of space. Goursat, É. C. R. 106 (1888) 1786-; Par. Éc. Norm. A. 6 (1889) 9-.

- - (single alternate substitution). Taber, $H$. Am. J. Mth. 16 (1894) 123-.

- -, theory. Voss, A.E. Mth. A. 13 (1878) 320 -.

- - - - Rados, G. Mth. Termt. Éts. 10 (1892) 16-; Mth. Nt. B. Ung. 10 (1893) 95-. system in parallel arrangement. Voss, $A$. Mth. A. 24 (1884) 48-.

- systems. Kronecker, L. Berl. Ak. Sb. (1890) 525-, 601-, 691-, 873-, 1063-.

Powers and roots of linear substitutions. Sylvester, J. J. C. R. 94 (1882) 55-.

Quadratic form, automorphic transformation. Rosanes, J. Crelle J. Mth. 80 (1875) 52-. - - , - transformations. Jordan, C. Liouv. J. Mth. 4 (1888) 349-.

- - , - Loewy, A. Ac. Nt. C. N. Acta 65 (1896) 1-.

- -, orthogonal transformation to sum of squares. Kneser, $A$. Arch. Mth. Ps. 15 (1897) 225-.

- - , reduction. Timerding, H. E. Crelle J. Mth. 122 (1900) 172-.

- - - - to squares: equation $\left|a_{1} k+S\right|=0$. Walecki, -. N. A. Mth. 1 (1882) 401-, $556-$.

- - transformation. Enneper, A. Z. Mth. Ps. 9 (1864) 358-.

- - - - to sum of squares. Hesse, L. O. Crelle J. 45 (1853) 93-; 57 (1860) 175-.

- _, _ - _ - Gundelfinger, S. [1880] Crelle J. Mth. 91 (1881) 221-.

- - - - - - Z. Zajaczkowski, W. (xII) [1883] Krk. Ak. (Mt.-Prz.) Pam. 9 (1884) 1-; Fschr. Mth. (1884) 165-.

- - , - - - Presle, - de. Par. S. Mth. Bll. 14 (1886) 98-.

- _, _ _ _ _ - Gauss's method. Somoff, J. St. Pét. Ac. Sc. Bll. 18 (1873) 442-.

- forms and the discriminantal equation. Laurent, $H$. N. A. Mth. 10 (1891) 503-.

- - reduction to least number of terms. Jacobi, C. G. J. Berl. B. (1848) 414-.

- -, - and transformation. Jordan, $C$. Liouv. J. Mth. 19 (1874) 397-.

- -, removal of product terms from two. Jacobi, C. G. J. Crelle J. 12 (1834) 1-.

- - , simultaneous transformation. Cayley, $A$. Camb. and Dubl. Mth. J. 4 (1849) 47-; QJ. Mth. 2 (1858) 192-.

- - - - Siacci, F. Tor. At. Ac. Sc. 7 (1871-72) 758-.

- - theory. Jamet, $V$. Mars. Fac. Sc. A. 10 (1900) Fasc. 5, 18 pp.

- - , transformation. Smith, Arch. Camb. Mth. J. 1 (1839) 272-.

- _, - . Cayley, A. Crelle J. 50 (1855) 277-. 


\section{Linear Substitutions}

Quadratic forms, transformation. Trudi, N. Nap. Rd. 1 (1862) 113-.

- - - - Brioschi, F'. G. Mt. 1 (1863) 26-.

- - - - Lemonnier, H. [1874] Par. S. Mth. Bll. 3 (1875) 48-.

- - transformations. Kretkonsski, W. (xIr) Krk. Ak. (Mt.-Prz.) Pam. 7 (1882) 69-.

Quadratics, reduction to canonical form. Sardi, C. G. Mt. 5 (1867) 35-.

Quadric automorphic linear transformation. Muir, T. [1897] Edinb. R. S. T. 39 (1900) 209-.

Quaternary quadratic form, automorphic linear transformations. Bachmann, -. D. Nf. Tbl. (*1871) 138-.

Real linear transformations whose invariant is an alternant. Taber, $H$. Am. Ac. P. 31 (1896) 336-.

- _ - - - a real quadratic form. Taber, H. Am. Ac. P. 32 (1897) 75-.

- orthogonal substitution. Taber, H. Am. Ac. P. 28 (1893) 212-.

Roots of substitutions. Kirkman, T.P. B. A. Rp. (1860) (pt. 2) 4-; (1861) (pt. 2) 4-.

Solutions of the equation $S X=X S$. Voss, $A$. Münch. Ak. Sb. 26 (1897) 273-.

Special automorphic groups and quadratic forms. Fricke, $R$. Mth. A. 39 (1891) 62-.

Structure of linear groups with quadratic invariants. Dickson, L. E. L. Mth. S. P. 30 (1899) 70-

Substitutions permutable inter se. Cauchy, A. L. C. R. 21 (1845) 1188-.

- - - , and other systems of substitutions. Cauchy, A. L. C. R. 21 (1845) 1238-.

-, product of two. Miller, G. A. Am. J. Mth. 22 (1900) 185-

-, regular, irregular, and conjugate. Cauchy, A. L. C. R. 21 (1845) 835-, 895-, 931-, 972-, 1025-。

- of six letters. Betti, E. C. R. 63 (1866) 878.

- which do not alter value of a function. Cauchy, A. L. C. R. 21 (1845) 1234-.

_ _ - _ - - - of several variables, number and form. Cauchy, A. L. C. R. 21 (1845) 1287-.

Ternary substitutions of determinant unity with integral coefficients. Burnside, $W$. Mess. Mth. 24 (1895) 109-.

Type of successive linear substitutions. Veltmann, $W$. Arch. Mth. Ps. 58 (1876) 342-.

\section{General theory of quantics.}

Absolute invariants of integral linear transformation groups. Hurwitz, A. Mth. A. 20 (1882) 125-

Algebraic forms. Combescure, É. Par. Ec. Norm. A. 1 (1864) 269-.

- - in any number of variables. Deruyts, $J$. Brux. Ac. Bll. 15 (1888) 951-.

- -, geometrical applications. Le Paige, C. M. M. H. H. [1878] Brux. Mm. Cour. $4^{\mathrm{c}}, 42$ (1879) (No. 4) $71 \mathrm{pp}$.

- - with Hessian determinant vanishing identically. Noether, $M$. Erlang. Ps. Md. S. Sb. 8 (1876) 51 -。
Quantics 2040

Algebraic forms with Hessian determinant vanishing identically. Gordan, $P$., \& Noether, M. Mth. A. 10 (1876) 547-.

- - invariants of a class. Deruyts, J. Brux. Ac. Bll. 23 (1892) 152-.

- with $p$ variables, theory of. Perrin, $R$. C. R. 104 (1887) 108-.

- - , points in theory. Le Paige, C. M. M. H. H. Liége S. Sc. Mm. 9 (1882) (No. 4) $23 \mathrm{pp}$.

_- principle of classification. Rosanes, $J$. Crelle J. 76 (1873) 313-.

- -, property. Le Paige, C. M. M. H. H. Mth. A. 15 (1879) 206-.

- - with several series of variables. Le Paige, C. M. M. H. H. C. R. 94 (1882) 31-, 69-.

- - - - variables, development by polars. Capelli, A. Rm. R. Ac. Linc. Rd. 7 (1891) (Sem. 1) 161-; 1 (1892) (Sem. 1) 3-.

- - symbolic representation. Clebsch, $A$. [1860] Berl. Mb. (1860) 536-; Crelle J. 59 (1861) 1-.

- - theorem, with application to binary sextic. Ovidio, E. d'. Palermo Cir. Mt. Rd. 6 (1892) 225-.

- -, theory. Sylvester, J. J. C. R. 101 (1885) 1110 .

- - , , arithmetical. Kronecker, $L$. Crelle J. Mth. 93 (1882) 365-.

- - , -, general. Capelli, A. Rm. R. Ac. Linc. Mm. 12 (1882) 529-.

- - , - . Deruyts, J. Liège S. Sc. Mm. 17 (1892) No. 4, vI $+157 \mathrm{pp}$.

_ - new. Sylvester, J. J. C. R. 101 (1885) 1042-, 1225-, 1461.

- - , , operations in. Capelli, A. Mth. A. 37 (1890) 1-.

Anharmonic ratio and invariant theory of complex quantities. Schick, J. [1900] Münch. Ak. Sb. 30 (1901) 249-.

Associated forms. Kohn, G. Wien Ak. Sb. 100 (1891) (Ab. 2a) 865-.

- - general theory. Sylvester, J.J. Camb. and Dubl. Mth. J. 6 (1851) 289-.

- - , theory of Clebsch and Gordan. Sylvester, J. J. C. R. 86 (1878) 448-.

Asyzygetic invariants, extension of a theorem. Sylvester, J. J. C. R. 102 (1886) 1430-.

Boolian trinomial. Cockle, (Sir) J. [1868] Mess. Mth. 5 (1871) 33-.

Canonical forms, a certain class. Roberts, R.A. [1894] N. Y. Am. Mth. S. Bll. 1 (1895) $105-$.

- - and hyperdeterminants, discovery in. Sylvester, J. J. Ph. Mg. 2 (1851) 391-.

Chemico-algebraical theory. Frankland, $E$. Am. J. Mth. 1 (1878) 345-.

Chemistry and algebra. Sylvester, J. J. Nt. 17 (1878) 284.

Combinants. Betti, E. Tortolini A. 1 (1858) 344 .

-. Gordan, P. [1871] Mth. A. 5 (1872) 95-.

-, theory. Stroh, E. Mth. A. 22 (1883) 393-.

Contrariants, a new species of invariants. Sylvester, J. J. Ph. Mg. 18 (1884) 374-. 
Contravariants, invariants and covariants. Janni, G. G. Mt. 1 (1863) 174-, 194-, 240-, 340-.

Covariant, a definition. Keyser, C. J. N. Y. Am. Mth. S. Bll. 5 (1899) 468-.

- of system of $n$ quantics. Newson, H. B. N. Y. Am. Mth. S. Bll. 2 (1896) 272-.

Covariants. Cayley, A. [1852] Crelle J. 47 (1854) 109-.

-. Jordan, C. C. R. 80 (1875) 875-.

-, algebra applied to theory. Roberts, $M$. [1861] QJ. Mth. 5 (1862) 18-.

-, composition, theorem. Jordan, C. C. R. 81 (1875) 495-.

-, computation by transvection. McClintock, E. Am. J. Mth. 14 (1892) 222-.

-, etc. Bellavitis, G. Ven. At. (1858-59) 65-, 82-.

-, formula for number of, extension to independent linear transformations. Capelli, A. Rm. R. Ac. Linc. Mm. 15 (1883) 233-.

- of 4 th order and 4 th class of two conics, simultaneous. Björling, C. F.E. Stockh. Öfv. 33 (1876) No. 3, 21-.

- - given degree, number of, for forms of any species. Capelli, A. G. Mt. 20 (1882) 293-.

-, lists. McClintock, E. N. Y. Mth. S. Bll. 1 (1892) 85-, 236.

-, note on certain. Le Paige, C. M.M.H.H. Brux. Ac. Bll. 1 (1881) 490-.

-, primary. Deruyts, J. Brux. Ac. Bll. 20 (1890) 116-.

-, property of invariants of. Kohn, G. Wien Ak. Sb. 102 (1893) (Ab. 2a) 801-.

- of a system of quantics. Story, W. $E$. Mth. A. 41 (1893) 469-.

-, theorem. Cayley, A. Mth. A. 5 (1872) 625-.

-, -. Jordan, C. C. R. 80 (1875) 1160-.

-, - Cayley, A. [1878] Crelle J. Mth. 87 (1879) 82-.

-, -, general. Faà de Bruno, F. C. R. 90 (1880) 1203-.

-, theorems. Faà de Bruno, F. [1880] Crelle J. Mth. 90 (1881) 186-.

-, theory, etc. Brioschi, F. Mil. G. I. Lomb. 8 (1856) 329-; Tortolini A. 1 (1858) 158-, 296-, 549-; 2 (1859) 82-, 265-; 3 (1860) 160 -

Critical functions. Cockle, Jas. QJ. Mth. 4 (1861) 97-, 265-.

- -. Warren, J.W. [1863-64] QJ. Mth. 6 (1864) 231-, 372-.

Cubic forms with any number of variables, property. Voss, A. Mth. A. 27 (1886) 515-.

Decomposable forms, especially cubics. Meyer, A. Zür. Vjschr. 42 (1897) 149-.

Decomposition of forms, conditions. Hadamard, -. Par. S. Mth. Bll. 27 (1899) 34-.

Differences, products. Cayley, A. QJ. Mth. 15 (1878) 174-.

Differential equations satisfied by concomitants of quantics. Forsyth, A.R. L. Mth. S. P. 19 (1889) 24-.

Equation in $S$, applications of properties of quantics to. Brisse, C. N. A. Mth. 1 (1882) 193-.
Equivalence of forms. Jordan, C. C. R. 88 (1879) 906-; 90 (1880) 1422-; Par. Éc. Pol. J. 29 (=cah. 48$)(1880)$ 111-.

Factorisable forms, theory. Rados, G. Mth. Termt. Éts. 4 (1886) 198-.

Forms, calculus. Sylvester, J.J. Camb. and Dubl. Mth. J. 7 (1852) 52-, 179-; 8 (1853) 62-, 256-; 9 (1854) 85-.

- of higher grade. Kronecker, L. Berl. Ak. Sb. (1883) 957-.

-, theorem. Voss, A. Münch. Ak. Sb. 18 (1889) 15-.

-, theorems. Cazzaniga, T. G. Mt. 38 (1900) 321-.

Functions, 2nd order. Cayley, A. Crelle J. 38 (1849) 105-.

-, similar, of several groups of functions or elements. Reiss, M. Quetelet Cor. Mth. 5 (1829) 201-.

Fundamental law, algebraic. Jacobi, C. G.J. Crelle J. 53 (1857) 275-.

- - - , remarks on Jacobi's paper. Borchardt, C. W. Crelle J. 53 (1857) 281-.

Geometric significance of algebraic forms in theory of cubic curves. Gundelfinger, $S$. Mth. A. 3 (1875) 136-.

Ground-forms and syzygants. (Extracts from letters to Sylvester.) Hammond, J. (xII) J. H. Un. Cir. [2] (1883) 59, 84-, 150.

Hessians, theory. Gravelaar, N.L.W. A. N. Arch. Wisk. 3 (*1877) 193-.

Higher algebra, note on a theorem. Dawson, H. G. Mess. Mth. 17 (1888) 69-.

Homogeneity, principle, note. Serret, $P$. N. A. Mth. 14 (1855) 312-.

Homogeneous isobaric functions, some properties. Elliott, E. B. [1892] L. Mth. S. P. 24 (1893) 21-.

Homology of sets. Cayley, A. QJ. Mth. 1 (1857) 178.

Hyperdeterminant calculus, exercises. Salmon, G. Camb. and Dubl. Mth. J. 9 (1854) 19-.

- identity. Cayley, A. Mess. Mth. 21 (1892) 131-.

Hyperdeterminants. Cayley, A. [1846-51] Crelle J. 30 (1846) 1-; 34 (1847) 148-; 42 (1852) 368-.

- and canonical forms, discovery. Sylvester, J. J. Ph. Mg. 2 (1851) 391-.

- , conditional, scholium on theorem. Sylvester, J. J. Am. J. Mth. 1 (1878) 370-.

Independent concomitants of simultaneous forms, number of. Sylvester, J.J. C. R. 84 (1877) 1359-, 1427-。

Inertia of quadratic form. Hermite, $C$. Crelle J. 53 (1857) 271-.

- - - Borchardt, C. W. Crelle J. 53 (1857) 281-.

- _ - - Buchwald, E. (xx) Ts. Mth. 2 (1872) 20-.

- - forms, demonstration of law. Presle, - de. Par. S. Mth. Bll. 15 (1887) 179-.

- - - law. Frobenius, G. Berl. Ak. Sb. (1894) 241-, 407-.

Invariant, definition. Elliott, E. B. Mess. Mth. 16 (1887) 5-. 
Invariant formation by operator $\Omega$. Note on Macmahon's theory. Sylvester, J.J. Mess. Mth. 13 (1884) 163-.

- functions associated with a transformable system. Deruyts, J. Brux. Ac. Bll. 32 (1896) 82-.

- - law of formation. Deruyts, J. Brux. Mm. Cour. $4^{\circ}, 51$ (1889) No. 10, 16 pp.

- -, mutual differentiation. Deruyts, $J$. Brux. Ac. Bll. 16 (1888) 207-.

- - number. Deruyts, J. Brux. Ac. Bll. 21 (1891) 437-.

- - property. Deruyts, J. Brux. Ac. Bll. 26 (1893) 258-.

- - reduction. Deruyts, $J$. Brux. Ac. Bll. 20 (1890) 265-; 24 (1892) 286-.

-,- - in the system of geometric variables. Deruyts, J. Brux. Ac. Bll. 24 (1892) $558-$.

- operations, a law of reciprocity. Capelli, $A$. Nap. Rd. 27 (1888) 189-.

- - which permit permutation. Capelli, A. Nap. Rd. 26 (1887) 236-; 27 (1888) 45-.

- permutative character. Capelli, $A$. Nap. Rd. 25 (1886) 135-.

- properties of system of a linear form and an alternate bilinear form. Morera, $G$. [1883] Tor. Ac. Sc. At. 18 (1882) 383-.

- sum of products of coefficients of pair of quantics. Rankine, $W . J . M$. QJ. Mth. 1 (1857) 357-.

- systems, finite character. Maurer, $L$. Münch. Ak. Sb. 29 (1900) 147-.

- - general. Maurer, L. Münch. Ak. Sb. 18 (1889) 103-.

- theory. Meyer, F. D. Mth. Vr. Jbr. 1 (1892) 79-.

- - and chemistry, relation of formulae. Gordan, P., \& Alexejeff, $W$. [1900] Erlang. Ps. Md. S. Sb. 32 (1901) 107-.

Invariantive differential operators. Capelli, $A$. Nap. Rd. 26 (1887) 110-.

Invariants. Brioschi, $F$. Tortolini A. 5 (1854) 207-, 409-.

-. Bellavitis, G. Ven. At. (1858-59) 65-, 82-; Tortolini A. 2 (1859) 137-.

- Blerzy, -. N. A. Mth. 17 (1858) 301-; 18 (1859) 420-.

-. Sylvester, J. J. C. R. 85 (1877) 992-, 1035-, 1091-.

- of algebraic forms, conception of. Study, E. Leip. Mth. Ps. B. 39 (1887) 137-.

- - - functions of forms. Wölffing, $E$. Mth. A. 43 (1893) 26-.

-, arithmetical. Poincaré, H. As. Fr. C. R. (1881) 109-.

- calculus, test operators, properties; and application to development of any power of a logarithm. Sylvester, J.J. Ph. Mg. 32 (1866) 461-.

- of a certain orthogonal transformation with reference to theory of strain and stress. Sharp, W. J. C. L. Mth. S. P. $13(1881-82)$ 216-.

- common to two quadratic forms in 2, 3 , or 4 variables. Picquet, $H$. As. Fr. C. R. (1874) 1205-.
Invariants, complete systems of. Hilbert, $D$. D. Mth. Vr. Jbr. 1 (1892) 61-; Mth. A. 42 (1893) 313-.

-, covariants, and contravariants. Foglini, G. Rm. N. Linc. At. 31 (1878) 249-.

,,---- , theory. Janni, G. G. Mt. 2 (1864) 135-, 161-, 211-.

and covariants as criteria for roots of an equation. Schramm, H. A. Mt. 1 (1867-68) 259-; 3 (1869-70) 41-.

- - - definition. Mansion, P. Brux. S. Sc. A. 12 (1888) (Pt. 1) 47-.

- - - of function transformed by a quadratic substitution. Spottiswoode, W. Rm. R. Ac. Linc. T. 7 (1883) 218-

- - - - system of three conics. Burnside, W. S. [1869] QJ. Mth. 10 (1870) 239-.

-, generation by integration. Hurwitz, $A$. Gött. Nr. (1897) 71-.

- of homogeneous forms. Mainardi, G. [1857] Mil. G. I. Lomb. 9 (1856) 208-.

-, identical equation in theory. Cayley, $A$. QJ. Mth. 12 (1873) 115-.

- of integral polynomials, certain. Laisant, C. A. Palermo Cir. Mt. Rd. 8 (1894) 249-.

-, principles deduced from theory of linear formations. Gram, J.P. (vII) [1873] Mth. A. 7 (1874) 230 -

- of quadratic forms. Kleiber, J. Z. Mth. Ps. 37 (1892) 79-.

- - - and higher geometry. Study, E. Leip. Mth. Ps. B. 49 (1897) 443-.

- - quantics. Deruyts, J. Brux. Mm. Cour. $4^{\circ}, 52(1890-93)$ No. $2,28 \mathrm{pp}$.

- in special mathematics. Campoux, P. de. N. A. Mth. 8 (1869) 395-; 9 (1870) 113-.

-, theorem, fundamental. Ovidio, E. d'. G. Mt. 15 (1877) 187-.

-, -,$\frac{-}{-}$, proof. Christoffel, E. B. [1867] Crelle J. 68 (1868) 246-.

-, -, -, -. Aronhold, -. Crelle J. 69 (1868) 185-.

-, - of Hilbert. Gordan, P. Mth. A. 42 (1893) 132-.

-, 一, proof. Dahl, C. Ts. Mth. 4 (1880) 154-.

-, theory. Spottiswoode, W. R. S. P. 7 (1854-55) 204-.

-, -. Combescure, E. Liouv. J. Mth. 20 (1855) 337-.

-, -. Faà de Bruno, F. Tortolini A. 6 (1855) 328-.

-, - Aronhold, S. H. Crelle J. 62 (1863) 281-.

-, - Cayley, A. [1870] Mth. A. 3 (1871) 268-.

, -. Christoffel, E. B. [1881] Mth. A. 19 (1882) 280-.

-, - Veltmann, -. Z. Mth. Ps. 34 (1889) $321-$

-, - Maurer, L. Crelle J. Mth. 107 (1891) 89 -

-, - Hilbert, D. Gött. Nr. (1891) 232-;

(1892) 6-, 439-.

-, - Weber, H. Gött. Nr. (1893) 109-.

-, -. Hurwitz, A. Mth. A. 45 (1894) 
Invariants, theory, elementary development of principles. Gram, J.P. (xII) Ts. Mth. 3 (1873) 1-.

- - -, foundations. Veltmann, W. Z. Mth. Ps. 22 (1877) 277-.

-, -, fundamental problem. Clebsch, R.F.A. Gött. Ab. 17 (1872) 62 pp.; Mth. A. 5 (1872) 427-.

-, - , new application. Fuchs, L. Crelle J. Mth. 81 (1876) 97-.

Involution, theory. Cayley, A. [1864] Camb. Ph. S. T. 11 (1871) 21-.

-, - , in connection with algebraic forms. Deruyts, $F$. Brux. Ac. Bll. 14 (1887) 650 -.

Involutions. Gerbaldi, $\boldsymbol{F}$. Palermo Cir. Mt. Rd. 9 (1895) 167-.

- with ordinary singularities. Guccia, G. B. Palermo Cir. Mt. Rd. 7 (1893) 49-; 8 (1894) $227-$.

Irrational covariants. Klein, $F$. Gött. Nr. (1888) 191-.

Isobaric function, properties. Amanzio, $D$. Nap. Ac. Pont. At. 17 (1887) 85-.

- functions. Faì de Bruno, F. Tortolini A. 7 (1856) 76-. - -. Stassano, P. G. Mt. 24 (1886)

- homogeneous functions. Cesáro, E. G. Mt. 22 (1884) 33-, 166.

Jacobians and polar opposites. Clifford, $W . K$. (I) Mess. Mth. 2 (1864) 229-.

Joachimsthal, theorem, remark. Lebesgue, V. A. N. A. Mth. 7 (1848) 225-

Kronecker's modular systems, and inertia of quadratic forms. Netto, $E$. Crelle J. Mth. 110 (1892) 184-.

Linear forms, theory. Hensel, $K$. Crelle J. Mth. 107 (1891) 241-.

- transformations and theory of covariants. Deruyts, J. Brux. Mm. Cour. 4 51 (1889) No. 9, $22 \mathrm{pp}$.

Multiple forms, theorem. Peano, G. Tor. Ac. Sc. At. 17 (1881) 73-.

- involutions. Vályi, G. Mth. Termt. Éts. 12 (1894) 394-; 13 (1895) 408-; 14 (1896) 381-; Mth. Nt. B. Ung. 13 (1897) 244-, 259-, 343-.

- points in systems of equations and of their Jacobian. Bareroft, D. [1886] J. H. Un. Cir. [6] (1886-87) 30.

Multiplicity, new theory. Sylvester, J.J. $\mathrm{Ph}$. Mg. 3 (1852) 460-.

Operator that produces all the covariants and invariants of any system of quantics. Story, W. E. L. Mth. S. P. 23 (1892) 265-.

Perpetuants, applications to theory of finite quantics. Hammond, J. Am. J. Mth. 8 (1886) 104-.

Projective invariants, report. White, H. S. [1898] N. Y. Am. Mth. S. Bll. 5 (1899) 161-.

Quadratic forms, formulae. Day, R. H. G. QJ. Mth. 14 (1877) 184-.

- - geometric interpretation of invariants. Hesse, L. O. Crelle J. 45 (1853) 82-.

- -, non-homogeneous. Cremona, L. Mil. I. Lomb. Rd. 4 (1867) 199-.

voL. I.
Quadratic forms, physical theories involving. Maxwell, J. C. L. Mth. S. P. 4 (1871-73) 334 .

- - in $n$ variables. Cotterill, J. H. (II) Mess. Mth. 2 (1864) 210-.

- -, reduction. Jordan, C. C. R. 93 (1881) 113-, 181-, 234-.

- - theorem, and application to small oscillations. Weierstrass, C. T. W. Berl. Mb. (1858) 207-; Berl. Ak. Mb. (1879) 430-

- - - of Weierstrass. Brioschi, F. (vr Adds.) Tortolini A. 1 (1858) 250-.

Quadrilinear forms. Le Paige, C. M.M.H.H. [1882] Tor. Ac. Sc. At. 17 (1881) 299-.

Quadriquadric function, two invariants of a. Cayley, A. Mess. Mth. 20 (1891) 68-.

Quantic. Catalan, E. C. C. R. 47 (1858) 1073-.

- with $n$ variables, reduction to one with $n-1$. Hesse, L. O. Crelle J. 42 (1851) 117-.

Quantics. Lejeune-Dirichlet, G. Berl. B. (1841) 280 -.

-. Genocchi, A. N. A. Mth. 12 (1853) 393-. - Cayley, A. Phil. Trans. 144 (1854) 245-; 146 (1856) 101-, 627- ; 148 (1859) 415-, 429-; 149 (1860) 61-; 151 (1862) 277-; 157 (1867) 513-; 161 (1871) 17-; 169 (1878) 603-; R. S. P. 23 (1875) 496 (corr.).

-. Laisant, -. Par. S. Mth. Bll. 20 (1892) 117-.

- of alternate numbers used for determining invariants and covariants of quantics in general. clifford, $W . K$. L. Mth. S. P. 10 (1878-79) 124-.

-, decomposition into linear factors. Painvin, L. C. R. 50 (1860) 84-; Liouv. J. Mth. 6 (1861) 209-.

-, general properties. Sylvester, J.J. Camb. and Dubl. Mth. J. 6 (1851) 1-.

-, - theorems. Gundelfinger, S. Mth. A. 4 (1871) 164-.

-, - theory and application to curves and

surfaces. Reiss, M. Mth. A. .2 (1870) 385-.

-, index symbol. Carmichael, R. Camb. and Dubl. Mth. J. 6 (1851) 277-; 8 (1853) 81-.

-, method. Terquem, O. N. A. Mth. 7 (1848) 5 -.

-, note. Aelt, -. N. A. Mth. 3 (1864) 289-.

-, properties. Malmsten, C.J. Ts. Mt. Fys. 4 (1871) 225-.

-, property. Gukovskij, A. N. Rs. S. Nt. Mm. (Mth.) 11 (1890) 145-.

-, theory. Hesse, L. O. Crelle J. 56 (1859) 263-.

Reciprocity, Hermite's law. Gordan, P. Gött. Nr. (1897) 182-.

-, - - extension. Deruyts, J. Brux. Ac. Bll. 22 (1891) 11-.

- laws. Igel, B. Wien Ak. D. 54 (1888) (Ab. 2) 75-.

- in theory of algebraic forms. Brioschi, F. Tortolini A. 7 (1856) 303-.

Reduced invariant functions, characteristic equations. Deruyts, J. Brux. Ac. Bll. 25 (1893) 450-.

\section{$\mathbf{K}$}


2050 Quantics

Reducibility of every quadratic form to sum of $n$ squares. Sylvester, J.J. (vi Adds.) $\mathrm{Ph}$. Mg. 4 (1852) 138-.

Representation of definite forms as sums of squares. Hilbert, D. Mth. A. 32 (1888) 342-.

Reproduction of forms. Poincaré, $H$. C. R. 97 (1883) 949-.

Resolution into linear factors. Walker, $J . J$. QJ. Mth. 6 (1864) 328-.

- - - of a form of $m$ th order and $n$th degree. Schendel, L. Z. Mth. Ps. 32 (1887) 83 -

Roots of solvable quantics as symmetric functions of homologues. Kummell, $C$. $H$. Am. J. Mth. 18 (1896) 74-.

Secondary invariants. Rogers, L. J. L. Mth. S. P. 20 (1889) 161-.

Semi-combinants as concomitants of affiliants. White, H. S. Am. J. Mth. 17 (1895) 235-.

Seminvariants. Petr, $K$. Prag České Ak. Fr. Jos. Rz. (Trída 2) 6 (1897) Art. 38, 22 pp.; Fschr. Mth. (1897) 108.

-, generalisation. Deruyts, J. Brux. Mm. Cour. 4ㅇ 51 (1889) No. 8, 20 pp.

Symbolic invariants in general theory of algebraic forms, relations. Pascal, E. Rm. R. Ac. Linc. Mm. 5 (1888) 375-.

Systems, finite, of forms in theory of rational functions. König, J. [1880?] Mth. A. 18 (1881) 69-.

Syzygetic relations among powers of linear quantics. Clifford, W. K. [1869] L. Mth. S. P. 3 (1869-71) 9-.

_ _ _ _ _ _ Clifford's paper. Cayley, A. [1891] L. Mth. S. P. 23 (1892) 99-.

Syzygies. Cayley, A. (xI) J. H. Un. Cir. [2] (1883) 150.

-, irreducible, of quantics, tables. Sylvester, J.J. Am. J. Mth. 4 (1881) 41-.

- among perpetuants, expression by means of partitions. MacMahon, (Capt.) P.A. Am. J. Mth. 10 (1888) 149-.

Transformation principle of Clebsch, and application. Gundelfinger, S. [1872] Mth. A. 6 (1873) 16-.

Trilinear forms. Le Paige, C. M. M. H. H. [1881] C. R. 92 (1881) 1048-, 1103-; 93 (1881) 264-, 509-; Rm. N. Linc. At. 35 (1882) 140-.

- - a system of two. Le Paige, C. M. M. H. H. Rm. N. Linc. At. 35 (1882) 54-。

Universal invariants. Rabut, -. C. R. 115 (1892) 926-, 1028.

Variables of different kinds, forms in several series. Deruyts, J. Brux. Ac. Bll. 28 (1894) $157-$.

\section{Binary Forms.}

Absolute invariant and covariants of a biquadratic form, geometrical representation. Lacour, -. N. A. Mth. 17 (1898) 341-.

Algebraic forms, theorem, with application to binary sextic. Ovidio, E. d'. Palermo Cir. Mt. Rd. 6 (1892) 225-.

Anharmonic ratio and absolute invariant of biquadratic. Wedekind, L. Mth. A. 17 (1880) 1-.
Binary Forms 2050

Apolarity, applications of principle. Igel, B. Wien Ak. Sb. 92 (1886) (Ab. 2) 1153-.

Asyzygetic covariants of the third degree of a binary form. Stroh, E. Mth. A. 31 (1888) 444-.

Binary cubic form, automorphic transformation. Cayley, A. L. Mth. S. P. 14 (1883) 103-.

- - geometrical representation. Ger baldi, -. Palermo Cir. Mt. Rd. 3 (1889) 276-.

_ - - relations between roots and those of first covariants. Mainardi, G. Rm. At. N. Linc. 26 (1873) 391-.

- - forms. Eisenstein, $G$. Crelle J. 27 (1844) 89.

- - Arndt, F. Grunert Arch. 17 (1851) 1-; Crelle J. 53 (1857) 309-.

- - - Hermite, C. QJ. Mth. 1 (1857) 20 -

- - - Cayley, A., \& Hermite, -. QJ. Mth. 1 (1857) 85-.

- - Battaglini, G. Nap. Rd. 3 (1864) 109-, 163-.

- - Hermite, C. Crelle J. 63 (1864) 30 -

- Beltrami, E. Bologna Ac. Sc. Mm. 9 (1869) 607-.

- - Brioschi, F. [1876] Mth. A. 11 (1877) 111-.

- - Tannery, J. Bll. Sc. Mth. As. 1 (1877) (Pt. 1) 260-.

- - Pepin, T. Rm. N. Linc. At. 37 (1884) 227-.

- - classification. Pepin, T. Rm. N. Linc. At. 39 (1886) 23-.

- - - complete form-system for three. Gall, (Frhr.) - von. Mth. A. 45 (1894) 207-.

- - - geometrical application. Torelli, G. G. Mt. 24 (1886) 270-.

- - - - interpretation. Retali, V. Palermo Cir. Mt. Rd. 2 (1888) 25-.

- - - , - representation of system of two, and their associated forms. Roberts, W.R.W. [1881] L. Mth. S. P. 13 (1881-82) 16-.

- - - imaginary elements. Pittarelli, G. G. Mt. 23 (1885) 368-.

- - , reduction. Hermite, C. C. R. 48 (1859) 351-.

- - - simultaneous. Clebsch, R. F. A. Crelle J. 67 (1867) 360-

- - - - system of two. Gundelfinger, S. Mth. A. 7 (1874) 452-.

$17(1879)$ 373-.

- - - - - three. Igel, B. Wien Ak. D. 49 (1885) (Ab. 2) 277 -

_- - system of two. Caporali, $E$. Nap. Rd. 22 (1883) 95-.

(1890) 330 -

- - - - several. Torelli, G. Nap. Rd. 24 (1885) 258-.

- -, geometrical representation. Gerbaldi, - Palermo Cir. Mt. Rd. 3 (1889) 276-.

- - and quartic forms, geometry. Nevoson,

H. B. [1893] Kan. Un. Q. 2 (1894) 85-. 


\section{Binary Forms}

Binary cubies, geometric interpretation. Retali, $V$. [1887] Palermo Cir. Mt. Rd. 2 (1888) 25-.

- decimic, reduction to canonical form. Spottiswoode, W. B. A. Rp. (1861) (pt. 2) 11-.

- evectant form. Roberts, S. QJ. Mth. 11 (1871) 42-.

- expressions, canonical form. Lerch, $M$. Prag Sb. (1883) (Mth. Vortr.) 447-.

- form, determination from initial members of covariants. Gordan, $P$. Mth. A. 40 (1892) 503-.

- - of $n$th order with a sum of powers $n^{e}$. Paolis, R. de. Rm. R. Ac. Linc. Mm. 12 (1882) 405-.

- - - - conditions for factor of $m$ th order. Igel, B. [1880] Wien Ak. Sb. 82 (1881) (Ab. 2) 943-.

- forms. Betti, E. Tortolini A. 7 (1856) 60-.

- - Brioschi, F. Tortolini A. 7. (1856) 231-.

- - Gundelfinger, S. [1871] Crelle J. 74 (1872) 87-.

- - Cassani, P. G. Mt. 10 (1872) 230 -

- -. Mollo, C. G. Mt. 27 (1889) 327-.

- - absolute invariants, partial differential equations satisfying, in higher transformations. Clebsch, R. F.A. Gött. Ab. 15 (1870) (Mth.) 65-.

- of alternate variables. Clifford, $W . K$. L. Mth. S. P. 10 (1878-79) 214-.

- -, any degree. Battaglini, G. [1866] Nap. At. Ac. Sc. 3 (1866-68) (No. 10) 34 pp.

- application to differential equation. Battaglini, G. G. Mt. 24 (1886) 128-.

-, - - plane geometry. Laguerre, $E$. C. R. 78 (1874) 744-.

- , canonical form. Cayley, A. Crelle J. 54 (1857) 48-.

- - , - - new, of groups of. Meyer, F.W. Mth. A. 21 (1883) 434-

- - class. Klein, [C.] F. [1874] (viri) Erlang. Sb. Ps. Md. S. 6 (1874) 160-; (x) 7 (1875) 49-.

- -, -. Brioschi, F. [1876] A. Mt. 8 (1877) 24-.

- -, properties. Brioschi, F. Rm. R. Ac. Linc. Rd. 2 (1886) (Sem. 1) 302-.

- -, classes, two. Maisano, G. Rm. R. Ac. Linc. Mm. 15 (1883) 44-.

- - Clebsch's theory, two points in. Ovidio, E. $d$ '. 'Tor. Ac. Sc. At. 22 (1886-87) 427-.

- - combined, invariants. Bessel, A. St. Pet. Ao. Sc. Mm. (Rs.) 14 (1868) (Suppl.) No. 3, $88 \mathrm{pp}$.

- - common factor of two. Noether, $M$. Erlang. Ps. Md. S. Sb. 27 (1896) 110-.

- - , - - - Noether's paper. Lüroth, J. Erlang. Ps. Md. S. Sb. 27 (1896) 119.

- - , construction. Thieme, H. Mth. A. 23 (1884) 597-.

- - and correlations in $n$ dimension space. Waelsch, E. Mh. Mth. Ps. 6 (1895) 261-, 375-.

- forms and elimination. Stephanos, $C$.

Par. Éc. Norm. A. 1 (1884) 329-.

\section{Binary Forms 2050}

Binary forms of equal degree, systems of, and complete system of cubics. Peano, G. Tor. Ac. Sc. At. 17 (1881) 580-.

- - and equation of 6th degree. Brill, $A$. Mth. A. 20 (1882) 330-.

- - 1st four degrees. Battaglini, G. G. Mt. 2 (1864) 170-, 193-, 243-, 340; 3 (1865) 24-, 51-, 218-.

- - _ - - pertaining to a ternary quadratic form. Battaglini, G. Nap. Rd. 4 (1865) 351-; 5 (1866) 35-, 141-.

- - - - and 2nd degrees. Battaglini, G. Nap. Rd. 3 (1864) 76-.

- -, 3rd and 4th degrees. Bessel, A. A. (xII) Rec. Mth. (Moscou) 3 (1868) (Pt. 1) 123-.

- - geometric interpretation. Wedekind, $L$. Mth. A. 9 (1876) 209-.

(1884) $111-$.

- with integral coefficients, reduction. Hermite, C. Crelle J. 36 (1848) 357-.

- - linear automorphic transformations. Klein, C. F. [1875] Mth. A. 9 (1876) 183-.

- - mixed, 3rd and 4th degrees. Battaglini, G. Nap. Rd. 3 (1864) $282-$.

- -, mutual transformation, possibility of. Clebsch, R. F. A. [1869] Mth. A. 2 (1870) 373-.

- - with prescribed discriminant. Hilbert, $D$. Mth. A. 31 (1888) 482-.

- - - functional determinant. Hilbert, D. Mth. A. 33 (1889) 227-.

- - representation. Clebsch, R. F.A., \& Gordan, P. [1867] A. Mt. 1 (1867-68) 23-.

,--- , application to hyperelliptic integrals. Gordan, P. A. Mt. 2 (1868-69) 346-.

- - - in plane and space. Laguerre, $E$. (x) Par. S. Phlm. Bll. 8 (1872) 83-.

- - - as sums of powers. Rosanes, J. [1872] Crelle J. 75 (1873) 172-.

-,$-\ldots-$ - especially $2 n$-ic by $n+1$ powers. Bauer, G. Münch. Ak. Sb. 22 (1893) 3-.

- - represented on curve of genus zero. Maisano, G. Palermo Cir. Mt. Rd. 1 (1887) 14 -.

- - with several series of variables. Le Paige, C. M. M. H. H. Brux. Ac. Bll. 2 (1881) 40-; Lisb. J. Sc. Mth. 9 (1883) 211-.

- - simultaneous, system of four. Perrin, $R$. Par. S. Mth. Bll. 15 (1887) 45-.

- - , some. Brioschi, F. [1876] Erlang. Ps. Md. S. Sb. 9 (1877) 30-.

- - , system derivable from a single form. Astis, $F$. de. G. Mt. 36 (1898) 161-.

- - systems, simultaneous. Gordan, $P$. Mth. A. 2 (1870) 227-.

- - theorem. Arcais, $F$. $d$ '. Bologna Ac, Sc. Mm. 9 (1878) 173-.

- - - - Ocagne, M. d'. C. R. 102 (1886) 916-.

- -, - and application to biquadratic. Arndt, $F$. Grunert Arch. 17 (1851) 409-.

,--- of Hilbert's. Brioschi, F. Palermo Cir. Mt. Rd. 10 (1896) 153-.

- - - , and Jacobian. Faà de Bruno, $F$. Mth. A. 18 (1881) 280-. 


\section{Binary Forms}

Binary forms, theorem on $m$ th differentials. Dawson, H. G. Mess. Mth. 18 (1889) 145-。

- - theorems, and application to multiple roots of sextic equation. Maisano, G. $\mathrm{Rm}$. R. Ac. Linc. T. 7 (1883) 231-.

- - - -, extensions. Ovidio, E. d'. Tor. Ac. Sc. At. 14 (1878) 963-.

- - theory. Hermite, C. Camb. and Dubl. Mth. J. 9 (1854) 172-.

- - - Clebsch, R. F. A. Gött. Nr. (1870) 405-

- - Noether, M. Erlang. Ps. Md. S. Sb. 13 (1881) 41-.

-, 一. Gundelfinger, S. Gött. Nr. (1883) 115-; Crelle J. Mth. 100 (1887) 413-

- -, - Deruyts, J. Brux. Ac. Bll. 14 (1887) 53-.

- - - Waelsch, E. Wien Ak. Sb. 100 (1891) $(A b .2 a)$ 574-.

- -, -, notes. Pittarelli, G. G. Mt. 15 (1877) 362-.

- - - , point in. Capelli, A. G. Mt. 16 (1878) 217-.

- - transformations,' geometric interpretation. Clebsch, R. F. A. Gött. Nr. (1871) 335-. - - with two series of variables, new symbol in theory. Pascal, E. Nap. Rd. 26 (1887) 200 -

- - vanishing covariants. Gordan, P. Mth. A. 12 (1877) 147-.

- which are complete powers, conditions for. Hilbert, D. Mth. A. 27 (1886) 158-. - - - polars of a form. Wiederhold, $P$. Mth. A. 8 (1875) 444-

- - whose variables are fundamental integrals of a linear differential equation. Vernier, $P$. Par. S. Mth. Bll. 22 (1894) 133-.

- groups with special property of combinants. Hilbert, D. Mth. A. 30 (1887) 561-

- nonic, complete system of groundforms. Sylvester, J. J. Am. J. Mth. 2 (1879) 98-.

- octavic, complete system of forms. Gall, (Frhr.) - von. Mth. A. 17 (1880) 31-, 139-, 456.

- - proof of impossibility of its possessing groundform of deg.-order 10.4. Sylvester, J.J. Am. J. Mth. 4 (1881) 62-.

- - properties. Brioschi, F. C. R. 96 (1883) 1689-.

- quadratic forms, system of three. Astis, $F$. de. G. Mt. 37 (1899) 148-.

- quantic, $a, b, c$ form. Hammond, J. L. Mth. S. P. 27 (1896) 393-.

- quantics. Brioschi, F. Tortolini A. 7 (1856) $5-, 15-, 69-$.

- -, derivatives. Cayley, A. QJ. Mth. 15 (1878) 157-.

- - reduction to canonical form. Faà de Bruno, F. Liouv. J. Mth. 17 (1852) 193-.

- quartic form, Legendre's reduction to canonical form. Warren J. [1865] QJ. Mth. 7 (1866) 338-.

- - properties of a. Brioschi, F. G. Mt. 22 (1884) 130-

- - theory. Igel, B. Mh. Mth. Ps. 10 (1899) 102-.

- forms. Arndt, F. Grunert Arch. 18 (1852) 111-.

\section{Binary Forms 2050}

Binary quartic forms. Battaglini, G. Nap. Rd. 3 (1864) 201-, 234-

- - Clebsch, R. F. A. Crelle J. 67 (1867) 371-.

- - - and elliptic functions, connection between. Study, E. Am. J. Mth. 17 (1895) 216-.

- - in involution. Battaglini, G. Nap. Rd. 3 (1864) 263-.

_ _ _ simultaneous system of two. Bertini, E. [1875] G. Mt. 14 (1876) 1-.

- - , system derivable from same quintic. Stasi, F. G. Mt. 37 (1899) 160-.

- _ and ternary cubics. Brioschi, F. [1875] A. Mt. 7 (1876) 52-.

- quintic. Battaglini, G. Rm. R. Ac. Linc. At. 2 (1875) 582-.

- -, application of theorem to. Ovidio, E. $d$ '. Palermo Cir. Mt. Rd. 7 (1893) 1-.

- -, canonical form. MacMahon, P.A. QJ. Mth. 19 (1883) 337-.

- and sextic, new canonical forms. Growe, B. E. Kan. Un. Q. 6 (1897) 201-.

- quintics. Ovidio, E. d'. Tor. Ac. Sc. At. 15 (1879) 591-.

- Maisano, G. Rm. R. Ac. Linc. Mm. 14 (1883) $97-$.

- septic. Sylvester, J. J. C. R. 87 (1878) 899-.

- , complete form-system of the. Gall, (Frhr.) - von. Mth. A. 31 (1888) 318-.

- sextic. Perrin, R. C. R. 96 (1883) 1717-, 1776-, 1842-.

-. Maisano, G. Rm. R. Ac. Linc. Mm. 19 (1884) 9-.

- - formulae relative to. Ovidio, $E$. d'. Tor. Ac. Sc. At. 27 (1892) 535-; 28 (1893) 133.

- - its syzygies and their relations. Hammond, J. Am. J. Mth. 7 (1885) 327-.

- sextics. Clebsch, R. F. A., \& Gordan, P. C. R. 64 (1867) 582-.

- - Clebsch, R. F.A. Gött. Ab. 14 (1869) $75 \mathrm{pp}$.

- - Bolza, O. Mth. A. 30 (1887) 546-.

- - with linear transformations into themselves. Bolza, O. Am. J. Mth. 10 (1888) 47 -.

Canonic roots of binary quantic of odd order. Cayley, A. Ph. Mg. 25 (1863) 206-.

- - , sequel to Cayley's theorems. Sylvester, J. J. Ph. Mg. 25 (1863) 453-.

Canonical forms, point in Sylvester's theory. Morley, F. [1900] N. Y. Am. Mth. S. Bll. 7 (1901) 202, 206.

Catalecticant is an invariant, proof. Humbert, -. As. Fr. C. R. (1888) (Pt. 2) 163-.

Combinants of algebraic binary forms. Le Paige, C. M. M. H. H. Brux. Ac. Bll. 48 (1879) 530 -

- - binary associated with curves of 4 th order. Berzolari, L. A. Mt. 20 (1892-93) 101-.

- - form-systems, etc. Gross, W. Mth. A. 32 (1888) 136-.

- linear system of quintics. Berzolari, $L$. Palermo Cir. Mt. Rd. 7 (1893) 5-. 


\section{Binary Forms}

Combinants of two binary biquadratic forms, complete system. Stephanos, C. C. R. 97 (1883) 27-.

- - forms, complete system. Stroh, E. Mth. A. 34 (1889) 321-.

Concomitant binary forms in terms of the roots. MacKinnon, (Miss) A. L. A. Mth. 9 (189495) $95-$; 12 (1898-99) 95-.

Concomitants, irreducible, of any number of binary quartics. Young, A. L. Mth. S. P. 30 (1899) 290-.

Condition for involution of two quartics. Alagna, R. Palermo Cir. Mt. Rd. 5 (1891) 301-.

Conditions that binary octavic should have 4 double points. Alagna, $R$. Palermo Cir. Mt. Rd. 4 (1890) 25-.

- form $F\left(x^{2}, y^{2}\right)$ may be expressed in terms of an exact square. Giordano, G. G. Mt. 37 (1899) 367-.

Conjugate binary forms and their geometric construction. Schlesinger, O. [1882] Mth. A. 22 (1883) 520 .

Covariant formula. Cayley, A. QJ. Mth. 16 (1879) 224-.

Covariants of binary cubic, geometrical interpretation. Cayley, A. QJ. Mth. 10 (1870) $148-$.

- _ - forms. Cayley, A. Crelle J. 50 (1855) $277-$.

- - - Betti, E. Tortolini A. 1 (1858) $129-$.

- - Jordan, C. C. R. 82 (1876) 269-; 87 (1878) 202-; Liouv. J. Mth. 2 (1876) 177-; 5 (1879) 345-

- - Le Paige, C. M. M. H. H. Brux. Ac. Bll. 49 (1880) 113-.

- - - Petersen, J. P. C. (xir) Ts. Mth. 4 (1880) 177-; 5 (1881) 33-; 6 (1889) 152-; Fschr. Mth. (1888) 117-.

- - - development. Bonsdorff, $E$. [E.J.] [1877] St. Pét. Ac. Sc. Bll. 24 (1878) 320 -

- - - geometric representation. Lindemann, C. L. F. Par. S. Mth. Bll. 5 (1877) 113-; 6 (1878) 195-。

- - reduction of two. Stroh, E. Mth. A. 22 (1883) 290-.

- - quantic. Cayley, A. B. A. Rp. 41 (1871) (Sect.) 9-.

- - - - finite number of. Cayley, $A$. QJ. Mth. 17 (1881) 137-; Mth. A. 34 (1889) $319-$

- - of $n$th degree. Roberts, M. QJ Mth. 4 (1861) 168-, 324-; 5 (1862) 144-.

- - quintic. Roberts, MI. Tortolini A. 3 (1860) 340-.

-, biquadratic (Cayley's formula). Brioschi, F. Crelle J. 53 (1857) 377-.

- of a biquadratic form, geometrical representation. Le Paige, C.M.M.H.H. Brux. Ac. Bll. 50 (1880) 115-.

- - cubo-biquadratic system. Le Paige, C. M. M. H. H. Brux. Ac. Bll. 46 (1878) 765-

-, double, of binary forms. Laguerre, $E$. (x) Par. S. Phlm. Bll. 8 (1872) 48-.

\section{Binary Forms 2050}

Covariants, elementary, of system of two biquadratic binary forms, number. Sylvester, J. J. C. R. 84 (1877) 1285-.

- fundamental, of binary cubo-quadratic system. Sylvester, J. J. C. R. 87 (1878) 242-, 287-.

-, - - - - system of 2 cubics, true number. Sylvester, J. J. C. R. 89 (1879) 828-.

-, independent, of binary forms, number. Stroh, E. Mth. A. 31 (1888) 441-.

- and invariants of binary form, theorem. Gordan, $P$. Crelle J. 69 (1868) 323-.

- - - - Brioschi, F. Tortolini A. 1 (1858) 296-, 549-; 2 (1859) 82-, 265-; 3 (1860) 160-.

Helsingf. Acta 11 (1880) 167-.

- - - - relations. Perrin, R. C. R. 96 (1883) 426-

- - - - - , residues. Perrin, R. Par. S. Mth. Bll. 11 (1883) 88-

- - - - - theory. Dienger, J. [1870] Prag Ab. 4 (1871) 47 pp.

- - - - even character of binary sextic relations. Stephanos, C. C. R. 96 (1883) 232-, 1564-.

- _ - - same binary form, relations be-

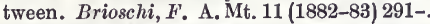
- irrational, of certain binary forms. Study, $E$. Am. J. Mth. 17 (1895) 185-.

-, irreducible, of binary octavic. Sylvester, J. J. C. R. 93 (1881) 192-, 365-.

-, - - - septic. Sylvester, J.J. C. R. 87 (1878) 505-.

-, -, - cubo-biquadratic binary system, exact number. Sylvester, J.J. C. R. 87 (1878) 477-

- linear fundamental, of two binary cubics. Ovidio, E. d'. Tor. Ac. Sc. At. 15 (1879) 267 -.

_, simultaneous, of two quartics and of two quintics. Pascal, E. A. Mt. 16 (1888-89) 85-.

-, special mode of forming. Wiltheiss, $E$. Mth. A. 35 (1890) 433-; 36 (1890) 134-; 37 (1890) 229-.

- of system of three binary forms, principle for obtaining. Igel, B. [1882] Wien Ak. D. 46 (1883) ( $A b .2) 350$ -

_, two simultaneous, of two biquadratic binary forms. Ovidio, E. $d$ '. 'Tor. Ac. Sc. At. 15 (1879) 301-.

Cubi-quadric system. Hammond, J. Am. J. Mth. 8 (1886) 138-

Cubic resolvent of a binary quartic. White, H. S. N. Y. Am. Mth. S. Bll. 3 (1897) 250 - transformation of a binary cubic form. Torelli, G. Nap. Ac. Pont. At. 18 (1888) 215-.

Determinant, functional, of any number of binary forms. Le Paige, C. M. M. H. H. C. R. 92 (1881) 688 -.

Differential operators, certain linear, interchange of variables in. Elliott, $E . B$. [1889] Phil. Trans. (A) 181 (1891) 19-.

Discriminant of binary cubic, arithmetical theorem connected with. Sylvester, J.J. C. R. 60 (1865) 1011-, 1121-。 


\section{Binary Forms}

Discriminant of binary cubic, theorem. Eisenstein, G. Crelle J. 27 (1844) 105-.

- - - quantic. Cayley, A. QJ. Mth. 10 (1870) 23.

- - calculation. Bauer, $G$. Münch. Ak. Sb. 16 (1887) 183-.

- - septic $f=a_{x}{ }^{7}$. Gordan, P. Mth. A. 31 (1888) 566-.

- - septics. Brioschi, F. A. Mt. 26 (1897) 255-.

_- - sextic. Maisano, G. [1884-85] Palermo Cir. Mt. Rd. 1 (1887) 7-; Mth. A. 30 (1887) $442-$.

- - Gordan, -. Erlang. Ps. Md. S. Sb. 17 (1885) 40-.

- - sextics. Brioschi, F. A. Mt. 1 (186768) 159.

Discriminants of binary forms. Bonsdorff, $E$. [E. J.] [1876] Helsingf. Acta 11 (1880) 191-.

- and resultant of two binary quantics, geometrical relation. Cayley, A. L. Mth. S. P. 5 (1874) 31-.

- - resultants of binary forms. Meyer, $F$. Gött. Nr. (1895) 119-, 155-; Acta Mth. 19 (1895) 385-.

Divisors of binary cubics. Pepin, T. C. R. 92 (1881) 173-.

Eliminant of two binary quantics. MacMahon, (Capt.) P. A. QJ. Mth. 23 (1889) 139-.

Factions, definition. Cayley, A. B. A. Rp. (1875) (Sect.) 10.

Finite character of number of invariants of binary forms. Mertens, $F$. Crelle J. Mth. 100 (1887) 223-.

- - - system of binary forms. Study, Erlang. Ps. Md. S. Sb. [19] (1888) 31-.

_ - Gordan, - Erlang. Ps. Md. S. Sb. [19] (1888) 35-.

- _ - _ - invariants of a binary fundamental form. Hilbert, $D$. Mth. A. 33 (1889) 223-.

Mth. A. 35 (1890) 110-.

Form system of binary forms. Gordan, $P$. Rpm. Mth. 1 (1877) 12-.

Generating functions and tables of groundforms for binary quantics, calculation. Franklin, $F$. Am. J. Mth. 3 (1880) 128-

Geometrical property of invariant II $(a b)^{2}$ of a binary $2 n$-ic, etc. Schoute, $P . H^{n+1}$ Amst. Ak. Vs. 7 (1899) 379-; Amst. Ak. P. 1 (1899) 313-.

Graphical representation of invariants and covariants, application of new atomic theory. Sylvester, J. J. Am. J. Mth. 1 (1878) 64-.

Graphs, Clifford's. Spottiswoode,W. L. Mth. S. P. 10 (1878-79) 204-.

-, - . Buchheim, A. L. Mth. S. P. 17 (1887) 80 -

-, -, application to ordinary binary quantics. Kempe, A. B. [1885] L. Mth. S. P. 17 (1887) $107-, 419$.

-, Sylvester-Clifford, application to ordinary binary quantics. Kempe, A. B. L. Mth. S. P. 24 (1893) 97-.
Binary Forms 2050

Harmonic and equianharmonic polars of binary forms. Schoute, P. H. Amst. Ak. Vs. M. 6 (1889) 277-; As. Fr. C. R. (1889) (Pt. 2) 100 -

Hessian of a binary cubic. Fontene, $G . \mathrm{N}$. A. Mth. 18 (1899) 459-.

- - - - form, geometrical illustration. Schoute, P. H. Amst. Ak. Vs. M. 6 (1889) 190 -

- - - - theorem on. Gerbaldi, $F$. Palermo Cir. Mt. Rd. 3 (1889) 22-.

- _ _ - _ - . Schoute, P. H. Palermo Cir. Mt. Rd. 3 (1889) 160-.

- - - - quantic in terms of the roots. McMahon, J. A. Mth. 5 (1889-90) 17-.

- - - - sextic, and discriminant of octavic. Maisano, G. Palermo Cir. Mt. Rd. 3 (1889) 53-; 4 (1890) 1-; 5 (1891) 152-.

- covariant of a binary quintic. Lindemann, C. L. F. [1882] Mth. A. 21 (1883) 71-

- covariants of binary biquadratic forms, geometric hints for construction. Wedekind, L. Erlang. Ps. Md. S. Sb. 7 (1875) 93-.

-, Jacobian, etc., in one dimension geometry. Newson, H. B. [1894] Kan. Un. Q. 3 (1895) 103-.

- of a product of linear functions. Franklin, F. J. H. Un. Cir. [9 (1889-90)] 31; A. Mth. 5 (1889-90) 103-.

- _ - _ _ - - identical vanishing of. Franklin, F. J. H. Un. Cir. [9 (1889-90)] 77-.

Higher algebra, theorem in (binary quantics). Russell, $R$. QJ. Mth. 21 (1886) 373-.

Identities, linear, between squares of binary forms. Meyer, F. W. Mth. A. 21 (1883) 441-.

Indefinite binary forms with conjugate indeterminates. Picard, É. C. R. 97 (1883) 745-.

Invariant of binary cubic, expression in terms of complete system. Pascal, $E$. Nap. Rd. 26 (1887) 245- ; 27 (1888) 67-.

- conditions that two binary quintics may have four common roots. Berzolari, $L$. A. Mt. 19 (1891-92) 269-.

- configuration of a binary form. Mertens, $F$. Wien Ak. Sb. 98 (1890) (Ab. 2a) 73.-

- derived forms, mutual actions. Sylvester, J. J. Crelle J. Mth. 85 (1878) 89-.

-, direct computation. Chapman, C. H. [1891] J. H. Un. Cir. [11 (1891-92)] 41-

- forms of a system of binary biquadratics. Pascal, E. Nap. Rd. 27 (1888) 402-.

- operations admitting of permutation. Capelli, A. Nap. Ac. At. 1 (1888) No. 1, 17 pp.

- processes, on binary fields of higher genus. Pick, G. Gött. Nr. (1894) 311-.

-, simultaneous, of a binary quadratic and binary biquadratic form. Clebsch, R. F.A. [1870] Mth. A. 3 (1871) 262-.

-, - quadratic, of two binary forms. Le Paige, C. M. M. H. H. Brux. Ac. Bll. 44 (1877) 365-.

- of 10th order of a sextic. Le Paige, C. M. M. H. H. Liége S. Sc. Mm. 9 (1882) (No. 3) $12 \mathrm{pp}$. 
2050 Binary Forms

Invariant of 12th order of quintic. Fà̃ de Bruno, F. QJ. Mth. 1 (1857) 361-。

- - 18th order of quintic. Le Paige, C. M. M. H. H. C. R. 92 (1881) 241-.

- $-\ldots-\ldots$ and solution of quintic equation. Hermite, C. Crelle J. 59 (1861) 304-.

- theory applied to solution of algebraic equations. Berger, A. Stockh. Öfv. (1890) 165-; Fschr. Mth. (1890) 140.

- of binary forms. Schapira, $H$. D. Mth. Vr. Jbr. 2 (1893) 63-.

- - generating functions. MacMahon, (Capt.) P. A. Am. J. Mth. 9 (1887) 189-.

Invariants of binary biquadratic. Jamet, $V$. N. A. Mth. 19 (1900) 419-.

- - forms. Capelli, A. Palermo G. Sc. Nt. 15 (1882) 59-.

---1 Hilbert, D. Leip. Mth. Ps. B. 37 (1885) 427-; Mth. A. 28 (1887) 381-.

- - Gordan, -. Liouv. J. Mth. 6 (1900) 141-.

- - - ete. (letter to $M$. Hermite). Hilbert, $D$. Liouv. J. Mth. 4 (1888) 249-.

- - _ - for higher transformations. Gordan, P. [1868] Crelle J. 71 (1870) 164-.

- - , representation. Hilbert, D. Mth. A. 30 (1887) 15 -

- - - - in two series of variables, geometrical interpretation. Kohn, G. D. Nf. Vh. (1896) (Th. 2, Hälfte 1) 5-; D. Mth. Vr. Jbr. 5 (1901) (Heft 1) 58-.

- - octavic, relation between. Alagna, $R$. Palermo Cir. Mt. Rd. 6 (1892) 77-.

- - sextic. Richmond, H.W. QJ. Mth. 31 (1900) 57-

- - - system, relations between. Torelli, $G$. Nap. Rd. 25 (1886) 126-.

-, complete systems, application of symmetric functions. Mertens, $F^{\prime}$. Krk. Ak. (Mt.-Prz.) Rz. 2 (1892) 141-; Fschr. Mth. (1892) 117 -

- and covariants of binary forms. Novi, $G$. G. Mt. 2 (1864) 306-, 321-.

- - - - - especially of 3 rd and 4 th degree. Siacci, F. A. Mt. 7 (1865) 73-.

- - - - , law of reciprocity. Sylvester, J. J. C. R. 86 (1878) 446-

- - - - quartic considered as two ternary quadrics. Burnside, W. S. [1869] QJ. Mth. 10 (1870) 211-.

- - - , fundamental, of binary forms, method for obtaining ensemble of. Sylvester, $J . J$. C. R. 84 (1877) 1113-, 1211-.

- - - irreducible, of binary forms, higher limit to number. Sylvester, J. J. C. R. 86 (1878) 1437-, 1491-, 1519-.

- - - , - to binary quintic, synoptical table. Sylvester, J. J. Am. J. Mth. 1 (1878) 370 -

$--\rightarrow$, rule for calculating number. Sylvester, $J . J .[1878]$ Mess. Mth. 8 (1879) 1-.

- of equation of $n$th degree. Studnička, F. U. Č́asopis 10 ("1881) 208-; Fschr. Mth. ("1881) 130 .

-, fundamental, of binary octavic. Sylvester, J. J. C. R. 84 (1877) 240-, 532-.

\section{Binary Forms 2050}

Invariants, fundamental, of binary quantics, limits to order and degree. Sylvester, J. J. R. S. P. 27 (1878) 11-.

- of given order and degree of binary decimic, table of numbers of. Sylvester, J.J. C. R. 89 (1879) 395-.

- - hyperelliptic equations. Brun, F. de. Stockh. Öfv. (1897) 173-; Fschr. Mth. (1897) $400-$.

- - integral polynomials, certain. Laisant, C. A. Palermo Cir. Mt. Rd. 8 (1894) 249 -.

-, irreducible, of the binary quintic and sextic. Elliott, E. B. Mess. Mth. 26 (1897) 105-.

- , - , - degrees 20 and 30 for binary septic. Hammond, J. Mth. A. 36 (1890) 255-.

-, method of research for. Elliott, E. B. B. A. Rp. (1891) 568 .

- of octavic, irreducible relations among. Alagna, R. Palermo Cir. Mt. Rd. 10 (1896) 41-.

-, Prof. Malet's, identification with Sir James Cockle's criticoids. Harley, (Rev.) R. R. S. P. 38 (1885) 45-.

-, - - - - - Nalet, J. C. R.

S. P. 38 (1885) 211-

, rational, of binary form. Alexejero, $W . G$. Fschr. Mth. (1899) 110-.

-, representation by $\theta$ functions. Bolza, $O$. Gött. Nr. (1887) 418-; Mth. A. 30 (1887) 478-.

- of simplest simultaneous binary forms. Bessel, A. [1868] Mth. A. 1 (1869) 173-.

- simultaneous, of binary forms. Gordan, $P$. Mth. A. 5 (1872) 595-.

- , - , and resultant of two binary forms of orders 6 and 3 . Ovidio, $E$. $d^{\prime}$. Tor. Ac. Sc. At. 28 (1893) 20-.

- , - of two binary forms of orders 5 and 4 , and their resultant. Ovidio, $E$. d'. Rm. R. Ac. Linc. Mm. 4 (1887) 607-.

-, theorem concerning number of. Sylvester, J. J. Ph. Mg. 5 (1878) 178-.

- of two binary forms having a common factor. Brioschi, F. Erlang. Ps. Md. S. Sb. 27 (1896) 116-.

- of orders 5 and 2 , or 5 and 3 . Ovidio, E. d'. [1881] Rm. S. It. Mm. 4 (1882) No. 2, 19 pp.

- - quartic forms, relations between. Ovidio, E. d'. Tor. Ac. Sc. At. 15 (1879) 471-.

Involution. Hesse, L. O. [1863] Crelle J. 63 (1864) 179-.

Involutionsdetermined by quartic, corresponding points. Walker, J. J. [1870] L. Mth. S. P. 3 (1869-71) 69-.

- equations, recurring formula in. Weyr, $E$. Časopis 9 ("1880) 279-; Fschr. Mth. (*1880) 544 -.

—, cubic. Le Paige, C. M. M. H. H. Wien Ak. Sb. 81 (1880) ( $A b .2)$ 845-; 83 (1881) ( $A b$. 2) $351-$.

- , higher, and invariants of binary forms. Le Paige, C. M. M. H. H. Brux. Ac. Bll. 44 (1877) 231-.

-, _, multiple points. Le Paige, C.M.M.H.H. Brux. Ac. Bll. 46 (1878) 247-. 


\section{Binary Forms}

Irreducible forms of cubo-biquadratic system, true number of. Sylvester, J.J. C. R. 87 (1878) 445-.

Isobaric functions, binary and modified. Trudi, N. G. Mt. 12 (1874) 110-

Jacobian of a biquadratic and a quadratic. Giordano, G. G. Mt. 35 (1897) 349-.

Jerrard's transformation and combinants. Igel, B. Wien Ak. D. 53 (1887) (Ab. 2) 155-。

Linear substitution, formulae for. Elliott, E. B. B. A. Rp. (1894) 581.

- transformations, theory of. Boole, G. Camb. and Dubl. Mth. J. 6 (1851) 87-.

Modular transformation of 5 th order. Invariant theory of its sextic equation. Gordan, $P$. A. Mt. 1 (1867-68) 367-.

Octahedron, forms connected with. Pittarelli, G. Rm. R. Ac. Linc. Rd. 4 (1888) (Sem. 1) $509-$.

Orthogonal concomitants. Vries, J. de. Amst. Ak. Vs. 8 (1900) 562-; Amst. Ak. P. 2 (1900) 485-.

Pencils (faisceaux) of binary cubic forms. Berzolari, L. Mth. A. 51 (1899) 473-.

- (-) - - forms with same functional determinant. Hilbert, D. Leip. Mth. Ps. B. 39 (1887) 112-.

- (-) - - - having same Jacobian. Stephanos, C. C. R. 93 (1881) 994-; Par. Mm. Sav. Étr. 27 (1883) No. 7, 138 pp.

Peninvariants of binary forms. Ocagne, $M$. $d$ '. Brux. S. Sc. A. 11 (1887) (Pt. 2) 314-; C. R. 104 (1887) 961-.

- - - Perrin, R. C. R. 104 (1887) 1097-, 1258-.

- - - Ocagne, M. d'. C. R. 104 (1887) 1364-.

- - Perrin, R. [1887] Par. S. Mth. Bll. 16 (1888) 82-.

- - - principal. Ocagne, M. d'. Par. S. Mth. Bll. 16 (1888) 183-.

Perpetuants. MacMahon, P. A. Am. J. Mth. 7 (1885) 26-, 259-; L. Mth. S. P. 26 (1895) 262-.

Point covariant with five points, construction by ruler. Morley, F. Mth. A. 49 (1897) $596-$.

Polynomials with 2 variables. Collins, $E$. St. Pét. Ac. Sc. Bll. 2 (1837) 321-.

Projective involutions. Paolis, $R$. de. Rm. R. Ac. Linc. Rd. 2 (1886) (Sem. 2) 335-.

Quadratic and biquadratic forms, system. Harbordt, F. Mth. A. 1 (1869) 210.

Quadric transformations. Spottiswoode, $W$. L. Mth. S. P. 16 (1884-85) 148-

Quartic, $a U+6 \beta H=0$, solution. Cayley, $A$. [1868] Mth. A. 1 (1869) 54-.

Quintic, the R. G. F. for the. Hammond, $J$. J. H. Un. Cir. [5] (1885-86) 37.

Regular graphs, theory. Petersen, $J$. Acta Mth. 15 (1891) 193-.

Resultant of a binary quadric and $n$-ic. White, H. S. N. Y. Am. Mth. S. Bll. 1 (1895) 11-.

- - two binary biquadratic forms, reduction to fundamental invariants. Warren, $J$. [1865] QJ. Mth. 7 (1866) 67-.
Binary Forms 2050

Resultant of two binary biquadratic forms, reduction to fundamental invariants. Ovidio, E. $d$ '. Tor. Ac. Sc. At. 15 (1879) 385-.

$-\ldots \ldots-\ldots$, and relation between their simultaneous invariants. Brioschi, $F$. Tor. Ac. Sc. At. 31 (1895) 299- or 441-

- - - cubics. Cayley, A. QJ. Mth. 6 (1864) 380 -

- - - forms, various representations. Schendel, L. Z. Mth. Ps. 33 (1888) 1-, 65-.

Resultants. Vaněcek, M. N. Prag Sb. (1888) (Mth.-Nt.) 152-.

- of binary forms, structure. Netto, E. Gött. Nr. (1895) 209-.

Semi-invariant tables. Cayley, A. Am. J. Mth. 7 (1885) 59-.

- - for the weights 11 and 12 , corrected. Cayley, A. Am. J. Mth. 14 (1892) 195-

Seminvariants. Cayley, $A$. QJ. Mth. 19 (1883) 131- ; 20 (1885) 212-.

- MacMahon, P. A. Am. J. Mth. 8 (1886) 1-.

- Ocagne, M. d'. Brux. S. Sc. A. 10 (1886) (Pt. 2) 75-.

-. Deruyts, J. Liège S. Sc. Mm. 15 (1888) No. 4, 11 pp., No. 6, 8 pp.; Brux. Ac. Bll. 19 (1890) 255-.

-. Roberts, S. [1890] L. Mth. S. P. 21 (1891) 219-.

-. Cayley, -. QJ. Mth. 26 (1893) 66-.

- of a binary quantic, etc. Elliott, E. B. Mess. Mth. 23 (1894) 91-.

- exact differential expressions connected with. Elliott, E. B. Mess. Mth. 25 (1896) 173-.

- of a given type, Cayley's number of. Elliott, E. B. L. Mth. S. P. 23 (1892) 298-.

- of infinite form, memoir. Cayley, A. Am. J. Mth. 7 (1885) 1-.

-, operators in theory. MacMahon, P.A. QJ. Mth. 20 (1885) 362-.

-, some properties. Deruyts, J. Brux. Ac. Bll. 13 (1887) 226-

- and symmetric functions. MacMahon, P.A. Am. J. Mth. 6 (1884) 131-.

一, theory. Cayley, A. QJ. Mth. 21 (1886) 92-.

Semi-(sub) invariants to binary quantics of unlimited order. Sylvester, J. J. Am. J. Mth. 5 (1882) 79-.

Sextic perpetuants for any weight $w$, proof that number of $=0$. Cayley, A. [1883] (xII) J. H. Un. Cir. [3] (1884) 13.

Steiner's covariant of binary sextic. Maisano, G. Mth. A. 31 (1888) 493-.

- _ - - Ovidio, E. d'. Tor. Ac. Sc. At. 24 (1889) 164-.

Sylvester's canonical form of binary quantics, degree $2 n-1$. Booth, $W$. QJ. Mth. 20 (1885) 270 -.

Symbol of operations in the theory of forms. Brioschi, F. Mil. I. Lomb. Rd. 22 (1889) 117-.

Symbolic calculus in theory of binary forms. Pascal, E. G. Mt. 26 (1888) 33-, 102-.

- notation of Aronhold and Clebsch. Osgood, W. E. Am. J. Mth. 14 (1892) 251-. 


\section{Binary Forms}

Symbolic notation of binary forms. Kluyver, J. C. N. Arch. Wisk. 20 (1893) 97-.

- representation of syzygants and covariants. Stroh, E. Mth. A. 36 (1890) 262-.

Syzygants of binary sextic forms. Stroh, $E$. Mth. A. 34 (1889) 306-.

-, fundamental, of binary quintic. Stroh, E. Mth. A. 34 (1889) 354-.

-, irreducible, of binary sextic. Gall, (Frhr.) - von. Mth. A. 35 (1890) 63-.

-, -, - two simultaneous cubic forms. Gall, (Frhr.) - von. Mth. A. 31 (1888) 424-.

- of two simultaneous binary biquadratic forms. Gall, (Frhr.) - von. Mth. A. 33 (1889) 197-; 34 (1889) 332-.

(1890) 154 .

- - - - - Gall, (Frhr.) - von. Mth. A. 43 (1893) 550-

Syzygies for the binary sextic, new, obtained by Aronhold's operation. Ovidio, E. d'. Tor. Ac. Sc. At. 28 (1893) 118-.

-, some classes of binary. Ovidio, E. d'. Tor. Ac. Sc. At. 28 (1893) 447-.

Tables of binary cubic forms. Cayley, A. QJ. Mth. 11 (1871) 246-.

- generating functions and groundforms of binary duodecimic. Sylvester, J.J. Am. J. Mth. 4 (1881) 41-.

- - - - - - for binary quantics of first ten orders. Sylvester, J. J. Am. J. Mth. 2 (1879) 223-.

- - - - - - - simultaneous binary quantics of first four orders, taken two and two together. Sylvester, J.J. Am. J. Mth. 2 (1879) 293-, 324-.

$-\ldots-\ldots$, reduced and representative for certain ternary systems of binary forms. Sylvester, J. J. Ä. J. Mth. 5 (1882) 241-. -, syzygy, for binary quantic. Hammond, $J$. Am. J. Mth. 8 (1886) 19-.

Tamisage, case of failure in Sylvester's theory. Hammond, J. [1882] L. Mth. S. P. 14 (1882-83) 85-.

-, - - - - Cayley, A. L. Mth. S. P. 14 (1883) 88- ; (xII) J. H. Un. Cir. [2] (1883) 85-.

Transformation of algebraic equations by covariants. Brioschi, F. Mth. A. 29 (1887) $327-$.

- - binary biquadratic form to form containing only even powers. Hesse, L. $O$. Crelle J. 41 (1851) 243-.

- - cubic form into sum of cubes. Hesse, L. O. Crelle J. 38 (1849) 262-.

- - - form, higher. Cayley, A. Mth. A. 4 (1871) 359-.

- - - forms and of the corresponding integrals. Brioschi, -. Rm. R. Ac. Linc. Rd. 4 (1895) (Sem. 1) 363-.

- - - of order not prime. Meyer, F. W. Mth. A. 21 (1883) 528-.

- - - quantics. Grow, (Miss) B. E. [1900]

N. Y. Am. Mth. S. Bll. 7 (1901) 3, 12-.

- - elliptic integral to Weierstrass's form.

Pittarelli, G. Rm. R. Ac. Linc. Rd. 4 (1888)

(Sem. 1) 703-.
Ternary Forms 2060

Transformation and reduction of binary forms. Silva, D. A. da. Lisb. Mm. 3 (1851) (Sc. Ex.) 1-.

Transvectants from powers of quadratic. Berzolari, L. Palermo Cir. Mt. Rd. 12 (1898) 258-.

Transvection of binary forms, fundamental property. Stroh, E. Mth. A. 33 (1889) 61-。

- - - - and its geometric significance. Pittarelli, G. G. Mt. 16 (1878) 225-; 17 (1879) 160-.

Twisted cubic curve, representation of binary cubic and quartic forms by. Berzolari, $L$. Palermo Cir. Mt. Rd. 5 (1891) 9-, 33-.

- - forms of fifth order on. Waelsch, E. Wien Ak. Sb. 100 (1891) (Ab. 2a) 803-.

Weierstrassian formulæ applied to binary quartic and ternary cubic. Baker, H. F. QJ. Mth. 24 (1890) 1-.

\section{Ternary forms.}

Aronhold, theorems, generalisation. Gundelfinger, S. [1870] Crelle J. 73 (1871) 175-.

Biternary forms with contragredient variables. Clebsch, R. F. A., \& Gordan, P. [1868] Mth. A. 1 (1869) 359-.

Characteristics of conic sections. Study, E. Mth. A. 27 (1886) 58-.

- Cremona's formula for. Study, E. Mth. A. 27 (1886) 102-.

-, revision of Study's theory. Zeuthen, H. G. Mth. A. 37 (1890) 461-。

Combinant $N$ of the ternary cubic. Maisano, $G$. Palermo Cir. Mt. Rd. 4 (1890) 153-.

Combinants of three ternary quadrics. Gerbaldi, F. Tor. Ac. Sc. At. 25 (1890) 390-.

Common tangents of Hessian and Cayleyan of curve. Maisano, G. [1885] Palermo Cir. Mt. Rd. 1 (1887) 46-.

Concomitants of ternary cubic, the 34. Cayley, A. Am. J. Mth. 4 (1881) 1-.

- - cubics. Dingeldey, $F$. Mth. A. 31 (1888) 157-.

- - forms, the associated. Brunyate, W.E. QJ. Mth. 25 (1891) 155-.

- - three ternary quadrics. Baker, H. F. Camb. Ph. S. T. 15 (1894) 62-.

Conditions that a quadric may be one-signed. Nanson, E. J. Mess. Mth. 25 (1896) 157-; 26 (1897) 57-.

Covariant conics and cubics of plane cubic. White, H. S. N. Y. Am. Mth. S. T. 1 (1900) 1-.

- of 9 th order of ternary cubic form, geometric significance. Gerbaldi, $F$. Tor. Ac. Sc. At. 15 (1879) 707-.

$-S$ of plane quartic, theorem on. Ciani, $E$. Palermo Cir. Mt. Rd. 14 (1900) 16-.

Covariants of plane cubic. Gordan, $\dot{P}$. N. Y. Am. Mth. S. T. 1 (1900) 9-.

- - sixth degree of ternary quartic. Maisano, G. Palermo Cir. Mt. Rd. 1 (1887) 54-.

Curves analogous to Hessians, Steinerians, and Cayleyans. Maisano, G. Palermo Cir. Mt. Rd. 1 (1887) 66-. 


\section{Ternary Forms}

Cyclicants or termary reciprocants, and allied functions. Elliott, E. B. L. Mth. S. P. 19 (1889) 377-.

Differential equations of pure ternary reciprocants. Elliott, E. B. L. Mth. S. P. 18 (1886-87) 142-.

- operators, certain linear, interchange of variables in. Elliott, E. B. [1889] Phil. Trans. (A) 181 (1891) 19-.

Discriminant of ternary cubic forms. Mangeot, S. N. A. Mth. 12 (1893) 421-.

- - form. Gordan, P. Münch. Ak. Sb. 17 (1888) 477-.

- _ - order of. Roberts, S. [1869] L. Mth. S. P. 3 (1869-71) 14-.

Eliminant of set of general ternary quadrics. Muir, T. [1899] Edinb. R. S. T. 39 (1900) 667-; 40 (1905) 23-.

Generating functions of ternariants, essay towards the. Forsyth, A. R. L. Mth. S. P. 29 (1898) 487-.

Hessian covariant of a ternary form. Wölffing, E. Mth. A. 36 (1890) 97-.

- of product of two ternary forms. Gerbaldi, $F$. Palermo Cir. Mt. Rd. 3 (1889) 60-, 280.

Hessians and Cayleyans of cubic curves. Gordan, P. N. Y. Am. Mth. S. T. 1 (1900) 402-.

- of ternary quantics, identical vanishing. Manning, H. P. J. H. Un. Cir. [9 (1889-90)] 78.

Invariant of a quadratic bi-ternary form. Cayley, A. Crelle J. 57 (1860) 139-.

- system of a ternary cubic form. Mertens, $F$. Wien. Ak. Sb. 97 (1889) (Ab. 2a) 437-.

- - - ternary forms. Mertens, $F$. Wien Ak. Sb. 95 (1887) (Ab. 2) 942-.

- of trilinear ternary form. Pasch, M. Mth. A. 52 (1899) 127-。

Invariants and covariants of ternary forms, properties. Bernardi, G. [1880] G. Mt. 19 (1881) 136-, 258-.

-, simultaneous, from which resultant of 3 ternary quadratic forms is composed. Igel, B. Wien Ak. Sb. 77 (1878) (Ab. 2) $783-$.

- of 3 termary quadratic forms. Mertens, $F$. Wien Ak. Sb. 93 (1886) ( $A b .2)$ 62-.

Involution $A F+B G+G H=0$, where the letters denote ternary quadrics. Cayley, A. Mess. Mth. 22 (1893) 182-.

Irrational covariant cubics of plane cubics. White, H. S. N. Y. Am. Mth. S. T. 1 (1900) 170-, 508.

Jacobian of net of quadrics. Schoute, P. H. Kazan S. Ps.-Mth. Bll. 9 (1900) 1-, (Prot.) $33-$.

- 3 ternary forms. Gerbaldi, F. G. Mt. 27 (1889) 33-.

Number of proper ternary $n$-ics. Cunningham, (Lt.-Col.) A. Mess. Mth. 23 (1894) 1-.

Plane cubic curves. Gerbaldi, $F$. Palermo Cir. Mt. Rd. 7 (1893) 19-.

Quadratic forms, reduction, with proof of Seeber's theorem on reduced ternary forms. Lebesgue, $V$. A. Liouv. J. Mth. 1 (1856) 401-.

Resultant of three ternary quadratic forms. Cayley, A. Crelle J. 57 (1860) 139-.

\section{Ternary Forms 2060}

Resultant of three ternary quadratic forms. Hermite, C. Crelle J. 57 (1860) 371-.

- - - - Radan, R. N. A. Mth. 8 (1869) 358-.

3 (1897) 195-.

Resultants of ternary forms. Gordan, $P$. Mth. A. 50 (1898) 113-.

Ternariants, algebraically complete systems. Forsyth, A. R. Am. J. Mth. 12 (1890) 1-, 115-.

Termary cubic form. Tanner, H. W. L. L. Mth. S. P. 27 (1896) 187 -.

- - - resolution into factors, conditions. Walker, J. J. [1865] QJ. Mth. 7 (1866) 272-.

- - - - - linear factors. Spottiswoode, W. QJ. Mth. 7 (1866) 281-.

- - - , - 3 linear factors, conditions. Brioschi, F. A. Mt. 7 (1876) 189-.

- _ - transformation to canonic form. Spottiswoode, W. [1863] Crelle J. 63 (1864) 244-.

- - - - - Brioschi, F. C. R. 81 (1875) 590

- forms. Cayley, A. Camb. and Dubl. Mth. J. 1 (1846) 97-.

- - Aronhold, S. H. Crelle J. 39 (1850) 140- ; 55 (1858) 97-.

- - Hermite, $C$. Liouv. J. Mth. 3 (1858) 37-.

- - Brioschi, F. C. R. 56 (1863) 304-. - - Clebsch, R. F. A., \& Gordan, P. [1868] Mth. A. 1 (1869) 56-.

- Gundelfinger, S. Mth. A. 4 (1871) 144 .

- - Clebsch, R. F. A., \& Gordan, P. Mth. A. 6 (1873) 436-.

- - - arising from division of circle. Eisenstein, G. Crelle J. 28 (1844) 289-; 29 (1845) 19-.

- - - canonic transformation, property of parameter. Brioschi, F. L. Mth. S. P. 12 (1880-81) 58-.

- _-, relation between 2. Cayley, $A$. Ph. Mg. 20 (1860) 512-.

- - - theory. Harnack, A. Mth. A. 9 (1876) 218-.

- and quartic forms. Hesse, L. O. Crelle J. 41 (1851) 285-.

- definite forms. Hilbert, D. Acta Mth. 17 (1893) 169-.

- form, conditions of decomposition. Hadamard, -. Bordeaux S. Sc. PV. (1896-97) 100-.

- - , representation by linear combination of 2 others. Bertini, E. Mil. I. Lomb. Rd. 24 (1891) 1095-.

- forms. Eisenstein, $G$. Liouv. J. Mth. 17 (1852) 473-.

- - Šimerka, W. Wien SB. 38 (1859) 390-.

- - Brioschi, F. A. Mt. 15 (1887-88) 235-.

- -, any degree. Battaglini, G. Nap. At. Ac. 4 (1869) (No. 3) 38 pp.

- and Aronhold's invariants. Sylvester, J. J. Ph. Mg. 5 (1853) 299-, 367-. 


\section{Ternary Forms}

Ternary forms, conditions for linear factors of. Brill, A. [1896-97] D. Mth. Vr. Jbr. 5 (1901) (Heft 1) 52-; Mth. A. 50 (1898) 157-.

- - of $4 \mathrm{th}, 5 \mathrm{th}, 6 \mathrm{th}, 7 \mathrm{th}$ orders, equations arising from certain. Brioschi, $F$. Mil. I. Lomb. Rd. 17 (1884) 401-.

- - Hilbert's method of deriving invariants, etc. White, H. S. Am. J. Mth. 14 (1892) 283-.

- -, property. Brioschi, F. G. Mt. 1 (1863) $65-$.

- -, reduced systems. Bonsdorff, E. Helsingf. Acta 14 (1885) 397-.

- with several series of variables. Capelli, A. G. Mt. 18 (1880) 17-.

- - , simultaneous, equivalent to given ternary. Forsyth, A. R. QJ. Mth. 23 (1889) 102-.

- linear forms. Study, E. Mth. A. 30 (1887) 120 -.

- quadratic forms. Battaglini, $G$. [1867] Nap. At. Ac. Sc. 3 (1866-68) (No.17 e No. 26) $26+32$ pp.

- - - automorphic transformation. Hermite, $C$. Crelle J. Mth. 78 (1874) 325-.

- - - complete invariantive system of three. Fischer, E., \& Mumelter, $K$. Mh. Mth. Ps. 8 (1897) 97-.

- _ - indefinite. Picard, É. C. R. 97 (1883) $845-$.

- - linear syzygetic relations between coefficients. Genese, R.W. B. A. Rp. (1882) 452-.

- - - positive, reduction. Charve, L. Par. Éc. Norm. A. 9 (1880) (Suppl.) 156 pp.

$-\ldots$, representation by squares. Igel, $B$. [1874] A. Mt. 6 (1875) 141-.

- - - simultaneous reduction of 2 , to canonical form. Vogt, H. N. A. Mth. 15 (1896) 441-.

- - - system of 2. Perrin, R. Par. S. Mth. Bll. 18 (1890) 1-.

,$----\frac{-}{-}$ simultaneous. Osgood, W. F. Am. J. Mth. 14 (1892) 262-.

- - - - - 3. Ciamberlini, C. G. Mt. 24 (1886) 141-.

- - - - - simultaneous. Gundelfinger, S. [1874] Crelle J. Mth. 80 (1875) 73-. - - theory. Meyer, A. Crelle J. Mth. 98 (1885) 177-; 112 (1893) 87-.

- quartic $f=x_{1}^{3} x_{2}+x_{2}^{3} x_{3}+x_{3}{ }^{3} x_{1}$, complete form system of. Gordan, P. Mth. A. 17 (1880) 217-; 20 (1882) 487-.

- - $f=x_{1}{ }^{3} x_{2}+x_{2}{ }^{3} x_{3}+x_{3}{ }^{3} x_{1}$, typical representation. Gordan, P. Mth. A. 17 (1880) 359-.

- quartics. Joubert, P. C. R. 56 (1863) 1045-, 1088-, 1123-.

- - Scherrer, F. R. A. Mt. 10 (1880-82) $212-$

- - invariant systems of. Maisano, $G$. G. Mt. 19 (1881) 198-.

- and quaternary cubic forms. Poincaré, $H$. Par. Éc. Pol. J. 31 (=Cah. 50) (1881) 199-; Cah: 51 (1882) 45-.

Vanishing determinants of 3rd degree, in ternary linear forms. Pasch, M. Mth. A. 44 (1894) 89-.
Algebraic Forms 2070

Vanishing functional determinants, ternary forms with. Pasch, M. Mth. A. 18 (1881) 93-.

Weierstrassian formulæ applied to binary quartic and ternary cubic. Baker, H.F. QJ. Mth. 24 (1890) 1-.

\section{Special developments asso- ciated with forms in more than three variables.}

Algebraic forms, finite invariant system. Hilbert, D. Gött. Nr. (1888) 450-; (1889) 25-, 423-; Mth. A. 36 (1890) 473-.

- - - - - Gordan, P. Gött. Nr. (1899) 240 -.

Biquadratic (quadri-quadric) forms. Frobenius, G. Crelle J. Mth. 106 (1890) 125-.

Characteristic determinant of any $n$ quantics, property. MacMahon, $P$. A. B. A. Rp. (1900) 644.

Covariants of quadratics. Mertens, F. Krk. Ak. (Mt.-Prz.) Pam. 12 (1886) 1-; Fschr. Mth. (1886) 103-.

Equation connected with quadrics. Sauvage, $L$. N. A. Mth. 14 (1895) 369-.

Hermite's form, transformation. Loewy, $A$. Gött. Nr. (1900) 298-.

Hessian of composite quaternary cubic form. Walker, J. J. [1865] QJ. Mth. 7 (1866) $347-$.

- - a quaternary function. Cayley, A. QJ. Mth. 12 (1873) 193-.

- - - manifoldness in space of 4 dimensions. Ascione, E. G. Mt. 31 (1893) 210-.

Invariant systems of quaternary forms. Mertens, $F$. Wien Ak. Sb. 98 (1890) (Ab. 2a) $691-$.

- theory of $n$-ary forms. Pascal, E. Rm. R. Ac. Linc. Rd. 4 (1888) (Sem. 1) 119-.

Invariants, application of a known theorem to. Rosanes, J. Mth. A. 23 (1884) 412-.

-, simultaneous, of two quadratic forms. Segre, C. Mth. A. 24 (1884) 152-.

- of 3 quaternary quadratic forms. Mertens, F. Wien Ak. Sb. 99 (1891) (Ab. 2a) 367-.

Jacobian of 4 algebraic surfaces, its singularities. Gerbaldi, $F$. Palermo Cir. Mt. Rd. 10 (1896) 158-.

Quadratic form in $m$ variables, decomposition into sum of $m-n$ squares. Benoit, -. N. A. Mth. 5 (1886) 30-.

- - with non-vanishing determinant, characteristic of a real. Loewy, A. Mth. A. 52 (1899) 588-.

- - in $n$ variables, conditions for reduction of number. Lévy, $M$. C. R. 86 (1878) 463-.

- - - - - transformation to $n$ squares. Studnickka, F. J. Prag Sb. (1888) (Mth.-Nt.) 256-.

- forms, adjoint. Rados, G. Mth. Termt. Ets. 14 (1896) 26-; Mth. Nt. B. Ung. 14 (1898) 85-.

- - algebraic theory. Kronecker, L. Berl. Mb. (1872) 490-. 


\section{Algebraic Forms}

Quadratic forms, algebraic theory. Darboux, $G$. Liouv. J. Mth. 19 (1874) 347-。

- - , families, algebraic reduction. Kronecker, L. Berl. Ak. Sb. (1890) 1375- ; (1891) 9-, 33-.

_- Gauss's method for canonisation. Somov, O. I. [1873] St. Pet. Ac. Sc. Mm. (Rs.) 23 (*1874) 26-.

- - symmetric. Schapira, H. D. Mth. Vr. Jbr. 3 (1894) 99-.

- - systems (priority question). Jordan, C. C. R. 78 (1874) 1763-.

- -, theory. Morera, $G$. Mil. I. Lomb. Rd. 19 (1886) 552-.

- - - Jamet, V. Mars. Fac. Sc. A. 10 (1900) Fasc. 5, $18 \mathrm{pp}$.

- - with 2 series of variables. Le Paige, C. M. M. H. H. C. R. 94 (1882) 424-.

- and Hermite forms, families of real. Loewy, A. Crelle J. Mth. 122 (1900) 53-.

- n-ary forms. Cauchy, A. L. C. R. 44 (1857) $361-, 416$.

$-\ldots-$ criteria for reduction to $m$-ary $(m<n)$. Valyi, G. Mth. Termt. Ets. 5 (1887) 226- ; Mth. Nt. B. Ung. 5 (1886-87) 16-.

- - - multiplication. Hurwitz, A. Gött. Nr. (1898) 309-.

Quadrilinear form, and surfaces of the 3rd order. Le Paige, C. Brux. Ac. Bll. 8 (1884) 555-.

Quaternariants, algebraically complete system. Mair, D. B. [1894] Camb. Ph. S. T. 16 (1898) 1-.

-, - - systems. Forsyth, A.R. Camb. Pb. S. T. 14 (1889) 409-.

Quaternary cubic forms. Salmon, G. Phil. Trans. (1860) 229-.

_- - transformation. Clebsch, A. Crelle J. 58 (1861) 109-.

- forms with 2 series of variables. Autonne, -. Brux. Mm. Cour. $4^{\circ}, 59$ (1901) (Pt. 2) $254 \mathrm{pp}$.

- $n$-ics, number of proper. Cunningham, (Lt.-Col.) A. Mess. Mth. 23 (1894) 8-.

- quadratic forms, positive. Korkine, A., \& Zolotareff, G. Mth. A. 5 (1872) 581-.

$-\ldots,-$, reduction. Charve, L. Par. Éc. Norm. A. 11 (1882) 119-.

- quartic forms, representation as sums of 10 biquadratics. Reye, T. Crelle J. Mth. 78 (1874) 123-.

Reduction of $2 u=\left(u_{11}, u_{12}, \ldots \times x_{1}, x_{2}, \ldots\right)^{2}$ to a sum of squares. Hathaway, A. S. J. H. Un. Cir. [3]'(1884) 57-.

Semi-covariants of $n$-ary forms. Gallucci, $G$. G. Mt. 35 (1897) 206-.

Sums of 8 squares, products. (Extension of theorem of Euler's.) Young, J. R. Ir. Ac. P. 4 (1850) 19-.

Sylvester's canonical form of quaternary cubic. Reye, T. Crelle J. Mth. 78 (1874) 114-.

System $(s \phi-\psi)$ of quadratic forms. Weierstrass's theorem on multiple roots of discriminant. Hovestadt, $H$. Z. Mth. Ps. 26 (1881) 392-.

Ternary and quaternary linear transformations, generating systems. White, H.S. Am. J. Mth. 14 (1892) 274-.

\section{Theory of Equations 2400}

\section{Theory of Equations. 2400 General.}

Addition-substitutions in algebraic equations. Bertelsen, B. P. N. Ts. Mth. 9 (B) (1898) 84-; Fschr. Mth. (1898) 73.

Algebraic equation, depression of, owing to linear relation between roots. Sayre, H. $A$. [1891] A. Mth. 6 (1891-92) 45-.

- and its derivative, relation between roots. Berg, F. J.van den. N. Arch. Wisk. 9 ("1882) $1-, 60 ; 11$ (1884) 153-; 15 (1888) 100-; Fschr. Mth. ("1882) 51; (1884) 76; (1888) 75-.

- equations. Siebel, A. Arch. Mth. Ps. 56 (1874) 422-; 57 (1875) 73-, 350-; 58 (1876) $127-; 60$ (1877) 138-; 61 (1877) 122-; 65 (1880) 394-; 67 (1882) 375-.

_-. West, É. C. R. 91 (1880) 598-, 664-. - - application of form theory to. Szabó, P. Orv.-Termt. Éts. (Termt. Szak) (1894) 153-.

- and an arithmetical progression, theorem. Volpicelli, P. N. A. Mth. 14 (1855) 120-。 - _ - Cotes's theorem. Beltrami, E. G. Mt. 1 (1863) 123-.

- -, rationalisation. Förstemann, W. A. Crelle J. 14 (1835) 236-.

- - - - Cayley, A. Camb. and Dubl. Mth. J. 8 (1853) 97-.

- - theorems. Mathieu, É. N. A. Mth. 15 (1856) 409-.

- -, -. Lucas, F. C. R. 78 (1874) 431-.

- - -, general. Laguerre, $E$. N. A. Mth. 19 (1880) 241-.

- - theory. Brassinne, E. Toul. Mm. Ac. 1 (1863) 23-.

_- - - König, J. Mth. A. 21 (1883) 424-. _- _, developments. Kronecker, L. Berl. Ak. Mb. (1879) 205-.

_ _, an extension. Sylvester, J.J. C. R. 58 (1864) 689-.

- - whose highest powers have small coefficients. Morton, W. B. QJ. Mth. 31 (1900) 247-.

- (repeated) transformation, principle of. Schapira, H. Heidl. Nt. Md. Vh. 4 (1892) 25-.

- transformation. Horner, W. G. [1823] Mathematician 1 (1845) 108-, 136-, 311-; 2 (1847) 32-, 129-.

Alternate functions and cyclotomy. Cauchy, A. L. C. R. 10 (1840) 178-.

Coefficients in a transformed equation. Lodge, A. Mess. Mth. 12 (1883) 178-.

Complex magnitudes applied to solution of equations. Lerch, M. Časopis 20 (1891) 265-, 302-; Fschr. Mth. (1891) 83.

Construction of equations, historico-critical notice. Favaro, A. Mod. Ac. Sc. Mm. 18 (1878) 127-; 19 (1879) 233-.

Cubic and higher involutions. Vries, J. de. Mh. Mth. Ps. 5 (1894) 1-.

- involutions. Vries, J. de. Palermo Cir. Mt. Rd. 5 (1891) 289-; Amst. Ak. Vs. 3 (1895) 64-. 
Differential operators, use in theory of equations. Hammond, J. L. Mth. S. P. 14 (1882-83) 119-.

Equation whose roots are functions of roots of 2 equations. Mackenzie, J. L. Edinb. Mth. S. P. 5 (1887) 59-.

- $-1-\ldots-1$ roots of given equation. Amigues, E. N. A. Mth. 11 (1892) 245-.

Equations, Adeler, -. Bordeaux Ac. Sé. Pbl. (1834) 50-.

-, algebraic theory. Pellet, A. E. Par. S. Mth. Bll. 15 (1887) 61-.

-, clearing surds from. Allman, W. [1813] Phil. Trans. (1814) 23-.

-, congeneric surd. Horner, W. G. Ph. Mg. 8 (1836) 43-.

-, identities in theory. Brioschi, F. Tortolini A. 5 (1854) $301-$.

_, impossible. Cockle, Jas. Ph. Mg. 36 (1850) 281-.

-, - Finlay, R. Manch. Ph. S. Mm. 9 (1851) 236-.

-, - and surd. Harley, $R$. Manch. Ph. S. Mm. 9 (1851) 207-

- infinitesimal theory of, and implicit functions. Pellet, A. Par. S. Mth. Bll. 23 (1895) 7-.

- with integral coefficients. Kronecker, $L$. Crelle J. 53 (1857) 173-.

- - - Runge, C. Gött. Nr. (1899) 89-.

-, metaphysics of. Cossali, P. Padova N. Sag. 1 (1817) 159-.

- with rational coefficients. Dedekind, $R$. D. Mth. Vr. Jbr. 1 (1892) 33-

-, rationalisation, direct method. Scheffler, $H$. Grunert Arch. 13 (1849) 389-.

- in tenth book of Euclid's elements. Christensen, S. A. Ts. Mth. 6 (1889) 161-; Fschr. Mth. (1888) 5 .

-, theory. Abbati, P. Mod. Mm. S. It. 10 (1803) 385-

-, -. Cockle, Jas. Ph. Mg. 27 (1845)

292-.

-, - Catalan, E. C. C. R. $47^{\circ}$ (1858)

$797-$.

-, -. Becker, J. K. Grunert Arch. 34 (1860) 288-

-, -. Buys-Ballot, C. H. D. Amst. Vs. Ak. 13 (1862) 430-.

- -. Cockle, (Sir) J. [1863] Manch. Lt. Ph. S. P. 3 (1864) 171-.

-, - Brassinne, E. [1864] Toul. Mm. Ac. Sc. 3 (1865) 190-

- - I'etersen, J. P. C. (xII) Ts. Mth. 1 (1865) 57-; 3 (1867) 46; 5 (1869) 174-; 3 (1879) 170-; 6 (1882) 3-。

-, -. Laurent, H. [Herm.] C. R. 62 (1866) 140-

-, -, algebraic transformation, and quadruple algebra. Cockle, Jas. Ph. Mg. 3 (1852) 436-.

_, - analysis. Cockle, Jas., \& Davies, Ph. Mg. 32 (1848) 351-

-, _, - - Cockle, Jas. Ph. Mg. 37 (1850) 493-.

-, - , application of rational mechanics to. Lucas, F. $\quad$ C. R. 89 (1879) 224-
Equations, theory, formulæ. Hudson, C. QJ. Mth. 18 (1882) 74-.

-, -, general, note. Vieille, J. N. A. Mth. 6 (1847) 174-

-, -, historical note. Bertrand, J. N. A. Mth. 14 (1855) 30-.

,,--- - Tortolini, B. C. R. 47 (1858) $598-$.

-, - illustration of theorem. Cayley, $A$. Mess. Mth. 11 (1882) 111-.

-, -, synopsis. Cayley, A. Mess. Mth. 5 (1876) 39-.

- whose roots have their real parts negative. Hurwitz, A. Mth. A. 46 (1895) 273-.

Equivalent equations, some. Taylor, C. QJ. Mth. 10 (1870) 93-.

Fourier's "Analysis," error in. Murphy, $R$. Ph. Mg. 11 (1837) 38-

Homogeneous functions of 2 polynomials of the same degree. Mirman, L. N. A. Mth. 4 (1885) 173-.

"Invention nouvelle en l'algèbre" (reproduction of 16th century work by Albert Girard). Bierens de Haan, D. N. Arch. Wisk. 11 (1884) 83-.

Irrationality in solution of $f(x y z)=0 . \$$ Enriques, F. Rm. R. Ac. Linc. Rd. 4 (1895) (Sem. 2) 311-; Mth. A. 49 (1897) 1 -.

Irrationals, algebraic, theory. Capelli, A. Nap. Rd. 30 (1891) 61-.

Isobaric calculus. Cesáro, $E$. N. A. Mth. 3 (1884) 561-; 4 (1885) 59-, 578.

Logic of characteristics. Cayley, $A$. QJ. Mth. 1 (1857) 257-.

Number of variations of a polynomial whose coefficients depend on a parameter. André, $D$. Par. Éc. Norm. A. 2 (1885) 75-.

Progressions, geometrical, Euler-Pfaff theorem. Grunert, J. A. Grunert Arch. 2 (1842) 220 -

-, - - - Luchterhandt, A.R. Grunert Arch. 3 (1843) 305-.

-, - , - - -. Göpel, A. Grunert Arch. 3 (1843) 394-.

Quadri-quadric porism. Mathews, G. B. QJ. Mth. 30 (1899) 16-.

Quintic surds, 2, rationalisation of expression. Tortolini, B. (viII) A. Mt. 4 (1861) 285-.

Radicals, removal from equations. Mooney, $D$. [1796] Ir. Ac. T. 6 (1797) 221-.

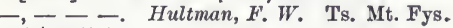
4 (1871) 1-

Rational functions of $n$ elements, and general theory of equations. König, J. [1878] Mth. A. 14 (1879) 212-.

Reviews of books by Fourier and Drobisch. Gartz, -. Grunert Arch. 1 (1841) 225-

Roots of derived equation, property. Legebeke, G. J. [1881] N. Arch. Wisk. 8(*1882) 75-; Arch. Néerl. 16 (1881) 273-.

System of trigonometrical equations, with applications to porisms of coaxal conics. Wolstenholme, J. QJ. Mth. 10 (1870) 356-; 11 (1871) 66-.

Systems of equations with peculiarities. Krey, H. [1881] Mth. A. 19 (1882) 497-. 


\section{Elements of the theory of algebraic equations; existence of roots, symmetric functions; rational fractions; partial frac- tions.}

Algebra, fundamental theorem, proof. Mertens, F. Wien Ak. Sb. 97 (1889) (Ab. 2a) 1505-.

,$- \ldots$, and solution of equations by square roots. Vahlen, K. T. Acta Mth. 21 (1897) 287-.

Algebraic equation, roots of derivative. Berloty, -. C. R. 99 (1884) 745-, 832.

,$---1-$ Cayley, A. C. R. $110(1890)$ 174-, 215-.

- equations, a law of inertia for. Meyer, $F$. Gött. Nr. (1891) 279-.

Cauchy's theorem. ZZbikovskij, A. K. Kazan S. Nt. (Ps.-Mth.) P. 6 (1888) 240 -.

Coefficients in expansions invol ring symmetric expressions. Anglin, A. H. Liouv, J. Mth. 2 (1886) 139-.

- expressed as functions of sums of like powers of roots. Janni, V. Nap. Rd. 18 (1879) 199-.

- and sums of powers of roots, relations between. Cauchy, A. L. C. R. 12 (1841) 698-, 813-.

Decomposition of certain expressions into 2 squares. Réalis, S. N. A. Mth. 4 (1865) 451-.

Development of an algebraic fraction. MacMahon, P. A. Am. J. Mth. 6 (1884) 287-。

Diaphoric functions. Tanner, H.W.L. Mth. Gz. 1 (1900) 152-.

- - (Tanner). Genese, R. W. Mth. Gz. 1 (1900) 184.

Differences of roots, analytic use. Minich, S.R. [1875] Rm. R. Ac. Linc. At. 3 (1876) (Pt. 2) 303-.

Differential equations in theory of asymmetric algebra. Forsyth, A. R. Camb. $\mathrm{Ph}$. S. T. 16 (1898) 291-.

Discriminant of cubic equation and quadratic expression $Q^{2}-3 P P^{\prime}$, relation. Young, J. $R$. [1876] Ir. Ac. P. 2 (1875-77) 744-.

Equation of differences. Cayley, A. Tortolini A. 2 (1859) 365-; Phil. Trans. (1860) 93-.

- - nth order, and system of $n$ equations satisfied by roots. Antaev, S. N. [1897-1900] Rec. Mth. (Moscou) 20 (1899) 33-; 21 (1901) 1-; Fschr. Mth. (1897) 92 ; (1900) 94.

- for product of differences of (all but one) roots of an equation. Cayley, A. Phil. Trans. (1861) 45-.

- of quotients of roots. Terquem, $O$. N. A. Mth. 4 (1845) 516-.

- with roots in arithmetical progression. Viaggi, $F$. G. Mt. 15 (1877) 376-.

- roots of which are diminished by $p$, relations between coefficients. Lobatto, $R$. Amst. Vs. Ak. 4 (1856) 208-.

-, similar functions of roots. Kronecker, $L$. Liouv. J. Mth. 19 (1854) 279-.
Equation of squares of differences. Desmarest, E. N. A. Mth. 1 (1842) 169-

- - Tarnier, -. N. A. Mth. 3 (1844) 410-.

$--1-$ Pegado, M. Lisb. A. 2 (1858) 19-.

- - - progressive development. Minich, S. R. Ven. At. (1858-59) 343-.

- - _ - - of roots, existence of relation among coefficients. Ellis, R.L. (vr Adds.) Camb. Mth. J. 1 (1839) 256-.

- - - - - and geometrical application to tangents to a curve from one point. Joachimsthal, $F$. N. A. Mth. 9 (1850) 98-.

- - sums of roots taken 2 together. Gerono, G. C. N. A. Mth. 2 (1843) 128-.

- with 2 roots rationally expressible in terms of others (Brioschi). Genocchi, A. N. A. Mth. 16 (1857) 95-.

Equations with equal differences of roots. Gatti, S. [1876] G. Mt. 15 (1877) 28-.

- - - - Viaggi, $F^{\prime}$. G. Mt. 15 (1877) 376-.

-, formulae in theory. Sharp, W. J. C. Mess. Mth. 9 (1880) 188-.

-, irreducible, relations of roots. Schönemann, T. [1852] Wien D. 5 (1853) 143-.

- , - - - - (Schönemann). Ettingshausen, A. von. Wien SB. 8 (1852) 442-.

-, proof that roots have form $p+q \sqrt{-1}$. Crelle, A. L. Berl. Ab. (1829) 1-.

-, resolvability into factors of 1 st or $2 \mathrm{nd}$ degree, Laplace's proof, etc. Hube, $K$. (vi Adds.) Krk. Roczn. Uniwers. 3 (1818) 92-.

- with roots expressible as linear functions of one root. Pellet, A. E. Bll. Sc. Mth. As. 4 (1880) (Pt. 1) 262-.

- - in geometrical progression. Terquem, O. N. A. Mth. 5 (1846) 210 -

- - - progression. Guilmin, A. N. A. Mth. 8 (1849) 242-.

-; theorem that complex roots occur in pairs, Gauss's proof. Combette, E. (xII) Brest S. Ac. Bll. 7 (1871) 28 (bis)-.

-, theory. Gauss, C. F. Gött. Ab. 4 (184850) 3-.

-, - , fundamental principle. Du Bourguet, -. Gergonne A. Mth. 2 (1811-12) 338-.

- - - - - Bret, - . Gergonne A. Mth. 3 (1812-13) 31-.

-, - , - - Du Bourguet, -. Gergonne A. Mth. 3 (1812-13) 94-.

-,,$--\ldots$ Bret, - Gergonne A. Mth. 3 (1812-13) 369-.

,,---1 . Encontre, D. Gergonne A. Mth. 4 (1813-14) 201-.

-, - , - - Liouville, J. Liouv. J. Mth. 4 (1839) 501-; 5 (1840) 31-.

_, - _ - - Woodhouse, L. G. Teix. J. Sc. 6 (1885) 177-.

-, -,- theorem. Hocks, H. Z. Mth. Ps. 28 (1883) 123-.

-, - - - Cauchy's. Rudio, F. Zür. Vjschr. 39 (1894) 345-.

,,$-- \ldots-$ Mourey's proof. Grunert, J. A. Grunert Arch. 1 (1811) 81-. 


\section{Existence of Roots}

Equations, theory, fundamental theorem, proof. Stern, M. A. Crelle J. 23 (1842) 370 -

11 (1848) $218-$.

Every equation in one unknown can be satisfied by a complex value of this unknown, geometric proof. Wittstein, T. Grunert Arch. 6 (1845) 225-.

- rational integral function vanishes for some real or complex value of variable, proof. Stieltjes, T. J. (jun.) N. Arch. Wisk. 9 (*1882) 196-.

\section{EXISTENCE OF ROOTS.}

Murphy, R. Ph. Mg. 2 (1833) 60-, 220-.

Henry, J. (vi Adds.) Ph. Mg. 3 (1833) 417. (proof.) Clausen, T. As. Nr. 17 (1840) 325(-) Deahna, F. Crelle J. 20 (1840) 337-.

(-.) Eisenstein, G. Crelle J. 29 (1845) 96.

- Staudt, G. K. C. von. Crelle J. 29 (1845) 97-.

(-.) Goodwin, H. Camb. and Dubl. Mth. J. 2 (1847) 224-

(一.) Airy, (Sir) G. B. [1858] Camb. Ph. S. T. 10 (1864) 283-, 327-

(一) Cayley, A. Ph. Mg. 18 (1859) 436-.

-. Challis, J. Ph. Mg. 19 (1860) 46-

- Foscolo, G. G. Mt. 2 (1864) 13-

-. Young, G. P. Cn. J. 9 (1864) 26-

-. De Morgan, A. L. Mth. S. P. 1 (1866) No. 6, 1 p.; Camb. Ph. S. P. 1 (1866-67), 24 -

(-.) Laurent, $H$. Les Mondes 11 (1866) 438-. -.) Walton, $W$. [1869] QJ. Mth. 11 (1871) 178-.

(-.) Clifford, W. K. [1870] Camb. Ph. S. P. 2 (1876) 156-

(-.) Gordan, P. Erlang. Ps. Md. S. Sb. 8 (1876) 138-

(-.) Macnie, J. Des Moines Anal. 5 (1878) $80-$.

(-.) Malet, J. C. [1878] Ir. Ac. T. 26 (1879) 453 -

(-) Mansion, P. (xI) Brux. S. Sc. A. 4 (1880) (Pt. 2) 99-.

(-.) Netto, E. Crelle J. Mth. 88 (1880) 16-. -.) Dutordoir, H. [1882] (xII) Brux. S. Sc. A. 7 (1883) (Pt. 1) 52-; (Pt. 2) 437-.

(-.) Mansion, $P$. (xII) Mathesis 3 (1883) (Suppl.) 14 (bis)-.

(-.) Maksimovīch, V. P. (xII) Kazan S. Nt. (Ps.-Mth.) P. 1 (1883) [No. 7] 49-.

(一.) Walecki, - . N. A. Mth. 2 (1883) 241-. - Holst, E. Christiania F. (1886) No. 1, 8 pp.; Acta Mth. 8 (1886) 155-.

(-.) Juel, C. Ts. Mth. 5 (1887) 161-; Fschr. Mth. (1887) 70.

(-.) Laisant, C. A. Par. S. Mth. Bll. 15 (1887) 42-.

(-.) Zeuthen, H. G. N. Ts. Mth. 1 (B) (1890) 65-; Fschr. Mth. (1890) 105-.

(一.) Amigues, E. C. R. 112 (1891) 212-.

-. Brunel, -. Bordeaux S. Sc. Mm. 2 (1891) i-.

(-.) Carvallo, E. N. A. Mth. 10 (1891) 109-.
Existence of Roots 2410

(proof.) Loria, G. Bb. Mth. (1891) 99-; (1893) 47-; Rv. Mt. 1 (1891) 185-; 2 (1892) 37-; 3 (1893) 105-.

(-) Méray, C. Bll. Sc. Mth. 15 (1891) 236-.

(-.) Phragmén, E. Stockh. Öfv. (1891) 113-.

(一.) Weierstrass, K. Berl. Ak. Sb. (1891) 1085-.

(一) Mertens, F. Mh. Mth. Ps. 3 (1892) 293-.

(-) Mansion, P. Brux. S. Sc. A. 17 (1893) (Pt. 1) 65-.

(-.) Bôcher, M. N. Y. Am. Mth. S. Bll. 1 (1895) 205-.

(-.) Carnoy, J. Rm. N. Linc. At. 53 (1900) $167-$.

(一) Lamb, H. Manch. Lt. Ph. S. Mm. \& P. $43(1900)$ No. 7, 4 pp.

d'Alembert's theorem. Brisse, C. Par. Éc. Pol. J. 56 (1886) 163-.

- - Catalan, E. Rv. Sc. 43 (1889) 265-.

- - . Durand-Grêville, E. Rv. Sc. 43 (1889) 266-.

- -. Vivanti, J. Rv. Sc. 43 (1889) 267.

- - Lougraire, L. de. Rv. Sc. 43 (1889) $267-$.

- -. L. Rv. Sc. 43 (1889) 268-.

- -. Delbœuf, J. Rv. Sc. 43 (1889) 269-.

- - Jamet, $V$. Mathesis 14 (1894) 5-; N. A. Mth. 14 (1895) 437-.

Cauchy's proof, simplification. Sussmann, $J$. Crelle J. 44 (1852) 57-.

(proof) and Cauchy's theorem on imaginary roots. De Morgan, A. [1857] Camb. $\mathrm{Ph}$. S. T. 10 (1864) 261-.

Gordan's proof. Dalwigk, F. von. Mth. A. 34 (1889) 158-.

of higher equations. Burg, A. Wien Jb. Pol. I. 17 (1832) 141-; 19 (1837) 155-.

_-, 2 proofs. Uulherr, J. C. Crelle J. 31 (1846) 231-.

proof that every equation has root of form $p+q \sqrt{-1}$. Ullherr, J. C. D. Nf. Vsm. B. (1845) 85-.

of a rational integral equation. Elliott, E. B. L. Mth. S. P. 25 (1894) 173-.

$n$ roots in every equation of $n$th degree. Scheffler, H. Grunert Arch. 15 (1850) 390-.

_ _ _ _ - Valeriani, $V$. G. Mt. [13 (1875)] 33-.

24 (1887) 257-.

Mg. 17 (1859) 112-.

G. B. Ph. Mg. 17 (1859) 176-

- _- - - - - - (reply to Airy).

Challis, J. Ph. Mg. 17 (1859) 283-.

trinomial and quadrinomial equations. Pineto, $Z$. St. Pet. Ac. Sc. Mm. (Rs.) 9 ("1866) 254-, 306.

Expression of $x^{n}+y^{n}$ in powers of $(x+y)$ and xy. Gruson, J. P. Berl. Ab. (1814-15) (Mth.) 30- 
Factors, resolution of integral functions into. Bretschneider, C. A. Arch. Mth. Ps. 46 (1866) 422-.

- --- - - Mandl, M. D. Nf. Vh. (1894) (Th. 2, Hälfte 1) 7.

-, resolvability into real simple and quadratic. Gauss, C. F. Gött. Cm. 3 (1814-15) 107-, 135-.

-, - - - Collins, E. St. Pét. Ac. Sc. Bll. 2 (1837) 377-; Crelle J. 18 (1838) 119-.

Mth. 99 (1886) 141-.

Formula

$$
\begin{aligned}
&(x-a)(x-b) f_{1}(x)=-\mathbf{F}(x)+f(x) \\
&\left\{\frac{\mathrm{F}(a) x-b}{f(a) a-b}+\frac{\mathrm{F}(b) x-a}{f(b) b-a}\right\} .
\end{aligned}
$$

Lemonnier, H. Par. S. Phlm. Bll. 2 (1878) 97-.

Fractions, a theorem on. Catalan, $E$. Brux. Ac. Bll. 19 (1890) 742-.

Function aleph, its properties. Dickstein, $S$. Krk. Ak. (Mt.-Prz.) Pam. 12 (1886) (35)-; Fschr. Mth. (1886) 121.

- -, some theorems. Dickstein, S. Krk. Ak. (Mt.-Prz.) Pam. 16 (1889) 53-.

Functions of differences, fundamental fact as to. Elliott, E. B. Mess. Mth. 29 (1900) 180-.

- - - of roots of given equation. Selivanov, D. T. Rec. Mth. (Moscou) 15 (1891) 581-.

- roots, and coefficients, relations. Dickson, J. D. H. Edinb. R. S. T. 29 (1880) 535-.

- _ - of an equation, Newton's identities concerning. Saalschütz, - Königsb. Schr. 36 (1895) [5]-.

Generating function, Borchardt's. Faà de Bruno, F. [1875] Crelle J. Mth. 81 (1876) 217-.

- -, - Kostka, C. [1876] Crelle J. Mth. 82 (1877) 212-.

$-\frac{-}{-}$ - Rehoŕovský, $V$. Časopis 11 (* 1882) 111-; Fschr. Mth. (*1882) 105-.

- of symmetric functions. Meier, $F$. Brux. Ac. Bll. 10 (1860) 608-.

- -, symmetric relations found by. Borchardt, C. W. [1855] Crelle J. 53 (1857) 193-.

Homogeneous products, sums, and roots of equations, theorems. Horner, J. QJ. Mth. 6 (1864) 360-.

Infinite partial fractions, reduction of exponential fractions to. Cisa de Gresi, -. Tor. Mm. Ac. 31 (1827) 225-.

Integral symmetric functions. Mertens, $F$. Krk. Ak. (Mt.-Prz.) Rz. 1 (1891) 333-; Fschr. Mth. (1891) 154-.

Irrational fractions, rationalisation of denominator. Sommer, B. Grunert Arch. 18 (1852) 44-.

Isobaric functions, use in recurring series. Pietrocola, C. Nap. Ac. At. 9 (1899) No. 8, 23 pp.

Jacobi, theorem, general analysis of formula $\Sigma \frac{F_{1}(a)}{f_{1}(a)}=0$. Liouville, J. C. R. 13 (1841) 467-.
Logarithmic derived function of an integral polynomial. Laisant, C. A. As. Fr. C. R. (1894) (Pt. 2) 208-.

MacMahon's generalisation of the NewtonGirard formulæ. Gegenbauer, L. [1900] Amst. Ak. Vs. 9 (1901) 332-; Amst. Ak. P. 3 (1901) 347-.

Newton, a formula of. Cesáro, $E$. Mathesis 6 (1886) 172-.

Number of roots, theorem of d'Alembert. Delbœuf, J. Rv. Sc. 42 (1888) 823-.

Numerical equations, theory. Anon. (xI 53) N. A. Mth. 17 (1878) 104-.

Partial fractions. Franchini, $P$. [1829] Lucca At. Ac. 6 (1830) 173-.

- - Eisenstein, G. Crelle J. 32 (1846) 71-。 - - Meyer, U. H. Grunert Arch. 7 (1846) 316-, 386-.

- - , identities concerning. Schmidt, J. R. Amst. Mengelwerk 2 (1816) 153-.

- - reduction to. Serooskerken, R. C. van T. Utr. A. Ac. (1817-18) 67 pp.

- - - - De Wit, Ae. Utr. A. Ac. (181718) $54 \mathrm{pp}$.

- - - - Dirksen, E. H. Crelle J. 1 (1826) 53-.

- - - -. Crelle, A. L. Berl. Ab. (1831) 1-.

,--- - Oettinger, $L$. Crelle J. 32 (1841) 63-, 148-.

17 (1881) $145-$.

,$---\frac{1}{-}$ Hoepflingen-Bergendorf, H. von. Arch. Mth. Ps. 66 (1881) 314-.

- - - - Stephanos, C. [1883] Bll. Sc. Mth. As. 8 (1884) 120 -.

$-\ldots, \ldots-$, with application to trigonometry. Saalschütz, L. Mh. Mth. Ps. 9 (1898) 132-, 268.

$-{ }_{-},-$, geometrical meaning of. Reuschle, C. Z. Mth. Ps. 41 (1896) 103-.

- - , and their integration, theory. Sauvage, L. Mars. Fac. Sc. A. 2 (1892) 3-.

- - theorems. Mainardi, G. Tortolini A. 1 (1850) 342-; 2 (1851) 505-.

Polynomials approximating to fractional functions. Čebyšev, P. [1892] St. Pet. Ac. Sc. Mm. (Rs.) 72 (1893) (Suppl.) No. 7, 13 pp.

一, Jacobi's. Stieltjes, -. C. R. 100 (1885) 620 -.

Power of polynomial, formula for. Piarron de Mondésir, E. S. As. Fr. C. R. (1877) 154-.

Powers of roots, Newton's theorems combined in one formula. Torriani, J. E. Lisb. Ac. Sc. Mm. (Cor.) 3 (pte. 2) (1814) 1-.

Rational fraction $\frac{f(x)}{\left[(x-a)^{2}+\beta^{2}\right]^{n}}$, decomposition.

Gerono, G. C. N. A. Mth. 18 (1859) 346.

- - formula of Laplace's.

$$
\begin{aligned}
\frac{Y}{\left(y-p_{1}\right)^{\mu_{1}+1} \ldots\left(y-p_{r}\right)^{\mu_{r}+1}} \\
\quad={ }_{m=1}^{m=r} \frac{\delta^{\mu_{m}}\left(\frac{\lambda_{m}\left(p_{m}\right)}{y-p_{m}}\right)}{1.2 .3 \ldots \mu_{m} \cdot \partial p_{m}^{\mu_{m}}} .
\end{aligned}
$$

Jürgensen, C. Crelle J. 11 (1834) 136-. 
Rational fractions. Stainville, - de. Gergonne A. Mth. 3 (1812-13) 279-.

- - algebraic integrals in. Bouniakowsky, $N$. St Pét. Ac. Sc. Mm. 3 (1833) 205-.

- -, approximation to functions of one variable by. Laguerre, E. Par. S. Mth. Bll. 5 (1877) 78-.

- - decomposition. Stainville, - de. Par. Ec. Pol. Cor. 3 (1814-16) 25-.

- - - - Crelle, A. L. Crelle J. 9 (1832) 231-; 10 (1833) 42-.

- -, 一. Finck, B. N. A. Mth. 4 (1845) 295-.

- - - Rawson, R. Mathematician 1 (1845) 265-; 2 (1847) 6-.

- -, -. Liouville, J. Liouv. J. Mth. 11 (1846) 462-.

- - - - Terquem, O. N. A. Mth. 6 (1847) 127-.

- - - Montojo, S. Cadiz Period. M. Ci. 1 (1848) 54-.

- - - - Brioschi, F. Crelle J. 50 (1855) 239-, 318-. 540-. -. Rouché, E. C. R. 46 (1858)

——, 一. Vieille, J. C. R. 49 (1859) 746-. (1862) 39 -.

- -, 一. Moch, S. N. A. Mth. 1 (1862) 339 -

438

,--- Trudi, N. G. Mt. 2 (1864) 225-, $257-$.

- -, - . St Germain, A. de. N. A. Mth. 8 (1869) 369-

- - Hermite, C. (xII) Brux. S. Sc. A. 2 (1878) (Pt. 2) 157-.

- - - Gomes Teixeira, $F$. G. Teix. J. Sc. 1 (1878) 5-, 17-, 33-, 49-, 97-, 113-; 2 (1880) 33-.

- -, - Jamet, V. N. A. Mth. 8 (1889) 228

- - , - and theory of residues. Rouché, E. C. R. 49 (1859) 863-.

- -, - - transformation. Rangoni, $L$. [1834] Mod. Mm. S. It. 21 (1836) 65-.

- -, development in partial fractions. Anon. (vi 536) Gergonne A. Mth. 10 (1819-20) 255-.

- -, expansion. Trudi, N. (xI) Nap. Ac. At. 2 (1865) No. 17, 68 pp.; (vir) Nap. Rd. 5 (1866) 441-.

- - Factorials, ratios of reciprocal. Cauchy, A. L. C. R. 17 (1843) 921-, 1159-.

- - - - , - - - , and transformation of logarithms into definite integrals. Cauchy, A. L. C. R. 17 (1843) 779-.

- - geometric properties. Lucas, F. C. R. 78 (1874) 140-, 180-, 271-.

- - index. Hermite, C. Par. S. Mth. Bll. 7 (1879) 128-.

- - theorems by Hermite. Laguerre, $E$. Crelle J. Mth. 89 (1880) 339-.

- -, theory. Picquet, H. Par. Éc. Pol. J. Cah. 53 (1883) 79-.

- functions of the cubic. Savin, A. A. Mth. 9 (1894-95) 158-.

VOL. I.
Rational functions of roots, form. Réalis, $S$. G. Mt. 9 (1871) 206-.

- - - of integral equation. Blutel, Par. S. Mth. Bll. 20 (1892) 92-.

Rationalisation of algebraic expressions. Scheibmer, W. Leip. B. 15 (1863) 63-.

- - - consisting of sums of square roots (Fermat's problem). Lehmann, J. W. $H$. Grunert Arch. 35 (1860) 207-.

- - any binomial denominator. Decher, $G$. Grunert Arch. 20 (1853) 245-.

- - fractions. Unferdinger, $F$. Grunert Arch. 33 (1859) 104-.

- - a sum of $2^{q}$ th roots. Rychlicki, S. Arch. Mth. Ps. 68 (1882) 180-.

Recurring functions of 3rd degree. Gohierre de Longchamps, -. As. Fr. C. R. 9 (1880) 115-.

Regular difference terms. Kempe, A. B. L. Mth. S. P. 25 (1894) 343-, 396.

Roots of equations, dimensions. De Morgan, $A$. QJ. Mth. 1 (1857) 1-, 80, 232-.

_ _ - expression by transcendental functions impossible. Wantzel, L. Par. S. Phlm. PV. (1845) 114-.

- - - form of. Olivier, L. Crelle J. 1 (1826) 97-

- - - forms of. Casinelli, A. (s. L.) Bologna N. Cm. 8 (1846) 317-.

- _ - geometrical representation. Goodwin, H. [1846] Camb. Ph. S. T. 8 (1849) 342-. - - - properties. Cockle, Jas. QJ. Mth. 5 (1862) 291-.

- -, property. Brioschi, F. N. A. Mth. 15 (1856) 366-.

- _ - - , with geometrical applications. Laisant, C. A. As. Fr. C. R. (1888) (Pt. 2) $1-$.

- _ - square roots of. Terquem, O. N. A. Mth. 6 (1847) 456-.

- - - transformation. Gelder, J. de. Amst. Mengelwerk 1 (1816) 46-.

- - quadratic with complex coefficients. Johnson, -. N. Y. Mth. S. Bll. 2 (1893) 171.

Successive powers of root, Anglin's formula. Cayley, A. QJ. Mth. 19 (1883) 223-.

Sum of like powers of roots. Faure, $H$. N. A. Mth. 14 (1855) 94-.

- - - _ - as function of coefficients. Pellet, A. E. N. A. Mth. 14 (1875) 259-.

- - - - - - - - and vice-versa. Farkas, J. Arch. Mth. Ps. 65 (1880) 433-.

Sums of like powers of roots of equations, and Brioschi's theorem. Genocchi, A. N. A. Mth. 12 (1853) 260-; 14 (1855) 246-.

- powers of roots of general quadratic. Kapteyn, $W$. N. Arch. Wisk. $3\left(^{*} 1877\right)$ 203-.

_- Newton's formula, generalisation. Lachlan, R. Mess. Mth. 13 (1884) 155-.

- - _ - - - theorem. Collins, E. [1837] St Pét. Ac. Se. Bll. 3 (1838) 52-.

- - - - - - - - and application to infinite products. Stockler, $F^{\prime}$. de $B . G$. Lisb. Mm. Ac. Sc. 2 (1799) 1-.

$-{ }_{-}-\ldots,-$, proof. Schubert, $F . T$.

[1808] St Pét. Ac. Sc. Mm. 2 (1810) 124-. 


\section{Symmetric Functions}

Sums of powers of roots to $(2 n-1)^{\text {th }}$ power, to construct polynomial from. Laguerre, $E$. Par. S. Mth. Bll. 5 (1877) 26-.

- - - Waring's formula. Janni, $V$. Nap. Rd. 17 (1878) 27-.

$--\dot{-}-\frac{1}{-},-$ Cayley, A. Mess. Mth. 21 (1892) 133-.

- - - - - Almeida Arez, J. B. d' G. Teix. J. Sc. 14 (1900) 117-; Fschr. Mth. (1900) 162.

_ _ _ _ - and Wronski's aleph function, Crocchi's proposition. Dickstein, S. Krk. Ak. (Mt.-Prz.) Pam. 12 (1886) (41)-; Fschr. Mth. (1886) 121.

\section{SYMMETRIC FUNCTIONS.}

Cauchy, A. L. [1824] Par. Mm. Ac. Sc. 9 (1830) 104-.

Conti, C. Padova N. Sag. 3 (1831) 90-.

Pioch, A. Brux. Ac. Bll. 2 (1835) 252-.

Transon, (Prof.) A. N. A. Mth. 9 (1850) 75-.

Dienger, $J$. Grunert Arch. 16 (1851) 471-.

Jaufroid, B. N. A. Mth. 12 (1853) 116-.

Brioschi, F. Tortolini A. 5 (1854) 313-, 422-.

Borchardt, C. W. N. A. Mth. 14 (1855) 26-.

Faù de Bruno, F. Tortolini A. 6 (1855) 338-, 412-, 476-.

Kronecker, L. Liouv. J. Mth. 1 (1856) 385-.

Betti, E. Crelle J. 54 (1857) 98-.

Cayley, A. Phil. Trans. (1857) 489-.

Roberts, $M$. Tortolini A. 3 (1860) 172-; QJ. Mth. 4 (1861) 57-; (vII) A. Mt. 4 (1861) 50 -.

Matthiessen, L. Z. Mth. Ps. 12 (1867) 322-.

Bonolis, A. G. Mt. 11 (1873) 321-.

Faà de Bruno, F. C. R. 76 (1873) 163-.

Isely, J. P. [1874] Neuch. S. Sc. Bll. 10 (1876) $59-$.

Malet, J. C. [1874] Ir. Ac. T. 25 (1875) 337-.

Kapteyn, $W$. N. Arch. Wisk. 2 (*1876) 73-.

Boschi, P. Bologna Ac. Sc. Mm. 7 (1876) $569-$.

Kronecker, L. Berl. Ak. Mb. (1880) 936-.

Forsyth, A. R. Mess. Mth. 10 (1881) 44-.

Durfee, W. P. [1882] (xI) J. H. Un. Cir. [2] (1883) 23.

Biehler, C. N. A. Mth. 3 (1884) 218-.

Ocagne, M. d'. G. Teix. J. Sc. 7 (1886) 133-.

Allardice, R. E. Edinb. Mth. S. P. 7 (1889) 41-.

Worontzoff, - . N. A. Mth. 10 (1891) 325- ; 12 (1893) 116-.

Kohn, G. Wien Ak. Sb. 102 (1893) (Ab. 2a) 199-.

Brunel, -. Bordeaux S. Sc. Mm. 4 (1894) vi-.

Junker, F. Mth. A. 45 (1894) 1-.

Metzler, W. H. L. Mth. S. P. 28 (1897) 390 -

Gordan, P. Mth. A. 52 (1899) 501-; D. Mth. Vr. Jbr. 8 (1900) (Heft 1) 178-.

algebraic theorem. Mertens, $F$. Wien Ak. Sb. 106 (1897) (Ab. 2a) 422-.

and alternating functions, genesis. Frisiani, $P$. Mil. Effem. As. (1846) 97-.

application to tetrahedra. Reiss, $M$. Quetelet Cor. Mth. 10 (1838) 229-.
Symmetric Functions 2410

associated quantities. Sylvester, J.J. C. R. 98 (1884) 779 -

and binomial theorem. Bret, -. Gergonne A. Mth. 4 (1813-14) 25-.

Brioschi's theorem. Sylvester, J. J. C. R. 98 (1884) 858-.

calculation. Abbati, P. [1804] Mod. Mm. S. It. 12 (1805) 8-.

- Hammond, J. L. Mth. S. P. 13 (1881-82) 79-.

- Laurent, H. N. A. Mth. 6 (1887) 416-.

Cayley's proof of rule for degree. Sylvester, J. J. Ph. Mg. 5 (1853) 199-.

class. Kapteijn, $W$. $[1881]$ Arch. Mth. Ps. 67 (1882) 102-.

- of permanent. Muir, T. Edinb. R. S. P. 11 (1882) 409-.

classes. Garbieri, G. [1882] Ven. I. At. 1 (1883) 33-.

classification. Mathews, G. B. QJ. Mth. 25 (1891) 127-.

complete. Torelli, G. G. Mt. 5 (1867) 110-

-, and coefficients of an equation, correspondence between. Crocchi, L. G. Mt. 20 (1882) 301-.

"critical" and Spencian. Cockle, Jas. QJ. Mth. 4 (1861) 97-.

and a determinant of common occurrence. Trudi, N. Nap. Rd. 3 (1864) 121-

determinant occurring in calculation. Gubler, E. Zür. Vjschr. 35 (1890) 79-.

determination from coefficients. Kostka, C. [1875] Crelle J. Mth. 81 (1876) 281-.

development by sums of like powers. Janni, $V$. G. Mt. 23 (1885) 34-.

of differences of roots of an equation. Tannery, J. C. R. 98 (1884) 1420 .

elementary, relations between. Junker, $F$. Mth. A. 38 (1891) 91-.

-, and sums of powers. Junker, $F$. Z. Mth. Ps. 41 (1896) 199-.

form $\left(x_{1}-x_{2}\right)^{2 p}, x_{1}, x_{2}$ being roots. Roberts, M. N. A. Mth. 19 (1860) 23-.

formula concerning. Pesci, G. Bologna Rd. (1886-87) 49-.

formulae, 2. Petersen, J. P.C. (xn) Ts. Mth. 6 (1876) 9-.

fundamental principle. Gergonne, J. $D$. Gergonne A. Mth. 3 (1812-13) 238-

- -. Bret, -. Gergonne A. Mth. 4 (181314) 25-.

- systems for. Vahlen, K. T. Acta Mth. 23 (1900) 91-.

geometric representation. Spitzer, S. [1850] Haidinger Ab. 4 (1851) (Ab. 3) 1-.

and inverse operators. MacMahon, P.A. L. Mth. S. P. 15 (*1883-84) 20-.

maximal and minimal values, proof of theorems of Cauchy. Terquem, $O$. Liouv. J. Mth. 5 (1840) 37.

multiplication. Babczynski, T. Z. Mth. Ps. 17 (1872) 147-

-. MacMahon, P. A. Mess. Mth. 14 (1885) 164 .

of $n$ variables, general theorem. Jung, G. C. R. 82 (1876) 988-.

new theorem. MacMahon, $P$. A. QJ. Mth. 20 (1885) 365-. 


\section{Symmetric Functions}

new theory. MacMahon, P. A. Am. J. Mth. 11 (1889) 1-; 12 (1890) 61-; 13 (1891) 193-; 14 (1892) 15-.

Newton's propositions, proof. Steen, A. Ts. Mth. 3 (1885) 30-; Fschr. Mth. (1885) 111.

- theorem. Tweedie, C. Edinb. Mth. S. P. 11 (1893) 61.

operators. Lachlan, R. L. Mth. S. P. 18 (1886-87) 39- ; 19 (1889) 294-.

of pairs of variables. Brill, $A$. Gött. Nr. (1893) 757-.

partition problem. Lemoyne, G. G. Mt. 10 (1872) 93-.

problem. Holmboe, B. M. Mg. Ntvd. 2 (1836) 279-.

properties of a complete table. MacMahon, $P$. A. Am. J. Mth. 10 (1888) 42-.

rational, calculation with. Hoppe, $R$. Schlömilch Z. 4 (1859) 353-.

relation between forms. Kostka, C. [1881] Crelle J. Mth. 93 (1882) 89-.

- - and invariants. Gambioli, D. G. Mt. 29 (1891) 41-.

representation by sums of powers. Hankel, $H$. [1865] Crelle J. 67 (1867) 90-.

of roots and combinations of coefficients of weight 11 and 12. Rehořovský, $W$. [1882] Wien Ak. D. 46 (1883) (Ab. 2) 53-.

- common to several equations. Betti, $E$. Tortolini A. 1 (1858) 193-.

$--\ldots-2$ equations. Dewulf, -. N. A. Mth. 19 (1860) 18-

---2 equations, representation. Hess, $E$. Z. Mth. Ps. 15 (1870) 325-.

- - of a pair of cubic equations. Walker, J.J. [1865] Mess. Mth. 3 (1866) 107-.

- - and resultants of simultaneous equations. Escherich, G. von. Wien Ak. D. 36 (1876) (Ab. 2) 251-.

- of systems of equations. MacMahon, P.A. [1890] Phil. Trans. (A) 181 (1891) 481-.

- _ _ - 2 equations. Cayley, A. Phil. Trans. (1857) 717-.

- - tables to the degree 10. Cayley, A. Am. J. Mth. 7 (1885) 47-.

satisfying partial differential equations. Netto, E. Z. Mth. Ps. 38 (1893) 357-.

and seminvariants. Cayley, A. Am. J. Mth. 15 (1893) 1-.

of several series of variables. Junker, $F$. Mth. A. 43 (1893) 225-.

simple and complete, relation. Crocchi, $L$.

[1879] G. Mt. 18 (1880) 377-.

of sines of angles $\left(a_{0}+\frac{2 k \pi}{n}\right)$. Johnson, W.W.

Des Moines Anal. 6 (1879) 105-.

- specified number of roots. Murphy, R. (vI Adds.) Ph. Mg. 10 (1831) 413-.

symbolical representation and invariant characters. Gambioli, D. G. Mt. 30 (1892) 192-.

tabulation. Durfee, W. P. Am. J. Mth. 5 (1882) 348-.

in terms of coefficients. Mainardi, G. Rm. At. N. Linc. 20 (1867) 163-.

theorem. Cockle, Jas. Ph. Mg. 28 (1846) 132-.
Symmetric Functions 2420

theorem of Cauchy. Bertrand, J. C. R. 45 (1857) 1-.

theorems. Anglin, A. H. H. [1880-83] Ir. Ac. P. 3 (1883) 289-; Edinb. R. S. P. 12 (1884) 236-.

theory. Schönemann, T. Crelle J. 19 (1839) 231-, 289-.

- Cockle, Jas. Mathematician 3 (1850) 176-, 246-; Suppl. 27-.

- Borchardt, C. W. Berl. B. (1855) 165-.

- Mertens, F. Crelle J. 69 (1868) 289-; Wien Ak. Sb. 81 (1880) (Ab. 2) 988-.

- Anglin, A. H. Crelle J. Mth. 98 (1885) 175-.

—. König, G. Mth. Termt. Ets. 8 (1890) 9-; Mth. Nt. B. Ung. 8 (1891) 83-.

- Mertens, F. Wien Ak. Sb. 108 (1899) (Ab. 2a) 473-.

and theory of distributions. MacMahon, P.A. L. Mth. S. P. 19 (1889) 220-.

of $12^{\text {ic }}$, tables. Durfee, W. P. Am. J. Mth. 5 (1882) 45-.

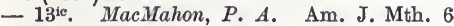
(1884) 289-.

- 14 ${ }^{\text {ic }}$. Durfee, W. P. Am. J. Mth. 9 (1887) 278-

- two ternary forms. Junker, $F$. Wien Ak. D. 64 (1897) 439-.

Waring's formula. MacMahon, P. A. Mess. Mth. 14 (1885) 8-.

- formulae. Terquem, O. N. A. Mth. 8 (1849) 76-.

$\Sigma \frac{a^{p}}{f^{\prime}(a)}, a$ being a root of equation $f(x)=0$. Vieille, J. C. R. 44 (1857) 1311-.

$\Sigma \frac{a^{n-1}+\mu}{f^{\prime}(a)}$ and differentials of sums of $k$ th powers of roots. Serret, J. A. C. R. 45 (1857) 60-.

Symmetry of a certain algebraic expression, proof. Allardice, R. E. Edinb. Mth. S. P. 4 (1886) 37. -, law. MacMahon, P. A. QJ. Mth. 22 (1887)

Teleological method. Dickstein, S. Prace Mt.-Fiz. 3 (1892) 126-; Fschr. Mth. (1892) 97-.

- - Wronski's. Krauze, W. Prace Mt.-Fiz. 3 (1892) 110-; Fschr. Mth. (1892) 97.

Wronski's formulæ, proof of two. Dickstein, $S$. Krk. Ak. (Mt.-Prz.) Pam. 12 (1886) (87)- ; Fschr. Mth. (1886) 121.

\section{Reality, multiplicity, separation, of roots.}

Algebra, fundamental theorem and Newton's approximation method. Mertens, $F$. Wien Ak. Sb. 101 (1892) (Ab. 2a) 415-.

Algebraic equations and Campbell's quadratic functions. Poulain, A. C. R. 106 (1888) 470-, 562, 1479.

- - general theorems. Poulain, A. N. A. Mth. 6 (1867) 21-. 
Algebraic equations, theorems, 2. Myasoyedov, A. N. [1883] (xII) Rec. Mth. (Moscou) 11 (1883-84) 616-.

Budan and Fourier's theorem. Fouret, G. N. A. Mth. 11 (1892) 82-, 352.

Budan's method of extracting roots. Nicholson, P. Tilloch Ph. Mg. 60 (1822) 173-.

- theorem concerning roots of an algebraic equation. Genocchi, A. Tortolini A. 7 (1856) 462-.

Complex roots. Canterzani, S. Verona Mm. S. It. 2 (1784) 720 -

- - of equations, existence, Hermite's proposition, generalisation. Studnička, F.J. Časopis 26 (1897) 95-; Fschr. Mth. (1897) 95. - - - - , , proof. Kinkelin, H. Mth. A. 1 (1869) 502-.

- _ - - - , theorem. Kolbe, J. Wien Sb. 67 (1873) (Ab. 2) 188-.

_ _ - , and factors of polynomials. Wantzel, L. N. A. Mth. 3 (1844) 325-

- - - numerical equations. Deladéréêre, A., \& Finck, - . N. A. Mth. 3 (1844) 41-.

Conditions that an equation of degree $n$ may have only $p$ distinct roots. Antomari, $X$. N. A. Mth. 16 (1897) 63-.

Continuity of roots. Rouquet, V. N. A. Mth. 15 (1876) 154-

--- , proof. Bonnet, O. Bll. Sc. Mth. As. 2 (1871) 215-.

Derived equations. Merlieux, É. N. A. Mth. 3 (1844) 178-.

- polynomials, property. Young, J. R. $\mathrm{Ph}$. Mg. 31 (1866) 369-.

Descartes, Rolle, Fourier, Sturm, and Cauchy, theorems of, deduced from one principle. Terquem, O. N. A. Mth. 3 (1844) 188-, 209-, 555-, 577-.

Descartes's rule, proof. Tebay, S. (vi Adds.) Ph. Mg. 24 (1844) 24-.

- of signs. Terquem, $O$. N. A. Mth. 2 (1843) 248-.

- - - Finck, B. N. A. Mth. 3 (1844) 316-.

$--1-$ Guilmin, A. N. A. Mth. 5 (1846) 239-, 334.

- Z - Zehfuss, G. Grunert Arch. 34 (1860) 400-.

$--1-$ Walton, $W$. QJ. Mth. 5 (1862) $153-$

- - - Parpaite, -. N. A. Mth. 8 (1869)

- Laguerre, E. N. A. Mth. 18 (1879) 5-。 1418-. - Poincaré, H. C. R. 97 (1883)

- - - Jonquières, E. de. Rm. N. Linc. At. 38 (1885) 55-.

$--\ldots-$ Borel, $\hat{E}$. Bll. Sc. Mth. 20 (1896) $327-$.

- (Harriot's) rule of signs, demonstration. Grunert, J. A. Crelle J. 2 (1827) 335-.

- rule of signs, demonstration (Gauss). Gergonne, J. D. Gergonne A. Mth. 18 (1827-28) 352 .

---- , extension. Laguerre, E. C. R.
Descartes's rule of signs, generalisation. Gegenbauer, $L$. Wien Ak. Sb. 83 (1881) (Ab.2) 321-.

- _ _ - lowering limits furnished by. André, D. 'C. R. 98 (1884) 212-.

- - - - theorem in complete algebraic polynomials applied to. Jonquières, $\boldsymbol{E}$. de. C. R. 99 (1884) 1143-.

- theorem, extension. Lucas, É. Par. S. Mth. Bll. 8 (1880) 187-.

Distribution of roots, theorems of Laguerre and Myasoyedov. Klark, N.I. (xח) Kazan S. Nt. (P8.-Mth.) P. 1 (1883) [(No.8)] 11-.

Double roots. Guldberg, A. S. Ts. Mth. 4 (1886) 97-; Fschr. Mth. (1887) 86.

Electrical determination of roots. Lucas, $F$. C. R. 106 (1888) 195-, 268-, 645-, 1072-; 111 (1890) 965-.

Equal roots. Vachette, A. N. A. Mth. $6(1847)$ 213-.

- - Ostrogradsky, M. A. [1849] St Pét. Ac. Sc. Bll. 8 (1850) 193-.

- - Hudson, C. QJ. Mth. 18 (1882) 215-, $327-$.

- condition for. Salmon, G. Camb. and Dubl. Mth. J. 5 (1850) 159-.

- -, theory. Rouché, E. N. A. Mth. 16 (1857) 66-.

- - - Mansion, - - Brux. S. Sc. A. 16 1892 (Pt. 1) 54-.

,--- , and application to tangents to curves. Roger, É. N. A. Mth. 3 (1844) 51-.

Equalities among roots, conditions for existence of. Cayley, A. Phil. Trans. (1857) 727-.

Equality of a given number of roots. Gerono, $G$. $C$. N. A. Mth. 1 (1842) 90-; 6 (1847) 75-, 113-.

- 2 roots, condition. Joachimsthal, $F$. Crelle J. 33 (1846) 371-.

Equation $f^{2}(z)+k^{2} f^{\prime 2}(z)=0$ has all roots imaginary. Cesáro, $E$. N. A. Mth. 4 (1885) 321-.

Equations with all roots imaginary. Catalan, E. C. Liége S. Sc. Mm. 15 (1888) No. 1, 224-.

- - - real. Gegenbauer, L. [1881] Wien Ak. Sb. 84 (1882) (Ab. 2) 1102-.

- - - Kraus, L. Časopis 15 (1886) 63 ; Fschr. Mth. (1886) 70.

- - - Ocagne, M. d'. C. R. 106 (1888) 731-.

- - - Selivanov, D. Th. Kazan S. Ps.-Mth. Bll. 9 (1900) 51-; Fschr. Mth. (1900) 94-.

- - - class. Clebsch, A. [1861] Crelle J. 62 (1863) 232-.

- - - - - Biehler, C. N. A. Mth. 19 (1880) 149-; 6 (1887) 9-.

- - - - properties. Laguerre, $E$. N. A. Mth. 19 (1880) 224-.

- - - - - - of coefficients. Florow, P. S. Fschr. Mth. (1898) 70 .

- - - - property. Sylvester, J. J. Crelle J. Mth. 87 (1879) 217-.

- - , source. Fouret, G. C. R. 106 (1888) 1135-.

--- -, types. Fouret, G. C. R. 106 
Equations, difference product in case of equal roots. Franke, E. [1876] Crelle J. Mth. 83 (1877) 65-.

- with a given number of complex roots. Gegenbauer, L. [1882] Wien Alk. Sb. 87 (1883) (Ab. 2) 264-.

- no positive root, conditions. Andre, $D$. C. R. 98 (1884) 561-。

-, 3rd \& 4th orders, nature of roots, criteria. Gaines, R. E. A. Mth. 1 (1900) 111-.

Fourier and Sturm, theorems. Crelle, A. L. Crelle J. 13 (1835) 119-.

,----- , graphic representation. Tanner, H. W. L. Mess. Mth. 18 (1889) 95-.

- theorem on roots of equations and recurring series. Stern, M. A. Crelle J. 9 (1832) 305-.

Fourier's rule, proof. Young, J.R. $\mathrm{Ph} . \mathrm{Mg}$. 23 (1843) 6-.

- - for real roots. Hill, C. J. D. Lund . Acta Un. 8 (1871) (Mth.) No. 2, 8 pp.

- theorem on equations. O'Kinealy, $J . \quad \mathrm{Ph}$. Mg. 48 (1874) 95-.

-1 - Sharp, W. J. C. QJ. Mth. 17 (1881) 132-.

Functions whose roots separate each other. Markow, W. A. Fschr. Mth. (1899) 100.

- - - - - Iwanow, J. J. Fschr. Mth. (1899) 100.

$--1-1-$ Markow, W. A. Fschr. Mth. (1899) 100-.

Imaginary and equal roots, criteria. Horner, $J$. [1867] QJ. Mth. 9 (1868) 57-.

- roots. Cauchy, A.L. Par. S. Phlm. Bll. (1817) 5-, 161-; Par. Éc. Pol. J. 11 (1820) (18e cah.) 411-.

- Bonnet, $\dot{0}$. N. A. Mth. 4 (1845) 236-.

- - Terquem, O. N. A. Mth. 5 (1846) 141-.

- - Prouhet, E. N. A. Mth. 14 (1855) 199-.

- Buys-Ballot, C. H. D. Amst. Vs. Ak. 7 (1858) 316-.

- - Heawood, P.J. Mth. Gz. No. 4 (1895) 31-.

- - Vaes, F.J. N. Arch. Wisk. 3 (1898) 117-.

- - Budan's criterion. Christie, J.R. $\mathrm{Ph}$. Mg. 21 (1842) 96-.

- -, Cauchy's theorem, extension. De Morgan, A. [1857] (Ix) Camb. Ph. S. P. 1 (1866) 194.

- - , - , proof. Liouville, J., \& Sturm, C. Liouv. J. Mth. 1 (1836) 278-.

,---- , proofs. Sturm, J. C. F. Liouv. J. Mth. 1 (1836) 290-.

- -, criteria. Young, J. R. Ph. Mg. 22 (1843) 186-, 252-; 23 (1843) 450-; 29 (1846) $32-$.

- - - Colombier, P. A. G. N. A. Mth. 7 (1868) 308-, 501-.

- - of equations of order higher than 4th. Carandino, G. G. Arcad. 32 (1826) 90-.

- - existence. Dupré, A. Gergonne A. Mth. 18 (1827-28) 68-.

,--- Gergonne, J.D. Gergonne A. Mth. 19 (1828-29) 124-.

,--- , test. Serret, $P$. N. A. Mth. 6 (1847) 200-.

- - , limiting values of coefficients for given number. Schramm, $H$. Arch. Mth. Ps. 45 (1866) 325-.
Imaginary roots, Newton's rule. Sylvester, J. J. [1865] L. Mth. S. P. 1 (1866) No. 2, 16 pp. ; Ir. Ac. T. 24 (1871) 247-.

-,$- \ldots-$, invariantive criteria for quintic. Sylvester, J.J. Phil. Trans. 154 (1864) 579-.

- - - - for number. Lobatto, $R$. Amst. Vs. Ak. 9 (1859) 92-.

- - - - - Minich, S. R. Ven. At. 11 (1865-66) 127-.

- - - - - - proof. Young, J.R. $\mathrm{Ph}$. Mg. 30 (1865) 113-, 289-, 363-; 31 (1866) 369-; 32 (1866) 121-; Ir. Ac. P. 10 (1870) 343-.

- - , - - - - Sylvester's theorem. Poulain, A. Les Mondes 11 (1866) 16-.

- - - - - - - Genocchi, A. N. A. Mth. 6 (1867) 5 -

- _, - _ and Sylvester's theorem. Jonquières, E. de. C. R. 99 (1884) 62-, 111-, 165-, 269-.

- - , - - theorem connected with. Sylvester, J. J. [1879] Mess. Mth. 9 (1880) 71-.

- -, number. Bérard, -. Gergonne A. Mth. 9 (1818-19) 345-.

,--- between limits. Dienger, $J$. Grunert Arch. 21 (1853) 361-.

,----1 , test for. Lobatto, R. Liouv. J. Mth. 9 (1844) 295-.

- - of numerical equations, number. Anon. (vi 529) Gergonne A. Mth. 9 (1818-19) 60- - - - test for. Lavernède, T. Gard. Not. Tr. Ac. (1809) 195-

- - - - - Faà de Bruno, F. Liouv. J. Mth. 15 (1850) 363-.

- - Rolle's theorem extended to. Liouville, J. Liouv. J. Mth. 9 (1864) 84-.

- _, - - - - Mansion, -. Brux. S. Sc. A. 13 (1889) (Pt. 1) 42-.

- - rules analogous to Newton's. Minich, S. R. Ven. At. 11 (1865-66) 211-.

- - , - for inferior limit to number. Minich, $S . R$. Ven. At. 11 (1865-66) 309-.

-- , theorem. Sturm, J. C. F. N. A. Mth. 5 (1846) 115-.

Infinite roots of equations. Gerono, G. C. N. A. Mth. 3 (1844) 32-, 85-

,--- and asymptotes. Gerono, $G$. $C$. N. A. Mth. 4 (1845) 33-.

Integral functions $Y_{n}=\prod_{\kappa=1}^{\kappa=1}\left(y-2 \cos \frac{2 \kappa \pi}{n+1}\right)$, and Sturm's functions. Vries, $J$. de. Amst. Ak. Vs. 4 (1896) 133-.

Interlacing of functions. Sylvester, J. J. Crelle J. Mth. 88 (1880) 1 -.

Laguerre's polynomials, property. Laurent, $H$. N. A. Mth. 19 (1880) 380-.

Least root, general expression for. König, $J$. Mth. A. 9 (1876) 530-.

- roots. Dickson, J. D. H. [1877] Edinb. R. S. T. 28 (1879) 119-.

Limit, inferior, of roots of an algebraic equation. Mrasojědov, A. N. Rec. Mth. (Moscou) 12 (1885) 22-.

S. Mth. Bll. 20 (1892) $\overline{4}$. 
Limit, superior, of commensurable roots. Montebello, G. de. N. A. Mth. 18 (1859) 256-.

$-, \ldots,-$ negative roots deduced from Newton's formula. Vitasse, - . N. A. Mth. 18 (1859) 213-.

-, 一, - positive roots. Mourgues, - N. A. Mth. 9 (1850) 108-.

,,----- Gisclard, L. N. A. Mth. 11 (1852) 107-.

-,,$--1-$ Gohierre de Longchamps, N. A. Mth. 19 (1880) 71-.

,,$---\ldots$ in numerical equations. Lenthéric, -. Gergonne A. Mth. 20 (1829-30) 297-.

-,- - roots, determination. Candèze, $G$. N. A. Mth. 20 (1881) 49-.

- - - - - Laguerre's rule. Candèze, $G$. N. A. Mth. 19 (1880) 307-.

$-, \ldots, \ldots-$ of numerical equations, Petersen's criterion proved erroneous. Giudice, Palermo Cir. Mt. Rd. 6 (1892) 157-

Limits of equations. Piani, D. Bologna N. Cm. 3 (1839) 237-.

- to moduli of roots. Dini, U. A. Mt. 1 (1898) 77-.

$--1-$ - Petrovitch, M. C. R. 129 (1899) 583-, 873-.

- of roots. Véne, A. A. Rouen Tr. Ac. (1821) 97-; Férussac Bll. Sc. Mth. 4 (1825) 206-.

- - Tillot, J. N. A. Mth. 4 (1845) 224 -

- - . Toussaint, - N. A. Mth. 18 (1859) 310 .

- Réalis, S. N. A. Mth. 3 (1864) $477-$

- - Gy"ry, S. (xiI) Mag. Tud. Ak. Éts. 1 (No. 6) (1867) 125-.

- Transon, A. N. A. Mth. 11 (1872) 254-.

- - Fouret, -. Par. S. Mth. Bll. 20 (1892) 35-.

- _ - Descartes's theorem applied. Fourier, J. B. J. Par. S. Phlm. Bll. (1820) 156-, 181-.

- - - and determination of commensurable roots. Thibault, -. N. A. Mth. 2 (1843) 517-.

- - - of equation with only real roots. Pleskot, A. Prag Sb. (1897) (Mth.-Nt.) No. 37, 9 pp. ; N. A. Mth. 18 (1899) 301-; 19 (1900) 144.

- - - literal equations. Bouvier, L. C. Gergonne A. Mth. 16 (1825-26) 54-.

- - - numerical equations. Bret, Gergonne A. Mth. 6 (1815-16) 112-.

- - - - - approximate. Maizière, de. Gergonne A. Mth. 3 (1812-13) 41-

- _ - - _ - of higher order. Row botham, J. Tilloch Ph. Mg. 63 (1824) 369-.

-, superior, of roots. Rachmanov, P.N. Kharkov Mth. S. Com. 4 (1895) 141-; Fschr. Mth. (1893-94) 140.

-, variation, in algebraic equations. Chevillard, A.J. N. A. Mth. 2 (1843) 343-.

Multiple roots of algebraic equations. Brioschi, -. C. R. 121 (1895) 582-

- - - - , theorem. Sondat, P. N. A. Mth. 19 (1900) 25-.
Multiple roots, theory. Sylvester, J. J. $\mathrm{Ph}$. Mg. 18 (1841) 249-.

Multiplicity of roots. Beynac, F. A. N. A. Mth. 9 (1850) 317-.

_ - , amount and distribution. Sylvester, J. J. Ph. Mg. 18 (1841) 136-.

- - - in the octavic equation. Alagna, $R$. Palermo Cir. Mt. Rd. 4 (1890) 287-.

Nature of roots, Borchardt's proposition. Studnicka, F.J. Časopis 27 (1898) 237-; Fschr. Mth. (1898) 73.

Newton and Descartes, rule of signs. Orzabal, A. Arg. S. Ci. A. 22 (1886) 39-, 97-.

Newton-Fourier imaginary problem. Cayley, A. [1879] Camb. Ph. S. P. 3 (1880) 231-.

- method applied to imaginary roots. Cayley, A. QJ. Mth. 16 (1879) 179-.

Newton's and Laguerre's criteria for positive roots. Vénard, C. N. A. Mth. 19 (1880) 261-.

Number of different roots of an algebraic equation. Baur, L. Mth. A. 50 (1898) 241-; 52 (1899) 113-.

- positive and negative roots. Gauss, C.F. Crelle J. 3 (1828) 1-.

- - roots between limits. Moigno, F. Liouv. J. Mth. 5 (1840) 75-.

- - - Grunert, J.A. Grunert Arch. 1 (1841) 19-, 126-.

- - - - Hermite, C. Crelle J. 52 (1856) 39-.

- _ _ _ - logarithmic criteria. Laguerre, E. C. R. 93 (1881) 1061-.

- - , theorem. Myasoyedov, A. N. [1882] (xiI) Rec. Mth. (Moscou) 10 (1882-83) (Pt.1) $56-$.

Real and imaginary roots of equations of 5 th order, theory. Sylvester, J. J. C. R. 59 (1864) 749-, 944-; 60 (1865) 759-.

- roots. Bolzano, B. Prag Ab. 5 (1818) $60 \mathrm{pp}$.

- - Terquem, O. N. A. Mth. 10 (1851) 180 -

-, absence of. Bellavitis, G. Crelle J. 50 (1855) 263-.

- - geometrical investigation of number. Pincherle, S. Rv. Mt. 3 (1893) 54-.

- - between given limits. Réalis, $S$. G. Mt. 9 (1871) 355-.

- -, limit. Gohierre de Longchamps, N. A. Mth 18 (1879) 49-.

,--- , superior. Paque, A. Liège Mm. S. Sc. 10 (1855) 199-.

- - - - of number. Olivier, L. Crelle J. 1 (1826) 223-.

- - _- - Sylvester, J. J. C. R. 60 (1865) 1261-.

$-\ldots,-\ldots$ in a class of equations. Sylvester, J. J. C. R. 58 (1864) 494-.

- - limits, method of finding. Sylvester, J. J. N. A. Mth. 12 (1853) 329-.

,--- , theorem. Sylvester, J.J. N. A. Mth. 12 (1853) 286-.

- - number. Cauchy, A. L. [1813] Par. S. Phlm. Bll. (1814) 95-; Par. Ec. Pol. J. 10 (1815) $\left(17^{\mathrm{e}} \mathrm{cah}.\right)$ 457-.

- -,- between limits. Bonnet, O. N. A. Mth. 6 (1847) 464-. 
Real roots, number between limits. Petr, $K$. Prag České Ak. Fr. Jos. Rz. (Tř̌ida 2) 6 (1897) Art. 8, 11 pp.; Prag Fr. Jos. Ac. Sc. Bll. (Mth.-Nt.) 4 (1897) (Pt. 2) 61-.

- _ _ _ - Sturm's theorem. Joachimsthal, $F$. Crelle J. 48 (1854) 386-.

- - , - for equation $x^{m}-p x+q=0$. Regis, D. G. Mt. $8(1870) 226-$.

- - series for number. Brioschi, F. N. A. Mth. 15 (1856) 264 -.

- - their upper and lower limits and separation. Steklov, $V$. A. Kharkov Mth. S. Com. 3 (1893) 103-; Fschr. Mth. (1891) 94-.

Reality of roots. Björling, C. F.E. [1868] Stockh. Öfv. 25 (1868-69) 241-; Arch. Mth. Ps. 48 (1868) 363-.

- - Favero, G. B. G. Mt. 13 (1875) 249

- - -, conditions. Griveau, A. Les Mondes 17 (1868) 214-.

- - of discriminating cubic. Gerono, G. C. N. A. Mth. 11 (1872) 305-

- - - equation of 3rd order. Grunert, J. A. Grunert Arch. 29 (1857) 442-.

- - - - - in transformation theory. Saalschütz, - - Königsb. Schr. 38 (1897) [17].

$-----\left(\frac{1+i x}{1-i x}\right)^{m}=A+B i$. Laisant, C. A. As. Fr. C. R. (1882) 102-.

- - - - important equation, new proof. Gravelaar, N. L. W. A. [1875] Arch. Mth. Ps. 58 (1876) 301-.

- - - quintic, invariant criteria. Harris, R. A. A. Mth. 5 (1889-90) 217-.

- - - - - - Petr, K. Prag Ceské Ak. Fr. Jos. Rz. (Trüida 2) 8 (1899) Art. 23, 12 pp. ; Fschr. Mth. (1899) 103.

_ _ - - secular equation, proof by Fourier's method. Sylvester, J.J. Crelle J. Mth. 88 (1880) 4-.

- - - theorem. Korteweg, D.J. N. Arch. Wisk. 4 (1899) 46-.

- and signs of roots. Du Bourguet, - Par. S. Phlm. Bll. (1815) 14-

Rolle's theorem. Collin, J. N. A. Mth. 20 (1881) 132-.

- - , and an application. Biehler, C. N. A. Mth. 6 (1887) 75-, 190-.

- -, extension. Boer, F. de. Amst. Ak. Vs. M. 19 (1884) 384-; Arch. Neérl. 19 (1884) 207-.

- -, -. McCulloch, J. F. A. Mth. 4 (1888) 5-.

- generalisation, Lucas, F. C. R. 106 (1888) 121-.

- - Lagrange's, and Cauchy's, geometrically equivalent. Mansion, $P$. [1875] Mess. Mth. 5 (1876) 34.

Root-limitation, Cauchy's theorems, geometrical representation. Cayley, A. [1874] Camb. Ph. S. T. 12 (1879) 395-.

Roots, commensurable, criteria. Mathieu, $\bar{E}$. N. A. Mth. 16 (1857) 145-.

-, - fractional, criteria. Terquem, 0. N. A. Mth. 4 (1845) 397-.
Roots of cubic, criteria. Vyvyan, T. G. Mth. Gz. 1 (1900) 39.

-, equal numerically, of opposite sign, criteria. Müller, F. Arch. Mth. Ps. 46 (1866) $32-$.

- of an equation, limits to. Gob, A. Mathesis 9 (1889) 269-.

_ _ _ _ multiplicitity of, and a limit to. Demoulin, A. Mathesis 9 (1889) 268-

- equations. Spitzer, S. Grunert Arch. 25 (1855) 137-.

- - - analysis. Murphy, R. Phil. Trans. (1837) 161-.

_ - - with complex coefficients. Hermite, C. Crelle J. 53 (1857) 182-。

- _ - elementary representation and inspection. Björling, C. F. E. Ts. Mt. Fys. 1 (1868) 119-.

- - with integral coefficients. Cauchy, A. L. C. R. 24 (1847) 407-.

_ _ - n th degree, with application to biquadratic. Mossbrugger, L. Grunert Arch. 28 (1857) 205-.

- - - surd. Moon, R. Camb. Mth. J. 3 (1843) 20-.

- - theorems. Fleury, L. L. Cherb. Mm. S. Sc. 8 (1861) 373-.

-, new formulæ relative to. Silva, J. A.M. da. [1882] G. Teix. J. Sc. 4 (1883) 3-.

- number satisfying a given condition. Cauchy, A. L. C. R. 40 (1855) 1329-.

-, theory. Bourget, J. N. A. Mth. 9 (1870) 505-.

-, - Björling, C. F. E. Stockh. Ak. Hndl. 10 (1871) (No. 3) $53 \mathrm{pp}$.

Rule of signs, new theory. Meech, L. W. Des Moines Anal. 2 (1875) 81-, 97-.

Rules of signs, combinations of four. Sylvester, J. J. C. R. 61 (1865) 282-.

Separation of nearly equal roots. Christie, J.R. Ph. Mg. 21 (1842) 96-.

- real roots of equation. Schramm, $H$. Grunert Arch. 36 (1861) 420-.

----- equations. Ovejero, J. Arg. S. Ci. A. 27 (1889) 169-.

- roots. Dirksen, E. H. Berl. Ab. (1835) 337-.

- - Umpfenbach, Dr. Crelle J. 20 (1840) 60-.

_- - Transon, A. N. A. Mth. 7 (1868) 25-, $57-$.

- - Björling, C. F. E. Ups. N. Acta S. Sc. 7 (1870) (No. 7 ) 35 pp.

- - Maleyx, L. N. A. Mth. 11 (1872) 404-; 13 (1874) 385-.

- - Laurent, H. N. A. Mth. 14 (1875) 37-. - - Mrasojědov, A. N. [1884] Rec. Mth. (Moscou) 12 (1885) 433-; Fschr. Mth. (1885) 63.

_ - - of certain equations. Laguerre, $E$. C. R. 92 (1881) 178-.

_- - improved statement of rule, and new theorem. Sylvester, J.J. Ph. Mg. 31 (1866) 214-.

- - - Laguerre's theorem. Lucas, $\dot{E} . \quad$ N. A. Mth. 19 (1880) 145-.

- - - - Lévy, L. N. A. Mth. 19 (1880) 148. 
Separation of roots by method of differences. Desboves, A. $H$. N. A. Mth. 13 (1854) 60 -

33 (1894) 191-, 214-.

Capelli, A. Nap. Rd.

- - - method, and Lagrange's formula. Pellet, A. E. Bll. Sc. Mth. As, 5 (1881) (Pt. 1) 393-.

- - of numerical equations. Zinna, $A$. G. Mt. 6 (1868) 344-.

$-\ldots-1$ Laguerre, $E$. C. R. 89 (1879). 635- ; 92 (1881) 1146-.

- - - some equations. Markov, A. A. Kharkov Mth. S. Com. (1885) 89-; Fschr. Mth. (1886) 161.

- - - and superior limit. Laguerre, $E$. N. A. Mth. 19 (1880) 49-, 97-.

Signs and nature of roots. Anon. (vr 537) Gergonne A. Mth. 10 (1819-20) 341-.

Sturmians, class of. Chrystal, $G$. [1881] Edinb. R. S. T. 30 (1883) 161-.

Sturm's functions. Cayley, $A$. Liouv. J. Mth. 11 (1846) 297-; 13 (1848) 269-.

- -. Brioschi, F. N. A. Mth. 13 (1854) 71-.

- - Gilbert, P. Liouv. J. Mth. 12 (1867)

87-.

- -. Brioschi, F. C. R. 68 (1869) 1318-

- -. Kronecker, L. Berl. Ak. Mb. (1878) 95-.

- - auxiliary, relation to roots of an equation. Sylvester, J.J. B. A. Rp. (1841) (pt.2) 23-.

- - calculation by determinants. Lemonnier, H. C. R. 80 (1875) 252-.

- - coefficients expressed as determinants. Heal, W. E. A. Mth. 2 (1885-86) 85-.

- - determination of a series from a single determinant. Vleck, E. B. van. [1899] A. Mth. 1 (1900) 1-.

- -, formulæ for equations of 2nd, 3rd, and 4th orders. Koralek, P. N. A. Mth. 11 (1852) 333-.

- - functions analagous to. Lemonnier, $H$. Par. S. Mth. Bll. 6 (1878) 149-.

- - - - - Miasojědov, A. N. [1884] Rec. Mth. (Moscou) 12 (1885) 461-; Fschr. Mth. (1885) 63-.

- and their reciprocal relations. Kronecker, L. Berl. Mb. (1873) 117-.

- of 2nd species. Wendlandt, $H$. Arch. Mth. Ps. 62 (1878) 1-.

- - series of. Gegenbauer, L. Amst. Ak. Vs. 5 (1897) 185-.

- - _ - Vries, J. de. N. Arch. Wisk. 3 (1898) 40 -.

- - , substitutes for. Mollame, $V$. Nap. Rd. 22 (1883) 256-.

- - , Sylvester's theorem on. Sturm, J. C. F. Liouv. J. Mth. 7 (1842) 356-.

- - - - - Turazza, D. Ven. At. 11 (1865-66) 419-.

- - and syzygies between rational integral functions. Sylvester, J.J. Phil. Trans. (1853) 407-.

- - , tables for equations of $2 \mathrm{nd}, 3 \mathrm{rd}, 4 \mathrm{th}$, and 5th orders. Cayley, A. Phil. Trans. (1857) 733-.

-- , theorem of Cayley's, proof. Zeipel, $E$.

V. von. QJ. Mth. 3 (1860) 108-
Sturm's method, application. Biehler, C. N. A. Mth. 19 (1880) 76-; 6 (1887) 421-.

- - of finding real roots, expressions for quotients in. Sylvester, J. J. B. A. Rp. (1853) $(p t, 2)$ 1-.

_ _ - separation of roots, modification. Nazimov, P. [1885] Rec. Mth. (Moscou) 13 (1886) 119-; Fschr. Mth. (1886) 71-.

- quotients, explicit values. Sylvester, J.J. Ph. Mg. 6 (1853) 293-.

- remainders. Heilermann, -. Crelle J. 43 (1852) 43-; 48 (1854) 190-.

- Faà de Bruno, F. Tortolini A. 7 (1856) 313-.

- series. Gegenbauer, L. Wien Ak. Sb. 81 (1880) $(A b .2)$ 576-.

- theorem. Terquem, 0 . N. A. Mth. 2 (1843) 97-.

-. Badon-Ghijben, J. (vII) Amst. N. Ws. Ntk. Vh. 1 (1844) 89-.

- - Mourgues, -. N. A. Mth. 9 (1850) 278-.

(1852) 403-.

- Hermite, C. C. R. 36 (1853) 294-.

- Bertrand, $\dot{J}$. C. R. 43 (1856) 1108-

- - Kronecker, L. C. R. 68 (1869) 1078-.

- -. Hattendorf, $K$. Gött. Nr. (1873) 779-.

- -. Darboux, G. Bll. Sc. Mth. As. 8 (1875) 56-, 92-.

- -. Janni, V. G. Mt. 20 (1882) 166-.

- Niewenglowski, B. N. A. Mth. 9 (1890) 181-.

-. Willotte, H. Toul. Ac. Sc. Mm. 3 (1891) 44 .

-, application. Spiller, -. Mth. Misc. 2 (1839) 133-.

,--- Candioti, M. R. Arg. S. Ci. A. 27 (1889) 201-.

- - - to transformations of binomial equations. Sturm, J. C. F. Liouv. J. Mth. 7 (1842) 132-.

- - , determination of $X_{3}$. Young, J.R. $\quad \mathrm{Ph}$. Mg. 7 (1835) 454-.

-, extension. Phragmén, E. C. R. 114 (1892) 205-, 440.

- - - - to system of equations. Bendixson, $I$. [1892] Stockh. Ak. Hndl. Bh. 18 (Afd. 1) (1893) No. 2, $18 \mathrm{pp}$.

- and limits of roots of equation. Sylvester, J. J. Ph. Mg. 5 (1853) 446-; 6 (1853) 14-, 138-, 210-.

- - - Ostrogradski's. Desboves, A.H. N. A. Mth. 6 (1867) 348-.

- -, proof. Ettingshausen, A, von, Baumgartner Z. 7 (1830) 444-.

- - - - Trudi, N. G. Mt. 1 (1863) 59-.

- - remarks. Candèze, G. N. A. Mth. 20 (1881) 193-.

- - , simplification of calculations. Housel, -. N. A. Mth. 6 (1867) 351-.

- theorems of comparison. Bôcher, M. N. Y. Am. Mth. S. T. 1 (1900) 414.

Variations, number gained or lost in multiplication of $f(x)$ by $x^{n} \pm a$. André, D. C. R. 99 (1884) 182-.

- - - in multiplication by $x-a$. André, $D$. C. R. 98 (1884) 292-. 
2430 Equations of the second, third and fourth orders: other particular equations.

Abelian cubics and symmetrical equations. Cockle, Jas. QJ. Mth. 5 (1862) 237-.

Algebraic equations. Jonquières, E. de. C. R. 99 (1884) 345-, 450, 469-, 483-, 546.

- - Lalanne, L. C. R. 99 (1884) 463-.

- - especially irreducible case of Cardan's formula. Gegenbauer, L. Liège S. Sc. Mm. 2 (1900) No. 10, 6 pp.

- - pair. Mansion, P. Mess. Mth. 7 (1878) 57-.

- - , solution by radicals and by series. Favero, G. B. Rm. R. Ac. Linc. Mm. 6 (1889) 415-.

- - whose first member satisfies a linear differential equation of the 2 nd order. Laguerre, E. C. R. 90 (1880) 809-; 94 (1882) 412-, 508-.

- resolution of equations, theory. Giudice, $F$. Rv. Mt. 2 (1892) 193-.

Anharmonic-ratio sextic. Cayley, A. QJ. Mth. 10 (1870) 56-.

Binary equations of 4 th or 5 th order, conditions for 3 equal roots, etc. Cayley, $A$. Phil. Trans. 158 (1868) 577-.

Continued roots. Dixom, T. S.E. Des Moines Anal. 5 (1878) 20-.

De Moivre, class of equations solved by, and their derivatives. Réalis, S. N. A. Mth. 4 (1865) 209-, 289-.

De Moivre's formula. Barsotti, $G$. Lucca At. Ac. 17 (1860) 149-.

- - theorem and Cardan's formula applied to equations. Barsotti, G. Pisa A. Un. Tose. Sc. Cosm. 5 (1858-61) 99-.

Equation, roots of which are products in pairs of roots of another equation. Malet, $J . C$. QJ. Mth. 13 (1875) 30-.

,$--\ldots-\ldots-\ldots-2$ given equations. Malet, J. C. [1878] A. Mt. (1878-79) 306-.

,$----\left(\frac{n}{r}\right) ; r=0,1,2 \ldots n$. Siacci, F. G. Arcad. 18 (1860) 166-.

- for secular inequalities of planetary motion, Cauchy's theorem that roots are essentially real. Hermite, C. C. R. 41 (1855) 181-.

- with variable parameter, roots. Cauchy, A. L. C. R. 12 (1841) 1133-.

Equations, certain reducible types. Spitzer, $S$. Wien SB. 8 (1852) 422-; Grunert Arch. 22 (1854) 1-.

- depending on numerical functions. [Bougaieff, N. V.] Bugaev, N. V. [1874] (xm) Rec. Mth. (Moscou) 7 (1874-75) (Pt. 1) 424-; (Ix) Bll. Sc. Mth. As. 10 (1876) 102-.

- determining functions of double argument. Hill, C. J. D. Crelle J. 11 (1834) 241-.

- first 4 orders, solution. Eisenstein, $G$. Crelle J. 27 (1844) 81-.

- of form $x^{3}-b x=1$. Lockhart, J. Mathematician 2 (1847) 253-, 319-.

$---x^{4}+a x^{2}+b x+c=0$, solution. Leire, P. (xII) Ts. Mth. 1 (1877) 117-.

$---\frac{A_{0}}{x-a_{0}}+\frac{A_{1}}{x-a_{1}}+\& c_{0}=0$. Laguerre, $E$. C. R. 93 (1881) 890 -.
Equations of form $\left(x^{p}-a^{p}\right) \psi(x)=0$. Berloty, -. N. A. Mth. 1 (1882) 173-.

$---(a, b, c, d)=\left(a^{2}, b^{2}, c^{2}, d^{2}\right)$, solution. Cayley, A. Mess. Mth. 15 (1886) 59-

$--(a, b, c-)=\left(a^{p}, b^{p}, c^{p}-\right)$, solution. Vivanti, G. G. Mt. 27 (1889) 229-.

$---(-)=(-),-$ Tanner, H.W.L. Mess. Mth. $19(1890) 118$.

$-\quad-\sqrt{(x-a)(x-b)}+\sqrt{(x-c)(x-d)}=e$, solution. Dicksom, J. D. H. Edinb. Mth. S. P. 9 (1891) 13-.

-, general theory and application to curves. Söderblom, A. Ups. Årsk. (1879) 64 pp.

-, roots of which are of form $\cos \frac{2 k \pi}{n}$ or $\sin \frac{2 k \pi}{n}$. Johnson, W. W. Des Moines Anal. 6 (1879) 17-.

- soluble by Cardan's method. Guldberg, A. S. Ts. Mth. 3 (1885) 39-; Fschr. Mth. (1885) 74.

-, solution of certain. Allardice, R.E. Edinb. R. S. P. 17 (1891) 139-.

\section{Equations, 2nd order.}

Mattheroson, R. E. Camb. (M.) Mth. M. 2 (1860) 232-.

Marrecas Ferreira, L. F. G. Teix. J. Sc. 2 (1880) 77-.

adfected, solution. . Seers, J. Ph. Mg. 4 (1828) 125-.

- - by goniometry. Mollweide, C. Zach M. Cor. 22 (1810) 43-

depression. Noel, J. N. Liège Mm. S. Sc. 8 (1853) 94-.

general. De Morgan, A. Mathematician 3 (1850) 154-; Suppl. 4-.

C, reduction. S., W. J. Camb. and Dubl. Mth. J. 5 (1850) 286-.

with imaginary coefficients, solution. Grunert, J. A. Grunert Arch. 8 (1846) 65-.

invariants. Routh, E. J. QJ. Mth. (v) 6 (1864) 270-, (viri) 308-.

maxima and minima, process for determining. Shreve, S.H. V.Nost.Eng. Mg. 15(1876)530-. numerical. Bugaev, N. V. (xII) Rec. Mth. (Moscou) 8 (1876) (Pt. 1) 239-.

paradox. Anon. (vi 556) Gergonne A. Mth. 15 (1824-25) 118-.

rational solutions, expression. Tist, - Les Mondes 23 (1870) 770-.

reality of roots. Cayley, A. [1862] Crelle J. 61 (1863) 367-.

relation between roots and coefficients. Baehr, G. F. W. [1869] Amst. Vs. Ak. 4 (1870) (Ntk.) 197-.

solution. Mathervs, G. B. Nt. 57 (1897-98) 463-.

- by trigonometry. Grunert, $\mathcal{J}$. A. Grunert Arch. 1 (*1841) 12-.

- - - (Grunert). Mensing, -. Grunert Arch. 1 (1841) 189 .

theorem. Lebesgue, $\dot{V}$. A. N. A. Mth. 13 (1854) 412-.

theory. Gay, J. Laus. Bll. S. Vd. 1 (184245) 388 -

-. Macfarlane, A. Am. As. P. (1897) 54. 


\section{Equations, 3rd order}

Equations, 2nd, 3rd and 4 th orders.

applications of theory of forms. Tortolini, B. Tortolini A. 6 (1855) 433-.

with complex coefficients. Denzler, $W$. Zür. Vjschr. 4 (1859) 37-.

- rational roots. Sinram, T. Arch. Mth. Ps. 66 (1881) 94-.

root-forms, general. Matthiessen, H. F. L.

Z. Mth. Ps. 24 (1879) 32-.

solution. Machonvetz, $J$. Böhm. Gs. Ab. 12 (1863) 325-.

-. Matthiessen, L. Schlömilch Z. 8 (1863) 133-.

- Sinram, T. Arch. Mth. Ps. 64 (1879) 296-.

-. Ravut, -. As. Fr. C. R. (1894) (Pt. 2) 285-.

- by function $\frac{n}{\mathbf{x}}(\mathbf{x})$. Guldberg, A.S. Chris tiania F. 14 (1872) 144-.

symmetric solutions. Levänen, $S$. Helsingf. Acta 19 (1893) No. 9, 14 pp.

trigonometric solution. Cagnoli, -. N. A. Mth. 9 (1850) 374-.

\section{Equations, 3rd order.}

Horner, W. G. Thomson A. Ph. 9 (1817) 378-. Grunert, J. A. Grunert Arch. 5 (1844) 417-; 6 (1845) 1-, 428-

Lebesgue, V. A. N. A. Mth. 8 (1849) 219-.

Grunert, J. A. Grunert Arch. 22 (1854) 347- ; 30 (1858) 231-

Catalan, E. C. C. R. 54 (1862) 659-.

Eisenlohr, F. C. R. 55 (1862) 64-.

Faure, H. N. A. Mth. 3 (1864) 116-.

Sivering, J. N. A. Mth. 5 (1866) 356-.

$D-g$. Ts. Mt. Fys. 2 (1869) 19-.

Laguerre, -. N. A. Mth. 9 (1870) 343-.

Le Besgue, V. A. N. A. Mth. 9 (1870) 529 -.

André, $D$. N. A. Mth. 14 (1875) 556-.

Liebrecht, E. Arch. Mth. Ps. 59 (1876) 217.

Barbour, L. G. Des Moines Anal. 5 (1878) 73-.

Realis, S. N. A. Mth. 17 (1878) 178-.

Sinram, T. Arch. Mth. Ps. 68 (1882) 106-.

Barbour, L. G. Des Moines Anal. 10 (1883) 115-.

Sawin, A. M. Science $2\left(^{*} 1883\right) 44$.

Cayley, A. Mess. Mth. 15 (1886) 62-.

Giudice, $F$. Rv. Mt. 3 (1893) 146-.

Sidler, G. Bern Mt. (1898) 57-.

Sochocki, J. Wiad. Mt. 3 (1899) 119- ; Fschr. Mth. (1899) 102-.

automorphic transformation. Aubry, A. N. A. Mth. 6 (1887) 175-.

Cardan's formula. Grunert, J.A. Grunert Arch. 40 (1863) 246-.

- - Bouniakowsky, V. Crelle J. 3 (1828) $347-$.

- - expansion in series for irreducible case. Voll, $W$. Oken Isis (1826) 678 -

- generalised. Miiller, J.H.T. Grunert Arch. 22 (1854) 16-.

- - (Halcken). Grunert, J. A. Grunert Arch. 14 (1850) 132-.

- - , historic note. Forestier, $C$. Toul. Ac. Sc. Mm. 6 (1874) 254-.

\section{Equations, 3rd order 2430}

Cardan's formula, imaginaries in. Bernardi, G. Padova N. Sag. 3 (1831) 184-.

- - transformations. Berndtson, B. Stockh. Ac. Hndl. 36 (1815) 183-.

- rule, and general solution of equations. Gy'rry, S. (xII) Mag. Ak. Ets. (Mth.Term.) 2 (1861-62) 81-.

- - new deduction. Szokol, P. (xir) Orv. Term. Êts. 8 (1883) (Term. Szak) 273-.

Chasles's homography, problem. Hesse, $L . O$. [1862] Crelle J. 62 (1863) 188-.

and Delian problem; trisection of an angle. Nissen, $H$. N. Ts. Mth. 1 (A) (1890) 2-, 40 ; Fschr. Mth. (1890) 111-.

equation for squared differences of roots. Cayley, A. QJ. Mth. 3 (1860) 307-.

expression for remaining roots. Young, J. R. Ph. Mg. 34 (1849) 193-.

extension of theorem of Grunert's. Toeplitz, J. Grunert Arch. 31 (1858) 222-.

general. Stainville, - de. Gergonne A. Mth. 9 (1818-19) 197-.

-. Borden, J. Des Moines Anal. 5 (1878) 110 -.

geometric representation. Thiele, T. N. Ts. Mth. 2 (1884) 11-; Fschr. Mth. (1884) 86.

giving principal planes of conicoids, etc. Humbert, $E$. As. Fr. C. R. (1888) (Pt. 2) 145-.

and higher, solution. Kulik, J. N. Böhm. Gs. Ab. 11 (1861) 21-.

integral roots. Realis, $S$. N. A. Mth. 14 (1875) 289-, 424-.

Kramp's treatment. Müller, J.H.T. Grunert Arch. 8 (1846) 107-.

literal, limits of roots. Rees, R. van. Quetelet Cor. Mth. 5 (1829) 30-.

for principal axes of quadric surface. Kummer, $E . E$. G. Arcad. 98 (1844) 71-.

- - - - - condition for 2 equal roots. Jacobi, C. G. J. G. Arcad. 99 (1844) 3-.

problem of Tortolini. Matthiessen, L. Arch. Mth. Ps. 47 (1867) 460-.

with rational coefficients. Kronecker, L. Crelle J. 56 (1859) 188-.

rational roots. Liebrecht, $E$. Arch. Mth. Ps. 60 (1877) 216-

real roots. Sawin, A. M. Science $2\left({ }^{*} 1883\right)$ 158 -

reality of roots. Roche, E. N. A. Mth. 1 (1842) 511-.

- - . Tarnier, -. N. A. Mth. 3 (1844) 161-. relations between roots of certain. Berger, $A$. Ups. S. Sc. N. Acta 18 (1900) No. 3, 14 pp.

residual. Bouniakowsky, $V$. St Pét. Ac. Sc. Mm. 2 (1833) 373-; 3 (1838) 13-.

roots. Barbarin, -. As. Fr. C. R. (1887) (Pt. 2) 126-.

- criteria. Kerz, $F$. Arch. Mth. Ps. 41 (1864) 68-; 42 (1864) 121-; 44 (1865) 1-, 129-, 379-; 45 (1866) 14-; 47 (1867) 363-.

- of which are the sides of a triangle. $\check{Z} b i$ kovskij, A. K. Kazan S. Nt. (Ps.-Mth.) P. 5 (1887) 182-; Fschr. Mth. (1887) 78.

solution. Meredith, T. [1791] Ir. Ac. T. 7 (1800) 69-.

-. Ivory, J. [1799] Edinb. R. S. T. 5 (1805) 99-. 
solution. Berndtson, B. Stockh. Ac. Hndl. 27 (1806) 269-; 28 (1807) 72 .

-. Cockle, Jas. Camb. Mth. J. 2 (1841) 248-; 3 (1843) 28-

-. Bretschneider, C. A. Grunert Arch. 4 (1844) 410-.

- Dippe, M. C. Grunert Arch. 7 (1846) 149-.

-. Schlesicke, W. Grunert Arch. 11 (1848) 345-.

-. Rutherford, $W$. Mathematician 3 (1850) $257-$.

-. Campbell, T. J. Ir. Ac. P. 6 (1853-54) 352 .

- Azzarelli, M. Tortolini A. 6 (1855) 467-.

-. Spitz, C. Grunert Arch. 32 (1859) 435-.

-. Veriot, E. C. R. 60 (1865) 556-.

- Talbot, W. H. F. Edinb. R. S. T. 24 (1867) 573-.

-. Thiele, T. N. (xп) Ts. Mth. 4 (1868) 65 -.

- Alexandre, R. N. A. Mth. 9 (1870) 293-.

- Gundelfinger, S. [1870] Mth. A. 3 (1871) $272-$.

-. Dahl, C. (xu) Ts. Mth. 4 (1874) 135-.

-. Azzarelli, M. Rm. N. Linc. At. 31 (1878) $355-$.

-. Scholtz, A. N. A. Mth. 20 (1881) 220 -.

-. Zurria, G. Catania Ac. Gioen. At. 8 (1895) Mm. 3, 24 pp.

- Macfarlane, A. As. Fr. C. R. (1897) (Pt. 2) 147-.

-. Pleskot, A. N. A. Mth. 18 (1899) 65-.

- by aid of a jointed system. Saint-Loup, L. C. R. 79 (1874) 1323-.

- circular and hyperbolic functions. Matzka,W. Grunert Arch. 37 (1861) 399-.

-, Cockle's. Cayley, A. Camb. and Dubl. Mth. J. 6 (1851) 186.

- by differences of roots. Eisenlohr, O. Crelle J. 42 (1852) 236-.

-, expressions occurring in. Eisenstein, $G$. Crelle J. 27 (1844) 319-.

—, formulæ for. Young, J. R. [1874] Ir. Ac. P. 2 (1875-77) 26-.

-, general. Graves, J. T. (vi Adds.) $\mathrm{Ph}$. Mg. 13 (1838) 217 -

-, -, and irreducible case. Penny, W. G. Atlantis 3 (1860) 156-.

-, Gierman's. Sintzow, D. Fschr. Mth. (1899) 102.

-, indirect (Posselt). Encke, J. F. As. Nr. 3 (1825) 395-.

- by introducing a perfect cube. Alexandre, $R$. N. A. Mth. 5 (1866) 358-.

- logarithm tables, \&c. Veriot, E. C. R. 66 (1868) 619-, 730-.

-, peculiar algebraic. Münster, E. N. Mg. Ntvd. 19 (1873) 23-.

-, and roots in functions of discriminant of cubic. Mollame, V. G. Mt. 16 (1878) 341-.

- by trigonometric and hyperbolic functions. Gronau,J.F.W. Danzig Schr.6 (Hft. 2u. 3) (1861) $68 \mathrm{pp}$.

- when one root is known. Lobatto, $R$. Liouv. J. Mth. 9 (1844) 177-. solution when one root is known (Lobatto). Serret, J. A. Liouv. J. Mth. 14 (1849) 47-.

- without removing 2 nd term. Rutherford, $W$. Mathematician 3 (1850) 180 -.

symmetries and irreducible case. Kummell, C. H. A. Mth. 6 (1891-92) 179-.

theorem. Prouhet, E. N. A. Mth. 11 (1852) 324-.

theory. Oekinghaus, $E$. Arch. Mth. Ps. 3 (1886) 92-.

-, contribution to. Müller, J. H. T. Grunert Arch. 25 (1855) 73-.

trigonometric expressions for roots. Björling, E. G. Stockh. Öfv. 7 (1850) 193-; Grunert Arch. 19 (1852) 228-.

- solution. Demongeot, A. N. A. Mth. 20 (1861) 143-.

- -. St Germain, A. de. N. A. Mth. 10 (1871) 63-.

two. Cayley, A. Mess. Mth. 22 (1893) 69-.

Irreducible case.

Lorgna, A. M. Verona S. It. Mm. 1 (1782) 707-.

Stainville, - de. Par. Éc. Pol. Cor. 3 (181416) 58

Gretschel, H. Arch. Mth. Ps. 42 (1864) 431-.

Meyer, J. Lux. S. Sc. Mm. 8 (1865) 359-.

Hermann, A. N. A. Mth. 6 (1867) 270 .

Unferdinger, $F$. Arch. Mth. Ps. 49 (1869) 484-.

Reidt, F. Z. Mth. Ps. 17 (1872) 430-

Benthem, A. N. Arch. Wisk. 1 ("1875) 67-.

Privat, F. C. R. 103 (1886) 774.

Azzarelli, M. Rm. N. Linc. At. 40 (1887) 67-.

Mollame, V. Nap. Rd. 29 (1890) 167-.

Hölder, $O$. Mth. A. 38 (1891) 307-.

Pokrowsky, P. M. Fschr. Mth. (1892) 95.

Gegenbauer, L. Mh. Mth. Ps. 4 (1893) 155-.

Kneser, A. Mth. A, 41 (1893) 344-.

Mollame, V. Nap. Rd. 34 (1895) 111-.

Hamilton, A. A. Mth. 1 (1900) 41-.

Cardan's formula, application of trigonometry. Zampieri, J. Arch. Mth. Ps. 41 (1864) 60-. solution. Nordmark, $Z$. Ups. N. Acta S. Sc. 6 (1799) 203-.

-. Kendall, $\dot{O}$. H. Am. J. Mth. 1 (1878) 285-.

-, non-transcendental. Grunert, J.A. Grunert Arch. 30 (1858) 135-.

- when roots are all real, and at least one is rational. Weichold, G. Liouv. J. Mth. 5 (1879) 293-.

- without imaginaries. Peche, $F$. Haidinger Ab. 4 (1851) (Ab. 3) 125-.

- - series. Root extraction. Valz, B. C. R. 58 (1864) 1186-

treatment, Clausen's. Meyer, G. F. Grunert Arch. 39 (1862) 235-.

Equations, 3rd and 4th orders.

Franke, E. [1880] Crelle J. Mth. 90 (1881) 102-.

Halphen, G. H. N. A. Mth. 4 (1885) 17-. 
Sochocki, J. Prace Mt.-Fiz. 10 (1899-1900) 135-; Fschr. Mth. (1899) 102-.

limit of roots. Fournier-Vannson, -. N. A. Mth. 11 (1852) 60-.

literal, solution. Jourdain, -. Liouv. J. Mth. 4 (1859) 205-.

- - Matthiessen, L. Arch. Mth. Ps. 45 (1866) 415-.

problem, solution. Tortolini, B. Rm. At. N. Linc. 22 (1869) 166-.

roots of which can be expressed without cubic radicals. Realis, $S$. N. A. Mth. 18 (1879) 296-.

solution. Twining, A. C. Silliman J. 9 (1825) 86-.

- Sturm, J. B. Grunert Arch. 24 (1855) $112-$.

-. Tortolini, B. Tortolini A. 1 (1858) 310-.

-. Hill, G. W. Des Moines Anal. 2 (1875) 4-.

- by construction. Chasles, M. Liouv. J. Mth. 20 (1855) 329-; C. R. 41 (1855) $677-$.

-, Lagrange's method. Candioti, M. R. Arg. S. Ci. A. 46 (1898) 223-.

- by linear substitution $x=\frac{m y+n}{y+1}$. Azzarelli, $M . \quad$ Rm. At. N. Linc. 24 (1871) 83-.

- - same principle. Sommer, B. Grunert Arch. 27 (1856) 354-.

solutions of which by radicals are rational. Kummer, E. E. Berl. Ak. Mb. (1880) 930 -.

Sturmian constants. Cayley, A. QJ. Mth. 4 (1861) 7-.

theorem. Desboves, A. C. R. 90 (1880) 1069-.

theory. Cockle, Jas. Camb. Mth. J. 3 (1843) 104 -.

-, elementary. Pokrowsky, P. M. Fschr. Mth. (1893-94) 152-.

values of roots when coefficient of first term is zero. Faure, $H$. N. A. Mth. 14 (1855) 194-.

\section{Equations, 4th order.}

Bret, -. Par. Ec. Pol. Cor. 2 (1809-13) 217-.

Ampère, A. M. Quetelet Cor. Mth. 9 (1837) 144-.

Roberts, M. N. A. Mth. 18 (1859) 87-.

Catalan, E. C. . N. A. Mth. 2 (1863) 341-.

Pokorny, M. Z. Mth. Ps. 10 (1865) 320-.

Ball, R. S. QJ. Mth. 7 (1866) 6-, 358-.

Enneper, A. Z. Mth. Ps. 18 (1873) 93-.

Rawson, R. Manch. Lt. Ph. S. P. 19 (1880) 127-.

Oekinghaus, E. Arch. Mth. Ps. 69 (1883) 169 .

Gordan, P. Mth. A. 29 (1887) 318-.

Darbi, G. G. Mt. 38 (1900) 153-.

analytical solution. Valeriani, V. G. Mt. [13 (1875)] 99-.

case of reduction to canonical form. Gruzintsev, A. P. (xI) Kharkov Mth. S. Com. (1881) 116-.

class. Brioschi, F. C. R. 57 (1863) 106-. double system of values in solving. Valles, $F$. C. R. 47 (1858) 30-.

_ _ _ _ _ - Ollive-Meinadier, _.: Gard Mm. Ac. (1859) 171-.

equation of anharmonic ratios corresponding to roots. Painvin, L. N. A. Mth. 19 (1860) 407-.

- - squares of differences of roots. Roberts, M. Tortolini A. 2 (1859) 330-.

_ _ C. Casey, J. [1874] Ir. Ac. P. 2 (1877) 40 -.

2 (1883) 209-.

factors. Elliott, E. B. Mess. Mth. 25 (1896) 170-.

and of 5th. Autonne, L. Par. S. Mth. Bll. 28 (1900) 90-.

- - - when $J_{18}=0$, solution. Faà de Bruno, F. Am. J. Mth. 3 (1880) 154 .

- - - and 6 th, solution by trigonometric and hyperbolic functions. Heymann, $W$. Crelle J. Mth. 113 (1894) 267-.

final formulø for algebraic solution. Merriman, M. Am. J. Mth. 14 (1892) 237-; N. Y. Mth. S. Bll. 1 (1892) 202-, 236.

general. Boer, F. de. Amst. Ak. Vs. M. 20 (1884) 413-; Fschr. Mth. (1884) 79.

-, root formulæ. Unferdinger, $F$. Wien Sb. 50 (1865) (Ab. 2) 225-.

-, solution. Pilatte, - Gergonne A. Mth. 2 (1811-12) 152-.

-, -. Gergonne, J. D. Gergonne A. Mth. 13 (1822-23) 385-.

, - Grassmann, H. Arch. Mth. Ps. 51 (1870) 93-.

-,- Fitz, N. Des Moines Anal. 3 (1876) 50 -

-, 一. Weichold, G. C. R. 82 (1876) 1093-. -, - - Lodge, A. QJ. Mth. 19 (1883) 257-.

literal, case. Pagani, G. M. Quetelet Cor. Mth. 4 (1828) 291-.

nature of roots. Lucas, F. Par. S. Mth. Bll. 18 (1890) 145-.

with no real root, calculation of roots. Koralek, P. N. A. Mth. 11 (1852) 117-.

reality of roots. Desboves, A. H. N. A. Mth. 3 (1844) 387-.

reduction. Schlömilch, O. Schlömilch Z. 8 (1863) 223-.

- to equation of 3rd order. Hoppe, R. Arch. Mth. Ps. 69 (1883) 111-。

- - reciprocal equation. Brocard, H.P.P.B. (xII) Mathesis 2 (1882) 180-.

- _ - of same order. Alexandre, R. N. A. Mth. 5 (1866) 477-.

- -1 - 2nd order. Ligowski, W. [1879] Arch. Mth. Ps. 65 (1880) 426-.

root forms, domains of the 3. Hoppe, R. Arch. Mth. Ps. 14 (1896) 398-.

roots, Aronhold's formula. Janni, V. G. Mt. 21 (1883) 213-.

- related to sides of cyclic quadrilateral. Matthiessen, L. Z. Mth. Ps. 9 (1864) 453-; 10 (1865) 331-.

and of $6 \mathrm{th}$, in theory of conics and quadrics. Joachimsthal, $F$. Crelle J. 53 (1857) 149-. solution. Berndtson, B. Stockh. Ac. Hndl. 28 (1807) 173-. 
Equations, 4th order, continued.

solution. Anon. (vi 1231) Tilloch $\mathrm{Ph} . \mathrm{Mg}$. 52 (1818) 378-.

-. Greatheed, S. S. [ascribed to Gregory, D. F., in vol. IIr]. Camb. Mth. J. 1 (1839) 254-.

- Graves, C. Ir. Ac. P. 3 (1847) 111-.

- Peche, F. Haidinger B. 3 (1847) 19-.

-. Grunert, J. A., \& Schlesicke, $W$. Grunert Arch. 12 (1849) 166-.

-. Lindman, C. F. Grunert Arch. 23 (1854) 435-.

-. Aronhold, S. H. Crelle J. 52 (1856) 95-.

- Grunert, J.A. Grunert Arch. 31 (1858) 476-.

-. Hermite, C. C. R. 46 (1858) 715-.

-. Spitz, C. Grunert Arch. 33 (1859) 442-.

-. Schlömilch, O. Schlömilch Z. 6 (1861) 49-; Liouv. J. Mth. 8 (1863) 99-.

- Matthiessen, L. Schlömilch Z. 8 (1863) 133-, 140-; Arch. Mth. Ps. 41 (1864) 231-.

-. Bellavitis, G. N. A. Mth. 3 (1864) 121-.

-. Darboux, G. Liouv. J. Mth. 18 (1873) 220-.

-. Dixon, T. S. E. Am. J. Mth. 1 (1878) 283-.

- Briot, F. N. A. Mth. 20 (1881) 225-.

- Borenius, G. [1882-83] Helsingf. Acta 12 (1883) 653-; Stockh. Ak. Hndl. Bh. 9 (188485) No. 4, $18 \mathrm{pp}$.

-. Sawin, A. M. A. Mth. 1 (1884-85) 14.

-. Rastorguev, D. Kazan S. Nt. (Ps.-Mth.) P. 4 (1886) 236-.

-. McClintock, E. N. Y. Am. Mth. S. Bll. 3 (1897) 389-; 4 (1898) 283.

-. Starkweather, G. P. N. Y. Am. Mth. S. Bll. 4 (1898) 524-.

-. Valentiner, $H$. N. Ts. Mth. 9 (B) (1898) 36-

-, Ampère's. Grunert, J.A. Grunert Arch. 1 (1841) 16-.

- by elliptic functions. Lebesgue, $V . A$. Liouv. J. Mth. 3 (1858) 391-.

-, Euler's. Strehlke, F. Crelle J. 12 (1834) 358-.

-, general. Schlesicke, $W$. Grunert Arch. 16 (1851) 58-.

-, -. Grunert, J. A. Grunert Arch. 40 (1863) 394-.

-, -. Nell, A. M. Arch. Mth. Ps. 56 (1874) 407-.

- by homographic relation between roots. Imšeneckij, $V$. G. Kharkov Mth. S. Com. 3 (1893) 257-; Fschr. Mth. (1893-94) 153.

-, method, and application to equations of higher orders. Perrin, R. Par. S. Mth. Bll. 10 (1882) 139-.

-, Schloemilch's. Meyer, G. F. Grunert Arch. 39 (1862) 230-.

-, and some algebraic theorems. Hermite, C. C. R. 46 (1858) 961-.

- without removal of 2 nd term. Grunert, J.A. Grunert Arch. 39 (1862) 198-

-, Wronski's. Gergonne, J. D. Gergonne A. Mth. 3 (1812-13) 137-, 206-.

solutions, various. Dippe, M. C. Grunert Arch. 7 (1846) 334-. and Sturm's theorem. König, J. F. Grunert Arch. 34 (1860) 101-.

trigonometric expressions for roots. Björling, E. G. Stockh. Öfv. 7 (1850) 233-; Grunert Arch. 19 (1852) 297-.

- solution. Oekinghaus, $E$. Arch. Mth. Ps. 70 (1884) 133-.

Equations, order above 4 th, solution in particular cases. Ruffini, P. [1801] Mod. S. It. Mm. 9 (1802) 444-.

- of $n$th order with only 2 real roots. André, D. C. R. 98 (1884) 417-.

C. R. 98 (1884) 550 .

Irrationality $\sqrt{ }(a+\sqrt{b})$. Azzarelli, $M$. Rm. N. Linc. At. 38 (1885) 227-.

Particular equations of various orders, solution. Steen, A. Mth. Ts. 2 (1860) 45-

Planets, mutual distance of 2 , roots of equation obtained by equating to zero. Cauchy, A. L. C. R. 19 (1844) 1228-.

"Problem of the lights." Parker, W. H. Camb. (M.) Mth. M. 1 (1859) 160-.

- - - (Parker). Runkle, J. D. Camb. (M.) Mth. M. 1 (1859) 178-

Reciprocal equations. Garnier, J. G. Quetelet Cor. Mth. 2 (1826) 153-.

- -. Comberousse, C. de. N. A. Mth. 8 (1889) 27-.

- - Gianni, L. G. Mt. 30 (1892) 206-.

- and equations of 4 th order. Pellet, A.E. N. A. Mth. 13 (1894) 108-.

- - , equations reducible to. Fischer, F. W. Arch. Mth. Ps. 55 (1873) 294-.

- - lowering degree of. Biehler, C. N. A. Mth. 6 (1887) 251-.

- -, theory. Vogt, A. Arch. Mth. Ps. 44 (1865) 50-.

_- , - Studnicka, F. J. Ćasopis 19 (1890) 1-; Fschr. Mth. (1890) 110-

Resolvents, first, of equation of 4 th order

$$
y^{4}+a y^{2}+x_{1} y-\frac{a^{2}}{12}=0
$$

and of equation of 3 rd order

$$
a y^{3}+3 b y^{2}+3 c y+m=0 \text {. }
$$

Rawson, $R$. Manch. Lt. Ph. S. P. 20 (1881) 144-.

Roots of equation $x^{4}+2=0$. Terquem, $O$. N. A. Mth. 4 (1845) 518-

"Rule of false" of the Arabians, and direct solution of literal equations of 2 nd and 3rd orders. Matthiessen, L. Z. Mth. Ps. 15 (1870) 41-.

" - _ -" and equations of 2 nd order. Castro, M. Méx. Reg. Trim. 1 (1832) 125-.

Trigonometrical solution of certain equations. Pellet, A. As. Fr. C. R. (1889) (Pt. 2) 164-.

\section{Trinomial Equations.}

Marrecas Ferreira, L. F. G. Teix. J. Sc. 5 (*1883) 50-.

Catalan, E. Brux. Ac. Bll. 13 (1887) 414-. 
Trinomial equations, continued.

Nekrassoff, $P$. Mth. A. 29 (1887) 413-.

class. Besso, $D$. Rm. R. Ac. Linc. Mm. 19 (1884) 631-.

of form $x^{2 n+1}-x-k=0, n$ prime. Berndtson, $B$. Gergonne A. Mth. 11 (1820-21) 373-.

$--x^{n} \pm A x \pm B=0$. Casinelli, A. Bologna N. Cm. 7 (1844) 331-.

$-x^{2 n+1}-x=k$. Genocchi, A. Tortolini A. 2 (1859) 253-.

$--x^{r}+a x+b=0$. Thiele, T. N. Mth. Ts. 1 (1859) 115-.

$--y^{n}+p y+q=0$. Borden, J. Des Moines Anal. 5 (1878) 41-.

- - $u^{m}-p u^{n}-q=0$. Nekrasov, P.A. [187982] (xII) Rec. Mth. (Moscou) 11 (1883-84) 1-.

$-1 t^{n}+a t^{n-8}+b=0$, a solution. Heymann, W. Z. Mth. Ps. 31 (1886) 223-.

$-u^{m}-p u^{n}-q=0$, theory. Nekrasov, P.A. [1894] Rec. Mth. (Moscou) 17 (1895) 439-; Fschr. Mth. (1893-94) 162.

Gauss's method of lowering. Montucci, $H$. C. R. 70 (1870) 445-.

- solution. Nell, A. M. Arch. Mth. Ps. 1 (1884) 311-.

general, roots. Farkas, G. (xiI) Mag. Tud. Ak. Éts. 12 (No. 5) (1878) 116.

nature of roots. Bonnet, O. N. A. Mth. 4 (1845) 293-.

of odd order $X^{m} \pm X=R$, solution by means of new algebraic sign. Piarron de Mondésir, E. S. As. Fr. C. R. (1877) 168-.

and quadrinomial, elementary treatment. Heymann, $W$. Z. Mth. Ps. 37 (1892) 90-.

of 7 th order in particular. Besso, D. Rm. R. Ac. Linc. Rd. 1 (1885) 237-.

solution. Garnier, J. G. Brux. Ac. Bll. 6 (1839) 474-.

-. Tetmajer, J. (xп) Krk. Ak. (Mt.-Prz.) Pam. 5 (1880) 1-.

- by algorithms resembling continued fractions. Hoffmann, K. E. [1880] Arch. Mth. Ps. 66 (1881) 33-.

theory. Heymann, $W$. Mth. A. 28 (1887) 61-.

\section{Numerical solution of equa- tions (including graphical me- thods).}

Numerical solution of equations in general.

Caluso, (l'abbé) T. V. de. Turin Mm. Ac. 6 (1792-1800) 159-.

Cassella, G. [1803] Mod. Mm. S. It. 11 (1804) 203-.

Berard, -. Gergonne A. Mth. 10 (1819-20) 61-.

Dandelin, G. P. Brux. Mm. Ac. Sc. 3 (1826) 7-, 153-.

Timmermans, J. A. Quetelet Cor. Mth. 2 (1826) 218-

Galois, E. Férussac Bll. Sc. Mth. 13 (1830) 413-.
Vincent, A. J. H. Lille Mm. S. (1833) 1-; (1838) 5-.

Dirksen, E. H. Crelle J. 14 (1835) 316-.

Sturm, J. C. F. Par. Mm. Sav. Etr. 6 (1835) 271-.

Kupfer, $K$. (xп) Mosc. Un. Mm. 12 (1836) 256-.

Cauchy, A. L. C. R. 11 (1840) 829-.

Encke, J. F. Berl. As. Jb. (1841) 281-.

Libri, G. C. R. 17 (1843) 93-.

Martynowski, J. Liège Mm. S. Sc. 1 (1843) 290-; 2 (1845-46) 445-; 8 (1853) 20 -.

Adeler, -. Bordeaux Ac. Act. (1844) 449-.

Galois, E. Liouv. J. Mth. 11 (1846) 397-.

Vallas, A. (xп) [Mag. Ak. Éts. (1850)] 39-.

Bellavitis, G. Ven. At. 3 (1852) 120-.

Möbius, A. F. Leip. B. (1852) 1-.

Valz, B. C. R. 41 (1855) 685-

Bellavitis, G. (vi Adds.) Ven. Mm. I. 6 (1856) 357-.

Dupré, A. C. R. 45 (1857) 585-.

Bellavitis, G. Ven. Mm. I. 9 (1860) 177 -.

Denzler, $W$. Zür. Vjschr. 5 (1860) 384- ; 6 (1861) 68-.

Oliveira, C. B. Rv. Brazil. 3 (1860) 185-.

Janni, $V$. G. Mt. 5 (1867) $182-$.

Woolhouse, W. S. B. [1868-70] L. Mth. S. P. 2 (1869) 75- ; I. Act. J. 15 ( $\left.^{*} 1870\right)$ 313-.

De Morgan, A. [1868-70] L. Mth. S. P. 2 (1869) 84- ; I. Act. J. 15 ( $^{*} 1870$ ) 327-.

Schröder, E. [1869] Mth. A. 2 (1870) 317-.

Vecchi, S. Il Polit. 20 (1872) 370-.

Siebel, $A$. Arch. Mth. Ps. 56 (1874) 422- ; 57 (1875) 73-, 350-; 58 (1876) 127-; 60 (1877) $138-; 61$ (1877) 122-; 65 (1880) 394-; 67 (1882) 375-.

Collignon, É. As. Fr. C. R. 4 (1875) 200-.

Houzeau, J. C. Brux. Ac. Bll. 40 (1875) 455-.

Janni, $V$. Nap. Rd. 17 (1878) 138-.

Laguerre, E. N. A. Mth. 17 (1878) 20-, 97-.

Amstein, $H$. Laus. S. Vd. Bll. 20 (1885) 201-.

Netto, E. Mth. A. 29 (1887) 141-.

Carvallo, E. Toul. Fac. Sc. A. 3 (1889) O, $40 \mathrm{pp}$.

Phragmén, E. Stockh. Öfv. (1892) 179-.

Söderblom, A. Göteb. Hndl. 2 (1899) 105-.

Approximate determination of roots.

Fourier, J. B. J. Par. S. Phlm. Bll. (1818) 61-.

Horner, W. G. Phil. Trans. (1819) 308-.

Gilbert, D. QJ. Sc. 14 (1823) 353-.

Crelle, A. L. Berl. Ab. (1835) 263-.

Ostrogradsky, M. A. [1835] St Pét. Ac. Sc. Bll. 1 (1836) 1-; St Pét. Ac. Sc. Mm. 3 (pte. 1) (1838) 233-.

Libri, G. C. R. 4 (1837) 168-.

Frisiani, $P$. Mil. Effem. As. (1847) 3-.

Grunert, J. A. Grunert Arch. 20 (1853) 337-.

Sornin, J. Toul. Mm. Ac. 1 (1857) 26-.

Grunert, J. A. Grunert Arch. 30 (1858) 54-.

Genocchi, A. N. A. Mth. 20 (1861) 48-.

Michaelis, J. P. Lux. S. Sc. Mm. 7 (1864) 143-.

Sang, E. [1864] Edinb. R. S. P. 5 (1866) 162-. 
Approximate determination of roots, continued. A. Q. G. C. Mess. Mth. 4 (1868) 132-.

$D \perp g$. Ts. Mt. Fys. 4 (1871) 201-.

Laguerre, -. N. A. Mth. 3 (1884) 113-.

Bugaev, N. V. [1894] Rec. Mth. (Moscou) 18 (1896) 289-; Fschr. Mth. (1896) 70-.

Laisant, C. A. As. Fr. C. R. (1895) (Pt. 2) 220 -.

Bugaev, N. V. [1896] Rec. Mth. (Moscou) 19 (1897) 421 (bis)-; Fschr. Mth. (1897) 96.

Lêmeray, E. M. N. A. Mth. 17 (1898) 534-.

of cubic equations by continued fractions. Sang, E. Edinb. R. S. P. 32 (1887) 311-.

- equation $A=\frac{(1+p)^{n}-1}{p}$. Ryley, E. Assur. $\mathrm{Mg}$. $\left[1\left({ }^{*} 1851\right)\right]$ 332-.

- with real roots only. Laguerre, E. C. R. 79 (1874) 522-; N. A. Mth. 19 (1880) 16i-, 193-.

- - - - - (Laguerre). Hermite, $C$. Rm. N. Linc. Mm. 3 (1888) 155-.

- _ - - (-). Borel, É. Bll. Sc. Mth. 22 (1898) 11-.

imaginary roots. Bonnet, O. N. A. Mth. 4 (1845) 164-, 388-; 12 (1853) 243-.

- - Lagrange's method. Cavalieri SanBertolo, N. Rm. At. 4 (1850-51) 1-.

incommensurable roots. Fauché-Prunelle, $A$. Grenoble Ac. Delph. Bll. 1 (1846) 563-.

irrational roots. Voll, $W$. Oken Isis (1826) 490 .

real roots. Piobert, - N. A. Mth. 10 (1851) 174-.

- - Buchwaldt, $F$. Mth. Ts. 2 (1860) 73-. - -. Janni, G. G. Mt. 8 (1870) 157-.

- of equation $x^{n}+p x+q=0$. Kjeldgaard, A. (xII) Ts. Mth. 4 (1880) 135-.

- - methods of Cauchy and Newton. Moigno, F. N. A. Mth. 10 (1851) 14-.

in series of aliquot parts. Horner, $J$. QJ. Mth. 3 (1860) 251-.

Simpson's method. Pessuti, G. [1806] Mod. S. It. Mm. 13 (1807) 193-.

singular case. Gergonne, J. D. Gergonne A. Mth. 10 (1819-20) 122-.

Budan's method. Gergonne, J. D. Gergonne A. Mth. 4 (1813-14) 115-.

- - Ferroni, P. [1823] Mod. S. It. Mm. 20 (1828) 17-.

Cauchy's modification of Newton's method. Housel, -. N. A. Mth. 15 (1856) 244 -.

Continued fractions applied to determining two roots simultaneously. Matthiessen, $L$. Schlömilch Z. 6 (1861) 51-.

- - solution by. Horner, W. G. QJ. Sc. 21 (1826) 72-, 282-; 22 (1827) 67-, 285-.

- - - - Grebe, E. W. Grunert Arch. 10 (1847) 345-; 16 (1851) 261-.

- - - - Gomes Teixeira, F. (x) Lisb. J. Sc. Mth. 4 (1873) 89-.

A. 7 (1874) 262-.

Convergence of certain series in numerical solution of equations. Graefe, C. H. Crelle J. 10 (1833) 288-.
Convergence of series of approximations to roots of equations. Lémeray, $E . M$. As. Fr. C. R. (1894) (Pt. 2) 252-.

- - - for root by Lagrange's theorem. Cauchy, A. L. C. R. 23 (1846) 493-.

Definite integrals, expression of roots of trinomial equations by. Lachtin, L. [1889] Rec. Mth. (Moscou) 15 (1891) 61-

- -, solution by. Jacobi, C. G. J. Crelle J. 2 (1827) 1-.

- - - - Hoppe, R. Schlömilch Z. 3 (1858) 173-.

,---1 . Kretkowski, W. [1881] (xrr) Krk. Ak. (Mt.-Prz.) Pam. 7 (1882) 158-.

Derived functions and numerical solution of equations. Mrasojědov, A. N. Rec. Mth. (Moscou) 12 (1885) 757-; Fschr. Mth. (1886) 71.

Differential equations, solution by. Heymann, W. Z. Mth. Ps. 31 (1886) 102-, 129-.

Eisenstein's theorem on series for root of algebraic equation. Hermite, C. L. Mth. S. P. 7 (1875-76) 173-.

- - - - - - Gomes Teixeira, F. Arch. Mth. Ps. 3 (1886) 315-.

Equation $A=(1+x)^{m}(1+b x)$, numerical solution, $x$ being a small fraction. Rädell, Grunert Arch. 2 (1842) 122-.

$--=(-)(-),-1,---1$. Lind man, C. F. Grunert Arch. 23 (1854) 445-.

Equations $a^{2}+b c=16, b^{2}+a c=17, c^{2}+a b=18$, approximate solution. Buée, - Thomson A. Ph. 5 (1815) 53-.

- in Bangma's "Algebra voor de Scholen." Schmidt, J.R. Amst. Mengelwerk 2 (1816) 153-.

-, continued fractions for whose roots terminate in same quotients. Serret, J. A. Liouv. J. Mth. 15 (1850) 152-.

-, order of which is power of prime number. Betti, E. Tortolini A. 6 (1855) 260-; C. R. 48 (1859) 182-.

-, - - - - - - Mathieu, $\dot{E}$. Tortolini A. 4 (1861) 113-.

- with secondary (imaginary) roots. Zielinski, A. Des Moines Anal. 3 (1876) 52-.

-, 2nd order, exhibition of both roots in one series of converging fractions. Sang, $E$. Edinb. R. S. P. 4 (1862) 70-.

- - - - solution by continued fractions. Lebesgue, V. A. Liouv. J. Mth. 5 (1840) 281-.

,,---- - successive approximation, cases. St Germain, A. de. N. A. Mth. 13 (1874) 401-.

-,- , where coefficient of $x^{2}$ is small. Gerono, G. C. N. A. Mth. 16 (1857) 436-.

-, 3rd order, development of roots in continued fractions. Sarrus, $F$. Gergonne A. Mth. 10 (1819-20) 189-.

-, - - - irreducible case, development of roots in continued fraction. Clausen, $T$. As. Nr. 19 (1842) 241-; Grunert Arch. 2 (1842) 446- (with additions by Grunert).

-, - -, numerical solution. Maulbon d'Arbaumont, - Épinal (Vosg.) A. 2 (1834) 637-. 
Equations, 3rd order, numerical solution. Lindman, C. F. Stockh. Öfv. 11 (1854) 333-; Grunert Arch. 25 (1855) 290-.

,,$----\ldots$ Burnier, $F$. Laus. Bll. S. Vd. $10(1868-70) 591-$.

- $,-\ldots,-\ldots$. Catania, S. Rv. Mt. 5 (1895) 81-.

,,--- roots as continued fractions. Märcker, -. Grunert Arch. 39 (1862) 39-.

,,$--- x^{3}-2 x-5=0$, solution to 153 figures. Hicks, J. P. [1850] Camb. Ph. S. T. 10 (1864) 337.

- below 4 th order, numerical solution. Warner, J. D. Am. As. P. (1880) 267-.

- 4 th order, numerical solution. Choquet, $C$. N. A. Mth. 3 (1844) 439.

-, - - - - Vidal, V. N. A. Mth. 17 (1878) 367-.

-, 5th order, and some others, numerical solution. Montucci, H. C. R. 60 (1865) 440-; 61 (1865) 31-.

Form of roots. Hill, C.J.D. Ups. N. Acta S. Sc. 2 (1856) $221-$.

Four roots of numerical equation, finding. Riedl von Leuenstern, J. Tortolini A. 6 (1855) 481-.

Gräffe's solution. Hansen, H. J. Ts. Mth. 5 (1887) 169-.

Horner's method. De Morgan, A. Mathematician 3 (1850) 289-.

- - Matzka, W. [1871] Prag Ab. 5 (1872) $47 \mathrm{pp}$.

- - proof. Jenkins, M. Mth. Gz. No. 6 (1895) 55-.

Imaginary roots, determination. Poletti, $G$. [1823-28] Tor. Mm. Ac. 30 (1826) 49-; 35 (1831) 77-.

,--- Bellavitis, G. [1845] Ven. Mm. I. 3 (1847) 109-.

- - - - Buchwaldt, F. Mth. Ts. 2 (1860) 177-. - -, -. Bellavitis, G. [1864] (vII) Ven. Mm. I. 11 (1862) 461-.

- -, -. Yvon-Villarceau, A. J. F. C. R. 86 (1878) 1427-.

- - - Farkas, J. C. R. 87 (1878) 791-, 1027-; 88 (1879) 273-, 565-.

- - - - by recurring series. Frisiani, P. Mil. Effem. As. (1850) 3-.

Infinite series, solution by. Multedo, -. Genova Mm. Ac. 3 (1814) 108-.

- - , - .. Jacobi, C. G. J. Crelle J. 6 (1830) 257-.

- -, - -.'Heegmann, A. [1861-64] (xII) Lille S. Mm. 8 (1862) 217-; 2 (1866) 1-.

,$---\ldots$. Dietrich, R. Arch. Mth. Ps. 69 (1883) 337-.

Integer roots of equation with integral coefficients. Gerono, G. C. N. A. Mth. 15 (1856) 449-.

Lagrange's treatise. Raabe, J.L. Crelle J. 17 (1837) 94-; 20 (1840) 57-.

Logarithmic solution. Garnett, J. Am. Ph. S. T. 6 (1809) 391-.

- Zenger. C. V. As. Fr. C. R. (1885) (Pt. 2) 91-.

Method of differences. Matrot, A. [1875] (xII) Lille S. Mm. 2 (1876) 7-.
Method, new. Weddle, T. R. S. P. 4 (1841) 300-.

-, - Lalanne, L. L. C. C. R. 81 (1875) 1186-, 1243-; 82 (1876) 1487-.

-, - Eggers, H. Des Moines Anal. 3 (1876) 149 -

Methods, two simple. Giesen, A. Z. Mth. Ps. 23 (1878) 35-.

Newton's method of approximation. Gergonne, J. D. Gergonne A. Mth. 20 (1829-30) 196-. - - Anne, L. N. A. Mth. 5 (1846) 116- - - Lemonnier, H. N. A. Mth. 1 (1862) 188-, 243-.

- - -. Genocchi, A. G. Mt. 2 (1864) $27-$. - - - - Thomson, F. D. [1864] Mess. Mth. 3 (1866) 40-

- - Piani, D. Bologna Ac. Sc. Mm. 6 (1866) 69-.

- - - Darboux, G. N. A. Mth. 8 (1869) 17-.

- - Franklin, F. Am. J. Mth. 4 (1881) 275-.

- Fouret, G. N. A. Mth. 9 (1890) 567-.

- - - Berg, F. J. van den. Amst. Ak. Vs. M. 9 (1892) 53-; Fschr. Mth. (1892) 103-.

169

- - - Tauber, A. Mh. Mth. Ps. 6 (1895) 291-.

- - - - and that of proportional parts, comparison. Maleyx, L. N. A. Mth. 18 (1879) 218-.

- solution. Baltzer, R. Leip. B. 18 (1866) 358-.

Numerical and algebraic solution of equations. Anon. (vr 564) Gergonne A. Mth. 20 (1829 30) 352-.

- - Biasi, G. [1876] Rpm. Mth. 1 (1877) 315-.

- equation, problem. Koralek, P. N. A. Mth. 13 (1854) 36-.

equations. Vallas, A. Camb. (M.) Mth. M. 3 (1860) 10 .

- --. Laguerre, E. C. R. 79 (1874) 996-.

- - and analytical geometry in space. Żmurko, L. [1869] Wien D. 30 (1870) (2te Ab.) 217-。 - - depending on expressions of 1st degree. Serdobinskǐ, V. E. [1874] (xII) Rec. Mth. (Moscou) 7 (1874-75) (Pt. 1) 263-.

- of even degree, analysis, improvement. Young, J. R. Ph. Mg. 34 (1849) 413-.

- - with given number of imaginary roots, determination. Laguerre, E. C. R. 90 (1880) 180 -.

- - having integer roots. Talbot, W. H. F. [1875] Edinb. R. S. T. 27 (1876) 303-.

- - , higher order, one or more unknowns, theorems. Spitzer, S. [1849] Haidinger Ab. 3 (1850) (Ab. 2) 143-.

- - and quadratic factors. Finck, B. Liouv. J. Mth. 10 (1845) 171-.

- - theory. Lepriol, C. J. Rouen Tr. Ac. (1812) 41-.

- -, -. Laguerre, E. C. R. 78 (1874) 278-; Liouv. J. Mth. 9 (1883) 99-; Acta Mth. 4 (1884) 97-. 
Principle of proportional parts. Biffignandi, A. Acireale Ac. At. 2 (1891) 62-.

Quadrinomial equations, solution by series. Ananzio, D. G. Mt. 14 (1876) 153-, 306-.

- equations, calculation of real roots. Wiener, A. Z. Mth. Ps. 31 (1886) 65-, 192.

Rational roots of numerical equations, new method of finding. Moon, R. [1849] Camb. Ph. S. P. 1 (1866) 79-.

Real and imaginary roots of numerical equation of higher order. Spitzer, S. Haidinger Ab. 3 (1850) (Ab. 2) 109-.

- root of equation, series expressing. Bras sinne, E. Toul. Mm. Ac. 6 (1849) 10-.

- roots, calculation. Buchoaldt, $F$. Mth. Ts. 3 (1861) 185-.

$-\frac{-}{-}$ - Margerie, C. N. A. Mth. 3 (1884) 33-.

- - - - by infinite series. Wallinowsky, Crelle J. 33 (1846) 164-.

- - development in series. Yvon-Villarceau, A. J. F. Liouv. J. Mth. 4 (1878) 119-.

- of equation with real coefficients. Giudice, $F$. Palermo Cir. Mt. Rd. 1 (1887) 69-.

- - Moth's determination. Stampfer, S. Wien Sb. 1 (1848) 194 -

- - , simplest method for obtaining. Bellavitis, G. [1845] Ven. Mm. I. 3 (1847) 109-.

Recurring series, solution by. Perrin, $R$. C. R. 119 (1894) 990-, 1190-, 1257-.

- - - - C. Capelli, A. Nap. Rd. 34 (1895) $194-$.

- - - - - extension of D. Bernoulli's theorem. Mainardi, $G$. A. Sc. Lomb. Ven. 9 (1839) 273-; 10 (1840) 113-.

Reversion of series, application. Hagen, J. G. Am. Ph. S. P. 21 (1884) 93-.

Roots of equation, development in series. Cauchy, A. L. C. R. 38 (1854) 1105-.

- - - - Amanzio, D. Nap. Rd. 16 (1877) 122-, 143-.

- - - - - Runge, C. Acta Mth. 6 (1885) 305-.

- _ - - - Madsen, N. Ts. Mth. 5 (1887) 129-; Fschr. Mth. (1887) 74.

- - - - - Fabre, A. As. Fr. C. R. (1894) (Pt. 2) 149-.

- - - - - - of powers. Heine, H. E. Crelle J. 48 (1854) 267-.

- - $x^{n}+a x+b=0$, development in series. Madsen, N. Ts. Mth. 6 (1889) 33-; Fschr. Mth. (1888) 86.

$---e^{x^{2}} \frac{d^{m}}{d x^{m}}\left(e^{-x^{2}}\right)=0$. Markoff, A. St.

Pét. Ac. Sc. Bll. 9 (1898) 435-.

- - equations, considered as functions of a variable parameter. Puiseux, V. C. R. 30 (1850) 171.

- - criteria for determination. Sylvester, J.J. Ph. Mg. (1844) 442-.

- - - as determinants of coefficients. Fïrstenau, E. Marb. Schr. 9 (1872) 19-.

- - _ - _ - Fürstenau's method. Nägelsbach, $H$. Arch. Mth. Ps. 59 (1876) 147-; 61 (1877) 19-.

voL. I.
Roots of equations given by recurring expressions. Cohn, F. Mth. A. 44 (1894) 473-. - - - method for simultaneous calculation. McClintock, E. [1894] Am. J. Mth. 17 (1895) 89-.

- _ - nature and approximate value. Malfatti, G. F. Verona S. It. Mm. 4 (1788) 206-.

-, expression by continued fractions. Cioojd zinski, $K$. Arch. Mth. Ps. 17 (1900) 29-.

- and powers of roots expressed as series. Euler, L. [1778] St. Pet. Ac. Sc. N. Acta 12 (1801) 71-.

Series and definite integrals, solution by. Lorenz, L. $V$. (xrr) Ts. Mth. 3 (1867) 71-。 - logarithms, solution by. Valz, B. C. R. 49 (1859) 705-.

-, solution by. Possou, -. Mntp. Rec. Bll. 2 (1805) 146-.

- - Fergola, $E$. Nap. Ac. Sc. Mm. 2 (1855-57) 207-; Tortolini A. 8 (1857) 204-.

Square and approximate rectangle, paradox concerning. Schlegel, S. F. V. [1878] Z. Mth. Ps. 24 (1879) 123-.

Successive substitutions, solution by. Sancery, L. N. A. Mth. 1 (1862) 305-, 384-.

- - - - Laska, $W$. Arch. Mth. Ps. 5 (1887) 199-; 6 (1888) vr.

Superior limit to roots of numerical equations, Petersen's criterion proved erroneơs. Giudice, -. Palermo Cir. Mt. Rd. 6 (1892) 157-.

"Teleologic" method. Dickstein, S. Krk. Ak. (Mt.-Prz.) Rz. 19 (1889) 167-; 20 (1890) 287-; Crc. Ac. Sc. Bll. (1889) No. 5, xxIx; (1890) 118-.

Transcendental functions, solution by. Lindemann, F. Gött. Nr. (1884) 245-; (1892) 292-.

Trinomial equations. Marrecas Ferreira, $L$. $F^{\prime}$. G. Teix. J. Sc. 5 ("1883) 50-.

- - imaginary roots, approximation. Weddle, $T$. Mathematician 3 (1850) 285-

- - numerical solution. Piobert, - N. A. Mth. 10 (1851) 174-.

- - - - . A (1877) No. 7, 49-; Arch. Mth. Ntvd. 6 (1882) 448-.

- - - - Farkas, J. [1878] Arch. Mth. Ps. 64 (1879) 24-.

- - - - M'Laren (Lord). Edinb. R. S. P. 17 (1891) 270-.

- -, real roots. Drobisch, M. W. Leip. B. 8 (1856) 21-; 10 (1858) $82-$.

- - , - - Geelmuyden, H. Christiania F. 15 (1873) 481-.

- - , series involving binomial coefficients for solution of. Schröder, E. Z. Mth. Ps. 25 (1880) 196-.

- - , solution by continued radicals. Astrand, J. J. As. Nr. 89 (1877) 347-.

- - - - series. Fergola, E. Nap. Rd. 1 (1862) 39-.

Weddle's method. Popper, J. Böhm. Gs. Ab. 11 (1861) 531-.

\section{Graphical methods.}

Geometric construction. Magistrini, $G . B$. Bologna N. Cm. 1 (1834) 194-. 
Geometric solution. Corancez,-- Par. J. Ec. Pol. 10 (1815) (17' cah.) 212-.

- - Lalanne, L. L. C. C. R. 87 (1878) 157-.

- solutions by means of conic or higher curves. Bucca, F. Palermo Cir. Mt. Rd. 14 (1900) 126-.

Graphic solution. Lill, E. C. R. 65 (1867) 854-; Les Mondes 16 (1868) 325-.

- - Hammer, E. Civing. 31 (1885) 275-.

- - Reuschle, C. Z. Mth. Ps. 31 (1886) 12-.

- - Mehmke, $R$. Civing. 35 (1889) 617-.

Imaginary roots, geometric construction. Ettingshausen, A. von. Wien SB. (1850) $(A b .2)$ 119 .

,--- Scheffler, $H$. Grunert Arch. 15 (1850) 375-.

2nd order, graphic solution. Davis, R. $F$. Mess. Mth. 5 (1876) 17-.

3rd order, geometric constructions involving. London, $F$. D. Mth. Vr. Jbr. 4 (1897) 163-.

- - , - solution, and properties. Paucker, M. G. von. St. Pét. Ac. Sc. Mm. 10 (182122) 159-.

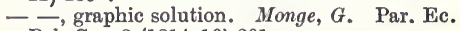
Pol. Cor. 3 (1814-16) 201-.

- - - - - Phillips, A.W. Des Moines Anal. 1 (1874) 113-. Nt.) 6--

- - , hydrostatical solution. Demanet, -. Mathesis 18 (1898) 81-.

- - solution by parabola. Gergonne, J. D. Gergonne A. Mth.9 (1818-19) 204-.

- or 4th order, construction of imaginary roots by means of a fixed parabola. Hoppe, R. Arch. Mth. Ps. 69 (1883) 216-.

- - -,- - - real roots by means of a fixed parabola. Hoppe, R. Arch. Mth. Ps. 56 (1874) 110-.

- and 4 th orders, mechanical and graphic solution. Bartl, C. Arch. Mth. Ps. 1 (1884) 1-.

4 th order, construction of roots. Gerono, G.C. N. A. Mth. 3 (1844) 533-.

,$---1-$ by intersection of parabola and circle. Vieille, J. N. A. Mth. 16 (1857) 453-. - - geometric solution. Hofmann, $F$. N. A. Mth. 7 (1888) 120-.

- - graphic solution. Mathews, G. B. [1899] Nt. 61 (1899-1900) 55.

- - interpreted by parabola. Heppel, $G$. L. Mth. S.'P. 22 (1891) 416-.

Higher order. Spitzer, $S$. Haidinger Ab. 4 (1851) (Ab. 3) 1-; Grunert Arch. 22 (1854) 21-.

Real roots, mechanical means for obtaining. Williams-Ellis, J. C. [1873] Camb. Ph. S. P. 2 (1876) 317.

Solution by hyperbolas. Hinton, C. H. N. Y. Am. Mth. S. Bll. 3 (1897) 309-

- - logarithmic coordinates. Nijland, A. A. N. Arch. Wisk. 19 (1892) 35-; Fschr. Mth. (1892) 104-.

Trinomial equations, solution by aid of cubocycloid $x^{\frac{2}{3}}+y^{\frac{2}{3}}=\lambda^{\frac{2}{3}}$. Montucci, H. C. R. 69 (1869) 525-, 757.
2450 General resolution of equations; theory of Galois. (See also 1210.)

Svanberg, J. Ups. N. Acta S. Sc. 10 (1832) 289 -

Murphy, $R$. Camb. Ph. S. T. 4 (1833) 125-.

Cauchy, A. L. C. R. 4 (1837) 362-.

Lubbock, J. W. Ph. Mg. 15 (1839) 165-.

Casinelli, A. (8. L.). Bologna N. Cm. 5 (1842) 65-, 433-; 6 (1844) 391-; 7 (1844) 509-.

Malmsten, C. J. Stockh. Öfv. 2 (1845) 226-; Crelle J. 34 (1847) 46-.

Badano, G. (vi Adds.) Majocchi A. Fis. C. 24 (1846) 111-.

Betti, E. Tortolini A. 3 (1852) 49-.

Weiler, A. Grunert Arch. 18 (1852) 194-.

Betti, E. Tortolini A. 5 (1854) 10-.

Cauchy, A. L. C. R. 38 (1854) 1104-.

Betti, E. QJ. Mth. 1 (1857) 91-.

Cauchy, A. L. C. R. 44 (1857) 268-.

Lobatto, R. Amst. Vs. Ak. 7 (1858) 335-.

Borden, J. Camb. (M.) Mth. M. 1 (1859) 373-. Györy, S. (xII) Mag. Ak. Éts. (Mth. Term.) 3 (1862) 101-.

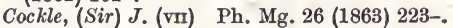

Guldberg, A. S. (xII) Ts. Mth. 2 (1866) 167-.

Mainardi, $G$. Rm. At. N. Linc. 20 (1867) 160 -

Kirkman, T. P. Manch. Lt. Ph. S. P. 7 (1868) 141-, 221-; Ph. Mg. 36 (1868) 169-, 264-.

Jablonowski, N. Les Mondes 42 (1877) 629-.

Meyer, $F$. Mth. A. 33 (1889) 511-.

Abelian equations. Kronecker, L. Berl. Ak. Mb. (1877) 845-.

- - Netto, E. Mth. A. 18 (1881) 247-.

- - Pellet, A. C. R. 112 (1891) 1196-, 1249-.

- class. Igel, $B$. Wien Ak. D. 45 (1882) (Ab. 2) 373-.

- - , roots. Hermite, C. Crelle J. 32 (1846) $277-$.

- - theorems. Abel, N.H. N. A. Mth. 4 (1845) 536-.

- - , theory. Schwering, $K$. Crelle J. Mth. 93 (1882) 334-.

- - - - (Schwering). Kronecker, L. Crelle J. Mth. 93 (1882) 338-

- - - - Netto, E. Mth. A. 42 (1893) 436-. - , 3rd order of region $(\sqrt{-31})$. Kronecker, L. Berl. Ak. Sb. (1882) 1151-.

- - 6th order, soluble by aid of equation of 4th order. Weis, $M$. Arch. Mth. Ps. 68 (1882) 304-.

Abel's theorems. West, É. C. R. 91 (1880) 759-.

- theory concerning equations above 4 th order. Hamilton, (Sir) W. R. [1837] Ir. Ac. T. 18 (1838) 171-.

- - Cockle, Jas. QJ. Mth. 5 (1862) 130-.

Algebraic and differential equations with relations between roots. Libri, $G$. [1830] Crelle J. 10 (1833) 167-.

- solubility of equations. Krey, H. Z. Mth. Ps. 15 (1870) 381-. 
Algebraic solution of certain equations. Tardy, $P$. Tortolini A. 2 (1851) 197-.

- - equations. Galois, E. Férussac Bll. Sc. Mth. 13 (1830) 271-.

- - - Pacinotti, L. Pisa A. Un. Tose. Sc. Cosm. 3 (1854) 77-.

- - Bellavitis, G. Ven. At. 4 (185859) 55-.

$-\frac{1}{-}$-. Sylow, [L.] Sk. Nf. F. 8 (1860) $536-$

(1867) - Jordan, C. Liouv. J. Mth. 12 105-.

Anal. 9 (1882) 1 - Dixon

- - - Tognoli, O. G. Mt. 28 (1890) 315-.

- Sawin, A. M. A. Mth. 6 (1891-

92) 169 .

$28(1893) \cdot 349$

--- Rodrigues, J. M. As. Fr. C. R. (1893) (Pt. 2) 289-.

- - - Jaggi, E. N. A. Mth. 13 (1894)

Anharmonic algebraic equations. Autonne, C. R. 130 (1900) 313-, 390-.

Arithmetical demonstration of fundamental theorems in algebra. Kneser, A. Crelle J. Mth. 102 (1888) 20-.

Coresolvents. Cockle, (Sir) J. [1865-75] Manch. Lt. Ph. S. P. 5 (1866) 13-; Vict. R. S. T. 7 (1866) 176-; B. A. Rp. 37 (1867) (Sect.) 3-; N. S. W. R. S. T. 1 (1867) 27-; QJ. Mth. 10 (1870), 35-; 14 (1877) 63-.

Depression of equations. Györy, S. (xII) Mag. Ak. Éts. (1847) 12.

- - - Györy's method. Vallas, A. (xII) Mag. Ak. Éts. (1847) 12-.

Differential resolvent. Rawson, $R$. Mess. Mth. 4 (1875) 108-.

- Harley, (Rev.) R. B. A. Rp. (1891) 566-.

- - of sextic equations with group of order 360. Lachtin, L. K. Rec. Mth. (Moscou) 20 (1899) 353-; Fschr. Mth. (1898) 78-; Mth. A. 51 (1899) 463-.

- resolvents. Cockle, (Sir) J. [1882] L. Mth. S. P. 14 (1883) 18 -.

- Rawson, R. Manch. Lt. Ph. S. P. 21 (1882) 59-.

- - of equations, method of determining. Rawson, R. L. Mth. S. P. 9 (1877-78) 202-. $---\frac{1}{-}--$ (Rawson). Harley, (Rev.) R. L. Mth. S. P. 9 (1877-78) 216-.

- - theory. Harley, (Rev.) R. L. Mth. S. P. 1 (1866) No. 4, 10 pp.

Discriminants of Galois's resolvents. Brioschi, F. C. R. 108 (1889) 878-.

Equation of Lagrange's in "Connaissance des Temps," 1821, lowering of degree. Cauchy, A. L. C. R. 26 (1848) 27-.

$-\frac{x^{n}-1}{x-1}=0$, irreducibility when $n$ is prime.

Serret, J. A. Liouv. J. Mth. 15 (1850) 296.

Equations, coefficients of which are functions of one variable, algebraic solubility. Frobenius, $G$. [1871] Crelle J. Mth. 74 (1872) 254-.
Equations every root of which is rational function of one. Bendixson, I. Stockh. Öfv. (1891) 131-; Toul. Fac. Sc. A. 7 (1893) C, 7 pp.

- of form $x^{5}+u x+v=0$, algebraically resolvable. Runge, $C$. Acta Mth. 7 (1885) 173-.

-, general, of order above 4th irresolvable by radicals. Margiochi, $F$. S. Lisb. Ac. Sc. Mm. 7 (1821) 317-.

-, higher, impossibilty of algebraic solution. Kneser, A. Crelle J. Mth. 106 (1890) 48-.

-, - Jerrard's method. Hamilton, (Sir) $W$. R. B. A. Rp. (1836) 295-.

-, - principles of solution. Young, G, P. Am. J. Mth. 6 (1884) 65-.

-, -, solution. Gyóry, S. (xп) Mag. Ak. Éts. (1847) 48-.

-, 一, - - Studler, A. Dunkerque Mm. S. Encour. 11 (1866) 345-

-, -, - Sinram, T. Arch. Mth. Ps. 68 (1882) 223-; 69 (1883) 111.

- irresolvable by radicals. Wantzel, L. N.A. Mth. 4 (1845) 57-.

- of prime degree, algebraic resolution. Dolbnia, J. Bll. Sc. Mth. 19 (1895) 27-.

- $-\ldots$, soluble by radicals. Betti, $E$. Tortolini A. 2 (1851) 1-.

- - - - Pellet, A. E. As. Fr. C. R. (1879) 245-.

-, primitive, degree $p^{2}$ ( $p$ an odd prime) Jordan, C. Liouv. J. Mth. 13 (1868) 111-.

-, property of a class. Brioschi, $F$. [1864] Mil. I. Lomb. Mm. 10 (1867) 21 pp.

-, roots not expressible as functions of coeffi. cients. Liouville, J. [1835] Liouv. J. Mth. 2 (1837) 56-; 3 (1838) 523-.

-, - of which are algebraic functions of radicals of 2nd degree. Chevillard, A.J. N. A. Mth. 4 (1845) 467-

- soluble algebraically. Kronecker, L. Berl. B. (1853) 365-; (1856) 203-.

- - - Despeyrous, C. Les Mondes 5 (1864) 191-; Liouv. J. Mth. 11 (1866) 9-.

_ - Boldt, G. G. [1878] Crelle J. Mth. 87 (1879) 1-.

- - , class. Abel, N.H. Crelle J. 4 (1829) 131-.

$--1,-$ Cordone, G. Palermo Cir. Mt. Rd. 10 (1896) 161-.

- _ by radicals. Söderberg, J. T. Ups. Årsk. (1886) (Mt. Nt.) $38 \mathrm{pp}$.

- - - -, form. Dolbnïa, I. P. [1894] Rec. Mth. (Moscou) 17 (1895) 801-; Bll.' Sc. Mth. 18 (1894) 130-.

- - - groups. Jordan, C. C. R. 58 (1864) 963-.

solution, and resolution into factors. Györy, S. (xII) Mag. Tud. Ak. Éts. 2 (No. 16) (1868) 291-.

-, symbolic, non-linear, resolution. Cauchy, A. L. C. R. 22 (1846) 235-.

-, theorems. Jordan, C. Liouv. J. Mth. 14 (1869) 139-.

theory. Galois, E. Liouv. J. Mth. 11 (1846) 408-.

-, -. Gegenbauer, L. Mh. Mth. Ps. 6 (1895) 12-. 
2450 Equations, 5th order

Equations, theory, recent researches. Harley, R. Manch. Ph. S. P. 3 (1862-63) 173-.

-, - of resolution and separation of roots. Jacobi, C. G. Crelle J. 13 (1835) 340-.

Crelle J. Mth. 107 (1891) 349-

-, - and solution. Lobatto, $R$. Arch. Wisk. Gn. 2 (1860-63) 235-.

- 4th order, in relation to group theory. Schumacher, - D. Nf. Tbl. (1887) 110.

,$- \ldots-$, solution by radicals, viz. 4 th roots. Minich, S. R. Ven. At. (1858-59) 19-.

\section{Equations, 5th order.}

Hamilton, (Sir) W. R. [1837-42] Ir. Ac. P. 1 (1841) 76-; Ir. Ac. T. 19 (1843) 329-.

Hermite, C. N. A. Mth. 1 (1842) 326-.

Casinelli, A. (s. L.) Bologna N. Cm. 6 (1844) 391-.

Jerrard, G. B. Ph. Mg. 26 (1845) 545-.

Cockle, Jas. Ph. Mg. 27 (1845) 125-.

Jerrard, G. B. Ph. Mg. 28 (1846) 63.

Bromwin, B. Ph. Mg. 31 (1847) 341-.

Cockle, Jas. Ph. Mg. 32 (1848) 50-.

Jerrard, G. B. Ph. Mg. 3 (1852) 112-.

Cockle, Jas. [1856-60] Ph. Mg. 12 (1856) $124-$; 13 (1857) 354- ; 17 (1859) 356-; 18 (1859) 50-, 342-, 508-; 19 (1860) 197-; Manch. Ph. S. Mm. 15 (1860) 131-; 1 (1862) 108 .

Hermite, C. C. R. 46 (1858) 508-.

Brioschi, $F$. Tortolini A. 1 (1858) 256-, 326-.

Kronecker, $L$. C. R. 46 (1858) 1150-.

Joubert, P. C. R. 48 (1859) 290 -

Harley, R. QJ. Mth. 3 (1860) 343-.

Jerrard, G. B. Ph. Mg. 19 (1860) 272-; 21 (1861) 39-.

Cockle, Jas. Ph. Mg. 19 (1860) 331-; 21 (1861) 90 -.

Cayley, A. Ph. Mg. 21 (1861) 210-.

Jerrard, G. B. (vi Adds.) Ph. Mg. 21 (1861) 348 .

Cockle, Jas. QJ. Mth. 4 (1861) 5-.

Kronecker, L. Berl. Mb. (1861) 609- ; Crelle J. 59 (1861) 306-.

Jerrard, G. B. Ph. Mg. 23 (1862) 146-.

Cayley, A. Ph. Mg. 23 (1862) 195-.

Cockle, Jas. Ph. Mg. 23 (1862) 196-.

Jerrard, G. B. Ph. Mg. 23 (1862) 469- ; 24 (1862) 193-, 457-.

Cockle, Jas. Ph. Mg. 24 (1862) 288-.

Cayley, A. Ph. Mg. 24 (1862) 290 -

Harley, $R$. QJ. Mth. 5 (1862) 248-.

Grunert, J. A. Grunert Arch. 40 (1863) 394-.

Jerrard, G. B. (vir) Ph. Mg. 26 (1863) 294-.

Hermite, C. C. R. 61 (1865) 877-, 965-, 1073-; 62 (1866) 65-, 157-, 245-, 715-, 919-, 959-, 1054-, 1161-, 1213-.

Tychsen, C. Ts. Mth. 1 (1865) 77-.

Roberts, M. A. Mt. 7 (1865) 257-; 1 (1867-68) 135-.

Brioschi, F. A. Mt. 1 (1867-68) 222-; C. R. 73 (1871) 1470-; 81 (1875) 753-, 815-; 85 (1877) 1000-; Mth. A. 13 (1878) 109.

Gordan, P. Mth. A. 13 (1878) 375-.

Klein, C. F. [1878] Mth. A. 14 (1879) 111-.

\section{Equations, 5th order 2450}

Kiepert, L. Crelle J. Mth. 87 (1879) 114-.

Brioschi, F. A. Mt. 9 (1878-79) 124-.

Minich, S. R. [1881] Ven. I. At. 8 (1881-82) 307-, 893-.

Besso, D. Rm. R. Ac. Linc. Mm. 19 (1884) 232 .

McClintock, E. Am. J. Mth. 6 (1884) 301-.

Glashan, J. C. Am. J. Mth. 7 (1885) 178-.

Gordan, $P$. Liouv. J. Mth. 1 (1885) 455-; Erlang. Ps. Md. S. Sb. 18 (1886) 81-; Mth. A. 28 (1887) 152-.

Biermann, O. Lotos 37 (1889) 25-.

Schumacher, J. D. Mth. Vr. Jbr. 2 (1893) 69-.

Sélivanoff, D. Par. S. Mth. Bll. 21 (1893) 97-.

Heymann, W. Z. Mth. Ps. 39 (1894) 162-, 193-, 257-, 321-.

Giudice, F. [1895] Tor. Ac. Sc.Mm. 46 (1896) 31-.

Abel, theorem. Cayley, A. Ph. Mg. 21 (1861) 257-; Camb. Ph. S. P. 3 (1880) 155-.

Abel's forms for roots in soluble case. Young, G. P. Cn. R.S. P. \& T. 4 (1887) (Sect. 3) 93-.

Analysis. McClintock, E. Am. J. Mth. 8 (1886) 45-.

Application of quadratic substitution. Clebsch, R. F. A. Mth. A. 4 (1871) 284-.

Auxiliary equation. Cayley, A. Phil. Trans. (1861) 263-.

Bezout's formula. Berndtson, B. Stockh. Ac. Hndl. 39 (1818) 1-.

Bring's reduction. Hill, C. J. D. Stockh. Öfv. 18 (1861) 317-.

Class. Brioschi, F. Par. Éc. Norm. A. 12 (1895) 337-.

- of resolvents. Brioschi, F. C. R. 63 (1866) 685-, 785-.

Determinantal resolvents. Schläfi, $L$. A. Mt. 3 (1869-70) 171-.

Equation determining anharmonic ratios of roots. Hill, M. J. M. L. Mth. S. P. 14 (1882-83) 179-.

- of squares of differences of roots. Roberts, M. QJ. Mth. 4 (1861) 234-.

Euler's researches. Cockle, Jas. Ph. Mg. 15 (1858) 389-

Expression of functions of differences of roots. Roberts, M. [1868] A. Mt. 2 (1868-69) 224-

Formation of Lagrange's sextic and quartic resolvents. Plana, $G$. Tor. Mm. Ac. 16 (1857) 1-.

General, Bring's reduction to trinomial form. Harley, R. Manch. Ph. S. P. 3 (1862-63) 69-.

-, reduction to Jerrard's form. Rahts, $J$. Mth. A. 28 (1887) 34-.

-, - trinomial form. Harley, R. QJ. Mth. 6 (1863) 38-.

-, solution. Schulenburg, A. von der. Des Moines Anal. 3 (1876) 141-, 173-; 4 (1877) 9-, 49-.

-, transformation. Tychsen, C. Mth. Ts. 5 (1863) 160

Guldberg's solution. Thiele, T. N. (xI) Ts. Mth. 6 (1870) 90-.

- - Guldberg, A.S. (xII) Ts. Mth. 6 (1870) $125-$.

Hargreave's solution a failure. Kirkman, T.P. Manch. Lt. Ph. S. P. 7 (1868) 133-. 


\section{Equations, 5th order}

Hermite's solution. Krey, H. Z. Mth. Ps. 25 (1880) 129-.

and higher, existence of finite solutions. Cockle, Jas. $\mathrm{Ph}$. Mg. 28 (1846) 190-, 395-.

History of theory. Pierpont, J. Mh. Mth. Ps. $6(1895)$ 15-.

Irreducible, solution. Young, G. P. Am. J. Mth. (1885) 170-.

-, - , criteria. Luther, $E$. Crelle J. 34 (1847) 244-.

Klein's results. Brioschi, $F$. [1876] Rm. R. Ac. Linc. T. 1 (1877) 31-.

Kronecker's method. Brioschi, F. Mil. At. I. Lomb. 1 (1858) 275-

Malfatti's method. Ruffini, P. Mod. S. It. Mm. 12 (1805) 321-.

- resolvent. Brioschi, F. Mil. Mm. I. Lomb. 9 (1863) 215-

- $\quad$ Giudice, $F$. Tor. Ac. Sc. At. 27 (1892) 817 -

Reduction to Bring's form by 'Tschirnhausen transformation. Sievers, J. [1867] As. Nr. 70 (1868) 353-.

Removal of terms. Heal, W. E. Des Moines Anal. 6 (1879) 78-.

Sextic resolvent. Minich, S. R. Ven. At. 8 (1862-63) 799-.

and 6th, nature of roots. Bonati, T. [1798] Mod. Mm. S. It. 8 (1799) 231-.

- - , solution. Cockle, Jas. QJ. Mth. 4 (1861) 49-; 5 (1862) 1-.

- - - - by construction. Grunert, J.A. Grunert Arch. 27 (1856) 245-.

- - , theory. Clebsch, R. F.A. Gött. Nr. (1871) 103-.

- - and 7th, a class of algebraically resolvable. Mandl, $M$. Wien Ak. Sb. 94 (1887) (Ab. 2) 246-.

Solubility by radicals, case. Perrin, $R$. [1882] Par. S. Mth. Bll. 11 (1883) 61-.

Soluble by radicals. Bugaev, N. V., \& Lachtin, L. K. [1890] Rec. Mth. (Moscou) 15 (1891) 83-.

- - - Factorisation of certain equation of order $\frac{p(p-1)}{2}[p$ prime]. Antaev, S. N. [1894] Rec. Mth. (Moscou) 17 (1895) 544-; Fschr. Mth. (1893-94) 156-.

Solvable cases. Cayley, A. QJ. Mth. 18 (1882) 154-; Am. J. Mth. 13 (1891) 53-.

-, with commensurable coefficients. Young, G. P. Am. J. Mth. 10 (1888) 99-.

- form, new. Cockle, Jas. QJ. Mth. 2 (1858) 144 .

— forms. Young, G. P. QJ. Mth. 5 (1862) 212 .

-, solution. Young, G. P. [1883] Cn. I. P. 2 (1884) 127-.

Synthesis. Beitler, G. T. F. [1802] (vI Adds.) St Pet. Ac. Sc. N. Acta 15 (1806) 191-.

Theorem on solvability. Hamilton, (Sir) W. R. Ph. Mg. 8 (1836) 538-; 9 (1836) 28-.

Theorems. Roberts, M. (vIII) A. Mt. 4 (1861) 50-

Theory, further researches in. McClintock, E. [1897] Am. J. Mth. 20 (1898) 157-.

\section{Equations, 6th order 2450}

Equations, 5th order, continued.

Transformation. Brioschi, F. A. Mt. 16 (1888-89) 181-.

- and solution. Schlömilch, O. [ascribed to Jerrard and Hermite in Vol, iii]. Schlömilch Z. 4 (1859) 77-.

- _ - elementary exposition. Heymann, $W$. Z. Mth. Ps. 42 (1897) 81-, 113-.

Transformations connected with solution. Jerrard, G. B. (vI Adds.) Ph. Mg. 7 (1835) 202 .

-, Jerrard's. Tortolini, B. A. Mt. 6 (1864) 33-.

Tschirnhausen's transformation applied. Lavagna, G. M. Tortolini A. 1 (1858) 238-.

\section{Equations, 6th order.}

Joubert, - . C. R. 64 (1867) 1025-, 1081-, 1237-.

Brioschi, F. Acta Mth. 12 (1889) 83-.

Certain. Hesse, L. O. Crelle J. 41 (1851) 264-.

Class. Brioschi, F. Par. Éc. Norm. A. 12 (1895) 343-.

- solvable by hypergeometric series. Besso, D. [1882] Rm. R. Ac. Linc. Mm. 14 (1883) 30-. with five equal roots. Cayley, A. QJ. Mth. 1 (1857) 257-

General. Cole,F.N. Am. J. Mth. 8 (1886) 265-. Irreducible, application of group theory. Glage, $F$. Mh. Mth. Ps. 11 (1900) 155-.

-, solvable algebraically, factors and resolvents. Luther, E. Crelle J. 37 (1848) 193-.

Jacobi and Kronecker, sextic resolvent equations. Cayley, A. Crelle J. Mth. 113 (1894) 42 .

Jacobian sextic equation. Cayley, A. QJ. Mth. 18 (1882) 52-; Mth. A. 30 (1887) 78-.

_- - . Brioschi, —. QJ. Mth. 28 (1896) 382-.

Lagrange's sextic. Sawin, A. M. [1891] A. Mth. 6 (1891-92) 1-.

Normal form. Brioschi, F. Rm. R. Ac. Linc. Rd. 4 (1888) (Sem. 1) 301-, 485-.

Numerical. Vincent, A.J.H. N. A. Mth. 10 (1851) 275-

and 7th. Klein, $F$. Mth. A. 28 (1887) 499-.

Solution. Maschke, H. Rm. R. Ac. Linc. Rd. 4 (1888) (Sem. 1) 181-.

-. Brioschi, $F$. Rm. R. Ac. Linc. Rd. 4 (1888) (Sem. 1) 183-.

Klein, F. Rm. R. Ac. Linc. Rd. 8 (1899) (Sem. 1) 324.

Theory, contribution to. Reichardt, W. Leip. Mth. Ps. B. 37 (1885) 419-.

Equations, 7th order. Kronecker, L. Berl. Mb. (1858) 287-.

-, - - Klein, C. F. Erlang. Ps. Md. S. Sb. 10 (1878) 110-, 119-.

_, _ _ , with group of 168 substitutions. Gordan, P. Mth. A. 20 (1882) 515-; 25 (1885) 459-.

_, _ - , solution. Radford, E.M. QJ. Mth. 30 (1899) 263-.

-, - - , , nomographic. Ocagne, M. $d^{\prime}$. C. R. 131 (1900) 522-.

, - and 8th orders, certain. Klein, C. F, Mth. A. 15 (1879) 251-.

- , - _ - - general. Moore, E. H. Mth. A. 51 (1899) 417 - 
Equations, 8th order. Armenante, A., \& Jung, G. G. Mt. 7 (1869) 98-.

,$--\ldots$, and theory of curves of 4 th degree. Noether, M. Mth. A. 15 (1879) 89-.

-, 9th order, class, solution. Hesse, L. O. Crelle J. 34 (1847) 193-.

-, 10th order, of Jacobi. Faure, H. N. A. Mth. 3 (1864) 116-.

-, 12th order. Kronecker, L. Berl. Mb. (1861) 609-.

-, - Klein, C. F. Erlang. Ps. Md. S. Sb. 7 (1875) 101-.

-, $n$th order, algebraic solution of certain classes. Björling, E. G. Stockh. Öfv. 9 (1852) 40-; Grunert Arch. 21 (1853) 17-.

_, - _ - equation of squares of differences of roots. Roberts, $M$. N. A. Mth. 17 (1858) 440-.

-, - - reduction. Boole, G. Camb. and Dubl. Mth. J. 6 (1851) 106-.

-, - - solution. Franchini, P. [1797] Turin Mm. Ac. 6 (1801) (pte. 2) 115-.

- $-\ldots,-$ Wilder, C. Silliman J. 16 (1829) 271-.

-, - - 一. Cauchy, A. L. C. R. 4 (1837) $773-, 805-; 5$ (1837) 301-; 29 (1849) 250 -.

-, - -, 一. Jerrard, G. B. Ph. Mg. 3 (1852) 457-.

-, - - - - Théremin, F. [1853] Crelle J. 49 (1855) 187-.

,,---- , method. Pronier, -. Fr. Cg. Sc. 20 (1853) 48 -

- - - whose roots are of form $\sqrt[n]{R_{1}}+\sqrt[n]{R_{2}}$. Guldberg, A. S. [1869-70] N. Mg. Ntvd. 16 (1869) 76-; Christiania F. 13 (1871) 431-

, - - - - - - Sylow, L. N. Mg. Ntvd. 17 (1870) 17-

Factorisation of integral algebraic functions in natural rationality domain. Kronecker, $L$. Crelle J. Mth. 94 (1883) 344-.

Formulæ for solution of equations. Brioschi, F. Tortolini A. 5 (1854) 416-.

Functions of 4 and 5 letters. Cockle, Jas. Liouv. J. Mth. 1 (1856) 383-.

Galois, commentary on. Jordan, C. C. R. 60 (1865) 770-; Mth. A. 1 (1869) 141-.

- field, existence. Dickson, L. E. N. Y. Am. Mth. S. Bll. 6 (1900) 203-.

- fields. Dickson, L. E. [1900] N. Y. Am. Mth. S. Bll. 7 (1901) 80, 84-.

- group of equation, reduction by adjunction of arbitrary irrationalities. Bucca, $F$. Palermo Cír. Mt. Rd. 14 (1900) 122-.

Galois's criterion of resolvability of equations by radicals. Dolbmra, I. P. [1888] Kazan S. Nt. (Ps.-Mth.) P. 7 (1889) 223-; N. A. Mth. 7 (1888) 467-.

- fundamental theorem on algebraic solution, proof. Söderberg, J. T. Acta Mth. 11 (1887-88) 297-.

--- , Söderberg's proof. Hölder, O. Mth. A. 34 (1889) 454-.

- moduli, 2nd to 5th rank, modular equations. Friedrich, G. Arch. Mth. Ps. 4 (1886) 113-. - theory. Janni, G. G. Mt. 12 (1874) 277-.

- - . Bachmann, P. Mth. A. 18 (1881)
Galois's theory. Pierpont, J. A. Mth. 1 (1900) 113-; 2 (1900-01) 22-.

- - general foundations. Weber, $H$. Mth. A. 43 (1893) 521-.

- -, history. Pierpont, J. N. Y. Am. Mth. S. Bll. 4 (1898) 332-, 560.

Group of numerical equations, determination. Maillet, E. C. R. 127 (1898) 1004-; Liouv. J. Mth. 5 (1899) 205-.

- theory and algebraic equations. Foglini, $G$. Rm. N. Linc. Mm. 3 (1888) 3-; 7 (1891) 175-.

(1891) 59 -.

- - Söderberg, J. T. Ups. S. Sc. N. Acta 15 (1895) No. 2, 38 pp.

Hamilton's numbers. Sylvester, J. J. As. Fr. C. R. (1887) (Pt. 2) 164-; Nt. 36 (1887) 557-. - - Sylvester, J. J., \& Hammond, J. Phil. Trans. (A) 178 (1888) 285-; (A) 179 (1889) 65-.

- -. Studnicka, F. J. Časopis 19 (1890) 119-; Fschr. Mth. (1890) 110.

- -. Mathews, G. B. QJ. Mth. 27 (1895) 184 .

Icosahedral irrationality. Vivanti, G. Palermo Cir. Mt. Rd. 9 (1895) 202-.

- - Bucca, F. Palermo Cir. Mt. Rd. 14 (1900) 130-.

- - , analogous irrationalities. Vivanti, $G$. Mh. Mth. Ps. 6 (1895) 393-.

- - solution of equation of 5 th degree by. Giudice, $F$. Tor. Ac. Sc. At. 28 (1893) 664 -

Icosahedron, Klein's researches, and equations of 5 th order. Gordan, P. Erlang. Ps. Md. S. Sb. 9 (1877) 183-.

Insolubility of equations of order above $4 \mathrm{th}$. Ruffini, P. [1802] Mod. S. It. Mm. 10 (1803) 410-

- - - Malfatti, G. F. Mod. S. It. Mm. 11 (1804) 579-.

- - - - - Ruffini, P. Mod. S. It. Mm. 12 (1805) 213-; Bologna Mm. I. It. 1 (pte. 2) (1806) 433-.

- - - - - Caccianino, A. Mil. Mm. I. Lomb. Ven. 1 (1812-13) 3-.

- - - - - Abel, N. H. Crelle J. 1 (1826) 65-.

- - - - - - proof. Hadamard, Bordeaux S. Sc. PV. (1896-97) 84-.

- - _ - - - - - , and methods for particular cases. Young, G.P.[1859] Cn. J.5 (1860) 20-, 127-.

- - - - - - -, new. Netto, $E$. Crelle J. Mth. 83 (1877) 86-.

Inverse functions applied to theory of equations. Guldberg, A. S. N. Mg. Ntvd. 15 (1868) 135-.

Irrational quantities, algebraic. Jordan, $C$. C. R. 63 (1866) 1063-.

Irreducibility of algebraic equations, and development of algebraic functions. Koenigsberger, L. Berl. Ak. Sb. (1898) 735-; Crelle J. Mth. 121 (1900) 320-

- _ - Eisenstein's law. Koenigsberger, L. Berl. Ak. Sb. (1894) 1135-; Crelle J. Mth. 115 (1895) 53-. 
Irreducibility of algebraic functional equations and linear differential equations. Koenigsberger, L. Berl. Ak. Sb. (1899) 672-.

- - equations. Kronecker, L. Berl. Ak. Mb. (1880) 155-.

$-\ldots-$, Kronecker's conception. Valle, $G$. Rm. N. Linc. Mm. 11 (1895) 67-.

Irreducible equations of prime degree, solvable. Young, G. P. Am. J. Mth. 7 (1885) 270 -

- - - - - by radicals. Giudice, $F$. Palermo Cir. Mt. Rd. 1 (1887) 227-.

Kirkman's method of solution. Harley, $R$. [1868] Manch. Lt. Ph. S. P. 8 (1869) 4 -.

Kronecker's definition of the group of an equation. Bolza, O. Mth. A. 42 (1893) 253-.

Lagrange's method of solution, examination. West, É. C. R. 91 (1880) 718-.

Literal equations, solution. Suremain-de-Missery, A. Gergonne A. Mth. 7 (1816-17) 257-.

,--- (Heger's paper). Petzval, J. Wien SB. 14 (1854) 201-.

- - - - method. Heger, I. Wien D. 12 (1856) (Ab. 2) 109-; 13 (1857) (Ab. 2) 143-.

Numerical equations, irresolvable by radicals. Pellet, A. E. As. Fr. C. R. (1881) 160-.

Rational functions of roots and group theory. Antaev, S. N. [1898] Rec. Mth. (Moscou) 20 (1899) 472-; Fschr. Mth. (1898) 70.

Reciprocal Abelian equations with roots of form $x, \theta(x), \theta^{2}(x), \ldots \theta^{n-1}(x)$. Mollame, $V$. Tor. Ac. Sc. Mm. 44 (1894) 293-; Nap. Ac. At. 7 (1895) No. 2, $40 \mathrm{pp}$.

Reducibility of algebraic expressions. Sélivanoff, $D$. Acta Mth. 19 (1895) 73-.

- - equations. Capelli, A. Nap. Rd. 36 (1897) 243-; 37 (1898) 84-, 243-.

_ - - especially those of 5 th order, with linear parameters. Meyer, $\boldsymbol{F}$. D. Nf. Tbl. (1885) 174; Münch. Ak. Sb. 15 (1886) 415-.

Reduction of equation to chain of equations. Hölder, O. Mth. A. 34 (1889) 26-.

- equations. Pellet, A.E. C. R. 97 (1883) $85-$.

- - Gelim, P. P. Kazan S. Nt. (Ps.Mth.) P. 3 (1885) 2-.

Representation of equations by their derivatives, and constants. Mossbrugger, $L$. Grunert Arch. 22 (1854) 447-.

Resolution, exact, in all soluble cases. Young, G. P. QJ. Mth. 4 (1861) 341-.

Resolvent of equation $x^{m}+p x+q=0$. Pujet, $A$. C. R. 91 (1880) 611-.

- _ - Pujet's treatment of problem. Lachtin, L. K. [1893] Rec. Mth. (Moscou) 17 (1895) 334-.

- equations. Pellet, A. E. C. R. 88 (1879) 638.

-, Lagrange's, for equations of prime degree soluble by radicals. Bianchi, L. A. Mt. 11 (1882-83) 255-.
Resolvents of algebraic equations. Minich, $S . R$. Ven. At. (1858-59) 127-.

- _ - geometric representation. Klein, $F$. Mth. A. 4 (1871) 346-.

- - equations soluble by radicals, theorem. Betti, E. Tortolini A. 2 (1851) 102-.

- No cubic or biquadratic can be resolvent of general equation of order higher than 4 th. Jeřabek, A. Časopis 21 (1892) 1-; Fschr. Mth. (1892) 94.

-, theory. König, J. [1880] Mth. A. 18 (1881) 78-.

Roots of equation, expression for, by inversion of algebraic integral. Dillner, $G$. Stockh. Öfv. 40 (1883) No. 6, 3-.

- - - general expression for. Borenius, $G$. Stockh. Ak. Hndl. Bh. 8 (* 1883-84) No. 20, $11 \mathrm{pp}$.

- - in terms of coefficients. Dirksen, E.H. Berl. Ab. (1834) 577-.

- - equations, analytical construction. Dantscher von Kollesberg, $V$. Innsb. Nt. Md. B. 17 (1887) 6-.

- - - representation by repeating function of one root. Minding, E. F.A. Crelle J. 20 (1840) 168-.

- - general algebraic equation, recent formula to express. Favero, G. B. Rm. R. Ac. Linc. Rd. 7 (1891) (Sem. 1) 373-.

- - higher equations as multipliers of 1st degree. Gy"̈ry, S. (xII) Mag. Tud. Ak. Ets. 1 (No. 9) (1867) 169-.

Ruffini-Abelian theorem. Pierpont, $J$. N. Y. Am. Mth. S. Bll. 2 (1896) 200 -

Solubility of algebraic equations. Falck, $H$. Svea 13 (1831) 426-.

- - - - Minich, S. R. Ven. At. (1857-58) 629-; Ven. I. At. 7 (1880-81) 905-.

Solution by algebraic and geometric theory of operations. $\dot{Z}$ murko, L. [1881] Wien Ak. D. 44 (1882) (Ab. 2) 59-.

- of equations by one another. Jordan, $C$. Liouv. J. Mth. 16 (1871) 1-.

- by Gauss's scheme for complex quantities. Raabe, $A$. Wien Sb. $63(1871)(A b, 2)$ 733-.

- - irrational functions. Mainardi, $G$. Rm. At. N. Linc. 20 (1867) 153-.

- - the normal form. Lipps, G. F. Z. Mth. Ps. 38 (1893) 321-; 39 (1894) 1-, 65-.

- partial differential equations. Raabe, J. L. [1850] Crelle J. 48 (1854) 167-.

- - radicals. Galois, $E$. Liouv. J. Mth. 11 (1846) 417-.

- - Minich, S. R. Ven. At. 11 (186566) $243-$.

- - Cayley, A. Ph. Mg. 36 (1868) $386-$

15 (1878) 65-

- - - , form of expression. Mossbrugger, $L$. Grunert Arch. 14 (1850) 113-.

- - of irreducible equations. Sylow, $L$. [1868] Sk. Nf. F. 10 (1869) 257-.

- roots of higher order. Guldberg, A. S. Christiania F. (1877) No. 3, 40 pp. 
2450 General resolution

Solution, transcendental and algebraic. Cockle, Ja8. Ph. Mg. 21 (1861) 379-; 23 (1862) 135-

- by transcendents. Pievani, (Dr) A. (viri) A. Mt. 5 (1863) 57[61]-.

Symmetric products involving prime roots of unity. Forsyth, A. R. Mess. Mth. 14 (1885) 40 -

- -, method. Cockle, Jas. Ph. Mg. 4 (1852) 492-; 5 (1853) 170-; 7 (1854) 130-.

$-\ldots,-$, application to algebraic solution. Harley, $R$. Manch. Ph. S. Mm. 15 (1860) 172-; Phil. Trans. (1861) 327-.

Theory of Lagrange and Vandermonde. Minich, $S . R$. Ven. At. (1858-59) 19-.

Transformation, Bring-Tschirnhausen. Sylvester, J.J. Crelle J. Mth. 100 (1887) 465-.

- of equations. Jerrard, G. B. Ph. Mg. 7 (1835) 478-

- - - Cockle, Jas. Ph. Mg. 26 (1845) 383-; Mathematician 1 (1845) 82-, 113-, 194-, 297-; 3 (1850) 178-.

- - J Jerrard, G. B. Ph. Mg. 5 (1853) 354-, 493-.

- - Brioschi, F. Mil. At. I. Lomb. 1 (1858) 231-.

- - Bring, E. S. Arch. Mth. Ps. 41 (1864) 105-.

- - Biehler, C. N. A. Mth. 3 (1884) 209-.

- - Brioschi, F. Mil. I. Lomb. Rd. 20 (1887) 364-; L. Mth. S. P. 20 (1889) 127-; C. R. 124 (1897) 661-.

- _ , limits of method. Cockle, Jas. $\mathrm{Ph}$. Mg. 29 (1846) 181-; 30 (1847) 29 -.

- - - principles of theory. Brioschi, F. A. Mt. 16 (1888-89) 329-.

- - - theorem (Hermite). Combescure, $\dot{E}$. C. R. 64 (1867) 174-.

- and finding roots, Bring's method. Tegman, P. Stockh. Ak. Hndl. 22 (1801) 228-

- - lowering. Prouhet, E. N. A. Mth. 3 (1864) 122-, 433-, 508-.

Tschirnhausen's coefficientș, property. Bournáz, J. Orv. Termt. Ets. (Termt. Szak) (1898) 167-.

- and Jerrard's transformations. Grunert, J. A. Grunert Arch. 40 (1863) 214 -

- transformation. Cayley, A. [1861-65] Phil. Trans. 152 (1862) 561-; 156 (1866) 97-. - -, coefficients, extension to quintic. Lavagna, G. M. Tortolini A. 1 (1858) 238-.

- -, equations invariant under. La Motte, F. A. [1900] N. Y. Am. Mth. S. Bll. 7 (1901) 244, 250-.

- -, J. Hammond's series of numbers connected with. Sylvester, J.J. C. R. 104 (1887) 1228-.

Uni-serial Abelian equations. Young, G. P. Am. J. Mth. 9 (1887) 225-.

Wronski's formulæ for general solution. Torriani, J. E. Lisb. Ac. Sc. Mm. 6 (1819) 33-.

- method for general solution. Gergonne, J.D. Gergonne A. Mth. 3 (1812-13) 51-, 206-; 9 (1818-19) 213-.

It. Mm. 18 (1818) 56-.
Simultaneous equations

2460

\section{Simultaneous equations; including linear equations.}

Algebraic systems and their relations to systems of partial differential equations. Delassus, É. C. R. 123 (1896) 546-.

Approximate determination of roots of several equations. Biermann, O. Mh. Mth. Ps. 11 (1900) 148-.

Common solutions of simultaneous equations, number. Mantel, $W$. N. Arch. Wisk. 16 (1889) 203-; Fschr. Mth. (1889) 132.

- zero values of 2 algebraic equations, mode of representing. Zatuski, J. Prace Mt.-Fiz. 8 (1897) 129-; Fschr. Mth. (1897) 118.

Conditions of co-existence of equations, order. Roberts, S. Crelle J. 67 (1867) 266-; L. Mth. S. P. 6 (1874-75) 101-.

Dependence of equations. Borden, J. Camb. (M.) Mth. M. 3 (1860) 7-

Equation, 2nd order, general, with 2 variables. Rodrigues, A. N. A. Mth. 4 (1845) 24-.

$-,-1,-,-\ldots-$ Grunert, J. A. Grunert Arch. 25 (1855) 146-.

_ Lenthéric, -. Mntp. Ac. Sc. Mm. 4 (185860) 46-.

-, 4th order, with 2 variables, factorisation. Janni, V. G. Mt. 7 (1869) 28.

,,----4 variables, solution. Le Besgue, V. A. C. R. 59 (1864) 1067-.

-, $m$ th order, with $n$ variables. Gergonne, J. D. Gergonne A. Mth. 4 (1813-14) 115-.

Equations, 2nd order, condition for a common root of 2. Gelin, $E$. Mathesis 13 (1893) 265-.

-, - - numerical solution of 2. Transon, A. (Prof.) N. A. Mth. 19 (1860) 414-.

,$- \ldots$, in $x, y, z$, solution of 3. Lemonnier, $H$. [1878] Par. S. Mth. Bll. 7 (1879) 16-.

-, - - with 2 variables. Bret, - Gergonne A. Mth. 2 (1811-12) 218-.

Albitzky, W. J. Fschr. Mth. (1890) 111.

,-----7 , solution. Anne, L. N. A. Mth. 2 (1843) 195-.

- , - - - - Unferdinger, $F$. Arch. Mth. Ps. 49 (1869) 474-.

- - - - - or 3 variables. Lemonnier, $H$. N. A. Mth. 3 (1864) 518-; 4 (1865) 5-.

-, 3rd order, 2, question. Roberts, M. N.A. Mth. 15 (1856) 76-; 16 (1857) 366-.

-, higher order, graphic solution. Spitzer, $S$. [1850] Haidinger Ab. 4 (1851) (Ab. 3) 1-.

- with 2 variables. Piani, D. Bologna $R d$. (1854-55) 33-.

- - - - approximate solution of 2. Grunert, J. A. Grunert Arch. 20 (1853) 337-.

- $-\ldots$, numerical solution of 2 . Zimmermann, $H$. Z. Mth. Ps. 20 (1875) 71-。

- - - , solution of 2. Lefébure de Fourcy, L. Par. Éc. Pol. Cor. 2 (1809-13) 276-.

$--\ldots,-$ - Bonnet, O. N. A. Mth. 6 (1847) 54-, 135-, 243-, 375-.

- _ - system of 2 , theorems. Jacobi, C. G. J. Crelle J. 14 (1835) 281-. 


\section{Simultaneous equations}

Equations with 2 variables, systems of 2 . Sivering, J. N. A. Mth. 2 (1863) 372-.

-3 variables,

$x+y+z=A ; \quad a x y+\beta x z+\gamma y z=B ; \quad x y z=C$, solution. Tortolini, $B$. (vrI) A. Mt. 4 (1861) 202-.

- - - - solution of 3, at least two being linear. Grunert, J. A. Grunert Arch. 37 (1861) 422-.

- $n$ variables, system. Mollame, $V$. Catania Ac. Gioen. At. 18 (1885) 53-.

- - - , theorems. Plücker, J. Crelle J. 16 (1837) 47-.

- - several variables. Liouville, $J$. Liouv. J. Mth. 12 (1847) 68-.

- - - Betti, E. [1857] Tortolini A. 1 (1858) 1-.

- - solution. Laurent, H. C. R. 67 (1868) 491-.

- - Enriques, $F$. Mth. A. 51 (1899) 134-.

,---- of a class. Drot, -. N. A. Mth. 5 (1846) 389.

$-a x+b y+c z=0, a_{1} x y+b_{1} y z+c_{1} z x=0$, solution. Grunert, J.A. Grunert Arch. 28 (1857) 110-.

$-x^{3}+y^{3}=a, x^{2} y+x y^{2}=b$, solution. Strehlke, F. Arch. Mth. Ps. 48 (1868) 1-.

Euler's equation, solution. Gyldén, $H$. St Pét. Ac. Sc. Bll. 10 (1866) 59-.

Homogeneous equations with 2 variables. Kaiser, H. Grunert Arch. 25 (1855) 76-.

- linear equations, system important in differential equations. Heffter, $L$. Crelle J. Mth. 111 (1893) 59-.

- simultaneous equations. Catalan, E. C. A. Mt. 7 (1865) 66-.

- - C, Catalan's memoir. Tortolini, B. A. Mt. 7 (1865) 70-.

Implicit equations with 2 variables, $x$ and $y$, to express $y$ in terms of $x$. Schubert, $F . T$. [1823] St Pét. Ac. Sc. Mm. 11 (1830) 138-.

- functions defined by infinite number of simultaneous equations. Koch, H. von. Par. S. Mth. Bll. 27 (1899) 215-.

Lagrange and Newton's method of greatest and least coefficients extended to 2 equations. Nasimow, P. S. Fschr. Mth. (1898) 365.

Lagrange's method of multipliers. Rutherford, $W$. Mathematician 1 (1845) 32-.

Line construction for solution of system of linear equations, regarded as a configuration. Berg. F. J. van den. Amst. Ak. Vs. M. 5 (1889) 267-; Fschr. Mth. (1888) 76.

\section{LINEAR EQUATIONS.}

Valat, -. Bordeaux Act. Ac. Sc. (1846) 1-.

Lebon, J. N. N. A. Mth. 8 (1849) 59-.

Biehler, C. N. A. Mth. 19 (1880) 311-, 356-.

Rouché, E. Par. Ec. Pol. J. 29 (=Cah. 48) (1880) 221-.

Meyer, A. N. Ts. Mth. 5 (B) (1894) 4-; Fschr. Mth. (1893-94) 163-.

Bez's method. Jakimov, $V . P$. Kazan S. Nt. (Ps.-Mth.) P. 5 (1887) 2-.

- -. Porěckij, P. S. Kazan S. Nt. (P8.Mth.) P. 5 (1887) 3-.

\section{Linear equations 2460}

class. Liouville, J. Liouv. J. Mth. 11 (1846) 466-

- (Chelini). Terquem, O. N. A. Mth. 6 (1847) 129-

-, solution. Terquem, O. N. A. Mth. 5 (1846) 67-, 162-.

compatibility of a system. Capelli, A. Rv. Mt. 2 (1892) 54-.

discussion. Rouché, E. C. R. 81 (1875) 1050-.

formula for values of variables. Molins, $H$. Liouv. J. Mth. 4 (1839) 508-

- - - Chelini, D. G. Arced. 85 (1840) 3-.

graphic solution. Isé, E. G. Mt. 14 (1876) 180 -

- - of system. Berg, F. J. van den. Amst. Ak. Vs. M. 4 (1888) 196-; Fschr. Mth. (1887) 89-.

- Klingatsch, A. Mh. Mth. Ps. 3 (1892) 169-.

- - - - Griend, J. van der (jun.). N. Arch. Wisk. 4 (1899) 22-; Fschr. Mth. (1899) 108.

- - - Vaes, F. J. N. Arch. Wisk. 4 (1899) 42-; N. A. Mth. 18 (1899) 74-

- - - of two. Crocchi, L. [1876] G. Mt. 15 (1877) 86-.

when indeterminate. Suremain-de-Missery, $A$. Gergonne A. Mth. 1 (1810-11) 204-.

$n$ variables expressed as multiple integrals. Jacobi, C. G.J. Crelle J. 14 (1835) 51-.

peculiar set. Muir, T. [1900] Edinb. R. S. P. 23 (1902) 248-.

- -, Muir's paper. Tweedie, C. [1900] Edinb. R. S. P. 23 (1902) 261-.

problem. Gauss, C. F. N. A. Mth. 10 (1851) 359-.

with real coefficients. Gordan, $P$. Mth. A. 6 (1873) 23-.

solution. Grunert, J.A. Grunert Arch. 10 (1847) 284-.

-. Prediger, C. Arch. Mth. Ps. 70 (1884) 319-.

- Veltmann, W. Z. Mth. Ps. 31 (1886) $257-, 384$.

-, method and application to determinants. Clasen, (l'abbé) B. I. Brux. S. Sc. A. 12 (1888) (Pt. 2) 251-.

system. Painvin, L. Liouv. J. Mth. 3 (1858) 41-.

-. Versluys, J. Arch. Mth. Ps. 52 (1871) 257-.

-, general solution. Hansen, P.A. Leip. B. 1 (1846-47) 333-; Leip. Ab. Mth. Ps. 1 (1852) 83-.

- of infinite number, solution. Kötteritzsch, T. Z. Mth. Ps. 15 (1870) 1-, 229-.

-, solution. Ettingshausen, A von. Baumgartner Z. 5 (1829) 209-.

一, 一. Farkas, J. C. R. 87 (1878) 523-.

-, standard solutions. Cayley, A. QJ. Mth. 19 (1883) 38-.

systems, a geometric interpretation. Nicoli, $\boldsymbol{F}$. Mod. Ac. Sc. Mm. 16 (1875) 141-.

-, solution by roots of unity. Cauchy, A. L. C. R. 25 (1847) 285-.

theory. Lichtenfels, $V$. (Frhr.) vom. Wien SB. 12 (1854) (Ab. 2) 935-. 
with trigonometric coefficients. Crelle, A. L. N. A. Mth. 11 (1852) 383-.

- 3 variables. Amiot, B. Rouen Ac. Tr. (1842) 41-.

- $n$ variables. Koch, H. von. Stockh. Öfv. (1890) 109-; Fschr. Mth. (1890) 111.

- large number of variables. Nekrasov, P.A. St Pet. Ac. Sc. Mm. (Rs.) 69 (1892) (Suppl.) No. $5,18 \mathrm{pp}$.

- - - _ - , solution by successive approximation, Seidel's process. Mehmke, $R$. Rec. Mth. (Moscou) 16 (1893) 342-.

Linear simultaneous equations. Soulé, Bordeaux S. Sc. Mm. 2 (1891) x-.

_ _ cases of insolubility or indeterminance. Farcy, A. N. A. Mth. 4 (1845) 474-

- - - solution, theorems. Meyer, U. H. Grunert Arch. 12 (1849) 336-.

Malfatti's problem, system of equations. Cayley, A. [1875] $\quad$ L. Mth. S. P. 7 (1876) 38-.

- _ , _ _ - and another algebraic system. Cayley, A. Camb. and Dubl. Mth. J. 4 (1849) 270 -.

Multiple solutions of several equations. Combescure, É. C. R. 62 (1866) 383-.

- - 2 equations, criteria. Perrin, $R$. C. R. 107 (1888) 22-.

- - 3 equations, criteria. Perrin, $R$. C. R. 107 (1888) 219-.

Newton's formulæ for sums of like powers of roots, extension. Méray, C. C. R. 61 (1865) 710-; Par. Éc. Norm. A. 4 (1867) 159-.

Nine quantities contained in a certain nine equations. Hearn, G. W. Camb. and Dubl. Mth. J. 4 (1849) 265-.

- - - - - - (Hearn). F., L.J. Camb. and Dubl. Mth. J. 5 (1850) 179-.

Nomographic method, application. Ocagne, M. d'. Par. S. Mth. Bll. 26 (1898) 16-.

- representation of equations of second order with 3 variables. Ocagne, M. $d^{\prime}$. Par. S. Mth. Bll. 24 (1896) 81-.

Normal equations, general solutions. Flint, A. S. A. Mth. 4 (1888) 182-

- - with 3 variables, Jacobi's solution of a system. Seeliger, $H$. As. Nr. 82 (1873) 249 .

Numerical equations, one or more variables, solution. Sarrus, F. Liouv. J. Mth. 6 (1841) 171-.

Order of certain systems of equations. Cayley, A. Camb. and Dubl. Mth. J. 4 (1849) 132-.

- _ - _ - Salmon, G. QJ. Mth. 1 (1857) 246-.

- - - Roberts, S. [1867] L. Mth. S. P. 2 (1869) 8-.

Porismatic equations. Davis, R. F. Mth. Gz. 1 (1900) 252-, 273-.

Problem, to find $x$ and $y$ given

$$
x-y=\frac{x}{y}=x^{2}+y^{2}
$$

Schlömilch, O. Grunert Arch. 9 (1847) 456. - of 3 simultaneous quadratics in Wallis's Algebra. Degen, $C . F$. (vi Adds.) Kiöb. Ov. $(1816-17) 2-$.
Problems with 2 unknowns, solution by successive approximations. [Barré de] SaintVenant, -. C. R. 54 (1862) 845-.

Quantities associated in systems, and application to theory of simultaneous equations. Méray, C. Par. Éc. Norm. A. 8(1879) 81-, 327-.

Real roots of 2 numerical algebraic equations with two variables, finding the. Mehmke, $R$. Z. Mth. Ps. 35 (1890) 174-.

Resolvent of 2 equations of 2 nd order. SaintGermain, A. de. (xr) Par. S. Mth. Bll. 1 (1873) 142-.

Roots common to several equations. Dyck, $W$. C. R. 120 (1895) 34-.

- - - simultaneous equations, number. Picard, É. N. A. Mth. 8 (1889) 5-; C. R. 113 (1891) 356-, 669-, 1012-.

$-\frac{1}{-}--,-$ Kronecker, -. C. R. 113 (1891) 1006-. Mth. 8 (1892) 5-.

- - - system of simultaneous equations, number, and sum of values of a function at these points. Dyck, W. C. R. 119 (1894) 1254-.

- of simultaneous equations. Cauchy, A.L. C. R. 4 (1837) 672-; 5 (1837) 6-.

$-\ldots+\ldots$, Cauchy's theorem. Liouville, $J$., \& Sturm, C. C. R. 4 (1837) 720-.

Separation of roots of simultaneous equations. Vasil'ev, A. V. (xп) Kazan Un. Mm. [10] (1874) 631-.

Simultaneous equations deducible from one another by substitution, system. Cauchy, A. L. C. R. 22 (1846) 159-, 193-.

- - solution by determinants. Bianchi, $G$. Mod. Mm. S. It. 22 (1839) 184 -

- - systems, solution, general method. Cauchy, A. L. C. R. 25 (1847) 536-.

- - and theory of combinations. Bouniakowsky, V. [1844] St Pét. Ac. Sc. Mm. 6 (1850) 109-.

- , three, $a= \pm \frac{u-v w}{\sqrt{\left(1-v^{2}\right)\left(1-w^{2}\right)}}$, etc., etc. Unferdinger, $F$. Grunert Arch. 35 (1860) $32-$.

- - verification of solution of system. Merrifield, C. W. [1870] Mess. Mth. 5 (1871) 206-.

- - 2nd order, graphic solution. Allardice, R. E. Edinb. R. S. P. 17 (1891) 139-.

- - m m order with two variables, system of 2. Escary, -. N. A. Mth. 20 (1881) 227-.

- - , higher order, application of determinants. Simmons, T. C. Fschr. Mth. (1895) 127-.

Sturm's theorem, extension to system of simultaneous equations. Hermite, C. C. R. 35 (1852) 52-.

System of equations $\sum_{8=1}^{8=n} a_{8}^{r} x_{8}=b_{r} ;(r=1,2 \ldots n)$. Šimerka, W. Wien SB. 33 (1858) 277-.

- - - occurring in Darboux's "Théorie générale des surfaces," solution. Craig, $T$. A. Mth. 11 (1896-97) 48-. 
System of equations, one quadratic, the rest linear, solution. Baur, C. W. [1868] Z. Mth. Ps. 14 (1869) 129-, 426-.

,$----\frac{1}{-}--\frac{-}{-}$ Versluys, $J$. Arch. Mth. Ps. 60 (1877) 128-.

- - - _ - , - - - Nanson, E. J. Mess. Mth. 28 (1899) 107-.

$\ldots \ldots-, 2$ quadratic, the rest linear, solution. Gundelfinger, S. Z. Mth. Ps. 18 (1873) 543-.

- - - solution. Sylvester, J. J. Ph. Mg. 37 (1850) 370-.

,,--- Scheibner, $W$. Leip. B. 8 (1856) 65 -.

- - $n$ equations with $n$ variables, number of solutions. Fouret, $G$. Par. S. Mth. Bll. 2 (1874) 127-.

- - - linear equations, graphic solution. Fouret, G. Par. S. Mth. Bll. 3 (1875) 93-; As. Fr. C. R. 4 (1875) 154-.

$--\frac{-}{-}$ with $n$ variables. Valeriani, $V$. G. Mt. 9 (1871) 371-.

- - - - Fontené, $G$. N. A. Mth. 14 (1875) 481-.

- — - relations between 2 systems of $n$ quantities. Fontené, G. N. A. Mth. 17 (1898) 317-.

- $-2 n$ equations with $2 n$ variables occurring in mechanics. Bonolis, A. G. Mt. 11 (1873) 38-.

- - 3 equations, goniometric solution. Grunert, J. A. Grunert Arch. 6 (1845) 370-.

- -6 equations in 9 quantities, solution. Frattini, G. [1879] G. Mt. 18 (1880) 174-.

Systems of equations. Cayley, A. Ph. Mg. 20 (1860) 341-; QJ. Mth. 11 (1871) 132-; Mess. Mth. 7 (1878) 17-; 12 (1883) 191-.

- - - solution without using determinants. Cockle, Jas. Ph. Mg. 2 (1851) 289-.

- - - , theory, with application to curves. Saltel, L. N. A. Mth. 20 (1881) 546-; 2 (1883) 554-.

Transformation of equations with 3 variables. Genocchi, A. G. Mt. 5 (1867) 106-.

- - linear equations by change of independent variable. Duclos, L. Mntp. Mm. Ac. Sect. Sc. 6 (1864-66) 281-.

- 2 simultaneous equations. Walton, $W$. QJ. Mth. 11 (1871) 262-.

- - - C. Cayley, A. QJ. Mth. 11 (1871) $266-$

\section{Transcendental equations.}

Algebraic analysis, application. Fourier, J.B.J. [1829] Par. Mm. Ac. Sc. 10 (1831) 119-.

Analysis of some equations. Frisiani, P. Mil. Effem. As. (1845) 3-.

Compound interest and annuities: to find $p$ from relation $E=A(1+p)^{n}+\frac{(1+p)^{n}-1}{p}$. Simony, O. Z. Mth. Ps. 22 (1877) 190-.

Elimination of intervals, solution of algebraic and transcendental equations by. Hermann, A. N. A. Mth. $9(1870) 180$ -
Transcendental equations 2470

Equal roots. Turquan, L. V. C. R. 59 (1864) 701-.

Equation allied to $J_{0}(z)=0$, least root. Freeman, A. Camb. Ph. S. P. 3 (1880) 375-.

- $a^{x}=b$, solution. Belli, S. Siena At. Ac. 9 (1808) 255-.

$-x^{y}=y^{x}$, solution in real quantities. Wittstein, $T$. Grunert Arch. 6 (1845) 154-.

$-u-\tan u=\zeta$, imaginary roots. Serret, J.A. C. R. 42 (1856) 1182-.

- - - - Catalan, E. C. (vi Adds.). C. R. 42 (1856) 1184-.

- $a^{x}+b^{x}=c^{x}$, solution. Dubois, $E$. Les Mondes 4 (1864) 735-.

$-1,-$ Q. Qâher-Bey. N. A. Mth. 5 (1866) 129-.

- $\tan x=\lambda x$, expression of roots in series. Michaëlis, J. P. Lux. S. Sc. Mm. 7 (1864) 167-.

$-0=\frac{1}{2}-\frac{x}{x+1}+\frac{x(x-1)}{(x+1)(x+2)} \ldots$, roots (Bour guet). Catalan, E. C. N. A. Mth. 16 (1877) 416-.

$-y^{x}=x^{y}$. Schwering, $K$. Z. Mth. Ps. 23 (1878) 339-.

$-x^{y}=y^{x}$. Luxemberg, M. [1880] Arch. Mth. Ps. 66 (1881) 332-.

- - Marrecas Ferreira, L. F. G. Teix. J. Sc. 2 (1880) 165-.

$-\cos x=x$, numerical value of roots. Miller, T. H. Edinb. Mth. S. P. 9 (1891) 80-.

Equations,

and

$$
\int_{0}^{\pi} \cos (x \cos \omega) d \omega=0
$$

$$
\int_{0}^{\pi} \cos (x \cos \omega) \sin ^{2} \omega d \omega=0,
$$

roots. Baehr, G. F. W. Amst. Vs. Ak. 6 (1872) 325-; Arch. Néerl. 7 (1872) 351-.

Number of solutions of transcendental equation. Bidone, G. "Turin Mm. Ac. (1805-08) (pte. 2) 35-.

Numerical solution by successive approximations. Sommerfeld, A. Gött. Nr. (1898) 360-.

Properties of algebraic equations applicable to transcendental equations. Laguerre, E. C. R. 94 (1882) 160 -

Real roots of algebraic or transcendental equations, calculation. Buchwaldt, $F$. Mth. Ts. 3 (1861) 185-.

- _ _ _ _ - - - practical method. Mehmke, R. Z. Mth. Ps. 36 (1891) 158-. - - - _ - , determination. Cauchy, A. L. C. R. 5 (1837) 357-.

$---1-1-$, exact separation. Siebel, A. Arch. Mth. Ps. 5 (1887) 279-; 6 (1888) vI.

Repeated functional operations, solution of algebraic or transcendental equations by. Isenkrahe, C. Mth. A. 31 (1888) 309-.

Roots. Poisson, S. D. Par. S. Phlm. N. Bll. (1826) 145-; Par. Mm. Ac. Sc. 9 (1830) 89-.

- of certain equations. Hurwitz, A. Hamb. Mth. Gs. Mt. 2 (1890) (Festschr., Tl. 2) 25-. 
Roots of certain equations. Lémeray, E. M. N. A. Mth. 16 (1897) 540-.

$--x=a^{x}$. Lémeray, E. M. N. A. Mth. 15 (1896) 548-; 16 (1897) 54-.

-, theory. Cockle, Jas. Ph. Mg. 20 (1860) 145-, 368-.

Solution. Stern, M. A. Crelle J. 22 (1841) 1-.

-. Spitzer, S. Wien D. 3 (1852) 155.

-. Terquem, O. (vi Adds.) N. A. Mth. 14 (1855) 295-, 384-; 15 (1856) 17-.

-. Schjellerup, H. C. F. C. Mth. Ts. 1 (1859) 33-.

- of a class. Boussinesq, J. C. R. 72 (1871) 480-.

Sturm's method, application. Stern, M. A. Crelle J. 33 (1846) 363-.

Theorem. Gerono, G. C. N. A. Mth. 17 (1858) 201-.

Theory. Liouville, J. Liouv. J. Mth. 3 (1838) 337-.

Transcendental equation arising from partial fractions. Hermite, C. Bll. Sc. Mth. As. 4 (1873) 61-.

- - satisfied by no complex number. Schlömilch, O. Grunert Arch. 12 (1849) 293-.

$--\ldots-$ Clausen, T. Grunert Arch. 13 (1849) 334-

- - - - - - Baltzer, R. Grunert Arch. 16 (1851) 243.

- equations. Vecchio, A. G. Mt. 7 (1869) $42 ; 10$ (1872) 171-.

- - and algebraic equations of higher order, solution. Nowikow, P. M. Fschr. Mth. (1899) 101.

- - with no roots. Seidel, L. [1869] Crelle J. 73 (1871) 297-.

- solution of equations. Harley, R. QJ. Mth. 5 (1862) 337-.

\section{Theory of Numbers. \\ 2800 General.}

$\mathrm{A}^{\alpha}+\mathrm{B}^{\beta}$ a prime, conditions. André, D. N. A. Mth. 12 (1873) 521-.

Addition, generation of integers by. Daublebsky von Sterneck, $R$. Wien Ak. Sb. 105 (1896) (Ab, 2a) 875-.

Additive functions. Laporte, $M$. As. Fr. C. R. (1895) (Pt. 2) 63-.

- theory of numbers. Daublebsky von Sterneck, R. Wien Ak. Sb. 109 (1900) (Ab. 2a) 28-.

- - - , proposition. Daublebsky von Sterneck, R. Wien Ak. Sb. 106 (1897) (Ab. 2a) 115-

Agreeable numbers. Seelhoff, $P$. Arch. Mth. Ps. 70 (1884) 75-, vII-

- - Cunningham, (Lt.-Col.) A. B. A. Rp. (1893) 699.

Arithmetical theorem connected with discriminant of binary cubic. Sylvester, J.J. C. R. 60 (1865) 1011-, 1121-.

Bisection, repeated. Bouniakonsky, V. [1866] St Pét. Ac. Sc. Bll. 11 (1867) 97-.

British Association Reports. Smith, H. J. S. B. A. Rp. (1859) 228-; (1860) 120-; (1861) 292-; (1862) 503-; (1863) 768-; 35 (1865)322-.
Calculus of finite differences, and theory of numbers. Busche, E. Mth. A. 53 (1900) 243-.

Cards puzzle and a law of numbers. Steen, $A$. (xII) Ts. Mth. 1 (1865) 49-; 2 (1866) 1-; 3 (1867) 1-; 6 (1870) 1-, 181-; 3 (1873) 156-. - - - Hertzsprung, S. (xII) Ts. Mth. 3 (1867) 97-.

Mth. 1 (1871) 53-.

Cauchy's "Exercices," error in vol. 2. Clausen, T. [1859] St Pét. Ac. Sc. Bll. 1 (1860) 145-.

- theorem : $\quad \Sigma[a ! \beta ! \ldots \lambda ! a a, b \beta \ldots l \lambda]^{-1}=1$ generalised. Sylvester, J.J. C. R. 53 (1861) 644-, 722-.

Complements, arithmetical. Nicholson, $P$. Tilloch Ph. Mg. 52 (1818) 210-.

,-- , new form. Hill, T. Am. As. P. (1857) 82.

Composite numbers and factor tables. Niegemann, A. Arch. Mth. Ps. 45 (1866) 203-.

- - observations. Laisant, C. A. Par. S. Mth. Bll. 16 (1888) 150-.

- - relation to components. Bianchi, $G$. Mod. Mm. S. It. 1 (1862) 1-, 207-.

Cubic numbers, property. Polignac, A. (Prince de). N. A. Mth. 8 (1849) 215.

Cycles. Hathaway, A. S. J. H. Un. Cir. [3] (1884) 123-.

Cyclic numbers. Dickson, L. E. QJ. Mth. 27 (1895) 366-.

"Derivatives" of numbers, theory. Daublebsky von Sterneck, $R$. Mh. Mth. Ps. 4 (1893) 53-.

Diatomic series, "median" series $\left\{\Sigma\left(\overline{2^{n}-1}\right)\right\}$ in. Polignac, A. (Prince de). N. A. Mth. 10 (1851) 308-.

Difference and mean of 2 integers, theorem. Gergonne, J. D. Gergonne A. Mth. 21 (1830-31) 83-.

- between $p$ th roots of two numbers, superior limit. Trébert, A. N. A. Mth. 3 (1844) 76-.

Divisibility, theorems mainly on. Catalan, $E$. Brux. Ac. Bll. 7 (1884) 448-.

Double equalities, impossibility of some. Genocchi, A. C. R. 78 (1874) 433-.

- -, problem of Fermat's. Coste, L. M. P. Gergonne A. Mth. 10 (1819-20) 101-.

- - in theory of numbers. Genocchi, $A$. [1881] Rm. S. It. Mm. 4 (1882) No. 3, $31 \mathrm{pp}$.

Dyadic coordination of primes up to 100,000 to series of odd numbers. Suchanek, $E$. Wien Ak. Sb. 103 (1894) (Ab. 2a) 443-.

Elementary theory (Euler, Legendre, Gauss and Cauchy). Terquem, $O$. N. A. Mth. 3 (1844) 204-, 214-, 337-.

- _, some results. Leudesdorf, $C$. L. Mth. S. P. 20 (1889) 199-.

- treatise. Jimenez, E. (xII) Madrid Ac. Ci. Mm. 7 (1877) 687 pp.

Equation, 6-period. Smith, H.J.S. As. Fr. C. R. (1880) 190-.

$-x y=x^{2}-y^{2}$ insoluble in integers. Vachette, A. N. A. Mth. 5 (1846) 68-. 
Equation $a x^{2}+b x+c=E\left(a_{1} x^{2}+b_{1} x+c_{1}\right)$, solution. Serdobinskir, V.E. (xII) Rec. Mth. (Moscou) 7 (1874-75) (Pt. 1) 437-; (xI) Bll. Sc. Mth. As. 10 (1876) 103-.

Euclidean numbers, so-called. Studnicka, $F$.J. Prag Sb. (1899) (Mth.-Nt.) No. 30, 4 pp.

- proofs of theorems. (Product of two primes by a third number is prime to the third, etc.) Genocchi, A. N. A. Mth. 13 (1854) 426-.

Euler, theorem. Integers are of form $4 m n-m-n$

or

$$
x^{2}+y^{2}+y \text {. }
$$

Genocchi, A. N. A. Mth. 12 (1853) 235-.

Even numbers. Studnicka, F. J. Casopis 26 (1897) 207-; Fschr. Mth. (1897) 164.

- - "Is every even number the sum of two odd primes?" Lionnet, E. N. A. Mth. 18 (1879) 356-.

Every cube is difference of 2 squares, etc. Tait, P. G. [1870] Edinb. R. S. P. 7 (1872) 144.

- integer divides a number expressed by 9's followed by 0's. Grunert, J. A. Crelle J. 5 (1829) 185-.

- - power $\mu$ of an integer $l$ can be obtained by taking the sum of $l^{k}$ consecutive odd numbers. Lemoine, E. $\quad$ N. A. Mth. 9 (1870) 368 -

- number is composed of 4 square numbers or less, proof without primes. Pollock, (Sir) $F$. [1851] R. S. P. 6 (1854) 132-.

Farey's series. Lucas, É. [1877] Par. S. Mth. Bll. 6 (1878) 118-.

- - , series of fractions analogous to. Halphen, G. H. Par. S. Mth. Bll. 5 (1877) 170-.

Fermat, some problems. Pepin, T. Rm. N. Linc. Mm. 8 (1892) 85-.

Fermat's, Euler's, Wilson's, von Staudt's and Clausen's theorems. Lucas, É. Mathesis 11 (1891) 9-.

- 4th porism. Ofterdinger, $(D r)$-. Arch. Mth. Ps. 46 (1866) 1-.

-numbers. Pollock, (Sir) F. [1866-68] R.S.P. 15 (1867) 115-; Phil. Trans. 158 (1868) 627-.

- -. Cunningham, (Lt.-Col.) A. B. A. Rp. (1899) 653-.

- theorem, identity with fundamental property of periodic fractions. Mansion, $P$. [1875] Mess. Mth. 5 (1876) 33.

Fifteen girl problem, cyclic solution. Peirce, $B$. Gould As. J. 6 (1861) 169-。

Formula, new. Cesáro, $E$. (xI) Mathesis 2 (1882) 148-.

$-2^{2 n}+1$. Pepin, T. C. R. 85 (1877) 329-.

$-2^{n}-1$. Pepin, T. C. R. 86 (1878) 307-.

Formulæ, general. Liouville, $J$. Liouv. J. Mth. 3 (1858) 143-, 193-, 201-, 241-, 273-, 325- ; 4 (1859) 1-, 73-, 111-, 195-, 281- ; 5 (1860) 1-; 9 (1864) 249-, 281-, 321-, 389-; 10 (1865) 135-, 169-.

一, various. Cauchy, A. L. C. R. 12 (1841) 698-, 813-.

Fourier's series and a formula of Gauss. Hermite, C. Am. J. Mth. 9 (1887) 381-.

Fraction, division into sum of fractions with given denominators. Tirelli, $F$. G. Mt. 16 (1878) 88-.
Fractions arranged in order of magnitude, systematic interruption. Airy, G. B. $\mathrm{Ph}$. Mg. 12 (1881) 175-.

-, denominators of which $>n$, theorem. Cauchy, A. L. Par. S. Phlm. Bll. (1816) 133-. _, irreducible, periods ; theorems of Lionnet's. Meyer, G. F. [1868] Arch. Mth. Ps. 49 (1869) 168-.

-, -, series. Ocagne, M. d'. Brux. S. Sc. A. 10 (1886) (Pt. 2) 90-

- number of, in any "Farey series" of which limiting number is given. Sylvester, $J$. $J$. Ph. Mg. 15 (1883) 251-; 16 (1883) 230-.

_, _ - denominators and numerators of which do not exceed certain number. Sylvester, J. J. (xII) J. H. Un. Cir. [2] (1883) 44-.

- $-\ldots$, formed with integers less than given number. Sylvester, J.J. C. R. 96 (1883) 409-; Mess. Mth. 27 (1898) 1-.

-, property. Farey, J. Tilloch Ph. Mg. 47 (1816) 385-.

-, -. Glaisher, J. W. L. Ph. Mg. 7 (1879) 321 -

-, tabulation of all, between given limits. Sang, E. [1878] Edinb. R. S. T. 28 (1879) $287-$.

Function $E(f(x))$, modification ; quadratic and non quadratic residues of form $4 k+1$. Bunıakovskij, V. Ja. [1885] St Pet. Ac. Sc. Mm. (Rs.) 52 (1886) 124-; Fschr. Mth. (1886) 144 .

Functions arising in theory of numbers. Berger, A. Stockh. Öfv. (1898) 579-.

- of even numbers, Laplace's method. Jacobi, C. G.J. As. Nr. 28 (1849) 257-.

- in theory of numbers, general questions. Bervi, N. V. [1894] Rec. Mth. (Moscou) 18 (1896) 519- ; Fschr. Mth. (1896) 156-.

_ _ - - , present state of knowledge. Bervi, N. V. Rec. Mth. (Moscou) 19 (1897) 182-; Fschr. Mth. (1896) 156.

General aspects of theory of numbers. Bourdat, -. Grenoble Ac. Delph. Bll. 3 (1850) 37-.

- remarks. Sludskix, T.A. [1864] (xII) Rec. Mth. (Moscou) 2 (1867) (Pt. 2) 195-.

Geometrical illustrations of some theorems. Davis, E. W. Am. J. Mth. 15 (1893) 84-.

Geometry, application to theory of numbers. Busche, E. Crelle J. Mth. 104 (1889) 32-.

- of numbers. Minkowski, H. D. Mth. Vr. Jbr. 1 (1892) 64-.

Goldbach's odd numbers. Stern, M. A. N. A. Mth. 15 (1856) 23-.

- theorem, Euler's extension. Terquem, $O$. N. A. Mth. 11 (1852) 326-.

Hamiltonian differences, Sylvester's tables. Glashan, J. C. QJ. Mth. 27 (1895) 242-.

History, remarks. Henry, C. As. Fr. C. R. (1880) 201-.

Identities, numerical, etc. Serdobinskǐ, $V . E$. [1872] (xII) Rec. Mth. (Moscou) 6 (1872-73) (Pt. 1) 107-.

_, - , method of finding and application to theory of numerical functions. Baskakov, S. I. [1881] (xII) Rec. Mth. (Moscou) 10 (1882-83) (Pt. 1) 313-. 
Inequalities relating to prime numbers. Sylvester, J.J. Nt. 38 (1888) 259-.

Infinite series, use in theory of numbers. Lejeune-Dirichlet, G. Crelle J. 18 (1838) 259-.

Infinitesimal analysis, applications to theory of numbers. Lejeune-Dirichlet, $G$. Crelle J. 19 (1839) 324-; 21 (1840) 1-, 134-.

Integers of certain form. Rocquigny, $F$. [G.] de. Les Mondes 53 (1880) 410-.

-, property of a certain series. Sylvester, J.J. C. R. 88 (1879) 1297-.

-, solutions by. Liouville, J. Liouv. J. Mth. 4 (1859) 271-.

-, successions that cannot be indefinitely continued. Sylvester, J. J. [1882] (xII) J. H. Un. Cir. [2] (1883) $2-$.

-, systems. Cesáro, $E$. Rm. R. Ac. Linc. Rd. 4 (1888) (Sem. 1) 457-.

-, theorem. Serret, J. A. Liouv. J. Mth. 13 (1848) 12-.

-, -. Durège, -. Grunert Arch. 30 (1858) 163 .

- - (Durège). Lottner, C. L. E. Grunert Arch. 32 (1859) 111-.

-, theory. Binet, J. P. M. Liouv. J. Mth. 6 (1841) 449-.

Integral calculus, applications. Lucas, $\dot{E}$. C. R. $82(1876)$ 1303-.

- - - - Weber, H. Gött. Nr. (1896) 275-.

Latent integer, formal property. Sylvester, J.J. QJ. Mth. 1 (1857) 185.

Laws of numbers, problems. Marchand, $D$. Les Mondes 38 (1875) 460-; 39 (1876) 26-, 346-, 693-.

Legendre's functions, application. Bauer, $G$. Münch. Ak. Sb. 24 (1895) 343-.

Linear modular system, reduction. Pund, $O$. Hamb. Mth. Gs. Mt. 3 (1900) 423-.

$m$ th root of product of $m$ numbers. Boutroux, -. N. A. Mth. 1 (1842) 368-.

Maximum product of components. Derbè s, $J$. N. A. Mth. 19 (1860) 117-.

Mean values in theory of numbers. LejeuneDirichlet, G. Berl. Ab. (1849) (Mth.) 69-.

- - - - Berger, A. Ups. S. Sc. N. Acta 14 (1891) No. 2, $130 \mathrm{pp}$.

Miscellaneous notes. Catalan, $E$. As. Fr. C. R. (1891) (Pt. 2) 194-.

$n$th powers that are sums of $n$th powers. Martin, A. Smiths. Misc. Col. 33 (1888) Art. 4, 107-. (Wash. Ph. S. Bll. 10 (1888).)

Nasik cubes. Frost, A.H. QJ. Mth. 8 (1867) 74.

- -, general properties. Frost, A.H. [1877] QJ. Mth. 15 (1878) 93-, 366-.

- squares, general properties. Frost, A. H. [1877] QJ. Mth. 15 (1878) 34-.

Natural system of numbers. Pujals de la Bastida, V. Madrid Rv. 4 (1854) 567-; 5 (1855) 436-.

Nature and properties of numbers. Jamard, Rouen Tr. Ac. (1804) 75-.

New basis for a general theory. Kraus, $J$. Z. Mth. Ps. 37 (1892) 321-; 39 (1894) 11-.

Number equal to sum of $p$ squares, certain. Catalan, E. C. (xI) Mathesis 3 (1883) 199.
Number, prime or composite, test. Koch, $H$. von. Stockh. Öfv. (1900) 789-.

- of times a prime is contained in product of $n$ primes. André, D. N. A. Mth. 13 (1874) 185-.

- 3, properties. Gegenbauer, $L$. Wien Ak. Sb. 97 (1889) (Ab. 2a) 271-.

-5 and number 7, characteristic properties. Lucas, $\hat{E}$. N. A. Mth. 18 (1879) 74-.

- 60, characteristic. Wasserschleben, - von. Arch. Mth. Ps. 54 (1872) 411-.

- 107, property. Schwering, $K$. Acta Mth. 11 (1887-88) 119-.

Numbers, class. Collignon, E. As. Fr. C. R. (1895) (Pt. 2) 6-.

-, classes, two. Debacq, C. Les Mondes 29 (1872) 308-.

, elementary theorems. Chabert, -. N. A. Mth. 3 (1844) 250-.

of form $4 m+1$, theorem. Liouville, $J$. Liouv. J. Mth. 15 (1870) 133-.

- - - - - (Liouville). Zolotareff, $G$. [1870] St. Pét. Ac. Sc. Bll. I6 (1871) 85-.

,$-----(-)$. Pepin, T. Rm. N. Linc. Mm. 5 (1889) 131-.

-, four, in arithmetical progression of which 3 are squares. Guibert, $A$. N. A. Mth. 1 (1862) 249-.

-, problems. Noel, J. N. Liège Mm. S. Sc. 4 (1847) 55-.

-, series and equations. Grolous, J. (x) Par. S. Phlm. Bll. 8 (1872) 119-.

- with a single arbitrary function, general theorem. Bugaev, N. V. [1865] (xII) Rec. Mth. (Moscou) 2 (1867) (Pt. 1) 10-.

-, succession of $2 n+1$, so that sum of squares of first $n+1=$ that of last $n$. Dostor, G. Arch. Mth. Ps. 64 (1879) 350-.

--, three, such that sum or difference of any two is square. Euler, L. [1780] 'St. Pét. Ac. Sc. Mm. 6 (1818) 54-.

Odd and even numbers. Martin, Art. Des Moines Anal. 2 (1875) 20-.

- - - theorem of Gauss. Stern, M. A. Gött. Nr. (1869) 330-.

- numbers, theorems on two. Catalan, E.C. Liége S. Sc. Mm. 6 (1877) No. 4, 4 pp.

- -, Turner's property. Grunert, J. A. Grunert Arch. 1 (1841) 59-.

_ - _ - - Hellerung, - . Grunert Arch. 1 (1841) 318-.

Origin and development of theory of numbers. Studnička, F.J. Časopis $4\left({ }^{*} 1874\right) 1-$.

Partition analysis applied to properties of any system of consecutive integers. MacMahon, P. A. [1899] Camb. Ph. S. T. 18 (1900) $12-$.

- of even number into two primes. Sylvester, J. J. [1871] L. Mth. S. P. 4 (1871-73) 4 -.

- $\phi\left(p^{n}\right)$ and $\phi\left(2 p^{n}\right), p$ being a prime. Sancery, L. [1875] Par. S. Mth. Bll. 4 (1876) 17-.

Perfect cubes. Henkle, $W$. D. Des Moines Anal. 1 (1874) 75-; 2 (1875) 65-.

- Sensenig, D. M. Des Moines Anal. 3 (1876) 104-. 
Perfect number, odd, cannot have less than five distinct prime divisors. Sylvester, J.J. C. R. 106 (1888) 522-.

- numbers. Lebesgue, V.A. N. A. Mth. 3 (1844) 552-.

- - Lionnet, E. N. A. Mth. 18 (1879) 306-.

- - Lucas, É. Mathesis 6 (1886) 145-.

- -. Novarese, H. G. Teix. J. Sc. 8 (1887) 11-.

- - Sylvester, J. J. C. R. 106 (1888) 403-, 641-; Mathesis 8 (1888) 57-.

- - Servais, C. Mathesis 8 (1888) 92-, 135.

- - Catalan, E. Mathesis 8 (1888) 112-.

- Lucas, É. Mathesis 10 (1890) 74-.

- - Bourlet, C. N. A. Mth. 15 (1896) 297-.

- - Pepin, T. Rm. N. Linc. Mm. 13 (1897) 345-.

-- , history. Curtze, M. Bb. Mth. (1895) 39-.

- - properties of analogous numbers. Minin, A. P. [1882] (xm) Rec. Mth. (Moscou) 10 (1882-83) (Pt. 1) 87-.

- - theory. Carvallo, J. C. R. 81 (1875) 73-.

Periods, law. Jonquières, E. de. C. R. 96 (1883) 1020-, 1129-, 1210-.

Permutations, circular, applications. MacMahon, P. A. L. Mth. S. P. 23 (1892) 305-.

Powers, difference of consecutive. Tromsens, A. N. A. Mth. 19 (1860) 310-.

- of number. Anon. (vi 886) N. A. Mth. 13 (1854) 424-.

- - - new laws. Coninck, G. de. N. A. Mth. 13 (1874) 568-.

- - - properties. O., D. QJ. Sc. 5 (1818) 55-.

--- , relations. Prouhet, E. C. R. 33 (1851) 225.

- - - remainders. Buttel, $P$. Grunert Arch. 26 (1856) 241-.

- - - theorem. Frégier, - Gergonne A. Mth. 9 (1818-19) 211-.

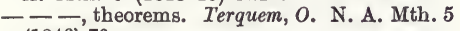
(1846) 70 -.

- - - uses in mechanics. Noel, J. N. Quetelet Cor. Mth. 1 (1825) 124-, 199-.

- - whose sum is same power of some number. Martin, Art. QJ. Mth. 26 (1893) 225-.

-, relations. Marchand, $D$. Les Mondes 44 (1877) 424-

- and their differences. Kirwan, R. [1809] Ir. Ac. T. 11 (1810) 131-.

- of 2 and 3. Sludskǐ, T. A. [1869] (xII) Rec. Mth. (Moscou) 4 (1869-70) (Pt. 2) 171-. - 5 and their multiples. Berdellé, C. As. Fr. C. R. 8 (1879) 176-.

Prime of form $8 n+1$, theorem. Stern, M. A. Crelle J. 32 (1846) 89-.

-, formation from lower primes. Scherk, H. F. Crelle J. 10 (1833) 201-.

- greater than given prime. Boije af Gennäs, C. O. Stockh. Öfv. (1893) 469-.

-, theorem of Legendre. Pepin (le père), $T$. Liouv. J. Mth. 5 (1879) 21-.
Primes. Laurent, H. N. A. Mth. 18 (1899) 234 . -, characteristic relations for. Hacks, $J$. Acta Mth. 17 (1893) 205-.

- of definite forms. Bang, A. S. N. Ts. Mth. 2 (B) (1891) 73-; Fschr. Mth. (1891) 183.

-, determination of certain. Lawrence, $F, W$. L. Mth. S. P. 28 (1897) 465-.

- equal to sums or differences of squares or multiples of squares. Vallès, $F$. (xI) Par. S. Phlm. Bll. 7 (1871) 191-.

-, expression in terms of preceding primes. Isenkrahe, C. Mth. A. 53 (1900) 42-.

-, forms arising from $(a+\sqrt{b}) k$. Genocchi, $A$. A. Mt. 2 (1868-69) 256-.

-, general formula. Dormoy, $\dot{E}$. C. R. 63 (1866) 178-.

-, geometrical interpretation. Cassani, $P$. Ven. I. At. (1896-97) 103-.

-, roots. Oltramare, G. Crelle J. 45 (1853) 303-.

-, theorems. Levi, G. Ven. I. At. (189293) 1816-.

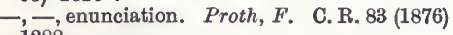
1288-.

-, theory. La Vallée-Poussin, C. J. de. Brux. S. Sc. A. 20 (1896) (Pt. 1) 100-, (Pt. 2) 183-, 281-; 21 (1897) (Pt. 1) xxiii, 1-, 60-, (Pt. 2) 251-.

Primitive numbers (not containing square factors), distribution. Bougaev, - C. R. 74 (1872) 449-.

- properties. Maria, P. C. G. Mt. 4 (1866) 345-.

Principles, first. Skrivan, $G$. Wien Jbr. Ober-Realsch. Inn. Stadt 2 (1860) 1-.

-, fundamental. Poinsot, L. C. R. 12 (1841) 803-; Liouv. J. Mth. 10 (1845) 1-; Grunert Arch. 7 (1846) 367-.

-, - (Poinsot). Grunert, J. A. Grunert Arch. 7 (1846) 168-.

Problem: to find several consecutive cubes whose sum is a square. Catalan, $E$. C. Brux. Ac. Bll. 22 (1866) 339-.

Problems, numerous. Gelin, E. Mathesis 16 (1896) (Suppl.) 34 pp.

Products of digits of certain numbers. Sanctis, P. de. Rm. N. Linc. At. 52 (1899) 58-; 53 (1900) 57-.

- and series involving prime numbers only. Glaisher, J. W. L. QJ. Mth. 27 (1895) 270-; 28 (1896) 1-.

Progressions, application. Volpicelli, $P$. Tortolini A. 5 (1854) 449-.

Properties of integers derived from space intuitions. Minkowski, $H$. N. A. Mth. 15 (1896) 394-.

- - numbers. Vince, S. [1810] Ir. Ac. T. 12 (1815) 34-.

- - - Anon. (vi 319) Dingler 34 (1829) 68. (1841) 112-.

- Whitworth, W. A. QJ. Mth. 6 (1863) 163-.

- - Valat, J. P. F. Bordeaux Ac. Act. 38 (1877) 73-.

- - Torelli, G. G. Mt. 16 (1878) 152-. 
Properties of numbers. Dostor, G. Arch. Mth. Ps. 63 (1879) 221-.

- - Pepin, T. C. R. 120 (1895) 1254-. $-{ }_{-}$, theorem. Ingleby, C. M. Mess. Mth. 3 (1866) 30-.

Property of numbers. Heider, $M$. Haidinger B. 1 (1846) 186.

- - Midy, -. N. A. Mth. 5 (1846) 640- - Dostor, G. J. N. A. Mth. $8(1849)$ 101-. 631 . Volpicelli, P. Rm. At. 6 (1852-53) (1853) (pte. 2) 397-.

- - Booth, J. R. S. P. 7 (1854-55) 42-. - - - Anon. (vi 314) Crelle J. 50 (1855) $187-$.

- - - Boncompagni, B. Tortolini A. 6 (1855) 371-.

- in theory of numbers. Earnshaw, $S$. (vI Adds.) Camb. Mth. J. 2 (1841) 79-.

Propositions, miscellaneous. Cauchy, A. L. C. R. 24 (1847) 996-; 25 (1847) 132-, 177-, 242-.

-, - Catalan, E. C. N. A. Mth. 13 (1874) 518 .

-, - . Thue, A. Arch. Mth. Ntvd. 16 (1893) 255-; 19 (1897) No. 4, 27 pp.; Fschr. Mth. (1897) 165.

Real representation of imaginary configurations, and application to theory of numbers. Busche, E. Crelle J. Mth. 122 (1900) 227-.

Reciprocals of primes. Goodwyn, $H$. Nicholson J. 4 (1801) 402-.

Remainder as function of divisor and dividend. Schl̈̈milch, O. Grunert Arch. 10 (1847) 424-.

Representation of number in form $\Sigma a_{r}{ }^{n}$.

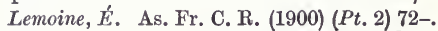

Residues of recurring series with regard to prime modulus. Mantel, $W$. N. Arch. Wisk. 1 (1895) 172-; Fschr. Mth. (1893-94) 423-.

Rhythmomachy, an ancient game. Terquem, O. N. A. Mth. 5 (1846) 662-.

Roots of numbers. Sanchis-Barrachina, $E$. N. A. Mth. 8 (1869) 265-.

Series involving inverse even powers of subeven and supereven numbers. Glaisher, J. W. L. Mess. Mth. 23 (1894) 176-.

- of natural numbers. Collignon, É. As. Fr. C. R. (1896) (Pt. 2) 17-.

- - - number occupying given place. Barbier, E. $\quad$ C. R. 105 (1887) 795-, 1238-. 106 (1888) 190 . - Ocagne, M. $d^{\prime}$. C. R.

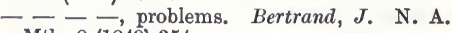
Mth. 8 (1849) 354-.

- - odd numbers, theorems. Selle, (le vicomte) - de. Aix Ac. Mm. 18 (1900) 75-.

- primes, and resolution of number into factors. Gough, J. Nicholson J. 1 (1802) 1-.

- in which coefficients are sums of divisors of exponents, powers of. Glaisher, J.W.L. Mess. Mth. 15 (1886) 33-. Glaisher, J. W. L. Mess. Mth. 14 (1885) 156-.
Series $\frac{1}{2}+\frac{1}{3}+\frac{1}{8}+\frac{1}{7}+\frac{-1}{11}+\ldots$ (primes). Glaisher, J.W. L. QJ. Mth. 25 (1891) 369-.

$-\frac{1}{3}-\frac{1}{8}+\frac{1}{7}+\frac{1}{11}-\frac{1}{1}-\ldots$ (primes). Glaisher, J. W. L. QJ. Mth. 25 (1891) 375-.

$-\frac{1}{3^{2}}-\frac{1}{5^{2}}+\frac{1}{7^{2}}+\frac{1}{11^{2}}-\frac{1}{13^{2}}-\ldots$. Glaisher, J. W. L. QJ. Mth. 26 (1893) 33-.

$-\frac{1}{2}+\frac{1}{5}-\frac{1}{4}+\frac{2}{11}-\frac{1}{13}+\ldots$ Glaisher, J.W. L. QJ. Mth. 26 (1893) 48-.

Sets of integers, determinant of which is $>$ or $<1$. Hermite, $C$. Liouv. J. Mth. 14 (1849) 21-.

Square array of 16 numbers satisfying 22 conditions. Lebesgue, $V . A$. N. A. Mtb. 15 (1856) 403-.

- numbers, analysis. Wheeler, A. D. Silliman J. 25 (1834) 87-.

- - , and biquadrates, ascertaining by inspection. Saint, $W$. Nicholson J. 22 (1809) 291 -. - , properties. Hoyt, D. H. Camb. (M.) Mth. M. 3 (1860) 2-

- - tables giving sets of final digits. Schady, -. Crelle J. Mth. 84 (1878) 85-.

- that are sums of squares. Martin, A. Wash. Ph. S. Bll. 11 (1892) 580-.

Squares, theorems of $2,4,8,16$. Cayley, $A$. QJ. Mth. 17 (1881) 258-.

Sum and difference of two squares. Claude, R. P. L. N. A. Mth. 2 (1863) 88-.

- of $n \mathrm{th}$ powers of numbers < and prime to $n$. Thacker, A. Crelle J. 40 (1850) 89-.

- - reciprocals of like powers of primes, theorem. Glaisher, J.W.L. As. Fr. C. R. 6 (1877) 172-.

- - series $\Sigma \frac{1}{p^{2}}, p$ being prime number. Glaisher, J.W.L. Mess. Mth. 7 (1878) 190 Sums of inverse powers of primes. Glaisher, J.W. L. QJ. Mth. 25 (1891) 347-.

- like powers of prime numbers. Bour, J. E. É. Par. S. Phlm. Bll. 2 (1865) 8-.

- - series of reciprocals of primes and of their powers. Merrifield, C. W. [1881] R. S. P. 33 (1882) 4-.

- - squares, relations. Dostor, G. Arch. Mth. Ps. 67 (1882) 265-.

- - theorems. Réalis, S. N. A. Mth. 12 (1873) 212-; 18 (1879) 500-.

- - three squares. Dainelli, $U$. G. Mt. 15 (1877) 378-.

-, value of some. Stern, M. A. Gött. Ab. 17 (1872) (Mth.) 63-.

Tables of uniform reversible functions of two variables on the simplest number-domains. Schröder, E. Mth. A. 29 (1887) 299-.

Theorems, Berger's, proof and generalisation. Cesáro, E. (xII) Mathesis 1 (1881) 99-.

-, various. Talbot, W. H. F. [1862] Edinb. R. S. T. 23 (1864) 45-.

-, -. Gegenbauer, $L$. Wien Ak. D. 49 (1885) (Ab. 1) 1-, (Ab. 2) 105-; 57 (1890) 497-; 60 (1893) 25-.

-, - Catalan, E. Brux. Ac. Mm. 46 (1886) No. 1, $16 \mathrm{pp}$.

Theory of numbers and automorphic functions, relations. Fricke, $R$. D. Mth. Vr. Jbr. (1899) (Heft 1) 94-. 
Transformation, numerical. Davies, T. S. (vi Adds.) Ph. Mg. 34 (1849) 347-.

Treatise on numbers. Crelle, A. L. Crelle J. 27 (1844) 6-, 107-, 330-; 28 (1844) 111-; 29 (1845) 58-, 103-.

Triads of once-paired elements. Horner, $J$. [1867] QJ. Mth. 9 (1868) 15-.

Wronski's theory of numbers. Dickstein, $S$. Krk. Ak. (Mt.-Prz.) Rz. 4 (1893) 73-, 396; Cre. Ac. Sc. Bll. (1892) 64-.

\section{Divisibility; linear con- gruences.}

Arithmetical "lines" (series), arrangement. Tarry, G. N. A. Mth. 18 (1899) 149-, 292.

Bugaev's "order", i.e. sum of exponents of prime factors of a number. Čistiakov, I. I. Rec. Mth. (Moscou) 20 (1899) 595-; Fschr. Mth. (1899) 194.

Chinese problem of remainders. Matthiessen, H. F. L. [1880-81] Crelle J. Mth. 91 (1881) 254-; Z. Mth. Ps. 26 (1881) (H.-lt. Ab.) 33-. --- , solution. Rocquigny, F. $[G] d$.$e .$ Les Mondes 54 (1881) 304-.

- - - - - Domingues, A. Les Mondes 55 (1881) 62-.

Congruence $487^{2} \equiv 486$. Shanks, W. R. S.P. 25 (1877) 551-.

- theorem relating to sums of binomial coefficients. Glaisher, J.W.L. QJ. Mth. 30 (1899) 361-.

Congruences. Lebesgue, V. A. N. A. Mth. 9 (1850) 436-; C. R. 51 (1860) 9-.

-. Hathaway, A. S. Am. J. Mth. 6 (1884) 316-.

- relating to sums of products of first $n$ numbers, etc. Glaisher, J.W.L. QJ. Mth. 31 (1900) 1-.

Congruent numbers. Woepcke, $F$. Tortolini A. 3 (1860) 206-.

- - Günther, S. Prag Sb. (1878) 289-.

- - multiplication. Woepcke, F. (vIII) A. Mt. 4 (1861) 247-.

Decomposition of fraction into quantièmes (fractions with unity for numerator). Bobynin, V. V. Z. Mth. Ps. 44 (1899) (Suppl.) 1-.

Difference of two powers divisible by power of prime. Bouniakowsky, $V .[1829]$ St. Pét. Ac. Sc. Mm. 1 (1831) 139-.

- -product, divisibility. Segar, H.W. Mess. Mth. 23 (1894) 31-.

\section{DIVISIBILITY.}

Gergonne, J. D. Gergonne A. Mth. 5 (1814-15) 170-.

Joubin, -. L'I. 2 (1834) 230.

Transon, A. (Prof.) N. A. Mth. 4 (1845) 173-.

Elliott, E. B. Camb. (M.) Mth. M. 1 (1859) 45-.

Zbikowski, A. [1860] St. Pét. Ac. Sc. Bll. 3 (1861) 151-.

Niederrist, - . Grunert Arch. 38 (1862) 384-.

Folie, $\boldsymbol{F}$. Liége Mm. S. Sc. 3 (1873) 85-。

Buguev, N. V. (xII) Rec. Mth. (Moscou) 8 (1876) (Pt. 1) $501-$.

VOL. I.
Ponte Horta, F. da. G. Teix. J. Sc. 1 (1878) $57-$.

Broda, K. Arch. Mth. Ps, 63 (1879) 413-.

Kessler, $O$. Z. Mth. Ps. 28 (1883) 60-.

Durfee, W. P. J. H. Un. Cir. [4] (1885) 23.

Catalan, E. Rv. Sc. 38 (1886) 508.

Bougon, - . Rv. Sc. 38 (1886) 508.

Dickstein, S. Fschr. Mth. (1886) 136.

Perrin, R. As. Fr. C. R. (1889) (Pt. 2) 24-.

Gelin, E. Mathesis 12 (1892) 65-, 93-.

Levänen, S. Helsingf. Öfv. 34 (1892) 109-; Fschr. Mth. (1892) 164 .

Fontès, -. As. Fr. C. R. (1893) (Pt. 2) 240 -.

Bělohlavek, F. Časopis (23) (1894) 59-; Fschr. Mth. (1893-94) 273.

Bourgerel, G. L. Rv. Sc. 1 (1894) 411-.

by any number, criterion. Fontès, - C. R. 115 (1892) 1259-.

- prime. Mpller, C. F. C., \& Holten, C. (xII) Ts. Mth. 5 (1875) 177-.

(1876) 15-.

- - character. Loir, - C. C. R. 106

(1888) 1070-, 1194.

- - criterion. Christie, R. W. D.

Nt. 40 (1889) 247.

of certain expressions by $p$, proof. Cauchy,

A. L. Par. Éc. Pol. J. 9 (1813) (16 cah.) 99-。 - numbers. Šimerka, $V$. Časopis 8 (*1879) 187-; Fschr. Mth. ( $\left.{ }^{*} 1879\right)$ 125-.

- - Mackay, J. S. Edinb. Mth. S. P. 4 (1886) 55-.

- numerical expression. Weyr, E. Časopis 11 (*1882) 39-; Fschr. Mth. (" 1882) 120.

- quotient by powers of factorial. André, D. C. R. 94 (1882) 426-

criteria. Unferdinger, $F$. Wien Sb. 59 (1869) (Ab. 2) 465-.

-. Delbouf, J. Rv. Sc. 38 (1886) 377-.

-. Noël, -. Rv. Sc. 38 (1886) 378-

- Greenstreet, W. J. Mth. Gz. 1 (1900) 186-.

-, history. Fontès, - Toul. Ac. Sc. Mm. 5 (1893) 459-.

criterion. Dupain, J. C. N. A. Mth. 6 (1867) 368-.

-. Burgess, H. T. [1897] Nt. 57 (1897-98) 8-. -, and Eratosthenes' sieve. Valerio, $P$. Rv. Sc. 52 (1893) 764 .

- for prime numbers and all odd numbers (except multiples of 5). Riess, L. T. Fschr. Mth. (1898) 137.

of faculties, geometric analogy. Pincherle, S. Bologna Rd. (1892-93) 17-.

Fergola's theorem. Torelli, G. G. Mt. 5 (1867) 250-.

of fraction with denominator $k !$ Thue, $A$. Arch. Mth. Ntvd. 14 (1890) 247-.

general characters. Lubin, J. N. A. Mth. 13 (1874) 528-.

of integers. Lapparent, - de. Cherb. Mm. S. Sc. 4 (1856) 235-.

- - Jonquières, - de. C. R. 120 (1895) 534 -

law. Burgess, H. T. [1897] Nt. 57 (1897-98) 30,55 .

-. Börgen, C. [1897] Nt. 57 (1897-98) 54 - 


\section{Divisibility}

by number 7. Brooks, $E$. Des Moines Anal. 2 (1875) 129-.

- - - Evans, A. Des Moines Anal. 10 (1883) 134-.

- - - , and conversion of fractions. Reyer, A. P. Grunert Arch. 25 (1855) 176-.

- - criteria. Heilmann, J. Rv. Sc. 38 (1886) 187.

- -9 , criterion, generalisation. Wilbraham, H. Camb. and Dubl. Mth. J. 6 (1851) $32-$.

- 13. Breton, P. Rv. Sc. 38 (1886) 444 .

445.

- 19. Badoureau, A. N. A. Mth. 18 (1879) 35-.

- numbers 7, 11, 13, tests. Grunert, J. A. Arch. Mth. Ps. 42 (1864) 478-.

- - and 13 especially. Pinaud, A. Toul. Mm. Ac. 1 (1844) 341-, 347-.

- - - - tests. Lindman, C. F. Grunert Arch. 26 (1856) 467-.

of numbers of form $2^{2^{m}}+1$, new case (Pervouchine). Bouniakowsky, $V$. St Pét. Ac. Sc. Bll. 24 (1878) 559; 25 (1879) 63-.

- one given number by another. Schlegel, S. F. V. Z. Mth. Ps. 21 (1876) 365-.

- - product of factorials by another, conditions. Landau, E. N. A. Mth. 19 (1900) 344-.

by power of 2, theorem. Frégier, - . Gergonne A. Mth. 9 (1818-19) 285-.

- prime numbers. Sensenig, D. $M$. Des Moines Anal. 3 (1876) 25-.

- a prime to 10 . Dietz, -, \& Vincenot, Metz Mm. Ac. 33 (1851-52) 37-.

problem. Elefanti, $F$. R. S. P. 10 (1859-60) 208-.

of product, distribution of primes. Catalan, E. C. Liège S. Sc. Mm. 12 (1885) No. 2, 20-, 119-.

question: Polignac, C. (Prince) de. C. R. 96 (1883) 485-.

simple properties. Elliott, E. B. Mess. Mth. 27 (1898) 12-.

of sum of products of first $n$ numbers taken $r$ together. Glaisher, J. W. L. Mess. Mth. 28 (1899) 184-.

theorem. Crelle, A. L. Crelle J. 5 (1830) 296 ; Gergonne A. Mth. 20 (1829-30) 349-.

- R., O. N. A. Mth. 4 (1845) 81-.

- Bauer, M. Mth. Nt. B. Ung. 15 (1899) 41-.

- of Euler, correction. Chevillier, I. N. A. Mth. 14 (1855) 433-; 15 (1856) 260-.

theorems. Catalan, $E$. Brux. Ac. Bll. 7 (1884) 448-.

- Allardice, R. E. Edinb. Mth. S. P. 8 (1890) 16-.

of $\prod_{i=0}^{i=n-1}(r+i)$ by $n !$, proof. Greatheed, S. S. (vI Adds.) Camb. Mth. J. 1 (1839) 102-, 112.

- $n !$, theorem. Gomes Teixeira, F. Arch. Mth. Ps. 2 (1885) 265-.
Division of a number by 9 or 11. Dodgson, C. L. Nt. 56 (1897) 565-.

- numbers, general law. Bugaev, N. Rec. Mth. (Moscou) 12 (1885) 283-; Fschr. Mth. (1885) 145-.

- by a prime, theorem. Mantel, W. N. Arch. Wisk. $4\left({ }^{*} 1878\right) 57-$.

- - - - Oskamp, G. A. N. Arch. Wisk. 4 (*1878) 83-

-, theorem. Lejeune-Dirichlet, G. Berl. B. (1851) 20-.

-, - on remainders (Lejeune-Dirichlet). Ter quem, $0 . \quad$ N. A. Mth. 13 (1854) 396-.

Divisors in case of two arbitrary functions, theorem. Bugaev, N. V. [1867] (xIr) Rec. Mth. (Moscou) 3 (1868) (Pt. 1) 69-.

-, construction of tables. Davis, W. B. L. Mth. S. P. 4 (1873) 416-

- of first $n$ numbers, relations. Glaisher, J. W. L. L. Mth. S. P. 22 (1891) 359-.

- - an integer. Lionnet, $E$. N. A. Mth. 7 (1868) 68-.

_ _ _ - , which exceed an assigned limit. Gegenbauer, L. Wien Ak. Sb. 98 (1890) $(A b, 2 a)$ 28-.

- - integers. Gegenbauer, L. Wien Ak. Sb. 91 (1885) (Ab. 2) 600-.

- - Egorov, D. T. [1891] Rec. Mth (Moscou) 16 (1893) 236-; Fschr. Mth. (1892) 188.

- number. Crelle, A.L. Berl. Ab. (1832) 33-.

- - Hain, E. Arch. Mth. Ps. 55 (1873) 290 -

- - - , formula of Euler, proof. Lebesgue, $V$. A. N. A. Mth. 12 (1853) 232-.

- - - method of finding. Canterzani, $S$. Bologna Mm. I. It. 2 (pte. 2) (1810) 445-.

$--\frac{\psi}{-} \psi$ functions. Gegenbauer, $L$. Wien Ak. Sb. $93(1886)(A b .2) 447-$

- - - - - - Lerch, M. Prag Sb. (1887) (Mth.-Nt.) 683-; (1894) (Mth.-Nt.) No. 11, 11 pp., No. 32, 20 pp.

_ - - theorems. Glaisher, J.W. L. [1875] Mess. Mth. 5 (1876) 52.

- - - - - Gegenbauer, $L$. Wien Ak. Sb. 91 (1885) (Ab. 2) 1194.

- - - Cesáro, E. G. Teix. J. Sc. 7 (1886) 3-.

- - - - . Lerch, M. Bll. Sc. Mth. 12 (1888) 100-; Ćasopis 21 (1892) 90-, 185-; Fschr. Mth. (1892) 186-.

- - -, -. Bugaev, N. V. [1893-1898]

Rec. Mth. (Moscou) 17 (1895) 720-; 20

(1899) 549-; Fschr. Mth. (1895) 218-; (1899) 194.

- - - - two. Nachtikal, $F$. Časopis 25 (1896) 344-; Fschr. Mth. (1896) 139.

- numbers, graphic representation. SaintLoup, L. C. R. 107 (1888) 24-; Par. Éc. Norm. A. 7 (1890) 89-.

- - - and products of factors. Roberts, $S$. QJ. Mth. 20 (1885) 370-

- - prime, theorem concerning remainders. Gauss, C. F. Gött. Cm. 16 (1804-08) 69-.

-, reduction of systems. Hensel, $K$. Crelle J. Mth. 118 (1897) 234-; 119 (1898) 114-. 
Divisors of series of natural numbers. Busche, E. [1896] Hamb. Mth. Gs. Mt. 3 (1900) 234 .

- $2 m$, Liouville's theorem. Mathews, G. B. $[1893]$ L. Mth. S. P. 25 (1894) 85-.

$E(\phi x)$ applied to determine integral part of quotient of two polynomials. Bugaev, N. V. [1897] Rec. Mth. (Moscou) 20 (1899) 247-; Fschr. Mth. (1898) 70.

Equation $(\phi) m(\phi) n=(\phi) m n$. Hammond, $J$. Mess. Mth. 24 (1895) 17-.

Factorial $N$, divisors. Weill, $M$. C. R. 93 (1881) 1066-

Factorials, property. Lerch, M. Prag Sb. (1898) (Mth.-Nt.) No. 2, 4 pp.

Factorisation. Kausler, C. F. [1801] St Pét. Ac. Sc. N. Acta 14 (1805) 268-

- Bouniakowsky, V. [1839] St Pét. Ac. Sc. Mm. 4 (1841) 447-.

Collins, E. [1839] St Pét. Ac. Sc. Bll. 6 (1840) 84-.

- Catalan, E. C. As. Fr. C. R. 9 (1880) 228-.

-. Busk, C.J. Nt. 39 (1889) 413-.

- Hudson, W. H. H. Nt. 39 (1889) 510 -.

- Cunningham, (Lt.-Col.) A. Mess. Mth. 20 (1891) 37-.

-. Thaarup, F. N. Ts. Mth. 2 (A) (1891) 49-; Fschr. Mth. (1891) 190.

- Selivanov, D. T. Rec. Mth. (Moscou) 15 (1891) 789-; 16 (1893) 469-; Fschr. Mth. (1891) 191-; (1892) 164.

- Lawrence, F. W. Mess. Mth. 24 (1895) 100-; QJ. Mth. 28 (1896) 285-.

. Biddle, D. Mess. Mth. 28 (1899) 116-, 192.

- Seliwanow, D. F. Fschr. Mth. (1899) 172-.

- of certain product (of integers). Gmeiner, J. A. Mh. Mth. Ps. 1 (1890) 159-.

- large numbers. Lucas, É. C. R. 83 (1876) 1286-.

-, method. Winter, G. K. Madras J. (188687) $13-$

-, problem. Rados, $G$. Mth. Termt. Ets. 3 (1885) 178-.

- 'Tesanek's method. Studnicka, F. J. Časopis 14 (1885) 120-; Fschr. Mth. (1885) 125.

Factorisations of an integer in two factors of prescribed form, mean number. Gegenbauer, L. Wien Ak. Sb. 93 (1886) (Ab. 2) 90 -

Factors of certain numbers. Workman, $W . P$. Mess. Mth. 24 (1895) 67.

- - composite numbers. Hill, C.J.D. Crelle J. 11 (1834) 251-。

- Möbius. Koch, H. von. Stockh. Öfv. (1900) 659-.

- - numbers. Cunningham, (Lt.-Col.) A. Nt. 39 (1889) 559-.

- - Speckmann, G. Arch. Mth. Ps. 12 (1894) 435-

- - - general rule for. Zeipel, E. V. von. Stockh. Öfv. 18 (1861) 425-.

_- - plan for table. Crelle, A. L. Crelle J. 51 (1856) 61-.
Factors, table, geometric properties. Möbius, A. F. Crelle J. 22 (1841) 276-.

Faculties. Mathieu, E. N. A. Mth. 17 (1858) 235-.

-. Lebesgue, V. A. N. A. Mth. 19 (1860) 112-, 135-.

-. Guibert, A. N. A. Mth. 1 (1862) 102-.

—, problem. Berton, -. N. A. Mth. 18 (1859) 191.

-, - - Hill, G. W. Des Moines Anal. 1 (1874) 27-.

Generalised Euler $\phi$ function, geometrical proof of theorems. Daublebsky von Sterneck, $R$. Mh. Mth. Ps. 5 (1894) 255-.

Greatest common divisor. Gegenbauer, L. Wien Ak. Sb. 97 (1889) (Ab. 2a) 420-.

_ - of all numbers represented by integral function of $n$ variables. Hensel, $K$. Crelle J. Mth. 116 (1896) 350-.

- - - , asymptotic laws. Gegenbauer, $L$. Wien Ak. Sb. 92 (1886) (Ab. 2) 1290-.

- divisors. Gegenbauer, $L$. Wien Ak. Sb. 94 (1887) (Ab. 2) 714-.

- prime divisor of $n^{2}+1$, theorem. Markoff, A. C. R. 120 (1895) 1032 -

- square divisor. Cesáro, E. A. Mt. 13 (1885) 251-.

Harmonic series, divisibility. Birkenmajer, $L$. Prace Mt.-Fiz. 7 (1896) 12-; Fschr. Mth. (1896) 141.

Identity, Clausen's, arithmetical proof. Glaisher, J. W. L. [1875] Mess. Mth. 5 (1876) 83.

Integers divisible by sum of their divisors. Minin, A. P. [1893] Rec. Mth. (Moscou) 17 (1895) 240-; Fschr. Mth. (1893-94) 277.

Kronecker's principle of equivalence. Busche, E. [1891] Hamb. Mth. Gs. Mt. 3 (1900) 3-.

Linear congruence, several unknowns. Lebesgue, V. A. Liouv. J. Mth. 4 (1859) 366. congruences, formuli for resolution. Zehfuss, G. Grunert Arch. 32 (1859) 422-.

- - homogeneous. Steinitz, E. [1896] D. Mth. Vr. Jbr. 5 (1901) (Heft 1) 87.

_ - modular system. Kronecker, L. Crelle J. Mth. 99 (1886) 329-.

- - , system. Burnside, $W$. Mess. Mth. 24 (1895) 51-.

-, -, solution. Studnička, F.J. Prag Sb. (1875) 114-

- - , systems. Smith, H.J.S. L. Mth.S.P. 4 (1871-73) 241-.

- - transformation of moduli. Bouniakowsky, $V$. N. A. Mth. 18 (1859) 168-.

- forms and congruence groups, composition. Stouff, X. C. R. 119 (1894) 993-.

- with integral coefficients. Frobenius, $G$. [1878-79] Crelle J. Mth. 86 (1879) 146-; 88 (1880) 96-.

- - - variables. Hurwitz, A. Gött. Nr. (1897) 139-.

- - representing primes. Vahlen, T. Königsb. Schr. 38 (1897) [47].

"Manifoldness" of a number (number of distinct prime divisors). Sylvester, J. J. Nt. 37 (1888) 152-, 179.

Moduli, 11, 9, 13, 101, tests. Anton, H. Arch. Mth. Ps. 49 (1869) 241-. 
Non-equivalent rectangular arrays, enumeration. Landsberg, G. Crelle J. Mth. 111 (1893) 87-.

Number of congruent divisors of a number. Traub, C. Grunert Arch. 37 (1861) 277-.

- - divisors of all numbers from 1 to $n$. Busche, E. [1894] Hamb. Mth. Gs. Mt. 3 (1900) 167-.

- - - , application of geometry to theory of numbers. Schröder, J. [1894] Hamb. Mth. Gs. Mt. 3 (1900) 177-.

- - - generalisation of theorem. Schröder, J. [1895] Hamb. Mth. Gs. Mt. 3 (1900) 219-.

- - - of given number, etc. Ettingshausen, A. von. Baumgartner Z. 5 (1829) 287-

_ - - integer. Stieltjes, T.J. (jun.) C. R. 96 (1883) 764-.

- - MSS. of Michael Stifel. Hoppe, E. Hamb. Mth. Gs. Mt. 3 (1900) 411-.

- - - of odd number having form $4 h \pm 1$, $8 h \pm 1,8 h \pm 3$. Andreevskǐ, M. A. [1869] (xII) Rec. Mth. (Moscou) 6 (1872-73) (Pt. 1) 97-.

- - form $2^{2 p-1} p-a$, theorem. Gomes Teixeira, P. G. Teix. J. Sc. 3 (1881) 105-.

- - integers prime to $m$ and $<m / k$. Laguerre, E. (x) Par. S. Mth. Bll. 1 (1873) 77-.

- - numbers in a set not divisible by a prime. Lipschitz, R. O. S. C. R. 96 (1883) 327-.

Numbers divisible by 7 and by $2,3,4,5,6$, with remainder 1 . Wiedemann, E. Erlang. Ps. Md. S. Sb. 24 (1892) 83.

-, geometrical representation according to modulus $p$. Laisant, C. A. As. Fr. C. R. (1887) (Pt. 2) 218-

- not divisible by squares, series of. Lipschitz, R. O. S. C. R. 89 (1879) 948-, 985-.

- prime to given number. Rocquigny, $F$. [G.] de. Les Mondes 52 (1880) 516-.

- - one another. Guilmin, A. N. A. Mth. 10 (1851) 23-.

_ - _ - - Schemmel, $V$. [1868] Crelle J. 70 (1869) 191-.

- - - - Goldschmidt, L. Z. Mth. Ps. 39 (1894) 203-.

- _ - - given number and less than another. Rocquigny, $F$. [G.] de. Les Mondes 50 (1879) 604-.

- - - - _ - - - Minine, $A$. Les Mondes 51 (1880) 333-; 53 (1880) 526-.

- - - - - - - Nazīmov, P.S. [1881] (xII) Rec. Mth. (Moscou) 11 (188384) 603-.

-, various theorems. Dienger, $J$. Grunert Arch. 12 (1849) 425-; 16 (1851) 120-.

Numerical divisors of polynomials. Borel, $\dot{E}$. Bll. Sc. Mth. 24 (1900) 75-.

- factors. Birch, (Rev.) J. G. Mess. Mth. 22 (1893) 52-.

- - of $a^{n}-1$. Bickmore, C. E. Mess. Mth. 25 (1896) 1-; 26 (1897) 1-.

Periodic division series, properties. Poselger, $F$. T. Berl. Ab. (1827) 21-.

Prime factors, determination. Stouff, -. N. A. Mth. 10 (1871) 104.

$-\cdots,-$, Ganss's tables. Pepin, T. Rm. N. Line. At. 42 (1889) 135-.
Prime factors of integers. Loir, A. As. Fr. C. R. (1898) (Pt. 2) 144-.

- - , resolution of large numbers into. Pepin, T. Rm. N. Linc. At. 43 (1890) 163-; Rm. N. Linc. Mm. 17 (1900) 321-.

- - - - _ - B. Barbette, E. Mathesis 19 (1899) 241-.

- - - - - - - Euler's method. Pepin, T. Rm. N. Linc. Mm. 9 (1893) (Pt. 1) 47-. - - - numbers into. Lucas, É. N. A. Mth. 14 (1875) 523-.

- - - - L Landry, F. As. Fr. C. R. (1880) 185-.

,$-----2^{n} \pm 1$ into. Gohierre de Longchamps, - C. R. 85 (1877) 950-

- number divides certain product of differences. Grunert, J. A. Grunert Arch. 10 (1847) 302-. -7, properties. Tucker, R. Nt. 40 (1889) 115-. - $p$, numerator of $\sum_{1}^{p-1} \frac{1}{r}$ is divisible by $p^{2}$. Wolstenholme, J. QJ. Mth. 5 (1862) 35-.

- - theorem on sum of products divisible by $p$. Steiner, J. Crelle J. 13 (1835) 356-.

- - - - - - - - - (Steiner). Jacobi, C. G. J. Crelle J. 14 (1835) 64-.

Primes. Tuxen, O. (xII) Ts. Mth. 5 (1881) 16-. - Petersen, J. P. C. (xII) Ts. Mth. 6 (1882) 138-.

in arithmetical progression. Cantor, M. Schlömilch Z. 6 (1861) 340-.

-, criteria. Thaarup, F. (xII) Ts. Mth. 5 (1881) 77-.

-, -. Zsigmondy, K. Mh. Mth. Ps. 5 .(1894) 123 -

- of form $2 n+1$, falsity of theorem in Gerg. Ix 320. Sarrus, $F$. Gergonne A. Mth. 10 (1819-20) 184.

- - $-6 m+1$. Krafft , W. L. [1798] St Pét. Ac. Sc. N. Acta 12 (1801) 217-.

- $-100 n+1$. Daublebsky von Sterneck, $R$. Wien Az. 31 (1894) 2-.

- - $-8 m+3,8 m+7,12 m+5,12 m+7$, etc. Iwanow, J. J. Fschr. Mth. (1899) 173.

- functions analogous to sine and cosine.

Lucas, É. Tor. Ac. Sc. At. 11 (1875-76) 928-. occurring in arithmetical progressions. Guibert, A. Liouv. J. Mth. 7 (1862) 414-. -, properties. Lionnet, E. N. A. Mth. 1 (1842) 175-.

, property. Bouniakowsky, V. St Pét. Ac. Sc. Bll. 4 (1838) 65-.

-, theorems. Laisant, C. A. Bordeaux S. Sc. Mm. 1 (1876) 399.

- - - Ferrers, N. M. Mess. Mth. 23(1894) 56-. - - (Ferrers). Rawson, R. Mess. Mth. 24 (1895) 68-.

-, theory. Baudrimont, A. Bordeaux Mm. S. Sc. 3 (cah. 2) (1865) 418-.

- of various linear forms. Liouville, $J$. Liouv. J. Mth. 3 (1858) 84-; 4 (1859) 399-; 5 (1860) 103-, 119-, 139-, 300, 301-, 303-, 309-, 311-, 387-, 389-, 391-; 6 (1861) 1-, 7-, 28-, 31-, 55-, 93-, 97-, 101-, 103-, 105-, 107-, $109-, 147-, 150-, 185-, 187-, 189-, 191-$, 193-, 195-, 197-, 199-, 201-, 203-, 205-, 207-, 219-; 7 (1862) 17-, 18-, 21-, 23-, 136; 8 (1863) 85-, 102-, 137-; 9 (1864) 135-, 137-. 
Primitive series of $k$ integers, determination. Mertens, F. Wien. Ak. Sb. 106 (1897) (Ab. 2a) 132-.

Quotients and residues. Ciccolini, $L$. Tortolini A. 3 (1852) 232-.

Remainders in division. Gorini, P. (vi Adds.) Majocchi A. Fis. C. 1 (1841) 235-.

- - Ingleby, C. M. B. A. Rp. 35 (1865) (Sect.) 7 .

- - - Walenn, W. H. Ph. Mg. 2 (1876) 345- ; 4 (1877) 378-.

-, problem. Kaiser, $H$. Grunert Arch. 25 (1855) 76-.

一, theorem. Walenn, W. H. B. A. Rp. 40 (1870) (Sect.) 16-.

Repetends, properties. Saint, W. Nicholson J. 24 (1809) 124-.

-, -. Heal, W. E. A. Mth. 3 (1887) 97-.

Residues, numerical, properties. Beaujeux, É., \& Laisant, A. N. A. Mth. 9 (1870) 221-, 271-, 302-, 354-.

- and sums, properties. Bugaev, N. $V$. [1878] (xII) Rec. Mth. (Moscou) 10 (1882-83) (Pt. 1) 30-.

Series of numbers prime to composite number. Binet, J. P. M. C. R. 32 (1851) 918 .

Smallest integer having given number of divisors. Mininn, A. P. [1882] (xI) Rec. Mth. (Moscou) 11 (1883-84) 632-.

Square number has odd number of divisors. Pujo, T. L. Les Mondes 27 (1872) 653-.

Successive division, remainders. Marchand, $D$. Les Mondes 54 (1881) 437-.

Sum of cubes of divisors of number. Glaisher, J. W. L. Mess. Mth. 21 (1892) 47-.

Sums of divisors. Bouniakowsky, $\bar{V}$. [1848] St Pét. Ac. Sc. Mm. 6 (1850) 259-.

-- Liouville, J. Liouv. J. Mth. 1 (1856) 349-; 2 (1857) 56.

- - - (Liouville). Bouniakowsky, V. St Pét. Ac. Sc. Bll. 15 (1857) 267-.

- - Liouville, J. Liouv. J. Mth. 7 (1862) 375-.

-- -. Sardi, C. G. Mt. 7 (1869) 112-.

- - Catalan, E. C. As. Fr. C. R. 6 (1877) 127-.

- - - Lipschitz, -. C. R. 100 (1885) 845-.

- - Stern, M. A. Acta Mth. 6 (1885) $327-$

- - - Glaisher, J. W. L. Camb. Ph. S. P. 5 (1886) 108-.

-- Hacks, J. Acta Mth. 9 (1887) 177-.

- - of $a, b$, where $a+b=n$, theorem. Besge, V. A. Liouv. J. Mth. 7 (1862) 256.

- - - number and its partitions. Buniakovskij, V. Ja. [1870] St Pet. Ac. Sc. Mm. (Rs.) 18 (*1871) 20 -

- - - , recurring formulæ. Halphen, G. $H$. Par. S. Mth. Bll. 5 (1877) 158-; 6 (1878) 119-, 173-.

$--\frac{1}{-}-$ involving. Glaisher, J.W.L. Ph. Mg. 33 (1892) 54-.

- - even and odd divisors, difference between. Glaisher, J. W. L. [1877] Mess. Mth. 7 (1878) 66-.

- - odd divisors, Glaisher, J. W. L. QJ. Mth. 19 (1883) 216-.
Sums of powers of divisors. Liouville, $J$. Liouv. J. Mth. 3 (1858) 63-.

- - _ - - recurring relations involving. Glaisher, J. W. L. Mess. Mth. 20 (1891) 129-, 177-; 21 (1892) 49-.

$-\div-\ldots$, relations between. Rogel, $F$. Prag Sb. (1897) (Mth.-Nt.) No. 7, 9 pp.

- - - - numbers prime to given number. Liouville, $J$. C. R. 44 (1857) 753-.

- products of quantities depending on divisors of number. Glaisher, J.W. L. Mess. Mth. 15 (1886) 1-.

\section{Continued fractions and indeterminate equations.}

\section{CONTINUED FRACTIONS.}

Euler, L. [1779] St Pét. Ac. Sc. Mm. 4 (1813) $52-$.

Viscovatoff, B. St Pét. Ac. Sc. Mm. 1 (1803-06) 226-.

Bret, - Gergonne A. Mth. 9 (1818-19) 37-:

Gergonne, J. D. Gergonne A. Mth. 9 (1818-19) 261-.

Wafelaer, P. F. Liège A. Ac. (1820-21) 27 pp.

Stern, $\boldsymbol{M}$. A. [1829-31] Crelle J.8 (1832) 192-; Quetelet Cor. Mth. 7 (1832) 36-.

Möbius, A. F. Crelle J. 6 (1830) 215-.

Ampère, A. M. Quetelet Cor. Mth. 9 (1837) 144-.

Stern, M. A. Crelle J. 18 (1838) 69-.

Gerono, G. C. N. A. Mth. 1 (1842) 1-.

Catalan, E. C. N. A. Mth. 4 (1845) 257-, 405-.

Frost, P. Camb. Mth. J. 4 (1845) 237-.

Schultén, N. G. af (fil.) [1845] Helsingf. Acta 2 (1847) 861-.

Cerquero, J. S. Cadiz Period. M. Ci. 1 (1848) 1-.

Terquem, $O$. N. A. Mth. 8 (1849) 48 -.

Catalan, E. C. N. A. Mth. 8 (1849) 154-, 294.

Vincent, A. J. H. N. A. Mth. 8 (1849) $292-$.

Eisenstein, G. N. A. Mth. 8 (1849) 341-.

Bourgoin, L. Liouv. J. Mth. 15 (1850) 71-.

Schlömilch, O. Grunert Arch. 18 (1852) 416-.

Gy̋̈ry, S. (xIr) Mag. Ak. Éts. (1854) 220 -

Tchebicheff, P. [1855] Liouv. J. Mth. 3 (1858) 289-.

Heine, H. E. Crelle J. 53 (1857) 284-.

König, J. F. Grunert Arch. 33 (1859) 369-.

Heine, E. Berl. Mb. (1866) 436-.

Laurent, $H$. N. A. Mth. 5 (1866) 540 -

Thiele, $T . N$. (xIr) Ts. Mth. 5 (1869) 144-; 6 (1870) 145-.

Eugenio, V. G. Mt. 9 (1871) 358 .

Gïnther, S. Arch. Mth. Ps. 55 (1873) 392-.

Christoffel, E. B. [1874] A. Mt. 6 (1875) 148-.

Smith, H.J.S. [1875] Mess. Mth. 6 (1877) 1-.

Glaisher, J. W. L. [1877] Mess. Mth. 7 (1878) 67-.

Gegenbauer, L. [1879] Wien Ak. Sb. 80 (1880) (Ab. 2) 763-.

Hoffmann, K. E. [1882] Arch. Mth. Ps. 69 (1883) 205-.

Bruno, G. Tor. Ac. Se. At. 21 (1885) 273-.

Hermite, -. Bll. Sc. Mth. 9 (1885) 11-。

Cesáro, E. N. A. Mth. 6 (1887) 29-. 


\section{Continued fractions}

Koppe, -. Mth. A. 29 (1887) 187-.

Veltmann, $W$. Z. Mth. Ps. 32 (1887) 193-.

Gambioli, D. [1889] Bologna Rd. (1889-90) 33-.

Hurwitz, A. Acta Mth. 12 (1889) 367-.

Pincherle, S. Rm. R. Ac. Linc. Rd. 5 (1889) (Sem. 1) 640-; 7 (1891) (Sem. 1) 604-.

Gegenbauer, L. Wien Ak. Sb. 98 (1890) (Ab. 2a) 673- ; 100 (1891) (Ab. 2a) 635-.

Amicis, E. de. G. Mt. 30 (1892) 217-

Markoff, A. [1894] St Pét. Ac. Sc. Bll. 2 (1895) 9-.

Stieltjes, T. J. Toul. Fac. Sc. A. 8 (1894) J, 122 pp. ; 9 (1895) A, 47 pp.

Klein, F. [1895] D. Mth. Vr. Jbr. 4 (1897) 153-.

Koch, H. von. Stockh. Öfv. (1895) 101-.

Lehmer, D. N. A. Mth. 11 (1896-97) 64.

Saalschütz, - . Königsb. Schr. 38 (1897) [37]-.

Candioti, M. R. Arg. S. Ci. A. 46 (1898) 149-.

Muirhead, R. F. Edinb. Mth. S. P. 17 (1899) 39-.

Lewicki, W. Wiad. Mt. 4 (1900) 52-; Fschr. Mth. (1900) 215.

Padé, -. Nt. 62 (1900) 419.

Steggall, -. Mth. Gz. 1 (1900) 39.

Macaulay, F. S. Mth. Gz. 1 (1900) 39-.

algebraic. Borchardt, C. W. N. A. Mth. 13 (1854) 157.

- Tchébicheff, P. de. Liouv. J. Mth. 10 (1865) 353-.

-. Pincherle, S. Par. Ec. Norm. A. 6 (1889) $145-$.

-, application. Pincherle, S. C. R. 108 (1889) 888-.

-, convergence. Halphen, G. H. C. R. 100 (1885) 1451-; 106 (1888) 1326-.

-, -. Bortolotti, E. Rm. R. Ac. Linc. Rd. 8 (1899) (Sem. 1) 28-.

-, generalisation. Humbert, G. Par. S. Mth. Bll. 8 (1880) 191-; 9 (1881) 24 -.

-, - Pincherle, $S$. Bologna Ac. Sc. Mm. 10 (1889) 513-; 4 (1894) 297-; A. Mt. 19 (1891-92) 75-.

一, 一. Hermite, C. A. Mt. 21 (1893) $289-$.

-

generalised. Cordone, G. Palermo Cir. Mt. Rd. 12 (1898) 240-.

algorithm, Euler's, development. Klug, $L$. [1881] Arćh. Mth. Ps. 67 (1882) 337-.

-, extension. Pincherle, S. Mil. I. Lomb. Rd. 22 (1889) 555-.

-, fundamental rule. Sylvester, J. J. $\mathrm{Ph}$. Mg. 6 (1853) 297-。

and anakolouthic sums. Sylvester, J.J. $\mathrm{Ph}$. Mg. 8 (1854) 187-

for analytic functions. Slešinskij, I. N. Rs. S. Nt. Mm. (Mth.) 7 (1886) 33-; Fschr. Mth. (1886) 160-.

application. Stern, M. A. Crelle J. 10 (1833) $1-, 154-, 241-, 364-; 11$ (1834) 33-, 142-, 277-, 311-

-. Lieblein, J. Z. Mth. Ps. 13 (1868) 63-.

- Stieltjes, -. C. R. 118 (1894) 1315-,
Continued fractions 2815

application to change wheels. Glörfeld, A. Cztg. Opt. 19 (1898) 31-.

- - equations. Oettinger, L. [1851] Crelle J. 49 (1855) 66-, 95-.

applications. Studnička, $F$. J. Časopis 1

("1872) 32-; Fschr. Mth. (" 1872) 82.

-. Seiliger, D. N. Kazan S. Ps.-Mth. Bll. 2 (1893) (Prot.) 54-.

—, new. Markoff, A. Mth. A. 47 (1896) 579-. ascending. Günther, - . D. Nf. Tbl. ( $\left.{ }^{*} 1875\right)$ 207-; Z. Mth. Ps. 21 (1876) 178-.

-. Čzuber (or Čuber), E. Arch. Mth. Ps. 60 (1877) 265-.

- Gerevich, E. Orv.-Termt. Ets. (Termt. Szak) (1889) 107-, 187-.

-, application. Gerevich, E. Orv.-Termt. Éts. (Termt. Szak) (1890) 155-.

for binomial powers (Lagrange). Euler, $L$.

[1780] St Pét. Ac. Sc. Mm. 6 (1818) 3-. calculation of $E \frac{2 p}{q}+E \frac{4 q}{p}+\ldots+E \frac{(p-1)}{p} q$ by.

Tatarinov, I. Rec. Mth. (Moscou) 14 (1890) 668-.

Čebyšev's extension. Hermite, C. [1879] Crelle J. Mth. 88 (1880) 10-.

and certain series. Catalan, E. C. [1883]

Brux. Ac. Mm. 45 (1884) (No. 6) 82 pp.

class. Osengu, G. Tortolini A. 2 (1851) 317-. - of functions. Humbert, G. Par. S. Mth. Bll. 8 (1880) 182-.

combined fractions, finite and purely periodic, value. Sylvester, J. J. C. R. 108 (1889) 1195-.

for complex quadratic surds. Mathews, $G, B$. L. Mth. S. P. 20 (1889) 237-.

conjugate recurrence. Walton, W. QJ. Mth. 4 (1861) 331-.

with constant quotients. Studnička, F. J. Časopis 17 (1888) 200-; Fschr. Mth. (1888) 200.

for continued products. Glaisher, J. W. L. (x) L. Mth. S. P. 5 (1873-74) 78-.

convergence. Schultén, N. G. af (fil.) [1846] Helsingf. Acta 3 (1852) 397-.

Malmsten, C. J. Stockh. Ak. Hndl. (1848) 91-; Camb. and Dubl. Mth. J. 5 (1850) 282-.

Stern, M. A. Crelle J. 37 (1848) 255-; Gött. Nr. (1863) 136-

-. Falk, M. Ts. Mt. Fys. 3 (1870) 197-.

-. Oppermann, L. H. F. (xII) Ts. Mth. 1 (1883) 163-; Fschr. Mth. (1884) 167.

Markov, A. A. Kharkov Mth. S. Com. (1885) 29-; Fschr. Mth. (1885) 171. Scheibner, $W$. Leip. Mth. Ps. B. 40 (1888) 7-.

Slešinskij, I. N. Rs. S. Nt. Mm. (Mth.) 8 (1888) 97-; 10 (1889) 201-; Rec. Mth. (Moscou) 14 (1890) 337-, 436-; Fschr. Mth. (1888) 200 ; (1889) 186 ; (1890) 220 .

-. Markoff, A. Acta Mth. 19 (1894) 93-.

-. Saalschütz, -. Königsb. Schr. 40 (1899) [8]-.

. Pringsheim, A. Münch. Ak. Sb. 29 (1900) 261-

convergents. Guilmin, A. N. A. Mth. 4 (1845) 205-, 311-; 5 (1846) 56-. 
2815 Continued fractions

convergents. Bartholomäi, F. Grunert Arch. 18 (1852) 328 .

- Heine, H. E'. Crelle J. 57 (1860) 231-.

-. Christoffel, E. B. Crelle J. 58 (1860) $90-$.

- Muir, T. B. A. Rp. (1876) (Sect.) 27-.

-. Vahlen, K. T. Crelle J. Mth. 115 (1895) 221-.

-, abnormal, of a function, distribution. Padé, H. C. R. 130 (1900) 102-.

-, differences. Dormoy, H. N. A. Mth. 5 (1846) 132-.

-, errors of principal and subsidiary. Hargreaves, $R$. Mess. Mth. 27 (1898) 152-。

-, generalisation. Barbier, E. C. R. 98 (1884) 1531-.

, law of formation. Minding, E. F.A. St Pét. Ac. Sc. Bll. 13 (1869) 524-

- - - - Euler's. Schlegel, S. F. V. Z. Mth. Ps. 22 (1877) 402-

-, resultants. Gegenbauer, L. Amst. Ak. Vs. 5 (1897) 289-.

and convergents, relation. Seidel, L. Münch. Ab. 7 (1855) 559-.

converging, application of determinants. Ramus, C. Kiöb. Ov. (1856) 106-.

- fractions and converging multiples. Heawood, P. J. Mess. Mth. 26 (1897) 79-.

for cube roots. Seeling, $P$. Grunert Arch. 8 (1846) 69-.

- - C. Cordone, G. G. Mt. 32 (1894) 183-.

- definite integral $\int_{-1}^{+1} F(x) d x$. Stieltjes, C. R. 99 (1884) $508^{-1}$.

- — integrals. Stieltjes, - . C'. R. 108 (1889) 1297-

- descending power series. Stieltjes, T. J. Toul. Fac. Sc. A. 3 (1889) H, 17 pp.

- - - Čebyšev, P. [1892] St Pet. Ac. Sc. Mm. (Rs.) 71 (1893) (Suppl.) No. 3, 73 pp.; Fschr. Mth. (1892) 192-.

development. Schendel, L. Crelle J. Mth. 80 (1875) 95-.

-, geometrical view. Klein, $F$. Gött. Nr. (1895) 357-.

doubly periodic. $K a h l, E$. Grunert Arch. 19 (1852) 158-.

for $e$. Hurwitz, - Königsb. Schr. 32 (1891) (Sb.) 59 -

- - and $e^{e}$. Sundman, K. F. Helsingf. Öfv. 38 (1896) 57-.

- $e^{F(x)}$. Laguerre, $E$. Liouv. J. Mth. 6 (1880) 99-.

$e^{\tan ^{-1}\left(\frac{1}{x}\right)}$. Laguerre, E. Par. S. Mth. Bll. 5 (1877) 95-.

Eisenstein's. Muir, T. [1877] Edinb. R. S. T. 28 (1879) 135-

equal. Heilermann, - Schlömilch Z. 5 (1860) 362-.

Euler's; and Wallis's theorem. Bauer, G. [1872] Münch. Ab. 11 (1873) (Ab. 2) 97-. expansion in series by means of. Chebuishev, P. L. [1865] (xII) Rec. Mth. (Moscou) [1] (1866) 291-.

forms of numbers determined by. Roberts, $S$. [1878] L. Mth. S. P. 10 (1878-79) 29-.
Continued fractions 2815

for fraction $1.3 .5 \ldots(2 n-1) / 2.4 .6 \ldots 2 n$. Stieltjes, - . QJ. Mth. 25 (1891) 198-.

- function $(1+x)^{n}$. Padé, H. C. R. 129 (1899) 753-, 875-.

Gauss's theorems, proof. Clausen, $T$. Crelle J. 3 (1828) 311.

generalisation. Lejeune-Dirichlet, G. Berl. B. (1842) 93-.

-. Jacobi, C. G. J. Crelle J. 69 (1868) 29-.

- (Jacobi). Bachmann, P. [1872] Crelle J. 75 (1873) 25-.

-. Poincaré, H. C. R. 99 (1884) 1014-.

- Minkowski, H. Par. Éc. Norm. A. 13 (1896) 41-.

geometric application. Léger, $\hat{E}$. Quetelet Cor. Mth. 9 (1837) 483-.

- interpretation. Lieblein, J. Z. Mth. Ps. 12 (1867) 185-.

indefinite, transformation to vulgar fractions. Pezzi, F. Mod. S. It. Mm. 11 (1804) 410-.

infinite. Schultén, N. G. af (fil.) [1844-47] Helsingf. Acta 2 (1847) 661-; 3 (1852) 427-.

-. Spitzer, S. Grunert Arch. 33 (1859) 418-.

-. Studnicka, F. J. Prag Sb. (1892) (Mth. Nt.) 254-; Časopis 22 (1893) 15-.

-, convergence. Pringsheim, A. Münch. Ak. Sb. 28 (1899) 295-; 30 (1901) 233.

-, evaluation. Spitzer, S. Grunert Arch. 30 (1858) 331-; 33 (1859) 474-.

for infinite series. Buzengeiger, K. H.J. Zach M. Cor. 22 (1810) 513-.

- - - Muir, T. Edinb. R. S. T. 27 (1876) $467-$.

- irrational numbers. Gyldén, H. C. R. 106 (1888) 1584-, 1777-. $-\int_{(1834) 346-\text {. }}^{\infty} e^{-x^{2}} d x$. Jacobi, C. G. J. Crelle J. 12

Jacobi's formula

$$
\frac{d^{n}\left(1-x^{2}\right)^{n+1}}{d x^{n}}=C \sin \left[(n+1) \cos ^{-1} x\right] .
$$

Hermite, C. [1875] Liége S. Sc. Mm. 6 (1877) No. $7,7 \mathrm{pp}$.

law of formation. Günther, S. [1875] Wien Ak. D. $36(1876)(A b .2) 187-$.

for $n$th roots. Seeling, $P$. Arch. Mth. Ps. 46 (1866) 80-.

for $n$th surds. Hoffmann, K. E. Arch. Mth. Ps. 64 (1879) 9-.

with negative quotients. Saalschiitz, L. Crelle J. Mth. 120 (1899) 132-, 242-, 354.

negative, for square roots. Stern, M. A. [1864] Gött. Ab. 12 (1866) 48 pp.

numerical values. Glaisher, J. W. L. [1874] QJ. Mth. 13 (1875) 255-.

periodic. Kramp, C. Gergonne A. Mth. 1 (1810-11) 261-, 319-.

-. Galois, E. Gergonne A. Mth. 19 (182829) 294-.

- Lebesque, V. A. Férussac Bll. Sc. Mth. 15 (1831) 155-.

-. Ramus, C. Kiöb. Ov. (1836-37) 1-; Kjøb. Dn. Vd. Selsk. Afh. 7 (1838) 197-; Crelle J. 20 (1840) 13-.

一. Broager, P. D. Kjøb. Dn. Vd. Selsk. Afh. 8 (1841) 145-. 


\section{Continued fractions}

periodic. Bianchi, G. [1844] Mod. Mm. S. It. 23 (1846) 219-.

-. Rodrigues, O. N. A. Mth. 4 (1845) 109-.

- Catalan, E. C. N. A. Mth. 4 (1845) 126-.

-. Galois, E. Liouv. J. Mth. 11 (1846) 385 -

-. Siebeck, H. Crelle J. 33 (1846) 68-.

-. Le François, (Prof.) -. Brux. Ac. Bll. 16 (1849) 338-.

-. Stern, M. A. [1854] Crelle J. 53 (1857) 1-.

- Simon, O. E. Grunert Arch. 33 (1859) 448 -

-. Günther, S. Z. Mth. Ps. 22 (1877) 31-.

- Le Paige, C. M. M. H. H. Brux. Ac. Bll. 43 (1877) 337-.

-. Appell, P. Arch. Mth. Ps. 62 (1878) 183-.

-. Jonquières, E. de. C. R. 96 (1883) 568-, 1297-.

-. Muir, T. Edinb. R. S. P. 12 (1884) 389-, $578-$.

- Selivanov, D. T. Rec. Mth. (Moscou) 15 (1891) 635-; Fschr. Mth. (1891) 221-.

-. Landsberg, G. Crelle J. Mth. 109 (1892) 231-.

- (Landsberg). Netto, E. Crelle J. Mth. 110 (1892) 349-.

-, algebraic. Bortolotti, E. Palermo Cir. Mt. Rd. 9 (1895) 136-.

-, - generalisation. Bortolotti, E. Palermo Cir. Mt. Rd. 6 (1892) 1-.

-, arithmetical theorem. Sylvester, J. J. Mess. Mth. 19 (1890) 63-

-, calculation. Anon. (vi 552) Gergonne A. Mth. 14 (1823-24) 337-.

-, closed form. Hoffmann, K. E. Arch. Mth. Ps. 62 (1878) 310-.

-, composition of periods. Jonquières, E. de. C. R. 96 (1883) 694-, 832-.

-, convergence. Thiele, T. N. (xII) Ts. Mth. 3 (1879) 70-.

-, -. Stolz, -. Innsb. Nt. Md. B. 15

(1886) xxi-; 17 (1887) xviii-.

-, -. Pringsheim, A. [1900] Münch. Ak. Sb. 30 (1901) 463-.

-, convergents. Oettinger, L. Arch. Mth. Ps. 43 (1865) 301-

-, - (Oettinger). Strehlke, F. Arch. Mth. Ps. 48 (1868) 1-.

-, -. Ocagne, M. d'. C. R. 108 (1889) $499-$

-, equivalent to square-roots of integers. Ely, G. S. A. Mth. 1 (1884-85) 72 .

-, given number of quotients. Seeling, $P$. Arch. Mth. Ps. 49 (1869) 4-

-, identities between convergents. Jonquières, E. de. C. R. 96 (1883) 1351-, 1420-, 1490-, 1571-, 1721-.

- and indeterminate analysis. Tédénat, Gard Not. Tr. Ac. (1811) 254-.

-, nth convergent. Strehlke, $F$. Arch. Mth. Ps. 42 (1864) 343-.

-, with one term. Clausen, T. Crelle J. 3 (1828) 87-; N. A. Mth. 5 (1846) 203-.

-, - symmetric period. Musso, G. G. Mt. 33 (1895) 1-。
Continued fractions 2815

for $\pi$. Sylvester, J.J. . Ph. Mg. 37 (1869) 373-. - - Jung, $V$. Casopis $9\left({ }^{*} 1880\right)$ 157-; Fschr. Mth. (" 1880$)$ 149-.

- - Sylvester's and other forms. Muir, $T$. Ph. Mg. 47 (1874) 331-.

primes of form $4 \lambda+1$, as sum of 2 squares. Smith, H. J. S. Crelle J. 50 (1855) 91-.

probability. of large quotients. Gyldên, $H$. Stockh. Öfv. (1888) 349-.

problem. Clausen, T. St Pét. Ac. Sc. Bll. 9 (1851) 353-.

for quadratic surds. Anon. (vi 551) Gergonne A. Mth. 14 (1823-24) 324-

- -. Schultén, N. G. af (fil.) [1846-47]

Helsingf. Acta 2 (1847) 1023-; 3 (1852) 435-.

- Schaar, M. Brux. Ac. Bll. 19 (1852) 16-

- - Hoffmann, K. E. Arch. Mth. Ps. 64 (1879) 1-.

_- Hansted, B. G. Teix. J. Sc. 2 (1880) 154 .

- -. Sylvester, J. J. C. R. 108 (1889) 1037-, 1084-.

__- Dolbnia, J. N. A. Mth. 10 (1891) 134-.

- - - Boutin, A. Mathesis 17 (1897) 8-.

- -. Crelier, -. C. R. 128 (1899) 229 .

-, convergence. Falk, M. Ts. Mt. Fys. 4 (1871) 249-.

- - , periodicity. Schultén, N. G. af (fil.) [1846] Helsingf. Acta 2 (1847) 1063-.

__ - _. Charves, _. Bll. Sc. Mth. As. 1 (1877) 41-

quotients in arithmetical progression. Hurwitz, A. Ziir. Vjschr. $4 \mathrm{I}$ (1896) (Festschr., Th. 2) 34-

for rational fractional power. Matthiessen, $L$. Z. Mth. Ps, 10 (1865) 315-.

reduction to series. De Morgan, A. Ph. Mg. 24 (1844) 15-.

- - - Heilermann, -. Crelle J. 46 (1853) 88 .

regular. Gegenbauer, $L$. Wien Ak. D. 58 (1891) 177-; Mh. Mth. Ps. 6 (1895) 209-

relation to astronomical cycles. Brunel, - . Bordeaux S. Sc. Mm. 5 (1890) xxv-.

_ _ - Tannery, P. Bordeaux S. Sc. Mm. 5 (1890) xxix-.

representation as definite integrals. Spitzer, S. Grunert Arch. 30 (1858) 81-.

- of numbers. Schultén, N. G. af (fil.) [1845] Helsingf. Acta 2 (1847) 1009-.

for roots. Collins, E. (vII) St. Pét. Ac. Sc. Bll. 7 (1840) 357-.

- - of equations. Montessus, R. de. Brux. S. Sc. A. 21 (1897) (Pt. 2) 71-

- - numbers. C. Camb. Mth. J. 3 (1843) 120 .

roots and their convergence. Doppler, $C$. Wien Jb. Pol. I. 17 (1832) 175-

for series. Kausler, C. F. [1802] St Pét. Ac. Sc. Mm. 1 (1809) 156-.

- Viscovatoff, B. [1802] St Pet. Ac. Sc. N. Acta 15 (1806) 181-.

-. Schubert, F. T. [1815] St Pét. Ao.

Se. Mm. 7 (1820) 139- 


\section{Continued fractions}

for series. Eytelwein, J. A. Berl. Ab. (182021) $15-$

- Le Barbier, -. Gergonne A. Mth. 21 (1830-31) 262-.

- Heine, H. E. Crelle J. 32 (1846) 205-.

-. Heilermann, -. Crelle J. 33 (1846)

$174-$.

- - Hankel, H. Leip. B. 14 (1862) 17-

- - Bromian, K. E. Ts. Mt. Fys. 4 (1871) 139 .

- Mollame, V. [1882] Catania Ac. Gioen. At. 17 (1883) 129-.

- - Gambioli, D. Bologna Rd. (1890-91) $43-$

- - Ćebyšev, P. St. Pet. Ac. Sc. Mm. (Rs.) 64 (1891) (Suppl.) No. 7, $67 \mathrm{pp}$.

and similar functions. Meyer, $F$. Königsb. Schr. 38 (1897) [57]-

simple, convergence. Padé, H. C. R. 112 (1891) 988-

solution of continued fraction equation. Mol-

lame, $V$. Rv. Mt. 2 (1892) 212-.

for solution of linear differential equation. Laguerre, E. [1878-79] C. R. 87 (1878) 923-; Par. S. Mth. Bll. 8 (1880) 21-.

- square roots. Ivory, J. [1800] Edinb. R. S. T. 5 (1805) 20 -

- - Booth, J. Camb. Mth. J. 2 (1841) 226-.

- - De Morgan, A. (vr Adds.) Camb. Mth. J. 2 (1841) 239-.

- - Schlömilch, O. Grunert Arch. 6 (1845) 147-.

- - - (Schlömilch). Müller, R. Grunert Arch. 6 (1845) 151-.

- - - Arndt, F. Crelle J. 31 (1846) 343-; Grunert Arch. 12 (1849) 211-.

- - -. Serret, J. A. Liouv. J. Mth. 12 (1847) 518-.

- - Badon-Ghijben, J. [1864] Amst. Vs. Ak. 17 (1865) (Ntk.) 121-。

- - - Weyr, Emil. Prag Sb. (1869) 18 -

- -. Schlömileh, O. Z. Mth. Ps. 17 (1872) 70-

- - - Weyr, Ed. Prag Sb. (1877) 65-.

- - Hermes, J. [1879] Arch. Mth. Ps. 65 (1880) 438-

- - -. Günther, S. [1882] Bordeaux S.

Sc. Mm. 5 (1883) 91-.

- -. Muir, T. Mess. Mth. 14 (1885) 115-.

- - Thaarup, F. N. Ts. Mth. 11 (A) (1900) 33-

- - , and factors. Kausler, C. F. [1807] St Pét. Ac. Sc. Mm. 2 (1810) 95-.

- - periodicity. Crelier, L. Arch. Sc. Ps. Nt. 6 (1898) 366-.

"step" fractions. Gohierre de Longchamps, -. G. Mt. 15 (1877) 299-.

successive approximations. De Morgan, $A$. Camb. Mth. J. 4 (1845) 97-.

summation. Stern, M. A. Crelle J. 8 (1832) $42-$.

- of series by. Horner, W. G. Thomson A. Ph. 11 (1826) 416-; 12 (1826) 48-.

symmetric, convergents. Musso, $G$. N. A. Mth. 14 (1895) 70-.

in "Theoria Motus," pp. 97-98. Ramus, $C$. Mth. Ts. 1 (1859) 129-.

\section{Indeterminate equations 2815}

and theory of numbers. Catalan, E. Brux.

Ac. Mm. 52 (1893-94) No. 1, 28 pp.

Wallis's. Euler, L. [1780] St Pét. Ac. Sc. Mm. 5 (1815) 24-.

for $\left(\frac{x+1}{x-1}\right)^{\omega}$. Laguerre, E. [1879] Par. S.

Mth. Bll. 8 (1880) 36-

- $(x-a)^{\alpha}(x-b)^{\beta}$. Fuchs, L. Acta Mth. 4 (1884) 89-.

- $(x-a)^{\alpha}(x-b)^{\beta}$. Hermite, C. Acta Mth. 4 (1884) 91-.

$\frac{m}{m+1}-\frac{m^{\prime}}{m^{\prime}+1}-\frac{m^{\prime \prime}}{m^{\prime \prime}+1}$

af (fil.) [1844]

1. Helsingf. Acta 2 (1847) 629-.

$\frac{1}{1+} \frac{1}{2+} \frac{1}{3+}$ etc. Amoretti, F. N. A. Mth. 14 (1855) 40-.

$\frac{1}{1+} \frac{1}{2+} \frac{1}{3+}$ etc. Spitzer, $S$. Grunert Arch. 25 (1855) 137-.

$\frac{a}{z+} \frac{a}{z+1+} \frac{a}{z+2+}--$ - (Legendre). Cata-

lan, E. C. Liége S. Sc. Mm. 13 (1886)

205-.

\section{INDETERMINATE EQUATIONS.}

Bouniakowsky, V. [1829] St Pét. Ac. Sc. Mm. 1 (1831) 139-.

Poselger, F. T. Berl. Ab. (1832) 1-.

Cauchy, A. L. C. R. 12 (1841) 698-, 813-.

Bouniakowsky, V. [1847] St Pét. Ac. Sc. Bll. 6 (1848) 196-.

Lebesgue, $V$. A. N. A. Mth. 8 (1849) 81-, 347-.

Wheeler, A. D. Camb. (M.) Mth. M. 2 (1860) 21-, 55-, 193-, 398-.

Šimerky [Šimerka], V. Prag Ab. 12 (1863) 193-.

Lebesgue, $V$. A. N. A. Mth. 8 (1869) 452-.

Catalan, E. Brux. Ac. Bll. 9 (1885) 531-.

Weill, -. N. A. Mth. 4 (1885) 189-.

Jonquieres, E. de. G. Mt. 24 (1886) 1-.

Cordone, G. G. Mt. 33 (1895) 106-, $218-$.

Maillet, E. Toul. Ac. Sc. Mm. 7 (1895) 179.

binary form, least integral value. Paoli, $P$. [1801] Mod. S. It. Mm. 9 (1802) 85-.

class. Palmstrdm, A. Christiania Skr. (Mth.Nt. Kl.) (1899) No. 7, $10 \mathrm{pp}$.

Diophantine analysis, advance. Euler, $L$. St Pét. Ac. Sc. Mm. 11 (1830) 1-.

- - elliptic and Abelian integrals, use in. Jacobi, C. G. J. Crelle J. 13 (1835) 353-.

- - geometric problem solved by. Euler, $L$. [1782] St Pét. Ac. Sc. Mm. 7 (1820) 3-.

- by integral calculus. Bouniakowsky, V. [1852] St Pét. Ac. Sc. Bll. 11 (1853) 65-

- - problems. Fuss, N. [1820] St Pét. Ac. Sc. Mm. 9 (1824) 151-,

- equation. Davis, R. F. Edinb. Mth. S. P. 3 (1895) 179-

- equations. Hilbert, D. Gött. Nr. (1897) 48-.

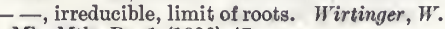
Mh. Mth. Ps..1 (1890) 47-. 


\section{Indeterminate equations}

Diophantine equations, solutions. Catalan, E. C. Liége S. Sc. Mm. 15 (1888) No. 1, 73-, 75-, 120-, 211-, 259.

- problem before Diophantus. Biot, J. B. C. R. 28 (1849) 576-

- - Fermat's solution. Roberts, S. As. Fr. C. R. (1886) (Pt. 2) 43-.

- -, solution. Kausler, C. F. [1806] St Pét. Ac. Sc. Mm. 1 (1809) 271-.

- -, 一. Strong, T. Silliman J. 1 (1818) 426-

- - - Wheeler, A. D. Silliman J. 20 (1831) 295-.

- - - - Bouniakowsky, V. [1841] St Pét. Ac. Sc. Mm. 5 (1844) 1-.

- - - Boije af Gennäs, C. O. N. A. Mth. 19 (1880) 278-.

- problems. Euler, L. St Pét. Ac. Sc. Mm. 9 (1824) 3- ; 11 (1830) 46-.

- -, solution. Euler, L. [1778] St Pét.

Ac. Sc. Mm. 4 (1813) 3-.

- - solutions of two. Strong, T. Silliman J. 31 (1837) 156-

- speculations. Lenhardt, $W$. Mth. Misc. 1 (1838) 263-, 330-; 2 (1839) 127-.

examples. Steen, A. (xII) Ts. Mth. 1 (1865) 20 -

impossibility of some. Lebesgue, $V . A$. N.A. Mth. 2 (1863) 68-.

mechanical solution. Tarry, H. As. Fr. C. R. (1897) (Pt. 1) 177.

Paoli's formula for number of solutions. Lucas, É. Mathesis 10 (1890) 129-.

- - - - (Lucas). Catalan, E. Mathesis 10 (1890) 197-.

power of polynomial, law of coefficients. Teixeira, J. P. G. Teix. J. Sc. 13 (1897) 65-; Fschr. Mth. (1897) 167.

problems, class, with application to decomposition of fractions. Gergonne, J.D. Gergonne A. Mth. 10 (1819-20) 271-.

—, solution. Leslie, J. [1788] Edinb. R. S. T. 2 (1790) 193-.

- - Kausler, C. F. [1800] St Pet. Ac. Sc. N. Acta 15 (1806) 116-.

- - - Perkins, G. R. Des Moines Anal. 1 (1874) 40-, 101-, 151-.

-, two. Bouniakowsky, V. [1864] St Pét. Ac. Sc. Bll. 8 (1865) 163-.

quadratic duplicate equalities. Collins, $M$. B. A. Rp. (1855) (Pt. 2) 2-.

series derived from. Oliveira Ramos, J. C. d', \& Faria, C. J. de. G. Teix. J. Sc. 7 (1886) 41-; Fschr. Mth. (1885) 129-.

solution. Libri, G. Crelle J. 9 (1832) 277 -

- Pepin, T. Rm. N. Linc. At. 38 (1885) 20 .

- Thue, A. Thrond. Skr. (1896) No. 7, 14 pp.

- of problem of Behâ-Eddin's. Lucas, $\vec{E}$. N. A. Mth. 15 (1876) 359-.

- by series. Libri, G. Crelle J. 9 (1832) 313-.

system. Lebesgue, V.A. N. A. Mth. $9(1850)$ 46-.

tetrahedron problem. Chabanel, C. N. A. Mth. 13 (1874) 289-.

theorem. Liouville, J. C. R. 10 (1840) 381-.

\section{Indeterminate equations 2815}

theorem of Liouville. Gegenbauer, $L$. Wien Ak. Sb. 95 (1887) (Ab. 2) 606-.

theorems. Pepin, T. C. R. 78 (1874) 144-; 88 (1879) 1255-.

and transformation of plane figures. Ruffini, F. $P$. Bologna Ac. Sc. Mm. 9 (1878) 199-.

triangle with bisectors of sides rational. Euler, L. [1779] St Pét. Ac. Sc. Mm. 2 (1810) 10 .

- - distances of centre of gravity from vertices rational. Euler, L. [1778] St Pet. Ac. Sc. N. Acta 12 (1801) 101-.

2 or 3 variables. Maillet, E. Liouv. J. Mth. 6 (1900) 261-.

3 variables, solution by slide-rule. San Roberto, P. di. Tor. At. Ac. Sc. 2 (1866-67) 454-

several variables. Bianchi, G. Mod. Mm. S. It. 24 (1850) 265-.

- - number of solutions. Berger, A. Stockh. Öfv. (1886) 355-; Fschr. Mth. (1886) 149-.

virgins problem. Sylvester, J. J. Ph. Mg. 16 (1858) 371-.

1 st order.

Pilatte, - Gergonne A. Mth. 2 (1811-12) 230-.

Gergonne, J.D. Gergonne A. Mth. 3 (181213) $147-$.

Libri, G. Gergonne A. Mth. 16 (1825-26) 297-.

Crelle, A. L. Berl. Ab. (1836) 1-.

Brassinne, E. Toul. Mm. Ac. 4 (1837) 189-.

Crelle, A. L. Berl. Ab. (1840) 1-.

Chevillard, A. J. N. A. Mth. 2 (1843) 471-.

Grunert, J. A. Grunert Arch. 3 (1843) 203-.

Catalan; E. C. N. A. Mth. 3 (1844) 97-.

Midy, -. N. A. Mth. 4 (1845) 146-.

Grunert, J. A. Grunert Arch. 7 (1846) 162-.

Jaufroid, B. N. A. Mth. 11 (1852) 158-.

Reuschle, C. (jun.) Z. Mth. Ps. 19 (1874) 272.

Méray, C. C. R. 94 (1882) 1167-; Par. Éc. Norm. A. 12 (1883) 89-.

Catalan, E. Mathesis 10 (1890) 220-, 241-, 275-.

Brocard, H. Mntp. Ac. Mm. 11 (1892) 139-.

Gram, J.P. N. Ts. Mth. 3 (B) (1892) 57-, 73-; Fschr. Mth. (1892) 174

Giudice, $F$. G. Mt. 36 (1898) 225-.

approximate solution. Kronecker, L. Berl. Ak. Sb. (1884) 1179-, 1271-.

solution. Pezzi, F. Mod. S. It. Mm. 11 (1804) 410-.

- Casterman, L. Liège A. Ac. (1819-20) 36 pp.

-. Binet, J. P. M. [1827] Par. Éc. Pol. J. 13 (1831) $\left(20^{\circ}\right.$ cah.) 289-.

-Paoli, G. de. Opusc. Mt. Fis. 1 (1832) 262-, 327-.

- Binet, J. P. M. Liouv. J. Mth. 6 (1841) 449-.

- Heger, $I$. Wien SB. 21 (1856) 550-; Wien D. 14 (1858) 1-.

-. Slavik, J. Časopis 14 (1885) 137-; Fschr. Mth. (1885) 122. 


\section{Indeterminate equations}

solution. Ayza, R. Fschr. Mth. (1897) 168. -, forms. Weihrauch, K. Z. Mth. Ps. 19 (1874) 53-.

systems. Smith, H. J. S. B. A. Rp. (1860) (pt. 2) 6; Phil. Trans. (1861) 293-.

-. Ovidio, E. d'. 'Tor. Ac. Sc. At. 12 (1876) 334 .

2 or more variables, solution by continued fractions. Pleskot, A. Časopis 22 (1893) 71-; Fschr. Mth. (1893-94) 278.

4 variables, solutions, number. Weihrauch, $K$.

Z. Mth. Ps. 22 (1877) 234-

$n$ variables. Sylvester, J.J. Ph. Mg. 16 (1858) $369-$.

\section{2nd order.}

Minding, E. F. A. Crelle J. 7 (1831) 140-

Arndt, F. Grunert Arch. 12 (1849) 211-.

Göpel, A. Crelle J. 45 (1853) 1-.

Realis, S. N. A. Mth. 3 (1884) 305-, 448.

Desboves, A. N. A. Mth. 5 (1886) 226-

Marcolongo, $R$. G. Mt. 25 (1887) 161-; 26 (1888) 65-.

Jonquières, 一de. C. R. 126 (1898) 863-, 990, 991.

Euler's formula. Chasles, M. Liouv. J. Mth. 2 (1837) 37-.

and fourth. Pepin, T. Rm. N. Linc. At. 32 (1879) 79-

Lagrange's and Gauss's methods. Jonquières, - de. C. R. 127 (1898) 694-.

solution. Kunerth, A. Wien Ak. Sb. 75 (1877) (Ab. 2) 7-

- by continued fraction, criteria. Kunerth, $A$. [1880] Wien. Ak. Sb. 82 (1881) (Ab. 2) 342 .

- in rational numbers. Kunerth, A. [1878] Wien Ak. Sb. 78 (1879) (Ab. 2) 327-.

\section{$3 r d$ order.}

Realis, S. N. A. Mth. 17 (1878) 454-.

Jonquières, E. de. Rm. N. Linc. At. 37 (1884) 183-.

or higher. Desboves, A. C. R. 87 (1878) 159-, 321-.

impossibility. Lucas, $\dot{E}$. Par. S. Mth. Bll. 8 (1880) 173-.

and problem of Sylvester's. Lucas, É. N. A. Mth. 17 (1878) 507-; Am. J. Mth. 2 (1879) 178 .

reduction to 2 nd order. Le Besgue, V.A. C.R. 64 (1867) 1267-.

4 th order.

Pepin, T. Rm. N. Linc. At. 31 (1878) 397-; Rm. N. Linc. Mm. 4 (1888) 227-; 9 (1893) (Pt. 1) 247-; 11 (1895) 223-.

with 4 variables, solution. Eisenstein, $G$. Crelle J. 27 (1844) 75-.

\section{5 th order.}

impossibility of some equations. LejeuneDirichlet, $G$. Crelle J. 3 (1828) 354-.

\section{Indeterminate equations 2815}

$a b\left(m a^{2}+n b^{2}\right)=c d\left(m c^{2}+n d^{2}\right)$. Euler, L. [1778] St Pet. Ac. Sc. N. Acta 13 (1802) 45-.

$0=1+b x+c y+d x^{2}+c x y+f y^{2}+g x^{2} y+h x y^{2}$ $+i x^{2} y^{2}$. Euler, L. St Pét. Ac. Sc. Mm. 11 (1830) 58-

$4\left(x^{n}-1\right)=(x-1)\left(Y^{2} \pm n Z^{2}\right)$. Legendre, A. MI. [1830] Par. Mm. Ac. Sc. 11 (1832) 81-.

$4\left(x^{p}-1\right)=(x-1)\left(y^{2} \pm p z^{2}\right)$. Germain, (Mlle.) $S$. Crelle J. 7 (1831) 201-.

$z^{2 n}-y^{2 n}=2 x^{n}$. Liouville, J. Liouv. J. Mth. 5 (1840) 360 .

$x^{m}-y^{n}=1$. Catalan, E. C. Crelle J. 27 (1844) 192.

$y=\cos \pi x$, solution in rational numbers. Schultén, N. G. af (fil.) [1844] Helsingf. Acta 2 (1847) 617-.

$a x+b y+c z=0$. Cauchy, A. L. Grunert Arch. 7 (1846) 305-

$A x^{3}+B y^{3}+C z^{3}=D x y z . \quad$ Sylvester, J. J. $\quad \mathrm{Ph}$. Mg. 31 (1847) 189-, 293-.

$a x+b y=c$. Dieu, T. N. A. Mth. 9 (1850) 67-. $a x-(a+b) y=m$. Grebe, E. W. Grunert Arch. 14 (1850) 333-.

$a_{1} x_{2}=a_{2} x_{1}+1$, smallest integers satisfying. Crelle, A. L. Berl. B. (1850) 141-; Crelle J. 42 (1852) 299-.

$x^{m}=y^{2}+1$, impossibility of solution in integers. Lebesgue, $V . A$. N. A. Mth. 9 (1850) 178 .

$a^{x}-b^{y}=1, a$ and $b$ being primes. Gerono, G. C. N. A. Mth. 16 (1857) 394-

$a x+b y+c z+$, etc. $=n$, number of solutions in positive integers. Hermite, C. QJ. Mth. 1 (1857) 370 -

$a_{1}+2 a_{2}+3 a_{3}+\ldots+n a_{n}=n$. Fergola, $E$. Nap. Rd. 2 (1863) $262-$.

$n s\left[s^{2}+\left(n^{2}-1\right) r^{2}\right]=8 y^{3}$. Le Besgue, V. A. (vIII) A. Mt. 5 (1863) 328.

$-=-$. Genocchi, A. (vII) A.Mt. 5 (1863) 329-. $(x+h)^{n}-x^{n}=z^{n}$, impossibility. Gaudin, C. R. 59 (1864) 1036-.

$A x+B y=S$. Clasen, B. I. Par. Éc. Norm. A. 4 (1867) 347-.

$4\left(x^{p}-1\right)=(x-1)\left(y^{2} \pm p z^{2}\right)$. Staudt, G. K. C. von. Crelle J. 67 (1867) 205-.

$\Sigma a_{r} x_{r}=f . u$. Jacobi, C. G. J. Crelle J. 69 (1868) 1 .

$x^{m}=y^{n}+1$. Gerono, G. C. N. A. Mth. 9 (1870) $469-$; 10 (1871) 204-.

$a x+b y+c z=m$. Gambardella, F. G. Mt. 9 (1871) 262-.

$a x+b y=c$, solution in integers, graphic representation. Polignac, C. (Prince) de. [1877] Par. S. Mth. Bll. 6 (1878) 158-.

$x=u^{2}, x+1=2 v^{2}, 2 x+1=3 w^{2}$. Gerono, C. C. N. A. Mth. 17 (1878) 381-.

$x=4 y^{2}+1, x^{2}=z^{2}+(z+1)^{2}$. Gerono, C. C. N. A. Mth. 17 (1878) 521-.

$A x+B=\phi(C x+D)$. Serdobinnskǐ, V. E. [1878]

(xII) Rec. Mth. (Moscou) 9 (1878-81) (Pt. 1) $557-$.

$\sum_{i=1}^{i=n} \lambda_{i}=r ; \sum_{i=1}^{i=n} i \lambda_{i}=n$. Sadun, E. A. Mt. 15 $(1887-88)^{i=1} 209$.

$b x+1=y^{2}$, posthumous problem of Leibnitz.

Studnička, F. J. Časopis 18 (1889) 97-;

Fschr. Mth. (1889) 30-. 
$a x+b y=c . \quad$ Spelta, C. G. Mt. 33 (1895) 125 -.

$m \tan ^{-1}\left(\frac{1}{x}\right)+n \tan ^{-1}\left(\frac{1}{y}\right)=\kappa \frac{\pi}{2}$. Stprmer, C. Christiania Skr. (Mth.-Nt. Kl.) (1895) No. 11, 21 pp.

$u^{m}+v^{n}=w^{k}$. Ermakov, V. [1898] Rec. Mth. (Moscou) 20 (1899) 293-.

$A I_{1}^{x_{1}} M_{2}^{x_{2}} \ldots B N_{1}^{y_{1}} N_{2}^{y_{2}} \ldots=C$. St Sẗrmer, $C$. C. R. 127 (1898) 752-.

$y^{2}=a x+b . \quad$ Besant, W. H. Mth. Gz. 1 (1900) 130.

\section{Quadratic residues.}

Gauss, C. F. Gött. Cm. 4 (1816-18) 3-. Lebesgue, V. A. Liouv. J. Mth. 5 (1840) 186-. Stern, M. A. Brux. Mm. Cour. 15 (1840-41) $38 \mathrm{pp}$.

Schaar, M. [1849-50] Brux. Ac. Sc. Mm. (1850) xxiv-.

Genocchi, A. Brux. Mm. Cour. 25 (1851-53) $55 \mathrm{pp}$.

Stern, M. A. [1862] Crelle J. 61 (1863) 334 .

Candido, Z. A. G. Teix. J. Sc. 1 (*1878) 171-.

Schering, E. Acta Mth. 1 (1882) 153-.

Nazimov, P. Bll. Se. Mth. 9 (1885) 15-.

Bumiakovskij, V. Ja. [1886] St Pét. Ac. Sc. Mm. (Rs.) 55 (1887) (Suppl.) No. 5, 16 pp. ; Fschr. Mth. (1887) 173.

Lipschitz, R. C. R. 108 (1889) 489-.

Matrot, A. As. Fr. C. R. (1890) (Pt. 2) 82-.

Frolov, M. As. Fr. C. R. (1892) (Pt. 2) 149-.

Reich, K. Arch. Mth. Ps. 11 (1892) 176-, vII-.

Pepin, -. C. R. 128 (1899) 1553-.

Congruence $x^{2} \equiv c\left(\bmod . p^{\lambda}\right)$, resolution. Tonelli, A. Rm. R. Ac. Linc. Rd. 1 (1892) (Sem. 1) 116-; 2 (1893) (Sem. 1) 259-.

Congruent division and quadratic residues. Stern, M. A. [1861] Crelle J. 61 (1863) $66-$

Continued fractions and quadratic forms. $L a$ Vallée-Poussin, C. J. de. Brux. S. Sc. A. 19 (1895) (Pt. 1) 111-.

Density of series of prime numbers. König, $J$. Mth. Nt. B. Ung. 14 (1898) 322.

Eisenstein's geometrical proof. Cayley, A. QJ. Mth. 1 (1857) 186-.

Historical study. Pepin, T. Rm. N. Linc. Mm. 16 (1899) 229-.

Prime modulus, square roots of unity for a. Tanner, H. W. L. Mess. Mth. 21 (1892) 139 .

Problems on numbers (Fermat). Pepin, $T$. Rm. N. Linc. Mm. 8 (1892) 85-.

Quadratic and cubic residues. Meyer, $G$. Arch. Mth. Ps. 63 (1879) 1-.

- reciprocity, formula of Gauss, extension. Le Besgue, V. A. C. R. 59 (1864) 1067-.

- residue-character. Gegenbauer, L. Wien Ak. Sb. 100 (1891) (Ab. 2a) 1072-.

-- - determination. Schering, $\dot{E}$. Gött. Ab. 24 (1879) (Mth.) $47 \mathrm{pp}$.
Quadratic residue-character, determination by continued fractions. Zeller, C. Gött. Nr. (1879) 197-.

- _-, Gauss's criterion. Schering, E. Berl. Ak. Mb. (1876) 330-.

- - _ - Kronecker, L. Berl. Ak. Mb. (1876) 331-。

- - of number 2. Stieltjes, T. J. (jun.) N. Arch. Wisk. 9 ("1882) 193-; Toul. Fac. Sc. A. 11 (1897) A, 4 pp.

- 3 for any prime. Bricard, $R$. N. A. Mth. 16 (1897) 546-.

- residues and non-residues. Cauchy, A. L. C. R. 10 (1840) 437-.

- - - - Lebesgue, V. A. Liouv. J. Mth. 7 (1842) 137-.

- - - of a prime, distribution. Aladov, N. S. Rec. Mth. (Moscou) 18 (1896) 61-; Fschr. Mth. (1896) 146.

- - - - - - _.. Sterneck, R. von.

[1898] Rec. Mth. (Moscou) 20 (1899) 269-; Fschr. Mth. (1898) 153-.

- - - - - primes, combination. Bounia. kowsky, V. [1868] St Pét. Ac. Sc. Bll. 13 (1869) 25-.

- , periods and primitive roots. Arndt, C.F. Grunert Arch. 2 (1842) 1-.

- of primes of form $4 m+3$. Woronoj, $G . F$. Fschr. Mth. (1899) 184.

-, trigonal, and bitrigonal residues. Stern, M. A. [1869] Crelle J. 71 (1870) 137-.

Reciprocity law. Eisenstein, G. Crelle J. 28 (1844) 41-.

- -. Liouville, J. Liouv. J. Mth. 12 (1847) 95-.

- - Mansion, P. Mess. Mth. 5 (1876) 140 -

- Petersen, J. P. C. (xII) Ts. Mth. 3

(1879) 86-; (x) Am. J. Mth. 2 (1879) 285-.

-. Kronecker, L. Bll. Sc. Mth. As. 4 (1880) 182-.

- Sylvester, J. J. C. R. 90 (1880) 1053-, 1104.

- Gegenbauer, L. Wien Ak. Sb. 90 (1885) (Ab.2) 1026-; 97 (1889) (Ab. 2a) 427-. - - Kronecker, L. Crelle J. Mth. 104 (1889) 348-.

- - Lucas, É. St Pét. Ac. Sc. Bll. 33 (1890) 495-.

- - Gegenbauer, L. Wien Ak. Sb. 103 (1894) (Ab. 2a) 285-.

-. Hasenöhrl, F. Wien Az. 31 (1894) 74-.

- -. König, G. Mth. Term. Ets. 13 (1895) 429-; Acta Mth. 22 (1899) 181-.

- for complex numbers. Lejeune-Dirichlet, G. Crelle J. 9 (1832) 379-.

- and Gauss's sums. Mertens, F. Wien Ak. Sb. 103 (1894) (Ab. 2a) 1005-.

- , history. Kronecker, $L$. Berl. Ak. Mb. (1875) 267-.

_- Legendre's, extension. Jenkins, M. [1867] L. Mth. S. P. 2 (1869) 29.-.

- - - - - to numbers not prime. Genocchi, A. C. R. 90 (1880) 300-.

- - - - and Gauss's sums. Sochocki, J.

Par. T. Nauk Śc. Pam. 10 ("1878) Art. 1, 37 pp.; Fschr. Mth. (*1878) 315. 


\section{Quadratic residues}

Reciprocity law, Legendre's, proof. · Eisenstein, G. Crelle J. 27 (1844) 322-.

- - - - - Lebesgue, $V . A$. Liouv. J. Mth. 12 (1847) 457-。

(1872) 354 -

- - - - - Pepin, T. Rm. N. Linc. At. 43 (1890) 192-; 51 (1897) 123-.

- - - - -, Gauss's 3rd. Lerch, -. G. Teix. J. Sc. 8 (1887) 137-.

- - , - theorem of Zolotarev. Lerch, $M$. Prag České Ak. Fr. Jos. Rz. (Tř́da 2) 5 (1896) Art. 17, 8 pp.; Fschr. Mth. (1896) 141.

- proof. Schaar, M. Brux. Ac. Bll. 14 (1847) 79

189-.

- - - - Stern, M. A. Gött. Nr. (1870) 237-.

- - - - Schering, E. Gött. Nr. (1879) $217-$.

- - - Kronecker, L. Berl. Ak. Sb. (1884) $519-641$.

- - - - Genocchi, A. C. R. 101 (1885) 425 -

- - - B Busche, E. Crelle J. Mth. 99 (1886) $261-$

(1893) 190-

- _. Busche, E. [1896] Hamb. Mth.

Gs. Mt. 3 (1900) 233 -

- - - , Eisenstein's. Fischer, E. Mh. Mth. Ps. 11 (1900) 176-.

- - , - elementary. Lange, - Leip. Mth. Ps. B. 48 (1896) 629-; 49 (1897) 607-.

- - - , Gauss's 1st. Lejeune-Dirichlet, G. Crelle J. 47 (1854) 139-; Liouv. J. Mth. 4 (1859) 401-.

- - - - 3rd. Thomae, C. J. Z. Mth. Ps. 26 (1881) 134-.

- - - - - - Kronecker, L. Berl. Ak. Sb. (1884) 645-; Crelle J. Mth. 97 (1884) 93-.

,,$---\ldots$ Schering, E. Berl. Ak. Sb. (1885) 113-

- - - - (Schering). Kronecker, L. Berl Ak. Sb. (1885) 117-

- - - - - 4th. Kronecker, L. Berl. Ak. Mb. (1880) 686-, 854-.

- - - by inversion. Hermes, J. Arch. Mth. Ps. 5 (1887) 190-, vIIr.

_ - _, Schering's. Hacks, J. Acta Mth. 12 (1889) 109-.

- - , proofs. Hermite, C. Crelle J. Mth. 100 (1887) 51-.

-, - Schmidt, H. Crelle J. Mth. 111 (1893) 107-.

- for Zeller's quadratic residues. Kronecker, L. Berl. Mb. (1872) 846-.

- laws. Eisenstein, G. Crelle J. 28 (1844) $53-$.

- - Kummer, E. E. Crelle J. Mth. 100 (1887) 10-.

- - Stouff, $\boldsymbol{X}$. 126 (1898) 812-.

- theorem in field $(1, i \sqrt{2})$. Bonaventura, $P$. G. Mt. 30 (1892) 221-.
Quadratic binary forms 2830

Residues and non-residues of primes of form $4 n+3$, distribution. Götting, R. [1868] Crelle J. Mth. 70 (1869) 363-

- of polynomials, form. Collins, E. St Pét. Ac. Sc. Bll. 1 (1836) 113-.

- - primes, properties. Osborn, G. Mess. Mth. 25 (1896) 45-.

Series of quadratic residues. Weihrauch, $K$. Z. Mth. Ps. 32 (1887) 1-.

Sums of quadratic residues and non-residues, numbers. Fields, J.C. Crelle J. Mth. 112 (1893) 247-.

Symbol, Legendre's, $\left(\frac{a}{p}\right)$. Bouniakowsky, $V$. St Pét. Ac. Sc. Bll. 14 (1870) 497-.

,$--(-)$, new propositions. Bouniakowsky, $V$. [1876] St Pét. Ac. Sc. Bll. 22 (1877) 358-. - , - generalised. Gegenbauer, L. [1881] Wien Ak. Sb. 84 (1882) (Ab. 2) 1089-.

-,$- \quad-$, algorithms for determination. Gegenbauer, L. [1880] Wien Ak. Sb. 82 (1881) (Ab. 2) 931-.

- - - and new function in number theory. Bock, W. Hamb. Mth. Gs. Mt. 1 (1889) 187-, 201-。

-, - , series involving. Berger, A. Ups. S. Sc. N. Acta 12 (1885) No. 7, 31 pp.

-, Legendre-Jacobi. Lebesgue, $V . A$. Liouv. J. Mth. 12 (1847) 497-; 15 (1850) 215-; C. R. 59 (1864) 940 -; 60 (1865) $377-$.

- - - - Gegenbauer, $L$. Wien Ak. Sb. 91 (1885) (Ab. 2) 11-; 96 (1888) (Ab. 2) 491-; 100 (1891) (Ab. 2a) 855-.

-, - - Scheibner, W. Leip. Mth. Ps. Ab. 25 (1899) 367-.

- - - , theorem involving. Mandl, M. QJ. Mth. 25 (1891) 227-.

$-\left(\frac{m}{n}\right)$. Dedekind, R. C. R. 91 (1880) 154-. - (-). Gegenbauer, L. Wien Ak. Sb. 92 (1886) (Ab. 2) 876-.

- (-), evaluation. Eisenstein, G. Crelle J. 27 (1844) 317-.

Theory, and Euler's summation formula. Genocchi, A. Tortolini A. 3 (1852) 406-.

- of Gauss, generalisation. Mandl, M. Mh. Mth. Ps. 1 (1890) 465-.

\section{Quadratic binary forms.}

Kausler, C. F. [1801] St Pet. Ac. Sc. N. Acta 15 (1806) 156-.

Wezel, J. L. Liège A. Ac. (1821-22) 48 pp.

Lejeune-Dirichlet, G. Berl. Ab. (1833) 101-; Berl. B. (1840) 49-.

Scheffler, $H$. Crelle J. 45 (1853) 349-.

Mainardi, G. Mil. At. I. Lomb. 1 (1858) 106-。

Cantor, G. Z. Mth. Ps, 13 (1868) 259-.

Grube, F. Z. Mth. Ps. 19 (1874) 492-.

Jonquières, E. de. C. R. 98 (1884) 1358-, 1515. Pellet, A. E. C. R. 98 (1884) 1482.

Cajöri, F. J. H. Un. Cir. [4] (1885) 122.

Gegenbauer, $L$. Wien Ak. Sb. 96 (1888) (Ab.2) 476-.

Lachtin, L. K. [1889-91] Reo. Mth. (Moscou) 14 (1890) 487-; 15 (1891) 573-; Fschr. Mth. (1890) 215. 


\section{Quadratic binary forms}

Preobraženskij, P. V. [1890] Rec. Mth. (Moscou) 15 (1891) 118-; Mosc. S. Sc. Bll. 65 (No. 1) (1890) 62-; Fschr. Mth. (1890) $215,215-$

Tano, $F$. Bll. Sc. Mth. 14 (1890) 215-.

Pepin, 一. C. R. 119 (1894) 397-; 120 (1895) 494.

Bauer, $M$. Mth. Termt. Éts. 13 (1895) 316-; Mth. Nt. B. Ung. 13 (1897) 37-.

Jonquières, - de. C. R. 126 (1898) 991-, $1077-, 1177$.

Automorphs. Tanner, H. W. L. Mess. Mth. 24 (1895) 180-.

Classification. Pepin, T. Rm. N. Linc. At. 33 (1880) 354-.

Class-number. Arndt, $F$. Crelle J. 56 (1859) $72-$.

- - Liouville, J. Liouv. J. Mth. 14 (1869) 1-.

- - (Liouville). Stieltjes, T. J. (jun.) C. R. 97 (1883) 1358-, 1415-.

- - Dirichlet's formula. Arndt, $F$. Crelle J. 56 (1859) 100.

- - - - . Lerch, M. Prag České Ak. Fr. Jos. Rz. (TÝ̛da 2) 7 (1898) Art. 5, 51 pp.; Prag Fr. Jos. Ac. Sc. Bll. (Mth. Nt.) 5 (1898) 33-.

- -, formulæ. Lerch, _. Bll. Sc. Mth. 21 (1897) $290-$.

- -, Legendre's symbol and Moebius's numbers. Lerch, M. Prag České Ak. Fr. Jos. Rz. (Trída 2) 7 (1898) Art. 6, 12 pp.; Prag Fr. Jos. Ac. Sc. Bll. (Mth. Nt.) 5 (1898) 33-.

- - relations. Gegenbauer, $L$. Wien Ak. Sb. 93 (1886) (Ab. 2) 288-.

_ _ _ and modular correspondences of prime degree. Hurwitz, A. Leip. Mth. Ps. B. 37 (1885) 222-.

- -numbers, Dirichlet's functions

$F(s)=\Sigma\left(\frac{D}{n}\right) \cdot \frac{1}{n^{8}}$. Hurwitz, A. Z. Mth. Ps. 27 (1882) (H.-lt. Ab.) 86-.

Complex forms. Smith, H.J. S. R. S. P. 13 (1864) 278-.

Composition. Lejeune-Dirichlet, G. Crelle J. 47 (1854) 155-; Liouv. J. Mth. 4 (1859) 389-.

-. Schläfli, L. Crelle J. 57 (1860) 170-.

-. Pepin, T. [1879] Rm. N. Linc. At. 33 (1880) 6-.

-. Klein, $F$. Gött. Nr. (1893) 106-.

-. Mertens, $F$. Wien Ak. Sb. 104 (1895) (Ab. 2a) 103-

-. La Vallée Poussin, C. .T. de. Brux. Mm. Cour. $8^{\circ}, 53$ (1895-96) No. 3, 59 pp.

-, Dirichlet's method. Levänen, S. Helsingf. Öfv. 35 (1893) 57-.

Concordant and discordant numbers. Euler, L. [1780] St Pét. Ac. Sc. Mm. 8 (1822) 3-.

- - - Euler's view. Degen, C. F. [1823] St Pét. Mm. Sav. Etr. 1 (1831) 29-

Congruence $x^{2}+y^{2}=1(\bmod . p)$. Schönemann, T. Crelle J. 19 (1839) 93-.

- $x^{2}-D y^{2} \equiv \lambda$ (mod. p). Frattini, G. [1884] Rm. R. Ac. Linc. Rd. 1 (1885) 136-.

Congruence, 2nd degree, number of roots. Berger, A. Stockh. Öfv. (1887) 127-; Fschr. Mth. (1887) 181-.
Quadratic binary forms

2830

Congruences, 2nd degree, numerical solution for every simple modulus. Kunerth, $A$. [1878] Wien Ak. Sb. 78 (1879) (Ab. 2) 338-. _, - - with prime modulus, resolution. Bugaev, N. V. [1881] (xII) Rec. Mth. (Moscou) 10 (1882-83) (Pt. 1) 103-.

Conjugate forms, arithmetical theory. Mathews, G. B. QJ. Mth. 27 (1895) 230-

Connexion. Cunningham, (Lt.-Col.) A. [1896] L. Mth. S. P. 28 (1897) 289-.

Decomposition of number into difference of 2 squares. Sykora, A. Arch. Mth. Ps. 61 (1877) 446-.

- numbers into quadratic binary forms. Jonquières, E. de. As. Fr. C. R. (1878) 40-; C. R. 87 (1878) 399-; N. A. Mth. 17 (1878) 419-, 433-.

- - - sum of 2 squares. Kausler, C. F. [1800] St Pet. Ac. Sc. N. Acta 14 (1805) 232 .

- - - Colpicelli, P. Palomba Rac. 5 (1849) 263, 313, 392, 402 ; Rm. At. 4 (1850-51) 508-.

P. 8 (1876-77) 215-.

S. P. 9 (1877-78) 187-.

QJ. Mth. 19 (1883) 212-

- - - - - McDonald, J. H. Cn. R. S. P. \& T. 6 (1900) (Sect. 3) 77-.

- - - - - - Gauss's formulæ. Volpicelli, P. Tortolini A. 1 (1850) 527-; Rm. At. 4 (1850-51) 22-, 71-.

N. A. Mth. 13 (1854) 158-

_ Volpicelli, P. [1853] Tortolini A. 5 (1854) 176-.

- - product into sum of two squares. Unfer dinger, $F$. Arch. Mth. Ps. 49 (1869) 116-.

- - square into two others. Volpicelli, $P$. N. A. Mth. 9 (1850) 305-.

Determinants, irregular, formation. Perott, $J$. Crelle J. Mth. 95 (1883) 232-; 96 (1884) 327-.

-, and subtriplicate forms. Nathews, $G$. B. Mess. Mth. 20 (1891) $70-$.

-, irregularity. Lejeune-Dirichlet, G. Berl. B. (1853) 194-

Diophantine relation $y^{2}+y^{\prime 2}=$ square. Cayley, A. L. Mth. S. P. 20 (1889) 122 .

Diophantus and quadratic forms. Jacobi, C. G. J. Berl. B. (1847) 265-.

Dirichlet's investigation, extension. Bach. mann, P. Mth. A. 16 (1880) 537-.

"Disquisitiones Arithmetice" (Gauss) No. 162, extension. Jonquières, - de. C. R. 127 (1898) 596-.

Divisor of sum of two squares. Euler, $L$. [1778] St Pet. Ac. Sc. N. Acta 15 (1806) 29-. - _ - _ - Fermat's theorem. Catalan, E. C. Liége S. Sc. Mm. 15 (1888) No. 1, 11-. - - of same form. Eugenio, $V$. G. Mt. 8 (1870) 162

Divisors of form $y^{2}+A z^{2}$, number of classes. Jacobi, C. G. J. Crelle J. 9 (1832) 189 -.

- - numbers of form $m x^{2}+n y^{2}$. Euler, $L$.

[1778] St Pét. Ac. Sc. Mm. 5 (1815) 3-. 


\section{Quadratic binary forms}

Divisors of numbers of form $x^{2}-c y^{2}$. Wantzel, L. Par. S. Phlm. PV. (1848) 19-.

- $x^{2}+A y^{2}$. Hermite, $C$. Liouv. J. Mth. 14 (1849) 451-.

Equations. $x+y=c^{2}, x^{2}+y^{2}=d^{4}$, solution. Euler, L. [1780] St Pét. Ac. Sc. Mm. 10 (1826) 3 -.

-. $x^{2}+y^{2}=z^{2}$. Saorgio, (le père) -

Turin Mm. Ac. 6 (1800) 239-.

-. $x^{2}-A y^{2}= \pm 1$. Pezzi, F. [1806] Mod.

S. It. Mm. 13 (1807) 342-.

- $y^{2}-A x^{2}=B$. Tédénat, - Gergonne A. Mth. 1 (1810-11) 349-.

-. $a y^{2}+b=x^{2}$. Kramp, C. (vi Adds.) Gergonne A. Mth. 1 (1810-11) 351-.

-. $a x^{2}+b=y^{2}, x^{2}+y^{2}=z^{2}$. Du Hays, Liouv. J. Mth. 7 (1842) 325-.

-. $x^{2}+y^{2}=z^{2}$, property. Binet, J. P. M. C. R. 28 (1849) 686-, 755 .

一. $x^{2}+y^{2}=z^{2}$. Liouville, J. C. R. 28 (1849) 687.

-. $x^{2}+y^{2}=z$ or $z^{2}$. Volpicelli, $P$. G. Arcad. 119 (1849-50) 20-.

- $x^{2}+y^{2}=N$. Bellavitis, G. Tortolini A. 1 (1850) 422-.

- $\quad x^{2}+y^{2}=z^{2}$. Volpicelli, $P$. Tortolini A. 1 (1850) 369-, 443-; Rm. At. 4 (1850-51) 124-, 346 -

- $x^{2}-D y^{2}=-N$. Tchebicheff, P. Liouv.

J. Mth. 16 (1851) 257-.

一. $x^{2}+y^{2}=\left(a^{2}+b^{2}\right)^{k}$. Volpicelli, P. Rm. At. 5 (1851-52) 315-.

-. $x^{2}+y^{2}=$ N. Chelini, D. Tortolini A. 3 (1852) 126-.

$x^{2}-y^{2}=e . \quad$ Volpicelli, $P$. Rm. At. 6 (1852-53) 77-。

-. $x^{2}-y^{2}=c$. Volpicelli, P. Tortolini A. 6 (1855) 120-.

$x^{2}+h=y^{2}, x^{2}-h=z^{2}$. Boncompagni, $B$.

Tortolini A. 6 (1855) 135-.

-. $x^{2}-D y^{2}= \pm 4, D \equiv 5(\bmod .8)$. Cayley, $A$. Crelle J. 53 (1857) 369-.

-. $a x^{2}+\beta x+\gamma=\zeta \gamma^{2}+\eta \gamma+\theta . \quad$ Cayley, A. N.

A. Mth. 16 (1857) 161-.

$x^{2}-n y^{2}=1$. Gerono, G. C. N. A. Mth. 18 (1859) 122-, 153-.

-. $x^{2}-f g y^{2}= \pm 1$, factors. König, J. $F$. Grunert Arch. 33 (1859) 1-.

-. $x^{2}+y^{2}=z^{2}$. Sturm, J. B. Grunert Arch. 33 (1859) $92-$.

一. $A^{\mu}=x^{2}+a y^{2}$. Besge, V. A. Liouv. J. Mth. 6 (1861) 239-.

- $x^{2}-N y^{2}=-1$. Richaud, C. Liouv. J.

Mth. 9 (1864) 384-; 10 (1865) 235-; 11 (1866) 145-.

- $x^{2}-A y^{2}= \pm 1$. Richaud, C. Rm. At. N. Linc. 19 (1866) 177-. $A x^{2} \pm B y^{2}=u^{2} . \quad$ Calzolari, L. [1869] G. Mt. $8(1870) 28$ -

—. $A+B t^{2}=u^{2}$. Khanikof, N. de. C. R. 69 (1869) 185-

$x^{2}+c y^{2}=$ A. Petersen, J. P. C. (xIr) Ts. Mth. 1 (1871) 76-.

-. $x^{2}-A y^{2}= \pm 1$. Seeling, $P$. Arch. Mth. Ps. 52 (1871) 40-.

$Y^{2}-(-1)^{\frac{p-1}{2}} p Z^{2}=4 X . \quad$ Zolotareff, $G$.

N. A. Mth. 11 (1872) 539-.

\section{Quadratic binary forms 2830}

Equations. $a x^{2} \pm 1=y^{2}$. Matthiessen, L. Z. Mth. Ps. 18 (1873) 426.

-. $x^{2}+y^{2}=z^{2}, \quad x^{2}-y^{2}=u^{2}$. Destournelles, G. Le S. Fr. Cg. Sc. 40 (1874, v. 1) 167-.

- $t^{2}-D u^{2}= \pm 4$ where $D$ is a positive odd number and not square, solution. Schmidt, W. Z. Mth. Ps. 19 (1874) 92-.

-. $x^{2}+y^{2}=N$. Chavannes, F. L. F. Laus. S. Vd. Bll. 13 (1874-75) 474-

- $x^{2}+y^{2}=z^{2}$. Genocchi, A. Tor. Ac. Sc. At. 11 (1876) 811-.

. $y^{2}-a x^{2}=b z$. Günther, S. Liouv. J. Mth. 2 (1876) 331-.

$x^{2}-6 y^{2}=u^{2}, x^{2}+6 y^{2}=v^{2}$. Lucas, E. . N.

A. Mth. 15 (1876) 466-.

$2 v^{2}-u^{2}=w^{2}$ and $2 v^{2}+u^{2}=3 z^{2}$. Lucas, $\hat{E}$. N. A. Mth. 16 (1877) 409-.

- $x^{2}-A y^{2}=u^{2}, x^{2}+A y^{2}=v^{2}$. Lucas, É. N.

A. Mth. 17 (1878) 446-.

-. $x^{2}+k=y^{2}$. Motta Pegado, L. P. da. G. Teix. J. Sc. 1 (1878) 150-.

- $\quad x^{2}+P y^{2}=u^{2}, x^{2}-P y^{2}=v^{2}$, solution. Roberts, S. [1879] L. Mth. S. P. 11 (187980) 35-.

-. $x^{2}-2 P y^{2}=-z^{2}$ or $\pm 2 z^{2}$. Roberts, S. L. Mth. S. P. 11 (1879-80) 83-

- $a x^{2}-y^{2}=-1$, properties of numerical solutions. Durfee, W. P. [1881] (xII) J. H. Un. Cir. [1] (1882) 178.

-. $X^{2}+Y^{2}=Z^{2}$. Piuma, C. M. G. Mt. 19 (1881) 311-.

-. $2 y^{2}-1=x, 2 z^{2}-1=x^{2}$, Fermat's theorem. Pepin, T. [1882] Rm. N. Linc. At. 36 (1883) 23-.

- $A x^{2}=y^{2}+1$, etc. Catalan, E. C. As. Fr. C. R. 12 (1883) 98-.

$2 y^{2}-1=x, 2 z^{2}-1=x^{2}$, Fermat's theorem. Genocchi, A. N. A. Mth. 2 (1883) 306-

- $x^{2}+k=y^{2}$. Realis, S. N. A. Mth. 2 (1883) 289-

-. $x^{2}+n x y-n y^{2}=1$. Realis, S. N. A. Mth. 2 (1883) 494-.

-. $(n+4) x^{2}-n y^{2}=4$. Realis, S. N. A. Mth. 2 (1883) 535-.

- $n=x^{2}+y^{2}$, number of solutions, theorems on function expressing. Stieltjes, T.J. (jun.) C. R. 97 (1883) 889-.

一. $x^{2}-K y^{2}=z^{n}$. Ocagne, $M$. d'. C. R. 99 (1884) 1112.

-. $x^{2}+y^{2}=z^{2}$. Airy, G. B. Nt. 33 (1886) 532.

- $x^{2}+y^{2}=n$, solutions and formulæ. Berger, A. Acta Mth. 9 (1887) 301-.

-. $t^{2}-D u^{2}=-1$. Perott, J. Crelle J. Mth. 102 (1888) 185-.

. $\quad a x y+x^{2}-y^{2}= \pm 1$. Studnička, F. J. Prag Sb. (1888) (Mth.-Nt.) 92-.

-. $x^{2}-D y^{2}= \pm N$, geometrical meaning. Frattini, G. Rm. R. Ac. Linc. Rd. 1 (1892) (Sem. 1) 51-.

- $x^{2}-D y^{2}=-N$ (Čebyšev). Frattini, $G$. Rm. R. Ac. Linc. Rd. 1 (1892) (Sem. 2) 85-.

-. $x^{2}+1=2 y^{2}, x^{2}-1=2 y^{2}$. Lemoine, E. G. Teix. J. Sc. 11 (1892) 68-.

- $(a+2) x^{2}-(a-2) y^{2}=4$, solutions. Palmstrøm, A. Berg. Ms. Aarb. (1896) No. 14, $11 \mathrm{pp}$. 


\section{Quadratic binary forms}

Equations. $t^{2}-D u^{2}=-1$. Jonquières, $-d e$. C. R. 126 (1898) 1837-。

-. $x^{2}+y^{2}=z^{2}$. Graeber, -. Arch. Mth. Ps. 17 (1900) 36-.

Equivalent substitutions. Hermes, J. [1882] Crelle J. Mth. 95 (1883) 165-.

Extended system of forms. Gordan, P. Mth. A. 33 (1889) 372-

Factorisation by binary quadratic forms. Levänen, S. Helsingf. Öfv. 34 (1892) 334-; Fschr. Mth. (1892) 165.

Form $x^{2}+y^{2}$. Genocchi, A. Tortolini A. 5 (1854) 491-.

Forms, binary and ternary. Selling, E. [1874] Liouv. J. Mth. 3 (1877) 21-, 153-.

- with complex coefficients. Mathews, G. B. QJ. Mth. 25 (1891) 289-.

- - - determinant, class-number, Dirichlet's formula. Mathews, G. B. L. Mth. S. P. 23 (1892) 159-.

- - conjugate indeterminates. Picard, $\dot{E}$. C. R. 96 (1883) $1567-$.

___ - indefinite. Picard, É. Par. Éc. Norm. A. 1 (1884) 9-.

- - given determinant, class-number. Pepin,

T. Par. Éc. Norm. A. 3 (1874) 165-.

- negative determinant. Kronecker, L. Berl. Ak. Mb. (1875) 223-.

- - - Hermite, -. Bll. Sc. Mth. 10 (1886) 23-.

_- Aladow, J. S. Fschr. Mth. (1899) 201-.

- - - - class-number. Kronecker, L. Crelle J. 57 (1860) 248-.

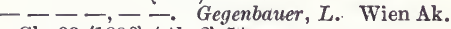
Sb. 93 (1886) (Ab. 2) 54-.

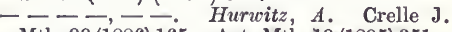
Mth. 99 (1886) 165-; Acta Mth. 19 (1895) 351-.

- - - - -. Lerch, M. C. R. 121 (1895) 878-.

- - - - - - , function expressing. Liouville, J. C. R. 62 (1866) 1350-.

- - - - - of primitive. Hacks, J. Acta Mth. 14 (1890-91) 321-.

- - - - - - relations. Gierster, $J$. [1879-83] Mth. A. 17 (1880) 71-, 74-; 21 (1883) 1-; 22 (1883) 190-.

- - - - - - - Hurwitz, A. Leip. Mth. Ps. B. 36 (1884) 193-; Mth. A. 25 (1885) 157-.

- - - - numbers. Berger, A. [1882] Ups. S. Sc. N. Acta 11 (1883) (No. 7) $22 \mathrm{pp}$.

- - - - mean class-number. Gegenbauer, L. Wien Ak. Sb. 92 (1886) (Ab. 2) 1307-.

- - - , property. Kronecker, L. Berl. Mb. (1862) 302-.

- - - determinants, periods. Šimerka, $W$. Wien SB. 31 (1858) 33-

- - discriminant, class-number. Lerch, M. Prag České Ak. Fr. Jos. Rz. (Třida 2) 7 (1898) Art. 4, 16 pp.; Fschr. Mth. (1898) 174.

$-\ldots$ - representation of whole numbers by. Gegenbauer, L. Wien Ak. Sb. 92 (1886) (Ab. 2) 380-.

\section{Quadratic binary forms 2830}

Forms with negative discriminant, theory of elliptic functions applied to. Petr, $K$. Prag České Ak. Fr. Jos. Rz. (Tř́ida 2) 9 (1900) Art. 38, 17 pp. ; Fschr. Mth. (1900) 212-. - positive determinant. Lejeune-Dirichlet, G. Berl. Ab: (1854) (Mth.) 99-; Liouv. J. Mth. 2 (1857) 353-.

- - - Mertens, $F$. Crelle J. Mth. 89 (1880) 332-

(1886) 273-; 32 (1887) 287-.

$-\ldots+$, equivalence of reduced. Mertens, $F$. Wien Ak. Sb. 103 (1894) (Ab. 2a) 995-.

- _ _ - property. Lejeune-Dirichlet, $G$. Berl. B. (1855) 493-.

- reducible to $(a, o, c),\left(a, \frac{1}{2} a, c\right)$, and $(a, b, a)$. Fields, J. C. [1885] J. H. Un. Cir. [5] (188586) 38.

- representing same numbers. Schering, $E$. Liouv. J. Mth. 4 (1859) 253-.

-, semi-definite. Rados, $G$. Mth. Termt. Éts. 13 (1895) 191-.

Functions $Y$ and $Z$ satisfying

$$
4\left(x^{p}-1\right) /(x-1)=Y^{2} \pm p Z^{2},
$$

$p$ prime of form $4 k \pm 1$. Rogers, $L$. J. L. Mth. S. P. 30 (1899) 23-.

Gauss's sums. Séguier, - de. C. R. 123 (1896) 166-.

- - Lerch, M. C̆asopis 28 (1899) 1-; Fschr. Mth. (1898) 168.

- - analogues. Lerch, M. Prag Sb. (1897) (Mth.-Nt.) No. 43, 16 pp.

- - Series $\sum_{\theta}^{n-1} e^{\frac{s^{2} 2 \pi t}{n}}$. Mertens, F. Berl. Ak. Sb. (1896) 217-.

- - with several variables. Jordan, C. C. R. 73 (1871) 1316-.

Geometrical representation of results. Baraniecki, M. A. Par. T. Nauk Śc. Pam. 8 (*1876) Art. 9, 8 pp.

Indefinite forms. Markoff, A. Mth. A. 15 (1879) 381-; 17 (1880) 379-.

Large primes, criteria; application of binary quadratic forms. Seelhoff, $P$. Am. J. Mth. 7 (1885) 264-; 8 (1886) 26-.

Linear forms of the divisors of $x^{2} \pm A$. Pepin, -. C. R. 123 (1896) 683-, 737-.

Malmsten's functions and invariants of quadratic forms. Lerch, M. Prag České Ak. Fr. Jos. Rz. (Třida 2) 2 (1893) Art. 4, 12 pp.; Fschr. Mth. (1893-94) 790-.

Number with prime factors $4 n+1$, as sum of two squares. Jonquières, E. de. N. A. Mth. 17 (1878) 241-, 289-.

Numbers whose squares are sums of two squares. Egidi, G. Rm. N. Linc. At. 50 (1897) 103-, 126-.

Numerical forms. Thiele, T. N. (xIr) Sk. Nt. Mød. F. (1873) 192-.

odd forms. Liouville, J. Liouv. J. Mth. 11 (1866) 221-.

Pellian equation. Frischauf, $J$. Wien Sb. 55 (1867) $(A b .2)$ 121-.

$-\cdots$ Roberts, S. I. Mth. S. P. $15\left(^{*} 1883-\right.$ 84) $247-$. 


\section{Quadratic binary forms}

Pellian equation. Aubel, H. van. As. Fr. C. R. (1885) (Pt. 2) 135-.

- - Frattini, G. G. Mt. 33 (1895) 371-; 34 (1896) 98-.

- - and connected problems. Oettinger, $L$. Arch. Mth. Ps. 49 (1869) 193-.

- - , and connexion with rational triangles. Müller, R. Arch. Mth. Ps. 5 (1887) 111-.

- - , correction of error in Barlow. Martin, Art. Des Moines Anal. 2 (1875) 140-.

-- , extension of Degen's tables. Brit. Ass. Comm. (Cayley). B. A. Rp. (1893) 73-.

- - property. Meyer, A. Zür. Vjschr. 32 (1887) 363-.

- - - Stфrmer, C. N. Ts. Mth. 7 (B) (1896) 49-; Fschr. Mth. (1896) 166.

- - and quadratic binary forms of positive determinant. Smith, H. J. S. L. Mth. S. P. 7 (1875-76) 196-.

- - solution. Luce, J. B. Silliman J. 8 (1849) 55-.

$-\frac{1}{619}$ - Minnigerode, B. Gött. Nr. (1873) 619-.

- - - - by elliptic functions. Kronecker, $L$. Berl. Mb. (1863) 44-.

- -, theorems. Lejeune-Dirichlet, G. Berl. Ab. (1834) 648-.

- - - - (Dirichlet). Tano, F. Crelle J. Mth. 105 (1889) 160-.

- - - - Stфrmer, C. Christiania Skr. (Mth. Nt. Kl.) (1897) No. 2, 48 pp.

Pell's equation $y^{2}-3 x^{2}=1$. Boutin, $A$. Mathesis 18 (1898) 159-.

Prime factors of numbers of form $1+4 x^{2}$. Markov, A. St Pét. Ac. Sc. Bll. 3 (1895) 55-; Fschr. Mth. (1895) 201.

$--1---A+x^{2}$. Ivanov, $I$. St Pét. Ac. Sc. Bll. 3 (1895) 361-; Fschr. Mth. (1895) 201.

- number $4 n+1$ is sum of two squares. Hermite, C. N. A. Mth. 12 (1853) 45-

- - - - Göpel, A. N. A. Mth. 12 (1853) 136-, 170-. Mth. Ps. 66 (1881) 327-. Harmuth, T. Arch.

Mth. 4 (1885) $\overline{367-}$

- - - - - Bock, J.W. Hamb.

Mth. Gs. Mt. 1 (1889) 101-

- numbers expressible by $m x^{2}+n y^{2}$. Euler, $L$. [1778] St Pet. Ac. Sc. N. Acta 12 (1801) 22 .

- - of form $A^{2}+\lambda B^{2}$ ( $B$ being odd). Liouville, J. Liouv. J. Mth. 10 (1865) 281-, 285-, 289-, 293-, 295-.

$--\frac{1}{4}+5 B^{2}$ ( $A$ being odd). Liouville, J. Liouv. J. Mth. 11 (1866) 41-.

$----m^{2}+k n^{2}$. Küpper, $K$. Časopis 10 (*1881) 10-; Fschr. Mth. (*1881) 135.

- as sum of two squares. Harmuth, $T$. [1881] Arch. Mth. Ps. 67 (1882) 215-.

Primitive forms, whose minus determinant is $>0$ and $\equiv 3$ (mod. 8). Liouville, J. Liouv. J. Mth. 11 (1866) 191-。

Problem. Miller, T. H. Edinb. Mth. S. P. 9 (1891) 23-.

Pythagorean numbers, generalisation. Züge, - Arch. Mth. Ps. 17 (1900) 354-.

VOL. I.

\section{Other quadratic forms 2840}

Quadratic forms and certain "greatest integer" formulæ. Lerch, $M$. Prag České Ak. Fr. Jos. Rz. (Trida 2) 7 (1898) Art. 7, 8 pp. ; Prag Fr. Jos. Ac. Sc. Bll. (Mth. Nt.) 5 (1898) 33-. - giving primes. Oltramare, G. Gen. I. Nt. Mm. 5 (1857) (pte. 2) 1-.

- and special automorphic groups. Fricke, R. Mth. A. 39 (1891) 62-.

- moduli, forms. Young, J.R. Ph. Mg. 33 (1848) 45-.

Reduction. Hurwitz, A. Mth. A. 45 (1894) 85-.

-, continual. Picard, É. C. R. 96 (1883) 1779-.

Series $\Sigma \frac{1}{a x^{2}+2 b x y+c y^{2}}$, asymptotic value. Mertens, $F$. Wien Ak. Sb. 106 (1897) (Ab. 2a) 411-.

Sums of squares equal to a square, method of finding. Euler, L. St Pét. Ac. Sc. Mm. 11 (1830) 12-.

Theorem of Dirichlet. Cayley, A. Camb. and Dubl. Mth. J. 9 (1854) 163-.

- - Meyer, A. Crelle J. Mth. 103 (1888) 98-

- Mertens, F. Wien Ak. Sb. 109 (1900) (Ab. 2a) 415-.

- - Gauss, demonstration. Perott, J. de. [1889] J. H. Un. Cir. [9 (1889-90)] 30.

\section{Quadratic forms of three or more variables; bilinear forms. (See also 2060, 2070.)}

Bouniakowsky, V. [1849] St Pét. Ac. Sc. Mm. 7 (1853) 303-.

Arndt, $F$. Grunert Arch. 13 (1849) 105-, 410-; 15 (1850) 429-.

Hermite, C. Crelle J. 47 (1854) 313-, 343-.

Liouville, J. Liouv. J. Mth. 2 (1857) 424.

Lipschitz, R. Crelle J. 54 (1857) 193-.

Hermite, C. C. R. 55 (1862) 681 .

Skrivan, G. Grunert Arch. 38 (1862) 259-.

Bachmann, P. Z. Mth. Ps. 16 (1871) 181-.

Siacci, $F . \quad$ Rm. At. R. Ac. 25 (1872) 339-.

Bachmann, P. Crelle J. Mth. 76 (1873) 331-.

Korkine, A., \& Zolotareff, G. Mth. A. 6 (1873) 366-.

Frobenius, G. C. R. 85 (1877) 131-.

Jordan, C. Par. Éc. Pol. J. Cah. 51 (1882) 1-.

Minkowski, $H$. Acta Mth. 7 (1885) 201-.

Algorithm for treatment. Hermes, J. [1881] Arch. Mth. Ps. 68 (1882) 432-.

Application to complex variables. Preobraschensky, P. W. Fschr. Mth. (1898) 176.

Composition. Bazin, -. Liouv. J. Mth. 16 (1851) 161-.

-. Arndt, F. Crelle J. 56 (1859) 64-.

Decomposition of numbers into sums of squares.

Malfatti, G. F. Mod. S. It. Mm. 12 (1805) 296-.

Plana, G. [1859] Tor. Mm. Ac. 20 (1863) 113-. 


\section{Quadratic Forms of three or more Variables; Bilinear Forms 2840}

Lionville, J. Liouv. J. Mth. 6 (1861) 233-; 13 (1868) 1-.

Glaisher, J. W. L. Ph. Mg. 1 (1876) 44 .

Catalan, E. C. Rm. N. Linc. At. 35 (1882) 103-; 37 (1884) 49-.

Glaisher, J. W. L. QJ. Mth. 20 (1885) 80-.

Matrot, A. As. Fr. C. R. (1890) (Pt. 2) 79-.

Catalan, E. Brux. Ac. Bll. 27 (1894) 10-.

Lemoine, É. As. Fr. C. R. (1896) (Pt. 2) 73-.

2 or 3 squares. Catalan, E. C. As. Fr. C. R. 12 (1883) 98-

3 squares. Lejeune-Dirichlet, G. Liouv. J. Mth. 4 (1859) 233-.

- - Liouville, $J$. Liouv. J. Mth. 5 (1860) 141 .

4 squares. Jacobi, C. G. J. Crelle J. 3 (1828) 191 ; 12 (1834) 167-.

- Hermite, C. C. R. 37 (1853) 133-.

- - Lebesgue, V.A. Liouv. J. Mth. 2 (1857) 149 .

- -. Sylvester, J. J. QJ. Mth. 1 (1857) $196-$

- - Liouville, J. Liouv. J. Mth. 3 (1858) 357-

- - Zehfuss, G. Grunert Arch. 30 (1858) 465-

- -. Liouville, J. Liouv. J. Mth. 13 (1868) 1-.

- - Glaisher, J.W.L. QJ. Mth. 19 (1883) $212-$

- - Weill, -. Par. S. Mth. Bll. 13 (1885) $28-$

- - Liouville's theorem (1858). Stern, $M$. A. Crelle J. Mth. 105 (1889) 250-.

- - relations among roots. Pollock, (Sir) $F$. [1858] (vI Adds.) Phil. Trans. (1859) 49-.

5 squares. Eisenstein, G. Crelle J. 35 (1847) 368 -

- Pepin, T. [1883] Rm. N. Linc. At. 37 (1884) 9-.

- - Stieltjes, T. J. (jun.) C. R. 97 (1883) $981-, 1545-$.

- - Hurwitz, A. C. R. 98 (1884) 504-.

- -. Smith, H. J. S. Par. Mm. Sav. Étr. 29 (1887) No. 1, 72 pp.

10 squares. Liouville, $J$. Liouv. J. Mth. 11 (1866) 1-.

12 squares. Liouville, $J$. Liouv. J. Mth. 5 (1860) 143-; 9 (1864) 296-.

Catalan's theorem, corollary. Moureaux, $M$. C. R. 118 (1894) 700-

Dirichlet's méthod. Kronecker, $L$. Berl. Mb. (1864) 285-.

Division of prime $m n+1$ into $m$ squares. Lebesgue, V. A. C. R. 39 (1854) 593-.

Equations, 2nd order, 3 variables. Poletti, $G$. [1826] Tor. Mm. Ac. 31 (1827) 409-.

,$--\frac{1}{-}-$ - Gerono, G. C. N. A. Mth. 15 (1856) 322-, 386-.

$-,-\frac{-}{5}, 5$ variables. Cockle, Jas. $\mathrm{Ph} . \mathrm{Mg}$. 1 (1851) 413-.

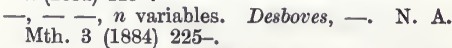

- $x^{2}+y^{2}=z^{2}+t^{2}$. Lebesgue, V.A. N. A. Mth. 7 (1848) 37-.

-. $t^{2}+u^{2}+v^{2}+w^{2}=4 m$. Lejeune-Dirichlet, $G$. Liouv. J. Mth. 1 (1856) 210-.
Equations.

$$
\begin{aligned}
f=a x^{2}+a^{\prime} x^{\prime 2}+a^{\prime \prime} x^{\prime \prime 2} & +2 b x^{\prime} x^{\prime \prime}+2 b^{\prime} x x^{\prime \prime} \\
& +2 b^{\prime \prime} x^{\prime} x=0 .
\end{aligned}
$$

Smith, H. J. S. R. S. P. 13 (1864) 110-.

-. $t^{2}=x^{2}+y^{2}+z^{2}$. Le Besgue, V. A. C. R. 66 (1868) 396-.

- $y^{2}=x_{1}^{2}+x_{2}^{2}+\ldots+x_{n}{ }^{2}$. Calzolari, L. G. Mt. 7 (1869) 313-.

-. $y=x^{2}+(x+1)^{2}$ and $y^{2}=z^{2}+(z+1)^{2}$. Jonquières, E. de. N. A. Mth. 17 (1878) 241-, 289 -.

- $2 v^{2}=u^{2}+t^{2}, 2 w^{2}=t^{2}+2 u^{2}$. Pepin, T. Rm. N. Linc. At. 32 (1879) 281-.

- $a x^{2}+b y^{2}+c z^{2}+d u^{2}=0$. Meyer, A. Zür. Vjschr. 29 (1884) 209-.

- $a x^{2}+b y^{2}+c z^{2}+d u^{2}+e v^{2}=0$. Meyer, $A$. Zür. Vjschr. 29 (1884) 220-.

Every primitive quadratic form can represent infinite number of prime numbers. Weber, H. Mth. A. 20 (1882) 301-.

Forms with complex coefficients and variables. Lejeune-Dirichlet, G. Crelle J. 24 (1842) 291-.

- - - - Bianchi, L. Rm. R. Ac. Linc. Rd. 5 (1889) (Sem. 1) 589-.

$---\frac{-}{-}$, classification. Minnigerode, B. Gött. Nr. (1873) 160-.

- conjugate variables. Picard, $\dot{E}$. Mth. A. 39 (1891) 142-.

- - integral coefficients. Minkowski, - Par. Mm. Sav. Étr. 29 (1887) No. 2, 180 pp.

- more than 3 variables, orders and genera. Smith, H. J. S. R. S. P. 13 (1864) 199-; 16 (1868) 197-.

Formulæ. Liouville, J. Liouv. J. Mth. 18 (1873) 142-.

-, system. Cayley, A. Crelle J. 39 (1850) 14-. Gauss's formula $4 \frac{x^{p}-1}{x-1}=Y^{2}-p Z^{2}$, theorem derived from. Catalan, E. C. Brux. Ac. Bll. 6 (1883) 264.

Genus, extension of arithmetical notion. Poincaré, $H$. C. R. 94 (1882) 67-, 124-.

Geometric representation, new method. Poincaré, $H$. Par. Éc. Pol. J. 28 (=Cah. 47) (1880) 177-.

Hermite's forms. Loewy, A. C. R. 123 (1896) 168-.

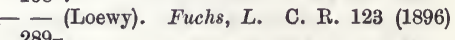
289-.

Integral power of sum of 3 squares is sum of 3 squares. Catalan, E. C. Brux. Ac. Bll. 6 (1883) 264.

Multiplication. Genocchi, A. Tortolini A. 3 (1860) 202-.

$n$-ary forms, indefinite. Meyer, A. Zür. Vjschr. 36 (1891) 241-.

Non-homogeneous forms and quadratic surfaces, classification. Hensel, $K$. Crelle J. Mth. 113 (1894) 303-.

Partitions of any integer into sum of 10 triangular numbers. Liouville, J. C. R. 62 (1866) 771-.

Positive forms. Korkine, A., \& Zolotaref, $G$. Mth. A. 11 (1877) 242-.

- - Minkowski, H. Crelle J. Mth. 99 (1886) $1-$; 101 (1887) 196-; 107 (1891) 278-. 


\section{Quadratic Forms of three or more Variables; Bilinear Forms 2840}

Prime number, sum of four squares, demonstrations of theorem. Catalan, E. C. Liége S. Sc. Mm. 15 (1888) No. 1, 159-, 259.

Primes of form $8 n+3,7 n+2,7 n+4$, quadratic resolution. Eisenstein, G. Crelle J. 37 (1848) 97-.

- $-3 n+1$, quadratic resolution. Stieltjes, T. J. (jun.) Amst. Ak. Vs. M. 19 (1884) 105-; Toul. Fac. Sc. A. 11 (1897) D, 6 pp.

Products of sums of squares. Degen, C. $F$. St Pét. Ac. Sc. Mm. 8 (1817-18) 207-.

$-\ldots$ Graves, J. T. Ir. Ac. P. 3 (1847) 527-.

- _ Cayley, A. Ph.Mg.4(1852) 515- - Liouville, J. Liouv. J. Mth. 2 (1857) 351-.

- - Souillart, C. N. A. Mth. 19 (1860) 320-.

60 (1862) 35i-

- - - Genocchi, A. G.Mt. 2 (1864) 47-

- Unferdinger, $F$. Wien Sb. 59 (1869) (Ab. 2) 455-.

- - - Thomson, J. J. [1877] Mess. Mth. 7 (1878) 73-.

- - - - Studnička, F. J. Prag. Sb. (1883) (Mth. Vortr.) 475-.

- Antomari, X. C. R. 104 (1887) 566-.

- _ - Euler's theorem. Young, J.R. Ph. Mg. 30 (1847) 424-; 31 (1847) 123; Ir. Ac. T. 21 (1848) 311-; Ir. Ac. P. 4 (1850) 19 -. $--1-1-,-1$. Roberts, S. QJ. Mth. 16 (1879) 159-; 17 (1881) 276-.

Properties. Poincaré, H. C. R. 89 (1879) 344-, 897-.

-, with tables.' Pollock, (Sir) F. [1853] Phil. Trans. (1854) 311-.

Quadratic or bilinear forms, simultaneous transformation of two. Lipschitz, R. Berl. Ak. Sb. (1890) 485-.

- form of irreducible factors of binomial equations. Trudi, N. A. Mt. 2 (1868-69) 150-.

- and linear form, simultaneous reduction. Poincaré, H. C. R. 91 (1880) 844-; Par. Éc. Pol. J. 56 (1886) 79-.

- forms and group-theory. Lipschitz, $R$. Acta Mth. 10 (1887) 137-.

- - of prime numbers and their powers. Cauchy, A. L. C. R. 9 (1839) 473-, 519-; 10 (1840) 51-, 85-, 181-, 229-.

- and linear forms of prime numbers. Oltramare, G. Crelle J. 49 (1855) 151-.

- representations, theorem of Euler, Brioschi and Genocchi. Puchta, A. Wien Ak. Sb. 96 (1888) (Ab. 2) 110-.

Quaternary forms. Bazin, - Liouv. J. Mth. 19 (1854) 215-.

- -. Pepin, —. Liouv. J. Mth. 6 (1890) 5-. - , positive. Korkine, A., \& Zolotareff, $G$. Mth. A. 5 (1872) 581-.

-- , - reduced, determinant $\leq 20$, table. Charve, L. C. R. 96 (1883) 773-.

- -, -, reduction. Charve, L. Par. Ec. Norm. A. 11 (1882) 119-.

Reduction, with proof of Seeber's theorem on reduced ternary forms. Lebesgue, $V$. A. Liouv. J. Mth. 1 (1856) 401-.
Representations of integer by certain forms, number. Gegenbauer, L. Wien Ak. Sb. 103 (1894) (Ab. 2a) 115-.

mean number. Gegenbauer, L. Wien Ak. Sb. 93 (1886) (Ab. 2) 215-.

$\Sigma\left(a_{i}-a_{j}\right)^{2}$ expressed as sum of $n$ squares. Glaisher, J. W. L. [1878] Mess. Mth. 8 (1879) 48.

Square numbers, problem. Hart, D. S. Des Moines Anal. 3 (1876) 81-.

- - as sums of squares. Avillez, J. F. d' (Visconde de Reguengo). Lisb. J. Sc. Mth. 5 (1898) 90-.

- - , sums, theorem. Studnicka, F.J. Prag Sb. (1894) (Mth.-Nt.) No. 15, 8 pp.

- of odd number is difference of two triangular numbers, generalisations. Henry, C. N. A. Mth. 19 (1880) 517-.

Sums of three squares. Catalan, E. C. Rm. N. Linc. At. 34 (1881) 63-, 135-.

Ternary forms. Hermite, C. Crelle J. 40 (1850) 173-.

_- Bachmann, P. Crelle J. 70 (1869) 365-.

- - Picard, 上. C. R. 94 (1882) 1241-.

- with complex coefficients. Fricke, $R$. Gött. Nr. (1895) 11-.

_ _ - different determinants, comparison. Eisenstein, G. Berl. B. (1852) 350-.

- - indefinite. Hermite, C. Crelle J. 47 (1854) 307-.

- - - Meyer, A. Crelle J. Mth. 108 (1891) 125-; 113 (1894) 186-; 114 (1895) 233-; 115 (1895) 150-; 116 (1896) 307-.

- - orders and genera. Smith, H. J. S. Phil. Trans. 157 (1867) 255-.

- - positive. Markov, V. A. Kharkov Mth. S. Com. 4 (1895) 1-; Fschr. Mth. (1893-94) 321-.

- - - , criticism of Seeber. Anon. (vi 311) Crelle J. 20 (1840) 312-.

- - - , reduction. Lejeune-Dirichlet, $G$. Berl. B. (1848) 285-; Crelle J. 40 (1850) 209-.

,,---- Charve, L. Par. Ec. Norm. A. 9 (1880) (Suppl.) 156 pp.

,,---- . Minkowski, H. C. R. 96 (1883) 1205 -

,--- , theorems. Eisenstein, G. Crelle J. 35 (1847) 117-.

- - , reduction. Hermite, C. [1874] Crelle J. Mth. 79 (1875) 17-.

_- transformation. Bachmann, P. [1869] Crelle J. 71 (1870) 296-.

- $(3)=$ squares, problem. Matteson, $J$. Des Moines Ânal. 2 (1875) 46-.

- and quaternary forms, indefinite. Fricke, $R$. Gött. Nr. (1893) 705-.

Theorem. Brioschi, F. Tortolini A. 5 (1854) 201-.

- Lejeune-Dirichlet, $G$. Liouv. J. Mth. 2 (1857) 273-.

- Andre, D. Par. S. Mth. Bll. 15 (1887) 188-.

Theorems. Lipschitz, R. Crelle J. 53 (1857) 238-.

Theory of numbers, especially quadratic forms. Hermite, C. Crelle J. 40 (1850) 261-. 


\section{Quadratic Forms of three or more Variables; Bilinear Forms 2840}

Transformation of linear forms of primes into quadratic forms. Oltramare, G. Gen. I. Nt. Mm. 14 (1879) 66 pp.

- - sum of 2 or 3 squares into itself by real substitution. Lipschitz, - . Liouv. J. Mth. 2 (1886) 373-.

- - two quadratic forms into each other. Minkowski, H. Crelle J. Mth. 106 (1890) 5-.

Triangles, right-angled, series. Pagnini, G. $M$. Firenze Opusc. Sc. 3 (1807) 3-.

$x^{2}+y^{2}+z^{2}+t^{2}, x^{2}+2 y^{2}+3 z^{2}+6 t^{2}$. Liouville, $J$. Liouv. J. Mth. 10 (1845) 169-.

$x^{2}+a y^{2}+b z^{2}+a b t^{2}$. Liouville, J. Liouv. J. Mth. 1 (1856) 230.

$x^{2}+y^{2}+5\left(z^{2}+t^{2}\right)$. Liouville, J. Liouv. J. Mth. 4 (1859) $47-$.

$x^{2}+y^{2}+3\left(z^{2}+t^{2}\right)$. Liouville, J. Liouv. J. Mth. 5 (1860) 147-.

$x^{2}+y^{2}+2\left(z^{2}+t^{2}\right)$. Liouville, J. Liouv. J. Mth. 5 (1860) 269-, 305-.

$x^{2}+3 y^{2}+4 z^{2}+12 t^{2}$. Liouville, J. Liouv. J. Mth. 6 (1861) 135-.

$x^{2}+y^{2}+z^{2}+2 t^{2}, x^{2}+2\left(y^{2}+z^{2}+t^{2}\right)$.

$J$. Liouv. J. Mth. 6 (1861) 225-.

Liouville,

$X^{2}+Y^{2}+Z^{2}+8 T^{2}$. Lionville, J. Liouv. J. Mth. 6 (1861) 324-.

$x^{2}+2 y^{2}+4 z^{2}+8 t^{2}$. Liouville, J. Liouv. J. Mth. 6 (1861) 409-.

$X^{2}+Y^{2}+Z^{2}+4 T^{2}, X^{2}+4 Y^{2}+4 Z^{2}+4 T^{2}$. Liouville, $J$. Liouv. J. Mth. 6 (1861) 440-.

$X^{2}+2 Y^{2}+2 Z^{2}+4 T^{2}$. Liouville, J. Liouv. J. Mth. 7 (1862) 1-.

$X^{2}+8\left(Y^{2}+Z^{2}+T^{2}\right)$. Liouville, J. Liouv. J. Mth. 7 (1862) 5-.

$X^{2}+4 Y^{2}+4 Z^{2}+8 T^{2}$. Liouville, $J$. Liouv. J. Mth. 7 (1862) 9-.

$X^{2}+8 Y^{2}+8 Z^{2}+16 T^{2}$. Liouville, J. Liouv. J. Mth. 7 (1862) 13-.

$x^{2}+2 y^{2}+4 z^{2}+4 t^{2}$. Liouville, J. Liouv. J. Mth. 7 (1862) 62-.

$x^{2}+2 y^{2}+8 z^{2}+8 t^{2}$. Liouville, J. Liouv. J. Mth. 7 (1862) 65-.

$x^{2}+8 y^{2}+16 z^{2}+16 t^{2}$. Liouville, J. Liouv. J. Mth. 7 (1862) 69-.

$x^{2}+4 y^{2}+4 z^{2}+16 t^{2}$. Liouville, J. Liouv. J. Mth. 7 (1862) 73-.

$x^{2}+16\left(y^{2}+z^{2}+t^{2}\right)$. Liouville, J. Liouv. J. Mth. 7 (1862) 77-.

$x^{2}+y^{2}+2 z^{2}+4 t^{2}$. Liouville, J. Liouv. J. Mth. 7 (1862) 99-.

$x^{2}+y^{2}+4 z^{2}+8 t^{2}$. Liouville, J. Liouv. J. Mth. 7 (1862) 103-.

$x^{2}+4 y^{2}+16 z^{2}+16 t^{2}$. Liouville, J. Liouv. J. Mth. 7 (1862) 105-.

$x^{2}+y^{2}+8 z^{2}+8 t^{2}$. Liouville, $J$. Liouv. J. Mth. 7 (1862) 109-.

$x^{2}+4 y^{2}+8 z^{2}+8 t^{2}$. Liouville, J. Liouv. J. Mth. 7 (1862) 113-.

$x^{2}+y^{2}+16 z^{2}+16 t^{2}$. Liouville, J. Liouv. J. Mth. 7 (1862) 117-.

$x^{2}+4 y^{2}+8 z^{2}+16 t^{2}$. Liouville, $J$. Liouv. J. Mth. 7 (1862) 143-.

$x^{2}+2 y^{2}+16 z^{2}+16 t^{2} \quad$ Liouville, J. Liouv. J. Mth. 7 (1862) 145-.

$x^{2}+2 y^{2}+2 z^{2}+8 t^{2}$. Liouville, J. Liouv. J. Mth. 7 (1862) 148-.

$x^{2}+2 y^{2}+4 z^{2}+16 t^{2}$. Liouville, $J$. Liouv. J. Mth. 7 (1862) 150-. $x^{2}+2 y^{2}+8 z^{2}+16 t^{2}$. Liouville, J. Liouv. J. Mth. 7 (1862) 153-.

$x^{2}+y^{2}+2 z^{2}+8 t^{2}$. Liouville, J. Liouv. J. Mth. 7 (1862) 155-

$x^{2}+y^{2}+4 z^{2}+16 t^{2}$. Liouville, J. Liouv. J. Mth. 7 (1862) 157-

$x^{2}+2 y^{2}+2 z^{2}+16 t^{2}$. Liouville, J. Liouv. J. Mth. 7 (1862) 161-.

$x^{2}+y^{2}+z^{2}+16 t^{2}$. Liouville, J. Liouv. J. Mth. 7 (1862) 165-.

$x^{2}+y^{2}+8 z^{2}+16 t^{2}$. Liouville, J. Liouv. J. Mth. 7 (1862) 201-.

$x^{2}+y^{2}+2 z^{2}+16 t^{2}$. Liouville, J. Liouv. J. Mth. 7 (1862) 205-.

$x^{2}+8 y^{2}+8 z^{2}+64 t^{2}$. Liouville, J. Liouv. J. Mth. 7 (1862) 246-.

$x^{2}+8 y^{2}+16 z^{2}+64 t^{2}$. Liouville, J. Liouv. J. Mth. 7 (1862) 249-.

$x^{2}+y^{2}+z^{2}+3 t^{2}$. Liouville, J. Liouv. J. Mth. 8 (1863) 105-.

$x^{2}+y^{2}+2 z^{2}+2 z t+2 t^{2}$. Liouville, J. Liouv. J. Mth. 8 (1863) 115-.

$x^{2}+y^{2}+z^{2}+z t+t^{2}$. Liouville, J. Liouv. J. Mth. 8 (1863) 120-.

$x^{2}+y^{2}+2 z^{2}+6 t^{2}$. Liouville, J. Liouv. J. Mth. 8 (1863) 124.

$x^{2}+y^{2}+2 z^{2}+3 t^{2}$. Liouville, J. Liouv. J. Mth. 8 (1863) 129-.

$x^{2}+2 y^{2}+4 z^{2}+6 t^{2}$. Liouville, J. Liouv. J. Mth. 8 (1863) 134-.

$x^{2}+x y+y^{2}+z^{2}+z t+t^{2}$. Liouville, J. Liouv. J. Mth. 8 (1863) 141-.

$x^{2}+y^{2}+z^{2}+12 t^{2}$. Liouville, J. Liouv. J. Mth. 8 (1863) 161-.

$x^{2}+2 y^{2}+2 z^{2}+12 t^{2}$. Liouville, J. Liouv. J. Mth. 8 (1863) 169-.

$x^{2}+y^{2}+4 z^{2}+12 t^{2}$. Liouville, J. Liouv. J. Mth. 8 (1863) 173-.

$x^{2}+4 y^{2}+4 z^{2}+12 t^{2}$. Liouville, J. Liouv. J. Mth. 8 (1863) 177-.

$3 x^{2}+4 y^{2}+4 z^{2}+4 t^{2}$. Liouville, J. Liouv. J. Mth. 8 (1863) 179-.

$x^{2}+y^{2}+3 z^{2}+4 t^{2}$. Liouville, J. Liouv. J. Mth. 8 (1863) 182-.

$x^{2}+3 y^{2}+4 z^{2}+4 t^{2}$. Liouville, $J$. Liouv. J. Mth. 8 (1863) 185-.

$2 x^{2}+2 y^{2}+3 z^{2}+4 t^{2}$. Liouville, J. Liouv. J. Mth. 8 (1863) 189-.

$x^{2}+y^{2}+z^{2}+3 t^{2}$. Liouville, J. Liouv. J. Mth. 8 (1863) 193-.

$x^{2}+4 y^{2}+12 z^{2}+16 t^{2}$. Liouville, J. Liouv. J. Mth. 8 (1863) 205-.

$x^{2}+3 y^{2}+6 z^{2}+6 t^{2}$. Liouville, $J$. Liouv. J. Mth. 8 (1863) 209-.

$2 x^{2}+3 y^{2}+3 z^{2}+6 t^{2}$. Liouville, J. Liouv. J. Mth. 8 (1863) 214-.

$x^{2}+3\left(y^{2}+z^{2}+t^{2}\right)$. Liouville, J. Liouv. J. Mth. 8 (1863) 219-.

$2 x^{2}+2 x y+2 y^{2}+3\left(z^{2}+t^{2}\right)$. Liouville, J. Liouv. J. Mth. 8 (1863) 225-.

$x^{2}+x y+y^{2}+3\left(z^{2}+t^{2}\right)$. Liouville, J. Liouv. J. Mth. 8 (1863) 227-.

$3 x^{2}+3 y^{2}+3 z^{2}+4 t^{2}$. Liouville, J. Liouv. J. Mth. 8 (1863) 229-.

$3 x^{2}+3 y^{2}+4 z^{2}+12 t^{2}$. Liouville, J. Liouv. J. Mth. 8 (1863) 239-.

$3 x^{2}+4 y^{2}+12 z^{2}+12 t^{2}$. Liouville, J. Liouv. J. Mth. 8 (1863) 241-. 


\section{Quadratic Forms of three or more Variables; Bilinear Forms 2840}

$x^{2}+3 y^{2}+3 z^{2}+12 t^{2}$. Liouville, J. Liouv. J. Mth. 8 (1863) 243-.

$x^{2}+3 y^{2}+12 z^{2}+12 t^{2}$. Liouville, J. Liouv. J. Mth. 8 (1863) 249-.

$x^{2}+12 y^{2}+12 z^{2}+12 t^{2}$. Liouville, J. Liouv. J. Mth. 8 (1863) 253-.

$3 x^{2}+4 y^{2}+12 z^{2}+48 t^{2}$. Liouville, J. Liouv. J. Mth. 8 (1863) 255-.

$x^{2}+y^{2}+3\left(z^{2}+t^{2}\right)$. Liouville, J. Liouv. J. Mth. 8 (1863) 296.

$x^{2}+x y+y^{2}+2 z^{2}+2 z t+2 t^{2}$. Liouville, $J$. Liouv. J. Mth. 8 (1863) 308-.

$x^{2}+y^{2}+z^{2}+5 t^{2}$. Liouville, J. Liouv. J. Mth. 9 (1864) 1-.

$x^{2}+y^{2}+2 z^{2}+2 z t+3 t^{2}$. Liouville, J. Liouv. J. Mth. 9 (1864) 13-.

$x^{2}+5\left(y^{2}+z^{2}+t^{2}\right)$. Liouville, J. Liouv. J. Mth. 9 (1864) 17-.

$2 x^{2}+2 x y+3 y^{2}+5 z^{2}+5 t^{2}$. Liouville, J. Liouv. J. Mth. 9 (1864) 23-

$x^{2}+y^{2}+z^{2}+t^{2}+u^{2}+3 v^{2}$. Liouville, $J$. Liouv. J. Mth. 9 (1864) 89-

$x^{2}+3\left(y^{2}+z^{2}+t^{2}+u^{2}+v^{2}\right)$. Liouville, J. Liouv. J. Mth. 9 (1864) 105-

$x^{2}+y^{2}+z^{2}+t^{2}+2 u^{2}+2 u v+2 v^{2}$. Liouville, J. Liouv. J. Mth. 9 (1864) 115-.

$2 x^{2}+2 x y+2 y^{2}+3\left(z^{2}+t^{2}+u^{2}+v^{2}\right)$. Liouville, J. Liouv. J. Mth. 9 (1864) 119-.

$x^{2}+y^{2}+2 z^{2}+2 z t+2 t^{2}+3 u^{2}+3 v^{2}$. Liouville, $J$. Liouv. J. Mth. 9 (1864) 123-.

$x^{2}+2 y^{2}+2 y z+2 z^{2}+3 t^{2}$. Liouville, J. Liouv. J. Mth. 9 (1864) 160.

$x^{2}+y^{2}+z^{2}+t^{2}+u^{2}+2 v^{2}$. Liouville, $J$. Liouv. J. Mth. 9 (1864) 161-

$x^{2}+2\left(y^{2}+z^{2}+t^{2}+u^{2}+v^{2}\right)$. Liouville, J. Liouv. J. Mth. 9 (1864) 175-

$x^{2}+x y+y^{2}+6 z^{2}+6 z t+6 t^{2}$. Liouville, J. Liouv. J. Mth. 9 (1864) 181-.

$2 x^{2}+2 x y+2 y^{2}+3 z^{2}+3 z t+3 t^{2}$. Liouville, $J$. Liouv. J. Mth. 9 (1864) 183-.

$x^{2}+x y+y^{2}+3 z^{2}+3 z t+3 t^{2}$. Liouville, J. Liouv. J. Mth. 9 (1864) 223-.

$x^{2}+y^{2}+z^{2}+t^{2}+2\left(u^{2}+v^{2}\right)$. Liouville, J. Liouv.

J. Mth. 9 (1864) 257-.

$x^{2}+y^{2}+2\left(z^{2}+t^{2}+u^{2}+v^{2}\right)$. Liouville, J. Liouv. J. Mth. 9 (1864) 273-.

$x^{2}+2 y^{2}+3 z^{2}+6 t^{2}$. Liouville, J. Liouv. J. Mth. 9 (1864) 299-.

$x^{2}+2\left(y^{2}+z^{2}+t^{2}+u^{2}\right)+4 v^{2}$. Liouville, J. Liouv. J. Mth. 9 (1864) 421-.

$x^{2}+y^{2}+5 z^{2}+5 t^{2}$. Liouville, J. Liouv. J. Mth. 10 (1865) 1-.

$2 x^{2}+2 x y+3 y^{2}+2 z^{2}+2 z t+3 t^{2}$. Liouville, J. Liouv. J. Mth. 10 (1865) 9-.

$x^{2}+y^{2}+9 z^{2}+9 t^{2}$. Liouville, J. Liouv. J. Mth. 10 (1865) 14-.

$2 x^{2}+2 x y+5 y^{2}+2 z^{2}+2 z t+5 t^{2}$. Liouville, J. Liouv. J. Mth. 10 (1865) 21-

$x^{2}+y^{2}+a\left(z^{2}+t^{2}\right)$. Liouville, J. Liouv. J. Mth. 10 (1865) 43-.

$x^{2}+2 y^{2}+a z^{2}+2 a t^{2}$. Liouville, J. Liouv. J. Mth. 10 (1865) 49-

$x^{2}+4 y^{2}+4 z^{2}+4 t^{2}+4 u^{2}+4 v^{2}$. Liouville, $J$.

Liouv. J. Mth. 10 (1865) 65-

$x^{2}+y^{2}+4 z^{2}+4 t^{2}+4 u^{2}+4 v^{2}$. Liouville, J.

Liouv. J. Mth. 10 (1865) 71-

$x^{2}+2 y^{2}+2 z^{2}+4 t^{2}+4 u^{2}+4 v^{2}$. Liouville, J.

Liouv. J. Mth. 10 (1865) 73-. $x^{2}+y^{2}+z^{2}+4 t^{2}+4 u^{2}+4 v^{2}$. Liouville, J. Liouv. J. Mth. 10 (1865) 77-.

$x^{2}+y^{2}+2 z^{2}+2 t^{2}+4 u^{2}+4 v^{2}$. Liouville, $J$. Liouv. J. Mth. 10 (1865) 145-.

$x^{2}+y^{2}+z^{2}+t^{2}+4 u^{2}+4 v^{2}$. Liouville, $J$. Liouv. J. Mth. 10 (1865) 151-.

$x^{2}+y^{2}+z^{2}+2 t^{2}+2 u^{2}+4 v^{2}$. Liouville, J. Liouv. J. Mth. 10 (1865) 155-.

$x^{2}+y^{2}+z^{2}+t^{2}+u^{2}+4 v^{2}$. Liouville, $J$. Liouv. J. Mth. 10 (1865) 161-.

$x^{2}+4 y^{2}+4 z^{2}+4 t^{2}+4 u^{2}+16 v^{2}$. Liouville, J. Liouv. J. Mth. 10 (1865) 203-.

$x^{2}+y^{2}+6 z^{2}+6 t^{2}, \quad 2 x^{2}+2 y^{2}+3 z^{2}+3 t^{2}$. Liou-

ville, J. Liouv. J. Mth. 10 (1865) 359-.

$x^{2}+2 y^{2}+2 y z+2 z^{2}+15 t^{2}$,

$2 x^{2}+2 x y+3 y^{2}+3 z^{2}+3 t^{2}$.

Liouville, J. Liouv. J. Mth. 11 (1866) 39-. $3 x^{2}+5 y^{2}+10 z^{2}+10 z t+10 t^{2}$, $2 x^{2}+2 x y+3 y^{2}+15 z^{2}+15 t^{2}$.

Liouville, J. Liouv. J. Mth. 11 (1866) 103 . $x^{2}+2 y^{2}+2 y z+2 z^{2}+6 t^{2}, \quad x^{2}+2 y^{2}+3 z^{2}+3 t^{2}$. Liouville, J. Liouv. J. Mth. 11 (1866) 131-. $x^{2}+3 y^{2}+a z^{2}+3 a t^{2}$. Liouville, J. Liouv. J. Mth. 11 (1866) 211-.

$2 x^{2}+3 y^{2}+4 z^{2}+4 z t+4 t^{2}, \quad x^{2}+2 y^{2}+6 z^{2}+6 t^{2}$. Liouville, J. Liouv. J. Mth. 11 (1866) 280 -. $x_{1} x_{2}+x_{2} x_{3}+x_{3} x_{4}+x_{4} x_{5}$. Liouville, J. C. R. 62 (1866) 714.

$x^{2}+2 y^{2}+3 z^{2}$. Liouville, J. Liouv. J. Mth. 14 (1869) 359-.

\section{BILINEAR FORMS}

Kronecker, L. Berl. Mb. (1866) 597-.

Christoffel, E. B. [1867] Crelle J. 68 (1868) 253-

Beltrami, E. G. Mt. 11 (1873) 98-.

Jordan, C. [1873] Liouv. J. Mth. 19 (1874) 35-.

Voss, A. Gött. Nr. (1887) 424-.

Cosserat, E. Toul. Fac. Sc. A. 3 (1889) M, $12 \mathrm{pp}$.

Weyr, E. Mh. Mth. Ps. 1 (1890) 163-, 201-.

Pasch, M. Mth. A. 38 (1891) 24-.

Taber, H. N. Y. Am. Mth. S. Bll. 3 (1897) 156-.

Application to transformation group of half plane. Picard, É. Par. S. Mth. Bll. 12 (1884) 43-.

Adjoint forms. Rados, $G$. Mth. Termt. Ets. 14 (1896) 165-; Mth. Nt. B. Ung. 14 (1898) 116-.

Automorphic linear transformation. Taber, $H$. Am. Ac. P. 29 (1894) 178-, 371-; 31 (1896) 181-.

- - of an alternate form. Taber, $H$. Mth. A. 46 (1895) 561-

Bilinear and quadratic forms. Weierstrass, C. Berl. Mb. (1868) 310

- _ - - Kronecker, L. Berl. Mb. (1868) 339-.

_- - _, families. Kronecker, L. Berl. Ak. Mb. (1874) 59-, 149-, 206-; C. R. 78 (1874) 1181-.

- - - - - Stickelberger, L. Crelle J. Mth. 86 (1879) 20-. 
2840 Bilinear Forms

Binary forms. Battaglini, $G$. Rm. R. Ac. Linc. Rd. 1 (1885) 691-; Nap. Ac. At. 2 (1888) No. 6, 14 pp.

Cogredient and adjoint automorphic transformations. Voss, A. [1896] Münch. Ak. Sb. 26 (1897) 211-.

- automorphic transformations. Voss, A. Münch. Ak. Ab. 17 (1892) 235-; Münch. Ak. Sb. 26 (1897) 1-, 281.

- transformations. Frobenius, $G$. Berl. Ak. Sb. (1896) 7-.

Congruent transformations. Kronecker, $L$. Berl. Ak. Mb. (1874) 397-.

Conjugate automorphic transformations. Voss, A. Münch. Ak. Sb. 19 (1890) 175-.

- - - Löwy, A. Münch. Ak. Sb. 26 (1897) 25-.

Elementary divisors in bilinear forms. Kantor, S. Mh. Mth. Ps. 11 (1900) 193-.

Families. Frobenius, G. Zür. Vjschr. 41 (1896) (Festschr., Th. 2) 20-.

-. Weber, E. von. Münch. Ak. Sb. 28 (1899) 369-.

-, algebraic reduction. Kronecker, L. Berl. Ak. Sb. (1890) 1225-.

Forms with conjugate complex variables. Loewy, A. Ac. Nt. C. N. Acta 71 (1898) 377-; Mth. A. 50 (1898) 557-.

- permutable into given bilinear form. Voss, A. Münch. Ak. Sb. 19 (1890) 283-.

Fundamental systems and bilinear forms. Landsberg, G. Crelle J. Mth. 116 (1896) 331-.

Invariant, skew, of bilinear or quadratic form. Frobenius, G. [1878] Crelle J. Mth. 86 (1879) 44-.

Invariants of different classes of bilinear forms. Rosenow, $H$. Crelle J. Mth. 108 (1891) 1-.

- - one and two bilinear forms. Mertens, $F$. Krk. Ak. (Mt.-Prz.) Pam. 10 (1885) 26 (bis)-; Fschr. Mth. (1885) 85.

Linear $\infty^{\lambda}$-groups of bilinear forms, equivalence. Kantor, S. Münch. Ak. Sb. 27 (1898) 367-.

- substitutions and bilinear forms. Frobenius, G. [1877] Crelle J. Mth. 84 (1878) 1-.

Pairs of forms. Del Re,A. Rm. R. Ac. Linc. Rd. 6 (1890) (Sem. 1) 237 -.

Quaternary forms. Battaglini, G. [1881] Rm. R. Ac. Linc. Mm. 12 (1882) 233-.

- - Kronecker, L. Berl. Ak. Ab. (1883) 60 pp.

Reduction. Jordan, C. C. R. 78 (1874) 614-.

- to sum of products by orthogonal substitution. Sylvester, - Mess. Mth. 19 (1890) $42-$.

Relations with reciprocal forms. Segre, $C$. G. Mt. 22 (1884) 29-.

Representation, typical. Frahm,W. Erlang. Ps. Md. S. Sb. 6 (1874) 74-.

Similar forms. Sforza, G. G. Mt. 32 (1894) 293-; 33 (1895) 80-; 34 (1896) 252-.

Ternary forms. Muth, P. Mth. A. 42 (1893) 257-.

- - geometric representation. Battaglini, G. [1880] Rm. R. Ac. Linc. Mm. 9 (1881) 3-

- of Hermite. Kollros, L. C. R. 131 (1900) 173-.
Higher Congruences 2850

Ternary forms, pairs. $\operatorname{Del} R e, A$. Rm. R. Ac. Linc. Rd. 7 (1891) (Sem. 2) 88-.

Transformation. Jacobi, C. G.J. Crelle J. 53 (1857) 265-.

-. Koch, H. von. Stockh. Öfv. (1900) 335-.

Weierstrass's theorem. Calo, B. A. Mt. 23 (1895) 159-

theorems on two forms, generalisation. Christoffel, E. B. Crelle J. 63 (1864) 255-.

\section{Congruences other than linear; cubic and higher resi- dues.}

Associated numbers of Euler, and a property of primes. Binet, J. P. M. C. R. 13 (1841) 210-.

- - Fermat's and Wilson's theorems. Lejeune-Dirichlet, $G$. Crelle J. 3 (1828) 390

- - Lejeune-Dirichlet's theory generalised. Terquem, $O$. N. A. Mth. 4 (1845) 379-.

- - Wilson's theorem generalised. Prouhet, E. N. A. Mth. 4 (1845) 273-。

Bimodular congruences. Dina, C. G. Mt. 21 (1883) 234-.

Binomial congruences. Serret, J. A. N. A. Mth. 14 (1855) 261-.

- - Pepin, T. Rm. N. Linc. At. 38 (1885) 201-.

- - Mirimanoff, D. Crelle J. Mth. 109 (1892) 82-.

- - exponential to base 3. Bonniakowsky, $V$. [1869] St Pét. Ac. Sc. Bll. 14 (1870) 356-.

- - extension of Fermat's theorem. Horner, W. G. Thomson A. Ph. 11 (1826) 81-.

- _ - - _ - and Wilson's theorems. Mitchell, O. H. Am. J. Mth. 3 (1880) 294-.

- - Indices relative to prime modulus $p$. Stern, M. A. Crelle J. 6 (1830) 147-.

- - - - - - - Frattini, G. G. Mt. 18 (1880) 369-.

- - modulus $p$ or $p^{n}$, where $p$ is a prime. Alagna, $R$. Palermo Cir. Mt. Rd. 13 (1899) 99-.

- - primitive roots. Oltramare, $G$. Crelle J. 49 (1855) 161-.

- -, - - Gegenbauer, L. Wien Ak. Sb. 95 (1887) (Ab. 2) 843-.

- - , - - theorem by Čebyšev. Allégret, A. N. A. Mth. 16 (1857) 309-.

- - solutions of certain, table. Jacobi, C. G. J. Crelle J. 3 (1828) 301-.

- - theorem. Bugaev, N. V. [1897] Rec. Mth. (Moscou) 20 (1899) 171-; Fschr. Mth. (1897) 166.

- $n$th degree, solution. Speckmann, G. Arch. Mth. Ps. 17 (1900) 110-.

- -, (mod. $p, f(x)$ ). Mitchell, O. H. [1881] (xII) J. H. Un. Cir. [1] (1882) 132.

- equations with prime modulus, composition of roots. Le Besgue, V.A. C. R. 62 (1866) 20 .

Biquadratic residue-character. Gmeiner, J.A. Wien Ak. Sb. 100 (1891) (Ab. 2a) 1093-. 
Biquadratic residue-character of number two. Lejeune-Dirichlet, G. [1857] Crelle J. 57 (1860) 187-. $-\frac{-1}{(1868)} 190-$ Halphen, G. C. R. 66

- residues. Gauss, C. F. Gött. Cm. 6 (182327) $27-; 7$ (1828-31) 89-. - - - Mathieu, É. Liouv. J. Mth. 12 (1867)

- Stieltjes, T. J. (jun.) Bll. Sc. Mth. As. 7 (1883) (Pt. 1) 139-.

- - fundamental theorem. Eisenstein, $G$. Crelle J. 28 (1844) 223-.

"Canon arithmeticus," Jacobi's, note. Lebesgue, $V . A$. C. R. 39 (1854) 1069-.

Circulating decimal, number of figures in period. Contejean, L. Par. S. Phlm. Bll. 4 (1892) 64-.

- decimals and Fermat's theorem. Genese, R. $W$. B. A. Rp. (1888) 580-.

- -, rule of remainders. Goodwyn, $H$. (vI Adds.) Nicholson J. 1 (1802) 314-.

- - theory. Brogtrop, A. J. M. N. Arch. Wisk. $3\left({ }^{*} 1877\right) 58$-.

Congruence, condition for existence of definite number of roots. Gegenbauer, $L$. Wien Ak. Sb. 95 (1887) (Ab. 2) 165-.

- of Fermat. Gruber, N. Mth. Termt. Ets. 14 (1896) 22-; Mth. Nt. B. Ung. 13 (1897) 413-.

- prime modulus. Piuma, C. $M$. A. Mt. 11 (1882-83) 237-.

-, 3rd degree. Ivanov, I. St Pét. Ac. Sc. Bll. 5 (1896) 137-; Fschr. Mth. (1896) 149.

-, - - , with simple modulus, number of roots. Woronoj, G. T. Fschr. Mth. (1898) 156.

-, 4th degree, with prime modulus. Cordone, G. Palermo Cir. Mt. Rd. 9 (1895) 209-.

$-\frac{r^{p}-r}{p} \equiv a(\bmod . p)$. Stern, - Crelle J. Mth. 100 (1887) 182-.

- $z^{n}-a y^{n} \equiv 0$ (mod. $M$ ), resolution. Dickstein, S. [1892] Krk. Ak. (Mt.-Prz.) Rz. 6 (1893) 155-; Crc. Ac. Sc. Bll. (1892) 372-.

$-x^{n}+A_{1} x^{n-1}+A_{2} x^{n-2}+\ldots+A_{n} \equiv 0$ (mod. $\left.p\right)$, resolution. Snopek, $E$. Prace Mt.-Fiz. 4 (1893) 63-; Fschr. Mth. (1893-94) 289.

- $x^{m} \equiv b\left(\bmod .2^{\eta}\right)$, resolution. Amici, $N$. Palermo Cir. Mt. Rd. 8 (1894) 187-.

$-2^{4 n} \equiv(-)^{n}(2 n) ! /(n !)^{2}$ where $2 n+1$ is a prime. Morley, $F$. A. Mth. 9 (1894-95) 168-.

- $2^{x} \equiv R$ (mod. $p$, mod. $m$ ). Cunningham, (Lt.-Col.) A. B. A. Rp. (1895) 613.

$-\left(r^{p-1}-1\right): p \equiv q_{r}(\bmod . p)$. Mirimanoff, $D$. Crelle J. Mth. 115 (1895) 295-.

$-x^{2 k} \equiv b$ (mod. $p^{\lambda}$ ), resolution. Amici, $N$. Palermo Cir. Mt. Rd. 11 (1897) 43-.

$-x^{p-1}-1 \equiv 0$ (mod. $\left.p^{2}\right)$, upper limit of number of roots. Aladow, J. S. Fschr. Mth. (1899) 185.

Congruences. Raussnitz, G. (xII) Mth. Term. Ets. 1 (1883) 296-; Mth. Nt. B. Ung. 1 (1882-83) 266-.

- Gegenbauer, $L$. Wien Ak. Sb. 95 (1887) (Ab. 2) 610-.
Congruences. Demeczky, M. Mth. Termt. Éts. 7 (1889) 131-; Mth. Nt. B. Ung. 8 (1891) $51-$.

-. Gegenbauer, L. Wien Ak. Sb. 98 (1890) (Ab. 2a) 652-.

-. Rogel, F. Arch. Mth. Ps. 10 (1891) 84-.

- and application to indeterminate analysis.

Bie, .L. H. (xII) Ts. Mth. 2 (1878) 161-.

- - cubic residues. Stern, M. A. Crelle J. 9 (1832) 97-.

, employment of roots in theory of numbers. Cauchy, A. L. C. R. 25 (1847) 37-.

- and equations, similarity between, and its significance. Hathaway, A. S. [1880] (xII) J. H. Un. Cir. [1] (1882) 97.

- having no roots with respect to prime modulus. Zsigmondy, K. [1894] D. Mth. Vr. Jbr. 4 (1897) 109-; Mh. Mth. Ps. 8 (1897) 1 -.

-, modulus $p^{n}$ where $p$ is prime. Schönemann, T. Crelle J. 32 (1846) 93-.

-, number of figures in periods of reciprocals of integers. Muir, T. Mess. Mth. 4 (1875) 1-.

-, - - roots. Gegenbauer, L. Wien Ak. Sb. 102 (1893) (Ab. 2a) 549-.

- and residues. Arndt, $F$. Grunert Arch. 6 (1845) 380-.

-, resolution of class. Pellet, A. E. C. R. 88 (1879) 417-.

-, - , logarithmic tables for various moduli. Bellavitis, G. Rm. R. Ac. Linc. T. 1 (1877) 778-.

- with respect to prime modulus. Schönemann, T. Crelle J. 31 (1846) 269-.

- - - - - Stankevich, B. V. [1882] (xII) Rec. Mth. (Moscou) 10 (1882-83) (Pt. 1) $112-$.

Ps. 5 (1894) 230-.

_ _ _ _ and to irreducible modular function. Serret, J. A. Par. Ac. Sc. Mm. 35 (1866) 617-.

-, resultant of two. Sylvester, J. J. [1881] (xiI) J. H. Un. Cir. [1] (1882) 131.

- with several variables. Gegenbauer, $L$. Wien Ak. Sb. 99 (1891) (Ab. 2a) 790-.

- theorems. Poinsot, L. [1817-18] Par. Mm. de l'I. (1813-15) 381-; Par. Mm. Ac. Sc. 4 (1819-20) 99-.

-, - Libri, G. [1825] Par. Mm. Sav. Etr. 5 (1838) 1-.

- - Cauchy, A. L. [1829-31] Férussac Bll. Sc. Mth. 12 (1829) 205-; 15 (1831) 137-; Par. Mm. Ac. Sc. 17 (1840) 249-.

-, 一. Galois, $E$. Férussac Bll. Sc. Mth. 13 (1830) 428-.

-, -. Stern, M. A. Crelle J. 12 (1834) 288-.

77-

Cauchy, A. L. C. R. 44 (1857)

- in $x$ of $n$th order with prescribed number of roots. Zsigmondy, $K$. Wien Ak. Sb. 103 (1894) (Ab. 2a) 135-.

Cubic and biquadratic characters. Pellet, A.E. C. R. 108 (1889) 609-. 


\section{Congruences other than Linear; Cubic and Higher Residues 2850}

Cubic and biquadratic residues. Stieltjes, T.J. (jun.) Amst. Ak. Vs. M. 17 (1882) 338-; Arch. Néerl. 18 (1883) 358-.

- - - with respect to prime modulus. Pellet, A. E. Par. S. Mth. Bll. 10 (1882) 157-.

- - other residues with respect to prime modulus. Lucas, É. As. Fr. C. R. (1886) (Pt. 2) 101-.

- residues. Jacobi, C. G. J. Crelle J. 2 (1827) 66-.

- Kummer, E. E. Crelle J. 23 (1842) 285-; 32 (1846) 341-.

- - Meyer, G.F. Arch. Mth. Ps. 43 (1865) 413-.

- - Pepin, T. C. R. 79 (1874) 1403-; Liouv. J. Mth. 2 (1876) 313-.

Decomposition of numbers, Fermat's propositions, extension. Realis, S. N. A. Mth. 5 (1886) 113-.

Determinantal congruences to prime modulus. Sardi, C. G. Mt. 5 (1867) 371-.

Divisibility of periodic numbers and determination of decimal periods. Barillari, $G$. [1870] G. Mt. 9 (1871) 125-.

Divisors of functions of primitive roots of unity. Sylvester, J. J. C. R. 92 (1881) 1084-.

Fermat's theorem, generalisation. Dickson, L. E. A. Mth. 1 (1900) 31-.

- -,$x^{\phi(m)} \equiv 1$ (mod. $m$ ), generalisation. Lucas, Eै. C. R. 84 (1877) 439-.

$--,-(-),-$ Picquet, H. C. R. 96 (1883) 1136-, 1424. $-\frac{1}{1300-}-(-),-$ Lucas, É. $\quad$ C. R. 96. (1883)

$--,-(-),-$ - Pellet, A. E. C. R. 96 (1883) 1301-.

$--,-(-),-$ - Kantor, S. C. R. 96 (1883) 1423.

$--,-(-),-$ Grandi, A. Ven. I. At. 1 (1883) 809-.

- - - (-), -. Cordone, G. Rv. Mt. 5 (1895) 25-.

$-\frac{1}{-}(-)$, - . Dickson, L. E. C. R. 128 (1899) 1083-.

,$---(-)$, theorem resembling. Horner, W. G. Ph. Mg. 11 (1837) 456-.

,$-- a^{p-1} \equiv 1$ (mod. $\left.p\right)$ first demonstration. Vacca, G. Bb. Mth. (1894) 46-.

- - - - (-), generalisation. Koenigs, G. Bll. Sc. Mth. As. 8 (1884) 286-.

,$---(-)$, new demonstration. Garibaldi, C. G. Mt. 26 (1888) 197-.

Functions irreducible with respect to prime modulus. Pellet, [A.] E. C. R. $70(1870)$ 328- ; 90 (1880) 1339-; 93 (1881) 1065-.

- reduced with respect to prime modulus. Pellet, A. E. Par. S. Mth. Bll. 17 (1889) 156-.

Higher reciprocity, function connected with. Stern, M. A. Crelle J. 55 (1858) 193-.

Indices appertaining to given number. Laisant, C. A. Bordeaux S. Sc. Mm. 1 (1876) 400 -

- with complex modulus. Hill, C. J. D. Lund Acta Un. 1 (1864) (Mth.) No.6, 18 pp.; Crelle J. 70 (1869) 282-.
Integral functions irreducible with respect to a prime modulus, when degree is equal to modulus. Serret, J. A. Liouv. J. Mth. 18 (1873) 301-.

- - - - - - - - - - power of modulus. Serret, J. A. Liouv. J. Mth. 18 (1873) 437-.

Integrals, any order, residues. Marie, $M$. C. R. 75 (1872) 1475-.

-, double, Cauchy's method extended to. Marie, M. C. R. 75 (1872) 865-, 937-; Par.

Ec. Pol. J. 27 (=Cah. 44) (1874) 51-.

Irreducible congruences. Dickson, L. E. N. Y. Am. Mth. S. Bll. 3 (1897) 381-.

- - Eisenstein's theorems. Schönemann, $T$. Crelle J. 40 (1850) 185-.

Period-length of decimal corresponding to $1 / p, p$ being one of first 1500 primes. Kessler, $E$. Arch. Mth. Ps. 3 (1886) 99-.

- lengths of circulates. Cunningham, (Lt.Col.) A. Mess. Mth. 29 (1900) 145-.

Periods of circulating decimals, method of forming. Johnson, $W . W$. Mess. Mth. 14 (1885) 14-.

- - pure circulates, frequency of occurrence of digits. Reynolds, $B$. Mess. Mth. 27 (1898) 177-.

Polynomials, application in theory of numbers. Crelle, A. L. Berl. Ab. (1843) 49-.

Power residues. Skrivan, G. Wien Jbr. OberRealsch. Inn. Stadt 3 (1861) 1-.

- - Jonquières, - de. C. R. 124 (1897) $334-, 428$.

- - of complex numbers. Kronecker, $L$. Berl. Ak. Mb. (1880) 404-.

_ - - numbers for composite modulus. Bennett, G. T. [1892] Phil. Trans. (A) 184 (1894) 189-.

- - proof of double theorem, and extension of idea of congruence. Hofmann, $F$. Mth. A. 20 (1882) 471-.

- -, theory. Zsigmondy, K. Mh. Mth. Ps. 3 (1892) 265-.

Prime numbers, property connected with Fermat's theorem. Sylvester, J.J. C. R. 52 (1861) 161-, 212-, 307-.

- -, theorems. Proth, F. C. R. 87 (1878) 926. - of which a given number cannot be a primitive root. Jonquières, - de. C. R. 123 (1896) 405-.

Primitive roots. Murphy, $R$. $\quad$ Ph. Mg. 19 (1841) 369.

-. Frolov, -. Par. S. Mth. Bll. 21 (1893) 113-; 22 (1894) 241-.

- - , comparative number. Szily, $K$. Mth. Termt. Éts. 9 (1891) 264-; 10 (1892) 19-; Mag. Tud. Ak. Éts. 2 (1891) 478.

- - of congruence $x^{p-1} \equiv 1$ (mod. $\left.p\right)$. Keferstein, H. Hamb. Mth. Gs. Mt. 1 (1889) 256-. - - for odd prime modulus. Perott, J. Bll. Sc. Mth. 17 (1893) 66-; 18 (1894) 64-. Bll. Sc. Mtb. 9 (1885) 21 -.

- - period, Jacobi's formation, proof. Le Besgue, V. A. C. R. 70 (1870) 1243-.

- - of primes of certain type. Wertheim, $G$. Acta Mth. 20 (1897) 143-. 


\section{Congruences other than Linear; Cubic and Higher Residues 2850}

Primitive roots of primes, criteria. Zsigmondy, K. Mh. Mth. Ps. 4 (1893) 79-.

- - - - properties. Jonquières, - de. C. R. 122 (1896) 1451-。

- - - and their residues. Forsyth, A.R. Mess. Mth. 13 (1884) 169-.

- - , solution of question proposed by Fermat. Lipschitz, $R$. Bll. Sc. Mth. 22 (1898) 123-.

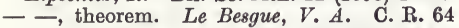
(1867) 1268-.

- - , two theorems of Gauss generalised. Arndt, F. Crelle J. 31 (1846) 326-.

- and secondary roots of primes. Jonquières, - de. C. R. 123 (1896) 374.

Pure circulates in various scales of notation. Reynolds, B. Mess. Mth. 28 (1899) 88-.

Quadratic, cubic and biquadratic residues. Pepin, T. Rm. N. Linc. At. 38 (1885) 197-.

- and cubic residues, theory. Meyer, $G$. Arch. Mth. Ps. 63 (1879) 1-.

Quintic, octavic, and duodecimic residues, complex primes in theory of. Jacobi, C. G.J. Crelle J. 19 (1839) 314-.

- residues. Pepin, -. C. R. 76 (1873) 151-.

- - and integers formed from 5th roots of unity. Schwering, K. Z. Mth. Ps. 27 (1882) 102-.

Reciprocals of primes, to determine prime itself from number of figures in reciprocal. Shanks, W. R. S. P. 22 (1874) 381-。

- _ n number of figures in period. Shanks, W. [1873-75] R. S. P. 22 (1874) 200-, 384-; 23 (1875) 260-, 261.

- - - periods in. Shanks, $W .[1872]$ Mess. Mth. 2 (1873) 41-; 3 (1874) 52-.

- - - - - Salmon, G. [1872] Mess. Mth. 2 (1873) 49-.

Reciprocity, bicubic, between real and complex numbers. Gmeiner, J. A. Mh. Mth. Ps. 3 (1892) 179-, 199-.

- law, bicubic. Gmeiner, J. A. Wien Ak. Sb. 100 (1891) (Ab. 2a) 1330-; 101 (1892) (Ab. 2a) 562-.

-_, biquadratic, theorem. Dintzl, E. Mh. Mth. Ps. 10 (1899) 88-

- -, cubic. Eisenstein, G. Crelle J. 27 (1844) 289-; 28 (1844) 28-.

- - - - Gegenbauer, L. Wien Ak. Sb. 81 (1880) (Ab. 2) 436-.

- - -, analytic proofs. Dantscher, $V$. Mth. A. 12 (1877) 241-.

- - -, theorem. Dintzl, E. Mh. Mth. Ps. 10 (1899) 303-.

- laws, general, of power residues. - Kummer, E. E. Berl. B. (1850) 154-; Berl. Mb. (1858) 158-; Crelle J. 44 (1852) 93-; 56 (1859) 270-; Berl. Ab. (1859) (Mth.) 19-; (1861) (Mth.) 81-.

- higher, and laws connected with them. Eisenstein, G. Crelle J. 39 (1850) 351-.

- -, of power residues. Pepin, T. [1877] C. R. 84 (1877) 762-; Rm. N. Linc. At. 31 (1878) 40-

Residue of a binomial coefficient divisible by $p^{n}$, with respect to $\left(p^{n+1}\right)$. Glaisher, J.W.L. QJ. Mth. 30 (1899) 349-.

- _ _ - - with respect to a prime modulus. Glaisher, J. W. L. QJ. Mth. 30 (1899) 150-.
Residue, two as a 16-ic. Cunningham, (Lt.Col.) A. [1895] L. Mth. S. P. 27 (1896) 85-.

Residues. Lebesgue, $V$. A. C. R. 3 (1836) 439-.

-. Arnat, $F$. Crelle J. 31 (1846) 333-.

- of binomial coefficients with respect to $p^{3}$. Glaisher, J. W. L. QJ. Mth. 31 (1900) 110 -

- in geometric progressions. Prouhet, $E$. N. A. Mth. 5 (1846) 175-, 652-, 675-.

- of higher orders. Gazzaniga, $P$. Ven. I. At. (1885-86) 1271-.

- - index-sums for primes of form $p=2^{2 \mu}+1$, symmetrical and complementary distribution. Hermes, J. Arch. Mth. Ps. 4 (1886) 207-.

- $(\bmod . k)$, of symmetric functions. Mitchell, O. H. Am. J. Mth. 4 (1881) 25-.

- and primitive roots, theorems. Bouniakonsky, V. [1869] St. Pét. Ac. Sc. Bll. 14 (1870) 356-, 432-.

- of sums of products of first $p-1$ numbers, modulus $p^{2}$ or $p^{3}$. Glaisher, J. W. L. QJ. Mth. 31 (1900) 321-.

Roots of unity, properties. Ruffini, $P$. Mil. Mm. I. Lomb. Ven. 3 (1816-17) 67-.

Secondary roots of prime numbers, properties. Jonquières, - de. C. R. 122 (1896) 1513-.

Septimic residues. Pepin, T. C. R. 80 (1875) 811-.

Wilson's and Fermat's theorems derived from common principle. Grunert, J.A. Crelle J. 8 (1832) 187-.

Arch. 32 (1859) -104 . Toeplitz, J. Grunert - - - -, divisibility of faculties, etc. Oettinger, $L$. Arch. Mth. Ps. 48 (1868) 159-.

- - - proofs. Petersen, J. P. C. (xп) Ts. Mth. 2 (1872) 64-.

- theorem. Brennecke, $(D r)$ - Crelle J. 19 (1839) 319-.

- - Sylvester, J.J. Camb. and Dubl. Mth. J. 9 (1854) 84 -

- - Liouville, J. Liouv. J. Mth. 1 (1856) $351-$.

- Catalan, E. C. Liège S. Sc. Mm. 15 (1888) No. 1, 139-.

- - Daublebsky von Sterneck, R. Mh. Mth. Ps. 7 (1896) 145-.

- -, extension. Bouniakowsky, V. [1856] St Pét. Ac. Sc. Bll. 15 (1857) 202-。

- - - to all numbers. Sylvester, J. J. (vI Adds.) Ph. Mg. 13 (1838) 454; 14 (1839) 47-。

- - Gauss's extension. Pollock, (Sir) F. [1847] R. S. P. 5 (1851) 664.

- - - treatment. Terquem, O. N. A. Mth. 2 (1843) 193-.

- - - - Arndt, F. Crelle J. 31 (1846) 329 .

- - generalisation. Babbage, C. Edinb. Ph.'J. 1 (1819) 46-.

- - - C. Crelle, A. L. [1839] Berl. B. (1839) 133-; Crelle J. 20 (1840) 29-.

- - Scherk, H. F. D. Nf. Vsm. B. (1846) 204-.

,--- Del Beccaro, T. Rm. R. Ac. Linc. Mm. 1 (1894) 344-. 
Wilson's theorem, generalisation. Lognon, - . N. A. Mth. 15 (1896) 503.

- - Lagrange's proof. Lista, A. Cadiz Period. M. Ci. 1 (1848) 63-.

- - point arising in. Liouville, J. Liouv. J. Mth. 5 (1860) 127-, 267-.

\section{Forms of higher degree} which cannot be considered as products of linear factors. (See also 2060, 2070.)

Algebraic forms with arithmetical connexions. Cesáro, E. Rm. R. Ac. Linc. Rd. 2 (1886) (Sem. 2) 56-.

Biquadratics, pairs of sums of two. Euler, $L$. St Pét. Ac. Sc. Mm. 11 (1830) 49-.

Congruences, theorems. Libri, $\dot{G}$. Crelle J. 9 (1832) 54-, 169-, 261-.

-, -. Lebesgue, V. A. Liouv. J. Mth. 2 (1837) 253-; 3 (1838) 113-; 4 (1839) 9-.

Cube $=$ sum of three cubes, Euler's solution. Binet, J. P. M. C. R. 12 (1841) 248-.

Cubic classes belonging to determining quadratic form, number. Arndt, $F$. Grunert Arch. 19 (1852) 408-.

— forms. Eisenstein, G. CrelleJ. 27 (1844) 75 -.

- and quartic forms, reduction to squares. Euler, L. [1780] St Pét. Ac. Sc. Mm. 11 (1830) 69-.

Decomposition of number into four positive cubes, theorem of Euler. Lucas, É. N. A. Mth. 19 (1880) 89-.

- - - its maximal $n$th powers. Lemoine, E. C. R. 95 (1882) 719-.

Diophantine equations of class zero. Hilbert, D., \& Hurwitz, A. Acta Mth. 14 (1890-91) 217-.

- problem, geometrical solution. Burhenne, H. Grunert Arch. 20 (1853) 466-.

- triangles, problem of Fermat. Tannery, $P$. [1885] Par. S. Mth. Bll. 14 (1886) 41-.

Equations between two variables, integral solutions. Runge, C. Crelle J. Mth. 100 (1887) 425-.

- $x^{5}+y^{5}=a z^{5}$. Lebesgue, $V . A$. Liouv. J. Mth. 8 (1843) 49-.

-. $x^{3}+y^{3}+A z^{3}=$ Mxyz. Sylvester, J. J. $\mathrm{Ph}$. Mg. 31 (1847) 467-.

-. $z^{2}=x^{4} \pm 2^{m} y^{4}$ and $2^{m} z^{2}=x^{4} \pm y^{4}$. Lebesgue, V. A. Lioav. J. Mth. 18 (1853) 73-.

-. $a x+b k y=z\left(x^{2}+k y^{2}\right)$. Oltramare, $G$. Crelle J. 49 (1855) 142-.

-. $x^{3}+y^{3}=z^{2}$, solution in relative primes. Hoppe; R. Schlömilch Z. 4 (1859) 304-.

-. $x^{3}+y^{3}=x-y$, solution in rational numbers. Hoppe, R. Schlömilch Z. 4 (1859) 359-.

-. $x^{3}+y^{3}+z^{3}=v^{3}$. Buniakovskij, V. [1864] St Pet. Ac. Sc. Mm. (Rs.) 6 ("1865) 142-.

- $x^{3}+y^{3}+z^{3}=u^{2}$, solution by toroid. Catalan, E. C. Brux. Ac. Bll. 22 (1866) 29-.

-. $x^{3}+y^{3}+z^{3}=u^{3}$. Richaud, C. Rm. At. N. Linc. 19 (1866) 183-

- $x^{3}+(x+r)^{3}+(x+2 r)^{3}$

Richaud $C, \quad \mathrm{Rm}+\ldots+\{x+(n-1) r\}^{3}=y^{2}$. Richaud, C. Rm. At. N. Linc. 20 (1867) 91-.
Equations. $x^{3}+y^{3}+z^{3}+u^{3}=0$. Grassmann, $H$. Arch. Mth. Ps. 49 (1869) 49-.

-. $x^{3}+y^{3}=z^{3}+u^{3}$. Hermite, C. N. A. Mth. $11(1872) 5-$.

-. $x^{3}=y^{2}+17$. Gerono, C. C. N. A. Mth. 16 (1877) 325-.

- $a x^{4}+b y^{4}=c z^{2}$. Desboves, A. C. R. 87 (1878) 522-, 598-, 925.

-. $x^{3} \pm a=y^{2}$. Jonquieres, $E$. de. N. A. Mth. 17 (1878) 374-, 514-.

- $4 \frac{z^{p}-1}{z-1}=Y^{2}-\left(\frac{-1}{p}\right) p Z^{2}$. Lucas, É. As. Fr. C. R. (1878) 164-.

-. $X^{3}+Y^{3}=A Z^{3}$. Lucas, É. N. A. Mth. 17 (1878) 425-.

- $a X^{4}+b Y^{4}+d X^{2} Y^{2}+f X^{3} Y+g X Y^{3}=c Z^{2}$. Desboves, A. C. R. 88 (1879) 638-.

-. $A x^{4}+B y^{4}=C z^{2}$. Lucas, E. N. A. Mth. 18 (1879) 67-. $a X^{m}+b Y^{m}=c Z^{n}$. Desboves, A. N. A. Mth. 18 (1879) 265-, 398-, 433-, 481-.

-. $7 x^{4}-5 y^{4}=2 z^{2}$. Pepin, T. Liouv. J. Mth. 5 (1879) 405-.

-. $U^{n} \pm V^{n}=S^{n}+W^{n}$, solution in integers, real or complex. Desboves, $A$. As. Fr. C. R. 9 (1880) 239-.

-. $x^{3}+y^{3}=A z^{3}$. Lucas, É. N. A. Mth. 19 (1880) 206-.

-. $a x^{4}+b y^{4}=z^{2}$. Pepin, T. C. R. 91 (1880) 100 ; 94 (1882) 122-.

-. $x^{n}+y^{n}+z^{n}=U^{n}$. Schier, O. [1880] Wien Ak. Sb. 82 (1881) (Ab.2) 883-.

-. $x^{3}+y^{3}=A z^{3}$. Pepin, T. Rm. N. Linc. At. 34 (1881) 73-.

-. $a x^{4}+b y^{4}=c z^{2}$. Pepin, T. [1882] Rm. N. Linc. At. 36 (1883) 34-.

. $a X^{4}-b Y^{4}=2 Z^{2}$. Desboves, A. As. Fr. C. R. (1887) (Pt. 1) 175.

-. $a X^{4}+b Y^{4}=c Z^{2}$. Desboves, A. C. R. 104 (1887) 846-, 1602-

-. $a X^{4}+b Y^{4}=c Z^{2}$, and $a X^{4}+b Y^{4}+d X^{2} Y^{2}=c Z^{2}$. Desboves, A. C. R. 104 (1887) 1832-.

-. $U^{4}+V^{4}=S^{4}+W^{4}$. Fauquembergue, $E$. Mathesis 9 (1889) 241-.

- $x^{2}+c Y^{2}=z^{3}$. Pepin, T. Rm. N. Linc. Mm. 8 (1892) 41-.

-. $x^{5}+y^{5}=2^{m} z^{5}$. Levänen, S. Helsingf. Öfv. 35 (1893) 69-.

-. $X^{4}+35 Y^{4}=Z^{2}$. Pepin, 一. Liouv. J. Mth. 1 (1895) 35j-.

-. $a x^{\lambda^{i}}+b y^{\lambda^{i}}=c z^{\lambda^{i}}$. Maillet, E. As. Fr. C. R. (1897) (Pt. 2) 156-.

-. $x^{4}-8 x^{2} y^{2}+8 y^{4}=z^{2}$. Pepin, T. Rm. N. Linc. Mm. 14 (1898) 71-.

-. $x^{4}+4 h x^{2} y^{2}+(2 h-1)^{2} y^{4}=z^{2}$, where $4 h-1$ and $2 h-1$ are primes. Pietrocola, $C$. G. Mt. 36 (1898) 77-.

-. $x^{\lambda}+y^{\lambda}=c z^{\lambda}$. Maillet, E. C. R. 129 $198-$

\section{Fermat's theorem on $x^{n}+y^{n}=z^{n}$.}

Barlow, $P$. Nicholson J. 27 (1810) 193-.

Legendre, A. M. Par. Mm. Ac. Sc. 6 (1823) $1-$.

Lamé, G. [1839] C. R. 9 (1839) 45-; Par. Mm. Sav. Etr. 8 (1843) 421-. 
(Lamé's memoir.) Ćauchy, A. L. C. R. 9 (1839) 359-.

Paulet, F. Quetelet Cor. Mth. 11 (1839) 307-.

Drach, S. M. Ph. Mg. 27 (1845) 286-.

Lamé, G. (III \& vIII). C. R. 24 (1847) 310-, 352, 569-; Liouv. J. Mth. 12 (1847) 172-.

(Lamé's demonstration.) Terquem, O. N. A. Mth. 6 (1847) 132-.

Cauchy, A. L. C. R. 24 (1847) 469-, 516-, 578-, 633-, 661-.

Kummer, E. E. [1847-49] Berl. B. (1847) 132-, 140-, 305-; Crelle J. 40 (1850) 130-.

Talbot, W. H. F. [1856] Edinb. R. S. T. 21 (1857) 403-.

Calzolari, L. Tortolini A. 8 (1857) 339-

Tait, P. G. [1864] Edinb. R. S. P. 5 (1866) 181.

Calzolari, L. [1865] A. Mt. 6 (1864) 280 -.

Lukas, F. C. Arch. Mth. Ps. 58 (1876) 109-.

Liouville, R. C. R. 89 (1879) 1108 -

Korkine, A. C. R. 90 (1880) 303-.

Lefébure, $A$. C. R. 90 (1880) 1406-

Pepin, T. C. R. 91 (1880) 366-.

Schier, O. Wien. Ak. Sb. 81 (1880) (Ab. 2) $392-$.

Jonquières, E. de. $\quad$ Rm. N. Linc. At. 37 (1884) 146-.

Catalan, E. C. Liège S. Sc. Mm. 12 (1885) No. 2, 179-, 403; 13 (1886) 387-.

Mathews, G. B. Mess. Mth. 15 (1886) 68-.

Borletti, E. Mil. I. Lomb. Rd. 20 (1887) $222-$.

Mansion, P. Brux. Ac. Bll. 13 (1887) 16-, 225.

Varisco, D. G. Mt. 27 (1889) 371-.

Tafelmacher, A. Santiago de Chile Un. A. 82 (1892) 271-, 415-.

Dutordoir, -. Brux. S. Sc. A. 17 (1893) (Pt. 1) 81-.

Wendt, E. Crelle J. Mth. 113 (1894) 335-.

Korneck, G. Arch. Mth. Ps. 13 (1895) 1-, 263 -

Mathew8, G. B. Mess. Mth. 24 (1895) 97-.

Gram, I. P. Sk. Nf. F. (1898) 182.

$n=2 \lambda$. Kummer, E. E. Crelle J. 17 (1837) 203-.

$n=3$. Tait, P. G. [1870] Edinb. R. S. P. 7 (1872) 144.

$n=3$. Günther, $S$. Prag Sb. (1878) 112-.

$n=3$. Perrin, R. Par. S. Mth. Bll. 13 (1885) 194 .

$n=4$. Tafelmacher, $A$. Santiago de Chile Un. A. 84 (1893) 307-.

$n=5$. Lamé, $G$. Liouv. J. Mth. 12 (1847) 137-.

$n=6$. Tafelmacher, $A$. Santiago de Chile Un. A. 97 (1897) 63-.

$n=7$. Lamé, G. Liouv. J. Mth. 5 (1840) 195-.

$n=7$. Lebesgue, V. A. Liouv. J. Mth. 5 (1840) 276-, 348-.

$n=7$. Genocchi, A. A. Mt. 6 (1864) 287-.

$n=7$. Pepin, T. C. R. 82 (1876) 676-, 743-.

$n=7$. Genocchi, A. C. R. 82 (1876) 910-. $n=14$. Lejeune-Dirichlet, G. Crelle J. 9 (1832) 390 -

$n=37$. Mirimanoff, $D$. Crelle J. Mth. 111 (1893) 26-.

application of complex numbers. Kummer, E. E. Berl. Ab. (1857) (Mth.) 41-.

and Arabian MS. Kummer, E. E. N. A. Mth. 9 (1850) 386-.

equation connected with. Jonquières, - de. C. R. 120 (1895) 1139-, 1236.

$-x^{2 n}+y^{2 n}=z^{2 n}$. Lebesgue, V. A. Liouv. J. Mth. 5 (1840) 184-.

Form $x^{3}+n y^{3}+n^{2} z^{3}-3 n x y z$, arithmetical theory. Mathews, G. B. L. Mth. S. P. 21 (1891) 280-.

Reduction of biquadratic form to a square. Pepin, T. Rm. N. Linc. At. 32 (1879) 166-.

- - quartic to a square, formulæ for. Pepin, T. Rm. N. Linc. At. 30 (1877) 211-.

Square formed by adding cube to given number. Pepin, (le rév. père) J. F. T. (хाז) Brux. S. Sc. A. 6 (1882) (Pt. 2) 86-.

- values of quartic form $x^{4}+m x^{2} y^{2}+y^{4}$. Euler, L. [1782] St Pét. Ac. Sc. Mm. 7 (1820) 10Sum of cubes, decomposition of integer into. Maillet, E. As. Fr. C. R. (1895) (Pt. 2) 242-.

- - - - number into. Elefanti, $F$. QJ. Mth. 4 (1861) 339-.

- _ - (2), decomposition of number into. Pepin, -. Liouv. J. Mth. 15 (1870) 217-.

- or difference of 2 biquadratics cannot be biquadratic. Kausler, C. F. [1799] St Pet. Ac. Sc. N. Acta 13 (1802) 237-.

- _ - - cubes cannot be cube. Kausler, C. F. [1799] St Pet. Ac. Sc. N. Acta 13 (1802) 245-.

- - cubo-cubes cannot be a cubocube. Kausler, C. F. [1801] St Pet. Ac. Sc. N. Acta 15 (1806) 146-.

- of 4 th powers, decomposition of numbers into. Lucas, E. N. A. Mth. 17 (1878) 536-.

$--r\left(\frac{p-1}{\lambda}\right)^{\text {th }}$ powers $(\lambda=2,3,4)$ not divisible by $p$. Gegenbauer, $L$. Wien Ak. Sb. 95 (1887) (Ab. 2) 838-

Sums of cubes, numbers as. Zornow, Crelle J. 14 (1835) 276-.

- - - J Jacobi, C. G. J. Crelle J. 42 (1851) 41-.

- powers, representation of integers as. Mathews, G. B. Mess. Mth. 25 (1896) 69-.

Ternary cubic form. Sylvester, J. J. Tortolini A. 7 (1856) 398-; Am. J. Mth. 2 (1879) 280-, 357-; 3 (1880) 58-, 179-.

- - Sawin, A. M. [1884] A. Mth. 1 (1884-85) 58-.

- - - resolution in integers. Desboves, $A$. N. A. Mth. 5 (1886) 545-.

Values of $x, y, v, z$ for which

$$
u v z^{2}\left(a x^{2}+b y^{2}\right)^{2}+\Delta x^{2} y^{2}\left(a v^{2}+b z^{2}\right)^{2}
$$

is a square. Euler, $L$. St Pét. Ac. Sc. Mm. 9 (1824) 14-. 


\section{Forms of higher degree which can be considered as products of linear factors; algebraic numbers; ideals. (See also 0820.)}

Abelian number-fields (Zahlkörper). Weber, $H$. Acta Mth. 8 (1886) 193-; 9 (1887) 105-.

- _ K Kronecker's theorem. Hilbert, D. Gött. Nr. (1896) 29-.

Algebraic composite fields. Hensel, $K$. Crelle J. Mth. 120 (1899) 99-.

- congruences and universal arithmetic. Kronecker, L. Crelle J. Mth. 100 (1887) 490 -

- fields, groups of numbers in. Weber, $H$. Mth. A. 48 (1897) 433-; 49 (1897) 83-; 50 (1898) 1-.

- number-field, unimodular substitutions in. Hurwitz, A. Gött. Nr. (1895) 332-.

- - fields, historical and bibliographical account. Hilbert, D. D. Mth. Vr. Jbr. 4 (1897) 175-

_- , units in. Minkowski, H. Gött. Nr. (1900) 90 -

- numbers. Dedekind, $R$. Bll. Sc. Mth. As. 11 (1876) 278-; 1 (1877) 17-, 69-, 144-, 207-.

- - Hurwitz, A. Gött. Nr. (1895) 324-.

- - , analogue of Riemann-Roch theorem in theory of. Landsberg, G. Mth. A. 50 (1898) 577 .

- -, arithmetical theory. Hurwitz, A. Gött. Nr. (1895) 230-.

- - , class of lowest order containing given classes. Kneser, A. Mth. A. 30 (1887) 179-.

- -, criterion. Minkowski, $H$. Gött. Nr. (1899) 64-.

- depending on cube root of given number. Markoff, $A$. St Pét. Ac. Sc. Mm. 38 (1892) No. 9, 37 pp.

- - derived from irreducible cubic equation. Mathews, G. B. L. Mth. S. P. 24 (1894) $327-$

- - and function theory. Grömwall, $H$. Stockh. Öfv. (1897) 199-.

- - , fundamental equation of a class-domain (Gattungsbereich). Mertens, $F$. Wien Ak. Sb. 103 (1894) (Ab. 2a) 5-.

- - of given class-domain. Hensel, $K$. Crelle J. Mth. 101 (1887) 99-.

- - greatest common divisor. Sochocki, $J$. Prace Mt.-Fiz. 4 (1893) 95-; Fschr. Mth. (1893-94) 297-.

- -, logarithms. Störmer, C. C. R. 130 (1900) 1603-.

- - , real, property of whole system. Cantor, G. [non M.] [1873] (vII) Crelle J. Mth. 77 (1874) 258-

Analytical loci. Cauchy, A. L. C. R. 24 (1847) 885-.

Arithmetical demonstration of algebraic theorems. Kneser, A. Crelle J. Mth. 102 (1888) 20 -
Aurifeuillians. Cunningham, (Lt.-Col.) A. L. Mth. S. P. 29 (1898) 381-.

Binary cubic forms, calculation and classification for negative determinants. Arndt, $F$. [1851] Grunert Arch. 31 (1858) 335-.

- forms, which cannot represent powers of primes. Gegenbauer, L. Wien Ak. Sb. 97 (1889) (Ab. 2a) 368-.

Biquadratic forms, class, prime divisors. Lejeune-Dirichlet, G. Crelle J. 3 (1828) 35-.

Class-number divisible by prime $\lambda$. Kummer, E. E. Berl. Ak. Mb. (1874) 239-.

- - for numbers formed from 11th and 13th roots of unity. Wolfskehl, P. Crelle J. Mth. 99 (1886) 173-.

Classes of resolvable forms. Schering, $E$. [1868] Gött. Ab. 14 (1869) 16 pp.

- - numbers and Hilbert's theorem. Capelli, A. Nap. Rd. 35 (1896) 198-.

Complex factorisation of numbers. Cauchy, A. L. C. R. 24 (1847) 1022-; 25 (1847) 46 -

- factors of prime numbers. Zignago, $I . \mathrm{Rv}$. Mt. 4 (1894) 151-.

- number systems, irreducible. Formenti, C. Mil. I. Lomb. Rd. 17 (1884) 6-.

- numbers. Schwering, $K$. Crelle J. Mth. 102 (1888) 56-.

- - , advantages of geometric representation. Vivanti, G. Rv. Mt. 2 (1892) 167-.

- -, class. Markoff, A. C. R. 112 (1891) 780-, 1049-, 1123-, 1288.

- connected with equation $\theta^{3}-2=0$. Mathews, G. B. L. Mth. S. P. 24 (1893) $319-$.

- -, extension. Vahlen, T. Königsb. Schr. 38 (1897) [72]-

- - of form $a+b i$. Gegenbauer, $L$. Wien Ak. Sb. 91 (1885) (Ab. 2) 1047-

- - formed from fourth roots of unity. Gegenbauer, $L$. Wien Ak. D. 50 (1885) (Ab. 1) 153-.

- - - - - - - , probabilities in connexion with. Gegenbauer, $L$. Wien Ak. Sb. $98(1890)(A b .2 a) 635-$.

- $-\ldots$ - _ _ -, quadratic reciprocity law for. Busche, E. Hamb. Mth. Gs. Mt. 2 (1890) (Festschr., Tl. 2) 80-.

- - ideal prime factors. Kummer, $E . E$. Berl. Ab. (1856) (Mth.) 1-.

- - norms. Schwering, $K$. Acta Mth. 11 (1887-88) 265-.

- - , systems of, and bilinear groups. Cartan, $E$. Toul. Fac. Sc. A. 12 (1898) B, 99 pp.

$--, \ldots,-$ transformation groups. Study, E. Mh. Mth. Ps. 1 (1890) 283-.

- -, theory. Ivanov, I. St Pet. Ac. Sc. Mm. (Rs.) 72 (1893) (Suppl.) No. 9, 14 pp.; Fschr. Mth. (1893-94) 302.

- primes. Gegenbauer, L. Wien Ak. Sb. 98 (1890) $(A b .2 a)$ 1036-.

- - formed from fifth roots of unity. Tanner, $H . W . L$. L. Mth. S. P. 24 (1893) 223-.

Cubic number-fields, number of ideal classes in. Dedekind, $R$. Crelle J. Mth. 121 (1900) 40 -

- - table of class numbers. Reid, L. W. N. Y. Am. Mth. S. Bll. 6 (1900) 268, 277-. 
Decomposition of numbers $f^{12}-9 g^{12}$ into two cubes. Henry, C. N. A. Mth. 20 (1881) 418-.

Dirichlet's biquadratic number-field. Hilbert, D. Mth. A. 45 (1894) 309-.

Divisors of certain polynomials and existence of certain primes. Genocchi, A. C. R. 98 (1884) 411-.

- complex numbers, theorems. Gegenbauer, L. Mh. Mth. Ps. 1 (1890) 39-.

-, elementary, of compounded matrices. Hensel, $K$. Crelle J. Mth. 114 (1895) 109-.

,,-- theory and applications. Sauvage, $L$. Par. Éc. Norm. A. 8 (1891) 285-; 10 (1893) 9-.

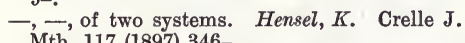
Mth. 117 (1897) 346-.

-, fundamental, of algebraic systems. Hensel, $K$. Crelle J. Mth. 117 (1897) 333-.

- , - _ - - system with reference to two different rational fields. Hensel, $K$. Crelle J. Mth. 118 (1897) 173-.

Dual group generated by three moduli. Dedekind, R. Mth. A. 53 (1900) 371-.

Equation $F(x, y, z)=\phi(a) \phi(\beta) \ldots=1$. LejeuneDirichlet, G. C. R. 10 (1840) 285-.

$-F(x, y, z)=\phi(a) \phi(\beta) \ldots=1$ (Lejeune-Dirichlet). Libri, G. C. R. 10 (1840) 311-.

Equivalences, theory. Grunert, J.A. Arch. Mth. Ps. 44 (1865) 443-; 45 (1866) 454-.

Factorisation of function with respect to prime modulus $p$. Pellet, A. E. C. R. 86 (1878) 1071-.

-, complex, of primes, theorem. Cellérier, $C$. Gen. S. Ps. Mm. 32 (1894-97) No. 7, 61 pp.

$-x^{n}-a$ in arbitrary field. Wendt, $E$. Mth. A. 53 (1900) 450 -.

Functions of several variables, irreducibility. Bendixson, I. Stockh. Öfv. (1892) 189-.

---1 , matrices. Kronecker, L. Berl. Mb. (1869) 159-, 688-.

-, two biquadratic, of 4 variables, whose product is a similar function. Tortolini, $B$. Tortolini A. 2 (1859) 9-.

Fundamental equation of a class for a real prime as modulus. Hensel, $K$. Crelle J. Mth. 113 (1894) 61-.

- system and discriminant of classes of algebraic numbers. Landsberg, $G$. Crelle J. Mth. 117 (1897) 140-.

Galoisian number-fields. Hilbert, D. Gött. Nr. (1894) 224-.

Galois's imaginaries, operation symbols involving. Le Vavasseur, -. Toul. Ac. Sc. Mm. 9 (1897) 247-.

Greatest common divisor. Gegenbauer, $L$. Wien Ak. Sb. 101 (1892) (Ab. 2a) 1143-.

--- , theorem. Mertens, $F$. Wien Ak. Sb. 101 (1892) (Ab. 2a) 1560-.

Groups of functions, representation by a basis. Ritter, E. Gött. Nr. (1895) 158-.

Ideal complex numbers, properties of classnumber. Kronecker, L. Berl. Mb. (1870) 881-.

- division of algebraic quantities by real primes. Hathaway, A. S. J. H. Un. Cir. [4] (1885) 79 .
Ideals. Hathaway, A. S. Am. J. Mth. 9 (1887) 162-.

- in algebraic number-field, existence of primitive numbers. Wiman, A. Stockh. Öfv. (1899) 879-.

-, Dedekind's. Baur, L. Mth. A. 32 (1888) 151-.

-, Dedekind-Weber, in a hyper-elliptic field. Baur, L. Mth. A. 41 (1893) 491-.

- and higher congruences, connection of theories. Dedekind, R. Gött. Ab. 23 (1878) 5-.

-, theory. Dedekind, R. Gött. Nr. (1894) 272-; (1895) 106-.

-, - Hurwitz, A. Gött. Nr. (1894) 291-. -, -. Furtwängler, $P$. Gött. Nr. (1895)

Integral functions. Heine, H. E. [1852] Crelle J. 48 (1854) 237-, 243-.

- - with binomial divisors. Hill, C. J. D. Crelle J. 70 (1869) 103-.

- - of integers, irreducible factors. Runge, $C$. Crelle J. Mth. 99 (1886) 89-.

$--1-,-\ldots$. Mandl, $M$. Crelle J. Mth. 113 (1894) 252-.

- - modular factors. Cauchy, A. L. C. R. 24 (1847) 1116-.

- polynomials with prime divisors of assigned form. Lefébure, $A$. C, R. 98 (1884) 293-, 413-, 567-, 613-; Par. Ec. Norm. A. 1 (1884) 389-; 2 (1885) 113-.

- - - several variables, resolution into factors. Hancock, $H$. Par. Éc. Norm. A. 17 (1900) 89-. Par. Éc. Norm. A. 2 (1885) 289-. Méray, $C$.

Modular systems, decomposition. Moore, E. H. N. Y. Am. Mth. S. Bli. 3 (1897) 372-.

- - and general complex numbers, theory. Kronecker, L. Berl. Ak. Sb. (1888) 429-, 447-, 557-, 595-, 983-.

- - Kronecker's, canonical forms for. Hancock, H. Crelle J. Mth. 119 (1898) 148-.

,--- , reduction. Hancock, $H$. QJ. Mth. 27 (1895) 147-; Crelle J. Mth. 122 (1900) 265-.

- - relations between. Pund, O. Hamb. Mth. Gs. Mt. 3 (1900) 325-.

- - of second rank, elementary properties of pure. Hensel, $K$. Crelle J. Mth. 119 (1898) 175-.

- ---- , and rings of numbers. Lands. berg, G. Gött. Nr. (1897) 277-.

Moduli, extension of symbol $(a, b)$ in theory. Dedekind, $R$. Gött. Nr. (1895) 183-.

-, theory. Steinitz, E. Mth. A. 52 (1899) 1-. Numbers, as sum of 2 cubes. Lenhart, $W$. [1836] Mth. Misc. 1 (1838) 114.

Pairs of forms $x y\left(x^{4}-y^{4}\right)$ whose product or quotient is square. Euler, L. St Pét. Ac. Sc. Mm. 11 (1830) 31-.

Period equation of numbers formed with $\lambda$ th roots of unity. Stouff, $X$. C. R. 125 (1897) 859-.

Polynomial of several variables, test for factorisability. Laurent, $H$. N. A. Mth. 12 (1893) 315-. 
Primary prime functions. Hancock, $H$. [1900] N. Y. Âm. Mth. S. Bll. 7 (1901) 202, 206-.

Prime factor of class-number $H$, divisibility by $\lambda$. Kronecker, L. Liouv. J. Mth. 1 (1856) 396 -

- factors of certain forms, Lejeune-Dirichlet's theorems. Kronecker, L. Berl. Ak. Sb. (1888) 417-

- ideals in relation to substitution groups. Frobenius, G. Berl. Ak. Sb. (1896) 689-.

- - separation of number-field into. Hilbert, D. Mth. A. 44 (1894) 1-.

- integral complex numbers formed from fourth roots of unity. Gegenbauer, $L$. Wien Ak. Sb. 101 (1892) (Ab. 2a) 984-.

Primes and prime ideals in domain of fifth roots of unity. Gmeiner, J. A. Mh. Mth. Ps. 11 (1900) 1-。

Primitive factors of $4\left(1-x^{n}\right) /(1-x)$, reduction of product to form $Y^{2} \pm n Z^{2}$, Cauchy's theorem. Genocchi, A. C. R. 67 (1868) 1035-。

Quadratic complex numbers, certain systems. W'estern, A. E. [1898] Camb. Ph. S. T. 17 (1899) 109-.

Representation of numbers of a class-domain. Hensel, $K$. Crelle J. Mth. 103 (1888) 230-.

- - - by forms. Poincaré, H. C. R. 92 (1881) 777-; Par. S. Mth. Bll. 13 (1885) 162-. - - - - Gegenbauer, L. Wien Ak. Sb. 95 (1887) (Ab. 2) 618-

Symmetric matrices. Kronecker, L. Berl. Ak. Sb. (1889) 349-.

\section{Application of trigonometri- cal functions to arithmetic; cyclotomy.}

Absolutely smallest residues of real magnitudes. Kronecker, L. Berl. Ak. Sb, (1885) 383-, 1045-.

Arithmetical series, Dirichlet's theorem. Sylvester, J. J. As. Fr. C. R. (1888) (Pt. 2) 118 -

\section{Binomial equations.}

Liouville, J. Liouv. J. Mth. 2 (1857) 413-.

Trudi, N. [1866] Nap. At. Ac. 3 (1866-68) No. 6, 49 pp.

Rubini, R. G. Mt. 5 (1867) 184-.

and algebraic radicals. Valat, - Bordeaux Act. Ac. Sc. (1843) 321-

alternating sums formed with primitive roots. Cauchy, A. L. C. R. 10 (1840) 560-.

application of Sturm's theorem. Gascheau, $G$. Liouv. J. Mth. 7 (1842) 126-.

- to summation of series. Berger, A. Ups. S. Sc. N. Acta 13 (1887) No. 7, 36 pp.

depression of degree. Lobatschewsky, N. (vI Adds.) Kazan Mm. Un. (1834) 1-.

existence and characteristics of roots. Méray, C. Par. Éc. Norm. A. 2 (1885) 337-.

functions of roots. Schönemann, $T$. Crelle J. 17 (1837) 372-.

irreducibility. Mertens, $F$. Wien Ak. Sb. 99 (1891) (Ab. 2a) 907- reducibility. Bucca, $F$. Palermo Cir. Mt. Rd. 14 (1900) 136-.

reducible. Vahlen, $K$. Acta Mth. 19 (1895) 195-. roots. Tardy, P. A. Mt. 3 (1869-70) 331-.

-, extraction. Berkhout, J.J.T. van (vi Adds.) Arch. Wisk. Gn. 1 (1859) 54.

solution. Garnier, J. G. Brux. Ac. Bll. 6 (1839) 474-.

-, cases. Lenthéric, -. Gergonne A. Mth. 21 (1830-31) 101-.

-, trigonometric. Tetmajer, J. (xII) Krk. Ak. (Mt.-Prz.) Pam. 5 (1880) 117-.

sums of powers of a primitive root. Cauchy, A. L. C. R. $10(1840) 594-$.

transformations. Gram, J. P. Ts. Mth. 5 (1887) 44-; Fschr. Mth. (1887) 72-.

$x^{257}=1$, solution. Richelot, $F$. J. Crelle J. 9 (1832) 1-, 146-, 209-, 337-.

$x^{257}-1=0$, solution. Fischer, $P$. A. Crelle J. 11 (1834) 201-.

$x^{p}=1$. Lebesgue, $V . A$. C. R. 5 (1837) 722-. $A^{B}=C$. Pagani, G. $M$. Brux. Ac. Bll. 4 (1837) 387 ; Brux. Ac. Sc. Mm. 11 (1838) $11 \mathrm{pp}$.

$x^{n}-1=0$, trigonometric series derived from. Cauchy, A. L. C. R. 10 (1840) 719-.

$x^{p}-1=0, p$ prime, solution. Realis, S. N. A. Mth. 2 (1843) 5-, 147-.

$x^{p}=1$, auxiliary equation of $\mathrm{mth}$ order $(p=m \pi+1)$. Lebesgue, V. A. C. R. 18 (1844) 696-.

$x^{p}-1=0$, modular indices of polynomials connected with. Cauchy, A. L. C. R. 25 (1847) 93-

$x^{p}-1=0, p$ prime, solution. Plana, $G .[1850]$ Tor. Mm. Ac. 11 (1851) 413-.

$x^{257}-1=0$. Cayley, A. Crelle J. 41 (1851) 81-.

$x^{n}-1=0$, primitive roots. Lebesgue, $V . A$. N. A. Mth. 11 (1852) 417-.

$z^{p}=1$, Lagrange's resolvent, expression analogous to. Kummer, $E$. $E$. Rm. At. 6 (1852-53) 237-.

$x^{p}=1, p$ prime, solution. Lebesgue, $V . A$. C. R. 38 (1854) 914-.

$w^{n}=1$, periods formed by roots when $n$ is not prime. Fuchs, L. [1862] Crelle J. 61 (1863) 374-.

$x^{m}-1=0$. Catalan, E. C. Brux. Ac. Bll. 29 (1870) 182-.

$x^{p}-1=0$, trisection and quartisection. Cayley, A. L. Mth. S. P. $11(1879-80) 4$ -

$x^{p}-1=0$, quinquisection. Cayley, $A$. L. Mth. S. P. 12 (1880-81) 15-; $16(1884-85)$ 61-.

$x^{p}-1=0 . \quad S c o t t, C . A . \quad$ Am. J. Mth. 8 (1886) 261-.

$x^{p}-1=0$, quinquisection. Tanner, H. W. L. L. Mth. S. P. 18 (1886-87) 214-.

$x^{p}-1=0, p$ prime, solution. Butters, J. $W$. Edinb. Mth. S. P. 7 (1889) 10-.

$x^{17}-1=0$. Cayley, A. Mess. Mth. 19 (1890) 184-.

$x^{p}-1=0$, solution. Pierpont, J. N. Y. Am. Mth. S. Bll. 2 (1896) 77-

$x^{n} \pm 1=0$, solution. Jarolimek, $V$. Casopis 27 (1898) 209-; Fschr. Mth. (1898) 75. 
Congruences, application of cyclotomy. Moesta, C. W. Grunert. Arch. 10 (1847) 98-. -, - - to divisors of cyclotomic functions. Hathaway, A. S. [1880] (xII) J. H. Un. Cir. [1] (1882) 67.

Congruent roots. Kummer, E. E. Crelle J. 53 (1857) 142-.

Cyclotomic equations, irreducibility. Dedekind, $R$. Crelle J. 54 (1857) 27-.

- $m$ prime, $m$-fold section. Glashan, J. C. Am. J. Mth. 21 (1899) 276-.

- -, quinquisection. Glashan, J. C. [1897] Am. J. Mth. 21 (1899) 270-.

- -, theory. Migotti, A. [1882] Wien Ak. Sb. 87 (1883) (Ab. 2) 7-.

- functions. Lucas, É. C. R. 90 (1880) 855-. - Tanner, H. W. L. L. Mth. S. P. 20 (1889) 63-, 258-.

- -, considered with respect to prime modulus $p$. Hathaway, A. S. [1881] (xII) J. H. Un. Cir. [1] (1882) 131.

- -, divisors. Sylvester, J. J. C. R. 90 (1880) 287-, 345-.

_C. R. 90 (1880) 526-.

Cyclotomy. Gauss, C. F. Par. S. Phlm. Bll. 3 (1802) 102-.

- Frischauf, J. Wien Sb. 55 (1867) (Ab. 2) 113 -.

-, application to theory of numbers. Jacobi, C. G. J. Berl. B. (1837) 127-; Crelle J. 30 (1846) 166-.

- - - - - (Jacobi). Lebesgue, V. A. Liouv. J. Mth. 19 (1854) 289-.

- derived from linear equations (for primes of form $\left.2^{m}+1\right)$. Hermes, $J$. [1878] Crelle J. Mth. 87 (1879) 84-.

-, generalisation. Stickelberger, L. Mth. A. 37 (1890) 321-.

- - , algebraic. Berger, A. Stockh. Öfv. (1891) 683-; Fschr. Mth. (1891) 190-.

-, new method. Quincke, G. A. Ps. C. 149 (1873) 270-.

-, primes of form $2^{2^{n}}+1$. Lucas, $\dot{E}$. C. R. 85 (1877) 136-.

-, proofs of theorems related to. Lanavicensis (Sylvester, J. J.). Ph. Mg. 18 (1859) 281-.

- by purely geometrical means; possible cases. Hessel, J. F. C. Grunert Arch. 37 (1861) $269-$.

Determinants arising in cyclotomy. Hermes, $J$. Arch. Mth. Ps. 6 (1888) 276-.

Divisibility of prime factor of class-number $H$ by $\lambda$. Kronecker, L. Liouv. J. Mth. 1 (1856) 396-.

Division of angle into $2^{n}+1$ equal parts. Kempe, A. N. Arch. Wisk. 1 (1895) 163-; Fschr. Mth. (1893-94) 1152-.

- - - any number of equal parts. Kempe, A. N. Arch. Wisk. 1 (1895) 215-; Fschr. Mth. (1893-94) 1153.

Divisors of Fermatians. Sylvester, J. J. C. R. 106 (1888) 446-; Nt. 37 (1888) 417-.

- - form $A x^{2}+B y^{2}+C z^{2}$, proof of theorem of Lagrange, and divisors of functions which multisect the roots of unity. Sylvester, J.J. Am. J. Mth. 3 (1880) 390-.
Divisors of functions of primitive roots of unity. Sylvester, J. J. C. R. 92 (1881) 1084-.

- resulting from cyclotomy. Kummer, E. E. Crelle J. 30 (1846) 107-.

Equation for primitive roots of unity, irreducibility. Lebesgue, V. A. Liouv. J. Mth. 4 (1859) 105-.

- $-n$th roots of unity. Bang, A.S. N. Ts. Mth. 6 (B) (1895) 6-; Fschr. Mth. (1895) 121.

- $t^{2}-p u^{2}=1, p$ prime, solution by circular functions. Lejeune-Dirichlet, $G$. Crelle J. 17 (1837) 286-.

$-x^{p-1}+x^{p-2}+\ldots+1=0$ is irreducible when $p$ is prime. Kromecker, $L$. Crelle J. 29 (1845) 280 ; Liouv. J. Mth. 1 (1856) 399-.

$-X=1+x+\ldots+x^{p-1}=0, p$ prime, irreducibility. Prouhet, E. N. A. Mth. 9 (1850) 348-.

Equations, resolubility, especially $x^{n}-1=0$. Allégret, $A$. C. R. 43 (1856) 860-.

Eulerian numbers, residues with respect to prime modulus. Lucas, $\hat{E}$. [1877] Par. S. Mth. Bll. 6 (1878) 49-.

Gauss, formula

$$
\left[\Sigma \omega^{a}-\Sigma \omega^{b}= \pm \sqrt{(-1)^{\frac{n-1}{2}} n,}\right.
$$

where $\quad \omega=\cos \frac{2 \pi}{n}+\sqrt{-1} \sin \frac{2 \pi}{n}$.

Kronecker, L. Liouv. J. Mth. 1 (1856) 392-. 一, theorem on $4 \frac{x^{p}-1}{x-1}$, extension. Lucas, É.

Tor. Ac. Sc. At. 13 (1877) 271-.

Gauss's sums. Séguier, - de. C. R. 123 (1896) 166-.

- Lerch, M. Časopis 28 (1899) 1-; Fschr. Mth. (1898) 168.

- - analogues. Lerch, M. Prag Sb. (1897) (Mth.-Nt.) No. 43, $16 \mathrm{pp}$.

- -, series $\sum_{0}^{n-1} e \frac{8^{2} 2 \pi i}{n}$. Mertens, $F$. Berl. Ak. Sb. (1896) 217-.

- with several variables. Jordan, C. C. R. 73 (1871) 1316-.

Imaginary cube roots. Horner, $W . G$. Thomson A. Ph. 8 (1816) 279-, 388-.

--- , every number has four. Lockhart, $J$. Thomson A. Ph. 5 (1815) 439-

Regular polygon, 17 sides, inscription. James, S. Ir. Ac. T. 13 (1818) 175-.

- - - - 一. Grunert, J. A. Arch. Mth. Ps. 42 (1864) 361-.

- - - - - Collignon, É. As. Fr. C. R. 8 (1879) 162-.

- polygons, number of inscriptible. Dickson, $L: E$. N. Y. Mth. S. Bll. 3 (1894) 123-. - 7 and 13 sides, construction. Affolter, F. G. Mth. A. 6 (1873) 592-.

$-\frac{1}{V}, 15$ and 17 sides, inscription. Lebesgue, V. A. N. A. Mth. 5 (1846) 683-.

-, 17 sides, construction. Staudt, G. K. C. von. Crelle J. 24 (1842) 251.

,,--- . Breton [de Champ], P. N. A. Mth. 5 (1846) 226-, 340.

$--,-1,-$ Hoïel, J. N. A. Mth. 16 
2880 Application of Trigonometry

Regular polygons, 17 sides, construction (von Staudt). Schröter, H. [1872] Crelle J. 75 (1873) 13-.

_- - - - - Richmond, H. W. QJ. Mth. 26 (1893) 206-.

-,,--- - Gérard, L. Mth. A. 48 (1897) 390-.

- - - - - Fontene, G. Mathesis 19 (1899) 179-.

-, 19 and 37 sides, construction. Amaldi, I. G. Mt. 30 (1892) 141-。

- - 257 sides. Baden-Ghijben, J. Amst. Vs. Ak. 2 (1868) (Ntk.) 1-.

_ , _ - Pascal, E. Nap. Rd. 26 (1887) 33-.

Roots of unity, equation of periods. Pellet, A. As. Fr. C. R. (1900) (Pt. 1) 125.

_ - , equations of three and four periods. Sylvester, J. J. As. Fr. C. R. (1880) 96-.

- - - multisection. Sylvester, J. J. [1881] (xII) J. H. Un. Cir. [1] (1882) 150-.

_- new system. Hamilton, (Sir) W. R. (vi Adds.) Ph. Mg. 12 (1856) 446.

_ _ _ , and operations relative to radicals. Wantzel, L. Férussac Bll. Sc. Mth. 16 (1831) 166-.

- - , primitive. Mollame, $V$. Nap. Rd. 31 (1892) 179-.

- _ -, proposition. Stewart, B. [1856] Edinb. R. S. T. 21 (1857) 407-.

- - - , - (Stewart). Todhunter, I. [1865] Edinb. R. S. P. 5 (1866) 517-.

- - , theorem. Schröder, E. Z. Mth. Ps. 22 (1877) 183-.

- _ - , and transformation of functions. Jürgensen, C. Crelle J. 6 (1830) 195-.

_ _ 9th. Cayley, A. Mess. Mth. 20 (1891) 63.

- - , nth, theorems. Glaisher, J.W.L. B. A. Rp. (1875) (Sect.) 13-.

Table of complex prime factors, formed from 5 th roots of unity of primes of form $5 \mu+1$. Reuschle, [C. G.] Berl. Mb. (1859) 488-.

- - - - - - 7th, 11th, 13th, 17th, $19 \mathrm{th}, 23 \mathrm{rd}$, and $29 \mathrm{th}$ roots of unity of primes $<1000$. Reuschle, [C. G.] Berl. Mb. (1859) 694-; (1860) 190-, 194-, 714-, 719-, 725-, 729 .

Trigonometric equations. Guilmin, A. N. A. Mth. 5 (1846) 49-.

- functions deduced from equation $x^{n}=1$. Svanberg, J. Stockh. Ak. Hndl. 33 (1812) 246-

- series, a special. Preobraženskij, P. V. Kazan S. Nt. (P8.-Mth.) P. 6 (1888) 10-; Fschr. Mth. (1888) 172-.

$\sin \frac{\pi x}{2}$, arithmetical rôle. Cesáro, E. A. Mt. 13 (1885) 315-.

$\cos \frac{2 \pi}{17}$, quadratic radical expression for. Steggall, -. Edinb. Mth. S. P. 7 (1889) 4 $x^{p}-A$, irreducibility. Mertens, $F$. Mh. Mth. Ps. 2 (1891) 291-.

$x^{n}-1$, irreducible divisors. Gravelaar, N.L. W. A. N. Arch. Wisk. 20 (1893) 7-; Fschr. Mth. (1893-94) 158-.
Transcendental functions 2890

2890 Application of other transcendental functions to arithmetic.

Asymptotic formulæ, note. Gegenbauer, $L$. Wien Ak. Sb. 93 (1887) (Ab. 2) 35-.

- laws. Lejeune-Dirichlet, C. Berl. B. (1838) 13-.

- Gegenbauer, L. Wien Ak. D. 49 (1885) (Ab. 1) 37-.

- value of series $\Sigma \frac{1}{a x^{2}+2 b x y+c y^{2}}$. Mertens, F. Wien Ak. Sb. 106 (1897) (Ab. 2a) 411-.

- values of some numerical functions. Hermite, C. Crelle J. Mth. 99 (1886) 324 .

_ _ _ _ - (Hermite). Gegenbauer, L.

Wien Ak. Sb. 99 (1891) (Ab. 2a) 387-.

Combinations of special kind. Bunžakovskij, V. Ja. [1871] St Pet. Ac. Sc. Mm. (Rs.) 20 (*1872) (Suppl.) No. 2, $71 \mathrm{pp}$.

Continuous variables in theory of numbers. Hermite, C. Crelle J. 41 (1851) 191-.

Decomposition of number into sum of squares. Lorenz, L. V. (xII) Ts. Mth. 1 (1871) 97-. - - - - - Mathews, G. B. L. Mth. S. P. 27 (1896) 55-.

- Pepin, $T$. Rm. N. Linc. At. 38 (1885) 139-.

Discontinuous factor, case. Lorenz, L. N. Ts. Mth. 1 (B) (1890) 76-; Fschr. Mth. (1890) 296-.

Division, theorem on remainders. LejeuneDirichlet, G. Berl. B. (1851) 20-.

- $-\ldots-$ (Lejeune-Dirichlet). Terquem, $O$. N. A. Mth. 13 (1854) 396-.

Divisors of a number, theorems. Cesáro, E. G. Teix. J. Sc. 7 (1886) 3-.

Elliptic functions, application. Kronecker, $L$. Liouv. J. Mth. 3 (1858) 265-

- - - - Hermite, C. C. R. 53 (1861) 214-; 55 (1862) 11-, 85-; Liouv. J. Mth. 7 (1862) 25-.

- -, -. Stieltjes, -. C. R. 98 (1884) 663-. - - - to quadratic forms, binary. Hermite. C. [1883] St Pét. Ac. Sc. Bll. 29 (1884) 325-, - - , - - - with negative discriminant. Petr, K. Prag České Ak. Fr. Jos. Rz. (Trída 2) 9 (1900) Art. 38, 17 pp.; Fschr. Mth. (1900) 212-.

- - - solution of equation $x^{3}+y^{3}=1$. Cayley, A. [1881] Camb. Ph. S. P. 4 (1883) 106-.

- _ , - theory of numbers. Nazimov, P. S. Mosc. Un. Mm. (Ps.-Mth.) 5 (1885) 67-; Par. Éc. Norm. A. 5 (1888) 23-, 147-. - _, - _ - Weber, H. Gött. Nr. (1893) 46-, 138-, 245-.

- - , - of transformation-theory to theory of numbers. Weber, $H$. Mth. A. 43 (1893) 185-.

- and other functional constants, numerical laws depending on. Bougaieff, H. B. Bll. Sc. Mth. 9 (1885) 89-.

Euler's product, and arithmetical results. Jacobi, C. G. J. Liouv. J. Mth. 7 (1842) 85-.

Fuchsian functions and arithmetic. Poincaré, $H$. Liouv. J. Mth. 3 (1887) 405-. 
Function $\phi(N)$, asymptotic expression. Mertens, F. Crelle J. Mth. 77 (1874) 289-.

$-\psi(m)$. Poréckij, P. S. Kazan S. Nt. (Ps.Mth.) P. 6 (1888) 52-; Fschr. Mth. (1888) 171.

$-F\left(\frac{x}{y}\right)=1$ or 0 as $y$ is $\gtreqless x$, formula con. cerning. Cavallin, C. B. S. N. Ts. Mth. 5 (B) (1894) 33-; Fschr. Mth. (1893-94) 266-.

Functions giving real prime numbers. Gegenbauer, L. Mh. Mth. Ps. 7 (1896) 73-.

- in theory of numbers. Daublebsky von Sterneck, R. Mh. Mth. Ps. 7 (1896) 37-.

- - - - calculation of certain. Daublebsky von Sterneck, R. Mh. Mth. Ps. 9 (1898) 43-.

$-\psi(p, q)$ and $\chi(p, q)$, series connected with. Lerch, M. Prag Sb. (1894) (Mth.-Nt.) No. $33,16 \mathrm{pp}$.

Infinitesimal analysis, applications. LejeuneDirichlet, G. Crelle J. 19 (1839) 324-; 21 (1840) 1-, 134-.

Integral calculus, applications. Lucas, $\dot{E}$. C. R. 82 (1876) 1303-.

- - - - Weber, $H$. Gött. Nr. (1896) 275-.

Lambert's series. Scherk, H. F. Crelle J. 9 (1832) 162-.

- - Schläflï,L. Grunert Arch.10 (1847) 332-. - - Cesáro, E. G. Teix. J. Sc. 6 (1885) 91-.

- - in asymptotic arithmetic. Cesáro, $E$. Nap. Rd. 32 (1893) 197-.

- - as definite integral. Schlömilch, $O$. Schlömilch Z. 6 (1861) 407-.

- - generalised. Cesáro, E. G. Teix. J. Sc. 6 (1885) 19-.

- - and law of primes. Curtze, M. [1867] A. Mt. 1 (1867-68) 285-.

- -, transformations. Cesáro, E. N. A. Mth. 7 (1888) 374-.

Law of prime numbers. Burhenne, $H$. Grunert Arch. 19 (1852) 442-.

Lemniscate functions and complex integers, theorem of reciprocity. Bonaventura, $P$. G. Mt. 30 (1892) 300-.

Linear forms, theorem on sum of $p$ th powers. Minkowski, H. C. R. 112 (1891) 209-.

Logarithms of large numbers. Le Barbier, Gergonne A. Mth. 20 (1829-30) 366-.

Numerical products in which exponents depend on numbers. Glaisher, J. W. L. Mess. Mth. 23 (1894) 145-; 25 (1896) 186.

Series connected with prime numbers. Rogel, F. Prag Sb. (1895) (Mth.-Nt.) No. 22, 11 pp.

- in theory of numbers, transformation. Rogel, F. Prag Sb. (1897) (Mth.-Nt.) No. 51, 31 pp.

-, trigonometric, functions in theory of numbers represented by. Rogel, $F$. Arch. Mth. Ps. 10 (1891) 62-.

Sums of powers of divisors, relations between. Rogel, $F$. Prag Sb. (1897) (Mth.-Nt.) No. 7, 9 pp.

\section{Distribution of prime numbers.}

Hargreave, C. J. Ph. Mg. 35 (1849) 36-.

Tchebicheff, $P$. [1850] Liouv. J. Mth. 17 (1852) 366-.

VOL. I.
Polignac, A. (Prince de). Liouv. J. Mth. 19 (1854) 305-; C. R. 45 (1857) 406-, 431-, 575-, 882-; 49 (1859) 350-, 386-, 624-, 724-. Lebesgue, $V . A$. N. A. Mth. 15 (1856) 130-, 236-. Mertens, $F$. Crelle J. Mth. 78 (1874) 46-.

Johnson, W. W. Des Moines Anal. 2 (1875) 9-.

Lucas, E. As. Fr. C. R. (1877) 159-.

Gegenbauer, $L$. Wien Ak. Sb. $89(1884)(A b .2)$ 841-.

Piltz, -. Jena. Sb. (1885) 42-.

Gegenbauer, L. Wien Ak. Sb. 97 (1889) (Ab. 2a) 374-.

Poincaré, H. C. R. 113 (1891) 819.

(Poincaré's theorem.) Stanievitch, $V$. C. R. 114 (1892) 109-; Fschr. Mth. (1899) 190-.

Foussereau, G. Par. Ec. Norm. A. 9 (1892) 31-. Phragmén, - . C. R. 114 (1892) 337-.

Cesáro, E. Nap. Rd. 35 (1896) 297-.

La Vallée-Poussin, C. J. de. Brux. S. Sc. A. 20 (1896) (Pt. 1) 100-, (Pt. 2) 183-, 281-; 21 (1897) (Pt. 1) xxm, 1-, 60-, (Pt. 2) 251-.

Koch, H. von. Stockh. Öfv. (1900) 669-.

Arithmetical progression containing infinity of primes. Lejeune-Dirichlet, $G$. Berl. B. (1837) 108-; Liouv. J. Mth. 4 (1839) 393-. - - - - Sylvester, J. J. L. Mth. S. P. 4 (1871-73) 7-; C. R. 106 (1888) 1278-, 1385-.

(1893) 47-.

$-\frac{1}{-}-$ Speckmann, G. Arch. Mth. Ps. 12 (1894) 439-.

115 (1895) 85-.

- - - - - (Dirichlet). La ValléePoussin, C. J. de. [1895] Brux. Mm. Cour. $8^{\circ}, 53$ (1895-96) No. 6, 32 pp.

- - - - - - $(-)$. Mertens, F. Wien Ak. Sb. 104 (1895) (Ab. 2a) 1093-, 1158-; 106 (1897) (Ab. 2a) 254-; 108 (1899) (Ab. 2a) 32-.

- progressions, with difference and first term mutually prime. Gegenbauer, $L$. Wien Ak. Sb. 100 (1891) (Ab. 2a) 1018-.

- series. Sylvester, -. Mess. Mth. 21 (1892) 1-, 87-, 192.

Complex primes, Dirichlet's theorem that linear complex function contains infinite number of. Mertens, $F$. Wien Ak. Sb. 108 (1899) (Ab. 2a) 517-.

- -, Tchebicheff's theorems extended to. Poincaré, H. Liouv. J. Mth. 8 (1892) 25-.

Composite numbers, long successions. Glaisher, J.W.L. [1877] Mess. Mth.7 (1878) 102-, 171-. - - - - Lucas, É. Mess. Mth. 8 (1879) 81.

Determination of primes. Bouniakowsky, $V$. [1839] St Pét. Ac. Sc. Mm. 4 (1841) 447-.

Divisibility of product, distribution of primes. Catalan, E. C. Liège S. Sc. Mm. 12 (1885) No. 2, 20-, 119-.

Enumeration of primes. Johnson, W. $W$. Des Moines Anal. 5 (1878) 7-.

Factors of Möbius. Koch, H. von. Stockh. Öfv. (1900) 659-.

Fermat's theorem $\left(2^{n}+1\right.$ prime $)$. Baltzer, $R$. [1878] Crelle J. Mth. 87 (1879) 172. 
Fermat's theorem $\left(2^{n}+1\right.$ prime), Cauchy's proof. Grunert, J. A. Grunert Arch. 30 (1858) 357-.

Formula for primes. Märcker, - C. Crelle J. 20 (1840) 350-.

- - Pervušin, I. M. Kazan S. Ps.-Mth. Bll. 1 (1891) (Prot.) 70-; Fschr. Mth. 1 (1892) 169.

- - - (Pervušin). Lachtin, L. K. [1892] Rec. Mth. (Moscou) 16 (1893) 460-; Fschr. Mth. (1892) 169-.

- - - (-). Cesáro, E. C. R. 119 (1894) 848-

Formulæ for primes. Lucas, É. As. Fr. C. R. (1877) 159-.

- - - Gegenbauer, L. Wien Ak. Sb. 95 (1887) (Ab. 2) 291-.

- - - approximate. Pervušin, I. Kazan S. Ps.-Mth. Bll. 4 (1895) (Prot.) 94-.

Function $\theta(n)$, development. Minin, A. $\boldsymbol{P}$. Mosc. S. Sc. Bll. 93 (No. 2) (1898) 22; Fschr. Mth. (1898) 165.

Glaisher's factor tables and the distribution of primes. Johnson, W. W. A. Mth. 1 (188485) $15-$.

Goldbach-Euler theorem. Sylvester, J.J. Nt. 55 (1896-97) 196-, 269.

Goldbach's theorem. Haussner, $R$. D. Nf. Vh. (1896) (Th. 2, Hälfte 1) 8-.

- - Stäckel, P. Gött. Nr. (1896) $292-$.

- - and Bertrand's postulate. Catalan, E.C. Liége S. Sc. Mm. 15 (1888) No. 1, 30-, 108-.

- - function $\phi(n)$ and its relation to. Landau, $E$. Gött. Nr. (1900) 177-.

- - tables. Haussner, R. [1897] Ac. Nt. C. N. Acta 72 (1899) 1-.

- - verification. Cantor, G. As. Fr. C. R. (1894) (Pt. 2) 117-.

Graphic determination of position. Wolf, $R$. Bern Mt. (1843) 28-.

- - primes. Kulik, J.P. Böhm. Gs. Ab. 2 (1843) (Sect. B. 1842) 47-.

Infinity of primes. Gegenbauer, $L$. Wien Ak. Sb. 95 (1887) (Ab. 2) 94-.

- - contained in $a x+b$. Proof for $a=8$ or 12. Serret, J.A. [1851] Liouv. J. Mth. 17 (1852) 186-.

- _- Euclid's theorem. Perott, J. Am. J. Mth. 11 (1889) 99-; 13 (1891) 235-.

- - - of form $2 p z-1, p$ being prime. Lebesgue, V. A. Liouv. J. Mth. 7 (1862) 417-.

- - proof. Kummer, E. E. Berl. Ak. Mb. (1878) $777-$.

- - the series of primes. Perott, J. Bll. Sc. Mth. As. 5 (1881) (Pt. 1) 183-.

Integral connected with distribution of primes. Preobraženskij, $P$. Kazan S. Nt. $\left(P_{8_{*}}-M t h.\right)$ P. 7 (1889) 5-; Fschr. Mth. (1889) 183-.

Large primes, determination. Euler, L. [1778] St Pet. Ac. Sc. N. Acta 13 (1802) 14-; 14 (1805) 3-, 11-.

- - - L Lucas, É. As. Fr. C. R. (1876) 61-. - - - Cunningham, (Lt.-Col.) A., \& Woodall, H.J. B. A. Rp. (1900) 646.

Law of distribution. Hargreave, C.J. $\mathrm{Ph}$. Mg. 8 (1854) 114-.

- - Glaisher, J. W. L. B. A. Rp. 42 (1872) (Sect.) 19-; Mess. Mth. 23 (1894) 97-.
Limits of prime of given form. Berton, $V . I$. C. R. 74 (1872) 1390-.

- - primes and a theorem of Legendre. Desboves, A. H. N. A. Mth. 14 (1855) 281-.

Mersenne's numbers. Lucas, É. Mess. Mth. 7 (1878) 185-.

- -. Ball, W. W. R. Mess. Mth. 21 (1892) $34-, 121$.

- - Cunningham, (Lt.-Col.) A. B. A. Rp. (1894) 563-; (1895) 614; Nt. 51 (1894-95) 533.

Number and form of primes. Sludskix, T. A. [1866] (xII) Rec. Mth. (Moscou) 3 (1868) (Pt. 1) 214-.

- of prime and composite numbers between given limits. Preobraženskij, P. V. Rec. Mth. (Moscou) 13 (1886) 707-; Fschr. Mth. (1888) 170-.

- - primes of form $k p+1$ infinite if $p$ is prime. Kraus, L. Casopis 15 (1886) 61-; Fschr. Mth. (1886) 134-.

- - below given limit. Kulik, J. P. Böhm. Gs. Ab. 2 (1843) (Sect. B. 1841) 17-. - - - Riemann, B. Berl. Mb. (1859) 671-.

Tortolini A. 3 (1860) 52-

- - - - Scheibner, W. Schlömilch Z. 5 (1860) 233-.

- Hoffmann, K. $\boldsymbol{E}$. Arch. Mth. Ps. 64 (1879) 333-

- - - - Jonquières, E. de. C. R. 95 (1882) 1144-, 1343-; 96 (1883) 231-.

- - - - - (de Jonquières). Lipschitz, R. O. S. C. R. 95 (1882) 1344-; 96 (1883) 58-, 114-

- - - - Gram, J. P. [1884] Kjøb. Dn. Vd. Selsk. Skr. 2 (1881-86) 183-. (With French résumê.)

- Laar, J. J.van. N. Arch. Wisk. 16 (1889) 209-; Fschr. Mth. (1889) 168. - Rogel, F. Arch. Mth. Ps. 7 (1889) 381-.

- Lorenz, L. Kjøb. Dn. Vd. Selsk. Skr. 5 (1889-91) 427-; Fschr. Mth. (1891) 201-.

- - - Baranowski, A. [1894] Krk. Ak. (Mt.-Prz.) Rz. 8 (1895) 192-; Crc. Ac. Sc. Bll. (1894) 280 -

- Koch, H. von. $\quad$ C. R. 118 (1894) 850-.

- - - - - (Riemann's memoir, 1859). Mangoldt, H. von. [1894] Crelle J. Mth. 114 (1895) 255-.

$--\frac{1}{-}-$ Wigert, S. Stockh. Öfv. (1895) 341-.

- - - Ajello, C. G. Mt. 34 (1896) 14-.

(1897) 235-.

- - Rogel, $F$. Prag Sb. (1899) (Mth.-Nt.) No. 22, 20 pp.

- - - Kluyver, J. C. Amst. Ak. Vs. 8 (1900) 672-; Amst. Ak. P. 2 (1900) 599 -

$-\overline{\text { Taff }}-\bar{P}-$ function determining. 1848] St Pét. Mm. Sav. Étr. 6 (1851) 141-. 
Number of primes below given limit, Legendre's formula. Glaisher, J.W. L. [1879] Camb. Ph. S. P. 3 (1880) 296-.

_- _ _ _ Riemann's formula, application. Mangoldt, $H$. von. Crelle J. Mth. 119 (1898) 65-.

- $-100,000,000$. Meissel, E. Mth. A. 3 (1871) 523-; 21 (1883) 304.

- _ - 1,000,000,000. Meissel, E. Mth. A. 25 (1885) 251 -

-_-_n. Lugli, A. G. Mt. 26 (1888) 86-.

- _ - - - determination when number under $\sqrt{n}$ is known. Graefe, $F$. Z. Mth. Ps. 39 (1894) 38-.

- _ - - - theorem of Legendre's. Sylvester, J. J. C. R. 96 (1883) 463-.

- - between given limits. Drach, S. M. Ph. Mg. 24 (1844) 192-.

- Meissel, E. [1869] Mth. A. 2 (1870) 636-.

- - - - O Oppermann, L. Kjøb. Ov. (1882) 169-; (Résumé, 9-).

$--1-$ Valle, $G$. Rm. N. Linc. Mm. 14 (1898) 143-.

- - - - - , analytical expression for. Levi-Civita, T. Rm. R. Ac. Linc. Rd. 4 (1895) (Sem. 1) 303-.

- - - - - Tchebycheff's theory. Sylvester, J.J. Am. J. Mth. 4 (1881) 230-.

- - _ - _ - theorem of Sylvester's. Gegenbauer, L. Mh. Mth. Ps. 10 (1899) 370-.

- _ - 100,000,001 and 100,001,699. Davis, $W$. Liouv. J. Mth. 11 (1866) 188-.

- -10 and $2 N$, formulæ for. Piarron de Mondésir, E. S. As. Fr. C. R. (1877) 79-. - - - n+1 and $2 n$, etc. Catalan, E.C. [1882] Liége S. Sc. Mm. 10 (1883) (No. 1), $12 \mathrm{pp}$.

- $-x$ and $(1+h) x$, theorem of Stieltjes. Cahen, -. C. R. 116 (1893) 490.

Numbers prime to and less than product of first $n$ primes, properties. Walla, F. Arch. Mth. Ps. 66 (1881) 353-.

Prime-pairs, enumeration. Glaisher, J.W.L. [1878] B. A. Rp. (1878) 470-; Mess. Mth. 8 (1879) 28-.

Primes in Burckhardt's and Dase's tables, enumeration. Glaisher, J.W.L. [1876-77] Camb. Ph. S. P. 3 (1880) 17-, 47-; B. A. Rp. (1877) (Sect.) 20-.

-, construction of a table. Laisant, C. A. As. Fr. C. R. (1891) (Pt. 2) 165-.

-, criteria. Montferrier, A. S. de. Quetelet Cor. Mth. 5 (1829) 94-.

一, - Warner, J. D. Am. As. P. (1890) 54-. ,-- , and factors. Seelhof, $P$. Am. J. Mth. 8 (1886) 39-.

-, distribution problems. Pandau, E. Par. S. Mth. Bll. 28 (1900) 25-.

- of form $4 n+1$ and $4 n+3$, distribution. Tchebichef, $P$. St Pét. Ac. Sc. Bll. 11 (1853) 208.

- - - - - - separate enumeration. Glaisher, J. W. L. B. A. Rp. (1878) 470-; R. S. P. 29 (1879) 192-.

- - 100n+1, enumeration. Daublebsky von Sterneck, $R$. Wien Az. 31 (1894) 2-.
Primes of forms $4 s+1,48-1$, theorems. Gegeubauer, L. Mh. Mth. Ps. 4 (1893) 89-, $98,184$.

-, formulæ, general. Polignac, A. (Prince de). Toul. Mm. Ac. 1 (1857) 308-.

-, properties and distribution. Polignac, $A$. (Prince de). C. R. 29 (1849) 397, 738-.

- in relation to an arithmetical progression. Polignac, A. (Prince de). C. R. 54 (1862) 158-.

-, report on calculations made by Bertelsen. Gram, J. P. Acta Mth. 17 (1893) 301-.

-, theorem. Laurent, $H$. C. R. 126 (1898) 809-.

Recurring series, application. Lucas, $\hat{E}^{\prime}$. C. R. 82 (1876) 165-; 83 (1876) 1286-.

Riemann's function, maximum value. Preobrazenskij, P. V. Mosc. S. Sc. Bll. 78 (No. 1) (1892) 27-; Fschr. Mth. (1892) 170.

$--\zeta(8)$. Bourget, -. C. R. 101 (1885) 304-. - - Jensen, J. L.W. V. C. R. 104 (1887) 1156-.

- - Gram, J. P. Kjøb. Ov. (1895) 303-.

- - - Landau, E. C. R. 129 (1899) 812-. - , and analogous functions. Cahen, $E$. Par. Éc. Norm. A. 11 (1894) 75-.

- $-\ldots,-$ number of primes below given limit. La Vallée-Poussin, C. J. de. Brux. Mm. Cour. 8०, 59 (1899-1900) No. 1, 74 pp.

- - for 8 odd and positive, values. Kluyver, -. Bll. Sc. Mth. 20 (1896) 116-.

- - , zeros. Hadamard, -. C. R. 122 (1896) 1470-; 123 (1896) 93; Par. S. Mth. Bll. 24 (1896) 199-.

- $f(x)$ and logarithmic integral. Phrag. mén, E. Stockh. Öfv. (1891) 599-.

$-\xi(t)$, arithmetical application. Franel, $J$. Zür. Vjschr. 41 (1896) (Festschr., Th. 2) 7-.

- $\zeta$, property. Mertens, $F$. Wien Ak. Sb. 107 (1898) (Ab. 2a) 1429-.

- prime number formula, calculation of some terms. Phragmén, E. Stockh. Öfv. (1891) 721-.

Series of primes. Smith, H. J.S. Ashmol. S. P. 35 (1857) 128-

- -. Lorenz, L. V. (xII) Ts. Mth. 2 (1878) 1-.

Sieve of Eratosthenes. Colombier, P. A. G. N. A. Mth. 2 (1843) $408-$

- - - extension. Buniakovskij, $V$. Ja. St Pet. Ac. Sc. Mm. (Rs.) 41 (*1882) (Suppl.) No. 3, 32 pp.

- - , historical notice. Polignac, $A$. (Prince de). N. A. Mth. 12 (1853) 429-.

_ - , six arithmological propositions deduced from. Polignac, A. (Prince de). N. A. Mth. 8 (1849) 423-.

- for primes. Speckmann, G. Arch. Mth. Ps. 11 (1892) 439-; 12 (1894) 431-.

- - Schapira, H. [1896] D. Mth. Vr. Jbr. 5 (1901) (Heft 1) 69-.

- - Christie, R.W.D. Nt. 56 (1897) 10-.

_ _ - ; number of primes; series of odd numbers. Terquem, O. N. A. Mth. 5 (1846) 607-.

Sum of logarithms of primes not exceeding $x$. Cahen -. C. R. 116 (1893) 85-. 


\section{Special Numbers, etc.}

Sum of $\nu$ th powers of primes less than $n$. Gegenbauer, L. Wien Ak. Sb. 94 (1887) (Ab. 2) 903-.

Tchebychef-de Polignac theorem. Cesáro, E. N. A. Mth. 4 (1885) 418-

- - - Gegenbauer, L. Mh. Mth. Ps. 3 (1892) 319-.

Tchebychef's theory of primes. Daublebsky von Sterneck, $R$. Wien Ak. Sb. 109 (1900) (Ab. 2a) 1137-.

\section{Special numbers and numerical functions.}

Arithmetical theorems. Gegenbauer, $L$. Wien Ak. Sb. 92 (1886) (Ab. 2) 1055-.

Asymptotic calculus, foundations. Cesáro, $E$. C. R. 106 (1888) 1651-; 107 (1888) 426-.

- - - Jensen, J. L.W. V. C. R. 107 (1888) 81-.

- -, proposition. Cesáro, E. A. Mt. 16 (1888-89) 178-.

- law in theory of numbers. Stieltjes, C. R. 101 (1885) 368-.

- laws of functions in theory of numbers. Lipschitz, $R$. Berl. Mb. (1865) 174-.

- - numbers. Cesáro, E. Rm. R. Ac. Linc. Rd. 4 (1888) (Sem. 1) 452-.

- values, formula relating to. Franel, $J$. Mth. A. 51 (1899) 369-.

\section{Bernoulli's numbers.}

Ohm, M. Crelle J. 20 (1840) 11-.

Eisenlohr, O. Crelle J. 28 (1844) 193-.

Bellavitis, G. Tortolini A. 4 (1853) 108-.

Fergola, E. Nap. Ac. Sc. Mm. 2 (1855-57) 315-. Kronecker, L. Liouv. J. Mth. 1 (1856) 385-.

Björling, E. G. $\quad$ Stockh. Öfv. 14 (1857) 107-. Catalan, E. C. C. R. 54 (1862) 1030-, 1059-.

Le Besgue, V. A. C. R. 58 (1864) 853-, 937-.

Catalan, E. C. C. R. 58 (1864) 902-, 1105-; 81 (1875) 441-.

Cayley, A. Mess. Mth. 4 (1875) 157-.

Hermite, C. [1875] Crelle J. Mth. 81 (1876) 93-.

Le Paige, C. M. M. H. H. C. R. 81 (1875) 966-; Brux. Ac. Bll. 41 (1876) 1017.

(Le Paige.) Catalan, E. C. Brux. Ac. Bll. 41 (1876) 1018-

Vorontsov, M. M. N. A. Mth. 15 (1876) 12-.

Berg, F. J. van den [1878] (xI) Amst. Ak.

Wet. P. (1878-79) (No. 5) 3-.

Stern, M. A. Crelle J. Mth. 84 (1878) 267-.

Gohierre de Longchamps, - . Par. Éc. Norm. A. 8 (1879) 55-

Küttner, W. Z. Mth. Ps. 24 (1879) 250 -

Stern, M. A. Crelle J. Mth. 88 (1880) 85-; 92 (1882) 349-.

Zhbikovskin, A. K. [1882] (xп) Rec. Mth. (Moscou) 10 (1882-83) (Pt. 1) 127-.

Kronecker, L. Crelle J. Mth. 94 (1883) 268-

Lipschitz, $R$. Crelle J. Mth. 96 (1884) 1-.

Gomes Teixeira, $F$. Am. J. Mth. 7 (1885) 288-.

Genocchi, A. Crelle J. Mth. 99 (1886) 315-.

Studnička, F.J. Časopis 15 (1886) 97-; Fschr. Mth. (1886) 224-.

\section{Bernoulli's Numbers}

Gegenbauer, $L$. Wien Ak. Sb. 96 (1888) (Ab.2) 491-.

Ocagne, M. d'. Par. S. Mth. Bll. 17 (1889) 107-.

Voronoj, G. T. Kharkov Mth. S. Com. 2 (1891) 129-; Fschr. Mth. (1890) 268.

Mangeot, S. Mars. Fac. Sc. A. 2 (1892) 63-.

Saalschiitz, L. Königsb. Schr. 33 (1892) [44]-.

Carda, $K$. Mh. Mth. Ps. 5 (1894) 185-.

Tschistiakow, J. J. Fschr. Mth. (1895) 283-.

Sincov, D. [1898] Kazan S. Ps.-Mth. Bll. 8 (1899) 104-; Fschr. Mth. (1898) 221.

Petr, $K$. Časopis 28 (1899) 24-; Fschr. Mth. (1899) 386.

Schwering, K. Mth. A. 52 (1899) 171-.

analytic expression. Plana, G. Tor. Mm. Ac. 25 (1820) 403-.

arithmetical developments concerning. Rogel, F. Arch. Mth. Ps. 11 (1892) 77-.

asymptotic representation of integral part. Lipschitz, - Bll. Sc. Mth. 10 (1886) 135-.

bibliography. Ely, G. S. Am. J. Mth. 5 (1882) 228-.

and binomial coefficients, relation. Hammond, $J$. [1875] L. Mth. S. P. 7 (1875-76) 9-.

calculation. Catalan, E. C. Liège S. Sc. Mm.12 (1885) No. 2, 86-, 91-, 97-, 103-, 320- up to $B_{62}$ by Staudt's theorem. Adams, J.C. B. A. Rp. (1877) (Sect.) 8-.

-, simplest method. Williot, - . Par. S. Mth. Bll. 16 (1888) 144-.

congruence theorem relating to. Glaisher, J. W. L. QJ. Mth. 31 (1900) 253-.

connected with fundamental discriminant, generalisation. Gegenbauer, $L$. Wien $\mathrm{Ak}$. Sb. 102 (1893) (Ab. 2a) 1059-.

connection of difference series with. Saalschütz, - . Königsb. Schr. 41 (1900) [14]-.

deduction from von Staudt's property. Glaisher, J. W. L. [1872] L. Mth. S. P. 4 (1871-73) 212 .

definite integrals connected with. Carda, $K$. Mh. Mth. Ps. 6 (1895) 121-.

determination. Presle, - de. Par. S. Mth. Bll. 14 (1886) 100-

and differences of $1^{p}$. Catalan, E.C. Tortolini A. 2 (1859) 239 -

errata in formulæ. Blissard, J. QJ. Mth. 9 (1868) $71-$.

and Euler's. Catalan, E. C. [1867] Brux. Ac. Sc. Mm. 37 (1869) 19 pp.

- -. Ely, G. S. Am. J. Mth. 5 (1882) $337-$.

- - Worpitzky, J. Crelle J. Mth. 94 (1883) 203-.

- - Cesáro, E. N. A. Mth. 5 (1886) 305-, 496.

- - Berg, F. J. van den. Amst. Ak. Vs. M. 5 (1889) 358-; 6 (1889) 265-; Arch. Néerl. 24 (1891) 99-.

- - Hermite, C. Prag Sb. (1894) (Mth.Nt.) No. 37, 4 pp.

- - and coefficients of power-series. Rogel, F. Arch. Mth. Ps. 10 (1891) 318-.

- - , - new theorem on primes. Sylvester, J. J. Ph. Mg. 21 (1861) 127-

- , new theory. Lucas, É. C. R. 83 (1876) 539-; A. Mt. 8 (1877) 56-. 


\section{Bernoulli's Numbers}

and Euler's, related expressions. Fields, J.C. Am. J. Mth. 13 (1891) 191-.

- - relations between. Sheppard, $W . F$. QJ. Mth. 30 (1899) 18-.

- -, theory. Stern, M. A. Gött. Ab. 23 (1878) (Mth.) 44 pp.; 26 (1880) (Mth.) 45 pp. - -, - Haussner, R. Gött. Nr. (1893) $777-$.

expression by definite integral. Hamilton, (Sir) W. R. Ph. Mg. 23 (1843) 360-.

formulø of Seidel and Stern. Lucas, É. Par. S. Mth. Bll. 8 (1880) 169-.

fractional part. Cesáro, E. A. Mt. 14 (188687) 221-.

function related to them, as $\Gamma n$ to $n$ ! Glaisher, J.W. L. B. A. Rp. 42 (1872) (Sect.) 17-.

and functions, generalisation. Berger, $A$. Stockh. Ak. Hndl. Bh. 13 (Afd. 1) (1888) No. 9, 43 pp.; Stockh. Öfv. (1888) 433-; Acta Mth. 14 (1890-91) 249-.

fundamental theorems relating to. Glaisher, J. W. L. Mess. Mth. 29 (1900) 49-, 129-.

general expression. Genocchi, A. Tortolini A. 3 (1852) 395-。

of higher orders. Grigorjev, $\boldsymbol{E}$. Kazan S. Ps.-Mth. Bll. 7 (1898) 146-; Fschr. Mth. (1898) 221-.

independent expression for. Berger, A. Stockh. Öfv. (1889) 129-; Fschr. Mth. (1889) 247.

- representation. Nägelsbach, H. Z. Mth. Ps. 19 (1874) 219-.

logarithms of first 40 . Thoman, $F$. C. R. 50 (1860) 905-.

method of finding. Canterzani, S. Mod. Mm. S. It. 11 (1804) 173-.

new expression for. Teixeira, J. P. Lisb. J. Sc. Mth. 3 (1895) 73-.

- recursion formula. Rogel, $F$. Prag Sb. (1895) (Mth.-Nt.) No. 26, 4 pp.

numbers $a_{n, m}$ in proof of Staudt's theorem. Ely, G. S. (xII) J. H. Un. Cir. [2] (1883) 47-.

- analogous to. Matter, K. Zür. Vjschr. 45 (1900) 238-.

and Pascal's arithmetical triangle, relations of. Lucas, É. $\quad$ N. A. Mth. 15 (1876) 497-.

properties. Stern, M. A. Crelle J. Mth. 81 (1876) 290-; Bll. Sc. Mth. 10 (1886) 280-.

-. Lipschitz, -. D. Nf. B. (*1883) 56-.

property, proof. Glaisher, J.W. L. Mess. Mth. 2 (1873) 190-

recurring determination. Schlömilch, $O$. Grunert Arch. 3 (1843) 9-.

- - (Schlömilch).

- Arch. 3 (1843) 64-.

- formulæ involving. Glaisher, J.W.L. Mess. Mth. 28 (1899) 36-.

and related functions. Le Paige, C.M.M.H.H. (xII) Brux. S. Sc. A. 1 (1877) (Pt. 2) 43-. - - series, simple method of deducing. Seidel, P. L. von. Münch. Ak. Sb. 7 (1877) 157-.

- secant coefficients. Schlömilch, O. Grunert Arch. 1 (1841) 360-.

(1860) $449-$

- - , formula for. Schlömilch,O. Crelle J. 32 (1846) 360-.
Special Numbers, etc. 2910

Bernoulli's Numbers, continued.

and secant coefficients, general expression for both. Scherk, H. F. Crelle J. 4 (1829) 299-. sum and difference formulø. Bauer, $G$. [1860] Crelle J. 58 (1861) 292-.

table of first 62. Adams, J. C. [1877] Crelle J. Mth. 85 (1878) 269-.

tables of 250, and their logarithms. Glaisher, J. W. L. [1871-72] Camb. Ph. S. T. 12 (1873) 384-.

and their composition from integers and reciprocal primes. Zeller, C. Bll. Sc. Mth. As. 5 (1881) (Pt. 1) 195-.

theorem. Staudt, G. K. C. von. Crelle J. 21 (1840) 372-.

- (Staudt). Schläfli, L. QJ.Mth. 6 (1863) 75-. -, Staudt-Clausen. Catalan, E. C. Bll. Sc. Mth. As. 4 (1880) 77-.

-, - - Lucas, É. Par. S. Mth. Bll. 11 (1883) 69-.

-, - Cesáro, E. Brux. Ac. Bll. 20 (1890) 280 -

-, - Kluyver, J. C. Mth. A. 53 (1900) $591-$.

- - and Binet's theorem. Lucas, É. N. A. Mth. 16 (1877) 157-

ultra-Bernoullian numbers. Trudi, N. Nap. At. I. Inc. 4 (1867) 105-.

Bernoulli's series. Sonin, N. St Pét. Ac. Sc. Bll. 7 (1897) 337-; Fschr. Mth. (1897) 224.

- - condition for first $n$ terms being a given function. Hill, C. J. D. Stockh. Öfv. 14 (1857) 259-.

- constants occurring in summations by. Glaisher, J. W. L. [1872] L. Mth. S. P. 4 (1871-73) 48-.

- - , series of differences of terms of. Markoff, A. Mth. A. 19 (1882) 27-.

Cauchy's numbers. Chessin, A. S. A. Mth. 10 (1895-96) 1-.

- - , and their application to astronomy. Bourget, J. Liouv. J. Mth. 6 (1861) 33-.

- - - Bessel's functions, relation between. Chessin, A. S. A. Mth. 12 (1898-99) 170-

Clapeyron's numbers, properties. Šolin, J.M. Prag Sb. (1878) 146-.

Constant which occurs in formula for $1^{1} \cdot 2^{2} \cdot 3^{3} \ldots n^{n}$. Glaisher, J. W. L. Mess. Mth. 24 (1895) 1-.

Dirichlet's series. Hadamard, - Bordeaux S. Sc. PV. (1896-97) 41-.

Discontinuity factors, arithmetical, Rogel, $\boldsymbol{F}$. Arch. Mth. Ps. 17 (1900) 147-.

Discontinuous function, Riemann's. Frischauf, J. Z. Mth. Ps. 34 (1889) 193-.

- functions. Sokolov, D. N. Rec. Mth. (Moscou) 16 (1893) 282-; Fschr. Mth. (1892) 187. - logarithmic functions. Bougaïef, -. C. R. 106 (1888) 1067-.

Divisors of number. Hermite, C., \& Lipschitz, $R$. Acta Mth. 2 (1883) 299-

- _ - formula of Hermite. Busche, $E$. Crelle J. Mth. 100 (1887) 459-; 103 (1888) 125. (1895) 432- 
Divisors of number, theorems (Bougaief). Gegenbauer, L. Mh. Mth. Ps. 7 (1896) 26.

- _ numbers, theorem of Bugaief. Gegenbauer, L. Wien Ak. Sb. 95 (1887) (Ab. 2) 219-.

Enumerative functions. Cesäro, E. A. Mt. 14 (1886-87) 141-.

Equation

$$
E \frac{1}{((x-a))}, \quad E \frac{1}{((x-b))} \cdots \quad E \frac{\phi(x)}{((x-h))}=0,
$$

residues and coefficients, analogy. Roselli, $E$.

Rm. Cor. Sc. 1 (1848) 373-.

$$
-\sum_{k=1}^{k=\infty} \frac{\mu(k)}{k}=0 \text {. Mangoldt, H. von. Berl. }
$$
Ak. Sb. (1897) 835-

Eulerian and other coefficients, congruence theorem relating to. Glaisher, J. W. L. L. Mth. S. P. 31 (1900) 193-.

- $\phi$-function. Busche, E. Mth. A. 31 (1888) 70 -.

- - Baker, H. F. [1889] . L. Mth. S. P. 21 (1891) 30-.

Euler's constant, calculation. Glaisher, J. W. L. R. S. P. 19 (1871) 514-.

- -, expression for. Franklin, F. (xII) J.H. Un. Cir. [2] (1883) 143.

- -, history. Glaisher, J. W. L. [1871] Mess. Mth. 1 (1872) 25- ; 2 (1873) 64.

- - $\operatorname{logs}_{e} 2,3,5,7,10$, to 260 places. Adams, J. C. R. S. P. 27 (1878) 88-.

- - , relation connecting constants analogous to. Glaisher, J. W. L. Mess. Mth. 24 (1895) 24-.

- - , series for. Nielsen, N. N. Ts. Mth. 8 (B) (1897) 10-; Fschr. Mth. (1897) 235.

- _, value to 260 places. Adams, J. C. B. A. Rp. (1877) (Sect.) 14-.

- functions. Rogel, $F$. Prag Sb. (1893) (Mth.-Nt.) No. 23, 52 pp.; (1896) (Mth.-Nt.) No. 2, $45 \mathrm{pp}$.

- numbers. Catalan, E. C. C. R. 66 (1868) 415-.

- - Stern, M.A. CrelleJ. Mth. 79 (1875) 67-。

- - Lucas, É. [1877] Mess. Mth. 7 (1878) 139 -

- - Radicke, A. Crelle J. Mth. 89 (1880) 257-.

- -, an analogue of. Studnička, F.J. Prag Sb. (1900) (Mth.-Nt.) No. 9, 8 pp.

- - coefficients analogous to. Glaisher, J. W. L. L. Mth. S. P. 31 (1900) 216-.

Expansion of numerical functions in series. Lerch, -. 'C. R. 108 (1889) 171-.

- - - - Rogel, F. Prag Sb. (1897) (Mth.-Nt.) No. 46 [44], 26 pp.; (1900) (Mth.-Nt.) No. 30, $7 \mathrm{pp}$.

$--\frac{1}{c e^{x}-1}$. Fergola, $E$. Nap. Ac. Sc. Mm. 2 (1855-57) 315-.

$--\left(\frac{z}{1-e^{-z}}\right)^{a}$ in series. Lucas, $\hat{E}$. [1877] Mess. Mth. 7 (1878) 82-.

$-\frac{x}{e^{x}-1}$ in powers of $x$, series resulting from. Oltramare, G. As. Fr.C.R.(1881)117-

Expansions, numerical. Cayley, $A$. QJ. Mth. 3 (1860) 366-.
Factorials, numerical, of 2 nd order. Collins, $E$. [1835] St Pét. Ac. Sc. Mm. 3 (1838) 225-.

Formula of Gauss, generalisation. Busche, E. C. R. 129 (1899) 952-.

Function connected with divisors of a number. Glaisher, J. W. L. [1890] L. Mth. S. P. 21 (1891) 395-.

- expressing $\left(n-2 E \frac{n}{2}\right)\left(n-3 E \frac{n}{3}\right)$. Mīnin A. P. [1874] (xII) Rec: Mth. (Moscou) 7 (1874-75) (Pt. 1) 485-.

- relating to $(4 m+1)$ and $(4 m+3)$ divisors of number. Glaisher, J. W. L. ' L. Mth. S. P. 15 ("1883-84) 104-.

Functions, numerical. Liouville, $J$. Liouv. J. Mth. 2 (1857) 141-, 244-, 377-, 408, 409425-.

-, - Bellavitis, G. Ven. At. (1858-59) 536-. -, -. Bouniakowsky, V. [1861] St Pét. Ac. Sc. Mm. 4 (1862) No. 2, 35 pp.

-, - Liouville, J. Liouv. J. Mth. 8 (1863) 347-.

-, - Bugaev, N. V. [1867] (xII) Rec. Mth. (Moscou) 3 (1868) (Pt. 1) 69-.

-, - Gegenbauer, L. Wien Ak. Sb. 89 (1884) (Ab. 2) 37-.

-, -, class. Cesáro, E. G. Mt. 25 (1887) 14-.

_, - , geometrical proof. Vries, J. de. Amst. Ak. Vs. 5 (1897) 218-

- - - obtained by differentiation. Rogel, $F$. Arch. Mth. Ps. 10 (1891) 209-.

-, -, problems. Bougaieff, N. V. N. A. Mth. 13 (1874) 381-.

,-- , series giving relation between. Minin, A. P. Mosc. S. Sc. Bll. 83 (No. 1) (1893) 23-.

,-- , theory. Cantor, G. Gött. Nr. (1880) 161-. Generating function, numerical, of binary seventhic. Cayley, A. Am. J. Mth. 2 (1879) 71-.

- functions in theory of numbers, class. MacMahon, P. A. [1893] Phil. Trans. (A) 185 (1895) 111-.

Greatest integer in certain series. Cesáro, $E$. [1882] Liége S. Sc. Mm. 10 (1883) (No.6) $355 \mathrm{pp}$.

- integers. Gegenbauer, $L$. Wien Ak. Sb. 94 (1887) (Ab. 2) 611-.

- - Busche, E. CrelleJ.Mth. 103 (1888) 118- -, sums. Hacks, J. Acta Mth. 10 (1887) 1-.

- - - - Mathews, G. B. L. Mth. S. P. 31 (1900) 355-.

- - - , in arithmetical progressions. Zeller, C. Gött. Nr. (1879) 243-.

- -, theorem. Schubert, H. [1895] Hamb. Mth. Gs. Mt. 3 (1900) 223-.

- -, - (Schubert). Busche, E. [1895] Hamb. Mth. Gs. Mt. 3 (1900) 225-.

Identities, numerical, application to theory of numerical functions. Baskakov, S. $I$. [1881] (xII) Rec. Mth. (Moscou) 10 (188283) (Pt. 1) 313-.

-, _, Liouville's, deduced from Bugaev's theorem. Sokolov, D. N. [1891] Rec. Mth. (Moscou) 16 (1893) 89-; Fschr. Mth. (1891) 197. -, a source of. Ocagne, M. d'. Par. S. Mth. Bll. 15 (1887) 133-. 
Inverse arithmetical functions. Cesaro, $E$. G. Mt. 23 (1885) 168-.

Laisant's function $l(1+\sqrt{2})$, calculation. Seydler, A. Časopis 20 (1891) 89-; Fschr. Mth. (1891) 442.

- - - - , new formula. Studnička, F.J. Časopis 20 (1891) 66-; Fschr. Mth. (1891) 441.

Mean and asymptotic expressions in arithmetic. Cesáro, E. G. Mt. 25 (1887) 1-.

- values of certain numerical functions. $L a$ Vallée-Poussin, - de. Brux. S. Sc. A. 22 (1898) (Pt. 1) 84-.

Möbius-Mertens function. Lerch's theorem. Dintzl, E. Mh. Mth. Ps. 11 (1900) 67-.

Number and sum of divisors of number. Hammond, J. Mess. Mth. 20 (1891) 158-, 182-.

Numbers with no square factors. Gegenbauer, L. Wien Ak. Sb. 100 (1891) (Ab. 2a) 1054-.

- representable by $\sum_{1}^{n} f_{r}\left(x_{r}\right)$. Pascal, E. G. Mt. 25 (1887) 45-.

- $\equiv 5$ (mod. 12), theorem. Liouville, J. Liouv. J. Mth. 14 (1869) 7-.

- $Q_{n}$ given by $n^{2} Q_{n}-2^{\alpha}\left(3 n^{2}-3 n+1\right) Q_{n-1}$ $+2^{2 \alpha+1}(n-1)^{2} Q_{n-2}=0$, theorem of de Jonquières, extension. Gegenbauer, $L$. Wien Âk. Sb. 97 (1889) (Ab. 2a) 82-.

- $K_{m}^{p}$, properties. Ocagne, $M$. $d^{\prime}$. Am. J. Mth. 13 (1891) 145-.

Numerical integrals according to divisors. Bougaieff, -. C. R. 106 (1888) 652-

- - - (Bougaieff). Cesáro, E. C. R. 106 (1888) 1340-.

- - - Bugaev, N. V. [1889-1900] Rec. Mth. (Moscou) 14 (1890) 169-; 18 (1896) 1-; 21 (1901) 335-, 499-; Fschr. Mth. (1889) 182-; (1896) 158; (1900) 197-; C. R. 119 (1894) 1259-.

- - - (Bougaieff, C. R. 1894). Gegenbauer, L. Mh. Mth. Ps. 6 (1895) 208.

- - , numerical series connected with. Mininn, A. P. [1877] (xIr) Rec. Mth. (Moscou) 9 (1878-81) (Pt. 1) 128-.

Partition of numbers, general law. Bougaieff, N. C. R. 100 (1885) 1123-.

- - - , - laws, application to numerical functions. Bougaieff, N. C. R. 100 (1885) 1159-.

Periodic numerical functions, simple. Lucas, É. [1877] Am. J. Mth. 1 (1878) 184-, 289-. Series $\sum_{i=1}^{i=\frac{q-1}{2}} E\left(i \frac{p}{q}\right)$, calculation. Sylvester, J. J. C. R. 50 (1860) 489-.

$-E\left(\frac{p}{q}\right)+E\left(\frac{2 p}{q}\right)+\ldots$ Sylvester, J. J. C. R. $50(1860) 650$ -

$-\sum_{r=1}^{r=n}\left(\frac{1}{r}\right)$. Govi, G. Tor. At. Ac. Sc. 5 $(1869-70) 753-$.

$-\sum_{x=1}^{x=n} \Phi\left(\left[\frac{n}{x}\right]\right)=\frac{n(n+1)}{2}$, theorem of Sylvester's, generalisation. Gegenbauer, $L$. Wien Ak. Sb. 94 (1887) (Ab. 2) 757-.
Series $\sum_{r=0}^{r=\mu} \frac{1}{x+r n}$, summation. Glaisher, J.W.L. Mess. Mth. 24 (1895) 124-.

- $\Sigma E p^{\frac{1}{3}}$, theorem. Ivanov, I. St Pét. Ac. Sc. Bll. 2 (1895) 253-; Fschr. Mth. (1895) 218.

Square roots, arithmetical theorems. Gegenbauer, L. Wien Ak. Sb. 90 (1885) (Ab. 2) 395-.

Sum of like powers of numbers in arithmetical progression, and coefficients analogous to Bernoulli's. Mollame, $V$. Catania Ac. Gioen. At. 15 (1881) 261-.

- values of $f(x)$ when $x$ takes $m$ successive values prime to $n$, theorem of Nasimof. Gegenbauer, L. Wien Ak. Sb. 102 (1893) (Ab. 2a) 1265-.

Summation of $\phi$ numbers. Perott, $J$. Bll. Sc. Mth. As. 5 (1881) (Pt. 1) 37-.

Symbol $\phi$. Rocquigny, $F$. $[G$.$] de. Les$ Mondes 48 (1879) 327.

Tangent and secant coefficients, independent representation. Rogel, $F$. Arch. Mth. Ps. 8 (1890) 295-

Totient sums, asymptotic evaluation of certain. Lehmer, D. N. Âm. J. Mth. 22 (1900) 293-.

Totients, two kinds of $k \mathrm{th}$, and notation for. Story, W. E. [1881] (xi1) J. H. Un. Cir. [1] (1882) 132, 151.

Transcendental numerical functions, new. Tagliaferro, N. As. Fr. C. R. (1878) 140-.

$\Lambda$ and $\Gamma$ (Kramp). Argand, - Gergonne $\mathbf{A}$. Mth. 5 (1814-15) 236-.

$\phi(n)$, theorems. Catalan, E. C. Liouv. J. Mth. 4 (1839) 7-.

-. Grunert, J. A. Grunert Arch. 3 (1843) 196-.

- Prouhet, E. N. A. Mth. 4 (1845) 75-.

-. Arndt, $F$. Crelle J. 31 (1846) 246-.

-. Oltramare, G. Gen. I. Nt. Mm. 4 (1856) 1-.

-. Liouville, J. Liouv. J. Mth. 2 (1857) 110-.

-. Sturm, J. B. Grunert Arch. 29 (1857) 448-.

-, theorem, proof. Crone, C. (xII) Ts. Mth. 4 (1880) 158-.

- Rocquigny, F. [G.] de. Les Mondes 51 (1880) 335-.

-, and notation of numbers. Rocquigny, F. [G.] de. Les Mondes 54 (1881) 160-.

- Catalan, E. C. Liége S. Sc. Mm. 15 (1888) No. 1, 21-

- = number of divisors of $N$, value of $N$. Minin, A.P. [1893] Rec. Mth. (Moscou) 17 (1895) 538-; Fschr. Mth. (1893-94) 277.

-, generalisation. Zsigmondy, $K$. Crelle J. Mth. 111 (1893) 344-.

-. Steggall, J. E. A. Edinb. Mth. S. P. 12 (1894) 23-.

- theorems. Moreau, C. N. A. Math. 17 (1898) 293-.

Seliwanow, D. F. Fschr. Mth. (1899) 172.

$E(x)$, and reversion of series. Meissel, $E$. Crelle J. 48 (1854) 301-.

-. Liouville, J. Liouv. J. Mth. 2 (1857) 280. 


\section{Special Numbers, etc.}

$E(x)$, properties. Stern, M. A. [1860] Crelle J. 59 (1861) 146-

-. Sylvester, J. J. C. R. 50 (1860) 732-.

一, theorems. Bouniakowsky, V. C. R. 94 (1882) 1459-; St Pét. Ac. Sc. Bll. 28 (1883) 257-, 411-; 29 (1884) 250-, 503-.

-. Hermite, -. Am. J. Mth. 6 (1884) 173-. - , representation by an infinite series. Pringsheim, A. Mth. A. 26 (1886) 193-.

-. Stern, M. A. Acta Mth. 8 (1886) 93-; 10 (1887) 53-; Crelle J. Mth. 102 (1888) 9-; $106(1890) 337-$.

-. Mansion, -. Brux. S. Sc. A. 13 (1889) (Pt. 1) 55-.

-. Giuliani, -. Palermo Cir. Mt. Rd. 5 (1891) 285-

- with complex argument. Busche, E. [189293] Crelle J. Mth. 110 (1892) 338-; Hamb. Mth. Gs. Mt. 3 (1900) 103-.

-, formulæ concerning. Lerch, _. G. Teix. J. Sc. 12 (1895) 129-.

-. Bertolani, G. G. Mt. 34 (1896) 1-.

$\phi^{-1}\left(\pi^{\prime}\right)$, finding. Cayley, A. Ph. Mg. 14 (1857) 539-.

$F(x)$. Liouville, J. Liouv. J. Mth. 5 (1860) 287-, 455-.

$N(n, p, q)$. Liouville, J. Liouv. J. Mth. 6 (1861) 369-.

$E$, numerical identities connected with. $B u$ gaev, N. V. (xII) Rec. Mth. (Moscou) [1] (1866) i-, 1-.

-, etc., equations depending on. Bugaev, N. V. [1874] (xII) Rec. Mth. (Moscou) 7 (1874-75) (Pt. 1) 424-; (rx) Bll. Sc. Mth. As. 10 (1876) 102-.

$E(a x+b)+1-(a x+b)$. Čeby̌ev, P. [1866] St Pet. Ac. Sc. Mm. (Rs.) 10 (*1867) (Suppl.) No. 4, 54 pp.

$F(k)$, theorem. Liouville, J. Liouv. J. Mth. 14 (1869) 1-, 260-

$\zeta_{1}(n)$, expressing sum of divisors of $n$. Liouville, $J$. Liouv. J. Mth. 14 (1869) 263-.

$\rho_{2}(n)$. Liouville, J. Liouv. J. Mth. 14 (1869) 302-.

$\phi(n)$ and $\phi(n, N)$. Smith, H. J. S. L. Mth. S. P. 7 (1875-76) 208-.

- - - (H. J. S. Smith). Mansion, P. (xI) Brux. S. Sc. A. 2 (1878) (Pt. 2) 211-.

$\psi(a)$ of Jacobi, theory. Schwering, K. Crelle J. Mth. 93 (1882) 334-.

$Q_{p}=\left(\frac{n}{1}\right)+\left(\frac{n}{2}\right)+\ldots\left(\frac{n}{p}\right)$, theorem of Stieltjes. Cesáro, E. C. R. 96 (1883) 1029-.

$u$, properties. Cesáro, E. N. A. Mth. 3 (1884) 431-.

$E\left(\frac{n}{x}\right)$. Gram, J. P. Ts. Mth. 2 (1884) 170-.

$z-[z]$. Cesáro, E. A. Mt. 13 (1885) 323-.

C (z). Cesíro, E. A. Mt. 13 (1885) 329-.

$\chi(n)$. Glaisher, J.W.L. QJ.Mth. 20 (1885) 97-.

$\chi_{k}(n)$. Gegenbauer, L. Wien Ak. Sb. 96 (1888) (Ab. 2) 607-.

$\psi(p, q)$, theorems. Lerch, M. Bll. Sc. Mth. 12 (1888) 121-.

$\sum_{x=1}^{x=\frac{q-1}{2}}\left[\frac{p x}{q}\right]$ (1890) 65 -

\section{Transcendental Numbers 2920}

(x). Kronecker, L. Crelle J. Mth. 106 (1890) $346-$.

-, applications. Hacks, J. Acta Mth. 14 (1890-91) 329-.

$N(n)$ and $L(n)$. Gram, J. P. [1890] Kjøb. Dn. Vd. Selsk. Skr. 7 (1890-94) 1-. (With French résumé.)

$\sigma(n)$, recurring formula for. Glaisher, J. W. L. Mess. Mth. 21 (1892) 122-.

- in theory of numbers (with tables). Mertens, F. Wien Ak. Sb. 106 (1897) (Ab. 2a) 761-.

- _ - - , course of. Daublebsky von Sterneck, $R$. Wien Ak. Sb. 106 (1897) (Ab. 2a) 835-.

$E(n)$, Cesáro's formula. Oltramare, G. As. Fr. C. R. (1893) (Pt. 2) 287-.

$\phi_{p}(n)=n^{p}\left(1-\frac{1}{a^{p}}\right)\left(1-\frac{1}{b^{p}}\right) \ldots . \quad$ Čistrakov, I. I. [1894] Rec. Mth. (Moscou) 17 (1895) 530-; Fschr. Mth. (1893-94) 269-.

$\psi_{n}(k, h)=\Sigma\left(\frac{s}{h}\right) e^{s \frac{2 k \pi i}{h}}$. Séguier, P. de. Liouv. J. Mth. 5 (1899) 55-.

2920. Irrationality and transcendence of particular numbers, such as $e$ and $\pi$. (See also 6810.)

Eisenstein's theorem on series for root of algebraic equation. Hermite, $C$. L. Mth. S. P. 7 (1875-76) 173-.

- Gomes Teixeira, $F$.

Arch. Mth. Ps. 3 (1886) 315-.

Exponential function. Hermite, C. C. R. 77 (1873) 18-, 74-, 226-, 285-.

- - irrationality and transcendence. Stieltjes, - . C. R. 110 (1890) 267-.

Irrational quantities, algebraic, theorem. Liouville, J. C. R. 18 (1844) 910-.

Transcendental numbers deduced from proba. bilities. Bouniakowski, V. St Pét. Ac. Sc. Bll. 1 (1836) 177; St Pét. Ac. Sc. Mm. 3 (1838) 457-, 517-.

- - two theorems. Grömwall, H. Stockh. Öfv. (1897) 623-; (1898) 153-.

$$
\text { e. }
$$

Leclerc, - . N. A. Mth. 14 (1855) 225-

Paque, A. Liège Mm. S. Sc. 10 (1855) 199-.

Realis, S. N. A. Mth. 6 (1867) 541-; 7 (1868) 16-, 158-, 191-.

arithmetical character. Borel, É. C. R. 128 (1899) 596-.

calculation. Bretschneider, C. A. Grunert Arch. 3 (1843) 27-.

Studnicka, F.J. Časopis 20 (1891) 61-; Fschr. Mth. (1891) 440-.

- from continued fraction. Glaisher, J.W.L. B. A. Rp. 41 (1871) (Sect.) 16-.

and continued fraction, relation between. Cailler, -. As. Fr. C. R. (1889) (Pt. 2) 158-. irrationality. Gruson, J. P. Berl. Ab. (181617) 18

-. Liouville, J. Liouv. J. Mth. 5 (1840) 192-. 


\section{Transcendental Numbers}

irrationality. Thue, A. [1888] Arch. Mth. Ntvd. 14 (1890) 251-; Fschr. Mth. (1888) 263. - and transcendence. Jamet, V. N. A. Mth. 10 (1891) 215-

limits for. Ligowski, W. Arch. Mth. Ps. 57 (1875) 220

new series for. Rados, G. Mth. Termt. Ets. 11 (1893) 358-.

transcendence. Cailler, C. As. Fr. C. R. (1891) (Pt. 2) 83-.

-. Hurwitz, A. Gött. Nr. (1893) 153-; C. R. 116 (1893) 788-.

- Gordan, - C. R. 116 (1893) 1040-.

-, change in Hermite's first proof. Venske, O. Gött. Nr. (1890) 335-.

value to 205 places. Shanks, $W$. R. S. P. 6 (1854) 397-; 20 (1872) 27-.

$e=\lim .\left(1+\frac{1}{n}\right)^{n}$. Liouville, J. Liouv. J. Mth. 5 (1840) 280.

- = - expansion when $n=\infty$. Spitzer, S. Grunert Arch. 23 (1854) 127-

- = - $n$ an integer. Donkin, W. F. [1862] QJ. Mth. 6 (1863) 1-

- = -, demonstration. Fort, $O$. Schlömilch Z. 7 (1862) 46-.

$e=\lim .(1+x)^{\frac{1}{x}}$, value when $x=0$. Schultên N. G. af ( $f i l$.$) [1842] Helsingf. Acta 2$ (1847) 81 -.

$-=-$, expansion. Wittstein, T. Grunert Arch. 3 (1843) 327-.

- = - - . Frost, P. QJ. Mth. 7 (1866) 381-. $e^{a}=\lim .(1+a x)^{\frac{1}{x}}$, expansion. Appell, $P$. Arch. Mth. Ps. 65 (1880) 171-.

$e^{x}$, irrationality. Hermite, C. B. A. Rp. 43 (1873) (Sect.) 22-.

$e$ and $\pi$, approximate numerical theorem. Glaisher, J. W. L. [1877] QJ. Mth. 15 (1878) 125-.

_ - irrationality. Pade, H. Bll. Sc. Mth. 12 (1888) 144-.

_ - transcendence. Gordan, P. Mth. A. 43 (1893) 222 -

- - - - Hilbert, D. Gött. Nr. (1893) 113-.

- - - . Mertens, $F$. Wien Ak. Sb. 105 (1896) $(A b .2 a) 839$

- - - - Jamet, V. Ens. Mth. 2 (1900) $355-$.

$\pi$.

Lindemann, C. L. F. Berl. Ak. Sb. (1882) 679-; Mth. A. 20 (1882) 213-.

(Lindemann). Weierstrass, $K$. Berl. Ak. Sb. (1885) 1067-.

approximation, Belli, G. Brugnatelli G. 3 (1820) 409-.

- Catalan, E. C. N. A. Mth. 1 (1842) 190-.

-. Caminati, P. As. Fr. C. R. (1887) (Pt.1) 173 .

approximations. Barrois, T. Gergonne A. Mth. 4 (1813-14) 360-.

calculation. Fanien, A. N.A.Mth. 9 (1850) 190-.

-. Dupain, J. C. N. A. Mth. 15 (1856) 82-.

-. Frisby, E. [1872] Mess. Mth. 2 (1873) 114-.

-. Glaisher, J. W. L. [1872] Mess. Mth. 2 (1873) 119-.

\section{Analysis. General 3190}

calculation, elementary. Farcy, A. N. A. Mth. 3 (1844) 582-.

construction. Efimov, M. Kazan S. Ps.-Mth. Bll. 5 (1896) (Prot.) 66.

convergency of Euler's developments. Grabowski, E. Prace Mt.-Fiz. 3 (1892) 130-; Fschr. Mth. (1892) 232.

error by method of isoperimeters. Hermann, A.

N. A. Mth. 5 (1866) 509-.

formula for. Grebe, E.W. Grunert Arch. 12 (1849) 181-.

geometric construction impossible. Saalschütz, -. Königsb. Schr. 35 (1895) [23]-.

hyperbolic analogue. Studnička, F.J. Liège S. Sc. Mm. 14 (1888) No. 6, 12 pp.; Prag Sb. (1892) (Mth.-Nt.) 250-.

- -, Laisant's, calculation. Studnička, F.J. Liège S. Sc. Mm. 17 (1892) No. 6, 7 pp.

- logarithm of. Glaisher, J.W. L. L. Mth. S. P. 14 (1882-83) 134-.

and its integral powers are incommensurable. Collignon, É. Par. S. Phlm. Bll. 3 (1879) 195-.

irrationality. Valat, - Bordeaux Act. Ac. Sc. 26 (1864) 269 -

- Lambert's proof, and irrationality of other quantities. Glaisher, J.W.L. B. A. Rp. 41 (1871) (Sect.) 12-.

- and transcendence, proof. Sylvester, C. R. 111 (1890) 866-.

- - - - (Sylvester). Markow, A.A. Fschr. Mth. (1899) 386.

and powers of $\pi$, series and products for. Glaisher, J. W. L. [1877] Mess. Mth. 7 (1878) 75 .

series for. Giaisher, J.W. L. [1872] QJ. Mth. 12 (1873) 232-.

- of Schwab for. Virieu, J. de. N. A. Mth. 18 (1859) 234-.

- - - Tannery, $J$. Bll. Sc. Mth. As. 5 (1881) 454-.

transcendence. Tognoli, O. G. Mt. 24 (1886) 354 .

-. Brunel, - . Bordeaux S. Sc. Mm. 2 (1891) xi-.

transformation of Leibnitz's series for. Polster, F. Arch. Mth. Ps. 63 (1879) 447-.

$\frac{\pi}{2}$, Nicholson's formula for, discussion. Strnad, A. Časopis 19 (1890) 144-; Fschr. Mth. (1890) 261-.

$M$ (Briggs' modulus), value to 205 places. Shanks, W. R. S. P. 6 (1854) 397-; 20 (1872) $27-$.

(For applications of arithmetical methods to algebraic functions see $\mathbf{4 0 1 0}$.)

\section{ANALYSIS 3190 General.}

La Place, P. S. (le marquis) de. Par. Ec. Pol. J. 8 (1809) (15 cah.) 229-.

Herschel, (Sir) J. F.W. Phil. Trans. (1814) 440 - 


\section{Analysis. General}

Libri, G. [1822-30] Tor. Mm. Ac. 28 (1824) 251-; Férussac Bll. Sc. Mth. 14 (1830) 1-.

Cauchy, A. L. [1827] Par. Mm. Ac. Sc. 8 (1829) 97-.

Ostrogradsky, M. A. Férussac Bll. Sc. Mth. 14 (1830) 249-.

Jacobi, C. G.J. C. R. 3 (1836) 59-.

Ostrogradsky, - . St Pét. Ac. Sc. Bll. 3 (1838) 209-.

Cauchy, A. L. C. R. 11 (1840) 933-.

Analysis, algebraic. Bellavitis, G. [1867] Ven. Mm. I. 14 (1868) 1-; 17 (1872) 189-.

-, application to problems. Quillet, -. N. A. Mth. 4 (1845) 249-.

-, applications. Gors, - Rouen Tr. Ac. (1835) 63-

-, controls. Cauchy, A. L. C. R. 44 (1857) 849 .

-, correlations. Cockle, Jas. Ph. Mg. 24 (1862) 531-.

-, elementary. Winckler, A. Wien Sb. 59 (1869) (Ab. 2) 356-.

-, general theorems and their applications. Prouhet, E. C. R. 34 (1852) 359-.

-, higher. Textor, - von. Zach M. Cor. 22 (1810) $202-$

-, -. Janni, G. G. Mt. 14 (1876) 321-.

-, - method, new. Schell, $W$. Grunert Arch. 24 (1855) 489 ; 25 (1855) 1-.

-, methods. Cauchy, A.L. Bb. It. 60 (1830) $202-$.

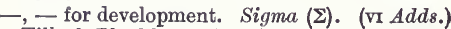
Tilloch Ph. Mg. 65 (1825) 344-.

-, proofs. Stern, M. A. Grunert Arch. 1 (1841) 57-.

-, pure, metaphysies. Piola, G. Bb. It. 97 (1840) 315-.

-, report on memoir by Stouvenel. Valat, - . Bordeaux Act. Ac. Sc. (1839) 639-.

- - - memoirs by Burmann. Lagrange, J. L., \& Legendre, - Par. Mm. de l'I. 2 (1799) (H.) 13-.

-, researches. Franchini, $P$. Mod. S. It. Mm. 11 (1804) 254-; 12 (1805) 160-; Lucca At. Ac. 5 (1829) 289-; 7 (1831) 97-.

-, 一. Brioschi, F. C. R. 56 (1863) 659-.

-, simplification. Eyres, E. S. Tilloch Ph. Mg. 43 (1814) 177-.

- and synthesis. Toplis, J. Tilloch Ph. Mg. 20 (1805) 193-.

- - Gergonne, J. D. Gergonne A. Mth. 7 (1816-17) 345-.

- - Franceschinis, F. M. Padova N. Sag. 1 (1817) 317-.

- - Z Zinelli, F. M. Poligrafo 12 (1832) 468-.

-, theory. Murphy, R. [1836] Phil. Trans. (1837) 179-.

-, transcendental. Galois, $\dot{E}$. Gergonne A. Mth. 21 (1830-31) 182-.

Analytical aphorisms. Schlömilch, O. Grunert Arch. 5 (1844) 90-.

Approximation, methods. Trembley, J. Berl. Mm. Ac. (1803) 50-

Approximations. Schulze, N.W. Crelle J. 13 (1835) 250-.
Foundations of Analysis 3200

Approximations. Winckler, A. [1875] Wien Ak. Sb. 72 (1876) (Ab. 2) 623-.

Conditional coefficients. Davies, T.S. Mathematician 1 (1845) 5-.

Functions, notation. Eneström, G. Bb. Mth. (1891) 89-.

Generic equations (expressing a general law of relation). Blissard, J. QJ. Mth. 4 (1861) 279-; 5 (1862) 58-, 185-.

Higher mathematics. Magistrini, G. B. Mod. S. It. Mm. 17 (1815) 445-.

Multiplicity of values. Cockle, Jas. $\mathrm{Ph} . \mathrm{Mg}$. 6 (1853) 444-.

Notation. Björling, E. G. Stockh. Ak. Hndl. (1845) 75-; Grunert Arch. 9 (1847) 383-; 11 (1848) 39-.

-, a universal analytical. Mansion, $P$. Mess. Mth. 4 (1875) 166-.

Polynomlals, two, approximate linear expressions. Tchebitchef, $P . L$. Bll. Sc. Mth. As. 1 (1877) (Pt. 1) 289-.

Pure mathematics, analysis, synthesis, definitions, bibliography. Bellavitis, G. (Ix) Ven. I. Mm. 15 (1870) 375-; Ven. I. At. 5 (1879) 673-.

Series, etc. Catalan, E. C. [1877-89] Brux. Ac. Sc. Mm. 42 (1878) (No. 3) 32 pp.; 48 (1892) (No. 1) $98 \mathrm{pp}$.

Synthesis, algebraic. Cauchy, A. L. C. R. 16 (1843) 867-, 967-, 1039-; 18 (1844) 803-.

Transcendental calculations. Paque, $A$. Liège Mm. S. Sc. 16 (1861) 145-.

\section{Foundations of Analysis}

\section{General.}

Analysis, Lagrange's elementary course. Prony, R. de. Par. Éc. Pol. J. 1 (1794) (2 $2^{\circ}$ cah.) 206-.

-, pure and applied. Prony, R. de. Par. Ec. Pol. J. 2 (1795) (6e cah.) 213-.

- and theory of numbers, applications. Bervi, N. V. Mosc. S. Sc. Bll. 92 (No. 1) (1896) 1-; Fschr. Mth. (1896) 157-.

Analytical expression, general, nature and signification. Raymond, G. M. Chambéry Mm. Ac. Sav. 1 (1825) 170-

"Calcul d'exposition." Gruson, J. P. Berl. Mm. Ac. (1799-1800) 157-.

Calculus, finite and infinitesimal. Lorgna, A. M. Turin Mm. Ac. 3 (1786-87) 409-

- of principal relations. Hamilton, (Sir) W. R. B. A. Rp. (1836) (pt. 2) 41-.

Function $y=\frac{1}{e^{x}-1}$. Catalan, E. C. Liége $\mathrm{S}$. Sc. Mm. 15 (1888) No. 1, 230-.

Functional systems, characteristics. Kronecker, L. Berl. Ak. Mb. (1878) 145-.

- - , - Kronecker's theory. Dyck, W. [1894] D. Mth. Vr. Jbr. 4 (1897) 94-

Functions, algebraic, of one variable. Hensel, $K$. D. Mth. Vr. Jbr. 1 (1892) 56-.

- , classification. H., C. Skandia 10 (1837) 
Functions, transcendental, Liouville's classification. Königsberger, $L$. Mth. A. 28 (1887) 483-.

- which vanish with their variables. Hamilton, (Sir) W. R. Ir. Ac. T. 16 (Pt. 1) (1830) 63-; 16 (Pt. 2) (1831) 129-.

- _ - Moore, A. A. [1837] Camb. Ph. S. T. 6 (1838) 317-

Indeterminate expressions, equivalents. Hargreave, C. J. B. A. Rp. (1857) 184-.

Induction, and symbolic formulæ. Cauchy, A.L. C. R. 39 (1854) 169-.

Infinitely small and infinitely great, two types. Stolz, O. Mth. A. 31 (1888) 601-.

Leibnitz's calculus. Arnoux, R. As. Fr. C. R. (1900) (Pt. 2) 1089-.

Limiting values, generalisation and application to curves. Schwarz, C. H. A. A. Mt. 10 (1880-82) 129-.

Line functions. Arzelà, C. Rm. R. Ac. Linc. Rd. 5 (1889) (Sem. 1) 342-.

Powers, theory. Crelle, A. L. Berl. Ab. (1829) 13-.

Principles. Lutter, N. [1860] (xII) Mag. Ak. Éts. (Mth. Term.) 2 (1861-62) 3-.

-, fundamental. Mansion, $P$. (xII) Brux. S. Sc. A. 3 (1879) (Pt. 2) 259-.

Products and functions, symbolic. Cauchy, A. L. C. R. 43 (1856) 169-.

Quantity, idea of, successive generalisation. Hoüel, J. [1882] Bordeaux S. Sc. Mm. 5 (1883) 149-.

Theorem on product $\stackrel{n}{I I}\left(x+a^{i} z\right)$. Gruson, J.P. Berl. Ab. (1814-15) ${ }^{1}$ (Mth.) 36-.

Theories, various, from one principle. Steen, $A$. Mth. Ts. 1 (1859) 145-.

Transcendental analysis, principles. Lamarle, E. Liege Mm. S. Sc. 2 (1845-46) 221-.

\section{Theory of functions of real variables.}

Cauchy, A. L. C. R. 43 (1856) 69-.

Bing, $F$. Mth. Ts. 4 (1862) 129-, 154-, 171-.

Schendel, L. Crelle J. Mth. 84 (1878) 80-.

Baire, R. C. R. 125 (1897) 691-; A. Mt. 3 (1899) 1-.

Stefano, A. de. G. Mt. 38 (1900) 178-.

Analytic functions. Lagrange, J. $L$. Par. Ec. Pol. J. 2 (1798) 232-; 3 (1800) Suppl., 277 pp.

- - , development for real value of the variables. Painlevé, - . C. R. 126 (1898) 385-.

- - principles and application. Ferroni, $P$. [1823] Mod. S. It. Mm. 20 (1828) 1-.

- -, problem. Collins, E. [1827] St Pét. Ac. Sc. Mm. 1 (1831) 345-

- - rational development. Kummer, E. E. Crelle J. 41 (1851) 368-.

Approximation of functions. Lebesgue, - Bll. Sc. Mth. 22 (1898) 278-.

Ascending and descending functions. Ceroni, $G$. A. Sc. Lomb. Ven. 11 (1841) 149-, 197-; 12 (1842) 123-, 137-.
Calculus of functions. Lagrange, J. L. [1799] Par. Sé. Éc. Norm. 10 (1801) 6-; Par. Éc. Pol. J. 7 (1808) (14\% cah.) Suppl., 90 pp.

- - indices of functions. Cauchy, A. L. Par. Ec. Pol. J. 15 (1837) (25॰ cah.) 176-.

Classification of functions. Schmidten, $H$. G. Kiöb. Ov. (1827-28) 2-.

Coefficients, limiting or restricting (which reduce to unity when certain conditions are fulfilled, and to zero when not). Cauchy, A. L. C. R. 37 (1853) 150 -

Constants of expression, conditions for independence. Maximovitch, $W$. de. Liouv. J. Mth. 6 (1880) 167-.

Continuity. Lüroth, J. [1872] Mth. A. 6 (1873) 319 -

- Lamb, H. Manch. Lt. Ph. S. Mm. \& P. 41 (1897) No. 10, 9 pp.

- and discontinuity, distribution of points in pointwise discontinuous functions of a real variable. Schönflies, A. Gött. Nr. (1899) $187 \rightarrow$.

- of functions. Schlömilch, O. Grunert Arch. 12 (1849) 430-.

- - criterion. Stringham, I. As. S. Pac. Pb. 2 (1890) 100-; 3 (1891) 112.

- - method of defining. Ruffini, F. P. (xi) Mod. Ac. Sc. Mm. 12 (1871) 3-.

- - - theorem. Darboux, G. Bll. Sc. - Mth. As. 3 (1872) 307-.

- principle, application. Young, J.R. Camb. Ph. S. T. 8 (1849) 429-.

Continuous and discontinuous functions, differentiation and representation. Cauchy, A. L. C. R. 40 (1855) 382-.

- - discrete quantities, difference. Toschi, $A$. Palomba Rac. 3 (1847) 216-.

- function with discontinuous differential coefficient. Lerch, M. G. Teix. J. Sc. 9 (1889) 97-.

- not admitting of differentiation, a. Schwarz, H. A. Arch. Sc. Ps. Nt. 48 (1873) 33-, 242 ; Sch. Nf. Gs. Vh. 56 (1873) 252-.

- - with oscillations in every interval. Köpcke, A. Mth. A. 34 (1889) 161; 35 (1890) 104-.

- functions. Cauchy, A. L. C. R. 18 (1844) 116- ; 20 (1845) 375-.

- - with extreme values in every interval. König, J. Mh. Mth. Ps. 1 (1890) 7-.

- - of one real variable. Scheeffer, $L$. Acta Mth. 5 (1884) 183-, 279-.

- - B Broden, T. Crelle J. Mth. 118 (1897) 1-.

- - - Kowalewski, G. Leip. Mth. Ps. B. 52 (1900) 214-.

- - two real variables. Tononsend, E.J. [1900] N. Y. Am. Mth. S. Bll. 7 (1901) 244, 251-.

- - $n$ variables. Riquier, -. Par. Ec. Norm. A. 7 (1890) 265-.

Demonstration of a lemma. Chavannes, $F$. Laus. Bll. S. Vd. 2 (1846-48) 284-.

Derived functions, class. Formenti, C. Mil. I. Lomb. Rd. 26 (1893) 330-, 382-, 482-. 
Discontinuity, curious examples. Plateau, J. A. F. Brux. Ac. Bll. 43 (1877) 84-, 255-.

- of functions, theorem. Casorati, F. Mil. I. Lomb. Rd. 1 (1868) 123-.

Discontinuous function. Lerch, M. G. Mt. 26 (1888) 375-.

- functions. Libri, G. Crelle J. 7 (1831) 224-; 10 (1833) 303-.

- - Boole, G. [1846] Ir. Ac. T. 21 (1848) 124 .

- - Cauchy, A. L. C. R. 28 (1849) 277-.

- -. Darboux, G. [1874] Par. Éc. Norm. A. 4 (1875) 57-; 8 (1879) 195-.

- -, application to analytic formulæ. Libri, G. C. R. 15 (1842) 401-.

- connected with continuous. Baire, $R$. C. R. 126 (1898) 1621-.

- - developable by continuous. Baire, $R$. C. R. 126 (1898) 884-.

- - real, of real variable, analytical representation. Severini, $C$. Tor. Ac. Sc. At. 33 (1897) 762- or 1002-; 34 (1898) 199-, 374or 325-, 518-.

- - representation. Rawson, R. [1846] Manch. Ph. S. Mm. 8 (1848) 235-.

- - sum, theorem of Abel's. Catalan, E. C. Liége S. Sc. Mm. 15 (1888) No. 1, 153-.

- -, theorem, proof. Baire, $R$. Par. S. Mth. Bll. 28 (1900) 173-.

- -, theory. Piola, G. [1830] Mod. Mm. S. It. 20 (1828) 573-.

- - - Haan, D. Bierens de. Amst. Vh. 7 (1859) $52 \mathrm{pp}$.

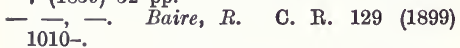

- and indeterminate functions. Wead, C. K. [1900] Wash. Ph. S. Bll. 14 (1906) 65-.

- punctuated functions. Volterra, V. G. Mt. 19 (1881) 76-.

Double functions, changes. Decher, G. Grunert Arch. 21 (1853) 423-; 27 (1856) 471-.

Elimination, a functional. Russell, W. H. L. Mess. Mth. 1 (1872) 102.

Empiric curves, deduction of true function from. Pucci, $E$. Rm. R. Ac. Linc. Mm. 6 (1889) 316-.

- functions, differentiation. Runge, C. Z. Mth. Ps. 42 (1897) 205-.

Evaluation of functions in indeterminate form. Unferdinger, F. Grunert Arch. 26 (1856) 224-.

Finite and continuous functions having no derivative. Dini, $U$. Rm. R. Ac. Linc. T. 1 (1877) 70-, 130-.

Fluctuating functions. Hamilton, (Sir) W. $R$. [1840] Ir. Ac. T. 19 (1843) 264-.

Fractional functions, decomposition and transformation. Rangoni, $L$. [non Gabba, A.] Brescia Cm. (1830) 150-.

Function satisfying no algebraic functional equation. Hölder, O. Gött. Nr. (1887) 662-.

Functional analysis with application to parallelogram of forces. Anon. (vi 553) Gergonne A. Mth. 14 (1823-24) 350-.

- indices. Liventsov, A.I. (xII) Rec. Mth. (Moscou) 8 (1876) (Pt. 1) 277-.
Functional powers. Collins, E. [1835] St Pét. Ac. So. Mm. 3 (1838) 313-.

- problems. Abel, N.H. Mg. Ntvd. 1 (1823) 216-; Crelle J. 2 (1827) 386-.

- - Decsy, S. (xII) Mag. Tud. Ak. Ets. 7 (No. 6) (1873) 80-.

- symbols. Cayley, A. QJ. Mth. 4 (1861) 225-.

Functions analogous to sine and cosine. Olivier, L. Crelle J. 2 (1827) 243-.

-, application to pure geometry. Holst, $E$. [1879] Par. S. Mth. Bll. 8 (1880) 52-.

-, approximate representation, application of method of least squares. Schlömilch, $O$. Schlömilch Z. 3 (1858) 124-.

- dependent on lines. Volterra, V. Rm. R. Ac. Linc. Rd. 3 (1887) (Sem. 2) 225-, 274-.

- determined by certain addition theorem. Zignago, I. Rm. N. Linc. At. 53 (1900) 139-.

-, differentiable, with infinitely close (dicht) maxima and minima. Brodén, T. Stockh. Öfv. (1900) 423-, 743-.

- expressible rationally in terms of one another. Woepcke, C. Liouv. J. Mth. 4 (1859) 339-.

-, having differential coefficient at every point, and oscillating infinitely often in every interval. Pereno, I. G. Mt. 35 (1897) 132-.

- - no derivative throughout one interval. Dini, U. A. Mt. 8 (1877) 121-.

- not expansible by Taylor's theorem. Pringsheim, A. Mth. A. 44 (1894) 41-.

- oscillating infinitely often, and discontinuous functions. Hankel, $H$. Mth. A. 20 (1882) 63-.

- of real arguments, classification by changes in least intervals. Du Bois-Reymond, $P$. [1874] Crelle J. Mth. 79 (1875) 21-.

- - one real variable. Burnside, $W$. Mess. Mth. 23 (1894) 39-.

- - - - Somigliana, C. Rm. R. Ac. Linc. Rd. 8 (1899) (Sem. 1) 4-, 618.

- - - - Straneo, P. Rm. R. Ac. Linc. Rd. 8 (1899) (Sem. 1) 438-, 618.

$\ldots-\ldots \ldots$, analytic representation. Weier. strass, $K$. Berl. Ak. Sb. (1885) 633-, 789-.

- - - - - - Mittag-Leffler, G. Palermo Cir. Mt. Rd. 14 (1900) 217-.

_ _ - _ - with derivatives of every order. Maurer, L. Mth. A. 41 (1893) 377-.

_ _ _ - and their differential coefficients. Harnack, A. Mth. A. 23 (1884) 244-; 24 (1884) 217-.

- - discontinuities. Bettazzi, $R$. Palermo Cir. Mt. Rd. 6 (1892) 173-.

- - - - geometric mean value. Lemoyne, G. G. Mt. 16 (1878) 209-.

$-{ }_{-}-$- special class. Study, E. Mth. A. 46 (1896) 298 - - - 8 - $1893-94)$ 65-.

- two real variables. Borel, $\mathscr{E}_{\text {. }} \quad$ Par. Ec. Norm. A. 13 (1896) 79-. 
Functions of two real variables. Biermann, $O$. Mth. A. 48 (1897) 393-.

- - - - - , and the idea of arbitrary function. Borel, É. C. R. 121 (1895) 811-.

_ _ - within a finite range. Ascoli, $G$. Mil. I. Lomb. Rd. 22 (1889) 317-, 438-, 686804-.

- - several real variables, differentiation and integration. Bettazzi, R. G. Mt. 22 (1884) 133-, 200.

— with recurring derivatives. Sang, E. [1866] Edinb. R. S. T. 24 (1867) 523-.

-, representation by a curve (strip). Klein, $F$. (vIII) Erlang. Sb. Ps. Md. S. 6 (1874) $52-$.

Harmonic functions of two real variables and functions of a complex argument, oscillations in values. Schottky, E. Crelle J. Mth. 117 (1897) 225-.

Homogeneous functions, extension of Euler's theorem. Philip, W. E. Edinb. Mth. S. P. 18 (1900) 101-.

Implicit functions of a real variable. Ingrami, G. Bologna Rd. (1888-89) 108-.

- - transformation into explicit. Cauchy, A. L. C. R. 38 (1854) 944-

- - , - - monodromic and monogenous. Cauchy, A. L. C. R. 40 (1855) 878-

Integral polynomials of one variable. Hermite, C. N. Ts. Mth. 5 (B) (1894) 1-.

Invariability of form. Maizière, - de. Gergonne A. Mth. 1 (1810-11) 368-.

Irrational functions. Cauchy, A. L. C. R. 32 (1851) 68-, 162-.

Isobaric calculus. Cesáro, E. N. A. Mth. 3 (1884) 561-; 4 (1885) 59-, 578.

Lagrange's "Théorie des Fonctions Analytiques," note. Poisson, S. D. Liouv. J. Mth. 2 (1837) 140-, 189-.

$-\frac{1}{-}-\frac{-}{-}$, Poisson's. Poinsot, $L$. C. R. 4 (1837) 559-.

Limits of functions. Matzka, W. Prag Sb. (1878) 262-.

-, superior, of functions, calculation. Cauchy, A. L. C. R. 15 (1842) 138-.

Mean value, theorem. Stringham, I. As. S. Pac. Pb. 2 (1890) 100-; 3 (1891) 112.

Ordered functions. Somigliana, $C$. Rm. R. Ac. Linc. Rd. 8 (1899) (Sem. 2) 125-.

Principle, fundamental, elementary proof. Mansion, P. B. A. Rp. (1876) (Sect.) 26-.

Properties, behaviour, etc. Scheffler, $H$. Grunert Arch. 28 (1857) 121-.

-, general. Ruffini, P. [1806] Mod. S. It. Mm. 13 (1807) 292-.

Rational functions, approximate square roots. Bugaev, N. V. [1881] (xir) Rec. Mth. (Moscou) 10 (1882-83) (Pt. 1) 524-.

- -, elements of a theory. Kraus, $L$. Časopis 14 (1885) 49-; Fschr. Mth. (1885) 369-

Real non-analytic functions, development. Painlevé, P. C. R. 126 (1898) 459-.

Reciprocal functions. Cauchy, A. L. Par. S, Phlm. Bll. (1818) 178-.
Reciprocal relation of functions. Raabe, J. $L$. [1852] Zür. Mt. 3 (1853-55) 198-.

Simple functions, gradation. Oettinger, $L$. Crelle J. 11 (1834) 75-, 173-; 12 (1834) 295-; 13 (1835) 292-.

Singularities, new general condensation principle. Cantor, G. Mth. A. 19 (1882) 588-.

Summatorial functions, properties. Hill, C. J. D. Sk. Nf. F. 8 (1860) 518-

Sums. (Björling.) Falk, M. Ts. Mt. Fys. 4 (1871) 71-.

Systems. Ricci, G. Rm. R. Ac. Linc. Rd. 5 (1889) ( $\mathrm{Sem} .1$ 1) 112-.

-. Stäckel, P. Crelle J. Mth. 112 (1893) 311-.

-, inversion. Kneser, A. Mth. A. 45 (1894) 446-.

Theorem. Grolous, J. Par. S. Phlm. Bll. 12 (1875) 95-.

-. Tonelli, A. A. Mt. 9 (1878-79) 173-.

- Poincaré, H. Par. S. Mth. Bll. 11 (1883) 112-.

-, applications. Krey, $H$. Z. Mth. Ps. 26 (1881) 357-.

- on determination of $u, v$ to satisfy $u f(x)+v F(x)=R$. Cauchy, A. L. Baumgartner Z. 1 (1826) 88-.

Theorems. Prym, F.E. Crelle J. 71 (1870) 223-.

-. Thomae, C. J. Gött. Nr. (1878) 466-.

-, two, of Tchebitchef's, generalisation. Minine, A. Les Mondes 55 (1881) 185-.

Transformation of functions. Deflers, Par. S. Phlm. Bll. (1819) 161-.

- - Nicholson, $P$. Tilloch $\mathrm{Ph}$. Mg. 62 (1823) 168-, 244-, 348-, 433-.

- - - Buquoy, G. von. Oken Isis (1827) 130-.

- - Collins, E. St Pét. Ac. Sc. Mm. 1 (1831) 583-.

- - Novi, G. Tortolini A. 1 (1850) 517-.

- - . Schlömilch, O. Liouv. J. Mth. 8 (1863) 99-.

- _ - general. Boole, G. Camb. and Dubl. Mth. J. 3 (1848) 112-.

- - into isotropic means. Cauchy, A.L. C. R. 34 (1852) 159-; 43 (1856) 261-.

- - - by substitution. Collins, E. [1826] St Pét. Ac. Sc. Mm. 1 (1831) 181-.

- and simplification, methods by interpolation. Richelmy, P. [1855] Tor. Mm. Ac. 17 (1858) 245-.

Weierstrass's function, $f(x)=\sum_{0}^{\infty} b^{n} \cos \left(a^{n} x \pi\right)$. Wiener, L. C. Crelle J. Mth. 90 (1881) 221-.

$z=f(x, y)$, graphic representation. Lalanne, $L$. Par. S. Phlm. PV. (1843) 71-.

$f(x) f(x+1) \ldots f(x+h)$, value. Gasparis, $A$. de. Nap. Rd. 1 (1852) 199-.

$F(x, y)$, when a function of $\phi(x, y)$, conditions. Dienger, $J$. Grunert Arch. 21 (1853) 219-.

$f(x)$, Wronski's expansion. Echols, W. $H$. N. Y. Mth. S. Bll. 2 (1893) 178-. 


\section{Infinite series; infinite pro- ducts and other infinite pro- cesses. (See also 5610, 5620.)}

\section{INFINITE SERIES.}

Fontana, G. Verona S. It. Mm. 2 (1784) 386-. Paoli, $P$. Verona S. It. Mm. 4 (1788) 429-.

Parseval, M. A. [1799] Par. Mm. Sav. Etr. 1 (1806) 638-

Bianchi, G. [1844] Mod. Mm. S. It. 23 (1846) 239-.

Malmsten, C. J. Crelle J. 38 (1849) 1-.

Dienger, J. Crelle J. 38 (1849) 266-, 331-.

Tremoil, - . N. A. Mth. 9 (1850) 66.

Björling, E. G. Stockh. Öfv. 10 (1853) 147-.

Schlömilch, O. Schlömilch Z. 1 (1856) 180-; 5 (1860) 132-.

Kinkelin, H. Bern Mt. (1858) 88-.

Greer, H. R. QJ. Mth. 3 (1860) 262-, 370-.

Isely, $M$. Neuch. Bll. 6 (1861-63) 356-.

Cockle, Jas. [1863] (II) Mess. Mth. 2 (1864) 196-.

Lindman, C. F. Arch. Mth. Ps. 50 (1869) 219 -

Enneper, A. Z. Mth. Ps. 15 (1870) 47-.

Schlömilch, O. X. Leip. Mth. Ps. B. 29 (1877) 101-.

Barbour, L. G. Des Moines Anal. 8 (1881) 191-.

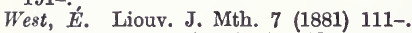

Catalan, E. C. Liege S. Sc. Mm. 12 (1885) No. 2, 148-, 152-, 206-, 282-, 294-.

Cesâro, E. G. Teix. J. Sc. 7 (1886) 171-; 8 (1887) 15-.

Biehler, C. N. A. Mth. 6 (1887) 243-.

Gutzmer, A. G. Teix. J. Sc. 8 (1887) 81-.

Studnička, F. J. Časopis 16 (1887) 81-; Fschr. Mth. (1887) 227.

Weyr, E. G. Teix. J. Sc. 8 (1887) 97-.

Catalan, E. Brux. Ac. Bll. 18 (1889) 41-.

Tauber, A. Mh. Mth. Ps. 8 (1897) 273-.

Absolutely summable series, series $(M)$ and analytical continuation. Borel, $k$. C. R. 131 (1900) 830-

Algebraic series. Sidler, G. Zür. Vjschr. 1 (1856) 180-; Bern Mt. (1899) 13-.

- -, new. Wallace, J. Silliman J. 7 (1824) 278-; 9 (1825) 98-.

- -, - (Wallace). B. (vr Add8.) Silliman J. 8 (1824) 131-; 9 (1825) 293-.

Analytic series. Svanberg, $J$. Stockh. Ak. Hndl. 22 (1801) 30-.

- - genetic explanation. Barfuss, $F, W$. Grunert Arch. 8 (1846) 387-.

Applications to arithmetic. Sardi, C. G. Mt. 7 (1869) 257-.

Approximation to geometric means by series of arithmetical and harmonic means. Mathieu, J. J. A. N. A. Mth. 18 (1879) 529-.

- series. Meyer, A. N. Ts. Mth. 5 (B) (1894) 81-; 6 (B) (1895) 41-, 73-; Fschr. Mth. (1893-94) 241.

Approximations and series. Houzeau, J. C. Brux. Ac. Bll. 41 (1876) 961-.
Asymptotic values of certain series. Le Roy, $E^{\prime}$. Bll. Sc. Mth. 24 (1900) 245-.

- - infinite approximations, and order of infinity in solution of equations. Du BoisReymond, P. Mth. A. 8 (1875) 363-, $574-$.

Bernoulli's functions. Baraniecki, M. A. Krk. Ak. (Mt.-Prz.) Rz. 13 (1886) 183-; Fschr. Mth. (1885) 416.

Binet's series, and products of a large number of factors. Gilbert, P. Brux. S. Sc. A. 10 (1886) (Pt. 2) 191-.

\section{Binomial theorem.}

Ampère, A. M. Gergonne A. Mth. 15 (1824-25) 369-.

Burg, A. Crelle J. 1 (1826) 367-; Wien Jb. Pol. I. 16 (1830) 171-.

De Morgan, A. Camb. Mth. J. 3 (1843) 61-.

Barfuss, F. W. Grunert Arch. 5 (1844) 155-; 7 (1846) 3-; 8 (1846) 387-.

(Barfuss.) Schlömilch, O. Grunert Arch. 5 (1844) 437-; 10 (1847) 321-.

Bianchi, G. [1844] Mod. Mm. S. It. 23 (1846) 239-.

Terquem, $O$. N. A. Mth. 9 (1850) 459-.

Heine, H. E. Crelle J. 55 (1858) 279-

Hinricks, G. Mth. Ts. 2 (1860) 38-.

Janni, V. G. Mt. 12 (1874) 229-, 312.

Lanza, G. Des Moines Anal. 1 (1874) 177-.

Gohierre de Longchamps, - . N. A. Mth. 17 (1878) 101-.

applications. Ferroni, P. [1801] Mod. S. It. Mm. 9 (1802) 291-.

demonstrations. Little, J. [1810] Ir. Ac. T. 12 (1815) 31-.

-. Knight, T. Phil. Trans. (1816) 331-; (1817) 245 -

-. Herapath, J. Thomson A. Ph. 13 (1819) $365-$.

- (Herapath). Ward, L. T. Tilloch Ph. Mg. 65 (1825) 432.

-. Colburn, W. Bost. J. Ph. 2 (1825) 474-.

-. Strong, T. Silliman J. 12 (1827) 132-.

-. Crelle, A. L. Crelle J. 4 (1829) 305-; 5 (1830) 187-.

-. Cerquero, J. S. Quetelet Cor. Mth. 10 (1838) 202-.

- Roche, - . N. A. Mth. 1 (1842) 42-, 118-.

Cox, H. Camb. and Dubl. Mth. J. 7 (1852) 98 .

-. Paque, A. Liège Mm. S. Sc. 10 (1855) 1-.

-. Macnie, J. Des Moines Anal. 7 (1880) $190-$.

—, direct. Gould, D. Silliman J. 19 (1831) 50 -.

- by fluxions. Evans, L. Tilloch Ph. Mg. 64 (1824) 270-.

- and generalisation. Eisenstein, G. Crelle J. 28 (1844) 44-; N. A. Mth. 8 (1849) $344-$.

.expansion. .André, D. N. A. Mth. 10 (1871) 74 . 
Binomial Theorem, continued.

expansion. Kluyver, J. C. Amst. Ak. Vs, 6 (1898) 421-.

-, approximate formulæ. Astrand, $J . J$. Grunert Arch. 12 (1849) 420-.

-, properties derivable from. Young, J. $R$. Ph. Mg. 33 (1848) 268-.

expression in form of a factorial which is always convergent. Kummell, C. H. A. Mth. 12 (1898-99) 60-.

fractional index, demonstration. Robertson, $A$. Phil. Trans. (1806) 305-.

- or negative index, demonstration. Anon. (vi 1170) Thomson A. Ph. 7 (1816) 346-.

---1, Rodrigues, $O$. Liouv. J. Mth. 3 (1838) 550-.

- - - Euler's demonstration. Muirhead, R. F. Edinb. Mth. S. P. 17 (1899) 38-.

- - - extension. Davidov, A. Y. (xI) Rec. Mth. (Moscou) 3 (1868) (Pt. 2) 1-.

- _ - , historical note. Terquem, 0 . N. A. Mth. 6 (1847) 85-.

generalised, expansion of factorial by. Cayley, A. Ph. Mg. 6 (1853) 182-; Mess. Mth. 5 (1871) 102-.

geometrical treatment. Koppe, $M$. Arch. Mth. Ps. 61 (1877) 113-.

and logarithmic theorem. Catlin, M. Mth. Misc. 1 (1838) 113-.

methods of demonstration. Davies, T. S. Tilloch Ph. Mg. 66 (1825) 115-.

negative index, demonstration. Trowbridge, $D$. Des Moines Anal. 2 (1875) 71-.

- integral index, demonstration. Grunert, J. A. Grunert Arch. 30 (1858) 336-.

particular case. Catalan, E. C. C. R. 45 (1857) 641-.

series analogous to binomial series. Jacobi, C. G. J. Crelle J. 32 (1846) 197-.

- - _ - - two groups. Weiss, $E$. Wien Ak. Sb. 91 (1885) (Ab. 2) 587-.

Calculation of series. Poncelet, J. V. [1833] Par. Mm. Sav. Étr. 6 (1835) 641-.

Class. Schlömilch, O. Schlömilch Z. 1 (1856) 21-.

Classification. Chessin, A. S. J. H. Un. Cir. [15 (1895-96)] 39.

Coefficients in a certain series. Björling, E. G. Crelle J. 28 (1844) 284-.

-, especially factorial coefficients, determination. Schlömilch, O. Grunert Arch. 18 (1852) 306-.

Conditionally convergent series. Schlömilch, $O$. Leip. B. 24 (1872) 327-.

- - multiplication. Pringsheim, $A$. Mth. A. 21 (1883) 327-.

$-\div-$, - Voss, A. Mth. A. 24 (1884) $42-$.

- - and products. Pringsheim, A. Mth. A. 22 (1883) 455-.

Continued identity, method. Kummell, C. H. A. Mth. 5 (1889-90) 85-, iv.

Continuity of sums, theorem of Cauchy. Du Bois-Reymond, P. Mth. A. 4 (1871) 135-.

- - - two theorems of Abel. Pringsheim, A. Münch. Ak. Sb. 27 (1898) 343-.

\section{Convergence.}

Olivier, L. Crelle J. 2 (1827) 31-.

(Olivier.) Abel, N. H. Crelle J. 3 (1828) 79-.

Cauchy, A. L. C. R. 9 (1839) 587-; 10 (1840) 640-; 11 (1840) 639-, 667-.

Binet, 'J. P. $M$. Liouv. J. Mth. 6 (1841) 495-.

Cauchy, A. L. . C. R. 19 (1844) 141-, 157.

Malmsten, C. J. Ups. N. Acta S. Sc. 12 (1844) 255-.

Schultén, N. G. af (fil.) [1844] Helsingf. Acta 2 (1847) 733-.

Cauchy, A. L. C. R. 20 (1845) 126-.

Lebesgue, V.A. N. A. Mth. 4 (1845) 66-.

Malmsten, C. J. Grunert Arch. 8 (1846) 419-.

Terquem, O. N. A. Mth. 5 (1846) 101-.

Timmermans, J. A. Brux. Ac. Bll. 13 (1846) 140 -

Schaar, M. Brux. Mm. Cour. $4^{\circ}, 22$ (1846-47) 9 pp.

Schlömilch, O. Grunert Arch. 14 (1850) 105-.

Arndt, F. Grunert Arch. 20 (1853) 43-, 461-; 21 (1853) 78-

Kinkelin, $H$. Bern Mt. (1858) 57-.

Ermakof, $V$. Bll. Sc. Mth. As. 2 (1871) 250-; 7 (1883) 142-.

Petersen, J. P. C. (xiI) Ts. Mth. 3 (1873) 49 -

Grolous, J. As. Fr. C. R. 6 (1877) 209-.

Kohn, G. Arch. Mth. Ps. 67 (1882) 63-.

Jensen, J. L. W. V. Ts. Mth. 2 (1884) 63-; Fschr. Mth. (1884) 192-.

Mansion, $P$. Mathesis 5 (1885) 270-.

Bugaev, N. V. [1888] Rec. Mth. (Moscou) 14 (1890) 279-; Fschr. Mth. (1889) 232.

Cesáro, E. C. R. 106 (1888) 1142-; N. A. Mth. 7 (1888) 49-, 190.

(Cesáro.) Jensen, J. L. W. V. C. R. 106 (1888) 1520-

Lerch, M. Prag Sb. (1890) 1 (Mth. Nt.) 219-.

Cahen, $E . \quad$ N. A. Mth. 10 (1891) 453-.

Gilbert, P. Brux. S. Sc. A. 15 (1891) (Pt. 1) 69-.

Bryant, R. [1895] L. Mth. S. P. 27 (1896) 69-.

Lerch, M. D. Nf. Vh. (1899) (Th. 2, Hälfte 1) 6-.

Sincov, D. M. Rec. Mth. (Moscou) 20 (1899) 616-; Fschr. Mth. (1899) 228.

and approximate value of functions of high numbers. Lobatschewsky, N. (vi Adds.) Kazan Mm. Un. (1835) (cah. 2) 211-.

characteristics. Andries, $F$. Brux. A. Un. (1846) 99-.

-. Doucet, -. N. A. Mth. 8 (1869) 266-. 844-.

- for series with positive terms; and functions increasing indefinitely. Hadamard, $J$. Acta Mth. 18 (1894) 319-, 421.

circle of convergence. Leau, - C. R. 127 (1898) 711-, 794. 
circle of convergence, convergence and divergence on. Thomé, L. W. Crelle J. Mth. 100 (1887) 167-.

- - - for reverted series. Stolz, O. Wien Ak. Sb. 104 (1895) (Ab. 2a) 485-.

- _ - , singular points on, and summation of divergent series. Leau, -. C. R. 127 (1898) 607-.

condensation test, Cauchy's. Hill, M. J. M. Mess. Mth. 26 (1897) 102-.

conditions. Liouville, J. C. R. 11 (1840) 615-.

- Dirksen, E. H. Berl. B. (1845) 349-. 222-.

Fouret, $G$. N. A. Mth. 9 (1890)

- (Fouret). Giudice, -. Palermo Cir. Mt. Rd. 4 (1890) 284-.

- Giudice, -. Palermo Cir. Mt. Rd. 4 (1890) 275-, 276-, 278-, 279, 279-, 280-, $281-, 283$.

of continued fractions. Markov, A. St Pet. Ac. Sc. Mm. (Rs.) 72 (1893) 8-; Fschr. Mth. (1893-94) 329-.

criteria. Schulz von Strasznitzki, L. C. Baumgartner Z. 5 (1829) 10-.

-. Schlömilch, O. Z. Mth. Ps. 11 (1866) 354-.

- Cajori, F. [1892] N. Y. Mth. S. Bll. 2 (1893) 1-.

-. Saint-Germain, A. de. C. R. 115 (1892) 1258-.

-, Bertrand's, generalisation. Petrini, $H$. Stockh. Öfv. (1899) 691-.

- connection. Zahradnik, $K$. Casopis 7 (*1878) 91-; Fschr. Mth. ( $\left.{ }^{*} 1878\right) 176$.

- dating from beginning of eighteenth century. Eneström, G. Stockh. Öfv. 36 (1879) No. 9, 71-.

-, Gauss's. Hornstein, K. Prag Sb. (1864) $52-$.

-, - , simple proof. Pranghofer, J. Wien Sb. 53 (1866) (Ab. 2) 503-.

-, general. Lerch, $M$. Časopis 20 (1891) 285-; Fschr. Mth. (1891) 248-.

-, Riemann's. Hurwitz, A. Mth. A. 44 (1894) 83-.

-, -, extension. Arzelà, C. [1900] Bologna Rd. 5 (1901) 25-.

and divergence. Burg, A. Wien Jb. Pol. I. 17 (1832) 112-.

- - Raabe, J. L. Baumgartner Z. 10 (1832) 41- ; Crelle J. 11 (1834) 309-.

- - Kummer, E. E. Crelle J. 13 (1835) $171-$.

- - Bonnet, O. [Bennet erroneously in C.S.P.] Liouv. J. Mth. 8 (1843) 73-.

- - Schlömilch, O. Z. Mth. Ps. 10 (1865) 74-.

- Aller, C. van. N. Arch. Wisk. 12 (1886) 204-.

- - conditions. Dirksen, E. H. Berl. Ab. (1832) 77-.

- criterion. Hoppe, R. Grunert Arch. 26 (1856) 217-.

- - limiting regions and so-called limit. Pringsheim, A. Münch. Ak. Sb. 26 (1897) 605-. and divergence of series with positive terms. Du Bois-Reymond, P. C. R. 107 (1888) 941-.

- Pringsheim, A. Mth. A. 35 (1890) 297-; 39 (1891) 125-.

- - - Petrini, H. Stockh. Ofv. (1900) 895-.

- - - - limits. Thomae, J. [1871] A. Mt. 5 (1871-73) 121-。

- - - - _ - new theory. Du BoisReymond, P. [1872] Crelle J. 76 (1873) 61-.

or divergence, simultaneous, of two series. Schlömilch, O. Z. Mth. Ps. 18 (1873) 425-.

of double series of Lamé. Escary, - C. R. 88 (1879) 558-.

- - - in theory of heat. Escary, - As. Fr. C. R. (1886) (Pt. 2) 149-; (1887) (Pt. 2) 1092.

in finite difference and in differential series. Crelle, A. L. [1839] Crelle J. 22 (1841) 249-.

of hypergeometric series. Horn, J. Mth. A. 34 (1889) 544-.

Lambert's series. Stolz, O. Wien Ak. Sb. 95 (1887) $(A b .2)$ 659-.

limit of convergence, behaviour of infinite series at. Lasker, E. [1900] Phil. Trans. (A) 196 (1901) 431-.

_ _ _, Du Bois-Reymond's, and a special form of conditions of convergence. Pringsheim, A. Münch. Ak. Sb. 27 (1898) 303-, 356-.

- - - of series representing integrals of differential equations. Picard, $\dot{E}$. C. R. 106 (1888) 1466-.

limits of convergence, theorem. Cauchy, A.L. C. R. 40 (1855) 162-.

of logarithmic series. Doppler, C. Wien Jb. Pol. I. 17 (1832) 172-

- multiple series. Cauchy, A. L. C. R. 19 (1844) 1433-.

- - conditions. Jordan, C. Par. S. Mth. Bll. 9 (1881) 113-.

- Newton's binomial series for case $x=1$. Broch, O.J. As. Fr. C. R. 7 (1878) 145-.

non-uniform. Chessin, A. S. Am. J. Mth. 18 (1896) 128-.

- - and integration of series term by term. Osgood, W. F. [1896] Am. J. Mth. 19 (1897) 155-.

regions of convergence. Dietrich, $R$. Arch. Mth. Ps. 69 (1883) 381-.

- - - continuity in. Meyer, A. (of Stockholm). Stockh. Ófv. 40 (1883) No. 9, 15-; Fschr. Mth. (1884) 203.

of reversions of series. Źorawski, $K$. Crc. Ac. Sc. Bll. (1899) 75-; Krk. Ak. (Mt.-Prz.) Rz. 17 (1900) 139-.

rule. Duhamel, J. M. C. Liouv. J. Mth. 4 (1839) 214-.

-. Genocchi, A. N. A. Mth. 6 (1867) 261-. - Gauss's, demonstration. Godefroy, -. N. A. Mth. 18 (1899) 157-.

-, -, generalisation. Saint-Germain, A. de. Bll. Sc. Mth. 14 (1890) 212-.

-, Raabe's. Longchamps, G. de. N. A. Mth. 19 (1900) 216-. 
rules. Bertrand, J. Liouv. J. Mth. 7 (1842) 35-. - Paucker, M. G. von [1849] Crelle J. 42 (1852) 138-.

of series arising from development of functions, Cauchy's theorem, extension. Timmermans, J. A. Brux. Ac. Bll. 13 (1846) (pte. 2) 17-.

- - with complex terms. Benthem, A. N. Arch. Wisk. 2 ( $\left.{ }^{*} 1876\right)$ 186-.

- - and continued products. Rees, R. van. Quetelet Cor. Mth. 6 (1830) 185-.

- - - definite integrals. Chartier, J. Liouv. J. Mth. 18 (1853) 201-.

(Ab. 2) 496-.

- - - - (Popper). Bugaev, N. V. (xII) Rec. Mth. (Moscou) 2 (1867) (Pt. 2) 81-.

- - - - Ermakov, V. P. [1872] (xir) Rec. Mth. (Moscou) 6 (1872-73) (Pt. 1) 39-.

- - formed from prime powers of natural numbers. Mertens, F. Gött. Nr. (1887) 265-.

- - general term of which is $n \times$ Laplace's coefficient $Y_{n}$. Plarr, G. C. R. 44 (1857) 835-.

- - for $p(u)$ and calculation of $u$. Söderblom, A. Ups. S. Sc. N. Acta 14 (1891) No. 5, 67 pp.

- - representing integrals of differential equations. Picard, $\dot{E}$. Bll. Sc. Mth. 12 (1888) 148(1898) 203-. $-\quad$ Staeckel, P. C. R. 126

- - - system of differential equations. Cauchy, A. L. C. R. 10 (1840) 939-; 11 (1840) 730-; 40 (1855) 330-, 373-.

- - roots of equations, etc. Cauchy, A.L. C. R. 4 (1837) 216-.

- - for solution of Kepler's problem. Carlini, F. As. Nr. 30 (1850) 197-.

- - in theory of planetary motion. Puiseux, V. Liouv. J. Mth. 14 (1849) 33-, 243-.

- - - - Serret, J. A. C. R. 42 (1856) 1134-.

theorem. Malmsten, C. J. Grunert Arch. 6 (1845) 38-

-. Delarue, D. M. (xIr) Kharkov Mth. S. Com. (1879) 19-.

-, general. Jensen, J. L. W. V. C. R. 106 (1888) 729-.

-, geometrical proof. Dixon, A. C. Camb. Ph. S. P. 8 (1895) 217-.

theorems. Scheibner, $W$. Leip. B. 25 (1873) 568 .

-, elementary. Oppermann, L. H. F. (xш) Ts. Mth. 3 (1873) 145-.

uniform. Cayley, A. Edinb. R. S. P. 19 (1893) 203-.

-. Bendixson, I. Stockh. Öfv. (1897) 605-.

-, application of notion. La Vallée-Poussin, C. J. de. Brux. S. Sc. A. 17 (1893) (Pt. 2) $323-$.

of variable series, degree. $D u$ Bois-Reymond, $P$. Crelle J. Mth. 100 (1887) 331-.

$-\frac{1.3 .5}{2.4 .6}+$ \&c. Fontana, G. Verona S. It. Mm. 3 (1786) 174 .

- $\sum_{n=1}^{\infty} \frac{x^{n}}{n-a}$. Lerch, M. Casopis 21 (1892) 65-; Fschr. Mth. (1892) 232-.
Convergent and divergent series. Garnier, J. G. Brux. Ac. Bll. 6 (1839) (Pte. 2) 113-, 262-.

- or divergent series, and continued fractions. Padé, H. Acta Mth. 18 (1894) 97-.

- series. Cauchy, A. L. C. R. 36 (1853) 454-, - C, continuity of values. Duhamel, J.M. C. C. R. 36 (1853) 643-.

- -, Euler's condensation formula. Studnička, F. J. Časopis $1\left({ }^{*} 1872\right) 33-;$ Bll. Sc. Mth. As. $6\left({ }^{*} 1874\right) 91$.

- - limit. Friedländer, J. Gould. As. J. 3 (1854) 129-.

Conversion into infinite products. Stern, M. A. Crelle J. 12 (1834) 353-; N. A. Mth. 6 (1847) 437-.

Deduction from infinite products. Glaisher, J. W. L. Mess. Mth. 2 (1873) 138-, 153-.

Derivation of negative powers (Eisenstein's theory). Sylvester, J. J. QJ. Mth. 1 (1857) 199-.

$-\frac{1}{-}-(-\rightarrow)$. Glaisher, J.W. L. [1875] QJ. Mth. 14 (1877) 79-.

- - - - $\left(-\frac{-}{)}\right.$. Cayley, A. [1875] QJ. Mth. 14 (1877) 83-.

- new infinite series from given series by change of sign. Mildner, $R$. [1882] Wien Ak. Sb. 86 (1883) (Ab. 2) 999-.

Derived series, theory. Werner, O. Grunert Arch. 22 (1854) 264-.

Differentiation of products of terms of a series. Collins, E. [1826] St Pét. Ac. Sc. Mm. 1 (1831) 3-.

- - series. Schlömilch, O. Grunert Arch. 10 (1847) 74-.

- - D. Dienger, $J$. Grunert Arch. 11 (1848) 38.

- - - Lerch, -. G. Teix. J. Sc. 11 (1892)

Diminishing series with alternating signs. Oltramare, G. Crelle J. 45 (1853) 345-.

Dirichlet's paradox. Unferdinger, $F$. [1868-69] Wien Sb. 57 (1868) (Ab. 2) 621-; 60 (1870) (Ab. 2) 591-.

- - Schlömilch, O. Z. Mth.,Ps. 15 (1870) 134-.

- series. Lerch, -. C. R. 128 (1899) 1310-.

- - multiplication and non-vanishing. Mertens, $F$. Crelle J. Mth. 117 (1897) 169-. - -, theory. Pringsheim, A. Mth. A. 37 (1890) 38-.

Discontinuity. Schlömilch, $O$. Schlömilch Z. 4 (1859) 161-.

- of values, tests. Duhamel, J.M. C. Liouv. J. Mth. 19 (1854) 112-.

Discontinuous series, case. Mansion, $P$. Mess. Mth. 7 (1878) 57-.

- -, Riemann's. Smith, H. J. S. [1881] Mess. Mth. 11 (1882) 1-.

Distribution of signs in a series. Cesáro, $E$. Rm. R. Ac. Linc. Rd. 4 (1888) (Sem. 2) 133-.

Divergence of a series. Unferdinger, $F$. Wien Sb. 55 (1867) (Ab. 2) 95-.

Divergent series, De Morgan, A. [1844] Camb. Ph. S. T. 8 (1849) 182-.

- - Young, J. R. B. A. Rp. (1844) (Pt. 2) 1. - Catalan, E. C. N. A. Mth. 17 (1859) $195-$ 
Divergent series, Chessin, A. S. [1895] N. Y. Am. Mth. S. Bll. 2 (1896) 72-, 177-.

- - Borel, É. Par. Ec. Norm. A. 16 (1899) 9 -.

- - (Borel's formulæ). Kluyver, J. C. Amst. Ak. Vs. 8 (1900) 331-; Amst. Ak. P. 2 (1900) 302-.

- Servant, M. Toul. Fac. Sc. A. 1 (1899) $117-$.

- - Le Roy, ל́. C. R. 130 (1900) 1293-, 1535-.

- -, comparison. Cesáro, E. Rm. R. Ac. Linc. Rd. 4 (1888) (Sem. 1) 115-.

- - discontinuity of arbitrary constants. Stokes, G. G. [1857-68] Ph. Mg. 16 (1858) 71-; Camb. Ph. S. T. 10 (1864) 105-; 11 (Pt. 2) (1869) 412-.

- - and errors connected therewith. Young, J. R. Ir. Ac. P. 3 (1847) 27-.

- - - functions defined by Taylor's series. Le Roy, É. $\quad$ C. R. 127 (1898) 654-; 128 (1899) 492-; Toul. Fac. Sc. A. 2 (1900) 317-.

- -, legitimate use. Cauchy, A. L. C. R. 17 (1843) 370-.

- - with positive terms. Appell, P. Arch. Mth. Ps. 64 (1879) 387-.

- - , possibility of defining function by. Padé, H. C. R. 116 (1893) 686-.

- - , significance, values, etc. Prehn, -. Crelle J. 41 (1851) 1-, 364-.

- -, sums. Hoppe, R. [1867] Ups. N. Acta S. Sc. 6 (1868) No. 7, 12 pp.

- summable series. Oldenburg, H. [1897] Stockh. Ȯfv. (1898) 157-.

- - - extension of Abel's theorem to, and generalisation of notion of limit. Borel, $\dot{E}$. C. R. 122 (1896) 73-.

- - - theory, applications. Borel, É. C. R. $122(1896) 805-$.

$--\frac{1}{-}$, bases. Borel, $\dot{E}$. Liouv. J. Mth. 2 (1896) 103-.

Division. Hagen, J. Am. J. Mth. 5 (1882) 236-.

Double series. Laurent, [Hermann non] Henry. C. R. 63 (1866) 296-.

- -. Schlömilch, O. Z. Mth. Ps. 11 (1866) 426-.

- Stolz, O. Mth. A. 24 (1884) 157-.

- - case. Catalan, E. C. Brux. Ac. Bll. 5 (1883) 709-.

- -, elementary theory. Pringsheim, $A$. Münch. Ak. Sb. 27 (1898) 101-.

- - of Kronecker's. Lerch, M. Prag Sb. (1893) (Mth.-Nt.) No. 9, 17 pp.

- and products. Biermann, O. Mh. Mth. Ps. 8 (1897) 115-.

- - - sequences. London, F. Mth. A. 53 (1900) 322-.

Doubly-infinite converging series. Forsyth, A. R. QJ. Mth. 21 (1886) 261-.

- - sequences of numbers. Pringsheim, $A$. Mth. A. 53 (1900) 289-.

- - series, case. Cayley, A. Camb. and Dubl. Mth. J. 6 (1851) 45-.

- - , transformation. Brioschi, F. Tortolini A. 7 (1856) 214-.
Elementary series. Igel, B. [1876] Wien Ak. Sb. 74 (1877) $(A b .2)$ 189-.

- - , theory deduced from principle of indeterminate coefficients. Oliveira, C. B. Rv. Brazil. 3 (1860) 194-.

Equalities between series. Du Bois-Reymond, $P$. Mth. A. 10 (1876) 576-.

Equivalence. Catalan, E. C. Liége S. Sc. Mm. 15 (1888) No. 1, 23-.

Euler's series, square of. Glaisher, J.W.L. [1889] L. Mth. S. P. 21 (1891) 182-.

\section{Expansion.}

Burja, -. Berl. Mm. Ac. (1801) 3-.

Sarrus, $\dot{F}$. Gergonne A. Mth. $12(1821-22)$ 289-.

Cauchy, A. L. Par. S. Phlm. Bll. (1822) 49-.

Paoli, P. Mod. S. It. Mm. 20 (1828) 293-.

Collins, E. [1828-31] St Pét. Ac. Sc. Mm. 2 (1833) 19-, 195-.

Crelle, A. L. Berl. Ab. (1830) 29-.

Cauchy, A. L. Férussac Bll. Sc. Mth. 16 (1831) 119-.

Conti, C. A. Sc. Lomb. Ven. 1 (1831) $257-$

Gordon, J. Ph. Mg. 9 (1831) 253-.

Liouville, J. Liouv. J. Mth. 3 (1838) 561-.

Cauchy, A. L. C. R. 13 (1841) 909-.

Dienger, $J$. Grunert Arch. 7 (1846) 430-; 8 (1846) 450-.

Brassinne, E. Toul. Mm. Ac. 2 (1852) 300 -.

Faà de Bruno, F. Tortolini A. 6 (1855) 479-

Friesach, $K$. Wien SB. 47 (Ab. 2) (1863) 264-.

Hermite, C. C. R. 58 (1864) 93-, 266-.

Sonin, N. Y. (xi) Rec. Mth. (Moscou) 5 (1870) (Pt. 1) 271-, 323-.

König, J. [1871] Mth. A. 5 (1872) 310-.

Lucas, É. [1877] Par. S. Mth. Bll. 6 (1878) $57-$.

Fà̀ de Bruno, F. Am. J. Mth. 3 (1880) 154-.

Hudson, E. C. QJ. Mth. 24 (1890) 233-.

Sochocki, J. Prace Mt.-Fiz. 2 (1890) 1-; Fschr. Mth. (1890) 260-.

Echols, W. H. Am. J. Mth. 15 (1893) 316-.

by aid of Bernoulli's numbers. Thoman, $F$. C. R. 64 (1867) 655-

- _ differentiation. Cerquero, J.S. Cadiz Period. M. Ci. 1 (1848) 35-, 116-.

of algebraic functions. Eisenstein, $G$. Berl. B. (1852) 441-.

- - - (Eisenstein). Heine, H. E. Crelle J. 45 (1853) 285-.

- - Hamburger, M. Z. Mth. Ps. 16 (1871) 461-.

- - David, (Lt.-Col.) -. C. R. 89 (1879) 219-.

- - - Rodrigues, J. M. As. Fr. C. R. (1893) (Pt. 2) 293-.

- analytic functions. Tadini, A. Mil. G. S. Inc. 7 (1809) 63-; 8 (1809) 286-.

- - Pincherle, $S$. Bologna Ac. Sc. Mm. 3 (1881) 151-; Mil. I. Lomb. Rd. 15 (1882) 224 -. analytic researches. Sarrus, $F$. Gergonne A. Mth. 10 (1819-20) 245-.

of any power of polynomial, general term. Gergonne, J. D. Gergonne A. Mth. 2 (1811-12) 197-. 
Appell's polynomials. Pincherle, $S$. Rm. R. Ac. Linc. Rd. 2 (1886) (Sem. 2) 214-.

application of logarithmic method and of successive approximations. Cauchy, A.L. C. R. 19 (1844) 51-, 699-.

- - method of successive approximations. Bugaev, N. V. [1895] Rec. Mth. (Moscou) 18 (1896) 471-; Fschr. Mth. (1896) 70, 71.

approximate. Hoppe, $R$. Arch. Mth. Ps. 68 (1882) 37-.

- value of coefficients of higher order. Féraud, A. Bordeaux Obs. A. 7 (1897) (Pt. 1) 37-.

in ascending power-series. Collins, E. [1830] St Pét. Ac. Sc. Mm. 1 (1831) 475-.

- _. Grunert, J. A. Grunert Arch. 1 (1841) 364-.

- - - Schlömilch, O. Grunert Arch. 7 (1846) 353-.

_- - Björling, E. G. Stockh. Ak. Hndl. (1852) 165-.

- powers of an increment, conditions. Fergola, E. Nap. Ac. Sc. Mm. 2 (1855-57) 197-. cases. Noel, J. N. Quetelet Cor. Mth. 10 (1838) 20-.

-. Schlömilch, O. Grunert Arch. 4 (1844) 431-; Schlömilch Z. 2 (1857) 420-.

-. Dienger, J. Grunert Arch. 31 (1858) 274-.

- Vachette, A. N. A. Mth. 20 (1861) 155-. - 87 -

-. Catalan, E. C. N. A. Mth. 9 (1870) 199-.

-. Dobiñski, G. [1877] Arch. Mth. Ps. 63 (1879) 108-.

- Picard, É. C. R. 88 (1879) 167-.

-. Marchand, J. N. A. Mth. 1 (1882) 450-.

-. Bucca, F. Palermo Cir. Mt. Rd. 12 (1898) 260-.

Cauchy's formula for expansion of function in powers of variable. Wantzel, L. Par. S. Phlm. PV. (1847) 15-.

class. Darboux, G. C. R. 82 (1876) 365-, 404-; Liouv. J. Mth. 4 (1878) 5-, 377-.

in continued fractions, functions arising from. Markov, A. [1893] St Pet. Ac. Sc. Mm. (Rs.) 74 (1894) (Suppl.) No. 2, 30 pp.; Fschr. Mth. (1893-94) 330 .

of continuous functions. Somine, N.J. Mth. A. 16 (1880) 1-.

Eisenstein's relation for coefficients in polynomial expansion, proof. Moreno, $G$. G. Mt. 16 (1878) 174-.

elementary expansion. Davies, T. S. Mathematician 1 (1845) 5-.

formula, general. Echols, W. H. N. Y. Mth. S. Bll. 2 (1893) 135-.

formulæ. Cauchy, A. L. C. R. 19 (1844) 1194-, 1331-.

一. Trudi, N. Nap. Rd. 1 (1862) 135-.

of four functions. Meyer, A. Brux. Ac. Sc. Mm. 21 (1848) 23 pp.

- function of binomial. [Greatheed, S. S. non Gregory, D. F.] $\iint$. Camb. Mth. J. 1 (1839). 67-.

- functions of multinomials. Knight, $T$. [1810] Phil. Trans: (1811) 49-.

_ - one variable. Jouvin, -. Gard Mm. Ac. (1852-53) 381-. of functions of one variable. Rodier de la Bruguière, -. Gard Mm. Ac. (1852-53) 394 -

$---1-$ Tchebicheff, P. [1859] St Pét. Ac. Sc. Bll. 1 (1860) 193-.

-. Darboux, G. Liouv. J. Mth. 2 (1876) 291-.

- Halphen, G.H. Bll. Se. Mth. As. 5 (1881) 462-; C. R. 95 (1882) 629-.

- - two variables. Hermite, C. Crelle J. 64 (1865) 294.

(1866) 225-.

- - - several variables. Hermite, C. C. $\mathbf{R}$. 60 (1865) 370-, 432-, 461-, 512-.

- - very small variables. Caldarera, $F$. G. Mt. 12 (1874) 348-

general coefficient. André, D. C. R. 85 (1877) 786-.

- - Anglin, A. H. H. Mess. Mth. 12 (1883)

Hermite's method. Hering, -. [1864] (x) Chemnitz B. 1 (1865) 23-.

Hoene-Wronski law. Dickstein, S. Prace Mt.. Fiz. 2 (1890) 145-; 5 (1894) 123-; Fschr. Mth. (1890) 43; (1893-94) 394-.

of implicit functions. Cauchy, A. L. C. R. 9 (1839) 587-.

- - Bellavitis, G. [1879] Rm. R. Ac. Linc. Mm. 5 (1880) 43-.

- - Gomes Teixeira, F. Liouv. J. Mth. 7 (1881) 277-.

- - - application to Wronski's problem. Bassani, A. G. Teix. J. Sc. 10 (1891) 81-. - intermediary function. Halphen, G. $H$. Par. S. Mth. Bll. 7 (1879) 92-.

- irrationals of second degree and their Napierian logs. Lucas, $\boldsymbol{E}_{\text {. }}$ Par. S. Mth. Bll. 5 (1877) 178-.

in limited series. Cauchy, A. L. C. R. 34 (1852) 8-, 70-, 121-.

method. Buquoy, G. von. Oken Isis (1826) 244 -.

- shortening astronomical calculations. Cauchy, A. L. C. R. 19 (1844) 1122-.

in periodic series. Cauchy, A. L. [1826] Par. Mm. Ac. Sc. 6 (1823) 603-.

of periodic series (Le Verrier). Encke, J. $F$. Berl. As. Jb. (1860) 313-.

- polynomial functions. Poselger, F.T. Berl. Áb. (1828) 33-.

in power series. Cauchy, A.L. C. R. 17 (1843) 193-, 940-; 19 (1844) 205-; Liouv. J. Mth. 11 (1846) 313-.

- - - Appell, P. Par. S. Mth. Bll. 11 (1883) 65-.

principle, general. Schlömilch, $O$. Schlömilch Z. 2 (1857) 289-.

of rational functions. Mittag-Leffler, $G$. Helsingf. Acta 11 (1880) 273-.

_- - Faà de.Bruno, F. Am. J. Mth. 5 (1882) 238-.

- real functions by method of least squares. Gram, J. P. [1881] Crelle J. Mth. 94 (1883) 41-.

and reversion of series. Français, J. F. Gergonne A. Mth. 6 (1815-16) 61-. 
Expansion, continued.

semi-convergent, case. Sonin, N, Ja. [1891] Vars. S. Nt. Tr. (1891-92) (C. R., Ps. C.) No. 3, 3-; Fschr. Mth. (1892) 232.

- - - - Lerch, M. Prag České Ak. Fr. Jos. Rz. (Trida 2) 5 (1896) Art. 18, 6 pp.; Fschr. Mth. (1896) 207.

- -, general form. Sonin, N. Ja. [1891]

Vars. S. Nt. Tr. (1891-92) (C. R., Ps, C.) No. 4, 2-.

and summation, cases. Winckler, A. Wien Ak. Sb. 64 (1871) (Ab. 2) 799-.

symbolic versus operative, case. Hathaway, A. S. Des Moines Anal. 5 (1878) 38-.

theorem of Cauchy. Lamarle, E. Brux. Ac. Bll. 13 (1846) 520-.

-, general. Schlömilch, O. Grunert Arch. 3 (1843) 400-.

theorems. Faà de Bruno, F. [1880] Crelle J. Mth. 90 (1881) 186-.

-. McClintock, $E$. Am. J. Mth. 4 (1881) 16-.

theory. Knar, J. Baumgartner Z. 2 (1827) 254-, 366-

-. Tetmajer, J. (xII) Krk. Ak. (Mt.-Prz.) Pam. 3 (1877) 155-; 4 (1878) 1-.

of transcendental functions, cases. Trudi, $N$. Nap. At. I. Inc. 4 (1867) 105-.

trigonometric. Rogel, $F$. Prag Sb. (1892) (Mth.-Nt.) 273-.

in two different series of powers, comparison. Göpel, A. Grunert Arch. 6 (1845) 25-

uniform, of all positive quantities less than unity. Lüroth, J. [1882] Mth. A. 21 (1883) 411-.

use of complementary variables. Cauchy, A.L. C. R. 20 (1845) 280-.

without use of derivatives. [1895] Echols, $W . H$. A. Mth. 10 (1895-96) 17-.

of $(1+x)^{m}$, Euler's. Catalan, E. C. Liouv. J. Mth. 9 (1844) 161-.

$-\left\{\left(x-x_{1}\right)^{2}+\left(y-y_{1}\right)^{2}+\left(z-z_{1}\right)^{2}\right\}^{-\frac{1}{2}}$. Plana, G. G. Arcad. 104 (1845) $182-$.

- $f(y)-f(x)$, in powers of $y-x$. Levänen, $S$. Helsingf. Öfv. 17 (1875) 1-.

$F(x)$, expansion of derivatives in powers of index of derivative. Grünwald, A. C. Prag Sb. (1880) 276-; Prag Ab. (1882) No. 2, $63 \mathrm{pp}$.

of $\left(\sum_{0}^{n} a_{r} x^{r}\right)^{-1}\left(a_{0}=1\right)$, in powers of $x$. Gam. bioli, D. [1891] Bologna Rd. (1891-92) 39-.

Finite expressions for infinite series. Dienger, J. Crelle J. 34 (1847) 209-.

Formulø. Glaisher, J. W. L. Mess. Mth. 5 (1876) 164-.

Functions "differing as little as possible from zero." Tchebitchef, P. L. Liouv. J. Mth. 19 (1874) 319-.

General problem, solution. André, D. C. R. 92 (1881) 697-.

- solution of algebraic problems by series. Marron, $F$. [1850] Madrid Mm. 2 (1853) 233-.

Geodetic positions on spheroid, formulø, expansion. Andra, - . Kjöb. Ov. (1859) 27-; As. Nr. 50 (1859) 161-; 53 (1860) 369-.
Geometric representation. Popper, J. Wien Az. 1 (1864) 34-.

- series of second order. Hochheim, A. Magdeb. Nt. Vr. Jbr. u. Ab. (1886) 127-; (1887) 25-.

Harmonic series. Kinkelin, H. [1861] Basel Vh. 3 (1863) 18-.

- Knar, J. Arch. Mth. Ps. 41 (1864) 297-; 43 (1865) 134-.

- -. Simon, H. Arch. Mth. Ps. 8 (1890) 113-; 9 (1890) vII.

- -, properties, with table. Hill, C.J. D. Lund. Acta Un. 3 (1866) (Mth.) No. 1, $16 \mathrm{pp}$.

- - and Stirling's formula. Mansion, $P$. (xII) Mathesis 1 (1881) 169-.

Higher series, new forms. Lukas, F. C. [1881] Arch. Mth. Ps. 67 (1882) 327-.

Identities. Glaisher, J. W. L. [1875] Mess. Mth. 5 (1876) 111-.

Indeterminate coefficients. Wittstein, $T$. Grunert Arch. 3 (1843) 300-.

- -. Schlömilch, O. Grunert Arch. 3 (1843) 269-.

- - (Schlömilch). Barfuss, F.W. Grunert Arch. 4 (1844) 225-.

- - (Barfuss). Schlömilch, O. Grunert Arch. 5 (1844) 374-.

- - (Schlömilch). Barfuss, F.W. Grunert Arch. 7 (1846) 29-.

- - extension of theory. Pexider, J. Časopis 28 (1899) 277-; Fschr. Mth. (1899) 240.

Integrability, case. Arzelà, $C$. Rm. R. Ac. Linc. Rd. 1 (1885) 321-.

Integral and integro-geometric series. Roe, E. D. [1896] A. Mth. 11 (1896-97) 184-.

- series. Hadamard, J. [1897] Acta Mth. 22 (1899) 55-.

- - with several variables. Lemaire, $E$. Bll. Sc. Mth. 20 (1896) 286-.

Integration. Schlömilch, $O$. Grunert Arch. 3 (1843) 278-.

-. Du Bois-Reymond, P. Berl. Ak. Sb. (1886) 359-.

- Fiske, T. S. N. Y. Am. Mth. S. Bll. 3 (1897) 223-

Inversion. Cesáro, $E$. A. Mt. 13 (1885) 339-.

-. Ježek, O. Wien Ak. Sb. 99 (1891) (Ab.2a) 191-.

-. Żorawski, $K$. Prace Mt.-Fiz. 5 (1894) 146-; Fschr. Mth. (1893-94) 393-.

Inversions of terms of given infinite series, and analytic representation of result. Pringsheim, A. Mth. A. 38 (1891) 153-.

Irrationality. Stern, M. A. Crelle J. Mth. 95 (1883) 197-.

- of value, cases. Stern, M. A. Crelle J. 37 (1848) 95-.

Limit of $\sum_{m+1}^{2 m} \frac{1}{r}$ for $m=\infty$. Unferdinger, $F$. Arch. Mth. Ps. 47 (1867) 231-; Wien Ak. Sb. 55 (1867) (Ab. 2) 93-.

- $\sum^{r=m(n-1)}(m+r \partial)^{-e}$ for $m=\infty$. Unfer. dinger, $\stackrel{r=1}{F}$. Wien Sb. 57 (1868) (Ab. 2) 621-.

Limited series, remainders, Cauchy, A.L. C. R. 34 (1852) 156-. 
Limiting values. Hoppe, $\boldsymbol{R}$. Arch. Mth. Ps. 68 (1882) 37-.

- - of series, Appell's theorem. Cesáro, $E$. Mathesis 13 (1893) 241-.

- - - on circle of convergence. Hölder, O. Mth. A. 20 (1882) 535-.

- - - - for infinite values of variables, cases. Denzler, W. Zür. Vjschr. 1 (1856) 333-.

Mixed periodic series. Oltramare, G. Gen. I. Nt. Mm. 5 (1857) 1-.

- series. Studnicka, F.J. Prag Sb. (1892) (Mth.-Nt.) 98-.

Moduli of series. Cauchy, A. L. C. R. 17 (1843) 1220-.

Multiple and divergent series, formulø resulting from. Cauchy, A. L. C. R. 20 (1845) 329-.

- modular series. Cauchy, A. L. C. R. 19 (1844) 1375-.

- -valued series. De Morgan, A. [1836] Camb. Ph. S. T. 6 (1838) 185-.

Multiplication. Cesáro, E. Bll. Sc. Mth. 14 (1890) 114-.

-. Cajori, F. N. Y. Mth. S. Bll. 1 (1892) 184 -.

- of two series. Stieltjes, T. J. N. A. Mth. 6 (1887) 210-.

- -1 , rule for. Mertens, $F$. Crelle J. Mth. 79 (1875) 182-.

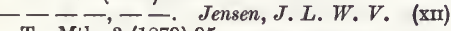
Ts. Mth. 3 (1879) 95-.

Neutral series. Marrat, $W$. Thomson A. Ph. 3 (1822) 417-.

- -, theorem. De Morgan, A. [1864-68] Camb. Ph. S. T. 11 (1871) 190-, 239447-.

New class. Babbage, C. [1824] Camb. Ph. S. T. 2 (1827) 218-.

Numerical calculation. Lerch, M. D. Mth. Vr. Jbr. 8 (1900) (Heft 1) 217-.

- series and their geometrical applications. Collignon, É. As. Fr. C. R. (1888) (Pt. 2) 4-.

- value of a series. Glaisher, J.W.L. L. Mth. S. P. 8 (1876-77) 200-; Mess. Mth. 7 (1878) 190-.

Paradox. Glaisher, J. W. L. [1871] Mess. Mth. 1 (1872) 97-.

Periodic aggregates, transformation. Gyldén, H. St Pét. Ac. Sc. Bll. 1 (1894) 379-.

- series, multiple, theorem. Enneper, A. QJ. Mth. 1 (1857) 280-.

Permanence of equivalent forms. Anon. A. Mth. 6 (1891-92) 81-.

Power of a series. Caldarera, $G$. Acireale Ac. At. 5 (1894) (Sc.) 1-.

\section{Power series.}

Appell, P. C. R. 87 (1878) 689-.

Kronecker, L. Berl. Ak. Mb. (1878) 53-.

Stieltjes, - C. R. 103 (1886) 1243-.

Tognoli, O. G. Mt. 25 (1887) 155-.

Pincherle, S. A. Mt. 21 (1893) 138-

Vivanti, G. A. Mt. 21 (1893) 193-.

Fleuri, G. Aust. As. Rp. (1895) 217-.
Puzyna, J. Krk. Ak. (Mt.-Prz.) Rz. 11 (1897) 270 - ; Crc. Ac. Sc. Bll. (1896) 295-.

abbreviated, expansion. Jordan, W. As. Nr. 85 (1875) 73-.

always divergent. Pincherle, S. C. R. 128 (1899) 407-.

and arithmetical series of $r \mathrm{th}$ rank, combination. Mildner, $R$. Wien Ak. Sb. 87 (1883) (Ab. 2) 532-.

ascending. André, D. Par. Éc. Norm. A. 12 (1883) 287-.

asymptotic determination. Cesáro, $E$. Nap. Rd. 32 (1893) 187-.

behaviour on circle of convergence. Pringsheim, A. [1885-1900] Mth. A. 25 (1885) 419-; Münch. Ak. Sb. 30 (1901) 37-.

on circle of convergence, and Fourier's series. Pringsheim, A. [1895] Münch. Ak. Sb. 25 (1896) 337-.

with coefficients depending on one variable. Vivanti, G. A. Mt. 21 (1893) 25-.

coefficients of $m$ th power. Hansted, $B$. (xIr) Ts. Mth. 5 (1881) 12-.

convergence and continuity. Arndt, $F$. Grunert Arch. 25 (1855) 211-.

- - limit (Abel's theorem). Catalan, $\boldsymbol{E}$. Mathesis 4 (1884) 25-.

converging, with rational numerical coefficients and prescribed zeros. Hurvitz, A. Acta Mth. 14 (1890-91) 211-.

differentiation. Schlömilch, $O$. Schlömilch Z. 5 (1860) 292-.

generalisation. Mittag-Leffler, G. Stockh. Öfv. (1898) 135-; Fschr. Mth. (1898) 358-.

- of a theorem. Seidel, L. Münch. Sb. 2 (1862) 91-.

of given function. Gomes Teixeira, F. Crelle J. Mth. 122 (1900) 97-.

- integers and their reciprocals, transformations. Rogel, $F$. Arch. Mth. Ps. 10 (1891) 169-.

linear criteria of convergence. Van Vleck, $E$. B. N. Y. Am. Mth. S. T. 1 (1900) 293-, 509.

and 'majorant' functions. Delassus, É. Bll. Sc. Mth. 20 (1896) 73-.

of a number. Hennessy, J. P. B. A. Rp. (1858) (pt. 2) 4 .

property. König, G. [1882] (xII) Mth. Term. Éts. 1 (1883) 60-; Mth. Nt. B. Ung. 1 (1882-83) 73-.

radius of convergence. Hadamard, - $\quad$ C. $R$. 106 (1888) 259-.

relations between approximate convergents. Frobenius, G. [1879] Crelle J. Mth. 90 (1881) 1-.

representing algebraic functions, Eisenstein's criterion. Suták, J. Mth. Termt. Ets. 11 (1893) 362-; Mth. Nt. B. Ung. 12 (1895) 1-. and their remainders. Schlömilch, $O$. Schlömilch Z. 1 (1856) 129-.

of two variables. Vivanti, $G$. Mh. Mth. Ps. 5 (1894) 99-.

- - (Vivanti). Zindler, K. Mh. Mth. Ps. 5 (1894) 287-.

- _ - , regions of convergence. Phragmén, E. Stockh. Öfv. 40 (1883) No. 10, 17-; Fschr. Mth. (1884) 203. 
Power Series, continued.

of two variables, regions of convergence. Zindler, $K$. Mh. Mth. Ps. 4 (1893) 115-. unique development of function. Mounier, G. J. D. N. Arch. Wisk. 19 (1892) 100-; Fschr. Mth. (1892) 360.

Problem. Petrovitch, M. N. A. Mth. 15 (1896) 58-.

Product of two series of form

$$
1+a \frac{z}{1}+a(a+k) \frac{z^{2}}{1 \cdot 2}+\& c .
$$

is of same form. Stainville, - de. Gergonne A. Mth. 9 (1818-19) 229-.

Products and sums, determination of real numbers by. Giudice, $F$. Tor. Ac. Sc. At. 29 (1894) 188-.

Progression, geometrical, theorem. Meech, L. W. Gould As. J. 4 (1856) 65-.

Progressions. Toledo, L. O. de. Fschr. Mth. (1899) 244.

-, geometrical. Heis, E. Grunert Arch. 6 (1845) 104-.

-, 一, of higher order. Retali, $V$. G. Mt. 11 (1873) 349-.

-, -, solution of problem supposed indeterminate. Rotheram, J. Manch. Ph. S. Mm. 3 (1790) 330-.

-, -, theorems. Blackburne, C. $\mathrm{Ph} . \mathrm{Mg} .6$ (1835) 196- ; B. A. Rp. (1837) (pt. 2$) 2$.

- of higher order. Bonolis, A. G. Mt. 12 (1874) 179-, 231-.

- - various orders. Cauchy, A. L. C. R. 20 (1845) 2-.

- Le Cointe, J. L. A. [1863] Mt. 6 (1864) 124-.

Properties. Nielsen, N. N. Ts. Mth. 3 (B) (1892) 91-; Fschr. Mth. (1892) 231-.

- of a class of series. Lipschitz, $R$. Crelle J. Mth. 105 (1889) 127-; 106 (1890) 27-

Property. Schlömilch, O. Schlömilch Z. 3 (1858) 130-.

-. Königsberger, L. Mth. A. 27 (1886) 397-.

Rational relations between certain series. Nielsen, N. N. Ts. Mth. 8 (B) (1897) 7-; Fschr. Mth. (1897) 228-.

Recurring relations in coefficients of series, especially Bernoulli's numbers. Berg, F.J. van den. [1880?] Amst. Ak. Vs. M. 16 (1881) 74-; Arch. Néerl. 16 (1881) 387-.

Reduced forms (réduites) of exponential function, convergence. Padé, H. C.R. 127 (1898) 444-.

Reduction, general method. Haton de la Goupillière, J. N. C. R. 44 (1857) 1145-; Par. Éc. Pol. J. 37 cah. (1858) 97-.

Remarkable class, Björling, E. G. Stockh. Ak. Hndl. (1846) 17-; Liouv. J. Mth. 17 (1852) 454-.

- series. Hessel, J.F.C. Grunert Arch. 5 (1844) 287-.

Representation by integrals. Kronecker, $\boldsymbol{L}$. Crelle J. Mth. 105 (1889) 157-, 345-.

Residue, Jacobi's method for determination. Scheibner, $W$. Leip. Mth. Ps. B. 45 (1893) 432-.

Reversion. Canterzani, S. Verona Mm. S. It. 5 (1790) 88-.
Reversion. Brinkley, J. [1798] Ir. Ac. T. 7 (1800) 321-.

- Degen, C. F. As. Nr. 2 (1824) 243-.

-. Collins, E. [1831] St Pét. Ac. Sc. Mm. 2 (1833) 31-.

- Möbius, A. F. Crelle J. 9 (1832) 105-.

-. Sylvester, J. J. R. S. P. 7 (1854-55) 219

-. Mainardi, G. Mil. At. I. Lomb. 1 (1858) 90 -.

- (Mainardi). Bellavitis, G. Ven. At. (185758) 623-; (1858-59) 334-.

- Glaisher, J. W. L. Mess. Mth. 6 (1877) 142-; Ph. Mg. 18 (1884) 518-.

-. McMahon, J. N. Y. Mth. S. Bll. 3 (1894) 170 .

—, formula. Cayley, A. [1855-57]. Crelle J. 52 (1856) 276-; 54 (1857) 156-.

-, general coefficient. Martynowski, J. Brux. Ac. Bll. 4 (1837) 135.

Secant series, coefficients. Stern, $M . A$. Crelle J. 26 (1843) 88-.

Semi-convergent series. Glaisher, J.W. L. [1872] QJ. Mth. 12 (1873) 52-.

- - Stieltjes, T. Par. Ec. Norm. A. 3 (1886) 201-.

- - -; arbitrary constants as multipliers. Stokes, (Sir) G. G. Camb. Ph. S. P. 6 (1889) 362-.

- - - multiplication. Cajori, F. Am. J. Mth. 15 (1893) 339-; N. Y. Am. Mth. S. Bll. 1 (1895) 180-.

$--\ldots,-$ and involution. Cajori, F. Am. J. Mth. 18 (1896) 195-.

- - - order of terms. Manning, H. B. Am. J. Mth. 16 (1894) 151-.

- - - - - , change. Borel, É. Bll. Sc. Mth. 14 (1890) 97-

- - - use of Bernoulli's function in expansion. Schlömilch, $O$. Schlömilch Z. 1 (1856) 193-.

Series for any binomial power. Euler, $\boldsymbol{L}$. [1779] St Pét. Ac. Sc. Mm. 4 (1813) 75-.

-, coefficients of which are in arithmetical progression. Grunert, J.A. Grunert Arch. 9 (1847) 322-.

_, - _ - inverses of binomial coefficients. Roberts, S. Mess. Mth. 9 (1880) 166-.

- , _ - - involve powers of Bernoullian numbers. Glaisher, J. W. L. Mess. Mth. 19 (1890) 138-.

-, _ - - proceed by sines and cosines of multiple angles. Tralles, J. G. Berl. Ab. (1820-21) 137-.

- of continuous functions, continuity of sum. Arzelà, C. Bologna Rd. (*1883-84) 79-.

- cubes. Simon, O. E. Grunert Arch. 27 (1856) 313-.

- and definite integrals. Kummer, E. E. Crelle J. 17 (1837) 210-, 228-.

- - (Kummer). Giuliani, G. G. Mt. 26 (1888) 234 -。

$22(1893)$ 97-. 37 (1890) $591-$. 
Series derived from indeterminate equation. Oliveira Ramos, J. C. d', \& Faria, C. J. de. G. Teix. J. Sc. 7 (1886) 41-; Fschr. Mth. (1885) 129-.

-, each term $=$ sum of two preceding. Clausen, P. A. Mth. Ts. 2 (1860) 168-.

-, exponents of which are contained in two quadratic forms. Jacobi, C. G.J. Crelle J. 37 (1848) 61-, 221-.

— of factorials. Schlömilch, O. Leip. B. 11 (1859) 109-.

-, finite or infinite, and series for inverted functions, powers. Buchwaldt, F. Ts. Mth. 3 (1885) 65-, 192.

- of functions. Arzelà, C. Bologna Rd. (1883) 142-.

- - Pincherle, S. G. Teix. J. Sc. 11 (1892) 129-.

- - Arzelà, C. Bologna Ac. Sc. Mm. 8 (1899-1900) 701-.

- -, theorem. Arzelà, C. Rm. R. Ac. Linc. Rd. 1 (1885) 262-.

-, general term $A_{n}\left(x-a_{1}\right)\left(x-a_{2}\right) \ldots\left(x-a_{n}\right)$, historical note. Eneström, $G$. C. R. 103 (1886) 523-.

- of geometric means, case. Jarrige, $A$. [1874] (xII) Isère S. Bll. 5 (1876) 409-.

- - integral polynomials representing real functions. Severini, C. Palermo Cir. Mt. Rd. 14 (1900) 157-.

- involving Bernoulli's numbers. $O h m, M$.

[1825] St Pét. Mm. Sav. Etr. 1 (1831) $109-$.

- of irreducible fractions. Ocagne, $M$. $d$ '. Par. S. Mth. Bll. 14 (1886) 93-; 15 (1887) 33-.

- - Kronecker : $\Sigma \Sigma\left(a m^{2}+2 b m n+c n^{2}\right)^{-8}$. Franel, -. Sch. Nf. Gs. Vh. (1896) 58-.

- and limits. Cockle, (Sir) J. [1875] Mess. Mth. 5 (1876) 25-.

- for $\log x$, a certain. McClintock, $E$. [1891] Am. J. Mth. 14 (1892) 67-.

- of polynomials. Poincaré, H. C. R. 96 (1883) 637-.

- - - case. Catalan, E. C. [1880] Brux. Ac. Sc. Mm. 43 (1882) (No. 10) 40 pp.

- positive terms. Dini, U. G. Mt. 6 (1868) 166-. 6 (1869) 185-.

- - - Giudice, F. G. Mt. 28 (1890) 283-.

- - - Jamet, V. C. R. 114 (1892) $57-$ 16 (1893) 195-.

- _ - including Euler's. Giudice, $F$. G. Mt. 27 (1889) 345-.

- - - - sums of coefficients. Tchebycheff, P. Acta Mth. 9 (1887) 182-.

- - - - - - - Sonin, N. Ja. [1894] St Pét. Ac. Sc. Bll. 2 (1895) 15-; Fschr. Mth. (1895) 275-.

- - - -, two theorems. Giudice, -. Palermo Cir. Mt. Rd. 4 (1890) 64-.

- proceeding by inverse factorials. Genocchi, A. Tortolini A. 2 (1859) 367-.
Series and products. Liouville, J. Liouv. J. Mth. 2 (1857) 433-.

- _ - association law. Hočevar, $F$. Mh. Mth. Ps. 5 (1894) 307-.

- - - - - (Hočevar). Peano, -. Mh. Mth. Ps. 6 (1895) 204.

- - - theorems and proofs. Jensen, J. L. W. V. (xII) Ts. Mth. 5 (1881) 65-.

- of quantities representing number of values of function of $n$ variables, first terms. Cauchy, A. L. C. R. 21 (1845) 1093-。

- reciprocal binomial coefficients. Rogel, F. Arch. Mth. Ps. 11 (1892) 412-.

- representing continuous function without derived function (Weierstrass). La ValléePoussin, C. de. Brux. S. Sc. A. 16 (1892) (Pt. 1) 57-.

- - discontinuous functions, property. Seidel, L. Münch. Ab. 5 (1847) 379-.

- - real quantity in given interval. Mollame, V. Catania Ac. Gioen. At. 19 (1886) 129-.

- - reciprocal of polynomial of $n$th degree, coefficients. Studnička, F. J. Casopis 15 (1886) 170-; Fschr. Mth. (1886) 215-.

- simultaneously convergent and divergent. Schlömilch, O. X. Z. Mth. Ps. 26 (1881) 63-。 - solving differential equations. Picart, $A$. N. A. Mth. 2 (1883) 109-.

- of successive derived functions of function. Pincherle, S. Palermo Cir. Mt. Rd. 11 (1897) 165-.

- summable by definite integrals. Schlömilch, O. Grunert Arch. 4 (1844) 23-.

- , terms of which are products of corresponding terms of two given series. Durán Loriga, $J$. Fschr. Mth. (1893-94) 409.

- , - - - contain binomial coefficients as factors. Werner, 0 . Grunert Arch. 9 (1847) 219-.

- for $\frac{1}{\pi}$. Forsyth, A. R. Mess. Mth. 12 (1883) 142-.

Slowly converging series, transformation into rapidly converging series. Markoff, $A$. St Pét. Ac. Sc. Mm. 37 (1890) No. 9, 18 pp.

Special type of series. Rogel, $F$. Arch. Mth. Ps. 7 (1889) 372-.

- - Ocagne, M. d'. N. A. Mth. 11 (1892) 526-.

Sum of all negative powers of all integers except $1=1$ (Steiner), etc., etc. Stern, M. A. N. A. Mth. 8 (1849) 250 -.

- fractions of form $\frac{1}{(2+x)^{2+y}+1}$. Stern, M. A. Crelle J. 25 (1843) 280.

- - geometrical progression. Young, J. R. $\mathrm{Ph}$. Mg. 28 (1846) 10-

- $301-$

301-. - - reciprocals of square numbers. Eneström, G. Bb. Mth. (1890) 22-.

- - series with terms of unlike sign. Studnička, F. J. Čsopis 17 (1888) 256-; Fschr. Mth. (1888) 256.

Summation of numerical functions, approximate. Halphen, G. H. C. R. 96 (1883) 634-. 


\section{Summation.}

Kausler, C. F. [1808] St Pét. Ac. Sc. Mm. 3 (1811) 137-.

Littrow, J. J. von. [1814] St Pét. Ac. Sc. Mm. 7 (1815-16) 110-.

Poisson, S. D. Par. S. Phlm. Bll. (1822) 134-; Par. Éc. Pol. J. $19^{\circ} \mathrm{cah}$. (1823) 404-.

Schubert, F. T. [1824] St Pét. Ac. Sc. Mm. 11 (1830) 158-.

Phillips, G. Ph. Mg. 11 (1832) 339-, 435-.

Bessel, F. W. As. Nr. 16 (1839) 1-.

Rawson, $R$. [1844-47] Manch. Ph. S. Mm. 7 (1846) 464-; Mathematician 1 (1845) 265-; 2 (1847) 6-.

Oettinger, L. Grunert Arch. 26 (1856) 1-, 212-.

Frisby, E. Des Moines Anal. 1 (1874) 121-.

Buchwaldt, F. (xп) Ts. Mth. 2 (1878) 76-.

André, D. C. R. 86 (1878) 1017-; 87 (1878) 973-; Par. Ec. Norm. A. 8 (1879) 239-; 9 (1880) 209-; 12 (1883) 191-.

by approximative fractions. Adcock, R. J. Am. J. Sc. 4 (1872) 505.

based on differentials and integrals of generating functions of series. Oettinger, $L$. Crelle J. 16 (1837) 131-.

Bernoulli's method, Arndt, $F$. Crelle J. 31 (1846) 253-.

cases. Gauss, C. F. Gött. Cm. 1 (1808-11) $40 \mathrm{pp}$.

- Tralles, J. G. Berl. Ab. (1814-15) (Mth.) 179 .

- Fuss, N. [1816-21] St Pét. Ac. Sc. Mm. 7 (1820) 194-; 10 (1826) 115-; 11 (1830) 230 -

- Lamla, K. Baumgartner Z. 3 (1827) 27-, 312-.

-. Stern, M. A. [1831] Crelle J. 10 (1833) 209-.

-. Noel, J. N. Quetelet Cor. Mth. 7 (1832) 184-.

-. Liouville, J. Liouv. J. Mth. 2 (1837) 107-

-. Lebesgue, V. A. Liouv. J. Mth. 5 (1840) 42-.

-. Stern, M. A. Crelle J. 20 (1840) 321-

-. Catalan, E. C. Liouv. J. Mth. 7 (1842) 1-.

- Arndt, F. Grunert Arch. 6 (1845) 187-; Crelle J. 31 (1846) 235-.

-. Wolfers, J.P. Grunert Arch. 11 (1848) 419-.

- (Gauss, 1808). Heine, H. E. N. A. Mth. 9 (1850) 414-.

- Catalan, E. C. N. A. Mth. 15 (1856) 293-.

- Cayley, A. QJ. Mth. 2 (1858) 167-.

-. Zehfuss, G. (xI) Z. Mth. Ps. 3 (1858) 247-.

-. Besge, V. A. Liouv. J. Mth. 5 (1860) 367-.

- Lindman, C. F. Stockh. Öfv. 20 (1863) 467-

-. Unferdinger, $F$. Arch. Mth. Ps. 41 (1864) 145-. cases. Escher, P. Arch. Mth. Ps. 44 (1865) 374-.

- Grunert, J. A. Arch. Mth. Ps. 47 (1867) 359-.

- André, D. N. A. Mth. 10 (1871) 368-.

-. Wolstenholme, J. L. Mth. S. P. 4 (187173) 283-.

-. Falk, M. Bll. Sc. Mth. As. 10 (1876) 204-.

- Hoppe, R. Arch. Mth. Ps. 61 (1877) 224.

-. Sykora, A. Arch. Mth. Ps. 61 (1877) 445-.

-. Croker, J. M. Mess. Mth. 7 (1878) 60-.

- Hoppe, R. Arch. Mth. Ps. 62 (1878) 165-.

-. Trowbridge, D. Des Moines Anal. 5 (1878) 168-.

-. Ocagne, M. d'. N. A. Mth. 1 (1882) 171-.

-. Mandl, M. Wien Ak. Sb. 94 (1887) (Ab. 2) 947-.

-. Lindman, C. F. Stockh. Öfv. (1888) 69-;

Fschr. Mth. 20 (1888) 425-.

-. Cayley, A. Mess. Mth. 19 (1890) 29-

- Segar, H. W. Mess. Mth. 20 (1891) 142-; 21 (1892) 145-.

-. Petersen, J. Sk. Nf. F. (1892) 354-

- Weyr, E. Casopis 21 (1892) 161-; Fschr. Mth. (1892) 230-.

-. Schou, E. N. Ts. Mth. 8 (B) (1897) 1-; Fschr. Mth. (1897) 228.

- Hermite, -. Kazan S. Ps.-Mth. Bll. 8 (1899) 107-.

-. $\frac{a}{1}+\frac{a^{2}}{4}+\frac{a^{3}}{9}+\frac{a^{4}}{16}+\ldots$ Euler, L. [1779] St Pét. Ac. Sc. Mm. 3 (1811) 26-.

- $\quad \Sigma \frac{1}{r^{3}}$ and $\Sigma \frac{1}{(6 r+1)^{2}}$. Clausen, $T$. Crelle J. 5 (1829) 380 -.

-. $\Sigma(b+r a)^{-(\theta+p)}$. Heine, H. E. Crelle J. 31 (1846) 133-.

- $\Sigma \frac{1}{\log \left(1+\frac{1}{x}\right)} \cdot$ Binet, J. P. M. C. R. 27 (1848) 199-.

一. $F(x)=1+\frac{x}{1}+\frac{x^{2}}{1.3}+\frac{x^{3}}{1.3 .5}+\ldots$

Schlömilch, O. Schlömilch Z. 3 (1858) 180-. -. $\quad \Sigma \frac{x^{p}}{a_{o} p^{n}+\ldots+a_{n}} \cdot$ Am Ende,-. Grunert Arch. 35 (1860) 220-.

一. $\Sigma\left(\frac{p \sin p u}{h^{2}+p^{2}}\right)$. Du Bois-Reymond, $P$. Mth. A. 5 (1872) 399-.

-. $\sum_{0}^{\infty} \frac{n^{m}}{n !}$. Ligowski, W. [1877] Arch. Mth. Ps. 62 (1878) 334-.

-. $\Sigma x^{r}$. Ligonoski, W. [1879] Arch. Mth. Ps. 65 (1880) 329-.

-. $\sum_{i=0}^{i=\infty} \frac{2^{i} \cdot x}{e^{k} \cdot x+1}$. Gomes Teixeira, F. G. Teix. J. Sc. $5\left(^{*} 1883\right)$ 185-.

of class of series. Querret, - Gergonne A. Mth. 13 (1822-23) 361-. 
of class of series. Frenet, F. [1856] Lyon Ac. Sc. Mm. 7 (1857) 59-.

- - - André, D. C. R. 88 (1879) 740-. - compound series and application to probabilities. Terrot, C. H. Edinb. R. S. T. 20 (1853) 541-.

by continued fractions. Horner, W. G. Thomson A. Ph. 11 (1826) 416-; 12 (1826) 48-.

of convergent series, cases. Verniory, Mathesis 12 (1892) 265-.

by development of $\left(1-a x-c x^{2}\right)^{-m}$. Dienger, J. [1847] Crelle J. 43 (1852) 88-.

- differential formulø. Euler, $L$. [1780] St Pét. Ac. Sc. Mm. 5 (1815) 45-.

of divergent series. De Morgan, A. Assur. Mg. 12 ( $\left.{ }^{*} 1866\right)$ 245-.

- - Borel, É. C. R. 121 (1895) 1125-. elementary. Bode, J. Grunert Árch. 34 (1860) 397-.

of elementary series, cases. Nielsen, $N$. N. Ts. Mth. 7 (B) (1896) 63-; Fschr. Mth. (1896) 208.

equation of coefficients occurring in summation. Eytelwein, J. A. Berl. Ab. (1820-21) 1-.

and expansion. Dienger, $J$. Grunert Arch. 7 (1846) 430-; 8 (1846) 450-.

formula. Frenet, F. C. R. 44 (1857) 1274-.

- Perrin, R. [1876] Par. S. Mth. Bll. 5 (1877) 47-.

-. Laisant, C. A. As. Fr. C. R. (1878) 179-.

-, Dorna's. Curtze, M. Arch. Mth. Ps. 46 (1866) 357-; 47 (1867) 238-.

-, Euler's. Franel, J. Mth. A. 47 (1896) 433-.

-, - or Maclaurin's. Malmsten, C.J. Acta Mth. 5 (1884) 1-.

- involving Bernoulli's numbers. Spitzer, $S$. Grunert Ärch. 23 (1854) 457-.

- for 3rd power of terms. Hellerung, Grunert Arch. 2 (1842) 198-.

- - reciprocal power series. Meyer, G. $F$. Arch. Mth, Ps. 41 (1864) 220-.

-, universal. Meech, L. W. Gould As. J. 2 (1852) 121-.

formulæ. Young, J. R. Ph. Mg. 10 (1837) 121-; 11 (1837) 41-.

-. Genocchi, A. Tortolini A. 6 (1855) 70-.

-, and Bernoulli's numbers. Bauer, G. [1860] Crelle J. 58 (1861) 292-。

- of Euler and Stirling, complementary terms. Somin, N. C. R. 108 (1889) 725-.

一, new. Lucas, É. N. A. Mth. 14 (1875) 487-. of four algebraic series. Glaisher, J.W. L. [1877] Mess. Mth. 7 (1878) 119-.

- geometric series, 2nd and higher orders, by definite integrals. Glaisher, J. W. L. QJ. Mth. 11 (1871) 328-.

- harmonic series. Malfatti, G. F. Turin Mm. Ac. 4 (1788-89) 53-.

investigations. Lorgna, $A . M$. Verona S. It. Mm. 1 (1782) 268-.

Kummer's method, auxiliary functions. Bresse, -. C. R. 64 (1867) 1023-, 1138-.

memoir by Goldbach, 1718. Eneström, $G$. Bb. Mth. (1887) 23-.

method. Schlömilch, O. Grunert Arch. 11 (1849) 130 -. of negative powers of natural numbers. Schlömilch, O. X. Leip. Mth. Ps. B. 29 (1877) 106-.

- neutral series. Young, J.R. Ph. Mg. 27 (1845) 362-, 437-.

- - Moon, R. Ph. Mg. 28 (1846) 136-.

new methods. Babbage, $C$. Phil. Trans. (1819) 249 .

peculiarity. Schlömilch, O. Grunert Arch. 10 (1847) 45-

of power series. Steen, A. (xII) Ts. Mth. 2 (1878) 183-.

- powers of reciprocals of numbers. Warming, E. Kjøb. Dn. Vd. Selsk. Skr.8(1895-98) 395-.

- series of ares. Dobinski, G. [1878] Arch. Mth. Ps. 63 (1879) 393-.

- - circular arcs. Grunert, J.A. Arch. Mth. Ps. 47 (1867) 361-.

- with simple generating functions. Oettinger, L. Crelle J. 14 (1835) 262-, 330-; 15 (1836) 264-, 317-.

- - of sines or cosines of multiple arcs. Clausen, T. Crelle J. 4 (1829) 281-.

- whose law is known. Gruson, J. P. Berl. Mm. Ac. (1804) 83-.

slowly converging and diverging series. Young, J. R. Ph. Mg. 6 (1835) 348-; 7 (1835) 25-.

- - - series. Kummer, E. E. Crelle J. 16 (1837) 206-

- - Lubbock, J. W. As. S. M. Not. $10(1849-50)$ 180-.

- - - Fabian, O. (xm) Krk. Ak. (Mt.Prz.) Pam. 2 (1876) 37-.

- - - (alternate harmonic series), rapid. Janet, A. C. R. 118 (1894) 239-.

solution of two problems. Trowbridge, $D$. Des Moines Anal. 3 (1876) 154-.

of terms very far on. Cauchy, A. L. C. R. 32 (1851) 389-.

- trigonometric and other series. Rogel, $F$. Arch. Mth. Ps. 12 (1894) 37-.

- - series. Adams, Jas. Thomson A. $\mathrm{Ph}$. 15 (1820) 335-; 4 (1822) 21-.

- - Macully, J. F. Mth. Misc. 2 (1839) 118 -

- - - Dirksen, E. H. Berl. B. (1842) 20- ; (1843) 83-.

- - Müller, J. H. T. Grunert Arch. 11 (1848) 439-.

- - - Blissard, J. Mess. Mth. 2 (1863) 50 -.

- cases. Schultén, N. G. af (fil.) Stockh. Ak. Hndl. 40 (1819) 184.

- - - Talbot, W. H. F. Gergonne A. Mth. 14 (1823) 88-, 187-.

-- - - Catalan, E. C. N. A. Mth. 3 (1844) 570 -

- - - - Dienger, J. Crelle J. 41 (1851) 48-

- - - - Reinemund, F. Brux. Ac. Bll. 40 (1875) 801-; 43 (1877) 356-.

- - - - Størmer, C. Christiania F. (1892) No. 17, $21 \mathrm{pp}$.

- - - - - Nielsen, N. Kjøb. Ov. (1896) 348-.

$(1879)$ 269-. 
Summation, continued.

of trigonometric series, class. Rogel, F. Arch. Mth. Ps. 15 (1897) 255-.

- - -, geometrical. Croker, J. M. QJ. Mth. 3 (1860) 181.

$--\tan ^{-1} \alpha+\sum_{k=1}^{k=\infty} \tan ^{-1} \frac{2 a}{1+a^{2}\left(k^{2}-1\right)}$. Alexéief, -. Bll. Sc. Mth. As. 1 (1877) 44.

- two series, by "representative notation." Blissard, J. QJ. Mth. 6 (1863) 167-.

Syntagmatic and power series. Cauchy, A.L. C. R. 20 (1845) 463-.

Terms deduced from given products of $n$ contiguous terms. Fuss, N. [1813] St Pét. Ac. Sc. Mm. 6 (1818) 118-; 7 (1820) 214-.

Theorem. Lejeune-Dirichlet, G. Liouv. J. Mth. 1 (1856) 80-.

-. Laurent, H. N. A. Mth. 1 (1862) 126-.

- Lerch, M. Acta Mth. 10 (1887) 87-.

- Gutzner, A. Mth. A. 32 (1888) 596-.

- of Abel. Saint-Germain, A.de. N. A. Mth. 4 (1885) 159-.

- - - generalisation. La Maestra, A. C. R. 111 (1890) 782-.

- Baschwitz. Catalan, E. Brux. Ac. Bll. 18 (1889) 770 .

- Dirichlet. Phragmén, E. Stockh. Öfv. (1892) 199-.

- Gauss, proof. Rouché, E. N. A. Mth. 5 (1866) 10-.

- 一, 一. Brisse, C. N. A. Mth. 9 (1870) 36-.

- Kummer. Cesáro, E. C. R. 106 (1888) 1791-.

Theorems. Schlömilch, O. Leip. B. (1854) 127-.

-. Winckler, A. Wien SB. 41 (1860) 675-.

- Sinram, T. Arch. Mth. Ps. 63 (1879) 103-

- Stieltjes, T. J. (jun.) N. Arch. Wisk. 9 (*1882) 98 -

一.Jackson, F. H. Edinb. Mth. S. P. 13 (1895) 115-, 160-.

-, two. Saalschütz, L. Königsb. Schr. 36 (1895) 67-.

Theory. Clausen, $T$. Crelle J. 3 (1828) 92-.

- Björling, E. G. Ups. N. Acta S. Sc. 13 (1847) 61-, 143-.

- Bonnet, O. Brux. Mm. Cour. $4^{\circ}, 23$ (1848-50) $116 \mathrm{pp}$.

-. Schlömilch, O. Grunert Arch. 14 (1850) 146-.

-. Catalan, E. C. C. R. 43 (1856) 626-.

- (Catalan). Cauchy, A. L. C. R. 43 (1856) 637-.

- Catalan, E. C. L'I. 26 (1858) 121.

-. Schendel, L. Z. Mth. Ps. 16 (1871) 211-.

- Meissel, E. [1875] Arch. Mth. Ps. 60 (1877) 337-.

- Lerch, $M$. Prag Sb. (1885) (Mth.-Nt.) 174-; G. Teix. J. Sc. 7 (1886) 79-.

- Cesáro, E. N. A. Mth. 9 (1890) 353-, 528.

-. Lerch, M. Prag Sb. (1891) (Mth.-Nt.) 250-.

- Franel, J. Mth. A. 52 (1899) 529-.

-, application of numerical identities. Sonine, N.J. [1885] Fschr. Mth. (1886) 214-.
Theory, elementary. Grunert, J.A. Grunert Arch. 23 (1854) 1-.

-, general principle. Schmidten, H. G. Kiöb. Ov. (1828-29) 2-; Crelle J. 5 (1830) 388-.

-, new mode of analysis. Herschel, (Sir) J. F. W. Edinb. Ph. J. 2 (1820) 23-。

Transformation. Gruson, J. P. Berl. Mm. Ac. (1804) 121-.

-. Cauchy, A. L. C. R. 11 (1840) 639-, 667-.

- Catalan, E. C. [1864-65] C. R. 59 (1864) 618-; Brux. Mm. Cour. 4\%, 33 (1867) 50 pp. -. Winckler, $A$. Wien Sb. 51 (1865) (Ab.2) 291-.

- (Baschwitz). Catalan, E. Brux. Ac. Bll. 18 (1889) 666-.

-, case. Euler, L. [1778] St Pet. Ac. Sc. N. Acta 12 (1801) 58-.

-, -. Eisenstein, G. Crelle J. 27 (1844) 193-.

_, -. Schlömilch, O. Leip. B. 13 (1861) 120 -

-, - Walton, W. [1862] QJ. Mth. 6 (1863) $22-$. -, - Catalan, E. C. N. A. Mth. 2 (1863)

2 (1888) 225-

,,$-- a x+b x^{2}+c x^{3}$ etc. Strong, $T$. Am. Ph. S. P. 3 (1843) 139-.

,,$-- \Sigma\left( \pm \frac{{ }^{n} C r}{r+1}\right)$. Grunert, J.A. Grunert Arch. 26 (1856) 107-.

- of functions by continued fractions. Čeby $P$. St Pet. Ac. Sc. Mm. (Rs.) 9 (* 1866) $^{*}$ (Suppl.) No. 1, 26 pp.

-, Leclert's method. Catalan, E. C. Bll. Sc. Mth. As. 1 (1877) 356-.

- by Stirling. Dietrich, M. [1860] Crelle J. 59 (1861) 163-.

\section{Trigonometric series.}

Herschel, (Sir) J. F. W. Camb. Mm. Anal. S. (1813) 33-.

Dippe, M. C. Grunert Arch. 7 (1846) 108-.

Heine, $E$. Crelle J. 71 (1870) 353-.

Cantor, G. [1871] Mth. A. 4 (1871) 139-; 5 (1872) 123-; Acta Mth. 2 (1883) 329-.

Ascoli, G. [1872] Mth. A. 6 (1873) 231-.

Cantor, G. [1879] Mth. A. 16 (1880) 113-, 267-.

Hoppe, R. Arch. Mth. Ps. 64 (1879) 435-.

Poincaré, H. C. R. 95 (1882) 766-; 97 (1883) 1471-; 101 (1885) 1131-.

Hölder, O. Mth. A. 24 (1884) 181-.

for arbitrary function between limits, convergence. Lejeune-Dirichlet, G. Crelle J. 4 (1829) 157-; 17 (1837) 35-.

cases. Frisiani, $P$. Mil. G. I. Lomb. 5 (1853) 446-.

-. Trowbridge, D. Des Moines Anal. 4 (1877) 4-.

-. Taylor, H. M. Mess. Mth. 7 (1878) $22-$.

-. Beltrami, E. Mil. I. Lomb. Rd. 13 (1880) $402-$.

- Catalan, E. Brux. Ac. Bll. 23 (1892) 143-. 


\section{Trigonometric Series}

cases. Barbette, $E$. Mathesis 15 (1895) 135-. -. $\sin \phi+\frac{1}{2^{2}} \sin 2 \phi+\frac{1}{3^{2}} \sin 3 \phi+\ldots$ Clausen,

T. Crelle J. 8 (1832) 298-.

- $\sum_{1}^{\infty} \frac{1}{2 n} \tan \frac{x}{2 n}$. Catalan, E. C. Liége S.

Sc. Mm. 15 (1888) No. 1, 227-.

-. $\sum^{\infty} \sin (n ! x \pi)$. Köpcke, H. A. Hamb. Mth. Gs. Mt. 1 (1889) 106-.

class. Rogel, F. Prag Sb. (1893) (Mth.-Nt.)

No. 33, $25 \mathrm{pp}$.

coefficients from particular values. Bronwin,

B. Ph. Mg. 34 (1849) 260-, (vi Add8.) 374-.

convergence. Lobatschevsky, $N$. (vi Add8.)

Kazan Mm. Un. (1834) cah. 2, 226 pp.

-. Holmgren, H. Liouv. J. Mth. 16 (1851) 186-.

-. Poincaré, H. Bll. As. 1 (1884) 319-.

-, method of increasing. Charlier, C. V.L.

Bll. As. 3 (1886) 378-; Stockh. Öfv. (1886) 157-.

_, _ - _. Poincaré, H. Bll. As. 3 (1886) $521-$.

of cosines of multiple angles. Fuss, N. [1809]

St Pét. Ac. Sc. Mm. 5 (1815) 115-.

deduced from algebraic expansions of $\frac{1}{2} l \frac{1+y}{1-y}$

and $e^{y}-1$. Studnitka, F. J. Časopis 20 (1891) 173-; Fschr. Mth. (1891) 264.

determination of constants. Hunyady, $J$. (xII)

Mag. Tud. Ak. Éts. 5 (No. 14) (1871) 256-.

differentiation. Lerch, $M$. C. R. 119 (1894)

725-; Par. Éc. Norm. A. 12 (1895) 351-;

Tor. Ac. Sc. At. 35 (1900) 54-.

double. Arzelà, C. Bologna Ac. Sc. Mm. 4 (1894) 373-.

extension of theorem. Cantor, G. [1871] Acta Mth. 2 (1883) 336-.

- - Arzelà, C. Rm. R. Ac. Linc. Rd. 1 (1885) 637-.

multiplication. Pringsheim, A. Mth. A. 26 (1886) 157-.

-. Bourlet, C. Bll. Sc. Mth. 13 (1889) 55-. with positive coefficients. Lerch, M. Prag Sb. (1898) (Mth.-Nt.) No. 23, $19 \mathrm{pp}$.

series for $\theta$ in terms of sines or tangents of $\frac{\theta}{a^{r}}$.

Rodrigues, $O$. Liouv. J. Mth. 8 (1843) 225-. of sines and cosines. Le Cointe, L. A. N. A. Mth. 3 (1844) 518 .

- - - of multiple angles. Dirksen, E. H. As. Nr. 1 (1823) 405-, 409-; Berl. Ab. (1827) 85-; (1829) 169-; Crelle J. 4 (1829) 170-

theorem. Cantor, G. Crelle J. 72 (1870) 130-. -. Appell, P. Arch. Mth. Ps. 64 (1879) 95-. theorems of Cantor and Du Bois-Reymond, extension to spherical functions. Neumann, C. G. Leip. Mth. Ps. B. 33 (1881) 1-; 35 (1883) 18-; Mth. A. 22 (1883) 406-.

theory of convergence applicable to other series. Boussinesq, J. Liouv. J. Mth. 7 (1881) 147-. - fundamental formula. Bonnet, O. Bll. Sc. Mth. As. 3 (1879) 480-

in two variables. Ascoli, $G$. Rm. R. Ac. Linc. Mm. 8 (1880) 263-.
Infinite Series

3220

Trigonometric Series, continued.

for $y\left(=\tan ^{-1}[m \tan x]\right)$, in sines of multiples of $x$. Saporetti, A. Bologna Ac. Sc. Mm. 8 (1887) 57-.

Triple series. Retali, V. G. Mt. 9 (1871) 266-. Unequally convergent series. Maggi, G. A. Mil. I. Lomb. Rd. 26 (1893) 368-.

Utility of series in finding fluent. Young, (Dr) T. Nicholson J. 22 (1809) 213-.

Various series. Sarrus, $F$. Gergonne A. Mth. 10 (1819-20) 217-.

- - Tchebicheff, P. Liouv. J. Mth. 16 (1851) 337-.

- Lebesgue, V. A. N. A. Mth. 18 (1859) $433-, 460$ -

- - Torelli, G. G. Mt. 10 (1872) 129-.

- - Genocchi, A. Tor. Ac. Sc. At. 10 (1875) 985

$\sum_{r=1}^{r=n}\left(\frac{1}{a k^{r} \pm b}\right)$. Lorgna, A. M. Verona S. It. Mm. 2 (1784) 210-.

$1+\frac{m}{1} x+\frac{m(m-1)}{1.2} x^{2}+\ldots$. Abel, N.H. Crelle J. 1 (1826) 311-.

$1^{n}-2^{n}+3^{n} \ldots$ and $1^{n}-3^{n}+5^{n} \ldots$ Clausen, $T$. Crelle J. 7 (1831) 112-.

$\Sigma e^{x} y$. Malmsten, C. J. Ups. N. Acta S. Sc. 12 (1844) 293-.

$\Sigma\left(u_{0}+u_{1}+\ldots\right)=\Sigma u_{0}+\Sigma u_{1}+\ldots$, conditional proof. Dienger, $J$. Grunert Arch. 13 (1849) 281-.

$\sum_{\sum}^{p=\infty} p^{n} x^{p}$. Lindman, C. F. Grunert Arch. 27 (1856) 291-

$\Sigma \frac{1}{(a+n d)^{k}}$. Lebesgue, V. A. N. A. Mth. 18 (1859) $82-$.

$1+\frac{u}{1}+\left(\frac{u}{2}\right)^{2}+\left(\frac{u}{3}\right)^{3}+$ etc., calculation by

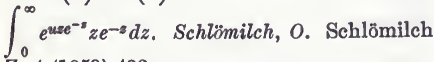
Z. 4 (1859) 433-.

$\frac{1}{a \cdot b}+\frac{1}{b \cdot c}+\frac{1}{c \cdot d}+$ etc., $a, b, c$, etc. being in arithmetical progression. Clausen, P. A. Mth. Ts. 3 (1861) 69-.

$\frac{1}{1^{r}}+\frac{1}{2^{r}}+$ etc. Frost, P. [1865] QJ. Mth. 7 (1866) 370-

$\sum_{0}^{\infty} \frac{(-1)^{n}}{(2 n+1)^{\mu}}$. Besso, D. G. Mt. 10 (1872) 160 . $(2 n+1)^{\mu}$

$\frac{1}{n+1}+\frac{1}{n+2}+\ldots+\frac{1}{2 n}$, and elliptic functions. Catalan, E. C. Brux. Ac. Bll. 34 (1872) 26-, 424-.

$\Sigma \Sigma[(x+i)(x+j)]^{-1}$ and other series. Glaisher, J.W. L. [1875] Mess. Mth. 5 (1876) 21.

$\Sigma^{n} u_{n} z^{n}$. Ascoli, G. Rm. R. Ac. Linc. At. 3 (1876) (Pte. 2) 156-.

$\frac{1}{n+1}+\frac{1}{n+2}+\ldots+\frac{1}{2 n}$, sum (Catalan'sformula), generallsation. Tchebitchef, $P . L$. As. Fr. C. R. (1876) 114-. 
$\frac{1}{2}-\frac{x}{x+1}+\frac{x(x-1)}{(x+1)(x+2)}-$ etc., note. Brisse, C. N. A. Mth. 16 (1877) 418-

$\left(l_{2}\right)^{-k}+\left(l_{3}\right)^{-k}+$ etc. Hermite, $C$. (xiI) Mathesis 1 (1881) 37.

-. Baehr, G. F. W. (xir) Mathesis 1 (1881) 58.

-. Catalan, E. C. (xII) Mathesis 1 (1881) 58.

$f(x)=f(0)+\ldots \frac{x(x-n \beta)^{n-1}}{n !} f^{(n)}(n \beta) \ldots$ (Abel).

Halphen, G. C. R. 93 (1881) 1003-; Par. S. Mth. Bll. 10 (1882) 67-.

$\Sigma E \sqrt{u}$, Buniakoffsky's formula. Petr, $K$. Casopis 16 (1887) 169-; Fschr. Mth. (1887) $172-$.

$\sum_{n=1}^{n=\infty} \frac{x^{n}}{n^{8}}$. Jonquière, A. Par. S. Mth. Bll. 17 (1889) 142-.

$\Sigma \frac{1}{k^{2}}$ and $\Sigma \frac{1}{k^{3}}$. Markoff, A. C. R. 109 (1889) 934 .

$\zeta(8)=\sum_{n=1}^{n=\infty}\left(\frac{1}{n}\right)^{8}$. Piltz, - . D. Nf. Vh. (1891) (Th. 2) 15-.

$\sum_{1}^{\infty} n^{-8}$. Lafay, A. Toul. Fac. Sc. A. 6 (1892) I, $6 \mathrm{pp}$.

$x^{n}+(x-q)^{n}+(x-2 q)^{n}+\ldots+r^{n}$ and $x^{n}-(x-q)^{n}$ $+(x-2 q)^{n}-\ldots \pm r^{n}$, values. Glaisher, J.W. L. QJ. Mth. 31 (1900) 193-.

$1^{n}(x-1)^{n}+2^{n}(x-2)^{n}+\ldots$ Glaisher, J. W. L. QJ. Mth. 31 (1900) 241-.

\section{INFINITE PRODUCTS.}

Racagni, G. Mil. Mm. I. Lomb. Ven. 1 (181213) 59-, 341-.

Le François, (Prof.) - Brux. Mm. Cour. 4\%, 14 (1841) $71 \mathrm{pp}$.

Schlömilch, O. [1851] Crelle J. 44 (1852) 344-. Weierstrass, C. [1854] Crelle J. 51 (1856) 1-.

Dini, U. [1868] A. Mt. 2 (1868-69) 28-.

Rodrigues, J. M. G. Teix. J. Sc. 3 (1881) 87-. Arzelà, C. Bologna Ac. Sc. Mm. 4 (1882) 419-; Bologna Rd. (1885-86) 92-.

Giudice, F. G. Mt. 28 (1890) 305-, 380.

Chessin, A.S. J.H. Un. Cir. [15(1895-96) ]38-

Anissimow, $W$. A. Fschr. Mth. (1897) 221.

Pasquale, $V$. de. G. Mt. 35 (1897) 259-.

Application of calculus of residues. Anon. Férussac Bll. Sc. Mth. 12 (1829) 202-.

- - - Cauchy, A. L. C. R. 17 (1843) 572-.

Case. Gill, C. Mth. Misc. 2 (1839) 140-.

Constant $G$ and some products. Catalan, $E$. Brux. Ac. Mm. 51 (1893) (No. 2) 28 pp.

Convergence. Arndt, $F$. Grunert Arch. 21 (1853) 78-.

- Pringsheim, A. Mth. A. 33 (1889) 119-.

- Bruno de Cabedo, J. G. Teix. J. Sc. 10 (1891) 138-.

-, conditional. Pringsheim, A. Mth. A. 44 (1894). 413-.

Decomposition of functions into factors. Cauchy, A. L. C. R. 19 (1844) 1069-; 30 (1850) 186-

- numbers into infinite products. Cantor, $G$. Z. Mth. Ps. 14 (1869) 152-.
Decomposition of numbers into infinite products (Cantor). Gegenbauer, L. Mh. Mth. Ps. 3 (1892) 87-.

Elementary theory. Genocchi, A. N. A. Mth. 8 (1869) 121-.

Expansion. Rogers, L. J. [1893-94] L. Mth. S. P. 24 (1893) 337-; 25 (1894) 318-; 26 (1895) 15-.

- by Euler. Glaisher, J.W. L. [1879] Mess. Mth. 9 (1880) 45-.

- of functions in infinite products. Bock, J.W. Hamb. Mth. Gs. Mt. 1 (1889) 76-.

- in series, proof. Sylvester, J.J. (xII) J. H. Un. Cir. [2] (1883) 46.

Finite product and infinite product, relation. Schlömilch, O. Grunert Arch. 3 (1843) 442-. Formula of Cauchy. Cazzaniga, P. Mil. I. Lomb. Rd. 15 (1882) 273-.

Holomorphic function, special mode of resolution into infinite product. Kotelov, $K, I$. Kazan S. Nt. (P8.-Mth.) P. 7 (1889) 407-; Fschr. Mth. (1890) 396.

Interpolation of two products. Ivanov, I. I. Kharkov Mth. S. Com. 1 (1889) 78-; Fschr. Mth. (1888) 423-.

Numerical product, case. Glaisher, J. W. L. [1876] Mess. Mth. 6 (1877) 71-, 189-.

Power series for a certain product. Schröder, J. [1897] Hamb. Mth. Gs. Mt. 3 (1900) $302-$.

Products arising from linear equation. Cauchy, A. L. C. R. 17 (1843) 523-, 567-.

- developable in powers of $\frac{1}{n}$. Giudice, Palermo Cir. Mt. Rd. 5 (1891) 287-.

- and divisors of numbers, theorem derived from Euler's. Dienger, J. Crelle J. 40 (1850) 235-.

- expressing real functions with infinitely close zeros. Broden, $T$. Mth. A. 51 (1899) 299-.

- roots of integers, properties. Schaar, $M$. Brux. Ac. Bll. 16 (1849) 580-.

- - a number. Schaar, M. Brux. Ac. Bll. 13 (1846) 228-.

- - - and powers of a number. Le Francois, (Prof.) -. Brux. Ac. Bll. 19 (1852) 23-.

- and series, changes in values due to changes in order of terms. Casorati, $F$. Mil. I. Lomb. Rd. 1 (1868) 126-.

- - - evaluation. Mildner, R. [1883] Wien Ak. Sb. 88 (1884) (Ab. 2) 591-.

- of series of factors. Dobinski, $G$. Arch. Mth. Ps. 59 (1876) 98- ; 61 (1877) 434-.

Properties and calculation. Savinov, A. (xII) Kazan Un. Mm. (1851) (Bk. 4) 43-.

Reduction in series. Novi, G. G. Mt. 2 (1864) 1-, 40-.

Relation between two products. Eisenstein, $G$. Crelle J. 27 (1844) 285-; 28 (1844) 36-.

Semi-convergent products. Catalan, E. C. [1871-86] Brux. Ac. Sc. Mm. 40 (1873) (No. 2) 127 pp.; Liège S. Sc. Mm. 13 (1886) 151-, 254-.

- - Beaupain, J. Brux. Mm. Cour. 4, 53 (1893-94) No. 7, 8 pp. 
Theorem of inequality. Schlömilch, O. N. A. Mth. 19 (1860) 280-.

- - - (Schlömilch). Prouhet, E. N. A. Mth. 19 (1860) 281-.

- Jacobi. Hathaway, A. S. J. H. Un. Cir. [2] (1883) 143-.

Transcendent functions connected with

$$
\prod_{0}^{\infty}\left(1+q^{n} x\right)
$$

Mellin, H. Stockh. Öfv. (1884) No. 5, 125-; Fschr. Mth. (1884) 375.

Transformations. Cauchy, A. L. C. R. 17 (1843) 523-.

Value. Bečka, B. Časopis 5 (* 1876) 37-; Fschr. Mth. (* 1876) 251.

- of $(3.5 .9 .17 \ldots) /(2.4 .8 .16 . .$.$) . Vachette,$ A. N. A. Mth. 5 (1846) 188-

values. Glaisher, J. W. L. [1873-75] Mess. Mth. 2 (1873) 189-; 5 (1876) 122.

- and geometrical series of $n$th order. Glaisher, J. W. L. [1875] L. Mth. S. P. $7(1875-76)$ 23-.

$\varpi(x, t)=(1+x)(1+t x)\left(1+t^{2} x\right) \ldots$ Cauchy, A. L. C. R. 17 (1843) 693-.

$(1-x)\left(1-x^{2}\right)\left(1-x^{3}\right) \ldots$, development. Franklin, $F$. [1880] C. R. 92 (1881) 448-.

-, Euler's theorem, Franklin's proof. $S y l$ vester, J. J. (xII) J. H. Un. Cir. [2] (1883) 42.

-. Sylvester, J. J. C. R. 96 (1883) 674.

-. Pepin, T. Rm. N. Linc. At. 41 (1888) 67-.

\section{OTHER INFINITE PROCESSES.}

Infinite processes, measure of convergence. Schimpf, E. [1899] D. Mth. Vr. Jbr. 8 (1900) (Heft 1) 216-.

Power expression $x^{x^{x *}}$ to infinity, limiting values. Seidel, L. [1871] Münch. Ab. 11 (1873) 1-.

\section{Principles and elements of the differential calculus.}

Algebraic differentials. Harnack, A. A. Mt. 9 (1878-79) 302-.

- - in homogeneous coordinates. Harnack, A. Mth. A. 9 (1876) 371-.

Algorithm for differential and integral operations, identity with algebraic. Tralles, J. G. [1809] Berl. Ab. (1804-11) (Mth.) 188-.

"Analyse des infiniment petits," Jean Bernoulli's part in. Eneström, G. Bb. Mth. (1894) 65-.

Calculus, analogy in. Conti, C. A. Sc. Lomb. Ven. 2 (1832) 179-.

-, elements. Boucharlat, - Rouen Tr. Ac. (1820) 6-.

-, - Ampère, A. M. N. A. Mth. 4 (1845) 105-, 161-, 209-, 278-.

-, formulæ. Spitzer, $S$. Grunert Arch. 24 (1855) 97-.

-, geometrical exposition. Lamarle, $E$. Brux. Mm. Cour. 8\%, 11 (1861) 170 pp. ; 15 (1863) $676 \mathrm{pp}$.
Calculus, higher, application of method of indeterminates. Wright, J.M. F. Silliman J. 17 (1830) 74-.

-, Lacroix's. Adams, Jas. Thomson A. $\mathrm{Ph}$. 12 (1818) 204-.

"- of Primaries" new branch of infinitesimal calculus. Sang, E. [1866] Edinb. R. S. T. 24 (1867) 515-.

-, principles. Brasseur, J.B. Liége Mm. S. Sc. 3 (1873) 113-.

-, 一, and applications. Brunacci, V. Bologna Mm. I. It. 1 (1806) (pte. 2) 79-.

- , - first. Cook, $H$. Des Moines Anal. 2 (1875) 185-.

-, relations of continuity to. Drobisch, M. W. Leip. B. (1853) 155-.

-, true basis. Werneburg, J. F. C. Oken Isis (1819) 281-

Change of independent variable. Poinsot, $L$. Par. Ec. Pol. Cor. 3 (1814-16) 124-.

- - - Greatheed, S. S. [ascribed to Gregory, D. F., in Vol. III.] Camb. Mth. J. 1 (1839) 236-.

- - - Hess, E. Z. Mth. Ps. 17 (1872)

- - - Ferreira dos Sanctos, R. G. Teix. J. Sc. 6 (1885) 24 -.

- - - - in a differential coefficient. Gallop, E. G. Camb. Ph. S. T. 16 (1898) 116-.

- - - - differentials. Schlömilch, 0 . Liouv. J. Mth. 3 (1858) 385-.

---- differentiation. Liouville, $J$. Par. Ec. Pol. J. 24 cah. (1835) 17-.

$-{ }_{-}$, formulæ. Liouville, J. Liouv. J. Mth. 5 (1840) 311-.

- - - in higher differential. Martini, $E$. G. Mt. 2 (1864) 353-

- _ - - - multiple differentiation and integration. Dienger, $J$. Grunert Arch. 10 (1847) 417-.

- - a system of independent variables. Gallop, E. G. Camb. Ph. S. T. 17 (1899) 231-.

- - systems of independent variables. Sylvester, J. J. QJ. Mth. 1 (1857) 42-, 126-.

- - variables. Marchand, E. Par. Éc. Norm. A. 3 (1886) 137-, 343-.

- - Leudesdorf, C. [1886-87] L. Mth. S. P. 17 (1887) 329-; 18 (1886-87) 235-.

- - L Lévy, L. N. A. Mth. 13 (1894) 5 -.

- - - of higher derivatives. Bochow, Z. Mth. Ps. 32 (1887) 346-.

Continuous functions, determination of differentials. Conti, C. Padova N. Sag. 3 (1831) 208-.

- - differentiation and representation. Köpcke, A. Mth. A. 29 (1887) 123-.

- - and their differentials. Franke, $T$. Grunert Arch. 15 (1850) 227-.

- - - - (Franke). Schlömilch, $O$. Grunert Arch. 16 (1851) 235-.

- - differentials, Cauchy's theorem. Theorell, A. G. [1867] Stockh. Öfv. 24 (1868) 75-. 
Continuous functions, existence of derived function. Gilbert, P. [1872] Brux. Ac. Bll. 33 (1872) 498-; Brux. Mm. Cour. $8^{\circ}, 23$ (1873) (No. 3) vi +31 pp. ; (Correction) Brux. Ac. Bll. 35 (1873) 709-.

$d$ and $\delta$, explanation. Adams, Jas. Thomson A. Ph. 10 (1817) 336-.

Derivations calculus. Paoli, P. [1806] Mod. S. It. Mm. 13 (1807) 23-.

- - of Arbogast and Lagrange, and comparison with differential calculus. Weingärtner, $F$. C. Erfurt N. Acta 2 (1802) 22 pp.

Derivative of power of variable, transformation. Whitlock, G. C: Camb. (M.) Mth. M. 2 (1860) 287-.

Derivatives. Hill, T. Camb. (M.) Mth. M. 1 (1859) 35-.

- of composite functions. Gomes Teixeira, $F$. G. Teix. J. Sc. 8 (1887) 120-; Fschr. Mth. (1887) 254.

- $-e^{\sqrt{x}}$, relation connecting. Glaisher, J. W. L. [1876] Mess. Mth. 6 (1877) 85-.

- - functions of functions. Cesáro, E. N. A. Mth. 4 (1885) 41-.

-, higher, of certain composite functions, zero values. Rogel, $F$. Arch. Mth. Ps. 11 (1892) 14-.

-, numerical. Bugaev [Bougaieff], N.V. (xII) Rec. Mth. (Moscou) 5 (1870) (Pt. 1) 1-; 6 (1872-73) (Pt. 1) 133-, 201-, 309-; (Ix) Bll. Sc. Mth. As. 6 (1874) 314-; 10 (1876) 13-.

-, - (Bugaev). Andreevskir, M. A. [1872] (xII) Rec. Mth. (Moscou) 5 (1870) (Pt. 1) 420 -

Derived function, definition. Peano, $G$. Mathesis 12 (1892) 12-.

- - of any order, of product of functions of several variables. Trudi, $N$. Nap. Rd. 18 (1879) 181-, 299-.

- - proof of existence. Interpretation of $\frac{0}{0}$. Faure, $H$. Fr. Cg. Sc. 33 (1866) 321-.

- functions of an algebraic function. Sintsof, D. N. A. Mth. 17 (1898) 411-.

- - algebraic functions. Ostrogradsky, M. A. [1850] St Pét. Ac. Sc. Bll. 11 (1853) 337-.

- - , and application to mechanics. Mathieu, E. L. (x) Par. S. Mth. Bll. 1 (1873) 157-.

- , class. Lobatto, R. Amst. Vs. Ak. 10 (1860) 255-.

- - Lagrange's methods. Caluso, (l'abbé) T. V. de. Mod. Mm. S. It. 14 (1809) 201-.

- - limits, Mendelěev's problem. Markov, A. [1889] St Pet. Ac. Sc. Mm. (Rs.) 62 (1890) 1-; Fschr. Mth. (1890) 283-.

- - of any order of $y$ when $f(x, y)=0$. Pereira da Silva, D. S. G. Teix. J. Sc. 4 (1883) 109-.

- -, theory. Piffet, A. (vir) A. Cond. Pon. Chauss. 4 (1860) 121-, 153-, 193-, 237-.

Difference and differential coefficient of same order, relation between. Genocchi, A. Arch. Mth. Ps. 49 (1869) 342-.
Differential calculus. Caluso, (l'abbé) T.V. de. Turin Mm. Ac. 3 (1786-87) 489-.

- - Bordoni, A., \& Conti, -. A. Sc. Lomb. Ven. 1 (1831) 419-.

- L La Casa, V. de. A. Sc. Lomb. Ven. 9 (1839) 49-, 139-, 149-.

- -. Foscolo, G. Ven. Aten. Esercit. 6 (1847) 189-.

- - Schlömilch, O. Grunert Arch. 18 (1852) 381-.

- - Cockle, (Sir) J. [1864] Mess. Mth. 3 (1866) 42-, 247-.

- -. Studnička, F. J. Prag Sb. (1877) 393-.

- - application. Minding, E. F. A. [1878] St Pét. Ac. Sc. Bll. 25 (1879) 225-

- - , - to solution of differential equations. Kelland, P. [1849] Edinb. R. S. T. 20 (1853) 39-.

- _, Du Bourguet's. Cockle, (Sir) J. [1879-81] Manch. Lt. Ph. S. P. 19 (1880) $9-, 181$ - 20 (1881) 119-

- - , elements. Conti, C. A. Sc. Lomb. Ven. 1 (1831) 5-.

- - Euler's principle. Ofterdinger, $L . F$. Grunert Arch. 5 (1844) 201-.

- - exposition, methods compared. Conti, C. Padova N. Sag. 3 (1831) 208-

- - formula. Faà de Bruno, $F$. QJ. Mth. 1 (1857) 359-.

- - , formulæ. Fergola, E. Tortolini A. 1 (1858) 370.

- - - - Kretkowski, W. [1881] (xrr) Krk. Ak. (Mt.-Prz.) Pam. 7 (1882) 164-

- - - - elementary proof. Berger, $A$. Stockh. Öfv. 37 (1880) No. 10, 39-.

- - Francour's, addenda to. Osorio, R. G. Coimbra I. 3 (1855) 323-.

- - fundamental problem. Echols, W. H. A. Mth. 11 (1896-97) 61-.

- - incorrect application to chemistry. Gïnther, S. [1873] Erlang. Ps. Md. S. Sb. 6 (1874) 66-

- -, law of derivation. Conti, C. A. Sc. Lomb. Ven. 1 (1831) 173-.

- -, metaphysics. Mazzola, A. A. Sc. Lomb. Ven. 13 (1844) 73-.

- -, principle. Conti, C. A. Sc. Lomb. Ven. 3 (1833) 295-.

,--- , history. Gerhardt, C. I. Grunert Arch. 2 (1842) 200-.

- - principles. Curten, P. Rot. N. Vh. 5 (1810) 59-.

,--- Lamarle, E. Brux. Ac. Sc. Mm. 29 (1855) 118 pp.

- - - establishment. Schultén, N. G. af (fil.) [1839] Helsingf. Acta 1 (1840) 413-, 687-.

- - - , exposition. Gergonne, J. D. Gergonne A. Mth. 20 (1829-30) 213-.

,,,---- methods compared. Servois, J. F. Gergonne A. Mth. 5 (1814-15) 141-.

,,---- new. Servois, J. F. Gergonne A. Mth. 5 (1814-15) 93-.

- - - and general laws. Poinsot, $L$. Par. Ec. Pol. Cor. 3 (1814-16) 111-.

- - reciprocal methods. Boole, G. Camb. and Dubl. Mth. J. 7 (1852) 156-; 8 (1853) 1-. 
Differential calculus, theorem. Genocchi, $A$. Tor. At. Ac. Sc. 4 (1868-69) 327-.

- , theory. Schubert, F. T. St Pét. Ac. Sc. Mm. 6 (1818) 153-.

- - use of new symbol. Lamarle, $E$. Liouv. J. Mth. 11 (1846) 254-.

- coefficient and continuity. Steinitz, $E$. Mth. A. 52 (1899) 58-.

- of function of function, etc. Fields, J. C. Am. J. Mth. 11 (1889) 388-.

- _ - - in terms of differential coefficients of inverse function. Fergola, E. Nap. Ao. Sc. Mm. 2 (1855-57) 200-.

- - - - of two variables. Genocchi, A. Tor. At. Ac. Sc. 4 (1868-69) 323-.

- -, 1st order. Binet, J. P. M. Par. S. Phlm. Bll. 1 (1808) 275-.

- - $n$th order, $f(\sin x)$. Malmsten, C. J. Stockh. Öfv. 3 (1846) 36-.

,---- , of $y=e^{m x^{2}}$. Spitzer, S. Grunert Arch. 30 (1858) 79-.

- coefficients. Cohen, A. Phil. Trans. (1862) 469-.

- - of function of function. Meyer, U. H. Grunert Arch. 9 (1847) 96-.

- - - - one variable. Stieltjes, T.J. (jun.) N. Arch. Wisk. 9 (" 1882) 106-

- - - two functions, remarkable relation between. Steen, A. (xII) Ts. Mth.5 (1881) 101-.

- with general index, and integration of differential equations. Most, $R$. Z. Mth. Ps. 16 (1871) 190-.

- 一, higher. Most, R. Mth. A. 4 (1871) 499- - - Engel, F. Leip. Mth. Ps. B. 45 (1893) 468-

- - - - of composite functions. Tardy, $P$. G. Mt. 2 (1864) 73-.

,,---- functions of functions. Fais, A. G. Mt. 13 (1875) 47-

- -, 一, - some functions. Schlömilch, $O$. Grunert Arch. 4 (1844) 364-.

,,$----\ldots$, and definite integrals connected with them. Lindman, C. F. [1865] Stockh. Ak. Hndl. 5 (1866) No. 8, 29 pp.

,--- , independent representation. Hoppe, R. Mth. A. 4 (1871) 85-.

,,----- Jensen, J. L. W. V. (xח) Ts. Mth. 3 (1879) 90-.

- - , - , - - , and summation symbols. Hoppe, R. Crelle J. 33 (1846) 78-.

$-\ldots, \ldots$, of logarithmic functions. Schlömilch, o. Grunert Arch. 8 (1846) 427-.

- - , - - quotients. Meyer, $F$. Mh. Mth. Ps. 1 (1890) 33-.

,,---- tangent. Schlömilch, O. Grunert Arch. 12 (1849) 297-

- - - , theory. Malmsten, C. J. Stockh. Öfv. 3 (1846) 324-.

- - - - - Schlömilch, O. Grunert Arch. 7 (1846) 204-; Leip. B. 9 (1857) 163-.

,--- , of

$$
\begin{aligned}
& P=\frac{\sin x}{1+2 y \cos x+y^{2}}, \\
& Q=\frac{y+\cos x}{1+2 y \cos x+y^{2}} .
\end{aligned}
$$

Grunert, J. A. Grunert Arch. 3 (1843) 41-.
Differential coefficients, higher, of

$$
\left(x^{2}+a x+b\right)^{-(\mu+1)} \text {. }
$$

Schlömilch, O. Grunert Arch. 8 (1846) 357-. ,,$---\cos ^{n} \theta$. Schlömilch, O. Grunert Arch. 9 (1847) 313-.

-, any order, calculation. Laurent, $H$. N. A. Mth. 3 (1884) 240 -

- of unknown functions, determination. Nörrenberg, - . Baumgartner Z. 6 (1829) 437-.

$--e^{\alpha x} \cdot \cos (\alpha+\beta x)$, etc. Unferdinger, $F$. [1869] Wien Sb. 60 (1870) (Ab. 2) 605-.

-, to find, without use of infinitesimals or limits. Ficklin, $J$. Des Moines Anal. 4 (1878) 177-.

- formulø, theory. Liouville, J. Liouv. J. Mth. 17 (1852) 478-.

- of function of two variables. Johnson, $W$. W. Mess. Mth. 4 (1875) 132-.

- higher, of a power, base and exponent of which are functions of same variable. Studnička, F. J. Prag Sb. (1877) 368-.

- of $\log x$. Cerquero, J. S. Quetelet Cor. Mth. 10 (1838) 31-.

- - Verhulst, P. F. Brux. Ac. Bll. 7 (1840) 53-.

- $n$th order of implicit function $y$ defined by $f(x, y)=0$. Preobrazhenskix, $V$. $V$. (xII) Kazan S. Nt. (Ps.-Mth.) P. 1 (1883) [(No. 9)] 1-.

- relations in finite terms. La Casa, V. de [Casa, V. della]. A. Sc. Lomb. Ven. 7 (1837) 178-, 205-; 10 (1840) 255-; 13 (1844) 117-.

---1 (de la Casa). Minich, S. R. A. Sc. Lomb. Ven. 9 (1839) 216-; 11 (1841) 63-.

- identical. Glaisher, J. W. L. [1876] L. Mth. S. P. 8 (1876-77) 47-.

Differentials, common divisors and multiples. Heffer, L. D. Nf. Vh. (1895) (Th. 2, Hälfte 1) 8-.

- containing quadratic surd, rationalisation. Mertens, $F$. Mh. Mth. Ps. 2 (1891) 217-.

-, elimination of radicals. Schlömilch, $O$. Leip. B. 20 (1868) 151-.

- employed as alternate numbers (clefs algébriques). Cauchy, A. L. C. R. 37 (1853) $38-, 57-$.

-, exact. Sarrus, F. C. R. 1 (1835) 115-.

- of exponentials with variable base, etc. Oettinger, L. Grunert Arch. 22 (1854) 401-.

- - a function of two variables. Falk, M. (xiI) Ts. Mth. 6 (1882) 164-.

- as functions of finite differences, and vice versa. Lemonnier, $H$. N. A. Mth. 20 (1861) 197-.

- of functions, new method of obtaining, with especial reference to Newtonian conception. Johnson, W. W., \& Rice, J. M. Am. Ác. P. 8 (1873) 488-.

-, higher, of fractional functions of one variable, representation. Studnička, F.J. Prag $\mathrm{Sb}$. (1874) 1-.

- - - - a function of functions. Kömigsberger, L. Mth: A. 27 (1886) 473-. 
Differentials, higher, of $y=\sqrt{a^{2}-b^{2} x^{2}}$. Grunert, J. A. Grunert Arch. 3 (1843) 236-

,,$----=-$, law of coefficients. Luchterhandt, A. R. Grunert Arch. 4 (1844) 87-.

-, new method of finding. Wilson, $W . D$. Des Moines Anal. 1 (1874) 195-.

-, - proof for. Ficklin, J. [1883] Sid. Mess. 2 (*1883-84) 209-.

-, any order. Tardy, $P$. Tortolini A. 1 (1858) 135-.

-, - - Rutgers, A. Arch. Néerl. 7 (1872) $27-$.

(1880) 301-

-, - - , of certain general functions, theorems. Schlömilch, O. Crelle J. 32 (1846) 1-.

-, 2nd and higher orders. Miller, $E$. Kan. Ac. Sc. T. 12 (1890) 65-.

-, successive, of a function of one variable, geometrical representation. Fontené, $G$. Ens. Mth. 1 (1899) 458-.

,,$-----\frac{-}{-}$ several variables. Moutard, - C. R. 124 (1897) 603-.

,,$-----1-1$ Goursat, É. C. R. 124 (1897) 676.

-, -, sum. Genocchi, A. Tortolini A. 8 (1857) 289-.

-, transformation. Jürgensen, C. Mth. Ts. 2 (1860) 1-.

- versus differential coefficients. Magistrini, G. B. [1846] Bologna Mm. Ac. Sc. 1 (1850) 297-.

$-\frac{d^{n-1}}{d z^{n-1}}\left(1-z^{2}\right)^{n-\frac{1}{2}}$. Liouville, J. Liouv. J. Math. 6 (1841) 69-.

$-\frac{d^{i-1}}{d z^{i-1}}\left(1-z^{2}\right)^{i-\frac{1}{2}}$. Grunert, J.A. Grunert Arch. 4 (1844) 104-.

Differentiation, application of factorials. Langsdorf, $W$. Grunert Arch. 21 (1853) 249-.

- , - Fourier's formula. Liouville, $J$. Crelle J. 13 (1835) 219-.

-, - - fundamental formulø to summation of infinite series. Florov, $P$. S. Kharkov Mth. S. Com. (1886) 3-; Fschr. Mth. (1886) 245.

-, to periodic series. Young, J.R. $\mathrm{Ph}$. Mg. 28 (1846) 213-.

-. Case where $\frac{d \phi}{d x} \frac{d \psi}{d y}=\frac{d \psi}{d x} \frac{d \phi}{d y}$ is an identity for values of $y$ given by $\phi(x, y)=0$. Spitzer, S. Grunert Arch. 25 (1855) 137-.

- by finite differences and vice versa. Lobatto, R. Crelle J. 16 (1837) 11-.

-, formulø for. Cayley, A. R. S. P. 9 (1857-59) 93-; Tortolini A. 2 (1859) 214-.

-, fractional. Greer, H.R. QJ. Mth. 3 (1860) 327-.

-, - Zachartchenxo, W. [VashchenkoZakharchenko, M.] QJ. Mth. 4 (1861) 237-.

-, - (Riemann). Cayley, A. [1879] Mth. A. $16(1880) 81-$.

-, -, theory and application. Liouville, $J$. Par. Ec. Pol. J. 21 cah. (1832) 1-.
Differentiation of functions of functions. Anne, T. (vI $A d d 8$.$) N. A. Mth. 9$ (1850) 119-; 11 (1852) 376-.

- _- - Gomes Teixeira, F. G. Teix. J. Sc. 7 (1886) 150-; 9 (1889) 137-; Fschr. Mth. (1885) 242-; (1889) 261-.

general. Greatheed, S. S. [ $\left.\iint.\right]$ Camb. Mth. J. 1 (1839) 11-, 109-.

-, - Kelland, P. [1839] Edinb. R. S. T. 14 (1840) 567-, 604-; 16 (1849) 241-.

-, - Hammond, J. Am. J. Mth. 3 (1880) $164-$.

-, 一. Nekrasov, P. A. [1888] Rec. Mth. (Moscou) 14 (1890) 45-; Fschr. Mth. (1888) 275-.

-, imaginaries in. Gilbert, $P$. Brux. Ac. Bll. 33 (1872) 108-.

- to any index. Liouville, J. Par. Éc. Pol. J. 21 cah. (1832) 71-.

- - - - Holmgren, H. [1865] Stockh. Ak. Hndl. 5 (1866) (No. 11) 83 pp.

- - - Somin, N. Y. [1869] (xII) Rec. Mth. (Moscou) 6 (1872-73) (Pt. 1) 1-.

- - - new method. Buchwaldt, $F$. Kjöb: Ov. (1876) 51-, 248; (Résumé, 14-). - - - , theory. Lyetnikov [Letnikow], A. V. (xII) Rec. Mth. (Moscou) 3 (1868) (Pt. 1) 1-, 85-; 6 (1872-73) (Pt. 1) 413-; (x) Bll. Sc. Mth. As. 6 (1874) 316-.

- and integration. Gosiewski, W. Par. T. Nauk Śc. Pam. 12 ("1882) Art. 3, $15 \mathrm{pp}$.

-, introduction to. Macnie, $J$. Des Moines Anal. 7 (1880) 152-.

-, metaphysics. Nieuport, C. F. F. de. [1822] Brux. Ac. Sc. Mm. 2 (1822) 45-.

-, method independent of expansion. Francais, J. F. Gergonne A. Mth. 2 (1811-12) 325-.

-, multiple, of certain expression. Glaisher, J. W. L. Ph. Mg. 2 (1876) 208-, 522-.

-, order indifferent, assumptions required for proof. Schwarz, H. A. Sch. Nf. Gs. Vh. 66 (1873) 259-.

-, - -, proofs. Lindelöf, L. L. [1866] Helsingf. Ácta 8 (1867) 205-.

- of a power. Schlömilch, O. Grunert Arch. 10 (1847) 42-.

-, problem. Jacobi, C. G. J. Crelle J. 6 (1830) 212.

-, successive, of composite functions. Mossa, F. G. Mt. [13 (1875)] 175-.

-, -, formulæ. Scott, G. QJ. Mth. 4 (1861) 77-.

- of symmetrical determinant, theorem. Glaisher, J. W. L. [1876] QJ. Mth. 14 (1877) 245-.

- - transcendental functions. Unger, E.S. Oken Isis (1825) 1162-.

- - unknown function, error to be avoided. Buquoy, G. von. Oken Isis (1824) 93-.

- $x^{n}$. Matzka, W. Grunert Arch. 6 (1845) 335-.

- - $\{f(x)\}^{k}$. Götting, R. [1870] Mth. A. 3 (1871) 276-.

Euler's theorem, proof. Orlov, T. E. [1866] (xII) Rec. Mth. (Moscou) 2 (1867) (Pt. 1) 321-.

Exponential calculus. Curten, $P$. Rot. N. Vh. 5 (1810) 59-. 
Expression $\left(x^{2}+a x+b\right)-(p+1)$, higher derivatives. Björling, E. G. Grunert Arch. 9 (1847) 233-.

Fluxional ratio, applications. Wright, $E$. Silliman J. 24 (1833) 298-; 25 (1834) 93-.

Fluxions. Christison, (Prof.) A. Thomson A. Ph. 5 (1815) 327-; 6 (1815) 420-; 10 (1817) 417-.

-, invention. Weld, C. R. Ph. Mg. 31 (1847) 35-.

- method, principles. Stockler, F. de B. G. Lisb. Mm. Ac. Sc. 1 (1797) 200-.

-, problems, solutions. Strong, T. Silliman J. 16 (1829) 283-; 17 (1830) 69-, 329-; 18 (1830) 67-.

—, theory. Wright, E. Silliman J. 14 (1828) 330-; 15 (1829) 196-; 16 (1829) 53-.

-, two, proposed by Simpson. Andrew, $J$. Tilloch Ph. Mg. 39 (1812) 383-.

Functions possessing no differential coefficient. Hertz, K. Par. T. Nauk Śc. Pam. 11 ( $\left.{ }^{*} 1879\right)$ Art. 1, 24 pp. ; Fschr. Mth. (*1879) 275.

-, successive differentials of which are in arithmetical progression. Weyr, E. A. Mt. 4 (1870-71) 212-.

-, variability. Marroquín y Rivera, M. Méx. S. "Alzate" Mm. 1 (1887) 27-.

Identity $\quad \phi D^{\mu} \psi=D^{\mu}(\phi \psi)-\frac{\mu}{1} D^{\mu-1}(\psi D \phi)+$ $\frac{\mu(\mu-1)}{1.2} D^{\mu-2}\left(\psi D^{2} \phi\right)-\ldots+(-1)^{\mu} \psi D^{\mu} \phi$, proof. Posse, K. A. Rec. Mth. (Moscou) 13 (1886) 489-; Fschr. Mth. (1887) 256.

Implicit functions, determination. Cauchy, A. L. C. R. 34 (1852) 265-.

$n$ independent variables, each expressed as function of $n$ others, theorem. Minchin, G. M. [1871] QJ. Mth. 12 (1873) 172-.

Indeterminate expressions. Seliwanow, D. $F$. Fschr. Mth. (1892) 253.

- forms, values. Olivier, L. Crelle J. 1 (1826) 308-.

- ratios with several variables. Autonne, $L$. N. A. Mth. 16 (1897) 420-.

Infinite, employment of the. Transon, A. C.R. 73 (1871) 367-.

- quantities. Hoppe, R. Arch. Mth. Ps. 55 (1873) 49-.

Infinitesimal analysis. Cauchy, A. L. C. R. 17 (1843) 275-.

- - fundamental questions. Casorati, $F$. Rm. R. Ac. Linc. T. 5 (1881) 216-.

- calculus. Franchini, P. Brugnatelli G. 10 (1827) 79-.

- -. Cauchy, A. L. Bb. It. 61 (1831) 321-.

- - Bergasse, - Rouen Ac. Tr. (1849-50) 111-.

- - Noel, J. N. Liège Mm. S. Sc. 10 (1855) 25-; 16 (1861) 73-, 410-.

- -. Méhay, A. Mon. Sc. 12 (1870) 148-.

- -, applications. Bellavitis, G. A. So. Lomb. Ven. 1 (1831) 347-.

Mm. 4 (1890) 293-.

- - extension of some conceptions. Viterbi, A. Rm. R. Ac. Linc. Rd. 6 (1897) (Sem. 1) 267-.

VOL. I.
Infinitesimal calculus, formation of new algorithms. Guldberg, A. S. [1868] Sk. Nf. F. 10 (1869) 165-.

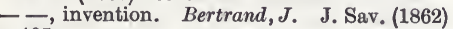
465-.

- -, - Eneström, G. Bb. Mth. (1898) 50 -.

- -, metaphysics. Saporetti, A. Bologna Ac. Sc. Mm. 5 (1895-96) 309-.

- , origin. Milhaud, G. [1900] N. Y. Am. Mth. S. Bll. 7 (1901) 157, 158.

- - - - Cantor, M. [1900] N. Y. Am. Mtb. S. Bll. 7 (1901) 158, 159-.

- - - - and principles. Gomes Teixeira, F. G. Teix. J. Sc. 3 (1881) 21-.

- -, paradoxes. Du Bois-Reymond, P. Mth. A. 11 (1877) 149-.

- -, principles. Gomes Teixeira, F. [1879] Bordeaux S. Sc. Mm. 4 (1882) 41-.

,--- and difficulties. Hoppe, $R$. N. Mg. Ntvd. 18 (1871) 321-.

- , problem. Gilbert, L. P. (xII) Mathesis 2 (1882) 17-.

- - problems. Domenico, D. Brugnatelli G. 3 (1820) 229-, 319-.

-- , questions. Strebor [Roberts, W.] N. A. Mth. 9 (1850) 181-.

- - theory. Carnot, L. N. M. Tilloch Ph. Mg. 8 (1800) 222-, 335-; 9 (1801) 39-.

-, conception, history. Vivanti, $G . \mathrm{Bb}$. Mth. (1894) 1-.

-, -, logical analysis. Fischer, E. G. Berl. Ab. (1829) 29-.

-, definition. Vivanti, G. Rv. Mt. 1 (1891) 135-, 248-.

-, - Bettazzi, R. Rv. Mt. 1 (1891) 174-; 2 (1892) 38-.

- method, independent of metaphysics and of limits method. Manilius, J. A. Gén. Civ. 2 (1863) 251-.

Infinitesimals. Debacq, C. Les Mondes 16 (1868) 484-; 17 (1868) 238-.

-. Houiel, J. Les Mondes 16 (1868) 567-; 17 (1868) 287-.

-. Debacq, C. Les Mondes 18 (1868) 608-; 19 (1869) 66-, 156-, 255-, 485-; 20 (1869) 77-, 173-, 368-, 761-.

-. Stolz, O. Innsb. Nt. Md. B. 14 (1884) 21-, 37-.

-, class considered by Newton. Vivanti, $G$. Bb. Mth. (1891) 97-.

-, principle. Inghirami, G. Inghirami Opusc. 2 (1821) 494 .

-, principles, establishment. Marianini, $P . D$. [1858] (x) Mod. Ac. Sc. Mm. 3 (1861) 17-.

-, ratio finite, geometrical illustration. Terquem, $O$. N. A. Mth. 3 (1844) 580-.

-, theory. Hoppe, R. Arch. Mth. Ps. 64 (1879) 444-.

Inverse differentiation. Buquoy, G.von. Oken Isis (1825) 37-; 21 (1828) 1090-.

- processes of calculus, failures in. Gregory, D. F. Camb. Mth. J. 2 (1841) 73-.

Inversion of variables in $\frac{d^{n} u}{d x^{n}}$, Burmann's law. Sylvester, J.J. Ph. Mg. 8 (1854) 535.

Irrational functions, fluents. Bromhead, (Sir) E. F. Phil. Trans. (1816) 335-. 
Law of mean, and limits $\frac{0}{0}, \frac{\infty}{\infty}$. Osgood, W. F. A. Mth. 12 (1898-99) 65-.

Leibnitz's infinitesimal calculus, compensation of error. Guldberg, A. S. N. Mg. Ntvd. 16 (1869) 46-.

- infinitesimals, and first principles of calculus. Debacq, C. Les Mondes 29 (1872) 486-.

- theorem. Genocchi, A. Tor. At. Ac. Sc. 4 (1868-69) 263-.

- - Tardy, P. N. A. Mth. 8 (1869) 69-.

- Frobenius, G. [1879] Crelle J. Mth. 89 (1880) 262-.

- Mansion, P. Mathesis 13 (1893) 36-.

- - generalisation. Hoüel, J. Bordeaux Mm. S. Sc. 5 (1867) 379-.

- - - - Genocchi, A. Tor. Mm. Ac. Sc. 26 (1871) 61-.

Limit of a function, definition. Peano, $G$. Rv. Mt. 2 (1892) 77-; Am. J. Mth. 17 (1895) 37-.

- $x^{x}$ is 1 , when $x=0$, Pfaff's proof. Möbius, A. F. Crelle J. 12 (1834) 134-, 292-.

$--x-\sqrt{x^{2}-1}$ when $x=\infty$. Grunert, J. A. Arch. Mth. Ps. 43 (1865) 116-.

- $-x^{x}$ when $x$ approaches zero. Grunert, J. A. Arch. Mth. Ps. 53 (1871) 510-

- ${ }_{n=\infty}\left(\cos ^{n} x\right)$, Tait's problem. Muir, $T$. [1877] Edinb. R. S. P. 9 (1878) 359-.

- of $\frac{1}{n} f(n)$, extension of Cauchy's theorem. Jensen, J. L. W. C. R. 106 (1888) 833-.

"Limited" differentiation and its applications. Grïnwald, A. K. Z. Mth. Ps. 12 (1867) 441-.

Limiting value of fraction $\frac{\infty}{\infty}$. Bertrand, $J$. Liouv. J. Mth. 6 (1841) 14-.

- -1 - Blanchet, P. H. Liouv. J. Mth. 6 (1841) 65-.

- - - - Liouville, J. Liouv. J. Mth. 7 (1842) 160-.

(1846) 259 .

$--\frac{1}{-}$. Rouqet, V. N. A. Mth. 16 (1877) 113-.

$----\frac{0}{0}$. Jerrard, G. B. Ph. Mg. 12 (1838) 345-. $-\frac{1}{(1853)}-\frac{1}{381-}$. Wiener, C. Grunert Arch. 21

(1873) 479-.

,$---- 0^{0}$. Terquem, O. N. A. Mth. 6 (1847) 391-.

- $-0^{0}$. S. (vI Adds.) Crelle J. 11 (1834) 272 .

_ _ - - Johnson, W. W. Des Moines Anal. 3 (1876) 118-

- - Sprague, T. B. Edinb. Mth. S. P. 3 (1885) 70 -

$-\frac{1^{m}+2^{m}+\ldots+s^{m}}{s^{m+1}}=\frac{1}{m+1}$. Schlömilch, $O$.

Grunert Arch. 14 (1850) 452-.
Limiting value of $\frac{\sqrt{1}+\sqrt{2}+\ldots+\sqrt{r}}{r \sqrt{r}}$ when $r=\infty$. Schlömilch, O. Grunert Arch. 14 (1850) 454-. $\underline{1}$

- - $\frac{1}{n}\left(a^{n}-1\right)$ when $n=\infty$. Schlbmilch, $O$. Schlömilch Z. 3 (1858) 387-.

- $-f(x, y)$ when $x$ and $y$ are zero. $D u$ Bois-Reymond, P. Crelle J. 70 (1869) 10- $-f^{\prime}(x)=\lim . \frac{f(x)}{x}$, theorem. Du BoisReymond, P. Mth. A. 16 (1880) 550.

- values, calculus. Thieme, F.E. Arch. Mth. Ps. 58 (1876) 185-.

- - of fractions. Mansfield, J. [1816] Am. Ph. S. T. 1 (1818) 200-

$--\frac{\text { - }}{-}$ Galbraith, $W$. Edinb. Ph. J. 3 (1820) 69-.

_ Marianini, P. D. Mod. S. It. Mm. 25 (1855) (pte. 2) 347-.

_- Young, J.R. Ph. Mg. 32 (1866) 121-.

- - -, L'Hôpital's formula for $\frac{0}{0}$.

Forestier, C. Toul. Ac. Sc. Mm. 10 (1878) $482-$.

- - - , theory (Woolhouse). Young, J.R. Ph. Mg. 8 (1836) 295-, 515-; 9 (1836) 92-. - _ - , - (reply to Young). Woolhouse, W. S. B. Ph. Mg. 8 (1836) 393-; 9 (1836) 18-, 209-.

$---\frac{0}{0}$ and $\frac{\infty}{\infty}$. De Morgan, A. QJ.

Mth. 1 (1857) 204-.

$---\frac{0}{a}, \frac{a}{0}, \frac{0}{0}$. Sexe, S. A. N. Mg.

Ntvd. 17 (1870) 1-.

_ - indeterminate forms. Johnson, $W . W$. Mess. Mth. 4 (1875) 132-; Des Moines Anal. 4 (1877) 177-.

- - - Marianini, P. D. Mod. Ac. Sc. Mm. 4 (1886) 379-.

- - quotients. Stolz, O. Mth. A. 14 (1879) 231-; 15 (1879) 556-.

- - , theory. Cesáro, E. Palermo Cir. Mt. Rd. 1 (1887) 224-.

Limits. Cockle, (Sir) J. [1867] Mess. Mth. 4 (1868) 116-.

-. Sexe, S. A. N. Mg. Ntvd. 18 (1871) (pt. 2) 81-.

-. Wood, DeV. Des Moines Anal. 9 (1882) 79-.

-, Berkeley's Analyst and its critics. Gibson, G. A. Bb. Mth. (1899) 65-.

-, calculation. Cesáro, E. Mathesis 17 (1897) $177-$.

-, calculus. Kirrov, N. (xII) Mose. Un. Mm. 8 (1835) 151-.

-, -, new. Cauchy, A.L. (vi Adds.) Opuse. Mt. Fis. 2 (1834) 1-, 133-, 261-.

- Cauchy's theorems, extension. Peano, $G$. Tor. Ac. Sc. At. 30 (1895) 20 -

and continuity, conceptions. Cesáro, E. Rm. R. Ac. Linc. Rd. 4 (1888) (Sem. 1) 12-, 866.

-, doctrine. Newcomb, S. Des Moines Anal. 9 (1882) 114-. 
Limits, method. Pollet, - Amiens Mm. Ac. Sc. (1841) 363-.

-, proof of existence. Slešinskij, I. N. Rs. S. Nt. Mm. (Mth.) 8 (1888) 129-; Fschr. Mth. (1888) 200, 201.

-, questions. Laisant, C. A. As. Fr. C. R. (1881) 184-.

- related to $\operatorname{Lim} \cdot \frac{n}{\sqrt[n]{n !}}$ for $n=\infty$. Unferdinger, F. Wien Sb. 67 (1873) (Ab. 2) 355-.

-, theory. Cesaro, E. Bll. Sc. Mth. 13 (1889) 51-.

-, -. Fubini, G. G. Mt. 38 (1900) 72-.

-, -, with examples. Leclercq, $D$. Liège A. Ac. (1820-21) $25 \mathrm{pp}$.

-, -, - - Mareska, J. D. B. Liège A. Ac. (1823-24) 42 pp.

Logarithmic function, its $n$th differential. Tichomandrickij, $M$. A. Kharkov Mth. S. Com. (1886) 42-; Fschr. Mth. (1886) 373.

Mean, arithmetical and geometrical. Dostor, G. J. N. A. Mth. 10 (1851) 88-.

Meaning of $\frac{d^{n}}{d x^{n}}$, where $n$ is complex. Fransen, A. E. Stockh. Öfv. (1895) 481-; Fschr. Mth. (1895) 307.

Method applicable to same purposes as Newton's fluxions. Tredgold, T. Tilloch Ph. Mg. 57 (1821) 177-, 200-, 325-.

Multiplication of partial differential operators. Sylvester, J.J. Ph. Mg. 33 (1867) 48-.

Operation $x \frac{d}{d x}$. Walton, W. [1866] QJ. Mth. 9 (1868) 18-.

- $e^{x} \frac{d}{d x}$. Walton, W. QJ. Mth. 9 (1868) 355-.

Operative symbols in differential calculus. Crofton, M. W. L. Mth. S. P. $12(1880-81)$ 122-, 213.

- - method, applications. Crofton, $M$. W. [1890] Ir. Ac. P. 1 (1889-91) 600-.

Operators $V \frac{d}{d x}, Z \frac{d}{d x}$ etc., transformations. Amanzio, D. G. Mt. 21 (1883) 110-.

Partial or apparent differentials. Ascoli, $G$. Rv. Mt. 4 (1894) 22-.

- differentials, error in theorem. Fontana, $G$. Verona S. It. Mm. 2 (1784) 123-.

-, property. $M$. Camb. Mth. J. 1 (1839) 252-.

- - of $\frac{x}{x^{2}+y^{2}}$. Lobatto, R. Crelle J. 11 (1834) 169-.

- differentiation, proof that $\frac{\partial^{2} u}{\partial x \partial y}=\frac{\partial^{2} u}{\partial y \partial x}$. Whittemore, J. K. N. Y. Am. Mth. S. Bll. 4 (1898) 389-.

- differentiations, reversibility of order. Peano; G. Mathesis 10 (1890) 153-.

Poisson's determinants. Albeggiani, $M$. $L$. Palermo Cir. Mt. Rd. 1 (1887) 6-.

- - generalisation. Albeggiani, M. L. Palermo Cir. Mt. Rd. 1 (1887) 9-, 12-, 314-.

Prime and ultimate ratios, application to fluxionary calculus. Marrat, $W$. Tilloch Ph. Mg. 36 (1810) 186-.
Principles, doubtful points. Györy, S. (xir) Mag. Ak. Éts. (Mth. Term.) 1 (1860) 287-.

Relative magnitude of infinities of functions. Du Bois-Reymond, P. A. Mt. 4 (1870-71) 338-; Crelle J. 74 (1872) 294-.

Rolle's theorem, forms. Glashan, J. C. Am. J. Mth. 4 (1881) 282-.

Second differentials and equicrescent variables. Webb, J. B. Am. As. P. (1886) 69-.

Symbolic formula, new. Faà de Bruno, $F$. C. R. 43 (1856) 725-.

Theorem of Cauchy. Jensen, J. L. W. V. Ts. Mth. 2 (1884) 81-; Fschr. Mth. (1884) 193.

- - generalisation. Stolz, O. Mth. A. 33 (1889) 237-.

- Hesse, demonstration. Jacobi, C. G.J. Crelle J. 40 (1850) 318.

Uniform function of $x$ and its first differential, relation. Schultén, N. G. af (fil.) [1843] Helsingf. Acta 2 (1847) 317-.

Vandermonde's theorem and application to Jacobi's theorem. Lebesgue, $V . A$. Liouv. J. Mth. 6 (1841) 17-.

Vector differentiation. MacFarlane, A. [1900] Wash. Ph. S. Bll. 14 (1906) 73-.

\section{Taylor's series. Maxima} and minima; other analytical applications of the differential calculus.

Analysis, formula. Gomes Teixeira, $F$. N. A. Mth. 5 (1886) 36-.

-, - for $\frac{d^{n}}{d x^{n}}\left[f(x) \phi\left(\frac{1}{x}\right)\right]$. Halphen, G. H. Par. S. Mth. Bll. 8 (1880) 62-.

-, theorems. San Martino, A. [1843] N. A. Sc. Nt. 4 (1845) 353-.

-, - (San-Martino). Barsotti, G. [1844] Lucca At. Ac. 13 (1845) 275-.

Approximate representation of experimental functions between given limits. Vallier, C. R. 116 (1893) 712-.

- - - a function by rational fractions. Padé, H. Par. Éc. Norm. A. 9 (1892) (Suppl.) 93 pp.

Arbogast's expansion formulæ. De Morgan, $A$. Camb. and Dubl. Mth. J. 1 (1846) 238-; Assur. Mg. 12 (*1866) 206-.

- method of derivations. Donkin, W. $F$. Camb. and Dubl. Mth. J. 6 (1851) 141-.

- - - Roberts, S. QJ. Mth. 4 (1861) 193-; 7 (1866) 10-.

- Cayley, A. Mess. Mth. 7 (1878) 158.

3 (1887) 53-.

- - - Tanner, H. W. L. Mess. Mth. 20 (1891) 81-.

_ _ - _ - application to partition of numbers and other problems. David, - Liouv. J. Mth. 8 (1882) 61-.

- - - extension. Cayley, A. Phil. Trans. (1861) 37-. 
Arbogast's method of derivations, extension. Thomson, J. J. Mess. Mth. 7 (1878) 142-. - _ - - - (Cayley). Jenkins, M. Am. J. Mth. 10 (1888) 29-.

- - explained by combinations. De Morgan, A. Camb. and Dubl. Mth. J. 6 (1851) 35-.

- theory of separation of symbols of operation. Harvey, G. Thomson A. Ph. 16 (1820) 178-

Building and restoration expenses, mathematical theory. Launhardt, -. Hann. Archt.-Vr. Z. 33 (1887) 367-.

Calculation of function from its derivatives. Riquier, C. Mars. Fac. Sc. A. 10 (1900) (Fasc. 1) $60 \mathrm{pp}$.

Coefficient of general term in certain expansions. Anglin, A. H. Par. S. Mth. Bll. 15 (1887) $192-$.

Derivatives of composite functions, combinatorial representation. Vollers, $J$. Arch. Mth. Ps. 1 (1884) 64-

- - function of function, application to Bernoulli's numbers. Presle, - de. Par. S. Mth. Bll. 16 (1888) 157-.

-, higher, algebraic relations between coefficients. Meyer, F. Mth. A. 36 (1890) 453-. — of sec $x$. Stieltjes, - . C. R. 108 (1889) 605-.

Differential calculus, theorem. Hobson, E. W. Mess. Mth. 23 (1894) 115-.

- coefficients compared with Bernoulli's numbers. Eytelwein,J.A. Berl. Ab. (1816-17) 28-

Differentiation of certain infinite expressions. Kretkowski, W. Fschr. Mth. (1888) 280.

Eisenstein's theorem. Gomes Teixeira, $F$. Par. Ec. Norm. A. 3 (1886) 389-.

Euler-McLaurin summation formula and its applications. Pokrovsky, P.M. Fschr. Mth. (1898) 216.

Expansion coefficients of $(1-x)^{k} \log ^{p}(1-x)$. Féraud, A. Bordeaux S. Sc. PV. (189697) 93-.

- of Didon's, an. Escary, - C. R. 86 (1878) 1014-.

- - explicit algebraic functions in series, mode. Mangeot, S. Par. Ec. Norm. A. 14 (1897) 247-.

- - function. Echols, W. H. A. Mth. 8 (1893-94) 176-.

- - - in given interval, according to mean values. Leauté, $H$. Liouv. J. Mth. 7 (1881) 185-.

- functions. Corey, S. A. A. Mth. 1 (1900) 77-.

- - - application of method of least squares. Gilman, $F$. Franklin I. J. 137 (1894) 438-. - - of form $F(z+x)$. Roberts, S. Ph. Mg. 32 (1848) 284-.

- - functions, theorem. McClintock, $E$. Am. J. Mth. 2 (1879) 348-; 3 (1880) 173.

- - particularly integrals, new formula. Lagrange, $C$. Brux. Ac. Bll. 9 (1885) 114-.

- - - in power series of determinant. Capelli, A. Palermo Cir. Mt. Rd. 1 (1887) 58-, 133-.

- - - - sines and cosines. Gomes Teixeira, F. Bll. Sc. Mth. 14 (1890) 200-; G. Teix. J. Sc. 10 (1891) 35-; Crelle J. Mth. 116 (1896) 14-.
Expansion of functions in series. Gomes Teixeira, F. Madrid Ac. Ci. Mm. 18 (Pt. 1) (1897) $116 \mathrm{pp}$.

- _ - of several variables by McLaurin's series. Ptasickij, I. L. Kharkov Mth. S. Com. (1884) 73-; Fschr. Mth. (1885) 211.

-, general form. Hill, C. J. D. Lund Acta Un. 6 (1869) (Mth.) No. 1, 36 pp.

- of implicit functions. David, - . Par. Éc. Pol. J. 57 (1887) 147-.

- - Gomes Teixeira, F. Liouv. J. Mth. 5 (1889) 67-.

- - - in series. Worontzoff, -. N. A. Mth. 13 (1894) 167-.

- Léauté's polynomials in trigonometric series. Appell, P. N. A. Mth. 16 (1897) 265-.

- $f(x), f(x, y)$, etc. Adams, Jas. Thomson A. Ph. 1 (1821) 359-.

- $f(z)$ in ascending powers of $h$, when $z-x-h \pi(z)=0$. Cauchy, A.L. Par. Mm. Ac. Sc. 8 (1829) 130 -

- $F(x+h)$. Powers, - \& Pike, - . [1841] Am. Ph. S. T. 8 (1843) 155-.

- $f\left(z, z^{\prime}\right)$ in ascending powers of $y, y^{\prime}$ where $z=x+y f(z)$. Raabe, $J$. L. Zür. Mt. 1 (Heft 2) (1847-48) 36-; Crelle J. 48 (1854) 143-.

- $\dot{H}(x+h)$. Whitcom, A. W. Am. J. Mth. 3 (1880) 344.

$-e^{-1 /(1-x)}$, with applications. Saalschuitz, $L$. Arch. Mth. Ps. 6 (1888) 305-, vii.

Expansions of rational functions in series. Biehler, C. N. A. Mth. 6 (1887) 485-.

-, theory. Boole, G. Camb. Math. J. 4 (1845) 214-.

Explicit representation of $\frac{d^{k}}{d x^{k}}(x-a)^{p}(x-b)^{q}$. Oppermann, L. H. F. (xir) Ts. Mth. 5 (1881) 184 .

Factorial derived functions. Laisant, A. As. Fr. C. R. (1898) (Pt. 2) 76-.

Finite difference formula. $D-g$. Ts. Mt. Fys. 2 (1869) 215-.

Forestry, felling periods. Dienger, J. Arch. Mth. Ps. 41 (1864) 191-.

Formula including binomial theorem. Abel, N. H. Crelle J. 1 (1826) 159-.

- - - Gerono, G. C. N. A. Mth. 16 (1857) 237-, 398-.

- $n F(n x)=f(x)+f\left(x+\frac{1}{n}\right)+$ etc. Kinkelin, H. Grunert Arch. 22 (1853) 189-.

Formulæ, general. Libri, G. Crelle J. 7 (1831) 57-。

Function of functions, $n$th differential. Arez, J. G. Teix. J. Sc. 12 (1895) 110-; Fschr. Mth. (1895) 307-.

"Functiones inexplicabiles," i.e. not expansible by Taylor's theorem. Euler, L. [1780] St Pét. Ac. Sc. Mm. 4 (1813) 88-.

Functions, analytical, abrupt changes of value. Du Bois-Reymond, P. [1873] (vII) Mth. A. 7 (1874) 241-.

General theorems, two, including Lagrange's and Laplace's. Johnson, A. R. Mess. Mth. 14 (1885) 76-. 
Indeterminate algebraic expressions. Studnička, F. J. Časopis 22 (1893) 321-; Fschr. Mth. (1893-94) 464.

Jacobian of functions, extension of theory. Gomes Teixeira, $F$. Mh. Mth. Ps. 1 (1890) 481-.

\section{Lagrange's Series.}

Cayley, A. Camb. Mth. J. 3 (1843) 283-.

Ménabréa, L. F. [1843] Tor. Mm. Ac. 8 (1846) 91-.

Caraffa, A. Palomba Rac. 1 (1845) 43-.

Chì, F. [1846] Par. Mm. Sav. Etr. 12 (1854) 340 -

Dienger, J. [1847] Crelle J. 42 (1852) 287-.

Cayley, A. Camb. and Dubl. Mth. J. 6 (1851) 37-.

Cauchy, A. L. C. R. 34 (1852) 309-, 316-, 345-.

Heine, H. E. Crelle J. 54 (1857) 388.

Tchebicheff, P. St Pét. Ac. Sc. Bll. 15 (1857) 289-.

Oliveira, C. B. de. [1860] Rv. Brazil. 4 (1861) 3 -.

Rouché, E. [1861] C. R. 52 (1861) 295-; Par. Mm. Sav. Étr. 18 (1868) 457-.

(Rouché.) Bertrand, J. C. R. 52 (1861) 1301-.

Laurent, [Herm. non] Henry. C. R. 60 (1865) 25-.

Chio, $F$. Tor. At. Ac. Sc. 7 (1871-72) 647-.

Zolotarev, E. I. N. A. Mth. 15 (1876) 422-.

Rodrigues, J. M. G. Teix. J. Sc. 4 (1883) 121-; 8 (1887) 59-.

Hamy, M. Bll. Sc. Mth. 20 (1896) 213-.

analogue. Cauchy, A. L. [1824] Par. Mm. Ac. Sc. 9 (1830) 104-.

approximate determination of $m$ th term where $m$ is very great. Nekrasov, P. A. [1900] Rec. Mth. (Moscou) 21 (1901) 439-; Fschr. Mth. (1900) 268.

and approximate expressions for functions of large numbers. Nekrasov, $P$. A. Rec. Mth. (Moscou) 12 (1885) 49-, 315-, 432, 483-, 643-; Fschr. Mth. (1885) 212-.

- Burmann's series. Dienger, J. [1868] Prag Ab. 3 (1870) 25 pp.

controversy. Genocchi, A. Tor. At. Ac. Sc. 8 (1872-73) 18-.

convergence, Cauchy's and Lagrange's conditions. Ménabréa, L. F. C. R. 77 (1873) 1358-.

-, - - - (Ménabréa). Genocchi, A. C. R. 77 (1873) 1541-.

demonstration. Miller, T. H. Edinb. Mth. S.P. 4 (1886) 45-

- and forms, new. Meech, L. W. Des Moines Anal. 3 (1876) 33-.

demonstrations, three. Rothe, H. A. Kastner Arch. Ntl. 12 (1827) 1-.

expansion derived from. Glaisher, J. W. L. [1877] Mess. Mth. 7 (1878) 118-.

extension. Peirce, B. Gould As. J. 5 (1858) 164.

generalisation. Moth, F.X. Baumgartner Z. 7 (1830) 64-. generalisation. Gomes Teixeira, $F$. [1876-79] Lisb. J. Sc. Mth. 5 (1876) 203-; 7 (1880) 247-. -. Cesáro, E. N. A. Mth. 4 (1885) 316-.

-. Stieltjes, T.J. Par. Ec. Norm. A. 2 (1885) 93-.

-. Bassani, A. Ven. I. At. (1886-87) 1163-. -,Laplace's. Schläfi, L. QJ. Mth.2(1858) 24-. - requiring proof. Schläfi, L. Bern Mt. (1848) 97-.

interpretation. Ménabréa, L. F. [1846] Tor. Mm. Ac. 10 (1849) 111-.

Lagrange-Cauchy formula. Zinin, $N . N$. Vars. S. Nt. Tr. (1897) (P8. C.) Fasc. 2, No.6. remainder. Ramus, $C$. Kiöb. Dn. Vd. Selsk. Afh. 9 (1842) 365-

-. Popof, A. C. R. 53 (1861) 795-.

successive differentiations connected with.

Knar, I. Baumgartner Z. 3 (1827) 175-.

summation by definite integrals. Parseval, M. A. [1804] Par. Mm. Sav. Étr. 1 (1806) 567-.

- Gruson, J.P. Berl. Ab. (1812-13) (Mth.) 31-.

and summation of series. Dienger, $J$. Crelle J. 34 (1847) 75-.

symbolic theorems derived from. Glaisher, J. W. L. QJ. Mth. 16 (1879) 263-.

Laplace's series. Mainardi, G. Rm. At. N. Linc. 20 (1867) 164-.

- -. Darboux, G. C. R. 68 (1869) 324-.

- - Saint-Germain, A. L. de (xII) Caen Ac. Mm. (1880) 112-.

- -, remainder. McClintock, E. Am. J. Mth. 4 (1881) 96.

- -, summation. Dirksen, E. H. Berl. Ab. (1839) (Mth.) 49-.

Maclaurin's series, Adams, Jas. Thomson A. Ph. 1 (1821) 98-.

- - Hermite, C. [1877] Crelle J. Mth. 84 (1878) 64-.

- -. Genocchi, A. C. R. 86 (1878) 466-.

- - Callandreau, O. C. R. 86 (1878) 589-;

Par. S. Mth. Bll. 15 (1887) 23-.

-- , convergence. Timmermans, J.A. Brux. Ac. Bll. 13 (1846) 53-.

- - demonstration, direct. Dienger, $J$. Grunert Arch. 12 (1849) 93-; 13 (1849) 333-. - -, legitimate use. Jacobi, C. G.J. Crelle J. 12 (1834) 263-.

- - limits. Craufurd, A.Q. G. Camb. Mth. J. 3 (1843) 84-, 159-.

- - for real variable. Callandreau, O. C. R. 104 (1887) 38-.

- - - - and application to potential of a homogeneous body. Callandreau, $O$. C. R. 103 (1886) 864-, 954.

- - , symbolic form, applications. Walton, W. Camb. Mth. J. 4 (1845) 176-.

- - theory. Scheibmer, W. Leip. B. 9 (1857) 190-.

- theorem, demonstration. Hayes, J. $S$. Des Moines Anal. 8 (1881) 149-; 9 (1882) 12-.

- - expansion of circular functions by. Rutherford, $W$. Mathematician 3 (1850) 35-. - - remainder. Veneziani, C. Am. J. Mth. 7 (1885) 168-. 


\section{MAXIMA AND MINIMA.}

Slee, T. Thomson A. Ph. 13 (1819) 193-.

Verdam, G.J. Leijd. A. Ac. (1823-24) 100 pp. Verhulst, P. F. Leijd. A. Ac. (1823-24) 30 pp. Marianini, P. D. Mod. S. It. Mm. 25 (1855) (pte. 2) 347-.

Gibbes, L. R. Camb. (M.) Mth. M.1 (1859)335-. Kleinfeller, -. Z. Mth. Ps. 13 (1868) 515-.

Genese, R. W. [1871] Mess. Mth. 1 (1872) 33-. of algebraic functions. Bary, É. (vII) Gergonne A. Mth. 22 (1831) 37-.

- - - Valat, _. Bordeaux Act. Ac. Sc. (1848) 625-.

- - - new method. Cassani, P. Ven. Aten. At. 1 (1878) 55-.

- - polynomials. Trowbridge, D. Des Moines Anal. 2 (1875) 1-.

analytic theory. Schultên, N. G. af (fil.) [1839] Helsingf. Acta 1 (1840) 171-.

application to central conics and quadrics. Bérard, -. Gergonne A. Mth. 3 (1812-13) 105-.

- of Horner's and Budan's substitutions method. Spitzer, S. Grunert Arch. 23 (1854) 100-.

- - symbol of discontinuity. Walton, $W$. Camb. and Dubl. Mth. J. 3 (1848) 201-.

of arc of curve. Euler, L. [1779] St Pét. Ac. Sc. Mm. 2 (1810) 3-.

_- - - Steiner, J. Crelle J. 17 (1837) 83-. case. Frigelius, A. Stockh. Ak. Hndl. 37 (1816) 1-.

conditional. Cauchy, A. L. C. R. 24 (1847) 757-.

conjugate, Elliott, $E$. B. Mess. Mth. 14 (1885) 182-.

criteria. Brioschi, F. Mil. G. I. Lomb. 3 (1851) 400-; Tortolini A. 3 (1852) 322-.

of 2nd degree. Noel, J. N. Quetelet Cor. Mth. 2 (1826) 71-, 135-.

determination. Guldberg, C. M. Mth. Ts. 1 (1859) 113-.

- by interpolation, formulø. Lehmann, J. W. H. Grunert Arch. 25 (1855) 237-。

distance problem. Reiss, $M$. Quetelet Cor. Mth. 6 (1830) 368.

distances of point from curve. Bedetti, $G$. Bologna N. Cm. 9 (1849) 455-.

- _ - - - Brenner, -. Grunert Arch. 13 (1849) 167-.

duplication of results. Elliott, E. B. Mess. Mth. 9 (1880) 121-.

elementary. Holmgren, $H$. Ts. Mt. Fys. 1 (1868) 31-, 73-.

-. Phragmén, L. Ts. Mt. Fys. 2 (1869) 201-. of explicit function. Gaudard, J. A. Gén. Civ. 2 (1873) 476-.

Fermat's method, application to tangents and centres of gravity. Duhamel, J. M. C. C. R. 50 (1860) 741-.

of figures, on plane or sphere, or in space. Steiner, J. Crelle J. 24 (1842) 93-, 189-.

- function on closed surface. Stieltjes, T. $\dot{J}$. As. Fr. C. R. (1887) (Pt. 1) 168.

- - of radii vectores from moving point to fixed centres. Lindelöf, L. L. [1866] Helsingf. Acta 8 (1867) 189-. of function $y=\frac{a x^{2}+b x+c}{a^{\prime} x^{2}+b^{\prime} x+c^{\prime}}$. Catalan, E. C. (xiI) Mathesis 2 (1882) 5-

- functions. Turazza, D. A. Sc. Lomb. Ven. 10 (1840) 68-.

- - Lindman, C. F. Stockh. Öfv. 10 (1853) 98-.

- - Björling, E. G. Stockh. Öfv. 10 (1853) 161-.

- - Vashchenko-Zakharchenko, M. E. (xп)

Rec. Mth. (Moscou) 3 (1868) (Pt. 1) 217-.

- - Haluschka, F. Wien Ak. Sb. 83 (1881) (Ab. 2) 1092-

- of 1 real variable. Arzela, C. Palermo G. Sc. Nt. 14 (1879) 55-.

- - - variable. Ermakov, V. P. [1892] Kharkov. Mth. S. Com. 3 (1893) 155-; Fschr. Mth. (1892) 253-.

- - or 2 variables. Walton, W. [1882] Mess. Mth. 12 (1883) 14-.

$\div-1-1$ more variables. Gergonne, J.D. Gergonne A. Mth. 20 (1829-30) 317-.

- - 2 variables. Fisher, A. M. Silliman J. 5 (1822) $82-$

8 (1824) 133-.

B. (v1 Adds.) Silliman J.

- - - - Bouniakowsky, V. [1829]

St Pét. Ac. Sc. Mm. 1 (1831) 463-.

- - - D. D. Camb. Mth. J. 2 (1841) 138 .

- - - - Aldis, W. S. QJ. Mth. 6

(1864) 301-.

- $-1--$ Scheeffer, L. Leip. Mth. Ps. B. 38 (1886) 102-.

- - - - Posse, K. A. Rec. Mth.

(Moscou) 14 (1890) 591-; Fschr. Mth. (1890) 286.

- - - Ermakov, V. P. [1892] Rec.

Mth. (Moscou) 16 (1893) 415-; Fschr. Mth. (1892) 254 .

- - - - Bukrěev, B. Ja. Rec. Mth. (Moscou) 16 (1893) 813-.

- _ - - Dantscher, V. von. Mth. A. 42 (1893) 89-.

- - Vivanti, G. Rm. R. Ac.

Linc. Rd. 7 (1898) (Sem. 1) 240-.

- - - criteria. Walton, W. [1861] QJ. Mth. 5 (1862) 20 -

- - 3 variables. Cayley, A. Camb. and Dubl. Mth. J. 1 (1846) 74-.

- _ - - Bukréev, B. Ja. [1897] Rec. Mth. (Moscou) 20 (1899) 92-; Fschr. Mth. (1897) 250.

- - $n$ variables. Francais, J. F. Gergonne A. Mth. 3 (1812-13) 132-, 197-.

- - - Schubert, F. T. [1815] St Pét. Ac. Sc. Mm. 6 (1818) $267-$.

- - - Brashmann, N. D. (xiI) Mose. Un. Mm. 8 (1835) 131-.

8 (1843) 155.

- - - - Bouniakowsky, V. [1857]

St Pét. Ac. Sc. Mm. 9 (1859) 153-.

_ _ - _. Żmurko, L. [1866] Wien D. 27 (1867) (2te Ab.) 63-.

- - - Williamson, B. QJ. Mth. 12

(1873) 48 . 
of functions of $n$ variables. Walton, $W$. [1882] Mess. Mth. 12 (1883) 42-.

$--\ldots-$ Novikov, P.M. (xII) Kharkov Mth. S. Com. (1883) 43-.

- - - Stolz, O. Wien Ak. Sb. 99 (1891) (Ab. 2a) 495-; 100 (1891) (Ab. 2a) 1167-; 102 (1893) (Ab. 2a) 85-.

- - - Mayer, A. Leip. Mth. Ps. B. 44 (1892) 54-.

- - - Pierpont, J. N. Y. Am. Mth. S. Bll. 4 (1898) 535-.

- - Dantscher, V. von. Mth. A. 51 (1899) 227-.

- _ - - - , application of Hessian determinants. Studnička, $F$. Prag Sb. (1868) 67-.

- - - characteristics. Studnička, $F$. Prag Sb. (1870) 12-.

- $-\ldots-$, tests for distinction. Stolz, 0 . Wien Sb. 58 (1868) ( $A b .2$ ) 1063-.

geometrical application. Schlömilch, $O$. Grunert Arch. 9 (1847) 448-

-, cases. G. Camb. Mth. J. 3 (1843) 237-.

- methods. Alexandrov, I. Fschr. Mth. (1892) 256.

-, and normals to curves and surfaces. Peano, G. Palermo Cir. Mt. Rd. 2 (1888) 189 .

of implicit functions, and laws of reciprocity in ordinary maximum and minimum. Mayer, A. Leip. Mth. Ps. B. 41 (1889) 308-.

indeterminate. Walton, $W$. Camb. Mth. J. 4 (1845) 253-.

interpretation of imaginary roots. Alvord, $B$. Camb. (M.) Mth. M. 2 (1860) 237-.

maxima maximorum, and minima minimorum. Cauchy, A. L. C. R. 17 (1843) 1215-.

maximum solid of given surface and minimum solid of given volume. Babinet, J. C. R. 63 (1866) 361-.

method. Dirksen, E. H. Berl. B. (1838) 145-; Berl. Ab. (1841) 105-.

- ascribed to Fermat. Mansion, $P$. (xII) Mathesis 2 (1882) 193-.

neutral case. Korn, A. [1900] Münoh. Ak. Sb. 30 (1901) 235-.

paradox. Euler, L. [1779] St Pét. Ac. Sc. Mm. 3 (1811) 16-

problems. Canterzani, S. [1808-14] Mod. Mm. S. It. 14 (1809) 167-; 17 (1815) 241-.

-. Mossbrugger, L. Grunert Arch. 2 (1842) 400 -

-. Bonnet, O. N. A. Mth. 2 (1843) 417-; 3 (1844) 64-.

-. Mossbrugger, L. Grunert Arch. 4 (1844) 373-.

-. Grunert, J. A. Grunert Arch. 31 (1858) 476-; 38 (1862) 475-.

- Battaglini, G. Nap. Rd. 2 (1863) 56-.

- Grunert, J.A. Arch. Mth. Ps. 49 (1869) 68 -.

-. Enneper, A. Z. Mth. Ps. 25 (1880) 41-.

-. Langley, E. M. Nt. 37 (1888) 605.

- Markov, A. A. Kharkov Mth. S. Com. 1 (1889) 250-; Fschr. Mth. (1889) 267-.

- to find $x$ so that $\sin x^{2} \sin (\theta-x)$ is maximum or minimum. Grunert, $J_{.}$. . Grunert Arch. 26 (1856) 354-. problems, geometrical. Sturm, J. C. F. Gergonne A. Mth. 14 (1823-24) 108-.

-,-.. Liouville, J. Liouv. J. Mth. 7 (1842) 163-.

-, - Thue, A. Sk. Nf. F. (1892) 352-; Fschr. Mth. (1892) 259.

-, -, class. Schell, W. Grunert Arch. 19 (1852) 450-.

-, sphere touching cone. Alvord, - . Smiths. Misc. Col. 33 (1888) Art. 1, 149. (Wash. Ph. S. Bll. 6 (1884).)

-, - - - Farquhar, H. Smiths. Misc. Col. 33 (1888) Art. 1, 152. (Wash. Ph. S. Bll. 6 (1884).)

- in turning and planing. Tobell, J. Civing. 35 (1889) 569-.

question propounded by Čebyšev. Markoff, $A$. Acta Mth. 9 (1887) 57-.

questions. Gelin, (l'abbé) E. (xII) Mathesis 2 (1882) 164-.

relative, application of Euler's principle. Zimmermann, W. A. Fschr. Mth. (1899) 344-.

-, - - - Sleszinski, J.W. Fschr. Mth. (1899) 344, 345.

-, Euler's and Lagrange's methods. Strauch, G. W. Grunert Arch. 4 (1844) 39-.

-, new point of view. Kummell, C. $H$. A. Mth. 4 (1888) 33-.

of stationary values. Nanson, $E$. J. Mess. Mth. 25 (1896) 129-.

Steiner's theorems. Sturm, $R$. Crelle J. Mth. 96 (1884) 36-.

of sum of powers of distances of point from system of points, lines, or planes. Wetzig, $F$. Crelle J. 62 (1863) 346-.

- sums of function and its derivatives. Čeby̌̌ev, P. [1867] ' St Pet. Ac. Sc. Mm. (Rs.) 12 ("1868) (Suppl.) No. 3, 74 pp.; Liouv. J. Mth. 13 (1868) 9-.

- symmetric function of several variables. Bouniakowsky, $V$. St Pét. Ac. Sc. Bll. 12 (1854) 353-.

without Taylor's theorem. Hill, E. Mess. Mth. 5 (1876) 84-.

theorem. Purkiss, H.J. Mess. Mth. 1 (1862) 180-.

-. Walton, W. [1869] QJ. Mth. 10 (1870) 253-.

-. Cayley, A. QJ. Mth. 10 (1870) 262-.

-, demonstration. Adams, Jas. Thomson A. Ph. 10 (1817) 430-.

theory. Spitzer, S. Grunert Arch. 22 (1853) 183-.

-. Björling, E. G. Ștockh. Öfv. 13 (1856) 83-.

-. Richelot, F.J. As. Nr. 48 (1858) 272-.

-. Brenner, -. Grunert Arch. 35 (1860) 157-.

Transon, A. Par. Éc. Pol. J. Cah. 42 (1867) 205-.

-. Unferdinger, $F$. Arch. Mth. Ps. 53 (1871) 15-.

-. Gruber, J. Arch. Mth. Ps. 60 (1877) 415-.

-. Mayer; A. Leip. Mth. Ps. B. 41 (1889) $122-$. 
Maxima.

circle, point and diameter. Lanavicensis (Sylvester, J. J.). Mess. Mth. 2 (1863) 100-, 168.

elementary method. Grillet, H. N. A. Mth. 9 (1850) 70 -

of factorial functions. Cayley, A. Mess. Mth. 2 (1873) 129-.

geometrical question. Vachette, A. N. A. Mth. 5 (1846) 45-.

maximum maximorum of function $\psi\left(r c^{\phi i}\right)$ in reference to $\phi$. Nekrassoff, $P$. Mth. A. 31 (1888) 337-.

problem. Dienger, $J$. Grunert Arch. 14 (1850) 221-.

-. Rodenberg, C. Z. Mth. Ps. 24 (1879) 63-.

-. Gilbert, L. P. (xir) Mathesis 3 (1883) 200 -

of a product. Deladérềre, A. N. A. Mth. 3 (1844) 165-.

- - Tychsen, C. Mth. Ts. 1 (1859) $22-$.

question. Serret, $P$. N. A. Mth. 2 (1863) 79-.

questions, two. Lebergue, V.A. N. A. Mth. 2 (1863) 433-.

relative, question. Ostrogradsky, - - (vIII) St Pét. Ac. Sc. Bll. 8 (1841) 327-.

theorem. Terquem, $O$. N. A. Mth. 5 (1846) 207-.

value of $\sqrt[x]{x}$ and theorems in connection. Oettinger, L. Arch. Mth. Ps. 42 (1864) 106-.

of $\frac{\sin x}{x}$. Bacaloglo, E. Grunert Arch. 36 (1861) 12-.

Minima.

case. Korkine, A., \& Zolotareff, G. N. A. Mth. 12 (1873) 337-.

geometrical, determination of remarkable. Cellerier, C. Bll. Sc. Mth. 15 (1891) 167-.

of linear function of irrational quantity, Lagrange's theorem, demonstration. Sylvester, J. J. C. R. 53 (1861) 1267-; 54 (1862) $53-$.

minimum area cut off by normal to ellipse. Lemoine, E. Fschr. Mth. (1893-94) 470.

- - of polygon circumscribing re-entering curve. Goodwin, $H$. Camb. and Dubl. Mth. J. 3 (1848) 181-.

- distance. Cinger, V. Ja. [1891] Rec. Mth. (Moscou) 16 (1893) 317-.

- - Fermat's problem. Callegari, P. Bologna N. Cm. 4 (1840) 309-.

- quantities. Posse, K. [1880] St Pet. Ac. Sc. Mm. (R8.) 38 (*1881) (Suppl.) No. 3, $31 \mathrm{pp}$.

- straight line, problem. Roche, - N. A. Mth. 2 (1843) 488-.

problems. Dilettant (Pseud.). Mth. Ts. 5 (1863) 17-.

- Tychsen, C. (xir) Ts. Mth. 1 (1871) problems. Hoppe, $R$. Arch. Mth. Ps. 62 (1878) 215-.

-. Bruns, $H$. Mth. A. 20 (1882) 455-.

-. Markov, A. A. Kharkov Mth. S. Com. (1884) 83-; Fschr. Mth. (1885) 369.

-. Millar, W. J. Edinb. Mth. S. P. 7 (1889) 27 -

-, elementary solution. Tychsen, C. Mth.

Ts. 4 (1862) 106-.

- geometrical. Gruson, J. P. Berl. Ab. (1816-17) 11-.

-, - Neovius, $E$. Mth. A. 31 (1888) 359-.

-, railway construction. Pollack, $V$. Förster Al. Bauztg. 51 (1886) 45-, 53-.

-, sum of distances from vertices of tetrahedron. Lindelöf, L. L. Les Mondes 10 (1866) 691-.

questions. Puissant, L. Par. Ec. Pol. Cor. 1 (1804-08) 430-.

-. Liouville, J. Liouv. J. Mth. 1 (1856) 7-.

-. Posse, C. Mth. A. 26 (1886) 593-.

- in connection with approximate representation of functions. Tchebicheff, $P$. [1857] St Pét. Ac. Sc. Mm. 9 (1859) 201-.

- of transport. Dupain, J. C. N. A. Mth. 13 (1854) 141-.

theorem for straight lines in space. Hossard, P., \& Poudra, -. N. A. Mth. 9 (1850) 241-.

in triangle, theorem. Poudra, -. N. A. Mth. 7 (1848) 407-.

Mean value, theorem. Bruno de Cabedo, J. G. Teix. J. Sc. 9 (1889) 129-; Fschr. Mth. (1889) 234

Numerical identities discovered by means of differentiation and integration. Minin, A.P. Mose. S. Sc. Bll. 78 (No. 1) (1892) 22-; Fschr. Mth. (1892) 187.

Partial differences calculus applied to analytical problems. Sarrus, F. Gergonne A. Mth. 10 (1819-20) 33-.

Prices, laws. Launhardt, - Hann. Archt.Vr. Z. 32 (1886) 293-.

Quadratic irrationals for representation of function. Pincherle, S. Mil. I. Lomb. Rd. 23 (1890) 373-.

Railway fares, best scale. Launhardt, Hann. Archt.-Vr. Z. 37 (1891) 113-.

Remainder in expansion of $(1+x)^{-n}$, and theorem on products of squares. Young, J.R. Ph. Mg. 34 (1849) 113-.

Repeated differentiation, formulæ. Chrystal, G. Edinb. Mth. S. P. 3 (1885) 94-.

Rodrigues's theorem. Hudson, W. H. H. [1877] Mess. Mth. 7 (1878) 117.

- - Glaisher, J. W. L. [1879] Mess. Mth. 9 (1880) 55-.

- - two demonstrations. Walton, W. QJ. Mth. 15 (1878) 335-.

Series, new, for $f\{a+\phi(x+h, y)\}$. Murphy, R. Ph. Mg. 10 (1837) 28-.

Sum of $n$th powers, in powers of $n$. Arndt, $F$. Crelle J. 31 (1846) 249-.

Summation, approximate, of series of differential coefficients. Hill, C.J.D. Crelle J. 5 (1830) 319-. 


\section{TAYLOR'S SERIES.}

S., W. Tilloch Ph. Mg. 29 (1807) 211-. Adams, Jas. Thomson A. Ph. 15 (1820) 99-. Wilmot, E. QJ. Sc. 17 (1824) 74-.

De Morgan, A. (vi Adds.) Ph. Mg. 7 (1835) 188-.

Liouville, J. Liouv. J. Mth. 2 (1837) 483-. Smith, Arch. Camb. Mth. J. 1 (1839) 143-. Caqué, J. Liouv. J. Mth. 10 (1845) 379-. Schlömilch, O. N. A. Mth. 11 (1852) 177-. Mainardi, $G$. Mil. At. I. Lomb. 1 (1858) 90-. (Mainardi.) Bellavitis, G. Ven. At. (1857-58) 623-; (1858-59) 334-.

Roche, É. Mntp. Ac. Sc. Mm. 4 (1858-60) 125-.

Schlömilch, O. Liouv. J. Mth. 3 (1858) 384-. Turquan, -. N. A. Mth. 2 (1863) 19-.

Roche, $\hat{E} . \quad$ C. R. 58 (1864) 379-.

Wilkinson, M. M. U. Mess. Mth. 1 (1872) 36-, 135-.

Picart, A. N. A. Mth. 13 (1874) 15-, 353-.

Amigues, É. N. A. Mth. 19 (1880) 105-.

Bruno de Cabedo, J. G. Teix. J. Sc. 7 (1886) 145-.

Peano, G. Tor. Ac. Sc. At. 27 (1892) 40-.

Borel, É. C. R. 123 (1896) 1051-; Acta Mth. 21 (1897) 243-.

Fabry, E. C. R. 124 (1897) 142-; 125 (1897) 1086-.

analogue with operator $K=x d y / y d x$. Zehfuss, $G$. Grunert Arch. 30 (1858) 465-.

analytic continuation. Borel, -. Par. S. Mth. Bll. 28 (1900) 200.

and analytic functions with restricted existence domain. Pringsheim, A. [1892] Mth. A. 42 (1893) 153-.

application. Poisson, S. D. Par. Ec. Pol. Cor. 2 (1809-13) 81-.

- Bergsten, N. J. Stockh. Ac. Hndl. 35 (1814) 135-.

-, and some definite integrals. Mildner, $R$. Arch. Mth. Ps. 9 (1890) 285-.

and application to resolution of algebraic fractions. Schellbach, C. H. Crelle J. 11 (1834) 274-.

applications, etc. Buquoy, G. von. Gilbert A. 44 (1813) 106-; Oken Isis (1826) 244-, 666-; (1827) 801-.

with approximation of third degree. Bougaief, N. C. R. 122 (1896) 369-

as basis of calculus of functions. Poselger, F. T. Berl. Ab. (1835) 311-.

and binomial expansion, with other applications. Gourieff, S. [1799] St Pet. Ac. Sc. N. Acta 14 (1805) 306-.

conditions for functions of a real variable. Pringsheim, A. Mth. A. 44 (1894) 57-.

convergence. Tchebicheff, $P$. Crelle J. 28 (1844) 279-.

-. Lamarle, E. Brux. Ac. Bll. 13 (1846) 724-. -, boundary, determination. Marie, $M$. C. R. 75 (1872) 469-; Liouv. J. Mth. 18 (1873) 68-, 185-.

- region. Marie, M. C. R. 60 (1865) 1085-; 74 (1872) 1485-; Liouv. J. Mth. 18 (1873) $53-$. convergence region, report on Marie's memoirs. Puiseux, V. C. R. 76 (1873) 618-.

deduction. Ettingshausen, A. von. Wien SB. (1848) 238-.

-. Echols, W. H. A. Mth. 8 (1893-94) 62-

- and demonstration. Echols, W. H. Am. J. Mth. 15 (1893) 283-.

deductions. Fais, A. G. Mt. 12 (1874) 148-.

demonstration. Poisson, S. D. Par. Éc. Pol. Cor. 1 (1804-08) 52-.

- Schubert, F. T. St Pét. Ac. Sc. Mm. 7 (1820) 71-.

-. Wilmot, E. QJ. Sc. 16 (1823) 228-.

-. Cox, H. Camb. and Dubl. Mth. J. 6 (1851) 80 -

-. König, J. N. A. Mth. 13 (1874) 270-.

-. Maggi, G. A. Mil. I. Lomb. Rd. 19 (1886) 217-, 888.

-. Jekel, A. N. Arch. Wisk. 1 (1895) 201-; Fschr. Mth. (1893-94) 389-.

-, Carpenter's. Muirhead, R. F. Edinb. Mth. S. P. 12 (1894) 114-.

-, elementary. Mansion, P. Mess. Mth. 8 (1879) 17-.

-, Lagrange's. Cayley, A. Mess. Mth. 1 (1872) 22-, 105-, 137.

-, simplified. Hatzidakis, J. N. Ens. Mth. 2 (1900) 447-.

demonstrations. Johnson, W. W. Mess. Mth. 2 (1873) 180-

-, criticism. Bonnycastle, C. [1840] Am. Ph. S. T. 7 (1841) 217-.

derivation from a transcendental function. Buquoy, G. von. Oken Isis (1826) 666-

expansions of uniform functions. Borel, ${ }^{\prime}$. C. R. 127 (1898) 751.

extension. Glashan, J. C. Am. J. Mth. 1 (1878) 287-.

of Hadamard's theorem to. Leau, -. Par. S. Mth. Bll. 26 (1898) 267-.

formulø derived from. Buquoy, G. von. Gilbert A. 44 (1813) 106-.

for functions of complex variable. Mansion, $P$. [1878] Mess. Mth. 8 (1879) 17-.

- - - - Ź Zmurko, W. Par. T. Nauk Śc. Pam. 11 ( $\left.{ }^{*} 1879\right)$ Art. 4, 150 pp.

functions defined by. Leau, L. C. R. 128 (1899) 804-.

- - Hadamard, J. Liouv. J. Mth. 8 (1892) 101-.

generalisation. Roche, $\dot{E}_{\text {. }}$ (vi Adds.) Mntp. Ac. Sc. Mm. 5 (1861-63) 419-; (vII) Liouv. J. Mth. 9 (1864) 129 -

-. Mansion, $P$. Mess. Mth. 4 (1875) 161-.

-. Sylvester, J. J. Ph. Mg. 4 (1877) 136-

-. Mittag-Leffler, G. Gött. Nr. (1900) 194 . generalised. Schlömilch, $O$. Schlömilch Z. 2 (1857) 269-; Leip. Mth. Ps. B. 31 (1879) 27-.

history. Pringsheim, A. Bb. Mth. 1 (1900) 433-.

illustration. Forbes, G. Mess. Mth. 2 (1873) 106-.

impossibility of continuation in certain cases. Méray, C. Bll. Sc. Mth. 12 (1888) 248-. with infinite number of singular points. Fabry, E. Acta Mth. 22 (1899) 65-. 
investigation. Strong, T. Silliman J. 45 (1843) 269-.

and Lagrange's series. Picart, A. N. A. Mth. 13 (1874) 69 -

- _ - , successive approximation. Bugaev, N. V. [1895] Rec. Mth. (Moscou) 18 (1896) 586-; Fschr. Mth. (1896) 70, 71-.

- its limit. Whitcom, A. W. Des Moines Anal. 4 (1877) 137-.

limits. Poisson, S. D. Liouv. J. Mth. 3 (1838) 4-.

and Maclaurin's series. Bourget, J. N. A. Mth. 9 (1870) 537-.

- - - analogues to. Clapeyron, E., \& Lamé, G. Crelle J. 6 (1830) 40-.

--- , combination. Young, J.R. Ph. Mg. 32 (1848) 98-.

- - - , convergence. Lamarle, $E$. Liouv. J. Mth. 12 (1847) 305-.

- - -, limits. Garnier, J. G. Quetelet Cor. Mth. 2 (1826) 89-.

phoronomic interpretation. Möbius, $A . F$. Leip. B. 1 (1846) 79-.

practical forms and nautical applications. Rouyaux, J. A, Rv. Mar. et Col. 56 (1878) 127-, 440-.

- reduced forms. Rouyaux, J.A. C. R. 84 (1877) 1014-.

property of coefficients. Harnack, A. Mth. A. 13 (1878) 555-.

region of possible summation. Borel, $E_{\text {. }}$ C. R. 123 (1896) 548-.

remainder. Ampère, A. M. Par. Éc. Pol. J. 6 (1806) 148-; Gergonne A. Mth. 17 (1826-27) $317-$.

-. Jürgensen, C. Crelle J. 17 (1837) 291-.

-. Stamkart, F. J. Amst. Vs. Ak. 8 (1858) 65-. - ${ }_{291-}$

-. Jürgensen, C. N. A. Mth. 19 (1860) 308-.

-. Roche, É. N. A. Mth. 19 (1860) 311-.

-. Winckler, A. [1867] Wien D. 28 (1868) (Ab. 1) 243-.

-. $D-g$. Ts. Mt. Fys. 2 (1869) 74-.

- Glashan, J. C. Am. J. Mth. 4 (1881) $282-$.

- Mansion, P. Brux. Ac. Bll. 10 (1885) 846-.

- Bruno de Cabedo, J. G. Teix. J. Sc. 10 (1891) 13-; Fschr. Mth. (1890) 253.

-. Ocagne, M. d'. Mathesis 11 (1891) 19-.

- Morera, G. Rv. Mt. 2 (1892) 36.

- Amigues, E. N. A. Mth. 12 (1893) 88-.

-, and binomial theorem. Mansion, $P$. Brux. Ac. Bll. 8 (1884) 183-.

-, development. Cauchy, A. L. C. R. 13 (1841) 842-.

-, form. Reynaud, -. N. A. Mth. 2 (1863) 271-.

-, - , for functions of imaginary variable. Mansion, $P$. Mathesis 6 (1886) 101-.

-, new form. Peano, G. Mathesis 9 (1889) 182-.

-, - - Somin, N. Ja. Vars. S. Nt. Tr. (Mm.) 2 (1892) No. 4, 17 pp.

remainders. Crelle, A.L. Berl. Ab. (1828) 1-. and series of Cayley and Lagrange, simple method of obtaining. Glashan, J. C. Am. J. Mth. 4 (1881) 277-.

singular points. Fabry, $E$. Liouv. J. Mth. 4 (1898) 317-.

- - and Euler's transformation of a function defined by. Lindelöf, E. C. R. 126 (1898) 632 .

- of a function defined by. Le Roy, - . C. R. 127 (1898) 948-.

-----1 Servant, -. C. R. 128 (1899) 80-.

singularities. Borel, $E_{\text {. }} \quad$ Par. S. Mth. Bll. 26 (1898) 238-.

- of a function defined by. Borel, $\dot{E}$. C. R. 127 (1898) 1001-.

- - - - - Leau, L. Liouv. J. Mth. 5 (1899) 365-.

summation, and definite integrals. Vernier, $H$. Gergonne A. Mth. 15 (1824-25) 165-.

- , - - Gergonne, J. D. Gergonne A. Mth. 15 (1824-25) 360-.

symbolic statement, generalisation. Graves, $C$. Ir. Ac. P. 5 (1853) 285-.

Taylor-Maclaurin series, conditions, Pascal, E. Rv. Mt. 5 (1895) 37-.

transformation. Jourjon, -. C. R. 78 (1874) 493.

transformed. Bougaief, N. U. C. R. 121 (1895) 1127-.

treating the circle of convergence as section. Borel, $\dot{E}$. Liouv. J. Mth. 2 (1896) 441-.

trigonometric series deduced from. Pfaff, $J$. W. [1808] St Pét. Ac. Sc. Mm. 3 (1809-10) 108-. validity, conditions. König, G. [1883] (xI) Mth. Term. Ets. 2 (1884) 42-; Mth. A. 23 (1884) 450-.

-, -, for real variables. Pringsheim, $A$. D. Mth. Vr. Jbr. 3 (1894) 82-.

-, range. Du Bois-Reymond, $P$. Münch. Ak. Sb. 6 (1876) 225- ; Mth. A. 21 (1883) 253-.

with zero convergence-radius. Borel, $\dot{E}$. C. R. 128 (1899) 1281-.

Tisserand's theorem (expansion of

$$
\begin{aligned}
T=\left[\left(x_{1}-c_{1}\right)^{2}\right. & +\left(x_{2}-c_{2}\right)^{2} \\
& \left.\left.+\left(x_{3}-c_{3}\right)^{2}+\left(x_{4}-c_{4}\right)^{2}\right]^{-1}\right) .
\end{aligned}
$$

Stieltjes, T. J. (jun.). C. R. 95 (1882) 901-, 1043-.

$n$th total derivative of function of any number of variables, general value. Branford, B.B.P. Mess. Mth. 28 (1899) 169-.

Total derivative of functions. Bettazzi, $R$. G. Mt. 26 (1888) 21-.

Transcendental functions, approximation - formulæ. Buzengeiger, K. H.J. Zach M. Cor. 24 (1811) 583-.

Transformation of system of independent variables. Fields, J. C. Am. J. Mth. 14 (1892) 230-.

Utility equation, form. Francke, A. Hann. Archt.-Vr. Z. 38 (1892) 547-.

Wronski's problem. Lagrange, C. Brux. Obs. A. $($ As.) 7 (1896) $40 \mathrm{pp}$. theorem. Cayley, A. QJ. Mth. 12 (1873)
221-. 
Wronski's theorem. Transon, A. É.L. N. A. Mth. 13 (1874) 305-; Par. T. Nauk Śc. Pam. 8 (*1876) Art. 8, 8 pp.

- - Cayley's demonstration. Transon, A.É.L. N. A. Mth. 13 (1874) 161-.

- -, demonstration. Źurakowski, S. Krk. Ak. (Mt.-Prz.) Pam. 14 (1888) 56-; Fschr. Mth. (1888) 250.

$\frac{F(x)+f(x+h)-f(x)}{F_{1}(x)+f_{1}(x+h)-f_{1}(x)}=\frac{F(x)+h f^{\prime}(x+\theta h)}{F_{1}(x)+h f_{1}^{\prime}(x+\theta h)}$, where $0<\theta<1$. Arez, J. G. Teix. J. Sc. 11 (1892) 187-; Fschr. Mth. (1893-94) 463.

$f\left(e^{a x}\right)$, general term of series for. Petrovitch, $M$. Palermo Cir. Mt. Rd. 14 (1900) 22-.

\section{Principles and elements of} the integral calculus. Approximate integration. Mechanical quadrature.

Algebraic integrals. Nicolai, F.B. G. As. Nr. 5 (1827) 376- ; 6 (1828) 79-.

- - of algebraic differentials, determination. Zeuthen, H. G. C. R. 90 (1880) 1114-.

Amsler's planimeter, theory. Thiré, A. A. Mines 11 (1887) 121-.

Analysis, formula. Bassani, A. G. Mt. 25 (1887) 225-.

-, formulæ, two. Bruno de Cabedo, J. G. Teix. J. Sc. 9 (1889) 129-; Fschr. Mth. (1889) 234.

Approximate calculation of integrals of higher orders. Bellavitis, G. (vi Adds.) Ven. Mm. I. 6 (1856) $91-$.

- formula for measurement of area. PetitBois, G. Rv. Un. Mines 27 (1894) 135-.

- value of area of segment of curve. Hermite, Kazan S. Ps.-Mth. Bll. 8 (1899) 109.

Approximation formula. Thiele, T. N. (xir) Ts. Mth. 3 (1873) 22-.

Asymptotic functions. Sonin, N. Ja. [1889] Vars. S. Nt. Tr. (1889-90) (C. R., Ps. C.) No. 3, 1-.

Change of variables. Cauchy, A. L. C. R. 23 (1846) 382-.

- - - in calculation of integrals. Saltel, $L$. [1881] Bordeaux S. Sc. Mm. 4 (1882) 451-.

- _ - integrals. Schlömilch, O. Grunert Arch. 18 (1852) 391-.

Convergence of integrals with non-vanishing arguments. Du Bois-Reymond, P. [1877] Mth. A. 13 (1878) 251-.

Differential equation for Milner's lamp. Tait, P. G. Edinb. Mth. S. P. 5 (1887) 97-.

- - - - Cayley, A. Edinb. Mth. S. P. 5 (1887) 99-.

- and integral calculus, notations. $J ., J$. Ph. Mg. 24 (1844) 25-.

Differentiation of integral with respect to upper limit. Thomae, J. Gött. Nr. (1893) 696-.

- and integration of real function with one independent variable. Gosiewoski, $W$. Par. T. Nauk Śc. Pam. $12\left({ }^{*} 1882\right)$ Art. 3, 15 pp. -, repeated, under sign of integration. Schlömilch, O. Z. Mth. Ps. 15 (1870) 207-.
Differentiation under sign of integration. Werner, O. Grunert Arch. 18 (1852) 39-. $--1-$ Kapteyn, W. N. Arch. Wisk. 4 (1899) 245-.

Difficult integrals. Euler, L. [1780] St Pét. Ac. Sc. Mm. 6 (1818) 30-.

- - soluble by other means. Euler, $L$. [1777] St Pet. Ac. Sc. N. Acta 14 (1805) 62 .

Discontinuous function $(x)$ and applications. Sonin, N. Ja. Vars. S. Nt. Tr. (Mm.) 1 (1891) No. 5, $78 \mathrm{pp}$.

Distributive operations ; successive integration. Pincherle, S. Rm. R. Ac. Linc. Rd. 5 (1896) (Sem. 1) 236-.

Euler's "Integral Calculus," problems in. Coutinho; R. de S. [1806] Turin Mm. Ac. 2 (1805-08) 95-.

_"—_." Vol. Iv, Suppl. 4, Sec. 2. Farini, G. Padova N. Sag. 2 (1825) 249-.

Exponential integral $\frac{x e^{x}}{(1+x)^{2}}$. Wolfers, J.P. Grunert Arch. 20 (1853) 247-.

- - (Wolfers). Lindman, C. F. Grunert Arch. 23 (1854) 445-.

Expressions, integrals of which are algebraic. Liouville, J. [1832-37] Par. Ec. Pol. J. 22 cah. (1833) 124-, 149-; Crelle J. 10 (1833) 347-; C. R. 5 (1837) 330-.

Farm, mean distance from farm yard. Wasmund, C. Grunert Arch. 13 (1849) 96-.

Finite integral of rational algebraic function. Orchard, W. Assur. Mg. [1 ("1851)] 9 (bis)-.

Formula, Brinkley's, for Lagrange's summation. Herschel, (Sir) J. F.W. Phil. Trans. (1860) 319-.

- of Euler,

$$
\begin{aligned}
\int \phi x d x=h \Sigma \phi x & +\frac{h}{2} \cdot \phi x-\beta_{1} \phi^{\prime} x \cdot \frac{h^{2}}{1.2} \\
& +\beta_{2} \phi^{\prime \prime \prime} x \cdot \frac{h^{4}}{1.2 .3 .4}-\ldots
\end{aligned}
$$

Rodrigues, J. M. G. Teix. J. Sc. 3 (1881) 157-.

-, general, of Cauchy. Kapteyn, W. Bll. Sc. Mth. 16 (1892) 270-.

Fresnel's integrals, theory. Lindstedt, $A$. A. Ps. C. 17 (1882) 720-.

Functional relation

$$
\left.\begin{array}{l}
u=x \sin x+\cos x \\
v=\sin x-x \cos x
\end{array}\right\}
$$

Strekalov, V. Kazan S. Ps.-Mth. Bll. 3 (1893) (Prot.) 58-.

- - Zejliger, D. Kazan S. Ps.-Mth. Bll. 3 (1893) (Prot.) 64-.

Functions, first derivative of which is of zero class. Ascoli, G. Mil. I. Lomb. Rd. 12 (1879) 337-.

Hohmann-Coradi integrator. Reitz, $F$. $H$. Hamb. Mth. Gs. Mt. 1 (1889) 48-.

Integrability in finite form of

$$
\sqrt[m]{\left(x-a_{1}\right)^{n_{1}} \ldots\left(x-a_{\lambda}\right)^{n_{\lambda}}}
$$

Ptaszycki, -. Bll. Sc. Mth. 12 (1888) 262-. - of functions. Peano, G. [1883] Tor. Ac. Sc. At. 18 (1882) 439-. 
Integrability of functions, altered form of condition for. $D u$ Bois-Reymond, P. [1874] Crelle J. Mth. 79 (1875) 259-.

- - conditions. Mainardi, G. Mod. S. It. Mm. 1 (1862) 79-.

- - criteria. Brioschi, F. [1853] Mod. Mm. S. It. 25 (1855) 205-.

- - of integrable functions, theorem. Du Bois-Reymond, P. [1881] Münch. Ak. Sb. 12 (1882) 240-.

-, general theorem. Du Bois-Reymond, $P$. [1879] Mth. A. 16 (1880) 112.

- of a series of functions. Giuliani, G. G. Mt. 24 (1886) 44-, 333.

\section{INTEGRAL CALCULUS.}

Lorgna, A. M. Verona S. It. Mm. 2 (1784) 177-.

Soldner, J. Gilbert A. 39 (1811) 231-.

Cauchy, A. L. [1824] Par. Mm. Ac. Sc. 22 (1850) 39-.

Franchini, $P$. Lucca At. Ac. 4 (1828) 141-.

Talbot, W. H. F. Phil. Trans. (1836) 177-; (1837) 1 -

De Morgan, A. [1851] Camb. Ph. S. T. 9 (1856) 107-.

Brassinne, E. Toul. Ac. Sc. Mm. 6 (1874) 599-.

Stolz, O. Innsb. Nt. Md. B. 5 (1875) 24-.

Lerch, M. Časopis 22 (1893) 298- ; Fschr. Mth. (1893-94) 488.

algebraic analogies. Brassinne, E. Toul. Mm. Ac. 6 (1850) 14-; 1 (1851) 290-; N. A. Mth. 12 (1853) 82-.

application of inequalities. La Vallée-Poussin, C. de. Brux. S. Sc. A. 16 (1892) (Pt. 1) 8-. applications, geometric. Roberts, $W$. Liouv. J. Mth. 15 (1850) 209-

- of geometry to. Laguerre, E. (x) Par. S. Phlm. Bll. 4 (1867) 81-.

arithmetical and geometrical, difference between. Unferdinger, $F$. Wien Sb. 56 (1867) (Ab. 2) 272-。

Condorcet's treatise. Ferroni, $P$. Verona $\mathbf{S}$. It. Mm. 5 (1790) 130-.

direct method. Simonoff, I. Zach Cor. 11 (1824) 438-.

Du Bourguet's theorem, confutation. Sarmento, J. L. Coimbra I. 6 (1858) 179-.

Euler's approximate formula. Imšeneckij, $V . G$. St Pet. Ac. Sc. Mm. (Rs.) 62 (1890) 45-.

exercises in. Cockle, (Sir) J. [1870-81] Mess. Mth. 1 (1872) 5-, 64-; 3 (1874) 108-; 4 (1875) $11-, 25-, 150-$; 5 (1876) 150-; 6 (1877) 116-; 10 (1881) 15-.

extensions. Grunert, J.A. Grunert Arch. 18 (1852) 241-.

formula. Brioschi, F. Nap. Rd. 3 (1864) 63-. -. Didon, F. Par. Ec. Norm. A. 2 (1873) 31-.

- in attraction theory. Morera, G. Palermo Cir. Mt. Rd. 10 (1896) 75-.

formulœ. Serret, J. A. Liouv. J. Mth. 8 (1843) 1-.

-. Cayley, A. Liouv. J. Mth. 12 (1847) formulæ. Dantscher von Kollesberg, $V$. Mh. Mth. Ps. 1 (1890) 92-.

fundamental principle, want of generality. Gosiewski, W. [1882] (xI) Krk. Ak. (Mt.Prz.) Rz. \& Sp. 10 (1883) 141-, LII.

- theorem $\int_{a}^{b} F^{\prime}(x) d x=F(b)-F(a)$, demonstration. Du Bois-Reymond, P. [1879] Mth. A. 16 (1880) 115-.

paradoxes. Brougham and $\operatorname{Vaux},($ Lord $)$. C. R. 44 (1857) 1134-, 1177-.

principles. Volterra, V. G. Mt. 19 (1881) 333-. problems. Fuss, $N$. St Pét. Ac, Sc. Mm. 5 (1815) 177.

-. Catalan, E. C. Liouv. J. Mth. 6 (1841) 340-, 419-.

-. Le François, (Prof.) -. Brux. Mm. Cour. 4 ', 22 (1846-47) 24 pp.

- Roberts, W. Tortolini A. 2 (1851) 150-.

-. Serret, J. A. Par. Éc. Norm. A. 6 (1869) 177-

-. Petit-Bois, G. (xm) Mathesis 2 (1882) 233-.

- Mansion, P. (xII) Mathesis 2 (1882) 236-.

-. Ball, L. de. As. Nr. 126 (1891) 333-.

relations to geometry. Meyer, $W . F$. Ens. Mth. 1 (1899) 343-.

Talbot's researches, and more general solution. Peirce, B. Mth. Misc. 1 (1838) 404-.

theorems. Fuss, N. [1806-22] St Pét. Ac. Sc. Mm. 4 (1813) 205-; 11 (1830) 268-.

- Cauchy, A. L. C. R. 13 (1841) 1087-, 1130- ; 21 (1845) 407-

- Brassinne, E. C. R. 43 (1856) 1190-.

-. Sylvester, J.J. (vIr) Ph. Mg. 26 (1863) 293-.

-. Winckler, A. Wien. Az. 1 (1864) 92-.

- Bierens de Haan, D. B. A. Rp. 37 (1867) (Sect.) 4-.

-. Crofton, M. W. C. R. 68 (1869) 1469-.

- Besge, V. A. Liouv. J. Mth. 19 (1874) 423-.

-. Ascoli, G. Mil. I. Lomb. Rd. 12 (1879) 215-.

-, arithmetical proof. Smith, H.J.S. L. Mth. S. P. 4 (1871-73) 249-.

-, elementary. Fergola, E. Nap. Rd. 3 (1864) 256-.

-, extension. Liouville, J. C. R. 42 (1856) 985-.

- required in. Grunert, J. A. Arch. Mth. Ps. 45 (1866) 279-.

use of continued fractions. Kausler, $C . F$. [1803] St Pét. Ac. Sc. Mm. 1 (1809) 181-.

- - imaginaries. Euler, L. [1777] St Pet. Ac. Sc. N. Acta 12 (1801) 3-.

Integral of Euler. Lerch, - Bll. Sc. Mth. 16 (1892) 337-.

- in Euler's "Theoria motus corporum." Wolfers, J. P. Grunert Arch. 14 (1850) 111-. - forms. Schlömilch, O. Schlömilch Z. 6 (1861) 205-; 7 (1862) 262-.

- formula. Silva, J. A. Martins da. [1878] G. Teix. J. Sc. 2 (1880) 167-.

- Beltrami, E. Mil. I. Lomb. Rd. 12 (1879) 421-. 
Integral formula, general. Tralles, $J . G$ [1805] Berl. Ab. (1804-11) (Mth.) 85.

- formulæ. Frullani, G. A. Sc. Lomb. Ven. 2 (1832) 257-.

- - general. Schlömilch, O. Z. Mth. Ps. 10 (1865) 152-.

- - values of which can be expressed by quadrature of circle. Euler, L. Bll. Sc. Mth. As. 4 (1880) 207-.

- logarithm, constant. Popper, J. Wien Sb. $52(1866)(A b .2) 527-$.

- of product of rational function and logarithm of such function. Besso, D. G. Mt. 25 (1887) 356-.

- - rational fraction, transcendent part. Duarte Leite, -. G. Teix. J. Sc. 7 (1886) 180 -.

- in theory of trigonometric series, limiting value. Du Bois-Reymond, P. Bll. Sc. Mth. As. 3 (1879) 343-.

Integrals. Tait, P. G. QJ. Mth. 3 (1860) 364-.

- Lommel, E. Grunert Arch. 38 (1862) 206-.

-. Skrivan, G. Schlömilch Z. 8 (1863) 303-.

-. Lindman, C. F. Stockh. Öfv. 39 (1882) No. 8, 3-.

-, application of geometry. Lobatschewsky, N. A. (vi Adds.) Kazan Mm. Un. (1836) cah. 1, 1-.

- with closed curve of integration. Pochhammer, L. Mth. A. 37 (1890) 500-

- common to several dynamical problems. Bertrand, J. [1851-56] Liouv. J. Mth. 17 (1852) 121-; C. R. 43 (1856) 829-.

- - - - Brioschi, F. Mil. G. I. Lomb. 8 (1856) 413-; 9 (1856) 110-.

- _ - - - Mainardi, G. Mil. G. I. Lomb. 9 (1856) 222-.

- - - Padova, E. Ven. I. At. 1 (1882-83) 1005-.

-, conversion. Cockle, (Sir) J. Ph. Mg. 33 (1867) 537-; 34 (1867) 442-.

-, definition. Ascoli, G. A. Mt. 23 (1895) 67-.

-, -. Peano, G. A. Mt. 23 (1895) 153-.

-, derivatives of which contain implicit functions of one variable. Cauchy, A. L. C. R. 12 (1841) 1029-.

- of differential systems. Riquier, C. Acta Mth. 23 (1900) 203-.

- and differentials, sums and differences, relations. Hansen, $P$. A. Leip. Ab. Mth. Ps. 7 (1865) 505-.

-, evaluation. Cockle, (Sir) J. [1867] Manch. Lt. Ph. S. P. 7 (1868) 67-.

-, expansion in series. Schulze, N.W. Grunert Arch. 1 (1841) 257-.

-, formulæ. Gaudard, J. A. Gén. Civ. 2 (1873) 269-.

- of functions containing other variables. Arzelà, C. Bologna Rd. (1888-89) 16-.

_ - of sine and cosine found by converging series. Gudermann, C. Crelle J. 14 (1835) 182-; 15 (1836) 100 .

- - general form. Schlömilch, O. Z. Mth. Ps. 18 (1873) 315-.
Integrals, higher, of composite functions. Oettinger, L. Grunert Arch. 20 (1853) 321-. -, improper, absolute convergence, explanation. Stolz, O. Wien Ak. Sb. 107 (1898) (Ab. 2a) 207-; 108 (1899) (Ab. 2a) 1234-.

-, indefinite. Realis, S. N. A. Mth. 1 (1882) 343-.

-, - . [Duarte Leite] Pereira da Silva, [1882-83] G. Teix. J. Sc. 4 (1883) 87-; 5 (*1883) 21-.

-, -, determination. Resal, H. A. N. A. Mth. 20 (1881) 529-.

-, - , evaluation of some of Euler's. Pánek, A. Časopis 28 (1899) 97-, 177-; Fschr. Mth. (1899) 271.

-, - , reduction. Andréiewsky, -. Bll. Sc. Mth. As. 2 (1878) 246-.

- involving trigonometric functions. Schlömilch, O. Grunert Arch. 6 (1845) 200-.

-, irrational algebraic, class. Piuma, C. $M$. (vIII) Tortolini A. 4 (1861) 1-.

_, -, formulæ rationalised. Fuss, N. [1826] St Pét. Ac. Sc. Mm. 11 (1830) 305-.

- of irrational functions. Aronhold, S. $H$. Crelle J. 61 (1863) 95-.

- - - - general formulæ. Stringham, W. I. Am. J. Mth. 2 (1879) 188-

-, limiting values. Tchebitchef, P.L. Liouv. J. Mth. 19 (1874) 157-.

-, - -. Posse, K. A. Kharkov Mth. S. Com. (1885) 35-; Fschr. Mth. (1885) 171-. -, - - Markov, A. A. St Pét. Ac. Sc. Bll. 2 (1895) 195- ; Fschr. Mth. (1895) 322-. ,$- \ldots-$, representation by integral residues. Čeby sev, P. St. Pet. Ac. Sc. Mm. (Rs.) 51 (1885) (Suppl.) No. 4, 25 pp.; Acta Mth. 9 (1887) 35-

-, - -, two. Stolz, O. Wien Ak. Sb. 106 (1897) (Ab. 2a) 453-.

-, maxima and minima. Poisson, S. D. Par. S. Phlm. Bll. 3 (1804) 219-.

- , - - Gergonne, J. D. Gergonne A. Mth. 12 (1822-23) 1-.

- - - -, criteria. Brunacci, V. [1805] Bologna Mm. I. It. 1 (1806) (Pte. 2) 191-; 2 (1810) (Pte. 2) 121-。

- $-1-1$, - Hesse, L. O. Crelle J. 54 (1857) 227-。

-, - - -, -. Mayer, A. Crelle J. 69 (1868) 238-.

_, _ _ - values. Löfler, A. Wien SB. 34 (1859) 227-.

-, mean value and Rolle's theorem. Schiff, $P$. Mosc. S. Sc. Bll. 78 (No. 2) (1893) 45-; 83 (No. 1) (1893) 21-; Fschr. Mth. (1893-94) 461-.

- partial. Delassus, É. Bll. Sc. Mth. 19 (1895) 37-.

- and their periods, elementary theory. Marie, M. C. R. 75 (1872) 524-; Par. Éc. Pol. J. Cah. 44 (1874) 1-.

-, reciprocal relation of certain. Kausler, C. F. [1808] St Pét. Ac. Sc. Mm. 3 (1811) 114-.

- reduction. Newman, F.W. Mathematician 2 (1847) 28-, 87-.

- - to more simple forms. Bronwin, $B$. Mathematician 1 (1845) 303-; 2 (1847) 75-. 
Integrals, remarkable. Pomey, E. N. A. Mth. 7 (1888) 191-.

- and sums, value. Popov, A.T. (xIr) Kazan Un. Mm. (1851, Bk. 4) 1-.

- transformation. Bouniakowsky, V. St Pét. Ac. Sc. Bll. 2 (1860) 136-.

—, - Čebyšev, P. [1883] St Pet. Ac. Sc. Mm. (Rs.) 47 (1884) (Suppl.) No. 4, 17 pp.

-, -, formulæ. Schlömilch, $O$. Grunert Arch. 10 (1847) 440-.

,-- , method depending on square roots, application to geodesy. Callandreau, $O$. C. R. 85 (1877) 664-, 1062-.

-, - of sums. Cauchy, A. L. C. R. 13 (1841) 181-.

-, transformations. Bronwin, B. Camb. Mth. J. 2 (1841) 263-; 4 (1845) 233-; Mathematician 3 (1850) 135-.

-, value. Stern, M. A. Crelle J. Mth. 78 (1874) 340-; 79 (1875) 263-.

-. $\int \frac{d x}{\log x}$, theory. Plana, G. Gergonne A. Mth. 12 (1821-22) 145-.

- -, - (Plana). Anon. (vr 544) Gergonne A. Mth. 12 (1821-22) 365-.

- $\int \frac{d x}{x}$. Verdam, G. J. Grunert Arch. 4 (1844) 221-.

- - Schlömilch, O. Grunert Arch. 6 (1845) $326-$.

Integrals $\int e^{\sin x} \cos (n x-\cos x) d x, \& c$. Unferdinger, F. Wien Sb. 57 (1868) (Ab. 2) 611-. $-\int x^{n} \cdot \cos \{m \lg (a+b x)\} d x, \& c$. Unferdinger, $F$. Wien Sb. 59 (1869) (Ab. 2) 437-. $-\int_{(1874)} \cos ^{2 m+1} x d x$. Realis, S. N. A. Mth. 13

$-\int_{-\infty}^{\infty} \phi\left(x-\frac{a}{x}\right) d x=\int_{-\infty}^{\infty} \phi(x) d x$, proof. Glaisher, J. W. L. Mess. Mth. 4 (1875) 186-.

$-\int_{0}^{1}(u)_{n} d u$, infinite values. Schröder, E. Z. Mth. Ps. 25 (1880) 106-.

- - , - - (Schröder). Schlömilch, O.X. Z. Mth. Ps. 25 (1880) 117-.

$-\int f(z) d z$ over a surface containing no singularity of $f(z)$. Rados, $I$. [1884] Mth. Termt. Éts. 3 (1885) 46-; Mth. Nt. B. Ung. 3 (188485) $186-$.

- $\int f(x) \cos x d x$, and the equation $\frac{d^{2} y}{d x^{2}}$ $+y=f(x)$. Studnicka, F. J. Časopis 13 (1884) 12-; Fschr. Mth. (1884) 235.

$-\int e^{\omega x} f(x) d x$. Gomes-Teixeira, F. Rm. R. Ac. Linc. Rd. 1 (1885) 278 -

11-. Young, J. R. Ph. Mg. 32 (1848)

$-\int x^{-1} d x$, symbolical value. Center, $W$. Camb. and Dubl. Mth. J. 4 (1849) 261-.

$-\int^{\frac{1}{2}} \frac{(d x)^{\frac{1}{2}}}{\sqrt{\bar{x}}}$, fractional integration. Oettinger, $L$. Grunert Arch. 15 (1850) 424-.

$-\sqrt{x+2 \sqrt{x}}$. Schulze, N.W. Grunert Arch. 19 (1852) 181-.

- - (Schulze). Lindmun, C. F. Grunert Arch. 23 (1854) 445-.

$-\int d \phi \sin ^{2 n} \phi\left(1-k \sin ^{2} \phi\right)^{m}$, solution. Schönbichler, $K$. Wien SB. 16 (1855) 447-.

$-\iint^{2} \frac{x^{2}-y^{2}}{\left(x^{2}+y^{2}\right)^{2}} d x d y$. Grunert, J.A. Grunert Arch. 27 (1856) 362-.

$-\int \frac{d x}{\sqrt{a+b x+c x^{2}}}$, evaluation. Gauss, C. $F$. Grunert Arch. 30 (1858) 229-.

$-\int \sqrt{a^{2}-x^{2}} d x$ in connection with other integrals. Fischer, - - Grunert Arch. 38 (1862) 151-.

$-\int \frac{d x}{(x-\alpha)^{p}(x-\beta)^{q}} \cdot$ Spitzer, S. Grunert Arch. 40 (1863) 168-

$-\int \frac{x^{m} d x}{\sqrt{1-x^{2}}}$. Hermite, C. A.Mt. 1 (1867-68) $-e^{z^{2}} \int_{z}^{\infty} e^{-z^{2}} d z$, semi-convergent series for. Schols, C. M. Delft Éc. Pol. A. 1 (1885) 213-; Amst. Ak. Vs. M. 2 (1886) 40-

$-\int \frac{d x}{\sqrt{X}}$ where $X$ is reciprocal cubic or quartic. Toropov, K. A. Kharkov Mth. S. Com. (1885) 3-; Fschr. Mth. (1885) 258.

$-\int_{6}\left(1+z^{2}\right)^{-n} d z$. Balitrand, -. N. A. Mth. 6 (1887) 45-.

$-\int \frac{\sin z}{z^{\alpha}} d z$ and $\int \frac{\cos z}{z^{\alpha}} d z$. Linhardt, $E$. Arch. Mth. Ps. 5 (1887) 91-.

$-\int d x / \sqrt[4]{x^{4}+p x^{2}+q} . \quad$ Dolbnia, J. Bll. Sc. Mth. 19 (1895) 76-.

- $\int_{0}^{x} \log \sec x d x$, proposed tabulation. $M c$ Mahon, J. Science 4 (1896) 345. $-\int_{J . J .} d x \cdot\left[\Pi\left(x-\cos \frac{2 x+1}{2 m} \pi\right)\right]^{-\frac{1}{m}}$. Fschr. Mth. (1899) 271.

Integrating equation. Jong, J.de. Arch. Néerl. 7 (1872) 140 -

\section{INTEGRATION.}

$155-$.

Le Barbier, - . Gergonne A. Mth. 20 (182930) 117-; 21 (1830-31) 73-. 
Carmichael, R. Camb. and Dubl. Mth. J. 9 (1854) 227-.

Encke, J. F. Camb. (M.) Mth. M. 2 (1860) 276Macloskie, G. A. Mth. 5 (1889-90) 106-.

of algebraic differentials by logarithms. Weier. strass, C. Berl. B. (1857) 148-.

- - functions, with mechanical illustrations. Russell, W. H. L. R. S. P. 23 (1875) 279. approximate. Gauss, C. F. [1814] Gött. Cm. 3 (1814-15) 39-.

Kramp, C. Gergonne A. Mth. 6 (181516) 281-; 7 (1816-17) 241- ; 9 (1818-19) 373-. - (Kramp). Gergonne, J. D. Gergonne A. Mth. 6 (1815-16) 303-.

-. Mainardi, G. [1845] Mod. S. It. Mm. 23 (1846) 309-.

-. Stieltjes, T. J. (jun.) C. R. 97 (1883) 740-, 798-.

-, Gauss's method. Jacobi, C. G. J. Crelle J. 1 (1826) 301-.

-, - - generalisation. August, F. Arch. Mth. Ps. 66 (1881) 72-

-, of irrational functions by rational substitu. tions. Merrifield, C. W. Ph. Mg. $20(1860)$ 446-.

- , of $\int_{-\infty}^{+\infty} F(x) d z$. Gourier, G. C. R. 97 (1883) 79 -

by auxiliary integrals. Stille, $W . A$. Des Moines Anal. 10 (1883) 81-, 108-, 137-.

of binomial differentials. Perevošikov, D.M. [1870] St Pet. Ac. Sc. Mm. (Rs.) $18\left({ }^{*} 1871\right)$ $123-$.

and calculus of iterative functions. Ferber, N. A. Mth. 17 (1898) 509-.

cases. Strekalov, $V . N$. Kazan S. Nt. (Ps.Mth.) P. 5 (1887) 125-.

continuous, of numerical function. Cellérier, G., \& Pictet, $R$. Arch. Sc. Ps. Nt. 64 (1878) 185 -

depending on rectification of conics. Malfatti, G. F. Verona S. It. Mm. 2 (1784) 749-.

of differential expressions involving angles. Fuss, N. [1800] St Pét. Ac. Sc. Mm. 1 (1809) 138-.

- - of physical astronomy. Woodhouse, $R$. Phil. Trans. (1840) 219-

- - functions involving sines and cosines. Melainderhjelm, $D$. Ups. N. Acta S. Sc. 6 (1799) 211-.

- differentials, cases. Martin, Art. Mess. Mth. 5 (1876) 145-.

- - - (Martin). Glaisher, J.W. L. Mess. Mth. 5 (1876) 147-.

,$---(-)$. Cayley, A. Mess. Mth. 5 (1876) 163 ; 6 (1877) 82-.

- - with several independent variables. Hansen, P. C. V. (xII) Ts. Mth. 3 (1879) 165-.

- - involving radicals. Lagrange, J. L. Turin Mm. Ac. 2 (1784-85) 218-.

by differentials of negative index. Maksimovich, V. P. [1882] (xII) Kazan S. Nt. (Ps,-Mth.) P. 1 (1883) [No. 7] 6-.

and differentiation of indeterminate fractions. Du Bois-Reymond, P. [1878] Mth. A. 14 (1879) 498-. direct, of $\sin ^{p} x \cos ^{q} x d x$. Studnička, $F . J$. Časopis 21 (1892) 180-; Fschr. Mth. (1892) 260.

of discontinuous functions. Smith, H. J.S. L. Mth. S. P. 6 (1874-75) 140-.

- exact differentials. Steen, $A$. Mth. Ts. 1 (1859) 176-.

extension of Leibnitz's theorem to. Young, J. R. Ph. Mg. 33 (1848) 335-.

- - summation theorem of Abel's to. Gibson, G. A. Edinb. Mth. S. P. 6 (1888) 40 -.

formula. Degen, C. F. Kiöb. Dn. Vd. Selsk. Skr. 3 (1803-04) 79-.

-. Aronhold, S. H. [1862] Crelle J. 61 (1863) 95-.

- (Aronhold). Cayley, A. Mess. Mth. 5 (1876) 88-.

-. Cayley, A. C. R. 78 (1874) 1624-

- Nicholson, J.W. Des Moines Anal. 10 (1883) 53-.

- Hill's, demonstration. Ramus, C. Crelle J. 19 (1839) 117-

formulæ. Kramp, C. Gergonne A. Mth. 6 (1815-16) 372-.

-. Buquoy, G. von. Oken Isis (1825) 155-.

- for two first orders. Sivering, J. Lux. I. R. Pb. 18 (1881) 33-.

of functions containing radical of second degree. Koehler, C. N. A. Mth. 6 (1867) 448-.

graphical methods. Saviotti, C. Cuyper Rv. Un. 13 (1883) 483-.

by help of trigonometric functions. Rodde, $L$. Hann. Z. Archt.-Vr. 3 (1857) 359-.

- infinite series, advantages. Gherardi, $S$. [1849] Bologna Mm. Ac. Sc. 2 (1850) 417-. of irrational differential formulæ. Ettingshausen, A. von. Wien SB. (1850) (Ab. 2) 31-. - differentials. Tchebicheff, $P$. Liouv. J. Mth. 18 (1853) 87-; C. R. 51 (1860) 46-; Liouv. J. Mth. 9 (1864) 242-.

- - expressions. Gebhard, $H$. Arch. Mth. Ps. 63 (1879) 334-.

- - and trigonometric differential formulæ. Zmurko, L. Wien SB. (1849) (Ab. 2) 40-. - irrationals of second degree. Alexéeff, $N$. C. R. 89 (1879) 403-.

- logarithmic functions. Perevošcikov, D. M. St Pet. Ac. Sc. Mm. (Rs.) 21 (*1872) 87-.

- -rational differentials. Hill, C.J.D. Crelle J. 3 (1828) 101-.

methods. Radicke, G. Crelle J. 36 (1848) 183-.

-. Carmichael, R. Ph. Mg. 14 (1857) 507-.

-. Helmling, $P$. St Pét. Ac. Sc. Mm. 29 (1881) No. 2, 39 pp.

partial, application. Steen, A. (xII) Ts. Mth. 6 (1882) 51-.

of partial differential coefficients. Euler, $L$. [1781] St Pét. Ac. Sc. Mm. 11 (1830) 92-. - - equations of second order. Melnīkov, M. (xII) Kazan Un. Mm. (1841) (Bk. 1) 208- ; (1841) (Bk. 2) 167-.

by partial fractions. Baltzer, $R$. (Ix) Leip. Mth. Ps. B. 25 (1873) 523-.

partial, generalisation. Worpitzky, J. Z. Mth. Ps. 23 (1878) 407-. 
by parts. Gibson, G. A. Edinb. Mth. S. P. 4 (1886) 88-.

- - repeated, development of function by. Stolp, C. N. Arch. Wisk. 10 (1884) 81-. - - , theorems. Rogel, $F$. Prag Sb. (1892) (Mth.-Nt.) 185-

- -, useful formula. Kronecker, L. Berl. Ak. Sb. (1885) 841-.

from point of view of real variables. Baire, $R$. C. R. 126 (1898) 1700-.

of polynomial differentials, reduction formulæ

for. Greene, $D$. Des Moines Anal. 1 (1874) 137-.

practical. Schläfi, L. Bern Mt. (1899) 83-.

problem. Lindeloef, L. L. Les Mondes 3 (1863) 492-.

process. Cayley, A. Mess. Mth. 4 (1875) 149 -.

of rational differentials. Catalan, E. C. N. A. Mth. 12 (1873) 423-.

- - - Weyr, E. Časopis 11 ( $\left.{ }^{*} 1882\right)$ 125-; Fschr. Mth. ("1882) 208-.

- fraction with imaginary roots in denominator. Starkov, A. N. Rs. S. Nt. Mm. (Mth.) 6 (1885) 87-; Fschr. Mth. (1885) 257-.

- - fractions. Ostrogradsky, M. A. St Pét. Ac. Sc. Mm. 2 (1833) 569-, 657-.

- - Poisson, S. D. Liouv. J. Mth. 2 (1837) 224-.

- - -. Ostrogradsky, M. A. [1844] St

Pét. Ac. Sc. Bll. 4 (1845) 145-, 286-.

- - De Morgan, A. Camb. and Dubl. Mth. J. 3 (1848) 238-; Mathematician 3 (1850) 242-.

- - - Perevoščikov, D. [1867] St Pet. Ac. Sc. Mm. (Rs.) 11 (*1867) 112 ; $12\left({ }^{*} 1868\right)$ 23-.

- - Hermite, C. N. A. Mth. 11 (1872) $145-$.

- - Holst, E. Arch. Mth. Ntvd. 10 (1886) 290-.

- - - by finite differences, by means of algebraic functions when possible. Beyer, E. I. von. (xII) Rec. Mth. (Moscou) 4 (1869-70) (Pt. 1) 297-; 5 (1870) (Pt. 1) 64-, 145-.

- - functions. Kapteyn, W. N. Arch. Wisk. 10 (1884) 177-.

- - homogeneous function. Stephanos, $C$. C. R. 97 (1883) 1290-.

repeated, of rational function, transcendents arising from. Jonquière, A. Stockh. Öfv. (1888) 522-.

-, Starkov's formula. Iznoskov, I. A. Kazan S. Nt. (Ps.-Mth.) P. 2 (1884) 37-.

results without process of integration. Raabe, J. L. Zür. Mt. 2 (1850-52) 466-.

by series. Arzelà, $C$. Rm. R. Ac. Linc. Rd. 1 (1885) 532-, 566-; 6 (1897) (Sem. 2) 290-. - - Boole on defects of Euler's method. Rubini, R. Nap. Rd. 19 (1880) 132-. - -, formula. Tetmajer, J. (xII) Krk. Ak. (Mt.-Prz.) Rz. \& Sp. 6 (1880) 231 -.

of simpler differentials containing a cube root. Chebuishev, P. L. (xu) Rec. Mth. (Moscou) 2 (1867) (Pt. 1) 71-. by substitution. $D-g$. Ts. Mt. Fys. 2 (1869) 253-.

- -. Arzelà, C. Bologna Rd. 4 (1900) 82-. successive, arranged to yield ordinates for scale of areas. Merrifield, C. W. Nv. Archt. T. 6 (1865) 51-.

symmetrical. Carmichael, $R$. Ph. Mg. 20 (1860) 348-.

theorems. Malet, J. C. [1873] (x) A. Mt. 6 (1873-75) 252-.

Lindman, C. F. Stockh. Ófv. (1893) 563-; Fschr. Mth. (1893-94) 481.

- (potential). Watson, (Rev.) H. W. QJ. Mth. 21 (1886) 225-.

- (analogous to moments of inertia). Routh, E. J. QJ. Mth. 21 (1886) 281-

of trigonometric expressions. Encontre, $D$. Mntp. Rec. Bll. 1 (1803) 151-.

- - Perevošcikov, D. M. St Pet. Ac. Sc. Mm. (Rs.) 19 (*1871) 154-.

- - series. Du Bois-Reymond, P. Mth. A. 22 (1883) 260-

virtual, performed by Kepler. Eneström, $G$. Bb. Mth. (1889) 65-.

volume of cylinder. Petersen, J.P. C. (xII) Ts. Mth. 3 (1867) 24- ; 5 (1869) 140-; 2 (1878) 178-.

of $\int x^{m-1} d x\left(\Delta+x^{n}\right)^{\lambda}$ in convergent series. Fuss, N. [1797] St Pet. Ac. Sc. N. Acta 15 (1806) 55-.

of $d y=\frac{d x}{(1+x) \sqrt[4]{2 x^{2}-1}} \cdot \quad$ Kausler, C. F. [1810] St Pét. Ac. Sc. Mm. 4 (1813) 253-.

$-\frac{d z}{a+b \cos z}$. Lobatto, R. Crelle J. 9 (1832) 259-.

$-\frac{1}{x}$. Matzka, W. Grunert Arch. 20 (1853) 1-.

$-\int \frac{x+A}{\sqrt{x^{4}+a x^{3}+\beta x^{2}+\gamma x+\delta}} d x$. Tchébicheff, $P$. de. Liouv. J. Mth. 9 (1864) 225-.

- - (T'chébicheff's method). Zolotareff, $G$. Mth. A. 5 (1872) 560-

Integrators, two mechanical. Fraser, $A . Y$. Edinb. Mth. S. P. 4 (1886) 29-.

"Interest" questions, applications of infinitesimal calculus. Farren, E. J. Assur. Mg. $5\left({ }^{*} 1855\right)$ 254-.

Leibnitz's formula for $\int u d z$, historic note. Boncompagni, - Les Mondes 18 (1868) $687-$.

Length of curve as limit of polygon, independent of manner in which polygon approaches curve. Ascoli, G. Mil. I. Lomb. Rd. 16 (1883) 851-.

Limited and integrable functions. Fejer, [non Tejer] L. C. R. 131 (1900) 984-; 132 (1901) 48.

Limits, inverse method, to find law of all series of which $F x$ can be limit of expression. Stockler, F. de B. G. QJ. Sc. 15 (1823) 357-. 
Logarithmic integral $\int_{0}^{x}(\log x)^{-1} d x$. Amstein, H. Laus. S. Vd. Bll. 31 (1895) 203-; 36 (1900) 1-.

Mean area of rectangle with varying sides. Breton [de Champ], P. Liouv. J. Mth. 9 (1844) 373-.

- limits of two numbers. Ocagne, M. $d$ '. G. Teix. J. Sc. 7 (1886) 19-, 27.

- _ - - Cesáro, E. G. Teix. J. Sc. 7 (1886) 28-.

- value of function of several variables, analytical determination. Philbert, $H$. Liouv. J. Mth. 5 (1879) 115-.

- values. Hayes, E. Science 21 (1893) 333. - -, determination. Kronecker, L. C. R. 103 (1886) 980-.

- - of functions of a real variable. Maurer, L. Mth. A. 47 (1896) 263-.

- - theorem. Du Bois-Reymond, P. Crelle J. 69 (1868) 65-.

- - - (Du Bois-Reymond). Meyer, G. F. [1872] Mth. A. 6 (1873) 313-.

- - - condition, new. Du Bois-Reymond, P. Mth. A. 7 (1874) 605-.

_- - for finite sums and integrals, second. Pringsheim, A. [1900] Münch. Ak. Sb. 30 (1901) 209-.

- - theorem, second. Mansion, $P$. Mathesis 5 (1885) 97-.

- - - - Tikhomandritzky, -. N. A. Mth. 18 (1899) 173-.

- -, various orders. Schlömilch, O. Leip. B. 10 (1858) 77-; Schlömilch Z. 3 (1858) 301-.

Mechanical quadrature. Clausen, T. Crelle J. 6 (1830) 287-.

- - Ligowski, $W$. [1875-76] Arch. Mth. Ps. 58 (1876) 49-; 59 (1876) 329-.

- - Vogel, R. Fschr. Mth. (1887) 283.

- - Lampe, E. [1893] D. Mth. Vr. Jbr. 3 (1894) 102-.

- - Poincaré, H. Bll. As. 16 (1899) 382-. - - and interpolation. Grunert, J. A. Grunert Arch. 20 (1853) 361-.

- - law of numerical coefficients. Oppolzer, T. von. As. Nr. 91 (1878) 329-.

- - pairs of formulæ in. Skutsch, R. Arch. Mth. Ps. 13 (1895) 78-.

- - Simpson's method. Hočevar, $F$. Wien Ak. Sb. 90 (1885) (Ab. 2) 908-.

- -, theory. Mehler, F. G. [1863] Crelle J. 63 (1864) 152-.

- - - - Gegenbauer, L. [1878] Wien Ak. Sb. 78 (1879) (Ab. 2) 768 -

- - , - generalisation. Stieltjes, - C. R. 99 (1884) 850-.

Minimum value of $\int \sqrt{ }\left(d x_{1}{ }^{2}+d x_{2}{ }^{2}+\ldots+d x_{n}{ }^{2}\right)$, the $x$ 's being connected by equation of second degree. Schläfli, L. [1849] Crelle J. 63 (1852) 23-.

Numerical differential of a numerical integral. Tomashevich, E. [1879] (хтI) Rec. Mth. (Moscou) 9 (1878-81) (Pt. 1) 546-.

Partial fractions, extension of theorem relating to. Neumann, C. Gött. Nr. (1869) 9-. voL. I.
Periodic functions, theory based on integrals between imaginary limits. Toeplitz, $J$. Grunert Arch. 23 (1854) 241-.

Points, distribution on a line. (Demonstration of Peirce's propositions, Camb. (M.) Mth. M. 1 (1859) 16-18.) Adcock, R.J. Camb. (M.) Mth. M. 1 (1859) 82-.

Pseudoelliptic integral $\int \frac{x d x}{\left(x^{3}+c\right) \sqrt{x^{3}+d}}$. Markov, A. A. [1894] St Pet. Ac. Sc. Mm. (Rs.) 75 (1895) 111-.

Pyramid, triangular, $\int x y d m, \int x^{2} d m$, etc. Lobatto, R. Grunert Arch. 31 (1858) 249-.

Quadrature, approximate. Maggi, G. A. Rv. Mt. 3 (1893) 60-.

Quadratures, formula. Woolhouse, W. S. B. Assur. Mg. $13\left({ }^{*} 1867\right) 119$.

Ratio of two integrals taken between same limits. Ćeby̌̌ev, P. [1882] St Pet. Ac. Sc. Mm. (Rs.) 44 (" 1883) (Suppl.) No. 2, 33 pp.

Rational fractions. Cockle, (Sir) J. [1867] Mess. Mth. 4 (1868) 168-.

Reduction formula. Mertens, $F$. Krk. Ak. (Mt.-Prz.) Rz. 8 (1895) 53-; Crc. Ac. Sc. Bll. (1893) 344.

- Gibson, G. A. Edinb. Mth. S. P. 14 (1896) 27-.

- - for $\int \frac{C_{0}+C_{1} x+C_{2} x^{2}+\ldots+C_{n} x^{n}}{(x-a)^{p}(x-\beta)^{q}} d x$. Spitzer, S. Schlömilch Z. 7 (1862) 123-. - formulæ. Moth, F.X. Baumgartner Z. 6 (1829) 158-.

- -. Winckler, A. Wien SB. 47 (1863) (Ab. 2) 146-.

- - Watson, W. Mess. Mth. 3 (1866) 54-.

Relation of two integrals expanded to one and same value of variable. Chebuishev, $P . L$. (xII) Kazan S. Nt. (Ps.-Mth.) P. 1 (1883) [No. 9] 43.

Representative functions. Du Bois-Reymond, P. C. R. 92 (1881) 915-, 962-; Mth. A. 18 (1881) 593-.

Richard planimeter. Anon. Sc. Abs. 1 (1898) 635.

Summation function. Kronecker, L. Berl. Ak. Sb. (1889) 867-.

Surface and volume integrals of ellipsoid, cases. Hogg, E. G. Aust. As. Rp. (1900) 191-.

Symmetrical integrals, formula. Zucchetti, $F$. Tor. At. Ac. Sc. 4 (1868-69) 333-.

Theorem of Čebyšev. Arone, G. d'. G. Mt. 26 (1888) 61-.

- - Cesáro, E. G. Mt. 27 (1889) 48-.

- - Frullani, generalisation. Lerch, $M$. Prag Sb. (1893) (Mth.-Nt.) No. 30, 6 pp.

-, geometrical. Béliankin, I. [1900] Rec. Mth. (Moscou) 21 (1901) 461-.

- of Stokes. Fleuri, G. Aust. As. Rp. (1893) 297-.

- - - Gosiewski, W. Wiad. Mt. 2 (1898) 213-; Fschr. Mth. (1898) 247.

--- , generalisation. Maggi, G. A. Catania Ac. Gioen. At. 4 (1892) (Mm. 8) 12 pp. 
Transcendents, derivatives of which depend on cubic equations. Minding, E.F.A. Crelle J. 11 (1834) 373-.

Transformation of a function, Heine's. Pincherle, S. Palermo Cir. Mt. Rd. 4 (1890) 143-.

-, method, applications. Collignon, $\dot{E}$. As. Fr. C. R. (1894) (Pt. 2) 14-.

- of plane figures. Freuchen, $P$. Mth. Ts. 2 (1860) 33-.

Variation of parameters, method. Andréievssky, -. N. A. Mth. 16 (1877) 61-.

$\int \frac{\rho d x}{\sqrt[8]{R}}$. Ceby̌ev, $P$. St Pet. Ac. Sc. Mm. (Rs.) 7 ("1865) (Suppl.) No. 5, 55 pp.

\section{Definite integrals (simple).}

Legendre, A. M. Par. Mm. de l'I. (1809) 416-.

Poisson, S. D. Par. S. Phlm. Bll. 2 (1811) 243-, 375-.

Bidone, G. Turin Mm. Ac. (1811-12) 231-.

Poisson, S. D. Par. Éc. Pol. J. $16^{\mathrm{e}} \mathrm{cah}$. (1813) 215-; $17^{\circ} \mathrm{cah}$. (1815) 612-.

Cauchy, A. L. [1814] Par. S. Phlm. Bll. (1814) 185-; Par. Mm. Sav. Etr. 1 (1827) $599-$.

Plana, G. Tor. Mm. Ac. 23 (1818) 7-.

Poisson, S. D. Par. J. Éc. Pol. $18^{\mathrm{e}} \mathrm{cah} .(1820)$ 295-.

Cisa de Gresi, -. Tor. Mm. Ac. 26 (1821) 209-.

Sarrus, $F$. Gergonne A. Mth. 12 (1821-22) 36-.

Schmidten, H. G. von. Gergonne A. Mth. 12 (1821-22) 205-.

Abel, N. H. Crelle J. 2 (1827) 22-.

Paoli, P. [1827] Mod. S. It. Mm. 20 (1828) 161-.

Frullani, G. Mod. S. It. Mm. 20 (1828) 448-. Ostrogradsky, M. A. [1828] St Pét. Ac. Sc. Mm. 1 (1831) 117-.

Paoli, P. [1831] Mod. S. It. Mm. 21 (1836) 55-.

Svanberg, A. F. Ups. N. Acta S. Sc. 10 (1832) 231-.

Plana, G. Brux. Ac. Bll. 3 (1836) 364 ; Brux. Ac. Sc. Mm. 10 (1837) 23 pp.

Svanberg, A.'F. Crelle J. 18 (1838) 55-.

Liouville, $J$. Liouv. J. Mth. 4 (1839) 225-; C. R. 8 (1839) 626.

(Bechelot). Jürgensen, C. Crelle J. 23 (1842) 142-.

Boncompagni, B. Crelle J. 25 (1843) 74-. Cayley, A. Camb. Mth. J. 3 (1843) 138-. Goodwin, H. Camb. Mth. J. 3 (1843) 168Bronwin, B. Ph. Mg. 24 (1844) 491-. Schlömilch, O. Grunert Arch. 5 (1844) 204 -. Arndt, $F$. Grunert Arch. 6 (1845) 187-, 434-. Bromwin, B. Mathematician 1 (1845) 197-. Roberts, W. Liouv. J. Mth. 10 (1845) 453-. Piola, G. At. Sc. It. (1846) 238-.

Schlömilch, O. Crelle J. 33 (1846) 316-.

Arndt, F. Grunert Arch. 10 (1847) 225-.
Moesta, C. W. Grunert Arch. 10 (1847) 447-.

Roberts, $W$. Ir. Ac. P. 3 (1847) 305-.

Bonnet, O. Liouv. J. Mth. 14 (1849) 249-.

Meyer, A. Brux. Ac. Bll. 16 (1849) 334-.

Lindman, C. F. Stockh. Ak. Hndl. (1850) 343-.

Cayley, A. Camb. and Dubl. Mth. J. 6 (1851) 136-.

Lindman, C. F. Grunert Arch. 16 (1851) 94-.

Lobatschewsky, N. (vr Adds.) Kazan Mm. Un. (1852) 34 pp.

Lindman, C. F. Grunert Arch. 23 (1854) 448-.

Dienger, J. Grunert Arch. 30 (1858) 250-.

Lindman, C. F. Stockh. Öfv. 15 (1858) 457-; Grunert Arch. 34 (1860) 17- ; 35 (1860) 475-.

Ostrogradsky, M. A. [1860] St Pét. Ac. Sc. Bll. 3 (1861) 65-.

Steen, A. Mth. Ts. 2 (1860) 17-.

Sylvester, J. J. Tortolini A. 3 (1860) 254 -.

Zehfuss, G. Grunert Arch. 34 (1860) 486-.

Enneper, A. Schlömileh Z. 6 (1861) 405-.

Sylvester, J. J. QJ. Mth. 4 (1861) 319-.

Volpicelli, P. Rm. At. 15 (1861-62) 143-.

Enneper, A. Schlömilch Z. 7 (1862) 346-.

Lindman, C. F. Grunert Arch. 38 (1862) 251-.

Oettinger, L. Grunert Arch. 39 (1862) 121-, 241-, 425- ; 40 (1863) 355-, 474-.

Heine, H. E. Crelle J. 61 (1863) 356-.

Oettinger, L. [1863] Crelle J. 63 (1864) 252-.

Popov, A. T. (xm) Kazan Un. Mm. (1863) (P8.-Mth. No. 2) 168 pp.

Stefan, J. Schlömilch Z. 8 (1863) 229-.

Catalan, E. C. C. R. 59 (1864) 618-.

Oettinger, L. Arch. Mth. Ps. 41 (1864) 1-.

Catalan, E. C. [1865] Brux. Mm. Cour. $4^{\circ}$, 33 (1867) 50 pp.

Schlömilch, O. Z. Mth. Ps. 10 (1865) 76-, $500-$.

Enneper, A. Z. Mth. Ps. 11 (1866) 251-; 13 (1868) 250-.

Weber, H. Crelle J. 69 (1868) 222-.

Hill, C.J. D. Lund Acta Un. 6 (1869) (Mth.) No. 1, $36 \mathrm{pp}$.

Grube, F. Z. Mth. Ps. 15 (1870) 464-.

Glaisher, J.W.L. B. A. Rp. 41 (1871) (Sect.) 10 -.

Pendlebury, R. Ph. Mg. 42 (1871) 437-.

Walton, W. QJ. Mth. 11 (1871) 289-, 373-.

Besso, D. G. Mt. 10 (1872) 119-.

Glaisher, J. W. L. [1872] Mess. Mth. 2 (1873) 71-; QJ. Mth. 12 (1873) 165-.

Enneper, A. Mth. A. 6 (1873) 360-; Z. Mth. Ps. 18 (1873) 407-.

Gegenbauer, L. Wien Sb. 67 (1873) (Ab. 2) 202-; 70 (1874) (Ab. 2) 433-; $72(1876)(A b$. 2) 343-.

Graindorge, $J$. Liége Mm. S. Sc. 3 (1873) 77-; Liouv. J. Mth. 18 (1873) 129-.

Walton, W. QJ. Mth. 12 (1873) 181-.

Hermite, C. Par. Éc. Pol. J. Cah. 44 (1874) 181-.

Liouville, J. Liouv. J. Mth. 19 (1874) 55-.

Liebrecht, E. Arch. Mtb. Ps. 59 (1876) 218 -

Enneper, A. Z. Mth. Ps. 22 (1877) 129-, 195 -.

Kostka, C. Z. Mth. Ps. 22 (1877) 258-. 
Russell, W. H. L. R. S. P. 25 (1877) 176, 507-; 26 (1878) 359-; 27 (1878) 129-; 29 (1879) 361-; 30 (1880) 100-, 103-; 31 (1881) 330 ; 32 (1881) 450-; 33 (1882) 258-; 34 (1883) 470-; 38 (1885) 62- ; 39 (1886) 20- ; 42 (1887) 477-; 44 (1888) 311-.

Liventsov, A. I. [1878] (xII) Rec. Mth. (Moscou) 9 (1878-81) (Pt. 1) 565-.

Tait, P. G. Edinb. R. S. P. 9 (1878) 594-.

Thomae, C.J. Z. Mth. Ps. 23 (1878) 67-.

Bachmann, P. Mth. A. 15 (1879) 424-.

Callandreau, O. C. R. 89 (1879) 90-.

Cayley, A. Mess. Mth. 8 (1879) 126-.

Catalan, E. C. [1880] Brux. Ac. Sc. Mm. 43 (1882) (No. 10) $40 \mathrm{pp}$.

Cockle, (Sir) J. [1880] Mess. Mth. 10 (1881) 98-.

Gilbert, L. P. (xII) Brux. S. Sc. A. 4 (1880) (Pt. 2) 141-.

Lindman, C. F. Stockh. Öfv. 37 (1880) No. 9, 13-.

Hermite, C. (xII) Mag. Tud. Ak. Éts. 15 (No. 8) (1881) 198-.

Mansion, P. (xir) Mathesis 1 (1881) 67-.

Bourguet, L. Acta Mth. 1 (1882) 363-.

Malet, J. C. [1882] Ir. Ac. T. 28 (1880-86) 197-; Crelle J. Mth. 92 (1882) 342-.

(Russell.) Spottiswoode, $W$. R. S. P. 33 (1882) 341-.

Wolstenholme, (Rev.) J. L. Mth. S. P. 13 (1881-82) 185-.

Laisant, C. A. As. Fr. C. R. (1883) 181.

Mildner, $R$. Wien Ak. D. 48 (1884) (Ab. 2) 317-.

Catalan, E. C. Liège S. Sc. Mm. 12 (1885) No. 2, 171-, 201-, 211-, 310-, 387-; 13 (1886) 399- ; 15 (1888) No. 1, 26-, 60-, 138, 156-, 237-.

Enneper, A. Gött. Nr. (1885) 169-.

Pincherle, S. Acta Mth. 7 (1885) 381-.

Catalan, E. Brux. Ac. Mm. 46 (1886) (No.6) $24 \mathrm{pp}$.

Stieltjes, T. J. Amst. Ak. Vs. M. 2 (1886) 210-.

Lindman, C. F. Stockh. Öfv. (1887) 235-; (1888) 421-; (1889) 331-; (1892) 5-; Fschr. Mth. (1887) 271; (1888) 426; (1892) 265-.

Malet, J. C. A. Mt. 16 (1888-89) 277-.

Pincherle, S. Rm. R. Ac. Linc. Rd. 4 (1888) (Sem. 1) 100-.

Catalan, E. Brux. Ac. Mm. 47 (1889) (No. 2) $8 \mathrm{pp}$.

Mertens, F. Krk. Ak. (Mt.-Prz.) Rz. 19 (1889) 204- ; Fschr. Mth. (1889) 283-.

Ciemniewski, M. Prace Mt.-Fiz. 2 (1890) 228-; Fschr. Mth. (1890) 290-.

Mukhopádhyây, A. Beng. As. S. P. (1890) 165-.

Somin, N. Ja. Vars. S. Nt. Tr. (1890-91) (C.R., Ps. C.) No. 6, 2-.

Jordan, C. Liouv. J. Mth. 8 (1892) 69-.

Lerch, M. G. Mt. 31 (1893) 171-; Prag České Ak. Fr. Jos. Rz. (Tŕida 2) 2 (1893) Art. 9, 40 pp. ; Fschr. Mth. (1893-94) 482-.

Studnička, F.J. Časopis 22 (1893) 228-; Fschr. Mth. (1893-94) 474.
-Ocagne, M. d'. N. A. Mth. 13 (1894) 198-.

König, G. Mth. Termt. Éts. 15 (1897) 380-.

Nielsen, $N$. Kjøb. Ov. (1897) 197-.

Kapteijn, $W$. Amst. Ak. Vs. 6 (1898) 329-; Fschr. Mth. (1898) 247.

Genese, -. N. A. Mth. 18 (1899) 273-.

Lerch, $M$. Časopis 28 (1899) 32- ; 29 (1900) 28-; Fschr. Mth. (1898) 245-; (1899) 390-, 391.

Application of function theory. Hurwitz, $A$. Mth. A. 53 (1900) 220-.

Approximate expression of certain integrals by others taken between same limits. Chebuishev, P.L. (xII) Kharkov Mth. S. Com. (1882) 93-.

- - - - - - Chebuishev's formula, complementary term. Posse, K.A. Bll. Sc. Mth. As. 7 (1883) (Pt. 1) 214-.

- values, series for. Smith, Arch. Camb. Mth. J. 2 (1841) 167-.

Approximation of functions of high numbers. Cauchy, A. L. C. R. 20 (1845) 481-, 552-, 691 - 29 (1849) 42-.

- - - - D. Darboux, G. C. R. 82 (1876) 365-, 404-; Liouv. J. Mth. 4 (1878) 5-, 377-.

- - - - - Hamy, M. C. R. 114 (1892) 993-; 125 (1897) 926-.

- - - - —. Nekrasov, P. A. [1900] Rec. Mth. (Moscou) 21 (1901) 68-, 225-; Fschr. Mth. (1900) 315-.

Approximations to integrals. Cauchy, A. $L$. C. R. 20 (1845) 907-.

Arbitrary constants, nature, when value becomes indeterminate, etc. Cauchy, A. $L$. Par. S. Phlm. Bll. (1822) 161-.

- - , variation. Schlömilch, O. Crelle J. 33 (1846) 268-.

Asymptotic values. Cesaro, E. Mathesis 14 (1894) 57-.

Auxiliary theorem. Hankel, H. Z. Mth. Ps. 14 (1869) 436-.

Binet's integrals. Lerch, $M$. Časopis 23 (1894) 274- ; Fschr. Mth. (1893-94) 482.

Calculation. Piola, G. Opusc. Mt. Fis. 1 (1832) 73-, 169-, 273-, 345- ; 2 (1834) 105-, 345-.

-, case. Anger, C. T. As. Nr. 27 (1848) $239-$.

-, 一. Radau, R. C. R. 97 (1883) 157-.

-, general formulæ. Petrovitch, M. Palermo Cir. Mt. Rd. 11 (1897) 247-.

-, higher orders, extension of Gauss's method to. Minich, S. R. Ven. At. 4 (1853) 39-.

-, numerical. Poisson, S. D. [1826] Par. Mm. Ac. Sc. 6 (1823) 571-.

-, - Baillaud, B. $[1880-82]$ C. R. 90 (1880) 974-; Toul. Ac. Sc. Mm. 5 (1883) (Sem. 1) 167-; Toul. Obs. A. 2 (1886) B. 1-.

-, 一. Kapteyn, W. N. Arch. Wisk. 3 (1898) 283-.

,-- , use of unequal intervals. Turazza, $D$. [1850] Ven. Mm. I. 5 (1855) 277-.

,,$---\frac{B}{-}-$ Bellavitis, G. Ven. At. 1 (1850) 74-. 
Cauchy's formula. Pendlebury, R. Mess. Mth. 5 (1871) 30-.

- methods, modification. Richelot, $F . J$. Crelle J. 42 (1851) 32-.

- theorem, demonstration. Goursat, $\dot{E}$. Stockh. Ak. Hndl. Bh. 9 (1884-85) No. 5, $6 \mathrm{pp}$.

- -, -, elementary. Falk, M. Bll. Sc. Mth. As. 7 (1883) 137-.

Cebyšev, P., last mathematical discussion with. Grave, D. St Pét. Ac. Sc. Bll. 2 (1895) 131-.

Circular definite integrals. Lommel, $E$. Grunert Arch. 37 (1861) 349-.

Class. Cellérier, C. Liouv. J. Mth. 8 (1843) 255-.

-. Arndt, F. Grunert Arch. 10 (1847) 240-

-. Glaisher, J. W. L. Ph. Mg. 42 (1871) 294-, 421-.

- Elliott, E. B. [1876] Mess. Mth. 6 (1877) 66-.

-. Rawson, R. [1877] Mess. Mth. 7 (1878) 65-.

- Terry, T. R. [1879] L. Mth. S. P. 11 (1879-80) 29-.

-. Tujisawle, R. Mess. Mth. 15 (1886) 79-.

-. Gibson, G.A. Glasg. Ph. S. P. 18 (1887) 167-.

-. Pochhammer, L. Mth. A. 41 (1893) 167-.

Classes, two, theory. Mellin, $H$. Helsingf. Acta 22 (1897) No. 2, 75 pp.

Collection. Haan, D. Bierens de. Crelle J. 47 (1854) 222-.

Comparison of transcendents. Boole, G. Phil. Trans. (1857) 745-.

Conception. Ascoli, G. Rm. R. Ac. Linc. At. 2 (1875) 863-.

Convergence. La Vallée-Poussin, C. J. de. Liouv. J. Mth. 8 (1892) 421-.

-. Whittemore, J. K. A. Mth. 1 (1900) 189-.

Convergent definite integrals. Cavallin, C.B.S. Stockh. Ak. Hndl. Bh. 13 (Afd. 1) (1888) No. 3, 13 pp.

Cubic integral, $u=\int_{\beta}^{a} \frac{d x}{\sqrt{(a-x)(b-x)(c-x)}}$. Rawson, R. Manch.Lt.Ph.S. Mm.6 (1879) 201-.

Definite integral connected with trinomial integrals. Liouville, J. Liouv. J. Mth. 20 (1855) 133-.

- - expressing function. Liventsov, $A . I$. (xII) Rec. Mth. (Moscou) 8 (1876) (Pt. 1) 285-.

- - " "range" of conditioned variables. Monro, C.J. [1866] QJ. Mth. 8 (1867) 278-.

- - sum of $p+1$ terms of $(\alpha+\beta)^{m}$. Catalan, E. C. Brux. Ac. Bll. 23 (1867) 402-.

- - of Gompertz's function, with tables. Makeham, W. M. I. Act. J. 17 (*1873) 305-, 445-.

- - involving the function $[x]$ of the theory of numbers. Sonine, N.J. [1885] Fschr. Mth. (1886) 257-.

- - occurring in attraction of spheroids. Ostrogradsky, M. A. [1828] St Pét. Ac. Sc. Mm. 1 (1831) 39-.

- integrals with circular functions in numerator, and powers of variable only in denominator. Glaisher, J. W. L. L. Mth. S. P. 4 (1871-73) 291-.
Definite integrals connected with Bernoullian function. Glaisher, J. W. L. Mess. Mth. 26 (1897) 152-; 27 (1898) 20-.

- - - - numbers. Catalan, E. C. Liège S. Sc. Mm. 12 (1885) No. 2, 387-; 13 (1886) 188-.

6 - Carda, K. Mh. Mth. Ps.

- Taylor's series. Elliott, E. B. Mess. Mth. 12 (1883) 144-; 13 (1884) 103-.

- containing hyperbolic functions, class. Hardy, G. H. Mess. Mth. 29 (1900) 25-.

- - - trigonometric functions, expansion. Schlömilch, O. Crelle J. 33 (1846) 353-.

- - deduced from theory of orthogonal surfaces. Lame, G. Liouv. J. Mth. 3 (1838) $552-$.

- - and differentials, dependence. Frullani, G. [1818] (rx) Mod. S. It. Mm. 18 (Mt.) (1820) 458-.

- - and differentiation. Cayley, A. Camb. and Dubl. Mth. J. 2 (1847) 122-.

- of discontinuous functions. Cauchy, A. L. C. R. 23 (1846) 537.

- - and discontinuous functions. Steen, $A$. (xiI) Ts. Mth. 6 (1882) 65-

- - - - Escher, R.J. N. Arch. Wisk. 18 (1891) 187- ; Fschr. Mth. (1891) 428-

- - elliptic functions, infinite series. Lerch, M. Prag České Ak. Fr. Jos. Rz (Tř́da 2) 1 (1892) Art. 8, 11-, Art. 25, 6 pp. ; 2 (1893) Art. 23, 42 pp.; 4 (1895) Art. 1, 55 pp. ; Fschr. Mth. (1892) 445; (1893-94) 791-; (1895) 501-.

- - expressed rationally by $\pi$ and $\log 2$. Schröder, E. D. Nf. Vh. (1890) (Th. 2) 8-. - - extending to every point of a closed curve. Cauchy, A. L. C. R. 23 (1846) 251-.

- - with factor $e^{q x^{p}}, \cos \left(q x^{p}\right), \sin \left(q x^{p}\right)$. Bierens de Haan, D. (Ix) Arch. Néerl. 8 (1873) 135-.

- - finite differences. Libri, G. Crelle J. 12 (1834) 240-.

- - given by Schlömilch, corrections. Unferdinger, $F$. Arch. Mth. Ps. 52 (1871) 252-.

- with indefinite limits. Padé, H. Par. Ec. Norm. A. 6 (1889) 349-.

- - infinite limits. La Vallée-Poussin, C. de. Brux. S. Sc. A. 16 (1892) (Pt. 1) 6-, (Pt. 2) 150-.

$--\frac{-}{-}$, convergence. Gilbert, P. Bll. Sc. Mth. 12 (1888) 66-.

$---1--$ Hočevar, $F$. Mh. Mth. Ps. 4 (1893) 177-.

- - and infinite series. Kummer, $\boldsymbol{E}$. $\boldsymbol{E}$. Crelle J. 17 (1837) 210-, 228-.

- - - - Malmsten, C. J. Crelle J. 38 (1849) 1-.

- - - (Kummer, 1837). Giuliani, $G$. G. Mt. 26 (1888) 234-.

- _ - class, calculation. Stokes, G. G. Ph. Mg. 37 (1850) 68-; Camb. Ph. S. T. 9 (1856) 166-.

_- _ - , theory. Pringsheim, A. Mth. A. 37 (1890) 591-.

- integrable by quadrature or division of circle. Richelot, $F . J$. Crelle J. 21 (1840) 293-. 
Definite integrals involving exponential integral. Glaisher, J.W.L. QJ. Mth. 18 (1882) 370-.

- - in mechanics of motion of point on surface. Rouché, E. Liouv. J. Mth. 3 (1858) 337-.

- - obtained by symbolic methods. Bromwin, B. Camb. and Dubl. Mth. J. 3 (1848) 243-. - with periodic factor. Chartier, $J$. Liouv. J. Mth. 18 (1853) 201-.

- - in planetary motion, numerical expression. Lefort, $F$. Liouv. J. Mth. 11 (1846) 142-.

- - of products of functions, inequalities limiting value of. Imshenetskin, V.G. (xII) Kharhov Mth. S. Com. (1882) 99-.

$-{ }_{-}-{ }_{-}$, relation between. Andréief, C. Bordeaux S. Sc. Mm. 2 (1886) 1-.

- - _ - - - theorems of Čebyšev and Imšeneckij, Andreev, K.A. (xII) Kharkov Mth. S. Com. (1882) 110-.

- reducible to $\int_{\infty}^{p} \frac{e^{-x} d x}{x}$ or $\int_{\infty}^{p} \frac{\cos x}{x} d x$. Arndt, F. Grunert Arch. 10 (1847) 233-.

- - representing functional operations. Pincherle, S. Acta Mth. 10 (1887) 153-.

$---1-$ Viterbi, $A$. Rm. R. Ac. Linc. Rd. 6 (1897) (Sem. 1) 247-

- _ - logarithms and Euler's constant. Sonin, N. Ja. Vars. S. Nt. Tr. (Mm.) 1 (1891) No. 6, $15 \mathrm{pp}$.

- - and series. Glaisher, J.W.L. Mess. Mth. 22 (1893) 97-.

- - - summation of series. Graindorge, $J$. Liouv. J. Mth. 18 (1873) 129-.

- _ - sums deduced from Bernoulli function. Raabe, J. L. Crelle J. 42 (1851) 348-.

- - in theory of heat. Russell, W. H. L. Camb. and Dubl. Mth. J. 8 (1853) 157-.

- _ - - Meyer, G. F. Mth. A. 3 (1871) 157-.

- - uniformly convergent. Selivanoff, Par. S. Mth. Bll. 10 (1882) 147-.

- - used for expanding functions in series. Deflers, -. Par. S. Phlm. Bll. (1819) 161-. - integration. Rawson, R. [1844] Manch. Ph. S. Mm. 7 (1846) 464-.

- - as element of operation in mathematics. Viterbi, A. A. Mt. 26 (1897) 261- ; 3 (1899) 299-.

- -, paradox. Walton, W. QJ. Mth. 6 (1864) 319-.

- - theorem. Glaisher, J.W.L. QJ. Mth. 10 (1870) 347-.

- - - , general. Boole, G. Liouv. J. Mth. 13 (1848) 111-; Camb. and Dubl. Mth. J. 4 (1849) 14-.

_- theorems. Glaisher, J. W. L. [1878] Mess. Mth. 8 (1879) 63-.

Definition of definite integral. Fouché, $M$. N. A. Mth. 15 (1896) 207-.

- - - Burali-Forti, C. N. A. Mth. 15 (1896) 495-.

- - - as limiting value of a sum. Thomae, C. J. Z. Mth. Ps. 21 (1876) 224 -. Derivation of two definite integrals. Zehfuss, $G$. Schlömilch Z. 7 (1862) 445-.

Determinant of definite integrals. Tissot, $A$. Liouv. J. Mth. 17 (1852) 177-.
Determinant of definite integrals. Enneper, $A$. Z. Mth. Ps. 11 (1866) 69-.

Determinantal and generating functions of Abel. Bassani, A. Nap. Ac. At. 7 (1895) No. 9, 19 pp.

Determination of limits to values. Winckler, A. Wien Ak. Sb. 90 (1885) (Ab. 2) 528-.

- by series. Frullani, G. Mod. S. It. Mm. 20 (1828) 663-.

Differentiation. Lindman, C. F. Stockh. Öfv. 38 (1881) No. 6, 3-.

- Lerch, M. Prag České Ak. Fr. Jos. Rz. (Třda 2) 2 (1893) Art. 34, 15 pp. ; Fschr. Mth. (1893-94) 479.

- Hallgren, E. Göteb. Hndl. 32 (1897) 83-; Fschr. Mth. (1897) 255.

Dirichlet's integral. Kronecker, L. Berl. Ak. Sb. (1885) 641-.

Discontinuous integrals connected with series for $\left(1-2 p x+x^{2}\right)^{-\frac{1}{2}}$. Hamilton, (Sir) W. R. Ph. Mg. 20 (1842) 288-.

Division into elements, mode. Petrovitch, $M$. C. R. 122 (1896) 27-.

\section{Evaluation.}

Ellis, R. L. Camb. Mth. J. 3 (1843) 185-; Liouv. J. Mth. 9 (1844) 422-.

Lobatschewsky, $W$. Erman Arch. Rs. 14 (1855) 232-.

Gegenbauer, L. Wien Ak. Sb. 64 (1871) (Ab.2) 703-.

Todhunter, I. R. S. P. 23 (1875) 300-.

Hočevar, $F$. [1876] Wien Ak. Sb. 74 (1877) (Ab. 2) 155-.

Appell, P. C. R. 87 (1878) 874-.

Spitzer, S. Arch. Mth. Ps. 62 (1878) 221-.

approximate. Dirksen, E.H. Berl.Ab. (1831) 117-.

-. Schlömilch, O. Grunert Arch. 9 (1847) 215-.

-. Grunert, J.A. Grunert Arch. 14 (1850) 225-.

- Araldi, A. [1859] (Ix) Mod. Ac. Sc. Mm. 3 (1861) 65-, 137.

-. Andra, C. C. G. Kjöb. Ov. (1867) 165-; Résumé 26-.

-. Deruyts, $J$. Brux. Ac. Bll. 11 (1886) 307-.

- Berger, A. Ups. S. Sc. N. Acta 16 (1893) No. 4, $52 \mathrm{pp}$.

- Moors, B. P. N. Arch. Wisk. 20 (1893) 129-; 3 (1898) 285-; Fschr. Mth. (1893-94) 493.

-. Bugaev, N. V. Kazan S. Ps.-Mth. Bll. 7 (1898) 95-; Fschr. Mth. (1897) 258-.

- Trchebyschew, P.L. Fschr. Mth. (1899) 279.

- formulæ. Radau, R. Liouv. J. Mth. 6 (1880) 283-.

, and functions derived therefrom. Somine, N. J. Fschr. Mth. (1887) 282-.

_, Gauss's formulø. Schellbach, C. H. Crelle J. 16 (1837) 192-.

_, integral for compound survivorship annuity. Todhunter, R. I. Act. J. 33 (1898) 311-. -, method, Cotes-Gauss. Minich, S. R. Ven. At. 3 (1852) 7-. 
Evaluation, continued.

approximate, method, Gauss's. Ettingshausen, A. von. Baumgartner Z. 7 (1830) 429.

-, 一, - Markoff, A. Mth. A. 25 (1885) 427-.

-, -, - , exposition and generalisation. Pujet, A. C. R. 84 (1877) 1071-.

-, by quadratures. Raabe, J. L. [1836] Crelle J. 18 (1838) 75-.

case. Krantz, H.J. N. Arch. Wisk. 7 (*1881) 207-.

by double integration. Schlömilch, $O$. Grunert Arch. 4 (1844) 71-.

formula. Cauchy, A. $L$ Gergonne A. Mth. 16 (1825-26) 97-; 17 (1826-27) 84-.

-, Cauchy's. Glaisher, J. W. L. [1876] Camb. Ph. S. P. 3 (1880) 5-.

formulæ. Lommel, E. Grunert Arch. 37 (1861) 433-.

-. Winckler, A. Wien Sb. 52 (1866) (Ab. 2) 57-.

-. Stieltjes, -. Bll. As. 1 (1884) 568-.

-, value of residues. Deruyts, J. Par. S. Mth. Bll. 14 (1886) 151-.

in series. Glaisher, J. W. L. B. A. Rp. 42 (1872) (Sect.) 15-.

theorem. Raabe, $J . L$. Zür. Mt. 1 (Heft 3) (1848-49) 256-.

Expansion. Grunert, J.A. Crelle J. 8 (1832) 146 -

-. Arndt, F. Grunert Arch. 11 (1848) 70-.

- in continued fractions. Stieltjes, T.J. QJ. Mth. 24 (1890) 370-.

- - series. Worontzoff, - . N. A. Mth. 10 (1891) 158-.

First principles developed without the notion of differentials. Hoppe, $R$. Arch. Mth. Ps. 61 (1877) 270-.

Formula of Abel, generalisation of a. Sonine, N. Acta Mth. 4 (1884) 171-.

-, Gauss's, remainder in. $\left\{y=\int_{t}^{T} F(t) d t\right.$, $\left.t=t_{1}+\frac{T-t_{1}}{2}+\& \mathrm{c}.\right\} \quad$ Sabinin, E. T. [1866] (xiI) Rec. Mth. (Moscou) 2 (1867) (Pt. 1) $52-$.

-, general. Dawidoff, A. Liouv. J. Mth. 8 (1882) 389-.

-, new. Glaisher, J. W. L. Ph. Mg. 48 (1874) 53-.

-, -. O'Kinealy, J. Ph. Mg. 48 (1874) 295-.

- of Poisson's for $\frac{1}{2} f(-n h)+f(-n h+h)$ $+f(-n h+2 h)+\ldots+f(n h-h)+\frac{1}{2} f(n h)$. Schaar, M. Brux. Mm. Cour. 4', 23 (184850) $17 \mathrm{pp}$.

$-\int_{0}^{\infty} \phi(x+\alpha) a^{\mu-1} d a=(-1)^{\mu} \Gamma(\mu) \int^{\mu} \phi x d x^{\mu}$. Liouville, J. Liouv. J. Mth. 20 (1855) 115-.

formulæ, Bierens de Haan's. Lindman, C. F. Stockh. Öfv. (1890) 353-; Fschr. Mth. (1890) 293.

-, development. Schlömilch, O. Grunert Arch. 1 (1841) 263-. formulæ, inverse method. Nieuport, C.F.F.de. [1817] Brux. Ac. Sc. Mm. 1 (1820) 3-.

- and methods. Winckler, A. Wien Sb. 60 (1870) $(A b .2)$ 857-.

Fourier-Bessel function $\frac{1}{\pi} \int_{0}^{\pi} e^{i x \cos a} d a$. Lerch, M. Mh. Mth. Ps. 1 (1890) $105-$.

Fourier's integrals, and analogues. Frombeck, $H$. Wien Sb. 65 (1872) (Ab. 2) 133-.

Fresnel's integrals. Jamet, $V$. N. A. Mth. 15 (1896) 372-.

- -. Godefroy, -. N. A. Mth. 17 (1898) 205-

Frullani's formula, extension. Lerch, M. [1891] Prag České Ak. Fr. Jos. Rz. (Tř́ida 2) 1 (1892) Art. 8, 1-.

Function under definite integral, to find. Liouville, J. Par. Éc. Pol. J. 24 cah. (1835) 55-.

Functions, cotan. integral, $H(a)=\int_{0}^{\frac{\pi}{2}} x \cot a x d x$.

Lindman, C. F. Ups. N. Acta S. Sc. 1 (1854) 137-.

- differing very little from zero. Čeby̌sev, $P$. [1873-80] St Pet. Ac. Sc. Mm. (Rs.) 22 ("1873) (Suppl.) No. 1, 32 pp.; 40 ("1882) (Suppl.) No. 3, 29 pp.

Hermite's integral $\int x^{2}(a u+b v)^{-2} d x$. Sincov, D. Kazan S. Ps.-Mth. Bll. 4 (1895) (Prot.) 76-; Fschr. Mth. (1895) 314.

Historical note. Escher, R.J. N. Arch. Wisk. 18 (1891) 142-.

Inequalities. Sonin, N. Ja. St Pét. Ac. Sc. Mm. 6 (1898) No. 6, 54 pp.; Fschr. Mth. (1898) 254.

- of Čebyšev, proof. Markoff, A. Mth. A. 24 (1884) 172-.

Integral residues giving approximate values of definite integrals. Čeby̌sev, P. [1886] St Pet. Ac. Sc. Mm. (Rs.) 55 (1887) (Suppl.) No. 2, 50 pp.; Acta Mth. 12 (1889) 287-.

- sine and integral cosine. Schlömilch, $O$. Grunert Arch. 11 (1848) 389-; Schlömilch Z. 5 (1860) 294-.

- - - Besso, D. G. Mt. 6 (1868) 313-.

- - related definite integrals. Stefan, $J$. Wien Jbr. Ober-Realsch. Inn. Stadt 4 (1862) 41-.

Integrals involving cubic radicals, identity. Thomae, C. J. Gött. Nr. (1877) 223-.

-, numerical values, accuracy. Sonin, N. Ja. St Pet. Ac. Sc. Mm. (Rr.) 69 (1892) 1-; Fschr. Mth. (1892) 272-.

Integration under finite forms. Genocchi, A. [1864-72] Tor. Mm. Ac. Sc. 23 (1866) 299-; 28 (1876) 1-.

- formula of Jacobi. Kronecker, L. Berl. Ak. Sb. (1884) 539-.

- by means of selected values of function. Woolhouse, W. S. B. I. Act. J. 27 (1889) 122-.

- of simplest integrals containing cube-roots. Sonin, N. St Pét. Ac. Sc. Bll. 12 (1900) 411-; Fschr. Mth. (1900) 308. 
Integration of $\int_{0}^{\infty} \frac{x^{a-1}}{1+x} d x$ by integration of differential equations. Veltmann, $W$. Grunert Arch. 38 (1862) 337-.

Inverse calculus. Boole, G. Camb. Mth. J. 4 (1845) 82-.

- - Bromwin, B. Ph. Mg. 31 (1847) 12-.

- - Rouché, E. C. R. 51 (1860) 126-.

- - Laurent, H. Liouv. J. Mth. 4 (1878) 225-.

- method. Murphy, R. [1832-35] Camb. Ph. S. T. 4 (1833) 353-; 5 (1835) 113-, 315-.

Inversion. Pincherle, $S$. Mil. I. Lomb. Rd. 20 (1887) 376-.

-. Levi-Civita, T. Tor. Ac. Sc. At. 31 (1895) 25-.

- Volterra, V. Tor. Ac. Sc. At. 31 (1895) 231-, 286-, 389-, 429- or 311-, 400-, 557-, 693-; Rm. R. Ac. Linc. Rd. 5 (1896) (Sem. 1) 177-; A. Mt. 25 (1897) 139-.

- Holmgren, E. [1899-1900] Stockh. Ak. Hndl. Bh. 25 (1900) (Afd. 1) No. 3, 19 pp.; Ups. S. Sc. N. Acta 20 (1904) No. 2, 32 pp.

- (Volterra's theorem). Holmgren, E. Tor. Ac. Sc. At. 35 (1900) 384- or 570-.

Law of reciprocity in, cases. Cauchy, A. L. Par. S. Phlm. Bll. (1817) 121-.

Legendre's formula, generalisation. Lerch, $M$. [1891] Prag České Ak. Fr. Jos. Rz. (Třida 2) 1 (1892) Art. 8, 37-.

Limit given by an inequality. Gomes Teixeira, F. Bll. Sc. Mth. 12 (1888) 288-.

Limiting ratio of two integrals. Cesáro, $E$. Bll. Sc. Mth. 17 (1893) 321-.

Limits, two. Hermite, C. (xi) Mathesis 3 (1883) 82.

-0 to $\infty$. Malmsten, C. J. Ups. N. Acta S. Sc. 12 (1844) 177-.

Line and surface integrals, new demonstration of theorem. Morera, G. Rv. Mth. 6 (189699) 19-.

Maxima and minima. Zimmerman, $V$. A. Rec. Mth. (Moscou) 17 (1895) 229-.

- - -, convergence. Cavallin, B. B. S. [1889] Stockh. Ak. Hndl. Bh. 15 (Afd. 1) (1890) No. 3, $17 \mathrm{pp}$.

- - criteria. Żmurko, L. [1875-76] D. Nf. Tbl. ( $\left.{ }^{*} 1875\right)$ 177-; Wien Ak. D. 37 (1877) (Ab. 2) 43-.

- - - - Mertens, F. Z. Mth. Ps. 21 (1876) 142-

- - , relative. $\dot{Z}$ murko, L. Wien Ak. D. 36 (1876) ( $A b .2)$ 235-.

- - - values. Liventsov, A. I. [1879] (xI) Rec. Mth. (Moscou) 10 (1882-83) (Pt. 1) 70-. - - - - , determination. Markov, A. A. (xII) Kharkov Mth. S. Com. (1883) 95-; Fschr. Mth. (1885) 168, 171.

Mean values. Hauber, C. F. Baumgartner Z. 8 (1830) 25-, 147-, 295-, 443-; 9 (1831) 302-; 10 (1832) 425-.

- Pleskot, A. Prag České Ak. Fr. Jos. Rz. (Trida 2) 2 (1893) Art. 6, 6 pp. ; Fschr. Mth. (1893-94) 475-.

Methods. Tait, P. G. [1879] Edinb. R. S. P. 10 (1880) 271.

Multiplication. Russell, W. H. L. [1874] R.S. P. 23 (1875) 120-.
Peano's concept. Stolz, O. Mh. Mth. Ps. 7 (1896) 291-; 8 (1897) 95-.

Poisson's integral, applications. La ValléePoussin, C. J. de. Brux. S. Sc. A. 17 (1893) (Pt. 2) 18-.

- - and its conjugate. Tauber, A. Mh. Mth. Ps. 6 (1895) 109-, 302.

Proof of equality. Heine, H. E. Crelle J. 50 (1855) 323-.

Properties. Winckler, A. Wien SB. 43 (1861) $(A b .2) 315$ -

- deduced from elliptic coordinates. Roberts, W. Liouv. J. Mth. 16 (1851) 1-.

-, general. Murphy, $R$. Camb. Ph. S. T. 3 (1830) 429-.

Reduction. Roberts, (Rev.) W. R. [1900] Ir. Ac. P. 6 (1900-02) 93-.

-, formula. Schlömilch, O. Grunert Arch. 9 (1847) 379-.

-, formulæ. Bierens de Haan, D. Arch. Néerl. 5 (1870) 447-; Amst. Vh. Ak. 12 (1871) 65 pp. - method and applications. Haan, $D$. Bierens de. Amst. Vh. 2 (1855) 54 pp.

Resolution into other definite integrals with narrower limits. Schlömilch, O. Grunert Arch. 4 (1844) 316-.

Simultaneous representation. Kowalewski, $G$. Crelle J. Mth. 117 (1897) 267-.

Singular definite integrals. Cauchy, A. L. C. R. 16 (1843) 422-.

- values. Schlömilch, O. Grunert Arch. 11 (1848) 63-.

Solution of equations by definite integrals. Du Bois-Reymond, $P$. [1871] Crelle J. 74 (1872) 281-.

- - problems by definite integrals. Abel, N.H. Mg. Ntvd. 2 (1823) 55-, 205-.

Substitution of non-periodic for periodic functions. Cauchy, A.L. C. R. 18 (1844) 1072-.

Summation of Gaussian series by definite integrals. Kronecker, L. Crelle J. Mth. 105 (1889) 267-.

- series by definite integrals. LejeuneDirichlet, G. Berl. Ab. (1835) 391-; Crelle J. 17 (1837) 57-.

- - - - Dienger, J. [1847] Crelle J. 46 (1853) 119-.

J. 42 (1852) 222 .

- $-1-1-$ Dienger, J. Crelle J. 38 (1849) 266-, 331-; 41 (1851) 137-.

- _ - - -. Du Bois-Reymond, $P$. [1871] Crelle J. 74 (1872) 281-.

- - Petrovitch, -. C. R. 120 (1895) 819-.

- - - Rogel, $F$. Prag Sb. (1895) (Mth.-Nt.) No. 39, 33 pp.

Synectic functions. Dillner, $G$. Ts. Mt. Fys. 3 (1870) 257-; 4 (1871) 33-, 121-.

Tables. Haan, D. Bierens de. Amst. Vh. 5 (1858) $572 \mathrm{pp}$.

- (Bierens de Haan). Bellavitis, G. Ven. At. (1858-59) 423-.

- (- - - Lindman, C. F. Stockh. Öfv. 33 (1876) No. 9, 7-; 35 (1878) No. 1, 3-; Stockh. Ak. Hndl. Bh. 10 (1885) No. 3, 268 pp.; Stockh. Ak. Handl. 24 (1890-92) No. 5, 231 pp. 


\section{Definite Integrals (simple)}

Tabulation of $\log \sin \frac{a \pi}{4}$. Lindman, C. F. Ups. S. Sc. N. Acta 14 (1891) No. 1, 15 pp. Theorem. Ramu8, C. Crelle J. 24 (1842) 257-. -. Grunert, J. A. Grunert Arch. 7 (1846) 358-.

-, attempt at proof. Mittag-Leffler, G. Stockh. Öfv. 30 (1873) (No. 8) 35-.

- of Cebyšev. Usov, N.A. (xII) Rec. Mth. (Moscou) 2 (1867) (Pt. 2) 93-.

- - demonstration. Franklin, F. Am.J. Mth. 7 (1885) 377-.

- - on integral $\int_{0}^{1} \phi(x) \cdot \psi(x) d x$. Korkine, A. C. R. 96 (1883) 326-.

Theorems. Winckler, A. Wien SB. 21 (1856) 389-.

-. Gegenbauer, L. Wien Ak. Sb. 97 (1889) (Ab. 2a) 1053-.

-, connection. Walton, W. [1872] QJ. Mth. 12 (1873) 126-.

- of Liouville, demonstrations. Glaisher, J. W. L. Mess. Mth. 4 (1875) 170-.

- - generalisation. Rawson, R. Mess. Mth. 5 (1876) 30-.

Theory. Schlömilch, O. Grunert Arch. 1 (1841) 417-.

-. Grunert, J. A. Grunert Arch. 2 (1842) 266- ; 4 (1844) 113-.

- (Grunert). Meyer, U. H. Grunert Arch. 5 (1844) 216-.

-. Russell, W. H. L. [1854] Phil. Trans. (1855) 157-.

-. [Bierens de] Haan, D. Amst. Vs. Ak. 4 (1856) 332-; 16 (1864) 28-; 1 (1866) (Ntk.) 117-; 3 (1869) (Ntk.) 323-; 5 (1871) (Ntk.) 65-; 7 (1873) 12-; Arch. Néerl. 2 (1867) 349-.

—. Enneper, A. Schlömilch Z. 6 (1861) 289 -.

- Gomes Teixeira, F. G. Teix, J. Sc. 9 (1889) 39-; Fschr. Mth. (1889) 276.

-. Schiff, P. Rec. Mth. (Moscou) 17 (1895) 607-; Fschr. Mth. (1895) 321-.

-, elementary. Meyer, A. Liége Mm. S. Sc. 7 (1851) $510 \mathrm{pp}$.

—, formulæ. Cauchy, A. L. [1815] Par. Ec. Pol. J. $28^{\circ} \mathrm{cah}$. (1841) 147-.

-, 一. Andréiewsky, -. Mth. A. 4 (1871) 550 -. -, foundations. Haan, D. Bierens de. Amst. Arch. Wisk. Gn. 1 (1856-59) 343-.

Transcendents, functional, reduction. Glaisher, J. W. L. Mess. Mth. 1 (1872) 153-.

-, squares of. Pendlebury, R. [1871] Mess. Mth. 1 (1872) 131-.

\section{Transformation.}

Bassani, A. G. Mt. 25 (1887) 223-

Nielsen, N. Kjøb. Ov. (1896) 335-.

Kapteyn, $W$. N. Arch. Wisk. 4 (1899) 243-. and calculation. Blažek, G. [1864] Wien Śb. 50 (1865) (Ab. 2) 60-.

cases. Schaar, M. Brux. Ac. Bll. 13 (1846) (pte. 2) 30-.

-. Schlömilch, O. Crelle J. 36 (1848) 271-.

- Hoppe, R. Crelle J. 40 (1850) 139-.

-. Besge, V. A. Liouv. J. Mth. 18 (1853) 112-
Transformation

3260

cases. Schlömilch, O. Leip. B. 9 (1857) 181-; Schlömilch Z. 3 (1858) 115-

-. Unferdinger, F. Arch. Mth. Ps. 53 (1871) $27-$

-. Glaisher, J. W. L. [1876] Mess. Mth. 6 (1877) 155-.

Silva, J. A. Martins da. G. Teix. J. Sc. 3 (1881) 177-.

by Cebyšev's formula, generalisations. Andreev, $K$. A. (xII) Kharkov Mth. S. Com. (1883) 19-.

and determination. Cauchy, A. L. C. R. 12 (1841) 1145-.

and evaluation. Haan, D. Bierens de. Amst. Vh. 8 (1862) 702 pp.

formula. Jacobi, C. G. J. Crelle J. 15 (1836) 1-. general. Kummer, E. E. Crelle J. 20 (1840) 1 -.

theory. Dirksen, E. H. Berl. B. (1848) 152-. in variation. Sabinin, E. T. [1890] Rec. Mth. (Moscou) 15 (1891) 99-; Fschr. Mth. (1890) 380 .

of $D_{k=0}^{n}\left(1-2 k \cos \gamma+k^{2}\right)^{-\frac{1}{2}}$ into definite integrals. Dirksen, E. H. Berl. B. (1848) 120-.

Transition from indefinite to definite integrals. Heine, H. E. Crelle J. 51 (1856) 382 .

Use of multiform functions in finding definite integrals. Raabe, J. L. Crelle J. 37 (1848) 356-.

$\int_{0}^{1} x^{n} d x e^{-\alpha x \sin \beta x} \cos \beta$ Fuss, N. [1810] St Pét. Ac. Sc. Mm. 11 (1830) 238-.

$\int_{0}^{\infty} e^{-x^{2}} d x=\frac{1}{2} \sqrt{\pi}$. Buzengeiger, K. H. J. Zach M. Cor. 24 (1811) 169-.

$\int \frac{d x}{\left(1+2 q x \cos \phi+q^{2} x^{2}\right)^{n-1}}$. Frullani, G. Mod. S. It. Mm. 19 (1821) 223-.

$\int_{0}^{\pi} z^{i} d z F(\cos z)$. Frullani, G. Mod. S. It. Mm. 20 (1828) 712-.

$\int_{0}^{\infty} \frac{x^{2 m} d x \cos a x}{1+x^{2 n}}$ and $\int_{0}^{\infty} \frac{x^{2 m} d x \cos a x}{1-2 x^{2 n} \cos \theta+x^{4 n}}$. Plana, G. [1836] Quetelet Cor. Mth.9 (1837) 47-.

$\int_{0}^{\infty} \phi(\sin a x, \cos b x) d x$, reduction. Raabe,J.L. Crelle J. 15 (1836) 355-.

$\int_{a}^{b} \phi(x) d x$ when $\phi(x)$ is infinite or discontinuous. Raabe, J. L. [1837] Crelle J. 20 (1840) 173-. $\int_{0}^{\pi} \log \left(1-2 a \cos x+a^{2}\right) d x$. Delaunay, $C$.
Liouv. J. Mth. 3 (1838) 355-. $\int_{0}^{\infty} \frac{\cos a x d x}{\left(1-x^{2}\right)^{n}}$. Catalan, E. C. Liouv. J. Mth.

$\int \frac{d M}{r}=V$, for potential of ellipsoid. Plana, $G$. Crelle J. 20 (1840) 271-. 
$\int_{a}^{y} y^{2 i} d y e^{-\frac{p}{y^{2}}-q y^{2}}$. Binet, J. P. M. C. R. 12 (1841) 958-.

$\int_{0}^{\pi} \cos i(u-x \sin u) d u$. Liouville, J. Liouv. J. Mth. 6 (1841) 36.

$\int_{0}^{\infty} \frac{x^{a-1}}{1+x} d x$. Bonnet, O. Liouv. J. Mth. 6 (1841) 238-.

$\int_{a}^{b} d x \cdot \sin \{\pi \cdot f(x)\}$, Fresnel's approxima-

tion method. R. Camb. Mth. J. 2 (1841) 81-. $\int_{0}^{\frac{\pi}{2}} \log (\sin \theta) d \theta$. Ellis, R. L. Camb. Mth. J.

$\int_{0}^{\infty} \frac{\cos a x d x}{\left(1+x^{2}\right)^{n}}$. Malmsten, C.J. Stockh. Ak. Hndl. (1841) 65-.

$\int_{0}^{\infty} \phi(\sin a x, \cos b x) \frac{d x}{x}$, reduction. Raabe, J. L. Crelle J. 23 (1842) 105-; 25 (1843) 160-.

$\int_{0}^{1} \frac{\log (1+x) d x}{1+x^{2}}$. Bertrand, J. Liouv. J. Mth. 8 (1843) 110-.

and

$$
\int_{0}^{m} \frac{(1-p \cos t x) \phi(x) d x}{1-2 p \cos t x+p^{2}}
$$

$$
\int_{0}^{m} \frac{p \sin t x f(x) d x}{1-2 p \cos t x+p^{2}} \text {. }
$$

Malmsten, C. J. Ups. N. Acta S. Sc. 12 (1844) 155-.

$\int_{0}^{1} \frac{\log (1+x)}{1+x^{2}} d x$. Serret, J.A. Liouv. J. Mth. 9 (1844) 436.

$\int_{0}^{1} \frac{\log (1+x)}{1+x^{2}} d x: \quad$ Ellis, R. L. $\quad$ (vI Adds.) Camb. Mth. J. 4 (1845) 143-. $-\int_{30}^{x} \frac{\log (1-a)}{a} d a . \quad$ Schäffer, -. Crelle J.

$\int_{0}^{2 \pi} \frac{d \phi}{1-A \cos \phi-B \sin \phi}$, value for imaginary values of $A$ and $B$. Jacobi, C. G. J. Crelle J. 32 (1846) 8-.

$\int_{0}^{\frac{1}{2} \pi} \frac{\log \left(1+n \sin ^{2} \phi\right) d \phi}{\sqrt{1-k^{2} \sin ^{2} \phi}}$. Roberts, W. Liouv.

J. Mth. 11 (1846) 471-.

$\int_{0}^{\infty} \frac{d \theta}{\theta^{2}+a^{2}} e^{-x \theta}$. Schlömilch, O. Crelle J. 33 (1846) 325-. $\int_{0}^{\infty} e^{-a x} \sin ^{m} x d x$. Schlömilch, O. Grunert
Arch. 7 (1846) 38-.

$\int_{0}^{\infty} \frac{\cos b x}{x^{2}-a^{2}} d x$ and $\int_{0}^{\infty} \frac{x \sin b x}{x^{2}-a^{2}} d x$. Schlömilch, O. Grunert Arch. 7 (1846) 270-.

$$
\begin{gathered}
\int_{0}^{\infty} \frac{e^{-\beta x} \cdot x^{m-1} d x}{1+x^{2}}, \int_{0}^{\infty} \frac{\cos \beta x \cdot x^{m-1} d x}{1+x^{2}}, \\
\int_{0}^{\infty} \frac{\sin \beta x \cdot x^{m-1} d x}{1+x^{2}} .
\end{gathered}
$$

Svanberg, A.F. Liouv. J. Mth. 11 (1846) 197-.

$$
\int_{0}^{\infty} \frac{e^{-b x} d x}{x^{2}-a^{2}} \text { and } \int_{0}^{\infty} \frac{x e^{-b x} d x}{x^{2}-a^{2}}
$$

Arndt, F. Grunert Arch. 10 (1847) 247-. $\int_{0}^{1}\left(\frac{x^{a-1}}{1-x}-\frac{n x^{n a-1}}{1-x^{n}}\right) d x$, two developments. Arndt, $F$. Grunert Arch. 10 (1847) 253-. $\int_{0}^{\infty} \frac{\sin x}{x} d x$, value. Newman, F.W. Camb. and Dubl. Mth. J. 2 (1847) 75-.

$\int \arctan f(x), f(x)$ being unique function of $x$. Raabe, J. L. Zür. Mt. 1 (Heft 1) (1847) 61-. $\int_{0}^{\infty} \frac{z^{a-1} d z}{1+z^{n}+z^{2 n}}$. Dienger, J. N. A. Mth. 7 (1848) 201-.

$\int_{0}^{\infty} \frac{\sin a x}{x} d x$. Besge, V.A. Liouv. J. Mth. 14 (1849) 31-.

$\int_{0}^{\pi} \log \left(1-2 r \cos x+r^{2}\right) \cos n x d x$. Haan, $D$. ${ }^{0}$ Bierens de. Grunert Arch. 13 (1849) 193 -.

$\int_{0}^{\infty} \frac{x^{\mu} d x}{r^{2}+2 r x \cos u+x^{2}}$. Schlömilch, O. Grunert Arch. 12 (1849) 198-.

$\int_{0}^{\infty}\left\{\frac{a^{n}}{[n]}-\frac{a^{n-1}}{[n-1]} \frac{1}{\phi}+\frac{a^{n-2}}{[n-2]} \frac{1}{\phi^{2}}-\&\right.$ c. $\mp \frac{a^{2}}{[2] \phi^{n-2}}$ $\left.\pm \frac{a}{\phi^{n-1}} \mp \frac{1}{\phi^{n}} \pm \frac{1}{\phi^{n}} e^{-a \phi}\right\} \times \frac{\sin \phi}{\phi} d \phi$.

Smaasen, $W$. Amst. Ts. Wis. Nt. Wet. 2 (1849) 251-.

$\int_{0}^{2 \pi} f\left(r e^{\phi i}\right) e^{-n \phi i} d \phi$. Dienger, J. Grunert Arch. 15 (1850) 119-. $\int_{0}^{\infty} \frac{\sin ^{n} x}{x^{m}} d x$. Lindman, C. F. Grunert Arch.

$\int \arctan f(x), f(x)$ being unique function of $x$. Raabe, J.L. Crelle J. 41 (1851) 54-.

$\int_{0}^{1} \frac{1}{(a-x z)^{r+1}} \cdot \frac{d z}{(1-z)^{1-n} z^{n}} \cdot$ Dienger,J. Crelle J. 42 (1852) 283-.

$\int_{0}^{\infty} \frac{e^{-c x^{n}}}{a+x^{n}} d x$. Lindman, C. F. Grunert Arch. 21 (1853) 113-.

$\int_{0}^{\infty} \frac{e^{-g x} \sin a x d x}{1+x}$. Popoff [Popov], A. T. Mosc. S. Nt. Bll. 27 (1854) (Pte. 2) 206-; (xIr) Kazan Un. Mm. (1855) (Bk. 1) 57-. $\int_{0}^{1} \frac{1-\phi^{a}}{1-\phi} d \phi$. Lebesgue, $V . A$. Liouv. J. Mth. 1 (1856) 377-.

$\int_{a}^{b} \frac{d x}{\sqrt{B^{\prime} x^{3}+C^{\prime} x^{2}+D^{\prime} x+E^{\prime}}} \cdot$ Lindman, C. $F$. Grunert Arch. 27 (1856) 1-. 
$\int_{0}^{1} \frac{t^{\mu+\frac{1}{2}}(1-t)^{\mu-\frac{1}{2}} d t}{\left(a+b t-c t^{2}\right)^{\mu+1}}$. Liouville, J. Liouv. J. $\int_{-1}^{+1} \frac{d x}{(a-x) \sqrt{1-x^{2}}}$. Hermite, C. A. Mt. 3 Mth. 1 (1856) 421-.

$\int_{0}^{\infty} \frac{e^{-p x} \sin q x \sin r x \ldots}{x^{a}} d x$. Haan, D. Bierens (1869-70) 83.

de. Amst. Arch. Wisk. Gn. 1 (1856-59) 177-, 288-.

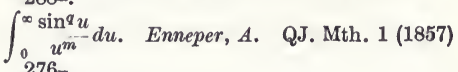

$\int_{0}^{\infty} F(x) \frac{\cos p x d x}{q^{2}+x^{2}}, \int_{0}^{\infty} F(x) \frac{\sin p x d x}{q^{2}+x^{2}}$, reduction and applications. Haan, D. Bierens de. Amst. Vh. 5 (1857) 117 pp.

$\int_{0}^{1} \frac{t \mu+\frac{1}{2}(1-t)^{\mu-\frac{1}{2}}}{\left(a+b t-c t^{2}\right)^{\mu+1}}$. Liouville, J. Liouv. J. Mth. 2 (1857) 47-.

$\int_{0}^{1} \frac{t^{\mu+\frac{l}{2}}(1-t)^{\mu-\frac{1}{3}} d t}{\left(a+b t-c t^{2}\right)^{\mu+1}}$. Cayley, A. Liouv. J. Mth. 2 (1857) 49 -.

$\int_{0}^{1} \frac{x^{p-1}(1-x)^{q-1} d x}{(1+\sqrt{1+g x})^{2 p+2 q}}$. Liouville, J. Liouv. J. Mth. 2 (1857) 279.

$\int_{0}^{\infty} e^{-a x} e^{\left(x^{2}+b x\right) \sqrt{ }-1} d x$. Popoff, A. St Pét. Ac. Sc. Bll. 15 (1857) 307-.

$\int_{0}^{\infty} \frac{\sin x^{m}}{x^{n}} d x$. Minding, E. F. A. Grunert Arch. 30 (1858) 171-.

$\int_{0}^{\infty} e^{u z e^{-z}} z e^{-z} d z$ for calculation of series

$$
1+\frac{u}{1}+\left(\frac{u}{2}\right)^{2}+\left(\frac{u}{3}\right)^{3}+\text { etc. }
$$

Schlömilch, O. Schlömilch Z. 4 (1859) 433-. $\int_{0}^{\left(\frac{a}{b}\right)^{\frac{1}{n}}}\left(a-b x^{n}\right)^{\frac{p}{q}} x^{m-1} d x$. Bacaloglo, E. Gru$\int_{0}^{\infty} \frac{\sin ^{p} x}{x^{q}} d x$. Schlömilch, O. Schlömilch Z.

$\int_{0}^{1} \frac{\left(z^{m}-1\right) d z}{\log z}$. Wolfers, J. P. Grunert Arch. 37 (1861) 245-.

$\int_{0}^{\infty} \frac{1-\cos ^{m} x}{x^{2}} d x$. Stefan, J. Schlömilch Z. 7 (1862) 356-.

$\int_{0}^{\pi} \cos ^{a+b} \frac{1}{2} x \cos \frac{1}{2} a x \cos \frac{1}{2} b x d x$. Lindman, C.F. [1864] Stockh. Öfv. 21 (1865) 373-.

$\int_{0}^{\beta} \frac{\sin ^{m} x}{x} d x$. Besso, D. G. Mt. 7 (1869) 210-. $\int_{0}^{1} \frac{\operatorname{arctang} x d x}{1+x}$. Liouville, J. Liouv. J. Mth. 14 (1869) 298-.

$\int \sin x^{n} d x, \int \cos x^{n} d x$, etc., between limits. Oettinger, L. Arch. Mth. Ps. 49 (1869) 51-.

$\int_{-1}^{+1} \frac{\sin \alpha d x}{1-2 x \cos \alpha+x^{2}}$. Hermite, C. Bll. Sc. Mth. As. 1 (1870) 320-.

$A=\int_{0}^{\infty} f\left(x+\frac{1}{x}\right) \operatorname{arctang} x \frac{d x}{x}$. Liouville, J. Liouv. J. Mth. 15 (1870) 7-.

$\int_{0}^{\infty} \frac{\cos b x}{a^{2}+x^{2}} d x$. Glaisher, J.W.L. [1871] Mess. Mth. 1 (1872) 35.

$\int_{0}^{\infty} \sin \left(x^{n}\right) d x$ and $\int_{0}^{\infty} \cos \left(x^{n}\right) d x$. Glaisher, J.W. L. [1871] Mess. Mth. 1 (1872) 106-. $\int_{0}^{\infty} \frac{x^{a-1} d x}{1+x}$. Walton, W. [1871] QJ. Mth. 12 (1873) 39-

$\int_{0}^{1} \frac{x^{m-1}-x^{-m}}{(1+x) \log x} d x$. Walton, W. [1872] QJ. Mth. 12 (1873) 184-.

$\int_{b}^{c} \phi(x, a) d x, n$th differentiation. Walton, $W$. [1872] QJ. Mth. 12 (1873) 215-.

$\int_{a}^{x}(x-u)^{p-1} f(u) d u$. Lyetnikov [Letnikowv], ${ }_{A}$. V. [1872-73] (xII) Rec. Mth. (Moscou) 7 (1874-75) (Pt. 1) 5-; (x) Bll. Sc. Mth. As. 7 (1874) 233-.

$\int_{0}^{\infty} \sin x^{2} d x$ and $\int_{0}^{\infty} \cos x^{2} d x$. Glaisher, J.W.L. [1872-75] QJ. Mth. 13 (1875) 343-.

$\int_{0}^{\infty} \cos \left(x^{2}\right) d x$ and $\int_{0}^{\infty} \sin \left(x^{2}\right) d x$. Cayley, $A$. QJ. Mth. 12 (1873) 118-.

$\int_{0}^{2 \pi} \frac{d \phi}{A+B \cos \phi+C \sin \phi}$. Worpitzky, $(D r)$-. Arch. Mth. Ps. 55 (1873) 59-.

$\int F(x) \log x d x$ between real and positive limits,

$F(x)$ being rational function. Besso, $D$. G. Mt. 12 (1874) 1-.

$\int_{0}^{\infty} \frac{\cos b x}{a^{2}+x^{2}} d x$. Glaisher, J.W. L. Mess. Mth. 5 (1876) 168-.

$\int_{0}^{\frac{\pi}{2}} \frac{x F(x) d x}{1+p \sin ^{2} x \cos ^{2} x}, F$ being a goniometrical function. Bierens de Haan, D. Amst. Ak. Vs. M. 12 (1878) 325-; Arch. Néerl. 13 (1878) 389-.

$\int_{0}^{1} \frac{z^{a-1}-z^{-a}}{1-z} d z$. Hermite, C. Tor. Ac. Sc. At. 14 (1878) 91-.

$\int_{0}^{1}(u)_{n} d u$, infinite value. Schröder, E. Z. Mth. Ps. 25 (1880) 106-.

$\int_{0}^{\infty} \frac{\phi(a x)-\phi(b x)}{x^{n}} d x$, expansion for. Elliott, E. B. Mess. Mth. 10 (1881) 118-。 
$\int_{a}^{\beta} \frac{y d z}{x-z}$. Jürgens, E. [1881] Mth. A. 19 (1882) 435-.

$\int_{0}^{1}(\log x)^{2 n-2} \log \left(\frac{1+x}{1-x}\right) \frac{d x}{x}$ etc. Scott, R. F. Mess. Mth. 10 (1881) 142-.

$\int_{0}^{\pi} \frac{f\left(e^{x i}\right)+f\left(e^{-x i}\right)}{\sqrt{1-2 \alpha \cos x+a^{2}}} d x$, reduction. Silva, J. A. Martins da. G. Teix. J. Sc. 3 (1881) 65 -.

$\int_{0}^{\infty} \frac{\cos s x}{\left(a^{2}+x^{2}\right)^{\frac{1}{2}(2 p+1)}} d x$. Thomson, J.J. QJ. Mth. 18 (1882) 377-.

$\int_{0}^{\infty} \frac{\cos a x}{\left(1+x^{2}\right)^{n}} d x$. Catalan, E. C. Liège S. Sc. Mm. 12 (1885) No. 2, 26-.

$\int_{0}^{2 \pi f(\sin \phi, \cos \phi) d \phi} \frac{\text { Laguerre, } E \text {. }}{F(\sin \phi, \cos \phi)}$. R. 100 (1885) 624-.

$\int_{0}^{\infty} \frac{\sin a x}{\sin b x} \frac{d x}{1+x^{2}}$ and related integrals. Schläfi, L. Acta Mth. 7 (1885) 187-. $\int_{0}^{\infty} e^{-x}(1+x / a)^{-a-b} d x$. Stieltjes, - . Bll. Sc.
Mth. $9(1885) 306-$.

$\int_{0}^{\infty} \phi(x) x^{\frac{1}{2} s-1} d x$. Lerch, M. J. Prag Sb.

(1886) (Mth.-Nt.) 588-; Fschr. Mth. (1886) $258-$.

$\int_{0}^{a} e^{x^{2}} d x$, expansion. Stieltjes, T.J. Acta Mth. 9 (1887) 167-.

$\int_{0}^{r} e^{-t^{2}} d t$, approximate calculation. Mansion, P. Brux. S. Sc. A. 12 (1888) (Pt. 1) 63-. $\int_{a}^{b} f(x) G(x) d x$. Stieltjes, T. J. N. A. Mth.
$7(1888)$ 161-.

$\cos ^{p} \phi \cos q \phi$ etc., integration. Beaupain, $J$.

Brux. Mm. Cour. $4^{\circ}, 51$ (1889) No. 4, 60 pp.; 52 (1890-93) No. 4, $62 \mathrm{pp}$.

$\int_{0}^{\pi} \cot (x-a) d x$. Gomes Teixeira, F. G. Teix.

J. Sc. 9 (1889) 113-; Fschr. Mth. (1889)

$\int_{0}^{\infty} e^{-x^{2}} d x$. Gomes Teixeira, F. Prag Sb.

(1889) 1 (Mth.-Nt.) 118-.

$\int_{0}^{\infty} \frac{e^{-b x} \sin a x}{x} d x$. Martins da Silva, J. A.

[1890] Lisb. J. Sc. Mth. 6 (1902) 14-.

$\int_{0}^{2 \pi} \log \left\{(x-a \cos \theta)^{2}+(y-b \sin \theta)^{2}\right\}{ }_{\sin }^{\cos } m \theta d \theta$.

Gibson, G. A. Edinb. Mth. S. P. 10 (1892) 83 -.

$\int_{0}^{\infty} \frac{e^{a i \phi} z^{a i} d z}{1-z e^{i \phi}}$, formulø deduced from. Lerch, M. Časopis 21 (1892) 218-; Fschr. Mth. (1892) 265-.

$\int_{a}^{b} F(x) \frac{d x}{z-x}$. Sonin, $N$. St Pét. Ac. Sc. Mm. 38 (1892) No. 14, $44 \mathrm{pp}$.

$\int_{0}^{2 \pi} \sin ^{p} x \cos ^{q} x \frac{\sin }{\cos }(m x) d x$, integration. Studnička, F.J. Casopis 23 (1894) 67-; Fschr. Mth. (1893-94) 481.

$\int_{0}^{\pi} e^{a \theta} \sin ^{m} \theta d \theta$. Forsyth, A. R. QJ. Mth. 27 (1895) 216-.

$\int \frac{\cos y x}{\left(a^{2}+b^{2} x^{2}\right)^{n}} d x$. Oltramare, G. As. Fr. C. R. (1895) (Pt. 1) 182.

$\int_{0}^{\infty} \frac{\sin a x}{x} d x=\frac{\pi}{2}$, generalisation. Stфrmer, $C$. Christiania F. (1895) No. 4, $11 \mathrm{pp}$.

$\frac{2}{\pi} \int_{0}^{\infty} \arctan \frac{a}{x} \arctan \frac{b}{x} d x=\log \frac{(a+b)^{a+b}}{a^{a} b^{b}}$, proof. Lerch, M. [1899] Casopis 29 (1900) 39-; Fschr. Mth. (1899) 391. $\int_{0}^{\frac{\pi}{2}}(10$ [1899] N. Y Am. Mth S. Bll 7 (1901) 390-.

$\frac{2}{\sqrt{\pi}} \int_{0}^{t} \epsilon^{-t^{2}} d t$, with tables of values. Burgess, $J$. Edinb. R. S. T. 39 (1900) 257-.

\section{Multiple integrals.}

Lejeune-Dirichlet, G. 'Crelle J. 4 (1829) 94-; Berl. B. (1839) 18-, 61-; C. R. 8 (1839) 156-.

Cauchy, A. L. C. R. 11 (1840) 1000-, 1019.

Cayley, A. Liouv. J. Mth. 10 (1845) 158-, 242-.

Ellis, R. L. Camb. Mth. J. 4 (1845) 64-, 116-.

Bronwin, B. Ph. Mg. 28 (1846) 373-.

Roberts, $W$. Liouv. J. Mth. 11 (1846) 201-.

Schlömilch, O. Tortolini A. 3 (1852) 327-.

Winckler, A. [1869] Wien Sb. 60 (1870) (Ab. 2) 379-.

Scott, R. F. [1875] Mess. Mth. 5 (1876) 22-.

Zinine, N. N. Fschr. Mth. (1887) 281.

Sauvage, L. Mars. Fac. Sc. A. 2 (1892) 3 -.

Puzyna, J. Prace Mt.-Fiz. 4 (1893) 1-; Fschr. Mth. (1893-94) 491-.

Abel's theorem $\pi f(x)=\int_{0}^{x} \frac{d y}{\sqrt{x-y}} \int_{0}^{y} \frac{f^{\prime}(\xi) d \xi}{\sqrt{y-\xi}}$. Tait, P. G. [1874] Edinb. R. S. P. 8 (1875) 440-.

Application of Gauss's integration method. Löwenstern, S. St Pét. Mm. Sav. Etr. 3 (1837) 279-.

- Stokes's formula. Vogt, - N. A. Mth. 19 (1900) 97-.

Approximate multiple integration between limits by summation. Maxwell, J.C. [1877] Camb. Ph. S. P. 3 (1880) 39-.

Boole's theorem. Cayley, A. Liouv. J. Mth. 13 (1848) 245-.

Cases. Boole, G. [1846] Ir. Ac. T. 21 (1848) 140-. 


\section{Multiple Integrals}

Cases. Liouville, J. Liouv. J. Mth. 4 (1859) 155-. 29-.

-. Sersawy, V. Arch. Mth. Ps. 64 (1879) 30-.

-. Staeckel, -. Bll. Sc. Mth. 21 (1897) 31-.

Change of variables. Catalan, E. C. Par. S. Phlm. PV. (1839) 123-.

- - Baehr, G. F.W. Arch. Mth. Ps. 41 (1864) 453-.

Class. Tchebicheff, $P$. Liouv. J. Mth. 8 (1843) 235-.

- Liouville, J. Liouv. J. Mth. 1 (1856) 82-.

-. Lipschitz, R. Crelle J. Mth. 101 (1887) 214-.

-, numerical values. Hamilton, (Sir) W. $R$. Ph. Mg. 14 (1857) 375-.

Classes. Elliott, E. B. [1876-77] L. Mth. S. P. 8 (1877) 35-, 146-.

Convertent functions. Cockle, (Sir) J. [1868] Manch. Lt. Ph. S. P. 8 (1869) 2-; 9 (1870) 86-; 10 (1870) 1-.

Curvilinear coordinates, Stokes's theorem. Padova, E. Mil. I. Lomb. Rd. 25 (1892) 1021-.

Determination by transformation of coordinates. Cauchy, A. L. C. R. 13 (1841) 34-.

Differentiation with regard to parameter on which limits also depend. Zindler, $K$. Wien Ak. Sb. 106 (1897) (Ab. 2a) 359-.

\section{Double integrals.}

Schlömilch, O. Grunert Arch. 11 (1848) 174 Dienger, J. Grunert Arch. 13 (1849) 286-.

Steen, A. Kiöb. Dn. Vd. Selsk. Skr. 1 (1849) 333-.

Besge, V. A. Liouv. J. Mth. 3 (1858) 324.

Le Cordier, P. C. R. 66 (1868) 707-.

Besso, D. G. Mt. 10 (1872) 79-.

Du Bois-Reymond, P. [1882] Crelle J. Mth. 94 (1883) 273-.

Goursat, É. C. R. 96 (1883) 1304-.

Arzeld, C. Bologna Ac. Sc. Mm. 2 (1891) 133-.

Pochhammer, L. Mth. A. 41 (1893) 179-.

of algebraic functions, reduction. Picard, $\hat{E}$. C. R. 126 (1898) 1116-

apparently with two values. Puzyna, J. [1883] (xII) Krk. Ak. (Mt.-Prz.) Pam. 9 (1884) 48-.

and binary intervals, theory. Méray, C. Par. Éc. Norm. A. 16 (1899) 193-.

calculation, approximate. Minding, E. F.A. Crelle J. 6 (1830) 91-. - case. Callandreau, O. C. R. 96 (1883)

Cases. Roberts, $W$. A. Mt. 3 (1869-70) $327-$

-. Didon, F. Par. Ec. Norm. A. 7 (1870) 89-.

-. Orlow, G. A. N. A. Mth. 1 (1882) 311-.

Cauchy's theorem. Kluyver, J. C. Fschr. Mth. (1897) 258.
Double Integrals 3270

class. Goursat, É. Acta Mth. 5 (1884) 97-.

- including Fourier's. Du Bois-Reymond, $P$. Crelle J. 69 (1868) 65-.

Crofton's theorem, corollary. Hoppe, $R$. C. R. 70 (1870) 1394-; Arch. Mth. Ps. 55 (1873) 426-

differentiation and integration. Bierens de Haan, D. Amst. Vs. Ak. 5 (1871) (Ntk.) 53-, 65-; Arch. Néerl. 6 (1871) 329-.

of explicit irrational functions, theory. Bier. mann, $O$. Wien Ak. Sb. 98 (1890) (Ab. 2a) 340 -

formulø, integration. Fuss, N. [1826] St Pét. Ac. Sc. Mm. 11 (1830) 294-

Fourier's theorem, Boole's and other proofs. Alexander, P. Edinb. Mth. S. P. 3 (1885) 45-.

geometric demonstration. Chasles, M. Liouv. J. Mth. 3 (1838) 10-.

- evaluation. Collignon, É. As. Fr. C. R. 3 (1874) 1193-.

integration. Sylvester, J. J. Ph. Mg. 14 (1839) 298-.

inversion. Appell, P. Am. J. Mth. 19 (1897) $377-, 382$.

-. Le Roux, J. C. R. 130 (1900) 882-.

of 2nd kind in theory of algebraic surfaces.

Picard, É. [1897] Liouv. J. Mth. 5 (1899) 5 -.

maxima and minima. $K o b b, G$. Stockh. Öfv. (1890) 385-; Fschr. Mth. (1890) 382 ; Acta Mth. 16 (1892-93) 65-; 17 (1893) 321-.

and their periods, elementary theory. Marie, M. C. R. 75 (1872) 576-, 614-, 660-.

Poisson's theorem, proof. Roberts, $W$. Liouv. J. Mth. 11 (1846) 210-.

properties. Rodrigues, $(D r)$-. Par. S. Phlm. Bll. (1815) 34-.

of rational functions, residues. Picard, $\dot{E}$. C. R. 124 (1897) 433-.

reduction, and new invariant in theory of algebraic surfaces. Picard, É. C. R. 126 (1898) 297-.

- to products of simple integrals. Schlömilch, $O$. Z. Mth. Ps. 9 (1864) $205-$.

- - quadratures. Winckler, A. [1851] Crelle J. 45 (1853) 102-.

residues. Poincaré, H. C. R. 102 (1886) 202-; Acta Mth. 9 (1887) 321-.

- (Poincaré). Marie, M. N. A. Mth. 10 (1891) 77-.

-, theory. Marie, M. C. R. 75 (1872) 695-, 751-.

returning to same form after transformation of variables. Jacobi, C. G. J. Crelle J. 15 (1836) 193-.

theory. Harnack, A. Mth. A. 26 (1886) 566-. -. Pringsheim, A. Münch. Ak. Sb. 28 (1899) $59-$.

- Green's and Cauchy's theorems. Pringsheim, A. Münch. Ak. Sb. 29 (1900) 39-, 268-.

- and invariant of two triangles. Le Roux, -. Par. S. Mth. Bll. 28 (1900) 168-.

transformation. Jacobi, C. G. J. [1827-32] Crelle J. 2 (1827) 234-; 8 (1832) 253-, 321-; 10 (1833) 101-. 
transformation. Cayley, A. Mess. Mth. 4 (1875) 92-.

—. Berger, A. Stockh. Ófv. (1887) 153-.

-. Stolz, O. Mh. Mth. Ps. 9 (1898) 316-, $371 ; 10$ (1899) 96.

- formula. Scheibmer, $W$. Leip. Mth. Ps. B. 36 (1884) 185-

- - Euler's. Plana, G. Brux. Ac. Bll. 10 (1843) 303-.

-, general. Winckler, A. Wien D. 20 (1862) 97-.

- by Green's theorem. Gmeiner, J.A. Mh. Mth. Ps. 4 (1893) 277-

and triple integrals, simultaneous substitutions, theory. Unferdinger, $F$. Wien Sb. 63 (1871) $(A b .2)$ 773-.

- - - transformation. Piola, G. Mod. Mm. S. It. 20 (1828) 272-.

- - - - Tait, P. G. [1873] Edinb. R.

S. P. 8 (1875) 209-.

$$
\begin{aligned}
& \int_{0}^{\infty} \int_{0}^{\infty} \phi\left(a x^{m} \pm b y^{n}\right) x^{p-1} y^{q-1} d x d y, \\
& \int_{-\infty}^{+\infty} \int_{-\infty}^{+\infty} \phi\left(a x^{m} \pm b y^{n}\right) x^{p-1} y^{q-1} d x d y .
\end{aligned}
$$

Raabe, J. L. Crelle J. 37 (1848) 345-.

$\int_{b}^{c} \int_{0}^{b} \frac{\log \left(\mu^{2}-\nu^{2}\right) d \mu d \nu}{\sqrt{\left(c^{2}-\mu^{2}\right)\left(\mu^{2}-b^{2}\right)\left(c^{2}-\nu^{2}\right)\left(b^{2}-\nu^{2}\right)}}$.

Roberts, $W$. Liouv. J. Mth. 15 (1850) 238-.

Equality. Besge, $V . A$. Liouv. J. Mth. 3 (1858) 416.

Evaluation. Ellis, R. L. Camb. Mth. J. 4 (1845) 1-。

-, case. Gregory, D. F. Camb. Mth. J. 2 (1841) 215-.

-, process. Arndt, F. Grunert Arch. 5 (1844) 443-.

Expansibility of quotient of two definite integrals of form $\int d x d y \ldots d z$. Schläfli, $L$. Crelle J. 67 (1867) 183-.

Expansion. Schlömilch, O. Schlömilch Z. 1 (1856) 75-.

Formula, demonstration. Thomson, (Sir) $W$. Liouv. J. Mth. 10 (1845) 137-.

-, - (Thomson). Liouville, $J$. Liouv. J. Mth. 1 (1856) 445-.

-, Fourier's, generalised by Liouville. Popov, A. T. (xII) Kazan Un. Mm. (1848) (Bk. 4) 3-.

Frullanian integrals. Elliott, $E, B$. L. Mth. S. P. 15 (*1883-84) 12-.

Integral expressing area of circle and of spherical triangle. Schläfi, $L$. Liouv. J. Mth. 20 (1855) 359-.

Integrals of area and volume, transformations and values. Tortolini, B. G. Arcad. 80 (1839) 13-; 82 (1840) 97-.

- with 3 complex variables. Picard, $\dot{E}$. C. R. 108 (1889) 132-.

- of Jacobi. Zinin, N. N. Vars. S. Nt. Tr. (1897) (P8. C.) Fasc. 1, No. 3, ii.

- - any order, and their periods, elementary theory. Marie, M. C. R. 75 (1872) 1078-, 1247-.
Integrals in probability theory, two. Czuber, $E$. Mh. Mth. Ps. 2 (1891) 119-。

- representing products and powers of simple definite integrals. Raabe, J.L. [1847] Zür. Mt. 1 (Heft 2) (1847-48) 29-; Crelle J. 48 (1854) 137-.

- in theory of attractions. Cayley, A. Camb. and Dubl. Mth. J. 2 (1847) 219-; 7 (1852) 174-.

- unaltered in form by transformation of the variables. Gundelfinger, S. [1880] Crelle J. Mth. 91 (1881) 215-.

-, value of which is affected by order of integration. Decher, G. Grunert Arch. 19 (1852) 403-; 22 (1854) 413-.

Inversion. Volterra, $V$. Rm. R. Ac. Linc. Rd. 5 (1896) (Sem. 1) 289-.

-, mode. Appell, P. C. R. 124 (1897) 213-.

Limits. MacColl, $H$. L. Mth. S. P. $16(1884$ 85) $142-$

Line and surface integrals. Peirce, B. O. Am. Ac. P. 26 (1891) 20 -

-, surface and volume integrals, relation among. Tait, - Edinb. R. S. P. 16 (1890) 257.

Maxima and minima, criteria. Sabinine, $G$. [1869] St Pét. Ac. Sc. Bll. 15 (1871) 70-.

Mean values of functions. Cauchy, $A . L$. C. R. 18 (1844) 558-; 20 (1845) 119-, 212-; 26 (1848) 549-, 624-, 666-; 27 (1848) 6-, 37-; 29 (1849) 341-.

Minimum. Lebesgue, $H$. C. R. 131 (1900) 935-.

Multiple Gaussian sums. Weber, H. [1871] Crelle J. 74 (1872) 14-.

- and single integrals. Nanson, E. J. Mess. Mth. 26 (1897) 119-.

Ostrogradskii's formulæ. Zinin, $N . N$. Rec. Mth. (Moscou) 15 (1891) 645-; Fschr. Mth. (1891) 304-.

Problem. Zinine, N. N. Fschr. Mth. (1888) 308-.

Reduction. Catalan, E. C. Liouv. J. Mth. 4 (1839) 323-; 6 (1841) 81-; 8 (1843) 239-; Brux. Mm. Cour. 4\%, 14 (1841) 47 pp.

- (error in Catalan's paper, 1841). Boole, $G$. Camb. Mth. J. 3 (1843) 277-.

-. Chelini, D. G. Arcad. 94 (1843) 49-.

-. Liouville, J. Liouv. J. Mth. 1 (1856) 289-.

-. Enneper, A. Gott. Nr. (1867) 164-; Z. Mth. Ps. 15 (1870) 121-.

- Létnikov, A. V. Rec. Mth. (Moscou) 14 (1890) 303-; Fschr. Mth. (1889) 288.

-. Nekrasov, P. A. [1891] Rec. Mth. (Moscou) 16 (1893) 81-; Fschr. Mth. (1891) 305.

-. La Vallée-Poussin, C. J. de. C. R. 127 (1898) 950 .

-, application of general differentiation. Nekrasov, P. A. Rec. Mth. (Moscou) 14 (1890) 410-; Fschr. Mth. (1889) 288-.

-, cases. Raabe, J. L. Crelle J. 28 (1844) 19 -

-, -. Schaar, M. Brux. Ac. Bll. 15 (1848) (pte. 2) 501-.

-,- Genocchi, A. Tortolini A. 8 (1857) 284 .

_, - Schlömilch, O. Leip. B. 9 (1857) 67-; Liouv. J. Mth. 2 (1857) 206-. 


\section{Multiple Integrals}

Reduction, cases. Catalan, E. C. Liège S. Sc. Mm. 12 (1885) No. 2, 12-, 17-.

-, 一. Maggi, G. A. Mil. I. Lomb. Rd. 19 (1886) 689-.

-, -. Sonine, N. J. Fschr. Mth. (1889) $287-$.

-, 一. Zinin, N. N. Vars. S. Nt. Tr. (188990) (C. R., P8. C.) No. 6, 9-

-, -. Sonin, N. Ja. Rec. Mth. (Moscou) 14 (1890) 527-; Vars. S. Nt. Tr. (Mm.) 1 (1891) No. 4, $7 \mathrm{pp}$.

-, 一. Black, A. [1897] Camb. Ph. S. T. 16 (1898) 219-.

-, direct, of class. Silva, J. A. Martins da. G. Teix. J. Sc. 3 (1881) 49-.

-, formula. Sonin, N. Ja. Vars. S. Nt. Tr. (1889-90) (C. R., Ps. C.) No. 2, 13-; Fschr. Mth. (1889) 287.

- of generalised. La Vallée-Poussin, C.J.de. Liouv. J. Mth. 5 (1899) 191-.

-, quaternion formulæ. Carvallo, $E$. Par. S. Mth. Bll. 18 (1890) 80-.

-, Zinin's treatment of problem. Nekrasov, P. A. Rec. Mth. (Moscou) 16 (1893) 823-. Space and surface integrals. Fiske, T. S. [1891] A. Mth. 6 (1891-92) 61-, 131.

Surface and curvilinear integrals. Cazzaniga, T. Mil. I. Lomb. Rd. 33 (1900) 567-.

- integrals, condition of dependence on boundary alone, new interpretation. Méray, $C$. C. R. 128 (1899) 913-.

- - definition. Lebesgue, H. C. R. 131 (1900) 867-.

- - evaluation, and application to expansion, in series, of potential of ellipsoids. Hobson, E. $W$. L. Mth. S. P. 24 (1893) 80-.

- - in problem of 3 bodies. Weiler, A. As. Nr. 74 (1869) 85-.

- - transformation into multiple line integrals. Larmor, J. Mess. Mth. 17 (1888) 23-.

Mth 18 (1889) -. McAulay, A. Mess.

Theorem. Volterra, $V$. Tor. Ac. Sc. At. 32 (1896) 597- or 859-.

- of Fourier. Glaisher, J.W. L. [1876] Mess. Mth. 6 (1877) 30-

- Frullani, extensions. Leudesdorf, C. L. Mth. S. P. 9 (1877-78) 118-.

- Hadamard. Hurwitz, A. C. R. 128 (1899) 350-.

- - Kronecker. Koenigs, G. Liouv. J. Mth. 2 (1896) 41-.

Theorems. Jacobi, C. G. J. Crelle J. 12 (1834) 1-.

-, general. Ellis, R. L. Camb. and Dubl. Mth. J. 1 (1846) 1-.

- of Green and Cauchy. Chessin, - C. R. 128 (1899) 604-.

Theory. Andrade, J. C. R. 119 (1894) 1192-.

- of numbers, application to integral calculus. Gegenbauer, L. Wien Ak. Sb. 102 (1893) (Ab. 2a) 927-.

Transformation. Haedenkamp, $H$. Crelle J. 22 (1841) 184-.

-. Boole, G. Camb. Mth. J. 3 (1843) 216-; 4 (1845) 20 -

\section{Calculus of Variations}

Transformation. Svanberg, A. F. Stockh. Öfv. 2 (1845) 228-; Ups. N. Acta S. Sc. 13 (1847) $1-$.

-. Chelini, D. G. Arcad. 106 (1846) 127-.

-. Genocchi, A. Tortolini A. 4 (1853) 401-

-. Russell, W. H. L. QJ. Mth. 4 (1861) 163-, 316-.

-. Holmgren, H. [1864] Stockh. Ak. Hndl. 5 (1866) (No. 6) $40 \mathrm{pp}$.

-. Tannenberg, $W$. de. Bordeaux S. Sc. PV. (1897-98) 109-.

-, case. Haedenkamp, H. Crelle J. 20 (1840) 97-.

- formula. Cayley, A. Camb. and Dubl. Mth. J. 4 (1849) 50-.

-. Brioschi, F. Rm. R. Ac. Linc. Rd. 2 (1886) (Sem. 2) 111-.

- theorem. Minchin, G.M. [1871] QJ. Mth. 12 (1873) 172-.

- theorems of Gauss and Stokes. Predella, P. Mil. I. Lomb. Rd. 20 (1887) 215-.

- of variables. Ostrogradsky, M. A. [1836] St Pét. Ac. Sc. Mm. 3 (1838) 401-.

Triple integrals, case. Le Paige, C.M.M. H. H. Liége S. Sc. Mm. 9 (1882) (No. 3) 4 pp.

- - embracing potential of homogeneous ellipsoid as particular case. Mertens, $F$. Gött. Nr. (1887) 269-.

- - , reduction to quadratures. Winckler, A. [1853] Crelle J. 50 (1855) 1 -.

- - , transformation by changing order of integration. Winckler, $A$. Crelle J. 45 (1853) 168-.

- - , - and determination, case. Unferdinger, $F$. Wien Sb. 61 (1870) $(A b .2)$ 105-, 417-.

- - variation. Abbott, $R$. R. S. P. 4 (1838) 42.

$\int^{n} d x d y \ldots d z$, whose limits are $p_{1}=a_{1} x+b_{1} y+$ $\ldots+h_{1} z>0, p_{2}>0, \ldots p_{n}>0$, and $x^{2}+y^{2}+$ ... $+z^{2}<1$. Schläfi, L. QJ. Mth. 2 (1858) 269-; 3 (1860) 54-, 97-.

$\int^{n} d x d y \ldots d z$, whose limits are $p_{1}=a_{1} x+b_{1} y+\ldots$ $+h_{1} z>0, p_{2}>0, \ldots p_{n}>0$, and $x^{2}+y^{2}+\ldots$ $+z^{2}<1$, expansibility. Schläfli, L. QJ. Mth. 8 (1867) 370-

$\iiint \frac{d x d y d z}{r^{1+a}}$, extended over the interior of ellipsoid. Bigler, $U$. Bern Mt. (1887) 52-.

\section{Calculus of variations.}

Bossut, C. Thomson A. Ph. 9 (1817) 445-.

Verhulst, P. F. Gand A. Ac. (1823-24) $30 \mathrm{pp}$.

Walsh, J. Tilloch Ph. Mg. 64 (1824) 330-.

Cankrein, H. K. Ph. Mg. 8 (1830) 321-.

Cauchy, A. L. Bb. It. 62 (1831) 373-.

Poisson, S. D. [1831] Par. Mm. Ac. Sc. 12 (1833) 223-.

Müller, A. Crelle J. 13 (1835) 240-.

Jacobi, C. G. J. [1836] Crelle J. 17 (1837) 68

(Jacobi.) Liouville, J. Liouv. J. Mth. 3 (1838) 1-.

Bertrand, $J$. Liouv. J. Mth. 7 (1842) 55-. 
Delaunay, C. [1842] Par. Éc. Pol. J. Cah. 29 (1843) 37-.

Cauchy, A. L. C. R. 17 (1843) 275-

Goodwin, H. Camb. and Dubl. Mth. J. 3 (1848) 225-.

Sarrus, F. Par. Mm. Sav. Étr. 10 (1848) 1-.

Mainardi, G. Tortolini A. 3 (1852) 149-.

Blanken, H. van. (vII) Amst. N. Ws. Ntk. Vh. 2 (1854) 1-.

(Jacobi, 1837.) Heine, H. E. Crelle J. 54 (1857) 68-.

Meyer, A. Liége Mm. S. Sc. 11 (1858) 125-.

Lindeloef, L. L. B. A. Rp. (1859) (pt. 2) 5-.

Steichen, - . Brux. Mm. Cour. $8^{\circ}, 14$ (1862) $126 \mathrm{pp}$.

Sabinin, E. T. [1868] (xir) Rec. Mth. (Moscou) 4 (1869-70) (Pt. 1) 94-.

Heine, E. Mth. A. 2 (1870) 188-.

Żmurko, W. [L.] Krk. Ak. (Mt.-Prz.) Pam. 2 (1876) $57-$.

Méray, C. Par. Éc. Norm. A. 6 (1877) 187-.

(Sarrus.) Sabinin, E. T. [1889] Rec. Mth. (Moscou) 14 (1890) 451-; Fschr. Mth. (1890) 384.

Ermakov, V. P. [1892] Rec. Mth. (Moscou) 16 (1893) 369-; Fschr. Mth. (1892) 336-.

Hancock, H. A. Mth. 9 (1894-95) 179-; 10 (1895-96) 81-.

Dixon, A. C. Mess. Mth. 26 (1897) 54-.

Tannenberg, $W$. de. Bordeaux S. Sc. PV. (1897-98) 105-.

Kneser, A. Mth. A. 50 (1898) 27 -

Duhem, P. Toul. Fac. Sc. A. 2 (1900) 115-.

Sommerfeld, A. D. Mth. Vr. Jbr. 8 (1900) (Heft 1) 188-.

Application to determination of equation of propeller surface. Martin, $L$. (xII) Mag. Tud. Ak. Étk. (Mth.) 5 (1879) (No. 7) 30 pp. - Double and triple integrals. Strauch, G. W. Wien D. 16 (1859) 19-.

- - geodesics on rotation ellipsoid of $n$ dimensions. Rudio, F. Zür. Vjschr. 43 (1898) 340-.

- - geometrical problems. Starkoff, $A$. Par. S. Mth. Bll. 13 (1885) 132-; Kazan S. Nt. (P8.-Mth.) P. 4 (1886) 7-.

- of Hamilton's operator $\nabla$. Joly, C. J. [1899] Ir. Ac. P. 5 (1898-1900) 666.

- to integration of differential equations. Binet, J. P. M. C. R. 14 (1842) 654-.

- of integration of differential equations by factors and differentiation. Challis, $J$. Ph. Mg. 46 (1873) 388-.

- to mechanics. Ampère, A. M. [1803] Par.

Mm. Sav. Étr. 1 (1806) 493-.

Constants, determination. Stern, M. A. [1867] Gött. Ab. 13 (1868) (Mth.) 53-.

Criteria. Mainardi, G. Tortolini A. 3 (1852) 379 .

Differential equations. Richelot, F.J. C. R. 49 (1859) 641-.

- -. Hirsch, A. Mth. A. 49 (1897) 49-.

- - arising from the calculus. Volterra, $V$. Rm. R. Ac. Linc. Rd. 6 (1890) (Sem. 1) 43-.

- - partial, arising from the calculus, class. Kürschák, J. Mth. Termt. Éts. 8 (1890) 60-; Mth. Nt. B. Ung. 8 (1891) 35-.
Differential equations, partial, arising from double integrals. Kürschík, J. Mth. Termt. Éts. 7 (1889) 296-; Mth. Nt. B. Ung. 7 (1890) 263-.

Discontinuity. Challis, J. Ph. Mg. 24 (1862) 196-.

- curves. Sabinin, E. T. [1898] Rec. Mth. (Moscou) 20 (1899) 285-; Fschr. Mth. (1898) 328.

-lines. Zimmermann, W. Fschr. Mth. (1896) 294-.

Failure, supposed. Airy, G. B. Ph. Mg. 22 (1861) 12-.

Geometrical theory of milling machines. Pregél, —. Dingler 296 (1895) 254-, 265-.

History. Strauch, G. W. N. A. Mth. 10 (1851) 433-.

-, previous to Lagrange. Guiraudet, $P$. [1862] (xiI) Lille S. Mm. 9 (1863) 525-.

Isoperimeters. Trembley. J. Berl. Mm. Ac. (1804) 25-.

-. Eneström, G. Ups. Årsk. (1876) 77 pp.

-, differential equations. Ostrogradsky, M. A. [1848] St Pét. Ac. Sc. Mm. 6 (1850) 385-.

-, history. Eneström, G. Bb. Mth. (1888) 38.

—, method. Léger, É. N. A. Mth. 5 (1846) 204-.

-, -. Bellion, -. N. A. Mth. 6 (1847) 27-.

-, -. Ancion, -. (xIr) Mathesis 3 (1883) 145-, 161-, 176.

Isoperimetric integrals, maxima and minima, criteria. Mayer, A. C. G. Leip. Mth. Ps. B. 29 (1877) 114 .

- problem, distinction of maxima and minima. Lundström, C. E. [1869] Ups. N. Acta S. Sc. 7 (1870) (No. 4 ) 39 pp.

- problems. Minding, E. F. A. [1877] St Pét. Ac. Sc. Bll. 24 (1878) 398-.

- - Kelvin, (Lord). R. I. P. 14 (1896) 111-.

- products, maxima. Dillner, G. Ts. Mt. Fys. 1 (1868) 102-.

Isoperimetry on curved surfaces. Delaunay, $C$. Liouv. J. Mth. 8 (1843) 241-.

Jacobi-Hamilton process, extension. Volterra, $V$. Rm. R. Ac. Linc. Rd. 6 (1890) (Sem. 1) 127-.

Lagrange's method of multipliers. Mayer, $A$. [1885] Mth. A. 26 (1886) 74-; Leip. Mth. Ps. B. 47 (1895) 129-.

- - - Turksma, B. Mth. A. 47 (1896) 33-.

Maxima and minima, concepts. Scheeffer, $L$. Leip. Mth. Ps. B. 37 (1885) 92-.

- - , criteria. Delaunay, C. Liouv. J. Mth. 6 (1841) 209-.

-,$- \ldots$. Spitzer, S. Wien SB. 12 (1854) 1014-; 14 (1854) 41-.

,---- Culverwell, E. P. B. A. Rp. (1884) 649-; (1887) 598-.

,--- . Winckler, A. Wien Ak. Sb. 97 (1889) $(A b .2 a)$ 1065-.

- - - Culverwell, E. P. L. Mth. S. P. 23 (1892) 241-; 25 (1894) 361-; 26 (1895) $345-$.

- - - (Jacobi). Bertrand, J. Par. Éc. Pol. J. $28^{\circ}$ cah. (1841) 276-. 
Maxima and minima of integrals. Markoff, Par. Ec. Norm. A. 3 (1886) 81-.

- - - - simple integrals between fixed limits. Scheeffer, L. Mth. A. 25 (1885) 522-. - _ - _ - with variable limits, criteria. Mayer, A. Leip. Mth. Ps. B. 36 (1884) 99-. $-\ldots-$, solutions, criteria. Culverwell, E.P. [1886] Phil. Trans. (A) 178 (1888) 95-.

- _ - - - for finite variations, conditions. Culverwell, E. P. [1899] Ir. Ac. P. 5 (18981900) 377-.

Minimum of simple integrals with variable limits, criteria. Mayer, A. Leip. Mth. Ps. B. 48 (1896) 436-.

Multiple integrals. Poisson, S. D. Par. S. Phlm. Bll. (1816) 82-.

- - Ostrogradsky, M. A. [1834] St Pét. Ac. Sc. Mm. 3 (Sc. Mth. Ps. pte. 1) (1838) 35-. - - (Ostrogradsky's formula). Sabinine, Crelle J. 59 (1861) 185-.

- - Sabinine, G. A. Mt. 2 (1899) 203-.

Osculation function. Żmurko, W. [L. ] (xII) Krk. Ak. (Mt.-Prz.) Pam. 3 (1877) 24-, 94-.

Principles. Ampère, A.M. Gergonne A. Mth. 16 (1825-26) 133-.

-. Crelle, A. L. Berl. Ab. (1833) 1-.

-, elementary. Du Bois-Reymond, $P$. Mth. A. 15 (1879) 283-, 564-.

-, extension. Challis, $J_{\text {. }}$ Ph. Mg. 32 (1866) 45-.

Problems. Pagani, G. M. Crelle J. 15 (1836) 84-.

-. Strauch, G. W. Grunert Arch. 3 (1843) 119-.

-. Schellbach, C. H. Crelle J. 41 (1851) 293-.

- Challis, J. [1866-71] Ph. Mg. 31 (1866) 218-; 32 (1866) 278-; 42 (1871) 28-, 302-; 43 (1872) 52-.

-. Todhunter, I. Ph. Mg. 31 (1866) 425-; 32 (1866) 199-; 42 (1871) 440-.

-. Cayley, A. 'L. Mth. S. P. 3 (1869-71) 221-.

- Walton, W. QJ. Mth. 10 (1870) 72-.

-. Wilkinson, M. M. U. Mess. Mth. 1 (1872) 175-.

-. Somin, N. N. Rs. S. Nt. Mm. (Mth.) 6 (1885) 1-, 93-; Fschr. Mth. (1885) 359-.

- Starkov, A. N. Rs. S. Nt. Mm. (Mth.) 6 (1885) 23-; Fschr. Mth. (1885) 359-; Palermo Cir. Mt. Rd. 2 (1888) 116-.

- containing problem of mechanics. Lipschitz, R. [1871] Crelle J. 74 (1872) 116-.

- corresponding to straight lines of space. Koenigs, G. C. R. 121 (1895) 1122-.

-, double integrals. Koenigs, G. C. R. 122 (1896) 126-

-, fundamental, generalisation. Whittemore, $J . K$. N. Y. Am. Mth. S. Bll. 6 (1900) 96, 101-.

-, one independent variable. Clebsch, $\boldsymbol{A}$. Crelle J. 55 (1858) 335-.

-, - - - Mayer, A. C. G. Leip. Mth. Ps. B. 30 (1878) 16-.

-, partial differential equation. Kurschak, $J$. Mth. A. 44 (1894) 9-.

-, solution. Challis, J. Ph. Mg. 22 (1861) 108 .
Problems, solution. Spitzer, S. Arch. Mth. Ps. 42 (1864) 301-.

- - - by theory of quantics. Warren, $J$. [1865] QJ. Mth. 7 (1866) 171-.

-, solutions, discontinuous. Maxwell, J.C. [1873] (vII) Camb. Ph. S. P. 2 (1876) 294-. -, - - - Erdmann, G. [1875] Crelle J. Mth. 82 (1877) 21-.

Simple integrals, theory. Lipschitz, R. [1864] Crelle J. Mth. 65 (1866) 26-.

Surfaces of minimum area. Bonnet, $O$. C. R. 40 (1855) 1107-; 41 (1855) 1057-; 42 (1856) $532-$.

Theorems. Boole, G. Camb. Mth. J. 2 (1841) 97-.

-. Roberts, R. A. Mess. Mth. 15 (1886) 148-.

- corresponding to principle of least action in mechanics. Mayer, A. Mth. A. 2 (1870) 143-; Leip. Mth. Ps. B. 38 (1886) 343-.

- extension to differential equations. Ermakov, V. P. [1889] Fschr. Mth. (1890) 381.

-, fundamental, demonstration. Gourieff, $S$. [1797] St Pét. Ac. Sc. N. Acta 13 (1802) 154-.

-, - 一. Lindeloef, L. L. C. R. 50 (1860) 85-.

Theory, new. Picart, A. N. A. Mth. 2 (1883) 49-.

Todhunter's "History of the calculus of variations." Mainardi, G. Mil. At. I. Lomb. 3 (1862) 414-.

- "Researches," review. Wilkinson, M.M.U. Mess. Mth. 3 (1874) 184-.

Trajectory of a point for which a certain integral is minimum. Bresse, - C. R. 74 (1872) 1562-.

Transformations. Walton, W. [1863] QJ. Mth. 8 (1867) 222-.

- of differential equations. Kurschák, J. Mth. Termt. Ets. 17 (1899) 457-; Mth. Nt. B. Ung. 17 (1901) 29-.

- showing maxima and minima. Minding, E. F. A. Crelle J. 55 (1858) 300-.

Variation of differential coefficient of function of $n$ variables. Stanley, A.D. Silliman J. 3 (1847) 415-.

-, second, criteria for maximum or minimum of simple integrals. Kneser, A. Mth. A. 51 (1899) 321-.

$-,-,-\frac{\text { sign. Strauch, G. W. Grunert }}{}$ Arch. 4 (1844) 39-.

-, - , of multiple integrals. Clebsch, A. Crelle J. 56 (1859) 122-.

-,,$--\ldots-$ Sabinine, G. Bll. Sc. Mth. As. 2 (1878) (Pt. 1) 100-.

-, - , reduction. Clebsch, A. Crelle J. 55 (1858) 254-.

,,--- - Dixon, A. C. Mess. Mth. 26 (1897) 65-.

-, - , - Jacobi's. Horner, J. QJ. Mth. 14 (1877) 218-.

,-- , of simple integrals. Erdmann, G. Z. Mth. Ps. 23 (1878) 362-.

-, - - - - Escherich, G. von. Wien Ak. Sb. 107 (1898) (Ab. 2a) 1191-, 1267-, 1381-; 108 (1899) (Ab. 2a) 1269-. 
Variation, second, simple integrals, theory. Kneser, A. D. Mth. Vr. Jbr. 6 (1899) (Heft 1) 95-.

-, -, theory. Escherich, G. von. Wien Ak. Sb. 97 (1889) (Ab. 2a) 1416-; 98 (1890) ( $A b$. 2a) 1463-.

,,-- - Kneser, A. D. Nf. Vh. (1897) (Th. 2, Hälfte 1) 5 -.

Variations of coefficients, necessary relations. Potier, A. J. de Ps. 2 (1873) 328-.

-, higher, simple integrals. Erdmann, $G$. [1876] Z. Mth. Ps. 22 (1877) 324-.

- of $n$th order. Erdmann, G. Z. Mth. Ps. 26 (1881) 73-.

Weierstrassian formulæ. Hancock, $H$. A. Mth. 11 (1896-97) 20-; 12 (1898-99) 33-.

\section{Theory of functions of complex variables.}

\section{General.}

Cauchy, A.L. C. R. 23 (1846) 271-; 32 (1851) $160-; 43$ (1856) 69-.

Marie, M. Liouv. J. Mth. 3 (1858) 361-; 4 (1859) 121-, 305-, 369-; 5 (1860) 43-, 393-, $457-; 6$ (1861) 57-, 153-, 377-; 7 (1862) 81-, 425-; 17 (1872) 337-.

Riemann, B. Tortolini A. 2 (1859) 288-, 337-.

Roch, G. Schlömilch Z. 8 (1863) 12-, 183-; 10 (1865) 169-.

Casorati, $F$. Mil. I. Lomb. Rd. 3 (1866) 337-.

Darboux, G. Bordeaux Mm. S. Sc. $8(1870)$ 291-; 9 (1873) 1-.

Dini, $U .[1870]$ A. Mt. 4 (1870-71) 159-.

Frombeck, $H$. Wien Sb. 64 (1871) (Ab. 2) 495-.

Heine, E. Crelle J. 74 (1872) 172-.

Marie, M. Par. Éc. Norm. A. 2 (1873) 417-.

Puchewicz, $W$. Par. T. Nauk Śc. Pam. 4 (*1874) Art. 5, $131 \mathrm{pp}$.

Benthem, A. (Gz.) N. Árch. Wisk. $1\left({ }^{*} 1875\right)$ 124-; 2 (* 1876) 1-, 113-; 3 ( $^{*} 1877$ ) 113-.

(Marie.) Bierens de Haan, $D$. N. Arch. Wisk. $2\left({ }^{*} 1876\right) 150-; 3\left({ }^{*} 1877\right) 21-; 4$ (*1878) 95-.

Hermite, C. [1880] Crelle J. Mth. 91 (1881) 54 .

Weierstrass, C. T. W. Berl. Ak. Mb. (1880) 719-; (1881) 228-.

Mittag-Lefler, G. Helsingf. Öfv. 23 (1881) 95-; Bll. Sc. Mth. As. 5 (1881) (Pt. 1) 388-.

Gomes Teixeira, F. G. Teix. J. Sc. $6(1885)$ 33-, 129-; Fschr. Mth. (1885) 362-.

Mansion, P. Brux. S. Sc. A. 9 (1885) (Pt. 2) 1-.

Lerch, M. Prag Sb. (1886) (Mth.-Nt.) 571-; (1887) (Mth.-Nt.) 423-.

Beltrami, E. Mil. I. Lomb. Rd. 20 (1887) 624-; 24 (1891) 1188-; 27 (1894) 337-.

Pasch, M. Mth. A. 30 (1887) 132-.

Saporetti, A. Bologna Ac. Sc. Mm. 8 (1887) 555-.

Henrici, O. Nt. 43 (1891) 321-, 349-.

Echols, W. H. A. Mth. $8(1893-94)$ 45-, $137-$.

Poenisch, R. Santiago de Chile Un. A. 84 (1893) 405-, 595-, 899-.

VOL. I.
Borel, É. C. R. 118 (1894) 340-; Par. Éc. Norm. A. 12 (1895) y-.

Brill, J. Mess. Mth. 23 (1894) 108-, 185-.

Brodén, $T$. Lund. Un. Acta 33 (1897) ( $S$. Psgr. No. 3) $45 \mathrm{pp}$.

Osgood, W. F. [1898] N. Y. Am. Mth. S. Bll. 5 (1899) 59-.

Lerch, $M$. Prag České Ak. Fr. Jos. Rz. (Třida 2) 9 (1900) Art. 8, 5 pp.; Fschr. Mth. (1900) 402.

Algebraic equations and Cotes's theorem. Beltrami, E. G. Mt. 1 (1863) 123-.

$-\frac{1}{\text {, theorems. Lucas, F. }}$ C. R. 78 (1874) 431-.

- - , general. Laguerre, $E$. N. A. Mth. 19 (1880) 241-.

- functions. Brun, $F$. de. Stockh. Öfv. (1896) 315-; Fschr. Mth. (1896) 322.

- - development of theory. Brill, A., d Noether, M. D. Mth. Vr. Jbr. 3 (1894) 107-.

Analytic expressions for functions with infinite number of singularities. Pincherle, $S$. $\mathrm{Rm}$. R. Ac. Linc. Rd. 3 (1887) (Sem. 1) 370-.

- with removable discontinuities. Pringsheim, A. Mth. A. 26 (1886) 167-.

- - representing portions of analytic functions. 'Arcais, F. d'. Rv. Mt. 5 (1895) 186-. - - validity and continuity. Christoffel, E. B. Mth. A. 53 (1900) 465-。

- functions. Runge, C. Acta Mth. 6 (1885) 245-.

- - Arzelà, C. Bologna Rd. (1887-88) 25-. - Vivanti, G. Palermo Cir. Mt. Rd. 3 (1889) 38-.

- Timčenko, I. N. Rs. S. Nt. Mm. (Mth.) 12 (1892) 256 pp.; 16 (1899) 216 pp.; 19 (1899) xv + 183 pp.; Fschr. Mth. (1899) 48-.

- -. Slešinskij, I. V. [1893] Rec. Mth. (Moscou) 17 (1895) 223-; Fschr. Mth. (189394) 680 .

- Krygowski, Z. Wiad. Mt. 3 (1899) 147-; Fschr. Mth. (1899) 375.

- - Lindelöf, E. Helsingf. Acta 24 (1899) No. 7, $39 \mathrm{pp}$.

- definition, Cauchy's. Goursat, ḱ. N. Y. Ám. Mth. S. T. 1 (1900) 14-.

- differential operations. Pincherle, $S$. G. Mt. 22 (1884) 62-.

- - existence domain. Lerch, $M$. Prag České Ak. Fr. Jos. Rz. (Tŕida 2) 9 (1900) Art. 9, $8 \mathrm{pp}$.

- expansion. Dillner, G. Ups. S. Sc. N. Acta 12 (1885) No. 10, 22 pp. ; 15 (1895) No.5, 64 pp.

_- generalisation. Scheffers, G. C. R. 116 (1893) 1114-.

- limits for $n=\infty$ of the $n$th derivatives. Vitali, G. Palermo Cir. Mt. Rd. 14 (1900) 209-.

- - properties. Poincaré,H. Palermo Cir. Mt. Rd. 2 (1888) 197-.

,--- , arithmetical. Staeckel, P. C. R. 128 (1899) 725-, 805-.

- - representation, approximate. Arzelà, $C$. Bologna Rd. 2 (1898) 139-.

- - - general, of any kind. Desaint, $L$. C. R. 130 (1900) 999-. 
Analytic functions with restricted existence domain. Lerch, $M$. Prag Ab. 2 (1888) No. 9, 20 pp.

- - theorem of Hadamard. Pincherle, $S$. Bologna Rd. 3 (1899) 67-.

-- , zeros. Desaint, L. C. R. 124 (1897) 276-.

$-\sum_{0}^{\infty} f_{n}(x), \sum_{0}^{\infty} f(x, y)$, systematic formation. Puzyna, J.von. Mh. Mth. Ps. 5 (1894) 67-.

Application to geometry. Goy, P. de. Metz Ác. Mm. 67 (1889) 71-.

- - Stolz, O. Innsb. Nt. Md. B. 19 (1891) 3-.

- - - Bôcher, M. A. Mth. 7 (1892-93) 70-.

- - Macfarlane, A. N. Y. Am. Mth. S. Bll. 5 (1899) 326, 327-.

- - integrals. Dirksen, E. H. Berl. B. (1841) 4-.

- mechanics. Stankevic, I. V. Rec. Mth. (Moscou) 19 (1897) 586 (bis)-.

Calculus, extensions. Hagen, J. G. N. Y. Am. Mth. S. Bll. 6 (1900) 381-.

-, new, including quaternions and complex variable theory. Lipschitz, R. O.S. C. R. 91 (1880) 619-, 660-.

Cauchy's formula for non-monogenic functions. David, -. Toul. Ac. Sc. Mm. 7 (Sem. 1) (1885) 193-.

- integral, in simply or multiply connected space. Harnack, $A$. Leip. Mth. Ps. B. 37 (1885) 379-.

- integration theorem, validity of proofs, discussion. Pringsheim, A. Münch. Ak. Sb. 25 (1896) 39-, 295-.

- lemma, elementary demonstration. Méray, C. Bll. Sc. Mth. 15 (1891) 86-.

- - - proposition to replace. Meray, $C$. Bll. Sc. Mth. 19 (1895) 154-.

Cauchy-Goursat theorem, demonstration. Moore, E. H. N. Y. Am. Mth. S. T. 1 (1900) 499-.

Circuit integration over straight line. Echols, W. H. A. Mth. 12 (1898-99) 175-.

Circular coordinates. Johnson, W. W. Des Moines Anal. 10 (1883) 129-.

Complementary functions. Cauchy, A. L. C. R. 19 (1844) 1377-.

- -, theorem. Liouville, J. Crelle J. 11 (1834) 1-.

Complex integrals, proof of existence. Stolz, O. Mh. Mth. Ps. 11 (1900) 64-.

-- , theorem of Cauchy. Bôcher, M. N. Y. Am. Mth. S. Bll. 2 (1896) 146-

- point series. Spiro, B. $V$. Mosc. S. Sc. Bll. 73 (No. 1) (1891) 33-; Fschr. Mth. (1891) 726 .

- quantities and their applications. Despeyrous, C. Toul. Mm. Ac. 4 (1866) 255-; 6 (1868) 159-; 1 (1869) 174-; 2 (1870) 274-.

- - roots. Saalschütz, L. Königsb. Schr. 37 (1896) [24]-.

- variables and anharmonic ratios. Schröter, H. D. Nf. Tbl. (*1876) (Beil.) 64-.

- in higher dimension space. Volterra, $V$. Rm. R. Ac. Linc. Rd. 5 (1889) (Sem. 1) 158-.
Conception of fundamental domain. Klein, $F$. Mth. A. 40 (1892) 130-.

"Condensation-points" and their application. Puzyna, J. Fschr. Mth. (1888) 410.

Conjugate functions of Cartesians and other quartics. Greenhill, A. G. [1881] Camb. Ph. S. P. 4 (1883) 77-.

- - properties. Carlini, $F$. Wien SB. 13 (1854) 357-.

- - theory, partial differential equations in. Volterra, $V$. Palermo Cir. Mt. Rd. 3 (1889) 260-.

Continuation of a function. Mittag-Leffer, $G$. [1900] N. Y. Am. Mth. S. Bll. 7 (1901) 65-.

Continuity. Litroth, J. [1872] Mth. A. 6 (1873) 319-.

- of functions on change of fundamental domain. Ritter, $E$. Mth. A. 45 (1894) 473-; 46 (1895) 200-.

- and limit, notions. Riquier, -. Par. Ec. Norm. A. 12 (1895) 197-.

Critical points. Safford, $F$. $H$. A. Mth. 1 (1900) 46-.

Curves cutting plane into squares, systems. Tait, P. G. Edinb. Mth. S. P. 7 (1889) 2-.

Definite integrals round a closed curve and between imaginary limits. Cauchy, A. L. C. R. 23 (1846) 689-.

- - of complex functions, class. Niemöller, F. Arch. Mth. Ps. 66 (1881) 225-.

- - with complex limits, interpretation. Raabe, J. L. Zür. Mschr. 1 (1856) 483-.

- - evaluation by application of free path of integration, case. Bigler, $U$. Arch. Mth. Ps. 9 (1890) 60-.

- -, -, case. Pell, A. [1899] A. Mth. 1 (1900) 144-.

- - of functions of complex variable. Dillner, G. [1880] Stockh. Ak. Hndl. 18 (1882) No. 6, 23 pp.

- - and Hermite's sections. Albeggiani, M. L. Palermo Cir. Mt. Rd. 1 (1887) 59-, 398.

- - Hermite's theorem, proof by curvilinear integrals and by Cauchy's theorem. Goursat, $\dot{E}$. Acta Mth. 1 (1882) 189-.

- - between imaginary limits. Malmsten, C. J. [1865] Stockh. Ak. Hndl. 6 (1865-66) (No. 3) $18 \mathrm{pp}$.

Determinant of four points in plane with respect to a fifth. Laisant, C. A. Lisb. J. Sc. Mth. 5 (1898) 205-.

Determination by boundary and discontinuity conditions. Riemann, B. Crelle J. 54 (1857) 111-.

Development and objects. Krause, - Civing. 34 (1888) 639-.

Discontinuities, non-polar. Durège, $H$. Wien Ak. Sb. 73 (1876) (Ab. 2) 173-.

-, polar. Hadamard, -. C. R. 108 (1889) 722-.

Discontinuous function. Weyr, $E$. Prag České Ak. Fr. Jos. Rz. (Trida 2) 2 (1893) Art. 12, 21 pp.

- functions, zeros. Desaint, L. C. R. 119 (1894) 364-.

Examples. Bocher, M. A. Mth. 1 (1900) 37-. 
Expansion. Gomes Teixeira, F. G. Teix. J. Sc. 8 (1887) 17-.

- of functions in series. Cauchy, A. L. Férussac Bll. Sc. Mth. 16 (1831) 119-.

_ _ _ _ _ - Puiseux's theorem. Kronecker, L. Berl. Ak. Sb. (1884) 543-.

Factorial functions. Landfriedt, $E$. Toul. Fac. Sc. A. 9 (1895) G, 18 pp.

Formula of Hermite. Pincherle, S. Rm. R. Ac. Linc. Rd. 1 (1885) 267-.

- $i \log i=-\frac{1}{2} \pi$, origin. Eneström, G. Bb. Mth. (1899) 46.

Formulæ, elementary. Moseley, H. Ph. Mg. 14 (1857) 260-.

Function of analytic point $(x, y)$ describing cycle, relations between residues. Appell, $P$. C. R. 95 (1882) 714-.

-, definition. Osgood, W. F. N. Y. Am. Mth. S. Bll. 2 (1896) 296-.

Functions of analytic point, with rational periods or exponential factors. Lacour, $E$. Par. Éc. Norm. A. 12 (1895) 3-.

- complex variables, geometric signification. Friesach, K. Steierm. Mt. (1872) 15-.

- convergent inside Cassini's oval. Puzyna, $J$. Prace Mt.-Fiz. 5 (1894) 21-; Fschr. Mth. (1893-94) 710-.

- dependent on other functions. Volterra, $V$. Rm. R. Ac. Linc. Rd. 3 (1887) (Sem. 2) 97-, 141-, 153-.

- of functions, properties. Fabri, C. Tor. Ac. Sc. At. 25 (1890) 654-.

- with indeterminate differential coefficients. Kretkonski, W. [1892] Krk. Ak. (Mt.-Prz.) Rz. 6 (1893) 142- ; Crc. Ac. Sc. Bll. (1892) 339-.

- - infinitely quick oscillations. Brodén, $T$. Stockh. Öfv. (1895) 763-.

- - lacunæ. Poincaré, $H$. C. R. 96 (1883) 1134-; Helsingf. Acta 12 (1883) 341-.

- - Lerch, M. G. Teix. J. Sc. 10 (1891) 27-

- - - analytic representation. Homén, $T$. Helsingf. Acta 12 (1883) 445-.

- of large numbers, approximation. Flamme, -. Bordeaux S. Sc. Mm. 3 (1887) xix-.

- not susceptible of differentiation. Lerch, $M$. Crelle J. Mth. 103 (1888) 126-.

- regular within a connected area within finite distance. Ascoli, G. A. Mt. 20 (1892-93) 243-.

- satisfying no algebraic differential equation. Grönwall, H. Stockh. Öfv. (1898) 387-.

- of one variable. Bugaev, N. V. Rec. Mth. (Moscou) 12 (1885) 579-, 725-; 13 (1886) 1-, 167-.

Generalisation. Volterra, $V$. Acta Mth. 12 (1889) 233-.

- of equations. Picard, É. C. R. 112 (1891) 1399-; Liouv. J. Mth. 8 (1892) 217-.

Geometric interpretation of $i$. Sprague, $T . B$. Edinb. Mth. S. P. 12 (1894) 34-.

Geometry, higher. Dillner, $G$. Ts. Mt. Fys. 1 (1868) 27-, 124-, 169-, 209-, 270-; 2 (1869) 82-, 137-; 3 (1870) 30-, 111-, 176-; Ups. N. Acta S. Se. 8 (1873) No. 9, 136 pp.

Graphic imaginaries. Baker, A. L. [1894] Rochester (N. Y.) Ac. Sc. P. 3 (1900) 151-.
Green's function, existence in plane simply connected region. Osgood, $W . \vec{F}$. N. Y. Am. Mth. S. T. 1 (1900) 310-.

Hamiltonian pairs and generalised theory of complex variable. Maksimovich, $V$. $P$. [1883-84] (xII) Kazan S. Nt. (P8.-Mth.) P. 2 (1884) I-, 95-.

Imaginary in function theory and in analytic geometry. Schiller, W. F. D. Nf. B. ( $\left.{ }^{*} 1877\right)$ 109-.

- points, representation. Neovius, E. $R$. Helsingf. Öfv. 29 (1887) 154-.

- - - -, geometric. Coolidge, J. L. N. Y. Am. Mth. S. T. 1 (1900) 182-.

- - in space, representation. Molenbroek, $P$. N. Arch. Wisk. 19 (1892) 113-; Fschr. Mth. (1892) 757-.

- variables, representation by correspondence of two planes. Cayley, A. L. Mth. S. P. 9 (1877-78) 31-.

Implicit functions, and equations. Pellet, $A$. C. R. 117 (1893) 719-; 118 (1894) 182-.

- -, existence. Lindelöf, $E$. Bll. Sc. Mth. 23 (1899) 68-.

- - and infinitesimal theory of equations. Pellet, A. Par. S. Mth. Bll. 23 (1895) 7-.

Integrals round contours, application of Kronecker's theorem. Czuber, E. Mh. Mth. Ps. 4 (1893) 364-.

Integration. Stäckel, P. Bb. Mth. 1 (1900) 109-.

- along closed paths. Schlömilch, O. Z. Mth. Ps. 17 (1872) 347-.

Isogonal relations, theory. Holzmiller, $G$. Z. Mth. Ps. 18 (1873) 227-.

Isotherms connected with $z=\sqrt[n]{\bar{Z}}$ and $z=\sqrt[m]{\frac{a Z^{n}+b}{c Z^{n}+d}} \cdot$ Holzmilller, F. G. [1880] Z. Mth. Ps. 26 (1881) 231-.

Isotropic functions. Cauchy, A. L. C. R. 26 (1848) 549-, 624-, 666-; 27 (1848) 6-.

Lateral curves, theory. Thieme, F.E. Arch. Mth. Ps. 58 (1876) 185-.

Linear functions of complex variable. Cole, $F$. N. A. Mth. 5 (1889-90) 121-, iv.

Logarithmic potential. Tatarinov, I. V. Mosc. S. Sc. Bll. 96 (No. 1) (1899) 7-.

Maximum and minimum. Scheibner, $W$. Leip. Mth. Ps. B. 40 (1888) 4 -.

Operations, cases. Pincherle, S. Bologna Ac. Sc. Mm. 7 (1886) 393-.

Orders of infinity of functions. Pincherle, $S$. Bologna Ac. Sc. M.m. 5 (1883) 739-.

Oscillations of harmonic functions of two real variables and of functions of one complex variable. Schottky, $F$. Crelle J. Mth. 117 (1897) 225-.

Periodic function of one variable with more than two periods impossible, theorem of Jacobi. Casorati, $F$. Mil. I. Lomb. Rd. 15 (1882) 623-.

- functions. Berger, A. Bll. Sc. Mth. As. 6 (1874) 72-.

Periods of integral of any order. Marie, $M$. C. R. 46 (1858) 738-.

Plane $n$-line, metric geometry. Morley, $F$. N. Y. Am. Mth. S. T. 1 (1900) 97-. 
Plane $n$-line, metric theorems. Loud, $F$. $H$. N. Y. Am. Mth. S. T. 1 (1900) 323-.

Point involutions in plane of complex variable. Vries, J. de. Mh. Mth. Ps. 3 (1892) 285-

Principles. Riquier, - Par. Éc. Norm. A. 8 (1891) 59-, 141-; 9 (1892) 281-.

-, fundamental. Cellérier, C. Bll. Sc. Mth. 14 (1890) 142-.

-, generalisation. Scheffers, G. Leip. Mth. Ps. B. 45 (1893) 828-; 46 (1894) 120-.

- of Weierstrass. Pincherle, S. G. Mt. 18 (1880) 178-, 317-.

Problem. Puzyna, $J$. Fschr. Mth. (1888) 411.

Properties. Trzaska, $W$. Par. T. Nauk Śc. Pam. $\left.1{ }^{*} 1871\right)$ 109-; Bll. Sc. Mth. As. 6 $\left({ }^{*} 1874\right) 152$-.

Rational functions. Beke, E. Mth. A. 47 (1896) 441-.

Real functions of complex variables. Murphy, R. Ph. Mg. 2 (1833) 287-.

- - given over any aggregate of points, analytic representation. Phragmén, $E$. Palermo Cir. Mt. Rd. 14 (1900) 256-.

- part of any function of complex variable. Bougaieff, -. Bll. Sc. Mth. 11 (1887) 61-.

Representation. Fuchs, L. [1872] Crelle J. 75 (1873) 177-; 76 (1873) 175-.

-, approximate forms for. Pincherle, $S$. Bologna Ac. Sc. Mm. 10 (1889) 77-.

-, conformal. Wagner, H. A. Hamb. Mth. Gs. Mt. 1 (1889) 64-.

- , - given by quadratic fractional relation. Holzmiller, F. G. Mth. A. 18 (1881) 289-.

- by definite integrals, cases. Somigliana, $C$. Mil. I. Lomb. Rd. 21 (1888) 431-.

-, theory of images. Harris, R. A. A. Mth. 4 (1888) 65-, 128.

Roots considered as functions of a variable parameter. Puiseux, V. C. R. 30 (1850) 171.

Series representing arbitrary functions in separate regions of convergence. Pringsheim, A. Mth. A. 22 (1883) 109-.

- terms of which are functions of complex variable. La Vallée Poussin, C. de. G. Teix. J. Sc. 11 (1892) 77-.

Simplifications, Pringsheim, A. Mth. A. 47 (1896) 121-.

Singular functions, representation, analytic. Tonelli, A. Rm. R. Ac. Linc. Rd. 1 (1885) 124 -

- lines of analytic functions. Painlevé, $P$. Toul. Fac. Sc. A. 2 (1888) B, 130 pp.

- points of a certain function. Dell' Agnola, C. A. Ven. I. At. (1898-99) (Pt. 2) 525-, $669-$.

Singularities of analytic functions, class. Pringsheim, A. Mth. A. 50 (1898) 442-.

- - - (two), comparison. Pincherle, $S$. Rm. R. Ac. Linc. Rd. 3 (1887) (Sem. 2) 310-.

- - - - especially functions defined by differential equations. Painlevé, $P$. C. R. 131 (1900) 489-.

$--\ldots-$, single-valued and general. Chessin, A. S. A. Mth. 11 (1896-97) 52-.

- function depending on two given functions. Pincherle, S. Rm. R. Ac. Linc. Rd. 8 (1899) (Sem. 1) 228-.
Theorem. Lerch, M. Prag Sb. (1885) (Mth.Nt.) 351-; Fschr. Mth. (1886) 347.

- Abel's, demonstration of non-existence of any other. Königsberger, L. [1885] Münch. Ak. Sb. 15 (1886) 462-.

- Cauchy's, on roots, two deductions from. Mittag-Leffler, G. Stockh. Öfv. 31 (1874) No. 7, 23-.

-, fundamental. Ascoli, G. [1870] A. Mt. 4 (1870-71) 31-.

-, new. Jensen, J. L. W. V. Acta Mth. 22 (1899) 359-.

Theorems, two. San-Martino, A. N. A. Sc. Nt. 4 (1845) 353-.

Total variation in describing contour. Cauchy, A. L. C. R. 40 (1855) 651-, 713-, 804-.

Transcendental functions, class. Picard, É. C. R. 123 (1896) 1035-.

- - genus zero and genus one. Laguerre, $E$. C. R. 95 (1882) 828-

- - integrals. Koenigsberger, L. Gött. Nr. (1884) 116-; Crelle J. Mth. 98 (1885) 97-. - - - , reduction. Koenigsberger, L. Am. J. Mth. 11 (1889) 221-.

- - new, class. Picard, É. Acta Mth. 18 (1894) 133-; 23 (1900) 333-.

- - , roots being all transcendental numbers. Gegenbauer, L. Wien Ak. Sb. 108 (1899) (Ab. 2a) 423-.

Transformation, orthomorphic, of circle into itself. Cayley, A. Edinb. Mth. S. P. 8 (1890) 91-.

Transformations, algebraic. Pagani, G. M. Brux. Ac. Bll. 7 (1840) (pte. 2) 50-.

Weierstrass's function $\sum_{\nu=1}^{\Sigma} b^{\nu} \cos \left(a^{\nu} \pi x\right)$. Tauber, A. Mh. Mth. Ps. 8 (1897) 330-.

Wronskians, fundamental property. Demoulin, A. Mathesis 17 (1897) 62-.

$\phi(x, y)+i \psi(x, y)=F(x+i y)$, conditions. Dienger, $J$. Grunert Arch. 10 (1847) 422-.

$\operatorname{Tan}^{-1}(\xi+i \eta)$, reduction to form $x+i y$. Unferdinger, $F$. Arch. Mth. Ps. 49 (1869) 478-.

- value. Unferdinger, $F$. Z. Mth. Ps. 14 (1869) 77-.

$\phi(x+y i)+\phi(x-y i)$, Abel's expression for. Glaisher, J. W. L. [1872] Mess. Mth. 2 (1873) 12-.

${ }_{\Pi-1}^{m-1}(x+i y+r)$, properties. Schlömilch, O. Leip. B. 24 (1872) 26-; Z. Mth. Ps. 17 (1872) 248-. $\phi(x+y \sqrt{-1})+\phi(x-y \sqrt{-1})$, value. Oltramare, G. As. Fr. C. R. (1885) (Pt. 2) 127-.

\section{Uniform functions of one variable.}

Bouquet, J., \& Briot, -. Par. Ec. Pol. J. cah. 36 (1856) 85-, 133-, 199-.

Bertrand, J. C. R. 48 (1859) 427-.

Imshenetskir, $V . G$. (xII) Kharkov Mth. S. Com. (1880) 173-.

Farkas, J. C. R. 96 (1883) $1646-$

Vivanti, G. G. Mt. 25 (1887) 54-, 232-, 312.

Additions to work of Weierstrass and MittagLeffler. Casorati, F. A. Mt.10 (1880-82) 261-. 
Analytic functions, Runge, C. Acta Mth. 6 (1885) 229-.

- - Stäckel, P. Crelle J. Mth. 112 (1893) 262-.

- -, arithmetical properties. Stäckel, $P$. Mth. A. 46 (1895) 513-.

- - expansion. Painlevé, P. C. R. 128 (1899) 1277-.

- - extension of Taylor's series. Mittag. Leffler, $G$. Nt. 62 (1900) 419.

- -, representation. Herz, N. [1879] Z. Mth. Ps. 25 (1880) 125-.

- -, - Painlevé, P. C. R. 126 (1898) 200-. - - - - Mittag-Leffler, G. C. R. 128 (1899) 1212-.

- - , series. Gerbaldi, F. Rv. Mth. 6 (189699) 24 -.

- - theorems. Mittag-Leffer, G. Bll. Sc. Mth. As. 3 (1879) (Pt. 1) 269-.

- - theory, conception of derived function. Vivanti, G. Palermo Cir. Mt. Rd. 13 (1899) 263-.

_ - , values at periphery of convergence circle. Puzyna,J. Krk. Ak. (Mt.-Prz.) Rz. 6 (1893) 311-; Crc. Ac. Sc. Bll. (1893) 200-.

- point $(x \cdot y)$. Appell, P. Acta Mth. 1 (1882) 109-.

$--(-)$, nature of algebraic relation between two uniform functions. Goursat, $E_{\text {. }}$ C. R. 97 (1883) 1048-.

Approximation, method. Jablonski, E. C. R. 116 (1893) 19-.

Arbitrary functions, representation. Runge, $C$. Acta Mth. 7 (1885) 387-.

Arithmetical means. Gutzmer, A. G. Teix. J. Sc. 8 (1887) 147-; N. A. Mth. 8 (1889) 101-. Case. Stieltjes, - C C. R. 101 (1885) 153-.

- (Stieltjes). Hermite, C. C. R. 101 (1885) 112-.

Cauchy's formula $\int \frac{F(x)}{x-a} d x=2 i \pi F(a)$, application. Schönholzer, $J$. Bern Mt. (1874) (Ab.) 255-.

- integral. Morera, G. Mil. I. Lomb. Rd. 22 (1889) 191-.

- theorem. Kronecker, L. Berl. Ak. Sb. (1885) 785-.

- - demonstration. Goursat, $\tilde{E}$. Acta Mth. 4 (1884) 197-.

- - - - Bruno de Cabedo, J. G. Teix. J. Sc. 9 (1889) 7 -.

- - extension. Ravut, L. N. A. Mth. 16 (1897) 365-.

- - on integral of function, demonstration. Falk, M. Ups. S. Sc. N. Acta 12 (1885) No. 3, $18 \mathrm{pp}$.

- - value of complex function at any point of area, deduction from. Grandi, $A$. G. Mt. 7 (1869) 374-.

- $-\int f(x) d x$ round a closed curve. MittagLeffler, G. Gött. Nr. (1875) 65-.

Characteristic conditions. Volterra, $V$. A. Mt. 11 (1882-83) 1-.

Class, determination. Sparre, - de. C. R. 102 (1886) 740-.
Coefficients and roots of an integral transcen. dental function, relations. Pincherle, $S$. Mil. I. Lomb. Rd. 11 (1878) 391-。

Construction. Ovidio, E. $d$ '. 'Tor. Ac. Sc. At. 28 (1893) 812-.

Continuation. Picard, É. C.R. 128(1899) 193-.

- of analytic functions. Borel, $\hat{E}$. C. R. 128 (1899) 283-.

-, analytic, of functions representing interior of conic on half plane. Lindemann, $F$. Münch. Ak. Sb. 26 (1897) 401-.

,-- , generalisation. Fabry, E. C. R. 128 (1899) 78-.

-, -, -. Borel, É. C. R. 130 (1900) 1115-. - of monogenic functions. Mittag-Leffler, $G$. Stockh. Ofv. (1898) 247-, 263-, 375-; Fschr. Mth. (1898) 358-.

- - - Viterbi, A. Palermo Cir. Mt. Rd. 12 (1898) 95-.

- - single-valued function represented by double infinite series. Kluyver, J.C. Amst. Ak. Vs. 8 (1900) 40-; Amst. Ak. P. 2 (1900) 24 .

Curves represented by imaginary equation. David, - Toul. Ac. Sc. Mm. 6 (Sem. 2) (1884) 239-.

Differential equations, class. Picard, $\dot{E}$. Bll. Sc. Mth. As. 4 (1880) (Pt. 1) 416-.

Equality of two products, theorem. Cauchy, A. L. C. R. 16 (1843) 886-.

Expansion in any domain. Painlevé, $P$. C. R. 126 (1898) 318-.

- - power series. Pringsheim, A. Münch. Ak. Sb. 25 (1896) 75-.

Expressions representing distinct functions in different parts of plane. Lerch, J. Bll. Sc. Mth. 10 (1886) 45-.

Factorial in complex quantities, and some definite integrals. Raabe, J. L. Sch. Gs. N. D. 8 (1847) 19 pp.

- $m_{k} \div k !$, limiting value when $k$ increases indefinitely. Raabe, J. L. Crelle J. 48 (1854) 130-.

Formula. Hermite, C. Am. J. Mth. 6 (1884)60-.

Function corresponding to given continuum. Osgood, W. F. N.Y. Am. Mth.S. Bll. 5 (1899) 14-.

- existing only in interior of circle. Stieltjes, T. J. Bll. Sc. Mth. 11 (1887) 46-.

- with isolated singularities, expansion in series. Bucca, $F$. Palermo Cir. Mt. Rd. 11 (1897) 90 -

- natural boundary. Osgood, W. $F$. N. Y. Am. Mth. S. Bll. 4 (1898) 417-; 5 (1899) 17-.

- a certain singular point. Arcais, F. $d$ '. Ven. I. At. (1897-98) 1048-.

- - two singular points expressed in a series, converging for all values of the variables except along the arc of a circle or a straight line. Ermakov, V. P. Rec. Mth. (Moscou) 16 (1893) 518-; Fschr. Mth. (1892) 354-.

Functions of complex variable, special form. Seidel, L. [1869] Crelle J. 73 (1871) 297-. - connected by algebraic relation. Picard, $\dot{E}$. Bll. Sc. Mth. As. 4 (1880) (Pt. 1) 416-; 7 (1883) (Pt. 1) 107-. 
Functions connected by algebraic relation, theorem. Picard, É. Acta Mth. 11 (1887-88) 1-.

- with "coupures" (sections). Picard, $\dot{E}$. C. R. 94 (1882) 1405-

- - (-). Appell, P. C. R. 96 (1883) 1018-.

- - line of essential singularities, decomposition into prime factors. Picard, $\hat{E}$. C. R. 92 (1881) 690-.

- - - - - - - - - (Picard). Dautheville, S. Mntp. Ac. Mm. 11 (1892) 45-.

- in neighbourhood of a singular point.

Picard, É. C. R. 89 (1879) 745-.

- presenting lacunæ. Goursat, É. C. R. 94 (1882) 715-.

- represented by series convergent within certain limits. Ascoli, G. Mil. I. Lomb. Rd. 19 (1886) 173-.

Holomorphic functions. Cesáro, E. $\quad$ C. R. 99 (1884) 26-; G. Mt. 22 (1884) 191-.

- - Hermite, C. Liouv. J. Mth. 1 (1885) 9-.

- - Bassi, A. G. Mt. 36 (1898) 100-; 37 (1899) 326-.

- - in an elliptic field. Pascal, E. Rm. R. Ac. Linc. Rd. 5 (1896) (Sem. 1) 319-.

$-{ }_{-}-{ }_{-}$Cazzaniga, $T$. Tor. Ac. Sc. At. 33 (1897) 574- or 808-.

- - expansion in series in area bounded by circular arcs. Appell, P. Acta Mth. 1 (1882) 145-.

- - genus zero or unity, roots of derivative. Bassi, A. Mil. I. Lomb. Rd. 28 (1895) 979-.

- - quotient of two, and recurring series. Presle, - de. Par. S. Mth. Bll. 19 (1891) 114-.

- - roots of derivative. Bassi, A. Mil. I. Lomb. Rd. 28 (1895) 1119-.

- and meromorphic functions in rational and in elliptic field. Cazzaniga, $T$. Tor. Ac. Sc. At. 33 (1897), 743- or 983-.

Integrability of function of one variable, new form of condition for. Stolz, $O$. Wien Ak. Sb. 107 (1898) (Ab. 2a) 736-.

Integrals between imaginary limits. Cauchy, A. L. Férussac Bll. Sc. Mth. 3 (1825) 214-. - - - Dienger, J. Crelle J. 37 (1848) $363-; 39$ (1850) 62-.

- - - - Cauchy's theorem. Lerch, $\boldsymbol{M}$. Prag Sb. (1887) (Mth.-Nt.) 673-.

Integration, application of free path. Christen, T. Arch. Mth. Ps. 16 (1898) 1-.

Irrational function of an imaginary variable. Cayley, A. [1876] L. Mth. S. P. 8 (1877) 212-.

Isogonal transformation, statics of polynomials. Harris, R. A. A. Mth. 6 (1891-92) 77-.

Laguerre's rank of uniform function with infinite number of zeros. Puzyna, J. von. Mh. Mth. Ps. 3 (1892) 1-.

Laurent's theorem, demonstration. Scheeffer, L. Acta Mth. 4 (1884) 375-.

- _, - for monogenic function with $n$ singular points. Mittag-Leffler, $G$. Stockh. Öfv. 40 (1883) No. 9, 5-; Acta Mth. 4 (1884) 80 -.
Laurent's theorem, extension. Hermite, $C$. Crelle J. Mth. 116 (1896) 85-.

Linear dependence. Bôcher, $M . \dot{0}[1900]$ N. Y. Am. Mth. S. Bll. 7 (1901) 120-, 234.

Maximum of $f(x),\left(=\sum_{-m^{\prime}}^{m} a_{\mu} x^{\mu}\right)$. Puzyna, $J$. Prace Mt.-Fiz. 6 (1895) 1-; Fschr. Mth. (1895) 444-.

Meromorphic functions. Borel, É. C. R. 120 (1895) 303-.

Monodromic functions having a characteristic equation. Pincherle, S. Mil. I. Lomb. Rd. 12 (1879) 536-.

- - important class. Pincherle, S. G. Mt. 18 (1880) 92-.

- and monogenic functions. Cauchy, A. L. C. R. 43 (1856) 13-.

Monogenic functions. Craig, $T$. [1893] N.Y. Mth. S. Bll. 3 (1894) 78-.

- - , representation, analytic. Mittag-Leffer, G. Acta Mth. 4 (1884) 1-.

$-\ldots,-, \ldots$ Volterra, V. Tor. Ac. Sc. At. 34 (1898) 348- or 492-.

- -, -, 一. Mittag-Leffler, G, [1898-1900] Camb. Ph. S. T. 18 (1900) 1-.

- -, singular points of which make an aggregate of first kind, analytic representation. Mittag-Leffler, G. Stockh. Ofv. 39 (1882) No. 2, 11-, No. 4, 21-.

- and holomorphic functions, inversion, cyclic. Rodrigues, (le capit.) J.M. As. Fr. C. R. (1893) (Pt. 1) 174; Rv. Mt. 5 (1895) 52-.

- monotypical functions. Cauchy, A. L. C. R. 32 (1851) 484-.

Number of periods. Milhll, $K$. von der. Basel Vh. 9 (1893) 78-.

- - -. Astor, A. N. A. Mth. 15 (1896) 227-.

Partial fractions found by complex functions. Hankel, $H$. Z. Mth. Ps. 9 (1864) 425-.

Power series, non-continuable. Vivanti, $G$. Rv. Mt. 3 (1893) 111-, 127.

- - relation between real and imaginary parts. Tauber, A. Mh. Mth. Ps. 2 (1891) 79-.

Products of variable factors. André, D. Par. S. Phlm. Bll. 2 (1890) 151-.

Property. Poincaré, H. C. R. 92 (1881) 1335-.

Proposition of Hermite. Raffy, $L$. Par. Éc. Norm. A. 2 (1885) 99-.

Radius of convergence. Fuchs, L. Crelle J. Mth. 106 (1890) 1-.

Rational functions with arbitrary limiting point, representation, analytic. Mittag-Leffler, $G$. Stockh. Öfv. 34 (1877) No. 1, 33-.

- - class, representation, analytic. MittagLeffler, G. Stockh. Öfv. 33 (1876) No. 6, 3-.

- - with finite number of limiting points, representation, analytic. Mittag-Leffler, $G$. Stockh. Öfv. 34 (1877) No. 2, 31-.

- - , representation, analytic. Mittag-Leffler, G. Stockh. Öfv. 34 (1877) No. 1, 17-.

- - - - by quotient of two converging power series. Mittag-Leffler, $G$. Stockh. Öfv. 34 (1877) No. 3, 5-. 
Rational integral function of a complex variable. Stieltjes, T. J. (jun.) Arch. Néerl. 18 (1883) 1-.

Representation, approximate, by rational fractions. Padé, H. C. R. 111 (1890) 674-.

- by infinite products and series of partial fractions. Frenzel, C. Z. Mth. Ps. 24 (1879) 316-.

Residues. Tortolini, B. G. Arcad. 63 (183435) 86-; 67 (1836) 179-.

-. Gregory, D. F. Camb. Mth. J. 1 (1839) 145-.

-. Oltramare, G. C. R. 12 (1841) 953-.

- (Oltramare). Cauchy, A. L. C. R. 13 (1841) 296-.

-. Cauchy, A. L. C. R. 41 (1855) 41-.

-. Oltramare, G. Gen. I. Nt. Mm. 3 (1855) 13 pp.

-, application. Amigues, E. N. A. Mth. 12 (1893) 142-.

-, - in analysis. Cauchy, A. L. C. R. 32 (1851) 207-.

-, - to integrals. Cauchy, A. L. C. R. 23 (1846) 321-.

-,- - mathematical physics. Cauchy, A. L. Par. Mm. Ac. Sc. 7 (1827) 463-.

- , - - resolution of transcendental functions. Cauchy, A. L. C. R. 32 (1851) 267-, 354-.

-, applications. Heine, H. E. [1879] Crelle J. Mth. 89 (1880) 19 -

- and calculus of limits, relations. Cauchy, A. L. [1831-37] Férussac Bll. Sc. Mth. 16 (1831) 116-; Mod. Mm. S. It. 22 (1839) 91-.

-, Cauchy's formula, demonstration. Mansion, -. Brux. S. Sc. A. 24 (1900) (Pt. 1) 88-.

-, - formulæ, generalisation. Amanzio, $D$. G. Mt. 15 (1877) 257-.

-, - work on. Kummell, C. H. Des Moines Anal. 6 (1879) 1-, 41-, 173-.

-, extension to space. Carvallo', E. Par. S. Mth. Bll. 24 (1896) 180-.

—, formulæ. Cauchy, A. L. C. R. 12 (1841) 871-.

-, - and their application. Roselli, E. G. Arcad. 114 (1848) 1-, 121-; 115 (1848) 3-.

-, functions. Falk, M. Ups. S. Sc. N. Acta Vol. extra ord. (1877) (No. 7) 32 pp.

- of functions, theory, application. Dini, $U$. Rm. R. Ac. Line. Mm. 2 (1895) 495-; A. Mt. 1 (1898) 39-.

-, principles (Cauchy). Radicke, G. Crelle J. 25 (1843) 216-

-, - and uses (Cauchy). Piola, G. Opusc. Mt. Fis. 2 (1834) 237-.

-, propositions, fundamental. Cauchy, A.L. C. R. 19 (1844) 1337-.

-, theory. Cauchy, A. L. C. R. 44 (1857) 406-.

Series, two, representing same function in one part of plane. Kapteyn, $W$. N. Arch. Wisk. 3 (1898) 225-.

Singular points of function given by series, and impossibility of analytic continuation. Fabry, E. Par. Éc. Norm. A. 13 (1896) 367-.
Singularities of analytic function, operations adding or removing. Pincherle, S. A. Mt. 4 (1900) 219-.

-, arrangement of infinite number. Veltmann, W. Z. Mth. Ps. 27 (1882) 176-.

- on convergence circle of analytic functions. Pincherle, S. Bologna Rd. 2 (1898) 77-.

- essential. Vivanti, G. Palermo Cir. Mt. Rd. 3 (1889) 223-.

- of $F(x)=\sum_{0}^{\infty} a^{n} x^{n^{2}}$. Fredholm, I. Stockh. Ofv. (1890) 131-; Fschr. Mth. (1890) 410.

Symmetrical expressions formed from values of modulus of function. Lewicki, $W$. Prace Mt.-Fiz. 6 (1895) 5-; Fschr. Mth. (1895) 445. Synectic functions. Pringsheim, $A$. Münch. Ak. Sb. 26 (1897) 167-.

Theorem. Lerch, M. Prag Sb. (1885) (Mth.Nt.) 400 -.

- of Bertini, demonstration. Liiroth, J. Mth. A. 42 (1893) 457-; 44 (1893) 539-.

-, fundamental. Morera, G. Mil. I. Lomb. Rd. 19 (1886) 304-.

- of Mittag-Leffler, extension. Phragmén, E. C. R. 128 (1899) 1434 .

- - Puiseux, on different paths leading to same value. Cauchy, A. L. C. R. $42(1856)$ 663-.

-. Uniform function near singular point can approach infinitely near to any value. Proof. Hölder, O. Mth. A. 20 (1882) 138. - of Weierstrass in an elliptic field. Cazzaniga, T. Mil. I. Lomb. Rd. 31 (1898) 1065-.

Theory. Weierstrass, C. T. W. Berl. Ak. Ab. (1876) (Mth.) 11-.

-. Mittag-Leffler, G. C. R. 94 (1882) 414-, 511-, 713-, 781-, 938-, 1040-, 1105-, 1163-; 95 (1882) 335-.

- Goursat, $\dot{E} . \quad$ C. R. 96 (1883) 565-

-. Stäckel, P. Crelle J. Mth. 106 (1890) 189 .

-. Desaint, L. Par. Ec. Norm. A. 14 (1897) 311-。

-. Hallgren, E. Göteb. Hndl. 32 (1897) 53-; Fschr. Mth. (1897) 355.

Transcendental function discovered by Fredholm. Mittag-Lefler, G. C. R. 110 (1890) 627-.

- functions, class. Poincaré, H. C. R. 103 (1886) 862- ; Liouv. J. Mth. 6 (1890) 313-.

- - - Craig, T. Am. J. Mth. 16 (1894) $207-$.

- - determination of genus. Laguerre, $E$. C. R. 94 (1882) 635-.

- -, resolution into partial fractions. Bukrejeff, B. [1886] Fschr. Mth. (1887) 381-.

Transformation of imaginary trigonometric binomials into exponentials. Matzka, W. Prag Sb. (1864) (pt. 2) 115-.

Uniform substitutions, convergence. Lémeray, E. H. C. R. 123 (1896) 793-; 124 (1897) 1220-.

Zeros, distribution. Cesáro, E. N. A. Mth. 6 (1887) 36-.

$\sum_{p=1}^{\infty} e^{-\sqrt{p}} \sin p x . \quad$ Lerch, M. Mh. Mth. Ps. 8 $p=1$ (1897) 377-. 


\section{Multiform functions of one variable; Riemann surfaces. (See also 4060, 6420, 8075.)}

Algebraic configurations admitting involution. Fuchs, L. Berl. Ak. Sb. (1886) 797-.

- - - uniform automorphic transformations. Hurwitz, A. Gött. Nr. (1887) 85-.

- functions, application of a theorem in, to partial differential equations. Fuchs, $L$. Berl. Ak. Sb. (1887) 159-.

- -, branches. Ascoli, G. Mil. I. Lomb. Rd. 18 (1885) 279-.

- - contours round singular points. David, -. Toul. Ac. Sc. Mm. 8 (1886) 295-.

- -, theory. Rados, G. Mth. Termt. Éts. 3 (1885) 185-.

- - - Klein, F. D. Mth. Vr. Jbr. 2 (1893) 61-.

Analytic point describing cycle, relations between residues. Appell, $P$. C. R. 95 (1882) 714-.

-- , theorems. Appell, P. C. R. 95 (1882) 624 -

- polydromic functions. Volterra, $V$. $\mathrm{Rm}$. R. Ac. Linc. Rd. 4 (1888) (Sem. 2) 355-.

Arbitrary function, class, determination. Thomé, L. W. Crelle J. Mth. 108 (1891) 335-.

Cauchy's theorem. Tonelli, A. Rm. R. Ac. Linc. Rd. 1 (1885) 785-.

Class. Picard, É. $\quad$ C. R. 88 (1879) 852-.

Coinciding points in ditropic $(2,2)$ correspondence. Brodén, T. Stockh. Öfv. (1893) 45-.

Continuity. Neumann, C. Leip. Mth. Ps. B. 40 (1888) 120-.

- or discontinuity, simple method of investigating. Neumann, C. G. Leip. Mth. Ps. B. 35 (1883) 85-.

Contours traced round given points. David, Toul. Ac. Sc. Mm. 9 (1887) 179-.

Cross-cuts. Laguerre, E. C. R. 99 (1884) 1065-.

Curves, points of which correspond to several values of the parameters. Thomae, J. Leip. Mth. Ps. B. 41 (1889) 365-

Doubly periodic functions. Pick, $G$. Wien Ak. Sb. 92 (1886) (Ab. 2) 893-.

- - with-lacunæ. Niccoletti, O. G. Mt. 32 (1894) 364-.

Function theory and differential equations. Königsberger, L. D. Nf. Vh. (1894) (Th. 2, Hälfte 1) 5 .

Functions belonging to a group of linear substitutions. Wilczynski, E. J. Am. J. Mth. 21 (1899) 85-.

- of complex variable, investigation. Riemann, B. Crelle J. 54 (1857) 101-。

-, especially integrals of differential equations, representation near singular points. Hamburger, M. [1876] Crelle J. Mth. 83 (1877) 185-.

- of quintuply connected surface. Thomae, $J$. Z. Mth. Ps. 11 (1866) 427-.
General equation of 4 th degree. Boer, $F$. de. Amst. Ak. Vs. M. 20 (1884) 413-; Fschr. Mth. (1884) 79 .

Integral with double circuit. Pochhammer, $L$. Mth: A. 35 (1890) 470-.

Integrals of complete differentials with two terms, analytic theorems. Riemann, $B$. Crelle J. 54 (1857) 105-.

- - differential equations of 1st order. Vivanti, G. A. Mt. 19 (1891-92) 29-.

- - solutions of differential equations, 2nd order, with three singular points. Kepiñski, S. Krk. Ak. (Mt.-Prz.) Rz. 17 (1900) 112-; Crc. Ac. Sc. Bll. (1899) 67-.

Laplace's transformation and applications. Pincherle, S. Bologna Ac. Sc. Mm. 8 (1887) 125-.

Linearly related functions, theory. Heun, $K$. Acta Mth. 11 (1887-88) 97-; 12 (1889) 103-.

Multiformity, Dühring's conception, applications. Wessely, $K$. Arch. Mth. Ps. 16 (1898) 225-.

Periodic functions, generalisation. Floquet, $G$. Nancy S. Sc. Bll. (1897) 260-.

Periodicity. Benthem, A. N. Arch. Wisk. 3 (* 1877) 186-.

Polynomials, geometry. Lucas, $F$. Par. Ec. Pol. J. Cah. 46 (1879) 1-.

Relations of reality in the normal curve of $\phi$ 's, belonging to any genus. Klein, $F$. Mth.A. 42 (1893) 1-.

Representation. Desaint, L. C. R. 130 (1900) 1296-.

-, conformal, by rational functions. Neovius, E. R. Helsingf. Acta 17 (1891) 1-.

Riemann integrals, theory. Baker, H.F. Mth. A. 45 (1894) 118-.

- -, - (Baker). Hensel, $K$. Mth. A. 45 (1894) 598-; 46 (1895) 160.

\section{RIEMANN SURFACES.}

Bertini, E. Rm. R. Ac. Linc. Rd. 3 (1894) (Sem. 1) 106-.

algebraic configurations on, moduli. Fricke, R. D. Mth. Vr. Jbr. 3 (1894) 93-.

analysis situs of. Durège, $H$. Wien Ak. Sb. 69 (1874) $(A b .2)$ 115-.

- - , theorems. Thomae, J. Z. Mth. Ps. 12 (1867) 361-

analytic functions on. Vitali, $G$. Palermo Cir. Mt. Rd. 14 (1900) 202-.

with branch points given. Hurwitz, A. Mth. A. 39 (1891) 1-.

- - variable. Hoyer, P. Mth. A. 47 (1896) 47 -.

canonical cross-cuts. Liuroth, J. Erlang. Ps. Md. S. Sb. 15 (1883) 24-.

- form and dissection. Clifford, W. K. L. Mth. S. P. 8 (1877) $292-$.

case. Burnside, $W$. L. Mth. S. P. 22 (1891) 410 -

class non-transformable into simply connected surfaces. Puchta, A. [1880] Wien Ak. Sb. $82(1881)(A b .2) 260$ -

connection of Bernoulli's functions. Jonquière, A. Stockh. Ak. Hndl. Bh. 16 (Afd. 1) (1891) No. 6, $28 \mathrm{pp}$. 


\section{Riemann Surfaces}

in connection with curve $\lambda^{3} \mu+\mu^{3} \nu+\nu^{3} \lambda=0$. Haskell, M. W. Am. J. Mth. 13 (1891) 1-.

construction for inverse of rational functions by conformal representation. Bouton, $C$. $L$. A. Mth. 12 (1898-99) 1-.

- of monogenic analytic functions on. Ott, $K$. Mh. Mth. Ps. 4 (1893) 367-.

"coupures," and cross-cuts, and concepts of integration. Casorati, F. A. Mt. 15 (188788) 223-; 16 (1888-89) 1-.

cross-cuts, transformation. Wellstein, $J$. Mth. A. 52 (1899) 433-.

deficiency. Baker, H. F. Mth. A. 45 (1894) 133-.

function constructed on. Wilczynski, E. J. N. Y. Am. Mth. S. Bll. 5 (1899) 388-.

for Galois's resolvent of certain modular equations. Dyck, W. Mth. A. 18 (1881) 507-.

given, algebraic functions appertaining to. Thomae, C. J. Mth. A. 18 (1881) 443-.

-, integration of $\nabla^{2} u=0$ on. Ascoli, G. [1887] Stockh. Ak. Hndl. Bh. 13 (Afd. 1) (1888) No. 2, 83 pp.

,$---\frac{1}{-}=--$ (Ascoli). Eneström, G. Stockh. Öfv. (1887) 99-; Fschr. Mth. (1887) 29.

integration of $\nabla^{2} u+k^{2} u=0$ on. Sommerfeld, $A$. Gött. Nr. (1895) 267-.

linearly bounded. Schönflies, A. Gött. Nr. (1892) 257-.

models for. Durège, $H$. Prag Sb. (1864) (pt. 2) $112-$.

new kind. Klein, F. (vm) Erlang. Sb. Ps. Md. S. 6 (1874) 102-, 133-; (x) Mth. A. 7 (1874) 558-; 10 (1876) 398-.

order of branch points. Hensel, $K$. Berl. Ak. Sb. (1895) 933-.

prime form on, Ritter's. Fricke, R. Gött. Nr. (1900) 314-.

- forms on. Klein, F. C. R. 108 (1889) 134-. - - - Wellstein, J. Gött. Nr. (1900) 380-. regular, group and irrationality. Dyck, $W$. Mth. A. 17 (1880) 473-.

-, of 3rd species, and corresponding " normal curve" of 4 th order. Dyck, W. Mth. A. 17 (1880) 510-

representation of function in, with variable multipliers of periodicity. Suchar, P. I. [Bucarest S. Sc. Bll. 7 (1898)] 143-.

sections. Litroth, J. Mth. A. 4 (1871) 181-. symmetrical, and periodicity moduli of corresponding Abelian normal integrals. Weichold, G. Z. Mth. Ps. 28 (1883) 321-.

theory. Clebsch, R. F. A. [1872], Mth. A. 6 (1873) 216-.

three-leaved. Baur, L. Crelle J. Mth. 119 (1898) 171-.

- and four-leaved. Hensel, $K$. Berl. Ak. Sb. (1895) 1103-.

treatment of unicursal curves by. Osgood, W. F. N. Y. Am. Mth. S. Bll. 2 (1896) 168-.

use in applied mathematics. Carslaw, H. S. B. A. Rp. (1900) 644 .

Riemann theorem, demonstration. Prym, F.E. Crelle J. Mth. 83 (1877) 251-.

\section{Expansions in Series 3630}

Riemann theorem, demonstration. Croullebois, $M$. C. R. 93 (1881) 719-.

- theory. Klein, C. F. [1882] Mth. A. 21 (1883) 141-.

- - (Klein). Noether, M. Z. Mth. Ps. 27 (1882) (H.-lt. Ab.) 201-.

- -, extension. Volterra, V. Rm. R. Ac. Linc. Rd. 3 (1887) (Sem. 2) 281-; 4 (1888) (Sem. 1) 107-, 196-, (Sem. 2) 485.

- note. Lippich, F. Wien Ak. Sb. 69 (1874) $(A b .2)$ 91-.

-Roch theorem. Landsberg, $G$. [1897] Mth. A. 50 (1898) 333-.

-- -. Fields, J. C. [1900] N. Y. Am. Mth. S. Bll. 7 (1901) 244, 254-.

- _ - extension to linear systems of forms. Ritter, E. Gött. Nr. (1894) 328-.

Rolle's theorem, extension. Veneziani, $C$. J. H. Un. Cir. [4] (1885) 78-.

Surface of two sheets, theory of functions in. Prym, F. E. [1866] Zür. N. D. Sch. Gs. 22 (1867) $47 \mathrm{pp}$.

Surfaces, relationship, in Riemann's sense. Lippich, F. [1873] Mth. A. 7 (1874) 212-.

Symmetric and periodic functions derived from algebraic function. Petrovitch, M. Bll. Sc. Mth. 20 (1896) 108-.

Theta functions, representation of quotient of two. Thomae, J. Mth. A. 6 (1873) 603-.

Transformation of functions, theory. Weber, $H$. Crelle J. 76 (1873) 345-.

$\phi(t)=\int \frac{R_{1}(z, w) d z}{R_{2}(z, w)-t}$ where $R_{1}$ and $R_{2}$ belong to one Riemann's surface. Krieg von Hochfelden, F. (Frhr.) Wien Ak. Sb. 94 (1887) (Ab.2) 221-.

\section{Expansions in series of func- tions, other than powers of the variable.}

Gomes Teixeira, F. Bll. Sc. Mth. 12 (1888) $272-$.

Algebraic expressions, expansion in a class of. Allé, $(D r) M$. Wien Ak. Sb. 52 (1866) (Ab. 2) 453-.

- functions, cases. Deruyts, $J$. Brux. Ac. Mm. 49 (1890-93) (No. 5) 20 pp.

Analytic functions, expansion in given functions. Frobenius, G. [1870] Crelle J. 73 (1871) 1-.

- - , - integral rational functions, case. Hilbert, D. Gött. Nr. (1897) 63-.

- - systems, and series formed with them. Pincherle, S. A. Mt. $12\left({ }^{*} 1883-84\right)$ 11-, $107-$.

Arbitrary functions, expansion in given functions. Lorenz, L. V. (xir) Ts. Mth. 6 (1876) 129-.

- powers of trigonometric functions. Sincov, D. M. Kazan S. Ps.-Mth. Bll. 4 (1895) 199-; Fschr. Mth. (1895) 471.

Bernoulli's functions, expansion in. Rogel, $F$. Prag Sb. (1896) (Mth.-Nt.) No. 31, 48 pp.

- with fractional indices. Sincov, D. Kazan S. Nt. (Ps.-Mth.) P. 8 (1890) 291-; Fschr. Mth. (1890) 443-. 


\section{Expansions in Series}

Bernoulli's functions, generalised, applications. Imšeneckij, V. G. [1885] St Pet. Ac. Sc. Mm. (Rs.) 52 (1886) (Suppl.) No. 2, 62 pp. ; Fschr. Mth. (1886) 373-.

- polynomials and their applications. Sonine, N.J. Fschr. Mth. (1888) 424.

Cases. Deruyts, J. Brux. Mm. Cour. $4^{\circ}, 47$ (1886) No. 6, $18 \mathrm{pp}$.

Convergence-subtrahends for trigonometric functions expressed in infinite sums. Bohannan, R. D. A. Mth. 1 (1884-85) 49-. Deduction of $C, S$, from

$$
C+i S=\sum_{x=0}^{\infty} \frac{n^{x, 1}}{x !} \frac{a^{x}}{c^{x}} e^{i \beta x} .
$$

Pánek, A. Časopis 17 (1888) 227-; Fschr. Mth. (1888) 263.

Determination of coefficients in

$$
f(n)=B_{1} \psi(n, 1)+B_{2} \psi(n, 2)+\ldots
$$

where $f(n)=A_{1} \phi(n, 1)+A_{2} \phi(n, 2)+\ldots$

and $\psi(n, k)=a_{1} \phi(n, 1)+a_{2} \phi(n, 2)+\ldots$.

Minin, A. P. Rec. Mth. (Moscou) 13 (1886) 535-; Fschr. Mth. (1887) 173-.

Effective validity, cases. Pincherle, S. Rm. R. Ac. Linc. Rd. 5 (1896) (Sem. 1) 27-.

Expansion in continued fractions. Hurwitz, $A$. Acta Mth. 11 (1887-88) 187-.

- - denominators of convergents of continued fraction. Rouché, E. Par. Éc. Pol. J. $37^{\mathrm{e}} \mathrm{cah}$. (1858) 1-.

- Euler's functions. Rogel, F. Prag Sb. (1896) (Mth.-Nt.) No. 42, 9 pp.

- factorials. Schlömilch, O. Leip. B. 15 (1863) 58 .

- of the variable. Graves, C. Ir. Ac. P. 3 (1847) 455-.

- - harmonic functions. Steklov, V. A. Kharkov Mth. S. Com. 5 (1896?) 60-; Fschr. Mth. (1896) 203-; C. R. 128 (1899) 279-.

- - infinite series. Morera, $G$. Tor. Ac. Sc. At. 21 (1885) 892-.

- - inverse factorial series. Kluyver, J. C. N. Arch. Wisk. 4 (1899) 74-; Fschr. Mth. (1899) 372.

- - other functions. Hugoniot, - C. R. 95 (1882) 907-.

- - - (Hugoniot). Du Bois-Reymond, $P$. C. R. 96 (1883) 61-.

- - - - form of residue. Lagrange, $\boldsymbol{C}$. C. R. 98 (1884) 1422-.

- _ - - any number of variables. Lagrange, C. Brux. Mm. Cour. $4^{\circ}, 48$ (1886) No. 2, $16 \mathrm{pp}$.

- polynomials. Pincherle, S. C. R. 107 (1888) 986-.

- - Leau, L. Par. S. Mth. Bll. 27 (1899) 194-.

- powers of $n$ algebraic functions from $n$ equations. Arzelà, C. G. Mt. 11 (1873) 368 -

- - - a polynomial. Jacobi, C. G. J. [1847] Crelle J. 53 (1857) 103-.

- - - - - Laguerre, $E$. [1878-79]

C. R. 86 (1878) 383-; Crelle J. Mth. 88 (1880) 35-.

Bll. $8-7-1$. Humbert, G. Par. S. Mth.

\section{Multivariable Functions 3640}

Expansion in trigonometric functions of imaginary arcs. Clapeyron, E., \& Lame, G. Crelle J. 6 (1830) 45-.

- trigonometric functions $\sin ^{p} x, \cos ^{p} x, x^{p}$ when $p$ is irrational. Yakovlev, N. Y. (xח) Kazan Un. Mm. (1857) (Bk. 4) 84-.

Formula of Darboux. Mansion, $P$. Brux. S. Sc. A. 13 (1889) (Pt. 2) 108-.

- - Hermite. Pincherle, S. Rm. R. Ac. Linc. Rd. 1 (1885) 267-.

Functions of fractional powers of sine and cosine. Stein, J. P. Gergonne A. Mth. 15 (1824-25) 150-.

- one real variable, expansion in Jacobian functions. Dini, $U$. A. Mt. 10 (1880-82) 145 -.

Generating functions, fundamental theorem. Lerch, M. Prag České Ak. Fr. Jos. Rz. (Trida 2) 1 (1892) Art. 33, 7 pp.; Fschr. Mth. (1892) 363-.

Holomorphic function within a given contour, expansion in polynomials. Renaux, - C. R. 129 (1899) 473-, 626.

Integral function, expansion in denominators of certain regular continued fractions, property of. Gegenbauer, $L$. Wien Ak. Sb. 98 (1890) (Ab. 2a) 867-.

Lagrange's series, generalisation. Amigues, $E$. C. R. 116 (1893) 368-, 429.

- - , series analogous to. Amigues, E. C. R. 99 (1884) 1149-.

- - Bougaïev, N. C. R. 131 (1900) 793-.

Series with imaginaries. Denzler, $W$. Zür. Vjschr. 1 (1856) 333-.

- of rational functions. Borel, $\dot{E}$. C. R. 130 (1900) 1061-.

Uniform functions, expansion in polynomials. Painleve, P. C. R. 102 (1886) 672-; 129 (1899) 27-.

Wronski's law, elementary demonstration. Lagrange, $C$. Brux. Mm. Cour. $4^{\circ}, 47$ (1886) No. 2, 8 pp.

$(x-z)^{m}$, expansion in powers of $\left(z^{2}-1\right)$. Laguerre, E. C. R. 86 (1878) 956-.

$f(x)$, expansion in series

$$
\sum_{1}^{\infty} \frac{a_{n} x^{n}}{1-x^{n}}, \sum_{1}^{\infty} \frac{a_{n} x^{n}}{1-x^{2 n}}
$$

Rogel, F. Prag Sb. (1892) (Yth.-Nt.) 3-. $x^{k}$, expansion in powers of $\sin x$. Gomes Teixeira, F. N. A. Mth. 15 (1896) 270-.

\section{Functions of several variables.}

Trzaska, W. Par. T. Nauk Śc. Pam. $2\left({ }^{*} 1872\right)$ 27-; Bll. Sc. Mth. As. 6 (*1874) 156.

Mittag-Lefler, G. Stockh. Öfv. 34 (1877) No. 10, 3-, 17-.

(Mittag-Leffler.) Weierstrass, C. T. W. Berl. Ak. Mb. (1880) 707-.

(- - ) Hermite, C. [1881] Crelle J. Mth. 92 (1882) 145-.

Combescure, É. C. R. 96 (1883) 235-, 483-.

Cousin, P. Acta Mth. 19 (1895) 1-.

Lebesgue, H. C. R. 128 (1899) 811-. 
3640 Multivariable Functions

Algebraic functions of 2 variables. Hensel, $K$. D. Mth. Vr. Jbr. 7 (1899) (Heft 1) 58-.

- - - new theory. Hensel, $K$. D. Mth. Vr. Jbr. 8 (1900) (Heft 1) 221-.

- - several variables. Nöther, M. Gött. Nr. (1869) 298-.

Analytic continuation. Goursat, É. C. R. 128 (1899) 591-.

- functions in $n$ dimensions, theorems. Scheffers, G. C. R. 116 (1893) 1242-.

- of several variables. Osgood, W. F. Mth. A. 52 (1899) 462-; 53 (1900) 461-.

- - - - expansion. Painlevé, P. C. R. 129 (1899) 92-.

Conjugate functions in $n$ variables. Volterra, $V$. Rm. R. Ac. Linc. Rd. 5 (1889) (Sem. 1) 599-.

Differential quotients. Czuber, $E$. Wien Ak. Sb. 101 (1892) (Ab. 2a) 1417-.

Differentials. Goursat, É. Par. Ec. Norm. A. 2 (1885) 255-.

-, successive. Darboux, G. C. R. 93 (1881) 1123-; 94 (1882) 575-; Bll. Sc. Mth. As. 5 (1881) 376-, 395-.

Expansion, conditions of reality. Schultén, N. G. af (fil.). [1843] Helsingf. Acta 2 (1847) $347-$.

- in series, form when curtailed. Riquier, C. R. 126 (1898) 1558-.

Functions of two real variables, Schönflies's theorem. Osgood,W.F. Gött. Nr. (1900) 94-. Nr. (1900) $-\frac{-}{98-}-$ - Bernstein, F. Gött.

- variables. Poincaré, $H$. Acta Mth. 2 (1883) 97-.

- - - - behaviour near a zero point. Brill, A. Münch. Ak. Sb. 21 (1892) 207-.

- - - - boundary conditions for value. Ascoli, G. Mil. I. Lomb. Mm. 16 (1891) 197-.

--- , class. Appell, P. Acta Mth. 2 (1883) 71-.

- _ - - divisibility by another function, case. Berry, A. L. Mth. S. P. 30 (1899) 271-. - _ - , graphical representation. Klejber, I. A. Rs. Ps.-C. S. J. 19 (Ps.) (1887) 18 -

- - - inversion. Fuchs, L. Berl. Ak. Sb. (1887) 99-.

_ _ _ _ limiting values, two theorems. Du Bois-Reymond, P. [1876] Mth. A. 11 (1877) 145-.

- $n$ real variables. Giuliani, G. G. Mt. 29 (1891) 234-.

- - - variables arising from inversion of integrals. Fuchs, L. Berl. Ak. Sb.(1883) 507-.

Gauss's formula for interlaced curves and extension to higher configurations. Dyck, $W$. Münch. Ak. Sb. 25 (1896) 447-.

Hyperspaces, complex variables in. Volterra, $V$. Rm. R. Ac. Linc. Rd. 5 (1889) (Sem. 1) 291-; 6 (1890) (Sem. 2) 241-.

-, functions of, and their differential parameters. Volterra, V. Rm. R. Ac. Linc. Rd. 5 (1889) (Sem. 1) 630-.

Integral function of $n$ elements, number of values. Netto, $E$. Crelle J. Mth. 85 (1878) 327-.
Algebraic Functions 4000

Integrals and expansions in series, cases. Bucca, F. PalermoCir. Mt.Rd. 12 (1898) 260-.

Kronecker's characteristic. $D y c k, W$. Münch. Ak. Sb. 25 (1896) 261-.

Limiting values. Stolz, O. Mth. A. 26 (1886) 83-; Innsb. Nt. Md. B. 17 (1887) xxvI-.

Periodic functions of two variables. Appell, $P$. Liouv. J. Mth. 7 (1891) 157-.

Potential theory. Dyck,W. Münch. Ak. Sb. 25 (1896) 261-, 447-; 28 (1899) 203-.

Rational functions of $n$ variables, classes. Kronecker, L. Berl. Ak. Sb. (1886) 251-.

- - - - conjugate values. Netto, E. Crelle J. Mth. 100 (1887) 436-.

Relation between $p$ integral functions of $p-1$ variables. Perrin, R. C. R. 106 (1888) 1789-.

Series integral with reference to several variables. Dautheville, S. Par. Éc. Norm. A. 2 (1885) (Suppl.) $59 \mathrm{pp}$.

Theorem, demonstration. Trzaska, $W$. Par. T. Nauk Śc. Pam. $2\left({ }^{*} 1872\right)$ 39-; Bll. Sc. Mth. As. 6 (*1874) 156.

Theory. Baker, H. F. Camb. Ph. S. T. 18 (1900) 408-.

-, formula. Pincherle, S. Stockh. Ofv. (1886) $51-$.

- - , and integration of total differentials. Jaggi, E. N. A. Mth. 16 (1897) 297-.

Transcendental functions, property of class. Volterra, $V . \quad$ Rm. R. Ac. Linc. Rd. 2 (1886) (Sem. 2) 211-.

Undeveloped functions, theory. Schwarz, H.A. Berl. Ak. Sb. (1897) 948-.

Uniform analytic functions of $n$ variables, theory. Biermann, $O$. Wien Ak. Sb. 89 (1884) (Ab. 2) 266-.

- functions defined by inversion of total differentials. Painlevé, P. C. R. 122 (1896) $660-, 769$ -

- - expressible as quotient of two power series, theorem. Hurwitz, A. [1882] Crelle J. Mth. 95 (1883) 201-.

- - of 2 variables, class. Picard, É. C. R. 95 (1882) 594-.

_ _ _ - connected by an algebraic equation. Gunther, P. Crelle J. Mth. 109 (1892) 199-.

- _ _ _ - and a group of linear substitutions. Picard, É. C. R. 94 (1882) 579-, 837-. - - _ - poles. Autonne, L. Palermo Cir. Mt. Rd. 10 (1896) 196-.

- - - several independent variables, poles. Autonne, L. C. R. 124 (1897) 139-; Acta Mth. 21 (1897) 249-.

Zeros. Dyck, W. Münch. Ak. Sb. 28 (1899) 203-.

\section{Algebraic functions and their integrals.}

\section{General.}

Rydberg, $J$. $R$. Lund. Un. Acta 15 (1878-79) (Mth.) (No. 2) $18 \mathrm{pp}$.

Algebraic functions. Cockle, Jas. Camb. and Dubl. Mth. J. 2 (1847) 267-; 3 (1848) 179-. 
Algebraic functions. Puiseux, $V$. Liouv. J. Mth. 15 (1850) 365-; 16 (1851) 228-.

- - (Puiseux). Cauchy, A. L. C. R. 32 (1851) 276 -

- - Hermite, C. C. R. 32 (1851) 458-.

- - , application in geometry. Brill, A., \& Nöther, M. [1873] (vII) Mth. A. 7 (1874) 269-.

- - class. Schlömilch, O. Grunert Arch. 10 (1847) 67-.

- - continuity and discontinuity, conditions. Gascheau, -. Toul. Ac. Sc. Mm. 2 (1880) (Pt. 2) 122-.

- and curves, property. Picard, É. C. R. 91 (1880) 214-, 724-.

- - fundamental theorem. Voss, A. Mth. A. 27 (1886) 527-.

- - number of arbitrary constants. Roch, $G$. [1864] Crelle J. 64 (1865) 372-.

- -, property. Darboux, G. C. R. 94 (1882) 575-.

- - rationalisation. Polewski, S. Arch. Mth. Ps. 69 (1883) 149-.

- - , representation, approximate, by means of rational functions. Bortolotti, $E$. Rm. R. Ac. Linc. Rd. 8 (1899) (Sem. 1) 57-.

- - , invariant. Noether, $M$. Mth. A. 17 (1880) 263-.

- - Riemann's theorem, demonstration. Herz, N. [1881] Arch. Mth. Ps. 68 (1882) 14-.

- - singular points, application of Newton's polygon to theory. Baker, H. F. Camb. Ph. S. T. 15 (1894) 403-.

- - theorem. Noether, M. Crelle J. Mth. 92 (1882) 301-.

- -, theory, development. Brill, A., $\&$ Noether, M. D. Mth. Vr. Jbr. 3 (1894) 107-.

- - values, connexion between. Runge, $C$. Crelle J. Mth. 97 (1884) 337-.

- integrals, theorem. Ptašickij, I.L. Kharkov Mth. S. Com. 1 (1889) 74-; Fschr. Mth. (1888) 296-.

Arbitrary function, determination. Cellérier, $C$. Liouv. J. Mth. 8 (1843) 245-.

- functions, elimination. Hermite, C. B. A. Rp. 42 (1872) 233-.

Class of functions connected with

$$
s^{3}=\left(z-a_{1}\right)\left(z-a_{2}\right) \ldots\left(z-a_{6}\right),
$$

theory. Wellstein, J. Mth. A. 52 (1899) 440-.

Coincidence of function with algebraic functions in infinite number of places. Schering, $E$. [1879] Gött. Ab. 27 (1881) (Mth.) 62 pp.

Continued fractions, new applications. Markov, A. St Pét. Ac. Sc. Mm. 3 (1896) No. 5, $50 \mathrm{pp}$.

Differentials, integrals of which are algebraic functions, form. Kapteijn, W. Amst. Ak. Vs. M. 17 (1882) 93-.

Expressions, integrals of which are algebraic. Liouville, J. [1832-37] Par. Éc. Pol. J. 22 cah. (1833) 124-, 149-; Crelle J. 10 (1833) 347-; C. R. 5 ' (1837) 330-.

Formula of Jacobi, demonstration. Brioschi, F. Tortolini A. 1 (1858) 117-.
Fractional functions, decomposition and transformation. Rangoni, L. [1827] (vri) Mod. Mm. Ac. Sc. 1 (1833) (pte. 2) 254-.

Functions in general, fundamental theories established without reduction to the canonical form. Tikhomandrītskit, M. A. (xII) Kharkov Mth. S. Com. (1883) 79-; Fschr. Mth. (1885) 423.

- with multiple periods. Casorati, $F$. C. R. 57 (1863) 1018-.

Goniometric functions, relation to certain algebraic expressions. Studnička, F.J. Časopis 19 (1890) 249-; Fschr. Mth. (1891) 444.

Identities, generalisation. Oltramare, $G$. Gen. I. Nt. Mm. 16 (1886) No. 4, 109 pp.

Integrals of algebraic differentials. Ramus, $C$. Crelle J. 24 (1842) 69-.

- - - functions. Ostrogradsky, M. A. [1842] St Pét. Ac. Sc. Bll. 1 (1843) 113-.

- - - Pellet, A.E. C. R. 90 (1880) 676-. - _ _ - , reduction to integrals of rational functions. Formenti, C. Mil. I. Lomb. Rd. 14 (1881) 667-; 15 (1882) 165-.

- - - theorems. Poisson, S. D. Crelle J. 12 (1834) 89-.

- - - - theory. Jurgensen, $C$. Kiöb. Ov. (1839) 6-; Crelle J. 23 (1842) 126-; N. Mg. Ntvd. 3 (1843 ?) 85-.

- - and transcendental formulæ. Tortolini, B. G. Arcad. 56 (1832) 81-.

- $\int \frac{G(x) d x}{\sqrt{R(x)}}$. Guichard, C. Par. Ec. Norm. A. 5 (1888) 193-.

Integration in finite explicit functions, determination of algebraic part. Piuma, C. $M$. (vIII) Tortolini A. 4 (1861) 154-.

- - - form of cubics and quartics symmetrical in $x$ and $\frac{1}{x}$. Toropov, $K$. A. Kharkov Mth. S. Com. (1885) 3-; Fschr. Mth. (1885) 258.

-, Jacobi-Mayer method, improvement. Lie, S. Christiania F. 15 (1873) 282-.

Inverse derived functions. Buquoy, G. von. Gilbert A. 67 (1821) 202-.

Inversion of system of functions. Lipschitz, $R$. Gött. Nr. (1870) 439-.

Isoperimetric equations and integrability. Brioschi, F. [1853] Mod. Mm. S. It. 25 (1855) 205-.

Periodic function, calculation of coefficients from given mean values. Krilger, A. As. Nr. 82 (1873) 333-.

Periods of integrals. Marie, M. C. R. 36 (1853) 437-.

Powers of functions, expansions, cases. André, D. [1877] Par. S. Mth. Bll. 6 (1878) 120-.

Product of several integrable and finite functions. Ascoli, G. Mil. I. Lomb. Rd. 12 (1879) 372-.

Resolution of a class of functions. Jïrgensen, C. [1838] Kiöb. Dn. Vd. Selsk. Afh. 8 (1841) 1-; Crelle J. 19 (1839) 84-.

Sums depending on positive values of any function. Čebyrev, $P$. St Pét. Ac. Sc. Mm. 1 (1895) No. 7, 20 pp.; Fschr. Mth. (1895) 441-. 
Theorem, fundamental. Noether, $M$. Mth. A. 6 (1873) 351-; 30 (1887) 410-; 34 (1889) 450 -; 40 (1892) 140-.

-, - (Noether). Baker, H. J. Mth. A. 42 (1893) 601-.

,$--(-)$. Scott, C. A. Mth. A. 52 (1899) $593-$.

,-- , demonstration. Bohannan, R. D. Am. J. Mth. 19 (1897) 192.

Transcendental functions, classification. Liouville, J. [1835] Liouv. J. Mth. 2 (1837) 56-; 3 (1838) 523-.

Undeveloped functions, new proof of fundamental theorem. Grave, D. Fschr. Mth. (1899) 379.

\section{Algebraic functions of one variable.}

Betti, E. Pisa A. Un. Tose. 7 (Sc. Cosm.) (1862) 101-.

Hensel, K. D. Mth. Vr. Jbr. 1 (1892) 56-.

Algebraic configuration of $n$th rank in domain of $n+1$ magnitudes. Biermann, $O$. Wien Ak. Sb. 95 (1887) (Ab. 2) 802-.

- differentials, integration. Ptašickij, I. L. Kharkov Mth. S. Com. 1 (1889) 61-; Fschr. Mth. (1888) 296-.

- functions of one and two variables. Nöther, M. Gött. Nr. (1871) 267-.

- integrals. Cremona, L. [1869] Bologna Ac. Sc. Mm. 10 (1870) 3-.

- logarithmic integrals of algebraic functions, extension of Abel's theorem. Königsberger, L. Mth. A. 17 (1880) 560-.

- part of integral of rational functions, determination. Gomes Teixeira, $F$. Rm. R. Ac. Linc. Rd. 1 (1885) 187-.

- systems, reduction to canonical form. Hensel, K. Crelle J. Mth. 117 (1897) 129 -.

Application of theory of linear differential equations. Thomé, L. $W$. Crelle J. Mth. 104 (1889) 1-; 112 (1893) 165-.

Canonical systems. Fischer, $K$. Crelle J. Mth. 117 (1897) 1-.

Circular are polygons. Schönflies, A. Mth. A. 42 (1893) 377-.

44 (1894) 105-.

- quadrilaterals and principle of symmetry. Fricke, R. Mth. A. 44 (1894) 565-.

Definition by cubic equation. Betti, E. G. Mt. 3 (1865) 143-.

$--1-$ Floquet, G. Par. S. Mth. Bll. 23 (1895) 76-.

Factorisation of integral function of one variable into factors without multiple roots. Engel, F. Leip. Mth. Ps. B. 49 (1897) 603-.

Function $\phi(x)=\frac{a x+b}{c x+d}$. Tanner, H. W. L. [1879] Mess. Mth. 9 (1880) 109-.

$-(x-1)^{a}$. Humbert, $G$. Par. S. Mth. Bll. 9 (1881) 56-.

$-f(x)=(x+1)(x-1)(x-2)$. Bigler, U. Arch. Mth. Ps. 14 (1896) 337-.
Functions analogous to Legendre's polynomials. Hugoniot, -. C. R. 95 (1882) 983-.

- of form $f(x)=\frac{a x+b}{c x+d}$, symbolic powers and roots. Johnson, W. W. Mess. Mth. $9(1880)$ 99-.

$--\frac{a x+b}{c x+d}$, powers. Tanner, H.W. L. Mess. Mth. 9 (1880) 139-.

Fundamental system for given class domain, determination. Mertens, $F$. Wien Ak. Sb. 102 (1893) (Ab. 2a) 497-.

- systems for. Baker, H. F. L. Mth. S. P. 26 (1895) 107-.

Geometrical study. Tognoli, O. G. Mt. 22 (1884) 308-; 23 (1885) 247-, 345-.

Hyperelliptic integrals. Chessin, A. S. [1897] N. Y. Am. Mth. S. Bll. 4 (1898) 93-.

Implicit function defined by algebraic equation, values. Marie, M. C. R. 47 (1858) 145-.

Integral algebraic functions, representation. Hensel, K. Crelle J. Mth. 111 (1893) 139-.

- rational functions with integral coefficients, irreducibility. Hilbert, $D$. Crelle J. Mth. 110 (1892) 104-.

$-\int \frac{x^{2 m} d x}{\left(1+x^{* n}\right) \sqrt{1+x^{2}}}$, value. Popov, A.T. (xп) Kazan Un. Mm. 6 (1871) 236-.

$-\int \frac{x^{a-1} d x}{x+\mu}$, evaluation. Worpitzky, J. Z. Mth. Ps. 19 (1874) 90-.

Integrals of algebraic expressions with several variables. Grönwall, $H$. Stockh. Öfv. (1896) 67-.

- value, theory. Bertrand, J. Par. S. Phlm. PV. (1843) 126.

- - factorial functions, inversion. Picard, $\hat{E}$. Am. J. Mth. 16 (1894) 111-.

- - form $\int \frac{z^{m-1} d z}{\left(z^{n}-c^{n}\right) \sqrt{z^{n}-b^{n}}}$, reduction. Stuart, G. H. QJ. Mth. 18 (1882) 245-.

Integration of differentials containing square root of polynomial of $3 \mathrm{rd}$ or $4 \mathrm{th}$ degree. Tchebicheff, P. [1854] Liouv. J. Mth. 2 (1857) 1-.

- - - - - - - 4th degree or cube root of polynomial of 3rd degree. Aleksyeev, N. N. (xII) Rec. Mth. (Moscou) [1] (1866) 187-.

- in finite series, cases. Pezzi, F. Verona S. It. Mm. 4 (1788) 577-; 6 (1792) 256-.

-, graphical treatment, and applications. Massau, J. Rv. Un. Mines 16 (1884) 243-, 579-; 17 (1885) 1-; 20 (1886) 1-, 475-; 21 (1887) 85-, 308-, 572-; 22 (1887) 1-.

- of an irrational formula. Maurice, $F$. Bb. Brit. 17 (1801) 103-.

- - irrational functions. Casorati, $\boldsymbol{F}$. Tortolini A. 7 (1856) 223-.

-, reduction formulæ. Azzarelli, $M$. $\mathrm{Rm}$. N. Linc. Mm. 1 (1887) 247-.

- by substitution, case. Luchterhandt, A. R. Crelle J. 17 (1837) 248-. 
4010 Functions of One Variable

Integration by use of imaginaries. Martin, $H$. N. A. Mth. 2 (1863) 57-.

- of $\quad \frac{d x(1-z z)^{2}}{(1+z z) \sqrt[4]{\left(1+6 z z+z^{4}\right)^{3}}}$

and

$$
\frac{d z(1+z z)^{2}}{(1-z z)^{4} \sqrt{\left(1-6 z z+z^{4}\right)^{3}}} \text {. }
$$

Rumovski, S. [1807] St Pét. Ac. Sc. Mm. 2 (1807-08) 84-.

$--\int \frac{x^{m} d x}{\left(1+a_{(1)} x+a_{(2)} x^{2}+a_{(3)} x^{3}+\ldots+a_{(\theta)} x^{\theta}\right)^{n}}$.

Nicolai, F. B. G. As. Nr. 5 (1827) 371-.

$-\frac{x+A}{\sqrt{x^{4}+\alpha x^{3}+\beta x^{2}+\gamma x+\delta}} d x$. Tchebicheff, P. $[1860]$ St Pét. Ac. Sc. Bll. 3 (1861) $1-$.

$-\frac{a+b x+c x^{2}+\ldots+p x^{n}}{A+B x+C x^{2}+\ldots+P x^{\mathrm{N}}} \quad \frac{d x}{\sqrt{\left(\alpha+\beta x+\gamma x^{2}\right)}}$. Grelle, F. Z. Mth. Ps. 16 (1871) 254 -.

Isodynamic lines of a polynomial, electric determination. Lucas, $F$. C. R. 106 (1888) 587-.

Limiting values of integrals. Markov, A. A. St Pét. Ac. Sc. Mm. 10 (1900) No. 9, 34 pp.; F'schr. Mth. (1900) 313-.

Linear functions. Pellet, A. E. C. R. 90 (1880) 1111-.

- _ of one variable. Christoffel, E. B. Crelle J. 55 (1858) 281-.

Normal basis and absolute fundamental system, connexion between. Baur, L. Mth. A. 49 (1897) 73-.

Periodicity, multiple, in functions of one variable. Casorati, $F$. Mil. I. Lomb. Rd. 16 (1883) 815-.

Polynomials, statics. Lucas, F. Par. S. Mth. Bll. 17 (1889) 17-.

Rational fractional functions, representation by. Biermann, $O$. Wien Ak. Sb. 89 (1884) (Ab. 2) 84-.

- integral functions, type. Laguerre, C. R. 98 (1884) 79-.

- operations. Noether, M. Mth. A.23(1884) 311-.

Reciprocity theorem. Study, E. Leip. Mth. Ps. B. 42 (1890) 153-.

Simple transcendental functions, elementary proof of periodicity. Studnicka, $F$. $J$. Časopis 22 (1893) 209-; Fschr. Mth. (189394) 737 .

Theorem of Abel. Stickelberger, L. Crelle J. Mth. 82 (1877) 45-.

一, fundamental. Bertini, E. Mth. A. 34 (1889) 447-.

-, - Hensel, K. Crelle J. Mth. 115 (1895) 254.

- of Hadamard, application. Borel, $\dot{E}$. Bll. Sc. Mth. 18 (1894) 22-.

- - Netto. Bertini, E. Mth. A. 35 (1890) 456-.

- Noether. Stickelberger, L. Mth. A. 30 (1887) 401-.

- Weierstrass. Noether, M. Crelle J. Mth. 97 (1884) 224-.
Algebraic Functions 4020

Theory. Dedekind, R., \& Weber, H. [1880] Crelle J. Mth. 92 (1882) 181-.

-. Kneser, A. Mth. A. 29 (1887) 171-.

-. Hensel, $K$. Crelle J. Mth. 109 (1892) 1-; Acta Mth. 18 (1894) 247-.

-. Baur, L. Crelle J. Mth. 116 (1896) 167-.

-, arithmetical. Landsberg, G. Gött. Nr. (1895) 407-.

Transcendental functions, arising from repeated integrations. Kummer, E. E. Crelle J. 21 (1840) 74-, 193-, 328.

Trinomial integrals. Besge, $V . A$. Liouv. J. Mth. 4 (1859) 194.

Values assumed by a rational integral function between assigned limits of the variable. Laguerre, - C C. R. 98 (1884) 136-.

- of $0 \times \infty$, etc. Nievengloski, G. H. N. A. Mth. 7 (1848) 425-.

$\frac{\left(1-x^{m}\right)\left(1-x^{m-1}\right) \ldots\left(1-x^{m-\mu+1}\right)}{\text { is an integral }}$ $(1-x)\left(1-x^{2}\right) \ldots\left(1-x^{\mu}\right)$

function of $x$ when $m$ and $\mu$ are integers, and $m>\mu$. Perott, J. Par. S. Mth. Bll. 10 (1882) 87 -.

\section{Algebraic functions of several variables.}

Convergence and double limits. Osgood, W. F. N. Y. Am. Mth. S. Bll. 3 (1897) 59-.

Formula of Léauté, extension. Karagiannidès, -. N. A. Mth. 17 (1898) 539-.

Theorem of Kronecker on functional determinant of 3 equations. Marenghi, C. Ven. I. At. (1897-98) 1688-.

Theory. Vessiot, E. Toul. Fac. Sc. A. 10 (1896) D, 14 pp.

$\left(\frac{x}{a}\right)^{p}+\left(\frac{y}{b}\right)^{q}+\left(\frac{z}{c}\right)^{r}+\ldots=1$, threeintegrations within. Most, R. Z. Mth. Ps. 14 (1869) 422-.

\section{2 variables.}

Landsberg, G. Berl. Ak. Sb. (1900) 296-. change of independent variables. Gergonne, J. D. Gergonne A. Mth. 1 (1810-11) 251-. class. Picard, ḱ. C. R. 90 (1880) 1119-. first differentials being given implicitly. Woisard, J. L. Par. Mm. Sav. Étr. 3 (1832) $1-$.

polynomials of two variables and approximate calculation of double integrals. Appell, $P$. Toul. Fac. Sc. A. 4 (1890) H, 20 pp.

representability by double trigonometric series. Ascoli, G. Rm. R. Ac. Linc. Mm. 4 (1879) 253-; Mil. I. Lomb. Rd. 15 (1882) 543-.

resolution into partial fractions. Hoyer, $P$. Mth. A. 47 (1896) 113-.

and theorem of Noether. Brill, A. Mth. A. 39 (1891) 129-.

theory. Picard, É. Liouv. J. Mth. 5 (1889) 135 -.

-. Kobb, G. Liouv. J. Mth. 8 (1892) 385-; Par. S. Mth. Bll. 21 (1893) 76-.

-. Grave, D. Fschr. Mth. (1899) 378-.

-, new. Hensel, $K$. Acta Mth. 23 (1900) 339-.

transformation. Euler, L. [1779] St Pét. Ac. Sc. Mm. 3 (1811) 43-. 
3 or more variables.

approximation. Didon, F. C. R. 70 (1870) 749-.

functions analogous to sine and cosine. Glaisher, J. W. L. [1878] QJ. Mth. 16 (1879) 15-.

integrability. Brunn, H. [1848] St Pét. Ac. Sc. Bll. 7 (1849) 126-.

integration. Minich, S. R. At. Sc. It. (1844) $62-$.

-, formulæ. Minich, S. R. Ven. Mm. I. 6 (1856) 473-.

rational functions, representation, analytic. Mittag-Leffler, G. Stockh. Öfv. 34 (1877) No. 10, 3-, 17-.

theory. Allé, M. [1875] Wien Ak. Sb. 72 (1876) $(A b .2)$ 289-.

-. Capelli, A. Nap. Rd. 29 (1890) 297-.

\section{Logarithmic, circular, exponential functions.}

\section{CIRCULAR FUNCTIONS.}

Raabe, J. L. $\quad$ Crelle J. 16 (1837) 219-.

Calculation of tables. Olivier, L. Crelle J. 2 (1827) 252-.

Circular ares, reduction to imaginary logarithms. Calandrelli, $G$. [1825] Mod. Mm. S. It. 20 (1828) 45-.

- functions, analogues. Appell, P. C. R. 84 (1877) 1378-.

- - , analysis. Rees, R. van. Quetelet Cor. Mth. 6 (1830) 277-.

- - analytic derivation. Tralles, J. G. Berl. Ab. (1812-13) (Mth.) 161-.

- - , - theory. Chessin, A. S. Am. J. Mth. 19 (1897) 217-.

- - , approximate formulæ and application. Fein, A. (Ix) Förster Al. Bauztg. 38 (1873) $34-$.

- - , approximation by means of algebraic functions. Laguerre, E. C. R. 90 (1880) 304-.

- - astronomical formulæ. Le Verrier, U. J. J. (x) Par. Obs. A. 1 (1855) 73-; 2 (1856) $301+$ [167] pp.; 3 (1857) 199-, [1]-; 4 (1858) 1-, [1]-; 5 (1859) 1-; 6 (1861) 435 pp.; 10 (1874) 1-, [1]-; 11 (1876) $560+[136]$ pp.; 12 (1876) $76+[178]+\mathrm{A}$. $80+[$ A. 286] pp. ; 13 (1876) 1-, [1]-; 14 (1877) Pt. 1, A. 1-, [A. 1]-, Pt. 2, 1-, [1]-.

- - differential, integration in finite form. $\mathrm{Du}$ Bourguet, - Gergonne A. Mth. 4 (1813-14) 72-. $-\frac{-}{\text { Ab. }(1820-21)}-\frac{14}{44}$.. Gruson, J. P. Berl.

- - differentiation, successive. Colombier, P. A. G. N. A. Mth. 9 (1850) 29-; 1 (1862) 11-.

- , formula. Catalan, - As. Fr. C. R. (1889) (Pt. 1) 232.
Circular functions, fundamental principles. Sarrus, F. Gergonne A. Mth. 11 (1820-21) 323-.

- - - properties, new proof. Fleck, (l'abbé) J. M. (xII) Metz Ac. Mm. 54 (1874) 337-.

- integration. Hermite, C. [1872] L. Mth. S. P. 4 (1871-73) 164.

- - inverse, differential coefficients. Whatley, F. Mth. Gz. 1 (1900) 83-.

- -, 2nd order, integrals. Meyer, U. H. Grunert Arch. 17 (1851) 426-.

- - principal values. Stolz, $O$. Innsb. Nt. Md. B. 17 (1887) 67-.

- - resolution into partial fractions. Oliveira Ramos, J. C. d'. G. Teix. J. Sc. 7 (1886) 7-; Fschr. Mth. (1885) 231-.

- - de Stainville's theorem. Gergonne, J. D. Gergonne A. Mth. 13 (1822-23) 270-.

- -, summation. Jarrett, T. B. A. Rp. (1847) (pt. 2) 5.

- and hyperbolic functions. Imshenetskit, V. G. (xII) Kazan Un. Mm. (1862) (P8.Mth.) $19 \mathrm{pp}$.

- _ - Giudice, F. G. Mt. 27 (1889) 124 .

- - - analogy. Mossotti,O.F. Tortolini A. 2 (1851) 474-; Pisa A. Un. Tosc. 6 (1861) 7-.

- - - - logarithmic tables. Forti, A. (vi Adds.) N. Cim. 17 (1863) 243-.

- - - , relations. Realis, S. Mathesis 6 (1886) 7-.

Concepts $\cos x$ and $\sin x$, extension. Beyssell, -. Grunert Arch. 31 (1858) 299-.

Cosines, sums of series of like powers. Radicke, A. Mathesis 4 (1884) 161-.

Cotangent and cosecant, fractional series for, algebraic proof. Glaisher, J. W. L. [1880] QJ. Mth. 17 (1881) 211-.

Definite integrals containing circular functions. Haan, D. Bierens de. Haarl. Ntk. Vh. Mtsch. 17 (1862) $199 \mathrm{pp}$.

\section{Expansion.}

Oliveira, C. B. Rv. Brazil. 3 (1860) 214-.

of are in terms of tangent. Schlömilch, $O$. Grunert Arch. 16 (1851) 230-.

- circular and exponential functions. Ampère, A. M. Gergonne A. Mth. 15 (1824-25) $369-$.

- - - - Demoulin, A. Brux. Ac. Bll. 19 (1890) 541-.

- cosines and sines of multiple arcs. Malden, H. Ph. Mg. 20 (1842) 113-.

- - - in powers of arc. Schellbach, C. H. Crelle J. 16 (1837) 363-.

_ _ _ - terms of arc, and of are in terms of tangent. Gergonne, J.D. Gergonne A. Mth. 3 (1812-13) 344-.

- exponential in powers of sine and cosine of are. David, - . [1882] Toul. Ac. Sc. Mm. 5 (1883) (Sem. I.) 148-.

- fractional powers of cosines. Crelle, A. L. Gergonne A. Mth. 13 (1822-23) 213-; Crelle J. 7 (1831) 253-, 314-. 
of fractions contrining sines and cosines of multiple arcs. Trembley, J. Berl. Mm. Ac. (1802) 31-.

in indefinite products. Gergonne, J. D. Gergonne A. Mth. 1 (1810-11) 116-.

of infinite products for trigonometric functions. Schlömilch, O. Schlömilch Z. 3 (1858) 389 .

- logarithmic, exponential and trigonometric functions. Bangma, O. S. Amst. Mengelwerk 2 (1816) $92-$.

- powers of trigonometric ratios in series of ascending powers of variable. Jeffery, H.M. [1860] QJ. Mth. 5 (1862) 91-.

- sines of multiple angles. Krafft, W. L. [1802] St Pet. Ac. Sc. N. Acta 14 (1805) 290 .

- in products. Padé, H. N. A. Mth. 17 (1898) 312-.

- trigonometric functions. Young, J. R. $\mathrm{Ph}$. Mg. 5 (1834) 198-.

- - as infinite products. Dienger, $J$. Arch. Mth. Ps. 43 (1865) 474-.

- - - in infinite series. Burg, A. Wien Jb. Pol. I. 19 (1837) 147-.

- - - products of binomial factors. Rodrigues, $O$. Liouv. J. Mth. 8 (1843) 217-.

in trigonometric series. Walton, W. [1871] QJ. Mth. 12 (1873) 146-.

of $\sin n \theta$ and $\cos n \theta$. Euler, L. [1780] St Pét. Ac. Sc. Mm. 5 (1815) 57-.

- - in terms of $\sin \theta$ and $\cos \theta$, demonstration. Kausler, C. F. [1810] St Pét. Ac. Sc. Mm. 4 (1813) 256-.

- - - (Euler). Fuss, N. [1813] St Pét. Ac. Sc. Mm. 6 (1818) 100-.

$-\ldots, \cos n \theta$ and $\sin ^{n} \theta, \cos ^{n} \theta$. Crelle, A. L. Crelle J. 5 (1830) 197-.

- - and $\cos n \theta$ in ascending powers of $\sin \theta$ and $\cos \theta$. Gregory, D. F. Camb. Mth. J. 2 (1841) 129-.

- - - - - terms of powers of $\sin \theta$ and $\cos \theta$, by de Moivre's theorem. Sylvester, J. J. QJ. Mth. 4 (1861) 159-.

- - - - - series of sines and cosines of multiples of $\theta$. Poisson, S. D. Par. Éc. Pol. Cor. 2 (1809-13) 212-; Férussac Bll. Sc. Mth. 4 (1825) 140-, 344-.

- - - Kummer, E. E. Crelle J. 14 (1835) 110-.

- - - in terms of multiples of $\theta$. Simony, O. Arch. Mth. Ps. 55 (1873) 64-.

- $(a+b \cos x)^{n}$, general term. Degen, C. $F$. (vi Adds.) Kiöb. Ov. (1819-20) 3-.

- $\cos ^{n} \theta$ in cosines of multiples of $\theta$. Plana, $G$. Gergonne A. Mth. 11 (1820-21) 84-.

- - in sines and cosines of multiples of $\theta$. Michel, P. Gergonne A. Mth. 13 (1822-23) 94-.

- - cosines of multiples of $\theta$. Olivier, $L$. Crelle J. 1 (1826) 16-

- $\tan \theta$ in powers of $\theta$, law. Bouvier, L. C. Gergonne A. Mth. 14 (1823-24) 347-.

- $(\cos x)^{n}$, etc. Ohm, M. Férussac Bll. Sc. Mth. 5 (1826) 250-.

- $\theta^{n}$ in terms of $\sin \theta$. Scholtz, E.J. Crelle J. 3 (1828) 70 -. of $\theta^{a}$ in powers of $\sin \theta$. Scherk, H. F. Crelle J. 11 (1834) 101-.

- $\left(l+2 l^{\prime} \cos \phi+2 l^{\prime \prime} \cos \phi^{\prime}\right)^{-n}$ in series of cosines of multiples. Jacobi, C. G.J. Crelle J. 15 (1836) 205-.

- $\cos n(\theta+\Delta \theta)$ in powers of $\cos \theta$. Cauchy, A. L. C. R. 15 (1842) 411-.

- $\sin ^{-1} x$. Raabe, J. L. Crelle J. 25 (1843) $169-$.

- - Schlömilch, O. Schlömileh Z. 1 (1856) 181-.

- - by Maclanrin's theorem. Chevilliet, N. A. Mth. 13 (1874) 209-.

- $\cos n x / \cos ^{n} x$ and $\sin n x / \cos ^{n} x$ in powers of $\tan x$. Arndt, F. Grunert Arch. 4 (1844) 441-.

- sine and cosine in powers of arc. Schlömilch, O. Grunert Arch. 5 (1844) 326-

- $\cos ^{n}$ ax. Dienger, $J$. Grunert Arch. 11 (1848) 331-.

- $\cos ^{n} x$ in series of cosines for all values of $n$. Newman, F. W. Camb. and Dubl. Mth. J. 3 (1848) 61-.

$-\frac{1}{1-\mu \cos \phi}$ in series of even multiples of $\phi$. Wolfers, J. P. Grunert Arch. 21 (1853) 190 -.

- sine and cosine. Grunert, J.A. Grunert Arch. 29 (1857) 452-.

- $\sin n x$ and $\cos n x$ in powers of $\sin x$ and $\cos x$. Baehr, G. F. W. Amst. Vs. Ak. 10 (1860) 86-.

- - - Yvon-Villarceau, A. J. F. C. R. 82 (1876) 1469-.

- - - Catalan, E. C. N. A. Mth. 2 (1883) 529-.

- $\sin x$ and $\cos x$. Almqvist, P.W. Ts. Mt. Fys. 3 (1870) 121-.

$--\frac{1}{-}$ in infinite series. Meusburger, $K$. Mh. Mth. Ps. 6 (1895) 256-.

- - in series of exponential functions with real exponents. Popov, A.T. (xII) Kazan Un. Mm. 11 (1875) 661-.

- $\cos n \theta$ in powers of cosines, etc. Walton, W. [1872] QJ. Mth. 12 (1873) 168-.

- sine and cosine of arc. Sang, E. [1877] Edinb. R. S. P. 9 (1878) 400-.

- - - - David, -. Par. S. Mth. Bll. 11 (1883) 72-.

- $\sec x$ and $\tan x$. André, D. C. R. 88 (1879) 965-.

- $\cot x$. Le Paige, C. M. M. H. H. C. R. 88 (1879) 1075-.

$-\left(1+b \cos \beta+b^{\prime} \cos \beta^{\prime}+\ldots\right)^{n}$. Oppolzer, $T$. von. As. Nr. 105 (1883) 367-.

$-\frac{x}{\sin x}$. Catalan, E. C. Liége S. Sc. Mm. 15 (1888) No. 1, 82-.

- $\sin n \phi$ and $\cos n \phi$ in powers of $\cos \phi$. Ocagne, $M . d$ '. G. Teix. J. Sc. 9 (1889) 161-. - $\tan ^{-1} x$, in convergent series. Saalschiltz, Königsb. Schr. 40 (1899) [28]-.

Function of 3 angles, first differentials of which are also to be regarded as angles. Schläfi, L. [1852] Crelle J. 48 (1854) 292-.

Functions of small angles, method of finding from five-figure tables. Pfeil, L. von. Arch. Mth. Ps. 58 (1876) 147-. 
Gregory's series. Chartres, R. Nt. 42 (1890) 341.

Hyperbolic base, analytical value. Hill, $T$. Am. As. P. 19 (1870) 21-.

- logarithms of first $n-1$ numbers, formula for. Tilly, J. M. de. Brux. Ac. Bll. 35 (1873) $30-$.

Logarithmic series, and series for $\tan ^{-1} \theta$, with alternating signs, sum. Unferdinger, $F$. Wien Sb. 55 (1867) (Ab. 2) 75-, 332.

Parabolic trigonometric functions. Egidi, $G$. Rm. N. Linc. At. 47 (1894) 16-.

- trigonometry. Günther, -. D. Nf. Tbl. (*1881) [20]-.

Periodic functions, real, derived from equation $\left(\frac{d x}{d t}\right)^{2}=F(x)$. Weierstrass, C. Berl. Mb. (1866) 97-, 185.

- - , simplest. Schellbach, C. H. Crelle J. 48 (1854) 207-.

Periodicity of $\sin x$ and $\cos x$. Studnička, - . Palermo Cir. Mt. Rd. 13 (1899) 378-.

Powers of sines, series, summation. Rittenhouse, D. [1792] Am. Ph. S. T. 3 (1793) 155-.

- - - - - Nulty, O. [1816] Am. $\mathrm{Ph}$. S. T. 1 (1818) 395-.

Problems. Adams, Jas. Thomson A. Ph. 13 (1819) 188-.

Product series for $\cos x$ and summation for sec $x$. Weyr, E. Bll. Sc. Mth. 12 (1888) $25-$.

Representation of sine and cosine as infinite products. Hoppe, $R$. Grunert Arch. 27 (1856) 170 -

Secant and tangent coefficients, formulæ for determination. Schlömilch, $O$. Grunert Arch. 16 (1851) 411-.

Series, value. Glaisher, J. W. L. [1878] Mess. Mth. 8 (1879) 127-.

Side of regular $n$ gon, series for finding. Oppert, J. As. Fr. C. R. (1896) (Pt. 1) 133.

Sines of fourth order $\left[\frac{z^{\mu}}{\mu !} \pm \frac{z^{\mu+n}}{\mu+n !}+\right.$ $\left.\frac{z^{\mu+2 n}}{\mu+2 n !} \pm \ldots(n=4)\right] . \quad$ Kapteyn, J. C., $\&$ Kapteyn, W. Amst. Ak. Vh. 24 (1886) 98 pp.

Tangent and cotangent series, new deduction of Euler's. Studnička, F. J. Prag Sb. (1887) (Mth.-Nt.) 103-.

Transcendental functions, method of treatment differing from Euler's, and theorem. Pezzi, F. Verona S. It. Mm. 5 (1790) 417-.

Transformation involving de Moivre's theorem. Garnier, J. G. Brux. Ac. Bll. 6 (1839) 151-.

Trigonometric and astronomical infinite series. Grunert, J. A. Grunert Arch. 18 (1852) 420 -. $56(1874) 328$.

- developments, and integration of trigonometric functions. Richardson, J.M. Franklin I. J. 32 (1856) 409-.

VOL. I.
Trigonometric functions, integration of general classes. Philbrick, P.H. Des Moines Anal. 9 (1882) 177- ; 10 (1883) 9-.

- -, new researches. Lyetnikov, A. V. [1882] (xII) Rec. Mth. (Moscou) 10 (1882-83) (Pt. 1) 227-.

- - periodicity. Mantel, W. N. Arch. Wisk. 3 (1898) 279-; Fschr. Mth. (1898) 376.

- - properties deduced from addition theorems. Muirhead, R. F. Edinb. Mth. S. P. 14 (1896) 127-.

- - , two reciprocal relations, and application to 4 dimensions. Hoppe, $R$. Arch. Mth. Ps. 67 (1882) 412-.

- and hyperbolic functions, multiplication. Trzaska, W. Par. T. Nauk Śc. Pam. 10 (*1878) Art. 8, 7 pp.

- series. Dobinski, G. [1876] Arch. Mth. Ps. 63 (1879) 380-.

- -, Abel's transformation. Lerch, M. Prag České Ak. Fr. Jos. Rz. (Tř̂̀da 2) 5 (1896) Art. 24, 5 pp.; Fschr. Mth. (1896) 203.

Trigonometry, Dr Stewart's theorems, analytical demonstration. Ellis, R. L. Camb. Mth. J. 2 (1841) 271-.

$\left(l+2 l^{\prime} \cos \phi+2 l^{\prime \prime} \cos \phi^{\prime}\right)^{-n}$ in series of cosines of multiples. Jacobi, C. G.J. Crelle J. 15 (1836) 205-.

$\int \frac{d x}{a+b \cos x+c \sin x} \cdot$ Björling, E. G. Stockh. Öfv. 6 (1849) 171-; Grunert Arch. 21 (1852) 26-.

-. Dienger, J. Grunert Arch. 12 (1849) 409-.

$\sin \infty$ and $\cos \infty$. Glaisher, J. W. L. [1870] Mess. Mth. 5 (1871) 232-.

$U \sin x+V \cos x+W$. Hermite, $C$. Crelle J. 76 (1873) 303-.

are $\sin (x+i y)$. Cayley, A. QJ. Mth. 15 (1878) 171-.

$\int_{0}^{2 \pi} f(\sin x, \cos x) d x$. Hermite, C. [1879 ?] G. Teix. J. Sc. 2 (1880) 65-.

$\cos \theta+i \sin \theta$, and its application to some equations. Airy, (Sir) G. B. [1887] Camb. Ph. S. P. 6 (1889) 104-.

$\Sigma x^{k-1} \cos k a$ and similar series deduced from $\int_{0}^{x} \frac{e^{i a} d x}{1-x e^{i a}}$. Studnička, F. J. Casopis 19 (1890) 113-; Fschr. Mth. (1890) 261.

$\frac{\sin (x \pi)}{\pi}$, continued product for. McClintock, - N. Y. Mth. S. Bll. 2 (1893) 147-.

$\Sigma x_{n}$ are tan $K_{n}{ }^{-1}=k \pi / 4$, integral solutions. Störmer, C. C. R. 122 (1896) 175-, 225-. $\sin \theta$, expression as an infinite product. Dixon, A. C. Mth. Gz. 1 (1900) 130-.

\section{LOGARITHMIC AND EXPONENTIAL FUNCTIONS.}

Compound interest, approximate calculation of $r$ Lemonnier, $H$. N. A. Mth. 3 (1864) 337-. 
Compound interest and current accounts. Starkov, A. N. Rs. S. Nt. Mm. (Mth.) 3 (*1881).

Discontinuous logarithmic functions. Bugaev, N. $V$. [1887-88] Rec. Mth. (Moscou) 13 (1886) 757-; 14 (1890) 1-; Fschr. Mth. (1888) 186.

Equations of form $\Sigma \int_{a}^{b} e^{-x z} F(z) d z=0$. Laguerre, E. C. R. 93 (1881) 1000-.

Expansion. Bangma, O. S. Amst. Mengelwerk 2 (1816) 92-.

- Cerquero, J. S. Quetelet Cor. Mth. 10 (1838) 383-.

- in convergent series. Oliveira, C. B. Rv. Brazil. 3 (1860) 205-.

- - series. Gergonne, J. D. Gergonne A. Mth. 5 (1814-15) 363-.

Exponential equations, reduction to algebraic form. Leyommark, G. A. Stockh. Ak. Hndl. 29 (1808) 284-; 31 (1810) 101-.

- - solution. Arnott, G. A.W. Tilloch Ph. Mg. 49 (1817) 321-.

- expressions, imaginary. Roy, A. Mathematician 3 (1850) 13-.

- forms, indeterminate. Franklin, F. Am. J. Mth. 1 (1878) 368-.

- functions. Hermite, C. C. R. 77 (1873) 18-, 74-, 226-, 285-.

- - Laguerre, E. [1879] Par. S. Mth. Bll. 8 (1880) 11-.

- - Gegenbauer, L. Wien Ak. Sb. 95 (1887) (Ab. 2) 846-; Mh. Mth. Ps. 5 (1894) 303-.

- - application, case. Hilgard, J.E. Am. As. P. 20 (1871) 61-.

- case. Pagani, G. M. Brux. Ac. Bll. 13 (1846) (pte. 2) 347-.

- - expansion in continued fractions. Padé, H. Par. Ec. Norm. A. 16 (1899) 395-.

- -, Lindemann's theorem. Vahlen, $T$. Mth. A. 53 (1900) 457-.

- -, periodicity. Studnička, F. J. Časopis 23 (1894) 1-; Fschr. Mth. (1893-94) 737.

- - , representation by infinite product. Lipschitz, R. C. R. 99 (1884) 701-.

- - - - - Bassani, A. G. Teix. J. Sc. 11 (1892) 93-.

- -, table, 12 place. Newman, $F . W .[1876]$ Camb. Ph. S. T. 13 (1883) 145-.

- functions, tables. Glaisher, J.W.L. [1877] Camb. Ph. S. T. 13 (1883) 243-.

- - , theorems. Glaisher, J.W. L. Mess. Mth. 5 (1876) 164-.

- , theory. Graves, J.T. B. A. Rp. (1834) 523-.

- - - , arithmetical propositions derived from. Lipschitz, -. Liouv. J. Mth. 2 (1886) $219-$.

- and imaginary quantities, theory. Woolhouse, W. S. B. Mth. Misc. 1 (1838) 336-.

- - logarithmic quantities. Toblini, $G$. Poligrafo 1 (1830) 21-.

- - theorems, method. Strong, T. Silliman J. 48 (1845) 36-.

- - other functions, treatment by equiangular spiral. Schellbach, C. H. Crelle J. 17 (1837) 321-.
Exponential series. Barfuss, F. W. Grunert Arch. 5 (1844) 155-.

- - error in theorem. Fontana, $G$. Verona S. It. Mm. 2 (1784) 123-.

- - inequalities. Tweedie, C. Edinb. Mth. S. P. 17 (1899) 33-.

- - - connected with. Gibson, G. A. Edinb. Mth. S. P. 18 (1900) 84-.

- - and other expansions. Dupuis, N. F. Cn. R. S. P. \& T. 7 (1890) (Sect. 3) 16-, 21-. -, sine, etc., series with alternating signs, sum. Unferdinger, $F$. Wien Sb. 56 (1867) (Ab. 2) 257-.

- symbolic operations. Scott, R. F'. Mess. Mth. 10 (1881) 142-.

- - operators, theorems. Glaisher, J.W. L. QJ. Mth. 15 (1878) 266-.

- theorem. Schaeberle, J. M. A. Mth. 3 (1887) 156.

- transformation. Laisant, C.A. As. Fr. C. R. (1879) 206-.

- and trigonometric functions, differentiation. Martin, Art. Des Moines Anal. 6 (1879) 23-.

Exponentials, expansion in a series of. Popoff, A. [1874] Crelle J. Mth. 80 (1875) 204.

-, imaginary. Tarry, G. N. A. Mth. 14 (1895) 269-.

-, -, expansion. Davies, T. S. Mathematician 3 (1850) 66-.

- and logarithms, differentiation. Schlömilch, $O$. Grunert Arch. 11 (1848) 386-.

-- - expansion. Ocagne, M. $d$ '. N. A. Mth. 1 (1882) 241-.

- - as limiting values of $\left(1+\frac{b}{n}\right)^{n}$, $(\sqrt[n]{a}-1) n(n=\infty)$. Wulf, T. Mh. Mth. Ps. 8 (1897) 43-.

- - -, theory, application of geometry. Schultén, N. G. af. (vm) Helsingf. Acta 5 (1858) 437-.

-, successive, of Euler. Grillet, J. H. Liouv. J. Mth. 10 (1845) 233-.

-, theory. Laurent, H. N. A. Mth. 13 (1874) 5-.

Hyperbolic areas, and natural logarithms. Grunert, J. A. Grunert Arch. 25 (1855) $82-$.

- and cyclic sine and cosine, functions representing generalisation. Hellwig, C. Grunert Arch. 35 (1860) 186-.

- functions. Fuss, N. [1810] St Pét. Ac. Sc. Mm. 11 (1830) 220-.

- Hoilel, J. N. A. Mth. 3 (1864) 416-.

- -. Azzarelli, M. Rm. At. N. Linc. 24 (1871) 112-.

- Laisant, C. A. Bordeaux S. Sc. Mm. 10 (1875) 233-.

- Gallatly, W. Mth. Gz. No. 5 (1895) 40 -

- Haskell, M. W. N. Y. Am. Mth. S. Bll. 1 (1895) 155-.

- - in connection with the hyperbola. Crawford, L. Edinb. Mth. S. P. 13 (1895) 153-.

- - functions of. Nicodemi, R. G. Mt. 15 (1877) 193-. 
Hyperbolic functions, tables. Jordann, -. Crelle J. 16 (1837) 196.

- - - , new. Forti, A. [1881] Z. Mth. Ps. 27 (1882) (H.-lt. Ab.) 1-.

- - in teaching. Barbarin, $P$. Ens. Mth. 2 (1900) 443-.

- - theorems. Cantor, M. Grunert Arch. 19 (1852) 88-.

- - theory. Barsotti, G. Lucca At. Ac. 15 (1855) 441-.

- - - and application. Grunert, J.A. Grunert Arch. 38 (1862) 48-.

- - , - , - - to resistance coefficients. Gromau, J. F. W. Danzig Schr. 1 (1865) (Heft 2) 79 pp.

- -, -, geometrical. Thomson, $W . L$. Edinb. Mth. S. P. 17 (1899) 2-.

- logarithms. Formula $\int d x(-L x)^{ \pm \frac{1}{2}}, \& c$. Ferroni, P. [1794] Siena At. Ac. 8 (1800) 1-.

Indices in higher arithmetic and application to logarithmic tables. Agardh, J. M. Stockh. Ac. Hndl. (1838) 253-.

Infinite products, cases. Stern, M. A. Crèlle J. 27 (1844) 279-.

Integral in dynamics, Euler. Dostor, G. $J$. N. A. Mth. 11 (1852) 45-.

- transcendentals (resolvable into factors of form $e^{P(x)}\left(1-\frac{x}{a}\right), P(x)$ being a polynomial). Poincaré, H. C. R. 95 (1882) 23-.

Integrals, class, expressible by logarithms alone. Piuma, C. M. A. Mt. 7 (1875-76) 18-.

- of irrational algebraic functions expressible by logarithms alone. Piuma, C. M. (vII) Tortolini A. 4 (1861) 1-.

- - - - - - - - Aleksyeev, N. N. (xII) Rec. Mth. (Moscou) [1] (1866) 173-.

Irrationals $\sqrt{2}$ and $\sqrt{3}$ cannot be Napierian logarithms of rational numbers. Schultén, N. G. af (fil.) [1844] Helsingf. Acta 2 (1847) 681-.

Logarithm, common, method of finding. Ritten. house, D. [1795] Am. Ph. S. T. 4 (1799) 69-.

-, expansion, case. Schlömilch, O. Leip. Mth. Ps. B. 39 (1887) 53-.

-, expression for integral power. Walton, $W$. Camb. Mth. J. 2 (1841) 102-.

-, isolated, to many decimal places. Lefort, $F$. N. A. Mth. 6 (1867) 308-.

- of $n !$, when $n$ is large. Lerch, $M$. Casopis 24 (1895) 129-; Fschr. Mth. (1895) 472.

-, Napierian, of a number and its integral powers are incommensurable with number, etc. Pollak, $(D r)-$ [1849] Haidinger B. 6 (1850) 95-.

Logarithmic and algebraic functions giving logarithmic integrals. Hill, C.J.D. Lund Acta Un. 4 (1867) (Mth.) No. 1, 28 pp.
Logarithmic calculation, errors from using proportional parts. Vincent, A.J.H. Gergonne A. Mth. 16 (1825-26) 19-.

- circular and exponential functions, differentials. Gruson, J.P. Berl. Ab. (182021) 49 -.

- and circular functions. Lambert, $C . J . \quad \mathrm{N}$. A. Mth. 12 (1853) 302-.

- _ - - development of properties from definite integral. Entleutner, A. F. [1878] Arch. Mth. Ps. 63 (1879) 225-.

- _ - - formulæ relating to. Terquem, $O$. N. A. Mth. 5 (1846) 79-, 151-, 221-, 349-, 411- ; 7 (1848) 269-.

- - - series. Adams, Jas. Thomson A. Ph. 4 (1822) 261-.

- - exponential series, summation. Chessin, A. Am. J. Mth. 16 (1894) 164-, 290.

- formulæ derived from a differential equation. Durège, -. Schlömilch Z. 3 (1858) 241-.

- -, rectification. Graves, J.T. [1828] Phil. Trans. (1829) 171-.

- functions, integration by. Dolbnia, I. $P$. Rec. Mth. (Moscou) 18 (1896) 150-.

- integral and allied functions, calculation. Bretschneider, C. A. Schlömilch Z.6 (1861) 126-.

- -, constant, numerical determination. Arndt, F. Grunert Arch. 11 (1848) 315-.

- -, -, value. Lindman, C. $F$. Grunert Arch. 29 (1857) 238-

-,,$- ;-$ O Oettinger, L. [1861] Crelle J. 60 (1862) 375-.

- - $r$ th integral. Glaisher, J. W. L. [1882] Mess. Mth. 12 (1883) 120-.

- - theory. Bretschneider, C. A. Crelle J. 17 (1837) 257-.

- - - - Schlömilch, O. Grunert Arch. 9 (1847) 5-, 307-.

$-\frac{1}{-}-$ Beez, R. Grunert Arch. 19 (1852) 419-.

- integrals, 2nd order. Newman, $F$. $W$. Camb. and Dubl. Mth. J. 2 (1847) 77-, 172-.

$--\int l X \frac{d x}{1+x^{2}}$. Bierens de Haan, D. Amst. Vs. Ak. 1 (1866) (Ntk.) 117-.

- and other series, connection. Grunert, J.A. Grunert Arch. 8 (1846) 272-.

- problems. Thiele, T.N. Mth. Ts. 3 (1861) 145-; 4 (1862) 17-, 93-, 145-.

- progressions. Barbette, $E$. Mathesis 18 (1898) 135-.

- series. Servois, J. F. Gergonne A. Mth. 2 (1811-12) 178-.

- Dantscher, V. von. Mh. Mth. Ps. 6 (1895) 390-.

- - interpolation. Meikle, J. Assur. Mg. 6 (*1857) 200-.

- - of Wronski.. Dickstein, S. Prace Mt. Fiz. 4 (1893) 88-; Fschr. Mth. (1893-94) 422.

sines, construction of canon. Sang, $E$. Edinb. R. S. P. 12 (1884) 601-. 
Logarithmic systems (three). Hessel, J, F.C. Grunert Arch. 14 (1850) 97-.

- -, classification. Stringham, I. [1891] Am. J. Mth. 14 (1892) 187-.

\section{Logarithmic tables.}

Bresson, -. (vir) Rouen Bll. S. Ém. (1841) $162-$.

Shortrede, R. Beng. J. As. S. 11 (1842) 40-.

Galbraith, W. Edinb. N. Ph. J. 39 (1845) 169-.

Riese, (Prof.) - von. Rheinl. Westphal. Sb. 14 (1857) xxviii-.

Haan, D. Bierens de. Amst. Vs. Ak. 14 (1862) 15-.

Tennant, J. F. As. S. M. Not. 33 (1873) $563-$.

August's. Lehmann, J. W. H. As. Nr. 43 (1856) 225-.

Bremiker's, errata. Fabritius, $W$. As. Nr. 85 (1875) 319-.

Callet's, errata. Secretan, - C. C. 44 (1857) 1276-.

compact, use. Templeton, $R$. Ph. Mg. 30 (1865) 221-.

construction. Knight, T. Phil. Trans. (1817) 217-.

-. Serret, J. A. N. A. Mth. 11 (1852) 2 2-.

-, formulæ. Lavernède, T. Gard Not. Tr. Ac. (1807) 179-

early. Bierens de Haan, D. Ph. Mg. 45 (1873) 371-.

errata. Stanley, A. D. Silliman J. 5 (1848) 398-.

of 1849 , errata. Shortrede, R. As. S. M. Not. 27 (1867) 272-.

errors, common to. Babbage, C. [1827] As. S. Mm. 3 (1829) 65.

exercise on. Tait, P. G. Edinb. Mth. S. P. 5 (1887) 101-.

and factor tables. Drach, S. M. [1873] Mess. Mth. 3 (1874) 6.

5 figures. Bouché, A. [1858] M.-et-L. Mm. S. Ac. 5 (1859) $23-$.

7 figures, smallest number of entries necessary in. Steggall, J. E. A. Edinb. Mth. S. P. 10 (1892) 35-.

10 figures. Carlini, $F$. Wien. Jb. Pol. I. 10 (1827) 77-.

12 figures, from 1 to 120,000 , by Thomson. Glaisher, J.W. L. As. S. M. Not. 34 (1874) 447-; 35 (1875) 135.

20 figures, Callet's, Hermann's corrections. Stampfer, S. (vir) Wien Sb. 1 (1848) 126-.

27 figures, Thoman's. Bertrand, J. J. Sav. (1870) 750 -

Köhler's. Lehmann, J. W. H. As. Nr. 44 (1856) 209-.

proof that large errors arise from use in certain cases. Voll, W. Oken Isis (1826) $677-$

and trigonometric tables, of Bureau du Cadastre. Prony, R. de. Par. Mm. de l'I. 5 (1802) (H.) 49-. and trigonometric tables, of Bureau du Cadastre. Delambre, J. B.J. Par. Mm. de l'I. 5 (1804) (H.) 56 -

- - - - - Lefort, $F$. C. R. 46 (1858) 994-; (vi Adds.) Par. A. Obs. 4 (1858) [123]-.

R. S. P. 8 (1875) 421-

- - - - - - - (Sang). Lefort, P. A. F. Edinb. R. S. P. 8 (1875) 563-.

Vega's. Mollweide, C. Zach M. Cor. 21 (1810) 284-

-, errata. Bremiker, (Prof.) -. As. Nr. 85 (1875) 373-

to 200,000 . Sang, E. [1871] Edinb. R. S. T. 26 (1872) 521-.

Logarithmic theorem. Catlin, M. Mth. Misc. 1 (1838) 113-.

- transcendents. Glaisher, J.W.L. Mess. Mth. 1 (1872) 138-.

Logarithms. Laurent, $H$. Ens. Mth. 2 (1900) 450 -.

- of algebraic numbers, arithmetical property. Störmer, C. Par. S. Mth. Bll. $28(1900)$ 146-.

- and antilogarithms. Gram, J. P. Ts. Mth. 4 (1886) 1-; Fschr. Mth. (1886) 1122.

- - - calculation. Weddle, T. Mathematician 1 (1847) 17-.

- $-\ldots$, - to 12 or 16 places, improved bimodular method, with brief tables. Ellis, A.J. R. S. P. 31 (1881) 381-.

-, approximate formulø for. Buquoy, G. von. Oken Isis 21 (1828) 1203-.

-, arithmetical solutions. Guilmin, A. N. A. Mth. 5 (1846) 429-.

-, base, expression. Baltzer, R. Grunert Arch. 18 (1852) 405-.

-, calculation. Manning, T. Phil. Trans. (1806) 327-

- - Transon, (Prof.) A. N. A. Mth. 10 (1851) 71-.

-, - . Tagert, -. Grunert Arch. 27 (1856) 132-.

一, 一. Oliveira, C. B. Rv. Brazil. 3 (1860) 181-.

-, - Wace, H. B. A. Rp. 43 (1873) (Sect.) 24-; Mess. Mth. 3 (1874) 66-.

- - by algebraic fractions. Murphy, $R$. Ph. Mg. 19 (1841) 479-.

-, -, best methods. Franchini, $P$. Lucca At. Ac. 6 (1830) 349-.

-, - of characteristics. Holten, C. Mth. Ts. 5 (1863) 65-.

,-- , elementary. Paugger, F. Arch. Mth. Ps. 42 (1864) 197-.

,,--- , of common. Sturm, J.B. Grunert Arch. 24 (1855) 228-.

-, -, formula. Du Bourguet, - Gergonne A. Mth. 2 (1811-12) 69-, 286-.

_, - , formulæ. Lavernède, $T$. Gergonne A. Mth. 1 (1810-11) 18-, 78-

-, - to many figures. Burnier, F. [1871] Laus. Bll. S. Vd. 11 (1873) 147-.

- - - - - easy method. Stille, W. A. Des Moines Anal. 10 (1883) 143-. 
Logarithms, calculation, potential radix as means. Ellis, A. J. R. S. P. 31 (1881) 398-; 32 (1881) 377-.

-, -, series for. Aliman, W. [1796] Ir. Ac. T. 6 (1797) 391-.

- - _, - - (Halley's). Murray, R. [1801] Ir. Ac. T. 9 (1803) 3-.

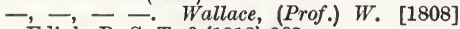
Edinb. R. S. T. 6 (1812) 269-.

-, - , - - Thomson, (Prof.) J. [1838] Edinb. R. S. T. 14 (1840) 217-。

-, canon. Kulik, J. P. As. Nr. 4 (1826) 47-.

-, criticism of Thiele. H., E. L. Mth. Ts. 4 (1862) 113-, 161-.

一, definition. Haskell, M. W. N. Y. Mth. S. Bll. 2 (1893) 164-.

-, - , geometrical (polygonal spirals). Barbarin, P. As. Fr. C. R. (1895) (Pt. 2) 257-.

- of different orders of numbers. Grillet, J.H. Liouv. J. Mth. 10 (1845) 233-.

- and exponentials, definitions. Stringham, $I$. N. Y. Mth. S. Bll. 2 (1893) 168-.

-, 4 figure Gaussian, in new arrangement. Wittstein, T. As. Nr. 51 (1859) 125-.

-, hyperbolic and Napierian. Wackerbarth, $A$. D. As. S. M. Not. 31 (1871) 263-.

-, - - - Dubois, E. Les Mondes 27 (1872) 651-.

-, imaginary, Graves's theory. Young, J. R. Ph. Mg. 25 (1844) 401-.

- of imaginary quantities. Cayley, A. [1867] L. Mth. S. P. 2 (1869) 50-

-, impossible. Gregory, D. F. Camb. Mth. J. 1 (1839) 226-.

- with large number of places. Perott, $J$. Bll. Sc. Mth. 11 (1887) 51-.

- of large numbers. Le Barbier, - Gergonne A. Mth. 20 (1829-30) 366-.

- Napier and Briggs. Blanken, H. van. Arch. Wisk. Gn. 1 (1856-59) 66-.

-, natural, hyperbolic and Napierian, discussion of terms. $D-g$. Ts. Mt. Fys. 4 (1871) 28-.

-, 一, of numbers 1-25, to 81 figures. Vietz, - As. Nr. 4 (1826) 53-.

-, -, systematic treatment. Matzka, W. Prag Sb. (1878) 206-.

- of negative quantities. Vincent, $A . J . H$. Gergonne A. Mth. 15 (1824-25) 1-; 16 (182526) $92-$.

$--\frac{-}{-}$ (Vincent). Stein, J.P. Gergonne A. Mth. 15 (1824-25) 230-.

-, new kind. Hill, C. J. D. Crelle J. 70 (1869) 282-.

- of numbers, calculation. Huygens, _. C. R. 66 (1868) 565-.

- - - - - Huygens' method. Bertrand, $J$. N. A. Mth. 7 (1868) 229-.

- - - - - - Thoman, F. C. R. 66 (1868) 662-.

- - order $N$, application of logarithmic and Archimedes' spirals. Cabreira, A. Lisb. J. Sc. Mth. 5 (1898) 226-.

-, origin, geometrical, and trigonometry of parabola. Booth, J. B. A. Rp. (1856) 68-.
Logarithms and powers, principal. Björling, E. G. Stockh. Öfv. 9 (1852) 100-; Grunert Arch. 21 (1853) 1-.

-, proportional, table. Shortrede, $R$. Beng. J. As. S. 10 (1841) 713-.

-, representation, geometrical. Fischer, E. G. [1804] Berl. Ab. (1804-11) (Mth.) 1-.

of sines and tangents of small angles. Burnier, $F$. Laus. Bll. S. Vd. 8 (1864-65) 271-.

- sum and difference of two numbers, calculation from logarithms of the numbers. Seeling, $P$. Arch. Mth. Ps. 45 (1866) 451-.

- - or difference, calculation, tables. Matthiessen, E. A. As. Nr. 2 (1824) 103-, 121-.

-, theory. Bouvier, L. C. Gergonne A. Mth. 14 (1823-24) 275-.

-, -. Stein, J. P. Gergonne A. Mth. 15 (1824-25) 105-.

-, -. Pagani, G. M. Brux. Ac. Bll. 6 (1839) 259-.

-, - Matzka, W. Grunert Arch. 15 (1850) 121-.

-, -. Mourgues, -. N. A. Mth. 11 (1852)

109 .

-, - Cayley, A. Ph. Mg. 11 (1856) 275-; 12 (1856) 354-.

- - Berdellé, C. As. Fr. C. R. (1889) (Pt. 2) 38-.

-, -, elementary. Jamet, $V$. Mathesis 8 (1888) 89-.

- and trigonometric functions, expansion in series. Jack, J. Edinb. Mth. S. P. 13 (1895) 132-.

- of trigonometric quantities, formulæ for finding from one another. Wallace, (Prof.) W. [1823] Edinb. R. S. T. 10 (1826) 148-.1 - - - ratios, tables. Elphinstone, H.W. QJ. Mth. 2 (1858) 222-.

-, trigonometric, tables. Escher, P. Grunert Arch. 23 (1854) 264-.

- of unity. Graves, J.T. Ph. Mg. 8 (1836) 281-.

$M$, Briggs' modulus, value to 205 places. Shanks, W. [1854-71] R. S. P. 6 (1854) 397-; 20 (1872) 27-.

$M$, fractions for value. Templeton, $R$. $\mathrm{Ph}$. Mg. 30 (1865) 434-.

Napierian logarithms of commensurable numbers or of algebraic irrationals. Lindemann, C. L. F. C. R. 95 (1882) 72-.

- - and exponential functions, analytical theory. Méray, C. Toul. Fac. Sc. A. 4 (1890) Q, 35 pp.

Natural logarithm of number, method of finding without logarithmic tables. Spiegler, G. (xII) Mag. Ak. Éts. (1858) 299-.

Notation for printing complicated exponents. Glaisher, J. W. L. [1872] Mess. Mth. 2 (1873) 107-.

Number whose logarithm is equal to itself, form. Farren, E.J. Assur. Mg. 3 (*1853) 323-.

Numbers whose logarithms are rational. Arndt, F. Grunert Arch. 6 (1845) 57-。 
Potential expression $((1))^{x}$. Kinkelin, $H$. Grunert Arch. 26 (1856) 304-.

Reciprocal series of sines and cosines of arcs in arithmetical progression. Lorgna, A. M. Turin Mm. Ac. 3 (1786-87) 215-.

Series formed from series for $\log (1+x)$ etc. Hellwig, C. Grunert Arch. 21 (1853) 43-.

- for roots and logarithms. Bouvier, L. C. Gergonne A. Mth. 16 (1825-26) 294-.

$-1-\frac{1}{2}+\frac{1}{3}-\frac{1}{4} \ldots$, note. Lionnet, $E$. N. A. Mth. 18 (1879) 509-.

Stirling's approximation to $n$ ! Allardice, R. E. Edinb. Mth. S. P. 6 (1888) 22-.

- formula, proof by logarithmic inequalities. Mansion, $P$. Mathesis 20 (1900) 265-.

- theorem, elementary proof. Franklin, $F$. J. H. Un. Cir. [3] (1884) 57.

Transcendental functions arising from repeated integration of rational functions. Jonquière, A. Stockh. Ak. Hndl. Bh. 15 (Afd. 1) (1890) No. 1, 50 pp.

- - class, integration. Winckler, A. [1874] Wien Ak. Sb. 70 (1875) (Ab. 2) 17-.

- integrals of form $\int_{0}^{x} e^{-x^{2}} f x d x$. Thomson, (Sir) W. QJ. Mth. 1 (1857) 316-.

Wronski, formula,

$\left[\int F(x) d x=l\left\{\left[e^{\psi x_{0}}\right]^{x / 1}\right\}+\right.$ constant,

where

$\left.\psi x=F x+\frac{1}{1.2} \cdot \frac{d F x}{d x}+\frac{1}{1.2 \cdot 3} \cdot \frac{d^{2} F x}{d x^{2}}+\ldots\right]$.

Rodrigues, J. M. G. Teix. J. Sc. 3 (1881) 55 -.

$\int e^{n x} x^{m} X_{1} X_{2}^{p} X_{3}^{q} d x$, where $X_{1} X_{2} X_{3}$ are poly-

nomials in $x$. Melanderhjelm, D. [1798]

St Pet. Ac. Sc. N. Acta 12 (1801) 114-.

$\int \frac{d z}{\log z}$. Bidone, G. Turin Mm. Ac. (1805-08) (pte. 2) 19-.

- Caluso, (l'abbé) T.V. de. Mod. Mm. S. It. 12 (1805) 268-.

$\int \frac{d x}{\log x}$. Bessel, F.W. Zach M. Cor. 22 (1810) 395-; Königsb. Arch. 1 (1812) 1-, 239-.

- Buzengeiger, K. H. J. Zach M. Cor. 28 (1813) 373-.

-. Casinelli, A. (s. L.). Bologna N. Cm. 3 (1839) 97-.

$x^{x}=A$, solution. Leyonmark, G. A. Stockh. Ak. Hndl. 31 (1810) 161-.

$x^{x^{n}}$. Carlini, F. Mil. Mm. I. Lomb. Ven. 1 (1812-13) 167-.

$e^{2 i \phi}$, trigonometric expression for. Busse, F. G. von. Oken Isis (1825) 394-, 528-.

$\log (-x)$ and Bouvier. Busse, F. G. von. Oken Isis (1825) 1048-

$0^{0^{x}}$. Libri, G. Crelle J. 6 (1830) 67-. $\pi=\frac{2}{i} \log i . \quad$ Schellbach, C. H. Crelle J. 9 (1832) 404-. $\int e^{x} y d x$, integration where $y$ is algebraic.

Liouville, J. Crelle J. 13 (1835) 93-.

$\log (1+x)^{m}$. Casinelli, A. (s. L.). Bologna N. Cm. 4 (1840) 147-.

$\frac{\log _{e} x}{x^{n}}$, limiting value for $x=\infty$. Schultén, N. G. af (fil.) Helsingf. Acta 1 (1840) 741-.

$\log x$ and $a^{x}$, differentials. Grunert, J.A. Grunert Arch. 1 (1841) 204-.

$\log x d x$, higher integrals. Arndt, $F$. Grunert Arch. 4 (1844) 436-.

$e^{\sqrt{-1} \theta}$, use in transformations. Goodwin, $H$. Camb. Mth. J. 4 (1845) 177-.

$\left[\frac{(1+u)^{\mu}-1}{\mu u}\right]^{\kappa}$, expansion. Meyer, U. H. Grunert Arch. 9 (1847) 101-.

$e^{x}$, expansion in series. Brassinne, $E$. N. A. Mth. 7 (1848) 30-.

$a^{x}>x$, condition. Hessel, J. F. C. Grunert Arch. 14 (1850) 93-.

$x^{y}$ and $\log \beta(x)$. Björling, E. G. Stockh. Ak. Hndl. (1852) 121-.

$\int \frac{e^{-x^{2}} d x}{\sqrt{a+\beta x^{2}}}, \int \frac{e^{-x^{2}} x^{2} d x}{\sqrt{a+\beta x^{2}}}, \int \frac{e^{-x^{2}} d x}{\left(\gamma+\delta x^{2}\right) \sqrt{a+\beta x^{2}}}$. Roberts, $W$. Liouv. J. Mth. 17 (1852) 117-.

$x^{a}$ and $a^{x}$, differentials, calculation. Schlömilch, O. N. A. Mth. 12 (1853) 31-.

$\log x$ and $\tan ^{-1} x$, differentials, and expansions in series. Soufflet, - N. A. Mth. 12 (1853) 438-.

$\log _{e} 2, \log _{e} 3, \log _{e} 5, \log _{e} 10$ to 205 places (Napierian). Shanks, W. [1854-71] R. S. P. 6 (1854) 397-; 20 (1872) 27-.

$\int \frac{e^{-x}-e^{-a}}{x^{2}-a^{2}} d x=\frac{1}{2 a}\left[e^{a} l i \cdot e^{-a}-e^{-a} l i \cdot e^{a}\right]$.

Zehfuss, G. Grunert Arch. 30 (1858) 465-.

$e^{a+b i}$. Dienger, J. Grunert Arch. 33 (1859) 481-.

$\{(z)\}^{y}, \log \beta\{(z)\}$, theory. Almqvist, P.W. Stockh. Öfv. 20 (1863) 203-.

$y=e^{\lambda x^{r}}$, representation in form $y=S\left[A_{m} e^{m x}\right]$. Spitzer, S. Arch. Mth. Ps. 42 (1864) 104-.

$e^{x}$, limits. Steen, A. (xII) Ts. Mth. 3 (1867) 29 -

$\int e^{x} \frac{f(x)}{\sqrt[n]{\bar{X}}}$, integration. Tissot, A. Par. Ec. Pol. J. $43^{\circ} \mathrm{cah}$. (1870) 201-.

$y=x^{n} e^{\lambda x^{2}}$, representation in form $y=S\left[A_{m} e^{m x}\right]$.

Spitzer, S. Arch. Mth. Ps. 52 (1871) 364-.

$y=x^{n} e^{a x^{y}}$, representation in form $y=S\left[A_{m} e^{m x}\right]$.

Spitzer, S. Arch. Mth. Ps. 52 (1871) 368-.

$\log M=\log \cdot \log e$, value to 24 places, also $\log M \pi$. Gray, $P$. Mess. Mth. 5 (1876) $172-$.

$x^{n} e^{\lambda x^{2}}$, transformation. Spitzer, S. Arch. Mth. Ps. 58 (1876) 431-.

$e^{z}$, period. Yvon-Villarceau, A. J. F. C. R. 83 (1876) 594-。 
$\log (1+x)$, expansion. Fuhrmann, W. Arch. Mth. Ps. 62 (1878) 220.

$\int_{0}^{z} z^{n} e^{-\frac{z^{2}}{2}+z x} d z$. Laguerre, E. [1878] Par. S.

Mth. Bll. 7 (1879) 12-.

$\log 2$, \&c., calculation (Gauss). Cayley, $A$. Mess. Mth. 8 (1879) 125-.

$\int_{x}^{\infty} \frac{e^{-x} d x}{x}$. Laguerre, E. Par. S. Mth. Bll. 7 (1879) 72-.

$\int_{0}^{\frac{\pi}{2}} \log \sin x d x$, determination. Ligonoski, $W$.

[1879] Arch. Mth. Ps. 65 (1880) 110-

$1^{x}$ and $(-1)^{x}$. Laisant, C. A. Par. S. Mth. Bll. 8 (1880) 109-.

$e^{x}, \log _{e}(1 \pm x),(1+x)^{m}$, series for. Hill, M. J.M. Camb. Ph. S. P. 5 (1886) 415-

$e^{a z}:\left(e^{z}-1\right)$, resolution into partial fractions. Stolz, O. Innsb. Nt. Md. B. 15 (1886) 84-.

$\cos ^{p} x \sin ^{p} x d x$, integration. Laisant, $C . A$. As. Fr. C. R. (1889) (Pt. 2) 225-.

$e^{x}$, regular combined fractions for. Padé, $H$. C. R. 112 (1891) 712-.

$\log x$ and $e^{x}$, independent definitions. McClintock, E. Am. J. Mth. 14 (1892) 72-.

$\int_{0}^{\infty} \cos y x d x /\left(a^{2}+b^{2} x^{2}\right)$. Oltramare, G. As. Fr. C. R. (1895) (Pt. 2) 167-.

$\int \frac{d x}{\sqrt{x^{4}+p x^{2}+q}}$, expression by logarithms. Dolbmia, I. P. Bll. Sc. Mth. 19 (1895) 76-; Rec. Mth. (Moscou) 18 (1896) 108-.

$f\left(e^{a x}\right)$, series for. Petrovitch, $M$. Palermo Cir. Mt. Rd. 14 (1900) 22-.

\section{General properties of elliptic functions and single theta func- tions; addition-theorem. (See also 8050, 8060.)}

\section{Addition.}

of arguments in elliptic functions. Hermite, $C$. G. Teix. J. Sc. 11 (1892) 65-.

- - periodic functions, 2 nd order. Fontené, G. C. R. 122 (1896) 172-.

- elliptic functions. Cayley, A. Crelle J. 41 (1851) 57-.

- - Darboux, G. Par. Éc. Norm. A. 4 (1867) 81-. - 1479-. Catalan, E. C. C. R. 78 (1874)

[1879] Crelle J. Mth. 88 (1880) 146-.

___. Cayley, A. Mess. Mth. 14 (1885) 56 -

- - formula. Gilbert, L. P. (xII) Brux. S. Sc. A. 4 (1880) (Pt. 2) 87-.

- - formulæ. Björling, E. G. [1866] Arch. Mth. Ps. 47 (1867) 399-. of elliptic functions, formulæ of Hermite. Novarese, E. [1882] Tor. Ac. Sc. At. 17 (1881) 607-.

(1864) 999

- - 1st kind. Catalan, E. C. Brux. Ac. Bll. 27 (1869) 145-.

,----1 . Catalan, E. Brux. Ac. Mm. 45 (1884) (No. 3) 20 pp.

,----- , and Euler's integral. Iaggi, E. N. A. Mth. 19 (1900) 443-.

- - integrals, geometrical application. Hoppe, R. Arch. Mth. Ps. 64 (1879) 274.

equation. Thomson, J. J. [1879] Mess. Mth. 9 (1880) 52-.

- for elliptic integrals, 1st kind, geometric construction. Hermite, C. Bll. Sc. Mth. As. 2 (1871) 21-.

- _ - - 2nd kind, proof by $q$-series. Glaisher, J.W. L. [1882] Mess. Mth. 12 (1883) 43-.

- - 3rd kind. Glaisher, J.W. L. [1880-81] Mess. Mth. 10 (1881) 124-.

-, spherical triangle proof. Johnson, W. W. QJ. Mth.18 (1882) 365-.

equations for elliptic and theta functions of sum of $n$ arguments. Wilkinson, M.M.U. L. Mth. S. P. 13 (1881-82) 110-.

\section{ADDITION-THEOREM.}

Story, W. E. Am. J. Mth. 7 (1885) 364-.

Mukhopadhyay, A. QJ. Mth. 21 (1886) 212-.

Günther, P. Crelle J. Mth. 109 (1892) 213-.

Teixeira, J. P. G. Teix. J. Sc. 11 (1892) 136-; Fschr. Mth. (1893-94) 767.

for elliptic integrals. Olsson, $O$. Ts. Mth. 5 (1887) 33-; Fschr. Mth. (1887) 455.

- - , construction, geometric. Morera, $G$. [1880] Tor. Ac. Sc. At. 15 (1879) 649-. - - demonstration. Gwyther, R. $F$. Manch. Lt. Ph. S. P. 22 (1883) 81-.

-- , derivation from equation $\frac{d a}{\Delta a}+\frac{d b}{\Delta b}=0$.

Graefe, F. [1880] Crelle J. Mth. 90 (1881) 83-.

- - Euler's. Richelot, F. J. Crelle J. 44 (1852) 277-.

- $-\ldots,-$, Degen's attempt to generalise. Bjerknes, C. A. Bb. Mth. (1888) 1-.

- _ - 1st kind, demonstration, Sturm's. Schröter, H. Z. Mth. Ps. 17 (1872) 508-. $-\ldots-,-\ldots$, demonstrations. Bigler, $U$. Arch. Mth. Ps. 7 (1889) 401-.

- _ _ - _ derivation, method of Sturm. Much, - Z Z. Mth. Ps. 26 (1881) 333-.

$--\ldots,-$, 2nd and 3rd kinds. Dolbria, $I$.

Kazan S. Nt. (Ps.-Mth.) P. 7 (1889) 364-; N. A. Mth. 8 (1889) 204-.

___ 2nd kind, demonstrations. Walker, J. J. L. Mth. S. P. 13 (1881-82) 172-。

- - - - and 3rd kinds. Forsyth, A, R. Mess. Mth. 15 (1886) 49-.

--1 , theory. Schellbach, C. H. Crelle J. 54 (1857) 59-. 
extension. Winckler, A. Wien SB. 44 (1861) (Ab. 2) 477-.

and inversion problem. Brill, A. Mth. A. 17 (1880) 87-.

of Jacobi and of Weierstrass. Study, E. Am. J. Mth. 16 (1894) 156-.

proof. Griffiths, J. L. Mth. S. P. 13 (188182) 177-.

for theta functions. Caspary, F. C. R. 104 (1887) 1255-.

- transcendental functions. Pokrovskij, P. M. Rec. Mth. (Moscou) 18 (1896) 121-; Fschr. Mth. (1896) 360.

_ _ _ integration of differential equations leading to. Steen, $A$. Kjöb. Skr. 8 (1870) 1-; Résumé, 15-.

$-\zeta(u)$ and for cotangent. Schottky, F. Crelle J. Mth. 110 (1892) 324-.

- $\rho(u)$, elementary deduction. Anissimow, [1893] Fschr. Mth. (1893-94) 767.

- -. Stäckel, P. Mth. A. 47 (1896) 604.

Arithmetico-geometric mean. Borchardt, C.W. [1858] Crelle J. 58 (1861) 127-.

- - Frisby, E. Des Moines Anal. 6 (1879) 10-.

- - Lohnstein, T. Z. Mth. Ps. 33 (1888) $129-, 318$.

- - - of 4 elements (Borchardt). Schering, K. Crelle J. Mth. 85 (1878) 115-.

Class $d x / U_{x}^{\frac{2}{n}}+\ldots$ where $U_{x}=(a, b, c, d, e)(x, 1)^{n}$. Russell, $R$. Q̈J. Mth. 20 (1885) 179-.

$-d x / U_{x}^{\frac{1}{2}}+\ldots$ where $U_{x}=(a, b, c, d, e)(x,-1)^{4}$. Russell, R. QJ. Mth. 20 (1885) 265-.

Closed polygons in- and circum-scribed to conics. Oekinghaus, E. Arch. Mth. Ps. 6 (1888) 186-.

Complete elliptic integrals. Smith, H. J.S. Rm. R. Ac. Linc. T. 1 (1877) 42-.

- - - functions derivable from, and connected with Legendre's functions. Kleibner, J. Mess. Mth. 22 (1893) 1-.

_ _ - 1st kind, formula relating to. Besso, D. Rm. R. Ac. Linc. Rd. 4 (1895) (Sem. 1) 229-.

- - - - - - , and Legendre's coefficients, connection. Stuart, G. H. Mess. Mth. 13 (1884) 161-.

,----- , quotient, extension of theorem of Riemann. Torelli, $G$. Nap. Rd. 29 (1890) 238-.

- - - , - and 2nd kinds, approximations. Lodge, A. Mess. Mth. 28 (1899) 17-.

- - - , - - - continued fraction for ratio. Baraniecki, M. A. Par. T. Nauk Sc. Pam. 7 (*1875) Art. 5, 8 pp.

- - - , - - - - representation in simple form. Radicke, $A$. Z. Mth. Ps. 21 (1876) 442-.

- _ - 2nd kind, expression by sines of multiples of modular angle. Glaisher, J. W. L. Ph. Mg. 19 (1885) 504-.

- - - - , - - reduction to complete elliptic integrals of 1st kind for same modulus. Meissel, E. Arch. Mth. Ps. 56 (1874) 337-.
Conjugate functions of Cartesians and other quartics. Greenhill, A. G. [1881] Camb. Ph. S. P. 4 (1883) 77-.

Definite theta-function integrals, cases. Rogers, L. J. [1894] L. Mth. S. P. 26 (1895) 145-.

Differential equation in elliptic functions, geometrical illustration of solution. Hart, $H$. Mess. Mth. 4 (1875) 125-.

- - Euler's. Halphen, G. H. Palermo Cir. Mt. Rd. 2 (1888) 40-.

- - 2nd order, for $\rho(u)$. Mittag-Leffler, $G$. C. R. 117 (1893) 92-.

- remarkable, integration. Picart, $A$. C. R. 66 (1868) 1192-.

- - satisfied by $1 \pm 2 q+2 q^{4} \pm 2 q^{9}+\ldots$. Jacobi, C. G. J. Liouv. J. Mth. 14 (1849) 181-.

- equations, class. Catalan, -. As. Fr. C. R. (1886) (Pt. 1) 85 .

- - and elliptic series. Liouville, J. Liouv. J. Mth. 14 (1849) 225-.

- expression, reduction. Manning, $H . P$. Am. J. Mth. 13 (1891) 185-.

- - $d t \div \sqrt{t-a \cdot t-\beta \cdot t-\gamma \cdot t-\delta}$, reduction to standard form. Griffiths, J. L. Mth. S. P. 14 (1882-83) 196-.

\section{Doubly periodic functions.}

Liouville, J. Crelle J. Mth. 88 (1880) 277-. arising from $x^{3}+y^{3}-3 a x y=1$. Dixon, $A$. C. QJ. Mth. 24 (1890) 167-.

considered as limits of algebraic functions. Biehler, C. [1879] Crelle J. Mth. 88 (1880) 185-.

with essential singular points. Picard, $\dot{E}$. C. R. 89 (1879) 852-.

expansion in Fourier's series. Charlier, C.V.L. Stockh. Ak. Hndl. Bh. 12 (Afd. 1) (1887) No. 9, 24 pp.; Fschr. Mth. (1887) 448.

- - trigonometric series. Krause, $M$. D. Nf. Tbl. (1889) 188-.

expression by definite integrals and arithmetical invariants. Poincare, $H$. As. Fr. C. R. (1881) 109-.

Fourier's expansion for, new method. Mohrmann, G. Arch. Mth. Ps. 12 (1894) 1-.

Hermite's memoir. Cauchy, A. L. C. R. 32 (1851) 442-.

of 2 nd kind. Mittag-Leffler, G. C. R. 90 (1880) 177-.

- - - Gegenbauer, L. [1882] Wien Ak. Sb. 86 (1883) (Ab. 2) 969-.

- - - Goursat, $\bar{E}$. C. R. 98 (1884) 35-.

- Teixeira, J. P. G. Teix. J. Sc. 9 (1889) 103-; Fschr. Mth. (1889) 471.

_ - , application. Hermite, - . Par. Éc. Norm. A. 2 (1885) 303-.

- - - differential equations satisfied by. Krause, M. Leip. Mth. Ps. B. 42 (1890) 55-, 268-, 430-; 43 (1891) 32-, 289-, 597-. - and 3rd kinds. Krause, M. Leip. Mth. Ps. B. 41 (1889) 347-.

- 3rd kind. Appell, $\dot{P}$. Par. Éc. Norm. A. 1 (1884) 135-; 2 (1885) 9-; 3 (1886) 9-; C. R. 101 (1885) 1478-. 


\section{Doubly Periodic Functions}

of 3rd kind, differential relations in reference to. Krause, M. Leip. Mth. Ps. B. 41 (1889) 110-.

- - - resolution into simple elements. Appell, P. C. R. 97 (1883) 1419-.

monodromic and monogenic. Méray, C. C. R. 40 (1855) 787-.

periods. Dantscher von Kollesberg, $V$. Innsb. Nt. Md. B. 15 (1886) 79-.

resolution into simple elements. Hermite, Toul. Fac. Sc. A. 2 (1888) C, 12 pp.

- - - Weyr, E. Bll. Sc. Mth. 12 (1888) 246-.

and singly periodic functions, analogy between formulø. Glaisher, J. W. L. B. A. Rp. (1881) 548-.

theorem. Jordan, C. C. R. 70 (1870) 1128-

一, Liouville's. Rink, H. J. Z. Mth. Ps. 28 (1883) 48-.

-, proof. Schumann, A. Z. Mth. Ps. 27 (1882) 125-.

uniform, differential relations. Jahnke, $E$. Crelle J. Mth. 112 (1893) 265-.

Weierstrass's. Forsyth, A. R. Mess. Mth. 15 (1886) 165-; QJ. Mth. 22 (1887) 1-.

Doubly periodic series, examples. Appell, $P$. N. A. Mth. 15 (1896) 126-.

Elliptic differential equation. Battaglini, $G$. [1879] Rm. R. Ac. Linc. Mm. 5 (1880) 50-. _ - - application of binary quadratic forms to. Battaglini, G. [1885] Nap. Ac. At. 2 (1888) No. 4, 11 pp.

- $-\longrightarrow$ multiplier, Despeyrous's. Du BoisReymond, P. [1882] Mth. A. 21 (1883) 255-.

- - - - of general. Winckler, A. Wien Ak. Sb. 95 (1887) (Ab. 2) 209-

- differentials, general condition for finite integrability. Bugaev, N.V. St Pet. Ac. Sc. Mm. (Rs.) 69 (1892) (Suppl.) No. 8, 16 pp.; Fschr. Mth. (1892) 422-.

- double series. Scheibner, $W$. Leip. Mth. Ps. B. 42 (1890) 130-.

\section{ELLIPTIC FUNCTIONS.}

Abel, N. H. Crelle J. 2 (1827) 101-; 3 (1828) 160 -.

(Abel.) Jacobi, C. G. J. Crelle J. 3 (1828) 88.

Jacobi, C. G. J. Crelle J. 3 (1828) 192-, 303-, 403-; 4 (1829) 185-.

Legendre, A. M. As. Nr. 6 (1828) 205-.

Abel, N.H. As. Nr. 7 (1829) 33-; Crelle J. 4 (1829) 85-, 194-; 5 (1829) 336-; 6 (1830) $73-$.

Jacobi, C. G. J. Crelle J. 4 (1829) 371-; 6 (1830) 397-; 7 (1831) 41-.

Gudermann, C. Crelle J. 16 (1837) 78-.

Verhulst, P. F. Brux. Ac. Bll. 7 (1840) $322-$

Bronwin, B. Camb. Mth. J. 3 (1843) 123-.

Liouville, J. [1844-51] Liouv. J. Mth. 20 (1855) 203-.

Bromwin, B. Ph. Mg. 26 (1845) 75-.
Elliptic Functions 4040

(Bronwin.) Cayley, A. Ph. Mg. 26 (1845) 208-.

Bronwin, B. Ph. Mg. 27 (1845) 42-.

Hermite, C. Nancy Mm. S. Sc. (1845) 201-.

Jacobi, C. G. J. Liouv. J. Mth. 11 (1846) 97-.

Roberts, $W$. Liouv. J. Mth. 12 (1847) 449-.

Despeyrous, C. Dijon Mm. Ac. (1849) (pte. 2) 41-.

Cayley, A. Camb, and Dubl. Mth. J. 5 (1850) 201-.

Malmsten, C. J. Crelle J. 39 (1850) 116-.

Meyer, M. $H$. Grunert Arch. 16 (1851) 365-.

Peche, $F$. Haidinger Ab. 4 (1851) (Ab. 3) 19-.

Sturm, J. C. F. C. R. 42 (1856) 988-.

Röthig, O.' [1857] Crelle J. 56 (1859) 197-.

Strebor [Roberts, W.] N. A. Mth. $19(1860)$ 185-.

Mathet, G. Liouv. J. Mth. 6 (1861) 329-.

Mathieu, E. [1862] Par. Éc. Pol. J. Cah. 42 (1867) 265-.

Catalan, E. C. Rm. At. N. Linc. 20 (1867) 171-.

Klein, $F$. D. Nf. B. (*1877) 104.

Königsberger, L. Rpm. Mth. 1 (1877) 191-, 340 -

Gruss, G. Prag Sb. (1878) 246-.

Farkas, J. C. R. 90 (1880) 1482-.

Glaisher, J. W. L. [1880] QJ. Mth. 17 (1881) 50-.

Ferrers, N. M. Mess. Mth. 10 (1881) 102-.

Glaisher, J.W.L. [1881-83] Mess. Mth. 11 (1882) 81-, 120-; 12 (1883) 174-.

Sparre, (le comte)-de. Brux. S. Sc. A. 10 (1886) (Pt. 2) 129-; 11 (1887) (Pt. 2) 200-; 12 (1888) (Pt. 2) 1-.

Teixeira, J. P. G. Teix. J. Sc. 9 (1889) 132-; Fschr. Mth. (1889) 471-.

Appell, P. C. R. 110 (1890) 32-.

Grave, P. Kazan Un. Mm. 1897 (Pt. 3) 61-, (Pt. 4) 127-; Fschr. Mth. (1897) 388.

Hölder, $O$. Königsb. Schr. 38 (1897) [53]-.

Pierpont, J. N. Y. Am. Mth. S. Bll. 5 (1899) 490 -.

Abelians, and transcendentals of higher orders, geometric origin and representation. YvonVillarceau, A. J. F. Liouv. J. Mth. 4 (1878) 305-.

actually multiplied together. Kiepert, $L$. [1872] Crelle J. 76 (1873) 21-.

algebraic relations. David, - Toul. Ac. Sc. Mm. 6 (Sem. 2) (1884) 81-.

and alternants, identities connected with. Muir, T. [1900] Edinb. R. S. T. $40(1905)$ 187-.

- anharmonic properties of cubics. Ktodnicki, G. Wiad. Mt. 4 (1900) 232-.

applications. Clebsch, A. [1855] Crelle J. 53 (1857) 292-.

- Hermite, C. C. R. 85 (1877) 689-, 728-, $821-, 870-, 984-, 1085-, 1185-; 86$ (1878) $271-, 422-, 622-, 777-, 850-; 89$ (1879) 1001-, 1092- ; 90 (1880) 106-, 201-, 478-, 643-, 761- ; 93 (1881) 920-, 1098- ; 94 (1882) 186-, 372-, 477-, 594-, 753-. 
applications. Picard, É. C. R. 89 (1879) 74 .

-. Stouff, $X . \quad$ N. A. Mth. 15 (1896) 262-.

-. Sparre, (le comte) - de. Par. S. Mth. Bll. 28 (1900) 52-.

- of Abel's theorem. Vries, J. de. Amst. Ak. Vs. 4 (1896) 96-.

- to celestial mechanics. Liouville, J. Liouv. J. Mth. 1 (1836) 445-.

- - discontinuous functions. Bugaev, N. V. (xII) Rec. Mth. (Moscon) 11 (1883-84) 201-, 415-, 515-; 12 (1885) 3-.

- of formulæ of spherical trigonometry. Böklen, O. Grunert Arch. 40 (1863) 27-.

- to functions differing very little from zero. Zolotarev, E. St Pet. Ac. Sc. Mm. (Rs.) 30 (1877) (Suppl.) No. 5, 71 pp.

-, geometric. Paci, P. G. Mt. 12 (1874) 93-, 97-.

-, - Ahlborn, H. Hamb. Mth. Gs. Mt. 1 (1889) 63.

- to maxima and minima. Zolotarev, E. I. [1877] St Pét. Ac. Sc. Bll. 24 (1878) 305-.

- mechanics. Padova, E. Ven. I. At. (1889-90) 547-.

- - motion of body. Padova, E. Ven. I. At. $(1889-90)$ 437-.

- - problem in geometry. Boyd, J. H. A. Mth. 6 (1891-92) 93-, 163-.

- problems of mean values. Mukhopadhyay, A. [1888] Beng. As. S. J. 58 (Pt. 2) (1890) 199-, 213-.

- of theory of binary forms. Faà de Bruno, $F$. Am. J. Mth. 5 (1882) 1-.

- to theory of equations of higher degree. König, G. (xII) Mag. Tud. Ak. Etk. (Mth.) [1] (No. 8) (1871) $34 \mathrm{pp}$.

- - - numbers. Joubert, P. C. R. 50 (1860) 774-, 832-, 907-, 1040-, 1095-, 1145 -.

- - - Nazimov, P. S. Mosc. Un. Mm. (Ps.-Mth.) 5 (1885) 67-; Par. É. Norm. A. 5 (1888) 23-, 147 -.

- - - perturbations. Gyldén, J. A. H. Liouv. J. Mth. 2 (1876) 411-.

- - twisted quartics. Mathews, G. B. L. Mth. S. P. 19 (1889) 507-.

- - trajectory. Gossot -. Rv. Mar. et Col. 105 (1890) 225-, 417-.

and binary quartics, connection. Study, E.

Am. J. Mth. 17 (1895) 216-.

calculation, approximate. Verhulst, $P, F$. Brux. Ac. Bll. 6 (1839) (pte. 2) 424-.

cases. Schlömilch, O. Schlömilch Z. 2 (1857) 49-; Liouv. J. Mth. 2 (1857) 43-.

- of St Venant's problem integrable by. Nocciòli, G. N. Cim. 33 (1893) 208-.

and circular functions, continued fractions for. Malmsten, C. J. Camb. and Dubl. Mth. J. 4 (1849) 286.

class of integrals related to. Ramus, C. Kiöb. Ov. (1835-36) 4-.

and complex variable. Herrmann, O. Z. Mth. Ps. 28 (1883) 193-, 257-.

construction for amplitudes. Chasles, $M$. C. R. 19 (1844) 1239-. corresponding to function $\cos x+i \sin x$. Lemonnier, $H$. Par. Éc. Norm: A. 4 (1875) 371-; 6 (1877) 79-.

deduction of $q$-series from $q$-products. Glaisher, J. W. L. Mess. Mth. 16 (1887) 133-.

and definite integrals, and infinite series. Lerch, M. Prag České Ak. Fr. Jos. Rz. (Tŕ̌ida 2) 1 (1892) Art. 8, 11-, Art. 25, 6 pp.; 2 (1893) Art. 23, 42 pp.; 4 (1895) drt. 1, 55 pp.; Fschr. Mth. (1892) 445; (1893-94) 791-; (1895) 501-.

definition. Varicak, V. N. A. Mth. 14 (1895) 14-.

of 2nd degree. Falk, M. Ups. S. Sc. N. Acta 16 (1893) No. 1, 30 pp.

derivation. Verdam, G. J. Amst. I. (1842) $57-$.

differential equation in. Cayley, A. Mess. Mth. 4 (1875) 69-, 110-; 6 (1877) 29.

- - involving. Palmstrøm, A. Berg. Ms. Aarb. (1894-95) No. 14, 16 pp.

-- new. Brioschi, F. Mil. I. Lomb. Rd. 10 (1877) 417-.

- equations in. Genocchi, A. [1864] Tor. Mm. Ac. 23 (1866) 223-.

- - for periods. Brioschi, F. A. Mt. 14 (1886-87) 238-

- - satisfied by $K, E$, etc. Kleiber, $J$. Mess. Mth. 18 (1889) 167-.

- relations. Martins da Silva, J.A. G. Teix. J. Sc. 6 (1885) 169-.

differentiation with regard to modulus. Hermite, C. [1879] As. Nr. 96 (1880) 321-.

___ _ _ , formulæ arising from. Wilkinson, M. M. U. L. Mth. S. P. 13 (1881-82) $212-$.

- - - or function of modulus. Bierens de Haan, D. Amst. Ak. Vh. 18 (1879) 33 pp.; Arch. Néerl. 15 (1880) 225-

- - - periods and invariants. Frobenius, G., \& Stickelberger, L. [1881] Crelle J. Mth. 92 (1882) 311-.

and equation of 4 th order. Pellet, A. E. Par. S. Mth. Bll. 14 (1886) 90-.

especially elliptic arc, relations of curve of elasticity. Bender, C. Arch. Mth. Ps. 60 (1877) 117-.

and Eulerian integrals. Lerch, M. Prag Sb. (1897) (Mth.-Nt.) No. 28, 11 pp.

evaluation of definite integrals by. Roberts, $W$. Liouv. J. Mth. 11 (1846) 157-.

existence domain, determination. Lerch, M. Mh. Mth. Ps. 11 (1900) 107-.

expansion. Nicolls, W. [1883] Ir. Ac. T. $28(1880-86) 267-$.

-. Jack, J. Edinb. Mth. S. P. 14 (1896) 35-.

- as continued fractions. Rogers, L. J. L. Mth. S. P. 19 (1889) 550-.

- of imaginary period when modulus is small enough. Schlïfi, L. A. Mt. 3 (1869-70) 243-.

- in increasing powers of variable. Hermite, $C$. [1875] Crelle J. Mth. 81 (1876) 220-.

- power series. Krause, M. Leip. Mth. Ps. B. 46 (1894) 30- 
expansion of

$$
\begin{aligned}
\Delta^{ \pm r} \text { am } & \frac{2 K}{\pi} x \cdot \cos ^{ \pm 8} \mathrm{am} \frac{2 K}{\pi} x \cdot \sin ^{ \pm t} \frac{2 K}{\pi} x \\
& \times \int_{0}^{x} \Delta^{e} \mathrm{am} \frac{2 K}{\pi} x \cdot d x
\end{aligned}
$$

in sines and cosines of multiple angles. Meyer, C. O. Crelle J. 37 (1848) 273-, 281-.

expansions, new. Gyldén, $H$. Stockh. Öfv. (1886) 197-; Fschr. Mth. (1886) 387-.

- in trigonometric series. Appell, P. Par. S. Mth. Bll. 13 (1885) 13-.

- - - (Appell). Poincaré, H. Par. S. Mth. Bll. 13 (1885) 19-.

14 (1886) 131-.

- - - Krause, - $\&$ Mohrmann, Mth. A. 32 (1888) 331-.

- Lerch, M. Acta Mth. 12 (1889) $51-$.

expressing definite integrals. Bronwin, $B$. Mathematician 2 (1847) 297-.

expression for, by ratio of two series. Appell, $P$. Am. J. Mth. 14 (1892) 9-.

formula. Glaisher, J. W. L. Mess. Mth. 7 (1878) 144-.

-. Cayley, A. Mess. Mth. 8 (1879) 126-.

-, Cayley's. Forsyth, A. R. Mess. Mth. 14 (1885) 23-.

formulæ. Jacobi, C. G.J. Crelle J. 30 (1846) 269-.

- Dillner, G. [1863] Stockh. Ak. Hndl. 5 (1866) No. 3, 19 pp.

-. Sylow, L. [1864] Christiania F. 7 (1865) 68-.

-. Brioschi, F. Rm. R. Ac. Linc. T. 2 (1878) 115-.

- Glaisher, J. W. L. [1879-82] B. A. Rp. (1879) 269 ; QJ. Mth. 16 (1879) 382-; 19 (1883) 22-.

-. Craig, T. Am. J. Mth. 5 (1882) 62-.

- Wilkinson, M. M. U. L. Mth. S. P. 13 (1881-82) 106-.

- Martins da Silva, J. A. G. Teix. J. Sc. 5 ( ${ }^{*}$ 1883) 75-.

-. Cayley, A. Mess. Mth. 14 (1885) 21-.

-. Stieltjes, T. J. (jun.). Amst. Ak. Vs. M. 2 (1886) 101-.

- Albeggiani, M. L. Palermo Cir. Mt. Rd. 1 (1887) $350-, 398$.

- Glaisher, J. W. L. Mess. Mth. 16 (1887) 67-.

-. Brioschi, F. Tor. Ac. Sc. At. 26 (1891) 586-.

- Goursat, É. Par. S. Mth. Bll. 23 (1895) 18-.

-, application to geometric problem. Richelot, F. J. Crelle J. 38 (1849) 353-.

-, class derivable from $q$-series in. Glaisher, J. W. L. Mess. Mth. 14 (1885) 102-.

-, connection. Cayley, A. Mess. Mth. 9 (1880) 23-.

-, and deduction of fundamental theorem. Richelot, F. J. [1854] Crelle J. 50 (1855) 41-. - deduction of trigonometric formulæ from. Glaisher, J. W. L. B. A. Rp. (1880) 477-. formulæ, derivation from confocal conics. Griffiths, J. [1882] L. Mth. S. P. 14 (188283) 46-.

-, -, method. Glaisher, J. W. L. [1881] Camb. Ph. S. P. 4 (1883) 186-.

-, geometric interpretation. Cayley, A. (xII) J. H. Un. Cir. [1] (1882) 238.

-, new. Brun, F. de. Stockh. Öfv. (1897) 309-; (1898) 523-.

-, systems. Glaisher, J. W. L. [1880-81] Mess. Mth. 10 (1881) 104-; 11 (1882) 78-.

functions connected with. Cauchy, A. L. C. R. 17 (1843) 640-.

and geodesic lines. Braunmühl, A, von. Mth. A. 26 (1886) 151-.

geometric considerations. Küpper, C. Crelle J. 63 (1864) 40-.

- construction. Essen, E. Grunert Arch. 22 (1854) 241-.

- interpretation. Janssen van Raaij, W. H.L. Haarl. Ms. Teyl. Arch. 6 (1900) 151-.

- investigations. Tortolini, B. Tortolini A. 3 (1860) 179-; (vIII) 4 (1861) 204-.

- origin and fundamental formulæ. Despeyrous, C. Toul. Mm. Ac. 5 (1873) 211-.

- properties relative to. Serret, J. A. Liouv. J. Mth. 8 (1843) 495-

- - Roberts, $W$. Liouv. J. Mth. 10 (1845) 297-

and hyperbolic and cyclic functions, numerical calculation of arguments. Runge, C. Acta Mth. 15 (1891) 221-.

- - functions, relations. Griffiths, J. L. Mth. S. P. 6 (1874-75) 98-.

- - - - Sincov, D. Kazan S. Ps.-Mth. Bll. 9 (1900) 27 -

- hyperelliptic functions, approximation method. Merrifield, $C . W$. Phil. Trans. (1860) 223-; (1862) 417-.

- - , reports on progress. Russell, W. H.L. B. A. Rp. 39 (1869) 334-; 40 (1870) 102-; 42 (1872) 334-; 43 (1873) 307-.

- - - representation, geometric. Serret, J. A. Par. Mm. Sav. Etr. 11 (1851) 103-. identities. Glaisher, J.W.L. [1874-76] $\mathrm{Ph}$. Mg. 47 (1874) 437-; 50 (1875) 539-; Phil. Trans. 165 (1876) 489-; L. Mth. S. P. 7 (1875-76) 61-; Mess. Mth. 6 (1877) 102-.

-. Cayley, A. QJ. Mth. 16 (1879) 281-.

- Glaisher, J. W. L. As. Fr. C. R. 9 (1880) 223-.

-, case. Rogers, L. J. [1893] L. Mth. S. P. 25 (1894) 44-.

imaginary period. Johnson, W. W. Am. J. Mth. 6 (1884) 246-.

integrals, calculation. Steen, A. (xII) Ts. Mth. 3 (1867) 153-

-, with regard to modulus. Salvert, $F$. de. C. R. 124 (1897) 1008-, 1186 ; Brux. S. Sc. A. 21 (1897) (Pt. 1) 119-.

- related to. Lerch, M. Acta Mth. 22 (1899) 365-.

-, relations. Herz, N. [1881] Arch. Mth. Ps. 67 (1882) 343-.

and integrals, and Jacobian functions, theory. Bolzani, J.F. (xr) Kazan Un. Mm. (1856) (Bks. 3 \& 4) $170 \mathrm{pp}$. 
integration by. Bouquet, $J_{.}, \&$ Briot, C. R. 61 (1855) 1229-.

- Dolbnia, I. Kazan S. Ps.-Mth. Bll. 1 (1891) 46-; Fschr. Mth. (1891) 463-. - of equation connected with. MacMahon, $P$. A. B. A. Rp. (1882) 450-

interpolation problem. Appell, $P$. Bll. Sc. Mth. 10 (1886) 109-.

- - Schottky, F. Crelle J. Mth. 107 (1891) 189 -.

inverse. Cayley, A. Camb. Mth. J. 4 (1845) 257-.

—, integrals expressed by, Greenhill, A. G. Camb. Ph. S. P. 3 (1880) 361-.

investigations. Klein, F. Mth. A. 26 (1886) 455-.

involved in definite integrals. Glaisher, J. W. L. R. S. P. 29 (1879) 331-.

- - system of integrals. Glaisher, J.W.L. QJ. Mth. 19 (1883) 145-.

Jacobian. Stringham, I. A. Mth. 8 (1893-94) $105-$.

of 1st kind. Serret, J. A. Liouv. J. Mth. 8 (1843) 145-

- - Iaggi, E. N. A. Mth. 17 (1898) 367-。

- - - fundamental properties, geometric explanation. Lémeray, $E . M$. N. A. Mth. 19 (1900) 255-, 289-.

- _ - geometric demonstration of two theorems. Schläfli, L. Grunert Arch. 12 (1849) 188-.

- - - - - study. Léauté, H. Par. Ec. Pol. J. Cah. 46 (1879) 65-.

- - -, Jacobi's 1st and 2nd theorems, supplements to. Essen, E. Grunert Arch. 21 (1853) 241-, 418-.

- - - representation by ares of curves. Allégret, [A.] C. R. 70 (1870) 1032-.

- - - - - gauche biquadratics. Léauté, H. C. R. 83 (1876) 527-.

$--\ldots,-$, geometric. Roberts, $W$. Liouv. J. Mth. 8 (1843) 263-.

,,----- Serret, J. A. Camb. and Dubl. Mth. J. 1 (1846) 187-.

- - - 一, 一. Davidov, A. Y. (xir) Rec. Mth. (Moscou) 2 (1867) (Pt. 1) 129-.

- and 2nd kinds. Lobatto, $R$. Amst. N. Vh. 12 (1846) 119-.

- - - Bellavitis, G. Padova Rv. Period. 1 (1851-53) 149-.

- - - - Küpper, C. Crelle J. 55 (1858) 89-.

- - - - - as functions of amplitude. Liouville, J. [1833] Par. Éc. Pol. J.23 cah. (1834) 37-.

,----- modulus. Liouville, $J$. Liouv. J. Mth. 5 (1840) 34-, 441-.

- 2nd kind. Frobenius, G. [1881] Crelle J. Mth. 93 (1882) 53-.

- - and 3rd kinds, expansion in trigonometric series. Krause, $M$. Mth. A. 30 (1887) 425-, 516-; 33 (1889) 108-; 35 (1890) 577-. P. $38(1885)$, 206-, 316-. Griffiths, J. R. S.

- 3rd kind. Salvert, (le vicomte) - de. Brux. S. Sc. A. 21 (1897) (Pt. 2) 131-

- - calculation, method. Somoff, $J$. of $3 \mathrm{rd}$ kind, reduction to functions of two arguments. Verhulst, P. F. Quetelet Cor. Mth. 11 (1839) 225-.

_- - representation, geometric. Tortolini, B. G. Arcad. 100 (1844) 257-.

- - - - - - Drobisch, M.W. Grunert Arch. 24 (1855) 320-.

logarithm, behaviour. Schottky, $F$. Crelle J. Mth. 108 (1891) 342-.

method, short. Meech, L. W. Des Moines Anal. 4 (1877) 129-, 161-; 5 (1878) 9-.

- - - (Meech). Kummell, C. H. Des Moines Anal. 5 (1878) 17-, 97-.

modulus, formulæ relative to. Hermite, $C$. Liouv. J. Mth. 9 (1864) 313-.

- as function of quotient of two periods. Hermite, C. A. Mt. 3 (1869-70) 81-.

numerators and denominators satisfying partial differential equation. Jacobi, C. G.J. Crelle J. 36 (1848) 81-.

of 2nd order. Bricard, R. Par. S. Mth. Bll. 25 (1897) 212-; 26 (1898) 91-.

$-{ }_{-}$, properties. Berger, $A$. Ups. S. Sc. N. Acta 14 (1891) No. 12, 50 pp.

- 3rd order, differential equation. Pfannenstiel, E. Ups. S. Sc. N. Acta 15 (1895) No. 3, 18 pp.

- nth order. Rados, $G$. Mth. Termt. Éts. 6 (1888) 353-.

and orthogonal transformations. Wilkinson, M. M. U. L. Mth. S. P. 14 (1882-83) 203-.

periodicity. Benthem, A. N. Arch. Wisk. 3 ( 1877) 186-.

-, double. Franklin, $F$. Am. J. Mth. 11 (1889) 283-.

periods, existence of two. Méray, C. Par. Éc. Norm. A. 1 (1884) 177-.

and their powers, expansion. André, D. C. K. 83 (1876) 135-.

- - - - in series. André, D. Par. Éc. Norm. A. 6 (1877) 265-.

property, demonstration. Walton, W. [1870] QJ. Mth. 11 (1871) 177-.

and $q$-series. Glaisher, J.W.L. Mess. Mth. 13 (1884) 126-.

- quadriquadric curve. Cayley, A. Mth. A. 25 (1885) 152-.

of $n$th rank. Klein, $F$. Leip. Mth. Ps. B. 36 (1884) 61-.

rational combinations. Meyer, C. O. [1885] Crelle J. 56 (1859) 314-.

and reciprocal factorials. Cauchy, A. L. C. R. 17 (1843) 825-.

recurring relation. Jonquières, $E$. de. C. R. 101 (1885) 415-.

reduction of some definite integrals to, and applications, Tortolini, B. G. Arcad. 116 (1848) 137-, 265-.

- integrals to. Dienger, J. Grunert Arch. 11 (1848) 94-; 13 (1849) 424-.

- _ - Tortolini, B. Tortolini A. 3 (1860) 183-.

- - - cases. Sparre, (le comte) - de. Brux. S. Sc. A. 8 (1884) (Pt. 2) 97-; 9 (1885) (Pt. 2) 205-; 21 (1897) (Pt. 2) 45-.

representation. Liouville, J. C. R. 21 (1845) 1255-. 
representation, conformal, of curvature lines of ellipsoid by. Neovius, E. R. Helsingf. Acta 15 (1888) 363-.

- by cubic curve. Juel, C. Sk. Nf. F. (1892) 344-; Fschr. Mth. (1892) 455.

- of curves of 3rd degree by. Picquet, Par. Éc. Pol. J. 54 (1884) 31-.

- by doubly infinite products. Fiske, T. S. [1891] N. Y. Mth. S. Bll. 1 (1892) 61-.

- - elastic curve. Greenhill, A. G. Mess. Mth. 5 (1876) 180-; 6 (1877) 182-.

-, geometric. Strnad, A. Arch. Mth. Ps. 61 (1877) 321-.

-, - of equation, and applications. Trudi, N. Nap. Ac. Sc. Mm. 1 (1852-54) 63-.

- - , equations relative to. Serret, J. $A$. Liouv. J. Mth. 10 (1845) 351-.

-, 一 of three. Roberts, W. Liouv. J. Mth. 9 (1844) 155-.

-, -,- - (Roberts). Serret, J.A. Liouv. J. Mth. 9 (1844) 160 .

-, graphic, and binodal quartic. Cayley, $A$. Camb. Ph. S. T. 14 (1889) 484-.

- by power series. Jacobi, C. G.J. Crelle J. 54 (1857) 82-.

- of any quantity by $\sin \operatorname{am}(u+w, k)$. Richelot, F. J. Crelle J. 45 (1853) 225-.

- as quotient of convergent series. Kneser, $A$. Mth. A. 32 (1888) 309-.

- by rational functions of exponential function. Schwarz, H. A. Berl. Ak. Sb. (1894) 1187-.

Riemann's lectures. Stahl, $H$. Z. Mth. Ps. 45 (1900) 216-.

roots of unity occurring in. Molien, $T$. Leip. Mth. Ps. B. 37 (1885) 25-.

series connected with. Gutzmer, A. G. Teix. J. Sc. 8 (1887) 33-.

-, new. Faà de Bruno, F. C. R. 95 (1882) $22-$.

solution of $x^{3}+y^{3}-1=0$. Cayley, A. [1881] Camb. Ph. S. P. 4 (1883) 106-.

$---=-$ (Cayley). Greenhill, A. G. [1882] Camb. Ph. S. P. 4 (1883) 223-.

special. Thomae, C.J. Z. Mth. Ps. 27 (1882) 181-.

spherical triangle in. Johnson, W. W. QJ. Mth. 19 (1883) 185-.

and spherical trigonometry, connexion. Glaisher, J. W. L. QJ. Mth. 17 (1881) 353-. - - and orthogonal substitutions. Study, E. Leip. Mth. Ps. Ab. 20 (1893) 85-.

summation formula. Alexéief, -. Bll. Sc. Mth. As. 1 (1877) 44.

theorem. Jacobi, C. G. J. [1827] As. Nr. 6 (1828) 133-.

- (Jacobi). Plana, G. [1828] Tor. Mm. Ac. 33 (1829) 333-.

- (-). Poisson, S. D. Par. Mm. Ac. Sc. 10 (1831) 73-.

-. Richelot, F. J. Crelle J. 32 (1846) 219.

- Cayley, A. L. Mth. S. P. 10 (1878-79) 43-.

- Hermite, C. C. R. 90 (1880) 1096-.

- Glaisher, J. W. L. B. A. Rp. (1882) 456-.

- Phragmén, E. Stockh. Ófv. (1884) No.9, 199-; Acta Mth. 7 (1885) 33-. theorem. Falk, M. Acta Mth. 7 (1885) 197-.

- Lerch, M. G. Teix. J. Sc. 8 (1887) 3-.

- Moore, E. H. Palermo Cir. Mt. Rd. 4 (1890) 186-.

-, fundamental. Pringsheim, A. Mth. A.27 (1886) 151-.

- of Landen. Cayley, A. [1881] L. Mth. S. P. 13 (1882) 47-.

theorems, 2. Scheibmer, W. Leip. B. 11 (1859) 159-.

-. Falk, M. Stockh. Ak. Hndl. Bh. 14 (Afd. 1) (1889) No. 1, 30 pp.

-, geometric. Roberts, $W$. Ir. Ac. P. 3 (1847) 77-.

and trigonometric theorems. Glaisher, J.W. L. [1880] Mess. Mth. 10 (1881) 92-. theory. Jacobi, C. G.J. [1827-32] Par. Éc. Norm. A. 6 (1869) 127-.

- Ivory, $J$. Phil. Trans. (1831) 349-.

-. Jacobi, C. G. J. Crelle J. 8 (1832) 413-

- Bronwin, B. Ph. Mg. 22 (1843) 258-.

- (Bronwin). Cayley, A. Ph. Mg. 22 (1843) 358-.

- (Cayley). Bromwin, B. Ph. Mg. 23 (1843) 89-.

-. Jacobi, C. G. J. Crelle J. 26 (1843) 93-.

- Serret, J. A. Par. S. Phlm. PV. (1845) 81-.

- Eisenstein, G. Crelle J. 30 (1846) 185-;

32 (1846) 59-; 35 (1847) 137-, 185-.

-. Cayley, A. Camb. and Dubl. Mth. J. 2 (1847) 256- ; 3 (1848) 50-.

Malmsten, C. J. Stockh. Ak. Hndl. (1847) 71-.

- Hermite, C. Camb. and Dubl. Mth. J. 3 (1848) 54-; C. R. 29 (1849) 594.

_. Krusemarck, R. [1852] Crelle J. 46 (1853) 189-.

-. Richelot, F. J. C. R. 49 (1859) 641-.

-. Betti, E. Tortolini A. 3 (1860) 65-, 298-; 4 (1861) 26-, 57-, 297-.

-. Plana, G. [1860] Tor. Mm. Ac. 20 (1863) 189-, 422-.

-. Hermite, C. Liouv. J. Mth. 7 (1862) 25-;

C. R. 57 (1863) 613-, 993-.

. Rubini, R. G. Mt. 1 (1863) 33-, 118-, 140-, 291-.

- Thiele, T. N. (xir) Ts. Mth. 3 (1873) 65-.

- Weyr, E. Prag Sb. (1876) 172-

- Frobenius, G., \& Stickelberger, L. Crelle J. Mth. 83 (1877) 175-

-. Kronecker, L. Berl. Ak. Mb. (1881) 1165-.

-. Stolz, O. Innsb. Nt. Md. B. 12 (1882) xiv-.

- Kronecker, L. Berl. Ak. Sb. (1883) 497-, 525-.

- Brioschi, F. A. Mt. 12 ("1883-84) 49-.

-. Mansion, P. Brux. Ac. Bll. 8 (1884) 180-

- Schroeter, H. Acta Mth. 5 (1884) 205-.

-. Kronecker, L. Berl. Ak. Sb. (1885) 761-; (1886) 701-.

-. Lipschitz, R. Par. Éc. Norm. A. 2 (1885) 315-.

-. Mertens, F. Wien Ak. Sb. 91 (1885) (Ab. 2) 974-

Weber, $H . \quad$ Acta Mth. 6 (1885) 329-; 11 (1887-88) 333-. 
theory. Lerch, M. J. Prag Sb. (1886) (Mth.Nt.) 391-; Fschr. Mth. (1886) 388-.

-. Pick, G. Mth. A. 28 (1887) 309-.

- Kronecker, L. Berl. Ak. Sb. (1889) 53-, 123-, 199-, 255-, 309-; (1890) 99-, 123-, 219-, 307-, 1025-.

- Gomes Teixeira, F. G. Teix. J. Sc. 10 (1891) 150-; Fschr. Mth. (1892) 425-.

-. Giunther, P. Crelle J. Mth. 108 (1891) 256-; 109 (1892) 43-.

- Lerch, M. Prag České Ak. Fr. Jos. Rz. (Třida 2) 1 (1892) Art. 24, 18 pp.; Fschr. Mth. (1892) 442-.

-. Igel, B. Mh. Mth. Ps. 7 (1896) 149-.

- Mansion, - Brux. S. Sc. A. 22 (1898) (Pt. 1) 90-.

-. Vasiljev, A. V. St Pét. Ac. Sc. Mm. 6 (1898) No. 9, $42 \mathrm{pp}$.

-, algebraic investigations. Königsberger, [1869] Crelle J. 72 (1870) 176-.

-, arithmetical identities deduced from. Glaisher, J. W. L. Mess. Mth. 2 (1873) 177-.

- based on infinite double products. Schläfi, L. Grunert Arch. 14 (1850) 395-.

-, concepts. Björling, E. G. Arch. Mth. Ps. 48 (1868) 121-.

-, elementary. Laurent, $H$. N. A. Mth. 16 (1877) 78-, 211-, 361-, 385-, 433-, 481-; 17 (1878) 119-, 247-, 385-, 537-; 18 (1879) 126-, 145-.

-, equations in, and geometric theorems connected therewith. Fergola, E. Rm. S. It. Mm. 4 (1882) No. 6, 10 pp.

-, formula, fundamental, direct verification. Jacobi, C. G. J. Crelle J. 36 (1848) 75-.

-, formulæ, three. Martins da Silva, J.A. Bll. Sc. Mth. 10 (1886) 78-.

- Gauss. Pepin, T. Rm. N. Linc. Mm. 9 (1893) (Pt. 2) 1-; 10 (1894) 125-.

-, -. Giüther, $P$. Liouv. J. Mth. 3 (1897)

- of Legendre. Caldarera, $\boldsymbol{F}$. Palermo G. Sc. Nt. 1 (1866) 38-.

and theory of numbers. Kronecker, $L$. Liouv. J. Mth. 3 (1858) 265-.

theory, outline. Heine, H. E. Crelle J. 39 (1850) 122-.

-, partial fractions in. Gunther, $P$. Crelle J. Mth. 113 (1894) 262-.

-, point in. Lipschitz, R. O. S. C. R. 97 (1883) 1411-.

- - - - (Lipschitz). Hermite, C. C. R. 97 (1883) 1414-.

-, - - Martins da Silva, J. A. Brux. Ac. Bll. 10 (1885) 79-.

,--1 . Koenigs, G. Toul. Fac. Sc. A. 4 (1890) E, 4 pp.

-, points in. Krause, $M$. Dresden Isis $\mathrm{Sb}$. (1897) 9.

-, précis. Abel, N. H. Crelle J. 4 (1829) 236-, 307-.

-, relation of Cayley's. Hermite, C. Acta Mth. 1 (1882) 368-.

-, teaching. Mansion, - Brux. S. Sc. A. theory, use of cross-gratings. Sylvester, J. J. (xII) J. H. Un. Cir. [2] (1883) 43-.

- - - infinite products. Hermite, $C$. Acta Mth. 4 (1884) 193-. 4 (1884) -194 -. Lipschitz, $R$. Acta Mth.

-, - $\rho(u)$. Gomes Teixeira, F. Prag Sb. (1892) (Mth.-Nt.) 182-.

- of Weierstrass, simplification of exponentials. Puzyna, J. Prace Mt.-Fiz. 10 (1899-1900) 8-; Fschr. Mth. (1899) 401.

and trigonometric functions, analogy between. Dolbnia, I. P. [1889] Kazan S. Nt. (Ps.Mth.) P. 8 (1890) 129-; N. A. Mth. 8 (1889) 459-.

use in theory of plane quadrilateral. Darboux, G. C. R. 88 (1879) 1183-, 1252-; Bll. Sc. Mth. As. 3 (1879) 109-.

Weierstrass's. Söderblom, $A$. Ups. S. Sc. N. Acta 12 (1885) No. 12,-123 pp.

- Caspary, F. Liouv. J. Mth. 5 (1889) 73-

-. Bukreiew, B. J. [1893] Fschr. Mth. (1893-94) 767.

algebraic equations connected with. Pokrovskij, P. M. Mosc. S. Sc. Bll. 83 (No. 1) (1893) 25-; Fschr. Mth. (1893-94) $152,795$.

- _- - (Pokrovskij). Bukrěev, B. Ja. Mosc. S. Sc. Bll. 83 (No. 2) (1894) 1-.

-, applications. Greenhill, A. G. L. Mth. S. P. 17 (1887) 355-; 18 (1886-87) 263-. and Jacobi's, comparison. Cayley, A. Mess. Mth. 16 (1887) 129-.

- method. Daniels, A. L. Am. J. Mth. 6 (1884) 177-, 253; 7 (1885) 82-.

- ratio $\eta^{\prime}: \eta$ as function of $\omega^{\prime}: \omega$ in. Bigiavi, C. A. Mt. 19 (1891-92) 261-.

-, solution of cubic and quartic equation by. Greenhill, A. G. L. Mth. S. P. 17 (1887) 262-.

and zeta-functions, transformation and expansions. Glaisher, J.W. L. Mess. Mth. 17 (1888) 1-.

- - - of $K / 3$ and $K / 4, q$-series derived from. Glaisher, J. W. L. [1890] L. Mth. S. P. 22 (1891) 143-.

cos am $x$, expansion. Hermite, C. Liouv. J. Mth. 9 (1864) 289-.

$F(x)=\operatorname{sn}^{a} x \cdot \operatorname{cn}^{b} x \cdot \mathrm{dn}^{c} x$, expansion for integer exponents. Hermite, C. [1875] Stockh. Ak. Hndl. Bh. 3 (1876) No. 10, 10 pp.

$A l(x)$, expansion in series. Joubert, -. C. R. 82 (1876) 1259-, 1326-.

$S n^{\alpha} x$. Hermite, C. Helsingf. Acta 12 (1883) 437-.

$K, E, J, G, K^{\prime}, E^{\prime}, J^{\prime}, G^{\prime}$ in. Glaisher, $J . W$. L. QJ. Mth. 20 (1885) 313-.

$\rho(u)$, periods. Vivanti, $J$. Par. Éc. Norm. A. 2 (1885) 325-.

-. Mansion, - Brux. S. Sc. A. 13 (1889) (Pt. 1) 46-.

-, definite integral connected with. Pfannenstiel, E. Ups. S. Sc. N. Acta 14 (1891) No. 6, $24 \mathrm{pp}$.

-. Burnside, W. Mess. Mth. 21 (1892) 84-.

- Gomes Teixeira, -. Bll. Sc. Mth. 16 (1892) 76-. 
$\vartheta(u)$, application to Poncelet's polygons. Vivanti, G. Palermo Cir.Mt. Rd. 7 (1893) 61-. $\mathrm{Z}(u), \Theta(u)$, II $(u, a)$. Glaisher, J. W. L. L. Mth. S. P. 17 (1887) 152-.

$\rho\left(u: g_{2}, g_{3}\right)$. Söderblom, $A$. Ups. S. Sc. N. Acta 17 (1898) No. 6, 56 pp.

$\omega(x)=\frac{\operatorname{sn} x \cdot \operatorname{dn} x}{\operatorname{cn} x}$. Grave, D. Fschr. Mth. (1899) 402.

Elliptic and hyper-elliptic systems of differential equations, etc. Roberts, W. R. W. L. Mth. S. P. 26 (1895) 379-.

\section{Elliptic integrals.}

Lobatto, R. (vIr) Amst. N. Ws. Ntk. Vh. 1 (1844) 70-.

Genocchi, A. Schlömilch Z. 2 (1857) 414-.

Enneper, A. Z. Mth. Ps. 11 (1866) 74-; Mth. A. 11 (1877) 567-.

Thomae, C. J. Z. Mth. Ps. 23 (1878) 409-.

Enneper, A. Gött. Nr. (1884) 175-.

Heymann, $W$. Crelle J. Mth. 103 (1888) 87-.

Catalan, $E$. Brux. Ac. Mm. 49 (1890-93) (No. 6) $20 \mathrm{pp}$.

algebraic equations leading to. Johanson, A. $M$. Stockh. Öfv. (1887) 691-; Fschr. Mth. (1887) 448.

and arc and logarithm, representation by infinite products. Seidel, L. [1867] Crelle J. 73 (1871) 273-.

cases. Schlömilch, O. X. Z. Mth. Ps. 27 (1882) 62-.

class of integrals allied to. Ramus, C. Kiöb. Ov. (1834-35) 2-; (1835-36) 4-; Kiöb. Dn.

Vd. Selsk. Afh. 6 (1837) 249-, 265-.

complete, and gamma functions, definite integrals expressible by. Glaisher, J. W. L. L. Mth. S. P. 12 (1880-81) 92-.

condition that an algebraic equation shall lead to. Johanson, A. M. Stockh. Öfv. (1887) 703-; Fschr. Mth. (1887) 448.

elliptic functions for. Baraniecki, M. A. Par. T. Nauk Śc. Pam. $7\left({ }^{*} 1875\right)$ Art. 5, $8 \mathrm{pp}$.

and elliptic functions, connexion with ellipse. Imšeneckij, $V . G$. St Pet. Ac. Sc. Mm. (Rs.) 48 (1884) (Suppl.) No. 5, 43 pp.; Fschr. Mth. (1884) 652.

- _ - in connexion with theory of catenary, transformations. Oekinghaus, $E$. Arch. Mth. Ps. 2 (1885) 138-; 4 (1886) 225-.

- - - evaluation by transformation of 2 nd degree. Kluyver, J. C. Par. Éc. Norm. A. 11 (1894) 37-.

- - - , representation, geometrical. Grave, $P$. Kazan S. Ps.-Mth. Bll. 4 (1895) 43-, 159-; Fschr. Mth. (1893-94) 768.

equivalence of forms of quadratures of differentials reducible to. Salvert, $F$. de. C. R. 120 (1895) 1034-.

evaluation by arithmetico-geometric mean. Kowalczyk, J. Wiad. Mt. 2 (1898) 21-.

expansion in series, and comparisons. Schulze, N. W. Grunert Aroh. 19 (1852) 181-. expansion by zonal harmonics. Hargreaves, $R$. Mess. Mth. 26 (1897) 89-.

- of $\left(a^{2}+b^{2}-2 a b \cos \phi\right)^{n+\frac{1}{2}}$ by. Wallace, (Prof.) W. [1802] Edinb. R. S. T. 5 (1805) 253-.

expression as definite integrals. Zurria, $G$. Rm. S. It. Mm. 8 (1892) No. 3, 18 pp.

- in finite form. Bugaev, N. V. [1891] Rec. Mth. (Moscou) 16 (1893) 259-; Fschr. Mth. (1892) 421-.

- of line-element by. Selander, $E$. Helsingf. Öfv. 34 (1892) 203-.

formula $y=\sin (A+B+C+\ldots)$, deduction from. Griffiths, J. L. Mth. S. P. 15 (*1883-84) 78-.

- - = - - - (Griffiths). Cayley, A. L. Mth. S. P. $15(* 1883-84) 81$.

formulæ. Cayley, A. Mth. A. 12 (1877) 369-.

-. Torelli, G. A. Mt. 15 (1887-88) 67-.

as functions of modulus. Torelli, $G$. G. Mt. 12 (1874) 168-.

general forms. Scheibner, $W$. Leip. Mth. Ps. B. 43 (1891) 575-.

geometrical, analytical and dynamical significance. Oekinghaus, E. Arch. Mth. Ps. 1 (1884) 337-; 4 (1886) 279 -

and hyperelliptic integrals. Neumann, $C$. [1870] Mth. A. 3) 1871) 611-.

- - Oekinghaus, E. Arch. Mth. Ps. 11 (1892) 132-.

with imaginary modulus. Hermite, - [1890] Hamb. Mth. Gs. Mt. 3 (1900) 22-.

- parameters. Booth, J. Ph. Mg. 7 (1854) 213-.

in indeterminate form, two. Darboux, $G$. Bordeaux S. Sc. Mm. 3 (1880) 373-.

integrals formed from, and their applications. Torelli, G. G. Mt. 11 (1873) 17-.

integration in finite form. Ptaszycki, J. [1888] Bll. Sc. Mth. 13 (1889) 65-.

inversion. Pasch, M. Mth. A. 19 (1882) 155-.

一. Halphen, G. H. Par. Éc. Pol. J. 54 (1884) 171-.

-, Cauchy's method. Didon, $F$. Liouv. J. Mth. 14 (1869) 230-.

- and irreducibility of periods. Picard, $\dot{E}$. Bll. Sc. Mth. 14 (1890) 107-.

- problem. Tikhomandritskǐ , M. A. Kharkov Mth. S. Com. (xII) (1883) 47-; (1884) 187-; Mth. A. 22 (1883) 450-; 25 (1885) 197-.

- problems. Lindemann, $F$. Münch. Ak. Sb. 28 (1899) 37-.

of 1st kind, application of Abel's theorem. Burnside, $W$. Mess. Mth. 21 (1892) 164-.

- - , calculation. Radau, R. Bll. As. 4 (1887) 99-

- _ - , - Legendre's scale. Newman, $F . W$. B. A. Rp. (1876) (Sect.) 28-.

- - - expansion, and a series of integers. Catalan, $E$. Brux. Ac. Mm. 46 (1886) (No. 4) $24 \mathrm{pp}$.

- - - - in trigonometric series. Beaupain, J. Liège S. Sc. Mm. 1 (1899) No. 4, 22 pp.

$-\ldots$, Gauss's approximation. Freeman, $A$. Mess. Mth. 5 (1871) 245-.

- - - illustration by certain link-work. Emch, A. A. Mth. 1 (1900) 81-. 
of 1st kind, infinitely many normal forms. Klein, C. F. Münch. Ak. Sb. 10 (1880) 533-. - - - interpretation, geometric. Mansion, - Brux. S. Sc. A. 13 (1889) (Pt. 1) 47-.

- - - inversion. Kluyver, J. C. N. Arch. Wisk. 4 (1899) 109-; Fschr. Mth. (1899) 400. - _ - new transcendental transforming into a circular function. Iaggi, E. N. A. Mth. 19 (1900) 537-.

- - - normal forms of 3rd and 5th degree. Bianchi, L. Mth. A. 17 (1880) 234-.

- _ - , representation, geometric. Morgan, $A$. Edinb. Mth. S. P. 15 (1897) 2-.

- - - transformation of integrals of irrational differentials to normal form. Steen, A. Kjöb. Skr. 8 (1870) 183-.

- — and 2nd kinds, differential equations satisfied by moduli. Fuchs, L. Crelle J. Mth. 112 (1893) 156-.

- - - - - , series for calculating, etc. Gudermann, C. Crelle J. 16 (1837) 366-; 17 (1837) 382-.

- -, 2nd and 3rd kinds, solution of

$$
\int \frac{x^{a} d x}{\sqrt[3]{\left(a+b x+c x^{2}\right) \beta}}
$$

by. Simony, $O$. Arch. Mth. Ps. 55 (1873) 193-. - 2nd kind. Thomae, C. J. Crelle J. Mth. 93 (1882) 69-; 94 (1883) 241-; Z. Mth. Ps. 27 (1882) 179-.

- - - functions inverse to. Glaisher, J.W. L. Camb. Ph. S. P. 5 (1886) 372-. - _ - representation, mechanical. Darwin, G. H. Mess. Mth. 4 (1875) 113-.

- - and 3rd kinds, expression by $\theta$ functions. Pokorny, M. Z. Mth. Ps. 10 (1865) 317-.

- 3rd kind. Newman, F. W. Camb. and Dubl. Mth. J. 8 (1853) 190-.

- - Hermite, C. C. R. 94 (1882) 901-.

- - - Kummell, C. H. A. Mth. 2 (188586) 73-, 104-.

- - Weyr, E. [1891] Prag České Ak. Fr. Jos. Rz. (Tŕida 2) 1 (1892) Art. 6, 9 pp. - - - Mansion, -. Brux. S. Sc. A. 21 (1897) (Pt. 1) 15-.

$--\frac{1}{-}$ formula. Cayley, A. L. Mth. S. P. 13 (1882) 175-.

- - -, formulæ, complementary moduli. Salvert, F. de. C. R. 120 (1895) 1208-.

- - - reducible to 1st kind. Saalschiltz, $L$. Z. Mth. Ps. 34 (1889) 199-.

- - - reduction. Gudermann, C. Crelle J. 14 (1835) 169-, 185-.

linear transformation. Burnside, W. Mess. Mth. 21 (1892) 170-.

of 1 st and 2nd order, relation between. Childe, (Rev.) G. F. Mess. Mth. 19 (1890) 155-. periods. Bruns, H. Mth. A. 27 (1886) 234 -. Jamet, V. N. A. Mth. 10 (1891) 193-. and polynomials of 4 th degree. Tannenberg, $W$. de. Bordeaux S. Sc. Mm. 3 (1899) 277-. properties, geometric. Booth, J. Phil. Trans. (1852) 311-; (1854) 53-.

in real form, reduction. Scheibner, $W$. [1879-80] Leip. Mth. Ps. Ab. 12 (1883) 57-, i-.

reduction. Genocchi, A. [1864] A. Mt. 6 (1864) 5-; Tor. Lav. Sc. Fis. Mt. (1869) 39-. reduction. Kluyver, J. C. C. R. 116 (1893) 48- to. Aronhold, S. $H$. Berl. Mb. (1861) 462-.

- - canonical form. Heine, H. E. [1855] Crelle J. 53 (1857) 199-.

- of definite integrals to. Lindman, C. $F$. Stockh. Öfv. 36 (1879) No. 6, 9-.

- - integral, containing square root of quartic, and calculation of modulus. Alexéeff, $N$. C. R. 59 (1864) 244 .

- to Jacobi's functions. Greenhill, A. G. QJ. Mth. 18 (1882) 66-.

- normal form. Vorsteher, E. Z. Mth. Ps. 32 (1887) 145-.

$-\frac{1}{651-}$.

651 - Stieltjes, T. J.

relating to continued fractions. Steen, A. (xII) Ts. Mth. 3 (1873) 151-.

representation by curvilinear arcs. M'Covan, J. Edinb. Mth. S. P. 9 (1891) 55-.

- of functions by

$F(x)=\Sigma\left(A_{p} \cos p\right.$ am $\frac{2 K x}{\pi}+B_{p} \sin p$ am $\left.\frac{2 K x}{\pi}\right)$. Gyldén, J. A. H. C. R. 92 (1881) 213.

-, geometric. Catalan, E. C. Liége S. Sc. Mm. 15 (1888) No. 1, 68-

and series. Catalan, E. C. Liège S. Sc. Mm. 13 (1886) 119-, 399.

system, as functions of absolute invariant. Pincherle, S. Rm. R. Ac. Linc. Rd. 7 (1891) (Sem. 1) 74-.

theory. Unferdinger, $F$. Arch. Mth. Ps. 54 (1872) 459-.

-. Cayley, A. Mth. A. 12 (1877) 143-.

-, elementary. Lerch, $M$. Par. Éc. Norm. A. 6 (1889) 263-

-, new. Weiler, A. Grunert Arch. 35 (1860) 408-.

transformation. Cayley, A. Camb. and Dubl. Mth. J. 5 (1850) 204-; Crelle J. 55 (1858) 15-.

- to normal form, and substitutions of 1st order. Richelot, $\boldsymbol{F} . J$. Crelle J. 34 (1847) 1-.

- - Weierstrass's form. Pittarelli, G., \& Brioschi, -. Rm. R. Ac. Linc. Rd. 4 (1888) (Sem. 1) 703-.

Weierstrass's. Fiske, T. S. [1891] A. Mth. 6 (1891-92) 7-.

$\int \frac{y d y}{\left(y^{3}+8\right) \sqrt{y^{3}-1}} \cdot$ Clausen, T. As. Nr. 19 (1842) 177-.

$\int d x \div \sqrt{(x+a)(x+b)}(x+c)$, geometrical representation. Cayley, A. Ph. Mg. 5 (1853) 281-.

$\int d x \div \sqrt{(m-x)(x+a)(x+b)(x+c)}$. Cayley, $A$ Ph. Mg. 6 (1853) 103-. $\int(\sin a m u)^{2 r} d u$, reduction. Thomae, C. J.
Crelle J. Mth. 81 (1876) 81-.

$\int \frac{\mathrm{dn}^{4} u}{\left(1-n \operatorname{sn}^{2} a\right)^{2}} \mathrm{~d} u$. Hermite, C. [1880] As. Nr. 101 (1882) 17-。 
$\int \frac{\sqrt{1+x^{4}}}{1-x^{4}} d x$, Euler's reduction to integral of rational function. Hermite, $C$. Liouv. J. Mth. 6 (1880) 5-.

,-----1 - (Hermite). Bounia. kowsky, V. St Pét. Ac. Sc. Bll. 26 (1880) 188-.

$E$ and $F$, indefinite integrals containing. Bierens de Haan, D. B. A. Rp. (1883) 440-.

$d x / \sqrt{X}$, transformation. Stieltjes, T.J. Toul. Fac. Sc. A. 2 (1888) K, 26 pp.

Elliptic integration. Rawson, R. B. A. Rp. (1849) (Pt. 2) 4-.

- series, approximate evaluation by algebraic means. Cesáro, E. Mathesis 14 (1894) 177-.

- -transcendent identities, theorems in theory of numbers deduced from. Glaisher, J.W.L. B. A. Rp. 43 (1873) (Sect.) 10-.

- - relations. Glaisher, J. W. L. B. A. Rp. (1874) (Sect.) 15-.

- transcendents, representation of functions by. Hermite, C. [1880] A. Mt. $10(1880-82)$ 137-.

Equations algebraically integrable. Fuler, $L$. [1778] St Pet. Ac. Sc. N. Acta 13 (1802) 3-.

- containing both algebraic and transcendental functions, principle for simplification. Zehfuss, G. Schlömilch Z. 5 (1860) 210 -

Euler's equation. Laguerre, $E$. (x) Par. S. Phlm. Bll. 7 (1871) 270.

- -. Stieltjes, T. J. Bll. Sc. Mth. 12 (1888) 222-; C. R. 107 (1888) 617-.

- - Jamet, V. Bll. Sc. Mth. 19 (1895) 208-.

- -. Lacour, E. N. A. Mth. 18 (1899) 293-.

- - forms of integral. Lagierre, E. Par. S. Mth. Bll. 3 (1875) 101-.

- - - - - case. Hadamard, J. Bll. Sc. Mth. 20 (1896) 263-.

- - integration of an equation analogous to. Picard, É. C. R. 92 (1881) 506-.

- - , - by lines of curvature of ruled hyperboloid. Floquet, G. N. A. Mth. 14 (1875) 120 .

- - , - - - - - - - (Floquet). Escary, -. N. A. Mth. 15 (1876) 61-.

- _ and lines of curvature of ellipsoid. Adam, P. Par. S. Mth. Bll. 22 (1894) 205-.

Expansion of $\sin x$ and $\cos x$ in infinite series of factors. Duhamel, J. M. C. Liouv. J. Mth. 19 (1854) 121-.

- $-\sin$ and $\cos$ of $n \mathrm{am} \frac{2 K}{\pi} x$ in trigono. metric series. Lindeberg, $K$. $M$. Stockh. Öfv. 32 (1875) No. 8, 31-.

- $\operatorname{sn} x$. Harris, R. A. A. Mth. 4 (1888) 87-.

- $G / K, K / G, I / E, \ldots$ in ascending powers of $k^{2}$. Glaisher, J. W. L. Mess. Mth. 19 (1890) 164-.

Expansions in powers of $k^{2}-k^{2}$. Glaisher, J. W. L. Mess. Mth. 22 (1893) 109-.

VOL. I.
Expansions in series and application to elliptic functions. Schlömilch, O. [1854] Leip. Ab. Mth. Ps. 2 (1855) 395-.

- of theta functions in powers of argument. Glaisher, J. W. L. Camb. Ph. S. P. 6 (1889) 96-.

- $\quad \lambda(x), \mu(x)$ and their powers, with regard to modulus. André, $D$. Par. S. Mth. Bll. 6 (1878) 163-; Par. Éc. Norm. A. 8 (1879) 151-; 9 (1880) 107-.

- - $K^{\prime}, E^{\prime}, J^{\prime}, G^{\prime}$ in powers of modulus. Glaisher, J. W. L. Camb. Ph. S. P. 5 (1886) 184-, 232-.

- $K, I, G, E$ in powers of $k^{2}-k^{2}$. Glaisher, J. W. L. Mess. Mth. 19 (1890) 146-.

Formula of G. Fontené. Hermite, C. Bll. Sc. Mth. 20 (1896) 218-.

- - Hermite. Lipschitz, - . Crelle J. Mth. 100 (1887) 66-.

$-K E^{\prime}+K^{\prime} E-K K^{\prime}=\frac{\pi}{2} . \quad$ Glaisher, J. W. L. [1874] Mess. Mth. 4 (1875) 95-.

Formulæ of Jacobi's on functions of $k, k^{\prime}, K, K^{\prime}$ in $q$ series. Glaisher, J.W.L. Mess. Mth. 5 (1876) 174-.

-, systems for sn, en, dn of $u+v+w$. Johnson, W. W. L. Mth. S. P. 13 (1881-82) 97-.

- for sn $8 u$, en $8 u$, dn $8 u$, in terms of sn $u$. Glaisher, E. H. R. S. P. 32 (1881) 444; 33 (1882) 480-.

- $\mathrm{sn}, \mathrm{cn}, \mathrm{dn}$ of $u+v+w$. Glaisher, J. W. L. [1881] Mess. Mth. 11 (1882) 45-.

Functional images in Cartesians. Greenhill, A. G. QJ. Mth. 18 (1882) 231-, 346-.

Functions having algebraic addition theorem. Pszeborski, A. G. Fschr. Mth. (1896) 341-.

$G$-function, theory. Barnes, $E . W$. QJ. Mth. 31 (1900) 264-.

General elliptic element, transformations. Russell, $R$. L. Mth. S. P. 18 (1886-87) 48-.

- integral, integration. Lobatto, $R$. Crelle J. 10 (1833) 280-.

Geometrical application of a theorem of Jacobi. Humbert, G. Liouv. J. Mth. 1 (1885) 347-.

Gudermannian of $u$. Cayley, A. Ph. Mg. 24 (1862) 19-.

Hermite's $H$-product theorem. Cayley, $A$. Mess. Mth. 18 (1889) 104-.

Higher transcendents in mechanical problems. Russell, W. H. L. Mess. Mth. 7 (1878) 18-, 136- ; 8 (1879) 8-; 9 (1880) 40-.

Infinite series, exponents of which are contained in two quadratic forms. Jacobi, C. G. J. Crelle J. 37 (1848) 61-, 221-.

Integral of

$$
\begin{aligned}
\overline{\left(A+3 B x+3 C x^{2}+D x^{3}\right)^{\frac{2}{3}}} & d y \\
& +\frac{d y}{\left(A+3 B y+3 C y^{2}+D y^{3}\right)^{\frac{2}{3}}}=0 .
\end{aligned}
$$

MacMahon, P. A. C. R. 95 (1882) 831-.

Integrals of differential equations, satisfied by moduli of elliptic integrals. Fuchs, L. [1876] Crelle J. Mth. 83 (1877) 13-.

- - form $\sqrt{1+p \sin ^{2} x \cdot \cos ^{2} x}$ etc. Bierens de Haan, D. Amst. Ak. Vh. 21 (1881) (No. 6) 50 pp. 
Integrals, reduction to elliptic forms. Martin, Art. Mess. Mth. 4 (1875) 179-; 6 (1877) 28-; 7 (1878) 24-.

Integration, complete, of

$$
\frac{x+C_{1}}{x+C_{2}} \cdot \frac{d x}{\sqrt{x^{4}+A x^{3}+B x^{2}+C x+D}},
$$

special cases. Buniakovskij, $V$. St Pet. Ac. Sc. Mm. (Rs.) 3 ("1863) (Suppl.) No. 2, 32 pp.

- of irrational fractions. Perevošcikov, D. M. St Pet. Ac. Sc. Mm. (Rs.) $16\left(^{*} 1870\right)$ 1-.

Integrations over surface of ellipsoid. Tallqvist, H. Helsingf. Acta 18 (1891) 329-。

Invariants of $\rho(u)$ when $\frac{2 \omega_{2}}{2 \omega_{1}}=\sqrt{-7}$. Kluyver, J. C. N. Arch. Wisk. 3 (1898) 36-.

Inversion of algebraic integral. Dillner, $G$. Stockh. Öfv. (1885) No. 4, 3-; Fschr. Mth. (1885) 378.

Jacobian equation. Sterba, J. Crelle J. Mth. 122 (1900) 198-.

- functions with 1 argument, theory. - Brioschi, F'. G. Mt. 2 (1864) 8-, 33-, 129-. - -, expansion in powers of variable. Brioschi, F. Tortolini A. 1 (1858) 41-.

Kleiber's functions $K$ and $G$. Glaisher, J. W. L. Mess. Mth. 22 (1893) 71-.

Kronecker's double series $(\xi, \eta, u, v, w)$. Lerch, M. Mh. Mth. Ps. 5 (1894) 367-.

Lambert's series. La Vallée Poussin, C. J. de. Brux. S. Sc. A. 20 (1896) (Pt. 1) 56-.

Lamé's equation. Brioschi, F. C. R. 85 (1877) 1160-; 86 (1878) 313-.

-- Hermite, C. [1877] A. Mt. 9 (1878-79) 21-.

- - Lindemann, C. L. F. B. A. Rp. (1883) 351-.

- - Greenhill, A. G. [1888] L. Mth. S. P. 20 (1889) 213-.

- - Floquet, G. C. R. 121 (1895) 805-, 920.

- -. Markoff, A. Mth. A. 47 (1896) 598-.

- - integration. Hermite, C. [1879] Crelle J. Mth. 89 (1880) 9-.

- -, new application. Gyldén, J.A. H. C. R. 93 (1881) 537-.

- -, solution. Crawford, L. QJ. Mth. 27 (1895) 93-.

- - - factors. Crawford, L. QJ. Mth. 29 (1898) 196-.

- -, special case. Stenberg, E. A. Acta Mth. 10 (1887) 339-.

,--- - Jamet, V. C. R. 111 (1890) 638 -

- theorems. Brioschi, F. C. R. 92 (1881) 325-.

Lamé-Hermite differential equation. Brioschi, F. St Pét. Ac. Sc. Bll. 35 (1894) 449-.

Legendre's " Fonctions elliptiques," revision of chaps. xxiv and xxvi. Greenhill, A.G. QJ. Mth. 19 (1883) 225-.

Lemniscate function, representation, geometric. Krimphoff, $W$. Crelle J. Mth. 110 (1892) 73-.

- - sn $u$, multiplication. Schwering, $K$. Crelle J. Mth. 107 (1891) 196-.
Lemniscate functions, expansion coefficients. Hurwitz, A. Gött. Nr. (1897) 273-; Mth. A. 51 (1899) 196-.

Linear differential equations. Stenberg, E. A. Helsingf. Acta 16 (1888) 333-.

- - - with doubly periodic coefficients. Malmborg, M. Ups. Årsk. (1897) (Mt. Nt.) $31 \mathrm{pp}$.

- homogeneous differential equations, with doubly periodic coefficients. Stenberg, E. A. Helsingf. Acta 16 (1888) 493-, 555-; 19 (1893) No. 11, $7 \mathrm{pp.}$

Periodic equation. Rodrigues, J.M. G. Teix. J. Sc. 6 (1885) 191-.

- function of one variable with more than two periods impossible, theorem of Jacobi. Casorati, $F$. Mil. I. Lomb. Rd. 15 (1882) 623-.

- functions. Laurent, $H$. N. A. Mth. 6 (1867) 267-.

- -, generalisation. Picard, Ĺ. C. R. 89 (1879) 140-.

- - of 2 nd kind. Rausenberger, O. Mth. A. 20 (1882) 550-.

- - theory, fundamental theorem. Pringsheim, A. [1900] Münch. Ak. Sb. 30 (1901) 541-.

- - uniform, of not more than two periods. Gade, $K$. (xII) Ts. Mth. 5 (1881) 181-.

Problem of Abel, complement to. Bougaief, C. R. 113 (1891) 1025-.

proposed by Hermite, solution. Le Vavasseur, - Toul. Fac. Sc. A. 8 (1894) $\mathrm{G}, 3 \mathrm{pp}$.

Pseudo-elliptic integrals. Goursat, $\dot{E}$. Par. S. Mth. Bll. 15 (1887) 106-.

- Halphen, G. H. C. R. 106 (1888) 1263-.

_-. Burnside, W. Mess. Mth. 22(1893) 83-. - - Abel's. Dolbnia, I. P. Kazan S. Nt. (P8.-Mth.) P. 8 (1890) 252-; Liouv. J. Mth. 6 (1890) 293-.

- - - depending on cube root of trinomial. Dolbnia, J. Bll. Sc. Mth. 17 (1893) 125-.

_-_ _ - - radicals of 4 th and 6 th degrees. Dolbnia, J. Bll. Sc. Mth. 17 (1893) 288-.

- _ - and their dynamical applications. Greenhill, A. G. [1893] L. Mth. S. P. 25 (1894) 195-.

- evaluation. Gunther, S. Par. S. Mth. Bll. 10 (1882) 88.

$q$-formula leading to expression for $E_{1}$. Cayley, A. Mess. Mth. 6 (1877) 63-.

- for sine-amplitude, method of obtaining. Glaisher, J. W. L. [1879] L. Mth. S. P. 11 (1879-80) 45-.

$q$-series. Heine, H. E. Crelle J. 32 (1846) 210-; 34 (1847) 285-.

- Catalan, E. Par. S. Mth. Bll. 19 (1891) 145-.

-, formula due to Jacobi. Catalan, E. C. Liége S. Sc. Mm. 15 (1888) No. 1, 32-.

- formulæ. Catalan, $E$. C. Liége S. Sc. Mm. 15 (1888) No. 1, 197-, 241-。

-, relation of Kronecker's. Igel, B. Mh. Mth. Ps. 8 (1897) 354-.

- for $\pi / 2 K$ and $2 G / \pi$, coefficients. Glaisher, J. W. L. QJ. Mth. 21 (1886) 60-. 
$q$-series for $k p$ sn $u, k p$ cn $u, p$ dn $u$, process for squaring. Glaisher, J. W. L. Mess. Mth. 16 (1887) 145-.

Quadratic forms, Kronecker's theorems. Hermite, C. Liouv. J. Mth. 9 (1864) 145-.

Quadrature of the ellipsoid. Carda, $K$. Mh. Mth. Ps. 7 (1896) 129-.

Quadri-quadric porism. Mathews, G. B. QJ. Mth. 30 (1899) 16-.

Rational functions of elliptic form. Pokrovskij, P. M. [1900] Rec. Mth. (Moscou) 21 (1901) 387-; Fschr. Mth. (1900) 445.

Rectangular transformation, Wilkinson's. Cayley, A. L. Mth. S. P. 14 (1883) 222-. Reduction of $\frac{d u}{\sqrt{U}}$, when $U$ is of fourth order. Cayley, A. Camb. and Dubl. Mth. J. 1 (1846) 70 -.

Relation of Jacobi, arithmetical deduction. Lipschitz, R. Acta Mth. 7 (1885) 95-.

- Legendre. Kronecker, L. Berl. Ak. Sb. (1891) 323-, 343-, 447-, 905-.

Rotation of body, theory. Marcolongo, $R$. Palermo Cir. Mt. Rd. 7 (1893) 26-.

Segner's numbers. Catalan, E. C. Liége S. Sc. Mm. 15 (1888) No. 1, 113-.

Series representing the twelve elliptic and the four zeta functions. Glaisher, J. W. L. Mess. Mth. 18 (1889) 1-.

Sigma function. Tognoli, 0 . G. Mt. 25 (1887) $367-$.

- - general function satisfying equation for. Delisle, A. Mth. A. 30 (1887) 91-.

- - Weierstrass's fundamental theorem. Caspary, F. Crelle J. Mth. 96 (1884) 182-.

- functions. Loria, G. G. Mt. 31 (1893) 179-.

- - of several arguments, new method. Jahnke, E. Z. Mth. Ps. 37 (1892) 178-.

- - elliptic, as special case of hyperelliptic $\sigma$-functions. Bolza, O. N. Y. Am. Mth. S. T. 1 (1900) 53-.

- - even. Pascal, E. A. Mt. 23 (1895) 181-.

- - , - Brioschi's recursion formula for expansion. Bolza, O. Am. J. Mth. 21 (1899) 175-.

- - expression of orthogonal system by, and application to rotation. Jahnke, $E$. Liouv. J. Mth. 5 (1899) 155-

- - general, formation. Krazer, A. Mth. A. 33 (1889) 591-.

- functions and theta functions, Weierstrass's, properties. Lerch, M. Prag Sb. (1887) (Mth.-Nt.) 426-.

— series, Weierstrass's, numerical coefficients in. Haussner, R. Gött. Nr. (1894) 299-.

$\sigma(u)$, Weierstrass's, and allied functions. Kluyver, J. C. N. Arch. Wisk. 3 (1898) 80-; Fschr. Mth. (1897) 392.

Sine of amplitude, inverse and square, expansion in powers of the variable. Hermite, C. As. Fr. C. R. (1875) 131-.

Subsidiary elliptic function pm $(u, k)$. Griffiths, J. L. Mth. S. P. 15 ("1883-84)
Symmetric functions of $I, G, E$ in terms of Q. Glaisher, J. W. L. Mess. Mth. 21 (1892) 65-.

Theorem of Dirichlet. Cayley, A. Camb. and Dubl. Mth. J. 9 (1854) 163-.

- Poncelet on $\sqrt{a+b x+c x^{2}}$. Sylvester, J. J. Ph. Mg. 20 (1860) 307-.

\section{THETA FUNCTIONS.}

attached to plane algebraic curve without singularities. Pick, G. Wien Ak. Sb. 94 (1887) (Ab. 2) 739-.

breaking up into products of such functions, after transformation. Wiltheiss, E. Mth. A. 26 (1886) 127-.

of class 3. Thomae, J. Leip. Mth. Ps. B. 39 (1887) 100-.

connexion with elliptic integrals. Scheibner, $W$.

Leip. Mth. Ps. B. 41 (1889) 86-, 245-.

constant factor in expression of $\Theta(x)$ as an infinite product. St Germain, A. de. [1873] Par. S. Mth. Bll. 2 (1874) 62-.

differential equation and expansion. Wiltheiss, E. Mth. A. 29 (1887) 272-.

elliptic constant $\theta(0)$. Thomae, $J$. Z. Mth. Ps. 11 (1866) 247-.

and eta functions, expansion by products. Presle, - de. Par. S. Mth. Bll. 15 (1887) 216-.

evaluation, and integrals of 2nd kind. Roch, G. Z. Mth. Ps. 11 (1866) 53-.

expression as definite integrals. Glaisher, $J$. W. L. [1877] Camb. Ph.S. P. 3 (1880) 61-。 - - - - series summation leading to. Glaisher, J. W. L. B. A. Rp. (1876) (Sect.) 15-; Camb. Ph. S. P. 6 (1889) 129-.

- of direction-cosines of two orthogonal systems of coordinates by. Kötter, $F$. Berl. Ak. Sb. (1895) 807-; Crelle J. Mth. 116 (1896) 213-. - - elliptic integrals of 2nd and 3rd kinds by. Roch, G. Z. Mth. Ps. 10 (1865) 317-.

- Euler's angles by. Caspary, F. Bll. Sc. Mth. 13 (1889) 89-.

- $\Theta(x)$ as definite integral. Glaisher, $J$. W. L. Mess. Mth. 5 (1876) 173; 17 (1888) $152-$

formula. Craig, T. Am. J. Mth. 5 (1882) 350-. -, fundamental, new demonstration. Kapteyn, W. Bll. Sc. Mth. 15 (1891) 125-.

- of Hermite. Humbert, G. [1880] Par. S. Mth. Bll. 9 (1881) 42-.

-, Jacobi's. . Gutzmer, A. Crelle J. Mth. 110 (1892) 177-.

-, -, geometrical proof. Baker, H. F. Mth. A. 43 (1893) 593-

formulæ, general. Krazer, A. Mth. A. 52 (1899) 369-.

-, Jacobi's. Kronecker, L. Crelle J. Mth. 102 (1888) 260-.

with fractional characteristics. Krause, $M$. Mth. A. 26 (1886) 569-.

integration by. Appell, P. C. R. 90 (1880) 1207-.

Jacobi's. Frenet, $F$. Bordeaux Mm. S. Sc. 8 (1870) 177-.

, theory. Herstowski, F. Mth. A. 11 (1877) 1-. 
logarithmic derivatives of higher order. Bertolani, G. G. Mt. 34 (1896) 135-.

products of 3 and 4 . Scheibner, $W$. Crelle J. Mth. 102 (1888) 255-.

representation of invariants. Bolza, $O$. Gött. Nr. (1887) 418-; Mth. A. 30 (1887) 478-.

- - motion of top. Marcolongo, R. G. Teix. J. Sc. 14 (1900) 169-.

- - quotient of two. Thomae, J. Mth. A. 6 (1873) 603-.

resolution into factors. Humbert, G. C. R. 126 (1898) 394-.

resultants and discriminants. Schlesinger, $O$. Mth. A. 33 (1889) 411-.

and sigma functions, logarithmic derivatives of higher order than 2nd. Bertolani, $G$. G. Mt. 33 (1895) 139-.

- - - and orthogonal systems. Caspary, F. Liouv. J. Mth. 6 (1890) 376-.

theorem, fundamental. Craig, T. [1891] J. H. Un. Cir. [11 (1891-92)] 42.

- -, Jacobi's. Caspary, F. C. R. 104 (1887) 1094-.

theorems. Lerch, M. Mh. Mth. Ps. 9 (1898) 177-.

theory. Enneper, A. Z. Mth. Ps. 12 (1867) 79-. —,exposition. Caspary,F. C.R.111 (1890) 225-.

-, illustration. Cayley, A. Mess. Mth. 7 (1878) 27-.

triple. Cayley, A. Mess. Mth. 7 (1878) 48-.

vanishing of. Riemann, [G. F.] B. [1865] Crelle J. 65 (1866) 161-.

- - even. Frobenius, G. Gött. Nr. (1888) 67-.

of one variable, algebraic identities used to obtain relations for. Caspary, $F$. Mth. A. 28 (1887) 493-.

- - - expression of orthogonal systems by. Caspary, F. $\quad$ C. R. 107 (1888) 859-, 934.

- - with fractional characteristics. Vos8, R. Arch. Mth. Ps. 4 (1886) 385-.

zero value. Cayley, A. Crelle J. Mth. 100 (1887) 87-.

for zero values, relations of derivatives. Pascal, E. A. Mt. 24 (1896) 23-

and zeta functions, values for certain values of argument. Glaisher, J.W.L. R. S. P. 29 (1879) 351-.

Theta products, relations. Gordan, $P$. [1865] Crelle J. 66 (1866) 185-.

- - , linear. Krazer, A. Acta Mth. 17 (1893) 281-.

- series, analogy, and some special Heine's hypergeometric series. Lerch, M. [1893] Prag České Ak. Fr. Jos. Rz. (Třida 2) 3 (1894) Art. 5, 10 pp.; Fschr. Mth. (1893-94) 794.

- - , and connexion with double integrals. Lipps, G. F. Leip. Mth. Ps. B. 44 (1892) 340-, 473-.

- - constant factors. Frobenius, $G$. Crelle J. Mth. 98 (1885) 244-.

- Klein, $F$. L. Mth. S. P. 20 (1889) 235-.

--series, convergence. Krazer, A. Mth. A. 49 (1897) 400-.
Transcendental functions. Bidone, $G$. Tor. Mm. Ac. 23 (1818) 295-; 24 (1820) 255-.

- - Jacobi, C. G. J. Ás. Nr. 6 (1828) 33-; Crelle J. 3 (1828) 376-; 15 (1836) 199-.

- Clausen, T. As. Nr. 19 (1842) 181-.

- -, calculation. Petersen, J.P.C. (xII) Ts. Mth. 6 (1876) 1-.

- -, comparison, theory. Ellis, R. L. B. A. Rp. (1846) 34-.

- - and their Fourier developments. Kry gowski, Z. Prace Mt.-Fiz. 5 (1894) 70-.

- - having property

$$
f(z+\omega)=f(z), f\left(z+\omega^{\prime}\right)=f(z), S(z),
$$

$S(z)$ denoting a doubly periodic function. Picard, $\dot{E}$. C. R. 86 (1878) 657-.

- -, representation by arcs of curves. Allégret, $[A]$. Par. Ec. Norm. A. 2 (1873) 149-.

- space-coordinates, class. Staude, 0 . Acta Mth. 10 (1887) 183-.

Transformation formula

$$
y=\sin (L+A+B+C+\ldots),
$$

some consequences. Griffiths, $J$. L. Mth. S. P. 17 (1887) 11-.

Values of integrals in terms of $k^{2}$. Glaisher, J. W. L. Mess. Mth. 22 (1893) 145-.

Weierstrass's equation of three terms, generalisation. Morley, F. N. Y. Am. Mth. S. Bll. 2 (1896) 21-.

- formula. Picard, É. Bll. Sc. Mth. 24 (1900) 30-.

- formulæ applied to binary quartic and ternary cubic. Baker, H. F. QJ. Mth. 24 (1890) 1-. al $(x)$ functions. Cayley, $A$. Liouv. J. Mth. 7 (1862) 137-

- - - expansion in powers of variable. André, D. Liouv. J. Mth. 5 (1879) 31-.

Zeta-function. Glaisher, J. W. L. [1885-86] Camb. Ph. S. P. 5 (1886) 221-; Mess. Mth. 15 (1886) 92-.

_ - complex, map; a condenser problem. Michell, J. H. Mess. Mth. 23 (1894) 72-.

- - , process of squaring. Glaisher, J.W.L. Mess. Mth. 16 (1887) 150-. $\int \frac{f x d x}{\sqrt{ \pm\left(1-x^{8}\right)}}$, reduction to elliptic integrals.
Richelot, A. Crelle J. 32 (1846) 213-.

$\int_{0}^{\frac{\pi}{2}} \log \left(\frac{1}{\Delta}\right) \frac{d \phi}{\Delta^{3}}, \int_{0}^{\frac{\pi}{2}} \frac{E(k \phi) \sin \phi \cos \phi d \phi}{1-k^{2} \sin ^{2} \phi}$ etc. Roberts, $W$. Liouv. J. Mth. 11 (1846) 343-. $V=\int \frac{T d x}{\sqrt{X}}$, reduction to elliptic integral of trigonometric form. Plana, G. Crelle J. 36 (1848) 1-.

$\frac{d x}{\sqrt{\bar{X}}}+\frac{d y}{\sqrt{\bar{Y}}}=0$. Cayley, A. I. Mth. S. P. 8 (1877) 184-.

- = - integration formulø. Cayley, A. [1878] Mess. Mth. 8 (1879) 60-.

$-=-$, Richelot's integral. Cayley, A. Mess. Mth. 23 (1894) 42-.

$\int \frac{d x}{\left(1-x^{3}\right)^{\frac{2}{3}}}$, reduction to elliptic integral.

Cayley, A. Mess. Mth. 11 (1882) 142-. 
$p$ dn $u$, squaring. Fierrers, N.M. Mess. Mth. 16 (1887) 189-.

$\left(\frac{d u}{d y}\right)^{2}=G^{2}(u-a)(u-b)(u-c)(u-d)$, integration, new method. Kapteyn, $W$. Par. Éc. Norm. A. 9 (1892) 35-.

$\int \frac{d x}{\sqrt[4]{x^{4}+p x^{2}+q}}$, expression by logarithms. Dolbnia, I. A. Bll. Sc. Mth. 19 (1895) 76-; Rec. Mth. (Moscou) 18 (1896) 108-.

$\theta\left[u^{(i)}(z)-G_{i}\right]$, resolution into factors. Lacour, E. Par. Éc. Norm. A. 13 (1896) 415-.

\section{Multiplication, division, transformation of elliptic functions; modular functions. (See also 4440.)}

Algebra, transcendental, researches. Franchini, P. Mod. S. It. Mm. 16 (1813) 223-; 17 (1815) 262-.

Bisection. Igel, B. Crelle J. Mth.119(1898) 50-. Class-invariants. Mathews, G. B. [1890] L. Mth. S. P. 21 (1891) 234-.

- numbers of binary quadratic forms, relations. Hurwitz, A. Mth. A. 25 (1885) 157-.

Congruence groups of 6 th order. Fricke, $R$. Mth. A. 29 (1887) 97-.

Determinants formed from elliptic functions. Lerch, M. Prag České Ak. Fr. Jos. Rz. (Třida 2) 2 (1893) Art. 5, 5 pp.; Fschr. Mth. (1893-94) 790.

\section{DIVISION.}

Abelian relations for. Engel, $F$. Leip. Mth. Ps. B. 36 (1884) 32-.

algebraic equations determining. Kronecker, $L$. Berl. Ak. Mb. (1875) 498-

of argument. Emmanuel, D. [Bucarest S. Sc. Bll. 6 (1897)] 20-.

differential equation satisfied by roots of equations for. Migotti, A. Wien Ak. Sb. 94 (1887) (Ab. 2) 748-.

of lemniscate. Liouville, J. C. R. 17 (1843) 635-.

- - Kohl, E. Wien Ak. Sb. 98 (1890) (Ab. 2a) 364-.

- Mathews, G. B. L. Mth. S. P. 27 (1896) 367-.

- - Ripa, P. G. Mt. 38 (1900) 7-.

- - equation, irreducibility, etc. Eisenstein, G. Crelle J. 39 (1850) 160-, 224-.

,--- , separation into four factors. Schwering, K. Crelle J. Mth. 110 (1892) 42-; 112 (1893) 37-.

- - equations, resolution. Schwering, $K$. Crelle J. Mth. 111 (1893) 170-.

- periods. Brioschi, F. Mil. I. Lomb. Rd. 26 (1893) 727-; 27 (1894) 186-.

- - algebraic equations connected with. Palmstr $\phi m$, A. Berg. Ms. Aarb. (1897) No.4, $17 \mathrm{pp}$.

- - , resolvent of algebraic equation determining. Kiepert, L. Gött. Nr. (1885) 257-. of periods by 7, forms of equation determining. Palmstr $\phi$, A. Arch. Mth. Ntvd. 19 (1897) No. $5,17 \mathrm{pp}$.

- _ - Galois's resolvent. Halphen, G. $H$. C. R. 108 (1889) 476-.

,---- - Brioschi, F. C. R. 109 (1889) 520-.

- by 9 . Burnside, $W$. Mess. Mth. 22 (1893) 89-.

- theta-function. Klein, C. F. Mth. A. 17 (1880) 565-.

Doubly periodic functions. Cayley, A. Liouv. J. Mth. 10 (1845) 385-; 19 (1854) 193-.

- - - rational functions analogous to. Vasīl'ev, A. V. (xII) Kazan Un. Mm. [16] (1880) (Pt. 2) 121-.

Elliptic formulæ of Jacobi, demonstration. Somoff, J. [1851] St Pét. Ac. Sc. Bll. 10 (1852) 65-

一 functions. Klein, F. D. Nf. B. (*1877) 104. - -, expansion, method. Schlömilch, $O$. Leip. B. (1853) 25-.

,--- in series. Broch, O.J. [1863] Sk. Nf. F. 9 (1865) 146-.

- -, theory. Krause, M. Mth. A. 12 (1877) 1-. - - - - Rausenberger, $O$. Crelle J. Mth. 93 (1882) 328- ; 94 (1883) 251-.

- - - - Weierstrass, C. T. W. Berl. Ak. Sb. (1882) 443-; (1883) 193-, 265-, 1271-.

- - sn nu. Caylcy, A. Crelle J. 37 (1848) $58-$.

- - of $\frac{1}{3} K$. Forsyth, A. R. Mess. Mth. 12 (1883) 134-.

- - - Glaisher, J. W. L. Mess. Mth. 20 (1891) 191-.

- -1 , etc. Burnside, W. Mess. Mth. 12 (1883) 154-.

- integral, 1st kind, expansion, cases. Catalan, E. C. As. Fr. C. R. 6 (1877) 214-.

- integrals, 1st and 2nd kinds, expansion in recurring series. Farkas, J. C. R. 92 (1881) 181-.

A. Mt. 2 (1868-69) 97-.

- -, theory. Newman, F. W. R. S. P. 9 (1857-59) 704-.

- normal curves and connected modular functions. Klein, F. Leip. Mth. Ps. Ab. 13 (1887) 337-.

Equation between 2 transcendental functions, demonstration. Enneper, A. QJ. Mth. 1 (1857) 272-.

Equations, 5th order, equations of icosahedron in solution of. Klein, C. F. Mil. I. Lomb. Rd. 10 (1877) 253-.

Formulæ for sn $9 u$. Sudo, O. Tōk. Coll. Sc. J. 7 (1895) 283-.

- - sn 10u, en $10 u, \operatorname{dn} 10 u$ in terms of sn $u$. Sakai, E. Tök. Coll. Sc. J. 7 (1895) 285-.

Functions with several periods not interchangeable. Rausenberger, O. Mth. A. 20 (1882) 47-.

Gauss's pentagramma mirificum. Schlesinger, L. Mth. Termt. Éts. 17 (1899) 526-; Mth. Nt. B. Ung. 17 (1901) 20-. 
Hermite's $\phi$-function, linear transformation. Königsberger, -. Mth. A. 3 (1871) 1-.

Integral occurring in Cauchy's transformation of elliptic integral. Lerch, $M$. D. Nf. Vh. (1894) (Th. 2, Hälfte 1) 8-.

Jacobian functions. Frobenius, G. Crelle J. Mth. 97 (1884) 16-, 188-.

- generalisation of formula. Hoppe, $R$. Arch. Mth. Ps. 70 (1884) 400-.

Kleinian functions of 3rd family, generalisation. Cassel, G. Stockh. Öfv. (1890) 237-; Fschr. Mth. (1890) 410.

Landen's substitution. Koláček, $F$. Časopis 18 (1889) 21 ; Fschr. Mth. (1889) 495.

Linear equation connecting complete function of 1 st kind with modulus. Tannery, J. C. R. 86 (1878) 811-.

Par. S. Mth. Bll. $\overline{10} \overline{(1882)} \overline{44}$.

Modular correspondences of 7 th kind. Fricke, R. Gött. Nr. (1892) 272-.

\section{Modular equations.}

Schröter, H. E. Crelle J. 58 (1861) 378-.

Jordan, C. C. R. 66 (1868) 308-.

Smith, H. J. S. Rm. R. Ac. Linc. Mm. 1 (1877) 136-.

Klein, C. F. Mil. I. Lomb. Rd. 12 (1879) $21-$. Pick, G. Wien Ak. Sb. 91 (1885) (Ab. 2) 138-. Russell, R. [1890] L. Mth. S. P. 21 (1891) 351-. Brioschi, F. Rm. R. Ac. Linc. Rd. 2 (1893) (Sem. 2) 185-; 5 (1896) (Sem. 2) 333-.

Pierpont, J. N. Y. Am. Mth. S. Bll. 3 (1897) 279 .

and application to theory of numbers. Krause, M. Mth. A. 12 (1877) 419-.

class. Brioschi, F. C. R. 112 (1891) 28-. and curves, singularities. Smith, H.J.S. L. Mth. S. P. 9 (1877-78) 242-.

of 8th degree, new form. Brioschi, F. Am. J. Mth. 13 (1891) 381-.

- - - property of reduced form. Brioschi, F. Rm. R. Ac. Linc. Rd. 1 (1885) 514-, 583-

- - - reduction. Hermite, C. Tortolini A. 2 (1859) 59 .

- 12th degree, resolvent of 11 th degree. Klein, C. $F$. Rm. R. Ac. Linc. T. 3 (1879) 177-.

discriminants. Krause, $M$. Mth. A. 8 (1875) 539-; 9 (1876) 554-.

equations analogous to. Brioschi, F. C. R. 47 (1858) 337-.

- - Joubert, P. C. R. 47 (1858) 341-.

form in transformation of 3rd order. Cayley, A. Mess. Mth. 12 (1883) 173-

Galois's group. Gierster, $J$. Leip. Mth. Ps. B. 37 (1885) 291-; Mth. A. 26 (1886) 309-.

- - sub-groups in case of a transformation of prime degree. Gierster, J. Mth. A. 18 (1881) 319-

irrational. Fiedler, $E, W$. Zür. Vjschr. 30 (1885) 129-

reduction: Betti, E. Tortolini A. 4 (1853) 81-.

- of degree. Sylow, L. [1870] Christiania F. 13 (1871) 387-.

resolution, cases. Morera, G. Mil. I. Lomb. Rd. 18 (1885) 629-. theory. Schröter, H. E. Liouv. J. Mth. 3 (1858) 258-.

- Hermite, C. C. R. 48 (1859) 940-, 1079-, 1095-; 49 (1859) 16-, 110-, 141-.

-, algebraic proofs. Igel, $B$. Mh. Mth. Ps. 3 (1892) 349-.

$\kappa \lambda-\kappa^{\prime} \lambda^{\prime}$. Russell, R. [1887] L. Mth. S. P. 19 (1889) 90 -.

\section{MODULAR FUNCTIONS.}

Klein, C. F. [1879] Münch. Ak. Sb. 10 (1880) 89-.

Rausenberger, $O$. Mth. A. 20 (1882) 45-.

Wirtinger, $W$. Mh. Mth. Ps. 1 (1890) 429-.

Cayley, A. Mth. A. 47 (1896) 1-.

Weber, $H$. Mth. A. 47 (1896) 6-.

Lewicki, $W$. Prace Mt.-Fiz. 8 (1897) 5-.

demonstration of Hermite's transformation tables. Schläfi, L. Crelle J. 72 (1870) 360-.

functions akin to. Stouff, $X$. Toul. Fac. Sc. A. 5 (1891) C, 16 pp.

and Gauss's theory of arithmetico-geometric mean. Schlesinger, L. Berl. Ak. Sb. (1898) 346-.

- integrals. Gudermann, C. Crelle, J. 18 (1838) 1-, 142-, 220-, 303-; 19 (1839) 45-, $119-, 244-; 20$ (1840) 62-, 103-;21 (1840) 240-; 23 (1842) 301-; 25 (1843) 281-; 41 (1851) 93-; [Latin version with preface and annotations by J. F. Stader] Tortolini A. 2 (1851) 557-; 3 (1852) 442- ; 5 (1854) 81-. - invariants, differential equations. Scheibner, $W$. Leip. Mth. Ps. B. 41 (1889) 331-.

of lowest degrees. Klein, F. Leip. Mth. Ps. B. 37 (1885) 70 -

and multiplicator equations of 1st degree, foundations of theory. Hurwitz, A. Mth. A. 18 (1881) 528-.

representation by infinite products. Mangoldt, H. von. Gött. Nr. (1885) 313-.

_- - and extension to their functions. Mangoldt, H. von. Gött. Nr. (1886) 1-.

special subgroups. Fricke, $R$. Mth. A. 31 (1888) 227-.

theory. Dienger, J. Grunert Arch. 11 (1848) 395- ; 13 (1849) 1-; 21 (1853) 344-; 22 (1854) $362-$.

-. Dedekind, $R$. Crelle J. Mth. 83 (1877) 265-. $\chi \omega . \quad$ Cayley, A. C. R. 116 (1893) 1339-.

Modular group. Lewicki, W. Mh. Mth. Ps. 11 (1900) 118-.

- - sub-groups of 11th class. Rougier, $J$. Mars. Fac. Sc. A. 6 (1897) Fasc. 1, 112 pp.

- groups, automorphic. Fricke, $R$. Gött. Nr. (1896) 91-.

- relations, new. Brioschi, F. [1866] Nap. At. Ac. 3 (1866-68) (No. 2) 16 pp.

- transformation of degree $p$. Frattini, $G$. Rm. R. Ac. Linc. Rd. 1 (1885) 142-, 166-, 884.

- - - 5th order. Invariant theory of its sextic equation. Gordan, P. A. Mt. 1 (1867-68) 367-.

- transformations, application of a theorem of Abel to solution of a geometrical problem. Roberts, W. C. R. 58 (1864) 709-. 
Moduli, Cauchy's theory, and two theorems. Piola, G., \& Frisiani, $P$. Opusc. Mt. Fis. 2 (1834) 49-, 173-, 301-.

- of functions, principal. Cauchy, A. $L$. C. R. 20 (1845) 546-.

Modulus of elliptic functions, and invariants of biquadratic binary forms, relations. Milller, $(D r) F^{\prime}$. Z. Mth. Ps. 18 (1873) 280 - - - integrals, variation. Bierens de Haan, D. B. A. Rp. (1877) (Sect.) 23-.

\section{MULTIPLICATION.}

Laguerre, E. [1877] Par. S. Mth. Bll. 6 (1878) 68-.

Frobenius, G., \& Stickelberger, L. [1879] Crelle J. Mth. 88 (1880) 146-.

Halphen, G. H. C. R. 88 (1879) 414-.

Novarese, $E$. Tor. Ac. Sc. At. 17 (1881) 723-.

Kronecker, L. Berl. Ak. Sb. (1883) 717-, 949-.

Fujisawa, R. Tōk. Coll. Sc. J. 6 (1894) 151-. and addition, geometric. Trudi, N. Nap. Rd. 2 (1853) 66-.

of argument, two partial differential equations relating to. Halphen, G. $H$. C. R. 88 (1879) 698-.

complex. Kronecker, L. Berl. Mb. (1857) 455-; (1862) 363-; Par. Éc. Norm. A. 3 (1866) 295-.

-. Greenhili, A. G. [1882] Camb. Ph. S. P. 4 (1883) 271-.

一. Pick, G. [1885] Mth. A. 25 (1885) 433-; 26 (1886) 219-.

-. Stuart, G. H. QJ. Mth. 20 (1885) 18-, 221 -

- Greenhill, A. G. Camb. Ph. S. P. 5 (1886) 4-; QJ. Mth. 22 (1887) 119-, 174.

-. Sylow, -. Liouv. J. Mth. 3 (1887) 109 -

- Weber, H. Mth. A. 33 (1889) 390-.

- Kiepert, L. Mth. A. 39 (1891) 145-.

-, geometric application. Kiepert, L. [1871] Crelle J. 74 (1872) 305-

-, with imaginary modulus. Cayley, A. L. Mth. S. P. 19 (1889) 300-.

-, moduli. Greenhill, A. G. [1888] L. Mth. S. P. 19 (1889) 301-.

-, -. Mathews, G. B. [1889] L. Mth. S. P. 21 (1891) 215-.

-, -, table. Greenhill, A. G. [1890] L. Mth. S. P. 21 (1891) 403-.

-, and quadratic forms. Franel, $J$. Mth. A. 48 (1897) 595-.

-, of theta functions. Scheibner, $W$. Leip. Mth. Ps. B. 40 (1888) 154-.

一, by $\sqrt{-23}$. Brioschi, F. A. Mt. 24 (1896) $335-$.

-, - - especially. Halphen, G. H. Liouv. J. Mth. 5 (1889) 5-.

and division of elliptic integrals. Küpper, $C$. Schlömilch Z. 7 (1862) 239-.

of doubly periodic functions of 2 nd kind. Krause, M. Leip. Mth. Ps. B. 42 (1890) 24 .

of elliptic functions of first kind. Baehr, G. F. W. Grunert Arch. 36 (1861) 125-.

formula, Jacobi's. Brioschi, F. Mil. I. Lomb. Rd. 1 (1864) 344 . formulæ. Cayley, $A$. Crelle J. 39 (1850) $16-$; 41 (1851) 85-.

- Hoffmann, H. Crelle J. 48 (1854) 332-.

- Brioschi, F. C. R. 59 (1864) 769-.

-, deduction of infinite double products from. Mittag-Leffler, G. Stockh. Ófv. 33 (1876) No. 6, 17-.

for integers, in connection with Poncelet's problem, etc. Simon, $M$. Crelle J. Mth. 81 (1876) 301-.

of 4 theta functions, formula for. Smith, H. J. S. L. Mth. S. P. $10(1878-79) 91-$.

and transformation. Sanio, J.T. Crelle J. 14 (1835) 1-.

- - new formulø in. Brioschi, F. A. Mt. 23 (1895) 73-.

of cn $u$, algebraic derivation. Runge, $C$. Crelle J. Mth. 94 (1883) 349-.

Multiplicator equations. Klein, C. F. [1878] Mth. A. 15 (1879) 86-.

- - of higher degree. Biedermann, P. Leip. Mth. Ps. B. 37 (1885) 201-.

- - Biedermann, $P$. Arch. Mth. Ps. 5 (1887) 1-.

Octavic equation having group of modular transformation, 7 th order. Brioschi, F. Mil. I. Lomb. Rd. 1 (1868) 68-.

Poncelet's polygons. Lelieuvre, M. Ens. Mth. 2 (1900) 410-.

Substitution, double, in elliptic functions, principle. Raabe, J. L. Crelle J. 15 (1836) 191-.

- groups for roots of $k^{2}(\omega)$. Fricke, R. Mth. A. 28 (1887) 99-.

-, linear, finite groups, in theory of elliptic transcendents. Hurwitz, A. Mth. A. 27 (1886) 183-.

,-- , for reduction of elliptic functions of 1 st kind. Tortolini, B. Tortolini A. 1 (1858) 57 -

Theorem of Legendre, extension. Malet, J.C. A. Mt. 11 (1882-83) 246-, 312-.

Theta-characteristics, groups. Frobenius, $G$. Crelle J. Mth. 96 (1884) 81-.

- - theory. Ameseder, A. Wien Ak. Sb. 93 (1886) ( $A b .2$ ) 618-.

- functions with complex characteristics. Craig, T. Am. J. Mth. 6 (1884) 337-.

\section{TRANSFORMATION.}

Cayley, A. Ph. Mg. 25 (1844) 352-; 27 (1845) 424.

Brioschi, F. Tortolini A. 8 (1857) 70-, 125-。

Casorati, F. Tortolini A. 8 (1857) 209-.

Brioschi, F. Tortolini A. 1 (1858) 175-.

Bjerknes, C. A. Christiania F. (1860) 152-.

Hertz, K. [1869] (xII) Rec. Mth. (Moscou) 4 (1869-70) (Pt. 1) 139-.

Cayley, A. [1873] Phil. Trans. 164 (1874) 397-.

Clifford, W. K. $[1875-76]$ L. Mth. S. P. 7 (1876) 29-, 225-.

Laguerre, E. [1876-77] $\quad$ C. R. 82 (1876) 1257-; Par. S. Mth. Bll. 6 (1878) 72-. 
Cayley, A. Phil. Trans. 169 (1878) 419-.

Klein, C. F. L. Mth. S. P. 9 (1877-78) 123-; $11(1879-80)$ 151-; Mth. A. 14 (1879) 111-.

Krause, M. Acta Mth. 3 (1883) 93-; Leip. Mth. Ps. B. 38 (1886) 39-.

Cayley, A. Am. J. Mth. 9 (1887) 193-; 10 (1888) 71-; QJ. Mth. 24 (1890) 259-.

Hermite, C. Palermo Cir. Mt. Rd. 5 (1891) 155-; Toul. Fac. Sc. A. 6 (1892) L, 13 pp.

Brodén, $T$. Lund. Un. Acta 30 (1893-94) ( $S$. Psgr., No. 2) 24 pp.

Brioschi, F. A. Mt. 22 (1894) 313-.

Beer, F. Arch. Mth. Ps. 14 (1896) 113-.

Krause, $M$. D. Mth. Vr. Jbr. 4 (1897) 121-.

Valle, G. Rm. N. Linc. Mm. 17 (1900) 81-.

algebraic, of doubly periodic functions. Veltmann, W. Z. Mth. Ps. (Suppl.) 29 (1884) 73-.

annihilator, $G$-function. Griffiths, J. L. Mth. S. P. 20 (1889) $248-$.

annihilators. Griffiths, J. L. Mth. S. P. 18 (1886-87) 164-, 377-.

case. Malet, J. C. [1873] QJ. Mth. 13 (1875) 278-.

-. Valle, G. Tor. Ac. Sc. At. 26 (1891) 236-.

of compound degree, modular equations in. Gierster, J. [1878] Mth. A. 14 (1879) 537-.

cubic. Cayley, A. Ph. Mg. 15 (1858) 363-; C. R. 64 (1867) 560-.

-, geometrical illustration. Cayley, A. QJ. Mth. 13 (1875) 211-.

-, Jacobian formula for. Faà de Bruno, (l'abbé) -. Am. J. Mth. 10 (1888) 169-.

where degree is composite. Kiepert, $L$. Mth. A. 32 (1888) 1-.

of 5 th degree, moduli and their complements useful for, relations between. Pokrovskir, P. M. [1881] (xII) Rec. Mth. (Moscou) 10 (1882-83) (Pt. 1) 63-

diagram. Cayley, A. B. A. Rp. (1881) 534.

differential equations satisfied by modulus and multiplier in. Valle, $G$. Tor. Ac. Sc. At. 25 (1890) 114-.

and division. Morera, $G$. Leip. Mth. Ps. B. 37 (1885) 302-; Mth. A. 25 (1885) 203-.

- - Kiepert, L. Mth. A. 26 (1886) 369-

- -. Greenhill, A. G. [1894] L. Mth. S. P. 27 (1896) 403-.

- - of 3rd order. Bolza, O. Mth. A. 50 (1898) 68-.

of doubly periodic functions, discriminant. Biermann, $O$. Wien Ak. Sb. $109(1900)(A b$. 2a) 849-.

- elliptic functions with imaginary modulus. Lacour, E. Par. Éc. Norm. A. 15 (1898) 455-.

- - - of 1st kind. Baehr, G. F. W. Grunert Arch. 33 (1859) 354-.

- $-\ldots$ 2nd and 3rd kinds, general theorem. Abel, N. H. Crelle J. 3 (1828) 402.

- - 3rd order. Hermite, C. [1861] Crelle J. 60 (1862) 304.

- - integrals. Brioschi, F. Tortolini A. 3 (1860) 216-. of elliptic integrals. Lerch, M. Casopis 13 (1884) 140-; Fschr. Mth. (1884) 402.

_- - application to spherical trigonometry. Russell, $R$. QJ. Mth. 20 (1885) 378-.

_- - of 2nd kind. Hermite, - Prag Ab. 2 (1888) No. 12, 7 pp.

- - new. Brioschi, F. Mil. I. Lomb. Rd. 1 (1864) 46-.

- _ - to Weierstrass's form. Pittarelli, $G$. Rm. R. Ac. Linc. Rd. 4 (1888) (Sem. 1) 703-.

equations, class. Westlund, J. Am. J. Mth. 21 (1899) 339-.

expression for number of classes. Seguier, - de. C. R. 118 (1894) 1407-.

formula. Brioschi, F. C. R. 79 (1874) 1065-; 80 (1875) 261-.

formulæ. Hermite, C. C. R. 46 (1858) 171-; Liouv. J. Mth. 3 (1858) 26-.

integral. Cayley, $A$. Camb. and Dubl. Mth. J. 3 (1848) 286-; QJ. Mth. 1 (1857) 4-.

invariant, of elliptic integrals. Raffy, $L$. Par. S. Mth. Bll. 12 (1884) 51-.

of Jacobi of elliptic functions $F$ when $p=3$, geometric construction. Roberts, $W$. (vir) A. Mt. 5 (1863) 225-.

Kiepert's $L$-equations in. Cayley, A. Mth. A. 30 (1887) 75-.

linear. Cayley, A. L. Mth. S. P. 8 (1877) 226-.

-. Rausenberger, O. Crelle J. Mth. 91 (1881) 335-.

-, of theta functions. Cayley, A. Mess. Mth. 13 (1884) 54-; QJ. Mth. 21 (1886) 77-.

-, - - - Kinn, G. A. [1900] Mth. Nt. B. Ung. 18 (1903) 52-.

-, - - constants. Thomae, C.J. Gött. Nr. (1883) 194-.

,---1, determination of constant factors in. Sochocki, J. Par. T. Nauk Śc. Pam. 10 ( $\left.{ }^{*} 1878\right)$ Art. 1, 37 pp. ; Fschr. Mth. $\left({ }^{*} 1878\right)$ 315.

-,- - and Gaussian sums. Landsberg, $G$. Crelle J. Mth. 111 (1893) 234-.

modular equation in. Valle, $G$. Tor. Ac. Sc. At. 24 (1889) 374-.

- equations for. Sohncke, L. A. Crelle J. 12 (1834) 178 ; 16 (1837) 97-.

-, Lagrange's. Brassinne, E. Toul. Mm. Ac. 6 (1850) 252-.

of modulus, deductions from formulæ. Meyer, U. H. Grunert Arch. 17 (1851) 85-.

and numerical calculation of elliptic integrals of 1st, 2nd, and 3rd kinds. Weiner, $I$. Brünn Vh. 5 (1866) (Ab.) 82-.

of 3rd order, modular equation for. Smith, H. J. S. L. Mth. S. P. $10(1878-79)$ 87-.

$-\ldots-,-\ldots$, geometrical interpretation. Humbert, G. Par. S. Mth. Bll. 26 (1898) 233-.

- 7th order. Klein, C. F. [1878] Mth. A. 14 (1879) 428-.

(Pt. 2) 211-.

- - - formation of reduced form of multiplicator equation. Joubert, (le père) C. (xII) Brux. S. Sc. A. 3 (1879) (Pt. 2) 157-. 
of 7 th order, modular equation for. Gützlaff, $C$. E. Crelle J. 12 (1834) 173-.

- - , - - , algebraic solution. Ely, G.S. L. Mth. S. P. 13 (1882) 153-

- 11th order. Klein, C. F. Mil. I. Lomb. Rd. 12 (1879) 629-; Mth. A. 15 (1879) 533-. - -. Brioschi, F. A. Mt. 21 (1893) 309

- - - modular equation for. Cayley, A. C. R. 111 (1890) 447-.

- periodic functions. Teixeira, J. P. Lisb. J. Sc. Mth. 3 (1895) 1-.

principle of Jacobi, extension. Königsberger, $L$. [1878] Crelle J. Mth. 87 (1879) 173-.

problem for elliptic function of 2nd kind, four connected solutions. Salvert, F. de. C. R. 118 (1894) 1181-.

- _ - 3rd kind, four connected solutions. Salvert, F. de. C. R. 118 (1894) 1403-.

- in representation leading to. Thomae, $J$. Z. Mth. Ps. 18 (1873) 401-.

-, solution. Abel, N. H. As. Nr. 6 (1828) 365-.

quadratic, of elliptic function. Gundelfinger, $S$. [1873] (VII) Mth. A. 7 (1874) 449-.

quadric, of elliptic integrals. Kummell, C. H. Smiths. Mise. Col. 33 (1888) Art. 2, 102-; (Wash. Ph. S. Bll. 7 (1885).)

quartic. Cayley, A. QJ. Mth. 12 (1873) 266-.

to reciprocal and to complementary modulus. Johnson, $W . W$. Mess. Mth. 11 (1882) 138-. simplification of equations. Kiepert, $L$. Mth. A. 37 (1890) 368-.

simultaneous, of ellipticintegrals. Kuschniriuk, $M$. Mh. Mth. Ps. 8 (1897) 281-.

by substitution of a given function of 1st degree, number. Abel, N. H. Crelle J. 3 (1828) 394 -

theory. Kiepert, L. Crelle J. Mth. 87 (1879) 199-; 83 (1880) 205-; 95 (1883) 218-.

-. Hurwitz, A. [1881] Mth. A. 19 (1882) 67-.

-. Smith, H. J. S. [1882-83] Mess. Mth. 12 (1883) 49-; 13 (1884) 1-.

- Grifiths, J.' L. Mth. S. P. 15 ("1883-84) $152-$.

-. Fricke, R. Mth. A. 40 (1892) 469-.

-, algebraic. Griffiths, J. [1891] L. Mth. S. P. 23 (1892) 275-.

- and application to theory of numbers. Weber, $H$. Mth. A. 43 (1893) 185-.

-, Jacobi's. Griffiths, J. L. Mth. S. P. 15 $\left({ }^{*} 1883-84\right)$ 58-

-, results from. Griffiths, J. L. Mth. S. P. 16 (1884-85) 83-.

of theta functions. David, - Liouv. J. Mth. 6 (1880) 187-.

-. Miiller, $F$. Arch. Mth. Ps. 1 (1884)

- Rohde, F. Arch. Mth. Ps. 3 (1886) 138 .

- - - Krause, M. Leip. Mth. Ps. B. 45 (1893) 99-, 349-, 523-, 805-; 48 (1896) 291-. - - Jacobian equations of 40th degree. Burkhardt, H. Gött. Nr. (1890) 376-.

,-- problem, special. Krazer, A. Crelle J. Mth. 111 (1893) 64-. of theta functions, quadratic. Krazer, $A$. Mth. A. 46 (1895) 442-.

- - of one variable. Krause, M. Mth. A. 25 (1885) 319-. $-\frac{1}{(1893)}-\frac{113}{41}-$. Krazer, A. Mth. A. 43

verification. Chevallier, J. Camb. Ph. S. P. 5 (1886) 381-.

Trigonometric and elliptic functions, expansion in partial fractions and infinite products. Tichomandrickij, M. A. Kharkov Mth. S. Com. 2 (1891) 167-; Fschr. Mth. (1890) 450 .

Weierstrass's elliptic functions, trisection of periods for. Burnside, $W$. Mess. Mth. 16 (1887) 177-.

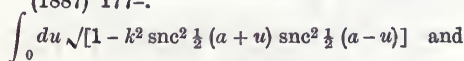

$\int_{0}^{0} d u \sqrt{ }\left[1-k^{2} \operatorname{sn}^{2} \frac{1}{2}(a+u) \operatorname{sn}^{2} \frac{1}{2}(a-u)\right]$.

Gudermann, C. Crelle J. 39 (1850) 50-.

Arg. sn $a$ and (arg. sn $a)^{2}$, expression as definite integrals. Glaisher, J. W. L. [1882] QJ. Mth. 19 (1883) 71-.

\section{Abelian integrals. (See also 8050, 8060.)}

Abelian differential equations in case of reduction of number of periods. Picard, $\hat{E}$. C. R. 95 (1882) 898-.

- equation, and theorem of Parseval. Prang. hofer, J. Wien Sb. 57 (1868) (Ab. 2) 29-.

\section{Abelian functions.}

Jacobi, C. G. J. Crelle J. 9 (1832) 394-; 13 (1835) 55-; 30 (1846) 183-; Liouv. J. Mth. 11 (1846) 97-.

Steen, A. Kiöb. Dn. Vd. Selsk. Skr. 1 (1849) 323 .

Rosenhain, G. Crelle J. 40 (1850) 319-.

Dienger, J. Grunert Arch. 16 (1851) 67-

Davidov, A. Y. [1868] (xII) Rec. Mth. (Moscou) 4 (1869-70) (Pt. 1) 287-.

Clebsch, R. F. A. [1869] Gött. Ab. 14 (1869) 75 pp.; Mth. A. 2 (1870) 193-.

Roberts, M. A. Mt. 3 (1869-70) 70-.

Poincaré, H. C. R. 92 (1881) 958-.

Appell, P. C. R. 94 (1882) $1702-$.

Poincare, H. C. R. 100 (1885) 785-; Am. J. Mth. 8 (1886) 289-.

Appell, P. C. R. 103 (1886) 1246-.

Poincaré, H. C. R. 120 (1895) 239-; Liouv. J. Mth. 1 (1895) $219-$.

Ermakow, W.P. Fschr. Mth. (1897) 402.

addition formulø for, explicit form. Salvert, $F$. de. C. R. 116 (1893) 304-, 412 .

- theorem. Weber, H. Crelle J. 70 (1869) 193-.

application to continued fractions. Borchardt, C. W. Crelle J. 48 (1854) 69-.

- in geometry. Clebsch, R. F. A. [1863] Crelle J. 63 (1864) 189-. 
application to Weddle quartic surface. Caspary, F. Bll. Sc. Mth. 15 (1891) 308-.

bisection. Brioschi, F. C. R. 70 (1870) 504-; Rm. At. R. Ac. 24 (1870-71) 47-.

- problem. Tauber, A. Mh. Mth. Ps. 7 (1896) 97-.

calculation by repeated transformations. Riche. lot, F. J. As. Nr. 13 (1836) 361-.

of 1st class. Pokrovskij, P. M. Rec. Mth. (Moscou) 13 (1886) 245-, 409-.

- class p. Ahlborn, F. H. H. Hamb. Mth. Gs. Mt. 1 (1889) 80-.

class, and a hyperfuchsian group. Picard, $E^{\prime}$. C. R. 98 (1884) 289-.

classification. Burkhardt, H. Mth. A. 35 (1890) 198-.

complex multiplication. Humbert, G. C. R. 127 (1898) 857-.

connected with characteristic equations of symmetrical plane surfaces. Schottky, F. Crelle J. Mth. 106 (1890) 199-.

differential equations integrable by. Minich, $S . R$. Ven. Mm. I. 3 (1847) 269-.

and differential expressions, theory. Noether, M. Mth. A. 37 (1890) 417-, 465-.

division. Hermite, C. [1843] Par. Mm. Sav. Étr. 10 (1848) 563-.

- (Hermite). Liouville, J. Liouv. J. Mth. 8 (1843) 502-

-. Jordan, C. C. R. 70 (1870) 1028-.

-, equations. Jordan, C. Mth. A. 1 (1869) $583-$.

-, priority claim. Libri, G. C. R. 17 (1843) 431-, 546-.

and elliptic functions. Eisenstein, $G$. Crelle J. 27 (1844) 185-.

or elliptic functions, class of differential equations integrable by. Fuchs, L. [1877] Gött. Nr. (1878) 19-

especially of 3rd order, theory. Pringsheim, $A$. Mth. A. 12 (1877) 435-.

expansion in trigonometric series, application of integrals of factorial functions to. Appell, $P$. Acta Mth. 13 (1890) $174 \mathrm{pp.}$

generalisation. Picard, E. C. R. 98 (1884) $665-$.

of genus $p=2$, Borchardt's moduli. Reichardt, W. Mth. A. $28(1887) 84$.

- 3. Amstein, H. Laus. S. Vd. Bll. 24 (1889) 99-; 25 (1890) 133-.

- - Wirtinger, W. Mth. A. 40 (1892) 261-.

- - - algebraic relations between. Brunel, G. E. A. Par. Ec. Norm. A. 12 (1883) 199-.

historical development to Riemann's time. Hancock, H. B. A. Rp. (1897) 246-

inversion problem. Noether, $M$, Mth. A. 28 (1887) 354-

1st kind, algebraic relations between. Caspary, F. Par. Eo. Norm. A. 10 (1893) 253-.

- - for $p=2$. Pascal, E. A. Mt. 18 (1890) 131-.

2nd kind, reduction. Goursat, É. C. R. 100 (1885) 622-.

linear relations between $2 p$ circuits of 1 st and 2nd kinds. Schläfi, L. Crelle J. 76 (1873) 149-. mechanical problems depending on. Charlier, C. V. L. [1888] St Pét. Ac. Sc. Bll. 33 (1890) 9-.

1st order. Richelot, F.J. Crelle J. 12 (1834) 181-.

- - Krause, M. Crelle J. Mth. 98 (1885) 148 .

241-

Brioschi, F. A. Mt. $14(1886-87)$

-, algebraic relations. Brioschi, F. $\mathrm{Rm}$. R. Ac. Linc. T. 7 (1883) 137-.

- - differential equation of periods. Königs berger, - . [1868] Mth. A. 1 (1869) 165-.

- _, modular equations. Krause, M. [1881] Mth. A. 19 (1882) 423-, 489-

- - - - for transformation of 3rd degree. Krause, M. [1881] Mth. A. 19 (1882) 103-. - - , - - - - 5th degree. Krause, $M$. Mth. A. 20 (1882) 226-.

- -, multiplication. Krause, M. Mth. A. 17 (1880) 448-.

- - multiplicator. Krause, M. Acta Mth. 3 (1883) 283-.

,--- equations. Krause, M. Mth. A. 20 (1882) 54-.

- -, theory. Göpel, A. Crelle J. 35 (1847) $277-$

- - transcendents of 2 nd and $3 \mathrm{rd}$ kinds. Weber, $H$. Crelle J. Mth. 82 (1877) 131-.

- -, transformation. Königsberger, [1864-66] Crelle J. 64 (1865) 17-; 65 (1866) 335-; 67 (1867) 58-, 97-.

- - - Brioschi, F. Rm. R. Ac. Linc. Rd. 1 (1885) 315-.

- - - - of 2nd degree. Pringsheim, A. Mth. A. 9 (1876) 445-.

- - - - 5th degree. Krause, M. [1879] Mth. A. 16 (1880) 83-

- - - linear. Krause, M. Mth. A. 17 (1880) 435-.

any order, Göpel's relation. Brioschi, $F$. A. Mt. 10 (1880-82) 161-.

order $n$, algebraic relations between. Brioschi, -. C. R. 99 (1884) 889-, 951-, 1050 .

$p=2$, periods, differential relations between. Brioschi, F. Crelle J. Mth. 116 (1896) $326-$.

- =-, transformation, and importance for Kummer's surface. Rohn, K. Mth. A. 15 (1879) 315-.

properties. Brioschi, F. [1857] Tortolini A. 1 (1858) 12-, 20-.

questions of reality regarding. Klein, F. Gött. Nr. (1892) 310-.

of 4 th rank, special. Schottky, $F$. Crelle J. Mth. 103 (1888) 185-.

relations between derivatives with parameters and arguments. Wiltheiss, E. Crelle J. Mth. 99 (1886) 236-.

Riemann's lectures, 1861-62. Noether, $M$. D. Mth. Vr. Jbr. 8 (1900) (Heft 1) 177-.

series for. Wiltheiss, E. Mth. A. 31 (1888) 410-

singular. Humbert, G. Liouv. J. Mth. 5 (1899) 233-; 6 (1900) 279-.

theorems. Minich, S. R. Ven. At. 6 (1847) 130 . 
theorems concerning complementary indices. Richelot, A. Crelle J. 29 (1845) 281-.

theory. Weierstrass, C. Crelle J. 47 (1854) 289-; 52 (1856) 285-.

-. Riemann, B. Crelle J. 54 (1857) 115-.

- Prym, F.E. Wien D. 24. (1865) (2 $\left.2^{\text {to }} A b.\right)$ $1-$.

- Clebsch, R. F. A., \& Gordan, P. C. R. 62 (1866) 183-, 227-.

- Thomae, J. [1872] Crelle J. 75 (1873) 224-.

- Pick, G. Mth. A. 29 (1887) 259-.

-. Klein, F. Gött. Nr. (1889) 179-, 376-; Mth. A. 36 (1890) 1-.

-. Dolbnia, J. N. A. Mth. 10 (1891) 478-.

- Hurwitz, A. Gött. Nr. (1892) 247-.

- Fuchs, L. Berl. Ak. Sb. (1897) 608-; (1898) 477-.

-, exceptional cases in. Weber, H. Mth. A. 13 (1878) 35-.

-, point in. Halphen, G. H. C. R. $78(1874)$ 1833-.

-, Riemann's. Capelli, A. Nap. Rd. 28 (1889) 236-

-, - explanation of 3rd section. Ascoli, G. Mil. I. Lomb. Rd. 18 (1885) 783-, 806-.

transformation. Gordan, P. C. R. 60 (1865) 925-.

- Humbert, G. C. R. 129 (1899) 955-.

- of 3rd degree in. Brioschi, F. Mth. A. 28 (1887) 594-. - - K97-. - Krause, M. Mth. A. 28 (1887)

-, theory. Hermite, C. C. R. 40 (1855) 249-, 304-, 365-, 427-, 485-, 536-, 704, 704-, 784-.

-, -. Brioschi, F. C. R. 47 (1858) 310-.

transformations of, form and number of representatives of non-equivalent classes. Dorn, E. Mth. A. 7 (1874) 481-.

-, singular. Humbert, G. C. R. 126 (1898) 882-.

- which, applied twice, produce duplication. Borchardt, C. W. C. R. 88 (1879) 885-, 955-.

trisection. Clebsch, R. F. A. [1869] Gött. Ab. 14 (1869) 75 pp. ; Mth. A. 2 (1870) 193-.

-. Jordan, C. C. R. 68 (1869) 865-.

and $\log \Gamma$. Clausen, $T$. Grunert Arch. 30 (1858) 166-

$\sigma$-functions. Klein, F. Mth. A. 27 (1886) 431- ; 32 (1888) 351-.

-. Brioschi, F. Rm. R. Ac. Linc. Rd. 3 (1887) (Sem. 1) 245-, 311-.

- Burkhardt, $H$. Mth. A. 32 (1888) 381-.

- Pascal, E. A. Mt. 17 (1889-90) 257-.

-. Baker, H. F. Am. J. Mth. 20 (1898) 301-.

-, derivatives. Bolza, O. Am. J. Mth. 17 (1895) 11-.

-, elliptic $\sigma$ functions as special case. Bolza, O. N. Y. Am. Mth. S. T. 1 (1900) $53-$.

-, expansion in series. Brioschi, F. Rm. R. Ac. Linc. Mm. 6 (1889) 471-.

-, - - - second limit. Pascal, E. A. Mt. 24 (1896) 193-. $\sigma$-functions, expansions. Bolza, O. Am. J. Mth. 22 (1900) 101-.

and $\theta$-functions. Cayley, A. Am. J. Mth. 5 (1882) 137-; 7 (1885) 101-.

$\theta$-functions, algebraic characteristics. Staude, O. Mth. A. 25 (1885) 363-.

-, differential equations. Wiltheiss, E. D. Mth. Vr. Jbr. 1 (1892) 72-.

- partial differential equations. Wiltheiss, $E$. Mth. A. 33 (1889) 267-.

- and their periods, partial differential equations. Wiltheiss, E. Mth. A. 31 (1888) 134-.

-, points in theory. Schröder, J. Hamb. Mth. Gs. Mt. 3 (1900) 73-.

- and $\sigma$-functions, connection. Schröder, $J$. Hamb. Mth. Gs. Mt. 2 (1890) (Festschr., Tl. 2) 162-.

- _ - partial differential equations. Bolza, o. Am. J. Mth. 21 (1899) 107-.

\section{ABELIAN INTEGRALS.}

Minding, E. F. A. Crelle J. 22 (1842) 255-.

Haedenkamp, $H$. Crelle J. 25 (1843) 178-

Rink, H.J. QJ. Mth. 19 (1883) 374-.

Biermann, O. Mh. Mth. Ps. 3 (1892) 21-.

Seiliger, D. N. Kazan S. Ps.-Mth. Bll. 2 (1893) (Prot.) 57.

Roberts, W. R. W. B. A. Rp. (1894) 557-.

Epstein, P. Acta Mth. 20 (1897) 1-.

Thomae, J. Leip. Mth. Ps. B. 52 (1900) 105-.

addition-theorem, demonstration. Schumann, A. Mth. A. 7 (1874) 623-.

algebraic part. Stoliarow, N.A. Fschr. Mth. (1899) 405.

- -, separation. Tichomandrickij, M. A. Kharkov Mth. S. Com. (1885) 99-; Fschr. Mth. (1886) 252-.

- researches. Raffy, L. Par. Ec. Norm. A. 12 (1883) 105-.

analytical exercises. Rosenhain, $G$. Crelle J. 28 (1844) 249- ; 29 (1845) 1-.

application of representation of binary forms. Gordan, P. A. Mt. 2 (1868-69) 346-.

calculation. Söderblom, A. Göteb. Hndl. 2 (1899) 127-.

canonical periods. Lüroth, J. Münch. Ak. Ab. 15 (1886) 329-; 16 (1888) 197-.

class, and certain differential equations. $P i$ card, É. C. R. 92 (1881) 398-.

of 1st class, periods. Roberts, W. R.W. [1880] Ir. Ac. T. $28(1880-86) 31-$.

- 2nd class. Thomae, J. Crelle J.Mth. 101 (1887) 326-.

complete. Winckler, A. Wien Sb. 58 (1868) (Ab. 2) 976-.

-, of different species, relations. Winckler, A. Wien Sb. 62 (1870) (Ab. 2) 49-.

connected with a binomial irrationality. Biermann, O. Wien Ak. Sb. 87 (1883) (Ab. 2) 934-.

for curves of 4 th degree, march. Klein, C. F. Mth. A. 10 (1876) 365-; 11 (1877) 293-.

on curves in hyperspace. White, H.S. Ac. Nt. C. N. Acta 57 (1892) 41-. 


\section{Abelian Integrals}

depending on binomial algebraic equations, reduction. Dolbnia, I. $P$. Rec. Mth. (Moscou) 18 (1896) 647-; Bll. Sc. Mth. 20 (1896) 156-.

and elliptic and hyperelliptic integrals, reduction. Scheibner, W. Leip. Mth. Ps. B. 41 (1889) 31-.

- - integrals, use in Diophantine analysis. Jacobi, C. G. J. Crelle J. 13 (1835) 353-.

evaluation, method. Richelot, F.J. C. R. 2 (1836) 622-

(and similar integrals), expansion in continued fractions. Vleck, E. B. van. Am. J. Mth. 16 (1894) 1-.

expansions in series for. Schlömilch, O. X. Leip. Mth. Ps. B. 34 (1882) 1-.

expressible by logarithms. Goursat, É. C. R. 118 (1894) 515-.

expression in terms of set of fundamental integrals. Bolza, O. [1900] N. Y. Am. Mth. S. Bll. 7 (1901) 244, 252-.

extension of Legendre's relation to. Vahlen, K. T. Crelle J. Mth. 114 (1895) 47-.

and functions of 1st class, transformations. Pokrovskij, P. M. Rec. Mth. (Moscou) 15 (1891) 397-; Bll. Sc. Mth. 17 (1893) 85-.

fundamental loci of functions inverse to. Casorati, F. Acta Mth. 8 (1886) 360-.

most general relations among. Königsberger, $L$. [1875] Crelle J. Mth. 81 (1876) 193-.

genus of certain class, determination. Dolbnia, J. Bll. Sc. Mth. 19 (1895) 272-; 21 (1897) $243-$

- 1, direct study. Dolbnia, J. Par. Ec. Norm. A. 15 (1898) 393-.

geometrical representation. Cayley, A. $\mathrm{Ph}$. Mg. 6 (1853) 414-.

and higher integrals of 3rd kind, interchange of parameter and argument. Jacobi, C. G.J. Crelle J. 32 (1846) 185-.

imaginary numbers resulting from. Efimov, M. T. Kazan S. Ps.-Mth. Bll. 4 (1895) (Prot.) 72-.

independence of zeros in Jacobian function. Martins da Silva, J. A. G. Teix. J. Sc. 5 ("1883) 3-.

integration in finite form, general theorems for. Ptaszycki, J. Prace Mt.-Fiz. 11 (1900) 23-; Fschr. Mth. (1900) 452.

inversion. Weber, H. Crelle J. 70 (1869) 314-.

- Appell, P. Liouv. J. Mth. 1 (1885) 245-

- Goursat, É. C. R. 115 (1892) 787-.

- Hallgren, $E$. Göteb. Hndl. 30 (1895) $77 \mathrm{pp}$.

inversions, representation. Dillner, $G$. Bordeaux S. Sc. Mm. 5 (1883) 291-.

and Jacobi's equations. Cayley, A. Camb. and Dubl. Mth. J. 3 (1848) 51-.

1st kind, number. Elliot, ZZ. Par. Ec. Norm. A. 5 (1876) 399-

- -, 一. Picard, É. Bll. Sc. Mth. 14 (1890) 131-

- - periodicity moduli, fundamental relations. Casorati, F. A. Mt. 3 (1869-70) 1-. - and 2nd kinds, expansion in series. Königs. berger, L. [1875] Mth. A. 9 (1876) 487-.
Abelian Integrals 4060

3rd kind. Emmanuel, D. Par. Éc. Norm. A. 8 (1879) 299-

- - interchange of argument and parameter paths. Graf, J. H. Bern Mt. (1877) 64 -

of different kinds, forms. Appell, P. Toul. Fac. Sc. A. 7 (1893) A, 4 pp.

mechanical application. Neumann, C. Crelle J. 56 (1859) 46-.

moduli, choice. Borchardt, C. W. C. R. 88 (1879) 834-.

1st order. Cayley, A. Crelle J. Mth. 98 (1885) 95-.

- - expressible as sum of two elliptic integrals. Burnside, $W$. L. Mth. S. P. 23 (1892) 173-.

- - , reduction to normal form. Jacobi, C. G. J. Crelle J. 55 (1858) 1-.

- - 3rd species. Roch, G. [1865] Crelle J. 65 (1866) 42 -.

- - transformation. Richelot, F.J. Crelle J. 16 (1837) 221-, 226-, 285-.

- - - Krause, M. Acta Mth. 3 (1883) 153-; Crelle J. Mth. 95 (1883) 256-.

- - - of 2nd order. Richelot, F.J. Berl. Mb. (1866) 412-

$p=3$, reduction to elliptic integrals. Gilespie, W. Am. J. Mth. 22 (1900) 259 -

periodicity moduli. Keppinski, S. Krk. Ak. (Mt.-Prz.) Rz. 17 (1900) 63-; Crc. Ac. Sc. Bll. (1898) 270-

- - differential equations for. Fuchs, $L$. Crelle J. 73 (1871) 324-

- - as functions of a branch-point. Fuchs, $R$. Crelle J. Mth. 119 (1898) 1-。

- - - - parameter. Fuchs, L. [1869] Crelle J. 71 (1870) 91-, 128-.

- - relations. Königsberger, $L$. Gött. Nr. (1875) $327-$

- - - Herz, N. [1881] Arch. Mth. Ps. 68 (1882) 196-.

periods, differential equations for. Wiltheiss, $E$. Mth. A. 34 (1889) 150-

properties, general. Abel, N. H. [1826-29] Par. Mm. Sav. Étr. 7 (1841) 176-; Crelle J. 3 (1828) 313-; 4 (1829) 200 -.

-, -. Mainardi, G. Mil. G. I. Lomb. 7 (1855) 426-.

1st rank, inversion. Dolbnia, J. P. Fschr. Mth. (1898) 391-.

reducible. Kluyver, J. C. Amst. Ak. Vs. 7 (1899) 425-; Amst. Ak. P. 1 (1899) 449-.

reduction. Malet, J. C. Crelle J. 76 (1873) 97-.

-. Picard, É. C. R. 93 (1881) 696-.

-. Kotanyi, L. [1883] (x) Wien Ak. Sb. 88 (1884) (Ab. 2) 401-.

- Hermite, C. Edinb. R. S. P. 12 (1884) $642-$.

一. Poincaré, H. C. R. 99 (1884) 853-; Par. S. Mth. Bll. 12 (1884) 124-.

-. Goursat, É. Par. S. Mth. Bll. 13 (1885) 143-.

- Gomes Teixeira, F. G. Teix. J. Sc. 8 (1887) 164.

-. Pick, G. Mth. A. 32 (1888) 443-.

- to algebraic logarithmic functions. Königsberger, L. Mth. A. 11 (1877) 119-. 


\section{Abelian Integrals}

reduction to algebraic logarithmic functions, inexactitude of Koenigsberger's rule for value of integral. Ptaszycki,J. Mth. A. 16 (1880) 264 .

- of class, to elliptic integrals. Kowalevski, S. Acta Mth. 4 (1884) 393-.

- to elliptic functions. Cayley, A. C. R. 85 (1877) 265-, 373-, 426-, 472-.

- - cases. Brioschi, F. C. R. 85 (1877) 708-

- - - example. Hermite, C. (xII) Brux. S. Sc. A. 1 (1877) (Pt. 2) 1-.

- - - and hyperelliptic integrals. Königs berger, $L$. Mth. A. 15 (1879) 174-.

- - - integrals. Königsberger, $L$. Crelle J. Mth. 85 (1878) 273-.

- - - - Picard, É. C. R. 93 (1881) 1126-; 94 (1882) 1704-.

- - Ungar, M. Wien Ak. Sb. 83 (1881) (Ab. 2) 759-.

_- - Picard, É. Par. S. Mth. Bll. 12 (1884) 153-.

- _ - Bolza, O. Freiburg B. $8\left(^{*} 1882\right.$ 85) 330 -

- - Biermann, O. Wien Ak. Sb. 105 (1896) $(A b .2 a)$ 924-

_- Brun, F. de. Stockh. Öfv. (1897) 413-.

_ _ _ - , relation to complex multiplication of elliptic integrals. Königsberger, $L$. [1878] Crelle J. Mth. 86 (1879) 317-.

- $-\ldots$ by transformation of 3rd degree. Brioschi, F. Par. Éc. Norm. A. 8 (1891) 227-.

- _ - _ - - Bolza, O. Mth. A. 50 (1898) 314-; 51 (1899) 478-.

- - functions of 1st, 2nd and 3rd kinds. Hermite, C. Bll. Sc. Mth. As. 7 (1883) 36-.

- of group. Alth, G. von. Wien Ak. Sb. 95 (1887) (Ab. 2) 702-.

- to lower integral forms, especially elliptic. Königsberger, L. [1879] Crelle J. Mth. 89 (1880) 89-.

- - normal form, cases. Ptaszycki, J. Mth. A. 33 (1889) 600-; Prace Mt.-Fiz. 2 (1890) 57-.

- - - , demonstration. Malet, J.C. [1874] Crelle J. Mth. 79 (1875) 176-.

- - integrals. Ungar, $M$. [1882] Wien Ak. Sb. 86 (1883) (Ab. 2) 893-.

- of number of periods, in particular for curves of 2nd genus. Picard, É. [1882] Par. S. Mth. Bll. 11 (1883) 25-.

- by transformation of 4th degree. Bolza, $O$. Mth. A. 28 (1887) 447-.

-, and transformation of Fuchsian functions. Poincaré, $H$. C. R. 102 (1886) 41-.

related to intersection curve of two surfaces, covariants. White, H. S. Mth. A. 36 (1890) $597-$.

Riemann's theory (Neumann). Bertini, $\boldsymbol{E}$. Palermo Cir. Mt. Rd. 6 (1892) 165-.

1st species. Rink, H. J. Z. Mth. Ps. 29 (1884) 272-.

- - plane curves rectifiable by. Brodén, $T$. Stockh. Ak. Hndl. Bh. 15 (Afd. 1) (1890) No. 5, $26 \mathrm{pp}$.

\section{Abel's Theorem 4060}

2nd and 3rd species. Staude, O. Acta Mth. 8 (1886) 81-.

- - Krause, M. A. Mt. 15 (1887-88) 187-.

- - addition theorems. Jacobi, C. G.J. Crelle J. 30 (1846) 121-.

- _ - _ and 1st order, representation by integrals of 1st species. Schirdewahn, $G$. Z. Mth. Ps. 34 (1889) 355-, 385.

3rd species. Roch, G. [1866] Crelle J. 68 (1868) 170-.

- -, attached to algebraic curves without singularities. Pick, G. [1886] Wien Ak. Sb. 94 (1887) (Ab. 2) 367-.

theorem of Weierstrass, demonstration. Běljankin, I. Kazan S. Ps.-Mth. Bll. 9 (1900) 35-.

theorems. Malet, J. C. [1874] Ir. Ac. T. 25 (1875) 279-.

theory. Bouquet, -. C. R. 67 (1868) 989-; Par. Mm. Sav. Etr. 21 (1875) 675-.

-. Goursat, É. C. R. 97 (1883) 1281-.

-. Dolbnia, I. Kazan S. Ps.-Mth. Bll. 3 (1893) 1-, 57-, 109-.

- of a class. Pick, G. A., \& Ungar, M. [1880] Wien Ak. Sb. 82 (1881) (Ab.2) 893-. transformation. Elliot, $Z$. Par. Éc. Norm. A. 9 (1880) 167-.

-. Heymann, W. Z. Mth. Ps. 33 (1888) 31-. -, case. Toropov, K. A. [1887] Kharkov Mth. S. Com. 1 (1889) 82-; Fschr. Mth. (1887) 477-.

- equation of 2nd order and coefficients of linear equations. Eisenstein, G. Crelle J. 44 (1852) 261-.

- problem, reduction. Königsberger, L. Mth. A. 13 (1878) 540-

Abelian system of differential equations. Roberts, E. R. W. R. S. P. 57 (1895) 301-.

Abel's formula. Roberts, W. Liouv. J. Mth. 16 (1851) 143-.

- Bertrand, J. Tortolini A. 1 (1858) 156-.

- - demonstration. Ovidio, E. d'. G. Mt. 6 (1868) 37 -

- - generalisation. Somin, N. N. Rs. S. Nt. Mm. (Mth.) 5 (1884).

- and Jacobi's theorem, demonstration. Mainardi, G. [1844] Mod. S. It. Mm. 23 (1846) 171-.

- problem, Lětnikov's solution. Liventsov, A. I. [1878] (xII) Rec. Mth. (Moscou) 9 (1878-81) (Pt. 1) 114-.

- problems. Formenti, C. Mil. I. Lomb. Rd. 8 (1875) $276-$.

Abel's theorem.

Jacobi, C. G. J. Crelle J. 9 (1832) 99.

Liouville, J. C. R. 17 (1843) 720.

Cremona, L. Tortolini A. 7 (1856) 99-.

Cayley, A. Tortolini A. 8 (1857) 201-.

Genocchi, A. Tortolini A. 1 (1858) 33-.

Heine, H. E. [1862] Crelle J. 61 (1863) 276-.

Königsberger, L. [1880] Crelle J. Mth. 90 (1881) 109-. 


\section{Abel's Theorem}

Rowe, R. C. [1880] Phil. Trans.172(1882)713-. Craig, T. $\quad$ L. Mth. S. P. 12 (1880-81) 89-.

Humbert, G. C. R. 103 (1886) 919-.

Dixon, A. C. QJ. Mth. 22 (1887) 200-.

Koenigsberger, L. Crelle J. Mth. 100 (1887) 121-; 101 (1887) 1-.

Pszeborsky, A. P. Fschr. Mth. (1898) 369-.

and Abelian functions. Forsyth, A. R. [1882]

Phil. Trans. 174 (1884) 323-.

application. Brioschi, F. C. R. 94 (1882) 686-. and some applications. Beltrami, E. Mil. I. Lomb. Rd. 13 (1880) 327-.

applications, elementary. Lubbock, J. W. Ph. Mg. 6 (1835) 116-.

- to geometry. Rink, H.J. Arch. Néerl. 17 (1882) 460 -.

and some applications to geometry. Humbert,

G. Liouv. J. Mth. 3 (1887) 327-; 5 (1889) 81-; 6 (1890) 233-.

demonstration. Jacobi, C. G. J. Crelle J. 24 (1842) 28-.

-. Lejeune-Dirichlet, G. Liouv. J. Mth. 7 (1862) 253-.

- Harnack, A. Erlang. Ps. Md. S. Sb. 7 (1875) 96-.

-. Weber, $H$. Mth. A. 8 (1875) 49-.

differential equations. Landsberg, G. [1898]

D. Mth. Vr. Jbr. 7 (1899) (Heft 1) 56-.

extension. Brioschi, F. Mil. G. I. Lomb. 8 (1856) 333-.

formulæ, development. Dillner, $G$. Gött. Nr. (1876) 29-.

generalisation to skew curve. Poincaré, $H$. C. R. 100 (1885) 40 -.

generalised, existence. Koenigsberger, $L$. Crelle J. Mth. 102 (1888) 224.

geometrical interpretation of. Klein, $F$. Mth. A. 28 (1887) 533-.

in a new form. Pokrovskij, P. M. [1898] Rec. Mth. (Moscou) 20 (1899) 299-.

and surface generated by translation of curves. Lie, S. C. R. 114 (1892) 277-.

and trigonometric formulæ deduced therefrom. Baehr, G. F. W. [1880] Amst. Ak. Vs. M. 16 (1881) 195-; Arch. Néerl. 16 (1881) 116-.

Algebraic differentials. Harnack, A. A. Mt. 9 (1878-79) 302-.

- - in homogeneous coordinates. Harnack, $A$. Mth. A. 9 (1876) 371-.

- integrals, class. Minding, E. F.A. Crelle J. 10 (1833) 195-, 292.

- - inversion, case. Dillner, G. Stockh. Öfv. (1885) No. 4, 3-; Fschr. Mth. (1885) 378.

Arithmetico-geometric mean of 4 elements. Borchardt, C. W. Berl. Ak. Ab. (1878) (Mth.) 33-. 89 (1880) 221-.

Binomial integrals, theory. Pick, G. [1886] Wien Ak. Sb. 94 (1887) (Ab. 2) 372-.

Differential expressions, reduction to normal form. Noether, $M$. Erlang. Ps. Md. S. Sb. 16 (1884) 18-, 23-, 84-; 18 (1886) 18-.

\section{Abelian Integrals 4060}

Double-point, accessory reduction in number of periods due to. Marie, $M$. C. R. 76 (1873) 865-.

- planes of genus 1. Enriques, F. Rm. S. It. Mm. 10 (1896) 201-.

Doubly periodic functions given by

$$
\frac{d u}{d z}=\sqrt[m]{F^{\prime}(u)}
$$

Bouquet, J., \& Briot, - C. R. 40 (1855) 342-.

- - of 2nd kind, generalisation. Appell, $P$. Liouv. J. Mth. 9 (1883) 5-.

Elliptic and hyper-elliptic functions, approximation method. Merrifield, $C$. $W$. Phil. Trans. (1860) 223-; (1862) 417-.

Forms connected with algebraic configuration. Pick, G. Mth. A. 50 (1898) 381-.

Functions analogous to theta functions. Elliot, Z. C. R. 93 (1881) 1008-; Par. Éc. Norm. A. 11 (1882) 79-, 425-.

- having an Abelian integral as logarithmic derivative. Fulco, $P$. Catania Ac. Gioen. At. 13 (1900) Mem. 14, 24 pp.

- whose logarithms are sums of Abelian integrals of 1 st and 3rd kinds. Appell, P. C. R. 92 (1881) 960-.

- with multiple periods. Casorati, F. C. R. 58 (1864) 127-, 204-.

- of one variable, with any number of periods. Casorati, $F$. Acta Mth. 8 (1886) 345-.

Hyperabelian functions. Picard, $\hat{E}$. Liouv. J. Mth. 1 (1885) 87-.

$-\frac{-}{1}$, class. Bourget, H. C. R. 124 (1897) 1428-.

Hyperelliptic analogue, general, of function $\rho(u)$. Bolza, O. Gött. Nr. (1894) 269-.

- curves. Bobek, $K$. Prag Ab. 1 (1886) No. 1, 27-.

- - genus $>1$. Küpper, $K$. Prag Sb. (1896) (Mth.-Nt.) No. 43, 11 pp.

- - order $3^{n}$, genus arbitrary. Kupper, C.J. Prag Ab. 1 (1886) No. 1, 26 pp.

- - $C^{n} p$ with special systems $g_{2}^{(1)}, g_{3}^{(1)}$. Küper, $K$. Prag Ab. 3 (1890) No. 4, 18 pp. $-\ldots, C_{8}^{8}$ with 13 given double points, system. Kupper, C. Prag Sb. (1898) (Mth.-Nt.) No. 1, 7 pp.

- differential equations, integration. Jacobi, C. G. J. Crelle J. 32 (1846) 220 -

$--\ldots,-$ Weierstrass, C. Berl. Mb. (1862) 127-.

- differentials, integration by logarithms. Pick, G. A. Wien Ak. Sb. 85 (1882) (Ab. 2) 643-.

- equations, integration. Brioschi, F. Crelle J. 55 (1858) 56-.

- - invariant. Brun, F. de. Stockh. Öfv. (1897) 173-; Fschr. Mth. (1897) 400-

- formulæ. Brioschi, F. C. R. 102 (1886) 239-, 297-.

- modular functions. Burkhardt, H. Mth. A. 36 (1890) 371-; 38 (1891) 161-; 41 (1893) 313-.

- multiplicator equation. Burkhardt, $H$. Gött. Nr. (1889) 553-. 
Hyperelliptic surfaces, general theory. Humbert, G. Liouv. J. Mth. 9 (1893) 29-, 361-.

- system of general form, explicit expression of algebraic integral. Salvert, $F$. de. C. R. 116 (1893) 243-.

- systems of barriers. Thompson, H.D. Am. J. Mth. 15 (1893) 91-.

Integral equations, new, of Jacobi's system of differential equations. Richelot, $F$. J. Crelle J. 25 (1843) 97-.

Integrals, class, involving roots of polynomials. Minding, E. F. A. Crelle J. 10 (1833) 195-, 292.

- of solutions of 2nd order of differential equations, with 3 singular points. Kepinski, S. Krk. Ak. (Mt.-Prz.) Rz. 17 (1900) 112-; Crc. Ac. Sc. Bll. (1899) 67-.

Inversion problem. Jacobi, C. G. J. Crelle J. 32 (1846) 176-.

_- Jacobi's. Goursat, É. Bll. Sc. Mth. 19 (1895) 24-.

- - - , generalisation. Lindemann, C. L.F. [1878] Freiburg B. 7 (1880) 273-.

- - - , solution. Stahl, $H$. Crelle J. Mth. 89 (1880) 170- ; 111 (1893) 98-.

Kleinian prime forms and automorphic functions. Ritter, E, Mth. A. 44 (1894) 261-.

Minding's integral $\int d x\left(1-x^{3}\right)^{-3}$, reduction to elliptic integral of 1st kind. Richelot, $F . J$. Crelle J. 9 (1832) 407-.

Multiply-tangential curves to algebraic curves. Weis8, W. Mh. Mth. Ps. 7 (1896) 370-.

Number of periods of uniform function. Astor, A. N. A. Mth. 15 (1896) 227-.

- - - - functions. Muhil, K. von der. Basel Vh. 9 (1893) 78-.

Parameters, representation of algebraic curves by. Enriques, $F$. Palermo Cir. Mt. Rd. 10 (1896) 30-.

Quadruple theta functions, relations satisfied by. Craig, T. Am. J. Mth. 6 (1884) 205-.

Representation of function in Riemann surface, with variable multipliers of periodicity. Suchar, P. I. [Bucarest S. Sc. Bll. 7 (1898)] 143-.

Riemann's integrals of Ist species, canonical form. Christoffel, E. B. A. Mt. 9 (187879) 240 -

Theorem in geometry of $n$ dimensions. Segre, C. Mth. A. 30 (1887) 308.

Theorems. Abel, N.H. N.A.Mth.4 (1845)536-.

Theta functions, Jacobian, partial values, and Gauss's posthumous papers. Göring, $W$. Mth. A. 7 (1874) 310-.

- - with $p$-series, characteristics formed of thirds of integers. Braunmilhl, A. von. Erlang. Ps. Md. S. Sb. 18 (1886) 37-; Münch. Ak. Ab. 16 (1888) 325-; Mth. A. 32 (1888) 513-.

- - - - - - - - nths of integers. Braunmihl, A. von. Mth. A. 37 (1890) 61-。 -- , theorems. Bock, $W$. Hamb. Mth. Gs. Mt. 2 (1890) (Festschr., Tl. 2) 74.

Transcendental functions. Abel, N. H. (vi Adds.) Norske Vd. Skr. 2 (1824-27) (2d Heft) 177-.
Transcendental functionswith algebraic differen. tials, summation. Jurgensen, C. Kiöb. Dn. Vd. Selsk. Afh. 8 (1841) 17-; Crelle J, 19 (1839) 113-.

Transcendents, of which hyperelliptic functions are special case. Laguerre, $\boldsymbol{E}$. C. R. 84 (1877) 643-.

Univocal correspondences on elliptic curves (application of Abelian integrals). Patrassi, P. G. Mt. 34 (1896) 195-.

- - hyperelliptic curves. Kantor, $S$. $\mathrm{Pa}$ lermo Cir. Mt. Rd. 9 (1895) 65-.

$\frac{\rho d x}{\sqrt{R}}$, integration. Abel, N. H. Crelle J. I (1826) 185-.

-, - Abel's theorems, demonstration. Dolb. nia, I. P. Kazan S. Nt. (P8.- Mth.) P. 6 (1888) 307-; Fschr. Mth. (1888) 486.

$\int \sqrt[3]{1+a x^{3}} \cdot d x$. Minding, E. F. A. Crelle J. 9 (1832) 295-.

$\frac{F}{E \sqrt[3]{\psi}}$, integration, $E, F, \psi$, being rational integral functions of same variable. Mainardi, G. Ven. Mm. I. 2 (1845) 401-.

$A l(x), A l_{1}(x), A l_{2}(x)$, expansion in powers of modulus. André, $D$. Liouv. J. Mth. 5 (1879) 131-.

$\Sigma \frac{U}{\frac{\partial(f, \phi)}{\partial(x, y)}}$ where $f(x, y)=0$ and $\phi(x, y)=0$, and theorem of Jacobi. Forsyth, A. R. QJ. Mth. 18 (1882) 313-.

$\int \frac{G(x)}{\sqrt{R(x)}} d x . \quad$ Guichard, C. C. R. 104 (1887) 1494-.

\section{Periodic functions of several variables; general theta func- tions.}

Abelian functions. Poincaré, H. C. R. 124 (1897) 1407-.

- - addition. Salvert, (le vicomte) - de. Brux. S. Sc. A. 17 (1893) (Pt. 1) 70-; 18 (1894) (Pt. 2) 41-.

- -, generalisation. Picard, ḱ. C. R. 98 (1884) 665-.

- - integration by. Poincare, H. C. R. 92 (1881) 913-.

- - Ist order, modular equations for transformation of 3rd degree. Königsberger, [1868] Mth. A. 1 (1869) 161-.

- with 4 periods. Roberts, M. [1870] A. Mt. 4 (1870-71) 95-.

- - and quadratic forms of $n$ differentials. Lipschitz, R. [1871] Crelle J. 74 (1872) 150-. - , singular. Humbert, G. C. R. 126 (1898) 508-.

- -, transformation. Humbert, G. C. R. 126 (1898) 814-.

- - of 2 variables. Pokrovsky, P. Bll. Sc. Mth. 20 (1896) 86-.

- - - - addition theorem. Pokrowsky, P. [1895] D. Mth. Vr. Jbr. 4 (1897) 137-. 
Abelian functions of 2 variables, complex multiplication. Wiltheiss, E. E. Mth. A. 21 (1883) 385-.

- - - - - differential equations for periods. Brioschi, $F$. Rm. R. Ac. Linc. Rd. 4 (1888) (Sem. 2) 341-, 429-.

$--1-1$, expression in series. Brioschi, F. Rm. R. Ac. Linc. Rd. 2 (1886) (Sem. 1) 199-, 215-.

- $-\ldots \ldots$, modular equations in transformation of 3rd order. Brioschi, - $\mathrm{Rm}$. R. Ac. Linc. Rd. 1 (1885) 769-.

- _ _ _ new moduli for. Brioschi, $F$. Rm. R. Ac. Linc. Rd. 2 (1886) (Sem. 1) 159-. - _ - - , transformation. Wiltheiss, Crelle J. Mth. 96 (1884) 17-.

- -4 variables. Schottky, F. Crelle J. Mth. 102 (1888) 304-.

- - - any number of variables. Klein, $F$. Gött. Nr. (1887) 515-.

$-\sigma$ functions. Pascal, E. A. Mt. 17 (1889-90) 81-, 197- ; 18 (1890) 1-.

- - - term in expansion. Schröder, $J$. [1891] Hamb. Mth. Gs. Mt. 3 (1900) 7-.

- $\theta$ functions of 3 variables, partial differential equations. Wiltheiss, E. Gött. Nr. (1889) 381-; Mth. A. 38 (1891) 1-.

- integrals, reduction. Poincaré, $H$. C. R. 102 (1886) 915-.

Algebraic surfaces, class. Humbert, G. [1889] Palermo Cir. Mt. Rd. 4 (1890) 54-.

Characteristics, $p$-rowed, theorem. Moore, E.H. N. Y. Am. Mth. S. Bll. 1 (1895) 252-.

Convergence of Jacobi's $\theta$ series with Riemann's moduli. Christoffel, E. B. Zür. Vjschr. 41 (1896) (Festschr., Th. 2) 3-.

Differential equations, integrals of which are Abelian functions of 1st kind, two systems. Caspary, F. C. R. 112 (1891) 1305-.

- - of theta functions, formation. Caspary, F. C. R. 112 (1891) 1120-.

Doubly periodic functions, uniform, behaviour at essential singularities. Appell, P. C. R. 94 (1882) 936-.

Elliptic Abelian functions of 4 variables. Schott$k y, F$. Crelle J. Mth. 108 (1891) 147-, 193-.

Function, single-valued, of $n$ variables cannot have more than $2 n$ periods. Riemann, [G. F.] B. Crelle J. 71 (1870) 197-.

Functions of 3rd kind. Tichomandrickij, $M$. A. Kharkov Mth. S. Com. 5 (1896?) 182-; Fschr. Mth. (1896) 360.

- with 4 pairs of periods. Humbert, G. C. R. 130 (1900) 483-.

- -2 real periods, class. Staude, $O$. Mth. A. 29 (1887) 468-.

- of 2 variables analogous to modular functions. Picard, É. Acta Mth. 2 (1883) 114-.

- - - - - Weierstrass's elliptic functions. Pokrovskij, P. M. Rec. Mth. (Moscou) 18 (1896) 598-; Fschr. Mth. (1896) 360.

- - - with 3 or 4 pairs of periods. Appell, P. C. R. 90 (1880) 174-.

- - - - several pairs of periods. Appell, P. C. R. 110 (1890) 181-.

_ _ _ _ and 4 periods, inverses of Abelian integrals of 1st class. Rosenhain, $G$. [1846] Par. Mm. Sav. Étr. 11 (1851) 361-.
Functions of $n$ variables admitting $2 n$ systems of periods, Riemann's theorem. Picard, É., \& Poincaré, H. C. R. 97 (1883) 1284-

Hyperabelian functions and surfaces representable by them. Humbert, G. . C. R. 129 (1899) 667-.

Inversion problem. Elliot, $Z$. C. R. 90 (1880) 1466-.

- - Jacobi's. Noether, M. Erlang. Ps. Md. S. Sb. 16 (1884) 88-.

Jacobian functions of $k$ th order of 2 variables. Witting, A. Mth. A. 29 (1887) 157-.

- - 3 variables. Frobenius, $G$. Crelle J. Mth. 105 (1889) 35-.

- - several variables. Weierstrass, C. T. W. Bll. Sc. Mth. As. 6 (1882) (Pt. 1) 136-.

Lamé's differential equation, generalisation. Grönwall, H. Stockh. Öfv. (1898) 357-.

Linear equations integrable with the help of $\chi_{m}(x, y)$. Appell, $P$. Par. Éc. Norm. A. 5 (1888) 211-.

Periodic functions, $2 n$-ply. Wirtinger, $W$. Mh. Mth. Ps. 6 (1895) 69-; 7 (1896) 1-.

,--- , application to total differential equations. Grönwall, $H$. Stockh. Öfv. (1896) 295-; Fschr. Mth. (1896) 285-.

,--- , of $r$ variables. Weierstrass, C. T. W. Crelle J. Mth. 89 (1880) 1-.

- -, special 4-ply. Roch, G. Z. Mth. Ps. 11 (1866) 463-.

- of 2 variables. Appell, P. C. R. 111 (1890) 636- ; Liouv. J. Mth. 7 (1891) 157-.

,$---n$ variables. Puiseux, $V$. C. R. 43 (1856) 321-.

-,$--\ldots-$, , demonstration of a leading theorem. Weierstrass, C. T. W. Berl. Ak. Mb. (1876) 680-.

- _ , - _ - most general uniform and $2 n$ ply. Wcierstrass, C. Berl. Mb. (1869) 853-. Periodicity, general, theory. Rausenberger, $O$. Mth. A. 18 (1881) 379-.

Periods of double integrals. Picard, É. C. R. 102 (1886) 349-.

--1 - Poincaré, H. C. R. 125 (1897) 995-.

- of algebraic functions. Picard, $\hat{E}$. C. R. 125 (1897) 1068-.

$-\ldots--$, calculation. Picard, É. C. R. 102 (1886) 410-.

- - - - and two-dimensional cycles on algebraic surfaces. Picard, É. C. R. 126 (1898) 1457-.

- - uniform $2 n$-ply periodic functions. Hur witz, A. [1882] Crelle J. Mth. 94 (1883) 1-.

Potential, properties; and Abelian functions. Poincaré, $H$. Acta Mth. 22 (1899) 89-.

Quadruply periodic expressions. Picard, É. Par. S. Mth. Bll. 17 (1889) 131-.

- - - depending on 2 variables. Picard, É. C. R. 108 (1889) 557-.

(1889) 607-.

- - functions of 2nd kind, systems of differential equations satisfied by. Krause, $M$. C. R. 126 (1898) 1086-, 1489-, 1618-; 127 (1898) 91-; A. Mt. 1 (1898) 265-. 
Quadruply periodic functions of 2 nd and 3rd kinds. Krause, $M$. Liouv. J. Mth. 3 (1887) 87-.

- - - satisfying systems of differential equations. Krause, M. N. Y. Am. Mth. S. T. 1 (1900) 287-.

- - - of 2 variables. Picard, $\mathcal{E}^{\circ}$

C. R. 124 (1897) 1490-.

- - - - - of ord kind. Appell, P. Par. Éc. Norm. A. 7 (1890) 143-.

- - series. Picard, É. C. R. 108 (1889) $659-$.

Rosenhain's fundamental formulæ, simple demonstration. Caspary, F. Mth. A. 30 (1887) 571-。

Sigma functions of 2 variables, expansion in series. Brioschi, F. Gött. Nr. (1890) 236-.

Theta characteristics. Noether, $M$. Erlang. Ps. Md. S. Sb. 11 (1879) 198-.

- - , demonstration of theorem of Riemann's. Stahl, $H$. Crelle J. Mth. 88 (1880) 273-.

- constants, 2 variables, certain relations among. Hutchinson, J. I. N. Y. Am. Mth. S. T. 1 (1900) 391-.

- formula, general, derivation. Prym, F.E. Acta Mth. 3 (1883) 216-.

- - Riemann's, demonstration. Prym, F.E. [1882] Acta Mth. 3 (1883) 201-.

-,-- , derivation. Prym, F. E. Crelle J. Mth. 93 (1882) 124-.

,-- , generalisation. Krazer, C. A. J., \& Prym, $F . E$. Acta Mth. 3 (1883) 240.

- function, infinite variety of forms. Weber, H. Crelle J. 74 (1872) 57-.

- functions. Enneper, A. Gött. Nr. (1883) 175-, 261-.

- -, characteristics. Jordan, C. C. R. 88 (1879) 1020 - 1068-; Par. Éc. Pol. J. Cah. 46 (1879) 35-.

- - - - of which are formed from thirds of integers. Krazer, C. A. J. Mth. A. 22 (1883) 416-.

- -, double. Cayley, A. [1877-78] Crelle J. Mth. 83 (1877) 210-, 220-; 85 (1878) 214-; 87 (1879) 74-.

_ - _, addition. Cayley, A. [1879] Crelle J. Mth. 88 (1880) 74-

- _, _, formulæ connected with. Thomae, J. 'Jena. Z. 20 (1887) 581-.

- - - -, transformation. Cayley, A. QJ. Mth. 21 (1886) 142-.

- - equation between. Enneper, A. Gött. Nr. (1878) 550-.

- - equations for $\theta(0,0, \ldots 0)$. Fuchs, $L$. Crelle J. 73 (1871) 324 .

- - , form of arguments, and determination of $\theta(0,0 \ldots)$ as function of class moduli. Fuchs, L. Crelle J. 73 (1871) 305-.

- -, function analogous to. Appell, $P$. Mars. Fac. Sc. A. 1 (1892) 47-.

- -, general. Noether, M. [1879] Erlang. Ps. Md. S. Sb. 12 (1880) 1-.

$-\frac{-}{-}$. Wirtinger, $W$. Wien Az. 32 (1895) 58-.

- - on a general Riemann's surface. Klein, F. C. R. 108 (1889) 277-.

VOL. I.
General Theta Functions

4070

Theta functions with multiple arguments. Roch, G. Crelle J. 66 (1866) 177-.

- - multiple, theorem. Cayley, A. Mth. A. 16 (1880) 115-.

- - multiplication of four, formula. Smith, H. J. S. L. Mth. S. P. 1 (1866) No. 8, $14 \mathrm{pp}$.

- -, particularly those of 2 variables. Forsyth, A. R. [1881] Phil. Trans. 173 (1883) 783-.

- -, quadruple. Craig, T. Am. J. Mth. 6 (1884) 14-, 183-.

_- , single and double. Cayley, A. [1879] Phil. Trans. 171 (1880) 897-.

- -, theorems, generalisation of two. Elliot, Z. C. R. 90 (1880) 352-.

- - transformation. Frobenius, G. [1879] Crelle J. Mth. 89 (1880) 40-.

- - - - theory, especially for 3 variables. Weber, H. A. Mt. 9 (1878-79) 126-.

- - triple. Cayley, A. [1878] Crelle J. Mth. 87 (1879) 134-, 165-, 190-.

- - vanishing. Neumann, C. G. Leip. Mth. Ps. B. 35 (1883) 99-.

- - of 2 variables. Caspary, F. [1881] Crelle J. Mth. 94 (1883) 74-.

- - - Darboux, G. C. R. 92 (1881) 685-, 1493-.

- - - - Schleiermacher, L. Erlang. Ps. Md. S. Sb. 18 (1886) 22-.

- _ - - and corresponding Kummer's surface. Schleiermacher, L. Mth. A. 50 (1898) 183-.

- _ _ _ - , derivatives in terms of squares of theta functions. Jahnke, E. C. R. 126 (1898) 1083-.

- _ - - - , differential relations for. Jahnke, E. Leip. Mth. Ps. B. 52 (1900) 140-. _ - _ - - - connected with. Krause, M. Mth. A. 26 (1886) 1-; A. Mt. 15 (188788) 173 -.

- _ _ - - expressions for elements of orthogonal system. Jahnke, E. C. R. 126 (1898) 1013-.

- $-1-\ldots$, Fourier's series in domain of. Krause, M. Mth. A. 27 (1886) 419-.

- _ - - - and generalised Kummer's surface. Wirtinger, $W$. Mh. Mth. Ps. 1 (1890) 113-.

- - _ - - orthogonal system formed from. Jahnke, E. Berl. Ak. Sb. (1896) 1023-; Crelle J. Mth. 119 (1898) 234-.

- _ - _ - - - systems for derivatives. Jahnke, E. C. R. 125 (1897) 486-.

- - - - - , reduction to theta functions of one variable. Appell, P. C. R. 94 (1882) 421-.

- - - - relations. Staude, O. Mth. A. 24 (1884) 281-.

(1891) 157-.

- - - - - - between the 16. Schottky, F. Crelle J. Mth. 105 (1889) 233-.

- - - - transformation. Krause, $M$. Mth. A. 25 (1885) 323-.

- - - - - , , application to arithmetico. geometric mean of 4 elements. Hettner, $G$. Crelle J. Mth. 112 (1893) 89-. 
Theta functions of 3 variables. Borchardt, C. W. [1878] Crelle J. Mth. 87 (1879) 169-. - Krause, M. C. R. $131(1900)$ 1188-.

- - - - algebraic investigations. Schottky, F. Crelle J. Mth. 105 (1889) 269-。 - - 4 variables. Noether, $M$. Erlang. Ps. Md. S. Sb. 10 (1878) 87-; Mth. A. 14 (1879) 248-.

- $n$ variables. Noether, M. Mth. A. 16 (1880) 270-.

- - - Poincaré, H. Par. S. Mth. Bll. 11 (1883) 129-.

-. Frobenius, G. Crelle J. Mth. 96 (1884) 100-

- - - - , addition theorem. Frobenius, G. Crelle J. Mth. 89 (1880) 185-.

- -1 - C - Caspary, F. Crelle J. Mth. 97 (1884) 165-.

- - - - definition. Schottky, F. Crelle J. Mth. 107 (1891) 117-.

_ _ _ _ - , formation of orthogonal systems by. Caspary, F. C. R. 104 (1887) 490-.

- - - - - , reduction to theta functions of fewer variables. Appell, $P$. Par. S. Mth. Bll. 10 (1882) 59-.

- - - - , theory. Caspary, F. Crelle J. Mth. 96 (1884) 324-.

- - - - -, transformation, principal. Frobenius, G. Crelle J. Mth. 95 (1883) 264-. - _ - - vanishing. Tichomandritzky, M. A. [1900] N. Y. Am. Mth. S. Bll. 7 (1901) 64-.

- - - p variables. Dalwigk, $F$. von. Ac. Nt. C. N. Acta 57 (1892) 221-.

$-\ldots-1$, addition theorem. Stahl, H. Crelle J. Mth. 88 (1880) 117-.

- series, convergence. Thomae, C. J. Z. Mth. Ps. 25 (1880) 43-

- -, n-tuple, infinite, theory. Meissel, $E$. Crelle J. 48 (1854) 324-.

Uniform functions with several non-interchangeable periods. Rausenberger, $O$. Mth. A. 20 (1882) 187-; 21 (1883) 59-; 25 (1885) $222-$

$d \log \theta(0,0, \ldots 0)$, determination by classmoduli. Thomae, J. [1865] Crelle J. 66 (1866) 92-.

$\theta(0,0, \ldots 0)$, determination by class-moduli of algebraic functions. Thomae, $J$. [1869] Crelle J. 71 (1870) 201-.

$\phi(x, y, z)=\stackrel{n=+\infty}{\sum} e^{a n^{4}+4 x n^{3}+6 y n^{2}+4 z n}$, zeros.

Rivereau, $\left(l^{\prime} a b b e ́\right)$-. Mars. Fac. Sc. A. 2 (1892) 59-.

$p\left(u_{1}+u_{2}+\ldots u_{2 n}\right)$, expression by means of a Pfaffian. Fontené, G. Par. Ec. Norm. A. 13 (1896) 469-.

\section{0ther special functions. 4400 General.}

Bernoullians, Raabe's. Dickson, J. D. $H$. [1888] L. Mth. S. P. 20 (1889) 14-.

Bernoulli's function. Hermite, C. [1874] Crelle J. Mth. 79 (1875) 339-.
Bernoulli's function. Glaisher, J.W.L. QJ. Mth. 29 (1898) 1-.

- - Raabe's monograph. Saalschittz, L. Z. Mth. Ps. 42 (1897) 1-.

- - , and its use in semi-convergent series. Schlömilch, O. Schlömilch Z. 1 (1856) 193-. - functions. Imschenetsky, $V$. G. G. Mt. 9 (1871) 87-.

- - Schroder, 一. D. Nf. Vh. (1890) (Th. 2) 5 -.

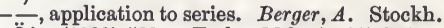
Öfv. (1891) 523-; Fschr. Mth. (1891) 274.

- with arbitrary indices. Sincov, $D$. Kazan S. Ps.-Mth. Bll. 1 (1891) 234-; Fschr. Mth. (1892) 404-.

- - class of functions related to. Beaupain, J. Brux. Mm. Cour. 4, 59 (1901) (Pt. 1) 33 pp.

- - connection with Riemann's surface. Jonquière, A. [1890] Stockh. Ak. Hndl. Bh. 16 (Afd. 1) (1891) No. 6, 28 pp.

- -, definitions. Renfer, $H$. Bern Mt. (1900) 1-.

- expansion in. Rogel, $F$. Arch. Mth. Ps. 17 (1900) 129-.

- - generalisation. Imshenetsky, $V . G$. St Pét. Ac. Sc. Mm. 31 (1883) No. 11, $58 \mathrm{pp}$.

- - and numbers. Berger, A. Stockh. Ak. Handl. Bh. 13 (Afd. 1) (1888) No. 9, 43 pp.; Stockh. Öfv. (1888) 433-; Acta Mth. 14 (1890-91) 249-.

- - resolution into partial fractions. Worpitzky, J. Z. Mth. Ps. 29 (1884) 45-. polynomial. Platner, G. Mil. I. Lomb. Rd. 25 (1892) 1179-.

- polynomials. Somin, -, \& Hermite, -. Crelle J. Mth. 116 (1896) 133-.

- -, approximate values. Appell, P. N. A. Mth. 6 (1887) 547-.

- - and numbers. Pietrocola, C. G. Mt. 34 (1896) 48-.

Functional operations represented by definite integrals. Pincherle, $S$. Acta Mth. 10 (1887) 153-.

Functions allied to trigonometric functions. Knar, J. Grunert Arch. 27 (1856) 365-.

- analogous to algebraic functions, property. Picard, É. C. R. 89 (1879) 1106-.

- - functional determinants. Rosanes, $J$. [1872] Crelle J. 75 (1873) 166-.

- with lacunæ. Goursat, É. Bll. Sc. Mth. 11 (1887) 109-.

- - Poincaré, H. Am. J. Mth. 14 (1892) 201-.

- - C. Cayley, -. QJ. Mth. 26 (1893) 279-. - - Krygowski, Z. Par. S. Mth. Bll. 25 (1897) 240-; Prace Mt.-Fiz. 9 (1898) 213-; Fschr. Mth. (1898) 352.

- - Pscheborsky, A. P. Fschr. Mth. (1897) 357.

- - - case. Goursat, $\not$. Bll. Sc. Mth. 17 (1893) 247-.

-- , Goursat's theorem. Pszeborsky, A.P. Fschr. Mth. (1897) 356-.

- lacunary circle. Gomes Teixeira, F. Bll. Sc. Mth. 17 (1893) 29-. 
Functions representable by Heine's series. Thomae, J. [1868] Crelle J. 70 (1869) 258-. - of real variables possessing distributive property. Volpi, R. G. Mt. 35 (1897) 104-. - -2 variables arising from inversion of integrals of two given functions. Fuchs, $L$. Gött. Ab. 27 (1881) 39 pp.

Higher sines of 4 th order

$$
\left[\frac{z^{\mu}}{\mu !} \pm \frac{z^{\mu+n}}{\mu+n !}+\frac{z^{\mu+2 n}}{\mu+2 n !} \pm \ldots(n=4)\right] \text {. }
$$

Kapteyn, J. C., \& Kapteyn, W. Amst. Ak. Vh. 24 (1886) 98 pp.

- - nth order. Kapteyn, J. C., \& Kapteyn, $W$. Wien Ak. Sb. 93 (1886) (Ab. 2) 807-.

Infinite group of rational integral functions

$$
\left[R_{n} x=(-1)^{n} e^{x} \frac{d^{n}}{d x^{n}}\left(e^{-x} \cdot x^{n}\right)\right] .
$$

Berger, A. Stockh. Öfv. (1899) 175-.

Integral derived from continued fraction, theorem of Hermite. Gegenbauer, $L$. Wien Ak. Sb. 92 (1886) (Ab. 2) 995-.

- transcendental functions, distribution of roots of class. Bukrěev, B. Ja. [1893] Rec. Mth. (Moscou) 17 (1895) 217-; Fschr. Mth. (1893-94) 678.

Iterative functions. Farkas, $J$. Liouv. J. Mth. 10 (1884) 101-.

- - Netto, E. Mth. A. 29 (1887) 148-.

_- differential coefficients, at limiting point. Lémeray, - . Par. S. Mth. Bll. 25 (1897) 51-. - - theorem. Lémeray, -. Par. S. Mth. Bll. 23 (1895) 255-.

- - in theory of integral transcendental functions. Hill, C. J. D. Crelle J. 11 (1834) 193-.

Prym's function, generalisation. Nielsen, $N$. N. Ts. Mth. 11 (B) (1900) 73-; Fschr. Mth. (1900) 440-.

Pseudo- and hyper-Bernoullian functions and their applications. Longchamps, $G$. de. Brux. Mm. Cour. 4\%, 52 (1890-93) No. 1, 41 pp.

Recurring function $c_{n}=\sum_{\nu=0}^{n}(-1)^{\nu}\left(\begin{array}{l}n \\ \nu\end{array}\right) c_{\nu}$. Lerch, M. Časopis 22 (1893) 31-; Fschr. Mth. (1893-94) 409-.

Repeated exponentials. Gravé, D. N. A. Mth. 17 (1898) 80-.

Riemann's $\zeta(s)$ function, generalisation. Mellin, $H$. Helsingf. Acta 24 (1899) No. 10, 50 pp.

- - - for odd values of argument. Lerch, $M$. G. Teix. J. Sc. 14 (1900) 65-.

Theorem of Stieltjes, and functions defined by continued fractions. Koch, H. von. Par. S. Mth. Bll. 23 (1895) 33-.

Transcendental function, new. Soldner, $J$. Zach M. Cor. 23 (1811) 182-.

$-\ldots,-$, in relation with functions $\Gamma$ and $\mathbf{Z}$. Blaserna, P. Rm. R. Ac. Linc. Mm. 1 (1894) 499-.

- functions. Broch, O. J. Crelle J. 20 (1840) 178-; N. Mg. Ntvd. 2 (1840) 297-.

- with algebraic differentials. Hermite, $C$. C. R. 18 (1844) 1133-.
Transcendental functions, arithmetical property. Hurwitz, A. Mth. A. 32 (1888) 583-. - -, case. Hölder, $O$. Gött. Nr. (1886) 514 . - - classification; and general theory of integration. Drach, J. Par. Ec. Norm. A. 15 (1898) 243-.

- -, comparison. Boole, G. Phil. Trans. (1857) 745-.

- -, theorem of Sturm. Liouville, J. Liouv. J. Mth. 1 (1836) 269-.

- - $Z^{\prime}(a)$ and $G a$, and calculation of their values for several values of $a$. Lindman, C. F. [1863] Stockh. Ak. Hndl. 5 (1866) No. 2, $18 \mathrm{pp}$.

- quantities. Liouville, J. Liouv. J. Mth. 16 (1851) 133-.

Transcendentally transcendental functions. Moore, E. H. Mth. A. 48 (1897) 49-.

Trigonometric null-representations, special class. Rogel, $F$. Arch. Mth. Ps. 15 (1897) 431-.

$\int x^{s-\gamma p-1} F\left(x^{p}\right)\left(R\left(x^{p}\right)\right)^{ \pm \frac{s}{r p}} d x . \quad$ Broch, O.J. Crelle J. 23 (1842) 145-, 201-.

$a^{a}$;, development. Eisenstein, G. Crelle J. 28 (1844) 49-.

and corresponding invera? functions. Woepcke, $F$. Crelle J. 42 (1851) 83-.

$x^{x}$. Le Cointe, J. L. A. (vII) A. Mt. 5 (1863) $106-$.

$\Sigma e^{2 k \pi i x} /(w+k)^{8}, k=0$ to $\infty$. Lerch, M. Acta Mth. 11 (1887-88) 19-.

$C_{n}^{\nu}(x)$. Gegenbauer, $L$. Wien Ak. Sb. 97 (1889) (Ab. 2a) 259-.

-, addition theorem. Gegenbauer, $L$. Wien Ak. Sb. 102 (1893) (Ab. 2a) 942-.

$y$ defined by $\Sigma a_{r g} x^{r} y^{s}=0$. Melander, $K$. Fschr. Mth. (1890) 409.

$\Sigma\left(\frac{w^{n \sigma}}{\sin n \sigma \pi}+(-w)^{m} \frac{1}{\sigma} \cot \frac{m \pi}{\sigma}\right)$, new form of analytical expression. Lerch, $M$. Prag Ceské Ak. Fr. Jos. Rz. (Trída 2) 9 (1900) Art. 6, 17 pp.; Fschr. Mth. (1900) 401-.

\section{Eulerian functions.}

Bourguet, L. C. R. 96 (1883) 1307-.

Analogues to Eulerian functions. Appell, $P$. [1879-81] C. R. 86 (1878) 953-; 89 (1879) 841-, 1031-; Mth. A. 19 (1882) 84-.

_- - Ciani, V. G. Mt. 29 (1891) 68-. Analytic factorials, coefficients. Gambardella, F. G. Mt. 11 (1873) 49-, 86-.

Approximate value of $n !$ Malfatti, $G . F$. Verona S. It. Mm. 4 (1788) 206-.

Asymptotic value of $\sqrt{(a)}$ when $a$ is great. Hermite, -. Am. J. Mth. 17 (1895) 6-.

Bernoulli's theorem, gamma-function and Laplace's integral. Eggenberger, $J$. Bern Mt. (1893) 110-, 236.

Binet's formula. Genocchi, A. Brux. Ac. Bll. 20 (1853) (pte. 2) 392-; Tortolini A. 5 (1854) 150-.

- for $\omega(\mu)$, generalisation. Tilly, J.M. de. Brux. Ac. Bll. 38 (1874) 67-. 


\section{Eulerian Integrals}

Coefficients in differential formulæ, determination and application to Eulerian integrals. Zurria, G. Catania At. Ac. Gioen. 10 (1854) 265-.

Dirichlet's formula, application to mechanics. Clariana Ricart, L. Fschr. Mth. (1900) 687.

Eulerian functions, and Bernoulli's functions. Brioschi, F. (vr Adds.) Tortolini A. 1 (1858) 260-.

- - of 2nd kind, properties. Gasparis, A. de. Nap. Rd. 1 (1852) 97-

\section{Eulerian integrals.}

Bellavitis, G. A. Sc. Lomb. Ven. 4 (1834) 10 -.

Lejeune-Dirichlet, G. Crelle J. 15 (1836) 258-.

Stern, M. A. Crelle J. 21 (1840) 377-.

Cauchy, A. L. C. R. 17 (1843) 376-; 19 (1844) 67-.

Zhbikovskiu, A. K. [1867] (xI) Kazan Un. Mm. 3 (1866) 212-; (App. 3) I-.

Bourguet, L. Acta Mth. 1 (1882) 295-; C. R. 96 (1883) 1487-.

Tannery, J. C. R. 94 (1882) 1698-; 95 (1882) 75.

application of binomial theorem. Catalan, E.C. C. R. 47 (1858) 545-.

- to series. Binet, J. P. M. Par. Éc. Pol. J. $27^{\mathrm{e}} \mathrm{cah}$. (1839) 123-.

calculation by common algebraic substitution. Pánek, A. Prag Sb. (1893) (Mth.-Nt.) No. 21, 5 pp.; Fschr. Mth. (1893-94) 474.

cases. Dedekind, $R$. Crelle J. 45 (1853) 370 -. Walton, W. [1872] QJ. Mth. 12 (1873) 192.

-. Enneper, A. Gött. Nr. (1885) 163-.

- Pánek, A. Prag Č̀eské Ak. Fr. Jos. Rz. (Třida 2) 2 (1893) Art. 17, 7 pp.; Fschr. Mth. (1893-94) 474.

and the constant G. Catalan, E. C. [1882] St Pét. Ac. Sc. Mm. 31 (1883) No. 3, 51 pp.

- elliptic functions. Lerch, M. Prag Sb. (1897) (Mth.-Nt.) No. 28, 11 pp.

expansion in series. Bourguet, L. Par. Éc. Norm. A. 10 (1881) 175-.

expressions related to. Lerch, $M$. Prag Sb. (1890) 1 (Mth.-Nt.) 137-.

formulæ. Catalan, E. C. [1877] Brux. Ac. Sc. Mm. 42 (1878) (No. 4) 20 pp.; As. Fr. C. R. (1884) (Pt. 2) 11-.

fundamental properties. Lerch, $M$. Prag Sb. (1889) 2 (Mth.-Nt.) 188-; Fschr. Mth. (1889) 447-.

1st kind. Realis, S. G. Mt. 9 (1871) 345-.

- - Beaupain, J. Par. Ec. Norm. A. 9 (1892) 309-; Brux. Mm. Cour. 4, 53 (1893-94) No. 1, 18 pp.

- - fundamental property, new demonstration. Lerch, - . Par. S. Mth. Bll. 15 (1887) 173-.

- and 2nd kinds. Matthiessen, L. A. Mt. 2 (1868-69) 21-.

2nd kind. Gohierre de Longchamps, - Par.

Ec. Norm. A. 9 (1880) 419-.

- - Gyldén, J. A. H. C. R. 92 (1881) $897-, 942-$
Gamma Functions 4410

2nd kind. Hermite, C. Crelle J. Mth. 90 (1881) 332-.

- - expansion in series of expressions depending on. Winckler, A. Wien Ak. Sb. $85(1882)(A b .2)$ 1039-.

- - formula, demonstration. Hermite, C. Prag Sb. (1888) (Mth.-Nt.) 364-.

multiple integral reducible to. Pochhammer, $L$. Crelle J. Mth. 107 (1891) 246-.

relation between the two. Mansion, - Brux. S. Sc. A. 19 (1895) (Pt. 1) 1-.

and series. Catalan, E. C. Brux. Ac. Bll. 17 (1889) 129-.

- sinusoid spirals $\left[\rho^{n}=\frac{(2 a)^{n}}{2} \cos n \phi\right]$. Loria, G. Prag Sb. (1897) (Mth.-Nt.) No. 18, 6 pp. tables. Bellavitis, G. [1873] Ven. I. Mm. 18 (1874) 125-.

theorem, demonstration. Hoppe, $R$. Arch. Mth. Ps. 41 (1864) 65-.

theorems. Catalan, E. Brux. Ac. Bll. 22 (1891) 459-.

theory. Schaar, M. Brux. Mm. Cour. 4, 22 (1846-47) $16 \mathrm{pp}$.

-. Stern, M. A. Gött. Stud. (1847) 283-.

-. Pochhammer, L. Mth A. 35 (1890) 495-.

-, explanation. Preobraženskij, P.V. Mosc. S. Sc. Bll. 65 (No. 2) (1890) 55-.

-, formula, fundamental. Tortolini, $B$. Tortolini A. 5 (1854) $292-$.

-, formulæ. Serret, J. A. Liouv. J. Mth. 8 (1843) 489-.

and other uniform functions. Bourguet, $L$. Acta Mth. 2 (1883) 261-.

with unlimited argument. Hankel, $H . \quad \mathrm{Z}$. Mth. Ps. 9 (1864) 1-.

Euler's constant. Lerch, M. G. Teix. J. Sc. 13 (1897) 129-

- - and Binet's function. Catalan, E. C. C. R. 77 (1873) 198-; Liouv. J. Mth. 1 (1875) 209-.

- -, expressions for. Lerch, M. Prag Sb. (1897) (Mth.-Nt.) No. 42, 5 pp.

- E, calculation. Shanks, $W$. [1867-71] R. S. P. 15 (1867) 429-; 16 (1868) 154, 299-; 18 (1870) 49 ; 20 (1872) 29 -.

- sum formula, representation of functions by. Lipschitz, $R$. Crelle J. 56 (1859) 11-.

Factorials, Stirling's theorem

$$
1.2 .3 \ldots n=\sqrt{2 n \pi} \cdot n^{n} \cdot e^{-n},
$$

proof. Glaisher, J.W.L. [1877] QJ. Mth. 15 (1878) 57-.

Formula of Raabe, on $\int_{0}^{1} \log F(x+u) d x$. Lerch, M. G. Mt. 26 (1888) 39-

\section{Gamma functions.}

Enneper, A. Schlömilch Z. 7 (1862) 189-. Mellin, $H$. Stockh. Öfv. 40 (1883) No. 5, 3-. Brunel, G. Bordeaux S. Sc. Mm. 3 (1887) 1-. Mansion, P. Brux. S. Sc. A. 17 (1893) (Pt. 1) 4 -.

Baker, H. F. Mess. Mth. 25 (1896) 125-. 
Lerch, M. Prag České Ak. Fr. Jos. Rz. (Tř́da 2) 5 (1896) Art. 14, 37 pp.; Fschr. Mth. (1896) 333.

analogues to. Alekséevskij, V. P. Kharkov Mth. S. Com. 1 (1889) 169-; Fschr. Mth. (1890) 439-; Leip. Mth. Ps. B. 46 (1894) 268-.

and analytical factorials. Hoppe, R. [1845] Crelle J. 40 (1850) 152-.

with arbitrary parameter. Bigler, - Crelle J. Mth. 102 (1888) 237-.

and beta functions, relations between. Spitzer, S. D. Nf. Tbl. ("1876) (Beil.) 63.

- - - series connected with. Lerch, $M$. G. Teix. J. Sc. 10 (1891) 103-.

of a complex. Sharpe, H.J. Mess. Mth. 13 (1884) 111-.

deduced from $\Sigma(w+n)^{-8}$. Lerch, $M$. Prag České Ak. Fr. Jos. Rz. (Tř́da 2) 8 (1899) Art. 35, 9 pp.; Fschr. Mth. (1899) 392. and definite integrals. Matthiessen, L. Z. Mth. Ps. 12 (1867) 302-.

derivatives. Jeffery, H. M. [1862] QJ. Mth. 6 (1863) 82-.

double. Barnes, E. W. [1900] Phil. Trans. (A) 196 (1901) 265-.

-, genesis, Barnes, E. W. L. Mth. S. P. 31 (1900) 358-.

evaluation of infinite factorials by. Weyr, $E$. Časopis 22 (1893) 161-; Fschr. Mth. (189394) 750 .

expansion embracing series of Stirling and Kummer. Landsberg, G. Brux. Mm. Cour. $8^{\circ}, 55$ (1896-98) No. 4, 28 pp.

- and integrals depending on it. Gilbert, L.P. [1873] Brux. Ac. Mm. 41 (Pt. 1) (1875) (No.3) $60 \mathrm{pp}$.

expression of definite integrals by. Graf, J.H. Bern Mt. (1884) (Heft 2, Ab.) 46-.

expressions in terms of complete elliptic integrals. Glaisher, J.W. L. Mess. Mth. 24 (1895) 27-.

and factorials. Ohm, M. Crelle J. 36 (1848) 277-.

- figurate numbers, binomial coefficients in relation to. Matthiessen, -. D. Nf. Tbl. (*1868) 128-.

formula. Lerch, M. Mh. Mth. Ps. 8 (1897) 187-.

$-\int_{0}^{\infty} \phi(x+a) a^{\mu-1} d a=(-1)^{\mu} \int^{\mu} \phi(x) d x^{\mu}$.

Liouville, J. Crelle J. 12 (1834) 273-.

formulæ. Genocchi, A. Brux. Ac. Bll. 21 (1854) 84-.

-. Lindhagen, A. Stockh. Öfv. 40 (1883) No. 2, 27-.

function related to. Schlömilch, O. X. Z. Mth. Ps. 25 (1880) 335-.

Gauss's expression for. Arndt, F. Grunert Arch. 10 (1847) 250-.

$---\Gamma(\mu)$. Zehfuss, G. Grunert Arch. 30 (1858) 441.

generalisation of equation

$$
\Gamma(1+x) \Gamma(1-x)=\pi x / \sin \pi x .
$$

Mellin, $H$. Acta Mth. 3 (1883) 102-. and hypergeometric functions, application of Cauchy's theorem. Mellin, H. Helsingf. Acta 21 (1896) No. 1, $115 \mathrm{pp}$.

incomplete. Hočevar, $F$. Z. Mth. Ps. 21 (1876) 449-

-, continued fraction for. Schlömilch, $O$. Z. Mth. Ps. 16 (1871) 261-.

and infinite products. Bauer, $G$. Crelle J. 57 (1860) 256-.

- - - Mellin, $H$. Acta Mth. 3 (1883) 322-; Helsingf. Öfv. 26 (1884) 170-.

integrals expressible by. Schlömilch, $O$. Z . Mth. Ps. 9 (1864) 256-.

2nd kind. Serret, J.A. Liouv. J. Mth. 7 (1842) 114-.

- -. Schlömilch, O. Grunert Arch. 4 (1844) 167-.

- - Liouville, J. Liouv. J. Mth. 11 (1846) 464-.

- -, general properties. Schlömilch, $O$. Grunert Arch. 6 (1845) 213-.

- - negative minima. Glaisher, J.W.L. B. A. Rp. 43 (1873) (Sect.) 13.

- - theorem. Liouville, J. Liouv. J. Mth. 20 (1855) 157-.

- -, -, Legendre's. Schlömilch, O. Grunert Arch. 7 (1846) 348-.

Legendre's. Liouville, J. Liouv. J. Mth. 17 (1852) 448-.

-, Gauss's formula, demonstration. Liouville, J. C. R. 42 (1856) 501-.

logarithm. Binet, J. P. M. C. R. 9 (1839) 156-:

- Hermite, C. Am. J. Mth. 17 (1895) 111-.

of negative quantity. Newman, $F, W$. Camb. and Dubl. Mth. J. 3 (1848) 57-.

new notation in connexion with. Lindner, $P$. Arch. Mth. Ps. 70 (1884) 96-, vir.

with a power as argument, property. Eggenberger, J. Bern Mt. (1895) 8-.

property of satisfying no algebraic differential equation. Hölder, $O$. Mth. A. 28 (1887) 1-. properties. Blissard, J. QJ. Mth. 9 (1868) 280-.

quotient of two. Schlömilch, O. X. Z. Mth. Ps. 25 (1880) 351-.

reciprocal value. Schlömilch, O. X. Z. Mth. Ps. 25 (1880) 103-.

series for. Schlömilch, $G$. Schlömilch Z. 4 (1859) 431-.

and Stirling's series. Genocchi, A. Rm. S. It. Mm. 6 (1887) No. 2, 24 pp.

theorem, Gauss's, demonstration. Sonine, N.J. Par. S. Mth. Bll. 9 (1881) 162-.

theory. Kummer, E. E. Crelle J. 35 (1847) 1-. -. Prym, F. E. Crelle J. Mth. 82 (1877) $165-$.

-. Mellin, H. Acta Mth. 8 (1886) 37-.

-. Pringsheim, A. Mth. A. 31 (1888) 455-.

- Barnes, E. W. Mess. Mth. 29 (1900) 64-.

-, definite integrals from. Lerch, $M$. Prag České Ak. Fr. Jos. Rz. (Tŕida 2) 8 (1899) Art. 37, 5 pp.; Fschr. Mth. (1899) 391.

- elementary exposition. Jensen, J. L. W. V. N. Ts. Mth. 2 (B) (1891) 33-, 57-, 83-; Fschr. Mth. (1891) 445-. 
theory, formulæ, generalisation. Berger, $A$. Stockh. Öfv. 38 (1881) No. 9, 13-.

-, Gauss's equation. Lerch, M. Prace Mt.-Fiz. 10 (1899-1900) 1-, 269-; Fschr. Mth. (1899) 390.

-, point in. Lerch, $M$. Prag Sb. (1893) (Mth.-Nt.) No. 26, 8 pp.

transcendental function connected with, and application to integration. Kinkelin, $\boldsymbol{H}$. Crelle J. 57 (1860) 122-.

- functions related to. Mellin, $H$. Helsingf. Acta 14 (1885) 353-; 15 (1888) 1-; Fschr. Mth. (1886) 441-.

transformations connected with. Catalan, E.C. Liége S. Sc. Mm. 15 (1888) No. 1, 64-, 103-.

Gamma integral, property. Björling, E. G. Stockh. Öfv. 13 (1856) 181-.

Generalisation of Eulerian functions. Pincherle, S. C. R. 106 (1888) 265-.

Interpolation of some products. Steklov, V.A. Kharkov Mth. S. Com. 1 (1889) 239-; Fschr. Mth. (1890) 439.

Malmsten series. Lerch, $M$. Prag České Ak. Fr. Jos. Rz. (Tř́da 2) 3 (1894) Art. 28, 1-; Fschr. Mth. (1893-94) 484-.

- - Hermite, $C$. Prag České Ak. Fr. Jos. Rz. (Tŕida 2) 3 (1894) Art. 28, 62-; Fschr. Mth. (1893-94) 484-.

Periodic functions. Scheibner, $W$. Leip. B. 14 (1862) 64-.

Prym's and Hermite's functions. Genocchi, $A$. Brux. Ac. Bll. 4 (1882) 438-; 5 (1883) 421-. Rapidly converging series for $\frac{\Gamma^{\prime}(w)}{\Gamma(w)}$. Lerch, $M$. Prag České Ak. Fr. Jos. Rz. (Třida 2) 8 (1899) Art. 36, 9 pp.; Fschr. Mth. (1899) 390-.

Reduction of certain integrals to Eulerian functions. Beaupain, $J$. Brux. Mm. Cour. $4^{\circ}, 51$ (1889) No. 6, 40 pp.

Stirling's formula. Rouché, E. C. R. 110 (1890) 513-.

- -, demonstration. Catalan, E. C. Liège S. Sc. Mm. 12 (1885) No. 2, 221-.

Theorem of Legendre, demonstration. Stern, M. A. [1866] Crelle J. 67 (1867) 114-.

- Vandermonde, extension. Jackson, F.H. L. Mth. S. P. 26 (1895) 285-.

Theorems of Fermat. Studnicka, F.J. Časopis 29 (1900) 257-; Fschr. Mth. (1900) 438.

$\Gamma a \Gamma b$

$\overline{\Gamma(a+b)}$, demonstration. Jacobi, C.G.J. Crelle J. 11 (1834) 307.

$\int_{0}^{1} x^{p-1} d x\left(1-x^{n}\right)^{q}$. Plana, G. Crelle J. 17 (1837) 1-, 163-, 338-.

$\int_{0}^{1} x^{p-1} d x(1-x)^{q-1}, p$ or $q$ being rational. Binet, J. P. M. C. R. 16 (1843) 377-.

$\Gamma(n+1)$, approximation when $n$ is large. Raabe, J. L. Crelle J. 25 (1843) 146-; 28 (1844) 10 -.

$\Gamma(a)$, theory, supplement. Gudermann, $C$. Crelle J. 29 (1845) 209 -.

$\Gamma(x) \Gamma\left(x+\frac{1}{2}\right)=2^{1-2 x} \sqrt{\pi} \cdot \Gamma(2 x)$. Liouville, $J$. Liouv. J. Mth. 20 (1855) 161-.
$\Gamma(x)$, fundamental equations. Kinkelin, $H$. [1856] Bern Mt. (1857) 1-.

$G_{m}=\frac{1.2 .3 \ldots m}{(m+1)(m+2) \ldots 2 m} \cdot$ Oltramare, G. Gen.

I. Nt. Mm. 4 (1856) (pte. 2) 33-.

II $(z)$ complex variable. Enneper, A. QJ. Mth. 1 (1857) 393-.

$\log \Gamma x$, differentials. Clausen, $T$. Grunert Arch. 30 (1858) 166-. $\int_{0}^{\infty} \frac{x^{k-n-1} \cdot d x}{1+2 x^{k} \cos \theta+x^{2 k}}=$ etc. Plana, G. Tor.

$\log \Gamma(1+a)$, approximation. Limbourg, $H$. Brux. Mm. Cour. 4, 30 (1861) 21 pp.

$\Sigma \frac{1}{\Gamma(x)}$, calculation. Gasparis, A. de. Nap. Rd. 6 (1867) 276-.

$\Pi(x)$, and imaginary triangles, etc. Grunert, J. A. Arch. Mth. Ps. 51 (1870) 423-.

$\int_{a}^{b} \log \Gamma x(d x)$. Bierens de Haan, D. Amst. Vs. Ak. 7 (1873) 26-; Arch. Néerl. 8 (1873) 148-. $\log \Gamma(x)$, expansions. Gilbert, $P$. Brux. Ac. Bll. 36 (1873) 541-.

-, - . Genocchi, A. Brux. Ac. Bll. 36 (1873) 546 -

-. Genocchi, A. Arch. Mth. Ps. 61 (1877)366-. $\Gamma\left(\frac{n}{6}\right)$, calculation. Kummell, C. H. Des Moines Anal. 4 (1877) 156-, 182-.

$\Gamma(x)$, etc., applications. Appell, $P$. C. R. 86 (1878) 953-. $\int_{0}^{1} \log \Gamma(x+u) d u . \quad$ Stieltjes, T. J. (jun.). N.

$\Gamma(x)$, maxima and minima. Bourguet, $L$. Tor. Ac. Sc. At. 16 (1881) 758-.

$\Gamma(x)-P(x)=Q(x)$. Mellin, $H$. Acta Mth. 2 (1883) 231-.

$\log \Gamma(a)$, expansion. Stieltjes, T. J. Liouv. J. Mth. 5 (1889) 425-.

$\log \Gamma(x)$ represented as a definite integral. Gomes Teixeira, F. Fschr. Mth. (1891) 450 . Г (a). Pochhammer, L. Mth. A. 41 (1893) 157-. $\Gamma(1+i)$, numerical value. Cayley, $A$. Mess. Mth. 23 (1894) 36-.

$\phi(\gamma)=\frac{2}{\sqrt{\pi}} \int_{0}^{\gamma} e^{-t^{2}} d t$, table. Kämpfe, B. $\mathrm{Ph}$. Stud. 9 (1894) 145-.

$\log \Gamma(a)$. Hermite, $C$. Crelle J. Mth. 115 (1895) 201-.

$\Gamma(n)$. Dixon, A. C. [1898] Mth. Gz. 1 (1900) 189-.

$1 / \Gamma(\mu)$, integral expression for. Jørgensen, $N$. N. Ts. Mth. 11 (B) (1900) 34-; Fschr. Mth. (1900) 440 .

\section{Legendre's functions; Bes- sel's functions; hypergeometric functions.}

Algebraic functions, class, special, arising from expansion of $\left(1-2 x z+z^{2}\right)^{\frac{1}{2}}$. Jacobi, C.G.J. Crelle J. 2 (1827) 223-. 
Associated functions, especially those of spherical shell. Beltrami, $E$. Bologna Ac. Sc. Mm. 4 (1882) 211-.

Bessel's equation, general solution. Chessin, A. Am. J. Mth. 16 (1894) 186-.

- - and Riccati's equation. Greenhill, A. G. QJ. Mth. 16 (1879) 294-.

- - tables of roots. Willson, R. W., $\&$ Peirce, B. O. N. Y. Am. Mth. S. Bll. 3 (1897) 153-.

\section{BESSEL'S FUNCTIONS.}

Schlömilch, O. Schlömilch Z. 2 (1857) 137-. Rayleigh, (Lord). Ph. Mg. 44 (1872) 328-.

Gegenbauer, $L$. Wien Ak. Sb. 70 (1874) (Ab.2) 6-; 74 (1877) (Ab.2) 124-; 75 (1877) (Ab.2) 218-; 88 (1884) (Ab. 2) 975-; 95 (1887) (Ab. 2) 409-.

Hall, $A$. Des Moines Anal. 1 (1874) 81-。

Kapteyn, W. N. Arch. Wisk. 20 (1893) 116-; Fschr. Mth. (1893-94) 849.

Gegenbauer, L. Mh. Mth. Ps. 8 (1897) 383-.

Macdonald, H. M. [1897] L. Mth. S. P. 29 (1898) 110-.

Nielsen, N. Kjøb. Ov. (1899) 661-; (1900) 55-.

addition and subtraction of arguments. Graf, J. H. Mth. A. 43 (1893) 136-.

and Cauchy's numbers, relation between. Chessin, A. S. A. Mth. 12 (1898-99) 170-.

connection with hyperspherical and spherical harmonics. Hobson, E. W. [1893] L. Mth. S. P. 25 (1894) 49-.

convergence of expansion of arbitrary function $f(x)$ in. Schläfli, L. Mth. A. 10 (1876) 137-.

definite integrals connected with. Basset, $A . B$. Camb. Ph. S. P. 8 (1895) 122-.

development for effect of periodic forces. Schott, C. A. U. S. Coast Sv. Rp. (1862) 232-.

developments. Meissel, E. As. Nr. $129(1892)$ 281-; 130 (1892) 363-.

expansion of function with 2 variables in. Cantor, G. [1871] Mth. A. 5 (1872) 133-.

- - any functions by. Neumann, C. Crelle J. 67 (1867) 310 -

- in series by. König, J. Mth. A. 17 (1880) 85-.

expression of function in terms of. Sheppard, W. E. QJ. Mth. 23 (1889) 223-.

- in semi-convergent series for large values of variable. Callandreau, $O$. Bll. Sc. Mth. 14 (1890) 110-.

formula, demonstration. Giuliani, G. G. Mt. 22 (1884) 201-.

- involving. Graf. J.H. Sch. Nf. Gs. Vh. (1896) 59-.

function related to. Lommel, E. C. J. [1875] Mth. A. 9 (1876) 425-.

integration of equation $x^{m+\frac{1}{2}} \frac{\delta^{2 m+1} y}{\delta x^{2 m+1}} \pm y=0$ by. Lommel, E. Mth. A. 2 (1870) 624-.

- formulæ. Lerch, $M$. Prag České Ak. Fr. Jos. Rz. (Tř́̊a 2) 5 (1896) Art. 23, 16 pp.; Fschr. Mth. (1896) 233-. integration formulæ connected with. Gegenbauer, L. Mh. Mth. Ps. 5 (1894) 53-.

1st kind. Wagner, C. Bern. Mt. (1894) 204-.

- Gegenbauer, L. Mh. Mth. Ps. 10 (1899) 189-.

-, addition theorem, generalisation. Wendt, C. Mh. Mth. Ps. 11 (1900) 125-.

- and a continued fraction. Graf, I. H. A. Mt. 23 (1895) 45-.

- -, roots, new properties. Gegenbauer, L. Liège S. Sc. Mm. 2 (1900) No. 3, $7 \mathrm{pp}$.

- and 2nd kinds. Nicolas, J. Par. Ec. Norm. A. 11 (1882) (Suppl.), 90 pp.

_ _ _ , descending series for. McMahon, J. A. Mth. 8 (1893-94) 57-.

2nd kind. Gegenbauer, $L$. Wien Sb. 65 (1872) (Ab. 2) 33-; 66 (1872) (Ab. 2) 220-.

- -. Bôcher, M. A. Mth. 6 (1891-92) 85-.

- - Julius, V.A. Arch. Néerl. 28 (1895) 221-.

- - Crelier, L. Bern. Mt. (1897) 61-.

- - Otti, H. Bern Mt. (1898) 1-.

Neumann's researches. Schläfli, L. [1870] Mth. A. 3 (1871) 134-.

2nd order. Coates, C.V. QJ. Mth. 20 (1885) 250-; 21 (1886) 183-.

0th order, roots. Bôcher, M. N. Y. Am. Mth. S. Bll. 5 (1899) 385-

and $P$-functions, roots. Van Vleck, E. B. Am. J. Mth. 19 (1897) 75-.

product $J_{m}(x) J_{n}(x)$. Orr, W. McF. [1899] Camb. Ph. S. P. 10 (1900) 93-.

products and squares. Neumann, C. Mth. A. 2 (1870) 192.

properties. Hobson, E. W. L. Mth. S. P. 28 (1897) 370-.

- derived from continued fractions. Crelier, L. A. Mt. 24 (1896) 131-.

with purely imaginary index. Bôcher, M. A. Mth. 6 (1891-92) 137-.

and related functions, roots. McMahon, $J$. A. Mth. 9 (1894-95) 23-.

representation of arbitrary functions by. Weber, H. [1872] Mth. A. 6 (1873) 146-.

- by definite integrals. Gubler, $E$. Zür. Vjschr. 33 (1888) 130-.

- of definite integrals by. Wagner, C. Bern Mt. (1895) 115-; (1896) 53-.

roots. Bôcher, $M$. N. Y. Am. Mth. S. Bll. 3 (1897) 205-.

series, expression for a rational polynomial in. McMahon, J. Am. As. P. (1900) 42-.

solution of some differential equations by. Pearson, $K$. Mess. Mth. 9 (1880) 127-.

tables. Brit. Ass. Comm. B. A. Rp. (1889) 28-; (1893) 227-; (1896) 98-.

theory. Lommel, E. C. J. [1870-79] Mth. A. 3 (1871) 475-; 4 (1871) 103-; 14 (1879) 510-; 16 (1880) 183-.

-. Weber, H. Mth. A. 37 (1890) 404-.

-. Meissel, E. As. Nr. 128 (1891) 435-.

-, deduction of formulæ. Ball, L. de. As. Nr. 128 (1891) 1-.

transcendental equations formed by equating to zero. Rudzki, M. P. Prace Mt.-Fiz. 3 (1892) 69-; Fschr. Mth. (1892) 107-. 
4420 Bessel's Functions, etc.

transformation of origin of reference. Walker, G. T. Mess. Mth. 25 (1896) 76-

zeros. Hurwitz, A. Mth. A. 33 (1889) 246-. -. Macdonald, H. M. L. Mth. S. P. 29 (1898) 575-; 30 (1899) 165-.

-. Schafheitlin, P. Crelle J. Mth. 122 (1900) 299-

Bessel's and hypergeometric series, differential equations for. Schafheitlin, $P$. Crelle J. Mth. 114 (1895) 31-.

Catalan's theorem on roots of

$$
\int_{-1}^{x} P_{2 n+1}(x) d x=0,
$$

generalised. Gegenbauer, L. Wien Ak. Sb. 94 (1887) (Ab. 2) 801-.

Circular opening, intensity relationships of diffraction phenomenon. Steiner, L. Mth. Termt. Éts. 12 (1894) 44-; Mth. Nt. B. Ung. 11 (1894) 362-.

\section{Cylindrical functions.}

Ermakof, V. Mth. A. 5 (1872) 639-.

Gïther, S. Arch. Mth. Ps. 56 (1874) 292-.

Beltrami, E. Tor. Ac. Sc. At. 16 (1880) 201-.

Sonine, N.J. Mth. A. 16 (1880) 1-; 30 (1887) $582-$.

analogues to. Giuliani, G. G. Mt. 25 (1887) 198-.

application of theorem of Jacobi's to. Schläfi, L. [1871] A. Mt. 5 (1871-73) 199-.

definite integrals with. Hankel, $H$. [1869] Mth. A. 8 (1875) 453-.

expansions in terms of. Nielsen, N. N. Ts. Mth. 9 (B) (1898) 73-; 10 (B) (1899) 73-; Fschr. Mth. (1898) 361-; (1899) 374-.

history. Maggi, G. A. Rm. R. Ac. Linc. T. 4 (1880) 259-.

1st kind, theorems. Marcolongo, $R$. Nap. Rd. 28 (1889) 96-

- and 2nd kinds. Hankel, H. [1868] Mth. A. 1 (1869) 467-.

2nd kind, for small arguments. Morton, $W . B$. [1900] Nt. $63(1900-01) 29$.

product of two. Nielsen, N. Mth. A. 52 (1899) 228-.

representation of a function of two variables by. Mehler, F. G. (1871] Mth. A. 5 (1872) 135-.

Differential equation of functions of elliptic cylinder. Lindemann, C. L. F. Mth. A. 22 (1883) 117-.

- equations for series $F(\rho, \sigma ; x), F(\rho, \sigma, \tau ; x)$. Pochhammer, L. Mth. A. 41 (1893) 197-.

Discontinuous integral. Gubler, E. Mth. A. 48 (1897) 37-.

Expansion of potential in morphometry of earth's surface. Kövesligethy, $R$. Mth. Termt. Éts. 13 (1895) 167-; Mth. Nt. B. Ung. 13 (1897) 365-.

Expansions in series of $X_{n}$ functions of functions of one variable, uniformity. Dini, $U$. [1873] A. Mt. 6 (1875) 216-.

$F$-series, generalised, reduction of differential equation. Pochhammer, L. Crelle J. Mth. 112 (1893) 58-
Hypergeometric Functions 4420

$F$-series, 3rd order, differential equations. Pochhammer, L. Mth. A. 46 (1895) 584-.

-, 4th order, differential equations. Pochhammer, L. Mth. A. 50 (1898) 285-.

Fluctuating functions, numerical calculation of roots. Rayleigh, J. W. Strutt (Lord). (xI) L. Mth. S. P. 5 (1873-74) 119-.

Fourier-Bessel functions. Heine, E. Crelle J. 69 (1868) 128-.

_ - Kapteyn, W. Par. Éc. Norm. A. 10 (1893) 91-.

- - _ expansion of function in squares and products of. Neumann, C. Leip. B. 21 (1869) 221-.

Functions arising in continued fraction for $e^{a x i}$, addition theorem. Gegenbauer, $L$. Wien Ak. Sb. 85 (1882) (Ab. 2) 491-.

- from expansion of $\left(1-2 a x+a^{2} \alpha^{2}\right)^{-\frac{2 l+1}{2}}$. Escary, -. C. R. 86 (1878) 114-, 1451-.

- "differing as little as possible from zero." Tchebitchef, P. L. Liouv. J. Mth. 19 (1874) 319 .

Gaussian function $F(a, 1, \gamma, x)$, expansion as continued fraction. Thomé, W. L. Crelle J. 66 (1866) 322-.

- $F^{\prime}(\alpha, \beta, \gamma, x)$, differential equation satisfied by. Mathieu, E. L. Liouv. J. Mth. 8 (1882) 357-.

- quotient $\frac{F(a, \beta+1, \gamma+1, x)}{F(a, \beta, \gamma, x)}$, expansion as continued fraction. Thomé, W. L. Crelle J. 67 (1867) 299-.

Gauss's polynomials $X_{n}$. Catalan, $E$. Brux. Ac. Mm. 49 (1890-93) (No. 1) 34 pp.

- series. Thomae, J. Gött. Nr. (1884) 493-.

- $F(\alpha, \beta, \gamma, x)$, functions representable by, theory. Riemann, B. Gött. Ab. 7 (Mth.) (1856-57) 3-.

Geometrical series, 2nd order. Hochheim, A. Magdeb. Nt. Vr. Jbr. u. Ab. (1886) 137-; (1887) 25-.

Harmonics, tesseral, functions of more than 2 variables analogous to. Hill, M. J. M. Camb. Ph. S. T. 13 (1883) 273-.

Hypergeometric differential equation. Besso, D. Rm. R. Ac. Linc. T. 7 (1883) 230.

- - equations. Besso, D. Rm. R. Ac. Linc. Rd. 3 (1894) (Sem. 2) 393-.

- equation. Humbert, G. Par. S. Mth. Bll. 8 (1880) 112-.

- - derivation. Pochhammer, L. Crelle J. 73 (1871) 85-

- -, special cases. Boyd, J.H. A. Mth. 7 (1892-93) 145-.

- expansions, notation suitable for. Radau, R. C. R. 98 (1884) 39-.

\section{HYPERGEOMETRIC FUNCTIONS.}

Pincherle, S. G. Mt. 32 (1893) 209-, 375. algebraic equations soluble by. Lachtin, L. K. [1892] Rec. Mth. (Moscou) 16 (1893) 597-; 17 (1895) 1-; Fschr. Mth. (1893-94) 753-.

- transformation. Papperitz, E. [1885-86] Mth. A. 27 (1886) 315-. 
analogues to. Goursat, É. Par. Ec. Norm. A. 3 (1886) 107-.

arising from Gauss's equation. Halphen, G.H. C. R. 92 (1881) 856-.

and Bessel's functions, roots. Porter, $M$. B. Am. J. Mth. 20 (1898) 193-.

with branchings $(2,3,7),(2,4,7)$, arithmetical theory. Fricke, R. Mth. A. 41 (1893) 443-. case. Markov, A. A. [1892] St Pét. Ac. Sc. Mm. 41 (1893) No. 2, 37 pp.

last element of which has special value. Hey. mann, W. Z. Mth. Ps. 44 (1899) 280-.

expression as definite integrals. Mellin, $H$. Helsingf. Acta 20 (1895) No. 7, 39 pp.; Fschr. Mth. (1895) 481.

extension of Riemann's problem to functions of 2 variables. Picard, É. [1880] Par. Éc. Norm. A. 10 (1881) 305-.

_- Goursat, É. C. R. 95 (1882) 903-, 1044-; 96 (1883) 185-. of form

$$
1+\frac{p p^{\prime} p^{\prime \prime}}{1 \cdot q^{\prime} q^{\prime \prime}}+\frac{p \cdot p+1 \cdot p^{\prime} p^{\prime}+1}{1 \cdot 2 \cdot q^{\prime} \cdot q^{\prime}+1} \frac{p^{\prime \prime} \cdot p^{\prime \prime}+1}{q^{\prime \prime} \cdot q^{\prime \prime}+1} .
$$

Thomae, C. J. Crelle J. Mth. 87 (1879) 26-. and gamma functions, application of Cauchy's theorem. Mellin, $H$. Helsingf. Acta 21 (1896) No. 1, $115 \mathrm{pp}$.

generalisation. Pincherle, $S$. Rm. R. Ac. Linc. Rd. 4 (1888) (Sem. 1) 694-, 792-.

history. Papperitz, $E$. Dresden Isis Sb. (1889) (Ab.) 61-.

2nd kind and higher order. Beaupain, $J$. Brux. Mm. Cour. 4, 54 (1896) No. 7, 46 pp., No. 8, 46 pp.

higher order. Goursat, É. Par. Éc. Norm. A. 12 (1883) 261-, 395-.

- L. Lětnikov, A. V. Rec. Mth. (Moscou) 14 (1890) 216-; Fschr. Mth. (1890) 443.

- Beaupain, J. Brux. Mm. Cour. $4^{\circ}, 56$ (1897-98) No. 2, 52 pp.

$n$th order. Pochhammer, L. [1869] Crelle J. 71 (1870) 316-.

- - Fuchs, L. Crelle J. 72 (1870) 255-

simultaneous linear differential equations integrable by. Heymann, $W$. Z. Mth. Ps. 32 (1887) 176-.

with singularity. Ritter, $E$. Mth. A. 48 (1897) 1-.

theorem, fundamental. Baraniecki, $M$. $A$. Par. T. Nauk Śc. Pam. $8\left({ }^{*} 1876\right)$ Art. 2, 19 pp.; Bll. Sc. Mth. As. 1 (*1877) (Pt. 2) 223-.

2 variables. Goursat, $\dot{E}$. C. R. 95 (1882) 717-. - -. Appell, P. Liouv. J. Mth. 10 (1884) 407-. - - formulæ. Appell, P. Liouv. J. Mth. 8 (1882) 173-.

several variables. Lauricella, G. Palermo Cir. Mt. Rd. 7 (1893) 111-.

Hypergeometric integral, general, fundamental values. Radicke, A. Z. Mth. Ps. 22 (1877) 87-. - integrals, $n$th order. Pochhammer, $L$. [1870] Crelle J. 73 (1871) 135-.

- quantities, calculation. Multedo, -. Genova $\mathrm{Mm}$. I. Ligure 1 (1806) 1-; Genova Mm. Ac. 2 (1809) 230-.

\section{Hypergeometric series.}

Gauss, C. F. Gött. Cm. 2 (1811-13) 46 pp.

Kummer, E. E. Crelle J. 15 (1836) 39-, 127-. Cayley, A. Ph. Mg. 16 (1858) 356-.

Dronke, A. Schlömilch Z. 8 (1863) 401-.

Schläfli, L. [1870] Mth. A. 3 (1871) 286-.

Gegenbauer, L. Arch. Mth. Ps. 55 (1873) 284-.

Meissel, E. [1874] Arch. Mth. Ps. 57 (1875) 446-.

Markoff, A. C. R. 114 (1892) 54-; Mth. A. 40 (1892) 313-.

Graf, I. H. G. Mt. 36 (1898) 233-.

case. Cayley, A. QJ. Mth. 16 (1879) 268 -.

class of equations of 6th order soluble by. Besso, D. [1882] Rm. R. Ac. Linc. Mm. 14 (1883) 30-.

in connexion with observation polygons. Pear. son, $K$. Ph. Mg. 47 (1899) 236-.

definite integrals connected with. Rajewski, $J$. Krk. Ak. (Mt.-Prz.) Rz. 20 (1890) 272-; Crc. Ac. Sc. Bll. (1890) 61-.

and definite integrals, theorem. Catalan, E.C. Liége S. Sc. Mm. 15 (1888) No. 1, 46-.

definite integrals satisfying differential equation. Torelli, G. Rm. S. It. Mm. 7 (1890) No. 4, $18 \mathrm{pp}$.

differential and difference equations integrable by. Heymann, $W$. Crelle J. Mth. 122 (1900) 164-.

- equation. Jacobi, C. G.J. Crelle J. 56 (1859) 149-.

- - Spitzer, S. Crelle J. 57 (1860) 78-. - (Spitzer). Borchardt, C. W. (vI Adds.) Crelle J. 57 (1860) 81.

- Markov, A. A. Kharkov Mth. S. Com. (1886) 51-, 95-; Mth. A. 28 (1887) 586-; 29 (1887) 247-. 586-.

- - 2nd solution. Johnson, W. W. Mess. Mth. 17 (1888) 35-.

- equations integrable by. Heymann, $W$. Z. Mth. Ps. 29 (1884) 144-.

- - satisfied by. Pochhammer, L. Mth. A. 33 (1889) 353-.

divergent (or semiconvergent). Orr, W. McF. [1898] Camb. Ph. S. T. 17 (1899) 171-, 283-.

elementary treatment. Thomae, C. J. Z. Mth. Ps. 26 (1881) 314-; 27 (1882) 41-. extension of theorem. Jackson, (Rev.) F. H. L. Mth. S. P. 28 (1897) 475-.

finite, discriminant. Hilbert, $D$. Crelle J. Mth. 103 (1888) 337-.

form of square. Clausen, T. Crelle J. 3 (1828) 89-.

more general integral representation. Schafheitlin, P. Crelle J. Mth. 103 (1888) 89-.

Heine's, analogy of $\theta$ series. Lerch, $M$. [1893] Prag České Ak. Fr. Jos. Rz. (Tř́ida 2) 3 (1894) Art. 5, 10 pp.; Fschr. Mth. (1893-94) 794.

integral function equal to product of two. Markov, A. A. Kharkov Mth. S. Com. 3 (1893) 252-; Fschr. Mth. (1892) 408-. 
and Jacobi's polynomials. Appell, $P$. C. R. 89 (1879) 31-.

3rd order, special case. Saalschitt, L. Z. Mth. Ps. 36 (1891) 278-, 321-.

higher order, especially $1+\frac{a_{0} a_{1} a_{2}}{1 . b_{1} b_{9}} x+\ldots$

Thomae, J. [1869] Mth. A. 2 (1870) 427-.

- -, relations between. Thomae, J. Leip.

Mth. Ps. B. 43 (1891) 459-.

- orders. Mellin, H. Helsingf. Acta 23 (1897) No. 7, $10 \mathrm{pp}$.

with 5 parameters, differential equation. Markov, A. A. St Pét. Ac. Sc. Mm. 5 (1897) No. 5, 23 pp.; Fschr. Mth. (1897) 288-.

product of two, theorems. Orr, W. McF. [1898] Camb. Ph. S. T. 17 (1899) 1-.

representation by definite integral. Schafheitlin, $P$. Mth. A. 30 (1887) 157-.

roots. Gegenbauer, L. Mh. Mth. Ps. 2 (1891) 125-.

- Porter, M. B. N. Y. Am. Mth. S. Bll. 3 (1897) 274-; 6 (1900) 280-.

with two singular points, more general, differential equation. Pochhammer, $L$. Crelle J. Mth. 102 (1888) 76-.

special case, identities. Blissard, J. [1864] QJ. Mth. 7 (1866) 155-, 223-.

theory. Gegenbauer, L. Wien Ak. Sb. 100 (1891) (Ab. 2a) 225-.

transformation into continued fraction. Muir, T. L. Mth. S. P. 7 (1875-76) 112-.

2 variables. Appell, P. C. R. 90 (1880) 977-.

- -. Picard, É. C. R. 104 (1887) 896-.

- -, contiguous functions relative to. Levavasseur, -. C. R. 115 (1892) 1255-.

- - , and linear partial differential equations. Appell, P. C. R. 90 (1880) 296-, 731-.

- -, uniform functions arising from. Goursat, Æ.. C. R. 104 (1887) 893-.

zeros. Hurwitz, A. Gött. Nr. (1890) 557-.

-. Klein, $F$. Mth. A. 37 (1890) 573-.

$F(\alpha, \beta, \gamma, x)$, cases where it is algebraio function of its fourth element. Schwarz, H. A. [1871-72] Sch. Gs. Vh. 54 (1871) 74-; Crelle J. 75 (1873) 292-.

$F_{1}\left(\alpha, \beta, \beta^{\prime}, \gamma ; x, y\right)$, partial differential equations satisfied by. Le Vavasseur, $R$. Toul. Fac. Sc. A. 7 (1893) F, 205 pp.

for $x^{n}$. Jackson, (Rev.) F. H. Edinb. Mth. S. P. 15 (1897) 90-

Hypergeometric theorems

$$
\begin{aligned}
0 & =F^{\prime}(-\overline{2 m-1},-n,-2 n, 2) \\
(-1)^{m} & =\frac{n_{m}}{(2 n)_{2 m}}=F(-2 m,-n,-2 n, 2) .
\end{aligned}
$$

Bretschneider, C. A. Grunert Arch. 13 (1849) 223.

- transcendentals, representation by uniform functions. Papperitz, $E$. Mth. A. 34 (1889) $247-$.

Hyperspherical functions, and development of any function in series of. Létnikov, $A . V$. Rec. Mth. (Moscou) 12 (1885) 205-.
Icosahedra, regular. Klein, $C . F$. Erlang. Ps. Md. S. Sb. 9 (1877) 16-, 70-, 179-; Mth. A. 12 (1877) 503-.

Integral $\int P_{n}^{2} d x$ and allied forms between arbitrary limits. Hargreaves, $R$. [1897] $\mathrm{L}$. Mth. S. P. 29 (1898) 115-.

Kleiber's functions $K_{i}$ and $G_{i}$. Glaisher, $J$. W. L. Mess. Mth. 22 (1893) 71-.

Kummer's equation. Goursat, E. Helsingf. Acta 15 (1888) 45-.

- - integration. Lindelöf, $E$. Helsingf. Acta 19 (1893) No. 1, 31 pp.

Lamé's, Laplace's and Bessel's functions, connexion between. Raentzschel, $\boldsymbol{E}$. Z. Mth. Ps. 31 (1886) 25-.

Laplace's and Bessel's functions, relation between. Rayleigh, J. W. Strutt (Lord). L. Mth. S. P. 9 (1877-78) 61-.

- coefficients, expansion of integral functions in series of. Cayley, A. Camb, and Dubl. Mth. J. 3 (1848) 120-

,$---F(\mu \omega)$ in series of. Goodwin, $H$. Camb. Mth. J. 2 (1841) 223-.

- $b_{s}^{i}$ coefficients. Radau, R. Bll. As. 13 (1896) 5-.

- equation. Tychsen, C. (xس) Ts. Mth. 6 (1876) 165-.

- functions, equation, solution. Graves, $C$. Ir. Ac. P. 6 (1853-54) 162-, 186-, 221-.

- - - - - Hamilton, (Sir) W. R. Ir. Ac. P. 6 (1853-54) 181-.

Legendre's coefficients. Todhunter, I. R. S. P. 27 (1878) 381-.

- - Webb, R. R. [1879] Mess. Mth. 9 (1880) 125-.

_ - and complete elliptic integral, connexion between. Stuart, G. H. Mess. Mth. 13 (1884) 161-

- - product of any two as series of Legendre's coefficients. Adams, J. C. [1877] R. S. P. 27 (1878) 63-.

- -, theorems. Zanotti Bianco, O. Tor. Ac. Sc. At. 22 (1886-87) 225-; 23 (1887-88) 5 -.

- - value for $n$ great. Sharpe, H. J. QJ. Mth. 24 (1890) 383-.

- equation, solution in a particular case. Forsyth, A. R. Mess. Mth. 16 (1887) 168-.

\section{LEGENDRE'S FUNCTIONS.}

Rouché, E. C. R. 47 (1858) 917-.

Laurent, $H$. Liouv. J. Mth. 1 (1875) 373-.

(Laurent.) Heine, H. $E$. Liouv. J. Mth. 2 (1876) 155-.

Catalan, E. C. As. Fr. C. R. 5 (1876) 68-; Brux. Mm. Cour. $8^{\circ}, 31$ (1881) (No. 3) 64 pp. ; Brux. Ac. So. Mm. 43 (1882) (No. 9) 10 pp.; 44 (1882) (No. 1) 103 pp.; 46 (1886) (No. 5) 28 pp.; 47 (1889) (No. 1) 12 pp., (No. 4) 12 pp., (No. 5) 24 pp.

Laguerre, E. C. R. 91 (1880) 849-.

Deruyts, J. Liège S. Sc. Mm. 11 (1885) No. 8, $22 \mathrm{pp}$.

analogues to. Didon, $F$. Par. Éc. Norm. A. 5 (1868) 229-. 
analogues to. Deruyts, J. Liège S. Sc. Mm. 14 (1888) No. 2, $15 \mathrm{pp}$.

calculation for high values of $n$. Callandreau, O. Bll. Sc. Mth. 15 (1891) 121-.

expansion of function in series of functions similar to. Andreev, $K$. A. St Pet. Ac. Sc. Mm. (Rs.) 51 (1885) 1-; Fschr. Mth. (1885) 500.

functions connected with, and derivable from complete elliptic integrals. Kleiber, J. Mess. Mth. 22 (1893) 1-.

- similar to. Čebyšev, P. [1869] St Pet. Ac. Sc. Mm. (Rs.) 16 (*1870) 131-.

- - - Posse, K. A. Kharkor Mth. S. Com. (1885) 155-; Fschr. Mth. (1886) 430. generalisation. Hansted, B. [1881] G. Teix. J. Sc. 4 (1883) 53-.

- arising from development of

$$
\left[1-2 a x+a^{2}\right]^{ \pm \frac{2 l+1}{2}} \text {. }
$$

Escary, -. Liouv. J. Mth. 5 (1879) 47-.

for $n$ infinite. Giuliani, G. G. Mt. 22 (1884) 236-.

and Lamé's functions. Schmit, N.C. Liége Mm. S. Sc. 13 (1858) 289-.

new form for. Catalan, $E$. Par. S. Mth. Bll. 16 (1888) 128-.

polynomials analogous to. Didon, $F$. Par. Ec. Norm. A. 7 (1870) 247 -.

properties, general. Biervliet, A. van. (xix) Brux. S. Sc. A. 7 (1883) (Pt. 1) 68-, (Pt. 2) 402-.

-, new. Catalan, E. C. Liége S. Sc. Mm. 15 (1888) No. 1, 122-.

- of series involving. Plarr, G. C. R. 44 (1857) 984-.

transformation into definite integral. Silva, J. A. Martins da. G. Teix. J. Sc. 3 (1881) 17-.

\section{Legendre's polynomials.}

Hermite, C. G. Teix. J. Sc. 6 (1885) 81-; Palermo Cir. Mt. Rd. 4 (1890) 146-.

Stieltjes, T.J. Toul.Fac.Sc. A. 4 (1890) G, 17 pp.

Hermite, C. Crelle J. Mth. 107 (1891) 80-.

Morera, G. Palermo Cir. Mt.Rd. 11 (1897) 176asymptotic expression of. Lerch, M. [1891] Prag České Ak. Fr. Jos. Rz. (Třida 2) 1 (1892) Art. 8, 27-.

class of associated polynomials analogous to. Deruyts, J. Liège S. Sc. Mm. 14 (1888) No. 3, 14 pp.

generalisation. Clariana Ricart, L. [1892] Barcel. Ac. Bl. 1 (1892-1900) 73-.

- of formulæ. Gegenbauer, L. Palermo Cir. Mt. Rd. 12 (1898) 21-.

and Hermite's and Polignac's. Catalan, E.C. Brux. Ac. Mm. 49 (1890-93) (No. 4) 48 pp.

limit. Gomes Teixeira, $F$. Prag Sb. (1886) (Mth.-Nt.) 19-.

theorem. Stieltjes, - - C. R. 100 (1885) 439-.

theory. Hilbert, D. Acta Mth. 18 (1894) 155-.

Legendrian coefficient, formulæ for $r$ th integral. Glaisher, J. W. L. [1882] Mess. Mth. 12 (1883) 120-.
Polygons, area, theorem. Tardy, P. Tortolini A. 3 (1852) 116-.

Poly-harmonic functions, i.e. solutions of

$$
\left(\frac{d^{2}}{d x^{2}}+\frac{d^{2}}{d y^{2}}\right)^{n} u=0
$$

in simply connected plane space. Almansi, $E$. Palermo Cir. Mt. Rd. 13 (1899) 225-

Polynomials $A_{m, n}$ of 2 variables analogous to Jacobi's. Appell, P. [1880] Arch. Mth. Ps. 66 (1881) 238-.

$-P_{m, n}$ of Didon, generating function. Orlow, G. A. N. A. Mth. 20 (1881) 481-.

Reduction of a certain integral. Zinin, N.N. Rec. Mth. (Moscou) 14 (1890) 549-.

Riemann's $P$-function, mathematical representation. Radicke, A. Rpm. Mth. 1 (1877) 50 -.

- functions of 2nd order with four branchpoints. Heun, K. Mth. A. 33 (1889) 161-.

Rodrigues's theorem (formula for

$$
\begin{aligned}
& \left(\frac{d}{d x}\right)^{i-m}\left(x^{2}-1\right)^{i} \\
& \left.=\left(x^{2}-1\right)^{m} \frac{i-m}{i+m}\left(\frac{d}{d x}\right)^{i+m}\left(x^{2}-1\right)^{i}\right) .
\end{aligned}
$$

Hudson, W. H. H. [1877] Mess. Mth. 7 (1878) 117.

- - demonstration. Hogg, R. W. Mess. Mth. 14 (1885) 20.

Series of Gauss. Tano, F. G. Mt. 9 (1871) 60-. -, generalised form, cases. Glaisher, J.W.L. L. Mth. S. P. 9 (1877-78) 197-.

- of Heine. Tano, F. G. Mt. 9 (1871) 60- - - elements, threefold symmetry in. Rogers, L. J. L. Mth. S. P. 24 (1893) 171-. - - -, higher. Thomae, J. A. Mt. 4 (1870-71) 105-.

- - - transformation. Rogers, L. J. Mess. Mth. 23 (1894) 28-.

$$
\begin{aligned}
-1+\frac{x}{z}+\frac{x(x-1)}{z(z-1)} & +\frac{x(x-1)(x-2)}{z(z-1)(z-2)} \\
& +\frac{x(x-1)(x-2)(x-3)}{z(z-1)(z-2)(z-3)}+\ldots,
\end{aligned}
$$

summation. Grunert, J. A. Crelle J. 2 (1827) 358-.

$-1+\frac{\beta}{\alpha+1}+\frac{\beta(\beta+1)}{(\alpha+1)(\alpha+2)}+\ldots$, summation. Schlömilch, $O$. Grunert Arch. 3 (1843) 442 - $F(v, x, k, \sigma)$ with relation to Euler's formula (also expressed as sum of definite integrals). Lipschitz, $R$. Crelle J. 54 (1857) 313-.

$-\sum_{n} A_{n} X_{n}$. Ascoli, G. A. Mt. 7 (1876) 258-. Solution of

$$
\left(1-x^{2}\right) \frac{d^{2} u}{d x^{2}}-2 x \frac{d u}{d x}+n(n+1) u=0 .
$$

Steinthal, A. E. QJ. Mth. 18 (1882) 330-.

\section{Spherical harmonics.}

Betti, E. [1867] A. Mt. (1867-68) 81-. Sylvester, J. J. Ph. Mg. 2 (1876) 291-, 400. Beltrami, E. Mil. I. Lomb. Rd. 20 (1887) 469-. 
Frischauf, J. Crelle J. Mth. 107 (1891) 87-.

Caspary, F. Crelle J. Mth. 107 (1891) 137-; Par. S. Mth. Bll. 19 (1891) 11-.

Bes8o, D. G. Teix. J. Sc. 12 (1895) 65-; Fschr. Mth. (1893-94) 818.

Gegenbauer, L. Mh. Mth. Ps. 7 (1896) 35-.

Rogel, $F$. Prag Sb. (1898) (Mth.-Nt.) No. 30, 3 pp.

Tallqvist, $H$. Helsingf. Acta 26 (1900) No. 4, $87 \mathrm{pp}$.

application. Niemöller, $\boldsymbol{F}$. Z. Mth. Ps. 24 (1879) 57-.

- to Mittag-Leffler's theorem, and to determination of functions with lacunæ. Pincherle, S. Bologna Rd. (1883) 100-.

- of principle of maximum and minimum exponents to determination. Mazing, $A$. [1891] Rec. Mth. (Moscou) 16 (1893) 173-.

- to Segmer's numbers. Caspary, $F$. Brux. Mm. Cour. 4, 52 (1890-93) No. 8, 15 pp.

- of theorem in differentiation. Hobsom, $E$. W. [1892] L. Mth. S. P. 24 (1893) 55-.

and associated functions. Basset, A. B. Mess. Mth. 13 (1884) 147-.

- cylindrical functions. Olbricht, R. Ac. Nt. C. N. Acta 52 (1888) 1-.

definite integrals connected with. Frost, $P$. QJ. Mth. 19 (1883) 242-.

as determinants. Heun, $K$. Gött. Nr. (1881) 104 .

- - Kronecker, L. Gött. Nr. (1881) 271-.

determination of Green's function. Dougall, $\boldsymbol{J}$. Edinb. Mth. S. P. 18 (1900) 33-.

differentiation. Gallop, E. G. [1896] L. Mth. S. P. 28 (1897) 183-.

expression for. Dougall, $J$. Edinb. Mth. S. P. 8 (1890) 81-.

extension. Pincherle, S. Bologna Ac. Sc. Mm. 1 (1890) 337-.

formula. Fujisawa, R. Mess. Mth. 21 (1892) 40-. functions analogous to. Giuliani, G. G. Mt. 25 (1887) 203-.

general form. Dedekind, $R$. Zür. Vjschr. 4 (1859) 346-; 5 (1860) 66-.

generalisation. Pincherle, $\dot{S}$. Bologna Rd. (1891-92) 31-.

identities in connexion with. Vivanti, $G, \mathrm{Z}$. Mth. Ps. 36 (1891) 1-.

any index, expression and expansion. Lyetnikov, A. V. (xII) Rec. Mth. (Moscou) 10 (1882-83) (Pt. 1) 383-.

2nd kind, roots. Hermite, $C$. Toul. Fac. Sc. A. 4 (1890) I, 10 pp.

- - - Stieltjes, T. J. Toul. Fac. Sc. A. 4 (1890) J, 10 pp.

- - - Hermite, C. Prag Ab. 4 (1892) No. 2, $10 \mathrm{pp}$.

logarithms of 1st five orders for every 5 degrees. Schmidt, A. Terr. Mag. 1 (1896) 73-.

higherorder. Giuliani, G. G. Mt.26(1888) 155-. property. Clebsch, A. [1861] Crelle J. 60 (1862) 343-.

representation by an integral. Walter, $A$. Steierm. Mt. (1887) 25-.

roots of equations occurring in. Markoff, $A$. Mth. A. 27 (1886) 143-, 177-.

series. Dini, U. [1873] A. Mt. 6 (1875) 112-, 208-. series, convergence. Frischauf, J. Steierm. Mt. (1886) 3-.

-, and their differentials, tables for approximate evaluation. Schmidt, A. Terr. Mag. 4 (1899) 59-.

theorems. Pizzetti, - Palermo Cir. Mt. Rd. 13 (1899) 89-.

- generalisation. Gegenbauer, L. Palermo Cir. Mt. Rd. 13 (1899) 92-.

theory. Schendel, L. Crelle J. Mth. 80 (1875) 86-; 82 (1877) 158-.

- Hermite, C. C. R. 86 (1878) 1515-

- Frischauf, J. Steierm. Mt. (1886) 19-.

-, extension. Frombeck, H. Wien Ak. Sb. 70 (1874) (Ab. 2) 61-.

transformation of coordinates. Schmidt, $A$. Z. Mth. Ps. 44 (1899) 327-.

one variable, coefficients. Bauer, G. Crelle J. 56 (1859) 101-.

- - , and coefficients of series of spherical functions. Winckler, A. [1860] Wien D. 21 (1863) 37-.

$P_{n}^{m}(\mu)$, regarded as a function of $n$, zeros. Macdonald, H. M. [1899] L. Mth. S. P. 31 (1900) 264-.

Theory of potential, expansion in series in. Johanson, A. M. Ups. Arsk. (1893) (Mt. Nt.) 55 pp.

Zonal harmonics, table. Perry, J. [1890] L. Ps. S. P. 11 (1892) 221-; Ph. Mg. 32 (1891) $512-$.

- - - corrections to Perry's. Farr, C. C. Ph. Mg. 49 (1900) 572.

$\left[1-a \cos \phi+a^{2}\right]^{\lambda} d \phi$, integration. Moth, F.X. As. Nr. 4 (1826) 539-.

$\left(1-2 a \cos \phi+a^{2}\right)^{-\frac{1}{2}}$, expansion in powers of $a$, coefficients. Plana, G. Zach Cor. 14 (1826) 125-.

$\left(1-2 x z+z^{2}\right)^{-\frac{1}{2}}$, expansion. Ivory, J. Liouv. J. Mth. 2 (1837) 105-.

-, - Liouville, $J$. Liouv. J. Mth. 2 (1837) 135-.

- - in powers of $z$. Schaar, M. Brux. Ac. Bll. 15 (1848) (pte. 2) 506-.

$Y^{(n)}$, application to phenomena which are functions of latitude and longitude. Neumann, F. E. As. Nr. 15 (1838) 313-.

$\left(1-2 a t+a^{2}\right)^{-\frac{1}{2}}$, expansion by definite integrals. Dirksen, E. H. Berl. B. (1843) 111-.

$\left[a^{2}-2 a a^{\prime}(\cos \omega \cos \phi\right.$ $\left.\left.+\sin \omega \sin \phi \cos \left(\theta-\theta^{\prime}\right)\right)+a^{\prime 2}\right]^{-\frac{1}{2}}$, expansion. Jacobi, C. G. J. Crelle J. 26 (1843) 81-.

$\sin \theta \frac{d}{d \theta}\left(\sin \theta \frac{d \phi}{d \theta}\right)+\frac{d^{2} \phi}{d \omega^{2}}+n(n+1) \sin ^{2} \theta \cdot \phi=0$, transformation. Liouville, $J$. Liouv. J. Mth. 11 (1846) 458-.

$1+\frac{x}{y} \cdot v+\frac{x(x+1)}{y(y+1)} v^{2}+\& c$. ad inf. Schäffer, Crelle J. 37 (1848) 127-.

$Y_{n}$, series, convergence. Bonnet, $O$. C. R. 28 (1849) 349-, 378-.

- and $X_{n}$, expansion in series of. Bonnet, $O$. Liouv. J. Mth. 17 (1852) 265-. 
$\left(1-2 a H+a^{2}\right)^{-\frac{1}{2}}$, expansion in powers of $a$. Hansen, P. A. Leip. Ab. Mth. Ps. 1 (1852) 123-.

$\sqrt{ }\left\{r^{2}+r^{\prime 2}-2 r r^{\prime}\left(\cos U \cos U^{\prime}+\sin U \sin U^{\prime} \cos J\right)\right\}$, expansion of negative odd powers. Hansen, P. A. [1854] Leip. Ab. Mth. Ps. 2 (1855) 283 -

$e^{h x+\frac{\mu}{x}}$, expansion in infinite series. Spitzer, $S$. Schlömilch Z. 3 (1858) 244-.

$\left(1-2 q x+q^{2}\right)^{-\frac{m}{2}}$, and $\left\{1-2 q\left[\cos \theta \cos \theta^{\prime}\right.\right.$ $\left.\left.+\sin \theta \sin \theta^{\prime} \cos (\psi-\psi)\right]+q^{2}\right\}^{-\frac{m}{2}}$,

expansion. Alle, $(D r) M$. Wien Ak. Sb. 51 (1865) (Ab. 2) 429-.

$P\left(\begin{array}{lll}\alpha, & \beta, \gamma \\ \alpha^{\prime}, & \beta^{\prime}, & \gamma^{\prime}\end{array}\right)$, theory. Thomae, J. [1867] Z. Mth. Ps. 14 (1869) 48-.

$E_{n}$. Hermite, C. A. Mt. 3 (1869-70) 83.

$Y_{n}^{m}$ and $X_{n}^{m}$. Gegenbauer, $L$. Wien $\mathrm{Sb} .65$ (1872) $(A b .2) 373-; 66$ (1872) (Ab. 2) 55-; 68 (1873) (Ab. 2) 357-.

- integral expressions for. Gegenbauer, $L$. Wien Sb. 66 (1872) (Ab. 2) 374-.

$X_{n}^{2 r+1}$, expansion in terms of. Gegenbauer, $L$. Wien Sb. 66 (1872) (Ab. 2) 415-.

$\sqrt{1-2 \eta U+\eta^{2}}$, expansion of negative odd powers. Backlund, J. O. [1877] St Pét. Ac. Sc. Bll. 24 (1878) 509-.

$C_{n}^{\nu}(x)$. Gegenbauer, $L$. Wien Ak. Sb. 75 (1877) (Ab. 2) 891-.

$Y_{n}$, final value for infinitely increasing values of $n$. Escary, 一. As. Fr. C. R. 8 (1879) 273-.

-, expansions in series whose terms are these functions. St Germain, A. de. C. R. 88 (1879) 1186-, 1313-.

$P^{n}(\cos \gamma), n$ infinite. Heine, H. E. [1880] Crelle J. Mth. 90 (1881) 329-.

$P_{n}(\cos \gamma)$, development. Pleskot, A. Prag Sb. (1893) (Mth.-Nt.) No. 17, 7 pp.; Fschr. Mth. (1893-94) 820.

$\int_{-1}^{1} P_{i} \mu^{m} d \mu, \quad \int_{-1}^{1} P_{i} \cos m \theta d \mu, \int_{-1}^{1} P_{i} \nu^{m} d \mu$, $\int_{-1}^{1} P_{i} \sin m \theta d \mu$. Forsyth, A. R. QJ. Mth. 17 (1881) 37-.

$C_{n}^{\nu}(x)$ where $\left(1-2 z x+x^{2}\right)^{-\nu}=\sum_{n=0}^{n=\infty} C_{n}^{\nu}(x) z^{n}$.

Gegenbauer, L. Wien Ak. D. 48 (1884) (Ab. 2) 293-.

-, theorems. Gegenbauer, L. Wien Ak. D. 57 (1890) 425-.

-, - on roots. [Coefficient of $z^{2}$ in expansion of $\left(1-2 z x+z^{2}\right)^{-\nu}$.] Gegenbauer, $L$. Amst. Ak. Vs. 8 (1900) 250-; Amst. Ak. P. 2 (1900) 196-.

$Y^{m}(x)$, addition theorem. Gegenbauer, $L$. Wien Ak. Sb. 92 (1886) (Ab. 2) 1340-.

$T_{n}^{m}(x)$. Gegenbauer, $L$. Wien Ak. Sb. 95 (1887) (Ab. 2) 274-.

$X_{n}=0$, roots. Stieltjes, T. J. Acta Mth. 9 (1887) 385-.
$J_{0}(k)$ and $J_{1}(k)$ (or $I_{k}^{0}$ and $\left.I_{k}^{1}\right)$ from $k=0$ to $k=15 \cdot 5$, table. Meissel, $E$. Berl. Ak. Ab. (1888) (Anh. Mth.) 23 pp.

$I$ functions, expansions. Meissel, $E$. As. Nr. 127 (1891) 359-.

$I_{\kappa}^{(h)}$, theory. Meissel, E. As. Nr. 128 (1891) 145-.

$J_{n-\frac{1}{2}}(x)$, new formulæ. Kapteyn, $W$. Bll. Sc. Mth. 16 (1892) 41-.

$J_{n+\frac{1}{2}}(x)=0$ ( $n$ integral), place of roots. Rudski, M. P. Liège S. Sc. Mm. 18 (1895) No. 3, 29 pp.

$O^{n}(x)$ and $S^{n}(x)$. Crelier, L. C. R. 125 (1897) 421-, 860-.

$J_{n}(-\mathrm{ic})=\frac{(-1)^{n}}{\pi} \int_{0}^{\pi} \cos ^{c \cos \phi} \cos n \phi d \phi$. Gubler, E. Mth. A. 49 (1897) 583-.

$Y_{0}$ and $Y_{1}$, table. Smith, B. A. Mess, Mth. 26 (1897) 98-.

$G_{0}(x), G_{1}(x)$, and $J_{n}(x \sqrt{ } i)$, numerical computation. Aldis, W. S. R. S. P. 66 (1900) 32-.

4430 Polymorphic functions. Other functions which may be defined by definite integrals. (See also 4860.)

Abelian generating functions. Minine, $A$. Les Mondes 2 (1882) 126-.

- - Pareto, V. Crelle J. Mth. 110 (1892) 290-.

Associated functions and potential of spherical segment. Beltrami, E. N. Cim. 14 (" 1883$)$ 139-.

- polynomials $\left(\int_{0}^{\lambda} U_{\mu, \lambda} V_{\nu, \lambda} d x=0\right)$. Didon, F. Par. Éc. Norm. A. 6 (1869) 111-.

Conical functions, adjunctive, properties of integrals. Leonhardt, G. Mth. A. 19 (1882) 578-.

Definite integrals to express the roots of $u^{m}-p u^{n}-q=0$.

Nekrasov, P. A. Rec. Mth. (Moscou) 13 (1886) 739-; Fschr. Mth. (1888) 304.

- -, functions involved under. Gomes de Souza, J. R. S. P. 8 (1856-57) 146-.

Double gamma function. Barnes, E. W. [1900] Phil. Trans. (A) 196 (1901) 265-.

- functions, genesis. Barnes, $E . W$. L. Mth. S. P. 31 (1900) 358-.

Formula of Anger for

$$
\int_{0}^{2 \pi} \cos (h a-k \sin \alpha) d \alpha=2 \pi 1_{k}^{h}
$$

and analogous formulæ. Cauchy, A. L. C. R. 39 (1854) 129-.

Functions analogous to Laplace's. Routh, E.J. L. Mth. S. P. 11 (1879-80) 92-。

- - Legendre's. Aleksyeev, N. N. (xm) Rec. Mth. (Moscou) 5 (1870) (Pt. 1) 125-. , arbitrary, expressed by double integrals. Pioch, A. Brux. Mm. Cour. $4^{\circ}, 15(1841-42)$ 74 pp. 
Funtions, arbitrary, expressed by double integrals. Meyer, A. [1850] Crelle J. 43 (1852) 60 -.

-, class, represented by definite integrals. Goursat, E. [1882] Acta Mth. 2 (1883) 1-.

-, expansion, by definite integrals. Lorenz, $L$. Mth. Ts. 2 (1860) 161-.

- in form of definite integrals. Pochhammer, L. Crelle J. Mth. 104 (1889) 152-.

Gamma and related functions. Scheeffer, $L$. Crelle J. Mth. 97 (1884) 230-.

$G(r, v)$-integrals, calculation. Brit. Ass. Comm. (Pearson, K.). B. A. Rp. (1896) 70-.

- - tables. Brit. Ass. Comm. (Pearson, K.). B. A. Rp. (1899) 65-.

Integral and sum between same limits, difference. Imschenetsky, $V . G$. G. Mt. 9 (1871) 87-.

Laplace's transformation and applications. Pincherle, S. Bologna Ac. Sc. Mm. 8 (1887) 125-.

Toroidal functions. Hicks, W. M. Phil. Trans. 172 (1882) 609-.

Transcendental function $\int_{0}^{\frac{\pi}{2}} \frac{x d x}{\sin a x}=1$. Lindman, C. F. [1874] Ups. S. Sc. N. Acta 9 (1875) (No. 5) $48 \mathrm{pp}$.

- functions considered by Legendre, two. Lerch, M. Prag Sb. (1893) (Mth.-Nt.) No. 25, 5 pp.

Transcendents arising from integration of homogeneous functions. Winckler, A. [1864] Wien Sb. 50 (1865) (Ab. 2) 531-.

$P\left(u ; v_{1}, v_{2}, \ldots v_{p} ; c_{1}, c_{2}, \ldots c_{p} ; s\right)$. Lerch, $\boldsymbol{M}$. Prag Sb. (1893) (Mth.-Nt.) No. 24, 7 pp.

\section{Automorphic functions.}

\section{(See also 1210, 4050.)}

Burnside, W. [1891-92] L. Mth. S. P. 23 (1892) 49-, 281-.

Stäckel, P. Crelle J. Mth. 112 (1893) 287-.

Abelian functions, generalisation. Picard, $\vec{E}$. C. R. 98 (1884) 665-.

Algebraic surfaces, class. Humbert, G. Palermo Cir. Mt. Rd. 4 (1890) 54-.

Analogue to exponential function. Aleksěevskij, V.P. Kharkov Mth. S. Com. 4 (1895) 253-; Fschr. Mth.' (1895) 466.

Automorphic elementary forms. Fricke, $R$. Gött. Nr. (1900) 303-.

- forms of arbitrary class. Ritter, E. Gött. Nr. (1893) 120-.

- function groups, application of ideals to. Fricke, $R$. Gött. Nr. (1894) 106-.

- functions of two variables. Picard, É. Acta Mth. 1 (1882) 297-; C. R. 96 (1883) 320-; C. R. 97 (1883) 1045-.

- $-n$ variables, theory. Wirtinger, $W$. Wien Ak. Sb. 108 (1899) (Ab. 2a) 1239-.

- prime forms, representation in theta-series. Burkhardt, $H$. Mth. A. 42 (1893) 185-.

Connexion with algebraic functions. Whittaker, E. T. [1898] Phil. Trans. (A) 192 (1899) 1-.
Cyclic linear transformation. Ermakov, $V . P$. Rec. Mth. (Moscou) 14 (1890) 427-.

Differential equations of $3 r d$ order satisfied by automorphic functions. Hurwitz, $A$. Mth. A. 33 (1889) 345-.

Equations in 2 variables, automorphic for a system of rational uniform transformations. Schwarz, C. H. A. Crelle J. Mth. 87.(1879) 139-.

G. Gött. Nr. (1880) 386-.

Fuchsian functions.

Poincaré, H. C. R. 92 (1881) 333-, 395-, 957, 1198-, 1274-, 1484-; 93 (1881) 301-, 581- ; 94 (1882) 163-, 1038-, 1166-; 95 (1882) 626-; 96 (1883) 1485-; Acta Mth. 1 (1882) 193-.

Mittag-Leffler, G. B. A. Rp. (1894) 577.

application, and properties. Poincaré, $H$. C. R. 92 (1881) 859 -

of two complex variables. Kęinski, S. [1895] Krk. Ak. (Mt.-Prz.) Rz. 10 (1896) 211-; Mth. A. 47 (1896) 573-.

and equation $\nabla^{2} u=e^{u}$. Poincaré, H. C. R. 126 (1898) 627-.

existence, theorem. Bagnera, $F$. Rv. Mth. 6 (1896-99) 31-.

of first family, representation by infinite products. Biermann, O. Mh. Mth. Ps. 1 (1890) 49-; 3 (1892) 143-.

hyperfuchsian functions. Picard, É. C. R. 98 (1884) 563-.

- -, cases. Picard, É. C. R. 101 (1885) 1127-.

- - in hypergeometric series of two variables. Picard, É. C. R. 99 (1884) 852-; Par. Ec. Norm. A. 2 (1885) 357-; Par. S. Mth. Bll. 15 (1887) 148-.

- - - - - - Levavasseur, -. C. R. 115 (1892) 1006-:

- - and ternary quadratic forms. Picard, $E^{\prime}$. Acta Mth. 5 (1884) 121-.

and indefinite ternary quadratic forms. Poincaré, H. C. R. 102 (1886) 735-.

and quadratic forms. Picard, É. Am. J. Mth. 11 (1889) 187-.

- - Stouff, $X$. Toul. Fac. Sc. A. 8 (1894) D, 20 pp.

theory. Poincaré, J. H. (xII) Caen Ac. Mm. (1882) 3-.

- Biermann, O. Wien Ak. Sb. 92 (1886) (Ab. 2) 1137-.

Schlesinger, $L$. Mth. Termt. Ets. 6 (1888) 36- ; Mth. Nt. B. Ung. 6 (1889) 337-; Crelle J. Mth. 105 (1889) 181-; C. R. 114 (1892) 1100-, 1409-.

-, Poincaré's. Weber, H. Gött. Nr. (1886) 359-. - - (Weber). Cassel, G. Stockh. Ak. Hndl. Bh. 16 (Afd. 1) (1891) No. 2, 16 pp.; Fsehr. Mth. (1891) 438.

theta-fuchsian functions. Ovidio, E. $d$ '. Tor. Ac. Sc. At. 29 (1894) 741-.

transformation. Poincaré, $H$. C. R. 102 (1886)

—. Stouff, X. Par. Ec. Norm. A. 5 (1888) 219-. 


\section{Automorphic Functions}

zeta-fuchsian functions. Poincaré, $H$. Acta Mth. 5 (1884) 209-.

Fuchsian groups. Stouff, $X$. Toul. Fac. Sc. A. 4 (1890) P, 25 pp.

- - and quadratic forms. Stouff, X. Toul. Fac. Sc. A. 3 (1889) B, 28 pp.

- - reducible to modular groups. Bianchi, $L$. Rm. R. Ac. Linc. Rd. 6 (1890) (Sem. 1) 375-. -, theory. Poincaré, $H$. Acta Mth. 1 (1882) 1-.

- limiting circle. Nekrasov, P. A. [1889-91] Rec. Mth. (Moscou) 14 (1890) 537-; Fschr. Mth. (1890) 428; Mth. A. 38 (1891) 82-.

- - - Anisimov, V. A. [1891-92] Rec. Mth. (Moscou) 16 (1893) 234-; Fschr. Mth. (1892) 387 ; Mth. A. 40 (1892) 145-.

Fuchs's new rule for finding the radius of the discriminating circle, explanations to supply insufficiency. Nekrasov, P. A. Rec. Mth. (Moscou) 16 (1893) 219-; Fschr. Mth. (1892) 387-.

Functions unaltered by certain linear transformation of argument, class. Schottky, $F$. Crelle J. Mth. 101 (1887) 227-.

- - linear substitutions. Fuchs, L. Gött. Nr. (1882) 81-.

Group of 360 collineations in plane. Fricke, $R$. D. Nf. Vh. (1896) (Th. 2, Hälfte 1) 5.

- 504 operations. Fricke, R. Mth. A. 52 (1899) 321-.

Groups appearing in theory of analytic functions. Medolaghi, P. C. R. 126 (1898) 1188 .

Invariant of congruent figures. Andrade, $J$. N. A. Mth. 8 (1889) 150-.

- expression for Poincaré's substitutions. Brun, F. de. Stockh. Ófv. (1890) 265-; Fschr. Mth. (1890) 410.

Invariants of Schwarzian polygon of genus zero. Pick, G. Mth. A. 42 (1893) 489-.

Kleinian functions of third family, generalisation. Cassel, G. Stockh. Öfv. (1890) 237-; Fschr. Mth. (1890) 410.

Modular equation of 8 th degree. Brioschi, F. [1878] Rm. R. Ac. Linc. T. 3 (1879) 45-. 15 (1879) 241 - J

- equations, class. Brioschi, F. A. Mt. 9 (1878-79) 167-.

- -, depression. Klein, C. F. [1878] Mth. A. 14 (1879) 417-.

- - theory. Hurwitz, A. Gött. Nr. (1883) 350-.

Octahedron and equation of 4th order. Puchta, A. Wien Ak. D. 41 (1879) (Ab. 2) 57-.

- function. Cayley, A. QJ.Mth.16(1879)280-.

Polyhedral functions and Schwarzian derivative. Cayley, A. [1880] Camb. Ph. S. T. 13 (1883) 5-.

Quadratic quaternary forms and applications to Fuchsian groups. Stouff, $X$. Toul. Fac. Sc. A. 6 (1892) G, 19 pp.

- - - corresponding hyperabelian groups. Picard, É. C. R. 98 (1884) 904-.

Representation by infinite products. Stahl, $H$. Mth. A. 33 (1889) 291-, 604.

\section{Lamés and other Functions 4450}

Schwarzian triangles. Kapteijn, W. N. Arch. Wisk. 1 (1895) 185-; Fschr. Mth. (1893-94) 698 ; Bll. Sc. Mth. 18 (1894) 36-.

- with simple nodal point. Schilling, $F$. [1896] D. Mth. Vr. Jbr. 5 (1901) (Heft 1) 73-.

8 -function with branching $(2,3,7)$. Fricke, $R$. Gött. Nr. (1892) 279-

- - Schwarz's, with complex exponents, fundamental domain. Schilling, $F$. Gött. Nr. (1894) 261-.

- - - - - - - geometrical theory. Schilling, F. Mth. A. 46 (1895) 62-, 529-.

- - - - geometrical theory. Schilling, $F$. Mth. A. 44 (1894) 161-.

s-functions, related. Papperitz, E. Mth. A. 25 (1885) 212-; 26 (1886) 97-.

Substitutions of 2nd degree. Laurent, $H$. N. A. Mth. 16 (1897) 389-.

Symmetric $S$-functions with singularity. Schilling, F. Mth. A. 51 (1899) 481-.

Ternary automorphic transformations, infinite, theorem. Fano, G. Rm. R. Ac. Linc. Rd. 8 (1899) (Sem. 1) 562-

Theory. Dixon, A. C. L. Mth. S. P. 31 (1900) 297-.

-. Lindemann, F. [1899-1900] Münch. Ak. Sb. 29 (1900) 423- ; 30 (1901) 493-.

-, and theory of groups. Fricke, R. Gött. Nr. (1892) 453-; Mth. A. 42 (1893) 564-.

- - - - numbers, relations between. Fricke, R. D. Mth. Vr. Jbr. 6 (1899) (Heft 1) 94-.

Transformation. Fricke, R. Mth. A. 44 (1894) 97-.

- of 5 th and $7 \mathrm{th}$ orders of special automorphic functions. Fricke, $R$. Acta Mth. 17 (1893) 345-.

Uniform automorphic forms of zero class. Ritter, E. Gött. Nr. (1892) 283-; Mth. A. 41 (1893) 1-.

- - functions. Klein, C. F. Mth. A. 19 (1882) 565-; 20 (1882) 49-.

- - Schottky, F. Mth. A. 20 (1882) 299-.

- functions reproduced by linear substitutions. Poincaré, H. Mth. A. 19 (1882) 553-; 20 (1882) 52-.

\section{0ther functions which may be defined by linear differential equations. Lamé's functions.}

Analytical functions of one variable depending on two real arbitrary constants. Picard, $\boldsymbol{E}^{\prime}$. C. R. 114 (1892) 1310-.

Biharmonic functions. Arcais, $F . d$ '. Ven. I. At. (1898-99) (Pt. 2) 479-.

Complete functions, of 1st kind. Tannery, $J$. C. R. 86 (1878) 950 -

Dilogarithms. (Given by $\int_{0}^{x} \frac{d x}{x} \int_{0}^{x} \frac{d x}{1-x}$ ). Mantel, W. N. Arch. Wisk. 3 (1898) 292-; Fschr. Mth. (1898) 377.

Function analogous to modular functions. Poincarê, H. C. R. 93 (1881) 138-.

Functions arising from inversion of integrals of linear differential equations. Fuchs, L. Bll. Sc. Mth. As. 4 (1880) 278-, 328-. 
Functions defined by differential equation of 1st order. Vivanti, G. A. Mt. 16 (1888-89) 117-. - - - equations. Bouquet, J., \& Briot, C. R. 39 (1854) 368-.

$--1-$ (Bouquet \& Briot). Cauchy, A.L. C. R. 40 (1855) $557-$.

- - - Poincaré, H. Par. Ec. Pol. J. Cah. 45 (1878) 13-.

- - Fine, H. B. Am. J. Mth. 11 (1889) 317-.

- - - of 1st order. Petrovitch, $M$. Par. S. Mth. Bll. 24 (1896) 58-.

- - - two simultaneous differential equations. Guldberg, A. Mh. Mth. Ps. 2 (1891) 147-.

- - - a system of linear homogeneous differential equations of one or several variables, properties. Sauvage, L. Par. Éc. Norm. A. 11 (1882) 33-.

-, expansion in integrals of linear differential equations of 2nd order. Pochhammer, $L$. [1871] Crelle J. 74 (1872) 315-.

- of parabolic cylinder, differential equation. Haentzschel, E. Z. Mth. Ps. 33 (1888) 22-.

- with recurring derivatives. Glaisher, J.W.L. [1872] L. Mth. S. P. 4 (1871-73) 113-.

- satisfying $\frac{d^{n} y}{d x^{n}}=y$. Schapira, -. D. Nf. Tbl. (* 1879) 171-.

General co-functions, theory and application. Solutions of $\frac{d^{n} y}{d x^{n}}=y$. Šapiro, G. [Schapira, H.] N. Rs. S. Nt. Mm. (Mth.) 3 (*1881); D. Nf. Tbl. (1881) [1].

Integrals of solutions of differential equations of 2nd order, with 3 singular points. Kepinski, S. Krk. Ak. (Mt.-Prz.) Rz. 17 (1900) 112-; Crc. Ac. Sc. Bll. (1899) 67-.

Lamé-Hermite differential equation. Brioschi, F. St Pét. Ac. Sc. Bll. 35 (1894) 449-

Lamé's equation. Brioschi, F. C. R. 85 (1877) 1160-; 86 (1878) 313-.

- - Hermite, C. [1877] A. Mt. 9 (187879) 21-.

- Lindemann, C. L. F. B. A. Rp. (1883) 351-.

- - Greenhill, A. G. [1888] L. Mth. S. P. 20 (1889) 213-.

- - Floquet, G. C. R. 121 (1895) 805-, 920.

- - Markof, A. Mth. A. 47 (1896) 598-.

- - integration. Hermite, C. [1879] Crelle J. Mth. 89 (1880) 9-.

- -, new application. Gyldén, J.A.H. C. R. 93 (1881) 537-.

- -, solution. Crawford, L. QJ. Mth. 27 (1895) 93-.

- - - factors. Crawford, L. QJ. Mth. 29 (1898) 196-.

- -, special case. Stenberg, E. A. Acta Mth. 10 (1887) 339-.

- -, - - Jamet, V. C. R. 111 (1890) 638-

- - theorems. Brioschi, F. C. R. 92 (1881) $325-$.

\section{LAME'S FUNCTIONS.}

Heine, H. E. Crelle J. 56 (1859) 79-, 87-. Klein, C. F. Mth. A. 18 (1881) 237-.
Schläfi, L. Rm. R. Ac. Linc. Mm. 4 (1887) 37-.

Jaerisch, P. Hamb. Mth. Gs. Mt. 1 (1889) 212-.

Moser, C. Arch. Sc. Ps. Nt. 8 (1899) 377-.

and Hermite's function, zero values. Markov, A. A. Kharkov Mth. S. Com. 5 (1896 ?) 74-; Fschr. Mth. (1896) 334.

of 1st kind, existence and number. Heine, [H.] $E$. Berl. Mb. (1864) 13-。

- - any order. Heine, H. E. [1862] Crelle J. 62 (1863) 110-.

- 2nd kind. Bigler, U. Arch. Mth. Ps. 12 (1894) 113-, 225-.

Klein's theorem. Crawford, L. QJ. Mth. 30 (1899) 307-.

and Laplace's and Bessel's functions, connexion between. Haentzschel, E. Z. Mth. Ps. 31 (1886) 25-.

of 2nd order. Waelsch, E. Wien Ak. Sb. 105 (1896) (Ab. 2a) 741-.

different orders. Heine, H. E. Crelle J. 60 (1862) 252-.

and other functions. Stieltjes, T. J. Acta Mth. 6 (1885) 321-.

theory. Heun, $K$. Mth. A. 33 (1889) 180 -

-. Klein, F. Gött. Nr. (1890) 85-.

in "Theory of Heat." Escary, - C. R. 87 (1878) 646-; 88 (1879) 1027-; 90 (1880) 1341-. with given zeros. Johanson, A. M. Stockh. Öfv. (1900) 675-.

Lamé's numbers, generalisation. Berger, $A$. Ups. S. Sc. N. Acta 15 (1895) No. 10, 33 pp. - products. Safford, F. H. Am. J. Mth. 21 (1899) 1-.

Polyharmonic functions. Volterra, $V$. Ven. I. At. (1898) 233-.

- - i.e. solutions of $\left(\frac{d^{2}}{d x^{2}}+\frac{d^{2}}{d y^{2}}\right)^{n} u=0$, in simply connected plane space. Almansi, $\boldsymbol{E}$. Palermo Cir. Mt. Rd. 13 (1899) 225-.

Polynomials arising from $\left(1-2 x z+z^{2}\right)-\mu$. Amanzio, D. Nap. Ac. At. 7 (1895) No. 10, $35 \mathrm{pp}$.

-, Stieltjes'. Van Vleck, E. B. N. Y. Am. Mth. S. Bll. 4 (1898) 426-.

Potential functions in $n$ dimensional space. Biermann, $O$. Lotos 34 (1885) 93-.

Riemann's $P$-function, mathematical representation. Radicke, A. Rpm. Mth. 1 (1877) 50-.

Ring functions. Gegenbauer, $L$. Wien Ak. Sb. 100 (1891) (Ab. 2a) 745-.

Series and differential equations. Bervi, N. V. [1894] Rec. Mth. (Moscou) 17 (1895) 838-; Fschr. Mth. (1895) 275.

Specification of $n$ functions in plane, problem of Riemann. Schlesinger, L. C. R. 126 (1898) 723-.

Successive approximations, singular examples. Picard, É. C. R. 126 (1898) 497-.

Transcendental functions defined by differential equations of 2 nd order. Painleve, $P$. C. R. 116 (1893) 566-.

$\left(1-2 a z+a^{2}\right)^{-k}$, expansion. Radau, R. Par. Obs. A. (Mm.) 18 (1885) D, 20 pp. 


\section{Functions which may be de- fined by functional equations. (See also 6030.)}

Algebraic operations of higher order; e.g. $a e^{a \text { ete. }}$ Schubert, H. C. H. Hamb. Mth. Gs. Mt. 1 (1889) 62.

Formation of a function $F(x)$ having property $F[\phi(x)]=F(x)$. Appell, P. C. R. $88(1879)$ 807-.

Function given by $f(z)+\alpha \phi(z)=0$ expanded in infinite series; applications. Rodrigues, J.M. Fschr. Mth. (1884) 219-.

- $f(z)=\frac{\sin \pi z}{\pi}$, definition by a system of functional properties. Moore, E. H. A. Mth. 9 (1894-95) 43-.

Functional equations, solutions. Pick, $G$. Mth. A. 24 (1884) 590-.

- relation $\phi(u) \cdot \phi(v)=\phi(u+v)$. Wilson, E.B. A. Mth. 1 (1900) 47-.

Functions satisfying equation

$$
\Sigma \phi x \cdot F y=\Sigma F x \cdot \phi y,
$$

relations. Magnus, L. J. Crelle J. 5 (1829) 365-.

$$
\phi(x)+\phi(y)=\psi\left\{\frac{f y \cdot F x+f x \cdot F y}{\chi(x y)}\right\} .
$$

Lottner, C. L. E. [1850] Crelle J. 46 (1853) 367-.

- such that $F\left(\sin \frac{\pi}{2} x\right)=F(x)$. Appell, $P$. C. R. 88 (1879) 1022-.

Generalisation, ealculus. Oltramare, $G$. As. Fr. C. R. (1887) (Pt. 2) 285-.

Integral of function when only characteristic functional equation is given. Pexider, $J$. Časopis 29 (1900) 33-; Fschr. Mth. (1900) 306.

Iterative functions. Schröder, E. [1869] Mth. A. 3 (1871) 296-.

- - Lémeray, E. M. As. Fr. C. R. (1895) (Pt. 2) 149-

Transcendental function, Soldner's. Buzengeiger, K. H.J. Zach M. Cor. 26 (1812) 285-.

\section{Integral functions.}

Galois, É. Liouv. J. Mth. 11 (1846) 408-. Picard, É. C. R. 88 (1879) 1024-; 89 (1879) 662-; Par. Éc. Norm. A. 9 (1880) 145-; Par. S. Mth. Bll. 11 (1883) 136-.

Guichard, -. Par. Éc. Norm. A. 1 (1884) 427-. Vivanti, G. G. Mt. 26 (1888) 303-.

Laurent, H. Par. Éc. Pol. J. 60 (1890) 107-.

Desaint, -. C. R. 120 (1895) 548-.

Hadamard, - . C. R. 122 (1896) 1257-.

Laurent, $H$. N. A. Mth. 15 (1896) 23-.

Petersen, J. Acta Mth. 23 (1900) 85-.

Wigert, S. Stockh. Öfv. (1900) 1001-.

Divisibility. Koch, H. von. Stockh. Öfv. (1893) 449-.

VOL. I.
Divisibility of function of $n^{2}$ elements by their determinant. Mertens, $F$. Wien Ak. Sb. 81 (1880) $(A b .2)$ 260-.

- -properties of functions of higher derivatives. Meyer, F. Mth. A. 36 (1890) 435-.

Divisor, common, of two functions of one variable. Netto, $E$. Crelle J. Mth. 106 (1890) 81-.

Divisors. Mertens, $F$. Wien Ak. Sb. 97 (1889) (Ab. 2a) 618-.

Extension of property of polynomials to integral functions. Borel, $\hat{E}$. C. R. 123 (1896) 556-.

Factorisation. König, G. [1882] (xI) Mth. Termt. Éts. 2 (1884) 45-.

- and related elimination problems. König, J. [G.] Mth. A. 15 (1879) 161-.

Factors. Sélivanoff, D. Par. S. Mth. Bll. 13 (1885) 119-.

Function of 2 integral arguments. Muir, $T$. Edinb. Mth. S. P. 3 (1885) 19-. satisfying certain conditions, degree. Tchebitchef, P. L. Par. S. Mth. Bll. 3 (1875) 103.

Functions assuming given values at given points. Cazzaniga, $P$. A. Mt. 10 (188082) 279 -

- and continued fractions. Christoffel, E.P. [1876] A. Mt. 8 (1877) 1-.

- of form $e^{G(x)}$. Hadamard, -. C. R. 114 (1892) 1053-.

- of $m$ systems each of $n$ variables. Mertens, F. Mh. Mth. Ps. 4 (1893) 193-, 297-.

- and types of increase. Borel, $\hat{E}$. C. R. 126 (1898) 321-.

Hilbert's theorem, extension to infinite polynomials. Capelli, A. Nap. Rd. 35 (1896) 321-.

Homogeneous functions. André, D. Par. S. Phlm. Bll. 1 (1899) 124.

Irreducibility of functions of integral functions with integral coefficients. Netto, $E$. Giessen Oberh. Gs. B. 31 (1896) 113-; Mth. A. 48 (1897) 80-.

Irreducible polynomials. Kahan, W. F. Fschr. Mth. (1899) 100.

Polynomials satisfying relation of 3rd order. Weill, -. N. A. Mth. 4 (1885) 184 .

Properties. Hadamard, $J$. Liouv. J. Mth. 9 (1893) 171-.

-. Desaint, -. C. R. 124 (1897) 746-.

Rational function, transformation. Laisant, -. Par. S. Mth. Bll. 20 (1892) 6-.

- functions, theorems. Grunert, J. $A$. Grunert Arch. 31 (1858) 27-.

Reducible functions of $n$ variables. Meyer, $F$. Mth. A. 30 (1887) 30-.

Reduction. Pellet, A. E. Par. S. Mth. Bll. 19 (1891) 48-.

- to canonical form of functions of $n$ variables. Mirimanov, D. S. Rec. Mth. (Moscou) 19 (1897) 629 (bis)-; Fschr. Mth. (1897) 88.

Resultants of $n$ forms with $n$ variables, determining properties. Mertens, $F$. Wien Ak. Sb. 93 (1886) (Ab. 2) 527-.

System of functions $A \phi+B \psi$; theorem. Nöther, M. [1872] Mth. A. 6 (1873) 351-. 
Theorem of Picard, demonstration. Borel, $\dot{E}$. C. R. 122 (1896) 1045-.

- - - (Borel). Picard, É. C. R. 122 (1896) 1048.

Theorems. Vivanti, G. G. Mt. 22 (1884) 243-, 378.

Theory. Schou, E. C. R. 125 (1897) 763-.

Transcendental functions. Vivanti, $G$. G. Mt. 23 (1885) 96-; Mil. I. Lomb. Rd. 32 (1899) 569-.

Zeros. Borel, É. Acta Mth. 20 (1897) 357-.

-, position. Witting, A. Z. Mth. Ps. 30 (1885) 274.

$\psi(a, b)$ and $\chi(a, b)$, elementary properties. Lerch, M. Časopis 24 (1895) 25-, 118-, 228-; Fschr. Mth. (1895) 217-.

\section{Differential equations.}

\section{General. (See also 8080 Connexes.)}

Algebraic equations. Casorati, $F$. A. Mt. 9 (1878-79) 41-, 106-.

- - number of independent transcendentals belonging to. Koenigsberger, L. Mth. A. 30 (1887) 299-

Analogues. Bourlet, C. Par. Éc. Norm. A. 16 (1899) 333-.

Analytical remarks on Ouroussof's work. Minding, E. F. A. [1865] St Pét. Ac. Sc. Bll. 9 (1866) 48-.

Application of curve systems to graphic integration. Láska, T. Prag Sb. (1890) 1 (Mth.Nt.) 222-.

Asymptotic integrals. Boussinesq, J. C. R. 94 (1882) 208-.

Bibliographical note. Mansion, P. Brux. S. Sc. A. 15 (1891) (Pt. 1) 32-, 60, 105.

Case. Cayley, A. Manch. Ph. S. P. 2 (186062) 193.

Composition from given integral equation. Bierens de Haan, D. Amst. Ak. Vh. 18 (1879) $37 \mathrm{pp}$.

Conditions that primitive may have degree lower than normal. Casorati, F. Mil. I. Lomb. Rd. 10 (1877) 770-.

Conjoint differential equations, integration. Starkov, A. N. Rs. S. Nt. Mm. (Mth.) 2 ( $\left.{ }^{*} 1879\right)$.

Continuity of functions representing integrals of a system. Cauchy, A. L. C. R. 23 (1846) 702-.

Deduction from graphs. Warder, $R$. $B$. Science 4 (1896) 345.

Determination of four functions to satisfy a certain equation. Vivanti, $G$. Palermo Cir. Mt. Rd. 6 (1892) 100-.

Differential and difference equations, connexion between. Mellin, $H$. Acta Mth. 9 (1887) 137-.

- functions, equations of condition. Stockler, F. de B. G. Lisb. Mm. Ac. Sc. 2 (1799) 196-.

Elimination of arbitrary functions. Fais, $A$. Bologna Ac. Sc. Mm. 9 (1878) 649-.
Equation for confocal conic system, integration. Guimarães, R. Lisb. J. Sc. Mth. 5 (1898) 105-.

- of curve of any order. Sylvester, J. J. C. R. 103 (1886) 408-; Nt. 34 (1886) 365-.

Equations. Schmidten, $H$. G. Kiöb. Ov. (1825-26) 2-.

- and applied mathematics, historical sketch. Naetsch, E. Civing. 41 (1895) 655-.

- of conics. Monge, $G$. Par. Éc. Pol. Cor. 2 (1809-13) 51-.

- - Muir, T. Ph. Mg. 21 (1886) 143-.

- - Elliott, E. B. Mess. Mth. 19 (1890) 5-.

- determining form of roots of algebraic equations. Boole, G. Phil. Trans. 154 (1864) 733-.

-, general integral of which is algebraic, class. Picard, $\dot{E}$. C. R. 120 (1895) 402-.

- having fundamental solutions, theory. Guldberg, A. Crelle J. Mth. 115 (1895) 111-.

- obtained by elimination of 2 arbitrary functions. Worms de Romilly, $P$. Par. S. Mth. Bll. 8 (1880) 64-.

- with periodic integrals. Hill, G. W. A. Mth. 3 (1887) 145-.

- and their integrals. Raabe, J.L. Baumgartner Z. 5 (1829) $27-$.

Functional and differential equations, cases. Thiele, T. N. (xiI) Ts. Mth. 4 (1868) 109-, 180 - ; 5 (1869) 62-.

Functions defined by differential equations, residues. Petrovitch, $M$. Mth. A. 48 (1897) 75-; Prag Sb. (1898) (Mth.-Nt.) No. 6, 24 pp.

- satisfying algebraic equation, expansion. Hurwitz, A. Par. Éc. Norm. A. 6 (1889) $327-$.

- - differential equation, expansion. Gomes Teixeira, F. Par. Éc. Norm. A. 2 (1885) 321- ; 4 (1887) 107-.

Geometric meaning. Cunningham, A. QJ. Mth. 14 (1877) 226-; Nt. 38 (1888) 318-.

Imaginary integrals and their advantages. Cauchy, A. L. C. R. 23 (1846) 563-.

Integrable equation, derivation by Liouville's method. Thomae, C. J. Gött. Nr. (1874) 249-.

Integrals, algebraic, relations. Koenigsberger, L. Crelle J. Mth. 115 (1895) 23-.

- of equations about essential singularities (viz. " normal" series in Poincaré's sense). Rajewski, J. Krk. Ak. (Mt.-Prz.) Pam. 17 (1890) 166-; Crc. Ac. Sc. Bll. (1890) 62-.

-, rectilinear, and complete integrals of a system of equations, relations between. Cauchy, A. L. C. R. 23 (1846) 779-.

-, relations. Fuchs, L. Berl. Ak. Sb. (1887) 1077-.

-, 一, algebraic. Königsberger, L. [1877-80] Crelle J. Mth. 84 (1878) 284-; 91 (1881) 199-.

- of systems of ordinary or partial differential equations and expansions of these integrals in series. Cauchy, A. L. C. R. 15 (1842) 101-, 141-. 
Integrals, various kinds, of a system. Cauchy, A. L. C. R. 23 (1846) 729-.

Integrating factor. Anisimov, V.A. [1895] Vars. S. Nt. Tr. (1895-96) (C. R., P8. C.) No. 2, 2-.

Integration by approximation. Kramp, C. Gergonne A. Mth. 10 (1819-20) 317-, 361-. _, form of arbitrary constants. Brinkley, $J$. [1817] Ir. Ac. T. 13 (1818) 53-.

-, general problem. Riquier, - C. R. 116 (1893) 426-.

,$--\frac{1}{-}$, reduction. Riquier, - C. R. 124 (1897) 490-.

- by series. Picard, É. C. R. 124 (1897) 214-.

-, solution of algebraic equations by. Heymann, W. Z. Mth. Ps. 31 (1886) 102-, 129-.

Invariant operations admitting of permutation. Capelli, A. Nap. Ac. At. 1 (1888) No. 1, $17 \mathrm{pp}$.

Linear differential and difference equations, theory, and relations to theory of algebraic equations. Libri, $G$. Liouv. J. Mth. 1 (1836) 10 -

March of functions defined by differential equations. Borel, É. C. R. 128 (1899) 490-.

Monge's equation and its application to conics. Mukhopadhyay, A. Beng. As. S. P. (1888) $74-, 165-$.

- - of all conics. Mukhopadhyay, A. [1887] Beng. As. S. J. 56 (Pt. 2) (1888) 134-.

- - - - , geometric interpretation. Mukhopadhyay, A. Nt. 38 (1888) 173, 564-. - - - - - - - (Mukhopadhyay). $H[$ ayward?], R. B. Nt. 38 (1888) 197, 619 .

- - _ - - -. Mukhopadhyay, A. [1888] Beng. As. S. J. 58 (Pt. 2) (1890) 181-. - - geometry. Lie, S. Leip. Mth. Ps. B. 50 (1898) (Mth.) 1-.

Monodromic and monogenic integrals. Cauchy, A. L. C. R. 40 (1855) 511-.

Motion of particle, integrals that are linear (fractional) functions of velocities. Vivanti, G. Palermo Cir. Mt. Rd. 6 (1892) 127-.

"Numerical" differential equations, integration. Láska, W. Prag Sb. (1897) (Mth.Nt.) No. $35,10 \mathrm{pp}$.

Operators in connection with symmetric functions. Lachlan, R. L. Mth. S. P. 18 (188687) $39-$.

Order of a system. Jordan, M. E. C. (xII) Brux. S. Sc. A. 7 (1883) (Pt. 2) 127-.

Ordinary and partial differential equations, integration by separation of operative symbols. Grelle, $F$. Z. Mth. Ps. 15 (1870) 297-.

Partial differential equation of quadric surface. Scherck, -. [1883] Brem. Ab. 8 (1884) 366-.

- - equations, application of calculus of generalisation. Oltramare, G. As. Fr. C. R. (1889) (Pt. 2) 145-.

- - - problems leading to. Heine, H. E. Crelle J. 26 (1843) 185-.

Particular solution, relation between constants and those in the coefficients of the differential equation. Dillner, $G$. C. R. 92 (1881) 1498-; 93 (1881) 46-.

- solutions. Poisson, S. D. Par. Éc. Pol. J. $13^{\circ} \mathrm{cah}$. (1806) 60-.
Particular solutions. Hansen, C. (xII) Ts. Mth. 5 (1869) 119-.

- - (Hansen). Zeuthen, H. G. (хп) Ts. Mth. 5 (1869) 154-; 6 (1870) 33-.

Problems of differential calculus. Brown, $D$. W. Franklin I. J. 44 (1897) 348-.

Reciprocity. Orlov, T. E. (xII) Rec. Mth. (Moscou) 3 (1868) (Pt. 1) 167-.

Reduction of any system to a linear and integrable form of 1 st order. Riquier, -. C. R. 116 (1893) 866-; Par. Éc. Norm. A. 10 (1893) 359-.

- - system to partial differential equations in half as many variables. Painvin, $L$. C. R. 44 (1857) 787-.

Roots of algebraic equations, deduced from integration of linear or simultaneous differential equations. Raabe, J.L. Zür. Mt. 2 (1850-52) 248-.

Separation of variables. Piani, D. Bologna Mm. Ac. Sc. 4 (1853) 329-.

Simultaneous equations. Mackenzie, M. Mess. Mth. 5 (1871) 247-.

- - class, analogous to Lagrange's perturbation theorem. Brassinne, E. Liouv. J. Mth. 16 (1851) 283-.

- - with continuous transformation groups. Zindler, K. Mh. Mth. Ps. 11 (1900) 289-.

- - general integrals of certain systems. Zejliger, D. Kazan Un. Mm. (1897) (Pt. 1, Non-off. Sect.) 69-; Fschr. Mth. (1897) 295-.

- - integrating factors. Laurent, $H$. N. A. Mth. 19 (1880) 153-.

- -, integration. Gregory, D. F. Camb. Mth. J. 1 (1839) 173-.

- -, -. Painvin, L. C. R. 47 (1858) 693-.

- - - of a class. Bonnet, O. C. R. 53 (1861) 971-.

- - number of arbitrary constants in complete solution. Nanson, E.J. [1876] Mess. Mth. 6 (1877) 77-.

- - with one parameter. Severini, C. Mil. I. Lomb. Rd. 33 (1900) 825-.

- _, roots of fundamental determinant. Method of multipliers. Routh, E.J. L. Mth. S. P. 14 (1882-83) 144-.

- - singular points, Wigert, S. Stockh. Öfv. (1899) 697-; (1900) 47-.

- -, system. Webb, R. R. [1879] Mess. Mth. 9 (1880) 6-.

- + , theorems, demonstration. Lindelöf, $E$. Liouv. J. Mth. 6 (1900) 423-.

- -, theory. Anisimov, V.A. [1895] Vars. S. Nt. Tr. (1895-96) (C. R., Ps. C.) No. 3, 2-.

- - of type $\Sigma X d x=0$. Biermann, O. Z. Mth. Ps. 30 (1885) 234-.

- linear equations. Tilly, J. de. Mathesis 5 (1885) 121-.

- system, singular solutions, derivation from equations themselves. Mayer, A. C. G. Mth. A. 22 (1883) 368-.

$-\frac{d x}{X}=\frac{d y}{Y}=\frac{d z}{Z}$, singular points. Poincaré,

H. C. R. 94 (1882) 416-. 
Solution. De Morgan, A. [1856] Camb. Ph. S. T. 10 (1864) 21-。

-, influence of reality and continuity conditions on. Christoffel, E. B. Crelle J. 66 (1866) 1-.

System, degree of generality. Riquier, - . C. R. 130 (1900) 162-.

Systems and their connexion with partial linear equations. Jacobi, C. G. J. Crelle J. 23 (1842) 1-

- of infinite order. Koch, H. von. Stockh. Ofv. (1899) 395-.

-, theory. Stodolkievitz, A. J. C. R. 120 (1895) 36-, 595-.

-, transformations. Delassus, É. C. R. 123 (1896) 1246-.

Theory. Schmidten, H. G. Kiöb. Ov. (181819) 6-.

-. Jacobi, C. G. J. Crelle J. 17 (1837) 68-.

- Serret, J. A. C. R. 19 (1844) 910-.

- Anisimov, V. A. Vars. S. Nt. Tr. (1898) (C. R., Ps. C.) No. 6, 2-.

-, general. Picard, É. Bll. Sc. Mth. 11 (1887) 194-.

Tracing of curves from differential equations. Coriolis, G. Liouv. J. Mth. 1 (1836) 5-.

Transformation of the general integral of the characteristic equation; etc. Cauchy, A.L. C. R. 29 (1849) 103-.

\section{Existence theorems for ordi- nary and partial differential equations.}

Cauchy, A. L. C. R. 14 (1842) 1020-.

Jablonski, $E$. As. Fr. C. R. (1877) 198-.

Lerch, M. Prag Sb. (1889) 1 (Mth.-Nt.) 180-.

Lichtenfels, O. von. Mh. Mth. Ps, 1 (1890) 275-.

Sincov, D. Kazan S. Ps.-Mth. Bll. 3 (1893) 138-.

Osgood, W. F. N. Y. Am. Mth. S. Bll. 4 (1898) 291, 292-.

Cauchy's existence theorem. Koenigsberger, $L$. Crelle J. Mth. 104 (1889) 174-.

- - - Poincaré's extension. Koenigsberger, L. Crelle J. Mth. 113 (1894) 115-。

Complete integral for $f\left(x, y, y^{\prime}\right)=0$, demon. stration. Zeuthen, H. G. (xII) Ts. Mth. 4 (1880) 161-.

Differential relations soluble without integration. Combescure, É. [1870] Mntp. Mm. Ac. Sect. Sc. 7 (1867-71) 447-。

- resolvents. Cockle, (Sir) J. [1864] Manch. Lt. Ph. S. P. 4 (1865) 38-.

Equations having complete algebraic integrals, etc. Hill, C. J. D. Crelle J. 25 (1843) $38-$.

Existence of first integrals of partial equations, 2nd order. Brioschi, F. Tortolini A. 5 (1854) 268-; 6 (1855) 426-.

- - integrals of partial system, determined by initial conditions. Riquier, - . C. R. 126 (1898) 208-.

Finite number of singular points, Fuchs's theorem. Poincaré, H. C. R. 99 (1884) 75-.
Highest power of $\frac{d^{p} y}{d x^{p}}$ in $n$th derivative of $f(x, y)=0$. Zeuthen, H. G. Mth. Ts. 1 (1859) 40 -

Homogeneous linear equation. Hamburger, $M$. Crelle J. Mth. 118 (1897) 351-.

- - - (Hamburger). Fuchs, L. Crelle J. Mth. 118 (1897) 354-

- - - Gutzmer, A. Crelle J. Mth. 119 (1898) 82-.

\section{Integrability .}

conditions. C., B. D. (vi Add8.) Gergonne A. Mth. 14 (1823-24) 319-.

-. Joachimsthal, F. [1844] Crelle J. 33 (1846) 95-.

-. Letnikow, A. Z. Mth. Ps. 12 (1867) 223-.

- Laurent, H. N. A. Mth. 6 (1887) 19-.

- for differential and finite difference equations. Minich, S. R. Tortolini A. 1 (1850) 321-.

- - functions. Sarrus, $F$. Gergonne A. Mth. 14 (1823-24) 197-.

- _ - Bertrand, J. Par. Éc. Pol. J. $28^{\circ}$ cah. (1841) 249-; Liouv. J. Mth. 14 (1849) 123-.

- - - Sarrus, F. C. R. 28 (1849) $439-$.

- - - several variables. Dirksen, $E, H$. Berl. Ab. (1836) 79-.

-, equations which do not satisfy. Paoli, $P$. Verona S. It. Mm. 6 (1792) 501-.

- for linear equations, of 1st order, $n$ variables. Deahna, $F$. Crelle J. 20 (1840) 340-

- - partial equation, 1st order. Schultz, E. Arch. Mth. Ps. 13 (1895) 311-.

$--\phi_{3}(x) y^{\prime \prime \prime}+\phi_{2}(x) y^{\prime \prime}+\phi_{1}(x) y^{\prime}+\phi_{0} y=0$. Svanberg, A. F. Stockh. Öfv. 5 (1848) 155-.

- $\phi_{3}(x) y^{\prime \prime}+\phi_{2}(x) y^{\prime}+\phi_{1}(x) y=0$. Svanberg, A.F. Ups. N. Acta S. Sc. 1 (1851) 1-. criteria. Minich, S. R. Ven. At. 2 (1843) 377-.

-. Challis, J. Ph. Mg. 24 (1844) 94-.

- Volpicelli, P. Rm. At. 4 (1850-51) 622.

criterion, Euler's, reduction of conditions to single equation. Winckler, $A$. [1883] Wien Ak. Sb. 88 (1884) $(A b .2) 820$.

factor, means of determining. Maximovitch, $W$. C. R. 97 (1883) 1544 .

immediate, of differential expression, any order, conditions. Pujet, A. C. R. 82 (1876) 740 -

and integration. Combescure, $\dot{E}$. [1871] A. Mt. 5 (1871-73) 20-.

of ordinary equations. Peano, G. Mth. A. 37 (1890) 182-.

- - . Mie, G. Mth. A. 43 (1893) 553-

- Arzelà, C. Bologna Ac. Sc. Mm. ó

(1895-96) 257-.

Integrals, algebraic, of Kummer's equation. Goursat, $\hat{E}$. C. R. 103 (1886) 993-; Liouv. J. Mth. 3 (1887) 255-.

- of 1st class, representation by fundamental system. Hensel, K. Crelle J. Mth. 117 (1897) 29-. 
Integrals, continuous and discontinuous. Cauchy, A. L. C. R. 29 (1849) 548-.

- of equation, 1st order, degree. Autonne, -. C. R. 118 (1894) 1184-.

-, linear independent, of 1st class, number. Christoffel, E. B. A. Mt. 10 (1880-82) 81-.

- of ordinary equations, initial conditions determining. Niccoletti, $O$. Tor. Ac. Sc. At. 33 (1897) 514- or 746-.

- - - - system, unique determination by Cauchy's initial conditions. Painlevé, -. Par. S. Mth. Bll. 28 (1900) 191-.

- - partial equations, domains of convergence for. Koenigsberger, $L$. Crelle J. Mth. 112 (1893) 181-.

-, rational, of Kummer's equation. Goursat, É. Mth. A. 24 (1884) 445-.

- of system of total differential equations, convergence of expansions. Méray, - $\mathscr{E}$ Riquier, -. Par. Éc. Norm. A. 6 (1889) 355- ; 7 (1890) 23-.

- - $\frac{d^{2} y}{d x^{2}}+p y=0$, conditions for finiteness. Žukovskij, N. E. Rec. Mth. (Moscou) 16 (1893) 582-.

Integration, complete, of given system of ordinary equations, possibility. Lipschitz, $R$. [1868-76] A. Mt. 2 (1868-69) 288-; Bll. Sc. Mth. As. 10 (1876) 149-.

Irreducibility. Königsberger, L. Crelle J. Mth. 92 (1882) 291-.

Linear partial equation. Bendixson, I. Par. S. Mth. Bll. 24 (1896) 220-.

Ordinary equations. Picard, É. Par. S. Mth. Bll. 19 (1891) 61-.

- -. Peano, G. N. A. Mth. 11 (1892) $79-$

- - Arzelà, C. Bologna Ac. Sc. Mm. 6 (1896-97) 131-.

- - Lindelöf, $E$. Helsingf. Acta 21 (1896) No. $7,13 \mathrm{pp}$.

Orthoic systems. Riquier, - $\quad$ C. R. 125 (1897) 1159.

Partial equations. Mie, G. Z. Mth. Ps. 37 (1892) 151-, 193-.

- containing any number of functions and variables. Darboux, G. C. R. 80 (1875) $101-$

C. R. 80 (1875) 315-.

- - general demonstration. Méray, C. Liouv. J. Mth. 6 (1880) 235-.

- -, any order. Darboux, G. C. R. 80 (1875) 317-.

Any system. Méray, C. C. R. 80 (1875) 389-.

- - Riquier, -. C. R. 114 (1892) 731-; Par. Éc. Norm. A. 10 (1893) 65-, 123-, 167-.

System of ordinary equations, order. Jacobi, C. G. J. Crelle J. 64 (1865) 297-.

- - partial equations. Goursat, É. Par. S. Mth. Bll. 26 (1898) 129-.

Systems, cases. Riquier, - C. R. 125 (1897) 933-.

-, most general. Riquier, C. Par. Ec. Norm. A. 14 (1897) 99-.
Systems of partial equations. Koenigsberger, $L$. Mth. A. 42 (1893) 485-.

- - - Stäckel, P. Crelle J. Mth. 119 (1898) 339-.

-, priority claim. Delassus, É. Par. Éc. Norm. A. 14 (1897) 243-.

$\Delta u=k e^{u}$. Picard, É. C. R. 116 (1893) 1015-.

\section{Methods of solution and re- duction of ordinary differential equations.}

Adjoint equation, and systems of equations. Borel, É. Par. Éc. Norm. A. 9 (1892) 63-.

Algebraic equations. Königsberger, $L$. D. Nf. Tbl. (1889) 197-.

- -, 1st order. Vivanti, G. Palermo Cir. Mt. Rd. 4 (1890) 9-.

- - - - - and degree. Voss, A. Mth. A. 23 (1884) 157-.

- - , roots of which are integrals of Riccati's equation. Autonne, L. Liouv. J. Mth. 6 (1900) 157-.

- homogeneous equations. Appell, P. C. R. 104 (1887) 1776-.

Application of transformation groups. Page, $J$. $M$. A. Mth. 9 (1894-95) 59-.

Applications of calculus of symbols. Russell, W. H. L. Phil. Trans. (1861) 69- ; (1862) 253-; (1863) 517-.

Approximate methods. Durand, W. F. A. Mth. 12 (1898-99) 110-.

Behaviour near singular points. Dulac, $H$. C. R. 129 (1899) 276-.

Change of dependent and independent variables, applications. Niven, C. QJ. Mth. 10 (1870) 312-.

- - independent variable. Thomas, L. N.A. Mth. 9 (1850) 365-.

_- - - Spitzer, S. C. R. 49 (1859) 48-, $270-$.

- - - Glashan, J. C. Am. J. Mth. 3 (1880) 190-.

_ - variables, formulæ. Fais, A. Bologna Rd. (1877) 60-.

Clairaut's equation. Mansion, P. [1876] Mess. Mth. 6 (1877) 90 -

- , analogues of. Goursat, É. Par. S. Mth. Bll. 23 (1895) 88-.

- - , - - of higher order. Raffy, L. Par. S. Mth. Bll. 25 (1897) 71-.

- - generalised, and equations of 3 variables. Gasparis, A. de. Nap. Rd. 6 (1847) 149-.

Cremonian quadratic substitutions, application to equations, 1st order. Autonne, L. C. R. 105 (1887) 929- ; 106 (1888) 262-.

Depression of equations. Cunningham, (Lt.Col.) A. Mess. Mth. 17 (1888) 118-; 18 (1889) 122-.

Determination, explicit, of equations, 2nd order, with fixed critical points. Painlevé, P. C. R. 126 (1898) 1329-.

Differential resolvents. Spottiswoode, $W$. QJ. Mth. 6 (1864) 262-.

- - and partial differential resolvents. Raw. son, R. Manch. Lt. Ph. S. P. 21 (1882) 59-. 


\section{Equations.}

Fuss, N. [1818] St Pét. Ac. Sc. Mm. 9 (1824) 115-.

Besge, V. A. Liouv. J. Mth. 4 (1859) 72 ; 18 (1873) 139-.

Frahm, W. [1873] Mth. A. 8 (1875) 35-.

Le Paige, C. M. M. H. H. (xII) Brux. S. Sc. A. 1 (1877) (Pt. 2) 51-.

Tanner, H. W. I. [1877] Mess. Mth. 7 (1878) 107-.

Lindman, C. F. Stockh. Öfv. 37 (1880) No. 1, 3-.

Harley, R. QJ. Mth. 17 (1881) 352- ; 18 (1882) 41-.

Poincaré, H. C. R. 98 (1884) 793-.

Sharpe, H.J. Mess. Mth. 13 (1884) 66-.

Jamet, $V$. C. R. 104 (1887) 844-.

Guldberg, A. C. R. 117 (1893) 614-.

Chini, $M$. Tor. Ac. Sc. At. 34 (1898) 56-.

adjoint to equation, $n$th order. Grünfeld, $E$. Crelle J. Mth. 122 (1900) 43-, 88.

with algebraic integral. Brioschi, $F$. Mil. I. Lomb. Rd. 9 (1876) 786- ; 10 (1877) 48-.

- arbitrary parameter. Picard, É. C. R. 118 (1894) 760-.

class. Liouville, J. Liouv. J. Mth. 6 (1841) 448.

-. Boole, G. (vi Adds.) Ph. Mg. 30 (1847) 96-.

class. Rosanes, J. Z. Mth. Ps. 16 (1871) 263-.

-. Jablonski, E. As. Fr. C. R. (1877) 188-.

-. Haag, -. Par. S. Mth. Bll. 8 (1880) 80-.

- Catalan, E. Brux. Ac. Bll. 12 (1886) 17-.

- Vessiot, E. Par. Éc. Norm. A. 10 (1893)

53-; C. R. 116 (1893) 959-, 1112-.

-. Lindhagen, A. N. Ts. Mth. 10 (B) (1899) 35-; Fschr. Mth. (1899) 297.

- reducible to linear equations. Appell, C. R. 107 (1888) 776-.

with complete differentials, two systems. Stodótkiewicz, A. J. Krk. Ak. (Mt.-Prz.) Pam. 12 (1886) (93)-; Fschr. Mth. (1886) 307.

connected with orthogonal trajectories. Euler, L. [1781] St Pét. Ac. Sc. Mm. 11 (1830) $125-$.

of 1st degree. Trembley, $J$. Turin Mm. Ac. (1790-91) 1<.

- 2nd degree. Fuss, N. [1819] St Pét. Ac. Sc. Mm. 11 (1830) 258-

- - Enneper, A. Schlömilch Z. 8 (1863) $58-$.

- - general integral of which is uniform. Painlevé, P. C. R. 117 (1893) 211-.

- form $y^{m}=x^{m}\left(A x^{2} y^{\prime \prime}+B x y^{\prime}+C y\right)$. Spitzer, S. Arch. Mth. Ps. 58 (1876) 100-.

with fractional indices. Besge, $V . A$. Liouv. J. Mth. 9 (1844) 294.

general integral of which is linear fractional function of arbitrary constants. Wallenberg, G. Crelle J. Mth. 121 (1900) 210-

geometrical interpretation. Fouret, $G$. C. R. 102 (1886) 415-. having fundamental system of integrals. Guldberg, A. C. R. 116 (1893) 964-.

-- - - Lie, S. C. R. 116 (1893) 1233-.

integrated by differentiation. Raffy, $L$. Par. S. Mth. Bll. 23 (1895) 50-.

invariant under transformation $x x^{\prime}=1, y x^{\prime}=y^{\prime}$. Elliott, E. B. Mess. Mth. 19 (1890) 7-.

not containing the independent variable. Zehfuss, G. (xI) Z. Mth. Ps. 3 (1858) 247-.

occurring in Laplace's kinetic theory of tides. Ling, G. H. A. Mth. 10 (1895-96) 95-.

of multiplier. Genocchi, $A$. Mil. I. Lomb. Rd. 10 (1877) 553-.

- 1st order. Liouville, R. C. R. 103 (1886) 476-; 106 (1888) 1648-; Par. Éc. Pol. J. 62 (1893) 181-.

- - Vessiot, -. C. R. 116 (1893) 427-.

- - Korkine, A. C. R. 122 (1896) 1183-; 123 (1896) 38-, 139; Mth. A. 48 (1897) 317-. - - Petrovitch, M. C. R. 122 (1896) 1261-.

_- Painlevé, P. C. R. 122 (1896) 1319 - ; 123 (1896) 88-.

- - - Cahen, A. C. R. 127 (1898) 1196-.

- - Horn, J. Mth. A. 51 (1899) 360-.

- - class, and invariant formations attaching to them. Liouville, R. C. R. 105 (1887) 460-, 496.

- - , 2 variables. Ermakov, V.P. Fschr. Mth. (1887) 316-.

- - and 2 nd orders, 2 variables. Weiler, $A$. Grunert Arch. 29 (1857) 1-.

- 2nd order. Besge, $V$. A. Liouv. J. Mth. 9 (1844) 336.

- - Hazzidakis, J. N. [1880] Crelle J. Mth. 90 (1881) 74-.

- - - Guldberg, A. N. Ts. Mth. 7 (B) (1896) 1-; Fschr. Mth. (1896) 250.

- _ , with fixed critical points. Painlevé, $P$. C. R. 126 (1898) 1185-, 1697-.

- 3rd order. Goursat, É. C. R. 98 (1884) 419-.

-- - Klein, F. Mth. A. 23 (1884) 587-.

- Kummer, E. E. Crelle J. Mth. 100 (1887) 1-.

-4th order. Pochhammer, L. Crelle J. Mth. 104 (1889) 116-.

with periodic coefficients, series attached to theory of. Liapounoff, A. C. R. 123 (1896) 1248-.

possessing fundamental integrals. Lie, $S$. Leip. Mth. Ps. B. 45 (1893) 341-.

relating to breaking of railway bridges. Stokes, G. G. Camb. Ph. S. T. 8 (1849) 707-.

Euler's factor. Lorenz, L. V. (xII) Ts. Mth. 3 (1873) 174-; 4 (1874) 33-.

- - Heun, K. Mth. A. 31 (1888) 363-.

- - application. Peterson, J. Mth. Ts. 4 (1862) 139-.

- and Poisson's equations. Lévy, L. N. A. Mth. 8 (1889) 545-.

Exact differential. Autonne, L. N. A. Mth. 15 (1896) 232-. 
General equations reducible to quadratures. Maximovitch, W. C. R. 101 (1885) 809-.

Greatest and least exponents in theory of differential equations, complete particular integrals. Bugaev, N. V. [1891] Rec. Mth. (Moscou) 16 (1893) 39-; Fschr. Mth. (1891) 313.

Homogeneous equations. Lindelöf, E. Par. S. Mth. Bll. 24 (1896) 35-.

Integrability conditions for linear equations. Rusjan, C. K. Fschr. Mth. (1899) 314-.

-, formula, generalisation. Restellini, $L$. G. Mt. 36 (1898) 262-.

Integrable case of

$$
\begin{aligned}
x(1-x)(1-k x) y^{\prime \prime \prime} & +\left(u+v x+w k x^{2}\right) y^{\prime \prime} \\
& +\left(\tau+w^{\prime} k x\right) y^{\prime}+w^{\prime \prime} k y=0 .
\end{aligned}
$$

Thomae, C. J. Z. Mth. Ps. 21 (1876) 100 -

- cases of special equation. Heymann, $\dot{W}$. Crelle J. Mth. 113 (1894) 84-.

- class of differential and finite difference equations. Sylvester, J. J. [1881] (xI) J. H. Un. Cir. [1] (1882) 178.

- forms of linear equations, 2nd order. Liouville, $R$. C. R. 100 (1885) 235-.

- total differential equations, theory. Guldberg, A. Crelle J. Mth. 122 (1900) 34-.

\section{Integrals.}

algebraic, of Lamé's equation. Brioschi, $F$. Rm. R. Ac. Linc. Rd. 1 (1892) (Sem. 2) 327-. - particular. Bugaev, N.V. [1892-97] Rec. Mth. (Moscou) 17 (1895) 399-; 19 (1897) 662-; Fschr. Mth. (1893-94) 538.

cases. Herz, N. [1881] Arch. Mth. Ps. 67 (1882) 312-.

of equation, Ist order, at singular point, values. Fuchs, L. Berl. Ak. Sb. (1886) 279-.

- equations, 1st order, with limited number of values. Painlevé, P. C. R. 114 (1892) 107-, 280-.

- - with periodic coefficients, form. Anisimov, V. A. Rec. Mth. (Moscou) 20 (1899) 411-; Fschr. Mth. (1898) 286-.

- - - - - Anisimov, V. [1900] Rec. Mth. (Moscou) 21 (1901) 62-.

$---1-1$, Hermite, $C$. [1900] Rec. Mth. (Moscou) 21 (1901) 66-.

expansions in series. Picard, É. C. R. 128 (1899) 1363-. - 597 - , cases. Liouville, R. C. R. 111 (1890)

first, of systems. Painlevé, P. C. R. 124 (1897) 136-.

as functions of their initial values. Niccoletti, O. Rm. R. Ac. Linc. Rd. 4 (1895) (Sem. 2) 316-.

- - - - Holmgren, E. Stockh. Ak. Hndl. Bh. 25 (1900) (Afd. 1) No. 4, 19 pp.

general, of Bessel's equation, formulæ for numerical calculation. Niemöller, $F$. Z. Mth. Ps. 25 (1880) 65-.

-, - equation of 1st order, form. Fuchs, L. Berl. Âk. Sb. (1884) 1171-.

-, - certain systems of simultaneous differential equations. Seiliger, $D$. Kazan Un. Mm. (1897, Pt. 1) (Non-off. Sect.) 69-. particular, with fractional indices. Bugaev, N. V. [1891] St Pet. Ac. Sc. Mm. (Rs.) 67 (1892) (Suppl.) No. 4, 12 pp.; Fschr. Mth. (1891) 313.

- and general, connexion between. Bochow, Z. Mth. Ps. 33 (1888) 101-.

of special equation, form. Haentzschel, $E$. Crelle J. Mth. 112 (1893) 148-.

- system, calculation by Cauchy-Lipschitz method. Painlevé, -. Par. S. Mth. Bll. 27 (1899) 149-

- - of total differential equations. Sauvage, Liouv. J. Mth. 10 (1884) 387-.

- systems. Forsyth, A.R. [1899] Camb. Ph. S. T. $18(1900) 35-$.

- total differentials. Poincaré, H. C. R. 99 (1884) 1145-.

- - Picard, É. C. R. 100 (1885) 843-. - _ and class of algebraic surfaces. Picard, É. C. R. 99 (1884) 1147-.

uniform, of equations of 1st order and genus zero. Petrovitch, -. C. R. 118 (1894) 1190 -.

of $\frac{d z^{2}}{d x^{2}}=\frac{8 w}{R} z^{\frac{3}{2}}+C$. Wood, De V. Franklin I. J. 47 (1864) 28-.

$-f\left(y, x, \frac{d y}{d x}\right)=0$, representation in space. Autonne, L. C. R. 105 (1887) 850-.

Integrating equation. Bierens de Haan, $D$. Amst. Ak. Vs. M. 14 (1879) 162-.

- factor. Buchwaldt, $F$. Mth. Ts. 5 (1863) 33-, 82-.

- -, determination. Maksimovich, $V . P$. Kazan S. Nt. (Ps.-Mth.) P. 2 (1884) 19m.

- of elliptic equation. Sturm, $R$. Mth. A. 21 (1883) 446-.

- - - equations, 1st order. Winckler, $A$. Wien Ak. Sb. 99 (1891) (Ab. 2a) 457-, 875-. - - expressions, 1st order. Collet, C. R. 68 (1869) 799-.

- - - - _ - for any number of variables. Collet, -. Par. Éc. Norm. A. 7 (1870) 59-. - - homogeneous equations. Le François, (Prof.) -. Brux. Ac. Bll. 13 (1846) 159-.

_ _ - system of equations, new. Jacobi, C. G. J. Crelle J. 27 (1844) 199-; 29 (1845) 213-, 333-.

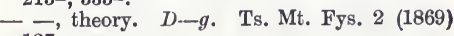
127-.

- - 3 variables. De Morgan, A. QJ. Mth. 2 (1858) 323-.

- factors of one or several differential forms. Chini, M. Mil. I. Lomb. Rd. 33 (1900) 601-. - - system of linear homogeneous equations. Escherich, G. von. Wien Ak. Sb. 101 (1892) (Ab. 2a) 1222-.

\section{Integration.}

Brassinne, E. Toul. Mm. Ac. 5. (1839) 139-. Bertrand, J. Liouv. J. Mth. 17 (1852) 175-. Hill, C.J. D. Sk. Nf. F. 8 (1860) 525-.

Steen, A. Ts. Mt. Fys. 3 (1870) 44 -

Spitzer, S. Z. Mth. Ps. 52 (1871) 1-, 16-; 53 (1871) 1-. 
Russell, W. H. L. Mess. Mth. 10 (1881) 38-.

Heymann, $W$. Z. Mth. Ps. 27 (1882) 1-; 29 (1884) 257-.

La Vallée-Poussin, C. de. Brux. S. Sc. A. 17 (1893) (Pt. 1) 8-.

Guldberg, A. C. R. 121 (1895) 49-; Crelle J. Mth. 118 (1897) 158-.

algebraic. Poincaré, H. C. R. 112 (1891) 761-.

-, of equations, 1st order and degree. Cazzaniga, $P$. G. Mt. 18 (1880) 72-.

- $-\ldots,-\ldots-$ Poincaré, H. Palermo Cir. Mt. Rd. 11 (1897) 193-.

-, - linear equations, 3rd order. Boulanger, $A$. C. R. 124 (1897) 1011-.

application of Leibnitz's formula. Tychsen, $C$. Mth. Ts. 6 (1864) 177-.

approximate, of equations, 1st order. $S e$ verini, C. Mil. I. Lomb. Rd. 31 (1898) 950 .

-,- of 1 variable. Heun, $K$. Z. Mth. Ps. 45 (1900) 23-.

- $-\ldots-2$ variables. Kramp, C. Gergonne A. Mth. 10 (1819-20) 1-.

-, - linear equations, cases. Sparre, (le comte) - de. Brux. S. Sc. A. 23 (1899) (Pt. 2) 176-.

- _ - - 2nd order, cases. Sparre, M. de. Rm. R. Ac. Linc. Rd. 7 (1898) (Sem. 2) 111 .

of Brassinne's equation. Longchamps, G. de. As. Fr. C. R. (1890) (Pt. 2) 146-.

cases. Favre-Rollin, A. M. Liouv. J. Mth. 1 (1836) 339-

-. Ellis, R. L. Camb. Mth. J. 2 (1841) 169-, 193-.

-. Bronwin, B. Camb. Mth. J. 3 (1843) 29-, 175-.

-. Huet, -. N. A. Mth. 5 (1846) 165-.

- Turquan, -. N. A. Mth. 5 (1846) 396-.

-. Spitzer, S. [1849] Haidinger B. 6 (1850) 86-.

-. Dupain, J. C. N. A. Mth. 10 (1851) 418

-. Björling, E. G. Stockh. Ak. Hndl. (1854) 149-; Liouv. J. Mth. 3 (1858) 417-.

- Spitzer, S. Schlömilch Z. 3 (1858) 106-.

-. Lorenz, L. Mth. Ts. 3 (1861) 102-

-. Steen, A. Mth. Ts. 5 (1863) 113-; (xII)

Ts. Mth. 2 (1866) 138-; 3 (1867) 56-.

-. Allégret, [A.] C. R. 66 (1868) 1144-.

-. Leffler, G. Mittag. Ts. Mt. Fys. 3 (1870) 28 -

-. Turquan, L. V. [1874] Par. S. Mth. Bll. 3 (1875) 40-.

- Lyetnikov, A. V. (xп) Rec. Mth. (Moscou) 8 (1876) (Pt. 1) 498-.

-. Halphen, G. H. C. R. 88 (1879) 562-.

-. Spitzer, S. Arch. Mth. Ps. 64 (1879) 393-.

- Toropov, K. A. Kharkov Mth. S. Com. (1884) 199-; Fschr. Mth. (1885) 309-.

Arneberg, A. Ts. Mth. 3 (1885) 168-; Fschr. Mth. (1885) 328.

of class of equations. Cauchy, A. L. Par. S. Phlm. Bll. (1818) 17-.

- - (Cauchy). Poisson, S. D. Par. S. Phlm. Bll. (1818) 19-. of class of equations. Woisard, J. L. Ger. gonne A. Mth. 15 (1824-25) 121-

- - - Liouville, J. Liouv. J. Mth. 3 (1838) 31-.

- -1, 2nd order. Laguerre, -. C. R. 67 (1868) 1130-; 69 (1869) 1125-.

- - - - - _ , in explicit finite quantities. Liouville, J. 'Liouv. J. Mth. 4 (1839) 423-.

- - linear equations. Spitzer, S. [1863] Arch. Mth. Ps. 43 (1865) 478-.

and classification of equations between $x$ and $y$ admitting of a group of transformations. Lie, S. Arch. Mth. Ntvd. 8 (1883) 187-, 249-, 371-; 9 (1884) 431-.

of complete differentials. Morera, $G$. Mth. A. 27 (1886) 403-.

definite, of a system of equations. Cauchy, A. $L$. C. R. 43 (1856) 497-

of differential equations or partial differential equations. Cauchy, A. L. Par. S. Phlm. Bll. (1822) 49-; C. R. 11 (1840) 1-.

by differentials to any index. Serret, $J . A$. Liouv. J. Mth. 9 (1844) 193-.

of equation in analysis of functions of 2 variables. Euler, L. [1777] St Pét. Ac. Sc. Mm. 11 (1830) 131-.

- equations, coefficients of which are algebraic functions of the independent variable. Spitzer, S. Wien SB. 29 (1858) 53-.

- with complete differentials. Stodotkiewicz, A. J. Prace Mt.-Fiz. 1 (1888) 78-; Fschr. Mth. (1888) 355.

- - in Euler's integral calculus, cases. Grunert, J. A. Grunert Arch. 32 (1859) 239-.

- with fractional index. Liouville, $J$. [1836] Par. Ec. Pol. J. 25 cah. (1837) 58-.

- -, 1st order. Bohlmann, G. Crelle J. Mth. 113 (1894) 207-; 115 (1895) 89-.

- -, - -. Severini, C. Mil. I. Lomb. Rd. 31 (1898) 657-.

-,- - — and degree, 3 variables. Volpicelli, P. Rm. At. 1 (1847-48) 108-

- _, - _, 2nd degree, several variables. Raabe, J. L. Baumgartner Z. 8 (1830) 425-; 9 (1831) 36-.

- - - - - - by equation of 2nd order, case. Dienger, $J$. Arch. Mth. Ps. 46 (1866) 317-.

- _, _ _, where independent variable does not appear. Raschke, $W$. Acta Mth. 14 (1890-91) 31-.

- - , - - 2 variables. Minding, E. F.A. [1845-61] St Pét. Ac. Sc. Bll. 4 (1845) 378-; St Pét. Ac. Sc. Mm. 5 (1863) No. 1, $96 \mathrm{pp}$.

- - 2nd order. Cayley, A. Ph. Mg. 42 (1871) 197-.

- _ _ _ - application of principle of last multiplier. Laguerre, -. Bll. Sc. Mth. As. 2 (1871) 246-.

- - - - cases. Brioschi, F. Tortolini A. 2 (1851) 497-.

- - - - - - Wolfers, J. P. Grunert Arch. 28 (1857) 271-.

-, - - - - Malmsten, C. J. Ts. Mt. Fys. 2 (1869) 71-.

Par. S. Phlm. Bll. 7 (1871) 158-. 
of equations, 2 nd order, cases. Steen, $A$. [1882] Kjöb. Dn. Vd. Selsk. Skr.1 (1880-85) 331-.

- - - - - - Gyldén, H. Stockh. Ak. Hndl. Bh. 12 (Afd. 1) (1887) No. 3, 15 pp.

$\ldots+, \ldots$, Challis's method, and application to calculus of variations. Ehrhorn, M. Arch. Mth. Ps. 66 (1881) 113-.

- - - - - and degree, cases. Lobatto, $R$. Grunert Arch. 30 (1858) 292-.

- - , - - - - geometrical significance of primitive. Tychsen, C. Mth. Ts. 5 (1863) 118-.

- - $n$th order, 1st degree. Malmsten, C.J. Stockh. Ak. Hndl. (1842) 145-.

-, 2 variables. Kramp, C. Gergonne A. Mth. 11 (1820-21) 97-, 345-.

,--- - Stodótkievicz, A.J. (xII) Krk. Ak. (Mt.-Prz.) Rz. \& Sp. 10 (1883) 225-.

under finite form. Genocchi, A. [1864-72]

Tor. Mm. Ac. 23 (1866) 299-; Tor. Lav. Sc.

Fis. Mt. (1869) 88-; Tor. At. Ac. Sc. 7 (1871-72) 682-.

formulæ. Pezzi, F. Turin Mm. Ac. (1790-91) 79-.

of functions, case. Dienger, J. Grunert Arch. 12 (1849) 203-.

geometric method, case. Crofton, $M$. $W$. Mess. Mth. 3 (1866) 106.

-, of $L(x d y-y d x)-M d y+N d x=0, L, M, N$ being linear functions of $x$ and $y$. Fouret, $G$. C. R. 78 (1874) 1837-.

of homogeneous equations. Cauchy, A. L. C. R. 13 (1841) 564-.

- - Minding, E. F. A. Liouv. J. Mth. 4 (1859) 273-.

method, Brisson's, for equations with constant coefficients. Mansion, $P$. N. A. Mth. 11 (1872) 118-.

-, general. Schmidten, H. G. [1826] Kiöb. Dn. Vd. Selsk. Afh. 3 (1828) 1-; Crelle J. 1 (1826) 137-.

methods. Anisimov, $V$. A. Vars. S. Nt. Tr. (1897) (Ps. C.) Fasc. 1, Mm. 1, 35 pp.; Acta Mth. 51 (1899) 181-.

problem, solution. Lagrange, $C$. Brux. Obs. A. (As.) 7 (1896) 40 pp.

by separation of symbols of operation. Francais, J. F. Gergonne A. Mth. 3 (1812-13) 244 -

- series. Cayley, A. Mess. Mth. 5 (1871) $77-$.

- -. Sabinine, G. Bll. Sc. Mth. As. 2 (1878) (Pt. 1) 284-.

- Poincaré, $H$. C. R. 94 (1882) 577-.

- - of $\frac{d^{2} y}{d x^{2}}-\frac{n-1}{x} \frac{d y}{d x}=y$. Bach, - Par. Éc. Norm. A. 3 (1874) 47-.

of simultaneous equations. Boole, $G$. R. S. P. 12 (1862-63) 13-.

- - - case. Steen, A. (xu) Ts. Mth. 1 (1865) 35-.

- - - 1st order. Bouquet, -. Bll. Sc. Mth. As. 3 (1872) 265-.

- symmetrical equations. Imchenetsky, $B$. St Pét. Ac. Sc. Mm. 37 (1890) No. 1, 38 pp.

- a system of equations. Richelot, $F . J$. Crelle J. 23 (1842) 354-. of a system of equations. Hill, C. J. D. Stockh. Ófv. 14 (1857) 267-.

- - - - - Imshenetski\%, V. G. (xII) Rec. Mth. (Moscou) 8 (1876) (Pt. 1) 254-.

- _- Eiesland, J. Am. J. Mth. 20 (1898) 245-.

- - _ - , and inversion of their integrals. Cauchy, A. L. C. R. 23 (1846) 617-.

- - - - - 2nd order, several variables. Binet, J. P. M. Liouv. J. Mth. 2 (1837) 457-. - - - - homogeneous equations. Catalan, E. C. Brux. Ac. Bll. 21 (1866) 25-.

- - - linear equations, $n$th order. Lebesgue, V. A. Crelle J. 15 (1836) 185-.

- $-1-$ simultaneous equations. Hočevar, F. [1878] Wien Ak. Sb. 78 (1879) (Ab. 2) 47-. - systems of equations. Cauchy, A. L. C. R. $10(1840)$ 957-.

_- Stodótkiewicz, A.J. (xII) Krk. Ak. (Mt.-Prz.) Pam. 8 (1883) 143-.

- _ - use of particular algebraic solutions. Darboux, G. C. R. 86 (1878) 1012-.

- - non-linear equations. Borchardt, At. Sc. It. (1843) 500-.

- - - total differential equations. Dixon, A. C. [1899] L. Mth. S. P. 31 (1900) 177-.

_- - linear differential equations with 2 independent variables. Horn, $J$. Mth. A. 42 (1893) 215-.

by theory of sines of higher orders. Farkas, $J$. C. R. 90 (1880) 1542-.

of total algebraic differentials. Picard, É. C. R. 99 (1884) 961-.

- - binary differential with 4 independent variables. Méray, C. Par. Éc. Norm. A. 16 (1899) 509-.

- differential equations. Kryger, C. (xII) Ts. Mth. 5 (1875) 147-。

and transformation. Bronwin, B. Ph. Mg. 29 (1846) 494-.

- - (Bronwin). Boole, G. Ph. Mg. 30 (1847) 6-.

- - (-). Hargreave, C. J. Ph. Mg. 30 (1847) 8-.

of $p^{3} v v d v=n v d p+n p d v(1-p)(2-p)$. Rumovski, S. [1797] St Pet. Ac. Sc. N. Acta 12 (1801) 192-.

$y d y+P y d x+Q d x=0 . \quad F u s s, N .[1803]$ St Pét. Ac. Sc. Mm. 3 (1811) 75-.

$-y=x F\left(\frac{d y}{d x}\right)+f\left(\frac{d y}{d x}\right)$. Monge, $G$. Par. Éc. Pol. Cor. 1 (1804-08) 73-.

$-d y+y y d x=\frac{A d x}{(a+2 b x+c x x)^{2}} \cdot$ Euler, L. St Pét. Ac. Sc. Mm. 3 (1811) 1-.

$-y d x-x d y=a\left(d x^{n}+d y^{n}\right)^{\frac{1}{n}}$ and $x y\left(d x^{2}-d y^{2}\right)-d x d y(x x-y y+a a)=0$. Fuss, N. [1825] St Pét. Ac. Sc. Mm. 11 (1830) 280 -

$-v d v+v(3 y+f) d y+\left(y^{3}+f y^{2}+g y+h\right) d y=0$. Fuss, N. [1826] St Pét. Ac. Sc. Mm. 11 (1830) 287-.

$-\left(A+A^{\prime} x+A^{\prime \prime} y\right)(x d y-y d x)$ $-\left(B+B^{\prime} x+B^{\prime \prime} y\right) d y+\left(C+C^{\prime} x+C^{\prime \prime} y\right) d x=0$. Jacobi, C. G. J. Crelle J. 24 (1842) 1-. 


\section{Ordinary Differential Equations, Solution and Reduction}

of $\left(A+A^{\prime} x+A^{\prime \prime} y\right)(x d y-y d x)$

$-\left(B+B^{\prime} x+B^{\prime \prime} y\right) d y+\left(C+C^{\prime} x+C^{\prime \prime} y\right) d x=0$.

Lebesgue, V. A. Liouv. J. Mth. 10 (1845) 316-.

$$
\begin{aligned}
& y^{\prime \prime}=x\left(A x^{2} y^{\prime \prime}+B x y^{\prime}+C y\right), \\
& y^{\prime}=x^{2}\left(A x^{2} y^{\prime \prime}+B x y^{\prime}+C y\right), \\
& y=x^{3}\left(A x^{2} y^{\prime \prime}+B x y^{\prime}+C y\right) .
\end{aligned}
$$

Spitzer, S. Arch. Mth. Ps. 42 (1864) 346.

$$
-\frac{\frac{d^{2} y}{d x^{3}}}{\left(1+\frac{d y^{2}}{d x^{2}}\right)^{\frac{3}{2}}}=2 f\left(x^{2}+y^{2}\right) \text {, method. Steen, } A \text {. }
$$

Ts. Mt. Fys, 3 (1870) 24 -.

$-y^{\prime \prime}=x\left(x y^{\prime}-n y\right)$. Spitzer, S. Arch. Mth. Ps. 53 (1871) 117 -

$-y^{(n)}=A x^{2} y^{\prime \prime}+B x y^{\prime}+C y$. Spitzer, S. Arch. Mth. Ps. 53 (1871) 385-.

$-f(x, y) d x+\phi(x, y) d y=0$, case. Harkerna, C. N. A. Mth. 13 (1874) 545-.

$-(x d y-y d x)(a+b x+c y)-d y\left(a^{\prime}+b^{\prime} x+c^{\prime} y\right)$

Allégret, A. C. R. 83 (1876) 1171. $+d x\left(a^{\prime \prime}+b^{\prime \prime} x+c^{\prime \prime} y\right)=0$.

$-y \frac{d^{2} y}{d x^{2}}-\frac{2}{3}\left(\frac{d y}{d x}\right)^{2}=6 f(x), f$ being a polynomial of 2nd degree. Laguerre, E. [1877] Par. S. Mth. Bll. 6 (1878) 121-.

- $\quad A y^{\prime 2}+E y y^{\prime}+C y^{2}+D y^{\prime}+E y+F=0$.

Alexéeff, N. C. R. 87 (1878) 641-.

$-f\left(\frac{d u}{d z}, u\right)=0$. Hansen, P. C. V. [1881]

Kjöb. Ov. (1881-82) 156- (\& Résumé, 7-).

$-L d x+M d y+N(x d y-y d x)=0$. Hočevar, $F$.

Wien Ak. Sb. 85 (1882) (Ab. 2) 848-.

$-\frac{d^{2} \rho}{d v^{2}}+\rho=\beta_{0}+\beta_{1} \rho+\beta_{2} \rho^{2} \ldots . \quad$ Lindstedt, $A$.

As. Nr. 103 (1882) 257-; 104 (1883) 145-.

$--=-$ (Lindstedt). Gyldén, J. A. H. As. Nr. 103 (1882) 321-.

$-\left(A_{1} x^{2}+B_{1} y^{2}+C_{1} x y\right) d x$

$\left.\begin{array}{l}+\left(A_{2} x^{2}+B_{2} y^{2}+C_{2} x y\right) d y \\ +\left(A_{3} x^{2}+B_{3} y^{2}+C_{3} x y+D_{3} x+E_{3} y+F_{s}\right)\end{array}\right\}=0$.

Heymann, $W$. Z. Mth. Ps. 28 (1883) 54-

$-\phi\left(x, y, \frac{d y}{d x}, \ldots \frac{d^{n} y}{d x^{n}}\right)$, when it is an exact differential coefficient. Foldberg, P. T. N. Ts. Mth. 1 (B) (1890) 23-; Fschr. Mth. (1890) 281-.

$-y^{\prime \prime}=A y^{3}+B y^{2}+C y+D+(E y+F) y^{\prime}$. Mittag . Leffler, G.' Acta Mth. 18 (1894) 233-.

Newton-Cotes method of quadratures. Jung, $V$. Prag České Ak. Fr. Jos. Rz. (Tř́ida 2) 8 (1899) Art. 17, 12 pp. ; Fschr. Mth. (1899) 279.

Non-linear equations, cases. Liouville, $R$. C. R. 103 (1886) 457-.

- - class. Liouville, R. C. R. 103 (1886) 520-, 571.

- _ - 2nd order, with doubly periodic coefficients. Gyldén, $H$. C. R. 122 (1896) $160-, 585-$.

Partial equations occurring in theory of conjugate functions. Volterra, $V$. Palermo Cir. Mt. Rd. 3 (1889) 260-.
Paths described by ships to perform certain evolutions. Bernotti, R. [1897] Rv. Mar. et Col. 137 (1898) 341-.

Reciprocal equations. Orloff, - - Brux. Ac. Bll. 33 (1872) 113-.

Reduction to canonical form. Pennacchietti, . G. Mt. 29 (1891) 239-.

- Riquier, C. Par. Éc. Norm. A. 14 (1897) 259-.

- of complete system to single system of ordinary equations. Mayer, A. Leip. Mth. Ps. B. 43 (1891) 448-.

- - - - Schur, $F$. Leip. Mth. Ps. B. 44 (1892) 177-

- - order. Königsberger, L. Mth. A. 26 (1886) 110-.

- - by known integrals. Koenigsberger, $L$. Mth. A. 31 (1888) 302-.

- to new variables. Baehr, G. F. W. Amst. Vs. Ak. 3 (1855) 233-.

Separation of variables in $M d x+N d y=0$. Harkema, C. N. A. Mth. 16 (1877) 215-.

\section{Solution.}

Roberts, S. QJ. Mth. 3 (1860) 218-.

asymptotic, cases. Picard, É. C. R. 115 (1892) 1030 -

- method, Petzval's. Spottiswoode, W. QJ. Mth. 5 (1862) 154-.

Boole's. Rawson, $R$. Mess. Mth. 5 (1876) 138-.

cases. Bromwin, B. Ph. Mg. 32 (1848) 256-.

- (Bronwin). Boole, G. Ph. Mg. 32 (1848) 413-.

-. Williamson, B. Ph. Mg. 11 (1856) 364-.

- Jackson, F. H. Edinb. Mth. S. P. 12 (1894) 109-.

of class of difference or differential equations. Sylvester, J. J. Am. J. Mth. 4 (1881) 260 .

common to two equations, orders $m+n, m$, Libri's theorem. Liouville, $J$. C. R. 8 (1839) 790 -

of differential resolvent. Russell, W. H. L. [1863] Manch. Lt. Ph. S. Mm. 2 (1865) 296-.

- equation, 2nd order, properties. Routh, E.J. L. Mth. S. P. 16 (1884-85) 245-.

- - of sources. Hammond, J. Am. J. Mth. 5 (1882) 218-.

- equations in series. Glaisher, J.W. L. [1878] Mess. Mth. 8 (1879) 20 -.

- homogeneous equation. Forsyth, A. R. Mess. Mth. 18 (1889) 136-.

and integration. West, $\dot{E}$. Liouv. J. Mth. 7 (1881) 5-; 8 (1882) 19-, 125-; 9 (1883) 301-. numerical. Runge, C. Mth. A. 46 (1895) 167 .

periodic, of linear equations. Bendixson, $I$. Stockh. Öfv. (1896) 193-.

regular, of a system. Sauvage, L. Par. Éc. Norm. A. 3 (1886) 391-; 5 (1888) 9-; 6 (1889) 157-.

singular. Fine, H. B. Am. J. Mth. 12 (1890) 295-.

-. Page, J. M. Am. J. Mth. 18 (1896) 95-. 
singular, of algebraic equations, 1st order. Hamburger, M. Crelle J. Mth. 112 (1893) 205-.

-, - equations, 1st order. Darboux, G. Bll. Sc. Mth. As. 4 (1873) 158-.

,,----- Kapteyn, W: Bll. Sc. Mth. 12 (1888) 135-.

-, - - - - Predella, L. G. Mt. 33 (1895) 31-, 183-.

, - - - - Maddison, I. QJ. Mth. 28 (1896) 311-.

- , - - - - Toussaint, H. Rm. N. Linc. Mm. 13 (1897) 283-.

-,--- - Petrovitch, M. Mth. A. 50 (1898) 103-.

- - - - higher order. Hamburger, M. Crelle J. Mth. 121 (1900) 265-.

-, - homogeneous equations. Johnson, W. W. Mess. Mth. 16 (1887) 186-.

-, - integrable equations, 1st order. Workman, $W^{\circ}$. P. QJ. Mth. 22 (1887) 175-, 308-.

-, - simultaneous equations. Goursat, $\hat{\boldsymbol{E}}$. Am. J. Mth. 11 (1889) 329-.

-, - - - and theory of congruencies. Dixon, A. C. [1894] Phil. Trans. (A) 186 (1896) 523-.

-, - a system. Biermann, O. Wien Ak. Sb. 90 (1885) (Ab. 2) 897-.

- , - - - of equations with $n$ dependent variables. Hamburger, $M$. Crelle J. Mth. 122 (1900) 322-.

symbolic and by definite integrals of $\frac{d^{n} y}{d x^{n}}=x^{m} y$. Fields, J. C. Am. J. Mth. 8 (1886) 367-. of $\frac{d y}{d x}=f(x, y)$ without Cauchy-Lipschitz condition. Osgood, W. F. Mh. Mth. Ps. 9 (1898) 331-.

Substitution of variables as method of finding integrating factor. Ermakov, $\boldsymbol{V} . P$. (xח) Kharkov Mth. S. Com. (1881) 3-.

Successive approximations. Picard, $\hat{E}$. C. R. 115 (1892) 543-.

- -. Bendixson, I. Stockh. Öfv. (1893) $599-$.

- - Lindelöf, E. Liouv. J. Mth. 10 (1893) 117-.

- Picard, É. Liouv. J. Mth. 9 (1893) 217-.

- - Bugaev, N. V. [1896] Rec. Mth. (Moscou) 19 (1897) 1-; Fschr. Mth. (1896) 70,72 .

- - Picard, É. Am. J. Mth. 20 (1898) 87-.

- Davidoglou, A. C. R. 130 (1900) 692-, 1241-.

- - application to equations, 1st order. Lindelöf, E. C. R. 118 (1894) 454.

-,$-\ldots$, - Picard, É. C. R. 118 (1894) 457-.

,,$-----\cdots$ Horn, J. Mth. A. 51 (1899) 346-.

- divergent approximations. Picard, É. C. R. 118 (1894) 899-.
Symbolic decomposition. Cockle, Jas. Ph. Mg. 24 (1862) 288-.

System of equations, determination of variables satisfying. Cauchy, A.L. C. R. 23 (1846) 485-, 529-.

Systems of equations. Combescure, $\dot{E}$. [1874] Crelle J. Mth. 80 (1875) 33-.

- - Halphen, G. H. C. R. 92 (1881) 1404-.

215-

- - case. Halphen, G. H. C. R. 92 (1881) 1101-.

- - - Brioschi, F. C. R. 92 (1881) $1389-$.

- - - 1st order, having fundamental systems of integrals. Vessiot, $E$. Toul. Fac. Sc. A. 8 (1894) B, 33 pp.

- - 2nd order, in involution. Goursat, É. Par. Éc. Pol. J. 3 (1897) 75-.

- integration of which is reducible to that of total differentials. Riquier, - . C. R. 127 (1898) 809-, 1194-.

Theorem of mean, extension to equations, 1st order. Petrovitch, M. C. R. 128 (1899) 981-.

- Poincaré on systems of 1st order. Bendixson, -. C. R. 118 (1894) 971-。

Total algebraic differentials. Picard's equation $P d x+Q d y=0$ when $F(x, y, z)=0$. Noether, M. Mth. A. 29 (1887) 339-.

Transformation, case. Hargreave, C.J. $\mathrm{Ph}$. Mg. 30 (1847) 8-.

- of equations, 1st order, case. Painlevé, $P$. C. R. 110 (1890) 840-.

- - - with 2 variables. Zeuthen, H. G. N. Ts. Mth. 1 (B) (1890) 1-; Fschr. Mth. (1890) 316.

- and integration of differential systems. Delassus, É. Par. Éc. Norm. A. 14 (1897) 195-.

- - - - simultaneous equations, several variables. Brassinne, $E$. Toul. Mm. Ac. 5 (1839) 129-; Liouv. J. Mth. 10 (1845) 194-. - of linear equations, 2nd order. Spottiswoode, W. Tortolini A. 3 (1852) 26-.

- - - - , - - with linear coefficients. Bolza, O. Am. J. Mth. 15 (1893) 264-.

$-\phi_{0} \frac{d y}{d x}+\phi_{1} y^{2}+\phi_{2} y+\phi_{3}=0$. Heymann, W. Z. Mth. Ps. 27 (1882) 374 .

Transformations. Bianchi, G. Tortolini A. 3 (1852) 347-.

Variation of arbitrary constants. Liouville, $J$. Liouv. J. Mth. 3 (1838) 342-.

- constants in solutions. Brunacci, $V$. Turin Mm. Ac. (1804-05) (pte. 2) 3-.

$\frac{d\left(k \frac{d V}{d x}\right)}{d x}+(g r-l) V=0$. Sturm, J. C. F. L'I. 1 (1833) 247-.

$y^{\prime \prime}+f(x) y^{\prime}+F(y) y^{\prime 2}=0$. Liouville, $J$. Liouv. J. Mth. 7 (1842) 134 -.

$\frac{d y}{d x}+f(x) \sin y+F^{\prime}(x) \cos y+\phi(x)=0$. Besge, V. A. Liouv. J. Mth. 11 (1846) 445. 
$\frac{d^{2} y}{d x^{2}}+\phi y \frac{d y^{2}}{d x^{2}}=\psi y$. Vieille, J. N. A. Mth. 5

(1846) 509-.

$d_{x}\left\{\left(x-x^{3}\right) y_{x}\right\}-x y=0 . \quad$ Liouville, $J$. Liouv.

J. Mth. 19 (1854) $151-$.

$\frac{d y}{d x}=f(x, y)$. Liouville, J. Liouv. J. Mth.

20 (1855) 143-.

$P \frac{d y}{d x} \frac{d^{2} y}{d x^{2}}+Q \frac{d^{2} y}{d x^{2}}+R\left(\frac{d y}{d x}\right)^{3}+S\left(\frac{d y}{d x}\right)^{2}+$

$T \frac{d y}{d x}+U=0$, where $P, Q$, etc. are func-

tions of $x$ and $y$. Tychsen, C. Mth. Ts. 6 (1864) 145-

$\frac{2}{\pi}\left\{\epsilon^{\frac{1}{2} \pi D}+\epsilon^{-\frac{1}{2} \pi D}\right\} \int_{0}^{\infty} \cos \left(l \frac{d}{8}+D \phi\right) \frac{d \phi}{8} Y$

$=\frac{\cos (x+l)}{x+l}$. Sharpe, H.J. [1881] Mess.

Mth. 11 (1882) 56-.

$\frac{d^{2} y}{d x^{2}}+\left[2 \nu \frac{k^{2} \operatorname{sn} x \operatorname{cn} x}{\operatorname{dn} x}+2 \nu_{1} \frac{\operatorname{sn} x \operatorname{dn} x}{\operatorname{cn} x}\right.$

$\left.-2 \nu_{2} \frac{\operatorname{cn} x d n x}{\operatorname{sn} x}\right] \frac{d y}{d x}=$, etc. Sparre (le comte)

-de. Acta Mth. 3 (1883) 105-, 289-.

$\frac{d y}{d x}+y^{2}+P y+Q=0 . \quad$ Johnson, W. W. A.

Mth. 3 (1887) 112-.

$\phi(x)\left(\frac{d y}{d x}\right)^{2}+\psi(x) y \frac{d y}{d x}+\chi(x) y^{2}+\theta(x)=0$.

Heymann, W. Crelle J. Mth. 119 (1898) 253-.

$\Sigma X_{k} d x_{k}$, two types of simplest forms. Rusjan,

C. K. Fschr. Mth. (1899) 315.

\section{Methods of solution and} reduction of partial differential equations of first order, including the differential equations of theoretical dynamics.

Algebraic equation and its complete primitive, discriminants. Torelli, G. A. Mt. 19 (1891-92) 254-.

- - in 4 variables, discriminants. Arcais, F. d'. A. Mt. 12 ("1883-84) 1-.

Application of complex variable. Imshenetsky, V. G. Bll. Sc. Mth. As. 11 (1876) $162-$.

- - determinant-relation. Hamburger, Crelle J. Mth. 100 (1887) 390-

- - methods analogous to Galois's. Drach, J. C. R. 120 (1895) 73-.

Cauchy's problem for equations with 2 independent variables. Delassus, $E^{\prime}$. Toul. Fac. Sc. A. 12 (1898) F, 9 pp.

Contact transformations. Lie, S. Christiania F. 14 (1872) 24 -

Discontinuities consistent with the holding of linear partial equations. Christoffel, $E . B$. [1876] A. Mt. 8 (1877) 81-。
Equations.

De Morgan, A. Camb. and Dubl. Mth. J. 7 (1852) 28 -

Malmsten, C. J. Stockh. Öfv. 16 (1859) 57-.

Hargreave, C. J. Ph. Mg. 27 (1864) 355-; 29 (1865) 121-.

Schläfli, L. A. Mt. 2 (1868-69) 89-.

Lie, S. Christiania F. 15 (1873) 16-.

Cayley, A. QJ. Mth. 14 (1877) 292-.

Hoćevar, F. [1877] Wien Ak. Sb. 76 (1878) (Ab. 2) 740-.

Mansion, P. (xI) Brux. S. Sc. A. 5 (1881) (Pt. 1) 58-; (Pt. 2) 17-; 6 (1882) (Pt. 1) 56-.

Garbieri, G. Ven. I. At. 1 (1883) 1173-.

Besso, D. Rm. R. Ac. Linc. Rd. 3 (1887) (Sem. 2) 158-.

Vivanti, G. Palermo Cir. Mt. Rd. 2 (1888) 53-.

Schur, F. Leip. Mth. Ps. B. 45 (1894) 38-.

Saltykow, N. N. A. Mth. 18 (1899) 533-.

admitting finite groups. Lie, S. Mth. A. 25 (1885) 71-.

canonical, occurring in mechanics. Mathieu, É. L. Liouv. J. Mth. 19 (1874) 265-.

class. Picard, É. C. R. 100 (1885) 231-.

- Pellet, A.E. As. Fr. C. R. (1890) (Pt. 2) 203-.

-. Guldberg, A. Christiania Skr. (Mth.-Nt. Kl.) (1899) No. 8, $17 \mathrm{pp}$.

- including those of dynamics. Donkin, W. F. Phil. Trans. (1854) 71-; (1855) 299-. of 1 st degree, 4 or more variables. Trembley, $J$. [1799] St Pet. Ac. Sc. N. Acta 14 (1805) 153-.

-2nd degree. Elliot, - Par. Ec. Norm. A. 9 (1892) 329-.

occurring in dynamics. Cayley, A. Camb. and Dubl. Mth. J. 2 (1847) $210-$.

- - - Boole, G. Phil. Trans. (1863) 485-. - - Hill, G. W. Des Moines Anal. 1 (1874) 200-

-- , class. Volterra, $V$. Tor. Ac. Sc. At. 33 (1897) 255- or 451-; 35 (1900) 118 or 192.

- - Helmholtz's paper on monocyclic systems. Kronecker, - . Crelle J. Mth. 97 (1884) 141-.

with pairs of orthogonal integrals. Levi-Civita, T. Rm. R. Ac. Linc. Rd. 8 (1899) (Sem. 1) 295-.

satisfied by absolute invariants of binary forms in higher transformations. Clebsch, R.F.A. Gött. Ab. 15 (1870) (Mth.) 65-.

and theory of surfaces. Enneper, A. Schlömilch Z. 7 (1862) 1-.

unknown contained explicitly. Lie, S. Christiania F. 15 (1873) 52-.

with 2 independent variables, admitting continuous group of transformations. Tannenberg, W. de. Toul. Fac. Sc. A. 5 (1891) B, $150 \mathrm{pp}$.

- several dependent variables. Tanner, H. W. L. L. Mth. S. P. 9 (1877-78) 41-.

- variables. Binet, J. P. M. C. R. 16 (1843) 1273-.

$-n$ variables. Frisiani, $P$. Mil. Effem. As. (1849) 1-. 
Hamiltonian methods, and general theory. König, G. [1880] (xm) Mag. Tud. Ak. Etk. (Mth.) 8 (1881) (No. 10) $71 \mathrm{pp}$.

Hamilton's equation, analogues. Pennacchietti, G. Catania Ac. Gioen. At. 3 (1891) 99-.

- - and relative motion. Neumann, C. Z. Mth. Ps. 11 (1866) 265-.

Insufficiency of general geometric definition of characteristics. Egorov, D. T. Rec. Mth. (Moscou) 20 (1899) 608-; Fschr. Mth. (1899) 320 .

Integrability conditions of simultaneous equations., Collet, J. C. R. 76 (1873) 1126-; Par. Ec. Norm. A. 5 (1876) 49-.

Integrals, algebraic, of systems of equations. Koenigsberger, L. Mth. A. 39 (1891) 285-.

- common to two equations. Königsberger, L. Mth. A. 20 (1882) 587-.

-, complete. Saltykow, N. C. R. 128 (1899) 166-.

-,- , of systems of equations. Koenigsberger, L. Mth. A. 44 (1894) 17-.

-, conservation of algebraic relation between. Königsberger, L. Crelle J. Mth. 111 (1893) 156-.

一, determination. Collet, J. C. R. 112 (1891) 1193-.

- of equations near singular points. Lindelöf, E. Helsingf. Acta 22 (1897) No. 7, 26 pp.

-, general, of equations of dynamies, and their evaluation by Lie's method. Mayer, A. C. G. Mth. A. 17 (1880) 332-.

- , independent, of linear homogeneous equation, Ist order, systems. Ricci, G. Rm. R. Ac. Linc. Rd. 2 (1886) (Sem. 2) 119-, 190-; A. Mt. 15 (1887-88) 127-.

- of linear equations. Gilbert, L. P. (xII) Brux. S. Sc. A. 4 (1880) (Pt. 1) 58-; (Pt. 2) 273-.

-, linear, of free motion, with independent characteristics. Volterra, V. Tor. Ac. Sc. At. 35 (1900) 112- or 186-.

-, particular. Lacroix, S. F. Par. S. Phlm. Bil. 1 (1797) 86-.

-, periodic, of linear equations. Levi-Civita, -. C. R. 128 (1899) 978-.

- of system of equations with several unknowns. Saltykow, N. Liouv. J. Mth. 3 (1897) 423-.

- - systems of equations not containing $z$. Gubkin, I. S. Mosc. S. Sc. Bll. 73 (No. 1) (1891) 31-; Fschr. Mth. (1891) 397.

\section{Integration.}

Brisson, B. [1804] Par. Éc. Pol. J. $14^{\ominus} \mathrm{cah}$. (1808) 191-.

Ampère, A. M. [1814] Par. Bll. S. Phlm. (1814) 107-, 163-; Par. Éc. Pol. J. $17^{\circ} \mathrm{cah}$. (1815) 549-; $18^{\circ} \mathrm{cah}$. (1820) 1 -.

Jacobi, C. G. J. Crelle J. 2 (1827) 317-.

Cauchy, A. L. C. R. 14 (1842) 740-, 769-, 881-, 952-, 1026-.

Kondor, G. (xIr) Mag. Ak. Ets. (Mth. Term.) 1 (1860) 141-.

Serret, J.A. C. R. 53 (1861) 598-, 734-.

Spitzer, S. Schlömilch Z. 6 (1861) 262-.
Bonnet, O. (vi Adds.) Mntp. Ac. Sc. Mm.5 (1861-63) 385-.

Bour, E. Par. Éc. Pol. J. cah. 39 (1862) 149-. Serret, J. A. Par. Éc. Norm. A. 3 (1866) 143-.

Imschenetsky, V. G. Arch. Mth. Ps. 50 (1869) 278 -

Graindorge, J. (x) Liége S. Sc. Mm. 5 (1873) No. 3, xII + $189 \mathrm{pp}$.

Mayer, A. Gött. Nr. (1873) 299 -.

Sersawy, V. Z. Mth. Ps. 18 (1873) 511-.

Allégret, A. Liouv. J. Mth. 1 (1875) 241-.

Boltzmann, L. [1875] Wien Ak. Sb. 72 ( $A b$. 2) (1876) 471-.

Collet, J. C. R. 91 (1880) 974-.

Nazimov, $P$. S. Mosc. Un. Mm. (Ps.-Mth.) 1 (*1880) 1-, 257-.

Stodótkiewicz, A.J. Prace Mt.-Fiz. 5 (1894) 175-.

Dickstein, S. Prace Mt.-Fiz. 5 (1894) 183-; Fschr. Mth. (1893-94) 610.

Weiler, A. Z. Mth. Ps. 39 (1894) 255-.

Antaev, D. N. [1898] Rec. Mth. (Moscou) 20 (1899) 443-; Fschr. Mth. (1898) 305.

Saltykow, N. N. Fschr. Mth. (1899) 320 ; Liouv. J. Mth. 5 (1899) 435-.

by Abelian functions, cases. Picard, $\dot{E}$. C. R. 94 (1882) 1036-.

approximate, of linear equation. Severini, $C$. Mil. I. Lomb. Rd. 32 (1899) 1427-.

cases. Hesse, L. O. Crelle J. 25 (1843) 171-. -. Steen, A. Mth. Ts. 2 (1860) 97-.

of class of dynamical equations. Volterra, $V$. Tor, Ac. Sc. At. 33 (1897) 342- or 542-.

- - equations. Levy, M. C. R. 95 (1882) $986-$

- - - in mathematical physics. Cauchy, A. L. Par. Éc. Pol. J. $20^{\circ}$ cah. (1831) 297-. by definite integrals. Spitzer, S. Grunert Arch. 25 (1855) 137-.

difficulty in. Poisson, S. D. Par. Éc. Pol. Cor. 3 (1814-16) 291-.

of equation of motion, general. Morrison, $J$. Des Moines Anal. 9 (1882) 110-.

- equations of analytic mechanics. Jacobi, C. G.J. Berl. B. (1836) 115-.

---- - Bour, E. [J.E.E.] C. R. 40 (1855) 524-; Par. Mm. Sav. Étr. 14 (1856) $792-$.

- - dynamics. Jacobi, C. G.J. C. R. 5 (1837) 61-. 2 (1837) 317-.

$-1-1$ - Serret, J. A. C. R. 26 (1848) 639-.

Mth. 20 (1855) 137-.

(1864) $61 \overline{7}$.

_ _ - _ algebraic demonstration of Hamilton's principle. Somoff, $J$. St Pét. Ac. Sc. Bll. 16 (1871) 87- ; St Pet. Ac. Sc. Mm. (Rs.) 19 ("1871) 119-.

--- , general. Sturm, J. C. F. C. R. 26 (1848) 658-.

- - including those of planetary motion. Cauchy, A. L. C. R. 29 (1849) 65-. 
of equations of mechanics. Bertrand, $J$. Liouv. J. Mth. 17 (1852) 393-.

- - motion of a particle. Serret, J.A. C. R. 26 (1848) 605-.

- - planetary motion. Cauchy, A. L. C. R. 9 (1839) 184-.

- - - theory. Liais, E. Cherb. S. Sc. Nt. Mm. 24 (1882) 41-.

- - problem of $n$ bodies. Dillner, $G$.

Stockh. Öfv. 39 (1882) No. 4, 13-; No. 8, 9-; A. Mt. 11 (1882-83) 56-.

- - with 2 independent variables and $n$ dependent variables. Weber, E. von. Crelle J. Mth. 118 (1897) 123-.

- -3 variables. Léauté, $H$. Toul. Ac. Sc. Mm. 7 (1875) 1-.

- - - several variables and constant coefficients. Alexéeff, N. [1880] St Pét. Ac. Sc. Bll. 27 (1881) 22-.

$---n$ independent variables. Lemonnier, H. Par. S. Mth. Bll. 10 (1882) 223-.

- _ - variables. Cauchy, A. L. Par. S. Phlm. Bll. (1819) 11-.

- $-1-$ Lavagna, G. M. At. Sc. It. (1845) 1081-.

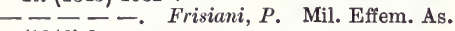
(1848) 3-.

$--\frac{1}{-}-$ Meyer, A. [1851] Brux. Ac. Sc. Mm. 27 (1853) 24 pp.

$--\frac{n+1)}{-}$ variables. Weiler, A. Schlömilch Z. 8 (1863) 264-.

geometric, of $L(p x+q y-z)-M p-N q+R=0$, $L, M, N, R$ being linear functions of $x, y, z$. Fouret, G. C. R. 83 (1876) 794 .

of Hamiltonian systems, etc. König, J. Mth. A. 23 (1884) 504-.

- Hamilton's equation. Staeckel, P. C. R. 121 (1895) 489-; Mth. A. 49 (1897) 145-.

- linear equations. Gilbert, $P$. Brux. S. Sc. A. 9 (1885) (Pt. 2) 41-.

-- , 3 variables. Lobatto, $R$. Amst. N. Vh. 6 (1837) 157-.

_ - _ 4 variables. Lobatto, $R$. Amst. Vh. 1 (1854) 30 pp.

- - homogeneous equations with 1 dependent variable. Żorawski, $K$. Prace Mt.-Fiz. 3 (1892) 1- ; Fschr. Mth. (1892) 327-.

- - system, by considering flow of fluid. Larmor, J. B. A. Rp. (1897) 562-.

method, Cauchy's. Zernov, S. N. (xII) Rec. Mth. (Moscou) 3 (1868) (Pt. 1) 309-.

-, - Hansen, P. C. V. (xII) Ts. Mth. 6 (1870) 25-, 73-, 132-.

- - Mansion, P. C. R. 81 790 .

-, general. Pfaff, J. F. Berl. Ab. (1814-15) (Mth.) 76-

-, graphic. Massau, J. Brux. A. Tr. Pbl. 5 (1900) 507-.

—, Jacobi-Hamilton. Mayer, A. [1870] Mth. A. 3 (1871) $435-$.

—, Jacobi's. Gilbert, L. P. [1880] (xII) Brux. S. Sc. A. 5 (1881) (Pt. 1) 52-; (Pt. 2) 1-.

-, - first. Darboux, G. C. R. 79 (1874) 1488-; 80 (1875) 160-.

一, - - Bertrand, J. C. R. 82 (1876) 641-. method, Jacobi's first, generalisation. Saltykow, N. C. R. 128 (1899) 225-, 274-; Fschr. Mth. (1899) 319.

_, _, and Pfaff's problem. Clebsch, A. Crelle J. 59 (1861) 190-.

-, Lagrange's. Mansion, - Brux. S. Sc. A. 15 (1891) (Pt. 1) 3-.

-, new. Lie, S. Gött. Nr. (1872) 321-.

-, - (Lie). Mayer, A. Mth. A. 6 (1873) 162-. -, 一. Kovaliskij, M. F. Kharkov Mth. S. Com. 5 (1896?) 125-.

-, - , for equations, $n$ variables. Jacobi, C. G.J. Crelle J. 60 (1862) 1-。

-, - , - - - Lie, S. Christiania F. 14 (1872) 28-.

-, Pfaff's. Morera, G. Mil. I. Lomb. Rd. 16 (1883) 637-, 691-.

-, Weiler's. Mayer, A. C. G. Mth. A. 9 (1876) 347-.

methods. De Morgan, A. [1848] Camb. $\mathrm{Ph}$. S. T. 8 (1849) 606-.

- Lie, M. S. Christiania F. (1875) 16-.

- of Cauchy, and of Jacobi and Mayer, comparison. Delassus, É. Bll. Sc. Mth. 21 (1897) 187-.

of non-linear equations, $n$ variables. Lavagna, G. M. Catania At. Ac. Gioen. 7 (1850) 17-. and a property of Poisson's function. Gilbert, L. P. As. Fr. C. R. 9 (1880) 61-; C. R. 91 (1880) 541-; (xI) Brux. S. Sc. A. (1881) (Pt. 1) 52-; (Pt. 2) 1-.

reduction to that of ordinary equation. Jacobi, C. G.J. Crelle J. 17 (1837) 97-.

simultaneous. Cauchy, A.L. C. R. 44 (1857) 805-.

-, of linear equations. Clebsch, R. F.A. [1865] Crelle J. 65 (1866) 257-.

-, - - -. Mayer, A. Gött. Nr. (1872) 315-; Mth. A. 5 (1872) 448-.

of simultaneous equations. Collet, -. Par. Éc. Norm. A. 7 (1870) 7-.

- - Mayer, A. Mth. A. 4 (1871) 88 .

- - Weber, E. von. C. R. 123 (1896) 292-.

- Bour's method. Schultz, E. Arch. Mth. Ps. 13 (1895) 316-.

- - -, two. Imshenetskir, V. G. [1874] (xir) Rec. Mth. (Moscou) 7 (1874-75) (Pt. 1) 206-.

- - - - with partial differentials of 2 functions of 2 independent variables. Tur quan, L. V. (xII) Brux. S. Sc. A. 4 (1880) (Pt. 2) 303-.

- $n$ simultaneous equations connecting $n$ functions of 2 independent variables and their partial differentials. Turquan, L. V. C. R. 91 (1880) 43-.

- a system of equations. Stodolkievitz, A.J. C. R. 120 (1895) 1037-.

- - - 5 equations. Carbonnelle, J. Brux. Ac. Bll. 22 (1855) (pte. 2) 652-.

$-\ldots-\ldots$ linear equations with 2 independent and $n$ dependent variables. Hamburger, M. [1875] Crelle J. Mth. 81 (1876) 243-.

- _ - non-linear equations with 2 independent and $n$ dependent variables. Ham burger, M. Crelle J. Mth. 93 (1882) 188-. 
of a system of simultaneous equations. Turquan, L. V. (xrr) Brux. S. Sc. A. 7 (1883) (Pt. 1) 70- ; (Pt. 2) 429-.

- systems of equations. Koenigsberger, L. Crelle J. Mth. 109 (1892) 261-.

- - - Riquier, -. C. R. 119 (1894) 324-.

_ - _ - with several unknowns. Beudon, J. C. R. 125 (1897) 156-.

theorem. Padova, E. N. Cim. 8 (1880) 89-. of total and partial differential equations. Shaposhnikov, N. A. [1879] (xI) Rec. Mth. (Moscou) 9 (1878-81) (Pt. 1) 627-.

use of calculus of limits. Cauchy, A.L. C. R. 15 (1842) 14-, 44-, 85-, 188-.

of $z=p x+q y+F(p, q)$

$F\left[x-\phi_{1}(p, q) y-\phi_{2}(p, q) z-\phi_{3}(p, q)\right]=0$. Steen, $A$. Schlömilch Z. 5 (1860) 427-.

$-\frac{d u}{d x}=\frac{d v}{d y}, \frac{d u}{d y}=-\frac{d v}{d x}$. Prym, F. E. Crelle J. 70 (1869) 354-.

Interpretation, geometric, case. Madsen,(Kapt.) V.H. O. (xII) Ts. Mth. 3 (1867) 18-.

Irreducibility of systems of algebraic equations. Königsberger, $L$. Münch. Ak. Sb. 21 (1892) 275-; Crelle J. Mth. 111 (1893) 1-.

Jacobi's multiplier theory, generalisation and application. Lie, M. S. Christiania F. (1874) 255-.

- theorem on Hamilton's equations, generalisation. Farkas, J. C. R. 98 (1884) 352-.

- -, proof. Steen, A. (xir) Ts. Mth.5 (1875) 33-.

Last multiplier. Jacobi, C. G. J. G. Arcad. 99 (1844) 129-.

- - (Jacobi). Pioch, A. Brux. Ac. Bll. 13 (1846) 151-.

$--(-)$. Woronetz, $P$. Fschr. Mth. (1898) 609.

- - (-). Chaplygin, S. A. [1900] Rec. Mth. (Moscou) 21 (1901) 479-; Fschr. Mth. (1899) 635; (1900) 696-.

Lie and Mayer, considerations on their researches. Saltykow, N. C. R. 128 (1899) 1550- ; 129 (1899) 34-.

Linear congruences. Guldberg, A. C. R. 125 (1897) 489-.

- equations. Nanson, E.J. [1877] Mess. Mth. 7 (1878) 111-.

- - Ermakov, V. P. Kharkov Mth. S. Com. 1 (1889) 104-; Fschr. Mth. (1890) 350.

- - Sokoloff, N. [1889] Fschr. Mth. (1890) 350.

- - Borel, É. C. R. 120 (1895) 677.

- - Delassus, É. C. R. 121 (1895) 46-.

- - Le Roux, J. Par. S. Mth. Bll. (1897) 63-.

- - application of vector operator. Tait, $P$. G. [1870] Edinb. R. S. P. 7 (1872) 190-

- and method of Riemann. Liouville, $R$. C. R. 131 (1900) 697-.

- - theory. Lipschitz, R. Crelle J. 69 (1868) 109-.

Multiplier of a Jacobian system. Mayer, A.C. G. Mth. A. 12 (1877) 132-.

Nature of equations. Favero, G.B. Rm. R. Ac. Linc. Mm. 8 (1880) 217-.
Pfaff's problem. Clebsch, A. [1860] Crelle J. 60 (1862) 193-; 61 (1863) 146-.

Poisson's theorem, and reciprocal concerning integrals of system $d x=\frac{d x_{1}}{X_{1}}=\frac{d x_{2}}{X_{2}}=$ etc.

Korkine, A. StPét. Ac. Sc. Bll.16(1871) 429-.

Reduction to canonical form of equations of equilibrium of flexible inextensible string. Appell, P. C. R. 96 (1883) 688-.

- - - - -, 2nd degree. Elliot, C. R. 113 (1891) 495-.

- of complete system to single system of ordinary equations. Mayer, A. Leip. Mth. Ps. B. 43 (1891) 448-.

- - - - - - - - Schur, F. Leip. Mth. Ps. B. 44 (1892) 177-.

- - equation, $n+1$ variables, to differential equation $n$th order, 2 variables. Weiler, $A$. D. Nf. Vsm. B. 34 (1858) 146-.

Simultaneous equations. Korkine, A. C. R. 68 (1869) 1460-

- - König, J. Mth. A. 23 (1884) 520-

- -, theorem. Combescure, É. C. R. 78 (1874) 1212-.

- - in one unknown. Laurent, $H$. Liouv. J. Mth. 5 (1879) 249-.

- - - several unknowns. Bourlet, C. Par. Éc. Norm. A. 8 (1891) (Suppl.) 63 pp.

- linear equations. Fuchs, L. Berl. Ak. Sb. (1898) 222-.

Singularities. Beudon, J. Par. S. Mth. Bll. 26 (1898) 77-.

- of equations of dynamics. Painlevé, $P$. C. R. 123 (1896) 636-.

- - - - - and application to problem of three bodies. Painleve, $P$. C. R. 123 (1896) 871-.

\section{Solution.}

cases. C., G. Camb. Mth. J. 1 (1839) 162 .

complete, and transformation. Mayer, A. Gött. Nr. (1872) 405-

of Euler's equations of motion by elliptic functions. Greenhill, A. G. QJ. Mth. 14 (1877) 265-.

Lagrange's rule, demonstration, and history of proofs. Chrystal, G. [1891] Edinb. R. S. T. 36 (1892) 551 -.

Lie's. Dixon, A. C. Camb. Ph. S. P. 9 (1898) 279-.

method, general, for equations with several dependent variables. Tanner, H. W. L. L. Mth. S. P. 11 (1879-80) 72-.

- of reciprocation. M'Cowan, $J$. Edinb. Mth. S. P. 11 (1893) 2-.

methods of Lagrange and Monge. Watson, $H$. $W$. QJ. Mth. 6 (1863) 146-.

particular, of $F^{\prime}(x, y, z, p, q)=0$. Madsen, (Kapt.) V.H. O. (xII) Ts. Mth. 2 (1866) 130-. of problems of mechanics by successive approximations. Picard, É. Tor. Ac. Sc. At. 34 (1898) 6-.

singular. Carmichael, R. Ph. Mg. 15 (1858) $522-$.

-. Weber, H. Crelle J. 66 (1866) 193-.

- Yaroshenko, S. P. (xII) Rec. Mth. (Moscou) 5 (1870) (Pt. 1) 383-. 


\section{Partial Differential Equations, First and Second Orders}

singular. Casorati, F. Mil. I. Lomb. Rd. 9 (1876) 522-.

-. Darboux, G. Par. Mm. Sav. Etr. 27 (1883) No. 2, 243 pp.

-. Möller, J. Stockh. Öfv. (1888) 463-.

- Egorow, D. T. Fschr. Mth. (1898) 305-.

-, of coincidence curve. Björling, C. F. E. Stockh. Ak. Hndl. Bh. 12 (Afd. 1) (1887) No. 7, 16 pp.

-, and envelopes. Cockle, (Sir) Jas. Manch. Lt. Ph. S. P. 24 (1885) 10-, 23-.

- of equations representing lines at infinity. Mansion, P. B. A. Rp. (1875) (Sect.) 19-.

- , - in 2 variables. Landré, C. L. As. Fr. C. R. (1879) 241-.

of system of simultaneous non-linear equations. Nanson, E. J. R. S. P. 23 (1875) 510 ; 24 (1876) 337-.

$-a\left(y \frac{d w}{d z}-z \frac{d w}{d y}\right)^{2}+$ etc. $=1 . \quad$ Schläfli, $L$. QJ. Mth. 8 (1867) 252-.

Substitution of complex values in equations with $2 n$ variables. Imshenetsky, $V . G$. Liége S. Sc. Mm. 7 (1878) (No. 2) 6 pp.

System of equations. Combescure, $\dot{E}$. C. R. 74 (1872) 977-。

- - Volterra, $V$. Tor. Ac. Sc. At. 30 (1895) 445-.

- - - equivalent to those in problem of $n$ bodies but admitting one more integral. Ébert, W. C. R. 131 (1900) 251-.

Systems, complete, of homogeneous linear equations. Schur, F. Crelle J. Mth. 108 (1891) 313-.

- of equations. Du Bois-Reymond, P. [1867] Crelle J. 68 (1868) 180-.

- - - Bäcklund, A. V. [1876] M.th. A. 9 (1877) 412-.

- - - analogous to equations of an involution. Beudon, J. Liouv. J. Mth. 5(1899) 351-.

- - - - - 1st order. Beudon, $J$. Par. Éc. Norm. A. 15 (1898) 229-.

- - deprived of integrals. Méray, C. C. R. 106 (1888) 648-.

- - - with one unknown function. Delassus, É. Par. Éc. Norm. A. 14 (1897) 109-.

- - - - several dependent variables. Tanner, H. W. L. L. Mth. S. P. $10(1878-$ 79) 55-.

- - involution. Weber, E. von. Mth. A. 49 (1897) 543-.

Theorem of Jacobi's. Bonnet, O. C. R. 45 (1857) 581-.

- Lie. Bendixson, I. Stockh. Öfv. (1892) 301-.

\section{Theory.}

Yanishevskix, E. P. (xII) Kazan Un. Mm. (1863) (Ps.-Mth., No. 2) $100 \mathrm{pp}$.

Darboux, G. C. R. 70 (1870) 746-.

Mansion, P. [1873] Brux. Mm. Cour. 8०, 25 (1875) No. 1, xvI + $282 \mathrm{pp}$.

Kowalevsky, S. von. [1874] Crelle J. Mth. 80 (1875) 1-.

Bäcklund, A. V. Mth. A. 17 (1880) 285-.

Czuber, E. Wien Ak. Sb.103 (1894) (Ab.2a) 295-.

Tannenberg, $W$. de. C. R. 120 (1895) 674-.
Saltykovo, N. C. R. 129 (1899) 195-.

analytic. Delassus, É. Liège S. Sc. Mm. 20 (1898) No. 3, 84 pp.

and classification. Lie, S. Gött. Nr. (1872)473-. direct application of first principles. Larmor, J. R. S. P. 43 (1888) 176-.

of equations with 3 variables. Du BoisReymond, $P$. Crelle J. 64 (1865) 271-.

general. Liouville, J. Liouv. J. Mth. 1 (1856) 345-.

- Lie, M. S. Christiania F. (1874) 198-; (1875) 1-; Mth. A. 9 (1876) 245-; 11 (1877) 464-.

Lie's. Biermann, O. Leip. Mth. Ps. B. 48 (1896) 665-.

of linear equations. Krankenhagen, $(D r)$ Gött. Nr. (1880) 197-.

Transformation of equations of dynamics. Radau, R. Par. Éc. Norm. A. 5 (1868) 311-. - _ - - Painlevé, P. C. R. 115 (1892) 714 -, 874 ; 123 (1896) 392-.

$-\ldots-$ motion of a particle. Cerruti, $V$. Rm. R. Ac. Linc. T. 3 (1879) 196-.

- - - - three bodies problem. Brioschi, F. C. R. 66 (1868) 710-.

- method of Jacobi, extension. Méray, C. Par. Éc. Norm. A. 7 (1890) 217-.

-, orthogonal, applicable to equations of $\mathrm{dy}$ namics. Radau, $R$. C. R. 67 (1868) 316-.

Transformations of equations of three bodies problem. Siacci, F. Tor. At. Ac. Sc. 6 (1870-71) 440-.

- - principal function. Cauchy, A. L. C. R. 13 (1841) 40-, 97-, 397-, 455-, 487-, 1127-; 14 (1842) 1-.

Variation of arbitrary constants in problems of dynamics. Brioschi, F. Tortolini A. 4 (1853) 298-.

- - definite integral and theory of equations. Marcolongo, $R$. Nap. Rd. 27 (1888) 500-.

\section{Methods of solution and re- duction of partial differential equations of the second and higher orders.}

Abel's theorem. Cayley, A. [1880-81] Phil. Trans. 172 (1881) 751-; Camb. Ph. S. P. 4 (1883) 119-.

- - equation connected with. Cayley, A. B. A. Rp. (1881) 534-.

Algebraic systems and their relations to systems of partial differential equations. Delassus, É. Par. Éc. Norm. A. 14 (1897) 21-.

Analogues of equations of 1st order. Beudon, J. C. R. 126 (1898) 324-, 388-.

Arbitrary functions, calculation. Beudon, $J$. C. R. 128 (1899) 1215-.

- -, elimination. Garbieri, G. Ven. I. At. (1886-87) 831-.

,--- , new method. Minich, S. R. C. R. 84 (1877) 1496-; 87 (1878) 161-.

Changes of variables. Beudon, J. Par. S. Mth. Bll. 28 (1900) 107-. 


\section{Partial Differential Equations. Second and Higher Orders 4840}

Change of variables, equations, 2nd order. Boussinesq, J. C. R. 74 (1872) 730-.

Characteristics. Beudon, J. Par. S. Mth. Bll. 25 (1897) 108-.

- of equations, 2nd order, theory. Bäcklund, A. $V$. Mth. A. 13 (1878) 411-.

- - - in 3 variables. Weber, E. von. Mth. A. 47 (1896) 229-.

-, and Huygens' principle. Coulon, J. C. R. 130 (1900) 1064-.

- of linear equations, 2 nd order, with constant coefficients. Coulon, J. Bordeaux S. Sc. PV. (1899-1900) 24-.

一, theory. Goursat, É. C. R. 126 (1898) 1332-.

\section{Equations.}

Falk, M. (xII) Ts. Mth. 5 (1881) 97-.

Moutard, T. $\quad$ Par. Éc. Pol. J. 64 (1894) 55-.

Nekrasov, P. A. Rec. Mth. (Moscou) 18 (1896) 468-; Fschr. Mth. (1896) 271, 272.

Duport, H. Liouv. J. Mth. 6 (1900) 41-.

and bilinear systems. Weber, E. von. Mänch. Ak. Sb. 29 (1900) 231-.

cases. Glaisher, J.W. L. B. A. Rp. (1882) 454-.

- Picard, É. C. R. 116 (1893) 454-.

-. Jamet, V. Par. Éc. Norm. A. 13 (1896) 95-.

- Goursat, É. Par. S. Mth. Bll. 25 (1897) 36 -

-. Schiff, P. A. Fschr. Mth. (1899) 332.

-, exceptional. Cockle, (Sir) J. L. Mth. S. P. 10 (1878-79) 105-.

class. Fredholm, I. C. R. 129 (1899) 32-.

-. Lauricella, G. A. Mt. 4 (1900) 281-.

-, invariants of which are equal. Guichard, C. Par. Éc. Norm. A. 7 (1890) 19-.

-, 3 variables. Poisson, S. D. Par. Éc. Pol. Cor. 2 (1809-13) 410-.

in conduction of heat. Liouville, $J$. Liouv. J. Mth. 1 (1836) 33-.

with constant coefficients and non-analytic functions. Borel, E. C. R. 121 (1895) $933-$.

and definite integrals. Sarrus, $F$. Gergonne A. Mth. 12 (1821-22) 254-

with equal characteristics. Kürschak, J. Mth. A. 37 (1890) 317-.

to a family of envelopes. Walton, $W$. Camb. Mth. J. 4 (1845) 152-.

of form $\frac{d^{2} z}{d x d y}=z f(x y)$, classes, 2 special. Medolaghi, P. G. Mt. 35 (1897) 267-.

group. Bohlin, $K$. Stockh. Ofv. (1887) 333-. integrable without use of arbitrary functions under the sign $\int$. Pistoye, - de. C. R. 78 (1874) 1102-

of light vibrations. Brill, A. [1868] Mth. A. 1 (1869) 225-.

linear. Appell, P. C. R. 90 (1880) 296-, 731-. - Petot, A. C. R. 111 (1890) 522-.

- Delassus, É. Par. Éc. Norm. A. 13 (1896) 339 .

- Le Roux, J. Liouv. J. Mth. 4 (1898) 359-.

VOL. I. linear. Vessiot, E. C. R. 128 (1899) 544-.

-, cases. Darboux, G. C. R. 95 (1882) 69-.

-, 一. Holmgren, E. Ups. Årsk. (1897) (Mt.

Nt.) 33 pp. ; Fschr. Mth. (1898) 314-.

-, with characteristics real. Delassus, $\hat{E}$. C. R. 119 (1894) 40-; Par. Éc. Norm. A. 12 (1895) (Suppl.) 53-.

-, class. Picard, É. C. R. 107 (1888) 476-.

-, -. Koch, H. von C. R. 121 (1895) 517-.

—, -. Drach, J. Bll. Sc. Mth. 21 (1897) 140-.

-, - with analytic integrals. Picard, É. C. R. 121 (1895) 12-.

-, - with constant coefficients and last term variable. Cauchy, A. L. [1822] Par. Éc. Pol. J. $19^{\circ} \mathrm{cah}$. (1823) 510-.

-, 3rd degree. Trembley, J. [1798] St Pet. Ac. Sc. N. Acta 13 (1802) 101-.

-, and their germ integrals. Earnshav, $S$. [1874] Camb. Ph. S. P. 2 (1876);360-

- - Laplace's method. Goursat, É. C. R. 122 (1896) 169-.

-, system. Horn, I. Acta Mth. 12 (1889) 113 -. -, - , characteristic surface corresponding to. Canchy, A. L. C. R. 13 (1841) 184-.

-, with 2 independent variables. Darboux, $G$. C. R. 105 (1887) 199-.

\section{2 nd order.}

Darboux, G. Par. Éc. Norm. A. 7 (1870) 163-. Mainardi, $G$. Rm. At. N. Linc. 26 (1873) 79-.

Poenisch, R. Santiago de Chile Un. A. 82 (1892) 675-.

Stouff, X. C. R. 118 (1894) 1320.

Chini, $M$. Tor. Ac. Sc. At. 30 (1895) 494-; 31 (1895) 400- or 568-.

Goursat, É. C. R. 120 (1895) 712-.

Koch, H. von. Stockh. Öfv. (1895) 721-.

Dini, U. Rm. R. Ac. Linc. Rd. 5 (1896) (Sem. 2) $381-, 421-; 6$ (1897) (Sem. 1) 5-, 45-; Rm. R. Ac. Linc. Mm. 3 (1899) 33-.

Forsyth, A. R. [1897] Camb. Ph. S. T. 16 (1898) 191-.

Dixon, A. C. B. A. Rp. (1899) 646.

Goursat, É. Toul. Fac. Sc. A. 1 (1899) 31-, 439-.

admitting an infinite group of point transformations. Medolaghi, $P$. A. Mt. 1 (1898) 229 -.

bilinear, geometrical interpretation. E'gorow, D. T. Fschr. Mth. (1898) 306.

case. Tonelli, A. Rm. R. Ac. Linc. Rd. 4 (1888) (Sem. 2) 384-.

-. Weingarten, J. C. R. 116 (1893) 493-.

characteristics, coalescence of 2 systems. Weber, E. von. C. R. 124 (1897) 1215-.

- - - - Goursat, E. C. R. 124 (1897) 1294-.

with characteristics real. Coulon, J. C. R. 128 (1899) 1386-.

class. Stephan, É. Par. Éc. Norm. A. 3 (1866) 7 .

-. Bäcklund, A. V. Erlang. Ps. Md. S. Sb. 8 (1876) 112-.

-. Picard, É. Palermo Cir. Mt. Rd. 5 (1891) 308-. 


\section{Partial Differential Equations. Second and Higher Orders 4840}

class. Kürschák, J. Mth. Termt. Éts. 15 (1897) 225-; Mth. Nt. B. Ung. 14 (1898) 285-.

- including Laplace's equation. Hearn, $G$. W. Phil. Trans. (1846) 133-.

- reduction. Oster, B. Arch. Mth. Ps. 17 (1900) 321-.

form $f(r, s, t)=0$. Guldberg, $A$. N. Ts. Mth. 10 (B) (1899) 82-; Fschr. Mth. (1899) 324.

having general first integral. Tanner, $H$. W. L. [1877] Mess. Mth. 7 (1878) 89-.

highest differential coefficient appearing in linear form. Liouville, R. C. R. 98 (1884) 216-.

integrable by ordinary differential equations. Weber, E. von.. Münch. Ak. Sb. 26 (1897) 425-.

linear. Engel, F. Leip. Mth. Ps. B. 34 (1882) 39-.

-. Liouville, R. C. R. 98 (1884) 569-, 704.

-. Bianchi, L. Rm. R. Ac. Linc. Rd. 5 (1889) (Sem. 2) 35-.

-. Du Bois-Reymond, P. Crelle J. Mth. 104 (1889) 241-.

-. Krukovskij, A. I. [1894] Rec. Mth. (Moscou) 17 (1895) 759-; Fschr. Mth. (1895) 393-.

- Petot, A., C. R. 119 (1894) 510-.

- Picard, É. BIl. Sc. Mth. 23 (1899) 150-.

-, caseș. Lêvy, L. C. R. 100 (1885) 98-; Par. Éc. Pol. J. 56 (1886) 63-.

- - -. Le Roux, J. C. R. 123 (1896) 1052-.

-, -. Guichard, C. C. R. 131 (1900) 100-

-, class. Picard, É. Acta Mth. 12 (1889) 323-.

-, with constant coefficients. Coulon, J. C. R. 130 (1900) 765-:

-, general. Paoli, $\dot{P}$. [1802] Mod. S. It. Mm. 10 (1803) 249-.

-, - theorem. Picard, É. C. R. 107 (1888) 939-.

-, invariants belonging to. Burgatti, $P$. Rm. R. Ac. Linc. Rd. 5 (1896) (Sem. 2) 433-.

-, one parameter, case. Horn, J. Mth. A. 52 (1899) 271-.

-, system. Liouville, R. . C. R. 104 (1887) 1496-.

-, with 2 independent variables. Le Roux, $J$. Par. Éc. Norm. A. 12 (1895) 227-.

- - - - - theory. Pfannenstiel, $E$. [1882] Ups. S. Sc. N. Acta 11 (1883) (No. 9) $86 \mathrm{pp}$.

-, - - v variables. Cotton, $E$.

C. R. 123 (1896) 936-; 124 (1897) 744-.

methods of Charpit and Lagrange. Mainardi, . G. Mil. G. I. Lomb. 9 (1856).94-.

point invariants. Medolaghi, $P$. Rm. R. Ac. Linc. Rd. 6 (1897) (Sem. 2) 247-, 313-.

simultaneous, with 3 variables. Weber, $E$. von. Münch. Ak. Sb. 25 (1896) 101-.

soluble by differentiation. Tanner, $H . W . L$. [1876] Mess. Mth. 6 (1877) 32-.

system. Niccoletti, $O{ }^{\prime} \mathrm{Rm}$. R. 'Ac. Linc. Rd. 4 (1895) (Sem. 1) 197-.

sýstems, in involution. Goursat, $\dot{E}$. C. R. 122 (1896) 1258-.

with 2 independent variables. Moutard, $\boldsymbol{T}$. C. R. 70 (1870) 834-. with 2 independent variables. König, $J$. Mth. A. 24 (1884) 465-.

- - - - theory.. Lévy, M. C. R. 75 (1872) 1094-.

- 3 independent variables. Vivanti, $G$. Mth. A. 48 (1897) $474-, 608$.

and $3 r d$ order, linear, with 2 independent variables. Picart, A. N. A. Mth. 2 (1883) 34-. - higher orders. Falk, M. Ups. Arsk. (1875) 40 pp.

\section{Higher orders.}

3rd order. Guldberg, A. Christiania Skr. (Mth.-Nt. Kl.) (1900) No. 5, $43 \mathrm{pp.}$

- - admitting an intermediate integral. Guldberg, A. C. R. 130 (1900) 1452-.

- -, case. Tonelli, A. Rm. R. Ac. Linc. Rd. 4 (1888) (Sem. 2) 458-.

- - linear, with 3 variables. Vries, J. de. N. Arch. Wisk. 10 (1884) 66-.

- - , of orthogonal systems of surfaces. Lévy, $M$. C. R. 77 (1873) 1435-.

- and higher orders, linear. Moon, $R$. Ph. Mg. 21 (1886) 63-.

4th order. Somigliana, C. A. Mt. 18 (1890) 59 -.

- - Fredholm, I. Stockh. Öfv. (1899) 935-. higher order. Gutzmer, A. Liouv. J. Mth. 6 (1890) 405-.

- - extension of Riemann's method to. Delassus, - C. R. 117 (1893) 510.

- - , with intermediate first integrals. Bäcklund, A. V. [1876] Mth. A. 9 (1877) 199-; 13 (1878) 69-.

any order. Davidov, A. Y. [1865] (xп) Rec. Mth. (Moscou) [1] (1866) 351-.

- - , and their reduction to linear system of 1st order. Cauchy, A. L. C. R. 15 (1842) 131-.

- - - - any number of variables. Bäcklund, A. $V$. Lund Un. Acta 12 (1875-76) (Mth., No. 2$) 4 \mathrm{pp}$.

- -, theory, general. Lie, S. Leip. Mth. Ps. B. 47 (1895) 53-.

$n$th order, class. Tonelli, A. Rm. R. Ac. Linc. Rd. 5 (1889) ( $\mathrm{Sem} .1$ ) 178-.

- - , formulw relative to. Tonelli, A. Rm. R. Ac. Linc. Rd. 5 (1889) (Sem. 1) 508-.

of potential. Picard, É. C. R. 90 (1880) 601-. satisfied by concomitants of quantics. Forsyth, A. R. L. Mth. S. P. 19 (1889) 24-. - - certain definite integrals. Torelli, $G$. Nap. Rd. 29 (1890) 123-.

similar to those of hydrodynamies, general properties. Craig, T. [1881] Am. Ac. Mm. 10 (Pt. 2) (1882) 362-.

simultaneous. Folkierski, W. Par. T. Nauk Śc. Pam. 3 ( $\left.{ }^{*} 1873\right)$ 1-; BH. Sc. Mth. As. 6 (*1874) 156-.

- Dixon, A. C. [1899] Phil. Trans. (A) 195 (1901) 151-.

—, linear: Painlevé, - C. C. R. 104 (1887)1497-. -, 一, cases. Appell, P., \& Picard, 一. C. R. 92 (1881) 692-.

_, theory. Campbell, J. E. [1898] - L. Mth. S. P. 31 (1900) 235-. 
system. Goursat, $\hat{E}$. C. R. 104 (1887) 1361-. 一. Picard, É.. C. R. 112 (1891) 685-. systems. Picard, É. C. R. 114 (1892) 805-. -. Beudon, J. C. R. 120 (1895) 304-.

-, characteristics of which depend on finite number of parameters. Beudon, J. Par. Éc. Norm. A. 13 (1896) (Suppl.) 51 pp.

- reducible to ordinary differential equations. Beudon, J. C. R. 127 (1898) 1003-.

-, symmetric. Somigliana, C. A. Mt. 22 (1894) 143-.

in theory of asymmetric algebra. Forsyth, A. R. Camb. Ph. S. T. 16 (1898) 291-.

and theory of surfaces, relation between. 'Stouff, X. Par. Éc. Norm. A. 13 (1896) 9-.

Existence of irreducible equations. Koenigs berger, L. Berl. Ak. Sb. (1894) 989-.

Extension of Cauchy's theorem to most genera] systems. Delassus, É. C. R. 122 (1896) 772-; Par. Éc. Norm. A. 13 (1896) 421-.

General equation, geometrical significance. Kolling, -. Mth. Ts. 5 (1863) 79-.

Generation. Minich, S. R. Ven. At: 4 (1845) 377-.

Integrability of Airy's equations. Moon, $R$. R. S. P. 15 (1867) 486-.

Integrable equations of 2 nd order, two. Goursat, É. C. R. 129 (1899) 31-.

- types of equations of $2 n d$ order. Goursat, É. C. R. 127 (1898) 854-.

\section{Integrals.}

Poisson, S. D, Par. S. Phlm. Bll. 3 (1804) 227-; Par. Éc. Pol. J. 19' cah. (1823) 215-.

Cauchy, A. L. C. R. 16.(1843) 572-.

analytic, of linear equations with 2 variables. Le Roux, J. C. R. 125 (1897) 1015-.

$-,-\frac{d^{n} z}{d y^{n}}=F(z), F(z)=\Sigma a_{i k} \frac{d^{i+k} z}{d x^{i} d y^{k}},(i+k<n)$. Delassus, - . C. R. 118 (1894) 968-.

,$--\frac{d^{2} z}{d y^{2}}=\frac{d z}{d x}$. Le Roux, $\dot{J}$. Bll. Sc. Mth. 19 (1895) 127-.

complete, of some classes of equations, any order. Pennacchietti, 'G. Mil. I. Lomb. Rd. 17 (1884) 279-.

- , and general solution of equations. Sersawy, $V$. Wien Ak. D. 53 (1887) (Ab. 2) 1-.

-, of homogeneous equations which do not contain explicitly the unknown function. Gubkin, I. S. Mosc. S. Sc. BHl. 73 (No. 1) (1891) 9-; Fschr. Mth. (1891) 396-.

of equations, 2nd order. Serdobinskij, $V . E$. Rec. Mth. (Moscou) 13 (1886) 511-; Fschr. Mth. (1887) 354.

- - , - cases. Picard, É. C. R. 111 (1890) 487-.

first, of equations of 2 nd degree in 4 or 5 variables. Trembley, $J$. [1805] St Pét. Ac. Sc. Mm. 4 (1811) 120-.

- $-\ldots-\ldots$ - and of 3 rd in 3 variables. Trembley, J. [1803] St Pet. Ac. Sc. N. Acta i5 (1806) 257-。 first, of equations, 2nd order. Boole [non Boldt], G. [1861] St Pét. Ac. Sc. Bll. 4 (1862) 198-.

- - - - cases. Tanner, H.W. L. Mess. Mth. 5 (1876) 133-.

forms of arbitrary functions. Challis, $J . \quad \mathrm{Ph}$. Mg. 6 (1829) 296-.

general, character. Forsyth, A. R. [1897] L. Mth. S. P. 29 (1898) 5-.

- , of equations with constant coefficients. Tortolini, B. [1854] Mod. S. It. Mm. 25 (1855) 310-.

,$--\frac{1}{d^{n}}$ of form

$\frac{d^{n} Z}{d \phi d \xi \ldots d \psi d \omega}=\Phi(\phi, \xi . . \psi, \omega) Z+\Phi(\phi, \xi . . \psi, \omega)$. Starkov, A. N. Rs. S. Nt. Mm. (Mth.) 2 ( $\left.{ }^{*} 1879\right)$.

for given initial conditions. Goursat, $\hat{E}^{\text {. }} \quad$ C. R. 125 (1897) 640-.

intermediate, of equations, 2nd order. Goursat, É. Acta Mth. 19 (1895) 285-; C. R. 127 (1898) 603-.

of linear equations. Cauchy, A. L. C. R. 16 (1843) 469-.

number of arbitrary functions. Gomes Teixeira, F. . Bordeaux S. Sc. Mm. 2 (1878) 315-.

of partial differential equations and difference equations, arbitrary functions. Oltramare, $G_{i} \quad$ As. Fr. C. R. (1895) (Pt. 2) 171-.

problem on determination. Goursat, É. C. R. 121 (1895) 671-.

singularities. Horn, J. Mth. A, 33 (1889) 310 .

of $\frac{d^{2} V}{d x^{2}}+\frac{d^{2} V}{d y^{2}}=0$. Matthiessen, L. Z. Mth. Ps. 16 (1871) 228-.

Integrating factor, theory. Lie, M. S. Christiania F. (1874) 242-.

- of $s+Q p q+R p+S q+T=0$, determination.. Johnsen, $S . N$. (xII) Ts. Mth. 2 (1878) 129-.

\section{Integration.}

Cauchy, A. L. [1823] ' Par. Mm. Ac. Sc. 9 (1830) 97-.

Pagani, G. M. Quetelet Cor. Mth. 6.(1830) 286-.

Bronwin, B. Camb. Mth. J. 2 (1841) 209-.

Weiler, A. Grunert Arch, 33 (1859) 171-。

Bour, E. Par. Éc. Pol. J. cah. 39 (1862) 149-.

Graindorge, $J_{*}$ (x) Liége S. Sc. Mm. 5 (1873) No. 3, xII + 189 pp.

Peterson, K. M. [1876-79] (xiI) Rec. Mth. (Moscou) 8 (1876) (Pt. 1) 291-; 9 (1878-81) (Pt. 1) 137-; 10 (1882-83) (Pt. 1) 169-.

Nazimov, P. S. Mosc. Un. Mm. (Ps.-Mth.) 1 ( $\left.{ }^{*} 1880\right)$ 1-, 257-.

Sersawy, $V$. Wien Ak. D. 49 (1885) (Ab. 2) 1 -.

Antomari, X. Par. S. Mth. Bll. 19 (1891) 154 -

application of contact-transformations. Brill, J. N. A. Mth. 10 (1891) 362-. 
application of differentio-differential relations. Šapošnikov, N. A. Mosc. S. Sc. Bll. 73 (No. 2) (1891) 20-; Fschr. Mth. (1891) 398. case. Zaremba, S. Par. Éc. Norm. A. 7 (1890) 135-.

cases. Christoffel, E. B. Gött. Nr. (1871) 435-.

- Steen, A. (xII) Ts. Mth. 6 (1876) 69 -

of class of equations. Duhamel, J.M.C. C. R. 3 (1836) 638-.

- - linear equations by definite integrals. Lie, M. S. [1881] Arch. Mth. Ntvd. 6 (1882) 328 -

complete, of homogeneous equations, by canonical system of variables. Imshenetskir, V. G. (xII) Kazan Un. Mm. 2 (1866) 5-. -, - linear equations, 2nd order, with constant coefficients. Parseval, M. A. [1799] Par. Mm. Sav. Étr. 1 (1806) 638-.

-, - - - - - - - - - Gruson, J. P. Berl. Ab. (1812-13) (Mth.) 23-.

of equation of lines of curvature of ellipsoid. Picart, A. N. A. Mth. 20 (1881) 145-.

- equations with constant coefficients by application of multiple definite integral formula. Tortolini, B. Tortolini A. 2 (1859) 260-.

- - of hydrodynamics. Fontaneau, -. As. Fr. C. R. (1899) (Pt. 2) 1-.

- - isostatic cylinders. Boussinesq, $J$. C. R. 74 (1872) 318-; 77 (1873) 667-.

- -, 2nd order. Weiler, A. Grunert Arch. 33 (1859) 171-.

- - - - - Graindorge, J. (x) Liége S. Sc. Mm. 5 (1873) No. 3, xII + 189 pp.

- - - - Picart, A. C. R. 78 (1874) $882-$.

- - - - Sonin, N. Y. (xiI) Rec. Mth. (Moscou) 7 (1874-75) (Pt. 1) 285-; (xI) Bll. Sc. Mth. As. 10 (1876) 96-.

- - - - Gomes Teixeira, F. G. Teix. J. Sc. 2 (1880) 138-; Brux. Ac. Bll. 3 (1882) 486-; C. R. 93 (1881) 702-; Par. S. Mth. Bll. 17 (1889) 125-

,---- Volterra, $V$. Rm. R. Ac. Linc. Rd. 1 (1885) 303-.

- - - - Beudon, J. C. R. 118 (1894) 1188-.

$-\frac{1}{-}-$. Sonin, N. J. Mth. A. 49 (1897) 417-.

- - , - - by Ampère's and Darboux's methods. Rudzki, T. Prace Mt.-Fiz.8(1897) 99-; Fschr. Mth. (1897) 307.

- - , - - case. Nicolaïdes, N. C. R. 81 (1875) 216-, 365-.

- - - - - (Nicolaïdes). Bonnet, $O$. C. R. 81 (1875) 259-.

,---- , class. Graindorge, J. Liouv. J. Mth. 17 (1872) 426-.

,---- , classes. Turquan, L. V. Brux. S. Sc. A. 8 (1884) (Pt. 2) 314-.

,$---\ldots$, no intermediary integral. Forsyth, A. R. [1897] Phil. Trans. (A) 191 (1898) 1 -.

- - , - - in 2 independent variables. Turquan, L. V. C. R. 92 (1881) 1200-.

- - , - - - - - - class. Picart, A. C. R. 97 (1883) 305-. of equations, 2nd and higher orders. Pellet, A. $E$. C. R. 89 (1879) 92-

- - 4 th order, class. Vaccaro, A. Bll. Sc. Mth. 22 (1898) 37-.

- -, higher orders. Raabe, J. L. Baumgartner Z. 7 (1830) 159-.

- -, - - Preobrazhenskiז, V. V. [1873] (xII) Rec. Mth. (Moscou) 7 (1874-75) (Pt. 1) 215-.

- -, nth order. Fabre, A. As. Fr. C. R. (1895) (Pt. 2) 136-.

- - - - one dependent, and 2 independent variables. Falk, M. [1871] Ups. N. Acta S. Sc. 8 (1873) No. 5, 40 pp.

- - with periodic coefficients, system. Potier, A. As. Fr. C. R. 1 (1872) 255-。

finite, of linear equations with constant coefficients. Earnshaw, S. Ph. Mg. 2 (1876) 47-. general and complete, of two equations in hydromechanics. Parseval, M. A. [1803] Par. Mm. Sav. Étr. 1 (1806) 524-.

of general linear equation, 2nd order. Moon, R. Ph. Mg. 35 (1868) 118-, 219-.

- linear equations. Poisson, S. D. Liouv. J. Mth. 3 (1838) 615-.

- - - Borel, É. Bll. Sc. Mth. 19 (1895) 122-.

- - Le Roux, J. Liouv. J. Mth. 6 (1900) 387-.

- - by calculus of residues. Tortolini, $B$. G. Arcad. 93 (1842) 3-; 94 (1843) 58-; 95 (1843) 3-.

- - - with coefficients constant. Vaschy, -. C. R. 116 (1893) 491-.

- - - - variable. Timmermans, J. A. Brux. Ac. Sc. Mm. 28 (1854) 10 pp.

- - - discriminant not zero. Le Roux, $J$. C. R. 130 (1900) 695-.

- - - Monge and Lagrange's methods. Walton, $W$. Camb. Mth. J. 3 (1843) 269-.

$\ldots+\ldots, 2$ nd order, with negative characteristics. Le Roy, - C. R. 122 (1896) 367-. - - - , - - , -4 or more independent variables. Fais, A. Bologna Ac. Sc. Mm. 3 (1881) 427-.

- - - - - , - $\boldsymbol{r}$ variables. Falk, M. Ts. Mt. Fys. 4 (1871) 230-.

_- _ - - and 3rd orders. Plana, G. [1809] Turin Mm. Ac. (1809-10) (pte. 2) 156-.

- - - higher orders. Gravé, D.A. Fschr. Mth. (1899) 332.

- - - - - - extension of Riemann's method to. Bianchi, L. Rm. R. Ac. Linc. Rd. 4 (1895) (Sem. 1) 8-, 89-, 133-.

$-{ }_{-},-{ }_{-},--\ldots-1 \quad$ Niccoletti, $O$. Rm. R. Ac. Linc. Rd. 4 (1895) (Sem. 1) 330-. - - with 3 variables. Frischauf, $J$. Wien Sb. 51 (1865) (Ab. 2) 317-.

- - and partial differential equations. Curtis, A. H. Camb. and Dubl. Mth. J. 9 (1854) 272-.

method. Bottomley, J. Manch. Lt. Ph. S. Mm. \& P. 7 (1893) 78-.

-, Darboux's, for equations, 2nd order. Goursat, É. C. R. 120 (1895) 542-.

- , - - non-linear equations, 2nd order. Speckman, H. A. W. Amst. Ak. Vs. M. 9 (1892) 441-; Arch. Néerl. 27 (1894) 303-. 
method, graphic. Massau, J. Brux. A. Tr. Pbl. 5 (1900) 507-.

-, Jacobi-Mayer, improvement. Lie, S. Christiania F. 15 (1873) 282-.

-, Lagrange's, special cases. Serdobinskij, V. E. [1889] Rec. Mth. (Moscou) 14 (1890) 439-; Fschr. Mth. (1890) 351.

—, Lie's, extension. Mayer, A. C. G. Mth. A. 8 (1875) 313-.

- for linear equations, 2nd order, with 2 independent variables. Winckler, A. [1883] Wien Ak. Sb. 88 (1884) (Ab. 2) 7-。

-, Monge-Ampère, extension. Vivanti, $G$. Mil. I. Lomb. Rd. 32 (1899) 506-.

-, Monge's, for linear equations, 2nd order. Laguerre, E. N. A. Mth. 15 (1876) 49-.

-, new, for Monge-Ampère equation. Lie, $M$. S. Arch. Mth. Ntvd. 2 (1877) 1-.

- for non-linear equations, 2nd order. Winckler, $A$. Wien Ak. Sb. 89 (1884) (Ab. 2) 614-.

-, Riemann's, for hyperbolic equations with application to vibration problems. Wirtinger, IV. Mth. A. 48 (1897) 365-.

methods for equations, 2nd order, of function of 2 variables. Imschenetsky, $V . G$. Arch. Mth. Ps. 54 (1872) 209-.

- - with 2 independent variables. Bur. gatti, P. Palermo Cir. Mt. Rd. 12 (1898) 210-

by multiple integrals. Mellin, $H$. Acta Mth. 22 (1899) 19-.

of simultaneous equations. Aorst, $L$. Crelle J. 47 (1854) 369-

_ - , 2nd order, with 2 independent variables. Vályi, J. Crelle J. Mth. 95 (1883) 99-. - - , systems. Koenigsberger, L. Mth. A. 41 (1893) 260 -

by symbolic method. Curtis, A. H. Camb. and Dubl. Mth. J. 9 (1854) 272-.

theory. Lie, M. S. Christiania F. (1880) No. 1, 4 pp.

- for higher partial differential problems. Weber, E. von. Leip. Mth. Ps. B. 49 (1897) 329-. of $\frac{d u}{d t}=\frac{d^{3} u}{d x^{3}}$. Liouville, J. [1836] Par. Éc. Pol. J. 25 cah. (1837) 85-.

$-a^{m} \frac{d^{m_{z}}}{d t^{m}}=x^{2 m} \frac{d^{m_{z}}}{d x^{m}}$. Spitzer, S. Grunert Arch. 30 (1858) 335 .

$-(a x+b y+c) \frac{d^{2} z}{d x d y}+a \lambda \frac{d z}{d y}+b \mu \frac{d z}{d x}=0$.

Spitzer, S. Grunert Arch. 33 (1859) 461.

$-(x+y)^{2} \frac{d^{2} z}{d x d y}+m_{1}(x+y) \frac{d z}{d x}+m_{2}(x+y) \frac{d z}{d y}$ $+n z=0$. Spitzer, S. Grunert Arch. 33 (1859) 476-; 38 (1862) 451-.

$-\frac{d^{2} z}{d x^{2}}\left[1+\left(\frac{d z}{d y}\right)^{2}\right]=\frac{d^{2} z}{d y^{2}}\left[1+\left(\frac{d z}{d x}\right)^{2}\right]$.

Fuchs, L. Crelle J. 58 (1860) 80-.

$--=-$ Hoppe, R. [1860] Crelle J. 58 (1861) 369-.

$-\frac{d^{2} \phi}{d x^{2}}+\frac{d^{2} \phi}{d y^{2}}=0 . \quad$ Neumann, C. Crelle J. 59 (1861) 335-.

$-x^{\frac{n}{2}} \frac{d^{n} z}{d x^{n}}=\frac{d^{n} z}{d y^{n}}$. Spitzer, S. Schlömilch Z. 7 (1862) 343-.

$$
\text { of } \begin{aligned}
\frac{d^{n} z}{d x^{n}} & +P_{1} \frac{d^{n} z}{d x^{n-1} d y}+P_{2} \frac{d^{n} z}{d x^{n-2} d y^{2}}+\ldots \\
& +P_{n-1} \frac{d^{n} z}{d x d x^{n-1}}+P_{n} \frac{d^{n} z}{d y^{n}}=Q .
\end{aligned}
$$

Tychsen, C. Z. Mth. Ps. 13 (1868) 441-. $-\frac{d^{2} u}{d x^{2}}+\frac{d^{2} u}{d y^{2}}+k^{2} u=0$. Weber, H. [1868] Mth. A. 1 (1869) 1-.

$-\frac{d^{2} u}{d x^{2}}+\frac{d^{2} u}{d y^{2}}=0$ under given conditions as to boundary and discontinuity. Schwarz, H.A. Berl. Mb. (1870) 767-.

$-\frac{1}{340}=-$ Prym, F.E. Crelle J. 73 (1871) $-\frac{d^{n} z}{d x^{n}}=x^{m} \frac{d^{m+n} z}{d y^{m+n}}+F_{1}(y)+x F_{2}(y)+\ldots$ $+x^{-1} F_{m}(y)$.

Spitzer, S. Arch. Mth. Ps. 51 (1870) 499 $-\Delta u=\frac{\partial}{\partial x}\left(x \frac{\partial u}{\partial x}\right)+\frac{\partial}{\partial y}\left(x \frac{\partial u}{\partial y}\right)=0$. Niemöller, F. Z. Mth. Ps. 28 (1883) 97-.

Laplace's equations with equal invariants, certain classes. Thybaut, A. C. R. 122 (1896) 834-.

- -, systems. Tzitzeica, G. C. R. 128 (1899) 601-.

$-\longrightarrow, \frac{d^{2} z}{d x d y}=a \frac{d z}{d x}+b \frac{d z}{d y}$, class. Tzitzéica, $G$. Bll. Sc. Mth. 24 (1900) 142-.

- - - $=-$ with quadratic solutions. Tzitzéica, G. C. R. 131 (1900) 487-.

Legendre's theory. Combescure, É. C. R. 74 (1872) 798-.

Majorant functions applied to some differential systems. Riquier, -. C. R. 125 (1897) 1018 -

Method, Cauchy's, extension to equations, any order. Beudon, J. C. R. 121 (1895) 808-.

- of characteristics, general system of equations integrable by. Tedone, O. A. Mt. 1 (1898) 283-.

-, Darboux's, application. Beudon, -. C. R. 120 (1895) 902-.

- - - - to $s=f(r, t)$. Boer, F. de. Amst. Ak. Vs. M. 8 (1891) 221-; Arch. Néerl. 27 (1894) 355-.

-, Laplace's, extension to equations, 3rd and higher orders. Le Roux, - Par. S. Mth. Bli. 27 (1899) 237-.

-, Picard's, extension to equations with 3 variables. Zaremba, S. Prace Mt.-Fiz. 9 (1898) 1 -.

- of successive approximations, and theory of equations. Picard, É. Liouv. J. Mth. 6 (1890) 145-, 231.

Methods of Riemann and Picard, extension to a class of equations. Niccoletti, $O$. Nap. Ac. At. 8 (1897) No. 2, 22 pp.

Monge-Ampère equation, equations of 2 nd order analogous to. Goursat, É. Par. S. Mth. Bll. 27 (1899) 1-.

-- equations, certain. Clairin, J. C. R. $130(1900)$ 997-. 
Monge's equation, special cases. Kapteyn, $W$. Amst. Ak. Vs. 8 (1900) 278-, 356-, 620-; Amst. Ak. P. 2 (1900) 241-, 326, 525-; Par. Éc. Norm. A. 17 (1900) 245-.

Moutard's theorem, geometrical interpretation. Bianchi, L. Rm. R. Ac. Linc. Rd. 3 (1894) (Sem. 1) 565-.

Schwarz's "alternate" process. Phragmén, $E$. Stockh. Öfv. (1892) 265-.

Singularities. Beudon, J. C. R. 124 (1897) 671-.

\section{SOLUTION.}

common to 2 equations, 2 nd order with 2 variables. Bianchi, L. Rm. R. Ac. Linc. Rd. 2 (1886) (Sem. 2) 218-, 237-, 307-.

- 3 equations, 3rd order with 2 variables. Pennacchietti, G. Palermo Cir. Mt. Rd. 7 (1893) 280-.

- - several equations with more than 2 variables. Pennacchietti, G. Palermo Cir. Mt. Rd. 7 (1893) 273-.

- - linear equations. Liouville, R. C. R. 101 (1885) 1134-.

definite integral, of linear equations. Russell, W. H. L. Camb. and Dubl. Mth. J. 9 (1854) $112-$.

of equations, 2nd order, with more than 2 independent variables. Tanner, $H . W . L$. [1875] L. Mth. S. P. 7 (1875-76) 43-.

- - - - - , - $n$ variables, when there is general first integral. Tanner, H.W. L. L. Mth. S. P. 7 (1875-76) 75-.

general. Moon, R. [1866] R. S. P. 15 (1867) 306-.

of linear equations, 2nd order. Parseval, M. A. [1798] Par. Mm. Sav. Etr. 1 (1806) 478-.

- - - - - Tanner, H.W. L. [1875-76] Mess. Mth. 5 (1876) 53-, 153-.

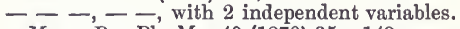
Moon, $R$. Ph. Mg. 40 (1870) 35-, 149.

method for equations having general first integral, application to equations of $3 \mathrm{rd}$ order with 2 independent variables. Tanner, H. W. L. L. Mth. S. P. 8 (1876-77) 229-. -, general. Tanner, H.W.L. L. Mth. S. P. 9 (1877-78) 76-.

一, new. Greatheed, S. S. Ph. Mg. 11 (1837) 239-.

-, -, for equations, 2nd order. Eastwood, G. Des Moinés Anal. 8 (1881) 154-.

-, particular, for linear equations, 2 nd order. Forsyth, A. R. Mess. Mth. 15 (1886) 44-, 64.

methods of Lagrange and Monge. Watson, H. W. QJ. Mth. 6 (1863) 146-.

-, symbolic. Gregory, D. F. Camb. Mth. J. 1 (1839) 123-.

Monge's, of non-integrable equation in 3 variables. Johnson, W. W. A. Mth. 4 (1888) 156-.

of non-linear equations, 2nd order. M'Convan, J. Edinb. Mth. S. P. 10 (1892) 63-

singular, of equations with 3 variables. Weber, E. von. Mth. A. 46 (1895) 1-, 160.
Successive approximations applied to equations, 2nd order. Picard, É. C. R. 110 (1890) 61-.

Symbolic operators, and application to solution of equations. Glaisher, J. W. L. L. Mth. S. P. 12 (1880-81) 201-.

System of equations defining periodic functions. Baker, H. F' Camb. Ph. S. P. 9 (1898) 513-.

Systems of equations, 2. Didon, $F$. Par. Éc. Norm. A. 6 (1869) 1-.

- - Ricci, G. A. Mt. 12 (*1883-84) $42-$.

- - parallel curves. Bukreiew, B. Fschr. Mth. (1891) 404-.

Theorem of Appell in conduction of heat, generalisation. Kobald, $E$. Wien Az. 31 (1894) 22-.

Theory. Liouville, J. Par. Mm. Sav. Etr. 5 (1838) 559-.

- Minich, S. R. Ven. At. 16 (1870-71) 2097-.

- Cayley, A. [1876] Mth. A. 11 (1877) 194.

- Minich, S. R. Ven. I. Mm. 22 (1882) 169-.

- Oster, B. Arch. Mth. Ps. 17 (1900) $102-$

- of equations, 2nd order. Bäcklund, A. V. [1878] Mth. A. 15 (1879) 39-.

-- - - - Goursat, É. C. R. 123 (1896) 680 .

$--\overline{-}-$ Vivanti, $G$. Mil. I. Lomb. Rd. 29 (1896) 777-.

-, general. Lie, S. Prace Mt.-Fiz. 7 (1896) 69 -.

Transformation of equations, 2 nd order, with 2 independent variables. Niccoletti, $O$. Tor. Ac. Sc. At. 33 (1897) 716- or 956-.

$-{ }_{-},-\ldots,-1-\ldots$ Burgatti, $P$. Rm. R. Ac. Linc. Rd. 7 (1898) (Sem. 1) 21-.

$--\ldots,-,-,-\ldots$, and integrations deduced therefrom. Liouville, R. C. R. 97 (1883) 836-.

- Laplace's. Vivanti, G. Palermo Cir. Mt. Rd. 14 (1900) 145-.

- -, and linear equations. Picard, É. C. R. 107 (1888) 594-.

- of linear equations, with constant coefficients, to fundamental forms. Earnshaw, S. Ph. Mg. 35 (1849) 24 .

- - - homogeneous equations with constant coefficients. Somigliana, C. A. Mt. 18 (1890) $265-$.

- - - - 2nd order. Niccoletti, $O$. Rm. R. Ac. Linc. Rd. 5 (1896) (Sem. 2) 94-; Tor. Ac. Sc. At. 32 (1896) 530-, 708-, or 790-, 970-.

- theory of $8^{2}-r t=\frac{\left(1+p^{2}+q^{2}\right)^{2}}{a^{2}}$. Lie, M. S. [1881] Arch. Mth. Ntvd. 6 (1882) 153-.

- of $s^{2}=4 p q f(x, y)$. Goursat, É. Par. S. Mth. Bll. 28 (1900) 1-.

Transformations. Stäckel, $P$. Crelle J. Mth. 114 (1895) 116-. 
Transformations, automorphic, of class of equations. Bianchi, L. Rm. R. Ac. Linc. Rd. 4 (1888) (Sem. 1) 445-.

-, new, of linear equations, 2nd order. Liouville, R. C. R. 100 (1885) 168-.

$\frac{d^{2} \phi}{d x^{2}}+\frac{d^{2} \phi}{d y^{2}}=0$. Liouville, J. Liouv. J. Mth. 8 (1843) 265-.

$\frac{d^{2} \log \lambda}{d u d \nu}= \pm \frac{\lambda}{2 a^{2}}$. Liouville, J. C. R. 36 (1853) 371-.

$R r+S s+T t+U\left(s^{2}-r t\right)=V . \quad$ Boole, G. [1862] Crelle J. 61 (1863) 309-.

- = - Cayley, -. QJ. Mth. 26 (1893) 1-. - $=$ - Dixon, A. C. L. Mth. S. P. 31 (1900) 332-.

$\frac{d}{d x}\left[\left(1-x^{2}\right) \frac{d z}{d x}\right]+\frac{d}{d \alpha}\left(a^{2} \frac{d z}{d \alpha}\right)=0 . \quad$ Didon, $F$. Par. Ec. Norm. A. 6 (1869) 377-.

$\frac{d^{2} z}{d x d y}+P \frac{d z}{d x}+Q \frac{d z}{d y}+Z=0 . \quad$ Tanner, H.W. L. L. Mth. S. P. 8 (1876-77) 159-.

$\frac{d^{2} z}{d x d y}=F(z)$. Lie, M. S. [1880] Arch. Mth. Ntvd. 6 (1882) 112-.

$\frac{\partial^{2} x}{\partial x \partial y}=\frac{m(1-m)}{(x-y)^{2}} z$. Appell, P. Bll. Sc. Mth. As. 6 (1882) 314-.

$r=q^{2 m} t$. Liouville, $R$. C. R. 98 (1884) 723-. $\frac{\partial u}{\partial x}+\frac{\partial v}{d y}+\frac{\partial w}{\partial z}=0$. Morera, G. Palermo Cir. Mt. Rd. 7 (1893) 288-.

$F(z)=8+\alpha x p+\beta y q+(\alpha \beta x y-\gamma) z=0$. Cotton, É. Bll. Sc. Mth. 24 (1900) 229 -

\section{General theory of ordinary linear equations. (See also 4450.)}

Euler, L. [1778] St Pet. Ac. Sc. N. Acta 14 (1805) 52-.

Liouville, J. Liouv. J. Mth. 3 (1838) 255-, 561 -.

Ostrogradsky, M. A. [1838] St Pét. Ac. Sc. Bll. 5 (1839) 33-.

Hill, C. J. D. Crelle J. 25 (1843) 1-.

Graves, C. Ir. Ac. P. 4 (1850) 88-.

Tardy, P. Tortolini A. 1 (1850) 136-

Brioschi, F. Tortolini A. 3 (1852) 269-.

Brassinne, E. Toul. Mm. Ac. 1 (1857) 175-.

Spitzer, S. Grunert Arch. 32 (1859) 127-.

Gilbert, P. Brux. Ac. Bll. 11 (1861) 200-.

Spitzer, $S$. Grunert Arch. 40 (1863) 212- ; Arch. Mth. Ps. 41 (1864) 234-; Z. Mth. Ps. 9 (1864) 60-.

Dienger, $J$. Arch. Mth. Ps. 46 (1866) 34-.

Russell, W. H. L. $[1869-72]$ R. S. P. 18 (1870) 118-, 210-; 19 (1871) 14-, 281-, 526-; 21 (1873) 14-, 20-.

Yvon-Villarceau, A. C. R. 71 (1870) 762-.

Thomé, L. W. [1871-83] Crelle J. Mth. 74 (1872) 193-; 75 (1873) 265-; 76 (1873) 273-; 78 (1874) 223-; 81 (1876) 1-; 83 (1877) 89-; 87 (1879) 222-; 91 (1881) 79-, 341-; 95 (1883) 44-.
Gegenbauer, L. Arch. Mth. Ps. 55 (1873) 258 -

Trudi, N. [1874] Nap. Ac. At. 6 (1875) No. 14, $2 \mathrm{pp}$.

Hermite, C. [1874-75] Bll. Sc. Mth. As. 3 (1879) 311-.

Klein, C. F. Erlang. Ps. Md. S. Sb. 8 (1876) 182-; Mth. A. 12 (1877) 167-.

Spitzer, S. Arch. Mth. Ps. 59 (1876) 334-.

Brioschi, F. Par. S. Mth. Bll. 7 (1879) 105-.

Floquet, $G$. Par. Ec. Norm. A. 8 (1879) (Suppl.) $132 \mathrm{pp}$.

Picard, É. C. R. 89 (1879) 140-.

Appell, P. [1880] Par. Éc. Norm. A. 10 (1881) 391-.

Brioschi, F. $\quad$ C. R. 91 (1880) 807-.

Floquet, G. Nancy S. Sc. Bll. 5 (13 ${ }^{\mathrm{e}}$ Ann. 1880) (1881) 112-.

Mittag-Leffler, G. C. R. 90 (1880) $218-$.

Spitzer, S. Arch. Mth. Ps. 65 (1880) 306-.

Casorati, F. A. Mt. 10 (1880-82) 154-.

Jïrgens, E. [1881] Mth. A."19 (1882) 435-.

Casorati, $F$. Rm. R. Ac. Linc. T. 6 (1882) 121-.

Pfannenstiel, E. Göteb. Hndl. 17 (1882) 1-.

Kapteijn, W. Amst. Ak. Vs. M. 18 (1883) 95-; Ârch. Néerl. 18 (1883) 105-.

Floquet, G. Par. Éc. Norm. A. 1 (1884) 405-.

Thomé, L. W. Crelle J. Mth. 96 (1884) 185-; 107 (1891) 79 .

Brioschi, F. A. Mt. 13 (1885) 1-.

Grïnfeld, $\dot{E}$. Crelle J. Mth. 98 (1885) 333-.

Lie, $S$. Christiania F. (1885) No. 21, 4 pp.

Starkov, A. P. Kazan S. Nt. (Ps.-Mth.) P. 3 (1885) 79-; Fschr. Mth. (1885) 283-.

Cayley, A. QJ. Mth. 21 (1886) 321-; Crelle J. Mth. 100 (1887) 286-.

Escherich, G. von. Wien Ak. D. 51 (1886) 1-.

Thomé, L. W. Crelle J. Mth. 99 (1886) 88-; 101 (1887) 203-.

Cayley, A. Crelle J. Mth. 101 (1887) 209-.

Painlevé, P. C. R. 105 (1887) 58-.

Volterra, V. Rm. R. Ac. Linc. Rd. 3 (1887) (Sem. 1) 393-; Rm. S. It. Mm. 6 (1887) No. 8, 104 pp.; Palermo Cir. Mt. Rd. 2 (1888) 69 .

Fuchs, L. Berl. Ak. Sb. (1888) 1115-, 1273-; (1889) 713-; (1890) 21-.

Anisimov, $V$. A. Mosc. Un. Mm. (Ps.-Mth.) 8 (1889) xiv $+200 \mathrm{pp}$.

Bigiavi, C. Rm. R. Ac. Linc. Rd. 5 (1889) (Sem. 1) 651-; 6 (1890) (Sem. 1) 86-.

Cels, J. C. R. 111 (1890) 98-; Par. Éc. Norm. A. 8 (1891) 341-.

Vessiot, E. C. R. 112 (1891) 778-.

Markoff, A. C. R. 113 (1891) 685-, 790-, 1024 .

Painlevé, -. C. R. 113 (1891) 739-.

Rudskij, M. N. Rs. S. Nt. Mm. (Mth.) 13 (1891) 1-; Fschr. Mth. (1891) 340.

Cels, J. C. R. 115 (1892) 1057-; 116 (1893) 176-.

Starkov, A. N. Rs. S. Nt. Mm. (Mth.) 14 (1892) 191-; Fschr. Mth. (1892) 291. 
Floridia, G. G. Mt. 31 (1893) 354-; 32 (1894) 86-; 33 (1895) 13-, 145-, 242-, 327-, 379-.

Metzler, G. F. A. Mth. 9 (1894-95) 171-; 11 (1896-97) 1-.

Anisimov, V. A. [1898] Rec. Mth. (Moscou) 20 (1899) 316-.

Igel, B. Mh. Mth. Ps. 9 (1898) 124-.

Grilnfeld, E. Mh. Mth. Ps. 9 (1898) 372.

Bôcher, M. A. Mth. 12 (1898-99) 45-.

Dini, U. A. Mt. 2 (1899) 297-; 3 (1899) 125-.

Horn, J. Mth. A. 53 (1900) 177-.

Adjoint and associated equations, $n$th order. Grilnfeld, E. Crelle J. Mth. 121 (1900) 218-.

- equations. Pick, G. Wien Ak. Sb. 101 (1892) (Ab. 2a) 893-.

- - Igel, B. Mh. Mth. Ps. 5 (1894) 163-; 10 (1899) 229-. - - Githther, $P$. Crelle J. Mth. 117 (1897)

Algebraically integrable equations. Frobenius, G. Crelle J. Mth. 80 (1875) 183-.

- - Fuchs, L. Berl. Ak. Sb. (1890) $469-$

- - in relation to Fuchsian functions. Viterbi, A. G. Mt. 35 (1897) 150-; 36 (1898) 55 -.

- - homogeneous equations. Boulanger, $\boldsymbol{A}$. Par. Éc. Pol. J. 4 (1898) 1-.

Analogies with algebraic equations. Mainardi G. Rm. At. N. Line. 26 (1873) 386-.

Application of determinant formula. Anisimov, $V$. A. Vars. S. Nt. Tr. (1897) (Ps. C.) Fasc. 3, Mm. 2, 16 pp.; Fschr. Mth. (1898) 263 ; Mth. A. 51 (1899) 388-.

- - infinite determinants. Koch, H. von. Acta Mth. 15 (1891) 53-; 16 (1892-93) 217-.

- - - to homogeneous equations. Koch, H. von. Stockh. Ófv. (1890) 225-, 499-; Fschr. Mth. (1890) 316.

- - integral calculus. Brassinne, E. Toul. Mm. Ac. 4 (1860) 510-.

- - theory of substitutions. Jordan, $C$. Par. S. Mth. Bll. 2 (1874) 100-.

Arbitrary constants, determination. Trudi, $N$. [1864] (xi) Nap. Ac. At. 2 (1865) No. 8, $25 \mathrm{pp}$.

Associated equations. Fuchs, L. Berl. Ak. Sb. (1899) 182-.

Binary forms, variables of which are fundamental integrals of equation, 2nd order. Vernier, P. Par. S. Mth. Bll. 22 (1894) 133-.

Binomial biordinal. Cockle, (Sir) J. L. Mth. S. P. 11 (1879-80) 123-.

- equations. Steen, A. (xir) Ts. Mth. 4 (1874) 104-.

- - Craig, T. [1886] J. H. Un. Cir. [6] (1886-87) 28-.

- - with algebraic coefficients, class. Appell, P. C. R. 94 (1882) 202-; Par. Ec. Norm. A. 12 (1883) 9-.

- - $n$th order, case. Pochhammer, L. Mth. A. 38 (1891) $247-$.
Case, and its application to trinomial equations. Heymann, $W$. Mth. A. 26 (1886) 534-.

- having hypergeometric series for integral. Goursat, É. Par. Ec. Norm. A. 10 (1881) (Suppl.) $142 \mathrm{pp}$.

-, 3rd order. Starkov, A. N. Rs. S. Nt. Mm. (Mth.) 6 (1885) 13-; Fschr. Mth. (1885) 323-.

-, - -. Markov, A. A. [1892] Rec. Mth. (Moscou) 16 (1893) 352-; Fschr. Mth. (1892) 313.

- satisfied by squares of $X_{2} y^{\prime \prime}+X_{1} y^{\prime}+X_{0} y=0$. Spitzer, S. Schlömilch Z. 4 (1859) 73-.

$---y=\int_{\alpha}^{\beta} e^{u(m+x)}(u-x)^{A-1}(u-\beta)^{B-1}$ $\log [(m+x)(u-a)(u-\beta)] d u$. Spitzer, $S$. Schlömilch Z. 3 (1858) 178-.

Cases. Cockle, Jas., \& Harley, R. Manch. Ph. S. P. 3 (1862-63) 16-.

-. Michaëlis, J. P. Lux. S. Sc. Mm. 8 (1865) 160-.

- Hermite, C. [1874] Crelle J. Mth. 79 (1875) 324-.

- Harley, $R$. B. A. Rp. (1878) 466-.

- Steen, A. Acta Mth. 3 (1883) 277-.

- Goursat, $\dot{E}$. C. R. 98 (1884) 1248-.

-. Brioschi, $F^{\circ}$. Mth. A. 26 (1886) 106-.

-. Gutzmer, A. [1891-95] G. Teix. J. Sc. 10 (1891) 3-; 13 (1897) 3-.

-. Jackson, F. H. Edinb. Mth. S. P. 14 (1896) 104-.

- Markov, A. A. St Pét. Ac. Sc. Mm. 3 (1896) No. 10, $17 \mathrm{pp}$.

Fano, $G$. Rm. R. Ac. Linc. Rd. 8 (1899) (Sem. 1) 285-.

Class. Picard, É. C. R. 90 (1880) 128-.

- Appell, P. C. R. 91 (1880) 972-; 96 (1883) 1018-.

-. Halphen, G. H. C. R. 92 (1881) 779-.

- Craig, T. Am. J. Mth. 7 (1885) 279 -.

-. Cels, J. C. R. 111 (1890) 879-; 112 (1891) 985-.

- Fuchs, L. Berl. Ak. Sb. (1896) 753-.

- Horn, J. Mth. A. 50 (1898) 525-.

- discussed by Mittag-Leffler. Brioschi, $F$. A. Mt. 11 (1882-83) 81-.

-, special. Hamburger, -. Crelle J. Mth. 103 (1888) 238-.

Common divisors and multiples of linear expressions. Heffter, L. D. Mth. Vr. Jbr. 4 (1897) 131-.

Complete cubic resolvent, explicit form. Harley, (Rev.) R. B. A. Rp. (1886) 439-.

Construction of equation from given element of integral function. Heun, K. Am. J. Mth. 11 (1889) 215-.

- - - particular integrals of which are products of those of 2 given equations. Spitzer, S. Arch. Mth. Ps. 42 (1864) 62-. - - - - _ - _ - squares of those of $X_{2} y^{\prime \prime}+X_{1} y^{\prime}+X_{0} y=0$. Spitzer, S. Arch. Mth. Ps. 42 (1864) 64.

- - satisfied by $y=e^{\lambda \int \sqrt{\frac{m+x}{n+x}} d x}$. S. Arch. Mth. Ps. 42 (1864) 345-. - equations of higher order, cases. Spitzer, S. Arch. Mth. Ps. 65 (1880) 321-.
Spitzer, 
Criticoids. Cockle, (Sir) J. [1869-71] Ph. Mg. 39 (1870) 201-; 41 (1871) 360-.

- Rawson, R. B. A. Rp. (1887) 604-.

-, inverse problem. Cockle, (Sir) Jas. $\mathrm{Ph}$. Mg. 12 (1881) 189-.

Decomposition, theory. Cayley, A. QJ. Mth. 21 (1886) 331-.

Determinantal relation in theory. Koenigsberger, L. Crelle J. Mth. 105 (1889) 170-.

- - - Igel, B. Mh. Mth. Ps. 4 (1893) 381-.

Differential resolvents. Harley, (Rev.) $R$. Manch. Lt. Ph. S. Mm. \& P. 5 (1892) 79-.

- - results connected with. Harley, (Rev.) $R$. B. A. Rp. (1896) 714-.

Distributive operations, non-homogeneous equations. Pincherle, $\mathrm{S}$. Rm. R. Ac. Linc. Rd. 5 (1896) (Sem. 1) 301-.

Divergent series in theory. Horn, J. D. Mth. Vr. Jbr. 8 (1900) (Heft 1) 219-.

Elementary divisors of determinants, application to homogeneous equations. Sauvage, L. Mars. Fac. Sc. A. 6 (1897) Fasc. 5, 9 pp.

Elimination. Favre-Rollin, A. M. Liouv. J. Mth. 1 (1836) 88-.

Equation with constant coefficients, equal roots, treatment. Routh, E.J. QJ. Mth. 19 (1883) 262-.

_ _ - having second member, application of Hermite's method. Darboux, G. Bll. Sc. Mth. As. 3 (1879) 325-.

- for hypergeometric series of $n$th order. Pochhammer, L. Crelle J. Mth. 108 (1891) 50 -.

- including Lamé's as special case. Darboux, G. C. R. 94 (1882) 1645-.

\section{Equations.}

admitting integrals (4) connected by quadratic relation. Goursat, É. Par. S. Mth. Bll. 25 (1897) 95-.

- - logarithmic differentials of which are doubly periodic functions. Fuchs, $L$. Liouv. J. Mth. 4 (1878) 125-

algebraic analogy. Mainardi, G. Mm. At. N. Linc. 26 (1873) 384.

with algebraic integrals. Jordan, $C$. [1877] Crelle J. Mth. 84 (1878) 89-.

- - Poincaré, H. C. R. 92 (1881) 698-

algebraic, 1st order and degree, singular points. Björling, C. F.E. Arch. Mth. Ps. 4 (1886) 358-.

automorphic by change of function and variable. Appell, P. Acta Mth. 15 (1891) 281-; C. R. 112 (1891) 34-。

belonging to same Riemann's class. Marotte, F. C. R. 125 (1897) 84-.

- - - type as their adjoint equations. Fano, G. Tor. Ac. Sc. At. 34 (1898) 260or 415-.

Mth. 121 (1900) 205 -.

with coefficients algebraic. Painlevé, P. C. R. 106 (1888) 535-.

- - - Guichard, C. Acta Mth. 12 (1889) 57-. with coefficients algebraic. Thomé, L. W. Crelle J. Mth. 122 (1900) 1-.

- - - functions of independent variable. Appell, P. C. R. 92 (1881) 61-.

- - , many-valued. Thomé, L. W. Crelle J. Mth. 115 (1895) 33-, 119-; 119 (1898) 131-; 121 (1900) 1-.

- constant. Lorenz, L. Mth. Ts. 4 (1862) 38-.

- Picquet, H. Par. Ec. Pol. J. Cah. 53 (1883) 79-.

- - - Méray, C. Bll. Sc. Mth. 12 (1888) 196-.

$-\frac{1}{47}$ Auric, 一. N. A. Mth. 13 (1894) - Franklin, F. Am. J. Mth. 19 (1897) 92-.

- - González, M. Arg. S. Ci. A. 47 (1899) 178-.

_ _ _ d'Alembert's method. Stodótkiewicz, A. J. Krk. Ak. (Mt.-Prz.) Rz. 6 (1893) 105-; Crc. Ac. Sc. Bll. (1892) 294-.

_ - - particular case. Ocagne, M. $d$ '. N. A. Mth. 3 (1884) 138-

- doubly periodic. Picard, É. C. R. 90 (1880) 293-. (1880) 299 -. Mittag-Leffer, $G$. C. R. 90

1005-

- - -. Picard, É. Crelle J. Mth. 90 (1881) 281-.

- - - Floquet, G. C. R. 98 (1884) 38-, 82-; Par. Éc. Norm. A. 1 (1884) 181-.

_ - - Bigiavi, C. Rm. R. Ac. Linc. Rd. 6 (1890) (Sem. 1) 339-.

- - - Malmborg, M. Ups. Arsk. (1897) (Mt. Nt.) $31 \mathrm{pp}$.

- - periodic. Floquet, G. Par. Ec. Norm. A. 12 (1883) 47-.

- - - , and their elementary integrals. Cauchy, A. L. Par. Mm. Ac. Sc. 22 (1850) $587-$.

- - rational. Schafheitlin, P. Crelle J. Mth. 106 (1890) 285-; 111 (1893) 44-.

- - Koch, H. von. C. R. 116 (1893) 91-.

--- , determination of groups. Poincaré, H. C. R. 96 (1883) 691-, 1302-.

- _ - relations due to definite integrals. Mellin, $H$. Helsingf. Acta 21 (1896) No. 6, 57 pp.

__ - solution of which depends on quadrature of algebraic irrational. Dillner, $G$. C. R. 91 (1880) 616-, 687-, 721-; 92 (1881) 117, 235-, 289-.

- real, singular points. Bôcher, M. N. Y. Am. Mth. S. Bll. 5 (1899) 275-:

- variable. Brassinne, E. Toul. Mm. Ac. 6 (1839-41) 50-.

68 (1868) 354-.

connected with algebraic surface. Klein, $F$. [1894] D. Mth. Vr. Jbr. 4 (1897) 91-.

- cubic equation. Cockle, J. Ph. Mg. 24 (1862) 37-.

equivalent to Lagrange's adjoint equations. Brioschi, F. A. Mt. 24 (1896) 339-. 
exact, symbolic treatment. Johnson, W. W. Am. J. Mth. 10 (1888) 94-.

of form $\left(m x^{2}+n x+p\right) y^{\prime \prime}+(q x+r) y^{\prime}+8 y=0$. Spitzer, S. Schlömilch Z. 4 (1859) 250-.

$-z^{(n)}=x^{m}\left(A x z^{\prime}+B z\right)$. Spitzer, S. Grunert Arch. 38 (1862) 458-.

$--x y^{(n)}-m y^{(n-1)}=a y$. Spitzer, $S$. Grunert Arch. 40 (1863) 232-.

$--\left(a_{2}+b_{2} x\right) y^{\prime \prime}+\left(a_{1}+b_{1} x\right) y^{\prime}+\left(a_{0}+b_{0} x\right) y=0$. Spitzer, $S$. Arch. Mth. Ps. 58 (1876) 361-.

and group-theory. Marotte, $F$. Toul. Fac. Sc. A. 12 (1898) H, 92 pp.

homogeneous. Catalan, E. C. Brux. Ac. Bll. 43 (1877) 335-.

-. Stenberg, E. A. Stockh. Öfv. (1884) No. 5, 143-

-. Lie, S. Leip. Mth. Ps. B. 43 (1891) 253-.

- Bendixson, I. [1892] Stockh. Öfv. (1892) 91-; Stockh. Ak. Hndl. Bh. 18 (Afd. 1) (1893) No. 7, 29 pp.

- Gutzmer, A. Crelle J. Mth. 115 (1895) 79-.

-, with algebraic relations between fundamental integrals. Wirtinger, W. Wien Ak. Sb. 98 (1890) (Ab. 2a) 66-.

,$--\frac{-}{-}-1$. Wallenberg, $G$. Crelle J. Mth. 111 (1893) 89-.

,$----\ldots$ Fano, G. Mth. A. 53 (1900) 493-

-, class, special. Guldberg, A. Christiania F. (1894) No. 9, 12 pp. ; Fschr. Mth. (189394) 545 -

-, with coefficients doubly periodic. Stenberg, E. A. Helsingf. Acta 16 (1888) 493-, 555-; 19 (1893) No. 11, 7 pp.

- - - common particular integrals, conditions for. Grünfeld, $E$. Z. Mth. Ps. 30 (1885) 210-.

-, general integral of which is uniform. Floquet, G. C. R. 121 (1895) 676-.

-, group-theory. Beke, E. Mth. A. 49 (1897) 573-.

-, with homogeneous relations between integrals. Fuchs, L. Acta Mth. 1 (1882) $321-$.

一, properties. Stenberg, E. A. Acta Mth. 8 (1886) 119-.

-, regularity of system. Sauvage, L. Mars. Fac. Sc. A. 4 (1895) Fasc. 3, 14 pp.

-, relations of two, to each other. Thomé, D. Nf. Tbl. ( $\left.{ }^{*} 1873\right) 50-$.

-, resolvents. Beke, M. Mth. Termt. Ets. 16 (1898) 407-.

-, special. Hurwitz, A. Mth. A. 26 (1886) 117-.

一, symmetric functions in. Beke, $E$. Mth. A. 45 (1894) 295-.

-, with symmetric integral determinant. Hazzidakis, J. N. Crelle J. Mth. 111 (1893) 315-.

-, systems, property. Hazzidakis, J. N. [1880] Crelle J. Mth. 90 (1881) 80-.

- $M+N \frac{d y}{d x}=0$, proof that $(M x+N y)^{-1}$ is an integrating factor. Stokes, G. G. Camb. Mth. J. 4 (1845) 241-. integrable, class, new. Halphen, G. H. C. R. 101 (1885) 1238-.

- by elliptic functions, class. Brioschi, $F$. Rm. R. Ac. Linc. T. 4 (1880) 241-.

,---- , generation. Brioschi, F. A. Mt. 10 (1880-82) 74-.

- - hyperbolic sine. Imšeneckij, V. G. [1891] Rec. Mth. (Moscou) 16 (1893) 177-; Fschr. Mth. (1891) 311-.

- - special functions. Schou, E. S. Kjøb: Ov. (1897) 433-.

- systems. Mayer, A. Mth. A. 5 (1872) 448 -. integrals having fixed branch-points. Fuchs, $L$. Berl. Ak. Sb. (1884) 699-.

- - one singularity at finite distance. Günther, P. Crelle J. Mth. 105 (1889) 1-; 119 (1898) 330-.

- regular at a singularity. Bendixson, I. Stockh. Öfv. (1892) 279-.

- satisfying relations of form

$$
F[\phi(x)]=\psi(x) F(x) .
$$

Appell, P. C. R. 93 (1881) 699-.

irreducible, property. Ratner, $E$. Mth. A. 32 (1888) 566-.

Laplace's method. Goursat, $\dot{E}$. Am. J. Mth. 18 (1896) 347-.

linearoid. Wilczynski, E.J. Am. J. Mth. 21 (1899) 354-.

non-homogeneous. Thomé, L.W. Crelle J. Mth. 107 (1891) 51-.

-, class. Floquet, $G$. Par. Ec. Norm. A. 4 (1887) 111-.

-, and their functional operations. Pincherle, S. Bologna Rd. (1895-96) 77-.

any order, with fixed critical points. Painlevé, P. C. R. 130 (1900) 1112-.

,---2 variables. Libri, G. C. R. 8 (1839) $732-$

(1839) 792-

1st order and degree, with 2 variables, singular points. Fouret, G. Par. S. Mth. Bll. 19 (1891) 128-.

- fundamental invariants of system. Grïnfeld, E. Z. Mth. Ps. 39 (1894) 237-.

- - , general integral of which is function with finite number of branches. Cahen, $A$. Toul. Fac. Sc. A. 1 (1899) 239-, 469-.

- - reduction of solution to continued fraction, case. Laguerre, (le capit.) $E$. C. R. 98 (1884) 209 -

\section{2nd order.}

Sturm, J. C. F. Liouv. J. Mth. 1 (1836) 106-. Svanberg, A. F. Stockh. Ak. Hndl. (1837) 207-.

Cockle, Jas. Mess. Mth. 1 (1862) 118-, 164-, 241-.

Heine, E. Berl. Mb. (1864) 13-.

Gegenbauer, L. Arch. Mth. Ps. 55 (1873) 252-.

Moutard, T. C. R. 80 (1875) 729-.

Pepin, T. C. R. 82 (1876) 1323-.

Tait, P. G. [1876] Edinb. R. S. P. 9 (1878) 93-.

Pepin, T. A. Mt. 9 (1878-79) 1-.

Brioschi, F. As. Fr. C. R. 8 (1879) 278 - 
Laguerre, E. [1879] Par. S. Mth. Bll. 8 (1880) 35-.

Tannery, J. Par. Ec. Norm. A. 8 (1879) 169 -. Gyldén, J. A. H. C. R. 90 (1880) 208-, 344-. Hermite, C. [1880] , A. Mt. 10 (1880-82) 101-. Humbert, G. Par. Éc. Pol. J. Cah. 48 (1880) 207-.

Picard, É. C. R. 90 (1880) 1479-.

Mittag-Lefler, G. C. R. 91 (1880) 978-.

Brioschi, F. C. R. 93 (1881) 941-.

Gyldén, H. Stockh. Ak. Hndl. Bh. 14 (Afd. 1) (1889) No. 6, 23 pp.

Petrovitch, M. Par. S. Mth. Bll. 25 (1897) 221-.

Thomé, L. W. Crelle J. Mth. 117 (1897) 185-.

Liapounoff, A. C. R. 128 (1899) 910-.

with algebraic integrals. Fuchs, $L$. Crelle J. Mth. 81 (1876) 97-; 85 (1878) 1-.

- - Jordan, C. C. R. 82 (1876) 605-.

- - Ermakov, V. P. [1894] Rec. Mth. (Moscou) 17 (1895) 500-.

allied to Hermite's equation. Vleck, E. B. van. Am. J. Mth. 21 (1899) 126-.

with arbitrary parameter. Picard, $\dot{E} . \quad$ C. R. 118 (1894) 379-.

arithmetical developments in theory. Fricke, $R$.

D. Mth. Vr. Jbr. 2 (1893) 59-.

cases. Molins, H. Toul. Mm. Ac. 2 (1858) 19-。

- Hoppe, R. Z. Mth. Ps. 9 (1864) 56-.

- Craig, T. Am. J. Mth. 8 (1886) 180 -

- Lohnstein, - Z. Mth. Ps. 38 (1893) 27-.

-. Igel, B. Mh. Mth. Ps. 5 (1894) 85-.

-, special. Pochhammer, L. Mth. A. 41 (1893) 174-.

class. Brioschi, F. A. Mt. 9 (1878-79) 11-; 10 (1880-82) 4-.

-. Fuchs, L. Crelle J. Mth. 100 (1887) 189-. -, and equation of 5th degree. Besso, D.

Rm. R. Ac. Linc. Rd. 2 (1886) (Sem. 1) 593-.

- integrable by elliptic functions. Krause, $M$.

Berl. Ak. Sb. (1900) 258-.

with coefficients binomial. $\dot{Z}$ murko, L. D. Nf. Tbl. (*1881) [28]-

- - doubly periodic, case. Elliot, Z. Acta Mth. 2 (1883) 233-.

- - - functions. Krause, M. Leip. Mth. Ps. B. 44 (1892) 15-, 238-.

- linear, case. Pochhammer, L. Mth. A. 36 (1890) 84-; 38 (1891) 225-.

- non-analytic, singular points. Bôcher, $M$.

N. Y. Am. Mth. S. T. 1 (1900) 40-, 507.

- periodic. Liapunov, A. M. Kharkov

Mth. S. Com. 5 (1896?) 190-; Fschr. Mth. (1897) 289.

- - transcendent. Cassel, G. Stockh. Öfv. (1890) 381-.

- variable. Tychsen, C. (xII) Ts. Mth. 1 (1871) 28-.

in connexion with plane curves of 4 th degree. Gordan, P. Mth. A. 46 (1895) 606-.

with fixed critical points. Painlevé, $P$. C. R. 117 (1893) 686-; 127 (1898) 541-, 945-; 129 (1899) 750-, 949-.

fundamental invariants. Vogt, $H$. Par. Éc. Norm. A. 6 (1889) (Suppl.) 71 pp.

Hermite's, application of doubly periodic functions of Weierstrass. Stenberg, $E$. A. Helsingf. Acta 16 (1888) 205-; Fschr. Mth. (1886) 296. homogeneous. Foldberg, $P$. Ts. Mth. 4 (1886) 81-; Fschr. Mth. (1887) 323.

-. Chini, M. Tor. Ac. Sc. At. 33 (1897) 505- or 737-.

-, functions satisfying. Floquet, $G$. Nancy S. Sc. Bll. (1895) 78-.

- integrable by doubly periodic functions of 2nd kind. Naetsch, E. Leip. Mth. Ps. B. 45 (1893) 538-.

-, properties, and algebraic equations. Besso, D. [1882] Rm. R. Ac. Linc. Mm. 14 (1883) 14-. -, system of solutions of theorem. Fuchs, L. Bll. Sc. Mth. As. 4 (1880) 334-.

of hyperbolic type, for which Laplace's series is finite in one sense only. Niccoletti, $O$. Rm. R. Ac. Linc. Rd. 6 (1897) (Sem. 1) 334-. - - - - - - - - both senses. Niccoletti, 0 . Rm. R. Ac. Linc. Rd. 6 (1897) (Sem. 1) 307-.

integrability by algebraic functions, cases. Hansen, P.C.V. (xm) Ts. Mth. 6 (1876) 144-. integrable, class. Grave, D. A. Rec. Mth. (Moscou) 14 (1890) 197-; Fschr. Mth. (1889) 325-.

integral forms. Liouville, $R$. Par. Éc. Pol. J. 56 (1886) 7-.

for which inverse function has finite multiformity. Schlesinger, L. Crelle J. Mth. 110 (1892) 265-.

non-homogeneous, properties. Besso, D. [1882] Rm. R. Ac. Linc. Mm. 14 (1883) 40-.

polynomials satisfying. Humbert, G. Par. S. Phlm. Bll. 4 (1880) 238-.

- - roots. Bôcher, M. N. Y. Am. Mth. S. Bll. 4 (1898) 256-.

primary forms occurring in. Schlesinger, $L$. Crelle J. Mth. 110 (1892) 130-.

property. Brioschi, F. A. Mt. 10 (1880-82) 1-. reduction to canonical form. Klein, $F$. Mth. A. 38 (1891) 144-.

refutation of Pepin's theorem. Fuchs, L. C. R. 82 (1876) 1494-; 83 (1876) 46-.

relation corresponding to. Winckler, A. [1877] Wien Ak. Sb. 76 (1878) (Ab. 2) 173-.

with relation between particular integrals. Winckler, A. Wien Ak. Sb. 92 (1886) (Ab. 2) 7-.

satisfied by function of independent variable of finite number of terms. Molins, $H$. Toul. Mm. Ac. 2 (1858) 357-.

series relative to. Liapounoff, A. C. R. 131 (1900) 1185-.

theorem of Hermite. Fuchs, L. C. R. 85 (1877) 947-.

theorems, generalisation. Casorati, $F$. A. Mt. 10 (1880-82) 224-.

and higher orders. Libri, G. [1833] Par. Mm. Ac. Sc. 14 (1838) 94-.

3rd and higher orders.

3rd order. Cockle, (Sir) Jas. [1864-76] QJ. Mth. 7 (1866) 316-; 8 (1867) 373-; 14 (1877) 340-.

- - Laguerre, E. C. R. 88 (1879) 116-.

- -. Combescure, É. C. R. 88 (1879) 275-. 


\section{Theory of Ordinary Linear Differential Equations (Ord. 3-n) 4850}

3rd order. Goursat, $\hat{E}_{\text {. }}$ C. R. 98 (1884) 609-. - - Halphen, - . Mth. A. 24 (1884) 461-. - - Painlevé, P. C. R. 104 (1887) 1829-. - - case. Malmsten, C. J. Stockh. Öfv. 5 (1848) 189-.

- - class integrable by hypergeometric series. Besso, D. Rm. R. Ac. Linc. Mm. 19 (1884) 251-.

- - with coefficients doubly periodic functions. Krause, M. Leip. Mth. Ps. B. 45 (1893) 35-. - - with fixed critical points. Painlevé, $P$. C. R. 130 (1900) 879-.

- - satisfied by automorphic functions. Hurwitz, A. Mth. A. 33 (1889) 345-.

- and 4th orders. Campbell, D. F. QJ. Mth. 31 (1900) 161-.

4th order. Halphen, G. H. C. R. 97 (1883) 247 .

- - class. Goursat, É. Par. S. Mth. Bll. 11 (1883) 144-.

- - , - , and equation of 5 th degree. Besso, D. Rm. R. Ac. Linc. Rd. 1 (1885) 183-, $233-, 884$.

- - , - integrable by hypergeometric series. Besso, D. Rm. R. Ac. Linc. Mm. 19 (1884) 245-.

- - determination of group of rationality. Marotte, F. C. R. 126 (1898) 715-.

- _, integrable when particular integral is known. Enriques, F. Mil. I. Lomb. Rd. 29 (1896) 257-.

- - invariants. Halphen, G. H. Acta Mth. 3 (1883) 325-.

- - , reduction, case. Goursat, É. C. R. 100 (1885) 233-.

5 th order, integral curves of which are contained in algebraic configurations. Fano, $G$. Mil. I. Lomb. Rd. 32 (1899) 843-.

- and 6th orders, integral curves of which are contained in a quadric. Fano, $G$. Tor. Ac. Sc. At. 34 (1898) 285- or 388-.

$n$th order, and their $n$ adjoint equations. Griunfeld, E. Crelle J. Mth. 117 (1897) 273-.

- _ , - _ _ _ connexion between fundamental determinants. Grünfeld, $\boldsymbol{E}$. Crelle J. Mth. 115 (1895) 328-.

- -, classes. Stodótkiewicz, A. J. Krk. Ak. (Mt.-Prz.) Rz. 6 (1893) 145-; Cre. Ac. Sc. Bll. (1892) 373-.

- -, non-essential singular points. Grünfeld, $E$. Wien Az. 26 (1890) 65-

- - possible forms. Starkov, A.P. Kazan S. Nt. (Ps.-Nth.) P. 3 (1885) 263-.

ordinary differential or with finite differences. Poincaré, H. Am. J. Mth. 7 (1885) 203-.

with parameter variable. Appell, $P$. As. Fr. C. R. 8 (1879) 253-.

- - Horn, J. Mth. A. 52 (1899) 340-

- particular integrals all of same form. Steen, A. Kjöb. Ov. (1866) 77-; Résumé, 10-; QJ. Mth. 8 (1867) 228-.

in particular that satisfied by series

$$
\begin{aligned}
& 1+\frac{a \beta \theta}{\gamma \epsilon} x+\frac{a \cdot a+1 \cdot \beta \cdot \beta+1 \cdot \theta \cdot \theta+1}{1 \cdot 2 \cdot \gamma \cdot \gamma+1 \cdot \epsilon \cdot \epsilon+1} x^{2}+\ldots . \\
& \text { Forsyth, A. R. QJ. Mth. } 19(1883) 292-.
\end{aligned}
$$

problem. Halphen, G. H. Liouv. J. Mth. 1 (1885) 11-.

reducible. Heffter, $L$. Crelle J. Mth. 122 (1900) 39-.

_, class. Bigiavi, C. A. Mt. 19 (1891-92) 97-.

reduction. Pochhammer, L. Crelle J. Mth. 110 (1892) 188-.

- to integrable forms. Halphen, M. Par. Mm. Sav. Étr. 28 (1884) No. 1, 301 pp.

simultaneous. Mainardi, $G$. Mil. At. I. Lomb. 1 (1858) 95-.

-. Picard, É. C. R. 90 (1880) 976-, 1065-, 1118 -

-. Tilly, J. de. Mathesis 5 (1885) 121-.

-, with coefficients variable, class. Grilnwald, A. K. Prag Sb. (1869) 63-.

- differential or difference equations with constant coefficients. Sylvester, J. J. Am. J. Mth. 4 (1881) 321-.

- or single, with absolute term. Franz, $J$. D. Nf. Vh. (1896) (Th. 2, Hälfte 1) 45.

singular point, fundamental equation. Gilnther, $P$. Crelle J. Mth. 106 (1890) 330-.

_ - , - , roots. Hanburger, ir. [1877] Crelle J. Mth. 84 (1878) 264-.

- points. Pochhammer, L. [1870] Crelle J. 73 (1871) 69-.

- - Bendixson, I. Stockh. Öfv. (1895) 81-. solutions of which satisfy quadratic relation. Halphen, G. H. C. R. 101 (1885) 664-.

with substitution-groups independent of parameters. Fuchs, L. Berl. Ak. Sb. (1892) 157-; (1893) 975-; (1894) 1117-.

- uniform integrals. Hirsch, A. Königsb. Schr. 33 (1892) [16]-.

- - - only. Stenberg, E. A. Helsingf. Acta 16 (1888) 333-.

-1 independent variable. Appell, $P$. C. R. 90 (1880) 1477-.

-2 variables, property. Ramus, C. Kiöb. Dn. Vd. Selsk. Afh. 9 (1842) 379-; Crelle J. 23 (1842) 372-.

_- - theorem. Ramus, C. Crelle J. 24 (1842) 260-

Equivalence of systems. Chrystal, G. Edinb. R. S. T. 38 (1897) 163-.

Euler's method demonstrated by the integrating equation. Bierens de Haan, D. Amst. Vs. Ak. 6 (1872) 122-; Arch. Néerl. 7 (1872) 273-.

- -, extension. Imšeneckij, $V . G$. St Pet. Ac. Sc. Mm. (Rs.) 42 ("1882) 1-.

Extension of Galois's ideas to theory. Picard, É. C. R. 121 (1895) 789-; Mth. A. 47 (1896) 155-.

Factorisation of operator. Pincherle, S. Bologna Rd. 4 (1900) 101-.

Fuchsian theory, extension to system of partial differential equations. Horn, I. Acta Mth. 14 (1890-91) 337-.

- of homogeneous equations. Zajacz kowski, W. Krk. Ak. (Mt.-Prz.) Pam. 13 (1887) (1)-; Fschr. Mth. (1887) 298-.

Fundamental equation. Casorati, $F$. Mil. I. Lomb. Rd. 13 (1880) 176-. 
Fundamental equation of homogeneous equations. Hamburger, M. Crelle J. Mth. 115 (1895) 343-.

- - theory. Schlesinger, L. Crelle J. Mth. 114 (1895) 143-.

- equations. Giinther, P. Crelle J. Mth. 107 (1891) 298-.

- - of homogeneous equations. Beke, $E$. [1898] Mth. Nt. B. Ung. 15 (1899) 273-.

- property, demonstrations. Mansion, $P$. Brux. Ac. Bll. 38 (1874) 578-; Mess. Mth. 4 (1875) 177-; 7 (1878) 188-; Arch. Mth. Ps. 58 (1876) 99 -.

General equation. Oliver, J. E. A. Mth. 3 (1887) 109-.

_-, 2nd order. Cockle, (Sir) Jas. L. Mth. S. P. 19 (1889) 257-.

Geometrical considerations. Fano, G. Rm. R. Ac. Linc. Rd. 4 (1895) (Sem. 1) 18-.

Groups. Picard, É. Bll. Sc. Mth. 9 (1885) 202-. Gyldén-Linstedt equation generalised. Picart, L. Bordeaux S. Sc. PV. (1896-97) 103-; (1897-98) 145-.

Hamburger sub-groups of canonical fundamental system. Schlesinger, L. Crelle J. Mth. 114 (1895) 159-, 309-.

Integrability conditions for $\frac{d^{3} u}{d x^{3}}+x^{m} u=0$. Florov, P. S. (xII) Kharkov Mth. S. Com. (1883) 129-; Fschr. Mth. (1884) 288.

\section{Integrals.}

Heffter, L. Crelle J. Mth. 109 (1892) 222-. algebraic. Goursat, $\hat{E}$. Bll. Sc. Mth. As: 6 (1882) 120-; C. R. 100 (1885) 1329-.

-, of equations with coefficients rational. Autonne, L. Par. Éc. Pol. J. 54 (1884) 1-. ,$--\frac{-}{2}$ 2nd order. Vernier, P. C. R. 118 (1894) 1317-.

-,$--- \ldots$, , method of obtaining. Pepin, $T$. Rm. N. Linc. At. 34 (1881) 243-.

-, form. Königsberger, L. [1882] Mth. A. 21 (1883) 454-.

- relations between. Koenigsberger, $L$. Mth. A. 31 (1888) 220-.

asymptotic representation. Kneser, A. Mth. A. 49 (1897) 383-.

—-. Horn, J. Mth. A. 49 (1897) 453-.

behaviour near singular point. Horn, $J$. Crelle J. Mth. 120 (1899) 1-; 122 (1900) 73-

cases. Graefe, F. [1880] Crelle J. Mth. 91 (1881) 262-.

complete integral from particular integrals with 2nd term zero. Minich, S. R. [1851] Ven. Mm. I. 5 (1855) 43-.

distinction in sub-groups. Casorati, F. C. R. 92 (1881) 175-, 238-.

of equations with coefficients constant. Mainardi, G. Rm. At. N. Linc. 26 (1873) 388-.

$--\ldots-$, introduction of algebraic functions of principal variable. Hincks, E. Ir. Ac. T. 13 (1818) 115-.

- - - - periodic. Anisimov, V. A. Vars. S. Nt. Tr. (Mm.) 3 (1896) 23 pp.; Fschr. Mth. (1896) 258; Vars. S. Nt. Tr. (1897) (Ps. C.) Fasc. 2, No. 6. of equations with coefficients variable, form. Hamburger, $M$. Crelle J. 76 (1873) 113-. $---1-$, properties. Tannery, J. Par. Éc. Norm. A. 4 (1875) 113-.

_- - 2nd order, bilinear relations between constants. Kęiñski, S. Krk. Ak. (Mt.-Prz.) Rz. 7 (1895) 384-; Fschr. Mth. (1895) 445-. - - - -, form. Steen, A. Kjöb. Ov. (1874) 1-.

- - - - infinite values. Gromeka, I. S. Kazan S. Nt. (Ps.-Nth.) P. 6 (1888) 14-; Fschr. Mth. (1887) 322.

- - , higher order. Schellbach, C. H. Crelle J. 16 (1837) 352-.

evaluation and reduction of principal function. Cauchy, A. L. C. R. 9 (1839) 637-.

exchange of argument and parameter. Frobenius, G. [1873] Crelle J. Mth. 78 (1874) 93-.

expansion in series. Fuchs, L. [1870] A. Mt. 4 (1870-71) 36-.

$-1-$ Fabre, A. As. Fr. C. R. (1894) (Pt. 2) 158-.

form. Jurgens, $E$. Crelle J. Mth. 80 (1875) 150-.

- Johanson, A. M. Stockh. Öfv. (1889) 583-; (1890) 283-; Fschr. Mth. (1890) 311-.

forms. Picard, É. C. R. 94 (1882) 418-.

fundamental, and their derivatives, algebraic relations for homogeneous equation, 2nd order. Koenigsberger, L. Mth. A. 31 (1888) 75-.

$-,-\ldots-,-\ldots--\longrightarrow, 3$ 3rd order. Koenigsberger, $L$. Crelle J. Mth. 103 (1888) 274-.

- , derivatives of same function. Craig, $T$. Am. J. Mth. 8 (1886) 85-.

- , of homogeneous equation, 2 nd order, relations. Königsberger, L. Mth. A. 22 (1883) 269-.

general, of equation, 3rd order. Starkov, A. P. Kazan S. Nt. (P8.-Mth.) P. 3 (1885) 86-; Fschr. Mth. (1885) 323, 324.

- and particular, connexion. Königsberger, L. Crelle J. Mth. 91 (1881) 265-.

of homogeneous equation connected by homogeneous relation $y_{1}^{p}-y_{2} y_{3} y_{4} \ldots y_{p+1}=0$. Wallenberg, G. Crelle J. Mth. 114 (1895) 181 -.

- - - expansion near singular point. Bucca, $F$. Palermo Cir. Mt. Rd. 14 (1900) 115-.

bauer, L. Wien Ak. Sb. 109 (1900) (Ab. 2a) 61-.

- - - 6th order, homogeneous quadratic relations between. Rosenkranz, M. Z. Mth. Ps. 35 (1890) 82-, 129-.

- _ - recurring formulæ for. Heffter, $L$. Crelle J. Mth. 106 (1890) 269-.

$--1,--1$ - (Heffter). Fuchs, L. Crelle J. Mth. 106 (1890) 283-.

and invariants of homogeneous equation, analytic representation. Mittag-Leffler, $G$. Acta Mth. 15 (1891) 1-.

irregular. Poincaré, $H$. C. R. 101 (1885) 939-, 990-; Acta Mth. 8 (1886) 295-; 10 (1887) 310-. 
irregular. Nekrasov, P. A. [1894] Rec. Mth. (Moscou) 17 (1895) 498-.

- Horn, J. C. R. 126 (1898) 205-.

-, of equations, 2 nd order. Horn, J. Acta Mth. 23 (1900) 171-.

logarithmic, of non-homogeneous equations. Königsberger, L. Crelle J. Mth. 90 (1881) 267- ; 94 (1883) 291-.

,---- , form. Koehler, C. ' Z. Mth. Ps. 33 (1888) 231- ; 34 (1889) 36-.

- - regular equations. Heun, $K$. Am. J. Mth. 10 (1888) 205-.

of non-homogeneous equations. Koenigsberger, L. Crelle J. Mth. 99 (1886) 10-.

between each pair of singular points, relations. Fuchs, L. Crelle J. 76 (1873) 177-.

- - - - - - - with constants of group substitutions. Fuchs, L. Berl. Ak. Sb. (1892) 1113-.

particular. Florov, P. S. Kharkov Mth. S. Com. (1886) 31-; Fschr. Mth. (1886) 303-.

-, common to two equations. Escherich, $G$. von. [1882] Wien Ak. D. 46 (1883) (Ab. 2) $61-$; 47 (1883) (Ab. 2) 1-.

-, of homogeneous equation, product. Besso, D. [1882] Rm. R. Ac. Linc. Mm. 14 (1883) 3-.

-, - non-homogeneous equations. Pincherle,

S. Rm. R. Ac. Linc. Rd. 6 (1890) (Sem. 1) $199-$ -

- , - reduced equation expressed by particular integrals of given equation. Starkov, A.P. Kazan S. Nt. (Ps.-Mth.) P. 3 (1885) 253-; Fschr. Mth. (1885) 284.

$n$th particular, of $y^{n}+P y^{(n-1)}+Q y^{(n-2)}+\ldots$ $S^{\prime} y^{\prime}+T y=0$. Malmsten, C. J. Crelle J. 39 (1850) 91-.

properties. Schlesinger, L. Orv.-Termt. Éts. (Termt. Szak) (1892) 341-.

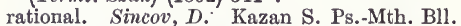
7 (1898) 1-, 137-; Kazan Un. Mm. (1898) (Pt. 4) 179-, (Pts. 5 \& 6), (Pts. 7 \& 8) 137-, (Pt. 9) ; Fschr. Mth. (1898) 273-; N. A. Mth. 17 (1898) 411-.

- algebraic, of equations with algebraic rational coefficients. Nekrasov, P. A. Kharkov Mth. Com. 4 (1895) 225-; Fschr. Mth. (1893-94) 534,536 .

- fractional, method of finding. Imšeneckij, V. G. St Pet. Ac. Sc. Mm. (Rs.) 55 (1887) (Suppl.) No. 9, 55 pp.; Fschr. Mth. (1887) 296-; St Pet. Ac. Sc. Mm. (Rs.) 58 (1888) 1-; Fschr. Mth. (1888) 317-.

,----- (Imšeneckij). Nekrasov, P.A. [1893] i Rec. Mth. (Moscou) 17 (1895) 341-, 391-; Fschr. Mth. (1893-94) 534-.

- - - - - (-). Posse, K. A. [1893] Rec. Mth. (Moscou) 17 (1895) 386-.

- - - - - Gilnther, N. Rec. Mth. (Moscou) 17 (1895) 680-; Fschr. Mth. (1893-94) 534, 537.

- - - - - (Imș̌eneckij). Nekrasov, P.A. Rec. Mth. (Moscou) 18 (1896) 337-; Fschr. Mth. (1896) 258.

-, method of finding. Ermukoff, W.P. Fschr. Mth. (1893-94) 534, 536-.

-, - - - (Ermakov). Nekrasov, P. A. Rec. Mth. (Moscou) 18 (1896) 275-; Fschr. Mth. (1896) 258. rational particular, investigation by integrating factor. Andreev, $K . A$. Kharkov Mth. S. Com. 4 (1895) 177-; Fschr. Mth. (1893-94) $534,535-$.

real, of equations, 3rd order, zeros. Davidoglou, - C. R. 130 (1900) 399-.

regular. Frobenius, G. Crelle J. Mth. 80 (1875) 317-.

-. Koch, H. von. Acta Mth. 18 (1894) 337-. representation. Fuchs, L. [1872] Crelle J. 75 (1873) 177- ; 76 (1873) 175-.

in series of binomial equations. Johnson, W. W. Am. J. Mth. 11 (1889) 37-.

of solutions of equations, 2nd order, with 3 síngular points. Kepiñski, S. Krk. Ak. (Mt.-Prz.) Rz. 17 (1900) 112- ; Crc. Ac. Sc. Bll. (1899) 67-.

- - - homogeneous equations, 2nd order. Kepiński, S. Krk. Ak. (Mt.-Prz.) Rz. 5 (1893) 264- ; Crc. Ac. Sc. Bll. (1893) 20 -

supplementary. Heymann, $W$. Crelle J. Mth. 98 (1885) 231-.

of system of equations, expansion near singular point. Bendixson, I. Stockh. Öfv. (1894) 141-.

- - - - - - points. Horn, J. Crelle J. Mth. 116 (1896) 265-; 117 (1897) 104-, $254-$.

- - - 1st order, with constant coefficients when characteristic equation has equal roots: Baehr, G. F. W. As. Fr. C.R. 11 (1882) 92-.

uniform. Koch, $H$ : von. C. R. 116 (1893) 365-.

-, of homogeneous equation with doubly periodic coefficients. Sjöblom, $E$. Stockh. Öfv. (1884) No. 5, 155- ; Fschr. Mth. (1884) 282.

-, - - equations with doubly periodic coefficients, general form: Stenberg, $E . A$. Acta Mth. 15 (1891) 259-.

zeros, real. Kneser, $A$. Mth. A. 42 (1893) 409-.

of $\frac{d^{2} y}{d x^{2}}+g x^{m} y=0$. Plana, G. Tor. Mm. Ac. 26 (1821) 519-, 557-.

$-I^{\prime \prime}+\frac{1}{x} I^{\prime}+I=0$. Lipschitz, R. Crelle J. 56 (1859) 189-.

$\int f(x) \cos x d x$, and the equation $\frac{d^{2} y}{d x^{2}}+y=f(x)$. Studnička, F. $J$. Časopis 13 (1884) 12-; Fschr. Mth. (1884) 235.

of $\frac{d^{2} y}{d x^{2}}+p y=0$, conditions for finiteness. $Z$ $u$ kovskij, N. E. Réc. Mth. (Moscou) 16 (1893) $582-$

Integrating factor. Florov, A. P. Kharkov Mth. S. Com. (1887) 47-; Fschr, Mth. (1887) 298.

- - of 'equations, 2nd order. Imshenetsky, $V$. G. Liége S. Sc. Mm. 9 (1882) (No. 10) 7 pp.

- factors. Halphen, G. H. C. R. 97 (1883) 1408-, 1541-; 98 (1884) 134-. 


\section{Integration.}

Schmidten, H. G. Gergonne. A. Mth. 11 (1820-21) 269-.

Rees, R. van. Quetelet Cor. Mth. 2 (1826) 332-. Conti, C. A. Sc. Lomb. Ven. 5 (1835) 131-.

Tortolini, B. G. Arcad. 67 (1836) 179-.

Lobatto, $R$. Amst. N. Vh. 6 (1837) 83-.

Cauchy, A. L. C. R. 8 (1839) 827-, 845-, 889-, 931-.

Bronwin, B. Mathematician 2 (1817) 204-; Camb. and Dubl. Mth. J. 3 (1848) 35-.

Malmsten, C. J. Camb. and Dubl. Mth. J. 4 (1849) 286-.

Mainardi, G. Tortolini A. 1 (1850) 50-, 251-.

Bronwin, B. Ph. Mg. 2 (1851) 477-; 3 (1852) 187-.

Russell, W. H, L. Camb. and Dubl. Mth. J. 9 (1854) 104-.

Petzval, $J$. Grunert Arch. 28 (1857) 300-.

Rouché, E. Mntp. Ac. Sc. Mm. 4 (1858-60) 195-.

Weiler, A. Crelle J. 55 (1858) 274-; Grunert Arch. 35 (1860) 440-.

Bruno, G. [1860] (vi Adds.) Tor. Ac. Sc. Mm. 21 (1864) 29-.

Lommel, E. Grunert Arch. 40 (1863) 101-.

Halphen, G. C. R. 58 (1864) 471-.

Waszmuth, A. Arch. Mth. Ps. 45 (1866) 70-.

Seydler, A. Časopis 1 (*1872) 195-; Fschr. Mth. (*1872) 152-.

Starkov, A. N. Rs. S. Nt. Mm. (Mth.) 1 (*1878).

Florov, P. S. Kharkov. Mth. S. Com. (1884) 143-; Fschr. Mth. (1885) 327-.

Vessiot, E. Par. Éc. Norm. A. 9 (1892) 197-.

Stodótkiewicz, A.J. Prace Mt.-Fiz. 6 (1895) 129- ; Fschr. Mth. (1895) 398.

algebraic. Poincaré, H. C. R. 97 (1883) 984-, 1189-.

-. Painlevé, P. C. R. 119 (1894) 37-.

-, of class of equations. Goursat, $\hat{E}$. C. R. 96 (1883) 323-.

-, - special equations. Hansen, P.C.V. (xI) Ts. Mth. 4 (1880) 148-.

application of co-functions. Schapira, $H$. D. Nf. Tbl. (1884) 57-.

by auxiliary equations. Imšeneckij, $V . G$. [1890] St Pet. Ac. Sc. Mm. (Rs.) 64 (1891) (Suppl.) No. 8, 47 pp. ; Fschr. Mth. (1891) 310-.

in case analogous to that of algebraic equation with equal roots. Minich, $S$. $R$. At. Sc. It. (1842) 415-.

cases. Dienger, J. N. A. Mth. 6 (1847) 124-. -. Spitzer, S. Wien SB. 26 (1857) 449-.

-. Seydler, A.J. Bll. Sc. Mth. As. 6 (1874) 94-

- Spitzer, 一. D. Nf. Tbl. $\left({ }^{*} 1875\right) 49$-.

- Winckler, A. Wien Ak. Sb. 71 (1875) (Ab. 2) 5-.

-. Spitzer, S. Crelle J.Mth. 88 (1880) 343-.

- Steen, A. Bll. Sc. Mth. As. 5 (1881) (Pt. 1) 30-.

of class of equations. Mittag-Leffler, G. Stockh. Öfv. 36 (1879) No. 3, 17-. of class of equations, 3rd order. Źorawski, $K$. Krk. Ak. (Mt.-Prz.) Rz. 14 (1899) 141-; Cre. Ac. Sc. Bll. (1897) 303-.

- complete equations. Studnička, F. Prag Sb. (1870) 76-.

- - - 1st order, with variable coefficients. Vállès, $F$. Gergonne A. Mth. 17 (1826-27) 72-. convergence of series occurring in. Kneser, $A$. Mth. A. 47 (1896) 408-

by doubly periodic functions. Goursat, É. Par. S. Mth. Bll. 12 (1884) 96-.

of equations with any coefficients. André, $D$. C. R. 84 (1877) 1018-.

- - coefficients constant. Boole, G. Camb. Mth. J. 2 (1811) 114-.

--- - Cauchy, A. L. C. R. 13 (1841) 109-.

- - - Daru, -. Liouv. J. Mth. 7 (1842) 266-.

- - Combescure, É. Gould As. J. 3 (1854) 52-.

(1867) 507-.

A. 4 (1887) 129-.

Tychsen, C. Z. Mth. Ps. 12 Collet, J. Par. Éc. Norm. Olt

(1887) (Pt. 2) 75-. 63 (1893) 39-. Vaschy, -. Par. Ec. Pol. J.

- - - and complete. Mainardi, $G$. Mil. G. I. Lomb. 7 (1855) 19-.

- - - - - and last term function of $x$. Herapath, J. $\quad$ Ph. Mg. 2 (1827) 419-; 3 (1828) 19-.

$-\ldots-1,-2$ variables. Minich, S. R. Padova N. Sag. 4 (1838) 402-.

- - - - - - - Tortolini, B. A. Mt. 6 (1864) 249-.

- - - containing $x$ to first power. Spitzer, S. Wien SB. 26 (1857) 479-.

- $-\ldots$ doubly periodic and integrals nonuniform functions of variable. MittagLeffler, G. Helsingf. Acta 12 (1883) 59-.

- - - periodic. Boltzmann, L. Wien Sb. 58 (1868) (Ab. 2) 54-.

- - - rational. Autonne, L. Par Éc. Pol. J. cah. 51 (1882) 93-.

- - - variable. Yur'ev, S. A. (xm) Rec. Mth. (Moscou) [1] (1866) 451-.

- - - equal roots. Lamla, $K$. Baumgartner Z. 4 (1828) 35-.

-, 1st order, 2 variables. Bouvier, L. C. Gergonne A. Mth. 15 (1824-25) 41-.

- - - - and 2nd orders. Tychsen, C. (xII) Ts. Mth. 4 (1868) 163-.

- -, 2nd order. Weiler, A. Grunert Arch. 32 (1859) 184-.

- -, - - Schlömilch, O. Schlömilch Z. 5 (1860) 323-.

- - - - De Jong, J. Amst. Ak. Vs. M. 8 (1874) 28-; Arch. Néerl. 9 (1874) 411-.

- - - - Winckler, A. Wien Ak. Sb. 75 (1877) (Ab. 2) 577-.

-, - -. Stodótkiewicz, A. J. Krk. Ak. (Mt.-Prz.) Rz. 15 (1887) 36-; Fschr. Mth. (1887) 323.

Cour. $8^{\circ}, 40$ (1887) No. 3, 98 pp. 
of equations, 2nd order, with coefficients linear. Żmurko, W. [L.] (xII) Krk. Ak. (Mt.-Prz.) Pam. 8 (1883) 74-.

- - - - - - - Stodótkiewicz, A. J. [1883] (xII) Krk. Ak. (Mt.-Prz.) Pam. 9 (1884) 113-; Fschr. Mth. ("1884) 286-.

- $-,-\ldots,--\ldots$ functions of the independent variables. Winckler, $A$. Wien Sb. 67 (1873) (Ab. 2) 143-.

$--,-1,-1-$ rational, in finite form. Pepin, -. [1862] (vII) A. Mt. 5 (1863) 185-. - -,-1 , by development in continued fractions with Abelian integrals. Heun, $K$. Mth. A. 30 (1885) 553-.

- - - - - on which evection depends. Gyldén, J. A. H. C. R. 93 (1881) 127-.

,---- , forms, theory. Brioschi, $F$. Mth. A. 11 (1877) 401-.

$--\frac{1}{23}-\frac{-}{1889},-$ - Forsyth, A. R. QJ. Mth. 23 (1889) 45-.

,$---\frac{1}{-}$ by Gauss's series. Thomae, C.J. Z. Mth. Ps. 19 (1874) 273-.

$-\ldots,--, 2$ variables. Buttel, P. Grunert Arch. 23 (1854) 410-.

$-{ }_{-},-\ldots,-$ or more variables. Weiler, $A$. [1854] Crelle J. 51 (1856) 105-.

- - 3rd order. Besso, D. Mod. Ac. Sc. Mm. 7 (1890) 245-.

- - , higher orders. Malmsten, C.J. Stockh. Öfv. 5 (1848) 135-.

- -, nth order. Stodótkiewicz, A.J. Crc. Ac. Sc. Bll. (1893) 241-.

,---- , case. Hossenfelder, $E$. Mth. A. 4 (1871) 195-.

$-\frac{1}{-}--$, - . Aleksěevskij, V. P. Kharkov Mth. S. Com. (1884) 222-; Fschr. Mth (1885) 328.

- - - - - , with constant coefficients and equal roots. $M ., F$. (vi $A d d s$.$) Gergonne$ A. Mth. 3 (1812-13) 46-.

- - particular integrals of which can be expressed in terms of one another. Libri, $G$. [1830] Crelle J. 10 (1833) 167-.

- - 2 variables. Canterzani, S. [1798] Mod. Mm. S. It. 8 (1799) 307-.

error attributed by Herapath to Lagrange. Alpha Beta (a. $\beta$.). (vi Adds.) Ph. Mg. 3 (1828) $96,262-$.

- - - - - - - F. R. S. (vi Adds.) Ph. Mg. 3 (1828) 97-.

$--1-1$ - Herapath, J. Ph. Mg. 3 (1828) 210-.

by finite explicit functions. Steen, A. Kjöb. Dn. Vd. Selsk. Skr. 10 (1875) 519-.

in finite form. André, D. C. R. 88 (1879) 230-; Liouv. J. Mth. 6 (1880) 27-; 7 (1881) 283-.

- - -. Stodótkievicz, A.J. [1892] Krk. Ak. (Mt.-Prz.) Rz. 6 (1893) 100-; Cre. Ac. Sc. Bll. (1892) 296-; Prace Mt.-Fiz. 4 (1893) 31-.

general, of equations of any degree and with any number of variables. Euler, $L$. [1779] St Pét. Ac. Sc. Mm. 4 (1813) 43-.

of general equations of order above 2 nd by finite number of quadratures, impossibility. Starkov, A. P. Kazan S. Nt. (Ps.-Mth.) P. 3 (1885) 256-; Fschr. Mth. (1885) 309. general formula for equation with 2 nd member and constant coefficients: Lyetnikov, A. V. [1879] (xII) Rec. Mth. (Moscou) 9 (1878-81) (Pt. 1) 550 .

graphic, of equations, 1st order. Czuber, E. Z. Mth. Ps. 44 (1899) 41-.

of Hermite's equations of 3rd and 4th orders, integrals having poles of 1st order. MittagLeffler, G. Helsingf. Acta 12 (1883) 409-; A. Mt. 11 (1882-83) 65-.

- homogeneous equation in annular space containing no singularities. Mittag-Leffer, G. Stockh. Öfv. (1889) 415-.

- - equations, classes. Mittag-Leffler, $G$. Stockh. Öfv. 37 (1880) No. 6, 59-.

,--- 2nd order, case. Besso, $D$. Mod. Ac. Sc. Mm. 7 (1890) 239-.

- - by quadratures. Schlesinger, $L$. Crelle J. Mth. 116 (1896) 97-.

- Kummer's equation. Lindelöf, $E$. Helsingf. Acta 19 (1893) No. 1, 31 pp.

mechanical, of equations, 2nd order, with variable coefficients. Thomson, (Sir) W. R. S. P. 24 (1876) 269-.

- - - general equation, any order, with variable coefficients. Thomson, (Sir) W. R. S. P. 24 (1876) 271-.

method. Woodward, R. S. N. Y. Am. Mth. S. Bll. 6 (1900) 366, 369-.

-, d'Alembert's. Brassinne, E. Toul. Mm. Ac. 1 (1844) 91-.

-, - , application to equations with constant coefficients. Stodótkiewicz, A.J. Krk. Ak. (Mt.-Prz.) Rz. 6 (1893) 105-; Crc. Ac. Sc. Bll. (1892) 294-.

-, Brisson's first, for linear and finite difference equations. Mansion, P. [1869] Brux. Mm. Cour. $8^{\circ}, 22$ (1872) (No. 1) 32 pp.

- for equations with one independent variable. Caque, $F$. Liouv. J. Mth. 9 (1864) 185-.

-, general, for special class of equations. Steen, A. Kjöb. Ov. (1866) 13-.

-, Laplace's, for linear and finite difference equations. Darboux, G. Bll. Sc. Mth. As. 3 (1879) 209-.

- for simultaneous equations, 1st order. Cauchy, d. L. C. R. 43 (1856) 127-.

of non-homogeneous equations. Heymann, $W$. Z. Mth. Ps. 30 (1885) 27-, 79-; 32 (1887) 22-. when polynomial has equal roots. Plana, $G$. [1826] Tor. Mm. Ac. 31 (1827) 377-.

of Riccati's equation $y^{\prime \prime}+2 z y^{\prime}+z^{2} y=0$ by expansion of $y e^{-\frac{1}{2} z^{2}}$ in infinite series. Clariana Ricart, L. Fschr. Mth. (1900) 352.

by series. Frobenius, $G$. Crelle J. 76 (1873) $214-$

- - Peano, G. Tor. Ac. Sc. At. 22 (188687) 437-; Mth. A. 32 (1888) 450-.

of simultaneous equations. Prouhet, $E . N$. A. Mth. 5 (1866) 323-.

- - - (2); d'Alembert's. Kryger, C. (xII) Ts. Mth. 5 (1875) 38-.

- - (-) in $n$ variables. Raabe, J. L. Zür. Mschr. 1 (1856) 130-.

by sines of higher orders. Yvon-Villarceau, A. J. F. C. R. 90 (1880) 721-, 767-; 91 (1880)13-; Par. Ing. Civ. Mm. (1880) (v.2) 18-. 
of system of equations. Sturm, J. C. F. Férussac Bll. Sc. Mth. 12 (1829) 313-.

- _ - 1st order. Grïnfeld, $E$. Wien Ak. D. 54 (1888) (Ab. 2) 93-; Wien Az. 25 (1889) 37-.

- - partial differential equations, 1st order. Zajaczkowski, W. Arch. Mth. Ps. 56 (1874) 163-.

- - simultaneous partial differential equations. Schiff, P. [1891] St Pét. Ac. Sc. Bll. 34 (1892) 407-.

- systems of simultaneous homogeneous equations. Spelta, C. G. Mt. 38 (1900) 217-.

$-\frac{d^{2} y}{d x^{2}}+\left(1-\frac{i(i+1)}{x^{2}}\right) y=0 . \quad$ Paoli, P. [1827]

Mod. S. It. Mm. 20 (1828) 183-.

$-x^{2}\left(a+b x^{n}\right) d^{2} y^{\prime}+x\left(c+e x^{n}\right) d y^{\prime} d x$ $+\left(f+g x^{n}\right) y^{\prime} d x^{2}=M d x^{2}$.

Sauer, C. G. Crelle J. 2 (1827) 93-.

$-\left(m x^{2}+n x+p\right) y^{\prime \prime}-(q x+r) y^{\prime}+8 y=0$.

Liouville, J. Par. Ec. Pol. J. 21 cah. (1832) 163-.

$-\frac{d^{n} y}{d x^{n}}=(a+\beta x) y . \quad$ Scherk, H. F. Crelle J. 10 (1833) 92-, 279.

$--=-$ (Scherk). Jacobi, C. G.J. Crelle J. 10 (1833) 279.

$-y^{\prime \prime}+\frac{r}{x} y^{\prime}=\left(b x^{m}+\frac{s}{x^{2}}\right) y . \quad$ Malmsten, C. J. Camb. and Dubl. Mth. J. 5 (1850) 180 -.

- $x y^{(n)}-y=0$. Spitzer, S. Grunert Arch. 26 (1856) 57-.

$-y^{(n)}=A x^{m} y^{\prime}+B x^{m-1} y$. Spitzer, S. Grunert Arch. 28 (1857) 254-.

$-y^{(n)}=d x^{m} y^{\prime \prime}+B x^{m-1} y^{\prime}+C x^{m-2} y$. Spitzer, $S$, Grunert Arch. 29 (1857) 403-; 30 (1858) 76-; 33 (1859) 118- ; 38 (1862) 77-.

- $x y^{\prime \prime}-y=0$. Spitzer, S. Schlömilch Z. 2 (1857) 165-.

$\left(a_{2}+b_{2} x\right) y^{\prime \prime}+\left(a_{1}+b_{1} x\right) y^{\prime}+\left(a_{0}+b_{0} x\right) y=0$.

Spitzer, $S$. Wien SB. 25 (1857) 31-.

$-x^{2}\left(a_{22}+b_{2} x\right) y^{\prime \prime}+x\left(a_{1}+b_{1} x\right) y^{\prime}$ $+\left(a_{0}+b_{0} x\right) y=0$.

Spitzer, S. Schlömilch Z. 3 (1858) 53-.

$-x^{3}\left(a_{3}+b_{3} x\right) y^{\prime \prime \prime}+x^{2}\left(a_{2}+b_{2} x\right) y^{\prime \prime}$ $+x\left(a_{1}+b_{1} x\right) y^{\prime}+\left(a_{0}+b_{0} x\right) y=0$.

Spitzer, S. Schlömilch Z. 3 (1858) 55-.

- $a^{m} \frac{d^{m} z}{d t^{m}}=x^{i m} \frac{d^{m} z}{d x^{m}}$. Weiler, A. Grunert Arch. 31 (1858) 44-.

$-x^{2 n} y^{(n)}=A x y^{\prime}+B y$. Spitzer, S. Grunert Arch. 33 (1859) 413-.

- $a_{2} y^{\prime \prime}+\left(a_{1}+b_{1} x\right) y^{\prime}+b_{0} x y=0$. Spitzer, $S$. Grunert Arch. 38 (1862) 462-.

$-\left(a_{n}+b_{n} x\right) y^{n}+\left(a_{n-1}+b_{n-1} x\right) y^{n-1}+\ldots$ $+\left(a_{1}+b_{1} x\right) y^{\prime}+\left(a_{0}+b_{0} x\right) y=0$.

Spitzer, S. Schlömilch Z. 7 (1862) 264-.

$-x y^{(r)}-y^{(r-1)}+m x^{2} y=0$. Spitzer, $S$. Grunert Arch. 40 (1863) 21-.

$-A_{1} \xi \frac{d^{n} y}{d \xi^{n}}+B_{1} \frac{d^{n-1} y}{d \xi^{n-1}}=\xi^{m}\left(A \xi \frac{d y}{d \xi}+B y\right)$.

Spitzer, S. Schlömilch Z. 8 (1863) 66-. $-s y^{\prime \prime}+(r+q x) y^{\prime}+\left(p+n x+m x^{2}\right) y=0$.

Spitzer, S. Schlömilch Z. 8 (1863) 123-.

VOL. I. of $\frac{d^{3} y}{d x^{3}}=3 m x^{2} \frac{d^{2} y}{d x^{2}}+6 m(\mu+2) x \frac{d y}{d x}$ $+3 m(\mu+2)(\mu+1) y$.

Spitzer, S. Arch. Mth. Ps. 42 (1864) 102-. - $x^{m} \frac{d^{2 m} y}{d x^{2 m}}=y, m$ being a negative integer.

Spitzer, S. Arch. Mth. Ps. 42 (1864) 328-.

- $\left(a+b x+c x^{2}\right)(b+2 c x) y^{\prime \prime}+A\left(a+b x+c x^{2}\right) y^{\prime}$ $+B(b+2 c x) y=0$.

Spitzer, S. Arch. Mth. Ps. 42 (1864) 330-.

- $(b+2 c x) y^{\prime \prime}+A\left(a+b x+c x^{2}\right) y^{\prime}$ $+B(b+2 c x)\left(a+b x+c x^{2}\right) y=0$.

Spitzer, S. Arch. Mth. Ps. 42 (1864) 331.

$-(b+2 c x) y^{\prime \prime}+A\left(a+b x+c x^{2}\right) y^{\prime}$ $+B(b+2 c x) y=0$.

Spitzer, S. Arch. Mth. Ps. 42 (1864) 332.

$-(m+x)(n+x) y^{\prime \prime}+(m-n) y^{\prime}-\lambda^{2}(m+x)^{2} y=0$. Spitzer, S. Arch. Mth. Ps. 42 (1864) 375-. $-\frac{d^{n} y}{d x^{n}}=x^{m} y+A_{1}+A_{2} x+A_{3} x^{2}+\ldots+A_{m} x^{m-1}$.

Spitzer, S. Z. Mth. Ps. 10 (1865) 155-.

$-x y^{(n)}+a y^{(n-1)}=b x y$. Spitzer, S. Z. Mth.

Ps. 10 (1865) 221-.

$-\frac{d^{2} y}{d x^{2}}=a y+\psi(x)$. Rogner, J. Arch. Mth.

Ps. 45 (1866) 315-.

$-x \frac{d^{n} y}{d x^{n}}+\lambda \frac{d^{n-1} y}{d x^{n-1}}=\kappa\left(x \frac{d y}{d x}+\mu y\right)$. Spitzer, $S$.

Arch. Mth. Ps. 46 (1866) 25-.

$-a_{m+n} y^{(m+n)}+a_{m+n-1} y^{(m+n-1)}+\ldots+a_{m+1} y^{(m+1)}$ $+\left(a_{m}+x\right) y^{(m)}+a_{m-1} y^{(m-1)}+\ldots$ $+a_{1} y^{\prime}+a_{0} y=0$.

Spitzer, S. Arch. Mth. Ps. 47 (1867) 110-. $-\left(a_{2}+b_{2} x+c_{2} x^{2}\right) \frac{d^{2} y}{d x^{2}}+\left(a_{1}+b_{1} x\right) \frac{d y}{d x}+a_{0} y=0$.

Holmgren, H. Stockh. Ak. Hndl. 7 (186768) (No. 9) $58 \mathrm{pp}$.

$-\left(a_{n}+b_{n} x\right) \frac{d^{n} y}{d x^{n}}+\ldots+\left(a_{0}+b_{0} x\right) y=0$. Lětnikov, A. V. [1876] Rec. Mth. (Moscou) 14 (1890) 205-; Fschr. Mth. (1889) 333-.

$-\frac{d^{k} y}{d x^{k}}=a x^{m} y$. Molins, H. Toul. Ac. Sc. Mm. 8 (1876) 167-.

$-\frac{d^{2} V}{d x^{2}}+\frac{\mu+1}{x} \frac{d V}{d x}+V=0$. Worms de Romilly, $P$. Liouv. J. Mth. 4 (1878) 177-.

$-y^{\prime \prime}+P y^{\prime}+Q y=0$. Alexéeff, $N$. As. Fr. C. R. 8 (1879) 190-.

$-\frac{d^{2} y}{d x^{2}}+2 \cot x \cdot \frac{d y}{d x}-y=0 . \quad$ Graindorge, $J$.

(xII) Kharkov Mth. S. Com. (1880) 46-.

$-x^{2} y^{\prime \prime \prime}+A x y^{\prime \prime}+B y^{\prime}+C x^{\mu} y=0$. Aleksyeevskit,

$V . P$. (xII) Kharkov Mth. S. Com. (1883)

115-; Fschr. Mth. (1884) 288.

$-x y^{\prime \prime}+m y^{\prime}+n y x=0 . \quad$ Stodótkiewicz, A. J. [1883] (xII) Krk. Ak. (Mt.-Prz.) Rz. \& Sp. 11 (1884) 155-, xLIX-; Fsehr. Mth. (1884) 287.

$\frac{d^{n} y}{d z^{n}}+\frac{a}{z} \frac{d^{n-1} y}{d z^{n-1}}+\beta y=0 . \quad$ Alekséevskij, V.P.

Kharkov Mth. S. Com. (1884) 41-; Fschr.

Mth. (1884) 289-. 
of $\left(c_{2} x^{2}+b_{2} x+a_{2}\right) y^{\prime \prime}+\left(b_{1} x+a_{1}\right) y^{\prime}+a_{0} y=0$, application of Żmurko's improvement of Laplace's method. Rajewski, J. Krk. Ak. (Mt.-Prz.) Pam. 9 (1884) 120-; Fschr. Mth. (1884) 287-.

$-\Sigma\left(a_{8}+b_{8} x\right) \frac{d^{8} y}{d x^{8}}=0$. Nekrasov, P.A. [1889] Rec. Mth. (Moscou) 14 (1890) 344-; Fschr. Mth. (1889) 334.

$-\frac{d y}{d x}=X\left(a y^{2}+b y+c\right)+X_{1}$, where $X$ and $X_{1}$ are functions of $x$ and $a, b, c$, are constants. Stodótkiewicz, A. J. Prace Mt.-Fiz. 3 (1892) 52-; Fschr. Mth. (1892) 300.

Invariants. Cayley, A. QJ. Mth. 21 (1886) 257-.

-. Brioschi, F. Acta Mth. 14 (1890-91) 233-.

-. Mittag-Leffler, G. Crelle J. Mth. 114 (1895) 306-.

- associated with equations. Platts, C. QJ. Mth. 25 (1891) 300-.

- of general equation, and relation to theory of continuous groups. Bouton, C. L. Am. J. Mth. 21 (1899) 25-.

Irreducibility. Koenigsberger, L. Gött. Nr. (1883) 390-; Crelle J. Mth. 96 (1884) 123-; Berl. Ak. Sb. (1899) 672-; Mth. A. 53 (1900) 49-.

-, concept. Frobenius, G. Crelle J. 76 (1873) 236-.

- of homogeneous equations. Beke, E. Mth. A. 45 (1894) 278-.

Iterated operators, equations arising from. Gutzmer, A. Prag Sb. (1892) (Mth.-Nt.) 54-.

Kummer's equation. Forsyth, A. R. QJ. Mth. 19 (1883) 125-.

_-, analogue of. Goursat, É. C. R. 99 (1884) 777-, 858-.

- - of hypergeometric series. Goursat, É. Helsingf. Acta 15 (1888) 45-.

- - 3rd order, particular case. Cayley, A. Mess. Mth. 20 (1891) 75-.

Lagrange's determinantal equation of small oscillations, occurrence of equal roots in. Purser, F. B. A. Rp. (1878) 463-.

Lamé-Hermite equation. Brioschi, F. St Pét. Ac. Sc. Bll. 35 (1894) 449-.

- - equations for two variables, generalised. Krause, M. Leip. Mth. Ps. B. 50 (1898) (Mth.) 192-, 231-.

Lamé's equation. Pick, G. A. Wien Ak. Sb. 96 (1888) (Ab. 2) 872-.

- - generalisation. Palmstrøm, A. Berg. Ms. Aarb. (1897) No. 11, 9 pp.

- - Hermite's case. Klein, $F$. Mth. A. 40 (1892) 125-

- - , second solutions. Crawford, L. Edinb. Mth. S. P. 16 (1898) 57-.

$--\frac{d^{2} y}{d u^{2}}-\{n(n+1) \delta u+B\} y=0$. Palmstr $\phi m$, A. Christiania Skr. (Mth.-Nt. Kl.) (1894) No. 6, $12 \mathrm{pp}$.
Laplace's equation and formula of Abel. Cailler, C. Bll. Sc. Mth. 23 (1899) 26-.

Multiform functions belonging to group of linear substitutions. Wilczynski, E.J. Am. J. Mth. 21 (1899) 85-.

Operations in general, and equations of infinite order. Bourlet, C. Par. Ec. Norm. A. 14 (1897) 133-.

Origin of some equations. Brioschi, F. [1881] Rm. R. Ac. Linc. T. 6 (1882) 42-.

Oscillation theorems of Sturm and Klein. Bôcher, M. N. Y. Am. Mth. S. Bll. 4 (1898) 295-, 365-; 5 (1899) 22-.

Pfaffian equation, 2nd order, linear. Stodótkiewicz, A.J. Krk. Ak. (Mt.-Prz.) Rz. 13 (1886) 160-; Fschr. Mth. (1885) 315.

Picard's equations, reduction and integration. Naetsch, E. Leip. Mth. Ps. B. 48 (1896) 1-. Product of $k$ integrals of $k$ equations, property. Dillner, G. C. R. 92 (1881) 290-.

Reducibility. Fabry, E. C. R. 106 (1888) 732-; Par. S. Mth. Bll. 16 (1888) 135-.

- of homogeneous equations. Hamburger, $M$. Crelle J. Mth. 111 (1893) 121-.

Reduction to set of 1 st order equations. Weinstein, B. Arch. Mth. Ps. 69 (1883) 225-.

Reversible symbolic factors. Cockle, (Sir) Jas. [1867] QJ. Mth. 9 (1868) 242-.

Riemann's equation, theory. Bolza, $O$. Mth. A. 42 (1893) 526-.

- form systems on arbitrary algebraic configuration. Ritter, E. Mth. A. 47 (1896) 157-.

Schwarzian derivatives. Sylvester, J.J. Mess. Mth. 15 (1886) 74-.

\section{Solution.}

Hargreave, C. J. [1847] Phil. Trans. (1848) 31-.

De Morgan, A. Camb. and Dubl. Mth. J. 4 (1849) 137-.

Bronwin, B. [1850] Phil. Trans. (1851) 461-.

Graves, C. Ir. Ac. P. 6 (1853-54) 1-.

Jordan, C. C. R. 73 (1871) 787-.

analogous to Riccati's, of equations of form $\frac{d^{n} y}{d x^{n}}=x^{m} y$. Fields, J. C. [1886] J. H. Un. Cir. [6] (1886-87) 29-.

application of calculus of finite differences. Russell, W. H. L. QJ. Mth. 1 (1857) 23-.

cases. David, C. A. [1862] (xII) - Lille S. Mm. 9 (1863) 409-.

conditions that two equations should have $p$ common solutions. Lemonnier, $H$. C. R. 95 (1882) 476-.

conjugate, in differential and difference equations. Pincherle, S. Rm. R. Ac. Linc. Rd. 4 (1895) (Sem. 2) 228-.

by continued fractions. Laguerre, E. [187885] C. R. 87 (1878) 923-; Par. S. Mth. Bll. 8 (1880) 21-; Liouv. J. Mth. 1 (1885) 135-.

dependence on certain parameters. Fuchs, L. Berl. Ak. Sb. (1895) 905-.

depending on multiple types. Routh, E.J. L. Mth. S. P. 14 (1882-83) 144-. 
of equation in perturbations. Motoda, $T$. As. J. 9 (1890) 124-.

- equations with constant coefficients. Gregory, D. F. (vi Adds.) Camb. Mth. J. 1 (1839) $22-, 278$.

_- 2nd order, with linear coefficients. Graf, J. H. Mth. A. 45 (1894) 235-.

- - - - product of two. Besso, D. Rm. R. Ac. Linc. Mm. 19 (1884) 219-.

- - 3rd and 4 th orders, relations between. Campbell, D. F. N. Y. Am. Mth. S. Bll. 5 (1899) 327, 332-.

expression of arbitrary constant in terms of initial conditions. Routh, E.J. QJ. Mth. 19 (1883) 262-.

history. Eneström, G. Bb. Mth. (1897) 43-. integral rational, of equation, 2 nd order. Berger, A. Ups. S. Sc. N. Acta 15 (1895) No. 4, 28 pp.

method for large class of equations. Graves, C. Ir. Ac. P. 6 (1853-54) 34-.

periodic. Bendixson, I. Stockh. Öfv. (1896) 193-.

regular, of system of equations. Sauvage, - . C. R. 108 (1889) 174-.

representation near singular point. Grünfeld, E. Z. Mth. Ps. 36 (1891) 21-.

second special, of equation, 2nd order. Hoppe, R. Arch. Mth. Ps. 64 (1879) 379-.

in series. Hammond, J. L. Mth. S. P. $6(1874$ 75) $67-$.

- -, arithmetical nature of coefficients. Pincherle, S. Crelle J. Mth. 103 (1888) 84-; Palermo Cir. Mt. Rd. 2 (1888) 153-.

by symbols. Graves, C. Ir. Ac. P. 3 (1847) 536.

synthetic, and deformation. Cockle, (Sir) Jas. QJ. Mth. 23 (1889) 1-.

of system of equations. Gunther, S. Arch. Mth. Ps. 57 (1875) 240-.

Sturm's "theorem of comparison." Bôcher, M. N. Y. Am. Mth. S. Bll. 6 (1900) 96, 100.

Successive approximations method. Picard, $E^{\prime}$. Par. S. Mth. Bll. 22 (1894) 52-.

- - Zaremba, S. Liouv. J. Mth. 3 (1897) 311-.

System of (2) equations of which the one gives integrating factors of the other. Ricci, $G$. G. Mt. 15 (1877) 135-.

- - simultaneous equations. Méray, C. C. R. 81 (1875) 1203-.

Systems of equations identical with their adjoint. Goursat, É. C. R. 106 (1888) 187-. - - , 1st order. Koch, H. von. C. R. 116 (1893) 179-.

- - - , - - admitting only regular solutions. Griufeld, E. Wien. Az. 26 (1890) 66-.

- - with 1 independent variable. Horn, J. Mth. A. 39 (1891) 391-; 40 (1892) 527-.

- formed from equations with 1 independent variable. Darboux, G. C. R. 90 (1880) 524-, 596 -.

- of homogeneous equations. Sauvage, $L$. Toul. Fac. Sc. A. 8 (1894); 9 (1895) $100+$ 76 pp.
Systems of simultaneous equations, class. Ibach, L. F. N. A. Mth. 3 (1884) 172-.

- - - - - (Ibach). Tardy, P. N. A. Mth. 3 (1884) 257-.

Theorem. Russell, W. H. L. B. A. Rp. (1879) 263.

-. Darboux, G. C. R. 94 (1882) 1456-.

- of Fuchs, point in Jordan's proof. Gerbaldi, F. Rv. Mt. 1 (1891) 125-.

- Malmsten. Joachimsthal, F. Crelle J. 40 (1850) 48-.

Theorems. Besso, D. Rm. R. Ac. Linc. Mm. 10 (1881) 252-.

-. Bôcher, M. N. Y. Am. Mth. S. Bll. 6 (1900) 279-.

Theory, analytical, of phenomena depending on linear differential equations. Ménabréa, L. $F$. Tortolini A. 6 (1855) 363-.

Transcendental equation and equations, 2nd order, with periodic coefficients. Liapounoff, A. C. R. 128 (1899) 1085-.

- functions arising from linear differential equation, arithmetical properties. Hurwitz, A. Mth. A. 22 (1883) 211 -.

Transformation. Bronwin, B. Mathematician 3 (1850) 9-.

- Appell, P. C. R. 91 (1880) 211-.

-. Pincherle, S. Mil. I. Lomb. Rd. 25 (1892) 633-.

- of a biordinal of Schwarz. Cockle, (Sir) Jas. Mess. Mth. 11 (1882) 49-.

-, case. Cayley, A. Ph. Mg. 23 (1862) 266-. - of equation, any order. David, -. Par. S. Mth. Bll. 12 (1884) 36-.

— groups. Picard, É. C. R. 96 (1883) 1131-; 119 (1894) 584-.

- in simultaneous equations. Heymann, $W$. Crelle J. Mth. 98 (1885) 241-.

Transformations, algebraic groups, and equations. Picard, É. Toul. Fac. Sc. A. 1 (1887) A, 15 pp.

- of equation with constant coefficients, by substitution of new variable. Hansted, $B$. G. Teix. J. Sc. 2 (1880) 183-; (xII) Ts. Mth. 5 (1881) 5-.

-, formula of Halphen. Torelli, G. Nap. Rd. 29 (1890) 233-.

-, group, determination. Marotte, $F$. C. R. 124 (1897) 608-.

- of ordinals, new. Cockle, (Sir) Jas. Ph. Mg. 13 (1882) 44-.

-, rational. Goursat, É. Par. Éc. Norm. A. 2 (1885) 37-

Transformed equation, Euler's. Pincherle, $S$. Crelle J. Mth. 119 (1898) 347-.

- - , - of homogeneous equations of Fuchs's class. Schlesinger, L. Crelle J. Mth. 117 (1897) 148-.

$(D+a)^{n} y=X$. De Morgan, A. Camb. Mth. J. 4 (1845) 60-, 96.

$\frac{d^{2} y}{d t^{2}}=\frac{y}{\left(e^{t}+e^{-t}\right)^{2}}$. Besge, V.A. Liouv. J. Mth. 11 (1846) 96.

$y^{\prime \prime}+\frac{m}{x} y^{\prime}+n y=0 . \quad$ Lebesgue, $V . A$. Liouv. J. Mth. 11 (1846) 338-. 
$\frac{d^{2} y}{d x^{2}}+F(x) \cdot y=0$, and other equations. Brassinne, E. Toul. Mm. Ac. 2 (1846) 326-; 3 (1847) 204-; 1 (1863) 483-. $x^{n-1}\left(a_{n}+b_{n} x\right) y^{(n)}+x^{n-2}\left(a_{n-1}+b_{n-1} x\right) y^{(n-1)}+\ldots$ $+\left(a_{1}+b_{1} x\right) y^{\prime}+b_{0} y=0$.

Malmsten, C. J. Crelle J. 39 (1850) 99-. $y^{\prime \prime}{ }_{x}+\frac{y_{x}^{\prime}}{x}+A x^{m} y=0$. Malmsten, C. J. Crelle J. 39 (1850) 108-.

$\sum_{r=0}^{r=m}\left(a_{r}+b_{r} x^{\rho}\right) x^{(m-r)} y^{(m-r)}=\sum_{r=0}^{r=p} c_{r} x^{r \rho} . \quad$ Most,$R$. Z. Mth. Ps. 15 (1870) 427-.

$x y^{\prime \prime}+k y^{\prime}-y=0$. Le Paige, C. M. M. H. H. Brux. Ac. Bll. 41 (1876) 1011-. $x(1-x) \frac{d^{2} y}{d x^{2}}+\left(\frac{2}{3}-\frac{7}{6} x\right) \frac{d y}{d x}+\frac{1}{48} y=0$, solution. Cockle, (Sir) Jas. QJ. Mth. 17 (1881) 293-.

$x \frac{d^{2} y}{d x^{2}}+\frac{d y}{d x}+y(x+A)=0$, from theory of reflexion of sound. Sharpe, H. J. Mess. Mth. 10 (1881) 174-.

$x \frac{d^{2} y}{d x^{2}}+\frac{d y}{d x}+y(x-A)=\frac{d}{d x} \cdot \frac{\cos (x+l)}{x+l}$, from theory of sound. Sharpe, H.J. [1881] Mess. Mth. 11 (1882) 41-.

$\frac{d^{2} y}{d x^{2}}-\left(a e^{x}+2\right) \frac{d y}{d x}+y=0$. Florov, P. S. (xI)

Kharkov Mth. S. Com. (1883) 127-; Fschr. Mth. (1884) 288.

$\left(a+b x+c x^{2}\right)^{2} \frac{d^{2} v}{d x^{2}}+\left(a+b x+c x^{2}\right)\left(a_{1}+b_{1} x\right) \frac{d v}{d x}$ $+\left(a_{0}+b_{0} x+c_{0} x^{2}\right) v=0$.

Heymann, $W$. Z. Mth. Ps. 28 (1883) 241 -.

$\frac{d^{n} u}{d x^{n}}=x^{m} u$. Florov, P. S. Kharkov Mth. S. Com. (1885) 131- ; Fschr. Mth. (1886) 302. $\frac{d^{n} \omega}{d \xi^{n}}=e^{\xi} \omega$. Florov, P.S. Kharkov Mth. S. Com. (1887) 81- ; Fschr. Mth. (1887) 337-.

\section{Integration of ordinary linear equations by definite integrals. (See also 4430.)}

Mainardi, G. A. Sc. Lomb. Ven. 7 (1837) 185-.

Wantzel, L. C. R. 17 (1843) 1191-.

Spitzer, S. Schlömilch Z. 3 (1858) 224-; 4 (1859) 37-.

Steen, A. [1868-80] Sk. Nf. F. 10 (1869) 101-; Kjöb. Ov. (1880) 237-.

Pincherle, S. Bologna Ac. Sc. Mm. 2 (1891) 523-.

Schlesinger, L. C. R. 120 (1895) 1396-.

Bilinear relations between hypergeometric integrals. Hirsch, A. Mth. A. 52 (1899) 130 -.

Cases. Lobatto, R. Crelle J. 17 (1837) 363-.
Cases: Hansted, B. (xII) Ts. Mth. 4 (1880) 121-.

Differential and difference equations with 1 or more independent variables. Brajtzew, J.R. Fschr. Mth. (1900) 356.

Equation, 2nd order. Hoppe, R. Grunert Arch. 27 (1856) 55-.

,$--\ldots$, with linear coefficients. Thomae, $J$. Z. Mth. Ps. 14 (1869) 349-.

- with rational coefficients, relations due to definite integrals. Mellin, $H$. Helsingf. Acta 21 (1896) No. 6, 57 pp.

Hypergeometric equation. Lětnikov, A. V. Rec. Mth. (Moscou) 11 ( $\left.^{*} 1883-84\right)$ 327- ; Fschr. Mth. (1884) 273-.

Integrable equations. Nekrasov, $P$. A. Rec. Mth. (Moscou) 15 (1891) 277-, 608-; Mth. A. 38 (1891) 509-.

Integral, general, of

$$
\begin{array}{r}
x^{n} \frac{d^{n} y}{d x^{n}}+A_{1} x^{n-1} \frac{d^{n-1} y}{d x^{n-1}}+A_{2} x^{n-2} \frac{d^{n-2} y}{d x^{n-2}}+\ldots \\
+A_{n} y=f(x),
\end{array}
$$

formula for, by multiple definite integral. Aoust, -. C. R. 96 (1883) 775-.

Integration by definite integrals of product of 2 arbitrary functions, cases. Boussinesq, $J$. C. R. 94 (1882) 33-.

-, method. Petzval, J. Haidinger Ab. 1 (1847) 177-.

Reduction of integrals of equations, 1st order, in mixed differences, to simple definite integrals. Grosso, (abbate) R. del. [1852] (xII) Nap. Ac. Pont. At. 6 (1854) 323-.

Riccati's equation. Kummer, E. E. Crelle J. 12 (1834) 144-.

Simultaneous equations. Mellin, $H$. Acta Mth. 22 (1899) 41-.

Solutions of systems of equations by means of multiple integrals, Jacobi's formulæ. Vasilǐev, A. V. Kazan S. Nt. (Ps.-Mth.) P. 4 (1886) 39-; Fschr. Mth. (1886) 264-.

Spitzer's priority claims. Petzval, J. [1857] Wien SB. 28 (1858) 253-.

Tissot's equation. Pochhammer, L. Mth. A. 37 (1890) 512-.

Total differential equations. Du Bois-Reymond, P. [1868] Crelle J. 70 (1869) 299-.

$\frac{d^{n} y}{d x^{n}}=x^{m} y . \quad$ Kummer, E. E. Crelle J. 19 (1839) 286-.

$8 y^{\prime \prime}+(r+q x) y^{\prime}+\left(p+n x+m x^{3}\right) y=0 . \quad$ Spitzer, S. Grunert Arch. 23 (1854) 121-.

$x^{m} \frac{d^{n} y}{d x^{n}}= \pm y$. Spitzer, S. C. R. 48 (1859) 746-; Crelle J. 57 (1860) 82-.

$x^{m} \frac{d^{n} y}{d x^{n}}=a y . \quad$ Spitzer, S. $\quad$ C. R. 48 (1859) 995-.

$y^{\prime \prime \prime}=x y^{\prime}-n y . \quad$ Spitzer, S. Schlömilch Z. 7 (1862) 113-.

$x^{2} y^{\prime \prime \prime}=y$. Spitzer, S. Schlömilch Z. 8 (1863) $292-$.

$y^{(n)}=A x^{2} y^{\prime \prime}+B x y^{\prime}+C y . \quad$ Spitzer, S. [1870] Mth. A. 3 (1871) 453-. 


\section{General theory of ordinary equations, not linear, of the first order.}

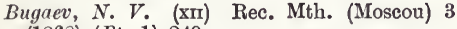
(1868) (Pt. 1) 249-.

Painlevé, P. C. R. 107 (1888) 221-, 320-, 724-; Par. Ec. Norm. A. 8 (1891) 9-, 103-, 201-, 267- ; 9 (1892) 9-, 101-, 283-.

Laurent, -. Par. Éc. Pol. J. 2 (1897) 1 -.

Algebraic equations. Rosanes, $J$. Mth. A. 3 (1871) 535-.

- - Vivanti, G. Palermo Cir. Mt. Rd. 4 (1890) 197-.

-. Wallenberg, G. Crelle J. Mth. 116 (1896) 1-; 122 (1900) 155.

- -, 1st degree. Darboux, G. Bll. Sc. Mth. As. 2 (1878) 60-, 123-, 151-.

$-\ldots, \ldots-$, with algebraic general primitive. Pincherle, S. Mil. I. Lomb. Rd. 10 (1877) 143-.

,-- 2nd degree, in 2 variables with general algebraic integrals. Casorati, F. Mil. I. Lomb. Rd. 7 (1874) 846-.

- - with general primitive. Casorati, $F$. Rm. R. Ac. Linc. T. 1 (1877) 185-.

- integrals. Autonne, L. C. R. 115 (1892) $587-$.

Approximations to solution. Trembley, $J$. Turin Mm. Ac. (1790-91) 53-.

Binomial equation. Petrovitch, $M$. C. R. 121 (1895) 632-.

Briot and Bouquet's equation, theory. Phragmén, $E$. Stockh. Öfv. (1891) 623-.

- - theory of equations. Horn, $J$. Crelle J. Mth. 113 (1894) 50-.

$---F\left(u, \frac{d u}{d z}\right)=0$. Cayley, $A$. L. Mth. S. P. 18 (1886-87) 314-.

Calculus of symbols, with application to nonlinear differential equations. Russell, W.H.L. R. S. P. 13 (1864) 423-.

Class of equations. Picard, É. Bll. Sc. Mth. As. 4 (1880) (Pt. 1) 416-.

- - Petrovitch, M. Palermo Cir. Mt. Rd. 14 (1900) 28-.

- - - 1st degree. Franklin, $F$. [1881] (xII) J. H. Un. Cir. [1] (1882) 165.

- - simultaneous equations. Guldberg, A. Christiania F. (1893) No. 18, 8 pp.

$p \bullet$ discriminant, and its connexion with general theory of envelopes. Chrystal, - Edinb. R. S. T. 38 (1897) 803-.

$c$ - and $p$-discriminants. Hill, M. J. M. [1888] L. Mth. S. P. 19 (1889) 561-.

Discriminants of algebraic differential equation, and its complete primitive. Torelli, $G$. A. Mt. 19 (1891-92) 254-.

Envelopes and singular solutions. Cockle, (Sir) J. [1877] Manch. Lt. Ph. S. P. 17 (1878) 13-; 21 (1882) 98-.

Equation of form $f\left(u, \frac{d u}{d z}\right)=0$. Fuchs, $L$. C. R. 93 (1881) 1063-.
Equation of form $\frac{d y}{d x}+P y^{m}+R y^{n}=Q$. Guld . berg, A. N. Mg. Ntvd. 31 (1890) 379-.

- Jacobi. Mansion, P. Brux. Ac. Bll. 44 (1877) 195-.

- - 1st order and Jacobi's equation. Elliot, - Par. Éc. Norm. A. 7 (1890) 101-.

- in any number of variables. Pittarelli, $G$. G. Mt. [13 (1875)] 323-.

Equations, 1st degree. Darboux, G. C. R. 86 (1878) 533-.

- with fixed branch-points. Wallenberg, $G$. Z. Mth. Ps. 35 (1890) 193-, 257-, 321-.

-, general integral of which is uniform, class.

Picard, É. C. R. 117 (1893) 603-.

- integral of which has form

$h(x)\left[y-g_{1}(x)\right]^{\lambda_{1}}\left[y-g_{2}(x)\right]^{\lambda_{2}} \ldots$

$$
\left[y-g_{n}(x)\right]^{\lambda_{n}}=C \text {. }
$$

Painlevé, P. Toul. Fac. Sc. A. 10 (1896) G, $37 \mathrm{pp}$.

-, and singularities of their algebraic integrals. Autonne, L. Par. Éc. Pol. J. 2 (1897) 51-; 3 (1897) 1-.

Eulerian factor and $M+N \frac{d y}{d x}=0$. Hansen, P. C. V. [1873] Kjöb. Dn. Vd. Selsk. Skr. 10 (1875) 435-.

General integrable forms of equations, and their criteria. Mayer, $A$. Leip. Mth. Ps. B. 42 (1890) 491-.

Homogeneous equations. Pullich, - - (xII) Ts. Mth. 4 (1874) 39-.

- - Frobenius, G. [1877] Crelle J. Mth. 86 (1879) 1-.

_- and Clairaut's equation. Mansion, $P$. Brux. Ac. Bll. 43 (1877) 169-.

- - - - C. Catalan, E. C. Brux. Ac. Bll. 43 (1877) 335-.

,$---\frac{1}{-}$. Folie, $F$. Brux. Ac. Bll. 43 (1877) 506-.

- functions of $n$ differentials. Lipschitz, $R$. Crelle J. 70 (1869) 71- ; 72 (1870) 1-.

Integrability. Peano, $G$. Tor. Ac. Sc. At. 21 (1885) 677-.

Integrable forms. Bugaev, N. V. [1869] (xI) Rec. Mth. (Moscou) 4 (1869-70) (Pt. 1) 61-.

Integral of equation in 2 variables; determination of properties. Woisard, J.L. Gergonne A. Mth. 14 (1823-24) 97-.

$-\frac{d y}{d x}=+\sqrt{y^{2}-\cos 2 x}$, property. Meissel, E. [1878] Arch. Mth. Ps. 65 (1880) 111.

Integrals, algebraic. Painlevé, P. C. R. 110 (1890) 945-; 112 (1891) 1190-.

-, - Automne, L. C. R. 113 (1891) 632- ; 114 (1892) 407-.

-, 一, degree, limitation. Autonne, L. C. R. 116 (1893) 132-, 1045-; Par. Éc. Pol. J. 63 (1893) 79-; 64 (1894) 1-.

- of algebraic equations, limits of extension. Lindelöf, E. Par. S. Mth. Bll. 27 (1899) 205-.

-, behaviour near singular point. Horn, $J$. Crelle J. Mth. 118 (1897) 257-; 119 (1898) 196-, 267-. 
Integrals, character. Fuchs, L. Berl. Ak. Sb. (1885) 5-.

-, form near critical points. Picard, É. Par. S. Mth. Bll. 12 (1884) 48-.

-, monogeneity. Bugaev, N. $V$. Kharkov Mth. S. Com. 5 (1896?) 89-; Fschr. Mth. (1896) 258.

-, multiform. Vivanti, G. A. Mt. 19 (189192) 29-.

-, particular. Cockle, (Sir) J. [1874] QJ. Mth. 13 (1875) 239-.

-, rational. Painlevé, P. C. R. 110 (1890) 34-. -, singular. Boon, A. J. Leijd. A. Ac. (1829-30) $66 \mathrm{pp}$.

-, - , criteria for. Veltmann, W. Arch. Mth. Ps. 58 (1876) 337-.

- of system of equations, nature. Cauchy, A. L. C. R. 40 (1855) 376-.

- - total algebraic differentials. Cayley, A. Bll. Sc. Mth. 10 (1886) 75-.

- - - - of 1st kind, $P d x+Q d y$; $f(x, y, z)=0$. Picard, E. Liouv. J. Mth. 1 (1885) 281-.

- - - differentials of 2 nd kind,

$$
P d x+Q d y ; f(x, y, z)=0 .
$$

Picard, É. C. R. 101 (1885) 734-; 102 (1886) 250-; Liouv. J. Mth. 2 (1886) 329 (bis)-.

Integrating factor. Laguerre, E. Par. S. Mth. Bll. 6 (1878) 124-.

- -. Stodótkiewicz, A. J. Krk. Ak. (Mt. Prz.) Rz. 7 (1895) 131-; Cre. Ac. Sc. Bll. (1893) 183-.

_. _, and form of integral of homogeneous equation of 1st order. Aleksyeev, N.N. (xII) Rec. Mth. (Moscou) 3 (1868) (Pt. 1) 260-.

Integration. Weyr, E. [1875] Prag Ab. 8 (1877) (Mth.) $43 \mathrm{pp}$.

- La Vallée Poussin, C. J. de. Brux. Mm. Cour. $8^{\circ}, 47$ (1892-93) No. 6, 82 pp.

-. Olearski, K. Krk. Ak. (Mt.-Prz.) Rz. 6 (1893) 131-, 434-; Crc. Ac. Sc. Bll. (1892) 371-.

-, algebraic, of equations of 1 st degree. Poincaré, H. Palermo Cir. Mt. Rd. 11 (1897) 193-.

- of algebraic equations of 1 st degree by linear functions. Casorati, F. Mil. I. Lomb. Rd. 11 (1878) 804-.

- - class of simultaneous equations. La guerre, E. (x) Par. S. Phlm. Bll. 6 (1869) 49-.

- - equations of 1st degree, theory. Steen, A. Kjöb. Ov. (1864) 45-.

- - not of 1st degree. Yanīshevskir, E. P. [1867] (xII) Kazan Un. Mm. 3 (1866) 149-. - - with rational coefficients of 2 nd degree. Winckler, $A$. Wien Sb. 64 (1871) (Ab. 2) 247-. - - - in several variables, complete in. tegral of which is single primitive equation. Minich, S. R. Ven. At. (1855-56) 794-.

- - a generalised Riccati's equation by continued fractions. Zimmerman, $V$. N. Rs. S. Nt. Mm. (Mth.) 10 (1889) 1-; Fschr. Mth. (1890) 325.

-, method. Mainardi, G. [1848] Mod. S. It. Mm. 24 (1850) 218-.
Integration, method of greatest and least exponents, additions to theory. Mazing, A. A. [1892] Rec. Mth. (Moscou) 16 (1893) 356-; Fschr. Mth. (1892) 280.

- of $Q d x+P y^{2} d x \pm d y=0$. Lorgna, $A$. $M$. Verona S. It. Mm. 3 (1786) 220 -

$-P \frac{d y}{d x}+Q=0, P$ and $Q$ being known functions of $x$ and $y$. Tychsen, C. (xII) Ts. Mth. 2 (1866) 17-.

- $\quad A(-d y)+B d x+C(x d y-y d x)=0$. Mansion, P. [1876] Mess. Mth. 6 (1877) 84-. Last multiplier of system of equations. Winckler, A. [1880] Wien Ak. Sb. 82 (1881) (Ab. 2) 628-.

Node- and cusp-loci enveloped by tangents at the cusps. Hill, M. J. M. L. Mth. S. P. 22 (1891) 216-.

Non-linear equations, cases. Liouville, $R$. Par. Éc. Pol. J. 57 (1887) 189-.

Polycritical points of equations of 1 st degree. Autonne, L. Lyon Un. A. 3 (Fasc. 1) (1892) $120 \mathrm{pp}$.

Primitive equations of derivatives of 1 st order. Hachette, J. N. P. (vi Adds.) Par. Éc. Pol. J. 5 (1804) 318-.

Reciprocal lines and surfaces. Bacaloglo, $E$. Grunert Arch. 36 (1861) 1-.

Reciprocity law between two solutions. Cauchy, A. L. C. R. 15 (1812) 200-.

Reduction to form $u d u+(R+S u) d \phi=0$. Gas paris, A. de. Nap. Rd. 7 (1848) 301-.

- - linear form, with reference to derivative of unknown function. Halphen, G. H. C. R. 87 (1878) 741-.

Riccatian equations. Florov, $P$. S. Kharkov Mth. S. Com. (1884) 5-; Fschr. Mth. (1884) 289.

- - cognate. Rawson, R. [1877] Mess. Mth. 7 (1878) 69-.

- - higher order. Wallenberg, G. Crelle J. Mth. 121 (1900) 196-.

\section{Riccati's equation.}

Liouville, J. [1833-40] Par. Éc. Pol. J. 22 cah. (1833) 1- ; Liouv. J. Mth. 6 (1841) 1-. Lommel, $E$. Grunert Arch. 40 (1863) 101-.

Hargreave, C.J. [1865] QJ. Mth. 7 (1866) 256-.

Cayley, A. Ph. Mg. 36 (1868) 348-.

Catalan, E. C. Brux. Ac. Bll. 31 (1871) 68-.

Glaisher, J. W. L. QJ. Mth. 11 (1871) 267-.

Genocchi, A. C. R. 85 (1877) 391-.

Meech, L. W. A. Mth. 1 (1884-85) 97-.

Fields, J. C. [1886] J. H. Un. Cir. [6] (188687) 29.

Cockle, (Sir) J. L. Mth. S.P.18 (1886-87) 180-. Meech, L. W. A. Mth. 3 (1887) 47-.

algebraic integrals. Autonne, L. C. R. 128 (1899) 410-.

-- , nature. Autonne, L. C. R. 96 (1883) 1354-.

analogues. Brassinne, $E$. Toul. Mm. Ac. 4 (1848) 234-; Liouv. J. Mth. 16 (1851) 255-. - Sincov, D. Kazan S. Ps.-Mth. Bll. 4 (1895) 1-; Fschr. Mth. (1893-94) 567. 
and its applications to chemistry. Petrowitch, M. Prag Sb. (1896) (Mth. Nt.) No. 39, $25 \mathrm{pp}$.

-Bessel's equation. Greenhill, A.G. QJ. Mth. 16 (1879) 294-.

differential equation allied to. Glaisher, J. W.L. [1872] QJ. Mth. 12 (1873) 129-.

extension of solution. Challis, H. W. [1864] QJ. Mth. 7 (1866) 51-.

generalisation. Aleksěevskij, $V . P$. Kharkov Mth. S. Com. (1884) 80-; Fschr. Mth. (1884) 290.

and its generalisation, double. Tilly, J. de. Brux. Ac. Bll. 9 (1885) 216-.

generalised form. Anisimov, $V$. A. Vars. S. Nt. Tr. (Mm.) 3 (1896) 33 pp. ; Fschr. Mth. (1896) 256.

integration. Zelinskij, I. I. Kazan S. Nt. (Ps.-Mth.) P. 8 (1890) 337-.

rational roots. Hill, C. J. D. Crelle J. 25 (1843) 22-.

solution, Cayley's, relations between particular integrals. Glaisher, J. W. L. Ph. Mg. 43 (1872) 433-.

- by continued fraction. Euler, L. [1780] St Pét. Ac. Sc. Mm. 6 (1818) 12-.

- of an equation allied to. Glaisher, J.W.L. B. A. Rp. (1878) 469-.

-, general, relations between definite integrals. Schläfli, L. A. Mt. 1 (1867-68) 232-.

theory, and properties of function satisfying. Feldblum, M. Fschr. Mth. (1898) 279-.

transformation, case. Rawson, R. [1882] Mess. Mth. 12 (1883) 34-.

and its transformations, and definite integrals satisfying them. Glaisher, J.W.L. Phil. Trans. 172 (1882) 759-.

Separation of variables. Russell, W. H. L. Camb. and Dubl. Mth. J. 9 (1854) 217-.

Simultaneous equations. Boole, G. Phil. Trans. (1862) 437-.

Singular points. Bendixson, I. Stockh. Öfv. (1898) 69-, 139-, 171-, 635-.

- - of algebraic equations. Möller, J. [1889] Stockh. Ak. Hndl. Bh. 15 (Afd. 1) (1890) No. 2, 31 pp.

- - theorem of Fuchs. Poincaré, H. Acta Mth. 7 (1885) 1-.

Singularities. Guldberg, A. N. Ts. Mth. 3 (B) (1892) 86-

-. Picard, ڤ́. Mth. A. 46 (1895) 521-.

Singularity, tests. Cockle, (Sir) J. [1875] QJ. Mth. 14 (1877) 146-.

\section{Solutions.}

complete, of equations in 2 variables, theory. Mayer, A. Mth. A. 37 (1890) 399-.

-, - - - - - - Hamburger, M. Mth. A. 41 (1893) 597-

decomposable. Cayley, A. Ph. Mg. 32 (1866) 379-.

of equations which can have equations of determined form for integrals. David, C. A. [1863] (xI) Lille S. Mm. 10 (1864) 165-. of Jacobi's equation

$$
L(x d y-y d x)-M d y+N d x=0 .
$$

Bes, K. [1894] N. Arch. Wisk. 1 (1895) 101-; Fschr. Mth. (1893-94) 556.

particular. Woisard, J.L. Metz Mm. Ac. 10 (1828-29) 310-.

-. Hansen, C. (xIr) Ts. Mth. 5 (1869) 119-.

-, of equations in 2 variables, elimination of foreign equations. Woisard, J. L. Gergonne A. Mth. 14 (1823-24) 365-.

-, use in search for general integral of equations of 1st degree. Darboux, G. C. R. 86 (1878) 584-.

singular. Timmermans, J.A. Brux. Ac. Sc. Mm. 15 (1842) 24 pp.

- Catalan, E. C. Par. S. Phlm. PV. (1846) 19-; Par. Ec. Pol. J. $31^{\ominus}$ cah. (1847) 271-.

- Houtain, L. Brux. A. Un. (1851-52) 971-

-. Cayley, A. QJ. Mth. 3 (1860) 36-.

-. Orlov, T. E. [1869] (XII) Rec. Mth. (Moscou) 4 (1869-70) (Pt. 1) 52-.

一. Darboux, G. (rx) Par. S. Phlm. Bll. 9 (1872) 180

- Mansion, P. Brux. Ac. Bll. 34 (1872) 149-

-. Zajaczkowski, W. Arch. Mth. Ps, 56 (1874) 175-

-. Jolnson, $W$. $W$. Des Moines Anal. 4 (1877) 1-.

-. Landré, C. L. N. Arch. Wisk. 3 ("1877) 1-.

- Delarue, D. M. [1879] (xп) Rec. Mth. (Moscou) 9 (1878-81) (Pt. 1) 501-.

Perry, H. M. (xII) J. H. Un. Cir. [1] (1882) 212.

- Rawson, R. [1882] (xI) Manch. Lt. Ph. S. Mm. 8 (1884) 170

-. Johnson, $W$. W. A. Mth. 3 (1887) 33-.

-. Amstein, $H$. Laus. S. Vd. Bll. 33 (1897) $22-$

-, of algebraic equations. Möller, $J$. Stockh. Öfv. (1887), 647-.

-, - - - Hamburger, M. Berl. Ak. Sb. (1899) 140-

- Cayley's theory, examples. Glaisher, $J$. W. L. [1882] Mess. Mth. 12 (1883) 1-.

-, of equations of 2 nd degree in 2 variables, theory. Casorati, $F$. Rm. R. Ac. Linc. At. 3 (1876) (Pte. 2) 160-; Rm. R. Ac. Linc. Mm. 3 (1879) 271-.

-, - - in 2 variables. Isely, J. P. [1878] Neuch. S. Sc. Bll. 11 (1879) 442-.

-, _ - _ - case. Raabe, J. L. [1848] Zür. Mt. 1 (Heft 3) (1848-49) 245-.

-, - _ _ - - theory, results. De Morgan, A. $[1850]$ (Ix) Camb. Ph. S. P. 1 (1866) 101-.

-, geometrically treated. Jïrgensen, C. Kiöb. Dn. Vd. Selsk. Afh. 5 (1832) $107-$.

-, and particular integrals. Walton, W. Camb. Mth. J. 2 (1841) 24-, 49-.

-, theory. Cayley, A. Mess. Mth. 2 (1873) 6-.

, Casorati, F. Mil. I. Lomb. Rd. 8 (1875) 962- 
singular, theory. Cayley, A. Mess. Mth. 6 (1877) 23-.

-, -. Hollman, P. J. N. Arch. Wisk. 7 (*1881) 59-, 150-.

special. Vasil'ev, A. V. (xIr) Kazan Un. Mm. [14] (1878) 309-.

Systems of equations. Escherich, G. von. Wien Ak. Sb. 108 (1899) (Ab. 2a) 621-.

Theorem. Radau, R. C. R. 66 (1868) 904-. - of Darboux, on equation

$$
P(x, y, z) d x+Q(x, y, z) d y=0 \text {. }
$$

Picard, $\dot{E}$. C. R. 100 (1885) 618-.

Theory. De Morgan, A. [1854] (Ix) Camb. Ph. S. P. 1 (1866) 136-.

- Bertrand, J. C. R. 45 (1857) 617-.

- of equations, 1st degree. Autonne, L. Par. Éc. Pol. J. 61 (1891) 35-; 62 (1892) 47-.

-, general. Peano, G. Tor. Ac. Sc. At. 33 (1897) 9-

-, paradox in. Tanner, H.W.L. Mess. Mth. 10 (1881) 158-.

Transcendental integrals of equations with coefficients of 2nd degree. Winckler, $A$. [1881] Wien Ak. Sb. 84 (1882) (Ab. 2) 940-.

Transcendents. Königsberger, $L$. Acta Mth. 3 (1883) 1-.

Transformation. Russell, W. H. L. Camb. and Dubl. Mth. J. 9 (1854) 81-.

-. Źorawski, K. Krk. Ak. (Mt.-Prz.) Rz. 6 (1893) 67-; Crc. Ac. Sc. Bll. (1892) $292-$.

$\frac{d u}{d z}=f(u, z)$, proof of theorem of Cauchy. Ascoli, G. [1871] A. Mt. 5 (1871-73) 14-. $x \phi\left(y^{\prime}\right)+y \psi\left(y^{\prime}\right)+\chi\left(y^{\prime}\right)=0$. Heymann, W. [1878] Z. Mth. Ps, 24 (1879) 252-.

$\frac{d y}{d x}=1+\frac{R(x)}{y}$. Sonin, N. Ja. [1894] St Pét. Ac. Sc. Bll. 2 (1895) 93-; 3 (1895) 339-.

\section{General theory of ordinary equations, not linear, of order higher than first.}

Abridged form of equations. Fais, A. G. Mt. 12 (1874) 320-.

Addition theorem. Mittag-Leffler, $G$. B. A. Rp. (1894) 561 .

Algebra and group theory of equations. Loewy, A. Mh. Mth. Ps. 8 (1897) 225-

Class of equations. Picard, É. C. R. 104 (1887) 41-.

- _ - including those of all geodesics. Liouville, $R$. C. R. 105 (1887) 1062-.

Construction of equations of form

$$
\frac{1}{z} \frac{d^{2} z}{d x d y}=\lambda(x, y),
$$

admitting an explicit general integral. Moutard, T. Par. Éc. Pol. J. Cah. 45 (1878) 1-.

Equation of algebraic curve. Chrystal, $G$. Edinb. Mth. S. P. 3 (1885) 95-.
Equation connected with $y^{n}-n y+(n-1) x=0$. Cayley, A. [1862-66] Manch. Lt. Ph. S. Mm. 2 (1865) 111-; Ph. Mg. 32 (1866) $379-$

-, 2nd order. Enneper, A. Z. Mth. Ps. 15 (1870) 56-.

-, $n$th order, 2 variables, conditions for derivation from equation of $(n-1)$ th order. Raabe, J. L. Crelle J. 31 (1846) 181-.

Equations, general integral of which is uniform. Painlevé, P. Par. S. Mth. Bll. 28 (1900) 201-.

- - - - - class. Picard, É. C. R. 110 (1890) 877-

-, 2 general, of 2 nd order, $n$th degree, derived from the known linear equation. Rawson, $R$. Mess. Mth. 2 (1873) 185-.

- integrable by meromorphic doubly periodic functions. Petrovitch, $M$. Acta Mth. 22 (1899) 379-.

- integral of which has finite number of determining values. Painlevé, P. C. R. 116 (1893) 88-, 173-.

- in lunar theory, two. Cayley, A. [1871] As. S. M. Not. 32 (1872) 31-, 201-.

,- 2nd order. Vessiot, E. Toul. Fac. Sc. A. 9 (1895) F, 26 pp.

- _ _, class. Wallenberg, $G$. Crelle J. Mth. 120 (1899) 113-.

_,$\ldots \ldots$, with fixed critical point and uniform correspondence of surfaces. Painlevé, $P$. C. R. 117 (1893) 611-.

-, - and 3rd orders, normal form. Pick, G. Mth. A. 38 (1891) 139-.

-, 3rd order, case. Brioschi, F. R. S. P. 27 (1878) 126-.

_- _ - Schwarzian derivative. Klein, C. F. Leip. Mth. Ps. B. 35 (1883) 1-.

- possessing fundamental first integrals. Guldberg, A. Christiania F. (1894) No. 1, 41 pp. ; Fschr. Mth. (1893-94) 544-.

Homogeneous equations, 2nd order. Steen, $A$. (xiI) Ts. Mth. 1 (1883) 11-.

- - - - Appell, P. Toul. Fac. Sc. A. 3 (1889) K, 12 pp.

- - , - - Wallenberg, G. Crelle J. Mth. 119 (1898) 87-.

Integrability. Bach, -, \& Stoffel, -. Liouv. J. Mth. 7 (1862) 49-.

-, conditions in quadratures of equation of 2nd order. Yurev, S. A. [1869] (xII) Rec. Mth. (Moscou) 4 (1869-70) (Pt. 1) 246-.

Integrals, asymptotic representation for large real values of argument. Kneser, A. Crelle J. Mth. 116 (1896) 178-; 117 (1897) 72-; 120 (1899) 267-

- common to 2 systems. Capelli, $A$. Nap. Rd. 39 (1900) 100-

- - - several systems. Pennacchietti, G. Catania Ac. Gioen. At. 3 (1891) 1-.

- form near critical points. Picard, $\dot{E}$. C. R. 87 (1878) 430-, 743-.

-, singular. Zajaczkovski, W. [1873]

Krk. Ak. (Mt.-Prz.) Pam. 1 (1874) 45-。

Integrating factor of equations of form $A+B y^{\prime}+C y^{\prime m}+D y^{\prime m+1}+E y^{\prime m-1} y^{\prime \prime}=0$. Andreevskǐ, M. A. (xII) Rec. Mth. (Moscou) 4 (1869-70) (Pt. 1) 143-. 


\section{Higher Orders}

Integration. Malmsten, $C . J$. Stockh. Ak. Hndl. 3 (1859-60) 94 pp.; Liouv. J. Mth. 7 (1862) 257-.

-. Schapira, Hälfte 1) 15-.

_, application of differential quotients with general index. Most, $R$. Z. Mth. Ps. 16 (1871) 190-.

- of equation of conics. Prévost, G. Bordeaux S. Sc. PV. (1897-98) 47-。

- equations, 2nd order. Svanberg, A. F. Stockh. Ak. Hndl. (1833) 1-; Ups. N. Acta S. Sc. 1 (1851) 261-.

- _ - _ - Guldberg, A. Christiania F. (1895) No. 6, 48 pp.; Fschr. Mth. (1896) 249-.

- - - cases. Winckler, A. [1874] Wien Ak. Sb. 70 (1875) $(A b .2)$ 149-.

- - - - - - - Pajak, S. Prace Mt.Fiz. 11 (1900) 32-; Fschr. Mth. (1900) 350-. - - - _ - class. Strong, $T$. Silliman J. 42 (1842) 273-.

- _ - _ - - by factors. Andréievosky, C. R. 68 (1869) 716-.

- $-\ldots, \ldots$, with 2 variables. Tychsen, $C$. Mth. Ts. 6 (1864) $1-$.

- - - of high order. Cardinali, F. [1806] Mod. Mm. S. It. 13 (1807) 381-.

$-P \frac{d^{2} y}{d x^{2}}+Q\left(\frac{d y}{d x}\right)^{2}+R \frac{d y}{d x}+S=0, P, Q, R$, $S$ being functions of $x, y$. Tychsen, $C$. Mth. Ts. 5 (1863) 173-.

$-\frac{d^{2} \rho}{d v^{2}}+\left(1-\beta_{1}\right) \rho=\beta_{0}+$ etc. Backlund, J. O. As. Nr. 103 (1882) 323-.

Irreducible functions, properties. Königsberger, L. Crelle J. Mth. 95 (1883) 171-.

Last multiplier. Winckler, A. [1879] Wien Ak. Sb. 80 (1880) (Ab. 2) 948-

Polynomials satisfying differential equation, 3rd order. Appell, P. As. Fr. C. R. 8 (1879) 253-.

Representation of functions, between certain limits, by series of values of function satisfying equation of 2 nd order. Picart, $A$. N. A. Mth. 2 (1883) 109-.

Singularities depending on integral constants. Fransén, A. E. Stockh. Öfv. (1897) 317-.

-, essential. Painlevé, P. C. R. 116 (1893) $362-$.

-, -. Picard, É. C. R. 116 (1893) 365; Acta Mth. 17 (1893) 297-.

Solutions, particular, of equations, 2nd order, in 2 variables. Weiler, $A$. Grunert Arch. 32 (1859) 286-.

-, rational fractional. Andreev, $K$. $A$. Kharkov Mth. S. Com. 4 (1895) 150-; Fschr. Mth. (1893-94) 537.

- _ - Markov, A. A. Kharkov Mth. S. Com. 4 (1895) 175-.

_, - - - Bugaev's method compared with others. Imšeneckij, $V$. G. Kharkov Mth. S. Com. 4 (1895) 60-; Fschr. Mth. (189394) 537.

-, - - - - - - -. Markov, A. A. Kharkov Mth. S. Com. 4 (1895) 146-; Fschr. Mth. (1893-94) 537.

\section{Differential Forms 5200}

Solutions, singular. Girault, C. Caen Ac. Mm. (1855) 211-.

-, - Cockle, (Sir) Jas. QJ. Mth. 12 (1873) $305-$.

, - Hollman, P.J. N. Arch. Wisk. 8 (*1882) 23-.

-, 一. Mitchell, H. Edinb. Mth. S. P. 16 (1898) 64-.

-, -, general theory. Zajuczkonoski, W. (xII) Krk. Ak. (Mt.-Prz.) Pam. 3 (1877) 1-.

-, -, geometric meaning. Fine, H. B. Am. As. P. (1887) 64.

Systems with fixed critical points. Painlevé, $P$. C. R. 130 (1900) 767-.

Theory, history, 1878-93. Craig, $T$. N. Y. Mth. S. Bll. 2 (1893) 119-.

Transformation. Cockle, (Sir) J. [1881] Mess. Mth. 11 (1882) 109-.

- of equation of even order to isoperimetric form. Imchenetsky, $V$. [1886] St Pét. Ac. Sc. Bll. 31 (1887) 283-.

$P \frac{d^{2} y}{d x^{2}}+a \frac{d P}{d y}\left(\frac{d y}{d x}\right)^{2}+R=0 . \quad$ Pendlebury, $R$. Mess. Mth. 5 (1871) 90-.

$\frac{d}{d r}\left(\frac{r^{2}}{\rho} \frac{d \rho}{d r}\right)=\frac{-4 \pi}{k} r^{2} \rho . \quad$ Tait, P. G. Edinb.

Mth. S. P. 4 (1886) 23-.

$y^{\prime \prime}=A y^{3}+B y^{2}+C y+D+(E y+F) y^{\prime}$. Fransén, A. E. Stockh. Öfv. (1895) 223-; Fschr. Mth. (1895) 374-.

\section{Differential forms and differential invariants.}

\section{General.}

Differential functions. Guérin, V. C. R. 60 (1865) 519-.

- operators, some families. Perrin, R. C. R. 106 (1888) 1131-.

- polynomials, theorem on class. Chini, M. Ven. I. At. (1893-94) 872-.

Exponential differences and differentials, Wronski's theory. Krauze, $W$. Prace Mt.Fiz. 5 (1894) 160-; Fschr. Mth. (1893-94) 459-.

- - - - - Dickstein, S. Prace Mt. Fiz. 5 (1894) 169-; Fschr. Mth. (1893-94) 459-.

Integrability conditions. Kronecker, $L$. Crelle J. 59 (1861) 311-.

Integration of algebraic differential expressions in finite form. Petersen, J. Gött. Nr. (1878) 68-.

_ - differential forms, five. Martin, Art. Des Moines Anal. 4 (1877) 175-.

_ - - formulæ, two. Malfatti, G. F. Turin Mm. Ac. 4 (1788-89) 53-.

- exact differentials. Combescure, É. Mntp. Ac. Mm. 11 (1892) 59-.

- method, Bertrand's, application to total differential equation in many variables. Stodótkiewicz, A. J. (xII) Krk. Ak. (Mt. Prz.) Pam. 8 (1883) 137-.

Invariant differential operators. Capelli, A. Nap. Rd. 26 (1887) 110-. 
Linear congruences. Guldberg, A. Christiania Skr. (Mth. Nt. Kl.) (1897) No. 10, 23 pp.

- differential expressions, adjoint. Frobenius, G. [1877] Crelle J. Mth. 85 (1878) 185-.

Primary forms. Cockle, (Sir) Jas. Ph. Mg. 49 (1875) 134-; 9 (1880) 348-.

Surfaces with infinitesimal and linear transformations. Lie, M. S. Arch. Mth. Ntvd. 7 (1882) 179-.

Transformation of variables. Gasparis, A. de. Nap. Ac. At. 7 (1878) No. 15, 8 pp.; 8 (1879) No. 2, 16 pp.; Rm. R. Ac. Linc. T. 2 (1878) $192-$.

\section{Linear differential forms; Pfaffians.}

Algebraic integral of two differential equations. Roberts, R. A. [1890] L. Mth. S. P. 22 (1891) 28-

- integrals of differential equations, 1st order and degree. Poincaré, H. Palermo Cir. Mt. Rd. 5 (1891) 161-。

Binomial differentials. Combescure, $\dot{E}$. Bll. Sc. Mth. 11 (1887) 245-.

- - Kapteyn, W. Bll. Sc. Mth. 12 (1888) 44-.

Common multiples of linear differential expressions. Heffer, L. Crelle J. Mth. 116 (1896) 157-.

Condition that total differential equation

$$
P d x+Q d y+R d z+\text { etc. }=0
$$

admit of single primitive. Van Velzer, C.A. [1879] (xII) J. H. Un. Cir. [1] (1882) 35.

- - - be integrable. Lovett, E. O. A. Mth. 1 (1900) 175-.

Differential expressions and Pfaff's problem. Cartan, É. Par. Éc. Norm. A. 16 (1899) 239-.

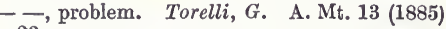
23-.

— forms. Combescure, É. C. R. 70 (1870) 1164-.

- parameters, 1st order. Banal, R. G. Mt. 30 (1892) 235-.

- problem, theory. Lie, S. Christiania F. 14 (1872) 132-.

- systems, with uniform general integral. Painlevé, P. C. R. 131 (1900) 497-, 534.

Equation $x d x+x_{1} d x_{1}+x_{2} d x_{2}+\ldots+x_{n} d x_{n}=0$ integrable by a primitive equation. Zajaczkovoski, $W$. Par. T. Nauk Śc. Pam. 6 ( $\left.{ }^{*} 1875\right)$ Art. 4, 14 pp.

Equations of form $d z=Z(U d x+V d y)$, where $U, V, z$, are functions of $x$ and $y$, and $Z$ a function of $z$. Hansen, C. (xח) Ts. Mth. 4 (1868) 188-.

Exact homogeneous differentials. Combescure, É. Bll. Sc. Mth. 11 (1887) 243-.

Integrability conditions. Laurent, $H$. N. A. Mth. 6 (1887) 274-, 305-.

- of certain differential expressions. Sincov, D. Kazan S. Ps.-Mth. Bll. 9 (1900) 38-.
Integrating factors. Saurel, $P$. J. Ps. C. 2 (1898) 116-.

Integration of equation, 1 st order. Petersen, $J$. Mth. Ts. 1 (1859) 90-.

- total differential equations. Bertrand, $J$. C. R. 83 (1876) 1191-.

_ - - - Du Bois-Reymond, P. Mth. A. 12 (1877) 123-.

- _ - 1st order and degree. Fais, A. G. Mt. 13 (1875) 344-.

Bologna Rd. (1893-94) 66-

$--d z=H d x+K d y+L d p+M d q+N d r+$ etc. Raabe, J. L. Crelle J. 14 (1835) 123-.

$--x_{1} d x+x_{2} d x_{1}+x_{3} d x_{2}+x d x_{3}=0$. Spitzer S. Grunert Arch. 23 (1854) 453-; 30 (1858) 83.

$--(A+C z) d x+(B+D z) d y+K d z=0$. Sonin, N. Y. $[1870]$ (xII) Rec. Mth. (Moscou) 6 (1872-73) (Pt. 1) 278-.

- $-d v=M d x+N d y+P d z$. Perevoščikov, D. M. St Pet. Ac. Sc. Mm. (Rs.) 22 (*1873) 201-.

$--X_{1} d x_{1}+X_{2} d x_{2}+\ldots+X_{n} d x_{n}=0$. Stodótkiewicz, $A$. J. Prace Mt.-Fiz. 7 (1896) 6-; Fschr. Mth. (1896) 271.

Linear differential forms. Pincherle, S. Rm. R. Ac. Linc. Rd. 1 (1892) (Sem. 1) 273-.

- - theory. Bortolotti, E. A. Mt. 23 (1895) 309-.

- equations with total differentials. Bianchi, L. Rm. R. Ac. Linc. Rd. 5 (1889) (Sem. 1) 312-.

MacMahon's differential equation. Cayley, $A$. QJ. Mth. 19 (1883) 182-.

Normals to surface. Bertrand, J. C. R. 17 (1843) 1277-.

Operation $\Omega$ having reference to Cayley's functions $A(x)$. Cayley, $A$. QJ. Mth. 13 (1875) 369-.

- - (Cayley's), reduction to polar operations. Capelli, A. Mth. A. 29 (1887) 331-.

Pfaffian equation, infinitesimal automorphic transformations. Vivanti, $G$. Palermo Cir. Mt. Rd. 12 (1898) 1-.

,----- Weber, E. von. Palermo Cir. Mt. Rd. 12 (1898) 133-.

- - - transformations. Engel, $F$. Leip. Mth. Ps. B. 51 (1899) (Mth.) 296-.

- - integration, Clebsch's 2nd method. Brill, J. L. Mth. S. P. 31 (1900) 315-.

- - -, general. Sauer, C. G. Oken Isis (1825) 1294.

- - , shortest integral curves. Lilienthal, $R$. von. Mth. A. 52 (1899) 417-.

- $\Sigma X d x=0$. Russjan, C. Fschr. Mth. (1896) 269-

- equations. Lovett, E. O. C. R. 129 (1899) 274 .

- - and line complexes. Weber, E: von. [1900] Münch. Ak. Sb. 30 (1901) 393-. - of special type, systems. Brill, J. Mess. Mth. 26 (1897) 183-

- -, system. Rusjan, C. K. Fschr. Mth. (1899) 315-.

- - - - normal number of complete integrals.' Rusjan, C. K. Fschr. Mth. (1900) 367. 
Pfaffian equations, systems. Weber, E. von. Münch. Ak. Sb. 25 (1896) 423-.

- - - - Brill, J. QJ. Mth. 30 (1899) 221-.

- -, - Kowalewski, G. Leip. Mth. Ps. B. 51 (1899) (Mth.) 265-.

,--- , invariant theory. Engel, $F$. Leip. Mth. Ps. B. 41 (1889) 157-; 42 (1890) 192-.

,,$----\ldots$. Weber, E. von. Leip. Mth. Ps. B. 50 (1898) (Mth.) 207-.

- expressions and equations. Lie, S. Leip. Mth. Ps. B. 48 (1896) 405-.

- , multilinear differential covariants. Brill, J. L. Mth. S. P. 30 (1899) 263-.

- having a vacant minor, development. Muir, T. [1900] Edinb. R. S. T. 40 (1905) 49-.

- system, reducibility to given number of terms. Weber, E. von. [1900] Münch. Ak. Sb. 30 (1901) 273-, 434.

- systems and line geometry. Weber, E. von. Leip. Mth. Ps. B. 52 (1900) 179-.

- theorem, extension. Hamburger, $M$. Crelle J. Mth. 110 (1892) 158-.

Pfaffians, functions allied to. Tanner, H.W. L. QJ. Mth. 16 (1879) 34-.

-, property. Baker, H. $F$. $\quad$ L. Mth. S. P. 29 (1898) 141-.

-, theorem. Tanner, H.W. L. [1878] Mess. Mth. 8 (1879) 56-.

-, -. Zajaczkowski, W. (xII) Krk. Ak. (Mt.Prz.) Rz. \& Sp. 7 (1880) 67-; (xI) Mess. Mth. 10 (1881) 36-.

Pfaff's integration method for equation in $2 n$ variables. Jacobi, C. G. J. Crelle J. 2 (1827) 347-.

------ Binet, J. P. M. C. R. 14 (1842) 1049-; 15 (1842) 74-.

- _ - , proof of theorem relative to. Zajaczkowski, L. Arch. Mth. Ps. 47 (1867) 106-.

- problem. Frobenius, G. [1876] Crelle J. Mth. 82 (1877) 230-.

- - Hamburger, M. [1876] Arch. Mth. Ps. 60 (1877) 185-.

- - Darboux, G. Bll. Sc. Mth. As. 6 (1882) 14-, 49-; C. R. 94 (1882) 835-.

- - Morera, G. [1883] Tor. Ac. Sc. At. 18 (1882) 521-.

- -. Stodótkiewicz, A. J. [1892] Prace Mt.Fiz. 4 (1893) 56-; C. R. 115 (1892) 592-; 119 (1894) 489-.

---. Engel, F. Leip. Mth. Ps. B. 48 (1896) $413-$

- Zantschewsky, J. Par. Éc. Norm. A. 13 (1896) 267-.

- - generalisation. Tanner, H. W. L. L. Mth. S. P. 11 (1879-80) 131-.

- - integration method. Lie, S. Christiania F. 15 (1873) 321-.

- - Pfaff's solution. Mayer, A. C. G. Mth. A. 17 (1880) 523-.

-, , theorem of Jacobi. Cayley, A. Crelle J. 57 (1860) 273-.

- -, theory. Lie, M. S. Arch. Mth. Ntvd. 2 (1877) 338-.

- transformation. Rusjan, C. Prace Mt.-Fiz. 8 (1897) 61-; 9 (1898) 61-; Fschr. Mth. (1897) 300-; (1898) 303.
Solutions, singular, of total differential equations. Guldberg, A. Christiania Skr. (Mth.Nt. Kl.) (1899) No. 4, 26 pp.

Theorems. Le Pont, H. G. Teix. J. Sc. 8 (1887) 37-, 65-, 175-.

Theory of system of equations. Stodolkievitz, A. J. C. R. 120 (1895) 825-.

Total differential (ternary) equations. Cockle, (Sir) Jas. [1876] Manch. Lt. Ph. S. P. 16 (1877) 66-.

- - (-) - Rawson, R. Manch. Lt. Ph. S. P. 16 (1877) 114 .

$--(-)-$ Cockle, (Sir) Jas. Manch. Lt. Ph. S. P. 20 (1881) 119-.

- - equations. Guldberg, A. Fschr. Mth. (1900) 368.

- - - , application of $2 n$-periodic functions to. Grömwall, H. Stockh. Öfv. (1896) 295-; Fschr. Mth. (1896) 285-.

- - - defining arithmetico-geometric mean of 4 elements. Borchardt, C. W. Par. S. Mth. Bll. 7 (1879) 124-.

- - - reduction from 3 to 2 variables. Minich, S. R. [1879] Rm. S. It. Mm. 4 (1882) No. 1, 47 pp.

- - _, singularities. Grönwall, $H$. Stockh. Ak. Hndl. Bh. 24 (Afd. 1) (1899) No. 9, $22 \mathrm{pp}$.

- - - system. Stodótkiewicz, A.J. Krk. Ak. (Mt.-Prz.) Rz. 2 (1892) 299-; Crc. Ac. Sc. Bll. (1891) 283.

- - - - - integration. Bendixson, $I$. Stockh. 'Öfv.' (1892) 271-.

- _ - systems. Grömwall, H. Stockh. Öfv. (1895) 729-; Fschr. Mth. (1895) 399.

- - - - - Hadamard, -. Bordeaux S. Sc. PV. (1894-95) 17-.

- - unconditionally integrable. Guldberg, A. Mh. Mth. Ps. 7 (1896) 332-.

- - - in 3 or more variables, primitive equation. Paoli, P. [1814] Mod. S. It. Mm. 17 (1815) 104-.

- differentials of 1 st kind. Noether, $M$. Erlang. Ps. Md. S. Sb. 18 (1886) 11-.

- - - - - integrals, quartic surfaces admitting. Berry, A. Camb. Ph. S. T. 18 (1900) 333-.

- and partial differential equations. Natani, L. [1860] Crelle J. 58 (1861) 301-.

Transformation of linear differential expression. Tanner, H. W. L. QJ. Mth. 16 (1879) 45-.

$X^{-\frac{3}{3}} d x+Y^{-\frac{2}{3}} d y+Z^{-\frac{2}{3}} d z=0$. MacMahon, $P$. A. [1882] QJ. Mth. 19 (1883) 158-.

$X_{1} d x_{1}+X_{2} d x_{2}+\ldots+X_{n} d x_{n}=0$, integrability conditions. Stodótkiewicz, A.J. Prace Mt.Fiz. 6 (1895) 20-; Fschr. Mth. (1895) 378.

$\Sigma a_{i} d x_{i}=0, \Sigma b_{i} d x_{i}=0 . \quad$ Duport, 一. Liouv. J. Mth. 3 (1897) 17-.

$X_{1} d x_{1}+X_{2} d x_{2} \ldots$, canonical forms. Roussiane, C. Mth. A. 50 (1898) 247-. 


\section{Differential forms of the second and higher orders. (See also 8450.)}

Clairautian functions and equations. Cunningham, A. [1876] R. S. P. 25 (1877) 43-.

Differential criticoid. Cockle, (Sir) Jas. Ph. Mg. 50 (1875) 440-.

- forms of infinitely high order. Zorawski, K. Krk, Ak. (Mt.-Prz.) Rz. 6 (1893) 419-; Fschr. Mth. (1893-94) 456-.

- parameter of 2 nd order, properties. Haton de la Goupillière, -. C. R. 101 (1885) 18-.

- parameters. Somigliana, C. Mil. I. Lomb. Rd. 22 (1889) 275-.

- - of functions. Combescure, $\boldsymbol{E}_{\text {. }} \quad$ Par. Ec. Norm. A. 7 (1878) 409-.

- _ _ - especially $\nabla_{2}$, definition. Boussinesq, J. C. R. 95 (1882) 479-.

- - theory. Beltrami, E. Bologna Ac. Sc. Mm. 8 (1868) 549-; Bologna Rd. (1869) 55-. - quadratic form, second parameter. Běljankin, I. [1900] Kazan S. Ps.-Mth. Bll. 10 (1901) 181-.

- - forms, classification. Ricci, G. Rm. R. Ac. Linc. Rd. 4 (1888) (Sem. 1) 203-.

- - , parameters and invariants. Ricci, $G$. A. Mt. 14 (1886-87) 1-.

- - , simultaneous transformation. Knoblauch, J. Crelle J. Mth. 115 (1895) 185-.

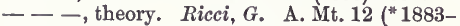
84) 135 -

,,--- Tannenberg, W. de. C. R. 119 (1894) 321-

- quadratics, invariants. Maschke, $H$. N. Y. Am. Mth. S. T. 1 (1900) 197-, 508.

Differentials of functions of several independent variables. Goursat, É. C. R. 101 (1885) 309-.

Homogeneous expressions of higher order in several variables. Andreevskǐ, M. A. [1869] (xII) Rec. Mth. (Moscou) 4 (1869-70) (Pt. 1) $105-$.

Invariable expressions. Padova, E. Rm. R. Ac. Linc. Mm. 4 (1887) 4 -.

Quadratic forms of $n$ differentials. Lipschitz, $R$. Crelle J. 71 (1870) 274-.

- - - - - , and Abel's transcendents. Lipschitz, R. [1871] Crelle J. 74 (1872) $150-$.

- and linear differential forms, invariants. Hessenberg, G. Acta Mth. 23 (1900) 121-.

Ternary quadratic differential forms, canonical types. Koenigs, G. C. R. 100 (1885) 789-.

Total differential equations. Guldberg, A. C. R. 126 (1898) 1335-; 127 (1898) 1199-.

- - - 2nd order. Guldberg, A. Christiania Skr. (Mth.-Nt. Kl.) (1898) No. 11, 24 pp.

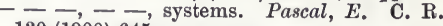
$130(1900) 645-$

- - 3rd order, theory. Pascal, E. Mil. I. Lomb. Rd. 33 (1900) 675-.

- - , any order. Pascal, E. Mil. I. Lomb. Rd. 33 (1900) 287 -

\section{Transformation of differen- tial forms, including tangential (or contact) transformations.}

Adjoint group, theory. Ahrens, $W$. Leip. Mth. Ps. B. 49 (1897) 358-.

- operation. Pincherle, S. Bologna Rd. 2 (1898) 130 -

Algebraic configurations with continuous integrable group of projective automorphic transformations. Fano, $G$. Ven. I. At. (1895-96) 1069-.

_ _ _ non-integrable group of projective automorphic transformations. Fano, G. Tor. Ac. Sc. Mm. 46 (1896) 187-.

- equations admitting infinitesimal transformations. Lie, M. S. Christiania F. (1881) No. 15, $6 \mathrm{pp}$.

Associated differential forms. Halphen, $G$. Liouv. J. Mth. 6 (1890) 211-.

Change of variable. Mertens, F. Krk. Ak. (Mt.-Prz.) Rz. 20 (1890) 267-; Fschr. Mth. (1890) 282.

Continuous projective groups, simply infinite, in ordinary space. Pittarelli, G. A. Mt. 22 (1894) 261-.

Differential parameters. Pennacchietti, G. Catania Ac. Gioen. At. 9 (1896) Mem. 1, 11 pp.

- quotients of higher order. Sonin, N. Ja. St Pét. Ac. Sc. Bll. 1 (1894) 321-; Fschr. Mth. (1893-94) 456.

Equations admitting a group of transformations. Lie, M. S. Arch. Mth. Ntvd. 7 (1882) 443-; 8 (1883) 187-, 249-, 371-.

-, and group theory. Lie, S. Leip. Mth. Ps. B. 47 (1895) 261-.

- of problems of dynamics admitting an infinitesimal transformation. Stäckel, $P$. C. R. 119 (1894) 508-, 723-.

- _ _ - - - - (Claim of priority.) Staude, O. C. R. 119 (1894) 903.

_ _ _ - _ _ _-. (Staude's claim of priority.) Stäckel, P. C. R. 119 (1894) 1189.

Infinitesimal adjoints of plane curve. Ocagne, M. d'. N. A. Mth. $19(1900)$ 219-

- deformations. Padova, E. Rm. R. Ac. Linc. Rd. 5 (1889) (Sem. 1) 174-.

Integrability factor, theory. Lie, M. S. Christiania F. (1874) 242-.

Lie, theorem of. Tannenberg, W. de. Bordeaux S. Sc. PV. (1897-98) 49-.

Lie's equations, double generalisation. Vessiot, E. C. R. 125 (1897) 1019-.

Linear homogeneous group. Maurer, L. Leip. Mth. Ps. B. 46 (1894) 215-.

Orbits of point on surface admitting infinitesimal transformations. Staude, $O$. Leip. Mth. Ps. B. 44 (1892) 429-; 45 (1893) 511-.

Orthogonal substitutions generated by repetition of infinitesimal substitution. Taber, $H$. L. Mth. S. P. 26 (1895) 364-.

Partial differential equations, 2nd order, admitting an infinite group of point transformations. Medolaghi, $P$. A. Mt. 1 (1898) 229 - 
Reciprocants, theory. Perrin, R. C. R. 102 (1886) 351-.

Reversion of partial differential expressions with two independent and two dependent variables. Elliott, E. B. [1890] L. Mth. S. P. 22 (1891) 79-.

Total differentials of first kind. Noether, $M$. Erlang. Ps. Md. S. Sb. 18 (1886) 11-.

\section{TRANSFORMATION.}

Sylvester, J. J. R. S. P. 7 (1854-55) 219-. Cockle, (Sir) Jas. Ph. Mg. 13 (1882) 357-. Stäckel, P. Crelle J. Mth. 111 (1893) 290-. automorphic, of $\nabla_{2} \nabla_{2}=0$. Levi-Civita, $T$. Ven. I. At. (1897-98) 1399-.

- infinitesimal, of sum of squares. Beez, Z. Mth. Ps. 43 (1898) 65-, 121-, 277-.

of Bäcklund. Clairin, J. Bll. Sc. Mth. 24 (1900) 281-.

- - for pseudospherical surfaces. Bianchi, L. Rm. R. Ac. Linc. Rd. 1 (1892) (Sem. 2) 3-.

- - - triply orthogonal pseudospherical systems. Bianchi, L. Rm. R. Ac. Linc. Rd. 1 (1892) (Sem. 2) 156-.

- biordinal of Schwarz's. Cockle, (Sir) Jas. Mess. Mth. 11 (1882) 49-.

continuous, discrete groups. Ahrens, $W$. Mth. A. 50 (1898) 518-.

Cremonian, continuous groups. Enriques, $F$., \& Fano, G. A. Mt. 26 (1897) 59-.

of curves and surfaces. Bäcklund, A. V. Lund Acta Un. 10 (1873) (Mth.) No. 2, 12 pp. - differential operator. Hathaway, A.S. [1883] (xII) J. H. Un. Cir. [3] (1884) 35-. - differentials of 1 st order and 2 nd degree by generalised elliptic coordinates. Henrici, $O$. [1865] Crelle J. 65 (1866) 1-.

dualistic differential. Elliott, E. B. B. A. Rp. (1891) 568; L. Mth. S. P. 23 (1892) 188-. of equations, 1st order. Zorawski, $K$. Krk. Ak. (Mt.-Prz.) Rz. 6 (1893) 67-; Cre. Ac. Sc. Bll. (1892) 292-.

group, finite continuous, functions representing. Schur, F. Mth. A. 41 (1893) 509-. groups, continuous. Maurer, L. Mth. A. 39 (1891) 409-.

-, -, equations defining. Engel, $F$. Mth. A. 27 (1886) 1-.

-, -, theory. Campbell, J. E. L. Mth. S. P. 28 (1897) 381-.

- of 4-dimensional manifoldness. Kowalew$s k i, G$. Leip. Mth. Ps. B. 50 (1898) (Mth.) 60-. -, elementary notions. Combebiac, - N. A. Mth. 18 (1899) 347-.

- finite continuous, composition. Killing, $W$. Mth. A. 31 (1888) 252-; 33 (1889) 1-; 34 (1889) 57-; 36 (1890) 161-.

-, 一, greatest sub-groups. Killing, $W$. Mth. A. 36 (1890) 239-.

-, -, theory. Schur, F. Mth. A. 35 (1890) 161-; 38 (1891) 263-.

- relating to certain differential equations. Picard, É. C. R. 106 (1888) 118-.

of homogeneous expressions. Christoffel, E. B. Berl. Mb. (1869) 1-; Crelle J. 70 (1869) 46-, 241-. infinitesimal. Saltykow, N. Liouv. J. Mth. 3 (1897) 429-.

-. Lovett, E. O. B. A. Rp. (1899) 648-.

-, of concentric conics. Lovett, E. O. N. Y. Am. Mth. S. Bll. 4 (1898) 520-

-, especially contact transformations of plane.

Mayer, A. Leip. Mth. Ps. B. 45 (1893) 697-。

-, of plane. Żorawski, $K$. Leip. Mth. Ps. B. 52 (1900) 77-.

- projective. Lovett, E. O. N. Y. Am. Mth. S. Bll. 4 (1898) 515-.

- - monomial group defined by. Torelli, $G$. Palermo Cir. Mt. Rd̃. 8 (1894) 41-.

$-\ldots,-\ldots-$, finite equations. Torelli, $G$. Nap. Rd. 33 (1894) 91-; 34 (1895) 56-

-, reduction to normal form. Engel, $\boldsymbol{F}$. Leip. Mth. Ps. B. 46 (1894) 73-.

-, of typical Cremonian groups. Fano, G.

Rm. R. Ac. Linc. Rd. 7 (1898) (Sem. 1) 332-. leaving equation $\left(\frac{\delta U}{\delta x}\right)^{2}+\left(\frac{\delta U}{\delta y}\right)^{2}+\left(\frac{\delta U}{\delta z}\right)^{2}=0$ unaltered. Campbell, J. E. Mess. Mth. 28 (1899) 97-.

lengths of arcs on surfaces unaltered. Campbell, J. E. L. Mth. S. P. 29 (1898) 249-.

Lie's, sub-groups of a group. Maillet, $E$. C. R. 130 (1900) 1449-.

linear homogeneous. Engel, $F$. Leip. Mth. Ps. B. 49 (1897) 249-

of linear partial differential operators by extended linear continuous groups. Elliott, E. B. L. Mth. S. P. 29 (1898) 439-.

- ordinals, new. Cockle, (Sir) Jas. Ph. Mg. 13 (1882) 44 .

point, infinitesimal elements of 2nd order in. Hadamard, -. Bordeaux S. Sc. PV. (189596) 11-.

of quadratic differential expressions. Voss, A. E. Mth. A. 16 (1880) 129-.

- - form. Lévy, M. C. R. 86 (1878) 463 -. tangential. Fouret, G. C. R. 85 (1877) 134-. -. Engel, F. Mth. A. 23 (1884) 1-.

- Lovett, E. O. [1898-1900] N. Y. Am. Mth. S. Bll. 4 (1898) 402- ; 7 (1901) 113, 115-. Levi-Civita, T. Ven. I. At. (1899-1900) (Pt. 2) 671-.

, analytic theory. Lie, S. Christiania F. 15 (1873) 237-.

-, of circles in plane, synthetic determination of all. Scheffers, G. Leip. Mth. Ps. B. 51 (1899) (Mth.) 145-.

-, class. Lovett, E. O. C. R. 127 (1898) 480-. -, -, determination. Scheffers, $G$. Acta Mth. 14 (1890-91) 111-.

-, of developable. Vivanti, $G$. Palermo Cir. Mt. Rd. 5 (1891) 205-.

-, - - surfaces. Lovett, E. O. Palermo Cir. Mt. Rd. 13 (1899) 210-.

-, and differential equations. Lie, S. Leip. Mth. Ps. B. 50 (1898) (Mth.) 113-.

-, generalised, theory. Liebmann, $H$. Leip. Mth. Ps. B. 51 (1899) (Mth.) 354-.

-, group of linear, groups of finite order contained in. Autonne, -. C. R. 102 (1886) 313-

-, infinitesimal, of mechanies. Lie, S. Leip. Mth. Ps. B. 41 (1889) 145-. 


\section{Differential Invariants}

tangential, infinitesimal, of optics. Lie, S. Leip. Mth. Ps. B. 48 (1896) 131-.

- , invariant theory. Lie, S. Christiania F. 14 (1872) 133-; Mth. A. 8 (1875) 215-.

-, leaving ratio of measures of curvature unaltered. Vivanti, G. Z. Mth. Ps. 37 (1892) 1-.

-, Lie's. Mayer, A. C. G. Gött. Nr. (1874) 317-.

-, - theory, and theory of perturbations. Lovett, E. O. Am. As. P. (1897) 58-

- and line geometry. Lie, S. Leip. Mth. Ps. B. 49 (1897) 687-

-, between line and sphere, infinite continuous group. Lovett, E. O. C. R. 129 (1899) 405-, 1296.

-, problem. Vivanti, G. Palermo Cir. Mt. Rd. 5 (1891) 269-.

-, properties in connexion with curvature. Mehmke, R. Z. Mth. Ps. 38 (1893) 7-.

-, of surfaces, theory. Bäcklund, A.V. Mth. A. 19 (1882) 387-.

—, theory. Lie, S. Leip. Mth. Ps. Ab. 14 (1888) 535-.

-, - Lilienthal, R. von. Mth. A. 50 (1898) 303-.

theory, general. Lie, S. Leip. Mth. Ps. B. 47 (1895) 494-; 48 (1896) 390-.

of $\frac{1}{2} \Sigma_{r 8} a_{r 8} \frac{d V}{d x_{r}} \cdot \frac{d V}{d x_{8}}$, reciprocity law. Beltrami, E. [1866] G. Mt. 5 (1867) 24-.

Uniform substitutions, Podetti, F. G. Mt. 35 (1897) 264-.

Universal invariants, application to point and contact groups in space of two dimensions. Rabut, -. Par. Éc. Pol. J. 4 (1898) 137-.

\section{Differential invariants. (See also 1230.)}

Combescure, É. [1870] Mntp. Mm. Ac. Sect. Sc. 7 (1867-71) 447-.

Lie, S. Mth. A. 24 (1884) 537-.

Sylvester, J. J. C. R. 102 (1886) 31-.

Appell, P. C. R. 105 (1887) 55-.

Papperitz, -. D. Nf. Tbl. (1887) 229.

Goursat, É. $\quad$ C. R. 107 (1888) 898-.

Appell, P. Liouv. J. Mth. 5 (1889) 361-.

Rivereau, $P$. Liouv. J. Mth. 8 (1892) 233 -.

Levi-Civita, $T$. Ven. I. At. (1893-94) 1447-, $1688-$

Bouton, C. L. N. Y. Am. Mth. S. Bll. 4 (1898) 313-.

Wallenberg, G. Crelle J. Mth. 121 (1900) 200-.

Application to linear homogeneous equations. Wallenberg, G. Crelle J. Mth. 113 (1894) 1-.

Binomial biordinals. Cockle, (Sir) Jas. L. Mth. S. P. 12 (1880-81) 63-; Ph. Mg. 24 (1887) 249-

Canonical expansions in series, coefficients being invariants of continuous group. Tresse, A. C. R. 114 (1892) 1256-.

Class. Malet, J. C. [1881] Phil. Trans. 173 (1883) 751-

-, relative to linear equations. Poincaré, $H$. C. R. 94 (1882) 1402-
Differential Invariants 5240

Configurations of three dimensions. Cotton, $\dot{E}$. Toul. Fac. Sc. A. 1 (1899) 385-.

Conjugate annihilators of homogeneous and isobaric differential expressions. Rogers, L. J. Mess. Mth. 18 (1889) 153-.

Connexion between elements of orthogonal 9 and 16 systems. Jahnke, E. Crelle J. Mth. 118 (1897) 224-.

Continuous groups. Lie, S. Christiania F. (1884) No. 8, 4 pp., No. 9, 4 pp.; (1885) No. 15,4 pp.

- transformation groups, extension. Żorawski, K. Krk. Ak. (Mt.-Prz.) Rz. 4 (1893) 34-; Fschr. Mth. (1893-94) 635.

Coresolvents. Cockle, Jas. [1862] QJ. Mth. 6 (1863) 9-, 151-, 226-.

Covariant belonging to quadratic invariant. Ricci, G. Rm. R. Ac. Linc. Rd. 3 (1887) (Sem. 1) 15-.

Covariants. Cockle, (Sir) Jas. [1863-64] Ph. Mg. 27 (1864) 225-; 28 (1864) 205-.

- of plane curves. Gwyther, R. F. [1893] Phil. Trans. (A) 184 (1894) 1171-.

Critical functions. Cockle, Jas. QJ. Mth. 4 (1861) 97-, 265-.

- - Warren, J. W. [1863-64] (vr) QJ. Mth. 6 (1864) 231-, (vir) 372-.

Deformation invariants. Żorawski, $K$. Acta Mth. 16 (1892-93) 1-.

Differential equation of most general substitution of 1 variable. MacMahon, P. A. Ph. $\mathrm{Mg} .23$ (1887) 542-.

- operators, certain linear, interchange of variables in. Elliott, E. B. [1889] Phil. Trans. (A) 181 (1891) 19-.

- - used to form system of invariants, etc. Elliott, -. L. Mth. S. P. 26 (1895) 185-.

- parameters, simple or simultaneous. Morin, $\bar{P}$. C. R. 66 (1868) 601-. resolvents. Cockle, (Sir) Jas. Manch. Lt. Ph. S. P. 4 (1865) 38-.

- - Spottiswoode, W. QJ. Mth. 6 (1864) $262-$.

- - of algebraic equations, higher degree. Lachtin, L. K. [1896-98] Rec. Mth. (Moscou) 19 (1897) 211-, 393-; 20 (1899) 260-; Fschr. Mth. (1897) 88-; (1898) 74.

- - and partial differential resolvents. Rawson, R. Manch. Lt. Ph. S. P. 21 (1882) 59-.

Functional invariants, class. Forsyth, A. R. [1888] Phil. Trans. (A) 180 (1890) 71-.

Function- and invariant-theory of binomial configurations. Wellstein, J. Mth. A. 52 (1899) 70-.

Fundamental invariants. Vogt, $H$. Par. Éc. Norm. A. 6 (1889) (Suppl.) $71 \mathrm{pp}$.

General quartine or incriticoid of 4 th degree. Harley, (Rev.) R. Ph. Mg. 26 (1888) 456-.

Group of motions and their invariants. Lie, S. Leip. Mth. Ps. B. 45 (1893) 370-.

- theory and its applications. Źorawski, $K$. Krk. Ak. (Mt.-Prz.) Rz. 6 (1893) 289-; Crc. Ac. Sc. Bll. (1893) 145-.

Homographic invariants and quotient derivatives, Forsyth, A.R. Mess. Mth. 17 (1888) 154-.

Indefinite quadratic invariants. Bianchi, $L$. Rm. R. Ac. Linc. Mm. 5 (1888) 539-. 
5240 Differential Invariants

Infinitesimal transformations of plane curve with corresponding differential invariants. Arvay, $W ., \&$ Komperda, $H$. Prace Mt.-Fiz. 10 (1899-1900) 113-; Fschr. Mth. (1899) 540.

Integral invariant of Poincaré. Picart, $L$. Bordeaux S. Sc. PV. (1898-99) 124-.

-invariants. Koenigs, G. C. R. 122 (1896) 25-.

- - application to reduction of differential equations to canonical type. Koenigs, $G$. C. R. 121 (1895) 875-.

- - of continuous transformation groups. Źorawski, K. Krk. Ak. (Mt.-Prz.) Rz. 8 (1895) 232- ; Crc. Ac. Sc. Bll. (1895) 127-

- - , theory, corollary of theory of differential invariants. Lie, S. Leip. Mth. Ps. B. 49 (1897) 342-.

- - , and their use in theory of differential equations. Lie, S. Leip. Mth. Ps. B. 49 (1897) 369-.

Invariant differential expressions. Hazzidakis, J. N. Crelle J. Mth. 104 (1889) 102-.

- formations attaching to class of differential equations, 1st order. Liouville, R. C. R. 105 (1887) 460-, 496.

- total differential equation

$$
P d x+Q d y+R d z=0 \text {. }
$$

Page, J. M. A. Nth. 12 (1898-99) 182-

Invariants and their applications. Liouville, R. C. R. 109 (1889) 560-; Par. Éc. Pol. J. 59 (1889) 7 -

- of class of equations of 1st order. Elliot, $Z$. C. R. 110 (1890) 629-.

- continuous finite groups. Lindelöf, $E$. Helsingf. Acta 20 (1895) No. 1, 62 pp.

- - - transformation groups. Tresse, $A$. Acta Mth. 18 (1894) 1-.

-, covariants and quotient-derivatives associated with differential equations. Forsyth, A.R. [1888] Phil. Trans. (A.) 179 (1889) 377-.

- of differential quadratics. Maschke, $H$. N. Y. Am. Mth. S. T. 1 (1900) 197-, 508.

-, form. Medolaghi, P. Rm. R. Ac. Linc. Rd. 7 (1898) (Sem. 1) 145-.

-, - with reference to certain groups. Cotton, É. C. R. 128 (1899) 495-.

- in general theory of surfaces. Zorawski, $K$. Krk. Ak. (Mt.-Prz.) Rz. 8 (1895) 1-; Crc. Ac. Sc. Bll. (1895) 91-.

- of Goursat and Painlevé. Lovett, E. O. Am. J. Mth. 22 (1900) 40-.

- - infinite continuous transformation group. Żorawski, $K$. Krk. Ak. (Mt.-Prz.) Rz. 4 (1893) 41-; Fschr. Mth. (1893-94) 635-.

- - linear differential equations. Laguerre, E. C. R. 88 (1879) 224 -

- - - Rivereau, -. Toul. Fac. Sc. A. $4(1890)$ M, 5 pp.

- - - homogeneous differential equation. Mittag-Leffler, G. C. R. 109 (1889) 637-; Stockh. Öfv. (1889) 427-.

- - partial equations. Le Roux, J. C. R. 126 (1898) 721-.

- C Cotton, É. Par. É. Norm. A. 17 (1900) 211-

-, Malet's, identification with Sir James Cockle's criticoids. Harley, (Rev.) R. R. S. P. 38 (1885) 45-.

\section{Differential Invariants 5240}

Invariants, Malet's, identification with Sir James Cockle's criticoids. Malet, J. C. R. S. P. 38 (1885) 211-.

- of motion of continuous material system. Źorawski, K. [1900] Krk. Ak. (Mt.-Prz.) Rz. 18 (1901) 353-; Crc. Ac. Sc. Bll. (1900) 367-.

- of skew curves. Halphen, G. H. Par. Ėc. Pol. J. Cah. 47 (1880) 1-.

- - surface with reference to conformal transformations of space. Tresse, $A$. C. R. 114 (1892) 948-.

- - system of $m$ points by projective trans. formation. Lovett, E. O. Am. J. Mth. 21 (1899) 168-; 22 (1900) 46-.

- $-1-m+1$ points with reference to projective transformations. Lovett, $E . O . C$. R. 127 (1898) 346-.

- - transformation groups, extension of concept. Killing, W. Mth. A. 35 (1890) 423-.

Linear partial differential equations with continuous transformation group. Campbell, J. E. N. Y. Am. Mth. S. T. 1 (1900) 243-, 509.

Multilinear partial differential operator. Mac Mahon, P. A. L. Mth. S. P. $18(1886-87) 61$.

Pfaff-invariants. Cayley, -. QJ. Mth. 26 (1893) 195-.

Point invariants of ordinary differential equation, 2nd order. Tresse, A. C. R. 120 (1895) 429-; Leip. Jablon. Preisschr. 32 (1896) $87 \mathrm{pp}$.

- - - partial differential equations, 2nd order. Medolaghi, P. Rm. R. Ac. Linc. Rd. 6 (1897) (Sem. 2) 247-, 313-.

Primary forms of linear differential equations, and order. Schlesinger, L. C. R. 115 (1892) 32-.

Projective cyclic concomitants. Elliott, E. B. L. Mth. S. P. 20 (1889) 131-.

- invariants for curves of hyperspace. Berzolari, L. A. Mt. 26 (1897) 1-.

Quantoids. Cockle (Sir) Jas. Ph. Mg. 30 (1865) 347-.

Reciprocants. Sylvester, J.J. Mess. Mth. 15 (1886) 88-.

-, homographic and circular. Rogers, L. J. L. Mth. S. P. 17 (1887) 220-, 344-; 18 (1886-87) 130-.

-, integrable, class. Hammond, J. L. Mth. S. P. 17 (1887) 128-.

- and invariants. Cayley, A. QJ. Mth. 26 (1893) 169-, 289-.

- method, as containing an exhaustive theory of singularities of curves. Sylvester, J. $J$. Nt. 33 (1886) 222-, 331-.

_, perpetuant. MacMahon, P. A. L. Mth. S. P. 17 (1887) 139-.

_, pure irreducible, of 4th order. Sylvester, J. J. C. R. 102 (1886) 152-.

_, _, tables to weight 8. Cayley, A. Am. J. Mth. 15 (1893) 75-

- - ternary, and allied functions. Elliott, E. B. L. Mth. S. P. 19 (1889) 6-.

-, - - , differential equations. Elliott, E.B. [1886] L. Mth. S. P. 18 (1886-87) 142-.

simultaneous. Berry, A. QJ. Mth. 23 (1889) 260- 
Reciprocants, ternary (cyclicants), and allied functions. Elliott, E.B. [1888] L. Mth. S. P. 19 (1889) 377-.

-, - and n-ary. Elliott, E. B. L. Mth. S. P. 17 (1887) 172-.

Reciprocants, theory. Sylvester, J.J. Am. J. Mth. 8 (1886) 196-; 9 (1887) 1-, 113-, 297-; 10 (1881) 1-.

-, - , results connected with. Leudesdorf, $C$. L. Mth. S. P. 17 (1887) 197-.

Resolvent of linear differential equations. Beke, E. Mth. A. 46 (1895) 557-.

Singularities of partial differential equations, 1st order. Marotte, F. C. R. 123 (1896) 933-.

Ternary quadratic forms. Ricci, G. Rm. R. Ac. Linc. Rd. 5 (1889) (Sem. 1) 643-.

Transformations with differential invariant. Haton de la Goupillière, J.N. Liouv. J. Mth. 2 (1876) 241-.

\section{Analytical methods connected with physical problems.}

\section{General.}

Applied mathematics. Franceschinis, F. M. Mil. G. S. Inc. 4 (1808) 1-.

- - Runge, C. Mth. A. 44 (1894) 437-.

Calculus, application to interpretation of natural phenomena and discovery of their laws. Pêrard, L. Brux. A. Un. (1849-50) 469-.

- of residues, application to mathematical physics. Cauchy, A. L. Par. Mm. Ac. Sc. 7 (1827) 463-.

Continuous functions with no derivative in elements of mechanics. Appell, P., \& Janaud, -. C. R. 93 (1881) 1005-.

Dirichlet's integral. Broden, T. Mth. A. 52 (1899) 177-.

- method for finding coefficients of Gauss's series. Kronecker, L. Hamb. Mth. Gs. Itt. 2 (1890) (Festschr., Tl. 2) 32-.

- - of transformation applied to

$$
\frac{\partial X}{\partial x}+\frac{\partial Y}{\partial y}+\frac{\partial Z}{\partial z} \text {. }
$$

Meyer, F. Mth. A. 26 (1886) 509-.

Expansion of arbitrary functions of 1 variable in trigonometric series, history. Sachse, $A$. Z. Mth. Ps. 25 (1880) (Suppl.) 229-.

- - functions in series of continuous functions with varying parameter. Pagani, G.M. [1828] Brux. Ac. Sc. Mm. 5 (1829) 5-

- - - - - periodic quantities, with applications. Poisson, S. D. Par. Éc. Pol. J. $18^{\mathrm{e}} \mathrm{cah} .(1820)$ 417-.

- - - - - terms of which satisfy same differential equation of 2 nd order with variable parameter. Liouville, $J$. [1835-37] Liouv.J.Mth. 1 (1836) 253-; 2 (1837) 16-, 418-

- - - - - - - linear differentia equation with variable parameter. Liouville, J., \& Sturm, C. Liouv. J. Mth. 2 (1837) 220 -

Green's function, existence in plane simply connected region. Osgood, W. F. N. Y. Am. Mth. S. T. 1 (1900) 310-.
Harmonic functions. Harris, R. A. [1898] N. Y. Am. Mth. S. Bll. 5 (1899) 89, 96-. - - class analogous to. Liouville, $J$. Liouv. J. Mth. 10 (1845) 327-.

Homogeneity in physics. Vaschy, A. C. R. 114 (1892) 1416-.

Line and surface integrals, generalisation of Stokes's theorem. Gilbert, - Brux. S. Sc. A. 16 (1892) (Pt. 1) 2-.

Mathematical expressions for physical quantities, permanence of form. Gwyther, R. F. Manch. Lt. Ph. S. Mm. \& P. 9 (1895) 119-.

- physics, application of linear systems. Larose, H. A. Tél. 23 (1896-97) 259-.

- - and pure analysis, relations. Poincaré, H. Acta Mth. 21 (1897) 331-。

Mathematics, application to natural science. Petzval, J. Wien Alm. (Anh.) (1858) 187-.

- , - physics. Liebermeister, C. Erdm. J. Pr. C. 84 (1861) 416-.

-, influence on physics. Collins, E. St Pét. Ac. Sc. Rec. (1837) 179-.

- and physics, relations. Örsted, H.C. Oken Isis (1831) 854-.

Operators in physical mathematics. Heaviside, O. R. S. P. 52 (1893) 504 ; 54 (1894) 105-。

Periodic series. Meyer, A. [1850] Crelle J. 43 (1852) 60-

Physical data applicable to mathematics. Rawson, R. [1847] Manch. Ph. S. Mm. 8 (1848) 329-.

Physics, principles of algebra of. Macfarlane, A. Am. As. P. (1891) 65-.

Solution, general, of Maxwell's equations. Birkeland, $K$. Arch. Sc. Ps. Nt. 34 (1895) 5-.

- of problems of electricity by transformation of conjugate functions. Maxwell, $J$. $C$. [1871] (vir) Camb. Ph. S. P. 2 (1876) 242-

Theory of matrices, application to linear differential equations. Brill, $J$. Camb. $\mathrm{Ph}$. S. P. 8 (1895) 201-.

Transformation of coordinates in heat problems. Bertrand, J. Liouv. J. Mth. 14 (1849) 1 -.

Use of Riemann surface in applied mathematics. Carslaw, H.S. B.A.Rp. (1900) 644-

Vector functions. Burkhardt, H. Mth. A. 43 (1893) 197-.

- - and their applications to physics. Élie, B. Bordeaux S. Sc: Mm. 3 (1893) 1-.

\section{Harmonic analysis; Fourier's series. (See also 3220.)}

\section{FOURIER'S SERIES.}

(Conduction of heat.) Fourier, $J . B . J$. [1807-11] Par. S. Phlm. N. Bll. 1 (1807) 112-; Par. Mm. Ac. Sc. 4 (1824) 185-; 5 (1826) 153-.

Thomae, J. Z. Mth. Ps. 17 (1872) 78-.

Du Bois-Reymond, P. Gött. Nr. (1873) 571-.

Ascoli, G. [1873-78] A. Mt. 6 (1875) 21-, 298-; Rm. R. Ac. Linc. Mm. 2 (1878) 584-. Jordan, C. C. R. 92 (1881) 228-.

Halphen, G. H. C. R. 95 (1882) 1217-.

Veltmann, W. Z. Mth. Ps. 27 (1882) 193-. 
Gilbert, P. Brux. S. Sc. A. 8 (1884) (Pt. 2) 1-.

Hermite, C. Am. J. Mth. 9 (1887) 381-.

Michelson, A. A. Nt. 58 (1898) 544 -

Love, A. E. H. Nt. 58 (1898) 569-.

Gibbs, J.W. [1898] Nt. 59 (1898-99) 200.

Michelson, A. A. [1898] Nt. 59 (1898-99) 200.

Love, A. E. H. [1898] Nt. 59 (1898-99) 200-

Hayward, R. B. Nt. 59 (1898-99) 271.

Baker, H. F. Nt. 59 (1898-99) 319-.

Gibbs, J. W. Nt. 59 (1898-99) 606.

Poincaré, -. Nt. 60 (1899) 52.

Love, A. E. H. Nt. 60 (1899) 100-.

Lerch, - . Bll. Sc. Mth. 24 (1900) 102-.

Application in optics. Wind, C. H. [1900] Ps. Z. 2 (1901) 189-.

- to theory of functions of complex variable. Harnack, A. [1882] Mth. A. 21 (1883) 305-.

Approximate representation by. Picard, $\dot{E}$. C. R. 112 (1891) 183-.

Behaviour at discontinuities. Lindemann, C. L. F. [1881] Mth. A. 19 (1882) 517-.

Calculation of Fourier and Bessel series. Dechevrens, M. Rm. N. Linc. Mm. 16 (1899) 149-; 17 (1900) 47-.

Case. Porěckij, P. S. Kazan S. Nt. (Ps.-Mth.) P. 6 (1888) 330-.

Coefficients. Bruno de Cabedo, J. G. Teix. J. Sc. 12 (1895) 81-; Fschr. Mth. (1893-94) 395.

- calculation. Macé de Lépinay, J. J. de Ps. 8 (1899) 137-.

-, harmonic analyser for graphic determination. Bervy, N. Mosc. Obs. A. 3 (Pt. 2) (1896) 109-.

Conduction of heat : imaginary roots of certain transcendental equations. Fourier, J. B.J. [1826] Par. Mm. Ac. Sc. 7 (1827) 605-.

- _ - mathematical theory, additions 1811-89. Gibson, G. A. Edinb. Mth. S. P. 7 (1889) 5-.

- - in solid, series connected with. Poisson, S. D. Crelle J. 16 (1837) 39.

$--\ldots-1,-\ldots i o u v i l l e, J$. Crelle J. 16 (1837) $41-$.

Convergence. Gibson, G. A. Edinb. Mth. S. P. 12 (1894) 39-.

-. Williams, $W$. Ph. Mg: 42 (1896) 125-.

- Ford, W. B. [1900] N. Y. Am. Mth. S. Bll. 7 (1901) 227-.

-, Cauchy's second proof. Harnack, A. Mth. A. 32 (1888) 175-.

- and divergence. Du Bois-Reymond, $P$. Münch. Ak. Ab. 12 (1876) (2te Ab.) 1-; Mth. A. 10 (1876) 431-.

Expansion of discontinuous functions. Berger, A. Ups. S. Sc. N. Acta 15 (1895) No. 13, $33 \mathrm{pp}$.

- - doubly periodic functions. Charlier, C. V. L. Stockh. Ak. Hndl. Bh. 12 (Afd.1) (1887) No. 9, 24 pp.; Fschr. Mth. (1887) 448.

-, graphic. Perry, J. Elect. 35 (1895) 285-.

- of uniform finite function of an angle by. Voigt, $W$. Rm. R. Ac. Linc. Rd. 8 (1899) (Sem. 1) 93-.

Extensions of Fourier's trigonometric series. Alexander, $P$. Mess. Mth. 16 (1887) 42-.
Finite and continuous functions not developable in Fourier's series at point $x=0$. Marolli, B. G. Mt. 37 (1899) 80-.

General form and application. Dienger, $J$. Grunert Arch. 39 (1862) 303-.

History. Gibson, G. A. Edinb. Mth. S. P. 11 (1893) 137-.

Logarithmic potentials and Fourier's series. Somigliana, C. Mil. I. Lomb. Rd. 20 (1887) 635-.

Machine for expansion of any function in. Sommerfeld, A. Königsb. Schr. 32 (1891) (Sb.) 28-.

Proof of law of coefficient formation. Du BoisReymond, $P$. [1875] Münch. Ak. Ab. 12 (1876) (1te $A b.)^{117-}$.

- for periodic functions. Alexander, $P$. Mess. Mth. 16 (1887) 23-.

Proofs, simplification. Harnack, A. [1881] Mth. A. 19 (1882) 235-, 524-.

Properties of Fourier's series and coefficients. Björling, C. F. E. [1865] Stockh. Öfv. 22 (1866) 511-.

Representation of functions by, sufficient condition for. Hölder, $O$. Berl. Ak. Sb. (1885) 419-.

- - - of 1 variable by Fourier's series and in other ways. Hermite, C. C. R. 91 (1880) 1018-.

Theorem, proof. Johnson, A. R. Mess. Mth. 16 (1887) 90 -.

"Théorie analytique de la chaleur," passage in. Stieltjes, T.J. N. A. Mth. 8 (1889) 472-.

Theory. Harnack, A. Bll. Sc. Mth. As. 6 (1882) 242-, 265-, 282-.

- Mansion, P. Brux. Ac. Mm. 45 (1884) (No. 5) $20 \mathrm{pp}$.

- Lerch, M. Prag České Ak. Fr. Jos. Rz. (Třida 2) 9 (1900) Art. 7, 15 pp.

Uniqueness. Cantor, G. Crelle J. 72 (1870) 139-; 73 (1871) 294-.

Approximately simple waves. Rayleigh, (Lord). Ph. Mg. 50 (1900) 135-.

Bessel's formula in meteorology. Schreiber, $P$. [1892] Ac. Nt. C. N. Acta 58 (1893) 137-.

Expansion of functions in periodic series. Hoilel, J. C. R. 52 (1861) 512-; (x) Par. Obs. A. 8 (1866) 83-.

- - - - series of cosines, case. Julius, V. A. N. Arch. Wisk. $3\left({ }^{*} 1877\right) 80$ -

- - - - - sines and cosines. Liouville, J. Liouv. J. Mth. 1 (1836) 14 -

- - - - trigonometric series, possibility. Riemann, [G. F.] B. Gött. Ab. 13 (1868) (Mth.) 87-; Bll. Sc. Mth. As. 5 (1873) 20-, 79-.

- - - of $\sin \theta$ and $\cos \theta$ in terms of sines and cosines of multiples of $\theta$. Cauchy, A.L. C. R. 12 (1841) 323-.

- - 2 summatory formulæ. Schlömilch, $O$. Crelle J. 42 (1851) 125-.

- in trigonometric series of functions having, between finite limits, an infinite number of maxima and minima. Lipschitz, $R$. Crelle J. 63 (1864) 296-。 
Expansion of $f\left(u, u^{\prime}\right)$ in series of sines and cosines of multiples of $u$ and $u^{\prime}$. Bessel, F.W. Berl. Ab. (1820-21) 55-.

- $-\phi(x)$ in sines and cosines of multiples of $x$. Bessel, F. W. As. Nr. 16 (1839) 229-.

Expansions of functions employed in physics, general reason for use. Boussines $q, J$. C. R. 92 (1881) 513-.

- - implicit functions in trigonometric series, and their transformation to isotropic means. Cauchy, A. L. C. R. 38 (1854) 910-.

- in series of multiple sines and cosines. Cayley, A. Camb. Mth. J. 3 (1843) 162-.

- _ - - particular solutions of equations of elasticity. Lauricella, G. Tor. Ac. Sc. At. 34 (1898) 11-.

-, trigonometric. Lerch, - Toul. Fac. Sc. A. 3 (1889) C, 11 pp.

,-- , of periodic solutions of $\nabla^{2} F=0$. Appell, P. C. R. 102 (1886) 1439-; Liouv. J. Mth. 3 (1887) 5-.

Fourier's double integral theorem. Glaisher, J. W. L. Mess. Mth. 2 (1873) 20 -.

- _ - Boole's and other proofs. Alexander, $P$. Edinb. Mth. S. P. 3 (1885) 45-.

- - _ - failing cases. Alexander, P. Edinb. Mth. S. P. 3 (1885) 12-.

- - integrals, more general theorem. Grinwald, F. K. Z. Mth. Ps. 9 (1864) 131-.

- expansion for doubly periodic functions, new method. Mohrmann, $G$. Arch. Mth. Ps. 12 (1894) 1-.

- expansions of functions in trigonometric series. Thomson, (Sir) $W$. Camb. Mth. J. 2 (1841) 258-.

- - - transcendent functions. Krygowski, $Z$. Prace Mt.-Fiz. 5 (1894) 70-

- formula and its analogues. Pellet, A. C. R. 106 (1888) 1062-.

- applied to differentiation. Liouville, $J$. Crelle J. 13 (1835) 219-.

- - generalised. Liouville, J. Liouv. J. Mth. 1 (1836) 102-.

- integrals. Du Bois-Reymond, P. Mth. A. 4 (1871) 362-.

- problem. Le Roy, E. C. R. 120 (1895) 179-.

- theorem. Gregory, D. F. Camb. Mth. J. 3 (1843) 288-.

- - La Vallée-Poussin, C. de. Brux. S. Sc. A. 15 (1891) (Pt. 1) 39-.

-- , general extension. Preston, T. Ph. Mg. 43 (1897) 281-.

- - generality of new theorem analogous to. Stoney, G. J. Ph. Mg. 43 (1897) 139-.

- - proof. Bonnet, O. N.A. Mth. 3 (1844) 119 -.

- - - Walton, W. [1865] QJ. Mth. 8 (1867) 136-.

- - - Cauchy's. Schouten, G. N. Arch. Wisk. 18 (1891) 146-.

- and $\sin \infty$ and $\cos \infty$. Moon, R. $\mathrm{Ph}$. Mg. 26 (1845) 483-.

- theorems. Hobson, E. W. Mess. Mth. 11 (1882) 11-.

- -, proof. Schlömilch, O. Crelle J. 36 (1848) 268-.

Functions expressible by series of periodic terms. Ivory, J. $\mathrm{Ph} . \mathrm{Mg} .9$ (1836) 161-.
Harmonic analyser, Henrici and Coradi's. Grabowski, L. Wiad. Mt. 4 (1900) 236-.

- components, computation (for yearly and daily periods). Strachey, (Lt.-Gen.) R. R. S. P. 42 (1887) 61-.

- - of periodic motion. Clifford, W. K. [1873] (vII) Mess. Mth. 3 (1874) 153-.

curves, graphic analysis. Wedmore, E. B. Elect. 35 (1895) 512-.

- decomposition of functions, and allied expansions. Johnson, A. R. L. Mth. S. P. 19 (1889) 75-.

- expansion of arbitrary function. Zaremba, S. Liouv. J. Mth. 6 (1900) 47-.

- series. Schlömilch, O. Z. Mth. Ps. 14 (1869) 250-.

- Cesáro, E. (xII) Mathesis 1 (1881) $51-, 80,143-$.

Integral formulæ representing arbitrary functions, range of application. Du Bois-Rey. mond, $P$. [1874] Crelle J. Mth. 79 (1875) 38-.

Neumann's potential function $\Sigma \frac{A e^{-a r}}{r} \cdot$ Bel.

trami, E. Rm. R. Ac. Linc. Rd. 4 (1895) (Sem. 2) 177-.

Periodic curves, analysis. Wedmore, $E . B$. I. Elect. E. J. 25 (1897) 224-.

- function $\cos x-\frac{1}{8} \cos 3 x+\frac{1}{5} \cos 5 x$ etc. Wilbraham, $H$. Camb. and Dubl. Mth. J. 3 (1848) 198-.

- - $A_{0}+A_{1} \cos \phi+B_{1} \sin \phi \ldots+A_{n} \cos n \phi$ $+B_{n} \sin n \phi$, transformation. Stieltjes, $T . J$. (jun.) N. Arch. Wisk. $9\left(^{*} 1882\right)$ 111-.

- series, summation. Raabe, J.L. Crelle J. 15 (1836) 355-.

- - value at certain limits. Newman, $F . W$. Camb. and Dubl. Mth. J. 3 (1848) 108-.

Periodicities, investigation of hidden, with application to supposed 26 day period. Schuster, A. Terr. Mag. 3 (1898) 13-.

-, lunar and solar, of earthquakes. Schuster, A. Nt. 56 (1897) 321.

- in series of observations, method of ascertaining. Nene, V. N. R. S. P. 36 (1884) 361-.

Phases of function, calculation. Cabreira, $A$. [1900] Lisb. J. Sc. Mth. 6 (1902) 55-.

Series of sines and cosines of multiple angles, numerical values of coefficients. Lubbock, J. $W$. Ph. Mg. 33 (1848) 106-.

Summation of periodic functions. Gyldén, J. A. H. (x) Stockh. Ak. Hndl. 11 (1872) No. 1, 15 pp.; Par. Ec. Norm. A. 8 (1879) 203-.

Trigonometric series, convergence for arbitrary function between given limits. LejeuneDirichlet, G. Crelle J. 4 (1829) 157-; 17 (1837) 35-.

- - , representation of arbitrary functions by. Lejeune-Dirichlet, G. Rpm. Ps. 1 (1837) 152-.

- Harnack, A. Mth. A. 17 (1880) 123-.

Wave motion, supposed proof of theorem in. Stoney, G. J. Ph. Mg. 43 (1897) 368-; 44 (1897) 98-, 206-.

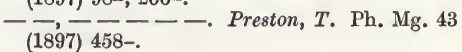




\section{Harmonic analysis; series other than Fourier's. Spheri- cal and ellipsoidal harmonics. (See also 3220.)}

Cylindrical functions, development of zero in series of. Nielsen, N. Mth. A. 52 (1899) $582-$.

- - integrals, and Fourier series. Hankel, $H$. [1869] Mth. A. 8 (1875) 471-.

Ellipsoidal harmonies. Niven, $W . D .[1890]$ Phil. Trans. (A) 182 (1892) 231-.

Expansion of given function in series according to harmonic functions. Steklov, $V . A$. Kharkov Mth. S. Com. 5 (1896?) 60-; Fschr. Mth. (1896) 203-; C. R. 128 (1899) 279-.

- $\left\{R^{2}-2 \operatorname{Rr}\left[\cos u \cos u^{\prime} \cos \left(x-x^{\prime}\right)\right.\right.$ $\left.\left.+\sin u \sin u^{\prime} \cos \left(y-y^{\prime}\right)\right]+r^{2}\right\}^{-1}$. Stieltjes, T. J. Liouv. J. Mth. 5 (1889) 55-.

Fourier-Bessel functions, representation of arbitrary function by. Harnack, $A$. Leip. Mth. Ps. B. 39 (1887) 191-.

Functional series, expansion of function in. Johnson, A. R. Mess. Mth. 15 (1886) 81-.

Functions $X, Y$, etc., possessing property that $\int X Y d \sigma=0$, and expansible in form $\alpha X+\beta Y$ $+\gamma Z+\ldots$, construction. Routh, E.J. L. Mth. S. P. 10 (1878-79) 146-.

Fundamental functions and expansion in terms of them. Renaux, -. C. R. 129 (1899) $545-$.

Harmonic analysis. Perry, $J_{.,}$\& Hunt, H. F. L. Ps. S. P. 14 (1896) 1-; Ph. Mg. 40 (1895) 506-.

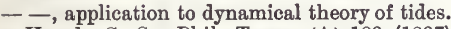
Hough, S. S. Phil. Trans. (A) 189 (1897) 201-.

- expression of daily variation of solar radiation, etc. Hargreaves, R. Camb. Ph. S. P. 9 (1898) 532-.

- functions for elliptic cone. Hobson, E. W. L. Mth. S. P. 23 (1892) 231-.

- - expansion of arbitrary function in series of. Zaremba, S. C. R. 128 (1899) 1088-.

- - of Poincaré. Stekloff, $W$. Toul. Fac. Sc. A. 2 (1900) 273 -

- -, theorem. Arone, G. D. d'. C. R. 114 (1892) 1055-.

- - of 3 variables, analogous to $\theta$-Fuchsian functions. Stouff, $X$. Toul. Fac. Sc. A. 4 (1890) A, $15 \mathrm{pp}$.

$--\frac{-}{-}$, new theorems. Sanctis, L. de. A. Mt. 4 (1900) 161-

- - several real variables. Arome, G.D.d'. C. R. 118 (1894) 342-.

- periodic infinite series, summation. Raabe, J.L. Crelle J. 23 (1842) 105-; 25 (1843) 160 -.

- series with infinitely small terms. Fontana, G. Verona S. It. Mm. 2 (1784) 123-.

Harmonics of a ring. Niven, $W . D$. L. Mth. S. P. 24 (1893) 272-.

Lamé's equation. Escary, - . C. R. 91 (1880) $40-, 102-$.
Lamés and spherical functions, expansion by. Lindemann, C. L. F. [1881] Mth. A. 19 (1882) 323-.

Laplace's coefficients. Glaisher, J.W.L. L. Mth. S. P. 6 (1874-75) 126-.

- - Hall, A. A. Mth. 3 (1887) 1-.

-, equation of. Moon, R. Ph. Mg. 40 (1870) 434-.

- - expansion, determination of numerical factors in. Tarleton, F. A. Ir. Ac. P. 1 (1889-91) 16-.

- - and functions. Heath, D. D. QJ. Mth. 7 (1866) 23-.

- - proof that $F(\mu, \omega)$ may be expanded into a series of. Procul. [Pseudon.] (vi Adds.) Ph. Mg. 25 (1844) 337-

- -, transformation. Plarr, G. Edinb. R. S. T. 36 (1892) 19-.

- , values of integral $\int_{0}^{1} Q_{n} Q_{n}^{\prime} d \mu, Q_{n}, Q_{n^{\prime}}$ being Laplace's coefficients of orders $n, n^{\prime}$, with application to theory of radiation. Rayleigh, (Lord). Phil. Trans. 160 (1870) 579 -.

-,$F(\theta, \psi)$ developable in only one series of. Pratt, J.H. Ph. Mg. 8 (1836) 474-.

$--b^{(i)}$, use of continued fractions in calculation. Callandreau, O. Par. Éc. Pol. J. cah. 45 (1878) 91-.

- dynamical equations, general integration. Hough, S. S. [1897] Phil. Trans. (A) 191 (1898) 139-

- functions. Cayley, A. Liouv. J. Mth. 13 (1848) 275-.

243-.

Schläfli, L. A. Mt. 1 (1867-68)

_- Dirichlet's proof of expansion possibility. Poincaré, H. C. R. 118 (1894) 497-

- - expansion of arbitrary function in. Echols, W. H. A. Mth. 12 (1898-99) 164 .

- -, fundamental principle, geometrical proof. Pratt, J. H. Ph. Mg. 24 (1862) 504.

- - of higher order, development of function of several variables according to. Mehler, F. G. Crelle J. 66 (1866) 161-。

- - property. Smith, Arch. Camb. Mth. J. 2 (1841) 192.

- - series expressing. Darboux, G. Liouv. J. Mth. 19 (1874) 1-.

- integrals, definite integrals allied to. Unferdinger, $F$. Wien Sb. 55 (1867) $(A b, 2)$ 90 -.

Legendre's functions, expansion of potential function $1 / R^{k-1}$ in. Routh, E.J. L. Mth. S. P. 26 (1895) 481-.

Linear, cylindric, spherical, and allied functions, expansion of functions in terms of. Alexander, $P$. Edinb. R. S. T. 33 (1888) 313-.

Periodic series, expansion of function. Encke, J. $F$. Berl. As. Jb. (1860) 313-.

- - , sums, critical values. Stokes, G. G. [1847] Camb. Ph. S. T. 8 (1849) 533-.

Potentials of ellipsoid and ellipse, expansion in series. Niven, $W . D$. Phil. Trans. 170 (1880) 379-. 


\section{Harmonic Analysis}

Series for function of elliptic coordinates. Escary, -. As. Fr. C. R. (1887) (Pt. 2) $63-$.

- of roots of transcendental equation, representation of arbitrary function by. Fujisawa, R. Tōk. Coll. Sc. J. 2 (1889) 1-.

Solar radiation, distribution on surface of earth, and dependence on astronomical elements. Hargreaves, $R$. [1896] Camb. Ph. S. T. 16 (1898) 58-.

Spherical analysis. Eastuood, G. Camb. (M.) Mth. M. 2 (1860) 163-, 186-, 271-, 301-; 3 (1861) 78-.

- function $P^{n}(\cos \theta)$, Dirichlet's integral expression for, and analogous form for cylin. drical function $J(x)$. Mehler, F. G. [1871] Mth. A. 5 (1872) 141-.

- functions, differentials. Most, R. [1868] Crelle J. 70 (1869) 163-.

- - geometric significance. Heine, E. Crelle J. 68 (1868) 386-.

- - , series, extension of theorem of Cantor's to. Neumann, C. G. Leip. Mth. Ps. B. 33 (1881) 1-; 35 (1883) 18-; Mth. A. 22 (1883) 406-.

- - , - proceeding by. Thomé, W.L. Crelle J. 66 (1866) 337-.

- _ , _ _ - with application to cylindrical functions. Bauer, G. Münch. Ak. Sb. 5 (1875) 247-.

- -, theory. Bruns, H. [1880] Crelle J. Mth. 90 (1881) 322-.

_-, - Sidler's. Schläfi, L. Zür. Vjschr. 8 (1863) 205-, 324-.

- harmonic analysis, definite integrals. Niven, W. D. Phil. Trans. 170 (1880) 379-。

- - , theorem. Darwin, G. H. Mess. Mth. 6 (1877) 165-.

- - of $n$th order, general canonical form. Thomson, (Sir) W. B. A. Rp. 41 (1871) (Sect.) 25-.

- harmonics. Tait, P. G. [1871] Edinb. R. S. P. 7 (1872) 589-.

- - Niven, W. D. [1877-78] Mess. Mth. 7 (1878) 1-, 131-.

23-.

- Beltrami, E. C. R. 110 (1890) 934-.

- -. Dyson, F. W. QJ. Mth. 26 (1893) 30 -.

- -, canonical form. Clifford, $W . K$. B. A. Rp. 41 (1871) (Sect.) 10.

- - cases. Burnside, W. Mess. Mth. 14 (1885) 122-.

- - of complex degree, with application to physical problems. Hobson, E. W. [1887] Camb. Ph. S. T. 14 (1889) 211-.

- - - 2nd kind, expression in finite form. Bryan, G. H. Camb. Ph. S. P. 6 (1889) 293-.

- - or Laplace's functions. Tallqvist, $H$. Helsingf. Acta 26 (1900) No. 4, 87 pp.

- representing magnetic potential of earth. Schuster, A. B. A. Rp. (1898) 752-.

- - systems. Hobson, E. W. L. Mth. S. P. 22 (1891) 431-.

- -, theory. Neumann, C. Leip. Mth. Ps. Ab. 13 (1887) 401-.
Equations of Physics 5630

Spherical harmonics, theory. Beltrami, $E$. C. R. 116 (1893) 181-; Mil. I. Lomb. Rd. 29 (1896) 793-.

- - of unrestricted degree, order, and argument, type. Hobson, E. W. [1896] Phil. Trans. (A) 187 (1897) 443-.

and hyper-spherical functions, theory. Neumann, C. Z. Mth. Ps. 12 (1867) 97-.

Tesseral harmonics, functions connected with. Leahy, A. H. R. S. P. 56 (1894) 45-.

Toroidal functions. Basset, A.B. Am. J. Mth. 15 (1893) 287-.

Zonal harmonics, expansion of elliptic integrals by. Hargreaves, $R$. Mess. Mth. 26 (1897) 89-.

\section{Generalities on the differen- tial equations of mathematical physics.}

Branched (verzweigte) potentials in space. Sommerfeld, A. L. Mth. S. P. 28 (1897) $395-$.

Conjugate potential functions, generalisation. Appell, $\bar{P}$. Mars. Fac. Sc. A. 2 (1892) 53-.

Differential alternating functions in astronomy. Cauchy, A. L. C. R. 11 (1840) 297-, 377-, $432-$.

Equation in mathematical physics, integration. Carvallo, E. Par. S. Mth. Bll. 22 (1894) 234-.

- of motion of heat in thin prism, complete integration. Pagani, G. M. Quetelet Cor. Mth. 3 (1827) 237-.

- in theory of propagation of electricity. Picard, É. Par. S. Mth. Bll. 22 (1894) 2-. Equations in conduction of heat. Sturm, J.C. F. Liouv. J. Mth. 1 (1836) 373-.

- of dynamics. Boole, G. Phil. Trans. (1863) 485-.

215-. Ball, (Sir) R. Aust. As. Rp. (1895) - - fundamental. Janaud, -. Arch. Mth. Ps. 67 (1882) 160-

- - mathematical physics. Poincaré, $H$. Palermo Cir. Mt. Rd. 8 (1894) 57 -

- - - Steklov, V.A. Rec. Mth. (Moscou) 19 (1897) 469 (bis) -.

- - mechanics. Grosso, R. del. G. Mt. 4 (1866) 243-.

- motion, property. Poisson, S. D. Par. S. Phlm. Bll. (1816) 109-.

- - physics, integration. Mathieu, É. Liouv. J. Mth. 17 (1872) 249 -.

- for problems of oscillation. Abraham, M. Mth. A. 52 (1899) 81-.

- - sound transmission through tube, Poisson's solution. Moon, R. [1866] R. S. P. 15 (1867) 306-.

- of thermodynamics, integrating factors. Fliegner, A. Zür. Vjschr. 40 (1895) 278-.

- - vibrator affected by its own reflected vibrations, and analogous problems. Waals, J. D. van der (jun.). Amst. Ak. Vs. 8 (1900) 638-; Amst. Ak. P. 2 (1900) 534-. 
Equilibrium of fluids, studies on Green's work. Büttner, $F$. Leip. Jablon. Preisschr. 36 (1900) $98 \mathrm{pp}$.

Existence proofs for potential in plane and in space. Harnack, A. Leip. Mth. Ps. B. 38 (1886) 144-.

Function $Z(x, y, z)$, applications to mathematical physics. Appell, $P$. Acta Mth. 8 (1886) 265-.

Functions of Laplace and Bessel, expression of velocity potential by. McMahon, J. N. Y. Am. Mth. S. Bll. 2 (1896) 173-.

- satisfying equation $\nabla F=0$. Appell, P. C. R. 96 (1883) 368-.

- of 3 variables satisfying equation $\nabla^{2} F=0$. Appell, P. Acta Mth. 4 (1884) 313-.

General equations of dynamical problems, particular integrals, use when general integration is impossible. Barnard, J. G. Am. As. P. 25 (1876) 49-.

Green's function. Ricci, $G$. Ven. I. At. (1884-85) 1439-.

Huygens' principle, demonstration. Tedone, $O$. Rm. R. Ac. Linc. Rd. 5 (1896) (Sem. 1) 357-, 483.

Integral of Laplace's equation in finite terms. Earnshaw, S. B. A. Rp. (1880) 486-.

$-\int \frac{\left(1-a^{2}\right) y^{\prime} d s}{f^{3}}$ in theory of heat. Ivory, $J$. Pैh. Mg. 1 (1827) 324-.

- Poisson, S. D. Ph. Mg. 2 (1827) 11-.

Integrals of differential equations, calculation by Cauchy-Lipschitz method. Painlevé, $P$. C. R. 128 (1899) 1505-.

- - partial differential equations, form. Poisson, S. D. Par. S. Phlm. Bll. (1817) 180-.

Kirchhoff's diffusion equations, reduction. Farkas, G. Mth. Termt. Éts. 16 (1898) 201-; Mth. Nt. B. Ung. 16 (1899) 97-.

Laplace's double equation, integration. $A l$ mansi, E. Rm. R. Ac. Linc. Rd. 9 (1900) (Sem. 1) 298-.

- equation. Boole, G. B. A. Rp. (1845) (pt. 2) 2 ; Camb. and Dubl. Mth. J. 1 (1846) 10 -

- Carmichael, R. Ir. Ac. P. 6 (1853) 216-.

- -. Donkin, W. F. [1856] Phil. Trans. (1857) 43-.

- - and analogues. Carmichael, R. Camb. and Dubl. Mth. J. 7 (1852) 126-; Ph. Mg. 6 (1853) 273-.

- - quaternion integration. Preobraženskij, $V . V$. Kazan S. Nt. (Ps.-Mth.) P. 2 (1884) 46-; Fschr. Mth. (1884) 308-.

- - most general solution of given degree. Hobson, E. W. L. Mth. S. P. 26 (1895) 492-.

- - in toroidal coordinates, solution by method of imaginary inversion. Safford, $F . H$. A. Mth. 12 (1898-99) 27-.

- - transformations leading to new solutions. Forsyth, A. R. L. Mth. S. P. 29 (1898) 165-.

- extended equation $\nabla^{2} \nabla^{2} u=0$, integration. Lauricella, G. Ven. I. At. (1898) 236-.
Linear equations occurring in mathematical physics. Macfarlane, A. Am. As. P. (1897) 54 -.

- partial differential equations. Järisch, $P$. Hamb. Mth. Gs. Mt. 2 (1890) (Festschr., Tl. 2) 110-.

- - - - especially general equation of motion of elastic fluids, integration. Poisson, S. D. Par. Mm. Ac. Sc. 3 (1818) 121-.

- - - - relative to motion of heat in solids, integration. Pagani, G. M. Brux. Ac. Sc. Mm. 8 (1834) $32 \mathrm{pp}$.

Maxwell's theory of electricity, mathematically considered. Planck, M. D. Mth. Vr. Jbr. 7 (1899) (Heft 1) 77-.

Molecular motion in gases, integration of equations. Boltzmann, L. [1876] Wien Ak. Sb. 74 (1877) (Ab. 2) 503-.

Operator $\nabla^{2}$, properties. Haton de la Goupillière, -. Brux. S. Sc. A. 9 (1885) (Pt. 2) 151-.

Partial differential equations of mathematical physics. Poincaré, $H$. Am. J. Mth. 12 (1890) 211-.

- - - - - - solutions, multiform. Carslaw, H. S. [1898] L. Mth. S. P. 30 (1899) 121-.

- - - - , simple. Mathieu, É. L. Liouv. J. Mth. 5 (1879) 5-.

- - - 4th order, $\nabla \nabla u=0$. Mathieu, $\dot{E}$. Liouv. J. Mth. 14 (1869) 378-.

- - systems, integration. Cauchy, A.L. C. R. 13 (1841) 1-.

- - and vibrating surfaces. Biot, J. B. Par. Mm. de l'I. 4 (1802) 21-.

_ - - of small vibrations of elastic bodies, integration. Ostrogradsky, M. A. [1832] St Pét. Ac. Sc. Mm. 2 (1833) 339-.

- - - - - - - medium, integration. Ostrogradsky, M. A. [1829] St Pét. Ac. Sc. Mm. 1 (1831) 455-.

Polynomials, conjugate, class. Deruyts, $J$. Brux. Mm. Cour. $4^{\circ}, 48$ (1886) No. 3, $20 \mathrm{pp}$.

Potential function, 3rd derivative. Paci, $P$. Genova Un. At. [11] (1892) 593-.

- theory. Dyck, W. Münch. Ak. Sb. 25 (1896) 261-, 447-; 28 (1899) 203-.

Schwarz's integrals of functions given by $\nabla^{2} V_{i+1}+V_{i}=0$. Andrade, - Par. S. Mth. Bll. 22 (1894) 50 -

Solution of $\frac{d\left(a^{2} \frac{d V}{d x}\right)}{d x}+$ etc. $=4 \pi \rho$, theorems. Thomson, (Sir) Wr. Camb. and Dubl. Mth. J. 3 (1848) 84-.

Stationary temperatures. Lauricella, G. Palermo Cir. Mt. Rd. 11 (1897) 189-.

Successive divergent approximations, example. Picard, É. Par. S. Mth. Bll. 28 (1900) 137-.

Transformation, application of Dirichlet's method. Meyer, F. Mth. A. 26 (1886) 509-.

Uniform functions $F$ satisfying $\nabla^{2} F=0$. Mittag-Lefler, G. As. Fr. C. R. (1888) (Pt. 1) 153. 


\section{Equations of Physics}

Variation of arbitrary constants in mechanics. Poisson, S. D. Par. Mm. Ac. Sc. 1 (1816) 1-.

. Lubbock, J.W. Ph. Mg. 11 (1837) 492-; 12 (1838) 254.

Vector conditions of integrability. Tait, $P$. G. [1877] Edinb. R. S. P. 9 (1878) 527-.

$\frac{d^{2} \phi}{d t^{2}}-a^{2} \nabla^{2} \phi=\psi(t, x, y, z)$, integration. Liouville, J. Liouv. J. Mth. 4 (1839) 1-.

" $\nabla$ " equal to positive unity (Lagrange). Plana, G. Brux. Ac. Bll. 8 (1841) (pte. 2) 68-.

$\frac{d\left(a^{2} \frac{d V}{d x}\right)}{d x}+$ etc. $=4 \pi \xi$.

Thomson, (Sir) W.

Liouv. J. Mth. 12 (1847) 493-

$\nabla^{2} u=0$, solution by method of quaternions. Boole, G. Ir. Ac. P. 6 (1853-54) 375-.

$-=-$, integration. Dini, U. A. Mt. 5 (187173) 305-

- =-, 一. Ascoli, G. Mil. I. Lomb. Rd. 19 (1886) 285-.

$-=-,-$ in non-Euclidean space. Bianchi, L. Rm. R. Ac. Linc. Rd. 9 (1900) (Sem. 2) 333-.

$\frac{d^{2} u}{d t^{2}}=a^{2} \frac{d^{2} u}{d x^{2}}$, integration. Mainardi, G. Tortolini A. 5 (1854) 161-, 273-, 459-.

$-=-$, applications in acoustics and optics. Klinkerfues, $W$. Gött. Nr. (1868) 469-.

$-=-$ in theory of sound. Amagat, [E. H.] J. de Ps. 9 (1880) 56-.

$\frac{d w}{d t}=\frac{d^{2} w}{d x^{2}}$. Schläfi, L. Crelle J. $72(1870)$ $263-$.

$\nabla^{2} v=0$, reduction to ordinary differential equations. Wangerin, F. H. A. Berl. Ak. Mb. (1878) 152-.

- =- $, \nabla^{2} v=-k^{2} v, \nabla^{2} v=c^{2} \ddot{v}$, new solutions. Forsyth, A. R. Mess. Mth. 27 (1898) 99-, 190.

$-=-$, solutions. Bromwich, T. J. I'A. L. Mth. S. P. 30 (1899) 281-.

$A \frac{d^{n} \phi}{d t^{n}}+\left(\frac{d^{2}}{d x^{2}}+\frac{d^{2}}{d y^{2}}+\ldots\right)^{n} \phi=0, \quad$ integration. Boussinesq, J. C. R. 94 (1882) 514-.

$\nabla^{2} f=0$ with $n$ variables. Giuliani, G. G. Mt. 25 (1887) 109-.

$\nabla^{2} z=0$. Du Bois-Reymond, P. Crelle J. Mth. 103 (1888) 204-.

$\nabla^{2} \phi=0$ in orthogonal coordinates. Petrini, $H$. Fschr. Mth. (1890) 982-.

$\nabla^{2} u+k^{2} u=0$. Bôcher, M. Am. J. Mth. 15 (1893) 78-

- = - in space of $\boldsymbol{n}$ dimensions. Marcolongo, R. A. Mt. 24 (1896) 301-.

$\frac{\partial u}{\partial x}+\frac{\partial v}{\partial y}+\frac{\partial w}{\partial z}=0$. Morera, G. Palermo Cir. Mt. Rd. 7 (1893) 288-.

$\nabla^{2} u=k e^{u}$ on closed Riemann surface. Picard, É. Liouv. J. Mth. 9 (1893) 273-.

$\nabla^{2} \Phi+k^{2} f(x y z) \Phi=0$. Petrini, H. Stockh. Ak. Hndl. Bh. 19 (Afd. 1) (1894) No. 4, 36 pp.

$\nabla^{2} u=F(u, x, y)$, integration. Picard, $\dot{E}$. C. R. 124 (1897) 1488-

\section{Integration by Series 5640}

$\nabla^{4} u=0$, integration. Almansi, $E$. Tor. Ac. Sc. At. 34 (1898) 68- or 92-.

- $=-$. Goursat, -. Par. S. Mth. Bll. 26 (1898) 236-.

- = - integration. Almansi, E. Rm. R. Ac. Linc. Rd. 8 (1899) (Sem. 1) 104-, 618. $-=-,-$ Boggio, T. Ven. I. At. (18991900) (Pt. 2) 497-.

$\nabla^{2} u=e^{u}$ and Fuchsian functions. Poincaré, $H$. Liouv. J. Mth. 4 (1898) 137-.

$\nabla^{2} \phi=\frac{d^{2} \phi}{d t^{2}}$ for space of $n$ dimensions. Tedone, O. A. Mt. 1 (1898) 1-.

$\nabla^{2} u+\xi u+f=0$. Zaremba, S. Par. S. Mth. Bll. 26 (1898) 70-.

$-=-$, and harmonic functions. Zaremba, $S$. Par. Éc. Norm. A. 16 (1899) 427-; Prace Mt.-Fiz. 11 (1900) 99-; Fschr. Mth. (1900) 375.

$\nabla^{2 n}=0$, integration. Almansi, E. A. Mt. 2 (1899) 1-.

$\nabla_{n}^{2} u$, integration. Brajcev, I. R. Rec. Mth. (Moscou) 21 (1901) 490-; Fschr. Mth. (1900) 376.

$\nabla^{2} u=k^{2} u$, problems relating to. Picard, Par. S. Mth. Bll. 28 (1900) 186-.

\section{Integration of the differen-} tial equations of mathematical physics by series.

Convergence of series in Kepler's problem (Carlini). Jacobi, C. G. J. As. Nr. 30 (1850) 197.

- - - serving as integrals of differential equations of 1st order. Nazimov, P.S. Kazan S. Nt. (Ps.-Mth.) P. 8 (1890) 145-; Fschr. Mth. (1890) 325.

Divergent series in differential equations. Horn, J. D. Mth. Vr. Jbr. 8 (1900) (Heft 1) 219-.

Expansions occurring in theory of conduction of heat. Poincaré, H. C. R. 118 (1894) 383-.

Formula for calculation of a certain potential. Lerch, - . Liouv. J. Mth. 5 (1899) 427-.

Functions satisfying the equation $\nabla^{2} u=0$. Ascoli, G. Mil. I. Lomb. Rd. 18 (1885) $474-, 546-, 599-, 617-$.

Gauss's series in experimental physics. Tammen, H. G. Carl Rpm. 18 (1882) 129-.

Heating of a cylinder. Laar, J.J.van. Haarl. Ms. Teyl. Arch. 6 (1900) 65-

Integrals of differential equations near essential singularities (viz. "normal" series in Poincaré's sense). Rajewski, J. Krk. Ak. (Mt.-Prz.) Pam. 17 (1890) 166-; Crc. Ac. Sc. Bll. (1890) 62-.

-, particular, of $\nabla^{2} F=F$, and their expansion in spherical harmonics. Neumann, C. Leip. Mth. Ps. B. 38 (1886) 75-.

Integration of equations of motion. Sokoloff, J. C. R. 55 (1862) 99-.

- _ - - sound with resistance terms. Boussinesq, J. C. R. 118 (1894) 162-, 223-, 271-. 
Integration by means of simple solutions. Somigliana, C. Mil. I. Lomb. Rd. 24 (1891) 1005 -.

- of $\nabla^{2} f=k^{2} f$ for elliptic and parabolic coordinates. Hartenstein, J. H. Arch. Mth. Ps. 14 (1896) 170-

Integrations relating to elastic equilibrium. Mathieu, É. L. Par. Éc. Pol. J. Cah. 48

- (1880) 163-.

Laplace's equation, form, special. Niven, $W$. D. $[1880]$ Mess. Mth. 10 (1881) 114-.

- - solution, particular. Jacobi, C. G.J. Crelle J. 36 (1848) 113-.

Maxwell's equations of electro-magnetic field, solution. Fitzgerald, $G . F$. B. A. Rp. (1890) 755-.

Propagation of electricity. Poincaré, $H$. C. R. 117 (1893) 1027-.

- - - , partial differential equation in. Picard, É. C. R. 118 (1894) 16-.

Solutions of $\left(V^{2}+k^{2}\right) \psi=0$ in elliptic coordinates, and physical applications. ( $V^{2}$ written for $\nabla^{2}$.) Maclaurin, R. C. [1898] Camb. Ph. S. T. 17 (1899) 41-.

$\nabla^{4} k=0$, solutions in series. Marcolongo, $R$. Nap. Rd. 28 (1889) 149-.

$\phi+\frac{2 k}{R} \frac{d \phi}{d t}=\frac{k}{R} \Theta(t)$, electric interpretation. Reignier, C., \& Bary, $P$. Cherb. S. Sc. Nt. Mm. 26 (1889) 65-.

\section{Integration of the differen- tial equations of mathematical physics by definite integrals.}

Duhamel, J. M. C. Liouv. J. Mth. 19 (1854) 337-.

Definite integrals suggested by problems in theory of electricity. Thomson, (Sir) W. Camb. and Dubl. Mth. J. 2 (1847) 109-.

Double definite integrals, employment in finding the form of waves. Cauchy, A. L. C. R. 13 (1841) 188-.

Finite integrals of partial differential equations occurring in physics. Earnshaw, S. Ph. Mg. 4 (1877) 213-.

Fresnel's integrals, geometric significance. Umov, N. N. Rs. S. Nt. Mm. (Mth.) 6 (1885) 57-; Fschr. Ps. (1885) (Ab. 2) 15-.

Integration of $\nabla^{2} u=0$ on given Riemann surface. Ascoli, G. [1887] Stockh. Ak. Hndl. Bh. 13 (Afd. 1) (1888) No. 2, 83 pp.

- - = - - - - (Ascoli). Eneström, G. Stockh. Öfv. (1887) 99-; Fschr. Mth. (1887) 29.

$--\nabla^{4} u=0$ (2 variables). Almansi, $E$. Tor. Ac. Sc. At. 31 (1895) 527- or 881-.

$---=-$ in a plane circular field. Lauri. cella, $G$. Tor. Ac. Sc. At. 31 (1895) 610-or 1010 -.

$---=-$ (Lauricella and Almansi). Volterra, $V$. Tor. Ac. Sc. At. 31 (1895) 618- or 1018-

- $-\nabla^{2} u+k^{2} u=0$ on Riemann surfaces. Sommerfeld, A. Gött. Nr. (1895) 267-.
Integration of $\nabla^{4} u=0$ (2 variables). LeviCivita, T. Tor. Ac. Sc. At. 33 (1897) 692or 932-.

\section{Dirichlet's problem and analogous problems affected by boundary conditions.}

Analytic theory of heat, problem in. Stekloff, W. C. R. 126 (1898) 1022-.

Boundary condition and discontinuities, functions determined from. Burnside, $W$. L. Mth. S. P. 22 (1891) 346-.

- conditions, determination of integrals of partial differential equations by. Picard, $\dot{E}$. Par. S. Mth. Bll. 22 (1894) 103-.

- - given, determination of function in a circle from. Veltmann, $W$. Z. Mth. Ps. 26 (1881) 1-.

-,$- \ldots$, solutions of certain differential equations satisfying. Brill, $J$. Camb. Ph. S. P. 6 (1889) 344-.

- value problem for continuous plane curve with no cusp. Noble, C. A. Gött. Nr. (1896) 191-.

Contour values, determination of integrals of differential equations by. Picard, É. C. R. 130 (1900) 447-.

- - , - - - linear equations, 2nd order, by. Picard, É. Liouv. J. Mth. 6 (1900) 129-.

- - , - - - partial differential equations by. Picard, É. C. R. 109 (1889) 499-; Par. Éc. Pol. J. 60 (1890) 89-; Liouv. J. Mth. 2 (1896) 295-.

- —, - - - - - - - 2nd order, by.

Picard, E. C. R. 128 (1899) 1487-.

Cooling of isotropic body, two theorems of Poincaré. Andradez, -. N. A. Mth. 8 (1889) 435-.

Dirichlet's principle. Weierstrass, $K$. [1870] Wiad. Mt. 2 (1898) 1-.

- -. Phragmén, E. Stockh. Öfv. (1891) $745-$.

- - Arzelà, C. Bologna Rd. 1 (1897) 71-. - Volterra, V. Palermo Cir. Mt. Rd. 11 (1897) 83-.

- - Hilbert, D. D. Mth. Vr. Jbr. 8 (1900) (Heft 1) 184-.

- -, application to function. Graf, J. $H$. Bern Mt. (1891) 95-.

- - in connection with $\nabla^{2} u=k e^{u}$. Schulz, $W$. N. Y. Am. Mth. S. Bll. 5 (1899) 219, 221-.

- - , demonstration, new. Phragmén, $\boldsymbol{E}$. Stockh. Öfv. (1892) 511-.

$-\ldots,-$, preliminary assumptions. Heine, $E$. Gött. Nr. (1871) 375-.

- - -, Riemann's. Weber, H. [1869] Crelle J. 71 (1870) 29-.

- -, generalisation. Riemann, -. C. R. 106 (1888) 123-.

- - , substitute for. Dalwigk, F. von. Gött. Nr. (1894) 160-. 
Dirichlet's principle, substitute for, in certain cases. Neumann, C. Leip. Mth. Ps. B. 47 (1895) 185-.

- problem. Riemann, J. Par. Ec. Norm. A. 5 (1888) 327-.

- -. Grave, D. A. As. Fr. C. R. (1895) (Pt. 2) 111-.

- Zaremba, S. Par. Éc. Norm. A. 14 (1897) 251-.

_-. Fredholm, I. Stockh. Öfv. (1900) 39-.

- for 2 dimensions, special case. Kluyver,

J. C. Acta Mth. 21 (1897) 265-.

- - in domains, with discontinuous boundaries. Schwarz, H. A. Zür. Vjschr. 15 (1870) 272-

- - and its extension to general linear equations, 2nd order. Paraf, A. Toul. Fac. Sc. A. 6 (1892) H, 75 pp.

- - generalisation, and linear partial differential equations, 2nd order. Picard, $\hat{E}$. C. R. 130 (1900) 1088-.

- - and harmonic functions belonging to closed surface. Le Roy, - . C. R. 123 (1896) 986-.

- - questions relating to. Liapounoff, $A$. C. R. 125 (1897) 808-; Liouv. J. Mth. 4 (1898) 241-.

- - , solutions by combination of Neumann's and Schwarz's methods. Kohn, A. Mth. A. 53 (1900) 593-.

Distribution of electricity, problem of. Poincaré, $H$. C. R. 104 (1887) 44-.

- - - _ - - and Neumann's problem. Stekloff, $W$. C. R. 125 (1897) 1026-.

Equation of vibrations of a membrane. Poincaré, $H$. C. R. 118 (1894) 447-.

Equations of conduction of heat, integration. Le Roy, É. Par. Ec. Norm. A. 14 (1897) 379-; 15 (1898) 9-.

Fundamental functions and Dirichlet's problem. Stekloff, W. C. R. 131 (1900) 870-.

-- , existence. Stekloff, W. C. R. 128 (1899) 808 -.

- 一, theory. Stekloff, W. C.R.128(1899) 984-. - problems of mathematical physics. Stekloff, W. C. R. 128 (1899) 588-

- - - - - general methods for solution. Stekloff, W. Toul. Fac. Sc. A. 2 (1900) 207-

Green's function, behaviour at limit of its region. Neumann, C. Leip. Mth. Ps. B. 40 (1888) 163-.

- - for system of non-intersecting spheres. Burnside, W. L. Mth. S. P. 25 (1894) 94-.

- -, theory. Zaremba, -. Par. S. Mth. Bll. 24 (1896) 19-.

- functions in 1 dimension. Burkhardt, $H$. Par. S. Mth. Bll. 22 (1894) 71-.

- second function. Arcais, $F$. d'. Ven. I. At. (1897-98) 1661-.

Integrals of Laplace's equation. Capelli, $\boldsymbol{A}$. G. Mt. 23 (1885) 123-.

Integration of $\frac{d^{2} \phi}{d x^{2}}+\frac{d^{2} \phi}{d y^{2}}=0$. Neumann, $C$. Crelle J. 59 (1861) 335-.

- $\frac{d^{2} u}{d x^{2}}+\frac{d^{2} u}{d y^{2}}+k^{2} u=0$. Weber, H. [1868] Mth. A. 1 (1869) 1-.
Integration of $\frac{d^{2} u}{d x^{2}}+\frac{d^{2} u}{d y^{2}}=0$ under given conditions as to boundary and discontinuity. Schwarz, H. A. Berl. Mb. (1870) 767-.

- - - - Prym, F. E. Crelle J. 73 (1871) 340-.

- $-\nabla^{2} u=0$ within a circular area. Ascoli, $G$. Mil. I. Lomb. Rd. 17 (1884) 627-.

$---=-$ for some simple areas. Ascoli, G. Mil. I. Lomb. Rd. 18 (1885) 252-.

$--\frac{-}{-}=$ in any Riemann area. Ascoli, G. Mil. I. Lomb. Rd. 18 (1885) 718-

$---=-$. Ohnesorge, 0 . Arch. Mth. Ps. 2 (1885) 53-.

$--\nabla^{4} u=0$ for plane regions. Venske, $O$. Gött. Nr. (1891) 27-.

$-\nabla^{2} u=f u$. Lindeberg, J. W. C. R. 130 (1900) 1539-.

$--\nabla^{2} u=e^{u}$ on a closed surface. Picard, $E^{\prime}$. Bll. Sc. Mth. 24 (1900) 196-.

Inverse square, actions following law of. Vaschy, -. As. Fr. C. R. (1894) (Pt. 2) $141-$.

Method of arithmetical mean. Neumann, $C$. Leip. Mth. Ps. Ab. 13 (1887) 705-; 14 (1888) 563-.

_ _ _ - Neumann's. Tauber, A. Mh. Mth. Ps. 5 (1894) 137-.

$---\frac{1}{-}$ - Stekloff, $W$. C. R. 131 (1900) $987-, 1182-$.

- - successive approximations, Picard's. Zaremba, S. C. R. 124 (1897) 554-.

Neumann's and Gauss's problems. Stekloff, $W$. C. R. 130 (1900) 480-.

- method and Dirichlet's problem. Poincaré, H. [1895] Acta Mth. 20 (1897) 59-.

- - - Stekloff, W. C. R. 130 (1900) 396-, 1599-.

--130 Korn, A. C. R. 130 (1900) $557,1238-; 131$ (1900) 26-.

- - - - (Korn). Stekloff, W. C. R. 130 (1900) 826-.

- of solving Dirichlet's problem. La ValléePoussin, C. de. Brux. S. Sc. A. 18 (1894) (Pt. 1) 11-.

Partial differential equation in theory of membranes. Picard, É. C. R. 117 (1893) 502-. - equations, 2nd order, with imaginary characteristics. Picard, É. C. R. $122(1896)$ 417-.

Poisson's integral. Bôcher, M. N. Y. Am. Mth. S. Bll. 4 (1898) 424-.

Potential, existence, proof. Kirchhoff, $G$. Acta Mth. 14 (1890-91) 179-.

- function in plane space bounded by two circles with given values at boundaries. Kluyver, J. C. N. Arch. Wisk. 3 (1898) 230-; Fschr. Mth. (1897) 363-.

- functions expressed in curvilinear coordinates. Painlevé, P. [1889] Lille Tr. Mm. 1 (1889-91) Mém. 1, 28 pp.

- theory, solution of fundamental problem in. Schütz, J.R. Gött. Nr. (1895) 1-.

Problem of hydrodynamics. Lejeune-Dirichlet, G. [1857] Gött. Ab. 8 (1858-59) (Mth.) 3-. - - Dirichlet's. Dedekind, R. [1860] Crelle J. 58 (1861) 217-. 
Problem of hydrodynamics, Dirichlet's. Brios chi, F. [1860] Crelle J. 59 (1861) 63-.

Residual integral in $\frac{d^{2} u}{d t^{2}}=a^{2} \nabla^{2} u$. Hadamard, -. Par. S. Mth. Bll. 28 (1900) 69-.

Solution of Laplace's equation, finite and continuous with normal deviation given at all points of bounding surface. Steklow, W. A. Fschr. Mth. (1897) 364-.

Steady flow of electricity on rectangular plate. Mathieu, É. As. Fr. C. R. (1886) (Pt. 2) 1-.

Stokes's theorem for hydrokinetic circulation, proof. Mukhopadhyay, A. Beng. As. S. J. 59 (Pt. 2) (1891) 59-.

Theorem of Picard. Kobb, G. C. R. 111 (1890) 726-.

_- Poincaré. Zaremba, S. C. R. 127 (1898) 215-.

Thermal equilibrium of closed radiating surface. Picard, É. C. R. 130 (1900) 1499-.

Values on closed surface, determination of integrals of partial non-linear equations by. Le Roy, E. C. R. 124 (1897) 1508-.

Vaschy's theorem on any vectorial distribution. Larose, -. Par. S. Mth. Bll. 24 (1896) 177-.

$\frac{d^{2} \phi}{d x^{2}}+\frac{d^{2} \phi}{d y^{2}}=0$. Liouville, J. Liouv. J. Mth. 8 (1843) 265-.

$\nabla^{2} u=e^{u}$. Picard, É. Liouv. J. Mth. 4 (1898) 313-.

\section{Difference equations and functional equations.}

\section{General.}

Analytic factorials, reduction in series. Novi, G. G. Mt. 2 (1864) 1-, 40-.

Difference equation, 2nd order. Cayley, $A$. QJ. Mth. 14 (1877) 23-.

Differential coefficients, calculation from tables. Sheppard, W. F. Nt. 60 (1899) 390-.

- - and finite differences. Makeham, W. M I. Act. J. 16 (*1872) 98-.

- equations, numerical solution. Láska, $W$. Prag Sb. (1897) (Mth.-Nt.) No. 35, 10 pp.

Euler's formula, deduction. Žbikovskij, $A$. [1870] St Pet. Ac. Sc. Mm. (Rs.) $18\left({ }^{*} 1871\right)$ 177 -

Finite differences. Lobatto, $R$. Amst. N. Vh. 6 (1837) 1-.

- increments, generalisation of formula for. Stieltjes, T. J. N. A. Mth. 7 (1888) 26-.

Generic equations, class. Blissard, $J$. QJ. Mth. 5 (1862) 325-.

Interpolation formula. Roe, E. D. [1900] N. Y. Am. Mth. S. Bll. 7 (1901) 202, 203-.

Lagrange's calculus of functions, superiority to infinitesimal calculus. Magistrini, G. B. [1832] Bologna Mm. Ac. Sc. 1 (1850) 93-.

Linear difference forms, algebra. Pincherle, $S$. Bologna Ac. Sc. Mm. 5 (1895-96) 87-.

Numbers, $\Delta^{m} 0^{n}$ class, properties. Blissard, $J$. QJ. Mth. 8 (1867) 85-; 9 (1868) 82-, $154-$.
Numbers, $K_{m}^{p}=\left[p^{m}-\left(\frac{p}{1}\right)(p-1)^{m}+\ldots\right.$

$$
\left.+(-1)^{r}\left(\frac{p}{r}\right)(p-r)^{m}+\ldots\right] \div p !
$$

Ocagne, M. d'. G. Teix. J. Sc. 8 (1887) $171-; 10$ (1891) 185-.

Progressions. Jadanza, N. G. Mt. 7 (1869) 117-.

Recurring systems of 3rd order, especially periodic systems. Bortolotti, E. Palermo Cir. Mt. Rd. 5 (1891) 129-.

Sum of functions of quantities in arithmetical progression. Richmond, H.W. Mess. Mth. 21 (1892) 29-.

Summation formula of Euler. Eneström, $G$. Stockh. Öfv. 36 (1879) No. 10, 3-.

- - Stirling. Sonin, N. Ja. [1889] Vars. S. Nt. Tr. (1889-90) (C.R., Ps. C.) No. 2, 1-。 - formulæ of Euler and Stirling. Somin, N. Ja. [1889] Vars. S. Nt. Tr. (1889-90) (C. R., Ps. C.) No. 1, 2-.

- - in finite differences. Glaisher, J.W.L. QJ. Mth. 29 (1898) 303-.

\section{Solution of equations of finite differences.}

Murphy, R. [1835] Camb.Ph. S.T.6(1838) 91-. Ellis, R. L. Camb. Mth. J. 4 (1845) 182-.

Siverly, $W$. Des Moines Anal. 1 (1874) 8-.

Pincherle, S. Rm. R. Ac. Linc. Rd. 3 (1894) (Sem. 1) 12-, 99-.

Case. Catalan, E. C. Liouv. J. Mth. 3 (1838) 508-; 4 (1839) 95̃-.

- Walton, W. [1867] QJ. Mth. 9 (1868) 108-.

-. Sylvester, J.J. Ph. Mg. 8 (1879) 120-.

Class of equations of integrable form. Sylvester, J. J. C. R. 54 (1862) 129-, 170-.

Difference and differential equations, connexion between. Mellin, $H$. Acta Mth. 9 (1887) 137-.

- - - - of integrals. Tortolini, B. G. Arcad. 97 (1843) 45-

- - - integrable class. Sylvester, J. J. [1881] (xII) J. H. Un. Cir. [1] (1882) 178.

- - - - law of reciprocity. Spitzer, $S$. Grunert Arch. 33 (1859) 415-.

Equation connected with $\frac{d^{n}}{d z^{n}}\left(e^{\frac{1}{3}}\right)$. Vivanti, $G$. G. Teix. J. Sc. 11 (1892) 167-.

—, 2nd order. Sylvester, J. J. Ph. Mg. 37 (1869) 225-.

-, - -, general. Muir, T. Ph. Mg. 17 (1884) 115-.

- of $n$th order has $n$ constants in its solution. Eneström, G. Stockh. Öfv. (1886) 247- ; Fschr. Mth. (1886) 303-.

Equations for approximating to differential equation. Coriolis, G. Liouv. J. Mth. 2 (1837) 230-.

_, 1st degree, in 1 variable. Herschel, (Sir) J. F. W. Camb. Mm. Anal. S. (1813) 65-. - of mixed differences. Biot, J. B. [1799] Par. S. Phlm. Bll. 2 (1799-1801) 86-; Par. Mm. Sav. Étr. 1 (1806) 296- 
Equations of mixed differences. Poisson, S. D. Par. Ec. Pol. J. $13^{\circ} \mathrm{cah}$. (1806) 126-.

- - Walton, W. [1869] QJ. Mth. 10 (1870) 248-.

- - Lecornu, L. Par. S. Mth. Bll. 27 (1899) 153-.

- - - case. Cesáro, E. N. A. Mth. 4 (1885) 36-.

- - - - Lémeray, E. M. Edinb. Mth. S. P. 16 (1898) 13-.

- possessing unique integral. Sylvester, J. J. Mess. Mth. 18 (1889) 113-.

-, new species. De Morgan, A. (vi Adds.) Camb. Mth. J. 4 (1845) 87-.

一, theory. Heymann, $W$. Crelle J. Mth. 109 (1892) 112-.

Finite difference summation of integral function. Hurwitz, A. Acta Mth. 20 (1897) 285-; 22 (1899) 179-.

- differences, development in functions of derivative and vice versa. Caldarera, $G$. Catania Ac. Gioen. At. 6 (1893) Mem. 9, 14 pp.

Functional calculus. Pincherle, S. Bologna Rd. (1894-95) 34-.

- equations, method in theory. Schröder, $E$. B. A. Rp. (1887) 621.

Integral calculus of equations. Lorgna, $A . M$. Verona S. It. Mm. 1 (1782) 373-

-, complete, of linear partial differential or difference equations with constant coefficients, arbitrary functions in. Oltramare, G. As. Fr. C. R. (1895) (Pt. 2) 171-.

- of general equation. Sylvester, J. J. $\mathrm{Ph}$. Mg. 24 (1862) 436-.

Integrals. Biot, J. B. Par. Ec. Pol. J. $11^{\circ}$ cah. (1802) 182-.

- report on Biot's memoir. Prony, R. de. Par. Mm. de l'I. 3 (1801) (H.) 12-.

Integrating factor of finite difference and differential equations. Urusov, (Prince) S. S. [1865] (xII) Rec. Mth. (Moscou) [1] (1866) 225-; 2 (1867) (Pt. 1) 227.

Integration, cases. Duhamel, J.M.C. Liouv. J. Mth. 4 (1839) 222-.

-, -. Bronwin, B. Camb. Mth. J. 3 (1843) 258-; Camb. and Dubl. Mth. J. 2 (1847) 42-. -, 一. Spitzer, S. Grunert Arch. 40 (1863) 25-.

-, - . Gohierre de Longchamps, -.. As. Fr. C. R. 6 (1877) 194-

-, determination of constants arising in. Zehfuss, G. Grunert Arch. 27 (1856) 12-.

- of equations involving circulating functions. Herschel, (Sir) J. F. W. Phil. Trans. (1818) 144-.

- - - reducible to linear equations. Oltra mare, G. As. Fr. C. R. (1893) (Pt. 2) 106-.

- by finite differences, first principles. Harvey, G. Thomson A. Ph. 10 (1817) 264-. - of linear equations. Mainardi, G. Rm. At. N. Linc. 20 (1867) 167-.

- - - Oltramare, G. As. Fr. C. R. (1891) (Pt. 2) 66- ; (1895) (Pt. 2) 175-

- - - by calculus of residues. Tortolini, B. G. Arcad. 90 (1842) 84-; 91 (1842) 3-; 92 (1842) 129-, 265-.

- - - - with constant coefficients. Mainardi, $G$. Tortolini A. 5 (1854) 5-。
Integration of linear equations, any order. Libri, G. Crelle J. 12 (1834) 234-.

- _ _ - with partial finite differences. Eytelwein, J. A. Berl. Ab. (1824) 53-.

- - - - variable coefficients. Binet, J. P. M. Par. Ac. Sc. Mm. 19 (1845) 639-.

- method, Brisson's first, for linear and finite difference equations. Mansion, $P$. [1869] Brux. Mm. Cour. $8^{\circ}, 22$ (1872) (No. 1) 32 pp. - - for equations, coefficients of which are algebraic functions of independent variable. Spitzer, S. Grunert Arch. 32 (1859) 334.

- - L Laplace's, for linear and finite difference equations. Darboux, G. Bll. Sc. Mth. As. 3 (1879) 209-.

- of partial finite and infinite difference equations. Paoli, P. Mod. S. It. Mm. 8 (1794) 575-.

$--f(x+n)=\phi(x) f(x)$. Spitzer, $S$. Grunert Arch. 38 (1862) 456-.

$-(n+\kappa+1)(n+\lambda+1) \Delta^{2} \phi(n)$ $+(a+b n) \Delta \phi(n)+c \phi(n)=0$. Thomae, J. Z. Mth. Ps. 16 (1871) 146-, 428-. $--\psi(n)=h \psi(n-1)+k \psi(n-2)+l$. Tagiuri, A. G. Mt. 31 (1893) 95-.

Inverse calculus of finite differences. Björling, E. G. Ups. N. Acta S. Sc. 12 (1844) 299-; 13 (1847) 14-.

Linear difference or differential equations. Poincaré, H. Am. J. Mth. 7 (1885) 203-

- - equations, transformation of linear differential equations into. Pincherle, S. Mil. I. Lomb. Rd. 19 (1886) 559-.

- equations. Lobatto, $R$. Amst. N. Vh. 6 (1837) 83-.

- - Tardy, P. Tortolini A. 1 (1850) 337-.

- - Cohen, A. QJ. Mth. 1 (1857) 10-.

- Brassinne, E. Toul. Mm. Ac. 2 (1870) 190 -

- West, É. As. Fr. C. R. (1884) (Pt. 2)

64-.

- - Torelli, G. Nap. Rd. 34 (1895) 225-. -, 1st order. Mellin, $H$. Acta Mth. 15 (1891) 317-.

- homogeneous equations, 2nd order, properties. Besso, $D$. Rm. R. Ac. Linc. Rd. 1 (1885) 381-.

- trinomial recurring equations. Caldarera, F. G. Mt. 35 (1897) 333-.

Partial equations. Paoli, $P$. Verona S. It. Mm. 2 (1784) 787-; 4 (1788) 455-.

- - case. Liouville, J. Liouv. J. Mth. 1 (1856) 295-

Particular equations. Herschel, (Sir) J. F.W. Camb. Mm. Anal. S. (1813) 65-

Plane surface, formation, law of progress of accuracy. Airy, (Sir) G. B. Ph. Mg. 42 (1871) 107-.

Series of Fuss's problem. Mention, $J$. St Pét. Ac. Sc. Bll. 1 (1860) 507-.

Simultaneous linear differential or difference equations with constant coefficients. Sylvester, J. J. Am. J. Mth. 4 (1881) 321-.

Solution, approximate. Pincherle, S. Rm. R. Ac. Linc. Rd. 7 (1898) (Sem. 1) 230-.

- of class of difference or differential equations. Sylvester, J.J. Am. J. Mth. 4 (1881) 260 - 
Solution, general, of equations, 1st order. Kramp, C. Erfurt N. Acta 1 (1799) 71-.

- of linear differential and difference equations by definite integrals. Brajtzew, J. $R$. Fschr. Mth. (1900) 356.

- - - or difference equations in series. Pincherle, S. Palermo Cir. Mt. Rd. 2 (1888) 153-.

- - - equation in most general form.

[. Sylvester, J. J. B. A. Rp. (1862) (pt. 2) 188.

- - equations. Hargreave, C. J. [1849] Phil. Trans. (1850) 261-.

- $\ldots$ - - of finite and mixed differences. Gregory, D. F. Camb. Mth. J. 1 (1839) 54-.

_ _ _ _ with variable coefficients. Zehfuss, G. Schlömilch Z. 3 (1858) 175-.

- $G(x+1)-G(x)=H(x)$. Guichard, $C$. Par. Éc. Norm. A. 4 (1887) 361-.

- $\sum_{n=2}^{n=\infty} \Delta^{2} a_{n} x^{n}=\sum_{n=2}^{n=\infty} \frac{c a_{n} x^{n}}{n}$. Lerch, M. С̌asopis 21 (1892) ${ }^{n=2}$ 69-; Fschr. Mth. (1892) $317-$.

Solutions, conjugate, in differential and difference equations. Pincherle, S. Rm. R. Ac. Linc. Rd. 4 (1895) (Sem. 2) 228-.

-, particular. Poisson, S. D. Par. Ec. Pol. J. $13^{\circ} \mathrm{cah}$. (1806) 60-.

- rational, of

$$
C \psi(n) \psi(n+1)+\left(A_{n}^{\prime \prime}+B^{\prime \prime}\right) \psi(n+1)
$$
$-\left(A^{\prime}(n+1)+B^{\prime}\right) \psi(n)+\left(A^{\prime}-A^{\prime \prime}\right)=0$. Saalschiitz, L. Crelle J. Mth. 119 (1898) 291-.

-, singular. Brunacci, V. [1808] Mod. Mm. S. It. 14 (1809) 175-.

$\psi^{2} x$, i.e. $\phi^{-1} f^{2} \phi x$. Horner, W. G. Thomson A. Ph. 11 (1826) 168-, 241-.

$P_{n+1}=n P_{n}+P_{n-1}$. Catalan, E. C. Liége S. Sc. Mm. 15 (1888) No. 1, 235-.

\section{Solution of functional equa- tions. (See also 4460.)}

Babbage, C. Gergonne A. Mth. 12 (1821-22) 73-.

Herapath, J. Tilloch Ph. Mg. 64 (1824) 197-, 333-; 66 (1825) 102-; 67 (1826) 442-.

Ellis, R. L. Camb. Mth. J. 3 (1843) 131-.

Schlömilch, O. Leip. B. (1852) 27-.

Koenigs, G. Par. Éc. Norm. A. 2 (1885) 385-.

Grévy, A. Bll. Sc. Mth. 16 (1892) 311-; Par. Ec. Norm. A. 11 (1894) 249-; 13 (1896) 295-.

Leau, -. C. R. 119 (1894) 901-; 120 (1895) 427-.

Pexider, J. V. Časopis 29 (1900) 153-; Fschr. Mth. (1900) 403.

Algebraic and functional equations, distinction. Ellis, R. L. Camb. Mth. J. 3 (1843) 92-.

- functional equations and linear differential equations, irreducibility. Koenigsberger, $L$. Mth. A. 53 (1900) 49-.

Algorithm, new. Lemeray, - C. R. 125 (1897) 524-.
Cases. Gregory, D. F. Camb. Mth. J. 3 (1843) 239-.

-. Ellis, R. L. Camb. and Dubl. Mth. J. 7 (1852) 103-.

- Cayley, A. QJ. Mth. 15 (1878) 315-, 338-.

-. Ježek, O. Prag Sb. (1885) (Mth.-Nt.) 63-.

-. Crofton, M. W. [1888] Ir. Ac. P. 1 (188991) 20 -.

- Giudice, F. Palermo Cir. Mt. Rd. 2 (1888) 28-.

- Lecornu, L. Par. S. Mth. Bll. 23 (1895) 102 -

-. Nielsen, N. Kjøb. Ov. (1897) 185- ; Fschr. Mth. (1897) 346-.

- Zejliger, D. N, [1900] Kazan S. Ps.Mth. Bll. 10 (1901) 187-.

Closed systems of operations. Brunel, Bordeaux S. Sc. Mm. 5 (1890) x-.

Convergent iterations. Źorawski, $K$. Krk. Ak. (Mt.-Prz.) Rz. 6 (1893) 271-; Crc. Ac. Sc. Bll. (1893) 144-.

Equation, fundamental, of Thiele's. Steen, $A$. (xII) Ts. Mth. 4 (1868) 129-; 5 (1869) 26-.

Expansion in series of repeated index function (4th "Rechnungsstufe"). Paugger, F. Grunert Arch. 35 (1860) 21-.

To find $f(x)$ from equations $f(x+y)=f(x)$ $+f(y), f(x+y)=f(x) \cdot f(y)$ by elementary means. Jensen, J.L. W. V. (xu) Ts. Mth. 2 (1878) 149-.

Form of function satisfying equation $F(x+z)+F(x-z)=a F(x) \cdot F(z)$.

Holmboe, B. M. Mg. Ntvd. 1 (1832 ?) 275-.

Formation of equations satisfied by given algebraic functions. Tortolini, B. [1848] Bologna N. Cm. 9 (1849) 283-.

Functional equation of Poisson. Andrade, Par. S. Mth. Bll. 28 (1900) 58-

$-\longrightarrow-f(x)-\frac{b}{k-c x} f\left(\frac{c-a x}{k-c x}\right)=h-\frac{g}{c-a x}$. Mainardi, G. Tortolini A. 4 (1853) 331-.

- - treated by Abel. Stäckel, P. Z. Mth. Ps. 42 (1897) 323-.

- equations characterising associative and distributive operations. Lémeray, - Par. S. Mth. Bll. 27 (1899) 130-.

- with 2nd member. Grévy, -. Par. S. Mth. Bll. 25 (1897) 57-.

- -, principle defective. Herschel, (Sir) J. F. W. Camb. Mm. Anal. S. (1813) 65-.

- with 1 or more variables. Leau, L. Toul. Fac. Sc. A. 11 (1897) E, 110 pp.

- linear equations. Lémeray, - C. R. 125 (1897) 1160-; 126 (1898) 949-; As. Fr. C. R. (1900) (Pt. 2) 208-.

relation of 2 variables $x, y$. Roselli, $E$. G. Arcad. 115 (1848) 129-.

Functions, calculus. Russell, W.H.L. Phil. Trans. (1862) 265-.

- determined by functional equation in 2 variables. Giudice, $F$. Palermo Cir. Mt. Rd. 2 (1888) 28-, 94-;- 3 (1889) 273-; 4 (1890) 67-.

Cir. Mt. Rd. 2 (1888) 94, 187-. 


\section{Functional Equations}

Functions determined by functional equation in 2 variables. Vivanti, - . Palermo Cir. Mt. Rd. 4 (1890) 66-, 69.

- satisfying certain functional equations. Wirtinger, $W$. Wien Ak. Sb. 99 (1891) (Ab. 2a) 918

Generating functions. Rangoni, L. Mod. Mm. S. It. 19 (1821) 241-, 659-.

- - Ostrogradsky, M. A. St Pét. Ac. Sc. Bll. 1 (1836) 73-.

- - and repeated differentiation. Jïrgensen, U. (vi Adds.) Kiöb. Ov. (1830-31) 2-.

Integrals. Koenigs, G. C. R. 99 (1884) 1016-; Par. Éc. Norm. A. 1 (1884) (Suppl.) 41 pp.

Iteration and general algorithms. Lémeray, Par. S. Mth. Bll. 26 (1898) 10-

-, problem. Leau, L. Par. S. Mth. Bll. 26 (1898) 5-.

-, , application of doubly periodic functions to solution. Lémeray, -. Par. S. Mth. Bll. 27 (1899) 282-.

Iterations and reversion series. Źorawski, $K$. Krk. Ak. (Mt.-Prz.) Rz. 9 (1895) 240-; Crc. Ac. Sc. Bll. (1894) 242.

Iterative and other functional equations, conditions of holomorphism for integrals. Koenigs, G. C. R. 101 (1885) 1137-.

Lagrange-Foncenex lever problem. Genocchi, A. Tor. At. Ac. Sc. 4 (1868-69) 323-; Brux. Ac. Bll. 36 (1873) 181-.

Linear difference forms equivalent to their adjoints. Bortolotti, E. Rm. R. Ac. Linc. Rd. 7 (1898) (Sem. 1) 257-, (Sem. 2) 46-.

Meaning of equation $U^{2}=V^{2}$, where $U$ and $V$ are products of linear functions. Kirkman, T. $P$. Camb. and Dubl. Mth. J. 5 (1850) 97-.

Reduction of functional equations to finite difference equations. Herschel, (Sir) J. F.W. [1820] Camb. Ph. S. T. 1 (1822) 77-.

Repeated functions. Tralles, J. G. Berl. Ab. (1814-15) (Mth.) 216-.

Reversion of series as connected with $\psi a^{-1} \psi a x=x$.

Horner, W. G. Thomson A. Ph. 11 (1818) 108-.

Solution with conditions. Jensen, J. L.W. V. N. Ts. Mth. 8 (B) (1897) 25-; Fschr. Mth. (1897) 346.

- of a functional equation and application to parallelogram of forces and to curves of equilibration. Wallace, W. (Prof.) [1839] Edinb. R. S. T. 14 (1840) 625-.

_ _ _ - used by Poisson for parallelogram of forces. Perkins, G. R. Silliman J. 42 (1842) 69-.

Symbolic equation, and its consequences. Pincherle, S. Rm. R. Ac. Linc. Rd. 8 (1899) (Sem. 1) 152-.

- equations. Pincherle, S. Bologna Ac. Sc. Mm. 5 (1895-96) 663-.

Theory of functional equations. Bugaev, N.V. [1878] (xiI) Rec. Mth. (Moscou) 9 (1878-81) (Pt. 1) 109-.

Transcendental functions, class. Winckler, $A$. Wien SB. 44 ( $A b .2)$ (1861) 477-.

Use of functional equations in elementary geometry. Sigma. (vi Adds.) Tilloch $\mathrm{Ph}$. Mg. 65 (1825) 101-.

\section{Geometry 6400}

$\psi^{n} x=x$. Horner, $W . G$. Thomson A. Ph. 10 (1817) 341-.

- $=$ - . Herapath, J. Thomson A. Ph. 8 (1824) 420-.

$f\{z, f(x, y)\}$ symmetrical in $x, y, z$, determination. Abel, N. H. Crelle J. 1 (1826) 11 -

$\phi(f x)=\phi x\left(\frac{d f x}{d x}\right) . \quad$ Ramus, C. Crelle J. 9 (1832) 359-.

$P(m)+E\left(\frac{m}{m-1}\right) P(1)+E\left(\frac{m}{m-2}\right) P(2)+\ldots$

$$
+E(m)=m \frac{m+1}{2} \text {. }
$$

Sylvester, J. J. QJ. Mth. 3 (1860) 186-.

$\Sigma h_{\nu} \phi\left(x+a_{\nu}\right)=f(x)$, coefficients constant. Pincherle, S. Bologna Ac. Sc. Mm. 9 (1888) 45-.

$-=-,-$ rational in $x$. Pincherle, $S$. Bologna Ac. Sc. Mm. 9 (1888) 181-.

\section{GEOMETRY.}

\section{General.}

Algorithms, geometry. Séguin, (aîné) Moigno An. Cosmos (1862) 451-.

Equation of the beautiful. Lagout, $E^{\prime}$. Les Mondes 31 (1873) 480-.

Geometrical investigation, modern tendency. Segre, C. Rv. Mt. 1 (1891) 46-, 154-.

- - , - (Segre). Peano, G. Rv. Mt. 1 (1891) 66-, 156-.

Geometry, linear and metric. Klein, F. [1871] Mth. A. 5 (1872) 257 -.

Reductio ad absurdum, origin and avoidance. Hennessy, J. P. B. A. Rp. (1857) (pt. 2) 3.

\section{Foundations.}

\section{General.}

Construction, geometrical, as proof of existence in Greek mathematics. Zeuthen, H. G. N. Ts. Mth. 3 (A) (1892) 105-; Fschr. Mth. (1892) 48-; Mth. A. 47 (1896) 222-.

Extension. Riccobelli, $\boldsymbol{F}$. Brescia Cm. (1829) 104-.

Functions, conception of, in teaching elementary geometry. Besso, D. G. Mt. 7 (1869) 131-.

Geometrical ideas, origins. Laquière, E. M. As. Fr. C. R. (1881) 162 -

- intuition and analysis, connection. Schlïfl, L. Mil. I. Lomb. Rd. 5 (1872) 290-.

- -, example. Monro, C. J. QJ. Mth. 2 (1858) 225-.

Method in geometry. Tannery, $J$. Ens. Mth. 1 (1899) 333-.

Morphology, analytic. Peirce, B. Am. As. P. (1855) 67-.

Perimeters and areas of circles, Legendre's demonstration. Breton [de Champ], P. N. A. Mth. 4 (1845) $415-$.

Ratio, principles of antecedental calculus. Glenie, J. [1794] Edinb. R. S. T.4 (1798)65-. 


\section{Principles of geometry ; non- Euclidean geometries; hyper- space.}

Absolute conic, Cayley's theory. Scott, C. A. N. Y. Am. Mth. S. Bll. 3 (1897) 235-.

- geometry. Bolyai de Bolya, J. Bordeaux Mm. S. Sc. 5 (1867) 206-.

- (Bolyai). Schmidt, F. D. Mth. Vr. Jbr. 4 (1897) 107-.

- - (-). Macaulay, $F$. S. Mth. Gz. 1 (1900) 25-, 49-.

$--(-)$. Stäckel, P. Mth. Termt. Éts. 18 (1900) 241-; Mth. Nt. B. Ung. 17 (1901) 1 -.

- - Thue, A. Arch. Mth. Ntvd. 10 (1886) 304-.

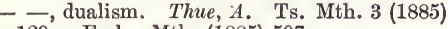
129-; Fschr. Mth. (1885) 507-.

- - parallel construction. Simon, M. Crelle J. Mth. 107 (1891) 84-.

Abstract geometry. Cayley, A. [1869] Phil. Trans. 160 (1870) 51-.

Analytical non-Euclidean geometry. Haus dorff, F. Leip. Mth. Ps. B. 51 (1899) (Mth.) $161-$.

- - Barbarin, P. Brux. Mm. Cour. $8^{\circ}, 60$ (1900-01) No. 1, $167 \mathrm{pp}$.

Angles in a triangle, Legendre's principle. Knight, T. Nicholson J. 27 (1810) 285-.

_ _ - - - second theorem, demonstration. Tilly, - de. Mathesis 19 (1899) 5-. - - - - - theorems on sum. Dehn, M. Mth. A. 53 (1900) 404-

- - - sum. Tilly, - de. Mathesis 19 (1899) 265-.

- - - - - Thibaut's demonstration. Germar, F. H. Grunert Arch. 15 (1850) 361-.

Angular geometry. Heatly, S. G. T. Beng. J. As. S. 11 (1842) 230-, 782-.

Anharmonies. Lovett, E. O. L. Mth. S. P. 29 (1898) 566-.

Aninvolutive metrics. Fontené, G. Par. S. Mth. Bll. 26 (1898) 176-.

Area of spherical triangle, generalisation. Stouff, $X$. C. R. 122 (1896) 303-.

and volume, concept. Hadamard, Bordeaux S. Sc. PV. (1896-97) 25-.

- _ - (Euclid's assumption). Thue, A. Arch. Mth. Ntvd. 10 (1886) 181-; Fschr. Mth. (1885) 507.

Asymptotes. Laurent, $H$. N. A. Mth. 7 (1868) 413-.

Bipolar geometry, principles. Leonardt, $G$. Z. Mth. Ps. 27 (1882) 346-.

Calculus of distances, areas, etc., and its relations to other forms of space. Cotterill, $T$. Camb. and Dubl. Mth. J. 7 (1852) 275-.

Circular points at infinity. Mannheim, $A$. As. Fr. C. R. 2 (1873) 82-.

Coincidence axiom and Euclid r. 4. Bogorodickij, V. Kazan Un. Mm. (1892) (Pt. 2) 129.

Commensurables, theory. Sang, E. Edinb. R. S. T. 23 (1864) 721-.
Compasses, Euclid's use of. Zeuthen, H. G. (xII) Ts. Mth. 6 (1882) 75-.

Content, theory. . Ball, (Sir) R. S. [1887] Ir. Ac. T. 29 (1887-92) 123-.

Continuity, geometrical, history. Taylor, C. [1880] Camb. Ph. S. P. 4 (1883) 14-.

, postulate. Veronese, G. Rm. R. Ac. Linc. Rd. 6 (1897) (Sem. 2) 161-.

- of space. Strong, W. M. N. Y. Am. Mth. S. Bll. 4 (1898) 443-.

Continuum, rectilinear, and axiom $\mathrm{V}$ of Archimedes. Veronese, $G$. Rm. R. Ac. Linc. Mm. 6 (1889) 603-.

Definitions. Olivier, L. Crelle J. 1 (1826) 241-.

- and axioms in Barrow's Euclid. Saint, W. Nicholson J. 23 (1809) 377-.

- propositions, fundamental, with reference to teaching. Newcomb, S. Nt. 21 (1880) 293-.

Differential equations of geometry. Stringham, I. B. A. Rp. (1899) 646-.

3-dimensional space of constant curvature, problems of mechanics. Francesco, D. de. [1900] Nap. Ac. At. 10 (1901) No. 4, 38 pp. No. $9,33 \mathrm{pp}$.

-, elementary remarks. Drobisch, M. W. Leip. Mth. Ps. B. 28 (1876) 268-.

- - geometry. Calinon, A. Nancy S. Sc. Bll. (1891) 35-.

- - - Newcomb, S. Crelle J. Mth. 83 (1877) 293-.

\section{4-Dimensional Space.}

Rodwell, G. F. Nt. 8 (1873) 8-.

Durège, 一. D. Nf. Tbl. (*1880) 175-.

Billson, C. J. Leic. S. T. 1 (1889) (Pt. 2) 11-.

Preble, W. P. Science 19 (1892) 304-.

De la Rive, 一. C. R. 120 (1895) 983-.

Analogue to quaternions. Wijthoff, W. A. Amst. Ak. Vs. 6 (1898) 520-.

Angles, calculation. Hoppe, $R$. Arch. Mth. Ps. 67 (1882) 269-; 69 (1883) 278-.

- between 2 planes. Schoute, P. H. N. Arch. Wisk. 3 (1898) 111-.

-, tetratops. Hoppe, R. Arch. Mth. Ps. 69 (1883) 287-, 297-, 439-.

Complex of first order. Ascione, E. Rm. R. Ac. Linc. Rd. 6 (1897) (Sem. 1) 162-.

Concept. Wake, C. S. Science 19 (1892) 331-. Crystals, types of regular system. Schoute, P. H. As. Fr. C. R. (1895) (Pt. 2) 278-

Figure of six points. Richmond, H.W. QJ. Mth. 31 (1900) 125-.

Figures. Stringham, W. I. [1880] (xп) J. H. Un. Cir. [1] (1882) 33.

-. Höppner, J. D. Edinb. Mth. S. P. 14 (1896) 121.

-, regular, linearly bounded. Hoppe, $R$. Arch. Mth. Ps. 67 (1882) 29-.

,,---- , internal angles. Hoppe, $R$. Arch. Mth. Ps. 68 (1882) 110-.

Geometry, descriptive. Veronese, $G$. Ven. I. At. 8 (1881-82) 987-.

-, and non-Euclidean geometry. Brückner, M. Zwick. Vr. Nt. Jbr. (1893) 1-. 
Geometry, proofs. Forchhammer, G. (xIr) Ts. Mth. 5 (1881) 157-.

-, theorem. Schlegel, S. F. V. As. Fr. C.R. (1887) (Pt. 2) 264-.

History. Haft, $F$. Arg. S. Ci. A. 30 (1890) 337-.

Intersection of straight lines and planes. Segre, C. Palermo Cir. Mt. Rd. 2 (1888) 45-.

Kinematics, 4-dimensional, applied to 3 dimensions. Oss, S. L. van. N. Arch. Wisk. 3 (1898) 165-; Fschr. Mth. (1897) 492.

Plane, place in. Hoppe, $R$. Arch. Mth. Ps. 68 (1882) 378-.

Prismoid. Schoute, P. H. Amst. Ak. Vh. (Sect. 1) 5 (1897) No. 2, 20 pp.

Projection of general locus into space of 3 dimensions. Craig, 7. Am. J. Mth. 2 (1879) 252-.

Projections, 3-dimensional, of 4-dimensional regular bodies or cells. Schlegel -. D. Nf. Tbl. (1884) 68-.

Quadratic spaces, special cluster. Schoute, $P$. $H$. D. Nf. Vh. (1900) (Th. 2, Hälfte 1) 6-.

Realisation. Hall, T. P. Science 19 (1892) 272

-. Sanford, E. C. Science 19 (1892) 332.

Rotation of rigid system. Stringham, I. Am. As. P. (1884) 55-.

Sections of regular hypersolids. Stott, $A . N$. [1900] Amst. Ak. Vh. (Sect. 1) 7 (1901) No. 3, $21 \mathrm{pp}$.

Solids. Durège, $H$. Wien Ak. Sb. 83 (1881) (Ab. 2) 1110 -

Systems of "spheres." Le Vavasseur, -. Toul. Ac. Sc. Bll. 2 (1899) 71-.

Volume of pentahedron. Liers, $E$. Arch. Mth. Ps. 12 (1894) 344-.

6-dimensional space, homogeneous coordinates, etc. Aschieri, $F$. G. Mt. 12 (1874) 368-.

\section{n-Dimensional Space.}

Betti, E. A. Mt. 4 (1870-71) 140-.

Lunde, J. L. Arch. Mth. Ntvd. 12 (1888) $364-$.

Badoureau, A. Rv. Sc. 46 (1890) 586-.

Mansion, P. Brux. S. Sc. A. 17 (1893) (Pt. 1) $12-$.

Chandler, C. H. Wisc. Ac. T. 11 (1898) 239-.

Analogue to maximum tetrahedron of given surface. Borchardt, C. W. Berl. Ab. (1866) (Mth.) 121-.

Angles. Hoppe, $R$. Arch. Mth. Ps. 66 (1881) 448 ; D. Nf. B. $\left({ }^{*} 1883\right) 55-$.

- Cassani, P. Rm. R. Ac. Linc. Rd. 1 (1885) 133-; Ven. I. At. (1894-95) 388-.

- between 2 spaces. Castelnuovo, G. Ven. I. At. (1884-85) 1331-

Complex variables. Volterra, $V$. Rm. R.Ac. Linc. Rd. 5 (1889) (Sem. 1) 158-.

Concept. Cassani, P. [1869] (Ix) Ven. Aten. At. 6 (1870) 63-.
Coordinates, hypotheses permitting introduction. Enriques, F. Palermo Cir. Mt. Rd. 12 (1898) 222-

Curvature. Folie, $F$. Leip. As. Gs. Vjschr. 35 (1900) 332-.

Curve, number of independent equations representing. Veronese, G. Mth. A. 18 (1881) 448.

Curves, class. Lasker, E. Nt. 52 (1895) 596.

Definition, and hypothesis of continuity in our space. Loria, G. G. Mt. 25 (1887) 97-.

Distance relations. Study, E. Z. Mth. Ps. 27 (1882) 140-.

Figures, regular, linearly bounded. Hoppe, $R$. Arch. Mth. Ps. 68 (1882) 151-.

Geometry. Cayley, A. Camb. Mth. J. 4 (1845) 119 -.

- Halphen, G. H. [1873] Par. S. Mth. Bll. 2 (1874) 34 -.

-. Jordan, C. Par. S. Mth. Bll. 3 (1875) 103-.

- Veronese, G. Rm. R. Ac. Linc. T. 5 (1881) 303-, 333-.

-. Schlegel, S. F. V. Par. S. Mth. Bll. 10 (1882) 172-.

-. Cassani, P. G. Mt. 23 (1885) 1-.

-. Dickstein, S. Prace Mt.-Fiz. 1 (1888) 129-; Fschr. Mth. (1888) 656.

- Veronese, G. Palermo Cir. Mt. Rd. 6 (1892) 42-

-. Cassani, P. Ven. I. At. (1893-94) 820 -

-. Vasiljev, A. V. Kazan S. Ps.-Mth. Bll. 4 (1895) (Prot.) 16-

- Dupuis, N. F. Cn. R. S. P. \& T. 3 (1897) (Sect. 3) 3-.

- Barbarin, P. As. Fr. C. R. (1898) (Pt.2) 111-.

-, history and state. Schlegel, $V$. Lpldina. 22 (1886) 92-, 108-, 133-, 149-, 160-; Ens. Mth. 2 (1900) 77-.

Lines, property. Lovett, E. O. Am. J. Mth. 22 (1900) 226-.

Manifoldness. Mlodzěevskij, B. K. Mosc. Un. Mm. (Ps.-Mth.) 8 (1889) xI +155 pp.; Fschr. Mth. (1891) 863-.

Maximum, geometrical question, extension. Geiser, C. F. Mil. I. Lomb. Rd. I (1868) 778-.

Means of arriving at conception of hyperspace. Farjon, F. N. A. Mth. 14 (1895) 101-.

Measures. Cesáro, E. G. Mt. 24 (1886) 49-.

Metric functions, fundamental, in spaces of $n$ dimensions and constant curvature. Ovidio, E. $d^{\prime}$. Rm. R. Ac. Linc. Mm. 1 (1877) 929-.

Philosophy of hyperspace. Newcomb, S. [1897] N.Y. Am. Mth. S. Bll. 4 (1898) 187-.

- (Newcomb). Halsted, G. B. Science 7 (1898) 212.

Plane spaces, metrical relations. Lasker, E. Nt. 52 (1895) 340-

Planet problem in curved space. Lipschitz, $R$. [1872] QJ. Mth. 12 (1873) 349-.

Points, associated groups. Castelnuovo, $G$. Palermo Cir. Mt. Rd. 3 (1889) 179-. 
Problem, solution. Kretkowski, $W$. Par. T. Nauk Śc. Pam. $12\left({ }^{*} 1882\right)$ Art, 4, 3 pp.

Projective geometry of hyperspace, postulates. Pieri, M. Rv. Mth. 6 (1896-99) 9-.

$-\overline{(1884)}-\overline{141}$.

Projections of regular "bodies" or cells. Schlegel, $V$. D. Mth. Vr. Jbr. 2 (1893) 66-.

Pythagorean theorem, generalisation. Klug, L. Mh. Mth. Ps. 10 (1899) 84-.

Quadrics, classification. Wythof, W. A. N. Arch. Wisk. 4 (1899) 162-.

Reciprocal relations. Schubert, $H$. D. Mth. Vr. Jbr. 4 (1897) 158-.

Representation by correlative figure of ordinary geometry. König, Jul. Gött. Nr. (1872) 157-.

- of figures by correlative figures of ordinary geometry. Spottiswoode, W. C. R. 81 (1875) 875-, 961-.

- on plane, of ordinary space by quadric. Aschieri, E. Mil. I. Lomb. Rd. 18 (1885) 494-.

- - - - regular solids. Schlegel, $V$. Palermo Cir. Mt. Rd. 5 (1891) 1-.

Six-hundred cell (hyper-polyhedron) and its self-corresponding displacements. Oss, S. L. van. [1899] Amst. Ak. Vh. (Sect. 1) 7 (1901) No. 1, 18 pp.; Nt. 61 (1899-1900) 304.

Sphere, potential. Giuliani, G. N. Cim. 21 (1887) 260-.

Spheres, $n$-fold linear systems. Del $R e, A$. Palermo Cir. Mt. Rd. 2 (1888) 124-.

Theory corresponding to curvature of ordinary space. Lie, S. Gött. Nr. (1871) 191-, 535-.

Volume calculation, three theorems. Hoppe, R. Arch. Mth. Ps. 69 (1883) 385-.

Direction. Blakslee, T. M. Des Moines Anal. 9 (1882) 15-, 51-.

-, arguments against disuse of term. Warren, S. E. $[1898]$ N. Y. Am. Mth. S. Bll. 5 (1899) 88, 94-.

-, geometry of. Laguerre, E. Par. S. Mth. Bli. 8 (1880) 196-

Distance, analytical theory. Beltrami, $E$. Mil. I. Lomb. Rd. 5 (1872) 294 .

- relations, theory. Dillner, G. Ups. Vet. S. Arsk. 2 (1861) 1-.

Divisibility of consecutive integer products, geometric analogy. Pincherle, S. Bologna Rd. (1892-93) 17-.

- - magnitude. MacWhorter, A. Silliman J. 6 (1848) 329-.

Equivalence of figures, proof of fundamental axiom. Veronese, $G$. Ven. I. At. (1894-95) 421-.

- in geometry, concept founded on number. Calinon, A. Nancy S. Sc. Bll. (1900) 85-.

- - - definition. Sbrana, $S$. Rv. Mt. 4 (1894) 147-.

-, group of, and its kinematic bases. Andrade, J. C. R. 126 (1898) 1775-.

Euclid, Bk. xII. Walsh, J. Tilloch $\mathrm{Ph} . \mathrm{Mg}$. 64 (1824) 181-.
Euclidean geometry, defence. Lagrange, $C$. Brux. Ac. Bll. (1899) 506-.

- without Euclid's postulate. Delbouf, $J$. Liège S. Sc. Mm. 19 (1897) No. 3, 116 pp.

- and non-Euclidean geometry. Andrade, $J$. Ens. Mth. 2 (1900) 298-.

Euclid's 3rd postulate, substitute for. Muirhead, R. F. Glasg. Ph. S. P. $12(1880)$ 112-.

- 5th postulate and principle of homogeneity. Flauti, $V$. Nap. Rd. 1 (1842) 61 (161)-.

Finite equality of two plane figures. Réthy, $M$. Mth. Termt. Ets. 12 (1894) 279-; Mth. Nt. B. Ung. 12 (1895) 72-.

Finitely equal surfaces. Réthy, M. Mth. Termt. Éts. 8 (1890) 176-; 11 (1893) 103-; Mth. Nt. B. Ung. 8 (1891) 170-; 11 (1894) 66-. (1893) 275-.

Functional notation, application to demonstration of fundamental propositions. $M ., F$. Bb. Un. 12 (1819) 85-.

- - , - - - geometrical theorems. Anon. (vi 534) Gergonne A. Mth. 10 (1819-20) 161-.

Fundamental figures in Euclidean space, measurement. Doehlemann, $K$. Z. Mth. Ps. 41 (1896) 265-.

- - of 1st rank, geometric definition of projectivity. Klein, C. F. Mth. A. 17 (1880) $52-$.

\section{Fundamental Principles.}

Nieuport, C. F. F. de, [1819] Brux. Ac. Sc. Mm. 1 (1820) 435-.

Price, B. Ashmol. S. P. 2 (1843-52) 177-.

Wedgwood, $H$. B. A. Rp. (1845) (pt. 2) 2-.

Matthes, C. J. Amst. Ts. Wis. Nt. Wet. 3 (1850) 35-.

Gardiner, M. Vict. T. Ph. I. 4 (1859) 76-.

Helmholtz, H. [1866] Gött. Nr. (1868) 193-.

Riemann, [G. F.] B. Gött. Ab. 13 (1868) (Mth.) 133-.

Lagout, É. Les Mondes 22 (1870) 13-.

König, G. (xIr) Mag. Tud. Ak. Ets. 6 (No. 5) (1872) 94-.

Cassani, P. G. Mt. 11 (1873) 331-; (Ix) Ven. Aten. At. 10 (1873) 222-.

Worpitzky, $(D r)$-. Arch. Mth. Ps. 55 (1873) 405-.

Zambelli, A. (xI) Ven. Aten. At. 11 (1873) 193-.

Helmholtz, H. L. F. von. V. Nost. Eng. Mg. 15 (1876) 501-; Rv. Sc. 12 (1877) 1197-.

Cassani, P. G. Mt. 15 (1877) 284-; 20 (1882) 143-.

Tilly, J. M. de. Bordeaux S. Sc. Mm.3 (1880) 1 -.

Krebs, C. (xir) Ts. Mth. 6 (1882) 81-.

Meyer, A. (xir) Ts. Mth. 6 (1882) 113-.

Zeuthen, H. G. (xir) Ts. Mth. 6 (1882) 119 -

Henrici, $O$. Nt. 29 (1884) 453-, 573.

(Henrici.) Geoghegan, E. Nt. 29 (1884) 551.

(Helmholtz's paper, 1866.) Lie, S. Leip. Mth. Ps. B. 38 (1886) 337-. 
Poincaré, H. Par. S. Mth. Bll. 15 (1887) 203-.

Brodén, $T$. Fschr. Mth. (1890) 540-.

Lie, S. Leip. Mth. Ps. B. 42 (1890) 284-, 355-; 44 (1892) 106-; C. R. 114 (1892) 461-.

Mansion, P. Brux. S. Sc. A. 14 (1890) (Pt. 2) 35-.

Killing, W. Crelle J. Mth. 109 (1892) 121-.

Wiener, H. D. Mth. Vr. Jbr. 1 (1892) 45-; 3 (1894) 70

Schmidt, H. Crelle J. Mth. 112 (1893) 112-, 319-.

Peano, G. Rv. Mt. 4 (1894) 51-.

Bonnel, J. Lyon Ac. Sc. Mm. 3 (1895) 241-, 351-; 4 (1896) 129-, 373-, 433-; 5 (1898) 401-.

Burkhardt, H. Gött. Nr. (1895) 114-.

Cassani, $P$. Ven. I. At. (1898) 42-.

Hilbert, D. Par. Éc. Norm. A. 17 (1900) 103-.

General geometry. Tarry, G. As. Fr. C. R. (1889) (Pt. 2) 60-; (1890) (Pt. 2) 152-; (1891) (Pt. 2) 90-; (1892) (Pt. 2) 132-; (1893) (Pt. 2) 217-; (1894) (Pt. 2) 184-.

- - or metageometry, Schering's researches. Mansion, P. Brux. S. Sc. A. 16 (1892) (Pt. 1) 51-.

- - - - and its three subdivisions. Mansion, P. [1895] Brux. Ac. Bll. 29 (1895) 495-; Wiad. Mt. 1 (1897) 1-, 68-.

Geometrical reasoning, facts in. Hennessy, J. P. Ph. Mg. 5 (1853) 127-.

Geometry, linear, superficial and solid. Heatly, S. G. T. I. Rv. 1 (1837) 755-; 2 (1838) 38-, 82-, 151-, 205-.

-, new elements, and proofs of laws of operation. Smith, S. Am. I. T. (1850) 207-.

-, - - founded on principle of analogues. Valat, - Bordeaux Act. Ac. Sc. 27 (1865) 61-; 28 (1866) 35-.

- as purely logical system. Pieri, M. [1900] N. Y. Am. Mth. S. Bll. 7 (1901) 158, 171-, 234.

-, system derived from ideas of direction and position. Wedgwood, H. (vi Adds.) Ph. Mg. 10 (1856) 459-.

- without axioms (Perronet Thompson). Newman, F. W. W. Eng. J. 1 (1836) 68-.

Gravity in Gaussian space. Schering, E. Gött. Nr. (1870) 311-.

- multiply extended Gaussian and Riemann -spaces. Schering, $E$. Gött. Nr. (1873) 149-.

Higher geometry, laws. Böklen, $O$. Grunert Arch. 33 (1859) 111-.

Imaginaries in pure geometry. Scott, $C . A$. N. Y. Am. Mth. S. Bll. 6 (1900) 163-.

,$- \sqrt{-1}$ in geometry. Shadow. (Pseudon.) Ph. Mg. 29 (1846) 171-.

Imaginary elements in geometry. McMahon, J. Am. As. P. (1888) 62 .

- forms in geometry. Mouchot, A. C. R. 103 (1886) 1110-.

- - - - realisation and use. Marie, $M$. N. A. Mth. 9 (1890) 60-, 161-, 375-, 435-, 508-; 10 (1891) 172-, 276-, 329-, 373-, $417-, 459-$ -
Imaginary forms in geometry, realisation and use (Marie). Boguslavsky, A. J. Fschr. Mth. (1891) 726-.

- points in space, geometrical representation. Molenbroek, $P$. [1890-91] N. Arch. Wisk. 18 (1891) 132-; Arch. Mth. Ps. 10 (1891) 261-; N. A. Mth. 10 (1891) 434-.

Indirect proof, admissibility. Sylvester, J.J. Ph. Mg. 4 (1852) 366-.

- _, - (Sylvester). Adamson, Jas. Ph.Mg. 5 (1853) 297-, 332-, 405-.

Infinity, concept, in elementary geometry. Ripert, L. Ens. Mth. 2 (1900) 370-. - Mth. 2 (1900) 205-.

Length of arc, concept, as depending on continuity. Ascoli, G. Mil. I. Lomb. Rd. 17 (1884) 564-.

-, concept, and the straight line. Bettazzi, $R$. A. Mt. 20 (1892-93) 19-.

- of curve, concept. Du Bois-Reymond, P. Acta Mth. 6 (1885) 167-.

- and space, concept. Wilkie, D. Queb. T. Lt. S. 2 (1831) 64-.

Lobačevskij's imaginary or pan-geometry. Lobatschewsky, N. (vr Adds.) Kazan Mm. Un. (1835) cah. 1, 3-; (1836) cah. 1, 3-; Crelle J. 17 (1837) 295-; (xII) Kazan Un. Mm. (1835, Bk. 3) 3-; (1836, Bk. 2) 3-; (1836, $B k$. 3) 3-; $(1837, B k .1)$ 3-; $(1838, B k .1)$ 3-; (1838, Bk. 3) 3-; Liège S. Sc. Mm. 2 (1900) No. 5, 101 pp.; (xII) Kazan Un. Mm. (1855, Bk. 1) 1-; (Iv) Erman Arch. Rs. 17 (1858) 397-.

- _ - Cayley, A. Ph. Mg. 29 (1865) 231-.

- - Bonnel, J. Lyon Ac. Mm. (Sc.) 29 (1888) 291-。

- K Kagan, W. Fschr. Mth. (1896) 373.

$28(1890)$ 181-, 240.

- $-\ldots$, construction of tangents to circles, and doubly infinite manifoldness of spheres. Simon, M. D. Mth. Vr. Jbr. 3 (1894) 80 -.

- - - equation of horicycles and horispheres, Mansion, P. Brux. S. Sc. A. 23 (1899) (Pt. 1) 32-.

- - - - intermediate planes (Zwischenebene) and limit surfaces (Grenzfläche). Simon, M. [1898] D. Mth. Vr. Jbr. 7 (1899) (Heft 1) 67-.

- - - - limit of a triangle. Dange, $F$. Mathesis 18 (1898) 267-.

- - - - parallels, construction. Engel, $F$. Kazan S. Ps.-Mth. Bll. 7 (1898) 118-.

- - - - - , theory, and nature of space. Levett, R. [1877] Birm. Ph. S. P. 1 (1879) (Pt. 2) 18-.

- _ - - and real linear transformations. Schwarz, A. Wien Ak. Sb. 99 (1891) (Ab. 2a) 153-.

- - , series, Efimov, M. N. A. Mth. 19 (1900) 28-.

- _ - - , space of five dimensions, properties. Mansion, P. Brux. S. Sc. A. 23 (1899) (Pt. 1) 5 . 
Lobačevskij's imaginary or pan-geometry, theorem. Zolt, A. de. G. Mt. 15 (1877) 336-.

Magnitude and direction, science of. Goodwin, H. [1845] Camb. Ph. S. T. 8 (1849) 278-.

Manifoldness of two dimensions and of finite extent, simplest continuous. Frankland, $F$. W. N. Z. I. T. 9 (1876) 272-.

- - - - - - - - - (Frankland). Monro, C.J. Nt. 15 (1877) 547; 22 (1880) 218.

- - - - - - (Monro). Frankland, F. W. Nt. 22 (1880) 170 -.

- - - - - - - - - - (Frankland). Skey, W. [1880] N. Z. I. T. $13\left({ }^{*} 1881\right)$ 100 -.

- - - - - - - - - (Skey). Frankland, F.W. [1884] N. Z. I. T. $18(1886)$ 58 .

- - - - - - - - - (Frankland). Hogben, G. [1886] N. Z. I. T. 19 (1887) 510 -

Maxima and minima, geometrical. Hurwitz, A. Sch. Nf. Gs. Vh. (1896) 57-.

Mechanical evaluation of plane surfaces. Cuénoud, S., \& Piccard, - [1860] Laus. Bll. S. Vd. 7 (1864) 93-.

- principle, problems solved by. Breton [de Champ], P. Liouv. J. Mth. 3 (1838) 488-.

Metaphysics of geometry. Dandelin, $G$. P. [1842] Brux. Mm. Ac. Sc. 17 (1844) 44 pp.

Minima, Fermat's problem, generalisation. Loria, G. Mathesis 19 (1899) 131-.

\section{NON-EUCLIDEAN GEOMETRY.}

Beltrami, E. G. Mt. 6 (1868) 284-.

Klein, [C.] F. Mth. A. 4 (1871) 573-; 6 (1873) 112-; 7 (1874) 531-.

(Klein.) Cayley, A. Mth. A. 5 (1872) 630-.

Ball, R. S. B. A. Rp. (1880) 476-.

Chrystal, G. Edinb. R. S. P. 10 (1880) 638-.

Reyes y Prósper, V. Mth. A. 29 (1887) 154-.

(Beltrami, 1868.) Commines de Marsilly, L. J. A. de. As. Fr. C. R. (1888) (Pt. 2) 121-.

Mansion, P. Brux. S. Sc. A. 13 (1889) (Pt. 1) $57-$.

Cayley, A. [1890] Camb. Ph. S. T. 15 (1894) 37-.

Klein, [C.] F. Mth. A. 37 (1890) 544-

Lindemann, - Königsb. Schr. 32 (1891) (Sb.) 20 -.

Miller, G. A. Science 20 (1892) 370-.

Poincaré, $H$. Nt. 45 (1892) 404-.

Gérard, -. N. A. Mth. 12 (1893) 74-.

MeClintock, E. N. Y. Mth. S. Bll. 2 (1893) $21-, 51$.

La Vallêe-Poussin, C. J. de. Brux. S. Sc. A. 19 (1895) (Pt. 2) 17-.

Neovius, $E$. R. Helsingf. Öfv. 37 (1895) 222-.

Dauge, -. Mathesis 16 (1896) 7-.

Isely, L. Neuch. S. Sc. Bll. 24 (1896) 137-.

VOL. I.
Mansion, P. Brux. S. Sc. A. 21 (1897) (Pt. 1) 117-; Mathesis 17 (1897) 112-, 134-, 158-; 18 (1898) 33-.

Engel, F. Leip. Mth. Ps. B. 50 (1898) (Mth.) 181-.

Villareal, F. Arg. S. Ci. A. 46 (1898) 23-, 67-, 129-.

Bianchi, L. A. Mt. 2 (1899) 95-.

Duport, H. Par. S. Mth. Bll. 27 (1899) 138-.

Frolov, (le gén.) $M$. As. Fr. C. R. (1899) (Pt. 2) 70-; Ens. Mth. 2 (1900) 179-, 293-.

Barbarin, P. Ens. Mth. 2 (1900) 306.

application to quadratic forms. Poincaré, $H$. As. Fr. C. R. (1881) 132-.

- - question in kinematics. Ball, R. S. B. A. Rp. (1881) 535-.

calculation in non-Euclidean forms of space. Killing, W. [1879] Crelle J. Mth. 89 (1880) 265-.

case. Johnson, W. W. N. Y. Mth. S. Bll. 2 (1893) 158-.

circles in. Battaglini, G. [1873] (Ix) Rm.R. Ac. Linc. At. 1 (1875) 53-.

circular affinity. Battaglini, $G$. Nap. Rd. 15 (1876) 219-.

conics, properties. Story, W. E. Am. J. Mth. 5 (1882) 358-; (xII) J. H. Un. Cir. 2 (1883) 71-.

constructions, singularities. Bouniakowsky, $\mathrm{V}$. St Pét. Ac. Sc. Mm. 18 (1872) (No. 7) 16 pp. cubics. Stecker, H. F. [1898-1900] N. Y. Am. Mth. S. Bll. 5 (1899) 3, 9-; Am. J. Mth. 22 (1900) 31-.

Foncenex's lever theory. Genocchi, A. [1877] Tor. Ac. Sc. Mm. 29 (1878) 365-.

formula. Janssen van Raay, W. H. L. N. Arch. Wisk. 3 (1898) 77-.

formulæ, elementary. Francesco, D. de. G. Mt. 37 (1899) 98-.

history. IIcClintock, E. N. Y. Mth. S. Bll. 2 (1893) 144-

-. Halsted, G. B. Am. As. P. (1899) 53-.

-. Monck, W. H. S. Pop. As. 8 (1900) 333-.

and hyperspace, bibliography. Halsted, G. B. Am. J. Mth. 1 (1878) 261-, 384-; 2 (1879) $65-$.

length, definition. Alexejewsky, W.P. Fschr. Mth. (1898) 411.

maxima problems. Bang, A. S. Ts. Mth. 5 (1887) 136-; Fschr. Mth. (1887) 515.

measurements, rational, in plane. Busche, $E$. Hamb. Mth. Gs. Mt. 3 (1900) 37-.

motion. Newson, H. B. N. Y. Am. Mth. S. Bll. 4 (1898) 2, 5-.

passage in Gauss. Mansion, P. D. Mth. Vr. Jbr. 7 (1899) (Heft 1) 156-.

9 -point theorem of Feuerbach, generalisation. Frattini, G. G. Mt. 16 (1878) 298-.

5 points, relation between distances. Mansion, P. Brux. S. Sc. A. 15 (1891) (Pt. 1) 8-; 20 (1896) (Pt. 1) 62-.

- or 6 points, relation between distances in Euclidean and non-Euclidean geometry. Mansion, P. Brux. S. Sc. A. 19 (1895) (Pt. 2) 189-.

Stokes's theorem, extension. Ricci, G. Ven. I. At. (1896-97) 1536-. 
straight lines. Petersen, $J$. Kjøb. Ov. (1900) 305-.

and teaching. Andrade, J. Ens. Mth. 2 (1900) 114-.

theorems. Simon, M. Mth. A. 48 (1897) 607. volume of a body, expression for. Mansion, $P$. Brux. S. Sc. A. 21 (1897) (Pt. 1) 118-.

Numbers and ratios in geometxy. Maizière, $A$. Lille Mm. S. (1833) 1-.

Paradoxes connected with mathematics of infinity. Domenech y Estapá, J. [1894] Barcel. Ac. Mm. 1 (1892-1900) 315-.

Plane angle, definition. Bonnel, $J$. Lyon Ac. Mm. (Sc.) 31 (1892) 39-.

-, concept, fundamental. Gerling, C. L. Crelle J. 20 (1840) 332-.

- definition, and axiom raised to theorem. Valeriani, $V$. G. Mt. 7 (1869) 376.

-, general theory. Crelle, A. L. Berl. Ab. (1834) 23-.

- in infinite dimension space, concept. Pincherle, S. Bologna Rd. 2 (1898) 71-.

- polygons and angles as system of absolute magnitudes. Stolz, O. Mh. Mth. Ps. 5 (1894) 233-; 7 (1896) 296.

- triangle, extension of elementary theorems to general space. Mehmke, R. Arch. Mth. Ps. 70 (1884) 210-.

Point pairs, and circles in space, treated by Grassmann's method. Miller, E. Mh. Mth. Ps. 7 (1896) 77-.

-, power of, with regard to five circles or six spheres. Lucas, É. Rm. R. Ac. Linc. Mm. 2 (1878) 449-.

-, straight line and circle, concepts. $R ., F$. Rv. Ligure 2 (1843) 132-.

Problems, methods of solution. Egidi, $G$. Rm. N. Linc. At. 40 (1887) 9-.

- $-\ldots$ not based on proportions. Airy, (Sir) G. B. [1857] Camb. Ph. S. T. 10 (1864) 166-.

Products of numbers, properties. Teixeira, J. P. G. Teix. J. Sc. 14 (1900) 179-.

Projections, areas of, applications of Tinseau's theorem. Cabreira, A. [1897] Lisb. J. Sc. Mth. 5 (1898) 93-.

Projective relations of space of various dimensions. Veronese, $G$. Mth. A. 19 (1882) 161-.

Proportion, equimultiples in ancient theory. Trudi, N. Nap. G. Mt. 1 (1863) 337-.

-, Euclid's theory. Hill, M. J. M. [1897] Camb. Ph. S. T. 16 (1898) 227-.

-, - - Gibson, G. A. Edinb. Mth. S. P. 16 (1898) 99-.

- - - filling a gap in. Nordmark, $Z$. Ups. N. Acta S. Sc. 6 (1799) 174-.

-, - - reduced to formulø. Peano, $G$. Mathesis 10 (1890) 73-.

-, geometrical. Marrat, $W$. Tilloch $\mathrm{Ph} . \mathrm{Mg}$. 33 (1809) 426-.

- and ratio, Euclid's theory; and nature of quantity. Powell, B. [1836] Ashmol. S. T. 1 (1838) No. 5, 64 pp.

-, theory. Chelini, D. G. Arcad. 73 (1837) 166-.
Proportion, theory. Gibson, G. A. Edinb. Mth. S. P. 18 (1900) (App.) 26 pp.

-, -, geometrical. Hoppe, $R$. Arch. Mth. Ps. 62 (1878) 153-.

Pseudospherical functions and their applications to pseudospherical geometry. Jakovkin, A. $F$. Kazan S. Ps.-Mth. Bll. 9 (1900) (Prot.) 45-.

- geometry. Cayley, A. R. S. P. 37 (1884) 82-.

-, theorem. Beltrami, E. G. Mt. 10 (1872) 53.

Quantities, geometrical and hyper-geometrical. Polignac, A. (Prince de). C. K. 52 (1861) 24-.

-, hyper-geometrical. Polignac, A. (Prince de). C. R. 56 (1863) 381-.

-, positive and negative, in geometry. Pourtalés, L. A. de. Neuch. Bll. 2 (1846-47) 127-.

-, principles applicable to geometry. Valat, -. Bordeaux Act. Ac. Sc. (1849) 535-.

Radical axis, existence demonstrated without Euclid's postulate. Reyes Prosper, V. Fschr. Mth. (1895) 571.

Ratio, Archimedes' principle. Terquem, $O$. N. A. Mth. 3 (1844) 586-

Ratios, arithmetical and geometrical. Bianchi, $G$. Palomba Rac. 3 (1847) 155-.

-, duplicate. Hain, $E$. Arch. Mth. Ps. 60 (1877) 404-.

Rectangle, geometrical existence. Collignon, E. As. Fr. C. R. (1899) (Pt. 2) 87-.

Riemann planes and Euclidean spheres, identity. Lechalas, G. Brux. S. Sc. A. 20 (1896) (Pt. 2) 167-.

- - - - -, non-identity. Mansion, $P$. Brux. S. Sc. A. 20 (1896) (Pt. 2) 178-.

Riemann's geometry, fundamental principle. Mansion, P. Mathesis 14 (1894) 180-; B. A. Rp. (1894) 579-.

- - interpretation of a theorem. Dauge, $F$. Mathesis 18 (1898) 5-.

- - and metageometry, principles. Mansion, P. Mathesis 15 (1895) 63-.

Segment, postulate of, deduced from that of angle. Sbrana, S. Rv. Mt. 3 (1893) 179-.

Signs of distances. Fontené, G. Ens. Mth. 1 (1899) 272-.

一, rule of. Laguerre, - . N. A. Mth. 9 (1870) 175-.

-, use. Fontené, G. Ens. Mth. 1 (1899) 123-.

- + and -. Haillecourt, A. N. A. Mth. 7 (1848) 83-.

- - - De Morgan, A. Camb. and Dubl. Mth. J. 6 (1851) 156-; 7 (1852) 242-.

Space and body, knowledge. Wedgwood, $H$. [1850] Camb. Ph. S. T. 9 (1856) 157-.

-, concept. Hoppe, -. D. Nf. Tbl. ("1875) 142-.

-, -, and application to natural science. Harnack, A. [1878] Dresden Isis Sb. (1879) 178-.

-, 一, empirical and ideal. Köpke, - . Hamb. Mth. Gs. Mt. 1 (1889) 274-.

-, - , an extension of concept of length. Graberg, F. Zür. Vjschr. 31 (1886) 339-. 
Space, concept, and laws of geometry. $\quad P ., R$. Kosmos (Lw.) 11 (1886) 530-.

- - - - - - - (R. P.). Raciborski, A. Kosmos (Lw.) 12 (1887) 27-.

-, concepts, fundamental. Graberg, F. Zür. Vjschr. 37 (1892) 274-.

-, correspondence between non-Euclidean, and Euclidean. Porchiesi, A. Bologna Ac. Sc. Mm. 5 (1883) 421-.

- and its dimensions. Whitmell, C. T. Card. Nt. S. T. 25 (Pt. 1) (1893) 6-.

-, elementary geometry theorems. Seipp, $H$. Arch. Mth. Ps. 12 (1894) 16-.

-, elliptic. Ball, (Sir) R. S. Nt. 33 (1886) 86-.

-, form. Hadamard, - Bordeaux S. Sc. PV. (1897-98) 83-.

-, functional, geometry. Pincherle, S. Bologna Rd. 1 (1897) 85-.

-, geometry. Randolph, R. V. Nost. Eng. Mg. 29 (1883) 137-.

- magnitude, successive developments. Graberg, $F$. Zür. Vjschr. 32 (1887) 191-.

- measurement, growth of ideas concerning. Graberg, F. Zü̈r. Vjschr. 37 (1892) 49-.

-, properties. Poncelet, J. V. [ascribed to Gergonne in vol. II. C. S. P.] Gergonne A. Mth. 17 (1826-27) 265-.

,-- (Poncelet). Gergonne, J. D. Gergonne A. Mth. 17 (1826-27) 272-.

-, recent researches. Becker, J. C. Z. Mth. Ps. 17 (1872) 314-.

- of spheres, Euclidean. Aschieri, F: Mil. I. Lomb. Rd. 19 (1886) 355-, 416-, 449-.

-, spherical, and figures therein. Porter, M. B. Texas Ac. Sc. T. 1 (No. 2) (1893) 45-.

Spaces of constant curvature, fundamental theory. Beltrami, E. [1868] A. Mt. 2 (1868 -69) 232-; Par. Éc. Norm. A. 6 (1869) 347-. - - - - - - (Beltrami). Schläfi, L. [1871] A. Mt. 5 (1871-73) 178-.

- - - - - (Schläfli). Beltrami, $E$. A. Mt. 5 (1871-73) 194-

- - - metrics of. Giudice, $F$. Tor. Ac. Sc. At. 35 (1900) 119- or 193-.

- - - negative. Bäcklund, A. V. Lund. Un. Acta 19 (1882-83) (Mth. No. 1) 48 pp.

- _ - - positive. Killing, W. [1877] Crelle J. Mth. 86 (1879) 72-.

-, parallel and perpendicular. Nicoli, $F$. Mod. Ac. Sc. Mm. 10 (1894) 257-; 11 (1895) 239-.

- various, comparability. Lechalas, $G$. [1900] N. Y. Am. Mth. S. Bll. 7 (1901) 158, $179-$.

Spheres of second species. Mannoury, $G$. N. Arch. Wisk. 4 (1899) 83-, 129.

Spherical geometry, fundamental theorems. Sikstel, V. Kazan S. Ps.-Mth. Bll. 2 (1893) 94-; 4 (1895) 18-; Arch. Mth. Ps. 15 (1897) 159-, 403-; 17 (1900) 337-.

Statical proofs in geometry. Brassinne, $E$. Toul. Mm. Ac. 2 (1858) 144-.

Straight line and curve, fundamental ideas. Lamarle, E. Brux. Ac. Sc. Mm. 30 (1857) 43 pp.
Straight line, Euclid's definition. Sikstel, V. A. Kazan S. Nt. (P8.-Mth.) P. 8 (1890) 288.

- joining two given points, construction. Burnside, $W$. [1897] L. Mth. S. P. 29 (1898) 125-.

- and plane, definitions. Bonnel, J. Lyon Ac. Mm. (Sc.) 30 (1889-90) 147-.

- - - generations. Cassani, P. Ven. Aten. At. 2 (1879) 46-.

- - plane and sphere. Calinon, A. Nancy S. Sc. Bll. (1887) 1-.

- - as shortest distance between two points. Hilbert, $D$. Mth. A. 46 (1895) 91-.

- lines and planes, unreal. Pasch, M. Mth. A. 32 (1888) 159-.

Superposition, geometry independent of. Pagnini, L. A. Firenze Opusc. Sc. 14 (1812) 3-.

Surfaces of constant curvature, two dimensional geometry. Calinon, A. Nancy S. Sc. Bll. (1895) 1-.

Symbolical geometry. Hamilton, (Sir) W. R. Camb. and Dubl. Mth. J. 1 (1846) 45-, 137-, 256-; 2 (1847) 47-, 130-, 204-; 3 (1848) 68-, 220- ; 4 (1849) 84-, 105-.

Symbols, algebraic, application to geometry. Gregory, D. F. Camb. Mth. J. 2 (1841) 1-.

\section{Theory of Parallels.}

Jeuffrain, A. Par. Tr. S. Amat. 2 (1808) 78-. Gergonne, J. D. Gergonne A. Mth. 3 (181213) 353-.

L. (I Adds.) Gilbert A. 52 (1816) 451-.

Hoffmann, J. J. I. Gilbert A. 54 (1816) 314-.

Blum, H. C. Gilbert A. 57 (1817) 222-.

Ivory, $J$. Tilloch Ph. Mg. 59 (1822) 161-.

Meikle, $H$. Tilloch Ph. Mg. 60 (1822) 423-.

Walsh, J. Tilloch Ph. Mg. 63 (1824) 100-, 271-.

Stein, J. P. Gergonne A. Mth. 15 (1824-25) $77-; 16$ (1825-26) 45-.

Servois, J. F. Gergonne A. Mth. 16 (1825-26) 233-.

Colburn, W. Bost. J. Ph. 3 (1826) 81-.

(Colburn.) Strong, T. Bost. J. Ph. 3 (1826) 371 [271]-.

(Strong.) Colburn, W. Bost. J. Ph. 3 (1826) 490-.

Knar, J. Baumgartner Z. 3 (1827) 414-; 4 (1828) 427-.

Lampredi, U. G. Arcad. 40 (1828) 19-.

R. ... r, J. F. Mitau Quatember 2 (1830) (Bd. 3) 35-.

Doppler, C. Wien Jb. Pol. I. 17 (1832) 167-.

Legendre, A. M. Par. Mm. Ac. Sc. 12 (1833) 367-.

Crelle, A. L. Crelle J. 11 (1834) 198.

Le Monnier, J. F. B. Cherb. Mm. S. Ac. (1838) 320 -

Amiot, B. Rouen Ac. Tr. (1839) 144-.

Powell, B. [1842] Ashmol. S. T. 2 (1838-52) 47 pp.

Schultén, N. G. af (fil.) [1842] Helsingf. Acta 3 (1852) 351-, 783-, 791-.

Bouniakowsky, V. [1843-51] St Pét. Ac. Sc. Mm. 6 (1850) 87-, 207-; St Pét. Ac. Sc. Bll. 9 (1851) 49-. 
Meikle, H. Edinb. N. Ph. J. 36 (1844) 310-; 43 (1847) 255-.

Minarelli, C. N. A. Mth. 8 (1849) 312-.

Lionnet, E. N. A. Mth. 9 (1850) 37.

Anon. (vi 840) Nap. At. Ac. 6 (1851) 1-.

Cabot, -. N. A. Mth. 14 (1855) 230-.

Hennessy, J. P. Ph. Mg. 12 (1856) 283-, 371-.

Osorio, R. G. Coimbra I. 4 (1856) 86-.

Schultén, N. G. af (fil.) Helsingf. Acta 4 (1856) 25-.

Stevelly, J. B. A. Rp. (1856) (Pt. 2) 8-; Ph. Mg. 12 (1856) 220-.

Vincent, A.J.H. C. R. 42 (1856) 1107-.

Day, A. Ph. Mg. 13 (1857) 156-.

Bouché, A. M.-et-L. Mm. S. Ac. 4 (1858) 162-.

Buniakovskij, V. Ja. St Pet. Ac. Sc. Mm. (Rs.) $2\left({ }^{*} 1862\right)$ 19-; St Pét. Ac. Sc. Bll. 5 (1863) 387-.

Thompson, T.P. R. S. P. 12 (1862-63) 268-.

Lobatchewsky, N. I. Bordeaux Mm. S. Sc. 4 (cah. 1) (1866) 83-.

Grunert, J. A. Arch. Mth. Ps. 47 (1867) 307-.

Baltzer, $R$. Leip. B. 22 (1870) 95-.

Dillner, $G$. Ts. Mt. Fys. 3 (1870) 1-.

Bunyakovskix, V. Y. [1872] (xII) Rec. Mth. (Moscou) 6 (1872-73) (Pt. 1) 77-.

Johnson, $W$. $W$. Des Moines Anal. 3 (1876) 103-.

Budden, E. Nt. 35 (1887) 92-.

(Budden's demonstration.) Henrici, O. Nt. 35 (1887) 100 .

Duclout, J. A.rg. S. Ci. A. 30 (1890) 308-, 351-, 467-; 31 (1891) 5-, 111-, 161-, 213-, 257-; 32 (1891) 72-, 132-.

Application of algebraic functions to proof of properties of parallels. Ivory, $J$. (vI Adds.) Tilloch Ph. Mg. 63 (1824) 161-, 246-.

- - law of logic. Matzka,W. Grunert Arch. 8 (1846) 320-.

Bolyai, letter of 1823. Szily, $K$. Mth. Termt. Ets. 5 (1887) 187-; Mth. Nt. B. Ung. 5 (1886-87) 189-.

Catalan's postulate. Breton [de Champ], $\boldsymbol{P}$. N. A. Mth. 7 (1848) 93-, 401-.

Definition of parallels. Bonnel, J. [1887] Lyon Ac. Mm. (Sc.) 29 (1888) 41-.

- - - importance. Hennessy, J. P. Ph. Mg. 12 (1856) 452-.

Dodgson's theory. Tucker, R. [1888] Nt. 39 (1889) 175.

Euclid's axiom. Olivier, L. Crelle J. 1 (1826) 151-.

- - Flye Sainte-Marie, C. [1870] (Ix) Par. S. Phlm. Bll. 7 (1871) 9-.

- - Lionnet, E. C. R. 70 (1870) 31-.

- - Stolz, O. [1885] Innsb. Nt. Md. B. 15 (1886) 25-.

- -. Simon, M. D. Mth. Vr. Jbr. 1 (1892) 39,41 .

- - Mansion, P. Mathesis 16 (1896) 109- ; 18 (1898) 188-

- - Frolov, (le gén.) M. Mathesis 16 (1896) 225-; As. Fr. C. R. (1898) (Pt. 2) $104-$

- Brassai, S. Mag. Tud. Ak. Éts. 9 (1898) 415-.
Euclid's axiom. Sincov, D. M. Kazan S. Ps.Mth. Bll. 9 (1900) (Prot.) 44.

- - Tikhomandritzky, M. Ens. Mth. 2 (1900) 385-.

- - Mansion, P. Ens. Mth. 2 (1900) 457.

- -, consequences. Sikstel, W. Fschr. Mth. (1896) 373-.

- -, demonstration. Limrick, P. As. Researches 7 (1801) 449-.

- - - - Anon. (vr 548) Gergonne A. Mth. 14 (1823-24) 269-.

- - - Bouvier, L. C. Gergonne A. Mth. 17 (1826-27) 152-.

- - - Wilkie, D. [1832] Queb. T. Lt. S. 3 (1837) $72-$.

- - - - Twining, A. C. Silliman J. 1 (1846) 89-.

-, - Hörlych, H. T. Grunert Arch. 18 (1852) 455-.

- - Lamarle, E. Brux. Ac. Bll. 23 (1856) (pte. 2) 408-, 637-.

- - - . Kulik, J.P. Prag Sb. (1860) 20-. -, - Murray, B. A. Ph. Mg. 33 (1867) 264-.

-, - Twining, A. C. Am. As. P. 18 (1869) 39- ; Am. J. Sc. 4 (1872) 333-.

-, - Rossi Re, V. de. Rm. N. Linc. At. 31 (1878) 461-.

- - - Bertrand's. Lüroth, J. Z. Mth. Ps. 21 (1876) 294-.

,,---- Becker, J. C. [1876] Z. Mth. Ps. 22 (1877) 60-.

- - - impossibility of, by plane construction. Hoüel, J. Bordeaux Mm. S. Sc. 8 (1870) xi-.

- -, -, possibility of, by stereometry. Günther, $S$. G. Mt. 14 (1876) 97-.

- - and the foundations of geometry. Commines de Marsilly, (le gen.) de. As. Fr. C. R. (1889) (Pt. 2) 88-.

- - L Lacroix's. Rérolle, -. Les Mondes 11 (1866) 55-; 12 (1866) 513-.

$-\ldots$, principles of mechanics and geometry in relation to. Genocchi, A. [1869] Mod. S. It. Mm. 2 (1876) 153-.

- - Saccheri's researches. Mansion, $P$. Brux. S. Sc. A. 14 (1890) (Pt. 2) 46-.

Geometrical theory. Lildicke, M.A.F. Gilbert A. 72 (1822) 423-.

Lambert's theory. Stäckel, P. Bb. Mth. (1899) 107-.

Legendre's proof of properties of lines. Young, G. P. Cn. J. 1 (1856) 519-.

Lobačevskij's views prior to 1826 . Wassiljef, $A$. D. Mth. Vr. Jbr. 4 (1897) 88-.

Metternich's theory. Wachter, - Lindenau Z. 2 (1816) 64-.

Non-Euclidean geometry and theory of parallels, opinions of Dutch mathematicians. Janssen van Raay, W. H. L. [1900] Kazan S. Ps.Mth. Bll. 10 (1901) 1-.

Plane geometry. Sexe, S. A. N. Mg. Ntvd. 19 (1873) 282-.

Point and line calculus. Graefe, F. Arch. Mth. Ps. 15 (1897) 34-, vII-.

Problems, celebrated. Franchini, $P$. Lucca At. Ac. 8 (1835) 91-. 
Twist and stretch, centres of finite. Genese, R. W. B. A. Rp. (1888) 579-.

Volume in any space, concept. Loria, G. G. Mt. 26 (1888) 96-.

\section{Topology of space and hyper- space. (See also 3620, 8075.)}

Simony, O. Mth. A. 19 (1882) 110-; 24 (1884) 253-.

(Listing.) Tait, P. G. [1883] $\quad$ Ph. Mg. 17 (1884) 30-.

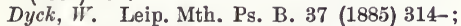
38 (1886) 53-; 39 (1887) 40-; Mth. A. 32 (1888) 45̃- ; 37 (1890) 273-.

Brunn, H. Z. Mth. Ps. 37 (1892) 106-.

Poincaré, H. C. R. 115 (1892) 633-; Par. Ec. Pol. J. 1 (1895) 121 pp. ; Palermo Cir. Mt. Rd. 13 (1899) 285-.

Dorsten, R. H. van. N. Arch. Wisk. 20 (1893) 113-.

Boguslavskij, A. I. Kharkov Mth. S. Com. 4 (1895) 86-.

Aspects of $n$ points in plane to observer in plane. Perrin, R. Par. S. Mth. Bull. 10 (1882) 103-.

Betti's numbers. Poincaré, H. C. R. 128 (1899) 629-.

Branched circuits. Wassmuth, A. Mh. Mth. Ps. 7 (1896) 49-.

Cayley's color-diagrams, representation of rotation groups. Maschke, H. Am. J. Mth. 18 (1896) 156-.

Cell-structure, natural, ideal geometrical form. Bryant, (Mrs) -. L. Mth. S. P. 16 (1884-85) 311-.

Close, partitions. Cayley, A. Ph. Mg. 21 (1861) 424-.

Closed contours, double points. Hadamard, -. Bordeaux S. Sc. PV. (1899-1900) 4 -

- curves. Schönflies, A. Gött. Nr. (1896) 79-.

- - general theorems. Tait, P. G. B. A. Rp. (1876) (Sect.) 29.

- -, plane, intersect an even number of times, applications. Tait, P. G. [1876] Edinb. R. S. P. 9 (1878) 237-.

- surfaces, number of maximum and minimum normals from point. Reech, $F$. Par. Éc. Pol. J. 37 $\mathrm{cah}$. (1858) 169 .

Combinations of resistances. Macmahon, (Major) P.A. Elect. 28 (1892) 601-.

Configuration of $2^{n}$ circles. Godt, $W$. Mth. A. 47 (1896) 564-.

Configurations on any surfaces. Brunel, $G$. Bordeaux S. Sc. Mm. 2 (1891) xxii-; (1897-98) 224.

- closed surfaces. Brunel, G. Bordeaux S. Sc. PV. (1896-97) 4-.

- closed surfaces having one summit and one face. Brunel, G. Bordeaux S. Sc. PV. (1894-95) 13-.

$-{ }_{-}-$, reciprocal configurations. Brunel, G. Bordeaux S. Sc. PV. (1894-95) 63-.

Congruency of plane surfaces. Dobriner, $H$. Mth. A. 42 (1893) 285-.
Connected surfaces, class. Emch, A. [1894] Kan. Un. Q. 3 (1895) 153-.

- -, simply. Schönfies, A. Gött. Nr. (1899) $282-$.

Connection of spaces. Tonelli, A. Rm. R. Ac. Linc. Rd. 2 (1875) 594-; 6 (1890) (Sem. 1) 139-.

- - - cyclomatic laws. Mannoury, $G$. N. Arch. Wisk. 3 (1898) 126-.

- surfaces. Klein, C.F. Mth. A. 7 (1874) 549- ; 9 (1876) 476-

-_- Bortolotti, E. Bologna Rd. (1889-90) $132-$.

- - Tonelli, A. Rm. R. Ac. Linc. Rd. 2 (1893) ( $\mathrm{Sem} .1$ 1) 15-; 4 (1895) (Sem. 1) 300-.

-, theorem. Bortolotti, E. Rm. R. Ac. Linc. Rd. 5 (1889) (Sem. 2) 229-.

Contours traced on surfaces. Brunel, $G$. Bordeaux S. Sc. PV. (1896-97) 128-.

Curves, algebraic. Hulburt, L.S. N. Y. Mth. S. Bll. 1 (1892) 197-.

Dissection of surfaces, Riemann's theorem. Koehler, C. Crelle J. Mth. 109 (1892) 118-.

Division, homogeneous, of space. Kelvin, (Lord). R. S. P. 55 (1894) 1-.

-, - - - V Vaes, F.J. N. Arch. Wisk. 4 (1899) 107- ; Fschr. Mth. (1899) 439-.

-, regular, of space. Schoenflies, $A$. Gött. Nr. (1888) 223-.

$-, \ldots,-\ldots$, non-Euclidean, into regular polyhedra. Bianchi, L. Rm. R. Ac. Linc. Rd. 2 (1893) (Sem. 2) 65-.

- - - - - and plane. Fedorow, E. von. Münch. Ak. Ab. 20 (1900) 465-.

,,---- by 14 -sided figures and cubes. Schoute, P. H. Amst. Ak. Vs. 3 (1895) 15-.

- of space, with minimum partitional area. Thomson, (Sir) W. (Lord Kelvin). Acta Mth. 11 (1887-88) 121-.

Divisions of space. Moore, E. H. (jun.), and Little, C. N. Am. J. Mth. 8 (1886) 127-.

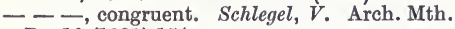
Ps. 10 (1891) 154-.

Double surfaces. Coar, H. I. Science 22 (1893) 328-.

Euclidean movements, general transformation of group. Page, J. M. A. Mth. 12 (1898-99) 87-.

Examples from thread manufacture. Hartig, E. Dresden Isis Sb. (1895) 34.

Folding rows and sheets of postage stamps. Schoute, P. H. Amst. Ak. Vs. M. 9 (1892) 226-; Fschr. Mth. (1892) 507-.

Four colours problem (of maps). Kempe, A.B. Am. J. Mth. 2 (1879) 193-.

$---(--)$. Story, W. E. Am. J. Mth. 2 (1879) 201-.

$--1-(-)$. Kempe, A. B. Nt. 21 (1880)

$-\frac{399-1}{-}(-\longrightarrow) . \quad$ Lucas, É. Rv. Sc. 6 (1883) $12-$

$---(--)$. Heawood, P. J. QJ. Mth. 24 (1890) 332-; 29 (1898) 270 -

Generation of lines and surfaces. Conti, $C$. [1825] Padova N. Sag. 3 (1831) 25-.

Hypothesis of Le Bel and Van't Hoff. Brown, A. C. Edinb. Mth. S. P. 2 (1884) 28 - 
Interlacing curves. Brunel, G. Bordeaux S. Sc. Mm. 5 (1895) xxxi-.

- -, Gauss's formula, and extension to higher configurations. Dyck, W. Münch. Ak. Sb. 25 (1896) 447-.

- surfaces, case. Brown, C. Edinb. R. S.P. 13 (1886) 382-.

Intersecting spaces, problem. Pieri, $M$. Mil. I. Lomb. Rd̃. 26 (1893) 534-; 27 (1894) 258-; 28 (1895) 441-.

Knot circles, examples of reading. Kirkman, (Rev.) T. P. Edinb. R. S. P. 13 (1886) 693-.

- of cravat. Lucas, $E_{\text {. (xII) Mathesis } 3}$ (1883) 54-.

-, insoluble, equation of curve, with solution in 4 th dimension. Hoppe, $R$. Arch. Mth. Ps. 64 (1879) 224.

-, linear section. Kirkman, (Rev.) T. P. Edinb. R. S. P. 13 (1886) 514-.

-, solution in 4 th dimension. Hoppe, $R$. Arch. Mth. Ps. 65 (1880) 423-.

Knots. Tait, P. G. [1877] Edinb. R. S. P. 9 (1878) 306-; Edinb. R. S. T. 28 (1879) 145-.

-. Little, C. N. Conn. Ac. T. 7 (1885-88) 27-.

- Tait, P. G. Edinb. R. S. T. 32 (1887) 327-, 493-.

-. Brunn, H. Münch. Ak. Sb. 22 (1893) 77-.

-, algebraic. Meyer, $F$. Edinb. R. S. P. 13 (1886) 931-.

-, alternate, of order eleven. Little, C. N. Edinb. R. S. T. 36 (1892) 253-.

-, amphicheiral forms. Tait, P. G. [1877] Edinb. R. S. P. 9 (1878) 391-.

-, classification according to connections. Simony, O. D. Nf. Vh. (1894) (Th. 2, Hälfte 1) 7-

-, - , topological apparatus. Simony, O. D. Nf. Tbl. (1887) 110-.

- and connections in certain closed surfaces. Dingeldey, $F$. Wien Ak. Sb. 98 (1890) ( $A b$. 2a) 79 -.

-, cruciform surfaces, figure obtained by joining ends in pairs. Simony, O. [1881] Wien Ak. Sb. 84 (1882) (Ab. 2) 237-.

-, double point of plane curve, resolution in 3-dimensional space, and related mechanical problem. Schlegel, S. F. V. Z. Mth. Ps. 28 (1883) 105-.

- on endless cords. Kempe, A. B. [1886] Edinb. R. S. P. 14 (1888) 36-.

- of fewer than ten crossings, enumeration, description and construction. Kirkman, (Rev.) T. P. Edinb. R. S. T. 32 (1887) 281-. - Listing and Trit. Kirkman, (Rev.) T.P. Edinb. R. S. P. 13 (1886) 363-.

-, measurement of beknottedness. Tait, $P . G$. [1877-79] Edinb. R. S. P. 9 (1878) 289-; 10 (1880) 48-.

-, and new theorems in higher arithmetic. Simony, O. [1887] D. Nf. Tbl. (1887) 229-; Wien Ak. Sb. 96 (1888) (Ab. 2) 191-.

-, non-alternate, with census for order ten. Little, C. N. Edinb. R. S. T. 39 (1900) 771-.

,-- , of orders eight and nine. Little, C.N. Edinb. R. S. T. 35 (1890) 663-

-, properties. Tait, P. G. [1877] Edinb. R. S. P. 9 (1878) 403 .
Knots, sevenfold knottiness. Tait, $P$. $G$. [1877] Edinb. R. S. P. 9 (1878) 363-

-, theorems, demonstration. Kirkman, (Rev.) T. P. Edinb. R. S. P. 13 (1886) 359-。

-, -, general. Koller, L. Wien Ak. Sb. 89 (1884) (Ab. 2) 250-.

-, unifilar, of ten crossings. Kirkman, T. P. Edinb. R. S. T. 32 (1887) 483-.

Königsberg bridges, Euler's problem. Coupy, É. N. A. Mth. 10 (1851) 106-.

- - - - - Saalschütz, L. [1875] Königsb. Schr. 16 (1876) (Sb.) 23-.

Labyrinths, problem. Wiener, $C$. [1872] Mth. A. 6 (1873) 29-

-, -. Tarry, G. As. Fr. C. R. (1886) (Pt. 2) 49-; N. A. Mth. 14 (1895) 187-.

Listing's theorem. Cayley, A. Mess. Mth. 2 (1873) 81-.

Looped line, possibility of traversing without repetition or interruption. Hierholzer, $C$. [1871] Mth. A. 6 (1873) 30-.

Manifoldness, $p$-dimensional, representation. Eberhard, V. Mth. A. 36 (1890) 121-.

"Nachbar" configurations and metacyclic groups. Heffter, L. Mth. A. 50 (1898) 261-.

- territories, problem. Heffter, L. Mth. A. 38 (1891) 477-.

Network, construction. Brunel, G. Bordeaux S. Sc. PV. (1895-96) 62-.

-, paths on. Brunel, -. Bordeaux S. Sc. Mm. 5 (1895) xxxix.

Networks. Lucas, É. As. Fr. C. R. (1887) (Pt. 1) 173-.

-. Brunel, G. Bordeaux S. Sc. Mm. 5 (1895) 165-.

- on closed surface. White, H. S. N. Y. Am. Mth. S. Bll. 4 (1898) 376-.

-, problems. Brunel, G. Bordeaux S. Sc. Mm. 4 (1894) ix-.

-, properties. Bugaev, N. V. (xII) Rec. Mth. (Moscou) 2 (1867) (Pt. 2) 87-.

-, regular. Brunel, G. Bordeaux S. Sc. PV. (1894-95) 3-.

- on surfaces of deficiency higher than 1 . White, H. S. [1896] N. Y. Am. Mth. S. Bll. 3. (1897) 116-.

- - - - - (White). Davis, E.W. N. Y. Am. Mth. S. Bll. 4 (1898) 529-

一, theorem. Thue, A. Ts. Mth. 3 (1885) 102-. One-sided surface, example. Hoppe, $R$. Arch. Mth. Ps. 57 (1875) 328-.

- _, "marrowbone." Schoute, P.H. Edinb. R. S. P. 19 (1893) 208-.

- - of Möbius. Maschke, H. N. Y. Am. Mth. S. T. 1 (1900) 39.

- surfaces. Brunel, G. Bordeaux S. Sc. Mm. 2 (1891) xxxvii.

- class. Feldblum, M. Wiad. Mt. 1 (1897) 101-; Fschr. Mth. (1897) 579.

- -, discovery. Stäckel, P. Mth. A. 52 (1899) 598-.

- - and singular points of plane curves. Delaunay, N. Par. S. Mth. Bll. 26 (1898) 43-. - - and spaces. Brunel, $G$. Bordeaux S. Sc. PV. (1895-96) 26-.

Packing of spheres. Hoppe, R. Arch. Mth. Ps. 56 (1874) 302-. 
Packing of spheres. Gilnther, S. Arch. Mth. Ps. 57 (1875) 209-.

- - Gwyther, R. F. Manch. Lt. Ph. S. P. 25 (1886) 35-.

- - - Reynolds, O. Manch. Lt. Ph. S. P. 25 (1886) 95-.

- - - Aldis, W. S. Nt. 39 (1889) 581-.

- - - Greenhill, A. G. Nt. 40 (1889) 10 -.

- - Liveing, G. D. Nt. 40 (1889) 55.

Point groups, regular, theorems. Rohn, $K$. Mth. A. 53 (1900) 440-.

- pairs, intervals between, theorems. Padoa, A. Rv. Mth. 6 (1896-99) 35-.

- systems, regular plane, of unlimited extent. Sohncke, L.A. (vin) Crelle J. 77 (1874) 47-.

Points in closed line, position relations. Vailati, G. Rv. Mt. 5 (1895) 75-.

- on line, position relations. Vailati, $G$. Rv. Mt. 5 (1895) 183-.

- in space, regular distributions. Bravais, $A$. [1848] C. R. 27 (1848) 601-; Par. S. Phlm. PV. (1848) 69-; Par. Ec. Pol. J. $33^{\circ} \mathrm{cah}$. (1850) 1-.

Polyhedra, classification. Davis, E. W. J. H. Un. Cir. [6] (1886-87) 122.

-, convex. Berger, A. Stockh. Öfv. (1898) 497-.

-, Euler's equation, generalisation for $n$-fold space. Stringham, W. I. [1880] (xп) J. H. Un. Cir. [1] (1882) 35.

- , - relation on $(p+1)$-ply connected surface. Kapteyn, W. Amst. Ak. Vs. 4 (1896) 199-; Fschr. Mth. (1896) 380.

-, - theorem and Cauchy's. Grunert, J. A. Crelle J. 2 (1827) 367.

_, _ - - Descartes's. Halsted, G. B. A. Mth. 1 (1884-85) 138-.

-, regular, elementary properties. Schoute, P. H. Fschr. Mth. (1891) 699-.

-, -, in 4-dimensional space. Schoute, P. H. Amst. Ak. Vh. (Sect. 1) 2 (1894) No. 2, 12 pp.; No. 4, 17 pp. ; No. 7, 26 pp.

,,----- , bluntness. Heyl, P.R. N. Y. Am. Mth. S. Bll. 4 (1898) 292, 293-.

-, - and semiregular, in $n$-dimensional space. Cesáro, E. Lisb. Ac. Sc. Mm. 6 (Pt. 2) (1887) No. 5, $75 \mathrm{pp}$.

Polyhedron, Kirkman's, problem connected with. Brunel, -. Bordeaux S. Sc. Mm. 5 (1895) xxix-.

- with trihedral summits, theorem. Tait, P. G. Edinb. R. S. T. 29 (1880) 657-.

Quincunx, application in science and weaving. Koturnitzky, P. [1893] Fschr. Mth. (1893 -94) 876-.

Region of $k$ th rank, division by $n$ regions of $(k-1)$ th rank. Pilgrim, L. [1878] Z. Mth. Ps. 24 (1879) 188-.

Regions and aspects relative to points in a plane. Laisant, C. A. Par. S. Mth. Bll. 10 (1882) 52-.

- - curves which fill them, analytical representation. Cesáro, E. Bll. Sc. Mth. 21 (1897) 257-.

-, number of, into which plane and space are divisible. Laisant, C. A. As. Fr. C. R. (1881) 71-.

—, theory. Cahen, E. N.A. Mth.16(1897)533-.
Relations, elementary. Möbius, A. F. Leip. B. 15 (1863) 18-.

Representation of points in $n$-dimensional space, groups which $r$ arbitrary points can represent. Schlegel, $V$. Arch. Mth. Ps. 10 (1891) 283-.

_ _ - - space by triplets of points on a line. Hirst, T. A. L. Mth. S. P. 2 (1869) 48.

- - ruled space by a system of conics in a plane. Aschieri, $\boldsymbol{F}$. Mil. I. Lomb. Rd. 12 (1879) 265-.

Ring formed closed knotless ribands, surfaces obtained from. Simony, O. [1880] Wien Ak. Sb. 82 (1881) ( $A b .2)$ 691-.

-, hollow flexible circular, results of dividing by closed cuts. Simony, O. [1882-83] Wien Ak. Sb. 85 (1882) (Ab. 2) 907-; 87 (1883) (Ab. 2) 556-; 88 (1884) (Ab. 2) 939-.

Straight line, definition. Brunel, G. Bordeaux S. Sc. Mm. 3 (1893) xxx-.

System of two tetrads of circles, and other systems of two tetrads. Cayley,- Camb. Ph. S. P. 8 (1895) 54-.

Tait's diagram. Polignac, - de. Par. S. Mth. Bll. 27 (1899) 142-.

Theorem. Klein, F. Gött. Nr. (1872) 290 .

-, and application to theory of numbers. Busche, E. [1898] Hamb. Mth. Gs. Mt. 3 (1900) 333-.

Three-dimensional spaces. Dyck, W. B. $\dot{\mathrm{A}}$. Rp. (1884) 648-.

Trigraphic fields of space. Russell, J.W. B. A. Rp. (1894) 578-.

Twists, class, figures formed by cutting. Schuster, J. L. Wien Ak. Sb. 97 (1889) (Ab. 2a) 217-.

- model of half-twist surface. Brovn, $C$. Edinb. R. S. P. 13 (1886) 513-.

\section{Methods of analytical geo- metry. (See also 0840.)}

Hachette, J. N. P., \& Monge, - Par. Éc. Yol. J. $11^{\circ} \mathrm{cah} .(1802) 143-, 170-$.

Fuss, N. [1808] St Pét. Ac. Sc. Mm. 4 (1813) 221-.

Monge, G. Par. Éc. Pol. J. 8 (1809) 68 -

Gergonne, J.D. Gard Not. Tr. Ac. (1810) 168-.

Binet, J. P. M. Par. Éc. Pol. J. $16^{\circ} \mathrm{cah}$. (1813) 280-.

Hachette, J. N. P. Par. Ec. Pol. Cor. 3 (1814-16) 132-.

Gergonine, J. D. Gergonne A. Mth. $8(1817-18)$ $156-$.

Hube, K. (vi Adds.) Krk. Roczn. Uniwers. 9 (1824) 76-.

Lloyd, H. Dubl. Ph. J. 1 (1825) 307-.

Plïcker, J. Crelle J. 6 (1830) 107-.

Conti, C. Padova N. Sag. 3 (1831) 90-.

Wilder, C. Silliman J. 20 (1831) 285-.

Bellavitis, G. Poligrafo 13 (1833) 53-.

Brunn, H. St Pét. Ac. Sc. Bll. 2 (1837) 275-; 6 (1840) 305-.

Chelini, D. G. Arcad. 75 (1838) 80-, 279-; 76 (1838) 3-, 257-.

D. Camb. Mth. J. 1 (1839) 74-. 
Greatheed, S. S. (vi Adds.) Camb. Mth. J. 1 (1839) 168-.

Boole, G. Camb. Mth. J. 2 (1841) 179-.

Doppler, C. Böhm. Gs. Ab. 2 (1841-42) 533-.

Padula, $F$. Nap. Rd. 2 (1843) 425-; 3 (1844) 241-, 321-, 401-; 4 (1845) 15-.

Rutherford, W. Ph. Mg. 22 (1843) 353-.

Bianchi, G. Palomba Rac. 1 (1845) 191-.

Cauchy, A. L. C. R. 21 (1845) 305-.

Dobson, $T$. Mathematician 1 (1845) 166-.

Finlay, $R$. Mathematician 1 (1845) 258-, 294-; 2 (1847) 1-, 84-, 143-, 237-; 3 (1850) 20-, 113-, 259-.

Luca, F. de. Nap. Ms. 6 (1845) 369-.

Briot, C. Lyon Mm. Ac. 2 (1847) 429-.

Ellis, A. J. B. A. Rp. (1855), (Pt. 2) 5-.

Brassai, S. (XII) Mag. Ak. Ets. (1857) 165-, 561-; (1858) 565-.

Grunert, J. A. Grunert Arch. 29 (1857) 235-.

Padula, $F$. Il Giamb. Vico 3 (1857) 133-; Nap. At. Ac. 1 (1863) No. 4, 14 pp.

Araldi, A. [1859] (Ix) Mod. Ac. Sc. Mm. 3 (1861) 113-.

Bellavitis, G. Ven. Mm. I. 8 (1859) 241-.

Fiedler, $W$. Schlömilch Z. 7 (1862) 53-.

Clayeux, -. C. R. 56 (1863) 788-.

Cassani, $P$. (Ix) Ven. Aten. At. 10 (1873) 114-.

Despeyrous, C. Toul. Ac. Sc. Mm. 7 (1875) 259-.

Frombeck, H. [1876] Wien Ak. Sb. 74 (1877) (Ab. 2) 399-.

Lucas, É. (xII) Mathesis 1 (1881) 65-.

Malcor, E. Rv. Mar. et Col. 81 (1884) 653- ; 82 (1884) 149-, 483-; 83 (1884) 376-, 880-.

Mukhopadhyay, A. [1887] Beng. As. S. J. 56 (Pt. 2) (1888) 288-.

Story, W. E. Am. J. Mth. 9 (1887) 38-.

Tilly, J. de. Brux. Mm. Cour. $8^{\circ}, 47$ (1892-93) No. 5, $80 \mathrm{pp}$.

Aragón, A. Méx. S. "Alzate" Mm.8(1894) 173-.

Brill, J. Mess. Mth. 25 (1896) 49-.

d'Alembert-Carnot geometrical paradox and its resolution. Turquan, L. V. N. A. Mth. 7 (1868) 437-.

Mth. 14 (1885) 92-.

Algebra, application to geometry, and vice-versâ. Schultén, N. G. af (fil.) [1853] Helsingf. Acta 4 (1856) 29-.

- of plane and of space. Boguslavskij, A. I. [1890-91] Rec. Mth. (Moscou) 14 (1890) 600-; 15 (1891) 683-; 16 (1893) 113-; Fschr. Mth. (1891) 734-.

Algebraic principle, single, deduction of geometric theorems. Zeuthen, H. G. [1879-80] L. Mth. S. P. 10 (1878-79) 196-; 11 (187980) 156-.

Analytical and geometrical methods, advantages of their separation. Woodhouse, $R$. Phil. Trans. (1802) 85-.

- operations, generalisation by help of geometry. Neovius, E. [1876] Helsingf. Acta 11 (1880) 111-。

Angle between two lines given by their equations. Heal, W. E. Des Moines Anal. 5 (1878) 148.

Area of polygon in terms of coordinates of vertices. Stainville, - de. Gergonne A. Mth. 1 (1810) 190-.
Area of polygon in terms of coordinates of vertices. Terquem, $O$. N. A. Mth. 9 (1850) 65.

Mth. 15 (1856) 373-

- - - - - - Veltmann, $W$. Z. Mth. Ps. 32 (1887) 339-.

- triangle. Lowry, W. L. [1874] Mess. Mth. 4 (1875) 129

- - and tetrahedron volume, in Cartesian, tetrahedral and quadriplanar coordinates. Grunert, J. A. Arch. Mth. Ps. 53 (1871) 317-.

Areas and centres of polygons. Padula, $F$. Nap. At. I. Inc. 1 (1864) 37-.

- of surfaces and volumes of solids, methods of approximation. Bugaev, N. V. [1897] Rec. Mth. (Moscou) 20 (1899) 451-; Fschr. Mth. (1898) 252.

Axes, conditions for system of three, being mutually rectangular. Dauge, $F$. Mathesis 14 (1894) 85- ; 15 (1895) 250-.

-, 3, problem. Puttkammer, J. N. van. Leijd. A. Ac. (1822-23) 25 pp.

-, rectangular, nine angles between two systems of 3 (Plana). Reiss, $M$. Brux. Ac. Bll. 9 (1842) 6-.

Barycentric calculus of Möbius. Allardice, $R$. E. Edinb. Mth. S. P. 10 (1892) 2-.

Binary forms, application to analytical geometry. Laguerre, $E$. Liouv. J. Mth. 1 (1875) 99-.

Cartesian geometry. Battaglini, G. G. Mt. 29 (1891) 3-, 93-, 195-, 298-.

- - , higher, foundations. Folie, F. [1869-79] Brux. Ac. Sc. Mm. 39 (1872) 142 pp. ; Brux. Ac. Bll. 36 (1873) 620-; Bll. Sc. Mth. As. 3 (1879) 278-.

- -, historical development and bases. Corzan-Avendano, G. [1864] (xm) Mag. Ak. Éts. (Mth. Term.) 6 (1865) 3-.

Circle and concentric rectangular hyperbola, connection. Godefroy, A. N. N. Arch. Wisk. 18 (1891) 119-.

Circular relation (Kreisverwandtschaft) in geometric terms. Möbius, A. F. Leip. Ab. Mth. Ps. 2 (1855) 529-.

- - of Möbius. Cayley, A. QJ. Mth. 2 (1858) 162.

- - Enneper, A. Z. Mth. Ps, 13 (1868) 261-.

- - -. Lucas, É. N. A. Mth. 15 (1876) 205-.

- (Lucas). Cassani, P. G. Mt. 14 (1876) 347-.

- Cayley, A. L. Mth. S. P. 8 (1877) 220 .

- - - equation. Grunert, J.A. Arch. Mth. Ps. 44 (1865) 376-.

- - - - Z. Zahradnik, $K$. Arch. Mth. Ps. 56 (1874) 15-.

Complex elements. Tognoli, O. G. Mt. 31 (1893) 137-.

- quantities, application to plane geometry. Brill, J. Mess. Mth. 16 (1887) 8-.

Condition that straight line should be normal to a conic in trilinear coordinates. Pinkerton, R. H. Edinb. Mth. S. P. 9 (1891) 2-. 


\section{Coordinates.}

anharmonic. Hamilton, (Sir) W. R. NH. Rv. 7 (1860) (P.) 242-, 325-, 506-.

areal. Hart, H. Mess. Mth. 11 (1882) 104-.

-, method connected with orthogonal projection. Sharpe, J. W. [1879] Mess. Mth. 9 (1880) $10-$.

axial. Esson, W. QJ. Mth. 10 (1870) 113-.

-. Ocagne, M. d'. N. A. Mth. 3 (1884) $545-$.

- (d'Ocagne). Cesáro, E. N. A. Mth. 4 (1885) 256-.

barycentric. Cesâro, E. Mathesis 10 (1890) 177 -

biangular. Walton, W. [1866] QJ. Mth. 9 (1868) 47 -.

-. Ford, P. [1873] (Ix) QJ. Mth. 13 (1875) 75-.

- Genese, R. W. [1881] QJ. Mth. 18 (1882) $150-$.

-. Biggin, T. QJ. Mth. 25 (1891) 237-.

-. Bertelsen, B. P. N. Ts. Mth. 8 (A) (1897) 21-.

-, and application to lines and conics. Azzarelli, M. Rm. N. Linc. At. 28 (1875) 443-.

bilinear tangential. Loud, F. H. Des Moines Anal. 10 (1883) 50-

bipolar. Barbarin, P. N. A. Mth. 1 (1882) $15-$

-. Vries, J. de. Amst. Ak. Vs. 4 (1896) 219-; Fschr. Mth. (1896) 491.

-, and geodesy. Grunert, J. A. Grunert Arch. 32 (1859) 444-.

bipunctual. Franklin, $F$. Am. J. Mth. 1 (1878) 148-.

3 circles and 4 spheres, coordinate system, formulæ. Lucas, É. [1876] A. Mt. 8 (1877) 187-; Par. S. Mth. Bll. 5 (1877) 136-.

circular. Stammer, $W$. Crelle J. 44 (1852) 295 -

-, applications. Franklin, F. Am. J. Mth. 12 (1890) 161-.

curvilinear. Combescure, $E_{\text {. }}$ Par. Éc. Norm. A. 4 (1867) 93-.

cyclic. Ocagne, M. $d$ '. Mathesis 7 (1887) 148-.

cylindrical. Brenner, - Grunert Arch. 13 (1849) 244 -

homogeneous. Terquem, $O$. N. A. Mth. 8 (1849) 113-.

-. Trudi, N. G. Mt. 1 (1863) 11-, 47-, 148-; Nap. At. I. Inc. 1 (1864) 77-.

-.Kohn, G. D. Mth. Vr. Jbr. 4 (1897) 162

- , in imaginary geometry, and application to systems of forces. Cox, H. QJ. Mth. 18 (1882) 178-.

-, metrical relations. Ovidio, E. d'. G. Mt. 11 (1873) 197-.

-, perpendicularity and parallelism in. Giu. dice, $F$. Tor. Ac. Sc. At. 34 (1898) 153- or 277 -

-, plane. Heger, R. Z. Mth. Ps. 15 (1870) 117-, 389-. homogeneous, points, planes, and lines in. Ovidio, E. $d^{\prime}$. G. Mt. 8 (1870) 241-.

-, in space. Heger, R. Z. Mth. Ps. 16 (1871) 1-.

linear, introduction into geometry of plane. Koehler, C. Z. Mth. Ps. 32 (1887) 152-.

multipolar. Vries, J. de. Haarl. Ms. Teyl. Arch. 5 (1898) 99-.

oblique. Baltzer, R. [1850] Crelle J. 46 (1853) 145-.

-. Silva, D. A. da. Lisb. Ac. Sc. Mm. 5 (Pt. 1) (*1875) No. 2, 20 pp.

-, 3, angle between 2 lines or 2 planes referred to. Whewell, $W$. [1823] Camb. Ph. S. T. 2 (1827) 197-.

-, at equal angles, and point, line, and plane in space. Schmidt, G. [1850] Haidinger Ab. 4 (1851) (Ab. 4) 13-.

-, straight line and plane. Français, J. F. Par. Éc. Pol. Cor. 1 (1804-08) 337-.

-, - - - Haedenkamp, H. Grunert Arch. 3 (1843) 67-.

orthogonal, new system. Thomson, (Sir) W. Liouv. J. Mth. 12 (1847) 256-.

parallel. Ocagne, M. d'. N. A. Mth. 3 (1884) $410-, 456-, 516-; 6$ (1887) 493-; 8 (1889) 568-; 9 (1890) 445-, 471-; Brux. S. Sc. A. 14 (1890) (Pt. 1) 47-; Par. S. Mth. Bll. 18 (1890) 108-.

pentahedral. Whitworth, W. A. Mess. Mth. 3 (1866) 146-.

peripolar. Neumann, C. G. [1877-80] Leip. Mth. Ps. B. 29 (1877) 134-; Leip. Mth. Ps. Ab. 12 (1883) 363-.

in plano, systems. Hill, T. Am. As. P. (1857) 42-; (1858) 1-.

point, and tangential, correlations between systems. Ocagne, M. $d$.. N. A. Mth. 11 (1892) 70-

of points, lines and planes. Casorati, F. Mil.

I. Lomb. Rd. 10 (1877) 11-, 40-

polar. Genese, R.W. Mess. Mth. 5 (1876) 14-.

-. Loria, G. Ens. Mth. 1 (1899) 357-.

-, equation of normals and tangents in. Jeřâbek, $V$. Časopis 18 (1889) 245-; Fschr. Mth. (1889) 694.

-, formula from figure. Unferdinger, $F$. Arch. Mth. Ps. 48 (1868) 106-.

- linear, in plano. Weinmeister, J. P. Z. Mth. Ps. 21 (1876) 301-

-, straight line and parabola. Davies, T. S. Ph. Mg. 21 (1842) 190-.

polygonal and polyhedric, etc. Sautreaux, $F$. Brux. Mm. Cour. $8^{\circ}, 36$ (1884) No. 3, 14 pp. principle, origin and development. Günther, A.W.S. (xII) Nürnb.Nt. Gs. Ab. 6 (1877) 1-. projective. Fiedler, W. Zür. Vjschr. 15 (1870) 152 -

quadrilinear. Whitworth, W. A. Mess. Mth. 1 (1862) 193-; 3 (1866) 71-, 173-.

quadriplanar. Salmon, G. QJ. Mth. 4 (1861) 231-, 271.

—, sphere in. Whitworth, W. A. Mess. Mth. 1 (1862) 162-.

- and tangential, principal radii of curvature of surface in. Jeffery, H. M. [1872] QJ. Mth. 12 (1873) 86-. 
quadriplanar tetrahedral volume, and triangular area in space. Fazzari, G. G. Mt. 29 (1891) 247-.

reciprocal to the Cartesian. Schlegel, S. F. V. Z. Mth. Ps. 23 (1878) 195-.

- - polar. Schlegel, V. As. Fr. C. R.' (1885) (Pt. 2) 156-.

rectangular. Bartlett, W. P. G. Camb. (M.) Mth. M. 1 (1859) 180-.

- axes, nine angles between two systems. Reiss, M. Quetelet Cor. Mth. 11 (1839) 119-.

- -, - - - - (Reiss). Plana, G. Brux. Ac. Bll. 8 (pte. 2) (1841) 68-.

- - , - - - (Plana). Reiss, M. Brux. Ac. Bll. 9 (1842) 6-.

- - variable system of three. Painvin, $L$. N. A. Mth. 10 (1871) 414-.

-, relation of cosines. Barré de Saint-Venant, -. Liouv. J. Mth. 9 (1844) 270-, $310-$.

-, - - - Schläfli, L. Bern Mt. (1848) 27-. relative signs. De Morgan, A. Ph. Mg. 9 (1836) 249-.

in space. Zeuthen, H. G. [1868] Sk. Nf. F. 10 (1869) 148-.

spherical. Greatheed, S. S. [ascribed to Gregory, D. F., in vol. III. C.S.P.] Camb. Mth. J. 1 (1839) 193-; 2 (1841) 37-.

- Cayley, A. Camb. and Dubl. Mth. J. 1 (1846) 22-.

system for circle in a plane and for sphere in space. Berg, F.J.van den. N. Arch. Wisk. 1 (1895) 11-; Fschr. Mth. (1893-94) 1106.

-, new. Plilcker, J. Crelle J. 5 (1829) 1-.

-, - Chasles, M. Quetelet Cor. Mth. 6 (1830) 81-.

-, - Frankenbach, $F$. W. Riga Cor.-Bl. 17 (1868) 117-.

-, - : theorems on conics. Bobillier, Gergonne A. Mth. 18 (1827-28) 320-.

-, - : - - - (Bobillier). Gergonne, J. D. Gergonne A. Mth. 19 (1828-29) 32-, 220-.

-, two parallel axes. Schwering, $K$. Z. Mth. Ps. 21 (1876) 278-.

systems. Fiedler, W. Schlömilch Z. 4 (1859) 91-.

- Chelini, D. Bologna Mm. Ac. Sc. 3 (1863) 3-.

-. Arcais, F.d'. [1877] G. Mt. 16 (1878) 18 -.

- Perrin, É. As. Fr. C. R. (1897) (Pt. 2) 90 -

-, simplest, to fix a point. Lemoine, E. Par. S. Mth. Bll. 16 (1888) 162-.

tangential. Booth, J. R. S. P. 9 (1857-59) 175-.

-. Routh, E. J. QJ. Mth. 8 (1867) 111-.

- Taylor, C. [1880] Mess. Mth. 10 (1881) 13-.

-. Ocagne, M. d'. N. A. Mth. 3 (1884) 410-, 456-, 516-, 545-; 4 (1885) 110-.

- Aubert, P. Edinb. Mth. S. P. 12 (1894) $55-$.

-, Brianchon's theorem, etc. Grunert, J. A. Arch. Mth. Ps. 53 (1871) 443-.

-, system. Balitrand, -. N. A. Mth. 12 (1893) 256-. tetrahedral. Fiedler, $W$. Schlömilch Z. 8 (1863) 45-.

-. Neuberg, J. [1869] Brux. Mm. Cour. $8^{\circ}$, 21 (1870) (No. 2) $52 \mathrm{pp}$.

- general equation of second degree referred to. Allen, A.J.C. L. Mth. S. P. 13 (1882) 165-.

- and quadriplanar. Ferrers, N. M. QJ. Mth. 5 (1862) 172-.

- - Grunert, J. A. Arch. Mth. Ps. 53 (1871) 193-.

-, system. Schlegel, V. Par. S. Mth. Bll. 23 (1895) 216-.

- and triangles, constant relations. Töplitz, J. Z. Mth. Ps. 14 (1869) 253-.

- - triangular. Torry, A. F. Mess. Mth. 1 (1862) 220-.

transformation. Livet, -. Par. Ec. Pol. J. $13^{\circ}$ cah. (1806) 270-.

- Francais, J. F. Par. Ec. Pol. J. 14 cah. (1808) 182-.

- Hachette, J. N. P. Par. Éc. Pol. Cor. 2 (1809-13) 6-, 247-.

- Guinard, J. B. (vi Adds.) Gand A. Ac. (1821-22) $41 \mathrm{pp}$.

Hube, $K^{\circ}$ (vi Adds.) Krk. Roczn. Uniwers. 13 (1829) 91-.

-. Löwenstern, S. As. Nr. 17 (1840) 369-.

-. Steczkowski, J. K. Grunert Arch. 22 (1854) 356.

- Dostor, G. J. Grunert Arch. 26 (1856) 121-.

-. Roberts, S. QJ. Mth. 2 (1858) 172-.

-. Lebesgue, $V$. A. N. A. Mth. 2 (1863) $392-$.

-. Walton, W. [1864] QJ. Mth. 7 (1866) 102-.

- Klein, F. [1869] Mth. A. 2 (1870) 366-.

-. Cayley, A. [1878] Camb. Ph. S. P. 3 (1880) 178-.

-. Fiedler, $W$. Zür. Vjschr. 24 (1879) 145-. - Reye, T. Crelle J. Mth. 94 (1883) $312-$.

-. Ziegel, R. Arch. Mth. Ps. 17 (1900) 263-.

-, Euler's formulæ. Jacobi, C. G. J. Crelle J. 2 (1827) 188-.

- - - Lobatto, $R$. Amst. Ts. Ws. Nt. Wet. 1 (1848) 254-.

-, - - Hällstén, K. Helsingf. Öfv. 20 (1878) 24-

-, - - demonstration, and deduction of Monge's. Grunert, J. A. N. A. Mth. 5 (1846) 414-.

-, formula containing symbol denoting given rotation. Graves, C. Ir. Ac. P. 5 (1853) 140-.

-, Monge's formulæ. Encke, J. F. Berl. As. Jb. (1832) 305-.

-, and signs of angles and distances in plano. Folie, F. Brux. Ac. Bll. 41 (1876) 86-.

- in space. Gergonne, J. D. Gergonne A. Mth. 7 (1816-17) 54-.

- - - Grunert, J. A. Crelle J. 8 (1832) $153-$.

$52-$ 
transformation in space. Wolf, $R$. Bern Mt. (1847) 24-; Grunert Arch. 13 (1849) 274-. - - Hesse, L. O. Crelle J. 63 (1864) 247-. (1869) 248 .

- - Le Paige, C. M. M. H. H. As. Fr. C. R. (1876) 384-.

- - Borletti, F. Mil. I. Lomb. Rd. 15 (1882) 252-.

triangular, and analytical geometry of plane in space. Loria, G. G. Mt. 24 (1886) 164-.

-, system. Sondat, P. N. A. Mth. 12 (1893) $360-, 503-$.

triaxial, in equations of first and second degree. Veltmann, $W$. Arch. Mth. Ps. 64 (1879) 113 -.

一, theorem. Dedekind, R. Crelle J. 50 (1855) $272-$.

trigonic. Walton, W. QJ. Mth. 9 (1868) 340-.

-. Levi, S. G. Mt. 14 (1876) 353-.

- Genese, R.W. L. Mth. S. P. $12(1880-81)$ 157-.

trilinear. Slesser, G. M. QJ. Mth. 4 (1861) 134-.

-. Esson, W. Mess. Mth. 1 (1862) 113-.

-. Grunert, J. A. Grunert Arch. 38 (1862) 289-.

- Faure, H. N. A. Mth. 2 (1863) 289 .

- C., J.B. Cn.J. 9 (1864) 249-; 10 (1865) 334

- Clifford, W.K. [1864] QJ. Mth. 7 (1866) $54-$; 8 (1867) 16-, 119-

-. Walker, E. (vi) Mess. Mth. 2 (1864) 40-.

-. Steen, A. (xII) Ts. Mth. 3 (1867) 129-; 4 (1868) 6-; 5 (1869) 33-; 6 (1870) 52-, 113-, 171-.

-. Pendlebury, R. Mess. Mth. 4 (1868) 138.

-. Loudon, .J. [1870] Cn. J. 13 (1873) 62-.

- Grave, P. Kazan S. Ps.-Mth. Bll. 3 (1893) 82-; Fschr. Mth. (1893-94) 1063.

-, angles and distances in. Vogt, -. N. A. Mth. 11 (1892) 148-.

-, and applications to straight lines and conics. Foglini, G. Rm. N. Linc. At. 30 (1877) 159-.

- , - - - - - points. Imshenetskix, V. G. (xII) Kazan Un. Mm. (1862) (P8.Mth.) $25 \mathrm{pp}$.

- - areal, results involving. Anglin, A. H. Edinb. Mth. S. P. 11 (1893) 8-.

-, circular points, use of coordinates. Faure, $H$. N. A. Mth. 3 (1884) 140

-, contact and curvature of curves, and theorems of conics. Grunert, J. A. Arch. Mth. Ps. 53 (1871) 443-.

-, equations for straight line. Whitworth, W. A. Mess. Mth. 1 (1862) 42-, 92-.

-, of focoids. Davis, R. F. Mth. Gz. 1 (1900) 336-.

- inscribed and circumscribed circles of triangle in. Grunert, J.A. Arch. Mth. Ps. 52 (1871) 331-.

-, principles of geometry in. Tillol, Toul. Mm. Ac. 5 (1867) 18-.

- and quadriplanar. Greer, H. R. QJ. Mth. 6 (1863) 237-.

$-{ }_{-}$, straight line and plane in. Esson, $W$. QJ. Mth. 5 (1862) 373-. trilinear, spherical ellipse. Jeffery, $H . M$. [1866] QJ. Mth. 8 (1867) 283-.

- and tetrahedral. Giudice, $F$. Palermo Cir. Mt. Rd. 12 (1898) 278-.

- - - fundamental equations. Zeuthen, H. G. (xII) Ts. Mth. 5 (1869) 129-

-, theorem. Mullins, W. E. Mess. Mth. 1 (1862) 111-.

-, transversals, seventy-two propositions. Martin, (Rev.) H. [1865] Edinb. R. S. T. 24 (1867) 37-; Mess. Mth. 4 (1868) 40-, 65-.

-, triangle, area. Walker, W. F. [1864] QJ. Mth. 7 (1866) 122-.

- - - fundamental, property. Turnbull, J.C. QJ. Mth. 5 (1862) 236.

trimetric point, first formulæ. Hain, E. [1881] Arch. Mth. Ps. 67 (1882) 425-.

tripolar. Lucas, É. Mathesis 9 (1889) 129-, 173-.

Curves as envelopes of a straight line. Dyrion, L. N. A. Mth. 7 (1868) 176-.

-, symmetrical equation. Holditch, H. QJ. Mth. 2 (1858) 38.

Demonstration of elementary geometry theorems. Reuschle, [C. G.] Crelle J. 24 (1842) 171-.

3-dimensional analytical geometry. Greatheed, S. S. [ascribed to Gregory in vol. III. C.S.P.] Camb. Mth. J. 1 (1839) 37-.

- - - formulæ. Bartels, - [1825] St Pét. Mm. Sav. Étr. 1 (1831) 77-.

- - - oblique axes. Weddle, T. Mathe matician 3 (1850) 169-, 233-; (Suppl.) 5-.

Direction, analytical designation. Wessel, $C$. Arch. Mth. Ntvd. 18 (1896) No. 1, 69 pp.

- cosine of straight line, expression in rational fractions. Hoppe, $R$. Arch. Mth. Ps. 61 (1877) 438-.

- cosines of line making given angles with given lines. Tallqvist, $H$. Helsingf. Acta 17 (1891) 465-.

Distance. Cayley, A. B. A. Rp. (1858) (pt. 2) 3 .

-, shortest, of point from straight line or plane, analytic expression. Prouhet, $E$. N. A. Mth. 3 (1844) 548-.

Distances of point attached to fixed centre from fixed plane. Cauchy, A.L. C. R. 16 (1843) 798-.

- - points from straight lines and planes. Schell, W. Grunert Arch. 19 (1852) 79-. Mth. Ps. 57 (1875) 225-

Equation of angle, rectangle, lozenge, etc. Neuberg, J.J.B., \& Plateau, J.A.F. (xII) Mathesis 1 (1881) 89.

- in geometry of straight lines. Muir, T. Mess. Mth. 1 (1872) 150-.

- of normal. Joachimsthal, F. Crelle J. 56 (1859) 280-.

- to a plane, Hessian normal form. Lilienthal, R. von. Mth. A. 42 (1893) 497-.

Equations represented by 3 systems of ciroles. Ocagne, M. d'. Z. Mth. Ps. 43 (1898) 269-. - - - systems of three points on curve. Ocagne, M. d'. Acta Mth. 21 (1897) 301-. 
Equations, tangential. Laguerre, - Liouv. J. Mth. 17 (1872) 1-.

-, -, new form. Casey, J. Phil. Trans. 167 (1877) 367-.

- between two variables, general interpretation. Walton, $W$. Camb. Mth. J. 2 (1841) 103-.

- - - , representation. Björling, C. F. E. [1874] Stockh. Ak. Hndl. 13 (1876) (No.4) $40 \mathrm{pp}$.

- - - - Cayley, A. (xir) J. H. Un. Cir. [1] (1882) 210.

Equipollences, calculus. Bellavitis, $G$. A. Sc. Lomb. Ven. 5 (1835) 244-.

-, - (Bellavitis). Hoilel, $J$. N. A. Mth. 8 (1869) 289-, 337-

Figures, geometrical, algebra. Jarosenko, $S$. N. Rs. S. Nt. Mm. (Mth.) 1 (*1878).

Formula for angles and distances, simplification. Ripert, L. N. A. Mth. 19 (1900) 409-.

Formulæ, fundamental. Stolz, O. Mth. A. 43 (1893) 591-.

Geometry as a branch of analysis. Heatly, S. G. T. Beng. J. As. S. 12 (1843) 110-

- higher, proofs of fundamental formulæ. Cotterill, T. Mess. Mth. 4 (1868) 98-.

"Harmonics" of triangle. Hain, E. [1874] Arch. Mth. Ps. 57 (1875) 316-

Hypercycle and theory of polar cycles. Dautheville, - . Par. S. Mth. Bll. 14 (1886) 45-.

Imaginary in function theory and in analytic geometry. Schüler, W. F. D. Nf. B. (*1877) 109 -.

- points. Feldblum, M. Wiad. Mt. 2 (1898) 130-; Fschr. Mth. (1898) 486.

Inclination of lines and planes in space, interpretation of formulæ. Painvin, L. N. A. Mth. 5 (1866) 337-.

- plane to horizontal, Lamé's theorem. Trzaska, W. Par. T. Nauk Śc. Pam. 10 (*1878) Art. 9, 3 pp.

Inverse figures. Bellavitis, G. A. Sc. Lomb. Ven. 6 (1836) 136-; Tortolini A. 3 (1860) 60-.

"Keys" (Cauchy), geometrical interpretation. Barré de Saint-Venant, —. C. R. 36 (1853) $582-$.

Leibnitz's " Characteristic." Grassmann, $H$. Leip. Jablon. Preisschr. (No. 1) (1847) 60 pp.

Lines, plane, generation. Conti, C. [1828] Padova N. Sag. 2 (1825) 255-.

一, theory. Miuller, G.W. Crelle J. 15 (1836) 229 -

Method, general, linking synthetic with analytic geometry. Caspary, $F$. Bll. Sc. Mth. 13 (1889) 202-.

Middle points of transversals. Valat, J. P.F. Bordeaux Ac. Act. 38 (1877) 49-.

Multiple centres, theory. Breton, $P h$. Liouv. J. Mth. 10 (1845) 430-.

Multipliers, indeterminate. Brassinne, E. N. A. Mth. 6 (1847) 381-.

Notation, new. O'Brien, M. [1846] Camb. Ph. S. T. 8 (1849) 415-.

-, Plücker's abridged. Finck, B. N. A. Mth. 3 (1844) 147-.

Numerical equations, and analytical geometry in space. Zmurko, L. [1869] Wien D. 30 (1870) (2te Ab.) 217-.
Object and image with respect to reflecting circle. Lehmus, -. Crelle J. 31 (1846) 85-.

Orthogonal axes, systematic treatment. Pelz, $C$. Wien Ak. Sb. 90 (1885) (Ab. 2) 1060-.

- coefficients, system, new relations. Hoppe, R. Arch. Mth. Ps. 2 (1885) 413-.

Parameter, variable, use in geometry. Brassinne, E. N. A. Mth. 6 (1847) 381-.

Plane algebra. Macfarlane, A . [1883] Edinb. R. S. P. 12 (1884) 184 -

-, analytical problem. Dienger, $J$. Grunert Arch. 11 (1848) 111-.

-, equation. Schell, $W_{\text {. }}$ Schlömilch Z. 1 (1856) 106-.

figures, representation and segmentation. Laisant, C. A. Par. S. Mth. Bll. 18 (1890) 123-.

Planes, angle between two, in oblique coordinates. Junghann, G. Z. Mth. Ps. 12 (1867) 350-.

-, bisecting, and axes in oblique system. Paterno, F.P. Palermo G. Sc. Nt. 18 (1887) 342-.

—, position. Littrow, J. J. von. [1813] St Pét. Ac. Sc. Mm. 8 (1817-18) 54-.

Points on a circle, 4 , relations between distances. Antomari, $X$. N. A. Mth. 1 (1882) 462-.

- - - , _ _ - Cayley, A. Mess. Mth. 17 (1888) 94-.

$-{ }_{-}-, \ldots,-\ldots$, extension of Cayley's theorem. Springer, J. F. J. H. Un. Cir. [9 (1889-90)] 78 .

- with integer coordinates in plane, joins of. Bravais, A. C. R. 5 (1837) 867-.

- in a plane, property of a system. Lucas, $F$. Par. S. Mth. Bll. 21 (1893) 109-.

- $-\ldots, 3$, condition for being in straight line. Terquem, $O$. N. A. Mth. 4 (1815) 462-.

,---- 4 (Euler). Grunert, J.A. Grunert Arch. 26 (1856) 335-.

- - - - - , coordinates and angles subtended at a 5th point, relation. Grunert, J. A. Grunert Arch. 1 (1841) 89-.

,----- , equation connecting mutual distances. Muir, T. Edinb. Mth. S. P. 3 (1885) 34-.

- - - - , problem. Lonvry, T.J. Des Moines Anal. 1 (1874) 146-.

- - - relation between distances. Ciamberlini, C. G. Mt. 31 (1893) 218-

- - - 5. Cayley, A. Ph. Mg. 29 (1865) 460-.

$-{ }_{-}-, 6$, and their areal product. Reiss, M. Mth. A. 2 (1870) 397-.

-, relations between distances. Ovidio, E.d'. G. Mt. 9 (1871) 211-.

- in space, 3, coordinates, relations (Lagrange). Terquem, O. N. A. Mth. 1 (1842) 387-.

$-\ldots, 5$, relation between distances. Salmon, G. QJ. Mth. 3 (1860) 282-.

- - , - , - - Cayley, A. Mess. Mth. 18 (1889) 100-.

S. P. $16, \overline{(1890)}$ 86-. Mt. $34, \frac{1}{(1896)}-\frac{1}{279}-$. Ciamberlini, C. G. 


\section{Analytical Geometry}

Points in space, 5, relation, general. Schumann, A, Z. Mth. Ps. 27 (1882) 368-.

- on a sphere, 5, distances. Antomari, $X$. N. A. Mth. 1 (1882) 462-.

Polar distances of points. Cabreira, A . [1900] Lisb. J. Sc. Mth. 6 (1902) 70-

- equations. Midy, -. N. A. Mth. 4 (1845) $597-$.

- reciprocation, multiple. Vályi, $G$. Mth. Termt. Éts. 16 (1898) 399-; Mth. Nt. B. Ung. 16 (1899) 50-.

- surfaces of point and of a plane, theory. Painvin, L. F. [1865] (xII) Lille S. Mm. 2 (1866) 345-.

Porisms, algebraical analysis. Davies, T. S. Mathematician 1 (1845) 42-.

-, analysis for discovering. Babbage, $C$. [1820] Edinb. R. S. T. 9 (1823) 337-

Problems. Lubbock,J.W. Ph. Mg. 10 (1831) 82-.

-. Sagajto, A. Par. T. Nauk Śc. Pam. 7 (*1875) Art. 8, $28 \mathrm{pp}$

-. Delarive, $L$. Arch. Sc. Ps. Nt. 5 (1881) 34-.

-, solution of two. Tortolini, B. Tortolini A. 1 (1850) 377-.

Projection of solid on three coordinate planes. Bottomley, J. Manch. Lt. Ph. S. P. 21 (1882) 188-.

Projections on axes of coordinates. Baltzer, $R$. (Ix) Leip. Mth. Ps. B. 25 (1873) 523-.

Projective geometry and analytical representation. Pasch, M. Mth. A. 30 (1887) 127-.

- - theorems. Plilcker, J. Crelle J. 10 (1833) 217-, 293-; 11 (1834) 26-, 117-, 219-, 356-.

Radicals of form $\sqrt{x^{2}-y^{2}}$, Poncelet's geometric approximation. Léauté, $H$. Par. S. Mth. Bll. 8 (1880) 106-.

Radii vectores, associated, advantages in mathematical physics. Cauchy, A. L. C. R. 38 (1854) 67-.

- - , and geometrical methods. Despeyrous, C. Toul. Mm. Ac. 3 (1871) 217-.

Rational polynomial $x^{m}+a x^{m-1}+\ldots+l$, graphic calculation of value by new coordinate system. Stamm, E. Mil. I. Lomb. Rd. I (1864) 203-.

Scalar and clinant coordinate geometry. Ellis, A. J. R. S. P. 10 (1859-60) 415-.

- - radical loci, application. Ellis, A.J. R. S. P. 11 (1860-62) 141-, 393-.

Signs in analytical geometry. Lucas, $\bar{E}$. Mathesis 10 (1890) 5-.

Similar figures, analytical treatment. Morley, $F$. N. Y. Am. Mth. S. Bll. 1 (1895) 235-.

Spherical geometry, analytical. Möbius, A.F . Leip. Ab. Jablon. Gs. (1846) 45-.

Stewart, Matthew, general theorems (1746). Davies, T. S. Edinb. R. S. T. 15 (1844) $573-$.

-, - , - - ( ) corrected. Breton 「de

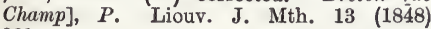
281-.

,,$----(\rightarrow)$ deductions. Bonsdorff, $E$. Helsingf. Öfv. 40 (1898) 36-.

\section{Elementary Geometry 6800}

Stewart, Matthew, general theorems (1746) demonstrations. Small, $R$. [1785] Edinb. R. S. T. 2 (1790) 112-. - 1 (1816) 6-. $\frac{-}{6-}$-. Babbage, C. QJ. Sc.

Stigmatics. Ellis, A. J. B. A. Rp. 34 (1864) (Sect.) 2; R. S. P. 14 (1865) 176-; 15 (1867) 192-.

Straight line, formulæ. Cayley, A. QJ. Mth. 15 (1878) 169-.

- - and plane, analytical theory. Bret, Gergonne A. Mth. 5 (1814-15) 329-

- - - expressions in theory of. Moth, F. X. Baumgartner Z. 4 (1828) 288-; 5 (1829) 17-.

- lines, imaginary, contained in every equation of first degree. Jiménez Rueda, C. Fschr. Mth. (1893-94) 1104.

- - in space, algebraic representation. Spottiswoode, S. C. R. 76 (1873) 1189-

- - - - equations derived directly from points. Rogner, $J$. Arch. Mth. Ps. 42 (1864) 95-.

Surfaces. Dietrich, M. Arch. Mth. Ps. 44 (1865) 200

Symbols and undetermined coefficients, use, and new geometric principle. Pliicker, $J$. Crelle J. 5 (1829) 268-.

Symmetrical relations. Böklen, O. Grunert Arch. 38 (1862) 198-.

Synopsis of analytical geometry. Spina, $P$. Méx. S. "Alzate" Mm. 7 (1893) 57-.

Triangle, problem. Grunert, J. A. Grunert Arch. 28 (1857) 344-.

- in space, area, determinant value. Dostor, G. Arch. Mth. Ps. 58 (1876) 289-.

\section{Elementary Geometry.}

\section{General.}

Gruson, J. P. Berl. Ab. (1814-15) (Mth.) $42-$.

Quetelet, L. A. J. Quetelet Cor. Mth. 1 (1825) 80 -

Gudermann, C. Crelle J. 6 (1830) 303-.

Gruson, J. P. Crelle J. 10 (1833) 275-.

Noel, J. N. Liège Mm. S. Sc. 2 (1845-46) 493-; 10 (1855) 461-.

Wolf, R. Grunert Arch. 7 (1846) 440-.

Smith, H. J. S. Ashmol. S. T. 2 (1852) 26 pp.

Csânyi, D. [1863] (xII) Mag. Ak. Éts. (Mth. Term.) 5 (1865) 292-.

Hoilel, J. Grunert Arch. 40 (1863) 171-.

Analogies of geometry of plane and space. Serret. P. N. A. Mth. 4 (1865) 145-, 193-, 433-.

Analogy in geometry. Noel, J.N. Liège Mm. S. Sc. 1 (1843) 1-.

Anaritius, commentary of, on Euclid's elements. Mansion, - ' Brux. S. Sc. A. 24 (1900) (Pt. 1) 47-.

Angles, notation. Peirce, J. M. Camb. (M.) Mth. M. 1 (1859) 168-.

Angular geometry. Shortrede, R. Beng. J. As. S. 11 (1842) 240-. 
Approximate square roots, methods of Greek geometers for. Buzengeiger, $K . H$. J. Lindenau Z. 5 (1818) 85-.

Area of a surface, definition. Lebesgue, $H$. C. R. 129 (1899) 870-.

Circle, division, apparatus. Anon. Gilbert A. $65\left({ }^{*} 1820\right) 329$ -

,,--- (Anon.). Liebherr, J. Gilbert A. 67 (1821) 109..

-, - - - (Liebherr). Reichenbach, G.R. von. Gilbert A. 68 (1821) 33-.

- , - - (Reichenbach). Liebherr, J. Gilbert A. 69 (1821) 320 -

- and sphere. Affolter, $F$. G. Arch. Mth. Ps. 57 (1875) 1-.

-, sphere, square, and equilateral triangle. Utting, J. Tilloch Ph. Mg. 59 (1822) 102-.

Circles, systems, inscribed in polygons, and spheres in solids. Lotteri, A. Brugnatelli G. 6 (1823) 115-, 361-.

Concepts and determinations. Sylvester, J.J. L. Mth. S. P. 16 (1884-85) 201-.

Converses of some theorems. Leudesdorf, $C$. Mess. Mth. 19 (1890) 14-.

Demonstrations. Hennessy, J. P. Ph. Mg. 4 (1852) 417-.

- Juel, C. S. (xir) Ts. Mth. 2 (1878) 4-.

Determinants, geometrical application. White, H. S. A. Mth. 1 (1900) 103-.

Electrostatic geometrical problem. Malagoli, R. Rv. Sc.-Ind. 29 (1897) 175-, 260-.

Equipollences calculus, application to problem. Françoise, É. N. A. Mth. 9 (1870) 66-.

Euclid and teaching of geometry. Wilson, J. M. G. Mt. 6 (1868) 361-.

- - - Magnus, $P$. Educ. Times 33 (*1880) 81-.

Euclidean teaching, improvement. Walmsley, J. Educ. Times 33 ("1880) 273-.

Exercises, geometrical. Riccardi, $P$. Mod. Ac. Sc. Mm. 16 (1875) 3-; 17 (1877) 3-.

Figures, geometrical, conditions necessary. Catania, S. Rv. Mt. 3 (1893) 101-.

,,,--- number determining. Fraser, A.Y. Edinb. Mth. S. P. 3 (1885) 22-.

Forms, geometrical, theory. Battaglini, $G$. Nap. Rd. 1 (1862) 220-; G. Mt. 1 (1863) 1-, 41-, 97-, 161-, 227-.

Geometrical constructions by compasses only. Delisle, G. N. A. Mth. 19 (1860) 35-.

- - - Colette, J. N. A. Mth. 8 (1889) 512-.

- - - - Schatunovsky, S. Fschr. Mth. (1891) 577.

- - Cesáro, $G$. Liège S. Sc. Mm. 1 (1899) No. 6, 11 pp.

- evolution. Degen, C. F. Kiob. Dn. Vd. Selsk. Skr. 2 (1801-2) 217-.

Geometrography. Lemoine, É. As. Fr. C. R. (1892) (Pt. 2) 36- ; (1893) (Pt. 2) 113-; N. A. Mth. 13 (1894) 155-; Edinb. Mth. S. P. 13 (1895) 19-.

- Balbin, V. Arg. S. Ci. A. 44 (1897) 110-; Fschr. Mth. (1897) 439.
Geometrography, application to anharmonic ratio. Lemoine, $\dot{E}$. As. Fr. C. R. (1894) (Pt. 2) 55-.

-, comparison of solutions of a problem. Lemoine, É. As. Fr. C. R. (1899) (Pt. 2) 102-; (1900) (Pt. 2) 74-.

- of Euclid's problems. Mackay, J.S. Edinb. Mth. S. P. 12 (1894) 2-.

- and measure of simplicity in construction. Lemoine, É. As. Fr. C. R. (1888) (Pt. 2) 75-; C. R. 107 (1888) 169-; Mathesis 8 (1888) 217-, 241-.

Geometry, applied, propositions. Noel, J. N. Liège Mm. S. Sc. 1 (1843) 208-.

- with constant radius circle, ancient. Kutta, M. Bb. Mth. (1896) 16.

- , elementary, as hypothetical deductive system. Pieri, M. [1899] Tor. Ac. Sc. Mm. 49 (1900) 173-.

-, 一, logic of. Adamson, J. (vi Adds.) S. Afr. QJ. 2 (1835) 353-.

-, -, new trend. Ciamberlini, C. Rv. Sc.Ind. 30 (1898) 99-, 113-, 153-.

-, modern, elementary exposition. Lenthéric, J. [1865-75] Mntp. Mm. Ac. Sect. Sc. 6 (1864-66) (PV.) 35-; 8 (1872-75) 507-.

-, note. Cesáro, E. (xII) Mathesis 3 (1883) 73-, 97-, 121-, 144, 176.

-, notes, miscellaneous. Matzka, $W$. Grunert Arch. 11 (1848) 432-.

,,--- . Bischoff, J. N. [1862] Crelle J. 61 (1863) 369-.

- - - - - Mackay, J. S. Edinb. Mth. S. P. 3 (1885) 38-.

,$--\frac{1}{-}$ Neuberg, J. As. Fr. C. R. (1894) (Pt. 2) 261-.

- of the ruler. Brianchon, C. J. Par. Ec. Pol. Cor. 2 (1809-13) 383-.

- - - Grunert, J. A. Grunert Arch. 27 (1856) 47-.

$---\frac{1}{-}$ metric relations. Möbius, A. F. Crelle J. 4 (1829) 101-.

- _ - -, problems, analytical solution. Gergonne, J. $D$. Gergonne A. Mth. 7 (1816-17) 325-.

Homogeneous body, geometrical concept. Popoff, A. Erman Arch. Rs, 14 (1855) 224-.

Imaginary intersections, geometrical representation. Suhle, -. Arch. Mth. Ps. 17 (1900) 244-.

- points, lines and planes, linear construction for joins and intersections. Grilnwald, $J$. Z. Mth. Ps. 45 (1900) 10-.

Inequalities, geometrical significance. Tsinger, V. Y. [1873] (xII) Rec. Mth. (Moscou) 7 (1874-75) (Pt. 1) 418 [408]-; (xi) Bll. Sc. Mth. As. 10 (1876) 100-.

Infinitesimals. Gergonne, J. D. Gergonne A. Mth. 5 (1814-15) 183-.

Lemmas and propositions. Adams, Jas. Thomson A. Ph. 11 (1818) 36-.

Line lying outside convex arc is longer than it. Uhde, A. Grunert Arch. 26 (1856) 43-.

Locus, class. Ruex, $P$. (xII) Mathesis 1 (1881) 53-.

Månsson, Peder, three small treatises attributed to. Eneström, G. Bb. Mth. (1888) 17-. 
Maxima and minima, geometrical methods. Genese, R. W. Mess. Mth. 5 (1871) 122-.

- - - problem. Chartres, R. Nt.37 (1888) 320.

Mean proportional. Grunert, J.A. Grunert Arch. 31 (1858) 476-.

- values, theorems. Crofton, $M$. W. L. Mth. S. P. 8 (1877) 304-.

Mensuration in one, two and three dimensions. Kummell, C. H. A. Mth. 4 (1888) 129-.

Organic bodies, representation. Vieth, G. U.A. Gilbert A. 53 (1816) 225-.

Pappus on progressions. Mackay, J. S. Edinb. Mth. S. P. 6 (1888) 48-.

Polyhedra and polygons, mensuration. Lhuilier, S. Bb. Un. 18 (1821) 85-.

- - - theorem by Carnot, correction. Grüson, J. P. [1826] Berl. Ab. (1831) 97-.

Problem. Jirgensen, $C$. Mth. Ts. 1 (1859) 81-.

Problems. Steichen, -. Quetelet Cor. Mth. 6 (1830) 302-.

-, Euclid's. Breton [de Champ], P. Liouv. J. Mth. 20 (1855) 209 -

,-- , solution with ruler and circle of fixed radius, by Italian geometers in 16 th century. Mackay, J. S. Edinb. Mth. S. P. 5 (1887) 2-.

- method, singular, for solution of many. Sixtel, W. Fschr. Mth. (1895) 561.

-, solution. Brianchon, C. J. Par. Ec. Pol. J. $10^{\mathrm{e}} \mathrm{cah}$. (1810) 1-.

-, - by calculation. Heller, H.J. Grunert Arch. 34 (1860) 6-.

-, - - ruler and compass. Petersen, J.P.C. (xiI) Ts. Mth. 4 (1874) 97-.

- solvable by ruler and compass, criteria for recognition. Wantzel, $L$. Liouv. J. Mth. 2 (1837) 366-.

-, treatment. Mensing, - Grunert Arch. 2 (1842) 341-.

-, two. Geisér, C. F. [1866] Crelle J. 67 (1867) 78 .

Puzzle, geometrical. Darwin, G. H. Mess. Mth. 6 (1877) 87.

Rates method, application to geometry. Johnson, $W . W$. Mess. Mth. 4 (1875) 72-.

Statical proofs in geometry. Brassinne, $E$. Toul. Mm. Ac. 2 (1858) 144 .

- - of some theorems. Alison, J. Edinb. Mth. S. P. 4 (1886) 58-.

Symbolical calculation with geometrical relations. Reye, T. Mth. A. 43 (1893) 145-.

Symmetrical figures. Möbius, A. F. Leip. B. (1851) 19-.

- - condition of equality. Weill, G. C. R. 105 (1887) 1237-.

- -, successive. Neuberg, J. As. Fr. C. R. (1890) (Pt. 2) 18-.

Symmetry. Fiedler, $W$. Zür. Vjschr. 21 (1876) 50-.

-, its place in elementary geometry. Redard, F. U. Neuch. S. Sc. Bll. 14 (1884) 69-.

-, plane and in space. Dostor, G.J. N. A. Mth. 6 (1847) 166-.

Theorem of Euler in elementary geometry. Fuortes, T. G. Mt. 7 (1869) 378.
Theorems, method for discovering. Magnus, L. J. Crelle J. 8 (1832) 51-.

\section{Planimetry; straight lines, and circles.}

Adams's hexagons and circles. Mackay, J. S. Edinb. Mth. S. P. 11 (1893) 104-.

Angles, conterminal. Bushell, W. D. [1873] Mess. Mth. 3 (1874) 12-.

-, curvilinear. Miquel, A. Liouv. J. Mth. 9 (1844) 20-; 10 (1845) 347-; 11 (1846) 65-.

Apollonius, three problems. Chasles, $M$. Mathematician 3 (1850) 201-.

Apparatus for drawing circles and other curves. Cerebotani, L. Rm. N. Linc. At. 46 (1893) 11-, 86-.

Arc of very large radius, construction by two given tangents. Breton, $P h$. Les Mondes (1880) $73-$

Ares, wide, parabolic or circular, tracing. Leclert, $\dot{E}$. Les Mondes 25 (1871) 18-.

Bisection, repeated. Bouniakowsky, V. [1866] St Pét. Ac. Sc. Bll. 11 (1867) 97-.

\section{CIRCLES. (See also 2880.)}

Crelle, A. L. Crelle J. 14 (1835) 66-.

Mehmke, R. Z. Mth. Ps. 24 (1879) 257-

Bessell, $F$. Arch. Mth. Ps. 67 (1882) 44-

Tarry, G. As. Fr. C. R. (1891) (Pt. 2) 90 -.

Approximations, rational. Rankine, $W . J . M$. Ph. Mg. 29 (1865) 421-.

Arc of given length, construction. Rankine, W. J. M. [1864-67] Ph. Mg. 29 (1865) 22-; 34 (1867) 284-.

-, rectification. Dostor, G. J. N. A. Mth. 6 (1847) $287-$

--, resolution. Fuss, N. [1804] St Pét. Ac. Sc. Mm. 2 (1810) 45-.

- whose tangent is rational. Prouhet, E. Liouv. J. Mth. 1 (1856) 215-.

Arcs, connecting two pairs of straight lines. Breton, Ph. Par. A. Pon. Chauss. 20 (1860) 135-.

Area of segments. Grunert, J. A. Grunert Arch. 27 (1856) 94-.

Chasles's circles. Barisien, E. N. Mathesis 15 (1895) 129-, 158-, 241-; 16 (1896) 265-.

- Droz Farny, A. Mathesis 16 (1896) 193-.

- properties. Brocard, H. Mathesis 18 (1898) 61-

Chords through given point. Büchner, $(D r)-$. Grunert Arch. 3 (1843) 388-.

Circle cutting 2 given circles at given angles. Ponte Horta, F. da. G. Teix. J. Sc. 1 (1878) $180-; 2$ (1880) 7-.

- - - - - [Schiappa] Monteiro, A. G. Teix. J. Sc. 2 (1880) 54-, 130-, 174-; 5 ("1883) 143-; 6 (1885) 103-.

- 3 circles at equal angles. Mannheim, $A$. N. A. Mth. 12 (1853) 113-. 
6810 Apollonian Problem

Circle cutting 3 circles isogonally. Wageningen, F. van. N. Arch. Wisk. $2\left({ }^{*} 1876\right) 180-$.

- - - at given angles. Griffiths, $J$. L. Mth. S. P. 3 (1869-71) 269-; 5 (1873-74) 33-; QJ. Mth. 11 (1871) 366-.

- derived from seven straight lines. Serret, P. C. R. 119 (1894) 474-, 493-; 123 (1896) $442-$.

- on diagonal of a rectangle as chord. Locus problem. Siacci, F. G. Arcad. 18 (1860) 166-.

- as figure of least perimeter for given area. Anon. (vi 525) Gergonne A. Mth. 4 (1813 -14) 338 -.

- $-1-1-1$ - Anon. (vi 545)

Gergonne A. Mth. 13 (1822-23) 132-.

- - Anon. (vi 550) Gergonne A. Mth. 14 (1823-24) 316-.

- - - - - - Anon. (vi 555) Gergonne A. Mth. 15 (1824-25) 257-

- - - - - - - - completion of Steiner's proof. Edler, F. Gött. Nr. (1882) 73-.

- orthotomic to three circles. Whitworth, W. A. [1864] Mess. Mth. 3 (1866) 26-

- through two points cutting another diametrally. Clausen, T. Grunert Arch. 15 (1850) 235-.

- to replace a given curve within a finite space, practical tracing. Léauté, $H$. C. $\mathrm{R}$. 85 (1877) 1049-.

- of similitude of two circles. Nöggerath, E. J. Grunert Arch. 33 (1859) 329-.

\section{Circle touching 3 given circles. (Apollonian problem.)}

Cauchy, A. L. Par. Éc. Pol. Cor. 1 (1804-8) 193-.

Gergonne, J. D. [1814-17] Tor. Mm. Ac. 22 (1816) $20^{*}-$; Gergonne A. Mth. 7 (1816-17) 289-.

Anon. (vi 547) Gergonne A. Mth. 13 (1822 -23) $193-$.

Badon Ghijben, J. (VII) Amst. N. Ws. Ntk. Vh. 1 (1844) 164 .

Kerz, F. Grunert Arch. 24 (1855) 211-; 26 (1856) 266-; 28 (1857) 402-; 35 (1860) 121-.

Grunert, J. A. Grunert Arch. 28 (1857) 354-.

Kurz, A. Grunert Arch. 37 (1861) 347-.

Serret, P. N. A. Mth. 2 (1863) 95-.

Barbier, E. N. A. Mth. 4 (1865) 313-; C. R. 60 (1865) 1076-.

Stoll, - . Mth. A. 6 (1873) 613-.

Cayley, -. QJ. Mth. 25 (1891) 104-.

Hioux, V. N. A. Mth. 10 (1891) 399-.

Langley, E. M. Mth. Gz. No. 5 (1895) 41.

Study, E. Mth. A. 49 (1897) 497-

analytical results. Crelle, A. L. Berl. Ab. (1841) 65- ; Berl. B. (1841) $162-$.

- solution. Strehlke, F. Crelle J. 12 (1834) 359 .

- - Cayley, A. Ph. Mg. 13 (1857) 507-.

- . Mertens, F. Z. Mth. Ps. 21 (1876) 443-.

Casey's equation and theorem. Cayley, $A$. QJ. Mth. 8 (1867) 334-; A. Mt. 1 (1867-68) $132-$ conjectured Apollonian solution. Flauti, V. Nap. At. Ac. 1 (1819) 71-.

construction. Turrell, I. $H$. Des Moines Anal. 5 (1878) 166-.

modern researches. Sagajto, A. Par. T. Nauk Śc. Pam. 8 ("1876) Art. 6, 7 pp.

nature of solutions. Muirhead, R. F. Edinb. Mth. S. P. 14 (1896) 135-.

tangencies, problems, solution (new). Fergola, N. [1809] Nap. At. Ac. 1 (1819) 3-.

Circle touching straight lines, equations. Cayley, A. N. A. Mth. 18 (1859) 222-.

Circles touching a common circle. Allardice, R. E. [1900] Edinb. Mth. S. P. 19 (1901) $7-$

- - each other in succession, radii of systems. Seitz, E. B. Des Moines Anal. 1 (1874) 173-; 2 (1875) 74-.

- - one circle, and orthogonal to another, system. Aubert, $P$. Edinb. Mth. S. P. 6 (1888) 33-.

- - semicircles, locus of centres. Dostor, $G$. Arch. Mth. Ps. 66 (1881) 17-

- - three circles which touch each other. Dostor, G. Arch. Mth. Ps. 51 (1870) 191-. - - - - - - , theorem $\left(T^{2}=4 d_{1} d_{3}\right)$. Turrell, I. H. Des Moines Anal. 1 (1874) 171-.

- - - given circles, centres and radii, to find. Prange, A.J. A. N. Arch. Wisk. 15 (1888) 165-; Fschr. Mth. (1888) 731.

- - - or cutting them at given angle. Fouché, M. N. A. Mth. 11 (1892) 227-, 331-, 404-.

- - - - - system treated by trilinear coordinates. Caldarera, F. Palermo Cir. Mt. Rd. 4 (1890) 43-.

- - - - , - - - (Caldarera). Albeggiani, M. L. Palermo Cir. Mt. Rd. 4 (1890) 50 -

-, 2, relation between. Cayley, A. QJ. Mth. 11 (1871) 82-.

- 3 , cutting at given angles, system. Cayley, A. Mess. Mith. 17 (1888) 60-.

$-,-,-\ldots,-$ of equations. Cayley, A. Mess. Mth. 17 (1888) 18-.

,-- , mutually tangent, theorems. Muir, T. Edinb. Mth. S. P. 3 (1885) 119-.

-, - , system, deduction from properties. Franklin, F. [1880] (xII) J. H. Un. Cir. [1] (1882) 85.

- - - with three common tangents, construction. Dewall, (Oberst) von. Arch. Mth. Ps. 41 (1864) 139-; 42 (1864) 65-, 80-.

,- 4 , in contact, radii. Baur, C. W. Schlömilch Z. 5 (1860) 365-.

-, - , in plano, and four powers relative to a point in plane, theorem. Lucas, $E^{\prime}$. N. A. Mth. 15 (1876) 205-.

(Lucas). Cassani, $\bar{P}$. G. Mt. 14 (1876) 347-. -, - pairs, through six common points of 3 circles. Griffiths, J. QJ. Mth. 9 (1868) 358- ; 10 (1870) 230-.

-, 8, touching 3. Casey, J. QJ. Mth. 5 (1862) 318-. 
Circumference, descriptive, segmentary, or metrical properties. Mouchot, - C. R. 105 (1887) 602-.

Circum-quadrilateral. Steiner, $J$. Crelle J. 32 (1846) 305-.

Coaxal circles. Casey, J. [1860] QJ. Mth. 5 (1862) 43-, 118-.

- . Schober, K. Mh. Mth. Ps. 5 (1894) 104 - - locus connected with. Holgate, $T$. $F$. N. Y. Am. Mth. S. Bll. 4 (1898) 63-; 5 (1899) 135-.

- - radical axes. Quidde, A. Grunert Arch. 23 (1854) 130-.

,---- . Hopkins, G. H. [1865] Mess. Mth. 3 (1866) 122.

- - , - - of certain circles in triangle. Tucker, R. [1892] Edinb. Mth. S. P. 11 (1893) 57-.

$-\ldots,-\ldots$, circle involved in extended definition of. Mack, L. Arch. Mth. Ps. 62 (1878) 405-.

- _, _ - line parallel to. Kösters, -. Grunert Arch. 19 (1852) 1-.

- -, systems. Lachlan, R. QJ. Mth. 26 (1893) 129-.

Concentric circles, two sets of three, having same common difference of radii. Tait, P. G. Edinb. R. S. P. 9 (1878) 533-.

- - , - systems, property. Brocard, $H$. Mathesis 4 (1884) 219-.

Condition that three circles should meet in a point. Enneper, $A$. Z. Mth. Ps. 16 (1871) 257-.

Conjugate circles. Möbius, A. F. Leip. B. 10 (1858) 1-.

- -, groups. Cunningham, (Lt.-Col.) A. B. A. Rp. (1892) 647 .

Constant ratio circle. Rump, F. H. Grunert Arch. 27 (1856) 30-.

Contacts. Paucker, M. G..von. [1827] St Pét. Mm. Sav. Étr. 1 (1831) 503-.

- and intersections. Steiner, J. Crelle J. 1 (1826) 161-, 252-.

- - Plilcker, J. Gergonne A. Mth. 18 (1827-28) 29-.

- of systems of circles. Larmor, A. L. Mth. S. P. 23 (1892) 135-.

Contraction caused by arranging circles of different diameters in rows. Kleiber, J. A. Fschr. Ps. (1890) (Ab. 1) 461-.

Coplanar circles, properties of triangle formed by. Lachlan, $R$. QJ. Mth. 21 (1886) 1-.

Diameter, to calculate from three contiguous chords. Voll, W. Oken Isis (1826) 670.

Division. Gauss, C. F. Par. S. Phlm. Bll. 3 (1802) 102-.

- Utenhove, J. M. C. van. Amst. Vh. 4 (1818) 92

-. Valat, -. Bordeaux Act. Ac. Sc. (1839) 433-.

-. Eisenstein, G. Crelle J. 27 (1844) 269-.

- Rammelman-Elsevier, W. J. C. (vur) Amst. N. Ws. Ntk. Vh. 1 (1844) 127-.

-. Küpper, C. Grunert Arch. 27 (1856) 62-.

-. Wenckebach, E. Gravenh. I. Ing. Ts. (1870-71) 79-.

-. Carey, F. S. QJ. Mth. 26 (1893) 322-.

voL. I.
Division into areas of given ratio and same perimeter. Gergonne, J. D. Gergonne A. Mth. 6 (1815-16) 57-.

- by compasses. Pfeil, L. von. Arch. Mth. Ps. 41 (1864) 153--.

- - [Schiappa] Monteiro, A. G. Teix. J. Sc. 4 (1883) 39-.

-, Gauss's theorems. Jung, G. G. Mt. 6 (1868) 67-.

-, irreducible equation. Arndt, $F$. Crelle J. 56 (1859) 178-.

-, new method. Pellet, A. E. C. R. 93 (1881) 838-.

, practical. Housel, -. N. A. Mth. 12 (1853) 77-.

-, - Tempier, -. N. A. Mth. 12 (1853) 345-; 13 (1854) 295.

-, problem. Arndt, F. Grunert Arch. 18 (1852) 461-.

-, series for. Huguenin, $U$. Amst. Vh. 5 (1820) 43-.

- into 5 equal parts. Barbour, L. G. Des Moines Anal. 5 (1878) 180-.

- 17 parts. A. B. (vi $A d d s$.) Tilloch Ph. Mg. 57 (1821) 172-.

- - , etc. Gruson, J. P. Berl. Ab. (1812-13) (Mth.) 15-.

- -66537 equal parts. Hermes, J. Gött. Nr. (1894) 170-.

Equations. Casey, J. [1878] R. S. P. 27 (1878) 417-; Ir. Ac. T. 26 (1879) 527-.

Faisceaux. Ribaucour, A. C. R. 76 (1873) 830 -.

Figures composed of circles, examination with a special system of coordinates. Crone, $C$. (xII) Ts. Mth. 6 (1876) 97-.

Grazing of two circles, best value of radii. Fontès, -. Toul. Ac. Sc. Mm. 4 (1892) 375 -.

Groups of circles, certain. Vries, J. de. Amst. Ak. Vs. 6 (1898) 418-.

Homocyclic points and circles in collineation, etc. Serret, P. C. R. 123 (1896) 396-.

Imaginary circles. Mobius, A.F. Leip. B. 9 (1857) 38-.

Intersections. Gufflet, G. N. A. Mth. 5 (1846) $352-$.

Inversion, point and tangential. Coelingh, $D$. N. Arch. Wisk. 16 (1889) 116-; Fschr. Mth. (1889) 614-.

Joachimsthal's circle. Longchamps, G. de. Mathesis 9 (1889) 153-.

Minimum circle enclosing $n$ given points. Chrystal, $G$. Edinb. Mth. S. P. 3 (1885) 30-.

Orthocentroidal circle. Prime, (Mme.) -. Mathesis 13 (1893) 33-.

Orthogonal systems. Lachlan, R. Mess. Mth. 16 (1887) 98-.

- trajectories of certain systems. Roberts, R. A. Mess. Mth. 14 (1885) 134-.

Plane system, coordinate. Schoute, $P$. $H$. Wien Ak. Sb. 94 (1887) (Ab. 2) 786-.

- - , and its representation in space. Thomae, J. Z. Mth. Ps. 29 (1884) 284-.

Polar system for circles. Meyer, $T$. Arch. Mth. Ps. 9 (1890) 18-, vII-.

- theorems, demonstrations. Zeuthen, H. G. (xII) Ts. Mth. 6 (1882) 75-. 
Polars of points with respect to two circles, theorem. Ploix, É. N. A. Mth. 8 (1849) $152-$.

- - - - - Gentil, -. N. A. Mth. 8 (1849) 290-.

Pothenot's problem. Vieth, G. U.A. Gilbert A. 54 (1816) 311-.

- - Grunert, J. A. Arch. Mth. Ps. 44 (1865) 184-.

- - Láska, V. Prag Sb. (1895) (Mth.-Nt.)

No. $17,5 \mathrm{pp}$.

- - algebraic aspect. Schlömilch, O. Z. Mth. Ps. 9 (1864) 433-.

- - analytical solution. Grunert, J. A. Grunert Arch. 1 (1841) 446-.

- - - - Fellini, D. Tor. Ac. Sc. At. 32 (1896) 214- or 320-.

- -, theory of errors in. Andra, -. As. Nr. 47 (185̃8) 193-.

Properties. Babbage, C. QJ. Sc. 1 (1816) 6-. - Laguerre, E. N. A. Mth. 2 (1883) 65-.

-, Grassmann's "Ausdehnungslehre" applied to. Cox, H. QJ. Mth. 25 (1891) 1-.

Property. August, E.F. Crelle J. 17 (1837) 387-.

-. Grunert, J. A. Grunert Arch. 1 (1841) 440-; 3 (1843) 259-; 25 (1855) 231-.

- (Grunert). Göpel, A. Grunert Arch. 3 (1843) 403-.

-. Darboux, G. C. R. 94 (1882) 1108-.

-, mechanical. Rispal, A. Liouv. J. Mth. 12 (1847) 225-.

Ptolemy's and Feuerbach's relations. Krahé, A. Mathesis 18 (1898) 83-.

- problem, application to astronomy. Hill, G. W. $[1900]$ As. J. 21 (1901) 33-.

- theorem. Oppermann, L. H.F. (xII) Ts. Mth. 1 (1871) 55-, 124 -

- - converse. Gerwien, -. Crelle J. 11 (1834) 264-.

- - demonstration. Schnell, (Dr.) -. Arch. Mth. Ps. 67 (1882) 225-.

- -, inversion. Förstemann, W. A. Crelle J. 13 (1835) 233-.

- - - - Strehlke, F. Grunert Arch. 2 (1842) $323-$

- - leads to Wallis's and others. Fergola, $N$. Nap. At. Ac. 1 (1819) 205-.

\section{Quadrature of the Circle.}

Delanges, $P$. At. Ac. It. 1 (1810) 157-.

Anon. (vi 215) Bb. Un. 3 (1816) 221-.

Anon. (vi 1171) Thomson A. Ph. 8 (1816) 13-.

Bitnicz, L. Evk. 2 (1832) 151-.

Lambert, C. J. N. A. Mth. 12 (1853) 298-.

Scheffler, H. Arch. Mth. Ps. 44 (1865) 84-

Pelnár, M. Časopis 6 ("1877) 89-; Fschr. Mth. (*1877) 406-.

approximate construction. Boswell, J. $W$. Nicholson J. 10 (1805) 151-; 13 (1806) 174-.

- - (Boswell). Gough, J. Nicholson J. 11 (1805) 29-.

$3 \overline{79}$. approximate method. Anon. (vi 312) Crelle J. 32 (1846) 91-.

approximations. Anon. (vI 275) Camb. and Dubl. Mth. J. 7 (1852) App. 1-.

-. Chase, P. E. Franklin I. J. 78 (1879) 45, $105 ; 79$ (1880) 409-; 81 (1881) 379.

arithmetical. Hill, C. J. D. Stockh. Ak. Hndl. (1854) 405-.

elementary. Schlömilch, O. Grunert Arch. 14 (1850) 101-.

Franco of Liége's "De Quadratura Circuli." Winterberg, C. Z. Mth. Ps. 27 (1882) (Suppl.) 135-.

historical notes, and proof of impossibility. Studnička, F. J. Čsopis 1 (*1872) 35-; Fschr. Mth. ( $\left.{ }^{*} 1872\right) 254$.

- - - - - Rudio, F. Zür. Vjschr. 35 (1890) 1-.

impossibility. Caluso, (l'abbé) T.V.de. [1801] Mod. Mm. S. It. 9 (1802) 558-.

-. Rouché, E. N. A. Mth. 2 (1883) 5-.

and rectification. Ruffini, $P$. [1801] Mod. S. It. Mm. 9 (1802) 527-.

- - Grunert, J.A. Grunert Arch. 6 (1845) 90-.

- - approximate. Specht, C. G. Crelle J. 3 (1828) 83, 405-; N. A. Mth. 4 (1845) 495-. - - graphic method. Schramm, A. Dingler 226 (1877) 17-.

- - impossible. Snart, $J$. Tilloch $\mathrm{Ph} . \mathrm{Mg}$. 62 (1823) 338-.

Viète (François) and his work. Ritter, F. As. Fr. C. R. (1879) 143-.

Radical and anti-radical circles. Durán Loriga, J. J. G. Teix. J. Sc. 13 (1897) 33-; Fschr. Mth. (1896) 398.

- circles. Durán Loriga, J. J. G. Teix. J. Sc. 12 (1895) 97-; Mathesis 16 (1896) 105-; Arch. Mth. Ps. 15 (1897) 117-, 232-.

Ratio of Diameter to Circumference.

(See also 2920.)

Lefaucheux, - [1811] Le Mans S. R. Tr. (1820) 19-.

Gergonne, J. D. Gergonne A. Mth. 6 (1815-16) 192-; 17 (1826-27) 148-.

Léger, $\hat{E}$. Quetelet Cor. Mth. 9 (1837) 479 -.

Lehmann, J. W. H. Grunert Arch. 21 (1853) 121-.

Didion, [(le gén.) I.]. C. R. 74 (1872) 36-; (xII) Metz Ac. Mm. 53 (1873) 257-.

(Didion.) Catalan, E. C. C. R. 74 (1872) 177. Lindemann, C. L. F. C. R. 95 (1882) 72-.

Sang, E. Edinb. R. S. P. 15 (1889) 348-.

Approximate construction. Grassmann, $H$. Arch. Mth. Ps. 49 (1869) 1-.

Approximation. Merrifield, C. W. Mess. Mth. 3 (1866) 105.

Calculation, and circular series. Mazzola, $G$. G. Mt. 2 (1864) 92-, 110-.

- to 8 decimal places. Drach, S. M. Ph. Mg. 27 (1864) 318-.

- $-200-$ Dahse [or Dase], Z. Crelle J. 27 (1844) 198; N. A. Mth. 9 (1850) 12-; 10 (1851) 198. 
Calculation to 200 decimal places (Dase). Schulz von Strasznitzki, L. C. Crelle J. 27 (1844) 198.

- $-250-$ Clausen, T. As. Nr. 25 (1847) 207-.

- $350-1$. Rutherford, W. N. A. Mth. 14 (1855) 209-.

- -400 - Richter, (Prof.) -. Grunert Arch. 22 (1854) 473.

- - - - Strehlke, F. Grunert Arch. 23 (1854) 475-; 24 (1855) 472.

- -500 - - Richter, (Prof.) -. Grunert Arch. 25 (1855) 471-.

- -707 - Shanks, W. R. S. P. 21 (1873) 318-.

-, elementary. Grunert, J. A. Arch. Mth. Ps. 46 (1866) 345-.

-, -, description. Matzka, W. Grunert Arch. 9 (1847) 74-.

- by logarithms. Chevillier, I. N. A. Mth. 15 (1856) 261-.

Determination by equivalent surfaces. Jubé, $E$. N. A. Mth. 7 (1848) 366-.

-, experimental. Hall, A. [1872] Mess. Mth. 2 (1873) 113-.

- by isoperimeters. Jubé, E. N. A. Mth. 5 (1846) 42-.

- - André, D. N. A. Mth. 13 (1874) 128 .

-, numerical. Girault, -. Rouen Tr. Ac. (1850-51) 63-.

- by perimeters. Huet, -. N. A. Mth. 4 (1845) 156-.

- - Fouché, M. Par. S. Mth. Bll. 18 (1890) 135-.

-, Ptolemy's method. Terquem, O. N. A. Mth. 13 (1854) 253-.

Dirichlet's formula, proof, etc. Lebesgue, $V$. A. Liouv. J. Mth. 11 (1846) 76-.

Discrepancies in published value of $\pi$. Shanks, $W$. [1873] R. S. P. $22(1873-74) 45-$.

Expression by rapidly convergent series. Lucas, F. C. R. 112 (1891) 1050 -

Numerical values of first 12 powers and their reciprocals, etc. Glaisher, J.W.L. L. Mth. S. P. 8 (1876-77) 140-.

Powers of $\pi$. Broch, O.J. Sk. Nf. F. 8 (1860) 571-.

Reciprocal of $\pi$, calculation to 208 decimal places. Rutherford, $W$. Phil. Trans. (1841) 281-.

Series for $\pi$, and general theorem. Gasparis, A. de. Nap. Rd. 7 (1848) 269-.

- - - new. Thomson, (Prof.) James. [183638] R. S. P. 3 (1836) 417-; Edinb. R. S. T. 14 (1840) 217-.

Theorem, of which Wallis's theorem is a case. Grunert, J.A. Grunert Arch. 38 (1862) 466-.

Van Ceulen's 35-decimal value and some of his works. Bierens de Haan, D. [1873] (vII) Mess. Mth. 3 (1874) 24-.

Wallis's and Brouncker's expressions, analytical investigation. Plana, G. Crelle J. 17 (1837) 1-, 163-, 338-.

- formula. Collignon, É. Par. Éc. Pol. J. $47^{\circ} \mathrm{cah}$. (1880) 103-.

- for $\pi$, and the area of the curve $y=\sin ^{k} x$. Mansion, P. Mathesis 20 (1900) 183-.
Wallis's fractions, analysis. Kramp, $C$. Erfurt N. Acta 1 (1799) 257-.

Rectification, approximate. Gergonne, J. $D$. Gergonne A. Mth. 8 (1817-18) 245-.

-, new method. Śruitek, $J$. Časopis 21 (1892) 83-; Fschr. Mth. (1892) 530-.

Segment, calculation. Grunert, J.A. Arch. Mth. Ps. 44 (1865) 363-.

- containing given angle, instrument for describing. Rollet de l'Isle, -. Rv. Mar. et Col. 128 (1896) 90-.

Segments, Greek approximate formulæ (Hero). Tannery, $P$. Bordeaux S. Sc. Mm. 5 (1883) 347-.

Self-polar circle. Grunert, J.A. Arch. Mth. Ps. 41 (1864) 132-.

Semicircles, Apollonius's problem. Sangro, G. Nap. At. Ac. 2 (1825) 41-.

Shoemaker's knife (á $\rho \beta \eta \lambda$ ○s). Mackay, J.S. Edinb. Mth. S. P. 3 (1885) 2 -.

Stewart's properties, demonstration. Strong, $T$. [1814] Conn. Mm. Ac. 1 (1816) 393-.

Systems of circles. Cayley, A. QJ. Mth. 2 (1858) 83-.

- - Gehrke, J. N. Ts. Mth. 9 (B) (1898) 11-; Fschr. Mth. (1898) 436.

- - - with application to Dupin's cyclides. Holst, E. Christiania F. (1885) No. 15, 4 pp.; Fschr. Mth. (1885) 574-.

- - - , new method of representation. Fiedler, $W$. As. Fr. C. R. 10 (1881) 127-.

Tangent circles. Guibal, -. Nancy Mm. S. Sc. (1843) 67-.

Tangents, trapeziums, etc. Hey, R. Phil. Trans. (1814) 348-.

Theorem. Miquel, A. Liouv. J. Mth. 3 (1838) 485-.

Theorems. Brioschi, F. N. A. Mth. 15 (1856) 462-.

- Jeřábek, W. [1880] Arch. Mth. Ps. 66 (1881) 325-.

Transformations. Larmor, A. B. A. Rp. (1887) 607.

Circular billiards problem. Transon, (Prof.) $A$. N. A. Mth. 9 (1850) 340-.

- - - Auric, -. N. A. Mth. 13 (1894) 215-

Congruent figures, propositions. Petersen, $J$. N. Ts. Mth. 9 (B) (1898) 1-; Fschr. Mth. (1898) 431.

- - relative positions. Zeuthen, H. G. Mth. Ts. 5 (1863) 97-.

Constructions. Smith, H. J. S. Camb. and Dubl. Mth. J. 7 (1852) 118-.

-, class. Feldblum, $M$. Wiad. Mt. 4 (1900) 1-. - of 2 nd degree, aids to. Adler, $A$. Wien Ak. Sb. 99 (1891) (Ab. 2a) 846-.

- founded on the circle. Study, - Bonn Niedr. Gs. Sb. (1897) 80-.

-, linear. Kirkman, T. P. Manch. Ph. S. Mm. 9 (1851) 279-.

-, Mascheroni's, theory. Adler, A. Wien Ak. Sb. 99 (1891) (Ab. 2a) 910-.

- in mensuration. Bangma, O. S., \& Keyser, J. $F$. Amst. Mengelwerk 2 (1816) 1-.

- - plane and in space with the straight line only. Tilly, J. de. Mathesis 5 (1885) 124-. 
Curves, pedal and inversion. Fleuri, $G$. N. S. W. R. S. J. 26 (1892) 60-.

- on railways, compound. Emch, A. Kan. Un. Q. 5 (1896) 99-.

- - - junction by two ares of circles. Ocagne, M. d'. N. A. Mth. 17 (1898) 314-.

Diagonals of figures. Dassen, C. C. Arg. S. Ci. A. 42 (1896) 165-, 198-.

Discontinuities, on removing certain. Rulf, $W$. Mh. Mth. Ps. 4 (1893) 173-.

Distances, sum of squares of, theorem. Grunert, J. A. Grunert Arch. 20 (1853) 473-.

Division of rhomboid. Fuss, N. [1799] St Pét. Ac. Sc. Mm. 3 (1811) 65-.

- - triangles and quadrilaterals, formulæ. Le Brun, - C. R. 38 (1854) 752.

Euclid, first three books. Wedgwood, H. [1853] Camb. Ph. S. P. 1 (1866) 127-.

- 1, 1 to 32 , demonstration. Traill, D. Edinb. Mth. S. P. 2 (1884) 40-.

- I, 4, Bain's theory. Smith, W. R. [1870] Edinb. R. S. P. 7 (1872) 176-.

- I, 4, 5, 6, 26. Wolff, $F$. QJ. Mth. 7 (1866) $76-; 8$ (1867) 301-.

- 1, 5. Kendall, E. K. Cn. J. 3 (1858) 318 -.

- 1, 32. Fischer, W. Grunert Arch. 28 (1857) 365-.

- vi, 7. Wolff, J. F. (jun.) QJ. Mth. 11 (1871) 76.

- vI, 7, simpler demonstration. Walton, $W$. [1832] QJ. Mth. 9 (1868) 241-.

x. Poselger, F. T. Berl. Ab. (1834) 341-.

- $x$, axiom, extension (continued subtraction of $\frac{1}{m}$ th). Miuller, G.W. Grunert Arch. 1 (1841) 211-.

Extreme and mean ratio, expression " to divide a line in", etc. Vincent, A.J.H. N. A. Mth. 3 (1844) 5-.

- - - - “- - - - ". Terquem, $O$. N. A. Mth. 12 (1853) 36-.

- _ - - "golden section" in square and cube. Hagen, $F$. W. Dingler 140 (1856) 42-.

- _ - , "Grunert Arch. 4 (1844) 15-.

Fermat's theorem of rectangle on diameter of semi-circle. Blindow, $R$. Grunert Arch. 31 (1858) 295-; 32 (1859) 124-.

- - - - - - - -, demonstration. Heinen, $F$. Grunert Arch. 30 (1858) 246-.

- - - - - - - generalisation. Krilger, A. Grunert Arch. 31 (1858) 61-.

Figure composed of a triangle and parallelograms on its sides. Paraira, M. C. N. Arch. Wisk. $9\left({ }^{*} 1882\right) 87-$.

- - - - - the squares on its sides, properties. Mackay, J. S. Edinb. Mth. S. P. 6 (1888) 2-

- geometrical, study. Aubert, P. Edinb. Mth. S. P. 5 (1887) 23-.

Figures variable in similar manner. Neuberg, $J$. L. Mth. S. P. 16 (1884-85) 184-.
Graduation, method in which curves are represented by straight lines. Kapteyn, A.P. Cuyper Rv. Un. 40 (1876) 136-.

Harmonic centres. Caldarera, $F$. Palermo Cir. Mt. Rd. 1 (1887) 2-.

Homology, centres of. Midy, - N. A. Mth. 3 (1844) 77-.

Imaginary solutions in ordinary geometry. Tarry, G. As. Fr. C. R. (1892) (Pt. 2) 132-.

Inversion. Liouville, J. N. A. Mth. 13 (1854) 227-.

-. Mannheim, A. L'I. 28 (1860) 413-.

-. Grassmann, H. E. Leip. Mth. Ps. B. 29 (1877) 133-.

-, application to surface envelope of sphere touching three spheres. Mannheim, A. N. A. Mth. 19 (1860) 67-.

-, circular, and Peaucellier cell. Baker, A.L. [1894] Rochester (N.Y.) Ac. Sc. P. 2 (1895) 256 -

-, demonstration of theorems by. Casey, $J$. [1864] QJ. Mth. 7 (1866) 378-.

-, new method. Coelingh, D. N. A. Mth. 7 (1888) 133-.

-, propositions obtained by. Bellavitis, $G$. Tortolini A. 4 (1853) 198-.

Isobaric points in space, properties. Ferrari, $F$. G. Mt. 34 (1896) 73-; 35 (1897) 273-.

Lengths, rational relations. Zeuthen, H. G. (xII) Ts. Mth. 6 (1882) 75-.

Loci of points, sum of whose distances from given lines or planes is constant. Heis, E. Grunert Arch. 31 (1858) 228-.

Locus of points from which two fixed lines subtend equal angles. Stammer, $W$. Arch. Mth. Ps. 68 (1882) 18-.

Lunes, quadrature, fragment of Eudemus. Tannery, $P$. Bordeaux S. Sc. Mm. 5 (1883) 211-.

-, - , and Hippocrates of Chio. Tannery, $P$. Bordeaux S. Sc. Mm. 2 (1878) 179-.

Mean distance, centres, analytic proofs. Rochat, -. Gergonne A. Mth. 3 (1812-13) 286-.

- - - , applications of theory. Gerono, G. C. Gergonne A. Mth. 17 (1826-27) $330-$

- - of field from homestead. Hill, C. J.D. Stockh. Ak. Hndl. (1849) 1-.

- - 2 figures on a plane. Maxwell, J. C. Edinb. R. S. T. 26 (1872) 729-.

- - - intersections of variable straight line with three fixed lines, centre. Greiner, $M$. Arch. Mth. Ps. 69 (1883) 323-.

- - - lines from a point. Wetzig, $F$. Z. Mth. Ps. 11 (1866) 15-.

- - point from system of points. Schlegel, -. As. Fr. C. R. (1887) (Pt. 2) 266-.

- line of system of lines in a plane. Ocagne, M. d'. Par. S. Mth. Bll. 12 (1884) 114-.

- position, centres and lines. Gorton, W.C.L. [1891] A. Mth. 6 (1891-92) 33-.

- proportionals, two, method of finding. Pendlebury, R. Mess. Mth. 2 (1873) 166-.

Medians. Quetelet, E. Brux. MIm. Cour. $4^{\circ}$, $25(1851-53) 32 \mathrm{pp}$. 
Minimum distance point. Steiner, J. Berl. B. (1837) 144-.

- - Woldstedt, F. [1858] (vm) Helsingf. Acta 6 (1861) 33-.

$-\frac{1}{-}$ Sturm, $\boldsymbol{R}$. Crelle J. Mth. 97 (1884) 49-.

_- - Cinger, V. Ja. [1891] Rec. Mth. (Moscou) 16 (1893) 317-.

- - - Solovjev, N. M. Mosc. S. Sc. Bll. 93 (No. 1) (1897) 27-; Fschr. Mth. (1897) 441-.

- - - properties. Seydewitz, F. Grunert Arch. 8 (1846) 174-.

- figures. Ocagne, M. d'. Par. S. Mth. Bll. 12 (1884) 168-.

- or maximum of sum of positive and negative squares of distances of point from three straight lines in plane. Wetzig, F. Z. Mth. Ps. 12 (1867) 281-.

- problem. Neovius, E. Gött. Nr. (1887) 407-.

- sum of distances from three points. Lindman, C. F. Grunert Arch. 27 (1856) 295-.

Mosaics, geometrical, algebraic representation. Mersch, C. F. Lux. I. R. Pb. 19 (1883) 153-.

Pappus's theorem. Zahradnik, K. Arch. Mth. Ps. 17 (1900) 79-.

Peaucellier cell. Peaucellier, A. N. A. Mth. 12 (1873) 71-.

- -. Lemoine, $\dot{E} . \quad$ As. Fr. C. R. (1874) 122-; Par. Ing. Civ. Mm. (1875) 247-.

Planimeter, Amsler's. Rateau, A. As. Fr. C. R. (1896) (Pt. 2) 130-.

-, -, "no-rolling" curves. Morley, $F$. A. Mth. 1 (1900) 21-.

-, description of new. Fessenden, C. Cn. I. P. 5 (1888) 27-.

-, polar. Barbarin, P. N. A. Mth. 19 (1880) 212- ; 20 (1881) 266-.

-, -, problem. Echols, W. H. [1889] A. Mth. 5 (1889-90) 9-.

- for rectangular coordinates. Schmidt, $J$. Civing. 26 (1880) 437-.

Planimeters, report. Henrici, O. B. A. Rp. (1894) 496-.

Planimetry, desiderata. Pfeil, L. von. Arch. Mth. Ps. 58 (1876) 369-.

-, theorems deduced from analogous linear theorems. Möbius, A. F. Leip. B. (1852) 41-.

Points, complementary. Vigarié, É. Mathesis 7 (1887) 6-, 57-, 84-, 105-.

-, -. Ocagne, M. d'. Mathesis 8 (1888) $62-$.

-, two unknown, found from two known. Clausen, T. As. Nr. 18 (1841) 367-.

\section{Polygons. (See also 7210.)}

Cauchy, A.L. [1812] Par. Ec. Pol. J. 16 cah. (1813) 87-.

Franchini, $\dot{P}$. [1819] Lueca At. Ac. 1 (1821) 97-.

Cauchy, A. L. C. R. 26 (1848) 489-.

Allardice, R. E. Edinb. Mth. S. P. 5 (1887) 78-.

Analytical principles. Cuyper, A.C.de. Liége Mm. S. Sc. 4 (1848-49) 387-.
Analytical theorem. Walton, W. Camb. and Dubl. Mth. J. 5 (1850) 182-.

Angles, sum. Liotard, - Gard Mm. Ac. (1847-48) 76-.

- - Unferdinger, $F$. Wien Sb. 57 (1868) (Ab. 2) 627-.

,-- Steinhauser, A. Arch. Mth. Ps. 52 (1871) 294-.

Areas. Hudson, E. C. Mess. Mth. 24 (1895) 171-.

-. Schur, F. Dorpat Sb. 10 (1895) 2-.

-, Mascheroni's theorem. Terquem, O. N. A. Mth. 7 (1848) 348-.

-, problem. Ellis, R. L. (vi Adds.) Camb. Mth. J. 2 (1841) 142-.

-, and sum of angles. Spitzer, S. Haidinger B. 5 (1849) 156-.

Circumscriptible polygons. Darboux, $G$. Bll. Sc. Mth. As. 3 (1879) 64-.

- - , radius and surface. Dostor, G. N. A. Mth. 5 (1866) 73-.

Classification. De Morgan, A. QJ. Mth. 2 (1858) 340-.

Derived polygons, property. Rubini, R. (vIII) A. Mt. 5 (1863) 5 -.

Determination from angles between sides and diagonals. Hoppe, $R$. Arch. Mth. Ps. 61 (1877) 439-.

Division into equal areas. Oettingen, A. von. Dorpat Sb. 8 (1889) 262-.

- $m$-gons. Liouville, $\dot{J}$. Liouv. J. Mth. 8 (1843) 391-.

- - - number of ways. Grunert, J.A. Grunert Arch. 1 (1841) 193-.

- $p$-gons, number of ways. Rowe, R. C., \& Taylor, H. M. L. Mth. S. P. 13 (1881-82) 102-.

- - triangles. Binet, J. P. M. Par. S. Phlm. PV. (1838) 127-; Liouv. J. Mth. 4 (1839) 79-.

- - Catalan, E. C. Liouv. J. Mth. 4 (1839) 91-.

- Faure, H. N. A. Mth. 17 (1858) 50 -

- - number of ways. Rodrigues, $O$. Liouv. J. Mth. 3 (1838) 547-.

Equality. Nievengloski, G. H. N. A. Mth. 11 (1852) 462-.

- Ponte-Horta, F. da. Lisb. J. Sc. Mth. 1 (1868) 6-.

Equiangular and equilateral polygons. Hess, E. Marb. Schr. 10 (Ab. 12, 1874) 611-.

- - Allardice, R. E. Edinb. Mth. S. P. 5 (1887) 28-.

Errors in calculating, estimation. Soulages, $E$. Arg. S. Ci. A. 36 (1893) 5-; Fschr. Mth. (1893-94) 1842.

Even polygons, and odd, property. Allardice, R. E. Edinb. Mth. S. P. 8 (1890) 22-.

- - properties. Cotterill, T. L. Mth. S. P. 1 (1866) No. 5, 13-.

Family formed with given polygon. Ibach, L. F. N. A. Mth. 2 (1883) 226-.

Harmonic polygons. Tarry, G., \& Neuberg, $J$. As. Fr. C. R. (1886) (Pt. 2) 12-.

- - Casey, J. Mathesis 10 (1890) 96-.

- new method. Simmons, (Rev.) T. C. L. Mth. S. P. 18 (1886-87) 289-. 
Heptagon chord, Dïrer's. Drach, S. M. (vII) Ph. Mg. 26 (1863) 408; 27 (1864) 320.

-, irregular, of Faulhaber. Gilnther, $S$. Erlang. Ps. Md. S. Sb. 6 (1874) 112-.

Hexagons. Dandelin, G. P. Brux. Mm. Ac. Sc. 3 (1826) $1^{*}$ -

Imaginaries in polygonometry. Spitzer, $S$. Haidinger B. 4 (1848) 96-.

In- and circum-polygon of polygon of same number of sides, construction. Nawrath, Arch. Mth. Ps. 50 (1869) 1-.

- - - polygons of circle. Casinelli, $A$. (s. L.) Bologna N. Cm. 2 (1836) 241-.

- _ _ - - Bouniakowsky, V. St Pét. Ac. Sc. Mm. 4 (1841) 423-.

- _ _- Kü̈rschák, J. Mth. Termt. Éts. 5 (1887) 153-; Mth. Nt. B. Ung. 5 (1886-87) 77-.

- polygon of circle. Glaisher, J.W.L. Mess. Mth. 9 (1880) 133.

- _ _ - with sides passing through fixed points, solution, etc. Mendthal, -. Arch. Mth. Ps. 59 (1876) 39-.

- - - - - - - given points, problem of Pappus. Malfatti, G. F. Verona S. It. Mm. 4 (1788) 201-.

- polygons of circle. Prouhet, E. N. A. Mth. 9 (1850) 130-.

_ _ _ - complete, remarkable straight lines and points. Kantor, S. [1878] Wien Ak. Sb. 78 (1879) (Ab. 2) 193-.

- $-\ldots$, radius and area in terms of sides. Möbius, A. F. Crelle J. 3 (1828) 5-.

_ _ - _ remarkable points in. Overeem, M. van (jun.). Amst. Ak. Vh. (Sect. 1) 3 (1896) No. 7, 29 pp.; Fschr. Mth. (1895) $575-$.

--- polygons. Gardiner, M. [1862] N. S. W. Ph. S. T. 1 (1866) 70-.

- - - Certo, L. G. Mt. 26 (1888) 46-.

Inscriptible polygons. Collignon, É. As. Fr. C. R. 9 (1880) 78-.

Maximum area with given sides. Umpfenbach, $(D r)$-. Crelle J. 25 (1843) 184-.

_ _ - - Fasbender, -. Crelle J. 26 (1843) 181-.

$--\frac{-}{-}-$ Molins, $H$. Toul. Mm. Ac. 2 (1852) 129-.

Mensuration. Lhuilier, S. Bb. Un. 25 (1824) 169-.

Number of polygons determined by $n$ points taken as vertices. Guibert, $A$. Liouv. J. Mth. 4 (1839) 392.

Pedal polygon of constant area, locus of points for. Combette, E. (xiI) Brest S. Ac. Bll. 7 (1871) 1 (bis)-.

- - point. Gardiner, M. [1862]

N. S. W. Ph. S. T. 1 (1866) 63-.

- polygons, successive. Schotten, H. Arch. Mth. Ps. 13 (1895) 65-.

Pentagon in and about circle. Grunert, J. A. Grunert Arch. 22 (1854) 357-.

-, Gauss's theorem, demonstration. Serret, $P$. N. A. Mth. 7 (1848) 28-.

-, plane. Clebsch, R.F.A. Mth. A. 4 (1871)
Perimeter of a polygon, double points on. Brunel, G. Bordeaux S. Sc. Mm. 4 (1894) 273-.

Perpendiculars on sides, given sum, problem. Mainardi, G. [1849] Mod. S. It. Mm. 25 (1852) 34-.

Polygon moving similar to itself with 3 sides passing through fixed points. Böklen, $O$. Grunert Arch. 36 (1861) 22-.

- with sides passing through $n$ fixed points, and divided by the points in the same given ratio. Lugli, A. Mil. I. Lomb. Rd. 14 (1881) $564-$.

Polygons in plane, problem. Moon, R. Camb. and Dubl. Mth. J. 5 (1850) 131-.

- - - - (Moon). Thomson, (Sir) W. Camb. and Dubl. Mth. J. 5 (1850) 137-. and quadrilaterals, new propositions. Sporer, B. Arch. Mth. Ps. 7 (1889) 389-.

Problem. Bérard, -. Gergonne A. Mth. 7 (1816-17) 61-.

Properties, Laisant, C. A. As. Fr. C. R. (1877) 142-.

\section{Regular polygons.}

Sturm, J. C. F. Gergonne A. Mth. 15 (182425) 250 -.

Burg, A. Wien Jb. Pol. I. 11 (1827) 99-

Amiot, B. N. A. Mth. 3 (1844) 264-.

Anon. (xr 9) A. Cond. Pon. Chauss, 12 (1868) (Pt. 1) 33-.

Ferron, E. Lux. I. R. Pb. 14 (1874) 36-.

Mathet, G. Par. Ec. Norm. A. 4 (1875) 303-.

Žbikovskij, A. K. Kazan S. Nt. (Ps,-Mth.) P. 2 (1884) 40-

and angles, graphic representation. Schlömilch, O. Civing. 5 (1859) 219-.

area. Cabreira, A. [1897] Lisb. J. Sc. Mth. 5 (1898) 7-; Fschr. Mth. (1897) 445.

and circle. Lhuilier, $S$. Bb. Un. 36 (1827) 159-.

circumscribed about a similar polygon. Kästner, A. G. [1796] Erfurt N. Acta 1 (1799) 241-.

construction. Steczkowski, J. K. Grunert Arch. 24 (1855) 311-.

- (Staudt-Schröter). Affolter, F. G. Mth. A. 6 (1873) 582-.

constructions. Bonsdorff, E. [E.J.] Helsingf. Acta 10 (1875) 457-.

- for three. Pascal, E. G. Mt. 25 (1887) 82-.

convex and stellate, of same number of sides, property. Muir, T. [1873] (vIII) Mess. Mth. 3 (1874) 47-.

covering a plane with. Wiuff-Petersen, A. Ts. Mth. 6 (1889) 182-; Fschr. Mth. (1888) 524. equation, general. Nieuport, C. F. F. de. [1802] Par. Mm. Sav. Etr. 1 (1806) 659-. equations for sides and diagonals. Buys-Ballot, C. H. D. [1863] Amst. Vs. Ak. 16 (1864) 293-; Arch. Néerl. 1 (1866) 97-; Grunert Arch. 40 (1863) 139-.

- - - - Lobatto, R. [1865] Arch. Néerl. 1 (1866) 115-; Amst. Vs. Ak. 1 (1866) (Ntk.) 33-. 
equations for sides and diagonals. BadonGhijben, J. [1865] Amst. Vs. Ak. 1 (1866) (Ntk.) 293-; Arch. Néerl. 1 (1866) 131-.

-, and decomposition of same into equations of lower degrees. Schönborn, $W$. Arch. Mth. Ps. 46 (1866) 425-

with half the number of sides odd, relations between sides and diameter. Schwarzer, $A$. Wien Sb. 53 (1866) (Ab. 2) 454 -

in- and circum-scribed.' Azzarelli, M. Rm. N. Linc. At. 50 (1897) 69-.

inscription. Dickson, L. E. A. Mth. 9 (189495) $73-$.

isoperimetrical. Rankine, W. J. M. Ph. Mg. 34 (1867) 364-.

-, and theorems of Chasles. Terquem, $O$. Liouv. J. Mth. 3 (1838) 97-.

linkage for describing. Everett, J. D. B. A. Rp. (1894) 559-.

mensuration. Hessel, J.F.C. Grunert Arch. 39 (1862) 279-.

paving an area with. Lévy, L. Par. S. Phlm. Bll. 3 (1891) 46-.

perimeter of, equal in area to given polygon. Čigir, G. I. Kazan S. Nt. (Ps.-Mth.) P. 2 (1884) 92-.

- - , - - - - - - (Čigir). Žbikovskij, A. K. Kazan S. Nt. (Ps.-Mth.) P. 4 (1886) 233-.

properties. Mensbrugghe, $G$. van der. Brux. Ac. Bll. 17 (1864) 153-.

sides and areas, method of finding. Cabreira, A. Lisb. J. Sc. Mth. 5 (1898) 175-.

theorem, general. Wallace, (Prof.) W. Edinb. Ph. J. 11 (1824) 377-.

theorems. Français, J. F. Gergonne A. Mth. 5 (1814-15̃) 341-.

- Reinemund, F. Brux. Ac. Bll. 40 (1875) $801-$; 43 (1877) 356-.

-. Meutzner, P. Mth. A. 13 (1878) 566-.

-. Sporer, B. Z. Mth. Ps. 37 (1892) 25-.

5-gon (Staudt). Barral, H. N. A. Mth. 11 (1852) 388-.

-, formula. Tortolini, B. A. Mt.6(1864) 191-.

- and "pentalpha" area. Möbius, A.F. As. Nr. 3 (1825) 131-.

-, theory. Clebsch, R. F. A. Mth. A. 4 (1871) 284.

-, 6-gon, and 10-gon in circle, relation between sides. Grunert, J. A. Grunert Arch. 40 (1863) 127-.

6-gon, in- and circumscribed of circle, etc. Durrande, J. B. Gergonne A. Mth. 14 (1823-24) 29-.

6-gonal facets within given circle. M., A. J. Mer. Sc. 6 (1858) 83-.

7-gon. Grunert, J. A. Grunert Arch. 17 (1851) 355-.

- and circle, fractional values for. Norton, $H$. Ph. Mg. 27 (1864) 281-.

-, Röber's construction. Hamilton, (Sir) W.R. [1863] Ph. Mg. 27 (1864) 124-.

and 9-gon, approximate construction. Efremoff, D. Fschr. Mth: (1892) 531-.

9-gon. Denys, A. Mathesis 10 (1890) 162-.

-, inscription in circle. Encontre, D. Mntp. Rec. Bll. 1 (1803) 293-. 15-gon, 30-gon, 60-gon, etc., inscription, and calculation of sides. Dostor, $G$. Arch. Mth. Ps. 62 (1878) 103-

20-gon, inscribed, perimeter compared with radius. Hamilton, (Sir) W. R. Ph. Mg. 23 (1862) 267-.

30-gons, inscription of four. Dostor, G. N.A. Mth. 17 (1878) 370-.

$n$ - and $2 n$-gons, relative number, for $n$ even and odd. Dostor, G. [1877] Arch. Mth. Ps. 62 (1878) 148-.

Revolving polygon, curve envelopes of sides, metrical relation. Olsson, $O$. Ts. Mth. 4 (1886) 120-; Fschr. Mth. (1886) 666.

Self-inscribed polygons. Brunel, G. Bordeaux S. Sc. PV. (1895-96) 35-.

-inscription, multiple, polygons with. Brunel, G. Bordeaux S. Sc. PV. (1897-98) 43-.

Semi-regular polygons, analytical researches. Pigeon, H. Par. Éc. Pol. J. Cah. 41 (1865) $165-$.

- -, area. Cabreira, A. [1897] Lisb. J. Sc. Mth. 5 (1898) 43-; Fschr. Mth. (1897) 446.

Series. Darboux, G. Bll. Sc. Mth. As. 2 (1878) 298-

-. Ocagne, M. d'. Brux. S. Sc. A. 9 (1885) (Pt. 2) 231-.

Star polygons. Lévy, $M$. Rouen Tr. Ac. (1824) 40-.

- - Saigey, J. F. Férussac Bll. Sc. Mth. 4 (1825) 201-.

- - Valat, - Bordeaux Act. Ac. Sc. (1840) 415-.

- - area. Hermes, O. Crelle J. 65 (1866) 174 .

- - , - Jacobi's rule extended to case of multiple points. Hermes, O. [1865] Crelle J. 65 (1866) 177-.

- -, general theory. Dostor, G. Liouv. J. Mth. 6 (1880) 343-.

- -, geometrical import. Schröder, $E$. Schlömilch Z. 7 (1862) 55-.

- - regular, with continuous outline. Azzarelli, M. Rm. N. Linc. At. 36 (1883) 316-.

- and rayed polygons. Dostor, $G$. Arch. Mth. Ps. 59 (1876) 375-.

Theorems. Fuss, N. [1810] St Pét. Ac. Sc. Mm. 11 (1830) 209-.

-, and application to arithmetic and trigonometry. Werner, O. [1857] St Pét. Ac. Sc. Bll. 16 (1858) 1-.

Porisms.

(See also 4050 and 7210.)

Playfair, J. [1792] Edinb. R. S. T. 3 (1794) 154-.

Wallace, (Prof.) W. [1796] Edinb. R. S. T. 4 (1798) 107-.

Breton [de Champ], P. C. R. 36 (1853) 1008-.

Housel, -. Liouv. J. Mth. 1 (1856) 193-.

(Breton de Champ.) Vincent, A.J.H. Liouv. J. Mth. 4 (1859) 9-. 
Breton [de Champ], P. Lionv. J. Mth. 4 (1859) 153-; C. R. 51 (1860) 1034-.

(- - - Bertrand, J., \& Serret, -. C. R. 53 (1861) 699-.

Circles, in- and circum-scribed, of polygon, relation between distance of centres and radii. Grunert, J. A. Grunert Arch. 32 (1859) 68-.

-, - or circum-scribed, of plane or spherical polygons, equations. Casey, J. As. Fr. C. R. $9(1880) 230$ -

- - - and e-scribed, of triangle, Beltrami's theorem for relation between centres, demonstration. Schmidt, C. Arch. Mth. Ps. 43 (1865) 238- ; 44 (1865) 120-.

- $-1-, \ldots-$, circum-circles of. Jeffery, H. M. QJ. Mth. 24 (1889) 180-.

-, porism. Lamnor, J. Mess. Mth. 13 (1884) 61-.

- porismatic systems. Lachlan, $R$. Mess. Mth. 16 (1887) 152-.

- , ring of, touching 2 circles, porism. Taylor, H. M. Mess. Mth. 7 (1878) 148-.

$-,-\ldots,-\ldots, \ldots$. Taylor, W. W. Mess. Mth. 7 (1878) 167-.

Euclid's porisms (Chasles), demonstration. Marianini, P. D. Mod. Mm. S. It. 2 (1866) 1-.

Fermat's fourth porism. Nagel, C. H. Arch. Mth. Ps. 46 (1866) 11-.

Functional symmetry in notation. Graves, J. T. Ph. Mg. 15 (1839) 129-.

Polygon porism of two circles. Enneper, $A$. Schlömilch Z. 7 (1862) 190-.

- - - - Weill, M. Liouv. J. Mth. 4 (1878) 265- ; N. A. Mth. 19 (1880) 57-.

- - - - Intrigila, C. G. Mt. 21 (1883) 323-.

--1 - Dixon, A. C. Mth. Gz. 1 (1900) 121-

- - - - , maxima and minima. Birnbaum, H. Grunert Arch. 29 (1857) 414-. - _ - - relations. Doležal, $\boldsymbol{E}$. Arch. Mth. Ps. 15 (1897) 172-.

Polygons, irregular symmetrical, both in- and circum-scribed to circle. Fuss, N. [1798] St Pet. Ac. Sc. N. Acta 13 (1802) 166-.

$\ldots, \ldots \ldots, \ldots \ldots \ldots$ - _ _ Fuss's problem. Mention, J. [1859] St Pét. Ac. Sc. Bll. 1 (1860) 15-, 33-

Problems. Clifford, $W . K$. [1868] Camb. Ph. S. P. 2 (1876) 120-

Triangles, in-, and circum-scribed, of circles. Garnier, J. G. Quetelet Cor. Mth. 1 (1825) 179 .

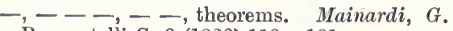
Brugnatelli G. 9 (1826) 119-, 181-.

- inscribed in ellipse and circumscribed to concentric circle. Barisien, $\boldsymbol{E}$. Mathesis 19 (1899) 224-, 247-, 269-; 20 (1900) 84-, 113-, 136-.

-, two, such that circum-circle of one is incircle of other, areal relation. Luchterhandt, A. R. Grunert Arch. 9 (1847) 262-.

Powers. Arndt, C. F. Grunert Arch. 5 (1844) 113-.

- of circles and spheres, theorems. Johnson, $W$. W. B. A. Rp. (1890) 743-.
Powers of points. Laisant, C.A. As. Fr. C. R. $(1875)$ 139-.

Problem. Martini, $M$. (vII) Bologna Opusc. Sc. N. Col. (1824) 251-.

- : through given point equidistant from two given lines to draw intercept of given length (Newton). Gergonne, J. D. Gergonne A. Mth. 10 (1819-20) 204-.

Fontés, -. N. A. Mth. 6 (1847) 180-.

(Newton). Transon, (Prof.) A. N. A. Mth. 6 (1847) 458-.

Problems, solutions of two. Mackay, J.S. Edinb. Mth. S.' P. 7 (1889) 42-.

- on triangle, circle, etc. Paque, A. Liège Mm. S. Sc. 10 (1855) 199-.

\section{Pythagorean theorem.}

Miller, H. K. F. von. Oken Isis (1826( $763-$. Gudermann, $C$. Crelle J. 42 (1852) 280 -

Vincent, A. J.H. N. A. Mth. 11 (1852) 5-.

De Morgan, A. QJ. Mth. 1 (1857) 236-.

Salmon, G. QJ. Mth. 1 (1857) 237-.

Richardson, J. M. Camb. (M.) Mth. M. 2 (1860) 45-.

Azzarelli, $\dot{M}$. [1873] Rm. N. Linc. At. 27 (1874) 66-.

Harvey, W. Edinb. Mth. S. P. 4 (1886) 17-.

Zahradnik, K. Časopis 25 (1896) 261- ; Fschr. Mth. (1896) 394.

Confirmation. Wittstein, T. Grunert Arch. 11 - (1848) 152-.

Demonstration. Buquoy, G. von. Oken Isis 21 (1828) 1-

- by dissection. Perigal, $H$. Mess. Mth. 2 (1873) 103-.

Extension to any triangle. Jelinek, $V$. Časopis 28 (1899) 79-; Fschr. Mth. (1899) 456.

Extensions. Anglin, A. H. Edinb. R. S. P. 12 (1884) 703-.

Figures for proving. André, H. $d^{\prime}$. N. A. Mth. 5 (1846) 324-.

Generalisation. Umpfenbach, (Dr) -. Crelle J. 26 (1843) 92.

Puzzles connected with theorem. Brand, $E$. Rv. Sc. 2 (1894) 274-, 809-.

Pythagorean triangles. Grunert, J.A. Grunert Arch. 31 (1858) 472-.

- - Cantor, M. Schlömilch Z. 4 (1859) 306-.

- -. Lewis, J. Am. Ph. S. P. 9 (1865) 415-.

- - Whitworth, W. A. Lpool. Lt. Ph. S. P. 29 (1875) 237-.

- - , and application to the division of the circumference. Graeber, - . Arch. Mth. Ps. 15 (1897) 337-

- -, nearly isosceles. Martin, Art. Des Moines Anal. 3 (1876) 47-.

- - properties. Breton, $\mathrm{Ph}$. Les Mondes 6 (1864) 401-.

Right-angled triangles. Bangma, O. S. Amst. Vh. 4 (1818) 28-

- - squares on sides (Hamett). Gergonne, J. D. Gergonne A. Mth. 14 (1823) 334-. 
Quadrangles, complete. Neuberg, $J$. Mathesis 11 (1891) 33-, 67-, 81-, 189-.

-, transformation. $G o b, A$. As. Fr. C. R. (1894) (Pt. 2) 294-.

\section{Quadrilaterals. (See also 7210.)}

Fraczkievicz, A. (vi Adds.) Krk. Roczn. Uniwers. 15 (1833) 123-.

Fenwick, S. Mathematician 2 (1847) 285-.

Baur, C. W. Schlömilch Z. 4 (1859) 236-.

Farjon, F. N. A. Mth. 11 (1892) 41-.

Area. Dostor, G. J. N. A. Mth. 7 (1848) 69-.

- with given sides, maximum. Enneper, $A$. Gött. Nr. (1885) 175-.

- - - , minimum. Sturm, R., \& Lampe, E. Crelle J. Mth. 96 (1834) 78-.

- in terms of vertices, determinant expression. Dostor, G. .N. A. Mth. 13 (1874) 559-.

Centroid. Commier, -. N. A. Mth. 2 (1843) $392-$.

-, when external. Matzka, W. Grunert Arch. 18 (1852) 352-.

Common or re-entrant quadrilateral, equations of condition. Shortrede, R. Beng. J. As. S. 11 (1842) 28-, 207-.

Complete quadrilateral. Schlömilch, $O$. Leip. B. (1854) 4-.

- - Mansion, P. [1875] Mess. Mth. 5 (1876) 158-.

- - Kantor, S. [1877-79] Wien Ak. Sb. 76 (1878) (Ab. 2) 774-; 78 (1879) (Ab. 2) 172-; 79 (1879) (Ab. 2) 757-.

- -, 6 circles of. Schlömilch, O. Schlömilch Z. 2 (1857) 274-.

- -, properties. Ehlert, A. [1882] Arch. Mth. Ps. 69 (1883) 332-.

- - - - relating to bisectors. Sancery, $L$. N. A. Mth. 14 (1875) 145-.

- - and quadrangle, involutory properties, generalisation. Loria, $G$. Mil. I. Lomb. Rd. 18 (1885) 491-.

- - theorem. Steiner, J. Gergonne A. Mth. 18 (1827-28) 302-.

Cyclic quadrilateral. Greiner, $M$. Arch. Mth. Ps. 60 (1877) 178-.

- - of two circles, properties. Dostor, $G$. N. A. Mth. 6 (1867) 57-.

- - (or rather tetrastigm), properties. Nager, J. Mh. Mth. Ps. 7 (1896) 325-.

Deltoid, plane or skew (2 isosceles triangles on common base). Reuschle, C. G. Z. Mth. Ps. 10 (1865) 506-.

Doubly-centric quadrilaterals. Schlömilch, $O . X . \quad Z$. Mth. Ps. 23 (1878) 193-.

- - Beyel, C. Z. Mth. Ps. 44 (1899) 237-.

Harmonic quadrilateral. Neuberg, $J$. Mathesis 5 (1885) 202-, 217-, 241-, 265-.

In- and circum-quadrilaterals. Schumacher, J. Arch. Mth. Ps. 2 (1885) 383-.

_ - Langr, J. Č́asopsis 28 (1899) 244-; Fschr. Mth. (1899) 458.

- - properties. Durrande, J. B. Gergonne A. Mth. 15 (1824-25) 133-.

In-quadrilateral with diagonals at right angles. Sancery, L. N. A. Mth. 10 (1871) 487-.

In-quadrilaterals. Grunert, $J$. A. Grunert Arch. 5 (1844) 428- ; 8 (1846) 335.
In-quadrilaterals, Girard's three, having equal sides and inscriptible in same circle. Dostor, G. Arch. Mth. Ps. 66 (1881) $27-$

-, metric relations. Zimmermann, O. Arch. Mth. Ps. 7 (1889) 64-.

Middle points of diagonals in straight line, Gauss's proof. Hall, A. Mess. Mth. 4 (1868) 137.

Miquel's theorem, generalisation to $n$ lines. Kantor, S. [1877-79] Wien Ak. Sb. 76 (1878) (Ab. 2) 753-; 78 (1879) (Ab. 2) 789-.

Orthogonal quadrilateral, 57 propositions. Beyel, C. Z. Mth. Ps. 34 (1889) 218-, 290-.

Parallelograms, most simple, symmetrical about an axis. Tchebitchef, $P$. L. As. Fr. C. R. (1878) 159-.

Properties. Dostor, $G$. Arch. Mth. Ps. 48 (1868) 245-.

Quadrilateral with opposite sides equal. Liidicke, M. A. F. Gilbert A. 56 (1817) 198-, 334-, 439-; 64 (1820) 341-.

Quadrilaterals derived from quadrilateral, metrical properties. Bretschneider, C. A. Grunert Arch. 3 (1843) 84-.

- and triangles, theorems. Vecten, - Gergonne A. Mth. 9 (1818-19) 293-.

Rational quadrilaterals. Kummer, E. E. Crelle J. 37 (1848) 1-.

Sides and diagonals, relation between. Grunert, J. A. Grunert Arch. 38 (1862) 373-.

Square with angles situated on four straight lines. Clausen, $T$. St Pét. Ac. Sc. Bll. 7 (1864) 177-.

- through four points, construction and generalisation. Crelle, A. L., \& Lehmus, 一. Crelle J. 34 (1847) 280-.

Squares connected with two circles, problems, analytical. Mack, L. Arch. Mth. Ps. 64 (1879) 225-.

-, decomposition into four superposable parts. Brunel, G. Bordeaux S. Sc. PV. (1896-97) 161-.

-, division into $n$ equal squares, problem. Stonvell, T. P. Des Moines Anal. 2 (1875) 73-.

-, properties. Westcott, O. S. Am. As. P. (1883) 94-.

Trapezium, centroid, construction. Walker, J. J. QJ. Mth. 9 (1868) 338-.

- properties. Davies, T. S. Tilloch $\mathrm{Ph}$. Mg. 68 (1826) 116-.

Rectilinear figures, cutting into equal portions. Gerwien, -. Crelle J. 10 (1833) 228-.

- - geometry. Neuberg, J. As. Fr. C. R. (1893) (Pt. 2) 26-.

- - logical order of treatment. Endrés, Toul. Mm. Ac. 4 (1860) 339-.

- - simpler than triangle, and their application in theory. Matzka, W. Grunert Arch. 8 (1846) 365-.

Set square, measurement with false. Grunert, J. A. Grunert Arch. 18 (1852) 477-; 25 (1855) 230 -.

Similar figures. Grouard, A. (x) Par. S. Phlm. Bll. 2 (1865) 68-, 104- ; 7 (1871) 4-, $51-, 74-, 115-; 10$ (1873) 34-. 
Similar figures, associated. Tarry, G. Mathesis 6 (1886) 97-, 148-, 196-.

- - (directly similar), theorems. Schoute, P. H. Delft Éc. Pol. A. 6 (1890) 51-.

- - in a plane, system. Laisant, C. A. As. Fr. C. R. (1883) 178-.

- -, theorems. Tarry, -. C. R. 112 (1891) 984-.

- - 3, with extension of Feuerbach's theorem. M'Cay, W. S. [1889] Ir. Ac. T. 29 (1887-92) 303-。

- - , - properties. Tarry, G. (xII) Mathesis 2 (1882) 73-.

- - - - - Neuberg, J. (xII) Mathesis 2 (1882) 76-, 144.

- - - - - Casey, J. Mathesis 7 (1887) $14-, 75$.

Similitude, general theory. Breton, Ph. [1877] (xII) Isère S. Bll. 8 (1879) 4-, 9, 12-.

- and inversion. Mackay, J.S. Edinb. Mth. S. P. 6 (1888) 69 -

Stewart's theorems, proof of two on circle and polygon. Davies, T. S. Camb. and Dubl. Mth. J. 1 (1846) 229-.

\section{STRAIGHT LINES.}

Pfeil, L. von. Arch. Mth. Ps. 49 (1869) 178-.

Hain, E. Arch. Mth. Ps. 64 (1879) 267-.

Tarry, G. As. Fr. C. R. (1890) (Pt. 2) 152-; (1894) (Pt. 2) 184-.

Description by link work. Aldis, W. S. N. Z. I. T. 21 (1889) 441-.

Drawing a straight line. Kempe, A. B. Nt. 16 (1877) 65-, 86-, 125-, 145-.

- - - approximately, by linkwork. Müller, R. D. Nf. Vh. (1897) (Th. 2, Hälfte 1) 34.

Finite lines in plane, number of cases as regards parallelism and situation. Rothe, H. A. Ac. Cæs. Leop. N. Acta 12 (1825) 399-.

2 lines making complementary angles with fixed axis, properties. Mensbrugghe, G. van der. Brux. Ac. Bll. 20 (1865) 60-.

$n$ lines in plane, metric geometry. Morley, $F$. N. Y. Am. Mth. S. T. 1 (1900) 97-.

- $-\ldots, \ldots$ Loud, F. H. N. Y. Am. Mth. S. T. 1 (1900) 323-.

Properties, descriptive, segmentary and metric. Mouchot, A. C. R. 104 (1887) 1053-.

Relation of distances from three given points. Ferrers, N. M. QJ. Mth. 2 (1858) 120-.

Section of a line. Gergonne, $J . D$. Gergonne A. Mth. 15 (1824-25) 93-.

- - - Wallace, W. Edinb. Mth. S. P. 12 (1894) 76-.

- Anderson, R. E. Edinb. Mth. S. P. 15 (1897) 65-.

System of concurrent lines, isometrics of a line with reference to. Ocagne, $M$. $d^{\prime}$. Par. S. Mth. Bll. 17 (1889) 171-.

- - straight lines, centre of least squares. Neuberg, -. Brux. S. Sc. A. 23 (1899) (Pt. 1) 27 -

- - - - - - - - construction. Rahusen, A. E. N. Arch. Wisk. 3 (1898) 33-. - - - in plane, intercepts. Roberts, $S$, L. Mth. S. P. 19 (1889) 405-.

Systems of straight lines, orientation. Hum. bert, G. Am. J. Mth. 10 (1888) 258-.
Theorems. Frankenheim, M. L. Crelle J. 8 (1832) 178-.

-. Brioschi, F. N. A. Mth. 15 (1856) 462-.

Superposition. Kelland, $P$. [1855-64] Edinb. R. S. T. 21 (1857) 271-; 23 (1864) 471-.

- (Kelland). Brodie, R. Edinb. R. S. T. 36 (1892) 307-.

- by means of dissection. Muirhead, R. $F$. Edinb. Mth. S. P. 14 (1896) 109-.

Theorems, geometrical, derived by inversion, reciprocation, etc. Bellavitis, $G$. A. Sc. Lomb. Ven. 2 (1832) 250-; Padova N. Sag. 4 (1838) 243-; Tortolini A. 5 (1854) 31-, $241-, 428-, 473-$.

Transformation of rectangle into square. Peddie, W. Edinb. Mth. S. P. 4 (1886) 24.

- by symmetrical straight lines, application to Steiner's problem. Schoute, P. H. Bll. Sc. Mth. As. 7 (1883) (Pt. 1) 314-.

Transformations of figures. Gob, A. As. Fr. C. R. (1890) (Pt. 2) 1-.

- by reciprocal radii, groups. Allardice, $R$. E. [1887] Edinb. Mth. S. P. 6 (1888) 14-.

- - - - - (Allardice). Alasia, C. Fschr. Mth. (1900) 530 .

- - vectors. Laguerre, $E$. N. A. Mth. 1 (1882) 542-

- _- Ocagne, M. d'. N. A. Mth. 2 (1883) 249-.

- - - (Laguerre's transformation). Juel, C. N. Ts. Mth. 3 (B) (1892) 10-; Fschr. Mth. (1892) 578-.

--- , properties of cycles. Laguerre, $E$. N. A. Mth. 2 (1883) 65-.

Translation, method dependent on principle. Maver, D. [1890] Edinb. R. S. P. 17 (1891) $188-$.

Triangles (including Circles related thereto). (For Conic Sections related to Triangles see 7210.)

(Euler.) Terquem, O. N. A. Mth. 1 (1842) 79-, 196-.

Möllmann, B. Grunert Arch. 17 (1851) 373-.

Hellwig, C. Grunert Arch. 19 (1852) 14 -

Unferdinger, $F$. Grunert Arch. 27 (1856) 327-; 29 (1857) 432- ; 33 (1859) 420-.

Grunert, J. A. Arch. Mth. Ps. 42 (1864) 229-; 45 (1866) 429-; 48 (1868) 465-.

Schubert, H. Z. Mth. Ps. 16 (1871) 83-.

Hain, E. Arch. Mth. Ps. 60 (1877) 290- ; 61 (1877) 417- ; 62 (1878) 422-.

Greiner, M. Arch. Mth. Ps. 61 (1877) 225-.

(Euler.) Mackay, J. S. Edinb. Mth. S. P. 4 (1886) 51-.

Lemoine, É., \& Neuberg, -. Mathesis 6 (1886) 55-, 73-.

Lemoine, $\dot{E}$. As. Fr. C. R. (1886) (Pt. 2) 83-; (1887) (Pt. 2) 13-; (1888) (Pt. 2) 165-; (1889) (Pt. 2) 197-; (1891) (Pt. 2) 130-; (1892) (Pt. 2) 101-; (1895) (Pt. 2) 186-; (1896) (Pt. 2) 58-.

Cesáro, E. N. A. Mth. 6 (1887) 215-. 
Lemoine, É. Edinb. Mth. S. P. 13 (1895) 2-.

Perrin, É. As. Fr. C. R. (1897) (Pt. 2) 90-.

Candido, G. N. A. Mth. 18 (1899) 31-; 19 (1900) 244-.

Analogies in the triangle, rule. Lemoine, $\hat{E}$. N. A. Mth. 12 (1893) 20-; G. Teix. J. Sc. 12 (1895) 105-.

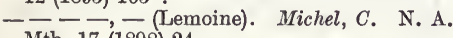
Mth. 17 (1898) 24-.

Angles, sum. Bertrand, J. C. R. 69 (1869) 1265-; 70 (1870) 17-.

-, 一, demonstration. Petersen, J.P.C. (xII) Ts. Mth. 1 (1883) 3-; Mth. A. 29 (1887) 238-.

,$--=180^{\circ}$, demonstration. Strong, T. Am. Ac. P. 1 (1848) 129-, 136-.

,$--=-,-$ Hedrick, B. S. Am. As. P. (1874) (Pt. 1) 104-.

,$--=-,-$, Legendre's. Levett, R. [1882] Birm. Ph. S. P. 3 (1883) 226-.

,$--=-,-$ without theory of parallels or infinity. Paulet, F. R. S. P. 3 (1836) 404.

- , - = - - - - - - Rosa, J. B. da. Coimbra I. 6 (1858) 115-.

Annex triangles, etc. Catalan, E. C. Brux. Ac. Bll. 4 (1882) 322-.

Antiparallels to sides of triangle. Lemoine, $\dot{E}$. Mathesis 4 (1884) 201-; Par. S. Mth. Bll. 14 (1886) 107-, 163.

-, use of word. Langley, E. M. Nt. 40 (1889) 460-.

Apollonian problem, special case. Hoffmann, K. E. [1880] Arch. Mth. Ps. 66 (1881) 246-.

Area. Studnička, F. Prag Sb. (1872) (pt. 2) 45 -.

-, and its dual analogue. Busche, E. Crelle J. Mth. 114 (1895) 1-.

-, equal division by perpendiculars drawn from a point. Laser, -. N. A. Mth. 3 (1884) 332-.

- of pedal triangle. Clausen, T. Crelle J. 3 (1828) 196-.

- of triangle formed by joining feet of bisectors of angles. Dostor, G. Arch. Mth. Ps. 64 (1879) 407-.

Bisectors of angles. Hain, E. [1875] Arch. Mth. Ps. 58 (1876) 90-.

- - - Mackay, J. S. Edinb. Mth. S. P. 13 (1895) 37-.

- - - construction of triangle from. Berg, F. J. van den. N. Arch. Wisk. 16 (1889) 179-; 17 (1890) 191-; Fschr. Mth. (1889) $576 ;(1890) 590$.

- - - - - Barbarin, -. Par. S. Mth. Bll. 22 (1894) 76-; Mathesis 16 (1896) 143-.

,$----1-\ldots$ Korselt, A. Z. Mth. Ps. 42 (1897) 304-.

- - - - - - - , and bibliographical notes. Baker, M. [1879] Smiths. Misc. Col. 20 (1881) Art. 3, 55-. (Wash. Ph. S. Bll. 3 (1880).)

- - , theorem. Mink, W. Grunert Arch. 15 (1850) 358-.

Brocard angle. Fuhrmann, W. Arch. Mth. Ps. 6 (1888) 1-.
Brocard angle and circle. Schoute, P. H. Amst. Ak. Vs. M. 3 (1887) 39-; Fschr. Mth. (1886) 477-.

- angles, triangles having equal. Neuberg, $J$. As. Fr. C. R. (1888) (Pt. 2) 135-.

- and Steiner angles. Avillez, J. F. d'. Lisb. J. Sc. Mth. 5 (1898) 85-

\section{Circles of the Triangle.}

Kücker, K. Arch. Mth. Ps. 47 (1867) 1-.

Duran-Loriga, J.J. As. Fr. C. R. (1897) (Pt. 2) 175-; (1898) (Pt. 2) 147-.

Brocard circle. Brocard, $\dot{H}$. As. Fr. C. R. 10 (1881) 138-.

- - and angle. Schoute, P. H. Amst. Ak. Vs. M. 3 (1887) 39-; Fschr. Mth. (1886) 477-.

- - and Brocard line, properties. Lemoine, $\underline{E}$. Mathesis 5 (1885) 103-.

- - generalised, properties. Griffiths, $J$. L. Mth. S. P. 26 (1895) 173-.

- - and Lemoine point, generalisation of properties. Lemoine, $k$. N. A. Mth. 4 (1885) 201-.

- - as locus. Langr, $J$. Časopis 29 (1900) 210-; Fschr. Mth. (1900) 499.

- - - - and related systems of conics. Müller, A. Arch. Mth. Ps. 8 (1890) 337-.

- _, variable seven-points circle analogous to. Griffiths, J. [1893] L. Mth. S. P. 25 (1894) 75-.

- circles of plane triangle generalised, inversion of system. Griffiths, J. L. Mth. S. P. 25 (1894) 376-.

- and other circles. M'Cay, W. S. [1885] Ir. Ac. T. $28\left(^{*} 1880-86\right)$ 453-.

Circle connected with triangle. Cwojdzinski, $K$. Arch. Mth. Ps. 17 (1900) 238-.

Circles connected with triangle formed by circular ares, properties of some. Lachlan, $R$. [1890] L. Mth. S. P. 21 (1891) 263-.

- cutting orthogonally in- and ex-circles of triangle. Hart, $H$. QJ. Mth. 18 (1882) 363-.

- touching circles described on sides of triangle. Rutherford, W. [1846] Mathematician 2 (1847) 69-.

- - sides. Noel, J. N. Quetelet Cor. Mth. 5 (1829) 22-.

Circum-circle, power of a point in relation to. Hain, E. [1881] Arch. Mth. Ps. 67 (1882) 106-.

-, property. Tucker, R. L. Mth. S. P. 22 (1891) 470-; 25 (1894) 315-.

-, relation to in- and ex-circles. Chrzaszczewski, S. Arch. Mth. Ps. 16 (1898) 119-.

-, - - inscribed conic. Laguerre, E. N. A. Mth. 18 (1879) 241-.

Groups of three circles related to. Berg, F.J. van den. N. Arch. Wisk. 7 ("1881) 78-.

In-circles, equation in trilinears. Russell, W. H. L. [1881-82] R. S. P. 33 (1882) 211-; 34 (1883) 35-.

-, etc., theorems. Lorenz, N. von. [1878] Arch. Mth. Ps. 63 (1879) 294-; 65 (1880) 212-. , circum-, and ex-circles. Matthes, C.J. Leijd. A. Ac. (1830-31) 66 pp. 
In- and ex-circles. Mackay, J. S. Edinb. Mth. S. P. 12 (1894) 86-; 13 (1895) 103-. - - Billups, H. B. Mth. Gz. 1 (1900) $177-$.

- - - (Billups). Mackay, —, \& Muirhead, -. Mth. Gz. 1 (1900) 209.

- - - of triangles formed by four straight lines, Steiner's theorem. Mention, J. N. A. Mth. 1 (1862) 16-, 65-.

Intersections of circle with triangle, relations. Taylor, H. M. L. Mth. S. P. $15\left({ }^{*} 1883-84\right)$ $122-$.

"Intimoscribed" circles. Anderson, $R, E$. Edinb. Mth. S. P. 10 (1892) 70-.

\section{Malfatti's problem.}

Gergonne, J. D., \& Lavernède, - Gergonne A. Mth. 1 (1810-11) 343-.

Tédénat, -. Gergonne A. Mth. 2 (1811-12) 165-.

Lechmütz, -. Gergonne A. Mth. 10 (1819-20) 289-.

Lavernède, T. Gard. Mm. Ac. (1832) 137-.

Catalan, E. C. N. A. Mth. 5 (1846) 60-.

Ghijben, J. B. Amst. Vs. Ak. 12 (1861) 189-.

Talbot, W. H. F. [1865] Edinb. R. S. T. 24 (1867) 127-.

Affolter, F. G. Mth. A. 6 (1873) 597-。

Simons, P. A. Brux. Ac. Bll. 38 (1874) 88-.

Catalan, E. C. Brux. Ac. Bll. 38 (1874) 480-.

Pelletreau, -. As. Fr. C. R. (1888) (Pt. 2) 99-.

Neumann, C. Leip. Mth. Ps. B. 41 (1889) $22-$

extension. Schellbach, C. H. Crelle J. 45 (1853) 186-.

history and complete analytical solution. Derousseau, $J$. Liège S. Sc. Mm. 18 (1895) No. 1, 52 pp.

and lemma on circles. Adams, C. N. A. Mth. 8 (1819) 62-

solution. Scheffer, H. Grunert Arch. 16 (1851) 424-.

-. Schellbach, C. H. [1852] N. A. Mth. 12 (1853) 131-.

- (Schellbach). Cayley, A. QJ. Mth. 1 (1857) 222-.

- Lebon, E. Palermo Cir. Mt. Rd. 3 (1889) 120 -

solutions, thirteen. Davids, C. Arch. Mth. Ps. 13 (1895) 10-; 14 (1896) 276-

Steiner's construction. Hart, A. S. QJ. Mth. 1 (1857) 219-

- -, demonstration. Quidde, A. Grunert Arch. 15 (1850) 197-.

- - - Mendthal, -. Arch. Mth. Ps. 55 (1873) 211-.

and Steiner's generalisation. Mertens, F. [187576] Wien. Ak. D. 36 (1876) (Ab. 2) 195-; Z. Mth. Ps. 21 (1876) 297-。

- - Godt, W. [1877] Crelle J. Mth. 84 (1878) 259-.

- - Mertens, F. [1893] Krk. Ak. (Mt.Prz.) Rz. 8 (1895) 67-; Prag Sb. (1894) (Mth.Nt.) No. 1, 21 pp.

Steiner's solution. Schrocter, H. E. Crelle J. Mth. 77 (1874) 230-
Steiner's solution. Petersen, J. Crelle J. Mth. 89 (1880) 127-.

- - demonstration. Zornono, - Crelle J. 10 (1833) 300-.

Neuberg's circles, Gob, A. Liège S. Sc. Mm. 16 (1890) No. 3, $14 \mathrm{pp}$.

New circle. Fuhrmann, W. Mathesis $10(1890)$ 105-.

\section{Nine-point or Feuerbach Circle.}

Trudi, N. G. Mt. 1 (1863) 29-.

Griffiths, J. N. A. Mth. 2 (1863) 339-; Mess. Mth. 2 (1864) 220-; QJ. Mth. 8 (1867) 15.

Besant, W. H. QJ. Mth. 7 (1866) 302-; Mess. Mth. 3 (1866) 222-.

Taylor, $C$. Mess. Mth. 4 (1868) 61-.

Hain, $E$. Arch. Mth. Ps. 59 (1876) 323-.

Zeuthen, H. G. (xI) Ts. Mth. 1 (1877) 113-.

Lemoine, $\boldsymbol{E} . \quad$ N. A. Mth. 5 (1886) 122-.

Wilson, (Rev.) J. Edinb. Mth. S. P. 6 (1888) 38-.

Cayley, A. Mess. Mth. 23 (1894) 23-, 25-.

Sporer, $B$. Wien Ak. Sb. 106 (1897) (Ab. 2a) 739-.

analytical treatment. Grunert, J.A. Arch. Mth. Ps. 41 (1864) 121-.

circle analogous to, about centroid of perimeter of triangle. Spieker, T. Arch. Mth. Ps. 51 (1870) 10-

Feuerbach's theorem. Lappe, J. [1869] Crelle J. 71 (1870) 387-.

- - Schroeter, H. E. Mth. A. 7 (1874) $517-$.

- -. Slawyk, R. Z. Mth. Ps. 35 (1890) 36 -.

- - demonstration. Leudesdorf, C. Mess. Mth. 13 (1884) 116-.

- - - Genese, R.W. L. Mth. S. P. 19 (1889) 216-

group of circles related to. Baker, $M$. Smiths. Misc. Col. 33 (1888) Art. 3, 45-. (Wash. Ph. S. Bll. 8 (1885).)

history. Mackay, J. S. Edinb. Mth. S. P. 11 (1893) 19-.

properties of triangle, generalisation. MacMahon, P. A. L. Mth. S. P. 14 (1882-83) 129 -.

Steiner's hypocycloid and the circumseribing hyperbolas of $\Delta$. Fiedler, $W$. Zür. Vjschr. 30 (1885) 390-.

and Steiner's quartic. Godt, $W$. [1895-96] D. Mth. Vr. Jbr. 4 (1897) 161-; Münch. Ak. Sb. 26 (1897) 119-.

Terquem's theorem, extension. Hart, A. S. QJ. Mth. 4 (1861) 260-.

theorems. Salmon, G. QJ. Mth. 4 (1861) 152-.

- Griffiths, J. QJ. Mth. 6 (1864) 357-; 7 (1866) 46-, 341-.

Pedal circle of a point with regard to given triangle. Vries, J. de. [1900] Amst. Ak. Vs. 9 (1901) 249-; Amst. Ak. P. 3 (1901) 323-. 
Polar circle, and Feuerbach's theorem, note on theorems. Roberts, S. Mess. Mth. 17 (1888) 57 -.

Relations of circle and triangle. Hain, $E$. [1876] Arch. Mth. Ps. 60 (1877) 78-.

Rings of circles connected with triangle. Taylor, W. W. L. Mth. S. P. 20 (1889) 397-。

"Scribed" circles, six, and triangle. Mackay, J. S. [1883] Edinb. Mth. S. P. 1 (1894) 4-, IV.

"Sine-triple-angle" circle (Tucker's circle). Tucker, R. Mess. Mth. 16 (1887) 125-.

Six circles and triangle. Evans, $A$. B. Des Moines Anal. 1 (1874) 189-.

- point circle. Taylor, H. M. Mess. Mth. 11 (1882) 177-.

- - - properties. Casey, J. QJ. Mth. 4 (1861) 245-.

Theorems. Sallabǎ̌ev, I. Prag Sb. (1875) 66-.

Tucker-circles, secondary. Griffiths, J. [1892] L. Mth. S. P. 24 (1893) 121-.

- or triplicate-ratio circles. Tucker, R. QJ. Mth. 19 (1883) 342-.

- - - - - group analogous to. Tucker, $R$. QJ. Mth. 20 (1885) 57-.

- - - - and others. Tucker, R. Edinb. Mth. S. P. 12 (1894) 17-。

- - - properties. Tucker, R. L. Mth. S. P. 25 (1894) 389-

- $-\ldots-$, systems analogous to. Third, J. A. Edinb. Mth. S. P. 17 (1899) 70-.

Circum-triangle on sides of triangle, construction for vertices. Clausen, T. Crelle J. 4 (1829) 391-.

Construction from radii of ex-circles. Grïson, J. P. (vi Adds.) Berl. Ab. (1818-19) (Mth.) $37-$.

Correlation. Callegari, $P$. [1843] Bologna Mm. Ac. 4 (1853) 179-.

Covariant geometry. Morley, F. QJ. Mth. 25 (1891) 186-.

Dissection of triangles. Muirhead, $R$. $F$. Edinb. Mth. S. P. 18 (1900) 5-, 100.

Division. Weiss, C. S. Berl. Ab. (1826) 93-. -. Dienger, $J$. Grunert Arch. 17 (1851) 300-. - in given ratio. Catalan, E. C. N. A. Mth. 4 (1845) 214-.

Equilateral triangles. Hain, E. [1882] Ärch. Mth. Ps. 69 (1883) 44-

- - on sides of triangle, theorem. Tucker, $R$. Edinb. Mth. S. P. 15 (1897) 98-.

Euler's line and circle. $G o b, A$. Liège $\mathrm{S}$. Se. Mm. 16 (1890) No. 2, 7 pp.

- -, hyperbola inverse to. Jeřábek, Mathesis 8 (1888) 81-.

_- _ _ - Neuberg, J. Mathesis 8 (1888) 84-, 115-.

- - , - - Fuhrmann, -. Mathesis 8 (1888) 115-

Formulæ. Catalan, E. Brux. Mm. Cour. $8^{\circ}$, 44 (1891) No. 4, $28 \mathrm{pp}$.

Geometry of triangle, bibliography. Vigarié, $E^{\prime}$. As. Fr. C. R. (1895) (Pt. 2) 50-.

$--\ldots$, history. Vigarié, $\dot{E}$. As. Fr. C. R. (1887) (Pt. 2) 87-; (1889) (Pt. 2) 117-.
Geometry of triangle, history. Fuhrmann, - . Königsb. Sehr. 40 (1899) [37]-.

Harmonic hexagon of a triangle. Casey, $J$. [1886] Ir. Ac. P. 4 (1884-88) 545-.

- triangle. Henry, C. Bll. Sc. Mth. As. 5 (1881) 96-

Homologous triangles. Neuberg, $J$. Mathesis 18 (1898) 155-.

In-triangle of circle with sides passing through fixed points, construction, etc. Giordano di Ottaiano, A. Verona Mm. S. It. 4 (1788) 4-.

- given form in given triangle. Allardice, R. E. Edinb. Mth. S. P. 9 (1891) 39-. - - - - inscription. Jenkins, $M$ QJ. Mth. 21 (1886) 84-.

- - - - - - Allardice, R. E Edinb. Mth. S. P. 6 (1888) 42-.

- least perimeter in triangle. Lindelöf, L. L. Helsingf. Öfv. 10 (1868) 31-; 11 (1869) 35-.

-, maximum equilateral, in given triangle. Malfatti, G. F. [1806] Mod. S. It. Mm. 13 (1807) 247-

- of similar triangle, inscription. Hoffmann, H. Grunert Arch. 9 (1847) 280-.

In-triangles of circle, special system. Strnad, A. Časopis 24 (1895) 136-; Fschr. Mth. (1895) 576-.

- in given triangle, group, etc. Tucker, $R$. [1892] L. Mth. S. P. 24 (1893) 131-.

Inversion of system of $n$ points, application to triangle. Laisant, $C$. $A$. As. Fr. C. R. (1887) (Pt. 2) 282-

Isogonal centres. Mackay, J. S. Edinb. Mth. S. P. 15 (1897) 100-.

- corresponding points. Hain, E. [1876] Arch. Mth. Ps. 60 (1877) 92-.

- systems in the triangle. Barbarin, $P$. As. Fr. C. R. (1896) (Pt. 2) 89-.

Isogonals of triangle. Mackay, J. S. Edinb. Mth. S. P. 13 (1895) 166-.

Isoperimetrical triangles having one side given and satisfying 3 other conditions. Chasles, M. C. R. 84 (1877) 471-, 627-, 1051-.

- - series satisfying 4 other conditions. Chasles, M. C. R. 84 (1877) 55-.

Isosceles triangle, property. Lange, $T$. Grunert Arch. 13 (1849) 337-; 15 (1850) 221-, 351-.

- - to be proved isosceles when bisectors of base angles are equal. Sylvester, J.J. Ph. Mg. 4 (1852) 366-.

- triangles. Pelišek, M. Časopis 26 (1897) 181-; Fschr. Mth. (1897) 442.

- - problems. Schiappa Monteiro, A. Lisb. J. Sc. Mth. 12 (1888) 57-, 121-.

Isoscelian hexagrams. Tucker, R. [1889] L. Mth. S. P. 21 (1891) 4-.

Isoscelians. Tucker, R. [1888-91] L. Mth. S. P. 19 (1889) 163-; 22 (1891) 178-.

Isostereans, group. Tucker, R. L. Mth. S. P. 19 (1889) 218-.

Least perimeter, triangle of, etc. Gregory, D. F. (vI Adds.) Camb. Mth. J. 1 (1839) 157-. 
Lehmus's theorem (angles of triangle equal, when bisectors are equal). Jerábek, $A$. Časopis 14 (1885) 20-; Fschr. Mth. (1885) 575.

Lines in triangle, class. Franke, - Crelle J. Mth. 99 (1886) 161-.

- - - - (Franke). Schroeter, H. Crelle J. Mth. 99 (1886) 233-.

Medians, generalisation. Azzarelli, $M . \mathrm{Rm}$. N. Linc. At. 42 (1889) 59-.

Menelaus's theorem, application to Euclid porisms. Serdobinskii, V. E. [1872] (xII) Rec. Mth. (Moscou) 6 (1872-73) (Pt. 2) 3-.

Orthocentric triangle. Tucker, R. Edinb. Mth. S. P. 12 (1894) 118-.

Orthologic triangles, triangles and conics. Lemoine, É. As. Fr. C. R. (1890) (Pt. 2) 111-.

Parallels and anti-parallels of a triangle, some properties. Lemoine, $\dot{E}$. Par. S. Mth. Bll. 12 (1884) $72-$.

- from point in plane to sides. Lemoine, $E$. As. Fr. C. R. (1882) 122-.

- and triangles, theorems. Denzler, W. Zür. Mt. 2 (1850-52) 74-.

Pedal triangle. Steggall, J. E. A. Edinb. Mth. S. P. 12 (1894) 85.

- -, theorem. Schoute, P. H. Palermo Cir. Mt. Rd. 1 (1887) 380-.

Perpendiculars and medians of a triangle, relations between. Pabst, C. Arch. Mth. Ps. 7 (1889) 10-.

Pole and polar. Greiner, $M$. Arch. Mth. Ps. 59 (1876) 351-.

Problem. Escher, P. Grunert Arch. 31 (1858) 46-.

-: In sides $A B, A C$ of triangle to find $E F$ so that $B E=E F=F C$. Edward, J. Edinb. Mth. S. P. 2 (1884) 5-.

$=-$ historical notes. Mackay, J.S. Edinb. Mth. S. P. 8 (1890) 93-.

$=-$, solution. Mackay, J. S. Edinb. Mth. S. P. 2 (1884) 27.

-: with given vertex to describe a triangle similar to given triangle and having its other angles on two given straight lines. Werebrussow, A. Fschr. Mth. (1897) 441.

Problems. Lorenz, N. von. Arch. Mth. Ps. 64 (1879) 253-.

-. Collignon, É. Edinb. Mth. S. P. 18 (1900) 88-.

Projections and contra-projections of fixed triangle. Neuberg, $J$. Brux. Mm. Cour. $8^{\circ}$, 44 (1891) No. 2, xvi +86 pp.

Properties. Schulz von Strasnitzki, L. C. Baumgartner Z. 2 (1827) 396-.

-. Schmidt, J. R. (vII) Amst. N. Ws. Ntk. Vh. 1 (1844) 30-.

- Pistoris, -de. N. A. Mth. 5 (1846) 451-.

- Azzarelli, M. Rm. N. Linc. At. 44 (1891) 49-.

- Molenbroch [? Molenbroek], -. N. A. Mth. 11 (1892) 121-, 179-.

-. Collette, M. L. Liège S. Sc. Mm. 19 (1897) No. 4, 12 pp.
Properties, demonstration. $V$. [Gregory, D. F.] Camb. Mth. J. 1 (1839) 157-.

-, extension. Schröter, H. [1867] Crelle J. 68 (1868) 208-.

-, mean orientation, equisegmentary points. Laisant, C. A. As. Fr. C. R. (1890) (Pt. 2) 23-.

-, new. Brocard, H. As. Fr. C. R. 12 (1883) 188 -

Property. Wallace, (Prof.) W. Camb. Mth. J. 1 (1839) 21-.

Proportionals to sides, etc. Albers, $J$. Arch. Mth. Ps. 68 (1882) 53-.

Pseudo-isosceles triangle. Emmerich, A. Mathesis 20 (1900) 129-.

Quadrilaterals and triangles in connection with progressions. Mikšić, M. Časopis $5\left({ }^{*} 1876\right)$ 134-; Fschr. Mth. (*1876) 325-.

- - - , new property. Brocard, H. [1874] Par. S. Mth. Bll. 3 (1875) 38-.

- - , series. Collignon, É. As. Fr. C. R. (1891) (Pt. 2) 38-.

Quadrisection. Euler, L. [1779] St Pét. Ac. Sc. Mm. 1 (1803-06) 26-, 49-.

Rational triangles. Grassmann, $H$. Arch. Mth. Ps. 49 (1869) 1-.

- -. Simerka, W. [1869] Arch. Mth. Ps. 51 (1870) 196-.

- Lehmer, D. N. A. Mth. 1 (1900) 97-.

- - complete development, with three tables. Rath, H. Arch. Mth. Ps. 56 (1874) 188-.

$--,-\ldots,---$ (Rath). Curtze, $M$. Arch. Mth. Ps. 57 (1875) 216-

Relation between area and sum of angles, supposing Euclid's 12th axiom false. Young, G. P. Cn. J. 5 (1860) 341-.

Relations between orthic and median triangles. Pressland, A. J. Edinb. Mth. S. P. 9 (1891) 36-.

-, metrical and of position. Bardelli, G. G. Mt. 14 (1876) 241-.

\section{Remarkable Points of the Triangle.}

Lange, J. [1880] Arch. Mth. Ps. 66 (1881) 220-; 67 (1882) 191-.

Lemoine, $E^{\prime}$. As. Fr. C. R. (1882) 108-.

Kapteyn, $W$. Amst. Ak. Vh. (Sect. 1) 3 (1896) No. 3, 33 pp.; Fschr. Mth. (1895) 665.

Associated points in the plane. Lemoine, $\hat{E}$. As. Fr. C. R. (1884) (Pt. 2) 49-.

Brocard points. Sweschnikow, P. Fschr. Mth. (1891) 599.

- - and angle. Davis, R. F. Edinb. Mth. S. P. 13 (1895) 28.

- - construction. Allardice, R. E. Edinb. Mth. S. P. 6 (1888) 88.

Centre of equal angular transversals. Brocard, G. Mathesis 16 (1896) 217-.

- - - - (Brocard). Neuberg, J. Mathesis 16 (1896) 221-.

Centres of similitude. Arndt, C. F. Grunert Arch. 5 (1844) 113-.

- - - of circles connected with triangle. Mackay, J. S. Edinb. Mth. S. P. 2 (1884) 2-. 
Centres of similitude of triangle of constant form circumscribed to given triangle. Griffiths, J. L. Mth. S. P. 24 (1893) 369-.

- - - - - - - inscribed in given triangle. Griffiths, J. L. Mth. S. P. 24 (1893) 181-.

Centroid. Hain, E. [1875] Arch. Mth. Ps. 58 (1876) 170-.

-, distances. Dostor, G. N. A. Mth. 2 (1883) 368 .

- and in-circle, distance. Grunert, J. A. Arch. Mth. Ps. 52 (1871) 247-.

-, intersection of medians and other theorems. Burg, A. Wien Jb. Pol. I. 12 (1828) 4-; 13 (1828) 223-.

-, Lemoine, and other points. Durán Loriga, J. J. G. Teix. J. Sc. 11 (1892) 161-; Fschr. Mth. (1893-94) 1104.

- of particular family of triangles, locus. Brocard, H. Mathesis 11 (1891) 153-.

-, points whose joins are trisected by. Reuschle, C. G. Z. Mth. Ps. 11 (1866) 475-.

Centroids, pedal and symmetrical. Neuberg, $J$. N. Arch. Wisk. 4 (1899) 192-.

"Cosine" orthocentres of a triangle, and a cubic through them. Tucker, $R$. Mess. Mth. 17 (1888) 97-.

Distances of point from vertices. Cayley, $A$. QJ. Mth. 5 (1862) 381-.

- - - Blazeievski, R. N. A. Mth. 13 (1894) 28-.

- points. Thiry, C. Brux. Ac. Bll. 21 (1891) 471-.

- - - from each other. Grunert, J.A. Grunert Arch. 36 (1861) 325-.

- _ - - Lemoine, $\dot{E}$. N. A. Mth. 9 (1870) 311-

Eighty-four special points, including Jerábek's. Jeřábek, V. Č́asopis 20 (1891) 141-, 237-; Fschr. Mth. (1891) 603-.

Fifth remarkable point. Hochheim, A. Arch. Mth. Ps. 52 (1871) 26-.

Four remarkable points (Euler). Grunert, $J . A$. Grunert Arch. 26 (1856) 343-.

- - - , analytically treated. Metzler, C. Arch. Mth. Ps. 47 (1867) 243-.

$G$ points of circle with respect to given triangle, finding. Griffiths, J. [1891] L. Mth. S. P. 23 (1892) 96-.

Loci. Lindman, C. $F$. Arch. Mth. Ps. 43 (1865) 350-.

-. Hochheim, A. Z. Mth. Ps. 15 (1870) 33-.

Locus of point whose pedal triangle has given area. Azzarelli, $M$. Rm. N. Linc. At. 27 (1874) 333-.

- points, sum of whose distances from sides is constant (Entfernungsort). Grunert, J.A. Grunert Arch. 17 (1851) 361-.

,$------\frac{-}{46}-(-)$. Emsmann, H. Arch. Mth. Ps. 46 (1866) 121-。

Middle points of triangles formed by in- and ex-centres of triangle. Nöggerath, $E . J$. Schlömilch Z. 8 (1863) 394-.

Nagel and Gergonne points. Harnischmacher, F. J. Arch. Mth. Ps. 42 (1864) 90-.

- - - Mink, W. Arch. Mth. Ps. 43 (1865) 1-.
Point, the sum of whose distances from vertices is a minimum. Gergonne, J.D. Gergonne A. Mth. 20 (1829-30) 299-.

-, Rm. N. Linc. At. 39 (1886) . Azzarelli, $M$.

Rm. N. Linc. At. 39 (1886) 95-

Reciprocal trilinear coordinates, properties of points with. Greiner, $M$. Arch. Mth. Ps. 1 (1884) 130-.

Series of points. Poulain, A. Mathesis 10 (1890) 246-

Spieker's point. Hain, E. [1875] Arch. Mth. Ps. 58 (1876) 164-.

Steiner's foci of a triangle. Neuberg, J., $\&$ Gob, A. As. Fr. C. R. (1889) (Pt. 2) 179-.

- point. Neuberg, J. As. Fr. C. R. (1885) (Pt. 2) 89-.

Symmedian or Lemoine point. Lemoine, $\dot{E}$. N. A. Mth. 12 (1873) 364-; As. Fr. C. R. 2 (1873) 90-; (1874) 1165-.

- Picquet, H. As. Fr. C. R. (1874) 1202-.

- - Hain, E. [1875] Arch. Mth. Ps. 58 (1876) 84-.

- - - - early history. Mackay, J. S. Edinb. Mth. S. P. 11 (1893) 92-.

- point axis of system of triangles. Tucker, R. QJ. Mth. 20 (1885) 167-.

Symmetry points. Hoppe, $R$. Arch. Mth. Ps. 57 (1875) 422-.

- -. Hain, E. Arch. Mth. Ps. 58 (1876) 176-, 385-, 394-; 59 (1876) 415-, 420-; 60 (1877) 71-; 64 (1879) 398-.

Tarry's point. Neuberg, J. Mathesis 6 (1886) $5-$.

Three points (Euler). Gentil, -. N. A. Mth. 5 (1846) 28-.

- -, certain groups. Kęiñski, S. Prace Mt.-Fiz. 2 (1890) 169-.

Two points. Grunert, J. A. Arch. Mth. Ps. 48 (1868) 37-.

- in plane of a triangle. Lemoine, $\mathfrak{E}_{\text {. }}$ As. Fr. C. R. (1885) (Pt. 2) 23-.

Remarkable triangle. Barisien, (le capit.) E.N. As. Fr. C. R. (1897) (Pt. 2) 107-.

Right angled isosceles triangle, calculation of angles. Voll, W. Oken Isis (1826) 490-.

- - triangles, properties. Dostor, G. Arch. Mth. Ps. 51 (1870) 103-.

- - - theorem. Ramus, C. Crelle J. 20 (1840) 28.

- - -, theorems, four. Lilienthal, Grunert Arch. 21 (1853) 99-.

Scalenity. Sharpe, H.J. [1864] Mess. Mth. 3 (1866) 52- ; 4 (1868) 177-.

Schroeter, theoremof, and Longchamps's line $\delta$. Droz-Farny, A. As. Fr. C. R. (1897) (Pt. 2) 136-.

Segmentary properties. Ocagne, M. d'. N. A. Mth. 2 (1883) 497-.

Segments of sides by concurrent lines through vertices. Bellavitis, G. A. Sc. Lomb. Ven. 2 (1832) 250-.

- _ _ - _ - (Bellavitis). Fusinieri, A. A. Sc. Lomb. Ven. 2 (1832) 254-

$-\ldots$, geometry of certain. Ocagne, M. $d$. G. Teix. J. Sc. 6 (1885) 125-. 
Segments of sides by straight line through fixed point. Durège, -. Grunert Arch. 30 (1858) 241-.

Similar triangles satisfying four conditions, number. Chasles, M. C. R. 78 (1874) 1599-.

- -, series. Chasles, M. C. R. 78 (1874) 1373-; 79 (1874) 877-, 1427-.

Similitude of triangles belonging to two series. Viaggi, $F$. G. Mt. 28 (1890) 113-.

Simson or Wallace pedal line. Durrande, J. B. Gergonne A. Mth. 7 (1816-17) 253-.

- _ - - Barbarin, P. (xII) Mathesis 2 (1882) 106-, 122-.

- - - - - Neuberg, J. J. B. (xI) Mathesis 2 (1882) 108-.

- - Alison, J. Edinb. Mth. S. P. 3 (1885) 77-.

- -1 - Strnad, A. Časopis 15 (1886) 114- ; Fschr. Mth. (1886) 471.

- _ - - Gibson, G. A. Edinb. Mth. S. P. 9 (1891) 50-.

- - - Grittner, A. Arch. Mth. Ps. 17 (1900) 318-.

- - - - of cyclic polygon. Quint, N. N. Arch. Wisk. 3 (1898) 153-.

- - - - extension of Wallace theorem. Quint, N. N. Arch. Wisk. 3 (1898) 180-.

- - - - generalisation. Cayley, $A$. QJ. Mth. 8 (1867) 264-.

- - - - - , - and application to tetrahedron. Hunyady, E. von. [1865] Z. Mth. Ps. 11 (1866) 64-.

- - - - - of polygon. Steggall, Edinb. Mth. S. P. 14 (1896) 122-.

- _ _ _ Steiner's theorem generalised. Beltrami, E. Bologna Ac. Sc. Mm. 7 (1876) 241-.

_ _ - _ - and the Wallace point. Mackay, J. S. Edinb. Mth. S. P. 9 (1891) 83-.

- - - lines. Tucker, R. Edinb. Mth. S. P. 14 (1896) 116-.

Squares of perpendiculars on sides from a point. Grebe, E.W. Grunert Arch. 9 (1847) 250-.

Statics and geometry of a triangle. SaintGermain, A. de. N. A. Mth. 3 (1884) 37-.

Steiner theorem on triangle and ellipse. Droz-Farny, A. Bern Mt. (1900) 135-.

- theorems, two, and the properties of triangles. Kantor, S. [1877] Wien Ak. Sb. 76 (1878) (Ab. 2) 758-.

Steiner's axes and Kiepert's hyperbola. Neuberg, J., \& Gob, A. As. Fr. C. R. (1889) (Pt. 2) 166-.

Straight line and triangle, relation. Hain, E. [1875] Arch. Mth. Ps. 59 (1876) 87-.

Symmedian. Ocagne, M. d'. N. A. Mth. 2 (1883) 450 - ; 3 (1884) 25- ; 4 (1885) 360-.

-. Azzarelli, M. Rm. N. Linc. At. 42 (1889) 255-.

Symmedians. Mackay, J. S. Edinb. Mth. S. P. 14 (1896) 37-.

Symmetrical lines in the triangle, and Steiner's curve. Bilcking, -. Arch. Mth. Ps. 16 (1898) 271-, vii-.

- properties. Davies, T. S. Ph. Mg. 2 (1827) 26-.
Symmetrical properties. Dobson, T. B. A. Rp. (1861) (Pt. 2) 2-.

Theorem of Beltrami. Grunert, J. A. Arch. Mth. Ps. 43 (1865) 102-.

- Mannheim. Catalan, E. Rm. N. Linc. Mm. 6 (1890) 223-.

Theorems on triangles, circles and conics. Lemoine, É. As. Fr. C. R. (1900) (Pt. 2) 79-.

Transformation. Lemoine, $\dot{E}$. Par. S. Mth. Bll. 19 (1891) 133-.

-, continuous. Lemoine, É. As. Fr. C. R. (1891) (Pt. 2) 118-; (1893) (Pt. 2) 132-; Par. S. Mth. Bll. 19 (1891) 136-; Mathesis 12 (1892) 58-, 81-, 264.

Transformations. Muirhead, R. F. Edinb. Mth. S. P. 13 (1895) 112-, 129-.

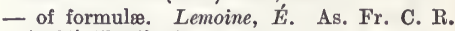
(1891) (Pt. 2) 118-.

Transversal $A D$ through angle $A$ of triangle $A B C$ such that $A D^{2}=B D . D C$. Jelinek, $V$. Časopis 21 (1892) 232-; Fschr. Mth. (1892) 526.

Transversals, equations relating to three. Kiechl, J. Arch. Mth. Ps. 12 (1894) 411-.

-, Matthew Stewart's theorem. Bretschneider, C. A. Arch. Mth. Ps. 50 (1869) 11-.

-, - - Mackay, J. S. Edinb. Mth. S. P. 10 (1892) 90-.

-, properties. Boyman, J. R. Grunert Arch. 13 (1849) 364-, 378-.

-, reciprocal. Neuberg, -. Brux. S. Sc. A. 24 (1900) (Pt. 1) 119-.

-, remarkable. $G o b, A$. Mathesis 18 (1898) $129-$.

一, 一. Zimin, M. Fschr. Mth. (1900) 492.

- through a remarkable point, characteristic property. Plamenevsky, J.J. Fschr. Mth. (1891) 599.

Triangle in which equal transversals cut base angles proportionally. Lange, $T$. Grunert Arch. 13 (1849) 337-; 15 (1850) 351-.

- - - - - - - Grunert, J. A. Grunert Arch. 13 (1849) 341-.

- _ - _ _ _ - Mink, W. Grunert Arch. 15 (1850) 358-.

Arch. 16 (1851) 201-.

- - - - - - - - lemma. August, E. F. Grunert Arch. 16 (1851) 259-.

- formed by medians. Grunert, J. A. Arch. Mth. Ps. 46 (1866) 340-.

_ - pairs of transversals through each angle of a triangle. Jelinek, $V$. Časopis 28 (1899) 88-, 145-; Fschr. Mth. (1899) 466.

- and point, sum of whose distances from sides is a minimum. Cohen, A. Camb. and Dubl. Mth. J. 8 (1853) 92-.

- - its reciprocal are in perspective, Cayley's proof. Muir, T. Edinb. R. S. P. 20 (1895) 298 -

- with side parallel to Euler's line. Avillez, J. F. d' (Vicomte de Reguengo). As. Fr. C. R. (1897) (Pt. 2) 131-.

Triangles of ares of circles. Weyr, E.J. [1873] Bll. Sc. Mth. As. 6 (1874) 89-. 
Triangles circumseribed in pairs by three circles. Tucker, R. [1892] L. Mth. S. P. 24 (1893) 162-.

- whose elements can be rationally expressed. Fuss, N. [1808] St Pét. Ac. Sc. Mm. 4 (1813) 240 .

- formed by common tangents of three circles. Barisien, E. N. Mathesis 16 (1896) 33-, 60-. - _ - joining points of contact of inscribed circles in turn, theorem. Lemoine, $\dot{E}$. As. Fr. C. R. (1880) 184.

- having in- and circum-circles common. Böklen, O. Grunert Arch. 38 (1862) 141-.

- with rational sides and areas. Breton, Ph. [1867] (xII) Isère S. Bll. 1 (1869) 299-.

- - Blichfeldt, H. F. A. Mth. 11 (1896-97) 57-.

- related to a triangle. Azzarelli, $M$. Rm. N. Linc. At. 40 (1887) 135-.

- whose sides are integers prime to one another, ratio of two angles being an integer. Weill, M. Par. S. Mth. Bll. 10 (1882) 55-.

- - - and medians are integers. Grebe, E. W. Grunert Arch. 17 (1851) 463-.

_ - - are multiples of radius of inscribed circle. Gerono, G. C. N. A. Mth. 17 (1858) 360-.

-, two, solution of problem of Frenicle's. Pepin, T. Rm. N. Linc. At. 33 (1880) 281-.

Trilinear figures, geometry. Petersen, J. N.Ts. Mth. 9 (B) (1898) 49-; Fschr. Mth. (1898) 473-.

\section{Stereometry; straight lines, planes, and spheres; polyhedra.}

Bees' cells. Terquem, O. (vI Adds.) N. A. Mth. 15 (1856) 176-.

- - construction. Sharpe, S. Ph. Mg. 4 (1828) 19-.

_- - - Powell, B. [1833] Ashmol. S. P. 1 (1844) No. 2, 10-.

- - - Wright, C. Am. Ac. P. 4 (1857-60) 432-; Camb. (M.) Mth. M. 2 (1860) 304-.

- - - - Willich, C. M. Ph. Mg. 18 (1859) 427- ; C. R. 51 (1860) 633-。

- - - - Hultman, $F$. W. Ts. Mt. Fys. 1 (1868) 197-.

- -, -. Glaisher, J. W. L. Ph. Mg. 46 (1873) 103-.

- -, -. Jonquière, A. Bern Mt. (1884) (Heft 1, Ab.) 71-.

- - - - Hennessy, H. R. S. P. 39 (1886) 253-; 41 (1887) 442-; 42 (1887) 176-.

- - - - Goodwin, (Rt. Rev.) H. Nt. 43 (1891) 295.

- - - - Busmann, - [1895] Westf. Vr. Jbr. (1895-96) 194-.

Cells, forms. Hennum, J. O. Arch. Mth. Ntvd. 9 (1884) 301-.

Centre of gravity in stereometry. I.andré, $C$. L. Grunert Arch. 39 (1862) 361-.

Cone, frustum. Grunert, J. A. Grunert Arch. 22 (1854) 343-.

VOL. I.
Cone, right. Bigourdan, E. N. A. Mth. 8 (1849) 9-.

, right circular. Woepcke, $F$. Liouv. J. Mth. 6 (1861) 231-.

- - - - (Woepcke's theorems). Steen, A. Mth. Ts. 4 (1862) 81-.

-, - -. Taylor, C. Mess. Mth. 4 (1875) 145-; 5 (1876) 189-.

-, - - , angle-property. Taylor, C. [1871] Mess. Mth. 1 (1872) 67-

-, - and frustum with spherical base. Milller, J. H. T. Grunert Arch. 16 (1851) 462-.

-, scalene. Taylor, C. [1879] Mess. Mth. 9 (1880) 33-.

Constructions in plane and in space with straight line only. Tilly, J.de. Mathesis 5 (1885) 124-.

Crystallographic derivations in the 1st system, table. Cesáro, E. Bll. Sc. Mth. 12 (1888) 270 -. Division of frustum of cone or pyramid. Flögl, J. Grunert Arch. 12 (1849) 423-.

- space into infinitesimal cubes. Tait, -. [1892] Edinb. R. S. P. 19 (1893) 193-.

- - surfaces and bodies, theorems. Olivier, T. Quetelet Cor. Mth. 5 (1829) 324-, 386-.

Duality, principle. Gergonne, J. D. Gergonne A. Mth. 19 (1828-29) 114-.

Euclid xr, 4. Grunert, J. A. Grunert Arch. 10 (1847) 260 - ; 26 (1856) 106-.

- XI, 28. Hoffmann, J. J. I. Grunert Arch. 10 (1847) 77-.

Figures, derivation by Bellavitis's method. Padula, F. Tortolini A. 5 (1854) 286-.

-, plane or spherical, of equal perimeter or surface. Thibault, -. N. A. Mth. 2 (1843) 480-.

Finite decomposition of two equivalent figures into superposable elements. Brunel, $G$. Bordeaux S. Sc. PV. (1896-97) 18-.

Gauss's pentagramma mirificum. Cayley, $A$. Ph. Mg. 42 (1871) 311-.

- - - Ahlborn, H. Hamb. Mth. Gs. Mt. 2 (1890) (Festschr., Tl. 2) 69-.

- - Schlesinger, L. Mth. Termt. Ets. 17 (1899) 526-; Mth. Nt. B. Ung. 17 (1901) 20-.

- point in system of $n$ planes. Neuberg, Brux. S. Sc. A. 17 (1893) (Pt. 1) 16-.

Geometrography in space. Lemoine, $\hat{E}$. As. Fr. C. R. (1900) (Pt. 2) 60-; C. R. 131 (1900) 937-.

Geometry of plane and space, analogies. Serret, $P$. Liouv. J. Mth. 7 (1862) 377-.

-, solid. Hachette, J. N. P. Par. S. Phlm. Bll. (1816) 201-.

-, -. Quetelet, L. A. J. [1826] Brux. Ac. Sc. Mm. 4 (1827) 51-.

_, -. Barsotti, G. [1829] Lucca At. Ac. 11 (1842) 113-.

-, -, combination with plane geometry in teaching. Candido, G. Ens. Mth. 1 (1899) 204 .

-, 一, problems. Prediger, C. Halle Z. Nw. 12 (1858) 24-.

Hexagonal pile of balls. Bach, - N. A. Mth. 4 (1845) 196-.

Infinitesimal mensuration of circle, pyramid, etc. Noël, J. N. [1865] Liége Mm. S. Sc. 1 (1866) 51-. 
Locus of intersection of 2 lines through given points, equally inclined to plane. Klug, $L$. [1881] Arch. Mth. Ps. 67 (1882) 330-.

Macles (twin crystals). Cesáro, G. Brux. Mm. Cour. $4^{\circ}, 53$ (1893-94) No. 5, $47 \mathrm{pp}$.

Mensuration of solids. Delanges, $P$. [1804] Mod. S. It. Mm. 12 (1805) 1-.

\section{Mensuration of Volumes.}

Rossi-Amatis, J. [1805] Turin Mm. Ac. (1809-10) (pte. 2) 98-.

Bordoni, A. Mod. Mm. S. It. 19 (1821) 527-.

Ligowski, $W$. Grunert Arch. 26 (1856) 204-; 32 (1859) 241-; 36 (1861) 181-.

Steen, A. Mth. Ts. 4 (1862) 1-.

Soufflet, - . Les Mondes 17 (1868) 110-.

Baillargé, $C$. Queb. T. 9 (1872) 73-.

Circle and inscribed square, ratio, applications to measurement of timber. Taylor, $C$. Madras J. 6 (1837) 301-.

Cone and cylinder, right circular. Frank, $A$. von. Arch. Mth. Ps. 59 (1876) 76-.

-, right, frustum, formula. Almeida, C. A. M. de. Lisb. J. Sc. Mth. 5 (1876) 208-.

-, segment. Grunert, J. A. Grunert Arch. 15 (1850) 356-.

Conicoid of revolution. August, E.F. Crelle J. 45 (1853) 239-.

Crystals, tesseral, cubic content of certain. Furst, J. Časopis 19 (1890) 20-; Fschr. Mth. (1890) 599-.

Earth and wall work, solid contents. Puller, Hann. Archt.-Vr. Z. 39 (1893) 549-.

Earthwork and cuttings. Wojciechowski, $L$. Par. T. Nauk Śc. Pam. 5 (*1874) Art. 3, 48 pp.; 9 (*1877) Art. 5, 15 pp.

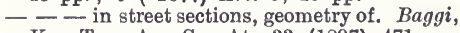
V. Tor. Ac. Sc. At. 33 (1897) 471- or 701-.

Formula. Lombard, C. N. A. Mth. 16 (1857) 131-.

Gauging of casks. Eytelwein, J.A. (vr Adds.) Berl. Ab. (1803) 93-.

- - Beeck-Calkoen, J. F.van. Rot. N. Vh. 5 (1810) 185-.

- - - Lubbock, J.W. Ph. Mg. 4 (1834) 326 -

- - - Stampfer, S. Wien SB. (1849) $227-$.

- - Grunert, J. A. Grunert Arch. 20 (1853) 301-; 23 (1854) 207-.

_ _ - and buoys, formula. Schrader, $C$. A. der Hydrog. 23 (1895) 459-.

- - -, doubly elliptic. Bruschetti, G. Brugnatelli G. 5 (1822) 165-.

- - - - - or elliptic. Cossali, P. [1814] Mod. Mm. S. It. 17 (1815) 237-.

- - , formulæ. Mansion, P. Mathesis 12 (1892) 14-.

- _ - problem by Kepler, general solution. Monteiro da Rocha, J. Lisb. Ac. Sc. Mm. 1 (1797) 1-.

- - - Prussian method. Stampfer, $S$. Wien SB. (1849) 291-.

-, Young's method. Wiseman, W. Tilloch $\mathrm{Ph}$. $\mathrm{Mg}$. 63 (1824) 415-。
Guldin's theorem, and truneated prism, demonstration. Lenthéric, -. Mntp. Ac. Sc. Mm. 2 (1851-54) 109-.

Obelisks. Grunert, J.A. Grunert Arch. 9 (1847) 82-.

Polygons, areas, and polyhedra, volumes. Bellavitis, G. A. Sc. Lomb. Ven. 4 (1834) 256-.

-, -, - -, -. Staudt, G. K. C. von. Crelle J. 24 (1842) 252-.

Polyhedra. Lhuilier, S. Bb. Un. 37 (1828) 256 -

-. Möbius, A. F. Leip. B. 17 (1865) 31-.

- bounded by triangles, volumes expressed by coordinates of vertices. Freuchen, $P$. (xII) Ts. Mth. 6 (1870) 189-.

-, regular. Schultze, F. Crelle J. 28 (1844) 108-.

-, - Dellmann, F. Schlömilch Z. 8 (1863) 460 -.

-, - - and Poinsot's, and their volumes by determinants. Löwe, $O$. Arch. Mth. Ps. 57 (1875) 392-.

-, symmetrical, equality of volumes. Ampère, A. $M$. Par. Éc. Pol. Cor. 1 (1804-08) 184-. Polyhedron with trapezoidal faces. Koppe, $C$. N. A. Mth. 8 (1849) 108-.

-, volume. Newson, H. B. A. Mth. 1 (1900) 108-.

Prismatoid. Grebe, E. W. Grunert Arch. 39 (1862) 93-.

Prismoidal formula. Wright, $C$. Camb. (M.) Mth. M. 1 (1859) 21-, 53-.

- - Davis, J. W. V. Nost. Eng. Mg. 20 (1879) 410-.

- Knibbs, G. H. N. S. W. R. S. J. 33 (1899) 129-.

- - limits. Hyde, E. W. Des Moines Anal. 3 (1876) 113-.

- formulæ. Taylor, T. U. Texas Ac. Sc. T. 1 (No. 5) (1897) 33-.

- -, two-term. Halsted, G. B. Texas Ac. Sc. T. 1 (No. 5) (1897) 19-.

- volumes. Grunert, J.A. Grunert Arch. 26 (1856) 341-; 30 (1858) 118-, 453-.

Prismoids. Kinkelin, H. Grunert Arch. 39 (1862) 181-.

- Sinram, T. Arch. Mth. Ps. 63 (1879) 440-.

Prisms. Matzka,W. Grunert Arch. 6 (1845) 113-.

-, truncated. Faà de Bruno, F. Tortolini A. 4 (1853) 71-.

Problem. Grunert, J. A. Arch. Mth. Ps. 43 (1865) 108-.

Pyramid and sphere. Havliček, T. V. Časopis 18 (1889) 76-; Fschr. Mth. (1889) 577.

-, triangular. Hessel, J.F.C. Grunert Arch. 14 (1850) 162-.

-, -. Grunert, J. A. Grunert Arch. 18 (1852) 239-.

- - Heinemann, P. G. K. Grunert Arch. 23 (1854) 361-.

-, -. Faà de Bruno, F. Tortolini A. 8 (1857) 77-.

-, truncated. Anon. (vI 565) Gergonne A. Mth. 20 (1829-30) 288-. 
Pyramid, truncated. Dietzel, $F$. Grunert Arch. 13 (1849) 447-.

,,$-- n$ sides, volume $=\frac{1}{8} h(B+b+\sqrt{B b})$. Flesch, J. Grunert Arch. 3 (1842) 444.

-, volume, theorem by Pappus extended to. Paraira, M. C. N. Arch. Wisk. $9\left({ }^{*} 1882\right)$ 96-.

-, 一, - - - - Boguslavskij, A. I. Mose. S. Sc. Bll. 73 (No. 1) (1891) 27-; Fschr. Mth. (1891) 614.

Simpson's rule. Lodge, A. Mth. Gz. No. 6 (1895) 58-.

- rules, and Woolley's rule, value in shipbuilding ealculations. Purkiss, H.J. [1865] QJ. Mth. 7 (1866) 235-.

Solids with two parallel faces, theorems. Steiner, J. Crelle J. 23 (1842) 275-.

-, volume, in terms of transverse sections. Knibbs, G. H. N. S. W. R. S. J. 34 (1900) 36-.

Sphere. Wittstein, T. Grunert Arch. 39 (1862) 1-.

- Saltel, L. [1880] Bordeaux S. Sc. Mm. 4 (1882) 375-.

-. Postnikov, M. V. Kazan S. Nt. (Ps.-Mth.) P. 5 (1887) 124-.

-, portion bounded by planes not passing through centre, volume and area. Somov, I. I. (xII) Rec. Mth. (Moscou) 3 (1868) (Pt. 1) 79-.

-, portions. Woodhouse, R. Phil. Trans. (1801) 153-.

- and portions of sphere, volume and area. Gergonne, J. D. Gergonne A. Mth. 13 (1822-23) 343-.

Spherical segment. Bary, - Gergonne A. Mth. 21 (1830-31) 326-.

- - Guimarães, R. Lisb. J. Sc. Mth. 5 (1898) 172-; Fschr. Mth. (1898) 444.

- - between two arbitrary planes, volume and area. Czuber, E. Crelle J. Mth. 105 (1889) 180.

Tetrahedra. Gergonne, J. D. Gergonne A. Mth. 19 (1828-29) 151-.

- Lhuilier, S. Bb. Un. 45 (1830) 208-.

-. Hunyady, E. von. Z. Mth. Ps. 11 (1866) 163-.

-. Dostor, G. N. A. Mth. 6 (1867) 410-

-. Günther, S. Arch. Mth. Ps. 56 (1874) 17-.

- Faure, H. N. A. Mth. 20 (1881) 338-.

-, class. Hill, M. J. M. [1895] L. Mth. S. P. 27 (1896) 39-.

-, polar reciprocal, volumes, relation. Luchterhandt, A. R. Grunert Arch. 10 (1847) 198-.

-, relation between volume, area of faces and area of projection on a plane, theorem by Roberts, generalisation. Lebesgue, $V . A$. N. A. Mth. 20 (1861) 63-.

-, volumes, from equations of bounding elements. Studnicka, F. Prag Sb. (1872) (pt. 2) 45-.

-, - and normal, relation. Sylvester, J. J. N. A. Mth. 13 (1854) 203-.

- , - - product of 16 algebraic values of surface. Sylvester, J. J. Camb. and Dubl. Mth. J. 8 (1853) 171-.
Tetrahedra, volumes, theorem. Grunert, J.A. Arch. Mth. Ps. 45 (1866) 66-.

,,--- , Staudt's. Gentil, -. N. A. Mth. 19 (1860) 218-.

- - -, and theorem by Steiner. Virieu, J. de. N. A. Mth. 5 (1866) 316-.

-, 5 (1866) - - - - Sartiaux, A. N. A. Mth. -, - , - triangles, areas, in terms of sides, Joachimsthal's formulæ. Droz, A. N. A. Mth. 20 (1881) 411-.

,,,----- , theorems. Gretschel, $H$. Arch. Mth. Ps. 45 (1866) 194-.

Tetrahedron, etc., theorems. Staudt, G. K. C. von. Crelle J. 57 (1860) 88-.

Theorems, elementary. Koppe, C. ․ A. Mth. 6 (1847) 480-.

- - , proof. Hessel, J. F. C. Grunert Arch. 7 (1846) 284-.

Tortoise, approximate valuation of volume and surface. Maurel, -, \& Rey-Pailhade, Toul. S. H. Nt. Bll. 33 (1900) 79-, 88.

Transformation, fundamental, in stereometry. Bordoni, A. (vi $A d d s$.$) Opuse. Mt. Fis, 1$ (1832) 14-.

Trapezoidal volumes. Giard, A. N. A. Mth. 6 (1867) 408-

Varignon's theorem, extension to volumes of three cones. Hočevar, $F$. Wien Az. 17 (1880) 196-.

Minimum distance problem. Talbot, W. H. F. Gergonne A. Mth. 13 (1822) 329-.

Obelisks, formation, etc. Grunert, J.A. Grunert Arch. 9 (1847) 87-.

,,--- (Grunert). Brix, A. F.W. Grunert Arch. 11 (1848) 339-.

-, Koppe's. Bretschneider, C. A. Grunert Arch. 36 (1861) 18-.

-, and prismatoid. Schlömilch, O. Z. Mth. Ps. 13 (1868) 160-

-, reality, problem. Schellen, - Grunert Arch. 11 (1848) 341-.

- 一, - Matzka, W. Grunert Arch. 11 (1848) 377-.

Perpendicular to given plane through two given straight lines. Grunert, J.A. Grunert Arch. 37 (1861) 445-.

Planes, seven, problem. Poudra, -. N. A. Mth. 15 (1856) 161-.

Point from which 3 other given points subtend given angles, determination. Bessel, F. W. Zach M. Cor. 27 (1813) 222-; As. $\mathrm{Nr} .3$ (1825) 193-, 221-.

- 2 straight lines and plane, problem. Flauti, V. Nap. At. Ac. 3 (1832) 35-.

Points in space, regular assemblages, properties. Bravais, A. C. R. 28 (1848) 601-; Par. S. Phlm. PV. (1848) 69-; Par. Éc. Pol. J. $33^{\circ} \mathrm{cah}$. (1850) 1-.

Polar trihedral angles and polar triangles. Harst, A. D. van der. N. Arch. Wisk. 17 (1890) 188-; Fschr. Mth. (1890) 600.

Polygons, skew and plane. Sturm, J. C. F. Gergonne A. Mth. 15 (1824-25) 309-.

-, plane and spherical, theorems. Bernardi, G. G. Mt. 29 (1891) 63-, 173-, 378. 


\section{POLYHEDRA.}

(See also 8075.)

Cauchy, A. L. Par. Éc. Pol. Cor. 2 (1809-13) 253-; Par. Éc. Pol. J. $16^{\circ} \mathrm{cah}$. (1813) 68-, 87-.

Anon. (vi 531) Gergonne A. Mth. 9 (1818-19) 321-.

Poinsot, L. C. R. 46 (1858) 65-.

(Descartes.) Prouhet, E. C. R. 50 (1860) 779-.

Catalan, E. C. [1862] Par. Éc. Pol. J. Cah. 41 (1865) 1-.

Kirkman, T. P. Phil. Trans. (1862) 121-; R. S. P. 12 (1862-63) 341-.

Jordan, $C$. C. R. 60 (1865) 400-; 61 (1865) 205-; 62 (1866) 1339-; Crelle J. 66 (1866) 22-; 68 (1868) 297-.

Becker, J. C. Z. Mth. Ps. 14 (1869) 65-, 337-; 18 (1873) 328-.

Landré, C. L. N. Arch. Wisk. 4 ( $\left.{ }^{*} 1878\right)$ 194-.

Lippich, F. [1881] Wien Ak. Sb. 84 (1882) (Ab. 2) 20-.

Bourlet, C. N. A. Mth. 8 (1889) 366-.

Brunel, -. Bordeaux S. Sc. Mm. 3 (1893) xxxvii-.

Bricard, R. N. A. Mth. 15 (1896) 331-.

Hermes, O. Crelle J. Mth. 120 (1899) 27-, 305-; 122 (1900) 124-.

Angles, new theorems. Brianchon, C.J. Par. Éc. Pol. J. 25e cah. (1837) 317-.

Archimedean polyhedra of higher kind, four. Hess, E. Marb. Schr. 11 (Ab. 4) (1878) $12 \mathrm{pp}$.

Articulated octahedron. Bricard, $R$. Liouv. J. Mth. 3 (1897) 113-

- - (Bricard). Mannheim, A. Liouv. J. Mth. 3 (1897) 149-.

Auto-polar polyhedra. Kirkman, T. P. Phil. Trans. (1857) 183-.

$-\ldots$, solution and constructions up to $p=10$. Kirkman, T. P. Lpool. Lt. Ph. S. P. 33 (1879) 133-.

Axial properties. Cayley, A. [1863] (vII) QJ. Mth. 7 (1866) 304-.

Axis of ternary symmetry. Viola, C. [1900] Pisa S. Tosc. At. (PV.) 12 (1899-1901) 75-, 138

Central polyhedron. Wolf, $R$. Bern Mt. (1847) 93-.

Classification. Breton, Ph. C. R. 51 (1860) 722 .

-. Fasci, A. Fr. Cg. Sc. 33 (1866) (pt. 2) 87-.

-. Eberhard, V. D. Mth. Vr. Jbr. 1 (1892) 50-.

Congruent polyhedra. Dehn, M. Gött. Nr. (1900) 345-.

Constants, number, and Euler's theorem. Schubert, H. C. H. Arch. Mth. Ps. 63 (1879) 97-.

Construction. Kirkman, T. P. Lpool. Lt. Ph. S. P. 32 (1878) 217-.

- of special polyhedra. Brunel, - - Bordeaux S. Sc. Mm. 5 (1895) xliv-.

Convex polyhedra. Minkowski, $H$. Gött. Nr. (1897) 198-

$-\rightarrow$, angular relations. Lalanne, L. C. $\mathrm{R}$. 74 (1872) 602-
Crystallography, and analytical theory of poly. hedra. Baptista, I. E. Lisb. A. 2 (1858) 257 -.

Deformable polyhedra. Pizzetti, P. Rm. R. Ac. Linc. Rd̃. 7 (1898) (Sem. 2) 19-.

Diagonals, number. Binder, $H$. Grunert Arch. 8 (1846) 221-

-, -. Prouhet, E. N. A. Mth. 2 (1863) 77-.

-, - in polygons and polyhedra. Vauthier, - Toul. Mm. Ac. 2 (1830) 95-.

Divisions, centroids, etc. Padula, $F$. Nap. At. I. Inc. 1 (1864) 37-.

Eberhard's hexagonoids. Schoenflies, A. Gött. Nr. (1894) 316-.

Equality of similar polyhedra. Cauchy, A. L. Par. S. Phlm. Bll. 3 (1812) 66-.

-, ete., theorems. Thibault, - . N. A. Mth. 2 (1843) 163-.

Equiangular polyhedra from crystallographic standpoint. Krejči, J. Prag Sb. (1885) (Mth.-Nt.) 120-; Fschr. Ps. (1885) (Ab.1) 178.

Euler's polyhedra. Bertini, E. [1869] Pisa A. Scuola Norm. 1 (1871) 89-.

- - Feil, M. Wien Ak. Sb. 93 (1886) $(A b .2)$ 869-.

- relation. De Morgan, $A$. (vi Adds.) $\mathrm{Ph}$. Mg. 12 (1838) 323-.

- theorem. Gergonne, J. D. Gergonne A. Mth. 19 (1828-29) 333-.

- - Baltzer, R. Berl. Mb. (1861) 1043-

- - Schäffer, H. Z. Mth. Ps. 9 (1864) $365-$.

- - Bugaev, N. V. (xII) Rec. Mth. (Moscou) 2 (1867) (Pt. 2) 87-.

-. Crone, C. Ts. Mth. 3 (1885) 44-; Fschr. Mth. (1885) 571-.

- - Jonquières, - de. C. R. 110 (1890) $110-, 169-$

- - Weili, $\dot{E}$. N. A. Mth. 17 (1898) 120-. - - apparent limitations. Natthiessen, L. Schlömilch Z. 8 (1863) 449-.

- - corollary. Hessel, J. F. C. Crelle J. 8 (1832) 13-.

- - demonstration. Rothe, H. A. Kastner Arch. Ntl. 4 (1825) 301-.

- -, - . Steiner, J. Crelle J. 1 (1826) 364 -

- - - - Pollak, $(\mathrm{Dr})$ - . [1849] Haidinger B. $6(1850) 93-$.

- - - - Thiel, J. M. N. Arch. Wisk. 19 (1892) 98-.

- - , - and exceptions. Lhuilier, S. St Pét. Ac. Sc. Mm. 3 (1811) 271-.

- - exceptions. Schulz von Strasznitzki, C. Crelle J. 14 (1835) 83-.

- - generalisation. Listing, J. B. Gött. Ab. 10 (1861-62) (Mth.) 97-; D. Nf. Tbl. (*1878) $77,224-$.

- - - Hoppe, R. D. Nf. Tbl. $\left({ }^{*} 1878\right) 39$; Arch. Mth. Ps. 63 (1879) 100-.

- - - - Perrin, R. C. R. 110 (1890) 273-.

$-\overline{144}$.

Existence of certain polyhedra. Cesáro, $E$. N. A. Mth. 2 (1883) 46 - 
General laws. Gergonne, J. D. Gergonne A. Mth. 15 (1824-25) 157-.

Harmonic polygons and polyhedra. Tarry, $G$., \& Neuberg, J. As. Fr. C. R. (1886) (Pt. 2) $12-$.

Hexahedron inscribed in sphere. Merrifield, C. W. Ph. Mg. 22 (1861) 382-.

In- and circum-scribed polyhedra. Forsyth, A. R. [1882] L. Mth. S. P. 14 (1883) 35-.

Inscriptible polygons and polyhedra, properties. Brassinne, $E$. Toul. Mm. Ac. 3 (1847) 209-.

Isosceles polyhedra. Badoureau, $A$. C. R. 87 (1878) 823-; Par. Éc. Pol. J. cah. 49 (1881) 47-.

Maxima, theorem. Berner, T. [1865] Z. Mth. Ps. 11 (1866) 81-.

Maximum and minimum, cubes and regular tetrahedra as. Sturm, $R$. Crelle J. Mth. 97 (1884) 1-.

- polyhedra. Lindelöf, $L$. Helsingf. Acta 24 (1899) No. 8, $47 \mathrm{pp}$.

Mensuration. Collalto, A. Padova Mm. Ac. (1809) 247-

-. Français, J. F. Gergonne A. Mth. 3 (1812-13) 189-.

-. Lhuilier, S. Bb. Un. 37 (1828) 249-.

-. Koppe, C. [1837] Crelle J. 18 (1838) 275-.

-. Becker, J. K. Grunert Arch. 38 (1862) 345- ; 40 (1863) 12-.

-, theorem, elementary proof. Martini, $M$. Palomba Rac. 1 (1845) 84 -

-, theorems. Lhuilier, S. [1799] Par. Mm. Sav. Étr. 1 (1806) 264-.

Möbius's theory. Reinhardt, C. Leip. Mth. Ps. B. 37 (1885) 106-.

Parallelepipedal system, conditions of perpendicularity. Smith, H.J.S. [1876] L. Mth. S. P. 8 (1876-77) 83-.

Parallelepipeds, diagonals. Smith, Arch. Camb. Mth. J. 1 (1839) 94-.

-, equality, demonstration. Matzka, W. Grunert Arch. 4 (1844) 363-.

- and parallelograms. Gergonne, J. D. Gergonne A. Mth. 9 (1818-19) 51-.

-, partition into tetrahedra. Brown, $C$. Edinb. R. S. T. 37 (1895) 711-.

-, rectangular, theorem. Mann, $F$. Grunert Arch. 34 (1860) 116-.

- skew. Littrow, J. J. von. Lindenau Z. 3 (1817) 42-.

Pentahedron. Le Paige, C. Brux. Ac. Bll. 13 (1887) 488-.

- of given volume and minimum surface. Sucksdorff, C. G. Liouv. J. Mth. 2 (1857) 91-.

Polar properties. Olivier, T. Quetelet Cor. Mth. 3 (1827) 187-.

Polyacrons, $\Delta$-faced. Cayley, A. Manch. S. Mm. 1 (1862) 248-

Polygons and polyhedra. Poinsot, L. [1809] Par. Mm. Sav. Étr. 2 (1811) 552-.

- - Timmermans, J. A. Gergonne A. Mth. 18 (1827-28) 217-。 - - - Cauchy, A. L. C. R. 26 (1848)
Polygons and polyhedra. Mainardi, G. Rm. At. N. Linc. 26 (1873) 80 -

-, polyhedra and polygrams. Hertzer, $(D r)$ Z. Mth. Ps. 11 (1866) 244-.

- and polyhedra, theorems. Bellavitis, $G$. A. Sc. Lomb. Ven. 2 (1832) 186-, 241-.

- - - - Sylvester, J. J. Ph. Mg. 4 (1852) 335-.

Polyhedra with both angles and faces equal. Hess, E. Marb. Schr. 11 ( $A b .1)$ (1876) $95 \mathrm{pp}$.

- congruent with their reflexions, but not symmetrical. Juel, C. N. Ts. Mth. 6 (B) (1895) 12-.

- with minimum surface for given volume. Kötter, E. Crelle J. Mth. 110 (1892) 198-.

- superposable on their images. Cesáro, $G$. [1896-97] Brux. Ac. Mm. 53 (1895-98) (No.3) 40 pp., (No. 5) 15 pp.

-, tetrahedron, and circum-sphere. Brassinne, E. Toul. Mm. Ac. 3 (1847) 209-.

- with trihedral summits and having neither triangle nor quadrilateral. Kirkman, T. P. [1882] Lpool. Lt. Ph. S. P. 37 (1883) $49-$.

- - - $n$ sides and $(n-1)$-gonal base. Kirkman, T. P. [1855] Phil. Trans. (1856) 399-.

Prismoids and trapezoids. August, E. F. Crelle J. 60 (1862) 377-.

Prisms, cones, and rhombs, acrometric relations. Riedl von Leuenstern, J. [1850] Haidinger Ab. 4 (1851) ( $A b .2) 49-$.

Problem, general solution. Kirkman, $T$. $P$. [1858] Manch. Ph. S. Mm. 15 (1860) 92-.

Properties. Gough, J. Nicholson J. 25 (1810) $321-$.

Property. Minich, S. R. A. Sc. Lomb. Ven. 2 (1832) 15-.

-, general, demonstration. Andreev, $K . A$. (xII) Rec. Mth. (Moscou) 6 (1872-73) (Pt. 1) 457-.

Pyramid of Cheops and geometrical structures. Zhbikovskir, A. K. [1882] (xII) Kazan S. Nt. (P8.-Mth.) P. 1 (1883) [No. 5] 15-.

- Great. Piani, D. Bologna Mm. Ac. Sc. 10 (1859) 391-.

-, - , angle of. Willich, C. M. Les Mondes 10 (1866) 479.

-, pentahedral, on trapezium as base. Terquem, 0 . N. A. Mth. 1 (1842) 396-.

Pyramids, analysis. Svanberg, A. F. Stockh. Ak. Hndl. (1829) 55-.

- centroid, and other theorems. Ferroni, $P$. [1812] Mod. S. It. Mm. 16 (1813) 347-.

- or cones, solid angle at vertex. Kramp, $C$. Gergonne A. Mth. 4 (1813-14) 187-.

-, corners, theorem. Muller, J. H. $F$. Grunert Arch. 2 (1842) 113-.

- with equal bases and heights. Hessel, J. F.C. Arch. Mth. Ps. 47 (1867) 433-.

- and frustum. Tortolini, B. (vin) A. Mt. 4 (1861) 175.

- polygons, analysis. Svanberg, A. $F$. Quetelet Cor. Mth. 9 (1837) 72-.

-, problem: to find 3 equal pyramids together equal to portion of earth. Moolenaar, $A$. Leijd. A. Ac. (1817-18) 9 pp. 
Pyramids, sum of solid angles. Leutenstern, J. R. von. [1849] Haidinger Ab. 3 (1850) (Ab. 2) 87-.

-, theorem. Catalan, E. C. N. A. Mth. 6 (1847) 353-.

\section{Regular Polyhedra.}

Legendre, A. M., \& Malus, - Par. S. Phlm. Bll. 2 (1811) 325-.

Lhuilier, $S$. Gergonne A. Mth. 3 (1812-13) 233-.

Garnier, J. G. Quetelet Cor. Mth. 1 (1825) 39-, 64-.

Gilbert, D. Ph. Mg. 3 (1828) 161-.

Fischer, - Grunert Arch. 11 (1848) 159-

Bertrand, J. C. R. 46 (1858) 79-.

Steichen, - . Brux. Mm. Cour. $8^{\circ}, 9$ (1859) 32 pp.

Björling, E. G. Ts. Mt. Fys. 3 (1870) 145-.

Cayley, A. QJ. Mth. 15 (1878) 127-.

Azzarelli, $M$. Rm. N. Linc. Mm. 4 (1888) 123-.

Steggall, - . Edinb. Mth. S. P. 7 (1889) 66.

Fiedler, $W$. Zür. Vjschr. 35 (1890) 343-.

Angles between faces, and volumes. Verdam, G. J. Groningen A. Ac. (1822-23) 79 pp.

Conjugate polyhedra. Dostor, G. [1877] Arch. Mth. Ps. 62 (1878) 285-.

Construction on one face. Krinicyn, $P . K$. Kazan S. Ps.-Mth. Bll. 1 (1891) (Prot.) 68.

- of the 5 regular solids. Sohncke, L. A. Arch. Mth. Ps. 47 (1867) 39-.

Convex polyhedra, new properties. Dostor, $G$. Arch. Mth. Ps. 59 (1876) 50-.

Group theory. Schoute, P. H. N. Arch. Wisk. 20 (1893) 100-.

In- and circum-spheres, radii. Sommer, $B$. Grunert Arch. 32 (1859) 289-.

Maxima. Babinet, J. C. R. 61 (1865) 827-, 973.

Mensuration. Žbikovskij, A. K. Kazan S. Nt. (Ps.-Mth.) P. 4 (1886) 80-

Properties. Cauchy, A.L. C. R. 26 (1848) 517-.

- analogous to Stewart's of regular polygons. Jeffery, H. M. [1882] R. S. P. 34 (1883) 105-.

Property. Todhunter, I. Mess. Mth. 3 (1866) 142-.

Regular polyhedra obtained by folding paper. Wiener, -. Z. Nw. 61 (1888) 631-.

Relations between angles. Drach, $S . \dot{M}$. Mess. Mth. 3 (1874) 6.

Space configurations, derivable. Vries, J. de. Wien Ak. Sb. 100 (1891) (Ab. 2a) 822-.

Sphere, property of, and regular polyhedra. Lhuilier, S. Bb. Un. 36 (1827) 260-.

Ten polyhedra, table of principal elements. Barbier, E. C. R. 101 (1885) 562-.

Theorems. Mollame, $V$. G. Mt. 9 (1871) 64-.

- Akerlund, J.R. N.Ts. Mth. 9 (A) (1898) 1-。 6-hedra, cube, division into 4 equal parts. Willich, C. M. C. R. 56 (1863) 100-.

-, 一, partitions, etc. Willich, C. M. $\mathrm{Ph}$. Mg. 33 (1867) 27-.

-, Ronayne's cubes (one passing through the other). Hennessy, $H$. B. A. Rp. (1894) 578.

8-hedra, Levy's theorem. Davies, T.S. Tilloch Ph. Mg. 67 (1826) 52-. 9-hedra, 9-acral, enumeration and construction. Kirkman, T. P. Lpool. Lt. Ph. S. P. 32 (1878) 177-.

12-hedra, description. Barbier, E. C. R. 95 (1882) 560-.

-, janal. Kirkman, T. P. Lpool. Lt. Ph. S. P. 29 (1875) 251-.

14-hedra, janal, 14-acral. Kirkman, T. $P$. Lpool. Lt. Ph. S. P. 30 (1876) 271-.

20-hedra, convex, sixteen nets of planes of. Hénard, E. C. R. 101 (1885) 232-.

- - , - - - B Barbier, E. C. R. 101 (1885) 304-, 540.

- and star 12-hedra, construction and representation. Schönemann, P. [1872] Z. Mth. Ps. 18 (1873) 387-.

- 12-hedra. Wicke, C. Grunert Arch. 25 (1855) 131-.

- - , relations in projections. Schubert, $J$. Z. Mth. Ps. 19 (1874) 460-.

- - - theorems. Akerlund, J. R. N. Ts. Mth. 8 (A) (1897) 17-.

Regular and semi-regular polyhedra. Babinet, J. C. R. 26 (1848) 530-.

Representation. Kirkman, T. P. [1855] Phil. Trans. (1856) 413-.

- and enumeration. Kirkman, T. P. [185̃3] Manch. Ph. S. Mm. 12 (1855) 47-.

Rhomboidal bodies, especially rhomboidal triacontahedra. Rothe, H. A. Kastner Arch. Ntl. 4 (1825) 129-, 257-.

Self-conjugate polygons and polyhedra. Johnson, A. R. QJ. Mth. 22 (1887) 158-.

Semi-regular polyhedra of Archimedes. Valat, -. Bordeaux Mm. S. Sc. 5 (1867) 319-.

- _ - - etc., series complementary to. Valat, -. Bordeaux Act. Ac. Sc. 28 (1866) 385-.

- - convex. Terrier, L. [1875] Neuch. S. Sc. Bll. 10 (1876) 201-.

- - résumé of theory. Catalan, E. C. As. Fr. C. R. 1 (1872) 147-.

- - , some. Hayward, R. B. [1897] Mth. Gz. 1 (1900) 73-.

- - with 6 squares and 8 triangles as faces or 12 pentagons and 20 triangles. Wezel, L. Louvain A. Ac. 6 (1826) 60 pp.

Seven-point polyhedron, theorem. Anon. (vi 310) Crelle J. 3 (1828) 199-.

Similitude of solid figures. Dellac, $H$. Mars. Fac. Sc. A. 9 (1899) Fasc. 1, 108 pp.

Star polygons and polyhedra. Poinsot, $L$. N. A. Mth. 8 (1849) 68-, 132-.

- - - (Poinsot). Dienger, J. Grunert Arch. 13 (1849) 434-.

- polyhedra. Cauchy, A. L. N. A. Mth. 8 (1849) 304-.

- (Poinsot). Cayley, A. Ph. Mg. 17 (1859) 123-, 209-.

- - Valat, -. As. Fr. C. R. 1 (1872) 155. - regular. Wiener, C. Z. Mth. Ps. 12 (1867) 174-.

- -, -, general properties. Dostor, G. Liouv. J. Mth. 5 (1879) 209-.

- - , - three spheres of. Dostor, G. [1877] Arch. Mth. Ps. 62 (1878) 78-. 
Surface area. Grunert, J. A. Arch. Mth. Ps. 53 (1871) 482-.

Surfaces, fundamental theorem, new proof, etc. Becker, J.C. Z. Mth. Ps. 19 (1874) 459-.

Symmetrical polyhedra. Bravais, A. Liouv. J. Mth. 14 (1849) 137-, 141-.

- -, division. Möbius, A. F. (vi Adds.) Crelle J. 4 (1829) 296-.

Symmetry of non-Eulerian polyhedra. Jordan, C. Crelle J. 66 (1866) 86-.

- - - - inverse. Jordan, C. Crelle J. 68 (1868) 350-.

- in polyhedra. Cesáro,G. Brux. Mm. Cour. $4^{\circ}, 53$ (1893-94) No. 4, $34 \mathrm{pp}$.

\section{Tetrahedra.}

L., J. (vI Adds.) Gergonne A. Mth. 1 (181011) $353-$.

(Joachimsthal.) Liersemann, $H$. Grunert Arch. 32 (1859) 107-.

Junghan, G. Grunert-Arch. 34 (1860) 369-.

Torry, A. F. Mess. Mth. 1 (1862) 156-.

Cayley, A. Mess. Mth. 3 (1866) 8-.

Transon, A. N. A. Mth. 12 (1873) 519-.

Pellet, A. E. As. Fr. C. R. (1881) 170-.

Thieme, H. Z. Mth. Ps. 27 (1882) 56-.

Intrigila, $C . \quad$ Nap. Rd. 22 (1883) 69-.

Neuberg, J. Brux. Mm. Cour. $8^{\circ}, 37$ (1886) No. 1, $72 \mathrm{pp}$.

Strnad, A. Časopis 29 (1900) 146-; Fschr. Mth. (1900) 506.

Angle, 6th, in terms of other five. Clausen, $T$. Crelle J. 8 (1832) 138-.

Application of symmetric functions. Reiss, $M$. Quetelet Cor. Mth. 10 (1838) 229-.

Cube in tetrahedron and square in triangle. Ferriot, -. Gergonne A. Mth. 2 (1811-12) 180 -.

Desmic systems of three tetrahedra. Stephanos, C. Bll. Sc. Mth. As. 3 (1879) (Pt. 1) 424-.

- tetrahedra. Caspary, F. Mth. A. 29 (1887) $581-$.

- - construction of three. Schroeter, ${ }^{\circ} H$. Crelle J. Mth. 109 (1892) 341-.

Dihedral angles. Clausen, $T$. N. A. Mth. 5 (1846) 374-.

Distance of summits from plane, theorem. Faure, $H$. N. A. Mth. 20 (1861) 222-.

Doubly homologous tetrahedra. Gallucci, $G$. [1898] G. Mt. 37 (1899) 1-.

Edges, opposite, inclination in terms of edges. Ferrers, N. M. QJ. Mth. 3 (1860) 145-.

Equilateral tetrahedra. Hoppe, R. Arch. Mth. Ps. 12 (1894) 327-.

- - and orthocentric tetrahedra. Hoppe, $R$. Arch. Mth. Ps. 16 (1898) 257-, 333-.

Expression by coordinates of vertices. Baltzer, $R$. Leip. B. 22 (1870) 97-.

Geometric principle of resultants, application. Chelini, D. Bologna Ac. Sc. Mm. 7 (1867) $79-$.

Irregular tetrahedron, problems. Kuijper [s], $T$. Leijd. A. Ac. (1835-36) 32 pp.

Locus problem. Maur, A. Grunert Arch. 19 (1852) 121-.
Maximum tetrahedron with faces of given area. Painvin, L. N. A. Mth. 1 (1862) 267-, 353-, 414-.

- - - - - Borchardt, C. W. Berl. Ab. (1865) (Mth.) 1-.

- - - - - Le Besgue, V. A. C. R. 66 (1868) 248-.

Mth. 83 (1877) 180 .

Minimum tetrahedra and sections of trihedra. Bermann, -. Arch. Mth. Ps. 6 (1888) 76-, vir.

- theorem. Steiner, J. N. A. Mth. 12 (1853) 47-.

Möbius's tetrahedra. Neuberg, $J$. Liège S. Sc. Mm. 11 (1885) No. 7, 14 pp.

Nine point circle, analogues in space of three dimensions. Roberts, S. L. Mth. S. P. 19 (1889) 152-.

Orthocentric tetrahedra. Longchamps, G. de. Mathesis 10 (1890) 49-, 77-.

- - with rational edges. Hoppe, R. Arch. Mth. Ps. 9 (1890) 434-.

Pairs of tetrahedra. Muth, P. Z. Mth. Ps. 37 (1892) 117-.

Parallelogram, property, analogues in space. Walker, J.J. [1866] Mess. Mth. 4 (1868) 144-.

Perpendicular planes bisecting edges, theorem. Flemming, C. G. Grunert Arch. 10 (1847) 326-.

Perpendiculars on faces from any point, relation between. Gilbert, $P$. Brux. Ac. Bll. 28 (1869) 535-.

Points. Hermes, O. [1865] Crelle J. 65 (1866) 293-.

- of symmetry. Hain, E. [1876] Arch. Mth. Ps. 60 (1877) 304-.

Polar tetrahedra, properties. Poudra, N. $G^{r}$. N. A. Mth. 4 (1865) 496-.

Positions, special, of two tetrahedra. Schur, $F$. Mth. A. 19 (1882) 429-.

Problems. Baltzer, $R$. Grunert Arch. 16 (1851) 125-.

-. Azzarelli, $M . \quad$ Rm. N. Linc. At. 29 (1876) 126-.

Properties. Lankeren-Matthes, D.van. Leijd. A. Ac. $(1835-36) 33 \mathrm{pp}$.

-. Ferrers, N. M. [1861] QJ. Mth. 5 (1862) 167-.

-. Gellenthin, H. Arch. Mth. Ps. 3 (1886) 52-. -, generalisation of certain. Brill, J. Camb. Ph. S. P. 9 (1898) 98-.

Property. Dostor, G. Arch. Mth. Ps. 56 (1874) 245-.

Pyramid, triangular. Monge, G. Par. Éc. Pol. Cor. 1 (1804-08) 440-; 2 (1809-13) 1-, 263-.

-, -. Hachette, J. N. P. Par. Éc. Pol. Cor. 2 (1809-13) 332-.

- - Schulz von Strasznitzki, L. C. Baumgartner Z. 2 (1827) 530-.

- - Bretschneider, C. A. Grunert Arch. 1 (1841) 1-.

-, -. Clausen, T. As. Nr. 19 (1842) 389-.

-, -. Grunert, J. A. Grunert Arch. 21 (1853) 352-; 23 (1854) 284

, - formula. Hoppe, $R$. Grunert Arch. 3 (1843) 213-. 
Pyramid, triangular, formulæ. Grunert, J. A. Grunert Arch. 36 (1861) 356-.

-, - , and inscribed spheres. Unferdinger, $F$. Grunert Arch. 28 (1857) 97-.

,-- , problem. Hachette, J.N.P. Quetelet Cor. Mth. 2 (1826) 211-.

,-- , problems. Flauti, V. Nap. At. Ac. 1 (1819) 51-.

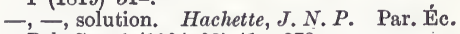
Pol. Cor. 1 (1804-08) 41-, 273-.

Quadrilaterals and tetrahedra, theorems, demonstration. Scheerer, $T$. Crelle J, 6 (1830) 98-.

Rational tetrahedra. Schwering, $K$. Crelle J. Mth. 115 (1895) 301-.

Reciprocal triangles and tetrahedra. Ferrers, N. $M$. QJ. Mth. 1 (1857) 191-.

Solid angles and tetrahedra. Hellwig, $C$. [1875] Arch. Mth. Ps. 58 (1876) 180-.

- _ _ - with application of determinants. Dostor, G. Arch. Mth. Ps. 57 (1875) 113-.

Species. Hill, M. J. M. B. A. Rp. (1895) 619-.

Spheres, circum-scribed. Brassinne, $E$. Toul. Mm. Ac. 3 (1847) 209-.

,- , radius in terms of edges. Dostor, $G$. N. A. Mth. 13 (1874) 523-.

-, e-scribed, radii. Unferdinger, $F$. Arch. Mth. Ps. 50 (1869) 110-.

-, in- and circum-scribed, radii. Dostor, $G$. N. A. Mth. 12 (1873) 367-, 370-

- , - - e-scribed, radii, Fermat's theorem. Stammer, W. Arch. Mth. Ps. 50 (1869) 111-.

-, inscription. Hermary, - Par. S. Mth. Bll. 7 (1879) 138-.

- tetrahedra always inscriptible in, theorem. Baudrimont, A. Bordeaux Mm. S. Sc. 3 (cah. 2) (1865) 445-.

Surfaces, tetrahedral, two classes of surfaces analogous to. Raffy, L. Par. S. Mth. Bll. 24 (1896) 2-.

Tetrahedra bounded by similar triangles. Schoute, P. H. Amst. Ak. Vs. M. 6 (1889) 462- ; Fschr. Mth. (1889) 578-.

- with 6 edges touching sphere. Junghan, $G$. Grunert Arch. 40 (1863) 447-.

- - - - Dostor, G. N. A. Mth. 13 (1874) 563-.

- in- and circum-scribed to each other. Möbius, A. F. Crelle J. 3 (1828) 273-.

- _ _ _ _. Bauer, $G$. Münch. Ak. Sb. 27 (1898) 359-.

- with opposite edges equal in pairs. Lemoine, É. As. Fr. C. R. (1875) 173-.

- $-1-\ldots$, and solution of problem. Lemoine, É. $\quad$ N. A. Mth. 19 (1880) 133-.

- - - perpendicular, theorem. Wolstenholme, J. QJ. Mth. 3 (1860) 89.

- - - at right angles. Lewis, T. C. Mess. Mth. 10 (1881) 36-.

- - - - - Temperley, E. [1881]

Mess. Mtb. 11 (1882) 114-.

- related to 4 spheres meeting in point. Roberts, S. L. Mth. S. P. $12(1880-81)$ 117-.

- of same base and altitude, equivalence. Gergonne, J. D. Gergonne A. Mth. 12 (1821-22) 362-.
Tetrahedra of same base and altitude, equivalence. Querret, - . Gergonne A. Mth. 12 (1821-22) 364-.

- with sides some or all of which are equal, etc. August, F. Arch. Mth. Ps. 17 (1900) 65-.

Tetrahedron of 4 congruent triangles. Schmidt, A. Z. Mth. Ps. 29 (1884) 321-.

- with 4 faces passing through 4 given lines, and 3 vertices on 3 other lines, theorem by Chasles, correction. Lange, E. Z. Mth. Ps. 26 (1881) 98-.

- - - - - (Lange). Schroeter, H. E. Z. Mth. Ps. 26 (1881) 270-

Theorem analogous to Pythagoras's. (Euc. I, 47.) Ensström, G. Bb. Mth. (1898) 113-.

Theorems. Clarke, A. R. Mathematician 3 (1850) 182-.

-. Heis, E. Grunert Arch. 31 (1858) 41-.

-. Staudt, G. K. C. von. N. A. Mth. 18 (1859) 441.

-. Marmier, G. N. A. Mth. 5 (1866) $268-$.

-. Schröder, -. D. Nf. Tbl. (*1879) 177.

-, two. Müller, J. H. T. Grunert Arch. 9 (1847) 319-.

Transformation, continuous, application. Lemoine, É. As. Fr. C. R. (1893) (Pt. 2) 146-.

Transversals in plane triangle or tetrahedron. Seipp, H. Arch. Mth. Ps. 9 (1890) 375-.

Triangles and tetrahedra, analogies. Ferriot, -. Gergonne A. Mth. 2 (1811-12) 133-; Fr. Cg. Sc. 24 (1857) 474-.

- _ — - ; 9-point circle, and 12-point sphere. Prouhet, E. N. A. Mth. 2 (1863) 132-.

- - - theorems. Frégier, - Gergonne A. Mth. 7 (1816-17) 167-.

Twelve-point sphere and quadries. Ripert, $L$. Mathesis 18 (1898) 218-.

Volume and angles in terms of edges. Unfer. dinger, $F$. Arch. Mth. Ps. 51 (1870) 353-.

Theorem analogous to Cotterill's on polygons. Clifford, $W . K$. [1872] L. Mth. S. P. 4 (1871-73) 178-.

- of Euclid generalised. Fedoroff, E. Bll. Sc. Mth. 18 (1894) 59-.

Theorems. Meyer, A. Brux. Ac. Bll. 15 (1848) 261-.

- of Descartes, etc. Minich, S. R. Ven. At. (1859-60) 939-.

Uniaxal polyhedra of minimum surface with constant volume. Hoppe, $R$. Arch. Mth. Ps. 13 (1895) 69-.

Polymerisation of carbon. Brunel, G. Bordeaux S. Sc. PV. (1894-95) 8-.

Propositions in plano, and analogues in three dimensions. Hearn, G. W. Mathematician 2 (1847) 117-.

Quadrilaterals, analogues in space to two properties of. Weddle, T. Camb. and Dubl. Mth. J. 5 (1850) 238-.

- and pentahedra, correspondence. Hermes, O. [1857] Crelle J. 56 (1859) 247-.

-, plane and spherical, inseribed in circle. Gueneau d'Aumont, -. Gergonne A. Mth. 12 (1821-22) 269-. 
Quadrilaterals, plane and spherical, inscribed in circle. Baur, C. W. Schlömilch Z. 6 (1861) 221-.

-, - - - theorems, two. Strehlke, $F$. Grunert Arch. 2 (1842) 323-.

-, skew. Studerende. (Pseud.) Mth. Ts. 1 (1859) 85-.

-, -, section and form. Beyel, C. Z. Mth. Ps. 32 (1887) 301-.

-, - , trigonometric properties. Wasteels, $J$. Mathesis 18 (1898) 85-.

- and tetragons, complete, analogous systems in space. Weddle, T. Camb. and Dubl. Mth. J. 7 (1852) 251-.

- theorem extended to space. Hermes, $O$. Crelle J. 56 (1859) 204-.

Relations in space, graphic representation. Ritter, C. Berl. Ab. (1828) (Ph.) 213-.

Resultant angle of two angles. Fontené, $G$. N. A. Mth. 18 (1899) 407-.

Rotation surface (urn), diameter of crosssection. Besch, C. Königsb. Schr. 14 (1873) 66-.

Shortest distance between two straight lines. Beltrami, E. G. Mt. 5 (1867) 351-.

- - - - adjacent. Cesáro, E. Mathesis 5 (1885) 196-, 248 (footnote).

- - - - - - application to surfaces. Lebesgue, $V . A$. N. A. Mth. 8 (1849) 236-, $381-$.

- - - - - - coordinates with oblique axes. Mollame, $V$. Nap. Rd. 17 (1878) 106 -

G. Mt. 5 (1867) 236-

- - - - - when they become parallel. St. Germain, A. de. N. A. Mth. 5 (1866) 346-.

Similarity and equality of figures. Baltzer, $R$. [1852] Crelle J. 52 (1856) 142-.

Solid angles, measurement. Leuenstern, J. $R$. von. [1847] Haidinger Ab. 2 (1848) (Ab. 2) 1 -.

- - and polyhedra. Gram, J.P. (xII) Ts. Mth. 4 (1874) 161-.

- - of prism, cone and rhomb. Leuenstern, J. R. von. [1850] Haidinger Ab. 4 (1851) (Ab. 2) 49-

- - and tetrahedra, rational. Hoppe, $R$. Arch. Mth. Ps. 61 (1877) 86-.

- - theorem. Sturm, J. B. Grunert Arch. 28 (1857) 364 .

- - , - Certo, -. Palermo Cir. Mt. Rd. 4 (1890) 286.

- -, theorems. Minich, S. R. Ven. At. 7 (1861-62) 823-.

- - triangle, etc., properties. Anon. (vi 532) Gergonne A. Mth. 9 (1818-19) 271-.

- -, trihedral. Hemming, J. J. Z. Mth. Ps. 17 (1872) 159-.

-,- , application of principle of duality. Isely, L. Neuch. S. Sc. Bll. 20 (1892) 118-. - - - , equality. Anon. (vr 560) Gergonne A. Mth. 17 (1826-27) 252-.

,--- , given section, problem. Lhuilier, $S$. [1819] Nap. At. Ac. 2 (1825) 13-.

- - - , - - - (Lhuilier). Flauti, $V$. Nap. At. Ac. 2 (1825) 21-.
"De Solidorum Elementis" (posthumous work with translation and annotations by Jonquières). Descartes, $R$. Par. Ac. Sc. Mm. 45 (1899) 325-.

Solids, ordered classification. Ehrenburg, Würzb. Ps. Md. Sb. (1890) 139.

- of revolution. Dostor, G. Arch. Mth. Ps. 60 (1877) 307-; 67 (1882) 254-.

\section{SPHERES.}

Euler, L. [1780] St Pét. Ac. Sc. Mm. 5 (1815) 96-.

Hachette, J. N. P. Par. Ec. Pol. Cor. 2 (1809 13) 337 -

Heegmann, A. Lille Tr. (1825) 106-.

Spiller, -. Mth. Misc. 1 (1838) 29-, 182-.

Cassani, P. G. Mt. 4 (1866) 15-, 131-, 223-, 373-.

Niewenglowski, B. N. A. Mth. 9 (1870) 26-.

Laguerre, E. (x) Par. S. Mth. Bll. 1 (1873) 241-.

Stephanos, C. C. R. 92 (1881) 1195-.

Allardice, R. E. Edinb. Mth. S. P.2 (1884) 8-.

Loria, G. Tor. Ac. Sc. At. 20 (1885) 505-.

Analogues to theorems in plane geometry. Chasles, M. Liouv. J. Mth. 1 (1836) 324-.

Analytical geometry of sphere. Graves, $C$. Ir. Ac. P. 2 (1844) 127-.

- - - Borgnet, -. Fr. Cg. Sc. 1 (1847) 316-; N. A. Mth. 7 (1848) 147-, 174 - _ - - fundamental formulæ. Fournier. Vannson, -. N. A. Mth. 17 (1858) 65-, 99-, 140-, 163-, 209-, 243-, 307-; 18 (1859) 1-.

Angles of 5 circles in plane, or of 6 spheres in space, relations. Lucas, $\mathfrak{E}_{\text {. }}$ [1877] Mess. Mth. 8 (1879) 37-.

Archimedes's theorem of sphere and cylinder, extensions. Piani, D. (viII) Bologna Opusc. Sc. N. Col. (1824) 228-.

Ares of great and small circles. Bond, G. $P$. Camb. (M.) Mth. M. 1 (1859) 342-.

Area between two circles on sphere. Steen, $A$. (xII) Ts. Mth. 1 (1883) 84-.

of figure formed by small circle arcs. Wasteels, C. E. Mathesis 12 (1892) 105-.

- portion of sphere between 3 arcs of small circles. Faure, $H$. N. A. Mth. 12 (1853) 446-.

Areas, spherical, properties. Humbert, $G$. C. R. 106 (1888) 477-; Liouv. J. Mth. 4 (1888) 313-.

-, - , simplification of measurement. Breton, $P h$. [1872] (xII) Isère S. Bll. 4 (1875) 243-, 247, 248-.

Axes, radical, in spherical geometry. Allardice, R. E. Edinb. Mth. S. P. 3 (1885̃) 59-

Box, rectangular, inside sphere. Baker, E. F. [1874] Amt. Mch. S. J. 2 (1877) 89-.

Brassinne's formula for radius of circumscribed sphere. Perrodil, - de. N. A. Mth. 6 (1847) 396-.

Centres of similitude of circles and spheres. Fuss, N. [1799] St Pet. Ac. Sc. N. Ácta 14 (1805) 139-.

Chords subtending right angle at given point. Juel, C. Ts. Mth. 5 (1887) 141-; Fschr. Mth. (1887) 660. 
Circle cutting given circles at given angles. Kretkowski, W. Krk. Ak. (Mt.-Prz.) Pam. 13 (1887) 97-; Fschr. Mth. (1887) 564.

Circles described about eight small circles. Jeffery, H. M. QJ. Mth. 24 (1889) 198-.

- passing through two points and meeting a line, locus of centres. Schiffner, $F$. Arch. Mth. Ps. 5 (1887) 442-.

- of sphere, geometry. Schumacher, F. Z. Mth. Ps. 34 (1889) 257-.

- and spheres with common radical axis and centre. Study, E. Crelle J. Mth. 94 (1883) 233-.

Condition that 4 spheres or 3 circles go through same point. Steen, A. (xII) Ts. Mth. 2 (1872) 56-.

Cone circumscribing sphere, maximum volume or surface. Snell, E. S. Camb. (M.) Mth. M. 1 (1859) 236-.

- - minimum volume. Snell, E. S. Silliman J. 5 (1848) 227-.

- and sphere, intersection. Chasles, $\boldsymbol{M}$. Quetelet Cor. Mth. 5 (1829) 44-.

- _ - theorems. Miller, W.J. Mess. Mth. 2 (1863) 11-.

- with vertex on sphere, intersection with sphere. Reiss, M. Quetelet Cor. Mth. 4 (1828) 355-.

Cones and cylinders, intersection by spheres, theorems. Baltzer, $R$. Crelle J. 54 (1857) $162-$.

Construction, graphical, from 4 conditions. Gaultier, L. Par. E.c. Pol. J. $16^{\circ}$ cah. (1813) 124-.

Constructions, geometrical, on sphere. Barbarin, $E$. Mathesis 19 (1899) 57-, 81-.

-, - - - - Bonnesen, T. N. Ts. Mth. 10 (B) (1899) 1-, 25- ; Fschr. Mth. (1900) 508.

\section{Contacts.}

Circle touching 3 circles on sphere. Olivier, $T$. Par. S. Phlm. N. Bll. 3 (1813) 312.

- - - - Gergonne, J. D. Gergonne A. Mth. 4 (1813-14) 349-.

- - - - - -. Trzaska, W. Par. T. Nauk Śc. Pam. $1\left({ }^{*} 1871\right)$ 123- ; Bll. Sc. Mth. As. 6 ( $\left.{ }^{*} 1874\right)$ 153-.

- - - - _ solution. Breton, $\mathrm{Ph}$. [1864] (xII) Isère' S. Bll. 7 (Livr. 3 \& 4) (1867) 458.

- 4 spheres. Barbier, E. C. R. 60 (1865) 1076-.

Circles, contact on sphere. Neumann, $F . E$. Oken Isis (1826) 349-, 466-.

-, - - - Fournier-Vannson, -. N. A. Mth. 14 (1855) 55-.

- and spheres, contacts. Alvord, B. Smiths. Ct. 8 (1856) 16 pp.

$-\frac{1}{-}$ Coaklay, G. W. Camb. (M.) Mth. M. 2 (1860) 116-.

-, spheres, cylinders and cones, contacts. Durrande, J. B. Gergonne A. Mth. 11 (1820-21) 1-。

- touching each its predecessor and two fixed circles. Gudermann, C. Crelle J. 39 (1850) 42-.
Sphere touching 3 or 4 spheres. Dupin, $C$. Par. Éc. Pol. Cor. 2 (1809-13) 420-.

- 4 spheres. Euler, L. [1779] St Pét. Ac. Sc. Mm. 2 (1810) 17-.

- - - Poisson, S. D. Par. S. Phlm. Bll. 3 (1812) 141-.

- - - Tucci, F. P. Nap. At. S. Pont. 2 (1812) 257-.

- - - Français, J. F. Gergonne A. Mth. 3 (1812-13) 158-

- - - Gergonne, J. D. [1814] Tor. Mm. Ac. 22 (1816) $20^{*}-$.

- - - Hachette, J. N. P. Par. Éc. Pol. Cor. 3 (1814-16) 21-; Par. Éc. Pol. J. $17^{\mathrm{e}} \mathrm{cah}$. (1815) 129-.

- - - Binet, J. P. M. Par. Éc. Pol. J. $17^{\circ}$ cah. (1815) $113-$.

- - - Bohnenberger, G. C. Lindenau Z. 6 (1818) 270 -.

- - - - Dandelin, G. P. Quetelet Cor. Mth. 2 (1826) 13-.

- - - Trébert, A. N. A. Mth. 3 (1844) $101-$.

- - - Serret, J. A. Crelle J. 37 (1848) $51-$.

1151-.

- - - - Frischauf, J. Wien Sb. 52 (1866) (Ab. 2) 222-.

- - - Apollonian problem. Flauti, $V$. Nap. At. Ac. 1 (1819) 369-.

- - - - - Hunyady, J. [1877] (xII) Mag. Tud. Ak. Etk. (Mth.) 5 (1879) (No. 5) $16 \mathrm{pp}$.

- _ - circle touching 3 circles on plane or sphere, and cone touching 3 cones. Poncelet, J. D. Gergonne A. Mth. 11 (1820-21) 317-.

- _ - - 2 constructions and associated theorem. Walker, G. F. Mess. Mth. 11 (1882) 173-.

$-{ }_{-}-$, magnitude. Fenwick, $S$. Mathematician 1 (1845) 27-.

- _ - in mutual contact. Rutherford, $W$. Mathematician 1 (1845) 21-.

Spheres, contact. Hachette, J. N. P. Par. Éc. Pol. Cor. 1 (1804-08) 17-.

- - , problems. Hlauti, V. [1809] Nap. At. Ac. 1 (1819) 21-.

- - , theorems. Orr, W. McF. Camb. $\mathrm{Ph}$. S. P. 9 (1898) 271-.

- touching central sphere of same diameter, maximum number. Tandel, $C$. Quetelet Cor. Mth. 2 (1826) 310-.

- - given sphere. Hoppe, $R$. Arch. Mth. Ps. 1 (1884) 148-.

- 4 planes. Catalan, E. C. N. A. Mth. 6 (1847) 253-; 9 (1850) 352-.

- _ - - and circles touching 3 lines. R., L. P. F. (vi Adds.) Gergonne A. Mth. 19 (1828-29) 211-.

- - - - - - , radii. Steiner, J. Gergonne A. Mth. 19 (1828-29) 85-.

- - - -, radii. Ĺ́ky, B. Orv.-Termt. Éts. (Termt. Szak) (1895) 265-.

- - skew quadrilateral. Vogt, $H$. Crelle J. Mth. 92 (1882) 328-. 
Spheres touching tetrahedron edges. Miller, J. H. T. [1854] Wien SB. 20 (1856) 225-.

- - faces. Klug, L. Arch. Mth. Ps. 61 (1877) 361-.

-, 5 in contact, radii. Baur, C. W. Schlömilch Z. 5 (1860) 365-.

,- 16 touching 4 , and circles touching 3 circles, centres. Stephan, É. Par. S. Phlm. Bll. 2 (1865) 133-.

, - - - - - - - - radii. Mention, J. N. A. Mth. 18 (1859) 438-.

-, - _ - , - _ - - on sphere. Casey, J. [1866] Ir. Ac. P. 9 (1867) 396-.

- _ _ - - geometric property. Schubert, H. Z. Mth. Ps. 14 (1869) 506-.

-, - - - metrical relations of radii. Schubert, H. Z. Mth. Ps. 14 (1869) 513-.

Steiner's series of spheres touching two spheres. Vahlen, K. T. Z. Mth. Ps. 41 (1896) 153-.

Coordinates in spherical geometry. Lupton, $W$. Ph. Mg. 14 (1857) 35-.

Cylinders and spheres, cylindrically pierced. Fuss, N. [1800] St Pet. Ac. Sc. N. Acta 14 (1805) 213-.

- - - mensuration problems. Ferroni, $P$. Mod. S. It. Mm. 10 (1803) 649-.

Diameters of sphere, number. Hessel, J.F.C. Grunert Arch. 24 (1855) 1-.

Division of sphere, equal, by concentric spheres. Davidson, G. [1873] Calif. Ac. P. 5 (187374) 5-, 98-

- - -, problem. Brinkley, J. [1801] Ir. Ac. T. 8 (1802) 513-.

- - - solutions, new. Hess, E. D. Nf. Tbl. (*1878) 38 .

Dodecahedron and icosahedron in sphere, relation between. Flaugergues, $H$. Gergonne A. Mth. 2 (1811-12) 357-.

Ellipse, spherical, properties. Strebor. [Pseud. of Roberts, $W$.$] N. A. Mth. 7$ (1848) 135-, 451- ; 9 (1850) 308-.

Ellipsoids and spheres, theorems. Kilbinger, $G$. Ens. Mth. 2 (1900) 196-.

Equal spheres, number touching given equal sphere. Bender, C. [1869] Arch. Mth. Ps. 55 (1874) 303-.

Excentric sector. Hoppe, R. Arch. Mth. Ps. 65 (1880) 176-.

Figures, which by inversion and reflection, fill up whole spherical surface. Holst, E. B. (xII) Ts. Mth. 5 (1875) 87-.

Frustum of sphere, surface. Escher, $P$. Grunert Arch. 32 (1859) 188-.

Gergonne's method, application to sphere. Barbarin, P. As. Fr. C. R. (1895) (Pt. 2) 43-.

Hemisphere penetrated by indefinite number of equal cylinders. Knight, $T$. Phil. Trans. (1812) 310-.

Homocentric spheres of Eudoxus, Calippus and Aristotle. Schiaparelli, G. V. [1874] Z. Mth. Ps. 22 (1877) (Suppl.) 101-.

In-quadrilateral. Collite, J. N. A. Mth. 8 (1849) 435-.
Inscribed regular polyhedra, volume. $B a x, D$. Leijd. A. Ac. (1815-17) 20 pp.

,,--- . Serooskerken, R. C. van T. Leijd. A. Ac. (1815-17) $12 \mathrm{pp}$.

- solids. Kumps, H.J. Louvain A. Ac. 4 (1822) $51 \mathrm{pp}$.

Intercepts on axes of coordinates of sphere or cone, proof of theorem of Steiner. Bermann, $O$. Arch. Mth. Ps. 53 (1871) 129-.

Intersections of circles, and intersections of spheres. Alvord, B. Am. J. Mth. 5 (1882) 25-.

- - - - - - - (Alvord). Kretkowski, W. Krk. Ak. (Mt.-Prz.) Pam. 13 (1887) 81-; Fschr. Mth. (1887) 563.

$--_{-}-{ }_{-}$, theorems. Miquel, A. Liouv. J. Mth. 3 (1838) 517-.

- - or spheres at given angles. Pellet, $A$. As. Fr. C. R. (1889) (Pt. 2) 161-.

Isodromes on a sphere. Réveille, $\dot{J}$. Rv. Mar. et Col. 100 (1889) 546-.

Lexell's theorem concerning " parallelograms" and triangles on a sphere, proof. Lebesgue, V. A. N. A. Mth. 14 (1855) 24-.

Maximum, property. Bonnet, O. N. A. Mth. 12 (1853) 433-.

Multiplication of matrices, theorem proved by. Chrystal, G. Edinb. Mth. S. P. 2 (1884) 45-.

Osculating surfaces of moving sphere of variable radius. Starkov, A. N. Rs. S. Nt. Mm. (Mth.) 4 (*1883).

Pentahedron and quadrilateral, circum- and inscribed. Jeffery, H. M. QJ. Mth. 25 (1891) 336-.

Perspective of spheres, problem. Bergh, J. G. van den. Leijd. A. Ac. (1818-19) 24 pp.

Plane and sphere, Ceva's theorem. Spitzer, $S$. [1848] Haidinger B. 5 (1849) 21-.

4 points, mutual distances. Scott, R. F. [1873] Mess. Mth. 3 (1874) 58-.

- - on sphere, ares joining two and two. Bérard, -. Gergonne A. Mth. 6 (1815-16) 253-.

5 points lie on sphere, condition. Luchterhandt, A. R. Crelle J. 23 (1842) 375-.

- - - - - Enneper, $A$. Z. Mth. Ps. 13 (1868) 261-.

- - _ - - - - from barycentric principle. Möbius, A. F. Crelle J. 26 (1843) 26-.

Polygons, angles, theorem. Lebesgue, $V . A$. N. A. Mth. 9 (1850) 327-.

-, convex, plane and spherical, angles. John, C. N. A. Mth. 5 (1846) 674-.

Poristic polygons on sphere. Enneper, A. Z. Mth. Ps. 14 (1869) 147-.

_-_-. Stoll, -. Z. Mth. Ps. 29 (1884) 91-.

Pothenot's problem extended. Grunert, J. A. Grunert Arch. 1 (1841) 238-.

- - to space. Plath, C. W. Grunert Arch. 35 (1860) 241-. |

- - - sphere. Grunert, J.A. Grunert Arch. 7 (1846) 104-; 47 (1867) 194-.

- - - - Friesach, K. Steierm. Mt. (1876) 85-.

- - graphical solution. Bauernfeind, C.M. Münch. Sb. (1871) 124-. 


\section{Spheres}

Pothenot's problem and the kumaïds. Vieth, G. U. A. Gilbert A. 54 (1816) 306-.

Powers of circles and spheres. Johnson, W. W. B. A. Rp. (1890) 743-

Problem solved graphically. B.,J. Nicholson J. 21 (1808) 201-.

Problems. Schubert, F. T. [1798] St Pet. Ac. Sc. N. Acta 12 (1801) 196-.

- by Steiner on circles and spheres. Aubert, $O$. G. D. Crelle J. 5 (1829) 163-.

Property of having least surface for given volume. Anon. (vi 525) Gergonne A. Mth. 4 (1813-14) 338-.

Anon. (vr 545) Gergonne A. Mth. 13 (1822-23) 132-.

- - - - - Anon. (vi 550) Ger. gonne A. Mth. 14 (1823-24) 316-.

- - - Anon. (vi 555) Ger. gonne A. Mth. 15 (1824-25) 257-.

—, new. Liebmann, H. Gött. Nr. (1899) 44 -.

Pythagorean theorem in spherical geometry. Eilles, J. Arch. Mth. Ps. 44 (1865) 440-.

Recurring series of circles and spheres, two. Serret, P. N. A. Mth. 1 (1862) 184-.

Section of sphere by cone. Schoute, P.H. N. Arch. Wisk. 4 (1899) 1-; Fschr. Mth. (1899) 470 -

Segment, spherical. Anon. (vr 894) N. A. Mth. 17 (1858) 438-.

- , - between any 2 planes. Crelle, A. L. Crelle J. 52 (1856) 175-.

-, -, theorem. Prouhet, E. N. A. Mth. 12 (1853) 208-.

Segments, spherical, Greek approximate formulæ (Hero). Tannery, $P$. Bordeaux S. Sc. Mm. 5 (1883) 347-.

- - - investigated by Humbert. Hoppe, $R$. Arch. Mth. Ps. 9 (1890) 53-.

Shortest path on sphere. Thomas, L. N. A. Mth. 8 (1849) 279-.

- - - Dienger, J. Grunert Arch. 14 (1850) 219-.

- - - Barbet, -. N. A. Mth. 10 (1851) 415-.

-. Mansion, $P$. Mathesis 9 (1889) $112-, 212$

Similar points of spheres of a Dupin's system. Kröber, (Dr) -. Z. Mth. Ps. 25 (1880) 279-.

Small circle on sphere, equation. Lachlan, $R$. Mess. Mth. 14 (1885) 145-.

Sphere cutting 4 spheres at given angles. Battaglini, G. Tortolini A. 2 (1851) 373-.

- - - - - - , and circles cutting 3 circles; cyclide. Laquière, E. M. As. Fr. C. R. (1880) 132-; N. A. Mth. 2 (1883) 272-, 348 .

- 5 spheres at same angle. Craig, T. Des Moines Anal. 7 (1880) 13-.

- equidistant from five points. Baker, $M$. [1882] Smiths. Misc. Col. 25 (1883) Art. 2, 107-. (Wash. Ph. S. Bll. 5 (1883).)

- given by general equation, centre and radius. Gerono, G. C. N. A. Mth. 18 (1859) 346-.

- in quadriplanar coordinates. Fazzari, $G$. G. Mt. 29 (1891) 257-.

Systems of spheres. Heger, $R$. Dresden Isis Sb. (1900) 27-.
Stereometry 6820

Systems of spheres and circles. Lachlan, $R$. [1886] Phil. Trans. 177 (1887) 481-.

_- Johnson, W. W. Am. J. Mth. 14 (1892) 97-.

- - - - - synthetic theory. Rupp, $O$. Wien Ak. Sb. 104 (1895) (Ab. 2a) 623-.

- - properties. Herz, N. Arch. Mth. Ps. 65 (1880) 385-.

- - and of straight lines. Cremona, $L$. B. A. Rp. (1876) (Sect.) 12-.

Theorems. Gerwien, -. Crelle J. 11 (1834) 130 -.

-. Roberts, S. L. Mth. S. P. 25 (1894) 306-.

- on circles and spheres. Broager, P.D. (xII) Ts. Mth. 1 (1865) 113-.

- and problems. Affolter, F. G. Mth. A. 4 (1871) 185-.

Transformation and division of spherical figures by construction. Steiner, J. Crelle J. 2 (1827) 45-.

Transversals, spherical. Jullien, (le Père) $\boldsymbol{M}$. N. A. Mth. $9(1850) 322-$.

Triangles formed by circles as projections of spherical triangles. Weyr, $E$. Časopis 1 (*1872) 24-; Fschr. Mth. ("1872) 269-

-, tri-rectangular, two, on sphere, theorem. Johnson, W. W. Mess. Mth. 4 (1875) 14

Zone, spherical. Biehringer, $(D r)-$. Z. Mth. Ps. 17 (1872) 255-.

--, - area. Grunert, J. A. Grunert Arch. 3 (1843) 56-.

- - - - Neovius, - [1850] Helsingf. Acta 3 (1852) 807-.

- - - centre of gravity. Grunert, J. A. Grunert Arch. 3 (1843) 61-.

Stereotomy. Monge, G. Par. Éc. Pol. J. (1794-95) 1-.

- Eisenman, - Par. Éc. Pol. J. $2^{\mathrm{e}}$ cah. (1796) 100-; $3^{\mathrm{e}} \mathrm{cah} .(1796)$ 440-; $4^{\mathrm{e}} \mathrm{cah}$. (1796) 619-.

- Sapalski, F. Krk. Roczn. Tow. Nauk. 5 (1820) 229-.

- (Leroy). Bruno, G. Tor. At. Ac. Sc. 2 (1866-67) 601-.

-, problem. Malfatti, G. F. [1802] Mod. S. It. Mm. 10 (1803) 235-.

-, - Brunel, G. Bordeaux S. Sc. PV. (1894-95) 10-.

Straight line cutting at given angles two straight lines in space defined by their projections, construction for. Petzold, M. Z. Mth. Ps. 27 (1882) 252-.

- lines and planes, general geometry. Tarry, G. As. Fr. C. R. (1894) (Pt. 2) 184-

- - - - problems. Hube, $K$. (vi Adds.) Krk. Roczn. Uniwers, 11 (1826) 23-.

- - - in space. Oliveira, C. B. Rio Arch. Palestra 1 (1858) 61-.

- - in space. Housel, -. N. A. Mth. 14 (1855) 228-.

- - - - 3 given, problem of cutting in a triangle with given angles. Salfner, $E$. Arch. Mth. Ps. 16 (1898) 347-.

_ _ _ _ 4, no two being in same plane. Garbinski, $H$. Crelle J. 5 (1829) 174-. 
Straight lines, vector, addition of. Matzka, W. [1868] Prag Ab. 2 (1869) $63 \mathrm{pp}$.

Triangle of given shape, one vertex at given point, others on given straight lines. Hachette, J. N. P. [1826] Brux. Ac. Sc. Mm. 4 (1827) 91-.

Vault, hemispherical, theorem. Bossut, $C$. Par. Mm. de l'I. 2 (1796) 226-.

Vaulting, cross-, of round tower. Hachette, $J$. N. P. Par. S. Phlm. Bll. (1833) 68-.

Vaults, mensuration, problems, Masetti, $G$. Bologna Opusc. Sc. 2 (1818) 263-.

\section{Trigonometry, plane and spherical.}

Mollweide, C. Zach M. Cor. 18 (1808) 394-. Heegmann, A. Lille Mm. S. (1849) 487-.

Fenwick, S. Mathematician 3 (1850) 250-

Isely, L. Neuch. S. Sc. Bll. 13 (1883) 230-.

Study, E. Leip. Mth. Ps. B. 47 (1895) $532-$.

Cwojdzinski, K. Arch. Mth. Ps. 17 (1900) 1-.

Addition theorems, general proof. Philip, W.E. Edinb. Mth. S. P. 18 (1900) 103-.

Analytical trigonometry. Strong, $T$. Am. Ph. S. P. 3 (1843) 49-.

- - Root, O. Camb. (M.) Mth. M. 2 (1860) 190 -.

Angles, composition. Roche, -

[1830] Quetelet Cor. Mth. 7 (1832) 39-

- determination from trigonometrical function, or of function from angle. Herrmann, -. Wien SB. (1848) 465-.

-, projection and measurement. Patterson, $R$. [1801] Am. Ph. S. T. 6 (1809) 29-.

-, reduction problems. Evans, T.S. Tilloch Ph. Mg. 28 (1807) 25-, 129 -

-, sums, trigonometrical functions of, and relations between angles of a polygon. Seipp, -. Arch. Mth. Ps. 7 (1889) 27-.

-, -, - ratios. Burckhardt, J. K. Erfurt N. Acta 1 (1799) 293-.

Angular compensation, general case. Venturi, A. Palermo Cir. Mt. Rd. 4 (1890) 269-.

- measurement, errors in. Marroquín y Rivera, $M$. Méx. S. "Alzate" Mm. 2 (1888) 132-.

- unit, calculation to many places. Glaisher, J. W. L. L. Mth. S. P. 4 (1871-73) 308-.

- - natural. Matzka, W. Grunert Arch. 8 (1846) 400-

$-\frac{1}{1387}$, se.

Anharmonic ratios and trigonometry. Möbius, A. F. Crelle J. 24 (1842) 85-.

Apparent enlargement of mountains. Fontès, - As. Fr. C. R. (1892) (Pt. 2) 966-.

Approximations, accurate determination of $\phi$ when $\sin \phi$ or $\cos \phi$ nearly $=1$. Grunert, J. A. Grunert Arch. 1 (1841) 73-.

-, ares in terms of sine or cosine. Yanishevskir, E. P. (xII) Kazan Un. Mm. (1855) (Bk. 4) 119-.

-, - - - - Lampe, E. Mathesis 17 (1897) 129-, 153-, 183-.
Approximations, circular sector, construction of triangle of nearly equal area. Collignon, $\dot{E}$. N. A. Mth. 13 (1874) 389-.

-, Euler's formula. Glaisher, J.W.L. Mess. Mth. 7 (1878) 190-.

- - - , demonstration. Realis, S. N.A. Mth. 9 (1870) 12 .

-, hyperbolic formulæ, algebraic signs. Macloskie, G. A. Mth. 8 (1893-94) 53-.

-, inaccuracy of formulæ in certain cases, correction. Nell, A. M. Arch. Mth. Ps. 49 (1869) 104 .

—, Poncelet's formulæ, elementary demonstration. Fort, O. Schlömilch Z. 2 (1857) 412-.

- $\sin \theta=\theta=\tan \theta$, error in assuming, when $\theta$ small. Matzka, W. Grunert Arch. 13 (1849) 138-.

-, $\sin \theta>\theta-\frac{1}{6} \theta^{3}$, demonstration. Ravson, $R$. Mess. Mth. 3 (1866) 101-.

,$- \sin \theta<\theta-\frac{\theta^{3}}{4}$, geometrical demonstration. Joffroy, J. N. A. Mth. 14 (1875) 171-.

Arc of ecliptic and equator, problem of Regiomontanus. Delambre, J. B. J. Con. des Temps (1818) 284-.

- and sine, difference. Deladérêere, A. N. A. Mth. 2 (1843) 494-.

,---- (Pappus). Terquem, O. N. A. Mth. 3 (1844) 49-.

,,---- limit of error. Lionnet, E. N. A. Mth. 2 (1843) 216-.

Arc $=\tan =\sin$ for small angles. Wolfers, J. P. Grunert Arch. 30 (1858) 359-.

Ares of first quadrant, lines, calculation. Gelin, E. Mathesis 8 (1888) (Suppl. 3) 1-.

-, multiplication, application of formula. Goursat, É. N. A. Mth. 14 (1895) 245-.

-, small, computation. Wurm, J.F. Lindenau Z. 5 (1818) 188-.

- trisecting circle, property of tangents. Maskelyne, N. Phil. Trans. (1808) 122-.

Area and sum of ungles for triangle and quadrilateral, geometrical theorems. Veltmann, $W$. Arch. Mth. Ps. 17 (1900) 442-.

Cayley's theorem. Airy, G. B. Ph. Mg. 17 (1859) 176-.

Chords in circle, theorem. Le Cointe, L. A. N. A. Mth. 1 (1842) 508-.

-, values (Ptolemy). Arnott, G. A.W. Tilloch Ph. Mg. 52 (1818) 454-.

Circle and trigonometry, general geometry. Tarry, G. As. Fr. C. R. (1891) (Pt. 2) 90-.

Circles in and about quadrilateral, radii. Milinowski, - . Z. Mth. Ps. 23 (1878) 139-. - tracing on uneven ground. Hinard, -. A. Cond. Pon. Chauss. 14 (1870) 117-.

Circular functions of $3 \theta$ and $5 \theta$. Anglin, A.H. Edinb. R. S. P. 21 (1897) 453-.

Circumference, approximate construction. Lemoine, É. $\quad$ Par. S. Mth. Bll. 23 (1895) 242-.

Clouds, heights, determination. Besson, $L$. Rv. Sc. 48 (1891) 58-.

Colour analogy, trigonometrical. Plašil, $J$. Časopis 5 ("1876) 32-; Fschr. Mth. ( $\left.{ }^{*} 1876\right)$ 333. 
Compass, variation, graphical method of determination. Salichon, (le lt.) $H$. Rv. Mar. et Col. 124 (1895) 341-.

Cotes's property. Brinkley, J. [1797] Ir. Ac. T. 7 (1800) 151-.

- theorem deduced from formula in cosines of multiple ares. Fergola, $N$. Nap. At. Ac. 1 (1819) 249-.

- - and De Moivre's. Arndt, F. Grunert Arch. 11 (1848) 181-.

- - theorems related to. Gregory, D. F. (vi Adds.) Camb. Mth. J. 3 (1843) 144-.

Decimal division of angles and time. YvonVillarceau, $A$. C. R. 70 (1870) 1233-; 71 (1870) 362-.

_____. Mendizábal Tamborrel, J. de. Méx. S. "Alzate" Mm. 10 (1896) 285-.

- - circles, etc., and remarks on tables. Sang, E. Edinb. R. S. P. 12 (1884) 533-.

- - quadrant. Abbadie, A. d'. C. R. 71 (1870) 335-.

- _ - right angle. L., O. Cztg. Opt. 21 (1900) 54-.

Degree, Fernel's measure. De Morgan, A. (vr Adds.) Ph. Mg. 19 (1841) 445-; 20 (1842) 116-, 408-; 21 (1842) 22-.

Distance of moving ship, determination. Wollin, L. Stockh. Ak. Hndl. 24 (1803) 231-.

,---- Bergsten, N.J. Stockh. Ak. Hndl. (1833) 193-

- -2 places whose situations relative to a third are known. Littrow, J.J. von. Crelle J. 1 (1826) 232-.

- - tower from observer at 3 points. Bretschneider, C. A. Grunert Arch. 2 (1842) 431-.

Division of Angles and Circular Arcs.

Nieuport, C. F. F. de. [1802] Par. Mm. Sav. Étr. 1 (1806) 659-.

Gough, J. Nicholson J. 12 (1805) 225-.

Cerquero, J. S. Cadiz Period. M. Ci. 1 (1848) $76-$.

Tate, T. Ph. Mg. 19 (1860) 261-.

Wasserschleben, (Maj.) - von. Arch. Mth. Ps. 56 (1874) 335-.

Radicke, A. [1878] Arch. Mth. Ps. 63 (1879) $328-$.

Horst, E. Z. Mth. Ps. 24 (1879) 407-.

Nicholson, J. W. Des Moines Anal. 10 (1883) 41-.

Murphy, I. J. Belfast NH. S. Rp. \& P. (1892-93) 47-.

Müller, $F$. Dresden Isis Sb. (1899) 24 -

Approximate division. Hill, C.J.D. Skandia 3 (1834) 268-.

-- , and hints for reducing error. Kummell, C. H. Des Moines Anal. 5 (1878) $172-$.

- - by rule and compass. Pellet, $A$. C. R. 105 (1887) 1119-; Par. S. Mth. Bll. 16 (1888) $113-$.

Bisection. Širjaev, V. A. Kazan S. Nt. (Ps.Mth.) P. 5 (1887) 1-.

Division of angles, and inscriptibility of polygons. Speelman, E. Brux. Ac. Bll. 25 (1868)
Division of angles, and multiplication of cube. Soymié, É. Les Mondes 22 (1870) 348-

- - given angle. Rauch, $F$. Kosmos (Lw.) 20 (1895) 77-.

Equations. Biehler, C. N. A. Mth. 8 (1889) 552 -

Formulæ. Ramus, C. Kiöb. Ov. (1856) 239-.

Multisection. Mariantoni, 一, \& Palatini, N. A. Mth. 18 (1899) 126-.

- by cycloid. Wilkie, D. E. Vict. T. R. S. 5 (1860) 217-.

- - epicycloid. Leeuven, J. H. van. N. Arch. Wisk. $7\left({ }^{*} 1881\right) 213$.

Practical methods. Michelotti, I. [1797] Turin Mm. Ac. 6 (1792-1800) 105-.

- - Glotin, _. Bordeaux Mm. S. Se. Ps. 2 (1861-63) 252-.

Right angle, division, mechanical. Herschel, A. S. QJ. Mth. 4 (1861) 315-.

\section{Trisection.}

Winthrop, J. Bost. Mm. Am. Ac. 2 (1793) 14-.

Mansfield, J. Conn. Mm. Ac. 1 (1810) 119 -.

Newton, P. Tilloch Ph. Mg. 62 (1823) 10-.

Fusinieri, $A$. Bb. It. 40 (1825) 285-

Querret, -. Gergonne A. Mth. 16 (1825-26) 108-.

Garnier, J. G. Quetelet Cor. Mth. 2 (1826) 271-.

Hill, C. J. D. Crelle J. 2 (1827) 304-; 7 (1831) 44-.

Masters, W. Beng. As. S. J. 1 (1832) 501-.

Barré, -. Lille Mm. S. (1833) 44.

Morrieson, N. Beng. J.As. S. 2 (1833) 71-.

Hudson, C. I. Rv. 2 (1838) 399.

Le Monnier, J. F. B. Cherb. Mm. S. Ac. (1838) 320-.

Otto, -. Grunert Arch. 4 (1844) 223-.

Fusinieri, A. [1845] Mod. S. It. Mm. 23 (1846) 294-.

Ferrari, S. Il Cim. 6 (1855) 536-.

Poudra, -. N. A. Mth. 15 (1856) 381-.

Tietz, J. Grunert Arch. 30 (1858) 114-.

Walter, $F$. Grunert Arch. 34 (1860) 295-.

Kuhlmey, - . Grunert Arch. 36 (1861) 123-.

Richter, -. Z. Bauw. 12 (1862) 284-.

(Richter.) Franz, -. Z. Bauw. 12 (1862) 287-.

O'Donoghue, J. Franklin I. J. 45 (1863) 382 .

Veriot, E. C. R. 66 (1868) 619-, 730-.

$D ., G$. Ts. Mt. Fys. 4 (1871) 199-.

Genese, R. W. Mess. Mth. 1 (1872) 103, 181.

Hillhouse, W. [U.S.A.] Des Moines Anal. 3 (1876) 151-.

Leeuwen, J. H. van. N. Arch. Wisk. $2\left({ }^{*} 1876\right)$ 177-.

Garbieri, G. G. Mt. 15 (1877) 111-.

Rusch, M. Arch. Mth. Ps. 68 (1882) 444-

Sporer, B. Arch. Mth. Ps. 69 (1883) 224.

Moser, C. Bern Mt. (1884) (Heft 3, Ab.) 50 - 
Collignon, É. As. Fr. C. R. (1887) (Pt. 2) 141-.

Câdiz, M. Chili S. Sc. Act. 3 (1893) Ixxxviii.

Candy, A. L. [1893] Kan. Un. Q. 2 (1894) 35-.

Obrecht, - Chili S. Sc. Act. 3 (1893) xevi-, exviii.

Crawford, L. Edinb. Mth. S. P. 16 (1898) $42-$.

Mardones, $\boldsymbol{F}$. Santiago de Chile Un. A. 101 (1898) 689-.

Durell, C. V., \& Beard, W. F. Mth. Gz. 1 (1900) 232.

apparatus. Schweder, G. (jun.). Riga Cor.Bl. 37 (1894) 46-.

application of property of equilateral hyperbola. Sørensen, S. J. (xII) Ts. Mth. 4 (1868) 1-.

approximate. Björling, C. F. E. Arch. Mth. Ps. 15 (1897) 223-.

-, by compasses and rule. Lampe, $E$. Crelle J. Mth. 100 (1887) 364.

Baratta's suggestion. Schiaparelli, G. V. Mil. I. Lomb. Rd. 2 (1869) 1083-.

classification of solutions. Baker, $M$. Smiths. Misc. Col. 33 (1888) Art. 4, 96-. (Wash. Ph. S. Bll. 10 (1888).)

by compasses. Lucas, É. C. R. 81 (1875) 368-.

compasses for, theorem leading to. Toscani, $C$. Tortolini A. 3 (1852) 276-.

and conchoids. Sidler, G. Bern Mt. (1873) (Ab.) 31-.

- cube, multiplication, etc., solutions. Werneburg, J. F. C. Oken Isis (1819) 1519-.

by curve of $3 \mathrm{rd}$ degree. Boyman, J. $R$. Grunert Arch. 15 (1850) 205-.

- - 4th degree. Bichot, -. Fr. Cg. Sc. (1835) 87-.

curves for. Allen, W. Silliman J. 4 (1822) 343-.

by fixed conics. Panzerbieter, $W$. Arch. Mth. Ps. 11 (1892) 408-

- - , arbitrary. Glaser, S. Arch. Mth. Ps. 12 (1894) 367-.

- - hyperbola. Panzerbieter, W. Arch. Mth. Ps. 10 (1891) 333-.

geometrical principles connected with. Walker, John. Thomson A. Ph. 7 (1824) 356-.

graphic. Sarrus, $F$. Gergonne A. Mth. 10 (1819-20) 189-.

- Anon. (vI 231) Bb. Un. 27 (1824) $162-$.

-. Lhuilier, S. Bb. Un. 28 (1825) 3-.

by hyperbola and circle. Teixeira, $A . J$. Coimbra I. 6 (1858) 121-, 133, 134-, 177-, 191-.

- -, equilateral. Neuberg, J. J.B. (xI) Mathesis 3 (1883) 130-.

and inscribed quadrilateral. Broager, $P, D$. (xII) Ts. Mth. 4 (1868) 113 -

mechanical. Laurent, $P$. Nancy Mm. S. Sc. (1833-34) 58.

- methods. Hudson, C. I. Rv. 2 (1838) 332-, 396-.

and multisection. Roche, - Quetelet Cor. Mth. 6 (1830) 375-. new curve for. Hillhouse, W. [U.S.A.] [1878]

Des Moines Anal. 9 (1882) 181-.

optical solution. Hill, C. J. D. Grunert Arch. 1 (1841) 215-.

radiatrix for. $M ., N$. (vi $A d d s$.) Thomson A. Ph. 6 (1815) 154-.

rectilinear geometric method. Lyesn...skir, $I$. (xII) Mosc. Un. Mm. 9 (1835) 40-.

without trigonometry. Dippe, M. C. Grunert Arch. 7 (1846) 108-.

$n$ - and $(n+1)$-division of angle and circle. Grinten, A. van der. Arch. Mth. Ps. 70 (1884) 393-.

Double-altitude problem, solution. Börgen, $C$. A. der Hydrog. 28 (1900) 84-.

Elimination of sines and cosines. MLaren, (Lord). Edinb. R. S. P. 20 (1895) 145-.

Eliminations in trigonometry. Meyer, $F$. D. Mth. Vr. Jbr. 4 (1897) 92-.

Equation giving $\tan \frac{a}{m}$, when $\tan a$ is known. Biehler, $C$. N. A. Mth. 6 (1887) 5-.

- $\tan x-x=a$, solution. Gough, J. Nicholson J. 21 (1808) 1-.

$-\stackrel{n-1}{\Sigma} \cos r \theta=0$, solution. Gregory, $D . \quad F$. (vi ${ }^{1}$ Adds.) Camb. Mth. J. 1 (1839) 44-.

- $\tan x=m \tan a$. Girault, C. Caen Ac. Mm. (1856) 22-.

$-\cos \alpha^{2} \sin \phi^{4}-2 \sin \alpha \cos \alpha \sin \phi \cos \phi$ $+\sin \alpha^{2} \cos \phi^{4}=0$.

Grunert, J. A. Arch. Mth. Ps. 45 (1866) 417-.

- $m \sin ^{4} z=\sin (z-q)$, table for numerical solution. Gasparis, A. de. Nap. Rd. 21 (1882) 94-.

- $\Sigma c$ arc tan $x=k \frac{\pi}{4}$, complex integers applied to solve in rational numbers $x, c, k$. Stфrmer, C. Arch. Mth. Ntvd. 19 (1897) No. 3, 96 pp.

Equations, trigonometrical, roots determined by ruler and compasses. Florow, P. Fschr. Mith. (1897) 455.

- - , solution by series. Chauvenet, $W$. Gould As. J. 3 (1854) 161.

- - transformation, cases. Hopf, L. As. Nr. 57 (1862) 137-.

Finite variations calculus, application. Lorgna, A. $M$. Verona S. It. Mm. 7 (1794) 346-.

\section{Formule of Plane Trigonometry.}

Zahradnik, $K$. Arch. Mth. Ps. 6 (1888) 415-.

Cosine of angle between lines. Grunert, J.A. Grunert Arch. 8 (1846) 194-.

Derivation from coordinate geometry. Riese, (Prof.) - von. Grunert Arch. 30 (1858) 143-.

- of formulæ. Matzka, W. Grunert Arch. 13 (1849) 73-. 
Extension to system of 3 non-intersecting straight lines. Grunert, J.A. Grunert Arch. 35 (1860) 1-.

Formulæ deduced from definite integrals. Girault, C. Caen Ac. Mm. (1856) 19-.

- - - regular polygons in-, or circum-scribed to circle. Kroutil, J. Časopis 19 (1890) 70-; Fschr. Mth. (1891) 265.

- - $a=b \cos C+c \cos B$. Egidi, G. Rm. N. Linc. At. 43 (1890) 147-.

Fundamental formulæ (Legendre). Osorio, R. G. Coimbra I. 3 (1855) 234-.

Half angles. Redl, $F$. Ens. Mth. 2 (1900) 201-.

Hero's polygon formulæ, are they trigonometrical? Schmidt, W. Bb. Mth. 1 (1900) 319-.

Multiple angles from equation of circle. Rudberg, F. Stockh. Ak. Hndl. (1823) 229-.

Ozanam's formula for approximate solution of triangle. Brocard, $H$. Mathesis 9 (1889) 161-.

- - Mansion, $P$. Mathesis 9 (1889) 162-, 181-, 265-.

- - - - - - ascribed to W. Snell. Le Paige, -. Mathesis 10 (1890) 34-.

Quadrilaterals, area, given sum of 2 opposite angles. Anglin, A. H. H. QJ. Mth. 19 (1883) 138-.

-, -, Lefèvre's formula. Verhulst, $P . F$. Quetelet Cor. Mth. 6 (1830) 120-.

Series derived from $\sin ^{2} x+\cos ^{2} x=1$. Lemonnier, $H$. N. A. Mth. 4 (1845) 606-.

- for tangent, cotangent, etc. Herschel, (Sir) J. F. W. Nicholson J. 31 (1812) 133.

$-\cos \theta-\frac{1}{2} \cos 2 \theta+$ etc. $=\log \left(2 \cos \frac{\theta}{2}\right)$ etc. Blissard, J. Mess. Mth. 1 (1862) 124-.

Substitution of $\frac{\pi}{2}-\frac{A}{2}$ for $A$, etc. Dostor, $G$. N. A. Mth. 19 (1880) 362-.

Sums and products. Wiegers, C. Grunert Arch. 33 (1859) 338-.

System connecting sines and cosines of $A, B, C$ and their differences. Glaisher, J.W. L. [1880] Mess. Mth. 10 (1881) 73-.

Systems, complete, in plane trigonometry. Meyer, $W . F$. D. Mth. Vr. Jbr. 5 (1901) (Heft 1) 61-.

Triangle, altitude. Dufour, C. [1877] Laus. S. Vd. Bll. 15 (1878) (PV.) 49-.

-, area. Wallace, (Prof.) W. Camb. Mth. J. 2 (1841) 35-.

-, - Baker, M. [1885] A. Mth. 1 (1884-85) 134-; 2 (1885-86) 11-.

-, -, geometric derivation of formulæ. Hultman, F. W. Ts. Mt. Fys. 2 (1869) 1 -.

-, formulæ, examination question. Gerono, G. C. N. A. Mth. 16 (1857) 76-.

Triangles, right angled, sides. Slop de Cadenberg, G. Mod. S. It. Mm. 13 (1807) 285-.

-, - - - Azzarelli, $M$. Rm. At. N. Linc. 26 (1873) 43-.

Wallis's formula, deduction. Herschel, (Sir) J. F. W. Nicholson J. 32 (1812) 13.
Wallis's formula, and Stirling's, proved by hyperbola. Mansion, -. Brux. S. Sc. A. 23 (1899) (Pt. 1) 1-.

$(2 \cos x)^{m}$, formulæ. Anon. (vi 558) Gergonne A. Mth. 16 (1825-26) 254-.

$1-\cos n \phi$, factorisation, and series for $\frac{\pi}{\sin 2 n \phi}$ etc. Huguenin, $U$. (vir) Amst. N. Ws. Ntk. Vh. 1 (1844) 1-.

$\operatorname{Cos}^{n} x$ in sines or cosines of multiples of $x$. Dirksen, E. H. Berl. B. (1845) 261-.

$\operatorname{Cos} \frac{\alpha}{n}$ in terms of $\cos \alpha$, note on equation. Guilmin, A. N. A. Mth. 4 (1845) 52-, 122-.

$x^{n}-2 \cos n \alpha+\frac{1}{x^{n}}$, factorisation. Airy, (Sir) G. B. [1868] Camb. Ph. S. T. 11 (pt. 2) (1869) 426-.

$x^{n}+\frac{1}{x^{n}}-2 \cos n^{\alpha}$, factorisation. Adams, (Prof.) J. C. [1868] Camb. Ph. S. T. $11(p t .2)$ (1869) 444-.

$\operatorname{Cos} \frac{360}{n}$, when rational. Hessel, J.F.C. Arch. Mth. Ps. 48 (1868) 81-.

$\operatorname{Cos} n \phi$ in powers of $\cos \phi$. Heine, $E$. Mth. A. 2 (1870) 187-.

$\operatorname{Cos} m a$, $\sin m a$ as functions of $\sin a$ or $\cos a$, demonstration. Desboves, A. N. A. Mth. 14 (1875) 385-.

$x^{n}-2 \cos n \theta+\frac{1}{x^{n}}$, factorisation. Ferrers, N. M. Mess. Mth. 5 (1876) 6 .

$\operatorname{Cos}^{2} \alpha+\cos ^{2} \beta+\cos ^{2} \gamma+2 \cos \alpha \cos \beta \cos \gamma-1=0$. Zahradnik, $K$. Arch. Mth. Ps. 62 (1878) 330-.

$a=b \cos C+c \cos B$, substitution of $\left(\frac{y}{w}+\frac{w}{y}\right)$ for $2 \cos B$, etc. Cayley, A. (xI) J. H. Un. Cir. [1] (1882) 241.

$\operatorname{Cos} \frac{2 \pi}{17}$, quadratic radical expression for. Steggall, -. Edinb. Mth. S. P. 7 (1889) 4-.

$\operatorname{Cos}^{n} \theta$ in terms of cosines of multiples of $\theta$. Candido, G. Par. S. Phlm. Bll. 1 (1899) 5-.

Sin $n A$ and other formulæ. Cagnoli, A. Verona Mm. S. It. 7 (1794) 1-.

$\operatorname{Sin}^{n} \theta, \cos ^{n} \theta$ and $\sin n \theta, \cos n \theta$, extension to hyperbolic areas. Brinkley, J. [1797] Ir. Ac. T. 7 (1800) 27-.

Sin $(A \pm B)$ and $\cos (A \pm B)$ deduced from Ptolemy's theorem. Mollweide, $C$. Zach M. Cor. 26 (1812) 601-.

- _ - method of obtaining formulæ. Strong, T. Silliman J. 1 (1818) 424-.

- - Thibault, -. N. A. Mth. 2 (1843) 309 -.

$--\frac{1}{-}$, construction. Midy, - . N. A. Mth. 3 (1844) 374-.

$-\frac{1}{-}$ Arndt, $F$. Grunert Arch. 6 (1845) $95-$.

325-. 
$\operatorname{Sin}(A \pm B)$ and $\cos (A \pm B)$, demonstration. Rispal, A. N. A. Mth. 5 (1846) 84-.

- - deduced from triangle. Grunert, J.A. Grunert Arch. 21 (1853) 237-.

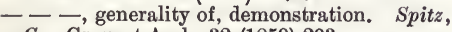
C. Grunert Arch. 32 (1859) 293-.

- - - demonstration. Schreder, E. Grunert Arch. 36 (1861) 447-.

- - Barbe, P. A. Cond. Pon. Chauss. 9 (1865) 182-.

- _ - deduced from Ptolemy's theorem. Tucker, R. [1865] Mess. Mth. 3 (1866) 123. - Tucker, R. [1865] Mess. Mth. 4 (1868) 31-.

- - Lyetnikov, A. V. (xп) Rec. Mth. (Moscou) 2 (1867) (Pt. 2) 96-.

- - Lemonnier, H. N. A. Mth. 10 (1871) 26-.

- - Gravelaar, A. W. N. Arch. Wisk. 5 (*1879) 187-.

- - Z Zejliger, D. N. Kazan S. Ps.-Mth. Bll. 2 (1893) (Prot.) 23.

- - - , and other formulæ, geometrical demonstration. Butters, J. W. Edinb. Mth. S. P. 15 (1897) 86-.

- - - deduced from triangle. Bochow, Arch. Mth. Ps. 17 (1900) 97-.

$\operatorname{Sin} x=2^{m-1} . \prod_{0}^{m-1} \sin \frac{r \pi+x}{m}$. Du Bourguet, Gergonne A. Mth. 3 (1812-13) 19-.

$\operatorname{Sin}^{m} \theta$ and $\cos ^{m} \theta$ in series of sines and cosines of multiples of $\theta$. Pagani, G. M. Férussac Bll. Sc. Mth. 5 (1826) 4-.

$\operatorname{Sin}^{m} x$ and $\cos ^{m} x$ for all values of $m$. Garnier, J. G. Brux. Ac. Bll. 6 (1839) (pte. 2) 375-, 488-.

$\operatorname{Sin} m \theta$ and $\cos m \theta$ in terms of $\sin \theta$ and $\cos \theta$. Moon, R. Camb. Mth. J. 4 (1845) 250-.

$\operatorname{Sin} x$ and $\cos x$, imaginary expressions for. $Y$. Mathematician 1 (1845) 232-.

$\operatorname{Sin}(x \pm y)$ or $\cos (x \pm y)$, simple demonstration. Astrand, J.J. Grunert Arch. 18 (1852) 479-. $\operatorname{Sin} \frac{A}{2}$ and $\cos \frac{A}{2}$. Sturm, J. B. Grunert Arch. 24 (1855) 112-.

$\operatorname{Sin}^{n} \theta$ and $\cos ^{n} \theta$, expansion in sines and cosines of multiples of $\theta$. Wolfers, J.P. Grunert Arch. 24 (1855) 303-.

$\operatorname{Sin} \frac{\pi}{3}, \frac{\pi}{6}, \frac{\pi}{5}$, etc., and $\cos \frac{\pi}{3}$, etc. Gerono, G.C. N. A. Mth. 17 (1858) 46-.

$\operatorname{Sin}\left(\theta+\theta_{1}+\theta_{2}+\ldots+\theta_{n}\right)$ and $\cos \left(\theta+\ldots+\theta_{n}\right)$, expansion, and related theorem of numbers. Unferdinger, $F$. Grunert Arch. 34 (1860) 72-. $\operatorname{Sin} \mu \theta$ and $\cos \mu \theta$, expansion. Gyldén, H. As. Nr. 69 (1867) 193-.

$\operatorname{Sin}(n a+z)$, and $\cos (n a+z), \sin ^{n} a$, and $\cos ^{n} \alpha$, expansion. André, D. N.A.Mth.10(1871) 359-.

$\operatorname{Sin}(A+B+\ldots)$ or $\cos (A+B+\ldots)$. Legge, $A$. di. G. Mt. 9 (1871) 377-.

Sin $\infty$ and $\cos \infty$. Walton, W. QJ. Mth. 11 (1871) 326-.

$\operatorname{Sin}^{-1}$ and $\cos ^{-1}(x+i y)$, values. Schlömilch, $O$. Z. Mth. Ps. 17 (1872) 245-.

Sin na and cosna, expansion in powers of $2 \cos a$ and $2 \sin a$. Le Besgue, V. A. N. A. Mth. 12 (1873) 425-.

VOL. I.
Sin $m a$ and $\cos m a$ in terms of $\sin a$ or $\cos a$. Mourgue, -. N. A. Mth. 12 (1873) 408-.

- - - - - - - - calculation. Ocagne, M. $d$ '. N. Arch. Wisk. 17 (1890) 229-.

$1 \pm \sin (2 p+1) u$. Cayley, A. Mess. Mth. 5 (1876) 7-.

$\frac{\phi}{2}=\frac{\sin \phi}{1}-\frac{\sin 2 \phi}{2}+\frac{\sin 3 \phi}{3}-\ldots$, generalisation. Störmer, C. Acta Mth. 19 (1895) 341-.

$\operatorname{Tan}\left(A_{1}+A_{2}+\right.$ etc. $)$ in terms of $\tan A_{1}, \tan A_{2}$, etc., demonstration. Stainville, - de. Par. Éc. Pol. Cor. 2 (1809-13) 432-.

Tan $z$, continued fraction for. Schlömilch, $O$. Z. Mth. Ps. 16 (1871) 259-.

Tan $n x$, continued fraction for. Glaisher, J. W. L. [1873-74] (vII) Mess. Mth. 3 (1874) 137 ; (x) 4 (1875) 65-.

$\operatorname{Tan}\left(\sum_{i}^{n} a_{r}\right)$, tan $n \theta$, and other formulæ. $A n-$ glin, A.H. [1884] Ir.Ac. P. 4 (1884-88) 259-. $\sum^{n} \arctan a_{r} . \quad$ Anglin, A. H. Arch. Mth. Ps. 2 1 (1885) 407-.

$\frac{1}{\sqrt{ } p}= \pm \Pi \cot .\left(\frac{2 \pi a}{p}\right)$, sign. Stern, M. A. Liouv. J. Mth. 5 (1840) 216-.

$\frac{b+c}{b-c}=\tan \frac{B+C}{2} / \tan \frac{B-C}{2}$, geometric demonstration. Vignal, -. N. A. Mth. 3 (1844) 456-.

Foundations of trigonometry. Brisse, C. N.A. Mth. 16 (1877) 49-.

Functions, developments and properties. Garnier, J. G. Quetelet Cor. Mth. 11 (1839) 283-.

Goniometry. Lindman, C. F. Arch. Mth. Ps. 53 (1871) 112-.

-, problem. Cotterill, T. [1865] QJ. Mth. 7 (1866) 259-.

-, - (Cotterill). Cayley, A. QJ. Mth. 15 (1878) 196-.

-, theorems. Schlömilch, O. Grunert Arch. 8 (1846) 453-; 9 (1847) 1-.

Graphic tables, use in solution of problems in cosmography, and spherical trigonometry. Collignon, É. N. A. Mth. 18 (1879) 179-; 1 (1882) 490-.

History. Suter, H. Bb. Mth. (1893) 1-.

Identities, algebraic, in plane trigonometry. Meyer, W. F. Fschr. Mth. (1898) 441.

-, trigonometrical. Fouret, G. N. A. Mth. 2 (1883) 262-.

Identity. Hermite, C. N. A. Mth. 4 (1885) 57-. -, case. Glaisher, J.W.L. As. Fr. C. R. 9 (1880) 222-.

- involving products of four sines. Glaisher, J. W. L. B. A. Rp. (1880) 484 -

,$- \arctan \frac{B}{A}=\sum_{1} \arctan \frac{\beta_{r}}{a_{r}}$ where

$$
A+i B=\stackrel{n}{I}\left(\alpha_{r}+i \beta_{r}\right) .
$$

Glaisher, J.W.L. [1877-78] As. Fr. C. R. 6 (1877) 211-; QJ. Mth. 15 (1878) 151-; 16 (1879) 329-. 


\section{Trigonometry}

Identity, $m \operatorname{arctang} \frac{1}{x}+n \operatorname{arctang} \frac{1}{y}=k \frac{\pi}{4}$, solution in integers. Störmer, C. Par. S. Mth. Bll. 27 (1899) 160 -

$\cos 36^{\circ}-\cos 72^{\circ}=\frac{1}{2}$, proof. Volpicelli, $P$. Rm. At. 16 (1862-63) 85.

- if $\sum_{1}^{n} \cos x_{r}=0, \sum_{1}^{n} \cos \left(x_{r}+a\right)=0$, then shall $\stackrel{n}{2} \cos \left(x_{r}+\theta\right)=0$. Laisant, C. A. Par. S.

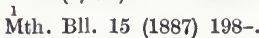

Inaccessible points, distance between 2 . Boyman, J. R. Grunert Arch. 18 (1852) 452-.

- - - - - Ladame, H. (jun.) Neuch. Bll. 7 (pt. 2) (1866) 403-

- - - 3. Lemoine, É. As. Fr. C. R. (1884) (Pt. 2) 47-.

- straight line, problem. Gelin, E. Mathesis 9 (1889) 93-.

Inequalities, trigonometrical. Fenwick, $E$. Mth. Gz. 1 (1900) 107-.

Inequality, case. Genese, R. Ir. As. Fr. C. R. (1894) (Pt. 2) 134-.

Isoperimetric figures, simple demonstrations. Steiner, J. Berl. Ab. (1836) 117-.

Landen's substitution, geometrical representation. Schellbach, C. H. Crelle J. Mth. 91 (1881) 347-.

Logarithmic sines and tangents of small arcs, finding. Burgess, J. Edinb. R. S. P. 22 (1900) 265-.

Logarithms, graphic notation. Haro, - As. Fr. C. R. (1887) (Pt. 2) 128-.

Maximum and minimum, problems. Burg, $A$. Wien Jb. Pol. I. 8 (1826) 213-.

- product of tangents of $n$ angles whose sum is given. $(a+b<90$, etc.) Gerono, G. $C$. N. A. Mth. 17 (1858) 360-.

de Moivre's theorem. Barfuss, F. W. Grunert Arch. 7 (1846) 3-.

- - analogue in plane point system. Hyde, E. W. Science 4 (1896) 344; A. Mth. 11 (1896-97) 129-.

- - demonstration, elementary. Davidov, A. Y. (xII) Rec. Mth. (Moscou) 2 (1867) (Pt. 2) 20-.

- -, - without imaginaries. Göpel, A. Grunert Arch. 6 (1845) 102-.

- - - - that $e^{x i}=\cos x+i \sin x$. Bauer, K.L. Arch. Mth. Ps. 46 (1866) 355-.

- - , symbolic use. Dupuis, N. F. [1891-95] Cn. R. S. P. \& T. 9 (1892) (Sect. 3) 43-; 2 (1896) (Sect. 3) 167-.

Navigation problems, graphical solutions. Anon. Nt. 47 (1892-93) 547-.

Negative quantities, use. Waille, J. [1876] (xII) Doubs S. Mm. 1 (1877) 33-.

Networks, trigonometrical, of low order. Zrzavy, F. Prag Sb. (1872) 107-.

Nomenclature. Björling, E. G. Stockh. Ak. Hndl. (1846) 275-, 299-.

Non-Euclidean trigonometry. Story, $\boldsymbol{W}$. $E$. Am. J. Mth. 4 (1881) 332-; 5 (1882) 180-.

- - fundamental equations derived by elementary method. Réthy, $M$. Arch. Mth. Ps. 58 (1876) 416-.

\section{Plane Triangles 6830}

Non-Euclidean trigonometry, plane, of 3-dimensional homogeneous space. Réthy, $M$. (xI) Mag. Tud. Ak. Étk. (Mth.) 4 (1877) (No. 7) $25 \mathrm{pp}$.

Parallelograms formed from any three elements. Čeby šev, P. [1879] St Pet. Ac. Sc. Mm. (Rs.) 36 (*1880) (Suppl.) No. 3, 40 pp.

- symmetric about axis. Čebyšev, P. [1878] St Pet. Ac. Sc. Mm. (Rs.) 34 ("1879) (Suppl.) No. 3, $16 \mathrm{pp}$.

Partial fraction and product, developments for trigonometric functions. Schröter, $H$. Z. Mth. Ps. 13 (1868) 254-.

\section{Plane Triangles.}

Ambiguous case. Grunert, J. A. Grunert Arch. 2 (1842) 333-.

Angles, construction for difference of two. Walker, J. J. QJ. Mth. 8 (1867) 323-.

-, property. Arndt, $F$. Grunert Arch. 6 (1845) 333-; 8 (1846) 111-.

-, relations between trigonometrical ratios. Dostor, G. Arch. Mth. Ps. 65 (1880) 188-.

- in terms of sides. Borenius, H. G. [1839] Helsingf. Acta 1 (1840) 159-.

- _ _ _ - Newton's Arithmetica Universalis, prob. xI., solution and generalisation. Leblais, A. Presse Sc. 2 (1863) 687-.

Area. Tweedie, C. [1900] Edinb. Mth. S. P. 19 (1901) 2-.

-, determinant value. Dostor, G. Arch. Mth. Ps. 57 (1875) 204-.

- of indeterminate triangle. Györy, S. [1860] (xir) Mag. Ak. Éts. (Mth. Term.) 2 (186162) 59 -.

- in terms of bisectors. Sacchi, G. N. A. Mth. 1 (1862) 332-.

- - - sides. Gerono, G. C. Gergonne A. Mth. 15 (1824-25) 305-.

A _ _ _ Hero's theorem. Hultsch, $F$. Z. Mth. Ps. 9 (1864) 225-.

_- - - _ - Panek, A. Časopis 20 (1891) 310-; Fschr. Mth. (1891) 607. _ _ _ , proof of the "Three Brothers." Kinkelin, $H$. Grunert Arch. 39 (1862) 187-.

Grunert Arch. 40 (1863) 134.

Grunert, J.A. $-1-1-$, synthetic demonstration. Degen, C. F. [1821] Kiob. Dn. Vd. Selsk. Afh. 1 (1824) 129-.

Hypotenuse, approximation to. Buquoy, $G$. von. Oken Isis (1826) 570-, 662-.

Mensuration, notes on. Durán Loriga, J. J. G. Teix. J. Sc. 12 (1895) 45-; Fschr. Mth. (1893-94) 928.

Pothenot's problem. Clausen, T. As. Nr. 20 (1843) 69-.

- - Lehmann's theorem. Hartner, Wien SB. (1849) (Ab. 2) 216-.

Problems, important, solution. Korselt, $A$. Arch. Mth. Ps. 17 (1900) 275-.

Rational right-angled triangles, incommensurability of angles. Berdellé, C. As. Fr. C. R. (1890) (Pt. 2) 186-. 
Sides and angles, elimination problem. Diekmann, J. Arch. Mth. Ps. 63 (1879) 267-.

- in terms of altitudes. Huet, - N. A. Mth. 5 (1846) 225-.

- - - bisectors of angles. Anon. (xr 54) N. A. Mth. 18 (1879) 311-. Mth. 18 (1879) 313-. Gerono, C. C. N. A.

Similar triangles, solution of difficulty in theory. Suremain-de-Missery, A. Gergonne A. Mth. 1 (1810-11) 129-

Solution. Franchini, P. [1831] Lucea At. Ac. 8 (1835) 1-.

-. Vervaet, P.J. Čsopis 5 (*1876) 57-; Fschr. Mth. (*1876) 327.

-, 3rd case. Seidelin, C. J. L. (xIr) Ts. Mth. 5 (1869) 8-.

-, given $a, b, C ; \quad c$ found more directly. Wallace, (Prof.) W. [1823] Edinb. R. S. T. 10 (1826) 168-.

--, - -, -, -, find c. Schultén, N. G. af (fil.). [1838-43] Helsingf. Acta 1 (1840) 151-; Grunert Arch. 3 (1843) 1-.

-, - - , - - - - C Czuber (or $\check{C} u b e r), E$. [1877] Arch. Mth. Ps. 62 (1878) 222-.

-, - 2 sides and contained angle. Girault, - Férussac Bll. Sc. Mth. 15 (1831) 159-. - without logarithmic tables. Olbers, $W$. Zach M. Cor. 16 (1807) 539-

Tangents of angles, property. Garrard, $W$. Phil. Trans. (1808) 120-

Theorems. Hain, E. Arch. Mth. Ps. 55 (1873) 331-.

Triangle with sides in arithmetical progression. Libický, A. Časopis 27 (1898) 141-, 220- ; Fschr. Mth. (1898) 442.

Vertex transversals. Helwig, P.I. N. Arch. Wisk. 17 (1890) 217-; 19 (1892) 216-; Fschr. Mth. (1890) 581 ; (1892) 527-.

Plane trigonometry, basis. Unferdinger, $F$. Grunert Arch. 33 (1859) 429-.

- - and spherical, analogies. Ferromi, $P$. Mod. S. It. Mm. 12 (1805) 106-.

Points, determination from measured directions. Zrzavy, F. Prag Sb. (1882) 12-.

-, 4 in plane, relation. Luchterhandt, A.R. Grunert Arch. 2 (1842) 62-.

Porismatic equations, systems. Wolstenholme, J. L. Mth. S. P. 4 (1871-73) 312-.

Porisms, trigonometrical. Bromvich, T.J. I'a. Mth. Gz. 1 (1900) 331-.

Powers of angular functions. Crelle, A. L. Crelle J. 7 (1831) 253-, 314-.

Problem, geometrical, solution. Aubert, $P$. Edinb. Mth. S. P. 5 (1887) 23-.

-, trigonometrical. Gardie, $V$. A. Cond. Pon. Chauss. 20 (1876) (1 $1^{\text {re }}$ Pt.) 52-, 113-.

Problems, solution by continued approximations. Andrews, H. (jun.). V. Nost. Eng. Mg. 31 (1884) 54-

Prosthapheric method instead of logarithms. Braunmilhl, A. von. Bb. Mth. (1896) 105-.

Ptolemy's problem. Hill, G. W. [1900] As. J. 21 (1901) 33-.
Ptolemy's theorem and formula for $\cos (A-B)$. Zanten, L. van. N. Arch. Wisk. 11 (1884) 188-

- - plane trigonometry. Quidde, A. Grunert Arch. 23 (1854) 238-

Quadrilateral with sines of angles in given ratio. Euler, L. [1780] St Pét. Ac. Sc. Mm. 5 (1815) 73-.

Quadrilaterals, eyclic, areas, simple investigation. Terquem, $O$. N. A. Mth. 3 (1844) $219-$.

-, trigonometry. Bretschneider, C. A. Grunert Arch. 2 (1842) 225-.

-, - Catalan, E. C. Liége S. Sc. Mm. 15 (1888) No. 1, 167-.

Radius of vision, calculation. Ramsauer, $F$. D. Alpvr. Z. 29 (1898) 81-.

Rational trigonometry. Caluso, (l'abbé) $T$. V. de. Turin Mm. Ac. (1809-10) 179-.

Ratios, generalised forms. Hathaway, A. S. [1880] (xII) J. H. Un. Cir. [1] (1882) 48.

Relations between sides of triangle whose angles satisfy a linear relation. Bang, A.S. Ts. Mth. 2 (1884) 53-.

- , trigonometrical, connected with cyclotomy. Sporer, B. Arch. Mth. Ps. 16 (1898) 68-.

Ship's position, application of altitude lines. Delafon, (le lt.) R. Rv. Mar. et Col. 120 (1894) 509-.

- - formulæ. Ocagne, M. d'. Rv. Mar. et Col. 100 (1889) 305-.

- - movements of current or another ship, calculation. Jaffré, P. Rv. Mar. et Col. 136 (1898) 461-.

Signs of trigonometrical lines (ratios). Horner, W. G. Ph. Mg. 6 (1835) 86-.

Sine and cosine of infinity. Earnshaw, $S$. [1844] Camb. Ph. S. T. 8 (1849) 255-.

- of half are. Aynard, -. N. A. Mth. 5 (1846) 399-.

Sines, calculation. Bach, - N. A. Mth. 12 (1853) 108-.

- and cosines of angle and half angles. Bouvier, L. C. Gergonne A. Mth. 15 (182425) $56-$.

- - - etc., calculation. Bakevell, T. $W^{\text {r }}$ Franklin I. J. 7 (1844) 221-.

- - - of irrational angles. Gyldén, $H$. [1867] Helsingf. Acta 9 (1871) 73-.

- - - multiple and sub-multiple angles. Pezzi, F. [1803] Mod. S. It. Mm. 11 (1804) 10 -

- - - products, expansion. Verdon, $R$. [1877] Mess. Mth. 7 (1878) 122-.

or cosines, products of four, theorems. Glaisher, J. W. L. Mess. Mth. 10 (1881) 26-.

- and cosines, tables, calculation. Grunert, J. A. Grunert Arch. 6 (1845) 205-.

- _ - in terms of arc, new approximations. Buquoy, G. von. Oken Isis 21 (1828) 1088.

- for every 3 degrees, table. Lambert, C.J. N. A. Mth. 12 (1853) 284-.

- of first quadrant from degree to degree, product. Laisant, C. A. As. Fr. C. R. (1875) 159-.

- fundamental theorem. Encontre, D. Mntp. Rec. Bll. 2 (1805) 219-. 
Sines of higher orders. Yvon-Villarceau, A.J.F. C. R. 86 (1878) 1160-, 1216-, 1287-; 91 (1880) 195-.

--1 - Farkas, J. C. R. 91 (1880) 209-, 278-, 544-.

---- West, É. C. R. 92 (1881) 1279-.

-, higher, of same order, algebraic relations. Rouyaux, J. A. C. R. 92 (1881) 1276-.

-, natural, limit of error. Finck, B. N. A. Mth. 2 (1843) 329-.

-, - tables, construction and degree of accuracy. Vincent, A. J. H. N. A. Mth. 1 (1842) 272-.

-, etc., relations. Brehmer, - . Grunert Arch. 4 (1844) 236-.

-, tangents, and secants, rational expressions for. Gould, D. Silliman J. 22 (1832) 392-.

Solid angles. Schultén, N. G. af (fil.). [1839] Helsingf. Acta 1 (1840) 101-.

- - Stammer, W. Grunert Arch. 27 (1856) $123-$.

- - four, formed by 4 straight lines from a point, relation between sines. Dostor, $P$. Arch. Mth. Ps. 58 (1876) 1-.

- -, measurement. Riedl von Leuenstern, $J$. [1847] Haidinger Ab. 2 (1848) (Ab. 2) 1-.

- - new solution by projection. Klekler, $K$. [1876] Arch. Mth. Ps. 61 (1877) 337-.

- of pyramid, sums. Riedl von Leuenstern, $J$. Haidinger Ab. 3 (1850) (Ab. 2) 87-.

- -, theorems, two. Sturm, J. B. Grunert Arch. 24 (1855) 112-.

- - trihedral. Barbet, - . N. A. Mth. 6 (1847) 104-.

,--- , and lines related to them. Grunert, J. A. Arch. Mth. Ps. 42 (1864) 377-.

$-{ }_{-},-$, sines, geometric significance. Thaer, A. Z. Mth. Ps. 28 (1883) 249-.

- - , - - , properties. Dostor, G. Arch. Mth. Ps. 56 (1874) 247-.

\section{SPHERICAL TRIGONOMETRY.}

Puissant, L. Par. Ec. Pol. Cor. 3 (1814-16) 60-.

Hube, $K$. (vi Adds.) Krk. Roczn. Unlwers. 5 (1820) 290-

Sorlin, A. N. J. Gergonne A. Mth. 15 (182425) $273-$.

Barsotti, G. [1829] Lucca At. Ac. 11 (1842) 113-.

Ribeiro de Sousa Pinto, R. Coimbra I. 3 (1855) 130 -, 185-.

Unferdinger, $F$. Grunert Arch. 29 (1857) 479-; 33 (1859) 14-; Arch. Mth. Ps. 42 (1864) 453-.

Meissel, E. Mth. A. 15 (1879) 380; 16 (1880) 529-; As. Nr. 95 (1879) 69-; 96 (1880) 139 -.

Allardice, R. E. Edinb. Mth. S. P. 2 (1884) 53-.

Study, E. D. Mth. Vr. Jbr. 2 (1893) $72-$.

Azimuth, calculation. Le Blanc, C. M. Rv. Mar. et Col. 118 (1893) 326-.

Concurrent quadrants, theorem. Lasala, A. Fschr. Mth. (1892) 551.

Equations. Heppel, G. Mth. Gz. No. 3 (1894) 20 .

\section{Formula of Spherical Trigonometry.}

Approximation formulæ. Klilgel, G. S. Bode As. Jb. (1803) 215-.

Cosine, etc. Matzka, W. Grunert Arch. 13 (1849) 88-.

-, - Franke, T. Grunert Arch. 17 (1851) 309 .

- formula, incorrect demonstration in Verdam's spherical trigonometry. Berg, $F$. J. van den. N. Arch. Wisk. 10 (1884) 193-

Deduction of chief formulæ. Cordeiro-Feyo, $J$. Lisb. Mm. Ac. Sc. 10 (1830) (pte. 2) 208-.

Delambre's or Gauss's analogies. Feldt, $L$. Crelle J. 7 (1831) 68-.

- - - . Arndt, $F$. Grunert Arch. 13 (1849) 159-.

- - - C. Chartres, R. Nt. 40 (1889) 644.

- - - - mnemonic rule. Dostor, G. J. Grunert Arch. 30 (1858) 467-; N. A. Mth. 5 (1866) 417-.

$-{ }_{-},-,-$, note. Sylvester, J. J. $\mathrm{Ph}$. Mg. 32 (1866) 436-

- - - and Napier's. Crelle, A. L. Crelle J. 12 (1834) 348-.

- - Crelle, A. L. N. A. Mth. 7 (1848) 232-.

- - - - - Grunert, J. A. Grunert Arch. 17 (1851) 259-.

- - - - - Crofton, M. W. [1869] L. Mth. S. P. 3 (1869-71) 13-.

- - - - - - Potier, A. Ens. Mth. 2 (1900) 303-.

$-1--,-1$, demonstration by construction. Essen, E. Grunert Arch. 27 (1856) 38 -

- - - , - other formulæ. Gergonne, $J$. D. Gergonne A. Mth. 3 (1812-13) 348-

- - - - - - Werner, 0 . Grunert Arch. 24 (1855) 55-.

- - - - - - Essen, E. Grunert Arch. 27 (1856) 158-.

Determination of signs. Stolz, O. [1870] Z. Mth. Ps. 16 (1871) 168-.

Formulæ derived from plane figure. Unferdinger, $F$. Grunert Arch, 26 (1856) 436-; 27 (1856) 300-.

- involving $\frac{S}{2}-45^{\circ}, 45^{\circ}-\frac{1}{2}(S-A)$, etc. Unferdinger, $F$. Wien Sb. 58 (1868) $(A b .2)$ 30 -

—, two. Arrest, H. d'. As. Nr. 35 (1853) 281-.

Fundamental formula and proof of polar triangle theorem. Schreder, E. Grunert Arch. 37 (1861) 438-.

- formulæ, easy demonstration. Grunert, $J$. A. Grunert Arch. 16 (1851) 194-.

Gauss's formulæ $\frac{\sin \frac{a-b}{2}}{\sin \frac{c}{2}}=\frac{\sin \frac{A-B}{2}}{\cos \frac{C}{2}}$, etc. Servois, J. F. Gergonne A. Mth. 2 (1811-12) 84-. 
General demonstration. Harst, A. D. van der. N. Arch. Wisk. 17 (1890) 176-; Fschr. Mth. (1890) 600.

Generalisation. Chauvenet, W. Gould As. J. 3 (1854) 50-, 137.

Interpretation of double sign in certain formulæ. Thomson, (Prof.) James. Ph. Mg. 10 (1837) 18-.

Lagrange's formulæ, deduction from theorem in determinants. Studnička, F.J. Prag Sb. (1875) 1-.

Napier's analogies. Gerono, G. C. N. A. Mth. 2 (1843) 222-.

- -. Grunert, J. A. Grunert Arch. 3 (1843) 104-.

- -. Cortazar, J. N. A. Mth. 6 (1847) 218 -

- - Werner, O. Grunert Arch. 24 (1855) 95-.

Ptolemy and formulæ of spherical trigonometry. Schubert, F. T. [1796] St Pet. Ac. Sc. N. Acta 12 (1801) 165-.

Significance in case of complex argument. Schilling, F. Gött. Nr. (1891) 188-.

Spherical excess. Grunert, J.A. Grunert Arch. 16 (1851) 483-.

_- formulæ, derivation. Werner, O. [1857] St Pét. Ac. Sc. Bll. 16 (1858) 11-.

- - Lhuilier's formula. Gent, -. Grunert Arch. 20 (1853) 358-.

- - - - Prouhet, E. N. A. Mth. 15 (1856) 91-.

- -, - -. Ligowski, W. [1875] Arch. Mth. Ps. 58 (1876) 96-.

- - - - demonstration. Lobatto, R. (vm) Arch. Mth. Ps. 39 (1862) 240.

- - of quadrilateral in small circle, and radius of small circle circumscribing quadrilateral. Hart, $H$. Mess. Mth. 4 (1875) 181-.

- polygon, area. Quetelet, L. A.J. [1820-32] Brux. Ac. Sc. Mm. 2 (1822) 105-; Quetelet Cor. Mth. 7 (1832) 278-.

- triangles. Bretschneider, C. A. Crelle J. 13 (1835) 85-, 145-.

- - Dienger, $J$. Grunert Arch. 7 (1846) 225-.

- -, cotangent formulø. Unferdinger, $F$. Arch. Mth. Ps. 50 (1869) 107-.

Supplements of angles, introduction. Grebe, E. W. Grunert Arch. 39 (1862) 226-.

$\sin A+\sin B+\sin C=4 \cos \frac{A}{2} \cos \frac{B}{2} \cos \frac{C}{2}$, generalisation. Arrest, H. $d$ '. Leip. B. (1852) 34-.

$\sin \frac{A}{2}, \cos \frac{A}{2}$, etc., geometric demonstration.

Barbier, E. N. A. Mth. 4 (1865) 421-.

$\sin a \cos B=\cos b \sin c-\sin b \cos c \cos A$.

Hertz, K. [1869] (xir) Rec. Mth. (Moscou)

4 (1869-70) (Pt. 2) 94-.

$\tan \frac{a}{2}(\cos B-i \sin B)$

$$
=\frac{\tan \frac{c}{2}-\tan \frac{b}{2}(\cos A+i \sin A)}{1+\tan \frac{c}{2} \tan \frac{b}{2}(\cos A+i \sin A)} .
$$

Cayley, A. I. Mth. S. P. 11 (1879-80) 48-.
Fundamental principle. Vincent, A. J. $H$. N. A. Mth. 1 (1842) 33-.

- - Crova, (Prof.) -. (vr Adds.) Perpignan Bll. S. Ag. Pyr. Orient. 7 (1848) 255.

- - Foucaut, C. N. A. Mth. 8 (1849) 58.

- - Patry, É. N. A. Mth. 17 (1858) 208-.

Great circle, relation between spherical distances from three points. Walton, W. QJ. Mth. 4 (1861) 328-.

Hexagon, rectangular skew, trigonometry. Burnside, W. Mess. Mth. 28 (1899) 92-.

Identity, case. Cayley, A. Mess. Mth. 1 (1872) 145.

Mechanical solution of problems. Rambaut, A. A. Dubl. S. Sc. P. 5 (1886-87) 642-.

Method, new. Pessuti, G. Mod. S. It. Mm. 15 (1811) 197-.

Mnemonics. Chasles, M., \& Forestier, -. N. A. Mth. 12 (1853) 312-.

Napier's rules of circular parts. Bowditch, $N$. Am. Ac. Mm. 3 (1809) 33-.

- - - - Hemert, W. J. J. van, d Schröder, J. C. Amst. Mengelwerk 2 (1816) 105-.

(1821) $255-$.

16 (1898) 40-

- - - Lovett, E. O. N. Y. Am. Mth. S. Bll. 4 (1898) 552-.

- - - - graphic proof. Herschel, A. S. Nt. 5 (1872) 24,141 -.

- - - - partly anticipated. De Morgan, A. Ph. Mg. 22 (1843) 350-.

Orthogonal substitutions, elliptic functions, etc. Study, E. Leip. Mth. Ps. Ab. 20 (1893) 85-.

Polar triangles, and polar trihedral angles. Harst, A. D. van der. N. Arch. Wisk. 17 (1890) 188-; Fschr. Mth. (1890) 600.

- - in spherical trigonometry, history of. Braunmühl, A. von. Bb. Mth. (1898) 65-.

Projection, stereographic, application to spherical trigonometry. Grunert, J.A: Grunert Arch. 39 (1862) 318-.

Quadratic binary forms, relation of system of three, to spherical trigonometry. Stephanos, C. Par. S. Mth. Bll. 10 (1882) 134-.

Rational normal configurations $M_{3}^{4}$ of $S_{6}$. Young, G. C. Tor. Ac. Sc. At. 34 (1898) 429- or 587-.

Resultants, concept. Meyer, $F$. Crelle J. Mth. 115 (1895) 209-.

Simplification of spherical trigonometry. Keogh, C., \& Lebesgue, $V . A$. N. A. Mth. 12 (1853) 304-.

Spherical excess. Grunert, J.A. Grunert Arch. 38 (1862) 220-.

- - Bacaloglo, E. Grunert Arch. 39 (1862) 237-.

- - construction. Terquem, O. N. A. Mth. 5 (1846) 17-.

- - Gauss's theorem. Clausen, T. As. Nr. 20 (1843) 33-.

- oblong, area. Gudermann, C. Crelle J. 42 (1852) 280-.

- polygonometry. Raabe, J. L. Crelle J. 2 (1827) 9 . 
Spherical polygons, area. Barbet, -. N. A. Mth. 9 (1850) 183-.

- - regular. Sornin, J. Toul. Mm. Ac. 6 (1856) 76-.

,--- Faure, H. N. A. Mth. $19(1860)$ 421-.

,--- , relations between sides and diameter. Schwarzer, A. Wiẹn Sb. 52 (1866) (Ab. 2) 363-.

- - , star, area. Dostor, G. Arch. Mth. Ps. 63 (1879) 433-.

- quadrangle, problem. Palmer, J.C. Mth. Gz. 1 (1900) 231-.

- quadrilaterals. Sohncke, L. A. Grunert Arch. 4 (1844) 447-.

- , area. König, J. F. Grunert Arch. 34 (1860) 12-, 355-.

- - - Strehlke, $F$. Grunert Arch. 35 (1860) 104-, 447-.

- -, birectangular. Kramp, C. Gergonne A. Mth. 1 (1810-11) 161-.

- - inscribed. Collète, J. N. A. Mth. 8 (1849) 435-.

- - - - with sides given. Kambly, Grunert Arch. 40 (1863) 440-.

Dijon Sé. Ac. (1821) 106-.

- - , theorem: if two diagonals are quadrants, so is third. Colson, C. G. Mess. Mth. 5 (1876) 161-.

\section{Spherical Triangles.}

Lagrange, J. L. (Comte) de. Par. Éc. Pol. J. $6^{\circ} \mathrm{cah} .(1799) 270$-.

Schmeisser, -. Crelle J. 10 (1833) 129-.

Maas, A.J. Brux. Ac. Bll. 6 (1839) (pte. 2) $271-$.

Angelitti, F. Nap. Ac. Pont. At. 25 (1895) No. 9, 24 pp.

Ambiguous cases. Lenthéric, - . N. A. Mth. 2 (1843) 32-.

- -. Carbonnelle, J. Brux. Ac. Bll. 19 (1852) (pte. 3) 71-.

- - Jenkins, M. Mess. Mth. 2 (1873) 150-.

Area. Gerling, C. L. Zach M. Cor. 27 (1813) 297-.

- Tédénat, - Gergonne A. Mth. 6 (181516) $46-$.

-. Buzengeiger, K. H. J. Lindenau Z. 6 (1818) 314-.

-.Lebesgue, V. A. N. A. Mth. 16 (1857) 319-.

-, and spherical ellipse. Fournier-Vannson, -. N. A. Mth. 7 (1848) 14, 51-.

-, theorem, notes. Juel, C. N. Ts. Mth. 10 (A) (1899) 13-.

- of two spherical triangles, equality. Grunert, J. A. Grunert Arch. 32 (1859) 118-.

Areas of plane and spherical triangles. Shortrede, $R$. Beng. J. As. S. 11 (1842) 776-.

----1 Tillot [Tillol], J. N. A. Mth. 9 (1850) 406-.

- - - - Lebesgue, V. A. N. A. Mth. 15 (1856) 352.

N. A. Mth. - , two theorems. Terquem, $O$.
"Associated" triangles, properties. Fenvick, S. Mathematician 1 (1845) 18 -

Cases. Crosswell, $W$. Bost. Mm. Am. Ac. 3 (1809) 38-.

Centre of mean distances. Lhuilier, S. Gergonne A. Mth. 2 (1811-12) 72-.

Centroid. Besge, $V . A$. Liouv. J. Mth. 7 (1842) 516.

-(Besge and Giulio). Grunert, J.A. Grunert Arch. 4 (1844) 75-.

- Matzka, W. Grunert Arch. 4 (1844) 359-.

-. Hansen, P. A. Leip. B. 22 (1870) 71-。

Chordal triangle and spherical triangle, Grunert's theorem, demonstration. Unferdinger, $F$. Grunert Arch. 33 (1859) 89-.

- - - - relations. Simonelli, $R$. Tortolini A. 5 (1854) 186-.

Circles, circum-, in- and e-scribed. Colson, C. G. Edinb. R. S. P. 8 (1875) 589-.

- - - and in-scribed. Clausen, T. Crelle J. 6 (1830) 84-, 90.

,- - _ - , radii. Unferdinger, $F$. Grunert Arch. 29 (1857) 238.

-, in- and e-scribed, circle touching. Salmon, G. QJ. Mth. 6 (1863) 67-.

Coexistence of three spherical triangles, with two sides equal in pairs. Berg, $F$. J. van den. N. Arch. Wisk. 14 (1888) 78-; Fschr. Mth. (1887) 565.

Congruence theorems for fully defined spherical triangles. Petersen, J. N. Ts. Mth. 11 (B) (1900) 77-; Fschr. Mth. (1900) 504.

Differentials of sides and angles, etc. Wolfers, J. P. Grunert Arch. 10 (1847) 431-.

Gauss's pentagramma mirificum, extension to any spherical triangle. Dziobek, - Arch. Mth. Ps. 16 (1898) 320-.

Graphical solution. Littrow, K. L. von. Wien Sb. 42 (1860) 203-.

- Penrose, F. C. As. S. M. Not. 37 (1877) 403-.

- - Janse, L. N. Arch. Wisk. 11 (1884) $1-$; 12 (1886) 113-.

- - by projection, history. Haller, $S . \mathrm{Bb}$. Mth. (1899) 71-.

Large geodetic triangles, resolution. Saija, $G$. Catania Ac. Gioen. At. 12 (1899) Mem. 3, $58 \mathrm{pp}$.

Legendre's theorem. Gauss, C. F. Crelle J. 22 (1841) 96.

- - (Gauss). Grunert, J. A. Grunert Arch. 1 (1841) 436-.

- -. Winckler, A. Liouv. J. Mth. 16 (1851) 375-; Crelle J. 44 (1852) 273-.

- - Catalan, E. C. N. A. Mth. 11 (1852) 406-.

- Caldarera, F. Palermo G. Sc. Nt. 1 (1866) 126-.

- - demonstration. Ivory, J. Tilloch $\mathrm{Ph}$. Mg. 58 (1821) 270 -.

- - - - Grunert, J. A. Grunert Arch. 23 (1854) 111-.

- -, -. Tissot, A. N. A. Mth. 1 (1862) 5-. - -, and Serret's. Rouché, E. N. A. Mth. 15 (1856) 354-.

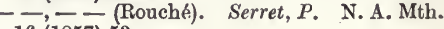
16 (1857) 53- 
Locus of vertex of spherical triangle of given base and area (Lexell's theorem). Barbier, $E$. Les Mondes 4 (1864) 42-.

Malfatti's problem for spherical triangle. Mertens, $F$. [1872] Crelle J. 76 (1873) 92-.

Nine-points circle of spherical triangle. Cayley, -. QJ. Mth. 27 (1895) 35-.

Perimeter, inner side of. Möbius, A. F. Leip. B. 12 (1860) 51-.

Perpendiculars. Grunert, J.A. Grunert Arch. 22 (1854) 478-.

Plane construction. Birkbeck, W.L. Thomson A. Ph. 1 (1821) 259-.

- and spherical triangles. Danione, D. Brugnatelli G. 5 (1822) 1-.

- - - , analogies. Domenico, D. Brugnatelli G. 4 (1821) 259-.

- - - - - , and analytical treatment. Tralles, J. G. Berl. Ab. (1816-17) 65-

- - - -, comparison. Grunert, J.A. Grunert Arch. 1 (1841) 110.

- $-\ldots$ - - , degree of approximation of results on taking one for the other. Valente do Couto, M. Lisb. Mm. Ac. Sc. 3 (pte. 2) (1814) 119-.

- - - problem, solution. Steiner, J. Crelle J. 28 (1844) 375-.

Problems. Meissel, E. Arch. Mth. Ps. 65 (1880) 429-

Rational spherical triangles. Bessell, $F$. Arch. Mth. Ps. 65 (1880) 363-.

Relations. Moth, F. X. As. Nr. 6 (1828) 217-.

- between two triangles, plane or spherical. Bretschneider, C. A. Grunert Arch. 2 (1842) 132-.

Right angled spherical triangles. Adams, Jas. Thomson A. Ph. 3 (1822) 422-.

- - Grunert, J. A. Grunert Arch. 11 (1848) 225-.

- - - problems solved by spherical 5-gon. Prestel, M. A. F. Grunert Ärch. 11 (1848) 56-.

- - - properties. Dostor, G. Arch. Mth. Ps. 51 (1870) 109-.

- - triangles, spherical and plane, analogies. Lhuilier, S. Gergonne A. Mth. 1 (1810-11) 197-.

Lindenau Z. 3 (1817) 198-.

Small-circle spherical triangle. Hudson, E.C. QJ. Mth. 27 (1895) 378-.

- spherical triangle compared with plane. Buzengeiger, $K$. $H$. $J$. Lindenau Z. 6 (1818) 264-.

- - - - - Grunert, J.A. As. Nr. 52 (1860) 49-.

- - triangles. Burg, A. Wien Jb. Pol. I. 7 (1825) 86-.

- - Grunert, J. A. Grunert Arch. 9 (1847) 8-.

- - Caldarera, $F$. Palermo At. 2 (1853) 14 pp.

Solution. Unferdinger, $F$. Arch. Mth. Ps. 41 (1864) 142-.

- Ragona, D. Mod. Ac. Sc. Mm. 5 (1887) 53-.
Solution of all triangles, spherical and plane, by one formula. Steffenelli, A. von. Münch. D. (1813) 343-.

-, case $(a, b, C)$. Mollweide, $C$. Lindenau Z. 1 (1816) 459-.

,$--(-,-,-)$. Sang, E. Edinb. N. Ph. J. 14 (1833) 311-.

-, constructive. Steinheil, C. A. von. Münch. Sb. (1869) (II) 369-.

- given altitudes. Unferdinger, $F$. Wien Sb. 51 (1865) (Ab. 2) 97-.

- - - Hunyady, E. von. Z. Mth. Ps. 12 (1867) 91-.

-, - perpendiculars. Skrivan, G. Grunert Arch. 28 (1857) 471-.

- , - side, opposite angle, and one other element. Matzka, W. Grunert Arch. 11 (1848) 300-.

-, - sides. Thomson, (Prof.) James. Edinb. J. Sc. 4 (1831) 124-,

- - - , in plane or spherical triangles. Bretschneider, C. A. Arch. Mth. Ps. 52 (1871) 371-.

- - $(a, b, A)$ or $(A, B, a)$. Lhuilier, $S$. Gergonne A. Mth. 2 (1811-12) 257-.

- by plane triangles on gnomonic projection. Glotin, P. Bordeaux S. Sc. Mm. 3 (1880) 395-.

-, special cases. Saporetti, A. Bologna Ac. Sc. Mm. 7 (1897) 213-.

-, and stereographic projection. Martinetti, $V$. Mil. I. Lomb. Rd. 24 (1891) 830-, 976-.

Spherical triangle, one side small, solution. Puissant, L. Par. Éc. Pol. Cor. 2 (1809-13) 236 -.

- - $A B C$, angles between $O A$ and chord $B C$. Grunert, J. A. Grunert Arch. 26 (1856) 113-.

- with $C=A+B$. Unferdinger, $F$. Arch. Mth. Ps. 53 (1871) 344-.

Substitution of chords for sides. Nagel, $A$. Schlömilch Z. 1 (1856) 257-.

Surface. Sornin, $J$. Toul. Mm. Ac. 5 (1855) 351-.

Transversals bisecting sides. Schäwen, P. von. Z. Mth. Ps. 27 (1882) 126-.

- joining mid-points of sides, and applications. Unferdinger, $F$. Wien Sb. 52 (1866) (Ab. 2) 323-.

Unlimited spherical triangles. Chaurenet, $W$. Gould As. J. 1 (1851) 39-, 41-.

Substitution groups, Napier's rules in particular. Pund, O. [1897] Hamb. Mth. Gs. Mt. 3 (1900) 290-.

Transformation of elliptic integrals, application to spherical trigonometry. Russell, R. QJ. Mth. 20 (1885) 378-.

Transversals in spherical triangles and quadrilaterals. Rogner, J. Arch. Mth. Ps. 45 (1866) 318-.

Spheroidal triangle, small, reduction of angles to angles of corresponding plane or spherical triangle. Hansen, P. A. Leip. B. 21 (1869) 138-; Leip. Ab. Mth. Ps. 9 (1871) 289-. 


\section{Trigonometry}

Spheroidal trigonometry. Oriani, B. Zach M. Cor. 10 (1804) 244-; 11 (1805) 551-; Bologna Mm. I. It. 1 (1806) 119-; 2 (1808) 1-; (pte. 2) (1810) 1-; Mil. Mm. I. Lomb. Ven. 4 (1832) 325-; Mil. Ephem. As. (1827) 3-; (1828) 3-; (1829) 3-.

- Cayley, A. [1876] As. S. M. Not. 37 (1877) 92.

- -, formulæ. Bonsdorf, A. [1885] St Pét. Ac. Sc. Bll. 30 (1886) 205-.

Stadia measurements, theory. Winslow, $A$. V. Nost. Eng. Mg. 30 (1884) 313-.

Straight lines, and plane trigonometry. Schröder, J. C. Mengelwerk Wisk. Vh. 2 (1816) 119 .

Sum $\sum_{0}^{x} \sin (p+x q)$ etc. Fuss, N. [1796] St Pet. Ac. Sc. N. Acta 12 (1801) 125-.

- - Reusch, E. Z. Mth. Ps. 11 (1866) $536-$.

Sums of like powers of sines and cosines of divisions of circumference. Lenthéric, Gergonne A. Mth. 16 (1825-26) 39-.

Surds, trigonometrical quadratic, values. Gray, P. Mess. Mth. 6 (1877) 105-.

Survey, trigonometrical, of Europe. Reitz, $F$. H. Hamb. Mth. Gs. Mt. 1 (1889) 39 .

Surveying, approximately concurrent lines, to find intersection. Sanchez, P.C. Méx. S. "Alzate" Mm. 13 (1900) 43-.

-, errors. Pérez, E. Méx. S. "Alzate" Mm. 8 (1894) 135-.

-, -, probable. Gauss, C. F. As. Nr. 1 (1823) 81-.

-, importance of verticality in instruments. Sanchez, P. C. Méx. S. "Alzate" Mm. 12 (1898) 467-.

-, plane, problem. Hoppe, R. Arch. Mth. Ps. 17 (1900) 269-.

- Pothenot's problem and modern geometry. Binder, $W$. Wien Ak. Sb. 83 (1881) (Ab. 2) 659-.

-, problem of 3 points in. Campbell, John. Madras J. 4 (1836) 321-.

-, simplification of formula in. Driencourt, -. A. Hydrog. 20 (1899) 68-.

-, trigonotelemeter, use. Antequeda, C. Arg. S. Ci. A. 45 (1898) 390-; 46 (1898) 5-.

Tables. Lindman, $C$. $F$. Stockh. Öfv. 11 (1854) 183-; Grunert Arch. 25 (1855) 284-.

- for calculating radius of an are, length, angle at centre, etc., when chord and height of the arc are given. Sigaud, - (vir) A. Cond. Pon. Chauss. 1 (1857) 132-.

Tachymetry (tacheometry or rapid surveying). Teischinger, E. Hann. Archt.-Vr. Z. 31 (1885) 215-.

$-\left(--\frac{-}{-}\right)$, methods. Jordan, W. Hann. Archt.-Vr. Z. 30 (1884) 453-.

$-(-\ldots--)$, theory, and logarithmic tachymeter. Fischer, G. Cztg. Opt. 6 (1885) 169-, 181-, 200-.

Tangent $\left(45^{\circ} \pm w\right)$. Parkhurst, H. M. Gould As. J. 6 (1861) 103.

Tangents and anti-tangents, calculation. Weddle, $T$. Mathematician 2 (1847) 135-.

\section{Descriptive Geometry}

6840

Teaching of trigonometry. Hoilel, $J$. G. Mt. 13 (1875) 72-.

- Aragón, A. Méx. S. "Alzate" Mm. 5 (1891) 69-

- - Fehr, H. Ens. Mth. 1 (1899) 45-. _ _ , various points. Pfeil, L. von. Arch. Mth. Ps. 58 (1876) 319-.

Theorem: $\sin a \sin b \sin c \sin d+\cos a \cos b$ $\cos c \cos d=\sin a^{\prime} \sin b^{\prime} \sin c^{\prime} \sin d^{\prime}+\cos a^{\prime}$ $\cos b^{\prime} \cos c^{\prime} \cos d^{\prime}$. Glaisher, J. W. L. Camb. Ph. S. P. 3 (1880) 319-, 383-.

Transformation, trigonometrical. Cayley, A. Camb. and Dubl. Mth. J. 9 (1854) 61-.

,-- , used by Hansen in theory of perturbations. Baillaud, B. Bll. Sc. Mth. As. 2 (1878) 292-.

Trapezium, calculation from sides. Bretschneider, C. A. Arch. Mth. Ps. 52 (1871) 24 -

Trigonometry in absolute geometry. Simon, $M$. Crelle J. Mth. 109 (1892) 187-.

Viète, François, trigonometry of. Ritter, F. As. Fr. C. R. (1892) (Pt. 2) 208-.

\section{Descriptive geometry; perspective.}

Analytical treatment of descriptive geometry and perspective. Doppler, C. Böhm Gs. Ab. 1 (1837-40) 78 pp.

_ _ _ _ _ _ _ , four lectures. Hesse, L. O. Z. Mth. Ps. 11 (1866) 369-.

\section{Anharmonic or Cross-Ratio.}

Davies, T. S. Mathematician 1 (1845) 169-, 243-; 2 (1847) 91-, 199-, 310-; 3 (1850) Suppl. 35-.

De Morgan, A. Ph. Mg. 35 (1849) 165 -.

Terquem, O. N. A. Mth. 12 (1853) 272-, 358-.

Clifford, W. K. [1866] L. Mth. S. P. 2 (1869) 3-.

Busche, E. Mth. A. 41 (1893) 591-.

Autonne, L. N. A. Mth. 18 (1899) 341-.

Analogues in solid geometry. Heis, E. Grunert Arch. 31 (1858) 37-.

Anharmonic configurations, interchangeableness of elements. Hofmann, $\boldsymbol{F}$. [1882] Arch. Mth. Ps. 69 (1883) 214-.

- ratio equation. Cayley, A. Mess. Mth. 17 (1888) 95-.

- - of four points in plane. Lucas, $F$. C. R. 77 (1873) 1463-.

- $-\ldots-\ldots$, three being fixed, variations in. Laisant, C. A. Par. S. Mth. Bll. 17 (1889) 169-.

- - and invariant theory of complex quantities. Schick, J. [1900] Münch. Ak. Sb. 30 (1901) 249-.

- - Pappus's problem. Fiedler, W. Schlömilch Z. 5 (1860) 377-.

_ - sextic. Walker, J. J. QJ. Mth. 10 (1870) 53-.

- - (Walker). Cayley, A. QJ. Mth. 10 (1870) 56 - 
Anharmonic ratio sextic of pair of conics. Walker, J. J. QJ. Mth. 10 (1870) 162-.

- - and sine generalisations. Folie, F., d Le Paige, C. Brux. Ac. Bll. 48 (1879) 41-.

- ratios of $m$ points in plane. Möbius, A. F. Leip. B. (1853) 14-.

Doubly anharmonic relation. Battaglini, $G$. G. Mt. 1 (1863) 321-.

Equi-anharmonic range,construction. Schroeter, H. E. Mth. A. 10 (1876) 420-.

- ranges, upon 2 straight lines, theorems. Spottiswoode, $W$. Camb. and Dubl. Mth. J. 8 (1853) 103-.

- relations. Battaglini, $G$. Nap. Rd. 2 (1863) 88-.

Geometrography, application of. Lemoine, $\dot{E}$. As. Fr. C. R. (1894) (Pt. 2) 55-.

Notation. Johnson, $W . W$. Des Moines Anal. 9 (1882) 185- ; 10 (1883) 76-.

Pencil of 5 straight lines in space, anharmonic ratio. Merrifield, $C$. $W$. Mess. Mth. 5 (1876) 94-

Pencils of straight lines, anharmonic properties. Terquem, $O$. N. A. Mth. 12 (1853) 90-.

Problem. Baltrusch, S. E. Grunert Arch. 16 (1851) 245-.

- Ponte Horta, F. da. [1881] Lisb. J. Sc. Mth. 8 (1882) 137-.

Projective ranges. Jacobi, [A. non] C. G. J. Crelle J. 31 (1846) 40-, 93-.

- - construction. Sacheri, G. [1873] (xI) Tor. Ac. Sc. At. 9 (1873-74) 76-.

- -, theorem. Pasch, M. Crelle J. Mth. 91 (1881) 349-; Z. Mth. Ps. 27 (1882) 124-.

Range of points in a straight line. Retali, $V$. [1882] Bologna Rd. (1883) 38-.

Relation of 1st degree. Battaglini, G. Nap. Rd. 2 (1863) 122-.

von Staudt's casts (Würfe), theory. Sturm, $R$. Mth. A. 9 (1876) 333-.

- - (-), -. Schröder, E. Mth. A. 10 (1876) 289-.

- - - $(-),-$, and its extension. Lilroth, J. Gött. Nr. (1873) 767-.

- - - (-), - , - -. Kohn, G. [1894] Mth. A. 46 (1895) 285-.

$--,(-),-,-,--$, and imaginaries in geometry. Litroth, J. [1874-76] Mth. A. 8 (1875) 145-; 11 (1877) 84-.

Theorem. Liouville, J. Liouv. J. Mth. 17 (1852) 391-.

- of higher geometry. Tsinger, V. Y. [1869] (xII) Rec. Mth. (Moscou) 4 (1869-70) (Pt. 2) 23-.

- Lie. Nazimov, P. Kazan S. Ps.-Mth. Bll. 7 (1898) 92-.

Theorems, extension. Heis, E. Grunert Arch. 31 (1858) 39-.

Axonometry.

Schlömilch, O. Civing. 2 (1856) 196-; 5 (1859) 221-.

Weisbach, J. Civing. 2 (1856) 236-; 3 (1857) 61-, 102-.

Mann, F. Schlömilch Z. 4 (1859) 284-.

Schlömilch, O. Schlömilch Z. 4 (1859) 361-.
Vecchi, S. Il Polit. 18 (1870) 857- ; 19 (1871) 466-, 541-, 592-, 717-; 20 (1872) 98-, 216-.

Reis, J. Förster Al. Bauztg. 37 (1872) 69-.

Hauck, G. Z. Mth. Ps. 21 (1876) 81-.

Seidelin, C. N. Ts. Mth. 1 (B) (1890) 49-.

Sobotka, J. Prag Sb. (1900) (Mth.-Nt.) No. 33, 20 pp.

Angles, locus of planes on which projection is constant. Delarive, L. [1882] Gen. S. Ps. Mm. 28 (1883-84) No. 2, 76 pp.

Axes of affinity, application to graphic determination of plane. Fiedler, $W$. Schlömilch Z. 6 (1861) 76-.

- projections of circle, direct determination. Morstadt, $R$. Wien Ak. Sb. 56 (1867) (Ab.2) $92-$.

Axis of homology of triangle and its Brocard's triangle. Tarry, G. Mathesis 15 (1895) 5-.

Change of coordinate planes. N., F. G. Mt. 12 (1874) 154-.

Clinogonal representation of surfaces of revolution. Pelz, C. Prag Sb. (1895) (Mth.-Nt.) No. 7, 15 pp.

Clinographic parallel perspective, graphic solution. Koutny, $E$. Civing. 18 (1872) 265-.

Drawing and axonometry, geometrical principles. Sella, Q. Arch. Mth. Ps. 43 (1865) 245-.

Oblique free projection. Peschka, G. A. von. Wien Ak. Sb. 75 (1877) (Ab. 2) 917-.

- and orthogonal parallel projection. Neelsen, C. Civing. 14 (1868) 461-.

- - - projection, identity of constructions. Staudigl, R. Wien Sb. 64 (1871) (Ab. 2) 490-.

- parallel projection. Burmester, L. Z. Mth. Ps. 16 (1871) 449-.

- - projections. Deschwanden, J. W. von. Zür. Vjschr. 6 (1861) 254-; 7 (1862) 159-.

Orthogonal axonometric foreshortened circle. Tesar̆, J. Wien Ak. Sb. 81 (1880) (Ab.2) 453-. - axonometry. Streissler, $J$. Arch. Mth. Ps. 65 (1880) 208-.

- - , scientific treatment. Pelz, C. Wien Ak. Sb. 81 (1880) (Ab. 2) 300-; 83 (1881) (Ab.2) 375-; Prag Sb. (1885) (Mth.-Nt.) 648-. - projection, axonometry as. Weiler, $A$. Z. Mth. Ps. 33 (1888) 257-.

- - on two planes not at right angles as foundation for perspective. Skuhersky, R. [1858] Böhm. Gs. Ab. 10 (1859) 51-.

- projections. Maszkowski, $K$. Par. T. Nauk Śc. Pam. 5 ( $\left.{ }^{*} 1874\right)$ Art. 1, 38 pp.

- - in metric-projective geometry. Ovidio, E. $d^{\prime}$. Tor. Ac. Sc. At. 11 (1875-76) 830-.

Pohlke's theorem. Schwarz, H. A. [1863] (vIII) Crelle J. 63 (1864) 309-.

- Deschwanden, J. W. von. Zür. Vjschr. 10 (1865) 384-.

- - Reye, T. Zür. Vjschr. 11 (1866) 350-.

- - Pelz, C. [1877] Wien Ak. Sb. 76 (1878) (Ab. 2) 123-.

_-. Peschka, G. A. von. [1878] Wien Ak. Sb. 78 (1879) (Ab. 2) 1043-.

- -. Schur, F. Mth. A. 25 (1885) 596-.

- Mandl, J. Wien Ak. Sb. 94 (1887) (Ab. 2) 60-. 
6840 Axonometry

Pohlke's theorem. Kulpper, C. Mth. A. 33 (1889) 474-.

- - Ruth, F. Wien Ak. Sb. 100 (1891) (Ab. 2a) 1088-.

-. Schur, F. Crelle J. Mth. 117 (1897)

Problem, fundamental. Beck, A. Crelle J. Mth. 106 (1890) 121-.

Problems, graphic solution. Deschwanden, J. W. von. Zür. Vjschr. 9 (1864) 223-.

Projection or shadow of a circle, solution of 2 problems. Motta Pegado, L. P. da. Lisb. J. Sc. Mth. 6 (1878) 217-

Bilinear forms, three pairs of principal triangles connected with. Igel, $B$. Mh. Mth. Ps. 5 (1894) 289-.

Centric figures, graphical properties. Reyes $y$ Prosper, V. Mth. A. 32 (1888) 157-.

Complex elements in geometry, analysis. Klein, $C . F$. Gött. Nr. (1872) 373-

Correspondence, elementary presentation of, etc. Essen, E. Grunert Arch. 29 (1857) 77-.

Drawing, compulsory laws of error. Haddon, A. L. Nt. 48 (1893) 402-.

-, geometrical. Paulus, C. Grunert Arch. 23 (1854) 364-

- from nature, instrument for. B., T. C. Nicholson J. 10 (1805) 111-.

-, programme for systematic teaching of. Gobin, A. Lyon S. Ag. A. 5 (1882) 131-.

-, solid and perspective, teaching. La Gournerie, - de. A. Cons. Arts et Mét. 10 (*1873) 260 -.

Figures, mutual dependence. Battaglini, $G$. Nap. Ac. Sc. Mm. 2 (1855-57) 175-.

-, rectilinear. Olivier, $L$. Crelle J. 1 (1826) 227 -

Forms, geometrical, of second species. Battaglini, G. G. Mt. 3 (1865) 298-; 4 (1866) 96-, 174-.

Harmonic axes and centres. Cassani, $\boldsymbol{P}$. (Ix) Ven. Aten. At. 3 (1866) 147-; (vII) 5 (1868) 207-.

- - of system of straight lines and planes. Cassani, P. G. Mt. 4 (1866) 128 .

- configuration 24. Vietor, A. [1884] Freiburg B. 8 (1882-85) 206-.

- division, generalisation. Laisant, C. A. As. Fr. C. R. (1878) 135-.

- mean-centres (Poncelet). Cauchy, A. L. Gergonne A. Mth. 16 (1825-26) 349-.

- pencils. Chevillard, A.J. N. A. Mth. 1 (1842) 312-.

- - Euler, J. Fschr. Mth. (1891) 584.

- plane of point with reference to tetrahedron. Hain, E. [1876] Arch. Mth. Ps. 60 (1877) 302-.

- pole of points in a line. Klug, L. Mh. Mth. Ps. 8 (1897) 361-.

- range, to find pair of points forming, with 2 given pairs. Grelle, $F$. Z. Mth. Ps. 13 (1868) 148-.

- ranges. Dostor, G. J. N. A. Mth. $9(1850)$ 118-; 10 (1851) 73-.

\section{Descriptive Geometry 6840}

Harmonic relation of two lines or points. Cayley, A. Ph. Mg. 6 (1853) 105-.

- section, illustrations. Browning, $H . H$. Edinb. Mth. S. P. 2 (1884) 6-.

- on straight line. Bohannan, R. D. A. Mth. 1 (1884-85) 121-.

-6-gon and 8-gon. Heger, R. Z. Mth. Ps. 18 (1873) 307-.

Homographic division, imaginary. Battaglini, G. G. Mt. 2 (1864) 142-

- - of 2 straight lines (de la Gournerie). [Schiappa] Monteiro, A. G. Teix. J. Sc. 1 (1878) 117-.

- - Bohannan, R. D. A. Mth. 1 (1884-85) 109-

- figures and projective systems of planes. Reye, T. Crelle J. Mth. 104 (1889) 211-; 106 (1890) 30-, 315-; 107 (1891) 162-; 108 (1891) 89-.

- - in space, properties. Jonquières, E. de. N. A. Mth. 17 (1858) 51-.

$-\div-\longrightarrow$, self-corresponding points. Devoulf, E. N. A. Mth. 17 (1878) 265-.

- , volumes of 2 . Amigues, $\hat{E}$. N. A. Mth. 12 (1873) 374-.

Homographies and rotations, correspondence. Cayley, A. Mth. A. 15 (1879) 238-.

Homography. Poudra, -. N. A. Mth. 19 (1860) 108-.

-, double elements of homographies and their relation to curves of 3rd order and class. Weyr, E. Prag Sb. (1869) 3-.

-, - - in projective systems. Brodén, $T$. Ts. Mth. 6 (1889) 6-; Fschr. Mth. (1888) 585.

- in geometry. Chasles, M. Brux. Mm. Cour. 11 (1837) 575-.

- and homology of figures in 3 dimensions. Dewulf, É. Bll. Sc. Mth. As. 1 (1877) 137-.

-, Newtonian transformation applied to. Lenthéric, -. (vi Adds.) Mntp. Ac. Sc. Mm. 5 (1861-63) 205-.

- and perspective. Housel, -. N. A. Mth. 8 (1869) 492-.

Homologous figures, theory. Seiliger, $D$. Kazan Un. Mm. (1894) (Pt. 4) 127-.

- tetrahedra. Hermes, O. [1857] Crelle J. 56 (1859) 218-.

- triangles. Muir, T. [1872] Mess. Mth. 2 (1873) 55-, 99-.

- -, similar. Jeřabek, $V$. Mathesis 16 (1896) 81-.

- - in study of pentahedron. Gallucci, $G$. Nap. Rd. 38 (1899) 131-.

- -, triply homologous. Aubel, H. van. Mathesis 17 (1897) 53-.

- - - - - related to conics. Ripert, $L$. As. Fr. C. R. (1900) (Pt. 2) 112-; Mathesis 20 (1900) 226-.

,,$---- \ldots-\ldots$ Third,J.A. Mathesis 20 (1900) 153-.

Homology. Cassani, $P$. G. Mt. 3 (1865) 150-.

-, centres, faisceaux, and envelopes of. Martin, H. Edinb. R. S. T. 24 (1867) 591-. -, principles, application to perspective. Regis, D. G. Mt. 8 (1870) 222- 
Homology, principles, application to perspective. Zona, T. Palermo G. Sc. Nt. 15 (1882) 152-

-, trihedral sheafs. Rocha Peixoto, A. F. da. G. Teix. J. Sc. 5 (*1883) 17-.

Imaginaries, geometry of. Tarry, G. As. Fr. C. R. (1887) (Pt. 2) 168-; (1888) (Pt. 2) 31-. - - - Retali, V. Bologna Ac. Sc. Mm. 9 (1888) 259-

- in projective geometry. Schur, F. Mth. A. 39 (1891) 113-.

- - - introduction of. Bonola, R. G. Mt. 38 (1900) 105-.

Imaginary elements, pairs of, in synthetic projective geometry. Segre, $C$. Tor. Ac. Sc. Mm. 38 (1888) 3-.

- projection. Servais, C. Brux. Mm. Cour. $8^{\circ}, 52$ (1894-95) No. 3, 51 pp.

- - of conic sections. Retali, $V$. Bologna Ac. Sc. Mm. 7 (1886) 601-.

Infinitely distant elements in geometry. Weyr, E. Časopis 1 (*1872) $161-$; $2\left(^{*} 1873\right) 105$; Fschr. Mth. (*1872) 328-; (*1873) 382.

Involution.

Terquem, $O$. N. A. Mth. 12 (1853) 24-.

Hesse, L. O. [1863] (vII) Crelle J. 63 (1864) 179 .

Hain, E. Arch. Mth. Ps. 63 (1879) 407-.

Korteveg, D.J. N. Arch. Wisk. 18 (1891) 131-.

Configurations, and application to conics. Seydewitz, F. Grunert Arch. 4 (1844) 246-; 5 (1844) 225-, 331-.

-, uniform, elements of rank. Paulus, C. Grunert Arch. 21 (1853) 175-.

Elementary property of involutions. Pomey, J. B. N. A. Mth. 4 (1885) 489-.

Hesse's theorem and adjoined involutions. Böger, R. [1899] Hamb. Mth. Gs. Mt. 3 (1900) 390-.

Hexagon, involutions connected with. Böger, R. [1899] Hamb. Mth. Gs. Mt. 3 (1900) $387-$.

Homography and involution. Fiedler, $W$. Schlömilch Z. 6 (1861) 1-.

Involution common to group of 5 lines and system of 9 planes. Serret, $P$. C. R. 113 (1891) 326-, 347-.

- of different orders in systems of 2 nd species. Battaglini, G. (Ix) Nap. Ac. At. 2 (1865) No. $19,15 \mathrm{pp}$.

Plane involution. Poudra, N. G. N. A. Mth. 3 (1864) 498-.

- - relatively to sphere. Poudra, N. G. N. A. Mth. 4 (1865) 493 -

Points. Möbius, A. F. Leip. B. 7 (1855) 33-.

- in plane. Möbius, A. F. Leip. B. (1853) 176-.

-,6, systems lying in three ways. Maschke, $H$. A. Mth. 10 (1895-96) 22-

,- 8 , in plane, involutions formed by. Le Pont, H. G. Teix. J. Sc. 7 (1886) 91-.

Poles of pencil in involution. Gribodo, $G$. [1880] Tor. Ac. Sc. At. 15 (1879) 521-.

Projective involutions. Paolis, $R$. de. Rm. R. Ac. Linc. Rd. 2 (1886) (Sem. 2) 335-.
Projective involutions, and projective, pyramidal, triangular and other relations. Terquem, $O$. N. A. Mth. 6 (1847) 68-.

Ranges. Budden, E. Mth. Gz. 1 (1900) 86.

Straight line, involution on, theorem. Jonquieres, $E$ de. $\quad$ N. A. Mth. 19 (1860) 316-.

Magnitude, theorems independent of. Seydewitz, $F$. Grunert Arch. 10 (1847) 59-.

Panoramas. Petit, G. Rv. Sc. 43 (1889) 558-.

Pencils, intersections of four line and two line. Cayley, A. Ph. Mg. 29 (1865) 501-.

- of lines, theorems. Spottiswoode, $W$. Camb. and Dubl. Mth. J. 6 (1851) 160-.

-, projective, determination of skew relation of two in plane. Consentius, R. O. Z. Mth. Ps. 25 (1880) 122-.

- and ranges. Ramisch, A. Arch. Mth. Ps. 69 (1883) 54-.

- _ - projective. Göpel, A. Grunert Arch. 3 (1843) 93-.

\section{PERSPECTIVE.}

Tacani, F. Bb. It. 40 (1825) 417-

Lermier, $J$. Bordeaux Ac. Sc. Sé. Pbl. (1829) 40 -

Fagnoli, G. Bologna N. Cm. 9 (1849) 553-.

Grunert, J. A. Grunert Arch. 32 (1859) 361-.

Larmor, A. QJ. Mth. 21 (1886) 339-.

Peližek, MI. Prag Sb. (1886) (Mth.-Nt.) 290-.

Rees, J. van. Mbl. Nt. (1888) 73-.

(Newton.) Graham, R. H. Nt. 41 (1890) 439-.

Pelǐek, M. Prag Sb. (1890) 1 (Mth.-Nt.) 175-.

Affinity of two spaces. Beck, A. Z. Mth. Ps. 44 (1899) 85-.

Analytical perspective. Mossbrugger, L. Grunert Arch. 11 (1848) 113-.

- - Kahrer, G. Wien Jbr. Ober-Realsch. Inn. Stadt 2 (1860) 84.

Anamorphosis and distortion. Hachette, J.N.P. Par. Éc. Pol. Cor. 1 (1804-08) 361-.

_ new constructions for reflexion images for plane and curved surfaces. Niemtschik, $R$. Wien Sb. 53 (1866) (Ab. 2) 577-.

Architectural plans, effects of perspective with reference to. Menzel, C.A. Dingler 58 (1835) 89-.

Barycentric perspective, application to transformation of structures. Rankine, W. J.M. (viri) Ph. Mg. 26 (1863) 387-.

Bounding plane in linear perspective. Anton, $H$. Wien Sb. 54 (1866) (Ab. 2) 230-.

Circle, perspective of. Lubbock, J.W. Ph. Mg. 5 (1829) 420-.

_, - -. Chapuis, -. J. Gén. Civ. $8(1830)$ 131-.

-, - - Sobotka, J. Mh. Mth. Ps. 10 (1899) 289-.

Wien Ak. Sb. 109 (1900) (Ab. 2a) 583-

- - - , theorems. Hey, R. Phil. Trans. (1814) 348-. 


\section{Perspective}

Conic, projection of, is a conic, demonstration. Leau, -. Par. S. Phlm. Bll. 1 (1899) 87-.

Construction, new. Leib, L. Arch. Mth. Ps. 11 (1892) 1-, vII.

Coordinate planes. De Morgan, A. Camb. Mth. J. 2 (1841) 92-.

Curved surfaces. Hachette, J. N. P. Par. S. Phlm. Bll. (1823) 147-; Crelle J. 1 (1826) 371-.

Distance relations of ground-lines, preservation. Chevillard, A. J. N. A. Mth. 13 (1854) 91-.

Distortion. Anger, C. T. Danzig N. Schr. 4 (1851) (Heft 4) $20 \mathrm{pp}$.

- in perspective representation of plane figures. Scheffers, G. Leip. Mth. Ps. B. 44 (1892) 162-.

Double perspective. Brasseur, J. B. Liége Mm. S. Sc. 3 (1873) 731-.

Eye, quality of, in relation to perspective. Hiern, W. P. (III) Mess. Mth. 2 (1864) 30-.

History of theory. Viehweger, - Civing. 35 (1889) 165-.

Interference curves depending on perspective. Mallock, A. [1899] Nt. 61 (1899-1900) 29-.

Isometric perspective. Farish, W. [1820] Camb. Ph. S. T. 1 (1822) 1-.

Linear perspective. Gergonne, J. D. Gergonne A. Mth. 13 (1822-23) 181-.

- Marstaller, R. Hann. Z. Archt. Vr. 14 (1868) 395-.

- - elements. Mazzetti, G. M. G. Arcad. 46 (1830) 272- ; 47 (1830) 32-, 300-; 48 (1830) 125-; 50 (1831) 1-.

- - by method of vanishing points. Hachette, J. N. P. Par. Éc. Pol. Cor. 1 (1804-08) 313-. - -, principles (Taylor). Uglieni, M. G. Mt. 3 (1865) 338-.

- - - Hauck, G. Z. Mth. Ps. 26 (1881) 273- ; 27 (1882) 236-.

- - - and application. Garnier, J. G. Quetelet Cor. Mth. 2 (1826) 3-.

Metric relations. Schlömilch, $O$. Grunert Arch. 7 (1846) 274-.

Monodimetric and anisometric methods. Weisbach, J. Pol. Mt. 1 (1844) 125-.

Orthographic perspective. Skuhersky, $R$. Wien SB. (1850) (Ab. 2) 326-.

Paintings, theory of perspective in. Donders, -. Amst. Ak. Vs. M. 1 (1885) 318-, 383-.

Perspective drawing, history. Rouché, $E$. A. Cons. Arts et Mét. 3 (1891) 357-.

- - methods. Williams-Ellis, J. C. [1872] Camb. Ph. S. P. 2 (1876) 276-.

- ellipse of sphere. Gergonne, J. D. Gard Not. Tr. Ac. (1807) 192-.

- projections. Hain, E. Arch. Mth. Ps. 70 (1884) 281-.

- Thornton, W. M. A. Mth. 1 (1884-85) 12-.

- - Echols, W. H. A. Mth. 4 (1888) 93-; 5 (1889-90) 15-.

- representations. $K l u g, L$. (xm) Mag. Tud. Ak. Étk. (Mth.) 9 (No. 11) (1882) 10 pp.

- -. Pelǐsek, M. Prag Sb. (1886) (Mth.Nt.) 360 -

- - Prochazka, B. Časopis 26 (1897) 45-; Fschr. Mth. (1897) 462.

Photographic perspective. Mallock, $A$. Nt. 43 (1891) 517-
Descriptive Geometry 6840

Plane point-systems. Voss, (Dr) A. Z. Mth. Ps. 17 (1872) 375-.

Proportional perspective. Stix, A. Förster Al. Bauztg. 35 (1870) 31-.

Relief-perspective (Poudra). Chasles, M. C. R. 37 (1853) 880-.

- - Anger, C. T. As. Nr. 38 (1854) 289-.

- - Burghardt, -. Grunert Arch. 36 (1861) 437-.

- -. Pelǐek, M. Prag Sb. (1886) (Mth.-Nt.) 434-; Časopis 18 (1889) 78-, 107.

- - especially of sphere. Morstadt, $R$. Z. Mth. Ps. 12 (1867) 326-.

Rows of trees, perspective of. Gergonne, J. D. Gard Not. Tr. Ac. (1807) 168-

Similarly divided lines. Skuhersky, $R$. Wien SB. (1851) (Ab. 2) 471-.

Stereoscopic drawing apparatus. Jensen, $J$. [1870] Arch. f. Ap. 4 (1871) 233-.

- perspective. Stevens, W. Le C. Am. As. P. (1894) 109.

Survey maps, perspective drawing in. Laussedat, (le col.) A. A. Cons. Arts et Mét. 2 (1890) 291-; 3 (1891) 313-; 5 (1893) 283-; 6 (1894) 1-, 81-.

Triangles in perspective. Rosanes, $J$. Mth. A. 2 (1870) 549-

- - - Schröter, H. Mth. A. 2 (1870) $553-$.

- - Hain, E. [1880] Arch. Mth. Ps. 66 (1881) 282-.

- _ - Desargues's theorem. Hunyady, E. von. [1879] Crelle J. Mth. 89 (1880) 79-.

- _ - , - proved by descriptive geometry. Sucharda, A. Časopis 29 (1900) 42-; Fschr. Mth. (1900) 517.

- - - - - , space analogies. Vályi, $G$. Mth. Termt. Éts. 11 (1893) 30-; Mth. Nt. B. Ung. 13 (1897) 166-.

- _ - multiple, groups. Vályi, J. $\mathrm{Mh}$. Mth. Ps. 9 (1898) 169-.

- - - plane, relations of position. Wedekind, L. Mth. A. 16 (1880) 209-.

- - -, triply. Third, J. A. [1900] Edinb. Mth. S. P. 19 (1901) 10-.

- and tetrahedra in perspective. Hess, E. Mth. A. 28 (1887) 167-

_ - with their reciprocals. Brioschi, F. QJ. Mth. 1 (1857) 368-.

Vanishing points. Benteli, A. Bern Mt. (1875) 229-.

Photogrammetric constructions. Procházka,B. Časopis 27 (1898) 1-, 101-; Fschr. Mth. (1898) 449.

Photogrammetry. Finsterwalder, $S$. D. Nf. Vh. (1897) (Th. 2, Hälfte 1) 32.

-, problem in photographic geometry. Prochazka, B. Časopis 25 (1896) 341-; Fschr. Mth. (1896) 416.

Polar quadrilateral. Brill, A. Mth. A. 20 (1882) 531-.

- systems, theorem of Cremona's. Jung, G. G. Mt. 14 (1876) 139-.

- triangles, of a circle, two are in perspective. Affolter, F. G. [1872] G. Mt. 11 (1873) 110-. 
Poristic tetrahedra. Bobillier, - Gergonne A. Mth. 18 (1827-28) 320-.

- - (Bobillier). Gergonne, J. D. Gergonne A. Mth. 19 (1828-29) 32-, 220-

\section{Practical Solid Geometry.}

Monge, G. Par. Sé. Éc. Norm. 1 (1800) 49-, 280-, 413-; (pte. 2) 28-, 63-, 144-; 2 (1800) 149-, 338-; 3 (1800) 61-, 332-; 4 (1800) 87-, 291-.

Hachette, J. N.P. Quetelet Cor. Mth. 7 (1832) 360 -

Davies, T. S. Camb. and Dubl. Mth. J. 1 (1846) 227-; 2 (1847) 252-.

Fiedler, $W$. Zür. Vjschr. 25 (1880) 217-.

Miller, E. Königsb. Schr. 40 (1899) [23]-.

Apparatus for illustrating descriptive geometry. Hoza, F. Arch. Mth. Ps. 53 (1871) 188-.

Applications. Brasseur, J. B. [1853] Brux. Ac. Sc. Mm. 29 (1855) 148 pp.

Cone, foci of section obtained by tangential spheres. Dandelin, G. P. Par. S. Phlm. N. Bll. (1826) 3 .

Constructions. Burali-Forti, C. Rv. Mt. 2 (1892) 96-.

-, execution. Bardin, -. N. A. Mth. 10 (1851) 32-; 11 (1852) 268-.

-, problem. Breton, Phil. Mathesis 9 (1889) 73-.

Cylinder, right, cut by another at right angles, surface area intercepted. Lommel, E. Grunert Arch. 34 (1860) 286-.

Domes. Hausding, A. Förster Al. Bauztg. 3334 (1868-69) 305-.

Families of curves, plane and "relief" representation. Graberg, $F$. Zür. Vjschr. 34 (1889) 209-.

Focal planes of plane curve with one or more axes of symmetry. Schoute, P. H. C. R. 125 (1897) 931-.

Forms, geometrical, representation. Nicodemi, R. Nap. Ac. Pont. At. 25 (1895) No. 3, 24 pp.; 28 (1898) No. 3, 14 pp.

Geometrography, application. Lemoine, $\dot{E}$. As. Fr. C. R. (1894) (Pt. 2) 82-.

History. Bache, L. Mth. Ts. 2 (1860) 88-, 129-.

Intersection of planes with cones and cylinders, etc., etc., general method of representation. Niemtschik, $R$. Wien Sb. 63 (1871) (Ab.2) 571-.

Maximum brilliancy, points of. Hachette, J. N. P., \& Monge, -. Par. Éc. Pol. Cor. 1 (1804-08) 295-.

- _ - _ Schoute, P. H. Wien Ak. Sb. 90 (1885) (Ab. 2) 983-.

Methods. Fiedler, $W$. Schlömilch Z. 8 (1863) 444-.

New projection method. Fiedler, W. Zür.

- Vjschr. 24 (1879) 205-.

Practical solid geometry and projective geometry. Fiedler, $W$. Wien Sb. 55 (1867) (Ab. 2) 659-.

Problem, note. Ruffini, F.P. (xı) Mod. Ac. Sc. Mm. 7 (1866) 17-.

Projection, estimation of errors. Burg, $A$. Wien Jb. Pol. I. 7 (1825) 99-; 8 (1826) 213-.
Projection, horizontal, etc., of system of surfaces, representation of points. Caron, $J$. N. A. Mth. 2 (1883) 161-.

Rectangle on sphere. Jochmann, E. Z. Mth. Ps. 14 (1869) 532-.

Revolution quadrics, projection of intersection curve. Gros, -. N. A. Mth. 19 (1860) 29-.

- surface on plane, best projection. Markov, A. A. St Pét. Ac. Sc. Bll. 2 (1895) 177-.

Shadow of fillet on base of column. Belli, $G$. Brugnatelli G. 10 (1817) 287-.

- - hemispherical vault. Bordoni, A. Brugnatelli G. 8 (1815) 60-

- theory, apparent contours, and separation of light and shadow. Hachette, J. N.P. Par. S. Phlm. Bll. (1833) 2-.

- -, application to machine-drawing. Hachette, J. N. P. Par. Ec. Pol. Cor. 2 (1809-13) 13-.

- - constructive. Dupuis, - Par. Éc. Pol. J. (1794-95) 167-.

- - - , formulæ. Fischer, F.W. N. Arch. Wisk. 10 (1884) 172-.

- - , - fundamental theorem. Streissler, J. Arch. Mth. Ps. 69 (1883) 144-.

,,$----\ldots$ (Streissler). Pelz, C. Arch. Mth. Ps. 69 (1883) 437-.

- - penumbra, contour. Bordoni, A. Brugnatelli G. 5 (1822) 255-, 341-.

- photometric bases in brushwork. Chrếtien, L. V. A. Cond. Pon. Chauss. 17 (1873) 29-, 41-.

- - , sphere, shading in brushwork. Breton, Phil. [1872] (xII) Isère S. Bll. 4 (1875) 259-, 321-.

Shadows, application of theorem. Bruno, $G$. (viI) A. Mt. 5 (1863) 18-.

- - - theory of envelopes. Meyer, C. T. Grunert Arch. 9 (1847) 45-.

- architectural ormaments. Bordoni, A. Brugnatelli G. 8 (1815) 284-.

-, and cast shadows. Bonnet, $O$. N. A. Mth. 5 (1866) 71-.

-, loci of centres of boundary curves. Moss brugger, L. Grunert Arch. 6 (1845) 7-.

- of quadrics, construction, for central illumination. Pelz, C. Prag Sb. (1879) 514-.

- rotation surfaces. La Gournerie, J. de. Par. Éc. Pol. J. $35^{\circ}$ cah. (1853) 29-.

- - - in parallel light. Koutny, $E$. Wien Sb. 55 (1867) (Ab. 2) 215-.

- screw surfaces. La Gournerie, J. de. Par. Éc. Pol. J. 34 cah. (1851) 1-.

- _ - screw of Saint-Gilles. Vialla, $B$. Par. Éc. Pol. J. cah. 37 (1858) 191-.

- _ - - triangular thread screw, contour. Francais, J. F. Par. Éc. Pol. Cor. 2 (180913) 69 -.

- - - - - - - in parallel light. Hachette, J. N. P. Par. Éc. Pol. Cor. 2 (1809-13) 447-.

Solution of problems by synthetic geometry. Cardinaal, $J$. Delft Éc. Pol. A. 3 (1887) 151-. Straight line meeting four lines. Chevillard, A. J. N. A. Mth. 15 (1856) 306-. 
Straight lines, plan and elevation of which coincide. Vries, H. de. N. Arch. Wisk. 1 (1895) 107-; Fschr. Mth. (1893-94) 950-.

- - , - - - - Waelsch, E. Mh. Mth. Ps. 3 (1892) 92-.

- - , - - - - Fiedler, W. Mh. Mth. Ps. 3 (1892) 193-.

Teaching of descriptive geometry. Verstraeten, T. (xII) Mathesis 3 (1883) 5-.

- - - - first lesson. Budelot, G. Ens. Mth. 1 (1899) 286-.

- - - lacuna in. Tilly, (le maj.) - de. Brux. S. Sc. A. 9 (1885) (Pt. 2) 95-.

Tetrahedron, construction from data. Gantier, -. Toul. Mm. Ac. 3 (1834) 111-.

Transformations, in geometry of representation (practical solid). Fiedler, $W$. Z. Mth. Ps. 9 (1864) 331-.

-, theory of, history. Torelli, G. G. Mt. 13 (1875) 352-.

Trihedral angle, section of given species. Bruno, $F$. Nap. At. Ac. 2 (1825) 29-.

- - - - - - (Bruno). Hachette, J.N.P. Par. S. Phlm. N. Bll. (1826) 81-.

Vaulted roofs for constructions with trapezoidal ground plan. Staudigl, R. Z. Mth. Ps. 14 (1869) 97-.

- - and lunes, construction. Riccati, G. Verona S. It. Mm. 5 (1790) 48-.

Vaults, figure and construction. Bossut, (l'abbé) C. (xII) Lille S. Mm. 6 (1860) 217-.

\section{Projective Geometry.}

Français, J. F. Gergonne A. Mth. 4 (1813-14) 61-.

Gueneau d'Aumont, -. Dijon Sé. Ac. (1816) 17-.

Vallot, J. N. Bordeaux Act. Ac. Sc. (1842) 1-.

Beazeley, A. Allelod. S. T. (1848) 15-.

Brioschi, F. Tortolini A. 6 (1855) 209-.

Mainardi, G. Mil. G. I. Lomb. 9 (1856) 89-.

Sattelberger, -. Schlömilch Z. 6 (1861) 81-.

Battaglini, $G$. [1873-75] Nap. Ac. At. 6 (1875) No. 6, 10 pp., No. 12, 21 pp.; 7 (1878) No. 5, 21 pp.

Ovidio, E. d'. (x) A. Mt. 6 (1873-75) 72-; 7 (1875-76) 25-.

Lucas, É. Rv. Sc. 17 (1879) 154-; 18 (1880) 948-; 19 (1880) 36-, 375-; 1 (1881) 408783-; 2 (1882) 365-

Veronese, G. G. Mt. 17 (1879) 172-.

Andreev, K. A. (xII) Kharkov Mth. S. Com. (1880) 139-.

Goedseels, - - Brux. S. Sc. A. 17 (1893) (Pt. 1) 50-.

Pieri, M. [1895-97] Tor. Ac. Sc. At. 30 (1895) 607-; 31 (1895) 267- or 381-, 315-or 457-; Tor. Ac. Sc. Mm. 48 (1899) 1-.

Pasch, M. Mth. A. 48 (1897) 111-.

(Von Staudt.) Scott, C. A. Mth. Gz. 1 (1900) 307-, 323-, 363-.

Algebraic theory and application to rotation of rigid body. Pagani, G. M. Brux. Ac. Sc. Mm. 7 (1832) 20 pp.

Analytical representation and projective geometry. Pasch, M. Mth. A. 30 (1887) 127-.
Analytical solution of conics, problem. Mossbrugger, L. Grunert Arch. 16 (1851) 138 .

- theory. Giorgini, G. [1819] Lucca At. Ac. 1 (1821) 29-.

Angle on horizontal plane, projection. Eudes, A. N. A. Mth. 4 (1845) $360-$.

Applications. Noel, J. N. Quetelet Cor. Mth. 8 (1834) 238-.

Central projection derived from orthogonal projection. Czuber (or Cuber), E. Arch. Mth. Ps. 62 (1878) 259-.

- - method in. Nicodemi, $R$. Nap. Ac. Pont. At. 24 (1894) No. 5, 7 pp.

- of 4 given points. Deschwanden, J. W. von. Zür. Vjschr. 10 (1865) 97-.

Chasles's “Géométrie Supérieure." Burghardt, -. Grunert Arch. 20 (1853) 431-.

Circle, orthographic projection. Strong, $T$. Mth. Misc. 1 (1838) 401-。

-, projections. Benteli, A. Bern Mt. (1878) $177-$.

Closed figure, projection of $=$ zero. $\mathrm{Ohm}, \boldsymbol{M}$. [1853] Crelle J. 50 (1855) 79-.

Coincidence problem in generalised Mongeian projection. Schmid, T. Mh. Mth. Ps. 4 (1893) 159-.

Collinear projections and fundamental laws of projection. Schlesinger, $J$. Wien Sb. 58 (1868) $(A b, 2)$ 658-.

Collineation and projections. Sucharda, $A$. Arch. Mth. Ps. 64 (1879) 105-.

Configurations, fundamental. Juel, $C$. Acta Mth. 14 (1890-91) 1-.

-, projective, certain relations between. Lipschitz, R. Crelle J. Mth. 66 (1866) 267-.

Constructions, some. Crone, $C$. Ts. Mth. 2 (1884) 13-; Fschr. Mth. (1884) 520.

Coordinate systems, interpretations in projective geometry yielded by. Graberg, $F$. Zür. Vjschr. 30 (1885) 55-.

Cyclical projective ranges. Weyr, E. Časopis 11 ( $\left.{ }^{*} 1882\right)$ 191-, 265-; Fschr. Mth. ( ${ }^{* 1882)}$ $508-$

- relations. Ameseder, A. Wien Ak. Sb. 98 (1890) (Ab. 2a) 290-.

Deductive development. Pieri, M. Mil. I. Lomb. Rd. 31 (1898) 780-.

Degenerate homographic relations. Battaglini, $G$. Nap. I. Inc. At. 1 (1882) No. 14, $4 \mathrm{pp}$.

Desargues (17th century), Dutch edition of his method of perspective. Le Paige, $C$. $\mathrm{Bb}$. Mth. (1888) 10-.

- and modern geometry. Mundi y Giró, S. [1898] Barcel. Ac. Mm. 2 (1892-1900) 415-.

Distance of 2 elements in a projective system of one dimension, formula. Suvorov, T. $M$. [1880] (xI) Kazan S. Nt. (Ps.-Mt.) P. 1 (1883) [No. 1] 9-.

Duality. Gergonne, J. D. Gergonne A. Mth. 16 (1825-26) 209-.

Elements, primitive, of abstract projective geometry. Pieri, M. Tor. Ac. Sc. At. 32 (1896) 231- or 343-.

Equipollences and projections. Conti, C. A. Sc. Lomb. Ven. 7 (1837) 66-.

Figures in one plane. Rädell, -. Grunert Arch. 1 (1841) 181-. 
Figures that can be traced continuously. Lemoine, É. As. Fr. C. R. (1881) 175-

Forms, geometrical. Fellini, D. Rv. Mt. 5 (1895) 170-.

-, _, simple, projectivity. Weyr, E. Prag Sb. (1889) 2 (Mth.-Nt.) 163-; Fschr. Mth. (1889) 605.

-, projective. Geiser, C.F. [1869] A. Mt. 4 (1870-71) 25-.

Foundations. Enriques, $\boldsymbol{F}$. Mil. I. Lomb. Rd. 27 (1894) 550 -.

Fundamental principle, extension. Koln, $G$. [1894] Mth. A. 46 (1895) 285-.

- problem. Küpper, K. Prag České Ak. Fr. Jos. Rz. (T'́rida 2) 6 (1897) Art. 21, 5 pp.; Prag Fr. Jos. Ac. Sc. Bll. (Mth.-Nt.) 4 (1897) (Pt. 1) 45-.

- theorem. Zeuthen, H.G. C. R. 126 (1898) 213-.

- -. Schur, F. Mth. A. 51 (1899) 401-.

- - von Staudt's. Darboux, G. Mth. A. 17 (1880) 55-.

- - - - Schur, F. Mth. A. 18 (1881) 252-.

- - - - Le Paige, C. Liège S. Sc. Mm. 15 (1888) No. 5, 8 pp.

- - , - - new demonstration. Zeuthen, H. G. C. R. 125 (1897) 638-, 858-; N. Ts. Mth. 8 (B) (1897) 73-; Fschr. Mth. (1897) 470.

- theorems. Paolis, R. de. Rm. R. Ac. Linc. Mm. 9 (1881) 489-.

- -. Le Paige, C., $\&$ Deruyts, E. Brux. Ac. Bll. 15 (1888) 335-.

- Fano, G. Palermo Cir. Mt. Rd. 9 (1895) 79-, 84-.

- Enriques, $F$. Palermo Cir. Mt. Rd. 9 (1895) 82-.

- -, two. Montesano, D. G. Mt. 23 (1885) 285-.

Gnomonic projection. Zona, T. Palermo G. Sc. Nt. 15 (1882) 79-.

Grassmann's method in projective geometry. Burali-Forti, C. Palermo Cir. Mt. Rd. 10 (1896) 177-, 254; 11 (1897) 54-.

Harmonic and general projection, new derivation of first law. Wiener, C. Z. Mth. Ps. 11 (1866) 1-.

Higher geometry, introduction. Nazimov, P.S. Kazan Un. Mm. (1891) (Pt. 3) App., (Pt. 5) App., (Pt.6) App.; (1892) (Pt. 6) App.; (1893) (Pt. 2) App.; Fschr. Mth. (1892) 566.

Imaginary points, geometrical definition. Stephanos, C. Bll. Sc. Mth. As. 7 (1883) (Pt. 1) 204-.

Invariants of a quadrangle by projective transformation. Lovett, $E$. O. A. Mth. 12 (1898-99) 79-.

Isotomic projection of quadrilateral, centres. Rueda, C.J. Fschr. Mth. (1897) 445.

Maclaurin's "Geometrica Organica." Jonquières, E. de. Liouv. J. Mth. 2 (1857) 153-.

Map projection, graphic processes for. Drien. court, -. A. Hydrog. 20 (1899) 78-.

Maximum projection of system of plane figures in space, and of spherical triangle. Lhuilier, $S$. Gergonne A. Mth. 2 (1811-12) 49-.
Mechanics, relation of projective geometry to. Stephanos, C. B. A. Rp. (1900) 644.

Metric properties of figures, projection. Faure, H. A. (xII) Isère S. Bll. 1 (1869) 243-; 2 (1870) 381-; 4 (1875) 129-.

- relations, projections, conic and orthogonal. Poncelet, P. V. [1831] Quetelet Cor. Mth. 7 (1832) 118-, 141-.

Mine, inclination of passages. Lefroy, (Ing.) - . J. Mines 9 (1798-99) 339-.

Modern geometry and projections. Schlömilch, O. Grunert Ärch. 1 (1841) 248-.

- methods. Brent, $(D r)$ - N. Z. I. T. 3 (1870) (P.) 59-.

$n$ dimension geometry, new theorem. Meyer, - . D. Nf. Tbl. (1884) 141.

Notation of geometric position. Henry, L. $d^{\prime}$. (xII) Lille S. Mm. 10 (1882) 5-.

Orthogonal projections, certain. Magistrini, $D$. [1846] Bologna N. Cm. 10 (1849) 195-.

Pappus's theorem on triangle, sides of which pass through fixed collinear points, two vertices move on fixed lines. Zahradník, $K$. Arch. Mth. Ps. 56 (1874) 11-.

Planes and lines. Koppe, C. Crelle J. 14 (1835) 70-.

Polygons with pairs of axial and parallel sides. Matzka, W. Prag Sb. (1864) (Pt. 2) 117-.

Polyhedra, projections. Chevillard, A. $J$. N. A. Mth. 15 (1856) 197-.

Problem: to place two projective straight lines so that they shall generate a circle (Steiner). Kramer, $A$. Crelle J. 18 (1838) 185-.

Projections of special dimensions, mathematical basis. Weisz, J.A. (xI) Mag. Ak. Éts. (Ith. Term.) 1 (1860) 67-.

Projective geometry in plane. Grassmann, $H$. [1851] Crelle J. 42 (1852) 193-, 204-.

Projectivity in space, problem. Sturm, $R$. Mth. A. 6 (1873) 513-.

- - - , simplification. Sturm, R. Mth. A. 15 (1879) 407-.

Quadrilateral, complete, mid-points of 3 diagonals are collinear. Collins, M. Arch. Mth. Ps. 52 (1871) 243-.

- theorem and extension. Sachse, A. Z. Mth. Ps. 27 (1882) 380-.

Skew projection. Transon, A. N. A. Mth. 4 (1865) 385-; 5 (1866) 63-.

- -, case. Fontené, G. N. A. Mth. 15 (1896) 369-.

- vault, section. Mannheim, A. N. A. Mth. 16 (1897) 404-.

Solutions by projections. Coste, L. M. P. Gergonne A. Mth. 7 (1816-17) 304-.

Synthetic geometry. Esson, W. L. Mth. S. P. 28 (1897) 491-.

- - development since Monge. Kötter, $\boldsymbol{E}$. [1898-1901] D. Mth. Vr. Jbr. 5 (1901) (Heft 2) $\mathrm{xxvIII}+486 \mathrm{pp}$.

- - theorem in, and some corollaries. Del Re, A. Palermo Cir. Mt. Rd. 2 (1888) 128 .

- and projective geometry, need of, in analytical geometry. Davidescu, D. A. Bucarest S. Sc. BIl. 9 (1900) 19-. 
6840 Projective Geometry

Systems of trilaterals and quadrilaterals. Folie, F. Liége S. Sc. Mm. 7 (1878) No. 3, 109.pp.

Teaching, introduction to projective geometry. Amodeo, F. G. Mt. 34 (1896) 22-.

Theorem. Amodeo, F. [1879] G. Mt. 18 (1880) 15-.

Theorems. Bauer, G. Crelle J. 19 (1839) 205-.

- of Cremona's, proofs of two. Dewulf, É. G. Mt. 13 (1875) 168-.

-, introductory. Asted, A. N. Ts. Mth. 6 (A) (1895) 1-.

-, various. Cayley, A. Camb. Mth. J. 2 (1841) 267-; Crelle J. 31 (1846) 213-; 34 (1847) 270-; 38 (1849) 97-; 41 (1851) 66-, 84.

-, 一. Jacobi, C. G.J. Crelle J. 31 (1846) 40-, 93-, 359-.

Topographical problems, solution by "one plane descriptive geometry." Richardson, J. M. Franklin I. J. 37 (1859) 148-.

Triangle traced by plane of projection on the coordinate planes, sides. Pantanelli, $D$. G. Mt. 8 (1870) 161.

Trilinear relationship, new constructions in perspective, etc. Hauck, $G$. Crelle J. Mth. 95 (1883) 1-.

Vectors. Kotelinikov, A. Kazan S. Ps.-Mth. Bll. 8 (1899) (App.); 9 (1900) (App.) $317+$ 40 pp.; Fschr. Mth. (1899) 501-.

Proportional axes. Tawaststjerna, C. G. Helsingf. Acta 1 (1840) 489-.

- planes. Tawaststjerna, C. G. [1844] Helsingf. Acta 2 (1847) 777-.

Relations, geometrical, generalisation of certain. T"öössy, B. von. Mth. Termt. Éts. 18 (1900) 233-; Mth. Nt. B. Ung. 18 (1903) 427.

- between segments got by dividing a line in $3,4,5 \ldots$ points. Teixeira, A. J. Fschr. Mth. (1892) 671 .

- of 2nd degree (between planes and pencils). Reye, T. [1865] Z. Mth. Ps. 11 (1866) 280 -

Representation, elementary methods, history and theory. Fiedler, $W$. Zür. Vjschr. 27 (1882) 125-.

- of planes on planes. Miihll, K. von der. Crelle J. 69 (1868) 264-.

Stereographic Projections, and other Projections of the Sphere.

(See also 8840 and Geography 83.)

Orthogonal projection in spherical geometry. Lodge, A. Mth. Gz. 1 (1900) 97-.

Sphere, perspective. Gergonne, J. D. Gergonne A. Mth. 7 (1816-17) 311-.

,-- , conical. Ocagne, M. d'. N. A. Mth. 17 (1898) 44-.

-, projection on plane. Landré, C. L. N. Arch. Wisk. 6 (*1880) 203-

-, - of 2/3. James, (Sir) H. (viII) R. E. Pp.7 (1858) 134.
Projections of Sphere

6840

Sphere, projective geometry. Allardice, R. E. Edinb. Mth. S. P. 4 (1886) 56-.

Stereographic projection. Heawood, P. J. Mth. Gz. 1 (1900) 102 .

- - of circle, proof of Francoeur's problem. Brocard, H. Fschr. Mth. (1892) 551.

- - converse. Jeffery, H.M. L. Mth. S. P. 17 (1887) 379-.

- - , equatorial, of sphere, application of Peaucellier cell. Phillips, A.W. B. A. Rp. (1884) 649.

- -, history. Gilnther, -. D. Nf. Tbl. (1887) 227-.

- - and theorems of Quetelet and Dandelin. Pelz, C. Prag Sb. (1898) (Mth.-Nt.) No. 31, $4 \mathrm{pp}$.

- - use in geometry. Dandelin, G. P. Gergonne A. Mth. 16 (1825-26) 322-; Brux. Mm. Ac. Sc. 4 (1827) 13-.

- projections of point and its inverse, relation between. Tweedie, C. Edinb. Mth. S. P. 10 (1892) 46-.

Straight lines joining vertices of triangles or of skew quadrilaterals, theorems. Steiner, $J$. Crelle J. 1 (1826) 38-.

Transformation of figures. Anger, C. T. Grunert Arch. 4 (1844) 281-.

- - - Lenthéric, -. (vI Adḋs.) Mntp. Ac. Sc. Mm. 5 (1861-63) 440-, 446-, 449.

- - metric properties of figures. Faure, $H$. N. A. Mth. 17 (1858) 276-, 381-; 18 (1859) 181-, 381-; 19 (1860) 189-.

- - plane figures, Newtonian. Lenthéric, - . Mntp. Ac. Sc. Mm. 4 (1858-60) 145-, 351-.

\section{Transversals.}

Ferriot, - . Gergonne A. Mth. 17 (1826-27) 141-.

Garnier, J. G. Quetelet Cor. Mth. 4 (1828) 143-.

Cousinery, - . N. A. Mth. 2 (1843) 66-.

Kulp, L. Arch. Mth. Ps. 56 (1874) 437-.

Cesáro, E. G. Mt. 22 (1884) 240 -

Candy, A. L. A. Mth. 10 (1895-96) 175-; 11 (1896-97) 10-

Analytical problems. Bergsten, N. J. Stockh. Ac. Hndi. (1820) 1-.

Application of geometrical theorem. Rump, F. H. Grunert Arch. 27 (1856) 332-.

Carnot's theorem, application to polygons. Lerch, M. Prag Sb. (1882) 132-.

- - , - tangents. Terquem, O. N. A. Mth. 18 (1859) 347-

- - , demonstration. Walton, $W$. Camb. and Dubl. Mth. J. 5 (1850) 92-.

Circle, transversals, theorems. Lefaivre, $P$. N. A. Mth. 2 (1843) 362-.

Complete polygons and polyhedra, tranversals. Vaněček, M. N. Prag Sb. (1881) 274-.

Metrical relations. Pelǐ̌ek, M. Časopis 27 (1898) 26-, 81-, 165-; Fschr. Mth. (1898) 438.

Parallel transversals, equal. Hain, E. [1874-77] Arch. Mth. Ps. 57 (1875) 441; 61 (1877) 177-. 
Parallel transversals of triangle. Hain, $E$. [1874] Arch. Mth. Ps. 57 (1875) 438-.

Polygons, transversals. Becker, J.K. Grunert Arch. 38 (1862) 342-.

Problems, F. Camb. Mth. J. 1 (1839) 87-.

Straight lines, 4, in space, determination of transversals. Schlömilch, $O$. Leip. B. 7 (1855) 39-.

System of 2 points. Hain, E. Arch. Mth. Ps. 66 (1881) 280.

Theorem. Delacour, V. N. A. Mth. 2 (1843) 444-.

- Terquem, O. N. A. Mth. 7 (1848) 459-.

- Hyde, E. W. Des Moines Anal. 5 (1878) $113-$

-. Lasala, A. Fschr. Mth. (1891) 584.

-. Krahe, A. Fschr. Mth. (1900) 499.

Theorems. Ferrari, $F$. N. A. Mth. 14 (1895) 41-.

- two, analytical proof. Sturm, J. C. F. Gergonne A. Mth. 14 (1823-24) 225-.

Transversals, two, cut proportionally. Chevillard, A. J. N. A. Mth. 1 (1842) 449-.

Triangles, plane, transversals. Skrivan, $G$. Wien Jbr. Ober-Realsch. Inn. Stadt 4 (1862) 1-

- and pyramid, transversals. Most, R. Arch. Mth. Ps. 50 (1869) 238-.

-, reciprocal transversals. Neuberg, - . Brux. S. Sc. A. 24 (1900) (Pt. 1) 119-.

-, transversals. Paque, A. Liége Mm. S. Sc. 10 (1855) 199-.

-, - Hain, E. [1873] (x) Arch. Mth. Ps. 56 (1874) 99-.

\section{Geometry of Conics and Quadrics.}

\section{General.}

Chasles, M. Par. Éc. Pol. Cor. 3 (1814-16) 11-.

Frégier, -. Gergonne A. Mth. 6 (1815-16) 229-, 321-; 7 (1816-17) 95-.

Chasles, M. Brux. Ac. Sc. Mm. 5 (1829) 81 pp. (Guinoddie.) Valat, - Bordeaux Act. Ac. Sc. (1839) 631-

Hesse, L. O. Crelle J. 20 (1840) 285-.

Ingram, J. K. Ir. Ac. P. 3 (1847) 502-.

Painvin, L. N. A. Mth. 17 (1858) 130-.

Berger, -. Mntp. Mm. Ac. 4 (1858-60) 31-.

Heilermann, -. Schlömilch Z. 5 (1860) 69-

Haillecourt, -. Metz Ac. Mm. 67 (1889) 131-.

Analytical theory. Raymond, G. M. Chambéry Mm. Ac. Sav. 5 (1831) 140-

Axes, principal, of conics or quadrics, practical determination. Serret, $P$. N. A. Mth. 7 (1868) 352-.

Central conics and quadrics. Bret, - Gergonne A. Mth. 5 (1814-15) 357-.

Classification of conics and quadrics. Poussart, A. Ens. Mth. 1 (1899) 279-.

- - - - Bromwich, T.J. I'A. Camb. Ph. S. P. 10 (1900) 358-.

Concomitants of systems of conics and quadrics, etc. Johnson, A.R. [1890] L. Mth. S. P. 21 (1891) 432-.

VOL. I.
Conditions for determining conic or quadric surface, number of. Anon. (vr 563) Gergonne A. Mth. 20 (1829-30) 185-.

Cone and sections, general properties. Chasles, M. Liouv. J. Mth. 3 (1838) 102-.

Confocal conics or quadrics, properties. Koenigs, G. N. A. Mth. 19 (1880) 74-.

Conics, description. Pelosi, A. Tortolini A. 3 (1852) 252-.

-, - . Kilpper, C. Grunert Arch. 28 (1857) $100-$.

-, - by homography. Cassani, P. Ven. Aten. At. 12 (1875) 119-.

-, - - tangents. Gergonne, J. D. Gergonne A. Mth. 5 (1814-15) 49-.

-, general method of solving questions on. Chasles, M. C. R. 59 (1864) 7-, 209-.

-, geometrical, elementary sketch. Zeuthen, H. G. (xII) Ts. Mth. 2 (1878) 33-, 65-, 109-, 132-; 6 (1882) 13-.

-, -, principles. Taylor, C. Mess. Mth. 5 (1871) 141-, 214-.

- with infinite branches. Page, C. E. N. A. Mth. 1 (1842) 21-, 61-.

-, new treatment. Horner, J. K. Zach Cor. 4 (1820) 440 -

- and perspective in the teaching of analytical geometry. Chelini, D. Bologna Rd. (1864) 152-.

- - quadrics dividing given segments harmonically. Fuortes, $T$. G. Mt. 10 (1872) 98-.

- - which reciprocate two given conics or quadrics. Ovidio, E. d'. G. Mt. 10 (1872) 313-.

- - revolution quadrics. Tucci, F. P. [1812] Nap. At. S. Pont. 3 (1819) 131-.

— in solid geometry. Quetelet, L. A.J. [1820] Brux. Ac. Sc. Mm. 2 (1822) 123-.

- _ - Bobillier, _. Quetelet Cor. Mth. 3 (1827) 270-

13 (1887) 58- ; Fschr. Mth. (1886) 754.

-, synthetic geometry. Ponte Horta, F. da. Lisb. Ac. Sc. Mm. 3 (Pt. 2) ( $\left.{ }^{*} 1865\right)$ No. 1, $59+8$ pp.

-, theorems derived from general equation. Giorgini, G. [1823] Lucca At. Ac. 3 (1827) $63-$.

-, use in geometrical problems. Penny, $W . G$. Atlantis 2 (1859) 435-.

Conjugate conics and quadrics. Cremona, $L$. Tortolini A. 3 (1860) 257-.

- diameters. Camus, -. N. A. Mth. I (1842) 300-.

- - Fenwick, S. Mathematician 1 (1845) 38-.

- -. Brassinue, E. N. A. Mth. 6 (1847) 381-.

Conjugation-point and its application to graphic constructions. Loud, F. H. A. Mth. 7 (1892-93) 77-.

Conoid circumscribed to conic, sections of. Motta Pegado, L. P. da. [1875] Lisb. J. Sc. Mth. 5 (1876) 65-.

Construction on cone for finding parameter of given section. Berkhout, J.J.T. van. (vI Adds.) Arch Wisk. Gn. 2 (1863) 299-. 
Construction of conics and quadrics. Dupin, $C$. Par. Éc. Pol. J. cah. 14 (1808) 45-.

- walls of parabolic shape. Conard, $J$. Brux. A. Tr. Pbl. 50 (1893) 359-.

Constructions by means of cone and cylinder. Staudigl, R. Wien Sb. 58 (1868) (Ab. 2) 960-.

Dandelin's theorem, extension, etc. Sauze, N. A. Mth. 17 (1858) 33-.

- - , sphere inscribed to cone touches plane of section at focus, etc. Transon, A. N. A. Mth. 12 (1873) 21-.

Determination of conics and quadrics. Hunyady, J. (хп) Mag. Tud. Ák. Étk. (Mth.) 7 (1881) (No. 18) 39 pp.

Discontinuity of constructions caused by imaginary elemenț. Wiener, C. Z. Mth. Ps. 12 (1867) 375-.

- in locus question connected with ellipse. Goodwin, H. Camb. Mth. J. 4 (1845) 190-.

Discriminants, application to conics and quadrics. Dostor, $G$. Arch. Mth. Ps. 58 (1876) 5-.

Ellipse and ellipsoid, inscribed. Girault, $C$. Caen Ac. Mm. (1884) 3-

- - - maximum inscribed triangle and tetrahedron. Unferdinger, $\boldsymbol{F}$. Arch. Mth. Ps. 51 (1870) 127-.

- - - - - and minimum polygons and polyhedra. Mainardi, $G$. Tortolini A. 1 (1850) 348-.

- $-\ldots$, minimum, round triangle and tetrahedron. Liourille, $J$. Liouv. J. Mth. 7 (1842) 190 -

Ps. 25 (1880) 59-.

- - - normals. Joachimsthal, $F$. Crelle J. 26 (1843) 172-.

- - - properties. Rochat, -. Gergonne A. Mth. 3 (1812-13) 25-

- - - D Durrande, J. B. Gergonne A. Mth. 12 (1821-22) 223-.

-, tracing. Maurin, H. (viri) A. Cond. Pon. Chauss. 4 (1860) 106-.

Equations relating to Conics and Quadrics.

Gergonne, J. D. Gergonne A. Mth. 5 (1814 -15) 61-.

Raabe, J. L. Crelle J. 2 (1827) 182-.

Lenthéric, -. Mntp. Ac. Sc. Mm. 2 (1851-54) 455-; 3 (1855-57) 449-.

Grunert, J. A. Arch. Mth. Ps. 51 (1870) 326-.

Versluys, J. Arch. Mth. Ps. 52 (1871) 278-.

Axes, equations, rapid notation. Dostor, G. J. Grunert Arch. 30 (1858) 202-

- in oblique coordinates. Bobillier, -. Quetelet Cor. Mth. 4 (1828) 216-.

Centres, equations for, in absolute geometry. Frank, A. von. Steierm. Mt. (1874) 47-.

Characteristics of conics given by equation. Ehlert, A. Arch. Mth. Ps. 1 (1884) 51-.

Conditions for circle or sphere. Ferrers, N.M. QJ. Mth. 2 (1858) 267-.

Conics, plane and spherical, in Boothian tangential coordinates. Jeffery, H. M. QJ. Mth. 9 (1868) 189-.

- - - - - - 3-point tangential coordinates. Jeffery, H. M. [1867] QJ. Mth. 9 (1868)
Coordinates, tetrahedral or triangular. Chelini, D. Bologna Mm. Ac. Sc. 3 (1863) 3-.

Determination of conics and quadrics. Halphen, G. H. (x) Par. S. Mth. Bll. 1 (1873) 130-, 226-; 2 (1874) 11-.

Geometrical construction. Bérard, - Gergonne A. Mth. 6 (1815-16) 157-.

- interpretation of coefficients. Faure, $H$. N. A. Mth. 5 (1866) 1-.

- significance. Schultén, N. G. af (fil.). [1832] St Pét. Mm. Sav. Étr. 2 (1835) 313-.

Reduction. Boole, G. Camb. Mth. J. 2 (1841) 64-.

Representation. Chambers, C. [1877] Manch. Lt. Ph. S. P. 17 (1878) 58-.

Tangential and quadriplanar coordinates, application of Salmon's notation. Versluys, $J$. Arch. Mth. Ps. 52 (1871) 377-.

Tangents to conics or quadrics, equation. Verdam, G. J. Grunert Arch. 2 (1842) 188-.

Transformation. Lemonnier, $H$. N. A. Mth. 14 (1875) 396-.

- of coordinates, factor. Greiner, M. Arch. Mth. Ps. 57 (1875) 337-.

Focal properties of conics and quadrics. Booth, J. Ph. Mg. 27 (1845) 538-

- _ - Walker, John. Camb. and Dubl. Mth. J. 7 (1852) 16-.

- - - Ravier, -. N. A. Mth. 9 (1890) 233-

Foci and poles of conics and quadrics. Cauchy, A. L. C. R. 16 (1843) 809-.

Generation of conics and quadrics. Bretom [de Champ], $P$. N. A. Mth. 3 (1844) 289-.

- - - - (Jacobi). Waille, J. [1874] (xII) Doubs S. Mm. 9 (1875) 123-.

Homographic ray systems, theorem of Chasles. Bohannan, R. D. A. Mth. 1 (1884-85) 139-.

Hyperbola and hyperboloid, rectangular. Kulpper, C. Schlömilch Z. 3 (1858) 119-.

Imaginary projection of conics, quadrics and twisted quartic curves. Wiener, - D. Nf. Tbl. (1885) 354-.

Indices (powers) of points with regard to conics and quadrics. Faure, $H$. N. A. Mth. 11 (1872) 261-, 385-.

Intersections of lines and surfaces. Lamé, $G$. Gergonne A. Mth. 7 (1816-17) 229-.

Invariants of conics and quadrics given by motion and similitude groups. Lovett, $E$. $O$. A. Mth. 11 (1896-97) 33-.

Inverse of conics and quadrics. Vaněček, J.S. Prag Sb. (1883) (Mth. Vortr.) 28-.

- - - - from centre. Rice, M. E. Kan. Ac. Sc. T. 14 (1896) 14-.

Involutions arising from conics and quadrics. Bischoff, J. N. [1874] A. Mt. 6 (1875) 232.

$-1-C_{0}$ Cardinaal, $J$. Amst. Ak. Vs. 8 (1900) 271-; Amst. Ak. P. 2 (1900) 234 .

Loci, some, derived from conics and quadrics, cases. Azzarelli, M. Rm. N. Linc. At. 27 (1874) 216-.

-, quadratic, absolute classification. Story, IV. C. Am. J. Mth. 6 (1884) 222-。 


\section{Conics and Quadrics}

Oblique circular cones, plane sections. Pistoye, - de. (xI) Par. S. Mth. Bll. 1 (1873) 117-.

Parameters, variation. Rutherford, $W$. Mathematician 1 (1845) 116-.

Plane sections of cone of revolution, theorem. Rocha Peixoto, A. F. G. Teix. J. Sc. 3 (1881) 46-.

- - method. Dostor, G. J. Grunert Arch. 30 (1858) 185-.

Polar triangle and tetrahedron for conics and quadrics. Fiedler, $\mathrm{W}$. Schlömilch Z. 6 (1861) 140-.

Mth. 2 (1883) - - Humbert, G. N. A.

Mth. Ps. 31 (1886) 321-.

Polars, theory for conics and quadries. Lenthéric, -. Mntp. Ac. Sc. Mm. 1 (1847-50) 279-, 427-.

Poles of conics and quadrics, theory. Gergonne, J. D. Gergonne A. Mth. 3 (1812-13) 293-.

Polygons and polyhedra, maximum and minimum, in quadric curves and surfaces. Lindelöf, L. L. Helsingf. Öfv. 11 (1869) 53-.

Porisms, etc. Brougham and Vaux, (Lord). Phil. Trans. (1798) 378-.

Projective geometry of conics and quadrics. Del Re, A. Rv. Mt. 2 (1892) 138-.

Properties of conics and quadrics. Picquet, $H$. (xI) Par. S. Phlm. Bll. 2 (1865) 196-.

- - - new. Booth, J. Ph. Mg. 27 (1845) 538-.

- - curves and surfaces of 1st and 2nd degree. Cauchy, A. L. C. R. 16 (1843) 805-.

Reciprocal polar of conic and of quadric. Terquem, $O$. N. A. Mth. 16 (1857) 264-.

- propositions. Serret, $P$. Liouv. J. Mth. 6 (1861) 9-.

Representation in space of the ellipses drawn by an ellipsograph. Blake, $E, M$. A. Mth. 11 (1896-97) 195-.

Segments of ellipse, ellipsoid, and hyperboloid. Unferdinger, $F$. Grunert Arch. 28 (1857) $52-$.

- parabola and elliptic paraboloid. Unfer. dinger, F. Grunert Arch. 29 (1857) 209-.

Simultaneous transformation of two quadratic functions. Campbell, J.E. Mess. Mth. 21 (1892) 78-.

System of related conics and quadrics. Heiler. mann, -. Schlömilch Z. 6 (1861) 353-.

Teaching conics. Grunert, J.A. Grunert Arch. 17 (1851) 54-.

- -, comments on syllabus. Taylor, C. Mth. Gz. No. 5 (1895) 37-.

Theorems on conics and quadrics. Steiner, $J$. Crelle J. 1 (1826) 38-.

--- Faure, $H$. N. A. Mth. 5 (1866) 299-.

- Catalan, E. Brux. Ac. Bll. 23 (1892) 500-

(1893) 60 -

Barbarin, -. Mathesis 13

Triangle, trihedral angle and tetrahedron. Chasles, M. Gergonne A. Mth. 19 (1828-29) 65-.
Properties of Conics 7210

\section{Metrical and Projective Properties of Conics.}

Sturm, J. C. F. Gergonne A. Mth. 16 (1825 -26) 265- ; 17 (1826) 173-

Franchini, $P$. [1831] Lucca At. Ac. 8 (1835) 1-.

Terquem, O. Liouv. J. Mth. 2 (1837) 36.

Page, C. E. N. A. Mth. 1 (1842) 223-.

Obici, P. Pisa A. Un. Tose. Sc. Cosm. 1 (1846) 361-.

Le Poivre, -. Hain. Mm. S. 1 (1853) 146-

Grunert, J. A. Grunert Arch. 24 (1855) 370-

Maurin, H. (vir) A. Cond. Pon. Chauss. 6 (1862) 29-, 53-.

Taylor, C. Mess. Mth. 1 (1862) 250- ; 2 (1864) 64-, 65-.

Cremona, L. G. Mt. 1 (1863) 225- ; 2 (1864) 17-.

Michaelis, J. P. Lux. S. Sc. Nt. 6 (1863) 65-.

Burnside, W. S. [1865] QJ. Mth. 7 (1866) 227 .

Chasles, $M . \quad$ Rm. At. N. Linc. 18 (1865) 55-.

Bauer, G. [1867] Crelle J. 69 (1868) 293-.

Ovidio, E. d'. G. Mt. 6 (1868) 46-, 190-, 259-; 7 (1869) 1-.

Bäcklund, A. V. Stockh. Öfv. 28 (1871) 323-.

Morel, A. N. A. Mth. 10 (1871) 269-, 305-, $537-$.

Mittelacher, C. Z. Mth. Ps. 18 (1873) 1-。

Taylor, C. Mess. Mth. 2 (1873) 97-.

Lucas, É. N. A. Mth. 14 (1875) 265-, 458-.

Gundelfinger, S. N. A. Mth. 3 (1884) 7-.

Maley $x, L$. N. A. Mth. 9 (1890) 240-, 318-, 424-, 481-, 596- ; 10 (1891) 37-, 91-, 125-, 163-.

Lerch, $M$. Časopis 23 (1894) 151-, 223-, 288-; Fschr. Mth. (1893-94) 1107-.

Algebraic identity with 2 geometrical applications. Bromwich, T. J. I'A. Mess. Mth. 29 (1900) 184-.

Analytical methods, and solution of some problems. Poncelet, $J . V$. Gergonne A. Mth. 8 (1817-18) 141-.

- theory. Grunert, J. A. Grunert Arch. 31 (1858) 67-.

- - Schaar, M. Brux. Ac. Bll. 6 (1859) 42-.

- - Cayley, A. Phil. Trans. (1862) 639-.

- - Genese, R. W. Mess. Mth. 1 (1872)

104.

- - Hesse, L. O. Z. Mth. Ps. 19 (1874) 1-; 21 (1876) 1-.

- -. Jarolimek, Č. Časopis 19 (1890) 14-, 76-; Fschr. Mth. (1890) 723.

Anharmonic property. Graves, C. B. A. Rp. (1838) (pt. 2) 1-.

- ratio, sectional and tangential. Battaglini, G. Rm. At. R. Ac. 26 (1873) 565-.

- - of 4 straight lines through a point and touching two conics. Weyr, E. [1871] Crelle J. 75 (1873) 67-.

Arches, parabolic and elliptic. Haase, $H$. Förster Al. Bauztg. 48 (1883) 75-, 89-.

Asymptotes, circular, as coordinate axes. Lerch, M. Prag Sb. (1884) (Mth.-Nt.) 90-. 
Axes of conics. Lolli, C. G. Mt. 28 (1890) 193-. - _ - Jeřábek, $V$. Časopis 28 (1899) 351-; Fschr. Mth. (1899) 481-.

- - , determination. Pelz, C. Wien Ak. Sb. 73 (1876) (Ab. 2) 379-.

-, formulæ. Rochat, -. Gergonne A. Mth. 2 (1811-12) 331-.

-, - (Rochat, addition to). Gergonne, J. D. Gergonne A. Mth. 2 (1811-12) 335-.

-, - (Rochat and Dubourquet). Bret, Gergonne A. Mth. 3 (1812-13) 31-.

-, minor axis, determination from major axis and perimeter. Clément, L. A. Cond. Pon. Chauss. 8 (1864) 305-.

Central conics, trigonometrical formulæ. Egidi, G. Rm. N. Linc. At. 37 (1884) 295-.

Centres of similitude and radical axes. Collins, M. Camb. (M.) Mth. M. 1 (1859) 268-

-, theorems. Terquem, $O$. N. A. Mth. 4 (1845) 260-, 304-, 322-, 371-.

Chasles, application of a theorem of. Balitrand, -. Mathesis 14 (1894) 62-, 81-.

Chords, common, of conic and circle of curvature. Grunert, J. A. Arch. Mth. Ps. 50 (1869) 69-.

-,,$----1-$ zero radius. Ocagne, M. $d$ '. Edinb. Mth. S. P. 5 (1887) 84-.

- of conics and of parabola. Amodeo, F. A. Mth. 4 (1888) 92.

- at right angles, note. Steiner, J. N. A. Mth. 9 (1850) 407-.

-, supplemental. Ponte-Horta, F. da. Lisb. J. Sc. Mth. 2 (1870) 169-.

Conics determined by given Points or Tangents, and Methods of Determination.

Conics enveloped by rays joining homologous points, discrimination. Chrystal, $G$. Edinb. Mth. S. P. 2 (1884) 47-.

-, given centre and in-, circum-, or polar triangle, properties. Schroeter, $H$. Z. Mth. Ps. 29 (1884) 160-.

- - by a diameter and conjugate chord, relation of straight lines to. Barchanek, $C$. Wien Ak. Sb. 79 (1879) (Ab. 2) 712-.

- - imaginary points and tangents, construction. Staudigl, $R$. Wien Sb. 61 (1870) $(A b .2)$ 607-.

- - points or tangents. Jonquières, $E$. de. N. A. Mth. 18 (1859) 215-.

- through certain points, theorem. Valeri, $D$. Mod. Ac. Sc. Mm. 7 (1890) 181-.

- touching 4 lines. Arndt, F. Grunert Arch. 8 (1846) 342-.

Criteria for species of conic. Hart, H. Mess. Mth. 10 (1881) 90-.

- - - - - determined from points or tangents. Hunyady, J. (xir) Mag. Tud. Ak. Étk. (Mth.) 7 (1881) (No. 25) $17 \mathrm{pp}$.

Determination of conics. Dillner, $G$. Ups. Arsk. (1863) (Mth.) $45 \mathrm{pp}$.

9 line conic. Ladd, (Miss) C. Des Moines Anal. 7 (1880) 147-.

- - and 9 point conic. Cassani, $P$. G. Mt. 7 (1869) 369-; 8 (1870) 374-.
Number determined by points, tangents and normals. Wiman, A. Z. Mth. Ps. 40 (1895) 296-.

1 point and 3 tangents given, conics with. Cayley, A. QJ. Mth. 8 (1867) 220-.

2 points and 2 tangents given, conics with. Cayley, A. QJ. Mth. 8 (1867) 211-.

3 points and centre given, Steiner's criterion. Hunyady, J. [E. von]. [1880] (xII) Mag. Tud. Ak. Etk. (Mth.) 7 (1881) (No. 24) 13 pp. (x) Crelle J. Mth. 91 (1881) 248 -

_- _- - - Halphen, G. H. [1881] B. A. Rp. (1881) 532-; Par. S. Phlm. Bll. 6 (1882) 19-.

_ - - focus given, construction (Halley's problem). Housel, -. N. A. Mth. 14 (1855) 425-.

- - - - Grunert, J. A. Arch. Mth. Ps. 54 (1872) 99-

- _ - _ - theorems, proof. Jonquières, E. de. N. A. Mth. 14 (1855) 440-.

- - 1 tangent given, conics with. Cayley, A. (x) QJ. Mth.6 (1864) 24-.

--12 tangents given, conics with. Bruno, G. Tor. Ac. Sc. At. 17 (1881) 29-.

4 points and 1 tangent given, Newton's method. Grunert, J. A. Arch. Mth. Ps. 65 (1880) 1-. ---2 tangents given, conics with. Cayley, A. [1863] (vII) QJ. Mth. 8 (1867) 162-.

5 point conic. Grunert, J.A. Grunert Arch. 9 (1847) 293-; 24 (1855) 330-; 27 (1856) 178-. - - - Schlömilch, O. Leip. B. 7 (1855) 1-. - - Newton's 1st method. Grunert, J. A. Arch. Mth. Ps. 64 (1879) 337-.

- - or 5 line conic, Möbius's criteria. Hunyady, J. [E. von]. [1879] (xII) Mag. Tud. Ak. Étk. (Mth.) 7 (1881) (No.6) 15 pp.; (x) Crelle J. 89 (1880) 70-.

- - - - - - - for species. Durège, H. [1880] Wien Ak. Sb. 82 (1881) (Ab.2) 123-.

- points, conic through any, theorem. Budden, E. Mth. Gz. 1 (1900) 145-.

9 point conic. Beltrami, $E$. Bologna Mm. Ao. 2 (1862) 361-; G. Mt. 1 (1863) 109-, 208-, 354-.

- Grunert, J. A. Arch. Mth. Ps. 43 (1865) 54-

- - - Bôcher, M. A. Mth. 6 (1891-92) $132,178$.

- - Böger, R. [1898] Hamb. Mth. Gs. Mt. 3 (1900) 346-

- - - generalisation of Feuerbach and Steiner's theorems. Beltrami, $\boldsymbol{E}$. Bologna Ac. Sc. Mm. 5 (1874) 543-.

_- - and its stereometric sequel. Gretschel, H. Arch. Mth. Ps. 43 (1865) 293-.

Conics, given, to obtain from given cone. Ponte Horta, F. da. Lisb. Ac. Sc. Mm. 3 (Pt. 2) ("1865) No. 2, 14 pp.

-, and quadratic and cubic binary forms. Pittarelli, G. G. Mt. 21 (1883) 19-.

- - right cone, contributions to theory of. Ruth, F. Arch. Mth. Ps. 8 (1890) 1-.

- satisfying a certain condition. Bauer, $G$. [1867] Crelle J. 68 (1868) 293-. 
Conics satisfying 5 conditions, construction. Lenthéric, -. (vi Adds.) Mntp. Ac. Sc. Mm. 5 (1861-63) 438.

_- Chasles, M. C. R. 58 (1864) 297-, 1167-.

- - - - - - ; and applications of higher geometry. Jonquières, $\boldsymbol{E}$. de. N. A. Mth. 16 (1857) 116-.

Conjoint lines. Chasles, M. Liouv. J. Mth. 3 (1838) 385-.

Conjugate diameters, determination. Vauthier, - Toul. Mm. Ac. 3 (1834) 117-

- - properties. Dostor, G. N. A. Mth. 8 (1869) 481-.

- - , theorems in perspective, and focal properties of oblique cone. Chasles, $M$. Liouv. J. Mth. 2 (1837) 388-.

- pairs of points. Rosanes, J. Z. Mth. Ps. 17 (1872) 174 -.

Constant angle, staight lines cutting conics at. Schoute, P. H. Mathesis 7 (1887) 38-.

Construction by circles of curvature. Meyer, M. H. Grunert Arch. 24 (1855) 3-.

- and general equation of conics. Schmitt, C. Arch. Mth. Ps. 43 (1865) 365-.

- from imaginary elements. Ruth, $F$. Mh. Mth. Ps. 3 (1892) 81-.

- - - , with ruler. Spath, F. Mh. Mth. Ps. 1 (1890) 237-.

-,$---\ldots$ Machovec, $F$. Mh. Mth. Ps. 4 (1893) 95-.

-, mechanical. Guest, J.J. Cn. R. S. P. \& T. 2 (1896) (Sect. 3) 25-.

-, - methods. Jiirges, $W$. Z. Mth. Ps. 38 (1893) 350-.

-, new method. Transon, A. N. A. Mth. 12 (1873) 5-.

- from points, by means of planaltimetry. Rossi Re, $V$. de. Rm. N. Linc. At. 29 (1876) 240 .

- - - and tangents. Koutny, E. Wien Sb. 57 (1868) (Ab. 2) 469-.

-, problem. Jonquières, E. de. N. A. Mth. 18 (1859) 404-.

Constructions. Fiedler, $W$. Zür. Vjschr. 35 (1890) 322-.

-, and solutions of graphic problems. Genty, -. N. A. Mth. 19 (1880) 216-.

\section{Contacts.}

Plïcker, J. Gergonne A. Mth. 17 (1826-27) 37-.

(Chasles.) Cayley, A. B. A. Rp. 34 (1864) (Sect.) 1.

Wasserschleben, (Maj.) - von. Arch. Mth. Ps. 60 (1877) 410-.

Apollonian problem, extension, and conics. Fiedler, $W$. Zür. Vjschr. 24 (1879) 190-; 25 (1880) 217-.

Circles bitangent to conic. Heilermann, Crelle J. 56 (1859) 365-.

- Le Besgue, V. A. N. A. Mth. 4 (1865) 161-. $-\frac{1}{210-}-$. Sporer, B. Z. Mth. Ps. 41 (1896)

- - conics, theorem, etc. Steiner, J. Crelle J. 45 (1853) 189-.
Circles, 2, and ellipse, tri-tangential system. Avillez, J. F. d'. I,isb. J. Sc. Mth. 4 (1897) 166-; Fschr. Mth. (1896) 434.

Conics having double contact. Steiner, $J$. Crelle J. 45 (1853) 212-

- - - Cayley, A. QJ. Mth. 3 (1860) 246 - - - with another conic. Lefébure de Fourcy, R. N. A. Mth. 13 (1874) 513-.

- - - - two conics. Amodeo, F. G. Mt. 24 (1886) 346-.

- - - - - fixed conics, locus of their foci. Roberts, R. A. N. Y. Am. Mth. S. Bll. 2 (1896) 98-.

- - - - - - systems. Bonnesen, $T$. N. Ts. Mth. 9 (B) (1898) 25-.

- and pencil of lines, contacts. Jonquières, E. de. Liouv. J. Mth. 4 (1859) 49 -.

- through 3 points and having double contact with given circle. Laguerre, E. N. A. Mth. 19 (1880) 347-.

- touching given conics. Lachlan, $R$. Mess. Mth. 16 (1887) 140-

- $-2,3$, or 4 conics, construction. Niemt. schik, $R$. Wien Ak. Sb. 69 (1874) (Ab. 2) 845-.

Contact of 2 nd and 3rd orders. Retali, $V$. Mathesis 12 (1892) 178-, 219-.

- - 3rd order, porismatic property of conics having. Sylvester, J.J. (vI Adds.) Ph. Mg. 37 (1850) 438-.

- property of eleven-point conic. Allardice, R. E. Edinb. Mth. S. P. 10 (1892) 37-.

- relations of systems of circles and conics. Orr, W. McF. [1891] Camb. Ph. S. T. 16 (1898) 95-.

Double and four-point contact of two conics. Retali, V. Liège S. Sc. Mm. 18 (1895) No. 8, $35 \mathrm{pp}$.

Generalised problem. Jeffery, H. M. QJ. Mth. 23 (1889) 358-

\section{Curvature of Conics.}

(See also 8430.)

Servais, C. Brux. Ac. Bll. 19 (1890) 231-, 529-; 23 (1892) 243-.

Centres of curvature. Boschi, P. Bologna Ac. Sc. Mm. 3 (1881) 579-.

- - Machovec, F. Prag Sb. (1884) (Mth.Nt.) 345-; Fschr. Mth. (1885) 625-.

- - construction. Schirek, C. Arch. Mth. Ps. 3 (1886) 318-.

- - - - Janisch, E. Mh. Mth. Ps. 10 (1899) 182-

_ _ _ parallels to tangent through, theorem. Siacci, $F$. G. Arcad. 18 (1860) 166-.

Circle of curvature of ellipse, theorems. Janni, $V$. (vIII) Tortolini A. 4 (1861) 199-.

- through 3 points of ellipse and circle of curvature. Grunert, J. A. Grunert Arch. 37 (1861) 255-.

Circles of curvature. Weiler, A. Z. Mth. Ps. 34 (1889) 1-, 177-, 282-.

- _ - Lamarle's construction. Grunert, J. A. Grunert Arch. 30 (1858) 296-

Concurrent chords of curvature. Servais, C. Mathesis 17 (1897) 222-. 
Conic through 5 points, radius of curvature, construction. Rohn, $K$. Leip. Mth. Ps. B. 52 (1900) 17-.

Conics inscribed in triangle of reference, curvature. Longchamps, G. de. As. Fr. C. R. (1891) (Pt. 2) 11-.

Curvature properties of conics. Sobotka, J. Prag Sb. (1894) (Mth.-Nt.) No. 42, 9 pp.

- treated dynamically. Collignon, $\dot{E}$. As. Fr. C. R. (1898) (Pt. 2) 34-.

Formula (Neuberg). Gob, A. As. Fr. C. R. (1893) (Pt. 2) 254-.

Radii of curvature. Strehlke, $F$. Crelle J. 2 (1827) 380-.

- - Transon, (Prof.) A. Liouv. J. Mth. 1 (1836) 191-.

- - Gregory, D. F. (vi Adds.) Camb. Mth. J. 1 (1839) 61-.

- - Schlömilch, O. Schlömilch Z. 2 (1857) 187-.

- - Ribaucour, A. N. A. Mth. 7 (1868) 171-.

- - - case. Mannheim, A. Par. S. Mth. Bll. 18 (1890) 155-

- - - - Gob, A. Mathesis 14 (1894) 133-.

- - - construction, geometrical. Azzarelli, M. Rm. N. Linc. Mm. 11 (1895) 87-.

,---- , simple. Brix, A.F.W. Grunert Arch. 9 (1847) 316-.

- - - constructions as corollaries of a theorem of Steiner's. Pelz, C. Prag Sb. (1879) 205-; (1882) 3-.

- - - evaluation. Taylor, C. Mess. Mth. 3 (1866) 97-; 2 (1873) 142-.

- - - property. Steiner, J. Crelle J. 30 (1846) 271-

Curve resulting from binocular union of straight line with circular arc, or of 2 circular ares, form. Rogers, W. B. Edinb. N. Ph. J. 3 (1856) 210-

Cylindrical projection of conics. Glaser, $S$. Arch. Mth. Ps. 13 (1895) 113-.

Degenerate conjugate conics. Retali, $V$. Bologna Rd. (1886-87) 88-.

- forms. Hirst, T. A. L. Mth. S. P. 2 (1869) 166-.

Demonstration, new, of properties. Bobillier, -. Gergonne A. Mth. 18 (1827-28) 359-. ,,$---\frac{1}{(1847)} 36$. Olivier, $T$. Par. S. Phlm. PV.

Derivation from any one conic. Azzarelli, $M$. Rm. N. Linc. At. 43 (1890) 103-.

Diameter, constructions for. Taylor, C. Mess. Mth. 3 (1866) 5-.

Diameters. Painvin, L. N. A.Mth.4(1865) 498 -

-, elementary geometrical proofs. Zeuthen, H. G. (xII) Ts. Mth. 4 (1868) 145-.

Director (or orthoptic) circle. Lemoine, $\dot{E}$. N. A. Mth. 17 (1858) 240-.

$-(--)$-. Laguerre, E. N. A. Mth. 18 (1879) 204-.

$-(--)$ - of inscribed conic. Rouse, E.P. Mth. Gz. No. 2 (1894) 12-.

$-(--)-$, locus of vertices of right angles circumscribed to ellipse. Forçade-Prunet, $G$. N. A. Mth. 13 (1854) 192-.
Director (or orthoptic) circles of linear system of conics. Vries, J. de. Amst. Ak. Vs. 7 (1899) 371-; Amst. Ak. P. 1 (1899) 305-.

Eccentric circle of Boscovich. Langley, E. M. Mth. Gz. No. 1 (1894) 1-; No. 3 (1894) 17-.

\section{Ellipses.}

Noel, J. N. Liège Mm. S. Sc. 2 (1845-46) 145-.

Weddle, T. Mathematician 2 (1847) 179-.

(Sinram.) Jeřábek, W. [1879] Arch. Mth. Ps. 65 (1880) 215-.

Axes, construction. Tucker, R. [1865] Mess. Mth. 3 (1866) 151-.

-, - from 2 conjugate diameters. Broch, $O$. J. Crelle J. 40 (1850) 233-.

-, - - - - Seidelin, C.J. L. (xII) Ts. Mth. 6 (1870) 20-, 22-; 6 (1876) 63-.

-, - - - - Millar, J. B. Manch. Lt. Ph. S. P. 14 (1875) 89, 112-.

-, - _ - - (Millar's method). Rawson, R. Manch. Lt. Ph. S. P. 14 (1875) 98-.

- $-\ldots-\ldots$ Mannheim, A. N. A. Mth. 17 (1878) 529-; Mess. Mth. 11 (1882) 175-.

-, - when considered as a Lissajou's curve. Januschke, H. Arch. Mth. Ps. 60 (1877) $222-$

-, determination. Fialkouski, N. Wien SB. 20 (1856) 167-.

- and radii-vectores. Crocchi, $L$. G. Mt. 12 (1874) 375-.

Brocard's ellipse. Catalan, E. [1890] Brux. Ac. Mm. 49 (1890-93) (No. 3) 20 pp.

Circle and ellipse. Kilbinger, $G$. Ens. Mth. 1 (1899) 452-

- through feet of 3 normals to ellipse. Weill, M. N. A. Mth. 19 (1880) 60-.

- 3 points of ellipse, theorem. Joachimsthal, $F$. Crelle J. 39 (1850) 138-.

-, theorem of Euler, generalisation and extension to ellipse. Dostor, G. Arch. Mth. Ps. 51 (1870) 106-.

Circles, system of 286 , connected with ellipse. Böklen, O. Z. Mth. Ps. 28 (1883) 300-.

Conjugate diameters, problem. Berg, $F . J$. van den. Amst. Arch. Wisk. Gn. 2 (186063) 129-, 152-, 189-.

- - , solution of problem (claim of priority for Le Poivre). Le Paige, $C$. Hain. S. Mm. 10 (1888) 587-.

Construction. Faconnet, - (vII) A. Cond. Pon. Chauss. 3 (1859) 381- ; 5 (1861) 187-.

- of ellipse and of circle. Fialkowski, $N$. Wien SB. 16 (1855) 9-.

Constructions. Staudigl, $R$. Wien Sb. 59 (1869) (Ab. 2) 189-.

Cotes's theorem extended to ellipse. Grunert, J. A. Grunert Arch. 30 (1858) 104-.

"Deviation" angle. Ocagne, M. d'. N. A. Mth. 7 (1888) 268-.

- in the ellipse. Ocagne, M. $d$ '. N. A. Mth. 5 (1886) 370 -.

- - Mannheim, A. N. A. Mth. 16 (1897) 249-.

Ellipses of equal probability. Bréger, $P$. Rv. Artl. 10 (1877) 82-. 
Ellipses, 2, or ellipse and parabola, with common focus. Bratt, (Prof.) - Stockh. Ac. Hndl. 24 (1803) 108-.

Envelope of perpendiculars to radii. Aynard, -. N, A. Mth. 5 (1846) 540-, 582-.

Fermat, theorem of, extended to ellipse. Schlömilch, O.X. Z. Mth. Ps. 19 (1874) 462.

In- or circum-triangles and quadrilaterals, area. Grunert, J. A. Grunert Arch. 30 (1858) 11-.

In-ellipse of circle with centre and tangent given, construction. Niemtschik, $R$. Wien Sb. 68 (1873) (Ab. 2) 377-.

Leonardo da Vinci's construction. Rulf, $W$. Mh. Mth. Ps. 9 (1898) 30-.

Maximum ellipse touching 4 lines. Seydervitz, F. Grunert Arch. 12 (1849) 44-.

- or minimum ellipse through 2 points and touching 2 lines. Seydevitz, F. Grunert Arch. 14 (1850) 364.

Mean radius vector. Dubois, $E$. A. Gén. Civ. 2 (1863) 193-; Les Mondes 3 (1863) 330-.

Minimum ellipse through 3 given points. Dantscher, V. von. Wien Ak. Sb. 99 (1891) (Ab. 2a) 10-; 104 (1895) (Ab. 2a) 301-.

- - 4 given points. Hearn, G.W. Mathematician 2 (1847) 315-.

$-\ldots$ Anger, C. T. As. Nr. 26 (1848) 35-.

New property. Auria, L. d'. Franklin I. J. 123 (1887) 27-

- - and its application to the "oval chuck". Leavitt, $F$. M. Franklin I. J. 81 (1881) 114-.

Parallel and different ellipses impossible, proof. Hyde, E. W. Des Moines Anal. 2 (1875) 144.

Parallels. Gomes Teixeira, F. Brux. Mm. Cour. $8^{\circ}, 58$ (1898-99) No. 5, 39 pp.

Point-circle and ellipse. Davis, R. F. Edinb. Mth. S. P. 13 (1895) 105-

Projection, application. Ferriot, - Gergonne A. Mth. 2 (1811-12) 240-

- of circle, ellipse considered as, etc. Jullien, A. N. A. Mth. 14 (1875) 324-, 359-

- - - - - - Kantor, S. Z. Mth. Ps. 23 (1878) 414-; 24 (1879) 54-; 25 (1880) $54-$.

Properties. Brasseur, L. N. A. Mth. 3 (1864) 111-.

- of ellipse, and trisection of an angle. Böklen, O. Grunert Arch. 30 (1858) 434-.

Ptolemy's theorem extended to ellipse. Grunert, J. A. Grunert Arch. 30 (1858) 109-.

Radial. Tucker, R. QJ. Mth. 18 (1882) 311-.

Radius of curvature, construction, Transon, (Prof.) A. Par. S. Phlm. PV. (1814) 85-.

Rectilinear figure, in- or circum-scribed. Lindman, $C$. F. Ts. Mt. Fys. 2 (1869) 119-.

Relation between points of intersection of tangents and of normals at ends of a chord. Bruno, $G$. Tor. At. Ac. Sc. 8 (1872-73) 90-.

Remarkable points, four. Osengu, $G$. Palomba Rac. 3 (1847) 353-.

Sectors and segments with reference to conjugate diameters. Zehme, $W$. Schlömilch Z. 3 (1858) 311-。

Singular case. Fuss, N. [1798] St Pet. Ac. Sc. N. Acta 15 (1806) 71-.
Theorems. Steiner, J. Crelle J. 32 (1816) 300-. - (Steiner). Clausen, T. Grunert Arch. 9 (1847) 259-.

Theory deduced from a transformation of circle. Lenthéric, -. (vi Adds.) Mntp. Ac. Sc. Mm. 5 (1861-63) 25-.

Trigonometry of ellipse. Malagoli, R., \& Mannei, E. G. Mt. 27 (1889) 60-.

Envelope, case. Wolstenholme, J. [1883] L.Mfth. S. P. 15 ("1883-84) 48-

- of chord of circle, conics as. Unferdinger, F. Grunert Arch. 34 (1860) 406-.

- - subtending constant angle. Breton [de Champ], P. N. A. Mth. 4 (1845) 300-.

- chords of constant length. Trudi, N. Nap. Rd. 1 (1862) 7-.

- - - Weyr, E. [1871] Z. Mth. Ps. 17 (1872) 164-

- circle whose diameter is focal radius of conic. Petersen, J. P. C. (xII) Ts. Mth. 3 (1867) 24-; 5 (1869) 140 ; 2 (1878) 178-.

- line cut by two circles harmonically. Cazamian, A. N. A. Mth. 13 (1894) 218-.

- - - cutting off intercepts whose sum is constant on two lines at right angles. Wolfers, J. P. Grunert Arch. 25 (1855) 109-.

- - of given length inscribed in angle. Joachimsthal, $F$. N. A. Mth. 6 (1847) 260-.

Envelopes of lines making constant angle with tangents to conic. Del Re, A. G. Mt. 22 (1884) 75-.

- variable lines. Ocagne, M. d'. N. A. Mth. 5 (1886) 88-.

Equations and Coordinates.

De Morgan, A. [1830] Camb. Ph. S. T. 4 (1833) 71-.

Ampère, A. M. Quetelet Cor. Mth. 10 (1838) 90-.

Finck, B. N. A. Mth. 3 (1844) 401-, 573-.

Finlay, R. [1851] Manch. Ph. S. Mm. 10 (1852) 33-.

Battaglini, G. Nap. Rd. 1 (1862) 24 .

Grunert, J. A. Arch. Mth. Ps. 51 (1870) 257-, 276-, 326-.

Mitchell, O. H. [1883] A. Mth. 1 (*1884-85) 78-, 103-.

Peed, M. T. [1886] J. H. Un. Cir. [6] (1886 -87) 81.

Weyr, E. Časopis 16 (1887) 97-, 145-, 202- ; Fschr. Mth. (1887) 796.

Axes, direction of, in oblique coordinates. Aller, C. van. Fschr. Mth. (1900) 577.

-, equation. Hensley, P. J. QJ. Mth. 5 (1862) 273-.

-, -. Whitworth, W. A. QJ. Mth. 5 (1862) $335-$.

-, - Thomson, F. D. (v) Mess. Mth. 2 (1864) 16-

280 -

Stolz, O. Wien Sb. 55 (1867) (Ab. 2)

-, C. C., J. B. Cn. J. 11 (1868) 388-

-, -. Thomson, F. D. Mess. Mth. 5 (1871)

65 -.

Walker, J.J. QJ. Mth. 15 (1878) 30-. 
Axes in space, equation. Geiser, C. F. [1876] A. Mt. 8 (1877) 113-.

Biangular coordinates. Jeffery, $H . M$. [1874] QJ. Mth. 13 (1875) 130-.

Chord, polar equation. Frost, $P$. Camb. and Dubl. Mth. J. 1 (1846) 68-.

Condition that 6 points lie on a conic, forms of equation. Hunyady, J. [E.von]. (xm) Mag. Tud. Ak. Étk. (Mth.) 4 (1877) (No. 6) 23 pp.; 5 (1879) (No. 4) 20 pp.; (x) Crelle J. Mth. 83 (1877) 76- ; 92 (1882) 307-.

Conic through 5 points, equation. Gaskin, $T$. Mathematician 3 (1850) 134-.

A. Mth. 16 (1857) 418-.

Conics, $(p, r)$ equation. Warren, J. W. QJ. Mth. 13 (1875) 16-.

- touching several straight lines, form of equations. Vieille, J. N. A. Mth. 20 (1861) 236-.

Determination of equation. Raymond, G. $M$. Gergonne A. Mth. 1 (1810-11) 180-, 233-.

Director circle, equation. Wolstenholme, (Rev.) J. L. Mth. S. P. 13 (1881-82) 185-.

Directrices and foci, equations. Eurenius, A. G.J. [1873] (Ix) QJ. Mth. 13 (1875) 198 .

- - - - Wolstenholme, (Rev.) J. L. Mth. S. P. 11 (1879-80) 102-.

- latera recta, and note on director circle. Genese, R. W. B. A. Rp. (1880) 480-.

Discussion from equations of axes. Guillaumet, -. N. A. Mth. 16 (1857) 59-.

Ellipse, area, general equation. Grunert, $J . A$. Arch. Mth. Ps. 53 (1871) 390-

-, equation to system of 4 normals. Crofton, -. N. A. Mth. 1 (1862) 449-.

Elliptic and hyperbolic sectors, analogous properties of coordinates. Wallace, (Prof.) W. [1839] Edinb. R. S. T. 14 (1840) 431-.

Equation, certain forms. Cayley, A. QJ. Mth. 2 (1858) 44-.

- of 4th order (Gerono). Tychsen, C. Mth. Ts. 1 (1859) 141-.

Equations, and relations of identity. Terquem, O. N. A. Mth. 1 (1842) 489-; 2 (1843) 26-, 106-, 300-, 425-, 532-; 3 (1844) 416-, 510-; 4 (1845) 14-; 5 (1846) 618-; 7 (1848) $308-, 409-$.

Focal coordinates and equations to axes in trilinear coordinates. Stoll, -. Z. Mth. Ps. 38 (1893) 282-.

Foci of conics in trilinears. Ferrers, N. $M$. QJ. Mth. 4 (1861) 235-.

Homogeneous co-ordinates and invariants. Weyr, E. Casopis 24 (1895) 1-, 81-; Fschr. Mth. (1895) 667-.

In-polygons of conics, analytical equations for sides. Härtenberger, $G$. Wien SB. 20 (1856) 541-.

Parabola and ellipse, polar equations. Grunert, J. A. Grunert Arch. 1 (1841) 322-.

-, equation, symmetrical form. $G$. Camb. Mth. J. 2 (1841) 14-.

Points of intersection and common tangents of two conics in trilinear coordinates. Versluys, J. Arch. Mth. Ps. 52 (1871) 377-.
Polar coordinates. Rubini, R. Tortolini A. 4 (1853) 1-.

- equation, deduction of properties from. Hittorf, $W$. Crelle J. 38 (1849) 89-.

Reciprocal of equation. Cayley, $A$. Mess. Mth. 1 (1872) 120.

Reduction. Cherriman, J. B. Cn. J. 1 (1856) 286-.

Straight lines drawn from point to intersections of conic with straight line, equation. Dostor, G. Arch. Mth. Ps. 69 (1883) 427-.

Tangential equation. Terquem, $O$. N. A. Mth. 7 (1848) 277-.

- C. Cayley, A. Crelle J. 39 (1850) 1-.

Tangents to conics, polar equation. Frost, $P$. (vi Adds.) Camb. Mth. J. 4 (1845) 112-.

- from a point to a conic, equation. Dostor, G. Arch. Mth. Ps. 57 (1875) 191-.

Transformations of equation. Martynonski, $J$. Liège Mm. S. Sc. 1 (1843) 177-.

Evolution (quasi involution). Folie, $F$. Brux. Ac. Bll. 43 (1877) 500-; 44 (1877) 181-.

Foci.

Morton, P. [1829] Camb. Ph. S. T. 3 (1830) 185-.

(Apollonius.) Terquem, O. N. A. Mth. 3 (1844) 412-.

Chasles, M. C. R. 22 (1846) 894-.

Humbert, $E$. N. A. Mth. 4 (1885) 138-.

Schoute, P. H. Mathesis 11 (1891) 129-

Cone of revolution, section of, construction for foci. Engler, E. A. [1892] St Louis Ac. T. 6 (1895) 49-.

Cones and cylinders, circular, locus of foci of sections through given point, etc. Schubert, E. Gould As. J. 4 (1856) 130-.

Conic with given focus and triple contact with given conic. Hofmann, F. [1881] Arch. Mth. Ps. 67 (1882) 332-.

- satisfying 3 conditions, formulæ connecting foci. Burnside, W. S. QJ. Mth. 8 (1867) $31-$.

Cormet's focal theorem. Dewulf, -. N. A. Mth. 17 (1858) 435-.

Determination. Hensley, P. J. [1861] QJ. Mth. 5 (1862) 177-.

- Salmon, G. QJ. Mth. 5 (1862) 307-.

- St. Germain, A. de. N. A. Mth. 8 (1869) 230 -.

- Dostor, G. Arch. Mth. Ps. 69 (1883) 432-.

- Lez, H. As. Fr. C. R. (1895) (Pt. 2) 267-.

-, analytical. Bret, -. Gergonne A. Mth. 8 (1817-18) 317-.

,-- , and definition. Emelen, L. van. Ens. Mth. 2 (1900) 423-.

- of foci and axes. Smith, C. A. QJ. Mth. 12 (1873) 240-

- - directrices, and axes (Plücker). Lac de Bosredon, V. Mathesis 10 (1890) 265-.

- - - - - - characteristics. Grunert, J. A. Grunert Arch. 25 (1855) 262-; 30 (1858) 474-. 
Determination of foci and lengths of axes. Serret, $P$. N. A. Mth. 7 (1848) 302-.

-, new. Dostor, G. Arch. Mth. Ps. 62 (1878) 289-.

- and properties. Letnikow, A. V. N. A. Mth. 20 (1881) 289-.

Distances from 4 points or 4 tangents. Antomari, X. N. A. Mth. 2 (1883) 193-, 337-, 385-.

Ellipse, foci, theorem. Serret, P. N. A. Mth. 4 (1845) 648-.

Focal circles. Mention, J. [1857] St Pét. Ac. Sc. Bll. 16 (1858) 29-.

- - angular properties. Barbarin, $P$. As. Fr. C. R. (1898) (Pt. 2) 132-.

- curves of families of conics. Bobek, $K$. Mh. Mth. Ps. 3 (1892) 309-.

- - Quetelet's, of conics in right cones. Pelz, C. Wien Ak. Sb. 97 (1889) (Ab. 2a) 1411-.

- line, parabolic. Dandelin, G. P. Brux. Mm. Ac. Sc. 2 (1822) 171-.

- properties. Grunert, J.A. Arch. Mth. Ps. 54 (1872) 183-.

- - Laisant, C. A. Par. S. Phlm. Bll. 2 (1890) 182-.

- - of conics in projective metrics. Ovidio, E. $d$ '. Tor. Ac. Sc. At. 26 (1891) 339-.

- - Dupin's space-extension of. Larmor, J. L. Mth. S. P. 18 (1886-87) 363-.

Foci and directrices. Davies, T. S. Ph. Mg. 22 (1843) 25-.

- - - of conies represented by general equation, etc. Dostor, G. Arch. Mth. Ps. 63 (1879) 113-.

- focal circles. Antomari, X. N. A. Mth. 1 (1882) 102-.

- - lines. Chasles, M. Brux. Ac. Bll. 2 (1835) 35-.

Generalisation of theory. Transon, (Prof.) $A$. Liouv. J. Mth. 4 (1839) 457-.

Imaginary foci of central conics, properties. Rogel, $F$. Arch. Mth. Ps. 13 (1895) 297-.

- line through circular point at infinity. McMahon, J. A. Mth. 4 (1888) 91.

In- and circum-conics of triangle, four groups of foci. Lemoine, E. As. Fr. C. R. (1883) $122-$.

Locus problem. Terquem, 0 . N. A. Mth. 9 (1850) 252-.

Parabola, focal property. Retali, V. G. Mt. 22 (1884) $217-$.

-, foci and directrices. Terquem, O. N. A. Mth. 8 (1849) 121-.

-, -, theorem. Serret, P. N. A. Mth. 4 (1845) 652-.

Pedal of conic referred to focus. Picquet, $H$. N. A. Mth. 5 (1866) 145-.

- curves, foci. Ruffini, F. Bologna Rd. (1891-92) 60-.

Points of contact of inscribed spheres, foci as, demonstration of theorem. Fresenius, $F$. $C$. Arch. Mth. Ps. 46 (1866) 321-.

- in which pairs of corresponding points of two systems in circular relationship are united, foci as. Siebeck, $H$. Grunert Arch. 33 (1859) 462-.
Trilinears, foci, axes and asymptotes in. Jeffery, H. M. QJ. Mth. 8 (1867) 348-.

Formulæ, elementary. Beltrami, E. G. Mt. 9 (1871) 341-.

General properties. Chasles, M. Quetelet Cor. Mth. 5 (1829) 6-.

- - Booth, J. Ir. Ac. P. 1 (1841) 53-.

- property. Molins, $H$. Toul. Mm. Ac. 4 (1854) 193-.

Generation of conics by circle or other conics, etc. [Schiappa] Monteiro, A. G. Teix. J. Sc. 4 (1883) 95-.

- - - elementary geometry. Derode, $V$. Lille Mm. S. (1838) 25-.

- - - - intersection of 2 straight lines. Raymond, G. M. Gergonne A. Mth. 2 (1811-12) 360-.

- method for conics, generalisation. Schlegel, S. F. V. Z. Mth. Ps. 23 (1878) 402-.

Harmonic middle points of second degree, series of. Slawyk, R. Z. Mth. Ps. (Suppl.) 29 (1884) 1-.

- and other properties of conics. Sollertinsky, -. Mathesis 11 (1891) 177-.

Homographic figures, theorem. Cayley, $A$. QJ. Mth. 3 (1860) 177-.

- theorem. Terquem, $O$. N. A. Mth. 7 (1848) 447- ; 8 (1849) 96-.

Homography and duality applied to metric properties of plane. Ripert, L. N. A. Mth. 18 (1899) 101-.

\section{Hyperbolas.}

Apollonian hyperbola, property deduced from analytical geometry. San Bertolo, N. Rm. At. N. Linc. 19 (1866) 101-.

Conjugate hyperbolas. T. (vi Adds.) Gleanings Sc. 1 (1831) 161-, 213-.

Construction. Seipp, H. Arch. Mth. Ps. 5 (1887) 172-.

Properties, general. Roberts, $W$. Ir. Ac. P. 4 (1850) 247-.

Property. De Morgan, A. (vi Adds.) Ph. Mg. 33 (1848) 546-.

Quadrature. Giannattasio, $F$. Nap. At. Ac. 1 (1819) 317-.

Rectangular hyperbola. Bobillier, -. Gergonne A. Mth. 19 (1828-29) 349-.

- - Mention, J. N. A. Mth. 4 (1865) 30-.

- -. Taylor, C. Mess. Mth. 1 (1872) 121-.

- - Servais, C. Brux. Ac. Bll. 19 (1890) $759-$.

- - and circle, analogies. Transon, (Prof.) A. N. A. Mth. 5 (1846) 535-.

- - determination from 4 conditions. Brianchon, C. J., \& Poncelet, - Gergonne A. Mth. 11 (1820-21) 205-.

,$---1-1-$ Seydevitz, F. Grunert Arch. 3 (1843) 225-.

- - and its inverses. Cazamian, A. N. A. Mth. 13 (1894) 265-.

- - position in cone. Weyer, G. D. E. Arch. Mth. Ps. 14 (1896) 139-. 
Imaginary points on conics. Schou, (Lt.) T. V. (xII) Ts. Mth. 1 (1865) 93-.

- projection. Retali, $V$. Bologna Ac. Sc. Mm. 7 (1886) 601-.

In- and circum-conies. Steiner, J. G. Arcad. 99 (1844) 147-.

- - (Steiner's theorems, proofs). Mathews, G. B. $[1890]$ L. Mth. S. P. 22 (1891) 18 -

- - - and conjugate conics. Schroeter, E. G. Mt. 22 (1884) 262-.

In-conics of conics, construction. Niemtschik, R. Wien Sb. 67 (1873) (Ab. 2) 295-, 505-; 71 (1875) (Ab. 2) 435-.

Indicatrix in conic sections. Haas, A. Z. Mth. Ps. 34 (1889) 65-.

Intersection of conics and circles, construction. Niemtschik, R. Wien Sb. 59 (1869) (Ab. 2) 39-.

- - - construction. Lazarski, M. (xп) Krk. Ak. (Mt.-Prz.) Rz. \& Sp. 8 (1881) 141-. - ellipse with circle and rectangular hyperbola. Vries, J. de. N. Arch. Wisk. 3 (1898) 160- ; Fschr. Mth. (1898) 528-.

- - straight line with conics, construction. Koutny, E. Wien Sb. 56 (1867) (Ab. 2) 303-.

- - - lines with conics, construction. Peschka, G. A. von. Arch. Mth. Ps. 59 (1876) 18-.

Ps. 66 (1881) 1 -

- _ _ _ - and integral formula. Cayley, A. [1862] (vII) QJ. Mth. 7 (1866) 1-.

Intersections of central conic with the 4 normals from any point in its plane. Bruno, $G$. Tor. Ac. Sc. At. 11 (1876) 597-.

- conic with 6 other conics. Lachlan, $R$. Mess. Mth. 15 (1886) 155-.

Involution of several points on a conic. Weill, M. N. A. Mth. 1 (1882) 62-.

Involutions on conics, construction of algebraic expressions by help of. Kotányi, $\mathbf{L}$. Z. Mth. Ps. 27 (1882) 248-.

- - Desargues's theorem. Poudra, N. G. N. A. Mth. 3 (1864) 202-.

- - - - Picquet, H. N. A. Mth. 5 (1886) 163-.

- - - - - demonstration. Weiler, A. Z. Mth. Ps. 24 (1879) 248 -

Isotomic figures, inscription. Gruson, $J . P$. Berl. Ab. (1824) 83-.

Latus rectum, or sections of given eccentricity, cutting from cone. Engler, E. A. St Louis Ac. T. 6 (1895) 183-.

Limiting cases of conics. Thomson, $F$. $D$. Mess. Mth. 4 (1868) 86-.

- - - . Salmon, G. Mess. Mth. 4 (1868) 129-.

- - Taylor, C. Mess. Mth. 4 (1868)

- Whitworth, W. A. Mess. Mth. 4 (1.868) 148-.

Loci and envelopes of 1 st and 2 nd degree, in projective geometry. Ovidio, E. $d^{\prime}$. Nap. Rd. 14 (1875) 103-.

- of poles of certain chords. Desboves, A. H. N. A. Mth. 19 (1860) 253-.

- - one conic with respect to another. Hermite, C. N. A. Mth. 1 (1842) 263-.
Loci of poles of variable circle etc., hyperbolas and parabolas as. Olivier, $T$. Quetelet Cor. Mth. 5 (1829) 51-.

Locus. Lhuilier, S. Gergonne A. Mth. 2 (1811-12) 173-.

- of centres of in-circles of triangles on line joining foci. Ferroni, $P$. Mod. Mm. S. It. 19 (1821) 377-.

- from 2 conics. Cayley, A. QJ. Mth. 8 (1867) 77-.

- of an eye which sees, (1) an ellipse (2) a hyperbola reflected in a perpendicular plane as a circle; problems of Steiner. Lazarski, M. Krk. Ak. (Mt.-Prz.) Rz. 18 (1888) 76-; Fschr. Mth. (1888) 806-.

- - points having constant chord of contact with reference to conic. Zahradnik, $K$. Arch. Mth. Ps. 61 (1877) 220-

- of vertex of angle with sides tangent to 2 conies. Caqué, J. N. A. Mth. 1 (1862) 402-.

- - - - triangle of constant perimeter formed by 2 tangents and chord of contact. $P ., L$. N. A. Mth. 12 (1873) 401-.

- - vertices of given ellipse and hyperbola, problem. Rispal, A. N. A. Mth. 2 (1843) $357-$.

Maxima and minima, certain. Duthie, $G$. Edinb. Mth. S. P. 12 (1894) 26-.

- - -, problems. Lehmus, -. Grunert Arch. 28 (1857) 249-.

Mean proportionals, two, Arabian construction for. Carra de Vanx, 一. Bb. Mth. (1898) 3-.

- - - , and other problems. Laab, C. Arch. Mth. Ps. 11 (1892) 262-.

Metrical properties. Dostor, G. Arch. Mth. Ps. 61 (1877) 171-.

- theorems. Poncelet, J. V. Gergonne A. Mth. 8 (1817-18) 1-.

Middle points of chords common to conic and its circle of curvature, locus. Zimmermann, J. Arch. Mth. Ps. 70 (1884) 38-.

- - - - through fixed point, locus. Wunder, C. G. Grunert Arch. 5 (1844) 361-.

- - - - - - [Am] Ende, $H$. Arch. Mth. Ps. 42 (1864) 98-.

- - - - - - - Lommel, E. Arch. Mth. Ps. 43 (1865) 231-.

_ _ - parallel chords, if collinear, curve is a conic, theorem. Bertrand, $J$. Liouv. J. Mth. 7 (1842) 215-.

Newton's influence on modern geometry. Graham, R. H. Nt. 42 (1890) 139-.

Non-Euclidean properties. Story, $\dot{W}$. $E$. Am. J. Mth. 5 (1882) 358-; (xm) J. H. Un. Cir. [2] (1883) 71-.

\section{Normals.}

Gerono, G. C. N. A. Mth. 2 (1843) 16-, 72-, 170 -

(Gerono.) Grunert, J. A. Grunert Arch. 6 (1845) 127-.

Pistoris, - de. N. A. Mth. 7 (1848) 246-.

Catalan, E. C. N. A. Mth. 7 (1848) 332-, 396-.

Cayley, A. Crelle J. 56 (1859) 182-. 
Grunert, J. A. Grunert Arch. 32 (1859) 129-.

Cremona, L. [1864] Mess. Mth. 3 (1866) 88Eckardt, $F$. E. Z. Mth. Ps. 11 (1866) 311-.

Geiser, C. F. Crelle J. 65 (1866) 381-.

Weyr, E. Z. Mth. Ps. 16 (1871) 440-.

Roberts, S. [1877] L. Mth. S. P. 9 (1877-78) 65-.

Gohierre de Longchamps, -. As. Fr. C. R. 7 (1878) 49-.

Pelišek, M. Prag Sb. (1883) (Mth. Vortr.) 126-.

Engler, E. A. St Louis Ac. T. 8 (1898) 137-.

Leau, - Par. S. Phlm. Bll. 2 (1900) 74-:

Apollonius, problem of, Legendre's solution. Drobisch, $M . W$. Leip. B. 8 (1856) 103-.

-, - -, - - Möbius, A. F. Leip. B. 8 (1856) 113-.

Construction. Martynonski, J. Liège Mm. S. Sc. 4 (1847) 1-.

-, geometrical. Painvin, L. N. A. Mth. 9 (1870) 348-.

Ellipse, normals. Lauermann, C. [1880] Wien Ak. Sb. 83 (1881) (Ab. 2) 92-; Z. Mth. Ps. 26 (1881) 387-.

-, - Anglin, A. H. Edinb. Mth. S. P. 15 (1897) 70 -

-, - , and equation of $4 \mathrm{th}$ degree, two cases. Waille, J. [1873] (xII) Doubs S. Mm. 8 (1874) 52-.

-, - Frégier's theorem, etc. Guimarães, $R$. As. Fr. C. R. (1893) (Pt. 2) 89-.

-, - , problem. Pelz, C. Wien Ak. Sb. 95 (1887) (Ab. 2) 481-.

-, - - - Lauermann, C. Wien Ak. Sb. 98 (1890) (Ab. 2a) 318-.

-,,- - of complete ellipse. Pelz, C. Wien Ak. Sb. 96 (1888) (Ab. 2) 387-.

-, 一, property. Böklen, O. N. A. Mth. 17 (1858) 356-.

,-- , theorems. Weill, M. N. A. Mth. 20 (1881) 73-, 110-.

Frégier point. Gérard, G. Mathesis 19 (1899) 136-.

Hyperbola, normals, problem. Lauermann, $K$. Wien Ak. Sb. 107.(1898) (Ab. 2a) 861-.

Joachimsthal's circle, equation. Guimaraes, R. Fschr. Mth. (1900) 586.

- theorems on normals, etc. Laguerre, $E$. Par. S. Mth. Bll. 5 (1877) 92-.

Normals common to 2 conics. Tucci, F.P. [1814] (xIr) Nap. S. Pont. At. 4 (1847) 253-.

- from point to conic. Laguerre, E. C. R. 84 (1877) 181-; Par. S. Mth. Bll. 5 (1877) 30 -

- - - construction. Joachimsthal, $F$. Crelle J. 48 (1854) 377-.

- - - - - Grunert, J.A. Arch. Mth. Ps. 43 (1865) 26-.

- - _ - - - (Joachimsthal). Pelz, C. Prag Sb. (1895) (Mth.-Nt.) No. 20, 4 pp.

Parabola, normal chords. Ocagne, M. d'. N. A. Mth. 15 (1896) 274 -

-, normals, problem. Zahradnik, K. Prag Sb. (1879) 98-.

Problem. Pelz, C. Wien Ak. Sb. 85 (1882) (Ab. 2) 169-.
Problem. Mertens, F. Wien Ak. Sb. 98 (1890) (Ab. 2a) 431-.

-. Schoute, P. H. Wien Ak. Sb. 98 (1890) (Ab. 2a) 1519-.

-. Schiappa Monteiro, A. [1900] Lisb. J. Sc. Mth. 6 (1902) 65-.

Properties, three. Zampieri, $J$. Grunert Arch. 32 (1859) 319-.

Orthotomic circle, Salmon's equation. Cayley, A. QJ. Mth. 1 (1857) 242-.

Osculating circle, construction. Plicker, $J$. Gergonne A. Mth. 17 (1826-27) 69-.

- circles of conics. Zimmermann, $J$. Arch. Mth. Ps. 70 (1884) 30-.

- - ellipse, 3, theorem of Steiner. Joachimsthal, F. Crelle J. 36 (1848) 95-.

- conics. Dewoulf, $\dot{E}$. Mathesis 10 (1890) 55-.

- - certain. Jonquières, E. de. N. A. Mth. 4 (1865) 504.

Osculation. Olivier, T. [1838] Par. S. Phlm. PV. (1838) 65-; Liouv. J. Mth. 4 (1839) 189-. -, triple. Zahradnik, $K$. Arch. Mth. Ps. 69 (1883) 419-.

Pappus's problem and conics. Brändli, $H$. Grunert Arch. 38 (1852) 1-.

- theorem generalised. Folie, F. Brux. Ac. Bll. 28 (1869) 87-.

\section{Parabola.}

Méray, C. N. A. Mth. 13 (1854) 41-.

Worthington, R. B. Mess. Mth. 5 (1871) 95-, 166-.

Apollonian parabola, inseription of circle in. Frigelius, A. Stockh. Ak. Hndl. 28 (1807) 229-.

Arc in terms of chord and sagitta. Portet, (vii) A. Cond. Pon. Chauss. 3 (1859) 121-.

Area of parabolic sectors, Lambert's theorem. Grunert, J. A. Crelle J. 16 (1837) 21-; Grunert Arch. 16 (1851) 439-.

- - triangle formed by chord and 2 secants. Dostor, G. Arch. Mth. Ps. 53 (1871) 100-.

Chord, common, of parabola and its circle of curvature. Graves, R.H. A. Mth. 3 (1887) 50.

Circle of curvature. Mack, L. Arch. Mth. Ps. 61 (1877) 385-.

Co-normal points of parabola. Tucker, $R$. Edinb. Mth. S. P. 13 (1895) 29-.

- - - - some properties. Tucker, R. [1890] L. Mth. S. P. 21 (1891) 442-

Construction from 4 conditions. Coste, L.M.P. Gergonne A. Mth. 8 (1817-18) 261-.

- - given points and tangents. Koutny, $E$. Wien Sb. 63 (1871) (Ab. 2) 760-.

- through 4 points. Umpfenbach, (Dr) -. Crelle J. 26 (1843) 183-.

Differential curve, polo-conic of 2nd degree. Hochheim, A. Arch. Mth. Ps. 57 (1875) 234 -

Miquel-Clifford theorem concerning parabolas touching three given lines, analogues. Serret, P. C. R. 123 (1896) 415-.

Miquel's theorem, demonstration. Clifford, W. K. Mess. Mth. 5 (1871) 124-.

- - - (Clifford). Ferrers, N. M. Mess. Mth. 5 (1871) 204 - 


\section{Properties of Conics}

Parabola connected with conic. Mannheim, Mess. Mth. 20 (1891) 45-.

- through 4 points, Möbius's theorem, exception. Clausen, $T$. As. Nr. 19 (1842) 375-.

Parabolas in space. Grunert, J.A. Grunert Arch. 3 (1843) 408-.

Parabolic arc of given span and height, construction. Thallmayer, $V$. Dingler 223 (1877) 148-

Properties. Degen, C. F. [1821] Kiob. Dn. Vd. Selsk. Afh. 1 (1824) 121-.

- Lubbock, J. W. Ph. Mg. 9 (1836) 100-.

- Ellis, R. L. Camb. Mth. J. 1 (1839) 204-; (vi Adds.) 2 (1841) 48.

- Davies, T. S. Ph. Mg. 21 (1842) 190-.

- Rutherford, $W$. Mathematician 1 (1845) 203-.

- Zeipel, E. von. Stockh. Öfv. 19 (1862) 429.

-. Anglin, - Edinb. R. S. P. 20 (1895) 35-.

- of hyperbola and parabola. Weddle, $T$. Mathematician 3 (1850) 14-.

Quadrature by pure geometry. Sasku,K. (xII) Mag. Ak. Ets. (Mth. Term.) 3 (1862) 218-

Relation between corresponding ordinates of two. Tait, P. G. [1871] Edinb. R. S. P. 7 (1872) 498-.

Tangents. Engler, E. A., \& Holman, M. L. Am. J. Mth. 1 (1878) 379-.

Theorems. Weill, M. N. A. Mth. 19 (1880) 367-, 442-.

-, analytical proofs. Mathieu, J.J.A. N. A. Mth. 2 (1863) 466-.

-, new, on parabola and hyperbola. Lucas, É. N. A. Mth. 15 (1876) 19-.

Triangle of tangents. Grunert, J.A. Arch. Mth. Ps. 47 (1867) 403-.

Trigonometry of parabola. Booth, J. Camb. and Dubl. Mth. J. 8 (1853) 65-; B. A. Rp. (1856) 68-.

- - - G Gilnther, -. D. Nf. Tbl. (*1881)

Values of elements. Dostor, G.J. Tortolini A. 7 (1856) 269-.

Wallace's property, analytical investigation. Young, J. R. Ph. Mg. 11 (1837) 302-.

Parameters. Jacob, -. N. A. Mth. 2 (1843) 138 -

Pascal's "Essais pour les coniques." Macdonald, W. J. Edinb. Mth. S. P. 2 (1884) 19-.

Pascal's hexagram, Brianchon's hexagon, and connected figures.

(See also 8075.)

Brianchon's hexagon. Brianchon, C.J. Par. Éc. Pol. Cor. 1 (1804-08) 307-.

- hexagon. Walton, $W$. Camb. Mth. J. 4 (1845) 163-.

- Frost, P. Camb. Mth. J. 4 (1845)
Pascal and Brianchon Figures 7210

Brianchon's theorem. Lubbock, J. W. Ph. Mg. 13 (1838) 83-.

- - Mention, J. [1857] St Pét. Ac. S. Bll. 16 (1858) 280 -.

- - application of abridged notation. Janni, $V$. G. Mt. 2 (1864) 253-.

-, generalisation. Brassinne, E. Toul. Ac. Sc. Mm. 9 (1877) 453-.

Conic and in-hexagon. Jacobi, C. G. $J$. Crelle J. 31 (1846) 178-.

In- and circum-hexagons of conic. Schlömilch, O. Grunert Arch. 3 (1843) 386-.

_- conics, and conic sections. Dandelin, G. P. Gergonne A. Mth. 15 (1824-25) 387-。

In-hexagon, complete. Kirkman, T.P. Camb. and Dubl. Mth. J. 5 (1850) 185-.

- of conic. Brioschi, F. N. A. Mth. 16 (1857) 269-.

Mystic hexagram. Lebesgue, $V$. A. N. A. Mth. 8 (1849) 139-.

- Cremona, L. Rm. R. Ac. Linc. T. 1 (1877) 142-.

-, theorems. Steiner, J. Gergonne A. Mth. 18 (1827-28) 339-.

,--- , new. Veronese, G. Rm. R. Ac. Linc. Mm. 1 (1877) 649-.

Octagram, conic. De Morgan, A. L. Mth. S. P. 2 (1869) 26-.

Pascal's hexagram. Brianchon, C. J. [1813] Par. Éc. Pol. Cor. 3 (1814-16) 1-.

- - Davies, T. S. Tilloch Ph. Mg. 68 (1826) 333-.

- - Lubbock, J. W. Ph. Mg. 6 (1829) 249-.

- Adams, C. Grunert Arch. 5 (1844) $323-$.

- Haillecourt, A. N. A. Mth. 6 (1847) 269-.

- Litroth, J. Z. Mth. Ps. 10 (1865) 390 -

(1879) 1 -

- Zanotti Bianco, $O$. Tor. Ac. Sc. At. 21 (1885) 686-.

- Richmond, H.W. Camb. Ph. S.T. 15 (1894) 267-.

- - Study, E. Leip. Mth. Ps. B. 47 (1895) $532-$.

- - and Brianchon's hexagon. Catalan, E. C. Brux. Ac. Bll. 46 (1878) 946-. (1882) 184-.

- - - correlation. Sondat, P. N. A. Mth. 13 (1894) 121-.

- - cases, two. Klug, L. Orv.-Termt. Ets. (Termt. Szak) (1899) 1-, (Rv.) 1-.

-, configuration. Klug, L. Orv.-Termt. Éts. (Termt. Szak) (1897) 101-, (Rv.) 67-.

- - deduced by stereometric theorems. Cremona, L. Rm. R. Ac. Linc. At. 1 (1877) 854-.

- - demonstrations. Weddle, T. Camb. and Dubl. Mth. J. 3 (1848) 285-; 4 (1849) 284-.

- properties. Martinetti, $V$. Catania Ac. Gioen At. 3 (1891) 31-.

- on sphere. Isely, L. Neuch. S. Sc. Bll. 12 (1880) 533-. 
7210 Properties of Conics

Pascal's hexagram, Steiner points of. Grass. mann, H. Crelle J. 58 (1861) 174-.

- - , - - Snyder, V. N. Y. Am. Mth. S. Bll. 4 (1898) 441-

- theorems. Lazzeri, G. Ven. I. At. (1884-85) 481-.

— theorem. Plücker, J. Crelle J. 34 (1847) 337-.

- -. Sylvester, J. J. Ph. Mg. 37 (1850)

$363-$

$26 \overline{9}^{\circ}$

Hesse, L. O. Crelle J. 41 (1851)

Cayley, A. QJ. Mth. 9 (1868)

348 .

- Bauer, G. Münch. Ak. Ab. 11 (1874) (Ab. 3) 109-.

- Dufau, $H$. N. A. Mth. 1 (1882)

99-.

- Heger, R. Z. Mth. Ps. 30 (1885)

279 .

- - Gordan, P. [1895] D. Mth. Vr. Jbr. 4 (1897) 155-.

- - analogues. clifford, W.K. QJ. Mth.6 (1863) 216-.

- - in analysis. Planck, - Grunert Arch. 18 (1852) 335-.

- --, analytical extension. Hesse, L. O. [1871] Münch. Ab. 11 (1873) 175-

- - and another. Ferrers, N. M. QJ. Mth. 1 (1857) 77-.

- - Brianchon's. Gergonne, J. D. Gergonne A. Mth. 4 (1813-14) 78-, 381-.

- - analogue, new. Serret, $P$. C. R. 82 (1876) 208-

- - - - and construction of conics. Koutny, E. Wien Ak. Sb. 71 (1875) (Ab. 2) 491-.

___ - elementary demonstration. Haase, K. Ts. Mth. 3 (1885) 23-.

- - - - graphic determination of conics by. Fiedler, $W$. Schlömilch Z. 6 (1861) 415-.

- _ - - synthesis of, priority claim for Catalan. Folie, F. Brux. Ac. Bll. 46 (1878) 379-.

- - Cayley's, reciprocity. Hesse, L. O. [1867] Crelle J. 68 (1868) 193-.

- - - deductions. Steiner, J. N. A. Mth. 11 (1852) 163-.

- -, demonstration. Rutherford, $W . \mathrm{Ph}$. Mg. 22 (1843) 168-

- - - - Cayley, A. Camb. Mth. J. 4 (1845) 18 -.

- - - (Cayley). Muir, T. Edinb. R. S. P. 17 (1891) 18-.

,--- , elementary. Zeuthen, H. G. (xп) Ts. Mth. 1 (1883) 78-.

- - , - by indeterminate coordinates. Sylvester, J. J. Ph. Mg. 37 (1850) 212.

- - and Desargues's, extension. Folie, $F$. Brux. Ac. Bll. 36 (1873) 620-.

- - extension to geometry of space. Petot, $A$. Par. Éc. Norm. A. 5 (1888) (Suppl.) 65 pp.

- -, generalisation. Möbius, A. F. Leip. B. 1 (1846) 170-.

,--- Hamilton, (Sir) W. R. Ir. Ac. P. 5 (1853) 100-.
Poles and Polars 7210

Pascal's theorem, generalisation. Serret, $P$. C. R. 119 (1894) 454-.

- - - giving nine points. Aubert, - $\mathrm{N}$. A. Mth. 8 (1889) 529-.

-, notation. Cayley, A. QJ. Mth. 9 (1868) 268-.

- and others, in trilinear coordinates. Grunert, J. A. Arch. Mth. Ps. 53 (1871) 395-, 443-.

- - , - pentagon, complete. Drach, C. A. von. Mth. A. 5 (1872) 404-.

6 points on conic, analytical relations. Hesse, L. O. Bll. S. Mth. As. 1 (1870) 33-.

- _ - and mystic hexagram. Scholtz, A. [1877] Arch. Mth. Ps. 62 (1878) 317-.

- - - - (Scholtz). Hunyady, J. (xII) Mag. Tud. Ak. Éts. 11 (No. 3) (1877) 30 -.

Spherical analogues. Thieme, F. E. Arch. Mth. Ps. 60 (1877) 43-.

Steiner's theorems. Göpel, $A$. Grunert Arch. 6 (1845) 87-

Substitutions of $n$ letters, particularly for $n=3,4,5,6$, in relation to groups of mystic hexagram, geometrical interpretation. Veronese, G. A. Mt. 11 (1882-83) 93-.

Point-reciprocation. Taylor, C. Mess. Mth. 1 (1872) 152.

Points, circular, at infinity. Roberts, S. QJ. Mth. 3 (1860) 90-.

,,---- . Whitworth, W. A. Mess. Mth. 4 (1868) 91-.

- of conic, situated on one circle. Cazamian, A. N. A. Mth. 13 (1894) 386-.

- special pairs on axes of central conics. Meyer, T. D. Nf. Vh. (1891) (Th. 2) 542-.

- 7, of conic, general relation. Tarry, $G$. C. R. 94 (1882) 941-.

-, 8, of conic. Cayley, A. Crelle J. 55 (1866) 180-; QJ. Mth. 11 (1871) 344-.

\section{Polars and Polar Reciprocals.}

Olivier, T. [1827] Férussac Bll. Sc. Mth. 15 (1831) 214-.

Lecointe, L. Liège Mm. S. Sc. 2 (1845-46) 36-. Kupper, C. Grunert Arch. 28 (1857) 261-.

Hain, E. [1880] Arch. Mth. Ps. 66 (1881) $274-$.

Applications. Dandelin, G.P. Quetelet Cor. Mth. 3 (1827) 277-.

Conics harmonic to given conics. Ovidio, $E$. $d$ '. G. Mt. 2 (1864) 58-.

- with imaginary elements. Schober, $K$. Mh. Mth. Ps. 2 (1891) 469-.

Conjugate (i.e. polar reciprocal) conics. Retali, V. Bologna Ac. Sc. Mm. 6 (1884) 189-.

- conics, properties. Ruffini, F.P. Bologna Ac. Sc. Mm. 9 (1888) 499-.

Inclined polars. Dewulf, - N. A. Mth. 18 (1859) 322-; 19 (1860) 175-.

Polar conics, certain. Ruffini, $F$. P. Bologna Ac. Sc. Mm. 7 (1886) 753-.

- constructions, some. Morley, E. Mth. A. 51 (1899) 410-. 
Polar groups, construction. Waelsch, E. Wien Ak. Sb. 100 (1891) (Ab. 2a) 315-.

Pole of given straight line, determination. Reggio, Z. Ven. I. At. 7 (1880-81) 1117-.

Poles and polars, theorems. Tychsen, C. Mth. Ts. 2 (1860) 19-.

Problem. Joachimsthal, $\boldsymbol{F}$. $\quad$ N. A. Mth. 6 (1847) 312.

Problems. Midy, -. N. A. Mth. 1 (1842) 336-.

Radii vectores and polars, theorem of Chasles. Terquem, $O$. N. A. Mth. 6 (1847) 162-.

Reciprocal polars. Poncelet, J. V. Gergonne A. Mth. 8 (1817-18) 201-; 18 (1827-28) 125-; Crelle J. 4 (1829) 1 -.

- - Terquem, O. N. A. Mth. 7 (1848) 308-, 409-.

- - Elliott, J. U. Mathematician 3 (1850) 305-.

- - applications, theorems. Godefroy, $R$. N. A. Mth. 12 (1893) 106-

- - , conics with respect to which 2 conics are reciprocal. Ruffini, F.P. (xI) Mod. Ac. Sc. Mm. 12 (1871) 49-; 13 (Pt. 1) (1872) 3-. - - - - - - - - - Siacci, $F$. Tor. At. Ac. Sc. 7 (1871-72) 758-

- - , - - - - - - Battaglini, G. Rm. At. R. Ac. 25 (1872) 195-.

- - - - - - - - Massarini, I. G. Mt. 37 (1899) 23-.

- - homologous curves. Ocagne, M. $d$ '. Par. S. Mth. Bll. 13 (1885) 204-.

- - theorems. Ruffini, F.P. Bologna Ac. Sc. Mm. 6 (1875) 383-.

Reciprocation. Hudson, R. W. H. T. Mess. Mth. 29 (1900) 191-.

-, cases. Tarry, G. N. A. Mth. 3 (1884) 270 Reciprocators of two conics. Russell, J.W. L. Mth. S. P. 26 (1895) 458-.

- - Jolliffe, A. E. L. Mth. S. P. 26 (1895) 466-.

Successive polars. Bobillier, - - Gergonne A. Mth. 19 (1828-29) 302-.

Triangle and its polar, relation of areas, extension to quadric. Walker, W. F. [1864] QJ. Mth. 7 (1866) 119-.

\section{Polygons related to Conics.}

(See also 6810.)

Circum-polygons, product property. Liouville, $J$. Liouv. J. Mth. 9 (1844) 350-.

-, theorem. Terquem, O. N. A. Mth. 12 (1853) 218-.

In- or circum-polygons, semidiameter through vertex of. Foscolo, $G$. Tor. At. Ac. Sc. 7 (1871-72) 338-

In-polygon, complete, extension of theorems on triangle to. Kantor, S. [1877] Wien Ak. Sb. 76 (1878) (Ab. 2) 768-.

- with sides through given points, inscription. Battaglini, G. Tortolini A. 2 (1851) 380-.

- - - - - - - - by aid of generating circle. Renshav, S. A. L. Mth. S. P. 7 (1875-76) 239-.

In-polygons. Gardiner, $M$. [?1862-64] N. S. W. Ph. S. T. 1 (1866) 88-; QJ. Mth. 7 (1866) 146-, 284-.
In-polygons. Lucas, $F$. Par. S. Mth. Bll. 20 (1892) 33-.

-, regular, of ellipse and circle. Terquem, 0 . Liouv. J. Mth. 3 (1838) 477-.

-, theorem. Molins, H. Toul. Mm. Ac. 6 (1862) 177-.

-, theorems. Serret, P. N. A. Mth. 6 (1847) 356-.

,-- (Serret). Catalan, E. C. N. A. Mth. 6 (1847) 425.

Maximum in-, and minimum circum-polygon of ellipse. Spitzer, S. Grunert Arch. 30 (1858) 352-.

- - , - - - Wanka, J. Lotos 39 (1891) 33

,-----1, , construction. Grunert, J. A. Grunert Arch. 30 (1858) 84-.

Minimum circum-polygon of ellipse. Mathews, G. B. Mess. Mth. 22 (1893) 68-.

Pentagon theorem. Serret, $P$. N. A. Mth. 6 (1847) 21-.

Polygon $m$ sides circumscribable to conic, conditions. Serret, $P$. N. A. Mth. 6 (1847) 21-.

Property common to three groups of two polygons inscribed, circumscribed or conjugate with respect to conic. Serret, P. C. R. 114 (1892) 1254-, 1343-.

Schröter's heptagon circumscribed by a conic. Andreev, K. A. Kharkov Mth. S. Com. 1 (1889) 277-; Fschr. Mth. (1891) 669.

Variable polygon and conics, theorem. Roberts, S. QJ. Mth. 4 (1861) 361-.

\section{Poncelet's Polygons, Porisms. \\ (See also 4050 and 6810.)}

Seydexitz, $F$. Grunert Arch. 4 (1844) 421-.

Bordoni, A. Tortolini A. 3 (1852) 5-.

Cayley, A. Ph. Mg. 6 (1853) 99-, 376-; 7 (1854) 339-.

Brioschi, $F$. N. A. Mth. 16 (1857) 421-.

Salmon, G. Ph. Mg. 13 (1857) 337-.

Mainardi, $G$. Mil. At. I. Lomb. 1 (1858) 117-.

Cayley, A. Phil. Trans. (1861) 225-.

Pasch, M., \& Rosanes, J. Crelle J. 64 (1865) 126-.

Cayley, A. QJ. Mth. 11 (1871) 83-.

Darboux, G. (Ix) Par. S. Phlm. Bll. 8 (1872) 100-, 128-.

Chelini, D. Bologna Ac. Sc. Mm. 5 (1874) 353-.

Czuber [or Čuber], E. Prag Sb. (1875) 156-.

Gundelfinger, S. Crelle J. Mth. 83 (1877) 171-.

Darboux, G. C. R. 90 (1880) 85-.

Andreev [Andréef], K. A. (xII) Kharkov Mth. S. Com. (1881) 91-; (Ix) As. Fr. C. R. 11 (1882) 96-.

Forsyth, A. R. [1882] Mess. Mth. 12 (1883) 100 -

Rogers, L. J. L. Mth. S. P. 16 (1884-85) 306-.

Berg, $F$. J. van den. N. Arch. Wisk. 16 (1889) 160-; Fschr. Mth. (1889) 719.

Frolov, (le gén.) $M$. As. Fr. C. R. (1894) (Pt. 2) 226-. 
Lelieuvre, M. Ens. Mth. 2 (1900) 410-.

Petr, K. Prag České Ak. Fr. Jos. Rz. (Tŕrida 2) 9 (1900) Art. 2, 23 pp.; Fschr. Mth. (1900) 582.

Application of function $\rho u$. Vivanti, $G$. Palermo Cir. Mt. Rd. 7 (1893) 61-.

Bibliographical and historical notes. Loria, $G$. Bb. Mth. (1889) 67-.

Characteristics, theory. Halphen, G. H. [1878] Par. S. Phlm. Bll. 3 (1879) 17-.

Conic of pentagon, porism. Terquem, O. N. A. Mth. 7 (1848) 106-.

Equations, porismatic, systems of. Wolstenholme, (Rev.) J. L. Mth. S. P. 4 (1871-73) 312-; (xI) 5 (1873-74) 35-.

-, trigonometrical, system, with application to porisms of coaxal conics. Wolstenholme, (Rev.) J. QJ. Mth. 10 (1870) 356-; 11 (1871) 66-.

Historical notes. Mackay, J. S. Edinb. Mth. S. P. 5 (1887) 62-.

Invariant relations. Kluyver, J. C. N. Arch. Wisk. 15 (1888) 37-; Fschr. Mth. (1888) 728-.

Irregular polygon, porism. Trudi, $N$. Nap. Rd. 2 (1843) 89-.

Metrical properties. Halphen, G. H. Liouv. J. Mth. 5 (1879) 285-

Parabola and circle, porismatic polygons of. Poncelet, J. V. Gergonne A. Mth. 8 (181718) 68 .

Poncelet's polygons of circles. Plank, Grunert Arch. 19 (1852) 7-.

- - - Puiseux, V.A. (xII) Brux. S. Sc. A. 3 (1879) (Pt. 2) 1-.

- theorem. Andreev, $K$. A. Kharkov Mth. S. Com. (1884) 123-; Fschr. Mth. (1884) 556.

- on 2 conics. Neuberg, J. Mathesis 15 (1895) 13-.

- - demonstration. Serret, P. C. R. 53 (1861) 507-.

- - Talayrach, F. N. A. Mth. 19 (1880) 280-.

- theorems. Trudi, N. G. Mt. 1 (1863) 81-, 125-.

- - , and elimination problem. Trudi, $N$. Nap. At. Ac. 1 (1863) No. 6, 53 pp.

Porismatic polygons in plane or sphere. Berg, F. J. van den. N. Arch. Wisk. 14 (1888) 95-, 125-; Fschr. Mth. (1887) 725-.

- problems. Juel, C. N. Ts. Mth. 1 (B) (1890) 11-; Fschr. Mth. (1890) 660-.

Projective properties, some. Koln, G. Wien Ak. Sb. 100 (1891) (Ab. 2a) 6-.

Relation, porismatic, between 2 conics, invariants. Wolstenholme, (Rev.) J. [1876] L. Mth. S. P. 8 (1876-77) 136-.

Steiner's and Poncelet's polygons, a connexion between. Thomae, J. Leip. Mth. Ps. B. 47 (1895) 352-.

Theorem of Jacobi. Thijn, A. van. N. Arch. Wisk. 1 (1895) 217-; Fschr. Mth. (1893-94) 1107.

Triangles, porism. Cayley, A. Ph. Mg. 9 (185.5) 513-; 13 (1857) 19-; QJ. Mth. 1 (1857) 344-; 2 (1858) 31-.
Triangles, porism. Salmon, G. Phil. Mg. 13 (1857) 190-, 267-.

-, -. Hart, A. S. QJ. Mth. 2 (1858) 143.

-, 一. Cayley, A. B. A. Rp. 34 (1864) (Sect.) 1 ; 40 (1870) (Sect.) 9-; Phil. Trans. 161 (1871) 369-.

- - Weill, M. N. A. Mth. 19 (1880) 253-.

,-- , new view of. Wolstenholme, (Rev.) $J$. [1874] L. Mth. S. P. 6 (1874-75) 9-.

-, porismatic relation for, proof. Lhuilier, $S$. Gergonne A. Mth. 1 (1810-11) 149-.

Power of a point with reference to central conic. Barbarin, P. (xII) Mathesis 1 (1881) 85-.

Projection, central, of conics. Moshammer, $K$. Wien Sb. 49 (1864) (Ab. 2) 372-.

- of conic is a conic, demonstration. Leau, Par. S. Phlm. Bll. 1 (1899) 87-.

- conics as curved figures. Göpel, $A$. Crelle J. 36 (1848) 317-.

- - ellipse, dependence on direction of projecting lines and position of plane of projection. Weinmeister, $P$. Arch. Mth. Ps. 10 (1891) 380-.

Projections of circle, construction of axes. Lazarski, M. [1882] (xII) Krk. Ak. (Mt.Prz.) Rz: \& Sp. 10 (1883) 148-, LIV-.

- - - and their diameter. Peschka, G. A. von. Arch. Mth. Ps. 57 (1875) 63-.

- conics. Binder, W. Wien Ak. Sb. 81 (1880) (Ab. 2) 648-.

- - intersecting conics. Olivier, $T$. Stockh. Ak. Hndl. (1823) 5-.

-, polyconic, note on error. Johnson, W.W. Des Moines Anal. 3 (1876) 15-.

- of sections of cones and cylinders. Koutny, E. Z. Mth. Ps. 12 (1867) 195-.

Projective properties (Poncelet's memoir). Cauchy, A. L. Gergonne A. Mth. 11 (1820-21) 69-.

- - Dandelin, G. P. Quetelet Cor. Mth. 3 (1827) 9-.

- - Wetzig, F. Schlömlich Z. 5 (1860) $63-$

-. Milinowski, -. Crelle J. Mth. 86 (1879) 290-.

- - Ponte Horta, $F$. da. G. Teix. J. Sc. 4 (1883) 65-

- - Aubert, P. Edinb. Mth. S. P. 7 (1889) 61-.

- -. Sondat, P. N. A. Mth. 14 (1895) 309-, $507-$.

- - Zahradnik, K. Časopis 28 (1899) 37-; Fschr. Mth. (1899) 481; Arch. Mth. Ps. 17 (1900) 89-.

- - Tresse, A. Par. S. Mth. Bll. 28 (1900) 131-.

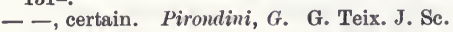
14 (1900) 75-.

- - new. Carnoy, J. (xi) Brux. S. Sc. A. 4 (1880) (Pt. 1) 60-; (Pt. 2) 260-.

- relation involving its correlative. Halphen, G. H. C. R. 83 (1876) 791-.

- theorem. Ascione, E. Palermo Cir. Mt. Rd. 9 (1895) 208. 
Projective theorems. Ovidio, E. $d$ '. Tor. Ac. Sc. At. 26 (1891) 525-.

Projectrices of a circle, successive. Bottomley, J. [1885-88] Manch. Lt. Ph. S. Mm. 10 (1887) 6-; 1 (1888) 89; Manch. Lt. Ph. S. P. 25 (1886) 233-.

Properties connected with integration of Euler's equation. Laguerre, -. N. A. Mth. 11 (1872) 156-.

- deduced from parallelogram. Ponte Horta, F. da. Lisb. J. Sc. Mth. 3 (1871) 1-.

- and loci. Zambelli, $A$. Ven. At. Aten. 7 (1872) 107-.

\section{Quadrilaterals, Quadrangles, and related Conics.}

(See also 6810.)

Terrier, $P$. N. A. Mth. 14 (1875) 514-.

Circum-conics, locus of centres. Lanavicensis [Sylvester, J.J.]. (III) Mess. Mth. 2 (1864) 169-.

-, Steiner's theorem. Gergonne, J. D. Gergonne A. Mth. 18 (1827-28) 100-.

Circum-parabolas, 2. Kluyver, - Mathesis 13 (1893) 106-.

Circum-quadrilaterals, Newton's theorem, demonstration. Poncelet, J. V. Gergonne A. Mth. 12 (1821-22) 109-.

-, theorem of Chasles. Housel, - N. A. Mth. 18 (1859) 352-.

Conjugate quadrilateral, theorem of Hesse, geometrical demonstrations. Hofmann, $F$. Crelle J. Mth. 102 (1888) 175-.

In- and circum-quadrilaterals, Steiner's theorem. Strnad, A. Časopis 17 (1888) 10-, 56-; Fschr. Mth. (1888) 548.

In-conics. Chasles, MI. Quetelet Cor. Mth. 4 (1828) 363-.

-. Battaglini, G. Nap. Rd. 1 (1862) 168-.

-. Painvin, L. N. A. Mth. 2 (1863) 156-.

--, anharmonic properties. Walker, J. J. [1869] QJ. Mth. 10 (1870) 317-.

-, centre lies on Newtonian. Grunert, J.A. Arch. Mth. Ps. 54 (1872) 361-.

- and circum-conics. Mathieu, J.J.A. N. A. Mth. 15 (1876) 354-.

,--- 120 theorems, etc. Terquem, $O . \mathrm{N}$. A. Mth. 4 (1845) 384-, 480-.

,- 4 , of same quadrilateral. Jonquières, $E$. de. N. A. Mth. 15 (1856) 312-.

In-quadrilateral of ellipse, theory. Mittelacher, C. [1869] Arch. Mth. Ps. 52 (1871) 206-.

- and 5-gon, theorems. Serret, P. N. A. Mth. 7 (1848) 214-.

Involutions of two quadrangles. Weyr, $E$. Wien Sb. 57 (1868) (Ab. 2) 449-.

Maximum in-ellipse. Gauss, C. F. Zach M. Cor. 22 (1810) 112-

- - Mollweide, C. Zach. M. Cor. 22 (1810) 227-, 296-.

- - or circum-ellipse. Serret, $P$. N. A. Mth. 4 (1865) 303-.

- - and minimum circum-ellipse. Fenvick, S. Mathematician 2 (1847) 229-.

Minimum circum-conic. Battaglini, G. Tortolini A. 5 (1854) 193-.
Minimum circum-ellipse. Seydevitz, F. Grunert Arch. 13 (1849) 54-.

14-point conic of quadrilateral. Cremona, $L$. Mess. Mth. 3 (1866) 13-.

- - - Ferrers, N. M. Mess. Mth. 3 (1866) 68-.

Quadrangle and conic. Sauve, $A$. Rm. N. Linc. Mm. 12 (1896) 249-.

-, moveable. Fontené, G. N. A. Mth. 17 (1898) 101-.

Quadrilateral formed by tangents from foci. Torry, A. F. [1881] Mess. Mth. 11 (1882) $54-$.

Relation between in-conic and circum-circle. Laguerre, E. N. A. Mth. 18 (1879) 246-.

Radii vectores and diameters. Chasles, $M$. C. R. 26 (1848) 531-.

- - of ellipse, mean. Baehr, G. F.W. Les Mondes 3 (1863) 409-.

- - peculiarity. Steiner, J. Crelle J. 30 (1846) 337-.

Ray systems of second order, condition for enveloping ellipse, parabola, etc. Meyer, $A$. (of Stockholm). Z. Mth. Ps. 28 (1883) 383-.

Secant, imaginary, of conic, Mainardi's theorems. Bellavitis, $G$. Ven. At. (185758) 623- ; (1858-59) 334-.

Sectors, quadrature (Leibnitz). Baltzer, $R$. Leip. B. 7 (1855) 62-.

-, - (Baltzer on Leibnitz). Möbius, A. F. Leip. B. 8 (1856) 19-.

Segments, theorems. Lescaze, A. N. A. Mth. 19 (1860) 225-.

Semiaxes of conics, some relations between. Fazzari, G. G. Mt. 23 (1885) 198-.

Similar arcs, Chasles's theorem, etc. Terquem, O. N. A. Mth. 3 (1844) 506-.

- conics, properties. Nieuport, C. F. F.de. [1817] Brux. Ac. Sc. Mm. 1 (1820) 39-.

Special forms of conics. Taylor, C. [1866] QJ. Mth. 8 (1867) 126-, 343-.

- - - Salmon, G. [1866] QJ. Mth. 8 (1867) 235-.

Straight lines conjugate to a conic and passing through same point, system. Gilles, L. N. A. Mth. 8 (1849) 87-.

- - connected with conics. Weisz,J.A. (xI) Mag. Tud. Ak. Éts. (1859) (Suppl., Mth. Term.) 312-.

- $-1-1$, characteristic points. Servais, C. Brux. Ac. Bll. 19 (1890) 519-.

Symbolic treatment of problem in conics. Schmidt, C. Z. Mth. Ps. 34 (1889) 365-, 385.

Systems of lines and surfaces, certain. Lazzeri, G. Palermo Cir. Mt. Rd. 2 (1888) 110-.

Tangents.

Woestyn, A. C. N. A. Mth. 4 (1845) 244 -

Steczkouski, J. K. Grunert Arch. 34 (1860) 302-.

Laisant, C. A. As. Fr. C. R. (1888) (Pt. 2) 113-. Common tangents of circle and conic, construction. Schirek, C. Arch. Mth. Ps. 69 (1883) 408-. 
Common tangents of circle and conic, intersection. Mister, 一, $\&$ Neuberg, - N. A. Mth. 2 (1863) 481-.

- - - - L - Le Cointe, -. N. A. Mth. 19 (1880) 122-.

- -2 conics, theorem. Cayley, A. Camb. (M.) Mth. M. 1 (1859) 130-.

Ellipse of eccentricity $e \cos \alpha$, tangent, problem. Ellis, R. L. (vI Adds.) Camb. Mth. J. 3 (1843) $94-$.

-, tangents, theorem. Gerono, G. C. N. A. Mth. 7 (1848) 68-.

General property. Gierman, S. Fschr. Mth. (1897) 526.

Locus of intersection of tangents containing constant angle. Tortolini, B. Rm. At. 1 (1847-48) 125-.

- _ - - at given angle. Ficklin, J. Des Moines Anal. 2 (1875) 50 -.

- - - - - right angles (orthocycle), Gaskin and Plücker's properties. Taylor, C. QJ. Mth. 17 (1881) 134-.

Minimum tangent between two fixed tangents. Serret, $P$. N. A. Mth. 11 (1852) 123-.

Perpendicular tangents to 2 conics. Terquem, O. N. A. Mth. 8 (1849) 282-.

- - , intersection (orthoptic curve). Greiner, M. Arch. Mth. Ps. 57 (1875) 343-.

Properties. Rochat, -. Gergonne A. Mth. 2 (1811-12) 225-.

- of tangent and normal. Dostor, G. Arch. Mth. Ps. 61 (1877) 160-.

Property. Pour, J.' Časopis 28 (1899) 209-; Fschr. Mth. (1899) 481.

Tangents to conic that are normal to another conic. Joly, $H$. Laus. S. Vd. Bll. 26 (1891) 1-.

- - fixed conic and to variable conics, intersection. Darboux, G. N. A. Mth. 19 (1880) 184-.

- from points, construction. Grunert, J.A. Grunert Arch. 32 (1859) 425-.

- -4 points, theorems. Grunert, J. A. Arch. Mth. Ps. 54 (1872) 375-.

- - same point. Dostor, G. Arch. Mth. Ps. 53 (1871) 90-, 98-.

Tetrahedra and conics, elementary theory. Chelini, D. Bologna Ac. Sc. Mm. 5 (1874) 223-.

Theorem of Pappus. Zahradnik, $K$. Casopis 28 (1899) 111-; Fschr. Mth. (1899) 516.

_ _ _, its correlative and corollaries which may be deduced from it. Mundi y Giró, $S$. Barcel. Ac. Bl. 1 (1892-1900) 569-.

Theorems. Zeuthen, H. G. Mth. Ts. 5 (1863) 69-.

- Bruno, G. Tor. At. Ac. Sc. 7 (1871-72) 783-.

-, derivation from circle. Milinowski, Crelle J. Mth. 86 (1879) 108-.

- obtained by inversion. Stubbs, J.W. $\mathrm{Ph}$. Mg. 25 (1844) 208-.

- of Steiner. Heinen, $F$. Crelle J. 23 (1842) 289-.

- - Jonquières, E. de. N. A. Mth. 15 (1856) 94-, 190-.

VOL. I.
Theorems of Steiner. Stoll, - Z. Mth. Ps. 33 (1888) 78-.

- - - and Newton. Godefroy, R. N. A. Mth. 12 (1893) 137-.

Transformation of angles, method of reversion applied to. Taylor, C. [1875] QJ. Mth. 14 (1877) 25-

- - conies into circles by projections. Lazar. ski, M. [1883] (xII) Krk. Ak. (Mt.-Prz.) Rz. \& Sp. 11 (1884) 145-, L.

- - - and curves, method. Ocagne, M. $d$ '. G. Teix. J. Sc. 8 (1887) 104 .

-, parabolic. Chasles, M. Quetelet Cor. Mth. 5 (1829) 281-; 6 (1830) 1-.

Transformations, application of comparative geometry. Mathieu, J. J.A. N. A. Mth. 4 (1865) 393-, 481-, 529-.

Transversals of conic, theorems. Mandl, $J$. Mh. Mth. Ps. 9 (1898) 117-.

Triangles, and related Conics.

(See also 6810.)

Neuberg, J. N. A. Mth. 9 (1870) 53-.

Brocard triangle, generalised, conics related to. Mitller, A. Arch. Mth. Ps. 9 (1890) 113-.

Carnot's theorem. Laisant, C. A. N. A. Mth. 9 (1890) 5-. - - Cazamian, A. N. A. Mth. 14 (1895)

Ceva's theorem deduced from Carnot's. Rueda, C. J. Fschr. Mth. (1898) 433.

Circum-conic, minimum. Greiner, M. Z. Mth. Ps. 28 (1883) 281-。

Circum-conics. Tortolini, B. Tortolini A. 8 (1857) 356-.

- conjugate to triangle. Salvatore-Dino, N. Nap. Rd. 14 (1875) 134 -.

- of triangles self-conjugate to another conic. Smith, H. J. S. [1868] L. Mth. S. P. 2 (1869) 85-.

Circum-ellipse, minimum. Anger, C. T. Grunert Areh. 10 (1847) 178-.

-, -. Tortolini, B. A. Mt. 6 (1864) 196-.

- - , and maximum in-ellipse. Hansen, $C$. Mth. Ts. 5 (1863) 37-.

- Steiner's and Lemoine's theorems. DrozFarny, A. Bern Mt. (1900) 135-.

Circum-hyperbolas, two triplets. Tucker, $R$. Edinb. Mth. S. P. 12 (1894) 69-.

Circum- and polar triangles, property. Mertens, F. Wien Ak. Sb. 94 (1887) (Ab. 2) 794-

Circum-triangle. Cayley, A. QJ. Mth. 1 (1857) 169-.

- formed by two fixed tangents and one variable, remarkable points of. Meyer, $T$. Arch. Mth. Ps. 8 (1890) 307-.

Conic, line, and triangle. Cayley, A. Ph. Mg. 25 (1863) 181-.

- in plane of triangle. Longchamps, G. de. As. Fr. C. R. 1886 (Pt. 2) 69-.

Conics conjugate with respect to triangle. Painvin, L. N. A. Mth. 6 (1867) 433-.

- related to triangle. Lemoine, $E$. Mathesis 14 (1894) 153-.

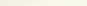


Conics related to triangle. Tucker, R. Edinb. Mth. S. P. 12 (1894) 51-.

- _ - - Neuberg, $J$. Mathesis 15 (1895) $60-$.

- - - system. Neuberg, J. Mathesis 16 (1896) 164-.

- - - - systems. Third, J. A. Edinb. Mth. S. P. 17 (1899) 99-.

- of symmetry. Hain, E. [1875] Arch. Mth. Ps. 59 (1876) 83-.

Conjugate triangle and circum-circle, Faure and Painvin's theorems, proofs. Serret, $P$. N. A. Mth. 20 (1861) 77-.

$--\ldots-$, Faure's theorem. Cazamian, $A$. N. A. Mth. 13 (1894) 324-.

$-{ }_{-}-,-{ }_{-}$and parallel curves. Salmon, G. N. A. Mth. 19 (1860) 345-.

Harmonic conic of triangle, Cayley's extension of Pollock's theorem. Ferrers, N. M. QJ. Mth. 1 (1857) 175-.

Hyperbola, Feuerbach's. Mandart, -. Mathesis 13 (1893) 81-.

-, Kiepert's. Cosserat, - As. Fr. C. R. (1887) (Pt. 1) 175.

-, -. M'Cay, —. Mathesis 7 (1887) 208-.

-, 一. Neuberg, $J$. Mathesis 12 (1892) 241-.

-, - asymptotes. Laisant, C. A. As. Fr. C. R. (1887) (Pt. 2) 113-.

Hyperbolas, "adjoint." Schiappa Monteiro, A. Lisb. J. Sc. Mth. 5 (1898) 213-.

In- and circum-ellipse, content. Greiner, $M$. Z. Mth. Ps. 29 (1884) 222-.

- - circum-triangle. Cayley, A. QJ. Mth. 3 (1860) 157-.

In-conics and circum-conics, theorem of Steiner. Terquem, $O$. N. A. Mth. 4 (1845) 480-

-, if Gergonne's point traverse a circum-conic, in-conic touches polar of point with respect to circum-conic. Krahe, $A$. Fschr. Mth. (1900) 583.

- passing through fixed point, locus of centres is a conic, etc., theorem of Faure. Intrigila, $C .$, \& Laudiero, $F$. G. Mt. 19 (1881) 245-.

-, reciprocal trilinear substitution. Cayley, $A$. QJ. Mth. 4 (1861) 131-.

-, Steiner's theorem, proof. Serret, P. N. A. Mth. 8 (1849) 453-.

In-ellipse, maximum. Schnmacher, $H . C$. Zach M. Cor. 22 (1810) 499 -.

-, - Moesta, C. W. Grunert Arch. 8 (1846) $59-$.

,-- , of triangle and of quadrilateral. Pfaff, $J$. $F$. Zach M. Cor. 22 (1810) 223-.

In- and e-scribed circles and conics. Milller, $A$. Arch. Mth. Ps. 10 (1891) 300-.

In- or circum-conic. Stuyvaert, - Mathesis 17 (1897) 63-, 81-.

- - circum-conics. Genocchi, A. Tortolini A. 3 (1852) 370-.

- - Battaglini, G. Nap. Rd. 1 (1862) 189 .

- - - having double contact with fixed conic. Weyr, E. Prag Sb. (1869) (pt. 2) 5-.

- - , system. Spijker, N. C. Mathesis 15 (1895) 105-.

- - systems, "descriptive" centres and lines of. Reggio, $Z$. Ven. I. At. 8 (1881-82)
In- or circum-conics of triangles and quadrilaterals. Helluig, C. Erfurt Ak. Jb. 20 (1894) 293-.

- _ - _ - self-conjugate with respect to given conic. Torry, A. F. Mess. Mth. 10 (1881) 161-.

- circum-triangle, perpendiculars, theorems. Salmon, G. QJ. Mth. 5 (1862) 362-.

In-triangle. Aubertin, - C Crelle J. 45 (1853) 246-.

-, equilateral. Wasserschleben, (Maj.) - von. Arch. Mth. Ps. 57 (1875) 302-.

-, maximum, of ellipse, and converse problem. Hansen, C. Mth. Ts. 4 (1862) 102-.

- - equilateral, of ellipse. Spiller, - . Mth. Misc. 1 (1838) 10-.

- with sides, 2, through fixed points, theorem of Poncelet. Serret, P. N. A. Mth. 7 (1848) 196-.

- - through given points. Clausen, $T$. Crelle J. 7 (1831) 55-.

In-triangles of ellipse. MacCullagh, J. N. A. Mth. 9 (1850) 296-.

- - - (MacCullagh's theorem, analytical proof). Mister, J., d Neuberg, -. N. A. Mth. 4 (1865) 458-.

- - , with centroid at given point. Piuma, C. $M$. G. Mt. 23 (1885) 20-.

- _ - circumscribed to concentric circle. Barisien, E. Mathesis 19 (1899) 224-, 247-, 269- ; 20 (1900) 84-, 113-, 136-.

- - - theorem. Grunert, J.A. Arch. Mth. Ps. 47 (1867) 462-.

-, Steiner's "Gegenpuncte," determined by two. Staudt, G.K. C. von. [1862] Crelle J. 62 (1863) 142-.

-, theorems. Terquem, O. N. A. Mth. 2 (1843) 186-; 4 (1845) 432.

Locus problem. Neuberg, $J_{\text {. }}$ \& Schoute, P. H. As. Fr. C. R. (1891) (Pt. 2) 168-.

Parabola touching 3 sides of triangle. Coltman, W.B. Camb. and Dubl. Mth. J. 7 (1852) 137-.

Parabolas connected with a triangle, three. Brocard, H. Mntp. Ac. Mm. 11 (1892) 51-. - $-\ldots \ldots$ - _. Mandart, _. Mathesis 13 (1893) 10.

-- - Tucker, R. Edinb. Mth. S. P. 12 (1894) 79-.

- - - - - - Avillez, J. F. de (Visconde de Reguengo). G.Teix. J. Sc. 12 (1895) 137-; Fschr. Mth. (1895) 677; As. Fr. C. R. (1897) (Pt. 2) 133-.

- - - _ - - Neuberg, J. Mathesis 18 (1898) 131-.

Theorems. Steiner, J. Gergonne A. Mth. 19 (1828-29) 37-.

- on triangles, circles and conics. Lemoine, $\dot{E}$. As. Fr. C. R. (1900) (Pt. 2) 79-.

Triangle formed by ares of 3 conics or confocal parabolas, Roberts's theorems. Terquem, $O$. N. A. Mth. 7 (1848) 397-.

- - - - - - - - - - , proof. Serret, P. N. A. Mth. 9 (1850) 320-.

- moving with two of its angles on fixed straight lines, locus of vertex. Stegmann, $F$. Grunert Arch. 7 (1846) 64-.

- in plane of conic. Terquem, O. N. A. Mth. 4 (1845) 419-. 


\section{Properties of Conics}

Triangle $A B C$, to find $D$ in $A B$ so that $C D^{2}$ : rect. $A D, B D$ in given ratio. Steiner, $J$. Berl. Ab. (1847) (Mth.) 41-.

Triangles, apolar, on conic. Morley, F. N. Y. Am. Mth. S. Bll. 1 (1895) 116-.

- connected with conics, properties. Bertrand, É. Mathesis 12 (1892) 130-; 13 (1893) 155-.

,- 2 inscribed, theorem of Poncelet, generalisation. Kantor, S. [1878] Crelle J. Mth. 86 (1879) 269-.

-, multiply projective, in conics. Vályi, $G$. Mth. Termt. Éts. 2 (1884) 170-; Mth. Nt. B. Ung. 2 (*1883-84) 232-.

-, orthologic, etc. Lemoine, É. As. Fr. C. R. (1890) (Pt. 2) 111-.

-, perspective, in conic. Klug, L. Arch. Mth. Ps. 1 (1884) 292-.

- , polar, of one conic and inscribed in another conic, property. Machovec, $F$. Časopis 16 (1887) 85-; Fschr. Mth. (1887) 625.

-, - , maximum ellipse has centre at centroid, etc. Fazzari, G. G. Mt. 25 (1887) 305-.

-, reciprocal. Cayley, A. QJ. Mth. 1 (1857) 7-.

-, -. Pasch, M. Z. Mth. Ps. 27 (1882) 122-.

-, - , properties. Pietrocola, C. G. Mt. 25 (1887) 183-.

- related to conics, properties. Bertrand, $\dot{E}$. Mathesis 12 (1892) 130-; 13 (1893) 155-.

-, self-conjugate, common, of 2 conics, area. Leudesdorf, C. [1876] Mess. Mth. 6 (1877) 151-.

-, -, MacCullagh's property. Casey, J. [1861] QJ. Mth. 5 (1862) 233-.

- whose sides contain poles conjugate with respect to four conics. Siebeck, H. A. Mt. (1868-69) 65-.

- triply-homologous. Ripert, L. As. Fr. C. R. (1900) (Pt. 2) 112-; Mathesis 20 (1900) 226-; Par. S. Mth. Bll. 28 (1900) 196-.

-, - . Third, J. A. Mathesis 20 (1900) 153-.

"Tripel" of conic, relation between theorems of Chasles and Steiner, and deductions. Schumann, A. Z. Mth. Ps. 27 (1882) 363-.

Two conics, common elements of, construction. Streissler, J. Arch. Mth. Ps. 68 (1882) 389-.

- - , - - - fundamental theorems on. Maccaferri, E. Palermo Cir. Mt. Rd. 9 (1895) 96-.

- - - points and tangents. Genese, R. W. L. Mth. S. P. 14 (1883) 235-.

- - - - - - reality. Gerbaldi, $F$. Palermo Cir. Mt. Rd. 1 (1887) 327-.

,$---\ldots-,-$, condition for. Sforza, $G$. Palermo Cir. Mt. Rd. 2 (1888) 172-.

- - , correlations in indeterminate coordinates. Sylvester, J. J. Camb. and Dubl. Mth. J. 5 (1850) 262-.

- - intersection. Rutherford, W. Mathematician 1 (1845) 13-.

- - - Joachimsthal, $F$. N. A. Mth. 9 (1850) 169-.

- - - - Sylvester, J. J. Camb. and Dubl. Mth. J. 6 (1851) 18-.

\section{Systems of Conics 7230}

Two conies, intersection. Amigues, E. N. A. Mth. 13 (1894) 81-

- - - , area of quadrilateral formed by 4 points of. Leudesdorf, $C$. L. Mth. S. P. 8 (1876-77) 205-.

,--- , construction. Nientschik, $R$. Wien Sb. 59 (1869) (Ab. 2) 481-。

- - - - - Tomes, $F$. Arch. Mth. Ps. 69 (1883) 307-.

- - - determination. Spitz, C. Grunert Arch. 32 (1859) 198-.

,--- , locus problem. Mention, J. N. A. Mth. 5 (1846) 136-.

- in a plane. Krammer, G. (xir) Krk. Ak. (Mt.-Prz.) Rz. \& Sp. 6 (1880) 143-

- , radical axis. Ferrers, N. M. QJ. Mth. 5 (1862) 312-.

- - , relation between coefficients of equation when 2 points of intersection are at infinity. Swellengrebel, J. G. H. Grunert Arch. 16 (1851) 321-.

- -, theory. Salmon, G. N. A. Mth. 17 (1858) 83-. 161-. - Gerbaldi, $F$. A. Mt. 17 (1889-90)

Unreal points and tangents. Paulus, C. Grunert Ârch. 22 (1853) 121-.

Values of elements. Dostor, G.J. Tortolini A. 7 (1856) 243-.

\section{Systems of conics. (See also 8070 and 8090.)}

Chasles, M. Gergonne A. Mth. 18 (1827-28) 277-; 19 (1828-29) 26-.

Battaglini, G. [1866] Nap. At. Ac. 3 (1866-68) (No. 8) $45 \mathrm{pp}$.

Zeuthen, H. G. C. R. 62 (1866) 177-

[Salvatore-] Dino, N. C. R. 65 (1867) 499-.

Rosanes, J. Mth. A. 6 (1873) 264-.

Zimmermann, H. E. M. O. Z. Mth. Ps. 28 (1883) 56-.

Del Pezzo, P. Nap. Rd. 23 (1884) 61-.

Burali-Forti, C. G. Mt. 24 (1886) 309-

Ser, J. N. A. Mth. 19 (1900) 126-.

Axes of conics touching 3 or 4 straight lines, product. Molenbrock [Molenbroek], P. Mathesis 12 (1892) 33-.

-, conjugate diameters, etc. of conics of a system. Mautner, J. [1879] Wien Ak. Sb. 80 (1880) (Ab. 2) 973-.

Centre, locus of. Poncelet, J. V. Gergonne A. Mth. 12 (1821-22) 233-.

-, - - (Poncelet). Gergonne, J. D. Gergonne A. Mth. 12 (1821-22) 249-

Centres of conics fulfilling certain conditions, loci. Ovidio, E. d'. G. Mt. 1 (1863) 265-.

Characteristics, analytical applications of method. Salvatore-Dino, N. Nap. Rd. 14 (1875) 95-.

- of systems of conics. Zeuthen, H. G. N. A. Mth. 5 (1866) 241-, 289-, 385-, 433-, 481-, 529-.

(1876) 537-。 
Characteristics, systems represented by 2, theorems. Chasles, M. C. R. 72 (1871) 511-.

Concentric ellipses and hyperbolas, property. Schlömilch, O. X. Z. Mth. Ps. 26 (1881) 135-. - rectangular hyperbolas, Roberts's theorems, demonstration. Serret, $P$. N. A. Mth. 8 (1849) 271-.

Conditions of perpendicularity among series of lines, systems with. Chasles, M. C. R. 72 (1871) 487-.

Conics with coefficients linear functions of two parameters. Rémond, A. N. A. Mth. 5 (1886) 424-.

\section{Conics with Common Focus or Foci.}

Confocal conics. Bobillier, -. Gergonne A. Mth. 18 (1827-28) 185-.

- -. Chasles, M. Gergonne A. Mth. 18 (1827-28) 269-; Quetelet Cor. Mth. 7 (1832) 295-; N. A. Mth. 8 (1849) 214.

_-. Cohn, S. Crelle J. 54 (1857) 329-.

-. Heilermann, -. Schlömilch Z. 3 (1858) 341-.

- Küpper, C. Schlömilch Z. 7 (1862) 239-.

- Casey, J. [1864] Mess. Mth. 3 (1866)

23-.

- Talbot, W. H. F. [1865] Edinb. R. S. T. 24 (1867) 53-.

- - Taylor, C. [1865] Mess. Mth. 3 (1866) 182- ; 4 (1868) 11-.

- - Wolstenholme, J. QJ. Mth. 10 (1870) 285-.

- Laguerre, E. Par. S. Mth. Bll. 7 (1879) 66-.

- - Hossfeld, C. Z. Mth. Ps. 28 (1883) 294.

- - and circles having common secants, reciprocity. Hesse, L. O. Münch. Ak. Ab. 11 (1874) (Ab. 3) 1-.

- - - derived cubic. Morley, F. Mess. Mth. 16 (1887) 181-。

- - differential equation for, integration. Guimarães, R. Lisb. J. Sc. Mth. 5 (1898) 105-.

- - - formula. Cayley, A. Mess. Mth. 2 (1873) 157-.

- - Graves's theorem in analytical mechanics, generalisations. Staude, -. Leip. Mth. Ps. B. 38 (1886) 199-.

$-\ldots, \ldots$, historical note. Roberts, S. L. Mth. S. P. 12 (1880-81) 120-.

-,-- and MacCullagh's, demonstration and extension. Griffiths, J. - L. Mth. S. P. 13 (1881-82) 177-.

- _, loci relative to parallel tangents. Volpicelli, $P$. Rm. At. N. Linc. 19 (1866) 26-, 53-, 249 [149]-, 219-, 268-.

- - in projective metrics. Ovidio, $E$. $d$ '. Tor. Ac. Sc. At. 26 (1891) 426-.

- - theorem by Chasles. Lucas, É. N. A. Mth. 19 (1880) 397-.

- - theorems by Chasles, demonstration. Reye, T. Zür. Vjschr. 41 (1896) (Festschr., Th. 2) 65-.

- - 2, theorems of Mannheim. Ovidio, E. d'. G. Mt. 7 (1869) 107-

,-- 3 , confocal in pairs. Lucas, $\dot{E} . \quad$ N. A. Mth. 19 (1880) 401-.
Conics with variable coefficients, especially confocal conics. Terquem, $O$. N. A. Mth. 4 (1845) 557-.

Mono-confocal conics. Keller, J. Zür. Vjschr. 27 (1882) 1-.

- - , orthogonal conjugate families. Keller, J. Zür. Vjschr. 32 (1887) 33-.

_ - radii vectores of, theorem of Chasles. Gerono, G. C. N. A. Mth. 6 (1847) 150-. - - system. Keller, J. Z. Mth. Ps. 39 (1894) 290-

Conics with 2 common points. Voss, $(D r) A$. [1872] Z. Mth. Ps. 18 (1873) 102-.

-, 3, with common points, invariant conditions. Walker, J. J. L. Mth. S. P. 4 (1873) 404-.

passing through 4 foci of given conic. Cayley, A. QJ. Mth. 5 (1862) 275-.

- - 2 points (fixed), systems. Porchiesi, A. Bologna Ac. Sc. Mm. 3 (1881) 681-.

- -3 points and touching given straight line, system. Hossfeld, $C$. Arch. Mth. Ps. 70 (1884) 253-.

- -4 points, envelope of axes. Laguerre, E. N. A. Mth. 18 (1879) 206-.

$-\ldots-$ - equation. Arndt, $F$. Crelle J. 35 (1847) 83-.

_ - _ - _, focal properties. Recoq, A. N. A. Mth. 5 (1866) 157-.

- $-\ldots$, , foci. Cayley, A. Ph. Mg. 32 (1866) 362-.

- _ - - minimum case. Trudi, N. Nap. Ac. Sc. Mm. 1 (1852-54) 237-.

- - - system. Jacobi, C. G. J. Crelle J. 23 (1842) 243-.

- - - - - - Cayley, A. QJ. Mth. 2 (1858) 206-.

- - - - - Ferrers, N. M. [1866] QJ. Mth. 8 (1867) 259-.

- - - - - Cardinaal, J. N. Arch. Wisk. 12 (1886) 149-; Fschr. Mth. (1885) 626. - _ _ _ _, 3, theorem of Chasles. Faure, $H . \quad$ N. A. Mth. 7 (1888) 31-. - - - - theorems. Wiener, C. Z. Mth. Ps. 9 (1864) 44-.

_ _ _ - or touching 4 lines, involutory theorems. Salmon, G. QJ.Mth.4 (1861) 152-. - of a plane, geometry of. Segre, C. Tor. Ac. Sc. At. 20 (1885) 487-.

- satisfying 4 conditions, Halphen's form of Chasles's theorem. Hirst, T. A. B. A. Rp. (1878) 464.

- in space. Olivier, T. Quetelet Cor. Mth. 3 (1827) 126-.

- - satisfying 7 conditions. Chasles, $M$. C. R. 61 (1865) 389-。

- touching 4 lines. Cayley, A. QJ. Mth. 3 (1860) 94-.

Construction for conics of pencil or other linear system. Wiener, -. D. Nf. B. $\left({ }^{*} 1883\right) 55$.

Contact problems of conics. Cayley, $A$. Crelle J. 39 (1850) 4 -。

Desargues's theorem, extension. Weyr, $E$. Wien $\mathrm{Sb} .58$ (1868) (Ab. 2) 223-.

Determination of conics. Zeuthen, $H . G$. Mth. A. 41 (1893) 539-. 
Envelope and locus of foci of family of conics. Kaufmann, G. Orv.-Termt. Éts. (Termt. Szak) (1898) 1-, (Rv.) 1-.

Equation $S-\lambda S^{\prime}=0$, discussion. Picart, $A$. N. A. Mth. 14 (1875) 31-.

Harmonically circuminscribed conics. Taylor, C. QJ. Mth. 18 (1882) 50-.

Homographies on a conic and their linear systems. Aschieri, F. Mil. I. Lomb. Rd. 22 (1889) 414-, 484-, 558-, 624-.

Homology of 2 conics in a plane. Laudiero, $F$. G. Mt. 21 (1883) 217-.

- 3 homographic figures, conic of. Tarry, G. C. R. 94 (1882) 941-.

Homothetic conics. Chasles, $M$. Gergonne A. Mth. 18 (1827-28) 305-

- - Woepcke, F. Crelle J. 53 (1857) 260-.

- - Lemonnier, $H$. N. A. Mth. 3 (1864) $461-$.

- - with same centre. Barbier, E. C. R. 66 (1868) 907-

- - , system through ends of chord of given conic. Serret, P. N. A. Mth. 3 (1864) 49-.

In-or circum-conics. Spijker, N.C. Mathesis 15 (1895) 105-.

- _ - "descriptive" centres and lines of. Reggio, $Z$. Ven. I. At. 8 (1881-82) 649-.

In-conics of quadric surface. Cayley, $A$. Crelle J. 41 (1851) 73-.

Intersection of fixed conic with variable conic through two fixed points. Appell, $P$. N. A. Mth. 8 (1889) 48-.

Invariants of two conics. Kluyver, J. C. As. Fr. C. R. (1887) (Pt. 2) 132-.

Involution, conics in, special kind. Weyr, E.J. Prag Sb. (1876) 42-.

-, 3 conics in. Weill, -. N. A. Mth. 3 (1884) 19-.

-, 6 conics in, groups. Gerbaldi, $\boldsymbol{F}$. Tor. Ac. Sc. At. 17 (1881) 566-.

Involutory position of conics touching one another. Weyr, E. J. Wien Ak. Sb. 83 (1881) (Ab. 2) 63-.

Joachimsthal's theorem, and properties of system of conics. Jamet, $V$. Mathesis 11 (1891) 105-.

Linear systems. Timerding, H. E. Gött. Nr. (1900) 103-.

Loci and envelopes, with reference to systems of conics. Heller, J. Arch. Mth. Ps. 7 (1889) 325-.

Locus of centres of conics touching two given lines and passing through two given points. Rossi, G. de'. G. Mt. 7 (1869) 174-.

- - intersections of ellipses with certain diametral relations. Faure, $H$. N. A. Mth. 4 (1845) 337-.

- - point of contact of tangents from point to system of conics. Greiner, $M$. Arch. Mth. Ps. 69 (1883) 30-.

Mixed ("gemischte") system of conics $S(3 l, 1 p)$ with imaginary pair of tangents. Tesar, $J$. [1881] Wien Ak. Sb. 84 (1882) (Ab. 2) 194-.

Net of conics, index 2. Vanéček, J. S. Prag Sb. (1886) (Mth.-Nt.) 281-, 314-.

- 4 conics, theorem. Hofmann, $F$. N. A. Mth. 1 (1882) 321-.
Nets of conics whose Jacobian or Hermitian form vanishes identically. Hahn, J. Mth. A. 15 (1879) 111-.

Normals, properties relative to series of. Chasles, M. C. R. 72 (1871) 419-.

Numerical characteristics. Aleksěev, V. G. Mosc. Un. Mm. (Ps.-Mth.) 10 (1893) 206 pp. ; Fschr. Mth. (1893-94) 1036-.

Orthoaxial conics. Salomon, A. Arch. Mth. Ps. 15 (1897) 1-.

Orthogonal conics. Taylor, H. M. QJ. Mth. 26 (1893) 148-.

- trajectories of families of conics. Ruffini, F. P. Bologna Rd. 1 (1897) 62-.

- - - family of conics. Blutel, -. Bll. Sc. Mth. 13 (1889) 255-.

- trajectory of all rectangular hyperbolas having same asymptotes. Durrande, $J$. B. (vi $A d d s$.$) Gergonne A. Mth. 13$ (1822-23) 319-.

- - - - - - - - Querret, Gergonne A. Mth. 13 (1822-23) 321-.

Osculating conics. Weyr, E. [1891] Prag České Ak. Fr. Jos. Rz. (Trída 2) 1 (1892) Art. 5, 14 pp.

Parabolic trajectories, geometry of a system. Habart, C. Lotos 38 (1890) 52-.

Pencil of conics. Meyer, M. N. A. Mth. 14 (1895) 291-.

- Wimain, A. Arch. Mth. Ps. 14 (1896) 149-.

_ _ - , conic having combinant property with reference to. Mertens, $\boldsymbol{F}$. Wien Ak. Sb. 91 (1885) (Ab. 2) 637-.

- - - curve connected with. Sporer, B. Z. Mth. Ps. 38 (1893) 34-.

- - - of index $2 n$. Vaněček, J. S. Prag Sb. (1886) (Mth.-Nt.) 451-.

_ _ _ with 4 real fundamental points cuts a straight line in a point involution. Hossfeld, C. [1882] Z. Mth. Ps. 28 (1883) 51-.

- - - - - - - - metric formulø. Kantor, S. [1878] Wien Ak. Sb. 78 (1879) (Ab. 2) 905-.

- - - , reduction to pencil of rays. Trebitscher, M. [1879] Wien Ak. Sb. 80 (1880) (Ab. 2) 913-.

__ _ _ symmetrical, to find the member degenerating into a pair of straight lines. Jarolimek, $V$. Prag České Ak. Fr. Jos. Rz. (Tŕida 2) 7 (1898) Art. 21, 7 pp.; Fschr. Mth. (1898) 466.

- - tangential, property. Ferrers, N.M. Mess. Mth. 1 (1862) 159-.

Pencils of conics. Gordan, P. Mth. A. 19 (1882) 529-.

- - - Thompson, H. D. Am. J. Mth. 9 (1887) 185-.

- - Chasles's theorem $\alpha \mu+\beta \nu$. Hurwitz, A., \& Schubert, H. Gött. Nr. (1876) 503-.

_- - constructions. Bergmann, F. [1881] Arch. Mth. Ps. 67 (1882) 177-; 68 (1882) 404-.

- - - generation, new method. Vaněček, J. S., \& Vaněcek, M. N. [1885] Prag Sb. (1885) (Mth.-Nt.) 180-; St Pét. Ac. Sc. Bll. 30 (1886) 153-. 
Pencils of conics, loci of centres. Hunyady, $J$. (xII) Mth. Term. Éts. 1 (1883) 205-; Mth. Nt. B. Ung. 1 (1882-83) 183-.

,- 2 , conics as 2.2 relation of. Janisch, $E$. Mh. Mth. Ps. 11 (1900) 170-.

Polar conics. Rubini, R. N. A. Mth. 14 (1855) 237-

- correspondence between conics as envelopes and as loci, defined by plane quartic. Ciani, E. Rm. R. Ac. Linc. Rd. 4 (1895) (Sem. 2) 274-.

- reciprocal conics in systems of conics. Crocchi, L. [1875] G. Mt. 14 (1876) 83-.

Properties. Chasles, M. C. R. 59 (1864) 93-. Quadrilateral and systems of conics. Klug, L. Arch. Mth. Ps. 1 (1884) 304-.

Similar conics. Weyr, E. Wien Sb. $62(1870)$ (Ab. 2) 261-.

- - theorem. Terquem, O. N. A. Mth. 2 (1843) 268-.

Singularities. Igel, B. Wien Ak. Sb. 75 (1877) (Ab. 2) 445-.

System. Mahler, E. [1881] Arch. Mth. Ps. 68 (1882) 78 -

-. Battaglini, G. G. Mt. 30 (1892) 287-.

- inscribed to conic, and touching 3 inscribed conics. Casey, J. [1866] Ir. Ac. P. 9 (1867) $396-$.

Systems cutting given conics at given angles, etc. Chasles, M. C. R. 58 (1864) 425-.

- projective with one another, and their generation. Mahler, $E$. Arch. Mth. Ps. 66 (1881) 358-.

Theorems on conics, circles, and confocal conics. Steiner, J. Crelle J. 66 (1866) 237-.

2 conics and Salmon's conic $F$. Ferrers, N.M. QJ. Mth. 7 (1866) 20-.

4 conics inseribed in same conic and passing through 3 points. Cayley, A. [1863] (vII) Ph. Mg. 27 (1864) 42-.

- -, system of, property. Woepcke, $\boldsymbol{F}$. Liouv. J. Mth. 19 (1854) 345-.

5 conics and 5 straight lines anharmonically corresponding. Jonquières, E. de. N. A. Mth. 15 (1856) 369-.

\section{Metrical and projective pro- perties of quadric surfaces.}

Livet, -. Par. Éc. Pol. Cor. 1 (1804-08) 28-.

Poisson, S. D. Par. Ec. Pol. Cor. 1 (1804-08) 237-.

Brianchon, C. J. Par. Éc. Pol. J. $13^{\circ}$ cah. (1806) 297-.

Monge, G. Par. Éc. Pol. Cor. 2 (1809-13) 313-.

Bret, - Gergonne A. Mth. 4 (1813-14) 93-.

(Monge's theorems, proof.) Chasles, M. Par. Éc. Pol. Cor. 3 (1814-16) 328-.

Lévy, A. Quetelet Cor. Mth. 4 (1828) 18 -.

Bobillier, -. Quetelet Cor. Mth. 4 (1828) 27-.

Chasles, M. Quetelet Cor. Mth. 4 (1828) 294-; 5 (1829) 173-; 6 (1830) 272-, 312-; 10 (1838)
Hesse, L. O. [1837] Crelle J. 18 (1838) 101-.

Chelini, D. G. Arcad. 94 (1843) 49-.

Grimaldi, $F$. Nap. Rd. 2 (1843) 169-.

Soufflet, -. Liouv. J. Mth. 15 (1850) 104-.

Marchesano, S. Tortolini A. 3 (1852) 517-.

Jouffet, E. N. A. Mth. 15 (1856) 440-.

Mention, J. N. A. Mth. 16 (1857) 207-.

Berger, -. [1858] Mntp. Mm. Ac. 4 (1858-60) 31-.

Fournier-Vannson, - - N. A. Mth. 17 (1858) 334 .

Painvin, L. N. A. Mth. 17 (1858) 370-, 403-, 457-; 18 (1859) 33-, 49-, 89-, 407-; 19 (1860) 144-; 20 (1861) 177-.

Méray, C. Tortolini A. 3 (1860) 30-.

Brassinne, E. N. A. Mth. 3 (1864) 248 -

Grunert, J. A. Arch. Mth. Ps. 45 (1866) 75-.

Hioux, $V$. N. A. Mth. 16 (1877) 303-.

Hunyady, J. (xIr) Mag. Tud. Ak. Éts. 13 (No. 6) (1879) 131-; Mag. Tud. Ak. Étk. (Mth.) 7 (1881) (No. 5) 36 pp.; (x) Crelle J. Mth. 89 (1880) 47-.

Le Paige, C. M. M. H. H. Brux. Ac. Bll. 5 (1883) 618-.

Biehler, C. N. A. Mth. 8 (1889) 573-.

Fleck, (l'abbé) -. Metz Ac. Mm. 68 (1890) 93-.

Aconic function of 6 vectors. Hamilton, (Sir) $W . R$. Ir. Ac. P. 5 (1853) 177-.

Analogue of Pappus's theorem relative to surfaces. Legoux, A. [1882] (xII) Isère S. Bll. 13 (1884) 11-.

- in space of Desargues's theorem. Valyi, $J$. Mh. Mth. Ps. 4 (1893) 121-.

- - - - Joachimsthal's circles. Schoute, P. H. Amst. Ak. Vs. 7 (1899) 6-; Amst. Ak. P. 1 (1899) 1-.

- - stereographic projection. Matheus, G. B. Mess. Mth. 17 (1888) 151-.

Analogues of theory of conics. Townsend, $R$. [1871] QJ. Mth. 11 (1871) 347-; Mess. Mth. 1 (1872) 113-.

Anharmonic ratio in which generator of ruled quadric cuts tetrahedron. Grave, $P$. Kazan S. Ps.-Mth. Bll. 7 (1898) 79-.

- ratios. Battaglini, G. G. Mt. 12 (1874) 266-.

\section{Axes of Quadric Surfaces.}

Axes, cyclic planes, etc. Painvin, L. N. A. Mth. 13 (1874) 113-.

- obtained by concentric sphere. Housel, N. A. Mth. 8 (1869) 260 -

Central projections, axes. Pelz, C. Wien Sb. 66 (1872) (Ab. 2) 481-.

- section of ellipsoid, axes. Lacour, - . N. A. Mth. 17 (1898) 272-.

Coaxial quadrics, criterion for. Finger, $J$. Wien Ak. Sb. 103 (1894) (Ab. 2a) 1061-.

- similar quadrics, constant volume cut off from one, by tangent plane to other. Minich, $S . R$. A. Sc. Lomb. Ven. 6 (1836) 169-.

Cones, axes, construction. Šolin, J. Prag Sb. (1885) (Mth.-Nt.) 164-; Casopis 16 (1887) 1-; Fschr. Mth. (1887) 573-.

-, -, determination. Pelz, C. Wien Ak. Sb. 92 (1886) (Ab. 2) 410- 
Cones, principal axes. Page, C. E. N. A. Mth. 2 (1843) 334-, 379-.

Conics on quadric, axes. Reye, T. A. Mt. 2 (1868-69) 1-.

Conjugate rectangular axes. Brassinne, $E$. Toul. Mm. Ac. 2 (1858) 406-.

Construction. Picquet, H. N. A. Mth. 7 (1868) 456-.

Determination. Pelz, C. Wien Ak. Sb. 69 (1874) (Ab. 2) 215-

Diametral section of ellipsoid, axes. Gilbert, $P$. Mathesis 8 (1888) 247-.

Paraboloid, hyperbolic, with two given generators, axes. Wilhelm, F. Mh. Mth. Ps. 2 (1891) 345-.

Plane sections, axes. Barbarin, - Mathesis 6 (1886) 25-, 49-.

Principal axes. Binet, J. P. M. Par. Ec. Pol. Cor. 2 (1809-13) 17-.

- -. Battaglini, G. G. Mt. 9 (1871) 38-.

- - Geiser, C. F. [1876] Crelle J. Mth. 82 (1877) 47-

- - Brückel, P. Crelle J. Mth. 119 (1898) 210-, 313-.

- - of conic on quadric, resolution of condition of equality. Hesse, L. O. Crelle J. 60 (1862) 305-.

- - determination. Bourdon, -. Par. Ec. Pol. Cor. 2 (1809-13) 189-, 250-.

- - lengths. Bret, - Gergonne A. Mth. 2 (1811-12) 33-.

- - position. Bret, - Gergonne A. Mth. 2 (1811-12) 144-.

Rectangular axes, Steiner's theorem. Hêment, $F$. N. A. Mth. 10 (1851) 119-.

Relation between synthetic and analytical methods of solution. Lolli, C. G. Mt. 29 (1891) 87-.

Section of $\frac{x^{2}}{a^{2}}+\frac{y^{2}}{b^{2}}+\frac{z^{2}}{c^{2}}=1$ by $l x+m y+n z=0$, axes. Taylor, S. QJ. Mth. 4 (1861) 167-.

Sections of quadrics, axes, determination. Drasch, $H$. Wien Ak. Sb. 87 (1883) (Ab. 2) 1226-.

Symmetry, axes of. Blâzek, G. Prag Sb. (1870) 10-.

Symptotic axis, surfaces with one. Escherich, G. von. Arch. Mth. Ps. 60 (1877) 22-.

- - , - - Czuber (or Čuber), E. Arch. Mth. Ps. 61 (1877) 351-.

Central surfaces, property. Minich, S. R. Ven. At. 9 (1864) 1211-.

- - space relations. Franke, T. Grunert Arch. 12 (1849) 378-

Centres, determination. Dienger, $J$. Grunert Arch. 16 (1851) 460-.

- of similitude. Cayley, A. Crelle J. 31 (1846) 227-.

Circle, geometry of, and quadrics. Müller, E. Crelle J. Mth. 122 (1900) 30-.

Concyclic quadries. Jeffery, H. M. QJ. Mth. 11 (1871) 225-.

Condition that a quadric should be developable, deduction from. Zeuthen, H. G. Ts. Mth. 4 (1886) 128-; Fschr. Mth. (1886) 760.
Condition that 4 straight lines lie on hyperboloid of one sheet. Strnad, A. Časopis 24 (1895) 34-; Fschr. Mth. (1895) 592.

- - - - quadric. Gergonne, J. D. Gergonne A. Mth. 19 (1828-29) 241-.

Cones. Chasles, M. Brux. Ac. Sc. Mm. 6 (1830) $58 \mathrm{pp}$.

-. Graves, C. Ir. Ac. P. 2 (1844) 54-.

- Torry, A. F. Mess. Mth. 1 (1862) $72-$.

-. Mirza-Nizam, -. N. A. Mth. 5 (1866) 105-.

-, analogue of nine-point conic. Holgate, $T$. F. A. Mth. 7 (1892-93) 73-.

-, hyperboloidal, graphic construction. Arzberger, F. A. Gén. Civ. 1 (1872) 259-.

- and hyperboloids connected with systems of forces. Cassani, P. G. Mt. 14 (1876) 146-.

-, locus of centres of sections. Dalican, d. Marquet, -. N. A. Mth. 17 (1858) 172-.

-, properties, two. Luchterhand, $A$. $R$. Grunert Arch. 4 (1844) 87-.

- right circular. Ruth, F. Wien Ak. Sb. 95 (1887) (Ab. 2) 240-.

- , - - theorems, and projection of section on base. Talbot, W. H. F. Gergonne A. Mth. 14 (1823) 123-.

- of rotation round trihedral angle, common generators. Klug, L. Mh. Mth. Ps. 8 (1897) 54-.

-, special class. Fiedler, W. Ziir. Vjschr. 36 (1891) 65-.

- and spheroconics. Lebesgue, V.A. N. A. Mth. 7 (1848) 150-.

Conic in space. Geer, P. van. Arch. Néerl. 22 (1888) 58-.

Conical and cylindrical surfaces in oblique projection. Suppan, V. [1879] (xII) Mag. Tud. Ak. Étk. (Mth.) 7 (1881) (No. 9) 14 pp.

Conjugate diameters. Mainardi, G. Brugnatelli G. 10 (1827) 333-.

- - Dahlander, G. R. Stockh. Öfv. 16 (1859) 387-.

- - Painvin, L. N. A. Mth. 20 (1861) 130-. - - properties. Göpel, A. Grunert Arch. 4 (1844) 202-.

- - - - deduced from Lagrange's relations. Terquem, $O$. N. A. Mth. 1 (1842) 497-.

- points. Brassime, E. Liouv. J. Mth. 7 (1842) 120-.

Construction and classification by projective configurations. Seydewitz, $F$. Grunert Arch. 9 (1847) 158-.

- from imaginary elements. Bobek, $K$. Prag Sb. (1882) 65-.

- of quadric. Cardinaal, J. Z. Mth. Ps. 27 (1882) 119-.

- _- given nine conjugate 2 nd class surfaces. Neumann, $H$. Mh. Mth. Ps. 11 (1900) 114.

Constructions of quadrics and plane cubies. Engel, J. H. Zür. Vjschr. 34 (1889) 299-.

Contacts of cones of rotation. Żmurko, W. [L.] [1873] (xII) Krk. Ak. (Mt.-Prz.) Pam. 1 (1874) 57-.

-: conic touching 3 conics on quadric. Durrande, J. B. Gergonne A. Mth. 7 (181617) $27-$. 
Contacts, problems, two. Mertens, F. Z. Mth. Ps. 25 (1880) 156-.

- : quadric having double contact with two given quadrics. Dixon, A. C. QJ. Mth. 26 (1893) 207-.

- of quadrics, 2. Carvallo, E. N. A. Mth. 9 (1890) 586-.

- - - with cones. Livet, - Par. Ec. Pol. Cor. 1 (1804-08) 75-.

- - - - Garnier, J. G. Quetelet Cor. Mth. 2 (1826) 17-.

- - and conics, enumeration. Sylvester, J. J. Ph. Mg. 1 (1851) 119-; 7 (1854) 331-。

- - criteria of Monge and Chasles. Hube, $K$. (vi Adds.) Krk. Roczn. Uniwers. 14 (1831) 189-.

- : spheres having double contact with quadrics. Servais, C. Brux. Ac. Bll. 26 (1893) 91-.

-: tangent cone cutting two segments homographically, locus of vertex. Mathews, G. B. QJ. Mth. 28 (1896) 190-

Cubature, elementary, of quadrics. Schlömilch, O. Grunert Arch. 14 (1850) 154-.

Denomination, new, of quadrics. Flauti, $V$. Nap. Rd. 1 (1842) 110-.

Developable quadrics through points, generation. Zimmermann, H. E. M. O. Z. Mth. Ps. 28 (1883) 255-.

Diameters, general theorems. Chasles, $M$. Quetelet Cor. Mth. 6 (1830) 255-.

-, 3 perpendicular, properties. Tillot [Tillol], $J$. Toul. Mm. Ac. 6 (1862) 365-.

-, - - sum of inverse square. Anon. (vi 561) Gergonne A. Mth. 18 (1827-28) 368-.

-, principal and conjugate. Hachette, J. N.P. Par. S. Phlm. Bll. 3 (1813) 310-.

—, semi-. Bobillier, -. Gergonne A. Mth. 19 (1828-29) 249-.

Diametral planes. Cayley, A. Camb. and Dubl. Mth. J. 1 (1846) 274-.

- -. Biehler, C. N. A. Mth. 8 (1889) 247-. - of hyperboloid. Tesch, J.W. N. Arch. Wisk. 1 (*1875) 194-.

- -, theory. Transon, (Prof.) A. N. A. Mth. 19 (1860) 182-.

Director sphere and connected theorems. Bobillier, - Gergonne A. Mth. 18 (182728) 230 .

\section{Ellipsoid.}

Courtois, - N. A. Mth. 2 (1843) 90-.

Michaëlis, J. P. (vm) Liége Mm. S. Sc. 18 (1863) 351-.

apparent size from any point of space. Schwarz, C. H. A. Gött. Nr. (1883) 39-.

central, and principal axes. Mylord, $H$. (xII) Ts. Mth. 5 (1869) 167-.

conjugate diameters. Gergonne, J. D. Gergonne A. Mth. 5 (1814-15) 29-.

- - equal. Gergonne, J. D. Gergonne A. Mth. 12 (1821-22) 157-.

- planes. Dahlander, G. R. Stockh. Öfv. 15 (1858) 207-

construction by 2 sliding spheres. Hamilton, (Sir) $W . R$. Ir. Ac. P. 4 (1850) 341-. - and tangent planes. Gudermann, $C$. Crelle J. 42 (1852) 282. contact boundary with 3 planes. Pelletreau, -. As. Fr. C. R. (1888) (Pt. 2) 103-.

- - - - determination of curve. Plarr, G. Edinb. R. S. T. 33 (1888) 465-; 35 (1890) 471-.

description by point in line. Booth, $J . \mathbf{P h}$. Mg. 21 (1842) 176-, 444-.

diameters. Chasles, M. Par. Ec. Pol. Cor. 3 (1814-16) 302-.

elongated, area. Peyronny, A. N. A. Mth. 3 (1814) 466-.

length and breadth, and reduced length and breadth. Grunert, J.A. Grunert Arch. 36 (1861) 79-.

mean radius. Schlömilch, O. Leip. B. 11 (1859) 87-.

minimum volume with area of several central sections given. Borchardt, C. W. Berl. Mb. (1872) 505-.

projection, stereographic. Grunert, J.A. As. Nr. 40 (1855) 297-.

properties. Weddle, T. Camb. and Dubl. Mth. J. 2 (1847) 13-.

property. Gilbert, L. P. (xII) Mathesis 3 (1883) 238-.

space-locus connected with. Glaisher, J.W.L. QJ. Mth. 16 (1879) 283-.

and sphere, Dirichlet's problem. Bjerknes, C. A. Gött. Nr. (1873) 439-.

sphere of surface most similar to given ellipsoid, radius. Anon. [? Lindenau, B. von.] (vi 1307) Zach M. Cor. 16 (1807) 424-.

spheres, osculating, of ellipsoids of revolution. Mugnaini, E. G. Mt. 16 (1878) 270-.

and spheres, theorems. Kilbinger, - Ens. Mth. 2 (1900) 196-.

- spherical trigonometrical formula. Grunert, J. A. Grunert Arch. 10 (1847) 156-.

surface, geometry. Clifford, W. K. [1872] L. Mth. S. P. 4 (1871-73) 215-

thread-construction. Staude, E. O. Mth. A. 20 (1882) 147-.

- -. Finsterwalder, S. Mth. A. 26 (1886) 546 -

and 3 unequal axes. Olivier, $T$. Liouv. J. Mth. 3 (1838) 145-.

volume. Martus, H. Arch. Mth. Ps. 46 (1866) 419-.

Ellipsoidal regular polyhedra. Breton [de Champ], P. C. R. 27 (1848) 154-.

Elliptic cone. Enneper, A. Schlömilch Z. 7 (1862) 354.

\section{Equations of Quadrics.}

(See also 2040 and 7200.)

De Morgan, - - [1832] Camb. Ph. S. T. 5 (1835) 77-.

Kitchener, F. E. (III) Mess. Mth. 2 (1864) 175-

Axes, equations. Plücker, J. Crelle J. 24 (1842) 60-, 283-.

-, lengths in tetrahedral coordinates. Ferrers, N. M. QJ. Mth. 4 (1861) 140-.

- of plane section in oblique coordinates. Pomey, É. N. A. Mth. 8 (1889) 88-. 
Central surface, equation. Graves, C. Ir.Ac. P. 4 (1850) 188-.

Circular sections. Frost, P. Camb. and Dubl. Mth. J. 1 (1846) 133-.

- - in tetrahedral coordinates. Ferrers, N. $M$. QJ. Mth. 5 (1862) 78-.

Cone circumscribing quadric surface, equation. Dostor, G. Arch. Mth. Ps. 57 (1875) 191-.

Curves on ruled quadrics, Plücker's coordinates. Plücker, J. Crelle J. 34 (1847) 341-, 360-.

Discussion of equation. Ripert, L. N. A. Mth. 17 (1898) 413-.

Ellipsoid and enveloping cone, equations referred to axes of cone. Scott, G. QJ. Mth. 6 (1864) 258-.

-, equation, Hamilton, (Sir) W. R. Ir. Ac. P. 4 (1850) 324-.

- and hyperboloid, equation as resolvent of biquadratic equation for focal distances. Staude, O. Leip. Mth. Ps. B. 49 (1897) 75-. General equation in oblique coordinates. Brisse, C. N. A. Mth. 1 (1882) 207-.

- - , reduction. Thomson, (Sir) W. Camb. Mth. J. 4 (1845) 227-.

- - tetrahedral. Hart, $H$. L. Mth. S. P. 12 (1880-81) 135-.

Grassmann's methods, applications to quadric curves and surfaces. Miller, $E$. Crelle J. Mth. 115 (1895) 234-.

Normals, equation determining. Caspary, $F$. Crelle J. Mth. 83 (1877) 72-.

Pairs of quadrics inscribed in quadrics. Casey, J. [1868] A. Mt. 2 (1868-69) 303-.

Partial differential equation. Scherck, -. [1883] Brem. Ab. 8 (1884) 366-.

Planes through 2 given points touching quadric, equation. Taylor, H. M. L. Mth. S. P. 11 (1879-80) 141-.

Principal planes, equation. Forestier, $C . \mathrm{N}$. A. Mth. 6 (1867) 355-.

Quadric through 9 points, equation. Poudra, -. N. A. Mth. 17 (1858) 297-.

Quadrics in Boothian tangential coordinates. Jeffery, H. M. QJ. Mth. 9 (1868) 309-.

- homogeneous linear coordinates. Aschieri, F. Mil. I. Lomb. Rd. 9 (1876) 222-.

- in- and circum-scribed to tetrahedron, tetrahedral equations. Haton de la Goupillière, J. N. N. A. Mth. 4 (1865) 241-.

- in plane coordinates. Pittarelli, G. [1874] G. Mt. 13 (1875) 204-, 298-.

- - 4-point tangential coordinates. Jeffery, H. M. [1868] QJ. Mth. 10 (1870) 97-.

- quadriplanar coordinates. Jeffery, H. M. QJ. Mth. 10 (1870) 1-.

- referred to oblique axes and analytical geometry in space. $\dot{Z}$ murko, L. [1865] Wien D. 26 (1867) (2te Ab.) 63-.

- - 3 tangent planes. Fenwick, S. Mathematician 2 (1847) 177-.

- touching 3 or 4 planes or inscribed in parallelepiped, equations. Weddle, $T$. Mathematician 2 (1847) 258-.

Reduction. Battaglini, G. Nap. Rd. 1 (1862) 79-.
Rotation surface, criterion for. Mondot, Par. Éc. Pol. Cor. 2 (1809-13) 205-.

Simplification by transformation of coordinates. Gerono, G. C. N. A. Mth. 15 (1856) 430 .

Solution. Poletti, G. Mod. Mm. S. It. 19 (1821) 30 -

Squares of principal axes, equation. Hachette, J. N. P. Par. Ec. Pol. Cor. 2 (1809-13) $324-$.

Stereometric equation. Grassmann, $H$. [1852] Crelle J. 49 (1855) 37-.

Systems of planes represented by an equation of 2nd degree, number of. Painvin, L. N. A. Mth. 10 (1871) 433-.

Tangent cone, equation referred to axes. Malet, J. C. [1882] Ir. Ac. P. 3 (1883) 594-

Transformations. Mossbrugger, L. Grunert Arch. 3 (1843) 430-.

Foci and Focal Properties of Quadrics.

Analogy of certain curves to foci. Chasles, $M$. Brux. Ac. Bll. 2 (1835) 35-.

Bifocal chords. Rutledge, J. Y. Camb. and Dubl. Mth. J. 5 (1850) 69-.

Cones, oblique, focal properties. Chasles, $M$. Liouv. J. Mth. 2 (1837) 388-.

Focal conics. Jellett, J. H. Ir. Ac. P. 2 (1844) 92-.

- generation of quadrics. Willock, W. A. Camb. and Dubl. Mth. J. 4 (1849) 149-.

- lines. Rees, R. van. Quetelet Cor. Mth. 4 (1828) 37-; 5 (1829) 361-.

- - of ellipsoid. Ionescu, B. G. [Bucarest S. Sc. Bll. 6 (1897)] 165-.

- - - tangent cone to quadric. Jacobi, C. G. J. Crelle J. 12 (1834) 137-.

- properties. Bobillier, -. Quetelet Cor. Mth. 3 (1827) 281-.

- -. Maccullagh, J. Ir. Ac. P. 1 (1836-40) 89-; 2 (1840-44) 446-.

- -. Booth, J. Ph. Mg. 17 (1840) 432-.

- - Heilermann, -. N. A. Mth. 17 (1858)

242-; Crelle J. 56 (1859) 345-.

- - (Heilermann). Weierstrass, -. Berl. Mb. (*1858) 270-.

$--(-)$. Dewulf, - . N. A. Mth. 18(1859) 46-.

- Oliver, J. E. [1865] Am. Ac. P. 7 (1868) 39-.

- -. Milinowski, -. Crelle J. Mth. 85 (1878) 88.

- - Staude, O. Mth. A. 27 (1886) 253-; 50 (1898) 398-.

- - and Jacobi's method of generating an ellipsoid. Townsend, $R$. Camb. and Dubl. Mth. J. 3 (1848) 1-, 97-, 148-.

— property of conics, analogue. Schroeter, H. E. Berl. Ak. Mb. (1877) 594-.

- spheres of quadrics, and focal circles of conics. Haillecourt, $A$. [1878] Bordeaux S. Sc. Mm. 3 (1880) xi-.

- or umbilical properties. Ingram, J. K. Ir. Ac. P. 3 (1847) 442-.

Focals. Cayley, A. Mess. Mth. 19 (1890) 113-. 
Focals, theory, and quadrics. Amiot, B. Liouv. J. Mth. 10 (1845) 109-.

Foci. Bobillier, - Quetelet Cor. Mth. 4 (1828) 157-.

-. Beccaro, T. del. Tortolini A. 2 (1859) 30-.

- Haillecourt, A. N. A. Mth. 17 (1878) 457-.

- of contours, general method of determining. Pelz, C. Wien Ak. Sb. 75 (1877) (Ab. 2) 175-, 320; 77 (1878) (Ab. 2) 259-.

- - curve of intersection of two quadrics. Catalan, E. C. N. A. Mth. 6 (1847) 421-. - and directrices. Crosnier, - N. A. Mth. 13 (1874) 266-.

- - Lemonnier, $H$. N. A. Mth. 14 (1875) 216-.

- - - , synthetic treatment. Hicks, W. M. [1873] Mess. Mth. 3 (1874) 122-.

- of quadrics and rotation surfaces, determination. Lemonnier, H. N. A. Mth. 13 (1874) 318 -

Locus of foci of central sections of quadric. Painvin, L. N. A. Mth. 5 (1866) 553-.

- - - - _ - (Painvin). Böklen, $O$. N. A. Mth. 18 (1899) 370-

- questions. Azzarelli, M. Rm. N. Linc. At. 27 (1874) 405-.

Paraboloid, focal properties. Staude, $O$. Leip. Mth. Ps. B. 47 (1895) 483-.

,$--\frac{1}{-}$, algebraic basis. Staude, $O$. Leip. Mth. Ps. B. 49 (1897) 172-.

-, pseudo-focus. Jung, G. Mil. I. Lomb. Rd. 15 (1882) 407-.

Plane section, foci, determination. Painvin, $L$. N. A. Mth. 3 (1864) 481-.

,,---- Saltel, L. N. A. Mth. $9(1870)$ 463-.

Surfaces without foci. Chasles, M. Liouv. J. Mth. 1 (1836) 187-.

Umbilical lines. Titeica, $G$. [Bucarest S. Sc. Bli. 6 (1897)] 401-.

- projection. Ascione, E. [1900] Nap. Ac. At. 10 (1901) No. 2, 33 pp.

Umbilici. Servais, C. Brux. Ac. Bll. 17 (1889) 366-.

- of ellipsoids. Stegmann, $F$. Grunert Arch. 8 (1846) 49-.

- - - Verdam, G. J. [1851] Amst. Ts. Ws. Nt. Wet. 5 (1852) 42-.

Formulæ relative to quadrics. Spottiswoode, $W$. Crelle J. 42 (1852) 169-.

Function $a t^{2}+b u^{2}+c t u$, geometrical interpretation, and applications. Weddle, $T$. Camb. and Dubl. Mth. J. 9 (1854) 67-.

\section{Generation and Generators of Quadrics.}

Generation. Dupin, C. Par. Ec. Pol. Cor. 1 (1804-08) 144-.

-. Salmon, G. Ir. Ac. P. 3 (1847) 536-.

-. Dienger, J. Grunert Arch. 16 (1851) 430-

-. Azzarelli, M. Rm. N. Linc. At. 37 (1884) 205-.

- by conics. Lenthéric, -. N. A. Mth. 6 (1847) 5-.
Generation and discussion. Amiot $[A m y o t], B$. Liouv. J. Mth. 8 (1843) 161-.

- - (Amyot). Cauchy, A. L. C. R. 16 (1843) 783-.

--1 - Chasles, M. C. R. 16 (1843) 828-.

- - (priority question). Amyot, B. C. R. 16 (1843) 938-.

$---(--)$. Poncelet, J. V. C. R. 16 (1843) 947-.

1105-. $(--)$. Chasles, M. C. R. 16 (1843) , double, by movement of circle. Brasseur, J. B. Liège Mm. S. Sc. 1 (1843) 157-.

- by homographic plane pencils. Jacobi, C. G. J. Crelle J. 73 (1871) 179-.

-, Jacobi's method. Hermes, O. [1870] Crelle J. 73 (1871) 209-.

-, - - Sturm, R. Crelle J. Mth. 122 (1900) 263-.

-, mechanical. Plücker, J. Crelle J. 34 (1847) 357-.

-, modular and umbilical, theory. Tillol, -. Toul. Mm. Ac. 2 (1864) 80-.

-, Salmon's and MacCullagh's methods. Newson, H. B. A. Mth. 6 (1891-92) 198-

Generator, condition that 2 quadrics should have one common. Bourlet, C. N. A. Mth. 13 (1894) 434-.

Generators. Hachette, J. N. P. Crelle J. 1 (1826) 339-.

Hyperboloid, generation, method. Olivier, $T$. Par. S. Phlm. N. Bll. (1833) 139-.

-, orthogonal, mode of generation. Ruth, $F$. [1879] Wien Ak. Sb. 80 (1880) ( $A b, 2)$ 257-.

- of 1 sheet and hyperbolic paraboloid, rectilinear generators. Gerono, G. C. N. A. Mth. 15 (1856) 388-.

Ts. 6 (1864) 38-.

Quadrics as generated by projective system of spheres. Röllner, F. Z. Mth. Ps. 24 (1879) 116-.

Homographic transformation of quadric into itself. Cayley, A. Ph. Mg. 6 (1853) 326-; 7 (1854) 208-.

Homographics, undevelopable uni-quadric. Gardiner, M. R. S. P. 16 (1868) 389-.

Homography and application to quadrics. Schoute, P. H. [1870] Arch. Néerl.6 (1871) 348-.

Homothetic conics on two quadrics. Jarolimek, V. Prag České Ak. Fr. Jos. Rz. (Tř̈ida 2) 7 (1898) Art. 20, 5 pp.; Fschr. Mth. (1898) 470.

Hyperbolas, rectangular, lying on quadric surface. Rupp, O. [1882] Wien Ak. Sb. 86 (1883) (Ab. 2) 909-.

\section{Hyperboloid and Hyperbolic Paraboloid.}

Conjugate diameters. Chasles, M. Quetelet Cor. Mth. 5 (1829) 137-.

Hyberboloid. Marrecas Ferreira, L. F. G. Teix. J. Sc. 7 (1886) 49-. 
Hyperboloid with 3 given generators, and resultant of 4 forces. Berg, F.J.van den. N. Arch. Wisk. 6 (*1880) 183-.

- given plane section, construction. Gra. berg, $F$. Zür. Vjschr. 10 (1865) 64-; 12 (1867) 391-.

- of 1 sheet. Magnus, L. J. Gergonne A. Mth. 16 (1825-26) 33-.

_ - Chasles, M. Liouv. J. Mth. 1 (1836) 324-; 4 (1839) 348-; C. R. 52 (1861) 1041-, 1094-.

_- _. Mannheim, A. C. R. 102 (1886) 253-.

_ _ - , and Cartesian ovals. Vries, J. de. Amst. Ak. Vs. 4 (1896) 252-; Fschr. Mth. (1896) 491.

- - - centre, construction. Seydewitz, F. Grunert Arch. 10 (1847) 59-.

- - - contrining parallelepipeds. Hachette, J. N. P. Par. S. Phlm. Bll. (1823) $71-$.

- _ - - curves connected with generators. Tovnsend, R. Camb. and Dubl. Mth. J. 4 (1849) 66-.

_ _ and hyperbolic paraboloid. Chasles, M. Quetelet Cor. Mth. 8 (1834) 128-; 11 (1839) 228-.

- - - - - Schoenflies, A. Z. Mth. Ps. 23 (1878) 269- ; 24 (1879) 62-.

_ _ _ _ _ _-, plane sections, and shadows. Niemtschik, $R$. Wien Sb. 61 (1870) (Ab. 2) 381-.

_ _ - - parameter of parabolic section. Lemonnier, $H$. N.A.Mth.14 (1875) 167-,169_ _ _ properties. Poudra, - N. A. Mth. 17 (1858) 158-.

$--1-\frac{1}{-}$, property. Glivier, T. Par. S. Phlm. PV. (1848) 39-.

- - - - Bauer, G. Münch. Ak. Sb. 10 (1880) 635-.

- _ - - - 3 of 3 pairs of parallel generators. Schroeter, H. E. Münch. Ak. Sb. 11 (1881) 238-.

_- - simple. Schroeter, H. E. Crelle J. Mth. 85 (1878) 26-.

- - - tangent plane. Hachette, J. N.P. Par. Ec. Pol. Cor. 2 (1809-13) 329-.

- -2 sheets. Unferdinger, $F$. Grunert Arch. 27 (1856) 476-.

Hyperboloidal straight lines derivable from tetrahedron and quadric. Doehlemann, $K$. Arch. Mth. Ps. 17 (1900) 160-.

Paraboloid, hyperbolic. Verdam, G.J. Amst. N. Vh. 10 (1844) 1-.

-, - mould-board of plough, description. Hachette, J. N. P. (xii) Fr. S. Ag. Mm. (1821) 525-.

-, rectangular, hyperbolic, locus of point equidistant from two straight lines, MottaPegado, L. P. da. [1867] Lisb. J. Sc. Mth. 1 (1868) 188-

Parallelepipeds determined by 3 generators of one system on hyperboloid. Mogni, A. G. Mt. 4 (1866) 321-.

Rectangular hyperboloids. Vogt, $H$. Crelle J. Mth. 86 (1879) 297-.

- - Schumann, A. Z. Mth. Ps. 26(1881) 136-. - -. Cazamian, A. N. A. Mth. 13 (1894)378-.
Ruled quadrics, analytical character. Haillecourt, A. [1879] Bordeaux S. Sc. Mm. 3 (1880) liv.

-, models, construction. Freeman, $A$. Mess. Mth. 4 (1868) 226-.

_ _, shortest path consisting of straight lines between two points on. Campbell, J. E. Mess. Mth. 23 (1894) 130-.

- -, surface ruled in two directions and undevelopable. Hachette, J. N. P. Par. S. Phlm. Bll. (1832-33) 36-.

- surfaces and congruences, theorems. Klug, L. Mh. Mth. Ps. 9 (1898) 110-.

Skew quadrics. Durrande, $H$. ' N. A. Mth. 6 (1867) 168-, 207-.

- -. Weyr, E. [1898] Prag České Ak. Fr. Jos. Rz. (Trida 2) 8 (1899) Art. 6, 8 pp.; Prag Fr. Jos. Ac. Sc. Bll. (Mth. Nt.) 5 (1898) 79.

- problems. Fouret, G. N. A. Mth. 8 (1889) 34-.

- , properties. Brasseur, J. B. Brux. Ac. Bll. 18 (1851) (pte. 2) 41-.

Tetrahedra and hyperboloids, "G. H." relation of straight lines. Casey, J. Mess. Mth. 3 (1866) 178-.

- - - - - - Routh, G. R. R. Mess. Mth. 26 (1897) 145-.

Triplet of straight lines on hyperboloid. Bauer, G. Münch. Ak. Sb. 11 (1881) 241-.

Hypercyclic planes (planes cutting quadrics in rectangular hyperbolas). Gillet, $J$. Mathesis 12 (1892) 153-, 180-, 223-.

Imaginary projection of quadric surfaces and twisted quartic curves. Retali, V. [1885] Bologna Rd. (1885-86) 21-.

Indices of points, straight lines, and planes with regard to quadric surface, theory. Neuberg, J. N. A. Mth. 9 (1870) 317-, 360-, 399-, 433-.

Inscribed, Circumscribed, and Conjugate Figures.

(See also 7210 and 8075.)

Circum-polygons. Voss, A. Mth. A. 25 (1885) 39-.

Circum-quadrilateral andits quadric. Mannheim, A. Par. S. Mth. Bll. 25 (1897) 78-.

-, skew. Bruno, G. Tor. Ac. Sc. At. 17 (1881) 35-.

Cone circumscribing trihedral angle. Hellwig, C. Arch. Mth. Ps. 63 (1879) 215-.

- enveloping elliptic spheroid. Friesach, $K$. Steierm. Mt. (1874) 57-.

Cones circumscribing quadric. Cardinaal, $J$. Haarl. Ms. Teyl. Arch. 5 (1898) 45-.

Cylinder circumscribing ellipsoid, sections. Catalan, E. C. Liége S. Sc. Mm. 15 (1888) No. 1, 3-.

Decagons, skew, class inscriptible in ellipsoid. Serret, $P$. C. R. 82 (1876) 162-.

Hexagon on hyperboloid. Hesse, L. O. Crelle J. 24 (1842) 40- 
Hexagon and hyperboloid of revolution. Dandelin, G. P. Brux. Mm. Ac. Sc. 3 (1826) $1^{*}$. -, parallel, on ruled hyperboloid. Schroeter, H. E. Mth. A. 18 (1881) 428-.

Hexagons, Brianchon's, in space. Hesse, $L$. O. Crelle J. Mth. 85 (1878) 304-.

,,$---\ldots$, extension of theorem of Hesse. Graefe, $F$. Crelle J. Mth. 93 (1882) 87-.

-, - theorem and analogue in space, proof. Weddle, T. Camb. and Dubl. Mth. J. 7 (1852) 10-.

In-conics of quadric surface. Cayley, $A$. Crelle J. 41 (1851) 73-.

In-polygon, closed, Hamilton's problem, solution. Gardiner, $M$. N. S. W. R. S. T. 3 (1869) 38-.

- with sides passing through given points. Battaglini, G. Tortolini A. 2 (1851) 20 -

- - - - - - - problem, solution by homographic division. Tovnsend, R. [1867] L. Mth. S. P. 2 (1869) 21-.

- $-\ldots \ldots n$ points, by Hamilton's process. Gardiner, M. [1868] L. Mth. S. P. 2 (1869) 63-.

In-polygons. Gardiner, M. [1863-64] (vII) N. S. W. Ph. S. T. 1 (1866) 105-; QJ. Mth. 7 (1866) 146-, 284-.

-, skew. Hamilton, (Sir) W. R. Ir. Ac. P. 4 (1850) 325-, 380-, 541-.

In-polyhedra, maximum, of ellipsoid. Spitzer, S. Grunert Arch. 32 (1859) 439-.

,,---- Tillot [Tillol], J. Toul. Mm. Ac. 5 (1861) 265-.

Inscribed and circumseribed figures in space. Roberts, R. A. QJ. Mth. 29 (1898) 179-.

Octahedron, hyperboloid circumscribing. Serret, P. C. R. 103 (1886) 867-.

Paraboloid, segment, series of in- and circumscribed solids. Martus, H. Grunert Arch. 38 (1862) 253-.

Pascal's theorem, analogues. Bordiga, G. A. Ven. I. At. 7 (1881) 1253-.

- - - - in space, two. Sautreaux, F. Brux. Ac. Bll. 45 (1878) 426-.

- and Brianchon's, analogues. Chasles, $M$. Brux. Ac. Bll. 6 (1839) 248-

,---- - Salmon, G. Ph. Mg. 24 (1844) 49-.

- - - - - in space. Weddle, T. Camb. and Dubl. Mth. J. 4 (1849) 26-; 5 (1850) 58-; 6 (1851) 114-.

$---\frac{1}{-}$, extension to quadrics. Petot, $A$. C. R. 98 (1884) 726-.

- -, Chasles's analogue to. Ellis, R. L. [1859] QJ. Mth. 9 (1868) 344-.

- - extension to space. Klein, $F$. (vir) Erlang. Sb. Ps. Md. S. 6 (1874) 10-.

$-\ldots,-\ldots-$ of 3 dimensions. Buchheim, A. Mess. Mth. 14 (1885) 74-.

Polygons in- and circum-scribed to ellipsoid. Darboux, G. [1870] (Ix) Par. S. Phlm. Bll. 7 (1871) 92-.

- inscribed in a quadric and circumscribed about two confocal quadrics. Roberts, R. A. L. Mth. S. P. 18 (1886-87) 202-.

Poncelet's polygons, movable polyhedra analogous to. Fontené, G. N. A. Mth. 18 (1899) 67-.
Poncelet's polygons in space. Kohn, G. Wien Ak. Sb. 106 (1897) (Ab. 2a) 481-.

Poristic tetrahedra in relation to two quadrics. Kohn, G. Mh. Mth. Ps. 11 (1900) 102-.

Skew polygons, inscriptible class. Serret, $P$. C. R. 82 (1876) 270 -

Tetrahedra, circum-quadrics. Bioche, C. N. A. Mth. 18 (1899) 38-.

-, conjugate, properties. Painvin, L. N. A. Mth. 19 (1860) 290.

- - to 1 quadric, and with edges tangential to another. Vogt, H. C. R. 118 (1894) 395-; Par. Ec. Norm. A. 12 (1895) 363-.

- - - quadrics of common tetrahedron. Meister, K. Z. Mth. Ps. 34 (1889) 6-, 73-.

-, inscribed. Sincov, D. Kazan S. Ps.-Mth. Bll. 6 (1896) 42-; 7 (1898) 82-.

-, - orthocentric polar. Machovec, $F$. Mh. Mth. Ps. 2 (1891) 451-.

,-- , regular. Hossfeld, C. Z. Mth. Ps. 29 (1884) 351-.

- - , theorems. Rubini, R. Nap. Rd. 3 (1854) 40-

, 12-point sphere and quadrics of. Ripert, $L$. Mathesis 18 (1898) 218-.

-, polar, spheres circumscribing. Reye, $T$. Crelle J. Mth. 78 (1874) 345-.

-, spherical polar system, and application. Meyer, K. T. Arch. Mth. Ps. 8 (1890) 363-:

Tetrahedron, 4 edges in 1 quadric, 3 faces touching another, envelope. Purser, F. [1866] QJ. Mth. 8 (1867) 149-.

- with edges touching quadric, relations with quadric. Weddle, T. Camb. and Dubl. Mth. J. 8 (1853) 105-.

- in ellipsoid, maximum. Spitzer, S. Grunert Arch. 32 (1859) 194-.

- - - Grelle, $F$. Z. Mth. Ps. 14 (1869) 372-.

- - volume determinant. Brioschi, $F$. Tortolini A. 4 (1853) 457-.

- - - - - (Brioschi). Genocchi, A. N. A. Mth. 13 (1854) 429-.

- - - - - extension of Joachimsthal's theorem. Mainardi, G. Mil. At. I. Lomb. 1 (1858) 119-.

- inscribed, maximum. Glaser, S. Arch. Mth. Ps. 14 (1896) 156-.

\section{Intersections of Quadrics.}

(See also 7660, 8440.)

Analogues of property of conics. Weddle, T. Mathematician 2 (1847) 66-.

Asymptotes. Caron, J. N. A. Mth. 12 (1873) 270 -

Cones, 3 , of 2 nd degree, with common vertex, intersecting in pairs, common lines lie on cone of $4 \mathrm{th}$ degree. Spottiswoode, W. (vr Add8.) Ph. Mg. 37 (1850) 289.

-, intersections of 2, problem of Steiner's on plane curves. Beck, A. Zür. Vjschr. 38 (1893) 199-, 266-.

- - - - projective. Fiedler, W. Zür. Vjschr. 36 (1891) 87-. 
Cones, right, intersections. Marrecas Ferreira, L. F. Lisb. J. Sc. Mth. 6 (1878) 175-.

Curve of intersection, developable along. Battaglini, G. [1857] (vII) Nap. At. I. Inc. 9 (1861) 241-.

- - - of 2 quadries. Beer, A. Grunert Arch. 16 (1851) 104-.

- L L La Gournerie, J. de. C. R. 62 (1866) 611-.

- _ _ _ osculating planes. Machovec, F. Prag Sb. (1890) 1 (Mth.-Nt.) 142-.

- - - - - - - construction. Drasch, $H$. Wien Ak. Sb. 81 (1880) (Ab. 2) 254-.

- - _ - - - , representation by algebraic parameters. Harnack, A. Mth. A. 15 (1879) 560-.

_- - , quadries having same. Fenwick, S. Mathematician 2 (1847) 80-.

- - - of rotation quadrics. Schüssler, $R$. Mh. Mth. Ps. 5 (1894) 241-.

_- _ _ - 2, projection. Jarolimek, $\check{C}$. Časopis 11 ( $\left.{ }^{*} 1882\right)$ 179-; Fschr. Mth. ( ${ }^{* 1882)}$ 494.

Descriptive geometry, note. [Schiappa] Monteiro, $A$. G. Teix. J. Sc. 3 (1881) 97-.

Ellipsoid and elliptic cylinder, intersection. Tortolini, B. Rm. At. N. Linc. 24 (1871) 181-.

Intersection, application of principle to quadrics. Meier, F. [1857] Brux. Mm. Cour. $4^{\circ}, 30$ (1861) $52 \mathrm{pp}$.

- with cone whose vertex is on surface. Fergola, $E$. Nap. At. Ac. 6 (1851) 105-.

- by plane, of quadric cones and cylinders in central projection. Fölser, I. (xII) Mag. Tud. Ak. Éts. 11 (No. 1) (1877) 9-.

- of 2 quadrics. Bérard, -. Gergonne A. Mth. 6 (1815-16) 276-.

- - - Painvin, L. N. A. Mth. 7 (1868) 481-, 529-; 8 (1869) 49-, 145-, 193-.

- - Salvatore-Dino, N. Nap. Rd. 20 (1881) 212-.

- - Lévy, L. N. A. Mth. 10 (1891) $65-$.

- - Tresse, A. N. A. Mth. 11 (1892) 216-.

(1896) 153-.

- 3 quadries, 8th point, construction. Hesse, L. O. Crelle J. 26 (1843) 147-.

- - - - _- - - Zeuthen, H. G. Kjöb. Ov. (1880) 227-; (Résumé, 28-).

Mth. 99 (1886) 128-

$---1,-,-$. Schroeter, $H$. Crelle J. Mth. 99 (1886) 131-

Mth. 99 (1886) 317-.

- - - - - - - Zeuthen, H. G. Crelle J. Mth. 99 (1886) 320-.

$-{ }_{-}-,-\ldots,-$, linear. Reye, T. Crelle J. Mth. 100 (1887) 487-.

- - - - , - - contribution to problem. Picquet, H. Par. S. Mth. Bll. 22 (1894) 19--18 points of. Hesse, L. O. Crelle J. 73 (1871) 371 ; 99 (1886) 110-.
Intersection of 3 quadrics, 8 points of. Dobriner, $H$. Acta Mth. 12 (1889) 339-.

- - - - - Zeuthen, H. G. Acta Mth. 12 (1889) 362-.

Mth. 14 (1890-91) $207-$.

- _ - _ - - - _ , analytical formulæ for "octad". Cayley, A. Mess. Mth. 18 (1889) 149 .

- - - - - - property of quadrics through same 8 points. Fenwick, S. Mathematician 2 (1847) 12-.

- yielding given conic. Krewer, M. Arch. Mth. Ps. 12 (1894) 185-.

Loci of intersection of 3 tangent lines and of 3 tangent planes. Wolstenholme, (Rev.) J. L. Mth. S. P. 14 (1882-83) 177-.

Problem, to fit together two quadrics so that their intersections shall be plane. Hyde, $E$. $W$. Des Moines Anal. 3 (1876) 97-.

Rotation surface, intersection with straight line. Caron, J. N. A. Mth, 1 (1882) $217-$.

Similarly situated quadrics, intersection. Molins, H. Toul. Mm. Ac. 5 (1855) 203-.

Sphere and hyperboloid, intersection. Brandes, $H$. W. Voigt Mg. 11 (1806) 422-.

- - quadric cone, intersections. Dandelin, G. $P$. Brux. Mm. Ac. Sc. 4 (1827) 3-. - - , intersection. Darboux, $G$. N. A. Mth. 3 (1864) 199-.

Straight line and quadric, intersection. Lebon, E. N. A. Mth. 1 (1882) 219.

- - - - Mansion, P. (xiI) Brux. S. Sc. A. 7 (1883) (Pt. 1) 64-.

- _ - rectangular hyperbolic paraboloid, intersections. Jeřábek, $V$. Casopis 27 (1898) 308-; Fschr. Mth. (1898) 471.

Invariants of binary forms in two series of variables, geometrical meaning. Kohn, G. D. Nf. Vh. (1896) (Th. 2, Hälfte 1) 5-.

Inverse of conics and quadrics from centre. Rice, $M . E$. Kan. Ac. Sc. T. 14 (1896) 14-.

Inversion. Cayley, A. QJ. Mth. 11 (1871) 283-.

Involution determined by three points. $L e$ Paige, C. M. M. H. H. Wien Ak. Sb. 87 (1883) (Ab. 2) 599-.

-, quadratic, problems. Bečka, G. Prag Sb. (1878) 272-.

Katoptric properties. Finsterwalder, S. Münch. Ak. Sb. 17 (1888) 33-.

- property of ellipsoid. Staude, O. Mth. A. 27 (1886) 412-.

Line-geometry as applied to quadrics. Voss, $A$. E. Mth. A. 10 (1876) 143-.

Loci. Mossbrugger, L. Grunert Arch. 27 (1856) 66-.

Locus from which cone subtends right angle. Böklen, O. Grunert Arch. 36 (1861) 22-.

- of point, distances of which from 2 nonintersecting straight lines are in constant ratio. Jarolimek, $\check{C}$. Časopis 20 (1891) 1-; Fschr. Mth. (1891) 688-.

- - - _ - - - 3 points satisfy certain relations. Jacobi, C. G.J. Crelle J. 12 (1834) 139-. 
Locus of point, distances of which from 3 points satisfy certain relations (Jacobi). Enneper, $A$. Z. Mth. Ps. 10 (1865) 416-.

- points, distances of which from two straight lines are in constant ratio. Ovidio, $E . d^{\prime}$. G. Mt. 1 (1863) 183-.

- - - dividing constant chords in given ratio. Roberts, S. QJ. Mth. 17 (1881) 280-.

- - vertex of cone dividing 6 or 7 lines harmonically. Chasles, M. C. R. 52 (1861) 1157-.

_ - vertices of solid angles circumscribing paraboloid etc., extension of Monge's theorem. Dubois, G. N. A. Mth. 2 (1863) 398-.

Metrical properties. Darboux, G. Par. S. Mth. Bll. 2 (1874) 144-.

Middle points of chords through fixed points, locus. Crova, (Prof.) —. (vi Adds.) Perpignan Bll. S. Ag. Pyr. Orient. 7 (1848) $252-$.

- - - - - Unferdinger, $F$. Arch. Mth. Ps. 42 (1864) 118-

- $-1-1-$ Ende, H. am. Arch. Mth. Ps. 42 (1864) 358-.

\section{Normals to Quadrics.}

Chasles, M. Liouv. J. Mth. 8 (1843) 215-. Desboves, A. H. N. A. Mth. 2 (1863) 228-. Joachimsthal, $k$. Crelle J. 73 (1871) 207-. Laguerre, E. N. A. Mth. 17 (1878) 163-.

Humbert, G. C. R. 111 (1890) 963-.

Machovec, $F$. Prag Sb. (1890) 1 (Mth.-Nt.) 119-.

Conditions for. Pinkerton, R. H. Edinb. Mth. S. P. 13 (1895) 126-.

Ellipsoid, normals. Geiser, C. F. [1867] A. Mt. 1 (1867-68) 317-.

-, 一. Böklen, O. N. A. Mth. 18 (1899) $121-$.

-, - making equal angles with two given normals. Grunert, J. A. Arch. Mth. Ps. 47 (1867) 204-.

-, -, number from point. Joachimsthal, $F$. Crelle J. 59 (1861) 111-.

-, shortest distance between 2 normals. Grunert, J.A. Grunert Arch. 21 (1853) 314-; 38 (1862) 228-.

Joachimsthal's theorem on circles and spheres through feet of normals. Schoute, P. H. Amst. Ak. Vs. 8 (1900) 744-; Amst. Ak. P. 2 (1900) 593-.

Normal surfaces to ellipsoid along an ellipse of principal system. Solin, J. M. [1868] Prag Ab. 2 (1869) 59 pp.

- - along plane sections. Koutny, E. Wien Ak. Sb. 75 (1877) (Ab. 2) 851-.

Normals and central surface of algebraic surfaces. Waelsch, $E$. Ac. Nt. C. N. Acta 52 (1888) 285-.

$-\frac{1}{-}$ - quadrics. Waelsch, E. Wien Ak. Sb. 95 (1887) (Ab. 2) 549-; 97 (1889) (Ab. 2a) 583-.

- from external point, simple method of drawing. Niemtschik, $R$. Wien Sb. 58 (1868) (Ab. 2) 831-.

- - given point. Clebsch, R. F. A. (vIr) Tortolini A. 4 (1861) 195-
Normals from given point. Laguerre, E. C. R. 78 (1874) 438-.

- to quadrics and conics. Clebsch, A. [1862] Crelle J. 62 (1863) 64 8 (1867) 66 -

Plane through certain point, analogy to conics. Mandl, J. Mh. Mth. Ps. 11 (1900) 183-.

Rectangular trihedra, edges of which are normals to given quadric. Bruno, G. Tor. Ac. Sc. At. 15 (1879) 617-.

- -,$-1-1-1-$ - quadric, locus of vertices. Painvin, L. N. A. Mth. 10 (1871) 337-.

Sum of squares of normals, locus when constant. Terquem, $O$. Liouv. J. Mth. 4 (1839) 241-.

QJ. Mth. 10 (1870) 167-.

Number of solutions of elementary questions. Housel, - . C. R. 60 (1865) 1072-.

Paraboloids, elliptic. Bazant, J. Fschr. Mth. (1899) 565.

-, - circular sections. Babinet, J. C. R. 43 (1856) 453-.

-, elliptic and hyperbolic. Colecchi, $O$. Il Progresso 22 (1839) 41-.

-, - - complanation. Schlömilch, $\mathrm{O}$. Grunert Arch. 11 (1848) 233-.

-, - - - generation. Bérard, -. Gergonne A. Mth. 6 (1815-16) 122-.

-, osculating. Olivier, T. Par. Ec. Pol. J. cah. 25 (1837) 230-; Liouv. J. Mth. 3 (1838) 249-, 335-.

-, properties. Allman, G.J. [1873] QJ. Mth. 13 (1875) 102-.

- of 8 straight lines. Mannheim, A. C. R. 84 (1877) 645-; 123 (1896) 983-.

-, surface. Böklen, O. [1882] Arch. Mth. Ps. 69 (1883) 222-.

-, vertex and principal axes. Housel, -. N. A. Mth. 17 (1858) 223-.

Parameters, invariable functions. Ledent, $J$. Liége Mm. S. Sc. 3 (1873) 21 -.

Plane enveloping' sphere, property and extension to any surface. Ocagne, M. $d^{\prime}$. L. Mth. S. P. 18 (1886-87) 361-.

\section{Plane Sections of Quadrics.}

Cayley, A. [1863] (vII) Ph. Mg. 27 (1864) 43-. Mertens, $F$. Crelle J. 74 (1872) 362-.

Central sections, locus of foci. Le Besgue, $V$. A. N. A. Mth. 5 (1866) 444-.

Centre, analytical determination. Saltel, $L$. N. A. Mth. 14 (1875) 271-.

Circular sections. Finlay, $R$. Mathematician 2 (1847) 173-.

- -. Weddle, T. Mathematician 3 (1850) $57-$.

- Tillot [Tillol], J. N. A. Mth. 10 (1851) 304-.

-. Walton, W. Camb. and Dubl. Mth. J. 7 (1852) 7-.

- Donkin, W. F., \& Spottiswoode, W. Crelle J. 47 (1854) 225-. 
Circular sections. Rubini, R. N. A. Mth. 14 (1855) 237-.

- -. Babinet, J. C. R. 43 (1856) 453-.

- -. Souillart, C. Crelle J. 65 (1866) $320-$

- -. Bauer, G. [1868] Crelle J. 71 (1870)

- 46-.

- - Crosnier, -. N. A. Mth. 13 (1874)

12-.

- 339 .

- - (Sourander). Souillart, C. Crelle J. Mth. 87 (1879) 220-

- - Graves, (Rt. Rev.) C. [1890] Ir. Ac. P. 1 (1889-91) 440 .

115-.

Servais, C. Brux. Ac. Bll. 22 (1891)

- - condition expressed as vanishing sum of squares. Henrici, $F$. C. [O.] [1864] Crelle J. 64 (1865) 187-.

- conditions for, and discriminant of quadric. Dingeldey, $F$. Crelle J. Mth. 122 (1900) 186-.

- - of ellipsoid, orthogonal trajectories. Catalan, E. C. Liouv. J. Mth. 12 (1847) 483-.

- -, existence. Gregory, D. F. (vi Adds.) Camb. Mth. J. 1 (1839) 100 -.

- - projection of quadrics on plane of. Pelz, C. Arch. Mth. Ps. 52 (1871) 313-.

- and umbilies. Lac de Bosredon, $V$. N. A. Mth. 5 (1886) 186-, 214-.

Cone and tangential sphere, section, extension of theorem. Walker, E. QJ. Mth. 4 (1861) 276-.

Cones, conical section of two. Dickl, $J$. Arch. Mth. Ps. 67 (1882) 219-.

-, plane sections. Gergonne, J.D. Gergonne A. Mth. 16 (1825-26) 361-.

(Pt. 2) 50 -

- , right circular, minor axis of plane section. Taylor, C. [1871] Mess. Mth. 2 (1873) 39-.

Cylinder, sections. Schubert, F. T. [1798] St Pet. Ac. Sc. N. Acta 13 (1802) 190-

Eccentricity. Nevson, H. B. [1889] A. Mth. 5 (1889-90) 1-.

Hyperbolic sections projecting into rectangular ones. Schober, K. Mh. Mth. Ps. 9 (1898) 233-.

Malfatti's problem, Steiner's extension (plane sections, three, each touching other two, and also two of three given sections). Cayley, $A$. Phil. Trans. (1852) 253-.

Parabolic section. Tweedie, C. Edinb. Mth. S. P. 13 (1895) 136-.

Parallel planes, sections of quadric by, ratio of magnitudes. Cayley, A. Camb. and Dubl. Mth. J. 7 (1852) 104.

Plane cutting quadric and plane, pole of line with respect to section. Cayley, $A$. QJ. Mth. 2 (1858) 140-.

- section and tangent cone, envelope and locus theorems. Bodemer, $\rightarrow$, $C$ Hioux, - N. A. Mth. 2 (1863) 426-, 488-.

- sections and their parallels. Veladini, $G$. Rm. Cor. Sc. 1 (1848) .57-.
Rectangular hyperbolic sections. Schlömilch, O. Schlömilch Z. 6 (1861) 418-.

- - of quadric cone. Rulf, $W$. Mh. Mth. Ps. 7 (1896) 93-.

- - - - surface. Schober, K. Mh. Mth. Ps. 7 (1896) 111-.

Rotation quadric, sections. Havliček, $\boldsymbol{V}$. Časopis 29 (1900) 81-; Fschr. Mth. (1900) 544.

Planes, tangent, polar, and diametral, and centres of quadrics. Cauchy, A. L. C. R. 16 (1843) 801-.

Points at infinity. Painvin, L. N. A. Mth. 4 (1865) 49-.

Polar plane, MacCullagh's theorem. Curtis, A. $H$. QJ. Mth. 1 (1857) 134 .

,---- Cayley, A. Ir. Ac. P. 6 (1858) 481-.

- planes and lines. Terquem, O. N.A. Mth. 16 (1857) 266-.

- of 4 quadrics. Spottiswoode, W. [1881] L. Mth. S. P. 13 (1881-82) 28-

- properties of 3 plane curves on surface. Olivier, T. Quetelet Cor. Mth. 4 (1828) 9-, 96-.

- - - sections. Olivier, T. Quetelet Cor. Mth. 4 (1828) 228-.

- - self-polar quadrics. Hioux, V. N. A. Mth. 13 (1894) 211-.

- quadrics. Zeuthen, H. G. A. Mt. 4 (187071) 331-.

- reciprocal quadrics. Ingram, J. K. $\mathrm{Ph}$. Mg. 23 (1844) 188-.

- - Del Pezzo, P. Nap. Rd. 24 (1885) 165-.

- Garbieri, G. Ven. I. At. (1885-86) 1149-.

(1888) $37-$

- - reciprocating quadric. Battaglini, $G$. Rm. At. R. Ac. 26 (1873) 5-.

$--\ldots,-$ - Thieme, H. Z. Mth. Ps. 22 (1877) 377-

- relation, and quadrics. London, $F$. Mth. A. 38 (1891) 334-.

- - tetrahedra. Vályi, G. Mth. Termt. Ets. 12 (1894) 93-; Mth. Nt. B. Ung. 13 (1897) 189-.

- tetrahedra, and curve of intersection of 2 quadrics. Lüroth, $J$. [1867] Z. Mth. Ps. 13 (1868) 404-.

Polars of algebraic surfaces. Mainardi, $G$. Mil. At. I. Lomb. 1 (1858) 109-.

- , theory, for conics and quadrics. Lenthéric, -. Mntp. Ac. Sc. Mm. 1 (1847-50) 279-, 427-.

Poles of conics and quadrics, proof of properties. Rochat, -. Gergonne A. Mth. 3 (1812-13) 302-.

Principal planes and chords. Vazeille, E. N. A. Mth. 10 (1871) 5-.

- - determination, to any axes. St Guilhem, P. D. Toul. Mm. Ac. 4 (1834-36) 67-.

Problem, solution. Aubert, $P$. Edinb. Mth. S. P. 4 (1886) 91-. 
Projection applied to problems. Staudigl, $R$. Wien Sb. 58 (1868) (Ab. 2) 811-.

- by application of theory of envelopes. Meyer, C. T. Grunert Arch. 12 (1849) 277-

- of ellipsoid. Cayley, A. Ph. Mg. 30 (1865) 50 -.

Projective figures on quadric, theory. Zeuthen, H. G. Mth. A. 18 (1881) 33-; 26 (1886) 247-.

- properties. Bobillier, -. Quetelet Cor. Mth. 4 (1828) 152-.

- surfaces. Kober, G. Mth. A. 33 (1889) 470 ; 40 (1892) 153-.

Projectivity, problem of, and application to quadrics. Sturm, R. Mth. A. 1 (1869) 533-.

3 quadrics, with common curve, theorem. Gardiner, $M$. N. S. W. R. S. T. 3 (1869) 41-.

Quadrics determined by Points, Lines and Tangent Planes, and Methods of Deter. mination.

Classification and discussion of quadrics. Méray, C. N. A. Mth. 11 (1892) 474-.

- of quadries. Vályi, $G$. Mth. Termt. Ets. 8 (1890) 218-; Mth. Nt. B. Ung. 8 (1891) 76-.

- - and non-homogeneous quadratic forms. Hensel, $K$. Crelle J. Mth. 113 (1894) 303-.

Criteria for species. Dieu, T. N. A. Mth. 4 (1865) 408-.

Determination of species. Cockle, Jas. Mathematician 2 (1847) 257-.

- - Hearn, G. W. Mathematician 3 (1850) 195-.

- _ - Plücker's method. Finck, B. Liouv. J. Mth. 3 (1838) 495-.

12-point quadric, and researches in connection therewith. Cassani, P. G. Mt. 17 (1879) 202-.

- -, - that of 12 planes. Del Re, A. G. Mt. 22 (1884) 221-.

50-point quadric. Whitworth, W. A. [1865] Mess. Mth. 3 (1866) 144-.

10 points on quadric surface, relation, etc. Weddle, T. Camb. and Dubl. Mth. J. 5 (1850) 226-.

Quadric through given points, construction, problem. Schröter, H. E. [1862] Crelle J. 62 (1863) 215-

- 9 póints, construction. Hesse, L. $O$. Crelle J. 24 (1842) 36-.

- - - - - (Hesse). Cayley, A. Camb. and Dubl. Mth. J. 4 (1849) 44-.

$-{ }_{-}-$, - Seydewitz, F. Grunert Arch. 17 (1851) 275-.

- - - - - Steiner, J. Crelle J. 68 (1868) 191-.

$---1-$. Salvatore-Dino, N. Nap. Rd. 18 (1879) 195-.

- - - - Heger, $R$, Z. Mth. Ps. 25 (1880) 98-. $46(1894),-760$. Rohn, $K$. Leip. Mth. Ps. B.

- - - - , linear. Thomae, J. Leip. Mth. Ps. B. 44 (1892) 543-; 49 (1897) 315-.
Quadric through 9 points, construction, linear, simple. Reye, T. Z. Mth. Ps. 13 (1868) 527-. - - - - determination. Tononsend, $R$. Camb. and Dubl. Mth. J. 4 (1849) 241-.

,---- -. Poudra, -. N. A. Mth. 15 (1856) 263-.

- - - - - of species. Jonquières, E. de. Liouv. J. Mth. 4 (1859) 81-.

_ - - 10th point, construction. Ravier, L. N. A. Mth. 11 (1892) 289-.

$-{ }_{-},-\ldots$, situation. Jonquières, E. de. Liouv. J. Mth. 3 (1858) 53-.

$--\ldots-, 2$ problems. Poudra, -. N. A. Mth. 15 (1856) 384-.

- - - - , and other problems, linear constructions. Picquet, $H$. Crelle J. 73 (1871) 365-; 99 (1886) 225-.

Quadrics through given conic sections, theorems. Petersen, J. P. C. (xII) Ts. Mth. 2 (1878) 107-.

- - curve. Battaglini, G. Nap. Rd. 2 (1853) 127-.

Quaternions applied to study of quadrics. $P a$. pelier, - . Par. S. Mth. Bll. 17 (1889) 182-. Reflecting and refracting surfaces with 2 conjugate foci. Quetelet, L. A.J. Quetelet Cor. Mth. 5 (1829) 1-.

Relations between quadrics. Schönherr, $H$. Schlömileh Z. 5 (1860) 153-.

Rotation ellipsoids. Fontana, G. Turin Mm. Ac. (1802-03) 263-.

- - generalisation of Chapuy's method. [Schiappa] Monteiro, A. G. Teix. J. Sc. 1 (1878) 129-.

- hyperboloids. Mister, J. N. A. Mth. 11 (1872) 352-.

- - and cone. Bazaine, P. D. St Pét. Mm. 7 (1815-16) 255-.

- - generated by straight line. Hachette, J. N. P. Par. Éc. Pol. Cor. 1 (1804-08) 242-. - - intersection with straight line. Rouché, E. N. A. Mth. 1 (1882) 97-。

- - penetration by parallel axes. Fiedler, $W$. Acta Mth. 5 (1884) 331-.

- - property. Laguerre, - Bll. Sc. Mth. As. 2 (1871) 279-.

- - tangency. Woodward, C.M. Des Moines Anal. 1 (1874) 106-.

- surfaces. Merle, -, \& Urban, -. Par. Éc. Pol. Cor. 2 (1809-13) 203-.

- -. Housel, -. Liouv. J. Mth. 5 (1860) 129-.

- Malmsten, C. J. Stockh. Öfv. 20 (1863) 3-.

- D. Dostor, G. N. A. Mth. 11 (1872) 362-; Arch. Mth. Ps. 55 (1873) 302-.

- -. Schoenflies, A. Crelle J. Mth. 99 (1886) 195-.

- -, conditions for. Genty, -. N. A. Mth. 20 (1881) 414.

- - determinants applied to. Dostor, $G$. Arch. Mth. Ps. 58 (1876) 17-, 285-.

- - in oblique coordinates. Anom. (vi 527) Gergonne A. Mth. 7 (1816-17) 174-.

- - , plane sections. Francœur, L. B. Rouen Tr. Ac. (1809) 18. 
Segments, volume. Mossbrugger, L. Grunert Arch. 27 (1856) 66-.

Self-conjugate quadrics. Sturm, R. Mth. A. 25 (1885) 236-.

Stereographic projection of quadrics, generalisation. Procházka, B. Ćasopis 14 (1885) 1-; Fschr. Mth. (1885) 587-.

Tangent plane of ellipsoid. Q. Camb. Mth. J. 1 (1839) 117-.

- planes. Delacour, $V ., \&$ Mayer d'Almbert, -. N. A. Mth. 5 (1846) 611-.

- to conic and quadric, theorem. Beer, A. Grunert Arch. 20 (1853) 202-.

- - orthogonal, complex of lines through which two may be drawn to quadric. Demoulin, A. Par. S. Mth. Bll. 20 (1892) 122-.

Tangents common to two quadries having skew quadrilateral in common. Schur, F. Z. Mth. Ps. 25 (1880) 414-.

- to lines of curvature of quadric, practical determination. Pieri, M. Fschr. Mth. (189394) 958 .

Transformation, automorphic, of sphere and ellipsoid. Geer, P. van. Z. Mth. Ps. 19 (1874) 82-

Vaultings, parabolic and elliptic. Haase, $H$. Förster Al. Bauztg. 47 (1882) 89-.

\section{Systems of quadric surfaces. (See also 8070, 8090.)}

\section{Confocal Quadrics.}

Bobillier, - Gergonne A. Mth. 18 (1827-28) 185-.

Chasles, M. [1847] Liouv. J. Mth. 13 (1848) 16.

Schläfi, L. Bern Mt. (1847) 96-.

Booth, J. Camb. and Dubl. Mth. J. 9 (1854) 115-.

Heilermann, - Schlömilch Z. 3 (1858) 341-.

Chasles, M. Liouv. J. Mth. 5 (1860) 425-; C. R. 50 (1860) 1055-, 1110-.

Laguerre, E. Bll. Sc. Mth. As. 3 (1879) (Pt. 1) 14.

Böklen, O. Z. Mth. Ps. 26 (1881) 204-.

Mannheim, A. R. S. P. 33 (1882) 322-.

Humbert, G. Par. S. Mth. Bll. 13 (1885) 95-.

Machovec, F. Prag České Ak. Fr. Jos. Rz. (Třida 2) 1 (1892) Art. 38, 48 pp.

Analogue in conics. Townsend, $R$. [1872] Mess. Mth. 2 (1873) 33-.

Centres of curvature, Salmon's theorem. Townsend, $R$. Camb. and Dubl. Mth. J. 5 (1850) 177-.

Confocal, conjugate, and other quadrics. Fiedler, $W$. Schlömilch Z. 7 (1862) 25-, 217-, 285-.

- and orthogonal surfaces. Legoux, A. N. A. Mth. 3 (1884) 161-.

- quadrics, 2, as loci of centres of surface. Liouville, J. Liouv. J. Mth. 16 (1851) 6-. - - of moments of inertia. Gardiner, $M$. Vict. R. S. P. 5 (1893) 200-

- surfaces, sphero-conics and lines of curvature. Casey, J. [1865] Mess. Mth. 3 (1866) 236-.

VOL. I.
Curves and cones connected with confocal ellipsoids. Rutledge, J. Y. Camb. and Dubl. Mth. J. 4 (1849) 139-; 9 (1854) 148 -

- on confocal quadrics. Rutledge, J. Y. Camb. and Dubl. Mth. J. 5 (1850) 110-.

Director sphere. Bobillier, - . Gergonne A. Mth. 19 (1828-29) 317-.

Distance between consecutive quadrics. Picart, A. N. A. Mth. 3 (1864) 532-.

Distance-correspondences for quadric surfaces, Ivory's and Jacobi's, extension. Larmor, $J$. L. Mth. S. P. 16 (1884-85) 189-.

Duplicate sets of confocal surfaces. Rutledge, J. Y. Camb. and Dubl. Mth. J. 6 (1851) 201-.

Edge of regression of imaginary circum-developable. Townsend, $R$. [1871] Mess. Mth. 1 (1872) 49-.

Ellipsoids, confocal. Zehfuss, G. (xI) Z.Mth. Ps. 4 (1859) 166-.

-, -. Mannheim, A. Mess. Mth. 11 (1882) 190 -, 一, Ivory's property. Haedenkamp, $H$. Grunert Arch. 3 (1843) 397-.

Equation. Mac Cullagh, J. Ir. Ac. P. 3 (184547) 429-

- (Mac Cullagh, priority question). Chasles, M. Liouv. J. Mth. 11 (1846) 120-

Extension to space of $n$ dimensions. Meyer, W. F. Königsb. Schr. 41 (1900) [24].

Focal lines and confocal quadrics. Ionescu, B. G. [Bucarest S. Sc. Bl. 6 (1897)] 159-。

- of tangent cone. Jacobi, C. G.J. Crelle J. 12 (1834) 137-.

- - - - Jacobi's theorem. Cayley, $A$. Camb. and Dubl. Mth. J. 3 (1848) 48-.

Locus of vertex of circum-cone. Breton [de Champ], P. N. A. Mth. 11 (1852) 369-.

Normal problem, two unicursal degenerate cubics connected with. Tesar, $J$. Wien Ak. Sb. 101 (1892) (Ab. 2a) 1248-.

Paraboloids, confocal. Böklen, $O$. Grunert Arch. 35 (1860) 81-.

-, 一. Greenhill, A. G. L. Mth. S. P. 19 (1889) 129-.

Property. Ferrers, N.M. QJ. Mth. 3 (1860) 155-. - of two quadries. Bruno, $G$. Tor. Ac. Sc. At. 14 (1878) 125-.

Synthetic theory. Maglioli, F. G. Mt. 16 (1878) 305-.

Tangent and normal plane at point, conjugacy of traces on diametral plane. Droz, A. N. A. Mth. 20 (1881) 305-.

- planes through line in axial plane. Picart, A. N. A. Mth. 4 (1865) 62-, 97-.

Tangents to confocal quadrics. Gilbert, $P$. N. A. Mth. 6 (1867) 529-.

- - - (Gilbert). Gibson, G. A. Edinb. Mth. S. P. 3 (1885) 61-.

Theorems of Lamé and Bertrand. Machovec, F. Mh. Mth. Ps. 4 (1893) 57-.

Director spheres of system of quadrics touching system of planes. Townsend, R. [1866] QJ. Mth. 8 (1867) 10.

Families of cones, $\frac{y^{2}}{x^{2} \tan ^{2} \psi}+\frac{z^{2}}{x^{2} \tan ^{2} \theta}=1$. Klug, L. Orv.-Termt. Éts. (Termt. Szak) (1900) 72-, (Rv.) 13-. 


\section{Systems of Quadrics}

Family of quadrics with common (quartic) curve. Cardinaal, J. N. Arch. Wisk. 13 (1887) 213-; Fschr. Mth. (1886) 603-.

_- - property. Cardinaal, $J$. N. Arch. Wisk. 10 (1884) 113-.

Homogeneous coordinates and invariants in theory of quadrics. Weyr, E. Časopis 26 (1897) 1-, 121-; Fschr. Mth. (1897) 563.

Homographic properties of conic and quadric systems. Battaglini, G. G. Mt. 1 (1863) 287-.

Hyperboloids, conjugate. Weddle, T. Camb. and Dubl. Mth. J. 2 (1847) 274.

- through 4 points. Valeri, $D$. Mod. Ac. Sc. Mm. 4 (1886) 355-.

Linear systems and nets of quadrics. Reye, $T$. Crelle J. Mth. 82 (1877) 54-.

Locus of centres of conics and quadrics. Picquet, H. N. A. Mth. 4 (1865) 66-.

- - - etc., of systems of conics and quadrics. Geiser, C. F. Zür. Vjschr. 10 (1865) 219-

Nets of quadrics. Sturm, R. Crelle J. 70 (1869) 212-.

- - Frahm, W. Mth. A. 7 (1874) 635-.

- - Vaněček, J. S. Liége S. Sc. Mm. 11 (1885) No. 17, 8 pp.

Orthogonal cones and hyperboloids, systems. Ruth, F. [1879] Wien Ak. Sb. 80 (1880) (Ab. 2) 257-.

- - touching quadric, locus of vertices. Koch, A. Arch. Mth. Ps. 9 (1890) 250-.

- quadries. Taylor, H. M. QJ. Mth. 26 (1893) 214 .

Parallel conjugate diameters in two surfaces. Janni, V. G. Mt. 1 (1863) 223-, 280-.

Pencils of quadrics. Vaněček, J.S. Liége S. Sc. Mm. 10 (1883) (No. 7) 16 pp.

- - on point or tangential, and extension of Chasles's theorem. Gardiner, M. [1868] QJ. Mth. 10 (1870) 132-.

-, synthetic treatment. Muller, Hub. Mth. A. 1 (1869) 627-.

5-point and 6-point quadrics, systems. Woepcke, F. Crelle J. 54 (1857) 285-.

6-point quadrics, equation. Mannheim, $A$. Bll. Sc. Mth. As. 2 (1871) 125-.

Polar reciprocal quadrics, systems. Montesano, D. A. Mt. 14 (1886-87) 131-.

Quadrics with common intersection. Aoust, (l'abbé) -. C. R. 64 (1867) 590-, 746-.

- conjugate with respect to fixed tetrahedron, system. Painvin, L. [1863] Crelle J. 63 (1864) 58-.

- having various contacts with given surface. Torroja, $E$. Fschr. Mth. (1895) 623.

- inscribed in same developable. Cazamian, $A$. N. A. Mth. 13 (1894) 395-.

- intersecting along two plane curves, or enveloped by two common cones. Stammer, W. G. Mt. 6 (1868) 153-.

- satisfying 8 conditions, and 9 conditions, etc. Chasles, M. C. R. 62 (1866) 405-.

- 9 conditions, number. Salmon, $G$. QJ. Mth. 8 (1867) 1-.

- touching four straight lines, properties. Cardinaal, J. N. Arch. Wisk. 10 (1884) 131-.
Higher Curves and Surfaces 7600

Rotation hyperboloids passing through skew cubic. Cremona, L. [1863] (vI) Crelle J. 63 (1864) 141-。

- 3 rectangular, of same system. Fiedler, W. Zür. Vjschr. 29 (1884) 343-.

- quadrics through 5 points, axes. Laguerre, E. N. A. Mth. 18 (1879) 206-.

- - - - - focal properties. Recoq, A. N. A. Mth. 5 (1866) 157-.

Self-reciprocal surfaces, system. Cayley, $A$. QJ. Mth. 15 (1878) 124-.

Similarity conditions for two quadrics not similarly placed. Weddle, T. Camb. and Dubl. Mth. J. 8 (1853) 35-.

Spheres, system. Timerding, $\dot{H} . E$. Crelle J. Mth. 121 (1900) 188-.

Straight lines and tangent-quadrics, systems. Bricard, R. Par. S. Mth. Bll. 25 (1897) 180 -.

Surfaces of symmetry common to several quadrics. Mangeot, S. As. Fr. C. R. (1891) (Pt. 2) 224-.

Symbolical calculus, application. Garbieri, $G$. Ven. I. At. (1885-86) 943-.

System of two quadrics, theorems. Chasles, $M$. Brux. Ac. Bll. 5 (1838) 561-.

Theorems. Ovidio, E. d'. Tor. Ac. Sc. At. 14 (1878) 452-.

$n$ times infinite systems. Burali-Forti, $C$. G. Mt. 24 (1886) 334-

\section{Algebraic curves and surfaces of degree higher than the second. (See also 8070.)}

\section{General.}

Gergonne, J. D. Gergonne A. Mth. 17 (182627) $214-; 18$ (1827-28) 149-; 19 (1828-29) 120 -

Bobillier, -. Gergonne A. Mth. 18 (1827-28) 89-, 157-, 253-; 19 (1828-29) 106-, 138-.

Poncelet, J. V. Gergonne A. Mth. 18 (182728) 125-.

Graves, C. Ir. Ac. P. 3 (1847) 151-.

Hunyady, J. (xH) Mag. Tud. Ak. Ets. 2 (No. 1) (1868) 13-.

Jonquières, E. de. A. Mt. 8 (1877) 312-.

Beck, A. [1878] Mth. A. 14 (1879) 207-.

Anharmonic ratio, extension of notion to $n \mathrm{th}$ order. Folie, F. Brux. Ac. Bll. 44 (1877) 469-; 45 (1878) 88-.

Billiard problem, circular. Tychsen, C. Mth. Ts. 3 (1861) 60-.

Centres, axes, and planes of symmetry in algebraic figures, determination. Mangeot, $S$. Par. Éc. Norm. A. 14 (1897) 9-.

- of curves and surfaces. Roberts, S. QJ. Mth. 9 (1868) 25-.

- - _ - - new method of finding. Mangeot, S. N. A. Mth. 17 (1898) 215-.

- - , and their polars. Jeffery, H.M. [1869] QJ. Mth. 10 (1870) 171-. 
Construction of curves and surfaces, given points. Escherich, G. von. Wien Ak. Sb. 85 (1882) (Ab. 2) 893-.

Contact of curves and surfaces. Chasles, $M$. Liouv. J. Mth. 2 (1837) 299-.

\section{Curves.}

Tellkampf, A. Crelle J. 14 (1835) 93-.

Steichen, -. Liège Mm. S. Sc. 1 (1843) 277-.

analysis. Valat, - Bordeaux Act. Ac. Sc. (1839) 411-.

-, barycentric. Cesáro, E. A. Mt. 15 (188788) 313-.

-, independent variable in. Cauchy, A. L. C. R. 14 (1842) 508-.

-, method. Walker, J.J. [1878-87] L. Mth. S. P. 9 (1877-78) 226-; 16 (1884-85) 215-; 19 (1889) 483-.

asymptotes, diameters, etc. Miller, A. [1858] Wien D. 19 (1861) 339-.

-, 一, - (Müller). Petzval, J. Wien SB. 29 (1858) 40-.

and circles, relations between. Genese, R. W. L. Mth. S. P. 18 (1886-87) 304-

classification. Ruffini, P. [1816] Mod. S. It. Mm. 18 (1818) 69-.

construction, application of geometric affinities. Andreev, K. A. (xII) Rec. Mth. (Moscou) 9 (1878-81) (Pt. 1) 193-, 361-; (Ix) Bll. Sc. Mth. As. 3 (1879) 35-.

-, equation being given by polar coordinates. Biehler, C. N. A. Mth. 3 (1884) 367-; 4 (1885) 153-, 223-, 249-

constructions. Schlömilch, $O$. Schlömilch Z. 6 (1861) 260 -.

一, résumé. Heger, R. Z. Mth. Ps. 31 (1886) 88-.

derivation. Bellavitis, G. Tortolini A. 3 (1852) 508-.

determination by points. Zeuthen, $H . G$. Ts. Mth. 5 (1887) 65-; Mth. A. 31 (1888) 235-.

equation, characteristic, interpretation. Mansion, $P$. (xII) Mathesis 2 (1882) 29-.

,,$---{ }_{0}$. Neuberg, J. (xII) Mathesis 2 (1882) 30 .

- of a curve, interpretation. De Morgan, $A$. Camb. and Dubl. Mth. J. 7 (1852) 242-.

equations to chords and tangents. Genese, R. W. [1897] Mth. Gz. 1 (1900) 110. -, reciprocal, relating to polars. Cauchy, A. L. C. R. 16 (1843) 1279-

-, symmetrical expression of constants. Roberts, S. QJ. Mth. 2 (1858) 39-.

higher, and conics, as generated by homographically related lines. Eckardt, $F$. E. Z. Mth. Ps. 9 (1864) 22-.

Noether's fundamental theorem $(F=K \Phi+M \Psi)$. Baker, H. J. Mth. A. 42 (1893) 601-.

$--(F=K \Phi+M \Psi)$. Scott, (Miss) C.A. Mth. A. 52 (1899) 593-.

notion of plane curve of given degree. Cayley, A. Mess. Mth. 20 (1891) 148-. parameter, apparent difficulty in theory. Cayley, A. Mess. Mth. 14 (1885) 12-.

plane, anonymous treatise. Loria, $G$. $\mathrm{Bb}$. Mth. (1899) 10-.

- general questions. Jonquières, $\boldsymbol{E}$. de. Crelle J. 59 (1861) 313-.

-, - theorems on simplest forms. Kneser, A. Mth. A. 41 (1893) 349-

-, history of investigation. Loria, G. Wiad. Mt. 2 (1898) 203-.

-, imaginary, representation. Saussure, R. de. J. H. Un. Cir. [15 (1895-96)] 39-.

一, intersection-figures. Study, E. Mth. A. 36 (1890) 216-.

- or skew, construction of tangents at multiple point. Olivier, T. Par. Éc. Pol. J. $21^{\circ} c a h$. (1832) 302-.

-, theory, general. Mahler, E. [1880] Arch. Mth. Ps. 66 (1881) 365-.

-, -, geometric. Kötter, E. Berl. Ak. Ab. (1887) (Anh., Mth.) 303 pp.

-, -, projective. Cremona, L. Bologna Mm. Ac. 12 (1861) 305-.

-, 3rd degree, in space, characteristics. Schubert, H. C. H. Gött. Nr. (1874) 267-. relation $\sum_{1}^{N} P^{n}=0$ for curve defined by $N-1$ conditions. Serret, P. C. R. 86 (1878) 39 -

similitude. Pirondini, G. A. Mt. 15 (188788) 53 -.

study of curve near one of its points. Cosserat, $E$. Toul. Fac. Sc. A. 4 (1890) 0, 16 pp.

synthetic geometry, Kötter's. Fraser, N. Edinb. Mth. S. P. 7 (1889) 46-.

theory. Jacobi, C. G.J. Crelle J. 14 (1835) 56-.

-. Miquel, A. Liouv. J. Mth. 3 (1838) 202-. 102-

Cayley, A. Camb. Mth. J. 4 (1845)

- Bu

15 (1891) 58-

-, analytical, principles. Hoppe, R. Arch. Mth. Ps. 56 (1874) 41-。

-, and elimination. Cayley, A. [1863-64] (vII) Crelle J. 63 (1864) 34-; 64 (1865) 167-.

-, general. Mainardi, G. Rm. At. N. Linc. 26 (1873) 86-.

-, - Puzyna, J. Krk. Ak. (Mt.-Prz.) Rz. 2 (1892) 1-; Crc. Ac. So. Bll. (1891) 62-.

-, geometric. Grassmann, H. Crelle J. 31 (1846) 111-.

Diameters. Le Cointe, J. L. A. C. R. 60 (1865) 1083-.

Equations, algebraic, geometric relations between two systems of points defined by. Mangeot, S. C. R. 129 (1899) 464.

-, curves with trinomial, and surfaces with quadrinomial. Euzet, -. N. A. Mth. 13 (1854) 193-.

-, differential, 1st order and degree, algebraic integration. Poincaré, $H$. Palermo Cir. Mt. Rd. 11 (1897) 193-. 
Equations, homogeneous and central, solutions. M'Laren, (Lord). [1888] Edinb. R. S. T. 35 (1890) 1043-.

- in $n$ variables, geometric interpretation. Péclet, E. Gergonne A. Mth. 14 (182324) 65-.

"Equipollence" method, application to finding certain loci. Minich, S.R. A. Sc. Lomb. Ven. 7 (1837) 57-, 171-.

- - - - - - - (Minich). Bellavitis, G. A. Sc. Lomb. Ven. 7 (1837) 165-.

Focal curves and surfaces. Schoute, $P$. $H$. Amst. Ak. Vs. 6 (1898) 404-.

Generation of forms of 3rd degree by projective forms of 1st and 2nd degree. Certo, $L$. G. Mt. 26 (1888) 41-.

-, mechanical, curves and surfaces capable of. Puchta, A. [1883] Wien Ak. Sb. 88 (1884) (Ab. 2) 571-.

- of polar elements for curves and surfaces by projective method. Geisenheimer, $L$. Z Z. Mth. Ps. 31 (1886) 193-; 32 (1887) 127-.

- by projective forms. Joerres, - Crelle J. 72 (1870) 327-.

- of surfaces and skew curves. - Vaněček, M. N. C. R. 94 (1882) 210 .

- - - - - - method analogous to MacLaurin's. Vaněček, M. N. Liouv. J. Mth. 9 (1883) 269-.

Intersection of curves and surfaces, centres of mean distances of points. Roberts, $S$. QJ. Mth. 9 (1868) 63-.

- - or surfaces, relations between points. Jacobi, C. G. J. Crelle J. 15 (1836) 285-.

- - - and surfaces by straight lines. Sharp, W. J. C. Mess. Mth. 9 (1880) 49-

Loci, classification. Clifford, $W . K$. Phil. Trans. 169 (1878) 663-.

Maximum and minimum distance of points on 2 curves or surfaces. Odin, A. A. Laus. S. Vd. Bll. 23 (1888) 108-.

- - - distances between points, curves, or surfaces, taken in pairs. Tucci, $F . P$. (xI) Nap. Ac. At. 2 (1865) No. 14, 13 pp.

Method, new, application. Stubbs, J. W. $\mathrm{Ph}$. Mg. 23 (1843) 338-.

Normals. Stuyvaert, —. Mathesis 18 (1898) 105-.

-, common, distance between lines and surfaces with. Bordoni, A. Brugnatelli G. 6 (1823) 1-.

- and normal planes, construction. Schönemann, $P$. (xI) Berl. Ak. Mb. (1855) 255-.

Plane enveloping sphere, property, and extension to any surface. Ocagne, M. $\boldsymbol{d}^{\prime}$. L. Mth. S. P. 18 (1886-87) 361-.

Polar groups of systems of points. Stuyvaert, Mathesis 18 (1898) 20-.

- reciprocal figures, transformation. Vaněček, J. S. Liege S. Sc. Mm. 11 (1885) No. 2, 21 pp.

- theory of curves and surfaces, 3rd degree. Milinowski, -. Crelle J. Mth. 89 (1880) 136-.

Polars of a straight line relative to curves and surfaces. Laguerre, E. Par. S. Mth. Bll. 3 (1875) 174-.
Polynomials, geometric application of a theorem. Laisant, C. A. As. Fr. C. R. (1898) (Pt. 2) 73-.

Priority claim for various theorems. Mainardi, G. Rm. At. N. Linc. 26 (1873) 88-.

Product of secants through 2 points. Jubé, $E$. N. A. Mth. 5 (1846) 340-.

Properties, central. Breton [de Champ], $\boldsymbol{P}$. Liouv. J. Mth. 11 (1846) 153-; N. A. Mth. 7 (1848) 187-.

-, general. Terquem, $O$. N. A. Mth. 16 (1857) 266-.

Quadric and cubic, polar $n$-hedra. Johnson, A. R. Mess. Mth. 16 (1887) 63-.

Segmentary theorems. Terquem, $O$. N. A. Mth. 18 (1859) 111-.

Similar curves and surfaces. Crelle, A. L. Berl. Ab. (1828) 21-.

Similarity of curves and solids. Brenner, Grunert Arch. 13 (1849) 182-.

Similarly variable figures, and loci derived from them. Réveille, J. N. A. Mth. 12 (1893) 297-.

Similitude and symmetry of 2 curves or surfaces. Mangeot, $S$. Par. Ec. Norm. A. 15 (1898) 385-.

Singularities, decomposition. Korteweg, D.J. Mth. A. 41 (1893) 286-.

Surface generated by conic doubly secant to given conic. Demartres, G. C. R. 106 (1888) 340-.

Surfaces and curves of form $\Sigma a_{r} x_{r}^{n}=0$. Pellet, A. C. R. 115 (1892) 498-.

- - given by equation $\sum_{1}^{n} \frac{a_{r}}{u_{r}}=0$, where $u_{r}$ denotes line or plane. Hunyady, E. von. C. R. 64 (1867) 218-; 65 (1867) 497-.

- with only one side, and singular points of plane curves. Delaunay, N. Par. S. Mth. Bll. 26 (1898) 43-.

Symmetric products in relation to curves and surfaces. Johnson, A. R. QJ. Mth. 22 (1887) 325-.

Symmetry of 2 algebraic figures with reference to a point. Mangeot, S. N. A. Mth. 19 (1900) 451-.

-, tetrahedral, curves and surfaces with. Jamet, V. Par. Ec. Norm. A. 4 (1887) (Suppl.) $78 \mathrm{pp}$.

Tangents of equidistant curves and surfaces, construction. Schirek, C. Z. Mth. Ps, 28 (1883) 183-.

Theorems, various. Frégier, - Gergonne A. Mth. 9 (1818-19) 241-.

-, - Serret, P. (xI) Par. S. Phlm. Bll. 3 (1866) 98-. 


\section{Metrical and projective} properties of algebraic plane curves of degree higher than the second. (See also 8030, 8075, 8430.)

Bergsten, N.J. Stockh. Ac. Hndl. 34 (1813) 22 .

Le François, (Prof.) -. Quetelet Cor. Mth. 6 (1830) 315-.

Reiss, M. Quetelet Cor. Mth. 9 (1837) 249-.

Steiner, J. [1848] Berl. B. (1848) 310-; Crelle J. 47 (1854) 1-。

Terquem, $O$. N. A. Mth. 9 (1850) 283-.

Schwarz, F. S. H. [1856] Halle Ab. Nt. Vr. 1 (1860) 149-.

Woepcke, F. Crelle J. 53 (1857) 260-.

Steen, A. Mth. Ts. 1 (1859) 65-.

Beltrami, E. G. Mt. 4 (1866) 76-

Olivier, A. [1868] Crelle J. 70 (1869) 156-.

Laguerre, E. (x) Par. S. Phlm. Bll. 5 (1868) 112-; 7 (1871) 241-; C. R. 80 (1875) 1218-

Azzarelli, $M . \quad$ Rm. N. Linc. Mm. 8 (1892) 7-.

Michell, J. H. Aust. As. Rp. (1892) 257.

Algebraic curve cannot have 'point d'arrêt.' Serret, P. N. A. Mth. 4 (1865) 311-.

Anallagmatic curves and surfaces, application of general properties of curves. Laguerre, $\boldsymbol{E}$. (x) Par. S. Phlm. Bll. 5 (1868) 112-.

Analytical geometry, new method. Story, W. E. Am. J. Mth. 9 (1887) 38-.

Anharmonic curves. Bioche, C. Par. S. Phlm. Bll. 5 (1893) (C. R.) (No. 10) 2.

Asymptote is always tangent to 2 parts of the curve, proof. Terquem, $O$. N. A. Mth. 8 (1849) 288-.

Asymptotes. Lenthéric, - Gergonne A. Mth. 17 (1826-27) 79-.

- Fournier-Vannson, -. N. A. Mth. 2 (1843) 398-.

-. Gregory, D. F. Camb. Mth. J. 4 (1845) 42-.

- Serret, P. N. A. Mth. 13 (1854) 144-; 1 (1862) 23-.

一. Stolz, O. Mth. A. 11 (1877) 41-。

- Casorati, F. Palermo Cir. Mt. Rd. 3 (1889) 49-.

-, curvilinear. Haskell, M. W. [1897] Calif. Ac. P. (Mth. Ps.) 1 (1903) 25-.

-, equations. Casorati, F. Mil. I. Lomb. Rd. 12 (1879) 117-.

-, Newton's theorem. Lebesgue, V. A. N. A. Mth. 7 (1848) 385-.

-, - - , proof, and theorem on multiple points. Terquem, O. N. A. Mth. 7 (1848) 422-.

-, property, and points at infinity. Transon, A. N. A. Mth. 12 (1873) 289-.

-, real, to imaginary branches of curves. Walton, W. Camb. Mth. J. 2 (1841) 236-.

-, rectilinear. Smith, E. B. A. Mth. 1 (1884-85) 11-, 72.
Asymptotes, rectilinear. Himstedt, A. Arch. Mth. Ps. 12 (1894) 357-.

Asymptotic curves. Serret, J. A. N. A. Mth. 6 (1847) 217-.

- points, theory. Schiffner, $F$. Arch. Mth. Ps. 67 (1882) 203-.

Axes, conditions that curve may have given number. Mangeot, S. Par. S. Mth. Bll. 25 (1897) 54-, 268.

-, harmonic. Chasles, $M$. C. R. 73 (1871) 229-, 1241-, 1289-, 1405-; 74 (1872) 21-.

- of symmetry, curves with several. St. Germain, A. de. N. A. Mth. 19 (1880) 350-.

Bicursal curves. Cayley, A. L. Mth. S. P. 4 (1871-73) 347-.

Bifocal curves. Onnen, $H$. N. Arch. Wisk. 17 (1890) 77-.

Bitangential curve. Heal, W. E. Des Moines Anal. 8 (1881) 171-.

Bitangents. Cayley, A. Phil. Trans. (1859) 193-.

-. Holditch, H. QJ. Mth. 3 (1860) 289-; 4 (1861) 28-.

- Retali, V. [1890] Bologna Rd. (189091) 35-.

-. Dowling, L. W. [1897] N. Y. Am. Mth. S. Bll. 4 (1898) 2,4 .

- with coordinates rational functions of a parameter, number. Schwering, $K$. Z. Mth. Ps. 21 (1876) 130-.

-, determination of points of contact. Salmon, G. QJ. Mth. 3 (1860) 317-.

Boundary curves, envelope of

$$
x n+y m=m n\left(m^{p}+n^{p}=s^{p}\right) .
$$

Meyer, C. W. Arch. Mth. Ps. 16 (1898) 150-.

Branches of curves, number. Harnack, $A$. Mth. A. 10 (1876) 189-.

-, imaginary, associated with possible magnitudes. Walton, $W$. [1864] QJ. Mth. 7 (1866) 232-.

-, infinite. Haillecourt, A. N. A. Mth. 6 (1847) 372-.

-, - Bellavitis, G. [1879] Rm. R. Ac. Linc. Mm. 5 (1880) 43-.

-, 一, forms. Frost, P. QJ. Mth. 3 (1860) 164 -. - and points, imaginary. Loud, $F$. H. A. Mth. 8 (1893-94) 29-.

-, real. Hilbert, D. Mth. A. 38 (1891) 115-. Carnot's theorem generalised. Gob, A. As. Fr. C. R. (1893) (Pt. 2) 258-.

Centre of curve. Taylor, H. M. QJ. Mth. 24 (1890) 55-.

-_- Baker, H. F. QJ. Mth. 24 (1890) 338-.

- mean distances. Liouville, J. Liouv. J. Mth. 9 (1844) 337-.

_ - - of points of rational curve. Weill, M. Par. S. Mth. Bll. 10 (1882) 137-.

- _ _ - theorem of Newton, generalisation. Delens, P. N. A. Mth. 12 (1893) 407-.

Centres. Roguet, C. N. A. Mth. 2 (1843) 210 -.

- of harmonic means. Poncelet, J.V. Crelle J. 3 (1828) 213-.

-, 2 theorems. Schoute, P. H. Par. S. Mth. Bll. 10 (1882) 219-. 
Centroid of certain points of curves. Ocagne, M. $d$ '. C. R. 99 (1884) 779 -

- - intersections of curves with circle of fixed centre. Ocagne, M. $d$ '. N. A. Mth. 5 (1886) 295-.

- - - - straight line with curve, Steiner's theorems. Sporer, B. Z. Mth. Ps. 37 (1892) 65-, 340-.

- points common to 2 curves. Sporer, $B$. Arch. Mth. Ps. 3 (1886) 84-.

Class of curve, lowering. Painvin, L. N. A. Mth. 6 (1867) 113-

- - - , - owing to presence of cusp. Painvin, L. Bll. Sc. Mth. As. 4 (1873) 131-.

Concentric parallel curves and complementary inverse curves. Chamonsset, (l'abbé) $F$. [1868] (xII) Sav. Ac. Mm. 11 (1869) Lxxxvi.

Conditions that 4 curves have 2 common points, order. Roberts, S. QJ. Mth. 9 (1868) 176-

Construction of curves. Bellavitis, G. [1878] Rm. S. It. Mm. 3 (1879) No. 4, 43 pp.

Contact of curves. Spottiswoode, W. [1861-66] Phil. Trans. (1862) 41-; QJ. Mth. 7 (1866) 114-.

-, double, curves having. Martin, J.W. Cn. J. 5 (1860) 331-.

- of higher order. Laurent, H. N. A. Mth. 16 (1877) 26-.

- point, lines making constant angle with tangent at. Chasles, M. C. R. 74 (1872) 1146-.

Coordinates, bi-polar. Vries, J. de. Amst. Ak. Vs. 4 (1896) 219-; Fschr. Mth. (1896) 491. - of plane curve in space. Tanner, H.W.L. L. Mth. S. P. 13 (1881-82) 125-.

-, polar, symmetry in. Lefevre, $J$. N. A. Mth. 11 (1892) 302-, 353-.

- tangential. Amstein, H. Laus. S. Vd. Bll. 15 (1878) 393-.

-, trilinear, of circular points at infinity. Mansion, P. [1875] Mess. Mth. 5 (1876) 158 -

-, -, and higher curves. Foglini, G. Rm. N. Linc. Mm. 1 (1887) 173-.

Cotangent theorems. Eckardt, F.E. Z. Mth. Ps. 10 (1865) 503- ; 12 (1867) 352-.

Cotes's theorem. Walker, J. J. L. Mth. S. P. $10(1878-79)$ 180-.

Covariant of 9th order of ternary cubic form, geometric significance. Gerbaldi, $F$. Tor. Ac. Se. At. 15 (1879) 707-.

Covariants, 2, applied to construction of certain curves. Mangeot, S. N. A. Mth. 16 (1897) 76-.

- of ternary forms. Gundelfinger, S. A. Mt. 5 (1871-73) 223-.

Cubic and biquadratic problems, solution by geometry. Smith, H. J.S. A. Mt. 3 (186970) 112-, 218-.

Curvature conics of plane curves. Berg, F.J. van den. Amst. Ak. Vs. M. 9 (1892) 85-; Fschr. Mth. (1892) 654-.

- of curves. Servais, C. Brux. Ac. Bll. 21 (1891) 587- ; 22 (1891) 512-.

Curve generated by 2 projective pencils of curves, degree. Olivier, A. [1869] Crelle J. 71 (1870) 195-.
Curve, polar of which with respect to given circle is congruent to curve itself. Genocchi, A. N. A. Mth. 14 (1855) 248.

- with tangents meeting at constant angle, problem. Ivory, J. Thomson A. Ph. 8 (1816) 272-.

Curves adjoint to algebraic curve. Biermann, O. Mh. Mth. Ps. 10 (1899) 373-.

- with branches in several planes, existence. (Geometrical interpretation of $\sqrt{-1}$ and fractional powers.) Gregory, D. F. Camb. Mth. J. 1 (1839) 259-.

- coordinates rational functions of 8 parameter. Clebsch, R.F.A. [1864] Crelle J. 64 (1865) 43-.

- generated by projective relations on curves. Schröter, H. E. Crelle J. 54 (1857) 31-.

- - section of cone by plane. Gergonne, J. D. Gergonne A. Mth. 12 (1821-22) 113-.

- in polar coordinates. Gourieff, $S$. [1797] St Pet. Ac. Sc. N. Acta 12 (1801) 176-.

- referred to centre as origin. Billelaut, N. A. Mth. 5 (1846) 228 .

- subtending right angle from points on circle. Levänen, $S$. Helsingf. Ofv. 34 (1892) 22-.

_, 3rd class. Laguerre, $E$. Liouv. J. Mth. 4 (1878) 213-.

_, - classification. Bellavitis, G. [1851] Mod. Mm. S. It. 25 (1855) 1-.

-, - - with given foci. Kapteyn, W. Amst. Ak. Vs. 5 (1897) 146-; Fschr. Mth. (1896) 487.

-, - - polar conics, theorems. Smith, T. St L. QJ. Mth. 2 (1858) 327-.

-, - -, rational. H., G. D. Ts. Mth. 6 (1889) 193-; Fschr. Mth. (1888) 715.

- - - types. Breyer, H. G. Amst. Ak. Vs. [2] (1894) 60-, 81.

- 4 th class, with 3 bitangents. Le Paige, $\boldsymbol{C}$. Prag Sb. (1884) (Mth.-Nt.) 45-.

- - - - - quadruple foci. Jeffery, $H . M$. B. A. Rp. (1880) 478-; QJ. Mth. 18 (1882) 1-.

-, - - - - - loops. Jeffery, H. M. [1881] QJ. Mth. 18 (1882) 158-

-, - - , singularities. Laguerre, E. Liouv. J. Mth. 1 (1875) 265-.

- , - - , with a triple and a single focus. Jeffery, H. M. B. A. Rp. (1883) 412-; QJ. Mth. 20 (1885) 273-.

-, 6th class, symmetric. Crone, C. Acta Mth. 2 (1883) 81-.

-, 9th class, adjoint to general curve of 4 th degree. Kohn, G. Wien Ak. Sb. 95 (1887) (Ab. 2) 338-.

-, $n$th class, foci. Serret, P. C. R. 86 (1878) 385-.

-, - -, orthoptic curve, theorem of Steiner. Dewulf, -. N. A. Mth. 18 (1859) 174-.

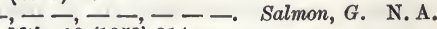
Mth. 18 (1859) 314-.

-, even degree, theory. Mahler, E. [1882] Arch. Mth. Ps. 69 (1883) 108-. 
Curves, 2nd and 3rd degrees.

Clausen, T. Grunert Arch. 15 (1850) 345-. canonical forms. Roberts, R. A. [1889] L. Mth. S. P. 21 (1891) 62-.

circum-polygons of conic, and in-polygons of cubic. Roberts, R. A. L. Mth. S. P. 17 (1887) 158-.

parametric determination of points. Juel, C. Mth. A. 47 (1896) 72-

tactinvariant of a conic and a cubic. Lindemann, F. B. A. Rp. (1884) 647-。

in trilinear coordinates. Jettmar, H. von. Arch. Mth. Ps. 10 (1891) 13-.

\section{Curves, 3rd degree.}

Clausen, T. Crelle J. 11 (1834) 402-; As. Nr. 21 (1844) 209-.

Cayley, A. Liouv. J. Mth. 9 (1844) 285-; 10 (1845) 102-

Chasles, M. N. A. Mth. 9 (1850) 382-.

Cotterill, T. Camb, and Dubl. Mth. J. 7 (1852) 14-.

Bjerknes, C. A. Crelle J. 55 (1858) 310-; N. Mg. Ntvd. 10 (1859) 191-.

Transon, (Prof.) A. N. A. Mth. 18 (1859) 266-.

[Salvatore-]Dino, N. G. Mt. 1 (1863) 187-, 334-.

Hunyady, J. (xп) Mag. Ak. Ets. (Mth. Term.) 4 (1863) 19-; 5 (1865) 66-

Sartiaux, A. N. A. Mth. 6 (1867) 68-.

Eckardt, F. E. Z. Mth. Ps. 13 (1868) 263-.

Bäcklund, A.V. Stockh. Öfv. 28 (1871) 715-; 29 (No. 2) (1872) 109-.

Koehler, C. N. A. Mth. 11 (1872) 21-, 66-, 122-.

Schröter, H. [1872] Mth. A. 6 (1873) 85-.

Milinowski, -. Crelle J. Mth. 78 (1874) 177-.

Sharp, W. J. C. QJ. Mth. 16 (1879) 186-, 298-.

Folie, F., \& Le Paige, C. [1880-81] Brux. Ac. Mm. 43 (1882) No. 7, 43 pp.; Brux. Ac. Bll. 1 (1881) 610-.

Arcais, F. d'. Ven. I. At. 8 (1882) 673-.

Boije af Gennäs, C. O. Des Moines Anal. 9 (1882) 108-.

Franklin, F. Am. J. Mth. 5 (1882) 212 -

Le Paige, C. M. M. H. H. [1882] Brux. Ac. Bll. 4 (1882) 334-; Prag Sb. (1882) 200-; Liége S. Sc. Mm. 10 (1883) (No.2) 103 pp.

Folie, F., \& Le Paige, C. Brux. Ac. Mm. 45 (1884) (No. 1) $45 \mathrm{pp}$.

Springer, J. F. [1889] J. H. Un. Cir. [9 (1889-90)] 31 .

Loud, F. H. [1891] A. Mth. 6 (1891-92) 5-.

Sauve, A. Rm. N. Linc. At. 53 (1900) 129-.

through 7 angular points of complete quadrilateral. Machovec, $F$. Casopis 16 (1887) 113-; Fschr. Mth. (1887) 629.

anharmonic property. Salmon, $G$. Crelle J. 42 (1852) 274-.

- ratio of a curve. Jamet, -. Par. S. Mth. Bll. 15 (1887) 35-.

axes, determination. Mangeot, S. Par. Éc. Norm. A. 10 (1893) 43-. branches, principal and secondary.

Grass mann, $H$. Gött. Nr. (1872) 505-。

circumscribed to complete quadrilateral. Ovidio, E. $d^{\prime}$. G. Mt. 10 (1872) 16-

classification. Plilcker, J. (vIII) Crelle J. 10 (1833) 98 .

-. Cayley, A. [1864] Camb. Ph. S. T. 11 (pt. 1) (1866) 81-.

-. Cremona, L. G. Mt. 2 (1864) 78.

-. Jeffery, H. M. [1878] QJ. Mth. 16 (1879) 98

- by nature of tangents from any point of curve. Durège, H. [1871] Crelle J. 75 (1873) 153-; 76 (1873) 59-.

-, Newton's. Ball, W. W. R. Bb. Mth. (1891) 35-.

through 3 collinear points, and touching pairs of lines. Roberts, R.A. Mess. Mth. 15 (1886) 152-.

and cones, 3rd degree. Cayley, A. [1864] Camb. Ph. S. T. 11 (pt. 1) (1866) 129-.

27 conics of closest contact. Sylvester, J. J. Ph. Mg. 11 (1856) 463-.

conics touching in 3 distinct points. Hesse, L. O. Crelle J. 36 (1848) 143-.

conjugate points, triangles, etc. Caporali, $E$. Rm. R. Ac. Linc. T. 1 (1877) 232-.

connected with isogonal triangles. Barbarin, $P$. As. Fr. C. R. (1896) (Pt. 2) 89-.

construction. Grave, $P$. Kazan Un. Mm. (1898) (Pt. 3) 121-, (Pt. 4) 63-, (Pts. 5 \& 6) 1-, (Pts. 7 \& 8) 63-, (Pt. 9) 51-, (Pt. 10) 1-, (Pt. 11) 1-; (1899) (Pt. 2) 1- ; Fschr. Mth. (1898) 508-.

- from conjugate points. Heger, R. Z. Mth. Ps. 25 (1880) 100-

contact, 6-point, with conic, 27 points of. Steiner, $\vec{J}$. Berl. B. (1845) 386-.

-, 9-point. Hart, A. S. Ir. Ac. T. 25 (1875) 559 -.

contacts, conics in 5, cubics in 8 consecutive points. Salmon, G. Phil. Trans. (1858) 535-.

coordinates. Hermite, C. [1876] Crelle J. Mth. 82 (1877) 343-.

covariant conics and cubics of plane cubic. White, H. S. N. Y. Am. Mth. S. T. 1 (1900) 1-.

- locus of vertex of pencil of tangents to cubic in involution. Walker, J.J. [1881] L. Mth. S. P. 13 (1881-82) 66-.

covariants. Gordan, P. N. Y. Am. Mth. S. T. 1 (1900) 9-.

curve as sum of 4 cubes. Walker, J. J. L. Mth. S. P. $10(1878-79)$ 180-.

- of 3rd class touching in 9 points. Halphen, G. H. [1880] Par. S. Mth. Bll. 9 (1881) 96-.

as curves of involution. Weyr, E. J. Prag Sb. (1877) 131-.

and curves of 3rd class. Hesse, L. O. Crelle J. 38 (1849) 241-.

cyclic polygons on, application of elliptic functions. Vries, J. de. Amst. Ak. Vs. M. 7 (1890) 430-; Arch. Néerl. 25 (1892) 1-.

decomposition of curve into conic and straight line. Brioschi, Fr. Rm. R. Ac. Linc. At. 3 (1876) (Pte. 2) 89-.

- _ - 3 straight lines. Thaer, A. [1878] Mth. A. 14 (1879) 545-. 
derivative points, algebraic theory. Sylvester, J. J. Ph. Mg. 16 (1858) 116-.

diameters. Walker, J. J. [1887] Phil. Trans. (A) 179 (1889) 151-.

with double point. Weyr, Em. [1870] Prag Sb. (1870) 43-; Mth. A. 3 (1871) 235-.

- - . Igel, B. Mth. A. 6 (1873) 633-.

- - . Anelli, P. G. Mt. 16 (1878) 364-.

- - - Pittarelli, G. Nap. Rd. 24 (1885)

$111-$. - $-\overrightarrow{272}$-. Dingeldey, $F$. Mth. A. 27 (1886)

- - - Torelli, G. G. Mt. 26 (1888) 172-. - - . Berzolari, L. Mil. I. Lomb. Rd. 25 (1892) 1025-.

- - , and curves of 4th degree with 3 double points. Juel, C.S. (xII) Ts. Mth. 1 (1877) 17 -

- - , equation. Walker, J.J. QJ. Mth. 14 (1877) 242-

- - - metrical properties. Stiner, $G$.

Mh. Mth. Ps. 4 (1893) 99-.

- - - series of tangents. Durège, $\boldsymbol{H}$. Mth. A. 1 (1869) 509-.

- - - Steiner's polygons in. Loria, $G$. Prag Sb. (1896) (Mth.-Nt.) No. 36, 4 pp.

- - - synthetic theory. Drasch, $H$. Wien Ak. Sb. 85 (1882) (Ab.2) 534-

- - - tangents drawn from any point to.

Roberts, R. A. L. Mth. S. P. 12 (1880-81) 99-.

_ _ - theorems. Weyr, Em. Wien Sb. 61 (1870) $(A b .2) 731-, 819-$.

- - - - Z Zimmermann, H. E. M. O. Z. Mth. Ps. 28 (1883) 56-.

-2 double points. Gordan, P. Mth. A. 3 (1871) 631-.

and ellipse, theorems. Steiner, J. Crelle J. 32 (1846) 300-.

- - , - (Steiner). Clausen, T. Grunert Arch. 9 (1847) 259-.

enumeration. Commines De Marsilly, (le gén.) A. de. As. Fr. C. R. (1886) (Pt. 2) 110-.

envelope derived from curve. Schoute, P. H. N. Arch. Wisk. 3 (1898) 30-.

as envelopes of conics. Küpper, C. Prag Sb. (1871) (pt. 2) 63-.

family, critical centres for. Borisov, E. V.

[1894] Rec. Mth. (Moscou) 17 (1895) 461-; Fschr. Mth. (1893-94) 1135.

figure formed by 16 cotangential chords of a curve. Robert8, S. QJ. Mth. 9 (1868) 232-.

figures. Disteli, M. Z. Mth. Ps. 36 (1891) 138 -

fundamental forms. Möbius, A. F. Leip. B. 2 (1848) 12-; Leip. Ab. Mth. Ps. 1 (1852) 1-. general properties, contravariant (Cayleyan), etc. Cayley, A. [1856] Phil. Trans. (1857) 415-.

generation. Weyr, Em. Wien Sb. 58 (1868) (Ab. 2) 633-.

-. Schröter, $\dot{H}$. [1871] Mth. A. 5 (1872) 50-.

-. Clebsch, R. F. A. Mth. A. 5 (1872) 422-.

-. Tognoli, O. G. Mt. 11 (1873) 378-.

- (Schröter). Hurwitz, A. Crelle J. Mth. 107 (1891) 141-.

-. Ioachimescu, A. G. [Bucarest S. Sc. Bl. 6 (1897)] 152-. generation by conic pencil and ray pencil, synthetic proof. Milinowski, - . Z. Mth. Ps. 23 (1878) 327-، Ps. 24 (1879) 119-. - -. Schur, F. Z. Mth.

-, linear. Grassmann, H. Crelle J. 36 (1848) 177-; 52 (1856) 254-.

-, - (Grassmann). Schroeter, H. Crelle J. Mth. 104 (1889) 62-.

- by symmetric elemental systems of 2 nd degree. Weyr, E. J. Wien Ak. Sb. 69 (1874) (Ab. 2) 784-.

Hessian and Cayleyan, construction. Scott, (Miss) C. A. [1893] Phil. Trans. (A) 185 (1895) 247 -.

Hessians and Cayleyans. Gordan, P. N. Y. Am. Mth. S. T. 1 (1900) 402-.

with infinitely distant stationary tangent, and their occurrence in statics. Solin, J. M. [1877] Prag Ab. 9 (1878) (Mth.) (No. 2) $32 \mathrm{pp}$.

inflexional lines, triplets and triangles. White, H. S. N. Y. Am. Mth. S. Bll. 4 (1898) $258-$.

- tangents. Clebsch, A. [1860] Crelle J. 58 (1861) 229-.

- - Wirtinger, $W$. Mh. Mth. Ps. 4 (1893) 395 -

- - (Wirtinger). Kohn, G. Mh. Mth. Ps. 4 (1893) 398.

- - of a curve, and conics touched by them. Ferrers, N. M. Mess. Mth. 24 (1895) 77-.

intersections. Chasles, M. C. R. 41 (1855) 1190 -

-. Hart, A. S. [1878] Ir. Ac. T. 26 (1879) 449-.

- of 2. Taylor, H. M. L. Mth. S. P. 29 (1898) 265-.

- with straight lines. Laguerre, E. [1875] Par. S. Mth. Bll. 4 (1876) 110-.

- 3 straight lines; if 6 lie on conic, other 3 are on straight line. Poncelet, J. $V$. Quetelet Cor. Mth. 7 (1832) 79-.

- of tangents through 2 points on a curve. Roberts, S. QJ. Mth. 3 (1860) 118-.

involution. Cayley, A. [1864] Camb. Ph. S. T. $11(p t .1)(1866) 39$ -

irrational covariant cubics of plane cubics. White, H. S. N. Y. Am. Mth. S. T. 1 (1900) $170-, 508$.

linear constructions. Harnack, A. Z. Mth. Ps. 22 (1877) 38-.

orthogonal invariants. Thomae, $J$. Leip. Mth. Ps. B. 51 (1899) (Mth.) 317-.

in pencil of 6 lines. Cayley, A. QJ. Mth. 9 (1868) 210 -

plane and skew. Mangeot, S. Par. S. Mth. Bll. 21 (1893) 44-.

9 th point determined by 8 . Weddle, $T$. Camb. and Dubl. Mth. J. 6 (1851) 83-.

- Hart, A.S. Camb. and Dubl. Mth. J. 6 (1851) 181-.

- - - -. Quetelet, E. Brux. Ac. Bll. 5 (1858) 15-.

-_- - Cayley, A. QJ. Mth. 5 (1862) $222-$.

- - - London, $F$. Mth. A. 36 (1890) $585-$. 
9th point determined by 8 . Kierboe, $T$. N. Ts. Mth. 7 (B) (1896) 53-; Fschr. Mth. (1896) 437-.

- - - - depending on system of conics. Smith, H.J.S. A. Mt. 2 (1868-69) 318-. - - - Salmon's theorem. Goursat, $\dot{E}$. N. A. Mth. 15 (1896) 20-

point, polar conic of which is a circle. Stuyvaert, -. N. A. Mth. 18 (1899) 275-.

through 9 points, condition for double point. Thomae, $J$. Leip. Mth. Ps. B. 47 (1895) 515-. -- , construction. Chasles, M. C. R. 36 (1853) 943-.

- - - - Tsinger, V. Y. [1867] (xrI) Rec. Mth. (Moscou) 3 (1868) (Pt. 1) 290-. $--\ldots$, - Beyel, C. Z. Mth. Ps. 40 (1895) 99-.

points of inflexion. Hesse, L. O. Crelle J. 28 (1844) 97-; 38 (1849) 257-.

- _ - Salmon, G. Crelle J. 39 (1850) 365-.

- - Sartiaux, A. [1867] (xII) Lille S.

Mm. 5 (1868) 429-.

- Clebsch, R.F.A. [1869] Mth. A. 2 (1870) 382-.

(1873) 356-.

453-.

- Wiman, A. N. Ts. Mth. 5 (B) (1894) $17-$

- - , and cusps of curves of 3rd class. Painvin, L. F. [1862] (xII) Lille S. Mm. 10 (1864) 103-.

- - - generalisation of theorem. Legoux, A. N. A. Mth. 2 (1883) 77-.

- - - position. Disteli, M. Zür. Vjschr. 35 (1890) 145-.

- _ - , and stationary tangents of curves of 3rd class. Hesse, L. O. Crelle J. 38 (1849) 257-.

- - , theorem. Russell, W. H. L. [1882] R. S. P. 34 (1883) 35-.

$--\frac{1}{-}-$ (Russell). Spottiswoode, W. [1882] R. S. P. 34 (1883) 37-.

9 points of intersection of 2 curves. Schottky, F. Crelle J. Mth. 119 (1898) 72-.

points, ruler construction for, given point $P$ on curve and points of contact of 4 tangents from P. Thomson, F. D. [1868] Mess. Mth. 5 (1871) 27 -

polar conics, theorems. Smith, T. St L. QJ. Mth. 2 (1858) 208-.

- figures. London, $\dot{F}$. Mth. A. $36(1890) 535-$. - -. Scorza, G. Mth. A. 51 (1899) 154 -.

- reciprocal of curve with respect to cubic. Russell, W. H. L. R. S. P. 44 (1888) 388-. - - - of syzygy $f-\rho \Delta=0$. Gerbaldi, $F$. Palermo Cir. Mt. Rd. 7 (1893) 19-.

- system of a curve. Bonsdorf, E. [E. J.] [1877] St Pét. Ac. Sc. Bll. 24 (1878) 409-.

polygon of $2 n$ sides inscribed to. Torelli, $G$. G. Mt. 26 (1888) 327-.

polygons in-and circum-scribed to same curve. Weyr, E. Mh. Mth. Ps. 4 (1893) 120, 154. -, Steiner's, on. Czuber, E. Crelle J. Mth. 114 (1895) 312-.

and poristic polygons. Steiner, J. Berl. B. (1845) 386-. problem on two. (Construction of conic through the 5 unknown points, 4 being given.) Poudra, -. N. A. Mth. 15 (1856) 24-.

projective generation, rational curves, etc. Kötter, E. Mth. A. 38 (1891) 287-.

rational. Weyr, Em. G. Mt. 9 (1871) 145-.

- Zahradn k, $K$. Arch. Mth. Ps. 56 (1874) 131-; 58 (1876) 23-; 61 (1877) 1-.

-. Astor, A. N. A. Mth. 11 (1892) 276-; 13 (1894) 184. 297-

Cazamian, A. N. A. Mth. 14 (1895)

-, application of polar reciprocals. Cazamian, A. N. A.Mth. 13 (1894) 300-

-, construction. Schoute, P. H. As. Fr. C. R. (1885) (Pt. 2) 169-.

-, -. Willig, H. Arch. Mth. Ps. 10 (1891) 1-.

-, - of Hessian curve. Dingeldey, F. Mth. A. 28 (1887) 81-.

-, projectivities and involutions on. Weyr, E. J. Wien Ak. Sb. 81 (1880) (Ab. 2) 169-.

referred to tetrad of corresponding points. Jeffery, H. M. QJ. Mth. 15 (1878) 198-.

relations on general curve. Weyr, $E$. $J$. Wien Ak. Sb. 87 (1883) (Ab. 2) 837-.

representation on circle. Durège, $H$. Z. Mth. Ps. 17 (1872) 433-.

- by elliptic functions. Picquet, - Par. Ec. Pol. J. 54 (1884) 31-.

ruler constructions in connexion with. Russell, $R$. [1893] Ir. Ac. T. 30 (1892-96) 295-.

satellite of line. Roberts, W. R. W. [1880] Ir. Ac. P. 3 (1883) 293-.

- - - Walker, J. J. [1890] L. Mth. S. P. 21 (1891) $247-$.

series of pentagons. Serret, P. C. R. 115 (1892) 406-, 436-.

- - (Serret). Rouquet, V. Toul. Ac. Sc. Mm. 5 (1893) 113-.

singularities. Gundelfinger, S. Mth. A. 4 (1871) 561-.

and surfaces of 2nd degree, geometrical constructions for. Engel, J.H. Zür. Vjschr. 34 (1889) 299-.

synthetic treatment. Milinowski, - Z. Mth. Ps. 21 (1876) 427-; 23 (1878) 343-.

system of linear equations in connexion with a curve. Rosanes, $J$. Mth. A. 36 (1890) 316-.

tangential of point on. Cayley, A. Phil. Trans. (1858) 461-.

6 tangents, conic through points of contact. Pliicker, J. Crelle J. 34 (1847) 329-.

tangents, forming pencil in involution. Roberts, R. A. $[1881]$ L. Mth. S. P. 13 (1881-82) 25-.

6 tangents from point, problem. Russell, W. H. L. [1881] R. S. P. 33 (1882) 211-. tangents, projective proof of anharmonic property. Dixon, A. C. Mess. Mth. 26 (1897) 53-.

-, real and imaginary. Sturm, $R$. Crelle J. Mth. 90 (1881) 85-.

'tertians,' diametral or centric. Newman, F. W. B. A. Rp. 39 (1869) (Sect.) 10- 
and theorem of Steiner on polygons. Clebsch, R. F. A. [1863] (vir) Crelle J. 63 (1864) 94-.

transformation of equation to canonical form. Dingeldey, $F$. Mth. A. 31 (1888) 177-.

triameters. Mainardi, $G$. A. Sc. Lomb. Ven. 7 (1837) 118-.

triangle of points of inflexion. Gundelfinger, S. [1871] Mth. A. 5 (1872) 442-.

triangles in- and ex-scribable to general curve. Sylvester, J. J. [1880] (xII) J.H. Un. Cir. [1] (1882) 49.

- of tangents. Reiss, M. Quetelet Cor. Mth. 9 (1837) 249-.

in trilinear coordinates. Whitworth, W.A. (vi) Mess. Mth. 2 (1864) 116-, 129-.

and 3rd class. Zahradnik, $K$. Prag Sb. (1873) 298-.

- - a geometrical relationship. Zahradník, $K$. Wien Ak. Sb. 75 (1877) (Ab. 2) 437-.

- - - with a single and a double focus. Jeffery, H. M. QJ. Mth. 14 (1877) 359-.

- _ - - 3 single foci. Jeffery, H. M. B. A. Rp. (1877) (Sect.) 26-; QJ. Mth. 16 (1879) 65-, 348-.

- - - - triple foci. Jeffery, H. M. [\& Cayley, A.] [1876] QJ. Mth. 14 (1877) 127-.

- 4th class. Zahradnik, $K$. Prag Sb. (1873) 310-.

$-\frac{1}{217}$. Pittarelli, G. Nap. Rd. 24 (1885) 217-.

$--\ldots$, conics of triple contact with. Weyr, E.J. [1879] Wien Ak. Sb. 80 (1880) (Ab.2) 1040-.

- 6th class. Vályi, G. Mth. Termt. Ets. 8 (1890) 23-; 9 (1891) 18-; 10 (1892) 2-; Mth. Nt. B. Ung. 8 (1891) 69-; 9 (1892) 143-; 10 (1893) 168-.

\section{Curves, 3rd and 4th degrees.}

Chasles, M. Quetelet Cor. Mth. 5 (1829) 231-; C. R. 37 (1853) 272-.

Küpper, C. Prag Ab. 5 (1872) 19 pp.

Zeuthen, H. G. (xII) Ts. Mth. 3 (1873) 97-; 4 (1874) 14-.

Amanzio, D. G. Mt. 14 (1876) 48-.

construction. Valentiner, $H$. N. Ts. Mth. 3

(B) (1892) 33-; Fschr. Mth. (1892) 599-.

constructions, Chasles's. Brioschi, F. Tortolini A. 6 (1855) 380-.

intersections of conics with. Rohn, $K$. Dresden Isis Sb. (1898) 24.

rational. Ameseder, $A$. [1879] Wien Ak. Sb. 80 ( $A b .2)(1880)$ 487-.

-, absolute systems of elements on. Binder, W. Z. Mth. Ps. 36 (1891) 78-.

-, generation. Bienaymé, A. N. A. Mth. 13 (1894) 144-.

reduction of equations to line coordinates. Hesse, L. O. Crelle J. 40 (1850) 316-.

systems of tangents to. Walker, J.J. L. Mth. S. P. $3(1869-71) 202-$.

Curves, 3rd and 5th degrees.

interpolation by. Dupin, C. C. R. 25 (1847) 3rd and 4th Degrees

\author{
Curves, 4 th degree.
}

Brioschi, Fr. A. Mt. 7 (1876) 202-.

Postnikov, M. V. Kazan S. Nt. (Ps.-Mth.) P. 4 (1886) 238; 7 (1889 118-); N. A. Mth. 13 (1894) 348-.

bitangents. Hesse, L. O. Crelle J. 49 (1855) 279-; 55 (1858) 83-.

- Cayley, A. Phil. Trans. (1861) 357-.

-. Aronhold, -. Berl. Mb. (1864) 499-.

-. Roch, G. [1864] Crelle J. 66 (1866) 97-.

- Cayley, A. [1867] Crelle J.68(1868)176-.

- Geiser, C. F. Mth. A. 1 (1869) 129-; Crelle J. 72 (1870) 370-.

-. Crone, C. (xI) Ts. Mth. 5 (1875) 161-; 1 (1877) 97-, 151-; (rx) Mth. A. 12 (1877) 561-.

- Cayley, A. Crelle J. Mth. 94 (1883) 93-.

- Frobenius, G. Crelle J. Mth. 99 (1886) 275-.

-. Hossfeld, L. Z. Mth. Ps. 31 (1886) 1-.

-. Ciani, E. Mil. I. Lomb. Rd. 31 (1898)310-.

-, algebraic solution. Aeschlimann, (Dr) $U$.

Zür. Vjschr. 25 (1880) 365-.

-, classification by. Zeuthen, H. G. C. R. 77 (1873) 270-; Mth. A. 7 (1874) 410-.

- and contact-conics. Kohn, G. Wien Ak. Sb. 97 (1889) (Ab.2a) 325-, 1381-; Crelle J. Mth. 107 (1891) 1-.

-, - double transformation of 3rd degree, first genus. Paolis, R. de. Rm. R. Ac. Linc. Mm. 2 (1878) 851-.

-, equation for point of contact. Freyberg, $J$. Mth. A. 17 (1880) 329-.

-, Galois group. Weber, H. Mth. A. 23 (1884) 489 .

-, grouping. Timerding, H. E. Crelle J. Mth. 122 (1900) 209-.

-, Hesse's method. Rosati, C. G. Mt. 38 (1900) 165-.

一, mutual relations. Steiner, J. C. R. 37 (1853) 121-.

-, properties relative to. Steiner, J. [1852] Crelle J. 49 (1855) 265-.

- studied through Kummer's configuration. Ciani, E. Mil. I. Lomb. Rd. 31 (1898) 312-; A. Mt. 2 (1899) 53-.

-, and surface of $4 \mathrm{th}$ degree. Humbert, $G$. Liouv. J. Mth. 6 (1890) 423-.

, systems of conics related to. Noether, $M$. Mth. A. 46 (1895) 545-.

classified according to nature of infinite branches. Plilcker, J. Liouv. J. Mth. 1 (1836) 229-.

- - shape. Cayley, A. [1864] Ph. Mg. 29 (1865) 105-.

conditions for curve being repeated conic. Brioschi, Fr. Rm. R. Ac. Linc. At. 3 (1876) (Pte. 2) 91-.

confocal, geometric generation. Berner, $T$. Z. Mth. Ps. 9 (1864) 369.

conis of 4-point contact. Zeuthen, H. G. (xII) Ts. Mth. 5 (1875) 190-.

conics inscribed in. Humbert, $G$. Toul. Fac. Sc. A. 4 (1890) L, 8 pp.

- intersections of which with quartic can be constructed by rule and compass. Crone, C. N. Ts. Mth. 7 (B) (1896) 81-; Fschr. Mth. (1896) 441. 
contact conics etc. Ameseder, A. [1882] Wien Ak. Sb. 86 (1883) (Ab. 2) 396-; 87 (1883) (Ab. 2) 15-.

- Jacobian covariants of. Frobenius, $G$. Crelle J. Mth. 103 (1888) 139-.

- - relations between systems. Kohn, G. Mh. Mth. Ps. 1 (1890) 71-, 129-.

- curves. Noether, M. Münch. Ak. Ab. 17 (1892) 103-.

covariant $S$, theorem. Scorza, $G$. Mth. A. 52 (1899) 457-.

- - - Ciani, E. Palermo Cir. Mt. Rd. 14 (1900) 16-.

determined by 14 points, description. Jonquières, E. de. Liouv. J. Mth. 1 (1856) 411-; 2 (1857) 267-.

with double point. Brill, A. Mth. A. 17 (1880) 103-, 517-.

- - , bitangents. Brioschi, $F$. Mth. A. 4 (1871) 95-.

- - - - (Brioschi). Cremona, L. Mth. A. 4 (1871) 99 -

- - - - Brill, A. [1872] Mth. A. 6 (1873) 66-.

double points coinciding with those of covariant curves. Jeffery, $H$. M. [1889] QJ. Mth. 24 (1890) 250 -

with 2 double points. Zeuthen, H. G. Kjöb. Ov. (1879) 89-; (Résumé, 15-).

- _ - Genese, R.W. B. A. Rp. (1881) 538.

- - Davis, E. W. (xII) J. H. Un. Cir. [2] (1883) 144.

- - - Humbert, G. C. R. 97 (1883) $1287-$

(1888) 193-; Fschr. Mth. (1887) 631.

- - Hjelmman, A.L. Helsingf. Ofv. 39 (1897) 222-

- - - - analytical proof of properties. Story, W. E. [1881] (xir) J. H. Un. Cir. [1] (1882) 178.

- - - classification. Jeffery, H. M. [1891] L. Mth. S. P. 23 (1892) 18-

- - - , generation by conics. Jeffery, H. M. [1890] L. Mth. S. P. 21 (1891) $287-$.

- - - - and graphical representation of elliptic functions. Cayley, A. Camb. $\mathrm{Ph}$. S. T. 14 (1889) 484-.

- 3 double points. Cayley, A. Camb. and Dubl. Mth. J. 5 (1850) 148-.

- - Ameseder, A. Wien Ak. Sb. 79 (1879) $(A b .2)$ 241-.

- - - Ferrers, N. M. QJ. Mth. 18 (1882) 73-

- Schoute, P. H. Amst. Ak. Vs. M. 19 (1884) 420-; Bll. Sc. Mth. As. 8 (1884) 278-.

- - -. Vaněček, J. S., \& Vaněček, M. N. Prag Sb. (1885) (Mth.-Nt.) 96-, 223-.

- - Vidal, -. [1885] I. Égypt. Bll. 6 (1886) 133-.

- - - Malet, J. C. Mathesis 9 (1889) $89-$.

- - Vries, J.de. [1899-1900] Amst. Ak. Vs. 7 (1899) 340-; Amst. Ak. P. 1 (1899) 263-; Haarl. Ms. Teyl. Arch. 7 (1902) 1-. with 3 double points, bitangents. Durège, $H$. [1875] Wien Ak. Sb. 72 (1876) (Ab. 2) 495-.

- - - - (Durège). Kantor, S. [1882] Wien Ak. Sb. 86 (1883) (Ab. 2) 1051-.

- - - construction. Ameseder, $A$. Prag Sb. (1880) 3-.

- double tangential node and double point. Beyel, C. Zür. Vjschr. 31 (1886) 178-.

equation of 24th degree giving inflexions of general curve. Gerbaldi, $F$. Palermo Cir. Mt. Rd. 7 (1893) 178-.

equations of form $\Sigma a_{i k} \xi_{i} \xi_{k}$, where $\xi_{i}=x_{i}{ }^{2}$. Schmidt, E. (xII) Ts. Mth. 5 (1881) 145-.

-, when one part is a double line. Zeuthen, H. G. C. R. 75 (1872) 950 .

generation, linear. Grassmann, H. [1851] Crelle J. 44 (1852) 1-.

- by 2 pencils of conics. Chasles, M. C. R. 37 (1853) 372-, 437-.

- - ray pencil and ray system of 2 nd class. Ameseder, A. Arch. Mth. Ps. 64 (1879) 109-.

genus 1. Liebmann, $H$. Z. Mth. Ps. 41 (1896) $85-$.

- - geometry. Fiedler, W. Zür. Vjschr. 29 (1884) 332-.

- 2. Bobek, K. Wien Ak. D. 53 (1887) (Ab. 2) 119-.

- - construction by ruler and compass. Björling, C. F. E. Stockh. Öfv. (1887) 19-. geometric theory. Cardinaal, $J$. Crelle $J$. Mth. 102 (1888) 160-.

group of coordinated conics. Pascal, G. Rm. R. Ac. Linc. Rd. 1 (1892) (Sem. 2) 385-, 417-; 2 (1893) (Sem. 1) 8-.

Hessian with double point. Geiser, $C, F$. A. Mt. 9 (1878-79) 35-.

intersections of curve with conic. Jung, $G$. Mil. I. Lomb. Rd. 8 (1875) 698-.

2 invariant curves. Ciani, $E$. A. Mt. 20 (1892-93) 257-.

multiple tangents of the Cayleyan. Bertini, $E$. Tor. Ac. Sc. At. 32 (1896) 32-.

pentagon not always inscribable. Lilroth, $J$. Mth. A. 13 (1878) 548-.

penultimate. Cayley, A. Mess. Mth. 1 (1872) 178-.

polar figures. Scorza, G. A. Mt. 2 (1899) 155-.

polars. Clebsch, A. [1860] Crelle J. 59 (1861) 125-.

problem. Jonquières, $E$. de. N. A. Mth. 15 (1856) 370 -

as projections of intersection of 2 conical surfaces. Hachette, J. N. P. Par. Éc. Pol. Cor. 1 (1804-08) 368-.

quadratic series of conics enveloping. Ciani, E. Mil. I. Lomb. Rd. 28 (1895) 659-.

quadritangential conics. Ameseder, $A$. Wien Ak. Sb. 80 (1880) (Ab. 2) 187-; 83 (1881) (Ab. 2) 829-.

- - Andoyer, -. Toul. Fac. Sc. A. 3 (1889) D, 6 pp.

- - degeneration of the 63 systems. Fontene, G. Par. S. Mth. Bll. 27 (1899) 229-.

'quartan,' with 1 diameter. Newman, $F$. W. B. A. Rp. 42 (1872) (Sect.) 23-. 
' quartan,' with 2 diameters. Newman, $F$. W. B. A. Rp. 41 (1871) (Sect.) 20-.

- -3 diameters. Newman, $F$. W. B. A Rp. 42 (1872) (Sect.) 22-.

- - - or 4 diameters. Newman, F. W. B. A. Rp. 42 (1872) (Sect.) 23.

rational. Weyr, Em. Wien Sb. 63 (1871) (Ab. 2) 493-.

一. Brill, A. Mth. A. 12 (1877) 90-.

- Bobek, $K$. [1879] Wien Ak. Sb. 80 (1880) (Ab. 2) 361-.

- Hossfeld, C. [1882] Z. Mth. Ps. 28 (1883) 296-.

-. Astor, A. N. A. Mth. 3 (1884) 181-.

- Roberts, R. A. L. Mth. S. P. 16 (188485) 44-.

-. Kohn, G. Wien Ak. Sb. 95 (1887) (Ab. 2) 318-.

-. Stahl, W. Crelle J. Mth. 101 (1887) 300-; 104 (1889) 302-.

-, algebraic generation. Meyer, $\boldsymbol{F}$. Mth. A. 31 (1888) 96-.

-, and associated conics. Roberts, R.A. L. Mth. S. P. 16 (1884-85) 9-.

-, bitangents. Binder, $W$. Z. Mth. Ps. 35 (1890) 25-.

-, double points. Nagel, K. [1881] Mth. A. 19 (1882) 433-.

-, with 2 double points and through 7 points. Dewulf, É., \& Schoute, P. H. As. Fr. C. R. 8 (1879) 249-; Bll. Sc. Mth. As. 3 (1879) 383-. _, - - - - - - Dewulf, É. [1880] N. A. Mth. 20 (1881) 401-.

- and new method of transformation. Leinekugel, G. N. A. Mth. 14 (1895) 391-.

-, system of tangential points. Binder, $W$. Z. Mth. Ps. 34 (1889) $272-$.

-, with tangents at double point becoming inflexional tangents. Weyr, Em. Wien Sb. $67(1873)(A b .2)$ 286-.

- $-1-1-1-1-$ Ameseder, $A$. Wien Ak. Sb. 79 (1879) (Ab. 2) 472-.

reciprocal of cubic whose $S$ invariant $=0$. Mathews, G. B. L. Mth. S. P. 22 (1891) 173-.

representation by 5 fourth powers. Liroth, $J$. [1868] Mth. A. 1 (1869) 37-.

_ _ _ _ - Clebsch's theorem, extension. Sylvester, J.J. C. R. 102 (1886) 1532-。

singularities. Beer, A. Grunert Arch. 14 (1850) 318-.

synthetic treatment. Milinowski, - Z. Mth. Ps. 23 (1878) 85-, 211-.

with tacnode at infinity at which line at infinity is multiple tangent. Jeffery, $H . M$. L. Mth. S. P. 13 (1881-82) 86-.

triangle in- and circum-scribed to curve. Cayley, A. Ph. Mg. 30 (1865) 340-

with triple point. Vidal, - - [1884] I. Egypt. Bll. 5 (1885) 102-

- - construction and properties. Eazarski, M. Krk. Ak. (Mt.-Prz.) Rz. 15 (1887) 224-; Fschr. Mth. (1887) 631-.

and 3rd class. Battaglini, $G$. Nap. Rd. 4 (1865) 399-.

- -, generation. Frahn [Frahm], W. Z. Mth. Ps. 18 (1873) 363-。 and 3rd class, generation by motion of a point. Siebeck, $H$. Crelle J. 66 (1866) 344-.

$\ldots \ldots \ldots$, Steiner's curve, and Feuerbach's circle. Godt, W. [1896] Münch. Ak. Sb. 26 (1897) 119-.

- 4th class, and porism of in- and circum. polygon. Roberts, R. A. L. Mth. S. P. 23 (1892) 202-.

\section{Curves, 5th degree.}

bitangential of. Heal, W. E. [1889-91] A. Mth. 5 (1889-90) 33-; 6 (1891-92) 64-.

bitangents. Maisano, G. Mth. A. 29 (1887) 431-.

- Cayley, A. A. Mth. 5 (1889-90) 109-.

- and inflexional tangents. Maisano, $G$. Palermo Cir. Mt. Rd. 1 (1887) 86-.

with 4 double points. Vries, J. de. [1894] Wien Ak. Sb. 104 (1895) (Ab. 2a) 46-.

---- , involutions. Vries, $J$. de. [1894] Amst. Ak. Vs. 3 (1895) 115-.

equation. Frattini, G. G. Mt. 16 (1878) 377. points of inflexion. Basset, A. B. B. A. Rp. (1900) 647-.

rational, simple linear construction. Rohn, K. Mth. A. 25 (1885) 598-

\section{Curves, 5th and 6th degrees.}

with multiple points, construction. Koehler, $C$. [1872] (x) Par. S. Mth. Bll. 1 (1873) 27-.

\section{Curves, 6th degree.}

in astronomy. Jonquières, E. de. Tortolini A. 1 (1858) 112-.

with 9 double points. Halphen, G. H. Par. S. Mth. Bll. 10 (1882) $162-$.

-2 triple points. Hjelmman, A. L. Helsingf. Öfv. 41 (1900) 26-.

and 4 th class, family. Longchamps, G. G. de. N. A. Mth. 5 (1866) 118-.

\section{Curves, 14th degree.}

cutting curve of 4 th degree, transformation. Hesse, L. O. Crelle J. 52 (1856) 97-。

\section{Curves, mth degree.}

$C^{m}$, projective generation. Kilpper, $C$. Prag Sb. (1896) (Mth.-Nt.) No. 1, 16 pp.; Mth. A. 48 (1897) 401-。

\section{Curves, nth degree.}

bitangents. Dersch, (Dr.) O. Mth. A. 7 (1874) 497-.

-, number. Jacobi, C. G. J. Crelle J. 40 (1850) 237- .

-, - (Jacobi). Rubini, R. Tortolini A. 2 (1851) 463-.

-, - (-). Clebsch, R. F. A. [1863] (vII)

Crelle J. Mth. 63 (1864) 186-.

with $\frac{n}{2}(n-3)$ double points. Clebsch, R. F.A. C. R. 60 (1865) 68 -

$\frac{(n-1)(n-2)}{2}$ double points. Haase, J.C.F.

Mth. A. 2 (1870) 515-. 
with $\frac{n-1 \cdot n-2}{1 \cdot 2}-2$ double points, expression of coordinates by parameter. Schwarz, C. H. A. Liouv. J. Mth. 6 (1880) 111-. and index $N$. Jonquières, $E$. de. Liouv. J. Mth. 6 (1861) 113-; 8 (1863) 71-.

with multiple point of order $n-1$. Niewenglowski, B. C. R. 80 (1875) 1067-; N. A. Mth. 15 (1876) 126-.

Fouret, G. C. R. 80 (1875) 1158-.

- - - - - construction with ruler, and reciprocal problem. Weyr, Em. Prag Sb. (1873) 198-.

$--1-n-2$, new property. Bertini, $E$. Rm. R. Ac. Linc. T. 1 (1877) 92-.

number of points of maximum and minimum curvature in. Brunel, G. Bordeaux S. Sc. Mm. 3 (1893) 167-.

- - needed to determine a curve. Machovec, F. Casopis 16 (1887) 225-; Fschr. Mth. (1887) 685 .

Curves, nth and pth degrees.

intersections, theorem. Schoute, P.H. Amst. Ak. Vs. [1] (1893) 53-, 62-.

Cusps. Schubert, F. T. [1818] St Pét. Ac. Sc. Mm. 8 (1822) 176-.

Degeneration of curves into straight lines. Gordan, P. Mth. A. 45 (1894) 410 -

Dependence, mutual, of curves, and general properties. Steiner, J. Liouv. J. Mth. 18 (1853) 309-.

Desargues's theorem, generalisation. Saltel, $L$. Brux. Ac. Bll. 41 (1876) 594.

Diameters. Wantzel, L. Par. S. Phlm. PV. (1847) 98-; Liouv. J. Mth. 14 (1849) 111-. 156-.

Fenwick, S. Mathematician 3 (1850) , bisecting. Breton [de Champ], P. N. A. Mth. 14 (1855) 7-.

-, properties. Chasles, M. C. R. 72 (1871) 794-.

一, theory. Lucas, F. Liouv. J. Mth. 8 (1863) 145-.

Diametrical curves. Walton, W. Camb. Mth. J. 2 (1841) 76-.

Directrices. Raabe, J. L. Crelle J. 2 (1827) 330-.

Discontinuities in curves. Vogel, $P$. Z. Mth. Ps. 26 (1881) 391-.

Discontinuous curves, geometry. Boidi, $G$. Bologna Opusc. Sc. 3 (1819) 276-.

Double point of plane curve, resolution in 3 dimensional space, and related mechanical problem. Schlegel, S.F.V. Z. Mth. Ps. 28 (1883) 105-.

- points. Oppenheimer, $H$. Z. Mth. Ps. 41 (1896) 305-.

- - curve with 6. Godefroy, A. N. N. Arch. Wisk. 18 (1891) 151-

- - group of curves with $m^{2}$. Vries, J. de. Amst. Ak. Vs. 3 (1895) 139-.

Elements at infinity in geometric figures, determination. Weyr, E. J. [1873] (xI) Bll. Sc. Mth. As. 6 (1874) 93-.
Elimination theory, with application to curves. Saltel, L. N. A. Mth. 20 (1881) 546-; 2 (1883) 554 -.

Elliptic curves, equation giving points of inflexion. Brioschi, F. Mil. I. Lomb. Rd. 2 (1869) 559-.

Envelope of diameters. Walker, J.J. L. Mth. S. P. $10(1878-79) 180$.

- - family of curves. Landré, C. L. N. Arch. Wisk. 5 (*1879) 205-.

Envelopes. Weill, -. N. A. Mth. 3 (1884) 376-.

Equation, algebraic, curves whose intersections give imaginary roots of. Sprague, $T . B$. [1882] Edinb. R. S. T. 30 (1883) 467-.

- in point coordinates obtained from tangential equation. Turquan, -. N. A. Mth. 4 (1845) 410 .

- of tangent. Purkiss, H.J. Mess. Mth. 3 (1866) 19-.

- - , magical. Hiern, W. P. QJ. Mth. 6 (1863) 31-.

-, tangential polar, of a curve. Ferrers, N.M. QJ. Mth. 1 (1857) 210-.

$-\sum_{1}^{n} \frac{X_{r} \phi_{r}(\lambda)}{\left(\lambda-a_{r}\right)^{m}}=\phi(\lambda)$, application. Beltrami, $E$. Bologna Ac. Sc. Mm. 10 (1879) 233-.

Equations of curve in polar coordinates, number for same axis. Forestier, C. Toul. Ac. Sc. Mm. 1 (1879) 250-.

-, intrinsic, of curves. Onnen, H. N. Arch. Wisk. 1 (*1875) 1-; 5 (*1879) 1-; (x) Arch. Néerl. 14 (1879) 1-.

,,$----\frac{1}{-}$ especially cycloids. Onnen, $H$. N. Arch. Wisk. 4 ( $\left.{ }^{*} 1878\right) 30$ -

-, higher, construction. Hill,C.J.D. Stockh. Öfv. 19 (1862) 307-.

,-- , geometric representation of numerical system. Spitzer, S. Wien SB. 6 (1851) 152-; 7 (1851) 455-.

Equidistant curves. Frolov, (le gén.) M. As. Fr. C. R. (1894) (Pt. 2) 235-.

Focal and normal properties. Laguerre, (le cap.) -. C. R. 60 (1865) 70-

Foci. Plücker, J. [1832] Crelle J. 10 (1833) 84-.

-. Roguet, C. N. A. Mth. 1 (1842) 131-.

- Vachette, A. N. A. Mth. 1 (1842) 417-.

Laguerre-Verly, E., \& Sacchi, J. N: A. Mth. 12 (1853) 225-.

-. Quetelet, E. Brux. Mm. Cour. $4^{\circ}, 26$ (1854-55) $44 \mathrm{pp}$.

-. Roberts, S. QJ. Mth. 2 (1858) 195-. 399-.

Terquem, $O$. N. A. Mth. 18 (1859)

- Gibert, E., \& Niewenglonoski, B. C. R. 82 (1876) $913-$.

-. Amigues, É. N. A. Mth. 11 (1892) 163-. - of curves and focals of cones. Laguerre, $E$. N. A. Mth. 18 (1879) 57-.

-, new analytic projective treatment. Siebeck, H. Crelle J. 64 (1865) 175-.

Generation of curves. Terquem, $O$. N. A. Mth. 12 (1853) 220.

- - - Jonquières, E. de. [1857] Par. Mm Sav. Étr. 16 (1862) 159-. 
Generation of curves (de Jonquières). Chasles, M. C. R. 45 (1857) 318-.

- - Härtenberger, $G$. Crelle J. 58 (1860) $54-$

-- - Olivier, A. [1869] Crelle J. 71 (1870) 1-.

- - Vaněček, J. S., \& Vaněček, M. N. Prag Sb. (1885) (Mth.-Nt.) 202-.

- - the curves determined by unknown points of intersection of given curves. Olivier, $A$. Z. Mth. Ps. 14 (1869) 209-.

- curves, linear. Grassmann, $H$. Crelle J. 42 (1852) 187-.

_ _ - by many valued elements. Weyr, Em. [1870] Prag Ab. 4 (1871) 32 pp.

- - -, mechanical. Schlegel, V. [1872] Mth. A. 6 (1873) 321-.

- - - projective. Bobek, $K$. Mth. A. 25 (1885) 448-.

- - - by projective involutions. Weyr, Em. [1870] Mth. A. 3 (1871) 34-.

- - projective curved configurations. Milinowski, -. Z. Mth. Ps. 18 (1873) 288-.

- - straight lines by curves. Steen, A. Mth. Ts. 4 (1862) 123-.

Geometrical transformation (pseudo-Newtonian). Brocard, H. Liège S. Sc. Mm. 1 (1899) No. 3, 24 pp.

$-(-)$ Retali, V. Liège S. Sc. Mm. 2 (1900) No. 4, 24 pp.

Hessian. Chrystal, - [1885] Edinb. R. S.T. 32 (1887) 645-.

- and Cayleyan of curve, common tangents. Maisano, G. [1885] Palermo Cir. Mt. Rd. 1 (1887) 46-.

- curve. Brill, A. [1877] Mth. A. 13 (1878) 175-.

- - Pezzo, P. del. Nap. Rd. 22 (1883) 203-. - - geometric treatment. Kötter, E. Mth. A. 34 (1889) 123-.

- and Steinerian. Steiner, J. [1848] Crelle J. 47 (1854) 1-.

- - (Steiner). Clebsch, R. F. A. [1864] Crelle J. 64 (1865) 288-.

Hessians, Steinerians, and Cayleyans, curves analogous to. Maisano, G. Palermo Cir. Mt. Rd. 1 (1887) 66-.

- and Steinerians of higher orders in 1-dimensional geometry. Newson, H. B. A. Mth. 11 (1896-97) 121-.

Intersection of curve with straight line. Routh, E. J. QJ. Mth. 24 (1890) 257-.

- - curves. Burrov, $R$. As. Researches 1 (1788) 330-.

- - Cayley, A. Camb. Mth. J. 3 (1843) 211-; Mth. A. 30 (1887) 85-.

- 2 curves. Painvin, L. Bll. Sc. Mth. As. 5 (1873) 138-.

- - in a singular point. Halphen, G. H. Par. Sc. Mth. Bll. 3 (1875) 76-.

-, curvilinear, theory of loci. Walton, $W$. Camb. Mth. J. 2 (1841) 85-.

- of lines with curves of odd degree. Dawson, H. G. Mess. Mth. 19 (1890) 108-.

- $n$ tangents where radius vector cuts curve $C_{m}^{n}$, problem of Steiner. Schoute, $P . H$. Bll. Sc. Mth. 10 (1886) 242-.
Intersection of $n$ tangents where radius vector cuts curve $C_{m}^{n}$, problem of Steiner. Zeuthen, H. G. Bll. Sc. Mth. 11 (1887) 82-.

Intersections of curves, theorems. Valentiner, $H$. (xII) Ts. Mth. 5 (1881) 1 -.

,$--- \frac{1}{-}$ of Humbert and Fouret. Borel, $E$. N. A. Mth. 9 (1890) 123-.

- 2 curves. Franklin, $F$. Am. J. Mth. 3 (1880) 192.

- _ - , theorem of Jacobi, proof. Petersen, J. P.C. (xII) Ts. Mth. 2 (1878) 14-.

Inversion, classification of curves with reference to. Johnson, $W . W$. Des Moines Anal. 4 (1877) 42-.

Kinematic determination of curves. Ocagne, M. $d$ '. N. A. Mth. 1 (1882) 40-; 2 (1883) 189-.

- geometry, applications. Mannheim, A. Prag Sb. (1888) (Mth.-Nt.) 87-.

Least chord through given point. Emsmann, $H$. Grunert Arch. 23 (1854) 460-.

Loci, applications of law of decomposition. Saltel, L. Brux. Ao. Bll. 42 (1876) 586-.

-, degree and class. Halphen, G. H. C. R. 83 (1876) 705-.

- and envelopes. Chasles, M. C. R. 82 (1876) 1463-; 85 (1877) 362-.

- - - theorem of Chasles, applications. Serret, $P$. C. R. 86 (1878) 116-.

-, general law. Saltel, L. Brux. Ac. Bll. 41 (1876) 595-.

-, node- and cusp-, enveloped by tangents at the cusps. Hill, M. J.M. L. Mth. S. P. 22 (1891) 216-.

-, orthoptic, degree. Taylor, C. Mess. Mth. 16 (1887) 1-.

-, - and isoptic, degree. Taylor, C. R. S.P. 37 (1884) 138-.

-, various. Brasseur, J. B. Brux. Ac. Bll. 13 (1846) 311-; 18 (1851) 372-; Brux. Mm. Cour. $4^{\circ}, 21$ (1846) 26 pp.

Locus of contact points of net of curves touching fixed curve. Faure, $H$. N. A. Mth. 18 (1859) 237-.

- - - - degree $k$, of curves of pencil with those of $\infty^{k}$ linear system. Franchis, M. de. Palermo Cir. Mt. Rd. 10 (1896) 118-; 11 (1897) 12-.

- defined by algebraic conditions, method of determining degree. Saltel, L. Brux. Ac. Bll. 42 (1876) 300-.

- - - - n number of double points. Saltel, L. C. R. 88 (1879) 329-.

-, determination of degree without calculation. Saltel, L. [1874] Brux. Mm. Cour. 8०, 24 (1875) No. 5, $33 \mathrm{pp}$.

- of harmonic centres on radii vectores. Palatini, F. G. Mt. 33 (1895) 210-.

- - intersection of tangents at ends of chord of constant length in a conic. Schlömilch, $O$. Z. Mth. Ps. 14 (1869) 158-.

- - point in chord of constant length in ellipse. Enneper, A. Schlömilch Z. 7 (1862) 200 .

- - points whose distances from curves fulfil given relation, symbolic formula for degree. Fouret, G. C. R. 83 (1876) 605-. 
Locus of points, tangents from which to 2 curves are equal. Laguerre, E. [1876] Par. S. Mth. Bll. 5 (1877) 25-.

- - - of 3-point contact of curve pencil with curve net. Bagnera, $G$. Palermo Cir. Mt. Rd. 10 (1896) 81-.

- problem. Gergonne, J. D. Gergonne A. Mth. 8 (1817-18) 312-.

- Schmitz, P. J. Louvain A. Ac. 7 (1825) $44 \mathrm{pp}$.

- of projections of point on tangents to curve. Richard, -. N. A. Mth. 2 (1843) 436-.

- - vertex of constant angle circumscribed to curve of $n$th class. Jonquières, E. de. N. A. Mth. 20 (1861) 206-.

$-y x^{2}+b x+c=0$. Bergis, -. N. A. Mth. 17 (1858) 118-.

Maclaurin's "Descriptio Linearum Curvarum." Vaněček, J. S., \& Vaněček, M. N. Prag Sb. (1884) (Mth.-Nt.) 374-.

- method for conics, curves obtained by exten. sion of. Roberts, S. L. Mth. S. P. 14 (188283) 110

Mechanical and geometrical relations of curves. Bardelli, G. Mil. I. Lomb. Rd. 13 (1880) 56-.

Mechanics, principles applied to properties of curves. Fabri, R. Rm. At. 6 (1852-53) 73-.

Metrical geometry, general theorems. Michel, C. N. A. Mth. 19 (1900) 169-.

- properties. Humbert, G. N. A. Mth. 6 (1887) 526-.

- , synthetic methods for study of. Holst, E. Christiania F. (1882) No. 11, 113 pp.; (1883) No. $13,6 \mathrm{pp}$.

- theorem in conies, and its generalisation. Schwarz, A. Mh. Mth. Ps. 9 (1898) 346-.

- theorems. Holst, E. [1876] Mth. A. 11 (1877) 341-, 575.

Motion on curves with separate branches, relation to motion on one branched curve. Staude, -. Dorpat Sb. 8 (1889) 336-.

Multiple points. Walton, $W$. Camb. Mth. J. 2 (1841) 155-.

- -, curves with 3 . Jonquières, E. de. Tortolini A. 1 (1858) 110-.

- - , determination of curves with. Chasles, M. C. R. 62 (1866) 1354 .

- - and equal roots, theorem. Sylvester, J.J. Ph. Mg. 3 (1852) 375-.

- at infinity. Painvin, L. N. A. Mth. 3 (1864) 145-, 193-, 241-.

- -1 , curves with 2. Michel, F. As. Fr. C. R. (1900) (Pt. 2) 250-.

- - number of, and rational curves. Pellet, A. E. N. A. Mth. 20 (1881) 444-.

Multiplicity, new theory. Sylvester, J.J. Ph. Mg. 3 (1852) 460-.

New properties. Mannheim, A. Par. S. Mth. Bll. 4 (1876) 158-.

Newton's organic description of curves. Taylor, C. Camb. Ph. S. P. 3 (1880) 381-.

- theorem, extension and applications. $G o b, A$. Brux. Ac. Bll. 28 (1894) 57-.

- - various consequences. Demoulin, $\boldsymbol{A}$. Brux. Mm. Cour. 8०, 45 (1891) No. 2, 18 pp.

Node-cusp. Cayley, A. (I) QJ. Mth. 6 (1864) 74-.
Normal, construction, in certain mode of generation of curves. Ocagne, M. $d^{\prime}$. N. A. Mth. 20 (1881) 197-.

Normals. Bäcklund, A. V. Lund Acta Un. 6 (1869) (Mth.) No. 2, 38 pp.

-, curve through feet of, theorem of Steiner. Dewulf, -. N. A. Mth. 18 (1859) 174-.

- to curves, and evolutes. Steiner, J. Crelle J. 49 (1855) 333-.

-, generalisation of theory by substituting for each normal a pencil of straight lines. Chasles, M. C. R. 80 (1875) 505-.

-, problems. Collignon, É. N. A. Mth. 19 (1900) 433-.

-, theorem. Dewulf, -. N. A. Mth. 16 (1857) 464.

-, - - Birkeland, K. Mh. Mth. Ps. 1 (1890) 417-.

Number of points common to 2 curves. Saltel, L. Brux. Ac. Bll. 43 (1877) 266-

- - - determining curves. Plilcker, J. Gergonne A. Mth. 19 (1828-29) 97-.

Obliques of curve, theorems. Chasles, $M$. C. R. 74 (1872) 1277-.

Parallel of curve, degree and class. Léauté, $H$. [1875] Toul. Ac. Sc. Mm. 8 (1876) 59-.

- - - - - singularities. Roberts, S. L. Mth. S. P. 3 (1869-71) 209-.

Penultimate forms of curves. Cayley, A. C. R. 74 (1872) 708-.

Perspectives, skew, of curves. Halphen, G. H. C. R. 80 (1875) 638-

Plane or skew curves whose points can be determined individually. Chasles, $M$. C. R. 62 (1866) 579-.

Point cluster of $n$th degree with base on curve of $m \mathrm{th}$ degree. Chasles, $M$. C. R. 45 (1857) 393-.

Points, collinear. Bellavitis, G. Ven. Mm. I. 8 (1859) 161-.

-, conjugate, theory. Young, J. R. Ph. Mg. 27 (1845) 91-.

- of inflexion. Eliot, $Z$. Bll. Sc. Mth. As. 2 (1878) 216-.

- - - curves with. Bammert, (Dr) - Z. Z. Mth. Ps. 10 (1865) 165-.

- - of curves in polar coordinates. Isely, J. $P$. Neuch. S. Sc. Bll. 18 (1890) 56-.

- _ - Hesse's equation. Lemonnier, $H$. N. A. Mth. 9 (1870) 531-.

$--\ldots,-\ldots$ Cretin, -. N. A. Mth. 20 (1881) 131-.

- _ - and other points. Cayley, A. Crelle J. 34 (1847) 30-.

- - intersection of 2 curves, multiplicity. Stolz, O. Innsb. Nt. Md. B. 8 (1879) (Heft 3) vii-; Mth. A. 15 (1879) 122-.

- and tangents, construction. Zeuthen, $H . G$. Mth. Ts. 3 (1861) 12-.

Polar curves, general theory. Paolis, R. de. Rm. R. Ac. Linc. Mm. 3 (1886) 265-.

- - theorem, proof. Jonquières, E. de. N. A. Mth. 16 (1857) 347-.

- properties, geometric derivation. Schur, $F$. Z. Mth. Ps. 22 (1877) 220-.

- reciprocal of curve, degree. Gergonne, J.D. Gergonne A. Mth. 19 (1828-29) 218-. 
Polar reciprocals, theory. Lenthéric, - N. A. Mth. 8 (1849) 252-.

- theory. Schwarz, A. Wien Ak. Sb. 95 (1887) (Ab. 2) 42-.

Polars. Gaertner, R. Arch. Mth. Ps. 7 (1889) 180 -

- 1st, condition for 2 curves of same degree having same. Bertini, E. Tor. Ac. Sc. At. 33 (1897) 23-.

-, generalised. Clifford, W.K. [1868] L. Mth. S. P. 2 (1869) 116-.

-, inclined, theory. Dewulf, $\mathfrak{E}^{\prime}$. Bll. Sc. Mth. As. 2 (1878) 41-, 372-.

- with respect to plane curves, construction. Andreev, K. A. (xiI) Kharkov Mth. S. Com. (1879) 51-.

- successive, theory. Lè Paige, C.M.M.H.H. Brux. Ac. Bll. 1 (1881) 134-

Poles of inversion, principal, curve having a geometric locus of. Fouret, G. N. A. Mth. 2 (1883) 259-.

- and polars. Jonquières, E. de. Liouv. J. Mth. 2 (1857) 249-.

- - Fiedler, W. Schlömilch Z. 4 (1859) 91-.

- - Polar reciprocals. Hunyady, J. (xII) Mag. Tud. Ak. Etk. (Mth.) [1] (No. 2) (1867) 30 pp.

Polygons in- or circum-scribed to curves. Chasles, M. C. R. 78 (1874) 922-.

- - and circum-scribed to same curve. Weill, M. Par. S. Mth. Bll. 10 (1882) 127-.

-, inscribed, 2 conjugate, generalisation of Pappus's theorem. Puzyna, J. Krk. Ak. (Mt.-Prz.) Pam. 17 (1890) 24-; Crc. Ac. Sc. Bll. (1889) No. 4, xxxvii-.

-, - , of maximum area. Hathaway, A. S. (xII) J. H. Un. Cir. [2] (1883) 48.

-, porismatic. Brioschi, F. Tortolini A. 8 (1857) 119-.

-, Steiner's, generalisation. August, F. [1875] Arch. Mth. Ps. 59 (1876) 1-.

Porismatic problems, especially polygons in curves. Hurwitz, A. [1878] Mth. A. 15 (1879) 8-.

"Power" of point with respect to curve. Petersen, J. P. C. (xII) Ts. Mth. 5 (1869) 106-.

Projective metrics. Ovidio, $E$. $d^{\prime}$. Tor. Ac. Sc. At. 28 (1893) 566-.

- properties. Juel, C. [1899] Kjøb. Dn. Vd. Selsk. Skr. 10 (1899-1902) 1-.

- - of curyes and surfaces. Jouquières, E. de. Crelle J. 66 (1866) 289-.

- - - - - application of transversals. Poncelet, J. V. Crelle J. 8 (1832) 21-, 117-, 213-, 370-.

- - elements of curves. Scholz, P. Z. Mth. Ps. 13 (1868) 267-, 355-.

Quadrilaterals with their 6 vertices on curve of 3rd class. Weill, -. N. A. Mth. 3 (1884) 401-.

Radii vectores, generalisation of theorems expressed in. Chasles, M. C. R. 82 (1876) 1463-.

Rational curves. Weyr, Em. Z. Mth. Ps. 16 (1871) 80-; Prag Sb. (1872) (pt. 2) 9-.

- - Painvin, L. C. R. 78 (1874) 1194-。
Rational curves. Pasch, M. Mth. A. 18 (1881) 91-.

- - Humbert, G. Par. S. Mth. Bll. 13 (1885) 49-, 89-.

- - Marin, E. Arg. S. Ci. A. 32 (1891) 15-

-. Laisant, C. A. As. Fr. C. R. (1892) (Pt. 2) 25-.

- - Houston, W. A. Mess. Mth. 28 (1899) 187-.

- - class. Mansion, P. (xII) Ts. Mth. 6 (1876) 64-.

- -, -. Darboux, G. C. R. 94 (1882) 930-.

- - construction by points and tangents.

Schoute, P. H. N. Arch. Wisk. 12 (1886)

1-; Arch. Néerl. 20 (1886) 49-.

- -, degeneration. Bissing, G. [1883] (xII) J. H. Un. Cir. [3] (1884) 58.

- - of even degree, generation by means of projective series of circles and spheres. Tognoli, O. G. Mt. 12 (1874) 161-.

- - double points and tangents. Weltzien, $C$. Mth. A. 26 (1886) 516-.

- - generation. Stahl, W. Mth. A. 38 (1891) 561-.

- -, 一, new mode. Fouret, G. C. R. 101 (1885) 1241-.

- - - , theorems, two. Tognoli, O. G. Mt. 12 (1874) 136-.

- - geometric constructions for, especially quintic with 5 given double points. Jonquières, $E$. de. Palermo Cir. Mt. Rd. 2 (1888) 118-

- - with imaginary double points, special class. Fuchs, L. Berl. Ak. Sb. (1900) 74-. - - linear constructions. Vanécek, M. N. Prag Sb. (1882) 75-.

- - for which multiple points can be assigned arbitrarily. Bertini, E. G.Mt.15 (1877) 329-. - , and new algebraic theorem. Garbieri, $G$. G. Mt. 16 (1878) 1-, 108-.

- - plane and skew. Roberts, R. A. L. Mth. S. P. 17 (1887) 25-.

- - , - - - in- and circum-polygons. Zecca, G. G. Mt. 25 (1887) 333-

- - systems. Story, W. E. J. H. Un. Cir. [6] (1886-87) 77 .

- - theorem, proof. Liroth, J. [1875] Mth. A. 9 (1876) 163-.

- - theory, application. Hermite, C. L. Mth. S. P. 4 (1871-73) 343-.

- - treated by inversion. Newson, H. B. [1892] Kan. Ün. Q. 1 (1893) 47-.

- -, 3rd class. Laguerre, E. [1877] Par. S. Mth. Bll. 6 (1878) 54-.

Reciprocal of curve, equation. Salmon, $G$. Crelle J. 42 (1852) 277-.

- curves of foci. Björling, C. F.E. Stockh. Öfv. 33 (1876) No. 1, 17-.

Reciprocity in plane, and application to plane curves. Beyel, C. Zür. Vjschr. 31 (1886) 161-.

Reducible curves. Noether, $M$. Erlang. Ps. Md. S. Sb. 17 (1885) 13-; Act\& Mth. 8 (1886) 161-.

Reflexions of points on circles, or inversion of normal problem. Röllner, $\boldsymbol{F}$. [1880] Wien Ak. Sb. 82 (1881) (Ab. 2) 1129-. 
Secants of curves, sum of squares. Minding, E. F. A. Crelle J. 11 (1834) 20-.

-, Maclaurin's theorem. Malet, J. C. Ir. Ac. T. 28 (*1880-86) 759-.

"Sectorial" curves. Ježek, O. Prag Sb. (1882) 117-; (1883) (Mth. Vortr.) 116-.

Segments of lines by curves. Ladd, (Miss) C. Am. J. Mth. 4 (1881) 272.

- on tangents and normals, loci derived from, and enumerative theorems. Chasles, $M$. C. R. 81 (1875) 253-, 355-, 643-, 993-, 1221-; 82 (1876) 1399-; 83 (1876) 97-, 467-, 495-, 519-, 589-, 641-, 757-, 867-, 1123-, 1195-.

Series of points given by recurring series, and connected curves. Segar, H. W. Mess. Mth. 22 (1893) 161-.

Singular points. Poisson, S. D. Par. Éc. Pol. J. $14^{\circ} \mathrm{cah} .(1808) 130$ -

- - Briot, C. Liouv. J. Mth. 10 (1845) $368-$

- - Choquet, C. N. A. Mth. 9 (1850) 260-. -. De Morgan, A. [1855] (Ix) Camb. $\mathrm{Ph}$. S. P. 1 (1866) 155-.

- Boussinesq, $\dot{J}$. Les Mondes 45 (1878) 575-.

- - Halphen, G. H. Par. Mm. Sav. Etr. 26 (1879) No. 2, $112 \mathrm{pp}$.

- - Biehler, C. N. A. Mth. 19 (1880) 492-; 20 (1881) 97-, 489-, 537-.

- - of algebraic functions and curves. Stolz, O. Mth. A. 8 (1875) 415-.

- - - curve, and singular systems of values of algebraic function. Noether, M. Mth. A. 9 (1876) 166-.

- - , new, discovered by Plateau. Mansion, $P$. (xII) Mathesis 3 (1883) 193-.

Singularities of curves. Clebsch, R. F'A . [1864] Crelle J. 64 (1865) 98-.

- - - Köstlin, W. Z. Mth. Ps. 41 (1896) 1-. - - , analytical determination. Brioschi, Fr. Rm. R. Ac. Linc. At. 2 (1875) xliv-.

- _ - discriminants and resultants of equations for. Meyer, F. Mth. A. 38 (1891) 369-. _ _ _ Plücker's first equation connecting. Knott, C. G. Edinb. Mth. S. P. 1 (1894) 130 -

—, higher. Cayley, A. Crelle J. 64 (1865) 369-; QJ. Mth. 7 (1866) 212-.

_, - La Gournerie, J. de. Liouv. J. Mth. 14 (1869) 425-; 15 (1870) 1-.

-, -. Scott, (Miss) C. A. Am. J. Mth. 14 (1892) 301-.

-, -, equivalents. Björling, C. $F . E$. Stockh. Öfv. 35 (1878) No. 7, 33-; Ups. S. Sc. N. Acta 10 (1879) (No. 17) 25 pp.

-, - , - (Björling). Zeuthen, H. G.

'Ts. Mth. 3 (1879) 74-.

Steiner's, Cayley's and other covariant curves, behaviour at singular points of original curve. Wollfing, E. Z. Mth. Ps. 40 (1895) 31-.

Straight lines cutting plane curve at same angle. Fouret, G. Palermo Cir. Mt. Rd. 5 (1891) 75-.

Supplementary curves, obtained by "ideal chords." Finlay, R. [1853] Manch. Ph. S. Mm. 11 (1854) 169-.

VOL. I.
System of 2 curves, properties. Demoulin, $A$. Brux. Ac. Bll. 23 (1892) 527-.

Tangent to certain curves, construction. Lisleferme, H. de. (xII) Mathesis 3 (1883) 206-.

- constructions. Hurwitz, A. Mth. A. 22 (1883) 230-.

- to curve, segments intercepted on line by it and a parallel. Cremona, $L$. Tortolini A. 3 (1860) 169-.

- property, curve determined from. Unferdinger, $F$. Arch. Mth. Ps. 52 (1871) 361-.

- - - - - Brocard, H. [1875] Par. S. Mth. Bll. 4 (1876) 42 .

Tangents. Bret, - Gergonne A. Mth. 8 (1817-18) 285-.

-. Reid, J. Mathematician 2 (1847) 83.

- Bischoff, J. N. Crelle J. 56 (1859) 166-.

-. Gundelfinger, S. [1870] Crelle J. 73 (1871) 171-.

-. Woodward, C, M. Am. As. P. (1886) 72-.

- and centres of curvature of curves, construction. Machovec, F. Prag Sb. (1888) (Mth.Nt.) 169-.

Linc. Mm. 14 (1898) 57 -.

- to certain curves, construction. Wiegers, C. Grunert Arch. 33 (1859) 166-.

-, conjugate. Hoza, $F$. Arch. Mth. Ps. 61 (1877) 218-.

- to curve from multiple point. Caporali, $E$. Nap. Rd. 20 (1881) 143-.

- - - 1 point, application of equation of squares of differences of roots. Joachimsthal, F. N. A. Mth. 9 (1850) 98-.

-, determination. Colard, H. N. A. Mth. 1 (1842) 268-.

-, formula. Joachimsthal, F. N. A. Mth. 12 (1853) 323-.

-, general and analytical method of drawing. Gruson, J. P. Berl. Ab. (1820-21) 39-.

- to polar curves. Rispal, A. 'N. A. Mth. 2 (1843) 511-.

- projections of curves, construction. Binet, J. P. M. Par. Ec. Pol. Cor. 3 (181416) 199-.

--1 - - - Chasles, M. Liouv. J. Mth. 2 (1837) 293-.

- and radii of curvature, new graphical construction. Chasles, M. [1833] Quetelet Cor. Mth. 8 (1834) 58-.

-, Roberval's method for. Geer, P. van. N. Arch. Wisk. 11 (1884) 28-; Fschr. Ps. (1884) (Ab. 1) 217-.

- and tangent planes, parallel, theorem of Chasles; point of mean distance. Terquem, $O$. N. A. Mth. 4 (1845) 153-, 178-.

-, theory. Doedes, L. Utr. A. Ac. (1829-30) $149 \mathrm{pp}$.

-, $n(n-1)$, from a point to curve of $n t h$ degree. Zeuthen, H. G. (xII) Ts. Mth. 2 (1872) 65-.

Theorems of Maclaurin and Jacobi. Terquem, $O$. N. A. Mth. 9 (1850) 440-.

-, various. Ascoli, G. G. Mt. 5 (1867) 358-, 365-.

Tracing of curve from its equation, asymptotes, etc. Biehler, C. N. A. Mth. 2 (1883) 354-, $397-$. 
Transversal, cotangent, theorem. Hunyady, E. von. [1865] Z. Mth. Ps. 11 (1866) 77-.

- directions corresponding to axes of conics. Stephanos, C. Par. S. Mth. Bll. 9 (1881) 49-.

- theory for plane curves. Gundelfinger, $S$. Z. Mth. Ps. 19 (1874) 68-.

Transversals. Steiner, J. Berl. B. (1851) 302-; Crelle J. 47 (1854) 7-.

Triangle, generalised form of analytical. Story, W. E. [1879] (xח) J. H. Un. Cir. [1] (1882) 34.

Triangles, 2, circumscribing conic, theorem extended to $n$-gons. Dixon, A. C. QJ. Mth. 26 (1893) 212-.

Undulations of curves $C_{6}^{4}$. Binder, $W$. Wien Ak. Sb. 106 (1897) (Ab. 2a) 295-; 107 (1898) (Ab. 2a) 23-.

$(m, n)$ homographies, and double elements. Weyr, Em. Prag Sb. (1869) (pt. 2) 33-.

\section{Special plane algebraic curves. (See also 8030.)}

Wesely, J. Arch. Mth. Ps. 9 (1890) 420-.

Aberrancy curve of plane cubic. Mukhopadhyay, A. Beng. As. S. J. 59 (Pt. 2) (1891) 61-.

Anallagmatic curves. Darboux, G. Par. Éc. Norm. A. 4 (1867) 81-.

- - Picquet, H. As. Fr. C. R. (1878) 95-; C. R. 87 (1878) 460 -.

- - Laquière, E. M. As. Fr. C. R. (1880) 121-.

Aplanatic lines. Chasles, M. Quetelet Cor. Mth. 5 (1829) 116-, 188-.

- - etc. Quetelet, L. A.J. Quetelet Cor. Mth. 5 (1829) 190-.

Araneids, division of angles by. Heymann, $W$. Z. Mth. Ps. 44 (1899) 263-.

Astroids derived from conics. Ameseder, $A$. Arch. Mth. Ps. 64 (1879) 177-.

"Atriphthaloid," $r^{2}(r-h)+k^{2} \sec ^{2} \omega=0$. Townsend, (Rev.) R. [1882] Ir. Ac. P. 4 (*1884-88) 62-.

Ballistic quatrefoil, $\left(x^{2}+y^{2}\right)^{3}=c^{2} x^{2} y^{2}$. Horta, F. Lisb. A. 1 (1857) 525-.

Barycentrides, locus of centroid of variable arc of curve. Borgnet, - Rouen Ac. Tr. (1839) 113-.

Cardioid. Purkiss, H. J. (v) Mess. Mth. 2 (1864) 241-.

-. Laguerre, E. N. A. Mth. 17 (1878) 55-.

- Weill, M. N. A. Mth. 20 (1881) 160-

-, connection between pole and centroid of triangle of contact. Zahradnik, $K$. Prag Sb. (1877) 188-.

-, generation by 2 unequal circles. Brändli, $H$. Bern Mt. (1858) 68-.

-, involutory angular relations. Weyr, Em. Prag Sb. (1871) (pt. 2) 69-.

-, locus of intersection of perpendicular tangents. Wolstenholme, J. L. Mth. S. P. 4 (1871-73) 327-.

- and Maclaurin's trisectrix, relation between. Longchamps, G. de. Prag Sb. (1887) (Mth.Nt.) $601-$.
Cardioid, and its mechanical construction. Pleyl, J. Arch. Mth. Ps. 68 (1882) 166-.

-, metrical property. Weyr, Em. Mil. I. Lomb. Rd. 5 (1872) 204-.

-, pole of constant triangle of contact. Zahradnik, $K$. Prag Sb. (1877) 184-.

-, relation to ellipse. Jopling, J. R. S. P. 6 (1852) 267-.

-, theory. Zahradník, K. Prag Sb. (1874)

180-; Arch. Mth. Ps. 59 (1876) 337-.

Cartesian ovals.

Genocchi, A. N. A. Mth. 14 (1855) 202-, 260Cayley, A. QJ. Mth. 1 (1857) 320-.

Strebor [Roberts, W.]. N. A. Mth. 17 (1858) 234-.

Roberts, S. [1870] L. Mth. S. P. 3 (1869-71) 106-.

Tait, P. G. [1871] Edinb. R. S. P. 7 (1872) 434-.

Cayley, A. QJ. Mth. 12 (1873) 16-.

Haentzschel, E. Arch. Mth. Ps. 69 (1883) 395-. Dixon, A. C. QJ. Mth. 28 (1896) 375-.

bibliography. Liguine, $V$. Bll. Sc. Mth. As. 6 (1882) (Pt. 1) 40-.

bipolar equations. Johnson, $W . W$. Des Moines Anal. 2 (1875) 106-.

confocal, correspondence with right lines of hyperboloid. Cayley, A. Mess. Mth. 18 (1889) 128-.

construction. Cayley, A. QJ. Mth. 15 (1878) 34. generation, Chasles's method. Ocagne, $M$. $d$ '. C. R. 97 (1883) 1424-

and hyperboloid of one sheet. Vries, $J$. de. Amst. Ak. Vs. 4 (1896) 252-; Fschr. Mth. (1896) 491.

with 2 imaginary axial foci. Cayley, $A$. [1870] L. Mth. S. P. 3 (1869-71) 181-.

mechanical description. Cayley, A. L. Mth. S. P. 6 (1875) 81-; QJ. Mth. 13 (1875) 328-.

- - Hammond, J. Am. J. Mth. 1 (1878) 283.

- - by string. Zeuthen, H. G. (xII) Ts. Mth. 6 (1882) 145-.

and other focal curves, mechanical description. Maxwell, J. C. [1846] Edinb. R. S. P. 2 (1851) 89-.

- - quartics, conjugate functions. Greenhill, A. G. [1881] Camb. Ph. S. P. 4 (1883) 77-. properties. Crofton, M. W. L. Mth. S. P. 1 (1866) No. 6, 5-.

regarded as conic intersections. Merrifield, C. W. Mess. Mth. 4 (1875) 148.

- projected intersections of quadric surfaces. Merrifield, C. W. Mess. Mth. 3 (1874) 141-. and related curves as sections of the anchorring. Brown, W. V. A. Mth. 6 (1891-92) 161-.

system through 4 points on circle. Roberts, R. A. L. Mth. S. P. 12 (1880-81) 200-.

triple focus. Engberg, C. C. [1898] A. Mth. 12 (1898-99) 54-.

Cassini's oval. Zeuthen, H. G. Mth. Ts. 5 (1863) 157-.

- -. Cayley, A. Mess. Mth. 4 (1875) 187-. 
Cassini's oval. Hart, H. Mess. Mth. 6 (1877) 169-.

- MacMahon, P. A. [1882] Mess. Mth. 12 (1883) 118-.

- - derived from section of rotation surface. Seidl, A. Wien SB. 18 (1855) 311-.

- ovals. Huber, G. Bern Mt. (1888) 93-.

- -. Bigler, U. Arch. Mth. Ps. 7 (1889) 311-.

-, centre of curvature. Friedrich, $J$. Časopis 27 (1898) 96-; Fschr. Mth. (1898) 511.

- - and lemniscates, generation. Garlin, N. A. Mth. 14 (1855) 305-.

-, plane and spherical. Laguerre, $E$. Par. S. Phlm. Bll. 5 (1868) 40 .

Catenaries through 2 fixed points. Hancock, $H$. A. Mth. 10 (1895-96) 159-

Cissoid. Schulz von Strasznitzki, L. C. Baumgartner Z. 8 (1830) 179-.

-. Greiner, M. [1881] Arch. Mth. Ps. 69 (1883) 313-.

-. Pánek, A. Časopis 16 (1887) 33-; Fschr. Mth. (1887) 734.

-, locus of points having a constant triangle of contact. Zahradnik, K. Prag Sb. (1877) 125-.

-, property. Petersen, J. Mth. Ts. 4 (1862) 40.

- and strophoid. Servais, C. Mathesis 10 (1890) 9-

-, tangents. Walker, J. J. L. Mth. S. P. 2 (1869) 161-.

-, theorem. Marcou, J. N. A. Mth. 2 (1843) 486-; 3 (1844) 472-.

-, theory. Zahradnik, $K$. Arch. Mth. Ps. 59 (1876) 335-

-, - based on a rational parameter. Zahradnik, K. Prag Sb. (1873) 221-.

Cissoidal curves. Zahradnîk, $K$. Časopis 2 (*1873) 183-; Arch. Mth. Ps. 56 (1874) 8 -

Cissoidometry. Werneburg, J. F. C. Oken Isis (1819) 209-.

Conchalic curves, trajectories, and cissoids of 4th degree. Huber, G. Mh. Mth. Ps. 6 (1895) 157-.

Conchoid. Midy, -. N. A. Mth. 2 (1843) 281-.

- with circular base applied to solution of Delian problem. Clausen, T. Grunert Arch. 2 (1842) 196-.

-, generalisation. Machovec, $F$. Časopis 14 (1885) 15-; Fschr. Mth. (1885) 714-.

- of R. de Sluse. Loria, G. Mathesis 17 (1897) 5-.

-, theory. Affolter, F. G. Arch. Mth. Ps. 55 (1873) 175-.

- traced by parabola. André, H. d'. N. A. Mth. 5 (1846) 122-, 208.

Conchoids, class. Kulp, $L$. Arch. Mth. Ps. 48 (1868) 97-.

- and conchoidal surfaces. Minich, S. R. Padova Rv. Period. 10 (1862) 135-

-, generation. Schoentjes, $H$. Mathesis 4 (1884) 145-.

Conics, curves depending on. Oddi, $G$. Bologna Opusc. Sc. 2 (1818) 293-.
Conjugate curves. Schlesinger, O. Mth. A. 30 (1887) 453-; 33 (1889) 315-.

Constructions in connection with special curves. Stuyvaert, - Mathesis 19 (1899) 33-.

Convolutes, unipolar. London, (Rev.) $H$. Manch. Lt. Ph. S. P. 24 (1885) 38-.

Critic centres. Morley, F. Am. J. Mth. 10 (1888) 141-.

Cubic centres of a line with respect to 3 lines and a line. Cayley, A. Ph. Mg. 20 (1860) 418-; 22 (1861) 433-.

Curve with 3 foci. Hachette, J. N. P. [1831] Quetelet Cor. Mth. 7 (1832) 32-.

- - - Reiss, M. Quetelet Cor. Mth. 7 (1832) 123-

-, 4th class, metric theorems. Loud, $F . H$. Am. As. P. (1900) 43.

Curves with centres on family of cubics. Schoute, P. H. N. Arch. Wisk. 13 (1887) 1-; Fschr. Mth. (1886) 692-.

- - - , and properties of curves generally. Steiner, J. Berl. B. (1851) 302-; Crelle J. 47 (1854) 7-.

_ _ - Steiner's. Bobek, $K$. Wien Ak. Sb. 98 (1890) (Ab. 2a) 5-, 394-, 526-.

- of constant "power" with respect to points in their plane. Ruffini, F. P. Bologna Ac. Sc. Mm. 10 (1889) 337-.

- containing foci and poles of surface of $2 \mathrm{nd}$ degree. Cauchy, A. L. C. R. 16 (1843) 885-.

- with harmonic pole and straight line, and deductions. Gilssfeldt, P. [1868] Mth. A. 2 (1870) 65-.

- - infinite branches. Bergsten, N. J. Stockh. Ac. Hndl. 31 (1810) 17-.

- - pedals having "power" at every point of plane. Ruffini, $F$. P. Bologna Ac. Sc. Mm. 3 (1892) 277-; 4 (1894) 235-.

- traced in plane of conic by foci and poles. Cauchy, A. L. C. R. 16 (1843) 818-

- with trinomial equations. Gilbert, $P$. N. A. Mth. 9 (1870) 370-

\section{Curves, 3rd degree.}

circular. Eckardt, F. E. Z. Mth. Ps, 10 (1865) 321-。 368-

- Consentius, R. O. Z. Mth. Ps. 25 (1880) 119 .

-. Jeřabek, V. Mathesis 18 (1898) 224-

- Retali, V. Mathesis 19 (1899) 87-.

-, with double point. Morley, F. Am. J. Mth. 11 (1889) 307-.

_, - - Servais, C. N. A. Mth. 8 (1889) $197-$.

_, - - Beyel, C. Z. Mth. Ps. 42 (1897) 281-

(1871) 259-.

-, - - - Pelz, C. [1880] Wien Ak. Sb. 82 (1881) (Ab. 2) 1207-. focal curve, construction from 6 points. Miller, R. Z. Mth. Ps. 40 (1895) 337-. 
circular, of genus 1 , foci. Schoute, Amst. Ak. Vs. 5 (1897) 261-.

-, inverses of lemniscate with respect to its vertices. Wolstenholme, (Rev.) J. L. Mth. S. P. 7 (1875-76) 91-.

-, involution system, and description by points when double focus is on curve. Cotterill, T. L. Mth. S. P. 1 (1866) No. 8, 14-.

-, metric properties, theorems of Jakob Steiner. Disteli, $M$. Zür. Vjschr. 36 (1891) 255-.

-, rational, construction. Longchamps, G. de. As. Fr. C. R. (1885) (Pt. 2) 131-.

-, - , points of inflexion on. Longchamps, G. de. As. Fr. C. R. (1886) (Pt. 2) 5-.

connected with reflexion of bright point in a sphere. Nicodemi, $R$. Nap. Ac. Pont. At. 17 (1887) 109-.

- - triangle. Steggall, 一. Edinb. Mth. S. P. 7 (1889) 66-.

- _ triangles in perspective. Roberts, S. L. Mth. S. P. 28 (1897) 448-.

and curves of 3rd class. Sporer, B. Z. Mth. Ps. 40 (1895) 159-.

with cusp. Greiner, $M$. Arch. Mth. Ps. 68 (1882) 1-.

- - Bioche, C. N. A. Mth. 12 (1893) 294-.

389.

- -, circum-polygons. Roberts, R. A. L. Mth. S. P. 13 (1881-82) 148-

- - construction by points and tangents. Ocagne, M. d'. N. A. Mth. 11 (1892) 386-.

- 13 degenerate forms and fundamental numbers of. Schubert, H. C. H. Gött. Nr. (1875) 359-.

- - properties. Drasch, H. Mh. Mth. Ps, 4 (1893) 81-.

cyclic. Andreasi, A. G. Mt. 30 (1892) 241-.

degeneration. Taylor, H. M. L. Mth. S. P. 28 (1897) 545-.

equianharmonic. Scott, (Miss) C. A. Mess. Mth. 25 (1896) 180-.

and hyperboloid, various theorems. Oekinghaus, E. Arch. Mth. Ps. 70 (1884) 370-.

inflecting on crossing their asymptotes. Morley, F. Mess, Mth. 17 (1888) 51-.

locus of foci of tangential pencil of conics. Schröter, H. [1871] Mth. A. 5 (1872) 50-.

Mth. A. 5 (1872) 83-.

- whence 2 lines subtend equal angles. Hermes, O. Crelle J. Mth. 97 (1884) 177-.

- - - - Schoute, P. H. Crelle J. Mth. 99 (1886) 98-

16-point, of triangle. Thomson, F. D. [1864] Mess. Mth. 3 (1866) 15-.

related to 2 circles. Mention, J. [1859] St Pét. Ac. Sc. Bll. 1 (1860) 233-.

through vertices of complete quadrilateral. Roberts, S. [1861] QJ. Mth. 5 (1862) 54-.

- - - - Roberts, M. QJ. Mth. 5 (1862) 365-.

and 4th class, "evolution" in. Weyr, E. J. Brux. Ac. Bll. 49 (1880) 7-.
Curves, 3rd and 4th degrees.

application of conic section coordinates. Oppenheimer, $H$. Arch. Mth. Ps. 13 (1895) 84-.

circular. Czuber, E. Z. Mth. Ps. 32 (1887) 257-.

-, mechanical description. Roberts, S. L. Mth. S. P. 2 (1869) 125-.

\section{Curves, 4 th degree.}

apolar to conics. Grassi, A. G. Mt. 38 (1900) 244-.

arising from quadratic transformation of conic. Leuch, A. Bern Mt. (1889) 1-.

Caporali's quartic. Ciani, E. Nap. Rd. 35 (1896) 126-.

case. Jeřabek, V. Mathesis 19 (1899) 15-. central, axes of symmetry. Schif, $V$. $O$.

Kharkov Mth. S. Com. 3 (1893) 163-; Fschr. Mth. (1892) 600.

circular. Casey, J. [1867] Ir. Ac. T. 24 (1871) 457-.

-. Cayley, A. Phil. Trans. 167 (1877) 441-. - Loria, G. QJ. Mth. 22 (1887) 44 -

-, with collinear foci. Jeffery, H. M. [188083] L. Mth. S.P. 12 (1880-81) 17-; 14 (188283) $239-$.

-, confocal. Franklin, F. Am. J. Mth. 12 (1890) 323-.

-, -, and "cyclides", analogy to Ivory's theorem. Dixon, A. L. L. Mth. S. P. 24 (1893) 306-.

-, construction. Cayley, A. L. Mth. S. P. 5 (1874) 29-

-, "cyclics"; and "cyclides", generation. Saltel, L. Par. S. Mth. Bll. 3 (1875) 95-, 99-.

-, -, foci. Saltel, L. Par. S. Mth. Bll. 3 (1875) 100-.

-, and "cyclides", curves connected with double normals of. Smyly, J. G. [1899] Ir. Ac. P. 5 (1898-1900) 370-.

-, double foci of, and nodal focal curves of a cyclide. Lachlan, $R$. L. Mth. S. P. 27 (1896) 71-.

-, with double point, description. Cayley, $A$. [1870] L. Mth. S. P. 3 (1869-71) 100-.

-, - 3 double points and surface of elasticity. Saltel, $L$. Brux. Ac. Bll. 35 (1873) 46-.

-, focal conics. Hart, $H$. L. Mth. S. P. 11 (1879-80) 143-.

-, geometrical treatment. Purser, F. B. A. Rp. (1878) 465.

-, mechanical description. Hart, H. L. Mth. S. P. 14 (1882-83) 199-.

-, proofs of theorems by elementary geometry. Crone, C. (xII) Ts. Mth. 3 (1879) 81-.

-, properties. Crofton, $M . W .[1867] \mathrm{L}$. Mth. S. P. 2 (1869) 33-.

-, property. Jessop, C. M. QJ. Mth. 23 (1889) 371-.

quadritangential conics. Richter, O. Z. Mth. Ps. 35 (1890) (Suppl.) 111 pp. and skew, theorem. Lachlan, R. [1890] L. Mth. S. P. 21 (1891) 274-.

-, special involution of 4 th order applied to. La Gournerie, J. de. C. R. 66 (1868) 832-. 
circular, theorems, Lachlan, R. Camb. $\mathrm{Ph}$. S. P. 7 (1892) 87-.

with 3 common double points, series. Taylor, H. M. L. Mth. S. P. 28 (1897) 316-.

- cusp. Richmond, H. W. QJ. Mth. 26 (1893) 5-.

- - at infinity at which line at infinity is a tangent. Jeffery, H. M. [1882] L. Mth. S. P. 14 (1882-83) 71-.

- 3 cusps, circum-polygons. Roberts, $R . A$. [1882] L. Mth. S. P. 14 (1882-83) 56-.

- - of 1st species. Padula, F. Nap. Mm. Ac. Sc. 1 (1852-54) 31-.

- double points of inflexion. Beyel, $C$. Z. Mth. Ps. 30 (1885) 1-, 65-.

- $-1-$ Schoute, P. H. G. Teix. J. Sc. 13 (1897) 10-.

- _ - - - - particularly the "Kreuzcurve". Balitrand, -. Mathesis 11 (1891) 241-; 13 (1893) 5-.

$---1-1-1$ lemniscate. Laguerre, E. N. A. Mth. 17 (1878) 337-。

- double tangential node and double point. Beyel, $C$. Zür. Vjschr. 31 (1886) 178-.

envelope derived from conic. Hall, A. [1871] Smiths. Misc. Coll. 20 (1881) Art. 1, 30-. (Wash. Ph. S. Bll. 1 (1874).)

Klein's quartic is its own S-covariant. Ciani, E. Mil. I. Lomb. Rd. 33 (1900) 565-.

locus of foci of certain plane sections of quadrics. Newson, H. B. Am. J. Mth. 14 (1892) 87-.

mechanical description. Cayley, A. [1872] L. Mth. S. P. 4 (1871-73) 186-.

multiply automorphic, possible types. Ciani, $E$. Palermo Cir. Mt. Rd. 13 (1899) 347-.

with 2 odd branches. Cayley, A. Mess. Mth. 6 (1877) 107-.

- points of inflexion. Masomi, U. Nap. Rd. 21 (1882) 45-.

- 3 points of inflexion. Schoute, P. H. Arch. Mth. Ps. 2 (1885) 113-; 3 (1886) 113-; 4 (1886) 308-; 6 (1888) 113-.

Steiner's quartic and 9 -point circle. Godt, $W$. [1895-96] D. Mth. Vr. Jbr. 4 (1897) 161-; Münch. Ak. Sb. 26 (1897) 119-.

with triangular symmetry. Nielsen, H. P. N. Ts. Mth. 4 (B) (1893) 30-; Fschr. Mth. (1893-94) 1145.

and 3rd class. Steiner, J. Berl. Mb. (1856) 1-.

\section{Curves, 5th degree.}

with 5 cusps, equation. Del Pezzo, P. Nap. Rd. 28 (1889) 46-.

non-singular, forms. Barcroft, D. Am. J. Mth. 10 (1888) 131-.

\section{Curves, 6th degree.}

Roberts, R. A. Mess. Mth. 15 (1886) 26-. bicursal. Cayley, A. L. Mth. S. P. 7 (1876) 166-.

double points. Valentiner, E. C. (xII) Ts. Mth. 5 (1881) 88-, 124-.

mechanical description. Cayley, A. [1872] L. Mth. S. P. 4 (1871-73) 105-.
Cyclotomic curves. Kempe, A. Liège S. Sc. Mm. 20 (1898) No. 8, 14 pp.

Ellipse, curves derived from. Dienger, $J$. Grunert Arch. 9 (1847) 90-, 335-; N. A. Mth. 6 (1847) 234-.

- and hyperbola, curves derived in similar manner to. Vieth, G. U.A. Gilbert A. 58 (1818) 187-.

Envelope of Simson's line. Greer, H. R. [1865] QJ. Mth. 7 (1866) 70-.

- - - Cayley, A. QJ. Mth. 9 1868) 31-, 175-.

346-.

Equilateral curves comprised in

$$
\begin{aligned}
& 0=\Sigma_{1}{ }^{2 n-2} l_{1} T_{1}^{n} \equiv H_{n}, \\
& 0=\Sigma_{1}{ }^{2 n-1} l_{1} T_{1}^{n} \equiv H_{n}+\lambda H_{1}^{\prime} .
\end{aligned}
$$

Serret, $P$. C. R. 121 (1895) 438-.

- hyperbola, curve derived from. Talbot, W. A. F. Gergonne A. Mth. 13 (1822) 242-.

- hyperbolas of any order. Serret, P. C. R. 121 (1895) 340-.

Evolutes of ellipse and hyperbola. Breton [de Champ], P. N. A. Mth. 2 (1843) 223-.

Focals of elliptic cylinders. Stähli, $F$. Bern Mt. (1894) 102-.

-, generation. Chasles, M. Quetelet Cor. Mth. 6 (1830) 207-.

-, points of intersection with circles and lemniscates. Weyr, Em. Prag Sb. (1873) 166 -

Foci of sections of cone, locus. Rees, R. van. Quetelet Cor. Mth. 4 (1828) 37-; 5 (1829) 361-.

Folium of Descartes. Midy, -. N. A. Mth. 3 (1844) 293-.

_- - Kolárìik, A. Časopis 7 (*1878) 113-, 146-; Fschr. Mth. $\left({ }^{*} 1878\right) 483$.

- - secants and tangents. Himstedt, $A$. Arch. Mth. Ps. 15 (1897) 129-.

- - - $\left(1+u^{3}\right) x=3 a u, y=u x . \quad Z a h r a d n i k$, K. Časopis 24 (1895) 282-; Fschr. Mth. (1895) 681.

"Fruiform" curve. Popoff, A. Mosc. S. Nt. Bll. 32 (1859) 283-.

Hinged rod in fluid of varying height, locus of point in surface. Collignon, $E_{\text {. As. Fr. C. }}$ R. (1890) (Pt. 2) 38-.

\section{Lemniscates.}

Verdam, G. J. Amst. N. Vh. 13 (1848) 81-. Arrest, H. d'. As. Nr. 37 (1854) 203-.

Torry, A. F. Mess. Mth. 4 (1868) 139-.

Weyr, E. J. [1873] (xI) Prag Ab. 6 (1874) (Mth.) 39 pp.

Schoute, P. H. [1883] (xr) Wien Ak. Sb. 88 (1884) (Ab. 2) 1252-.

Chini, M. G. Mt. 25 (1887) 51-.

Oekinghaus, E. Arch. Mth. Ps. 7 (1889) 337-; 8 (1890) 24-

Zahradnik, K. [1898] Arch. Mth. Ps. 16 (1898) 327-; Čsopis 28 (1899) 27-.

and aplanatic lines. Terquem, O. N. A. Mth. 4 (1845) 423-. 
ares. Chasles, M. C. R. 21 (1845) 199-.

- (Chasles). Roberts, $W$. Liouv. J. Mth. 10 (1845) 451-.

-, division. Tortolini, B. Palomba Rac. 1 (1845) 17-; Mod. S. It. Mm. 1 (1862) 91-.

Cassini's. Wangerin, A. Arch. Mth. Ps. 55 (1873) 19-.

chord of contact, construction. Cantor, $M$. Z. Mth. Ps. 12 (1867) 428-.

class of curves including. Tortolini, B. Tortolini A. 1 (1858) 178-.

division into 17 parts by ruler and compasses. Kiepert, L. [1872] Crelle J. 75 (1873) 255-, 348.

and elliptic curves of 1st class, geometric theory. Serret, J. A. Liouv. J. Mth. 11 (1846) 89-.

hyperbolic. Charpentier, $H$. N. A. Mth. 4 (1845) 142-.

mechanical construction. Haedenkamp, $H$. Grunert Arch. 3 (1843) 400.

- - Stegmann, F. Grunert Arch. 8 (1846) $49-$.

- property. Fuss, N. [1815] St Pét. Ac. Sc. Mm. 9 (1824) 91-.

- - (Fuss). Serret, J. A. Liouv. J. Mth. 9 (1844) 28.

new construction. Schultz, E. Arch. Mth. Ps. 12 (1894) 318-.

properties. Serret, J. A. Par. S. Phlm. PV. (1846) 20-.

- Hess, $W$. Z. Mth. Ps. 26 (1881) 143-。

-, and application to cubic equations, etc. Oekinghaus, E. Arch. Mth. Ps. 70 (1884) 113-.

property. Bonnet, O. Liouv. J. Mth. 9 (1844) 116.

-, generalisation. Roberts, W. Liouv. J. Mth. 12 (1847) 41-.

17-sided polygon. Clausen, $T$. As. Nr. 19 (1842) 243-.

tangents, construction. Steiner, J. Crelle J. 14 (1835) 80 -

theorem. Andrê, H. d'. N. A. Mth. 5 (1846) 331-.

theorems. Haan, D. Bierens de. Grunert Arch. 11 (1848) 1-.

-.Weyr, E.J. [1872] (xI) Par. S. Mth. Bll. 1 (1873) 18 .

-, proof. Sturm, J. C. F. Gergonne A. Mth. 14 (1823-24) 17-.

-, -. Schou, T. V. Mth. Ts. 4 (1862) $46-$

Limaçon. Weill, M. N. A. Mth. 20 (1881) 160 -.

-. Pittarelli, G. G. Mt. 21 (1883) 145-, 173-.

- Genocchi, A. C. R. 98 (1884) 81-.

- Sucharda, A. Arch. Mth. Ps. 4 (1886) 197-.

- and cardioid. Riggs, H. C. [1892] Kan. Un. Q. 1 (1893) 89-.

-, mechanical description and parallel motion deduced therefrom. Hart, $H$. Mess, Mth. 5 (1876) 35-.

-, Poncelet polygons. Morley, F. [1897] L. Mth. S. P. 29 (1898) 83-.
Line of force, construction for direction. Vincent, J. H. [1898] Nt. 59 (1898-99) 32.

- - - - - - Gray, A. [1898] Nt. 59 (1898-99) 32.

Lines of force due to 2 lines or points, construction for. Roever, $W . H$. [1896] St. Louis Ac. T. 7 (1894-97) 201-.

- - - - plane and line or plane and point. Roever, W. H. [1896] St. Louis Ac. T. 7 (1894-97) 273-.

Loci, certain. Piani, D. [1849] Bologna Mm. Ac. Sc. 4 (1853) 261-.

Locus problem $\left(t_{1} t_{2}=\right.$ constant, where $t$ is tangent to circle). Tortolini, B. A. Mt. 6 (1864) 169-.

- -, geometric. Grunert, J.A. Arch. Mth. Ps. 47 (1867) 477-.

Neoids. Stegmann, $F$. Grunert Arch. 8 (1846) 49-.

Ovals, class. Stampfer, S. Wien Jb. Pol. I. 19 (1837) 34-.

-, construction. Schlömilch, O.X. Z. Mth. Ps. 19 (1874) 263-.

- and ellipses of higher degrees. Burg, A. Wien Jb. Pol. I. 18 (1834) 259-.

Parabola and circle, curves depending on. Masetti, G. Bologna Opusc. Sc. 3 (1819) 159-.

-, cubic, mechanical construction. Foncaut, G. N. A. Mth. 17 (1858) 274-.

-, -, metrical properties. Schroeter, $H$. Mth. A. 25 (1885) 293-.

-, - , tables for location. Merfield, C. J. N. S. W. R. S. J. 34 (1900) 281-.

- locus of centres of circles through focus touching. Freund, E. Lotos 42 (1894) 160 -.

Parabolas, cubic, divergent. Cayley, A. [1865] QJ. Mth. 9 (1868) 185-.

,,--- , inflexions. Cayley, A. (I) QJ. Mth. 6 (1864) 199-.

-, 一, -, pedal curves. Ruffini, F. P. [1894] Bologna Âc. Sc. Mm. 5 (1895-96) 65-.

-, -, theorem. Collignon, É. Par. S. Phlm. Bll. 4 (1880) 180-.

- of higher degree. Himstedt, - Arch. Mth. Ps. 8 (1890) 210-, vi; 9 (1890) vii.

Parabolic curves, $n u=v^{n}$, ares. Talbot, W. H.F. R. S. P. 3 (1834) 287-.

,$-- e y=x^{n}$. Gibson, G. A. Edinb. Mth. S. P. 14 (1896) 31-.

Parallel curves of conics. Roberts, S. QJ. Mth. 12 (1873) 58-.

- to ellipse. Catalan, E. C. N. A. Mth. 3 (1844) 553-.

- - Grunert, J. A. Grunert Arch. 8 (1846) 375-.

- - Cayley, A. Tortolini A. 3 (1860) $311-$.

- - Fiedler, W. Grunert Arch. 39 (1862) 19-.

- - - and application to Diaphantine equation $x^{3}+y^{3}+z^{3}=u^{2}$. Catalan, E. C. Brux. Ac. Bll. 22 (1866) 29-.

Parallels to ellipse. Breton [de Champ], $P$. N. A. Mth. 3 (1844) 442-.

- - Forti, A. O. (xiI) Rv. Sc.-Ind. 14 (1882) 428-. 
Partially dotted curve $y=\sqrt[x]{x}$ Hessel, J. F.C. Grunert Arch. 14 (1850) 169-.

Pedal of circle. Centocroce, $M . B$. Rm. N. Linc. At. 42 (1889) 203-.

- - ellipse or hyperbola. Eckardt, F.E. Z. Mth. Ps. 10 (1865) 332-.

- - - negative. Tortolini, B. Palomba Rac. 2 (1846) 89-.

- parabola, foci, and forms of vector equation. Wolstenholme, (Rev.) J. L. Mth. S. P. 13 (1881-82) 77-.

Pedals of conics. Ameseder, A. Arch. Mth. Ps. 64 (1879) 143-, 145-.

- - central. Roberts, S. N. A. Mth. 10 (1871) 208-.

- - negative. Ameseder, A. Arch. Mth. Ps. 64 (1879) 170-.

Polar curves, harmonic, metrical properties. Bretschneider, C. A. Arch. Mth. Ps. 50 (1869) 475-.

- reciprocal of $y^{2} x=$ constant. Hochheim, $A$. [1875] Arch. Mth. Ps. 58 (1876) 423-.

Polars and asymptotic chords, curves enveloped by. Bermann, $O$. Grunert Arch. 14 (1850) 382-; 16 (1851) 179-; 17 (1851) 241-.

Polyzomal curves. Cayley, A. [1867] Edinb. R. S. T. 25 (1869) 1-.

Prunoid, $\rho=\frac{m+\cos \theta}{m^{2}-1}$. Barbour, L. G. Des Moines Anal. 7 (1880) 17-.

Quadri-quadric curve and elliptic functions. Cayley, A. Mth. A. 25 (1885) 152-.

- porism. Mathews, G. B. QJ. Mth. 30 (1899) 16-.

"Radial" curves, derived from curve and its evolute. Tucker, R. [1865] L. Mth. S. P. 1 (1866) No. 5, 1-.

Radical or equal "power" lines and points of curves. Ruffini, F. P. Bologna Rd. 4 (1900) 23-.

Rosettes. Brassinne, E. N. A. Mth. 7 (1848) 209 -

- Breton [de Champ], P. N. A. Mth. 7 (1848) 369-.

Self-polar curves. Berg, F. J. van den. N. Arch. Wisk. 19 (1892) 80-; Fschr. Mth. (1892) $652-$.

- 一. Appell, P. N. A. Mth. 13 (1894) 206-.

Shadow curve of screw, construction of tangent. Chemin, J. N. A. Mth. 5 (1866) 271-.

Spiral of Perseus $y=\sqrt{R^{2}-\left(l+\sqrt{x^{2}+c^{2}}\right)^{2}}$, foci. Gomes Teixeira, F. Fschr. Mth. (1900) 598-.

Stelloids, geometrical properties. Fouret, $G$. C. R. 106 (1888) 342-, 966.

Strophoid. Ritt, G. N. A. Mth. 5 (1846) 470 -

- Neuberg, J. Mathesis 6 (1886) 219-.

- Valdès, E. N. A. Mth. 13 (1894) 243-.

-, applications. Cazamian, A. N. A. Mth. 12 (1893) 387-.

- and cissoid. Balitrand, -. N. A. Mth. 12 (1893) 430-.

-, - conchoid of Nicomedes, curve connected with. Jeŕábek, $V$. Časopis 28 (1899) 122-; Fschr. Mth. (1899) 528-.

-, curves derived from. Reignier, C. Cherb. S. Sc. Nt. Mm. 26 (1889) 75-
Strophoid, properties. Loria, G. Mathesis 18 (1898) 265-.

-, -, application. Cazamian, A. N. A. Mth. 14 (1895) 192-.

-, - and geometric theorems. Maleyx, $L$. N. A. Mth. 13 (1874) 404-, 468-; 14 (1875) 193-, 241-.

Strophoidal curves of 3rd degree. Lagrange, N. A. Mth. 19 (1900) 66-.

Strophoids. Johnson, W. W. Am. J. Mth. 3 (1880) 320-.

- Barnes, E. (xII) J. H. Un. Cir. [2] (1883) 145

Theorems. Magnus, L. J. Crelle J. 9 (1832) 135-.

Three-bar curve, nodes and foci. Roberts, $S$. QJ. Mth. 15 (1878) 52-.

Trajectories, reciprocal. Brunel, - Bordeaux S. Sc. Mm. 5 (1890) lxviii.

Triangle, locus of 6 th order arising from. Rueda, C.J. Fschr. Mth. (1896) 434.

Trisectrix, Maclaurin's. Jeřábek, $V$. Mathesis 19 (1899) 61-.

-, mechanical description. Fusinieri, A. [1845] Mod. S. It. Mm. 23 (1846) 294-.

-, remarkable. Longchamps, G. de. Mathesis 8 (1888) 5-.

Versiera, visiera and pseudo-versiera,

$$
x^{2} y=a^{2}(a-y) \text {. }
$$

Loria, G. Bb. Mth. (1897) 7-, 33-.

- or " witch of Agnesi." Mister, $J$. Mathesis 7 (1887) 5-.

$x^{4}-y^{4}=4$ ay $x^{2}$. Tielemans, C. Louvain A. Ac. 9 (1827) pars 2,92 pp.

$x^{2} y^{2}-l^{2}\left(y^{2}+x^{2}-2 x y \cos \theta\right)=0$. Bouteiller, $E$. de. N. A. Mth. 6 (1847) 263-

$V=M+N X+P X^{2}+Q X^{3}$, application to curvature of general curve. Dupin, C. C. R. 25 (1847) 689-, 769-.

$\left(\frac{x}{a}\right)^{n}+\left(\frac{y}{b}\right)^{n}+\left(\frac{z}{c}\right)^{n}=0 . \quad$ Steen, A. Mth. Ts. 2 (1860) 101-.

$-=-(n=4)$. Schwering, K. Z. Mth. Ps. 21 (1876) 133-.

$-=-(n=2)$, and correlative quarties. Roberts, S. QJ. Mth. 15 (1878) 224-.

$-=-$. Roberts, R. A. [1885] Ir. Ac. T. 28 (1880-86) 709-.

$r^{2 n}=A \cos ^{2} \theta+B \sin ^{2} \theta$, properties. Tortolini, $B$. A. Mt. 6 (1864) 150-.

$y=x^{3}+a x^{2}+b x+c$, properties. Stöckly, $L$. Sch. Gs. Vh. 53 (1869) 105-; Arch. Mth. Ps. 56 (1874) 180-.

$y^{4}=a^{2} x^{2}-x^{2} y^{2}$. Azzarelli, $M$. Rm. N. Linc. At. 28 (1875) 234-.

$\rho=\frac{f(\varpi)}{\varpi+\phi(\varpi)}$, construction of tangent, $f(\varpi)$ and $\phi(\varpi)$ being functions of trigonometrical ratios of $\varpi$. Fouret, $G$. N. A. Mth. 19 (1880) 28-.

$y^{m}+\phi_{m-2}(x, y)=0$, properties. Weill, $M$. N. A. Mth. 20 (1881) 498 -

$$
\begin{aligned}
& x^{2}\left(4 a^{2}-y^{2}\right)=\left(y^{2}-2 a^{2}\right)^{2}, \\
& x^{2}(4 a-y)=y(3 a-y)^{2}
\end{aligned}
$$

Karliński, F. (xп) Krk. Ak. (Mt.-Prz.) Rz. \& Sp. 10 (1883) xI-. 
$x_{2}{ }^{3} x_{3}+x_{3}{ }^{3} x_{1}+x_{1}{ }^{3} x_{2}$, covariant theory. Brioschi, F. Mil. I. Lomb. Rd. 17 (1884) 401-; Rm. R. Ac. Linc. T. 8 (1884) 164 -.

$(2 y-3 b)\left(x^{2}+y^{2}\right)+b^{3}=0$. Almeida Lima, João $d^{\prime}$. G. Teix. J. So. 6 (1885) 13-; Fschr. Mth. (1884) 644-.

$a x^{3}+3 b y^{2} z+3 c z^{2} x=0$, concomitants. Walker, J. J. L. Mth. S. P. 20 (1889) 382-.

$r^{n}=a^{n} \sin n \theta . \quad$ Iversen, I. M. N. Ts. Mth. 4 (B) (1893) 59-; Fschr. Mth. (1893-94) 1154.

$\rho=2 a \cos n \theta$. Pirondini, $G$. Mathesis 14 (1894) 14-.

$\rho=R \sin \mu \omega$, algebraic if $\mu$ is integral, otherwise transcendental. Loria, $G$. Fschr. Mth. (1899) 515.

\section{Algebraic surfaces of degree higher than the second. (See also 8040.)}

(Müller.) Petzval, J. Wien SB. 41 (1860) 735-.

Cremona, L. Bologna Ac. Sc. Mm. 6 (1866) 91- ; 7 (1867) 29-.

Bäcklund, A.V. Stockh. Ak. Hndl. 9 (1870) No. $9,65 \mathrm{pp}$.

Mainardi, G. Rm. At. N. Linc. 26 (1873) 384 .

Beltrami, E. Bologna Ac. Sc. Mm. 10 (1879) 233-.

Ocagne, $M . d^{\prime}$. C. R. 99 (1884) 744 -

Asymptotes, curvature and singular points in surfaces of 2nd and 3rd degree. Beer, $A$. Grunert Arch. 17 (1851) 329-.

Asymptotic plane and cylinders of surface. Biehler, C. N. A. Mth. 8 (1889) 536-.

- planes and surfaces. Walton, $W$. Camb. and Dubl. Mth. J. 3 (1848) 28-.

- straight lines, planes, cones, and cylinders. Weddle, $T$. Ph. Mg. 31 (1847) 425-.

Axes, 3 rectangular, cutting surface, theorems. Haillecourt, A. N. A. Mth. 12 (1853) 398-.

Cones, algebraic, properties. Laguerre, $E$. [1870] (x) Par. S. Phlm. Bll. 7 (1871) 139-.

—, 3rd degree. Cayley, A. Ph. Mg. 18 (1859) 439-.

-, $m$ th degree, having vertices in straight line, and 2 plane intersection curves, theorem. Serret, P.' N. A. Mth. 1 (1862) 21-

Conical points on surfaces. Korteweg, - . N. Arch. Wisk. 18 (1891) 153-.

Conjugate lines analogous to conjugate points. Walton, $W$. Camb. and Dubl. Mth. J. 9 (1854) 238-.

Construction of surfaces from given points. Escherich, G. von. Wien Ak. Sb. 90 (1885) (Ab. 2) 1036-.

- - - by means of reciprocal pencils of surfaces. Escherich, G. von. Wien Ak. Sb. 85 (1882) (Ab. 2) 526-.

- - - plane sections. Cardinaal, $J$. Amst. Ak. Vs. M. 6 (1889) 198-; Fschr. Mth. (1889) 665-.
Contacts, and Tangent Lines and Planes.

(See also 8450.)

Contact of conics with surfaces. Spottiswoode, $W$. Phil. Trans. 160 (1870) 289-

- straight lines with surfaces. Salmon, $G$. QJ. Mth. 1 (1857) 329-.

- - - 4- and 5-point. Gordan, $P$. Z. Mth. Ps. 12 (1867) 495-.

- - various kinds. Clebsch, A. Crelle J. 58 (1861) 93-.

- surfaces. Spottiswoode, W. Phil. Trans. 162 (1872) 259-.

- - - (Spottiswoode's problems). Clifford, $W . K$. R. S. P. 21 (1873) 425.

- - Halphen, G. H. [1874] Par. S. Mth. Bli. 3 (1875) 28-.

- _ - 2, having common generator, fundamental principle. Mansion, $P$. Brux. Ac. Bll. 3 (1882) 753

- - - - order of condition for. Roberts, S. QJ. Mth. 12 (1873) 229-.

- - - - - criteria. Plücker, J. Crelle J. 4 (1829) 349.

Normals and tangents to algebraic surfaces and lines. Bardelli, $G$. Mil. I. Lomb. Rd. 5 (1872) 167-.

Points where straight line has 5-point contact. Clebsch, R. F. A. [1863] (vII) Crelle J. 63 (1864) 14-.

Problem : if $\frac{x}{x_{1}}+\frac{y}{y_{1}}+\frac{z}{z_{1}}=1$ is a tangent plane, and makes $f(x, y, z)$ a maximum or minimum, then $f(x, y, z)$ is constant. $\quad$. [Ellis, R. L.] (vi Adds.) Camb. Mth. J. 4 (1845) 47-.

Rotation surfaces, tangent planes, construction. Matzek, F. Wien Sb. 58 (1868) (Ab. 2) 44-.

Ruled surfaces, 2, touching along generator, condition. Ch., J. F. N. A. Mth. 2 (1863) $262-$.

Skew surfaces, method of drawing tangents to. Marchand, J. Par. S. Mth. Bll. 13 (1885) 34-.

Surfaces, 3rd degree, contact of 4 th order with surface. Moutard, T. (x) Par. S. Phlm. Bll. 2 (1865) 23-.

Tangent planes. Reid, J. Mathematician 2 (1847) 187-.

- - to algebraic surface through multiple line of surface, number. Fouret, G. Palermo Cir. Mt. Rd. 8 (1894) 202 -

- - certain surfaces, arrangement. Weyr, E. [1878] Bordeaux S. Sc. Mm. 3 (1880) 191-.

- - , higher, of algebraic surfaces. Tótössy, B. Mth. Termt. Éts. 16 (1898) 127-.

- and normals to skew surfaces. Chasles, M. Liouv. J. Mth. 2 (1837) 413-

- at point on surfaces. Laisant, A. N. A. Mth. 7 (1868) 116-.

- to skew surfaces. Monin, T. Prag Sb. (1888) (Mth.-Nt.) 210-.

- - - - theorems of Hachette and Chasles. Goedseels, E. (xII) Mathesis 3 (1883) 49-. 
Tangent planes to skew surfaces, theorems of Hachette and Chasles. Mansion, P. (xII) Mathesis 3 (1883) 52-.

- - touching surface along curve, condition for. Greatheed, S. S. (vI Adds.) Camb. Mth. J. 2 (1841) 22-.

- - - - - Salmon, G. Camb. and Dubl. Mth. J. 3 (1848) 44-.

Tangential hyperboloid of certain ruled quartic surface. Janisch, E. Mh. Mth. Ps. 10 (1899) 223-.

Tangents at multiple point of curve of intersection. Olivier, T. Par. S. Phlm. N. Bll. (1832) 189-.

- to surface or single system of surfaces, some numerical determinations for. Bonsdorff, $E$. [E.J.] [1881] Helsingf. Acta 12 (1883) 95-.

- touching surface in two points. Boyd, J. H. A. Mth. 5 (1889-90) 109.

- - - - - (Boyd). Loud, F. H. A. Mth. 5 (1889-90) 229.

Cylinders, oblique. Euler, L. [1778] St Pet. Ac. Sc. N. Acta 12 (1801) 91-.

Decomposition due to presence of multiple lines, and paradox. Saltel, L. Brux. Ac. Bll. 47 (1879) 184-.

Determination of degree. Cayley, A. [1882] Mess. Mth. 12 (1883) 29-.

Developable surfaces, double lines of. Künzler, G. Arch. Sc. Ps. Nt. 6 (1898) 370-.

Diametral planes of algebraic surfaces. Breton [de Champ], P. C. R. 34 (1852) 170-.

- surfaces. Binet, J. P. M. Par. Ec. Pol. Cor. 2 (1809-13) 74-.

Equation, homogeneous, of plane section of geometrical surface. Walker, J.J. L. Mth. S. P. 15 ( $\left.{ }^{*} 1883-84\right)$ 224-.

-, reduced, special forms. Dumont, $F$. Par. S. Mth. Bll. 26 (1898) 124 .

- of surface $P S-Q R=0$, number of constants. Cayley, A. (xII) Ts. Mth. 4 (1880) 145-.

Equations for lines on developable surfaces. Enneper, A. Gött. Nr. (1867) 73-.

- plane coordinates. Plücker, J. Crelle J. 9 (1832) 124-.

-, stereometric, of 3rd degree, and resulting surfaces. Grassmann, H. [1852] Crelle J. 49 (1855) 47-.

- as sum of homogeneous functions. Carmichael, R. Camb. and Dubl. Mth. J. 9 (1854) 55-.

- of surfaces, 2nd and 3rd degrees, geometric meaning of coefficients. Mossbrugger, $L$. Grunert Arch. 1 (1841) 337-.

-, tangential. Laguerre, $E$. (x) Par. S. Phlm. Bll. 8 (1872) 64-.

General properties. Lardner, D. [1824] Ir. Ac. T. 14 (1825) 69-.

- - Dietrich, M. Schlömilch Z. 7 (1862) 398 -

- survey of progress. Bertrand, J. J. Sav. (1867) 644 .

Generation, linear, of all algebraic surfaces. Grassmann, H. [1852-55] Crelle J. 48 (1854) 417-; 49 (1855) 1-, 21-.
Generation, projective, of general surface of 3rd, 4th, and any degree, by systems of surfaces of lower degree. Reye, T. Mth. A. 1 (1869) 455-

- of surfaces. Blavette, - Eure Rec. S. Ag. 5 (1834) 71-.

- - Vaněček, J. S., \& Vaněček, M. N. C. R. 97 (1883) 1473-, 1548-.

- - Jonquières, E. de. C. R. 105 (1887) 1203-.

(1898-99) 118-.

- _ - by quadrilinear sheaves of planes. Le Paige, C. Brux. Ac. Bll. 8 (1884) 238-

Generators, singular, in algebraic ruled surfaces. Björling, C. F. E. Stockh. Öfv. (1888) 587-. - , _, - bi-normal and principal normal sur. faces. Björling, C. F. E. Stockh. Ak. Hndl. Bh. 15 (Afd. 1) (1890) No. 8, 18 pp.

Harmonic pole and plane. Lebesgue, $V$. $A$. N. A. Mth. 8 (1849) 22-.

- - Tognoli, O. G. Mt. 8 (1870) 166-

Hessians of cubic and quartic, certain singularities. Heal, W. E. A. Mth. 4 (1888) 37-

_, nodes. Clebsch, A. Crelle J. 59 (1861) 193-.

- in relation to multiple points. Rohn, $K$. Mth. A. 23 (1884) 82-.

- of surfaces. Voss, $(\mathrm{Dr})$ A. Gött. Nr. (1873) 418-.

- - - degree, geometrical determination. Schubert, H. Z. Mth. Ps. 15 (1870) 126-.

Intersection curve of two surfaces, degree. Terquem, $O$. N. A. Mth. 9 (1850) 342-.

- of surface by plane, equation. Housel, N. A. Mth. 7 (1868) 277-.

- - 2 surfaces, construction by means of spheres and rotation surfaces. Niemtschik, $R$. Wien Sb. 64 (1871) (Ab. 2) 117-.

- 3 surfaces, points, and curve of intersection of 2 surfaces. Tognoli, $O$. G. Mt. 9 (1871) 68-.

Loci arising from three figures, projectively related. Del $R e, A$. Palermo Cir. Mt. Rd. 1 (1887) 272-, 398.

Locus of common apex of pyramids, surfaces as. Dufilhol, -. Rouen Ac. Tr. (1814) 44 .

- points, such that lines, joining each to 3 points, cut given plane in triangle of constant area. Sturm, J. C. F. Gergonne A. Mth. 14 (1823-24) 302-.

_ _ _ sum of whose distances from 2 lines is constant. Picart, A. Par. Éc. Norm. A. 1 (1864) 285-.

- - - - - - - - - - - - , and class of surfaces. Mannheim, A. Bll. Sc. Mth. As. 3 (1872) 119-.

Maximum and minimum distances of point from surface, etc. Mayer, A. C. G. Leip. Mth. Ps. B. 33 (1881) 28

Metrical properties. Craig, T. Am. J. Mth. 4 (1881) 297-.

Multiple points of surface. Umpfenbach, $(D r)$ -C Crelle J. 28 (1844) 380.

- - - specialising. Rohn, K. Leip. Mth. Ps. B. 36 (1884) 1-。 
Multiple points, surface with 2, theorem. Saltel, L. Brux. Ac. Bll. 34 (1872) 51-.

Newton's segment theorem. Terquem, $O$. N. A. Mth. 9 (1850) 342-.

Normals. Sturm, $R$. Mth. A. 7 (1874) 567-.

-. Schoute, P.H. N.Arch. Wisk.6(*1880) 19-.

- Reina, V. Nap. Rd. 29 (1890) 75-.

- common to two surfaces. Rindi, S. Palermo Cir. Mt. Rd. 5 (1891) 106-.

- - - - (Rindi). Pieri, -. Palermo Cir. Mt. Rd. 5 (1891) 323.

, double, to algebraic surfaces. Pieri, $M$. Rm. R. Ac. Linc. Rd. 2 (1886) (Sem. 2) 40-. -, 一, - - Rindi, S. As. Fr. C. R. (1887) (Pt. 2) 216-.

-, locus, when sum of squares is constant. Laisant, C. A. [1889] Par. S. Mth. Bll. 18 (1890) 141-.

- to surface from given point. August, $F$. [1867] Crelle J. 68 (1868) 242-.

- - - - - number. Terquem, $O$. Liouv. J. Mth. 4 (1839) 175-.

- - - - - - Salmon, G. Camb. and Dubl. Mth. J. 3 (1848) 46-.

-, theorems. Fouret, G. As. Fr. C. R. 6 (1877) 205-.

Paradox arising in construction of surface through given points, solution. Schur, F. Leip. Mth. Ps. B. 35 (1883) 59-.

Pedal and director surfaces, surfaces in relation to. Raabe, J.L. [1854] Crelle J. 50 (1855) 194-.

- surfaces. Mossbrugger, L. Grunert Arch. 22 (1854) 139-.

Plane sections, method of discussing. Dixon, A. C. Camb. Ph. S. P. 9 (1898) 198-.

Points at infinity. Painvin, L. C. R. 59 (1864) 666-; Crelle J. 65 (1866) 112-, 198-.

Polar reciprocal of surface with multiple points, determination of double curve. Saltel, $L$. Brux. Ac. Bll. 40 (1875) 27-.

- - surfaces, revision and extension of numerical formulæ in theory. Zeuthen, H.G. Mth. A. 10 (1876) 446-.

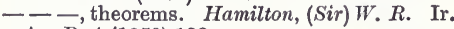
Ac. P. 4 (1850) 192-.

- surfaces of plane, theory. Painvin, L. F. [1865] (xII) Lille S. Mm. 2 (1866) 345-.

- theory, extension. Reye, $T$. Crelle J. Mth. 78 (1874) 97-.

Projective generation, points of intersection, and sections, of surfaces. Reye, T. [1869] Mth. A. 2 (1870) 475-.

- surface of centres. Voss, A. Münch. Ak. Ab. 16 (1888) 243-.

Relations and affinities of surfaces. Peterson, K. M. [1865] (xII) Rec. Mth. (Moscou) [1] (1866) 391-.

Rotation surfaces. Pirondini, G. N. A. Mth. 7 (1888) 486-; A. Mt. 18 (1890) 165-.

- - conditions for surfaces of any degree. Mangeot, S. N. A. Mth. 16 (1897) 408-.

- -, discrimination, and determination of axis. Mangeot, S. Par. Éc. Norm. A. 14 (1897) 191-.

- -, intersection, descriptive geometry. [Schiappa] Monteiro, A. G. Teix. J. Sc. 1 (1878) 177-.
Rotation surfaces, note. Steen, A. (xII) Ts. Mth. 1 (1871) 50-.

-, oblique. Walton, W. QJ. Mth. 6 (1863) 134-.

- - plane sections, Sobotka, J. Wien Ak. Sb. 105 (1896) (Ab. 2a) 371-.

- properties. Beltrami, E. A. Mt. 6 (1864) $271-$.

- , singularities. Workman, W. P. QJ. Mth. 25 (1891) 89-.

Ruled surfaces, Chasles, $M$. Quetelet Cor. Mth. 11 (1839) 49-.

- - Pirondini, G. G. Teix. J. Sc. 12 (1895) 19-.

Singular lines. Halphen, H. G. [1877] A. Mt. 9 (1878-79) 68-.

- points. Saltel, L. [1875] Brux. Mm. Cour. $8^{\circ}, 27$ (1877) No. 5, 66 pp.

- - class. Zeuthen, H. G. Mth. A. 9 (1876) 321-.

- - Newton's parallelogram generalised. Wölffing, E. Z. Mth. Ps. 42 (1897) 14-.

Singularities. Cayley, A. Camb. and Dubl. Mth. J. 7 (1852) 166-.

-. Jonquières, E. de. Liouv. J. Mth. 7 (1862) 409-; N. A. Mth. 3 (1864) 5-.

- Painvin, L. Bll. Sc. Mth. As. 4 (1873) 91-. - flecnodal and spinodal lines on surfaces, intersections. Korteweg, -. Amst. Ak. Vs. M. 5 (1889) 402-.

-, fleflecnodal planes. Cayley, A. QJ. Mth. 15 (1878) 49-.

- of Jacobian of 4 surfaces. Levi, A. Tor. Ac. Sc. At. 31 (1895) 358- or 502-.

- _ - - Gerbaldi, F. Palermo Cir. Mt. Rd. 10 (1896) 158-.

-, pinch points. Cayley, A. QJ. Mth. 9 (1868) 332-.

-, spinodal lines at conical point on surface. Korteweg, - . Amst. Ak. Vs. M. 8 (1891) 385-.

-, surfaces with any, property. Guccia, G. B. Rm. R. Ac. Linc. Rà. 5 (1889) (Sem. 1) 349-, 490-.

Skew surfaces. Olivier, T. Par. S. Phlm. N. Bll. (1832) 185-; Par. S. Phlm. PV. (1838) 84-.

- Catalan, E. C. Par. S. Phlm. PV. (1848) 67-.

_- Cayley, A. Camb. and Dubl. Mth. J. 7 (1852) 171-; Phil. Trans. 153 (1863) 453-; 154 (1864) 559-; 159 (1869) 111-.

- (Cayley, 1864). Cremona, L. Mil. I. Lomb. Rd. 1 (1868) 109-.

- - Vaněček, J. S., \& Vaněček, M. N. Prag Sb. (1883) (Mth. Vortr.) 449-.

- - Machov̌ec, F. Časopis 13 (1884) 32-; Fschr. Mth. (1884) 603.

- - Zejliger, D. Kazan S. Ps.-Mth. Bll. 7 (1898) 122-; Fschr. Mth. (1897) 553.

- -, classification. Fais, A. Bologna Rd. (1883) 170-.

- - construction of paper models. Schönemann, P. Z. Mth. Ps, 28 (1883) 243-.

- -, hyperbolic involutions of points on generators. Zajaczkowski, W. Krk. Ak. (Mt.-Prz.) Rz. 12 (1896) 279-; Crc. Ac. Sc. Bll. (1896) 111-. 
Skew surfaces, hyperboloid generated by tangent lines. Bruno, G. Tor. At. Ac. Sc. 7 (1871-72) 235-.

- - - - - - - (Bruno's theorem). Dewulf, É. Bll. Sc. Mth. As. 7 (1874) 142-. - and orthogonal cone. Beyel, C. Z. Mth. Ps. 32 (1887) 321-.

- - osculating hyberboloid to. Sobotka, $J$. Prag Sb. (1893) (Mth.-Nt.) No. 14, 16 pp.

Surface generated by solution of isotropic solid. Bottomley, J. Manch. Lt. Ph. S. Mm. \& P. 2 (1889) 154-, virr.

- with only one straight line, equation. Breton [de Champ], P. N. A. Mth. 8 (1849) 61-, 130-. Surfaces apolar to one another. Reye, $T$. Crelle J. Mth. 79 (1875) 159-.

- of even degree, theory. Mahler, E. Arch. Mth. Ps. 70 (1884) 313-.

- passing through certain number of points. Plilcker, J. Gergonne A. Mth. 19 (1828-29) 129.

- - - intersection of several surfaces. Delassus, É. Bll. Sc. Mth. 21 (1897) 59-.

Surfaces, 2nd and 3rd degrees.

Geiser, C. F. Crelle J. 69 (1868) 197-.

Surfaces, 2nd, 3rd, and 4th degrees.

Vaněček, J. S. [1882] St Pét. Ac. Sc. Bll. 28 (1883) 287-。

\section{Surfaces, 3rd degree.}

Sartiaux, A. [1867] (xII) Lille S. Mm. 5 (1868) 441-.

Cayley, A. [1868] Phil. Trans. 159 (1869) 231-. Cremona, L. Crelle J. 68 (1868) 1-.

Klein, $F$. Mth. A. 6 (1873) 551-; Erlang. Sb. Ps. Md. S. 5 (1873) 95-.

Zeuthen, H. G. (xII) Ts. Mth. 4 (1874) 53-.

Sturm, R. Crelle J. Mth. 88 (1880) 213-.

Caporali, E. Nap. Rd. 20 (1881) 122-.

Paolis, R. de. Rm. R. Ac. Linc. Mm. 10 (1881) 123-.

Le Paige, C. M. M. H. H. Acta Math. 3 (1883) 181-, 381; C. R. 98 (1884) 971-.

Vaněček, $M$. N. Liège S. Sc. Mm. 11 (1885) No. 3, 5 pp.

Ascione, $E$. Nap. Rd. 32 (1893) 39-.

Chasles's theorems for plane cubics, analogues. Dumont, F. Par. S. Mth. Bll. 25 (1897) 235-.

Classification. Rodenberg, $C$. Mth. A. 14 (1879) 46-.

-. Bobek, $K$. Wien Ak. Sb. 96 (1888) $(A b .2)$ 355-.

- with reference to singular points and reality of lines. Schläfli, L. Phil. Trans. (1863) 193-.

\section{Configurations.}

(See also 8075.)

Brioschi, F. Tortolini A. 6 (1855) 374-.

Double-sixers. Cayley, A: QJ. Mth. 10 (1870) 58-; Camb. Ph. S. T. 12 (1879) 366-

Hexad of lines on surface, case. Kohn, G. Mh. Mth. Ps. 2 (1891) 293-.
Lines which can be placed on surface of 3rd class or 3rd degree. $G_{.,} E$. N. A. Mth. 13 (1894) 138-.

Pascal's and Brianchon's theorems, extension to cubic surfaces. Folie, $\boldsymbol{F}$. Liége Mm. S. Sc. 3 (1873) 663-.

- theorem, extension to cubic surfaces. Petot, A. C. R. 102 (1886) 737-.

Pentad of co-tangential points, surface referred to. Jeffery, H. M. B. A. Rp. (1878) 491-.

Pentahedra. Gordan, P. Mth. A. 5 (1872) 341-. -, complete, inscribed in surface. Zeuthen, H. G. Acta Mth. 5 (1884) 203-.

Polar hexahedra. Cremona, L. D. Nf. B. (*1877) 93-; Mth. A. 13 (1878) 301-.

27 real straight lines, forms of surfaces containing. Blythe, W. H. Camb. Ph. S. P. 9 (1898) 6-.

— straight lines. Steiner, J. Berl. Mb. (1856) 50 -

- Jonquières, E. de. N. A. Mth. 18 (1859) 129-.

_- - Sylvester, J.J. C. R. 52 (1861) 977-.

- - Schröter, H. E. Crelle J. 62 (1863)

265 .

- Cremona, L. Mil. I. Lomb. Rd. 3 (1870) 209-.

- - Affolter, F. G. Arch. Mth. Ps. 56 (1874) 113-.

- - - Sturm, R. Mth. A. 23 (1884) 289-. - - , delineation. Caron, J. Par. S. Mth. Bll. 8 (1880) 73-.

- - - determination and classification of surfaces with respect to. Schläfli, $L$. QJ. Mth. 2 (1858) 55-, 110-

- - , equation. Jordan, C. Liouv. J. Mth. 14 (1869) 147-; C. R. 68 (1869) 865-.

- - - determining, resolution. Klein, $F$. Liouv. J. Mth. 4 (1888) 169-.

- - - groups of substitutions connected with. Pascal, E. A. Mt. 20 (1892-93) 269-; 21 (1893) 85-.

--- , new combination. Jordan, C. C. R. 70 (1870) 326-.

- _ - and parabolic curve. Bauer, $G$. Münch. Ak. Sb. 13 (1883) 320-.

_ - , representation on plane. Schoute, P. $H$. Amst. Ak. Vs. [1] (1893) 143-.

- - - and 45 triple tangent planes. Bertini, E. A. Mt. 12 (1883-84) 301-.

- - - - - - - and 36 double-sixers, construction of models showing lines. Frost, P. QJ. Mth. 18 (1882) 89-.

Triple tangent planes. Cayley, A. Camb. and Dubl. Mth. J. 4 (1849) 118-

- - Salmon, G. Camb. and Dubl. Mth. J. 4 (1849) 252-.

- - Spottiswoode, W. C. R. 77 (1873) 1181-.

- _ - Cayley's theorem, proof. Kohn, G. Mh. Mth. Ps. 2 (1891) 343-.

- - polyhedral configurations. Bertini, E. Mil. I. Lomb. Rd. 17 (1884) 478-, 712-.

- -, property. Brioschi, F. Rm. R. Ac. Linc. At. 3 (1876) (Pte. 2) 257-.

Construction, mechanical. Cayley, A. [1872] L. Mth. S. P. 4 (1871-73) 175-. 
Construction from 19 points. Pannelli, $\boldsymbol{M}$. A. Mt. 22 (1894) 237-.

- by projective rays. Jonquières, $E$. de. C. R. 106 (1888) 526-, 907-, 1038 ; 107 (1888) 209-.

一, synthetic. Thieme, H. Mth. A. 28 (1887) 133-.

Constructions, linear. Schroeter, H. Crelle J. Mth. 96 (1884) 282-.

Cubic surface of Grassmann, construction, and reciprocal of 3rd degree. Haft, $F$. Arg. S. Ci. A. 29 (1890) 157-.

Cubics, geometry of surfaces derived from. Russell, R. [1889] Ir. Ac. P. 5 (1898-1900) 462-.

Equation, general, special form, and diagram of 27 straight lines. Taylor, H. M. [1893] Phil. Trans. (A) 185 (1895) 37-.

-, pentahedral. Beltrami, E. Mil. I. Lomb. Rd. 12 (1879) 24-.

- of ruled surfaces. Godefroy, A. N. N. Arch. Wisk. 1 (1895) 137-; Fschr. Mth. (1893-94) 1259-.

Generation. Picquet, $H$. Par. S. Mth. Bll. 4 (1876) 128-.

-. Deruyts, F. Liège S. Sc. Mm. 14 (1888) No. 4, 12 pp.

-, homographic. Le Paige, $C$. Acta Mth. 5 (1884) 195-.

-, - (Le Paige). Schur, F. Leip. Mth. Ps. B. 36 (1884) 128-.

-, method. Deruyts, F. Brux. Ac. Bll. 22 (1891) 35-.

-, new mode. Kohn, G. Wien Ak. Sb. 99 (1891) (Ab. 2a) 683-.

Hessian of Hessian. Bauer, G. Münch. Ak. Ab. 14 (1883) (Ab. 3) 77-.

Hessians, cubic surfaces which form parts of Hessian of another cubic surface. Ciani, $E$. Mil. I. Lomb. Rd. 27 (1894) 222-.

-, determination of surfaces by. Dumont, $F$. N. A. Mth. 15 (1896) 312-.

- of surfaces. Le Paige, C. Wien Ak. Sb. 91 (1885) (Ab. 2) 981-.

- - Ciani, E. Mil. I. Lomb. Rd. 26 (1893) 498-, 523-, 557-.

- - property. Eckardt, F. E. Z. Mth. Ps. 19 (1874) 259-.

Invariants. Bobek, $K$. Wien Ak. Sb. 103 (1894) (Ab. 2a) 887-; Mh. Mth. Ps. 8 (1897) 145-.

Models, construction. Blythe, W. H. QJ. Mth. 29 (1898) 206-.

- by Rodenburg. Korteweg, D.J. N. Arch. Wisk. 20 (1893) 63- ; Fschr. Mth. (1893-94) 83.

Polar system, construction. Thieme, H. Mth. A. 20 (1882) 144-.

Portion not cut by any real plane. Schläfi, $L$. A. Mt. 5 (1871-73) 289-; 7 (1875-76) 193-.

Properties of position. Zeuthen, H. G. Mth. A. 8 (1875) 1-.

Quadrilinear form, and cubic surfaces. Le Paige, C. Brux. Ac. Bll. 8 (1884) 555-.

Representation on plane. Clebsch, R. F. A. Mth. A. 5 (1872) 419-.

Sections, parabolic and equilateral. Picquet, $H$. Par. S. Mth. Bll. 4 (1876) 153-.

Surface arising from pencil of quadrics. Cassani, P. G. Mt. 22 (1884) 51-.
Surfaces, 3rd and 4th degrees.

Congruences connected with. Schur, F. Crelle J. Mth. 95 (1883) 207-.

\section{Surfaces, 4th degree.}

Cayley, A. L. Mth. S. P. 3 (1869-71) 19-, 198-, 234-.

Durrande, $H$. N. A. Mth. 9 (1870) 410-, 440-.

Case. Hunyady, J. (xII) Mag. Tud. Ak. Etk. (Mth.) 8 (1881) (No. 12) 20 pp.

Developable, reciprocation. Cayley, A. [1865] QJ. Mth. 7 (1866) 87-.

Hessian. Cayley, A. QJ. Mth. 15 (1878) 141-.

Ruled surfaces. Rouyer, L. Toul. Fac. Sc. A. 2 (1900) 163-.

- - different kinds. Rohn, K. Mth. A. 28 (1887) 284-.

- - with double curve of 3rd degree, representation on plane. Clebsch, R. F.A. Mth. A. 2 (1870) 445-.

Section of surface by bitangent plane. JuhelRénoy, -. N. A. Mth. 7 (1888) 282-.

Surface and circumscribed sextic cones, theorem. Cayley, A. L. Mth. S. P. 3 (1869-71) 220-.

- derived from surface of $n$th degree. Painvin, L. N. A. Mth. 10 (1871) 481-; 11 (1872) 376-.

Surfaces with 8 to 16 nodes. Rohn, K. Leip. Mth. Ps. B. 36 (1884) 52-.

\section{Surfaces, 4th and 5th degrees.}

Recent researches. Cayley, A. [1870] L. Mth. S. P. 3 (1869-71) 186-.

\section{Surfaces, 5th degree.}

Developables, demonstration of theorems. Ovidio, E. d'. G. Mt. 3 (1865) 107-, 184-, 214 .

Feet of all axes of quadric through arbitrary point lie on quintic surface. Kloboǔek, $J$. Časopis 26 (1897) 156-; Fschr. Mth. (1897) 490.

Surfaces, $(m+n)$ th degree.

Degeneration when every plane section degenerates. Cayley, A. Ph. Mg. 25 (1863) 61-.

\section{Surfaces, nth degree.}

Cone circumscribing surface. Salmon, $G$. Camb. and Dubl. Mth. J. 4 (1849) 188-.

Construction. Schur, F. Mth. A. 23 (1884) 437-.

Singularities of general surface. Sturm, $R$. Crelle J. 72 (1870) 350-.

Tangent singularities. Schubert, H. C. H. Mth. A. 11 (1877) 347-.

Tangents, 5-pointic, and related problems, solution. Schubert, H. C. H. Gött. Nr. (1876) 89-.

Umbilici, number. Voss, A. E. Mth. A. 9 (1876) 241-. 


\section{Special algebraic surfaces.}

\author{
Anallagmatic surfaces. \\ (See also 8020.)
}

Circular sections. Laguerre, $E$. (x) Par. S. Phlm. Bll. 5 (1868) 48-.

Cyclides. Godart, A. N. A. Mth. 5 (1866) 218-.

-. Maxwell, J. C. QJ. Mth. 9 (1868) 111-.

- Darboux, G. C. R. 68 (1869) 1311-; Bordeaux Mm. S. Sc. 8 (1870) 291-; 9 (1873) 1 -.

-. Lemonnier, H. N. A. Mth. 9 (1870) $514-$.

- Laguerre, E. (x) Par. S. Phlm. Bll. 7 (1871) 209-.

Darboux, G. Par. Éc. Norm. A. 1 (1872) 273-.

-. Cayley, A. QJ. Mth. 12 (1873) 148-.

- Elliot, $Z$. Bll. Sc. Mth. As. 3 (1879) 238-

- Humbert, G. Par. Éc. Pol. J. 55 (1885) 127-.

-. Neuberg, J. As. Fr. C. R. (1885) (Pt. 2) 79-

-. Johnson, A. R. Mess, Mth. 16 (1887) 33-. Bortniker, (Mlle.) -. C. R. 106 (1888) 824 .

-, focal quadries. Hart, H. Mess. Mth. 14 (1885) 1-.

-, generation. Saltel, L. Par. S. Mth. Bll. 3 (1875) 95-, 99-.

-, plane sections. Laquière, E. M. As. Fr. C. R. (1880) 121-.

- and sphero-quartics. Casey,J. Phil. Trans. 161 (1871) 585-.

Dupin's cyclides. Enneper, A. Schlömilch Z. 7 (1862) 198-.

- - Neuberg, J. Liège S. Sc. Mm. 10 (1883) (No. 9) $10 \mathrm{pp}$.

- - Cosserat, E. Toul. Fac. Sc. A. 6 (1892) F, 7 pp.

- - Pellet, A. As. Fr. C. R. (1899) (Pt. 1) 138.

- - and Dupuis's theorem. Marchand, $E$. N. A. Mth. 9 (1890) 98-, 182-.

- - nodes in. Snyder, V. [1896] A. Mth. 11 (1896-97) 137-。

- - principal poles of inversion. Fouret, $G$. N. A. Mth. 7 (1888) 113-.

- - , systems of circles applied to. Holst, $E$. Christiania F. (1885) No. 15, 4 pp.; Fschr. Mth. (1885) 574-.

- - and theory of curvature, elementary account. Holzmuller, G. Z. Mth. Ps. 44 (1899) 194-.

"Girocyclides" of $4 \mathrm{th}$ degree. Jamet, $V . \mathrm{N}$. A. Mth. 20 (1881) 344-, 385-, 434-.

Properties. Laguerre, E. (x) Par. S. Phlm. Bll. 5 (1868) 17-.

Sphero-cyclides. Jeffery, H. M. [1884] L. Mth. S. P. 16 (1884-85) 109-.

Surfaces with infinite number of inversions. Hadamard, J. Bll. Sc. Mth. 12 (1888) 118-.

-, 4th degree. Moutard, T. N. A. Mth. 3 (1864) 536-.
Annular surfaces, case. Rossi, V. A. (xII) Nap. Ac. Pont. At. 5 (1853) 53-.

- with directrix cone. Rossi, V.A. [1849] (xir) Nap. Ac. Pont. At. 6 (1854) 53-.

- - enveloping ruled surfaces, etc. Rossi, V. A. Nap. Ms. 4 (1844) 19-.

- - 1st and 2nd classes. Rossi, V.A. Nap. Ms. 5 (1845) 56-, 322-; 6 (1845) 248-; 8 (1846) 174.

Apsidal surfaces. Cayley, A. QJ. Mth. 16 (1879) 109 -

- - Mannheim, A. C. R. 122 (1896) 1396-. Asymptotic lines, surfaces with skew cubics for. Blutel, 一. C. R. 117 (1893) 722.

- - - - - - Bioche, C. C. R. 125 (1897) 15-; Par. S. Mth. Bll. 26 (1898) 217-. -, - - - - 3rd degree. Bioche, $\boldsymbol{C}$. Par. S. Mth. Bll. 27 (1899) 96-.

Cardioido-hyperboloid surface. Vaněček, J.S. Prag České Ak. Fr. Jos. Rz. (Tř́ida 2) 4 (1895) Art. 30, 5 pp.

Cartesian ovals, analogues in space. Sylvester, J. J. Ph. Mg. 31 (1866) 287-, 380-.

Cassini's ovals, surface analogues. Roberts, $W$. (vIII) A. Mt. 4 (1861) 133-.

Centro-surface of ellipsoid. Cayley, A. [1870] Camb. Ph. S. T. 12 (1879) 319-.

- - Stahl, W. Crelle J. Mth. 101 (1887) 73-.

-, Rudio's inverse. Cayley, -. QJ. Mth. 22 (1889) 156-.

Clebsch's diagonal surface. Ciani, $E$. Rm. R. Ac. Linc. Rd. 7 (1891) (Sem. 1) 227-.

- - Bioche, C. N. A. Mth. 17 (1898) 111-.

Cono-cunei. Pabst, C. Arch. Mth. Ps. 2 (1885) 281-, 337-.

Cono-cuneus, Wallis's, plane sections. Rossi, V. A. [1835] Il Progresso 17 (1837) 74-.

Conoidal screw propellers, system of variants. Moshammer, $K$. Wien Sb. 51 (1865) (Ab. 2) 49-.

- surface with conic as curvilinear directrix. Bruno, G. [1866] Tor. Mm. Ac. Sc. 24 (1868) 327-.

- - - two straight lines as directrices. Bruno, G. [1866] Tor. Mm. Ac. Sc. 24 (1868) 317-.

- surfaces, section by plane. Fodor, L. Orv.Termt. Ets. (Termt. Szak) (1886) 113-.

Conoids circumscribing plane curve. Schiappa Monteiro, A. Lisb. J. Sc. Mth. 12 (1888) 19-.

-, and especially Plücker's. Mannheim, A. C. R. 106 (1888) 820-, 1038 .

-, plane sections. Poselger, F. T. Berl. Ab. (1825) 97-.

-, Plücker's. Picquet, H. Par. S. Mth. Bll. 14 (1886) 68-.

_, - new use. Mannheim, A. C. R. 119 (1894) 394-.

-, transformation. Michel, $F$. As. Fr. C. R. (1893) (Pt. 2) 184-.

-, - - - Mannheim, (le col.) A. As. Fr. C. R. (1894) (Pt. 2) 207.

-, shadow cast on themselves. Cahen, C. P. Par. S. Mth. Bll. 4 (1876) 88-. 
Conoids, 3rd and 4th degrees, certain. Seipka, E. Mh. Mth. Ps. 9 (1898) 1-.

Cylindrical surfaces, application of determinants. Dostor, G. Arch. Mth. Ps. 58 (1876) 293-.

Cylindroid. Lewis, T. C. Mess. Mth. 9 (1880) 1 -.

-, properties. Goebel, J. B. Z. Mth. Ps. 25 (1880) 281-.

-, 一. Ball, R. S. [1885] Ir. Ac. P. 4 (188488) 518-.

-, property, characteristic. Appell, P. Par. S. Mth. Bll. 28 (1900) 261-.

-, Wallis's. Flauti, V. [1828] Nap. At. Ac. 4 (1839) 1-.

,-- , problem, solution. Sangro, $G$. Nap. At. Ac. 1 (1819) 83-.

Desmian surface of 4 th degree. Humbert, $G$. Liouv. J. Mth. 7 (1891) 353-.

Developables arising from 2 quadrics. Cayley, A. Camb. and Dubl. Mth. J. 5 (1850) 46-.

- circumscribing 2 quadrics. Chasles, $M$. C. R. 54 (1862) 715-.

- - - Painvin, L. C. R. 67 (1868) 816-; (xII) Lille S. Mm. 9 (1871) 97-; 11 (1873) 81-; 13 (1874) 329-.

111-.

- - quadrics and rotation surfaces, construetion. Sobotka, J. Prag Sb. (1893) (Mth.Nt.) No. 2, $16 \mathrm{pp}$.

- depending on elliptic functions. Cayley, A. QJ. Mth. 14 (1877) 235-.

- derived from quartic, nodal curve. Cayley, A. Ph. Mg. 27 (1864) 437-

- - - quintic curve. Cayley, A. Camb. and Dubl. Mth. J. 5 (1850) 152-.

- Prohessians, etc., and note- on sextic. Cayley, A. (I) QJ. Mth. 6 (1864) 108-; (vII) 7 (1866) 105-.

-, singularities. Cayley, A. Liouv. J. Mth. 10 (1845) 245-; Camb. and Dubl. Mth. J. 5 (1850) 18-; QJ. Mth. 7 (1866) 110-.

-, 4th class, quadries inscribed to. Cremona, L. Tortolini A. 2 (1859) 65-.

-, - degree and 3rd class, conics inseribed to. Cremona, $L$. Tortolini A. 2 (1859) 201-.

-, 5th degree. Cremona, L. C. R. 54 (1862) 604-.

-

-, 6th degree, derived from quartic. Cayley, A. QJ. Mth. 9 (1868) 129-; A. Mt. 2 (186869) 99-, 219-.

,,-----1 , transformation. Cayley, A. QJ. Mth. 9 (1868) 373-.

-,$-\ldots$, with edge quadri-quadric curve. Cayley, A. QJ. Mth. 14 (1877) 229-.

,--- , edge of regression. Sucharda, $A$. Prag Sb. (1891) (Mth.-Nt.) 369-.

- - - - with excubo-quartic curve. Cayley, A. [1869] (Ix) Camb. Ph. S. T. 11 (1871) $507-$.

Ellipse revolving about straight line in its plane, problem. Böklen, $O$. Grunert Arch. 36 (1861) 22-.

Ellipsoid, negative pedal surface. Tortolini, $B$. Palomba Rac. 2 (1846) 140-.
Ellipsoid, negative pedal surface. Cayley, $A$. R. S. P. 9 (1857-59) 171-.

-, - - - Craig, T. Am. J. Mth. 4 (1881) 358-; 5 (1882) 76-.

Envelope of quadric revolving about straight line. La Gournerie, J. de. Liouv. J. Mth. 10 (1865) 33-.

- - sphere with centre on conic. Cayley, A. [1864] Ph. Mg. 29 (1865) 19-.

Equatorial surfaces, Plücker's models. Cayley, A. L. Mth. S. P. 3 (1869-71) 281-.

Flat ("aplatie") quartic. Cayley, A. C. R. 74 (1872) 1393-.

Glaisher's surface, construction of normal. Mannheim, A. QJ. Mth. 17 (1881) 147-.

Irrational quartic surfaces of genus zero. Franchis, M. de. Palermo Cir. Mt. Rd. 14 (1900) 33-.

Isogonal surfaces. Heffter, L. Crelle J. Mth. 115 (1895) 1-.

\section{Kummer's surface.}

(See also 8060 and 8080.$)$

Kummer, E. E. Berl. Mb. (1864) 246-, 495-.

Cayley, A. Crelle J. 73 (1871) 292-; 84 (1878) 238-; 94 (1883) 270-.

Reye, $T$. Crelle J. Mth. 86 (1879) 84-.

Brioschi, F. C. R. 92 (1881) 944-

Humbert, G. Liouv. J. Mth. 10 (1894) 473-.

Associated line complexes, etc. Klein, F. Gött. Nr. (1871) 44-.

Birational transformations. Hutchinson, J.I. [1900] N. Y. Am. Mth. S. Bll. 7 (1901) 211-.

Configurations, in- and circum-scribed. Klein, F. Mth. A. 27 (1886) 106-.

Contact sextics. Pascal, E. A. Mt. 19 (189192) $159-$.

Curves of principal tangents. Klein, $F ., \&$ Lie, S. Berl. Mb. (1870) 891-. (1885) 301-.

Elliptic surfaces. Humbert, G. Am. J. Mth. 16 (1894) 221-.

Equation, new form. Cayley, A. Camb. Ph. S. P. 4 (1883) 321.

-, rational. Pascal, E. A. Mt. 18 (1890) $227-$.

-, 16th degree, connected with Kummer's surface. Jordan, C. Crelle J. 70 (1869) 182-.

Forms, various. Rohn, K. Mth. A. 18 (1881) 99-.

Generation. Humbert, G. Palermo Cir. Mt. Rd. 11 (1897) 1-.

Models. Kummer, E. E. Berl. Mb. (1866) 216-.

Particular case. Segre, C. Leip. Mth. Ps. B 36 (1884) 132-.

- cases. Rohn, $K$. Leip. Mth. Ps. B. 36 (1884) 10-.

Projective generation of plane quartic curve with node, relation to surface. Wirtinger, $W$. D. Mth. Vr. Jbr. 4 (1897) 97-.

Properties, some. Paolis, - de. Rm. R. Ac. Linc. Rd. 6 (1890) (Sem. 2) 3-. 
Singular points and planes. Caporali, $E$. Rm. R. Ac. Linc. Mm. 2 (1878) 791-.

Skew hexagon and Kummer's surface. Geiser, C. F. Zür. Vjschr. 41 (1896) (Festschr., Th. 2) 24 .

16 straight lines of surface connected with Kummer's lines. Sucharda, A. Časopis 15 (1886) 149-; Fschr. Mth. (1886) 769-.

Surface with 9 portions, note. Klein, $C . F$. Mth. A. 18 (1881) 160.

$-S$ of 6 th degree connected with Kummer's surface. Humbert, G. C. R. 120 (1895) 365-, 425-.

- - - - - relation to Kummer's surface and curves of 4 th class. Humbert, G. C. R. 120 (1895) 863-.

Systems through lemniscate. Schjerning, $W$. Arch. Mth. Ps. 7 (1889) 113-.

Tetrahedroid, equation. Hofmann, $F$. Crelle J. Mth. 98 (1885) 264.

-, note. Hutchinson, J. I. N. Y. Am. Mth. S. Bll. 4 (1898) 327-.

- as particular case. Cayley, A. Crelle J. 65 (1866) 285-; 87 (1879) 161-.

Theta functions with 2 variables. Cayley, $A$. Crelle J. Mth. 83 (1877) 210-, 220-.

$--\ldots \ldots$ Weber, H. Crelle J. Mth. 84 (1878) 332-. $-\frac{-1}{685-, 1493-}-$. $\quad$ Darboux, G. C. R. 92 (1881)

_ _ - _ _ - Göpel's biquadratic relation between 4 , representation of surface by. Borchardt, C. W. Crelle J. Mth. 83 (1877) 234-.

Locus of centres of quadrics through 7 points of surface of $4 \mathrm{th}$ degree. Taylor, $H . M$. Mess. Mth. 13 (1884) 145-.

Frost, $P$. Mess. Mth. 14 (1885) 97-

_ - point, product of whose distances from 3 coplanar lines is constant. Czuber, E. Mh. Mth. Ps. 3 (1892) 217-.

Nodal points, biplanar and uniplanar, theory. Rohn, K. Mth. A. 22 (1883) 124-.

Normal developable of quadric cone. Jarolimek, $\check{C}$. Casopis 8 ( $\left.{ }^{*} 1879\right)$ 247-; Fschr. Mth. (*1879) 574-。

- surfaces, certain. Sucharda, A. Prag Sb. (1889) 2 (Mth.-Nt.) 385-.

One-sided surface ("marrowbone"). Schoute, P. H. Edinb. R. S. P. 19 (1893) 208-.

- - surfaces, class. Feldblum, M. Wiad. Mt. 1 (1897) 101-; Fschr. Mth. (1897) 579.

Parallel projection, and surfaces. Matzka, $W$. [1874] Prag Ab. 7 (1875) (Mth.) 70 pp.

- surface of ellipsoid. Cayley, A. Tortolini A. 3 (1860) 345-

- - - Fiedler, W. Grunert Arch. 39 (1862) 19-. (1882) 251 .

Craig, T. Crelle J. Mth. 93

- surfaces. Hoppe, R. Arch. Mth. Ps. 1 (1884) 280 -.

- - of conicoids and conics. Roberts, $S$. [1872] L. Mth. S. P. 4 (1871-73) 57-.
Parallel surfaces, cylindrical. Dienger, $J$. Grunert Arch. 10 (1847) 54-.

- - , and lines on surface. Bordoni, A. Mil. G. I. Lomb. 5 (1853) 70-.

Polar surfaces of certain ring-surfaces. Sucharda, A. Časopis 13 (1884) 1-, 161-; Fschr. Mth. (1884) 708-.

Quadricuspidal surfaces. La Gournerie, J. de. Liouv. J. Mth. 15 (1870) 264-.

Rational surfaces, case. Brambilla, A. Palermo Cir. Mt. Rd. 2 (1888) 176-.

- - connected with theory of complex variable. Pincherle, S. Mil. I. Lomb. Rd. 24 (1891) 354-.

- - , ruled, 4th degree, general properties. Ameseder, A. [1879] Arch. Mth. Ps. 65 (1880) 239-.

_-, skew. Genty, -. Par. S. Mth. Bll. 19 (1891) 70-.

- -, 4th degree. Noether, M. Mth. A. 33 (1889) 546-.

- _, 9th degree, double curve of special case. Brambilla, A. G. Mt. 32 (1894) 133-.

Reciprocal to surface of centres of central quadric. Roberts, S. QJ. Mth. 13 (1875) 188 -

- surfaces of same degree as their polars, and with same singularities. Kummer, E. E. Berl. Ak. Mb. (1878) 25-.

Reciprocity in central surfaces of $4 \mathrm{th}$ degree, case. Sucharda, A. Wien Ak. Sb. 101 (1892) (Ab. 2a) 585-.

Rotation surface with algebraic geodesics. Tannery, J. Par. S. Phlm. Bll. 4 (1892) 85-.

- - serving as type of pseudo-spherical surfaces. Beltrami, $E$. G. Mt. 10 (1872) 147-.

- surfaces. Mann, F. Grunert Arch. 29 (1857) 446-.

- - generated by conics. La Gournerie, J. de. Par. Éc. Pol. J. $40^{\circ} \mathrm{cah}$. (1863) 1-; Liouv. J. Mth. 8 (1863) 52-.

- _ - - - plane sections. Benedict, - . Silliman J. 31 (1837) 258-.

- - with generator known graphically, elements. André, L. Rv. Artl. 5 (1875) 546 -

- - - lemniscate for meridian. Levi, $D$. G. Mt. 21 (1883) 379-.

Ruled surfaces, general theorem. Darboux, $G$. Bll. Sc. Mth. As. 2 (1871) 301-.

- - generated by straight line resting on 3 directrix curves. Salmon, G. Camb. and Dubl. Mth. J. 8 (1853) 45-.

-- ,genus $p=0$. Clebsch, R. F.A. [1871] Mth. A. 5 (1872) 1-.

_-, new (La Gournerie). Chasles, M. C. R. 63 (1866) 254-.

- - 3rd degree. Bioche, C. Par. S. Phlm. Bll. 6 (1894) (C. R., No. 13) 1-.

- _ _ - case. Moshammer, $K$. Wien Ak. Sb. 73 (1876) (Ab. 2) 143-.

_ - _ -, model. La Gournerie, J. de. Par. A. Cons. 6 (1866) 205-.

- - - - and 4 th degrees, obtained from circle. Godefroy, A. N. N. Arch. Wisk. 20 (1893) 108-. 
Ruled surfaces, 4th degree, case. T'otonssy, $B$. [1882] (xII) Mth. Term. Éts. 1 (1883) 12-; Mth. Nt. B. Ung. 1 (1882-83) 25-.

$-\ldots, \ldots-$, generated by 2 projective sheaves of planes of 2nd degree. Holgate, T. F. Am. J. Mth. 15 (1893) 344-; 22 (1900) 27-.

- - , - - , with generators forming groups of "quadruples." Ameseder, $A$. Wien Ak. Sb. 81 (1880) (Ab. 2) 615-.

- -, - - - nodal conic. Ameseder, $A$. Wien Ak. Sb. 81 (1880) (Ab. 2) 271-.

,,-----2 nodal lines. Ameseder, $A$. Arch. Mth. Ps. 65 (1880) 73-.

,-- 6 th degree, classification. Bergstedt, $J$. Fschr. Mth. (1886) 775 .

- - , 8th degree, with generators all touching 4 quadric cones, case. La Gournerie, J.A. R. M. de. (x) Par. S. Phlm. Bll. 2 (1865) 124-.

- - , - - - 4 nodal conics, case. La Gournerie, J. de. C. R. 60 (1865) 1178-.

-,$- \ldots-, \ldots 5$ nodal quartics, case. La Gournerie, J. de. C. R. 61 (1865) 116-.

Self-reciprocal surface. Cayley, $A$. Berl. Ak. Mb. (1878) 309-.

- - generation. Cayley, A. [1868] L. Mth. S. P. 2 (1869) 61-.

Skew surfaces, with director plane. Catalan, E. C. Par. Éc. Pol. J. $29^{\circ}$ cah. (1843) 121-.

- -, level lines of which are conchoid. Catalan, E. C. Liége S. Sc. Mm. 15 (1888) No. 1, 17-.

- - with 2 rectilinear directrices and director conic. Drasch, H. Wien Ak. Sb. 101 (1892) (Ab. 2a) 171-.

- -, 3rd degree. Cremona, L. Mil. At. I. Lomb. 2 (1860) 291-; Crelle J. 60 (1862) 313-.

- - - - Cayley, A. Ph. Mg. 24 (1862) $514-$

,--- - (Cayley). Steen, A. Mth. Ts. 5 (1863) 86-; Ph. Mg. 25 (1863) 546-.

$-\frac{1}{-}-$ - Nicodemi, R. G. Mt. 21 (1883) 270 -

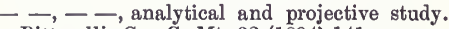
Pittarelli, G. G. Mt. 32 (1894) 141-.

,---- , delineation. Cayley, A. Ph. Mg. 25 (1863) 528-

- -, - - , first kind, geometry. Ferry, F. C. Arch. Mth. Ntvd. 21 (1899) No. 3, $60 \mathrm{pp}$.

- - - - , polar surfaces. Hochheim, A. Z. Mth. Ps. 23 (1878) 308-, 345-; 24 (1879) 18-.

- -, 4th degree. Cremona, L. Bologna Ac. Sc. Mm. 8 (1868) 235-.

- - - - - Cardinaal, J. Amst. Ak. Vs. M. 5 (1889) 447-; Fschr. Mth. (1888) 648.

- - - - , case. Nicodemi, $R$. (xII) Nap. Ac. Pont. At. 15 (1883) (Pt. 2) 179-

,---- , generated by two plane movements. Blake, E. M. N. Y. Am. Mth. S. T. 1 (1900) 421-.

- - - - , with 3 nodal lines. Segen, $D$. Crelle J. Mth. 112 (1893) 39-.

,$-- z=A y^{2} / x^{2}$. Hochheim, A. Arch. Mth. Ps. 53 (1871) 350-

- - $z=M y^{2} x$. Hochheim, A. Arch. Mth. Ps. 55 (1873) 38-.

\section{Steiner's surface.}

Kummer, E. E. Berl. Mb. (1863) 324-.

Weierstras8, C. Berl. Mb. (1863) 337-.

Schröter, H. E. Berl. Mb. (1863) 520-.

Cayley, A. [1864] Crelle J. 64 (1865) 172-.

Moutard, T. (x) Par. S. Phlm. Bll. 2 (1865) 66-.

Clebsch, R. F. A. [1866] Crelle J. 67 (1867) 1-.

Sturm, R. [1869] Mth. A. 3 (1871) 76-.

Eckardt, F. E. [1871] Mth. A. 5 (1872) 30-.

Laguerre, E. (x) Par. S. Phlm. Bll. 8 (1872) 75.

Cayley, A. [1873] (Ix) L. Mth. S. P. 5 (1874) 14-.

Amigues, É. C. R. 86 (1878) 38-.

Voss, A. Mth. A. 27 (1886) 357-.

Kïpper, K. Prag Áb. 2 (1888) No. 10, $11 \mathrm{pp}$.

Vahlen, K. T. Acta Mth. 19 (1895) 199-.

Schoute, P. H. Amst. Ak. Vs. 4 (1896) 224-; Fschr. Mth. (1896) 531.

Brambilla, A. Nap. Rd. 37 (1898) 19-.

Lacour, E. N. A. Mth. 17 (1898) 437-.

Montesano, $D$. Nap. Rd. 38 (1899) 88 -

Asymptotic lines. Darboux, $G$. (Ix) Par. S. Phlm. Bll. 10 (1873) 37.

Coordinates of point of, in terms of two parameters. Lacour, E. N. A. Mth. 17 (1898) 499-.

Curves on. Schorte, -. Amst. Ak. Vs. 4 (1896) 272-; Fschr. Mth. (1896) 531.

Locus of poles of fixed plane with reference to conics on, theorem. Lie, M. S. Arch. Mth. Ntvd. 3 (1878) 84-.

[1887] Par. S. Mth. Bll. 16 (1888) 15-.

Nodal cones of quadrinodal cubies, and zomal conics of tetrazomal quartics. Townsend, $R$. [1869] QJ. Mth. 10 (1870) 264-.

Plane sections, and three intersecting conics. Koenigs, G. Bll. Sc. Mth. 12 (1888) 28 -

Property, extension. Brambilla, A. Nap. Rd. 37 (1898) 300-.

Surface, 3rd degree, reciprocal of Steiner's surface. Laguerre, -. N. A. Mth. 11 (1872) 319-, 337-, 418-; 12 (1873) 55-.

Surfaces of Veronese and Steiner. Rosati, $C$. Tor. Ac. Sc. At. 35 (1900) 12-.

Theorem of Eckardt, extension. Brambilla, A. Nap. Rd. 38 (1899) 144-.

Surface of equal slope circumscribing conic. Cremona, L. N. A. Mth. 4 (1865) 271-.

- generated by apparent directions of star owing to aberration. Wittstein, A. Arch. Mth. Ps. 69 (1883) 195-.

- - circular are through ellipse and two straight lines. Camuri, A. (Ix) Mod. Ac. Sc. Mm. 10 (1869) 3-.

Surfaces of constant power with respect to points in space. Ruffini, $F$. P. Bologna Ac. Sc. Mm. 1 (1890) 235-.

- containing families of conics. Weyr, E. Mh. Mth. Ps. 2 (1891) 351-. 
Surfaces containing infinite number of conics, tangent planes of. Machovec, $F$. Prag České Ak. Fr. Jos. Rz. (Tř́ida 2) 2 (1893) Art. 10, 1-. - with continuous transitive group of transformations. Fano, G. Palermo Cir. Mt. Rd. 10 (1896) 1-.

- derived from given surface by composition of two projections, equations and properties. Bottomley, J. (Ix) Manch. Lt. Ph. S. Mm. 8 (1884) 218-.

- - quadrics. Andries, C. Brux. A. Un. (1845) 247-.

- - - case. Malet, J. C. [1878] Ir. Ac. T. 26 (1879) 456-.

- doubly generated by circles, and surfaces doubly enveloped by quadrics. Koenigs, $G$. C. R. 109 (1889) 364-

- with finite number of straight lines, especially of 4 th and 5 th degrees. Sturm, $R$. Mth. A. 4 (1871) 249-.

- - hyperelliptic canonical curves. Enriques, F. Rm. R. Ac. Linc. Rd. 5 (1896) (Sem. 1) 191-.

- inscribed in scalene cone, special cases. Wafelaer, P. F. Louvain A. Ac. 5 (1823) 45 pp.

- whose plane sections are all unicursal. Picard, É. Par. S. Phlm. Bll. 2 (1878) 127-; Crelle J. Mth. 100 (1887) 71-.

- - - - - (Picard). Guccia, G. B. Palermo Cir. Mt. Rd. 1 (1887) 165-.

- - sections are curves of genus three. Castelnuovo, G. Tor. Ac. Sc. At. 25 (1890) 695-.

_ - perpendicular to one axis are similar ellipses. Niemtschik, $R$. Wien Sb. 57 (1868) $(A b .2)$ 246-.

-, unicursal, transformation. Cayley, A. [1870] Mth. A. 3 (1871) 469-.

-, 6th class, two special. Kantor, $S$. Wien Ak. Sb. 79 (1879) (Ab. 2) 768-.

\section{Surfaces, 3rd degree.}

with axis of ternary symmetry, and surfaces with indicatrices of 3rd degree. Dumont, Par. S. Mth. Bll. 28 (1900) 117-.

case considered by Bertrand. Anon. (vr 885) N. A. Mth. 13 (1854) 413-.

with conical point, equations. Richmond, H.W. QJ. Mth. 23 (1889) 170-

equation, canonical form for. Dumont, N. A. Mth. 17 (1898) 503-.

Hessian of which breaks up. Ciani, E. Rm. R. Ac. Linc. Rd. 6 (1890) (Sem. 2) 55-. with infinite number of umbilici. SaintGermain, A. de. C. R. 101 (1885) 1246-.

- nodal points. Kohn, G. Wien Ak. Sb. 96 (1888) $(A b .2)$ 1298-

- 4 nodal points. Eckardt, F. E. [1871] Mth. A. 5 (1872) 30-.

_ 82-; Fschr. Mth. (1887) 645-

$-{ }_{-}-$, case. Thieme, H. Z. Mth. Ps. 40 (1895) 362-

self-polar with reference to quadric. Dumont, F. Par. S. Mth. Bll. 25 (1897) 74-.

3 straight lines on which meet in point. Eckardt, F.E. [1875] Mth. A. 10 (1876) 227-.
Surfaces, 3 rd and 4 th degrees.

with nodal conic. Kupper, - . Z. Mth. Ps. 34 (1889) 129-

related to tetrahedron. Jettmar, H. von. Arch. Mth. Ps. 10 (1891) 398-.

\section{Surfaces, 4th degree.}

case. Vaněček, J. S. Prag Sb. (1883) (Mth. Vortr.) 231-.

class generated by collineations. Schur, $F$. Mth. A. 20 (1882) 254-

connected with 2 tetrahedra. Bauer, $G$. Münch. Ak. Sb. 18 (1889) 337-.

with cuspidal conics. [Tötössy, B.] Béla, $T$. [1881] Mth. A. 19 (1882) 291-.

- - - Segre, C. Mth. A. 24 (1884) 313giving value of $\sqrt{x^{2}+y^{2}}$ approximately, case. Poncelet, J. V. N. A. Mth. 7 (1848) 39-.

with integral of total differential of lst kind. Berry, A. C. R. 129 (1899) 449-.

- 2 intersecting double pairs, construction. Korndörfer, G. Mth. A. 3 (1871) 496-.

- nodal conic. Clebsch, R. F.A. Berl. Mb. (1868) 284-; Crelle J. 69 (1868) 142-.

- - - Geiser, C. F. Crelle J. 70 (1869) 249(1871) 140-, 159-.

- Z. Zeuthen, H. G. [1879] A. Mt. 14 (*1886-87) 31-.

- - Berzolari, L. A. Mt. 13 (1885) 81-.

- Bobek, $K$. Wien Ak. Sb. 90 (1885) (Ab. 2) 923-, 1168-

- Del Re, A. Rm. R. Ac. Linc. Rd. 2 (1893) (Sem. 1) 211-.

- - - consisting of two indefinitely near straight lines, construction. Kornd̈̈rfer, $G$. Mth. A. 4 (1871) 117-.

- - - construction from quadric systems. Cardinaal, J. Amst. Ak. Vs. M. 8 (1891) 88-; Fschr. Mth. (1891) 847-.

- _ - and nodes, representation on plane. Korndörfer, G. [1868] Mth. A. 1 (1869) 592-; 2 (1870) 41-.

- - -, properties. Juel, C. S. (хп) Ts. Mth. 4 (1880) 81-, 113-

- - special case. Rudio, $F$. Crelle J. Mth. 104 (1889) 85-.

- - , and 16 straight lines. Kilpper, $K$. Prag Ab. 2 (1888) No. 3, 36 pp.

- - - - - - construction. Del Re, A. Mod. S. Nt. At. 12 (1893) 234-.

- - or cuspidal conic. Weiler, A. Z. Mth. Ps. 30 (1885) 170-

- - line consisting of circle at infinity. Loria, G. Tor. Ac. Sc. Mm. 36 (1885) 199-. - lines, generation from quadric surfaces. Cardinaal, J. Amst. Ak. Vh. (Sect. 1) 1 (1893) No. 6, 63 pp.; Fschr. Mth. (1892) 765-.

- 2 nodal lines. Fontené, G. N. A. Mth. 19 (1900) 400-.

_ node. Vries, J. de. N. Arch. Wisk. 3 (1898) 158-; Fschr. Mth. (1897) 532.

- nodes. Rohn, $K$. Leip. Jablon. Preisschr. 26 (1886) $58 \mathrm{pp}$.

- - construction. Jonquières, - de. C. R. 107 (1888) 430-.

VOL. I. 
with 4 nodes, case. Cayley, A. B. A. Rp. (1886) 540-.

- 12 nodes. Cayley, A. QJ. Mth. 14 (1877) 103-.

- 14, 15, and 16 nodes. Jessop, C. $M$. QJ. Mth. 31 (1900) 354-.

obtained by projection, case. Pannelli, $M$. G. Mt. 29 (1891) 133-.

passing through 8 points, generation by homographic ruled quadrics. Poudra, -. N. A. Mth. 17 (1858) 158-.

with properties similar to quadrics. $\dot{Z}$ murko, W. Krk. Ak. (Mt.-Prz.) Pam. 14 (1888) 208-; Fschr. Mth. (1888) 827.

special case. Sucharda, A. Časopis 24 (1895) 286-; Fschr. Mth. (1895) 627.

surface cut along 2 conics by each of its tangent planes. Cremona, L. Crelle J. 63 (1864) 315-.

- and 2 quadrics, giving points whence 2 conics project into circles. Schiappa Monteiro, A. Lisb. J. Sc. Mth. 5 (1898) 221-.

with triple point. Rohn, K. Mth. A. 24 (1884) 55-.

\section{Surfaces, 5th degree.}

with nodal cubic and triple point. $\operatorname{Del} R e, A$. Rm. R. Ac. Linc. Rd. 1 (1892) (Sem. 2) 170-, 203-, 343-, 378-, [464].

- - - 5 triple points. Del Re,A. Rm. R. Ac. Linc. Rd. I (1893) (Sem. 2) 99-, 138-; 3 (1894) (Sem. 2) 11-.

- quartic. Murer, V. Ven. I. At. (188687) 1223-.

- - quintic. Caporali, E. [1875] A. Mt. 7 (1876) 149-.

- - Del Re, A. Rm. R. Ac. Linc. Rd. 6 (1890) (Sem. 2) 221-.

- _ - extension of configuration of 10 straight lines. Pascal, E. Rm. R. Ac. Linc. Rd. 2 (1893) (Sem. 1) 65-.

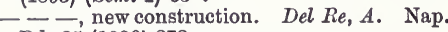
Rd. 25 (1886) 272-.

- - - projective generation. Del Re, A. Tor. Ac. Sc. At. 28 (1893) 420-.

- simple and nodal lines and one triple line. Del Re, A. Rm. R. Ac. Linc. Rd. 7 (1891) (Sem. 2) 11-, 111-.

- 2 or 3 triple points. Del Re,A. Mod. Ac. Sc. Mm. 9 (1893) 331-.

\section{Surfaces, 6th degree.}

with 9 nodal lines, case. Del Pezzo, P. Nap. Rd. 36 (1897) 196-.

- 6 straight lines. Bordiga, G. C. R. 102 (1886) 743-.

- triple cubic curve. Weyr, E.J. Wien Ak. $\mathrm{Sb} .85$ (1882) ( $A b .2)$ 513-.

triple tangents of certain. Pieri, M. Tor. Ac. Sc. At. 24 (1889) 514-.

\section{Surfaces, 7 th degree.}

three. Hill, J. E. [1896-97] N. Y. Am. Mth. S. Bll. 3 (1897) 2, 5-; Am. J. Mth. 19 (1897) 289-.
Surfaces, 8th degree.

case. Cayley, A. Mth. A. 4 (1871) 558-.

- Battaglini, $G$. Rm. R. Ac. Linc. At. 2 (1875) 244-.

cases. Cayley, A. QJ. Mth. 14 (1877) 249-.

family. Brambilla, A. G. Mt. 35 (1897) 1-.

remarkable case. Picquet, $H$. $[1875]$ Par. S. Mth. Bll. 4 (1876) 45-.

\section{Surfaces, nth degree.}

passing through given cubic curve. Cayley, $A$. Mess. Mth. 23 (1894) 79-.

with $(n-1)$-ple point, homaloidal system formed by. Paolis, R.de. G.Mt. 13 (1875) 226-, 282-. - $(n-2)$-fold straight line, generation. Murer,

V. Palermo Cir. Mt. Rd. 2 (1888) 107-.

$\pm \frac{x^{n}}{a^{n}} \pm \frac{y^{n}}{b^{n}} \pm \frac{z^{n}}{c^{n}}=1$. Steen, A. Mth. Ts. $2(1860)$ 101-.

\section{Surfaces, $(n+2)$ th degree.}

with double curve of degree $1+n(n-1) / 2$. Lo Piano, D. Nap. Rd. 39 (1900) 130-.

Symmetrical surfaces. Goursat, É. Par. Éc. Norm. A. 4 (1887) 159-, 241-, 317-.

- - Lecornu, L. Acta Mth. 10 (1887) 201-. - Ciani, E. Rm. R. Ac. Linc. Rd. 6 (1890) (Sem. 1) 399-.

-. Mangeot, S. C. R. 112 (1891) 1497-; Par. S. Mth. Bll. 20 (1892) 84-.

- -. Dumont, -. N. A. Mth. 16 (1897) 463 - _, 3rd degree, connected with quadric. Mangeot, S. N. A. Mth. 10 (1891) 235-.

Systems of conics and quadrics, locus of centres, etc. Geiser, C.F. Zür. Vjschr. 10 (1865) 219-.

Tangents common to two confocal surfaces. Chasles, M. Liouv. J. Mth. 11 (1846) 105-. Tetrahedral surfaces, umbilici. Lebon, $E$. Palermo Cir. Mt. Rd. 4 (1890) 115-.

- symmetrical surfaces. Hunyady, E. von. Z. Mth. Ps. 11 (1866) 356-.

- La Gournerie, J. de. $\quad$ C. R. 62 (1866) 78-.

- - (La Gournerie). Cayley, A. [1867] Camb. Ph. S. T. 11 (pt. 2) (1869) 277-.

- - - (Cayley). Sucharda, A. Wien Ak. Sb. 99 (1891) (Ab. 2a) 549-.

_ _ - 4th degree, with 48 straight lines, case. Schur, F. Liège S. Sc. Mm. 11 (1885) No. $12,7 \mathrm{pp}$.

Tore or anchor-ring.

Cayley, A. QJ. Mth. 13 (1875) 127-.

Vries, J. de. N. Arch. Wisk. 3 (1898) 72-.

and bitangent sphere. Monniot, - N. A. Mth. 13 (1874) 383-.

curve of intersection with sphere, and new property. Fuortes, T. G. Mt. 12 (1874) 146-. cylinder circumscribing rotation tore. Dunesme, M. C. R. 45 (1857) 527-.

intersection with quadric. Mangeot, S. N. A. Mth. 11 (1892) 519-.

inversion. Taylor, $\dot{H}$. M. L. Mth. S. P. 5 (1873-74) 105-. 
problem: to draw from given point lines doubly tangent to tore. Janin, - $\&$ Mannheim, A. (x) Par. S. Phlm. Bll. 2 (1865) 54-.

properties. Woodward, C. M. Am. As. P. (1886) 71-.

section by plane through centre and tangent at opposite sides. Hyde, $E$. $W$. Des Moines Anal. 3 (1876) 78-.

- - tangent plane through centre. Anon. (vi 896) N. A. Mth. 18 (1859) 258-.

sections. Garlin, -- N. A. Mth. 13 (1854) 415-; 18 (1859) 319-.

-. Cornu, A. N. A. Mth. 20 (1861) 101-.

-. Darboux, G. N. A. Mth. 3 (1864) 156-.

-, bicircular, theorem. Yvon-Villarceau, A. J. F. N. A. Mth. 7 (1848) 345-

-, - Yvon-Villarceau's theorem. Allocati, $A$. G. Mt. 1 (1863) 281-.

-,,-- - Barbier, E. Les Mondes 3 (1863) 675-, 703-.

- 1 (1878) - 84-.

- by bitangent plane. Godart, (Prof.) A. N. A. Mth. 3 (1864) 350-.

- - - Dyrion, L. N. A. Mth. 4 (1865) 348. - - - Gerono, G. C. N. A. Mth. 4 (1865) 349-.

- - - Eckardt, F. E. Z. Mth. Ps. 12 (1867) 183.

- - - - and by sphere. Nombel, J. N. A. Mth. 4 (1865) 346-.

一, circular. Godart, A. N. A. Mth. 4 (1865) 159-.

一, 一. Vicaire, E. Par. S. Mth. Bll. 19 (1891) 46-.

,-- , of tore, etc. Breton [de Champ], P. N. A. Mth. 15 (1856) 40-

-, plane. Fuortes, T. G. Mt. 10 (1872) 97.

,$- \ldots$, constructions for tangent and radius of curvature. Laquière, E. M. N. A. Mth. 1 (1882) 561-.

shadow cast on itself. Cahen, C. P. Par. S. Mth. Bll. 4 (1876) 87-.

\section{Wave surface.}

Cauchy, A. L. C. R. 13 (1841) 184-

Cayley, $A$. Liour. J. Mth. 11 (1846) 291-.

Prescott, J. E. QJ. Mth. 2 (1858) 1-.

Cayley, A. QJ. Mth. 3 (1860) 16-, 142-.

Durrande, $H$. N. A. Mth. 20 (1861) 456-; 2 (1863) 193-, 252-.

Mathieu, E. Liouv. J. Mth. 11 (1866) 298-

Turnbull, W. P. Mess. Mth. 3 (1866) 153-, 205-.

Catalan, E. C. [1868] Brux. Ac. Sc. Mm. 38 (1871) $64 \mathrm{pp}$.

Mannheim, A. As. Fr. C. R. (1874) 1168-; (1875) 167-; (1877) 167-; (1878) 63-; C. R. 88 (1879) 1248-; R. S. P. 32 (1881) 447-.

(Mannheim's researches.) Niven, C. QJ. Mth. 15 (1878) 242-.

Cayley, A. A. Mt. 20 (1892-93) 1-

Lacour, -. N. A. Mth. 17 (1898) 266-.

Böklen, O. Z. Mth. Ps. 44 (1899) 289-.

Iore, L. T. N. Y. Am. Mth. S. Bll. $6(1900)$ $3,12-$. asymptotic lines. Darboux, G. C. R. 97 (1883) 1039-.

Cartesian equation. Tait, P. G. QJ. Mth. 3 (1860) 269-.

Catalan's memoir on, and a geometrio transformation, report. Gilbert, P. Brux. Ac. Bll. 27 (1869) 129-.

corresponding surface of elasticity, etc. Lacour, E. N. A. Mth. 19 (1900) 362-.

definition, new. Darboux, G. C. R. 92 (1881) 446-.

equation in elliptic coordinates. Cayley, $A$. Mess. Mth. 8 (1879) 190-.

-, forms. Roberts, S. QJ. Mth. 17 (1881) 319-. equi-radial curve. Walton, $W$. QJ. Mth. 6 (1863) 144-.

- wave-cone. Walton, W. QJ. Mth. 6 (1863) 78-.

family of surfaces including. Walton, $W$. Camb. and Dubl. Mth. J. 7 (1852) 105-.

Fresnel's wave surface and related surfaces. Booth, W. [1896] Dubl. S. Sc. T. 6 (1898) 205-.

generation by intersection of 2 rotation surfaces. Walton, W. [1860] QJ. Mth. 4 (1861) 310 -.

-, new, and various constructions. Mannheim, A. C. R. 90 (1880) 1333-.

Hamilton's singular points. Booth, W. [1896] Dubl. S. Sc. P. 8 (1893-98) 381-.

modelling, practical method. Hicks, W. M. Mess. Mth. 5 (1876) 183-.

normals. Mannheim, A. As. Fr. C. R. (1877) 175-.

-, curvatures, and lines of eurvature. Mannheim, A. C. R. 64 (1867) 170-, 268-.

optical properties. Mannheim, A. As. Fr. C. R. (1875) 231-; C. R. 82 (1876) 368-; 122 (1896) 708-.

properties. Niven, C. QJ. Mth. 15 (1878) 257-. property. Townsend, R. [1872] Mess. Mth. 2 (1873) 28-.

-, generalisation. Anon. (xI 55) N. A. Mth. 1 (1882) 29-.

-, geometrical. Walton, W. QJ. Mth. 4 (1861) 151-.

representation by elliptic functions. Weber, $H$. Zür. Vjschr. 41 (1896) (Festschr., Th. 2) 82-.

as surface limit. Mannheim, A. C. R. 90 (1880) 971-.

tangent planes and radii, relation to ellipsoid of construction. Walton, W. QJ. Mth. 5 (1862) 285-.

_ - , singular, and sections by planes parallel to them. Mannheim, A. As. Fr. C. R. (1877) 125-.

theorems. Niven, C. QJ. Mth. 9 (1868) 22-. -, 2. Mannheim, A. C. R. 78 (1874) 839-.

triple system of confocal quartics comprising. Legoux, A. N. A. Mth. 4 (1885) 393-.

umbilici, geometric determination. Mannheim, A. C. R. 88 (1879) 902-

Weddle's quartic surface. Cayley, A. C. R. 52 (1861) 1216-.

- - - Poudra, -. N. A. Mth. 2 (1863) 307-. - - . Hierholzer, C. Mth. A. 4 (1871) 172-. 
Weddle's quartic surface. Laguerre, $E$. (x)

Par. S. Mth. Bll. 1 (1873) 71-.

- - Hunyady, E. von. [1881] Crelle J. Mth. 92 (1882) 304-.

- - Hutchinson, J. I. A. Mth. 11 (1896-97) 158-.

- - - analogue. Seydewitz, $F$. Grunert Arch. 10 (1847) 202-.

- - - generalisation. Cayley, A. [1872] L. Mth. S. P. 4 (1871-73) 11-.

$z y^{2}+B x y+C x^{2}+D y+E x+F=0$. Terquem, $O$.

N. A. Mth. 5 (1846) 370 -

$x^{n}+y^{n}+z^{n}=1$. Burhenne, H. Grunert Arch. 21 (1853) 35-.

$\frac{x^{n}}{a}+\frac{y^{n}}{b}+\frac{z^{n}}{c}=1$. Böklen, O. Grunert Arch. 35 (1860) 93-.

$\lambda r_{1}+\mu r_{2}+\nu r_{3}=0$. Cayley, $A$. Ph. Mg. 21 (1861) 491-.

$A x^{3}+B y^{3}+6 C R S T=0 . \quad$ Cayley, A. Ph. Mg. 27 (1864) 493-.

$\left(^{*}\right)(U, V, W)^{2}=0$. Cayley, A. QJ. Mth. 10 (1870) 24-; 11 (1871) 15-.

$\phi^{2}=\psi \chi$, etc. Kummer, E. E. Berl. Mb. (1872) 474-.

$\nabla=0$. Cayley, A. QJ. Mth. 14 (1877) 46-.

$x y z=l^{3}$, property. Floquet, G. C. R. 105 (1887) 854 .

$2 x y z-a x^{2}-a y^{2}-a z^{2}+a^{3}=0$. Allardice, R. E. Edinb. Mth. S. P. 10 (1892) 59-.

\section{Skew algebraic curves.}

\section{(See also 7240, 7660, 8030, 8050.)}

Bischoff, J. N. Crelle J. 58 (1861) 179-; 59 (1861) 394-.

Halphen, G. C. R. 70 (1870) 380-; 76 (1873) 558.

Eckardt, F. E. [1871] Mth. A. 5 (1872) 30-.

Weyr, E. [1873] (xi) Prag Ab. 6 (1874) (Mth.) $27 \mathrm{pp}$.

Schoute, P. H. Amst. Ak. Vs. M. 14 (1879) 251-.

Sturm, R. B. A. Rp. (1881) 440-

Noether, M. Berl. Ak. Ab. (1882) 120 pp.

Valentiner, $H$. Acta Mth. 2 (1883) 136-.

Rados, $G$. Mth. Termt. Éts. 8 (1890) 46-; Mth. Nt. B. Ung. 8 (1891) 90-

Anisimov, V. A. Rec. Mth. (Moscou) 17 (1895) 447-.

Chords of curve through given point. Vries, H. de. N. Arch. Wisk. 1 (1895) 127-; Fschr. Mth. (1893-94) 1232.

Classification. Salmon, G. Camb. and Dubl. Mth. J. 5 (1850) 23-.

-. Halphen, G. H. Par. Éc. Pol. J. Cah. 52 (1882) 1-.

- by monoid surfaces. Cayley, A. C. R. 54 (1862) 55-, 396-, 672-; 58 (1864) 994-.

Closed curves, Gaussian integral giving number of knots of 2. Thomae, C. J. Freiburg B. 7 (1880) 18-.

- -, parallel. Hoppe, -. D. Nf. Tbl.
Closed curves, representation on sphere. Flye Sainte-Marie, C. (Ix) Par. S. Mth. Bll. 1 (1873) 82-.

Common bisecants. Weyr, E. J. Wien Ak. Sb. 85 (1882) (Ab. 2) 840-.

Construction, linear. Seydewitz, $F$. Grunert Arch. 10 (1847) 203-.

Curves and developables. Cayley, A. Liouv. J. Mth. 10 (1845) 245-; Camb. and Dubl. Mth. J. 5 (1850) 18-; QJ. Mth. 7 (1866) 110-; 11 (1871) 294-.

- - polar planes. Bäcklund, A. V. Lund Acta Un. 8 (1871) (Mth.) No. 4, 50 pp.

- on quadric whose osculating planes touch a quadric. Stahl, W. Crelle J. Mth. 99 (1886) 154 -

\section{Curves, 3 rd degree.}

Cremona, L. Tortolini A. 1 (1858) 164-, 278-; 2 (1859) 19-; N. A. Mth. 19 (1860) 356-; 1 (1862) 287-, 366-, 436-; Crelle J. 60 (1862) 188-; Bologna Mm. Ac. Sc. 3 (1863) 385-; G. Mt. 1 (1863) 278-.

Drach, C. A. von. Z. Mth. Ps. 12 (1867) (Suppl.) 73-.

Beltrami, E. Mil. I. Lomb. Rd. 1 (1868) 130-, 407-.

Schröder, -. D. Nf. Tbl. (*1879) 176.

Catania, S. Catania Ac. Gioen. At. 19 (1886) 85-.

Cantone, A. Palermo Cir. Mt. Rd. 1 (1887) $72-$.

Cardinaal, J. Crelle J. Mth. 101 (1887) 142Caspary, F. Bll. Sc. Mth. 11 (1887) 222-.

Balitrand, -. N. A. Mth. 8 (1889) 520-.

Dixon, A. C. QJ. Mth. 23 (1889) 343-; 24 (1890) 30-

Bioche, C. Edinb. Mth. S. P. 13 (1895) 146-.

Stuyvaert, - . Brux. Ac. Bll. (1900) 820-.

analogue of helicoidal motion. Appell, $P$. C. R. 82 (1876) 70-; Par. Ec. Norm. A. 5 (1876) 245-.

application of binary cubic and quadratic forms. Pittarelli, G. G. Mt. 17 (1879) 260-.

- - - forms. Ovidio, E. $d^{\prime}$. [1879] Tor. Ac. Sc. Mm. 32 (1880) 1-.

- - imaginaries in geometry. Servais, C. Brux. Mm. Cour. $8^{\circ}, 49$ (1896) No. 3, 64 pp. - - quaternions. Hamilton, (Sir) W. R. [1863] Ir. Ac. P. 8 (1864) 331-.

- - resultant of 2 equations. Waelsch, $E$. Mh. Mth. Ps. 2 (1891) 421-.

automorphic for octahedral and ikosahedral groups, two. Kohn, G. Wien Ak. Sb. 108 (1899) $(A b, 2 a) 58$ -

binary cubic and quartic forms on. Berzolari, L. Palermo Cir. Mt. Rd. 5 (1891) 9-, 33-.

- forms on. Sturm, R. Crelle J. Mth. 86 (1879) 116-.

- - of order 5 on. Waelsch, $E$. Wien Ak. Sb. 100 (1891) (Ab. 2a) 803-.

case. Hurwitz, A. Mth. A. 30 (1887) 291-. centre.-Geisenheimer, L. Z. Mth. Ps. 27 (1882) 321-.

cones through a given curve. Cayley, A. $\mathrm{Ph}$. Mg. 12 (1856) 20-

congruences associated with. Krïger, $H$. Z. Mth. Ps. 40 (1895) 193-. 
48 coordinates. Spottiswoode, W. Phil. Trans. 172 (1882) 375-.

correspondence with conic. Weyr, Em. Mil. I. Lomb. Rd. 4 (1871) 636-.

and developables, class. Lelieuvre, - C. R. 117 (1893) 537-, 616-.

elimination problem. Pittarelli, G. G. Mt. 17 (1879) 244 .

equiangular curve. Krilger, $H$. Z. Mth. Ps. 38 (1893) 344-.

focal curve of skew parabola. Wirtinger, $W$. Wien Ak. Sb. 94 (1887) (Ab. 2) 302-.

- system. Servais, C. Brux. Mm. Cour. $8^{\circ}$, 52 (1894-95) No. 2, 11 pp.

generation, elements and characteristics. Sturm, $R$. Crelle J. Mth. 79 (1875) 99-; 80 (1875) 128-, 334.

through 5 given points. Koenigs, G. N. A. Mth. 2 (1883) 301-.

- 6 given points, graphic construction. Poudra, N. G. N. A. Mth. 5 (1866) 313-.

historical notes. Reye, $T$. Hamb. Mth. Gs. Mt. 2 (1890) (Festschr., Th. 2) 43-.

hyperboloidal projection. Cremona, $L$. (vII) A. Mt. 5 (1863) 227-.

inscribed tetrahedra. Gallucci, G. Nap. Rd. 37 (1898) 207-.

involution on a curve, and resulting complex. Weiler, A. Z. Mth. Ps. 24 (1879) 159-

metrical properties. Valeri, D. Mod. Ac. Sc. Mm. 8 (1892) 385-.

- - Heinrichs, E. Z. Mth. Ps. 39 (1894) 213-, 273-.

- - Sturm, R. Z. Mth. Ps. 40 (1895) 1-.

- -. Mehmke, R. Z. Mth. Ps. 40 (1895) 211-.

number of secants common to 2. Sturm, $R$. A. Mt. 3 (1869-70) 28-.

osculating plane. Tannery, J. Bll. Sc. Mth. As. 11 (1876) 183-.

- - at infinity, metrical properties. Loria, G. Nap. Rd. 24 (1885) 299-.

perspective relations to plane curves. Weyr, Em. Prag Sb. (1869) 22-.

plane and skew. Mangeot, $S$. Par. S. Mth. Bll. 21 (1893) 44-.

4, 5 and 6 point configurations. Guichard, $C$. Par. Éc. Norm. A. 3 (1886) 259-.

with 2 points, tangents and osculating planes common, pencil. Stuyvaert, - N. A. Mth. 19 (1900) 548-

polar reciprocal of cubic curve. Zeeman, $P$. (Gz.) Fschr. Mth. (1900) 626.

projections into conics. Bioche, C. N. A. Mth. 17 (1898) 541-.

- family of conics. Fabry, C. N. A. Mth. 8 (1889) 56-.

properties. Chasles, $M$. Liouv. J. Mth. 2 (1857) 397-.

- Lee, T. R. L. Mth. S. P. 24 (1893) 352-。 - Cazamian, A. N. A. Mth. 14 (1895) 108-. on quadric surfaces. Milller, Hub. Mth. A. 1 (1869) 407-.

- - -. Cayley, A. Mess. Mth. 14 (1885) 129

surfaces and curves circumscribing polyhedra formed by osculating planes. Cremona, $L$. Mil. I. Lomb. Rd. 12 (1879) 347-
4 tangents. Voss, A. E. Mth. A. 13 (1878) 168-.

theorem. Hurwitz, A. Mth. A. 20 (1882) 135-.

- Cantone, A. G. Mt. 25 (1887) 42-, 182.

- of Chasles. Cayley, A. Liouv. J. Mth. 10 (1845) 383-.

- - - Stuyvaert, - Mathesis 20 (1900) 181-.

theorems derived from involution. Cantone, $A$. Nap. Rd. 25 (1886) 181-.

touching tangent planes of skew cubic in 4,5 or 6 points. Kohn, G. Wien Ak. Sb. 105 (1896) (Ab. 2a) 1035-; Mth. A. 52 (1899) 293-.

and 3rd class. Schröter, H. E. Crelle J. 56 (1859) 27-.

- - (Schröter). Joachimsthal, F. Crelle J. 56 (1859) 44-.

- - C. Cremona, L. Crelle J. 58 (1861) 138 .

Curves, 3rd and 4th degrees.

description. Chasles, M. C. R. 53 (1861) 767-.

polarity in. Stuyvaert, - Brux. Ac. Bll. (1900) 87-.

theorems, new. Folie, F. Brux. Ac. Bll. 38 (1874) 65-, 465-.

\section{Curves, 4th degree.}

elliptic. Chasles, M. C. R. 54 (1862) 317-, 418-.

-. Laguerre, E. (x) Par. S. Phlm. Bll. 5 (1868) 65-.

-. Schroeter, H. E. Crelle J. Mth. 93 (1882) 132-.

-. Spottiswoode, W. [1882] L. Mth. S. P. 14 (1882-83) 15-.

-. Milinowski, -. Crelle J. Mth. 97 (1884) 277-.

-. Gegenbauer, L. Wien Ak. Sb. 93 (1886) (Ab. 2) 790 -

-, application of elliptic functions. Mathews, G. B. L. Mth. S. P. 19 (1889) 507-.

- $-1-$ Loria, G. Rm. R. Ac. Linc. Rd. 6 (1890) (Sem. 2) 179-.

_. - Valyi, G. Mth. Termt. Ets. 10 (1892) 244-; Mth. Nt. B. Ung. 10 (1893) 181-.

-, - - - Kluyver, J. C. Am. J. Mth. 19 (1897) 319-.

- Chasles's generation method. Laguerre, Liouv. J. Mth. 15 (1870) 193-.

-, configurations on. Ameseder, A. Wien Ak. Sb. 87 (1883) (Ab. 2) 1179-.

-, coordinates of tangent line. Roberts, $W$. R. $W$. L. Mth. S. P. $12(1880-81)$ 143-. , with cusp. Weyr, E.J. Wien Ak. Sb. 71 (1875) (Ab. 2) 400-.

- - double point. Weyr, E.J. Wien Ak. Sb. 75 (1877) (Ab. 2) 168-

- _ - - principal polygons. Brambilla, A. Nap. Ac. At. 9 (1899) No. 10, 33 pp.

-, double points in projection. Zeuthen, $H . G$. Ts. Mth. 2 (1884) 60-; Fschr. Mth. (1884) 520. 
elliptic, inflexion tangents. Kluyver, J. C. Amst. Ak. Vs. M. 8 (1891) 346-; Fschr. Mth. (1891) 849-.

-, intersection of sphere with quadric. Laguerre, E. (x) Par. S. Phlm. Bll. 4 (1867) 51-.

-, and intersections with quadric surface. Reye, T. [1868] A. Mt.2 (1868-69) 129-, 222-. -, - plane bicircular quartics, theorem. Lachlan, R. $\quad$ L. Mth. S. P. 21 (1891) 274-. -, properties. Duporcq, E. N. A. Mth. 15 (1896) 266-.

-, and 2 quadratic forms. Westphal, $G$. Mth. A. 13 (1878) 1-

-, - rational. Eberhard, V. Z. Mth. Ps. 32 (1887) 65-, 129-.

-, secant systems, representation by elliptic functions. Harnack, A. Mth. A. 12 (1877) 47-; 13 (1878) 49-.

-, 16 stationary points. Lange, E. Z. Mth. Ps. 28 (1883) 1-, 65-.

-, tetrahedra in multiple perspective on. Vályi, G. Mth. Termt. Éts. 11 (1893) 322-; Mth. Nt. B. Ung. 13 (1897) 183-.

models. Rohn, C. D. Mth. Vr. Jbr. 1 (1892) 43-.

through 8 points, intersections with plane through 2. Serret, P. C. R. 82 (1876) 322-, 370 -

and quadric surfaces, imaginary projection. Retali, V. [1885] Bologna Rd. (1885-86) 21-. rational. Cremona, $L$. (vII) A. Mt. 4 (1861) 71-.

-. Bertini, E. Mil. I. Lomb. Rd. 5 (1872) 622-.

-. Armenante, A. [1873] G. Mt. 11 (1873) 221-; 12 (1874) 250-.

-. Weyr, E.J. Prag Sb. (1874) 164-

-. Appell, P. C. R. 83 (1876) 1209-; Arch. Mth. Ps. 62 (1878) 175-

-. Adler, A. [1882] Wien Ak. Sb. 86 (1883) (Ab. 2) 919-, 1201-, 1212-.

-. Roberts, R. A. L. Mth. S. P. 14 (188283) 22-, 308-.

-. Brambilla, A. Ven. I. At. (1884-85) 1471-.

- Wirtinger, W. [1885] Wien Ak. Sb. 93 (1886) $(A b .2)$ 28-.

-. Study, E. Leip. Mth. Ps. B. 38 (1886) 3-.

- Meyer, F. Mth. A. 29 (1887) 447-.

-. Berzolari, L. Mil. I. Lomb. Rd. 23 (1890) 96-.

- Forsyth, A. R. QJ. Mth. 27 (1895) 247-.

- Grilnwald, J. Wien Ak. Sb. 108 (1899) (Ab. 2a) 1009-.

-, case. Cremona, L. Mil. I. Lomb. Rd. 1 (1868) 199-.

-, cases. Brambilla, A. Nap. Rd. 24 (1885) 279 .

-, combinants of binary forms associated with. Berzolari, L. A. Mt. 20 (1892-93) 101-.

-, with double point. Brambilla, A. Mil. I. Lomb. Rd. 17 (1884) 857-.

-, hyperboloids attached to. Berzolari, $L$. Mil. I. Lomb. Rd. 25 (1892) 950-.

- lines of striction of hyperboloids as. Migotti, A. [1879] Wien Ak. Sb. 80 (1880) (Ab. 2) 1023-. rational, number through 8 points. Cayley, $A$. As. Fr. C. R. 9 (1880) 135-.

-, point systems on. Weyr, E. J. Wien Ak. Sb. 75 (1877) (Ab. 2) 458-.

- stationary planes coinciding in pairs. Weyr, Em. Mil. I. Lomb. Rd. 4 (1871) 144 .

\section{Curves, 5th and higher degrees.}

5th degree, genus 1. Vries, J. de. Amst. Ak. Vs. 8 (1900) 451-; Amst. Ak. P. 2 (1900) 374-. - -, rational. Berzolari, L. Rm. R. Ac. Linc. Mm. 7 (1891) 305-.

6th degree, classification. Weyr, Ed. C. R. 76 (1873) 424-, 475-, 555-.

- -, genus 1. Petot, A. C. R. 102 (1886) $805-$.

- - - 4, triple tangent planes. Pascal, $E$. Rm. R. Ac. Linc. Rd. 2 (1893) (Sem. 1) 204-, 239 .

- -, rational, quadrisecants. Deruyts, $F$. Brux. Ac. Bll. 35 (1898) 421-

7th degree. Weyr, E. Wien Ak. Sb. 69 (1874) (Ab. 2) 399-.

- - case. Schoute, P. H. N. Arch. Wisk. 10 (1884) 54-.

$n$th degree, with $(n-2)$-fold secants. Bobek, $K$. Wien Ak. Sb. 95 (1887) (Ab. 2) 349-.

$p$ th and $q$ th degrees, intersections. Picquet, $H$. C. R. 77 (1873) 474-.

Cyclics. Darboux, G. C. R. 68 (1869) 1311-; Bordeaux Mm. S. Sc. 8 (1870) 291-; 9 (1873) 1-.

Degree, theorems. Saltel, L. Brux. Ac. Bll. 43 (1877) 29-.

Differential covariants. Gwyther, R. $F$. R. S. P. 56 (1894) 272-.

Double normals, number. Pieri, $M$. Rm. R. Ac. Linc. Rd. 2 (1886) (Sem. 1) 327-.

- points, apparent. Brunn, H. [1893] D. Mth. Vr. Jbr. 3 (1894) 84-.

Generation by variable figures. Caspary, $F$. Crelle J. Mth. 100 (1887) 405-.

- - - (Caspary). Carvallo, -. Par. S. Mth. Bll. 15 (1887) 158-.

Halphen's characteristic $n$. Cayley, A. Crelle J. Mth. 111 (1893) 347-.

Hyperboloid of one sheet, curves on. Chasles, $M$. C. R. 53 (1861) 985-.

Inflexional tangent planes. Clebsch, R. F. A. [1862] (vII) Crelle J. 63 (1864) 1-.

Inscribed polygons. Deruyts, $F$. Brux. Ac. Bll. 36 (1898) 553-.

Intersection of 2 cones, construction of tangents at double points. Juel, C. N. Ts. Mth. 8 (B) (1897) 28-; Fschr. Mth. (1897) 490.

- - - projects into Cartesian oval. Trenquelléon, $C$. de. N. A. Mth. 3 (1864) 539 .

Involutions on skew curves. Deruyts, $F$. Brux. Ac. Bll. 36 (1898) 194-

Isographic figures, and generation of skew curves. Jonquières, $E$. de. G. Mt. 23 (1885) 48-. 
Knotted curve. Hoppe, R: Arch. Mth. Ps. 64 (1879) 224.

- -, Hoppe's. Durège, H. [1880] Wien Ak. Sb. 82 (1881) (Ab. 2) 135-.

- curves. Brill, A. Mth. A. 18 (1881) 95-.

Line geometry of skew curves. Voss, A. E. Mth. A. 13 (1878) 232-.

Multiple secants of curve in 3- and 4-dimensional space. Berzolari, L. Palermo Cir. Mt. Rd. 9 (1895) 186-.

Number of constants involved. Rohn, $K$. [1896] D. Mth. Vr. Jbr. 5 (1901) (Heft 1) 84 .

- - points determining curves as intersection of $S_{m}, S_{n}$. Terquem, O. (vi Adds.) N. A. Mth. 15 (1856) 357-.

Numbers analogous to Plücker's for skew curves. Kluyver, J. C. Amst. Ak. Vs. M. 7 (1890) 121-; Fschr. Mth. (1890) 683-.

Osculating plane of intersection of 2 surfaces, reduction of degree by 2 . Hesse, L. $O$. Crelle J. 40 (1850) 316-.

- planes. Hesse, L. O. Crelle J. 41 (1851) $272-$.

Points on 2 curves, problem. Grunert, J. A. Wien SB. 17 (1855) 3-.

Projections. Wiener, -. D. Nf. Tbl. (*1879) 76-.

Properties. Steiner, J. Berl. B. (1839) 76-.

- Halphen, G. $H$. Par. S. Mth. Bll. 2 (1874) 69-.

Rational curves. Korndörfer, G. [1870] Mth. A. 3 (1871) 415-.

- Weyr, Em. G. Mt. 9 (1871) 217-; Z. Mth. Ps. 16 (1871) 354-.

- -. Tognoli, O. G. Mt. 12 (1874) 220 -

- - Genty, -. Par. S. Mth. Bll. 9 (1881) 115-.

-. Weyr, E. J. Mil. I. Lomb. Rd. 15 (1882) 250-; Prag Sb. (1882) 158-.

- Schoute, P. H. Amst. Ak. Vs. 8 (1900) 548-; Amst. Ak. P. 2 (1900) 421-. - and apolarity. Meyer, F. W. Mth. A. 21 (1883) 125-.

- - generation. Stahl, W. Mth. A. 40 (1892) 1-.

- - multiple secants. Deruyts, F. Brux. Ac. Bll. 35 (1898) 287-.

-, plane and skew. Roberts, R. A. L. Mth. S. P. 17 (1887) 25-.

- - - - - in- and circum-polygons. Zecca, G. G. Mt. 25 (1887) 333-.

- _ and ruled surfaces. Brill, A. Münch. Ak. Sb. 15 (1886) 276-.

- , theorem. Appell, P. Arch. Mth. Ps. 60 (1877) 125-.

Rectangular trihedra, whose edges are normals to quadric, locus of vertices. Painvin, $L$. N. A. Mth. 10 (1871) 337-.

Representation by cones. Cayley, A. QJ. Mth. 3 (1860) 225-; 5 (1862) 81-.

- of curves, and a formula of Halphen. Autonne, L. C. R. 119 (1894) 845-.

$--\ldots,-$ number of conditions determining them. Autonne, L. Lyon Un. A. [20] (1896) $37 \mathrm{pp}$.

Ruled surfaces of 3 rd and 4 th degrees, curves on. Chasles, M. C. R. 53 (1861) 884-.
Self-conjugate curves. Loria, $G$. Par. S. Mth. Bll. 23 (1895) 4-.

Singular points. Spottiswoode, $W$. Crelle J. 42 (1852) 372-.

- Halphen, G. H. As. Fr. C. R. 6 (1877) 132-.

- - classification. Chomé, $F$. Mathesis 18 (1898) 177-.

- - theory. Meder, A. Crelle J. Mth. 121 (1900) 230-.

-, types. Meder, A. Crelle J. Mth. 116 (1896) 50-, 247-.

Singularities. Halphen, G. H. [1877] Par. S. Mth. Bll. 6 (1878) 10-.

-. Björling, C. F. E. Arch. Mth. P8. 8 (1890) 83-; 10 (1891) vir.

-. Meyer, $F$. Gött. Nr. (1891) 88-; D. Mth. Vr. Jbr. 2 (1893) 62-.

-, discriminants of equations for. Meyer, $F$. Mh. Mth. Ps. 4 (1893) 229-, 331-.

-, reduction. Pannelli, $M$. Mil. I. Lomb. Rd. 26 (1893) 216-.

Spherical curve of 4 th degree. Gudermann, $C$. [1845] Crelle J. 43 (1852) 93-.

- curves. Davies, T.S. [1832] Edinb. R. S. T. 12 (1834) 259-, 379-

- - Möbius, A. F. N. A. Mth. 12 (1853) 238-.

- - with no singular points, form. Möbius, A. F. Leip. B. 2 (1848) 179-.

- - 3rd class, with double foci and double cyclic ares. Jeffery, $H . M$. B. A. Rp. (1876) (Sect.) 19-; QJ. Mth. 15 (1878) 131-. - _, - - with 3 single foci. Jeffery, H.M. [1880] QJ. Mth. 17 (1881) 104-.

- - , 4th class, with quadruple foci. Jeffery, H. M. [1881] QJ. Mth. 18 (1882) 270-.

- - 3rd degree, with triple foci. Jeffery, $H$. M. B. A. Rp. (1875) (Sect.) 16-.

- -, 4th degree. Casey, J. Phil. Trans. 161 (1871) 585-.

- - , - foci. Jeffery, H. M. QJ. Mth. 21 (1886) 130-.

- - - - - with quadruple cyclic arc, and triple focus. Jeffery, $H . M$. L. Mth. S. P. $12(1880-81) 168$.

- cyclics (intersections of quadric and sphere). Saltel, L. Par. S. Mth. Bll. 3 (1875) 95-, 99-.

- lemniscate arcs. Chasles, M. C. R. 21 (1845) 199-.

- - - (Chasles). Roberts, W. Liouv. J. Mth. 10 (1845) 451-.

- parabola. Arrest, H. $d^{\prime}$. Leip. B. (1853) 58 -

- and plane parabola, analogies. Mehmke, $\boldsymbol{R}$. [1876] Arch. Mth. Ps. 60 (1877) 215-.

- polygons and their polars, theorems. Löwenstern, S. Crelle J. 13 (1835) 79-, 159-.

Spherics, analytical. Gudermann, $C$. Crelle J. 6 (1830) 244-; 13 (1835) 262-.

-, elementary. Gudermann, C. Crelle J. 8 (1832) 363-.

Sphero-conic is locus of points equidistant from 2 small circles. Gudermann, C. Crelle J. 8 (1832) 160-.

Sphero-conics. Cayley, A. QJ. Mth. 3 (1860) 53. 
Sphero-conics. Cremona, L. N. A. Mth. 19 (1860) 269-.

-. Heilermann, -. Schlömilch Z. 6 (1861) 153-.

-. Sykes, G. S. [1877] Am. Ac. P. 13 (1878) 375-.

- Morley, $\dot{F}$. Mth. Gz. 1 (1900) 249-

-, application of elliptic functions. Enneper, A. Z. Mth. Ps. 22 (1877) 244-.

-, - - homogeneous spherical coordinates. Cassani, P. G. Mt. 6 (1868) 81-.

-, confocal. Chasles, M. Liouv. J. Mth. 5 (1860) 425-.

-, -. Fournier-Vannson, -. N. A. Mth. 19 (1860) 197-.

- - Heilermann, - Schlömilch Z. 6 (1861) 326-.

- and developable formed by tangents. Huber, G. Z. Mth. Ps. 45 (1900) 86-.

- as envelopes of systems of circles. Czuber, E. Arch. Mth. Ps. 7 (1889) 143-.

-, focal circles. Graves, (Rt. Rev.) C. [1889] Ir. Ac. T. 29 (1887-92) 285-.

- in- and circum-scribed to quadrangle. Jeffery, H. M. QJ. Mth. 13 (1875) 350-.

-, mechanical description. Hart, H. $\mathbf{L}$. Mth. S. P. 6 (1874-75) 136-.

-, properties. Chasles, $M$. Brux. Ac. Sc. Mm. 6 (1830) 46 pp.

Sphero- and plane conics, properties. Mehmke, R. Z. Mth. Ps. 23 (1878) 255-.

Surface of triple secants; number of quadruple secants, Picquet, H. (xI) Par. S. Mth. Bll. 1 (1873) 260-.

- - - - - - - - (Picquet). Guccia, G. B. Palermo Cir. Mt. Rd. 1 (1887) 26-.

Surfaces passing through skew curves, "excess." Kohn, G. [1880] Wien Ak. Sb. 82 (1881) (Ab. 2) 755-.

Tangent, construction. Anon. (vi 533) Gergonne A. Mth. 10 (1819-20) 89-.

Tangents and osculating planes of curves. Czuber, E. Mh. Mth. Ps. 3 (1892) 337-.

- to projections of curves. Hachette, J. N. P. Par. Ec. Pol. Cor. 3 (1814-16) 197-.

\section{Transformations and General Methods for Algebraic Con- figurations.}

(References to papers dealing with enumerative geometry may be found in sections 8000 to 8100 .)

\section{General.}

Centrals, theory. Grassmann, $H$. Crelle J. 24 (1842) 262-, 372-; 25 (1843) 57-.

Configurations, definition by construction of their polar systems. Thieme, $H$. Z. Mth. Ps. 24 (1879) 221-, 276-.

Continuous correspondences between the points of $r$ groups. Paolis, R. de. A. Mt. 18 (1890) 93-.
Envelopes of $\Sigma m_{i}\left(\alpha x_{i}+\beta y_{i}+\gamma z_{i}-p\right)^{2}=0$. Ruffini, F. $P$. Bologna Ac. Sc. Mm. 4 (1882) 123-.

Equations of geometry. Jordan, C. C. R. $68(1869) 656$ -

Higher geometry. Dillner, G. Ts. Mt. Fys. 1 (1868) 27-, 124-, 169-, 209-, 270-; 2 (1869) 82-, 137-; 3 (1870) 30-, 111-, 176-; Ups. N. Acta S. Sc. 8 (1873) No. 9, $136 \mathrm{pp}$.

Homogeneous projective coordinates in plane and space. Aschieri, $F$. Mil. I. Lomb. Rd. 25 (1892) 381-.

Imaginary points; antiprojective correspondence. Segre, C. Tor. Ac. Sc. At. 25 (1890) 276-, 430-, 592-; 26 (1891) 35-.

Locus theorems in which generating point divides variable linear segment in constant ratio, generalisation. Roberts, S. Am. J. Mth. 3 (1880) 336-.

Point system, harmonic mean point. Kohn, G. [1883] Wien Ak. Sb. 88 (1884) (Ab. 2) 424 .

- systems, equations representable by 3 . Ocagne, M. d'. Par. S. Phlm. Bll. 5 (1893) (C. R., No. 17) 2-; C. R. 123 (1896) 988-, 1098, 1254-.

Projective relations of geometric systems. Franke, J. N. Par. T. Nauk Śc. Pam. 1 ("1871) 101-.

Singular points on algebraic plane curves. Tichomandrickij, M. A. Kharkov Mth. S. Com. 2 (1891) 114-; Fschr. Mth. (1891) 757.

Transformation in geometry and in mathematical physics. Haton de la Goupillière, J. N. C. R. 58 (1864) 1001-; Par. Éc. Pol. J. 25 (1867) 153-.

- principle. Hesse, L. O. [1865] Crelle J. 66 (1866) 15-.

- -. Cayley, A. [1866] Crelle J. 67 (1867) 95-.

- sequence. Muirhead, R.F. Edinb. Mth. S. P. 16 (1898) 70-.

Transformations, circular. Emch, A. [1898] A. Mth. 12 (1898-99) 141-.

- of geometric propositions, examples. Zenthen, J. C. G. N. A. Mth. 3 (1864) 297 -

—, theory. Servais, C. Mathesis 8 (1888) $105-$

Trigraphy, applications. Russell, J.W. L. Mth. S. P. 26 (1895) 446-.

\section{Collineation; duality.}

Affinity of geometric figures, general kind. Möbius, A. F. Crelle J. 12 (1834) 109-

-, special case, applications. Korteweg, D. J. Z. Mth. Ps. 21 (1876) $28-$.

-, - type. Timerding, H. E. Arch. Mth. Ps. 17 (1900) 60-.

Anharmonic ratio, analogues. Brill, J. QJ. Mth. 29 (1898) 286-.

- - conical, of 10 points. Mozat, - C. R. 118 (1894) 790 - 
Automorphic curves and surfaces, family. Klein, F., \& Lie, S. C. R. 70 (1870) 1222-, 1275-.

- infinite transformations of a surface. Enriques, $F$. Ven. I. At. (1892-93) 1590-; (1893-94) 638-.

- transformations of space, groups. Schönfies, A. Mth. A. 34 (1889) 172-.

Birational transformations in geometry of position. Fiedler, $W$. Zür. Vjschr. 21 (1876) 369-.

Collinear figures. Frischauf, J. Steierm. Mt. 2 (Heft 1) (1869) 85-.

- forms, united elements. Nicoli, $F$. Mod. Ac. Sc. Mm. 7 (1890) 253-.

- fundamental configurations. Reye, $T$. [1870] Crelle J. 74 (1872) 1-.

- - - curves and surfaces generated by. Schur, F. Mth. A. 18 (1881) 1-.

- - forms of 2nd and 3rd species having united elements. Boschi, P. Bologna Ac. Sc. Mm. 2 (1880) 507-.

- planes, metrical relations between. Isè, $E$. Nap. Ac. Pont. At. 24 (1894) No. 3, 15 pp.

- ray-systems, 2, generation of curves of 4 th and 3rd degrees by. Heger, R. [1873] Z. Mth. Ps. 19 (1874) 170-.

- and reciprocal forms in ordinary geometry. Aschieri, $F$. [1879] Bologna Ac. Sc. Mm. 1 (1880) 145-.

- spaces, cyclic-projective point-quadruples in two. Schroeter, H. E. Mth. A. 20 (1882) 231-.

- - quintuple position. Ameseder, A. Wien Ak. Sb. 98 (1890) (Ab. 2a) 588-.

- - , series of points, planes, etc., in. Sturm, R. Mth. A. 28 (1887) 261-.

- systems. Genty, M. Par. S. Mth. Bll. 21 (1893) 131-.

- transformation of foci of conic. $K l u g, L$. Arch. Mth. Ps. 6 (1888) 88-.

Collineation. Sturm, R. Mth. A. 10 (1876) 117-.

- and affinity, identity of projection with. Spitzer, S. Grunert Arch. 9 (1847) 345-.

-, central, $n$th order, and plane collineation of $n$th class. Beyel, C. Zür. Vjschr. 31 (1886) 1-.

-, - , - - by similar points of circles. Beyel, C. Zür. Vjschr. 26 (1881) 297-

-, construction of conics from points and tangents by. Pelz, C. Prag Sb. (1875) 117-.

- and duality. Sturm, $R$. Mth. A. 22 (1883) 569-; 28 (1887) 268-.

- - Pasch, M. Mth. A. 23 (1884) 419-.

- - curvature and torsion in. Servais, C. Brux. Mm. Cour. $8^{\circ}, 58$ (1898-99) No. 2, $48 \mathrm{pp}$.

- of fundamental configurations of $2 \mathrm{nd}$ rank. Dantscher von Kollesberg, $V$. Innsb. Nt. Md. B. 18 (1889) 86-.

- - - - 3rd rank, special. Tötössy, B. [1883] (xII) Mth. Term. Éts. 2 (1884) 50-; Mth. Nt. B. Ung. 2 (1883-84) 237-.

- groups, discontinuity in certain. Fricke, $R$. Mth. A. 47 (1896) 557-.

- as mode of motion. Bôcher, M. N. Y. Mth. S. Bll. 1 (1892) 225-.
Collineation, new theory. Mehmke, R. Fschr. Mth. (1888) 861-.

- between non-ruled quadric and system of spheres, locus of centre. Jerábek, $W$. Arch. Mth. Ps. 65 (1880) 161-.

-, paradox in theory. Hofmann, F. Z. Mth. Ps. 28 (1883) 318-.

- of space, decomposition into 3 skew reflections. Wilson, E.B. N. Y. Am. Mth. S. T. 1 (1900) 193-.

- in space, like and unlike. Hauck, G. Z. Mth. Ps. 24 (1879) 381-.

- - - perspective and projective, axonometric theory. Hauck, G. Z. Mth. Ps. 21 (1876) 402-.

, spherical. Laroche, $F$. N. A. Mth. 8 (1849) 415.

of two 1-dimensional geometric forms. Neumayr, $E$. Innsb. Nt. Md. B. 9 (1879) 144 -.

Collineations, automorphic, cubic surfaces with. Bobek, $K$. Mh. Mth. Ps. 10 (1899) 122-, 307-.

-, class of quartic surfaces generated by. Schur, F. Mth. A. 20 (1882) 254-.

-, construction. Newson, H. B. Kan. Un. Q. 9 (1900) 65-.

-, cyclic, in plane and space. Schröter, D. Nf. Tbl. ( $\left.{ }^{*} 1874\right) 121$.

-, involutoric, in plane and space. Emch, $A$. Kan. Un. Q. 4 (1896) 205-.

- keeping 5 points fixed, invariant scrolls in. Snyder, V. Am. J. Mth. 22 (1900) 253-. - Klein's group of $(n+1) ! n$-ary. Moore, $E$. H. Am. J. Mth. $22(1900)$ 336-.

-, most general. Kantor, S. Wien Ak. D. 46 (1883) (Ab. 2) 83-.

-, perspective, projective groups. Emch, A. Kan. Un. Q. 5 (1896) 1-.

-, plane. White, H. S. [1897] N. Y. Am. Mth. S. Bll. 4 (1898) 17-。

-, -, covariants. Muth, P. Mth. A. 40 (1892) 89-.

-, - - finite groups with irreducible quartic invariant. Ciani, $E$. Mil. I. Lomb. Rd. 33 (1900) 1170-.

-, - - geometric interpretation of certain invariant in. Kraus, J. Mth. A. 29 (1887) 234 _, - , simple group of 360 . Wiman, A. Mth. A. 47 (1896) 531-.

- - - - - - Gerbaldi, $F$. Mth. A. 50 (1898) 473-; Palermo Cir. Mt. Rd. 12 (1898) 23-; 13 (1899) 161-; 14 (1900) 66-.

- and reciprocities, geometric theory. Ameseder, A. Mh. Mth. Ps. 1 (1890) 371-.

- - invariants. Muth, P. Mth. A. 33 (1889) 493-.

_ - - related to quadrics or skew cubic curves. Sturm, R. Mth. A. 26 (1886) 465-.

-, space, with automorphic quadric. Wood, (Miss) R. G. [1900] N. Y. Am. Mth. S. Bll. 7 (1901) 202, 207.

,- , invariable forms in. Mertens, $F$. Mh. Mth. Ps. 1 (1890) 13-.

Conic, transformation of circle into. Baraniecki, M. A. Krk. Ak. (Mt.-Prz.) Rz. 13 (1886) 172-. 
Conics as collinearly related to circles. Schlömilch, O. Schlömilch Z. 1 (1856) 1-.

Correlation of 2 planes. Hirst, T. A. L. Mth. S. P. 5 (1873-74) 40-; 8 (1876-77) 262-; Rm. R. Ac. Linc. T. 1 (1877) 86-.

-, simplest, between 2 regions of space. Richelot, F.J. [1868] Crelle J. 70 (1869) 137-.

- of 2 spaces. Hirst, T. A. [1874] L. Mth. S. P. 6 (1874-75) 7-.

Correlations, finite groups. Kantor, $S$. Crelle J. Mth. 116 (1896) 171-.

-, metrical properties. Segre, C. G. Mt. 25 (1887) 20-

-, polar, for which twisted cubic is self-conjugate. Montesano, $D$. Rm. R. Ac. Linc. Mm. 3 (1886) 105-.

Correlative pencils. Sturm, R. L. Mth. S. P. 7 (1875-76) 175-; Mth. A. 12 (1877) 254-.

- systems in plane. Le Paige, C. Brux. Ac. Bll. 12 (1886) 422-.

Correspondence of spaces, special. Biltzberger, F. Z. Mth. Ps. 38 (1893) 1-.

- between straight lines and points. Poulain, A. Mathesis 11 (1891) 88-.

Doubly harmonic linear elements, determination. Raffy, L. Liouv. J. Mth. 10 (1894) 331-.

Duality. Poncelet, J. V. Gergonne A. Mth. 18 (1827-28) 125-.

-. Booth, J. Lpool. Lt. Ph. S. P. 1 (pt. 4) (1844-45) 117-.

-. Gergonne, -. Mntp. Ac. Sc. Mm. 1 (1847-50) 61-

-. Zeuthen, H. G. (xII) Ts. Mth. 1 (1871) 1-, 129-, 161-; 2 (1872) 161-.

-. Study, E. Leip. Mth. Ps. B. 48 (1896) 199-.

-. Brill, J. Mess. Mth. 26 (1897) 134-.

- and collineation in space. Allé, $M$. Wien Ak. Sb. 85 (1882) (Ab. 2) 1021-.

- of figures in space. Möbius, A. F. Crelle J. 10 (1833) 317-

-, history. Miller, J.H.T. Grunert Arch. 34 (1860) 1-.

- and homography. Chasles, M. Brux. Mm. Cour. 11 (1837) 575-.

- - - applied to metric properties of space. Ripert, L. N. A. Mth. 18 (1899) 306-.

- in plane geometry. Ripert, L. N. A. Mth. 17 (1898) 446-.

-, a principle of, in geometry of space. Réthy, M. , Gött. Nr. (1873) 6-.

- of quadrilateral, spherical and plane. Beez, - Schlömilch Z. 7 (1862) 129-.

Equipollent figures. Tarry, G. N. A. Mth. 11 (1892) 251-.

Homogeneous projective coordinates for elements of ordinary space. Aschieri, F. Mil. I. Lomb. Rd. 18 (1885) 569-.

Homographic or correlative systems, relations between infinitesimal elements. Demoulin, A. C. R. 126 (1898) 390 -.

- correspondence between elements of 2 linear spaces. Deruyts, F. Brux. Mm. Cour. $4^{\circ}$, 52 (1890-93) No. 7, 40 pp.

- figures, focal properties. Smith, H. J.S. L. Mth. S. P. 2 (1869) 196-.
Homographic figures, focal properties. Reye, $T$. Mth. A. 46 (1895) 423-.

- - theorems. Petersen, L. (xiI) Ts. Mth. 5 (1875) 21-.

- pencils $H_{2}^{3}$, singularities of cubic surface generated by. Versluys, $W$. $A$. Liège $\mathrm{S}$. Sc. Mm. 20 (1898) No. 6, 61 pp.

- relation of 2 figures. Swellengrebel, J. G.H. Utr. Aant. Prv. Gn. (1853-54) 82-.

- transformation. Painvin, L. N. A. Mth. 9 (1870) 97 -

- - of any linear space, general. Aschieri, F. Mil. I. Lomb. Rd. 18 (1885) 989-.

- - - metric properties of plane figures. Brocard, G. N. A. Mth. 15 (1896) 426-; 16 (1897) 293-.

- - particular case. Antomari, X. N. A. Mth. 17 (1898) 489-.

- of plane figure, continuous. Mollame, $V$. G. Mt. 9 (1871) 269-.

- transformations, plane. Lucas, F. Liouv. J. Mth. 6 (1861) 137-.

- -, special kind. Bortniker, (Mlle.) L. C. R. 104 (1887) 771-.

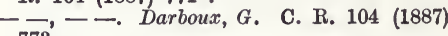
773-.

Homographies, binary, and their harmonic groups. Segre, C. Crelle J. Mth. 100 (1887) 317-.

- - - - products. Aschieri, F. Mil. I. Lomb. Rd. 24 (1891) 964-.

-, , systems of, and geometric representation of imaginaries. Amodeo, $F$. G. Mt. 26 (1888) 363-.

- and correlations, with special reference to 3-dimensional space. Segre, C. Tor. Ac. Sc. Mm. 37 (1886) 395-.

- of any linear space, construction. Bertini, $E$. Mil. I. Lomb. Rd. 20 (1887) 650-.

-, resolution into homologies. Bertini, $E$. Tor. Ac. Sc. At. 22 (1886-87) 865-.

- ternary, united real elements. Amodeo, $F$. G. Mt. 27 (1889) 40-

Homography. Battaglini, $G$. Giamb. Vico 1 (1857) 121-; 2 (1857) 272-.

-, general theory. Predella, P. Tor. Ac. Sc. At. 27 (1892) 270-.

一, problem. Weyr, E. Prag České Ak. Fr. Jos. Rz. (Třida 2) 8 (1899) Art. 24, 8 pp.; Fschr. Mth. (1899) 478.

- of 2 solid bodies. Sylvester, J. J. C. R. 101 (1885) 35-, 139-.

- - 3rd order. Le Paige, C. M. M. H. H. Rm. N. Linc. At. 36 (1883) 22; Brux. Ac. Bll. 5 (1883) 85-.

- - higher order. Appell, P. [1879] Par. S. Phlm. Bll. 4 (1880) 18-.

Homology and polar reciprocals, curves and surfaces corresponding by. Fouret, $G$. Par. S. Mth. Bll. 14 (1886) 18-.

- represented by space of conics in a plane. Certo, L. G. Mt. 20 (1882) 321-.

Involution, collinear, of pairs of points in plane and space. Möbius, A. F. Leip. B. 8 (1856) 143-.

-, generalisation of concept. Schroeter, D. Nf. Tbl. (*1868) 94 . 
Involution and homography, extension of theories. Le Paige, C. M. M. H. H. Brux. Ac. Bll. 44 (1877) 546-.

- of $k$ th rank and $(2 k+1)$ th degree, neutral group. Le Paige, C. M. M. H. H. [1882] Wien Ak. Sb. 86 (1883) (Ab. 2) 104-.

Involutions, harmonic, on plane and sphere. Vries, J. de. Haarl. Ms. Teyl. Arch. 4 (1896) 119-.

-, higher order. Möbius, A.F. Leip. B. 7 (1855) 123-.

Involutory automorphic substitutions of rational function, parametric representation. Hofmann, $F$. Aurch. Mth. Ps. 8 (1890) 225-. - configurations, projective. Stahl, W. Crelle J. Mth. 107 (1891) 179-.

- correspondence, system of conics in. Retali, $V$. Bologna Ac. Sc. Mm. 5 (1883) 373-.

- space transformations determined by line complex of Hirst. Pieri, M. Mil. I. Lomb. Rd. 25 (1892) 1037-.

- transformation of straight line. Emch, A. [1895] Kan. Un. Q. 4 (1896) 111-.

- transformations, 2, with applications. Stiner, G. Zür. Vjschr. 40 (1895) 317-.

Linear group of transformation in plane, fundamental property. Emch, A. [1895] A. Mth. 10 (1895-96) 3-.

- substitutions, new theory. Laurent, $H$. Liouv. J. Mth. 4 (1898) 75-.

- transformation of geometric figures. $E r$ makov, V.P. Rec. Mth. (Moscou) 13 (1886) 749 -.

- transformations. Kantor, $S .[1880]$ Wien Ak. Sb. 82 (1881) (Ab. 2) 34-.

- between 2 quadrics. Taber, $H$. L. Mth. S. P. 24 (1893) 290 -.

- -, successive. Kantor, S. [1880] Wien Ak. Sb. 82 (1881) (Ab. 2) 39-.

Lines and polar surfaces, theory. Lazzeri, $G$. Mil. I. Lomb. Rd. 24 (1891) 1021-.

Loci arising from 3 figures, projectively related. Del Re, A. Palermo Cir. Mt. Rd. 1 (1887) 272-, 398.

Metrical properties of involutions and conditions for double elements. Boguslavskij, A. I. Mosc. S. Sc. Bll. 65 (No. 2) (1890) 52-; Fschr. Mth. (1892) 572.

Null system, special case. Sucharda, $A$. Časopis 29 (1900) 89-; Fschr. Mth. (1900) 528.

Plane curves, polar transformation. Ocagne, M. $d$ '. G. Teix. J. Sc. 6 (1885) 3-.

- - transformable into one another by group of transformations. Klein, F., \& Lie, S. Mth. A. 4 (1871) 50-.

- transformations, conditions of rationality of double planes. Castelnuovo, G., \& Enriques, F. Palermo Cir. Mt. Rd. 14 (1900) 290-

Point plane systems, general. Voss, A. Mth. A. 23 (1884) 45-.

- - - rational algebraic. Voss, A. Mth. A. 23 (1884) 359-.

Polar plane systems; and ternary quadratic correspondences. Del Re, A. G. Mt. 28 (1890) 257-.

- reciprocal figures, contact of inverse figures with. Vaněček, J. S., \& Vaněček, M. N. [1883] St Pét. Ac. Sc. Bll. 29 ("1884) 281-.
Polar reciprocal figures, transformation. Vaněček, J. S. Liège S. Sc. Mm. 11 (1885) No. 2, $21 \mathrm{pp}$.

- - of surface with multiple points, determination of double curve. Saltel, L. Brux. Ac. Bll. 40 (1875) 27-.

- - surfaces, theorems. Hamilton, (Sir)W.R. Ir. Ac. P. 4 (1850) 192-.

- - - theory, revision and extension of numerical formulæ. Zeuthen, H. G. Mth. A. 10 (1876) 446-.

- reciprocals, transformation of barycentric properties by. Ocagne, M. $d$ '. Mathesis 5 (1885) 170-.

- surfaces of plane, theory. Painvin, L. F. [1865] (хп) Lille S. Mm. 2 (1866) 345-.

- systems. Romero, A. del. (xII) Barcel. Ac. Mm. 1 (1878) 133-.

- -. Jung, G. Mil. I. Lomb. Rd. 12 (1879) 169-, 218-, 535-.

- - (points, lines, planes), construction. Schröder, -. D. Nf. Tbl. ("1879) 176-.

- - in space, polar pentagons and hexagons of. Reye, T. Crelle J. Mth. 77 (1874) 269-. - theory of algebraic surfaces, extension. Reye, T. Crelle J. Mth. 78 (1874) 97-.

Poles and polars. Fenwick, $S$. Mathematician 1 (1845) 125-, 235-, 281-.

Projective connection of systems of curves. Fiedler, $W$. Zür. Vjschr. 24 (1879) 180-.

- construction of rational curves. Schoute, P. H. N. Arch. Wisk. 12 (1886) 1-; Arch. Néerl. 20 (1886) 49-.

- correspondence, plane, double points in. Brodén, $T$. Arch. Mth. Ps. 9 (1890) 225-.

- correspondences, degenerate. Del Prete, $G$. Mil. I. Lomb. Rd. 30 (1897) 400-, 464-.

- - in fundamental geometric forms of 1st kind. Paolis, R. de. Tor. Ac. Sc. Mm. 42 (1892) 495-.

- - between 2 planes and between 2 spaces. Loria, G. G. Mt. 22 (1884) 1-, 117.

- fundamental forms, surfaces arising from. Cardinaal, J. N. Arch. Wisk. 18 (1891) 123-.

- geometry. Killing, $W$. Mth. A. 43 (1893) 569 .

- metrics. Ovidio, E. d'. Tor. Ac. Sc. At. 28 (1893) 566-.

- properties of curves and surfaces. Jonquières, E. de. Crelle J. 66 (1866) 289-.

_ _ _ _ _ - application of transversals. Poncelet, J.V. Crelle J. 8 (1832) 21-, 117-, 213-, 370-.

- reflection, and space collineation. Bôcher, M. Mth. A. 43 (1893) 598-.

- relations given by 4 straight lines in space. Krieg von Hochfelden, F. (Frhr.) Wien Ak. Sb. 97 (1889) (Ab. 2a) 806-.

- relationships and theory of higher curves. Seydewitz, F. Grunert Arch. 7 (1846) 113-; 8 (1846) 1-.

- substitutions leaving a circle unchanged. Schlesinger, L. Orv.-Termt. Ets. (Termt. Szak) (1898) 14-, (Rv.) 15-; Crelle J. Mth. 121 (1900) 168-.

- transformation. Genese, R. W. B. A. Rp. (1886) 538-. 


\section{Collineation; duality}

Projective transformation. Lovett, E. O. A. Mth. 10 (1895-96) 5-.

- transformations, normal forms. Newson, H. B. Kan. Un. Q. 7 (1898) 125-.

- - in plane and space. Newson, H. B. Kan. Un. Q. 6 (1896) 63-.

- - of plane, 5 types. Newson, H. B. Kan. Un. Q. 8 (1899) 43-.

- - properties. Delaunay, N. B. Rec. Mth. (Moscou) 19 (1897) 387-; Fschr. Mth. (1896) 476.

Quadrilateral and associated quadrangle, fiveplane and associated pentagon. Kohn, $G$. Wien Ak. Sb. 93 (1886) (Ab. 2) 314-.

-, quadrangle, and projective correspondence in plane. Pasch, M. Mth. A. 26 (1886) 211-.

Reciprocal and allied relations. Sturm, $R$. Mth. A. 19 (1882) 461-.

- - - - (Sturm). Kantor, S. Mth. A. 20 (1882) 297-.

- coincident configurations. Schroeter, H.E. Crelle J. Mth. 77 (1874) 105-.

- of curve, equation. Salmon, $G$. Crelle J. 42 (1852) 277-.

- curves, property. Pasch, M. [1871] Crelle J. 74 (1872) 92-.

- and surfaces. Bacaloglo, E. Grunert Arch. 36 (1861) 1-.

- derivability of 2 functions by polars. Perna, A. G. Mt. 34 (1896) 110-

- plane systems. Sucharda, A. Časopis 29 (1900) 1-; Fschr. Mth. (1900) 529.

- relations, 2 , representation of curves of 3 rd degree and class by. Beyel, C. Z. Mth. Ps. 38 (1893) 65-

- relationship. Rosanes, J. Crelle J. Mth. 90 (1881) 303-.

- - and dependent point systems. Rosanes, J. Crelle J. Mth. 95 (1883) 247-.

- systems, elements doubly conjugate to one another. Keller, J. Zür.Vjschr. 25(1880) 1-. - -, plane. Krok, J. M. Lund Acta Un. 9 (1872) (Mth.) No. 5, 28 pp.

- transformations and theorems of Ceva and Menelaos. Emch, A. N. Y. Am. Mth. S. Bll. 5 (1899) 143-.

Reciprocity, geometrical. Cayley, A. Camb. and Dubl. Mth. J. 3 (1848) 173-.

-, plane, and application to theory of curves. Beyel, C. Z. Mth. Ps. 31 (1886) 147-.

Relation of spaces by projective plane pencils. Krieg, F. (Frhr.) von. Z. Mth. Ps. 29 (1884) (Suppl.) 38-.

Relationship, geometric, of systems in space. Silldorf, $(D r)$-. Z. Mth. Ps. 18 (1873) 523-.

- of plane figures, new. Möbius, A.F. Leip. B. (1853) 14-

Relationships in linear geometry. Hunyady, $J$. (xII) Mag. Tud. Ak. Ets. 16 (No. 2) (1882) 39-.

Ruled surfaces and envelopes of straight lines joining corresponding points of 2 plane curves. Chizzoni, $F$. Rm. R. Ac. Linc. Mm. 3 (1879) 69-.

Similar systems, and a cubic surface. Moshammer, $K$. [1876] Wien Ak. Sb. 74 (1877) (Ab. 2) 131-.

Similarly variable figures, and loci derived therefrom. Réveille, J. N. A. Mth. 12 (1893) 297-.

\section{Other Transformations}

8020

Similitude of 2 similar curves, centre. Monge, G. Par. Ec. Pol. Cor. 3 (1814-16) 4-.

Similitudes in real plane, group. Del Re, $A$. Rv. Mt. 2 (1892) 99-.

Skew curves on quadric, whose osculating planes touch a quadric. Stahl, W. Crelle J. Mth. 99 (1886) 154 .

Space transformation depending on quadric. Schoute, P. H. Palermo Cir. Mt. Rd. 1 (1887) 47, 379-.

- Capelli, A. Palermo Cir. Mt. Rd. 1 (1887) 48-, 53-.

Surface and polar reciprocal in given homography, condition. Del Re, A. Palermo Cir. Mt. Rd. 2 (1888) 37-.

Symmetry of 2 algebraic figures with reference to a point. Mangeot, S. N. A. Mth. 19 (1900) 451-.

System of 6 positions of plane figure. Stephanos, C. As. Fr. C. R. (1883) 175-.

Tangential correspondence, decomposition. Fontenê, G. Par. S. Mth. Bll. 25 (1897) 247 - transformation in plane. Naetsch, E. Dresden Isis Sb. (1896) 15-.

Trilinear relation between 3 fundamental configurations of 1st rank. Schubert, H. C. H. Mth. A. 17 (1880) 457-.

A. $44(1894) 375-$.

- of plane systems. Hauck, G. Crelle J. Mth. 97 (1884) 261-; 98 (1885) 304; 108 (1891) 25-; 111 (1893) 207-.

- - between 3 variables, reduced forms. Fontené, G. N. A. Mth. 19 (1900) 494-

Trilinearly related fields in space. Schmid, $T$. Mh. Mth. Ps. 6 (1895) 99-; 7 (1896) 180-

$(1,1)$ point transformation of plane. Czuber, $E$. Mh. Mth. Ps. 5 (1894) 267-.

\section{0ther algebraic transforma- tions. (See also 6810, 7650.)}

Adjoint curves. Scott, (Miss) C. A. QJ. Mth. 28 (1896) 377-.

Algebraic curves, transformation. Bertini, E. Rv. Mt. 1 (1891) 22-.

- -, -. Simart, 一, C. R. 116 (1893) 1047-.

- - - - Goursat, $\dot{E}$. Am. J. Mth. 16 (1894) 291-.

- and theorems of Steiner. Sporer, $B$. Z. Mth. Ps. 36 (1891) 339-.

Anallagmatic curves and surfaces. Picquet, $H$. As. Fr. C. R. (1878) 95-.

- $-\ldots$, application of general properties of curves. Laguerre, E. (x) Par. S. Phlm. Bll. 5 (1868) 112-.

- - , new species. Picquet, $H$. C. R. 87 (1878) 460

Arguesian curves and surfaces, properties derived from. Crocchi, L. G. Mt. 12 (1874) 378-.

- transformation. Saltel, L. [1870] Brux. Mm. Cour. 8, 23 (1873) (No. 4) 112 pp.

- - application to curves and surfaces. Saltel, L. [1870] Brux. Mm. Cour. $8^{\circ}, 22$ (1872) (No. 5) $53 \mathrm{pp}$.

- - extension to skew curves. Saltel, $L$. Brux. Ac. Bll. 46 (1878) 90-. 
Arguesian transformations, Saltel's. Mansion, P. Brux. Ac. Bll. 36 (1873) 625-

Arguesians, theorem. Saltel, L. Brux. Ac. Bll. 43 (1877) 265-.

Bifocal transformations. Burmester, L. Mth. A. 16 (1880) 89 .

Birational correspondence between 2 spaces, transformation by. Levi, B. Tor. Ac. Sc. At. 35 (1900) 20-.

- plane transformation, multiple transformations associated with every. Pannelli, $M$. G. Mt. 29 (1891) 154-.

- - , transformation of genus $p$ and degree $n+1$ associated with every. Jung, G. Mil. I. Lomb. Rd. 19 (1886) 812-.

- - transformations. Guccia, G. B. C. R. 101 (1885) 808-, 980.

- quadratic transformation. Ocagne, M. d'. N. A. Mth. 12 (1893) 337-.

--- , geometric interpretation. Servais, $C$. Mathesis 7 (1887) 129-, 187-.

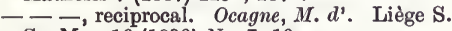
Sc. Mm. $16(1890)$ No. 7, 10 pp.

-_-, special cases. Third, J. A. Edinb. Mth. S. P. 18 (1900) 11-.

- - transformations. Mansion, $P$. Mathesis 7 (1887) 110-.

- reciprocities in plane. Lazzeri, G. $\mathrm{Rm}$. R. Ac. Linc. Rd. 2 (1886) (Sem. 2) 61-.

- - - null. Del Re, A. Nap. Rd. 28 (1889) 101-.

- - space. Lazzeri, G. Rm. R. Ac. Linc. Rd. 2 (1886) (Sem. 2) 73-.

- transformation, 2 multiple transformations associated with every. Jung, G. Rm. R. Ac. Linc. Rd. 2 (1886) (Sem. 2) 339-.

- - of 6th degree in 3-dimensional space. Cremona, L. L. Mth. S. P. 15 (1883-84) 242-.

- transformations of 3 geometric forms of 2 nd kind. Jung, G. Mil. I. Lomb. Rd. 19 (1886) 161-.

- - with integral coefficients. Kantor, $S$. C. R. 126 (1898) 946-.

- - surfaces generated by. Jung, G. Rm. R. Ac. Linc. Rd. 2 (1886) (Sem. 1) 85-.

- - of $n$th order. Jonquières, - de. C. R. 101 (1885) 720-, 848.

Central transformation, case. Longchamps, G. de. Mathesis 15 (1895) 186-.

- - - - Meurice, L. Mathesis 15 (1895) 191-.

- transformations of plane curves. Ocagne, M. d'. Mathesis 4 (1884) 73-, 97-.

Circles touching 4 isotropic planes, and surfaces with double circular generation. Cosserat, E. C. R. 130 (1900) 385-.

Conic, space transformations depending on. Pieri, M. Palermo Cir. Mt. Rd. 7 (1893) 296-.

Conical transformations of space. Del Pezzo, $P$. Nap. Rd. 35 (1896) 288-.

Conics, transformation theory. Verbessem, Mathesis 14 (1894) 184 -

Correspondence of 2 plane figures, quadratic and rational. Duporcq, E. C. R. 126 (1898) 1405-.

- - points collinear with fixed origin. Cotterill, T. L. Mth. S. P. 6 (1875) 200.

Correspondences, invariant formations of. Peano, G. G. Mt. 20 (1882) 79-.
Cremona cubic groups. Autonne, L. C. R. 101 (1885) 53-; 105 (1887) 267-; Liouv. J. Mth. 2 (1886) 49-.

- group with any number of variables, complete groups contained in. Del Re,A. Rm. R. Ac. Linc. Rd. 6 (1890) (Sem. 2) 271-.

- quadratic groups. Autonne, L. C. R. 98 (1884) 565-; 103 (1886) 1176-; 104 (1887) 767-, 1422-; 105 (1887) 929-; 106 (1888) 262-; Liouv. J. Mth. 1 (1885) 431-; 4 (1888) 177-, 407-.

- - transformation. Dickson, L. E. [1898] Calif. Ac. P. (Mth. Ps.) 1 (1903) 13-。

- - defined by conic. Dickson, L. E. Palermo Cir. Mt. Rd. 9 (1895) 256-.

- - transformations, cross-ratio group of 120 . Slaught, H. E. Science 8 (1898) 121-; Am. J. Mth. 22 (1900) 343-.

- transformation, involutions contained by. Doehlemann, $K$. Z. Mth. Ps. 36 (1891) 356-. - between 2 planes. Roberts, S. [1872] L. Mth. S. P. 4 (1871-73) 121-.

- - solution of equations of condition. Ruffini, F. P. Bologna Ac. Sc. Mm. 8 (1877) 457-.

- , - -- Palatini, $F$. Ven. I. At. (1896-97) 1555-.

- transformations. Clebsch, R. F. A. Mth. A. 4 (1871) 490 -

- - Guccia, G. B. C. R. 101 (1885) 866-.

- -. Sturm, R. Mth. A. 26 (1886) 304-.

- Guccia, G. B. Palermo Cir. Mt. Rd. 1 (1887) 17-, 20-, 24-, 50-, 56-, 119-, 398.

- - admitting fixed curve. Castelnuovo, $G$.

Rm. R. Ac. Linc. Rd. 1 (1892) (Sem. 1) 47-.

- -, continuous groups. Enriques, $F$. Rm. R. Ac. Linc. Rd. 2 (1893) (Sem. 1) 468-.

- - , - - Noether, M. D. Nf. Vh. (1896) (Th. 2, Hälfte 1) 11-.

- - - - Fano, G. Mh. Mth. Ps. 9 (1898) 17-.

- - , - - , and certain projective transformation groups. Fano, $G$. Palermo Cir. Mt. Rd. 10 (1896) 16-.

_ - indeterminate analysis relating to. Jonquières, (l'amiral) E. de. C. R. 101 (1885) 857-, 1082.

Cubic through 8 points, geometrical transformation by. Deruyts, $F$. Liege S. Sc. Mm. 14 (1888) No. 5, 14 pp.

- transformation and line complex arising therefrom. Machovec, $F$. Prag Sb. (1888) (Mth.-Nt.) 104-.

- - in space. Schoute, P. H. N. Arch. Wisk. 4 (1899) 90-.

transformations. Casey, $J$. Ir. Ac. Cunningham Mm. 1 ("1880) 140 pp.

Cyclic groups, number in a Cremona transformation. Kantor, S. [1880] A. Mt. 10 (1880-82) 71-.

-,$--1-$ quadratio plane transformation. Kantor, S. [1880] A. Mt. 10 (1880-82) 64-. ,---- space transformation. Kantor, $S$. C. R. 90 (1880) 1156-.

Double cubic transformation of 1st genus, and quartic curves. Paolis, R. de. Rm. R. Ac. Linc. Mm. 2 (1878) 851-. 
Double quadratic plane transformation, and application to non-Euclidean geometry. Paolis, R. de. [1877] Rm. R. Ac. Linc. Mm. 2 (1878) 31-.

- - space transformation, and application to non-Euclidean geometry. Aschieri, $\boldsymbol{F}$. Mil. I. Lomb. Rd. 14 (1881) 673-; 15 (1882) 66-, 147-, 247-.

- transformations of figures. Aoust, L. C. R. 56 (1863) 906-.

Enneads of lines and points. Cayley, A. B. A. Rp. 40 (1870) (Sect.) 10.

Figures generated by 2 fundamental forms of 2nd kind with (1.v) correspondence of $n$th degree. Visalli, $P$. Rm. R. Ac. Linc. Rd. 3 (1887) (Sem. 1) 124-.

-, transformation, application to construction of quadric through nine points. Darboux, G. Par. Éc. Norm. A. 6 (1869) 61-.

Generalised Jonquières groups. Fano, $G$. Tor. Ac. Sc. Mm. 48 (1899) 221-.

Generation of curves and surfaces by corresponding elements. Weyr, Em. [1871] Prag Ab. 5 (1872) 54 pp.

Geometric transformation with application in special case to kinematics. Dewulf, É. Par. Ec. Norm. A. 3 (1886) 405-.

Groups of transformation in 3-dimensional space, class. Blichfeldt, H. E. Am. J. Mth. 22 (1900) 113-.

Higher singularities of plane curves. Scott, (Miss) C. A. Am. J. Mth. 14 (1892) 301-.

Homaloidal transformation of quadrics. Michel, P. N. A. Mth. 12 (1893) 192-.

Homographies, higher. Saltel, L. [1875] Brux. Mm. Cour. 8, 27 (1877) No. 4, 32 pp.

Hyperbolic and other transformations. Schiaparelli, G. V. [1861] Tor. Ac. Mm. 21 (1864) 227-.

Inverse curves. Verdam, G.J. Amst. N. Vh. 12 (1846) 67-.

- - and surfaces, mutual relations. Booth, J. B. A. Rp. (1858) (pt. 2) 3.

- figures. Tortolini, B. Tortolini A. 2 (1859) 189-.

Inversion. Hart, A. S. Camb, and Dubl. Mth. J. 8 (1853) 47-.

-. Böklen, O. Grunert Arch. 32 (1859) 83-.

- Moutard, T. N. A. Mth. 3 (1864) 306-.

- Habich, E. N. A. Mth. 5 (1866) 399-.

-. Newenglowski, (Prof.) - Par. Éc. Norm. A. 2 (1873) 133 -

-. Bianchi, L. [1878] G. Mt. 17 (1879) 40-.

- Vaněček, J. S. As. Fr. C. R. (1883) 127-.

- Pirondini, G. G. Mt. 27 (1889) 168-.

- Laisant, -. Mathesis 10 (1890) 224-.

- Giacomini, A. G. Mt. 35 (1897) 125-.

- of curves. Ferrers, N. M. QJ. Mth. 1 (1857) 32-.

-, dual, of Cartesian and Boothian coordinates. Jeffery, H.M. [1880] QJ.Mth. 17 (1881) 311-.

-, general. Vaněček, J.S. [1882] C. R. 94 (1882) 1042-; Münch. Ak. Sb. 12 (1882) 463-; Prag Sb. (1882) 203-; R. S. P. 34 (1883) 29-

一, -.Vaněček, J. S., \& Vaněček, M. N. Prag Sb. (1883) (Mth. Vortr.) 345-; Fschr. Mth. (1884) 603-.
Inversion, general. Gaertner, $R$. Arch. Mth. Ps. 10 (1891) 337-.

- method. Taylor, H. M. Mess. Mth. 3 (1866) 228-.

quadratic, of plane curves. Hirst, T.A. R. S. P. 14 (1865) 91-。

- Series of quadrangles. Gob, A. As. Fr. C. R. (1891) (Pt. 2) 232-.

Involution in plane. Castelnuovo, $G$. Ven. I. At. (1885-86) 1559-.

Involutory birational transformation. Schoute, P. H. N. Arch. Wisk. $9\left(^{*} 1882\right)$ 117-; Bll. Sc. Mth. As. 6 (1882) (Pt. 1) 152-, 174-

multiple transformations of 2 spaces. Pannelli, M. Nap. Rd. 26 (1887) 153-.

- quadratic relation, type. Kilbinger, - . Z. Mth. Ps. 33 (1888) 14-.

- transformations. Schoute, P. H. As. Fr. C. R. (1884) (Pt. 2) 42-.

- transformations of space. Martinetti, $\boldsymbol{V}$. Mil. I. Lomb. Rd. 18 (1885) 132-.

- - Montesano, D. Mil. I. Lomb. Rd. 21 (1888) 688-; Rm. R. Ac. Linc. Rd. 5 (1889) (Sem. 2) 123-.

Isogonal transformation, Roberts's. Ocagne, M. $d$ '. G. Teix. J. Sc. 9 (1889) 109-.

Linear quasi-inversion, applications to osculating curves. Servais, C. Mathesis 8 (1888) 28-.

Linkwork and point transformations, simpler cases. Burgatti, P. Palermo Cir. Mt. Rd. 14 (1900) 192-.

Metamorphic methods of transformation, and corresponding points. Terquem, O. N. A. Mth. 5 (1846) 497-.

Method of transformation in geometry. Zeuthen, H. G. Mth. Ts. 5 (1863) 129-.

- - - - Gohierre de Longchamps, -. Par. Éc. Norm. A. 3 (1866) 321-.

- - - Allardice, R. E. Edinb. Mth. S. P. 6 (1888) 14-.

$-\frac{-}{-}$, miscellaneous applications. Collignon, É. As. Fr. C. R. (1894) (Pt. 2) 14-。

Multiform relation of 2 plane figures. Wiener, C. [1869] Mth. A. 3 (1871) 11-.

- transformation of plane. Björling, C.F.E. Stockh. Ak. Hndl. Bh. 13 (Afd. 1) (1888) No. 8, 19 pp.

Multiple correspondence between two 3-dimensional spaces. Tognoli, O. [1871] G. Mt. 10 (1872) 1-, 141-.

- plane transformations. Jung, G. Rm. R. Ac. Linc. Rd. 2 (1886) (Sem. 2) 302-.

- - Borghese, F. G. Mt. 32 (1894) 58 -. _ - of minimum order. Jung, G. Mil. I. Lomb. Rd. 20 (1887) 370-.

Non-isogonal plane transformations. Laisant, C. A. Par. S. Mth. Bll. 15 (1887) 103-.

Orthogonal transformation, geometrical meaning of a form. Hill, M. J.M. L. Mth. S. P. 26 (1895) 339-.

Orthotangential transformation in plane and space. Longchamps, G. de. Prag Sb. (1888) (Mth.-Nt.) 241-.

Periodic cubic transformations. Kantor, $S$. Am. J. Mth. 19 (1897) 1-.

- trunsformations. Kantor, S. C. R.100(1885) 95-. 
Periodic uniform transformations, method of treatment. Kantor, S. C. R. 100 (1885) 42-.

Plane curves, transformation. Cremona, $L$. B. A. Rp. 34 (1864) (Sect.) 3-.

- - - Cayley, A. [1865] L. Mth. S. P. 1 (1866) No. 3, 11 pp.

- - - Scott, (Miss) C. A. As. Fr. C. R. (1897) (Pt. 2) 50-; QJ. Mth. 29 (1898) 329-. - figures, transformation, and mode of generating skew curves. Jonquières, E. de. N. A. Mth. 3 (1864) 97-.

- - transformations. Cremona, L. [1864-73] Bologna Ac. Sc. Mm. 5 (1865) 3-; Bll. Sc. Mth. As. 5 (1873) 206-.

Point transformation of algebraic curves. Jamet, V. N. A. Mth. 19 (1900) 506-.

- transformations, general properties. Brill, J. QJ. Mth. 27 (1895) 356-.

- in plane. Brill, J. Mess. Mth. 24 (1895) 113-.

- - - Lovett, E. O. [1897] N. Y. Am. Mth. S. Bll. 4 (1898) 97-.

- - of space leaving volumes unaltered. Carda, K. Mh. Mth. Ps. 8 (1897) 170-.

Polar reciprocal of curve with respect to cubic. Russell, W. H. L. R. S. P. 44 (1888) 388-.

Polynomial $F(x, y)$ for which $x, y, \sqrt[n]{F}$ are rational functions of 2 parameters. Bottari, A. A. Mt. 2 (1899) 277-.

Porismatic $(2,2)$ relation, algebraic theory. Pasch, M., \& Rosanes, J. [1868] Crelle J. 70 (1869) 169-.

Projection, double, by means of quadric. Faure, H. A. (xII) Isère S. Bll. 2 (1870) 260 -.

-, stereoscopic. Cassani, P. Ven. I. At. (1884-85) 1835-.

-, - Emilio, R. d'. Ven. Aten. 2 (1886) 71-; 2 (1887) 75-; (1890) 607-.

Quadratic plane transformations. Retali, $V$. Bologna Ac. Sc. Mm. 10 (1889) 653-.

- - - construction by tri-homologous triangles. Ferrari, $F$. Par. S. Phlm. Bll. 1 (1899) 93-.

- - - degenerate forms of 1st rank. Schubert, H. C. H. Hamb. Mth. Gs. Mt. 1 (1889) 31-.

- _ - , successive. Kantor, S. [1880] Wien Ak. Sb. 82 (1881) (Ab. 2) 237 -.

- transformation. Hirst, T.A. N. A. Mth. 5 (1866) 213-.

- - Igel, B. Z. Mth. Ps. 17 (1872) 516-.

- - Paraira, M. C. N. Arch. Wisk. 2 (*1876) 62-.

- - Hirst, T. A. QJ. Mth. 17 (1881) 301-.

- - Rychlicki, S. Par. T. Nauk Śc. Pam. $12\left({ }^{*} 1882\right)$ Art. 2, 19 pp.

- - Petersen, $J$. Ts. Mth. 6 (1889) 1 -

- -. Neuberg, J. Liège S. Sc. Mm. 16 (1890) No. $8,12 \mathrm{pp}$.

- - of conic, quartic curves arising from. Leuch, A. Bern Mt. (1889) 1-.

- - between 2 planes, cases. Cayley, A. (xII) J. H. Un. Cir. [1] (1882) 178-.

- - of planes of space into system of quadrics. Timerding, H. E. A. Mt. 1 (1898) 95-.

- transformations. Amigues, $\stackrel{E}{E}$. N. A. Mth. 16 (1877) 422-, 451-, 496-, 529-.
Quadratic transformations by means of pencils in involution. Neuberg, $J$. Mathesis 8 (1888) 177-.

- - - - ranges, etc., in involution. Ripert, L. Mathesis 19 (1899) 185-, 217-. - and rational surfaces. Reye, $T$. Mth. A. 48 (1897) 113-.

- - reduction of point-singularities by. Levi, B. A. Mt. 26 (1897) 219-.

- -, transformations in $n$-dimensional space compounded of. Kantor, S. Am. J. Mth. 18 (1896) 219-.

Quartic transformation in 3-dimensional space. Cremona, L. [1884] Ir. Ac. T. 28 (1880-86) 279-.

Rational curves in 2 dimensions. Autonne, $L$. C. R. 121 (1895) 673-.

- - 3 dimensions. Autonne, - C. R. 121 (1895) 881-, 1129-.

and involutory space transformations. Montesano, D. G. Mt. 31 (1893) 36-; Mil. I. Lomb. Rd. 30 (1897) 563-.

- quadratic space transformation whose inverse is of 4 th degree. Cremona, L. Bologna Ac. Sc. Mm. 1 (1871) 365-.

- - transformation. Hofmann, F. Z. Mth. Ps. 31 (1886) 283-.

- - transformations. Haskell, M. W. [1898] Calif. Ac. P. (Mth. Ps.) 1 (1903) 1-.

- space transformations. Cremona, $L$. Mil. I. Lomb. Rd. 4 (1871) 269-, 315-; A. Mt. 5 (1871-73) 131-.

- - -, classification. Loria, G. Mil. I. Lomb. Rd. 23 (1890) 824.

- transformation between 2 planes. Cayley, A. L. Mth. S. P. 3 (1869-71) 196-.

- - - - spaces. Cayley, A. [1869] L. Mth. S. P. 3 (1869-71) 127-.

_ transformations in 3-dimensional space, class. Aschieri, F. [1879] Mil. I. Lomb. Mm. 14 (1881) 251-.

- - , theorem, and a transformation of Hirst. Saltel, L. Brux. Ac. Bll. 34 (1872) 51-.

Ray systems and focal surfaces of 2 related surfaces. Voss, A. Mth. A. 30 (1887) 227-.

Reciprocal Cremonian systems of degree $m$, surfaces generated by 2 . Jung, $G$. Rm. R. Ac. Linc. Rd. 1 (1885) 762-, 773-, 810-, 884. - directions, transformation in space by. Laguerre, E. C. R. 92 (1881) 71-.

- - - - - - (Laguerre). Smith, P. F. A. Mth. 1 (1900) 153-.

- radii vectores, interpretation of correspon. dences by. Amaldi, I. Nap. Rd. 30 (1891) 238-.

Relationship, geometric. Swellengrebel, J. G. H. [1850] Crelle J. 43 (1852) 245-.

-, quadratic. Geiser, C.F. Bern Mt. (1865) 97-.

-, -. Weyr, Ed. Z. Mth. Ps. 14 (1869) 445-.

-, - Cassani, $P$. [1897] Ven. I. At. (189798) 30 -.

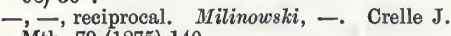
Mth. 79 (1875) 140-.

- of 5 th degree. Miller, Hub. Mth. A. 2 (1870) 281-.

Representable surfaces: algebraic surfaces uniformly representable on plane. Nöther, $\boldsymbol{M}$. Gött. Nr. (1870) 1-. 


\section{0ther Transformations}

Representable surfaces, equation. Brill, $A$. [1871] Mth. A. 5 (1872) 401-.

Representation, plane, of algebraic surfaces with cuspidal curves. Cremona, L. Bologna Ac. Sc. Mm. 2 (1872) 117-.

,,$-----\frac{1}{-}$ multiple curve. Tognoli, $O$. G. Mt. 14 (1876) 263-, 378-

-, -, - reciprocal of Steiner's surface. Laguerre, E. [1872] (x) Par. S. Mth. Bll. 1 (1873) 21-.

-,- , - ruled surfaces of 4 th degree, with double curve of 3rd degree. Clebsch, R.F.A. Mth. A. 2 (1870) 445-.

-, - - - surface of 3rd degree. Clebsch, $R$. F. A. Mth. A. 5 (1872) 419-.

$-, \ldots,--\ldots-\frac{1}{-}$, as affected by singularities. Diekmann, J. Mth. A. 4 (1871) 443-.

- of surface of $4 \mathrm{th}$ degree. Frahm,W. [1873] (Ix) Mth. A. 7 (1874) 512-.

Scalene transformation of plane curve. Cayley, A. QJ. Mth. 13 (1875) 321-.

Simply rational transformations of surfaces. Painlevé, P. C. R. 110 (1890) 184-

- - - - - and class of differential equations. Painlevé, P. C. R. 110 (1890) 226-.

Solid figures, transformation, 2 methods. Amigues, $E^{\prime}$. N. A. Mth. 18 (1879) 548-; 19 (1880) 433-, 481-.

Straight lines and circles through one point, systems. Fuortes, T. G. Mt. 16 (1878) 91-. - - transformations. Lovett, E. O. C. R. 129 (1899) 20-, 144-, 358.

Substitution, surfaces obtained from plane curves by. Eckardt, F.E. (vII) Mth. A. 7 (1874) 590 -.

Substitutions, rational, permitting rational inversion. Rosanes, J. [1870] Crelle J. 73 (1871) 97-.

-, regular non-linear. Autonne, -. C. R. 122 (1896) 1043-.

Surfaces, transformations by planes through normals. Enneper, $A$. Gött. Nr. (1877) 369- ; (1881) 305-; Mth. A. 21 (1883) 267-.

Symmetrical $(n, n)$ point transformations and Poncelet tetrastigms. Schober, $K$. Mh. Mth. Ps. 9 (1898) 89-.

- systems of elements. Weyr, E. J. [1874] Bordeaux S. Sc. Mm. 10 (1875) 329-.

System of quadrics with common generator and related projective system. Cardinaal, $J$. Crelle J. Mth. 111 (1893) 31-.

Tangential transformation. Genese, $R . W$. B. A. Rp. (1891) 571-。

Transformations which have no fundamental curves of 1 st kind. Kantor, $S$. Acta Mth. 21 (1897) 1-.

Transmutations of curves. Cayley, A. Liouv. J. Mth. 14 (1849) 40-; 15 (1850) 351-。

Uniform automorphic transformations, algebraic configurations with. Hurwitz, A. Mth. A. 32 (1888) 290-; 41 (1893) 403-.

- cubic space transformation, simple. Döhlemann, $K$. Münch. Ak. Sb. 24 (1895) 41-.

- - transformations, construction. Bertini, E. Mil. I. Lomb. Rd. 15 (1882) 154.

- plane and space transformations. Bianchi, L. G. Mt. 16 (1878) 263-.

\section{Point-groups 8030}

Uniform plane transformations. Nöther, $M$. Mth. A. 5 (1872) 635-.

- quadratic plane transformations. Timerding, H. E. Mth. A. 53 (1900) 193-.

- space transformations, and application to representation of algebraic surfaces. Nöther, M. [1870] Mth. A. 3 (1871) 547-.

- transformations, 2. Schoute, P. H. As. Fr. C. R. (1883) 131-.

,--- , geometric representation. Le Paige, C. M. M. H. H. Brux. Ac. Bll. 3 (1882) 760 - ; 4 (1882) 415-

$(1,2)$ plane transformations. Noether, $M$. Erlang. Ps. Md. S. Sb. 10 (1878) 81-.

$(-,-)$ relation between elements of funda. mental configuration of 1st rank. Schubert, H. C. H. Crelle J. Mth. 88 (1880) 311-.

$(-,-)-1$ fundamental configurations of 2nd rank. Schwarz, A. Wien Ak. Sb. 94 (1878) (Ab. 2) 310-.

$(-,-)$ space transformations. Paolis, R. de. Rm. R. Ac. Linc. Mm. 1 (1885) 576-

$(-,-)-$ - Wimmer, B. Z. Mth. Ps. 36 (1891) 214-.

$(1,3)$ representation of cubic surface. Kantor, S. [1881-83] Crelle J. Mth. 95 (1883) 147-. $(1,4)$ correspondence. Cotterill, $T$. [1868] L. Mth. S. P. 2 (1869) 119-.

$(-,-)$ relationship between 2 planes. Aleksěev, V. G. [1888] Rec. Mth. (Moscou) 14 (1890) 223-; Fschr. Mth. (1891) 647-.

$(2,2)$ relationship. Heger, $R$. Z. Mth. Ps. 17 (1872) 71-.

$(-,-,-$, extension of Poncelet's polygons. Fontené, $G$. N. A. Mth. 16 (1897) 437-。

$(-,-)$ transformation. Visalli, $P$. Palermo Cir. Mt. Rd. 3 (1889) 165-

$(-,-)$ transformations obtained by 2 quadric transformations. Burali-Forti, C. Palermo Cir. Mt. Rd. 5 (1891) 91-.

$(3,3)$ transformation. Ascione, E. G. Mt. 31 (1893) 55-.

$(m, n)$ relation on curve, Stephanos's theorem generalised. Kohn, G. Wien Ak. Sb. 90 (1885) (Ab. 2) 226-.

\section{Groups of points on an alge- braic curve; genus of curves; principle of correspondence. (See also 7610, 7630, 7660.)}

Algebraic functions. Brill, A., \& Nöther, $M$. [1873] Gött. Nr. (1873) 116-; Mth. A. 7 (1874) 269-.

- - Noether, M. Mth. A. 15 (1879) 507-.

- - (Noether). Weiss, W. Mh. Mth. Ps. 7 (1896) 321-.

- - development of theory. Brill, A., $d$ Noether, M. [1893] D. Mth. Vr. Jbr. 3 (1894) 107 -

- - and singularities of curves. Noether, $M$. Mth. A. 9 (1876) 166-.

- _ - - - - extension of theorem of Noether's. Del Pezzo, P. Palermo Cir. Mt. Rd. 2 (1888) 139-. 
Conics through several points of given curves. Berzolari, L. Mil. I. Lomb. Rd. 33 (1900) 664-, 809-.

Constructions of $3 r d$ and 4th degrees, by straight line and cubic curve. London, $F$. Z. Mth. Ps. 41 (1896) 129-.

Correspondence, Cremonian, in plane and space. Aschieri, F. Mil. I. Lomb. Rd. 14 (1881) 21-.

-, - quadratic, between elements of 2 line spaces. Aschieri, F. Mil. L. Lomb. Rd. 14 (1881) 219-.

- of 2 curves (Steiner's theorems, Crelle J. 1846). Padova, $\boldsymbol{E}$. G. Mt. 5 (1867) 240 -.

- on curves. Küpper, C. Prag Sb. (1892) (Mth.-Nt.) 257-.

-, formula. Brill, A. Mth. A. 7 (1874) 607-.

-, multiform, application to curves of $3 \mathrm{rd}$ degree. Vries, J. de. N. Arch. Wisk. 12 (1886) 82-; Fschr. Mth. (1885) 629.

- of 2 points on curve. Cayley, A. L. Mth. S. P. 1 (1866) No. 7, 7 pp.

- between points of 2 plane curves, special. Pirondini, G. G. Mt. 38 (1900) 92-.

- of points in relation to 2 tetrahedra. Cayley, A. L. Mth. S. P. 4 (1873) 396-.

- quadratic, between 2 simply infinite systems. Weyr, Em. A. Mt. 4 (1870-71) 272-.

- between straight line and curve, theorem of Lüroth and Gordan. Netto, E. Mth. A. 46 (1895) 310-.

- of straight lines; correspondence of points. Aschieri, $F$. G. Mt. 13 (1875) 328-.

- - systems of points on curve. Brill, $A$. Gött. Nr. (1871) 507-; Mth. A. 6 (1873) 33-.

- $(1,1)$ between focal cubics, metrical properties. Bricard, R. Par. S. Mth. Bll. 28 (1900) 39-. - $\left(-\frac{-}{\text { Rp. }}(1881)\right.$, involutional. Genese, $R . W$. B. A.

- $(1,2)$, algebraic and geometric study. Pittarelli, G. Km. R. Ac. Linc. Mm. 3 (1886) 375-.

$-\left(-, \frac{-}{-}\right)$ on cubies with double point. Pittarelli, G. Rm. R. Ac. Linc. Mm. 3 (1886) 401-.

- $(1,4)$ between points on curves of surfaces of 3rd degree. Waelsch, E. [1894] D. Mth. Vr. Jbr. 4 (1897) 113-.

- $(2,2)$. Cayley, A. QJ. Mth. 11 (1871) 83-; 12 (1873) 197-.

$-(-,-)$, and curves of genus 1. Weyr, E.J. Wien Ak. Sb. 87 (1883) (Ab. 2) 592-.

$-(-,-)$, form $f\left(x^{2} y^{2}\right)$ and its invariants and covariants. Capelli, A. G. Mt. 17 (1879) 69-.

$-(m, n)$. Retali, $V$. Mil. I. Lomb. Rd. 32 (1899) 1051-.

Correspondences, algebraic. Brill, A. Mth. A. 31 (1888) 374-; 36 (1890) 321-.

$\ldots, \ldots$, and the generalised principle. Hurwitz, A. Leip. Mth. Ps. B. 38 (1886) 10-; Mth. A. 28 (1887) 561-.

,-- , between $r$ points of a linear space. Berzolari, L. Rm. R. Ac. Linc. Rd. 4 (1895) (Sem. 2) 148-.

voL. I.
Correspondences on elliptic curves. Brodén, $T$. Stockh. Öfv. (1893) 45-, 213-.

--- Kantor, S. Tor. Ac. Sc. At. 29 (1894) 9-.

---1 , uniform. Segre, C. Tor. Ac. Sc. At. 24 (1889) 734-.

- between points of 2 curves. Halphen, G. H. [1876] Par. S. Mth. Bll. 5 (1877) 7-.

-, symmetrical, of odd degree. Kohn, G. Wien Ak. Sb. 106 (1897) (Ab. 2a) 488-.

-, uniform, between groups of $p$ points on curve of genus $p$. Castelnuovo, G. Mil. I. Lomb. Rd. 25 (1892) 1189-.

,-- , hyperelliptic curves having. Kantor, $S$. Palermo Cir. Mt. Rd. 9 (1895) 65-.

-, - , singular, of harmonic elliptic curves. Amodeo, $F$. A. Mt. 19 (1891-92) 145-.

- $(1,2)$ and $(1,3)$. Piazza, S. Tor. Ac. Sc. At. 17 (1881) 431-.

- $(p, p)$ on curves of genus $p$ with general moduli. Scorza, $G$. Tor. Ac. Sc. At. 35 (1900) 285- or 443-.

Corresponding points on 2 curves, theorems. Zeuthen, H. G. [1870] Mth. A. 3 (1871) 150-, 323-.

$--1-$, Zeuthen's theorem. Weiss, $W$. Mth. A. 29 (1887) 382-.

$---1-,-\frac{-}{-}$, deduction from. Brodén, T. Stockh. Ófv. (1893) 345-.

Cremona transformations with fixed curves. Doehlemann, $K$. Mth, A. 39 (1891) 567-.

Curve, genus 0 , binary forms represented on. Maisano, G. Palermo Cir. Mt. Rd. 1 (1887) 14-. ,--1 , cyclic relations of points on. Gegenbauer, L. Mh. Mth. Ps. 4 (1893) 330.

,--3 , and uniform functions of 2 variables. Picard, É. $\quad$ C. R. 93 (1881) 835-.

- $,-p, 2 q$ points on, problem. Ptaszycki, $J$. C. R. 130 (1900) 105-.

Curves, any genus, linear systems. Jung, $G$. [1887] A. Mt. 15 (1887-88) 277-.

-, genus 0 , algebraic forms in theory. Igel, $B$. Wien Ak. Sb. 89 (1884) (Ab. 2) 218-

-, - - , families. Lerch, M. Mh. Mth. Ps. 2 (1891) 465-.

-, - 1. Humbert, G. C. R. 97 (1883) 989-, 1042-, 1136-.

-, - - Schlesinger, O. C. R. 107 (1888)224-. -, _ _ involutions on. Weyr, E. Wien Ak. Sb. 101 (1892) (Ab. 2a) 1506-.

,,,----- , and Steiner's polygons. Weyr, E. Wien Ak. Sb. 101 (1892) (Ab. 2a) 1457-, 1695-.

-,- - parametric representation. Duarte Leite, - G. Teix. J. Sc. 9 (1889) 3-; Fschr. Mth. (1888) 716.

-, - - , symbolical calculus on. Czuber, $E$. [1894] D. Mth. Vr. Jbr. 4 (1897) 100-.

-, - - , - - Weyr, E. Wien Ak. Sb. 193 (1894) (Ab. 2a) 365-.

-, _ -, systems. Martinetti, V. Mil. I. Lomb. Rd. 20 (1887) 264-, [854].

,$-->1$, hyperelliptic. Kilpper, $K$. Prag Sb. (1896) (Mth.-Nt.) No. 43, 11 pp.

-, - 2, proof of theorem. Esclaibes, (le rév. père) - d'. (xII) Brux. S. Sc. A. 5 (1881) (Pt.2) 34-.

NN 


\section{Correspondence}

Curves, genus $p$, normal and subnormal, and birational and uniform transformation. Arme nante, A., \& Jung, G. G. Mt. 7 (1869) 235-

-, genera $4,5,6$, allowing automorphic transformation. Wiman, A. [1895] Stockh. Ak. Hndl. Bh. 21 (Afd. 1) (1896) No. 3, 41 pp.

- of maximum genus, plane and skew. Kilpper, K. Prag Sb. (1888) (Mth.-Nt.) 265-.

-, plane, decomposition of singularities, and genus. Bertini, $E$. Mil. I. Lomb. Rd. 21 (1888) 326-, 413-. -, -, general theorem. Guccia, G. B. C. R.

,- 2 , related point to point projectively, are of same genus $p$, proof. Bertini, $E$. G. Mt. 7 (1869) 105-.

- Riemann's surfaces in relation to. Del Pezzo, P. Palermo Cir. Mt. Rd. 6 (1892) 115-.

- and surfaces, points in theory. Humbert, $G$. Liouv. J. Mth. 10 (1894) 169-.

,$----\ldots-$ (Humbert). Painlevé, $P$. Liouv. J. Mth. 10 (1894) 203-.

-, 3rd degree, curves of $m$ th degree through $3 m-1$ points. Halphen, G. H. Mth. A. 15 (1879) 359-.

,--1, rational derivation on. Story, W. E. Am. J. Mth. 3 (1880) 356-.

- $-\ldots$, recurring series of pentagons in. Serret, P. C. R. 115 (1892) 406-, 436-

- , 4th degree, genus 3 , parametric expression of coordinates. Cayley, A. L. Mth. S. P. 6 (1875) 81-.

-, $n$th degree. Küpper, $K$. Prag Ab. 3 (1890) No. 7, 25 pp.

-, - - , maximum basis $B$ for $\mu$-fold variety. Kutpper, C. Mh. Mth. Ps. 6 (1895) $5-$.

"Curves," points on. Segre, C. A. Mt. 22 (1894) 41-.

Cyclic curves, curvature, and projective geometry. Schwarz, A. Mh. Mth. Ps. 10 (1899) 250-; 11 (1900) 71-.

Cyclic projective groups of points. Liiroth, $J$. Mth. A. 13 (1878) 305-。

- - systems. Bonsdorf, E. [E. J.] [1879] Helsingf. Acta 11 (1880) 327-.

Double planes which can be represented on single plane. Noether, $M$. Mth. A. 33 (1889) 525-.

Elliptic curyes, geometry on. Castelnuovo, $G$. Tor. Ac. Sc. At. 24 (1889) 4-.

Genus and adjoint curves of algebraic curve, method of determining. Tikhomandritzky, -. Bll. Sc. Mth. 17 (1893) 51-; Par. Ec. Norm. A. 10 (1893) 151-.

-, conservation in uniform transformations. Halphen, G. H. [1875] Par. S. Mth. Bll. 4 (1876) 29-.

Schubert, H. C. H. Mth. A. 16 (1880) 180-.

- of curves. Baltzer, R. (Ix) Leip. Mth. Ps. B. 25 (1873) 523-.

- - Halphen, G. H. As. Fr. C. R. 4 (1875) 237-.
Groups of Points 8030

Genus of curves corresponding to points in plane involutions of any class. Martinetti, $V$. Palermo Cir. Mt. Rd. 4 (1890) 30-, 126-

-, etc., of curves, Plücker's symbols for. Zahradnik, K. Ċasopis 2 (*1873) 172-, 266-; Fschr. Mth. ("1873) 414.

- of plane triangular curves. Jamet, $V$. Par. S. Mth. Bll. 16 (1888) 132-.

- - set of curves. Zeuthen, H. G. Palermo Cir. Mt. Rd. 3 (1889) 171-

- - skew curves. Salvatore-Dino, N. Nap. Rd. 18 (1879) 133-.

Groups of points.

Paolis, R. de. Rm. R. Ac. Linc. Mm. 1 (1877) 511-.

Castelnuovo, G. Tor. Ac. Sc. At. 24 (1889) 346-.

Bertini, E. Tor. Ac. Sc. At. 26 (1891) 118-.

Valentiner, $H$. Sk. Nf. F. (1892) 347-; Fschr. Mth. (1892) 660.

Küpper, C. Mh. Mth. Ps. 6 (1895) 127-.

Macaulay, F. S. L. Mth. S. P. 26 (1895) 495-.

Hardcastle, (Miss) F. B. A. Rp. (1900) 121-.

(de Paolis, 1877.) Scott, (Miss) C. A. [1900] N. Y. Am. Mth. S. Bll. 7 (1901) 24-

with bibliography. Hardcastle, (Miss) $F$. N. Y. Am. Mth. S. Bll. 4 (1898) 390-.

Cayley-Brill theorem, new proof. Sporer, $B$. Z. Mth. Ps. 39 (1894) 228-.

on circle. Kantor, $S$. [1878] Mth. A. 14 (1879) 323-.

- curves of 3rd degree. Weyr, Em. Z. Mth. Ps. 15 (1870) 344-.

_ - _ - , 8th point not determined by 7 . Cayley, A. Mess. Mth. 21 (1892) 132-.

- - - - - relation between 10 points. Weddle, T. Camb. and Dubl. Mth. J. 5 (1850) 236-.

Mth. A. $2(1870) 413$.

- - $m$ th degree, genus $p$, cut by $\infty^{2}$ lines. Kupper, $K$. Prag Sb. (1887) (Mth.-Nt.) 477-, 609-.

determination. Chasles, M. C. R. 73 (1871) 927-, 970-

harmonic, on rational curves of 3rd and 4th degrees. Zahradnik, K. Prag Sb. (1874) 12-.

involution triplets. Weyr, E.J. [1883] Wien Ak. Sb. 88 (1884) (Ab. 2) 436-.

involutory. Vries, $J$. de. Amst. Ak. Vs. M. 6 (1889) 92-; Arch. Néerl. 23 (1889) 355-.

- series, irrational. Amodeo, F. A. Mt. 20 (1892-93) 227-.

- of 1st order, anharmonic ratio of 4 pairs of points. Klein, B. Z. Mth. Ps. 28 (1883) 252-.

irrational series. Castelnuovo, G. Rm. R. Ac. Linc. Rd. 7 (1891) (Sem. 2) 294-.

linear. Rosanes, $J$. Crelle J. Mth. 88 (1880) 241-.

- series. Bertini, E. A. Mt. 22 (1894) 1-.

- multiples. Castelnuovo, G. Palermo Cir. Mt. Rd̃. 7 (1893) 89-. 
minimum abnormal, on curve $C^{m}$. Küpper, $C$. Prag Sb. (1892) (Mth.-Nt.) 403-.

primitive and imprimitive, on curve $C_{p}{ }^{n}$. Kiipper, K. Prag Sb. (1897) (Mth.-Nt.) No. 31,14 pp.

projective, on same straight line. Sidler, $G$. Zür. Vjschr. 9 (1864) 217-.

on rational curves. Weyr, Em. Prag Sb. (1873) 70 -.

and reduction of algebraic integral. Cayley, A. (xI) J. H. Un. Cir. [1] (1882) 209.

remarkable. Kraus, L. [1879] Mth. A. 16 (1880) 245-.

on skew curve intersected by surfaces, comparison with those of residual curves. Rohn, K. Leip. Mth. Ps. B. 49 (1897) 627-.

special systems, on particular type of basecurve. Hardcastle, (Miss) F. [1897] L. Mth. S. P. 29 (1898) 132-.

on straight line, and generation of plane curves. Schumacher, $R$. Crelle J. Mth. 110 (1892) 230-; 111 (1893) 254-.

$n$th order, with base on curve of $m$ th degree. Chasles, M. C. R. 45 (1857) 393-.

$G_{4}{ }^{1}$, on sextic curve with 5 double points. Cayley, A. [1874] Mth. A. 8 (1875) 359-. $g_{n}^{(2)}$ on curves $C_{p}{ }^{n}$. Küpper, $C$. Prag $\mathrm{Sb}$. (1892) (Mth.-Nt.) 264-.

$(m, n)$ homographies, and double elements. Weyr, Em. Prag Sb. (1869) (pt. 2) 33-.

Homography of 2nd kind. Castelnuovo, $G$. Ven. I. At. (1886-87) 1041-.

- - - on skew cubic. Deruyts, F. Brux. Ac. Bll. 17 (1889) 312-.

- - - - - - Aschieri, F. Mil. I. Lomb. Rd. 23 (1890) 312-.

Intersection of $\mathbf{2}$ cones, and problem of Steiner's on plane curves. Beck, $A$. Zür. Vjschr. 38 (1893) 199-, 266-.

- - curve with non-adjoint curves, systems of points. Noether, M. Mth. A. 15 (1879) $507-$.

- 2 curves at a singular point. Guccia, $G$. B. C. R. 107 (1888) 656-, 766.

- - curves, systems of points. Bacharach, J. [1879] Erlang. Ps. Md. S. Sb. 12 (1880) 12-.

Intersections of curves. Krey, H. Z. Mth. Ps. 22 (1877) 396-.

- - - of 3rd and 4 th degrees with conics. Rohn, K. Dresden Isis Sb. (1898) 24.

- - plane curves. Scott, (Miss) C. A. N. Y. Am. Mth. S. Bll. 4 (1898) 260-.

- - - Macaulay, F. S. N. Y. Am. Mth. S. Bll. 4 (1898) 540-.

$-{ }_{-}-$, multiplicities in. Segre, C. G. Mt. 36 (1898) 1-.

\section{Involutions.}

Hearn, G. W. Mathematician 2 (1847) 63-.

Terquem, $O$. N. A. Mth. 12 (1853) 24-.

any type. Genty, $M$. Par. S. Mth. Bll. 20 (1892) 106-.

biquadratic. Le Paige, C. C. R. 98 (1884) 285-.

-. Fairon, J. Brux. Ac. Bll. (1900) 950-. biquadratic, degenerate forms, and 7 systems of conics having 4-point contact with curve of $4 \mathrm{th}$ degree. Weyr, E.J. Wien Ak. Sb. 83 (1881) (Ab. 2) 807-。

-, 1st rank. Weyr, E. J. Wien Ak. Sb. 83 (1881) (Ab. 2) 300-.

, 2nd rank, and their typical curves. Weyr, E. J. Wien Ak. Sb. 81 (1880) (Ab. 2) 1007-.

-, 3rd rank, and application to curves of $4 \mathrm{th}$ degree. Le Paige, C. M. M. H. H. Prag Sb. (1881) 61-.

conjugate. Le Paige, C. M. M. H. H. [1881] Wien Ak. Sb. 84 (1882) (Ab. 2) 233-; 85 (1882) (Ab. 2) 844-.

cubic. Le Paige, C. M. M. H. H. Wien Ak. Sb. 81 (1880) (Ab. 2) 845-; 83 (1881) (Ab.2) 351-; Liege S. Sc. Mm. 11 (1885) No. 1, $19 \mathrm{pp}$.

-. Berzolari, L. Nap. Rd. 30 (1891) 35-, 71-.

-. Vries, J. de. Amst. Ak. Vs. 3 (1895) 64-.

-. Klug, L. Orv.-Termt. Éts. (Termt. Szak) (1898) 95-, (Rv.) 42-.

- and biquadratic. Milinowski, - Z. Mth. Ps. 19 (1874) 205-.

-, for conic. Kantor, S. Prag Sb. (1878) 312-.

-, conjugate. Servais, C. Brux. Ac. Bll. 20 (1890) 272-.

-, fundamental problem. Weyr, Em. Prag Sb. (1872) 28-.

- and higher. Vries, J. de. Mh. Mth. Ps. 5 (1894) 1-.

, involving anharmonic properties. Weyr, Em. Mil. I. Lomb. Rd. 4 (1871) 206-.

-, in plane of complex variable. Vries, J. de. Palermo Cir. Mt. Rd. 5 (1891) 289-.

- relation between singular elements. Le Paige, C. M. M. H. H. Wien Ak. Sb. 81 (1880) (Ab. 2) 159-.

-, - - - - (Le Paige). Weyr, E. J. Wien Ak. Sb. 81 (1880) (Ab. 2) 162-

- _ - - - projective. Weyr, E.J. Wien Ak. Sb. 73 (1876) (Ab. 2) 654-.

-, on skew curves of 3rd degree. Vries, J. de. N. Arch. Wisk. 4 (1899) 101-.

-, theory. Weyr, E. J. [1874] Prag Ab. 7 (1875) (Mth.) $54 \mathrm{pp}$.

on curves of 3rd degree. Weyr, E. Časopis 9 (*1880) 145-; Fschr. Mth. $\left({ }^{*} 1880\right) 484$.

- - - - Küper, C. Prag Ab. 12 (1885) (Mth.) No. 1, 18 pp.

$--1-$ Martinetti, V. G. Mt. 23 (1885) 37-.

- - 4th degree. Le Paige, C. Brux. S. Sc. A. 8 (1884) (Pt. 2) 87-.

fundamental, on rational curves. Stahl, W. Crelle J. Mth. 104 (1889) 38-.

generalisation of theory, geometrical applications. Jonquières, E. de. Tortolini A. 2 (1859) 86-.

higher. Weyr, Em. Crelle J. 72 (1870) 285-; Prag Sb. (1870) 14-; Wien Sb. 61 (1870) (Ab. 2) 600-; Z. Mth. Ps. 16 (1871) 353-. -. August, $F$. Arch. Mth. Ps. 55 (1873) 337-. -. Eggers, H. Arch. Mth. Ps. 55 (1873) 341-. 


\section{Involutions}

higher. Le Paige, C. M. M. H. H. [1877] (xII) Brux. S. Sc. A. 2 (1878) (Pt. 1) 54-; (Pt. 2) 25-.

-, groups of points common to 2. Le Paige, C. Brux. Ac. Bll. 11 (1886) 121-.

-, and invariants of binary forms. Le Paige, C. M. M. H. H. Brux. Ac. Bll. 44 (1877) 231-.

-, multiple points. Le Paige, C. M. M. H. H. Brux. Ac. Bll. 46 (1878) 247-.

-, represented on common basis. Weyr, $E$. J. Liége S. Sc. Mm. 10 (1883) (No. 3) $8 \mathrm{pp}$.

- on skew curves of genus 1. Weyr, $E$. Wien Ak. Sb. 100 (1891) (Ab. 2a) 589-.

and homographies, higher. Le Paige, $C$. G. Teix. J. Sc. 5 ( $\left.^{*} 1883\right)$ 27-, 77-.

multiple. Vályi, G. Mth. Termt. Ets. 12 (1894) 394-; 13 (1895) 408- ; 14 (1896) 381-; Mth. Nt. B. Ung. 13 (1897) 244-, 259-, 343-.

multiply infinite, belonging to curve, linearity. Castelnuovo, G. Tor. Ac. Sc. At. 28 (1893) 727-.

with ordinary singularities. Guccia, G. B. Palermo Cir. Mt. Rd. 7 (1893) 49-; 8 (1894) 227-.

plane. Bertini, E. [1882] Mil. I. Lomb. Rd. 16 (1883) 89-, 190-.

-, new theorem. Berzolari, L. Palermo Cir. Mt. Rd. 3 (1889) 145-.

quadruple, on curves of 4th degree. Vries, $J$. de. Amst. Ak. Vs. M. 4 (1888) 307-; Arch. Néerl. 23 (1889) 93-.

rational, number on curve of given genus. Castelnuovo, G. Rm. R. Ac. Linc. Rd. 5 (1889) (Sem. 2) 130-.

on skew curves of 4 th degree. Le Paige, $C$. C. R. 98 (1884) 353-.

surfaces. Weyr, E.J. Brux. Ac. Bll. 3 (1882) 472-.

theory. Cayley, A. [1864] Camb. Ph. S. T. 11 (1871) 21-.

-. Tognoli, O. G. Mt. 20 (1882) 270 -.

- Berzolari, L. Nap. Rd. 30 (1891) 35-.

various orders, in systems of 1st species. Battaglini, G. Nap. Ac. At. 1 (1863) No. 12, 14 pp.

,$---1-2$ 2nd species. Battaglini, $G$. Nap. Rd. 4 (1865) 226-.

3rd and 4th classes. Martinetti, V. A. Mt. 12 (*1883-84) 73-.

$n$th degree. Weyr, Em. G. Mt. 10 (1872) 165-. - - and kth rank. Weyr, E.J. Wien Ak. Sb. 79 (1879) (Ab. 2) 680-.

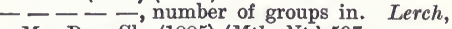
M. Prag Sb. (1885) (Mth.-Nt.) 597-.

2nd rank. Weyr, E. J. Wien Ak. Sb. 83 (1881) (Ab. 2) 349-.

Involutory pairs in multiform configurations, number. Weyr, Em. [1871] Crelle J. 74 (1872) 189-.

- plane transformations. Martinetti, $V$. A. Mt. 13 (1885) 53-.

- - - double. Bertini, E. Mil. I. Lomb. Rd. 22 (1889) 771-.

\section{Principle of Correspondence 8030}

Involutory plane transformations, uniform. Bertini, E. [1876] A. Mt. 8 (1877) 11-, 146, 244 .

- - - - Caporali, E. Nap. Rd. 18 (1879) $212-$.

- - - . Bertini, E. Mil. I. Lomb. Rd. 13 (1880) 443-.

- - - - Bobek, K. Wien Ak. Sb. 91 (1885) $(A b .2)$ 276-.

- _ - , - application to determine involutions of 5th class. Berzolari, L. A. Mt. 16 (1888-89) 191-, 335.

- relation arising from 2 curves. Fouret, $G$. C. R. 96 (1883) 1213-.

Moduli of class of algebraic equations. Brill, A. Mth. A. 1 (1869) 401-; 2 (1870) 471-.

- - equations or curves of given genus, number. Casorati, F., \& Cremona, L. Mil. I. Lomb. Rd. 2 (1869) 620-

Normal curves for $p=5,6,7$. Noether, $M$. Mth. A. 26 (1886) 143-.

- space and maximum genus for curves of $m$ th degree. Amodeo, F. Nap. Rd. 37 (1898) 433-.

- trigonal curves, plane. Amodeo, $F$. C. R. 130 (1900) 1744-; 131 (1900) 400.

Null system, significance for cubic involutions. Weyr, E. J. [1881] Wien Ak. Sb. 84 (1882) $(A b .2)$ 1264-.

- -, 2nd degree. Ameseder, A. Wien Ak. Sb. 83 (1881) (Ab. 2) 385-.

,$---\frac{1}{-}$ Ameseder's, applications. Oppenheimer, H. Arch. Mth. Ps. 13 (1895) 268-.

- -, - -, general. Ameseder, A. Crelle J. Mth. 97 (1884) 62-.

Polar curves. Plitcker, J. Crelle J. 12 (1834) 105-.

- groups. Weyr, E. J. Wien Ak. Sb. 81 (1880) (Ab. 2) 841-.

- - geometric representation of theory. Waelsch, E. [1883] Wien Ak. Sb. 88 (1884) (Ab. 2) 418-.

Polygonal curves. Amodeo, $F$. A. Mt. 21 (1893) 221-; 24 (1896) 1-; Nap. Ac. At. 9 (1899) No. 4, 21 pp.; Nap. Rd. 39 (1900) 174 .

- - , $n$th degree, genus $p$. Küpper, C. Prag Sb. (1895) (Mth.-Nt.) No. 25, 16 pp.; (1896) (Mth.-Nt.) No. 23, 9 pp.

,,$-----(p>1)$. Küpper, C. Mh. Mth. Ps. 8 (1897) 57-.

- and skew curves, relations between. Küpper, C. Prag Sb. (1896) (Mth.-Nt.) No. 4, $11 \mathrm{pp.}$

\section{PRINCIPLE OF CORRESPONDENCE.}

Chasles, M. C. R. 62 (1866) 579-.

Cayley, A. [1867] Phil. Trans. 158 (1868) 145-.

Chasles, M. C. R. 72 (1871) 577-.

Zeuthen, H. G. Bll. Sc. Mth. As. 5 (1873) 186-.

Cayley, A. QJ. Mth. 15 (1878) 32-.

Burkhardt, H. C. R. 126 (1898) 1854-.

application. Vahlen, K. T. Crelle J. Mth. 118 (1897) 251-. 
application to analytical solutions. Saltel, $L$. C. R. 82 (1876) 324-.

- to finding class of evolute and caustic of curve. Chasles, M. C. R. 72 (1871) 394 .

- - - degree of envelope of curve or surface. Saltel, L. C. R. 83 (1876) 608-.

- _ - - - locus defined by algebraic conditions. Saltel, L. C. R. 82 (1876) 63-; 83 (1876) 529-.

- - - number of points of intersection of 2 curves. Chasles, M. C. R. 75 (1872) 736-; 76 (1873) 126-.

- - - - - - - - 3 surfaces, or of skew curve with a surface. Fouret, $G$. (Ix) Par. S. Mth. Bll. 1 (1873) 122-, 258-.

- _ - _ - - plane in which 3 curves of 3 given systems touch. Fouret, G. Par. S. Mth. Bll. 5 (1877) 130-.

- _ _ - solutions of $n$ algebraic equations in $n$ unknowns. Fouret, G. C. R. 78 (1874) 183-.

-, new developments due to. Saltel, L. Brux. Ac. Bll. 45 (1878) 102-.

- to proof of Bezout's theorem. Saltel, $L$. C. R. 81 (1875) 884-.

- - segments. Chasles, M. C. R. 83 (1876) 467-, 495-, 519-.

- - on tangents. Chasles, M. C. R. 81 (1875) 253-.

Cayley and Brill's. Zeuthen, H. G. Mth. A. 40 (1892) 99-.

character. Chasles, M. C. R. 78 (1874) 577-. Chasles's, analytical extension. Saltel, L. C. R. 80 (1875) 1064 .

complementary theorem applied to determination, without calculation, of order of multiplicity of point on given locus. Saltel, $\boldsymbol{L}$. C. R. 81 (1875) 1047-.

definition, and applications. Saltel, L. [1879] Bordeaux S. Sc. Mm. 4 (1882) 1-.

degree of multiplicity of solutions, and application to Plücker's relations. Zeuthen, $H$. G. N. A. Mth. 6 (1867) 200 -

generalisation, applied to elimination. Saltel, L. N. A. Mth. 12 (1873) 565-.

generalised. Bobek, $K$. Wien Ak. Sb. 93 (1886) (Ab. 2) 899-.

for groups of $n$ points and $n$ rays. Schubert, H. C. H. Mth. A. $12(1877) 180$ -

and law of decomposition applied to skew curves, in particular to locus of centres of osculating spheres of given skew curve. Saltel, L. Brux. Ac. Bll. 43 (1877) 266-.

of plane and space. Zeuthen, H. G. C. R. 78 (1874) 1553-.

and systems of curves. Segre, C. Bb. Mth. (1892) 33-.

- theory of characteristics. Legoux, A. Toul. Ac. Sc. Mm. 8 (1886) 208-.

of 2 variables. Chasles, $M$. C. R. 41 (1855) 1097-.

Projective generation of groups of curves $C^{2 n+\nu}$. Kupper, K. Prag Sb. (1897) (Mth.Nt.) No. 5, 15 pp.
Projective properties of curves. Juel, C. [1899] Kjøb. Dn. Vd. Selsk. Skr. 10 (1899-1902) 1-. [With French résumé.]

Rational curve, plane, 3rd degree, representation on conic. Weyr, E.J. Wien Ak. Sb. 79 (1879) ( $A b .2$ ) 429-.

- -, skew, 4th degree. Rohn, $K$. Leip. Mth. Ps. B. 42 (1890) 208-; 43 (1891) 1-.

- -, -, - - , automorphic transformation. Brambilla, A. Mil. I. Lomb. Rd. 20 (1887) 780-.

- - - , - - - , harmonic centre of "quadruple" given by. Weyr, E.J. Wien Ak. $\mathrm{Sb} .81$ (1880) (Ab. 2) 1218-.

- - , - - - - representation on conic. Weyr, E. J. [1875-76] Wien Ak. Sb. 72 (1876) (Ab. 2) 686-; 73 (1876) (Ab. 2) 203-. curves. Davis, E. W. J. H. Un. Cir. [3] (1884) 123.

- with double point or cusp, Abel's theorem. Johansson, N. A. Fschr. Mth. (1885) 405.

- - in $n$-flat space. Bissing, G. J. H. Un. Cir. [3] (1884) 123.

- - generation. Jonquières, E. de. C. R. 105 (1887) 1148-.

- -, plane. Weyr, E. Casopis 8 (*1879) 193-; Fschr. Mth. (*1879) 488.

- - - - representations on one another. Weyr, E. J. Wien Az. 15 (1878) 228-

$-\ldots,-$, degrees $n$ and $n-3$, intersections. Fabry, E. Par. Éc. Norm. A. 13 (1896) 107-.

- - , skew, singularities of 2 nd order. Weyr, $E m$. A. Mt. 4 (1870-71) 328-

Reducible curves. Noether, $M$. Erlang. Ps. Md. S. Sb. 17 (1885) 13-; Acta Mth. 8 (1886) 161-.

Residuation in regard to curve of 3rd degree. Cayley, A. [1873] (vII) Mess. Mth. 3 (1874) 62-.

- theorem (intersections of plane curves at multiple points). Macaulay, F. S. L. Mth. S. P. 31 (1900) 381-.

_ - Noether's theorem, and the RiemannRoch theorem. Macaulay, F. S. [1899] L. Mth. S. P. 31 (1900) 15-.

Residues in canonical series of adjoint curves of degree $m-3-a$. Amodeo, F. Rm. R. Ac. Linc. Rd. 2 (1893) (Sem, 1) 528-.

- relative to asymptotes. Quadrature of curves dependent on genus. Marie, M. C. R. 76 (1873) 943-.

Singular points. Plilcker, J. [1836] Liouv.J. Mth. 2 (1837) 11-.

- - and tangents, influence on degree and class of plane curves. Eazarski, M. Krk. Ak. (Mt.-Prz.) Rz. 15 (1887) 278-; Fschr. Mth. (1887) 607.

Singularities of plane curves, higher. Smith, H. J. S. L. Mth. S. P. $6(1874-75)$ 153-.

- _ _ - , and new species of curve. Brill, A. [1879] Mth. A. 16 (1880) 348-.

Skew curve, 4th degree, with double point, representation on conic. Weyr, E.J. [1878] Wien Ak. Sb. 78 (1879) (Ab. 2) 891-.

,---- , property of 9 points, etc. Petot, A. C. R. 98 (1884) 1245-.

- -5 th degree, genus 1. Montesano, $D$. Nap. Rd. 27 (1888) 181-. 
Skew curve, 6th degree, genus 1, arising from 2 trilinear relations. London, $F$. Mth. A. 45 (1894) 545-.

- curves. Kilpper, K. Prag Ab. 4 (1892) No. 5, 16 pp., No. 7, 15 pp.

- - genus 0. Tognoli, O. G. Mt. 11 (1873) 180 -

$-1-$ - Saltel, L. C. R. 80 (1875) ,--- , double points. Brill, A. Mth. A. 3 (1871) 456-.

- - of given degree, maximum genus. Bobek, $K$. Wien Ak. Sb. 93 (1886) (Ab. 2) 13-.

- - , 5th degree, genus 1. Weyr, E. Wien Ak. Sb. 90 (1885) (Ab. 2) 206-; 92 (1886) (Ab. 2) 498-; 97 (1889) (Ab. 2a) 592-.

- - 6th degree, genus 1. Weyr, E. Wien Ak. Sb. 99 (1891) (Ab. 2a) 932-; 100 (1891) (Ab. 2a) 457-.

Steiner's polygons on curve of 3rd degree. Kitpper, C. [1873-84] Prag Ab. 6 (1874) 39 pp.; Mth. A. 24 (1884) 1-.

- _ - _ - Martinetti, V. Palermo Cir. Mt. Rd. 5 (1891) 109-

- porismatic polygons determined by $(2,2)$ relationship. Weyr, E. [1869] Crelle J. 71 (1870) 18-; 73 (1871) 87-.

System of 3 doubly quadratic relations between 3 variables. Fontené, $G_{.}, \&$ Bricard, $R . N$. A. Mth. 18 (1899) 437-.

Transformation of curve to one without multiple points. Pieri, $M$. Rv. Mt. 4 (1894) 40 .

Linc. Rd. 7 (1898) (Sem. 1) 111-.

_ - - systems in plane, uniform periodic. Kantor, S. Acta Mth. 19 (1895) 115-.

- method, reduction of singularities. Vessiot, E. Par. S. Mth. Bll. 22 (1894) 208-.

- of plane curves, uniform. Voss, (Dr) A. Gött. Nr. (1873) 414.

Transformations of curves, birational. Poincaré, H. C. R. 117 (1893) 18-.

- in plane, birational, finite groups. Wiman, A. Mth. A. 48 (1897) 195-.

- uniform automorphic, curves admitting. Noether, M. Mth. A. 20 (1882) 59-; 21 (1883) 138-.

- - - periodic, foundations for theory. Kantor, S. Nap. Ac. At. 1 (1888) No. 7, 264 pp.; 4 (1891) No. 2, $74 \mathrm{pp}$.

Trigonal curves. Cavazzoni, L. Mil. I. Lomb. Rd. 32 (1899) 777-.

Triplets of points on cissoid. Zahradnik, $K$. Casopis 12 (*1883) 105-; Arch. Mth. Ps. 6 (1888) 392-.

Uniform functions connected by an algebraic relation, property. Picard, É. Bll. Sc. Mth. As. 4 (1880) (Pt. 1) 416-.

Weierstrass's points on algebraic curve. Haure, M. Par. Ec. Norm. A. 13 (1896) 115-.

- - - - Segre, $C$. Rm. R. Ac. Linc. Rd. 8 (1899) (Sem. 2) 89-.
8040 Groups of curves and points on an algebraic surface; genus of surfaces. (See also 7640.)

Castelnuovo, G. Mil. I. Lomb. Rd. 24 (1891) 127-, 307-.

Enriques, $F$. [1893-96] Tor. Ac. Sc. Mm. 44 (1894) 171-; Rm. S. It. Mm. 10 (1896) 1-.

Castelnuovo, G., \& Enriques, F. Mth. A. 48 (1897) 241-.

Asymptotic curves on skew surfaces. Jung, $r$. Časopis 20 (1891) 294-; Fschr.Mth. (1891) 824.

Class of surfaces. Humbert, G. Palermo Cir. Mt. Rd. 3 (1889) 277-; 4 (1890) 54-, 71, 72.

- - (Humbert). Castelnuovo, G. Palermo Cir. Mt. Rd. 4 (1890) 69-, 71-, 195-.

_- - (Castelnuovo). Guccia, - Palermo Cir. Mt. Rd. 4 (1890) 71.

- - Noether, M. Palermo Cir. Mt. Rd. 4 (1890) 299-.

Collineation groups on Kummer's surfaces. Kantor, S. Am. J. Mth. 19 (1897) 86-.

Continuous series of curves on regular surface. Enriques, F. Palermo Cir. Mt. Rd. 13 (1899) 95-.

Correspondence, point to point, class of surfaces representable on plane by. Brambilla, A. Mil. I. Lomb. Rd. 21 (1888) 334-, 511-.

- between points of plane and those of elliptic paraboloid. Ciamberlini, C. G. Mt. 32 (1894) 69-.

-, projective, between forms of 2 dimensions. Paolis, R. de. Rm. R. Ac. Linc. Rd. 3 (1894) (Sem. 2) 225-.

-, uniform, of algebraic configurations. Noether, M. Mth. A. 2 (1870) 293-; 8 (1875) 495-.

-, - - - surfaces, 2 criteria. Nöther, $M$. Gött. Nr. (1873) 248-

$\ldots, \ldots,-\ldots$, and differential equations, 2nd order, with fixed eritical points. Painlevé, P. C. R. 117 (1893) 611-.

Correspondences, uniform, curves and surfaces admitting. Kantor, S. C. R. 100 (1885) 343-.

- $(1,4)$ between points on curves of surfaces of 3rd degree. Waelsch, G. [1894] D. Mth. Vr. Jbr. 4 (1897) 113-.

Curve of 4-point contact on surface. Voss, A. E. Mth. A. 9 (1876) 483-.

Curves on cones, genus. Sturm, R. Mth. A. 19 (1882) 487-.

- - surfaces. Brunel, -. Bordeaux S. Sc. Mm. 3 (1893) iv.

- and surfaces, points in theory. Humbert, $G$. Liouv. J. Mth. 10 (1894) 169-.

- - - - - (Humbert). Painlevé, P. Liouv. J. Mth. 10 (1894) 203-.

Developables, curves on. Künzler, G. Sch. Nf. Gs. Vh. (1898) 47.

Double planes of genus 1. Enriques, $F$. Rm. S. It. Mm. 10 (1896) 201-.

Envelope with $n$ parameters connected by $n-2$ relations, degree. Saltel, L. C. R. 83 (1876) 894-; Brux. Ac. Bll. 43 (1877) 24-, 31-.

Genus of certain surfaces. Cayley, A. Mth. A. 3 (1871) 526- 
Groups of points in involution marked on surface. Le Paige, C. M. M. H. H. C. R. 99 (1884) 537-.

- - _ - plane, and effect in determining curves. Macaulay, F. S. L. Mth. S. P. 29 (1898) 673-.

_ _ - on surface, impossibility of some series. Picard, É. C. R. 126 (1898) 1383-.

- - straight lines on surfaces of higher degree. Affolter, F. G. Mth. A. 27 (1886) 277-; 29 (1887) 1-.

Homaloidal surfaces containing groups of conics. Montesano, D. Mil. I. Lomb. Rd. 24 (1891) 889-.

- -, 4th and 5th degrees, with no multiple lines. Montesano, D. Nap. Rd. 39 (1900) 158-.

Hyperelliptic curves, surfaces containing net of. Castelnuovo, G. Rm. R. Ac. Linc. Rd. 3 (1894) ( $\mathrm{Sem}$. 1) 473-.

- surfaces, curves on. Humbert, $G$. Liouv. J. Mth. 9 (1893) 29-, 361-.

Intersection of $\mathbf{3}$ surfaces in a singular point. Guccia, G. B. Rm. R. Ac. Linc. Rd. 5 (1889) (Sem. 1) 456-.

Intersections of 3 surfaces. Berzolari, $L$. A. Mt. 24 (1896) 165-.

Invariant number in theory of surfaces. Picard, É. C. R. 116 (1893) 285-.

- numbers in theory of surfaces. Picard, $\boldsymbol{E}$. C. R. 119 (1894) 1169-; 122 (1896) 101-.

Involution, generalised. Cayley, $A$. Camb. and Dubl. Mth. J. 2 (1847) 52-.

- of higher dimensions. Vaněcek, J. S., \& Vuněcek, M. N. C. R. 99 (1884) 742-, 856-, 909-.

- and rational homography, theory. Deruyts, F. Liège S. Sc. Mm. 17 (1892) No. 3, 208 pp.

Involutions, plane. Chizzoni, $F$. Rm. R. Ac. Linc. Mm. 19 (1884) 301-.

- - , rationality. Castelnuovo, $G$. $\mathrm{Rm} . \mathbf{R}$. Ac. Linc. Rd. 2 (1893) (Sem. 2) 205-; Mth. A. 44 (1894) 125-.

Involutory transformations, groups. Montesano, $D$. Ven. I. At. (1887-88) 1425-.

Linear genus of surface and classification to which it leads. Castelnuovo, G. Rm. R. Ac. Linc. Rd. 6 (1897) (Sem. 1) 372-, 406-.

- systems of curves on surface. Castelnuovo, G. Rm. S. It. Mm. 10 (1896) 82-; A. Mt. 25 (1897) 235-.

- lines on surface. Picard, É. Palermo Cir. Mt. Rd. 13 (1899) 344-.

Moduli of class of surfaces, number. Noether, M. Berl. Ak. Sb. (1888) 123-.

Multiple curves and developables of certain surface. Lazzeri, $G$. Ven. I. At. (1887-88) 171-.

- - of surfaces. Nöther, M. [1871] A. Mt. 5 (1871-73) 163-.

Rational curves, surfaces with systems. Nöther, M. [1870] Mth. A. 3 (1871) 161-.

Reciprocal of surface, degree. Salmon, $G$. [1847-55] Camb. and Dubl. Mth. J. 2 (1847) 65-; Ir. Ac. T. 23 (1859) 461-.

- surfaces. Cayley, A. Ir. Ac. P. 7 (1858) 20-; Phil. Trans. 159 (1869) 201-; 162 (1872) 83-.
Reciprocal surfaces. Zeuthen, H. G. Mth. A. 4 (1871) 633-.

Riemann-Roch theorem extended to surfaces. Noether, M. C. R. 103 (1886) 734-.

Ruled surfaces, double curve on. Wiman, $A$. Acta Mth. 19 (1895) 63-.

- - of any genus. Segre, C. Tor. Ac. Sc. At. 22 (1886-87) 362-.

- - with rational double curves. Weyr, E. J. [1881] Wien Ak. Sb. 84 (1882) (Ab. 2) 691-. - - 4th degree, curves on. Williams, $F . B$. [1900] Am. Ac. P. 36 (1901) 17-.

- -, 5th degree. Schwarz, H. A. [1866] Crelle J. 67 (1867) 23-.

Singular lines of surfaces, generic points. Levi, B. A. Mt. 2 (1899) 127-。

- points. Guccia, G. B. C. R. 105 (1887) 741-.

- - Del Pezzo, P. Palermo Cir. Mt. Rd. 6 (1892) 139-.

- - decomposition. Segre, C. Tor. Ac. Sc. At. 32 (1896) 521- or 781-; 33 (1897) 19-; A. Mt. 25 (1897) 1-.

- - - (Segre). Del Pezzo, P. Nap. Ac. Pont. At. 27 (1897) No. 4.13 pp., No. 10, 7 pp. - - resolution. Levi, $\dot{B}$. Tor. Ac. Sc. At. 33 (1897) 56- or 66-.

Singularities, reduction by birational transformations of space. Pannelli, M. A. Mt. 25 (1897) 67-.

Skew curve, 4th degree, automorphic transformation, and correlation with developable of osculating planes. Del Re,A. Tor. Ac. Sc. At. 22 (1886-87) 901-.

- - , - - correlations changing into developable of bitangent planes. Del Re,A. Nap. Rd. 26 (1887) 167-.

_ - , - - developable quartics and sextics occurring in automorphic transformation of. Del Re, A. Nap. Rd. 27 (1888) 37-.

curves on hyperboloid of one sheet. Cremona, L. C. R. 52 (1861) 1319-.

- - - Chasles, $M$. C. R. 53 (1861) 1077-, 1203-.

- - surface of 3rd degree. Clebsch, $R$. F. A. C. R. 62 (1866) 1114 .

- - surfaces. Jonquières, E. de. [1862] (viii) A. Mt. 5 (1863) 24-.

_ - - of 3rd and 4th degrees. Rohn, $K$. Leip. Mth. Ps. B. 46 (1894) 84-; 49 (1897) 631-.

- surfaces of given degree, maximum genus. Bobek, $K$. Wien Ak. Sb. 96 (1888) (Ab. 2) 1024-.

- -, 2nd degree, homographic systems of points on. Townsend, R. [1867] QJ. Mth. 9 (1868) 249-, 296-.

Surface, 3rd degree, representation on plane. Dumont, F. N. A. Mth. 15 (1896) 318-.

-, 6th degree, genus -1 . Noether, M. Mth. A. 21 (1883) 399-.

-, 8th degree, genus 0 . Systems of straight lines passing through given straight line. Aschieri, F. G. Mt. 12 (1874) 129-.

,$-(n-4)$ th degree, passing through double curve of surface, constancy of number of coefficients. Clebsch, R. F. A. C. R. 67 (1868) 1238-. 


\section{Groups of Points}

Surface $z^{2}=f(x y)$, double planes with $p^{(1)}=1$. Enriques, $F$. Rm. R. Ac. Linc. Rd. 7 (1898) (Sem. 1) 234-, 253-.

Surfaces admitting doubly infinite system of plane reducible sections. Castelnuovo, $G$. Rm. R. Ac. Linc. Rd. 3 (1894) (Sem. 1) 22-. - and algebraic groups, theory. Picard, $\boldsymbol{E}$. C. R. 120 (1895) 658-.

- containing curves of certain genus. Castelnuovo, G., \& Enriques, F. C. R. 131 (1900) 739 .

- with double circular generation, determination. Cosserat, E. C. R. 130 (1900) 311-.

- on which families of conics lie. Weiler, $A$. Zür. Vjschr. 29 (1884) 223-.

-, genus 0. Tognoli, O. G. Mt. 11 (1873) 180 -

-, - - Castelnuovo, G. Rm. S. It. Mm. 10 (1896) 103-.

G. [1899] Bordeaux S. Sc. PV. (18991900) $2-$.

,$--p=0$, representation on plane. Armenante, A. [1869] A. Mt. 4 (1870-71) 50-.

-, linear genus $p^{(1)}=2$. Enriques, $F$. Rm. R. Ac. Linc. Rd. 6 (1897) (Sem. 1) 139-.

,$--p^{(1)}=3$. Enriques, $F$. Rm. R. Ac. Linc. Rd. 6 (1897) (Sem. 1) 169-.

- with plane sections elliptic curves. Castelnuovo, G. Rm. R. Ac. Linc. Rd. 3 (1894) (Sem. 1) 59-.

- - - - hyperelliptic curves. Castelnuovo, G. Palermo Cir. Mt. Rd. 4 (1890) 73-.

- - - rational. Picard, É. Par. S. Phlm. Bll. 2 (1878) 127-; Crelle J. Mth. 100 (1887) 71-。

- - - Guccia, G. B. Palermo Cir. Mt. Rd. 1 (1887) 165-

- - points corresponding, fundamental points of 2. Zeuthen, H. G. C. R. 70 (1870) 742- _ - properties. Zeuthen, H. G. Mth. A. 4 (1871) $21-$.

- - rational generators, class. Humbert, G. C. R. $116(1893) 1350$-.

-, representation on plane. Clebsch, R. F.A. Gött. Nr. (1870) 253-.

-, 2nd degree, curves on. Halphen, G. H. [1872] (x) Par. S. Mth. Bll. 1 (1873) 19-. _, - - Schoute, P. H. As. Fr. C. R. (1879) 180-.

$-, \ldots,-\cdots$, application of binary forms. Laguerre, E. [1872] (x) Par. S. Mth. Bll. 1 (1873) 31-。

-, - - , - - , hyperboloidal coordinates. Cayley, A. Ph. Mg. 22 (1861) 35-.

-, 3rd degree. Clebsch, R. F. A. [1865-69] Crelle J. 65 (1866) 359-; Mth. A. 1 (1869) 634-.

,--- , curves on. Sturm, R. Mth. A. 21 (1883) 457-.

- 3rd and 4th degrees, Steiner's surface. Kupper, $K$. Prag Ab. 2 (1888) No. 10, $11 \mathrm{pp}$.

Tacnodal points. Korteweg, D.J. Wien Ak. Sb. 98 (1890) (Ab. 2a) 1154-.

Transformation of figures in space, method. Vaněček, J. S. C. R. 94 (1882) 1463-, 1583-.

\section{Transcendental Functions 8050}

Transformation of figures in space, method. Vaněček, J. S., \& Vaněček, M. N. C. R. 95 (1882) 1049-, 1146-; 96 (1883) 1714-, 1773-. - rational surfaces. Cayley, A. [1870] Mth. A. 3 (1871) 469-.

- - surfaces, automorphic. Picard, $\dot{E}$. C. R. 103 (1886) 517-.

- - - , , and a fundamental number in theory of surfaces. Picard, $\hat{E}$. C. R. 103 (1886) 549-.

- - - and a class of differential equations. Picard, É. C. R. 103 (1886) 635-.

Transformations, birational automorphic, surfaces with continuous group of. Castelnuovo, $G_{.}, \&$ Enriques, $F$. C. R. 121 (1895) 242-.

-,$-----\cdots$ Painlevé, P. C. R. 121 (1895) 318-.

-, - , surfaces admitting double infinity. Picard, É. C. R. 103 (1886) 730-

-, bi-uniform, of surfaces. Painlevé, P. C. R. 122 (1896) 874-.

- rational, of space, determined by general surface of 3rd degree. Loria, $G$. Tor. Ac. Sc. At. 26 (1891) 275-

Uniform functions of 2 points, invariant by rational transformations. Appell, $P$. C. R. 96 (1883) 1643-.

\section{Application of transcendental functions to algebraic curves. (See also 4040, 4060, 7660.)}

Abelian functions and curves of any genus. Buchheim, A. L. Mth. S. P. 13 (1882) 180 -

- - genus 3. Amstein, H. Luus. S. $\nabla d$. Bll. 24 (1889) 99-.

- integrals and curves of 4 th degree with 1 double point. Roberts, W. R. W. L. Mth. S. P. 25 (1894) 151-.

- - genus $p=2$, periods. Kepiński, $S$. Prace Mt.-Fiz. 9 (1898) 139-; Fschr. Mth. (1898) 392.

- and plane curves. Lindemann, C. L. F. [1877] Crelle J. Mth. 84 (1878) 294-, 300-.

_- 1st species, plane curves rectifiable by. Brodén, T. Stockh. Ak. Hndl. Bh. 15 (Afd. 1) (1890) No. 5, $26 \mathrm{pp}$.

- - and uniform correspondences on elliptic curves. Patrassi, P. G. Mt. 34 (1896) 195-.

Abel's theorem, applications. Michel, C. C. R. 130 (1900) 885-.

- - - to conics. Léauté, H. C. R. 79 (1874) 93-, 602-; Toul. Ac. Sc. Mm. 8 (1876) 139-

- extension to skew curve. Poincaré, $H$. C. R. 100 (1885) 40-.

Conchalic curves, trajectories and cissoids of 4th degree. Huber, $G$. Mh. Mth. Ps. 6 (1895) 157-.

Conics, porism of 2. Gundelfinger, $S$. Crelle J. Mth. 83 (1877) 171-.

Curves whose corresponding Abelian functions are of class $p=2$. Clebsch, R. F. A. [1868] Mth. A. 1 (1869) 170-. 
Curves, 2nd and 3rd degrees, parametric determination of points. Juel, C. Mth. A. 47 (1896) 72-. -, 3rd degree, intersections with straight lines. Laguerre, E. [1875] Par. S. Mth. Bll. 4 (1876) 110-.

_, _ _ , and their representation on circle. Durège, H. Z. Mth. Ps. 17 (1872) 433-.

-, 6th degree, cases. Roberts, R. A. Mess. Mth. 15 (1886) 26-.

Elliptic curves, equation giving points of inflexion. Brioschi, F. Mil. I. Lomb. Rd. 2 (1869) 559-.

_-, plane. Schlesinger, O. Mth. A. 33 (1889) 444-; 34 (1889) 463-.

- - , reduction of linear systems, and general theorem on curves of genus $p$. Guccia, $G$. B. Palermo Cir. Mt. Rd. 1 (1887) 169-.

- function, 1st species, with any modulus, skew curves with arcs representing. Molins, H. Liouv. J. Mth. 4 (1878) 187-.

- functions. Hurwitz, A. [1881] Mth. A. 19 (1882) 56-.

- - , cubic transformation, and skew curves of 3rd degree. Dixon, A.C. Mess. Mth. 23 (1894) 94-.

- - and curves of 3rd degree. Harnack, A. (vII) Erlang. Sb. Ps. Md. S. 6 (1874) 163-; (x) Mth. A. 9 (1876) 1-.

(*1883) 1-; Fschr. Mth. ("1883) 407.

- - - - - Pick, G. Wien Ak. Sb. 97 (1889) (Ab. 2a) 711-.

- - - - Kötter, E. Crelle J. Mth. 114 (1895) 170-

- - - - 4th degree. Siebeck, H. Crelle J. 57 (1860) 359-; 59 (1861) 173-.

- - of one parameter, curves with coordinates representable as. Clebsch, R. F. A. [1864] Crelle J. 64 (1865) 210-.

$----\frac{-}{\text { Humbert }},-\frac{-}{\text { G. }}--\frac{-}{\text { Sar. Slebsch's }}$ 9 (1881) 166-.

- - - - expression of coordinates of bi-nodal quartic curve as functions of. Davis, E. W. Am. J. Mth. 5 (1882) 331-. - - and skew curves of 4 th degree, 1 st kind. Pick, G. Wien Ak. Sb. $98(1890)(A b .2 a)$ 536-. - - trisection, applied to points of inflexion of cubic. Heinze, L. Arch. Mth. Ps. 70 (1884) 1-.

- and hyperelliptic integrals, application to rational curves. Laguerre, $E$. C. R. 96 (1883) 769-.

Equation $y^{2}=x\left(x^{4}-1\right)$. Burnside, W. [1892] L. Mth. S. P. 24 (1893) 17-.

Eulerian integrals and sinusoid spirals

$$
\left[\rho^{n}=\frac{(2 a)^{n}}{2} \cos n \phi\right] \text {. }
$$

Loria, G. Prag Sb. (1897) (Mth.-Nt.) No. 18, $6 \mathrm{pp}$.

Fuchsian functions. Humbert, $G$. Liouv. J. Mth. 2 (1886) 239-.

- - of one parameter, expression of coordinates of curve by. Picard, $\dot{E}$. C. R. 92 (1881) 1332-.

Functional and invariant theory of binomial curves. Wellstein, J. Ac. Nt. C. N. Acta 74 (1899) 279-.
Hyperelliptic curves. Bobek, $K$. Prag Ab. 1 (1886) No. 1, 27-; Wien Ak. Sb. 93 (1886) (Ab. 2) 601-; 94 (1887) (Ab. 2) 861-; 95 (1887) (Ab. 2) 31-; Mth. A. 29 (1887) 386-. - -. Valentiner, $H$. N. Ts. Mth. 10 (B) (1899) 51-; Fschr. Mth. (1900) 572-.

- - class of curves akin to. Bobek, $K$. Wien Ak. Sb. 98 (1890) (Ab. 2a) 141-.

- - genus 3, allowing automorphic transformations. Wiman, A. Stockh. Ak. Hndl. Bh. 21 (Afd. 1) (1896) No. 1, 23 pp.

- - , special. Weltzien, C. Mth. A. 30 (1887) 535-.

- -, transformation. Cremona, L. Mil. I. Lomb. Rd. 2 (1869) 566-.

- $-C^{3 n}$, genus arbitrary: Kupper, C. J. Prag Ab. 1 (1886) No. 1, 26 pp.

- $C_{8}^{8}$ with 13 given double points, system. Küper, C. Prag Sb. (1898) (Mth.-Nt.) No.1, $7 \mathrm{pp}$.

- functions of one parameter, curves with coordinates representable as. Brill, A. [1865] Crelle J. 65 (1866) 269-.

Intersections of curves, coordinates. Blazeievski, R. N. A. Mth. 14 (1895) 49-.

Multiply tangential curves to algebraic curves. Weiss, W. Mh. Mth. Ps. 7 (1896) 370-.

Parameters, representation of curves by. Enriques, $F$. Palermo Cir. Mt. Rd. 10 (1896) $30-$.

Rational, elliptic, and hyperelliptic curves, linear systems. Kantor, S. Mh. Mth. Ps. 10 (1899) 18 -

- transformations of curves. Painlevé, P. C. R. 105 (1887) 792-.

Riemann's integrals of 1st species, canonical form. Christoffel, E. B. A. Mt. 9 (187879) 240-.

Spherical curves, 2 systems representable by elliptic functions. Lilienthal, R, von. Crelle J. Mth. 93 (1882) $237-$.

Steiner's polygons. Schoute, $P . H$. Crelle J. Mth. 95 (1883) 105-, 317-.

Theta functions and curves of 3rd degree. Schlesinger, O. Mth. A. 31 (1888) 183-.

$(2,2)$ correspondence, coincidence of elements. Brodén, T. Stockh. Öfv. (1893) 45-, 213-.

\section{Application of transcendental functions to algebraic surfaces. (See also 4040, 4060, 7650.)}

Enriques, F. [1893-96] Tor. Ac. Sc. Mm. 44 (1894) 171-; Rm. S. It. Mm. 10 (1896) 1-.

Abelian functions and a class of representations of surfaces. Clebsch, R. F.A. [1870] Mth. A. 3 (1871) $45-$.

- - genus 3, sextic surfaces associated with. Humbert, G. $\quad$ C. R. 120 (1895) 365-; Liouv. J. Mth. 2 (1896) 263-.

_ - of 2 parameters, surfaces with coordinates of any point expressible by. Picard, $\dot{E}$. C. R. 92 (1881) 1495-; Mth. A. 19 (1882) 569-. - - and quartic surfaces. Humbert, $G$. C. R. 129 (1899) 640-. 


\section{Transcendental Functions}

Abelian functions and Weddle quartic surface. Caspary, F. Bll. Sc. Mth. 15 (1891) 308-.

Abel's theorem and surface generated by translation of curves. Lie, S. C. R. 114 (1892) 277 -

- - - translation surfaces. Lie, S. Leip. Mth. Ps. B. 48 (1896) 141-.

- - - varieties. Lie, S. Leip. Mth. Ps. B. 49 (1897) 181-.

Addition theorem of hyperelliptic integrals and functions of 1st order, significance for confocal quadrics. Staude, E. O. Mth. A. 22 (1883) 1-, 145-.

Analysis situs, and integrals of total differentials. Picard, É. C. R. 124 (1897) 532-.

Cyclics and cyclides. Darboux, G. C. R. 68 (1869) 1311-; Bordeaux Mm. S. Sc. 8 (1870) 291- ; 9 (1873) 1-.

Elliptic functions, application to spherical polygons in one small circle and about another. Richelot, F.J. Crelle J. 5 (1829) 250 -.

- integrals, minimal surfaces representable by. Lilienthal, - von. Crelle J. Mth. 99 (1886) 179-.

- ruled surfaces in $n$-dimensional space. Segre, C. Tor. Ac. Sc. At. 21 (1885) 868-.

Groupsand surfaces, theory. Picard, É. Palermo Cir. Mt. Rd. 9 (1895) 244-.

Hessian of cubic surface. Hutchinson, J. I. N. Y. Am. Mth. S. Bll. 5 (1899) 282-; 6 (1900) 328-.

Hyperabelian functions and surfaces representable by them. Humbert, $G$. C. R. 129 (1899) 667-.

Hyperboloid of one sheet, and other ruled surfaces connected with it. Schoenfies, A. Z. Mth. Ps. 23 (1878) 269-; 24 (1879) 62-.

Hyperelliptic canonical curves, surfaces with. Enriques, F. Rm. R. Ac. Linc. Rd. 5 (1896) (Sem. 1) 191-.

- functions, reduction of problem of 27 lines of cubic surface to transformation of. Burkhardt, H. Gött. Nr. (1892) 1-.

- - , representation of Kummer's surface by. Reichardt, $W$. Ac. Nt. C. N. Acta 50 (1887) 373-.

- _ - - quartic surfaces with double conic by. Domsch, P.R. Arch. Mth. Ps. 2 (1885) 193-, 225-

- surfaces, general theory. Humbert, $G$. Liouv. J. Mth. 9 (1893) 29-, 361-.

Periods of doúble integrals, and 2-dimensional cycles on algebraic surfaces. Picard, É. C. R. 126 (1898) 1457-

Theta functions of 2 variables and corresponding Kummer's surface. Schleiermacher, $L$. Mth. A. 50 (1898) 183-.

- and Weddle quartic surface. Caspary, F. C. R. 112 (1891) 1356-.

Total differentials of 1st kind. Noether, $M$. Erlang. Ps. Md. S. Sb. 18 (1886) 11-.

- - - - - integrals, quartic surfaces admitting. Berry, A. Camb. Ph. S. T. 18 (1900) 333-.

Transformations of surfaces, automorphic. Poincaré, H. C. R. 103 (1886) 732-.

\section{Enumerative Geometry 8070}

Unicursal surfaces, general theory. Humbert, M. G. Mth. A. 45 (1894) 428-.

Uniform functions of 2 parameters, surfaces with coordinates expressed by. Picard, $\hat{E}$. Par. S. Mth. Bll. 28 (1900) 17-.

\section{Enumerative geometry.} (See also 7230, 7260, 7600-7660.)

Schubert, H. C. H. Mth. A. 10 (1876) 1-; 13 (1878) 429-; 15 (1879) 529-

Jonquières, E. de. C. R. 106 (1888) 234-, 437.

Kluyver, J. C. N. Arch. Wisk. 18 (1891) 125-.

Adjoint curves and special series. Amodeo, $F$. Nap. Rd. 35 (1896) 316-.

- minimal curves. Amodeo, $F$. Rm. R. Ac. Linc. Rd. 2 (1893) (Sem. 1) 460-, [601].

Algebraic form, certain, and its geometrical representation. Cotterill, T. [1872] L. Mth. S. P. 4 (1871-73) 139-.

Arbitrary points which can be double on curve or surface, maximum number. Campbell, $J$. $E$. Mess, Mth. 21 (1892) 158-

Bezout's theorem, geometrical extensions. Schubert, H. C. H. Gött. Nr. (1877) 401-.

Bitangents of curve of $n$th degree. Jacobi, $C$. G. J. Crelle J. 40 (1850) 237-.

$--1--$ (Jacobi). Rubini, R. Tortolini A. 2 (1851) 463-.

- - - - (-). Clebsch, R. F. A. [1863] (vir) Crelle J. 63 (1864) 186-.

- curves. Retali, V. [1890] Bologna Rd. (1890-91) 35-.

Branch points of any singularity of plane curve. Bertini, E. Mil. I. Lomb. Rd. 23 (1890) 307-.

Branches of curves, real. Hilbert, D. Mth. A. 38 (1891) 115-.

- - plane curves, real, number and arrangement. Hulburt, L. S. Am. J. Mth. 14 (1892) 246-.

Characteristic $n$, Halphen's, in theory of skew curves. Cayley, A. Crelle J. Mth. 111 (1893) 347-.

Characteristics, Chasles's theorem. Hurwitz, A., \& Schubert, $H$. Gótt. Nr. (1876) 503-.

- of conics. Halphen, G. H. L. Mth. S. P. 9 (1877-78) 149-.

- - Study, E. Mth. A. 27 (1886) 58 -

- - (Study). Zeuthen, H. G. Mth. A. 37 (1890) 461-.

- - and quadrics. Halphen, G. C. R. 76 (1873) 1074-

- Cremona's formula. Study, E. Mth. A. 27 (1886) 102-.

- of higher curves, determination. Saltel, $L$. [1873] (xr) Par. S. Mth. Bll. 2 (1874) $52-$

- - quadrics. Zeuthen, H. G. Kjöb. Ov. (1866) 91-; N. A. Mth. 7 (1868) 385-.

- - systems of conics and quadrics. Halphen, G. H. C. R. 83 (1876) 886-; Par. Éc. Pol. J. $45^{\circ}$ cah. (1878) 27-. 
Characteristics, theory. Schubert, H. Crelle J. 71 (1870) 366-.

-, -. Clebsch, R. F. A. [1872] Mth. A. 6 (1873) 1-.

-, -. Halphen, G. H. [1879] Par. S. Mth. Bll. 8 (1880) 31-.

-, - (Halphen). Schubert, H. C. H. Par. S. Mth. Bll. 8 (1880) 60-.

-, - (Kronecker). Dyck, W. [1894] D. Mth. Vr. Jbr. 4 (1897) 94-.

-, -, and bibliography. Painvin, L. Bll. Sc. Mth. As. 3 (1872) 155-.

Class and number of inflexions of curve. Guccia, G. B. Rm. R. Ac. Linc. Rd. 5 (1889) (Sem. 1) 18-.

Conditions that 4 curves have 2 common points, order. Roberts, S. QJ. Mth. 9 (1868) 176-.

Configurations arising from osculations of cubic curves. Vries, J. de. Amst. Ak. Vs. M. 6 (1889) 232- ; Fschr. Mth. (1889) 538, 541-.

Conical surfaces, numbers for. Krey, H. Acta Mth. 5 (1884) 83-.

Conics of 5-point contact with curve of 5th degree. Berner, T. N. A. Mth. 5 (1866) 17-.

- and quadrics, determination. Halphen, G. H. (x) Par. S. Mth. Bll. 1 (1873) 130-, 226- ; 2 (1874) 11-.

- satisfying 5 conditions, number. Jonquières, E. de. Liouv. J. Mth. 4 (1859) 49-.

---- , problem. Cayley, A. C. R. 63 (1866) 9-.

- double conditions, number. Cremona, $L$. C. R. 59 (1864) 776-.

--5 projective conditions, number. Halphen, G. $H$. L. Mth. S. P. $10(1878-79) 76-$

- in space, number cutting 8 straight lines. Liroth, J. [1867] Crelle J. 58 (1868) 185-. - of system satisfying simple condition, number. Schubert, H. C. H. Par. S. Mth. Bll. 8 (1880) 61 .

- - - $(\mu, \nu)$ satisfying a 5 th condition, formula for number. Saltel, $L$. Brux. Ac. Bll. 42 (1876) 617-.

- touching 5 curves, or satisfying other conditions, number. Chasles, $M$. C. R. 58 (1864) 222-.

- variously defined. Sporer, B. Z. Mth. Ps. 35 (1890) 237-, 293-.

Contact of curves with curve whose points can be individually determined. Chasles, $M$. C. R. 63 (1866) 670-.

- - 3-point. Schubert, H. C. H. Gött. Nr. (1880) 369-.

-, double, determination of curves and surfaces satisfying conditions. Zeuthen, $H . G$. C. R. 89 (1879) 899-, 946-.

- or intersection at given angle of system of curves with given curve. Fouret, $G$. [1876] Par. S. Mth. Bll. 5 (1877) 19-.

- of inverse with polar reciprocal figures. Vaněček, J. S., \& Vaněček, M. N. [1883] St Pét. Ac. Sc. Bll. 29 ( $\left.{ }^{*} 1884\right) 281-$.

- multiple, of conics with curve of any degree. Jonquières, E. de. N. A. Mth. 3 (1864) 218-.

-, - , and other multiple conditions. Chasles, M. C. R. 59 (1864) 345-。
Contact, points of tangents from point to $k$ curves, connectors. Guccia, G. B. Palermo Cir. Mt. Rd. 9 (1895) 268-.

- problem. Jonquières, E. de. Crelle J. 66 (1866) 289-.

- - (de Jonquières). Humbert, G. Palermo Cir. Mt. Rd. 4 (1890) 109-.

- problems, 2. Brill, A. Mth. A. 4 (1871) 527-.

- of system of curves with algebraic curve. Fouret, G. C. R. 82 (1876) 1328-.

- - - - surfaces (implex) with algebraic surface. Fouret, G. C. R. 82 (1876) 1497-; 84 (1877) 436-.

Coordinates of curves of $m$ th degree, genus $p$. Hermite, C. [1877] Crelle J. Mth. 84 (1878) 298 .

Correlation of two 3-dimensional spaces, enumeration. Hirst, T. A. [1890] L. Mth. S. P. 21 (1891) 92-.

Correlations in two 3-dimensional spaces, satisfying certain conditions. Visalli, $P$. Rm. R. Ac. Linc. Mm. 3 (1886) 597-.

$--\ldots-1,-$ twelve conditions. Visalli, P. Rm. R. Ac. Linc. Rd. 3 (1887) (Sem. 1) 118-.

Curves, application of enumerative geometry. Castelnuovo, G. Palermo Cir. Mt. Rd. 3 (1889) 27-.

-, enumerations concerning. Schubert, $H$. D. Nf. Tbl. (*1876) (Beil.) 65.

- of given system which cut given curve at given angle, etc. Jonquières, $E$. de. C. R. 58 (1864) 535-.

- - intersection of 2 surfaces, theorems. Valentiner, $H$. (xI) Ts. Mth. 3 (1879) $22-$.

-, lines making constant angle with tangent at contact point. Chasles, M. C. R. 74 (1872) 1146-.

-, mutual dependence and general properties. Steiner, J. Liouv. J. Mth. 18 (1853) 309-.

- satisfying given conditions. Jonquières, $E$. de. Crelle J. 66 (1866) 289-; C. R. 63 (1866) 423-, 485-, 522-.

$--\frac{-}{-}$ (de Jonquières). Cayley, A. C. R. 63 (1866) 666-.

- - Cayley, A. [1867] Phil. Trans. 158 (1868) 75-, 145-.

- , singly infinite series, and other theorems. Jonquières, E. de. N. A. Mth. 20 (1861) 83-; Liouv. J. Mth. 6 (1861) 113-; 8 (1863) 71-.

Cusps in curve of $n$th degree, genus $p$. Valentiner, E. C. Ts. Mth. 3 (1885) 179-.

Degree, class, etc., of algebraic surface and surfaces derived from it, determination. Sturm, R. Mth. A. 9 (1876) 573-.

- and class of curves formed under given conditions, determination. Chasles, M. C. R. 84 (1877) 971-; 85 (1877) 460-.

- $-1--1-$ Fouret, $G$. C. R. 85 (1877) 134-, 844-, 944-.

_ _ - extension to space of 2 laws given by Chasles. Fouret, G. C. R. 85 (1877) 216-.

- of surface, determination. Fouret, G. Par. S. Phlm. Mm. Cent. (1888) 77-. 
Desargues and enumerative geometry. Loria, G. Bb. Mth. (1895) 51-.

$n$-dimensional generalisations. Schubert, $H$. Mth. A. 26 (1886) 26-.

- geometry. Schubert, H. D. Nf. Vh. (1895) (Th. 2, Hälfte 1) 18-.

- -, problem. Pieri, M. G. Mt. 30 (1892) 133-.

- linear spaces. Schubert, H. Acta Mth. 8 (1886) 97-.

- quadrics. Schubert, H. Hamb. Mth. Gs. Mt. 2 (1890) (Festschr., Th. 2) 172-.

- - general enumerative functions for. Schubert, H. Mth. A. 45 (1894) 153-.

$p$-dimensional space, 1 st and 2 nd degrees. Schubert, H. [1891] D. Mth. Vr. Jbr. 1 (1892) 48-.

$q$-dimensional geometry, problem. Tanturri, A. Tor. Ac. Sc. At. 35 (1900) 269- or 427-.

Double points, apparent, of skew curves. Brunn, H. [1893] D. Mth. Vr. Jbr. 3 (1894) 84-.

- - of curve, maximum number. Jonquières, E. de. C. R. 105 (1887) 917-.

- - - surface, maximum number. Jonquières, E. de. C. R. 106 (1888) 19-.

Envelopes of surfaces, degree. Moutard, $T$. N. A. Mth. 19 (1860) 58-.

Excesses of systems of curves adjoint to algebraic curves, determination. Amodeo, F. Nap. Rd. 39 (1900) 224-.

Generation of curves and surfaces by corresponding elements. Weyr, Em. [1871] Prag Ab. 5 (1872) $54 \mathrm{pp}$.

Genus of certain set of curves. Zeuthen, H. G. Palermo Cir. Mt. Rd. 3 (1889) 171-.

Geometry, modern, enumeration as source of error in. Kupper, C. Mth. A. 32 (1888) $282-$.

Historical notes and bibliography. Loria, $G$. Bb. Mth. (1888) 39-, 67-; (1889) 23-.

Inflexions. Valentiner, $H$. (xII) Ts. Mth. 5 (1881) 167-.

Intersecting " "branches" of curve. Tanturri, A. A. Mt. 4 (1900) 67-.

Intersection of curves. Cayley, A. Camb. Mth. J. 3 (1843) 211-; Mth. A. 30 (1887) 85-.

- - - theorem (Cayley). Bacharach, I. Mth. A. 26 (1886) 275-.

,$----(-)$. Fine, H. B. Am. As. P. (1886) 73-.

Intersections of curves of $p$ th and $q$ th degrees. Picquet, H. C. R. 77 (1873) 474-.

-, inscriptions, etc., of curves. Estienne, J. E. N. A. Mth. 4 (1885) 87-, 131-, 297-.

- of 2 plane curves, multiplicity. Brill, $A$. Münch. Ak. Sb. 18 (1889) 81-.

Maximum class of curves and ruled surfaces. Kiipper, C. Mth. A. 31 (1888) 291-.

Multiple point common to 2 plane curves, number of points of intersection represented by. La Gournerie, J. de. C. R. 77 (1873) 573-.

- "points of curves. Coste, L. M.P. Liouv. J. Mth. 7 (1842) 184-

- - Transon, (Prof.) A. N. A. Mth. 10 (1851) 91- ; 18 (1859) 142-.
Multiple points of curves, maximum number. Schoute, P. H. Amst. Ak. Vs. M. 13 (1878) 96-.

105 (1887) 971 .

Jonquieres, $E$. de. C. R.

_ - - and surfaces, difference in relation to number. Jonquïeres, $E$. de. C. R. 106 (1888) 156-.

Normals common to 2 curves, 2 surfaces, or a curve and a surface, number. Fouret, $G$. [1877] Par. S. Mth. Bll. 6 (1878) 43-.

- _ - surfaces. Rindi, S. Palermo Cir. Mt. Rd. 5 (1891) 106-.

- - - - (Rindi). Pieri, M. Palermo Cir. Mt. Rd. 5 (1891) 323.

-, double, of skew curve, number. Pieri, $M$. Rm. R. Ac. Linc. Rd. 2 (1886) (Sem. 1) 327-.

Orders of varieties annulling minors of given matrix. Segre, $C$. Rm. R. Ac. Linc. Rd. 9 (1900) (Sem. 2) 253-.

Pencils of surfaces, oblique Jacobian, etc. Rindi, S. As. Fr. C. R. (1885) (Pt. 2) 123-.

Plückerian characteristics of curve, equation of which is resultant or discriminant. Roberts, S. QJ. Mth. 12 (1873) 281-.

- formulæ obtained in elementary manner. Beck, A. Zür. Vjschr. 33 (1888) 173-.

- numbers for curve of deviation. Bouwman, W. Mth. A. 49 (1897) 24-.

- - - and its evolute. Schoute, P. H. N. Arch. Wisk. 3 (1898) 236-.

- - of envelopes, determination. Zeuthen, H. G. C. R. 78 (1874) 274-, 339-.

- - for evolute. Zeuthen, H. G. Ts. Mth. 4 (1874) 129-.

Point aggregates on curves, theory of linear groups. Burkhardt, H. Gött. Nr. (1896) $267-$.

- groups, determination from their polars. Waelsch, E. [1883] Wien Ak. Sb. 88 (1884) (Ab. 2) 1039-.

- pairs in 2 planes or spaces, multiplicity. Segre, C. Palermo Cir. Mt. Rd. 5 (1891) 192-.

Points of inflexion of a $C^{n}$. Bischoff, J. N. A. Mt. 6 (1875) 144 .

- -2 surfaces, special case of uniform correspondence. Krey, H. [1880] Mth. A. 18 (1881) 82-.

Polygram, complete inscribed, determining involution on curve. Weyr, E.J. Wien Ak. Sb. 81 (1880) (Ab. 2) 80-.

Problems, generalisation. Schubert, H. [1891] Hamb. Mth. Gs. Mt. 3 (1900) 12-.

Quadrics, moduli of multiple conditions for. Schubert, H. C. H. Mth. A. 10 (1876) 318-.

-, number of solutions of elementary questions. Housel, -. C. R. 60 (1865) 1072-.

Ruled surface, geometry. Segre, C. Rm. R. Ac. Linc. Rd. 3 (1887) (Sem. 2) 3-, 406.

- surfaces, 4th degree, geometry. Williams, F. B. [1900] Am. Ac. P. 36 (1901) 17-.

Schubert's function $\psi\left(u_{0}, u_{1}, \ldots u_{q}\right)$. Schröder, J. [1899] Hamb. Mth. Gs. Mt. 3 (1900) 382-.

Sextactio points of plane curves. Gerbaldi, Palermo Cir. Mt. Rd. 4 (1890) 65-. 


\section{Enumerative Geometry}

Singular point of curve, characteristic combinations in transformation. Noether, $M$. Palermo Cir. Mt. Rd. 4 (1890) 89-, 299-.

- points of curves of higher degree. Chasles, M. C. R. 59 (1864) 209-.

- - plane curves. Halphen, G. H. C. R. 78 (1874) 1105-.

(1886) $594-\overline{708}$.

- - skew curves. Guccia, G. B. C. R. 120 (1895) 816-.

- tangents of surfaces. Krey, H. Mth. A. 15 (1879) 211-.

Singularities of curve of intersection of 2 surfaces having certain number of multiple points in common. Saltel, $L$. C. R. 80 (1875) 1285-.

- - - - - - - -4 or fewer multiple points in common. Saltel, L. Brux. Ac. Bll. 40 (1875) 22-.

- - relation. Klein, C. F. Erlang. Ps. Md. S. Sb. 8 (1876) 95-; Mth. A. 10 (1876) 199-.

- - locus defined by $k$ algebraic equations containing $k-1$ parameters. Saltel, $L$. Brux. Ac. Bll. 48 (1879) 632-.

- - plane curves. Zeuthen, H. G. Mth. A. 10 (1876) 210-.

- - - compound. Guccia, G. B. Palermo Cir. Mt. Rd. 3 (1889) 241-.

- - - , higher. Cayley, A. Crelle J. 64 (1865) 369-; QJ. Mth. 7 (1866) 212-.

- _ _ - - La Gournerie, J. de. Liouv. J. Mth. 14 (1869) 425-; 15 (1870) 1-.

- - - - nature and effect. Scott, (Miss) C. A. Am. J. Mth. 15 (1893) 221-.

$-\ldots-\ldots$, real, remarkable relation. Perrin, R. [1877] Par. S. Mth. Bll. 6 (1878) 84-.

- - rational plane curves, 2nd order. Weyr, Em. Prag Sb. (1872) 59-

- relating to multiple line of surface. Zeuthen, H. G. Mth. A. 4 (1871) 1-.

- of skew curve and developable surface. Zeuthen, H. G. A. Mt. 3 (1869-70) 175-.

- - - curves. Zeuthen, H. G. C. R. 67 (1868) 225-.

--1 - Fine, H. B. Am. J. Mth. 8 (1886) 156-.

- _ - - and characteristics of polar developables. Björling, C. F. E. Stockh. Öfv. 38 (1881) No. 4, 3-

Skew curves, complete representation. Vahlen, $K$. T. Crelle J. Mth. 108 (1891) 346-.

- - and developables. Cayley, A. Liouv. J. Mth. 10 (1845) 245-; Camb. and Dubl. Mth. J. 5 (1850) 18-; QJ. Mth. 7 (1866) 110-.

- - - - Bischoff, J. N. Crelle J. 59 (1861) 394-.

- - number of constants involved. Rohn, $K$. [1896] D. Mth. Vr.Jbr. 5 (1901) (Heft 1) 84-.

- _, numbers analogous to Plücker's for. Kluyver, J. C. Amst. Ak. Vs. M. 7 (1890) 121-; Fschr. Mth. (1890) 683-.

- -, properties. Deruyts, F. Brux. Ac. Bll. 36 (1898) 194-.

- - , secant conics. Severi, $F$. Tor. Ac. Sc. At. 35 (1900) 526- or 774-.

\section{Configurations 8075}

Skew curves, surface of triple secants, number of quadruple secants. Picquet, $H$. Par. S. Mth. Bll. 1 (1873) 260-.

- - , - - - - - - - - (Picquet) Guccia, G. B. Palermo Cir. Mt. Rd. 1 (1887) 26-.

Surfaces. Pieri, M. G. Mt. 35 (1897) 75-

-, genus 0. Castelnuovo, G. Rm. S. It. Mm. 10 (1896) 103-.

- with plane sections hyperelliptic curves. Castelnuovo, G. Palermo Cir. Mt. Rd. 4 (1890) 73-.

Systems of curves and surfaces, enumerative theorems. Chasles, M. C. R. 45 (1857) 1061-.

Tangent planes to surface passing through multiple line of the surface. Fouret, $G$. Palermo Cir. Mt. Rd. 8 (1894) 202-.

Tangents to curve at points collinear with fixed point, locus of intersection. Kluyver, J. C. N. Arch. Wisk. 17 (1890) 1-; Fschr. Mth. (1890) 683, 684-.

Theorems. Chasles, M. C. R. 81 (1875) 253-, 355-, 643-, 993-, 1221-; 82 (1876) 1399-; 83 (1876) 97-, 467-, 495-, 519-, 589-, 641-, 757-, 867-, 1123-, 1195-.

- and formulæ, group. Zeuthen, H. G. Acta Mth. 1 (1882) 171-.

-, geometric, application of enumerative geometry to deduction of. Zeuthen, H. G. (xII) Sk. Nt. Möt. F. (1880) 155-.

Transformation, conjugate. Schoute, $P . H$. As. Fr. C. R. (1879) 194-; (1880) 156-.

Transformations, Cremona. Guccia, G. B. Palermo Cir. Mt. Rd. 1 (1887) 17-, 20-, 24-, 50-, 56-, 119-, 398.

,-- , derivation of solutions in. Jonquières, (l'amiral) E. de. C. R. 101 (1885) 921-.

- of plane figures. Cremona, L. Bologna Rd. (1861-62) 88-; Bologna Mm. Ac. 2 (1862) 621-.

-, quadratic plane. Retali, $V$. Bologna Ac. Sc. Mm. 10 (1889) 653-.

Triangle. Schubert, H. C. H. Mth. A. 17 (1880) 153-.

Umbilics, number for surface of $n$th degree. Voss, A. E. Mth. A. 9 (1876) 241-.

8075 Special configurations of points, lines, planes or other elements. Space partitioning. (See also 3620, 6820, 7210, 7610, 7640.)

SPECIAL CONFIGURATIONS OF POINTS, LINES, PLANES OR OTHER ELE. MENTS.

Veronese, G. Rm. R. Ac. Linc. T. 4 (1880) 132-; Rm. R. Ac. Linc. Mm. 9 (1881) 265-.

Associated planes and points according to modulus 2. Le Pont, $H$. As. Fr. C. R. (1887) (Pt. 2) 119-.

Bitangents of curve of $4 \mathrm{th}$ degree. Hesse, $L . O$. Crelle J. 49 (1855) 279-; 55 (1858) 83-.

- - - - - Cayley, A. Phil. Trans. (1861) 357-. 
Bitangents of curve of 4 th degree. Geiser, $C$. $F$. Mth. A. 1 (1869) 129-; Crelle J. 72 (1870) 370 -

- - - - - studied through Kummer's configuration. Ciani, $E$. Mil. I. Lomb. Rd. 31 (1898) 312-; A. Mt. 2 (1899) 53-.

$--\frac{1}{-}-$, systems of conics related to. Noether, M. Mth. A. 46 (1895) 545-.

Characteristics of types 3 and 4 and their substitution groups. Pascal, E. A. Mt. 20 (1892-93) 163-; Rm. R. Ac. Linc. Rd. 2 (1893) (Sem. 1) 120-.

Class of configurations, certain. Schubert, $H$. Hamb. Mth. Gs. Mt. 1 (1889) 82-.

Configuration $(3,3)_{10}$. Kantor, $S$. [1881] Wien Ak. Sb. 84 (1882) ( $A b .2)$ 1291-.

- $(3,3)_{10}$, and rational curves of 4 th degree. Kantor, S. [1882] Mth. A. 21 (1883) 299 .

$-10_{3}$, formation and geometric construction. Schroeter, H. Gött. Nr. (1889) 193-.

- $\left(12_{6}, 16_{3}\right)$ and group of 2304 homographies and correlations. Feder, J. Mth. A. 47 (1896) 375-.

- $18_{3}$ harmonically inscribed in quadrilateral. Vries, J. de. Wien Ak. Sb. 97 (1889) (Ab. 2a) 1307-.

Configurations of index 3 , class. Jung, $G$. Mil. I. Lomb. Rd. 18 (1885) 231-.

-, 2nd and 3rd orders. Kalkmann, $G$. Mh. Mth. Ps. 9 (1898) 247-.

- $(3,3)$, indices 8,9 , and their connection with curves of 3rd degree. Kantor, S. [1881] Wien Ak. Sb. 84 (1882) (Ab. 2) 915-.

- $\left(8_{4}, 8_{4}\right)$ of points and planes. Martinetti, $V$. G. Mt. 35 (1897) 81-.

- $11_{3}$. Daublebsky von Sterneck, $R$. Mh. Mth. Ps. 5 (1894) 325-; 6 (1895) 255.

- 12. Daublebsky von Sterneck, $R$. Mh. Mth. Ps. 6 (1895) 223-.

- $n_{3}$, linear construction for. Schroeter, $H$. Gött. Nr. (1888) 237-。

- -, regular. Schönfies, A. Mth. A. 31 (1888) 43-.

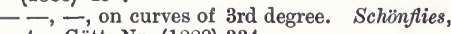
A. Gött. Nr. (1889) 334-.

- - untraceable as closed paths. Steinitz, E. Mh. Mth. Ps. 8 (1897) 293-.

Conic section configuration. Oppenheimer, $H$. Hamb. Mth. Gs. Mt. 3 (1900) 173-.

Curved configurations, 2 curves generated by. Milinowski, -. Crelle J. Mth. 78 (1874) 175-.

Curves of 3rd degree, inscribed configurations. Vries, J. de. Wien Ak. Sb. 98 (1890) (Ab. 2a) 446-.

- - - plane, configurations on. Vries, $J$. de. Wien Ak. Sb. 98 (1890) (Ab. 2a) 1290-.

- - 4th degree, configurations and polygons on. Ameseder, A. Wien Ak. Sb. 93 (1886) (Ab. 2) 357-.

- - - - skew, elliptic, configurations on. Ameseder, A. Wien Ak. Sb. 87 (1883) (Ab. 2) 1179-.

Derivation of one configuration from another. Zindler, $K$. Wien Ak. Sb. 105 (1896) (Ab. 2a) 311-.
Enneads of lines and points. Cayley, $A$. B. A. Rp. 40 (1870) (Sect.) 10.

Figure of 6 points in 4-dimensional space. Richmond, H. W. QJ. Mth. 31 (1900) 125-.

Hessian configuration $\left(12_{4}, 16_{3}\right)$. Schroeter, $H$. Crelle J. Mth. 108 (1891) 269-.

- - $(-,-)$ (Schroeter). Hess, E. Crelle J. Mth. 111 (1893) 53-.

Involution common to group of 5 lines and to system of 9 planes. Serret, $P$. C. R. 113 (1891) 326-, 347-.

Isodynamic and meta-harmonic configurations. Vries, J. de. Wien Ak. Sb. 101 (1892) (Ab. 2a) 66-.

Kummer configuration. Reye, $T$. Crelle $J$. Mth. 86 (1879) 209-.

- - Ciani, E. G. Mt. 34 (1896) 177-; 36 (1898) 68-; 37 (1899) 62-.

- - Martinetti, V. G. Mt. 34 (1896) 192-; 35 (1897) 235-.

- configurations multiply tetrahedroidal. Bertini, E. Mil. I. Lomb. Rd. 29 (1896) 566-.

Lines, configuration of 10 , regular rectangular. Morley, F. L. Mth. S. P. 29 (1898) 670-.

-, _ - 16, problem of Steiner. Schouite, P. H. Crelle J. Mth. 101 (1887) 154-.

,$--\ldots-$, of surface of 4 th degree with double conic. Pereno, I. A. Mt. 21 (1893) 57-.

-, groups. Jordan, C. Crelle J. 70 (1869) 185-

-, planes and points determined by given points, lines and planes, number. Bretschneider, C. A. Schlömilch Z. 6 (1861) 311-.

- and surfaces, systems. Lazzeri, G. Palermo Cir. Mt. Rd. 2 (1888) 110-.

Nets and configurations. Zindler, $K$. Wien Ak. Sb. 98 (1890) (Ab. 2a) 499-.

Normal curves of $n$th degree, elliptic, configurations on. Schönflies, A. Mth. A. 35 (1890) 527-.

Pascal's theorem, extension to geometry of space. Petot, A. Par. Éc. Norm. A. 5 (1888) (Suppl.) 65 pp.

Pentacycles, remarkable configuration of circles. Stephanos, C. C. R. 93 (1881) 578-, 633-.

Plane configuration of 24 points and 18 straight lines. Vries, J. de. Arch. Néerl. 25 (1892) 57-.

- configurations. Martinetti, $V$. A. Mt. 14 (1886-87) 161-.

-. Vries, $\dot{J}$. de. Amst. Ak. Vs. M. 5 (1889) 105-; 6 (1889) 8-, 45-; Fschr. Mth. (1888) 591-; (1889) 538, 539-, 540; Acta Mth. 12 (1889) 63-; N. Arch. Wisk. 18 (1891) 121-.

- - and corresponding substitution groups. Schoenflies, A. Gött. Nr. (1887) 410-.

- - : desmic configurations $9_{3}$ arising from triangles in triple perspective. Vries, $J$. de. Amst. Ak. Vs. M. 6 (1889) 171-; Fschr. Mth. (1889) 538, 540-.

- : harmonic configuration $\left(24_{3}, 18_{4}\right)$ Vries, J. de. Amst. Ak. Vs. M. 5 (1889) 210-; 7 (1890) 177-; Fschr. Mth. (1888) 591,592 ; $(1890) 560$-.

- - where each point lies on 2 lines. Vries, $J$. de. Amst. Ak. Vs. M. 6 (1889) 382-; Fschr. Mth. (1889) 538, 542; Palermo Cir. Mt. Rd. 5 (1891) 221-. 
Plane configurations, regular. Vries, J. de. Amst. Ak. Vs. M. 7 (1890) 75-; Arch. Néerl. 25 (1892) 33-; Mth. A. 35 (1890) 401-.

- - $\mu_{3}$. Martinetti, V. A. Mt. 15 (1887-88) 1-. - and space, class of configurations in. Kantor, S. [1879] Wien Ak. Sb. 80 (1880) $(A b .2) 715-$.

Planes, general systems. Eberhard, $V$. Crelle J. Mth. 106 (1890) 89-.

Point and line configuration. Brunel, Bordeaux S. Sc. Mm. 5 (1890) xciii-.

- _ - related to plane quartic. Ciani, $E$. Mil. I. Lomb. Rd. 31 (1898) 310-.

Points, $n$, in space, number of $n$-gons formed by joining. Grunert, J.A. Grunert Arch. 1 (1841) 108.

Point-groups in space, certain dependent. Rosanes, J. Crelle J. Mth. 100 (1887) 311-.

Polygons, regular, in modular network. Rogers, L. J. L. Mth. S. P. 29 (1898) 706-.

-, - star-shaped, of $m$ sides, theory of numbers applied to determine number. Minin, A. P. Mose. S. Sc. Bll. 93 (No. 1) (1897) 30-; Fschr. Mth. (1897) 167, 426.

-: 5-gon, decahedron. Kohn, G. [1879] Wien Ak. Sb. 80 (1880) (Ab. 2) 7-.

- : - - - , involutions of straight line cutting. Kohn, G. Mh. Mth. Ps. 2 (1891) 141-.

-: 6-gon, Clebsch's. Schroeter, H. Mth. A. 28 (1887) 457-.

-: -, skew, and hexahedron, involutory properties. Weyr, Em. Mil. I. Lomb. Rd. 6 (1873) 179-.

Polyhedra and polygons. Grunert, $J$. A. Crelle J. 5 (1829) 37-.

-, problem, general solution. Kirkman, $T$. $P$. [1858] Manch. Ph. S. Mm. 15 (1860) 92-.

-: 4-hedra, desmic systems. Klug, L. Mth. Nt. B. Ung. 17 (1901) 95-.

- : - in multiple perspective. Klug, L. Arch. Mth. Ps. 6 (1888) 93-.

- : - - - - desmic. Vályi, G. [1885] Mth. Termt. Ets. 4 (1886) 6-; Mth. Nt. B. Ung. 4 (1885-86) 1-.

_: - _ _ - on skew curves of 4 th degree. Vályi, G. Mth. Termt. Éts. 11 (1893) 322-; Mth. Nt. B. Ung. 13 (1897) 183-.

-: - - oblique perspective. Kohn, G. Wien Ak. Sb. 107 (1898) (Ab. 2a) 777-.

- : - - perspective. Vályi, G. [1885] Mth. Termt. Éts. 4 (1886) 55-.

-: 5-hedra. Le Paige, C. Brux. Ac. Bll. 13 (1887) 488-

-: -, complete. Ciani, E. Rm. R. Ac. Linc. Rd. 7 (1891) (Sem. 1) 209-.

-: 一, -. Ascione, E. Nap. Rd. 31 (1892) 147-.

-: - and 6-hedra and connected Kummer configuration. Schroeter, H. Crelle J. Mth. 100 (1887) 231-.

-: 6-hedra. Hermes, O. Crelle J. Mth. 100 (1887) 258-.

-_ - complete. Caporali, E. Nap. Rd. 20 (1881) 59-.

-: 8-hedra, and construction of associated straight line. Serret, $P$. C. R. 103 (1886) 999-.
Polyhedra : 8-hedra, diagonals, theorems of Steiner, demonstration. Bermann, O. Arch. Mth. Ps. 53 (1871) 129-.

-: - inscribed in cube. Brunel, -- Bordeaux S. Sc. Mm. 4 (1894) xlv.

-: 10-hedra, analysis of 4 autopolar. Kirk. man, (Rev.) T. P. Lpool. Lt. Ph. S. P. 43 (1889) 45-.

-: 24-hedra. Kirkman, (Rev.) T.P. Lpool. Lt. Ph. S. P. 38 (1884) 55-.

Polyhedral configurations. Vries, J.de. Mth. A. 34 (1889) 227-.

- - Brunel, G. [1897] Bordeaux S. Sc. PV. (1897-98) 20-

- - : 6-hedral and 8-hedral configurations $\left(12_{6}, 16_{3}\right)$. Reye, $T$. Acta Mth. 1 (1882) 97-.

Problem of configurations. Reye, $T$. Acta Mth. 1 (1882) 93-.

- - - Andreev, K. A. Kharkov Mth. S. Com. 2 (1891) 95-; Fschr. Mth. (1891) 564.

Projective configurations, Reye's geometry. Timerding, E. Gött. Nr. (1898) 317-.

Quadratio configuration, linear automorphic transformation. Lindemann, $F$. Münch. Ak. Sb. 26 (1897) 31-.

Quadric, regular configuration of 10 line pairs conjugate to. Morley, F. N. Y. Am. Mth. S. Bll. 5 (1899) 252-.

Solid figures, configurations arising by section and collineation of. Schönflies, A. [1891] D. Mth. Vr. Jbr. 1 (1892) 62-.

Space configuration of 140 straight lines. Maschke, H. Gött. Nr. (1889) 384-; Mth. A. $36(1890) 190$ -

- configurations, certain. Vries, $J$. de. Amst. Ak. Vs. 3 (1895) 154-.

- - homogeneous, composite. Schlegel, $S$. F. V. [1881] Ac. Nt. C. N. Acta 44 (1883) $337-$.

- - (Klein's and others). Hess, E. Ac. Nt. C. N. Acta 55 (1891) 97-; 75 (1899) 482 pp.

Symmetrical configurations on a surface. Brunel, - - Bordeaux S. Sc. Mm. 4 (1894) lvii-.

Triad elements of elementary configurations. Klein, B. A. Mt. 18 (1890) 213-; 19 (189192) 39-, 233-.

Triple systems, theory. Vries, J. de. Palermo Cir. Mt. Rd. 8 (1894) 222-.

- tangent planes of skew curve, 6th degree, genus 4, configuration. Pascal, $E$. Rm. R. Ac. Linc. Rd. 2 (1893) (Sem. 1) 204-, 239-.

\section{SPACE PARTITIONING.}

n-dimensional space, 3 regular divisions. Schoute, P. H. As. Fr. C. R. (1894) (Pt. 2) 257-.

Partition and reticulations of $r$-gon. Kirkman, T. P. Manch. Ph. S. Mm. 15 (1860) 220 -

Partitions, trihedral, of $x$-ace, and triangular partitions of $x$-gon. Kirkman, T.P. [1857] Manch. Ph. S. Mm. 15 (1860) 43-.

- $k$-, of $N$. Kirkman, T. P. [1854] Manch. Ph. S. Mm. 12 (1855) 129-.

- - , $-r$-gon and $r$-ace. Kirkman, T. P. [1856] Phil. Trans. (1857) 217-.

-, $k$-, $j$-nodal, of $r$-gon. Kirkman, T. $P$. Manch. Ph. S. P. 1 (1857-60) 113-. 
Partitions of $r$-pyramid. Kirkman, T.P. Phil. Trans. (1858) 145-.

Plane, division by circles and straight lines, and division of space by planes and spheres. Steiner, J. N. A. Mth. 4 (1845) 491-, 541-. - networks. Jordan, C. C. R. 63 (1866) 1061-. - and space, division, laws. Steiner, $J$. Crelle J. 1 (1826) 349-.

Planes, spheres, and space, homographic divisions, quaternion methods. Joly, C. J. [1897] Ir. Ac. P. 4 (1896-98) 515-.

Points about axis, most nniform distribution. Wright, C. Camb. (M.) Mth. M. 1 (1859) 244 -.

- in space, even distribution. Tait, -. Edinb. R. S. P. 14 (1888) 37.

Rotation composition and space partitioning. Rohn, $K$. Dresden Isis Sb. (1898) 9-.

Systems of surfaces, systematic division of space by. Graberg, $F$. Zïr. Vjschr. 35 (1890) 257-.

Triangles, chains, and nets, numbering. Berg, F. J. van den. N. Arch. Wisk. 19 (1892) 151-; Fschr. Ps. 1892 (Ab. 1) 31-.

\section{Line geometry. Connexes,} complexes, congruences; higher elements of space. (See also 7650.)

\section{LINE GEOMETRY.}

Silberstein, S. [1880] (xп) Mag. Tud. Ak. Etk. (Mth.) 7 (1881) (No. 23) 27 pp.

Segre, C. Tor. Ac. Sc. Mm. 36 (1885) 87-.

"Ausdehnungslehre," line geometry on principles of. Müller, E. Mh. Mth. Ps. 2 (1891) 267-.

Axial connections in space. Graberg, $F$. Zür. Vjschr. 35 (1890) 52-.

Conjugate straight lines, and polar planes. Fais, A. Bologna Ac. Sc. Mm. 7 (1876) 605-.

Coordinates, line, 6. Cayley, A. [1867] Camb. Ph. S. T. 11 (Pt. 2) (1869) 290-.

-, 一, formulæ. Ovidio, E. d'. G. Mt. 10 (1872) 33-.

-, -, homogeneous, geometric concept. Thaer, A. Z. Mth. Ps. 28 (1883) 315-.

-, -, in space. Zeuthen, H. G. Mth. A. 1 (1869) 432-.

-, - , theory and notation. Reuschle, C. D. Nf. Tbl. (1889) 191-.

Cremonian straight lines, systems. $\operatorname{Del} R e, A$. Rm. R. Ac. Linc. Rd. 2 (1893) (Sem. 1) 452-.

Desargues's theorem on triangles in perspective, space analogies. Valyi, $G$. Mth. Termt. Ets. 11 (1893) 30-; Mth. Nt.B. Ung. 13 (1897) 166-.

$n$-dimensional line geometry. Schubert, $H$. Hamb. Mth. Gs. Mt. 3 (1900) 86-.

Equivalence, collinear and reciprocal. Kalkmann, G. Mh. Mth. Ps. 9 (1898) 55-.

- of systems of linear elements. Kraft, $F$. Z. Mth. Ps. 39 (1894) 87-, 129-.

Fundamental configurations of line geometry. Frombeck, $H$. Wien Ak. Sb. 73 (1876) (Ab. 2) 475-.

Geometry and geomechanics. Fiedler, $W$. Zür. Vjschr. 21 (1876) 186-.
Geometry of space, new. Plilcker, J. Phil. Trans. (1865) 725-.

- - - Plücker's. Dronke, A. Z. Mth. Ps. 11 (1866) 46-; 12 (1867) 481-.

Hyperboloidal straight lines. Doehlemann, $K$. [1899] D. Mth. Vr. Jbr. 8 (1900) (Heft 1) 199-.

Invariant theory of line geometry. Waelsch, $E$. Mth. A. 37 (1890) 141-; Wien Ak. Sb. 98 (1890) (Ab. 2a) 1528-.

Invariants of quadratic forms and higher geometry. Study, E. Leip. Mth. Ps. B. 49 (1897) 443-.

Involution of axes of rotation. Sylvester, $J . J$. C. R. 52 (1861) 741-.

---1 (Sylvester). Chasles, M. C. R. 52 (1861) 745-.

- 6 lines in space. Sylvester, J.J. C. R. 52 (1861) 815-.

- - (Sylvester). Cayley, A. C. R. 52 (1861) 1039-.

-, mechanical. Sylvester, J.J. Am. J. Mth. 4 (1881) 336-; (xII) J. H. Un. Cir. [1] (1882) 242-.

Involutions in space. Ascione, $E$. Nap. Rd. 35 (1896) 13-.

Mechanics of rigid bodies and line geometry, relationship. Klein, $F$. Mth. A. 4 (1871) 403-.

Number of lines satisfying given conditions. Halphen, G. C. R. 68 (1869) 142-; 73 (1871) 1441-; 74 (1872) 41-.

Perpendicular on line from point, formula. Cayley, A. Ph. Mg. 30 (1865) 413-.

Plücker's geometry, fundamental problem. Voss, A. E. Gött. Nr. (1875) 101-.

- line-configurations. Vos8, (Dr) A. Gött. Nr. (1873) 544-.

- new geometry. Janni, G. G. Mt. 8 (1870) 302-.

Ray tetrads. Hermes, O. Crelle J. 67 (1867) 279 -.

Ruled space, representation by system of conics in a plane. Aschieri, $F$. Mil. I. Lomb. Rd. 12 (1879) 265-.

- spaces, 2, correspondence between. Demoulin, A. C. R. 129 (1899) 200-.

_ _ , _, Cremonian quadratic correspondence between. Aschieri, F. Mil. I. Lomb. Rd. 14 (1881) 123-.

- surfaces. Plilcker, J. [1867] A. Mt. 1 (1867-68) 160-

- - admitting homographic automorphic transformation. Bioche, C. Par. S. Phlm. Bll. 4 (1892) 130-.

Screws passing through given point or lying in given plane. Cardinaal, $J$. Amst. Ak. Vs. 7 (1899) 315-; Amst. Ak. P. 1 (1899) 258-.

-, theory. Kotelinikov, A. Kazan Un. Mm. (1895) (Pt. 9), (Pt. 11) 79-; (1896) (Pt. 1) 139-, (Pt. 2) 111-; Fschr. Mth. (1895) 804-. - uniform determination of cylindroid by. Somov, P. O. Vars. S. Nt. Tr. (1897) (Ps. C.) Fasc. 1, Mm. 2, 4 pp.

Skew curves, line geometry. Voss, A.E. Mth. A. 13 (1878) 232-.

Space-loci, class (Minding's theorem). Tait, P. G. [1879-81] Edinb. R. S. P. 10 (1880) 200; 11 (1882) 197-. 
Statics and line geometry. Bellavitis, $G$. [1879] Rm. R. Ac. Linc. Mm. 5 (1880) 29-. Straight lines in space, system. Maggi, P. G. A. Sc. Lomb. Ven. 8 (1838) 161-, 249-; 9 (1839) 187-.

- - and spheres, correspondence between. Lovett, E. O. C. R. 128 (1899) 83-; 129 (1899) 383-, 1296.

System of forces, Minding's. Chrystal, $G$. Edinb. R. S. T. 29 (1880) 519-.

Systems of forces (Dynamen), geometry. Study, E. [1899] D. Mth. Vr. Jbr. 8 (1900) (Heft 1) 204-.

- - - (-) in involution. Battaglini, $G$. Nap. At. Ac. 4 (1869) (No. 14) 15 pp.

Theorem. Klein, F. Gött. Nr. (1872) 164-.

Transformation, involutory, of 3rd order, family of surfaces occurring in. Chizzoni, $F$. Rm. R. Ac. Linc. Rd. 2 (1886) (Sem. 1) 470-.

Transformations, birational, surfaees generated by. Jung, G. Rm. R. Ac. Linc. Rd. 2 (1886) (Sem. 1) 85-.

-, geometric, of line systems. Lie, S. [1870] C. R. 71 (1870) 579-; Christiania F. 13 (1871) 506-.

-, involutory monoidal. Montesano, $D$. Mil. I. Lomb. Rd. 21 (1888) 579-, 684-.

\section{CONNEXES.}

(See also 2000-2070, and 4800-4880.)

Voss, A. E. Mth. A. 15 (1879) 355-.

Ahlborn, H. Hamb. Mth. Gs. Mt. 1 (1889) 41-.

Weber, E. von. Gött. Nr. (1896) 282-.

conjugate, theory. Stephanos, $C$. Bll. Sc. Mth. As. 4 (1880) (Pt. 1) 318-.

a new element of plane geometry. Clebsch, $R$. F. A. Gött. Nr. (1872) 429-.

particularly systems of straight lines of 2nd order. Masoni, $U$. Nap. Ru. 22 (1883) 145-. plane, representation of ruled space on. Lazzeri, $G$. Ven. I. At. (1884-85) 247-, 437-.

of planes and straight lines, surface connected with. Del $R e, A$. Nap. Rd. 27 (1888) 349-. quaternary, $(1,1)$, linear systems. Lazzeri, $G$. Rm. R. Ac. Linc. Mm. 4 (1887) 259-.

in space, new. Bonsdorff, $E$. [E.J.] St Pét. Ac. Sc. Bll. 27 (1881) 560-

- - , and partial differential equations of 1st order. Sincov, D. Kazan Un. Mm. (1894) (Pt. 6) 145-; (1895) (Pt. 1) 143-, (Pt. 3) 99-; Bll. Sc. Mth. 22 (1898) 221-.

ternary, 1st order and 1st class. Battaglini, $G$. [1880] Nap. Ac. At. 9 (1882) No. 4, 16 pp.

- $-\ldots \ldots$ - - geometric significance of invariants. Pannelli, M. G. Mt. 36 (1898) 81-.

-, 2nd order and 2nd class in double involution. Pannelli, M. G. Mt. 26 (1888) 1-.

,------ simple involution. Battaglini, G. Nap. Ac. At. 8 (1879) No. 6, 10 pp. 2nd order and 1st class, configuration corresponding to. Krause, R. Mth. A. 14 (1879) 294-.

- - 2nd class, generation. Armenante, $A$. Rm. R. Ac. Linc. At. 3 (1876) (Pte. 2) 123-.
$C(x, x, 0 ; u, u, 0)$, principal coincidence. Möller, $J$. Lund. Un. Acta 16 (1879-81) (Mth.) (No. 1) $16 \mathrm{pp}$.

$(1,2)$ and $(2,2)$, construction. Peano, G. Tor. Ac. Sc. At. 16 (1880) 497-.

$(1, n)$, special case of differential equation. Müllendorf, $E$. Arch. Mth. Ps. 69 (1883) 113-.

(2, 2), special connex. Amodeo, $F$. G. Mt. 25 (1887) 321-.

\section{COMPLEXES.}

Battaglini, G. [1868] Nap. At. Ac. 4 (1869) (No. 7) $27 \mathrm{pp}$.

Drach, C. A. von. Mth. A. 2 (1870) 128 -

Chelini, D. Bologna Ac. Sc. Mm. 1 (1871) 125-.

Aschieri, F. $\quad$ G. Mt. 10 (1872) 343-; 11 (1873) $107-, 246-, 340-; 12$ (1874) 15-; Mil. I. Lomb. Rd. 12 (1879) 409-.

Montesano, $D$. Nap. Rd. 25 (1886) 192-.

Jamet, V. C. R. 104 (1887) 567-.

Reye, T. Berl. Ak. Sb. (1889) 833-.

Waelsch, E. Wien Ak. Sb. 97 (1889) (Ab. 2a) 164.

Franel, J. Zür. Vjschr. 40 (1895) 104 -.

Petersen, $J$. Kjøb. Ov. (1898) 283-.

accelerations of any order. Castellano, $F$. Tor. Ac. Sc. At. 29 (1894) 306-

adjacent lines in. Küper, $C$. Mh. Mth. Ps. 1 (1890) 451-.

analogous configuration. Engel, $F$. Leip. Mth. Ps. B. 52 (1900) 63-, 220-.

application to surfaces and skew curves. Picard, É. Par. Ec. Norm. A. 6 (1877) 329-. arising from certain cubic transformation. Machovec, F. Prag Sb. (1888) (Mth.-Nt.) 104 .

associated with every birational space transformation. Pannelli, M. G. Mt. 28 (1890) 245-.

- Kummer's surface. Klein, F. Gött. Nr. (1871) 44 .

- - polar tetrahedron of 2 quadrics. Mertens, $F$. Wien Ak. Sb. 91 (1885) (Ab. 2) 519-.

of axes of central quadric. Huntington, $E, V$. [1900] A. Mth. 2 (1900-01) 8-.

- - quadric. Koenigs, G. N. A. Mth. 2 (1883) 267-.

$-1-1-, 2$ pedal surfaces. Bökle, C. Z. Mth. Ps. 39 (1894) 51-

- - quadrics. Schilke, E. Z. Mth. Ps. 19 (1874) 550-.

- - - Sucharda, A. Čsopis 29 (1900) 100-; Fschr. Mth. (1900) 543.

_ _ _ - , and general tetrahedral complex. Machovec, $F$. Prag Sb. (1886) (Mth.-Nt.) 501-.

- - Reye's. Weiler, Ad. Z. Mth. Ps. 28 (1883) 188-.

- - of screws. Emilio, R. d'. Ven. I. At. (1884-85) 1135-.

birational transformations connected with certain. Pieri, M. Palermo Cir. Mt. Rd. 6 (1892) 234.

Caporali's, motion of rigid body with 4 degrees of freedom in relation to. Cardinaal, $J$. [1898] D. Mth. Vr. Jbr. 7 (1899) (Heft 1) 61-. 
case. Schoute, P. H. Delft Éc. Pol. A. 3 (1887) 52-.

and circles and spheres. Grace, J. H. [1897] Camb. Ph. S. T. 16 (1898) 153-.

class. Cosserat, $E$. Toul. A. Sc. Mm. 4 (1892) 482-.

and congruences. Voss, A. E. Gött. Nr. (1874) 375-; Mth. A. 9 (1876) 55-.

- -. Jaggi, E. N. A. Mth. 4 (1885) 80-, 334-. - - . Küpper, K. Prag Ab. 4 (1892) No. 3, 11 pp.

- -, metrical properties. Ovidio, E. d'. Rm. R. Ac. Linc. At. 3 (1876) (Pt. 2) 260-.

- - projective methods applied to groups. Montesano, D. A. Mt. 1 (1898) 313-.

- - , representation of functions of 2 variables by. Vivanti, G. Palermo Cir. Mt. Rd. 9 (1895) 108-.

- - of 2nd degree. Caporali, $E$. Rm. R. Ac. Linc. Mm. 2 (1878) 749-.

- - 1st order. Heath, R. S. Birm. Ph. S. P. 4 ( $\left.{ }^{*} 1883-85\right)$ 334-.

- conics of a plane. Segre, C. Tor. Ac. Sc. At. 20 (1885) 487-

containing generators of systems of quadrics.

Stephanos, C. As. Fr. C. R. (1880) 150-.

covariant for 2 quadrics. Pick, $G$. Wien Ak. Sb. 100 (1891) (Ab. 2a) 561-.

curves. Zindler, K. [1899] Mh. Mth. Ps. 11 (1900) 28-.

- with tangents belonging to. Koenigs, $G$. Toul. Fac. Sc. A. 1 (1887) E, 9-.

- - - Appell, P. N. A. Mth. 11 (1892) 115-

1077-

determination of subgroups of projective group. Knothe, E. Arch. Mth. Ntvd. 15 (1892) 97-. determining systems of helicoidal axes. Somov, P. O. [1895] Vars. S. Nt. Tr. (1895-96) (C. R., Ps. C.) No. 1, 4 pp.

especially of line and sphere, with application to partial differential equations. Lie, $S$. [1871] Mth. A. 5 (1872) 144-

fundamental formulø. Seiliger, D. Fschr. Mth. (1897) 592.

- properties. Ovidio, E. d'. Tor. Ac. Sc. At. 16 (1880) 327-.

generated by 2 correlative planes. Hirst, $T, A$. L. Mth. S. P. $10(1878-79)$ 131-.

- - planes in reciprocal birational correspondence. Visalli, P. Rm. R. Ac. Linc. Rd. 4 (1895) (Sem. 1) 480-.

generation by double rotation. Zindler, $K$. [1894] D. Mth. Vr. Jbr. 4 (1897) 99-.

of generators on quadrics through seven given points. Kluyver, J. C. N. Arch. Wisk. 19 (1892) 1-; Fschr. Mth. (1892) 790-.

and their intersections, plane representations by conics. Aschieri, F. Mil. I. Lomb. Rd. 12 (1879) 341-.

investigations by pure geometry. Tognoli, $O$. [1870] G. Mt. 9 (1871) 19-.

involutory space transformations determining. Montesano, D. Rm. R. Ac. Linc. Rd. 4 (1888) (Sem. 1) 207-, 277-.

and linear systems of circles. Cosserat, $E$. C. R. 106 (1888) 1467-. lines common to 4. Snyder, V. N. Y. Am. Mth. S. Bll. 3 (1897) 247-.

of lines meeting a rational quartic curve. Cayley, A. L. Mth. S. P. 17 (1887) 232-.

metrico-projective theorems. Ovidio, $E$. $d$ '. Mil. I. Lomb. Rd. 14 (1881) 405-.

mutual moment of 2. Segre, $C$. Crelle J. Mth. 99 (1886) 169-.

6 , mutually in involution, groups $(P)_{360},(\Pi)_{360}$ arising from. Veronese, G. A. Mt. 11 (188283) 284 -

nets, in metrico-projective geometry. Ovidio, E. d'. Rm. R. Ac. Linc. At. 3 (1876) (Pt.2) $561-$.

of normals to series of plane curves. Bemporad, A. Tor. Ac. Sc. At. 34 (1898) 391or 535-.

orthogonal, of Reye. Thieme, H. Z. Mth. Ps. 36 (1891) 349-.

pencils (Büschel). Zindler, $K$. Mh. Mth. Ps. 3 (1892) 135-.

and Pfaffian equations. Weber, $E$. von. [1900] Münch. Ak. Sb. 30 (1901) 393-.

Plücker's. Clebsch, R. F. A. [1869] Mth. A. 2 (1870) 1-.

of polar lines with respect to system of surfaces. Veneroni, $E$. Mil. I. Lomb. Rd. 32 (1899) 536-.

- principal normals to geodesic movable on surface. Gebbia, M. Palermo Cir. Mt. Rd. 1 (1887) 25-.

principal surfaces, and asymptotic lines of their surface of singularities. Koenigs, G. C. R. 104 (1887) 1824.

and quadratic forms. Study, $E$. Leip. Mth. Ps. B. 42 (1890) 172-.

regular, and equilateral hyperbolas of order $n$. Serret, $P$. C. R. 121 (1895) 372 .

representation in ordinary space. Porchiesi, $A$. Rm. R. Ac. Linc. Mm. 1 (1885) 610-.

- point space. Aschieri, $F$. Mil. I. Lomb. Rd. 13 (1880) 531-.

resulting from involution on skew curve of 3 rd degree. Weiler, Ad. Z. Mth. Ps. 24 (1879) 159-.

Reye's, reciprocity relations. Lie, S. Gött. Nr. (1870) 53-.

ruled surfaces with generators forming part of. Picard, É. C. R. 84 (1877) 229-.

screw, 2nd order. Ball, R. S. B. A. Rp. (1875) (Sect.) 10.

singularities. Geisenheimer, $L$. Z. Mth. Ps. 18 (1873) 346-.

space generated by. Paolis, R. de. Rm. R. Ac. Linc. Mm. 1 (1885) 205-.

and spherical complexes, metrical geometry of, and mutual analogies. Segre, C. Tor. Ac. Sc. At. 19 (1883) 159-.

- surfaces, correspondence between theories. Cremona, L. [1875] Rm. R. Ac. Linc. At. 3 (1876) (Pte. 2) 285-.

surfaces with curves of principal tangents belonging to. Peter, A. Arch. Mth. Ntvd. 17 (1895) No. 8, $89 \mathrm{pp}$.

systems of forces and of rotations connected with. Franke, J. N. [1881] Wien Ak. Sb. 84 (1882) (Ab. 2) 570-. 
systems of skew curves of 3rd degree in connection with. Silldorf, $(D r)$-. Z. Mth. Ps. 19 (1874) 391-.

of tangents, rational space transformations specifying. Pieri, M. G. Mt. 33 (1895) 167-. tetrahedral. Aschieri, $F$. Mil. I. Lomb. Rd. 12 (1879) 426-.

Timerding, H. E. N. Y. Am. Mth. S. Bll. 6 (1900) 417-.

-, automorphic transformation. Ameseder, $A$. Wien Ak. Sb. 97 (1889) $(A b .2 a)$ 627-.

-, congruences in. Machovec, $F$. [1891] Prag České Ak. Fr. Jos. Rz. (Tř́ida 2) 1 (1892) Art. 3, 15 pp.

-, generated by 2 reciprocal nets of planes and lines. Aschieri, $F$. Mil. I. Lomb. Rd. 16 (1883) 988-.

- involutory space transformation determining. Montesano, $D$. Rm. R. Ac. Linc. Rd. 5 (1889) (Sem. 1) 497-.

-, representation in point space. Weiler, Ad. Z. Mth. Ps. 22 (1877) 261-.

-, surfaces with conjugate network of curves touching lines of. Demoulin, A. Par. S. Mth. Bll. 25 (1897) 83-.

tetrahedroidal, synthetic geometry. Hofmann, F. Arch. Mth. Ps. 5 (1887) 353-.

theorem analogous to Dupin's. Klein, $F$. Gött. Nr. (1871) 73-.

theory. Pasch, M. Crelle J. 75 (1873) 106-.

triple and quadruple series of, in metricoprojective geometry, Ovidio, E. $d^{\prime}$. Rm. R. Ac. Linc. At. 3 (1876) (Pt. 2) 723-.

and uniform and multiple correspondences of space. Aschieri, F. Mil. I. Lomb. Rd. 21 (1888) 216-, 285-, 446-.

1st degree. Battaglini, G. Nap. Rd. 5 (1866) 194-.

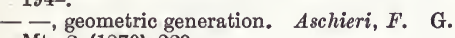
Mt. 8 (1870) 229-.

- and 2nd degrees. Klein, F. [1869] Mth. A. 2 (1870) 198-.

- - - - Schur, F. Mth. A. 15 (1879) 432-.

- - - Reye, T. Crelle J. Mth. 95 (1883) 330-.

- _ - - generation from line congruences. Weiler, Ad. Z. Mth. Ps. 27 (1882) 257-.

2nd degree. Aschieri, $F$. [1869-70] Pisa A. Scuola Norm. 1 (1871) 133-; G. Mt. 8 (1870) 35-, 229-.

- - Painvin, L. Bll. Sc. Mth. As. 2 (1871) 368-; N. A. Mth. 11 (1872) 49-, 97-, 202-, 289-, 481-, 529-.

- - Battaglini, G. [1878] Rm. R. Ac. Linc. Mm. 3 (1879) 35-.

- - Bertini, E. [1878] G. Mt. 17 (1879) 1-. - Aschieri, F. Bologna Ac. Sc. Mm. 10 (1879) 549-.

- - Segre, C., \& Loria, G. Mth. A. 23 (1884) 213-.

- -. Segre, C. Mth. A. 23 (1884) $235-$.

- - Deruyts, $F$. Brux. Ac. Bll. 24 (1892) 571-.

- - S., R. N. A. Mth. 14 (1895) 433-.

- - Snyder, V. N. Y. Am. Mth. S. Bll. 4 (1898) 144-. 2nd degree, Battaglini's, and application of an irrational transformation. Segre, C. G. Mt. 21 (1883) 355-.

- -, determined by a null polar system. Aschieri, F'. Mil. I. Lomb. Rd. 11 (1878) 612-. - -, general. Sturm, R. Berl. Ak. Sb. (1894) 697-.

- - , and geometry of circle. Snyder, $V . \mathrm{N}$. Y. Am. Mth. S. Bll. 6 (1900) 319-, 464.

- -, new properties. Reye, T. Mth. A. 49 (1897) 585-.

- - polar theory. Stahl, W. Crelle J. Mth. 94 (1883) 319-.

- - principal types. Reye, T. Crelle J. Mth. 98 (1885) 284-.

- - 2 quadrics connected with. Schur, F. Mth. A. 21 (1883) 515-.

- -, and quartic surfaces. Sucharda, $A$. Prag Sb. (1888) (Mth.-Nt.) 381-.

- - representation in point space. Aschieri, F. Mil. I. Lomb. Rd. 14 (1881) 500-

- - , simple generation of considerable num. ber. Weiler, Ad. Zür. Vjschr. 26 (1881) 41-; Crelle J. Mth. 95 (1883) 140-.

- -, synthetic construction. Stahl, W. Crelle J. Mth. 93 (1882) 215-.

- -, theory. Schur, F. Mth. A. $17(1880)$ 107-.

- - , uniform space transformations determining. Montesano, D. Mil. I. Lomb. Rd. 25 (1892) 795-.

- - , various species. Weiler, Ad. Erlang. Sb. Ps. Md. S. 5 (1873) 114-; Mth. A. 7 (1874) 145-.

3rd degree. Schoute, P. H. As. Fr. C. R. (1887) (Pt. 2) 189-.

- - Montesano, D. Bologna Ac. Sc. Mm. 3 (1892) 549-.

- - Veneroni, E. Mil. I. Lomb. Rd. 32 (1899) 1008-, 1403-.

6th degree. Rouquet, $\dot{V}$. Toul. Fac. Sc. A. 3 (1889) I, $20 \mathrm{pp}$.

$n$th degree, singularities. Schubert, H. C. H. Mth. A. 12 (1877) 202-.

3rd species, representation in point space. Aschieri, F. Mil. I. Lomb. Rd. 13 (1880) 182-.

Complex-surface. Veneroni, E. Mil. I. Lomb. Rd. 31 (1898) 257-.

-, Plücker's. Klein, F. [1873] (vm) Mth. A. 7 (1874) 208-

-, -, construction. Kleiber, J. Z. Mth. Ps. 33 (1888) 349-.

Complex-surfaces and surfaces of singularity. Clebsch, R. F. A. Gött. Nr. (1872) 33-.

-, 4th order and class. Klein, F. [1869] Mth. A. 2 (1870) 371-.

Conjugate axes of bodies. Binet, J. P. M. Par. S. Phlm. Bll. 2 (1811) 389-.

Coordinates, line, transformation and canonical form of general equation of 2 nd degree. Klein, $F$. Mth. A. 23 (1884) 539-.

Correspondence, algebraic, geometric forms generated by. Loria, G. G. Mt. 34 (1896) 354-.

Hyperboloid, problem. Gordan, P. Z. Mth. Ps. 13 (1868) 59-. 
Kummer's surface, mode of generating. Humbert, G. Palermo Cir. Mt. Rd. 11 (1897) 1-.

Lines with given moments with reference to fixed lines. Segre, C. Crelle J. Mth. 97 (1884) 95-.

Null spaces of a one-system and associated complexes. Young, W. H. [1898] L. Mth. S. P. 30 (1899) 33-.

- system, curves self-reciprocal in. Steinmetz, C. P. [1891] Am. J. Mth. 14 (1892) 161-.

- - and line complex, relation to polar system of paraboloid of revolution. Hauck, G. Z. Mth. Ps. 31 (1886) 362-.

- _, Möbius's, shortest and straightest lines in. Liebmann, $H$. Mth. A. 52 (1899) 120-.

- - quadratic. Timerding, H. E. A. Mt. 2 (1899) 239-.

- -, theory. Döhlemann, $K$. D. Mth. Vr. Jbr. 3 (1894) 96-.

- systems, higher. Sturm, R. Mth. A. 28 (1887) 277-.

- - invariant configurations. Mertens, $F$. Wien Ak. Sb. 97 (1889) (Ab. 2a) 519-.

Polar and null systems, comparison. Schmid, T. Mh. Mth. Ps. 8 (1897) 267-.

Ray systems with focal surfaces of 4 th degree having 16 singular points. Kummer, E. E. Berl. Mb. (1864) 495-.

Screw motion, null system and line complex of. Küpper, C. Mh. Mth. Ps. 1 (1890) 95-.

Skew curve, 3rd degree, condition that 4 straight lines should touch. Kluyver, J. C. Amst. Ak. Vs. M. 8 (1891) 41-; Arch. Néerl. 25 (1892) 70-.

- surfaces, genus 0 , asymptotic lines. Pittarelli, G. Rm. R. Ac. Linc. Rd. 7 (1891) (Sem. 1) 391-.

- - rational, Cayley's, asymptotic lines. Pittarelli, G. Rm. R. Ac. Linc. Rd. 7 (1891) ( $\mathrm{Sem} .1$ ) 452-.

Surfaces of same degree as their polar reciprocals, and with same singularities. Kummer, E. E. Berl. Ak. Mb. (1878) 25-.

Tetrahedral symmetrical curves. Demoulin, $A$. C. R. 115 (1892) 280-, 362 .

Transformation, Lie's, and surfaces which are envelopes of spheres. Demoulin, $A$. Bll. Sc. Mth. 22 (1898) 174-.

\section{CONGRUENCES.}

Monge, G. Par. Ec. Pol. Cor. 3 (1814-16) 152 .

Transon, (Prof.) A. Par. J. Ec. Pol. $38^{e}$ cah. (1861) 195-.

(Transon.) Chasles, $M$. C. R. 52 (1861) 1013-.

Frischauf, J. Z. Mth. Ps. 16 (1871) 159-.

Weiler, A. Z. Mth. Ps. 31 (1886) 18-.

Bianchi, L. Rm. R. Ac. Linc. Rd. 3 (1887) (Sem. 1) 369-; A. Mt. 15 (1887-88) 161-.

Gorton, W. C. L. Am. J. Mth. 10 (1888) 347-.

Cosserat, E. C. R. 115 (1892) 929-.

Palatini, F. Ven. I. At. (1894-95) 711-.

Guichard, C. C. R. 126 (1898) 1183-.
Cifarelli, $T$. A. Mt. 2 (1899) 139-.

Demoulin, A. C. R. 130 (1900) 1701-.

Zeeman, P. (Gz.) Fschr. Mth. (1900) 648.

algebraic, classification. Schumacher, $R$. Mth. A. 37 (1890) 100-.

arising in representation of surfaces. Caporali, E. Nap. Rd. 18 (1879) 244 -

associated. Guichard, C. C. R. 124 (1897) 669-.

asymptotic lines corresponding on 2 sheets of focal surface. Guichard, C. C. R. 110 (1890) 126-.

- - of ruled surfaces in. Pittarelli, G. Rm. R. Ac. Linc. Rd. 3 (1894) (Sem. 2) 111-, 148-, 229-, 264.

_ _ _ - - whose generators belong to. Bioche, C. Par. S. Mth. Bll. 19 (1891) 39-.

of central axes of complexes to which 3 straight lines belong. Demoulin, A. Par. S. Mth. Bll. 21 (1893) 92-.

class. Guichard, C. C. R. 112 (1891) 1424 -.

-. Petot, A. C. R. 113 (1891) 841-.

2 classes. Demoulin, A. Bll. Sc. Mth. 18 (1894) 233-.

of conics in space. Montesano, D. Mil. I. Lomb. Rd. 26 (1893) 589-; Nap. Rd. 34 (1895) 93-, 155-.

connected with hyperboloid. Waelsch, E. Wien Ak. Sb. 95 (1887) (Ab. 2) 781-.

- surfaces of 2nd degree. Rudio, $F$. Zür. Vjschr. 41 (1896) (Festschr., Th. 2) 76-.

- - - 3rd and 4 th degrees. Schur, $F$. Crelle J. Mth. 95 (1883) 207-.

- - theory of surfaces. Alexejeff, W. G. Fschr. Mth. (1899) 586.

Cremonian. Hirst, T. A. [1880] L. Mth. S. P. 14 (1882-83) 259-.

-, contained in linear complex. Hirst, T. A. L. Mth. S. P. 17 (1887) 287-.

cyclic, theorem of Cosserat. Tzitzéica, C. R. 127 (1898) 167-。

-, and triply conjugate systems. Tzitzéica, $G$. Par. Ec. Norm. A. 16 (1899) 137-.

deformation, and some classes of applicable surfaces. Bianchi, $L$. Rm. R. Ac. Linc. Rd. 9 (1900) (Sem. 2) 185-.

enumerative geometry. Martinetti, $V$. Rv. Mt. 3 (1893) 108-.

without focal surface. Sturm, $R$. Hamb. Mth. Gs. Mt. 2 (1890) (Festschr., Tl. 2) 61-. focal surfaces. Voss, (Dr) A. Gött. Nr. (1873) 611-.

and focal surfaces of 2 related surfaces. Voss, A. Mth. A. 30 (1887) 227-.

focal surfaces of, and surfaces of singularity of complexes. Pasch, M. [1872] Crelle J. 76 (1873) 156-.

2 focal systems cyclic. Guichard, C. C. R. 131 (1900) 1177-.

forming group of tangents to surface. Geisenheimer, L. Z. Mth. Ps. 18 (1873) 33-.

fundamental formulæ. Burgatti, P. Rm. R. Ac. Linc. Rd. 8 (1899) (Sem. 1) 515-.

and general complex of 3rd degree. Veneroni, E. Mil. I. Lomb. Rd. 31 (1898) 1072-.

generated by 2 planes in $(1,2)$ correspondence. Conti, I. Palermo Cir. Mt. Rd. 1 (1887) 230-; 2 (1888) 97-. 
generated by $(1, \nu)$ point correspondence in 2 planes. Visalli, P. Mil. I. Lomb. Rd. 28 (1895) 114-.

- -2 surfaces with uniform correspondence. Pannelli, $M$. Rm. R. Ac. Linc. Mm. 6 (1889) 216-.

homaloidal, 3rd degree. $\operatorname{Del} R e, A$. Mod. Ac. Sc. Mm. 1 (1898) 95-.

involutory space transformations giving rise to. Paolis, R. de. Rm. R. Ac. Linc. Rd. 1 (1885) 735-, 754-.

Kummer's representation. Weiler, A. Zür. Vjschr. 29 (1884) 366-.

of linear complexes, metrical relations. Bobek,

' $K$. Wien Ak. Sb. 83 (1881) (Ab. 2) 885-.

- lines and curves, parallelism. Guichard, $C$. C. R. 128 (1899) 723-.

- - cutting 3 projective pencils. Vries, J.de. N. Arch. Wisk. 3 (1898) 222-.

Mannheim and Hamilton's formula. Pelı̌̌ek, M. Prag Sb. (1900) (Mth.-Nt.) No. 2, 14 pp.

mean surface a plane. Guichard, C. C. R. 114 (1892) 729-.

metrical property common to 3 special classes. Demoulin, A. C. R. 118 (1894) 242-.

new principles in theory. Issaly, - [1887] Par. S. Mth. Bll. 16 (1888) 19-.

of normals. Goursat, E. C. R. 129 (1899) 669-.

- - , and curvature of surfaces. Mannheim, A. C. R. 70 (1870) 1074-; Liouv. J. Mth. 17 (1872) 109-.

- - - - cyclic systems. Guichard, C. C. R. 124 (1897) 1079-.

- - to surface. Bertrand, J. C. R. 17 (1843) 1277-.

-- - Laguerre, E. N. A. Mth. 17 (1878) 181-.

- _ - - and other congruences. Schläfi, L. Bern Mt. (1846) 89-, 111-.

_ _ - - of 2 nd degree, skew surface formed from. Nicodemi, $R$. Nap. Ac. Pont. At. 26 (1896) No. 5, 14 pp.

and orthogonal systems. Guichard, - C. R. 125 (1897) 564-.

of particular type, transformation with aid of. Brill, J. [1888] L. Mth. S. P. 20 (1889) 102-.

problem. Goursat, E. C. R. 129 (1899) 578-. of rays $(3,1)$ and $(1,3)$. Emch, A. A. Mth. 11 (1896-97) 148-.

referred to their developables, and surfaces to their asymptotic lines. Guichard, C. Par. Éc. Norm. A. 6 (1889) 333-.

and related functional problem. Hadamard, $J$. Bordeaux S. Sc. PV. (1894-95) 19-.

representation on plane. Loria, $G$. Tor. Ac. Sc. At. 21 (1885) 621-.

Roccella's, 3rd order and 3rd class. Hirst, $T$. A. Palermo Cir. Mt. Rd. 1 (1887) 64-.

ruled surfaces connected with. Emilio, R. $d$ '. Ven. I. At. (1884-85) 1265-, 1911-.

and ruled surfaces, theorems. Klug, L. Mh. Mth. Ps. 9 (1898) 110-.

- singular solutions of differential equations. Dixon, A. C. [1894] Phil. Trans. (A) 186 (1896) 523-. singular surfaces. Reye, $T$. Crelle J. Mth. 97 (1884) 242-.

of skew curves. Emch, A. [1900] N. Y. Am. Mth. S. Bll. 7 (1901) 243, 249.

special kind. Lilienthal, K. von. Mth. A. 31 (1888) 85-.

tangent, of a surface. Waelsch, $E$. Wien Ak. Sb. 102 (1893) (Ab. 2a) 757-.

of tangents to given surface, focal surfaces. Pell, A. Am. J. Mth. 20 (1898) 101-.

theorem, new applications. Halphen, G. $H$. (x) Par. S. Mth. Bll. 1 (1873) 253-.

theorems. Fouret, G. C. R. 85 (1877) 216-.

and theory of surfaces. Cosserat, $E$. Toul. Fac. Sc. A. 7 (1893) N, 62 pp.

volume generated by. Roberts, $R$. A. L. Mth. S. P. 19 (1889) 207-

2nd class. Reye, $T$. Crelle J. Mth. 86 (1879) 84-.

- - Visalli, P. Mil. I. Lomb. Rd. 28 (1895) 264-, 319-.

of like class and order. Sturm, R. Crelle J. Mth. 101 (1887) 162-.

$n$th degree, representable on plane. Visalli, P. Rm. R. Ac. Linc. Rd. 4 (1895) (Sem. 1) 33-.

- - , with singular skew curve of $n$th degree. Visalli, P. Rm. R. Ac. Linc. Rd. 4 (1895) (Sem. 1) 58-.

1st order and 1st class. Hermes, $O$. [1866] Crelle J. 67 (1867) 153-.

$---1-$ Fouret, -. Par. S. Mth. Bll. 19 (1891) 58-.

_- - - and line complex. Reye, $T$. Crelle J. 69 (1868) 365-.

- - - any class. Fouret, $G$. Palermo Cir. Mt. Rd. 6 (1892) 63-.

-, 2nd and other orders. Kummer, E. E. Berl. Ab. (1866) (Mth.) 1-.

2nd order. Stahl, $W$. Crelle J. Mth. 95 (1883) 297-.

- -, with focal or singular lines, classification. Sturm, R. Mth. A. 36 (1890) 467-.

- _, - - - - - . Schumacher, $R$. Mth. A. 38 (1891) 298-

-- , representation on plane. Montesano, $D$. Palermo Cir. Mt. Rd. 7 (1893) 159-.

- - , singular points, number and position. Sturm, R. Gött. Nr. (1888) 468-.

- - and 4th class. Montesano, D. Tor. Ac. Sc. At. 27 (1892) 1053-.

- - 6th class, 2. Montesano, D. Rm. R. Ac. Linc. Rd. 1 (1892) (Sem. 2) 77-.

_ - 6th class, 1st species, with focal surface. Bertini, E. Rm. R. Ac. Linc. T. 4 (1880) 30-. 3rd order, without singular line. Fano, $G$. Tor. Ac. Sc. At. 29 (1894) 474-; 31 (1895) 444- or 708-.

- - and 3rd class. Hirst, T. A. L. Mth. S. P. 16 (1884-85) 232-.

- - 6th class in ordinary space. Bordiga, G. Rm. R. Ac. Linc. Rd. 6 (1890) (Sem. 1) 8-. 4 th order and 2 nd class. Stahl, W. Crelle J. Mth. 97 (1884) 146-.

- - 4th class, etc. Painvin, L. Liouv. J. Mth. 19 (1874) 57-.

6th order, 2nd class, 1st species. Reye, $T$. Crelle J. Mth. 93 (1882) 81-. 
$(6,2)$ arising from 2 quadrics. Del $R e, A$. Palermo Cir. Mt. Rd. 1 (1887) 290-.

$(1,1)$ and line complex. Schoute, P. H. Amst. Ak. Vs. M. 5 (1889) 66-; Fschr. Mth. (1888) 849 .

$(3,4)$. Del Re, A. Rm. R. Ac. Linc. Rd. 2 (1893) (Sem. 1) 245-.

Paraboloid, hyperbolic rectangular, and raysystem derived from it. Schoenflies, $A$. Z. Mth. Ps. 23 (1878) 245-.

Ruled space, projective geometry. Aschieri, $F$. G. Mt. 16 (1878) 346-.

System of 4 lines in space. Franel, $J$. Zür. Vjschr. 40 (1895) 84-.

Systems of luminous rays, geometric properties. Del Re, A. Palermo Cir. Mt. Rd. 1 (1887) 284-.

Thin pencils. Schultén, N. G. af (fil.). [1836] St Pét. Mm. Sav. Étr. 4 (1845) 203-.

- - Möbius, A. F. Leip. B. 14 (1862) 1-.

- - Hensel, $K$. Crelle J. Mth. 102 (1888) 273-.

- - and affinity of plane systems. Zech, $P$. Z. Mth. Ps. 17 (1872) 353-.

Trajectory surfaces of figure of invariable form, all normals meet 2 straight lines. Mannheim, A. As. Fr. C. R. 2 (1873) 95-.

- - - - - - - normals, construction. Mannheim, A. As. Fr. C. R. (1878) 152 .

Transformation of pencil of normals. Mannheim, A. As. Fr. C. R. (1878) 132-; C. R. 88 (1879) 1179-.

Wave surface and transformation of ray-system. Mannheim, A. C. R. 88 (1879) 1248-.

\section{HIGHER ELEMENTS OF SPACE.}

Circle as generating element of space. Cosserat, E. Toul. Fac. Sc. A. 3 (1889) E, 81 pp.

- in space. Koenigs, $G$. Toul. Fac. Sc. A. 2 (1888) F, 19 pp.

Circles projecting into circles on given plane. Nicodemi, $R$. Nap. Ac. Pont. At. 18 (1888) 91- ; 21 (1891) 109-.

-, spheres, and points, groups. Darboux, $G$. Par. Éc. Norm. A. 1 (1872) 323-.

- and spheres, properties of systems. Guichard, C. C. R. 129 (1899) 944-。

Complex or congruence of curves, surface of singularities. Cosserat, E. C. R. 107 (1888) 653-.

- of conics, and surface of 3rd degree. Humbert, G. Par. Ec. Pol. J. 64 (1894) 123-.

-, linear spherical, envelopes. Smith, P.F. N. Y. Am. Mth. S. T. 1 (1900) 371-

-, spherical. Smith, P.F. [1900] N. Y. Am. Mth. S. Bll. 7 (1901) 3, 8-.

Complexes and congruences, spherical, 2nd degree. Reye, $T$. Crelle J. Mth. 99 (1886) 205-.

-, spherical, homaloidal. Del Re, A. Rm. R. Ac. Linc. Rd. 8 (1899) (Sem. 2) 100-.

-, -, theory. Bäcklund, A. V. Lund Acta Un. 9 (1872) (Mth.) No. 3, 24 pp.
Congruence of curves, general, property. Joly, C. J. [1899] Ir. Ac. T. 31 (1896-1901) 363-.

Congruences of circles in space. Stephanos, $C$. C. R. 93 (1881) 633-.

- - and spheres. Guichard, C. C. R. 129 (1899) 147-.

_ _ _ - - appearing in study of orthogonal and cyclic systems. Guichard, C. C. R. 129 (1899) 748-.

_ - _ - , several times cyclic. Guichard, C. C. R. 130 (1900) 1533-.

- conics. Pieri, $\boldsymbol{M}$. Tor. Ac. Sc. At. 28 (1893) 289-.

- - curves. Dall'Aequa, A. [1899] Ven. I. At. (1899-1900) (Pt. 2) 245-.

- - Levi-Civita, T. Rm. R. Ac. Linc. Rd. 8 (1899) (Sem. 1) 239-.

Conic in space, 18 coordinates. Spottiswoode, $W$. B. A. Rp. (1878) 462.

- - 21 coordinates. Spottiswoode, $W$. L. Mth. S. P. 10 (1878-79) 185-.

Conics in plane, representation on point-space. Study, E. Mth. A. 40 (1892) 551-.

- - space. Hierholzer, C. [1869] Mth. A. 2 (1870) 563-.

- - - with application to planet orbits. Grunert, J. A. Grunert Arch. 37 (1861) 1-. - - , generating surfaces. Lüroth, $J$. [1869] Mth. A. 3 (1871) 124-.

Families of transformations of straight lines into spheres. Lovett, E. O. Am. J. Mth. 22 (1900) 138-.

Null birational reciprocities in space. Montesano, $D$. Rm. R. Ac. Linc. Rd. 4 (1888) (Sem. 1) 583-.

\section{Systems (linear, and not linear) of curves and surfaces.}

Jonquières, E. de. Liouv. J. Mth. 10 (1865) 412-; C. R. 62 (1866) 293-; G. Mt. 4 (1866) 45-, 212-

Brill, A. [1874] Mth. A. 8 (1875) 534-.

Folie, $F$. Bll. Sc. Mth. As. 3 (1879) 278-.

Weyr, E. J. [1881] Wien Ak. Sb. 84 (1882) (Ab. 2) 884 .

Del Pezzo, P. Palermo Cir. Mt. Rd. 3 (1889) 236-.

Characteristics in systems. Jonquières, E. de. C. R. 63 (1866) 793-.

- of systems of conics and quadrics. Darboux, G. C. R. 67 (1868) 1333-。

-, theory. Clebsch, R. F. A. [1872] Mth. A. 6 (1873) 1-.

Curves and surfaces self-enveloping in similarly variable systems. Miller, R. Z. Mth. Ps. 22 (1877) 369-.

- - - touching infinite number of others. Gergonne, J. D. Gergonne A. Mth. 3 (181213) 361-.

Hyperjacobian surfaces and curves. Spottiswoode, W. [1877] Phil. Trans. 167 (1878) 351 -.

Infinity of conics or quadrics, curves or surfaces sibi-reciprocal with respect to. Fouret, $G$. Par. S. Phlm. Bll. 1 (1877) 42-. 
Intersections of systems, theorems. Woepcke, $F$. Liouv. J. Mth. 19 (1854) 407-; 20 (1855) 139 -.

Linear systems of conics and quadrics. Darboux, G. Bll. Sc. Mth. As. 1 (1870) 348-

- - curves and surfaces and their Jacobians. Doehlemann, $K$. Mth. A. 41 (1893) 545-.

Nets. Jonquières, E. de. C. R. 67 (1868) 1338-; Mth. A. 1 (1869) 424-.

Pencils. Pieri, M. G. Mt. 24 (1886) 13-.

\section{SYSTEMS OF CURVES.}

(See also 7230.)

Cremona, L. A. Mt. 6 (1864) 153-.

Chasles, M. C. R. 63 (1866) 816-; 64 (1867) 799-.

Jonquières, E. de. C. R. 63 (1866) 870-.

Dewulf, Ł́. [1880] N. A. Mth. 20 (1881) 391-.

Algebraic or transcendental plane curves defined by 2 characteristics. Fouret, G. Par. S. Mth. Bll. 2 (1874) 72-.

Characteristics of elementary systems of curves of 3rd degree. Zeuthen, H. G. C. R. 74 (1872) 521-, 604-, 726-.

- - - - - - 4th degree. Zeuthen, $H$. G. C. R. 75 (1872) 703-

-, relation between 2 in a system. Chasles, $M$. C. R. 62 (1866) 325-.

Circles and bicircular curves of $4 \mathrm{th}$ degree, vector analysis. $\operatorname{Cox}, H$. QJ. Mth. 19 (1883) 74-.

Circular coordinates. Gyurkovich, G. von. Z. Mth. Ps. 11 (1866) 494-; 12 (1867) 265-。

Conics, curves of 4 th degree arising from systems. Caporali, E. A. Mt. 20 (189293) 237-.

- of 4-point contact with curves of 4 th degree. Ameseder, $A$. Wien Ak. Sb. 80 (1880) $(A b .2)$ 187- ; 83 (1881) (Ab. 2) 829-.

-, surfaces containing systems. Weiler, A. Z. Mth. Ps. 30 (1885) 159-.

-, systems in connexion with curves of 3rd degree. Smith, H.J. S. [1868] L. Mth. S. P. 2 (1869) 67-.

-, - of, and rational curves of 3rd class, relation. Trebitscher, $M$. Wien Ak. Sb. 81 (1880) $(A b .2)$ 1080-

Contact curves, non-adjoint. Weiss, $W$. Wien Ak. Sb. 99 (1891) (Ab. 2a) 284-; 102 (1893) (Ab. 2a) 1025-.

- of systems of curves with straight lines. Gruss, G. [1881] Wien Ak. Sb. 84 (1882) (Ab. 2) 228 -

- _ - plane curves defined by 2 charac. teristics, transformations. Fouret, $G$. Par. S. Phlm. Bll. 13 (1876) 72-.

Continuous series of curves on regular algebraic surface. Enriques, $F$. Palermo Cir. Mt. Rd. 13 (1899) 95-.

Curves with common points. Woepcke, $F$. Crelle J. 54 (1857) 274-.

- dividing homographically generators of ruled surface. Bioche, C. Toul. Fac. Sc. A. 3 (1889) N, 41 pp.

- _ system of circles. Demartres, $G$. C. R. 106 (1888) 54 .
Curves of pencil, having double contact with given curve. Brill, A. Mth. A. 3 (1871) 459-.

- - 4-point contact with triply infinite system of curves. Spottiswoode, W. [1876] L. Mth. S. P. 8 (1876-77) 29-.

-, 3rd degree, through 7 fixed points. Geiser, C. F. [1866] Crelle J. 67 (1867) 78-

-, _ _, _ - - (Geiser). Milinowski, [1873] Crelle J. 77 (1874) 263-.

_, _ _ , inflexion point triangles of which have same orthocentre. Ameseder, A. Z. Mth. Ps. 28 (1883) 53-.

-, 6th degree, to which normal problem in curves of 2 nd degree leads. Bauer, $G$. Münch. Ak. Sb. 8 (1878) 121-.

-, $m$ th degree, involving $\mu$ th power of parameter, theorem of Jonquieres. Ascoli, $G$. G. Mt. 5 (1867) 377.

-, nth degree, under $\frac{n}{2}(n+3)-1$ conditions, degree of parametric equation. Battaglini, $G$. Nap. Rd. 2 (1863) 149-.

,--- , involution in. Serret, $P$. C. R. 87 (1878) 643-.

- $C^{4}$ of pencil through 13 given points, construction for 3 other common points. $B i$ schoff, J. N. A. Mt. 6 (1875) 144-.

Families of characteristic $\nu$, having principal multiple point of order $\nu$. Fouret, G. Par. S. Mth. Bll. 7 (1879) 177-.

- - curves, points specially related to. Brill, J. Camb. Ph. S. P. 7 (1892) 57-.

- - - projective, generation of curves by. Schoute, P. H. N. Arch. Wisk. $4\left({ }^{*} 1878\right)$ 182-.

\section{G. Mt. 9 (1871) 68-}

Family of curves. Timerding, H. E. N. A. Mth. 17 (1898) 351-.

- - - factors of $c$ - and $p$-discriminants in relation to fixed points on. Maddison, $I$. QJ. Mth. 26 (1893) 307-.

Flex-locus of system of plane curves with one arbitrary parameter. Hill, M.J.M. Mess. Mth. 23 (1894) 120-.

Involution properties of curves. Fouret, $G$. Palermo Cir. Mt. Rd. 3 (1889) 42-.

Jacobian of net of conics. Rosanes, J. Mth. A. 17 (1880) 21-

- - - curves of 3rd degree, generation. Cantor, S. Rm. R. Ac. Linc. T. 3 (1879) 93-.

- 3 plane curves, singularities. Gerbaldi, F. Palermo Cir. Mt. Rd. 8 (1894) 1-.

Limiting curves of given variety of curves. Ascoli, G. Rm. R. Ac. Linc. Mm. 18 (1883) 521-; Mil. I. Lomb. Rd. 21 (1888) 226-, 257-, 294-, 365-.

Linear system of conics in space. Montesano, D. Tor. Ac. Sc. At. 27 (1892) 660-.

_ - curves touching curve given by a contact of 3rd order. Lindemann, C. L. F. Par. S. Mth. Bll. 10 (1882) 21 -.

_ - - any genus, excess of fundamental elements in. Jung, G. Mil. I. Lomb. Rd. 21 (1888) 719-. 
Linear system of any genus, number of degenerate curves in. Jung, G. Mil. I. Lomb. Rd. 21 (1888) 723-.

- - plane curves. Martinetti, V. Palermo Cir. Mt. Rd. 1 (1887) 202-.

- systems. Jung, G. [1887] A. Mt. 15 (188788) 277-.

- - Scott, (Miss) C. A. N. Arch. Wisk. 3 (1898) 243-.

- belonging to algebraic surface, maximum dimension. Enriques, F. Tor. Ac. Sc. At. 29 (1894) 275-.

- - of curves of maximum genus with variable collinear intersections. Amodeo, $\boldsymbol{F}$. Nap. Rd. 35 (1896) 80-.

- - - elliptic curves, reduction, and general theorem on curves of genus $p$. Guccia, G. B. Palermo Cir. Mt. Rd. 1 (1887) 169-.

- - - plane curves. Guccia, G. B. Palermo Cir. Mt. Rd. 7 (1893) 193-; 9 (1895) 1-.

- - - Ciani, E. G. Mt. 33 (1895) 57-. - _ - - Castelnuovo, G. Rm. S. It. Mm. 10 (1896) 222-.

- - - - - fundamental curves in. Bertini, E. Palermo Cir. Mt. Rd. 3 (1889) 5-.

- - - - genus 1. Martinetti, $V$. Mil. I. Lomb. Rd. 20 (1887) 264-, [854].

$-{ }_{-}-1,-2$. Martinetti, V. Palermo Cir. Mt. Rd. 1 (1887) 205-.

$-{ }_{-}-,-,-$, reduction. Franchis, $M$. de. Palermo Cir. Mt. Rd. 13 (1899) 1-.

- - _ - - - p. Segre, C. Palermo Cir. Mt. Rd. 1 (1887) 217-.

,$------\ldots$. Guccia, G. B. Palermo Cir. Mt. Rd. 1 (1887) 386-.

- - - - - maximum degree. Jung, $G$. A. Mt. 18 (1890) 129-.

- - - - - dimension. Castelnuovo, $G$. A. Mt. $18(1890)$ 119-.

- _ - _ - and surfaces uniformly representable on plane. Jung, G. Palermo Cir. Mt. Rd. 4 (1890) 253-.

- - , reduction to minimum order. Jung, $G$. Mil. I. Lomb. Rd. 21 (1888) 488-; A. Mt. 16 (1888-89) 291-.

- - - - - - (Jung). Guccia, G. B. Palermo Cir. Mt. Rd. 3 (1889) 233-.

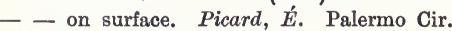
Mt. Rd. 13 (1899) 344-.

,$-- \infty^{k}$, of curves of $n$th degree and genus $p$. Guccia, G. B. Palermo Cir. Mt. Rd. 1 (1887) 139-.

- - , - , - plane curves, genus 3 , reduction for $k>1$. Franchis, M. de. Palermo Cir. Mt. Rd. 13 (1899) 130-

Linearity of systems belonging to algebraic surface. Enriques, $F$. Rm. R. Ac. Linc. Rd. 2 (1893) (Sem. 2) 3-.

Loci, geometric, for systems. Ekama, $H$. Arch. Mth. Ps. 12 (1894) 23-.

Locus of contact-points, order $k$, of curve of pencil, with those of $\infty^{k}$ linear system. Franchis, M. de. Palermo Cir. Mt. Rd. 10 (1896) 118-; 11 (1897) 12-.

- - points of 3-point contact of curve pencil with curve net. Bagnera, G. Palermo Cir. Mt. Rd. 10 (1896) 81-.
"Moirés" (systems of curves formed by intersections of 2 systems of lines on surfaces). Anon. (vi 562) Gergonne A. Mth. 19 (182829) 371-.

Net of curves, contact of curve with. Spottis woode, $W$. C. R. 83 (1876) 627-.

_- of 4 th degree. Sardi, C. G. Mt. 6 (1868) 217-.

- _ - - $n$th degree, involutory correspondences in plane determined by. Steinmetz, C. $P$. Am. J. Mth. 14 (1892) 39-.

Nets of curves cutting given curve at given angle. Fouret, G. C. R. 83 (1876) 633-.

- - of 3rd degree. Reye, T. Z. Mth. Ps. 13 (1868) 521-.

- - - - 4th degree. Küpper, C. Prag Ab. 1 (1886) No. 7, 25 pp.; 3 (1890) No. 5, 11 pp. - - - generated by homographic relations. Boguslavskǐ, A. I. [1881] (xII) Rec. Mth. (Moscou) 11 (1883-84) 313-.

-, geometry. Moore, E. H. (jun.) Am. J. Mth. 10 (1888) 243-.

- of plane curves. Castelnuovo, $G$. Tor. Ac. Sc. Mm. 42 (1892) 3-.

- - - Scheffers, G. Leip. Mth. Ps. B. 50 (1898) (Mth.) 261-.

- - - - genus 2, redundant. Franchis, M. de. Palermo Cir. Mt. Rd. 13 (1899) $200-$.

- - - singular. Laguerre, E. [1873] (x) Par. S. Mth. Bll. 6 (1878) 129-

, principle of theory. Folie, $F$. Brux. Ac. Bll. 46 (1878) 193-.

Noether's fundamental theorem $(F=K \phi+M \psi)$. Baker, H. J. Mth. A. 42 (1893) 601-.

$---(-=-)$. Scott, (Miss) C. A. Mth. A. 52 (1899) 593-.

Pencil of curves of 3rd degree, relation determined by. Aleksěev, V. G. [1891] Rec. Mth. (Moscou) 16 (1893) 256-.

-, differential equation. Casorati, F. Mil. I. Lomb. Rd. 10 (1877) 422-.

-, locus of points of inflexion. Bobek, $K$. Časopis 11 ( $\left.{ }^{*} 1882\right)$ 283-; Fschr. Mth. ("1882) 580.

- of plane curves, tangential, locus of foci. Humbert, G. C. R. 105 (1887) 54-.

- - rational curves, surfaces containing. Enriques, F. Rm. R. Ac. Linc. Rd. 7 (1898) (Sem. 2) 281-, 344-; Mth. A. 52 (1899) 449-.

Pencils. Woepcke, F. Liouv. J. Mth. 4 (1859) 329-.

- of curves determining involution on any straight line. Scheffers, G. Leip. Mth. Ps. B. 44 (1892) 269-.

- - , mutually tangential. Bischoff, J.N. Crelle J. 64 (1865) 185-.

(1879) 287-.

$--\ldots,-$, degree of curve generated by 2 . Olivier, A. [1869] Crelle J. 71 (1870) 195-.

- - - simple or double contact in. Berzolari, L. Tor. Ac. Sc. At. 31 (1895) 332- or 476-.

- - of 3rd degree. Caporali, $E$. Rm. R. Ac. Linc. T. 1 (1877) 236. 
8090 Systems of Curves

Pencils of curves of $3 \mathrm{rd}$ and $4 \mathrm{th}$ degrees. Kantor, S. Wien Ak. Sb. 79 (1879) (Ab. 2) 787-. - - - nth degree. Weyr, Em. Wien Sb. 61 (1870) (Ab. 2) 82-.

-, intersections. Dewulf, E. N. A. Mth. 11 (1872) 297-.

- and nets of plane curves, singularities. Ruffini, F. P. Bologna Ac. Sc. Mm. 1 (1880) 367-.

Pentagon, complete, and connected systems of curves. Kantor, S. [1878] Wien Ak. Sb. 78 (1879) (Ab. 2) 165-, 797-.

Plane curves. Beltrami, $E$. (vir) A. Mt. 4 (1861) 102-.

- -. Tognoli, O. G. Mt. 13 (1875) 359-.

- - Krey, H. Acta Mth. 7 (1885) 49-.

- - isometrics of straight line with reference to systems. Ocagne, $M$. $d$. Par. S. Mth. Bll. 13 (1885) 71-.

- - properties of systems, and characteristics of those of $4 \mathrm{th}$ degree. Zeuthen, H. G. [1873] (xi) Kjöb. Dn. Vd. Selsk. Skr. 10 (1875) 285-. (With French résumé.)

-- , singularities. Franchis, M. de. Palermo Cir. Mt. Rd. 11 (1897) 104-.

- - 3rd degree. Schoute, P. H. C. R. 101 (1885) 736-, 805-.

Polar curves, definition. Guccia, G. B. Palermo Cir. Mt. Rd. 7 (1893) 263-.

- -, - Kohn, G. Palermo Cir. Mt. Rd. 7 (1893) 307-

- - first. Koehler, C. (x) Par. S. Mth. Bll. 1 (1873) 124-.

- systems, real, having double contact with given real polar systems. $D e l R e, d$. Nap. Rd. 27 (1888) 424.

Property of systems. Weill, - Par. S. Mth. Bll. 16 (1888) 155-.

Rational curves, surfaces having non-linear system. Kantor, S. C. R. 131 (1900) 791-.

Representation of systems in plane. Vaněček, J. S., \& Vanéček, M. N. Prag Sb. (1884) (Mth.-Nt.) 354-; Fschr. Mth. (1885) 602-.

Skew curves. Jonquières, $E$. de. G. Mt. 4 (1866) 210-

- -, system dual or reciprocal to itself. Fiedler, W. Zür. Vjschr. 20 (1875) 173-.

- - 3rd degree, and developables of 3rd class. Gerbaldi, F. Tor. Ac. Sc. Mm. 32 (1880) 309-.

- - 4th degree, on cubic surface. Brambilla, A. Nap. Rd. 35 (1896) 171-; 36 (1897) 203-.

Stationary curves. Zeuthen, H. G. (xII) Ts. Mth. 6 (1882) 5-

Surfaces containing systems of curves. Hum. bert, G. C. R. 117 (1893) 361-.

Tangents and normals to systems. Schlömilch, O. X. Z. Mth. Ps. 23 (1878) 337-.

Triangles, parabolas, etc., systems generated by similar point-series. Stegemann, $W$. Arch. Mth. Ps. 10 (1891) 225-.

\section{SYSTEMS OF SURFACES.}

Algebraic or transcendental surfaces, systems defined by 2 characteristics. Fouret, G. C. R. 79 (1874) 467-.
Systems of Surfaces 8090

Algebraic or transcendental surfaces, systems, notion deduced from that of implexes. Fouret, G. C. R. 80 (1875) 167-.

Contact problem in theory of linear systems, solution. Bäcklund, A.V. (xI) Ts. Mth. 2 (1878) $97-$.

- of systems of surfaces, transformations. Fouret, G. C. R. 85 (1877) 1224-.

Families of surfaces. Merrifield, C. W. Mess. Mth. 1 (1872) 81-.

Family comprising new family of cyclides. Chizzoni, $F$. Rm. R. Ac. Linc. Rd. 2 (1886) (Sem. 1) 476-.

- of developable surfaces passing through given skew curve. Lévy, L. C. R. 97 (1883) 986-.

- homaloidal surfaces. Montesano, $D$. Palermo Cir. Mt. Rd. 2 (1888) 131-.

Generation of surfaces by projective nets. Padova, E. G. Mt. 9 (1871) 148-.

- - - and skew curves by systems of surfaces. Vaněeek, J. S., \& Vaněček, M. N. Rm. R. Ac. Linc. Rd. 1 (1885) 130-; A. Mt. 14 (1886-87) 73-.

Implex and system of surfaces. Fouret, $G$. C. R. 80 (1875) 805-.

Jacobian of 4 surfaces, singularities. Levi, A. G. Mt. 34 (1896) 215-.

Linear system of surfaces of $n$th degree, involution defined by. Steinmetz, C. Z. Mth. Ps. 35 (1890) 219-, 272-, 354-。

- systems. Enriques, $F$. Rm. R. Ac. Linc. Rd. 2 (1893) (Sem. 2) 281-; 3 (1894) (Sem. 1) 481-, 536-; Mth. A. 46 (1895) 179-.

- - of cones. Pieri, M. Rv. Mt. 3 (1893) 44 - - monoids. Pieri, M. G. Mt. 31 (1893) 151-.

- - - surfaces with certain singularities. Guccia, G. B. Palermo Cir. Mt. Rd. 1 (1887) 338-.

Locus of singular points and lines in connection with ultimate intersections of system of surfaces. Hill, M. J. M. [1891] Phil. Trans. (A) 183 (1893) 141-.

Method of deducing surface from plane figure, example. Cremona, L. Edinb. R. S. T. 32 (1887) 411-.

Net, conjugate, of surfaces derived from quadrics. Mangeot, S. C. R. 125 (1897) 1083-.

- of surfaces, locus of parabolic points. Lo Monaco-Aprile, L. Palermo Cir. Mt. Rd. 12 (1898) 141-.

- - - of 2nd degree. Toeplitz, E. [1876] Mth. A. 11 (1877) 434-

Normal variety formed by simple rational series of planes. Segre, C. Tor. Ac. Sc. At. 21 (1885) 95-.

Number of surfaces of net, having triple contact with intersection curve of 2 surfaces. Tognoli, O. G. Mt. 9 (1871) 188-.

Pencil of surfaces, double points. Guccia, $G . B$. C. R. 120 (1895) 896-

Pencils of surfaces, contact. Vaněček, J. S., \& Vaněcek, M. N. A. Mt. 15 (1887-88) 73-.

Problems relating to 2 systems. Combescure, $\dot{E}$. [1872] A. Mt. 5 (1871-73) 236-.

Properties. Jonquières, E. de. C. R. 58 (1864) 567-; 61 (1865) 440-. 
Properties of surfaces and systems of surfaces. Rindi, S. G. Mt. 24 (1886) 94-.

Rational systems. Murer, $V$. G. Mt. 24 (1886) 106-.

Reciprocal linear systems. Escherich, G. von. Wien Ak. Sb. 75 (1877) (Ab. 2) 523-.

- relationship of $F^{2}$-systems and $\Phi^{2}$-systems (Gewebe), and quadratic $F^{2}$-systems of $8 \mathrm{th}$ rank. Reye, $T$. Crelle J. Mth. 82 (1877) 173-.

Rotation surfaces, 2nd degree, with common curve of intersection. Gretschel, $H$. Arch. Mth. Ps. 44 (1865) 124-.

Series of surfaces (Gebüsche). Murer, $V$. G. Mt. 26 (1888) 178-

- - (pencils and nets). Murer, $V$. G. Mt. 25 (1887) 363-.

Surfaces with common curve. End, $W$. Mth. A. 35 (1890) 82-.

- through corners of given solid. Palatini, $F$. Ven. I. At. (1898) 187-.

- enveloped by quadric cones, where contact is along a circle. Blutel, E. C. R. 103 (1886) 687-.

- representable point for point on plane. Visalli, $P$. Rm. R. Ac. Linc. Rd. 2 (1886) (Sem. 2) 80-, 84-.

- several times generated by conics, determination of all. Koenigs, $G$. Par. Ec. Norm. A. 5 (1888) 177-.

- of various degrees of freedom. Reye, $T$. Crelle J. Mth. 82 (1877) 1-.

Systems depending on 1 parameter. Legoux, A. Toul. Ac. Sc. Mm. 9 (1887) 325-.

Translation-surfaces of 4 th degree, singularities of class. Sucharda, $A$. Wien Ak. Sb. 97 (1889) (Ab. 2a) 1083-.

Triple system. Legoux, A. Toul. Ac. Sc. Mm. 2 (1890) 440-.

$x^{\alpha} y^{\beta} z^{\gamma} u^{\delta}+k w^{\epsilon}=0 . \quad$ Legoux, A. N. A. Mth. 2 (1883) 233-.

\section{Algebraic configurations in hyperspace.}

Cassani, P. Ven. I. At. (1885-86) 227-.

Kühne, $H$. Arch. Mth. Ps. 11 (1892) 353-.

Del Pezzo, P. G. Mt. 31 (1893) 1-.

Abelian integrals and curves in hyperspace. White, H. S. Ac. Nt. C. N. Acta 57 (1892) 41-.

Algebraic theorem proved by geometry of $n$ dimensions. Veronese, $G$. [1883] Ven. I. At. (*1883-84) 137-.

Analytical geometry. Aschieri, F. Mod. Ac. Sc. Mm. 11 (1895) 301-.

Binary forms and correlations in hyperspace. Waelsch, E. Mh. Mth. Ps. 6 (1895) 261-, 375-.

Canonical curves of linear space and their quartic covariants. Scorza, G. Tor. Ac. Sc. At. 35 (1900) 517- or 765-.

"Characteristic" problem for linear space. Schubert, H. Hamb. Mth. Gs. Mt. 1 (1889) 134 .

Characteristics of linear spaces. Schubert, $H$. Mth. A. 38 (1891) 598-.
"Chords" of algebraic "curves." Levi, B. [1898] Tor. Ac. Sc. Mm. 48 (1899) 83-.

Clifford-Klein forms of space. Killing, $W$. Mth. A. 39 (1891) 257-.

Complexes and congruences. Bordiga, G. A. Ven. I. At. (1885-86) 163-.

-, line. Kantor, S. Crelle J. Mth. 118 (1897) 74-.

- $R_{i^{-}}$, in $R_{r}, n$ moments. Kantor, $S$. Münch. Ak. Sb. 26 (1897) 531-.

Congruences, orthogonal, in any variety. Ricci, G. Rm. R. Ac. Linc. Mm. 2 (1895) 276-.

"Conics" and "quadrics." Schubert, $H$. Hamb. Mth. Gs. Mt. 1 (1889) 290-.

Coordinates, homogeneous, interpretation. $K o h n, G$. Wien Ak. Sb. 104 (1895) ( $A b$. 2a) 1167-.

Correlation, involutory polar, in any linear space. Deruyts, $F$. Liège S. Sc. Mm. 17 (1892) No. 1, $16 \mathrm{pp}$.

Correspondence, algebraic, between 2 ruled spaces. Pieri, $M$. Tor. Ac. Sc. At. 25 (1890) 365-.

- between 2 hyperspaces. Giudice, $F$. G. Mt. 29 (1891) 163-.

- in linear space, principle. Pieri, M. Rm. R. Ac. Linc. Rd. 3 (1887) (Sem. 1) 196-.

Correspondences, class. $\operatorname{Del} R e, A$. G. Mt. 26 (1888) 348-.

-, linear reciprocal, in lineur space of any species. Aschieri, $F$. Mil. I. Lomb. Rd. 19 (1886) 167-.

Cross-ratio of certain Cremona transformations. Moore, E. H. [1896] Am. J. Mth. $22(1900)$ 279-.

- curves of $n$th degree. Schoute, P. H. [1900] Amst. Ak. Vs. 9 (1901) 268-; Amst. Ak. P. 3 (1901) 255-.

Curves. Jordan, C. C. R. 79 (1874) 795-.

- of given degree and maximum genus in any space. Fano, $G$. [1893] Tor. Ac. Sc. Mm. 44 (1894) 335-.

- in linear space. Piccioli, E. G. Mt. 36 (1898) 271-.

- and ruled surfaces. Segre, C. Mth. A. 30 (1887) 203-; 34 (1889) 1-.

"Curves," points on. Segre, C. A. Mt. 22 (1894) 41-.

Cyclical surfaces of 4 th degree. Snyder, $V$. N. Y. Am. Mth. S. Bll. 6 (1900) 194-.

Cylinder in homogeneous spaces. Quensen, $C$. Arch. Mth. Ps. 3 (1886) 45-.

Developable spaces, the most general. Puchta, $A$. Wien Ak. Sb. 101 (1892) (Ab. 2a) 355-.

Displacement, general. Schoute, $P$. $H$. Amst. Ak. Vs. M. 9 (1892) 416-; Delft Éc. Pol. A. 7 (1892) 139-.

Enumeration for "conics." Schubert, $H$. Hamb. Mth. Gs. Mt. 2 (1890) (Festschr., Tl. 2) 172-.

- quadrics. Schubert, H. Mth. A. 45 (1894) 153-.

Enumerative geometry, generalisations. Schubert, $H$. Mth. A. 26 (1886) 26-.

Figures with $n+2$ inversions. Schoute, $P$. $H$. Haarl. Ms. Teyl. Arch. 5 (1898) 159-, 241-; 6 (1900) 163-.

Fresnel surface. Vahlen, -. N. A. Mth. 14 (1895) 344-. 
Fresnel surface. Schoute, $P . H$. N. Arch. Wisk. 3 (1898) 239-; Fschr. Mth. (1897) 587-; Edinb. Mth. S. P. 16 (1898) 51-.

Homographic pencils, properties in linear spaces. Enriques, $F$. Rm. R. Ac. Linc. Rd. 6 (1890) (Sem. 2) 63-.

Homographies. Predella, P. A. Mt. 17 (188990) 113-.

-, binary and termary. Aschieri, $F$. Mil. I. Lomb. Rd. 24 (1891) 278-.

-, cyclic. Enriques, F. G. Mt. 30 (1892) 311-. -, harmonic, in linear spaces. Enriques, $F$. G. Mt. 30 (1892) 319-.

- and interchangeable relations. Del Prete, G. G. Mt. 37 (1899) 107-; 38 (1900) 40-. -, involutory, in linear space. Bertini, $E$. Mil. I. Lomb. Rd. 19 (1886) 176-.

- in linear space, theory and classification. Segre, C. Rm. R. Ac. Linc. Mm. 19 (1884) 127-.

Homography. Bordiga, G. Ven. I. At. (189697) 1091-.

-, fundamental spaces. Segre, C. Rm. R. Ac. Linc. Rd. 2 (1886) (Sem. 1) 325-.

$n+2$ hyperspheres, circumscribing prismatoids formed by $n+2$ hyperplanes, meet in point. Kuhne, H. Crelle J. Mth. 119 (1898) 186-.

Hypersurfaces of $S_{r}$ with same 1st polars, systems. Bertini, $\boldsymbol{E}$. Rm. R. Ac. Linc. Rd. 7 (1898) (Sem. 2) 217-, 275-, 357.

Indeterminate equations and $n$-dimensional geometry. Brunel, G. Bordeaux S. Sc. Mm. 2 (1886) 129-.

Intersection of 2 varieties, order. Pieri, $M$. G. Mt. 26 (1888) 251-.

_ _ _ _ in a simply infinite variety of space. Levi, B. Tor. Ac. Sc.At. 34 (1898) 581-or 745-.

Involutes of helices, generalisation. Piccioli, $H$. N. A. Mth. 18 (1899) 132 -

Involution. Castelnuovo, G. Ven. I. At. (188586) 1167-.

- in connection with algebraic forms. Deruyts, F. Brux. Ac. Bll. 14 (1887) 650-.

-, neutral elements. Deruyts, F. Brux. Ac. Bll. 36 (1898) 187-.

_, - - , and their application to skew curves. Deruyts, F. Brux. Ac. Bll. 35 (1898) 885-.

- on rational base, neutral groups with multiple elements in. Severi, $F$. Rm. R. Ac. Linc. Rd. 9 (1900) (Sem. 1) 379-.

Involutions, groups of elements common to 2 . Deruyts, F. Brux. Ac. Bll. 31 (1896) 664-.

_, - - points common to 2 . Le Paige, $C$. Brux. Ac. Bll. 11 (1886) 121-.

-, neutral elements. Le Paige, C. Brux. Ac. Bll. 14 (1887) 211-.

_, - common to 2, groups. Deruyts, $F$. Brux. Ac. Bll. 26 (1893) 232-.

_, - _ - - any number, groups. Deruyts, F. Brux. Ac. Bll. 27 (1894) 495-.

- unicursal, neutral groups of elements. Deruyts, F. Brux. Ac. Bll. 35 (1898) 196-. -, 一, representation. Deruyts, $F$. Brux. Ac. B11. 14 (1887) 322-.

Jacobian locus in hyper-geometry. Schoute, P. $H$. L. Mth. S. P. 30 (1899) 322-.

Kummer's surface, analogue for $p=3$. Wirtinger, W. Gött. Nr. (1889) 474-.
Line geometry. Schubert, H. Hamb. Mth. Gs. Mt. 3 (1900) 86-.

Linear spaces, geometry. Bertini, E. Mil. I. Lomb. Rd. 19 (1886) 855-.

- - of 4th species, spaces composed of. Aschieri, F. Mil. I. Lomb. Rd. 19 (1886) 614-, 698-.

— systems. Bertini, E. [1880] Mil. I. Lomb. Rd. 15 (1882) 24-.

- - order of variety arising from several. Pieri, M. Palermo Cir. Mt. Rd. 11 (1897) 58 .

- - of varieties in relation to birational transformations. Palatini, F. G. Mt. 38 (1900) 315-.

- varieties, complexes, congruences. Zindler, $K$. Wien Ak. Sb. 101 (1892) (Ab. 2a) 215-.

- -, synthetic construction. Zindler, $K$. Crelle J. Mth. 111 (1893) 303-.

Lines, surfaces, and higher configurations in multiply extended Gaussian and Riemannian spaces. Schering, $E$. Gött. Nr. (1873) 13-.

Multiple tangents. Schubert, $H$. Mth. A. 26 (1886) 52-

Normal curves of genus $p$ of various spaces. Segre, C. Mil. I. Lomb. Rd. 21 (1888) 523-.

- - linear spaces, property common to. Deruyts, F'. Brux. Ac. Bll. 17 (1889) 545-.

- lines of spaces of odd number of dimensions, general theorem. Cassani, $P$. Rm. R. Ac. Linc. Rd. 2 (1886) (Sem. 1) 482-.

Pencil of quadrics, linear spaces of maximum dimension associated with. Rosati, $C$. Mil. I. Lomb. Rd. 32 (1899) 1267-.

- - - of space $S_{n}$, quartic base, representation in $S_{n-2}$. Rosati, C. A. Mt. 1 (1898) 25-.

Pencils of quadrics. Bertini, $\boldsymbol{E}$. Rm. R. Ac. Linc. Rd. 2 (1886) (Sem. 2) 208 -

Point aggregates in continuous space. Cantor, G. Acta Mth. 2 (1883) 409-; 7 (1885) 105-. - groups of linear space. Chizzoni, $F$. Nap. Ac. At. 5 (1893) No. 1, 28 pp.

- - regular, and the appropriate linear substitutions. Biermann, O. Wien Ak. Sb. 95 (1887) (Ab. 2) 523-. pairs, $\infty^{n}$ algebraic series. Pieri, $M$. Palermo Cir. Mt. Rd. 5 (1891) 252-.

,$-- \infty(k+1)(r-k)$, coincidences in $r$-dimensional space. Severi, $F$. Rm. R. Ac. Linc. Rd. 9 (1900) (Sem. 2) 321-.

Polar properties of algebraic varieties, geometric method. Rodenberg, C. Mth. A. 26 (1886) 557 -.

- triangles, 2, lie in perspective, extension of theorem. Schläfi, L. [1865] Crelle J. 65 (1866) 189-.

Polarity. Bordiga, G. A. Ven. I. At. (188485) $2097-$.

Polars, theorem. Brambilla, A. Tor. Ac. Sc. At. 22 (1886-87) 787-.

Poncelet-Zeuthen polygons inseribed in $p$-ary quadric. Voss, A. Mth. A. 26 (1886) 231-.

Projection of 4 -fold figures upon a three-flat. Hall, T. P. Am. J. Mth. 15 (1893) 179-.

Projections of surface and variety of $n$ dimensions. Del Pezzo, P. Nap. Rd. 25 (1886) 205-. 
Projective geometry, fundamental postulates. Amodeo, F'. Tor. Ac. Sc. At. 26 (1891) 741-.

- - in linear space, fundamental postulates. Fano, G. G. Mt. 30 (1892) 106-.

- groups, classification. Marotte, $F$. C. R. 129 (1899) 580-.

Quadric in hyperspace, representation of ordinary space on plane by. Aschieri, $F$. Mil. I. Lomb. Rd. 18 (1885) 494-.

Quadrics, confocal. Meyer, W. F. Königsb. Schr. 41 (1900) [24]-.

-, differential equations. Berzolari, L. Rm. R. Ac. Linc. Rd. 5 (1896) (Sem. 1) 247-.

- of even number of dimensions. Bertini, $E$. Tor. Ac. Sc. At. 30 (1895) 350-.

-, generation by reciprocation. Da Porto, $A$. G. Mt. 37 (1899) 124-

- in linear space. Segre, C. [1883] Tor. Ac. Sc. Mm. 36 ( $\left.{ }^{*} 1885\right)$ 3-.

- - - foci. Concina, U. G. Mt. 38 (1900) 129-.

- reciprocally polar to themselves. Del Pezzo, P. Nap. Rd. 24 (1885) 186-.

Rational curves. Davis, E.W. J. H. Un. Cir. [3] (1884) 123.

- in n-flat space. Bissing, G. J. H. Un. Cir. [3] (1884) 123.

- - - - : curves of degree $(n+1)$. Bissing, G. J. H. Un. Cir. [3] (1884) 77.

- - linear space. Brambilla, A. Mil. I. Lomb. Rd. 19 (1886) 326-.

- - - - Berzolari, L. A. Mt. 21 (1893) 1-.

- normal curves. Loria, G. G. Mt. 26 (1888) 334-.

- surfaces and varieties, class. Brambilla, $A$. Nap. Ac. At. 9 (1899) No. 14, 28 pp.

Regular figures. Stringham, W. I. Am. J. Mth. 3 (1880) 1-.

- - Biermann, O. Wien Ak. Sb. 90 (1885) (Ab. 2) 144-.

- - convex, analytical determination. Puchta, A. Wien Ak. Sb. 90 (1885) (Ab. 2) 168-.

- - , and semi-regular. Gosset, T. Mess. Mth. 29 (1900) 43-.

Ruled space, geometry. Aschieri, F. Mil. I. Lomb. Mm. 15 (1885) 75-, 263-.

- surfaces. Bordiga, G. Ven. I. At. (188586) 1085-.

- - elliptic. Segre, $C$. Tor. Ac. Sc. At. 21 (1885) 868-.

Simplicissima. Sharp, W. J. C. L. Mth. S. P. 18 (1886-87) 325-; 19 (1889) 423-; 21 (1891) 316-.

Singular points of algebraic curves, obtained by projection from hyperspace. Del Pezzo, P. Nap. Rd. 32 (1893) 15-, 45-.

Singularities of curves of multiple curvature. Fine, H. B. Am. J. Mth. 9 (1887) 180-.

- - discriminant surface. Hilbert, D. Mth. A. 30 (1887) $437-$

Spaces cutting several lines, number. Castelnuovo, G. Rm. R. Ac. Linc. Rd. 5 (1889) (Sem. 2) 71-.

- touching surface or variety. Del Pezzo, $P$. Nap. Rd. 25 (1886) 176-.
Straight line, geometry, deduced from that of quadrics in $n$-dimensional space. Aschieri, F. Mil. I. Lomb. Rd. 17 (1884) 756-.

- lines, systems. Segre, C. Palermo Cir. Mt. Rd. 2 (1888) 148-

Surfaces, analysis situs, and integrals of total differentials. Picard, É. C. R. 124 (1897) $532-$.

- of 2 dimensions, new groups. Bordiga, $G$. C. R. 102 (1886) 1442-.

- general theory, extension to varieties. Mahler, -. D. Nf. Tbl. ("1882) 147-.

-, normal equation. Pellet, A. C. R. 128 (1899) 233-.

-, plane section unicursal. Moore, E.H. (jun.) Am. J. Mth. 10 (1888) 17-.

-, theorems, extension. Hoppe, R. Arch. Mth. Ps. 3 (1886) 277-.

- and varieties. Del Pezzo, P. Nap. Rd. 26 (1887) 40 -.

- - property. Segre, C. Tor. Ac. Sc. At. 31 (1895) 341- or 485-.

- - - sections of which are normal curves, genus $p$. Del Pezzo, P. A. Mt. 15 (188788) 115-.

- of zero curvature in elliptic geometry. Bianchi, L. A. Mt. 24 (1896) 93-.

- - _ - - spaces of constant curvature. Bianchi, L. Tor. Ac. Sc. At. 30 (1895) 743-. -, nth degree. Del Pezzo, P. Palermo Cir. Mt. Rd. 1 (1887) 241-.

Theorem, 3-dimensional, generalisations. Schubert, H. Hamb. Mth. Gs. Mt. 1 (1889) 87.

Theorems of Clifford and Cayley, extensions. Moore, E. H. [1885] Conn. Ac. T. 7 (188588) 9 -.

Transformations, birational, between 2 spaces. Carrone, C. Catania Ac. Gioen. At. 11 (1898) Mem. 8, 62 pp.

-, involutory linear, complete groups of 3 . Del Re, A. Rm. R. Ac. Linc. Rd. 6 (1890) (Sem. 1) 57-.

-, quadratic, characteristics. Kantor, $S$. Mil. I. Lomb. Rd. 27 (1894) 477-.

-, -, periodic. Kantor, S. Mil. I. Lomb. Rd. 27 (1894) 712-, 749-; 28 (1895) 249-, 298-.

- successive linear. Kantor, S. Par. S. Mth. Bll. 8 (1880) 208-.

- of surfaces. Newcomb, S. Am. J. Mth. 1 (1878) 1-.

-, aniform automorphic, of elliptic curves. Segre, C. Mth. A. 27 (1886) 296-.

Varieties composed of simply infinite series of spaces. Segre, C. Rm. R. Ac. Linc. Rd. 3 (1887) (Sem. 2) 149-.

- with continuous non-integrable group of projective automorphic transformations. Fano, G. Tor. Ac. Sc. Mm. 46 (1896) 187-. - cubic, Hessian vanishing identically. Perazzo, U. G. Mt. 38 (1900) 337-.

- of different dimensions, representation on each other. Luroth, J. Erlang. Ps. Md. S. Sb. 31 (1900) 87-.

-, unicursal. Autonne, L. Par. S. Mth. Bll. 27 (1899) 263-. 


\section{Hyperspace}

Variety, quadric, successive projection on itself. Del Re, A. Rm.R.Ac. Linc.Rd. 5 (1896) (Sem. 2) 365-.

\section{4-dimensional space.}

collinearities and correlations, ordinary and special. Visalli, $P$. Mil. I. Lomb. Rd. 29 (1896) 351-, 439-, 521-, 559-.

complex of 1st order. Ascione, E. Rm. R. Ac. Linc. Rd. 6 (1897) (Sem. 1) 162-. complexes. Bordiga, G. Ven. I. At. (188788) $919-$.

_. Palatini, F. Ven. I. At. (1899-1900) (Pt. 2) 861-.

condition that 5 straight lines should lie on quadric. Richmond, H.W. Camb. Ph. S. P. 10 (1900) 210-.

configuration of lines. Bordiga, $G$. Ven. I. At. (1896-97) 420-.

congruence, 3rd order and 6th class. Castelnuovo, $G$. Ven. I. At. (1886-87) 1249-; (1887-88) 525-

- 4 th order and 2 nd class. Bordiga, G. Ven. I. At. (1893-94) 1605-

Cremonian transformation. Del Pezzo, $P$. Nap. Rd. 35 (1896) 336-; 36 (1897) 33-.

cubic varieties. Segre, C. Tor. Ac. Sc. Mm. 39 (1889) 3-.

- - Enriques, F. G. Mt. 31 (1893) 31-.

- variety with 10 double points. Segre, $C$. Tor. Ac. Sc. At. 22 (1886-87) 791-.

elliptic curve of 5 th degree. Meyer, $F$. Mth. A. 26 (1886) 154-.

figure formed from 6 points. Richmond, H.W. Mth. A. 53 (1900) 161-.

focal properties of quadrics. Sommer, J. Mth. A. 53 (1900) 113-.

geometric interpretation of linear equation. Nicoli, F. Mod. Ac. Sc. Mm. 7 (1890) 205-; 8 (1892) 489-.

homaloidal systems of quadrics. Del Pezzo, $P$. Nap. Rd. 34 (1895) 133-.

hyperquadrics. Schoute, P. H. [1900] Amst. Ak. Vh. (Sect. 1) 7 (1901) No. 4, 66 pp.; Haarl. Ms. Teyl. Arch. 7 (1902) 117-.

line geometry. Castelnuovo, G. Ven. I. At. (1890-91) 855-.

linear, birational transformation of ordinary ruled space in. Pannelli, $M$. Rm. R. Ac. Linc. Rd. 6 (1890) (Sem. 1) 479-.

- transformations. Hathaway, A. S. [1898] N. Y. Am. Mth. S. Bll. 5 (1899) 88, 93-. normal curve. Aschieri, $F$. Rm. R. Ac. Linc. Mm. 4 (1887) 172-

- - of 4th degree. Bordiga, G. A. Ven. I. At. (1885-86) 503-.

plane, lines of triple curvature. Pirondini, $G$. G. Mt. 28 (1890) 219-.

point pairs. Frege, - Jena. Sb. (*1883) 98-. points of, and straight lines of ordinary space, one-one correspondence between. Chizzoni, F. Catania Ac. Gioen. At. 20 (1888) 273-.

primitive groups of transformations. Page, $J$. M. Am. J. Mth. 10 (1888) 293-.

regular convex figures. Puchta, A. Wien Ak. Sb. 89 (1884) (Ab. 2) 806-.

2 representations of ruled space on linear form. Loria, G. G. Mt. 27 (1889) 224-.

\section{Infinitesimal Geometry 8400}

ruled surface of 4 th degree. Caldarera, $G$. Acireale Ac. At. 7 (1896) (Sc.) 141-.

surface of 6th degree, with 10 straight lines, and its projections in ordinary space. Bordiga, G. Rm. R. Ac. Linc. Mm. 4 (1887) 182-.

surfaces whose triple secants form complexes of 1st order. Ascione, $E$. Rm. R. Ac. Linc. Rd. 6 (1897) (Sem. 1) 240-.

syzygies connecting quadratic relations of line coordinates. Young, W. H. Tor. Ac. Sc. At. 34 (1898) 438- or 596-.

tetratops (=4-dimensional angle bounded by four spaces) and polytops. Hoppe, $R$. Arch. Mth. Ps. 67 (1882) 412-.

use in study of surfaces including several series of conics. Cosserat, E. C. R. 124 (1897) 1004varieties with continuous integrable group of projective automorphic transformations. Fano, $G$. Ven. I. At. (1895-96) 1069-.

variety of 27th order. Brambilla, A. Nap. Ac. At. 9 (1899) No. 2, $37 \mathrm{pp}$.

5-dimensional space, homaloidal surface of 2 dimensions and 4 th degree. Veronese, $G$. Rm. R. Ac. Linc. Mm. 19 (1884) 344-.

- -, surface of 7 th degree. Bordiga, $G$. Ven. I. At. (1886-87) 1397-.

- - , systems of straight lines considered as surfaces. Fano, G. A. Mt. 21 (1893) 141-.

6-dimensional space, surfaces of $5 \mathrm{th}$ and $6 \mathrm{th}$ degrees. Bordiga, G. Ven. I. At. (1885-86) 1461-.

$n-1$ dimensional space, axes of quadric surface. Story, W. E. J. H. Un. Cir. [3] (1884) 122.

- _ - involutions of degree $n$ and species 1 . Morale, M. Palermo Cir. Mt. Rd. 13 (1899) 274-.

- - - quadrics having $n$-fold common polars. Burali-Forti, C. Palermo Cir. Mt. Rd. 4 (1890) 118-.

- - , rational curves of $n$th degree. Loria, G. Palermo Cir. Mt. Rd. 2 (1888) 201-

_ - _, uniform correspondences in elliptic curves of $n$th degree. Amodeo, $F$. A. Mt. 19 (1891-92) 1-.

$n+1$ dimensional space, lines in ruled surfaces of $n$th degree. Palatini, F. G. Mt. 36 (1898) 368-.

_ _ - projective invariants of system of $m+1$ points. Lovett, E. O. Bll. Sc. Mth. 23 (1899) 10-.

- - - strip elements and collections of elements. Konvalewski, $G$. Leip. Mth. Ps. B. 52 (1900) 91-.

- - , surfaces of $n$th degree. Del Pezzo, P. Nap. Rd. 24 (1885) 212-.

\section{Infinitesimal geometry; applica- tions of differentialand integral calculus to geometry. \\ 8400 General.}

Area of surface, definition. Peano, G. Rm. R. Ac. Linc. Rd. 6 (1890) (Sem. 1) 54-.

Consecutive surfaces. N., M. N. Camb. Mth. J. 1 (1839) 187-. 
Construction of tangents to plane curves. Godefroy, $R$. C. R. 102 (1886) 604-.

Contact of lines and surfaces. Chasles, $M$. Liouv. J. Mth. 2 (1837) 299-.

- lines of surfaces with circumscribed cones. Vallès, $F$. Gergonne A. Mth. 16 (1825-26) 315-.

Correspondence of tangent planes in transformation of surfaces by symmetry curve. Mangeot, S. C. R. 114 (1892) 1463-.

Curvature propositions. Seiliger, D. N. Fschr. Mth. (1892) 582.

Curved surfaces, general properties. Lilienthal, - von. Crelle J. Mth. 98 (1885) 131-.

- -, theory. Lipschitz, R. Acta Mth. 10 (1887) 131-.

- - - , general. Ribaucour, A. Liouv. J. Mth. 7 (1891) 5-, 219-.

Curves, properties demonstrated by theory of limits. Azzarelli, $M$. Rm. N. Linc. At. 42 (1889) 160-.

- and surfaces, theory. Catalan, E. C. [1874] Brux. Mm. Cour. 8 $8^{\circ} 24$ (1875) (No. 2) $48 \mathrm{pp}$.

Cylinders, cones, and developable surfaces. Tanner, H. W. L. Mess. Mth. 6 (1877) 145-.

Density of chords of surfaces and plane curves. Hoppe, R. Arch. Mth. Ps. 7 (1889) 165-.

Differential relations in geometry, and synectic integrals of differential equations. Cauchy, A. L. C. R. 40 (1855) 445-.

Essential equations of plane curves, especially cycloids. Onnen, $H$. N. Arch. Wisk. 4 (1878) 30-.

Formation of surfaces by straight line. Hube, K. (vr Adds.) Krk. Roczn. Uniwers. 11 (1826) 23-.

Geometrical increments, with applications to geodesics, orthogonal surfaces etc. Lafon, $A$. As. Fr. C. R. (1876) 104-.

- significance of $\frac{d^{2} z}{d x d y}=0$. Tychsen, C. (xII) Ts. Mth. 1 (1865) 127-.

Infinitesimal geometry. Mannheim, A. Tortolini A. 2 (1859) 208-.

- -, problem. Gilbert, L. P. (xII) Mathesis 1 (1881) 97-.

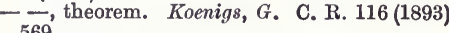
569.

Infinitesimals, theorem, in conics. M'Vicker, C. E. Mth. Gz. 1 (1900) 209-.

Integral calculus and geometry, reciprocal relations. Meyer, $\boldsymbol{F}$. Königsb. Schr. 39 (1898) [44]-.

Line and surface integrals, generalisation of Stokes's theorem. Gilbert, - Brux. S. Sc. A. 16 (1892) (Pt. 1) 2-.

Maximum and minimum, fallacy in certain proofs. Alison, J. Edinb. Mth. S. P. 15 (1897) 83-.

Mean direction of curved line in plane. Lindhagen, A. N. Ts. Mth. 1 (B) (1890) 80-; Fschr. Mth. (1890) 701-•

Method of tangents, inverse, problems. Euler, L. [1781] St Pét. Ac. Sc. Mm. 10 (1826) $16-$
Method of tangents, Roberval's. Duhamel, J. M. C. Par. Mm. Sav. Étr. 5 (1838) 257-. Multiple integrals in tangential and in ruled space, and principle of duality. Cartan, $\hat{E}$. Par. S. Mth. Bll. 24 (1896) 140-.

Orthogonal symmetry with reference to any cylinder. Pirondini, G. N. A. Mth. 19 (1900) 107-.

_- - line. Pirondini, G. G. Mt. 35 (1897) 181- ; 37 (1899) 212-.

- _ _ - surface of revolution. Pirondini, G. A. Mt. 22 (1894) 213-; 25 (1897) 61-.

Osculating circles and surfaces to conics and quadrics. Ducoroy, G. N. A. Mth. 19 (1860) 118-.

Spheres touching two surfaces. Pellet, $A$. As. Fr. C. R. (1887) (Pt. 2) 116-.

Sum of distances of point from given points. Schlegel, V. N. Y. Am. Mth. S. Bll. 1 (1895) 33-.

Surfaces referred to their lines of zero length. Cosserat, E. C. R. 125 (1897) 159-.

Transformation of coordinates from rectangular to polar in differential expressions. Smith, Arch. Camb. Mth. J. 1 (1839) 121-.

\section{Principles of infinitesimal geometry.}

Genty, -. N. A. Mth. 2 (1883) 237-.

Cesáro, E. Mathesis 7 (1887) 25-.

Analytic geometry. Bordoni, A. Mil. G. I. Lomb. 6 (1854) -287-, 420-.

Areas described in motion of plane figure. Koenigs, G. C. R. 118 (1894) 965-.

Circles or spheres related to envelope. Serret, P. C. R. 117 (1893) 400-, 435-, 480-.

Curvature, problem. Seiliger, $D$. Kazan S. Ps.-Mth. Bll. 10 (1901) 262-.

Curves, ternary operator in analysis. Walker, J. J. $[1878-87] \quad$ L. Mth. S. P. 9 (1877-78) 226-; 16 (1884-85) 215-; 19 (1889) 483-

Differential geometry, use of rectangular triheđron. Schoenflies, A. Gött. Nr. (1898) 71-.

Envelopes. Colard, H. N. A. Mth. 1 (1842) 281-.

Geometrical theorems connected with Poisson's equation $\frac{d^{2} z}{d t^{2}}=a^{2}\left(\frac{d^{2} z}{d x^{2}}-\frac{m z}{x^{2}}\right)$. Maclay, J. [1900] N. Y. Am. Mth. S. Bll. 7 (1901) 202, 208-.

Infinitesimal deformations, use of $\sqrt{ }-1$ and alternate numbers $i, j, k$ in. Perna, $A$. Palermo Cir. Mt. Rd. 12 (1898) 322-.

- elements, projective relation between. Oliver, J.E. [1884] A. Mth. 1 (1884-85) 40-.

- properties of curves, auxiliary curves. Ocagne, M. d'. Am. J. Mth. 11 (1889) 55-; 15 (1893) 389-.

- transformations, geometry. Źorawski, $K$. Krk. Ak. (Mt.-Prz.) Rz. 14 (1899) 218-; 17 (1900) 154-; Crc. Ac. Sc. Bll: (1897) 365-; (1899) 81-.

Intrinsic geometry, questions. Cesáro, $E$. Mathesis 20 (1900) 5-, 37-, 57-. 


\section{Kinematic Geometry}

Isogonal and isologic transformation of plane figures. Transon, A. N. A. Mth. 8 (1869) 222 -

Orthogonal trajectories on plane and sphere. Roberts, M. Liouv. J. Mth. 10 (1845) 251-.

Osculation function, Żmurko's. Mertens, $\boldsymbol{F}$. (xII) Krk. Ak. (Mt.-Prz.) Pam. 2 (1876) 124 -.

Question of limit in theory of surfaces. Lecornu, L. Bll. Sc. Mth. 16 (1892) 307-.

Real infinitesimals and infinite segments. Veronese, G. Mth. A. 47 (1896) 423-.

Surface elements of higher order in space of 3 dimensions. Weber, E. von. Mth. A. 44 (1894) 458-.

$d x^{2}+d y^{2}+d z^{2}=\lambda\left(d \alpha^{2}+d \beta^{2}+d \gamma^{2}\right) . \quad$ Liouville, J. Liouv. J. Mth. 15 (1850) 103.

\section{Kinematic geometry. (See also Mechanics 0420.)}

Mannheim, A. Camb. Ph. S. P. 6 (1889) 32-

Speckel, C. N. A. Mth. 11 (1892) 268-.

Nobile, $V . \quad$ N. A. Mth. 18 (1899) 218-.

Angle sliding along line, problem. Lamarle, $E$. Brux. Ac. Bll. 9 (1860) 127-, 361-.

- touching two curves, locus of vertex. Enneper, A. Z. Mth. Ps. 16 (1871) 342-.

Astroids, construction for tangents. Sucharda, A. Arch. Mth. Ps. 66 (1881) 321-

- - - - Stammer, W. Arch. Mth. Ps. 67 (1882) 222

- - - - Stoll, $(D r)$-. Arch. Mth. Ps. 67 (1882) 447-.

_, _- - Ameseder, A. Časopis 14 (1885) 46 ; Fschr. Mth. (1885) 713.

, - - Sucharda, A. Časopis 14 (1885) 138-; Fschr. Mth. (1885) 714.

-, curves parallel to. Loria, G. Mathesis 20 (1900) 244-.

-,-1 - Neuberg, J. Mathesis 20 (1900) 247 -

3-bar curves, triple generation. Clifford, $W . K$. L. Mth. S. P. 9 (1877-78) 26-.

- linkage, curves described by. Jensen, P.V. (xII) Ts. Mth. 6 (1882) 154-.

- motion in plane. Roberts, S. [1875] L. Mth. S. P. 7 (1875-76) 14-

7-bar pantograph of Delaunay. Somov, P.O. [1895] Vars. S. Nt. Tr. (1895-96) (C.R., P8. C.) No. 2, $5 \mathrm{pp}$

Cartesian oval treated kinematically. Cavalli, E. Tor. Ac. Sc. At. 20 (1885) 1143-.

Centre of curvature of Cassini's oval, exponential curve, catenary, tractrix, logarithmic and hyperbolic spirals, kinematic construction. Procházka, B. Prag České Ak. Fr. Jos. Rz. (Třida 2) 4 (1895) Art. 8, 8 pp.; Fschr. Mth. (1896) 794.

- _ - curves produced by motion of in. variable figure in a plane. Seidelin, C.J.L. (xiI) Ts. Mth. 6 (1876) 57-.

_ - - Descartes's oval, kinematic construction for. Prochazka, B. Časopis 24 (1895) 230-; Fschr. Mth. (1895) 621.

\section{Curve of Pursuit 8420}

Centres of curvature of curve rolling on straight line. Mannheim, A. Liouv. J. Mth. 4 (1859) 93-.

- curves described by points in displaced plane. Bobek, $K$. Prag Sb. (1880) 56-.

- - - and tangents of conic sections, kinematic construction for. Procházka, $B$. Prag České Ak. Fr. Jos. Rz. (Třrada 2) 3 (1894) Art. 19, 5 pp.; Fschr. Mth. (1893-94) 955.

- - of trajectories of points in moving figure. Marcolongo, $R$. Palermo Cir. Mt. Rd. 7 (1893) 29-.

Circle as roulette, curve represented by elliptic functions. Lieblein, J. [1869] Prag Ab. 3 (1870) 14 pp.

Compound and reverse curves. Thornton, W. $M$. A. Mth. 3 (1887) 39-.

Cone rolling on plane, curve traced by plane section. Schwering, $K$. Z. Mth. Ps. 25 (1880) 25-.

Coordinates, "ideal" (for moving systems). Hansen, P. A. Leip. B. (1851) 39-.

Curvature of paths of points of rigid system moving about fixed point. Minich, $S$. $R$. Ven. At. (1859-60) 183-.

- plane curves treated kinematically. Neuberg, J. Brux. Ac. Bll. 25 (1893) 374-, 848.

- - surface generated by point of solid moving under conditions. Réveille, $J$. C. R. 105 (1887) 159-.

_- surfaces. Suslow, G. Fschr. Mth. (1890) 868-.

Curve of flight. Lehmus, - . Crelle J. 1 (1826) 61-.

-, locus of intersection of 2 lines rotating about 2 fixed points. Le François, (Prof.) -. Quetelet Cor. Mth. 5 (1829) 379-.

\section{Curve of pursuit.}

Querret, -. Gergonne A. Mth. 13 (1822-23) 389 -.

Burg, $\dot{A}$. Wien Jb. Pol. I. 4 (1823) 508-

Gosselin, (le col.) -. Metz Mm. Ac. 40 (185859) 459-.

Tychsen, C. Mth. Ts. 3 (1861) 33-.

Ingalls, J. M. Des Moines Anal. 7 (1880) 89-, 117-.

Cesáro, E. N. A. Mth. 2 (1883) 85-.

application. Dienger, $J$. Grunert Arch. 15 (1850) 335-.

centre of curvature. Ocagne, M. $d^{\prime}$. Par. S. Mth. Bll. 11 (1883) 134-.

equation, ratio of velocities being constant. Anon. (vr 882) N. A. Mth. 8 (1849) 91-.

generalisation. Barbour, L. G. Des Moines Anal. 6 (1879) 108-.

length. Ficklin, J. Am. As. P. 20 (1871) 63-.

solution. Saint-Laurent, $T$. de. Gergonne A. Mth. 13 (1822-23) 145-.

-. Sturm, J. C.F., d Saint-Laurent, T. de. Gergonne A. Mth. 13 (1822-23) 289-. 
Curve rolling on straight line. Resal, H. A. Liouv. J. Mth. 6 (1880) 115-.

- traced by focus of ellipse rolling on straight line, property. Spitzer, S. Arch. Mth. Ps. 48 (1868) 235-.

Curves circumscribing rotating polygons with reference to drilled holes. Mallock, $A$. $\mathrm{R}$. S. P. 35 (1883) 319-.

- generated by motion of "reptation." Prouhet, E. N. A. Mth. 13 (1854) 274-, 335-.

- traced by end of moving bar, paradox. Terquem, O. N. A. Mth. 12 (1853) 107-.

- - - oval chuck. Wilkens, -. Kiöb. Òv. (1843) 61-.

Cyclic curves. Böklen, $O$. Grunert Arch. 37 (1861) 119-.

- - special kind. Durège, H. Zür. Vjschr. 8 (1863) 127-; Z. Mth. Ps. 9 (1864) 209-.

Cycloidal curves. Fabri, $R$. Rm. At. 10 (1856-57) 225-, 387-; 11 (1857-58) 399-.

- - Saussure, R. de. Am. J. Mth. 17 (1895) 269-.

- -, algebraic. Holst, E. [1881] Areh. Mth. Ntvd. 6 (1882) 125-.

- , common tangents. Morley, F. N. Y. Am. Mth. S. Bll. 2 (1896) 111-.

- -, plane and spherical. Ekama, H. Arch. Mth. Ps. 7 (1889) 207-.

- -, spherical. Ekama, $H$. N. Arch. Wisk. 16 (1889) 32- ; Fschr. Mth. (1889) 805-.

- and other curves, method of construction. Hele-Shaw, H. S. B. A. Rp. (1898) 792-.

- - trochoidal curves, adjustable. Morley, $\boldsymbol{F}$. Am. J. Mth. 16 (1894) 188-.

\section{Cycloids.}

Vincent, A. J. H. Lille Mm. S. (1841) 5-; Rouen Tr. Ac. (1853-54) 129-.

Gibbes, L. R. Camb. (M.) Mth. M. 1 (1859) 297-.

Weisz, J. A. (xس) Mag. Ak. Ets. (1859) (Suppl., Mth. Term.) 81-.

Holtz, - [1883] N.-Vorp. Mt. 15 (*1884) xiII-.

construction. Kracht, H. Dingler 289 (1893) 108-.

elementary demonstrations. Schulz von Strasznitzki, L. C. Grunert Arch. 13 (1849) 272 .

- treatment. Holzmuller, F. G. Z. Mth. Ps. 21 (1876) 128-.

and epicycloids and hypocycloids. Cardinal, -. Dingler 35 (1830) 329-.

- - rectification and quadrature. Lenthéric, -. Mntp. Ac. Sc. Mm. 2 (1851-54) 1-.

equilibrium curve in mining. Sanchez, $P$. $C$. Méx. S. "Alzate" Mm. 9 (1895) 107-.

evolutes. Lang, R. Grunert Arch. 30 (1858) 319-.

graphic solution of problems by. Guimarães, R. G. Teix. J. Sc. 6 (1885) 85-; Fschr. Mth. (1884) 492.

known in 16th century? Gilnther, $S$. Bb. Mth. (1887) 8-.

polar circle pairs. Vietor, A. Z. Mth. Ps. 25 (1880) 263-. on sphere. Fuss, N. [1817] St Pét. Ac. Sc. Mm. 8 (1822) 161-.

system having base-line and one cusp common, orthogonal trajectories. Baur, C.W. [1871] Z. Mth. Ps. 17 (1872) 424-.

as tautochrones. Mechanical discussion by ancient geometrical method. Lehmann, $J$. W. H. Crelle J. 6 (1830) 49-.

theory. Schjellerup, H.C.F.C. Mth. Ts. 1 (1859) 73-.

-, geometrical. Du Bourguet, -. Gergonne A. Mth. 6 (1815-16) 29-.

Deformation, infinitesimal, of a medium. Beltrami, E. C. R. 108 (1889) 502-.

Deformations due to elongation of rectangular parallelepiped in one direction only. Tresca, H. É. C. R. 97 (1883) 928-.

Determination of contour of skew helicoidal surface. Tesař, J. [1882] Wien Ak. Sb. 86 (1883) (Ab. 2) 377-.

\section{Displacement.}

continuous, of solid. Mannheim, A. C. R. 63 (1866) 1386-.

of curve touching fixed curve. Combescure, $\dot{E}$. Crelle J. 63 (1864) 332-.

- double cone. Mannheim, A. C. R. 111 (1890) 634-, 817-.

- figure of invariable form. Mannheim, $A$. C. R. 66 (1868) 591- ; Par. Mm. Sav. Étr. 20 (1872) 1-.

- D Darboux, G. C. R. 92 (1881) 118 .

- - - in its plane. Dewulf, É. C. R. 92 (1881) 1091-.

_ - _ - planes of which pass through fixed points. Mannheim, A. C. R. 112 (1891) 283-.

- figures. Brisse, C. Liouv. J. Mth. 15 (1870) 281-.

finite, conjugate axes in, generalisation of theorem of Chasles. Ligin, V. N. (xII) Rec. Mth. (Moscou) 6 (1872-73) (Pt. 1) 193-.

-, of figure of invariable form. Brisse, C. Liouv. J. Mth. 19 (1874) 221-; 1 (1875) 141-.

-, - - - - in space. Chasles, $M$. C. R. 51 (1860) 855-, 905-; 52 (1861) 77-, 189-, 487-.

-, - rigid system, reciprocal figures obtained by, and reciprocal plane diagrams deduced from them. Padelletti, D. Rm. R. Ac. Linc. T. 3 (1879) 120-.

helicoidal, of quadric touching fixed plane. Gontarev, D. A. Vars. S. Nt. Tr. (1895-96) (C. R., Ps. C.) No. 5, 6 pp.

infinitely small, of algebraic surface. Mannheim, A. C. R. 70 (1870) 1025-.

- - under 4 conditions. Mannheim, A. C. R. 73 (1871) 1096-.

- - of dihedron of invariable size. Mann. heim, A. C. R. 84 (1877) 1373-

$-\ldots,-\ldots$, proof of a theorem, and application. Mannheim, A. [1877] Par. S. Mth. Bll. 6 (1878) 5-. 
of a jointed hyperboloid. Bricard, R. Par. S. Mth. Bll. 25 (1897) 98-.

most general, of solid in space, treatment. Crofton, $M . W$. L. Mth. S. P. 5 (1874) 25-

- - , - straight line, points of which describe spherical trajectories. Duporc $q, E$. C. R. 125 (1897) 762-.

of plane figure, curvature of curves described during. Servais, C. Brux. Ac. Bll. 19 (1890) 241-.

- - in its plane. Chasles, M. C. R. 80 (1875) 346-.

- _ - - - theorem. Léauté, H. Par. S. Mth. Bll. 6 (1878) 170-

- -, 2 points of which slide on any 2 curves. Chasles, M. C. R. 82 (1876) 431-.

- - all points of which describe spherical lines. Bricard, R. C. R. 125 (1897) 1024-. remarkable case. Bricard, R. C. R. 123 (1896) 939-.

of rigid system in elliptic space, linear transformation corresponding to. Ball, $R$. S. [1885] Ir. Ac. P. 4 (1884-88) 532-.

- - systems. Küpper, C. Schlömilch Z. 6 (1861) 12-.

- solid of invariable form. Halphen, G. H. [1873] (x) Par. S. Mth. Bll. 2 (1874) 56-.

- - , new method of normals to curves or surfaces described during. Mannheim, A. (x) Par. S. Phlm. Bll. 3 (1866) 79-.

- - , singular cases. Halphen, G. H. [1879] Par. S. Mth. Bll. 8 (1880) 18-.

- - system in space, and resulting variation of coordinates. Rodrigues, $O$. Liouv. J. Mth. 5 (1840) 380 -

- straight line such that 3 of its points remain on faces of rectangular trihedron. Mannheim, A. Bll. Sc. Mth. 9 (1885) 137-.

- system of points, differential parameters connected with. Durrande, H. C. R. 78 (1874) 1036-.

theory. Halphen, G. H. N. A. Mth. 1 (1882) 296-.

Double cone rolling on 2 converging lines. Azzarelli, M. Tortolini A. 7 (1856) 317-.

Ellipse sliding against 2 straight lines, locus problem. Ferrier, L. N. A. Mth. 3 (1844) $352-, 438$.

Ellipse-glissette, eliminant of equations. M'Laren, (Lord). Edinb. R. S. P. 19 (1893) 89 -.

-, elimination problem. Muir, T. Edinb. R. S. P. 19 (1893) 25-.

_- _ - Nanson, E. J. Edinb. R. S. P. 22 (1900) 158-

-, - -, symmetric solution. M'Laren, (Lord). Edinb. R. S. P. 22 (1900) 379-.

Ellipses described by points in moving line. Breton [de Champ], $P$. N. A. Mth. 5 (1846) $591-$.

- as glissettes, and circular surface of 8 th order. Vaněček, J. S., \& Vaněcek, M. N. Par. S. Mth. Bll. 11 (1883) 76-.

Envelope of cone rolling on 2 developables. Blutel, - C. R. 108 (1889) 496-; Par. Éc. Norm. A. 7 (1890) 155-.

vol. I.
Envelope of sphere rolling on another sphere. Svěřnikov, P. Kazan S. Ps.-Mth. Bll. 5 (1896) 141-.

\section{Epicycloids.}

Johnson, E. F. Silliman J. 21 (1832) 280-. Anon. (vi 879) N. A. Mth. 4 (1845) 83-.

Drach, S. M. Ph. Mg. 35 (1849) 487-.

Dieu, T. N. A. Mth. 19 (1860) 125-.

Fouret, G. (Ix) Par. S. Phlm. Bll. 5 (1868) 80Collignon, É. As. Fr. C. R. 6 (1877) 92-.

Svěsnikov, P. I. Kazan S. Nt. (Ps.-Mth.) P. 8 (1890) 374-.

Morley, F. Am. J. Mth. 13 (1891) 179-.

and binomial theorem. Drach, S. M. Ph. Mg. 34 (1849) 444-, 520-.

- caustics by reflexion in circle etc. Quetelet, L. A. J. Quetelet Cor. Mth. 5 (1829) 190-. centre of curvature. Mannheim, A. N. A. Mth. 18 (1859) 371-.

contact of loops. Sang, E. [1865] Edinb. R. S. T. 24 (1867) 121-.

-, solution of Perigal's problem. Sang, E. [1865] Edinb. R. S. P. 5 (1866) 338-

double generation. Fouret, (le lt.) - N. A. Mth. 8 (1869) 162-.

general theory. Raabe, J. L. Crelle J. 1 (1826) 289-.

and hypocycloids. Eckardt, F. E. Z. Mth. Ps. 15 (1870) 129-; 18 (1873) 319-.

- - Wolstenholme, J. L. Mth. S. P. 4 (1871-73) 321-.

- - curves derived from. Barisien, $E . N$. G. Teix. J. Sc. 14 (1900) 121-

- - and derived curves. Kiepert, L. Z. Mth. Ps. 17 (1872) 129 -

- - , polar reciprocals. Jeřábek, $V$. Mathesis 19 (1899) 105-.

- -, quadrature. Baur, C.W. Schlömileh Z. 4 (1859) 311-.

- - tangential property. Jeffery, H. M. [1882] R. S. P. 34 (1883) 105-.

intersecting at constant angles, locus of vertices of tangents to. Loucheur, - . N. A. Mth. 11 (1892) 374-

multisection of angle by. Leeuwen, J. H. van. N. Arch. Wisk. $7\left({ }^{*} 1881\right) 213$.

plane and spherical, centres of curvature. Olivier, T. Par. Éc. Pol. J. cah. 23 (1834) 85-. in space. Vaněcek, J. S. [1880] Wien Ak. Sb. 83 (1881) $(A b .2)$ 69-.

spherical. Hachette, J. N. P. Par. Ec. Pol. Cor. 2 (1809-13) 22-.

-. Ruiz de Cardenas, A. G. Mt. 12 (1874) 313-.

-, rectifiable. Jeffery, $H$. M. B. A. Rp. (1882) 453-.

-, and their tangents. Hachette, J. N. P. Par. Éc. Pol. Cor. 2 (1809-13) 87-.

tangent problems. Binder, W. Wien Ak. Sb. 107 (1898) (Ab. 2a) 362-.

Epi-ellipside (analogue to epicycloid), equation. Biermann, O. Mh. Mth. Ps. 8 (1897) 87-.

Epi- and hypo-trochoids and allied curves, Plückerian characteristics. Roberts, S. L. Mth. S. P. 4 (1871-73) 353-.

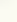


Epitrochoid. Cayley, A. Mess. Mth. 20 (1891) 150 -.

Epitrochoidal surfaces, and generalisation of their properties. Svěsnikov, $P$. Kazan S. Ps.-Mth. Bll. 1 (1891) 166-, 178-; Fschr. Mth. (1891) 861.

Equations of configurations in $n$-dimensional space. Hatzidakis, N. J. C. R. 130 (1900) $557-$.

- - curves, determination by kinematics. Verdam, G. J. Grunert Arch. 11 (1848) 13-.

Fan, construction and properties of new sort. Sylvester, J. J. L. Mth. S. P. 6 (1874-75) 196-.

Formation of figures in affinity by similarly variable systems. Geisenheimer, L. Z. Mth. Ps. 24 (1879) 345-.

Generation of surfaces and volumes. Barbier, E. N. A. Mth. 19 (1860) 463-。

Geometrical problem solved by mechanics. Breton, P. Les Mondes 24 (1871) 339-.

- and statical theorems on pyramid, etc. Grunert, J. A. Grunert Arch. 9 (1847) 353-.

- transformation with application in special case to kinematics. Dewulf, $\grave{E}$. Par. Ec. Norm. A. 3 (1886) 405-.

Glissette, centre of curvature. Mannheim, $A$. Par. Éc. Pol. J. 37 cah. (1858) 179-.

-, curvature. Chasles, M. Liouv. J. Mth. 10 (1845) 204-.

- derived from circle and straight line. Dewall, (Oberst) - von. Arch. Mth. Ps. 42 (1864) $65-$.

-, rod sliding against 2 fixed lines. Unferdinger, $F$. [1861] Wien SB. $45(A b .2)(1862)$ 251-.

- of sides of square with two angular points describing straight lines. Pomey, J. B. N. A. Mth. 5 (1886) 520-.

- - 2-term oval $\frac{x^{n}}{a^{n}}+\frac{y^{n}}{b^{n}}=1$. M'Laren, (Lord). Edinb. R. S. P. 18 (1892) 83-.

Glissettes of ellipse and hyperbola. Tait, Edinb. R. S. P. 17 (1891) 2-.

-, formula. Besant, W. H. [1870] QJ. Mth. 11 (1871) 38-。

Groups of movements. Jordan, C. C. R. 65 (1867) 229-; A. Mt. 2 (1868-69) 167-, 322-. - _ - continuous, of any configuration in 3 dimensions. Ricci, G. C. R. 127 (1898) $344-, 360-, 390$.

Helicoidal velocities of solid, connections of which are defined by inequalities. Somov, P. O. Vars. S. Nt. Tr. (1895-96) (C. R., P8. C.) No. 8, 5 pp.

Herpolhode in ease of quadric surface. Sparre, (le comte) - de. Brux. S. Sc. A. 9 (1885) (Pt. 2) 249-.

- of Poinsot, and an apparatus of Darboux and Koenigs. Gilbert, $P$. Brux. S. Sc. A. 14 (1890) (Pt. 2) 25-.

Hinged duplicator. Somov, P. O. Vars. S. Nt. Tr. (1900) (C. R., P8. C.) No. 2, 1-.

Hyperboloid of jointed rods, and de Sparre's theorem. Mannheim, A. C. R. 102 (1886)
"Hypercycles" (geometry of direction). Laguerre, $E$. C. R. 94 (1882) 778-, 832-, 933-, 1033-, 1160-.

\section{Hypocycloids.}

Root, O. Camb. (M.) Mth. M. 1 (1859) 133-. with 3 cusps. Steiner, $J$. Berl. Mb. (1856) 1 -. - - Cremona, L. [1864] Crelle J. 64 (1865) 101-.

_- Clebsch, R. F. A. [1864] Crelle J. 64 (1865) 124-.

- Painvin, L. [1865] N. A. Mth. 9 (1870) 202-, 256-.

_- Milinowski, -. Z. Mth. Ps. 19 (1874) 115-.

- Cahen, C. P. N. A. Mth. 14 (1875) 21-.

- Kantor, S. Bll. Sc. Mth. As. 3 (1879) (Pt. 1) 136-.

- - Laguerre, E. Par. S. Mth. Bll. 7 (1879) 108-.

_- -. MacMahon, P. A. [1882] Mess. Mth. 12 (1883) 151-.

- - Intrigila, C. G. Mt. 23 (1885) 263-.

- - Serret, P. C. R. 125 (1897) 404-, 423-, 445-, 459-.

- _ Ruffini, F. P. [1900] Bologna Rd. 5 (1901) 13-.

- - - connected with family of parabolas. Rupp, O. Mh. Mth. Ps. 4 (1893) 135 -.

- - construction. Stiner, G. Mh. Mth. Ps. 6 (1895) 372-.

- - - envelope of pedal line of triangle, Ferrers' theorem. Serret, P. N. A. Mth. 9 (1870) 73-.

- - - - Simson's line. Brocard, $H$. [1876] Par. S. Mth. Bll. 5 (1877) 18-.

- - - - Badoureau, A. N. A. Mth. 18 (1879) 33 .

- $-1,-\ldots-\ldots$, proof of Steiner's theorem. Brocard, H. (Ix) Par. S. Mth. Bll. 1 (1873) 224 .

- - - Feuerbach's circle, and the circumscribing hyperbolas of $\Delta$. Fiedler, $W$. Zür. Vjschr. 30 (1885) 390-.

- - - Steiner's problem, extension. Mac Mahon, P. A. [1882] Mess. Mth. 12 (1883) 138-.

- - - tangential geometry. Kantor, $S$. [1878] Wien Ak. Sb. 78 (1879) $(A b .2) 204$. -4 cusps. Breton [de Champ], P. N. A. Mth. 4 (1845) 135-.

- - Wolfers, J. P. Grunert Arch. 25 (1855) 109-.

- - . M., J. Mathesis 14 (1894) 129-.

- - , evolute. Graves, R. H. A. Mth. 4 (1888) 36.

- _ , and other curves. Böklen, O. Grunert Arch. 37 (1861) 105-.

Infinitesimal angle between adjacent normals and application to rolling of surfaces. Resal, H. C. R. 114 (1892) 381-, 700.

Instantaneous centre and its geometrical applications. Habich, E. Les Mondes 18 (1868) 709-, 750-; 19 (1869) 29-, 121-. 
Joints, simple. Chebuishev, P.L. [1879] (xr) Rec. Mth. (Moscou) 9 (1878-81) (Pt. 1) 340-.

Kinematic determination of curves. Ocagne, M. $d^{\prime}$. N. A. Mth. 1 (1882) 40-; 2 (1883) $189-$.

- geometry, applications. Mannheim, $A$. Prag Sb. (1888) (Mth.-Nt.) 87-.

- - of space, fundamental theorem in. Geiser, C. F. [1880] Crelle J. Mth. 90 (1881) 39-.

- and metrical properties of conjugate quadrilaterals. Stephanos, C. C. R. 95 (1882) 677-.

Kinematics applied to' modern geometry. Procházka, B. Prag České Ak. Fr. Jos. Rz. (Tríida 2) 5 (1896) Art. 40, 19 pp.; Fschr. Mth. (1896) 415-.

- of figures on surfaces. Lévy, M. C. R. 86 (1878) 812-.

- - jointed frameworks. Somov, P.O. Vars. S. Nt. Tr. (1900) (C. R., Ps. C.) No.5, $46 \mathrm{pp}$. - - plane. Cayley, A. QJ. Mth. 16 (1879) 1-.

-, reciprocal figures and quadratic transformation in. Cavalli, E. Nap. Ac. At. 9 (1899) No. 12, $28 \mathrm{pp}$.

- of variable configurations with relations of similarity or affinity. Schumann, A. Z. Mth. Ps. 26 (1881) 157-.

- - - or rigid systems, cases. Burmester, L. Z. Mth. Ps. 23 (1878) 108-.

Limacon, mechanical construction and parallel motion deduced therefrom. Hart, H. Mess. Mth. 5 (1876) 35-.

Lines, 2, revolving in same plane about 2 fixed points, curves of intersection. Mensbrugghe, G. van der. [1863] (vII) Brux. Mm. Cour. $8^{\circ}, 16(1864) 22 \mathrm{pp}$.

-, - - with uniform angular velocity about point, appearances. Plateau, J.A.F. Quetelet Cor. Mth. 4 (1828) 393-.

Linkage, every algebraic condition imposed on motion of body is realisable by. Koenigs, $G$. C. R. 120 (1895) 981-.

-, - - surface is describable by. Koenigs, $G$. C. R. 120 (1895) 861-.

Linkwork, expression of curves generated by, as irreducible determinant. Sylvester, J. J. L. Mth. S. P. 6 (1874-75) 196-.

Lissajous's curves. Braun, W. Mth. A. 8 (1875) 565-.

- -. Himstedt, -. Arch. Mth. Ps. 70 (1884) $337-$. -. Ekama, H. Arch. Mth. Ps. 6 (1888)

Mechanical construction of curves. Painvin, $L$. N. A. Mth. 15 (1856) 139-

- - Russell, W. H. L. [1869] R. S. P. 18 (1870) 72

- - - 4th degree. Cayley, A. [1872] L. Mtb. S. P. 4 (1871-73) 186-

- - sphero-conics, Hart, $H$. L. Mth. S. P. 6 (1874-75) 136-

Method of tangents. Johnson, W. W. A. Mth. 1 (1884-85) 131-.

\section{Motion.}

apparent, of heavy particle at earth's surface. Gilbert, L. P. [1877] (xiI) Brux. S. Sc. A. 2 (1878) (Pt. 1) 61-, (Pt. 2) 49-. of body remaining homothetic to itself during displacement and deformation. Fouret, $G$. C. R. 88 (1879) 227-.

conchoidal. Habich, E. Les Mondes 11 (1866) 307-.

under 5 conditions, case. Rouquet, $V$. Toul. Fac. Sc. A. 10 (1896) F, 23 pp.

- - curves and surfaces described in. Schoenfies, - Bll. Sc. Mth. 12 (1888) 18-.

of connecting rod, curves generated by, rule for substitution in given arc. Barbier, E. C. R. 96 (1883) 1648.

- continuous, material system, differential invariants. Źorawski, $K$. Krk. Ak. (Mt.Prz.) Rz. 18 (1901) 353-; Crc. Ac. Sc. Bll. (1900) 367-.

- 2 convicts chained together, problem. Collignon, É. As. Fr. C. R. (1893) (Pt. 2) 47-. - curve on another equal curve. Minozzi, A. G. Mt. 14 (1876) 190-.

- developable cylinders. Bottomley, J. Manch. Lt. Ph. S. P. 20 (1881) 128-.

elliptic, theorem of Möbius. Mansion, - - [1900] Brux. S. Sc. A. 25 (1901) (Pt. 1) 71-.

-, of unresisted projectiles, constructions connected with. Tait, P. G. Edinb. R. S. P. 5 (1866) 565-.

of figure in its plane. Ribaucour, A. (xI) Par. S. Phlm, Bll. 6 (1869) 12-.

- figures in plane and space. Jordan, C. (x) Par. S. Mth. Bll. 1 (1873) 144-.

- - remaining similar to themselves. Formenti, C. G. Mt. 17 (1879) 232-.

- - - - - - during displacement in space, case. Groward, A. (x) Par. S. Phlm. Bll. 10 (1873) 47-.

general, of solid. Seiliger, D. N. Kazan S. Ps.-Mth. Bll. 7 (1898) 131-.

of heavy spherical segment on inclined plane. Didion, -. C. R. 77 (1873) 167-.

helicoidal, of solid. Appell, $P$. Par. Ec. Norm. A. 5 (1876) 245-.

hyperbolic. Hall, A. [1867] Mess. Mth. 4 (1868) 106-.

of invariable figure, areas, arcs, etc. traced, properties. Darboux, G. Bll. Sc. Mth. As. 2 (1878) 333-.

in naval manœuvres. Tournier, (le lt.) $E . \quad R v$. Mar. et Col. 125 (1895) 430-; 130 (1896) 209-. - - . Réveille, J. Rv. Mar. et Col. 134 (1897) 465-.

- - Tournier, (le lt.) E. Rv. Mar. et Col. 135 (1897) 51-。

- - May, (Capt.) H. J. Rv. Mar. et Col. 136 (1898) 523-; 137 (1898) 77-.

_ - -. Réveille, J. Rv. Mar. et Col. 139 (1898) 186-.

non-Euclidean geometry. Newson, H. B. N. Y. Am. Mth. S. Bll. 4 (1898) 2, 5-

plane. Gilbert, $P$. Brux. Mm. Cour. $4^{\circ}, 30$ (1861) $37 \mathrm{pp}$.

of plane under certain conditions. Roberts, $S$. L. Mth. S. P. 3 (1869-71) 286-; 7 (1875-76) 216-.

plane, under conditions, ruled surfaces generated by. Blake, E. M. [1898] Am. J. Mth. 21 (1899) 257-. 
plane, curvature. Bresse, J. A. C. Par. Ec. Pol. J. 35 ${ }^{\circ}$ ah. (1853) 89-.

of plane figure, formulæ. Haton de la Goupillière, J. N. Par. Éc. Pol.J. Cah. 45 (1878) 105-.

- - in its plane. Habich, E. Les Mondes 14 (1867) 71-, 402-; 15 (1867) 24-; 16 (1868) $540-, 600-, 677-$. $-\frac{1}{1204}-\frac{1}{-}$. Pellet, A. C. R. 120 (1895)

- - remaining similar to itself. Laisant, C. A. As. Fr. C. R. (1882) 106-.

Mm. 1 (1883) 59-, 171-.

- - -, trajectories connected with. Procházka, B. Časopis 25 (1896) 81-, 161-; Fschr. Mth. (1896) 577.

plane, of 1 surface over another, problem. Eecen, A. N. Arch. Wisk. 15 (1888) 67-.

of plane variable systems with relations of affinity or collinearity. Burmester, L. Z. Mth. Ps. 19 (1874) 465-.

$-{ }_{-}-1-$ similarity. Burmester, $L$. Z. Mth. Ps. 19 (1874) 154-.

- point, diagrams, general method of obtaining. Padelletti, D. Mil. I. Lomb. Rd. 10 (1877) 134-.

- - on ellipsoid. Schellbach, E. H. Crelle J. 54 (1857) 380-.

- - given surface. Ruffini, F.P. Bologna Ac. Sc. Mm. 5 (1883) 211-.

- -, properties. Liguine, $V$. N. A. Mth. 1 (1882) 300-.

- - on surface. Haag, - Par. S. Mth. Bll. 5 (1877) 166-.

- - - - application of Hamilton's method. Padova, E. G. Mt. 7 (1870) 90-.

relative, expansion of coordinates of point in, and curvature of orthogonal lines. Bali. trand, -. Par. S. Mth. Bll. 23 (1895) 26-.

_, normals and centres of curvature found in case of. Gilbert, L. P. [1878] (xII) Brux. S. Sc. A. 3 (1879) (Pt. 1) 55-; (Pt. 2) 81-.

-, of ship and wind. Collignon, $\hat{E}$. As. Fr. C. R. (1885) (Pt. 2) 6-.

-, - surfaces of revolution touching one another. Zimmermann, $H$. Z. Mth. Ps. 19 (1874) 242-.

- to any systems. [Barré de] Saint-Venant, N. A. Mth. 8 (1849) 326-.

and reversions. Study, E. Mth. A. 39 (1891) 441-.

of rigid body, geometrical investigation. Neumann, C. Mth. A. 1 (1869) 195-.

- - or projectively variable systems, curves and surfaces described in. Rodenberg, $C$. Gött. Nr. (1888) 176-.

- rolling body. Beltrami, E. G. Mt. 10 (1872) 103-.

- ship towards given point in given direction. Corrard, A. Rv. Mar. et Col. 136 (1898) 433-.

- - in uniform current. Collignon, $E^{\prime}$. As. Fr. C. R. (1887) (Pt. 2) 42-.

- similarly varying systems. Geisenheimer, $L$. Z. Mth. Ps. 24 (1879) 129-. of skew quadrilateral, 2 angles of which describe circles. Pelisek, M. Prag České Ak. Fr. Jos. Rz. (Trida 2) 8 (1899) Art. 39, 13 pp.; Fschr. Mth. (1899) 492.

- solid remaining similar to itself, 2 cases. Nicoli, $F$. Mod. Ac. Sc. Mm. 1 (1883) 249-. - straight line. Halphen, G. H. (x) Par. S. Mth. Bll. 1 (1873) 114.

- - in plane. Habich, É. C. R. 89 (1879) 405-.

- surface about fixed point. Floquet, G. C. R. 105 (1887) 746-.

- always touching fixed surface. Gautero, G. G. Mt. 20 (1882) 168-.

- system of points collinearly variable. Somov, $P$. O. Vars. S. Nt. Tr. $(1890-91)(C . R$., Ps. C.) No. 8, 1-.

- tangent rolling on curve, properties. Chamousset, (l'abbé) F. [1868] (xII) Sav. Ac. Mm. 11 (1869) CVI-.

- triangle in plane, theorem. Serret, $P . N$. A. Mth. 12 (1853) 68-.

Moving curve, curves described by point of. Collins, E. [1813] St Pét. Ac. Sc. Mm. 6 (1813-14) 133-.

- curves, locus of intersections of 2. Nicolaïdè $s, N$. N. A. Mth. 3 (1864) 39-.

- line, orthogonal trajectories. Pirondini, $G$. N. A. Mth. 9 (1890) 297-.

- pencil of planes, properties. Mannheim, A. As. Fr. C. R. (1874) 1176-.

-- plane, orthogonal trajectories. Serret, J.A. C. R. 41 (1855) 1253-.

- sphere, orthogonal trajectories. Serret, J. A. C. R. 42 (1856) 105-.

- straight line, curves described by. La Gournerie, J. de. Liouv. J. Mth. 14 (1849) 417-.

- - - generation of curves by. Le Francois, (Prof.) -. Quetelet Cor. Mth. 4 (1828) 296-. Orthogonal web of jointed rods. Sylvester, $J$. J. L. Mth. S. P. 6 (1874-75) 197.

Plane kinematic geometry, applications. Ocagne, M. d'. N. A. Mth. 19 (1880) 264-, 289-.

- - trigonometry, theorem suggested by Gauss's curvatura integra. Thomson, (Sir) $W$. Ph. Mg. 32 (1891) 471-.

Polar reciprocal curves, and hodograph. Hirman, S. N. Vars. S. Nt. Tr. (1891-92) (C. R., Ps. C.) No. 7, 4 .

Problem. Geiser, C. F. Sch. Nf. Gs. Vh. 59 (1876) 236-.

-. Prochazka, B. Časopis 24 (1895) 291-; Fschr. Mth. (1895) 621.

-, application of non-Euclidean geometry to. Ball, R. S. B. A. Rp. (1881) 535-.

- on geometry of circle. Sobotka, J. Mh. Mth. Ps. 7 (1896) 347-.

Projective properties of surfaces generated by curves on moving plane. Schlesinger, $\vec{J}$. Wien Sb. 58 (1868) (Ab. 2) 435-。

- variable plane systems. Burmester, L. Mth. A. 14 (1879) 472-.

Pseudo-trochoids. Wölfing, E. Z. Mth. Ps. 44 (1899) 139-. 
Quadrangle, differential relation between sides. Cayley, A. Mess. Mth. 6 (1877) 99-.

"Radial" curves (derived from curve and its evolute). Tucker, R. [1865] L. Mth. S. P. 1 (1866) No. 5, 7 pp.

Radius of curvature of curve described by point of moving figure. Saint Loup, L. N. A. Mth. 12 (1873) 113-.

Real surfaces defined by $\frac{d^{4} \xi}{d^{2} u d u_{1}^{2}}=0$. Montcheuil, - de. Par. S. Mth. Bll. 27 (1899) 114-.

Rolling cone, envelope of connected cone. Hennessy, H. Ir. Ac. P. 7 (1861) 361-.

- motion of spheres, solution of problem. Siverly, $W$. Des Moines Anal. 2 (1875) 195-.

- - - - - - - Adcock, R. J. Des Moines Anal. 3 (1876) 61-, 129-.

$---\frac{-}{-}-\frac{1}{\text {. Hill, G.W. Des Moines }}$ Anal. 3 (1876) 92-.

- of plane curves. Lundberg, E. Ups. Årsk. (1874) (Mth.) (No. 3) $31 \mathrm{pp}$.

- solid bounded by cylinder on horizontal plane. Hoppe, $R$. Arch. Mth. Ps. 66 (1881) 213-.

- - - - tangent surface on horizontal plane. Hoppe, R. Arch. Mth. Ps. 66 (1881) 373-.

- - surfaces. Resal, $H$. C. R. 100 (1885) 260 -.

- - on one another. Hoppe, R. Arch. Mth. Ps. 60 (1877) 159-

- - - Cosserat, E. C. R. 121 (1895) 935-.

Rotation axes, loci in theory. Zančevskij, $I$. N. Rs. S. Nt. Mm. (Mth.) 14 (1892) i-, 1-.

- , geometrical theory. Donkin, W. F. Ph. Mg. 1 (1851) 187-.

_, infinitely small, proof of theorems by. Mannheim, A. C. R. 78 (1874) 633-.

-, representation without projecting on plane perpendicular to axis. Fialkonski, $N$. Wien SB. 21 (1856) 181-.

Rotations and homographies, correspondence. Cayley, A. Mth. A. 15 (1879) 238-.

-, spherical. Stephanos, C. Mth. A. 22 (1883) 299-.

\section{Roulettes.}

Holditch, H. [1838] Camb. Ph. S. T. 7 (1842) 61-.

Lenthéric, - Mntp. Ac. PV. (1850-51) 30-, 34-, 37-; Mntp. Ac. Sc. Mm. 2 (1851-54) 1-, 315-.

Sacchi, G. N. A. Mth. 2 (1863) 172-.

Purser, F. QJ. Mth. 7 (1866) 129-.

Aoust, (l'abbé) - C. R. 70 (1870) 978-.

Habich, É. (xII) Mathesis 2 (1882) 145-.

Gossot, (le capit.) F. Rv. Mar. et Col. 89 (1886) 319 .

case. Lane, A. V. Am. J. Mth. 8 (1886) 132-.

centres of curvature. Hadamard, -. Bordeaux S. Sc. Mm. 5 (1895) xxxvii-.

for conic on other curves. Ekama, H. Arch. Mth. Ps. 8 (1890) 388-.
Descartes's theorem. Frenet, $F$. N. A. Mth. 13 (1854) 299-.

described by conic. Ekama, $H$. N. Arch. Wisk. 3 (1898) 175-; Fschr. Mth. (1897) 525.

generalisation of theorem of Delaunay. SaintGermain, A. de. Bll. Sc. Mth. 17 (1893) 110 -.

generated by rolling circle, orthogonal trajectories. Breton, P. [1861] (xII) Isère S. Bll. 7 (Livr. 3 \& 4) (1867) 346-.

geometrical transformation applicable to. Demoulin, A. Brux. Mm. Cour. 8, 45 (1891) No. 3, $35 \mathrm{pp}$.

nomenclature for. Willson, $F$. N. Am. As. P. (1887) 59-.

and pedal curves. Catalan, E. C. Brux. Ac. Bll. 27 (1869) 144-.

plane. Hennig, R. [1864] Crelle J. 65 (1866) $52-$.

and polar paths of plane kinematic systems. Sellentin, R. Z. Mth. Ps. 28 (1883) 116-.

problems, inverse. Chrystal, $G$. Edinb. Mth. S. P. 5 (1887) 38-.

quadrature. Orlov, T. E. [1883] (xII) Rec. Mth. (Moscou) 11 (1883-84) 457-; Fschr. Mth. (1884) 764 .

rectification. Steiner, J. Berl. Ab. (1838) 19 -.

and rolling surfaces. Pirondini, G. G. Mt. 26 (1888) 352-.

skew. Laurent, $H$. Par. S. Mth. Bll. 2 (1874) 84-.

theorem. Lamarle, E. Brux. Ac. Bll. 4 (1858) 239-.

-. Serret, P. N. A. Mth. 18 (1859) 341-.

- attributed to de La Hire. Le Paige, C. Bb. Mth. (1887) 109.

$--\frac{1}{-}$ Curtze, M. Bb. Mth. (1888) 65-; (1895) 33-.

theory. Maxwell, J. C. Edinb. R. S. T. 16 (1849) 519-.

-. Catalan, E. C. N. A. Mth. 15 (1856) $102-$.

-. Mannheim, A. L'I. 26 (1858) 69.

-. Cesáro, E. N. A. Mth. 7 (1888) 209-.

Ruled mechanics. Saussure, R. de. C. R. 123 (1896) 796-.

- surface traced by line forming part of in. variable moving system. Rouquet, $V$. [1900] Toul. Ac. Sc. Mm. 1 (1901) 9-.

Runners, problem. Manderlier, -. Quetelet Cor. Mth. 2 (1826) 199-.

Skew curves, 3rd degree, analogue of helicoidal motion. Appell, P. C. R. 82 (1876) 70-; Par. Éc. Norm. A. 5 (1876) 245-.

Sphere sliding or rolling on horizontal plane. Resal, H. N. A. Mth. 11 (1872) 193-.

Spherical eycloidal and trochoidal curves. Jeffery, H. M. [1882] QJ. Mth. 19 (1883) 44-.

- loop line (tortuous curve). Schiffner, $\boldsymbol{F}$. Arch. Mth. Ps. 5 (1887) 160-.

- - - (- -). Janisch, E. Arch. Mth. Ps. 8 (1890) 184-, 334-; 9 (1890) vII; 10 (1891) vII. 


\section{Kinematic Geometry}

Straight line sliding against curves, locus of point in. Enneper, A. Z. Mth. Ps. 11 (1866) 434 .

Surface, 4th degree, with generators capable of moving normally to their trajectories. Chasles, M. C. R. 52 (1861) 1094-.

Surfaces capable of generating a Lamé system. Cosserat, E. C. R. 124 (1897) 1426-.

- generated by motion of lines, partial differential equations. Blaẑek, $G$. [1864] Wien Sb. 51 (1865) (Ab. 2) 186-.

- - moving curve. Pranghofer, $J$. Wien Sb. 53 (1866) (Ab. 2) 505-.

- - - plane curve when plane rolls on developable surface. Pirondini, G. G. Mt. 30 (1892) 188-.

- having generators of constant form, partial differential equation for. Gebbia, M. Palermo Cir. Mt. Rd. 1 (1887) 74-.

-, helicoidal, construction of parallel projection, and especially shadow. Burmester, $L$. Z. Mth. Ps. 18 (1873) 184-.

- related to curve (skew) rolling on another. Svéchnicoff [Svěšnikov], P. N. A. Mth. 14 (1895) 501-; Kazan S. Ps.-Mth. Bll. 6 (1896) 16 pp. ; Fschr. Mth. (1896) 537.

-, screw, with circumscribed cylinders, tangent curves. Sobotka, J. Prag Sb. (1893) (Mth.-Nt.) No. 22, 38 pp.

- susceptible of generation by motion of plane curve. Mainardi, G. Mod. S. It. Mm. 20 (1828) 482-.

System of 3 equal figures in same plane, properties. Stephanos, C. [1881] Par. S. Phlm. Bll. 6 (1882) 12-.

- 2 similar bodies. Chasles, M. Quetelet Cor. Mth. 7 (1832) 352-.

Theorem. Réveille, J. Par. S. Mth. Bll. 16 (1888) 130-.

- on description of areas. Johnson, W. W. B. A. Rp. (1881) 536-.

Theorems of Schooten and de La Hire. (1) Ellipse, kinematic description. (2) Hypocycloid degenerating to straight line. La Gournerie, J. de. Par. Éc. Pol. J. $36^{\circ}$ cah. (1856) 255-.

Trajectory of point on straight line displaced under conditions. Mannheim, A. C. R. 70 (1870) 1215-.

- surfaces of figure of invariable form, normals, construction. Mannheim, A. As. Fr. C. R. (1878) 152-.

- - - - - - - - meet two straight lines. Mannheim, A. Ás. Fr. C.R.2(1873) 95-.

- - - moving under 4 conditions. Mannheim, A. (x) C. R. 77 (1873) 752-; Par. Mm. Sav. Etr. 22 (1876) No. 12, 19 pp.

Transformation of demonstration in kinematic geometry. Mannheim, A. C. R. 112 (1891) 475-.

-, general, of group of Euclidean movements. Page, J. M. [1898] A. Mth. 12 (1898-99) 87-.

- method. Mannheim, A. C. R. 110 (1890) 220-, 270-.

- for ruled surfaces. Mannheim, A. C. R. 88 (1879) 1128-.

Transformations. Mannheim, A. C. R. 110 (1890) 391-.

\section{Plane Curves 8430}

Translation surface. Crelle, A. L. Crelle J. 51 (1856) 100-.

- surfaces. Sucharda, A. Wien Ak. Sb. 92 (1886) $(A b .2)$ 836-.

- - Pirondini, G. A. Mt. 17 (1889-90) 225-.

- -. Sanctis, P. de. N. A. Mth. 9 (1890) $552-$.

- - Lie, S. Leip. Mth. Ps. B. 44 (1892) 447-, 559-.

- - and Abel's theorem. Lie, S. C. R. 114 (1892) 277-; Leip. Mth. Ps. B. 48 (1896) $141-$.

- - arising in several ways. Scheffers, $G$. C. R. 116 (1893) 1352-.

- - construction of tangent rays. Sucharda, A. Prag České Ak. Fr. Jos. Rz. (Tř́ida 2) 6 (1897) Art. 24, 36 pp.; Prag Fr. Jos. Ac. Sc. Bll. (Mth. Nt.) 4 (1897) (Pt. 2) 63-.

- - determination of all. Lie, M. S. Arch. Mth. Ntvd. 7 (1882) 155-.

- - in general and biquadratic in particular.

Sucharda, A. Časopis 28 (1899) 257-, 336-; Fschr. Mth. (1899) 571-.

- "surfaces" and Abel's theorem. Lie, S. Leip. Mth. Ps. B. 49 (1897) 181-.

Trochoids. Studnička, F.J. Časopis 1 (*1872) 252-; Bll. Sc. Mth. As. 6 (1874) 97.

-, curvature. Gogu, C. [1890] Bucarest Ac. Rom. A. 13 (1890-91) (Pt. Admin.) 45-.

-, double generation. Wiener, L. C. Z. Mth. Ps. 26 (1881) 257-.

-, evolutes. Wiener, L. C. Z. Mth. Ps. 27 (1882) 129 .

-, foliate curves. Hyde, E.W. Des Moines Anal. 2 (1875) 12-.

-, history. Hoza, $F$. Časopis 1 ( $\left.{ }^{*} 1872\right)$ 54-. - of points on rolling conies. Ekama, $H$. N. Arch. Wisk. 16 (1889) 58-; Fschr. Mth. (1889) 719-.

Volumes generated by closed contour in any motion. Koenigs, G. C. R. 106 (1888) 1512-.

Watt's curve. Catalan, E. Mathesis 5 (1885) 154-, 222-.

- - theorem. Tchebitchef, P. L. Bll. Sc. Mth. As. 5 (1881) (Pt. 1) 216.

\section{Curvature of plane curves; other applications of the differen- tial calculus to plane curves. (See also 7210.)}

Aberration of curvature. Husquin de Rhêville, -. N. A. Mth. 9 (1890) 138-.

- - - Servais, C. Mathesis 12 (1892) 129-.

Antipedals, theorem. Catalan, E. C. Rm. At. N. Linc. 25 (1872) 349-.

Asymptotic chords. Bermann, O. Grunert Arch. 12 (1849) 323-.

Barycentre of curvature. Steiner, J. Berl. Ab. (1838) 19-.

- - of algebraic curves. Neumann, $C$. Z. Mth. Ps. 12 (1867) 172-, 425-. 
Boundary relations, and formula for radius of curvature. Voller, - . Grunert Arch. 32 (1859) 97-; 33 (1859) 350-.

Caustics, construction. Cornu, A. N. A. Mth. 2 (1863) 311-.

- identical with pedals. Weyr, E. Z. Mth. Ps. 14 (1869) 376-.

-, plane, application of coordinate system to. Habich, E. A. Mt. 2 (1868-69) 134-

Centre of curvature. Ocagne, M. d'. Par. S. Mth. Bll. 19 (1891) 31-.

- - - graphic construction. Anon. (v 541) Gergonne A. Mth. 12 (1821-22) 135-.

- - _ - - Gergonne, J. D. Gergonne A. Mth. 12 (1821-22) 137-.

- - of locus of points, distances of which from 2 given curves are in constant ratio. Mannheim, A. Tortolini A. 1 (1858) 364-.

- _ _ and normal of polytropic curves. Kosch, E. Z. Mth. Ps. 45 (1900) 161-.

_ _ - with polar coordinates. Husquin de Rhêville, -. N. A. Mth. 10 (1891) 411- - - of certain spiral curves. Rulf, $W$. Mh. Mth. Ps. 3 (1892) 211-.

Centres of curvature of integral curve. Machovec, F. Časopis 19 (1890) 67-; Fschr. Mth. (1890) 703 .

- - - isogonal curves. Seiliger, D. N. N. Rs. S. Nt. Mm. (Mth.) 14 (1892) 155-; Fschr. Mth. (1892) 582.

- - , successive. Haton de la Goupillière, J. N. C. R. 46 (1858) 930-; Liouv. J. Mth. 4 (1859) 183-.

- - - of $y^{n}=a^{r} x^{n-r}$. Machovec, $F$. Prag Sb. (1885) (Mth.-Nt.) 386-.

$--1-\lambda x^{n}+\mu y^{n}=d^{n}$. Machovec, $F$. Prag Sb. (1891) (Mth.-Nt.) 83-.

Circles of curvature. Schellbach, $\dot{C} . H$. Crelle J. 45 (1853) 263-.

- Tait, P. G. Edinb. Mth. S. P. 14 (1896) 26.

- - and asymptotes. Laisant, C. A. Par. S. Mth. Bll. 23 (1895) 95-.

- - - complex quantities. Petersen, $J$. Sk. Nf. F. (1892) 354; Fschr. Mth. (1892) 223-.

- - - of conics. Weiler, A. Zïr. Vjschr. 33 (1888) 119-.

- - - ellipse. Cesáro, E. Mathesis 5 (1885) 7-.

- - - - family of curves. Allégret, [A.] N. A. Mth. 9 (1870) 30 -

- - - nodal cubic. Janisch, E. Mh. Mth. Ps. 7 (1896) 142-.

- - - at point of curve of 4 th degree with 3 double points, of which 2 are circular points at infinity. Saltel, $L$. Brux. Ac. Bll. 34 (1872) 51-.

- - - radii. Pagani, G. M. Brux. Ac. Bll. 14 (1847) 185-.

_ _ _ relation to curve at point of contact. Mack, L. Arch. Mth. Ps. 64 (1879) 182-.

- having double contact with conic, point transformations in elliptic coordinates. Lovett, E. O. Am. J. Mth. 20 (1898) 242-.

Cochleoid. Falkenburg, C. Arch. Mth. Ps. 70 (1884) 259-.
Cochleoid, properties. Benthem, A. N. Arch. Wisk. 10 (1884) 76-.

Conics having higher order of contact with given curve. Ocagne, M. d'. N. A. Mth. 16 (1897) 252-.

- osculating curves of 3rd degree. Gent, $R$. Z. Mth. Ps. 17 (1872) 476-.

-, systems, differential equations. Roberts, R. A. [1895] N. Y. Am. Mth. S. Bll. 2 (1896) 11-.

- touching curves of any degree. Cayley, $A$. C. R. 59 (1864) 224 -

Contact. Christie, A. S. Smiths. Misc. Col. 33 (1888) Art. 1, 157-. (Wash. Ph. S. Bll. 6 (1884).)

- with conics or curves of 3 rd degree. Halphen, G. H. [1875] Par. S. Mth. Bll. 4 (1876) 59-

- and curvature, theory. Grunert, J. A. Grunert Arch. 30 (1858) 361-.

- of 2 curves, ratio of curvatures at point of. Wölffing, E. Z. Mth. Ps. 38 (1893) 237-.

- lines, successive intersections. Desmarest, $E$. N. A. Mth. 3 (1844) 220

- of given order, of conic with curve, conditions. Painvin, L. C. R. 78 (1874) 55-.

- 5 th order, of conic with curve, conditions. Painvin, L. C. R. 78 (1874) 436-, 835-.

- with parabola. Schell, W. Marb. Schr. 8 (1857) 135-.

- of plane cubic with curve of any degree, positions where closest. Disteli, M. Z . Mth. Ps. 38 (1893) 257-.

- 6-point, of curves of 3rd degree with conio, 27 points of. Steiner, J. Berl. B. (1845) 386-.

- points of parallel tangent lines or planes, centre of mean distances. Liouville, $J$. Liouv. J. Mth. 6 (1841) 345-; C. R. 13 (1841) 412-.

—, theory. Poselger, F. T. Berl. Ab. (1836) 55 -.

,-- , point in. Halphen, G. H. Par. S. Mth. Bll. 2 (1874) 94-.

Coordinates in plane, Watson's. Hill. $T$. Am. As. P. 22 (1873) 27-.

- - - , problems. Hill, T. Am. As. P. (1875) (Pt. 1) 41-.

Cubic parabolas, property. Cellérier, $G$. Arch. Sc. Ps. Nt. 11 (1884) 408-.

Curvature. Gergonne, J. D. Gergonne A. Mth. 21 (1830-31) 1-.

- Zimmermann, H. E. M. O. Z. Mth. Ps. 28 (1883) 115-.

- Demoulin, A. Brux. Mm. Cour. $8^{\circ}, 44$ (1891) No. 5, $48 \mathrm{pp}$.

- conics. Berg, F. J. van den. Amst. Ak. Vs. M. 9 (1892) 85-; Fschr. Mth. (1892) 654 -.

- curve of basis point of pencil of curves of $n$th degree. Zahradnik, K. Prag Sb. (1878) 250 -.

- at cusp. Anon. (xI 52) N. A. Mth. 10 (1871) 318.

- double point. Cayley, A. QJ. Mth. 3 (1860) 322-.

- formulø. Torry, A. F. Mess. Mth. 2 (1863) 81-. 
Curvature formulæ in trilinear coordinates. Walton, W. QJ. Mth. 8 (1867) 39-.

- at multiple point. Painvin, L. C. R. 68 (1869) 131-; A. Mt. 4 (1870-71) 215-.

- of 3rd order. Dupin, C. C. R. 26 (1848) 321-, 393-.

- properties of ellipse and hyperbola. Wright, C. Camb. (M.) Mth. M. 2 (1860) 198-.

-, question in. Cesáro, E. Mathesis 13 (1893) 177-.

- relations of pencil of curves at basis point. Weyr, E. Z. Mth. Ps. 15 (1870) 486-.

Curves.

(See also 7610, 7630.)

algebraic, curvature. Servais, C. Brux. Ac. Bll. 21 (1891) 587-; 22 (1891) 512-.

- -, etc. Ocagne, M. d'. Mathesis 12 (1892) 100-

-, equal to circular arcs $[p=r(a-r)]$. Fuss, N. [1823] St Pét. Ac. Sc. Mm. 11 (1830) $274-$.

-, - - hyperbolic ares. Fuss, N. [1798] St Pet. Ac. Sc. N. Acta 14 (1805) 111-.

- intersecting orthogonally, and their confocality. Kummer, E. E. N. A. Mth. 11 (1852) 426-.

analogous, intrinsically, to catenary of equal resistance. Cifarelli, T. G. Mt. 36 (1898) 183-.

angles with straight lines. Monteiro, A. S. G. Teix. J. Sc. 1 (1878) 81-.

angularly related, theorems. Holditch, $H$. QJ. Mth. 3 (1860) 271-.

apses. Longchamps, G. de. As. Fr. C. R. (1891) (Pt. 2) 23-.

arcs, Graves' and Chasles's theorem, generalisation. Humbert, G. C. R. 104 (1887) 1826-; N. A. Mth. 7 (1888) 5-.

with arcs between same radii vectores equal. Fuss, N. [1801] St Pét. Ac. Sc. Mm. 2 (1810) 29-.

asymptotes from polar equation. Nell, A. M. Grunert Arch. 15 (1850) 315-.

case where curve is its own evolute. Binet, $J$. P. M. Liouv. J. Mth. 6 (1841) 61-。

- - - - - pedal. Haton de la Goupillière, J. N. Liouv. J. Mth. 11 (1866) 329-.

- $s$ varies as $\tan \theta$. Fuss, N. [1818-26] St Pét. Ac. Sc. Mm. 9 (1824) 115-; 11 (1830) 314-.

cases where $\rho$ varies as $r^{2}$. Euler, $L$. St Pét. Ac. Sc. Mm. 9 (1824) 47-.

- - - - normal. Farcy, A. N. A. Mth. 3 (1844) 528-.

- - - - (1) $y^{n}$; (2) $r^{n}$; (3) $p^{n}$; (4) $s^{n}$. Hill, T. Am. As. P. 22 (1873) 31-.

- $-r$ is proportional to projection of $\rho$ upon r. Du Chatenet, M. N. A. Mth. 5 (1886) 233-.

- $s$ varies as $y / x$. Velde, $A$. Arch. Mth. Ps. 14 (1896) 200-.

characteristic equation, interpretation, cases. Brocard, H. P. P. B. (xII) Mathesis 2 (1882) 25-, 49-.

classes, two remarkable. Laisant, C. A. As. Fr. C. R. (1890) (Pt. 2) 74-. closed, belonging to same family, criterion. Crocchi, L. G. Mt. 10 (1872) 302-.

-, mean curvature, formulæ. Lindelöf, L. L. [1870] Helsingf. Acta 9 (1871) 361-.

-, parallels. Hoppe, $R$. Arch. Mth. Ps. 66 (1881) 46-.

with constant polar tangent. Giard, - N. A. Mth. 1 (1862) 70-.

continuous development, extension of theorem of Bernoulli. Mainardi, G. A. Sc. Lomb. Ven. 7 (1837) 29-.

convexity and concavity, theorem. Gerono, $G$. C. N. A. Mth. 18 (1859) 397.

cubic, curvature. Holst, $E$. Arch. Mth. Ntvd. 7 (1882) 109-, 177-.

with several cusps. Laurent, $H$. N. A. Mth. 19 (1860) 210-.

cutting at given angle. Hoppe, R. (vi Adds.) Berl. Pol. Gs. Vh. 20 (1859) 135-.

- given curve at angle which is given function of point. Franconi, E. G. Mt. 38 (1900) 232-.

cyclic, curvature, and projective geometry. Schwarz, A. Mh. Mth. Ps. 10 (1899) 250-; 11 (1900) 71-.

一, of direction. Humbert, G. Liouv. J. Mth. 4 (1888) 129-.

defined by differential equation, 1st order, between two variables, systems. Dyck, $W$. Mïnch. Ak. Sb. 21 (1892) 23-; 22 (1893)101-. - - intrinsic relation. Pirondini, G. G. Mt. 30 (1892) 326-; 31 (1893) 51.

- - law of rectification. Ocagne, $M . d$. N. A. Mth. 10 (1891) 82-, 133.

- - property of centre of gravity of area. Collignon, É. As. Fr. C. R. (1886) (Pt. 2) 53-. - - relation between ordinate area and subtangent. Schlömilch, $O$. Grunert Arch. 10 (1847) 215-.

"derived." Druckenmiller, N. Crelle J. 26 (1843) 1-.

"L," algebraic and differential equations. Tortolini, B. A. Mt. 6 (1864) 88-.

- from two plane curves. Laisant, C. A. Par. S. Mth. Bll. 16 (1888) 172-.

determination, application of method of parameters. Moseley, $H$. Thomson A. Ph. 11 (1826) 321-.

- by tangential distances from given curves. Ocagne, M. d'. N. A. Mth. 17 (1898) 115-. of direction of 3rd class. Laguerre, $E$. N. A. Mth. 2 (1883) 97-.

- - for which $\sqrt{\left(\frac{d f}{d x}\right)^{2}+\left(\frac{d f}{d y}\right)^{2}}$ is rational. Appell, P. N. A. Mth. 15 (1896) 491-.

dividing circular arcs through 2 fixed points into $n$ equal parts. Burali-Forti, $C$. G. Mt. 27 (1889) 153-.

double tangents. Cayley, A. Phil. Trans. (1859) 193-.

enveloped, theory. Heilermann, - Grunert Arch. 24 (1855) 438-.

equidistant, properties. Mahistre, G. A. [1855] (xII) Lille S. Mm. 3 (1857) 1-.

with evolutes and involutes equal inter se, integrals. Aoust, -. C. R. 84 (1877) 385-; Par. S. Mth. Bll. 7 (1879) 143-. 
and their evolutes, ratio of curvatures. Ruffini, F. $P$. Bologna Ac. Sc. Mm. 6 (1884) 715-.

families, remarkable. Cesáro, E. N. A. Mth. 7 (1888) 171-; 19 (1900) 489-.

family defined by algebraic differential equation of 1st order. Fouret, G. C. R. 86 (1878) 586 -

- represented by differential equation. Amigues, $\dot{E}$. As. Fr. C. R. 8 (1879) 117-. generalisation of Graves' theorem on confocal conics. Zehfuss, G. Schlömilch Z.5 (1860) 210 -

given by tangential equation, curvature. Pain. vin, L. Bll. Sc. Mth. As. 3 (1872) 174 .

having given relation between arc and area in polar coordinates. Collignon, $\hat{E}$. As. Fr. C. R. 11 (1882) 69-.

at infinity, curvature. Torroja, $E$. Fschr. Mth. (1893-94) 1077-.

intrinsic equation, and application. Whewell, $W$. Camb. Ph. S. T. 8 (1849) 659-; 9 (1856) 150 -.

- equations. Gergonne, J. D. Gergonne A. Mth. 4 (1813-14) 42-.

Lamé's, curvature. Fouret, G. C. R. 110 (1890) 843-.

limiting, of given manifoldness of curves. Ascoli, G. Rm. R. Ac. Linc. Mm. 18 (1883) 521-.

maximum and minimum inclinations to radius vector. Exner, $K$. Wien Sb. 57 (1868) ( $A b$. 2) $75-$.

2 , meeting tangents of 3rd at related angles, curvature. Habich, E. Par. S. Mth. Bll. 13 (1885) 201-.

neighbouring, 2, order of approach between finite limits. Léauté, $H$. C. R. 86 (1878) 1537-.

orthogonal, curvature, theory. Reech, F. Par. Éc. Pol. J. Cah. 41 (1865) 201-.

orthotomic, of system of lines in plane. Cayley, A. Mess. Mth. 22 (1893) 45-

osculating. Wolstenholme, J. QJ. Mth. 11 (1871) 210-.

-. Pirondini, G. G. Mt. 26 (1888) 257-, 380.

-, applications of linear quasi-inversion to. Servais, C. Mathesis 8 (1888) 28-

-, of triply infinite system. Krey, H. [1875] Mth. A. 10 (1876) 221-.

parabolic and other, curvatures. Machovec, $F$. Prag Sb. (1892) (Mth.-Nt.) 77-。

parallel. Petersen, J. P. C. (xir) Ts. Mth. 1 (1871) 154-.

-, theorems embracing those relating to. Steiner, J. Crelle J. 32 (1846) 75-.

-, theory. Hoppe, R. Crelle J. 55 (1858) 95-.

-

"periplegmatic", given by $y \frac{d y^{2}}{d x^{2}}=-X$. Gyldén, H. Stockh. Öfv. (1892) 69-.

polar reciprocal, and radius of curvature. Hir. man, C. N. Vars. S. Nt. Tr. (1891-92) (C. R., Ps. C.) No. 8, 6 . of "raccordement," bicircular for 2 straight lines. Fontès, - Toul. Ac. Sc. Mm. 4 (1892) 375-.

- -, with 3 centres. Fichera, $F$. Catania Ac. Gioen. At. 20 (1888) 323-.

- - - - several centres. Du Hays, Liouv. J. Mth. 15 (1850) 241-.

$\begin{aligned} & - \\ & -\end{aligned}-$ Revellat, J. P. $\quad$ C. R. 77 (1873) 434.

- , cubic parabola for easing. Merfield, C.J. N. S. W. R. S. J. 29 (1895) 51-。

- - - - as transition curve. Merfield, C.J. N. S. W. R. S. J. 31 (1897) lvi (bis)-.

- - for junctions. Brianchon, C. J. Par. Ec. Pol. J. $19^{e}$ cah. (1813) 187-.

- - lemniscate for junctions. Oostinjer, $H$. Brux. A. Tr. Pbl. 4 (1899) 335-.

- - method of easing. Shellshear, $W$. N. S. W. R. S. J. 22 (1889) 89-.

-- , parabolic. Verdugo,J.A. Santiago de Chile Un. A. $55\left({ }^{*} 1879\right)$ 123-.

- - , - curves for junction of 2 circular ares. Bernis, - . As. Fr. C. R. (1892) (Pt. 2) 212-. - - , - - junctions. Ménétrier, L. S. (vii) A. Cond. Pon. Chauss. 3 (1859) 14-. rectifiable, deduction of equation. Azzarelli, M. [1877] Rm. N. Linc. At. 31 (1878) 6-.

with relation of 2 nd degree between arc and coordinates. Hoppe, R. [1862] Crelle J. 62 (1863) 193-.

representing integral of $u^{2}+\left(\frac{d u}{d \theta}\right)^{2}=\omega^{2}$, property.

Hirst, T. A. Liouv. J. Mth. 2 (1857) 392.

rhizic (i.e. fulfilling condition $\frac{d^{2} \phi}{d x^{2}}+\frac{d^{2} \phi}{d y^{2}}=0$ ).

Rankine, W. J. M. R. S. P. 15 (1867) 468-.

-. Walton, W. QJ. Mth. 11 (1871) 91-.

-, curvature at multiple points. Walton, W. QJ. Mth. 11 (1871) 274-.

-, spoke asymptotes. Walton, W. [1870] QJ. Mth. 11 (1871) 200-.

satisfying certain condition of maximum or minimum, common properties. Vicaire, $E$. C. R. 106 (1888) 456 -.

scale increasing or diminishing indefinitely. Swellengrebel, J. G. H. Grunert Arch. 16 (1851) 419-.

with similar evolutes. Ettingshausen, A. von. Baumgartner Z. 9 (1831) 178-.

- - - Tucker, R. Mess. Mth. 3 (1866) 191-. and straight lines, agreement. Franciere, -

A. Cond. Pon. Chauss. 17 (1873) 137-.

- surfaces, curvature, and point-transformations. Mehmke, R. Z. Mth. Ps. 36 (1891) 206-.

symmetric, and images. Longchamps, G. de. N. A. Mth. 18 (1899) 373-.

systems, differential equations appertaining to. Czuber, E. Wien Ak. Sb. 102 (1893) (Ab. 2a) 1141-.

tangential, of conies. Hochheim, A. [1869] Z. Mth. Ps. 15 (1870) 377-.

tangents, lines cutting at constant angle. Böklen, O. Arch. Mth. Ps. 43 (1865) 14-.

-, theorems. Marchesano, S. Tortolini A. 4 (1853) 161-.

theory. Jelinek, C. Wien SB. (1849) (Ab. 2) 19-. 
triangular, and symmetric tetrahedral curves, curvature. Fouret, G. Par. S. Mth. Bll. 20 (1892) 60 -

$8=f\left(\frac{d y}{d x}\right)$, construction. Plana, G. Mod. Mm. S. It. 16 (1813) 361-.

$r^{m}=a^{m} \cos m \theta$. Allégret, [A.] N. A. Mth. 11 (1872) 162-.

$r^{m} \cos m \theta=a^{m}$. Bassani, A. G. Mt. 24 (1886) 23-.

$s=k \int\left[(\rho / a)^{n}-1\right]^{-\frac{1}{2}} d \rho$, and its evolute. Cesáro,
E. Fschr. Mth. (1892) 708 . $y=\frac{m}{f^{\prime}(x)}$ derived from $y=f(x)$. Azzarelli, $M$. Rm. N. Linc. At. 46 (1893) 186-.

Curvilinear angles. Miquel, A. Liouv. J. Mth. 9 (1844) 20-; 10 (1845) 347-; 11 (1846) 65-.

"Curvital" functions,

$$
\sin (y, x)=y-\int_{\text {avarni, - - }}^{2} y d x^{2}+\int_{\text {C. }}^{4} y d x^{4} \text { etc. } 60 \text { (1865) } 1079 \text {. }
$$

Gavarni, -. C. R. 60 (1865) 1079-.

Diameters, tangents at points on. Serret, $P$. C. R. 82 (1876) 67-.

Differential equation $L(x d y-y d x)-M d y+$ $N d x=0(\mu=1, \nu=1)$. Fouret, $G$. C. R. 78 (1874) 1693-.

Direction of curvature in polar coordinates. Schultén, N. G. af. [1843] (vm) Helsingf. Acta 6 (1861) 43-.

Dual curvature and duals of evolutes and involutes. Jeffery, H. M. [1869] QJ. Mth. 10 (1870) 321-.

\section{Envelopes.}

Fergola, E. Mod. S. It. Mm. 24 (1850) 290-. Cayley, A. Mess. Mth. 1 (1872) 3-.

Vecchio, A. G. Mt. 11 (1873) 318-.

Brunel, -. Bordeaux S. Sc. Mm. 4 (1888) xi. Aoust's problem. Hoppe, $R$. Arch. Mth. Ps. 66 (1881) 386-.

of carried curves, Savary's theorem. Resal, H. A. N. A. Mth. 1 (1882) 7-.

case. Walton, W. [1867] QJ. Mth. 9 (1868) $142-$.

-. Tognoli, O. G. Mt. 11 (1873) 376-.

- Fais, A. G. Mt. 12 (1874) 150-.

- Baehr,G.F.W. N. A. Mth. 20 (1881) 250-

-, special. Gilbert, - Brux. S. Sc. A. 15 (1891) (Pt. 1) 37-.

of circles. Ribaucour, A. (xI) Par. S. Phlm. Bll. 4 (1867) 88-; 5 (1868) 30-.

decomposition. Saltel, L. '[1881] Bordeaux S. Sc. Mm. 4 (1882) 443-.

determination of class. Chasles, M. C. R. 81 (1875) 757-.

c-discriminant as. Macdonald, J.A. Edinb. R. S. T. 39 (1900) 27-.

imaginary, of conjugates of plane locus. Marie, $M$. C. R. 75 (1872) 7-.

Lagrange's methods. Flauti, $V$. [1848] Mod. S. It. Mm. 24 (1850) 251-.

of line with ends on 2 straight lines, consequences. Terquem, $O$. N. A. Mth. 3 (1844) 182-. of line with 2 fixed lines cutting off constant intercept. Gomes Teixeira, F. Fschr. Mth. (1899) 530.

normals to, and envelopes with doubly infinite set of straight lines as normals. Elliott, E. B. [1879] Mess. Mth. 9 (1880) 85-.

osculating. Möller, J. Lund. Un. Acta 24 (1887-88) (Mth., No. 1) 4 pp.

of pedal line of triangle. Ferrers, N. $M$. [1866] QJ. Mth. 8 (1867) 209-; 9 (1868) 147-.

- - - - Besant, W. H. [1868] QJ. Mth. 10 (1870) 110-

- plane series of curves, order. Zimmermann, O. Crelle J. Mth. 116 (1896) 10-.

- polars of curve. Hoppe, R. [1860] Crelle J. 58 (1861) 374 .

singularities. Henrici, $O$. L. Mth. S. P. 2 (1869) 177-.

of straight line. Desmarest, E. N. A. Mth. 3 (1844) 154-.

- - Sacchi, G. N. A. Mth. 2 (1863) 31-.

- - - Genese, R. W. QJ. Mth. 13 (1875) 260-.

_- - application to centre of curvature of conics. Mannheim, A. N. A. Mth. 16 (1857) 322-.

- - - joining ends of hands of watch. Gilbert, L. P. (xIr) Brux. S. Sc. A. 4 (1880) (Pt. 2) 159-.

- - making equal chords in 2 circles. Taylor, H. M. Mess. Mth. 4 (1875) 163.

- _ - moving with ends on 2 other lines. Hyde, E. W. Des Moines Anal. 1 (1874) 60-.

- - - such that sum of squares of distances of number of points from it is constant. Sýkora, A. Prag Sb. (1881) 23-.

- lines, cases. Ocagne, M. d'. N. A. Mth. 2 (1883) 252-.

- system of curves. Laisant, C. A. N. A. Mth. 13 (1874) 571-

- - - Bosi, L. G. Mt. 35 (1897) $120-, 353$.

- tangent to 2 variable circles. Terré, $E$. N. A. Mth. 10 (1851) 340-.

theorem. Laurent, $H . \quad$ N. A. Mth. 13 (1874) 273-.

theorems. Magnus, L. J. Gergonne A. Mth. $16(1825-26) 80$ -

theory. Petersen, J. P. C. (xII) Ts. Mth. 2 (1872) 81-.

-. Genese, R. W. Mess. Mth. 7 (1878) 61-.

- Cayley, A. Mess. Mth. 7 (1878) 62-.

-, general, connexion with $p$-discriminant of differential equation of 1st order. Chrystal, G. Edinb. R. S. T. 38 (1897) 803-.

-, problem. Wagner, C. Arch. Mth. Ps. 56 (1874) 1-.

\section{Evolutes.}

Wolfers, J. P. Grunert Arch. 4 (1844) 135-。 Dunesme, M. C. R. 35 (1852) 518-.

Enneper, A. Schlömilch Z. 7 (1862) 120-

Nicolä̈ès, N. Les Mondes 8 (1865) 621-.

Bassani, A. G. Mt. 24 (1886) 371-.

Jamet, $V$. N. A. Mth. 9 (1890) 496-. 
of algebraic curves. Henneberg, L. Zür. Vjschr. 21 (1876) 71-.

analogues to. Halphen, G. H. Liouv. J. Mth. 2 (1876) 87-.

centres of curvature. Mannheim, A. L. Mth. S. P. 20 (1889) 241-.

- - Ocagne, M. d'. Par. S. Mth. Bll. 20 (1892) 49-.

and centres of curvature of conics. Machovec, F. Časopis 16 (1887) 235-; Fschr. Mth. (1887) 722-.

of conics. Terquem, $O$. N. A. Mth. 6 (1847) 205-.

- - centres of curvature. Machovec, $F$. Č́asopis 20 (1891) 97-; Fschr. Mth. (1891) 672.

- cubic curves. Jeffery, H. M. [1870] QJ. Mth. 11 (1871) 78-, 145-。

- curves of 4 th degree, equations. Tucci, F. $P$. Nap. Rd. 5 (1846) 34-.

- - $n$th degree, determination of degree and class. Milinowski, - Z Z Mth. Ps. 19 (1874) 182-.

- , identity. Pirondini, G. N. A. Mth. 5 (1886) 460-.

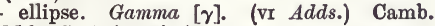
Mth. J. 1 (1859) 47-.

- - Lignières, - \& Trenquelléon, C. de. N. A. Mth. 2 (1863) 85-

- - Laguerre, E. C. R. 84 (1877) 224-.

- - equation. Taillier, L. N. A. Mth. 2 (1863) 143-.

- -, theorems. Pitt, G. N. A. Mth. 11 (1852) 412-.

elliptic. Amiot, B. C. R. 21 (1845) 348-.

imperfect, conjugate. Aoust, $L$. C. R. 30 (1850) 523-.

-, elongated or contracted. Levi, D. Tor. At. Ac. Sc. 4 (1868-69) 576-

and involutes. Maggi, P. G. A. Sc. Lomb. Ven. 8 (1838) 161-, 249-; 9 (1839) 187-.

- -. Puiseux, V. Liouv. J. Mth. 9 (1844) $377-$.

- - Brioschi, F. Tortolini A. 4 (1853) 50-. - (nth). Holditch, H. QJ. Mth. 3 (1860) 236-.

- - Krantz, H. J. N. Arch. Wisk. 10 (1884) 97-.

- -, theory. Beltrami, E. (vir) A. Mt. 4 (1861) 257-.

from involutes. Hoppe, R. Grunert Arch. 25 (1855) 125-.

and osculating curves and trajectories, reduced to a single problem. Toblini, $G$. Poligrafo 3 (1830) 14-, 265-.

of parabolas. Recoq, A. N. A. Mth. 4 (1865) 360 -

- -, equation. Turnbull, J. C. QJ. Mth. 5 (1862) 152.

and parallel curves. Cayley, A. QJ. Mth. 11 (1871) 183-.

- radii of curvature. Dubois-Aymé, -. [1807] Turin Mm. Ac. (1809-10) (pte. 2)

Azzarelli, $M$. Rm. N. Linc. At. 33 (1880) 323-. of reciprocal curves, relation between radii of curvature. Godefroy, $R$. Par. S. Mth. Bll. 19 (1891) 109-.

referred to oblique axes. Bary, -. Gergonne A. Mth. 21 (1830-31) 249-.

successive. Sharp, W. J. C. Mess. Mth. 9 (1880) 95-.

-. Pirondini, G. Nap. Rd. 30 (1891) 139-.

-, centre of mean distance of centres of curvature. Haton de la Goupillière, -. C. R. 115 (1892) 856-.

, - - - Fouret, G. C. R. 115 (1892) 1055-.

-, property. Anon. (vi 898) N. A. Mth. 2 (1863) 347-.

symmetric bicircular quartic. Hart, $H$. QJ. Mth. 18 (1882) 382-.

theory. Catalan, E. C. C. R. 45 (1857) 135-.

-. Cayley, A. Ph. Mg. 29 (1865) 344-.

of $a^{2} x^{2}+b^{2} y^{2}=\left(x^{2}+y^{2}\right)^{2}$. Perrodil, $-d e$. N. A. Mth. 6 (1847) 275-

Formula $\int_{0}^{2 \pi} p d \omega$ of Legendre, generalisation. Cavallin, C. B. S. (xII) Ts. Mth. 6 (1882) 1 -.

Hyperbolic, parabolic and elliptic curvature. Bergsten, N.J. Stockh. Ak. Hndl. 30 (1809) 11-.

Implexes of surfaces, defined by two characteristics, properties. Fouret, G. C. R. 79 (1874) 689-

"Infinitesimal analysis of plane curves," by Aoust, and a priority claim. Habich, $\dot{E}$. Les Mondes 50 (1879) 150-.

- geometry of curves. Sobotka, J. Prag Sb. (1898) (Mth.-Nt.) No. 26, 38 pp.

- _ - - auxiliary curves. Ocagne, M. d'. Lisb. J. Sc. Mth. 12 (1888) 193-; 2 (1892) $227-$.

- - - integral curves. Sobotka, J. Wien Ak. Sb. 107 (1898) (Ab. 2a) 569-.

Inflexions. Bordoni, A. Mil. G. I. Lomb. 3 (1842) 314-

Intrinsic equations. Wölffing, $E$. Bb. Mth. 1 (1900) 142-.

- , discontinuity. Walton, W. QJ. Mth. 5 (1862) 260-

_ - and properties of Cassini's ovals and conics. Cesáro, E. Mathesis 11 (1891) 51-.

- some curves. Cesáro, E. Mathesis 9 (1889) 209-.

- - use. Cesáro, $E$. N. A. Mth. 5 (1886) $127-, 352$.

Invariants of $n$ points. Lovett, $E . O$. [1897] N. Y. Am. Mth. S. Bll. 4 (1898) 58-.

Inverse method of tangents. Fuss, N. [1799] St Pét. Ac. Sc. Mm. 1 (1809) 88-, 119-.

_ _ - Tortolini, B. G. Arcad. 79 (1839) 32-; 80 (1839) 124.

_ _ - , problem. Minich, S. R. A. Sc. Lomb. Ven. 7 (1837) 57-, 171-. 
Inverse method of tangents, problems. Fuss, P. H. [1822] St Pét. Ac. Sc. Mm. 10 (1826) 130 .

- - - - - Collignon, É. $\quad$ N. A. Mth. 18 (1899) 488-; 19 (1900) 11-.

Involute of given curves. Rouquet, $V$. Toul. Fac. Sc. A. 1 (1899) 79-.

Involutes. Dienger, $J$. Grunert Arch. 20 (1853) 69-.

-, oblique, any order, integrals. Aoust, C. R. 85 (1877) 609 .

- of point. Cesáro, E. Mathesis 8 (1888) 36-.

-, problem. Pirondini, G. A. Mt. 17 (188990) 65 -.

-, successive. Anon. (vi 530) Gergonne A. Mth. 9 (1818-19) 73-.

—, theory. David, C. A. [1862] (xII) Lille S. Mm. 9 (1863) 499-.

Loci, general equations and applications. Callegari, $P$. Rm. At. 7 (1853-54) 179-, 261-.

Macrograph. Morgan, W. B. Franklin I. J. 48 (1864) 16-.

Maximum and minimum properties of curves. Sonine, N.J. Fschr. Mth. (1887) 359-.

Method of investigating curves, and application to evolutes and caustics. Watson, W. Am. As. P. (1859) 151-.

Multiple points. Droop, H. R. QJ. Mth. 2 (1858) 149-.

Multipolar coordinates, relations. Ghysens, $\boldsymbol{E}$. Brux. Ac. Bll. 45 (1878) 231-.

Networks with equal invariants, and their asymptotic lines. Koenigs, $G$. C. R. 114 (1892) 55-, 728-.

Normal and centre of curvature of "radial " of curve. Sucharda, A. Prag České Ak. Fr. Jos. Rz. (Tr̈ida 2) 6 (1897) Art. 25, 3 pp.; Prag Fr. Jos. Ac. Sc. Bll. (Mth.-Nt.) 4 (1897) (Pt. 2) 100.

- of curvature. Whitlock, G. C. Silliman J. 16 (1853) 231.

Osculating conics. Amiot, B. Par. S. Phlm. PV. (1846) 144-.

- - Lundberg, E. Ts. Mt. Fys. 4 (1871) 241-.

- - Enneper, A. Z. Mth. Ps. 19 (1874) 138-.

- -. Dewulf, $E$. Mathesis 10 (1890) 55-.

- Pirondini, G. G. Teix. J. Sc. 11 (1892) 9-.

- - of cubics. Durège, H. Prag Sb. (1870) (pt. 2) 47-.

- - - Servais, C. Brux. Ac. Bll. 23 (1892) 522-.

- - - etc., theorem of Steiner. August, F. [1867] Crelle J. 68 (1868) 235-.

- - and problem of Carnot's. Minich, S. $R$. Ven. Mm. I. 6 (1856) 111-.

- parabola. Hoppe, $R$. Arch. Mth. Ps. 12 (1894) 168-.

- - , construction at any point of curve. Koenigs, G. N. A. Mth. 20 (1881) 11-.

- - , - - - - given curve. Ocagne, M. 'd'. N. A. Mth. 11 (1892) 326-.

- parabolas and differential functions unaffected by change of axes. Ampère, A. $M$. [1803] Par. Éc. Pol. J. 7 (1808) 159-.
Osculation. Cesáro, E. N. A. Mth. 9 (1890) 143-.

-, formulæ. Busse, F. G. von. Gilbert A. 71 (1822) 91-.

-, 5-pointic. Cayley, A. Phil. Trans. (1859) 371-.

Osculations of higher orders. Dupin, C. C. R. 32 (1851) 569-.

Pedal coordinates. Purkiss, H. J. [1865] Mess. Mth. 3 (1866) 83-.

\section{Pedals.}

Drobisch, M. W. Leip. B. 9 (1857) 49-.

Bacaloglo, E. Grunert Arch. 35 (1860) 41 -

Cayley, A. (vir) Ph. Mg. 26 (1863) 20 .

Albrich, (Prof.) C. Arch. Mth. Ps. 52 (1871) 56-.

Juel, C. S. (xII) Ts. Mth. 3 (1873) 177-; 4 (1874) 9-.

Reuschle, C. (jun.) Z. Mth. Ps. 21 (1876) 139-.

Hicks, W. M. Mess. Mth. 6 (1877) 94-.

Johnson, W. W. Des Moines Anal. 4 (1877) 143-.

Laisant, C. A. As. Fr. C. R. (1882) $104-$.

Weinmeister, J. P. Z. Mth. Ps. 28 (1883) 256.

Ruffini, F. P. Bologna Ac. Sc. Mm. 2 (1891) 123-.

Petrini, H. Stockh. Öfv. (1893) 613-.

and anti-pedals. Azzarelli, $M$. Rm. N. Linc. At. 27 (1874) 127-.

of circle. Richter, $O$. Z. Mth. Ps. 34 (1889) 338 .

- conics. Wolf, R. Crelle J. 20 (1840) 88-.

- - Schilte, -. Grunert Arch. 20 (1853) $175-$.

- - Roberts, S. [1870] L. Mth. S. P. 3 (1869-71) 88-.

construction of circle of curvature. Weyr, $E$. Wien Sb. 59 (1869) (Ab. 2) 169-.

and director curves. Raabe, J. L. Crelle J. 50 (1855) 189-.

- _ - relations to original curve and solutions by singular integrals. Raabe, J.L. Crelle J. 48 (1854) 105-.

of ellipse. Jerabek, $V$. Mathesis 16 (1896) 15-.

- - determination of real foci and of vector equation. Wolstenholme, (Rev.) J. L. Mth. S. P. 13 (1881-82) 70-.

- - and hyperbola, curvature and evolute. Tortolini, $B$. Palomba Rac. 1 (1845) 278-. equations and properties. Tortolini, $B$. $\mathrm{Pa}$ lomba Rac. 3 (1847) 305-.

first, curvature, and polar reciprocal of curve. Servais, C. Mathesis 11 (1891) 84.

of hyperbola, construction of arcs with rectifiable difference. Wieger,$C$. Schlömilch Z. 3 (1858) 308-.

inverse. Dieu, T. N. A. Mth. 13 (1854) 259-.

law of formation, generalisation. Janisch, $E$. Arch. Mth. Ps. 8 (1890) 171-; 9 (1890) vii.

of logarithmic spiral. Amato, $V$. G. Mt. 38 (1900) 149-.

negative. Tortolini, B. N. A. Mth. 5 (1846) 365-. 
negative, first, of central conic, properties, demonstration. Malet, J. C. [1878] Ir. Ac. T. 26 (1879) 431-.

-, theory. Ameseder, A. Arch. Mth. Ps. 64 (1879) 164-.

oblique. Pellet, E. Bll. Sc. Mth. As. 3 (1872) 278 -

any order. Wetzig, F. Schlömilch Z. 4 (1859) $319-; 5$ (1860) 1-, 81-.

and orthogonal trajectories. Hochheim, A. Arch. Mth. Ps. 47 (1867) 121-.

- pedal surfaces. Roberts, $W$. C. R. 49 (1859) 742-.

- Bacaloglo, E. Grunert Arch. 35 (1860) 41-.

- _ - (Bacaloglo). Magener, A. Grunert Arch. 36 (1861) 375-.

property. Besant, W. H. [1870] QJ. Mth. 11 (1871) 38-.

-. MacMahon, P. A. Mess. Mth. 10 (1881) 190 -

and reciprocal curves, curvature. Steede, B. H. [1898] Ir. Ac. P. 5 (1898-1900) 124-.

successive. Barisien, E. N. A. Mth. 14 (1895) 89-, 157-, 207-, 233-, 463-.

-, radii of curvature. Niewenglowski, $B$. C. R. 84 (1877) 765-.

-, theory. Roberts, W. N. A. Mth. 3 (1864) 80 -.

of system of conics. Amstein, $H$. Laus. S. Vd. Bll. 22 (1887) 87-.

tangent constructions for. Janisch, E. Arch. Mth. Ps. 9 (1890) 196-, viii.

theorem. [Salvatore-]Dino, N. G. Mt. 1 (1863) 217-.

theory. Tortolini, B. [1870] Rm. At. N. Linc. 24 (1871) 63-.

Plane sections of cones, formula relating to. Guimarães, R. As. Fr. C. R. (1893) (Pt. 2) 93-.

- - surfaces, curvature. Exner, K. Z. Mth. Ps. 17 (1872) 416-.

Points of inflexion. Hesse, L. O. Crelle J. 41 (1851) 272-.

- - of cubics. Hesse, L. O. Crelle J. 28 (1844) 97-; 38 (1849) 257-

- - - curve corresponding to plane or skew curve, traced on developable surface. Olivier, T. Par. Ec. Pol. J. cah. 22 (1833) 78-.

- - - and cusps. Jeffery, H. M. [1869] QJ. Mth. 10 (1870) 232-.

- - - in development of plane section of cone. Balitrand, $F$. N. A. Mth. 15 (1896) 65 -

_ - infinitesimal space round. Long. champs, G. de. As. Fr. C. R. (1893) (Pt. 2) 277-.

- - - symmetrical investigation. Walton, W. Camb. Mth. J. 4 (1845) 13-.

- - visible, and curvature at multiple points. Walton, W. QJ. Mth. 6 (1863) 204-.

- - singular curvature. Walker, E. QJ. Mth. 2 (1858) 107-.
Polar coordinates, formulæ, $\left[\tan (u+n \alpha)=\frac{d\left(\rho^{n+1} \sin (n+1) \alpha\right)}{d\left(\rho^{n+1} \cos (n+1) \alpha\right)}\right]$ etc. Lucas, $F$. Les Mondes 18 (1868) 611-.

Polars of point of eurve of $n$th degree, curvature. Servais, C. Brux. Ac. Bll. 21 (1891) 362-.

Radii of curvature.

Moore, W. Nicholson J. 21 (1808) 256-.

Liouville, J. Liouv. J. Mth. 9 (1844) 435.

Schellbach, C. H. Crelle J. 45 (1853) 265-.

Schlïmilch, $O$. Schlömileh Z. 2 (1857) 273-.

Schlechter, - . Grunert Arch. 31 (1858) 327-.

Mannheim, A. N. A. Mth. 1 (1862) 123-.

Haag, -. (x) Par. S. Phlm. Bll. 3 (1866) 25-.

Soufflet, -. Les Mondes 17 (1868) 110-; 24 (1871) 337-.

Laisant, C. A. N. A. Mth. 13 (1874) 367-.

Wasteels, C. Mathesis 11 (1891) 268-.

application of differential calculus. Le Barbier, -. (vIII) Gergonne A. Mth. 22 (1831) 31-. of caustics at cusps. Breton (de Champ), N. A. Mth. 4 (1865) 25-.

and centres of curvature. Lamarle, E. Brux. Ac. Bll. 2 (1857) 33-, 307-, 528-; 3 (1857) 295-; 5 (1858) 5-; 6 (1859) 11-.

- circles of curvature. Schellbach, C. H. N. A. Mth. 12 (1853) 390-.

of class of curves. Godefroy, R. N. A. Mth. 5 (1886) 272-.

- - - - geometrical determination. Transon, (Prof.) A. Liouv. J. Mth. 10 (1845) 147-.

- collinear curves, relation between. Geisenheimer, L. Z. Mth. Ps. 25 (1880) 214-.

- - reciprocal, and inverse curves, relation between. Geisenheimer, L. Z. Mth. Ps. 25 (1880) 300-.

construction for symmetric triangular curves, etc. Fouret, G. C. R. 110 (1890) 778-, 876.

of curve and its polar reciprocal, relation. Chemin, J. N. A. Mth. 6 (1867) 49-.

determination. Laguerre, E. (x) Par. S. Phlm. Bll. 4 (1867) 15-.

- in parallel point-coordinates. Ocagne, $M . d^{\prime}$. Brux. Ac. Bll. 21 (1891) 220-.

expression in dipolar coordinates. Frost, $W$. Mess. Mth. 10 (1881) 18-.

- - polar coordinates. Smith, Arch. Camb. Mth. J. 2 (1841) 191-.

_ _ _ and curves, equation of which is $r^{k}=a^{k} \sin k \theta$. Unferdinger, $F$. Arch. Mth. Ps. 51 (1870) 72-

- for, in Salmon's Higher Plane Curves, p. 83, reduction by determinants. Story, $W . E$. [1880] (xII) J. H. Un. Cir. [1] (1882) 35. expressions for. Wolfers, J.P. Grunert Arch. 9 (1847) 60-.

- - in point- and tangential coordinates, relation between. Ocagne, M. d'. Par. S. Mth. Bll. 19 (1891) 26-.

of Lamé's curves. Godefroy, R. Par. S. Mth. B1l. 21 (1893) 20-. 
of normal section of given surface determined on mechanical considerations. Koláček, $F$. Casopis 24 (1895) 225-; Fschr. Mth. (1895) 698.

and normals (Chasles-Transon construction). Böklen, O. Schlömilch Z. 3 (1858) 252-.

- - and tangents of certain system of curves, construction. Stegmann, $F$. Grunert Arch. 7 (1846) 48-.

- _ , _ - - unknown curves, construction. Pressel, $W$. Grunert Arch. 4 (1844) 337-.

of plane sections of anallagmatic surfaces. Laguerre, E. (x) Par. S. Phlm. Bll. 7 (1871) 241-.

at point of inflexion, or at cusp. Breton [de Champ], P. N. A. Mth. 13 (1854) 127-.

and polar sub-normals. Ghysens, $\dot{E}$. Brux. Ac. Bll. 43 (1877) 544-.

properties, transformation by reciprocal polars. Mannheim, A. Liouv. J. Mth. 11 (1866) 193-.

of reciprocally polar curves, relation between. Ocagne, M. d'. Par. Éc. Norm. A. 4 (1887) 313-.

successive, of certain curves. Godefroy, $R$. C. R. 117 (1893) 1062-; Par. Éc. Pol. J. 2 (1897) 19-.

-, theory of curves deduced from. Timmermans, J. A. Lille Mm. S. (1827-28) 46-.

of systems of curves, relation between. Chemin, J. N. A. Mth. 7 (1868) 120-.

Radius vector and tangent, angle between, application to optics. Piani, D. [1850] Bologna Mm. Ac. Sc. 4 (1853) 309-.

Semicurvilinear coordinates, system. Petrovitch, M. Prag Sb. (1898) (Mth.-Nt.) No. 7, $21 \mathrm{pp}$.

Sextactic points. Cayley, A. Phil. Trans. 155 (1865) 545-.

- - Spottiswoode, W. Phil. Trans, 155 (1865) 653-.

- Battaglini, G. Rm. R. Ac. Linc. Rd. 4 (1888) (Sem. 2) 238-.

- - of algebraic curves. Gerbaldi, -. Palermo Cir. Mt. Rd. 4 (1890) 65-.

Singular point, motion. Jacobi, C. G. J. Crelle J. 24 (1842) 5-.

- points. Cremona, G.F. [1820] (vII) Mod. Mm. Ac. Sc. 1 (1833) (pte. 2) 79.

- -, form, etc. Matthiessen,L. Schlömilch Z. 8 (1863) 451-.

Tac-loci. Workman, W. P. [1882] Mess. Mth. 12 (1883) 21-.

Tangent and centre of curvature of certain curve, constructions. Sucharda, A. Prag České Ak. Fr. Jos. Rz. (Tř́ida 2) 8 (1899) Art. 40, 6 pp.; Fschr. Mth. (1899) 471.

- normal of curve, locus derived from. Bassani, A. G. Mt. 22 (1884) 211-.

Tangential triangle, infinitesimal ratio of segment. Voller, -. Grunert Arch. 31 (1858) 449-; 33 (1859) 433-.

,$-----\ldots$ - Weiler, A. Grunert Arch. 32 (1859) 418-.
Tangents, normals, curvature. Gaudard, $J$. A. Gén. Civ. 2 (1873) 484-.

,-- , etc., of curves in general, and of conics. Dostor, G. Arch. Mth. Ps. 51 (1870) 129-.

Theorem. If $\theta=x \cos \theta+y \sin \theta-f(\theta)$, then $\theta^{\prime}=0$ is normal to envelope of $\theta=0, \theta^{\prime \prime}=0$ is normal to evolute of envelope, etc. Tait, P. G. QJ. Mth. 3 (1860) 364-.

Theorems of Fouret and Jamet. Cesáro, $E$. Mathesis 13 (1893) 217-.

Theory of curvature. Tichomandrickij, M. A. Kharkov Mth. S. Com. (1886) 33-.

- _ - and kinematics of rigid body, connexion between: Landsberg, $G$. Crelle J. Mth. 118 (1897) 163-.

\section{Trajectories.}

of confocal conics, plane and spherical. Roberts, $W$. A. Mt. 6 (1864) 28-.

determination, so that the two radii of curvature at points of intersection are in given relation. Hansen, C. (xII) Ts. Mth. 2 (1866) 117-.

isogonal, areas, etc., of systems of curves. Ekama, H. N. Arch. Wisk. 1 (1895) 55-; Fscbr. Mth. (1893-94) 1079.

oblique, of system of confocal ellipses. Mukhopadhyay, A. [1887] Beng. As. S. J. 56 (Pt. 2) (1888) 117-。

orthogonal. Kummer, E. E. Crelle J. 35 (1847) 5-.

-. Kiepert, L. Z. Mth. Ps. 17 (1872) 420-. - in bipolar coordinates. Baur, C. W. Z. Mth. Ps. 12 (1867) 430-.

- - - -. Dariès, G. N. A. Mth. 13 (1894) 283-.

-, composed solely of conics, families. Appell, P. [1878] Arch. Mth. Ps. 63 (1879) 50-

-, example in Boole's "Differential equations." Glaisher, J. [1879] Mess. Mth. 9 (1880) 46-.

- and homofocal. Legoux, A. N. A. Mth. 20 (1881) 406-.

-, of surface. Euler, L. [1782] St Pét. Ac. Sc. Mm. 7 (1820) 33-.

of points and envelopes of movable lines in plane. Ocagne, M. d'. C. R. 109 (1889) 959-; 110 (1890) 60.

- - of moving figure, centres of curvature. Marcolongo, R. Palermo Cir. Mt. Rd. 7 (1893) 29-.

- - rigid body in motion, curvature. Schönflies, A. Liège S. Sc. Mm. 11 (1885) No. 6, 9 pp., No. 9, 15 pp.

- - in solid subject to 4 conditions, radius of curvature. Réveille, J. C.R. 104 (1887) 1827-. - on straight line. Ocagne, M. d'. As. Fr. C. R. (1889) (Pt. 2) 228-.

- - moving in space. Mannheim, $A$. C. R. 76 (1873) 551-, 635-; (x) Par. S. Mth. Bll. 1 (1873) 106-.

Transformation, orthotangential. Cesáro, $E$. N. A. Mth. 8 (1889) 116-.

$-x^{\prime}=x-\frac{f(x)}{f^{\prime}(x)}, y^{\prime}=\frac{C}{f^{\prime}(x)}$ of curves. Collignon, É. As. Fr. C. R. (1897) (Pt. 2) 7-. 
Transformations, isogonal, curvature in. Laisant, C. A. Par. S. Mth. Bll. 15 (1887) 39-.

-, laws of curvature in. Transon, A. N. A. Mth. 8 (1869) 114-.

Vector pedal equation of curve. Nanson, E.J. Mess. Mth. 28 (1899) 80-.

\section{Curvature of skew curves; other applications of the dif- ferential calculus to skew curves. (See also 7240, 8420.)}

Centre of curvature. Möller, $J$. Lund. Un. Acta 21 (1885-86) (Mth., No. 2) 18 pp.

Characteristic differential equation. Hoppe, $R$. Arch. Mth. Ps. 15 (1897) 244 .

Cone-loxodrome as osculator of curve. Tesar, J. Prag Sb. (1886) (Mth.-Nt.) 347-.

Contact and osculation. Pirondini, $G$. G. Teix. J. Sc. 10 (1891) 113-.

Contingence angle. Rodrigues, $(\mathrm{Dr})-$ - [1813] Par. Éc. Pol. Cor. 3 (1814-16) 36-.

- - Terquem, O. N. A. Mth. 4 (1845) 266 .

Coordinates in terms of arc. Mathews, G. B. QJ. Mth. 26 (1893) 27-.

Correspondence. Daug, H. T. Ups. Årsk. (1861) (Mth.) $56 \mathrm{pp}$.

\section{Curvature.}

Chelini, D. Palomba Rac. 1 (1845) 105-, 129140-, 156-; Tortolini A. 4 (1853) 337-.

Spottiswoode, $W$. Camb. and Dubl. Mth. J. 9 (1854) 234 -.

Böklen, O. N. A. Mth. 19 (1860) 136-.

Greer, H. R. QJ. Mth. 4 (1861) 183-.

Routh, E.J. QJ. Mth. 7 (1866) 37-.

Constable, E. C. Mess. Mth. 1 (1872) 50.

Hart, $H$. Mess. Mth. 4 (1875) 172-.

Stolz, O. Mh. Mth. Ps. 1 (1890) 433-.

Zur Kammer, A. Arch. Mth. Ps 15 (1897) 14-.

absolute, radius. Smith, Arch. Camb. Mth. J. 1 (1839) 92-.

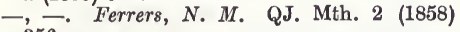
356.

of curve, and locus of centres of osculating spheres. Daug, H. T. Stockh. Öfv. 15 (1858) 1-.

- curves on spherical surface. Fuss, N. [1807] St Pét. Ac. Sc. Mm. 2 (1810) 73-.

- - surface. Beltrami, E. N. A. Mth. 4 (1865) 258.

- - - Issoly, P. N. A. Mth. 3 (1884) $522-$.

- - and surfaces. Stuyvaert, M. Brux. Mm. Cour. $8^{\circ}, 55$ (1896-98) No. 6, 19 pp.

- - - elements. Mangeot, S. Par. Éc. Norm. A. 10 (1893) 87-.

and deformation. Olivier, $T$. Par. Éc. Pol. J. cah. 24 (1835) 61-, 252-.

of intersection of 2 given surfaces. Binet, J. M. P. C. R. 19 (1844) 210-. of intersection of 2 quadrics. Tortolini, $B$. (vIII) A. Mt. 5 (1863) 305-.

kinematic analogy. Hoppe, -. D. Nf. Tbl. $\left({ }^{*} 1873\right) 118$.

- treatment. Szily, $K$. Mth. Termt. Éts. 7 (1889) 140-; Mth. Nt. B. Ung. 7 (1890) $220-$.

law, demonstration. Grunert, J. A. Arch. Mth. Ps. 42 (1864) 467-.

of orthogonal projections of curve. Catalan, E. C. Liége S. Sc. Mm. 15 (1888) No. 1, 208-.

radii, of curve and of its projection, relation. Peaucellier, -. N. A. Mth. 20 (1861) 427-. radius. Bammert, $(D r)-$ Z. Mth. Ps. 10 (1865) 163-.

- Hermite, C. N. A. Mth. 5 (1866) 297-.

-, of helicoidal curves on developable surfaces. Résal, H. A. [1856] (xII) Doubs S. Mm. 8 (1857) 100-.

- and osculating circle, determination. Fuss, N. [1810] St Pét. Ac. Sc. Mm. 6 (1818) 66-.

-, of projection of curve. Caron, J. N. A. Mth. 14 (1895) 138-.

- $=f(s)$, resulting differential equation. Goursat, É. Toul. Fac. Sc. A. 1 (1887) C, $26 \mathrm{pp}$.

at singular points. Wölffing, E. Arch. Mth. Ps. 15 (1897) 146-.

spherical. Sobotka, J. Wien Ak. Sb. 104 (1895) (Ab. 2a) 144-.

-, special kind. Grunert, J. A. Grunert Arch. 25 (1855) 301-.

systems of curves. Lilienthal, $R$. von. Mth. A. 32 (1888) 545-; 38 (1891) 429-.

and torsion. Hoppe, $R$. Arch. Mth. Ps. 15 (1897) 124-.

- - in collineation and reciprocity. Servais, C. Brux. Mm. Cour. $8^{\circ}, 58$ (1898-99) No. 2, $48 \mathrm{pp}$.

- - , determination of curves by relation between. Hoppe, $R$. Arch. Mth. Ps. 65 (1880) 287-.

$-\frac{-}{\text { of problem. Hoppe, } R \text {. Arch. Mth. Ps. } 9}$ (1890) 43-.

Tor. Ac. Sc. At. 32 (1896) 331- or 471-.

- - , radii, and spherical indicatrix. Paulli, H. (xI) Ts. Mth. 5 (1881) 86-.

- - representation of curves by. Hoppe, $R$. Crelle J. 60 (1862) 182-; 63 (1864) 12\%-.

total, and torsion of two curves, relations between. Rouquet, - Toul. Ac. Sc. Bll. 1 (1898) 39-.

\section{Curves.}

Lancret, M. A. [1802] Par. Mm. Sav. Etr. 1 (1806) 416-.

Barré de Saint-Venant, - . C. R. 19 (1844) 547-; Par. Ec. Pol. J. 18 (1845) 1-.

Trenet, F. [1847] Liouv. J. Mth. 17 (1852) 437-.

Enneper, A. Gött. Nr. (1866) 134-; Z. Mth. Ps. 12 (1867) 510-.

Bianchi, L. G. Mt. 21 (1883) 222-.

Pirondini, G. G. Mt. 26 (1888) 104-。 
algebraic, as evolutes. Stäckel, $P$. Mth. A. 45 (1894) 341-.

apparent singularities, when projected from arbitrary point. Kneser, A. Mth. A. 34 (1889) 204.

barycentric properties, use of intrinsic equation. Cesáro, E. Rv. Mt. 5 (1895) 90-.

Bertrand's. Demartres, G. C. R. 106 (1888) 1065-.

-. Bioche, C. Par. S. Mth. Bll. 17 (1889) 109-.

- Cesáro, E. Rv. Mt. 2 (1892) 153-; Mathesis 14 (1894) 265-.

-, generalisation. Demoulin, A. C. R. 116 (1893) 246-.

case with one asymptotic point, and its tangent surface. Schiffner, $F$. [1881] Arch. Mth. Ps. 67 (1882) 207-。

- given by relation between curvature and torsion angles. Hoppe, $R$. Arch. Mth. Ps. 2 (1885) 417-.

- with known properties as to $\rho$ and $\sigma$, formulæ for equations. Daug, H. T. [1878] Ups. S. Sc. N. Acta 10 (1879) (No. 12) 29 pp.

cases where radii of curvature, plane and spherical, have given relation. Molins, $H$. Toul. Ac. Sc. Mm. 5 (1883) (Sem. II) 175-. - $\rho$ and $\sigma$ are constant. Puiseux, $V$. Liouv. J. Mth. 7 (1842) 65-.

- - - - - in constant ratio. Puiseux, V. Liouv. J. Mth. 16 (1851) 208-.

- - - - - - Z Zeuthen, H. G. (xII) Ts. Mth. 5 (1875) 182-.

- - - - - - - - Molins, H. Toul. Ac. Sc. Mm. 6 (Sem. 2) (1884) 155-. - _ - - have algebraic relation. Molins, H. Toul. Ac. Sc. Mm. 4 (1892) 1-.

$-----\ldots$ given relation. Molins, $H$. Liouv. J. Mth. 19 (1874) 425-.

------ linear relation. Molins, $H$. Toul. Ac. Sc. Mm. 6 (1894) 394-.

- $\rho / \sigma$ is function of arc. Pirondini, $G$. A. Mt. 19 (1891-92) 213-.

- $\rho, \sigma$ and $s$ have any relation. Lie, M. S. Christiania F. (1882) No. 10,6 pp.

class, and simultaneous differential system. Picard, É. C. R. 90 (1880) 976-, 1065-, 1118-.

of constant curvature. Titeica [Tzitzéica], G. [Bucarest S. Sc. Bll. 6 (1897)] 31-.

- - - torsion, total curvature and ratio of curvature. Hoppe, $R$. Arch. Mth. Ps. 11 (1892) 101-.

- torsion, Koenigs, G. Toul. Fac. Sc. A. 1 (1887) E, 1-.

$-1-$ Molins, $H$. Toul. Ac. Sc. Mm. 5 (1893) 588-.

- _ -, algebraic. Fouché, M. Paris Ec. Norm. A. 7 (1890) 335-.

- - - - Fabry, E. Par. Ec. Norm. A. 9 (1892) 177-; C. R. 114 (1892) 158-; 123 (1896) 865-.

- - - spherical. Le Vavasseur, -. Toul. Ac. Sc. Bll. 1 (1898) 82-.

corresponding so that centroid of the one lies on chord of the other. Cesáro, $E$. Rv. Mt. 2 (1892) 43-.

determination by relation between $\rho$ and $\sigma$. Steen, A. (xII) Ts. Mth. 5 (1875) 129-. and developable surfaces, involutes. Lancret, M. [1806] Par. Mm. Sav. Etr. 2 (1811) 1-. differential invariants. Halphen, G. $H$. Par. Éc. Pol. J. Cah. 47 (1880) 1-.

elements, determination. St Germain, A. de. N. A. Mth. 12 (1873) 126-, 179-, 207-。

and their evolutes. Anisimov, $V$. [1883] Rec. Mth. (Moscou) 12 (1885) 42-; Fschr. Mth. (1885) 745-.

having common polar surface, integrals of equations. Aoust, -. C. R. 78 (1874) 1481-; A. Mt. 7 (1876) 1-.

- same principal normals. Fais, A. Bologna Ac. Sc. Mm. 8 (1877) 609-; 9 (1878) 657-.

- - Niewenglonoski, B. C. R. 85 (1877) 394-.

- - - - , and surface formed by these normals. Mannheim, A. C. R. 85 (1877) 212-

involutes of given. Rouquet, - Toul. Ac. Sc. Bll. 2 (1899) 34-.

isophotal, on revolution surfaces, singularities. Tesař, J. Prag Sb. (1888) (Mth.-Nt.) 355-.

locus of centres of curvature of curve when unwound on straight line. Aoust, - C. R. 88 (1879) 768-.

with locus of centres of similar osculating ellipsoids given, integrals of equations. Aoust, - C. R. 78 (1874) 1548-.

locus of helicoidal instantaneous axis. Demoulin, -. Par. S. Mth. Bll. 21 (1893) 8-.

maximum and minimum problems connected with. Euler, L. [1779] St Pét. Ac. Sc. Mm. 4 (1813) 18-.

plane or spherical, considered as envelopes of circles, arcs. Mannheim, A. Liouv.J. Mth. 7 (1862) 121-.

polar surfaces and evolutes. Christensen, S. A. (xII) Ts. Mth. 6 (1882) 17-.

principal normals of which make constant angle with each other. Hatzidakis, N.J. Bll. Sc. Mth. 24 (1900) 42-, 190.

problem regarding two. Koenigs, G. Am. J. Mth. 19 (1897) 259-.

properties. Bonnet, O. N. A. Mth. 12 (1853) 192 -

property. Beltrami, E. [1866] G. Mt. 5 (1867) 21-.

- (Beltrami). Chelini, D. G. Mt. 5 (1867) 190 -

- Jamet, V. C. R. 100 (1885) 1332-.

2 ruled surfaces connected with. Pirondini, $G$. G. Mt. 28 (1890) 92-.

with spherical involutes. Bobillier, - Gergonne A. Mth. 18 (1827-28) 57-.

and surfaces. Scheffers, $G$. Leip. Mth. Ps. B. 52 (1900) 1-.

- - theory. Pellet, A. Par. Éc. Norm. A. 14 (1897) 287-.

system, on developable of tangents. Hoppe, $R$. Arch. Mth. Ps. 12 (1894) 354-.

and torses, plane and spherical envelopes. Pirondini, G. Bologna Ac. Sc. Mm. 9 (1888) 641-.

Deviation. Transon, (Prof.) A. Par. S. Phlm. PV. (1848) 58-.

Direction, curvature and tortuosity. Staude, $O$. Dorpat Sb. 11 (1896) 1-. 
Distance from point of curve to osculating sphere at neighbouring point. Lecornu, $L$. C. R. 100 (1885) 1207-.

Envelopes. Collet, J. Liouv. J. Mth. 9 (1883) 257 -

Equation of curve such that $\rho$ subtends constant angle from given point. Lecocq, H. N. A. Mth. 19 (1860) 285-.

Evolutes. Weyr, E. Wien Sb. 62 (1870) ( $A b$. 2) 804-.

-. Adam, P. N. A. Mth. 10 (1891) 142-.

-. Biermann, O. Mh. Mth. Ps. 11 (1900) 59-. -, equations in form of integrals. Molins, $H$. Liouv. J. Mth. 8 (1843) 379-.

-, polar developable. Anissimow, W. A. Fschr. Mth. (1898) 517.

Family of skew curves, and lines of contact. Andrade, J. C. R. 122 (1896) 1110-.

Formulæ. Gilbert, P. C. R. 101 (1885) 52.

- 3. Hatzidakis, N. I. C. R. 128 (1899) 923-; Bll. Sc. Mth. 23 (1899) 118-.

-, and application to helix and curves on sphere. Serret, J. A. Liouv. J. Mth. 16 (1851) 193-.

-, Frenet's. Hermite, C. G. Teix. J. Sc. 1 (1878) 65-.

- _. Mannheim, -. Par. S. Mth. Bll. 25 (1897) 4 -.

-, Frenet-Serret, and the analytic distinction of right-and left-handed curves. Kneser, $A$. Crelle J. Mth. 113 (1894) 89-.

-, Molins's. Fais, A. G. Mt. 14 (1876) 219-.

-, Serret's. Weyr, E. Mh.Mth.Ps.5(1894) 346-.

Hyperosculating spheres of cubic. Sobotka, J. Mh. Mth. Ps. 5 (1894) 349-.

Intrinsic equation. Hoppe, -. D. Nf. Tbl. (*1874) 175-.

- geometry. Cesáro, $E$. Nap. Ac. At. 6 (1894) No. 17, 9 pp.

Involute and evolute in space. De Morgan, $A$. Camb. and Dubl. Mth. J. 6 (1851) 267-.

Line at angle $a$ with principal normal, locus. Cesáro, E. N. A. Mth. 7 (1888) 147-.

Locus of centres of curvature. Molins, $H$. Toul. A. Sc. Mm. 10 (1888) 400-; 1 (1889) 474-, 485.

Molins's problem, radius of osculating sphere $=f(\rho)$, to find curve. Hoppe, $R$. Arch. Mth. Ps. 2 (1885) 269-.

Motion of point and geometry of curve, analogy. Nicolaïdès, $N$. Les Mondes 9 (1866) 290-, 415-, 596-.

- - rectangular trihedron at each point. Demoulin, -. Par. S. Mth. Bll. 20 (1892) 43-.

Normal, principal, condition that variable straight line should be. Hoppe, R. Arch. Mth. Ps. 63 (1879) 369-.

Normals. Pellet, A. E. C. R. 104 (1887) 1501-. -, double, of rational curve, number. Weyr, E. Crelle J. 74 (1872) 279-.

-, principal. Serret, J.A. Liouv. J. Mth. 16 (1851) 499-.

- - - , of 2 curves, conditions. Serret, J.A. C. R. 85 (1877) 307-.

- - - surface of, use of its representative curve. Mannheim, A. C. R. 86 (1878) 1254-. - of rational curves. Weyr, E. [1871] Crelle J. 74 (1872) $277-$.

voL. I.
Osculating circle. Gourieff, $S$. St Pét. Mm. Ac. Sc. 2 (1807-08) 130-.

- - determination. Hunyady, E. [J.] von. N. A. Mth. 20 (1881) 53-.

- at any point on curve. Poncelet, J. V. Gergonne A. Mth. 15 (1824-25) 245-.

- cone. Voizot, - . Liouv. J. Mth. 15 (1850) 481- ; 17 (1852) 253-.

- helix. Ruchonnet, C. N. A. Mth. 10 (1871) 444-.

- - etc. Laurent, $H$. Par. Éc. Norm. A. 1 (1872) 219-

- - Cesáro, $E$. Mathesis 5 (1885) 32-.

- lines of cubic. Timerding, H. E. A. Mt. 4 (1900) 199-.

- plane and centre of curvature. Dupin, C. Gergonne A. Mth. 7 (1816-17) 18-.

- - equation. Studnička, F.J. Prag Sb. (1878) 37-.

- - at intersection of 2 confocal surfaces. Housel, -. N. A. Mth. 2 (1863) 400-.

- - - - - surfaces, anharmonic property. Imshenetsky, V. G. (x) Liége S. Sc. Mm. 5 (1873) (No. 7) $5 \mathrm{pp}$.

- - , proof that, if it goes through fixed point, curve is plane. Mylord, $H$. (xII) Ts. Mth. 1 (1871) 126-.

- - and sphere. Saltel, L. Par. S. Mth. Bll. 2 (1874) 64 .

- planes. Schoute, -. As. Fr. C. R. (1890) (Pt. 2) 191-.

- - and radii of curvature of intersection of 2 surfaces. Hachette, J. N. P. Gergonne A. Mth. 7 (1816-17) 24-

- - - - at multiple point. Painvin, $L$. C. R. 68 (1869) 796-; A. Mt. 4 (1870-71) 281-. - - , synthetic investigations. Kneser, $A$. Mth. A. 31 (1888) 507-.

- sphere, distance of curve from. Buchonnet, C. N. A. Mth. 9 (1870) 457-.

- - at point of intersection curve of 2 surfaces, construction. Mannheim, A. Par. S. Mth. Bll. 2 (1874) 140; C. R. 83 (1876) 1040 -. - - and spherical torsion. Schell, W. Grunert Arch. 19 (1852) 393-.

- spheres. Fuss, N. [1806] St Pét. Ac. Sc. Mm. 7 (1820) 61-.

- - of 2 curves with same principal normals, geometrical relation between. Mannheim, $A$. L. Mth. S. P. 16 (1884-85) 273-.

- - theory. Jamet, $V$. Toul. Fac. Sc. A. 4 (1890) F, 8 pp.

Pedals. Weyr, E. Prag Sb. (1871) 3-.

Perimorphy and Codazzi's formulø. Balitrand, - Par. S. Mth. Bll. 22 (1894) 97-.

Projection, dependence of stationary elements on those of curve. Wiener, L. C. Z. Mth. Ps. 25 (1880) 95-.

"Rectifying" straight line, propertiês. Ruchonnet, C. N. A. Mth. 12 (1873) 315-.

Ruled surfaces generated by instantaneous rotation axis of trikedron. Rouquet, $V$. Toul. Fac. Sc. A. 2 (1900) 71-.

Skew spirals. Horn, W. Crelle J. 2 (1827) 70-. Spherical and algebraic helix. Buffone, $A$. G. Mt. 34 (1896) 152-.

- indicatrices. Pirondini, G. Rv. Mt. 3 (1893) 27-. 
Sphero-conic, arc of. Burstall, H. F. W. [1886] L. Mth. S. P. 18 (1886-87) 58-.

Striction line of curve system. Brioschi, $\boldsymbol{F}$. Mil. G. I. Lomb. 9 (1856) 400-.

- lines and maximum distance lines of curve systems. Pirondini, $G$. Bologna Ac. Sc. Mm. 1 (1890) 631-.

Tangent to conical spiral. Vallès, $F$. Gergonne A. Mth. 16 (1825-26) 376-.

-, normal and binormal, straight lines connected with. Cesáro, E. N. A. Mth. 2 (1883) 129-.

- and osculating planes of curves and surfaces. Vanēěek, M. N. C. R. 96 (1883) 1562-.

Theorem of Gauss generalised. Jacobi, C. G.J. As. Nr. 20 (1843) 115-.

Theorems. Frenet, F. N.A. Mth.12(1853) 365-. - of Navier. Chì, F. Tortolini A. 3 (1860) 353-

Theory. Bertrand, J. Liouv. J. Mth. 15 (1850) 332- ; C. R. 31 (1850) 623- ; 63 (1856) 829-.

-. Hoppe, R. Arch. Mth. Ps. 60 (1877) 376-.

-. Enneper, A. Mth. A. 19 (1882) 72-.

-. Rouquet, $V$. Toul. Ac. Sc. Mm. 3 (1891) 117-; 4 (1892) 241-.

-, class of differential equations in. Serret, J.A. C. R. 35 (1852) 50-; Liouv. J. Mth. 18 (1853) 1-.

- extension of Aoust's problem. Hoppe, $\boldsymbol{R}$. Arch. Mth. Ps. 2 (1885) 129-

Torsion angle. Terquem, $O$. N. A. Mth. 4 (1845) 266-.

- Lemonnier, H. N. A. Mth. 4 (1845) 606-.

- of curve defined by its osculating plane. Demoulin, A. Par. S. Mth. Bll. 28 (1900) 180-

-, method of calculating. Steen, A. (xп) "Ts. Mth. 2 (1866) 178-.

-, radius. Mehmke, $R$. Arch. Mth. Ps. 62 (1878) 212-.

-, spherical, of curves, and geodesic torsion of lines on surface. Demartres, - Bll. Sc. Mth. 21 (1897) 182-.

Tortuosity at singular points. Staude, O. von. Am. J. Mth. 17 (1895) 359-.

Transformation of skew into plane curves. Enneper, A. Z. Mth. Ps. 12 (1867) 123-.

Transformations, infinitesimal, of rectangular trihedron. Catalan, E. C. Liége S. Sc. Mm. 6 (1877) No. 3, 79 pp.

Trihedron, given relation among direction cosines. Hoppe, R. Arch. Mth. Ps. 1 (1884) 46-.

\section{Curvature of surfaces; cur-} vilinear coordinates, and other applications of the differential calculus to surfaces. (See also $5220,8810,8840,8850$.)

\section{CURVATURE OF SURFACES.}

Hachette, J. N. P. Par. S. Phlm. Bll. (1823) 147-; Crelle J. 1 (1826) 371-.

Gergonne, J. D. Gergonne A. Mth. 21 (183031) $217-$.

Germain, (Mlle.) S. Crelle J. 7 (1831) 1-.

Poisson, S. D. Par. Ec. Pol. J. 21' cah. (1832)
Young, J. R. R. S. P. 4 (1838) $112-$.

Finck, B. Liouv. J. Mth. 9 (1844) 400.

Chelini, D. Palomba Rac. 1 (1845) 105-, 129-, 140-, 156-.

Sereni, C. Palomba Rac. 1 (1845) 353-.

Babinet, J. C. R. 25 (1847) 441-.

Molins, H. Toul. Mm. Ac. 6 (1850) 97-.

Bonnet, O. C. R. 33 (1851) 89-; 37 (1853) 529-.

Alings, A. W. Grunert Arch. 20 (1853) 423-.

Chelini, $D$. Tortolini A. 4 (1853) 337-.

La Gournerie, J. de. Liouv. J. Mth. 20 (1855) 145-.

Babinet, J. C. R. 49 (1859) 418-.

Bacaloglo, $E$. Schlömilch Z. 4 (1859) 312-.

Cayley, A. QJ. Mth. 3 (1860) 322-.

Ostrogradsky, M. A. St Pét. Ac. Sc. Bll. 1 (1860) 545-.

Beltrami, E. (vi) A. Mt. 4 (1861) 283-.

Aoust, L. C. R. 57 (1863) 217-.

Böklen, O. Arch. Mth. Ps. 41 (1864) 32-.

Minding, E. F. A. A. Mt. 6 (1864) 201-.

Nicolaïdès, N. C. R. 60 (1865) 634-.

Gilbert, $L . P$. (rx) Par. S. Phlm. Bll. 4 (1867) 226-; 5 (1868) 3-.

Aoust, (l'abbé) -. (rx) Par. S. ${ }^{\text {Phlm. Bll. }}$ 4 (1867) 240-; 5 (1868) 24-, 102-; (vII) C. R. 67 (1868) 768-.

Chelini, D. Bologna Ac. Sc. Mm. 8 (1868) 27-. Dini, U. Mod. Mm. S. It. 1 (pte. 2) (1868) 17-.

Roger, $\grave{E} . \quad$ A. Mines 14 (1868) 47-.

Mainardi, G. Rm. At. N. Linc. 23 (1869) 220-; 26 (1873) 77-.

Roger, É. C. R. 69 (1869) 1071-.

Enneper, A. Mth. A.2 (1870) 587-.

Zeuthen, H. G. (xII) Ts. Mth. 1 (1871) 125-.

Hansen, P. C. V. (xII) Ts. Mth. 2 (1872) 113-.

Azzarelli, $M$. [1875] Rm. N. Linc. At. 29 (1876) 16-

Serret, P. C. R. 84 (1877) 543-.

Böklen, O. Z. Mth. Ps. 27 (1882) 369-; 29

(1884) 129-; Crelle J. Mth. 96 (1884) 152-.

Lafon, A. Lyon Ac. Mm. (Sc.) 27 (1885) 331-.

Korkin, A. N. Rec. Mth. (Moscou) 13 (1886) 491-; Kharkov Mth. S. Com. (1887) 3-.

Le Pont, H. As. Fr. C. R. (1888) (Pt. 2) 25-。

Suslow, G. Fschr. Ps. (1890) (Ab. 1) 259.

Catalan, E. Acta Mth. 15 (1891) 191-.

Loria, G. Rv. Mt. 2 (1892) 84 -

Servais, C. Brux. Ac. Bll. 27 (1894) 896-.

Źorawski, K. Krk. Ak. (Mt.-Prz.) Rz. 13 (1898) 107-; Crc. Ac. Sc. Bll. (1896) 390-.

Bukrejew, B.J. Fschr. Mth. (1899) 534.

Angle between adjacent normals. Lamarle, $E$. C. R. 60 (1865) 851-.

Asymptotic lines. Enneper, A. Gött. Nr. (1870) 493-; (1871) 2-.

- - Goursat, É. C. R. 122 (1896) 593-; Par. S. Mth. Bll. 24 (1896) 43-.

- of algebraic surfaces, cases. Stouff, $X$.

Par. Éc. Norm. A. 10 (1893) 45-.

- - - - class. Brambilla, A. Tor. Ac. Sc. At. 20 (1885) 784-.

- on catenoid. Bacaloglo, E. Grunert Arch. 35 (1860) 40-

- - of class of surfaces. Jamet, V. As. Fr. C. R. (1886) (Pt. 2) 140-. 
Asymptotic lines, condition for representation by plane net. Koenigs, $G$. Par. S. Phlm. Bll. 4 (1892) 94.

- - of conoidal surfaces. Halphen, G. H. Par. S. Mth. Bll. 5 (1877) 134-.

- - - cubic surfaces, cases. Sucharda, $A$. Prag České Ak. Fr. Jos. Rz. (Trida 2) 5 (1896) Art. 9, 32 pp.; Mh. Mth. Ps. 8 (1897) 297-.

- - and curvature of skew surface. Catalan, E. C. Liege S. Sc. Mm. 13 (1886) 6-, 12-. - - projection. Bioche, - Par. S. Phlm. Bll. 6 (1894) (C. R., No. 10) 2.

- - ruled surface referred to. Koenigs, $G$. C. R. 106 (1888) 51-.

- - and their spherical representation. Lelieuvre, -. Bll. Sc. Mth. 12 (1888) 126-. - of surface of 4 th degree. Rouché, $E$. C. R. 84 (1877) 434-.

Axoids, general property. Mannheim, A. C.R. 120 (1895) 671.

- of 2 plane lines. Resal, H. C. R. 120 (1895) 484-.

Balloons, elongated, shape. Espitallier, Isère S. Bll. 25 (1887) 234.

Barycentre of curvature of algebraic surfaces. Neumann, C. Z. Mth. Ps. 12 (1867) 426-.

Capillary surfaces. Brunel, - Bordeaux S. Sc. Mm. 3 (1893) xlii-.

Centre of curvature of plane section, construction. Mannheim, A. C. R. 78 (1874) 959-.

Centres of curvature, principal, of confocal quadrics. Mannheim, A. R. S. P. 33 (1882) 421-.

,,----- helical surface of triangular thread, construction. Mannheim, A. As. Fr. C. R. (1878) 156-.

Conjugate lines of surface. Reina, $V$. Rm. R. Ac. Linc. Rd. 6 (1890) (Sem. 1) 156-, 203-.

Conoids, right, having $\rho_{1}=-\rho_{2}$ at every point. St. Germain, A. de. N. A. Mth. 10 (1871) 30 .

Contact transformations leaving $\rho_{1} / \rho_{2}$ unaltered. Vivanti, G. Z. Mth. Ps. 37 (1892) 1-.

Contour evolutes of axial screw surfaces. Tesaŕ, J. Wien Ak. Sb. 94 (1887) (Ab.2) 181-.

Convexity of portion of surface at every point, condition. Bassi, A. G. Mt. 34 (1896) 146-.

Curvature of apparent contour. Ocagne, $M . d^{\prime}$. Brux. S. Sc. A. 19 (1895) (Pt. 1) 99-.

- and Bonnet's functions. Stäckel, $P$. Leip. Mth. Ps. B. 48 (1896) 478-.

- at given point. Plïcker, J. Crelle J. 3 (1828) 324-.

- - - -. Lyetnikov, A. V. (xII) Rec. Mth. (Moscou) 2 (1867) (Pt. 2) 1-.

Curves on confocal quadrics. Rutledge, J. Y. Camb. and Dubl. Mth. J. 5 (1850) 110

- osculating surfaces. Tognoli, O. G. Mt. 9 (1871) 367-.

- and surfaces. Transon, (Prof.) A. Par. S. Phlm. PV. (1840) 43-; Liouv. J. Mth. 6 (1841) 191-.

_- Watson, H. W. QJ. Mth. 12 (1873) 318-.
Curves and surfaces, curvature treated by Grassmann's methods. Mehmke, $R$. Rv. Mt. 2 (1892) 65-.

- - - elements of curvature. Mangeot, $S$. Par. Éc. Norm. A. 10 (1893) 87-.

- - - infinitesimal theory. Brisse, C. Par. Ec. Norm. A. 3 (1874) 87-.

- - - new relation between curvatures. Hoppe, R. Arch. Mth. Ps. 16 (1898) 112.

Developoids, centres. Hachette, J. N.P. Par. S. Phlm. Bll. (1815) 32.

Ellipsoid of revolution and osculating sphere. Sanchez, P. C. Méx. S. "Alzate" Mm. 13 (1900) 405-.

Equations, 2 general. Hamilton, W. R. [1865] Ir. Ac. P. 9 (1867) 302-.

Euler's theorem, generalisation. Jordan, $C$. C. R. 79 (1874) 909-.

- - relations deduced from. Kummell, C. $H$. Des Moines Anal. 8 (1881) 93-.

Evolutes of surfaces. Ribaucour, A. C. R. 74 (1872) 1399-.

- - - curvature properties. Halphen, G. H. C. R. 80 (1875) 116-.

Expression $r t-s^{2}$, geometrical interpretation. Elphinstone, H. W. QJ. Mth. 2 (1858) 74-, 145-.

Extension of Joachimsthal's problem. Mainardi, G. Tortolini A. 3 (1852) 495-.

Form of surface at given point. Odin, A. A. Laus. S. Vd. Bll. 23 (1888) 59-.

Formula, $\tau_{0}= \pm \sqrt{-R R^{\prime}}$, and its correlative. Issaly, -. Par. S. Mth. Bll. 26 (1898) 114.

Formulæ. Stolp, C. N. Arch. Wisk. 14 (1888) 201-; Fschr. Mth. (1887) 754.

-, Gauss's. Baltzer, R. Leip. B. 18 (1866) 1-.

Geometrical errors in theory of functions. Jacobi, C. G. J. Crelle J. 16 (1837) 342-.

- memoirs. Dupin, C. Par. Éc. Pol. Cor. 2 (1809-13) 387-.

Hypercyclic surfaces, constant curvature. Nannei, E. Nap. Rd. 27 (1888) 119-.

Indeterminate curvature. Walton, $W$. Camb. and Dubl. Mth. J. 7 (1852) 1-.

\section{Indicatrix.}

Poincaré, H. N. A. Mth. 13 (1874) 449-.

Hoza, F. Arch. Mth. Ps. 60 (1877) 371-。

Źorawski, K. Krk. Ak. (Mt.-Prz.) Rz. 9 (1895) 250-; Crc. Ac. So. Bll. (1894) $243-$.

circular. Lamarle, E. C. R. 61 (1865) 249-.

Dupin's, generalisation. Barbier, E. C. R. 105 (1887) 516-.

of normals, determination of surface from. Hoppe, R. Arch. Mth. Ps. 59 (1876) 407-. - order of contact of 2 surfaces. Olivier, $T$. Par. Éc. Pol. J. cah. 25 (1837) 123-.

parabolic, and theory of singular points. Guiraudet, P. [1861] (xII) Lille S. Mm. 8 (1862) 457-.

and principal centres of curvature of quadric. Mannheim, A. Liouv. J. Mth. 2 (1896) 51-.

- - radii of curvature of quadric, determination. Laguerre, $E$. Liouv. J. Mth. 4 (1878) 247-. 
and principal radii of curvature of quadric, determination. Mannheim, A. Liouv. J. Mth. 8 (1882) 167-.

of quadric. Humbert, G. Par. S. Mth. Bll. 13 (1885) 142-.

Indicatrix-surface. Salvert, (le vicomte) - de. Brux. S. Sc. A. 8 (1884) (Pt. 2) 67-.

Intersection of 2 surfaces, curvature. Enneper, A. Gött. Nr. (1868) 181-.

Isogonal transformations, curvature in. Laisant, C. A. Par. S. Mth. Bll. 15 (1887) 39-.

Law. Dienger, J. Grunert Arch. 11 (1848) 328-.

Linear doubly harmonic elements (Liouville's form). Raffy, L. C. R. 109 (1889) 609-, 661-.

\section{Lines of curvature.}

Joachimsthal, F. Crelle J. 30 (1846) 347-; N. A. Mth. 9 (1850) 64-.

Brioschi, $F$. Tortolini A. 4 (1853) 129-.

Casey, J. [1865] Mess. Mth. 3 (1866) 236-.

Taylor, H. M. Mess. Mth. 5 (1876) 186-.

D'Estocquois, T. Dijon Ac. Sc. Mm. 4 (1877) 63.

Bissing, G. J. H. Un. Cir. [3] (1884) 124.

Catalan, E. C. Lì̀ge S. Sc. Mm. 13 (1886) 103-, 108-, 143-.

Pirondini, G. A. Mt. 16 (1888-89) 61-.

Catalan, E. Brux. Ac. Bll. 27 (1894) 240-.

Craig, T. C. R. 120 (1895) 672-.

Taliaferro, T. H. Am. J. Mth. 17 (1895) 273-.

Burali-Forti, C. Palermo Cir. Mt. Rd. 12 (1898) 111-.

of algebraic surface. Enneper, A. Z. Mth. Ps. 24 (1879) 180-.

- all surfaces of 4 th class, correlatives of cyclides. Darboux, G. C. R. 92 (1881) 29-.

- annular surfaces with two spherical directrices. Snyder, V. Am. J. Mth. 22 (1900) 96-.

and asymptotic lines. Le Pont, H. G. Teix. J. Sc. 8 (1887) 43 -

- - Lelieuvre, - . C. R. 106 (1888) 183cases. Enneper, A. Gött. Nr. (1870) 267-. -. Blutel, E. C. R. 128 (1899) 289-, 721-.

of central surfaces of 2 nd degree. Durrande, $H$. N. A. Mth. 2 (1863) 362-.

- class of surfaces of 4 th degree. Moutard, $T$. C. R. 59 (1864) $243-$

- _ _ _ - especially of Lamé's tetrahedra] surfaces. Darboux, G. C. R. 84 (1877) 382 -.

and confocal surfaces and sphero-conics. Casey, J. [1865] Mess. Mth. 3 (1866) 236-.

of conic, generators of which are parallel to tangents to given curve. Molins, H. Toul. Mm. Ac. 4 (1860) 49 -.

and conics, analogy. Laving, J. G. Mess. Mth. 1 (1862) 247-.

- curvature of surfaces. David, C. A. [1861] (xii) Lille S. Mm. 8 (1862) 383-. curvilinear coordinates of, expression of $(x, y, z)$. Enneper, A. Gött. Nr. (1874) 474-.

of cyclides. Humbert, G. C. R. 106 (1888) 257-.

- cylinders, character. Ahrendt, A. Arch. Mth. Ps. 9 (1890) 31-.

- developable surface with given edge of regression. Molins, $H$. Toul. Mm. Ac. 4 (1860) 493-.

- - surfaces. Falk, M. Ts. Mt. Fys. 3 (1870) 123-.

differential equations. Brasseur, J. B. Liège Mm. S. Sc. 1 (1843) 263-.

of doubly ruled surfaces. Picart, A. Par. Ec. Norm. A. 1 (1864) 289-.

- ellipsoid. Monge, G. Par. Ec. Pol. J. $2^{\circ} \mathrm{cah}$. $(1795-96)$ 145-.

- - Ellis, R. L. Camb. Mth. J. 2 (1841) 133-.

- - Cayley, A. Camb. Mth. J. 3 (1843) 264-.

- - Roberts, M. Ir. Ac. P. 3 (1847) 383-; Camb. and Dubl. Mth. J. 3 (1848) 159-.

- - Aoust, L. C. R. 48 (1859) 886-.

- - Dewulf, -. N. A. Mth. 20 (1861) 424-

- Böklen, O. Grunert Arch. 38 (1862) 158 .

294 .

- Catalan, E. C. Liege S. Sc. Mm, 12 (1885) No. 2, 226-; 13 (1886) 20-, 215-.

-. Salvert, (le vicomte) - de. N. A. Mth. 8 (1889) 214-.

- - Neuberg, J. Mathesis 13 (1893) 244 .

- - application of Abel's theorem to comparison of arcs. Roberts, $M$. A. Mt. 2 (1868-69) 13-.

- - conformal representation by elliptic functions. Neovius, E. R. Helsingf. Acta 15 (1888) 363-.

- -, foci. Böklen, O. Z. Mth. Ps. 26 (1881) 383-.

- - projection. Küpper, C. Schlömilch Z. 2 (1857) 223-.

- - , quadrilateral formed by. Plagemann, $W$. Grunert Arch. 33 (1859) 390-

- - and their relation to confocal spherical ellipses. Verdam, G. J. [1864] Amst. Vs. Ak. 1 (1866) (Ntk.) 64-.

- - of wave surface. Catalan, $E$. C. As. Fr. C. R. 7 (1878) 56-

- equilateral paraboloid. Barbarin, P. N. A. Mth. 3 (1884) 97-, 160-

especially shortest distance between two adjacent normals, one passing through an umbilic. Vieille, J. Liouv. J. Mth. 20 (1855) 121-.

generation of surfaces by. Amigues, É. N. A. Mth. 16 (1877) 337-.

- - - Hazzidakis, J. N. Crelle J. Mth. 98 (1885) 49-.

and geodesics on confocals. Chasles, M. Liouv. J. Mth. 11 (1846) 105-.

- - of developable surfaces, generators of which are parallel to those of any ruled surface. Molins, $H$. Liouv. J. Mth. 4 (1859) 347-. 
and geodesics on ellipsoid. Laing, J. G. QJ. Mth. 5 (1862) 367-.

- - - properties. Picart, A. N. A. Mth. 1 (1882) 49 -.

- - etc., on surfaces and pseudo-surfaces. Issaly, (l'abbé) -. Bordeaux S. Sc. PV. (1898-99) 38-.

of locus of points, sum of distances of which from two intersecting straight lines is constant. Catalan, E. C. Brux. Mm. Cour. $4^{\circ}, 32$ (1865) $34 \mathrm{pp}$.

modes of transformation retaining. Darboux, G. C. R. 92 (1881) $286-$.

on parallel surface to quadric. Johnston, J.P. Mess. Mth. 18 (1889) 88-

plane. Liouville, J. Liouv. J. Mth. 11 (1846) 87-.

-. Lebesgue, V. A. Liouv. J. Mth. 11 (1846) 336 -.

- or spherical. Bonnet, O. C. R. 36 (1853) 291-, 389-; Par. Ec. Pol. J. $35^{\circ}$ cah. (1853) 117-.

-- - Brioschi, F. Tortolini A. 8 (1857) $297-$.

principal, Joachimsthal's theorem. Maggi, $P$. G. Ven. At. 1 (1850) 64-

of quadric analogous to lines in plane. Aoust, L. C. R. 49 (1859) 729-.

- quadrics. Thomson, (Sir) W. Camb. Mth. J. 4 (1845) 279-.

- - Chasles, M. C. R. 22 (1846) 107-.

- - Bonnet, O. N. A. Mth. 7 (1848) 331-.

- - Aoust, L. C. R. 51 (1860) 640-; 52 (1861) 1150-.

- - Torry, A. F. Mess. Mth. 1 (1862) 69-.

- - Cremona, L. [1870] Bologna Ac. Sc. Mm. 1 (1871) 49-.

- - Ryew, M. L. G. Mt. 11 (1873) 111-.

- Laguerre, E. [1876] Par. S. Mth. Bll. 5 (1877) 24 .

- -. Mannheim, -. Par. S. Mth. Bll. 23 (1895) 1-.

- - , and confocal quadrics. Weyr, E. Wien Sb. 58 (1868) (Ab. 2) 60-.

- - osculation planes. Machovec, $F$. Prag Sb. (1889) 2 (Mth.-Nt.) 352-.

- -, theorems. Mannheim, A. R. S. P. 32 (1881) 447-.

and radii of curvature on surfaces, and transformation of double integrals connected therewith. Rodrigues, $(D r)$ - Par. Éc. Pol. Cor. 3 (1814-16) 162-.

in reciprocal surfaces, correspondences. Serret, P. C. R. 103 (1886) 1116-.

$--\frac{-}{-}$ (Serret). Lindelöf, L. L. C. R. 104 (1887) 43.

of ruled hyperboloid, integration of Euler's equation by. Floquet, $G$. N. A. Mth. 14 (1875) 120-.

- - - - - - (Floquet). Escary, N. A. Mth. 15 (1876) 61-。

- - snrfaces. Weyr, E. C. R. 78 (1874) 1649-.

- skew surfaces. Bioche, C. Par. S. Mth. Bll. 16 (1888) 119-.

- surface $z=L \cos y-L \cos x$. Saint Germain, A. de. N. A. Mth. 18 (1879) 201-。 and surfaces admitting common evolute. Pirondini, G. G. M.t. 22 (1884) 272-, 377; 23 (1885) 222-.

surfaces admitting two series of orthogonal geodesic circles for. Adam, P. Par. S. Mth. Bll. 22 (1894) 110-.

of surfaces generated by circles. Lelieuvre, C. R. 118 (1894) 967-.

theorem. Wangerin, A. D. Nf. Vh. (1900) (Th. 2, Hälfte 1) 6 .

theory. Grunert, J. A. Grunert Arch. 37 (1861) 205-.

-Ribaucour, A. C. R. 74 (1872) 1489-, 1570-.

of wave surface. Brioschi, F. Tortolini A. 2 (1859) 135-, 285-.

_ - Combescure, $\dot{E}$. Tortolini A. 2 (1859) 278-.

- - Darboux, G. C. R. 97 (1883) 1133-.

Lines of equal curvature on skew surface, construction. Olivier, T. Par. S. Phlm. N. Bll. (1833) 109-.

- and surfaces. Stuyvaert, M. Brux. Mm. Cour. 8०, 55 (1896-98) No. 6, 19 pp.

- - - elementary theory. Gergonne, J. D. Gergonne A. Mth. 9 (1818-19) 127-.

Mean curvature. Minding, E. F. A. [1874] St Pét. Ac. Sc. Bll. 20 (1875) 531-.

- - of ellipsoid, sphere. Grunert, J. A. Arch. Mth. Ps. 42 (1864) 256-; 43 (1865) 361-.

- - at point. Unferdinger, $F$. Wien Sb. 67 (1873) $(A b .2) 361-$

\section{Measure of curvature.}

Gauss, C. F. $\quad$ Gött. Cm. 6 (1823-27) 99-.

Chelini, D. Tortolini A. 2 (1851) 291-.

Grunert, J. A. Grunert Arch. 28 (1857) 285-.

Casorati, F. Tortolini A. 3 (1860) 363-; 4 (1861) 177-.

Mahler, E. [1881] Arch. Mth. Ps. 67 (1882) 96-.

Casorati, F. Mil. I. Lomb. Rd. 22 (1889) 335-, 842; Acta Mth. 14 (1890-91) 95-.

Lilienthal, R. von. Acta Mth. 16 (1892-93) 143-.

Gauss's. Liouville, J. Liouv. J. Mth. 12 (1847) 291-.

-. Bertrand, J. Liouv. J. Mth. 13 (1848) 80 -

-. Puiseux, V. Liouv. J. Mth. 13 (1848) 87-.

-. Frenet, F. Bordeaux Mm. S. Sc. 6 (1868) 385-.

-, extension for triangles on surfaces of continuous curvature. Schering, E. Gött. Nr. (1868) 389 .

- improved form of formula. Warren, $J . W$. QJ. Mth. 16 (1879) 219-.

-, theory. Stäckel, P. Crelle J. Mth. 111 (1893) 205-; Leip. Mth. Ps. B. 45 (1893) 163-.

-, - Wangerin, A. Leip. Mth. Ps. B. 45 (1893) 170-. 
general expression. Escherich, G. von. Arch. Mth. Ps. 57 (1875) 385-.

at point, Gauss's theorem. Williamson, $B$. QJ. Mth. 11 (1871) 362-.

in polar coordinates. Firth, $W$. Mess. Mth. 5 (1871) 66-.

theory. Grunert, J. A. Arch. Mth. Ps. 43 (1865) 437-.

一. Beltrami, E. Mth. A. 1 (1869) 575-.

$\frac{1}{\rho_{1} \rho_{2}}$, value. Liouville, J. C. R. 32 (1851) 533-.

Normal congruences and curvature of surfaces. Mannheim, A. C. R. 70 (1870) 1074-; Liouv. J. Mth. 17 (1872) 109-.

- planes to surface. Broca, $A$. As. Fr. C. R. (1898) (Pt. 2) 170-.

- section of surface, radius of curvature. Salvert, (le vicomte) F. de. (xII) Brux. S. Sc. A. 4 (1880) (Pt. 2) 91-.

- sections of ellipsoid, new expression for greatest and least curvatures. Grunert, J.A. Grunert Arch. 40 (1863) 259-.

- - at equal angles, $\Sigma(\rho)^{-p}$ constant. Brassinne, $E$. Toul. Mm. Ac. 4 (1848) 228-.

- - of surface, curvature. Genty, - N. A. Mth. 6 (1887) 24-.

Normals and surface of centres of algebraic surface. Roberts, S. L. Mth. S. P. 4 (1871-73) 302-.

Osculating circle at point of plane section of surface, variation. Saltel, L. [1880] Bordeaux S. Sc. Mm. 4 (1882) 383-.

- - of surface, generalisation of theory. Lipschitz, R. O. S. C. R. 82 (1876) 160-, 218-.

- conics of surface at point. Saint-Germain, A. L. de. (xII) Caen Ac. Mm. (1881) 93-.

- - surfaces, laws. Transon, A. N. A. Mth. 9 (1870) 193-.

- hyperboloids. Olivier, T. Par. S. Phlm. N. Bll. (1833) 52-.

- - to ruled surfaces. Machovec, $F$. Prag České Ak. Fr. Jos. Rz. (Tŕ̀ida 2) 2 (1893) Art. 10, 8-.

- - - skew surfaces, construction. Weyr, E. [1880] Wien Ak. Sb. 82 (1881) (Ab. 2) 7-.

- - - - - - Šolin, J. M. Prag Sb. (1883) (Mth. Vortr.) 11-.

- surfaces. Olivier, T. Liouv. J. Mth. 6 (1841) 296-.

- -. Spottiswoode, W. C. R. 79 (1874) 24-, 105 -

- -. Pepin, T. Liouv. J. Mth. 7 (1881) 71-.

- - contact. Hachette, J. N. P. Par. S. Phlm. Bll. (1822) 36.

$-\rightarrow$, degree. Jonquières, - de. C. R. 98 (1884) 1025-.

Paraboloids osculating surfaces. Picart, $A$. N. A. Mth. 1 (1882) 163-.

Plane sections of ellipsoid, curvature. Grunert, J. A. Grunert Arch. 28 (1857) 1-.
Principal curvatures, product. Brioschi, $F$. Tortolini A. 3 (1852) 273-.

"- tangent" curves on algebraic surface. Dyck, $W$. D. Mth. Vr. Jbr. 1 (1892) 60-.

Properties of contact transformations in connexion with curvature. Mehmke, $R$. Z. Mth. Ps. 38 (1893) 7-.

Pseudo-spherical surfaces. Genocchi, A. Brux. Ac. Bll. 36 (1873) 181-.

_- Bäcklund's transformation for. Bianchi, L. Rm. R. Ac. Linc. Rd. 1 (1892) (Sem. 2) 3-. Quadric, curvatures at a point. Zech, $P$. D. Nf. Vsm. B. 34 (1858) 145.

Quadrics, curvature. Servais, C. Brux. Ac. Bll. 24 (1892) 467-.

- - and polhodes. Servais, C. Brux. Ac. Bll. 25 (1893) 772-.

-, hyperosculation conics. Enneper, A. Gött. Nr. (1874) 127-.

- umbilically touching two spheres. Aoust, $L$. C. R. 54 (1862) 765-.

\section{Radii of curvature.}

Rodrigues, $(D r)$-. Par. S. Phlm. Bll. (1815) 34-.

of elliptic arcs of oblate spheroid. Adams, Jas. Thomson A. Ph. 14 (1819) 116-

- Lamé's and other, curves and surfaces. Godefroy, R. Par. Éc. Pol. J. 62 (1892) 37-.

mechanical determination from oscillation of bar placed on surface. Baehr, G. F.W. As. Fr. C. R. 6 (1877) 203-.

of orthogonal projection of curve traced on surface. Mannheim, A. C. R. 78 (1874) 1214-.

at any point of surface defined by its tangential equation. Painvin, L. C. R. 73 (1871) 902-; Liouv. J. Mth. 17 (1872) 219-.

principal. Borchardt, C. W. N. A. Mth. 14 (1855) 26-.

-. Enneper, A. Schlömilch Z. 8 (1863) 410-. -, construction. Weyr, E. [1870] Mth. A. 3 (1871) 228-.

-, ratio measured by angle of inflexional tangents. Dietrich, R. Z. Mth. Ps. 26 (1881) 57-.

properties. Hachette, J. N. P. Par. Ec. Pol. Cor. 1 (1804-08) 213-.

of quadrics. Dupin, C. Par. Ec. Pol. Cor. 1 (1804-08) 183-.

- Clausen, T. As. Nr. 25 (1847) 209-.

- surface given in homogeneous plane coordinates. Franz, J. Arch. Mth. Ps. 55 (1873) 105-.

synthetic proof of Euler's theorem. Marx, $W$. Mth. A. 17 (1880) 110-.

theorem. Haag, -. Par. S. Phlm. Bll. 3 (1866) 25-.

-, Haag's. Mannheim, A. (x) Par. S. Phlm. Bll. 3 (1866) 27-.

Reciprocal figures in space, relations between curvatures. Geisenheimer, L. Z. Mth. Ps. 30 (1885) 129.

- tangents to surface. Hachette, J. N.P. Par. S. Phlm. Bll. (1816) 162-. 
Ruled surface, line of striction for which is also line of curvature. Hoppe, $R$. Arch. Mth. Ps. 15 (1897) 251-.

- surfaces passing through given curve. Bioche, C. C. R. 110 (1890) 515-.

Section made in surface by tangent plane, curvature. La Gournerie, $J$. de. Liouv. J. Mth. 3 (1858) 73-.

Series of surfaces and lines, curvature. Hirst, T. A. Tortolini A. 2 (1859) 95-, 148-.

Skew hyperboloid, locus of points at which $\rho_{1}, \rho_{2}$ are equal. Bruno, $G$. Tor. At. Ac. Sc. 6 (1870-71) 133-.

Spherical lines on surfaces. Pirondini, $G$. G. Teix. J. Sc. 9 (1889) 65-.

- representation, Gauss's. Ruffini, $F$. $P$. Bologna Ac. Sc. Mm. 8 (1887) 661-.

Spheroids and surfaces generally, curvature. Naccari, G. Ven. Aten. 1 (1893) 237-; 2 (1893) 133-.

Sub-normals, polar, and curvature of surfaces. Ghysens, E. Brux. Ac. Bll. 44 (1877) 220-.

\section{Surface of centres.}

Codazzi, D. Tortolini A. 8 (1857) 129-.

Curtis, A. H. Liouv. J. Mth. 3 (1858) 79-

Darboux, G. C. R. 70 (1870) 1328-; 71 (1870) 267-.

Catalan, E. C. C. R. 71 (1870) 50-.

Bäcklund, A. V. Stockh. Öfv. 29 (No. 8) (1872) 3-.

Halphen, G. H. C. R. 80 (1875) 258-

Voss, A.E. Mth. A. 16 (1880) 560-.

developable. Hoppe, $R$. Arch. Mth. Ps. 63 (1879) 205-.

of ellipsoid. Darboux, G. Bll. Sc. Mth. As. 3 (1872) 122-.

- -, equation. Salmon, G. QJ. Mth. 2 (1858) 217-.

- elliptic paraboloid. Caspary, F. [1875] Crelle J. Mth. 81 (1876) 143-.

with lines of curvature plane. Bonnet, $O$. C. R. 36 (1853) 1046-.

projective. Voss, A. Münch. Ak. Ab. 16 (1888) 243-.

of quadric, bitangents to. Laguerre, $E$. C. R. 78 (1874) 556-.

- - - - Purser, F. QJ. Mth. 13 (1875) 338 -

- - expressed as contravariant of two quaternary forms. Björling, C. F. E. Stockh. Ófv. 34 (1877) No. 4, 3-.

relation between curvature elements of two sheets. Mannheim, A. C. R. 74 (1872) 458-; 79 (1874) 1328-.

\section{Surfaces.}

of buoyancy. Saint Germain, A. de. Par. S. Mth. Bll. 3 (1875) 37-.

canal. Bertrand, J. Liouv. J. Mth. 13 (1848) 73-.

of constant curvature. Minding, E. F.A. Crelle J. 19 (1839) 370-.

- - - Bukrejew, B. Fschr. Mth. (1897) 546. of constant curvature, application of calculus of variations to demonstration of sum of angles of geodesic triangles on. Sabinin, $E$. Rec. Mth. (Moscou) 21 (1901) 54-; Fschr. Mth. (1900) 476.

$--1,-\theta$ functions. Dobriner, $H$. Acta Mth. 9 (1887) 73-.

- - transformations. Bianchi, L. A. Mt. 3 (1899) 185-.

- - mean curvature. Jellett; J. H. Liouv. J. Mth. 18 (1853) 163-.

- - negative curvature. Bianchi, L. Mth. A. 16 (1880) 577-.

- positive curvature. Bianchi, L. G. Mt. 20 (1882) 287-.

containing centres of one curvature. Binet, J. P. M. Liouv. J. Mth. 6 (1841) 61-; C.R. 12 (1841) 435-.

determination of form by local measurements. Christoffel, E. B. [1864] Crelle.J. 64 (1865) 193-.

envelopes of planes cutting off constant volume. Padula, F. Nap. Mm. Ac. Sc. 2 (1855-57) 308-.

generation, cases. Curtis, A. H. (n) Mess. Mth. 2 (1864) 76-.

with given surface of centres, and curvature relation. August, $F$. Arch. Mth. Ps. 68 (1882) 315-.

inverse to Cayley's cylindroid. Janisch, $E$. Mh. Mth. Ps. 8 (1897) 278-.

-, relation between curvatures. Demoulin, $A$. Bll. Sc. Mth. 16 (1892) 268-.

loci of centres of curvature, theory. Lilienthal, R. von. Mth. A. 30 (1887) 1-.

with multiple points. Skew curve of 3rd degree, osculating sphere. Saltel, L. Brux. Ac. Bll. 35 (1873) 539-.

of negative curvature and their geodesics. $\mathrm{Ha}$ damard, -. Liouv. J. Mth. 4 (1898) 27-.

non-spherical, application of spherometer. Larmor, J. Camb. Ph. S. P. 7 (1892) 327-. parabolic points. Salmon, $G$. Camb. and Dubl. Mth. J. 2 (1847) 74-.

of positive curvature, minimum of angle between two conjugate tangents on. Hoppe, $R$. Arch. Mth. Ps. 69 (1883) 19-.

with radii of curvature equal and opposite. Padula, $F$. Nap. Rd. 1 (1852) 76-.

_- Catalan, E. C. C. R. 41 (1855) 35-, 274-, 1019-; Par. Éc. Pol. J. $37^{\circ} \mathrm{cah}$. (1858) 129-.

reciprocal, curvature. Franke, J. N. Liouv. J. Mth. 3 (1877) 415-.

of revolution, construction from fragment. Barbarin, P. As. Fr. C. R. (1887) (Pt. 2) 123-.

- - curvature of geodesics. Resal, H. N. A. Mth. 6 (1887) 57-.

ruled, theory. Bonnet, O. C. R. 46 (1858) 906-.

screw, constructions for indicatrix. Sobotka, $J$. Wien Ak. Sb. 102 (1893) (Ab. 2a) 1204-。

sections, curvature Grunert, J.A. Arch. Mth. Ps. 41 (1864) 241-.

and skew curves, theory, historic development. Christensen, S.A. (xiI) Ts. Mth. 1 (1883) 97-. 
skew, curvature. Weyr, E. Prag Sb. (1870) (pt. 2) 29-.

-, - at points of generating straight line. Buka, F. Z. Mth. Ps. 26 (1881) 15-.

-, osculation hyperboloid, construction. Weyr, E. Prag České Ak. Fr. Jos. Rz. (Tř́ida 2) 5 (1896) Art.5, 6 pp. ; Fschr. Mth. (1896) 415.

-, - -, 一. Procházka, B. Prag České Ak. Fr. Jos. Rz. (Trída 2) 6 (1897) Art. 15, 38 pp.; Prag Fr. Jos. Ac. Sc. Bll. (Mth. Nt.) 4 (1897) (Pt. 1) 14-.

- with radii of curvature in certain relation. Beltrami, E. A. Mt. 7 (1865) 139-.

theory, angle between adjacent normals. Lamarle, $E$. C. R. 60 (1865) 851-.

umbilical, 2nd order, properties derived from sphere. Booth, J. B. A. Rp. (1858) (pt. 2) 2-.

Symmetrical investigation of curvature, etc. Johnson, A. [1882] (xII) Cn. R. S. P. \& T. 1 (1883) (Sect. 3) 31-.

Tangent-plane. Bedetti, G. Bologna N. Cm. 5 (1842) 485-.

\section{Theorems.}

Dini, U. [1869] Mod. S. It. Mm. 2 (1876) 1-. Dupin's, demonstration. Gergonne, $J$. $D$. Gergonne A. Mth. 4 (1813-14) 368-.

-, -. Cayley, A. QJ. Mth. 12 (1873) 185-.

-, -. Le Pont, H. G. Teix. J. Sc. 7 (1886) $98-$.

- - Lichtenfels, O. von. Mh. Mth. Ps. 5 (1894) 380-.

-, - and application. Biffignandi, A. G. Mt. 28 (1890) 202-.

- - - elementary. Thomson, (Sir) W. Camb. Mth. J. 4 (1845) 62-.

-, generalisation. Cremona, $\boldsymbol{L}$. Tortolini A. 3 (1860) 325-.

Gauss's. Chelini, D. G. Arcad. 115 (1848) 257-; 116 (1848) 3-.

-. Bertrand, J. C. R. 42 (1856) 1088-.

-, analogue to. Sturm, R. Mth. A. 21 (1883) 379-.

-, industrial application. Lévy, M. C. R. 86 (1878) 111-.

Meunier's, analogue to. Mannheim, A. [1870] (x) Par. S. Phlm. Bll. 7 (1871) 138-.

- and Bonnet's. Brisse, C. Par. S. Mth. Bll. 4 (1876) 96-.

- , - curvature of skew curves. Besant, W. H. QJ. Mth. 6 (1863) 140-

-, demonstration. Bonnet, O. Par. S. Phlm. PV. (1848) 59-.

-, generalisation. Mannheim, A. C. R. 74 (1872) 372-.

$\frac{1}{\rho_{1}}+\frac{1}{\rho_{2}}=\frac{d l}{d x}+\frac{d m}{d y}+\frac{d n}{d z}$, applications. Webb, R. R. Mess. Mth. 9 (1880) 170-.

Theory. Bonnet, O. Par. Éc. Pol. J. $32^{\circ} c a h$. (1848) 1-; C. R. 33 (1851) 89-; 37 (1853) 529-.

-. Grunert, J.A. Grunert Arch. 28 (1857) 163-

- Lipschitz, R. O. S. Crelle J. Mth. 81 (1876) 230-.
Theory. Salvert, (le vicomte) F.de. (xII) Brux. S. Sc. A. 5 (1881) (Pt. 1) 63-, (Pt. 2) 291-.

- Lilienthal, R. von. Crelle J. Mth. 104 (1889) 341-.

Vos8, A. Mth. A. 39 (1891) 179-.

-, analytic. Amiot, $B$. Liouv. J. Mth. 12 (1847) 129-.

and Dupin's cyclides. Holzmuiller, $G$.

Z. Mth. Ps. 44 (1899) 194-.

fundamental formulæ. Laguerre, -

N. A. Mth. 11 (1872) 60-.

_, Gauss's. Cayley, A. L. Mth. S. P. 12 (1880-81) 187-.

-, - principle of least action in relation to its foundations. Beke, $M$. Mth. Termt. Ets. 2 (1884) 133-; Mth. Nt. B. Ung. 2 (*1883-84) 282-.

, general. Beltrami, E. Arch. Mth. Ps. 42 (1864) 117.

-, geometrical. Mannheim, A. C. R. 74 (1872) 598-.

- - interpretation of quantities of 2 nd order. Hoppe, R. Arch. Mth. Ps. 60 (1877) 65-.

-, use of direction cosines of normal. Gilbert, P. Brux. S. Sc. A. 18 (1894) (Pt. 2) 1-.

Total curvature, and application to double definite integrals. Tortolini, B. Rm. At. $4(1850-51) 53-$.

- of bounded portions of surfaces. Graves, C. Ir. Ac. P. 5 (1853) 339-.

- at edge of regression. Stouff, X. Par. Ec. Norm. A. 9 (1892) 91-.

Transformation to chief axes. Cayley, $A$. QJ. Mth. 12 (1873) 34-.

Umbilic, conditions for. Stone, E. J. QJ. Mth. 3 (1860) 146-.

-, - - Hall, A. A. Mth. 9 (1894-95) 42. -, lines of curvature near. Frost, $P$. QJ. Mth. 10 (1870) 78-.

,---- (Frost). Cayley, A. QJ. Mth. 10 (1870) 111-.

- - - - through. Bioche, C. Par. S. Mth. Bll. 18 (1890) 95-.

Umbilics. Bonnet, O. Par. Éc. Pol. J. $30^{\circ} \mathrm{cah}$. (1845) 165-; Liouv. J. Mth. 16 (1851) 191-.

—. Souillart, C. Crelle J. 65 (1866) 320-.

- Catalan, E. Mathesis 5 (1885) 73-.

- and differential equations. Cayley, $A$. (vII) Ph. Mg. 26 (1863) 373-, 441-.

-, lines of curvature at. Hoppe, R. Arch. Mth. Ps. 70 (1884) 289-.

- of surface of order $n$, problem comprising determination of number. Berzolari, $L$. Tor. Ac. Sc. At. 30 (1895) 756-.

- - tetrahedral surfaces. Lebon, E. Palermo Cir. Mt. Rd. 4 (1890) 115-.

Variation in contact transformation of surface. Mehmke, R. Rv. Mt. 2 (1892) 159-.

$\mu^{2} \sin ^{2} i^{\prime}+\nu^{2} \sin ^{2} i^{\prime \prime}=a^{2}$, demonstration. Chasles, $M$. C. R. 22 (1846) $517-$.

\section{CURVILINEAR COORDINATES.}

Lamé, G. C. R. 6 (1838) 43-; Liouv. J. Mth. 5 (1840) 313-; 16 (1851) 171-; (vim) C. R. 49 (1859) 341-.

Aoust, L. C. R. 48 (1859) 842-; Crelle J. 58 (1861) 352-. 
Böklen, O. Grunert Arch. 34 (1860) 26-.

Aoust, L. C. R. 54 (1862) 461-.

Combescure, É. C. R. 74 (1872) 1517-.

Frattini, G. G. Mt. 10 (1872) 235.

Roger, É. A. Mines 5 (1874) 110-; 7 (1875) 92-.

Darboux, G. [1877] Par. Ec. Norm. A. 7 (1878) 101-, 227-, 275-.

Mathieu, É. L. Liouv. J. Mth. 8 (1882) 5-.

Cayley, A. QJ. Mth. 19 (1883) 1-.

Catalan, E. C. Liège S. Sc. Mm. 12 (1885) No. 2, 234-, 403.

Williamson, B. [1890] Ir. Ac. T. 29 (1887-92) 515-.

Coordinates of point in relative motion, expansion, and curvature of orthogonal lines. Balitrand, -. Par. S. Mth. Bll. 23 (1895) 26-.

- reducing square of arc to form $d s^{2}=(U+V)\left(d u^{2}+d v^{2}\right)$. Ricci, G. Rm. R. Ac. Linc. Rd. 2 (1893) (Sem. 1) 73-.

Curvature and geodesic torsion. Reina, $V$. Rm. R. Ac. Linc. Rd. 6 (1890) (Sem. 2) 103-, 176.

- of system of plane curves in curvilinear coordinates, extension to 3 dimensions. Harst, A. D. van der. N. Arch. Wisk. 4 (1899) 226-.

Curves referred to any system of coordinates, analysis. Aoust, $\left(l^{\prime} a b b e ́\right)$-. Par. Éc. Norm. A. 6 (1869) 205-.

Curvilinear coordinates and curves on surfaces. Aoust, (l'abbé) -. C. R. 65 (1867) 814-.

_ - - geodesic lines of isothermic surfaces. Salvert, (le vicomte) - de. Brux. S. Sc. A. 10 (1886) (Pt. 2) 293-; 11 (1887) (Pt. 2) 1-.

- - in space and on surfaces. Codazzi,D. A. Mt. 1 (1867-68) 293-; 2 (1868-69) 101-, 269- ; 4 (1870-71) 10-; 5 (1871-73) 206-.

- - - - - - Chelini, D. Bologna Ac. Sc. Mm. 8 (1868) 483-.

- and normal coordinates. Warren, J. W. [1876-77] Camb. Ph. S. T. 12 (1879) 455-, 531-.

Development of surfaces, linear element of which is expressible by homogeneous function. Lévy, M. C. R. 87 (1878) 788-.

Differential parameters. Somigliana, $C$. Mil. I. Lomb. Rd. 22 (1889) 275-.

- Knoblauch, J. Crelle J. Mth. 111 (1893) 329-.

-. Pennacchietti, G. Catania Ac. Gioen. At. 9 (1896) Mem. 1, 11 pp.

- -, application to theory of surfaces. Bendixson, I. Stockh. Öfv. (1897) 331-.

- - and curvature of surface expressed in curvilinear coordinates. Somoff, $J$. [1864] St Pét. Ac. Sc. Mm. 8 (1865) No. 16, 45 pp.; St Pet. Ac. Sc. Mm. (Rs.) 8 (*1866) (Suppl.) No. 4, $66 \mathrm{pp}$.

- -, theory. Beltrami, E. Bologna Ac. Sc. Mm. 8 (1868) 549-; Bologna Rd. (1869) 55-.

Elliptic coordinates. Böklen, O. Grunert Arch. 34 (1860) 308-.

- -. Somov, O. I. St Pet. Ac. Sc. Mm. (Rs.) 9 (*1866) 295-.
Elliptic coordinates. Darboux, G. Bll. Sc. Mth. As. 3 (1872) 122-.

- - application to determination of oblique trajectories. Mylord, H. (xI) Ts. Mth. 1 (1871) 33-, 65-.

- - , development. Jacobi, C. G. J. As. Nr. 28 (1849) 257-.

- - , evaluation of surface of ellipsoid in. Obenrauch, $F$. J. Arch. Mth. Ps. 12 (1894) 155-.

- in plane. Grunert, J. A. Grunert Arch. 39 (1862) 377-.

- - - space. Grunert, J. A. Grunert Arch. 39 (1862) 402-.

- - of tangents common to two quadrics (Liouville's formula). Roberts, $W$. Ir. Ac. P. 4 (1850) 288-.

- - transformation of differentials by. Gundelfinger, S. [1877] Crelle J. Mth. 85 (1878) 80-.

,---1 rectangular rectilinear coordinates into. Somof, $J$. St Pét. Ac. Sc. Bll. 10 (1866) 41-.

Equations of motions of heat referred to curvilinear coordinates. Thomson, (Sir) W. Camb. Mth. J. 4 (1845) 33-.

Formulæ, analytical. Enneper, A. Schlömilch Z. 7 (1862) 75-, 313-, 365-; 8 (1863) 241-.

- on surface and volume integrals, in curvilinear coordinates, Gilbert, $P$. Brux. S. Sc. A. 14 (1890) (Pt. 2) 1-.

Geodesic coordinates, transformation. Láska, $V$. Prag Sb. (1894) (Mth.-Nt.) No. 12, 6 pp.

- polar coordinate system, properties, demonstration. Bonnet, G.O. C. R. 97 (1883) 1422-.

Isometric coordinates on sphere. Enneper, $A$. Z. Mth. Ps. 24 (1879) 256.

Lemniscate coordinates. Lommel, E. Z. Mth. Ps. 12 (1867) 45-.

- - nth order. Holzmüller, F. G. Crelle J. Mth. 83 (1877) 38-.

- geometry, etc., deduced by aid of function of complex argument. Holzmiiller, $F$. $G$. Z. Mth. Ps. 21 (1876) 325-.

Lines of zero length on surface as curvilinear coordinates. Burnside, $W$. Mess. Mth. 19 (1890) 99-.

Orthogonal coordinates. Darboux, G. C. R. 60 (1865) 560-.

- -. Enneper, A. Gött. Nr. (1867) 232-, 277-; (1868) 258-, 421-; (1874) 125-。

- - , application to curvature of curves on surfaces. Aleksyeev, N. N. (xII) Rec. Mth. (Moscou) 2 (1867) (Pt. 1) 79-.

- - equation $\nabla^{2} \phi=0$ in. Petrini, H. Ups. Årsk. (1890) (Mt. Nt.) Iv +104 pp.

_ - especially for conicoids. Lévy, M. [1867] Par. Éc. Pol. J. Cah. 43 (1870) 157-.

- - , system belonging to differential equation $\nabla^{2} \Phi+k^{2} f(x y z) \Phi=0$. Petrini, H. Stockh. Ak. Hndl. Bh. 19 (Afd. 1) (1894) No. 4, 36 pp. - - - - new, for problems in heat and electricity. Liouville, J. Liouv. J. Mth. 12 (1847) 265-.

- geodesic coordinates on ellipsoid. Láska, $V$. Prag České Ak. Fr. Jos. Rz. (Třida 2) 3 (1894) Art. 17, 8 pp. 
Orthogonal hyperboloids, families. Vaněček, J. S. Prag České Ak. Fr. Jos. Rz. (Trída 2) 4 (1895) Art. 28, 4 pp.

- projection on surface of revolution. Pirondini, G. N. A. Mth. 17 (1898) 246-.

- surfaces. Frenet, $F$. Lyon Ac. Sc. Mm. 3 (1853) 5-.

- trajectories of polhodes. Catalan, E. C. Liége S. Sc. Mm. 15 (1888) No. 1, 57-.

Parabolic coordinates, and application to paraboloids. Valson, C. A. N. A. Mth. 19 (1860) 298-.

Quadric surfaces, rotational, contour lines. Langer, $K$. Wien Az. 36 (1899) 3.

Radii of curvature of sections. Brioschi, $F$. Tortolini A. 4 (1853) 232-.

Surfaces referred to a conjugate azimuthal network. Raffy, L. Par. S. Mth. Bll. 24 (1896) 51-.

- with 2 sets of conjugate geodesics. Razzaboni, A. Bologna Rd. (1888-89) 66.

- and certain unicursal curves on them. Lelieuvre, -. C. R. 111 (1890) 568-.

System, particular, of curvilinear coordinates. Habich, E. N. A. Mth. 3 (1884) 353-.

Theorem. Issaly, (l'abbé) - Bordeaux S. Sc. PV. (1898-99) 38-.

- of Stokes. Webster, A. G. N. Y. Am. Mth. S. Bll. 4 (1898) 438-.

Theory. Codazzi, D. Tortolini A. 8 (1857) 129-.

- Aoust, (l'abbé) -. A. Mt. 6 (1864) 65-; 2 (1868-69) 39-, 268; 3 (1869-70) 55-; 5 (1871-73) 261-。

-. Brioschi, F. A. Mt. 1 (1867-68) 1-.

- Padova, E. Rm. R. Ac. Linc. Rd. 4 (1888) (Sem. 2) 369-, 454-.

-, application to integral calculus. Frattini, G. G. Mt. 15 (1877) 1-.

- of surfaces, and partial differential equations. Enneper, A. Schlömilch Z. 7 (1862) 1-.

Trajectories of generators on skew surface. Molins, H. Toul. Ac. Sc. Mm. 7 (1895) 421-.

Transformation of class of differential equations into curvilinear coordinates. Gundelfinger, S. Crelle J. Mth. 85 (1878) 295-.

Triply conjugate systems. Tzitzéica, -. Bll. Sc. Mth. 23 (1899) 330-.

Use in evaluation of surfaces, masses, etc. Cauchy, A. L. C. R. 16 (1843) 413-.

\section{OTHER APPLICATIONS OF THE} DIFFERENTIAL CALCULUS TO SURFACES.

Analysis applied to geometry. Beltrami, $\boldsymbol{E}$. G. Mt. 2 (1864) 267-, 297-, 331-, 355- ; 3 (1865) 15-, 33-, 82-, 228-, 311-.

Angle at which surfaces cut. Morin, $P$. C. R. 66 (1868) 741-.

Asymptotic lines of surface $X Y Z=T^{3}$. Appell, P. Arch. Mth. Ps. 61 (1877) 144-.

Conchoidal surfaces and conchoids. Minich, S. R. Padova Rv. Period. 10 (1862) 135-.
Conformal representation in configurations of three dimensions. Cotton, $\dot{E}$. C. R. 125 (1897) 225-

Contact of conics and surfaces. Darboux, $G$. C. R. 91 (1880) 969-.

- Moutard, T. C. R. 91 (1880) 1055-.

- - curves and surfaces. Collet, J. Lionv. J. Mth. 4 (1878) 315-.

- - - _. Darboux, G. Bll. Sc. Mth. As. 4 (1880) 348-.

-, multiple, of surfaces. Spottiswoode, $W$. [1875] Phil. Trans. 166 (1877) 227-.

, 3rd order, of two surfaces. Mannheim, A. C. R. 74 (1872) 856-, 928-.

- of quadrics with other surfaces. Spottiswoode, W. (xI) L. Mth. S. P. 5 (1873-74) 70 .

- surface of revolution and developable, construction of tangents to curve. Drasch, $H$. Wien Ak. Sb. 77 (1878) (Ab. 2) 174-.

Contacts of line and surface, Schubert's method. Cayley, A. QJ. Mth. 17 (1881) 244-.

Curve arising from illumination of revolution quadric. Enneper, A. Gött. Nr. (1868) 174-.

- of 4 th degree, bicircular. Cayley, A. Phil. Trans. 167 (1877) 441 .

Curves drawn from point on surface having contact of $n$th order, theorem. Mannheim, A. (x) Par. S. Phlm. Bll. 7 (1871) 228-.

- on surfaces, flexion. Demartres, - Par. S. Mth. Bll. 15 (1887) 129-.

Cyclide, envelope of planes perpendicular to radii vectores. Roberts, $W$. C. R. 53 (1861) 1118-.

Cylinder orthogonal to certain surfaces. Pirondini, G. G. Teix. J. Sc. 13 (1897) 77-.

Cylinders circumscribing surfaces of revolution, lines of contact and tracing. Nicoli, $F$. (x) Mod. Ac. Sc. Mm. 13 (Pt. 1) (1872) 39-.

Developable circumscribed to quadric or cubic. Roberts, M. N. A. Mth. 14 (1855) 268-.

-, sextic, edge of regression. Sucharda, $A$. Prag Sb. (1891) (Mth.-Nt.) 369-.

Developoids (envelopes of straight lines meeting any curve under a constant angle). Haton de la Goupillière, J. N. Par. S. Mth. Bll. 5 (1877) 126-; (xI) Brux. S. Sc. A. 2 (1878) (Pt. 2) 1-.

- (Haton de la Goupillière). Aoust, -. C. R. 85 (1877) 331-.

Diagonal curves, crossing two families of curves. Breton, $P$. Isère S. Bll. 25 (1887) 28-.

Duals of geodesies and lines of curvature on ellipsoid and on its pedal surfaces. Jeffery, H. M. QJ. Mth. 12 (1873) 322-.

Envelope of quadric surface, case. Cayley, $A$. QJ. Mth. 11 (1871) 244-.

Evolutes of surfaces, cases. Cals, B. A. Mt. 21 (1893) 195-.

Generating characteristics of curved surfaces. Minich, S. R. Ven. I. Mm. 22 (1882) 169-.

Geometry of surface of ellipsoid. Clifford, W. K. [1872] L. Mth. S. P. 4 (1871-73) 215-.

Homography, case in geometry of surfaces. Somoff, $J$. [1862] St Pét. Ac. Sc. Mm. 5 (1863) No. 9, 22 pp. 
Hyperboloid osculating ruled surface along generator. Duporcq, E. N. A. Mth. 17 (1898) 106-.

- skew surface along generator. Chasles, $M$. Brux. Ac. Bll. 5 (1838) 643-.

- touching skew surface along generator. Bruno, G. [1871] Tor. At. Ac. Sc. 7 (187172) 235-.

Hyperboloids of one sheet, construction of line of striction. Petersen, J. N. Ts. Mth. 10 (B) (1899) 39-; Fschr. Mth. (1900) 542.

Infinitely near surfaces. Maggi, P. G. [1850] Ven. Mm. I. 5 (1855) 299-.

Jacobian of three surfaces, applications. Arzelà, C. G. Mt. 9 (1871) 32-.

Normal curves and surfaces. Brenner, -. Grunert Arch. 13 (1849) 225-.

Normals. Bedetti, G. [1844] Bologna N. Cm. 10 (1849) 523-.

-. Bertrand, J. Liouv. J. Mth. 9 (1844) 133-.

-. Röthig, O. Crelle J. Mth. 85 (1878) 250 -

-, adjacent. Joachimsthal, $F$. Liouv. J. Mth. 13 (1848) 415-.

- and normal planes of certain curves and surfaces, construction. Schönemann, T. Berl. B. (1855) 255-.

- - - - pedal curves and surfaces. Sturm, R. [1872] Mth. A. 6 (1873) 241-.

-, proof of Bertrand's relation. Mannheim, A. Liouv. J. Mth. 17 (1872) 403-.

-, theory. Bertrand, J. Liouv. J. Mth. 12 (1847) 343-.

-, 3rd variation. Mayer, A. C. G. Leip. Mth. Ps. B. 33 (1881) 28-.

Orthogonal trajectories of systems of surfaces. Morera, G. Mil. I. Lomb. Rd. 19 (1886) 282-

Osculation problem. Clebsch, A. Liouv. J. Mth. 8 (1863) 297-.

Parallel lines and surfaces. Bordoni, A. [1811] Mod. Mm. S. It. 16 (1813) 72-.

- $-\ldots$, equations: Bordoni, A. Mil. At. I. Lomb. 1 (1858) 209-

Parallelism of curved lines and surfaces. Crelle, A. L. Gergonne A. Mth. 12 (182122) 1-.

Plane and spherical envelopes of curves and torses. Pirondini, G. Bologna Ac. Sc. Mm. 9 (1888) 641-.

Pseudo-surfaces, extension of Laguerre's formula to. Issaly, - . Par. S. Mth. Bll. 25 (1897) 243-.

-, triple systems. Issaly, $\left(l^{\prime} a b b e ́\right)$-. N. A. Mth. 9 (1890) 204-.

Quadric cones osculating developable surfaces. Whittemore, J. K. [1900] N. Y. Am. Mth. S. Bll. 7 (1901) 203, 209.

Quadrics, equation deduced from a polar relation. Jamet, $V$. Brux. Ac. Bll. 6 (1883) 885-.

Radius of geodesic curvature of a curve $\Sigma$, of constant normal curvature, theorem. Laguerre, $E$. C. R. 80 (1875) 822-

Reciprocal tangents. Dupin, C. Par. Éc. Pol. Cor. 3 (1814-16) 138-.
Ruled helicoid, the most general. Ocagne, $M$. d'. Par. S. Mth. Bll. 23 (1895) 114-.

Solid angle subtended by quadric at point. Beltrami, E. G. Mt. 1 (1863) 68-.

Spherical conic, and developable formed by its tangents. Huber, G. Z. Mth. Ps. 45 (1900) 86-.

- coordinates, transformation. Necker, $K$. As. Nr. 130 (1892) 49-.

Sphero-conjugate tangents. Cremona, $L$. Tortolini A. 6 (1855) 382-.

Surface envelope of planes cutting rational skew curve of 4 th degree in 4 concyclic points. Sturm, R. [1869] A. Mt. 4 (187071) $73-$.

- - - perpendicular to radii of ellipsoid. Cayley, A. Tortolini A. 2 (1859) 168-.

- - - sphere. Monge, G. Par. Éc. Pol. J. 6 (1806) 41-.

- envelopes of spheres. Ribaucour, A. (xI) Par. S. Phlm. Bll. 5 (1868) 30-.

- - - Lecornu, L. Par. Éc. Pol. J. Cah. 53 (1883) 135-.

- - - Jamet, V. Mars. Fac. Sc. A. 10 (1900) Fasc. 2, 18 pp.

- - - - property. Ribaucour, A. C. R. 67 (1868) 1334-.

- - - - touching two circles in plane. Niemtschik, R. Wien Ak. Sb. 73 (1876) (Ab. 2) 7-.

- , theory, characteristics, etc. Raabe, J.L. Crelle J. 15 (1836) 125-.

- - of variable quadric surface, edge of regression. Livet, - Par. Ec. Pol. Cor. 1 (1804-08) 422-.

\section{Surfaces.}

admitting systems of equal and similar lines. Pirondini, G. A. Mt. 23 (1895) 93-.

algebraic, theory, new invariant in, and reduction of double integrals. Picard, $\dot{E}$. C. R. 126 (1898) 297-.

capable of generating a Lamé system. Darboux, G. C. R. 124 (1897) 1428 .

- - - - - - by helicoidal movement, two classes, Bianchi, L. Toul. Fac. Sc. A. 11 (1897) H, 8 pp.

_ _ _ _ _ - screw displacement. Petot, A. C. R. 118 (1894) 1409-.

_ - 2 Lamé systems by 2 different movements. Medolaghi, $P$. Rm. R. Ac. Linc. Rd. 8 (1899) (Sem. 2) 304-.

conical and cylindrical, property. Merrifield, C. $W$. Mess. Mth. 1 (1872) 81-.

and curves. Scheffers, G. Leip. Mth. Ps. B. 52 (1900) 1-.

- - derived. Tortolini, B. Tortolini A. 2 (1859) 316-.

- - theory. Pellet, A. Par. Éc. Norm. A. 14 (1897) 287-.

- - thereon, general formulæe and special cases. Enneper, A. Z. Mth. Ps. 9 (1864) 96 -.

cyclic. Enneper, A. Gött. Nr. (1866) 243-; Z. Mth. Ps. 14 (1869) 393-. 
described by points of straight line and reciprocal problem. Mannheim, A. As. Fr. C. R. 2 (1873) 98-.

developable. Poisson, S. D. [1825] Baumgartner Z. 1 (1826) 110-.

-. Beltrami, E. Ven. At. Aten. 5 (1868) 535-.

-. Voss, A. E. Mth. A. 13 (1878) 232-.

- Procházka, F. Fschr. Mth. (1899) 492-.

-, characteristic property. Mangoldt, H. von. Mth. A. 18 (1881) 604-.

-, circumscribed to 2 conics. Regis, D. Tor. Ac. Sc. At. 11 (1875-76) 971-.

- - - given surfaces. Enneper, A. Gött. Nr. (1866) 321-; Z. Mth. Ps. 13 (1868) 322-; 15 (1870) 283-.

-, - - 2 quadrics. Painvin, L. F. [186874] (xII) Lille S. Mm. 9 (1871) 97-; 11 (1873) 81-; 13 (1874) 329-.

-, - - - Saint-Germain, A. L. de. (xII) Caen Ac. Mm. (1883) 99-

-, of conical screw (envelope of tangents). Schiffner, F. Arch. Mth. Ps. 69 (1883) 444-. - , edge of regression. Combescure, $\dot{E}$. Crelle J. 62 (1863) 174-.

- - - Lebon, E. [1879] Par. S. Mth. Bll. 8 (1880) 27-.

- , - - - defined by tangential equations, determination. Painvin, L. C. R. 71 (1870) 217-; Liouv. J. Mth. 17 (1872) 177-.

-, envelope of moving plane, curvature. Mannheim, A. C. R. 70 (1870) 1259-.

-, formed by refraction of pencil of parallel rays by a given curve. Lévy, $L$. Par. $\mathrm{S}$. Mth. Bll. 11 (1883) 186-

一, formulæ. Cayley, A. B. A. Rp. 41 (1871) (Sect.) 19-

-, generated by straight lines drawn through points of conic at $45^{\circ}$ to its plane. Fiedler, $W$. Zür. Vjschr. 29 (1884) 348-

-, isogonal trajectories of generators. Pirondini, G. Crelle J. Mth. 118 (1897) 61-.

-, properties. Pirondini, G. Liouv. J. Mth. 3 (1897) 405-.

-, theorem. Catalan, E. C. C. R. 17 (1843) 738 -

-, -. Saltel, L. [1881] Bordeaux S. Sc. Mm. 4 (1882) 443-.

—, theorems. Pirondini, G. A. Mt. 19 (189192) 247-.

- - - deduced by quaternions. Graefe, $F$. [1882] Arch. Mth. Ps. 69 (1883) 1-.

divided into equal parallelograms by their isogonal trajectories. Fibbi, C. Rm. R. Ac. Linc. Rd. 4 (1895) (Sem. 1) 413-.

of equal incidence. Lecornu, L. As. Fr. C. R. (1892) (Pt. 2) 172-

- - inclination. Regis, D. Tor. At. Ac. Sc. 6 (1870-71) 500-.

generated by line, equations of which contain arbitrary parameter. Tortolini, B. G. Arcad. 57 (1832) 110 -

- - motions of curve, determination of all. Lie, M. S. Arch. Mth. Ntvd. 7 (1882) 155-. - normals. Chasles, M. N. A. Mth. 18 (1859) 192-.

- - principal normals of skew curve. Curtis, A. $H$. Liouv. J. Mth. 1 (1856) 223-. with given properties, deduction of equation. Schlömilch, O. X. Z. Mth. Ps. 21 (1876) 75-.

having higher contact with surface along continuous line, characteristic. Bordoni, $A$. (vi Adds.) Opusc. Mt. Fis. 1 (1832) 22-.

infinitesimal geometry. Bukrejew, B.J. Fschr. Mth. (1900) 600.

with infinitesimal and linear transformations. Lie, M. S. Arch. Mth. Ntvd. 7 (1882) 179-. intrinsic differential equations. Ricci, $G$. Ven. I. At. (1894-95) 445-.

isostatic. Weingarten, J. L. G. J. Crelle J. Mth. 90 (1881) 18-.

maximum and minimum inclinations to radii vectores. Exner, $K$. [1870] Wien Sb. 63 (1871) (Ab. 2) 149-.

new system of coordinates for. Bonnet, $O$. Liouv. J. Mth. 5 (1860) 153-.

normal, of developable along intersection with surface. Peschka, G. A. von. Wien Ak. Sb. 83 (1881) (Ab. 2) 790-.

- to given surface. Colnet-d'Huart, - . Lux. S. Sc. Nt. 5 (1857-62) 69-.

-, along plane sections. Peschka, G. A. von. Wien Ak. Sb. 81 (1880) (Ab. 2) 1163-.

-, of quadrics along plane sections. Peschka, G. A. von. Wien Ak. Sb. 85 (1882) (Ab. 2) 381-.

-, - surface along intersection with another surface. Peschka, G. A. von. [1881] Wien Ak. Sb. 84 (1882) (Ab. 2) 30-.

-, theory. Peschka, G. A. von. Wien Ak. Sb. 81 (1880) (Ab. 2) 1128-.

normals of which at point cut off spherical triangle of constant area. Aoust, $\left(l^{\prime} a b b e ́\right)-$. C. R. 81 (1875) 963-.

- - - touch given surface. Braunmiühl, A. von. Münch. Ak. Ab. 14 (1883) (Ab. 3) 91-.

of oblique revolution. Walton, W. QJ. Mth. 6 (1863) 134-.

parabolic lines, and singular points of curves. Segre, $C$. Rm. R. Ac. Linc. Rd. 6 (1897) (Sem. 2) 168-.

parallel. Mannheim, A. L. Mth. S. P. 12 (1880-81) 177-.

- Craig, T. Crelle J. Mth. 94 (1883) 162-. -, of developables and skew curves. Roberts, S. (xI) L. Mth. S. P. 5 (1873-74) 90-.

-, extension of concept. Enneper, A. Gött. Nr. (1870) 70-.

pedal. Bacaloglo, E. Grunert Arch. 35 (1860) 41-; Schlömilch Z. 5 (1860) 67-.

- Hirst, T. A. QJ. Mth. 3 (1860) 210

-. Enneper, A. Schlömilch Z. 8 (1863) 53-. with rational generating lines. Lelieuvre, Par. Éc. Norm. A. 12 (1895) 57-.

reciprocal, a particular kind. Bonnet, $O$. C. R. 42 (1856) 485-.

of revolution, construction of tangent to shadow. Mannheim, A. (x) Par. S. Phlm. Bll. 2 (1865) 9-.

- - quartic, geodesics of which are algebraic. Tannery, -. Par. S. Phlm. Bll. 4 (1892) 85-. - - tangents of boundary of shadow, determination. Staudigl, $R$. Wien Sb. 68 (1873) $(A b .2)$ 228-. 
of revolution, tangents of boundary of shadow, determination. Pelz, C. Wien Ak. Sb. 79 (1879) $(A b .2)$ 447-.

ruled. Pirondini, G. G. Mt. 25 (1887) 25-, 115-.

-, 2nd asymptotic line. Hoppe, R. Arch. Mth. Ps. 60 (1877) 276-.

-, belonging to skew curve. Ciani, E. G. Mt. 27 (1889) 233-.

-, class. Rouquet, V. Toul. Ac. Sc. Mm. 7 (1895) 117-。

-, contact. Binet, J. P. M. Par. Éc. Pol. Cor. 2 (1809-13) 331-.

-, and curves with constant curvature and torsion. Allen, A. J. C. [1882] Mess. Mth. 12 (1883) 26-.

-, expression for $d s^{2}$ on. Bioche, C. [1889] Par. S. Mth. Bll. 18 (1890) 91-.

-, geodesic and line of striction, theorem. Mannheim, A. [1877] Par. S. Mth. Bll. 6 (1878) 7-.

-, intrinsic equations. Cesáro, E. N. A. Mth. 9 (1890) 294-.

- line of striction. Nicolaĩdès, N. Les Mondes 9 (1866) 146-.

- _ - - given, construction. Hoppe, R. Arch. Mth. Ps. 11 (1892) 345-.

-, - - - satisfying certain conditions. Amigues, É. $\quad$ N. A. Mth. 8 (1889) 77-.

-, orthogonal trajectories of generators. Fais, A. [1879] Bologna Ac. Sc. Mm. 1 (1880) 67-

-, paraboloid of normals. Mannheim, A. Par. S. Mth. Bll. 5 (1877) 190-.

-, passing through curve. Bioche, C. N. A. Mth. 12 (1893) 412-

-, and surface envelopes. Stammer, W. Liége Mm. S. Sc. 1 (1866) 137-.

-, tangential helicoid. Mannheim, A. Liouv. J. Mth. 4 (1878) 57-.

singular points. Gregory, D. F. Camb. Mth. J. 2 (1841) 252-.

- - Amiot, B. Brux. Mm. Cour. $4^{\circ}, 21$ (1846) 48 pp.

singularities. Maur, A. Grunert Arch. 25 (1855) 335-

-, pinch points. Cayley, A. QJ. Mth. 9 (1868) 332-.

skew. Catalan, E. C. [1865] Brux. Mm. Cour. $8^{\circ}, 18$ (1866) (No. 5) 80 pp.

- Dewulf, É. Par. Éc. Norm. A. 3 (1886) 189 .

-, asymptotic lines. Anon. (xr 19) Bll. Sc. Mth. As. 1 (1870) 228-.

- helicoidal, determination of contour. Pelz, C. Wien Ak. Sb. 87 (1883) $(A b .2) 473-$.

-, intrinsic equations. Cesáro, E. N. A. Mth. 8 (1889) 445-.

-, lines of striction, application to illumination. Procházka, B. Časopis 18 (1889) 1-; Fschr. Mth. (1889) 589-.

-, with plane line of striction. Molins, $H$. Toul. Ac. Sc. Mm. 9 (1887) 516-.

-, principal tangent curves. Voss, A. $E$. Mth. A. 12 (1877) 485-.

-, properties. Dini, U. G. Mt. 3 (1865) 281-. -, property. Chomé, $F$. (xII) Mathesis 2 (1882) 82-. skew, with same plane curve for line of striction and of curvature. Amigues, $\dot{E}$. N. A. Mth. 14 (1895) 491-.

一, theorems. Amigues, $\dot{E}$. C. R. 104 (1887) 1092-.

-, theory. Catalan, E. C. Par. S. Phlm. PV. (1848) 67-. -, $\rightarrow$. Lüroth, J. [1866] Crelle J. 67 (1867)

-, 一. Voss, A. E. Mth. A. 8 (1875) 54 -.

-, - Korteweg, D. J. N. Arch. Wisk. 3 (*1877) 66-.

theory. Pellet, A. C. R. 124 (1897) 451-; Par. S. Mth. Bll. 26 (1898) 138-.

- fundamental relations referred to movable trihedra. Issaly, - . N. A. Mth. 19 (1900) 49-, 240.

一, general. Padova, E. Bologna Ac. Sc. Mm. 10 (1889) 745-.

-, -. Pellet, A. C. R. 124 (1897) 739-.

-, infinitesimal, formulæ. Frattini, $G$. G. Mt. 13 (1875) 161-.

-, new element (Christoffel). Beltrami, $E$. Mil. I. Lomb. Rd. 2 (1869) 853-.

-, solutions of problems depending on small quantities of 3rd order. Mannheim, A. C. R. 80 (1875) 541-, 619-.

transformation method. Habich, E. N. A. Mth. 8 (1869) 253-.

transformations. Enneper, A. Z. Mth. Ps. 9 (1864) 126-.

triply asymptotic system, impossibility. Eisen. hart, L. P. [1900] N. Y. Am. Mth. S. Bll. 7 (1901) 184-.

useful formulæ for. Baehr, G. F. W. Grunert Arch. 32 (1859) 221-.

vectorial geometry. Genty, - As. Fr. C. R. (1888) (Pt. 2) 95-.

Tangent planes, parallel, centre of mean distances of their points of contact. Liouville, J. Liouv. J. Mth. 6 (1841) 345-.

Terrestrial ellipsoid, theorems. Angelitti, $F$. Nap. Ac. Pont. At. 28 (1898) No. 2, 124 pp.

Theorem of Betti and Weingarten. Pieri, $M$. G. Mt. 24 (1886) 290-.

Variation of straight lines in connexion with surface. Hoppe, R. Arch. Mth. Ps. 3 (1886) 290 -

\section{Differential geometry of congruences and other ap- plications of the differential calculus to elements of space.}

Congruences conjugate to systems $C$ ( $K$ congruences). Guichard, C. C. R. 126 (1898) 718-.

- which are $K$ congruences in several man. ners. Guichard, C. C. R. 126 (1898) 1011-. -, intrinsic analysis. Cifarelli, T. G. Mt. 36 (1898) 145-.

-, - geometry. Cesáro, E. Nap. Rd. 33 (1894) 141-. 
Congruences and nets of curves, spherical representation. Guichard, C. C. R. 116 (1893) 1238-

- of normals. Transon, (Prof.) A. N. A. Mth. 2 (1863) 138-.

- special type for transformation. Brill, J. L. Mth. S. P. 20 (1889) 102-.

Conjugate systems with equal invariants. Koenigs, G. C. R. 113 (1891) 1022-.

Contact transformations and optics. Lovett, E. O. Camb. Ph. S. T. 18 (1900) 256-.

Curves, tangents of which are part of line complex of 1st order, theorem. Appell, $P$. Arch. Mth. Ps. 60 (1877) 274-.

Cyclic system, in envelopes of spheres. Ribaucour, A. C. R. 92 (1881) 233-.

- systems. Ribaucour, A. C. R. 113 (1891) 304-, 324-.

Differential equations. Lie, $M . S$. Christiania F. (1882) No. 21,12 pp., No. 22,6 pp. ; (1883) No. 10, 4 pp., No. 18, 5 pp.

- forms and systems of elements. Koenigs, $G$. Acta Mth. 10 (1887) 313-.

Infinitesimal properties of circular complexes. Cosserat, E. C. R. 106 (1888) 1514-.

- - complexes. Bourget, H. C. R. 104 (1887) 1253-.

- - ruled space. Koenigs, G. Par. Éc. Norm. A. 11 (1882) 219-.

Kinematic geometry (ruled space). Saussure, R. de. Am. J. Mth. 18 (1896) 304-; 19 (1897) 329-.

Line congruences, first differential projective invariant. Waelsch, E. C. R. 118 (1894) 736-.

- - and problem of Ribaucour. Cosserat, $E$. C. R. 118 (1894) 335-

- - - surfaces, infinitesimal geometry. Waelsch, E. Wien Ak. Sb. 100 (1891) (Ab.2a) $158-$

- - - - theorem of Darboux on orthogonal systems. Cosserat, E. Toul. Fac. Sc. A. 8 (1894) B, 9 pp.

Normals to surface. Bonnet, $O$. C. R. 52 (1861) 1081-

- - Baehr, G. F. W. N. A. Mth. 2 (1863) 35-.

Osculatory transformations of surfaces. Bäcklund, A. V. [1875] Mth. A. 9 (1876) 297-.

Plane curves with factorable parallels. Snyder, V. [1900] N. Y. Am. Mth. S. Bll. 7 (1901) 299 .

Pseudospherical congruences. Candido, $G$. N. A. Mth. 17 (1898) 275-.

Quadratic forms in geometry of complexes. Koenigs, G. Toul. Fac. Sc. A. 1 (1887) E, 13-.

Ruled surfaces. Bouquet, $J$. Liouv. J. Mth. 11 (1846) 125-; Lyon Mm. Ac. 2 (1847) 385-.

Surfaces with circular generators. Demartres, G. Par. Éc. Norm. A. 2 (1885) 123-.

Translation surfaces. Lie, S. Leip. Mth. Ps. B. 44 (1892) 447-, 559-.

- and Abel's theorem. Lie, S. Leip. Mth. Ps. B. 48 (1896) 141-.

- -, special class. Wiegner, $G$. Arch. Mth. Ntvd. 16 (1893) 325-.

\section{Rectification and quadrature of curves; areas and volumes of surfaces. Other applications of the integral calculus to geometry.}

Arcs of algebraic curves, plane or skew, properties. Humbert, $G$. Liouv. J. Mth. 1 (1895) 181-.

- - conics, plane and spherical, and other curves, comparison. Maccullagh, J. Ir. Ac. P. $2(1840-44) 507-$

- - plane algebraic curves. Humbert, G. Par. Éc. Pol. J. 57 (1887) 171-.

- - - curves, approximate calculation, application of theorem of Poncelet's. Resal, H. A. C. R. 94 (1882) 1375-.

_ _ _ _ 2, Bertrand's proposition on curve defined by sum or difference. Sucharda, $A$. Časopis 18 (1889) 49-; Fschr. Mth. (1889) 269.

- with rectifiable, and zones with quadrable difference. Lebesgue, V. A. Liouv. J. Mth. 11 (1846) 331-.

Areas.

Amsler's theorems, as regards related variable systems. Kleiber, J. Arch. Mth. Ps. 14 (1896) 405-.

of anallagmatic curves. Liguine, $V$. As. Fr. C. R. (1881) 231-.

approximation, by seven equidistant ordinates. Weddle, T. Camb. and Dubl. Mth. J. 9 (1854) $79-$.

calculation. Lorenz, L. Mth. Ts. 6 (1864) 33-.

-, formula, Poncelot's, error in. Chevilliet, C. R. 80 (1875) 823-.

-, - Simpson's, degree of accuracy. Che villiet, -. C. R. 78 (1874) 1841-.

-, -, -, transformation. Saigey, J. $F$. N. A. Mth. 15 (1856) 291-.

-, Simpson's two rules, comparison, and Woolley's rule. Merrifield, $C$. W. Nv. Archt. T. 6 (1865) 40-.

- - - - - - - - - Purkiss, H.J. Nv. Archt. T. 6 (1865) 48-.

of circular ring, subdivision. Davidson, $G$. Calif. Ac. P. 4 (1868-72) 278-, 290-.

- closed skew curve. Laisant, C. A. As. Fr. C. R. (1899) (Pt. 2) 135-.

- cones and cylinders. Azzarelli, $M$. Tortolini A. 8 (1857) 441-.

- conic, from trilinear equation. Ferrers, N. M. QJ. Mth. 2 (1858) 247-.

- - - - - (Ferrers). Cayley, A. QJ. Mth. 2 (1858) 248-

conical, calculation, cases. Guimarães, $R$. As. Fr. C. R. (1892) (Pt. 2) 166-.

of conical wedge-surfaces. Schlömilch, $O$. Schlömilch Z. 8 (1863) 142-.

- convex contours. Petrini, $H$. Stockh. Ak. Hndl. Bh. 19 (Afd. 1) (1894) No. 5, 58 pp.; Fschr. Mth. (1893-94) 1079. 
of corresponding surfaces. Craig, $T$. (xr) J. H. Un. Cir. [1] (1882) 209-.

- curve and its in- and circum-scribed $n$-gons, Völler's theorem, and application to skew curves. Matthiessen, L. Schlömilch Z. 5 (1860) 146-.

between curve and rectilinear base, calculation, formula. Como, G. da. (xII) Brescia At. Cm. (1878) 131-, 279; (1880) 152-.

of curve $r=\frac{\gamma}{1+\tan \alpha}$. Bender, $C$. Arch. Mth. Ps. 47 (1867) 45-.

- curved surfaces. Borletti, F. G. Mt. 22 (1884) 207-.

- - - Maggi, G. A. Rm. R. Ac. Linc. Rd. 5 (1896) (Sem. 2) 440-.

cut off by spiral on hemisphere (theorem of Pappus). Resal, H. A. N. A. Mth. 20 (1881) 433-.

of cycloid. Walton, $W$. Camb. and Dubl. Mth. J. 9 (1854) 263-.

- cylindrical figures. Wasteels, C. E. Mathesis 19 (1899) 9-.

described by line moving in plane under given conditions. Bardelli, G. Mil. I. Lomb. Rd. 12 (1879) 290-.

division, historico-critical notice. Favaro, $A$. Ven. I. Mm. 22 (1882) 129-.

of ellipse. Mossbrugger, L. Grunert Arch. 6 (1845) 19-.

- - and hyperbola. Grunert, J.A. Grunert Arch. 23 (1854) 385-, 477-.

- , problems. Matzka, $\dot{W}$. Arch. Mth. Ps. 46 (1866) 300-.

- ellipsoid. Lobatto, R. Liouv. J. Mth. 5 (1840) 115-.

- - Catalan, E. C. Brux. Ac. Bll. 30 (1870) 97-.

- Zurria, G. Catania.At. Ac. Gioen. 5 (1871) 97-.

- - Stickelberger, -. D. Nf. B. ( ${ }^{* 1883)}$ 56.

- -, approximate values. Peano, G. $\mathrm{Rm}$. R. Ac. Linc. Rd. 6 (1890) (Sem. 2) 317-.

_ - , and contour of ellipse, approximate expressions for. Boussinesq, J. C. R. 108 (1889) 695-.

- - elongated. Peyronny, A. N. A. Mth. 3 (1844) 466-.

- ellipsoidal zone. Wolfers, J. P. Grunert Arch. 22 (1854) 473-.

- zones, cases. Humbert, G. C. R. 109 (1889) 611-, 734-.

- ellipsoids of revolution. Grillo, S. N. A. Mth. 17 (1858) 272-.

- elliptic cone. Dienger, J. Tortolini A. 2 (1851) 119-.

- paraboloid. Tortolini, B. (vir) A. Mt. 4 (1861) 293-.

on elliptic paraboloid, expression of. Humbert, G. Par. S. Mth. Bll. 21 (1893) 13-.

- - - - - new. Humbert, G. Par. S. Mth. Bll. 21 (1893) 17-.

of elliptic sectors. Grunert, J.A. Grunert Arch. 30 (1858) 472-.

extension of term. De Morgan, A. Camb. and Dubl. Mth. J. 5 (1850) 139-. extension of term, first, to autotomic plane circuit. Muir, T. Ph. Mg. 45 (1873) 450-.

of figure on sphere formed by small circle arcs. Wasteels, C. E. Mathesis 12 (1892) 105-.

formed by arc of curve and two consecutive normals. Dahlander, G. R. Stockh. Öfv. 14 (1857) 269-.

of lemniscate. Dippe, M. C. Crelle J. 16 (1837) 65-.

between 2 loxodromic curves and meridian of sphere. Berg, F. J. van den. Amst. Arch. Wisk. Gn. 2 (1860-63) 1-, 10-, 23-.

of loxodromic triangles on oblate spheroid. Grunert, J. A. Grunert Arch. 27 (1856) 143-. mean, of prismoid, and associated theorems. Echols, W. H. [1894] A. Mth. 9 (1894-95) 1-.

of oblique cone. Schönbichler, $K$. Wien SB. 16 (1855) 447-.

- parabola, geometric determination. Mathiot, O. L. Des Moines Anal. 9 (1882) 106-.

- parabolas of higher order. Schoute, P. H. [1896] C. R. 122 (1896) 1113-; Amst. Ak. Vs. 5 (1897) 35.

- - - - - theorem of Schoute. Korteneg, D. J. C. R. 122 (1896) 1399.

- - - - - Mannoury, G. C. R. 122 (1896) 1399-.

- pedal curves. Lecornu, L. As. Fr. C. R. (1894) (Pt. 2) 276-.

and perimeter of 6-faced surface formed by orthogonal quadrics. Hoppe, R. Arch. Mth. Ps. 56 (1874) 354-.

- - to 3 rd or 4 th order, of geodesic circle. Bonnet, G. O. C. R. 97 (1883) 1360-.

plane, approximate calculation. Mansion, $P$. (xII) Brux. S. Sc. A. 5 (1881) (Pt. 2) 231-. -, - - Harnack, A. Civing. 28 (1882) 257 -. -, - - Petit Bois, G. Mathesis 5 (1885) $5-, 27-$

-, - - Mansion, P. Mathesis 7 (1887) 77-。 - curvilinear, calculation. Leclert, E. A. Gén. Civ. 7 (1868) 630-.

of 3 plane sections of surface. Olivier, $T$. Férussac Bll. Sc. Mth. 15 (1831) 312-.

plane, of Simpsonian curves, approximate calculation. Petit Bois, G. Liège S. Sc. Mm. 18 (1895) No. 6, 19 pp.

- theorem, extension to curved surfaces. Elliott, E. B. Mess. Mth. 10 (1881) 156-. of portions of ellipsoid. Ghysens, $\dot{E}$. (xI) Brux. S. Sc. A. 2 (1878) (Pt. 2) 89-.

- projection of lemniscate on sphere. Delaire, -. N. A. Mth. 15 (1856) 53-.

- quadric surfaces, central. Schlömilch, $O$. Leip. B. 14 (1862) 23-.

- right cone cut obliquely. Horn, W. Crelle J. 2 (1827) 364-.

- segment of conic. Cremona, L. G. Mt. 1 (1863) 360-.

- sinusoids, and Wallis's formula for. Mansion, $P$. Mathesis 20 (1900) 183-.

- spherical surface between great and small circles which intersect. Edelmann, - Gergonne A. Mth. 3 (1812-13) 141-.

- - zone. Escher, P. (vr Adds.) D. Nf. Vsm. B. 34 (1858) 143-. 
of spheroidal triangle, sides of which are lines of shortest distance. Puissant, $L$. Par. Mm. Ac. Sc. 10 (1831) 530-.

- star-polygons. Jacobi, C. G. J. Crelle J. 65 (1866) 173-.

- surface of elasticity. Roberts, $W$. Liouv. J. Mth. 11 (1846) 81-.

- _ - ellipsoid, and surface of ellipsoidal vault on rectangular base. Plana, $G$. Crelle J. 17 (1837) 345-.

- -, expression for. Strebor [Roberts, W.]. N. A. Mth. 9 (1850) 310 .

,---- Faure, H. N. A. Mth. 11 (1852) 393-.

- - from perimeter, application to volumes. Linotte, L. Bologna Opusc. Sc. 2 (1818) 108-.

- surfaces. Essen, E. Grunert Arch. 22 (1854) 56-.

- - , analogies to Viviani's theorem. Crocchi, L. G. Mt. 13 (1875) 170

- - by coordinates determining direction of normal to an element. Schlömilch, $O$. Z. Mth. Ps. 11 (1866) 505-。

- - , and length of curves. Goedseels, Brux. S. Sc. A. 16 (1892) (Pt. 1) 79-.

- - in polar coordinates. Bing, $F$. Ts. Mth. 4 (1874) 164-.

- - various. Schlömilch, O. Leip. B. 18 (1866) 38-

theorem including Holditch's, and analogue in 3 dimensions. Elliott, E. B. Mess. Mth. 7 (1878) 150-.

of trajectories described in plane motion of figure of invariable form. Liguine, $V$. Bll. Sc. Mth. As. 2 (1878) (Pt. 1) 306-.

-2 triangles, problems in maximum. Dippe, M. C. Crelle J. 16 (1837) 65-

and volumes. Terquem, O. N. A. Mth. 5 (1846) 232-.

- - (shallow surfaces), approximate formulæ for. Geisenheimer, L. Z. Mth. Ps. 30 (1885) 325-.

volumes, centroids, etc., application of approximation formulæ for definite integrals. $L i$ gowski, $W$. Rv. Mar. et Col. 51 (1876) 159-.

and volumes of conical wedge. Monteiro, A.S. G. Teix. J. Sc. 2 (1880) 68-, 81-, 110-.

- - - cylindrical ungula. Schell, $W$. Grunert Arch. 19 (1852) 70-.

- - determination. Juel, C. N. Ts. Mth. 8 (B) (1897) 49-; Fschr. Mth. (1897) 497.

- of ellipsoid, reduction of integrals for. Lobatto, R.' Amst. Vs. Ak. 13 (1862) 193-. - , fundamental problem. Rausenberger, $O$. Mth. A. 43 (1893) 601-.

- - generated by motion of curves and surfaces. Nehls, C. Arch. Mth. Ps. 13 (1895) 225-, 337-.

- - and lengths related to regular polygons. Gelin, E. Mathesis 11 (1891) 160-.

- - measurement. Sang, J. [1836] Edinb. N. Ph. J. 22 (1837) 231-.

- - of surfaces of equation

$$
\left(\frac{x}{a}\right)^{2 m}+\left(\frac{y}{b}\right)^{2 m}=1 \text {. }
$$

Spitzer, S. D. Nf. B. (*1877) 111-. and volumes of surfaces generated by regular polygon rotating about a diameter. Sohncke, L. A. Arch. Mth. Ps. 48 (1868) 457-.

- - - tore (anchor ring). Gelin, $E$. Mathesis 10 (1890) 190-; 11 (1891) 66-.

- - in trilinear and quadriplanar coordinates. Slesser, G. M. QJ. Mth. 2 (1858) 357-.

- zone, etc. Stein, J. P. Gergonne A. Mth. 16 (1825-26) 257-.

of zone of pedal surfaces of quadrics. Schlömilch, O. Leip. B. 14 (1862) 51-.

Argument $\int d x / \frac{d F}{d y}$ of plane curve $F(x, y)=0$, properties. Roberts, R. A. L. Mth. S. P. 15 (1883-84) 4-; 16 (1884-85) 238-.

Boundary integral. Prym, F. E. [1869] Crelle J. 71 (1870) 305-.

Closed plane curves, theorem. Risteen, A.D. A. Mth. 3 (1887) 104.

Conical surfaces, integrals analogous to angle and its bisector. Humbert, G. C. R. 105 (1887) 739-.

Conics, pedals and inverses of division of arcs. Strebor [Roberts, W.]. N. A. Mth. 11 (1852) 182-.

Cono-cuneus of Wallis, problem. Berg, F.J. van den. Amst. Arch. Wisk. Gn. 2 (1860-63) 1-, 10-, 23-.

Curves, arc of which is elliptic or hyperelliptic integral of 1st kind. Schwering, $K$. Z. Mth. Ps. 25 (1880) 234-.

- , - - - - integral of 1st kind. Kiepert, L. [1874-76] Crelle J. Mth. 79 (1875) 304-; Freiburg B. 7 (1880) 1-.

- $-\ldots$ - equals elliptic arc. Euler, $L$. [1781] St Pét. Ac. Sc. Mm. 11 (1830) 95-.

-, - - - parabolic arc. Euler, L. [1781] St Pét. Ac. Sc. Mm. 11 (1830) 100 .

-, arcs of which can be expressed by circular arcs. Euler, L. [1781] St Pét. Ac. Sc. Mm. 11 (1830) 114-.

- - - - - - - - - Serret, J. A. [1852] Par. Ec. Pol. J. 33॰ cah. (1853) 69-.

-, - - - represent elliptic functions. Serret, J. A. Liouv. J. Mth. 10 (1845) 421-.

ist kind. Talbot, W. H. F. Gergonne A. Mth. 14 (1823) 380.

- - - conjugate hyperbolas, perimeters, two theorems. Roberts, $W$. Liouv. J. Mth. 13 (1848) 179-.

- on ellipsoid. Dienger, $J$. Tortolini A. 2 (1851) 104-.

- with equal arcs. Léauté, $H$. Toul. Ac. Sc. Mm. 6 (1874) 419-.

- quadrable by elliptic or circular functions, Clebsch's two theorems. Marie, M. C. R. 84 (1877) 227-.

Cycloidal arcs. Tortolini, B. A. Mt. 7 (1865) 211-.

Cylinder and sphere, problems. Berg, F. J. van den. Amst. Arch. Wisk. Gn. 2 (1860 63) $1-, 10-, 23-$. 
Division of surface of right cone on circular base. Lampe, E. Fschr. Mth. (1895) 736.

Ellipse, circumference. Brinkley, J. [1802] Ir. Ac. T. 9 (1803) 145.

-, -. Bakewell, T. W. Franklin I. J. 29 (1855) 369-.

-, -, approximate calculation. Schlömilch, $O$. Civing. 12 (1866) 31-.

-, -, - values. Cavallin, C. B. S. (хп) Ts. Mth. 1 (1883) 33-.

-, - expression by series. Hoppe, $R$. Grunert Arch. 3 (1843) 265-.

-, quadrant. Schlömilch, O. Z. Mth. Ps. 13 (1868) 530.

Elliptic arc. Morin, A. N. A. Mth. 2 (1843) 493.

- ares, calculation. Seewald, E. [1877] Wien Ak. Sb. 76 (1878) (Ab. 2) 365-.

- - comparison. Guimarães, R. G. Teix. J. Sc. 7 (1886) 111-.

- -, property. Buzengeiger, K. H. $J$. Lindenau Z. 2 (1817) 98-.

- -, Schmidt's tables. Verdam, G. J. Amst. I. (1842) 57-.

- functions, application to problems of mean values. Mukhopadhyay, A. [1888] Beng. As. S. J. 58 (Pt. 2) (1890) 199-, 213-.

Equal surfaces and lines cut off by common radii, systems. Hoppe, $R$. [1871] Ups. N. Acta S. Sc. 8 (1873) No. 4, 18 pp.

Extension of Woolley's rule to integration of moments, proof. Merrifield, $C . W$. Nv. Archt. T. 8 (1867) 218-.

Frustum of pyramid, divided in given ratio. Grunert, $J$. A. Grunert Arch. 36 (1861) 503-.

Geodesic circles, Bertrand's theorems, demonstrations. Bonnet, G. O. Par. Éc. Pol. J. 56 (1886) 143-.

Hyperbola, arc and asymptote. Roberts, $M$. Ir. Ac. P. 3 (1847) 385.

-, rectangular, property of arcs. Talbot, W. H. F. R. S. P. 3 (1834) 258.

Hyperbolic arc, Landen's theorem. Plana, $G$. Palomba Rac. 2 (1846) 233-.

,---- Griffiths, J. L. Mth. S. P. 11 (1879-80) 87-.

- - - - Cayley, A. [1881] L. Mth. S. P. 13 (1881-82) 47-.

- - - - (Cayley). Griffiths, J. [1881] L. Mth. S. P. 13 (1881-82) 49-.

- arcs, comparison. Merrifield, C.W. Phil. Trans. (1859) 171-.

Integration by approximation. Martini, $E$. G. Mt. 3 (1865) 91-.

- of $d x^{2}+d y^{2}+d z^{2}=d s^{2}$. Serret, J. A. Liouv. J. Mth. 13 (1848) 353-.

$--=-$. Darboux, G. Liouv. J. Mth. 3 (1887) 305-.

$--d x^{2}+d y^{2}=d s^{2}$ and analogous equations. Darboux, G. Liouv. J. Mth. 18 (1873) 236-.

$--\dot{d s^{2}}=E d u^{2}+2 F d u d v+G d v^{2}$. Legoux, A. Toul. Fac. Sc. A. 3 (1889) F, 2 pp.

Inverse of ellipse from centre, division of arcs. Strebor [Roberts, W.]. N. A. Mth. 11 (1852) 182-.

voL. I.
Length of arc and of asymptote, difference. Fuss, P. H. St Pét. Ac. Sc. Mm. 9 (1824) 175-.

- - - on sphere and spheroid, difference, approximate formula. Bruns, $H$. As. $\mathrm{Nr}$. 97 (1880) 73-.

- Cartesian oval arc, expression by elliptic functions. Roberts, S. [1873-75] (xI) L. Mth. S. P. 5 (1873-74) 6-; 6 (1874-75) 200.

- curve, first determination. Christensen, S. A. Ts. Mth. 5 (1887) 121-; Bb. Mth. (1887) 76-.

- - elliptic arc, geometrical researches. Camus, G. Ven. I. At. 1 (1883) 997-.

- - helix on cone. Dienger, $J$. Grunert Arch. 16 (1851) 454-.

- line. Goedseels, (le lt.) E. Brux. Ac. Bll. 16 (1888) 86-, [800].

Lengths of arcs. Ribaucour, A. (xI) Par. S. Phlm. Bll. 6 (1869) 12-.

- - - of curves, geometrical comparison. Mannheim, A. Par. Éc. Pol. J. $40^{\circ} \mathrm{cah}$. (1863) 205-.

Mean radius vector of curve, surface or solid. Drobisch, M. W. Leip. B. 10 (1858) 124-.

Measurement of arcs of curves, etc. Tarry, $G$. As. Fr. C. R. (1893) (Pt. 2) 217-.

- - convex surface of prism or truncated cylinder. Breton [de Champ], P. Liouv. J. Mth. 2 (1837) 133-.

- - curved surfaces by ordinates. Merrifield, C. $W$. Nv. Archt. T. 6 (1865) 64-.

- - prisms. Dieu, T. N. A. Mth. 16 (1857) 143-.

Perimeter of ellipse. Le François, (Ing.) A. Gén. Civ. 1 (1862) 206-.

- - Brocard, H.P.P.B. (xII) Mathesis 2 (1882) 180-.

- _-. Mansion, P. (xiI) Mathesis 2 (1882) 211-.

- - Muir, T. Mess. Mth. 12 (1883) 149-.

- - - and approximation of definite integrals. Mansion, $P$. [1883] (xII) Brux. S. Sc. A. 8 (1884) (Pt. 1) 51-; (Pt. 2) 11-.

- - - - certain geometric means. Cavallin, C. B. S. [1882-83] (xII) Mathesis 3 (1883) $56-$.

- - - limits. Peyronny, A. N. A. Mth. 3 (1844) 232-.

-, volume, centroid, etc. of certain spaces, relations. Dainelli, $U$. G. Mt. 16 (1878) 279 -

Perimeters of curves derived from conjugate hyperbolas, two theorems. Roberts, $W$. Liouv. J. Mth. 13 (1848) 179-.

Planimeter, Amsler's, theory. Thiré, A. N. A. Mth. 5 (1886) 353-.

-, polax, theory. Grunert, J. A. Arch. Mth. Ps. 51 (1870) 385-.

Polyhedra of maximum volume for given surface. Lindelöf, L. L. [1869] St Pét. Ac. Sc. Bll. 14 (1870) 257-.

Prismatoid, Wittstein's. Bretschneider, C. A. Grunert Arch. 36 (1861) 18-.

Quadrable cones. Sucksdorff, C. G. [1855] Helsingf. Acta 4 (1856) 617-. 
Quadrable figures on cylinders. Schlömilch, $O$. Grunert Arch. 9 (1847) 149-.

- lunes. Clausen, T. Crelle J. 21 (1840) 375-; N. A. Mth. 8 (1849) 395-.

Quadratrix of curve of $m$ th degree, conditions for disappearance of periods. Marie, $M$. C. R. 76 (1873) 757-.

- - - - - - , cyclic periods. Marie, $M$. C. R. 80 (1875) 872-; 84 (1877) 27-, 120-.

-, generalisation. Fouret, $G$. N. A. Mth. 5 (1886) 39-.

-, tangent, construction. Resal, H.A. N. A. Mth. 15 (1876) 337-.

\section{Quadrature.}

Servois, J. F. Gergonne A. Mth. 8 (1817-18) 73-.

Ménabréa, L. $F$. [1844] Tor. Mm. Ac. 8 (1846) 195-.

Hill, C.J. D. Stockh. Öfv. 13 (1856) 15-.

Tardy, P. [1865] Mod. Mm. S. It. 2 (1866) 133-.

Oppermann, L. H. F. (xII) Ts. Mth. 1 (1871) 11-.

Tchébicheff, P. de. As. Fr. C. R. 2 (1873) 69-.

Posse, K. A. N. A. Mth. 14 (1875) 49-.

of algebraic curves, classification of integrals. Marie, M. C. R. 76 (1873) 692-, 943-; Par. Éc. Pol. J. Cah. 44 (1874) 109-.

algebraic and logarithmic. Raffy, L. Par. Ec. Norm. A. 2 (1885) 185-.

approximate. Hall, $A$. Des Moines Anal. 3 (1876) 1-.

-. Liventsov, A. I. [1878] (xTi) Rec. Mth. (Moscou) 9 (1878-81) (Pt. 1) 569-.

-. Jadanza, N. Rv. Mt. 3 (1893) 15-.

-. Bardelli, G. Rv. Mt. 3 (1893) 16-.

- Peano, G. Rv. Mt. 3 (1893) 17-.

by approximation. Dupain, J.C. N. A. Mth. 17 (1858) 288-.

- - Bierens de Haan, D. Amst. Vs. Ak. 6 (1872) 185-; Arch. Néerl. 8 (1873) 113-.

and areas and volumes, simple formulø for. Francke, A. Hann. Archt.-Vr. Z. 21 (1875) 177-.

of conics. Bary, - Gergonne A. Mth. 19 (1828-29) 245-.

- -, elementary. Azzarelli, M. Rm. N. Linc. At. 32 (1879) 331-.

- - , series for. Wallace, (Prof.) W. [1808] Edinb. R. S. T. 6 (1812) 269-.

- curves. Bérard, -. Gergonne A. Mth. 7 (1816-17) 101-.

- - (Bérard). Ampere, A. M. Gergonne A. Mth. 8 (1817-18) 117-.

- - Cost Jordens, G. H. Leijd. A. Ac. (1821-22) 64 pp.

- - method, new, any function. Bérard, Gergonne A. Mth. 7 (1816-17) 101-.

,--- , unpublished, of Newton. De Morgan, A. Camb. and Dubl. Mth. J. 8 (1853) 93-.

- by polar coordinates, Lindman, C. $F$. Grunert Arch. 26 (1856) 471-.

-- , principle. Steiner, J. Berl. B. (1840) by elementary methods. Gretschel, $H$. Arch. Mth. Ps. 42 (1864) 424-.

of ellipsoid. Tortolini, B. G. Arcad. 78 (1839) 1-.

- - Carda, K. Mh. Mth. Ps. 7 (1896) 129- - of revolution. Tortolini, B. (vrir) A. Mt. 4 (1861) 170-.

- elliptic paraboloid. Laciani, F. Tortolini A. 6 (1855) 406-.

- - sectors. Grunert, J. A. Grunert Arch. 17 (1851) 313-; 20 (1853) 217-.

- envelope of negative pedal. Tortolini, $B$. Palomba Rac. 2 (1846) 339-.

exact, of certain curves. Breton [de Champ], $P$. N. A. Mth. 2 (1843) 351-.

-, - surfaces. Borchardt, C. W. Liouv. J. Mth. 19 (1854) 369-.

of geodesic triangle, small, Gauss's theorem. Jacobi, C. G. J. Crelle J. 16 (1837) 344-.

geometric. Hudson, C. I. Rv. 2 (1838) 206-. of hyperbola. Nizze, $E$. Grunert Arch. 26 (1856) 111-.

- Essen, E. Grunert Arch. 27 (1856) 40 .

- -, and hyperbolic natural ${ }^{\circ}$ logarithms. Grunert, J. A. Grunert Arch. 25 (1855) 82-.

- - by method of limits. Prudot, $H$. N. A. Mth. 3 (1844) 565-.

- hyperbolic paraboloid. Tucci, F.P. Nap. At. Ac. 6 (1851) 69-.

- hypocycloids. Baur, C. W. Schlömilch Z. 4 (1859) 311-.

from integrals of differentials in two variables, properties. Andréiewsky, -. Mth. A. 4 (1871) 391-.

of logarithmic spiral, $y=a^{x}$. Rispal, $A$. N. A. Mth. 4 (1845) 117-.

mechanical, theory. Stieltjes, T. J. Par. Éc. Norm. A. 1 (1884) 409-.

method. Catalan, E. C. Par. S. Phlm. PV. (1851) 16-.

-. Saviotti, C. Cuyper Rv. Un. 14 (1883) 584-. -, Gauss's. Callandreau, O. C. R. 84 (1877) 1225-; 90 (1880) 1067-

-, -. Radau, R. C. R. 90 (1880) 913-.

—, - Mansion, -. Brux. S. Sc. A. 15 (1891) (Pt. 1) 57-.

-, 一. Raffy, L. N. A. Mth. 15 (1896) 249-. - - , extension. Bourget, H. C. R. 126 (1898) 634-.

-, -, generalisation. Christoffel, E. B. Crelle J. 55 (1858) 61-.

-, -, remainder. Mansion, P. C. R. 102 (1886) 412-; Brux. Ac. Bll. 11 (1886) 293-. -, - , and solution of Kepler's problem. Strehlke, F. Grunert Arch. 32 (1859) 433-.

methods. Gaudard, J. A. Gén. Civ. 2 (1873) 480 -.

- comparison and new formulæ. Parmentier, T. N. A. Mth. 14 (1855) 370-; As. Fr. C. R. (1875) 179-; (1882) 64-.

-, formula of Parmentier. Anon. (vi 892) N. A. Mth. 16 (1857) 11-.

-, formulæ with equal coefficients. Radau, $R$. C. R. 90 (1880) 520-.

-, 一, practical. Piobert, -. N. A. Mth. 13 (1854) 323-. 
methods, Simpson's formula, elementary demonstration. Steen, A. N. A. Mth. 10 (1871) 301-.

-, - -, generalisation. Peano, G. Tor. Ac. Sc. At. 27 (1892) 608-

-, Stirling's and Wallis's formulæ, demonstration. Mansion, - Brux. S. Sc. A. 23 (1899) (Pt. 1) 1-.

of parabola. Völler, -. Grunert Arch, 32 (1859) 420-.

- - cycloid, and logarithmic spiral. Stainville, - de. Par. Éc. Pol. Cor. 2 (1809-13) 434-.

- parabolas of 3rd degree. Parmentier, $T$. As. Fr. C. R. (1879) 150-.

- parabolic curves. Catalan, E. C. [1880] Brux. Ac. Sc. Mm. 43 (1882) (No. 8) 9 pp.

- - segments cut off by focal chords. Grunert, J. A. Grunert Arch. 26 (1856) 351-.

- parallel surfaces. Roberts, $W$. Tortolini A. 1 (1850) 143-.

- plane curves. Carvallo, J. N. A. Mth. 1 (1842) 370 -

- polygons. Tessari, D. A. Gén. Civ. 3 (1864) 239-.

problems reducible to, approximate solution. Monteiro da Rocha, J. Lisb. Ac. Sc. Mm. 1 (1797) 218-.

of quadrics. Jellett, J. H. Camb. and Dubl. Mth. J. 1 (1846) 57-; Liouv. J. Mth. 12 (1847) 92-.

- Azzarelli, M. Rm. N. Linc. At. 29 (1876) 337-.

- - central. Schlömilch, O. Liouv. J. Mth. 8 (1863) 89-.

and rectification. Carmichael, $R$. Camb. and Dubl. Mth. J. 9 (1854) 266-.

- - reduction to "cubature" and quadrature respectively. Denzler, $W$. Zür. Mt. 3 (1853-55) 75-.

reduction to rectification. Plana, G. Palomba Rac. 2 (1846) 276-.

of simple curves, synthetic demonstration of rule. Young, $M$. [1786] Ir. Ac. T. 1 (1787) 31-.

- spherical curves, application of elliptic functions. Tortolini, B. [1849] Mod. S. It. Mm. 24 (1850) 337-.

- surface of cone. Sucksdorff, C. G. Helsingf. Acta 4 (1856) 121-.

- - ellipsoidal, and volume of surface of 8 th degree, similarity of expressions for. Tortolini, B. Tortolini A. 3 (1852) 530-.

- - reduction to rectification of curve, Euler's and Legendre's formulæ compared. Plana, G. Palomba Rac. 2 (1846) 169-, 254-.

- surfaces. Schubert, F. T. St Pét. Ac. Sc. Mm. 9 (1824) 277-.

- - Dirksen, E. H. Berl. Ab. (1833) 123-.

-. Bedetti, G. Bologna N. Cm. 6 (1844) 341-.

- Carmichael, R. Camb. and Dubl. Mth. J. 9 (1854) 266-

- -. Steen, A. (xir) Ts. Mth. 1 (1865) 38-. - - formula. Roberts, $W$. Tortolini A. 1 (1850) 318-. of surfaces parallel to ellipsoid. Tortolini, $B$. G. Arcad. 119 (1849-50) 3-.

- - - surface of elasticity. Tortolini, $B$. Tortolini A. 7 (1856) 373-.

- resulting from intersection of cylinders. Lanciani, F. A. Mt. 7 (1865) 169-.

- - , ruled, cases, and volumes of solids en. closed. Saladini, G. [1804] Bologn $\mathrm{Mm}$. I. It. 1 (1806) 69-.

- -, theorem. Jellett, J. H. Camb. and Dubl. Mth. J. 5 (1850) 176-.

- - , and volumes of solids. Tortolini, $B$. Palomba Rac. 1 (1845) 96-; Crelle J. 31 (1846) 12-; 34 (1847) 101-.

- vaults and lunes. Riccati, G. Verona S. It. Mm. 5 (1790) 48-.

and volumes. Carvallo, J. N. A. Mth. 1 (1842) 370

- -, approximate. Mansion, P. C. R. 95 (1882) 384-.

- - , equations being given implicitly in Cartesian coordinates. Azzarelli, $M$. $\mathrm{Rm}$. N. Linc. At. 28 (1875) 134-.

- by infinite series. Trowbridge, D. Des Moines Anal. 7 (1880) 47-.

- of surface of elasticity. Roberts, $W$. Liouv. J. Mth. 11 (1846) 124.

of $y^{2}=x^{3}-x^{4}$. Bonnet, O. N. A. Mth. 3 (1844) $75-$

$-\left(\frac{x}{a}\right)^{2 m}+\left(\frac{y}{b}\right)^{2 m}=1$. Spitzer, S. Arch. Mth. Ps. 61 (1877) 329-.

Ratio of triangle to sector of conic. Fabritius, $W$. As. Nr. 129 (1892) 49-.

- - _ - - B. Ball, L. de. As. Nr. 129 (1892) 283-

Rectifiable algebraic curves. Humbert, $G$. C. R. 104 (1887) 1051-.

- arcs of ellipse. Ocagne, M. d'. G. Teix. J. Sc. 7 (1886) 178-

- curves. Schlömilch, O. Z. Mth. Ps. 15 (1870) 124-.

- Koenigsberger,.L. Mth. A. 32 (1887) 589 -.

- finding. Doppler, C. Grunert Arch. 19 (1852) 236-.

- - on surfaces of revolution. Fuss, N. [1818] St Pét. Ac. Sc. Mm. 8 (1822) 198-. - plane algebraic curves. Humbert, $G$. Liouv. J. Mth. 4 (1888) 133-.

\section{Rectification.}

of algebraic curves. $K o b b, G$. Stockh. Öfv. (1887) 713-; Fschr. Mth. (1887) 504.

approximate. Pellet, A.E. Par. S. Mth. Bll. 19 (1891) 5-.

- of circular ares. Rankine, W. J. $M$. Ph. Mg. 34 (1867) 381-。

-, - - -, case. Berg, F. J.van den. N. Arch. Wisk. 4 (*1878) 200 -; 10 (1884) 186-. -, - circumference of circle. Barrachina, E. S. Fschr. Mth. (1897) 447.

-, - curves. Somof, J. [Somov, O. I.] [1870] St Pét. Ac. Sc. Bll. 15 (1871) 257-; St Pet. Ac. Sc. Mm. (Rs.) 18 ("1871) 25-. 
approximate, of ellipse. Schlömilch, O. Z. Mth. Ps. 10 (1865) 501-.

-, - Peano, G. C. R. 109 (1889) 960-, - - and trochoid. Rankine, W. J. M. [1864] Ph. Mg. 29 (1865) 22-.

of arc of meridian, means of shortening calculation. Puissant, L. C. R. 13 (1841) 53-.

- Cartesian ovals. Roberts, W. Liouv. J. Mth. 15 (1850) 194-.

- - Genocchi, A. C. R. 80 (1875) 112-. - - Darboux, G. C. R. 87 (1878) 595-, 741.

- - - and other curves. Genocchi, $A$. Mathesis 4 (1884) 49-.

- cassinoid, $n$ foci. Roberts, W. Liouv. J. Mth. 13 (1848) 38-.

- circular unicursal cubics. Longchamps, G. de. C. R. 104 (1887) 964-.

- class of curves of 4 th degree. Darboux, $G$. C. R. 87 (1878) 692-.

- - plane curves by elliptic functions. Roberts, $W$. Liouv. J. Mth. 10 (1845) 177- conics. Hellins, J. Phil. Trans. (1802) 448 -

- - geometrical theorems. Maccullagh, $J$. [1830] Ir. Ac. T. 16 (1831) (pt. 2) 79-.

- convex curve, Legendre's formula, generalisation. Cavallin, C. B. S. Ts. Mth. 2 (1884) 147-; Fschr. Mth. (1884) 623.

- curve cutting generators of cone at constant angle. Turquan, -. N. A. Mth. 4 (1845) 659 .

- curves. Beverley, T. Tilloch Ph. Mg. 67 (1826) 393-.

- - Gill, C. Ph. Mg. 9 (1831) 250

- -. Dieu, T. N. A. Mth. 12 (1853) 210-

- - Booth, J. A. Mt. 2 (1868-69) 81-.

- - Schlömilch, O. Z. Mth. Ps. 15 (1870)

215.

- -. Scheeffer, L. Acta Mth. 5 (1884) 49-.

- - cases. Roberts, W. Liouv. J. Mth. 12 (1847) 445-

- - - - Roberts, R. A. [1886] L. Mth. S. P. 18 (1886-87) 97-

- - - , remarkable. Longchamps, G. de. Mathesis 7 (1887) 127-, 170-

- - 4th degree, singular case. Sang, $E$. Edinb. R. S. P. 7 (1872) 613-.

- - difficulty. Poisson, S. D. Par. Ec. Pol. Cor. 3 (1814-16) 23-.

- - using projective measurement. Wirtinger, $W$. Mh. Mth. Ps. 5 (1894) 92-.

- cycloid. Peyronny, A. N. A. Mth. 3 (1844) 232-

- -. Dainelli, U. (xII) Rv. Sc.-Ind. 13 (1881) 369-.

- ellipse. Ivory, J. [1796] Edinb. R. S. T. 4 (1798) 177-.

- -. Wilder, C. Silliman J. 18 (1830) $38-$

Verdam, G.J. [1851] Amst. Ts. Ws. Nt. Wet. 5 (1852) 42-

- - Strehlke, $F^{\prime}$. Grunert Arch. 22 (1854) 444 -

- Grunert, J. A. Grunert Arch. 27 (1856)

99-; 30 (1858) 213-

- - Schlömilch, O. Schlömilch Z. 6 (1861) of ellipse. Gaudard, J. A. Gén. Civ. 2 (1873) 483-.

- - Williot, V. A. Pon. Chauss. 12 (1896) $22-$

- application of Taylor's theorem. Benz, O. Arch. Mth. Ps. 8 (1890) 378-.

- - calculation, mode. Vaudoncourt, C.F.G. de. (viri) A. Cond. Pon. Chauss. 5 (1861) 167-. - - given, table for finding. Vaudoncourt, C. F. G. de. (vir) A. Cond. Pon. Chauss. 3 (1859) 252-.

- _ and hyperbola. Bangma, O. S. Amst. N. Vh. 1 (1827) 55-.

- - - Lobatto, R. Amst. N. Vh. 4 (1833) 115-.

- -, resultant integrals. Fergola, N. [1828] Nap. At. Ac. 4 (1839) 13-.

- elliptic arc. Wallace, (Prof.) W. [1802] Edinb. R. S. T. 5 (1805) 253-.

- - - by series. Stainville, - de. Par. Ec. Pol. Cor. 2 (1809-13) 429-.

- - arcs. Nieuport, C. F. F. de. Amst. Vh. 4 (1818) 171-.

- - Guimarães, R. G. Teix. J. Sc. 8 (1887) 30-; Lisb. J. Sc. Mth. 12 (1888) 133-. by elliptic functions. Ramus, $C$. Kiöb. Ov. (1843) 83-; Kiöb. Dn. Vd. Selsk. Afh. 12 (1846) 95-.

Fagnanian arcs of ellipse, and 2 corresponding ares of hyperbola. Grifiths, J. (x) L. Mth. S. P. 5 (1873-74) 95-.

- - - - curves, arcs of which possess same property as. Griffiths, J. L. Mth. S. P. 11 (1879-80) 87-.

Fagnano's point. Andrien, - Mathesis 20 (1900) .105-.

- theorem. Grunert, J. A. Grunert Arch. 26 (1856) 198-.

- - Kitpper, C. Schlömilch Z. 1 (1856) 363-. - Talbot, W. H. F. Edinb. R. S. T. 23 (1861) 285-.

- Malmsten, C. J. Schlömilch Z. 8 (1863) 306-

- - , analogous theorem on associated ellipsoids. Besge, V. A. Bordeaux Mm. S. Sc. 2 (1861-63) 247-.

- - Arcs of conics with rectifiable difference. Chasles, M. C. R. 17 (1843) 838-.

-. - - - - - Serret, J. A. N. A. Mth. 3 (1844) 425-.

- - and associated points of ellipse. Lebesgue, V. A. N. A. Mth. 4 (1845) 573-.

- - for any conic. Azzarelli, M. Rm. At. N. Linc. 24 (1871) 336-; 25 (1872) 427-. - - proof. Walker, J. J. L. Mth. S. P. 13 (1881-82) 172-.

- - on sphere. Enneper, A. Schlömilch Z. 8 (1863) 231-.

- - stereometric analogues. Schlömilch, $O$. Leip. B. 13 (1871) 13-

- - ; and surface of oblique cylinder, etc. Brinkley, J. [1802] Ir. Ac. T. 9 (1803) 145.

first, and Torricelli. Loria, G. Rm. R. Ac. Linc. Rd. 6 (1897) (Sem. 2) 318-.

and Fontaine's theorem on triangle. Lecointe, (l'abbé) -. N. A. Mth. 10 (1851) 196-.

of geodetic curve. Young, (Dr) T. QJ. Sc. 21 (1826) 153-. 
graphic, of ellipse. Somoff, J. [1850] St Pét. Ac. Sc. Bll. 9 (1851) 97-.

of hyperbola. Hellins, J. Phil. Trans. (1811) 110 -

- - Küpper, C. Crelle J. 55 (1858) 89-.

- lemniscates and other curves. Roberts, $W$. Ir. Ac. P. 2 (1844) 180-.

- lines of curvature on cylinders. Ahrendt, A. Arch. Mth. Ps. 8 (1890) $442-$.

- - - - ellipsoid. Roberts, M. [1871] A. Mt. 5 (1871-73) 17-.

- - on surfaces. Böklen, O. Grunert Arch. 36 (1861) 32-.

- plane curves. Prouhet, E. N. A. Mth. 3 (1864) 403-.

- - cases. Tortolini, B. G. Arcad. 105 (1845) 193-.

- - unicursal cubics. Raffy, L. C. R. 107 (1888) 944-, 1030; Par. Éc. Norm. A. 6 (1889) 103-.

$-\frac{-1}{892}$.

and property of secondary caustics. Genocchi, A. A. Mt. 6 (1864) 97-

- quadrature. Cauchy, A. L. [1832-41] Par. Mm. Ac. Sc. 22 (1850) 3-; C. R. 13 (1841) 1060-.

- -. Grunert, J. A. Grunert Arch. 26 (1856) 48-.

- - of conics. Azzarelli, M. Rm. N. Linc. At. 28 (1875) 287-.

- - - intersections of quadrics. Azzarelli,

M. Rm. N. Linc. At. 29 (1876) 337-.

- - - involute of circle and of developable helicoid. Pressel, W. Grunert Arch. 4 (1844) 337-.

- - spherical ellipse. Booth, J. R. S. P. 4 (1842) 387-; Ph. Mg. 25 (1844) 18-.

- - toroid. Dienger, $J$. Grunert Arch. 9 (1847) 438-.

- - and volume. Dirksen, E. H. Berl. Ab. (1833) 123-.

with reference to complex roots of algebraic equations. Lebesgue, $V . A$. N. A. Mth. 3 (1844) 145-.

of roulettes. Steiner, $J$. Berl. Ab. (1838) 19-. same, pairs of curves having. Euler, $L$.

[1781] St Pét. Ac. Sc. Mm. 11 (1830) 102-.

of spherical curves. Tortolini, B. Tortolini A. 5 (1854) 71 -.

- - ellipse and division of arcs. Tortolini, B. G. Arcad. 109 (1846) 231-.

- sphero-conics. Strebor [Roberts, W.]. N. A. Mth. 9 (1850) 141-.

- trisectrix of Maclaurin by elliptic integrals.

Longchamps, G. de. C. R. 104 (1887) 676-.

Ruled surfaces, theorem. Thue, A. Thrond. Skr. (1896) No. 6, 6 pp.

Sectors in space on same base. Zeuthen, H. G. (xII) Ts. Mth. 2 (1866) 60-.

- - theorems. Oppermann, L. As. Nr. 66 (1866) 129-.

Segment of circle and Simpson's formula. Wittstein, $T$. Grunert Arch. 39 (1862) 12-.

Skew curves rectifiable in algebraic terms. Stäckel, P. Mth. A. 43 (1893) 171-.
Skew curves, theorems, general. Hurwitz, $A$. Mth. A. 25 (1885) 287-; 27 (1886) 162.

Solid bounded by sphere and lemniscate cone. Azzarelli, $M$. Tortolini A. 8 (1857) 372-.

Spherical curves analogous to Serret's. Roberts, W. Liouv. J. Mth. 12 (1847) 479-.

- - - - Serret, J. A. Liouv. J. Mth. 12 (1847) 480-.

Surface of body in terms of a volume-integral. Tait, $P$. G. Edinb. R. S. P. 9 (1878) 541-.

- - conoid with elliptic base. Korteweg, D. J. N. Arch. Wisk. 3 (*1877) 60-

-, 4th degree, $a^{3} z=x^{2} y^{2}$, cubature, etc. Schlömilch, O. Grunert Arch. 12 (1849) 193-.

- of ellipsoid, integrations over. Tallqvist, $H$. Helsingf. Acta 18 (1891) 329-.

- - - in terms of Weierstrass's elliptic functions, Boyd, J. H. [1892] A. Mth. 7 (1892-93) 1-。

- integrals reducible to contour, theory. Réthy, M. (xI) Mag. Tud. Ak. Étk. (Mth.) 3 (1875) (No. 7) 20 pp.

- of oblique cyclinder, Brinkley's theorem. Grunert, J. A. Grunert Arch. 10 (1847) $222-$.

Tetrahedrometry, differential formulæ. Miller, J. H. T. Schlömilch Z. 5 (1860) 49-。

Theorem. Mendeleef, -. C. R. 121 (1895) 421-.

- of Holditch, extension. Echols, W. H. A. Mth. 4 (1888) 47-.

- - Pappus. Henck, J. B. Camb. (M.) Mth. M. 1 (1859) 200-

- Viviani, generalisation. Weinberg, W. P. Fschr. Mth. (1900) 624.

Vaulted surfaces (generated by semicircles on radii vectores of plane curve). Schlömilch, O. Schlömilch Z. 8 (1863) 121-.

\section{Volumes.}

application of Simpson's rule. Isely, (Prof.) -. [1869] Neuch. Bll. 8 (1870) 262-.

- - - to cylindrical wedge. Graeber, Arch. Mth. Ps. 17 (1900) 401-.

and areas, determination. Ghysens, $\boldsymbol{E}$. Brux. Ac. Bll. 44 (1877) 532-.

bounded by ruled surface and parallel ends. Chelini, D. G. Arcad. 96 (1843) 3-.

calculation. Gaudard, $J$. A. Gén. Civ. 2 (1873) 483.

-. Maleyx, L. N. A. Mth. 19 (1880) 529-.

-, approximate. Daug, H.T. [1865] Stockh. Öfv. 22 (1866) 415-.

- - Mansion, P. (xII) Brux. S. So. A. 6 (1882) (Pt. 2) 228-

_, - , formulæ, application to ships. Grunert, J. A. Arch. Mth. Ps. 47 (1867) 176-.

-, formula. Latars, G. (xII) Isère S. Bll. 11 (1882) 341-; 13 (1884) 18.

- for pedal surface of ellipsoid. Magener, $A$. Grunert Arch. 34 (1860) 450-.

- solids. Dirksen, E. H. Berl. Ab. (1833) 123-.

- -, cases. Finck, B. N. A. Mth. 7 (1848) 241-. 
calculation for solids derived from ellipsoid. Dienger, J. Grunert Arch. 12 (1849) 81-. - - of revolution. Collignon, $\dot{E}$. As. Fr. C. R. 10 (1881) 196-.

- - wave surface. Roberts, $W$. (vIII) A. Mt. 4 (1861) 345-; (v) C. R. 55 (1862) 503.

and centroid of class of solids, formula for. Lobatto, R. (vir) Amst. N. Ws. Ntk. Vh. 2 (1854) 121-.

of class of solids. Grunert, J.A. Grunert Arch. 31 (1858) 481-.

- conical and cylindrical ungulæ. Timmermans, J. A. Quetelet Cor. Mth. 2 (1826) 149-.

- - heaps of ore. Heyse, G. Karsten Arch. 5 (1832) 511-

- - wedge. Hube, K. (vi Adds.) Krk. Roczn. Uniwers. 8 (1823) 115-.

- cylinder cut by plane oblique to base. Eilles, J. Arch. Mth. Ps. 42 (1864) 186-.

- Eilles). Lobatto, $R$. Arch. Mth. Ps. 43 (1865) 235-.

determination by help of centroid. Blazek, $G$. Wien SB. $46(A b .2)$ (1863) 342-.

-, in Lobačevskij's geometry. Simon, $M$. Mth. A. 42 (1893) 471-.

of ellipsoid, geometrical determination. Mathiot, $O$. L. Des Moines Anal. 8 (1881) 124-.

equal, division of ellipsoid into. Weddle, $T$. Mathematician 2 (1847) 302-.

generated by closed contour in any motion. Koenigs, G. Liouv. J. Mth. 5 (1889) 321-.

- - - - turning about all straight lines of space, distribution. Koenigs, G. C. R. 106 (1888) 927-.

- - contour rigidly connected with trihedron of curve. Koenigs, G. C. R. 107 (1888) 474-.

- - revolving arc of circle. Noel, J. N. Quetelet Cor. Mth. 6 (1830) 61-.

Guldin's rule. Grunert, J. A. Grunert Arch. 32 (1859) 348-.

- -, extension. Richter, P. B. Z. Mth. Ps. 37 (1892) 172-.

- theorem. Bordoni, A. [1826] Mod. Mm. S. It. 20 (1828) 86-.

- -, extensions. Jung, $G$. Rm. R. Ac. Linc. T. 7 (1883) 97-.

- - generalisation. Fabry, Mntp. Ac. Mm. 11 (1892) 257-.

- -, 一. Kúscow, -. N. A. Mth. 17 (1898) 209 -.

integrals, classification. Geometrical definition of surfaces capable of cubature. Marie, $M$. C. R. 80 (1875) 757-.

of loci of connected points. Elliott, E. B. [1882] L. Mth. S. P. 14 (1883) 62-.

- paraboloid, geometrical determination. Mathiot, O.L. Des Moines Anal. 10 (1883) 46-.

- - of revolution, extension of theorem of Archimedes to. Haedicke, $H$. Dingler 237 (1880) 165, 328.

- pedal surfaces. Hirst, T. A. C. R. 55 (1862) 572-; Phil. Trans. (1863) 13-. of portion of elliptic cone cut off by two planes. Unferdinger, $F$. Arch. Mth. Ps. 41 (1864) 178-.

- - right circular cone between a circular and a hyperbolic segment. Zambelli, $A$. Ven. Aten. At. 12 (1875) 267-.

- prismatoid, formula. Becker, J. C. [1877] Z. Mth. Ps. 23 (1878) 412-.

between ruled surface and two parallel planes. Terrier, L. [1874] Neuch. S. Sc. Bll. 10 (1876) (App.) 6-.

of ruled surfaces. Haillecourt, A. Bordeaux Ac. Act. $39-40$ (1877-78) 155-.

- segment of surface, 2nd degree, bounded by parallel planes. Booth, J. Ph. Mg. 20 (1842) 472-.

- segments, and frusta of quadrics. $U n$ ferdinger, $F$. [1869] Wien Sb. 60 (1870) (Ab. 2) 631-.

- ship-shaped bodies. Grunert, J.A. Grunert Arch. 13 (1849) 443-.

- skew surface. Boulanger, A. N. A. Mth. 16 (1897) 171-.

- solid bounded by hyperbolic paraboloid. Kawalki, W. [1899] Hamb. Mth. Gs. Mt. 3 (1900) 400-.

_ - , formula of "three levels" for. Goulard, -. Mathesis 17 (1897) 105-.

- generated by motion of plane in space. Christiansen, C. (xII) Ts. Mth. 1 (1865) 163 -.

- solids of revolution. Sinram, T. Hamb. Mth. Gs. Mt. 1 (1889) 20-.

- -, J. Wilson's method. Clark, T. Glasg. Ph. S. P. 2 (1844-48) 161-.

- spherical segment. Les Enfants, J. Les Mondes 3 (1882) 173-.

- surface, 4th degree, pedal of hyperboloid. Tortolini, B. [1847] Mod. S. It. Mm. 24 (1848) 378-.

- - of revolution given in polar coordinates. Boije af Gennäs, -. Ts. Mt. Fys. 3 (1870) 193-.

- surfaces and lengths of arcs of figures described by straight line. Schumann, $A$. Z. Mth. Ps. 25 (1880) 87-.

- - of revolution generated by conics, and areas of conics. Gaboardi, S. Brugnatelli G. 10 (1827) 266-.

- -, sections of which parallel to plane are quadratic functions of distance. Weinmeister, -. Arch. Mth. Ps. 17 (1900) 190 -.

and surfaces of 2 solids of revolution. Dostor, G. Arch. Mth. Ps. 68 (1882) 421-.

of truncated cone by indeterminate coefficients. Ivon, L. N. A. Mth. 2 (1843) 23-.

- ungulæ. Lehmus, (Dr) - (vIII) Z. Bauw. 4 (1854) 573-.

- various bodies. Stamkart, F. J. (viri) Amst. N. Ws. Ntk. Vh. 2 (1854) 187-.

- zones of equal height of solids of revolution, formulæ. Winkhaus, W. Crelle J. 44 (1852) 375. 
8470 Special transcendental curves. (For cycloids, epicycloids and trochoids see 8420.)

Catenary. Hill, T. Gould As. J. 2 (1852) 10-. -, areas and volumes related to. Wasteels, $C$. $E$. Mathesis 16 (1896) 241-.

-, centrifrugal. Webb,J.B. Am. As. P.(1889) 76.

-, constants. Löfler, A. Grunert Arch. 36 (1861) 323-.

construction, graphic method. Palmer, W. K. Kan. Un. Q. 7 (1898) 211-.

- of equal strength, involute. Azzarelli, $M$. Palomba Rac. 4 (1848) 9-, 17-, 35-, 57-.

- _ - _, - , identity of tractrix with. Calegari, P. (vII) Bologna Opusc. Sc. N. Col. (1824) 233-.

- _ - - and other transcendental curves, properties. Masetti, G. Bologna Opusc. Sc. 4 (1823) 179-.

-, evolute (Huygens' problem). Egger, $\boldsymbol{E}$.

A. Mt. 6 (1864) 19-.

-, mechanical description. Robillier, -. Angers Mm. S. Ag. 1 (1831) 41-.

-, by parabolic trigonometry. Booth, J. R. S. P. 8 (1856-57) 443-.

- problem, Huygens' solution. Korteweg, D. J. Bb. Mth. 1 (1900) 97-.

-, spherical. Greenhill, A. G. [1894] L. Mth. S. P. 27 (1896) 123-.

- on surface of revolution. Molins, H. Toul. Mm. Ac. 5 (1849) 261-.

-, vertical force on elements and distance of elements from horizontal plane. Molins, $H$. Toul. Mm. Ac. 4 (1848) 221-.

Closed class of transcendental curves. Schwering, $K$. Z. Mth. Ps. 20 (1875) 457-.

Curve of elliptic functions of 1st kind. Serdobinskij, V. E. [1893] Rec. Mth. (Moscou) 17 (1895) 337-; Fschr. Mth. (189394) 1158.

- logarithmic sines. Barbour, L. G. Des Moines Anal. 4 (1877) 114-.

- _ - evolute. Barbour, L. G. Des Moines Anal. 4 (1877) 169-.

- second sines and its variations. Sang, E. [1874] Edinb. R. S. P. 8 (1875) 356-.

$-r=\frac{a \sin \theta}{\theta}$ : Fontana, G. Verona S. It. Mm. 2 (1784) 123-.

Differential curve of parabola, foci. Hochheim, A. [1875] Arch. Mth. Ps. 58 (1876) 278-.

Elastic eurve. Halphen, G. Par. Éc. Pol. J. 54 (1884) 183-.

- - Isè, E. Nap. Ac. Pont. At. 18 (1888) 203-.

- Tedone, O. Rm. R. Ac. Linc. Rd. 3 (1894) (Sem. 1) 265-.

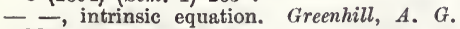
Mess. Mth. 8 (1879) 82.

Evolutes of circular arcs. Poinsot, L. Par. Éc. Pol. Cor. 1 (1804-08) 245-.

Exponential curve. Church, I. P. Des Moines Ánal. 1 (1874) 84-.
Helices. Tissot, A. N. A. Mth. 11 (1852) 454-.

-, characteristic property. Appell, P. Arch. Mth. Ps. 64 (1879) 19-.

- and helicoidal surfaces. Richardson, J. M. Franklin I. J. 33 (1857) 231-.

-, problem. Cesáro, E. G. Mt. 24 (1886) 46-. -, theory. Tos y Feitto, J. de. [1898] Barcel. Ac. Mm. 2 (1892-1900) 307-.

-, -, general. Schoelcher, (le col.) -. As. Fr. C. R. (1891) (Pt. 1) 160.

-, - of Torricelli. Ferroni, P. Mod. S. It. Mm. 15 (1811) 60-.

Helix, only curve with ratio of curvatures constant. Bertrand, J. Liouv. J. Mth. 13 (1848) 423-.

-, cylindrical, lines connected with. Pirondini, G. Crelle J. Mth. 121 (1900) 245-.

- projected into cycloid. Olivier, T. Par. S. Phlm. PV. (1847) 106-.

-, property. Reye, T. Z. Mth. Ps. 15 (1870) 64-.

-, sense. Anon. (vI 889) N. A. Mth. 14 (1855) 175-

Hirundo. Hill, (Rev.) T. Am. As. P. (1886) 67-.

Hypergeometric curves. Multedo, - Genova Mm. Ac. 3 (1814) 328-.

Involute of circle. Bakewell, T. W. Franklin I. J. 7 (1831) 99-

_- - Crofton, M. W. [1864] Mess. Mth. 3 (1866) 93-.

- - D'Auria, L. Franklin I. J. 80 (1880) 45-.

_- - Skinner, J. J. Franklin I. J. 80 (1880) 105-.

Involutes of circle. Hill, T. Am. As. P. 19 (1870) 21-.

- - - depending on Diophantine equation. Sylvester, J. J. L. Mth. S. P. 2 (1869) 137-.

- - and their relation to analytic functions. Thoman, F. C. R. 26 (1848) 498-.

- - - successive. Sylvester, J. J. $\mathrm{Ph}$. Mg. 36 (1868) 295-, 459-.

Kumaids. (Plane curves becoming ellipses on cylinder.) Vieth, G. U. A. Gilbert A. 54 (1816) 311-.

Logarithmic curvé, researches of Torricelli. Loria, G. Bb. Mth. 1 (1900) 75-.

- exponential curves. Vincent, A. J. H. Gergonne A. Mth. 15 (1824-25) 1-; 16 (182526) 92 .

parabola. Booth, J. Camb. and Dubl. Mth. J. 7 (1852) 35-.

Logocyclic curve. Booth, J. R. S. P. 9 (185759) 256-; QJ. Mth. 3 (1860) 38-, 127-.

- or strophoid. Gunther, A.W.S. (xI) Mathesis 1 (1881) 81-.

Loxodromes of cone. Piccioli, H. N. A. Mth. 18 (1899) 508-.

Mechanical properties of some curves. Heulen, J. van. N. Árch. Wisk. $7\left({ }^{*} 1881\right) 33-$.

Normals to several mechanical curves, construction. Chasles, M. [1829] (Ix) Par. S. Mth. Bll. 6 (1878) 208-.

Plane curves, Roberval's method. Geer, $\boldsymbol{P}$. van. N. Arch. Wisk. 11 (1884) 28-; Fschr. Ps. (1884) (Ab. 1) 217-. 


\section{Spirals}

Plane curves, theory. Enneper, A. Gött. Nr. (1884) 364-.

- transcendental curve, arc, representation of elliptic function, 1st species, by. Tortolini, $B$. Tortolini A. 4 (1853) 485-.

- - curves belong to system $(\mu, \nu)$. Fouret, G. Par. S. Mth. Bll. 2 (1874) 96-.

"Points de dédoublement." Mansion, P. Bll. Sc. Mth. As. 3 (1879) (Pt. 1) 514 -

Singular curves. Peano, G. Tor. Ac. Sc. At. 26 (1891) 299-.

Sinusoidal curves. Cesáro, E. N. A. Mth. 13 (1894) 102-.

\section{Spirals.}

Pagani, G. M. Brux. Mm. Cour. 5 (1826) $58 \mathrm{pp}$.

Olivier, T. Par. S. Phlm. N. Bll. (1833) 21-. Hill, T. Am. As. P. (1859) 158.

Schou, T. V. Mth. Ts. 2 (1860) 6-.

La Gournerie, J. de. C. R. 66 (1868) 283-, 832-; Liouv. J. Mth. 14 (1869) 9-, 103-.

approximate construction. Thallmayer, $V$. Dingler 226 (1877) 156-.

of Archimedes, minimal property. Janisch, E. Arch. Mth. Ps. 9 (1890) 445-.

class, properties. Laquière, E. M. N. A. Mth. 2 (1883) 118-

conical, graphic method for tangents. Garbinski, $H$. Gergonne A. Mth. 16 (1825-26) 167-.

equiangular, geometrical proofs. Whitworth, W. A. Mess. Mth. 1 (1862) 5-, 138-.

harmonic. Raffy, L. Par. Ec. Norm. A. 12 (1895) 145-.

hyperbolic or inverse. Palmer, W. K. Kan. Un. Q. 7 (1898) 155-.

-, tangents. Schiffner, F. Arch. Mth. Ps. 66 (1881) 334-.

logarithmic. Turquan, - N. A. Mth. 5 (1846) 88 -

-. Pirondini, G. Mathesis 19 (1899) 153-.

-, construction. Wiebe, F. K. H. Berl. Pol. Gs. Vh. 23 (1862) 106-.

-, history of applications. Favaro, A. Bb. Mth. (1891) 23-.

-, and involute of circle. Orlando, L. Mathesis 20 (1900) 110-.

-, new. Binet, J. P. M. Par. S. Phlm. PV. (1841) 15 .

new. Drach, S. M. Mathematician 3 (1850) 34 .

peculiar. Wolfers, J. P. Grunert Arch. 28 (1857) 114-.

properties. Laguerre, E. (x) Par. S. Phlm. Bll. 6 (1869) 39-.

system, intrinsic equations. Onnen, $H$. Arch. Néerl. 10 (1875) 361-。

$r=a \frac{\sin \phi}{\phi}$. Stöckly, L. Arch. Mth. Ps. 48 (1868) 109-.

where $\rho=r$. Schlömilch, $O$. Z. Mth. Ps. 14 (1869) 162-.

$\rho^{n}=A \sin n \omega . \quad$ Haton de la Goupillière, J. N. N. A. Mth. 15 (1876) 97-.

$r \theta=a \sin \theta$. Jung, G. Rm. R. Ac. Linc. T. 7 (1883) 97-

\section{Transcendental Surfaces 8480}

$\rho^{m}-\rho^{n}=\left(\alpha^{m}-\alpha^{n}\right) \frac{\theta}{\pi}$, properties. Cabreira, $A$. Lisb. J. Sc. Mth. 5 (1898) 1-; Fschr. Mth. (1897) 536.

$\rho=R \sin \mu \omega$, algebraic if $\mu$ is integral, transcendental otherwise. Loria, G. Fschr. Mth. (1899) 515.

Strophoidals. Longchamps, G. de. Mathesis 14 (1894) 138-.

Tractrix. Cesáro, E. (xIr) Mathesis 2 (1882) $217-$.

- of curve with planimetric application. Lehmer, D. N. A. Mth. 1 (1900) 14 -

-, polar. Webb, J. B. Am. As. P. (1889) 74-.

-, problem. Dubuat, -. Gergonne A. Mth. 4 (1813-14) 332-; 5 (1814-15) 210-.

Trajectories, isogonal, of co-axal circles. Lodge, A. Mth. Gz. 1 (1900) 213-

,,----- Macaulay, F. S. Mth. Gz. 1 (1900) 214

Transcendental curve, properties. Fuss, N. [1817] St Pét. Ac. Sc. Mm. 8 (1822) 147-.

,-- - Azzarelli, M. (vII) A. Mt.5 (1863) $72-$

- 一, 一. Egger, E. A. Mt. 6 (1864) 21-.

- curves, properties of two. Collins, E. [1812] St Pét. Ác. Sc. Mm. 6 (1813-14) 86-.

Trigonometric curves, circular and hyperbolic analogies. Anon. (vi 888) N. A. Mth. 14 (1855) 151-.

$y=\sqrt[x]{x}$. Muller, Hub. Arch. Mth. Ps. 44 (1865) 128.

$\sin y \sin m y=a \sin x \sin n x+b$. Nevoton, H.A., de Phillips, A. W. [1875] Conn. Ac. T. 3 (1874-78) 97-.

\section{Special transcendental surfaces.}

Curves and surfaces. Pirondini, G. G. Mt. 26 (1888) 352-.

Envelope of sphere, centre of which describes cycloid, edge of regression. Livet, - Par. Éc. Pol. Cor. 1 (1804-08) 195.

Helical surfaces, theory. Stäckel, $P$. Leip. Mth. Ps. B. 50 (1898) (Mth.) 3-.

Helicoid. Schiffner, $F$. Arch. Mth. Ps. 7 (1889) 50-.

-, general. Issaly, -. N. A. Mth. $19(1900)$ 499-.

-, principal radii of curvature. Paignon, $F$. M. N. A. Mth. 4 (1845) 413 -

- of revolution, properties of lines of shadow. Negri, C. Tor. Ac. Sc. At. 14 (1878) 116 .

Helicoids. Enneper, A. Gött. Nr. (1870) 335-.

-. Bianchi, L. [1878] G. Mt. 17 (1879) 9-.

- Fouret, G. As. Fr. C. R. 7 (1878) 173-.

-. Schiffner, F. Arch. Mth. Ps. 68 (1882) 72-.

-. Machovec, F. Časopis 13 (1884) 111-; Fschr. Mth. (1884) 519-. 


\section{Transcendental Surfaces}

Helicoids. Pirondini, G. N. A. Mth. 6 (1887) 87- ; A. Mt. 16 (1888-89) 137-.

-, curvature and indicatrix. Sobotka, $J$. Wien Ak. Sb. 101 (1892) (Ab. 2a) 899-.

- with director plane. Catalan, E. C. Par. S. Phlm. PV. (1843) 113-.

- - - , sections. Pécharman, P. C. R. 103 (1886) 987-

-, orthogonal and tangential. Pirondini, G. Rv. Mt. 2 (1892) 127-.

Skew helicoids. Bruno, G. Tor. At. Ac. Sc. 2 (1866-67) 601-; 3 (1867-68) 194-.

- - contact-curves and enveloping torses of. Schmid, $T$. Wien Ak. Sb. 99 (1891) (Ab. 2a) 952-.

- - developable. Lamarle, E. Brux. Ac. Bll. 11 (1861) 321-.

- - - surfaces connected with. Sobotka,J. Mh. Mth. Ps. 4 (1893) 59-.

- surfaces, asymptotic lines. Bioche, $C$. Toul. Fac. Sc. A. 2 (1888) A, 7 pp.

Spiral surfaces, characteristic property, and theorem of Ribaucour. Demoulin, A. Par. S. Mth. Bll. 23 (1895) 198-.

- - linear element. Demoulin, A. C. R. 118 (1894) 337-.

- with parallel lines of curvature, linear element. Raffy, L. Par. S. Mth. Bll. 20 (1892) 22-.

- -, theory. Ebner, - Arch. Mth. Ps. 14 (1896) 241-.

Surface, equation of which is $e^{r}=\frac{\cos x}{\cos y}$, Padula's work. Rubini, R. N. A. Mth. 15 (1856) 183-.

- generated by logarithmic curve. Bruno, $G$. [1879] Tor. Ac. Sc. At. 14 (1878) 735-.

Surfaces associated with Peterson's. Mlodzéevskij, B. K. Rec. Mth. (Moscou) 21 (1901) 450-; Fschr. Mth. (1900) 613.

Transcendental surfaces including cyclide as case. Holzmuller, F. G. [1882] Crelle J. Mth. 94 (1883) 239-.

\section{Hypergeometric configura- tions and higher elements of hyperspace.}

Curvature of "curves" in $n$ dimensions, Landsberg, G. Crelle J. Mth. 114 (1895) 338-.

- - higher varieties. Voss, A. E. Mth. A. 16 (1880) 129-.

- - - - measure. Beez, R. (vir) Mth. A. 7 (1874) 387-; (Ix) Z. Mth. Ps. 21 (1876) 373-; 24 (1879) 1-, 65-.

-, measure, Gauss's, theory. Allé, M. [1876] Wien Ak. Sb. 74 (1877) ( $A b .2)$ 9-.

-, -, Riemann's, theory. Voss, A.E. Mth. A. 16 (1880) 571-

- of varieties. Berzolari, L. Tor. Ac. Sc. At. 33 (1897) 462-, 527- or 692-, 759-.

Curves of triple curvature and their parallels. Hoppe, R. Arch. Mth. Ps. 65 (1880) 373-.

4th dimension, uniform positive curvature in. Nerocomb,$S$. Crelle J. Mth. 83 (1877) 293-.
Hypergeometry 8490

3-dimensional varieties. Cotton, $\dot{E}$. Toul. Fac. Sc. A. 1 (1899) 385-.

- - with one zero and two equal curvatures. Banal, R. A. Mt. 24 (1896) 213-.

3- and higher dimensional space, curvature. Hadamard, - . Bordeaux S. Sc. PV. (189798) 85-

4-dimensional geometry. Lovett, E. O. [1900] N. Y. Am. Mth. S. Bll. 7 (1901) 88-

$n$-dimensional curves, intrinsic treatment. Cesáro, E. Rm. R. Ac. Linc. Rd. 5 (1889) (Sem. 2) 165-

- Euclidean space, expansion of coordinates in powers of arc. Richmond, H. W. QJ. Mth. 31 (1900) 315-.

- rotation ellipsoid, geodesics on, application of calculus of variations. Rudio, $\bar{F}$. Zür. Vjschr. 43 (1898) 340-.

- space. Keyser, C. J. N. Y. Am. Mth. S. Bll. 4 (1898) 177, 181-.

- - differential geometry. Lovett, $E . O$. [1900] N. Y. Am. Mth. S. Bll. 7 (1901) 203,210 .

- - linear, metrical properties of skew curves. Brunel, G. E. A. [1881] Mth. A. 19 (1882) 37 .

- spheres, volumes and surfaces of. Story, W. E. [1880] (xII) J. H. Un. Cir. [1] (1882) 48.

- theory of curves. Hoppe, R. Arch. Mth. Ps. 6 (1888) 168-.

Elliptic space, helicoidal and ruled surfaces. Gigli, D. Mil. I. Lomb. Rd. 33 (1900) 717-.

Generalisation to $p$-dimensional space of trans. formation of line into surface integrals. Franchis, M. de. Palermo Cir. Mt. Rd. 12 (1898) 163-.

Hyperspace, Codazzi's formulø in. Cesáro, $E$. Nap. Rd. 33 (1894) 87-.

-, complex variables in. Volterra, $V$. Rm. R. Ac. Linc. Rd. 5 (1889) (Sem. 1) 291-; 6 (1890) (Sem. 2) 241-.

-, extension of Euler's and Meunier's theorems to Berzolari, L. Rm. R. Ac. Linc. Rd. 6 (1897) (Sem. 2) 283-; 7 (1898) (Sem. 1) 4-.

-, functions. Fabri, C. Ven. I. At. (1892-93) $283-$.

-, - , and their differential parameters. Volterra, V. Rm. R. Ac. Linc. Rd. 5 (1889) (Sem. 1) 630-

-, theory. Ricci, G. Rm. R. Ac. Linc. Rd. 4 (1895) (Sem. 2) 232-.

Hyperspheric lines of constant curvature. Piccioli, H. N. A. Mth. 19 (1900) 385-.

Knotted curve, equation, and solution in 4th dimension. Hoppe, $R$. Arch. Mth. Ps. 64 (1879) 224.

- - Hoppe's. Durège, H. [1880] Wien Ak. Sb. $82(1881)(A b .2)$ 135-

- - , solution in 4th dimension. Hoppe, $R$. Arch. Mth. Ps. 65 (1880) 423-

Lobačevskij's planimetry, concrete interpretation. Lachtin, L. K. Rec. Mth. (Moscou) 17 (1895) 767-; Fschr. Mth. (1895) 534-.

Non-Euclidean space, geodesic geometry of surfaces, Whitehead, A. N. L. Mth. S. P. 29 (1898) 275-. 


\section{Differential Geometry}

Osculating sphere of curve and analogues in $n$ dimensions. Hoppe, $R$. Arch. Mth. Ps. 12 (1894) 96-.

Parallel varieties, invariants. Forsyth, A. R. B. A. Rp. (1899) 640-.

Pseudospherical surfaces. Bianchi, L. A. Mt. 24 (1896) 347-.

- varieties of three dimensions. Brill, $A$. Mth. A. 26 (1886) 300-.

Pseudo-surfaces, geometrical study of curvature. Issaly, -. Par. S. Mth. Bll. 17 (1889) 84-.

Space of constant negative curvature, fundamental equations in geometry. Kagan, $H$. N. A. Mth. 14 (1895) 20-

Spaces of constant curvature. Banal, $R$. Rm. R. Ac. Linc. Rd. 7 (1898) (Sem. 1) 7-.

- _ - - connexion with projective spaces. Schur, F. $\quad$ Mth. A. 27 (1886) 537-.

$--\ldots$, metrics. Giudice, $F$. Tor. Ac. Sc. At. 35 (1900) 119- or 193-.

\section{Differential geometry; applications of differential equations to geo- metry.}

\section{General.}

Automorphic curves and surfaces, family. Klein, F., \& Lie, S. C. R. 70 (1870) 12221275-.

Curves represented by

$\frac{d x}{\sqrt{\left\{\left(1-x^{2}\right)\left(1-k^{2} x^{2}\right)\right\}}} \pm \frac{d y}{\sqrt{\left\{\left(1-y^{2}\right)\left(1-k^{2} y^{2}\right)\right\}}}=0$, and their envelopes. Turner, H. H. Mess. Mth. 10 (1881) 21-.

Developable surfaces. Kobb, $G$. Par. S. Mth. Bll. 19 (1891) 1-.

Differential equation of conics. Halphen, G. H. Par. S. Mth. Bll. 7 (1879) 83-.

- , 1st order, curves defined by. Picard, $k^{\prime}$. C. R. 120 (1895) 522-

- - , - - - - - asymptotes. Zeuthen, H. G. N. Ts. Mth. 4 (B) (1893) 73-.

- - of all parabolas: Mukhopadhyay, $A$. [1888] Beng. As. S. J. 57 (Pt. 2) (1890) 316 -

- - for points of curve or surface. Halphen, G. H. C. R. 81 (1875) 1053-.

- equations, curves defined by. Poincaré, $H$. C. R. 93 (1881) 951-; Liouv. J. Mth. 7 (1881) 375-; 8 (1882) 251-; C. R. 98 (1884) 287-; Liouv. J. Mth. 1 (1885) 167-; 2 (1886) 151-.

- representable by rational curves. Fano, G. Rm. R. Ac. Linc. Rd. 4 (1895) (Sem. 1) 51-.

- - of ruled surfaces and surfaces of revolution. Dolezal, $E$. Arch. Mth. Ps. 14 (1896) 1-.

- - trajectories. Mukhopadhyay, A. [1887] Beng. As. S. J. 57 (Pt. 2) (1890) 72-.

- geometry, question in. Piccioli, $H$. N. A. Mth. 18 (1899) 454-.

,- 2 theorems in. Lamioni, C. N. A. Mth. 19 (1900) 557-.

\section{Curves on Surfaces 8810}

Differential parameters in theory of surfaces. Frobenius, G. Crelle J. Mth. 110 (1892) 1-.

Envelopes. Lecornu, L. Caen Ac. Mm. (1890) (Pt. 1) 12-.

Fundamental magnitudes in theory of surfaces. Knoblauch, J. Crelle J. Mth. 103 (1888) 25-.

Geometry in 3-dimensional space. Calinon, $A$. Nancy S. Sc. Bll. (1891) 35-.

- on surfaces of constant curvature. Carda, $K$. Wien Ak. Sb. 107 (1898) (Ab. 2a) 44-.

Infinitesimal geometry of surfaces. Arvay, $W$. Prace Mt.-Fiz. 9 (1898) 164-.

Isometric curves. Ocagne, M. d'. Brux. S. Sc. A. 9 (1885) (Pt. 1) 56-.

Linear partial differential equations belonging to theory of surfaces. Craig, $P$. C. R. 123 (1896) 634-.

Partial differential equations derived from Codazzi's, applications. Craig, $T$. Crelle J. Mth. 120 (1899) 165-.

- _ - in geometry, and projectivity. Sanio, T. Arch. Mth. Ps. 1 (1884) 225-.

Points on curve satisfying algebraic differential equation, space analogue. Halphen, G. $H$. Liouv. J. Mth. 2 (1876) 257-, 371-.

Shortest line on sphere. Hoilel, J. N. A. Mth. 7 (1868) 73-.

Surfaces cutting lines at given angle. Borenius, H. G. [1838-39] St Pét. Mm. Sav. Etr. 4 (1845) 255-; Helsingf. Acta 1 (1840) 381-.

- on which point can move according to certain law. Saint Germain, A. de. Liouv. J. Mth. 2 (1876) 325-.

- of revolution with minimum line in given plane. Molins, H. Toul. Ac. Sc. Mm. 2 (1890) 360-.

Transformations, class. Clairin, J. C. R. 130 (1900) 309-.

Unique ellipsoid. Adan, E. [1878] Brux. Mm. Cour. $8^{\circ}, 29$ (1880) (No. 4) $10 \mathrm{pp}$.

\section{Determination of curves on surfaces.}

Algebraic curves of constant torsion, and algebraic minimal surfaces inscribed in sphere. Cosserat, E. C. R. 120 (1895) 1252-.

Arc element of surfaces, geodesic circles of which admit of contact transformation. Lie, S. Arch. Mth. Ntvd. 9 (1884) 40-.

Asymptotic lines and conjugate systems on surface. Lelieuvre, - C. R. 109 (1889) 792-.

- of Kummer surface. Hutchinson, J. I. N. Y. Am. Mth. S. Bll. 5 (1899) 465-.

- on ruled surfaces. Snyder, V. N. Y. Am. Mth. S. Bll. 5 (1899) 343-.

- - surface, condition that family of plane curves should be projection of. Koenigs, $G$. Par. S. Phlm. Bll. 4 (1892) 94.

_ - tore. Maschke, H. [1895] N.Y.Am. Mth. S. Bll. 2 (1896) 19-.

Bisectors of network of lines on any surface. Aoust, -. Liouv. J. Mth. 9 (1883) 43-. 
Characteristic lines and characteristic angle of surface. Pucci, E. Rm. R. Ac. Linc. Rd. 5 (1889) (Sem. 1) 501-.

- -, properties. Reina, $V$. Rm. R. Ac. Linc. Rd. 5 (1889) (Sem. 1) 881-.

Conjugate lines on surfaces, functions of angle between. Massimi, $P$. Rm. N. Linc. At. 52 (1899) 126-.

- systems, one of which is geodesic. Guichard, C. C. R. 128 (1899) 599-.

Contour and slope lines. Cayley, A. Ph. Mg. 18 (1859) 264-.

Contours on surfaces. Jordan, C. Liouv. J. Mth. 11 (1866) 110-

Curvature of isogonals. Seiliger, D. N. Fschr. Mth. (1895) 725-.

\section{Curves on surfaces.}

Spitzer, S. Wien SB. (1853) 435-.

Mainardi, $G$. Mil. G. I. Lomb. 9 (1856) 385-. Böklen, O. Grunert Arch. 39 (1862) 204-.

Peterson, K. M. [1866] (xII) Rec. Mth. (Moscou) 2 (1867) (Pt. 1) 17-.

Gilbert, P. [1868] Brux. Ac. Sc. Mm. 37 (1869) $47 \mathrm{pp}$.

(Gilbert.) Aoust, (l'abbé) - Brux. Ac. Bll. 26 (1868) 471-.

(Aoust.) Gilbert, P. Brux. Ac. Bll. 26 (1868) 480-.

Aoust, (l'abbé) -. C. R. 68 (1869) 526-.

Pirondini, G. Nap. Rd. 28 (1889) 87-.

on algebraic surfaces. Darboux, $G$. Bll. Sc. Mth. As. 2 (1871) 23-, 184-, 221-, 314-; 3 (1872) 221-, 251-, 281-.

_ - _, " normal segments." Laguerre, E. [1870] (x) Par. S. Phlm. Bll. 7 (1871) 69-. that are always geodesics of surfaces they generate. Hazzidakis, J.N. [1882] Crelle J. Mth. 95 (1883) 120-.

on cone or cylinder, developed on plane, equation. Sornin, J. Toul. Mm. Ac. 5 (1855) 301-.

of constant curvature on sphere. Zeuthen, H. G. N. Ts. Mth. 1 (B) (1890) 67-; Fschr. Mth. (1890) 774.

- contact of conic and surface. Hachette, J. N. P. Par. Éc. Pol. Cor. 1 (1804-08) 188-.

- - - cylinder circumscribed to helicoid with director plane. Verstraeten, $T$. (xII) Mathesis 1 (1881) 49.

(xII) Mathesis $\overline{1}$ (1881) $\overline{137-}$.

- - skew surface and its director surface, construction of tangents. Drasch, H. [1880] Wien Ak. Sb. 82 (1881) ( $A b .2$ ) 1140-.

- - surface of revolution and circum-cone or cylinder, construction of tangents. Seidelin, C. J. L. (xп) Ts. Mth. 6 (1882) 33-.

contained in algebraic surfaces, linear differential equations defining. Fano, G. Rm. R. Ac. Linc. Rd. 4 (1895) (Sem. 1) 232-, 292-, 322-.

curvature. Beltrami, E. N. A. Mth. 4 (1865) 258-. curvature (Beltrami). Bonnet, O. N. A. Mth. 4 (1865) 267-.

-, geodesic. Cayley, A. L. Mth. S. P. 12 (1880-81) 110-.

- and torsion. Pellet, A. E. As. Fr. C. R. (1890) (Pt. 2) 200-.

and curvilinear coordinates. Aoust, (l'abbé) -. C. R. 65 (1867) 814.

of $4 \mathrm{th}$ degree on sphere, and their osculating planes at point. Laguerre, E. (x) Par. S. Mth. Bll. 1 (1873) 101-.

determination. Enneper, A. Gött. Nr. (1873) 785-.

on developables. Codazzi, D. Tortolini A. 8 (1857) 165-.

- -. Michel, C. Bll. Sc. Mth. 24 (1900) 157-. - - cut by osculating planes at constant angle. Molins, $H$. Liouv. J. Mth. 12 (1847) 394-.

- -, properties. Olivier, T. Par. S. Pblm. N. Bll. (1832) 166-.

development. Minding, E. F. A. Crelle J. 6 (1830) 159-.

differential equation of class. Enneper, $A$. Gött. Nr. (1871) 577-.

- equations. Ricci, G. Ven. I. At. (189293) 1336-.

on ellipsoid, geodesic radius of curvature. Liouville, J. Liouv. J. Mth. 19 (1854) 368.

- - ' Poinsot's poloids. Allman, G. J. Camb. and Dubl. Mth. J. 3 (1848) 287-.

of equal illumination on algebraic ruled surface. Burali-Forti, C. Palermo Cir. Mt. Rd. 4 (1890) 57-.

- - - sphere. Chrétien, L. V. A. Cond. Pon. Chauss. 16 (1872) 45-.

- - - surfaces of revolution, construction by tangent cones. Matzek, F. Wien Sb. 58 (1868) (Ab. 2) 49.

equidistant, on sphere. Gudermann, C. Crelle J. 25 (1843) 119-.

-, - - Robert8, W. [1869] A. Mt. 4 (1870-71) 207-.

geodesic curvature. Pirondini, G. A. Mt. 21 (1893) 33-.

- - and normal curvatnre, loci of centres. Gattorno, G. G. Mt. 37 (1899) 41-.

- torsion. Burgatti, P. Palermo Cir. Mt. Rd. 10 (1896) 229-.

of given curvature on given surface. Hoppe, R. Arch. Mth. Ps. 11 (1892) 193-.

on given surfaces. Graves, C. Ir. Ac. P. 2 (1844) 576-.

having same polar surface, integrals of differential equations. Aoust, (l'abbé) -. C. R. 78 (1874) 1290-; A. Mt. 7 (1876) 1-. ,$-----\frac{-}{-}$ (Aoust). Serret, J. A. C. R. 78 (1874) 1329-.

- - Aoust, (l'abbé) -. C. R. 78 (1874) 1481-.

integral. Delassus, $\dot{E}$. Bll. Sc. Mth. 22 (1898) 318-

networks of, and congruences connected with them. Guichard, C. C. R. 128 (1899) 1149 -.

plane, on conical surfaces. San Bertolo, $N$. Rm. At. 12 (1858-59) 173-. 
plane, on spheres, shadows. Jeffery, $H$. $M$. [1875] QJ. Mth. 14 (1877) 53-.

of principal tangents of skew surfaces. Clebsch, R. F. A. [1867] Crelle J. 68 (1868) 151-.

properties. Raabe, J. L. Crelle J. 2 (1827) 368-.

一. Brioschi, F. Tortolini A. 5 (1854) 232-.

- Gilbert, P. Brux. Ac. Bll. 9 (1860) 46-.

- Ribaucour, A. C. R. 80 (1875) 642-.

- (Ribaucour). Mannheim, A. C. R. 80 (1875) 725-.

property. Laguerre, E. [1870] (x) Par. S. Phlm. Bll. 7 (1871) 49-.

on quadrics, formula. Laguerre, -. N. A. Mth. 9 (1870) 5-.

radii of curvature, demonstration of Bonnet's formulø. Beltrami, $E$. G. Mt. 4 (1866) 123 -.

on right cylinder with circular base. Azzarelli, $M$. [1876] Rm. N. Linc. At. 30 (1877) 1-.

- scroll, number of intersections. Story, W. E. (xII) J. H. Un. Cir. [2] (1883) 143.

skew, on sphere, absolute radius of osculating circle, and evolutes. Plana, G. Mod. S. It. Mm. 24 (1848) 343-.

on sphere. Bacaloglo, $E$. Grunert Arch. 35 (1860) 57-.

- -. Ginther, S. Arch. Mth. Ps. 56 (1874) 267-.

- - arcs. Newenglonski, (Prof.) - . Par. Ec. Norm. A. 2 (1873) 137-.

-- , class. Roberts, $W$. Ir. Ac. P. 2 (1844) 194-.

- -, evolutes. Mehler, F. G. [1863] (vII) Crelle J. 64 (1865) 183-.

- - and plane, transmutation method. Roberts, W. Liouv. J. Mth. 13 (1848) 209-。

- surface of right cone, involutes. Valles, $F$. Gergonne A. Mth. 17 (1826-27) 349-.

- surfaces of revolution. Biehringer, $(D r)$ Z. Mth. Ps. 18 (1873) 552-; 21 (1876) 229-; 22 (1877) 151-; 28 (1883) 157-.

- - - , transformation. Pirondini, $G$. Nap. Rd. 29 (1890) 155-.

- - touching one another, relation due to Laguerre. Mannheim, A. C. R. 82 (1876) 554 -.

theory. Cosserat, $E$. Toul. Ac. Sc. Mm. 7 (1895) 366-.

torsal, on ruled surface, determination of number. Schubert, H. C. H. Mth. A. 17 (1880) 575-.

transformation. Faure, $H$. Mntp. Ac. Sc. Mm. 2 (1851-54) 463-.

Darboux or $D$ lines. (Curves on surface, with osculating sphere touching surface at every point.) Darboux, G. C. R. 73 (1871) $732-$.

- - - Cosserat, E. C. R. 121 (1895) 43-.

- - - Hardy, J. G. Am. J. Mth. 20 (1898) 283-.

- - - on quadrics. Pell, A. N. Y. Am. Mth. S. T. 1 (1900) 315-.
Differential parameters, application in theory of surfaces. Bendixson, I. Stockh. Öfv. (1897) 331-.

Expression for $d s^{2}$ in three variables, new. Levi-Civita, -. C. R. 124 (1897) 1434-.

Extension to any surface of Euler's problem on $d s^{2}=d x^{2}+d y^{2}$. Koenigs, $G$. C. R. 108 (1889) 221-.

Fundamental equations, geometrical significance. Knoblauch, $J$. Acta Mth. 15 (1891) 249-.

Geodesic arcs on ellipsoid whose difference is algebraic. Staude, E. O. Mth. A. 20 (1882) 185-.

- circles. Ribaucour, A. [1870] (xi) Par. S. Phlm. Bll. 7 (1871) 22-, 110-, 124-.

- -, contact transformations. Oseen, C. W. Stockh. Öfv. (1900) 957-.

- - and parallel curves. Betti,E. Tortolini A. 3 (1860) 336-.

- curvature. Bonnet, O. C. R. 42 (1856) $1137-$.

-. Schlesinger, L. Orv.-Termt. Éts. (Termt. Szak) (1891) 267-.

-. Lilienthal, R. von. Mth. A. 42 (1893) $505-$.

- -. Staude, O. Dorpat Sb. 11 (1896) 72-. -. Vostokov, I. A. Vars. S. Nt. Tr. (1895-96) (C.R., Ps. C.) No. 8, 5 pp.

- centres. Caronnet, T. C. R. 115 (1892) 589-.

orthogonals on sphere and spheroid. Berardinis, G. de. G. Mt. 27 (1889) 127-, 318-; 28 (1890) 53-, 138-.

- polygons on quadries. Staude, E. O. Mth. A. 21 (1883) 219-.

- representation, most general, of geodesic circles of surface. Lie, S. Arch. Mth. Ntvd. 9 (1884) 62-.

- torsion. Mathews, G. B. QJ. Mth. 27 (1895) 145-.

- - angles, of intersection of two surfaces. Mannheim, A. Mess. Mth. 8 (1879) 122.

- triangle on spheroid. Puissant, L. Par. Mm. Ac. Sc. 10 (1831) 530-.

- - - surface and corresponding spherical triangle, ratio of areas. Clausen, T. As. Nr. 20 (1843) 13-; 21 (1844) 145-.

\section{Geodesics.}

Minding, E. F. A. Crelle J. 20 (1840) 323-.

Spitzer, S. Grunert Arch. 23 (1854) 125-.

Bonnet, O. C. R. 40 (1855) 1311-; 41 (1855) 32-.

Grunert, J. A. Grunert Arch. 37 (1861) 264-.

Anon. (xi 41) Mess. Mth. 5 (1871) 87-.

Enneper, A. Z. Mth. Ps. 18 (1873) 613-.

Hoppe, R. Arch. Mth. Ps. 64 (1879) 60-

Böklen, O. Z. Mth. Ps. 26 (1881) 264-.

Lie, M. S. Mth. A. 20 (1882) 357-.

Pirondini, G. G. Mt. 23 (1885) 288-.

Ermakov, V. P. Rec. Mth. (Moscou) 15 (1891) 576-; Fschr. Mth. (1890) 760.

Lechalas, G. N. A. Mth. 10 (1891) 527-.

Koenigs, G. [1892] Par. Mm. Sav. Étr. 31 (1894) No. 6, $318 \mathrm{pp}$. 
Sochocki, J. Prace Mt.-Fiz. 3 (1892) 82-; Fschr. Mth. (1892) 732.

(Koenigs.) Ricci, G. Rm. R. Ac. Linc. Rd. 2 (1893) (Sem. 2) 146-, 338-.

(Ricci.) Koenigs, G. Rm. R. Ac. Linc. Rd. 2 (1893) (Sem. 2) 336-.

Hadamard, -. Bordeaux S. Sc. PV. (189697) 115-, 131.

application of theta functions. Hoppe, $R$. Arch. Mth. Ps. 3 (1886) 75-.

approximate calculation. Nobile, $A$. Nap. Rd. 34 (1895) 139-.

cases. Albeggiani, M. L. Palermo Cir. Mt. Rd. 3 (1889) 80-.

-. Waelsch, E. C. R. 125 (1897) 521-.

complex of principal normals to. Gebbia, $M$. Palermo Cir. Mt. Rd. 1 (1887) 25-.

on cone. Antomari, X. Par. S. Mth. Bll. 17 (1889) 118-.

- - Piccioli, H. N. A. Mth. 17 (1898) 207-. conjugate system, surfaces having. Razzaboni, A. Bologna Ac. Sc. Mm. 9 (1888) 765-.

of constant curvature on pseudospherical surfaces. Reina, $V$. Rm. R. Ac. Linc. Rd. 5 (1889) (Sem. 1) 448-.

and other curves on surfaces. Kummell, C. H. Smiths. Misc. Col. 33 (1888) drt. 1, 123-. (Wash. Ph. S. Bll. 6 (1884).)

departure from normal sections of surface. Berardinis, G. de. Tor. Ac. Sc. Mm. 36 (1885) 159-.

on developable surface. Molins, $H$. Toul. Mm. Ac. 5 (1861) 401-.

differential equation. Issaly, -. N. A. Mth. 19 (1900) 392-.

and elliptic functions. Braunmïhl, A. von. Mth. A. 26 (1886) 151-.

envelopes. Braunmilhl, A. von. [1878] Mth. A. 14 (1879) 557-.

equation, and applications. Azzarelli, $\boldsymbol{M}$. Rm. N. Linc. At. 31 (1878) 327-.

-, general, discovery. Eneström, G. $\mathrm{Bb}$. Mth. (1899) 19-.

- integration. Brioschi, $F$. Tortolini A. 4 (1853) 133-.

generalisation of problem. Schlömilch, $O$. Z. Mth. Ps. 13 (1868) 156-.

and geodesic triangle. Brill, $A$. Münch. Ak. Ab. 14 (1883) (Ab. 2) 109-.

geometrical demonstrations. Hart, $A$. $S$. Camb. and Dubl. Mth. J. 4 (1849) 80-.

at infinity, and geodesics of ruled quadrics. Hadamard, -. Par. S. Mth. Bll. 26 (1898) 195-.

integrable, surfaces having. Laguerre, $E$. (x) Par. S. Mth. Bll. 1 (1873) 281.

Joachimsthal's theorem, geometrical proof. Graves, C. [1850] Crelle J. 42 (1852) 279.

length. Ivory, J. QJ. Sc. 21 (1826) 361-.

letters to Johann and Daniel Bernoulli. Clairaut, A. C. Rm. N. Linc. At. 45 (1892) 245-.

lines analogous to. Darboux, G. Par. Ec. Norm. A. 7 (1870) 175-.

and lines of curvature. Joyce, M. J. QJ. Mth. 5 (1862) 265-.

- - - - general equations. Dickson, $B$. Camb. and Dubl. Mth. J. 5 (1850) 166-. and Liouville's isothermic systems. Ricci, $G$. Ven. I. At. (1893-94) 643-.

- normal plane sections at two adjacent points, divergence between. Berg, F. J. van den. Amst. Ak. Vs. M. 10 (1876) 1-; Arch. Néerl. 12 (1877) 353-.

partial differential equation of 3rd order connected with. Lévy, M. C. R. 85 (1877) 904-.

- - - 4th order connected with. Lévy, M. C. R. 85 (1877) 938-, 1009-.

problem, and equations with mixed functions. Koenigs, G. C. R. 117 (1893) 683-.

-, intermediary integrals of equation. Lévy, M. C. R. 85 (1877) 1150-

-, rational integrals. Lévy, M. C. R. 85 (1877) 1065-

projection. Schols, C. M. Delft Éc. Pol. A. 5 (1889) 133-.

-, curvature. Schols, C. M. Delft Ec. Pol. A. 2 (1886) 179-.

properties. Young, (Dr) T. QJ. Sc. 21 (1826) 136 -

property, fundamental. Ferrers, N. M. QJ. Mth. 1 (1857) 245.

with quadratic integrals. Koenigs, G. Par. S. Phlm. Bll. 5 (1893) 26-

representing motion with 2 degrees of freedom. Kelvin, (Lord). B. A. Rp. (1892) 652-.

on ruled surfaces. Astor, A. As. Fr. C. R. (1887) (Pt. 2) 1-.

special, on harmonic surfaces. Raffy, $L$. Par. S. Mth. Bll. 22 (1894) 8-.

on special surfaces. Guldberg, $A$. N. Ts. Mth. 6 (B) (1895) 1-; Fschr. Mth. (1895) 704-.

and spheroidal trigonometry. Grunert, J.A. Grunert Arch. 22 (1853) 64-; 40 (1863) 33-.

on spiral surfaces, and their differential equa. tions. Hadamard, J. Bordeaux S. Sc. PV. (1895-96) 55-.

- any surface. Euler, L. [1779] St Pet. Ac. Sc. N. Acta 15 (1806) 44-

- surface of centres. Hoppe, $R$. Arch. Mth. Ps. 63 (1879) 81-.

- surfaces of constant curvature. Liouville, R. Am. J. Mth. 10 (1888) 283-.

- - - anticlastic curvature. Hadamard, Bordeaux S. Sc. PV. (1896-97) 60-; C. R. 124 (1897) 1503-.

- - - revolution. Jamet, -. Brux. Ac. Bll. 13 (1887) 421-

- - - Catalan, E. C. Brax. Ac. Bll. 13 (1887) 425-; Liége S. Sc. Mm. 15 (1888) No. 1, 213-.

$-\ldots-$ Clairaut's equation. Lorenzoni, G. Ven. I. At. 2 (1875-76) 591-.

- - - - - theorem. Emilio, R. d'. Ven. I. At. (1892-93) 1191-。

- - - - between 2 points of generator. Castet, - Bordeaux S. Sc. Mm. 2 (1878) 185 -

- - - theorem. Lommel, E. Grunert Arch. 38 (1862) 201-.

system, characteristic of differential equation. Liouville, R. C. R. 108 (1889) 495-.

- contained in cyclic systems. Guichard, C. C. R. 128 (1899) 1308-. 
theorem. Dini, U. G. Mt. 3 (1865) 65-.

-, fundamental. Fà̀ de Bruno, $F$. Les Mondes 8 (1865) 739-.

theorems of Jacobi, two. Paraf, -. C. R. 106 (1888) 1139-.

theory. Böklen, O. Grunert Arch. 39 (1862) 189-.

-. Beltrami, E. Mil. I. Lomb. Rd. 1 (1868) 708-.

- Jamet, V. Mars. Fac. Sc. A. 8 (1898) 117-.

-. Stäckel, P. D. Nf. Vh. (1900) (Th. 2, Hälfte 1) 5-.

\section{Geodesics on quadrics.}

(See also Geography 70.)

Hart, A. S. Camb. and Dubl. Mth. J. 4 (1849) 192-

Graves, C. Ir. Ac. P. 4 (1850) 283-.

Cayley, A. [1872] L. Mth. S. P. 4 (1871-73) 191-, 368-.

Laguerre, $E . \quad$ N. A. Mth. 15 (1876) 10 -

on confocals, and lines of curvature. Chasles, M. Liouv. J. Mth. 11 (1846) 105-.

- ellipsoid. Jacobi, C. G. J. Crelle J. 19 (1839) 309-.

- Liouville, J. Liouv. J. Mth. 9 (1844) 401-.

- -. Jacobi, C. G. J. Berl. B. (1846) 351-.

- Roberts, M. Liouv. J. Mth. 12 (1847) 491-.

- -. Schläfi, L. Bern Mt. (1847) 96-.

- - Besge, V. A. Liouv. J. Mth. 14 (1849) 247-.

- - Roberts, W. Liouv. J. Mth. 2 (1857) 213 .

- - Aoust, L. C. R. 50 (1860) 484-.

- - Böklen, O. Grunert Arch. 35 (1860) $101-$.

- Weierstrass, C. Berl. Mb. (1861) 986-.

- Cayley, A. [1871] Ph. Mg. 41 (1871) 534-; As. S. Mm. 39 (1872) 31-.

- - application of elliptic functions. Roberts, M. Liouv. J. Mth. 15 (1850) 275-

- - centres of maximum and minimum curvature, etc. Schläfli, L. Bern Mt. (1846) 145-.

- - equation, geometrical demonstration. Liouville, J. $\quad$ C. R. 22 (1846) 111-.

- - Jacobi's method. Tortolini, B. Rm. At. 4 (1850-51) 287-.

- - and lines of curvature. Roberts, $M$. Liouv. J. Mth. 11 (1846) 1-; 13 (1848) 1-. - - , - - - two theorems. Liouville, J. C. R. 22 (1846) 893-.

- , radii of two curvatures. Tortolini, $B$. Tortolini A. 2 (1851) 345-.

- - through umbilic. Hart, A, S. Ir. Ac. P. 4 (1850) 274 .

and their envelopes. Braunmuhl, A. von. Mth. A. 20 (1882) 557-.

geometrical demonstration. Liouville, $J$.

Liouv. J. Mth. 11 (1846) 21-.

and lines of curvature. Joachimsthal, $F$. Crelle J. 26 (1843) 155-. and lines of curvature. Chasles, $M$. Liouv. J. Mth. 11 (1846) 5-, 105-.

- - - (Chasles). Liouville, J. Liouv. J. Mth. 12 (1847) 255.

loci. Rutledge, J. Y. Camb. and Dubl. Mth. J. 6 (1851) 21-.

on non-central quadrics. Marcolongo, $R$. Nap. Rd. 30 (1891) 32-.

- paraboloids. Marcolongo, R. Rm. R. Ac. Linc. Rd. 6 (1890) (Sem. 1) 392-; G. Teix. J. Sc. 11 (1892) 145-.

- - , and lines of curvature. Chelini, D. G. Arcad. 106 (1846) 161-

- quadrics not of revolution. Forsyth, A. R. L. Mth. S. P. 27 (1896) 250 -

- right cone. Vész, J.Á. [1868] (xII) Mag. Tud. Ak. Etk. (Mth.) [1] (No. 5) (1869) 19 pp.

Czuber [Čuber], E. Arch. Mth. Ps. 69 (1883) 125-.

- spheroid. Soldner, J. Zach M. Cor. 11 (1805) 7-.

- - Baeyer, J. J. As. Nr. 71 (1868) 289-.

- Ciscato, G. Ven. I. At. (1891-92) 1087-, 1333-

- -, approximate calculation. Pizzetti, $P$. Nap. Rd. 35 (1896) 75-.

- ares. Halphen, G. H. C. R. 105 (1887) 583-.

- - Dalby's method of finding difference of longitude of 2 points. Tiarks, J. L. $\mathrm{Ph}$. Mg. 4 (1828) 364-.

- -, new geometrical representation. Schwering, $K$. Z. Mth. Ps. 24 (1879) 405-.

- - oblate. Cayley, A. Ph. Mg. 40 (1870) 329-.

- -, 一. Fergola, E. Nap. Rd. 33 (1894) 136-

- - Pizzetti, $P$. Tor. Ac. Sc. At. 30 (1895) 217-.

- - - Forsyth, A. R. Mess. Mth. 25 (1896) 81-.

,--- , conjugate points. Forsyth, A. R. Mess. Mth. 25 (1896) 161-.

- - , - properties. Ivory, J. Tilloch $\mathrm{Ph}$. Mg. 67 (1826) 241-, 340-.

- - between 2 points, latitude and longitude of which are given. Puissant, L. Par. S. Gg. Bll. 9 (1828) 184-.

- - prolate. Halphen, G. H. C. R. 105 (1887) 535-.

torsion at a point. Laguerre, E. Par. S. Mth. Bll. 4 (1876) 160-.

Geodesy, solution of fundamental question in. Chandrikov, M. T. Rec. Mth. (Moscou) 17 (1895) 575-.

Helix on cone. Junge, A. Civing. 4 (1858) 172-.

Integrals of dynamics. Painlevé, P. C. R. 114 (1892) 1168-.

Isoperimetric figures on any surface. Bordoni, A. (vr Adds.) Opusc. Mt. Fis. 1 (1832) 1-.

Latitude, longitude and azimuth. Andrae, Kjöb. Ov. (1858) 230-; As. Nr. 50 (1859) 161-. 
Latitude and longitude, formulæ. Oriani, $B$. Mil. Effem. As. (1808) 3-.

Line-element of surface, certain forms. Massimi, P. Rm. N. Linc. At. 52 (1899) 93-.

Lines of equal slope on given surfaces. Schlömilch, O. Schlömilch Z. 1 (1856) 250 -

- - greatest slope. Steen, A. (xII) Ts. Mth. 1 (1865) 123-.

- - - Breton (de Champ), - C. R. 70 (1870) 982-.

- - - differential equation. Breton (de Champ), -. C. R. 64 (1867) 407-.

- - - - ridge lines and channels. Boussinesq, J. C. R. 73 (1871) 1368-; 75 (1872) 198-, 835-.

- - shadow for some surfaces. Pirondini, $G$. G. Mt. 29 (1891) 356-.

- - striction. Larmor, J. QJ. Mth. 19 (1883) 381-.

- _ - of hyperboloid. Monteiro, A. S. G. Teix. J. Sc. 3 (1881) 131-.

- $-\ldots \ldots$ as curves generated by higher configurations. Schmid, T. [1881] Wien Ak. Sb. 84 (1882) (Ab. 2) 908-.

- - - - - - rational skew curves of 4 th order. Migotti, A. [1879] Wien Ak. Sb. $80(1880)(A b .2)$ 1023-.

_ - of one sheet. Bobek, K. Par. S. Mth. Bll. 11 (1883) 125-.

- - - - - - - and of hyperbolic paraboloids. Bauer, $M$. Z. Mth. Ps. 28 (1883) 274-.

- - - ruled surfaces. Nicolaĩdès, $N$. Les Mondes 9 (1866) 146-.

- - - - - - of 2 nd and 3rd degrees. Adler, A. Wien Ak. Sb. 85 (1882) (Ab. 2) 369-.

- - etc. of skew surfaces. Enneper, $A$. Gött. Nr. (1867) 454-.

\section{Loxodromes.}

on conical surfaces. Enneper, A. Gött. Nr. (1869) 459-.

- cylinder and cone. Grebe, E.W. Grunert Arch. 2 (1842) 127-.

- ellipsoid and sphere. Plagemann, $W$. Grunert Arch. 32 (1859) 1-.

equation and properties. Fournier-Vannson, N. A. Mth. 20 (1861) 31-, 225-.

and geodesics on tore. Puchta, $A$. Mh. Mth. Ps. 1 (1890) 443-.

- loxodromic figures. Friesach, $K$. Steierm. Mt. (1878) 78-.

on paraboloid of revolution. Boyman, J. $R$. Grunert Arch. 13 (1849) 375-.

projecting into logarithmic spirals, surfaces with. Molins, H. Toul. Âc. Sc. Mm. 7 (Sem. 2) (1885) 293-; 8 (1886) 426-.

properties. Arrest, H. d'. As. Nr. 36 (1853) 351-.

on quadrics. Boyman, J.R. Grunert Arch. 7 (1846) 337-.

and shortest paths on spheroid. Caluso, (l'abbé) T.V. de. Turin Mm. Ac. 4 (1788-89) 325-; 5 (1790-91) 100-.

- spherical catenary. Gudermann, C. Crelle J. 11 (1834) 394-. on any surface. Legoux, A. Toul. Ac. Sc. Mm. 1 (1889) 352-.

- surfaces. Dina, C. G. Mt. 19 (1881) 298-. - of revolution. Schubert, F. T. [1803] St Pet. Ac. Sc. N. Acta 15 (1806) 202-.

- - - Gergonne, J. D. Gergonne A. Mth. 8 (1817-18) 125-.

- - - Aoust, L. Liouv. J. Mth. 11 (1846) 184-.

- - - Junge, A. Schlömilch Z. 5 (1860) 296-.

- Laisant, C. A. N. A. Mth. 13 (1874) 573-

- - - -, equations. Grunert, J.A. Grunert Arch. 21 (1853) 304-.

Loxodromic arc between two points on sphere. Friesach, $K$. Steierm. Mt. (1880) 3-.

- and great circles on sphere, relation. Friesach, $K$. Steierm. Mt. (1876) 97-.

- triangles. Grunert, J.A. Grunert Arch. 16 (1851) 23-.

Maximum area on surface with given perimeter. Aelt, -. N. A. Mth. 19 (1860) 100-

Meridian curve of surface of revolution. Resal, H. A. C. R. 85 (1877) 5-.

Motion of point on surface. Enneper, A. Gött. Nr. (1869) 62-.

Number of conditions for existence of an $r$-fold straight line on surface of $n$th order is

$$
\frac{1}{6} r(r+1)(3 n-2 r+5) \text {. }
$$

Machovec, $F$. Časopis 16 (1887) 230-; Fschr. Mth. (1887) 780.

Orthodromic sailing. Glotin, P. [1876-79] Bordeaux S. Sc. Mm. 1 (1876) xlviii-; 2 (1878) 189-; 3 (1880) 377-.

Orthogonal lines on surface, and linear partial differential equations of 2nd order (elliptic type). Burgatti, P. A. Mt. 23 (1895) 225-.

Points on surfaces such that geodesics through them never cease to be shortest lines. Mangoldt, H. von. [1880] Crelle J. Mth. 91 (1881) 23-.

Projection, quasi-stereographic, due to Gauss. Forsyth, A. R. QJ. Mth. 21 (1886) 376-.

Pseudo-spheres, Viviani's theorem. Torelli, $G$. G. Mt. 10 (1872) 128-

Quadrable figures on surface of right cone. Magistrini, D. [1845] Bologna N. Cm. 8 (1846) 427-.

Ribaucour's problem. Guichard, C. C. R. 125 (1897) 1013-.

Ridge lines and channels. Barré de SaintVenant, -. Par. S. Phlm. PV. (1852) 24-.

- - - Jordan, C. C. R. 74 (1872) 1457-; 75 (1872) 625-, 1023-.

_- Breton (de Champ), P. Liouv. J. Mth. 3 (1877) 99-.

- _ - - geometrical characters. Breton [de Champ], P. C. R. 53 (1861) 808-.

_ - lines connected with them. McConvan, J. Ph. Mg. 37 (1894) 227-.

Spherical catenaries. Gudermann, C. Crelle J. 33 (1846) 189-, 281-.

- class-cubics with 3 single foci. Jeffery, H.M. B. A. Rp. (1878) 490-. 
Sphero-conics, confocal, differential equation. Burnside, W. Mess. Mth. 20 (1891) 60-

Spheroidal trigonometry. Puissant, L. [181329] Par. S. Phlm. Bll. 3 (1813) 273-; Par. Mm. Ac. Sc. 10 (1831) 457-.

- - especially geodesics. Gudermann, C. [1847] Crelle J. 43 (1852) 294.

Spiral, conical, development. Jubé, $E . \mathrm{N}$. A. Mth. 4 (1845) 454-

- on tore. Kinealy, J.H. St Louis Ac. T. 5 (1892) 1-.

Surfaces, reciprocally polar, relations between infinitesimal elements. Demoulin, $A$. C. R. 114 (1892) 1102-.

- of revolution cut by sphere in geodesics. Molins, H. Toul. Ac. Sc. Mm. 3 (1891) 296-

System of variables, new, of Bonnet. Tortolini, B. [1868] Rm. At. N. Linc. 22 (1869) 172-.

Trajectories cutting at given angle tangents to any curve. Molins, $H$. Toul. Mm. Ac. 2 (1846) 65-.

- ---- skew curve. Molins, $H$. Toul. Mm. Ac. 6 (1839-41) 33-.

- , on given surface or in space, rendering minimum $\int_{0}^{8} \phi(\nu) d s$, where $\nu$ is the velocity. Roger, É. C. R. 40 (1855) 1176-.

- - surfaces. Gilbert, P. Brux. Ac. Bll. 25 (1868) 288-.

Triangle on spheroid, reduction of angle to angle of plane or spherical triangle. Weingarten, $J$. As. Nr. 75 (1870) 91-.

Triangles formed by lines of system $(a)$ or by geodesics, sum of angles. Böklen, $O$. Arch. Mth. Ps. 43 (1865) 18-.

- on spheroid. Legendre, A. M. Par. Mm. de l'I. 6 (1806) (Sem. 1) 130-.

- - , problems. Proteus, P. Nicholson J. 8 (1804) 151-.

- - - solution. Baeyer, (Gen.-Lt.) J.J. As. Nr. 61 (1864) 225-; 71 (1868) 289-.

\section{Minimal surfaces.}

Gergonne, J.D. Gergonne A. Mth. 7 (1816-17) 143-, 283-.

Bonnet, O. C. R. 40 (1855) 1107-; 41 (1855) 1057-; 42 (1856) 532-.

Lamarle, E. Brux. Ac. Bll. 6 (1859) 329-.

Christoffel, E. B. Crelle J. 67 (1867) 218-.

Roger, É. C. R. 71 (1870) 351-.

Schwarz, C. H. A. Zür. Vjschr. 19 (1874) 243-.

Kiepert, L. [1875-77] Crelle J. Mth. 81 (1876) 337-; 85 (1878) 171-

Cayley, A. QJ. Mth. 14 (1877) 190-.

Lie, M. S. Arch. Mth. Ntvd. 4 (1879) 477-.

Schwarz, H. A. Helsingf. Acta 15 (1888) 315-

Vivanti, J. Z. Mth. Ps. 33 (1888) 137-.

Cayley, A. C. R. 111 (1890) 953-.

Carvallo, $E$. Bll. Sc. Mth. 18 (1894) 12 .

Paci, P. Tor. Ac. Sc. At. 29 (1894) 446-.

Stäckel, P. Leip. Mth. Ps. B. 48 (1896) 478-.

Guichard, C. C. R. 126 (1898) 1487-.

Bromwich,T.J.I'A. L.Mth. S.P.30(1899)276-.

Richmond, H.W. Camb.Ph.S. T.18(1900) 324-.

Algebraic surfaces. Geiser, C. F' [1870] Mth. A. 3 (1871) 530-.
Algebraic surfaces, determination of lowest class number. Henneberg, L. [1877] A. Mt. 9 (1878-79) 54-.

- - inscribed in algebraic developable. Lie, M. S. Arch. Mth. Ntvd. 3 (1878) 340-.

- - - - sphere, and algebraic curves of constant torsion. Cosserat, E. C. R. 120 (1895) 1252-.

- - inscriptible in cone, determination of all, Lie, M. S. Arch. Mth. Ntvd. 3 (1878) 224 -. - - projective and metrical researches. Lie, M.S. Mth. A. 14 (1879) 331-; 15 (1879) 465-. Apsidal or conjugate surfaces. Gilbert, $P$. Brux. Ac. Bll. 28 (1869) 31-.

Area, second variation, especially for helicoids. Schwarz, H. A. Berl. Mb. (1872) 718-.

Case. Catalan, E. C. Par. S. Phlm. PV. (1841) 59-.

-. Salvatore-Dino, N. Nap. Rd. 19 (1880) 148-.

-. Zeeman, P. Haarl. Ms. Teyl. Arch. 5 (1898) 299-.

-, construction of model. Tallqvist, H. Helsingf. Öfv. 31 (1889) 32-.

Cases, determination. Herzog, A. Zür. Vjschr. 20 (1875) 217 -

Catenoid as minimal surface, limits. Lindelöf, L. L. [1870] Helsingf. Acta 9 (1871) 353-.

Class. Weierstrass, C. Berl. Mb. (1867) 511-.

-. Stouf, X. Toul. Fac. Sc. A. 6 (1892) A, $8 \mathrm{pp}$.

Continuous surface bounded by skew quadrilateral. Tucci, F. P. [1852] Nap. Mm. Ac. Sc. 1 (1852-54) 49-.

Curve, rotation of which generates minimal surface. Saalschiltz, L. Arch. Mth. Ps. 5 (1887) 131-, vII.

Cyclic surface, determination of constants. Juga, G. Mth. A. 52 (1899) 167-.

Developable surface with known edge of regression. Monge, G. Par. Éc. Pol. Cor. 1 (1804-08) 209-.

- - passing through curve which developes into arc of circle of given radius. Molins, $\boldsymbol{H}$. Liouv. J. Mth. 1 (1856) 265-.

Division of space with minimum partitional area. Thomson, (Sir) W. Ph. Mg. 24 (1887) 503-.

Doubly connected surface. Kluyver, J. C. Amst. Ak. Vh. (Sect. 1) 3 (1896) No. 9, 42 pp.; Fschr. Mth. (1895) 746-.

Expression of line element. Soler, E. Palermo Ac. At. 4 (1897) (Sc. Nt.) $10 \mathrm{pp}$.

Family. Weingarten, J. Gött. Nr. (1887) 272 -

- Rouquet, V. Toul. Ac. Sc. Mm. 2 (1890) 127-.

- of spheres connected with minimal surfaces. Rouquet, V. Toul. Ac. Sc. Mm. 1 (1889) 56-.

Focal conics of confocals, construction of surfaces, with centres of curvature on. Roberts, W. C. R. 53 (1861) 799-.

General equation. Weiler, A. Grunert Arch. 38 (1862) 356-.

Generation. Mathet, G. Liouv. J. Mth. 8 (1863) 323-.

Geodesics. Lie, M. S. Arch. Mth. Ntvd. 6 (1882) 490-. 
Geometrical researches. Sturm, R. Crelle J. Mth. 105 (1889) 101-.

Helicoids. Falchi, $M$. 89 -.

Joachimsthal's theorem. Cayley, A. C. R. 106 (1888) 995-.

Minimal curves on surfaces. Minding, E. F.A. Crelle J. 5 (1829) 297-.

11 (1866) 81-. - - - - Minding, E. F. A. [1878-79] St Pét. Ac. Sc. Bll. 25 (1879) 190-; Crelle J. Mth. 86 (1879) 279-.

- - - of revolution. Minding, E. F.A. [1875] St Pét. Ac. Sc. Bll. 21 (1876) $252-$.

Minimum areas (alvéole). Jacob, -. N. A. Mth. 2 (1843) 160-.

- circumscribed and maximum inscribed polygons to or in ellipse, and analogous polyhedra to or in ellipsoid. Mainardi, G. Rm. At. N. Linc. 26 (1873) 86.

- property of square and cube. Bouvier, L.C. Gergonne A. Mth. 15 (1824-25) 115-.

- - - - - (Criticism of Bouvier's proof.) Gergonne, J.D. Gergonne A. Mth. 15 (1824-25) 265-.

Non-algebraic surfaces, containing system of algebraic curves. Schwarz, C.H.A. Crelle J. Mth. 87 (1879) 146-.

Non-Euclidean surfaces. Guichard, - Par. Éc. Norm. A. 13 (1896) 401-.

Periodic surfaces with octahedral symmetry, two, equations. Schoenflies, A. C. R. 112 (1891) 515-.

Problem. Darboux, G. C. R. 104 (1887) 728-.

Problems. Pincherle, S. Mil. I. Lomb. Rd. 9 (1876) 444-; G. Mt. 14 (1876) 75-, 192.

Properties. Mathet, G. C. R. 57 (1863) 868-. Beltrami, E. Bologna Ac. Sc. Mm. 7 (1867) 411-.

Property. Berdellé, -. Par. S. Mth. Bll. 17 (1889) 102-.

-, characteristic. Bianchi, L. G. Mt. 22 (1884) 374-.

Pseudo-spherical surfaces, applications of hyperbolic functions. Forti, $A$. (xII) Rv. Sc.-Ind. 15 (1883) 221-, 250-.

Real algebraic surfaces. Lie, M. S. Arch. Mth. Ntvd. 2 (1877) 157-.

Ruled surface. Bonnet, $O$. Bll. Sc. Mth. 9 (1885) 14-.

- surfaces. Catalan, E. C. Liouv. J. Mth. 7 (1842) 203-.

- - , and surfaces with plane lines of curvature. Demoulin, A. Brux. Mm. Cour. $8^{\circ}, 58$ (189899) No. 7, $38 \mathrm{pp}$.

Schwarz's formulæ, generalisation. Montcheuil, - de. Par. S. Mth. Bll. 28 (1900) 268-.

Singularities. Neovius, E. R. Helsingf. Acta 16 (1888) 529-.

Special surfaces. Schwarz, H. A. Berl. Mb. (1872) 3-.

Surface bounded by skew quadrilateral formed by 4 edges of regular tetrahedron. Schwarz, H. A. Berl. Mb. (1865) 149-.

- of 5th class. Schilling, C. Bll. Sc. Mth. As. 4 (1880) (Pt. 1) 395-.

voL. I.
Surface generated by straight line. Lewänen, $S$. Z. Mth. Ps. 18 (1873) 423-.

— with given boundary. Riemann, [G. F.] $B$. Gött. Ab. 13 (1868) (Mth.) 1-.

- between given limits. Poisson, S.D. Crelle J. 8 (1832) 361-.

- - - - Scherk, H. F. Crelle J. 13 (1835) 185-.

- having Neil's parabola for plane geodesic. Henneberg, L. Zür. Vjschr. 21 (1876) 66-.

- of revolution. La Vallée-Poussin, C.J. de. Brux. S. Sc. A. 24 (1900) (Pt. 1) 49-.

- between 2 straight lines in space. Serret, J. A. C. R. 40 (1855) 1078-.

Surfaces applicable to surfaces of minimum area. Dini, U. C. R. 60 (1865) 340-.

- - - - revolution or spiral surfaces. Demoulin, A. Bll. Sc. Mth. 21 (1897) 244 - - themselves. Sinigallia, L. G. Mt. 36 (1898) 172-; 37 (1899) 171-.

- bounded by 4 edges of skew quadrilateral. Schoenflies, -. C. R. 112 (1891) 478-, 548. - _ - 3 straight lines. Neovius, E. $R$. Helsingf. Acta 16 (1888) 573-; 19 (1893) No. $4,37 \mathrm{pp}$.

- - - straight lines, theory. Schwarz, H.A. Berl. Ak. Sb. (1894) 1237-.

-, 2-branched, of less area than others with same limits. Schwarz, H.A. Gött. Ab. 34 (1887) (Mth.) $48 \mathrm{pp.}$

- of 3 first classes of polyhedra. Hoppe, $R$. Arch. Mth. Ps. 58 (1876) 327-.

- enveloped by system of cones of 2 nd degree. Schwarz, C.H.A. CrelleJ.Mth. 80 (1875) 301-. - of given contour, Riemann's method for determination. Niewenglowski, B. Par. Ec. Norm. A. 9 (1880) 227-.

- with given plane or spherical curve as curvature line. Tallqvist, H. Helsingf. Acta 17 (1891) 473-.

- - principal curvatures equal and opposite. Roberts, $M$. Liouv. J. Mth. 11 (1846) 300-. - - - - - - Simon, O. E. Grunert Arch. 27 (1856) 322-.

- - - - _ - case. Björling, E. G. Grunert Arch. 4 (1844) 290 -

- - - - - - - Brunel, G. Bordeaux S. Sc. PV. (1896-97) 62-.

- _ - _ - - - - Hadamard, Bordeaux S. Sc. PV. (1896-97) 163-.

- _ - - _ - -, pair. Roberts, $M$. Liouv. J. Mth. 15 (1850) 323-.

_- - _ - _, ruled, case. Serret, J. A. Liouv. J. Mth. 11 (1846) 451-.

- related to minimal surfaces. Appell, $\boldsymbol{P}$. Am. J. Mth. 10 (1888) 175-.

- representable by elliptic integrals. Lilienthal, - von. Crelle J. Mth. 99 (1886) 179-.

- of revolution, to which helicoid can be applied. Lamarle, E. Brux. Ac. Bll. 19 (1865) $537-$.

- in spaces of constant curvature. Bianchi, $L$. Rm. R. Ac. Linc. Mm. 4 (1887) 503-.

- with system of lines of spherical curvature. Dobriner, $H$. Acta Mth. 10 (1887) 145-

- of zero mean curvature. Catalan, $E$. C. Liège S. Sc. Mm. 12 (1885) No. 2, 254-; 13 (1886) 180-; 15 (1888) 259. 
Theorems. Lie, M. S. Arch. Mth. Ntvd. 3 (1878) 166-.

Theory. Enneper, A. Gött. Nr. (1882) 34-, 89-.

-. Darboux, G. C. R. 102 (1886) 1513-.

- Goursat, $\hat{E}$. C. R. 105 (1887) 743-.

—, extension. Lipschitz, R. O. S. [1872-73] (x) Crelle J. Mth. 78 (1874) 1-.

Transcendental surface. Lichtenfels, O. von. Wien Ak. Sb. 94 (1887) (Ab. 2) 41-.

Transformation. Goursat, $\dot{E}$. Acta Mth. 11 (1887-88) 135-, 257-.

Transformations. Bianchi, L. Rm. R. Ac. Linc. Rd. 8 (1899) (Sem. 2) 151-.

\section{Surfaces determined by re- lations of curvature and by other differential properties.}

Condition that lines can be cut orthogonally. Cayley, A. [1876] L. Mth. S. P. 8 (1877) 53-.

Corresponding surfaces, two classes. Mangeot, S. Mathesis 14 (1894) 34-.

Determination of all algebraic integral surfaces of the differential equation $s=0$ inscribed in an algebraic developable. Lie, M. S. Arch. Mth. Ntvd. 4 (1879) 334-.

- - - surfaces admitting continuous system of projective transformations. Lie, S. Leip. Mth. Ps. B. 47 (1895) 209-.

- - ovaloids by measure of curvature for each normal section. Liebmann, $H$. Gött. Nr. (1899) 134-.

- - spiral surfaces by line element. Raffy, $L$. C. R. 112 (1891) 1421-.

- - surface by its 2 fundamental quadratic forms. Raffy, L. C. R. 126 (1898) 1852-.

- - - one of its two surfaces of centres. Hoppe, R. Arch. Mth. Ps. 68 (1882) 256-.

- - surfaces by curvature relations. Lipschitz, R. O. S. Berl. Ak. Sb. (1882) 1077-; (1883) 169-, 541-.

- - - with prescribed expression for line element. Lipschitz, R. Berl. Ak. Sb. (1884) 649-.

- - - - system of equal lines of curvature. Bricard, R. C. R. 130 (1900) 475-.

Developable, surface of centres. Petersson, $V$. Fschr. Mth. (1886) 748-.

- formed by tangent planes along curve on surface. Enneper, A. Gött. Nr. (1869) 207-.

- through 2 given curves, generation. Taylor, H. M. Mess. Mth. 5 (1876) 1-.

Developables. Řehořovský, $V$. Časopis $9\left({ }^{*} 1880\right)$ 31-, 60-, 161-, 223-; Fschr. Mth. ("1880) 569 -.

-, representation of geodesics on. Puchta, A. Wien Ak. Sb. 97 (1889) (Ab. 2a) 1269-.

Differential equations of some families of surfaces. Tanner, H. W. L. [1876-77] Mess. Mth. 6 (1877) 120-.

- geometry, theorem. Padova, E. Mil. I. Lomb. Rd. 23 (1890) 840-.

Envelope of a surface. Enneper, A. Mth. A. 5 (1872) 304-; Gött. Nr. (1873) 217-.
Enveloping surfaces. Garbieri, G. Ven. I. At. ("1883-84) 1201-.

Fuchsian surfaces. Bianchi, L. Rm. R. Ac. Linc. Rd. 4 (1888) (Sem. 2) 161-.

Geodesic representations of surfaces. Liouville, R. C. R. 108 (1889) 335-.

Geometrical applications of Picard's method of successive approximations. Bianchi, L. Rm. R. Ac. Linc. Rd. 3 (1894) (Sem. 1) 143-.

Harmonic ruled surfaces. Raffy, $L$. C. R. 110 (1890) 223-.

- spirals. Raffy, L. C. R. 112 (1891) 518-.

- surfaces. Raffy, L. Par. S. Mth. Bll. 22 (1894) 63-, 84-.

- -, class. Raffy, L. C. R. 112 (1891) 424-.

surfaces, properties. Raffy, L. Toul. Fac. Sc. A. 9 (1895) C, 44 pp.

Isometric surfaces. Voss, A. Mth. A. 46 (1895) 97-.

- -. Pellet, d. C. R. 124 (1897) 1337-.

Isometry of lines of curvature. Knoblauch, $J$. Crelle J. Mth. 103 (1888) 40-.

Kinematic classification of surfaces according to geodesic triangles. Mangoldt, $H$. von. [1882] Freiburg B. $8\left({ }^{*} 1882-85\right)$ 73-; (x) Crelle J. Mth. 94 (1883) 21-.

- Weingarten, J.L.G.J. Berl. Ak. Sb. (1882) 453-.

Liouville's surfaces, characteristic property. Stäckel, P. Mth. A. 35 (1890) 91-.

Locus of centres of curvature. Arndt, $F$. Grunert Arch. 9 (1847) 68-.

Orthogonal surfaces. Cayley, A. C. R. 75 (1872) 177-, 246-, 324-, 381-, 1800-.

- - Vanéček, J. S. Prag České Ak. Fr. Jos. Rz. (Třída 2) 4 (1895) Art. 16, 4 pp.

Parallel surfaces. Steiner, J. Berl. B. (1840) 114-.

- -. Roberts, W. C. R. 54 (1862) 797-.

-. Roberts, S. L. Mth. S. P. 4 (1871-73) 218

- etc. Combescure, É. Par. Éc. Norm. A. 7 (1878) 409-.

- - , and application to ellipsoid. Tortolini, B. Tortolini A. 1 (1850) 5-.

- - theorem, applications. Roberts, $W$. Ph. Mg. 24 (1862) 39-.

Partial differential equation, Cayley's, in theory of surfaces. Bianchi, L. Rm. R. Ac. Linc. Rd. 4 (1888) (Sem. 1) 442-.

- - of surfaces of constant curvature. Darboux, G. C. R. 97 (1883) 946-.

- - equations connected with theory of surfaces. Craig, T. Am. J. Mth. 19 (1897) 94-.

- - of cylinders. Tanner, H. W. L. [1876] Mess. Mth. 6 (1877) 113.

- - and representation of surfaces. Dar. boux, G. C. R. 96 (1883) 766-.

- _ _ - theory of surfaces, relation between. Stouff, $X . \quad$ Par. Éc. Norm. A. 13 (1896) 9-.

Ruled surface, dependence of characters on characters of guiding curves. Rupp, $O$. Mth. A. 18 (1881) 366-.

- surfaces. Hachette, J. N. P. Crelle J. 8 (1832) 358-. 
Ruled surfaces, differential equation. Sincov, D. [1894-95] Kazan S. Ps.-Mth. Bll. 4 (1895) 198 ; N. A. Mth. 14 (1895) 58-.

-, line element of which is reducible to Liouville's form. Demartres, - C. R. 110 (1890) 329-.

- - partial differential equation. Mansion, P. [1876] Mess. Mth. 6 (1877) 45-.

Skew helicoids developable on one another. Lamarle, E. Brux. Ac. Bll. 19 (1865) 407-.

- surface, locus of principal normals of 2 curves. Mannheim, A. Liouv. J. Mth. 17 (1872) 406-.

- surfaces, applicable on surfaces of revolution, and skew surfaces formed by principal normals of curve. Dini, $U$. G. Mt. 4 (1866) 298-.

- - one radius of principal curvature being a function of the other. Dini, $U$. A. Mt. 7 (1865) 205-.

_- satisfying partial differential equations of 2nd order. Dini, $U$. G. Mt. 3 (1865) 321-; 4 (1866) 305- ; C. R. 61 (1865) 1001-.

Surface of centres of curvature of surface of $n$th degree. Marcks, L. Mth. A. 5 (1872) $27-$.

- - 4th class, lines of curvature of which can be determined. Laguerre, $\boldsymbol{E}$. Liouv. J. Mth. 2 (1876) 145-.

- - least resistance. Armanini, E. A. Mt. 4 (1900) 131-.

-, tangent plane of which makes with radius vector an angle which is given function of radius vector. Turazza, D. Tortolini A. 2 (1851) 55-.

Surfaces admitting lines of spherical curvature and having same spherical representation for their lines of curvature. Blutel, -. C. $\mathbf{R}$. 116 (1893) 249-.

-, asymptotic lines of which in one system are curves of constant torsion. Bianchi, $L$. A. Mt. 18 (1890) 301-.

- with circular generator. Boulanger, $A$. N. A. Mth. 11 (1892) 159-

- class related to pseudo-spherical surfaces. Bianchi, L. Rm. R. Ac. Linc. Rd. 5 (1896) (Sem. 1) 131-.

- with common evolute. Sanctis, $P$. de. Rm. N. Line. At. 46 (1893) 90-.

- confocal quadrics as surfaces of centres. Rudio, F. Crelle J. Mth. 95 (1883) 240-.

- - 2 or more conics, traced on surface, passing through each point. Koenigs, $G$. C. R. 105 (1887) 407-.

- conjugate geodesics. Guichard, C. C. R. 110 (1890) 995-.

\section{Surfaces of constant curvature.}

Dini, U. G. Mt. 3 (1865) 241-; C. R. 60 (1865) 340 -.

Nicolaides, N. Bll. Sc. Mth. As. 9 (1875) 142-.

Enneper, A. Gött. Nr. (1876) 597-.

Lie, M. S. Arch. Mth. Ntvd. 4 (1879) 345-, 355-; 5 (1881) 282-, 328-, 518-.

Darboux, G. C. R. 97 (1883) 892-.

Kuen, T. [1884] Münch. Ak. Sb. 14 (1885) 193-.
Waelsch, E. Wien Ak. Sb. 102 (1893) (Ab. 2a) 1317-.

Beliankin, J. J. Fschr. Mth. (1898) 523.

Bianchi, L. Rm. R. Ac. Linc. Rd. 8 (1899) (Sem. 1) 223-, 371-, 484-.

Darboux, G. C. R. 128 (1899) 1018-.

mean. Folie, F. Brux. Ac. Bll. 30 (1870) 15-. Tilly, J. M. de. Brux. Ac. Bll. 30 (1870) 28-.

-. Chini, M. G. Mt. 27 (1889) 107-.

-.Vivanti, G. Mil. I. Lomb. Rd. 28 (1895) 353-.

negative. Nannei, E. G. Mt. 26 (1888) 201-. - Kahan, V. Kazan S. Ps.-Mth. Bll. 5 (1896) 111-, 145-; Fschr. Mth. (1895) 532, 533.

-, case. Sabinin, E. T. Rec. Mth. (Moscou) 18 (1896) 507-; Fschr. Mth. (1896) 537.

-, geometry on. Escherich, G. von. Wien Ak. Sb. 69 (1874) (Ab. 2) 497-

properties. Hazzidakis, J. N. [1878] Crelle J. Mth. 88 (1880) 68-.

- of line element. Weingarten, J. L. G. J. Crelle J. Mth. 94 (1883) 181-; 95 (1883) 325-. and surfaces with Liouville's line element. Waelsch, E. C. R. 116 (1893) 1435-.

total. Darboux, G. C. R. 97 (1883) 848-.

-. Genty, E. Par. S. Mth. Bll. 22 (1894) 106-.

- Guichard, C. C. R. 126 (1898) 1556-, 1616-.

-. Tzitzéica [Titeica], - . 288.

- and congruences formed of optical axes. Cosserat, E. Toul. Fac. Sc. A. 8 (1894) C, 3 pp.

- - related surfaces. Guichard, C. Par. Éc. Norm. A. 7 (1890) 233-

—, transformations. Darboux, G. C. R. 128 (1899) 953-.

Surfaces containing one set of curvature centres. Binet, J. P. M. C. R. 12 (1841) 435-.

- corresponding to given surfaces of centres. Enneper, A. Gött. Nr. (1871) 210-; (1872) 577-

- defined by property of straight lines or spheres touching them. Koenigs, G. C. R. 100 (1885) 847-.

- - - $R+R^{\prime}=F(u)+F_{1}\left(u_{1}\right)$. Montcheuil, - de. Par. S. Mth. Bll. 26 (1898) 103-.

_, 3rd degree, with parabolic umbilical line. Saint-Germain, A. de. Bll. Sc. Mth. 12 (1888) 177-.

- with difference of principal curvatures constant. Lipschitz, R. C. R. 104 (1887) $418,534$.

- divisible by their lines of curvature into squares. Cayley, A. [1872] L. Mth. S. P. 4 (1871-73) 8-, 120-; C. R. 74 (1872) 1445-.

_ _ _ _ _ _ _ _, differential equation. Weingarten, J. L. G. J. Berl. Ak. Sb. (1883) 1163-.

- generated by motion of lines, partial differential equations. Blâsek, G. [1864] Wien Sb. 51 (1865) (Ab. 2) 186-.

, generation, analytic expression. Monge, $G$. Turin Mm. Ac. 1 (1784-85) 19-. 
Surfaces of given curvature. Weingarten, $J$. Schlömilch Z. 3 (1858) 43-.

- with line element of form $d u^{2}+K^{2} \cos ^{2} u \cdot d v^{2}$. Soler, $\boldsymbol{E}$. Palermo Ac. At. 4 (1897) (Sc. Nt.) $7 \mathrm{pp}$.

- - - - reducible to form

$$
f(U+V)\left(d u^{2}+d v^{2}\right) \text {. }
$$

Petot, A. C. R. 110 (1890) 330-

_ _ - - reducible to Liouville's form in several ways. Koenigs, G. C. R. 109 (1889) 565-, 639-.

- - lines of curvature equal in 1 system. Demoulin, A. C. R. 130 (1900) 823-.

- - - - - forming network with equal tangential invariants. Thybaut, A. C. R. 121 (1895) 519-.

- - - - - helices on any cylinders. Petot, A. C. R. 106 (1888) 1517-.

- - - - isometric. Craig, T. C. R. 123 (1896) 794-.

- - - - plane. Dini, U. [1867] A. Mt. 1 (1867-68) 146-.

- - - - - Razzaboni, A. Bologna Ac. Sc. Mm. 10 (1879) 529-.

- - - - all. Bonnet, O. C. R. 36 (1853) 81-, 219-; 42 (1856) 1067-.

$---1-1$, Rouquet, $V$. Toul. Ac. Sc. Mm. 9 (1887) 233-; 10 (1888) 161-.

- - - - - - Burnside, W. Mess. Mth. 20 (1891) 49-, 148.

- - - - - - and equal in 1 system. Caronnet, T. C. R. 117 (1893) 842-.

- - - - - or spherical. Enneper, $A$. Gött. Ab. 23 (1878) 95 pp.; 26 (1880) 139 pp. - - - - Cayley, A. Am. J. Mth. 11 (1889) 71-, 293-

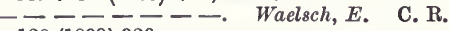
128 (1899) 920-.

- - - - - - all. Serret, J. A. Liouv. J. Mth. 18 (1853) 113-; C. R. 36 (1853) 432-.

- - - - in 1 system. Serret, J. A. C. R. 42 (1856) 194.

- - - - - Joachimsthal, $F$. Crelle J. 54 (1857) 181-.

- - - - - - - Combescure, $\hat{E}$. [1883] Mntp. Ac. Mm. 10 (1884) 401-。

- - - - - - - Pirondini, G. G. Mt. 22 (1884) 118-.

- - - - - - - - and isothermic. Darboux, G. C. R. 96 (1883) 1202-, 1294-; Bll. Sc. Mth. As. 7 (1883) 257-.

- - - - spherical. Blutel, E. C. R. 122 (1896) 301-.

- - - - - in 1 system. Serret, J. A. C. R. 42 (1856) 109-, 190-.

- - - - - - Dini, U. [1869] Mod. S. It. Mm. 2 (1876) 135-.

- - - - - - - Enneper, $A$. Gött. Nr. (1872) 17-, 80-.

- - - - - - - Dobriner, $H$. [1882] Crelle J. Mth. 94 (1883) 116-.

- Liouville's element of arc. Waelsch, $E$. Wien Ak. Sb. 106 (1897) (Ab. 2a) 323-.

- normals tangent to conic surface, case. Monge, G. Par. Ec. Pol. J. 4 (1801-02) 59 -.
Surfaces with normals tangent to developable surface, case. Monge, G. Par. Éc. Pol. J. 5 (1806) 1-.

$---1-$ given surface. Weingarten, $J$. [1861] Crelle J. 62 (1863) 61-.

- - - - sphere, all. Vályi, J. Arch. Mth. Ps. 68 (1882) 217-.

$----\frac{-}{-}$, case. Monge, $G$. Par. Éc. Pol. J. 4 (1801-02) 28-.

- - parallel sections cutting lines of curvature under a constant angle. Bianchi, $L$. Rm. R. Ac. Linc. Rd. 7 (1891) (Sem. 1) 4-.

- - every portion subtending solid angles in given ratio from two fixed points. Weyr, E. [1877] Wien Ak. Sb. 76 (1878) (Ab.2) 859-.

- - principal planes equidistant from fixed point. Guichard, -. C. R. 116 (1893) 487-.

- - - having ratio of distances from fixed point constant. Bianchi, $L$. Rm. R. Ac. Linc. Rd. 3 (1894) (Sem. 2) 77-.

- rational generators. Lelieuvre, -. C. R. 113 (1891) 635-.

- of revolution of constant mean curvature. Delaunay, C. Liouv. J. Mth. 6 (1841) 309-. - _ - - Sturm, J. C. F. Liouv. J. Mth. 6 (1841) 315-.

- _ - - - -. Lindelöf, L. L. [1862] (viII) Helsingf. Acta 7 (1863) 345-.

_ - - cut by sphere in geodesic line. Molins, H. Toul. Ac. Sc. Mm. 3 (1891) 296-.

- - -, loxodromes of which (or their analogues) project into logarithmic spirals. Molins, H. Toul. Ac. Sc. Mm. 7 (Sem. 2) (1885) 293-; 8 (1886) 426-.

- - with loxodromic relation. August, $F$. Z. Mth. Ps. 33 (1888) 154 .

- - - minimum line in given plane. Molins, H. Toul. Ac. Sc. Mm. 2 (1890) 360-.

- _ - theory. Jung, $V$. Časopis 9 ("1880) 216-; Fschr. Mth. ( $\left.{ }^{*} 1880\right) 569$.

- - with $R_{1} R_{2}$ constant. Metzler, G. F. Am. J. Mth. 20 (1898) 76-.

- with singular meridian curves. Enneper, $A$. Gött. Ab. 29 (1882) 87 pp.

- - system of umbilical lines, arising from system of envelopes. Gremigni, $M$. [1882] Rm. R. Ac. Linc. Mm. 15 (1883) 3-.

,- 2 systems of geodesics on which form a conjugate system. Voss, A. Münch. Ak. Sb. 18 (1889) 95-.

- of uniform slope, and proportional networks. Lecornu, $L$. Caen Ac. Mm. (1884) 65-.

- - - - - system of curves on them. Lecornu, L. C. R. 98 (1884) 972-.

- zero mean curvature. Weierstrass, C. Berl. Mb. (1866) 612-, 855-.

- - - - Ribaucour, A. [1880] Brux. Mm. Cour. $4^{\circ}, 44$ (1882) No. 1, vi $+236 \mathrm{pp}$.

- - - (Ribaucour). Calalan, E. C. Brux. Ac. Bll. 50 (1880) 333-.

- - - - case. Mensbrugghe, G. van der. Brux. Ac. Bll. 21 (1866) 552-.

- - - - - of which 4 straight lines enclose finite area. Schwarz, C. H. A. C. R. 96 (1883) 1011-. 
Surfaces with $R_{1}+R_{2}$ constant. Bonnet, $O$. C. R. 42 (1856) 110-.

- - -. Dini, U. A. Mt. 7 (1865) 5-.

- - proportional to distance of tangent plane from fixed point. Goursat, É. Am. J. Mth. 10 (1888) 187-.

G. Mt. 28 (1890) 349-.

- $R_{1} R_{2}$ constant. Codazzi, D. Tortolini A. 8 (1857) 346-.

- $R_{1}=f\left(R_{2}\right)$. Weingarten, J. [1862] Crelle J. 62 (1863) 160-.

$---=-$ Halphen, G. H. Par. S. Mth. Bll. 4 (1876) 94-.

$--=-$. Mannheim, A. C. R. 84 (1877) 932-; Par. S. Mth. Bll. 5 (1877) 163-.

$--=-$ Lie, M. S. Arch. Mth. Ntvd. 4 (1879) 507-.

--1 Weingarten, J. Crelle J. Mth. 103 (1888) 184-.

$--1=-$, cases. Raffy, L. Par. S. Mth. Bll. 19 (1891) 158-.

$---=-$, theory. Raffy, L. Par. S. Mth. Bll. 25 (1897) 147-.

$--R_{1}=R_{2}$. Forsyth, A.R. Mess. Mth. 27 (1898) 129-.

Uniformly illuminated lines of any surface. Pieri, M. Tor. Ac. Sc. At. 27 (1892) 347-.

Voss's surfaces. Guichard, C. C. R. 129 (1899) 23-, 188.

Weingarten's surfaces. Pellet, A. C. R. 125 (1897) 601-.

- - two surfaces that may be associated with. Demoulin, A. C. R. 131 (1900) 330-

$P d x+Q d y+R d z=0$, geometrical interpretation. Voss, A. E. Mth. A. 16 (1880) 556-.

\section{Conformal and other repre- sentations of surfaces on others. (See also 3600, 6840 and Geo- graphy 70-95.)}

Complex variables on any surface. Beltrami, E. [1867] A. Mt. 1 (1867-68) 329-.

Conformal relation of parallelism between two surfaces. Mlodzěevskij, B. K. [1898] Rec. Mth. (Moscou) 20 (1899) 620-; Fschr. Mth. (1899) 606.

\section{CONFORMAL REPRESENTATION.}

Eisenlohr, F. Crelle J. 72 (1870) 143-. Neumann, C. Leip. B. 22 (1870) 49-, 264 -. Klein, C. F. [1881] Mth. A. 19 (1882) 159-. Phragmén, E. Acta Mth. 14 (1890-91) 225-.

Escher, R.J. N. Arch. Wisk. 20 (1893) 111-. Stäckel, P. Mth. A. 44 (1894) 553-.

Lindemann, F. Königsb. Schr. 35 (1895) [27]-. Voss, A. Mth. A. 46 (1895) 133-.

Naetsch, E. Dresden Isis Sb. (1896) 33.

branch points of polynomial. Stolp, C. N. Arch. Wisk. 17 (1890) 235-; Fschr. Mth. (1890) 600 .

case. Amstein, H. [1877] Laus. S. Vd. Bll. 15 (1878) 175-. eases. Hentschel, O. Z. Mth. Ps. 17 (1872) 39-.

-. Ascoli, G. Mil. I. Lomb. Rd, 18 (1885) $349-, 390-, 440$ -

-, and their application to hydrodynamics. Amstein, H. [1879] Laus. S. Vd. Bll. 16 (1880) 249-.

by central projection. Weyr, E. Prag Sb. (1877) 273-.

of circle on interior of epicycloid. Huber, G. Bern Mt. (1891) 42-.

- configurations in three dimensions. Cotton, É. C. R. 127 (1898) 349-.

with conservation of area. Razzaboni, $A$. [1889] Bologna Rd. (1889-90) 21-.

of cyclide. Holzmuller, F. G. Crelle J. Mth. 94 (1883) 342-.

- on rectangle and unlimited plane. Holzmilller, F. G. [1882] Crelle J. Mth. 94 (1883) 237-.

depending on zeta-function. Kluyver, J. C. Z. Mth. Ps. 40 (1895) 129-.

of Earth by epicycloidal projection. August, $F$. Berl. Z. Erdk. 9 (1874) 1-.

by electricity. Kelvin, (Lord). Nt. 46 (1892) 490-, 541-.

of ellipsoid on plane. Jacobi, C. G. J. Crelle J. 59 (1861) 74-.

- elliptic area on circular area. Schwarz, H.A. A. Mt. 3 (1869-70) 166-.

extension to 3-dimensional space. Landsberg, G. D. Nf. Vh. (1896) (Th. 2, Hälfte 1) 7.

of half plane on polygon approaching limiting circle. Pick, G. Wien Ak. Sb. 100 (1891) (Ab. 2a) 1387-.

- _ - - simply connected surface bounded by algebraic curve. Lindemann, $F$. Münch. Ak. Sb. 24 (1895) 403-.

- - - - surface bounded by circular cubic or bicircular quartic. Goettler, J. [1900] Münch. Ak. Sb. 30 (1901) 165-.

- inner surface of circle on inner surface of regular polygon. Bigler, $U$. Arch. Mth. Ps. 14 (1896) 360-.

- multiply connected plane surfaces. Schottky, F. Crelle J. Mth. 83 (1877) 300-.

- paraboloid on plane. Koch,H. von. Stockh. Öfv. (1889) 181-; Fschr. Mth. (1889) 834.

- pentagon on half plane. Barwell, (Miss) M. E. L. Mth. S. P. 29 (1898) 695-.

- one plane on another. Wangerin, A. Arch. Mth. Ps. 55 (1873) 5-.

- plane area on circular area. Neumann, C. G. Leip. Mth. Ps. B. 29 (1877) 154-.

- - areas. Tallqvist, $H$. Helsingf. Acta 21 (1896) No. 3, 29 pp.

- figure bounded by straight lines in half plane, general possibility. Schläfi, $L$. Crelle J. Mth. 78 (1874) 63-.

- - on prism. Cassel, G. Stockh. Ak. Hndl. Bh. 16 (Afd. 1) (1891) No. 3, 11 pp.; Fschr. Mth. (1891) 893-.

on planes, theorem. Hoppe, R. Arch. Mth. Ps. 59 (1876) 59-.

of polygons. Painlevé, P. C. R. 106 (1888) 473-.

problem. Cassel, G. Acta Mth. 15 (1891) 33-. 
problem. Burnside, W. L. Mth. S. P. 24 (1893) 187-.

-. Źorawski, K. Krk. Ak. (Mt.-Prz.) Rz. 19 (1902) 218-; Crc. Ac. Sc. Bll. (1900) 325-.

of pseudosphere, and applications. Marcolongo, $R$. Nap. Rd. 27 (1888) 111-.

- quadrics. Hoppe, R. [1869] Mth. A. 2 (1870) 504-.

by rational function. Fuchs, L. Crelle J. Mth. 108 (1891) 181-.

of spherical triangles by algebraic functions. Fischer, $O$. Leip. Mth. Ps. B. 36 (1884) 17-. - one surface on another. Gauss, C. F. Schumacher As. Ab. 3 (1825) 1-.

- _ - Souslow [Suslov], G. K. C. R. 126 (1898) 30-; Mosc. S. Sc. Bll. 93 (No. 2) (1898) 12-.

- surface of rectangle on hemisphere. Schwarz, C. H. A. Gött. Nr. (1883) 51-.

- - tetrahedron on sphere. Schwarz, H.A. Crelle J. 70 (1869) 121-.

- surfaces of revolution on plane. Buchwaldt, F. Ts. Mth. 6 (1889) 73-; Fschr. Mth. (1888) 869-.

theorems of Poincaré. Brun, F. de. Stockh. Öfv. (1889) 677-.

theory. Painlevé, P. C. R. 112 (1891) 653-. - of Poincaré. Brun, F. de. Stockh. Öfv. (1890) 135-; Fschr. Mth. (1890) 410.

of trochoids. Amstein, $H$. Laus. S. Vd. Bll. 28 (1892) 67-.

Conformal transformation. Nanson, $E$. $J$. [1876] Mess. Mth. 6 (1877) 69-.

- - application of theory. Emmanuel, $D$. [Bucarest S. Sc. Bl. 7 (1898)] 14-.

- transformations in space, limitation. Capelli, A. A. Mt. 14 (1886-87) 227-.

Conformation of areas, special. Forsyth, A.R. QJ. Mth. 26 (1893) 145-.

Correlation between two plane figures, special case. Brill, J. Mess. Mth. 19 (1890) 57-, 151-.

Correspondence of surfaces, general theory. Egorov, D. T. Rec. Mth. (Moscou) 18 (1896) 86-; Fschr. Mth. (1896) 500.

- _ - geodesics. Busse, F. Berl. Ak. Sb. (1896) 651-.

Corresponding surface elements. Neumann, C. G. Leip. Mth. Ps. B. 28 (1876) $253-$.

Differential equation of second order with transcendental coefficients. Cassel, G. Stockh. Öfv. (1890) 381-.

Flamsteed's modified development, change of angles and distances. Tissot, $A$. Par. Éc. Pol. J. cah. 37 (1858) 217-.

Form of surface elements. Exner, $K$. Arch. Mth. Ps. 51 (1870) 7-.

Geodesy. Pizzetti, P. Méx. S. "Alzate" Mm. 4 (1890) 257-.

-, fundamental equations. Láska, W. Prag Sb. (1897) (Mth.-Nt.) No. 36, $13 \mathrm{pp}$.

Great circle on Mercator chart. Chauvenet, $W$. Camb. (M.) Mth. M. 1 (1859) 135.

- sailing. Janse, L. N. Arch. Wisk. 7 (*1881) 91-.
Isogonal relation of two plane spaces. Weyr, $E$. Časopis 3 (*1874) 1-; Fschr. Mth. ( $\left.{ }^{*} 1874\right)$ 532.

- transformation, use of supplementary curves. Harris, R. A. Am. J. Mth. 14 (1892) 291-.

Isothermic reflexion. Holzmiiller, F. G. [1880] Crelle J. Mth. 94 (1883) 179-.

Locus of centres of curvature and involutes. Fuss, N. [1804] St Pét. Ac. Sc. Mm. 3 (1811) 91-.

Maps, construction, Čebyšev's theorem. Grave, D. A. As. Fr. C. R. (1894) (Pt. 2) 196-.

-, - , development method. Obrecht, $A$. [1893] Santiago de Chile Un. A. 82 (1892) $871-$.

-, 一 on rotation ellipsoid. Vostokov, I. A. Vars. S. Nt. Tr. (1894-95) (C. R.) No. 11, 3-.

-, geographical. Korkine, $A$. Mth. A. 35 (1890) 588-.

-, - construction. Grave, D.A. Liouv. J. Mth. 2 (1896) 317-.

- , theory. Heger, $\dot{R}$. Civing. 36 (1890) 47-. Measurement on plane map of lines on terrestrial sphere. Collignon, É. As. Fr. C. R. (1884) (Pt. 2) 15-.

Normal curve $\Phi$ of genus $p=3$. Kęiński, $S$. Prace Mt.-Fiz. 11 (1900) 1-; Fschr. Mth. (1900) 455.

Orthogonality of elements, correspondence by. Demoulin, A. C. R. 116 (1893) 682-.

Orthomorphosis. Cayley, A. QJ. Mth. 25 (1891) 203-.

- of circle into parabola. Cayley, A. QJ. Mth. 20 (1885) 213-.

-, problems. Cayley, A. Crelle J. Mth. 107 (1891) 262-.

一, theory. Cayley, A. QJ. Mth. 26 (1893) 282-.

Point transformations leaving areas of all surfaces invariant. $\operatorname{Carda}, K$. Wien Ak. $\mathrm{Sb}$. 105 (1896) (Ab. 2a) 787-.

\section{Projection.}

adopted for "Carte de France," properties. Puissant, L. Par. S. Phlm. Bll. (1821) 17-. central. Mogni, A. G. Mt. 13 (1875) 186-.

conformal. Veltmann, $W$. As. Nr. 83 (1874) 225-.

of curved surfaces. Dienger, J. N. A. Mth. 11 (1852) 252-.

equivalent, with minimum deviation for limited circular area. Schols, C.M. Amst. Ak. Vs. M. 2 (1886) 130-; Arch. Néerl. 20 (1886) 388-. of figure of revolution. Codazzi, $D$. Tortolini A. 1 (1858) 89-.

gnomonic, notions of infinity derived from. Purkiss, H. J. [1865] Mess. Mth. 3 (1866) 171-.

horizontal, stereographic, of terrestrial hemisphere. Castrilli, C. G. Mt. 30 (1892) 31-, 105.

map. Bremiker, C. As. Nr. 41 (1855) 353-.

-. Collignon, É. Par. Éc. Pol. J. Cah. 41 (1865) 73-.

Markov, A. A. Kharkov Mth. S. Com. 1 (1889) 113-; Fschr. Mth. (1888) 870-. 


\section{Surfaces. Projection}

map, conformal. Wittstein, T. As. Nr. 71 (1868) 369-.

- - Grunert, J. A. Arch. Mth. Ps. 50 (1869) 176-.

-, graduation of instruments for. Driencourt, -. A. Hydrog. 20 (1899) 78-.

- for oblique cylinder and cone. Heger, $R$. Civing. 41 (1895) 401-.

-, problems. Rollet de L'Isle, -. A. Hydrog. 15 (1893) 58-.

-, with tables. Schols, C. M. N. Arch. Wisk. $8\left({ }^{*} 1882\right) 113$

Mercator's. Timerding, H. E. Z. Mth Ps. 43 (1898) 320-.

-, of skew hyperboloid. Cayley, A. Mess. Mth. 4 (1875) 17-.

-, for triangulation near equator. Schols, C. M. Delft Éc. Pol. A. 1 (1885) 1-。

methods, merits. Chelini, D. G. Arcad. 74 (1838) 47-.

oblique, axonometric. Kinkelin, $H$. Zür. Vjschr. 6 (1861) 358-.

orthographic. Avillez, J. F. de. Lisb. J. Sc. Mth. 3 (1895) 76-; Fschr. Mth. (1893-94) 1888.

orthomorphic, of ellipsoid on sphere. Craig, $T$. Am. J. Mth. 3 (1880) 114 .

plagiographic. Anger, C. T. Grunert Arch. 8 (1846) 235-.

problem. Cayley, A. QJ. Mth. 13 (1875) 19properties. Poisson, S. D. Par. Éc. Pol. Cor. 1 (1804-08) 389-.

quincuncial, of sphere. Pierpont, J. Am. J. Mth. 18 (1896) 145-.

of sphere, new system. Cornu, A. (Ix) Par. S. Phlm. Bll. 3 (1866) 111-.

- - - - Guyou, -. C. R. 102 (1886)

308-; Rv. Mar. et Col. 94 (1887) 228-

stereographic. Delambre, J.B.J. [1799] Par. Mm. de l'I. (1804) 393-.

-. Chasles, M. Gergonne A. Mth. 19 (182829) 157-.

-. Catalan, E. C. Liouv. J. Mth. 19 (1854) 132-.

-, analytic development. Grunert, $J . A$. Grunert Arch. 32 (1859) 250-

-, application. Zeuthen, H. G. (xII) Ts. Mth. 2 (1866) 97-.

-, - of theory of caustics. Quetelet, L.A.J. [1825] Brux. Ac. Sc. Mm. 4 (1827) 81-.

, construction of plane zonal arcs in. Websky, M. Berl. Ak. Sb. (1886) 33-.

-, history. Gilnther, -. D. Nf. Tbl. (1887) 227-.

-, property. Chasles, M. Liouv. J. Mth. 7 (1842) 272

-, of spherical conic. Cayley, A. Ph. Mg. 25 (1863) 350-

-, and theorems of Quetelet and Dandelin. Pelz, C. Prag Sb. (1898) (Mth.-Nt.) No. 31, 4 pp.

of straight line on spheroidal surface. Grunert, J. A. Grunert Ârch. 6 (1845) 293-.

on surface. Schoute, P. H. As. Fr. C. R. (1879) 155-.

of surface of revolution on plane. Grave, D.A. St Pét. Ac. Sc. Bll. 1 (1894) 73-.
Representation 8840

Projective relation between infinitesimal elements. Oliver, J. E. [1884] A. Mth. 1 (1884-85) 40-.

Reduction of altitude curves. Réveille, J. A. Hydrog. 15 (1893) 147-.

Relation between great circle and loxodrome joining two points on the Earth. Berg, F.J. van den. N. Arch. Wisk. 9 ("1882) 15-.

\section{Representation.}

by algebraic functions of whole plane on circle. Fuchs, L. Crelle J. Mth. 77 (1874) 339-; 78 (1874) 338-.

of algebraic surfaces. Cremona, $L$. Gött. Nr. (1871) 129-.

- - Darboux, G. Bll. Sc. Mth. As. 2 (1871) 40-, 155-.

- _ - especially those of 4 th and 5 th degrees. Clebsch, R. F. A. [1868] Mth. A. 1 (1869) 253-.

- - on plane. Clebsch, R. F. A. Mil. I. Lomb. Rd. 1 (1868) 794.

on circle, of plane areas with proportionality of small areas. Schellhammer, $F$. Z. Mth. Ps. 23 (1878) 69-.

- - - single sheeted simply connected plane surface. Christoffel, E. B. Gött. Nr. (1870) 283-.

- - , - n-sheeted simply connected plane surface. Christoffel, E. B. Gött. Nr. (1870) 359-.

of circular ring on rectangle, geometrical construction. Study, E. Crelle J. Mth. 97 (1884) 13-.

- class of skew surfaces on plane, and determination of their asymptotic curves. Cremona, L. A. Mt. 1 (1867-68) 248-.

- - surfaces of 5 th degree. Clebsch, R.F.A. Gött. Nr. (1869) 486-; Gött. Ab. 15 (1870) 64 pp.

on conic, of curve of 4th degree with cusp. Weyr, E.J. [1878] Wien Ak. Sb. 78 (1879) (Ab. 2) 396-.

with conservation of area. Wierzbicki, $E$. Prace Mt.-Fiz. 10 (1899-1900) 129-.

continuous, of line on surface. Hilbert, D. [1890] Mth. A. 38 (1891) 459-.

of continuous linear configuration on discontinuous one. Harnack, A. Mth. A. 23 (1884) 285-.

- curved surfaces, new principle. Voss, A.E. Mth. A. 19 (1882) 1-.

- curves on surface and application to maps. Du Chatenet, M. N. A. Mth. 5 (1886) 142-. equivalent, of rotation ellipsoid on sphere. Klingatsch, A. Mh. Mth. Ps. 8 (1897) 175-

of exterior of polygon of circular ares on circular surface. Sanio, T. Arch. Mth. Ps. 3 (1886) 1-.

geodesic. Luroth, J. Mth. A. 51 (1899) $161-$.

of geodesic circles on surface by tangential transformations. Oseen, C. W. Stockh. Öfv. (1900) 957-.

- half plane on polygon bounded by ares of confocal conics. Lindemann, $F$. Münch. Ak. Sb. 25 (1896) 219-. 


\section{Representation}

homocyclic, of sphere on plane. Andreev, K. A. Kharkov Mth. S. Com. 3 (1893) 35-; Fschr. Mth. (1891) 894 .

logarithmic, and orthogonal curve systems arising therefrom. Holzmüller, G. Z. Mth. Ps. 16 (1871) 269-.

of lunes. Igel, B. Z. Mth. Ps. 17 (1872) 251-.

orthogonal, representation of collinear projections in space in. Schlesinger, J. Wien Sb. 59 (1869) (Ab. 2) 636-.

-, of surfaces of revolution, relation between meridian and contour curve. Müller, $R$. Z Z Mth. Ps. 21 (1876) 265-.

of small part of ellipsoidal surface. Hammer, E. Ac. Nt. C. N. Acta 71 (1898) 447-.

- parts of curved surface on plane, principle. Weber, H. Crelle J. 67 (1867) 229-.

plane, of ruled surfaces. Mannheim, $\dot{A}$. C. R. 85 (1877) 788-, 847-, 941-.

-, - solid. Cayley, A. Ph. Mg. 41 (1871) 286-.

of polygons of circular ares. Pochhammer, $L$. Crelle J. 76 (1873) 170 -

problem. Weber, H. Mth. A. 2 (1870) 140-.

problems. Schwarz, H. A. Crelle J. 70 (1869) 105-.

on sphere. Dini, U. A. Mt. 4 (1870-71) 175-. of sphere on plane. Bianchi, L. Rm. R. Ac. Linc. Rd. 6 (1890) (Sem. 1) 226-.

- _ _ - Mlodzéevskij, B. K. Mosc. S. Sc. Bll. 65 (No. 1) (1890) 83-.

- - - rotation-cone. Klingatsch, A. Mh. Mth. Ps. 10 (1899) 75-.

spherical. Darboux, G. C. R. 68 (1869) 253 -.

-. Ribaucour, A. C. R. 75 (1872) 533-.

- Darboux, G. C. R. 94 (1882) 120-, 158-, 1290-, 1343-; 96 (1883) 366-; Par. Éc. Norm. A. 5 (1888) $79-$.

-. Adler, A. Wien Az. 37 (1900) 222-.

of Steiner's surfaces and of skew surfaces of 3rd degree on plane. Cremona, $L$. Mil. I. Lomb. Rd. 4 (1867) 15-.

- straight lines of space on plane. Scheffers, $G$. Leip. Mth. Ps. B. 47 (1895) 201-.

- one surface on another. Dini, U. A. Mt. 3 (1869-70) 269-.

Mth. As. 1 (1870) 383-.

- - - - Weyr, E. Časopis $9\left({ }^{*} 1880\right)$ 201-; Fschr. Mth. $\left({ }^{*} 1880\right) 628$.

- -1 - Somov, P. O. Vars. S. Nt. Tr. (1894-95) (C. R.) No. 11, 14-.

_ _ _ - in Gauss's manner. Razzaboni, A. G. Mt. 27 (1889) 274-.

- surface on plane so that geodesics are represented by straight lines. Beltrami, E. A. Mt. 7 (1865) 185-.

- - of revolution. Saija, G. Catania Ac. Gioen. At. 10 (1897) Mem. 17, $10 \mathrm{pp}$.

- surfaces of revolution with constant negative curvature on one another. Wangerin, A. D. Mth. Vr. Jbr. 1 (1892) 71-.

$x+y i=\sqrt[n]{X+Y i}$. Holzmuller, F. G. Crelle J. Mth. 83 (1877) 38-.

\section{Deformation of Surfaces 8850}

Schwarz's triangles. Kapteijn, W. N. Arch. Wisk, 1 (1895) 185-; Fschr. Mth. (1893-94) 698.

- - with simple nodal point. Schilling, $F$. [1896] D. Mth. Vr. Jbr. 5 (1901) (Heft 1) $73-$.

Skew surfaces and their application on one another. Enneper, A. Z. Mth. Ps. 9 (1864) 377 -.

Spheroidal trigonometry. Oriani, B. Zach M. Cor. 10 (1804) 244-; 11 (1805) 551-; Bologna Mm. I. It. 1 (1806) 119-; 2 (1808) 1-; (pte. 2) (1810) 1-; Mil. Ephem. As. (1827) 3-; (1828) 3-; (1829) 3-; Mil. Mm. I. Lomb. Ven. 4 (1832) 325-.

- -, formulæ. Bonsdorff, A. [1885] St Pét. Ac. Sc. Bll. 30 (1886) 205-.

Stereograms of surfaces, construction. Maxwell, J. C. $[1868]$ L. Mth. S. P. 2 (1869) 57-.

Sumner lines on Mercator chart. Greenhill, A. G. Mess. Mth. 16 (1887) 162-.

- - - and stereographic chart. Greenhill, A. G. Mess. Mth. 20 (1891) 4

Surface of revolution, tangents to boundary of shadow. Staudigl, $R$. Wien Sb. 68 (1873) (Ab. 2) 228-.

Surfaces of first 7 degrees developable on plane. Schwarz, H. A. [1864] Crelle J. 64 (1865) 1-.

-, development, case. Minding, E. F.A. Crelle J. 20 (1840) 171-.

- divided into squares by system of circles and their orthogonal trajectories. Demartres, $G$. Par. Éc. Norm. A. 4 (1887) 145-.

- representable on plane. Bonnet, O. [1875] A. Mt. 7 (1876) 61-.

- - - point for point. Guccia, J. As. Fr. C. R. 9 (1880) 191-.

- of revolution, applicable to sphere. Ocagne, M. $d^{\prime}$. As. Fr. C. R. (1894) (Pt. 2) 11-.

- - - homolographic development. La. marle, E. Brux. Ac. Bll. 10 (1860) 530-; 11 (1861) 42-.

- - - in orthogonal and perspective representations, construction of contours. Niemtschik, $R$. Wien Sb. $52(1866)(A b .2)$ 573-.

- with same spherical representation. Pellet, A. C. R. 124 (1897) 1291-.

Symmetric $S$ (Schwarzian) functions. Schilling, F. Ac. Nt. C. N. Acta 71 (1898) 207-.

Systems of maps with all circles of sphere represented by circles. $D u$ Chatenet, $M$. N. A. Mth. 5 (1886) 168-.

\section{Deformation of surfaces.}

Minding, E. F. A. Crelle J. 18 (1838) 365-. Maxwell, J. C. [1854] Camb. Ph. S. T. 9 (1856) 445-.

Bour, E. [J. E. E.] Par. Éc. Pol. J. cah. 39 (1862) $1-$

Jordan, C. Liouv. J. Mth. 11 (1866) 105-.

Moutard, T. (x) Par. S. Phlm. Bll. 6 (1869) $45-$.

Ribaucour, A. (xI) Par. S. Phlm. Bll. 6 (1869) 51-; (vir) C. R. 70 (1870) 330-. 
Daug, H. T. Stockh. Öfv. 33 (1876) 3-.

Laisant, C. A. As. Fr. C. R. (1878) 180-.

Weingarten, $J$. Berl. Ak. Sb. (1886) 83-.

Mlodzéevskij, B. K. Mosc. Un. Mm. (Ps.-Mth.) 7 (1887) 141 pp.; Bll. Sc. Mth. 15 (1891) 97-.

Bianchi, L. Rm. R. Ac. Linc. Rd. 1 (1892) (Sem. 2), 41-.

Goursat, E. Am. J. Mth. 14 (1892) 1-.

Cosserat, E. Toul. Fac. Sc. A. 8 (1894) E, $46 \mathrm{pp}$.

Adam, P. Par. S. Mth. Bll. 23 (1895) 106-, 219-; C. R. 121 (1895) 551-.

Guichard, -. Liouv. J. Mth. 2 (1896) 123-.

Staeckel, P. C. R. 123 (1896) 677-.

Weingarten, J. Acta Mth. 20 (1897) 159-.

Applicable figures, construction. Cayley, $A$. Am. J. Mth. 2 (1879) 186.

- -, theorem. Cayley, A. L. Mth. S. P. 10 (1878-79) 143-.

Application of invariants of linear and quadratic differential forms. Hessenberg, $G$. Acta Mth. 23 (1900) 121-.

Cases. Minding, E. F. A. Crelle J. 18 (1838) 297- .

-. Enneper, A. Gött. Nr. (1875) 129-.

Contact transformations. Lovett, E. O. N. Y. Am. Mth. S. Bll. 5 (1899) 113, 118-.

Covariants. Knoblauch, J. Crelle J. Mth. 111 (1893) 277-.

- and differential parameters. Stäckel, $P$. Crelle J. Mth. 113 (1894) 58-.

\section{DEFORMATION.}

of class of surfaces. Bianchi, L. G. Mt. 16 (1878) 267-.

- closed surfaces of positive curvature. Liebmann, H. Mth. A. 53 (1900) 81-.

and conjugate systems. Cosserat, E. C. R. 113 (1891) 460-.

- - - Stäckel, P. Mth. A. 49 (1897) 255-.

with conservation of principal curvatures. Hazzidakis, J. N. Crelle J. Mth. 117 (1897) $42-$.

continuous. Mair, D. B. QJ. Mth. 27 (1895) $1-$.

and curvature and representation of surfaces. Dienger, J. Grunert Arch. 19 (1852) 306-.

of curved surfaces under certain conditions. Stäckel, P. D. Mth. Vr. Jbr. 1 (1892) 70.

and cyclic systems. Cosserat, E. C. R. 113 (1891) 498-.

- equilibrium of skew surface. Cayley, $A$. L. Mth. S. P. 12 (1880-81) 103-.

of evolute of catenoid. Razzaboni, A. G. Mt. 28 (1890) 154-.

- extensible surfaces, mechanical theory. Finsterwalder, S. D. Mth. Vr. Jbr. 6 (1899) (Heft 2) 43-.

- flexible and inextensible surfaces. Volterra, $V$. Rm. R. Ac. Line. Rd. 1 (1885) 274-.

- geodesics. Allegret, $[A$.$] C. R. 66$ (1868) $342-$.

_ inextensible surfaces. Weingarten, J. Crelle J. Mth. 100 (1887) 296-. of inextensible surfaces, Gauss's theorem, simple demonstration. Boussinesq, V.J. (xII) Lille S. Mm. 8 (1880) 381-.

infinitesimal. Genty, E. Par. S. Mth. Bll. 22 (1894) 221-; Toul. Fac. Sc. A. 9 (1895) E, $11 \mathrm{pp}$.

-.Voss, A. [1895-97] D. Mth. Vr. Jbr. 4 (1897) 132-; Münch. Ak. Sb. 27 (1898) 229-.

-. Pszeborski, A. P. Fschr. Mth. (1900) 610-.

-, and Bianchi's associated surfaces. Cos. serat, $E$. C. R. 115 (1892) 1252-.

一, of curves. Perna, A. G. Mt. 36 (1898) 286-.

of infinitesimal element. Desmousseaux de Givré, F. X. É. Par. Mm. Ing. Civ. (1864) 92-.

intrinsic treatment. Cesáro, $E$. Nap. Rd. 33 (1894) 149-.

of model of hyperboloid. Cayley, A. Mess. Mth. 8 (1879) 51-. 9-.

- paraboloid. Thybaut, A. Par. Éc. Norm. A. 14 (1897) 45-.

- - cases, and Weingarten's theorem. Cosserat, $E$. C. R. 124 (1897) 741-.

- - of rotation in spaces of constant curvature. Bianchi, L. A. Mt. 4 (1900) 1-.

- plane closed polygon. Macaulay, $F$. S. L. Mth. S. P. 28 (1897) 442-.

- quadrics. Peterson, $K$. $M$. (xiI) Rec. Mth. (Moscou) 10 (1882-83) (Pt. 1) 476-.

- -. Guichard, C. C. R. 125 (1897) 596-.

- Darboux, G. C. R. 128 (1899) 760-, 854-, 1264-.

- - isothermal surfaces connected with. Darboux, G. C. R. 128 (1899) 1483-.

- of revolution. Guichard, C. C. R. 128 (1899) 232-.

- _ - Tzitzéica [Titeica], —. Bll. Sc. Mth. 23 (1899) 153-.

- ruled surfaces. Beltrami, E. A. Mt. 7 (1865) 105-.

- - Daug, H. T. Ups. S. Sc. N. Acta Vol. extra ord. (1877) (No.5) $18 \mathrm{pp}$.

- - Chini, M. Tor. Ac. Sc. At. 26 (1891) 20-.

- skew surfaces. Bonnet, O. C. R. 57 (1863) 805-.

small, of curves and surfaces (helix and circular ring). Michell, J. H. Mess. Mth. 19 (1890) $68-$.

of sphere. Soler, E. Palermo Cir. Mt. Rd. 8 (1894) 209-; Palermo Ac. At. 5 (1900) (Sc. Nt.) $35+23 \mathrm{pp}$.

- spherical surface. Cayley, A. Mess. Mth. 6 (1877) 88-.

- spiral surfaces. Raffy, L. C. R. 112 (1891) 850-, 976; Par. S. Mth. Bll. 19 (1891) 65-; Par. Ec. Norm. A. 9 (1892) 145-.

and strain of surfaces. Gosiewski, $W$. Par. T. Nauk Śc. Pam. 1 ("1871) 57-, 183-.

of one surface of revolution into another. Brodén, T. Fschr. Mth. (1886) 731-。

- surfaces connected with quadrics. Tzitzéica [Titeica], -. C. R. 128 (1899) 1276-. 
of surfaces of revolution. Rayleigh, J. $W$. Strutt (Lord). [1881] L. Mth. S. P. 13 (1881-82) 4-.

- - Ocagne, M. d'. Par. S. Mth. Bll. 21 (1893) 85-.

- - Bianchi, L. Rm. R. Ac. Linc. Rd. 8 (1899) (Sem. 1) 141-.

- - - case. Demoulin, A. Brux. Ac. Bll. 30 (1895) 61-.

- - - , and isometric networks. Picard, É. (xI) Par. S. Phlm. Bll. 4 (1867) 73-.

- - - translation. Adam, P. Par. S. Mth. Bll. 23 (1895) 204 -.

Dilatations of elements of small portion of elastic membrane. Boussinesq, V.J. (xII) Lille S. Mm. 8 (1880) 381-.

Gauss's theorem, product of radii constant. Bertrand, J. Liouv. J. Mth. 13 (1848) 80-.

Invariants. Źorawski, $K$. Krk. Ak. (Mt.Prz.) Rz. 3 (1891) 225-; Fschr. Mth. (1891) 805-; Krk. Ak. (Mt.-Prz.) Rz. 8 (1895) 1-; Crc. Ac. Sc. Bll. (1895) 91-.

-, and fundamental equations in theory of surfaces. Voss, A. Münch. Ak. Sb. 22 (1893) 247-.

Ivory's theorem and confocal quadrics. Mannheim, A. C. R. 102 (1886) 310-.

Partial differential equations. Serret, J. A. Liouv. J. Mth. 13 (1848) 361-.

Problem. Adam, P. Par. S. Mth. Bll. 24 (1896) 28-.

-, general. Raffy, L. C. R. 114 (1892) 1407-; Par. S. Mth. Bll. 22 (1894) 119-.

,,-- fundamental equations. Aoust, (l'abbé) -. C. R. 68 (1869) 1095-.

\section{Surfaces.}

applicability. Calo, B. A. Mt. 4 (1900) 123-. - on surfaces of revolution, conditions for. Lévy, $M$. C. R. 86 (1878) 947-.

applicable. Nicolaïdès, $N$. Les Mondes 8 (1865) 710-.

一. Amigues, E. C. R. 104 (1887) 564-.

-. Chini, M. G. Mt. 27 (1889) 265-.

-, class, new. Weingarten, J. Gött. Nr. (1887) 28-.

-, and curvilinear coordinates. Hoppe, $R$. Arch. Mth. Ps. 59 (1876) 225-; 68 (1882) 439-.

-, with distance of corresponding points constant. Demoúlin, A. Par. S. Mth. Bll. 23 (1895) 71-.

- on given surface. Dini, $U$. G. Mt. 2 (1864) 282-.

\section{(}

- - Weingarten, J. C. R. 112 (1891) 706-.

- - - theory. Bonnet, O. Par. Ec. Pol. J. Cah. 41 (1865) 209-; Cah. 42 (1867) 1-.

- one another, class. Weingarten, $J$. Crelle J. 59 (1861) 382-.

- - - criterion. Minding, E. F.A. Crelle J. 19 (1839) 370-.

-, pairs. Caronnet, T. Par S. Mth. Bll. 21 (1893) 134-. applicable, pairs. Genty, E. Par. S. Mth. Bll. 22 (1894) 36-.

,-- , and conjugate systems. Petot, A. C. R. 115 (1892) 1250-.

-, problem. Farkas, G. Orv.-Termt. Éts. (Termt. Szak) (1889) 260-.

-, 一. Raffy, L. C. R. 108 (1889) 493-.

-, ruled, with parallelism of generators. $A n$ tomari, X. Par. S. Mth. Bll. 22 (1894) 58-.

一, skew. Razzaboni, A. G. Mt. 21 (1883) 92-.

- on surface of revolution. Pellet, A. C. R. 125 (1897) 1159-; 126 (1898) 392-.

- - surfaces of minimum area. Dini, $U$. C. R. 60 (1865) $340-$.

- - - - revolution. Picart, A. N. A. Mth. 20 (1881) 113-.

- - - - Raffy, L. Bll. Sc. Mth. 13 (1889) 161-

, and theorem of Weingarten. Goursat, $\hat{E}$. Toul. Fac. Sc. A. 5 (1891) E, 34 pp.

一, theory. Dini, $U$. A. Mt. 7 (1865) 25-.

-, - Weingarten, J. C. R. 112 (1891) 607-.

-, -. Goursat, É. C. R. 112 (1891) 707-.

application. Combescure, É. C. R. 105 (1887) 434 .

-. Ricci, G. Ven. I. At. (1896-97) 1230-.

- on one another. Codazzi, D. Par. Mm.

Sav. Étr. 27 (1883) No. 6, 45 pp.

- - - - formulæ of Codazzi and Weingarten. Cayley, A. L. Mth. S. P. 24 (1893) 210-.

- - - - theory. Ribaucour, A. (xI) Par. S. Phlm. Bll. 6 (1869) 37-.

bent (umgestülpt). Deschwanden, J. W. von. Zür. Vjschr. 9 (1864) 1-.

with circles, for isothermal lines. Demartres, -. C. R. 104 (1887) 217-.

- conservation of lines of curvature after deformation. Delfino, C. Tortolini A. 7 (1856) 410-.

deformable, applicable on surfaces of revolntion, determination. Raffy, - . Par. S. Mth. Bll. 19 (1891) 34-.

-, with lines of equal curvature parallel. Raffy, -. Par. S. Mth. Bll. 19 (1891) 54-. of 3-dimensions, deformability. Banal, $R$. Rm. R. Ac. Linc. Rd. 8 (1899) (Sem. 2) 13-. finite simply connected, of negative curvature, theorem. Liebmann, $H$. Leip. Mth. Ps. B. 52 (1900) 28 -

inextensible. Jellett, J. H. [1853] Ir. Ac. T. 22 (1855) 343-.

with measure of curvature invariable. Brios. chi, F. Tortolini A. 3 (1852) 293-; 4 (1853) 232 -.

of revolution, applicable, families. Razzaboni, A. [1887] Bologna Rd. (1887-88) 19-.

-,$- \quad$ - on given surface of revolution. Adam, A. N. A. Mth. 10 (1891) 18-.

- - which by deformation are superposable in all their parts. Reech, F. C. R. 80 (1875) 1388-, 1442-.

- -, to which helicoid can be applied. Lamarle, E. Brux. Ac. Bll. 19 (1865) 537-. 
ruled, applicable on surface of revolution. Pellet, A. C. R. 106 (1888) 654.

- $-\ldots$ - - Bioche, C. C. R. 106 (1888) 829-.

skew, applicable on given skew surface. Cayley, A. L. Mth. S. P. 23 (1892) 217-.

susceptible of special infinitesimal deformations. Padova, E. Mil. I. Lomb. Rd. 24 (1891) 821-.

Theory. Weingarten, $J$. Acta Mth. 22 (1899) 193-.

-, continuous group of transformations with 28 parameters in. Staeckel, $P$. C. R. 121 (1895) 396-.

-, general differential equation in. Craig, $T$. Franklin I. J. 78 (1879) 289-.

\section{Orthogonal and isothermic surfaces. (See also 5630.)}

Lamé, G. (virI) C. R. 17 (1843) 338-.

Thomson, (Sir) W. Camb. Mth. J. 3 (1843) 286-; 4 (1845) 179-.

Tait, P. G. [1872] Edinb. R. S. T. 27 (1873) 105-.

Pellet, A. C. R. 124 (1897) 552-.

Bonnet's problem. Guichard, C. C. R. 125 (1897) 643-.

Condition that family of surfaces should belong to orthogonal system. Weingarten, J. L. G. J. Crelle J. Mth. 83 (1877) 1-.

- _ - - - - - - - Lilienthal, R. von. Mth. A. 44 (1894) 449-.

- for isogonal transformation of curvilinear coordinates in 3 dimensions. Maxwell, J. C. [1872] L. Mth. S. P. 4 (1871-73) 117-.

Confocals of surfaces cut them orthogonally. Lévy, M. C. R. 74 (1872) 176-.

Conjugate functions and equipotential curves. Brill, J. Camb. Ph. S. P. 6 (1889) 187-.

Curves and surfaces normal to one another, theory. David, C. A. [1864] (xII) Lille S. Mm. 1 (1865) 151-.

Cyclidal surfaces, application of physical methods to study. Darboux, G. C. R. 83 (1876) 1037-, 1099-.

Dirichlet's problem of stationary temperatures, and representation of given surface. Christoffel, E. B. A. Mt. 1 (1867-68) 89-; $4(1870-71) 1$-.

Equations of motions of heat referred to curvilinear coordinates. Thomson, (Sir) W. Camb. Mth. J. 4 (1845) 33-.

Equipotential cylindrical surfaces, class. Pomey, J. B. A. Tél. 21 (1894) 460-; 24 (1898) 150 -

- surfaces, class. Peirce, B. O. Am. J. Mth. 18 (1896) 130-.

- - plane. Burnside, $W$. Mess. Mth. 27 (1898) 138-.

- system of curves, singular points. Brill, $J$. Camb. Ph. S. P. 6 (1889) 313-.

- - - _ _ (Brill). Burnside, $W$. Camb. Ph. S. P. 7 (1892) 126-.
Isothermic coordinate systems. Kötteritzsch, $T$. Z. Mth. Ps. 19 (1874) 265-.

- curves, plane, property. Burnside, W. Mess. Mth. 20 (1891) 64-.

\section{ISOTHERMIC SURFACES.}

Pellet, A. C. R. 125 (1897) 291-.

Darboux, G. C. R. 128 (1899) 1299-, 1538.

Guichard, C. C. R. 130 (1900) 159-.

Thybaut, A. C. R. 131 (1900) 932-; Par. Éc. Norm. A. 17 (1900) 541-.

application of vectorial geometry. Genty, As. Fr. C. R. (1889) (Pt. 2) 53-.

class. Thybaut, A. C. R. 123 (1896) 295-.

and deformation of paraboloid. Thybaut, $A$.

C. R. 128 (1899) 1274-.

- "doubly cylindric" surfaces. Raffy, L. C. R. 128 (1899) 285-.

geodesic lines, use of curvilinear coordinates. Salvert, (le vicomte) - de. Brux. S. Sc. A. 10 (1886) (Pt. 2) 293-; 11 (1887) (Pt. 2) 1-.

and harmonic equations. Thybaut, A. C. R. 130 (1900) 387-.

in heterotropic media. Montier, J. Par. S. Phlm. Bll. 8 (1884) 184-.

- homogeneous solids in temperature equilibrium. Poisson, S. D. Liouv. J. Mth. 2 (1837) 147-.

paraboloidal. Lamé, $G$. [1843 or 1844] Liouv. J. Mth. 19 (1874) 307-.

with plane lines of curvature in one or both systems. Adam, P. C. R. 116 (1893) 1036-; Par. Éc. Norm. A. 10 (1893) 319-.

systems, algebraic. Haton de la Goupillière, J. N. C. R. 50 (1860) 307-.

- Liouville's, and geodesies. Ricci, $G$. Ven. I. At. (1893-94) 643-.

transformation. Guichard, C. C. R. 130 (1900) 477-.

triple systems. Betti, E. A. Mt. 8 (1877) 138-.

Isotherms, plane algebraic. Schwarz, C.H.A. Crelle J. Mth. 77 (1874) 38-.

Laplace's equation, transformations leading to new solutions. Forsyth, A.R. L. Mth. S. P. 29 (1898) 165-.

Non-ruled surfaces applicable on plane. Lebesgue, H. C. R. 128 (1899) 1502-.

Orthogonal and confocal curves, system. Legoux, A. Toul. Fac. Sc. A. 4 (1890) D, $7 \mathrm{pp}$.

- curves, two systems. Jamet, V. C. R. 106 (1888) 830-.

\section{Orthogonal surfaces.}

Bonquet, J. Liouv. J. Mth. 11 (1846) 446-. Serret, J. A. Liouv. J. Mth. 12 (1847) 241-.

Lebesgue, $V . A$. N. A. Mth. 10 (1851) 265-.

Bonnet, O. C. R. 54 (1862) 554-, 655-.

Darboux, G. C. R. 59 (1864) 240-; Par. Éc. Norm. A. 3 (1866) 97-; (Ix) Par. S. Phlm. Bll. 3 (1866) 16-.

Catalan, E. C. Brux. Ac. Bll. 26 (1868) 180 - 
Darboux, G. C. R. 67 (1868) 1101-.

Ribaucour, A. (xr) Par. S. Phlm. Bll. 6 (1869) 1-, 26-.

Tisserand, F. C. R. 72 (1871) 734-.

Enneper, A. Gött. Nr. (1872) 226-; (1873) 423-.

Darboux, G. C. R. 76 (1873) 41-, 83-, 160-.

Catalan, E. C. C. R. 79 (1874) 28-.

Tilly, J. M. de. C. R. 87 (1878) 361-.

application of elliptic coordinates. Roberts, $W$.

C. R. 53 (1861) 546-; Crelle J. 62 (1863) 50-.

Cayley's differential equation for. Johnson, $A$. $R$. Mess. Mth. 16 (1887) 27-, 111.

- - - extension. Johnson, A.R. QJ. Mth. 22 (1887) 81-.

and confocal surfaces. Legoux, A. QJ. Mth. 20 (1885) 1-.

- curvature. Cayley, A. Phil. Trans. 163 (1873) 229-.

Dupin's theorem and its converse, geometrical proof. Sommerfeld, A. D. Mth. Vr. Jbr. 6 (1899) (Heft 1) 123-.

and lines, formulæ. Beltrami, E. Mil. I. Lomb. Rd. 5 (1872) 474-.

represented by a partial differential equation of 1st order. Legoux, A. (xII) Isère S. Bll. $10(1880)$ 125, 132-.

system, elements, expressed by sigma functions, with application to rotation of connected solids. Jahnke, E. Liouv. J. Mth. 5 (1899) 155 -.

- formed by most general system of quadrics and two other systems. Schläfli, $L$. [1870] Crelle J. 76 (1873) 126-.

systems. Puiseux, V. Liouv. J. Mth. 8 (1863) 335-.

-. Enneper, A. (vII) Mth. A. 7 (1874) 456-.

-. Darboux, G. [1877] Par. Ec. Norm. A. 7 (1878) 101-, 227-, 275-.

-. Adam, P. C. R. 121 (1895) 812 -

-. Goursat, É. C. R. 121 (1895) 883-.

- Weyr, E. Časopis 25 (1896) 42-; Fschr. Mth. (1896) 506.

-.Tzitzéica [Titeica], 一. C. R. 127 (1898) $856-$ -

-. Pellet, A. C. R. 128 (1899) 284-.

-. Servant, - C. C. R. 130 (1900) 28-

- admitting continuous group of Combescure transformations. Egorov, $D . T$. C. R. 131 (1900) 668-.

- - - - Fouché, M. C. R. 131 (1900) 873-.

—, algebraic. Darboux, G. C. R. 69 (1869) $392-$.

-, associated. Guichard, C. C. R. 125 (1897) 929-.

-, class including isothermic systems as particular case. Darboux, G. C. R. 84 (1877) 298-.

- and congruences. Guichard, - C. R. 125 (1897) 564-.

- - cyclic systems. Guichard, C. C. R. 125 (1897) 519-; Par. Éc. Norm. A. 14 (1897) 467-; 15 (1898) 179-.

- including family of quadric surfaces. Darboux, G. C. R. 84 (1877) 336-. systems obtained by substitution with 4 parameters. Mehler, F. G. Crelle J. Mth. 84 (1878) 219-.

-, and orthogonal systems of curves. Brill, J. Camb. Ph. S. P. 6 (1889) 230-.

-, partial differential equation of 3 rd order for. Darboux, G. Acta Mth. 4 (1884) 93-.

- and partial differential equations. Bäcklund, A. V. Mth. A. 40 (1892) 194-.

theorem. Adam, P. C. R. 103 (1886) 996-.

theory, definite integrals deduced from. Ostrogradsky, - . (vir) St Pét. Ac. Sc. Bll. 7 (1840) 362-.

-, theorems demonstrated by. Lamé, G. C. R. 21 (1845) 112-.

triple system. Combescure, $\dot{E}$. [1862] (vII) A. Mt. 5 (1863) 39-.

- - Hoppe, R. Arch. Mth. Ps. 55 (1873) 362-; 56 (1874) 153-, 250-; 57 (1875) 89-, 255-, 366-; 58 (1876) 37-.

- - condition that family of surfaces should belong to. Hoppe, $R$. Arch. Mth. Ps. 63 (1879) 285-.

- - of developable. Molins, $H$. Toul. Ac. Sc. Mm. 1 (1879) (Sem. 1) 81-.

- - , elements of volume between. Chelini, D. Palomba Rac. 2 (1846) 109-, 126-.

- - formed from surfaces of 4th degree. Wangerin, $F$. H. A. Crelle J. Mth. 82 (1877) 145-, 348.

- - isothermic, theory. Salvert, (le vicomte) - de. Brux. S. Sc. A. 13 (1889) (Pt. 2) 117-; 14 (1890) (Pt. 2) 71-; 15 (1891) (Pt. 2) 201-; 16 (1892) (Pt. 2) 273-; 17 (1893) (Pt. 2) 103-; 18 (1894) (Pt. 2) 61-.

- -, new. Puchta, A. Wien Ak. Sb. 102 (1893) (Ab. 2a) 1197-

- systems. Betti, E. A. Mt. 8 (1877) 138-.

- - Kötteritzsch, T. Z. Mth. Ps. 23 (1878) 158-.

- - Lévy, L. Liouv. J. Mth. 8 (1892) 351-; C. R. 117 (1893) 477-; Brux. Mm. Cour. $4^{\circ}, 54$ (1896) No. 9, 92 pp.

- - . Geiser, C. $F$. Zür. Vjschr. 43 (1898) $317-$.

- of algebraic. Roberts, W. C. R. 58 (1864) 291-.

- - class. Morin, P. C. R. 67 (1868) 788- - - Bianchi, L. [1882] Rm. R. Ac. Linc. T. 7 (1883) 46-

- _, - including system of helicoids. Bianchi, L. A. Mt. 13 (1885) 39-.

- - - new. Bianchi, L. A. Mt. 18 (1890) 301-.

- - cyclic. Bianchi, L. G. Mt. 21 (1883) 275-; 22 (1884) 333-.

- - , cyclic systems. Ribaucour, A. C. R. 76 (1873) 478-.

- - - o Ribaucour. Bäcklund, A. V Stockh. Ak. Hndl. Bh. 16 (Afd. 1) (1891) No. 1, 32 pp., No. 8, 41 pp.; 17 (Afd. 1) (1892) No. 2, 41 pp.; Fschr. Mth. (1891) 395.

- Dupin's theorem. Hamilton, (Sir) W. R. Ir. Ac. P. $6(1853-54)$ 86-

- , equation of condition for parameters. Ricci, G. Rm. R. Ac. Linc. Rd. 3 (1894) (Sem. 2) 93-. 
triple systems, one family with same spherical representation for its lines of curvature. Fouché, M. C. R. 126 (1898) 210-.

- - including series of surfaces with one system of lines of curvature plane. Bianchi, L. $\quad$ A. Mt. 19 (1891-92) 177-.

- _ - system of pseudospherical surfaces. Bianchi, L. Rm. R. Ac. Linc. Rd. 2 (1886) (Sem. 1) 19-.

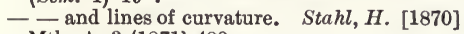
Mth. A. 3 (1871) 488-.

- - property. Catalan, E. C. Liége S. Sc. Mm. 15 (1888) No. 1, 90-.

- _, pseudospherical, Bäcklund's transformation for. Bianchi, L. Rm. R. Ac. Linc. Rd. 1 (1892) (Sem. 2) 156-.

- -, theorem. Bertrand, J. C. R. 121 (1895) 921-.

- - theory. Geiser, C. F. Arch. Sc. Ps. Nt. 6 (1898) 363-.

- - of Weingarten. Bianchi, L. Rm. R. Ac. Linc. Rd. 1 (1885) 163-, 243-; A. Mt. 13 (1885) 177-; 14 (1886-87) 115-; Palermo Cir. Mt. Rd. 8 (1894) 25-.

Surfaces cut orthogonally by spheres. Bonnet, O. C. R. 36 (1853) 1133-

- with lines of curvature isothermic. Burgatti, P. Rm. R. Ac. Linc. Rd. 9 (1900) (Sem. 2) 352-.

_ _ - _ - - and plane in both systems. Caronnet, T. C. R. 116 (1893) 1240-.

- orthogonal to $x y z=c^{3}$, integration of equation. Catalan, E. C. Liège $\mathrm{S}$. Sc. Mm. 13 (1886) 73-.

Systems of plane curves, orthogonals of which form a similar system. Tait, -. Edinb. R. S. P. 20 (1895) 497-.

- - spherical coordinates, and corresponding triply orthogonal systems. Petot, $A$. C. R. 112 (1891) 1426-.

Theorem in theory of functions, and application to isothermic systems of curves. Holzmüller, -. Z. Mth. Ps. 42 (1897) 217-.

\section{Hypergeometric configura- tions and higher elements of hyperspace.}

Applicability of 2 spaces with same Riemann's curvature constant. Bianchi, L. Rm. R. Ac. Linc. Rd. 7 (1898) (Sem. 2) 147-.

- - surfaces of spaces of constant curvature. Bianchi, L. Rm. R. Ac. Linc. Mm. 2 (1878) 479-.

Conditions that displacement of invariable system is possible in one or several directions. Lévy, M. C. R. 86 (1878) 875-.
Cubes of different dimensions, representation one on another. Schoenflies, A. Gött. Nr. (1896) 255-.

Curvature in hyperspace. Beez, R. Z. Mth. Ps. 21 (1876) 373-.

Deformation of 3-dimensional space in plane 4-dimensional space. Schur, F. Mth. A. 28 (1887) 343-.

- - spaces. Monro, C. J. L. Mth. S. P. 9 (1877-78) 171-.

- - - with Riemann's curvature constant. Schur, F. Mth. A. 27 (1886) 163-.

Deformations of figures in $n$ dimensions. Stäckel, P. Crelle J. Mth. 113 (1894) 102-.

Infinitesimal deformations of hyperspaces. Cesáro, F. Nap. Rd. 34 (1895) 47-.

- - - surfaces in spaces of constant curvature. Fubini, G. Rm. R. Ac. Linc. Rd. 8 (1899) (Sem. 1) 246-.

Loci of centres of hyper-spherical curvature of $n$-dimensional curves. Schoute, $P$. $H$. Amst. Ak. Vs. 8 (1900) 622-; Amst. Ak. P. 2 (1900) 527-.

Meunier's theorem in higher dimensional space. Brunel, -. Bordeaux S. Sc. Mm. 2 (1891) $\mathrm{xv}-$.

Non-Euclidean billiards. Hadamard, $J$. Bordeaux S. Sc. PV. (1897-98) 147-.

- surfaces of constant mean curvature. Servant, -. C. R. 131 (1900) 827-.

Orthogonal systems in any space. Ricci, G. C. R. 125 (1897) 810-.

$-\ldots-n$-dimensional space, and reduction of differential systems. Drach, J. C. R. 125 (1897) 598-.

$--\frac{n}{-}$ variables. Pellet, A. Toul. Fac. Sc. A. 2 (1900) 137-.

Quadric in 5-dimensional space, superlines. Cayley, A. QJ. Mth. 12 (1873) 176-.

- - n-dimensional space. Snyder, V. [1897] N. Y. Am. Mth. S. Bll. 4 (1898) 68-.

Real representation of imaginary configurations, and application to theory of numbers. Busche, E. Crelle J. Mth. 122 (1900) 227-.

Representation of $n$-dimensional surface in $(n-1)$-dimensional space. Vaes, $F . J$. Fschr. Mth. (1900) 642-.

Riemann's measure of curvature in hyperspace. Beez, R. Z. Mth. Ps. 24 (1879) 1-, 65-.

Spaces of constant curvature. Banal, $R$. Rm. R. Ac. Linc. Rd. 6 (1897) (Sem. 2) 357-.

- - - Tedone, O. Mil. I. Lomb. Rd. 32 (1899) 592-.

Surface images in 4-dimensional space. Mannoury, G. N. Arch. Wisk. 4 (1899) 112-.

Total curvature in hyperspace. Banal, $R$. Ven. I. At. (1894-95) 998-.

Weingarten's systems in spaces of constant curvature. Bianchi, L. Rm. R. Ac. Linc. Mm. 4 (1887) 221-. 


\section{APPENDIX.}

\section{0}

Limits of our knowledge respecting theory of parallels. Kelland, $P$. Edinb. R. S. T. 23. (1864) 433-.

Metaphysics of geometry. Dandelin, $G$. $P$. [1842] Brux. Mm. Ac. Sc. 17 (1844) 44 pp.

\section{0}

\section{HISTORY.}

Algebraic surfaces. Bertrand, J. J. Sav. (1867) 644-.

\section{Arabian Mathematics.}

Construction for 2 mean proportionals. Carra de Vaux, -. Bb. Mth. (1898) 3-.

Curvature of surfaces and skew curves, development of theory. Christensen, S. A. (xII) Ts. Mth. 1 (1883) 97-.

Desargues (17th century), Dutch edition of his method of perspective. Le Paige, C. Bb. Mth. (1888) 10-

Duality. Miller, J. H. T. Grunert Arch. 34 (1860) 1-.

Geometrical methods. Chasles, M. Brux. Mm. Cour. 11 (1837) $571 \mathrm{pp}$.

Geometry, priority claim for various theorems. Mainardi, G. Rm. At. N. Linc. 26 (1873) 88 .

- of triangle. Vigarié, É. As. Fr. C. R. (1887) (Pt. 2) 87-; (1889) (Pt. 2) 117-.

_ - Fuhrmann, - . Königsb. Schr. 40 (1899) [37]-.

\section{Greek Mathematics.}

Archimedes, "Arenarius." Rigaud, S. P. Ashmol. S. T. 1 (1835-37) No. 9, 32 pp.

Mathematics in Bohemia, 1848-98. Studnička, F. J. Prag České Ak. Fr. Jos. Pam. (1898) (II a) 3-.

Mensuration in Norway in 14th century. Eneström, G. Bb. Mth. (1898) 19-.

Plane curves, anonymous treatise. Loria, $G$. Bb. Mth. (1899) 10-.

Quadrature of circle. Studnička, F.J. Casopis 1 (*1872) 35-; Fschr. Mth. (*1872) 254.

_- - Rudio, F. Zür. Vjschr. 35 (1890) 1-.

Skew curves, 3rd degree. Reye, T. Hamb. Mth. Gs. Mt. 2 (1890) (Festschr., Tl. 2 ) 43-.

Various letters. Clairaut, A.C. Rm. N. Linc. At. 45 (1892) 233-.

\section{BIOGRAPHY.}

Bacaloglu, Emmanuel. [Bucarest S. Sc. Bl. 2 (1893)] 101-; [3 (1894) (Supl.)] 51-; 4 (1895) (Supl.) 97-.

Broch, Ole Jacob. Par. Poids et Mes. PV. (1889) 3-.

Claret y Parera, Felipe. Barcel. Ac. Bl. 1 (1892-1900) 28-.

Davidov, Avgust Julievič. Mosc. S. Sc. Bll. 51 (App.) (1887) 55 pp.

Henoch, Max. Fschr. Mth. (1888) 6 pp.

Hessel, Johann Friedrich Christian. N. Jb. Mn. (1896) (Bd. 2) 107-.

Kambly, Ludwig. Bresl. Sl. Gs. Jbr. (1887) 403-.

Kirchhoff, Gustav Robert. Bresl. Sl. Gs. Jbr. (1887) 172-.

Kletke, Caesar Albano. Bresl. Sl. Gs. Jbr. (1893) (Nek.) 6-.

Le Fébure de Fourcy, Luis. Santiago de Chile Un. A. 33 ("1869) 134-.

Lie, Marius Sophus. Christiania F. (1899) (Ov.) 4-.

Ostrogradskij, Michail Vasiljevič. . St Pet. Ac. Sc. Mm. (Rs.) 3 ("1863) 1-.

Raymond, Georges Marie. Sav. Ac. Mm. (1891) 19-.

Sadebeck, Benjamin Adolph Moritz. Bresl. Sl. Gs. Jbr. (1885) 432-.

Saint-Martin, Michel. Sav. Ac. Mm. (1891) 46-.

Somov, Osip Ivanovič. St Pet. Ac. Sc. Mm. (Rs.) 31 ("1877-78) 248-.

Wittiber, Gustav. Bresl. Sl. Gs. Jbr. (1886) 317-.

Zurria, Giuseppe. Catania Ac. Gioen. Bll. 46 (1897) 31-.

\section{2}

Cartesian ovals. Liguine, $V$. Bll. Sc. Mth. As. 6 (1882) (Pt. 1) 40-.

Theory of parallels. Stäckel, P. Bb. Mth. (1899) 47-.

\section{5}

Trigonometry, tables. Lindman, C. F. Stockh. Öfv. 11 (1854) 183-; Grunert Arch. 25 (1855) 284-.

\section{0}

Analysis, course applied to mechanics. Prony, R. de. Par. Éc. Pol. J. $1^{\text {er }}$ cah. (1794) 92-; $2^{\circ} \mathrm{cah}$. (1795) 1-; $3^{\circ} \mathrm{cah}$. (1796) 209-; $4^{\circ} \mathrm{cah}$. (1796) 459-. 
Mathematical study at Cambridge. Gautier, A. Scale, Marquoi's, improved. Marks, W. D. Bb. Un. 26 (1824) 161-, 243-.

\section{0}

\section{(For integrators and planimeters see also Mechanics 0120.)}

\section{INSTRUMENTS.}

Compasses, homographic. Koenigs, G. C. R. 131 (1900) 1179-.

-, universal. Weber, L. Carl Rpm. 16 (1880) 388.

Curve-tracing, use of flexible metallic strip in. Resal, H. A. C. R. 78 (1874) 709-; Par.Éc. Norm. A. 4 (1875) 191-.

Curves of 2nd degree of a continuous motion, apparatus for tracing. Uchard, A. Rv. Artl. 15 (1879) 496-.

Division of circle, apparatus for. Anon. Gilbert A. 65 ( $\left.{ }^{*} 1820\right)$ 329-.

,---- - (Anon). Liebherr, $J$. Gilbert A. 67 (1821) 109-.

- - - - (Liebherr). Reichenbach, G. R. Gilbert A. 68 (1821) 33-.

- _ - - - (Reichenbach). Liebherr, J. Gilbert A. 69 (1821) 320-.

Drawing instrument for circles and other curves. Cerebotani, L. Rm. N. Linc. At. 46 (1893) 11-, 86-.

- - - envelopes, and application to teeth of wheels, etc. Hele-Shaw, H. S. B. A. Rp. (1898) 619-.

- - parabolic curves. Inwards, R. L. Ps. S. P. 11 (1892) 337-; Ph. Mg. 34 (1892) 57-.

Graphometer for interpolating values in curves. Hensen, V. Z. Instk. 20 (1900) 357-.

Instrument for describing spiral of Archimedes. Quinby, A. B. Silliman J. 9 (1825) 316-.

Integrators. Abdank Abakanowicz, B. Lum. Élect. 27 (1888) 23-.

- and integral curve. Aubert, - . A. Cond. Pon. Chauss. 31 (1887) (Pt. 1) 165-.

Line divider. Marks, (Miss) S. L. Ps. S. P. 7 (1886) 1-; Ph. Mg. 19 (1885) 280 -.

Oval tracer. Rebiček, G. Z. Instk. 17 (1897) 289-.

Pantographs. Fischer, (Prof.) E. Carl Rpm. 1 (1866) 257-.

-, graduation. Woollgar, J.W. Tilloch $\mathrm{Ph}$. Mg. 59 (1822) 401-.

Planimeter, Amsler's, "no-rolling" curves. Morley, F. A. Mth. 1 (1900) 21-.

—, new. Fessenden, C. Cn. I. P. 5 (1888) 27-.

-, polar. Barbarin, P. N. A. Mth. 19 (1880) 212-; 20 (1881) 266-.

—, -, problem. Echols, W. H. [1889] A. Mth. 5 (1889-90) 9-.

- for rectangular coordinates. Schmidt, $J$. Civing. 26 (1880) 437-.

Reduction-compasses. Schiereck, $P . F$. Dingler 82 (1841) 265-.

-, improvement. Prony, R. de. Par. A. Pon. Chauss. 9 (1835) 81-.

Registering instruments. Gossier, J.F. Rouen Tr. Ac. (1833) 74-.

Franklin I. J. 75 (1878) 418-.

Spherical integrator, modifications of a form of. Ventosa, V. Ph. Mg. 23 (1887) 381.

\section{CALCULATING MACHINES.}

Buniakovskij, V. Ja. St Pet. Ac. Sc. Mm. (Rs.) 27 ("1876) (App.) No. 4, 28 pp.

Algebraic machines. Torres, L. C. R. 121 (1895) 245-.

Difference-calculating engine of Scheutz. $B a b$ bage, H. P. (vi Add8.) CE. I. P. 15 (185556) 497 -

Principle of moments. Auchincloss, W. S. Am. S. CE. T. 10 (1881) 135-.

Sonne's calculating machine. Hoyer, (Inspect.) - A. Gén. Civ. 5 (1866) 111-.

\section{MODELS.}

Cubic surface with 27 real lines. Frost, P. QJ. Mth. 18 (1882) 89-.

Equatorial surfaces, Plücker's models. Cayley, $A$. L. Mth. S. P. 3 (1869-71) 281-。

Peaucellier cell. Peaucellier, A. N. A. Mth. 12 (1873) 71-

- - Lemoine, $E_{\text {. }}$ As. Fr. C. R. (1874) 122-; Par. Ing. Civ. Mm. (1875) 247-.

- - and circular inversion. Baker, A. L. [1894] Rochester (N. Y.) Ac. Sc. P. 2 (1895) 256-.

Riemann surfaces. Durège, $H$. Prag Sb. (1864) (Pt. 2) 112

Skew curves of 4 th degree. Rohn, C. D. Mth. Vr. Jbr. 1 (1892) 43-.

- surfaces, construction of paper models. Schönemann, P. Z. Mth. Ps. 28 (1883) 243-

Vernier, model. Mannheim, $A$. J. de Ps. 2 (1873) 392-.

\section{0}

Abacus, logarithmic, for cable calculations. Bois, J. Lum. Elect. 39 (1891) 375-.

Graphic methods of solving electrical problems. Guerout, A. Lum. Élect. 9 (*1883) 113-.

Nomographic method. Ocagne, M. d'. C. R. 126 (1898) 397-.

- - application to equations with variables up to 10 . Ocagne, M. $d^{\prime}$. C. R. 117 (1893) $216-, 277-$.

Nomography. Ocagne, M. d'. Par. Ec. Pol. J. 4 (1898) 205-.

Periodic curves, analysis. Boucherot, P. Lum. Élect. 49 (1893) 251-.

- functions, instruments for analysis. Hess, A. Lum. Élect. 52 (1894) 551-.

Plane representation of equations with four variables. Ocagne, $M . d^{\prime}$. C. R. 112 (1891) 421-, 504.

\section{0}

Negative and imaginary quantities. Vidal, S. E. [1887] I. Egypt. Bll. 8 (1888) 31-.

\section{0}

Weights problem. Clausen, T. Crelle J. 7 (1831) 30 - 


\section{0}

Cube, duplication. Mansfield, J. Conn. Mm. Ac. 1 (1810) 119-.

-, -. Fontès, -. Toul. Ac. Sc. Bll. 1 (1898) 129-.

-, - , ancient methods. Mackay, J. S. [1885]

Edinb. Mth. S. P. 4 (1886) 2-.

- - - approximate constructions. Mayor, Gergonne A. Mth. 15 (1824-25) 90-.

-, - , and circle, quadrature, approximate solutions. Hayden, $W$. R. S. P. 20 (1872) 525 -.

-, multisection. Deladéréêre, A. N. A. Mth. 3 (1844) 231-.

Fractions approximating to square root of a variable. Tchébychef, P. L. Par. Éc. Norm. A. 15 (1898) 463-.

\section{0}

Continuous representation of line on surface. Hilbert, D. [1890] Mth. A. 38 (1891) 459-.

Curve covering plane area. Peano, $G$. Mth. A. 36 (1890) 157-.

Function, derivative of which is not integrable near certain points. Osgood, W. F. N. Y. Am. Mth. S. Bll. 5 (1899) 326, 328-.

Functions having no differential coefficients. Fambri, P. Ven. I. At. (1884-85) 823-.

Point aggregates. Veltmann, $W$. Z. Mth. Ps. 27 (1882) 313-.

- - in continuous space of $N$ dimensions. Cantor, G. Acta Mth. 2 (1883) 409-.

- - infinite linear. Cantor, G. Mth. A. 23 (1884) 453-.

- - theorem of Bendixson, proof. Phragmén, E. Acta Mth. 5 (1884) 47-.

\section{0}

Physical quantities, classification. Maxwell, J. C. L. Mth. S. P. 3 (1869-71) 224-.

Quaternion functions and astatics. Joly, C.J. [1899] Ir. Ac. P. 5 (1898-1900) 366-.

- operator $\omega+V \sigma()$ as motor, use in statics and kinematics. Everett, J. D. Mess. Mth. 4 (1875) 36-, 135-; 5 (1876) 72-.

Quaternions, application to skew curves, 3rd degree. Hamilton, (Sir) W. R. [1863] Ir. Ac. P. 8 (1864) 331-.

Stereometric multiplication. Grassmann, $H$. [1852] Crelle J. 49 (1855) 10-.

\section{0}

Couples, normal, and quadratic functions of 2 variables, connexion. Graves, J. T. $\mathrm{Ph}$. Mg. 26 (1845) 315-.

Directive calculus. Berdelle, C. As. Fr. C. R. (1886) (Pt. 2) 103-; (1891) (Pt. 2) 181-.

- - and calculus of position. Hyde, E.W. Am. J. Mth. 6 (1884) 1-.

Vector analysis of bicircular quartics and circles. Cox, H. QJ. Mth. 19 (1883) 74-.

- field, theory. Vaschy, A. A. Tél. 21 (1894) 97-.
Vectors, aconic function of six. Hamilton, (Sir) $W . R$. Ir. Ac. P. 5 (1853) 177-。

-, projective theory. Kotelinikov, A. Kazan S. Ps.-Mth. Bll. 8 (1899) (App.); 9 (1900) (App.) $317+40$ pp.; Fschr. Mth. (1899) 501-.

\section{0}

\section{(See also 2010.)}

Algebraic differential equations. Torelli, $G$. G. Mt. 24 (1886) 280-.

Differential systems, existence of integrals in. Riquier, C. C. R. 125 (1897) 933-.

- -, most general. Riquier, C. Par. Éc. Norm. A. 14 (1897) 99-

- - transformations. Delassus, É. C. R. 123 (1896) 1246-.

Linear systems. Laguerre, - . Par. Éc. Pol. J. Cah. 42 (1867) 215-

Polar umbræ. Sylvester, J. J. R. S. P. 12 (1862-63) 563-.

Systems of differential equations and their connexion with partial linear equations of 1st order. Jacobi, C. G. J. Crelle J. 23 (1842) 1-.

- - , theory. Stodolkievitz, A. J. C. R. 120 (1895) 36-, 595-.

- - infinite order of differential equations. Koch, H. von. Stockh. Öfv. (1899) 395-.

- - $n^{2}$ integral elements, reduction. Kronecker, L. Crelle J. Mth. 107 (1891) 135-.

\section{0}

Syllogism No. III, and logic in general. De Morgan, A. [1858] (vII) Camb. Ph. S. T. 10 (1864) 173-.

- - IV, and logic of relations. De Morgan, A. [1860] (vII) Camb. Ph. S. T. 10 (1864) 331-. - - V, and points of onymatic system. De Morgan, A. [1863] (vII) Camb. Ph. S. T. 10 (1864) 428-.

\section{0}

Finite screw motions, composition. Wiener, $H$. Leip. Mth. Ps. B. 42 (1890) 13-.

Geometrical analysis of motions. Wiener, $H$. Leip. Mth. Ps. B. 42 (1890) 245-; 43 (1891) 424 -

Groups of interchangeable two-reflexion relations. Wiener, H. Leip. Mth. Ps. B. 45 (1893) 555-.

- - motions. Schönflies, A. Mth. A. 28 (1887) 319-; 29 (1887) 50-.

- - - proof of a theorem. Schönflies, A. Gött. Nr. (1886) 497-.

Inversions, theory. Wiener, H. Leip. Mth. Ps. B. 42 (1890) 71-.

Relations compounded of two reflexions. Wiener, H. Leip. Mth. Ps. B. 43 (1891) 644-.

\section{0}

Cross-ratio group of 120 Cremona quadratic transformations. Slaught, $H . E$. Science 8 (1898) 121-; Am. J. Mth. 22 (1900) 343-. 
Cubic surfaces, polar hexahedra. Cremona, $L$. Mth. A. 13 (1878) 301-.

Cyclic groups, number in Cremona transformation. Kantor, $S$. [1880] A. Mt. 10 (188082) $71-$.

- - - - quadratic plane transformation. Kantor, S. [1880] A. Mt. 10 (1880-82) 64-.

- - , - space transformation. Kantor, $S$. C. R. 90 (1880) 1156-.

Equations of geometry. Jordan, C. C. R. 68 (1869) 656-.

Finite groups of plane collineations with irreducible quartic invariant. Ciani, $E$. Mil. I. Lomb. Rd. 33 (1900) 1170-.

Function, five times transitive, of twenty-four quantities. Mathieu, $E_{\text {. }}$ Liouv. J. Mth. 18 (1873) 25-.

Galois group of bitangents of curves of 4 th degree. Weber, H. Mth. A. 23 (1884) 489-.

Groups of class $n-u$ and degree $n$, at least $(n-1)$ times transitive. Maillet, $E$. C. R. 128 (1899) 277.

- - transformation in 3-dimensional space, class. Blichfeldt, H. E. Am. J. Mth. 22 (1900) 113-.

$-(\mathrm{P})_{360},(\mathrm{II})_{360}$ arising from 6 line complexes mutually in involution. Veronese, $G$. A. Mt. 11 (1882-83) 284-.

Hamiltonian groups. Miller, G. A. C. R. 126 (1898) 1406-.

Inflexion points of $3 \mathrm{rd}$ degree and stationary tangents of curves of 3rd class. Hesse, L.O. Crelle J. 38 (1849) 257-.

Kummer surface, equation, 16th degree, connected with. Jordan, C. Crelle J. 70 (1869) 182-.

Linear substitutions, new theory. Laurent, $H$. Liouv. J. Mth. 4 (1898) 75-.

Number of values assumable by function of $n$ letters, theorem of Bertrand. Cartan, C. R. 119 (1894) 902 .

Octahedral and ikosahedral groups, two skew cubics automorphic for. Kohn, $G$. Wien Ak. Sb. 108 (1899) (Ab. 2a) 58-.

Octahedron, equation. Brioschi, Fr. Rm. R. Ac. Linc. T. 3 (1879) 233-.

- and equation of 4 th order. Puchta, A. Wien Ak. D. 41 (1879) (Ab. 2) 57-.

Substitution group connected with 16 straight lines of quartic surface with double conic. Pereno, I. A. Mt. 21 (1893) 57-.

- groups connected with 27 straight lines of cubic surface. Pascal, E. A. Mt. 20 (189293) 269-; 21 (1893) 85-.

- -, Napier's rules in spherical trigonometry. Pund, O. [1897] Hamb. Mth. Gs. Mt. 3 (1900) 290 -.

\section{0}

Automorphic curves and surfaces, family. Klein, F., \& Lie, S. C. R. 70 (1870) 1222-, 1275-.

Continuous system of transformations, determination of all surfaces admitting. Lie, S. Leip. Mth. Ps. B. 47 (1895) 209-.

voL. I.
Discontinuity in certain homographies. Fricke, R. Mth. A. 47 (1896) 557-.

Infinitesimal transformations, geometry. Zorawski, $K$. Krk. Ak. (Mt.-Prz.)Rz. 14 (1899) 218-; 17 (1900) 154-; Crc. Ac. Sc. Bll. (1897) 365-; (1899) 81-.

Invariants of $n$ points. Lovett, $E . O$. [1897] N. Y. Am. Mth. S. Bll. 4 (1898) 58-.

Transformations, geometric, of line systems. Lie, S. $[1870]$ C. R. 71 (1870) 579-; Christiania F. 13 (1871) 506-.

\section{0}

Algebraic continued fractions, generalisation. Padé, -. C. R. 118 (1894) 848-.

Equalities in two degrees

$$
\Sigma a_{r}=\Sigma A_{r}, \quad \Sigma a_{r}^{2}=\Sigma A_{r}^{2} .
$$

Frolov, M. C. R. 107 (1888) 831-。

\section{0}

Chess.

Knight's move, problem. Chambure, - de. [1861] 1. Égypt. Mm. 1 ( $\left.{ }^{*} 1862\right) 67-$.

\section{COMBINATIONS.}

Lock, Bramah's, improved. Reuter, J. Wien Jb. Pol. I. 10 (1827) 32-.

- with 10,000 combinations. Nicholson, $W$. Nicholson J. 13 (1806) 158-.

- combinations, insecurity. Crivelli, $A$. Wien Jb. Pol. I. 5 (1824) 22-.

-, Egyptian. Crivelli, A. Wien Jb. Pol. I. 6 (1825) 1-.

\section{Partition of numbers.}

theorem. Clausen, T. As. Nr. 21 (1844) 367-.

\section{PERMUTATIONS.}

automatic tit-tat-to machine. Freeland, $F . T$. [1878] Franklin I. J. 77 (1879) 1-.

circular, sequences. André, D. C. R. 120 (1895) 714-.

of first $n$ numbers, distribution into 4 groups. André, D. C. R. 115 (1892) 872-.

\section{5}

Figurate numbers.

polygonal numbers. Marchand, D. Les Mondes 42 (1877) 164-.

- - in n-dimensional space. Harmuth, $T$. [1882] Arch. Mth. Ps. 69 (1883) 90-.

\section{0}

Curves of errors. Thiele, T.N. (xiI) Ts. Mth. 2 (1878) 54-.

Ellipses of equal probability. Bréger, $P$. Rv. Artl. 10 (1877) 82-.

Error triangles, Grunert, J. A. Grunert Arch. 4 (1844) 348-. 
Errors of observation arising from preconception of result. Dufour, C. Laus. Bll. S. Vd. 5 (1856) 99-.

\section{5}

Annuities, theory. Hinard, C. A. Cond. Pon. Chauss. 27 (1883) (Pt. 1) 250-.

\section{0}

\section{(See also 8080 Connexes.)}

Linear substitutions, extension of calculus. Stephanos, C. C. R. 128 (1899) 593-.

\section{0}

Determinant of Smith and Mansion. Cesáro, $E$. N. A. Mth. 5 (1886) 44-.

Determinants, theorems. Anglin, A. H. Par. S. Mth. Bll. 15 (1887) 120-

-, -. Studnička, F.J. Fschr. Mth. (1898) $121,122,123$; (1899) 150-.

\section{0}

Resultants in spherical trigonometry, concept of. Meyer, $\boldsymbol{F}$. Crelle J. Mth. 15 (1895) 209-.

\section{0}

Determination of points and planes after rotation about an axis. Hudson, R.W.H.T. Mn. Mg. 12 (1900) 343-.

Orthogonal coefficients, relation between systems. Hoppe, R. Arch. Mth. Ps. 61 (1877) 111-。

Rotatory transformations of space. Study, E. Leip. Mth. Ps. B. 42 (1890) 341-.

\section{0}

Algebraic forms, Hilbert's theory. Schönflies, A. Gött. Nr. (1891) 339-.

Concomitants of systems of conics and quadrics. Johnson, A. R. L. Mth. S. P. 21 (1891) 432-.

Configuration corresponding to connexes, 2nd order and 1st class. Krause, R. Mth. A. 14 (1879) 294-.

Invariants of conics and quadrics given by motion and similitude groups. Lovett, E. O. A. Mth. 11 (1896-97) 33-.

Polar constructions. Morley, F. Mth. A. 51 (1899) 410 -

- operations, numerical formulæ. Capelli, A. G. Mt. 21 (1883) 343-.

- rectilinear figures of quadric and cubic. Johnson, A. R. Mess. Mth. 16 (1887) 63-.

Quadratic equation, discriminant, conditions for circular sections. Dingeldey, $F$. Crelle J. Mth. 122 (1900) 186-.

- form, homographic transformation into itself. Cayley, A. Ph. Mg. 6 (1853) 326-; 7 (1854) 208-.

- functions, simultaneous transformation of two. Campbell,J.E. Mess. Mth. 21 (1892) 78-.

Quaternary connexes $(1,1)$, linear systems. Lazzeri, $G$. Rm. R. Ac. Linc. Mm. 4 (1887) 259-.
Reduction of general equation of 2 nd degree. Boole, J. Camb. Mth. J. 2 (1841) 64-.

\section{0}

Binary cubic and quadratic forms, application to skew cubic curves. Pittarelli, G. G. Mt. 17 (1879) 260-.

- - - and conics. Pittarelli, G. G. Mt. 21 (1883) 19-.

- forms, representation on skew cubic curves. Sturm, $R$. Crelle J. Mth. 86 (1879) 116-.

Covariant geometry of triangle. Morley, $F$. QJ. Mth. 25 (1891) 186-.

Discriminant surface, singularities. Hilbert, $D$. Mth. A. 30 (1887) 437-.

Equation, 4th order. Russell, R. [1887] L. Mth. S. P. 19 (1889) 56-.

Invariants and covariants of function transformed by quadratic substitution. Spottiswoode, $W$. Rm. R. Ac. Linc. T. 7 (1883) 218-. - _ - - - - - (Spottiswoode). Pittarelli, G. Rm. R. Ac. Linc. Rd. 1 (1885) 327-, 374-.

Octahedron, equation. Brioschi, Fr. Rm. R. Ac. Linc. T. 3 (1879) 233-.

Poncelet's theorem on $\sqrt{a+b x+c x^{2}}$. Sylvester, J. J. Ph. Mg. 20 (1860) 307-.

Solution of differential equation of sources. Hammond, J. Am. J. Mth. 5 (1882) 218-.

Symbolic notation of binary forms, study of skew cubic curves by. Ovidio, $E$. $d$ '. [1879] Tor. Ac. Sc. Mm. 32 (1880) 1-.

\section{0}

Analytical study of quartic curves. Brioschi, Fr. A. Mt. 7 (1876) 202-.

Conjugate connexes, theory. Stephanos, C. Bll. Sc. Mth. As. 4 (1880) (Pt. 1) 318-.

Connex, new element of plane geometry. Clebsch, R. F. A. Gött. Nr. (1872) 429-.

Connexes $(1,2)$ and $(2,2)$, construction. Peano, G. Tor. Ac. Sc. At. 16 (1880) 497-.

Contravariant (Cayleyan), etc. of cubic curves, general properties. Cayley, A. [1856] Phil. Trans. (1857) 415-.

Covariant curves, Steiner's, Cayley's, etc., behaviour at singular points of original curve. Wölffing, E. Z.Mth. Ps. 40 (1895) 31-.

- theory of quartic curves,

$$
x_{2}^{3} x_{3}+x_{3}^{3} x_{1}+x_{1}{ }^{3} x_{2} \text {. }
$$

Brioschi, F. Mil. I. Lomb. Rd. 17 (1884) 401-; Rm. R. Ac. Linc. T. 8 (1884) 164-.

Covariants, 2, applied to construction of certain curves. Mangeot, S. N.A.Mth. 16 (1897) 76-.

- of ternary forms. Gundelfinger, S. A. Mt. 5 (1871-73) 223-.

Curves, decomposition into straight lines. Gordan, P. Mth. A. 45 (1894) 410-.

Invariants of two conics. Kluyver, J. C. As. Fr. C. R. (1887) (Pt. 2) $132-$.

Tactinvariant of conic and cubic. Lindemann, F. B. A. Rp. (1884) 647-.

Ternary connexes, 1st order and 1st class. Battaglini, G. [1880] Nap. Ac. At. 9 (1882) No. $4,16 \mathrm{pp}$. 
Ternary connexes, 1st order and 1st class, geometrical significance of invariants. Pannelli, M. G. Mt. 36 (1898) 81-.

- - 2nd order and 2nd class, in double involution. Pannelli, $M$. G. Mt. 26 (1888) 1-.

- forms of 4 th, 5 th, 6 th, 7 th orders, equations arising from certain. Brioschi, F. Rm. R. Ac. Linc. T. 8 (1884) 164-.

\section{0}

Application of symbolic calculus to quadrics. Garbieri, G. Ven. I. At. (1885-86) 943-.

Quadratic form in $m$ variables, decomposition into sum of $m-n$ squares. Benoit, - C. R. 101 (1885) 869-.

- forms, 2, and skew quartic curve. Westphal, G. Mth. A. 13 (1878) 1-.

\section{0}

Algebraic functions, representation, theorem of Abel's. Königsberger, -. [1868] Mth. A. 1 (1869) 168-.

Resolution of functions into conjugate factors. Young, J. R. Ph. Mg. 34 (1849) 278-.

\section{0}

Sturm's functions. Vidal, - [1886] I. Égypt. Bll. 7 (1887) 400-.

Sylvester's theorem, new version. Horner, $J$. [1867] QJ. Mth. 9 (1868) 221-.

\section{0}

\section{Equations, $3 r d$ order.}

geometry. Russell, R. [1893] Ir. Ac. P. 3 (1893-96) 170-.

Polygonal and polyhedric coordinates, etc. Sautreaux, F. Brux. Mm. Cour. $8^{\circ}, 36$ (1884) No. 3, 14 pp.

\section{0}

Fluctuating functions, numerical calculation of roots. Rayleigh, John William Strutt (Lord). (xI) L. Mth. S. P. 5 (1873-74) 119-.

Numerical equations, separation of roots. Vidal, -. [1882] I. Egypt. Bll. 3 (*1883) 91-.

_-, solution. Vidal, -. [1880] I. Egypt. Bll. 1 (*1882) 3-.

\section{0}

Equations, 5th order.

Guldberg, A. S. [1869] Christiania F. 12 (1870) 308-.

Solution, equation of icosahedron in. Klein, C. F. Mil. I. Lomb. Rd. 10 (1877) 253-.
Icosahedron. Klein, C. F. Erlang. Ps. Md. S. Sb. 9 (1877) 16-, 70-, 179-; Mth. A. 12 (1877) 503-.

Irreducibility of equation in division of circle. Arndt, F. Crelle J. 56 (1859) 178-.

\section{0}

Equations representable by systems of three points on curve. Ocagne, M. $d$ '. Acta Mth. 21 (1897) 301-.

\section{0}

Bardwell, F. W. Kan. Ac. Sc. T. 3 (1874) (Reprint, 1896) 94-.

Application of theorem in analysis situs. Busche, E. [1898] Hamb. Mth. Gs. Mt. 3 (1900) 333-.

Function $\zeta(s)=\Sigma n^{-8}$, zeros. Piltz, -. D. Nf. Vh. (1891) (Th. 2) 15-.

Functional theorem of Lagrange, extension. Degen, C. F. (vI Adds.) Kiöb. Ov. (181819) $3-$.

Indeterminate equations and $n$-dimensional geometry. Brunel, $G$. Bordeaux S. Sc. Mm. 2 (1886) 129-.

Laws of reciprocity, and sub-groups of arithmetical group. Stouff, $X$. C. R. 116 (1893) 308-.

Products of numbers, properties. Teixeira, J. P. G. Teix. J. Sc. 14 (1900) 179-.

\section{0}

Congruence $x^{t} \equiv 1(\bmod . M)$, roots, etc. Arndt, $F$. Crelle J. 31 (1846) 259-.

$-n x \equiv n+1$ (mod. $p$ ). Graves, J. T. B. A. Rp. (1856) (pt. 2) 1-。

Congruences, linear, solution by binomial factorial. Bouniakowsky, V. [1842] St Pét. Ac. Sc. Mm. 5 (1844) 287-.

—, —, - of systems. Demeczky de Gyergyószentmiklos, D. C. R. 88 (1879) 1311-。

\section{DIVISIBILITY.}

of numbers of form of $2^{2^{m}}+1$, new case (Pervušin). Buniakovskij, $V$. Ja. St Pet. Ac. Sc. Mm. (Rs.) 31 (*1878) 223-.

Function entering into convergents of continued fractions, and roots of congruences of first degree. Porcelli, O. G. Mt. 10 (1872) 37-.

\section{5}

\section{INDETERMINATE EQUATIONS.}

2 or 3 variables, having only finite number of integral solutions. Maillet, $E$. C. R. 128 (1899) 1383-.

\section{0}

Congruences, 2nd degree. Bouniakowsky, $V$. [1830] St Pét. Ac. Sc. Mm. 1 (1831) 563-. _, - - solution. Tonelli, A. Gött. Nr. (1891) 344-。 
Quadratic binary forms, system of three, relation to spherical trigonometry. Stephanos, $C$. Par. S. Mth. Bll. 10 (1882) 134-.

\section{0}

Congruences, 2nd degree. Jordan, C. C. R. 62 (1866) 687-.

- , - _, canonical form and number of solutions. Jordan, C. Liouv. J. Mth. 17 (1872) 368-.

,--- , reduced forms. Jordan, C. C. R. 74 (1872) 1093-.

-, - - , with several unknowns, case. Le Besgue, V. A. C. R. 62 (1866) 868-.

Decomposition of numbers into sums of squares.

4 squares, Jacobi's theorem. Weill, M. C. R. 99 (1884) 859-.

Quadratic forms, application of non-Euclidean geometry to. Poincarê, $H$. As. Fr. C. R. (1881) 132-.

\section{0}

Binomial congruences, classification of roots, application to Canon Arithmeticus. Le Besgue, V. A. C. R. 63 (1866) 1100-.

- - Daniel's formula. Horta, F. Lisb. A. 1 (1857) 705-.

- - with prime modulus, theorem, and application to Canon Arithmeticus. Le Besgue, V.A. C. R. 61 (1865) 1041-.

Congruences, formula. Bellavitis, $G$. Ven. At. $(1859-60)$ 821-.

-, general theorems, and applications. Schönemann, T. Crelle J. 19 (1839) 231-, 289-.

-, higher, with real prime modulus. Dedekind, $R$. Crelle J. 54 (1857) 1-.

- - , theory. Rados, G. Crelle J. Mth. 99 (1886) 258-.

\section{0}

Cyclotomy. Eisenstein, G. Crelle J. 27 (1844) 269-.

Gauss's series, Dirichlet's method for finding coefficients. Kronecker, $L$. Hamb. Mth. Gs. Mt. 2 (1890) (Festschr., Tl. 2) 32-.

\section{0}

Automorphic functions, theory, and theory of numbers, relations between. Fricke, $R$. D. Mth. Vr. Jbr. 6 (1899) (Heft 1) 94-.

Fuchsian functions and quadratic forms. Picard, É. Am. J. Mth. 11 (1889) 187-.

---1 - Stouff, $X$. Toul. Fac. Sc. A. 8 (1894) D, $20 \mathrm{pp}$.

- - - indefinite ternary. Poincaré, $H$. C. R. 102 (1886) 735-.

- groups and quadratic forms. Stouff, $X$. Toul. Fac. Sc. A. 3 (1889) B, 28 pp.

Gamma function, applications to theory of numbers. Berger, A. [1880] Ups. S. Sc. N. Acta 11 (1883) (No. 1) 87 pp.
Hyperfuchsian functions and ternary quadratic forms. Picard, É. Acta Mth. 5 (1884) 121-.

\section{0}

Koch, H. von. C. R. 130 (1900) 1243-.

\section{0}

$\pi$.

converging series for. Ferrel, $W$. [1870] Smiths. Ct. 18 (1873) (Art. 5) 6 pp.

\section{0}

Functions having no differential coefficients. Fambri, $P$. Ven. I. At. (1884-85) 823-.

-, monomial, 2, property of primitives and derivatives of product. Sang, $E$. $\mathrm{Ph} . \mathrm{Mg}$. 6 (1829) 262-.

3220

\section{INFINITE SERIES.}

Integral series. Hadamard, - C. C. R. 124 (1897) 492; Bordeaux S. Sc. PV. (1896-97) 110-.

\section{0}

Analysis, variable parameters in. Cauchy, A. L. C. R. 40 (1855) 261-.

\section{0}

Expansion of functions satisfying an algebraic equation. Hurwitz, A. Par. Éc. Norm. A. 6 (1889) 327-.

\section{Lagrange's Series.}

and application to Kepler's equation. Weiss, $E$. Wien Ak. D. 49 (1885) (Ab. 1) 133-.

- - - - (Weiss). Stolz, O. Wien Ak. Sb. 95 (1887) (Ab. 2) 199-.

\section{0}

\section{INTEGRATION.}

graphical methods. Massau, J. Rv. Un. Mines 16 (1884) 243-, 579-; 17 (1885) 1-; 20 (1886) 1-, 475-; 21 (1887) 85-, 308-, 572-; 22 (1887) 1-.

Quadrature, Čebyšev's formula. Biles, J. H. [1898] Glasg. I. Eng. T. 42 (1899) 176-.

-, Gauss's method. Mansion, -. Brux. S. Sc. A. 15 (1891) (Pt. 1) 57-.

-, Gauss's method. Raffy, L. N. A. Mth. 15 (1896) 249-.

- -- , extension. Bourget, H. C. R. 126 (1898) 634-.

_, - , generalisation. Christoffel, E. B. Crelle J. 55 (1858) 61-.

-, - - remainder. Mansion, $P$. C. R. 102 (1886) 412-; Brux. Ac. Bll. 11 (1886) 293-. _, - _ , and solution of Kepler's problem. Strehlke, $F$. Grunert Arch. 32 (1859) 433-. 
Theorem of Cotes, new demonstration. Littrow, J. J. von. Zach Cor. 3 (1819) 22-.

Transcendental integrals of form

$$
\int_{0}^{x} e^{-x^{2}} f(x) d x \text {. }
$$

Thomson, (Sir) W. QJ. Mth. 1 (1857) 316-.

\section{0}

Ostrogradsky, M. A. [1839] St Pét. Ac. Sc. Mm. 4 (1841) 309-.

Poisson's integral. Mukhopadhyay, A. [1888] Beng. As. S. J. 57 (Pt. 2) (1890) 100-.

$\int_{0}^{\infty}[f(\sin \alpha x, \cos \alpha x)-f(\sin \beta x, \cos \beta x)] \frac{d x}{x}$.

$$
=\frac{1}{2} \log \left(\frac{\beta}{\alpha}\right)\left[f(\sin 0, \cos 0)-A_{0}\right] \text {. }
$$

Schlömilch, O. Grunert Arch. 5 (1844) 152-。 $\int_{0}^{\infty} \frac{x^{m-1}-1}{1-x^{2}} d x=\frac{\pi}{2} \cot \frac{m \pi}{2}$ if $2>m>0$.

Zehfuss, G. Grunert Arch. 30 (1858) 465-. $\int_{0}^{\frac{\pi}{2}}(2 \cos x)^{a+b} \cos (a-b) x d x$. Lipschitz, R. O.S. Bll. Sc. Mth. As. 5 (1881) (Pt. 1) 387-。

\section{0}

Curvilinear coordinates, Stokes's theorem. Webster, A. G. N. Y. Am. Mth. S. Bll. 4 (1898) 438-.

\section{Double integrals.}

of 2 nd kind in theory of algebraic surfaces. Picard, É. C. R. 125 (1897) 909-; 127 (1898) 579-; 129 (1899) 539-.

Integrals deduced from theory of orthogonal surfaces. Ostrogradsky, -. (vir) St Pét. Ac. Sc. Bll. 7 (1840) 362-.

\section{0}

Cuttings and embankments, problem. SaintGermain, A. de. Caen Ac. Mm. (1886) (Pt. 1) 23-.

Maximum or minimum, properties common to all curves satisfying certain condition of. Vicaire, E. C. R. 106 (1888) 456-.

Trajectories, on given surface or in space, rendering minimum $\int_{0}^{s} \phi(\nu) d s . \quad$ Roger, $\dot{E}^{\prime}$. C. R. 40 (1855) 1176-.

\section{0}

\section{(See also 8800-8840.)}

Analytic forces. Lecornu, L. Par. Éc. Pol. J. 55 (1885) 253-.

Conformal representation. Neumann, C. Leip. B. 22 (1870) 49-, 264-.
Curves along which the absolute value of a function is constant, use. Schläfli, L. [1873] (xI) A. Mt. 6 (1873-75) 1-.

Singular points, essential, theory. Guichard, C. Par. Éc. Norm. A. 12 (1883) 301-.

Transformation of polygons in $z$ plane. Lecornu, L. C. R. 92 (1881) 695-; Par. Éc. Pol. J. Cah. 49 (1881) 205-

\section{0}

Cubic corpus of functions, complete system of differentials of 1 st class in. Baur, $L$. Mth. A. 46 (1895) $31-, 160,608$.

- - functions, theory. Baur, L. Mth. A. 43 (1893) 505-.

Fundamental system for given class domain, determination. Mertens, $\boldsymbol{F}$. Prace Mt.-Fiz . 6 (1895) 106-.

Period systems of functions of real variables. Kronecker, L. Berl. Ak. Sb. (1884) 1071-.

\section{0}

Approximate equation $F^{1}(c)=\log \frac{4}{b}$. Verhulst, P. F. Brux. Ac. Bll. 10 (1843) (pte. 2) 378-.

Curves represented by

$\frac{d x}{\sqrt{ }\left\{\left(1-x^{2}\right)\left(1-k^{2} x^{2}\right)\right\}} \pm \frac{d y}{\sqrt{ }\left\{\left(1-y^{2}\right)\left(1-k^{2} y^{2}\right)\right\}}=0$, and their envelopes. Turner, H. H. Mess. Mth. 10 (1881) 21-.

Deformable frameworks. Dixon, A, C. Mess. Mth. 29 (1900) 1-.

\section{Doubly periodic functions.}

class of two. Farkas, J. C. R. 90 (1880) 1269-.

of 3rd kind, application of Mittag-Leftler's theorem. Appell, P. Par. Éc. Norm. A. 2 (1885) 67-.

\section{ELLIPTIC FUNCTIONS.}

applications to flexible cords. Marcolongo, $R$. Nap. Rd. 31 (1892) 71-, 89-.

of 1st kind, representation by arc of plane transcendental curve. Tortolini, B. Tortolini A. 4 (1853) 485-.

\section{0}

Curves, 4th degree, $x_{2}^{3} x_{3}+x_{3}^{3} x_{1}+x_{1}^{3} x_{2}$, covariant theory. Brioschi, $F$. Mil. I. Lomb. Rd. 17 (1884) 401-; Rm. R. Ac. Linc. T. 8 (1884) 164 .

\section{DIVISION.}

of lemniscate arcs. Tortolini, B. Palomba Rac. 1 (1845) 17-; Mod. S. It. Mm. 1 (1862) 91-.

\section{0}

\section{Abelian functions.}

in connexion with 2-dimensional fluid motion. Baker, H. F. Camb. Ph. S. P. 9 (1898) 381-。 
ABELIAN INTEGRALS.

inversion, Appell, P. C. R. 99 (1884) 1010-.

Algebraic differential equations, algebraic integrals. Jahnke, E. Z. Mth. Ps. 35 (1890) 148 -

- _- irreducibility. Koenigsberger, $L$. Berl. Ak. Sb. (1899) 783-.

- differentials, algebraic integrals. Humbert, G. Acta Mth. 10 (1887) 281-。

- - - - Ptaszychi, J. Acta Mth. 11 (1887-88) 395-; Prace Mt.-Fiz. 1 (1888) 81-; Kharkov Mth. S. Com. 1 (1889) 61-; Fschr. Mth. (1888) 296-.

Conditionally periodic functions in mechanics. Staude, O. Crelle J. Mth. 105 (1889) 298-.

\section{0}

Differential equations of tetrahedron, octa. hedron and icosahedron. Brioschi, $F$. A. Mt. 10 (1880-82) 104-

Greatest height of mast or tree consistent with stability. Greenhill, A. G. [1881] Camb. Ph. S. P. 4 (1883) 65-。

\section{HYPERGEOMETRIC FUNCTIONS.}

analogues, Goursat, ḱ. C. R. 102 (1886) 204-.

Kepler's problem and function

$$
I_{k}^{h}=\frac{1}{2 \pi} \int_{0}^{2 \pi} \cos h \epsilon \cdot \cos (k \sin \epsilon) d \epsilon .
$$

Anger, C. T. Danzig N. Schr. 5 (1855) (Heft 2) vi $+29 \mathrm{pp}$.

\section{0}

Conformal representation of half plane on polygon approaching limiting circle. Pick, G. Wien Ak. Sb. 100 (1891) (Ab. 2a) 1387-.

- _ - spherical triangles by algebraic functions. Fischer, O. Leip. Mth. Ps. B. 36 (1884) 17-.

Fuchsian surfaces. Bianchi,L. Rm. R. Ac. Linc. Rd. 4 (1888) (Sem. 2) 161-.

Surfaces with curve-symmetry of systems of planes. Langeot, S. C. R. 112 (1891) 1497-.

Symmetric $S$-functions with singularity. Schilling, F. Ac. Nt. C. N. Acta 71 (1898) 207 .

\section{0}

Integration, graphic methods. Massau, $J$. Rv. Un. Mines 16 (1884) 243-, 579-; 17 (1885) 1-; 20 (1886) 1-, 475-; 21 (1887) 85-, 308-, 572-; 22 (1887) 1-.

\section{0}

Equations.

Lie, M. S. Christiania F. (1882) No. 21, 12 pp., No. 22, 6 pp.; (1883) No. 10, 4 pp., No. $18,5 \mathrm{pp}$.
Solution.

of problem of 3 bodies, report on progress. Whittaker, E. T. B. A. Rp. (1899) 121-。

Surfaces of 4th degree admitting an integral of total differential of 1st kind. Berry, $A$. C. R. 129 (1899) 449-.

System of surfaces defined by linear equation, 1st order, fundamental points. Fouret, G. C. R. 86 (1878) 654-.

\section{0}

Connexes of line pairs. Weber, E. von. Gött. Nr. (1896) 282-.

\section{Integrals.}

intermediate, of equations, 2nd order. Goursat, É. C. R. 112 (1891) 1117-.

Integration.

case. Zaremba, -. C. R. 110 (1890) 127-。

Transformations, class. Clairin, J. C. R. 130 (1900) 309-.

\section{0}

\section{Equations.}

of tetrahedron, octahedron, and icosahedron. Brioschi, F. A. Mt. 10 (1880-82) 104-.

\section{0}

Algebraic equation, family of plane curves defined by. Fouret, G. C. R. 86 (1878) 586-.

Connex $C(x, x, 0 ; u, u, 0)$, principal coincidence. Möller, $J$. Lund. Un. Acta 16 (1879-81) (Mth.) (No. 1) $16 \mathrm{pp.}$

Equation between two variables, systems of curves defined by. Dyck, $W$. Münch. Ak. Sb. 21 (1892) 23-; 22 (1893) 101-.

\section{0}

Differential forms, and systems of elements. Koenigs, G. C. R. 104 (1887) 673-, 842-; Acta Mth. 10 (1887) 313-.

\section{0}

Equation of connex $(1, n)$, special case. Müllendorff, E. Arch. Mth. Ps. 69 (1883) 113-.

Partial differential equations. Duport, $H$. C. R. 130 (1900) 232-.

$P d x+Q d y+R d z=0$, geometrical interpretation. Voss, A. E. Mth. A. 16 (1880) 556-.

\section{0}

\section{TRANSFORMATION,}

infinitesimal, trajectories of motions of systems admitting. Painlevé, P. C. R. 116 (1893) 21-. 
5240

Covariants of skew curves, Guyther, $R$. $F$. R. S. P. 56 (1894) 272-.

\section{0}

Curves fulfilling the condition $\frac{d^{2} \phi}{d x^{2}}+\frac{d^{2} \phi}{d y^{2}}=0$ (rhizic curves), property. Rankine, $W . J . M$. R. S. P. 15 (1867) 468-.

Differential equation $\nabla^{2} \Phi+h^{2} f(x y z) \Phi=0$, system of orthogonal coordinates belonging to. Petrini, H. Stockh. Ak. Hndl. Bh. 19 (Afd. 1) (1894) No, 4, 36 pp.

\section{0}

Harmonic analysis by polar planimeter. Finsterwalder, S. Z. Mth. Ps. 43 (1898) 85-.

Rate of solution of solids. Bottomley, $J$. Manch. Lt. Ph. S. Mm. \& P. 3 (1890) 113-.

Surface generated by solution of isotropic solid. Bottomley, J. Manch. Lt. Ph. S. Mm. \& P. 2 (1889) 154-, viii.

\section{0}

Shells bounded by confocal quadrics. Klein, C. $F$. Mth. A. 18 (1881) 410-.

\section{0}

$\frac{1}{2} m \frac{d^{2} r^{2}}{d t^{2}}=m v^{2}+(X x+Y y+Z z)$. Mantel, W. N. Arch. Wisk. 18 (1891) 127-. $A^{2} \frac{\partial^{2} v}{\partial t^{2}}=\frac{\partial^{2} v}{\partial x^{2}}+\frac{\partial^{2} v}{\partial y^{2}}$, class of integrals. Levi. Civita, T. N. Cim. 6 (1897) 204-.

\section{0}

Dirichlet's problem. Zaremba, S. C. R. 124 (1897) 940 -

- - of stationary temperatures, and represen. tation of given surface. Christoffel, $E . B$. A. Mt. 1 (1867-68) 89-; 4 (1870-71) 1-。

Neumann's method and Dirichlet's problem. Poincaré, H. C. R. 120 (1895) 347-.

\section{0}

Calculus of functions. Spottiswoode, $W$. R. S. P. 11 (1860-62) 447-.

\section{0}

Tracing of line by point, paradox. Zehfuss, $G$. Grunert Arch. 30 (1858) 229.

\section{0}

Absolute geometry, trigonometry in. Simon, $M$. Crelle J. Mth. 109 (1892) 187-.

Area of triangle, imaginary geometry. Kagan, B. N. A. Mth. 14 (1895) 251-。

\section{NON-EUCLIDEAN GEOMETRY.}

trigonometry. Story, W. E. Am. J. Mth. 4 (1881) 332-; 5 (1882) 180-.

-, fundamental equations derived by elementary method. Réthy, $I$. Arch. Mth. Ps. 58 (1876) 416-.

-, plane, of 3-dimensional homogeneous

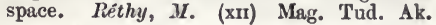
Etk. (Mth.) 4 (1877) (No. 7) 25 pp.

Space, elliptic, linear transformation corresponding to displacement of rigid system in. Ball, R. S. [1885] Ir. Ac. P. 4 (1884-88) 532-.

\section{0}

Knots of 2 closed skew curves, Gaussian integral giving number. Thomae, $C . J$. Freiburg B. 7 (1880) 18-.

-, skew curves of loop-form. Brill, A. Mth. A. 18 (1881) 95-.

Knotted curve, Hoppe's. Durège, H. [1880] Wien Ak. Sb. 82 (1881) (Ab. 2) 135-.

Links. Tait, P. G. [1877] Edinb. R. S. P. 9 (1878) 321-.

Ovals, exact foundations for theory. Brunn, H. Münch. Ak. Sb. 24 (1895) 93-.

Singularities of plane curves, real, remarkable relation. Perrin, R. [1877] Par. S. Mth. Bll. 6 (1878) 84-.

Straight lines, finite, in plane, number of cases as regards parallelism, non-parallelism and situation. Rothe, H. A. Ac. Caes. Leop. N. Acta 12 (1825) 399-.

\section{0}

\section{Coordinates.}

spherical, homogeneous, application to spheroconics. Cassani, P. G. Mt. 6 (1868) 81-。

\section{0}

Directly similar plane figures. Schoute, P. H. C. R. 111 (1890) 499-.

\section{0}

\section{CIRCLES.}

Circles on chords $A A_{0}, A A_{1}, A A_{2}$ of circle as diameter, theorem. Grunert, J. A. Arch. Mth. Ps. 47 (1867) 468-.

Properties. Glenie, J. [1805] Edinb. R. S. T. 6 (1812) 21-.

Property. Floryn, J. Amst. Vh. 2 (1816) 123-.

$(2 n+1)$-gon, property. Bangma, O. S. (vII) Amst. N. Ws. Ntk. Vh. 1 (1844) 51-.

\section{Quadrature of the Circle.}

Saint-Germain, A. de. Caen Ac. Mm. (1898) (Pt. 1) 24-.

Studnicka, F. J. Fschr. Mth. (1899) 386. 
erroneaus value. Lera, $L$. Bb. It. 2 (1816) 247 -

quadrature of circular àreas. Reggio, $Z$. Ven. I. At. 7 (1880-81) 1097-.

\section{Ratio of Diameter to Circumference.}

Vidal, S. E. [1888] I. Egypt. Bll. 9 (1889) 135 -.

Extension of value of $\pi$. Rutherford, $W . \quad R$. S. P. 6 (1853) $274-$.

Rectification, $\frac{\pi}{2}$ series for. Valat, - Bordeaux Act. Ac. Sc. 26 (1864) 276-.

Discontinuities, on removing certain (Rulf). Drasch, H. Mh. Mth. Ps. 4 (1893) 376-.

Figures variable with a certain affinity. Neuberg, J. Liège S. Sc. Mm. 16 (1890) No. 5, $12 \mathrm{pp}$.

\section{Polygons.}

Convex polygon, angles, theorem. Certo, Palermo Cir. Mt. Rd. 4 (1890) 285-.

Isoperimetric polygons, number of sides doubling continually. Rouché, $\boldsymbol{E}$. N. A. Mth. 1 (1882) 325-.

\section{Regular polygons.}

areas. Ghibaldan, I. Bucarest Ac. Rom. A. 17 (1894-95) (Pt. Admin.) 630-.

\section{Quadrilaterals.}

Complete quadrilateral. Schlömilch, $O . X$. Z. Mth. Ps. 23 (1878) 191-.

Property. Brune, -. Crelle J. 22 (1841) 379.

Square, maximum, in triangle. Brune, Crelle J. 15 (1836) 365-.

Squares, 3 , in arithmetical progression. Guibert, A. N. A. Mth. 1 (1862) 213-.

\section{Triangles.}

Bisection by line through any point. Lindman, C. F. Arch. Mth. Ps. 51 (1870) 247-.

Bisectors of angles, distances between feet. Dostor, G. Arch. Mth. Ps. 64 (1879) 426-.

\section{Circles of the Triangle.}

Circum-circle of triangle formed by three diagonals of complete quadrilateral. Möbius, A. F, Leip. B. (1854) 87-

In- and circum-circles, centres, relations of equilibrium. Nöggerath, $E . J$. Arch. Mth. Ps. 43 (1865) 89-.

- _ _ - collection of proofs of formula $r^{\prime}+r^{\prime \prime}+r^{\prime \prime \prime}-r=4 R$. Baker, $M$. Des Moines Anal. 5 (1878) 82-.

\section{Nine-point or Feuerbach Circle.}

and circles touching sides, points of contact. Gerono, G. C. N. A. Mth. 4 (1865) 220-. theorems. Fiedler, W. Schlömileh Z. 8 (1863) $390-$

Circum-triangles, equilateral, of given triangle. Fasbender, -. Crelle J. 30 (1846) 230-

Property. Lehmus, - . Crelle J. 50 (1855) 266-. Sides, raised to given powers, problem. Poudra, -. N. A. Mth. 15 (1856) 217-.

\section{0}

Polygons, spherical, transformation, and Lexell's theorem. 'Steiner, J. N. A. Mth. 4 (1845) 587-.

\section{SPHERES.}

Division of sphere in given ratio. Padula, $F$. Nap. Rd. 1 (1852) 45-.

Property of having least surface for given volume, demonstration. Schrarz, H.A. Gött. Nr. (1884) 1-.

Systems of spheres connected with tetrahedron. Third, J. A. Edinb. Mth. S. P. 17 (1899) 108-.

\section{0}

Busse, F. G. ron. Gilbert A. 19 (1805) 336-.

Inaccessible points, new method of surveying. Trouche, - A. Cond. Pon. Chauss. 38 (1894) 243-.

\section{SPHERICAL TRIGONOMETRY.}

Formula of Spherical Trigonometry.

Hoza , F. Časopis 11 ("1882) 212-; Fschr. Mth. ("1882) 473.

Legendre's theorem, analogue, geodesic applications. Grunert, J. A. Grunert Arch. 25 (1855) 197-.

Surveying. Koller, M. W. Brünn Vh. 3 (1864) (Ab.) 46-.

Topographic instrument (automatic). Gillet, C. A. Cond. Pon. Chauss. 28 (1884) (Pt. 1) 137-.

$b^{2} \cos ^{2} \beta+e^{2} \sin ^{2} \beta=1$ always entails $b=e= \pm 1$. Molluceide, C. Gilbert A. 25 (1807) 212-.

\section{0 \\ (See also 7240.) \\ Anharmonic or Cross-Ratio.}

Theorems. Spottiswoode, W. Camb, and Dubl. Mth J. 8 (1853) 103-.

\section{Axonometry.}

Cylinder orthogonal to certain surfaces. Pirondini, G. G. Teix. J. Sc. 13 (1897) 77-.

Rotation hyperboloids, penetration by parallel axes. Fiedler, $W$. Acta Mth. 5 (1884) $331-$.

\section{PERSPECTIVE.}

Gruber, T. Böhm. Gs. Ab. 1 (1804) 111 pp.

Calculus analogous to barycentric, plane and straight line as elements. Lippich, $F$. Steierm. Mt. 2 (Heft 2) (1870) 215-. 
Parallel to inaccessible line, tracing. Fépoux, O. A. Cond. Pon. Chauss. 33 (1889) 632-.

Poncelet's geometrical interpretation of $\sqrt{-1}$. Ghibaldan, I. Bucarest Ac. Rom. A. 17 (1894-95) (Pt. Admin.) 639-.

Quadrics, perspective contours for, new construction. Zipernovszky, $K$. Wien Ak. Sb. 71 (1875) (Ab. 2) 33-.

\section{Practical Solid Geometry.}

Illumination-constructions for surfaces whose sections perpendicular to one axis are similar ellipses. Niemtschik, $R$. Wien Sb. 57 (1868) (Ab. 2) 678-.

Intersection of surfaces of revolution. [Schiappa] Monteiro, A. G. Teix. J. Sc. 1 (1878) 177-.

Quarter cones (in breakwaters), drawing. Lanave, L. A. Cond. Pon. Chauss. 36 (1892) 17-, 33-

Shadow, and parallel projection generally, of screw surfaces. Burmester, L. Z. Mth. Ps. 18 (1873) 184-.

- tracing. Anon. A. Cond. Pon. Chauss. 38 (1894) 415-, 434-.

\section{Projective Geometry.}

Theorem of Schlömilch, demonstration. Quidde, A. Z. Mth. Ps. 28 (1883) 192.

- - - and desmic systems of 3 tetrahedra. Schroeter, H. E. Z. Mth. Ps. 28 (1883) 178-.

\section{0}

De Morgan, A. Camb. Mth. J. 2 (1841) 202-.

Axes of conics as central projections of circle. Pelz, C. Prag Sb. (1872) 32-.

Construction from points, general method. Azzarelli, M. Rm. N.Linc. At. 30 (1877) 64-.

Eccentric anomaly and conics. O'Brien, $M$. Camb. Mth. J. 4 (1845) 99-.

\section{Ellipses.}

Focal property of ellipse and hyperbola. Earnshaw, S. [Signed V.] (vi Adds.) Camb. Mth. J. 3 (1843) 196-.

Maximum in-ellipse. Caluso, (l'abbé) T.V.de. Turin Mm. Ac. 6 (1792-1800) 213-.

3 properties of ellipse and hyperbola deduced from property of circle. Pollock, (Sir) F. R. S. P. 4 (1843) 443-.

Envelope of chord subtending given angle at focus. Booth, J. Camb. Mth. J. 3 (1843) $87-$.

Foci.

Foci, directrices and eccentricities, quadruplex nature. Mitchell, O. H. (xI) J. H. Un. Cir. [2] (1883) 144 .

\section{Pascal's hexagram, Brianchon's hexagon, and connected figures.}

Pascal's theorem, analogues, extension to curves on surfaces. Folie, F. Brux. Ac. Bll. 37 (1874) 811-.
Quadrilaterals, Quadrangles, and related Conics.

Circum - quadrilaterals, Newton's theorem, demonstration. Terquem, $O$. N. A. Mth. 2 (1843) 378-.

In-quadrilateral, Newton's theorem, generalisation. Faure, H. N. A. Mth. 17 (1858) 368-.

Triangles, and related Conics.

Conjugate triangle of 2 conics. Cassani, $P$. G. Mt. 8 (1870) 200-.

Triangle and conic touching sides, solution of question of Cayley's. Jonquières, $E$. de. N. A. Mth. 16 (1857) 189-.

\section{0}

" Conchordal" conics, system. Story, W. E. [1881] (xII) J. H. Un. Cir. [1] (1882) 178.

Conics with Common Focus or Foci.

Confocal conics, etc. Bellavitis, G. Ven. At. (1859-60) 821-.

Special connex $(2,2)$. Amodeo, F. G. Mt. 25 (1887) 321-.

\section{0}

Double contact, 2 problems by Salmon, extension to surfaces. Anon. (xI 29) G. Mt. 7 (1869) 254

\section{Ellipsoid.}

as deformation of sphere. Blaẑek, G. Prag Sb. (1869) (pt. 2) 29-.

\section{0}

Singular points. Chasles, M. C. R. 59 (1864) 209-.

- - Halphen, G. H. C. R. 78 (1874) 1105-

- Tichomandrickij, M. A. Kharkov Mth. S. Com. 2 (1891) 114-; Fschr. Mth. (1891) 757.

Singularities of curve, relation. Klein, C. F. Erlang. Ps. Md. S. Sb. 8 (1876) 95-; Mth. A. 10 (1876) 199-

- curves, Zeuthen, H. G. Mth. A. 10 (1876) 210-.

- - compound. Guccia, G.B. Palermo Cir. Mt. Rd. 3 (1889) 241-.

- - nature and effect. Scott, (Miss) C. A. Am. J. Mth. 15 (1893) 221-.

\section{0}

Triangular potential curve. Cesáro, E. N. A. Mth. 7 (1888) 257-.

\section{0}

Singularities relating to multiple line of surface. Zeuthen, H. G. Mth. A. 4 (1871) 1-. Surface of elasticity, theorem. Strebor [Roberts, W.]. N. A. Mth. 10 (1851) 197-. 


\section{0}

Singular points. Guccia, G. B. C. R. 120 (1895) 816-.

Singularities. Zeuthen, H. G. C. R. 67 (1868) 225-.

- Fine, H. B. Am. J. Mth. 8 (1886) 156-. - of curve and developable surface. Zeuthen, H. G. A. Mt. 3 (1869-70) 175-.

- - - of intersection of 2 surfaces having certain number of multiple points in common. Saltel, L. C. R. 80 (1875) 1285-.

- - curves and characteristics of polar developables. Björling, C. F. E. Stockh. Öfv. 38 (1881) No. 4, 3-.

\section{0}

Connexes, 2nd order and 2nd class, generation. Armenante, $A$. Rm. R. Ac. Linc. At. 3 (1876) (Pte. 2) 123-.

Linear connexes. Voss, A. E. Mth. A. 15 (1879) 355-.

\section{0}

Characteristic combinations in transformation of singular point of curve. Noether, $M$. Palermo Cir. Mt. Rd. 4 (1890) 89-, 299-.

Substitutions, instability. Levi-Civita, C. R. 131 (1900) 103-.

\section{5}

SPECIAL CONFIGURATIONS OF POINTS, LINES, PLANES OR OTHER ELEMENTS.

9 point configuration. Clausen, T. Crelle J. 7 (1831) 36-.

\section{0}

Systems of forces (Dynamen), geometry. Study, E. Leip. Mth. Ps. B. 51 (1899) (Mth.) 29-.
8090

SYSTEMS OF CURVES.

Differential equations. Czuber, $E$. Wien Ak. Sb. 102 (1893) (Ab. 2a) 1141-.

\section{0}

Deformable frameworks. Dixon, A.C. Mess. Mth. 29 (1900) 1-.

\section{0}

Curves.

of "raccordement." Allaume, -. A. Cond. Pon. Chauss. 34 (1890) 76-.

- - parabolic. Fépoux, -. A. Cond. Pon. Chauss. 31 (1887) 233-.

\section{0}

Areas.

of hemisphere, calculation. Dumas, J. B. A. Cond. Pon. Chauss. 37 (1893) 505-.

\section{Rectification.}

of ellipse. Bronwin, B. Ph. Mg. 2 (1827) 378- - Moreau, A. A. Cond. Pon. Chauss. 38 (1894) 563-.

- - , extension of J. Bernoulli's rule. Magistrini, G. B. Bologna Opusc. Sc. 1 (1817) 244-.

\section{0}

Class of fluid motions connected with minimal surfaces, and particular integrals of $\nabla^{2} V=0$. Weingartèn, J. Gött. Nr. (1890) 313-. 
CAMBRIDGE:

PRINTED BY JOHN CLAY, M.A.

AT THE UNIVERSITY PRESS. 


University of Callfornia

SOUTHERN REGIONAL LIBRARY FACILITY 405 Hilgard Avenue, Los Angeles, CA 90024-1388

Return this material to the library from which it was borrowed.

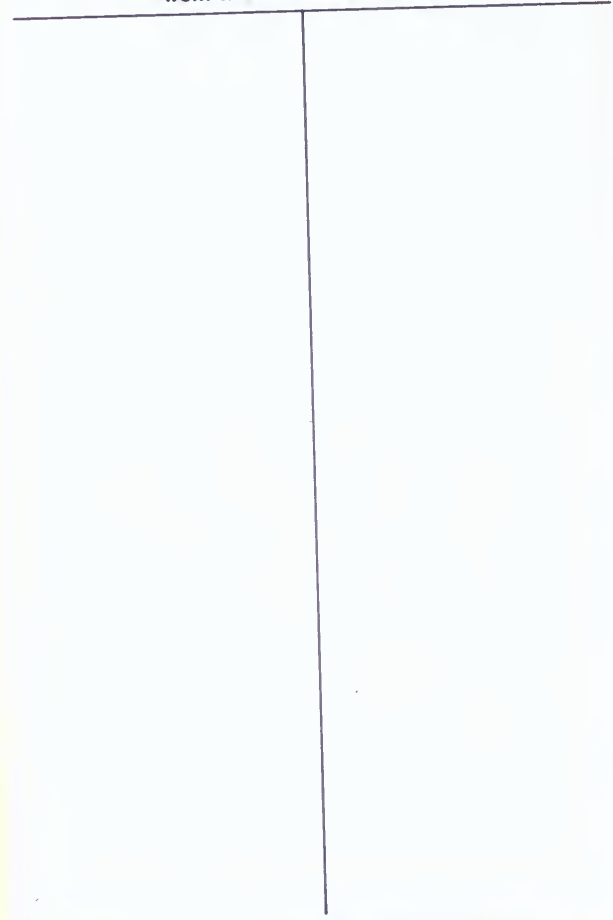


S. E. L .

UC SOUTHERN REGIONAL LIBRARY FACUITY A 0013579602

THIS BOOK IS NOT TO BE TAKEN FROM THE LIBRARY 


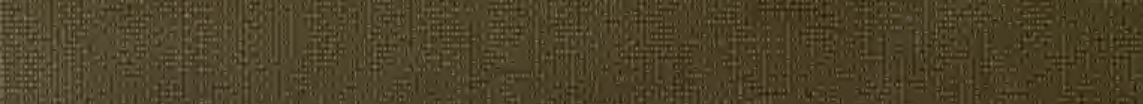

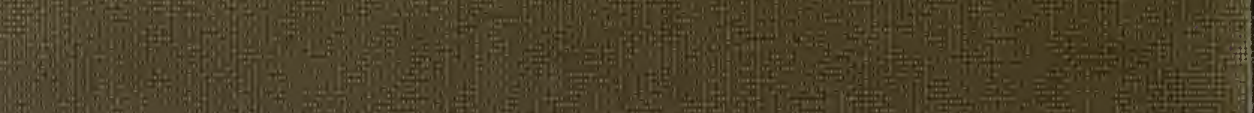

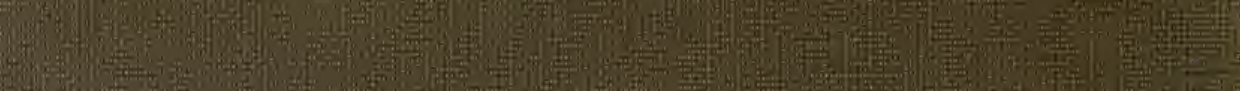
24. (1) (1) (5) 54 (5) a

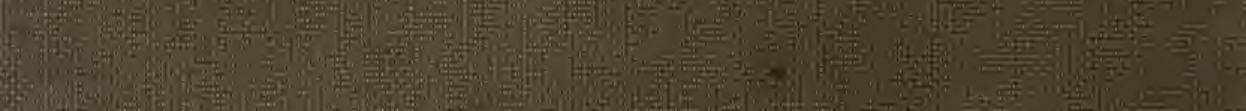
(6) 40 (5) 4 (3)

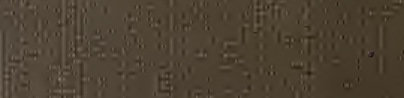

